\title{
SCIENTIFIC PROGRAM OF 35TH WORLD CONGRESS OF ENDOUROLOGY PROGRAM BOOK
}

Tuesday 12 September

Basic Research Poster Session 1: Bench to Bedside: BPH/LUTS/Reconstruction

13:00-15:00

Basic Science: BPH/LUTS

Convention Centre East, Meeting Room 2

BRPRS1-1 Withdrawn

BRPRS1-2 Withdrawn

BRPRS1-3 Withdrawn

BRPRS1-4 Effect of testosterone impregnated pellet therapy on lower urinary tract symptoms A Ostrowski, R Williams, G Broderick United States

BRPRS1-5 Effect of Hyoscine, Diclofenac, Solifenacin and Adrenaline on ureteral peristalsis. An in-vitro evaluation and animal model

V Panagopoulos, I Kyriazis, P Kallidonis, M Vasilas, P Ntasiotis, D Kotsiris, T Vrettos, E Liatsikos

Greece

BRPRS1-6 Insulin Receptor and Insulin-like growth factor expression increases and Insulin-like growth factor binding protein -3 expression reduces in association with prostate size in Benign prostatic hyperplasia

K Sreenivasulu, H Nandeesha, L Dorairajan, V Vinayagam India

BRPRS1-7 Comparison of transurethral incision of the prostate and silodosin in patients having benign prostatic obstruction in terms of retrograde ejaculation

E Arda, B Cakiroglu, i Hazar, O Sinanoglu, S Ekici

Turkey

BRPRS1-8 Nocturia Is Associated with Slipping and Falling

W Bang, H Kim, S Cho, M Choo, K Ko,

D Lee, K Koo, Y Yoon, S Lee, W Kim

Republic of Korea

BRPRS1-9 Analysis of the prevalence and associated factors of overactive bladder in adult Korean men

W Bang, H Kim, S Cho, M Choo, K Ko, D Lee, K Koo, Y Yoon, S Lee, W Kim Republic of Korea

BRPRS1-10 Withdrawn
BRPRS1-11 Role of Chaperon Proteins in Obstructed Renal Pelvis

H Sanguinetti, J Toblli, NO Bernardo, O Mazza

Argentina

BRPRS1-12 Re-do female genitoplasty for previously failed genitoplasty in male to female gender reassignment surgeries

A Ghoreifi, K Tavakkoli Tabassi, A Yarmohammadi, S Hajian Iran

BRPRS1-13 Midterm follow up of patients performed fold-back perineoscrotal flap plus penile inversion vaginoplasty for male-to-female gender reassignment surgery

K Tavakkoli Tabassi, A Yarmohammadi, A Ghoreifi

Iran

BRPRS1-14 Barbed polyglyconate versus polyglactin. An experimental model to compare pressure on renal suture

F Ferraris, F Silvano, E Kol, G Nevado Benzi, R Lobo, F Lacour, E Longo Argentina

BRPRS1-15 Gene Expression and Patterns of Scarring Response in Human Fibroblasts in Response to Mesh, Catheter Materials and Silca Using a 2D and Novel 3-D Collagen Model

R Kilani, L Yunyuan, L Stothers, A Ghahary Canada

BRPRS1-16 Withdrawn

BRPRS1-17 Does erectile function predicts outcome of second stage urethroplasty? A retrospective analysis

K Kaura, A Sokhal, S Sankhwar

India

BRPRS1-18 Single Surgeon Experience with Revision of Ureteroenteric Anastomosis for Benign Strictures after Urinary Diversion E Schommer, A Davidiuk, L Gundian, K Custer, P Young United States 
BRPRS1-19 Castration impairs erectile organ structure and function by inhibiting autophagy and promoting apoptosis of corpus cavernosum smooth muscle cells in rats

Z Shen, S Zhong, X Wang, T Xu, F Gao, M Zhang

China (People's Republic)
BRPRS1-20 In-Vivo trial of a Genuine Atraumatic Urinary Catheter Designed for Prevention of Catheter-Induced Trauma

R Azar, P Shadpour

Iran

BRPRS1-21 Bladder Stone and Urodynamics

J Sun

China (People's Republic)
BRPRS2-1 Non hemmorhagic Adrenal infarction in pregnancy

E Fernandes, S Gupte

India

BRPRS2-2 Is the stone density and location of kidney stones smaller than two centimeters a parameter that affects the surgery to be chosen? Preliminary findings of a prospective randomization study

A Gücük, B Yılmaz, S Gücük, U Üyetürk Turkey

BRPRS2-3 The Association between Osteopontin Gene Polymorphisms and Idiopathic Calcium Oxalate Stones

Z Guoxi, X Guancheng, Z Xiaofeng,

L Quanliang, X Yijun, W Gengqing

China (People's Republic)

BRPRS2-4 Two novel AGXT mutations identified in Chinese patients with sever bilateral multiple nephrolithiasis

Y Du, J Li, Y Tian

China (People's Republic)

BRPRS2-5 Erythropoietin receptor signalling in unilateral ureteral obstruction

E Park, D Lange

Canada

BRPRS2-6 The Drosophila melanogaster model of human uric acid nephrolithiasis as a novel in vivo high throughput drug screening platform

AN Ali, T Dayarathna, M Pignanelli, J Kim, RS Padda, P Spagnuolo, H Razvi, HS Leong Canada

BRPRS2-7 Probiotic supplementation to reduce stone burden assessed using a Drosophila model of nephrolithiasis

K Al, R Chanyi, H Razvi, J Burton

Canada

BRPRS2-8 Optimizing RNA extraction of renal papilla biopsy tissue in kidney stone formers: a new methodology for genomic study

K Taguchi, M Usawachintachit, DT Tzou,

S Hamamoto, BA Sherer, ML Stoller,

$\mathrm{T}$ Yasui, T Chi

United States
BRPRS2-9 Deficiency in butyrate metabolism and oxalate degradation in the gut microbiome of kidney stone patients

W Choy, C Morgan-Lang, E Gough, A Manges, S Hallam, BH Chew, D Lange Canada

BRPRS2-10 A novel laboratory model system for the evaluation of biofilm-induced urinary infection stone formation

T Hobbs, S Logan, E Lauchnor, E EspinosaOrtiz, D Lange, R Gerlach United States

BRPRS2-11 A hypercalciuric stone-forming rat model induced by active vitamin $D$

H Hu, S Wang, Y Lu, J Zhang, B Qin

China (People's Republic)

BRPRS2-12 Inflammatory Cytokines in Papillary Tips and Urine of Nephrolithiasis Patients

AY Sun, B Hinck, BR Cohen, K Keslar, R Fairchild, M Monga

United States

BRPRS2-13 Increased Urinary Oxalate Excretion in an Obese Mouse Model

K Wood, A Dowell, J Knight, D Assimos,

R Holmes

United States

BRPRS2-14 The Association Between the Gene Polymorphisms in the Calcium-sensing Receptor and Calcium Nephrolithiasis in Jiangxi Gannan Area

Z Guoxi, Z Qingming, Z Xiaofeng,

L Quanliang, X Yijun, W Gengqing

China (People's Republic)

BRPRS2-15 Use of the selective cholesterol absorption inhibitor ezetimibe and subsequent risk for urolithiasis

A Ostrowski, R Pak, I Porter

United States

BRPRS2-16 Evaluating the role of urine chemistry on the formation of infection stones

T Hobbs, E Espinosa-Ortiz, D Lange,

R Gerlach

United States 
BRPRS2-17 Protein modelling- A novel approach to risk stratify disease in patients with cystinuria

M Sahu, Z Kassam, K Wong, M Bultitude, M Wass, K Thomas

United Kingdom

BRPRS2-18 The effects of shockwave lithotripsy on the urinary microbiome and on bacterial dispersion using a phantom kidney stone model

AH Alathel, R Chanyi, J Denstedt, S Pautler, J Burton, H Razvi, Y Bao, D Mikhail Canada

BRPRS2-19 Impact of repeated extracorporeal shock wave lithotripsy on prepubertal rat kidney J Chung, S Lee, H Seo, S Lee Republic of Korea

BRPRS2-20 Sensitive Colorimetric Detection of Nitrite Ions Based on Aggregation of Gold Nanoparticles for Early Detection of Nitrate Reducing Bacteria in the Urinary Tract A Kulkarni, K Ziegler, VY Bird United States
BRPRS2-21 Colorimetric, pH-Responsive Membranes Allow for Immediate, Real-Time and Reversible Urine Monitoring in a Multipart System for Detection Elevated Urine pH secondary to Urease-Producing Bacteria M Fuchs, H Huda, CB French, N Patel, VY Bird United States

BRPRS2-22 Experimental study for a new medical adhesion-prevention agent after laparoscopic nephrectomy

Y Naitoh, J Ajiki, A Fujihara, M Kanazawa, S Ushijima, F Hongo, K Okihara, H Suong-Hyu, O Ukimura Japan

BRPRS2-23 Comparative evaluation of tissue damage induced by ultrasound and impact dual mode endoscopic lithotripsy versus conventional single mode ultrasound lithotripsy

MJ Bader, W Khoder, M Seitz, F Strittmatter, A Alghamdi, C Stief

Germany

Tuesday 12 September

Basic Research Poster Session 3: Bench to Bedside: Oncology

15:30-17:30

Basic Science: Oncology

Convention Centre East, Meeting Room 2

BRPRS3-1 LncLBCS inhibits the self-renewal of bladder cancer stem cells through hnRNPK-mediated Sox 2 repression

$\mathrm{X}$ Chen

China (People's Republic)

BRPRS3-2 Urinary proteomics may help identify men with higher likelihood of cancer detection on prostate biopsy

P Shrivastava, M Bhat, A Dinda, R Kumar India

BRPRS3-3 Gene silencing of SIRT7 by small interfering RNA influences the biological behaviors of renal cancer $786-O$ cells

Z Xiaofeng, Y Zengxiang, X Yijun,

L Quanliang, Z Guoxi, W Gengqing

China (People's Republic)

BRPRS3-4 Association of DNA Polymorphisms within CYP11B2 and CYP11B1 Locus with the Risk of Aldosterone Producing Adenoma

Z Guoxi, H Ruohui, Z Xiaofeng, L Quanliang, $X$ Yijun, W Gengqing

China (People's Republic)

BRPRS3-5 The effects of shRNA inhibiting TRPV2 gene expression on proliferation and invasion of human bladder cancer $E J$ cell line

L Quanliang, Z Guoxi, Z Xiaofeng,

W Gengqing, W Xiaoning, X Gang

China (People's Republic)
BRPRS3-6 The implication of survivin expression in dendritic cells from the mouse marrow

L Yanmin, Z Guoxi, Z Xiaofeng,

L Quanliang, X Tianpeng, W Gengqing

China (People's Republic)

BRPRS3-7 Withdrawn

BRPRS3-8 Withdrawn

BRPRS3-9 Management of Malignant Ureteric Obstruction: Are We Doing the Right Thing?

L Kerr, J Hendry, M Dupre, S Teahan United Kingdom

BRPRS3-10 Incidence of anaemia and blood transfusion in patients undergoing nephrectomy for urological malignancy

L Kerr, M Stephens, A Baker, G Oades

United Kingdom

BRPRS3-11 Does the Microphtalmia-Associated Transcription Factor (MITF) E318K mutation matter in the tumorigenesis of renal cell carcinoma?

Y Bodokh, L Mendel, I Bentellis, L Vignot,

P Treacy, P Regnier, F Roustan, R Haider,

A Mbeutcha, R Prader, B Tibi, J Fallot,

J Amiel, D Chevallier, F Pedeutour,

M Durand

France 
BRPRS3-12 ALK rearranged renal cell carcinoma: a literature review

Y Bodokh, L Mendel, I Bentellis, L Vignot, P Treacy, P Regnier, F Roustan, R Haider, A Mbeutcha, R Prader, B Tibi, J Fallot, J Amiel, D Chevallier, F Pedeutour, M Durand

France

BRPRS3-13 ALK-TPM3 rearrangement in adult renal cell carcinoma: an exceptional translocation renal carcinoma

Y Bodokh, L Mendel, I Bentellis, L Vignot, P Treacy, P Regnier, F Roustan, R Haider, A Mbeutcha, R Prader, B Tibi, J Fallot, J Amiel, D Chevallier, F Pedeutour, M Durand

France

BRPRS3-14 The role of 18F-FDG PET/CT in identifying inguinal nodal metastasis in patients on surveillance after primary treatment of penile squamous cell carcinoma: Single center prospective study

Y T B, S Waigankar, V Wagaskar India

BRPRS3-15 Correlation between pre-operative serum C-reactive protien, platelet count and calcium levels with the types and stages of renal cell cancer and its significance in predicting recurrence and mortality after curative surgery

B Nayak, P Shrivastava, P Singh,

P Dogra

India

BRPRS3-16 Should All UTUC Patients be Screened for Lynch Syndrome? A Single Center Analysis of the Role of Screening UTUC patients for Lynch Syndrome M Metcalfe, F Petros, P Rao, M Mork, L Xiao, R Broaddus, SF Matin United States

BRPRS3-17 Methylation signature for prediction of progression free survival in surgically treated clear cell renal cell carcinoma

H Kang, S Seo, H Jang, W Kim, Y Kim,

S Yun, S Lee, W Kim

Republic of Korea
BRPRS3-18 Expression of MHC class I chain-related protein A (MICA) and natural-killer group 2, member D receptor (NKG2D) predicts recurrence and progression of primary non-muscle invasive bladder cancer E Rijo, J Lloreta Trull, A Frances Comalat, J Lorente, N Juanpere Rodero, L Diaz Sanchez, M Lorenzo Perez, S Perez Fernandez, A Alcaraz Spain

BRPRS3-19 Kidney irreversible electroporation does not cause significant injury to adjacent ureter or bowel in a porcine model

I Sorokin, N Canvasser, E Lucas, BA Johnson, J Cadeddu

United States

BRPRS3-20 Pre-Clinical Assessment of Peri-operative Complications and Effect on Wound and Tissue Healing of sEphB4-HSA Administration Prior to Surgical Intervention S Chopra, C Ohe, A Bove, N Ahmadi, A Abreu, A Aron, M Aron, M Amin, P Gill, IS Gill United States

BRPRS3-21 Capability of Electrical Impedance Spectroscopy as a Sensor for Detecting Iatrogenic Injuries Caused by Veress Needle Insertion: Preliminary In Vivo Study Using Murine Models J Yun, K Lee, T Kim, J Lee Republic of Korea

BRPRS3-22 Preclinical ex-vivo experiment of photodynamic diagnosis using 5-aminolevulinic acid for upper urinary tract urothelial cancer

$\mathrm{R}$ Tanimoto, $\mathrm{K}$ Wada, M Ishizuka, K Murakami, T Ishii, Y Mitsui, T Sadahira, Y Kobayashi, M Araki, T Watanabe, Y Nasu Japan

BRPRS3-23 Automated and dynamic classification of bladder cancer using deep learning on real-time confocal laser endomicroscopy images

TC Chang, D Yi, D Rubin, JC Liao United States

BRPRS3-24 Assessment about heat injury of small intestine induced by surgical sealing devices in animal model

T Suzuki, T Minagawa, T Ogawa, T Uehara, R Hattori, O Ishizuka Japan 
BRPRS4-1 Holmium-YAG laser: Impact of pulse energy and frequency on local fluid temperature M Sourial, J Ebel, N François, G Box, B Knudsen United States

BRPRS4-2 Save time, save Nephrone....Emergency drainage for Urosepsis with upper tract calculi HG Thummar, M Dabhi, A Patel, N T, S D India

BRPRS4-3 Assessing irrigation flows influence on clearance of renal calculi fragments during percutaneous nephrolithotomy: a hydrodynamic computerized and practical model study

Y Barghouthy, S Shlain, G Aviram, A Liberzon, A Paster, M Sofer Israel

BRPRS4-4 Can Hounsfield unit (HU) measurement on non-contrast computed tomography scan (NCCT) predict urinary stone composition? A validation of 2 different techniques

Z Choo, A Yuwono, M Lim, Z Liu, C Tan, Y Tan, Y Lee Singapore

BRPRS4-5 Enhanced high rate fragmentation of model urinary stones using acoustic bubble coalescence with a commercial shockwave lithotripsy system TL Hall, WW Roberts United States

BRPRS4-6 Differences of metabolic evaluation in 24-hour urine between pre- and postmenopausal non-stone former women Z Mai, G Zeng China (People's Republic)

BRPRS4-7 Arbutin as a novel prevention and therapy for calcium oxalate nephrolithiasis

T Dayarathna, S Ali, AN Ali, P Spagnuolo, H Razvi, HS Leong

United States

BRPRS4-8 Vitamin D, Bone Turnover Markers and Bone Mineral Density in Recurrent Calcium Stone Formers

U Dhakad, B Singh, K Kaura, S Sankhwar, S Das India

BRPRS4-9 Metabolic characteristics and risks associated with stone recurrence in young adult stone patients

S Seo, H Kang, H Jang, W Kim, Y Kim, S Yun, W Kim, S Lee

Republic of Korea
BRPRS4-10 Staghorn Stone etiology paradigm Shift : Infective to metabolic !!! HG Thummar, N Zinto, S D, N T, J Vyas India

BRPRS4-11 Urine and serum fetuin-A levels in patients with urolithiasis R Arora, N Abrol, B Antonisamy, C Beri, S Kumar, N Kekre, A Devasia India

BRPRS4-12 Establishment of reference values for stone risk factors in 24-hour urine among healthy adult Han population across China

Z Mai, G Zeng

China (People's Republic)

BRPRS4-13 Prevalence of pediatric urolithiasis in Kashgar area of Xinjiang Province in China: an ultrasonography based cross-sectional study Z Mai, G Zeng China (People's Republic)

BRPRS4-14 The effect of Aged Vinegar on stone risk factors in 24-hour urine: a pilot randomized controlled trial

Z Mai, G Zeng

China (People's Republic)

BRPRS4-15 Percutaneous Treatment of Bladder Stones in Children: 10 Years Experience, Is Blind Access Safe?

H Ahmadnia, A Kamalati, M Younesi, A akhavan Rezayat, M Imani Iran

BRPRS4-16 Differential gene expression profile between papilla, medulla, and cortex leads to plaque and crystal formation

K Taguchi, DT Tzou, M Usawachintachit, BA Sherer, T Yasui, S Ho, ML Stoller, T Chi United States

BRPRS4-17 Initial Discovery of Spontaneous Randall's Plaque Stone Overgrowth in an Animal Model MS Borofsky, JC Williams, JP Lulich United States

BRPRS4-18 Canine Kidney Stones As A Comparable Human Stone Model in Lithotripsy Experimentation JS Ahn, A Randad, W Kreider, MR Bailey, AD Maxwell, JP Lulich, E Furrow, MS Borofsky, MD Sorensen, JD Harper United States

BRPRS4-19 Local and sustainable delivery of lidocaine/ ketorolac to the urethra in an animal model via an analgesic-eluting stent coated with biodegradable nanofibers

Y Lin, K Liu, T Tsai, T Hwang, S Liu

Taiwan (Republic of China) 
BRPRS4-20 Evaluation of in vitro burst wave lithotripsy exposure conditions

C Hunter, JS Ahn, W Kreider, AD Maxwell, BW Cunitz, Y Wang, B Dunmire, MR Bailey, JD Harper, MD Sorensen United States

BRPRS4-21 The Impact of Forces Applied during Ureteral Access Sheath Deployment on Ureteral Injury in a Porcine Model

KS Kaler, RM Patel, R Yoon, DJ Lama,

C Hwang, Z Okhunov, M Klopfer,

J Landman, RV Clayman

United States
BRPRS4-22 A Multi-centre cohort study evaluating the role of Inflammatory Markers In patient's presenting with acute ureteric Colic (MIMIC)

N Nkwam, T Shah, A O'Keefe, C Gao, T Manning, A Peacocke, S Cashman, A Nambiar, B Lamb, M Cumberbatch, R Pickard, P Erotocritou, D Smith,

V Kasivisvanathan

United Kingdom
MP1-1 Super-mini percutaneous nephrolithotomy (SMP) versus retrograde intrarenal surgery for the treatment of 1 to $2 \mathrm{~cm}$ lower pole renal calculi: a multinational multicenter randomized controlled trial

G Zeng, T Zhang, J Fan, W Zhang, S Yang, K Xiao, X Li, H Li, X He, C Xu, K Sarica, MS Agrawal

China (People's Republic)

MP1-2 Clinical effect of prone mini-percutaneous nephrolithotomy with two-step precise puncture method by ultrasound guidance under total paravertebral block anesthesia

H Yang, X Yu, S Wang China (People's Republic)

MP1-3 Prospective comparative study of the efficacy and safety of new-generation versus early-generation system for super-mini-percutaneous nephrolithotomy: a revolutionary approach to improving the irrigation and stone removal G Zeng, W Zhu, Y Liu China (People's Republic)

MP1-4 Application of super-mini percutaneous nephrolithotomy (SMP) in the treatment of upper urinary tract calculi

Z Guoxi, Z Xiaofeng, X Rihai, Y Yuanhu, W Gengqing, W Xiaoning China (People's Republic)

MP1-5 A Prospective Randomized Controlled Study of Instantly Phase-II Tubeless Percutaneous nephrolithotomy

L Folin, Z Xiaofeng, X Rihai, Y Yuanhu, W Gengqing, W Xiaoning China (People's Republic)

MP1-6 The role of super-mini percutaneous nephrolithotomy (SMP) in the treatment of symptomatic lower pole renal stones (LPSs) after the failure of shockwave lithotripsy (SWL) or retrograde intrarenal surgery (RIRS)

G Zeng, J Fan

China (People's Republic)
MP1-7 Prospective comparative study of Super-mini Percutaneous nephrolithotomy and Miniprec for the treatment of stone lager than $2 \mathrm{~cm}$ Y Liu, W Zhu, Y Liu, W Wu, W Zhong, J Fan, Y Lan, X Li, G Zeng China (People's Republic)

MP1-8 A comparison of fluoroscopy time for two different techniques of percutaneous nephrolithotomy tract dilation: one-shot vs metal telescopic dilation I Sedano-Portillo, G Ochoa-Leon, A GonzalezOjeda

Mexico

MP1-9 The Safety and Efficancy of Minimally Invasive Percutaneous Nephrolithotomy (MPCNL) in Treating Upper Ureteral Calculi of Elderly Patients

$$
\begin{aligned}
& \text { S Li, L He } \\
& \text { China (People's Republic) }
\end{aligned}
$$

MP1-10 Mini-Percutaneous Nephrolithotomy: Initial Experience S Moazami, L Cheuck, J Stern
United States

MP1-11 Supine Percutaneous Nephrolithotomy is safe and achieves higher stone free rates when combined with retrograde intrarenal surgery (Endoscopic Combined Intra Renal Surgery)

D Yong, S Koh, Y Tan Singapore

MP1-12 Which Way is Best for Stone Fragments and Dust Extraction During Percutaneous Nephrolithotomy B Kati, E Pelit, I Yagmur, Y Akin, H Ciftci, E Yeni Turkey

MP1-13 Minimally Endoscopic Combined Intrarenal Surgery for Staghorn Calculi in Breaststroke Position

D Lai, Y He, X Li, M Chen

China (People's Republic) 
MP1-14 Supracostal access tubeless percutaneous nephrolithotomy: Minimizing complications M Sourial, N François, G Box, B Knudsen United States

MP1-15 A novel method to re-position suboptimally preopertively placed nephrostomy tubes for PCNL without renal re-puncture S Dekalo, Y Barghouthy, J Ben-Chaim, S Conti, A Greenstein, M Sofer Israel

MP1-16 Single-session Bilateral Tubeless Percutaneous Nephrolithotomy in Supine and Prone Positions: A Comparison Study Y Barghouthy, S Dekalo, S Proietti, J Ben-Chaim, I Mintz, G Giusti, M Sofer Israel

MP1-17 Ultrasound guided paravertebral nerve block anesthesia of percutaneous nephrolithotomy: a Prospective Randomized, non-blind parallel controlled study

J Hu, X Yu, S Wang

China (People's Republic)

MP1-18 Comparative analysis of contact holmium laser and ultrasonic lithotripsy effectiveness in the case of minimally invasive percutaneous nephrolithotomy

D Merinov, A Artemov, S Gurbanov, E Valery, L Arustamov, O Merinova, D Dolotkazin Russia

MP1-19 Use of various entrance and exit strategies to facilitate percutaneous nephrolithotomy as an ambulatory procedure

T Zhou, K Mahagaokar, J Stern

United States
MP1-20 Ureteropelvic junction stent as an exit strategy for percutaneous nephrolithotomy: an updated series

T Zhou, J Stern

United States

MP1-21 Medically Facilitated Endoscopic Surgery: The Impact of One Week of Pre-operative Tamsulosin on Deployment of 16-French Ureteral Access Sheaths and Stone Free Rates

KS Kaler, S Safiullah, DJ Lama, RM Patel, Y Ko, Z Okhunov, J Landman, RV Clayman United States

MP1-22 The optical needle puncture followed by percutaneous nephrolithotomy using high definition image guide system K Wada, R Tanimoto, M Araki, K Sasaki, T Sadahira, T Iwata, A Takamoto, Y Kobayashi, T Watanabe, Y Nasu, H Kumon Japan

MP1-23 Papillary versus non papillary puncture in percutaneous nephrolithotomy:A prospective randomized trial P Kallidonis, I Kyriazis, D Kotsiris, $\mathrm{P}$ Ntasiotis, V Panagopoulos, A Koutava, W Kamal, E Liatsikos

Greece

MP1-24 Multi-Institutional Evaluation of MiniPercutaneous Nephrolithotomy: An Evaluation of Outcomes and Stone Free Rates for Stones up to $3 \mathrm{~cm}$

D Brooks, M Patel, S Moazami, K Basham, RB Pickens, J Stern, J Gutierrez United States

Wednesday 13 September

Moderated Poster Session 2: Shock Wave Lithoripsy

14:00-16:00

Clinical Stones: SWL

Convention Centre East, Meeting Room 2

MP2-1 Comparing the success of extracorporeal shock wave lithotripsy in the treatment ureteric calculi between the EDAP TMS Sonolith and the Storz Modulith SLX

$$
\text { K Manoharan, N Sharma, P Kumar }
$$$$
\text { United Kingdom }
$$

MP2-2 Does Mild Hydronephrosis Induced by full-Bladder Improve Outcomes in Patient Undergoing Shock Wave Lithotripsy for Lower Calyceal Stones?

E Arda, i Hazar, B Cakiroglu, O Sinanoglu Turkey

MP2-3 A prospective randomized study to investigate the effect of power ramping on treatment outcome in extracorporeal shockwave lithotripsy of renal calculi

AC Ng, J Yam, C Yee, W Chu, K Wong, S Leung, B Lau, J Teoh

Hong Kong
MP2-4 Extracorporeal shock wave lithotripsy in pediatric urolithiasis: A single-center 100 cases experience Y Tang, P Han China (People's Republic)

MP2-5 Tubeless versus Totally Tubeless Nephrolithotripsy A Randomized Study P Gyawali, S Chapagain, P Chalise Nepal

MP2-6 Does ESWL cause more injury to the kidneys than PNL for moderate size $(1.5-2 \mathrm{~cm})$ stones?

U Mete, A Mittal, B Mittal India

MP2-7 Emergency extracorporeal shock wave lithotripsy for upper ureteric stones with or without pre-stenting: A randomized clinical trial T El-Ghazaly, S Vita, S Hussain, A Al-Obaidy, A Al-Naimi Qatar 
MP2-8 Comparison of Fixed and Ramping Voltage Extracorporeal Shockwave Lithotripsy with Acute Renal Injury Biomarkers: Prospective Randomized Controlled Clinical Study A Atar, K Seker, A Kural, R Turkay, M Yenice, V Tugcu Turkey

MP2-9 Lower rate with a Storz lithotripter results in improved stone fragmentation both in vitro and in a clinical setting

K Schmitz, M Spencer, R Cleveland, B Turney United Kingdom

MP2-10 External validation of Triple D score and its modification to Triple D-Sscore for better prediction of stone free rates after extracorporeal shock wave lithotripsy

N Iqbal, M Imran Jamil, A Ur-Rehman, S Akhter

Pakistan

MP2-11 Clinical Efficacy and safety of anterograde ureterography for radiolucent ureteral stone localization in extracoporeal shock wave lithotripsy J You, H Kim, Y Shin, Y Kim Republic of Korea

MP2-12 Effects of shock wave lithotripsy, for the treatment of kidney stones, on routine blood tests, Neutrophil Gelatinase Associated Lipocalin (NGAL) and Interleukin-18 (IL-18) I Shergill, N Jones, SF Hughes, P Ella-Tongwiss

MP2-13 Long-term Risk of New-Onset Hypertension and Diabetes after Treatment of Shock Wave Lithotripsy: Evidence from a National Representative Cohort in Taiwan C Tsai, S Huang Taiwan (Republic of China)

MP2-14 Emergency Extracorporeal Shockwave Lithotripsy (ESWL) for acute ureteric colic: Outcomes from a large UK tertiary centre I Jubber, S Hori, S Elwakil, J Collie, M Wynn, S Isaacs, S Shah, O Wiseman, S Al-Hayek United Kingdom

MP2-15 Renal injury protection agents in patients undergoing extracorporeal shockwave lithotripsy (ESWL): A Systematic Review I Jubber, S Hori, S Al-Hayek United Kingdom

MP2-16 Withdrawn
MP2-17 Shock Wave Lithotripsy for Large Renal Calculi: Is Prophylactic Stenting Beneficial? T Kroczak, D Ghiculete, R D'A. Honey, M Ordon, J Lee, K Pace Canada

MP2-18 The Effect of Stone Dust and Collecting System Flow On Shockwave Lithotripsy JS Ahn, A Randad, W Kreider, MR Bailey, AD Maxwell, MD Sorensen, JD Harper United States

MP2-19 The efficacy and safety of and satisfaction with the transgluteal approach to extracorporeal shockwave lithotripsy via the supine position to treat distal ureter stones: A prospective, randomized, and multicenter study M Choo, J Han, S Lee Republic of Korea

MP2-20 The direction of Shockwave lithotripsy (SWL): Publication trends (PubMed) over the last 16 years (2000-2015)
A Pietropaolo, B Somani United Kingdom

MP2-21 Shock wave lithotripsy is more effective for residual fragments after percutaneous nephrolithotomy than for primary stones of the same size: A matched pair cohort study A Aminsharifi, D Irani, H Amirzargar United States

MP2-22 Extracoporeal shock wave lithotripsy treatment for radiolucent ureteral stone with the help of retrograde ureterography using a flexible cystoscopy

J You, H Kim, Y Shin, Y Kim

Republic of Korea

MP2-23 Mobile EWSL Treatment For 1-2cm Renal Stones Results and Complications

H Rooney, L Mokool, R Marri, SK Nalagatla United Kingdom

MP2-24 How to accelerate the upper urinary stone discharge after Extracorporeal Shockwave Lithotripsy (ESWL) : a Prospective Multi-center Randomized Controlled Trial about External Physical Vibration Lithecbole (EPVL)

G Zeng, W Wu, Z Yang, F Tang, C Xu, Y Wang, X Gu, X Chen, R Wang, J Yan, $X$ Wang, W Gao, C Hou, G Alberto, Z Ye China (People's Republic) 
MP3-1 "Stone-less" or "negative" ureteroscopy - a part of endourologic routine or avoidable source of frustration?

IM Sabler, A Isid, A Lorber, A Latke, G Hidas, E Landau, V Yutkin, D Pode, O Gofrit, M Duvdevani Israel

MP3-2 SWL vs. URS: Between indications and doctor's whim V Malkhasyan, I Semanyakin, V Ivanov, D Pushkar Russia

MP3-3 Ureteral stricture formation after removal of proximal ureteral stone: retroperitoneal laparoscopic ureterolithotomy versus ureteroscopy with holmium: YAG laser lithotripsy $\mathrm{C} \mathrm{Li}, \mathrm{H} \mathrm{Hu}, \mathrm{L} \mathrm{Xu}$ China (People's Republic)

MP3-4 Ureteroscopy in patients with ureteral duplication N Chertack, RK Jain, S Sivalingam, MJ Noble, M Monga United States

MP3-5 Indications for Stent Omission after Ureteroscopic Lithotripsy Defined: An Initial Analysis PE Bower, J Pereira, S Thavaseelan, G Pareek United States

MP3-6 CT Imaging: A Predictor of Impacted Ureteral Stones?

E Parkhomenko, T Tran, JN Thai, K Blum, S Purnell, M Gupta

United States

MP3-7 Withdrawn

MP3-8 Managing Lower calyceal renal stones, flexible ureteroscopy vs ESWL. Is it doctor or patient choice?

A hammady, M Elbadry

Egypt

MP3-9 Do Stone Characteristics Influence Avicenna Roboflex Robotic Assisted Retrograde Intra-Renal Surgery Outcomes?

A Patel, N Tokatli, A Kabakci, R Saglam United Kingdom

MP3-10 Defining the rate of encountering unacceptable reusable flexible ureteroscopes for immediate clinical use: 'Bad out of the Box.' SG Hubosky, KA Healy, DH Bagley United States

MP3-11 Is endoscopic evaluation at the end of retrograde intra-renal surgery a reliable predictor of post-operative significant residual fragments? E Alessandria, A Bosio, E Dalmasso, D Peretti, F Vitiello, A Bisconti, P Destefanis, P Gontero Italy
MP3-12 Effect of Dusting and Fragmentation Settings on Stone Models Using an Automated 3-D Gantry System

AH Aldoukhi, TL Hall, WW Roberts, KR Ghani United States

MP3-13 Non-Contact "Pop-corn" Holmium Laser Lithotripsy: What is the ideal duration for dusting effect?

AH Aldoukhi, TL Hall, WW Roberts, KR Ghani United States

MP3-14 Heat Generation During Holmium Laser Lithotripsy AH Aldoukhi, TL Hall, KR Ghani, WW Roberts United States

MP3-15 Laser Fiber Burnback: The role of Pulse Width on Tip Degradation AH Aldoukhi, TL Hall, WW Roberts, KR Ghani United States

MP3-16 Non-Contact Holmium Laser Lithotripsy: Which settings give the best Dusting effect? AH Aldoukhi, TL Hall, WW Roberts, KR Ghani United States

MP3-17 The impact of high-frequency positive-pressure ventilation (HFPPV) on operation time in retrograde intrarenal surgery for stones $>1 \mathrm{~cm}$ M Popiolek, D Al-Rammahi, R Swartz Sweden

MP3-18 The impact of stone density on outcome of ureteronephroscopy

A Shvero, N Kleinmann, Y Abu-Ghanem, A Eisner, D Zilberman, J Ramon, H Winkler Israel

MP3-19 Pelvicalyceal Anatomy Types Impact On The Success Of Procedure In Patients Who Performed Flexible Ureteroscopy S Kirecci, M Ilgi, C Kutsal, C Yesildal, H Demirel Turkey

MP3-20 A critical evaluation of Intra-renal pressures based on ureteral access sheath size and irrigation flow rate for safe and effective Robotic Assisted Retrograde Intra-renal Surgery N Tokatli, K Sarica, A Kabakci, R Saglam, A Patel Turkey 
MP3-21 Endoscopic Assessment Of Renal Papillae: Implications Of Compound Versus Simple Papillae Status On Stone Analysis BB Anderson, CU Nottingham, MA Adamsky, E Worcester, F Coe, GS Gerber United States

MP3-22 Reusable vs. disposable flexible ureterorenoscopy (fURS): How to strike a balance? Case-based CostBenefit Analysis

T Ozimek, MH Schneider, MC Hupe, JR Wiessmeyer, PL Chlosta, J Cordes, AS Merseburger, MW Kramer Germany
MP3-23 Effect of Ureteral Access Sheath Use on Postoperative Visual Analogue Scale and Verbal Rating Scale Levels During Retrograde Intrarenal Surgery

O Kilic, M Akand, E Altintas, G Yildiz, S Goktas

Turkey

MP3-24 Limitations of the Lithovue single use digital flexible ureteroscope S Bazargani, S Ghodoussipour, E Thompson, A Shah, A Mitra, M Dunn United States
MP4-1 Routine Urine Culture May Not Be Necessary Prior to Ureteroscopy

SK Bechis, S Patel, A Alagh, RL Sur,

DG Kronenberg

United States

MP4-2 Risk Factors of Readmission for Infection following pneumatic transurethral lithotripsy for Ureteral Stones

S Rabani, s rabani

Iran

MP4-3 Treatment of Non-Obstructive Urolithiasis is Effective in Treatment of Recurrent Urinary Tract Infections DK Agarwal, FJ Maldonado, ME Westerman, ME Rivera, AE Krambeck, JJ Knoedler United States

MP4-4 Early Discharge Following Decompression for Sepsis and an Obstructing Stone? A Multi-Institutional Study to Identify Predictors of Antibiotic Sensitivity

T Tran, M Cancian, E Parkhomenko, G Pareek, M Gupta United States

MP4-5 Impact of the timing of ureteral stent placement on outcomes in patients with obstructing ureteral calculi and presumed infection C Faw, J Hollingsworth, SN Ambani, KR Ghani, WW Roberts, CA Dauw United States

MP4-6 Withdrawn

MP4-7 Stone culture: Is it essential in the urolithiasis endoscopial approach?

A Rivero, C Manso, B de la Cruz, JM Hernández, JM Adot, J Montero, E Gutiérrez Spain

MP4-8 Risk and Protective Factors for Recurrent Urinary Tract Infection Following Percutaneous Nephrolithotomy

C Au, C Tsai, P Ku, S Chung, C Chang Taiwan (Republic of China)
MP4-9 Predictive Value of Preoperative Inflammatory Response Biomarkers for Metabolic Syndrome and post-PCNL systemic inflammatory response syndrome (SIRS) in Patients with Kidney Stones K Tang, H Liu, K Jiang, H Xu, Z Ye China (People's Republic)

MP4-10 Routine Complete Blood Count Has Limited Value Post Percutaneous Nephrolithotomy in Identifying Hemorrhagic or Infectious Complications T Kroczak, M Ordon, K Pace, R D'A. Honey, J Lee Canada

MP4-11 Preoperative urine culture analysis in 284 consecutive patients undergoing percutaneous nephrolithotomy: are current antibiotic prophylaxis recommendations adequate? A Garza-Gangemi, J Dominguez-Rodriguez, J Cruz-Ruíz, C Mendez-Probst Mexico

MP4-12 Prediction on infectious complications after percutaneous nephreolithotomy B Guliev, E Stetchik, A Zaikin Russia

MP4-13 The clinical yield of Stone culture in patients undergoing percutaneous nephrolithotripsy A Nevo, R Holland, R Gilad, D Lifshitz United States

MP4-14 Gentamicin and Ciprofloxacin resistance in PCNL Stone Cultures N Smyth, A McPhee, G Jones United Kingdom

MP4-15 What's growing in our renal stones? - Not Just the Usual Suspects N Smyth, A McPhee, G Jones United Kingdom

MP4-16 Is pre-operative automated cytometry adequate for the screening of pre-operative urine cultures before PCNL?

N Smyth, A McPhee, G Jones

United Kingdom 
MP4-17 Urinary tract infections in patients treated with percutaneous nephrolitotomy and their risk factors

E Hernández Méndez, C Martínez Arroyo, J Herrera Muñoz, I Calvo Vázquez, P Cortés Raygoza, M Ortega González, M Cantellano Orozco, G Fernández Noyola, J Morales Montor, C Pacheco Gahbler Mexico

MP4-18 Prospective Randomized Trial of Antibiotic Prophylaxis Duration for Percutaneous Nephrolithotomy in Low-Risk Patients: Preliminary Results

P Samson, G Gaunay, S Derisavifard, D Leavitt, B Morganstern, V Patel, M Elmasri, AD Smith, DM Hoenig, Z Okeke United States

MP4-19 Risk factor analysis of systemic inflammatory response syndrome (SIRS) after retrograde intrarenal surgery in patients with kidney stone

K Tang, H Liu, H Xu, Z Ye

China (People's Republic)
MP4-20 Tailored optimal perioperative antimicrobial prophylaxis in retrograde intrarenal surgery: evidence from a prospective randomized trail

G Zeng, J Fan, Z Zhao

China (People's Republic)

MP4-21 The role of various novel inflammatory biomarkers, following Flexible Ureterorenoscopy, for the treatment of kidney stones SF Hughes, AJ Moyes, I Shergill United Kingdom

MP4-22 Prophylactic Antibiotic Utilization Patterns and Postoperative Infectious Complications Following Percutaneous Nephrolithotomy SW Reese, TR McClintock, RE Krasnow, M Mossanen, SL Chang, R Korets United States

MP4-23 Stone Microbiology and Antibiotic Susceptibility based on Renal Calculi culture

R Galeana Ruiz, R Romero Aguilar, F Gonzalez Gonzalez, E Mendoza Villanueva, SA Victor, R Carvajal Garcia Mexico

MP4-24 Emergent double $J$ stenting: It's role in urosepsis with non-dilated pelvicalyceal systems

E Fernandes, A Thakarkar India
MP5-1 Skills Acquisition Trend and Outcomes in the transition from Laparoscopic to Robot Assisted Radical Cystectomy

SS Kommu, P Rouse, T Yeong, P Macneal, A Samateh, J Jaun, SH Garnett, PD Rimington, (S Academic Research Group United Kingdom

MP5-2 Upper body position analysis using optical motion capture during laparoscopic suturing maneuvers K Takayasu, K Yoshida, T Mishima, M Watanabe, T Matsuda, H Kinoshita Japan

MP5-3 The validation of a novel Robot-Assisted Radical Prostatectomy virtual reality module

P Harrison, N Raison, K Ahmed, W Watkinson

United Kingdom

MP5-4 Construct validity of laser fragmentation as a marker of expertise in ureteroscopy J Hendry, L Kerr, C McIlhenny United Kingdom

MP5-5 Evaluation of a 3D Virtual Reality Model for Surgical Planning and Education S Safiullah, DJ Lama, RM Patel, RC James, A Aljadaan, M Laughery, RV Clayman, J Landman United States
MP5-6 Assimilation of Skills: A Survey Among Percutaneous Nephrolithotomy Course Participants RM Patel, A Walia, KS Kaler, Z Okhunov, J Landman, RV Clayman United States

MP5-7 Prostate Dynamic Anastomotic Template On Robot (Pro-DATOR ${ }^{\text {TM }}$ ) - A Natural Simulation Platform for Optimal Skills Acquisition in Vesicourethral Anastomosis prior to Robotic Prostatectomy SS Kommu, L Samateh, RA Persad, P Rouse, SH Garnett, PD Rimington, (S Academic Research Group United Kingdom

MP5-8 Simulator Based Development of "Trans-Urethral Resection of Prostate" Skills and implementation of "Global Rating Scale" Evaluation System in Urology Postgraduate training K Soomro, Z Rajpar, I Memon, S Memon Pakistan

MP5-9 Analysis of the surgeon's posture during robotic simulator tasks using motion capture system K Yoshida, K Takayasu, T Matsuda Japan 
MP5-10 The Training Concept of Whole Procedure Equivalent in Robot Assisted Radical Prostatectomy - Results of a single surgeon in a Fellowship Training Programme P Rouse, SS Kommu, A Samateh, P Sturch, SH Garnett, PD Rimington, (S Academic Research Group United Kingdom

MP5-11 Predictors of trainees' robotic anastomosis competence evaluation (RACE) scores of a simulated da Vinci robot-assisted urethrovesical anastomosis on a novel 3D-printed bladder model NC Wong, J Hoogenes, U Blankstein, K Kim, Y Guo, B Shayegan, ED Matsumoto Canada

MP5-12 Development and validation of a 3D-printed bladder model for laparoscopic simulated urethrovesical anastomosis training for radical prostatectomy Y Guo, J Hoogenes, NC Wong, K Kim, B Shayegan, ED Matsumoto Canada

MP5-13 Skill Acquisition Trend (SATrend) - A Novel Approach for Redefining "The Learning Curve" in Minimally Invasive Urological Surgery SS Kommu, SH Garnett, PD Rimington, (S Academic Research Group United Kingdom

MP5-14 Comparison of 3D printed prostate models with standard radiological information to aid understanding of the precise location of prostate cancer: a construct validation study J Ebbing, F Jäderling, J Collins, O Akre, S Carlsson, J Höijer, M Olsson, P Wiklund Switzerland

MP5-15 A novel 3D-printed bladder model for training robot-assisted urethrovesical anastomosis J Hoogenes, NC Wong, Y Guo, K Kim, B Shayegan, ED Matsumoto Canada

MP5-16 Non-Technical Skills for Urological Surgeons (NoTSUS): Development and Validation of a Scenario-based Ureteroscopy Curriculum A Aydin, K Ahmed, O Brunckhorst, N Raison, F Dar, H Aya, C Lovegrove, M Iqbal, A Al-Jabir, T Abe, J Brewin, C McIlhenny, S Jain, M Shabbir, S Khan, P Dasgupta United Kingdom
MP5-17 Validation Measures of a Partial Nephrectomy Training Model

SM Monda, JR Weese, BG Anderson, R Venkatesh, B Cheng, RS Figenshau United States

MP5-18 The development of novel poly vinyl alcohol training model for robotic partial nephrectomy S Isotani, Y Noma, M Kurosawa, T China, T Ieda, K Kitamura, M Nagata, Y Wakumoto, S Muto, S Horie Japan

MP5-19 Construction and Assessment of An Innovative Indigenious Ultrasound Guided Puncture Simulator

AV Rawandale-patil, LG Patni, G Ladumor India

MP5-20 Comparing the K-Box and Advanced Scope Trainer for Flexible Ureterorenoscopy Training: A Randomised Controlled Trial F Nawab, A Aydin, B Smith, T Abe, S Khan, P Dasgupta, K Ahmed United Kingdom

MP5-21 A Novel Training Model for Laparoscopic Ureteroureterostomy Using Urechis Unicinctus Y Zhang
China (People's Republic)

MP5-22 Construction And Assessment Of An Innovative Virtual (Radiation Free) Fluoroscopy Optical Light Based Percutaneous Nephrolithotomy Simulator: An Indigenous Approach To Training AV Rawandale-patil, LG Patni, G Ladumor India

MP5-23 Hands on wet lab and live surgery training in PCNL: any impact to surgical skills of attending surgeons?

I Kyriazis, P Kallidonis, P Ntasiotis, D Kotsiris, V Panagopoulos, T Vrettos, E Liatsikos Greece

MP5-24 Preoperative simulation of PCNL using threedimensional printed model R Takazawa, S Kitayama, S Yoshida, T Tsujii Japan

Wednesday 13 September

Moderated Poster Session 6: PCNL II Techniques and Complications

16:30-18:30

Clinical Stones: PCNI

Convention Centre East, Meeting Room 1

MP6-1 Comparison of the Effect of Tolterodine and Gabapentin With Placebo in Reducing Catheter Related Bladder Discomfort After Percutaneous Nephrolithotomy: A Randomised Clinical Trial R Maghsoudi, M Etemadian, S Farhadi Niaki, A Kashi Iran
MP6-2 Bilateral percutaneous nephrolithotomy on the same procedure: A retrospective analysis of its safety and efficiency J Pelletier, I fugaru, F Soucy, B Laroche, J Cloutier

Canada 
MP6-3 Percutaneous Nephrolithotomy for Renal Calculi up to $15 \mathrm{~mm}$ : What should the ideal tract size be? RA Kukreja India

MP6-4 Supine Percutaneous Lithotomy for Partial and Complete Staghorn Renal Stones

L Mokool, Z Kratiras, M Alsawi, S Yallappa, SK Nalagatla

United Kingdom

MP6-5 Tubeless PCNL as daycare procedure: is it safe? A Rehman, W Rahim, I Khan, D Shohab, I Jamil, G Nawaz, S Muhammad, S Akhter Pakistan

MP6-6 Analysis of factors affecting Radiation Exposure during Percutaneous Nephrolithotomy procedures S Balaji, A Singh, A Ganpule, R Sabnis, M Desai India

\section{MP6-7 Withdrawn}

MP6-8 Percutaneous Nephrolithotomy Puncture and Tract Dilation: Evidence on the Safety of Approaches to the Infundibulum of the Calyx

P Kallidonis, I Kyriazis, P Ntasiotis, D Kotsiris, C Kalogeropoulou, D Apostolopoulos, P Kitrou, E Liatsikos Greece

MP6-9 Inferior renal displacement using a Ureteric Balloon Catheter- A Novel Renal Displacement Technique and A Systematic Review of the Literature

M Alsawi, L Mokool, S Yallappa, T Amer, SK Nalagatla

United Kingdom

MP6-10 Post-Operative Pain Management after Percutaneous Nephrolithotomy: A Randomized Double-Blind Controlled Study Assessing Electro-Acupuncture

E Parkhomenko, T Tran, R Chugh, JN Thai, K Blum, J Capodice, S Purnell, M Gupta United States

MP6-11 Randomized clinical trial of standard mini-percutaneous nephrolithotomy versus standard percutaneous nephrolithotomy R Haghighi Iran

MP6-12 Super-mini percutaneous nephrolithotomy (SMP) for renal stone $<25 \mathbf{~ m m}$ in pediatric patients: Could it be an alternative to shockwave lithotripsy (SWL) ?

K Sarica, B Eryildirim, A Tuerxun, A Batuer, K Erdem, A Buz, G Zeng

Turkey

MP6-13 Flank suspended supine position versus standard supine and prone position in percutaneous nephrolithotomy

M Mahmoud, A Abdel Aal, M Abdel Azim, Sa El Hamshary, E El-Barky

Egypt
MP6-14 Complications of upper-pole calyceal puncture in patients having supine percutaneous nephrolithotomy

L Mokool, S Yallappa, Z Kratiras, SK Nalagatla United Kingdom

MP6-15 Risk of complications with endoscopic renal papillary biopsy ME Rivera, J Lingeman, N York, JC Williams, AE Krambeck United States

MP6-16 Characterization of Thoracic Complications associated with Percutaneous Nephrolithotomy A Serrano, C Trujillo, A Bravo-Balado, M Echeverry, T Higuera, C Barco, A Díaz, D Robledo, J Caicedo, J Cataño, M Plata Colombia

MP6-17 Predictive Factors for Complications assoicated with Percutaneous Nephrolithotomy K Lee, S Choi, T Kim, T Oh, C Yoon, W LEE, $\mathrm{J}$ Lee Republic of Korea

MP6-18 Hydrothorax following supracostal PCNL is influenced by the type of post procedural drainage: A Prospective Randomized Study H Goldberg, A Nevo, Y Shtabholtz, MA Lubin, J Baniel, Y Ehrlich, D Lifshitz Israel

MP6-19 A Minimally Invasive Angioembolization Technique to Treat Delayed Hemorrhage After percutaneous Nephrolithotomy R Maghsoudi, M Etemadian, A Kashi, M Banaei Iran

MP6-20 Cessation of Tract Hemorrhage using the Dual Catheter Hemostatic Sandwich Technique Following Percutaneous Nephrolithotomy B Mattison, M Keheila, I Kelly, S Abourbih, J Cheng, M Pierce, H Wagner, D Baldwin United States

MP6-21 Effect of pelvicalyceal anatomy on perioperative bleeding during percutaneous nephrolithotomy SK Devana, R Patel, RS Mavuduru, A Lal, GS Bora, SK Singh, AK Mandal India

MP6-22 Tubeless Mini Percutaneous Nephrolithotomy: A Single Institution Experience with Outpatient Management of Renal Calculi S Fisher, M Jennings, CA Winter, K Basham, RB Pickens, WM White, B Waters United States

MP6-23 Effect of nephrostomy tube calibre (12F vs 24F) on the outcome of PCNL-A prospective randomized clinical study S Tiwari, S Gupta, I Singh India

MP6-24 Modified supine percutaneous nephrolithotomy with combined fluoroscopy \& ultrasound-guided renal puncture: Initial single-surgeon experience at a UK teaching hospital N Nkwam, M Khan United Kingdom 
MP7-1 The American Stent Epidemic: An International Survey of Ureteral Stenting Practices Following Routine Ureteroscopy J Pereira, PE Bower, G Pareek, S Thavaseelan United States

MP7-2 Office-based ureteric stent removal is achievable, cheaper and associated with reduced stent dwell time, stent-related symptoms and complications, compared with traditional removal methods E Baston, A Palmer, S Wellum, Z Bredow, G Storey, D Van Dellen, B Grey United Kingdom

MP7-3 Efficacy and cost comparisons of metallic versus polymer stents in the management ketamine induced ureteric strictures

D Yong, A Ismail, G Lee United Kingdom

MP7-4 Withdrawn

MP7-5 Experimental Study on the Effectiveness of an Anti-Reflux Ureteral Stent N Liu, Z Liu, J Yang China (People's Republic)

MP7-6 A prospective observational study about stentrelated symptoms after ureteroscopy assessed through a validated questionnaire

E Alessandria, A Bosio, E Dalmasso, D Peretti, A Bisconti, P Destefanis, P Gontero Italy

MP7-7 A multi-centre analysis of antibiotic susceptibility of urinary tract isolates in patients with ureteric stents and associated UTI

A McPhee, N Smyth, M Hinckley, W Ho,

D Clark, B Jones, L Cottom

United Kingdom

MP7-8 Parallel and Intraluminal Stenting M Eshghi, A Fathollahi, M Ferretti, SA Fullerton, JH Hillelsohn, NH Patel United States

MP7-9 Ureteric Stenting: Analysis Of Antegrade \& Retrograde Procedures And Timeliness Of Removal

P Parameshwaran, K Stephenson, N Sheikh United Kingdom

MP7-10 Comparison of polymer and Metallic ureteral stent in the management of benign and malignant ureteric obstructions: Six years experience

A Ismail, N Chia, G Lee

Malaysia

MP7-11 Risk factors for re-infection in patients drained with DJ stent for ureterolithiasis and sepsis

E Kord, G Goltsman, S Hirsh, Y Siegel, A Zisman

Israel
MP7-12 Forgotten Double J Catheter Management AM Cardenas Ortiz, LF Takano Araujo, PR Kawano, HA Yamamoto, R Guerra da Silva, P Pajolli, OE Hidetoshi Fugita Colombia

MP7-13 Two weeks too long: Optimal duration for ureteric pre-stenting and its physiological effects on the ureter in a porcine model

K Lim, Y Lim, L Ng, A Sim

Singapore

MP7-14 Retained Stents: who is at risk? RK Jain, H Chaparala, M Omar, S Sivalingam, MJ Noble, M Monga United States

MP7-15 Ureteral Stent Placement in Clinic Using Nitrous Oxide Analgesia: One Institution's Experience GL Machen, PA Milburn, PS Lowry, MM El Tayeb, L Tsai United States

MP7-16 Impact of loop-tail ureteral stents on ureteral stent-related symptoms immediately after ureteroscopic lithotripsy: Comparison with pigtail ureteral stents

M Taguchi, T Inoue, K Muguruma, T Murota, H Kinoshita, T Matsuda Japan

MP7-17 Long-term outcomes of double-layered polytetrafluoroethylene membrane-covered selfexpandable segmental metallic stents (Uventa) in patients with chronic ureteral obstructions: Is it really safe?

D Kim, M Kim, B Hong, H Park Republic of Korea

MP7-18 A swellable drug-eluting ureteral stent for the treatment of upper tract urothelial carcinoma - a proof of concept in-vivo porcine study K Chen, W Lim, G Xiong, Y Huang, $\mathrm{S}$ Venkatraman, T Chong Singapore

MP7-19 Uventa, a new covered metallic ureteric stent: initial experience in a United Kingdom centre A Pai, S Janardanan, P James, H Ni Raghallaigh, R Kulkarni United Kingdom

MP7-20 The ALLIUM $\AA$ ureteral stent in the management of ureteral disorders

F De Marco, C Corsi, L Grillenzoni, S Di Nicola, A Alfarone, M Pozza, U Parente, P Vicini, C Leonardo, G Ricciuti Italy

MP7-21 Withdrawn

MP7-22 Withdrawn 
MP7-23 The rendezvous technique of Ureteric stenting: when, how and why?

S Janardanan, P James, A Pai, R Kulkarni

United Kingdom
MP7-24 Malignant ureteral obstruction - Balloon dilatation and decompression using Tandem ureteral stents

A Shvero, N Kleinmann, M Laufer, ZA Dotan, D Zilberman, J Ramon, H Winkler Israel

Wednesday 13 September

MP8-1 Placement of ureteral access sheath without fluoroscopic guidance: $\mathbf{1 2 2}$ cases in a single center S Wang, X Zhang, B Xiao, W Hu, S Chen, J Li China (People's Republic)

MP8-2 Accuracy of Predicting Stone Composition During Endoscopic Evaluation

T Marien, A Darves-Bornoz, J Robles, N Miller

United States

MP8-3 Fluid Dynamic Analysis of Manual Pressure Infusor Irrigation

DJ Lama, RM Patel, S Safiullah, J Landman, RV Clayman

United States

MP8-4 Moses Technology from Stone Simulator to Early Clinical Experience

A Ibrahim, S Badaan, S Andonian, M Elhilali

Canada

MP8-5 Evaluating the Ergonomics of Flexible Ureteroscopy

WW Ludwig, G Lee, J Ziemba, BR Matlaga United States

MP8-6 Crowd Sourcing Evaluation of Ureteroscopic Videos using the Post-Ureteroscopic Lesion Scale (PULS) to Assess Ureteral Injury Following Ureteral Access Sheath Deployment KS Kaler, K Bettir, S Safiullah, DJ Lama, R Yoon, J Landman, RV Clayman United States

MP8-7 Reducing the Narcotic Burden: Comparison of Postoperative Outcomes of Opioids vs Non-opioids for Post-Ureteroscopy Pain Control

T Cisu, D Sobel, K Sternberg United States

MP8-8 A pilot study evaluating changes to haematological and biochemical tests after Flexible Ureterorenoscopy for the treatment of kidney stones

SF Hughes, AJ Moyes, I Shergill

United Kingdom
MP8-9 A prospective, observational study on the feasibility of a single-session endoscopic combined intrarenal surgery for ipsilateral renal stones and retrograde intrarenal surgery for contralateral renal stones: initial experiences

J Park, K Ko, W Bang, M Choo, S Lee, D Lee, H Kim, K Koo, S Yoo, M Cho, H Jeong, S Cho, T Choi, K Yoo Republic of Korea

MP8-10 Published (PubMed) trends and bibliometric analysis of Ureteroscopy (URS) for stone disease over a 16-year period (2000-2015)

A Pietropaolo, R Geraghty, B Somani United Kingdom

MP8-11 In Vitro Comparison of Stone Fragmentation When Using Various Settings with Modern Variable Pulse Holmium Lasers J Bell, KL Penniston, SY Nakada United States

MP8-12 Adverse Events Related to Holmium Laser Lithotripsy at the time of Ureteroscopy: Looking under the hood of the FDA

T Osumah, AH Aldoukhi, J Hollingsworth, WW Roberts, KR Ghani United States

MP8-13 Cost-Effective Semi-Rigid Ureteroscopy With Laser Dusting Is Effective For The Treatment Of Sizable Proximal Ureteral Calculi AH Gabr, ER Tawfiek Egypt

MP8-14 Primary Retrograde Intrarenal Surgery versus Excorporeal Shockwave Lithotripsy in High Risk Renal Stones

I Ab. Rashid, A Wahi, R Hassan, H Ghazali Malaysia

MP8-15 One surgeon active stone retrieval procedure in flexible ureteroscopy for urinary calculi S Okada, S Hamamoto, T Inoue, S Minagawa, H Morikawa, T Matsuda, H Miura Japan

MP8-16 Withdrawn

MP8-17 Withdrawn 
MP8-18 Micro-costing analysis demonstrates comparable costs for Lithovue versus reusable flexible ureteroscope use

K Taguchi, M Usawachintachit, DT Tzou, D Isaacson, BA Sherer, I Metzler, ML Stoller, T Chi

United States

MP8-19 Prospective Evaluation of Stone Free Rates by Computed Tomography After Aggressive Ureteroscopy

N Canvasser, I Sorokin, A Lay, E Kolitz, J Antonelli, M Pearle

United States

MP8-20 A Fluoroscopy-Free Retrograde Intrarenal Surgery for Renal Stones: A Feasibility and Efficacy Study Compared with Conventional Flexible Ureteroscopy

Y Tai, H Tai, Y Lee

Taiwan (Republic of China)
MP8-21 Pediatric Ureteropyeloscopy In Supine AbductedThigh Position

M Hosseini, A Aminsharifi, A Eslahi, R Haghpanah Iran

MP8-22 Does ureteral access sheath usage result in permanent damage in the ureter?

A Gücük, G Söyler, U Üyetürk, B Yılmaz, Nalbant, S Gücük, A Kükner, A Çetinkaya Turkey

MP8-23 Early experience of retrograde intra-renal surgery in Mongolia

D Batbold, A Sankjaajamts, T Zev, M Munkhbaatar, S Avirmed, J Jodov, J Bayarlakh Mongolia

MP8-24 Utilization of pressurized vs non-pressurized irrigation during ureteroscopy in the absence of ureteral access sheath: A comparative retrospective study

K Doersch, A El Mekresh, GL Machen, PA Milburn, K Hart, MM El Tayeb United States

Wednesday 13 September Moderated Poster Session 9: Non-Surgical \& Medical Management of Stones I 16:30-18:30

Clinical Stones: Medical Management

Convention Centre East, Meeting Room 11

MP9-1 Medical dissolution therapy for the treatment of uric acid nephrolithiasis

C Gridley, M Sourial, B Knudsen

United States

MP9-2 Do patients with non-alcoholic fatty liver disease have predictable 24-hour urine abnormalities?

E Ghiraldi, A Lee, C Tong, J Friedlander United States

MP9-3 A Prospective Randomized Controlled Trial Comparing the Efficacy of Low-oxalate Diet versus Vitamin B6 and Magnesium Supplementation versus Both in Idiopathic Hyperoxaluria

JN Thai, T Tran, E Parkhomenko, K Blum, M Gupta, S Purnell

United States

MP9-4 Does saturation index predict stone activity in patients with calcium oxalate nephrolithiasis? N Canvasser, I Sorokin, A Lay, E Kolitz, X Li, B Adams-Huet, J Poindexter, N Maalouf, O Moe, K Sakhaee, J Antonelli, M Pearle United States

MP9-5 Feasibility and Safety of Individual Renal Unit Urine Sampling for Metabolic Analysis JB Ziemba, R Gurnani, MA Gorin, WW Ludwig, BR Matlaga, JR Asplin United States

MP9-6 Mineral water in the prevention of renal stones P Sundaram, T Chong Singapore
MP9-7 Association between serum 25-OH-vitamin D and 24-hour urine calcium in urolithiasis patients S Tavasoli, M Taheri, F Bagheri Amiri, M Parvin, A Basiri Iran

MP9-8 Association between 24-Hour urine metabolic abnormalities and stone recurrence in patients with urolithiasis

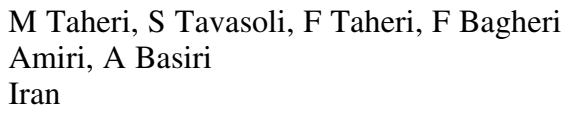

MP9-9 Dietary Assessment and 24 Hour Urine Collections in the Management of Hypercalciuria: Are Both Needed?

J Bell, M Ehlers, A Schweitzer, SY Nakada, KL Penniston United States

MP9-10 Association of subcutaneous adipose tissue with nephrolithiasis as measured by computed tomography

HS Alakrash, B Alharbi, J Hoogenes, K Kim, O Aljarallah, A Kapoor, B Shayegan,

ED Matsumoto

Canada

MP9-11 Relationship of Sodium Intake to Urinary Sodium Excretion in Stone Formers: Which Foods Contribute the Most?

J Bell, M Ehlers, A Schweitzer, SY Nakada, KL Penniston

United States 
MP9-12 Pharmaceutical Burden and Medication Compliance in Patients with Cystinuria BA Sherer, D Isaacson, Z Kornberg, M Usawachintachit, DT Tzou, K Taguchi, S Wiener, T Chi, ML Stoller United States

MP9-13 Long-term treatment of vitamin D insufficiency or deficiency in postmenopausal women with or without hypercalciuria does not increase urinary calcium excretion

KL Penniston, RA Jhagroo, AC Staples, SY Nakada, KE Hansen United States

MP9-14 Correlation between cystine concentration and cystine capacity in cystinuric patients N Canvasser, I Sorokin, J Friedlander, D Mollengarden, J Antonelli, M Pearle United States

MP9-15 Effect of Increased Fluid Intake on 24-hour Urinary pH in Stone Formers with Low Urine Volume

D Wollin, B Winship, W Tom, LG Davis, E Cone, C Scales, M Ferrandino, M Lipkin, GM Preminger

United States

MP9-16 The relationship between urinary stone composition and metabolic factors including obesity index, metabolic disease

J Kim, J Shim, S Kang, J Cheon, J Lee, J Kim, S Kang

Republic of Korea

MP9-17 Do Metabolic Factors Influence the Formation and Recurrence of Bladder Calculi?

JN Thai, T Tran, E Parkhomenko, K Blum, M Gupta, S Purnell

United States
MP9-18 Stone composition and lithogenic risk factors in patients with ureteropelvic junction and renal calculi

M Shahait, A Maganty, MJ Semins, T Tarin, S Jackman, T Averch, M Ost United States

MP9-19 Growing older, growing stones? Increasing health care use by older adults with stones

B Winship, T Hobbs, D Wollin, M Lipkin, K Schmader, GM Preminger, C Scales United States

MP9-20 Virtual Stone Clinic - The Future of Stone Management in the United Kingdom? T Smith, O Blach, K Guest, A Symes United Kingdom

MP9-21 Are Patients with Pure Uric Acid Stones Metabolically and Clinically Different than those with Mixed Uric Acid Stones?

JN Thai, T Tran, E Parkhomenko, K Blum, M Gupta, S Purnell United States

MP9-22 Impact of metabolic risk factors on stone recurrence in patients submitted to nephrectomy due to struvite stones

A Danilovic, IA Wei, T Ferreira, S Reis, A Brito, F Vicentini, F Torricelli, G Marchini, C Batagello, E Mazzucchi, W Nahas, M Srougi Brazil

MP9-23 Non Compliance in Recurrent Nephrolithiasis Undergoing Metabolic Evaluation E Kwenda, Z Chen, BK Carew, VG Bird, M Prosperi, VY Bird United States

MP9-24 Quality of life and satisfaction in a virtual stone clinic: a pilot study

J Hendry, L Kerr, C McIlhenny

United Kingdom

Wednesday 13 September

Moderated Poster Session 10: Epidemiology, Health Policy, Socioeconomics \& Outcomes

16:30-18:30

Epidemiology, Health Policy, Socioeconomics \& Outcomes

Convention Centre East, Meeting Room 19

MP10-1 Pre-Operative Nomograms for Predicting Renal Function at 2 Years after Donor Nephrectomy C Shum, CD Bahler, CP Sundaram Singapore

MP10-2 Stone Size at Initial Presentation at a Tertiary Care Center: Demographics MJ Wardenburg, J Brown, R Ruchi, X Wen, VG Bird, VY Bird United States

MP10-3 Post-Acute Care as a Driver of Episode Cost Variation for Ambulatory Stone Surgery J Hollingsworth, J San Juan, H Hou, J Dupree, KR Ghani United States
MP10-4 The "Acute" Stone Clinic Effect: Improving Healthcare Delivery by Reorganizing Clinical Resources

MA Asmus, S De, T Schuler, D Bochinski, T Wollin Canada

MP10-5 Modeling operating room costs for treatment of ureteral calculi using time-driven activity-based costing

TR McClintock, DF Friedlander, AY Feng, S Ganeshan, MA Shah, SL Chang, TW Feeley, AM Bader, GE Haleblian United States 
MP10-6 Improving value in urologic surgery episodes of care through time-driven activity-based costing TR McClintock, DF Friedlander, S Ganeshan, MA Shah, SL Chang, TW Feeley, GE Haleblian United States

MP10-7 Comparing the Utilization and Outcomes of Extracorporeal Shock Wave Lithotripsy between Inpatient and Outpatient Settings in New York State, 1990-2012

A Yang, KH Bilal, M Finkelstein, MA Palese United States

MP10-8 Nephrectomy for calculous disease: analysis of United States trends and outcomes from 2001 to 2014

V Dombrovskiy, E Olweny United States

MP10-9 Unplanned readmission rates and postoperative complications after day-case surgery in Urology: Retrospective study over 1 year

F Colomb, I Bentellis, P Regnier, P Treacy, L Vignot, R Haider, Y Bodokh, B Tibi, P Romain, J Fallot, J Amiel, D Chevallier, M Durand France

MP10-10 Trends and Sociodemographic Disparities in the Surgical Management of Benign Prostatic Hyperplasia, 1997-2012

A Yang, M Finkelstein, KH Bilal, MA Palese United States

MP10-11 Ethnicity and family history impact age of onset and number of episodes for urolithiasis:

a prospective cohort study from ReSKU

K Taguchi, DT Tzou, M Usawachintachit, BA Sherer, ML Stoller, T Chi United States

MP10-12 30 Day Unplanned Readmission After Minimally Invasive Urological Procedure at a Tertiary Care Academic Center AE Ward, SS Parikh, NH Patel, JH Hillelsohn, SA Fullerton, JL Phillips, MS Choudhury, M Eshghi United States

MP10-13 Prevalence of Kidney Stones Changing in Male to Female Ratio: National Health and Nutrition Examination Survey (NHANES) 2007-2014

Z Chen, M Prosperi, VY Bird United States

MP10-14 Kidney Stones Are Positively Associated With Sleep Disorders: National Health and Nutrition Examination Survey (NHANES) 2011-2014 Z Chen, M Prosperi, VY Bird United States

MP10-15 Withdrawn
MP10-16 Worldwide trends in the role of warmer climate on the incidence of renal colic and kidney stone disease (KSD): Evidence from a systematic review of literature

R Geraghty, S Proietti, M Archer, A Pietropaolo, BK Somani United Kingdom

MP10-17 Trends of Ambulatory Urological Surgery in the State of Florida: 2010-2014 HD Patel, BR Matlaga, JB Ziemba United States

MP10-18 Predicting Adverse Outcomes in Laparoscopic Nephrectomies Utilizing a Modified Frailty Index I Levy, M Finkelstein, MA Palese United States

MP10-19 Urologic Cancer Disparities among Hispanic Americans and Native Americans in Four U.S.-Mexico Border States

A Bergersen, K Batai, J Michalak, M Craig, E Price, BR Lee United States

MP10-20 Understanding the Time Course of Nephrolithiasis Management RL Sur, SK Bechis, RM Shapiro, DG Kronenberg United States

MP10-21 Care Pathway Variation and Surgical Cost Measurement for Percutaneous Nephrolithotomy with Time-Driven Activity-Based Costing I Metzler, D Issacson, M Usawachintachit, M Hudnall, DT Tzou, K Taguchi, T Chi United States

MP10-22 Defining the Costs of Reusable Flexible Ureteroscope Reprocessing Using Time-Driven Activity-Based Costing (TDABC)

D Isaacson, I Metzler, T Ahmad, S Rosenberg-Wohl, K Taguchi, M Usawachintachit, DT Tzou, BA Sherer, ML Stoller, T Chi United States

MP10-23 Impact of race/ethnicity and socioeconomic status on stone characteristics: results from ReSKU - the Registry for Stones of the Kidney and Ureter

M Usawachintachit, DT Tzou, K Taguchi, BA Sherer, BD Duty, JD Harper, MD Sorensen, RL Sur, RM Sweet, ML Stoller, T Chi United States

MP10-24 Feasibility of Performing MRI Prostate before Prostate Biopsy in a District General Hospital in the UK

Y Phan, A Loh, A Anandakumar, S Umranikar, N Lynn United Kingdom 


\section{MP11-1 Withdrawn}

MP11-2 Bilateral simultaneous ureteroscopic (BS-URS) approach in the management of Bilateral Urolithiasis is a safe and effective strategy in the contemporary era - Evidence from a Systematic Review

R Geraghty, BP Rai, P Jones, A Pietropaolo, BK Somani United Kingdom

MP11-3 The role of preoperative ureteral stenting in retrograde intrarenal surgery

S Yoo, H Yuk, S Lee, H Kim, C Jeong Republic of Korea

MP11-4 Surgical planning for the treatment of $2-3 \mathrm{~cm}$ kidney stones: Retrograde intrarenal surgery or mini-Percutaneous nephrolithotomy? A retrospective compared study G Zeng, Z Zhao, W Zhong, Y Liu China (People's Republic)

MP11-5 Withdrawn

MP11-6 Substantial Clinical Parameters (3s Rules) Affect The Outcomes of Flexible Ureteroscopy For Kidney Stone Retrieval M Ilgi, S Kirecci, E Abdullayev Turkey

MP11-7 Sequential balloon dilation Vs direct access sheath insertion pre flexible ureteroscopy in non pre-stented patients: Retrospective comparative study

\author{
AM Elmekresh, DM Masten, GL Machen, \\ PS Lowry, MM El Tayeb, L Tsai \\ United States
}

MP11-8 Ureterorenoscopy and Laser Lithotripsy for Upper Tract Stones Using "Stone Dusting" Technique MA Hussein, I Saad, A Abdalla, S Sadek Egypt

MP11-9 Endoscopic recognition of Kidney Stones: The new challenge for urologist?

V Estrade, I Jour, M Daudon, O Traxer France

MP11-10 Urolithiasis after bariatric surgery: a five-year follow-up study A Ostrowski, R Williams, D Thiel, R Pak United States

MP11-11 Ureteral Access Sheath Use and Incidence of Hydronephrosis

BJ Otto, R Terry, JM Shields, VG Bird United States

MP11-12 Analysis of ureter stone in ureteroscopic lithotripsy: A single institution experience T Oh, J Lee, S Park, I Seo Republic of Korea
MP11-13 Analysis of surgical complications of retrograde intrarenal surgery

B Kim, Y Lee, J Chung, Y Ha, S Choi, J Lee, H Kim, T Kim, E Yoo, T Kwon, S Chung Republic of Korea

MP11-14 Management of nephrolithiasis with a large ureteral access sheath is not associated with increased risk of surgical complications and stricture formation

A Khambati, B Jordan, S Yoo, K Perry, R Nadler

United States

MP11-15 Return for Unplanned Care after Ureteroscopy Does the Type of Pain Medication Matter? PA Milburn, A El Mekresh, GL Machen, MM El Tayeb, L Tsai United States

MP11-16 The feasibility of Poly FURS combined with ultrasound guided all seeing needle Micropen to treatment lower calyceal stones over $2 \mathrm{~cm}$ in size with Infundibular pelvic angle less than 60 degree Y Wenzeng China (People's Republic)

MP11-17 Flexible ureteroscopy with holmium laser lithotripsy completely fluoroless for treating kidney stones: feasibility, outcomes and complications. "Our experience" BO Manzo, E Lozada, J Sanchez, G Manzo, H Sanchez, R Maldonado, M Vanzzini Mexico

MP11-18 Does retrograde treatment of upper urinary tract stones necessitate postoperative upper urinary tract drainage? Conclusions from over 500 single center consecutive cases

IM Sabler, A Isid, A Lorber, A Latke, D Shurka, T Harhel, S Sfoungaristos, V Yutkin, G Hidas, E Landau, D Pode, O Gofrit, M Duvdevani Israel

MP11-19 Withdrawn

MP11-20 Upper Tract Imaging after Ureteroscopy: Risk Factors for Hydronephrosis

N François, M Sourial, J Cooper, H Miyagi, G Box, B Knudsen United States

MP11-21 Preliminary evaluation of High Frequency Jet Ventilation in RIRS from a tertiary center MN Hasan, M Brehmer, P Harbut Sweden 
MP11-22 Revisiting the $15 \mathrm{~mm}$ renal stone size threshold in the modern RIRS era

H Goldberg, D Golomb, Y Shtabholtz, S Tapiero, G Creiderman, A Shariv, J Baniel, Y Ehrlich, D Lifshitz

Israel

MP11-23 Flexible ureteroscopy in patients with nephrolithiasis associated with autosomal dominant polycystic kidney disease (ADPKD) P Geavlete, D Georgescu, R Multescu, C Ene, V Iordache, G Balan, B Geavlete Romania
MP11-24 Comparison of Two Novel Single-Use Flexible Ureteroscopes to Currently Existing Reusable and Single-Use Flexible Ureteroscopes D Wollin, B Winship, R Jiang, W Tom, D Radvak, C Scales, M Ferrandino, W Simmons, GM Preminger, M Lipkin United States

Thursday 14 September

Moderated Poster Session 12: Stones: Instrumentation

14:00-16:00

Clinical Stones: Equipment (Stents, Lasers, Guidewires, Sheaths) Convention Centre East, Meeting Room 2

MP12-1 Surgeon Assessment of the Dakota Stone Retrieval Device

SK Bechis, JE Abbott, RL Sur, DG Kronenberg United States

MP12-2 Holmium Laser Lithotripsy with Moses

Technology - Initial Evaluation

A Symes

United Kingdom

MP12-3 Optimizing Configuration of Thulium (Tm) Fiber Laser for Lithotripsy

P Glybochko, G Altshuler, A Vinarov, L Rapoport, D Enikeev, N Sorokin, A Dymov, V Vinnichenko, A Kovalenko, I Yaroslavsky Russia

MP12-4 Percutaneous nephrolithotomy assisted by a novel negative pressure sheath

D Lai, X Li, Y He, M Sheng

China (People's Republic)

MP12-5 Standardized Reporting of Device-Related Adverse Events During Percutaneous Nephrolithotomy: Review of the Manufacturer and User Facility Device Experience (MAUDE) Database

NH Patel, A Schulman, N Uppaluri, J Bloom, JL Phillips, S Konno, MS Choudhury, M Eshghi United States

MP12-6 A novel approach: simultaneous catch and blast Ureteroscopy lithotripsy with in situ dormia basket Vs standard stone fragmentation HG Thummar, K Thummar, N Zinto, J Vyas, N T, P Suthar India

MP12-7 Computed tomography Hounsfield parameters for discrimination of predominantly composed calcium oxalate mono- and di- hydrate stones: preliminary results

A Gallioli, M Fontana, E De Lorenzis, L Boeri, S Zanetti, F Palmisano, M Talso, F Longo, P Dell'Orto, M Ferruti, E Montanari Italy
MP12-8 In patients with obstructing ureteral calculi, the severity of perinephric stranding is predictive of creatinine elevation

D Papagiannopoulos, J Ebersole, M Farrell, G White, LA Deane United States

MP12-9 Is Limited Non-Contrast Pelvic CT really indicated for asymptomatic patients with small distal ureteric calculi?

N Hicks, W Finch, SO Irving, N Burgess United Kingdom

MP12-10 What Doses Are Our Patients Receiving? An audit examining the radiation dose associated with CT KUB, CT IVU and intra operative fluoroscopy during ureteroscopy and PCNL M Sahu, P Patel, N Lobo, J Makanjuola, I Honey, D Gallagher, D Phillips, G Rottenberg, S Willis, J Glass, K Thomas, M Bultitude United Kingdom

MP12-11 Furs Versus PNL; Total Stone Volume Measurement By Non-Contrast CT (NCCT) Could Be Defined Precise Cut-off Value For The Management Of Procedures M Ilgi, S Kirecci, C Kutsal, A Dalkilic Turkey

MP12-12 Imaging Patterns After Percutaneous Nephrolithotomy

JS Ahn, S Holt, P May, MD Sorensen, JD Harper United States

MP12-13 Stone management in patients with ureteropelvic junction obstruction is possible not only by lithotomy during laparoscopic pyeloplasty but also by secondary lithotripsy

M Nishi, K Matsumoto, D Ishii, T Hirayama, T Fujita, M Iwamura Japan 
MP12-14 Comparison of three different endoscopic techniques in treatment of bladder stones J Choi, Y Ko, P Song, K Moon, H Jung, J Kim, Y Ha

Republic of Korea

MP12-15 Impact of preoperative $\alpha$-adrenergic antagonists on ureteral access sheath insertion force and the upper limit of force to avoid ureteral mucosal injury: A randomized-controlled study

K Koo, J Yoon, Y Ha, B Chung, D Lee Republic of Korea

MP12-16 Effects of Laser Settings and Irrigation Rates on Ureteral Temperature During Laser Lithotripsy, an In Vitro Model

D Wollin, B Winship, W Tom, C Scales, M Ferrandino, W Simmons, GM Preminger, M Lipkin

United States

MP12-17 Vaporizing effect of popcorn technique for laser lithotripsy; comparing the different setting of high energy in calyceal model

K Jongjitaree, E Chotikawanich Thailand
MP12-18 How Do You Like Your Popcorn? An Evaluation of Laser Settings and Location in the Efficiency of the Popcorn Effect

D Wollin, B Winship, R Jiang, W Tom,

D Radvak, C Scales, M Ferrandino, W Simmons, GM Preminger, M Lipkin United States

MP12-19 Digital flexible ureteroscopy: Olympus vs Storz - technology differences

P Geavlete, D Georgescu, M Jecu, R Multescu, B Geavlete Romania

MP12-20 Dual Usage of a Stone Basket: Stone Capture and Retropulsion Prevention

T Kroczak, D Ghiculete, R Sowerby, K Pace, R D'A. Honey

Canada

MP12-21 A Multicenter, Prospective, Observational Study to Investigate the Feasibility of the Disposable Flexible Ureterorenoscopy (Lithovue ${ }^{\circledR}$ ) in Patients with Renal Stones S Cho, J Lee, D Shin, I Seo, H Park Republic of Korea

MP12-22 Laser versus pneumatic lithotripsy with semi rigid ureteroscope. A comparative randomized study

S Rabani, s rabani

Iran

Thursday 14 September

MP13-1 Short and long-term Quality of life (QoL) in patients with percutaneous nephrostomy (PCN), and comparision to other forms of urinary drainage: Systematic review of literature F New, B Somani United Kingdom

MP13-2 Outcomes of Retrogade Intrarenal Surgery in Shockwave Lithotripsy Failed Intermediate Size Lower Calyceal Calculi B Singh, K Kaura, S Sankhwar, A Goel India

MP13-3 Natural history, complications, and re-intervention rates of residual stone fragments following percutaneous nephrolithotomy AS Emmott, H Brotherhood, RF Paterson, D Lange, BH Chew Canada

MP13-4 Contemporary Trends In Utilization And Outcomes Of Percutaneous Nephrolithotomy In The United States From 2003 To 2014

S Harmouch, J Leow, C Meyer, Y Wang, SL Chang, B Chung, Q Trinh, R Korets, N Bhojani

Canada
MP13-5 Perioperative aspirin use during percutaneous nephrolithotomy (PCNL): our single center experience

J Ebel, M Sourial, B Knudsen

United States

MP13-6 Should MiniPercutaneous Nephrolithotomy (MiniPNL) be the ideal tract for Medium sized Renal Calculi (16-30mm)?

RA Kukreja

India

MP13-7 Continuing Aspirin Does Not Increase Blood Loss From Percutaneous Nephrolithotomy T Tran, E Parkhomenko, JN Thai, K Blum, M Gupta

United States

MP13-8 Is PCNL changing in the UK - Analysis of 9500 cases from the BAUS PCNL Registry

W Finch, JN Armitage, J Withington, SO Irving, S Fowler, N Burgess, O Wiseman United Kingdom 
MP13-9 How can we measure successful outcome for PCNL? Further planned treatment vs. traditional measurements of stone free rate: Analysis of a national registry

W Finch, JN Armitage, J Withington, SO Irving, S Fowler, N Burgess, O Wiseman United Kingdom

MP13-10 Effect of open surgery and shock wave lithotripsy (swl) on percutaneous nephrolithotomy in elderly patients

I Aridogan, V Izol, A Borekoglu, N Akdogan, I Sukur, M Deger

Turkey

MP13-11 Comparison of percutaneous nephrolithotomy results in different age groups

V Izol, I Aridogan, A Borekoglu, N Akdogan, O Yilmaz, F Ok

Turkey

MP13-12 Role of minimally invasive PCNL techniques: Micro and Ultra-mini PCNL $(<15 F r)$ in the paediatric population - A Systematic Review P Jones, A Pietropaolo, OM Aboumarzouk, B Somani United Kingdom

MP13-13 The Role of Frailty Assessment in the Prediction of Post-operative Complications for Percutaneous Renal Surgery

V Vasudevan, P Samson, G Gaunay, K Ren, Z Okeke, AD Smith, DM Hoenig United States

MP13-14 Does percutaneous nephrolitotomy affect renal function in the first year after surgery? E Hernández Méndez, C Martínez Arroyo, J Herrera Muñoz, I Calvo Vázquez, P Cortés Raygoza, M Ortega González, M Cantellano Orozco, G Fernández Noyola, J Morales Montor, C Pacheco Gahbler Mexico

MP13-15 Stone morphometry: tract (s) and procedure (s) predictor for percutaneous nephrolitotomy J Sedano Basilio, C Martínez Arroyo, U Sánchez Aquino, E Hernández Méndez, M Cantellano Orozco, G Fernández Noyola, J Morales Montor, C Pacheco Gahbler Mexico
MP13-16 Costs Variations For Percutaneous Nephrolithotomy In The United States From 2003 To 2015: A Contemporary Analysis Of An All-Payer Discharge Database M Meskawi, J Leow, SL Chang, Q Trinh, R Korets, N Bhojani, A Valiquette, B Chang Canada

MP13-17 Is there any difference in outcomes among variations of position in percutaneous nephrolithotomy? PA Melo, F Vicentini, C Murta, J Claro, R Perrella, M Hisano Brazil

MP13-18 Ambulatory Percutaneous Nephrolithotomy: 2 year 150 case experience JE Abbott, J Davalos United States

MP13-19 Withdrawn

MP13-20 Percutaneous Nephrolithotomy (PCNL): 15 Years Experience with Over 15,000 cases M Hosseini, A Aminsharifi, D Irani, A Eslahi, R Haghpanah, A Yousefi, R Inaloo Iran

MP13-21 The Clinical Safety and Efficacy of Upper Pole Access Percutaneous Nephrolithotomy (uPPCNL) for Inferior Pole Stones D Hurtado, JA Abraham Philippines

MP13-22 PCNL in kidney with borderline renal function: A single institution experience

M Shahait, O Ayyash, S Jackman, T Averch United States

MP13-23 Outpatient PCNL Outcomes: The UC San Diego Experience DS Han, SK Bechis, JE Abbott, RL Sur United States

MP13-24 Ambulatory Percutaneous Nephrolithotomy: A Cost-Reducing Innovation D Beiko, K Pace, S Andonian Canada

Thursday 14 September Moderated Poster Session 14: Pediatric Endourology, Laparoscopy and Robotics 14:00-16:00

Clinical Stones: Pediatrics

Convention Centre East, Meeting Room 11

MP14-1 Unique Combined Endoscopic Approach to Large Staghorn Calculus in a 2-Year-Old

N Jung, D Herz

United States

MP14-2 Combined use of flexible ureteroscopic lithotripsy with micro-percutaneous nephrolithotomy in pediatric multiple kidney stones

W Wang, J Li

China (People's Republic)
MP14-3 Staged ureteroscopic management of large bilateral renal calculi in prepubescent patients

EM Sebesta, AC Small, MJ Lipsky, GW Moran, OD Shah, P Casale United States 
MP14-4 A contemporary update of paediatric urinary stone composition in the United Kingdom \& the Republic of Ireland

PT Grice, N Nkwam, D Bodiwala, N Armugam, N Mamooowala, M Wells, V Lee, G Ball, P Gupta, P Patel

United Kingdom

MP14-5 Mini-percutaneous cystolithotripsy (PCCL) in pre-school children MS Agrawal, D Mishra India

MP14-6 Pre-Operative Tamsulosin and Ureteral Orifice Navigation in Pediatric Patients: Is there any benefit?

C Morley, O AL-Omar

United States

MP14-7 Post-Surgical Imaging Patterns in Pediatric Nephrolithiasis: Opportunities for Improvement J Ellison, P Merguerian, B Fu, S Holt, T Lendvay, J Gore, M Shnorhavorian United States

MP14-8 Renal Papillary Mapping and Quantification of Randall's Plaque in Pediatric Calcium Oxalate Stone Formers

A Darves-Bornoz, J Thomas, T Marien, $\mathrm{N}$ Miller United States

MP14-9 Outcomes of flexible ureteroscopic lithotripsy for treatment of kidney stones in infants W Wang, J Li China (People's Republic)

MP14-10 Subspecialty Practice Patterns in the Treatment of Pediatric Urolithiasis

R Davis, NJ Farber, A Kaplan, R Patel, R Steckler, S Elsamra United States

MP14-11 Trends of intervention for paediatric stone disease over the last two decades A Pietropaolo, B Somani United Kingdom

MP14-12 Emergency Department visits, readmissions, and complications after ureteroscopy for pediatric urolithiasis

RN Prasad, AC Strine, CW Concodora, ME Schulte, BA VanderBrink, PH Noh, E Minevich, PP Reddy, WR DeFoor United States

MP14-13 Radiologic evaluation of children prior to SWL: to what extent they are exposed to radiation ? B Eryildirim, O Turkoglu, C Goktas, O Kavukoglu, K Sarica Turkey
MP14-14 Outcomes of percutaneous nephrolithotomy (PCNL) in pediatric patients with spina bifida and/or non-ambulatory status: Are all PCNL patients created equally?

J Van Batavia, T Kawal, D Chu, A Shukla, A Srinivasan

United States

MP14-15 Percutaneous Nephrolithotomy(PCNL)

in Children under Age 15

M Hosseini, A Eslahi, A Derakhshan, M Basiratnia

Iran

MP14-16 Percutaneous Nephrolithotomy in Pediatric Patients with Solitary Kidney I Aridogan, V Izol, N Satar, N Akdogan, F Ok, B Kizilgok Turkey

MP14-17 Laparoendoscopic Reduced Port Pyeloplasty for Children and Young Patients

K Takei, M Yamasaki, K Iwasaki, M Hanada, Y Akita, K Hirai, T Ando, T Shin, T Nomura, F Sato, T Terachi, H Mimata Japan

MP14-18 Outpatient robotic extravesical ureteral reimplantation: short-term outcome assessment of safety

EJ Fichtenbaum, AC Strine, CW Concodora, ME Schulte, PH Noh

United States

MP14-19 Does side matter? The impact of laterality on pediatric laparoscopic pyeloplasty

D Bos, J Moore, M Rickard, A Lorenzo, R Romao, D D'Souza, L Braga Canada

MP14-20 The clinical application of laparoscopy combined flexible ureteroscope in the treatment of hydronephrosis associated with upper urinary tract calculi S Jie, Z Liang China (People's Republic)

MP14-21 Durability of pediatric robotic pyeloplasty: Should we follow long-term?

C Rowe, M Shapiro, J Ellison, T Lendvay United States

MP14-22 Establishment of a Pediatric Urology Robotic Program: Ancilllary and Technical Results in Robotic Assisted Pyeloplasty AC Keenan, TG Leffler, RR Su, SY Nakada United States

MP14-23 Paediatric Needleoscopic Pyeloplasty-Our initial experience in Advanced Centre of Kidney and Urology

M Naser, M Hossain, M Isam

Bangladesh 
MP15-1 Prostate cancer distribution and pathological stage in patients diagnosed by transrectal systemic random biopsy

$$
\begin{aligned}
& \text { K Wu } \\
& \text { Taiwan (Republic of China) }
\end{aligned}
$$

MP15-2 The Clinical Outcome of Prostate Ductal Adenocarcinoma: a matched cohort comparative study

NS Khan, Y Kiberu, O El Hage, M Suleman, A Chandra, S Morris, P Dasgupta United Kingdom

MP15-3 Early outcomes of focal brachytherapy for localized prostate cancer: comparison with whole gland brachytherapy

V Srougi, E Barret, I Nunes-Silva, F Rozet, M Galiano, R Sanchez-Salas, J Cosset, $\mathrm{X}$ Cathelineau France

MP15-4 Tumor Characteristics and Survival Outcomes of Prostate Cancer Diagnosed on Repeat Biopsies C Tseng, C Chen, I Chiang Taiwan (Republic of China)

MP15-5 Focal Therapy of Prostate Cancer May Lead to Undertreatment of Prostate Cancer: A Study on Laterality Agreement Between Clinial and Pathological Prostate Cancer A Chow, K Gallo, D Papagiannopoulos, M Montour, C Coogan, K Latchamsetty United States

MP15-6 Is PSA Density Still Relevant? Incidence and Predictive Factors of Prostate Cancer Upgrading A Chow, K Gallo, D Papagiannopoulos, M Montour, C Coogan, K Latchamsetty United States

MP15-7 The prognostic impact of downgrading and upgrading from biopsy to radical prostatectomy in a contemporary grading system for prostate cancer D Koh, W Jang, J Park, J Lee, Y Kim, I Cho, Y Choi Republic of Korea

MP15-8 Robotic, Laparoscopic, and Open Approaches in Radical Prostatectomy Effect on Mortality, Readmission, and Length of Stay M Finkelstein, KH Bilal, MA Palese United States

MP15-9 Withdrawn

\section{MP15-10 Withdrawn}

MP15-11 Lateral Bladder Neck Dissection Technique and Triple-layer Posterior Bladder Wall Reconstruction Lead to Early Removal of Foley Catheter and Improved Urinary Continence After Robotic-assisted Radical Prostatectomy G Sung, S Kim Republic of Korea
MP15-12 Retzius-sparing radical prostatectomy: a reversed evolution from robotic-assisted to laparoscopic methodology

$\mathrm{J} \mathrm{Hu}$, K Chiu

Taiwan (Republic of China)

MP15-13 Midterm outcomes of four-port extraperitoneal laparoscopic radical prostatectomy for high-risk prostate cancer within Asian population

RC Wu, V Lin

Taiwan (Republic of China)

MP15-14 Extended Thromboprophylaxis May Be Associated with Increased Symptomatic Lymphocele Formation Following Robotic Prostatectomy with Pelvic Lymph Node Dissection

J Pereira, H Shillan, C Tucci, B Gershman, G Pareek, D Golijanin, J Renzulli United States

MP15-15 Analysis of extended lymph node dissection during robot-assisted radical prostatectomy: External validation of preoperative nomograms

K Kiyoshima, J Inokuchi, K Imada, T Dejima, E Kashiwagi, A Takeuchi, M Shiota, $\mathrm{K}$ Tatsugami, M Eto Japan

MP15-16 Clinical outcomes of robot-assisted radical prostatectomy (RARP) with retroperitoneal approach

K Takahara, A Kawai, M Ito, M Hikichi, K Fukaya, M Ichino, N Fukami, H Sasaki, M Kusaka, R Shiroki Japan

MP15-17 Effects of Previous Hernia Repair on Robotic Assisted Laparoscopic Radical Prostatectomy; A Matched-Pair Analyse Study AH Al-Shareef Al-Shaibani, Y Akin, T Almouhissen, J Rassweiler, M SaadAlshehri, A SerdarGözen, D Teber Iraq

MP15-18 Comparison of two templates of lymphadenectomy in patients affected by high risk prostate cancer

R Sanseverino, G Napodano, U Di Mauro, O Intilla, G Molisso, T Realfonso Italy

MP15-19 Significance of erection hardness score as a diagnostic tool to assess erectile function recovery in Japanese men after robot-assisted radical prostatectomy

Y Okamura, J Furukawa, K Shigemura, K Harada, N Hinata, T Isihimura, M Fujisawa Japan 
MP15-20 Cytoreductive Laparoscopic Radical Prostatectomy for Men with Oligo-Metastatic Prostate Cancer S Liu, J Wang, T Li, B Hong, K Gong China (People's Republic)

MP15-21 Surgeon Technical Performance Impacts Patient Outcomes in Robotic-Assisted Radical Prostatectomy

M Goldenberg, L Goldenberg, T Grantcharov Canada

MP15-22 Utility of preoperative mpMRI for planning side-specific nerve sparing in patients undergoing robotic prostatectomy

MB Schaefer, J Piotrowski, P Langenstroer, W See, K Jacobsohn

United States
MP15-23 Narrow Bony Pelvic Dimensions are Associated with Symptomatic Lymphocele

A Hamada, W Devan, I Tuerk

United States

MP15-24 The Ben Stiller effect: The online and social media impact of the Ben Stiller essay and the ProtecT Study on public awareness of prostate cancer

T Cisu, K Sternberg

United States

Thursday 14 September

Moderated Poster Session 16: Stones: Epidemiology \& Clinical Outcomes

16:30-18:30

Clinical Stones: Equipment (Stents, Lasers, Guidewires, Sheaths)

Convention Centre East, Meeting Room 1

MP16-1 Holmium Laser Versus Pneumatic Lithotripsy for Bladder Calculi: Which is Faster?

P Budny, M Lacey, M Avallone, R O'Connor, C Davis

United States

MP16-2 A coast-to-coast descriptive analysis of discharge summaries for renal colic from emergency departments in the United States

T Cisu, A Pham, K Sternberg United States

MP16-3 The Effect of Distance on Health-Related Quality of Life for Patients with Urolithiasis

GL Narang, L Elizabeth Wiener,

KL Penniston, J Antonelli, T Averch, S Sivalingam, BH Chew, VG Bird, VM Pais, RL Sur, T Chi, SY Nakada, GG Koch, DP Viprakasit United States

MP16-4 Establishing the Cambridge Renal Stone Patient reported outcome measures reliability M Ragab, N Baldin, J Collie, M Tran, S Al-Hayek, JN Armitage, O Wiseman United Kingdom

MP16-5 Urolithiasis in the Elderly: Protocol driven care and outcomes

A Pai, S Janardanan, P James,

H Ni Raghallaigh, R Kulkarni

United Kingdom

MP16-6 Renal Forniceal Rupture: Is Conservative Management Safe? T Nikonow Morgan, J Bandari, M Shahait, A Nevo, T Averch United States

MP16-7 Evaluation of Ethnic Differences in Kidney Stone Composition in a United States Cohort R Patel, K Rhee, K Radadia, E Olweny United States
MP16-8 Impact of Day or Hour on Diagnosis, Admission and Treatment Outcomes of Acute Stone Episode in the Emergency Room Setting

C Tong, E Ghiraldi, A Lee, T Li, PC Dreher, J Brandt, J Friedlander United States

MP16-9 Incidence, Treatment and Risk of Adverse Birth Outcomes for Kidney Stones in PregnancyA Population-Based Study M Ordon, J Kroft, J Dirk, J Slater, E McArthur, S Dixon, B Welk Canada

MP16-10 Preoperative DJ stent placement vs. direct ureteroscopy: a retrospective comparative study AF Navetta, TD Durdin, A El Mekresh, GL Machen, MM El Tayeb, L Tsai United States

MP16-11 Correlation of Stress in Kidney Stone Patients with the Wisconsin Stone Quality of Life Questionnaire CJ Lundeen, BH Chew, T Chi, SY Nakada, KL Penniston Canada

MP16-12 Renal Colic in Pregnancy-From Prenatal to Postpartum Management W Chen, S Sivalingam United States

MP16-13 Does diabetes mellitus influence the surgical management of urinary tract stones among patients in the United Kingdom?

M Elhadi, J D'Costa, C De Vogel,

R Devarajan, U Otite

United Kingdom 
MP16-14 AiRSTRiP: An Acute Rapid Stone Treatment Pathway to Reduce Treatment Time M Sirohi, A Wang, SE Wason, P Muraki, CF McCammon, J Myers, P Venkataram, JW Lambert, JB Malcolm, MD Fabrizio United States

MP16-15 Characterization of Upper Tract Urolithiasis in Patients Following Urinary diversion M Shahait, O Ayyash, J Denk, H Taan, S Jackman, M Ost, T Averch, MJ Semins United States

MP16-16 Factors Predictive of Surgical Intervention in Patients Who Present to the Emergency Department with Renal Colic P Samson, W Wu, S Derisavifard, S Gurram, S Ahn, K Ren, J Chu, AD Smith, C Hartman United States

MP16-17 Managing Renal Colic in the Emergency Room Observation Unit: Predictors of Hospital Admission

P Samson, W Wu, S Gurram, S Derisavifard, D Friedman, K Ren, AD Smith, C Hartman United States

MP16-18 Preferences in Surgical Management in the Setting of Bilateral Stone Disease ME Rivera, K Heinsimer, J Lingeman, AE Krambeck United States
MP16-19 The Importance of Urologist Estimation of Stone Burden: Results from the Registry for Stones of the Kidney and Ureter (ReSKU) DT Tzou, D Isaacson, M Usawachintachit, ZJ Wang, K Taguchi, RS Hsi, BA Sherer, ML Stoller, T Chi United States

MP16-20 Effects of Hounsfield unit value on percutaneous nephrolithotomy outcomes H Moon, W Bae, U Ha, S Hong, J Lee, S Kim, H Cho Republic of Korea

MP16-21 Flexible ureteroscopy in anticoagulated patients P Geavlete, D Georgescu, C Ene, V Iordache, R Multescu, B Geavlete Romania

MP16-22 Withdrawn

MP16-23 Worldwide Trends of Urinary Stone Disease Treatment over the last two Decades: A Systematic Review

R Geraghty, P Jones, A Pietropaolo, BK Somani United Kingdom

MP16-24 Clinical outcomes of endoscopic management for $2 \mathrm{~cm}$ or more of large renal calculi K Tanabe, M Kobayashi, Y Kojima, H Kameoka Japan
MP17-1 Pathologic outcomes in patients affected by very low risk and low risk prostate cancer and eligible for active surveillance

R Sanseverino, G Napodano, O Intilla, U Di Mauro, G Molisso, T Realfonso Italy

MP17-2 Predictive factors of upstaging, upgrading and adverse pathological features in favourable GS $3+4$

R Sanseverino, G Napodano, U Di Mauro, O Intilla, G Molisso, T Realfonso Italy

MP17-3 Prostate-specific antigen density as a predictor of Gleason score upgrading

D Bos, J Hoogenes, K Kim, F Farrokhyar, B Shayegan

Canada

MP17-4 Comparison of surgical, oncological, and functional outcomes of robot assisted and laparoscopic radical prostatectomy

U Boylu, A Inkaya, A Adiguzel, TU Sen,

E Kucuk

Turkey
MP17-5 Secondary Procedures follow Open verses Robotic Radical Prostatectomy

E Fram, A Srivastava, J Fleischmann United States

MP17-6 Impact of Gleason score at the positive surgical margin site on biochemical recurrence following robot-assisted radical prostatectomy

K Kanao, M Saito, M Sugie, H Muramatsu, S Morinaga, K Kajikawa, I Kobayashi, G Nishikawa, Y Kato, M Watanabe,

K Nakamura, M Sumitomo Japan

MP17-7 A novel technique of of robotic radical prostatectomy: combining the best of anterior and retropubic approach

I Banerjee, A Mallya, S Arakere, T Jindal, A Mandhani, RK Ahlawat, FA Zafar India

MP17-8 The prediction of T3a disease on pre-operative 3T mp-MRI: a correlation study with the pathology of robotic assisted radical prostatectomy

K Lin, E Huang, S Shen, I Huang, C Lin, T Lin, H Chung, S Lu, W Huang, H Wu, Y Chang, A Lin

Taiwan (Republic of China) 
MP17-9 The factors influencing the performance of nerve sparing technique on sides of extraprostatic extension on 3T mp-MRI prior to robotic assisted radical prostatectomy

E Huang, K Lin, S Shen, I Huang, C Lin, T Lin, H Chung, S Lu, W Huang, $\mathrm{H} \mathrm{Wu}$, Y Chang, A Lin

Taiwan (Republic of China)

MP17-10 Perioperative, oncologic and functional outcomes of salvage robotic radical prostatectomy after focal therapy failure

I Nunes-Silva, E Barret, V Srougi, P Capogrosso, R Sanchez-Salas, F Rozet, M Galiano, X Cathelineau

France

MP17-11 Prevention Of Urethral Retraction With Stay Sutures (PURS) During Robot Assisted Radical Prostatectomy Improves Early Urinary Control: A Prospective Cohort Study OB Argun, MB Tuna, T Doganca, I Tufek, P Mourmoris, C Obek, AR Kural Turkey

MP17-12 Prostate Cancer in Men <55: Rates of Functional Outcomes Post Robotic Assisted Radical Prostatectomy

E Hernandez, G Ogaya-Pinies, H Palayapalayam, T Woodlief, T Rogers, V Patel United States

MP17-13 Artificial intelligence with machine learning: predicting outcomes following robotic prostatectomy NC Wong, L Patterson, B Shayegan, D Bos Canada

MP17-14 Impact of perineural invasion and tumor volume at prostate biopsy on long-term biochemical recurrence after radical prostatectomy J Kim, W Ham, S Park, J Ha, S Kang Republic of Korea

MP17-15 Efficacy of Liposomal Bupivicaine in Robotic Assisted Laparoscopic Prostatectomy: A Prospective Randomized Comparison I Kelly, S Abourbih, M Pierce, C Bautista, J Lee, B Mattison, J Cheng, H Wagner, M Keheila, B Hu, H Ruckle, D Baldwin United States
MP17-16 Basal Location of Prostatic Cancer on Multiparametric MRI as a New Predictor for Biochemical recurrence after Robotic Radical Prostatectomy A Mahran, R Abboud, C Buzzy, M Wang, M Glover, I Jaeger, V Gulani, L Ponsky United States

MP17-17 Retzius-sparing Robot-assited Laparoscopic Radical Prostatectomy: A Preliminary Result of First 20 cases

H Liu, T Zhou, T Lin, J Huang

China (People's Republic)

MP17-18 Salvage Robot-Assisted Radical Prostatectomy: 6-year Single Center Experience MW Mekhail, G Cacciamani, FZ Husain, PW Mekhail, S Chopra, L Medina, C Fay, M Oishi, A Berger, R Sotelo, H Djaladat, M Desai, IS Gill, A Abreu, M Aron United States

MP17-19 Robot-Assisted Laparoscopic Radical Prostatectomy In Coagulopathic Patients: Diagnosis And Management A Hsieh, J Stites, G Portman, M Degen, K Basralian, I Sawczuk, R Munver United States

MP17-20 Robot assisted radical prostatectomy in patients with oligometastatic prostate cancer W Jang, D Koh, J Park, J Lee, Y Kim, I Cho, Y Choi

Republic of Korea

MP17-21 A Rectal Swab Guided Prophylaxis Program on the Incidence of Infectious Complications Following Trans-Rectal Ultrasound Guided Prostate Biopsy and Fiducial Marker Placement A Van Hoof, N Ruhotina MD, C Pieczonka MD, B Omarbasha MD, Y Yang MD, $S$ Faisal, DM Albala United States

MP17-22 Clinical utility of gene panel associated with increased risk of hereditary prostate cancer: A pilot study H Palayapalayam Ganapathi, P Patel, G Ogaya-Pinies, E Hernandez, T Rogers, V Patel United States

MP17-23 Multivariate Analysis of Factors Affecting Biochemical Recurrence After Radical Prostatectomy JV Balingit, L Sapno, C Evangelista, D Lusaya, J Castillo, D Serrano, J So Philippines 

Convention Centre East, Meeting Room 8

MP18-1 Automated Radiographic Stone Measurement: An Improved Standardization for Stone Volume and Density

\author{
NM Posielski, J Bell, SY Nakada, \\ PJ Pickhardt \\ United States
}

MP18-2 Feasibility of Automating the Measurement of Kidney Stone Diameter, Volume, and Density on CT

WW Ludwig, P Li, J Ziemba, R Gurnani, S Lim, C Jun, S Kawamato, G Fung, BR Matlaga, D Stoianovici United States

MP18-3 Role of dual energy CT in the management of urinary uric acid calculi

O Levi, N Idkedek, A Sidi, A Tsivian, E Surin, E Tavdi, R Bass, D Khunovich Israel

MP18-4 CT software-based calculation of stone volume is accurate and reproducible compared to the ellipsoid formula in an in-vitro model RK Jain, M Omar, H Chaparala, A Kahn, L Kahn, S Sivalingam United States

MP18-5 Non-Invasive Diagnosis of Renal Cell Carcinoma Subtypes

A Bergersen, E Price, B Kalb, BR Lee United States

MP18-6 Conventional Versus Computer Assisted Stereoscopic Ultrasound Needle Guidance for Renal Access: A Randomized bench-top crossover trial

A Thomas, J Thomas, B Mattison, B West,

D Ruckle, M Keheila, S Abourbih,

D Baldwin, D Baldwin

United States

MP18-7 Correlation between Confocal laser endomicroscopy (Cellvizio ${ }^{\circledR}$ ) and histological grading of upper tract urothelial carcinoma: a step forward for a better selection of patients suitable for conservative management A Breda, A Territo, F Sanguedolce, G Basile, J Gaya, F Algaba, J Palou, H Villavicencio Spain

MP18-8 Gallium labelled-prostate-specific membrane antigen-Positron Emission Tomography/ Computed Tomography (GA-PSMA-PET/CT) vs. Choline-PET/CT in Malignant Prostate Cancer A Systematic Review

M Moghul, AR Rhudd, BP Rai

United Kingdom
MP18-9 The diagnostic outcomes of men with a negative Multi-Parametric Magnetic Resonance Imaging (mpMRI) for the diagnosis of Prostate cancer, in low-risk biopsy naive men AR Rhudd, M Moghul, J McDonald United Kingdom

MP18-10 Accuracy of High-Frequency Endoluminal Ultrasonography for Clinical Staging of Upper Tract Urothelial Carcinoma

JA Farnum, R Vikram, A Rao, D Bedi, C Dinney, SF Matin United States

MP18-11 Next-generation ultrasound methods for kidney stone characterization: Feasibility of non-linear beamforming techniques RS Hsi, J Tierney, S Schlunk, P Karve, R Duddu, B Byram United States

MP18-12 Change in nephrometry scoring in small renal masses $(<4 \mathrm{~cm})$ on active surveillance can predict grade of tumours: Tayside Active Surveillance Cohort (TASC) study W Zhang, C Wei, M Szewczyk-Bieda, C Paterson, G Nabi United Kingdom

MP18-13 The significance of bladder filling during computerized tomography in the diagnosis of bladder rupture

$\mathrm{T} \mathrm{Oh}$

Republic of Korea

MP18-14 Posture specific 3D pelvic reconstructions in pelvic organ prolapse derived from open magnetic resonance imaging MM Abdulaziz, L Stothers, AJ Macnab Canada

MP18-15 Withdrawn

MP18-16 Contrast-enhanced ultrasound as a radiation free alternative to fluoroscopic nephrostogram for evaluating ureteral patency T Chi, M Usawachintachit, S Weinstein, MP Kohi, A Taylor, DT Tzou, HC Chang, ML Stoller, J Mongan United States

MP18-17 Radiation Exposure from Computed Tomography for Urolithiasis: Results from the Registry for Stones of the Kidney and Ureter (ReSKU)

DT Tzou, M Usawachintachit, D Isaacson, K Taguchi, BA Sherer, ML Stoller, T Chi United States 
MP18-18 Radiation Exposure from KUB (kidney-ureterbladder) Radiographs for Nephrolithiasis is Not Trivial

J Kuebker, J Shuman, RS Hsi, S Herrell, N Miller

United States

MP18-19 Availability and Utilization of Reduced Dose Computed Tomography for Nephrolithiasis J Kuebker, J Robles, N Miller, S Herrell, RS Hsi United States

MP18-20 Identifying the Relationship between Fluoroscopy Pulse Rate and Radiation Absorbed Dose

T Yecies, MJ Semins, M Sheetz, M Duranko, H Taan, M Shahait, R Chaudhry

United States
MP18-21 The application of ImageJ in stone analysis on X-Ray images: A new modality for detecting changes in stone mass and density

ME Hassan, J Metcalfe, M Wilson,

K Subramonian

United Kingdom

MP18-22 Does Intermittent Fluoroscopic Screening During Endourological Procedures Reduce Exposure to Radiation?

L Mokool, T Amer, A Ramsay, SK Nalagatla United Kingdom

MP18-23 Diagnostic accuracy of low and ultra-low radiation dose CT (computerised tomography) of the urinary tract for identification of stones in patients with renal colic

FE Rodger, A Ng, N Santoni, OM

Aboumarzouk

United Kingdom
MP19-1 Impact of surgeon's awareness of radiation safety on fluoroscopy time during flexible ureteroscopy

$$
\text { B Becker, AJ Gross, C Netsch }
$$

Germany

MP19-2 The advantages of robotic-assisted laparoscopic surgery for huge adrenal tumors inferior vena cava and beside great vessels (Report of 58 Cases) S Zhong, Z Fang, Z Wu, S Chen, M Zhang, F Gao, X Wang, W He, T Xu, Y Zhu, Q Ding, Z Shen

China (People's Republic)

MP19-3 Long term outcome evaluation of laparoendoscopic single site (LESS) adrenalectomy

I LO, H Lee

Taiwan (Republic of China)

MP19-4 Robotic-assisted Adrenalectomies: An Initial Experience

S Saad, B Namdarian, M Ravindraanandan, B Challacombe, A Fernando United Kingdom

MP19-5 Transperitoneal Robotic Assistant Laparoscopic Resection of Large Adrenal Tumors Z Shen, S Chen, S Zhong, M Zhang China (People's Republic)

\section{MP19-6 Withdrawn}

MP19-7 Bilateral Simultaneous Laparoscopic Adrenalectomy: A Multicenter Experience A Tuncel, A Erkan, H Langenhuijsen, T Mikhaylikov, M Arslan, Y Aslan, D Berker, Y Ozgok, A SerdarGözen Turkey
MP19-8 Surgical management of pheochromocytoma: what are the indications for open surgery in the era of MIS?

P Prakash, R Kumar India

MP19-9 Initial experience of 243 cases in transperitoneal robotic-assisted laparoscopic surgery for adrenal diseases

$$
\begin{aligned}
& \text { Z Shen } \\
& \text { China (People's Republic) }
\end{aligned}
$$

MP19-10 Comparison of Minimally-Invasive Versus Open Adrenalectomy: An Institutional Review P Heller, P Fish, A Rawlings, N Pokala, K Murray United States

MP19-11 Role of open adrenalectomy in the laparoscopic era

$$
\begin{aligned}
& \text { J Kwok, A Lua, Y Chong } \\
& \text { Singapore }
\end{aligned}
$$

MP19-12 Natural Orifice Transluminal Endoscopic surgery for Donor Nephrectomy- outcomes in comparison with the laparoscopic Donor Nephrectomy

KM Ramaswami, H Pothiyedath, P Birud India

MP19-13 Complications of suprapubic-assisted laparoendoscopic single-site surgery (SA-LESS) in urology

Z Xiaofeng, X Gang, Z Guoxi, Y Yuanhu, $X$ Rihai, W Gengqing China (People's Republic) 
MP19-14 Application of Cumulative Summation (CUSUM) in learning curve analysis of Suprapubic-assisted laparoendoscopic single-site nephrectomy in one center

Z Xiaofeng, X Hui, Z Guoxi, Y Yuanhu, X Rihai, W Gengqing, W Xiaoning China (People's Republic)

MP19-15 A comparison of laparoendoscopic single-site (LESS) surgery versus conventional procedures in laparoscopic donor nephrectomy: a Japanese multi-institutional retrospective study H Tsuruta, T Inoue, M Miura, M Yanishi, K Harada, F Sato, M Nitta, K Yoshimura, J Hagiuda, K Shinoda, T Kobayashi, K Nakagawa, H Mimata, T Terachi, T Matsuda, T Habuchi Japan

MP19-16 Optimization of the key techniques in suprapubic-assisted laparoendoscopic single-site nephroureterectomy

Z Xiaofeng, Z Guoxi, W Gengqing, Y Yuanhu, X Rihai, W Xiaoning China (People's Republic)

MP19-17 The decline of LESS: a survey of the Endourological society to identify shortcomings and guidance for future directions I Sorokin, BA Johnson, N Canvasser, B Irwin, R Autorino, E Liatsikos, J Cadeddu, A Rane United States
MP19-18 Comparison of Robotic Laparo-Endoscopic Single Site (R-LESS) and Multiport Robotic Nephrectomy and Partial Nephrectomy Utilizing the Da Vinci-XI Platform A Bindayi, Z Hamilton, M Holden, M McDonald, K Yim, I Derweesh United States

MP19-19 Single Port Laparoscopic Repair of Post Retropubic Radical Prostatectomy Inguinal Hernia Via Previous Operative Scar: Case Series, Initial Experience

J Jo, Y Kim, S Park, H Choi, H Moon, K Kim, S Lee Republic of Korea

MP19-20 Laparoendoscopic single-site nephrectomy for children with an ectopic ureter associated with a hypoplastic kidney. Two cases report S Sejiyama, S Abe, K Takei, N Yamanaka, M Hanada, T Shibuya, M Yamasaki, T Shin, K Mori, T Ando, T Nomura, F Sato, H Mimata Japan

MP19-21 Laparoendoscopic Single-site Repair of Bladder Rupture Using a Home-made Single-port Device: Treatment for a Traumatic Intraperitoneal Bladder Rupture

J Jo, Y Kim, S Park, H Choi, H Moon, K Kim, S Lee

Republic of Korea

MP19-22 Usefulness of laparoendoscopic single site surgery varicocelectomy Y Yamanaka, S Takada Japan

Thursday 14 September

Moderated Poster Session 20: Urothelial Carcinoma I

16:30-18:30

Clinical Oncology: Outcomes \& Complications

Convention Centre East, Meeting Room 19

MP20-1 Percutaneous resection of upper urinary tract urothelial carcinoma: a single-surgeon screenshot about a controversial procedure in imperative cases

E De Lorenzis, A Gallioli, L Boeri, S Zanetti, G Sampogna, M Fontana, F Palmisano, M Talso, P Dell'Orto, F Longo, E Montanari Italy

MP20-2 Endoscopic treatment for upper urinary tract urothelial carcinoma larger than $1 \mathrm{~cm}$

A Shvero, T Aviv, M Laufer, ZA Dotan,

D Zilberman, Y Mor, O Porntoy, J Ramon,

$\mathrm{H}$ Winkler, N Kleinmann

Israel

MP20-3 Holmium and Thulium transurethral Laser En Bloc resection of bladder tumors

P Glybochko, Y Alyaev, G Altshuler,

A Vinarov, A Dymov, N Sorokin,

R Sukhanov, D Enikeev, I Yaroslavskyi,

V Vinnichenko, V Lekarev

Russia
MP20-4 Modified Frailty Index associated with Adverse Outcomes in Patients with Bladder Cancer in Open and Laparoscopic Cystectomies I Levy, M Finkelstein, MA Palese United States

MP20-5 Front-firing green-laser assisted laparoscopic partial cystectomy in selected patients with muscle-invasive bladder cancer: technique and initial outcome

K Wu, D He, J Fan

China (People's Republic)

MP20-6 Development and Validation of Cystectomy Assessment and Surgical Evaluation Scoring (CASE) for Male Radical Cystectomy

A Hussein, K Sexton, M Meng, A Hosseini, P Wiklund, D Eun, S Daneshmand, B Bochner, J Peabody, R Abaza, E Skinner, R Hautmann, K Guru United States 
MP20-7 Analysis of surgeon biometrics during open and robotic radical cystectomy T Patel, D Hardwick, J Robison, J Mayer United States

MP20-8 Robot-assisted radical cystectomy with intracorporeal urinary diversion in the setting of challenging patient factors N Ahmadi, D Freitas, C Fay, PW Mekhail, S Chopra, G Cacciamani, IS Gill, M Desai, M Aron

United States

MP20-9 Can Indocyanine Green Fluorescence Angiography Eliminate Ureteral Strictures After Robotic Radical Cystectomy?

N Ahmadi, D Freitas, C Fay, A Berger, M Desai, IS Gill, M Aron United States

MP20-10 Lymph node mapping in patients with bladder cancer undergoing laparoscopic radical cystectomy and extended lymphnode dissection K Minami, T Harabayashi, N Takada, S Nagamori Japan

MP20-11 Oncologic Outcomes and Predictive Factors for Recurrence following Robot-assisted Radical Cystectomy for Urothelial Carcinoma: Multicenter Study from Korea J Shim, T Kwon, K Rha, Y Lee, J Lee, B Jeong, J Choi, S Kang, S Kang, J Jung, J Jung Republic of Korea

MP20-12 Total intracorporeal robotic radical cystectomy: Does this method have a benefit when compared to the extracorporeal method in the patient outcome?

K Rha, Y Lee, J Lee, B Jeong, J Choi, S Kang, S Kang, J Jeong

MP20-13 Sequential robot-assisted bladder diverticulectomy and simple prostatectomy: Technique and surgical outcomes Z Shen, S Zhong, S Chen, M Zhang China (People's Republic)

MP20-14 The efficacy of hybrid laparoscopic procedures against bladder benign disease T Hamasaki, Y Endo, J Akatsuka
MP20-15 Trans-Urethral Resection of the Bladder Tumour (TURBT) surgery, for the treatment of Non-Muscle Invasive Bladder Cancer (NMIBC), results in changes to selective biochemical parameters SF Hughes, RM Lamb, P Ella-Tongwiis, I Shergill United Kingdom

MP20-16 Percutaneous Resection of Bulky Renal Pelvic Tumor

M Eshghi, RJ Yau, JH Hillelsohn United States

MP20-17 Multi-Institutional Evaluation of Quality of Upper Urinary Tract Biopsy Using BIGopsy Forceps, a 2.4F Nitinol Basket, and Piranha Forceps DJ Lama, S Safiullah, R Youssef, TK Lee, T McDonald, K Sheth, R Hutchinson, J Balani, K Flower, V Margulis, S Savage, J Landman, RM Patel United States

MP20-18 A novel transurethral resection technique for superficial bladder tumor: retrograde en bloc resection

$$
\begin{aligned}
& \text { K Zhang, J Xing, W Li, Z Wu } \\
& \text { China (People's Republic) }
\end{aligned}
$$

MP20-19 Intraoperative frozen section evaluation of the tumor and its base in patients with $\mathrm{T} 1$ urothelial bladder cancer underwent transurethral resection Y Shen, H Gan, Q Wang, Y Zhu, D Ye China (People's Republic)

MP20-20 Transurethral Laser Ablation of Non-Muscle Invasive Bladder Cancer with a Diode Laser: 5 year Outpatient Experience P James, A Pai, N Arumainayagam, S Agrawal, A Shamsuddin United Kingdom

MP20-21 Outcomes of Upper Urinary Tract Tumours Following Nephro-ureterectomy Over a 10 Year Period

A Carrera, A Johnstone, N Umez-Eronini, K Qureshi, G Oades, OM Aboumarzouk United Kingdom

MP20-22 The usefulness of transurethral resection in one piece (TURBO) for pathological staging of bladder tumor

S Ohtake, J Ohta, K Chiba, K Makiyama, M Yao Japan

MP20-23 En bloc transurethral resection with Hybrid Knife for treatment primary non-muscleinvasive bladder cancer: a single-center, randomized, controlled trial J Hu, X Song, X Yu, S Wang China (People's Republic) 


\section{MP21-1 Withdrawn}

MP21-2 Pure Transvaginal Natural Orifice Transluminal Endoscopic Surgery (NOTES) for Nephrectomy: Report of 19 Cases

Z Xiaofeng, Z Guoxi, W Xiaoning, L Dazhi, W Gengqing, X Rihai China (People's Republic)

MP21-3 The Incidnece of Adverse Pathological Outcome in Robotic Partial nephrectomy MW Salkini, N Idris, A Lamoshi United States

MP21-4 Robotic-assisted Laparoscopic Surgeries from the Team of Prof. Shen Zhoujun: 1001 Cases' Experience and Clinical efficacy analysis Z Shen, S Chen, S Zhong, Y Shen, M Zhang China (People's Republic)

MP21-5 A Prospective Randomized Trial of Clamped Versus Off-Clamp Robot-Assisted Partial Nephrectomy: Does It Make a Difference? BG Anderson, K Du, A Potretzke, J Vetter, RS Figenshau United States

MP21-6 The experience and skills of retroperitoneal laparoscopic nephron-sparing sugery for giant renal angiomyolipoma

$$
\begin{aligned}
& \text { M Qiu } \\
& \text { China (People's Republic) }
\end{aligned}
$$

MP21-7 Efficacy of laparoscopic simple nephrectomy for treatment of chronic pain in patients with recurrent ureteropelvic junction obstruction D Szabo, M Sourial, N François, C Dall, C Miller, DL Zynger, G Box United States

MP21-8 Minimally invasive versus open radical nephrectomy for clinical stage $\mathrm{T} 4$ renal masses ZL Smith, S Johnson, V Packiam, J Rodriguez, S Eggener United States

MP21-9 Retroperitoneal versus Transperitoneal RobotAssisted Laparoscopic Partial Nephrectomy: Operative, Oncologic and Late Outcomes M Lyons, E Stephenson, M Raynor United States

MP21-10 The impact of gender difference on the operative time of laparoscopic and robotic partial nephrectomy for $\mathrm{T} 1$ renal tumor, and the utility of retroperitoneal fat thickness as a predictor of operative time

K Makiyama, H Ito, K Osaka, Y Yokomizo, N Nakaigawa, M Yao Japan
MP21-11 Trifecta outcomes of robot-assisted partial nephrectomy in solitary kidney - A VCQI database analysis S Arora, RK Ahlawat, R Abaza, J Adshead, B Challacombe, P Dasgupta, G Gandaglia, D Moon, G Novara, F Porpiglia, U Capitiano, T Yuvaraja, J Porter, A Mottrie, M Bhandari, CG Rogers, H Gill United States

MP21-12 Laparoscopic partial nephrectomy for T1b or greater tumors: comparing the Trifecta outcome of T1a with T1b or greater tumors K Otsuka, A Fujimoto, K Hou, T Kato, T Suyama, K Araki, H Masuda, S Kojima, Y Naya Japan

MP21-13 Impact of selective renal artery clamp during robot-assisted laparoscopic partial nephrectomy on postoperative renal function

F Hongo, Y Inoue, M Kaneko, A Fujihara, Y Naitoh, K Okihara, O Ukimura Japan

MP21-14 The impact of the type of renal clamping on the surgical, functional and oncological outcomes of partial nephrectomy

U Boylu, U Yildirim, A Inkaya, B Simsek, E Kucuk Turkey

MP21-15 Intraoperative Ultrasonography in Laparoscopic Partial Nephrectomy for Intrarenal Tumors B Qin, H Hu, Q Wang, S Wang China (People's Republic)

MP21-16 Perinephric Drain Placement Is Not Required After Robotic-Assisted Pyeloplasty N François, M Sourial, C Dall, C Jaeger, G Box United States

MP21-17 Comparison of laparoscopic vs robotic partial nephrectomy: a retrospective single center analysis

A Araki, K Makiyama, K Osaka, K Kondo, N Nakaigawa, M Yao, S Ohtake Japan

MP21-18 ASA Score and the Charlson Comorbidity Index Fail to Discriminate Patients that Have Post-Operative Complications after Robotic Partial Nephrectomy M Greenstein, K Blum, D Paulucci, D Rosen, B Reddy, R Abaza, D Eun, A Bhandari, A Hemal, K Badani United States 
MP21-19 The Utility of "Composite Tumor Size" in Evaluating Perioperative and Functional Outcome for Multiple versus Solitary Ipsilateral Tumors During Robotic Partial Nephrectomy K Blum, R Cleary, D Paulucci, R Abaza, D Eun, A Bhandari, A Hemal, K Badani United States

MP21-20 Off Clamp Robotic Partial Nephrectomy Does Not Improve Renal Function Outcome for Patients With T1a Tumors Without Impaired Renal Function

D Rosen, K Blum, D Paulucci, R Abaza, D Eun, A Bhandari, A Hemal, K Badani United States

MP21-21 Comparison of Perioperative and Functional Outcomes of Robotic Partial Nephrectomy for cT1a versus cT1b Renal Masses CR Reynolds, JC Delto, D Paulucci, K Badani, D Eun, R Abaza, J Porter, A Bhandari, A Hemal United States
MP21-22 Negative impact of renal hypothermia with ice-cold saline on renal functional outcome for transperitoneal laparoscopic partial nephrectomy T Tachibana, T Fujita, M Nishi, D Ishii, K Tabata, K Matsumoto, K Yoshida, M Iwamura Japan

MP21-23 Renal function and perioperative outcomes of selective versus complete renal arterial clamping during robot-assisted partial nephrectomy using console-integrated real-time three-dimensional image overlay navigation

J Furukawa, N Hinata, K Shigemura, K Harada, T Isihimura, Y Nakano, M Fujisawa Japan

MP21-24 Prediction of surgical complications in laparoscopic nephrectomy caused by xanthogranulomatous pyelonephritis: how difficult will it be?

AM Cardenas Ortiz, PR Kawano, A Toledo, HA Yamamoto, R Guerra da Silva, JL Amaro, OE Hidetoshi Fugita

Colombia

Friday 15 September

MP22-1 Partial nephrectomy: Publication trends over the last 2 decades
A Pietropaolo, B Rai, C Lockyer, M Hayes, B Somani United Kingdom

MP22-2 Robotic versus Open Partial Nephrectomy for Patients with Pre-Existing Chronic Kidney Disease: A Multicenter Analysis A Bindayi, B Ristau, B Lane, S Berquist, S Dey, S Noyes, K Yim, F Wan, J Proudfoot, R Uzzo, Z Hamilton, I Derweesh United States

MP22-3 Multicenter comparison of robotic and open partial nephrectomy for quality measures controlling for clinical $T$ stage

A Bindayi, M Holden, Z Hamilton, A Larcher, S Dey, M Marshall, K Yim, DS Han, S Noyes, F Wan, U Capitiano, S Stroup, B Lane, I Derweesh, F Montorsi United States

MP22-4 Off-clamp Robot-Assisted partial nephrectomy offered renal functional advantage for selected renal masses

P Mouracade, J Chavali, J Dagenais, MJ Maurice, R Nelson, J Reese, J Kaouk United States

MP22-5 The Effect of Robotic Assistance on Outcomes for Partial Nephrectomies M Finkelstein, KH Bilal, MA Palese United States
MP22-6 5 Year Peri-operative Outcomes of Robot-Assisted Laparoscopic Partial Nephrectomy

A Rasmussen, H Evans, B St. Martin, M Hobart, T Schuler Canada

MP22-7 Initial experiences of Robotic Laparoendoscopic Single-Site Partial Nephrectomy by da-Vinci $\mathrm{Xi}{ }^{\circledR}$ surgical system

J Na, H Lee, Y Yoon, K Rha, Y Choi, W Han Republic of Korea

MP22-8 Safety and oncological outcomes of laparoscopic robot-assisted partial nephrectomy (RAPN) for pT1b/pT2a renal cell cancers S Vamadevan, J Klein, G Wirth, C Iselin Switzerland

MP22-9 Robotic-Assisted Partial Nephrectomy for Renal Angiomyolipoma Larger than $6 \mathrm{~cm}$ Y Lin, C Ho, T Wu, T Tsai, T Hwang Taiwan (Republic of China)

MP22-10 Development and Validation of an Objective Scoring tool for Minimally Invasive Partial Nephrectomy: Scoring for Partial Nephrectomy (SPaN)

A Hussein, R Abaza, CG Rogers, R Boris, J Porter, M Allaf, K Badani, M Stifelman, J Kaouk, K Guru, D Eun United States 
MP22-11 Impact of Positive Surgical Margins on Overall Survival after Partial Nephrectomy, a Matched Comparison Based on the National Cancer Database C Shum, CD Bahler, CP Sundaram Singapore

MP22-12 Comparison of Oncologic Results, Functional Outcomes and Complications after Partial Nephrectomy versus Percutaneous Radiofrequency Ablation in Small sized (4cm or less) Bosniak III or IV Cystic Renal lesions W Song, Y Choi, H Sung, H Jeon, B Jeong, S Seo, S Jeon, H Choi, H Lee Republic of Korea

MP22-13 Utilization of Renal Biopsy for a Small Renal Mass: A Survey Among American Urologists RM Patel, S Safiullah, KS Kaler, J Landman, RV Clayman United States

MP22-14 Impact of blood transfusions on oncological outcomes of surgically treated localized renal cell carcinoma

M Tsivian, E Tsivian, C Sze, A Schulman, M Abern, E Rampersaud, T Polascik United States

MP22-15 Frozen Sections for Margins During Partial Nephrectomy Do Not Influence Recurrence Rates J Dagenais, P Mouracade, MJ Maurice, O Kara, R Nelson, JS Chavali, J Kaouk United States

MP22-16 Avoiding Acute Kidney Injury: When to Employ Cold Ischemia

J Dagenais, MJ Maurice, P Mouracade, O Kara, J Li, R Nelson, E Malkoc, J Kaouk United States
MP22-17 How Much is Too Much? Exploring the Relationship between Volume Loss, Oncologic Outcomes, and Renal Dysfunction after Partial Nephrectomy

J Dagenais, P Mouracade, O Kara, JS Chavali, MJ Maurice, J Kaouk

United States

MP22-18 Current Trends in Cytoreductive Nephrectomy in the Era of Targeted Molecular Therapy Y Phan, S Segaran, J Bell, SY Nakada, A Rane

United Kingdom

MP22-19 Withdrawn

MP22-20 Predicting risk of pathological upstaging of clinical $\mathrm{T} 1 \mathrm{renal}$ masses in the era of renal mass biopsy

ZL Smith, S Johnson, S Eggener United States

MP22-21 Predictive value of preoperative BMI and total cholesterol in the renal cell carcinoma patients by gender - multicenter study

H Jeong, S Kang, H Moon, K Lee, S Choi, Y Park, W Bae, H Cho, U Ha, J Lee, S Kim, S Hong

Republic of Korea

MP22-22 Is There a Role for Nephron-Sparing Cytoreductive Partial Nephrectomy in Renal Cell Carcinoma?

P Heller, L Hockman, L McGuffey, K Murray, N Pokala

United States

MP22-23 Withdrawn

MP22-24 Impact of Hospital Volume on Intermediate to Long-term Patient Survival in Laparoscopic Radical Nephrectomy for Renal Cell Carcinoma R Hsu, M Barclay, G Lyratzopoulos, V Gnanapragasam, JN Armitage United Kingdom
MP23-1 Single-session primary high-intensity focused ultrasonography (HIFU) as a treatment for localized prostate cancer: predicting disease-free survival by risk stratification

B Shayegan, T Lee, J Hwang, J Hoogenes, W Orovan

Canada

MP23-2 Radiofrequency Ablation of Renal Tumors: 5 year follow-up

G Sung, S Kim

Republic of Korea
MP23-3 Interstitial Laser Coagulation of Localized Renal Cell Carcinoma. Seven Years Follow-up O Teodorovich, S Naryshkin, G Borisenko, E Rasshchupkina, A Ryazantsev, Y Osmanov, A Kudil, D Kochiev Russia

MP23-4 The course of recurrence following cryoablation for renal tumors

$\mathrm{R}$ van der Vijgh, A Montauban van Swijndregt, B Lagerveld, R Sandkuyl

Netherlands 
MP23-5 Percutaneous US-Guided Cryoablation of the Kidney under Local Anesthesia P Glybochko, Y Alyaev, L Rapoport, A Amosov, D Tsarichenko, M Enikeev, D Enikeev, D Chinenov, Z Dzhalaev, M Taratkin Russia

MP23-6 Increased Utilization of Ablation for T1b Renal Cancers with Improved Survival Relative to Observation M Farrell, J Tasse, S Madassery, B Arslan, S Vourganti United States

MP23-7 Usefulness of contrast-enhanced ultrasound in the diagnosis of renal masses and its concordance with CT Scan

G Del Pozo Jiménez, I Castillón Vela, G Rodríguez Reina, D Rengifo Abad, M Rodríguez Monsalve, T Fontanilla Echeveste, J Minaya Bernedo, J Carballido Rodríguez Spain

MP23-8 High Intensity Focused Ultrasound Kidney Ablation: Pre-Clinical Safety and Efficacy Evaluation in a Porcine Model using a 15mm Laparoscopic Probe S Chopra, IS Gill, A Bove, C Fay, T Shin, K King, V Duddalwar, A Aron, R Chaluisan, J Clanton, J Carr, C Johnson, B Ettinger, A Morris, R Carlson, N Sanghvi, M Carol, R Seip United States

MP23-9 Analysis of renal function after cryoablation of Small Renal Tumours (SRMs) in patients with solitary kidneys: a European Registry for Renal Cryoablation (EuRECA) multi-institutional study K Farrag, T Nielsen, B Lagerveld, F Keeley, G Lughezzani, N Barber, L Hansen, N Buffi, G Guazzoni, J van der Zee, M Ismail, A Emara, L Lund, Østraat, M Borre, S Sriprasad United Kingdom

MP23-10 Salvage Cryo-Ablation for localized radiorecurrent prostate cancer

A Breda, A Territo, G Basile, F Sanguedolce, P Gavrilov, J Palou, H Villavicencio Spain

MP23-11 Impact of MRI/Ultrasound Fusion-Guided Biopsy on Prostate Cancer Detection in a Community Based Large Urology Group Practice A Van Hoof, N Ruhotina MD, R Kronhaus MD, H James MD, C Pieczonka MD, B Omarbasha MD, DM Albala United States

MP23-12 Predictors of rectourethral fistula after primary, whole gland cryoablation for prostate cancer: Results from the Cryo-On-Line Database (COLD) Registry A Schulman, K Tay, A Elshafei, E Tsivian, C Sze, T Polascik, J Jones United States
MP23-13 Does African-American race impact oncologic outcomes following primary cryotherapy for prostate cancer? A matched study from the Cryo-On-Line Database (COLD) Registry

A Schulman, M Tsivian, E Tsivian, C Sze, K Tay, A Elshafei, T Polascik, J Jones United States

MP23-14 HIFU focal treatment for unilateral prostate cancer: a comprehensive study of pooled data S Albisinni, C Melot, K Limani, A Peltier, P Rischmann, RF van Velthoven Belgium

MP23-15 High Intensity Focused Ultrasound: zonal hemiablation for localized prostate cancer, a single center ten years experience RF van Velthoven, R Diamand, K Limani, S Albisinni, M Lemort, A Peltier, $\mathrm{T}$ Roumeguère Belgium

MP23-16 MRI Guided TRUS Fusion Prostate Biopsy OB Argun, C Obek, MB Tuna, T Doganca, I Tufek, S Keskin, AR Kural Turkey

MP23-17 Renal Mass Thermal Ablation: Cone Beam Computed Tomography, Alone or Combined with Pneumoperitoneum Under Surgeon Control- Description of Technique and Initial Results

EF Kelly, RJ Leveillee United States

MP23-18 Targeted MRI/US Fusion Prostate Biopsy: The First 212 Patients Without a History of Prostate Cancer - Relationship Between PIRADS Score and Prostate Cancer Detection Rate: A Single Urologist's Experience

A Kasraeian, J Cesaretti, J Yellin, A Brochert, B Noell, A Kasraeian United States

MP23-19 MR Targeted Prostate Biopsy against Standard Ultrasound Guided Prostate Biopsy. A Single Center Experience : First Results E Firat, Y ÖZLÜLERDEN, Tuncay, A YAĞCI Turkey

MP23-20 Geographical variation in the utilization of MRI/ Fusion prostate biopsies among 160 outpatient health care facilities in New York State between 2008-2012

KH Bilal, M Finkelstein, MA Palese United States

MP23-21 The Impact of mpMRI on Prostate Cancer Detection With Respect to Prostate Volume M Glover, R Abboud, A Mahran, C Buzzy, R Thawani, M Wang, V Gulani, L Ponsky, I Jaeger United States 
MP23-22 Modeling pre-biopsy and mpMRI variables: Avoiding unnecessary prostate biopsies R Abboud, C Buzzy, A Mahran, M Glover, R Thawani, M Wang, I Jaeger, V Gulani, L Ponsky United States
MP23-23 Prognostic grade group prostate cancer grading system: can multiparametric MRI accurately separate patients with PGG 1, PGG2 and high grade cancer?

K Tay, J Holtz, R Silverman, C Sze, R Gupta, T Polascik

United States

Friday 15 September

Moderated Poster Session 24: BPH/LUTS Outcomes

14:00-16:00

BPH/LUTS: Electrosurgery, Lasers \& Other Technoloy

Convention Centre East, Meeting Room 11

MP24-1 Relationship Between Intravesical Prostatic Protrusion (Ipp) And Postoperative Outcomes In Patients With Benign Prostatic Hyperplasia S Ramani India

MP24-2 Postoperative dysuria after high- and low-power en-bloc no-touch HoLEP CM Cracco, CM Scoffone Italy

MP24-3 Serum prostate specific antigen levels after green laser enucleation of the prostate (GreenLEP) E Rijo, V Misrai, S Tabatabaei, G Ferrari, F Gomez-Sancha Spain

MP24-4 Does a partial prostate resection improve voiding symptoms while shortening the learning curve for HOLEP?

S Bazargani, S Ghodoussipour, A Shah, E Thompson, A Mitra, M Dunn United States

MP24-5 The efficacy of fibrin glue injection in the prostatic fossa on decreasing postoperative bleeding following transurethral resection of prostate M KHORRAMI, K nouri mahdavi Canada

MP24-6 Holmium Laser Enucleation of Prostate v/s Thulium Laser Enucleation of Prostate for Benign Prostatic Obstruction : a Randomized Prospective study PK Pattnaik India

MP24-7 Bipolar plasma enucleation versus TURP in medium sized prostates- Is it a possible alternative?

B Geavlete, D Georgescu, R Multescu, C Ene, C Bulai, P Geavlete Romania

MP24-8 Report of transurethral resection of prostate in the past 20years:the experience of 3112 cases from single center
L Zuo, J Zou, S Lu, Z Zhou, X Wu
China (People's Republic)

MP24-9 The Clinical Effect of Bipolar Transurethral Resection in saline of the benign prostate hyperplasia with long term follow-up S Permpongkosol Thailand
MP24-10 Survey of Endourologists: Morcellator Preferences after Transurethral Prostate Enucleation K Heinsimer, ME Rivera, J Lingeman, AE Krambeck United States

MP24-11 Patient-Centered Goals for Holmium Laser Enucleation of Prostate: A Subjective Evaluation of Successful Surgical Outcome K Lee, C Yoon, S Choi, T Oh, T Kim, W LEE Republic of Korea

MP24-12 Withdrawn

MP24-13 Postoperative complications in 400 patients undergone endoscopic prostatic surgery with Thullium laser

L Carmignani, G Motta, S Nazzani, M Clementi, C Signorini, E Finkelberg, D Ratti, D Vizziello, S Nazzani Italy

MP24-14 For smaller resection weight of transurethral resection of prostate, could combined incision of bladder neck lead to less acute urinary retention rate after surgery? - a nationwide database study T Wei, E Huang, H Chung, A Lin Taiwan (Republic of China)

MP24-15 Risk factors for unimproved voiding symptoms after holmium laser enucleation of the prostate B Kim, Y Lee, J Chung, Y Ha, S Choi, J Lee, H Kim, T Kim, E Yoo, T Kwon, S Chung Republic of Korea

MP24-16 The introduction of TUEB (Transurethral enucleation with Bipolar) system for large BPH; Initial 40cases

T Yoshida, H Yoshinaga, N Fujimoto Japan

MP24-17 One out of three men undergoing transurethral resection of the prostate is lost to follow-up: a worrisome picture form the real-life setting L Boeri, M Fontana, A Gallioli, E De Lorenzis, F Palmisano, S Zanetti, G Sampogna, M Talso, F Longo, F Gadda, P Dell'Orto, E Montanari Italy 
MP24-18 Incidence and predictors of readmission within 30 days of transurethral resection of the prostate: which patients come back?

L Boeri, S Zanetti, G Sampogna, M Fontana,

A Gallioli, E De Lorenzis, F Palmisano,

F Longo, M Spinelli, F Gadda, M Ferruti, P Dell'Orto, E Montanari

Italy

MP24-19 Cost Savings Achieved through Introduction of HOLEP and Care Pathway

J Johannes, A Lai, A Baccala, J Feliciano,

C Georges, M Steinbook, M Voznesensky

United States
MP24-20 Robotic Transvesical Simple Prostatectomy :

USC early experience

PW Mekhail, A Berger, MW Mekhail,

A Abreu, N Ahmadi, A Monish

United States

MP24-21 Predictors and Recovery of Urinary

Incontinence after Holmium Laser Enucleation

of Prostate

B Singh, A Sokhal, S Sankhwar

India

\section{BPH/LUTS: Electrosurgery, Lasers \& Other Technoloy} Convention Centre East, Meeting Room 19

MP25-1 Comparison of tissue vaporization rate and efficiency between Lumenis Pulse 120H Holmium laser and AMS 180W Green Light KTP laser in an Ex-vivo model

SM Kumar

United States

MP25-2 Treatment and outcomes of patients diagnosed with prostate cancer and submitted to Holmium laser enucleation of the prostate (HoLEP) for concomitant benign prostatic hyperplasia JE Hunter, C Chemasle New Zealand

MP25-3 HoLEP performed at low power setting of $30 \mathrm{~W}$ with an en-bloc enucleation technique S Minagawa, S Okada, H Morikawa Japan

MP25-4 Metabolic syndrome is predictive of lower urinary tract symptom improvement after holmium laser enucleation of the prostate for benign prostatic obstruction

T Kwon, S Park, S Park, K Moon

Republic of Korea

MP25-5 En bloc enucleation technique with lateral median approach for safe and quicker operation when performing holmium laser enucleation of the prostate Y Shibata, S Arai, S Kurihara, T Miyao, Y Miyazawa, R Oki, H Nakayama, H Koike, K Ito, K Suzuki

Japan

MP25-6 Bipolar plasma enucleation of the prostate (B-TUEP) in Benign Prostate Hypertrophy Treatment. Medium-term results R GENTILE, B GENTILE, L Albanesi, G Rizzo, M Vermiglio, M Buscarini, P Tariciotti, G Mirabile Italy

MP25-7 Audit on UroLift- a novel minimally invasive technique to treat lower urinary tract symptoms due to benign prostatic enlargement W Akhter, A Habib, R Kavia, A Habib United Kingdom
MP25-8 Urolift- For the Managment of Obstructive BPH- 5 year data from LIFT study

S Gange, N Shore, D Bolton, B Cowan, A Cantwell, K McVary, A Te, S Gholami, P Rashid, W Moseley, P Chin, W Dowling, S Freedman, P Incze, K Coffield, S Heron, D Rukstalis, C Roehrborn, J Barkin Canada

MP25-9 Predictors of postoperative bacteriuria after Holmium Laser Enucleation of the Prostate (HoLEP)

AM Elmekresh, VH Villarreal, J Farewell, K Doersch, MM El Tayeb, L Tsai United States

MP25-10 Low-power versus high-power en-bloc no-touch Holmium Laser Enucleation of the Prostate (HoLEP): comparing feasibility, safety and efficacy

CM Cracco, CM Scoffone Italy

MP25-11 The bipolar transurethral enucleation of the prostate (BTUEP) is a safe and effective technique in patients under anticoagulation or antiplatelet therapy

L Boeri, M Fontana, G Sampogna, A Gallioli, E De Lorenzis, F Palmisano, S Zanetti, F Longo, G Albo, F Gadda, P Dell'Orto, E Montanari Italy

MP25-12 Comparative efficacy and safety of surgical treatments for benign prostate hyperplasia: an application of network meta-analysis S Huang, C Tseng, K Chien, C Tsai Taiwan (Republic of China)

MP25-13 Safety and effectiveness of high-power thulium laser enucleation of the prostate in patients with large glands

J Huang, C Chang, T Lin

Taiwan (Republic of China) 
MP25-14 How low can we go during holmium laser enucleation of the prostate (HoLEP)? 12-month results from a prospective low-power HoLEP series C Netsch, B Becker, TR Herrmann, AJ Gross Germany

MP25-15 Thulium vapoenucleation of the prostate versus holmium laser enucleation of the prostate for the treatment of benign prostatic obstruction: 12month results of a prospective randomized trial C Netsch, B Becker, TR Herrmann, AJ Gross Germany

MP25-16 Sexual outcomes of thulium laser ejaculationsparing surgery (TES) for benign prostatic hyperplasia

L Carmignani, S Nazzani, M Clementi, C Signorini, D Vizziello, G Motta, C Marenghi, P Acquati Italy

MP25-17 1000 Cases of Holmium Laser Enucleation of the Prostate: A Single Centre Case Series with 13 years of follow-up D Whiting, B Penev, M Cynk United Kingdom

MP25-18 Holmium laser enucleation of the prostate is a safe and effective procedure for patients with lower urinary tract symptoms in prostate cancer D Whiting, B Penev, M Cynk United Kingdom

MP25-19 Relationship between morcellation efficiency and resected volume of adenoma in holmium laser enucleation of the prostate for patients with benign prostatic hyperplasia H Kim, K Lee, D Shin, T Kim, S Choi, T Oh, C Yoon, W LEE, J Lee Republic of Korea
MP25-20 Clinical significance between resected prostate volume, serum prostate-specific antigen, and lower urinary tract symptoms after Holmium laser enucleation of the prostate

H Kim, K Lee, D Shin, T Kim, S Choi, T Oh, C Yoon, W LEE, J Lee Republic of Korea

MP25-21 A number of Laser procedures are available as effective alternatives to TURP entailing less morbidity and shorter hospital stays. our experience of Thulium Laser Vaporization $\&$ enucleation of prostate and intravesical morcellation

PK Pattnaik

India

MP25-22 Comparison Of Open And Robot Assisted Adenomectomy For The Treatment Of Large Prostate Glands Due To Benign Prostate Hyperplasia

S Keskin, P Mourmouris, T Doganca, A Skolarikos, I Tufek, OB Argun, MB Tuna, K Andreas, C Obek, AR Kural Turkey

MP25-23 Enucleation of the prostate and transurethral resection (B-TUEP) vs transvesical prostatectomy for enlarged prostate: initial experience in a single centre with 3 -year follow-up R GENTILE, B GENTILE, L Albanesi, C Falavolti, G Rizzo, M Buscarini, P Tariciotti, G Mirabile Italy
MP26-1 Diagnostic accuracy of CT angiography in evaluation of vascular anatomy in comparison with intraoperative findings an assessment of 392 patients

M Vijayakumar, A Ganpule, A Singh, R Sabnis, M Desai India

MP26-2 Robotic partial nephrectomy using soft coagulation may reduce the incidence of perioperative hemorrhagic complications

T Yoshida, S Kageyama, M Narita, A Kawauchi Japan

MP26-3 European Experience on Robotic Kidney Transplantation

A Breda, A Territo, L Gausa, G Basile, V Tugcu, A Alcaraz, K Decaestecker, M Stockle, P Fornara, G Siena, N Doumerc Spain
MP26-4 Utilization and Outcomes of Nephroureterectomy for Upper Tract Urothelial Carcinoma by Surgical Approach

J Rodriguez, ZL Smith, V Packiam, W Boysen, S Johnson, N Smith, A Shalhav, G Steinberg

United States

MP26-5 Robotic-Assisted vs. Open Partial Nephrectomy: A retrospective analysis of perioperative and early postoperative outcomes of patients recorded in a national UK registry AM Ahmed, A Tsiotras, GD Stewart, JN Armitage

United Kingdom 
MP26-6 Retroperitoneal vs. transperitoneal robot assisted partial nephrectomy - an propensity score weighted comparison in a multi-institutional database S Arora, R Abaza, J Adshead, B Challacombe, RK Ahlawat, P Dasgupta, G Gandaglia, D Moon, G Novara, F Porpiglia, T Yuvaraja, U Capitiano, A Mottrie, M Bhandari, CG Rogers, J Porter, H Gill United States

MP26-7 Subjective perception of nephrometric scores: red tape or clinical necessity V Malkhasyan, I Semanyakin, D Pushkar Russia

MP26-8 Investigation of the tissue stability of newly introduced polymeric clips for minimally invasive surgery: an ex vivo study

I Kyriazis, P Kallidonis, P Ntasiotis, D Kotsiris, L Amanatides, V Panagopoulos, E Liatsikos

Greece

MP26-9 Comparison of Perioperative Outcomes of Laparoscopic Versus Robotic Assisted Partial Nephrectomy for Patients with Complex Renal Cell Carcinoma: a Single Center Initial Experience from Northwest China G Zhu, D Wu, W Song, Z Yang, D He China (People's Republic)

MP26-10 Posterior Hilar Renal Tumors could be treated safely through the Trans-peritoneal Robotassisted Partial Nephrectomy Approach m koo, K Chang, A Ali, K Rha Republic of Korea

MP26-11 Laparoscopic Cytoreductive Nephrectomy is Associated with Significantly Improved Survival Compared to Open Cytoreductive Nephrectomy or Targeted Therapy Alone

EH Kim, J Vetter, K Du, R Grubb, RS Figenshau United States

MP26-12 Tumor-attaching surface area as the new parameter for extending warm ischemia time in robotic-assisted partial nephrectomy N Fukami, K Fukaya, M Kusaka, R Shiroki Japan

MP26-13 The Impact of Marijuana Intake Upon Outcomes of Hand-Assisted Laparoscopic Donor Nepherctomy D Ruckle, M Keheila, B West, B Mattison, J Thomas, S Abourbih, A Kore, M De Vera, P Baron, D Baldwin United States

MP26-14 Laparoscopic partial nephrectomy for upper pole tumors using pedicle rotation technique B Guliev, B Komyakov, X Yakubov Russia
MP26-15 Use of local anaesthetic infusion systems following laparoscopic nephrectomy and laparoscopic nephrouretectomy K Parekh, A Sultana, Y Goh, C Jellicoe, V Hariharan, HO Andrews United Kingdom

MP26-16 Does Strict Compliance to the Enhanced Recovery Programme (ERP) affect Outcomes during Laparoscopic Nephrectomy and Nephroureterectomy? A Sultana, K Parekh, Y Goh, S Ali, HO Andrews United Kingdom

MP26-17 Does Race Impact on Perioperative and Functional Outcomes in Patients Undergoing Robotic Partial Nephrectomy? CR Reynolds, K Badani, D Paulucci, A Bhandari, D Eun, R Abaza, J Porter, A Hemal United States

MP26-18 Comparison of Perioperative and Functional Outcomes in Elderly versus Non-Elderly Patients Undergoing Robotic Partial Nephrectomy: A Multi-Institutional Study CR Reynolds, K Badani, D Paulucci, D Eun, R Abaza, J Porter, A Bhandari, A Hemal United States

MP26-19 Feasibility of laparoscopically retrieved adult kidneys in pediatric renal transplantation S Balaji, A Singh, A Ganpule, R Sabnis, M Desai India

MP26-20 Bulb suction drainage is not necessary after routine robotic-assisted partial nephrectomy: A large case-control analysis CA Winter, RB Pickens, WM White United States

MP26-21 Outcomes of robot-assisted partial nephrectomy in high volume centers continue to improve for several years after the initial learning curve H Gill, T Quinn, R Cho, MJ Maurice, M Allaf, S Bhayani, M Stifelman, J Kaouk, CG Rogers United States

MP26-22 Laparoscopic Pyelolymphatic Disconnection for Refractory Chyluria B Singh, H Pathak, V Kumar, S Sankhwar India

MP26-23 Bibliometric trends of radical nephrectomy over the last 16 years (2000-2015): A systematic review A Pietropaolo, B Rai, C Lockyer, M Hayes, B Somani United Kingdom

MP26-24 Laparoscopic nephrectomy in children under 2 years of age

V Izol, N Akdogan, O Yilmaz, F Ok, E Ziyadov, K Karkin Turkey 
MP27-1 Kidney stone shadow measurements are more accurate than stone measurements with a commercial ultrasound unit

B Dunmire, J Thiel, BW Cunitz, JS Ahn, J Dai, JD Harper, K Sternberg, T Larson, MR Bailey, MD Sorensen United States

MP27-2 The efficiency of dual energy CT in determining urinary calculi composition

O Levi, N Idkedek, A Sidi, A Tsivian, E Surin, E Tavdi, R Bass, D Khunovich Israel

MP27-3 Robot-Assisted Percutaneous Nephrolithotomy Updates on Further Development M Oo, H Gandhi, K Chong, J Ng, A Goh, Y Tan Singapore

MP27-4 Towards intelligent laser lithotripsy: experimental evaluation of o novel laser system for automated stone/tissue analysis using autofluorescence in real-time

D Schlager, A Miernik, A Brandenburg,

J Schütz

Germany

MP27-5 Endoscopic clearance lithotripsy devices: bench comparison of stone elimination capacity and drilling speed

MJ Bader, F Strittmatter, A Alghamdi, T Pongratz, M Eisel, C Stief, R Sroka Germany

MP27-6 The safety and efficacy of a new micro-ultrasonic lithotriptor combined with $\mathrm{mPCNL}$ in the treatment of renal and upper ureteral stone (a preliminary report of the first 90 cases) L Xiong, X Ye, J Liu, X Huang, L Chen, K Ma China (People's Republic)

MP27-7 A Novel Technique For Access During Percutaneous Nephrolithotomy: First Clinical Experience

M Akand, O Kilic, E Altintas, K Bocu,

L Civcik, A Buyukaslan, M Koplay

Turkey

MP27-8 In Vitro Comparison of a Novel Single-probe Dual-energy Lithotripter to Current Generation Devices

D Wollin, B Winship, W Tom, D Radvak,

R Jiang, C Scales, M Ferrandino, W Simmons, GM Preminger, M Lipkin United States

MP27-9 A Novel Combination Stone Basket and Balloon Device To Treat Ureteral Stones

R Massoudi, TJ Metzner, B Bonneau, T Ngo, R Shinghal, J Leppert United States
MP27-10 Acoustic tracking of kidney stones in vitro for targeting during shock wave lithotripsy R Cleveland, K Shoar, B Turney United Kingdom

MP27-11 Current disposable ureteroscopes - performance and limitations in a standardized kidney model D Schlager, S Hein, A Miernik, M Schönthaler Germany

MP27-12 Linear focus extracorporeal shockwave therapy in the treatment of erectile dysfunction: a multicenter, prospective, randomized, double-blinded, placebo controlled study

L Yang, J Cai, D He China (People's Republic)

MP27-13 Real-Time Doppler Ultrasound Aided Nerve Sparing da Vinci ${ }^{\mathrm{TM}}$ Robot Assisted Radical Prostatectomy - Utility of Tandem Dissection of Neurovasular Bundle using a Mini Transducer SS Kommu, P Rouse, A Samateh, SH Garnett, PD Rimington United Kingdom

MP27-14 No Suture Technique of "Renorraphy" for Laparoscopic Partial Nephrectomy: Novel Use of a New Fibrin Sealant Patch C Bryson, D Singh United States

MP27-15 Investigation of the artery sealing efficacy of newly introduced polymeric clips for minimally invasive surgery: an in vivo study and histologic evaluation

I Kyriazis, P Kallidonis, M Ozsoy, P Ntasiotis, D Kotsiris, T Vrettos, P Ravazoula,

E Liatsikos

Greece

MP27-16 Utility of an Electronic Procedural Prostate Mapping Chart (e-PPMC ${ }^{\mathrm{TM}}$ ) in Robot Assisted Radical Prostatectomy

SS Kommu, A Samateh, P Rouse, J Wilson, SH Garnett, RA Persad, PD Rimington, (S Academic Research Group United Kingdom

MP27-17 Perineal Approaching Robotic Radical Prostatectomy Our Experience: First 15 cases V Tugcu, O Akca, A Simsek, I Yigitbasi, S Sahin, A Tasci Turkey

MP27-18 Usefulness of Endowrist One Vessel Sealer instrument and Endowrist Clip Applier for da Vinci Surgical System in robot-assisted radical prostatectomy K Tanaka, M Matsumoto, Y Takechi Japan 
MP27-19 Percutaneous Externally Assembled Laparoscopic Versus Traditional Laparoscopic Instruments: A Prospective Study of Cosmesis and Pain

I Kelly, M Pierce, S Abourbih, H Wagner, J Cheng, D Baldwin United States

MP27-20 A Pilot Experience in Using A Digital App To Follow-Up Prostate Cancer Patients in Shropshire, UK

Y Phan, A Loh, A Anandakumar, S Umranikar, A Elves

United Kingdom
MP27-21 Impact of a Novel Wireless Hands-Free Audio System on Communication during Robotic Surgery: A Prospective Randomized Comparison

A Thomas, M Keheila, J Thomas, B West, B Mattison, D Ruckle, M Pierce, P Hogue, J W. Cheng, H Wagner, S Abourbih, D Baldwin United States

MP27-22 A Robotic Machine-assisted Transperineal Prostate Biopsy System - Safety profile review J Kwok, D Yong, C Wong, K Chong Singapore

Friday 15 September

Moderated Poster Session 28: Laparoscopic and Robotic Surgery (Benign)

$16: 30-18: 30$

Laparoscopic/ Robotic: Other

Convention Centre East, Meeting Room 8

MP28-1 Robotic Surgery for Benign Urologic Conditions S Chopra, R Azhar, L Medina, R Sotelo, PW Mekhail, A Abreu, G Cacciamani, S Nagaraj, C Beckler, J Cai, M Desai, M Aron, IS Gill, A Berger United States

MP28-2 Ease of suturing during robotic assisted pyeloplasty and reimplantation impacts total operative time: Results of a prospective study on the learning curve of robotic pediatric urology procedures

$$
\text { N Stern, R Clark, A Shukla, S Dave }
$$

Canada

MP28-3 Laparoscopic management of complicated urachal remnants

H Jeong

Republic of Korea

MP28-4 Three Dimensional Laparoscopic Ureterolithotomy: New Treatment Option for Large Upper Ureteral Stones

J Kang, J Jeon

Republic of Korea

MP28-5 Comparison of Umbilicus-sparing laparoscopic approach Versus Open approach for treating symptomatic Urachal remnants in adults

$\mathrm{J} \mathrm{Hu}, \mathrm{Z}$ Liu

China (People's Republic)

MP28-6 Transperitoneal Laparoscopic Pyeloplasty for Retrocaval Ureter: Single Surgeon's Experience and Literature Review

T Oh, J Lee, S Park, I Seo

Republic of Korea

MP28-7 Clips offer a safe alternative to staples for vascular control of the renal vessels during laparoscopic donor nephrectomy

R Sowerby, T Kroczak, D Ghiculete, J Lee, R D’A. Honey, K Pace, M Ordon

Canada
MP28-8 Laparoscopic Donor Nephrectomy In Patients With A Circumaortic Left Renal Vein: Feasibility And Outcomes

A Hsieh, J Stites, G Portman, R Munver United States

MP28-9 Robotic Kidney Transplantation: Bakirkoy Experience V Tugcu, S Sahin, AH Yavuzsan, I Yigitbasi, FG Akbay, S Apaydın Turkey

MP28-10 Laparoscopic orchiopexy for bilateral high intra-abdominal testes in adult patients, single vs two stages
A hammady, M Elbadry
Egypt

MP28-11 Rectourinary Fistulas: Laparoscopic and Robotic Management and Outcomes R Sotelo, L Medina, FZ Husain, G Cacciamani, IS Gill United States

MP28-12 Report of pneumoscopic vesicovaginal fistula repair

H Choi, J Bae, H Chung, S Bae

Republic of Korea

MP28-13 Robot Assisted Transplant Allograft Nephrectomy Series: A Novel Approach for a Challenging Operation

R Nunez, S McAdams, HM Abdul-Muhsin, G Kelli, KL Stern, N Katariya, EP Castle United States

MP28-14 Prospective Evaluation of surgical and functional outcomes of Robot-assisted sacrocolpopexy and sacrocervicopexy in the management of vault prolapse

S Arakere, D Dubey, RK Ahlawat, A Jain India 
MP28-15 Comparing Traditional Three Port and Single Site Laparoscopic Inguinal Hernia Repair in Children

C Tong, J Van Batavia, D Chu, A Srinivasan United States

MP28-16 The Use of Intraureteral and Intraurinary diversion Indocyanine Green During Robotic Ureteroenteric Reimplantation for the Management of Benign Anastomotic Strictures Z Lee, A Asghar, B Waldorf, D Eun United States

MP28-17 Robotic Ureterolithotomy for Management of Large, Impacted Ureteral Calculi WR Lai, R Thomas United States

MP28-18 A novel technique of access of peritoneal cavity and creation of tunnel in Laparoscopic Orchidopexy with Points of Technique H Singh, A Kumar, S Singh, A Pal, J Kushwaha India

MP28-19 Critical Analysis Of Laparoscopic Donor Nephrectomy In Jehovah's Witnesses A Hsieh, J Stites, G Portman, R Munver United States
MP28-20 Migration of Left Renal Vein Endovascular Stents for Nutcracker Syndrome

S Chen, L Tian, K Yang, H Zhang, W Jin, $\mathrm{M} \mathrm{Li}$ China (People's Republic)

MP28-21 Withdrawn

MP28-22 Bilateral laparoscopic varicocelectomy associated with bilateral inguinal hernioplasty in the same procedure: a controlled prostective study

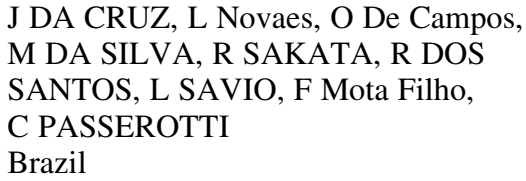

MP28-23 Case series study of transvesical and transurethral repair of vesicovaginal fistula C Huang Taiwan (Republic of China)

MP28-24 Robotic vs. Open Ureteroneocystotomy: The Novice Single Surgeon Experience B Shah, N Manimala, J Lockhart, L Wiegand United States

Friday 15 September

Moderated Poster Session 29: Functional Urology (BPH/Incontinence)

16:30-18:30

BPH/LUTS: Electrosurgery, Lasers \& Other Technoloy

Convention Centre East, Meeting Room 11

MP29-1 Is preoperative urinary flow a predictive value of postoperative acute urinary retention rate?

L Carmignani, G Motta, M Clementi,

D Vizziello, C Signorini, C Marenghi,

D Ratti, S Nazzani

Italy

MP29-2 Preoperative Voiding Efficacy Could Predict Postoperative Alpha blocker Usage after Transurethral Resection of The Prostate

C Hsu, W Cheng, C Chang, C Huang, Y Chiu, S Hong, A Chiu

Taiwan (Republic of China)

MP29-3 What are uro-geriatric factors that impact decision making in the elderly, with a refractory urinary retention, after a multidisciplinary team board?

C Rambaud, S Gonfrier, C Arlaud, B Tibi,

R Prader, O Guérin, M Durand

France

MP29-4 Patient Reported Outcomes via Online Questionnaire (PROVOQ) - A Novel, Automated Approach for tracking Patient reported 30 day continence following Robot-Assisted Radical Prostatectomy (RARP)

LM Huynh, TE Ahlering

United States
MP29-5 Analysis and Predictors of Urinary Retention for Idiopathic Overactive Bladder treated with Intravesical Onabotulinumtoxin A Injection Y Lu, T Kuo, L Ng Singapore

MP29-6 A change in UTI incidence in our intradetrusor Botox patient cohort following changes in preoperative protocol and antibiotic prophylaxis A Brewin, T Nitkunan, E White, $\mathrm{R}$ Walker United Kingdom

MP29-7 A prospective evaluation of a modified cervico-sacropexy /vagino-sacropexy procedure in treatment of female patients with refractory urge/ mixed urinary incontinence

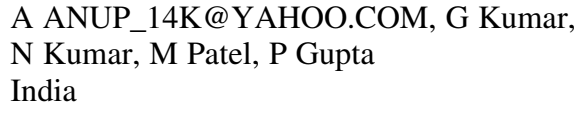

MP29-8 The efficacy of mid-urethral sling in female stress urinary incontinence with overactive bladder $\mathrm{H}$ Lee, J Seo Republic of Korea

MP29-9 Recurrent urinary retention: 6 months follow-up of elderly patients who benefited an alternative treatment to the indwelling catheter after a multidisciplinary team board

C Rambaud, S Gonfrier, C Arlaud, J Fallot, O Guérin, M Durand

France 
MP29-10 Efficacy and safety of OnabotulinumtoxinA in patients with overactive bladder according to site of injection: A systematic review and metaanalysis

J Jo, S Park, H Choi, H Moon, S Lee, K Kim, Y Kim

Republic of Korea

MP29-11 Relationship between pelvic muscle contractility and ability of immediate continence after robotassisted radial prostatectomy

K Park, J Huh, Y Kim, S Kim Republic of Korea

MP29-12 A Prospective, Multi-Institutional Assessment of 30-Day Pad-Free Continence Predicting Longterm Continence Following Robot Assisted Radical Prostatectomy (RARP)

LM Huynh, R Derderian, D Skarecky, TE Ahlering

United States

MP29-13 Outcomes of transobturator tape procedures in women with mixed urinary incontinence

K Oh, J Kim, S Kim, Y Cho, M Kim,

H Chung, E Hwang, S Jung, T Kang, K Park, D Kwon

Republic of Korea

MP29-14 Minisling single incision tape for treatment of female stress incontinence, is it better?!

M Elbadry, A essam, A hammady, m gamal, m abdelmalek

Egypt

MP29-15 Does Preoperative Prostate Volume Predictive For Medical Therapy Continuation After TURP? M Zor, E Kaya, T Ebiloglu, A Coguplugil, S Bedir, S Bedir Turkey

MP29-16 A Prospective, Multi-Institutional Assessment of 90-Day Percent Fullness - Predictive Modeling of Potency Recovery Post-Robot Assisted Radical Prostatectomy (RARP)

LM Huynh, D Skarecky, TE Ahlering United States
MP29-17 Urodynamic Testing in Patients with Lower Urinary Tract Symptoms Prior to Robotic Assisted Laparoscopic Prostatectomy Helps Guide Postoperative Use of Anticholinergic Therapy for Early Return of Continence S Razdan, LA Barrueto, JP Chiou, S Razdan United States

MP29-18 Withdrawn

MP29-19 Dehydrated Human Amniotic Membrane and Early Return of Erectile Function Post RoboticAssisted Laparoscopic Prostatectomy: Update from a High Volume Surgeon

S Razdan, JP Chiou, LA Barrueto, S Razdan United States

MP29-20 Improved urinary function following robotic radical prostatectomy with a concurrent retropubic vascularised fascial sling (RoboSling) S Leslie, R Thanigasalam, P Sved, A Vasilaras, N Jeffery, P Bergamin, G McClintock, E Lim, M Winter, N Ahmadi, M Desai, M Aron, IS Gill Australia

MP29-21 An Evaluation of Functional Outcomes after Robot-Assisted Radical Prostatectomy in patients with large prostates $(\geq 60 \mathrm{~g})$

Y Law, M Tan, J Du, H Huang, S Ho Singapore

MP29-22 Improvement of Immediate Continence Control after Minimally Invasive Radical Prostatectomy from anterior to posterior, from robotic-assisted to pure laparoscopic

K Chiu

Taiwan (Republic of China)

MP29-23 Robitic-Assisted Sacrocolpopexy For Pelvic Organ Prolapse: Technique And Early Outcomes PW Mekhail, FZ Husain, C Sevilla, MW Mekhail, IS Gill, A Monish, L Rodriguez United States

MP29-24 Technical development of a transvaginal probe using near infrared light to monitor pelvic floor muscle hemodynamics bilaterally EG Deegan, L Stothers, D Lazare, AJ Macnab Canada
MP30-1 The WATER Study - A Phase III Blinded Randomized Trial of Aquablation vs. Transurethral Resection of the Prostate for the treatment of Benign Prostatic Hyperplasia P Gilling, C Roehrborn New Zealand

MP30-2 Green laser (532-nm) enucleation of the prostate (GreenLEP) en bloc technique. initial experience, and short-term outcomes E Rijo, J Lorente, O Bielsa, F Gomez-Sancha Spain
MP30-3 Modern management of benign prostatic hyperplasia in large glands: a review of a single center with high volume open, robotic, and laser prostatectomy

S Mehta, G Gaunay, S Smith, Z Okeke,

L Richstone, P Samson

United States

MP30-4 Evaluating the learning curve for robotic assisted simple prostatectomy

I Sorokin, BA Johnson, N Singla,

C Roehrborn, J Gahan

United States 
MP30-5 Benign prostatic hyperplasia in octuagenarians: a thulium laser vaporization experience

L Carmignani, C Signorini, M Clementi, D Vizziello, G Motta, S Nazzani, S Picozzi, R Stubinski Italy

MP30-6 Safety and feasibility of thulium laser transrethral resection of prostate for benign prostatic enlargement surgery in overweight and obese patients

L Carmignani, D Vizziello, M Clementi, A Conti, E De Lorenzis, P Acquati Italy

MP30-7 Comparison of the urinary outcomes of thuvap in two groups of patients with a prostate volume $<80 \mathrm{ml}$ and $>80 \mathrm{ml}$ (preliminary data)

L Carmignani, M Clementi, D Vizziello, G Motta, E Finkelberg, D Ratti, S Picozzi Italy

MP30-8 Thulium laser prostate enucleation in refractory urinary retention: operative and functional outcomes in a large cohort of patients

L Carmignani, S Maruccia, D Vizziello, E Finkelberg, D Ratti, A Saccà, A Pastore, F Pisano, M Schirinzi Italy

MP30-9 Thulium laser vaporization: how many grams per minute?

L Carmignani, M Clementi, G Motta,

C Signorini, D Vizziello, E Finkelberg,

S Maruccia, S Nazzani

Italy

MP30-10 Can thulium vaporization of the prostate be considered as safe and effective as thulium vapoenucleation also for prostates larger than $80 \mathrm{ml}$ ? Preliminary results from a single institution

L Carmignani, M Clementi, S Nazzani,

C Signorini, D Vizziello, S Picozzi,

C Marenghi, D Ratti, R Stubinski, G Motta Italy

MP30-11 Simultaneous Percutaneous Cystolithotripsy with TURP is the gold standard for management of large prostates with big bladder stones M Raghavendran, K Kumar India
MP30-12 Does The Volume Effect of Prostatic Adenoma in Prostate Exist?: a Prospective Study

J Oh, K Kim, T Kim, C Kim, K Kim, H Jung, S Yoon

Republic of Korea

MP30-13 Laser Technologies in Surgical Treatment of Benign Prostatic Hyperplasia

D Enikeev, P Glybochko, G Altshuler,

A Vinarov, L Rapoport, M Enikeev,

N Sorokin, A Dymov, R Sukhanov,

O Khamraev, M Taratkin

Russia

MP30-14 Outcome and complications of Holmium Laser Enucleation of the Prostate in patients on anticoagulation

K Guest, T Smith, M Cynk

United Kingdom

MP30-15 A simple technique of tissue collection after morcellation during holmium laser enucleation of prostate

PN Maheshwari

India

MP30-16 Feasibility, Safety, and Efficacy of Transurethral Thulium Laser Prostatectomy in the Outpatient Setting: A Single Center Experience in the United States

T Cisu, D Sobel, RT Grunert

United States

MP30-17 Robotic assisted versus open simple prostatectomy for benign prostatic hyperplasia in large glands: a propensity score matched comparison

I Sorokin, BA Johnson, V Sundaram,

N Singla, V Margulis, C Roehrborn, J Gahan United States

MP30-18 Robot-assisted simple prostatectomy for large volume benign prostatic hyperplasia: Initial single-center experience in China Z Shen, S Chen, S Zhong, M Zhang China (People's Republic)

MP30-19 Holmium enucleation of prostate in patients taking anti platelets H Pothiyedath, KM Ramaswami India
MP31-1 Can the Use of Robotics Reduce the High

Morbidity of Inguinal Lymphadenectomy? R Patel, K Radadia, V Kosherkov, EA Singer, S Elsamra United States
MP31-2 Perioperative Outcomes And Complication Rates Following Robot Assisted Retroperitoneal Lymph Node Dissection For Testicular Cancer: A MultiInstitutional Update HM Abdul-Muhsin, M Marshall, KL Stern, S Stroup, M Woods, J L'esperance, EP Castle United States 
MP31-3 Development of a Robotic Surgery Program at Johns Hopkins Aramco Healthcare: An International Collaboration

T Tartir, K Taheini, M Johnson, J Ziemba, M Allaf

United States

MP31-4 “Video Endoscopic Inguinal Lymphadenectomy (VEIL) vs Conventional Inguinal Lymphadenectomy (CIL) - a comparative evaluation in cases of Carcinoma Penis" H Singh, S Jain, A Kumar, A Pal India

MP31-5 Minimally Invasive Inguinal Lymphadenectomy N Jung, H Smith, C Keel, A Singh United States

MP31-6 Salvage Robotic Retroperitoneal Lymphadenectomy for Prostate Cancer Nodal Recurrence: technical aspects and results F Mota Filho, J DA CRUZ, R Santos, M Nunes, R Sakata, C PASSEROTTI Brazil

MP31-7 Comparison of Surgical Outcomes of Laparoscopic Versus Robotic Assisted Repair of Vesicovaginal Fistula: Initial Experience From Northwest China G Zhu, D Wu, W Song, Z Yang, D He China (People's Republic)

MP31-8 Post-Chemotherapy Laparoscopic Retroperitoneal Lymph Node Dissection for Select Stage II and III Mixed Malignant Germ-Cell Testicular Tumors EH Kim, K Du, RS Figenshau United States

MP31-9 Robotic-assisted radical prostatectomy associated with robotic-assisted cholecystetomy in the same procedure: case series report and review of literature

J Queiroz, P Califano, M Belkovsky, J DA CRUZ, R SAKATA, M DA SILVA, M Srougi, W Nahas, C PASSEROTTI Brazil

MP31-10 Results of a Center for Robotic Surgery in Brazil: A nine years experience J Queiroz, P Califano, M Belkovsky, J DA CRUZ, R SAKATA, M DA SILVA, M Srougi, W Nahas, C PASSEROTTI Brazil

MP31-11 The utility of axial abdominal imaging after partial nephrectomy for $\mathrm{T} 1$ renal cell carcinoma surveillance

I Sorokin, BA Johnson, N Canvasser, V Margulis, Y Lotan, G Raj, A Sagalowsky, J Gahan, J Cadeddu United States
MP31-12 Effect of robot-assisted laparoscopic urological surgeries on the modern philosophy of nursing X Peng, X Wang, S Zhong, Z Shen, Q Ding China (People's Republic)

MP31-13 Repeat Robot Partial Nephrectomy: Initial Experience S Chopra, R Ahar, C Beckler, L Medina, R Sotelo, G Cacciamani, A Abreu, J Cai, M Desai, M Aron, IS Gill, A Berger United States

MP31-14 Robotic-Assisted Partial Nephrectomy for Hilar Tumors: Initial Experience M Santomauro, S Chopra, L Medina, R Sotelo, G Cacciamani, A Abreu, C Metcalfe, M Aron, M Desai, IS Gill, A Berger United States

MP31-15 The early experience of laparoscopic retroperitoneal nephrectomy in Mongolia A Sankjaajamts, D Batbold, T Zev, M Munkhbaatar, S Avirmed, J Jodov Mongolia

MP31-16 Transition from retroperitoneal to transperitoneal robotic-assisted laparoscopic partial nephrectomy :the learning curve for experienced surgeon in the robotic surgery era W Liao, C Zhang, L Diao, Q Yang, X Yao China (People's Republic)

MP31-17 The clinical application of retroperitoneal laparoscopic surgery combined with mini-flank incision "hybrid surgery" for partial nephrectomy of complex renal tumor $X$ Ye, J Liu, T Xu, X Huang China (People's Republic)

MP31-18 Cystic versus Solid Masses: Is There a Difference in Perioperative Outcome During Robotic Partial Nephrectomy?

K Blum, D Paulucci, R Abaza, A Bhandari, JC Delto, K Badani United States

MP31-19 Off-clamping robot-assisted partial nephrectomy and re-recognition of HuaShan standard Z Shen, S Zhong, Z Fang, Z Wu, S Chen, M Zhang, X Zhang, C Feng, S Mao, Q Ding China (People's Republic)

MP31-20 Outcome of near-infrared fluorescence imaging with indocyanine green in robot-assisted partial nephrectomy on different tumor morphology: preliminary 1st case series in Japan S Hisasue, H Takeuchi, S Ohta, T Kubota, Y Mitsui Japan 
Education, Simulation \& Virtual Reality

Convention Centre East, Meeting Room 2

MP32-1 Urology Residents' Experience and Attitude Towards Surgical Simulation: Presenting Our Four Year Experience With A Multi Institutional, Multi Modality Simulation Model

A Chow, BA Sherer, E Yura, S Kielb, E Kocjancic, S Eggener, T Turk, S Park, S Psutka, M Abern, K Latchamsetty, C Coogan United States

MP32-2 Assessment of Urology Postgraduate Trainees' Competencies in Flexible Ureteroscopic Stone Extraction
M Aloosh, F Couture, N Fahmy, M Elhilali, S Andonian
Canada

MP32-3 Impact of resident trainee involvement on robot assisted radical prostatectomy outcomes E Schommer, K Tonkovich, D Thiel United States

MP32-4 Learning curve associated with the WHO Surgical Safety Checklist: A simulation based evaluation F Dar, T Abe, P Amnattrakul, A Aydin, N Raison, S Khan, P Dasgupta, K Ahmed United Kingdom

MP32-5 Urology Resident Workload Profiles when Performing Live Porcine Nephrectomies and Robotic Surgery Simulator Training Modules: Update

T Rogers, G Ogaya-Pinies, E Hernandez, H Palayapalayam, V Patel

United States

MP32-6 Ureteroscopy simulation training under full immersion decreases mental workload in novice operators

T Abe, F Dar, P Amnattrakul, A Aydin, N Raison, N Shinohara, S Khan, K Ahmed, P Dasgupta Japan

MP32-7 Effectiveness of Procedural Virtual Reality Simulation in Surgical Training P Harrison, N Raison, K Ahmed United Kingdom

MP32-8 Acceptability of the Fundamentals of Laparoscopic Surgery (FLS) programme tasks for Urology trainees

J Hendry, L Kerr, C McIlhenny

United Kingdom

MP32-9 Evaluating the benefits of using objective benchmarks in a virtual reality training curriculum, for robotic and laparoscopic surgery W Watkinson, N Raison, K Ahmed, P Harrison United Kingdom
MP32-10 Use of Google Trends to Track Online Behavior and Interest in Kidney Stone Surgery

C Tong, PC Dreher, E Ghiraldi, J Friedlander United States

MP32-11 Construct and Content Validity of the Visual Prostate Symptom Score Following Inclusion of Pictograms for Urgency and Urgency Incontinence by men with Obstructive Lower urinary Tract Symptoms in a Low and Middleincome Country (LMIC)

L Stothers, AJ Macnab, R Mukisa

Canada

MP32-12 Safety of live surgery in urology. Propensity scored matched analysis

G Ogaya-Pinies, H Palayapalayam

E Hernandez, X Bonet, T Rogers, C Jenson,

V Patel

United States

MP32-13 Role of personality in postoperative healthrelated quality of life following ureteroscopic stone extraction with stent insertion R Davis, NJ Farber, P Modi, S Elsamra, E Olweny United States

MP32-14 A comparison of Twitter usage between general urologists and endourologists A Ludvigson, J Ingimarsson United States

MP32-15 Differences in practice patterns among LatinAmerican urologists with or without endourology training

BO Manzo, E Lozada, F Daels, M Bertacchi, C Vicentini, A Rasguido, M Cabrera,

P Gonzalez, W Pereyra, J Blasco, G Manzo,

C Velazquez, A Rodriguez, E Aleman,

A Etienne

Mexico

MP32-16 Comparison of Evidence Quality Underlying Surgical Subspecialty Guidelines in Urology, Orthopedics and Otolaryngology SG Antoine, AC Small, DJ Robins, MJ Lipsky, JM McKiernan, OD Shah United States

MP32-17 A critical analysis of evidence and opinions within the American Urological Association's nephrolithiasis Clinical Practice Guidelines AC Small, MJ Lipsky, DJ Robins, SG Antoine, BE Muller, JM McKiernan, OD Shah United States 
Clinical Stones: Medical Management

Convention Centre East, Meeting Room 1

MP33-1 Vascular Disease and Kidney stones: Abdominal Aortic Calcifications Are Associated with Low Urine pH and Hypocitraturia

N Patel, R Ward, J Calle, E Remer, M Monga United States

MP33-2 Diabetes and Stone Risk: A Matter of Glycemic Control?

SL Best, K Maciolek, KL Penniston United States

MP33-3 Current Use of Medical Expulsive Therapy Among Endourologists

DC Fedrigon, RK Jain, S Sivalingam United States

MP33-4 Assessment of Adherence to Metabolic Evaluation Guidelines in Patients with Nephrolithiasis Results of a National Multi-centric study S Harmouch, H Abou-Haidar, S Andonian, J Lee, T Schuler, A Lantz, BH Chew, N Bhojani Canada

MP33-5 Metformin Use and 24-hour Urine Abnormalities in Urinary Stone Formers MJ Wardenburg, BK Carew, BJ Otto, VY Bird, VG Bird United States

MP33-6 The Association of Hemoglobin A1C and Urinary Oxalate in Stone Formers K Wood, J Knight, R Holmes, D Assimos, R Oster, M Colaco United States

MP33-7 Medical expulsive therapy for distal ureteral stones: Tamsulosin versus silodosin in the Turkish Population E Arda, B Cakiroglu, E Akdeniz Turkey

MP33-8 Nephrolithiasis is Associated With Nonalcoholic Fatty Liver Disease A Weiss, E Ghiraldi, J Gaughan, J Friedlander United States

MP33-9 Patient Perceptions of Non-Obstructing Kidney Stones and Pain DW Smith, MS Borofsky United States
MP33-10 A pilot study comparing virtual and real consultation(out patient) for stone patients : Future of Endourology stone clinic HG Thummar, K Thummar, N Zinto, N T India

MP33-11 Conservative Management of Staghorn Calculi: Is it Safe?

T Nikonow Morgan, M Shahait, A Maganty, A Nevo, M Ost, S Jackman, T Averch, MJ Semins United States

MP33-12 A UK Snapshot of Loin Pain / Urolithiasis outcomes from June 2015 to June 2016

T Marsden, B Turney, M Bultitude, F Keeley, S Gordon, SO Irving, W Finch, B Grey, O Wiseman, A Rogers, N Shrotri, D Smith United Kingdom

MP33-13 Systemic Biomineralization in Kidney Stone Formers

BA Sherer, A Fernandez, DT Tzou, S Ho, ML Stoller United States

MP33-14 Rates of Spontaneous Ureteral Stone Passage After Ureteral Stent Placement J Kuebker, J Robles, N Miller, S Herrell, RS Hsi United States

MP33-15 Assessing the Need for Technology to Promote Fluid Consumption in Patients with Nephrolithiasis

N Streeper, K Lehman, D Conroy United States

MP33-16 Real practice results of medical expulsive therapy for symptomatic ureteral stones at 2 weeks after stone attack

S Pacik, W Choi, H Park, H Kim

Republic of Korea

MP33-17 Gender disparity in urolithiasis - fact or fiction? GN Tundo, VM Pais United States 
MP34-1 Positive Predictive Value of CT Urography for Upper Tract Urothelial Carcinoma Diagnosis using Diagnostic Ureteroscopy as the Reference Standard

TC Chang, I Mintz, J Ben-Chaim, S Conti, J Leppert, S Barnes, JC Liao, M Sofer United States

MP34-2 Isolated red patches seen during endoscopic surveillance of bladder cancer - how often should we biopsy?

N Nkwam, S Momcilovic, S Trecarten, A Bazo, G Mann, B Sherwood, R Parkinson United Kingdom

\section{MP34-3 Withdrawn}

MP34-4 Oncologic outcomes of kidney sparing surgery using endoscopic management of upper urinary tract urothelial carcinoma C Shen, Y Tsai, C Ou, W Yang Taiwan (Republic of China)

MP34-5 Ureteropelvic junction involvement does not predict advanced disease or clinical outcomes for patients with upper urinary tract urothelial carcinoma isolated to the ureter K Wymer, D Gearman, A Toussi, V Sharma, T Miest, B Leibovich, M Tollefson United States

MP34-6 Does retrograde Double-J stenting plays a role for tumor seeding in upper urinary tract in cases of bladder urothelial malignancy? A Sokhal, R Aeron, S Sankhwar, S Goel India

MP34-7 Tumor Location and Its Association with NonOrgan Confined Upper Tract Urothelial Carcinoma

D Gearman, A Toussi, V Sharma, T Miest, B Leibovich, M Tollefson United States

MP34-8 NBI re-transurethral resection ( reNBITURBT) for pT1HG non-muscle invasive bladder cancer : does it improve detection rate? Preliminary experience in single centre

R GENTILE, M Buscarini, M Vermiglio, B GENTILE, L Albanesi Italy

MP34-9 Arsenic Exposure and Temporal Trend of Incidence in Upper Urinary Tract Urothelial Carcinoma, 1979-2010: A Nationwide Populationbased Study in Taiwan

Y Tai, C Chen

Taiwan (Republic of China)
MP34-10 The impact of narrow band imaging (NBI) in the detection of bladder tumour in transitional cell carcinoma of the bladder: A randomized crossover controlled trial from a tertiary care centre in India P Mukherjee, R Manojkumar, C Beri, J L, S Kumar, N Kekre, A Devasia India

MP34-11 Observing the trends in treatment options for urothelial carcinoma in the inpatient setting of New York State hospitals - between 1994-2012 KH Bilal, DS Ali, M Finkelstein, MA Palese United States

MP34-12 Endoscopic Management of Upper Tract Urothelial Cancer: Improved Prediction of Invasive Cancer Using a Ureteroscopic Scoring Model

S Jeon, H Sung, Y Choi, H Jeon, B Jeong,

S Seo, H Lee, H Choi

Republic of Korea

MP34-13 Financial Benefit of TULA (Transurethral Laser Ablation) for Recurrent Non-Muscle Invasive Bladder Cancer

P James, A Pai, A Chetwood, T Smith, N Arumainayagam, A Shamsuddin,

S Agrawal United Kingdom

MP34-14 Is suitable today to use only the WLTURBT for a correct stadiation of a non muscle invasive bladder cancer. Preliminary experience in highflow centre with the use of WL TURBT plus repeat NBI TURBT

R GENTILE, M Vermiglio, M Buscarini, B GENTILE, L Albanesi, P Tariciotti Italy

MP34-15 Template-based laparoscopic lymphadenectomy combined with laparoscopic nephroureterectomy for renal pelvic and proximal ureter cancer: examination of safety and adequacy K Araki, K Ohotsuka, A Fujimoto, K Hou, T Kato, T Suyama, H Masuda, S Kojima, Y Naya, T Ichikawa Japan

MP34-16 Oncologic Outcomes Following MinimallyInvasive Radical Nephroureterectomy for Upper Tract Urothelial Carcinoma

A Toussi, T Miest, G Chow, B Leibovich, M Tollefson

United States

MP34-17 The use of homemade device for hand-assisted retroperitoneoscopic nephroureterectomy

T Tai

Taiwan (Republic of China) 
MP34-18 Does the Xi da Vinci robot reduce operative time of nephro-ureterctomy comparaired with the $\mathrm{Si}$ system?

L Vouga, G Wirth, C Iselin

Switzerland

MP34-19 Rigid Ureteroscopic resection of bulky ureteral tumor

JH Hillelsohn, M Eshghi, A Schulman,

NH Patel, SA Fullerton

United States

MP34-20 Clinical Assessment Of The Durability Of A Single-Use Digital Flexible Ureteroscope For The Treatment Of Large Stone Burden Or Extensive Upper Tract Urothelial Carcinoma

G Portman, J Stites, B Press, R Munver United States
MP34-21 Partial cystectomy for bladder urothelial cancer: single medical center experience L Tsai, P Hsiao, Y Chi-Rei, G Chen, S Chou Taiwan (Republic of China)

MP34-22 Initial experience with laparoscopic radical cystectomy and evaluation of operative and oncologic outcomes K Ohba, T Kondo, K Araki, H Nakanishi, Y Sagara, T Matsuo, Y Miyata, H Sakai Japan

MP34-23 Comparison of Monopolar and Bipolar Transurethral Resection of Bladder Tumor: A Randomized Controlled Trial SK Singh, M Pragatheeswarane, SK Devana, RS Mavuduru, N Kakkar, S Kumar, AK Mandal India
MP35-1 Laparoscopic assisted ileal ureter

B Komyakov, B Guliev, A Shipilov Russia

MP35-2 Five year outcomes data utilizing delayed barbed absorbable suture for robotic sacrocolpopexy

S Castellucci

United States

MP35-3 Minimally Invasive Renal Artery Aneurysm Repair: Initial Case Series

S Chopra, A Abreu, C Fay, A Aron, M Desai, M Aron, R Sotelo, IS Gill United States

MP35-4 Robotic uteretal reimplantation for ureteroenteric anastomotic strictures

N Ahmadi, C Fay, D Freitas, A Berger, L Misuraca, S Guaglianone, M Ferriero, G Simone, M Desai, A Goh, M Gallucci, M Aron United States

MP35-5 Management of Recto-Urethral Fistulas after Focal Treatment for Prostate Cancer R Sotelo, L Medina, D Hatcher, L Doumanian, IS Gill United States

MP35-6 Sexual dysfunction and abdominal aortic aneurysm surgical repair: incidence and consequences for clinical practice

P Regnier, F Lareyre, R Haider, F Roustan, Y Bodokh, P Treacy, B Tibi, R Prader, J Amiel, D Chevallier, M Durand France

MP35-7 The Early Outcomes of Ureteral Reconstruction in Iatrogenic Ureteral Injury and Non-Iatrogenic Ureteral Surgeries

C Tseng, P Chow, K Huang

Taiwan (Republic of China)
MP35-8 The Outcome of Robotic Boari Flap MW Salkini, N Idris United States

MP35-9 The outcome of conservative management of unilateral asymptomatic Pelvi-ureteric junction (APUJO) Obstruction

ME Hassan, M Smith, K Subramonian United Kingdom

MP35-10 Feasibility and safety of ileal neobladder with simplified catheterization and irrigation protocol K Kim, D Lee Republic of Korea

MP35-11 A Preoperative Mathematical Model to Estimate Available Tissue Length for Ureteral

Reimplantation by Different Methods of Bladder Mobilization

MA Perez, GL Lloyd

United States

MP35-12 Robotic reconstruction of ureteroenteric stricture after urinary diversion AC Weinberg, D Volkin, W Huang, L Zhao United States

MP35-13 Comparative outcomes of laparoscopic and open ureteroneocystoneostomy

B Guliev, B Komyakov, V Ochelenko,

A Shipilov

Russia

MP35-14 Perceptions and practice patterns of holmium laser goggles in endourological procedures: an unnecessary evil?

N Paterson, R Fitzpatrick, J Denstedt, B Blew, J Watterson

Canada 
MP35-15 Clinical Observation of Endoscopic Surgery for Hemorrhagic Cystitis after Hematopoietic Stem Cell Transplantation J Liu, B Yang, T Xu, X Wang, X Huang China (People's Republic)

MP35-16 Partial-tickness-endopyelotomy for failed pyeloplasty and factors effecting the success rate T Ebiloglu, E Kaya, M Zor, S Sarıkaya, B Topuz, B Kopru, S Bedir Turkey

MP35-17 Transperitoneal Laparoscopic Pyeloplasty Associated Rigid Nephroscopy in the Treatment of Ureteropelvic Junction Obstruction and Renal Calculi

J Liu, X Ye, L Xiong, X Huang, T Xu

China (People's Republic)

MP35-18 Percutaneous ureteroscopy laser unroofing-a minimally invasive approach for renal cyst treatment

J Hu, X Yu, S Wang, Z Ye

China (People's Republic)

MP35-19 Intracorporeal excisional tapering of megaureters: An easy task with Robot assistance SK Singh, RS Mavuduru, GS Bora, SK Devana, K Parmar, AK Mandal India
MP35-20 Cluster analysis identifies three urodynamic patterns in patients with orthotopic neobladder reconstruction

K Kim, D Lee

Republic of Korea

MP35-21 Percutaneous Nephroscopic Pancreatic Necrosectomy: A urologist's gift to pancreatic surgeon

V Jain, N Aggarwal, A Arora

MP35-22 The effect of hyaluronic acid/ carboxymethylcellulose instillation to prevent urethral stricture after transurethral bladder surgery

S Choi, S Yoon, J Do, S Lee, S Jeh, J Hyun, D Seo, C Lee, K Chung, S Kam, J Hwa Republic of Korea

MP35-23 Use of Guidewire during Placement of Prophylactic Ureteral Localization Stents (PULSe) for Colorectal Surgery (CRS) Cases Decreases Urologic-induced Operative Complications

R Pathak, G Broderick, R Pak, S Petrou, P Young, E Schommer, D Thiel United States

\section{VIDEO SESSIONS}

Wednesday 13 September

Video Session 1: Ureteroscopy

14:30-16:30

New Technology: Stones

Convention Centre East, Meeting Room 12

V1-1 Accept the challenge: A staged approach for impacted upper ureteric calculi making task difficult to Easy ! HG Thummar, N Zinto, N T, K Thummar, J Vyas India

V1-2 Ureteroscopic Management of a Large Upper Tract Urothelial Carcinoma

K Scotland, N Kleinmann, L Hubbard, SG Hubosky, DH Bagley

United States

V1-3 Vaporization of ureteral fibroepithelioma A Basiri, M Dadpour, A Vali Iran

V1-4 Malignant Ureteral Obstruction: Management with Tandem Ureteral Stenting G Gaunay, P Samson, AD Smith, Z Okeke United States

V1-5 Using flexible ureteroscopy to treat a complex biliary stone in a multidisciplinar aproach

RS Campos, G Guimarães, C Zurstrassen, R Oliveira, J Kawaoka Matushita, B Benigno, S Zequi Brazil
V1-6 Ureteroscopy Utilizing Coagulum and ClearPetra Access Sheath

ME Rivera, J Lingeman, AE Krambeck United States

V1-7 Robotic assisted retrograde intra-renal surgery for large kidney stone using Avicenna Roboflex A Patel, N Tokatli, V Tugcu, S Bedir, J Klein, A Atmaca, M Imamoglu, K Sarica, A Kabakci, J Rassweiler, R Saglam United Kingdom

V1-8 Ultrasound-assisted ureteroscopy with direct vision stent placement during pregnancy RJ Yau, A Schulman, NH Patel, C Fox, M Zhang, M Eshghi United States

V1-9 Flexible Ureteroscopic Management of Renal Caliceal Diverticular Stones Y Yoon, J Na, J Jung, J Jo, H Lee, W Han, S Park Republic of Korea

V1-10 Flexible ureteroscopic Management of Endocalyceal Diverticular Calculi

A Al Aown

Saudi Arabia 
Stones: PCNL

Convention Centre East, Meeting Room 13

\section{V2-1 Withdrawn}

V2-2 The new generation super-mini percutaneous nephrolithotomy (SMP) system: a step-by-step guide

G Zeng, W Zhu, Y Liu, J Fan

China (People's Republic)

V2-3 Complete Calcified ureteral stent: a combined one-session approach

F Torricelli, R Berjeaut, L Laferreira, F Vicentini, G Marchini, A Danilovic, W Nahas, M Srougi, E Mazzucchi Brazil

V2-4 Micro-Nephrolithotomy in a transplanted kidney: a peculiar indication for a niche procedure S Zanetti, G Sampogna, M Talso, E De Lorenzis, L Boeri, A Gallioli, F Palmisano, M Fontana, I Oliva, F Longo, C Beretta, M Ferraresso, E Montanari Italy

V2-5 Laser Assisted Retrograde Ureteroscopy for Percutaneous Nephrostomy: A New and Safer Technique for Percutaneous Nephrolithotomy CA Uribe, HD Osorio, JA Benavides, CH Martinez Colombia

V2-6 A novel modification of the ureteral access sheath for its use as a percutaneous access sheath with a pediatric cystoscope in minipercutaneous nephrolithotomy. 'Preliminary results' BO Manzo, J Sanchez, C Salazar, G Manzo, H Sanchez, R Maldonado, M Vanzzini, M Badillo Mexico

V2-7 Improvement of surgical outcomes after preoperative rehearsal using 3D printed patient specific simulation for percutaneous nephrolithotomy (PCNL)

A Ghazi, E Erturk

United States
V2-8 A New Twist on an Old Technique: Endoscopic-Guided Lawson Retrograde Nephrostomy Access for Percutaneous Nephrolithotomy in Prone Position KS Kaler, W Kim United States

V2-9 Supine Percutaneous Nephrolithotomy for Cystine Staghorn with Urolith Nanopulse

O Angerri, E Emiliani, F Sánchez-Martin, F Millan, H Villavicencio Spain

V2-10 Double negative pressure technique for stone clearance in MPCNL

D Lai, X Li, Y He, G Xu

China (People's Republic)

V2-11 Totally Tubeless Outpatient Minimally Invasive Percutaneous Nephrolithotomy (MIP)

J Davalos, JE Abbott, U Nagele United States

V2-12 Percutanous Exctraction Of Forgotten Calcified D-J Catheter At Supine Valdivia Position F ATEŞ, M Temel, BAŞAL, E malkoç, S Çakmak, T ŞENKUL Turkey

V2-13 Percutaneous Electrovaporization of Large Volume Upper Tract Transitional Cell Carcinoma T Tran, M Gupta United States

V2-14 ESUT educational video on fluoroscopic guided puncture in PCNL: All techniques step by step I Kyriazis, E Liatsikos, O Sopilidis, P Kallidonis, M Vasilas Greece 
V3-1 Robotic partial nephrectomy involving intracorporeal cold ischemia for post cryoablation failure Z Hamilton, M Holden, O Raheem, I Derweesh United States

V3-2 Minimally Invasive Nephron-Sparing Surgical Management of Multifocal Renal Masses in the Hereditary Kidney Cancer Syndrome Patient KL Stern, MA Zell, EP Castle United States

V3-3 Transperitoneal Robotic Assisted Laparoscopic Partial Nephrectomy in a Patient With Severe Scoliosis $\mathrm{C}$ Shen, $\mathrm{C} \mathrm{Hu}, \mathrm{C} \mathrm{Ou}$ Taiwan (Republic of China)

V3-4 Robot assisted radical nephrectomy and inferior vena cava thrombectomy: surgical technique, perioperative and oncologic outcomes G Simone, L Misuraca, G Tuderti, D Hatcher, M Ferriero, D Andre Luis, F Minisola, M Aron, S Guaglianone, M Desai, IS Gill, M Gallucci Italy

V3-5 Robotic Assisted Repair of Diaphragmatic Injury with Patch during Complex Partial Nephrectomy J Bloom, C White, M Stifelman United States

V3-6 Surgical Navigation using Intuitive Image-to-Patient Registration for Robot-Assisted Partial Nephrectomy: Clinical Feasibility study S Kobayashi, B Cho, K Tatsugami, M Hashizume, M Eto Japan

V3-7 Optimization of Blood Loss During Robotic IVC Thrombectomy - Is the Robotic Approach Feasible in the Jehovah's Witness?

P Heller, A Jones, C Johans, K Murray,

N Pokala

United States
V3-8 Da Vinci Robotic Assisted Laparoscopic Hemi-nephrectomy for Upper pole high risk tumors Y T B, S Waigankar, A Pednekar, T Kaushik India

V3-9 Robotic Radical Nephrectomy with Level II Inferior Vena Cava Thrombectomy

A Beksac, J Sfakianos, K Badani

United States

V3-10 Robotic Assisted Laparoscopic Right Radical Nephrectomy with Right Template Retroperitoneal Lymph Node Dissection

AB Edwards, J Messer

United States

V3-11 Robotic Assisted Laparoscopic Partial Nephrectomy on a complex upper pole posterior endophytic tumor AB Edwards, J Messer

United States

V3-12 Robotic Partial Nephrectomy: 4-Arm Retroperitoneal Approach

H Sawkar, N Patel, D Hatcher, FZ Husain, S Ghodoussipour, M Aron United States

V3-13 Robotic partial nephrectomy in complex renal tumors

S Arakere, RK Ahlawat, A Mandhani, A Mallya, I Banerjee, F Zafer, T Jindal India

V3-14 Robot-assisted Level II Inferior Vena Cava tumor thrombectomy with Metastasectomy for left sided renal tumor: Step-by-Step Technique

S Arakere, RK Ahlawat, A Mandhani, A Mallya, I Banerjee, F Zafer, T Jindal India
V4-1 Transurethral resection of prostate with ProstatoHemostatic Catheter

S Yang, D Kim, W Kim, J Kang

Republic of Korea

V4-2 Evaluation of the ProSac: a novel device to facilitate safety and efficiency of tissue morcellation during holmium laser enucleation of the prostate I Sorokin, H Beardsley, J Antonelli, BA Johnson, J Cadeddu, J Gahan United States
V4-3 The Efficacy and Safety of an Improved Transurethral Enucleation for the Treatment of Benign Prostatic Hyperplasia Y Xu, M Luo, T Xie, S Li, B Huang China (People's Republic)

V4-4 A novel technique for robotic prostatic adenomectomy: an evolution of transdouglas robotic prostatectomy B de Concilio, G Zeccolini, M Justich, F Vedovo, A Celia Italy 
V4-5 How to shorten your learning curve in transurethral diode laser enucleation of prostate? Y Zhang, M Liu, J Wang China (People's Republic)

V4-6 The role of Holmium laser enucleation of the prostate to alleviate PSA uncertainties

C Chemasle, JE Hunter, R Fong

New Zealand

V4-7 Aquablation of the Prostate for the Surgical Treatment of Benign Prostatic Hyperplasia

$$
\begin{aligned}
& \text { A Thomas } \\
& \text { United Kingdom }
\end{aligned}
$$

V4-8 Tips and Tricks for Safe Morcellation after Prostate Enucleation

D Whiting, M Cynk

United Kingdom

V4-9 The evolution of Green laser (532-nm) techniques in the treatment of benign prostatic obstruction: Not only for PVP

E Rijo, J Lorente, O Bielsa, F Gomez-Sancha Spain
V4-10 Thulium Laser Enucleation of the Prostate (ThuLEP): First Experience

P Glybochko, G Altshuler, A Vinarov, L Rapoport, M Enikeev, D Enikeev, N Sorokin, A Dymov, O Khamraev, R Sukhanov, M Taratkin, V Vinnichenko Russia

V4-11 Photoselective Vapoenucleation of the prostate using $180 \mathrm{~W}$ green laser (532-nm)

E Rijo, F Gomez-Sancha Spain

V4-12 En-bloc TURBT (E-TURBT) with collins loop: a new endoscopic treatment in the bladder tumour R GENTILE, B GENTILE, G Mirabile, L Albanesi, G Rizzo, P Tariciotti Italy

V4-13 Transurethral resection of a tumor in a Bladder Diverticulum

N Younes, K Al-rumaihi, A Badawi, K Khalafalla, T Ahmed Qatar

V4-14 A New Method of Suprapubic Cystotomy LM Wyner United States
V5-1 Retroperitoneal laparoscopic adrenalectomy for large adrenal pheochromocytoma

R Shimizu, Y Kimura, T Yumioka, N Yamaguchi, H Iwamoto, S Morizane, K Hikita, M Honda, A Takenaka Japan

V5-2 Robotic partial adrenalectomy for symptomatic aldosterone-secreting adenomas: technique and outcomes

G Simone, G Tuderti, L Misuraca, A Celia, B de Concilio, A Stigliano, F Minisola, M Ferriero, G Romeo, S Guaglianone, M Gallucci Italy

V5-3 Robot assisted Partial Adrenalectomy for adrenal tumours - Our initial experience Y T B, S Waigankar, A Padegaonkar, A Pednekar India

V5-4 Management of brisk hemorrhage during robotic right adrenalectomy for a $9 \mathrm{~cm}$ pheochromocytoma A Schulman, RJ Yau, NH Patel, MS Choudhury, M Eshghi, JL Phillips United States

V5-5 Robotic-assisted laparoscopic partial adrenalectomy for a unilateral pheochromocytoma in suspected von Hippel-Lindau disease

R Lee, L Giusto, B Waldorf, E Gewirtz, D Eun United States
V5-6 Transperitoneal Laparoscopic Left Adrenalectomy in Patients with Ectopic Pelvic Kidney: A Live Surgery Experience Y Aslan, O Guzel, I Aykanat, M Balci,
A Tuncel
Turkey

V5-7 robotic-assisted excision of a large Right Adrenal Cyst H Alenezi, A Albaghli, A Alenezi, F Abul Kuwait

\section{V5-8 Withdrawn}

V5-9 Double blessing - Laparoscopic partial nephrectomy with nephrolithotomy for a complex lower polar cyst with lower calyceal partial staghorn calculus S Kallappan, M Ramalingam, S Nachimuthu India

V5-10 Percutaneous Externally Assembled Laparoscopic Instruments to Decrease the Invasiveness of Hand-Assisted Laparoscopic Donor Nephrectomy J Thomas, S Abourbih, M Pierce, J Cheng, $\mathrm{H}$ Wagner, D Baldwin United States

V5-11 Laparo-endoscopic single site (LESS) right nephrectomy R Bass, O Levi, A Sidi, A Tsivian Israel 
V5-12 Da Vinci Si Robotic Assisted Laparoscopic Excision Of Retroperitoneal Mass (Paraganglioma) Y T B, S Waigankar, A Padegaonkar, K Chakradhar, S Sharma India
V5-13 Interdisciplinary Approach to Dermoid Cyst of the Spine: How Can Urologists Help Neurosurgeons? R Mauck, L Zhao, N Singla, C Hatchette United States
V6-1 Pediatric Robotic Pyeloplasty in a Duplex Kidney DY Yang, CF Granberg, R Avant, P Casale, PC Gargollo United States

V6-2 Robot Assisted Laparoscopic Bilateral Ureteral Reimplantation and Left Tailoring Procedure for a 2Year-Old Boy Patient: Right Ureterovesical Stricture and Left Ureterovesical Reflux

$$
\text { Y Ozgok, S Yalcin }
$$

Turkey

V6-3 Hybrid Lparoendoscopic Single Site Ureteroureterostomy With Transmesentric Approach For Congenital Miduretral Stricture K Kobayashi, K Johnin, M Narita, A Kawauchi Japan

V6-4 Laparoscopic Radical Nephrectomy for Prenatally Diagnosed Wilms Tumor

A Toussi, CF Granberg, PC Gargollo United States

V6-5 Robot-assisted left pyeloplasty in a pediatric patient with gastrostomy tube and severe scoliosis JP Joseph, CF Granberg, PC Gargollo United States

V6-6 Robotic Assisted Laparoscopic Pyelopyelostomy of a Pediatric Upper Pole UPJ Obstruction DK Agarwal, IJ Sobol, K Eeg, CF Granberg, PC Gargollo United States

V6-7 Second Stage Fowler-stephens Orchiopexy Using Only Novel Percutaneous Externally Assembled Laparoscopic (Peal) Instruments: a Video Presentation J Lee, C Bautista, I Kelly, S Abourbih, M T Chau, H Wagner, J Cheng, M Pierce, M Keheila, D Baldwin United States
V6-8 Hidden Incision Endoscopic Surgery (HIdES) Partial Cystectomy

D Gearman, CF Granberg, PC Gargollo United States

V6-9 Robotic Assisted Tapered Distal End-to-Side Uretero-Ureterostomy for Duplex Kidney with Upper Pole Obstruction L Ni, S Dave Canada

V6-10 Recurrent Bladder Stones in a Patient with Prior Bladder Exstrophy Repair J Han, JM Shields, A Rabley, VG Bird United States

V6-11 Robotic Kidney Transplant with grafts having multiple renal arteries - Technical nuances FA Zafar, A Mallya, S Arakere, I Banerjee, P Ghosh, A Mandhani, RK Ahlawat India

V6-12 A Simple Iced Renal Jacket during Renal Transplantation

FA Zafar, S Arakere, A Mallya, I Banerjee, T Jindal, A Gupta, A Mandhani, RK Ahlawat India

V6-13 Robotic kidney transplantation in a patient previously treated with robotic radical prostatectomy

A Breda, A Territo, G Basile, I Schwartzmann, L Gausa, O Rodriguez, J Gaya, J Ponce de Leon, H Villavicencio Spain

V6-14 Robot Assisted Ureteric Reimplantation of Transplanted Kidney

A Beksac, S Prasad, J Sfakianos, K Badani United States
V7-1 Robotic Pelvic Exenteration (Cystoprostatectomy and Combined Abdominoperineal Resection) with Double Barreled Wet Colostomy

N Velazquez, W Huang, M Bernstein, M Bjurlin

United States
V7-2 The Novel Use of a Magnetic Retraction Device in Robotic Urologic Surgery

P Samson, P Mistry, L Kavoussi,

L Richstone

United States 
V7-3 Retroperitoneal Robot-assisted Laparoscopic Partial Nephrectomy

M Lyons, J Vukina, M McKibben, M Raynor United States

V7-4 Retroperitoneal robotic partial nephrectomy for solitary horseshoe kidney

Z Hamilton, M Holden, O Raheem,

I Derweesh

United States

V7-5 Robot Assisted Laparoscopic Retroperitoneal lymph node dissection using the Da Vinci Si: Lateral and Anterior Approaches

M Ferretti, M Stifelman, G Lovallo, M Ahmed

United States

V7-6 Robotic Assisted Lymph Node Dissection in the Management of Penile Cancer J Bloom, M Ferretti, G Lovallo, M Ahmed United States

V7-7 Use of Immunofluorescence for Ureteral Identification During Robotic Surgery

JA Albersheim-Carter, WB Gaertner,

CA Dixon, CJ Weight

United States

V7-8 Robotic Assisted Extended Pelvic Lymph Node Dissection Including Presacral Area: tips and tricks RJ Yau, M Stifelman, G Lovallo, M Ahmed United States
V7-9 Robotic Assisted Laparoscopic Inguinal

Lymphadenectomy for Invasive Penile Cancer P Knoll, V Ramakrishnan, A Rogman, TM FitzGibbon, J Messer United States

V7-10 Robotic-assisted laparoscopic excision of a post chemotherapy, pelvic rhabdomyosarcoma in a child LF Sávio, R Ivanovic, J DA CRUZ, R Sakata, M Nunes, R Maia, C PASSEROTTI Brazil

V7-11 Novel Use of Fluorescence Lymphangiography During Robotic Groin Dissection for Penile Cancer M Bjurlin, L Zhao, D Volkin, A Kenigsberg, A Mass, S Taneja, W Huang United States

V7-12 Initial Report of Robotic Laparoendoscopic SingleSite Partial Nephrectomy by da-Vinci $\mathrm{Xi}{ }^{\circledR}$ surgical system for Renal Tumor $>4 \mathbf{c m}$ H Lee, Y Yoon, J Na, K Rha, Y Choi, W Han Republic of Korea

V7-13 Novel Robotic Approach to Inguinal and Pelvic Lymphadenectomy for Metastatic Melanoma N Jung, H Smith, A Hyde, A Valle, C Keel United States

V7-14 Robotic Metast Resection RJ Leveillee, D Diaz, G Leveillee, S Unsal United States Russia
V8-1 Clampless laparoscopic partial nephrectomy for hilar tumor

R Sanseverino, G Napodano, O Intilla, U Di Mauro, G Molisso, T Realfonso Italy

V8-2 Laparoscopic inferior vena cava thrombectomy with double loop tourniquet in $\mathrm{RCC}$

S Hong, H Jeong, S Cho, S Kang, H Moon, K Lee, S Choi, Y Park, W Bae, H Cho, U Ha, J Lee, S Kim

Republic of Korea

V8-3 Laparoscopic Partial Nephrectomy Concomitant with Isthmusectomy for Isthmus Renal Cell Carcinoma in the Horseshoe Kidney $\mathrm{RC} \mathrm{Wu}, \mathrm{V}$ Lin Taiwan (Republic of China)

V8-4 Clampless laparoscopic partial nephrectomy with tumor enucleation

P Kallidonis, D Kotsiris, P Ntasiotis, I Kyriazis, V Panagopoulos, E Liatsikos Greece
V8-5 3 -D Laparoscopic transperitoneal partial nephrectomy for clinical T1b renal tumors: A prospective evaluation A ANUP_14K@YAHOO.COM, G Kumar, N Kumar, M Patel, P Gupta India

V8-6 Laparoscopic partial nephrectomy for posterior hilar tumors: technique and clinical outcomes H Hirabayashi, R Hattori, H Kawanishi, J Nagayama, H Matsui, T Sano, S Suzuki, K Suzuki, K Kato Japan

V8-7 Percutaneous Renal Endoscopy and Resection of Urothelial Carcinoma of the Renal Pelvis Performed Under Local Anesthetic in High Risk Patient

JE Abbott, J Davalos

United States

V8-8 Complete laparoscopic approach for upper tract transitional cell carcinoma

M Vijayakumar, A Ganpule, A Singh, R Sabnis, M Desai India 
V8-9 Transvescicoscopic bladder cuff excision in laparoscopic nephroureterectomy: Initial Experience Y Yoon, S Kim, J Na, H Lee, S Park, W Han Republic of Korea

V8-10 Percutaneous Resection of Bulky Renal Pelvis Tumors

$$
\begin{aligned}
& \text { RJ Yau, M Eshghi } \\
& \text { United States }
\end{aligned}
$$

V8-11 A prospective evaluation of 3-D laparoscopic transperitoneal radical nephrectomy for large renal tumors(clinical T2N0M0)

A ANUP_14K@YAHOO.COM, G Kumar, N Kumar, M Patel, P Gupta India
V8-12 Renal Cell Carcinoma in a patient with total situs inversus

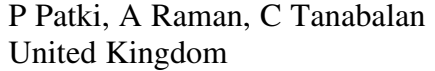

P Patki, A Raman, C Tanabalan United Kingdom

V8-13 Partial nephrectomy for bilateral synchronus renal cancer

R Sanseverino, G Napodano, O Intilla, U Di Mauro, G Molisso, T Realfonso Italy

V8-14 Laparoscopic nephroureterectomy for urothelial tumor in horseshoe kidney

G Del Pozo Jiménez, I Castillón Vela, J Diez Diez, A Souto Souto, J Carballido Rodríguez Spain
V9-1 Our Technique of Retzius sparing Robot assisted laparoscopic radical prostatectomy: initial results I Banerjee, A Mallya, S Arakere, FA Zafar, A Mandhani, RK Ahlawat, T Jindal India

V9-2 Anatomic robot assisted radical cystectomy in female: step by step technique

G Simone, S Guaglianone, L Misuraca, F Minisola, G Tuderti, M Ferriero, M Gallucci Italy

V9-3 Nerve Sparing Technique During Robot Assisted Retroperitoneal Lymph Node Dissection in Testicular Cancer

HM Abdul-Muhsin, M Marshall, J

L'esperance, M Woods, S Stroup, EP Castle United States

V9-4 Robot assisted radical cystectomy and cutaneous ureterostomy is a new paradigm in managing select cases of muscle invasive bladder cancers

S Arakere, RK Ahlawat, A Mandhani, A Mallya, I Banerjee, F Zafer, T Jindal India

V9-5 Robot assisted laparoscopic nerve sparing cystoprostatectomy: Intracorporeal Studer pouch formation

Y Ozgok, S Yalcin

Turkey

V9-6 Robot-assisted radical retrograde cystectomy with vaginal preservation and the ideal neobladder

T Yumioka, S Morizane, R Shimizu,

Y Kimura, N Yamaguchi, H Iwamoto, P Tsounapi, K Hikita, M Honda, A Takenaka Japan

V9-7 Robotic assisted en-bolic excision of tumor with prostate and rectum involvement

C Yang, L Tsai, P Hsiao, C Chang

Taiwan (Republic of China)
V9-8 A Novel Modified Urethro-vesical Anastomosis Technique: Prevention Of Urethral Retraction With Stay Sutures (PURS) During Robot Assisted Radical Prostatectomy

OB Argun, MB Tuna, T Doganca, I Tufek, C Obek, AR Kural Turkey

V9-9 Intracorporeal partly stapled 'Padua Ileal Bladder' using robotic staplers: surgical technique, perioperative and early functional outcomes of a prospective single center series

G Simone, G Salvatore, M Francesco, M Ferriero, L Misuraca, G Tuderti, G Romeo, M Gallucci Italy

V9-10 Video Demonstration: Robotic-assisted Laparoscopic Radical Cystoprostatectomy with Neobladder formation-Modified Karolinska Technique: Surgical technique \& Outcomes

Y T B, S Waigankar, A Pednekar,

V Wagaskar

India

V9-11 Robot assisted laparoscopic nerve sparing cystoprostatectomy: Experience on the patient with muscle invasive transitional cell carsinoma treated previously by trimodal therapy

S Yalcin, Y Ozgok, A Canda

Turkey

V9-12 Robot assisted radical prostatectomy for prostate cancer with more than 100 gram prostate: Technique and outcomes

H Palayapalayam Ganapathi, G Ogaya-Pinies, E Hernandez, T Rogers, V Patel United States 
V9-13 Robot assisted partial cystectomy with bilateral vesico-ureteric junction resection and reimplantation for a large paraganglioma involving urinary bladder trigone

J Stolzenburg, H Do, A Dietel, R Ganzer, I Kyriazis, E Liatsikos, P Kallidonis,

A Ravichandran-Chandra,

V Arthanareeswaran

Greece
V9-14 Experience of robot-assisted radical prostatectomy (RARP) in renal transplant recipients T Nishikimi, H Kobayashi, H Yamada, R Ishida, Y Yamauchi, K Hattori Japan
V10-1 An unusual case of bladder mass and its 3-D laparoscopic management

A ANUP_14K@YAHOO.COM, G Kumar, N Kumar, M Patel, P Gupta India

V10-2 Lessons learnt from 8 successful early laparoscopic repair of vesicovaginal fistula

S Kallappan, M Ramalingam, S Nachimuthu India

V10-3 Laparoscopic repair of vesicouterine fistula using TACHOSILTM sponge

R Sanseverino, G Napodano, O Intilla, U Di Mauro, G Molisso, T Realfonso Italy

V10-4 Transvesical laparoendoscopic single-site surgery to repair a vesicovaginal fistula in man after female to male sex reassignment M Borowik, M Przudzik, M Roslan Poland
V10-5 Trans-urethral laparoscopic suture of the bladder post major trauma

P Grange, C Kouriefs, J Makanjuola, A Khan United Kingdom

V10-6 Laparoscopic Repair of a Vesicosigmoid Fistula Secondary to Holmium Laser Enucleation of the Prostate

J Kang, J Jeon

Republic of Korea

V10-7 Amniotic Mesenchymal Stem Cells in the Management of Recurrent Urethral Stricture O Alalao, K Al-rumaihi, N Younes, A Alansari Qatar

V10-8 Robotic Assisted Repair of Parastomal Hernia PW Mekhail, MW Mekhail, D Hatcher, A Monish United States

V10-9 Laparoscopic radical cystectomy: delayed ureteral intersection

D Perlin, I Aleksandrov, V Zipunnikov, I Dymkov
V11-1 Robot assisted partial nephrectomy for multiple renal tumors - A Vattikuti Collective Quality Initiative database analysis

S Arora, RK Ahlawat, J Adshead, R Abaza,

B Challacombe, P Dasgupta, G Gandaglia,

D Moon, F Porpiglia, T Yuvaraja,

U Capitiano, J Porter, A Mottrie, M Bhandari, CG Rogers, H Gill

United States

V11-2 Concurrent robotic kidney and general surgery procedures

H Gill, M Franco, D Kwon, CG Rogers United States
V11-3 Robotic Minimal Margin Enucleo-resection Technique of Left Renal Tumor H tavukcu, O Aytac, H Kulaksızoglu, F Atug Turkey

V11-4 Robotically-assisted retroperitoneal lymph node dissection in stage IIa seminoma S Tadtayev, E Mayer, D Nicol United Kingdom

V11-5 'Hilum First': A Novel Technique to Radical Nephrectomy for Large Renal Masses M Ahmed, G Lovallo, C White United States 
V11-6 Single-Site Robotic Partial Nephrectomy M Holden, Z Hamilton, I Derweesh United States

V11-7 Serial Hem-o-lok Application for Robotic Partial (SHARP) Nephrectomy JS Hwong, SL Chang, R Korets United States

V11-8 Dilated Lumbar Veins due to Cement Application to lomber vertebrae in a metastatic renal cell cancer patient

O Aytac, H tavukcu, H Kulaksizoglu, F Atug Turkey
V11-9 Laparoscopic resection of retroperitoneal malignant tumors

$$
\begin{aligned}
& \text { D Perlin, I Aleksandrov, I Dymkov, } \\
& \text { V Zipunnikov } \\
& \text { Russia }
\end{aligned}
$$

V11-10 Robot Assisted Inferior Vena Cavoscopy HM Abdul-Muhsin, M Marshall, A Chau, RJ Fowl, S Stroup, J L'esperance, M Woods, EP Castle United States

V11-11 Marriage of Dyna-Computed Tomography Guidance During Laparoscopy for Needle Placement and Thermal Ablation: A Feasibility Study

EF Kelly, RJ Leveillee

United States

Friday 15 September

Video Session 12: Robotic Lower Tract Surgery for Benign Disease

16:30-18:30

Robotic Surgery: Lower Tract - Benign

Convention Centre East, Meeting Room 13

V12-1 Mullerian Duct abnormalities: A Robotic Approach to Surgical Excision

P Sturch, (S Academic Research Group,

A Samateh, S Mackie, PD Rimington

United Kingdom

V12-2 Robotic Hutch Diverticulectomy \& Ureteric Re-implant with Vas Preservation

R Catterwell, C Brown, R Thurairaja United Kingdom

V12-3 Robotic Assisted Laparoscopic Transvesical Repair of Rectovesical Fistula Following Open Radical Prostatectomy: Use of Allograft Human Dermis Interposition

E Capoccia, LA Deane, P Whelan United States

V12-4 Extravesical robotic-assisted bladder diverticulectomy with radical prostatectomy H Palayapalayam Ganapathi, G Ogaya-Pinies, E Hernandez, T Rogers, V Patel United States

V12-5 Purely intracorporeal robotic augmentation ileocystoplasty in ketamine cystitis

I Chen, J Lin, J Tsai, C Yu, T Wu, S Jeng

Taiwan (Republic of China)

V12-6 Robot assisted Boari flap ureteroneocystostomy using Politano-Leadbetter approach in a patient with distal stricture in duplex ureters

J Stolzenburg, H Do, A Dietel, R Ganzer, I Kyriazis, E Liatsikos, P Kallidonis,

V Arthanareeswaran

Greece

V12-7 Robot Assisted Transplant Ureteroneocystostomy HM Abdul-Muhsin, K Gross, KL Stern, N Katariya, EP Castle United States
V12-8 Robotic Assisted Extravesical Ureteric

Reimplantation with Psoas Hitch for Ureterovesical Junction Obstruction

L Ni, S Dave

Canada

V12-9 Combined Holmium Laser Enucleation of the Prostate and Robotic Diverticulectomy

KL Stern, WA Critchlow, MR Humphreys United States

V12-10 Robotic Assisted Ileal Ureter and Ileal Bladder Augmentation

TA Perkins, J Messer

United States

V12-11 New technologies for old procedures when FireFly technologie improves Robotic Bladder

Diverticulectomy

F Vedovo, B de Concilio, G Zeccolini,

M Justich, A Celia

Italy

V12-12 Intraureteral indocyanine green (ICG) application for ureter identification during robotic surgery I Tufek, OB Argun, MB Tuna, T Doganca, C Obek, S Keskin, AR Kural Turkey

V12-13 Robotic Salvage Prostatectomy for Radiation Induced Posterior Urethral Stenosis

D Volkin, AC Weinberg, L Zhao

United States

V12-14 Management of Complex Urinary Fistula

M Ahmed, L DiGiorgio

United States 
Robotic Surgery: Upper Tract - Benign

Convention Centre East, Meeting Room 18

V13-1 Robotic Pyelolithotomy For The Intact Removal of a Complete Staghorn Stone: A Feasible Approach Even After Previous Open Pyelolithotomy A Chow, R Yong, P Tsambarlis, LA Deane United States

V13-2 Technique of robot-assisted laparoscopic upper moiety partial nephrectomy in the management of complicated duplex collecting systems

$$
\text { D Yong, K Png }
$$

Singapore

V13-3 Robotic partial nephrectomy for renal congenital anomalies in adults: tips, tricks and outcomes M Sourial, N François, C Miller, DL Zynger, G Box United States

V13-4 Robot-assisted laparoscopic extravascular stent for nutcracker syndrome

I Sorokin, BA Johnson, J Nelson,

J Rectenwald, J Cadeddu

United States

V13-5 Surgical Management of Chronic Flank Pain in Autosomal Dominant Polycystic Kidney Disease: Robotic Cyst Decortication and Renal Denervation B Norris, J Borin United States

V13-6 Cutting for Stones: Robotic assisted Nephrolithotomy and Pyelolithotomy B Norris, J Borin United States

V13-7 Early Robotic Repair of a Catastrophic Iatrogenic Ureteral Injury

A Radtke, K Jacobsohn

United States

V13-8 Robotic Single Port Pielolithotomy: an alternative technique for stone burden calculi

LF Sávio, J DA CRUZ, F Mota Filho, R Sakata, M Nunes, R Maia, C PASSEROTTI Brazil
V13-9 Robotic Assisted Left Renal Vein Transposition HM Abdul-Muhsin, A Chau, C Martin, S Money, EP Castle United States

V13-10 Robot Assisted Partial Nephrectomies - 12 Tumors, 1 Kidney

KL Stern, G Grimbsy, C Chen, T Ho, EP Castle United States

V13-11 Robotic-assisted Laparoscopic Calyceal Diverticulectomy

N Jung, H Smith, D Turner, J Class, D Butler, C Keel, A Singh United States

V13-12 Robot Assisted Ureteroureterostomy for Management of Recurrent Proximal Ureteral Stricture

WR Lai, R Thomas

United States

V13-13 Expanding the spectrum of Robotic Pyeloplasty: bilateral ureteropelvic junction obstruction with multiple large calyceal stones on one side and crossing vessels in the other

A Mallya, I Banerjee, S Arakere, FA Zafar, A Mandhani, RK Ahlawat India

V13-14 Robotic Assisted Laparoscopic Ureteroplasty for a Recurrent Proximal Ureteral Stricture with Buccal Mucosal Graft

TM FitzGibbon, V Ramakrishnan, P Knoll, J Messer

United States 


\section{BRPS1: BENCH TO BEDSIDE: BPH/LUTS/RECONSTRUCTION}

\section{BRPRS1-1 Withdrawn}

\section{BRPRS1-2 Withdrawn}

\section{BRPRS1-3 Withdrawn}

BRPRS1-4 Effect of testosterone impregnated pellet therapy on lower urinary tract symptoms

A Ostrowski, R Williams, G Broderick

Mayo Clinic Florida

United States

Introduction \& Objective: Hypogonadism in men is commonly treated with testosterone replacement therapy (TRT). One such therapy is the subcutaneous testosterone impregnated pellet (STIP) delivery system that provides long-acting TRT. Given testosterone's growth-promoting effect on the prostate, concern exists for worsening of lower urinary tract symptoms (LUTS). In this study, we assessed the relationship between change in the American Urological Association Symptom Index Score (AUASIS) and use of STIP for treatment of hypogonadism.

Materials and Methods: Our patient population includes the 99 patients who have received STIP therapy for treatment of hypogonadism by a single surgeon in the Urology Department at Mayo Clinic Jacksonville. These patients were identified using the CPT code 11980. Patients were then further selected based on having completed AUASIS questionnaires for both pre and post STIP initiation.

Results: A total of 38 patients met the inclusion/exclusion criteria. The mean age $( \pm$ SD) at initiation of STIP therapy was 63.7 years $( \pm 9.3)$. Mean number of treatments per patient was 6.5 $( \pm 5.2)$ with a mean interval between treatments of 4.8 months $( \pm 1.5)$. Baseline mean total testosterone level was $206.8 \mathrm{ng} / \mathrm{dL}$ $( \pm 109.2)$ and the mean total testosterone level after starting STIP was $497.4 \mathrm{ng} / \mathrm{dL}( \pm 195.5)(\mathrm{p}<0.001)$. The mean pre-STIP AUA score was $9.2( \pm 6.0)$, and the mean post-STIP AUA score was 8.8 $( \pm 6.2)$, with a mean difference in AUASIS of -0.3 (p not significant). Of the study patients, 17 of $38(45 \%)$ had less than a $3-$ point change in their AUASIS after initiating STIP, with 11 of 38 (29\%) showing improvement and 10 of 38 (26\%) showing worsening of their AUASIS. For those patients with improving AUASIS, the mean total number of STIP treatments was 5.1 $( \pm 3.4)$, compared to $6.9( \pm 4.1)$ for those whose AUASIS worsened ( $\mathrm{p}$ not significant).

Conclusions: The average AUASIS in this cohort for both pre and post STIP treatment are in the moderate (8-19) category of voiding dysfunction. Based on the results of this study, when STIP therapy successfully elevates testosterone to eugonadal levels, there does not appear to be an association with any alteration of patient AUASIS. This suggests that worsening LUTS should not be a concern when recommending STIP therapy for hypogonadal men.
BRPRS1-5 Effect of Hyoscine, Diclofenac, Solifenacin and Adrenaline on ureteral peristalsis. An in-vitro evaluation and animal model.

V Panagopoulos, I Kyriazis, P Kallidonis, M Vasilas, P Ntasiotis, D Kotsiris, T Vrettos, E Liatsikos

Department of Urology, University of Patras Greece

Introduction \& Objective: The aim of the current study is to evaluate the effect of Hyoscine, Diclofenac, Solifenacin and Adrenaline on ureteral peristalsis in terms of force of contraction, frequency and amplitude of contraction.

Materials and Methods: Totally, 24 full-length porcine ureters were collected, after laparotomy, under general anesthesia from 12 female domestic pigs with average weight of $20 \mathrm{~kg}$. The ureters were further divided in upper, mid and lower ureter and dissected in $5 \mathrm{~mm}$ ureteral rings placed placed in 10ml chambers of an ML870B5/10 Panlab organ bath device. The chambers contained constantly gassed Krebs solution with $95 \% \mathrm{O}_{2}$ and $5 \% \mathrm{CO}_{2}$ under stable temperature of $37{ }^{\circ} \mathrm{C}$. The specimens were kept in place, during the experiment, between two hooks. Hyoscine, Diclofenac, Solifenacin and Adrenaline were separately applied into the chambers. After tissue equilibration (usually 30 minutes), each ureteral activity was recorded and analyzed by Labchart software (ADInstruments Ltd., Oxforshire, United Kingdom). Analysis of the results was conducted with the Microsoft Office Excel 2007 software.

Results: Based on our study, the presence of Hyoscine (1M) or Diclofenac (1M) into Krebs solution had no effect on porcine ureteral contractility. On the contrary, Solifenacin (1M) seemed to significantly decrease ureteral frequency whereas in terms of force or amplitude of contraction had minimal or no effect respectively. Lower concentrations of Solifenacin $(0.1 \mathrm{M})$ had no effect on ureteral contractility. Regarding Adrenaline $(0.01 \mathrm{M}$, $0.02 \mathrm{M}, 0.04 \mathrm{M})$, a significant increase of values was reported in all the tested parameters.

Conclusions: Hyoscine and Diclofenac showed no effect on ureteral contractility. Solifenacin only in greater concentrations seemed to decrease ureteral frequency. Whereas, adrenaline in every concentration, stimulates ureteral motility.

BRPRS1-6 Insulin Receptor and Insulin-like growth factor expression increases and Insulin-like growth factor binding protein -3 expression reduces in association with prostate size in Benign prostatic hyperplasia

K Sreenivasulu, H Nandeesha, L Dorairajan, V Vinayagam

Department of Biochemistry

India

Introduction \& Objective: Expression of Insulin receptor isoforms (IR-A and B), Insulin receptor substrates (IRS-1/2), and associated factors role in cell proliferation and survival was revealed in different type of cancer including prostate cancer. As 
there were no reports in Benign prostatic hyperplasia (BPH), the present study was designed to analyse the gene and protein expression of insulin receptor and downstream factors in benign prostate tissues.

Materials and Methods: Prostatic tissues were obtained from 27 males aged between 55 to 75 years who underwent transurethral resection of the prostate as surgical treatment of BPH. Based on the volume of Prostate, tissues were divided into two groups, Group A $(\leq 30 \mathrm{CC})$ and Group B $(>30 \mathrm{CC})$. RNA and protein expression levels of Insulin receptor and its related components were assessed by q-PCR and western blot respectively

Results: IR-A and B gene expression were significantly higher in group B ( $>30 \mathrm{CC}$ ), similarly protein expression of IR-B and IRS-1 both were high in group B ( $>30 \mathrm{CC})$. Insulin-like growth factors (IGF-1 and IGF-2) gene expression was increased, and its binding protein IGFBP-3 gene expression was reduced in association with prostate size. There were no difference in IRS-1 and IRS-2 gene expression were found between the groups.

Conclusions: The findings from the present study showed increased expression of IR, IRS, and IGF-1, 2 in BPH patients with larger prostate size indicating overexpression of components of insulin signaling pathway might be associated with the development of BPH.

BRPRS1-7 Comparison of transurethral incision of the prostate and silodosin in patients having benign prostatic obstruction in terms of retrograde ejaculation

E Arda, B Cakiroglu, i Hazar, O Sinanoglu, S Ekici

Trakya University

Turkey

Introduction \& Objective: To compare the functional outcomes and retrograde ejaculation (RE) after transurethral incision of the prostate (TUIP) or silodosin in bladder outlet obstruction (BOO) secondary to a small prostate.

Materials and Methods: Prospectively collected data from December 2011 through December 2014 of 192 LUTS patients having fertility concerns with prostate volume smaller than $40 \mathrm{ml}$ receiving either TUIP or silodosin treatment were prospectively reviewed. The treatment outcomes were evaluated and compared. Results: TUIP was performed in 96 cases and silodosin $8 \mathrm{mg}$ was prescribed in 96 cases. At 12th months after TUIP or con- tinuous silodosin treatment, the decrease in mean International Prostate Symptom Score (IPSS) and postvoiding residual urine (PVR) and the improvement of mean maximal flow rate (Qmax) were significant $(\mathrm{p}=0.000)$. The improvement in IPPS and Qmax was significantly higher in TUIP group compared to silodosin group $(\mathrm{p}=0.005, \mathrm{p}=0.000)$ with a lower rate of retrograde ejaculation (RE) in TUIP group. (11/96 vs 33/96) $(\mathrm{p}=0.000)$

Conclusions: Both TUIP and silodosin ensures comparable improvement in PVR, IPSS and Qmax with a lower rate of RE on the TUIP group in prostates weighing less than 40 grams suggesting that TUIP is a better choice in younger patiens seeking preservation of ejaculation with fertility concerns.

BRPRS1-8 Nocturia Is Associated with Slipping and Falling

W Bang, H Kim, S Cho, M Choo, K Ko, D Lee, K Koo, Y Yoon, S Lee, W Kim

Hallym Sacred Heart Hospital

Republic of Korea
Introduction \& Objective: Several reports have demonstrated associations between falls and nocturia in the elderly. However, little information is available regarding other age groups. This study evaluated the relationship between the frequency of nocturia and falls in men using a large, populationbased survey in Korea, and the results were adjusted for various confounding factors.

Materials and Methods: Data from a 2011 Korean community health survey (KCHS) were retrieved for 92,660 men aged 19 to 103 years. Information regarding the history of slips or falls in the past year was collected. The frequency of nocturia was classified as 0,1 , 2, 3, 4, and 5 instances a night. Walking during the day, education, income, body mass index (BMI), smoking, alcohol consumption, sleep time, stress level and medical histories of hypertension, diabetes mellitus, hyperlipidemia, cerebral stroke, angina or myocardial infarction, arthritis, and osteoporosis were adjusted using multiple logistic regression analysis with complex sampling. A subgroup analysis was conducted for young (19-30 years), middleaged (31-60 years), and elderly individuals (61+ years).

Results: Approximately $14.6 \%$ of the men had a history of falls. Their mean age was 42.9 years, which was significantly higher than that of the non-faller group $(\mathrm{P}<0.001)$. An increased frequency of nocturia was associated with increased adjusted odds ratio (AOR) for falls (AOR for 1 instance of nocturia/night $=1.41$ [95\% confidence interval, 1.33-1.50]; AOR for 2 instances = 1.41 [1.33-1.50]; $\mathrm{AOR}$ for 3 instances $=2.00[1.75-2.28]$; AOR for 4 instances $=2.12$ [1.73-2.61]; AOR for 5 instances $=2.02$ [1.74-2.36], $\mathrm{P}<0.001$ ).

Conclusions: In the subgroup analysis, the AORs for falls significantly increased in all age groups as the frequency of nocturia increased.

BRPRS1-9 Analysis of the prevalence and associated factors of overactive bladder in adult Korean men

W Bang, H Kim, S Cho, M Choo, K Ko, D Lee, K Koo, Y Yoon, S Lee, W Kim

Inje University

Republic of Korea

Introduction \& Objective: Overactive bladder $(\mathrm{OAB})$ is a prevalent condition characterized by lower urinary tract symptoms (LUTS). Age, education, income, marital status, sleep, and emotional problems have been associated with $\mathrm{OAB}$; however, conflicting results exist. The present study was conducted to estimate the prevalence of $\mathrm{OAB}$ and comprehensively analyze its associated factors in a large cross-sectional, population-based study. Materials and Methods: The data of 94,554 participants aged 19- 107 were analyzed from the Korean Community Health Survey (KCHS) of 2012. Data on marital status, physical activity, education level, occupation, body mass index (BMI), income level, sleep time, and stress level were retrieved for all enrolled participants. The overactive bladder symptom score (OABSS) was used to evaluate the presence and degree of OAB. Simple and multiple logistic regression analyses with complex sampling were used for the associations between various factors and the presence of OAB. Results: Overall, OAB was present in approximately $2.9 \%$ of the participants. The prevalence of OAB increased with age and steeply increased after 60 years of age (adjusted odds ratio [AOR] for each 10 years $=1.70,95 \%$ confidence interval $[\mathrm{CI}]=1.61-1.80, \mathrm{P}<0.001)$. Being underweight was correlated with $\mathrm{OAB}(\mathrm{AOR}=1.29,95 \% \mathrm{CI}=1.08-1.55, \mathrm{P}=0.018)$. Inadequate sleep showed a significant association with $\mathrm{OAB}$ (AOR $=1.13,95 \% \mathrm{CI}=1.02-1.25$ for 6 hours of sleep time and $\mathrm{AOR}=1.53,95 \% \mathrm{CI}=1.27-1.86$ for 9 hours of sleep, $\mathrm{P} 2.16$ $(1.88-2.48)>1.39(1.23-1.57)$ for severe stress $>$ moderate stress 


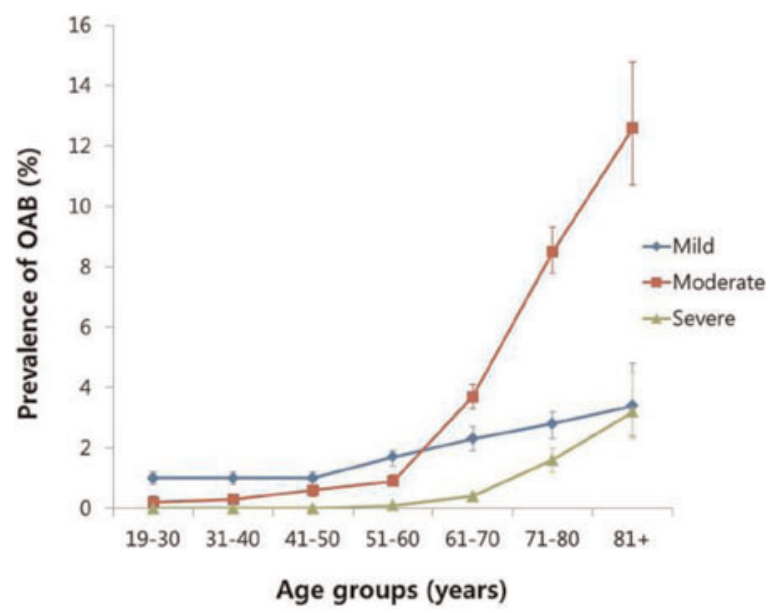

$>$ some stress, respectively, $\mathrm{P}<0.001]$. A medical history of diabetes mellitus, hyperlipidemia, and/or cerebral stroke was significantly related to $\mathrm{OAB}$.

Conclusions: Approximately $2.9 \%$ of adult Korean men experienced OAB based on the OABSS. Unmarried status; occupation; being underweight; inadequate sleep; stress; and medical history of diabetes mellitus, hyperlipidemia, or cerebral stroke were significantly correlated with OAB.

\section{BRPRS1-10 Withdrawn}

\section{BRPRS1-11 Role of Chaperon Proteins in Obstructed Renal Pelvis}

H Sanguinetti, J Toblli, NO Bernardo, O Mazza

Endourology Society

Argentina

Introduction \& Objective: The obstruction of the renal pelvis creates stress and hypoxia at the cellular level. As a protective mechanism, the cell synthesizes Heat Shock Proteins, also known as Chaperone Proteins, which bind to altered proteins, preventing them from folding and denaturalizing. Its expression is increased in other obstructed organs such as the bladder. The objective of this study is to evaluate the expression of these proteins in obstructed renal pelvis.

\begin{tabular}{|l|c|c|c|}
\hline Median \pm SD & $\begin{array}{c}\text { G1. UPJ } \\
(n=20)\end{array}$ & $\begin{array}{c}\text { G2. Hydro } \\
(n=14)\end{array}$ & $\begin{array}{c}\text { G3 Control } \\
(n=16)\end{array}$ \\
\hline $\begin{array}{l}\text { HSP27 } \\
\text { (Possitive stain/area) }\end{array}$ & $18.9 \pm 2.7^{*}$ & $5.5 \pm 1.8 \#$ & $11.4 \pm 2.7$ \\
\hline $\begin{array}{l}\text { HSP70 } \\
\text { (Possitive stain/area) }\end{array}$ & $16.2 \pm 2.1^{*}$ & $2.5 \pm 1.0 \#$ & $9.1 \pm 1.4$ \\
\hline
\end{tabular}

${ }^{\star} p<0.01$ versus $G 1$ y $G 2 . \quad \# p<0.01$ versus $G 1$

Table 1. Immunohystochemistry of renal pelvis.

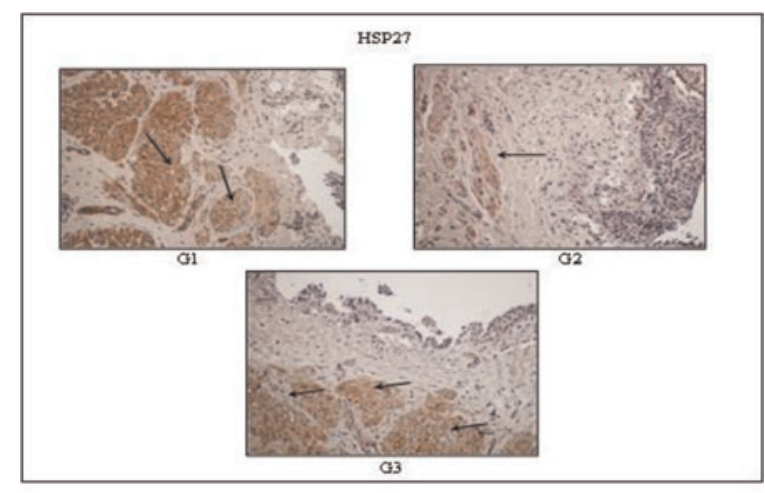

Figure 1. Immunohystocbemistry for HSP27. G1 UPJ. G2 Hydronephrosis G3. Control

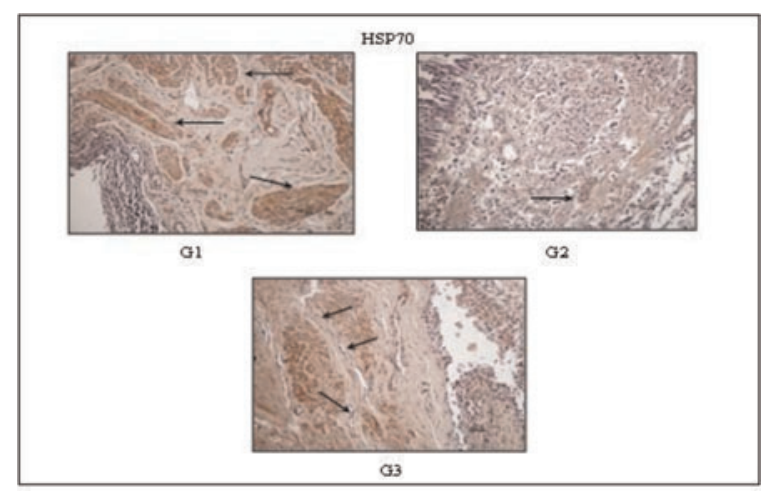

Figure 2. Immunohystochemistry for HSP7O G1 UPJ. G2 Hydronephrosis G3. Control

Materials and Methods: 20 samples of renal pelvis of patients with pyeloplasty for ureteropelvic junction (UPJ) obstruction, 14 obtained from nephrectomies for hydronephrosis and 16 controls were analyzed. HSP 27 and 70 were tested in all samples using immunolabelling.

Results: The percentage of positive immunolabeling for HSP27 was 18.9 for stenosis, 5.5 for hydronephrosis and 11.4 for controls. $(\mathrm{p}<0.01)$ The HSP 70 was 16.2 for stenosis, 2.5 for the hydronephrosis and 9.1 for controls. $(\mathrm{p}<0.01)$

Conclusions: The HSP 27 and 70 are increased in the obstructed renal pelvis, while their values are decreased in the renal pelvis of the kidney with hydronephrosis. These proteins could be a useful prognostic marker in obstructed kidneys.

BRPRS1-12 Re-do female genitoplasty for previously failed genitoplasty in male to female gender reassignment surgeries

A Ghoreifi, K Tavakkoli Tabassi, A Yarmohammadi, S Hajian

Mashhad University of Medical Sciences Iran

Introduction \& Objective: Various surgical procedures have been described for the correction of the external genitalia in male-to-female trans-sexuals. In this study, we described 
redoing reconstructive surgery techniques for previously failed genitoplasty in male to female gender reassignment surgeries.

Materials and Methods: In this study, 30 patients who had undergone male to female genitoplasty in other centers underwent re-do genitoplasty. All patients were referred to our center with the complaint of deformity or dysfunction of the external genitalia due to clitromegaly, inappropriate site of the clitoris, vaginal stenosis, inadequate depth of vagina, etc. All surgeries were performed by a single surgeon. For each patient the appropriate reconstructive surgery was planed based on the patient's complaint and complications. Follow-up visits were scheduled for 1 week, 1, 3 and 12 months, postoperatively.

Results: 17 patients had a history of vaginoplasty using the small intestine whereas in 13 patients it was done with penile and perineal skin. 4 cases needed clitoroplasty. Increased vaginal depth was indicated in 6 patients due to vaginal shrinkage which was repaired using amniotic membrane and a vaginal stent. Urethroplasty was performed in 7 patients as described before. Skin Removal was required in 6 cases. In 7 patients in whom their previous vaginoplasty had been performed as a superficial pouch at the perineal area, vaginoplasty was done with our classic method. None of the patients reported any major complications during this period and were generally satisfied with their sexual intercourse.

Conclusions: Using the various methods of redoing reconstruction of the external genitalia when the surgeon has enough experience might lead to desirable functional and aesthetic results.

BRPRS1-13 Midterm follow up of patients performed fold-back perineoscrotal flap plus penile inversion vaginoplasty for male-to-female gender reassignment surgery

K Tavakkoli Tabassi, A Yarmohammadi, A Ghoreifi

Iran

Introduction \& Objective: The use of the penile skin for vaginoplasty is a common method in male-to-female (MTF) transsexual surgery. We aim to describe the midterm follow up of a patients performed one-stage vaginoplasty using fold back perineoscrotal flap plus penile inversion flap.

Materials and Methods: In a prospective study, 208 previously circumcised MTF subjects underwent a modification of penile inversion vaginoplasty by adding a fold-back perineoscrotal flap to form the whole posterior vaginal wall and about half of the anterior vaginal wall (the proximal part). Patients follow-up, outcomes, and complications were recorded.

Results: The mean age of the subjects was $24.6 \pm 3.4$ years underwent feminizing genitoplasty. The success rate was $90.38 \%$, and the complication-free success rate was achieved in $72.11 \%$ of subjects in general. The need for repeated surgery (failure rate) was $9.62 \%$ (due to vaginal shrinkage in five patients, bulging in anterior vaginal wall in two cases and excessive labial skin in four cases). By adding the perineoscrotal flap to the penile skin flap, we were able to reach a mean vaginal depth of $13.3 \pm 1.5$. In a $24 \pm 4.3$ months follow up satisfaction level was $88.94 \%$.

Conclusions: Midterm follow up of patients performed foldback perineoscrotal flap plus penile inversion vaginoplasty showed acceptable results. This method of surgery is a suitable surgical approach for achieving adequate vaginal depth in cases of male-to-female (MTF) transsexual vaginoplasty when subjects have short penile skin flap because of circumcision.
BRPRS1-14 Barbed polyglyconate versus polyglactin. An experimental model to compare pressure on renal suture

F Ferraris, F Silvano, E Kol, G Nevado Benzi, R Lobo, F Lacour, E Longo

Churruca Hospital

Argentina

Introduction \& Objective: Laparoscopic partial nephrectomy is a technically complex procedure. Several refining techniques have been proposed to facilitate renorrhaphy during this procedure in order to reduce ischemic time. In parallel, novel suture materials have been developed, such as barbed polyglyconate $\left(\mathrm{V}-\mathrm{Loc}^{\mathrm{TM}}\right)$, turning reconstruction into a faster and safer technique. The objective of this study was to compare the pressure loss exerted over time on tissues with barbed polyglyconate versus polyglactin sutures in an experimental model using pig kidneys.

Materials and Methods: Ten pig kidneys were used. Perirenal fat was dissected. Saline solution was infused into the artery at $110 \mathrm{mmHg}$. Two equal cuts were made on each pole of each kidney. Cuts were $20 \mathrm{~mm}$ deep simulating a tumorectomy. A strength sensor (A101, Tekscan ${ }^{\mathrm{TM}}$ ) was placed between the edges of the sectioned kidney tissue. On each pole symmetrical stitches were made and closed with 0 barbed polyglyconate and 0 polyglactin respectively, using identical needles. Polymer clips were placed at the beginning and end of each suture to secure it. Strength was measured at 0, 30, 60, 120 and 180 seconds and calculated in grams. Wilcoxon test was used as the statistical method.

Results: Barbed polyglyconate yielded the following mean resistance: $0.43,0.88,1.01,1.11$, and $1.17 \mathrm{M} \Omega$, while polyglactin yielded: $0.54,1.28,1.43,1.52$, and $1.61 \mathrm{M} \Omega$, respectively. The differences between the 2 types of suture were statistically nonsignificant at time 0 , though significant at successive times $(\mathrm{p}=0.01)$.

Conclusions: According to our experimental model with pig kidneys, it can be concluded that barbed polyglyconate accounts for less pressure loss between tissues as compared to polyglactin over time.

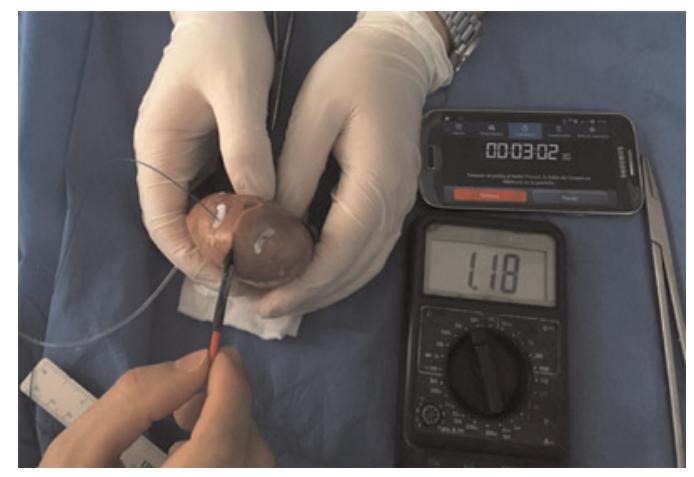

\begin{tabular}{|l|c|c|c|c|c|}
\hline & \multicolumn{2}{|c|}{ Polyglactin } & \multicolumn{2}{c|}{$\begin{array}{c}\text { Barbed } \\
\text { polyglyconate }\end{array}$} & $p$ \\
\hline & grams & $\mathrm{M} \Omega$ & grams & $\mathrm{M} \Omega$ & \\
\hline Time 0 & 191.8 & 0.54 & 197,5 & 0.43 & 0.18 \\
\hline $30 \mathrm{sec}$ & 180.0 & 1.28 & 183.9 & 0.88 & 0.0117 \\
\hline $60 \mathrm{sec}$ & 179.2 & 1.43 & 182.2 & 1.01 & 0.0116 \\
\hline $120 \mathrm{sec}$ & 178.8 & 1.52 & 181.2 & 1.11 & 0.0117 \\
\hline $180 \mathrm{sec}$ & 178.4 & 1.61 & 180.7 & 1.17 & 0.0117 \\
\hline
\end{tabular}


BRPRS1-15 Gene Expression and Patterns of Scarring Response in Human Fibroblasts in Response to Mesh, Catheter Materials and Silca Using a 2D and Novel 3-D Collagen Model

R Kilani, L Yunyuan, L Stothers, A Ghahary

Department of Urologic Sciences UBC and Peter Wall Institute of Advanced Studies

Canada

Introduction \& Objective: Scarring secondary to prosthetic materials is a serious clinical problem within the GU tract. Fibronectin is contained in fibrotic matricies and alpha-smooth muscle actin contributes to scar contraction. Metalloproteinases (MMPs) such as MMP-1 and -3 can modulate matrix protein accumulation through degradation. Our institution has shown that silica materials can induce scarring by interaction with tissue fibroblasts in vitro. We Hypothesis: synthetics induce fibrotic changes through cellular matrix gene expression. Objectives: 1) examine effects of Kynurenine and Kynurenic acid on scar formation in a 2D model 2) establish a 3D human fibroblast model to observe fibroblast response to synthetic materials and 3) measure gene expression in human fibroblasts exposed to synthetic materials compared to a control.

Materials and Methods: 3D model creation: Collagen gel was prepared using $3 \mathrm{mg} / \mathrm{ml}$ in final concentration with $0.5 \%$ of polyvinyl alcohol (PVA). Mesh or catheter materials and human dermal fibroblasts $(70,000 / \mathrm{ml})$ were added and seeded in a 24 -well plate $(0.5 \mathrm{ml}$ of gel in each well) to create the $3 \mathrm{D}$ environment for fibroblast response. After polymerization of collagen 250,000 cells in $0.5 \mathrm{ml}$ medium were added on the top of gel. Cells were cultured at $37{ }^{\circ} \mathrm{C}, 5 \% \mathrm{CO} 2$ for indicated time point. Reverse microscopy imaging was used to determine patterns of scarring contraction. Gel cell matrix was harvested and digested with $1 \mathrm{mg} / \mathrm{ml}$ of collagenase for 15 minutes, pelleted by centrifugation and RNA extracted. RTPCR was performed for 32 cycles to analyze gene expression.

Results: In the 2-D silica model kynurenine and kynurenic acid demonstrated an anti-fibrotic role based on a inhibition of nodule formation and collagen bundle formation. In the 3D model after 5 days, fibroblast contractions were identified surrounding prosthetic materials of mesh and catheters but not in the control. There were increases in type 1 collagen, a-smooth muscle actin and fibronectin expression in fibroblasts exposed to prosthetic materials compared to fibroblasts grown in collagen gel alone. In addition, MMP-1 and MMP-3 were detected.

Conclusions: Kynurenine and kynurenic acid demonstrated antifibrotic roles. Fibroblasts exposed to mesh and catheter materials responded with an increase in fibronectin, alpha smooth muscle actin and type 1 collagen compared to controls. This may signify why in vivo these materials induce fibrosis and scar formation. Since fibronectin, type 1 collagen and alpha smooth muscle actin are important components of scarring and their gene expression is was elevated, future directions indicate development of medical devices to induce downregulation of these genes may be possible.

\section{BRPRS1-16 Withdrawn}

BRPRS1-17 Does erectile function predicts outcome of second stage urethroplasty? A retrospective analysis.

K Kaura, A Sokhal, S Sankhwar

King George's Medical University

India

Introduction \& Objective: Complex, long segment anterior urethral strictures are still considered for staged repair. The higher recurrence rate and complications associated with two stage urethroplasty as compared to single stage repair can be attributed to the technique of repair and lichen sclerosis as etiology. Measuring outcome of urethral reconstruction, primarily focused on stricture recurrence, but postoperative erectile function is also an important aspect of overall patient satisfaction. We conducted this study to analyze the impact of sexual activity after first stage urethroplasty on the outcome of staged (second stage) urethral reconstruction.

Materials and Methods: We retrospectively reviewed medical records of 98 patients, who underwent 2 stage urethroplasty between January 2009 and December 2015. Decision of staged urethral repair was based on urethral stricture characteristics, etiology, the surgeon's experience and patient's preference. Modified Johansson's technique was adopted for performing staged urethroplasty. Patients were inquired about resumption of sexual activity after first stage procedure, sexual frequency and quality of erection and overall satisfaction.

Results: Ninety-eight patients were included in the study with the mean age of $40.1 \pm 9.3$ years. Patients were categorized in two groups based on the success or failure of urethroplasty within 1-year duration. Total 24 (24.5\%) patients had failure of the staged urethroplasty at the end of 1 year follow up. etiology of the stricture was similar between both groups. Mean stricture length was similar as $9.4 \pm 2.2$ and $9.6 \pm 1.8$ respectively. On univariate analysis, patients in the success group resumed sexual activity earlier (Median 4.2 vs 11.6 months) as compared to the failure group after the first stage procedure $(\mathrm{p}=0.001)$. Patients with sexual frequency of 1-3/ month, achieved near significant $p$ value $(\mathrm{p}=0.06 ; \mathrm{OR}=5.6)$. Patients, whose sexual frequency was in the range of 3-5 per month, secure 25.3 times higher probability of successful second stage urethroplasty.

Conclusions: The impact of stricture disease and urethroplasty on erectile function is well studied. Early resumption of sexual activity and frequency of sexual activity after first stage urethroplasty resulted in improved success of second stage urethroplasty. Based on this study, patient can be promoted early sexual activity for better urethroplasty outcomes.

BRPRS1-18 Single Surgeon Experience with Revision of Ureteroenteric Anastomosis for Benign Strictures after Urinary Diversion

E Schommer, A Davidiuk, L Gundian, K Custer, P Young

Mayo Clinic Florida

United States

Introduction \& Objective: Ureteroenteric anastomosis performed during UD are prone to stricture secondary to poor perfusion and scarring post-operatively. We sought to evaluate the frequency of ureteral obstruction after urinary diversion UD and rate of success after UEA revision (UAR) of benign strictures.

Materials and Methods: After IRB approval, we retrospectively reviewed 159 patients whom underwent UD by a single surgeon between January 2010 and November 2015. UD was performed for 3 indications including cystectomy (CX) for malignancy, CX for benign reasons, and UD without initial CX. Four types of UD were utilized including ileal conduit (IC), right colon pouch (RCP), Studer neobladder (SN), and transverse colon conduit (TCC). CX was performed robotically when appropriate and all UD were performed in open fashion. Data was gathered for UD indication, UD type, surgical approach, hydronephrosis present on the first outpatient 
computed tomography (CT) scan, ureteral stent placement, infection-related hospitalizations following stent placement, open UAR, and resolution of hydronephrosis following open UAR. The indication for stents placed in patients that did not ultimately undergo UAR was also reviewed.

Results: Overall, 114(71.7\%) patients underwent CX and UD for malignancy, 25(15.7\%) underwent simple CX and UD for benign reasons, and 20(12.6\%) underwent UD alone. In total, 106 IC, 29 $\mathrm{RCP}, 21 \mathrm{SN}$, and $3 \mathrm{TCC}$ were performed. Twenty five patients $(15.7 \%)$ had stents placed by Interventional Radiology postoperatively for reasons including stricture/hydronephrosis, anastomotic leak, conduit-cutaneous fistula, or persistent acute kidney injury. Of these patients, 20 underwent stent placement due to ureteral obstruction of benign or malignant cause. A total of 13 patients had open UAR of benign stricture and postoperatively, hydronephrosis resolved in 12 of the 13 (92.3\%). In the one patient with persistent hydronephrosis after UAR, reflux on loopogram was noted with continued elevation of creatinine. Prolonged ureteral stents accounted for 4 hospital admissions for urinary sepsis.

Conclusions: Open UAR is a challenging but successful procedure. $12.6 \%(20 / 159)$ of patients developed UEA stricture. Of the patients that underwent open UAR, hydronephrosis resolved in $92.3 \%$. Open revision is the most definitive way to manage ureteroenteric anastomotic stricture after urinary diversion.

BRPRS1-19 Castration impairs erectile organ structure and function by inhibiting autophagy and promoting apoptosis of corpus cavernosum smooth muscle cells in rats

Z Shen, S Zhong, X Wang, T Xu, F Gao, M Zhang

China (People's Republic)

Introduction \& Objective: The aim of this study was to determine the changes and underlying mechanisms of erectile organ structure and function in castrated rats. In addition, the regulatory effects of an androgen on autophagy and apoptosis in corpus cavernosum smooth muscle cells (CSMCs), especially the regulatory effect of androgen on the BECN 1-Bcl-2 interaction, were investigated.

Materials and Methods: Male Sprague-Dawley rats were divided into three groups (30/group): control group, castration group, and castration with testosterone supplementation group. The erectile function was examined both in vivo and in vitro, by electric stimulation of the cavernous nerve, and corpus cavernosum strip bath test, respectively. Transmission electron microscopy, TUNEL assay, Masson's trichrome staining, immunohistochemistry, and western blotting were performed to determine the levels of autophagy and apoptosis, and the structural changes in corpus cavernosum

Results: Compared with control group, the castration group showed (1) lower erectile function: lower intracavernosal pressure/ mean arterial pressure ratio, lower systolic and diastolic capability of corporal strips, and reduced expressions of eNOS and nNOS; (2) greater fibrosis: decreased smooth muscle/collagen ratio, lower expression of $\alpha$-SMA, and higher expression of TGF- $\beta 1$; (3) inhibited autophagy: decreased autophagosomes, lower expressions of BECN1 and LC3-II; (4) enhanced apoptosis: higher apoptotic index, and decreased $\mathrm{Bcl}-2 / \mathrm{Bax}$ ratio. Testosterone supplementation partially improved the effects of castration.

Conclusions: Castration attenuates erectile function and induces corporeal fibrosis by inhibiting autophagy and promoting apoptosis of CCSMCs in rats. Therefore, our study highlights the important role of androgens in maintaining the integrity of the structure and function of corpus cavernosum in rats through counter-regulation of autophagy and apoptosis, mainly by regulating BECN 1-Bcl-2 interaction.

BRPRS1-20 In-Vivo trial of a Genuine Atraumatic Urinary Catheter Designed for Prevention of CatheterInduced Trauma

\section{R Azar, P Shadpour}

Iran University of Medical Sciences

Iran

Introduction \& Objective: : Clinically significant urinary tract infection is the most familiar complication of urinary catheterization; however, catheter-associated trauma can entail even more significant morbidity. We have designed and patented a novel Atraumatic Urinary Catheter (AUC) and through this study, we compare its efficacy with a conventional Foley catheter (FC) in vivo.

Materials and Methods: 40 male rabbits were divided into two equal groups for FC and AUC. The animals were sedated prior catheterization by a lubricated catheter. After 4 days, the rabbits were reexamined following standardized forcible extraction of the catheter under sedation with the balloon still inflated. The results were confirmed by cystoscopy.

Results: Some catheters in both groups failed to drain urine effectively due to anatomical issues. Those were excluded from

\begin{tabular}{|c|c|c|c|c|}
\hline & \multirow{2}{*}{$\begin{array}{c}\text { No sign } \\
\text { post } \\
\text { extrusion }\end{array}$} & \multicolumn{3}{|c|}{ Positive findings post extrusion } \\
\hline & & $\begin{array}{c}\text { Urinary } \\
\text { Retention }\end{array}$ & $\begin{array}{c}\text { Gross } \\
\text { Hematuria }\end{array}$ & \begin{tabular}{|c|} 
Sustained \\
Urethrorrhagia
\end{tabular} \\
\hline $\begin{array}{c}\text { FC group (no. } \\
15 \text { ) }\end{array}$ & $2(13 \%)$ & $3(20 \%)$ & $6(40 \%)$ & $4(27 \%)$ \\
\hline $\begin{array}{l}\text { AUC group (no. } \\
14 \text { ) }\end{array}$ & $10(72 \%)$ & $2(14 \%)$ & 2 (14\%) & $0(0 \%)$ \\
\hline
\end{tabular}

Catheter

Table 2: Comparing cystoscopic findings upon forceful extrusion of a conventional Foley catheter vs. the Atraumatic Urinary Catheter from the rabbit bladder

\begin{tabular}{|c|c|c|c|c|c|}
\hline & \multicolumn{5}{|c|}{ Cystoscopic findings post extrusion } \\
\cline { 2 - 6 } & Normal & Hyperemia & $\begin{array}{c}\text { Superficial } \\
\text { mucosal tear }\end{array}$ & $\begin{array}{c}\text { Deep urethral } \\
\text { laceration }\end{array}$ & $\begin{array}{c}\text { Disruption into } \\
\text { perivesical fat }\end{array}$ \\
\hline $\begin{array}{c}\text { FC group (no. } \\
\text { 15) }\end{array}$ & $0(0 \%)$ & $2(13 \%)$ & $1(7 \%)$ & $7(47 \%)$ & $5(33 \%)$ \\
\hline $\begin{array}{c}\text { AUC group (no. } \\
\text { 14) }\end{array}$ & $0(0 \%)$ & $11(79 \%)$ & $3(21 \%)$ & $0(0 \%)$ & $0(0 \%)$ \\
\hline
\end{tabular}

Chi-square 16.39, P value 0.009; FC: Foley catheter, AUC: Atraumatic Urinary Catheter 


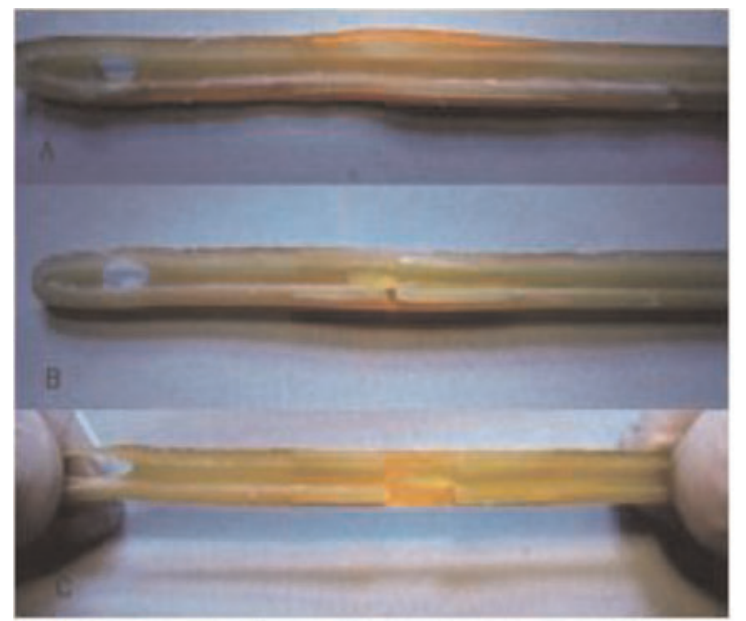

Figure 2: Cross sectional view of the Atraumatic UC (Patent No: 2939962) and the Foley catheter. D. $\mathrm{FC}$

E. AUC with aafety mechanism beginning to diatract

F. AUC with safety mechanism completely separated

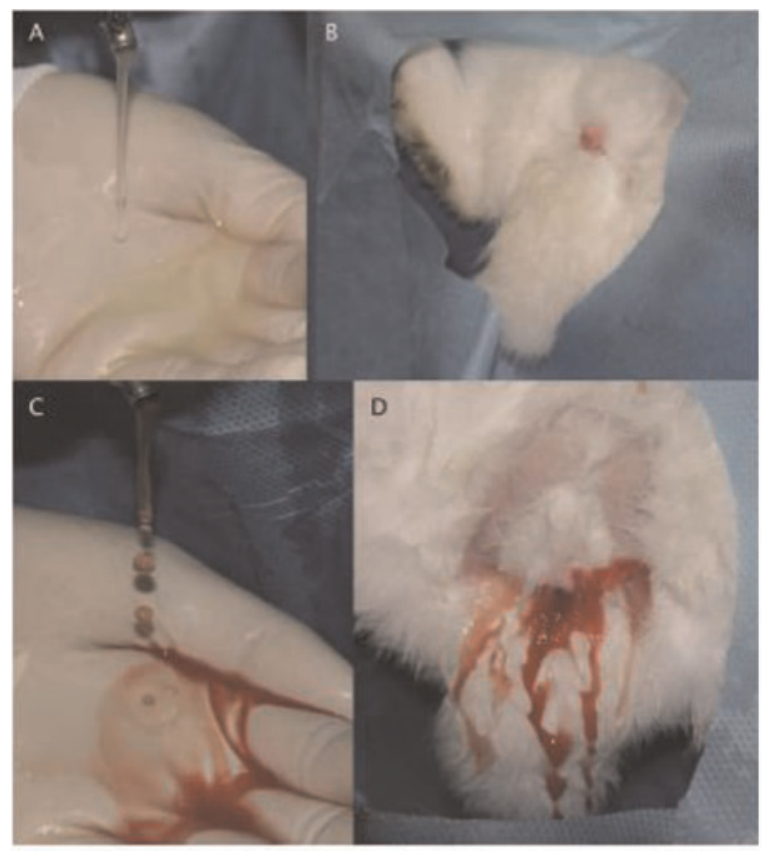

Figure 3: Visual grading of trauma from forceful catheter extraction in the rabbit

E. Normal urine (no change).

F. Urinary retention.

G. Gross hematuria.

H. Persistent urethrorrhagia.

the rest of the study. At the extraction phase, urethral trauma was encountered in 13 of 15 FC rabbits but only 4 of 14 in the AUC group $(\mathrm{P}=0.009)$. Major trauma, however, was exclusively seen in the FC group, with 12 of 15 subjects sustaining deep laceration or urethral disruption.

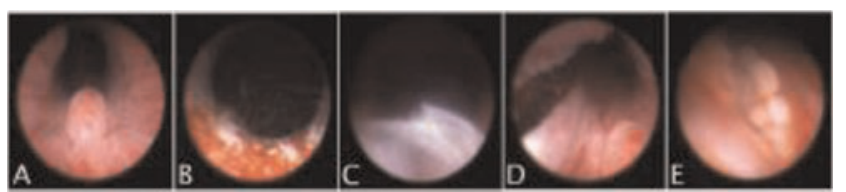

Figure 4: Oystoscopic grading of urethral trauma from forceful catheter extraction, compared to normal findings in the rabbit.

F. Normal cystoscopic image of the male rabbit urethra.

G. Mucoal hyperemia

H. Superficial mucosal tear

L. Deep urethral laceration

1. Disuption into perivesical fat

Conclusions: The two catheters bear similar efficiency for draining urine; however, the traumatic injury is significantly less common following forceful extraction of the atraumatic catheter than a conventional Foley.

\section{BRPRS1-21 Bladder Stone and Urodynamics}

J Sun

China (People's Republic)

Introduction \& Objective: Bladder stones (BS) can cause urinary retention, lower urinary tract dysfunction, and infection. We operated this experimental study, which is the first to create a rabbit $\mathrm{BS}$, to study micturition physiology based on the urodynamics that simulate clinical manifestations.

Materials and Methods: The study included 9 New Zealand adult male rabbits in the BS group and 9 controls. BS creation consisted of a lower midline laparotomy and bladder entry from the bladder wall, and then putting different diameter stones into the bladder. The sham group underwent bladder exposure with a same size midline incision. All rabbits underwent urodynamic study preoperatively and postoperatively, consisting of postmicturition residue (PMR), maximum bladder capacity (MBC), voiding detrusor pressure (VPdet), filling detrusor pressure (FPdet), compliance, and urine flow (Qflow).

Results: Preoperative MBC, Pdet, and Qmax were within reference ranges. No animals had PMR or urinary tract infections (UTIs). The BS group showed urodynamic changes, including decreased $(33 \%)$ cystometric bladder capacity and compliance (Sham: $25.4 \pm 0.3$; BS: $3.85 \pm 1.13, \mathrm{p}=0.0001$ ) and increased post-void residual PMR $(8.5 \pm 2.3 \mathrm{~mL})$. Urodynamics revealed intermittent BS bladder contraction during the filling phases. Eight BS group rabbits had UTIs.

Conclusions: In the literature, it has not been determined whether lower urinary tract disorders (LUTD) could cause stones, or if stones could be reason for LUTD. Anatomical or neurological reasons for a low-compliance bladder can cause stones. As demonstrated in our study with rabbits, stones could further reduce the compliance of the bladder. Further, a decrease in compliance logically correlates with the progressive decompensation of the bladder. All the information presented for this model is relevant to our clinical observations. We conclude that the rabbit bladder can be used for research into experimental stone-induced changes in the activity of the bladder and for experimental detrusor research. 


\section{BRPS2: BENCH TO BEDSIDE: THE SCIENCE OF STONES I}

\author{
BRPRS2-1 Non hemmorhagic Adrenal infarction in \\ pregnancy \\ E Fernandes, S Gupte \\ Vision Hospitals, Healthway Hospitals \\ India
}

Introduction \& Objective: Adrenal infarction is an infrequent cause of severe abdominal pain in pregnancy. We present a case of left(L) Adrenal infarction at 33weeks gestation mimicking a ureteric colic.

Materials and Methods: A 33yrs old pregnant lady, gravida $(\mathrm{G}) 4$, Para1 +1 (with a 9yr old child and two abortions with cervical incompetence) presented at 33 weeks gestation with severe colicky pain radiating from left loin to groin. She had colicks and renal calculus disease in the past. Pain was relieved only by injection diclofenac and not by injection hyoscine. Her abdomen was soft and there was minimal (L) renal angle tenderness. Her Urine analysis and serum(se) creatinine was normal. She has leucocytosis with Neutrophilia. Maternal Ultrasound showed (L) lower calyceal $4.5 \mathrm{~mm}$ calculus without hydronephrosis. A non contrast MRI showed a grossly enlarged (L) adrenal gland with features suggestive of infarct. Her coagulation profile, se cortisol and se Adreno corticotrophic hormone(ACTH) were normal. She was started on low molecular weight heparin. She refused to continue oral anticoagulation therapy.

Results: Her pain resolved in 4 days. She underwent an elective cesarian section at term. A followup MRI scan 6months after delivery showed complete resolution of infarct.

Conclusions: Unilateral, Non hemmorhagic Adrenal infarct is a rare event in pregnancy. The hypercoagulable state in pregnancy could contribute to the same. It could also be associated with syndromes like anti phospholipid antibody. It is more common on the right side and bilateral infarcts could lead to acute adrenal insufficiency. Anti coagulation therapy for nonhemmorhagic infarcts is the recommended treatment.

BRPRS2-2 Is the stone density and location of kidney stones smaller than two centimeters a parameter that affects the surgery to be chosen? Preliminary findings of a prospective randomization study

A Gücük, B Yılmaz, S Gücük, U Üyetürk

Abant Izzet Baysal Univercity Medical Faculty

Turkey

Introduction \& Objective: We aimed to evaluate whether there might be a parameter that could guide us in the selection of RIRS (retrograde intrenal stone surgery) or Mini PNL (percutaneous nephrolithotomy) in kidney stones smaller than $2 \mathrm{~cm}$.

Materials and Methods: Patients with kidney stones smaller than $2 \mathrm{~cm}$ in diameter and planned for surgery were prospectively randomized into 2 groups. The first group was dilatated to $16.5 \mathrm{~F}$ and treated with mini-PNL with $12 \mathrm{~F}$ nephroscopy while RIRS was performed using 7.5-F fiberoptic flexible ureterorenoscope to the other group. Both groups were compared in terms of preoperative characteristics (age, sex, BMI, stone load, stone density, stone location, etc.) and postoperative results (duration of operation, stone
Table 1 Comparison of groups in terms of postoperative values

\begin{tabular}{llll}
\hline & Mini PNL & RIRS & P value \\
\hline $\begin{array}{l}\text { Stone free rate } \\
\begin{array}{l}\text { Duration of } \\
\text { surgery (min) }\end{array}\end{array}$ & 105 & $\% 88$ & $\mathrm{P}>0.05$ \\
$\begin{array}{l}\text { Scopy duration } \\
\text { (sec) }\end{array}$ & 88 & 118 & $\mathrm{p}>0.05$ \\
$\begin{array}{l}\text { Hospitalization } \\
\text { period (days) }\end{array}$ & 2.4 & 20 & $\mathrm{P}<0.05$ \\
\begin{tabular}{l} 
VAS value \\
\hline
\end{tabular} & 2.3 & 1.2 & $\mathrm{P}<0.05$ \\
\hline
\end{tabular}

free rate, duration of scopy, hospitalization period, amount of bleeding, complication rate, pain scale). After that, the stone density was compared in terms of the HU value being higher or lower than 677 and the effect of the stone location on the results.

Results: The preliminary findings of the study consist of 25 patients for each group we have collected so far, and 50 patients in total. When the groups were compared, preoperative values were found to be similar ( $p>0.05)$. The comparative results in terms of postoperative values are shown in Table 1 . The duration of scopy and hospitalization was longer in the Mini PNL. In terms of stone densities, the HU value being lower than 677 or being multiple negatively affected the mini PNL (stone free rate $63 \%$ vs. $100 \%$ ), and the stone being in the lower calix negatively affected the RIRS results ( $94 \%$ vs. $62 \%$ ).

Conclusions: For kidney stones smaller than two centimeters, while both methods have similar success rates in total, stone density and location may be effective parameters in choosing treatment.

BRPRS2-3 The Association between Osteopontin Gene Polymorphisms and Idiopathic Calcium Oxalate Stones

Z Guoxi, X Guancheng, Z Xiaofeng, L Quanliang,

$\mathrm{X}$ Yijun, W Gengqing

Department of Urology, First Affiliated Hospital of Gannan Medical University

China (People's Republic)

Introduction \& Objective: To investigate the potential association between the osteopontin(OPN) gene polymorphisms and idiopathic calcium oxalate stones(ICS) in Jiangxi Gannan area. Materials and Methods: The 218 patients with ICS (ICS group) and 187 normal people (Control group) of OPN gene polymorphisms were detected by Real-time PCR TaqMan-MGB 5'nuclease assay. The correlation between the OPN of haplotype and genotype were analyzed by the SNPassoc 1.9-2 and Haplo .stats 2.17.0 software.

Results: The selected sites were successfully detected. The genotype distributions were consistent with Hardy-Weinberg equilibrium $(\mathrm{P}>0.05)$. The distribution frequencies of the delG and $\mathrm{G}$ alleles of rs 11439060 in ICS and control group were 69\%, 31\% and $57 \%, 43 \%$, respectively; and the difference was statistically 
significant $(\mathrm{P}<0.001)$. Also, there were significant differences in delGdelG, GG and delGG genotype between the two groups $(\mathrm{P}<0.001)$. OPN gene haplotype analysis showed that haplotype CGACCTAG, CGACCTGA was negatively correlated with the formation of the ICS $(\mathrm{OR}=0.861,95 \% \mathrm{CI}=0.762 \sim 0.974$; $\mathrm{OR}=0.833,95 \% \mathrm{CI}=0.713 \sim 0.973$ ).

Conclusions: OPN gene polymorphisms were significantly associated with the formation of ICS in Jiangxi Gannan area.

BRPRS2-4 Two novel AGXT mutations identified in Chinese patients with sever bilateral multiple nephrolithiasis

Y Du, J Li, Y Tian

Urology Department

China (People's Republic)

Introduction \& Objective: There are three types of primary hyperoxaluria $(\mathrm{PH})$, they are called as primary hyperoxaluria types 1,2 , and 3 . All the types are caused by different enzyme deficiency, and each affects a different intracellular organelle. More than 190 AGXT mutaitons have been reported in primary hyperoxaluria type 1 patients with Gly170Arg and c.33dupC occur across populations at a frequency of $30 \%$ and $11 \%$, respectively. PH1 (MIM\# 259900; gene AGXT, MIM\# 604285), the most frequent and most severe $\mathrm{PH}$, results from mutations in peroxisomal alanine:glyoxylate aminotransferase.

Materials and Methods: A Chinese child with early-oneset multi-nephrolithiasis was performed flexible ureteroscopy lithotrisy. Calculi analysis result is calcium oxalate monohydrate. A Chinese female with oneset multi-nephrolithiasis was performed bilateral percutaneous nephrolithotomy (PCNL). After PCNL for one year, the patient diagnosed chronic renal failure and starting hemodialysis. We suspected the child and the female having PH. We searched for AGXT, GRHPR and HOGA1 gene mutations in this patient and his younger brother and parents. All exon genes sequencing were analyzed using PCR followed by direct sequence analysis.

Results: Two novel mutations not previously described in the literature about AGXT were indentified. Two mutations were both frameshift mutation. Direct sequencing analysis failed to find these mutations in 100 unrelated in dbSNP, Hapmap, 1000genomes.

Conclusions: Two novel AGXT mutations were identified in association with PH1. This is the first description on two novel mutant genes PH1 in Asian families.

BRPRS2-5 Erythropoietin receptor signalling in unilateral ureteral obstruction

E Park, D Lange

University of British Columbia

Canada

Introduction \& Objective: The ureter transfers urine from the kidney to bladder via coordinated movement called peristalsis. Obstructing kidney stones impair ureteral peristalsis, reducing urine flow resulting in kidney dysfunction. Recovery of ureteral peristalsis following obstruction reversal is delayed despite resolved hydronephrosis, suggesting prolonged negative effects on kidney function than originally thought. Recent studies have shown that erythropoietin(EPO), the hormone that triggers hematopoeisis via erythropoietin receptor(EPOR) homodimer signalling, can provide protection from injury in different organs and accelerate recovery via signaling through an EPOR and $\beta$-common receptor $(\beta \mathrm{CR})$ heterodimer resulting in the activation of the STAT5 and anti-apoptotic Bcl-2 family. Previous results in our laboratory showed pre-obstruction EPO administration to decrease the degree of hydronephrosis and accelerate recovery of ureteral function. While these results suggest the use of EPO as a potential therapeutic to promote ureteral recovery following obstruction, its significant cost and side-effect profile significantly limit its use.

Materials and Methods: Unilateral ureteral obstruction using an atraumatic microclip was created for 24, 48 and 72 hours in CD-1 and C57BL/6 mice ( $n=10$ /group). We quantified the expression of EPO, EPOR, $\beta \mathrm{CR}$ and relevant downstream apoptotic targets in ureters and kidneys by qRT-PCR and western blots. Peristalsis before and after obstruction was determined microscopically.

Results: RNA expression of EPO and EPOR was decreased due to obstruction in kidneys and ureters of both mouse strains compared to contralateral tissues. Furthermore, expression of downstream anti-apoptotic targets, Bcl-2, Bcl-Xl, Nuclear factor kappa B(NFKB) and Stat5 was also decreased in the obstructed ureters. Interestingly, $\beta \mathrm{CR}$ expression in the kidney was increased due to the obstruction. The overall peristaltic activity was significantly decreased following obstruction compared to unobstructed contralateral ureters.

Conclusions: The results suggest that obstruction-induced ureteral dysfunction is in part mediated by increased apoptosis within the ureters as indicated by decreased $\mathrm{Bcl}-2, \mathrm{Bcl}-\mathrm{Xl}$, NFKB and Stat5 expression. Furthermore, the upregulation of $\beta \mathrm{CR}$ in the obstructed kidneys suggests the activation of EPO's "protective" pathway in response to injury, which may be impaired due to decreased EPO and EPOR expression.

Efficacy of prophylactic EPO in accelerating ureteral recovery may derive from EPO-induced effectors pre-obstruction, available to act in the low EPO/EPOR environment of ureteral obstruction.

BRPRS2-6 The Drosophila melanogaster model of human uric acid nephrolithiasis as a novel in vivo high throughput drug screening platform

AN Ali, T Dayarathna, M Pignanelli, J Kim, RS Padda, P Spagnuolo, H Razvi, HS Leong

Western University

Canada

Introduction \& Objective: Uric acid calculi account for approximately $10 \%$ of all kidney stones and are being observed with an increasing prevalence worldwide. The development of novel therapeutic agents for this multifactorial disorder has been hampered by the lack of a practical pre-clinical model, particularly in metabolic milieus where established treatments are poorly tolerated. Here, Drosophila melanogaster (DM), an emerging model for calcium oxalate nephrolithiasis, was investigated as a disease model and high throughput functional drug discovery platform for human uric acid nephrolithiasis.

Materials and Methods: Knockdown fly lines were generated by RNAi-mediated silencing of the Uro gene, encoding for the enzyme urate oxidase. Gene knockdown efficiency was quantified by qRT-PCR. Following a 7-day incubation period in purine rich lithogenic media ( $1 \% \mathrm{w} / \mathrm{v}$ yeast derived RNA), knockdown flies were dissected to recover intact malpighian tubules against wild-type (Canton $S$ ) controls $(n=20 /$ group). Fly fecal matter was collected on to coverslips suspended from fly vial closures. The presence of birefringent concretions in fly malpighian 
tubules and fecal matter was determined by confocal microscopy under polarized light. Formed tubule concretions were isolated by broad-spectrum protease dissolution of organic tubule material $(n=120)$ and characterized by SEM/EDX. A fecal birefringence assay was employed to evaluate the in vivo efficacy of standardized doses of candidate compounds added to fly media (200ul [1mM]) from a drug library of 360 small molecules via in silico birefringent particle analysis (ImageJ) versus controls. Results: Diffuse brilliant birefringence was visualized in Uro knockdown fly malpighian tubules and fecal matter, confirming the presence of crystals. The elemental composition of isolated concretions analyzed via SEM/EDX was consistent with uric acid. Quantification of birefringent areas in fly fecal matter via auto-thresholding (Yen) and particle analysis was seen to be a reliable method of approximating overall stone burden between groups. Drug library screening is currently in progress to identify compounds of interest for future study versus currently established treatments.

Conclusions: The DM model of human uric acid nephrolithiasis is a viable and cost-effective means for rapid drug candidate screening. This innovative platform has significant implications for the future of rational drug design for nephrolithiasis. Efficacies, drug-crystal interactions and mechanism of action of candidate "hits" can be further investigated by in vitro or in vivo methods in a mammalian system.

BRPRS2-7 Probiotic supplementation to reduce stone burden assessed using a Drosophila model of nephrolithiasis

K Al, R Chanyi, H Razvi, J Burton

Western University

Canada

Introduction \& Objective: The prevalence of nephrolithiasis in North America has risen to approximately $10 \%$. The gut microbiota is known to be an important factor in human health. It is thought that intestinal bacteria biotransform dietary and hostproduced toxic compounds. In this way, the microbiota has been implicated in preventing the formation of kidney stones; gut colonization with Oxalobacter formigenes appears to protect against formation of calcium oxalate $(\mathrm{CaOx})$ stones by reducing urinary oxalate. In stone-forming patients with low amounts of oxalate-degrading bacteria, probiotic supplementation to better tolerate and utilize dietary or host-derived oxalate may reduce plasma oxalate and potentially nephrolithiasis. Clinical trials with $O$. formigenes are limited and have not been successful, likely due to the strict anaerobic requirements of the bacterium. We sought to utilize the oxalate degrading potential of Bacillus subtilis 168 (BS168) a hardy spore-forming bacterium, that is starting to find favour as a probiotic species.

Materials and Methods: BS168 was grown in media conditioned with oxalate (up to $50 \mathrm{mM}$ ) to validate bacterial survival. A Drosophila melanogaster model of nephrolithiasis was utilized to evaluate how stone burden would be impacted upon exposure to the potential probiotic. Flies were administered BS168 weekly via a yeast paste and fed $1 \%$ sodium oxalate food (OF). Stone burden was assessed with a fecal crystal assay and survival curve analysis.

Results: BS168 preferentially grew in high oxalate, utilizing it before other available carbon sources. BS168 supplementation did not impact the survival of healthy flies fed normal food (NF). Preliminary results suggest that flies administered BS168 on OF developed fewer fecal crystals compared to flies without BS168. The BS168 flies had increased survival while consuming OF compared with non-BS168 flies. BS168 was viable in fly faeces. Conclusions: These findings suggest that supplementation with Bacillus subtilis 168, an organism potentially usable in humans, can reduce stone formation through degradation of oxalate.

BRPRS2-8 Optimizing RNA extraction of renal papilla biopsy tissue in kidney stone formers: a new methodology for genomic study

K Taguchi, M Usawachintachit, DT Tzou, S Hamamoto, BA Sherer, ML Stoller, T Yasui, T Chi

UCSF

United States

Introduction \& Objective: Endoscopic tools have provided versatile examination and treatment for kidney stone procedures. Despite endourologists performing translational research in urinary stone disease using endoscopes to collect tissue, this tissue collection method is limited. We investigated a new method of renal papilla biopsy and RNA extraction to establish a genomic research methodology for kidney stone disease.

Materials and Methods: We conducted a prospective multiinstitutional study between Japan and the United States and collected renal papilla specimens from consecutive percutaneous nephrolithotomy (PCNL) and ureteroscopy (URS) cases performed for removal of upper urinary tract stones. Renal papilla tissue was extracted using ureteroscopic biopsy forceps after stone removal. RNA was extracted using 2 different extraction kits, and their quantity and quality were examined. Additionally, the impact of biopsy on surgical complications was also compared between cases performed with and without papillary biopsy extraction by matched case control analysis adjusted for age, gender, BMI, bilaterality, and stone burden.

Results: A total of 90 biopsies from 49 patients were performed between September 2014 and August 2016. The median duration between specimen collection and RNA extraction was 61 days. One third of biopsies were performed during URS. Both univariate and multivariate analyses showed BIGopsy ${ }^{\circledR}$ forceps usage significantly increased the total yield $(\mathrm{p}=0.004)$ and quality $(p=0.001$ for A260/280, $p=0.004$ for A260/A230) of extracted RNA. Extraction using RNeasy Micro Kit ${ }^{\circledR}$ also improved A260/A230 of extracted RNA by univariate and multivariate analyses $(\mathrm{p}=0.002)$. (Table 1$)$ Moreover, matched case control study demonstrated that endoscopic renal papilla biopsy caused no significant surgical complications including bleeding, decreased stone clearance and hematocrit, and renal dysfunction. Biopsies during URS imparted an average of 20 minutes of procedure time over non-biopsy cases.

Conclusions: We demonstrate a safe methodology for optimal RNA extraction of renal papilla tissue. This technique will accelerate advanced genomic studies for kidney stone formers by facilitating larger tissue yields.

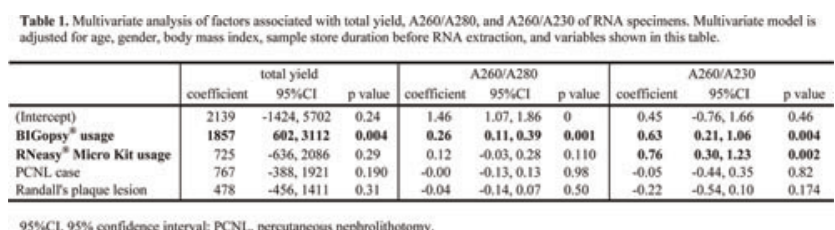


BRPRS2-9 Deficiency in butyrate metabolism and oxalate degradation in the gut microbiome of kidney stone patients

W Choy, C Morgan-Lang, E Gough, A Manges, S Hallam, $\mathrm{BH}$ Chew, D Lange

University of British Columbia

Canada

Introduction \& Objective: High urinary oxalate concentrations (hyperoxaluria) significantly increase the risk of calcium oxalate kidney stones. To reduce urinary oxalate, researchers are investigating oxalate excretion and breakdown in the gut, two processes that are driven by the gut microbiome. Previous studies have shown a role for Oxalobacter formigenes in reducing oxalate absorption across the intestinal epithelium by breaking it down in the intestine. We test the overall differences in the intestinal microbiome composition of recurrent stone formers to non stone-formers to identify bacterial determinants of overall oxalate homeostasis and any bacteria-induced metabolic deficiencies associated with recurrent calcium oxalate kidney stone disease.

Materials and Methods: Fecal samples were collected from 17 patients with calcium oxalate kidney stones and their non-stoneforming partners. DNA was extracted from the samples and shotgun-sequenced using Illumina HiSeq and the NexteraXT metagenomics library kit. The DNA sequences were then aligned to the KEGG gene database to obtain functional gene annotations. The DNA was also sequenced specifically for $16 \mathrm{~S}$ rRNA genes to identify the bacteria present.

Results: At the bacterial species level, we verified that the recurrent stone formers' microbiome lacked Oxalobacter formigenes, a well-known oxalate-degrading bacterium, as well as other bacterial species potentially involved in the regulation of oxalate absorption. At the metabolic level, the microbiomes of patients were found to have lower abundance of genes important for the production of butyrate, a fatty acid that plays a role in overall intestinal integrity and has been shown to upregulate the expression of oxalate transporters in the gut.

Conclusions: The present study verified a role for $O$. formigenes in recurrent calcium oxalate kidney stone disease. The abundance of additional bacterial species in the gut microbiome of non-stone-formers indicates a role for additional bacterial species in maintaining overall oxalate homeostasis. Furthermore, the overall lack of genes involved in butyrate metabolism within the gut microbiome of recurrent calcium oxalate kidney stone formers points to additional mechanisms related to the promotion of dietary oxalate uptake, thereby disrupting the overall oxalate homeostasis and causing hyperoxaluria associated with recurrent kidney stone disease.

BRPRS2-10 A novel laboratory model system for the evaluation of biofilm-induced urinary infection stone formation

T Hobbs, S Logan, E Lauchnor, E Espinosa-Ortiz, D Lange, R Gerlach

Montana State University

United States

Introduction \& Objective: Infection stones, comprised of struvite or its combination with calcium-based minerals, are induced by urinary tract infections with ureolytic pathogens. Association of struvite stones with bacteria presents significant treatment impediments. The role of biofilms and their influence on struvite formation and characteristics, and the effect of urinary chemistry on struvite formation are not well understood. In depth research into struvite stones is limited by the lack of models for the real-time observation of bacterial biofilm formation and struvite precipitation under varying conditions. Our goal was to develop a laboratory model capable of simulating the formation of struvite stones in the urinary tract system while allowing for real-time observations of biofilms, minerals and microbe-mineral interactions.

Materials and Methods: A laboratory model was constructed consisting of a media reservoir, a kidney analog reactor, ureter line (tubing), a bladder analog reactor, and a waste reservoir. The system was filled with artificial urine and inoculated with Proteus mirabilis in the bladder analog reactor. Flow of fresh artificial urine began after $24 \mathrm{~h}$. Periodic urination was simulated by emptying the bladder analog every $4 \mathrm{~h}$ during the day. Liquid samples were taken daily for chemical analysis. Coupons were harvested from the kidney analog reactor for characterization of biofilm formation (confocal microscopy) and mineral analysis (scanning electron microscopy and energy-dispersive X-ray spectroscopy).

Results: Ureolysis took place in the laboratory model; chemical and mineralogical analysis indicated precipitation of struvite and apatite. Stones obtained from laboratory experiments had similar elemental composition and mineral morphologies to clinically obtained stones. Progression of biofilm and mineral formation was observed over time, with biofilm formation and mineral accumulation being observed after 5 and 8 days, respectively.

Conclusions: An in vitro model that allows real time sampling of liquid and solid samples in a simulated kidney-ureter-bladder system was developed. Mineral and biofilm morphologies produced in the model were similar to those observed in stones obtained from patients. The model system developed in this study offers an opportunity to develop treatment and management strategies in vitro.

BRPRS2-11 A hypercalciuric stone-forming rat model induced by active vitamin D

H Hu, S Wang, Y Lu, J Zhang, B Qin

Department of Urology, Tongji Hospital, Tongji Medical College, Huazhong University of Science and Technology China (People's Republic)

Introduction \& Objective: Classic genetic hypercalciuric stone-forming rats and some gene-engineering mice with renal interstitial calcification are complex or expensive in cultivation. Our previous studies have confirmed the increased circulating active vitamin D in stone patients. Therefore, we try to administrate the SD rats with active vitamin D to establish a simple, economic and repeatable hypercalciuric stone-forming rats.

Materials and Methods: Six weeks old male SD rats were randomly divided into vitamin $D$ group $(n=24)$ and control group $(\mathrm{n}=18)$, and they received normal diet and drinking water. Vitamin D group were administered with $1,25(\mathrm{OH})_{2} \mathrm{D}_{3}$ intraperitoneally every other day. At the 4 th, 8 th and 12 th week, 8 rats were sacrificed and their blood samples were collected for determination of serum calcium and phosphorus, liver and renal function. The kidneys were stained with HE and Von Kossa to assess the microstructure, calcium deposition and renal stone. The scanning electron microscope was used to observe the ultrastructure of the deposition and stones, and micro CT scan and three-dimensional reconstruction was conducted to acquire the overall observation of renal calcium deposition and stone formation. The rats in the control group received an equivalent amount of solvent intraperitoneally every 2 days and the other 


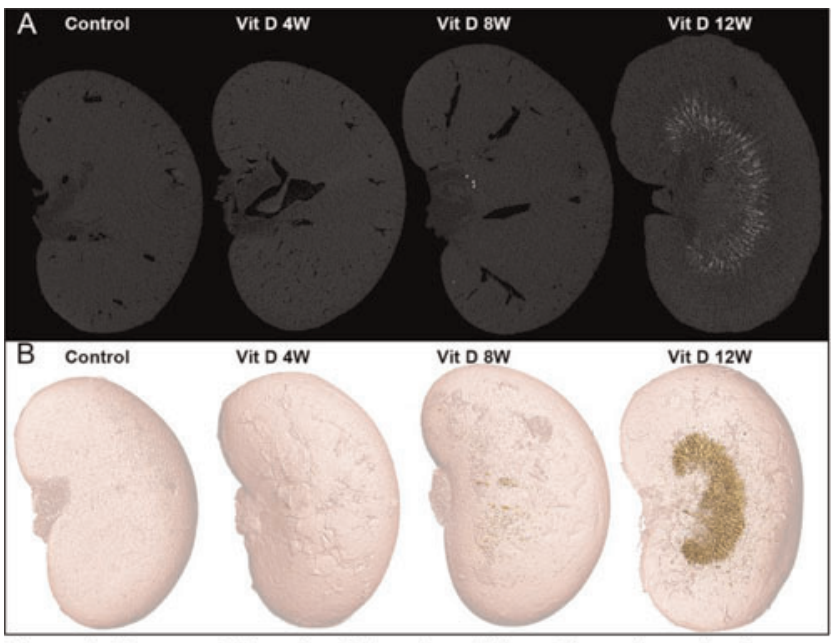

Figure 1. Representative micro CT sections (A) and three-dimensional reconstruction figures $(B)$ of kidneys of rats in the control group and Vitamin D group. processing and detection methods were same to the rats in the vitamin $\mathrm{D}$ group.

Results: The urinary calcium concentration and 24 hours urinary calcium were significantly higher in the vitamin D group. At the 4th week, micro CT could detected occasional renal calcium deposition in the rats receiving vitamin D; at 8th week, Von Kossa staining showed calcium deposition in the renal inner medulla and pelvic stones, and micro CT observed renal parenchyma calcification and renal stone formations; and the degree of calcification aggravated at the 12th week. Scanning electron microscopy showed that the renal interstitial calcifications were consisted of small plate-like or concentric mineral depositions, and further energy dispersive spectrometry showed that the calcification was composed primarily of calcium and phosphorus.

Conclusions: Administration of $1,25(\mathrm{OH})_{2} \mathrm{D}_{3}$ in SD rats could induce hypercalciuria, renal interstitial phosphate calcification and renal stones. This model can be used in the studies focusing on renal interstitial calcification and hypercalciuria stones.

BRPRS2-12 Inflammatory Cytokines in Papillary Tips and Urine of Nephrolithiasis Patients

AY Sun, B Hinck, BR Cohen, K Keslar,

R Fairchild, M Monga

Cleveland Clinic Foundation

United States

Introduction \& Objective: Intrarenal inflammation has been implicated in the pathogenesis of nephrolithiasis, with prior work showing increased urine levels of IL-6, IL-8, and CCL-2 in stone patients. However, no studies have yet assessed for inflammation in the renal papillae. We sought to characterize novel papillary tip and urinary biomarkers in stone patients.

Materials and Methods: 92 patients with nephrolithiasis undergoing percutaneous nephrolithotomy were enrolled. Papillary tip biopsies, kidney urine, and bladder urine were collected, as well as bladder urine from 8 healthy controls. QT-PCR was performed to examine for the presence of cytokines, and then compared across groups.

Results: Initial 84 gene PCR array revealed significant elevation of several cytokines in stone patients vs controls (fold change 2.3 - 694). 24 genes were selected for final analysis. In 41 matched pairs of urine samples, levels of CCL5, CD40, FasL, RIPK2, SELE, TLR3, and IL-15 were significantly elevated in kidney vs bladder urine (Figure 1, p0.0001 - 0.04). In 23 matched triplets of samples, these cytokines plus CCL2, CCL7, CCR2, CSF1, CXCL9, and CXCL10 were noted to be significantly greater in papillary tip biopsies versus urine samples (Figure 2, p0.001 $0.05)$. Regression analysis performed for each biomarker against maximum post-operative heart rate, respiratory rate, temperature, and white blood cell count did not show any significant correlations (p0.05). Biomarker levels did not vary by history of UTI in the past year, nor by whether the patient was on antibiotics at the time of surgery (p0.05).

Conclusions: Expression of CCL2, CCL5, CCL7, CXCL9, CXCL10, CCR2, CD40, CSF1, FAS-L, RIPK2, SELE, TLR-3, and IL-15 is markedly elevated in the papillary tips, kidney urine, and bladder urine of nephrolithiasis patients. Cytokine elevation was independent of signs of systemic inflammation. These findings further support the role of inflammation in nephrolithiasis, and imply that the inflammatory process likely begins at
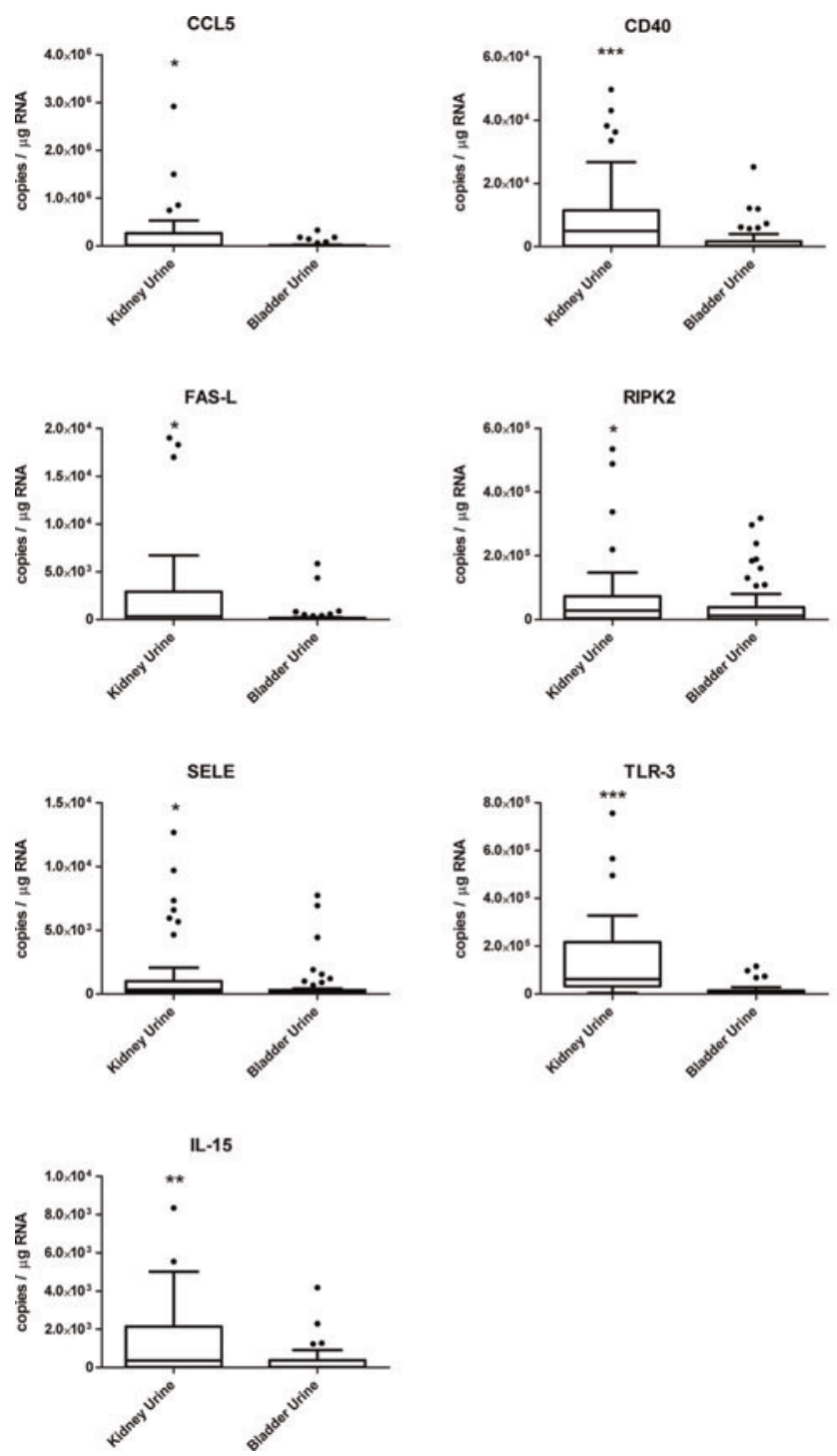

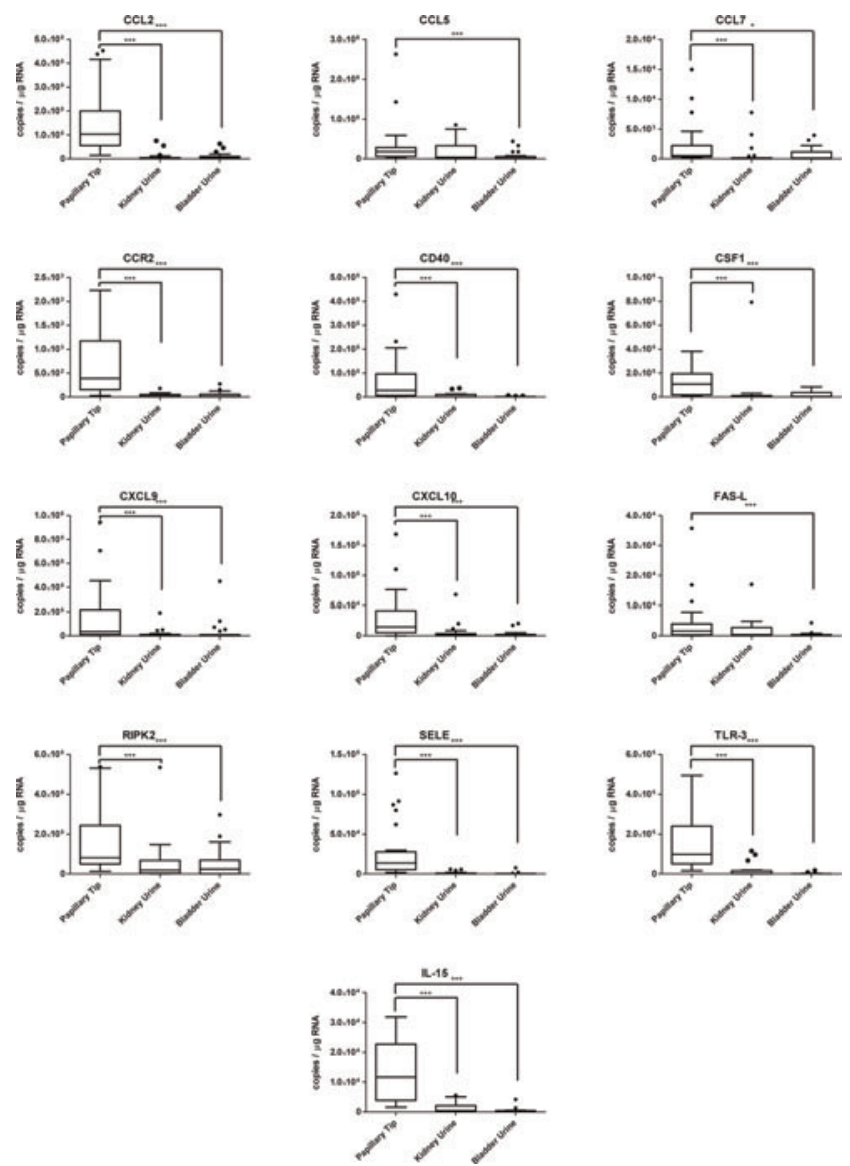

the renal papilae. These may represent novel biomarkers of stone disease which may be useful in basic nephrolithiasis research, disease diagnosis, and prognosis.

BRPRS2-13 Increased Urinary Oxalate Excretion in an Obese Mouse Model

\section{K Wood, A Dowell, J Knight, D Assimos, R Holmes}

University of Alabama at Birmingham

United States

Introduction \& Objective: Multiple epidemiological studies have demonstrated the correlation between increasing weight and urinary oxalate excretion. We hypothesize that this correlation is secondary to increased endogenous oxalate synthesis. This was studied in $o b / o b$ mice, a model of obesity.

Materials and Methods: Lean controls $(\mathrm{n}=3)$ and $o b / o b(\mathrm{n}=3)$ mice were fed a diet ultra-low in oxalate $(<10 \mu \mathrm{g} / \mathrm{g}$ diet $)$ and glycolate $(<3 \mu \mathrm{g} / \mathrm{g}$ diet $)$ and placed in metabolic cages. While on controlled diets, the mice were administered a subcutaneous bolus of $2 \mu$ moles ${ }^{13} \mathrm{C}_{2}$-glycolate and 24 -hr urine collected. After a wash period of two weeks, the mice were administered a subcutaneous injection of $2 \mu$ moles ${ }^{13} \mathrm{C}_{5}$-hydroxyproline and 24 -hr urine collected. Oxalate and glycolate isoptomers were measured in urine samples by ion chromatography coupled with mass spectroscopy. Glyoxylate was measured in liver tissue by HPLC. Results: Baseline 24 hour urinary oxalate and glycolate excretions were significantly higher in the $o b / o b$ mice compared to lean controls (303.3 vs $95.6 \mu \mathrm{g} / \mathrm{mg} \mathrm{Cr}(\mathrm{p}=0.01)$ and $289.5 \mathrm{vs} 105.0 \mu \mathrm{g} /$ $\mathrm{mg} \mathrm{Cr}(\mathrm{p}=0.0001)$, respectively). ob/ob mice produced more oxalate from glycolate following tail vein injection as measured by ${ }^{13} \mathrm{C} 2$-oxalate levels (19.8 vs $\left.1.7 \mu \mathrm{g} / \mathrm{mg} \mathrm{Cr} \mathrm{p}=0.003\right)$. ob/ob mice produce more oxalate from ${ }^{13} \mathrm{C}_{5}$-hydroxyproline following subcutaneous injection as measured by ${ }^{13} \mathrm{C} 2$-oxalate levels $(9.9$ vs $2.8 \mu \mathrm{g} / \mathrm{mg} \mathrm{Cr} \mathrm{p}=0.007)$. Liver glyoxalate was increased in the $o b /$ $o b$ compared to lean control ( $66.7 \mathrm{vs} 44.2 \mathrm{pg} / \mathrm{mg}$ protein, $\mathrm{p} 0.032$ ). Conclusions: In the $o b / o b$ obese mouse model, the increased urinary oxalate excretion is a result of increased endogenous oxalate synthesis. Blocking hydroxyproline and glycolate metabolism may reduce endogenous oxalate in this obese mouse model. Studies are needed to determine if obese humans have increased endogenous oxalate synthesis compared to lean individuals.

BRPRS2-14 The Association Between the Gene Polymorphisms in the Calcium-sensing Receptor and Calcium Nephrolithiasis in Jiangxi Gannan Area

Z Guoxi, Z Qingming, Z Xiaofeng, L Quanliang, $X$ Yijun, W Gengqing

Department of Urology, First Affiliated Hospital of Gannan Medical University

China (People's Republic)

Introduction \& Objective: To explore the association between the CaSR gene polymorphisms and calcium nephrolithiasis in Gannan area in Jiangxi province.

Materials and Methods: In all, 220 cases of patients with calcium nephrolithiasis (CN group) and 250 cases of normal people (control group) in Gannan area in Jiangxi province were recruited in this study. Rs9740, rs1501899, rs3749207, rs4678174, rs6776158, rs7652589, rs1042636, rs1801725, rs1801726, rs7632399 and rs9883981 single nucleotide polymorphisms (SNP) in CaSR gene were all detected by real-time fluorescent quantitative polymerase chain reaction TaqMan-MGB 5'-nuclease assay. The analysis of association between genotype or polymorphisms and calcium nephrolithiasis was performed with SNPassoc 1.9-2 and Haplo.stats 2.17.0 in R statistics program3.1.2.

Results: The frequency of the A allele of rs1501899 was higher than that in the Control group $(\mathrm{P}<0.001)$; but the frequency of the GG genotype of rs1501899 was higher than that in the Control group $(\mathrm{P}<0.001)$; CaSR gene haplotype analysis showed that haplotype AATCAGAGCAA, AGCTGAGGCGG was positively correlated with the formation of calcium nephrolithiasis $(\mathrm{OR}=1.57$, $95 \% \mathrm{CI}=1.46 \sim 1.69 ; \mathrm{OR}=1.55,95 \% \mathrm{CI}=1.46 \sim 1.64)$, but haplotype AATCGAAGCGG, AGCTAGGGCAA were negatively correlated with the formation of calcium nephrolithiasis $(\mathrm{OR}=1.16$, $95 \% \mathrm{CI}=1.05 \sim 1.29 ; \mathrm{OR}=0.87,95 \% \mathrm{CI}=0.79 \sim 0.96$ ).

Conclusions: CaSR gene polymorphisms were significantly associated with the formation of calcium nephrolithiasis in Gannan area in Jiangxi province.

BRPRS2-15 Use of the selective cholesterol absorption inhibitor ezetimibe and subsequent risk for urolithiasis

A Ostrowski, R Pak, I Porter

Mayo Clinic Florida

United States

Introduction \& Objective: Excessive urinary excretion of oxalate can manifest as recurrent urolithiasis, nephrocalcinosis and progression to ESRD. Ezetimibe is a selective cholesterol 
absorption inhibitor. A previous small report of two patients showed that the urinary oxalate levels decreased after the discontinuation of Ezetimibe. In the management of nephrolithiasis, patients are often encouraged to reduce their dietary intake of oxalate. We seek to find a relationship between the use of Ezetimibe and increased urinary oxalate levels and thus a reduction of oxalate with discontinuation of Ezetimibe.

Materials and Methods: Our patient population includes all patients following with the Department of Nephrology at Mayo Clinic Jacksonville for history of urolithiasis. Patients without the diagnosis of hyperlipidemia were excluded. Chart review of the remaining patients further narrowed the study population based on having documented use of Ezetimibe (including combination formulations) as well as having urinary oxalate studies both on and off of Ezetimibe.

Results: A total of 11 patients met the inclusion/exclusion criteria. Mean age $( \pm \mathrm{SD})$ was 67.4 years $( \pm 11.2)$. 2 of $11(18 \%)$ patients were documented with uric acid stones, and 7 of 11 (64\%) confirmed as calcium oxalate with an additional 2 of 11 (18\%) confirmed as simply non-uric acid. Mean urinary oxalate measured when Ezetimibe was being used at time of supersaturation test was $0.32 \mathrm{mmol} / \mathrm{spec}( \pm 0.13)$, while mean urinary oxalate measured when Ezetimibe was not being used at time of supersaturation test was $0.27 \mathrm{mmol} / \mathrm{spec}( \pm 0.10)(\mathrm{p}=0.078)$.

Conclusions: This study shows mean urinary oxalate values to be higher when Ezetimibe is being taken versus when not taken (approaches though does not reach statistical significance). Given that elevated urinary excretion of oxalate predisposes to formation of calcium oxalate stones, it stands to reason that a similar correlation could potentially exist between the selective cholesterol absorption inhibitor Ezetimibe and calcium oxalate stone formation. When working up a patient with calcium oxalate stones, Ezetimibe use should be discussed.

\section{BRPRS2-16 Evaluating the role of urine chemistry on the formation of infection stones}

T Hobbs, E Espinosa-Ortiz, D Lange, R Gerlach

Montana State University

United States

Introduction \& Objective: Urinary tract infections by ureolytic microbes can induce the formation of infection stones, consisting mainly of struvite and apatite. It is known that urine chemistry (presence and concentration of chemical species in urine) can play a major role in the precipitation of metabolic stones. However, the role of urine chemistry on the formation of infection stones is not well understood. Urine chemistry can vary widely due to kidney function, genetic and dietary factors. This study aimed to investigate the effect of different ion concentrations and $\mathrm{pH}$ values of urine on microbial growth, ureolysis kinetics and mineral precipitation.

Materials and Methods: 96 well plates and spectrophotometric analyses were used to measure microbial growth and mineral precipitation in artificial urine inoculated with Proteus mirabilis. Two sets of experiments were performed evaluating the effect of artificial urine composition: (1) calcium, magnesium, and phosphate concentrations were varied, (2) the $\mathrm{pH}$ was varied at given calcium, magnesium and phosphate concentrations. The 96 well plate experiments were scaled up to enable in depth analysis of urine chemistry (urea, calcium, magnesium, and phosphate concentrations) and produced minerals.
Results: Results suggest that initial calcium $\left(50-200 \mathrm{mg} \mathrm{L}^{-1}\right.$ ) and, to a lesser degree, magnesium (24-97 $\mathrm{mg} \mathrm{L}^{-1}$ ) concentrations influence the type and quantity of mineral precipitating, while initial phosphate $\left(664-1329 \mathrm{mg} \mathrm{L}^{-1}\right)$ concentration do not appear to have a significant effect. Slightly acidic $\mathrm{pH}$ values $(\mathrm{pH}$ 4-5) appear to slow down microbial growth and mineral precipitation initially, however after 24 hours there does not appear to be a significant difference relative to urine with initially neutral $\mathrm{pH}$. Slightly elevated initial $\mathrm{pH}$ values (8-9.5) in the artificial urine did not appear to affect microbial growth but appeared to decrease the total amount of mineral precipitated relative to treatments with circumneutral initial $\mathrm{pH}$ values.

Conclusions: Initial urine chemistry appears to have an effect on the rate and total amount of mineral precipitation induced by ureolytic microbes. The results of our work may allow for more individualized treatment and prevention of infection stones by matching treatment options to the specific urine chemistry of the patient.

\section{BRPRS2-17 Protein modelling- A novel approach to risk} stratify disease in patients with cystinuria

M Sahu, Z Kassam, K Wong, M Bultitude, M Wass, $\mathrm{K}$ Thomas

Guy's and St Thomas' NHS Foundation Trust United Kingdom

Introduction \& Objective: Cystinuria is a rare inherited disorder with patients suffering from recurrent stone disease. Clinical presentation is extremely variable and an understanding of why this occurs could enable a tailored approach to treatment. Mutations have been described in two genes, SLC3A1 and SLC7A9.

Materials and Methods: Structural analysis using several different computational models were used to suggest how these mutations may lead to cystinuria. The ability of automated predictors to assess the effect of known cystinuria associated mutations was matched with the phenotypic data available for a UK cohort of 74 patients. The available phenotypic data included urinary dibasic amino acid levels, age of disease presentation, number of stone episodes and the number of interventions over a three year period. Prediction scores were classified into two groups, mild or severe; giving them a score of 1 or 2 respectively. Depending on the type of mutation, an overall score was allocated to each patient $(1-4,1=$ mild, $4=$ severe $)$. Statistical analysis on each data set was performed. To investigate where in the protein structure the cystinuria variants occur and to analyse the effect they have on protein structure and function, protein structure modelling of the protein was performed.

Results: For SLC7A9, many mutations appear to swap hydrophobic amino acids for charged amino acids or vice versa, while others affect known functional sites. For SLC3A1, mutations often result in the loss of hydrogen bonds and largely appear to affect protein stability. All the automated methods used were unable to identify differences in the age of diagnosis, number of stone episodes and number of interventions but were better at finding differences in the urinary amino acid levels between the severity groups.

Conclusions: Categorising the severity of mutations and correlating this against phenotype is possible. However, existing methods have limitations and improvements are required before they can be used to classify cystinuria disease severity in order to provide individualized patient treatment. 
BRPRS2-18 The effects of shockwave lithotripsy on the urinary microbiome and on bacterial dispersion using a phantom kidney stone model

AH Alathel, R Chanyi, J Denstedt, S Pautler, J Burton, H Razvi, Y Bao, D Mikhail

Schulich School of Medicine \& Dentistry, Western University Canada

Introduction \& Objective: Introduction Historically, the bladder has been considered a sterile environment. Next-generation sequencing techniques have shown that the bladder has its "core" microbiome. Its role in health and disease is emerging, of interest is determining the role of bacteria in nephrolithiasis. Early studies have shown the presence of bacteria within kidney stones. Shockwave lithotripsy (SWL) may disrupt these communities and promote bacterial dissemination and may partially explain why these patients are at a greater risk of infection. Objectives Our aim was to determine if the urinary microbiome changes after SWL. Then to determine the extent of potential dissemination of bacteria using a gelatin model that contained kidney stone phantoms in the presence of bacteria.

Materials and Methods: For studies of the urinary microbiome, we recruited 10 patients planned for SWL (2F, 8M) and 6 controls $(3 \mathrm{M}, 3 \mathrm{~F})$. Urine samples were collected 21 days prior to SWL, before and after and then at 3 months post SWL. 2 samples were collected for the controls 21 days apart. Urine was processed by centrifugation and bacterial pellets were stored. DNA was extracted from the bacterial pellets, 16S rRNA gene PCR amplified and then Illumina sequenced. Analysis was done using QIIME and R statistical packages. The kidney model was prepared using $10 \%$ ordinance gelatin. Cylindrical wells were created for stone placement. Cylindrical phantom stones were prepared using Begostone plus plaster. Stones were soaked in phosphate buffered saline (PBS) overnight before Escherichia coli (E. Coli) containing green fluorescent protein (GFP) was added. They were subjected to 3000 Shocks.

Results: Patient urine samples collected after SWL had a different bacterial profile then prior to SWL. Post SWL, the samples had increased diversity suggesting that the stones themselves may harbor their own microbiome. Preliminary experiments have been performed on the in vitro SWL model. Efficient fractionation of the stone phantoms was achieved. Ongoing experimentation will focus on the visualization of bacteria into the surrounding area, away from the region being targeted by SWL. Conclusions: Using culture dependent analyses, we modified a SWL model developed in this department to determine how SWL alters bacterial survival and assess whether SWL will promote bacterial infiltration into surrounding tissue.

BRPRS2-19 Impact of repeated extracorporeal shock wave lithotripsy on prepubertal rat kidney

J Chung, S Lee, H Seo, S Chung

Pusan National University Yangsan Hospital Republic of Korea

Introduction \& Objective: The study was aimed to investigate the effects of repeated extracorporeal shock wave lithotripsy (ESWL) on the kidney of child and adult rats.

Materials and Methods: Thirty rats were used; 15 were child rats ( 3 weeks of age) with an average body weight of $72.3 \pm 3.3 \mathrm{~g}$ (range,
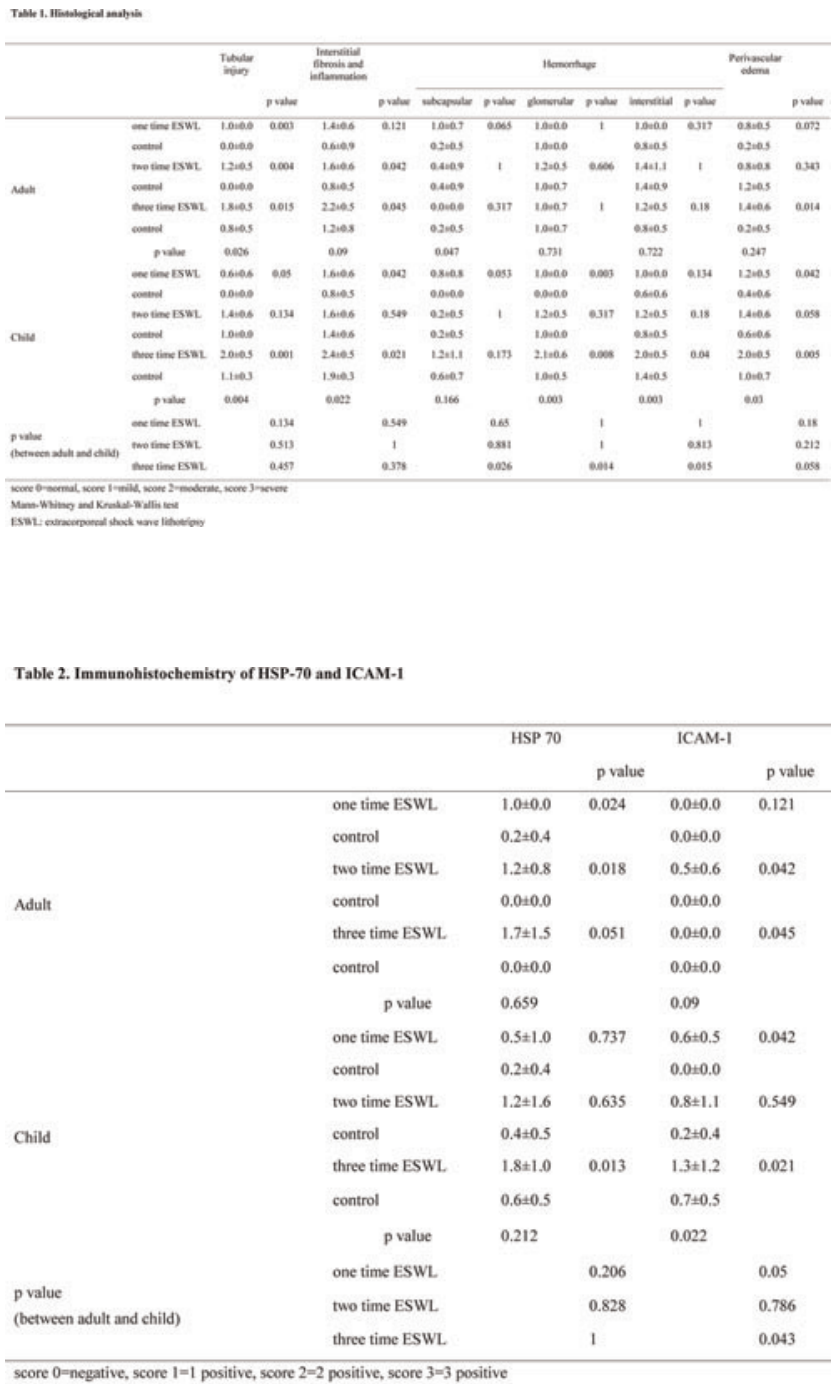

Mann-Whitney and Kruskal-Wallis test

ESWL: extracorporeal shock wave lithotripsy, HSP: heat shock proteins, ICAM: intercellular adhesion molecule

$65-75 \mathrm{~g}$ ) and 15 were adults with an average body weight of $265 \pm 11.3 \mathrm{~g}$ (range, 250-280g). The child and adult rats were separately and randomly allocated to three groups, each consisting of five rats. Following anesthetization, the left kidney of each rat in each group received $300015 \mathrm{kV}$ shock waves in one (group 1), two (group 2), or three (group 3) sessions. Sessions were interspersed by 72 hours. The rats in each group were killed 72 hours after the last ESWL session and both kidneys were harvested. The right kidney was used as the control. Renal injury was examined with histological analysis, immunohistochemistry and Western blot to detecting the expression of heat shock protein (HSP)70, tumor necrosis factor (TNF) $\alpha$, intercellular adhesion molecule (ICAM)1, and monocyte chemoattractant protein (MCP)1 as markers of renal damage.

Results: HSP70, ICAM1, MCP1, and TNF $\alpha$ were similarly increased with increased ESWL sessions in both age groups. Histological analysis revealed more serious fibrosis and inflammation in the ESWLtreated kidneys in both groups compared to both age controls, with the damage increasing with increasing numbers of sessions.

Conclusions: Kidney ESWL increased renal damage according to the number of sessions in both age groups of rats. The effect of ESWL on renal injury was similar in both groups. 
BRPRS2-20 Sensitive Colorimetric Detection of Nitrite Ions Based on Aggregation of Gold Nanoparticles for Early Detection of Nitrate Reducing Bacteria in the Urinary Tract

A Kulkarni, K Ziegler, VY Bird

United States

Introduction \& Objective: Bacteria that can reduce nitrates to nitrites or nitrite reducing bacteria can be detected in urine largely by using the Griess Reaction test. The concentration of nitrite to be detected by this method is $0.1 \mathrm{ug} / \mathrm{dL}$. This is an indirect measurement of nitrite reducing bacteria provided that enough dietary nitrates were available with at least a four hour indwelling time in bladder. At a $\mathrm{pH}$ of 5-5.5, nitrites are further reduced to nitric oxide, an indirect antimicrobial agent. Our test aims to detect nitrite at a very low concentration with the purpose of very early identification and possible prevention of a fully symptomatic urinary tract infection.

Materials and Methods: Nanoparticles can be functionalized using various ligands to increase their reactivity with the species to be detected. Functionalized nanoparticles of materials like gold enable colorimetric detection upon aggregation or antiaggregation based on localized surface plasmon resonance property. The aggregation of nanoparticles is affected by their bond strength with the cross-linker chemical that induces aggregation. Results: Aggregation of nanoparticles was achieved by altering the distance dependent surface plasmon resonance property of gold nanoparticles resulting in shift in the absorption spectrum that lies in the visible spectrum triggering a visible immediate colorimetric response. This was obtained by cross-linking agents that have functional groups possessing strong affinity for gold surface leading to aggregation of nanoparticles. Reaction of gold-disulfide bond with nitrite ions breaks the bond which results in loss of aggregated state (Figure 1). Thus when nitrites reacted with gold nanoparticles a clear to pink colorimetric responesed was observed. Senstivity of nitrites was obtained at the $400 \mathrm{nM}$ range.

Conclusions: Anti-aggregation of gold nanoparticles has been utilized to develop a colorimetric probe for detection of nitrite ions in urine. The assay has been tested over a wide range of nitrite concentrations and it is an order of magnitude sensitive when compared to current urine nitrite detectors. An ultrasensitive nitrite testing could aid in detecting UTI earlier allowing for preventive measures from developing fully symptomatic UTI by dietary modification for urine acidification.

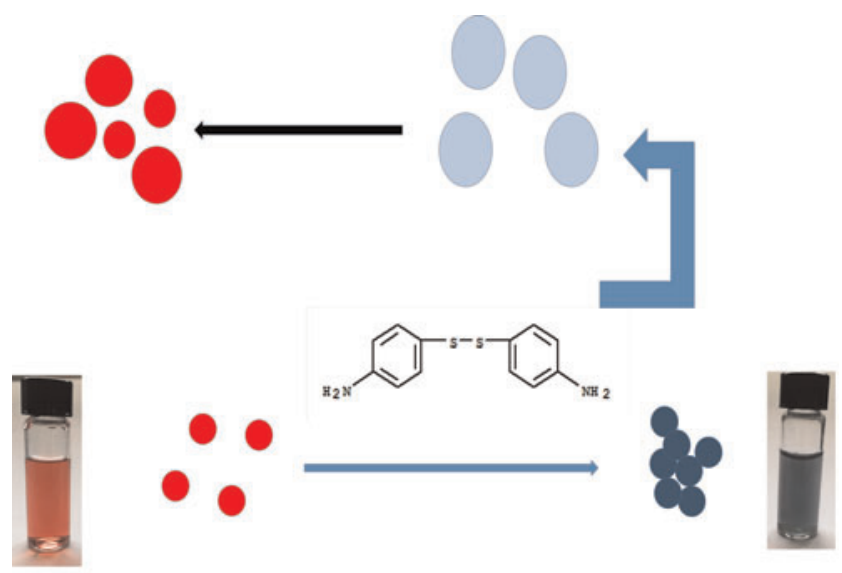

Reaction of gold-disulfide bond with nitrite ions breaks the bond which results in loss of aggregated state
BRPRS2-21 Colorimetric, pH-Responsive Membranes Allow for Immediate, Real-Time and Reversible Urine Monitoring in a Multipart System for Detection Elevated Urine pH secondary to Urease-Producing Bacteria

M Fuchs, H Huda, CB French, N Patel, VY Bird

UF Biomedical Engineering

United States

Introduction \& Objective: Within 4 weeks, 90\% of longterm catheterized patients will develop bacteriuria, and $50 \%$ will experience encrustation and marked elevated urine $\mathrm{pH}$. Ureaseproducing bacteria have been linked to elevated urine $\mathrm{pH}$, catheterassociated urinary tract infection (CAUTI), encrustation, and struvite renal calculi. We propose a method to monitor alkalinization of urine secondary to urease producing bacteria as part of a multimodal system which could play a role in prevention of catheter encrustation. Reversibility of the colorimetric membrane acts as a form of biofeedback to allow for modification of oral intake with acidifying agents and control urine $\mathrm{pH}$ to prevent encrustation.

Materials and Methods: To create a $\mathrm{pH}$-sensitive, reversible colorimetric porous membrane with immediate detection of alkaline urine, high molecular weight polymer was solvated in organic solvent. A quaternary ammonium salt and indicator dye were then added for $\mathrm{pH}$ sensitivity and electrostatic retention of the dye. Addition of plasticizer followed to modify the membrane mechanical properties. This solvated mixture was stored under $4^{\circ} \mathrm{C}$ refrigeration until casting.

Results: The solvated membrane blend was drop cast onto the inside of catheter tubing for demonstration. Basic solution ( $\mathrm{pH} 7.5$ / $\mathrm{NaOH}$ ) was passed through and a distinct and visible change of color to green (see Figure 1) was observed upon exposure. Dilute acidic solution was then passed through $\left(\mathrm{pH} 4 / \mathrm{H}_{2} \mathrm{CO}_{3}\right)$ and there was a rapid reversal ( $<5$ seconds) to original yellow color. Urine $\mathrm{pH}$ ranges from 4 to 8.5 can be monitored by this system.

Conclusions: The clear colorimetric indication afforded by this membrane provides a robust system for the reversible detection of real-time changes in urine $\mathrm{pH}$. As a component of a multipart system, this provides rapid biofeedback that allows for informed and directed intervention. This system will also assist in the detection of elevated urine $\mathrm{pH}$ secondary to urease-producing bacteria responsible for encrustation and recurrent struvite kidney stone formation.
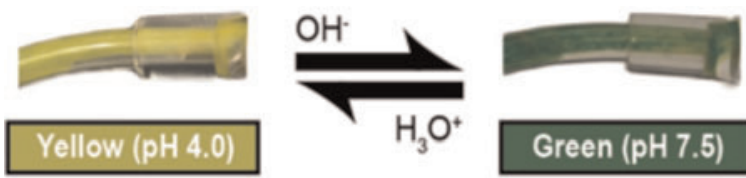

Figure 1. Rapid ( $<5 \mathrm{sec})$, easily-visible color change is observed within the physiolgic $\mathrm{pH}$ range of interest.

BRPRS2-22 Experimental study for a new medical adhesion-prevention agent after laparoscopic nephrectomy

Y Naitoh, J Ajiki, A Fujihara, M Kanazawa, S Ushijima, F Hongo, K Okihara, H Suong-Hyu, O Ukimura

Department of Urology, Kyoto Prefectural University of Medicine

Japan 


\section{The classification score system}

Grade 0 , no adhesions

Grade 1, loose filmy adhesions that could be separated by traction

Grade 2, adhesions requiring blunt dissection for separation

Grade 3, adhesions requiring sharp dissection for separation

Grade 4, serosal injury

Grade 5, tissue injury.
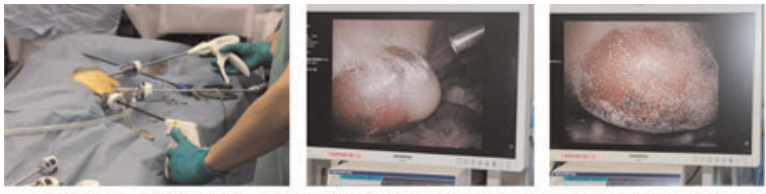

Experimental rabbits were placed under Ketalar anesthesia. Surgery was started with intra. abdominal pressure setting at $8 \mathrm{mmHg}$. Our new agent include aldehyde dextran and $\varepsilon$-poly (1lysine) in powder form

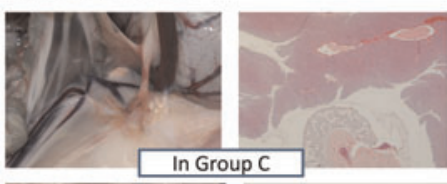

Adhesions requiring shear for detachment were observed between the nephrectomized region and the intestine/mesentery Infiltration of inflammatory cells was histologically noted at the adhesion site.

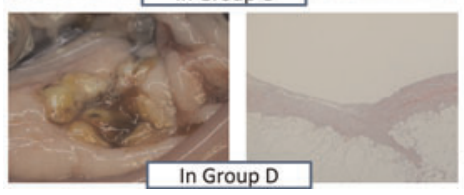

Neither adhesion nor infiltration of inflammatory cells was histologically observed.

Introduction \& Objective: Existing biodegradable and bioabsorbable adhesion-prevention agents used in clinical practice are sheet-like products, which require fixation onto tissues, and they are difficult to handle during laparoscopic surgery. Our new medical adhesion-prevention agent was powder form. It exerts its effect as an adhesive during fixation process, and after the fixation, its surface exerts its effect as an adhesion-prevention agent. Materials and Methods: Laparoscopic nephrectomy was performed in rabbits for evaluation of adhesion-prevention effect of the operation region with the intestine and the mesentery. In laparoscopic surgery, the frontal surface of the kidney was sprayed with saline in group A (control; $n=4)$; the adhesionprevention agent was sprayed without extirpating the kidney in group B $(n=4)$; after nephrectomy, saline was sprayed in group $C(n=4)$; and the adhesion-prevention agent was sprayed in group $D(n=4)$. A uniquely developed spraying device was used for spraying the adhesion-prevention agent and saline. We classified adhesion severity into six grades based on a modified version of the classification system.

Results: In the group A and B, no adhesion was observed in the abdominal cavity as well as in the frontal surface of the kidney with the intestine/mesentery both macroscopically and histologically. In the group $\mathrm{C}$, adhesions requiring shear for detachment were observed between the nephrectomized region and the intestine/mesentery. Infiltration of inflammatory cells was histologically noted at the adhesion site. In the group D, no adhesion was observed in the abdominal cavity as well as between the nephrectomized region and the intestine/mesentery. The score of group $\mathrm{C}$ was significantly higher than group $\mathrm{D}(P=0.03)$.

Conclusions: No adhesion was observed between the nephrectomized region and the intestine/mesentery when the nephrectomized region was covered with new adhesion-prevention agent. Based on these results, the adhesion-prevention effect of our new agent in laproscopic nephrectomy was confirmed.

BRPRS2-23 Comparative evaluation of tissue damage induced by ultrasound and impact dual mode endoscopic lithotripsy versus conventional single mode ultrasound lithotripsy

MJ Bader, W Khoder, M Seitz, F Strittmatter, A Alghamdi, C Stief

UroClinic München and Department of Urology, University of Munich

Germany

Introduction \& Objective: The purpose of this study was to investigate the tissue safety of a new ultrasound and impact dual mode lithotripsy transmitted through a single probe in an in vivo animal model. The EMS LithoClast Trilogy ${ }^{a}$ combined ultrasonic / impact lithotripter was compared to the established ultrasound lithotripter Storz Calcuson ${ }^{\hat{a}}$. The safety test simulated the accidental direct contact between lithotripter probes and the urothelium which can occur when sliding off a stone during lithotripsy or drilling through a stone.

Materials and Methods: Testing was performed in pigs on bladder tissues. 6 female pigs (German Landrace) were allocated into 2 groups. Cystoscopic access to the pig bladder was established through a conventional cystoscope with straight working channel. The bladder tissue was exposed to direct lithotripter probe contact at maximum power during 10 seconds to produce visible tissue lesions. Small and large diameter probes (1.5 / 3.4 re. $3.5 \mathrm{~mm}$ ) were tested. Acute tissue trauma was evaluated using a scoring model. After 7 days, all animals were sacrificed, necropsied and examined post mortem. Histological examinations of the urinary bladder were performed with each animal. The study was performed under a GLP compliant protocol and approved by the federal ethical committee of Bavaria, Germany. Results: Irrespective of the lithotripter used, no systemic signs of toxicity were observed up to 7 days after the intervention. All lesions were set successfully and the acute and 7 days' post-op scoring could be performed in all animals. The evaluation did not reveal any relevant differences between the lesions set with either the small or large probes. Lack of intense leucocyte and other inflammatory cells infiltration confirmed the absence of aggressive systemic reactions and was confirmed as well by the absence of effects on hematological parameters. The documented minimal to mild vascular congestion at surgery as well as 7 days post-operatively is to be considered a normal finding in response to trauma. Histologically, signs of normal ongoing healing were observed on the urinary bladder mucosa.

Conclusions: The single probe combined ultrasonic and impact lithotripsy application with the LithoClast Trilogy ${ }^{\hat{a}}$ device showed comparable tissue safety as conventional ultrasonic lithotripsy alone. 


\section{BRPS3: BENCH TO BEDSIDE: ONCOLOGY}

BRPRS3-1 LncLBCS inhibits the self-renewal of bladder cancer stem cells through hnRNPK-mediated Sox2 repression

$\mathrm{X}$ Chen

Sun Yat-sen Memorial Hospital

China (People's Republic)

Introduction \& Objective: Bladder cancer of poor differentiation is easier to progress, recur, metastasis and resist to chemotherapy and radiotherapy. Recent study indicated that this kind of bladder cancer had more cancer stem cells (CSCs), but the mechanism of bladder cancer stem cells is not well understood. This study aims to elucidate the function and mechanism of IncRNA in bladder CSCs, in order to discover new targets for bladder cancer stem cells targeting therapy.

Materials and Methods: The bladder CSCs related lncRNAs were identified by microarray and verified by RT-qPCR in several model. We analyzed lnc-LBCS expression and its correlation with clinicopathological characteristics in Sun Yatsen Memorial Hospital and TCGA dataset. The in vitro and vivo assays were performed to study the role of lnc-LBCS in the bladder cancer cells. The RNA pulldown and RNA immunoprecipitation (RIP) assay were used to investigate the protein binding with lnc-LBCS. The target genes of lnc-LBCS were identified by RT-qPCR and Western Blot. The region of lnc-LBCS binding to the target gene was identified by Chromatin Isolation by RNA Purification (ChIRP).

Results: Through microarray analysis of lncRNA, we identified a lncRNA which was down-regulated in bladder CSCs, but it increased in the process of differentiation. So we named it lncRNA- Low expressed in Bladder Cancer Stem cells (lnc-LBCS). In clinical analysis, lnc-LBCS was downregulated in bladder cancer tissue. It was negative related to tumor grade and recurrence, but was positively related to survival. Moreover, lnc-LBCS was an independent prognostic factor for overall survival of bladder cancer. Through in vitro and vivo assay, we found that lnc-LBCS dramatically inhibited bladder CSCs self-renewal and tumor initiation. We discover that lnc-LBCS locates in the nuclear and interacts with hnRNPK by RNA binding protein immunoprecipitation. Mechanistically, lnc-LBCS recruits the hnRNPK to the SOX2 promoter, a key stemness related gene, to repress the expression of SOX2, which is highly expressed in bladder CSCs and required for their self-renewal. The expression level of SOX2 correlates with clinical severity and prognosis of bladder cancer patients.

Conclusions: These data demonstrate for the first time that lncLBCS was down-regulated in bladder CSCs and cancer tissues, and correlated grade, recurrence and overall survival bladder cancer. Lnc-LBCS inhibited self-renewal and tumor initiation of bladder cancer cells by suppressing the Sox 2 expression through recruiting hnRNPK. Therefore, lnc-LBCS and SOX2 may serve as biomarkers for diagnosis and potential drug targets for bladder cancer.

BRPRS3-2 Withdrawn
BRPRS3-3 Gene silencing of SIRT7 by small interfering RNA influences the biological behaviors of renal cancer 786-O cells

Z Xiaofeng, Y Zengxiang, X Yijun, L Quanliang, Z Guoxi, W Gengqing

Department of Urology, First Affiliated Hospital of Gannan Medical University

China (People's Republic)

Introduction \& Objective: To explore the effect of silencing SIRT7 expression on the proliferation, apoptosis, migration and invasion of human 786-O renal cell carcinoma (RCC) cell lines by small interfering RNA in vitro.

Materials and Methods: The blank control (Bank Control), negative control (Negative Control) and siRNA interference (SIRT7siRNA) groups were designed in this study. The small interfering RNA (siRNA) was synthesized and transfected into 786-O cell lines with Lipofectamin 2000 for silencing the expression of SIRT7. The expression levels of SIRT7 were detected by RT-PCR, and the expression level of SIRT7 protein was detected by Western blotting. The cell proliferation was detected by MTT method. The cell apoptosis was detected by flow cytometry. The invasion and migration capabilities were evaluated by using transwell chamber migration assay and scratch wound healing assay, respectively.

Results: The expression levels of SIRT7 mRNA and protein in $786-0$ cell lines were effectively downregulated by siRNA. The proliferative rate was decreased $(\mathrm{P}<0.001)$ and the apoptosis rate was increased $(\mathrm{P}<0.001)$. The invasion and migration capabilities were both decreased significantly $(\mathrm{P}<0.001)$.

Conclusions: SIRT7-siRNA can efficiently inhibit the expressions of SIRT7 protein and mRNA, which results in decreased capacities of proliferation, migration and invasion and increased capacities of apoptosis of 786-0 cell lines. SIRT7 maybe become a new potential target in the treatment of renal cancer.

BRPRS3-4 Association of DNA Polymorphisms within CYP11B2 and CYP11B1 Locus with the Risk of Aldosterone Producing Adenoma

Z Guoxi, H Ruohui, Z Xiaofeng, L Quanliang,

$\mathrm{X}$ Yijun, W Gengqing

Department of Urology, First Affiliated Hospital of Gannan

Medical University

China (People's Republic)

Introduction \& Objective: To investigate the association of DNA polymorphisms within CYP11B2 and CYP11B1 locus with the risk of Aldosterone Producing Adenoma (APA).

Materials and Methods: The 80 patients with APA (APA group) and 101 normal people (Control group) of CYP11B2 and CYP11B1 gene polymorphisms were detected by Real-time PCR TaqMan-MGB 5'-nuclease assay. The correlation between the CYP11B2 and CYP11B1 of haplotype and genotype were analyzed by the SNPassoc 1.9-2 and Haplo.stats 2.17.0 software.

Results: The frequency of the A allele of rs5301, the A allele of rs3802228, the $\mathrm{G}$ allele of rs6433 in APA were higher than that in the Control group $(\mathrm{P}=0.015, \mathrm{P}=0.014, \mathrm{P}=0.011)$; but the 
frequency of the AG genotype of rs 4545 was less than that in the Control group $(\mathrm{P}=0.010)$; APA negatively correlated with haplotypes of GAACAAC, GCGAAGT $(\mathrm{OR}=0.73,95 \% \mathrm{CI}=0.57$ 0.93; $\mathrm{OR}=0.81,95 \% \mathrm{CI}=0.69-0.95)$.

Conclusions: CYP11B2 and CYP11B1 gene polymorphisms were associated with the the risk of Aldosterone Producing Adenoma.

BRPRS3-5 The effects of shRNA inhibiting TRPV2 gene expression on proliferation and invasion of human bladder cancer $\mathbf{E J}$ cell line

L Quanliang, Z Guoxi, Z Xiaofeng, W Gengqing, W Xiaoning, X Gang

Department of Urology, First Affiliated Hospital of Gannan Medical University

China (People's Republic)

Introduction \& Objective: To investigate the effects of sh-RNA inhibiting of the transient receptor potential vanilloid 2 (TRPV2) channel protein on the invasion and proliferation in human urothelial carcinoma (UC) EJ cell line.

Materials and Methods: EJ cells were divided into three groups: the blank control group, the negative control group, the shRNA-TRPV2 group. The vectors were transfected into EJ cells by lipofectamin 2000. The expression of TRPV2 mRNA and protein was detected by reverse transcription-polymerase chain reaction (RT-PCR) and Western blot, respectively. Cell proliferation was tested by MTT assay. Cell apoptosis was measured by flow cytometry with Annexin V-FITC/PI staining.

Results: In the sh-RNA TRPV2 group, the TRPV2 protein levels were down regulated obviously. The vectors were transfected into EJ cells successfully. Relative to controls, 24, 48 and $72 \mathrm{~h}$ after transfection of shRNA-TRPV2, he inhibitory rates of TRPV2 mRNA levels in EJ cells were 27.49\%, 53.65\%, 74.91\%, respectively. And those of TRPV2 proteins were $26.47 \%$, $54.89 \%$, and $73.74 \%$, respectively. The cell growth inhibition rate of at 24,48 and $72 \mathrm{~h}$ after transfection of shRNA-TRPV2, was $(4.34 \pm 0.76) \%,(9.87 \pm 1.54) \%$ and $(13.78 \pm 1.93) \%$, respectively. There was statistically significant difference between control blank groups at each time point $(\mathrm{P}<0.01)$. After $\mathrm{T} 24$ cells were transfected for 24,48 and $72 \mathrm{~h}$, the apoptosis rate of shRNA-TRPV2 was $(4.87 \pm 0.89) \%,(9.39 \pm 1.43) \%$ and (13. 46 \pm 1.68$) \%$, respectively $(\mathrm{P}<0.01)$ vs blank control. Compared to the blank control group and the negative control group, the proliferation abilities of the sh-RNA TRPV2 group were inhibited, also the motility and invasion were inhibited in vitro.

Conclusions: ShRNA-TRPV2 can inhibit the proliferation of bladder cancer EJ cells and promote the apoptosis in vitro. TRPV2 may serve as an important target for the treatment of bladder cancer.

BRPRS3-6 The implication of survivin expression in dendritic cells from the mouse marrow

L Yanmin, Z Guoxi, Z Xiaofeng, L Quanliang, $\mathrm{X}$ Tianpeng, W Gengqing

Department of Urology, First Affiliated Hospital of Gannan Medical University

China (People's Republic)

Introduction \& Objective: To discluss the significance of survivin expression on the mature process of dendritic cells from mouse marrow.
Materials and Methods: The cells in marrow as the precurosor of the dendritic cells (DC) were taken from mouse in bioclean condition. Then the immature dendritic cells (iDC) were induced by the precurosor of DC with GM-CSF and IL-4 in medium. The the mature dendritic cells ( $\mathrm{mDC}$ ) were induced by the precurosor of DCs with GM-CSF, IL-4, and TNF- $\alpha$ in medium. Survivin was compared by RT-PCR and Western-bolt among these three stages. Results: The RT-PCR showed that the expression ratios of survivin mRNA among the precurosor of $\mathrm{DC}, \mathrm{iDC}$, and $\mathrm{mDC}$ were $(0.735 \pm 0.081),(0.596 \pm 0.073)$ and $(0.393 \pm 0.052)$; Moreover, The expression of survivin mRNA in precurosor of DC was compared with the survivin mRNA in iDC $(\mathrm{P}<0.05)$; The expression of survivin mRNA in iDC was compared with the survivin mRNA in mDC $(\mathrm{P}<0.01)$. The Western-blot showed that the expression ratios of survivin proteinum among the abovementioned DC's three stages were $(0.767 \pm 0.045),(0.396 \pm 0.016)$ and $(0.168 \pm 0.009)$, respectively. Moreover, The expression of survivin proteinum in precurosor of DC was compared with the survivin proteinum in iDC $(\mathrm{P}<0.05)$; The expression of survivin proteinum in $\mathrm{iDC}$ was compared with the survivin proteinum in $\mathrm{mDC}(\mathrm{P}<0.01)$.

Conclusions: There was a correlation between survivin and differentiation of DC.

\section{BRPRS3-7 Withdrawn}

\section{BRPRS3-8 Withdrawn}

BRPRS3-9 Management of Malignant Ureteric Obstruction: Are We Doing the Right Thing?

L Kerr, J Hendry, M Dupre, S Teahan

NHS Forth Valley

United Kingdom

Introduction \& Objective: Malignant ureteric obstruction (MUO) is common in advanced malignancy with a reported incidence of $4.4 \%$. Mean survival after diagnosis is $120-140$ days and up to $20 \%$ of patients will not return home following diagnosis. Many of these patients will present to or be referred to urology units but there are no clear guidelines for management of this challenging group. The indications for intervention and likely prognosis may determine appropriateness of intervention,

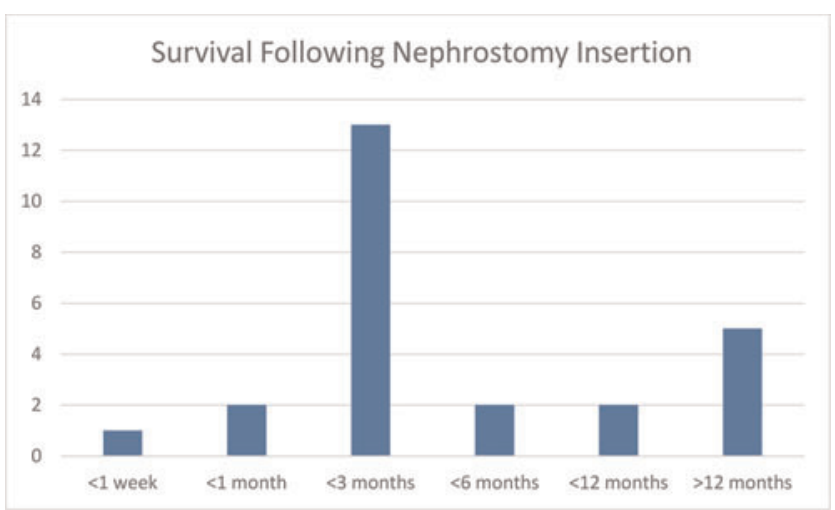


which typically is by means of nephrostomy tube (NT) or ureteric stent placement. We report a district general hospitals experience of managing MUO by nephrostomy drainage.

Materials and Methods: Radiology database was used to identify patients undergoing nephrostomy placement for MUO between 1/1/2015-14/10/2016. Patient records were reviewed to determine primary malignancy and survival following procedure Results: There were 237 NT episodes in 68 patients recorded in this period (60 insertions, 143 exchanges, 34 removals). The commonest indication for intervention was MUO in 43/68 patients (63\%). 25 of these patients $(58 \%)$ have died since initial intervention. Figure 1 illustrates survival after initial nephrostomy placed.

Conclusions: Malignant ureteric obstruction generates a significant workload in a district general. Patients presenting with MUO will have multiple comorbidities, and insertion of nephrostomy or ureteric stent has well documented morbidity. There is little data in the literature regarding quality of life after nephrostomies are inserted and no guidelines for management of MUO. Quality of life may deteriorate after nephrostomy insertion and should be a consideration during end of life care especially if the patient has poor prognosis. Further research is required to develop a guideline to help risk stratify and manage patients with MUO holistically.

BRPRS3-10 Incidence of anaemia and blood transfusion in patients undergoing nephrectomy for urological malignancy

L Kerr, M Stephens, A Baker, G Oades

NHS Forth Valley

United Kingdom

Introduction \& Objective: There is a consistent body of evidence linking perioperative blood transfusion with post operative complications and death. Transfusion can result in longer hospital stays and increased costs. We aimed to determine incidence of anaemia in patients undergoing nephrectomy and any relation to transfusion rates.

Materials and Methods: A retrospective audit of 468 sequential nephrectomies by a single surgeon was performed. The blood transfusion service provided a record of all blood transfusions in this cohort. Nephrectomy for benign disease was excluded.

Results: Of 420 patients, 219 male and 201 female, 98 patients were anaemic preoperatively (69 male, 29 female). There were 171 open and 249 laparoscopic procedures. Overall incidence of blood transfusion was $8.6 \%$. If anaemic preoperatively this figure was $33.7 \%$ vs $0.9 \%$ if not anaemic. Transfusion was more common with open procedures compared with laparoscopic (15.2\% vs $4 \%$ ) Mortality in these patients (follow up 6months - 5 years) was $13.8 \% .60 .3 \%$ of deceased patients were anaemic preoperatively. Conclusions: This data illustrates the association between preoperative anaemia and blood transfusion in patients undergoing nephrectomy. It suggests a link with overall mortality. Further audit will be done to relate this to other patient factors and stage of disease.

BRPRS3-11 Does the Microphtalmia-Associated Transcription Factor (MITF) E318K mutation matter in the tumorigenesis of renal cell carcinoma?

Y Bodokh, L Mendel, I Bentellis, L Vignot, P Treacy, P Regnier, F Roustan, R Haider, A Mbeutcha, R Prader, B Tibi, J Fallot, J Amiel, D Chevallier, F Pedeutour, M Durand

Departement of Urology, Andrology and Renal transplantation, Hospital Pasteur II, CHU Nice

France
Introduction \& Objective: Microphtalmia-Associated Transcription Factor (MITF) located on chromosome 3 in position 3p12.3-3p14.1 is a member of the MiT family. The latter was a transcription factor, involved in the stream of signals of hypoxiainducible factor $(\mathrm{HIF} 1-\alpha)$, which induce renal cell carcinoma (RCC). The mutation on the exon 9 codon 318 leads to deficient SUMOylation of MITF and to the over activation of way HIF1- $\alpha$. The MITF mutation p.E318K was described to major the risk of RCC by factor 5 . However, two recent studies refuted this theory. We dived into a large cohort of RCC in order to find MITF E318K mutation.

Materials and Methods: Genomic tumor DNA was extracted from formalin-fixed paraffin embedded RCC. The exon 9 codon 318 MITF mutation was researched first by Sanger sequencing and

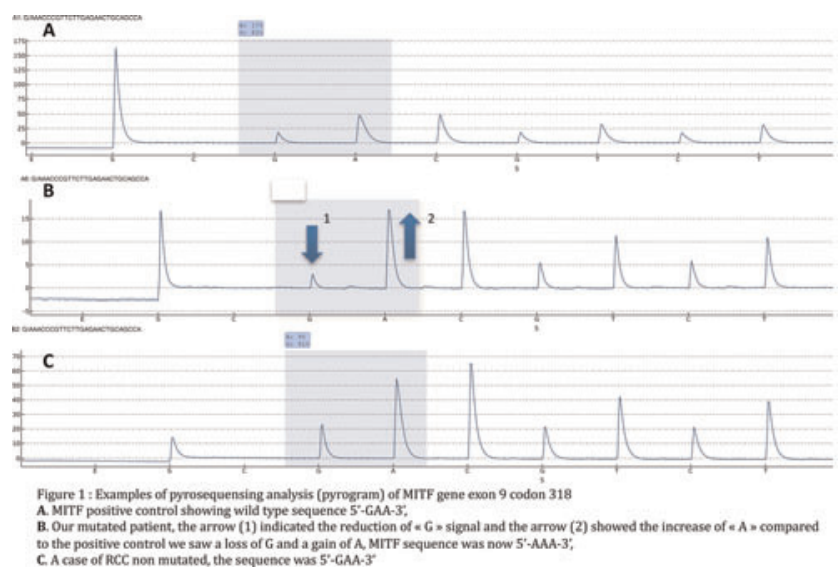

\begin{tabular}{|c|c|c|}
\hline \multicolumn{3}{|c|}{ Patients and renal cell carcinomas $(R C C)$ features $(n=148)$} \\
\hline & $\mathrm{n}$ & $\%$ \\
\hline \multicolumn{3}{|l|}{ Patient's features } \\
\hline Age (years) & 63 & - \\
\hline Range of years & $18-89$ & - \\
\hline Male & 107 & 72,3 \\
\hline Female & 41 & 27,7 \\
\hline \multicolumn{3}{|c|}{ Tumorals pathology (WHO 2016) } \\
\hline Clear cell RCC & 68 & 45,9 \\
\hline Papillary RCC & 35 & 23,6 \\
\hline Chromophobe RCC & 9 & 6,1 \\
\hline Translocation RCC & 9 & 6,1 \\
\hline Other RCC & 27 & 18,2 \\
\hline \multicolumn{3}{|l|}{ TNM (WHO 2016) } \\
\hline T1 & 28 & 18,9 \\
\hline $\mathrm{T} 2$ & 5 & 3,4 \\
\hline T3 & 24 & 16,2 \\
\hline T4 & 1 & 0,7 \\
\hline Unknown & 90 & 60,8 \\
\hline \multicolumn{3}{|l|}{ ISUP (WHO 2016) } \\
\hline 1 & 2 & 1,4 \\
\hline 2 & 13 & 8,8 \\
\hline 3 & 27 & 18,2 \\
\hline 4 & 18 & 12,1 \\
\hline Unknown & 88 & 59,4 \\
\hline
\end{tabular}

WHO : World Health Organization ; TNM : Tumor Nodes Metastasis ; ISUP : International Society of Urological Pathology 
then by Pyrosequencing. The normal sequence of MITF codon 318 was $5^{\prime}$-GAA-3' resulting in an acid glutamic. Afterwards, the E318K mutation was presented when a $\mathrm{G}$ was replaced by an $\mathrm{A}$ induced a lysine. The tumor was classified according to the World Health Organization's (WHO) 2016 RCC's classification.

Results: From 2011 to 2017, we screened 148 RCCs operated by laparoscopy or laparotomy surgery. The sample size was composed of 107 men and 41 women. We found 68 clear cell RCCs, 35 papillary RCCs, 9 chromophoba RCCs, 9 translocation RCCs and 27 other type of RCCs (Table 1). We found only 1 woman with MITF E318K mutation (Figure 1).

Conclusions: Our study reveals the lack of evidence on the link between MITF E318K mutation and RCC tumorigenesis. Nevertheless the 2016 classification of RCC shows an opening for new rare subpopulation of RCCs and possibly for the MITF RCC.

BRPRS3-12 ALK rearranged renal cell carcinoma : a literature review

Y Bodokh, L Mendel, I Bentellis, L Vignot, P Treacy, P Regnier, F Roustan, R Haider, A Mbeutcha, R Prader, B Tibi, J Fallot, J Amiel, D Chevallier, F Pedeutour, M Durand

Departement of Urology, Andrology and Renal transplantation, Hospital Pasteur II, CHU Nice

France

Introduction \& Objective: World Health Organization's (WHO) last classification of renal cell tumors published in 2016, describes thirteen types of renal cell carcinoma (RCC) and shows clear cell RCC to be the most frequent $(65-70 \%)$ and the most studied. WHO's work recorded three cases of ALK-translocation RCC (tRCC). Considering the scarcity of information available on ALK-tRCC, genetic diversity deserves to be further investigated. However, We did a review of literature to better understand this remarkable renal cell carcinoma.

Materials and Methods: A thorough research of literature was conducted using PubMed from 1990 to 2017 according to PRISMA criteria, focusing on ALK rearranged RCC diagnosis, prognosis, and genetics features. Keywords were ALK associated renal cell carcinoma, ALK translocation renal cell carcinoma and ALK fusion renal cell carcinoma.

Results: Overall, 63 studies were eligible, out of which 14 reported cases of ALK rearranged RCC, 13 cases reports and one review about papillary RCC. All these studies were included in our review and analyzed. 2653 renal cell carcinomas were screened during the 14 studies. Results show out of the 21 cases found, 9 were children, 12 adults and that all were diagnosed thanks to fluorescence in situ hybridization analysis (FISH). Thus, the pathology characteristics, TNM, fusion patterns, became of patients and risk factors of RCC were all listed in table 1. None of the patients had the same histological presentation. Only one patient presented metastasis, and 6 had nodes metastasis at the diagnosis. Finally, 3 patients were still alive 5 years after

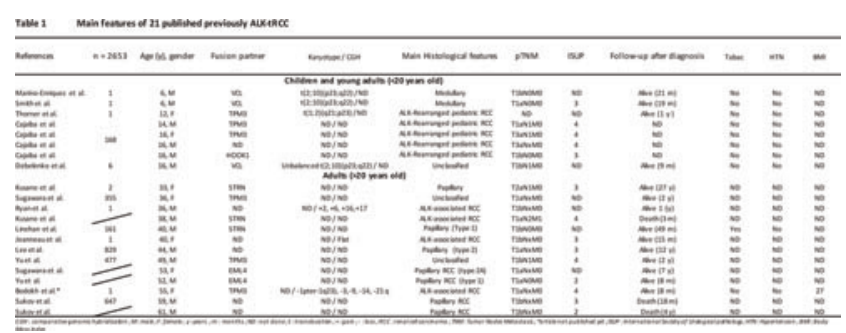

diagnosis, revealing the median survival to be 48 months, highlighting the prognosis to be positive.

Conclusions: ALK translocation associated RCC are an exceptional type of kidney cancers, which can be diagnosed with FISH analysis. We highlighted the necessity to genetically analyze patients when the pathology diagnoses were not established by histology.

BRPRS3-13 ALK-TPM3 rearrangement in adult renal cell carcinoma: an exceptional translocation renal carcinoma

Y Bodokh, L Mendel, I Bentellis, L Vignot, P Treacy, P Regnier, F Roustan, R Haider, A Mbeutcha, R Prader, B Tibi, J Fallot, J Amiel, D Chevallier, F Pedeutour, M Durand

Departement of Urology, Andrology and Renal transplantation, Hospital Pasteur II, CHU Nice

France

Introduction \& Objective: The last classification of renal cell tumors was published by the World Health Organization (WHO) in 2016, it describes thirteen types of renal cell carcinoma (RCC). Three cases of ALK-tranlocation RCC (tRCC) are recorded. We analyzed a large cohort of translocation RCC, unclassified RCC and histology ambivalent RCC for ALK translocation.

Materials and Methods: All the consecutive patients with RCC referred at the academic hospital were enrolled after informed consent. Hematoxylin and eosin staining and immunostaining were performed on formalin-fixed, paraffin embedded (FFPE) tissue sections. Immunohistochemical analyses were performed using antibodies against the following proteins. Genomic tumor DNA was extracted from formalin-fixed paraffin embedded RCC. ALK fluorescence in situ hybridization analysis (FISH) DNA Probe Split Signal (Dako) were used according to the manufacturer's recommendations. The tumor was classified according the WHO's 2016 RCC classification.

Results: Out of 390 renal cell carcinomas operated by laparoscopy or laparotomy surgery between 2011 and 2017, 34 RCCs were screened. Our sample was composed of 24 men and 10 women. We found 14 translocation RCCs, 8 unclassified RCCs and 12 ambivalent histology RCCs (Table 1). We discovered only 1 woman ALK rearranged RCC. Diagnosis were guided by pathologists who were certified by FISH. Fusion pattern was TPM3 (Figure 1) and the woman was 55 years old. She was still alive one year after being diagnosed, without relapses.
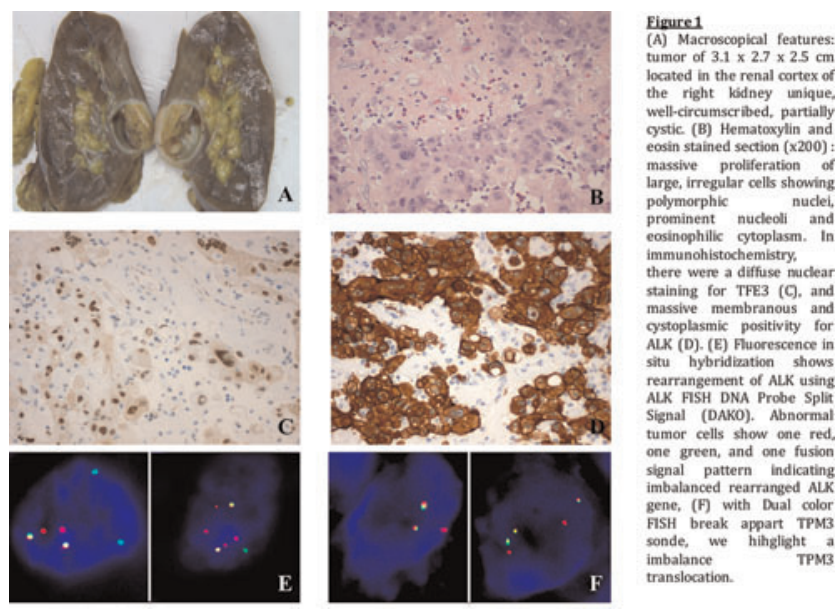


\begin{tabular}{|c|c|c|}
\hline \multicolumn{3}{|c|}{ Patients and renal cell carcinomas (RCC) features $(n=34)$} \\
\hline & $\mathrm{n}$ & $\%$ \\
\hline \multicolumn{3}{|l|}{ Patient's features } \\
\hline Age (years) & 59,7 & - \\
\hline Range of years & $18-88$ & - \\
\hline Male & 24 & 70,6 \\
\hline Female & 10 & 29,4 \\
\hline \multicolumn{3}{|c|}{ Tumorals pathology (WHO 2016) } \\
\hline Translocation RCC & 14 & 41,2 \\
\hline unclassified RCC & 8 & 23,5 \\
\hline Ambivalent RCC & 12 & 35,3 \\
\hline \multicolumn{3}{|l|}{ TNM (WHO 2016) } \\
\hline $\mathrm{T} 1$ & 6 & 17,6 \\
\hline $\mathrm{T} 2$ & 0 & 0 \\
\hline $\mathrm{T} 3$ & 12 & 35,3 \\
\hline $\mathrm{T} 4$ & 2 & 5,9 \\
\hline Unknown & 14 & 41,2 \\
\hline \multicolumn{3}{|l|}{ ISUP (WHO 2016) } \\
\hline 1 & 0 & 0 \\
\hline 2 & 4 & 11,8 \\
\hline 3 & 7 & 20,6 \\
\hline 4 & 11 & 32,3 \\
\hline Unknown & 12 & 35,3 \\
\hline
\end{tabular}

Conclusions: Our study highlighted one exceptional ALK translocation RCC out of a large cohort of renal cell carcinomas. These cases opened the door for targeted therapy by crizotinib such as cases of lung cancer, when this translocation was presented. Finally, it might be acceptable to look for this genetics translocation for RCC that are not clearly classified by histologist.

BRPRS3-14 The role of 18F-FDG PET/CT in identifying inguinal nodal metastasis in patients on surveillance after primary treatment of penile squamous cell carcinoma: Single center prospective study.

Y T B, S Waigankar, V Wagaskar

Kokilaben Dhirubhai Ambani Hospital and Research Institute India

Introduction \& Objective: Management of clinically normal groin nodes (N0 groin) in patients with carcinoma of penis is controversial. Aim of our study was to assess the role of ${ }^{18}$ F-FDG $\mathrm{PET} / \mathrm{CT}$ in detecting lymph node metastasis in carcinoma penis with clinically N0 groin during follow up.

Materials and Methods: 19 patients (38 groins) (14 low risk and 5 high risk patients, based on primary tumor histopathology) with PET-negative N0 groins who chose close observation after penectomy were analysed. Follow up protocol [Period: 12-48 months] included clinical examination, ultrasonography of groin (q3months) and PET scan [3, 6, 12, 18,24,36,48 months (high risk group) and 6, 12, 24, 36, 48 months (low risk group)].

Results: 6/19 patients were PET-positive on follow-up of which $3 / 5$ patients [5/10 groins] were high risk [Positive at 6 months (bilateral, palpable), 12 months \& 18 months (Right-palpable and left), 24 months (unilateral)] \& 3/14 patients [4/28 groins] were low risk [Positive at 12 months (Unilateral), 12 and 24 months (bilateral) \& 28 months (unilateral-had palpable disease)]. 1/9 PET positive groins were negative for metastasis post groin node dissection. One had PET-negative clinical node enlargement. ${ }^{18} \mathrm{~F}-\mathrm{FDG}$ PET/CT showed a sensitivity of $88 \%$, specificity of $96 \%$, positive predictive value of $88 \%$ and negative predictive value of $96 \%$ for detecting lymph node metastasis during follow up.

Conclusions: $18 \mathrm{~F}-\mathrm{FDG}-\mathrm{PET} / \mathrm{CT}$ is a promising follow up tool in picking early inguinal lymph node metastases in patients with penile carcinoma post-surgery. Further follow up of these patients is required to identify late recurrences.

BRPRS3-15 Correlation between pre-operative serum C-reactive protien, platelet count and calcium levels with the types and stages of renal cell cancer and its significance in predicting recurrence and mortality after curative surgery

P Shrivastava, P Singh, P Dogra, B Nayak

\section{AIIMS, NEW DELHI}

India

Introduction \& Objective: Renal cell carcinoma(RCC) is the most lethal of the common urologic cancers. C-reactive protein, an inflammatory mediator, represents a promising prognostic marker in RCC. The aim of the study was to correlate preoperative serum calcium, platelet and CRP levels with the type, grade and stage of RCC and development of recurrence and mortality in the first year following curative nephrectomy.

Materials and Methods: Patients with localised RCC who underwent curative nephrectomy between August 2014 and June 2016 were followed for the development of recurrence and mortality. Pre-operative serum calcium, platelet and CRP levels were measured in all the patients and post operatively CRP measured and correlated with the outcomes.

Results: 133 consecutive patients of localised RCC were included. The mean followup was 8.77 months. In 56(42.1\%) patients, a follow up of $1 \mathrm{yr}$. or more was available. Recurrence, local or systemic was seen in $17(13.8 \%)$ patients. A significant association was seen between the preoprerative CRP levels and histopathological stage $(p=0.001)$, grade $(p=0.00)$ of RCC and development of recurrence $(p=0.00)$ and cancer specific mortality $(p=0.04)$. On univariate analysis, statistical significance was observed for the clinical stage, histopathological stage, grade, $\mathrm{Hb}$ level, platelet counts and CRP levels for development of recurrence. On multivariate analysis, $\operatorname{CRP}(\mathrm{p}=0.04)$ and clinical stage $(\mathrm{p}=0.01)$ were found to be a significant predictor of recurrence.

Conclusions: Preoperative CRP level and clinical stage are strong predictors of recurrence and mortality after curative nephrectomy for localised RCC at a short term followup of 1 year. Preoperative CRP can be used to identify the subset of patients likely to develop metastasis, thereby help in prognosticating such patients and allowing early intervention with likely beneficial effect on survival.

BRPRS3-16 Should All UTUC Patients be Screened for Lynch Syndrome? A Single Center Analysis of the Role of Screening UTUC patients for Lynch Syndrome

MJ Metcalfe, F Petros, P Rao, M Mork, L Xiao, R Broaddus, SF Matin

MD Anderson Cancer Center

United States 
Introduction \& Objective: Lynch syndrome (LS) is an inherited syndrome that places patients at risk for upper tract urothelial carcinoma (UTUC) and other cancers. The goal of this study is to examine the role of point-of-care (POC) screening for LS in patients diagnosed with de novo UTUC.

Materials and Methods: Patient information was retrospectively collected in an IRB-approved protocol for patients treated for UTUC at our institution. From January 2013 through July 2016, 115 UTUC patients without a history of LS were universally screened for LS. Patients who had a known diagnosis of LS were used as positive controls $(n=7)$. We evaluated patients and family history (Amsterdam criteria I [AMS1] and II [AMS2]), tumor immunohistochemistry (IHC) for 4 mismatch repair proteins (MMRP), as well as for microsatellite instability (MSI). Patients who were AMS2, MSI, or IHC positive were classified as presumed Lynch syndrome (pLS) and referred for clinical genetic analysis and counseling (GAC) as confirmation. Results were compared with those for patients with known LS.

Results: A total of $19 / 115(17 \%)$ patients screened as pLS. Of these, 11/115 (10\%) patients met AMS2 criteria and 15/115 (13\%) had loss of at least one MMRP. There were two cases of high MSI, both in patients with MMRP loss. Five of 19 patients who were referred, underwent GAC; all had a confirmed germline mutation. Of the 7 UTUC patients with a known history of LS, all had positive AMS2 criteria, 5/5 had at least a single MMRP loss and 2/2 patients had high MSI.

Conclusions: We identified $17 \%$ of UTUC cases as pLS at the POC using IHC and AMS2 criteria.

BRPRS3-17 Methylation signature for prediction of progression free survival in surgically treated clear cell renal cell carcinoma

H Kang, S Seo, H Jang, W Kim, Y Kim, S Yun, S Lee, W Kim

Republic of Korea

Introduction \& Objective: Little is known about epigenetic silencing of genes by promoter hypermethylation, in renal cell carcinoma (RCC). The aim of study was to identify prognostic methylation markers in surgically treated clear cell RCC (ccRCC). Materials and Methods: Methylation patterns were assayed using the genome-wide Infinium HumanMethylation27 array on 12 primary surgically treated ccRCC and 12 matched tissue of surrounding normal kidney. Using quantitative PSQ analysis, tumor-specific hypermethylated genes were validated in 25 independent cohorts and their clinical relevance was also verified in 152 independent cohorts.

Results: Using genome-wide methylation array, Zinc finger protein 278 (ZNF278), Family with sequence similarity 155 member A (FAM155A) and Dipeptidyl peptidase 6 (DPP6) were selected for tumor-specific hypermethylated genes in primary ccRCC. The promoter methylation of these genes occurred more frequently in ccRCC than normal kidney in independent validation cohort (each $\mathrm{P}<0.001$ ). The hypermethylation of three genes were associated with advanced tumor stage and high grade tumor in ccRCC. During median follow-up of 39.2 (interquartile range, 15.4-79.1) months, 22 (14.5\%) patients experienced distant metastasis. Multivariate analysis identified the methylation status of these three genes, either alone, or in a combined risk score as an independent predictor of distant metastasis.

Conclusions: The promoter methylation of ZNF278, FAM155A and DPP6 genes are associated with aggressive tumor phenotype and early development of distant metastasis in patients surgically treated ccRCC. These potential methylation markers, either alone, or in combination, could provide novel targets for development of individualized therapeutic and prevention regimens.

BRPRS3-18 Expression of MHC class I chain-related protein A (MICA) and natural-killer group 2, member D receptor (NKG2D) predicts recurrence and progression of primary non-muscle invasive bladder cancer.

E Rijo, J Lloreta Trull, A Frances Comalat, J Lorente, N Juanpere Rodero, L Diaz Sanchez, M Lorenzo Perez, S Perez Fernandez, A Alcaraz

Department of Urology, Hospital Quiron Barcelona Spain

Introduction \& Objective: Major histocompatibility complex (MHC) class I chain-related protein A (MICA) are rarely detectable on the surface of healthy cells, but they can be upregulated by cellular malignant transformation, viral infection and cellular stress. MICA is the main ligand of Natural-Killer Group 2, member D (NKG2D) receptor expressed on natural killer, CD8+T cells and on $\gamma \delta$ T cells.

Expression of MICA is an innate immune response to oxidative DNA damage and its recognition by the NKG2D+ cell, generates a series of responses until the cell expressing MICA is destroyed.

Non muscle-invasive bladder cancer (NMIBC) depending on patient risk profile, rates of recurrence can be as high as $78 \%$ within
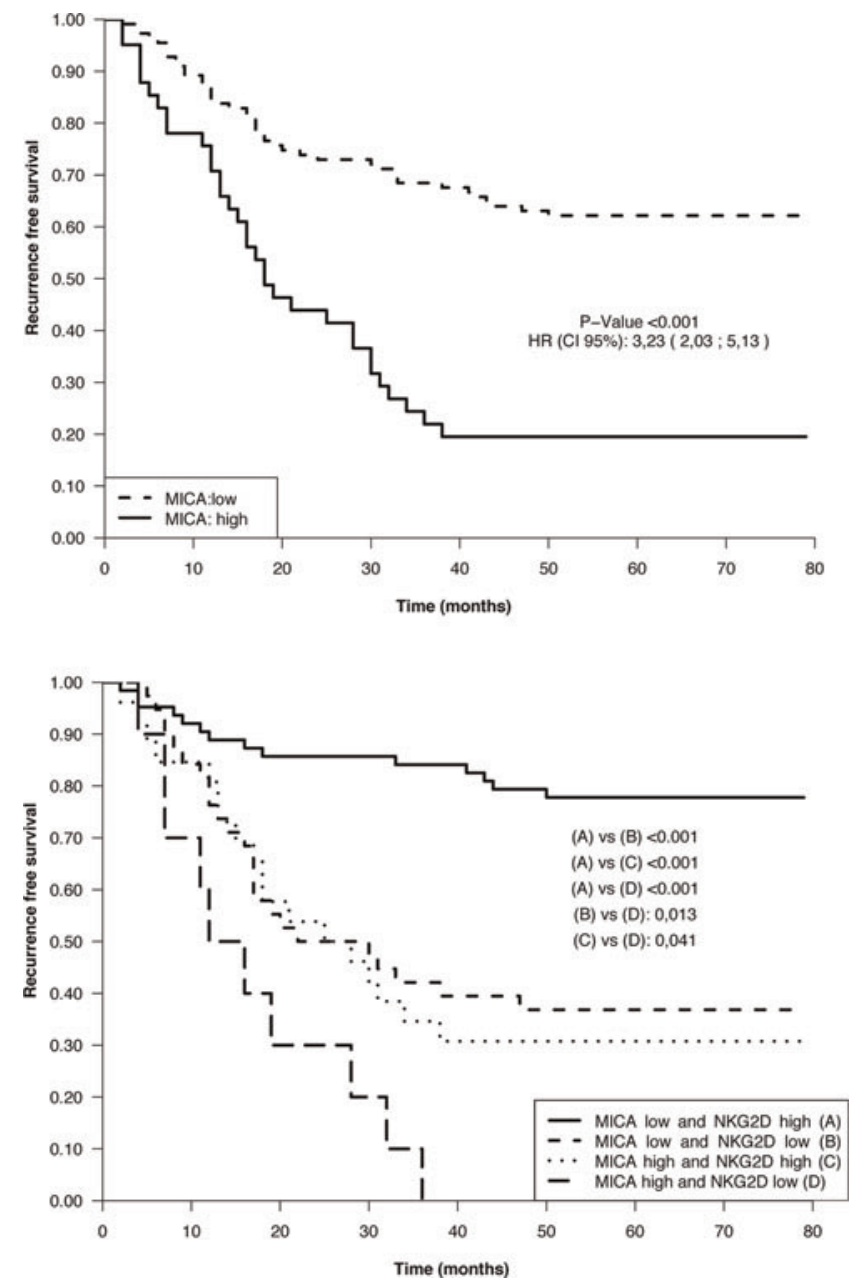
$5 \mathrm{yr}$, and rates of progression can be as high as $45 \%$, and several biomarkers have been studied as a recurrence prognostic tool.

To date, the relationship between the expression level of MICA in NMIBC have not been published.

The purpose of this study was to assess the role of the expression of MICA and NKG2D in the urotelial NMIBC, and the relationship with clinicopathological characteristics and its potential utility as an independent prognostic factor for recurrence and progression.

Materials and Methods: Retrospectively we analyzed the data of 175 consecutive patients (median age $68 \mathrm{yr}, 85 \%$ male) with primary urothelial NMIBC between 1998-2003. Representative paraffin blocks from each patient were used to construct three TMAs. IHC staining for MICA and NKG2D was performed using anti-human MICA monoclonal antibody (Immatics, clon AMO1) and anti-NKG2D rabbit polyclonal (NovusBio, clone CD314).

Univariate analysis and multivariate Cox regression were used to identify predictors of recurrence and progression. The recurrence-free survival rate and the progression-free survival rate were estimated using the Kaplan-Meier method.

Results: With a median follow-up of 78 months, the rate of recurrence was $50.9 \%$. High expression of MICA is associated with a significant increased risk of recurrence (OR:6.777 [2.86116.053]; pp <0.001).

There was no correlation between NKG2D expression and clinicopathological characteristics, except the age, gender and smoking.

High NKG2D expression was associated with lower recurrence rates (OR:3.86 [0.153-0.976]; $\mathrm{p}=0.044)$. NKG2D expression is a protective factor of progression (OR:0.161 [0.003-0.875]; $\mathrm{p}=0.034$ ).

Conclusions: High expression of MICA can predict recurrence in patients with NMIBC. Further studies are needed to assess the role of MICA-NKG2D as a therapeutic target.

BRPRS3-19 Kidney irreversible electroporation does not cause significant injury to adjacent ureter or bowel in a porcine model

I Sorokin, N Canvasser, E Lucas, BA Johnson, J Cadeddu

UT Southwestern

United States

Introduction \& Objective: Irreversible electroporation (IRE) is a novel ablative technology that works by passing pulsed, lowenergy direct current through target tissue. In theory, this is a non-thermal destruction of tissue, and therefore, the architecture of the surrounding supportive tissue structures is preserved. Thus, IRE may be potentially used safely in tumors adjacent to the ureter or bowel.

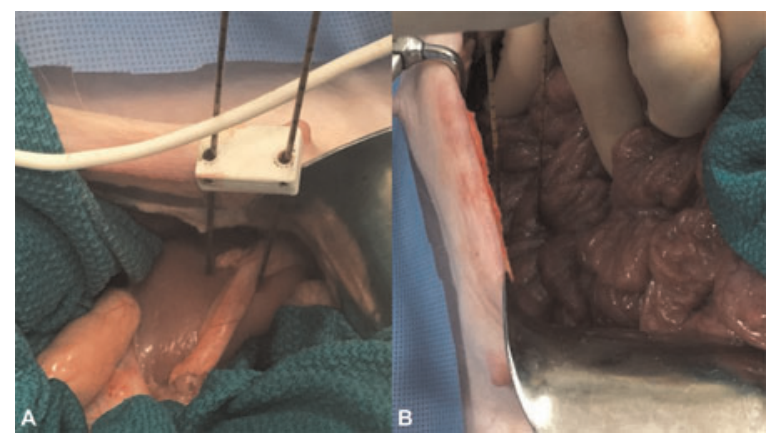

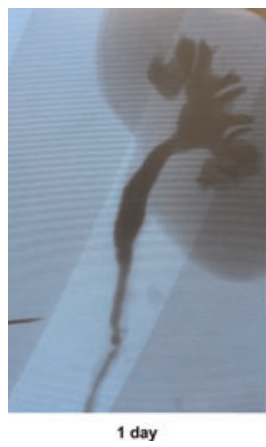
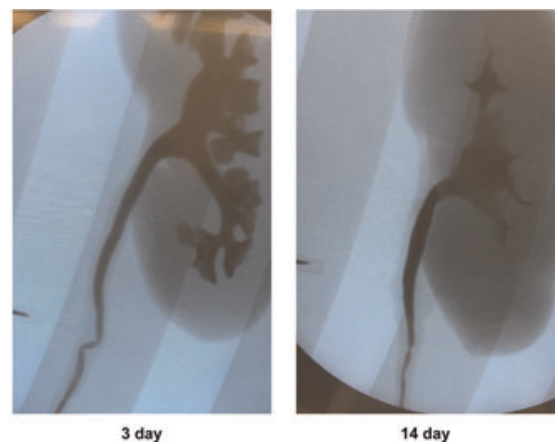

Materials and Methods: Six adult pigs each underwent open bilateral IRE of the anterior lower pole of the kidney. Prior to ablation, the left sided proximal ureter was tacked on to the left kidney and 2 IRE probes were placed bridging the ureter $1.5 \mathrm{~cm}$ apart. Small bowel was tacked on the right kidney with similar IRE probe configuration bridging the bowel. IRE was delivered at $2000 \mathrm{~V} / \mathrm{cm}$ for 70 pulses followed by another 70 pulses in reverse polarity. The animal was survived and euthanized at 1, 3, or 14 days. Histopathology was obtained for all potentially injured bowel and ureteral segments. Retrograde pyelograms (RPG) were performed on the left sided ureter.

Results: RPG did not reveal any ureteral strictures or urine extravasation in all pigs. Histologically, one day after IRE, sections of the ureters demonstrated focal hemorrhage and mild acute inflammation in the periureteral adipose tissue. Three days after IRE, the peripheral aspects of the periureteral adipose tissue demonstrated reactive stromal changes with early fibrosis, neovascularization, mild chronic inflammation and rare acute inflammatory cells. Fourteen days after IRE, focal fibrosis developed in the periureteral adipose tissue. Urothelial mucosa and surrounding smooth muscle layers remained unaffected with no histologic alterations identified. One day after ablation, sections of the bowel demonstrated focal fibrin deposition and acute inflammation in the serosa and subserosal tissue. Three days after IRE, acute inflammation, with neutrophils in the lamina propria, cryptitis and crypt abscesses were focally present in the deep aspects of the bowel mucosa. Fourteen days after IRE, no inflammation or pathologic alterations were seen in the mucosa or muscularis layers. The serosa demonstrated fibrosis in the area immediately adjacent to the ablation site.

Conclusions: In a porcine model of IRE, no significant injury was apparent after intentional ablation adjacent to the ureter and bowel. Inflammatory changes present in the early post-IRE period resolved by day 14 without any damage to mucosa or muscularis layers. IRE may be potentially used safely in tumors adjacent to the ureter or bowel.

BRPRS3-20 Pre-Clinical Assessment of Peri-operative Complications and Effect on Wound and Tissue Healing of sEphB4-HSA Administration Prior to Surgical Intervention

S Chopra, C Ohe, A Bove, N Ahmadi, A Abreu, A Aron, M Aron, M Amin, P Gill, IS Gill

USC Institute of Urology

United States

Introduction \& Objective: sEphB4-HSA is a novel cancer therapeutic that is beginning Phase II clinical trials. Its mechanism of 


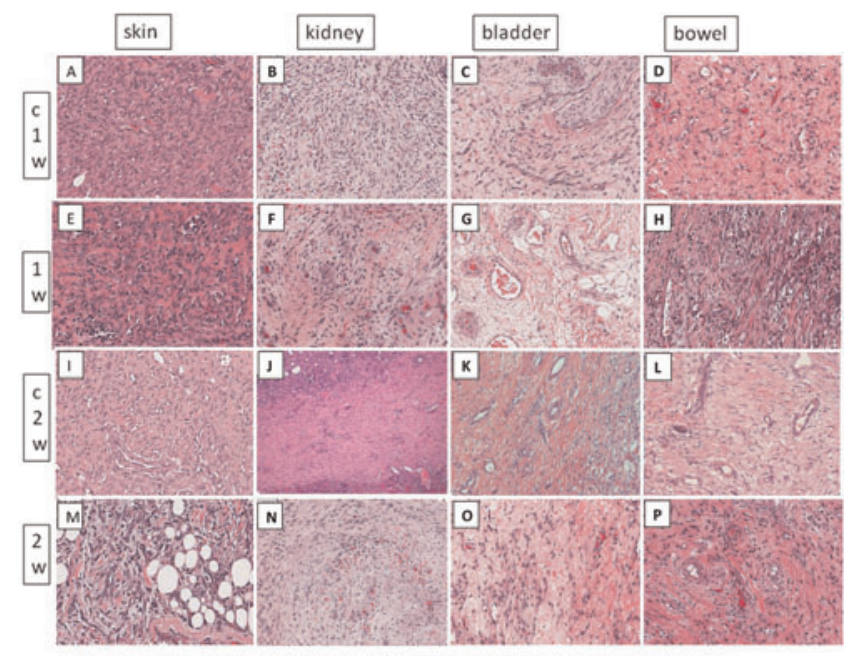

Histological features of wound healing areas. (A-D) 1 week control porcine $(E-H) 1$ week experimental porcine (I-L) 2 week control porcine (M-P) 2 week experimental porcine. A,E,I,M; skin, $B, F, J, N$ : kidney, C, G,K,O: bladder, D,H,L,P: bowel. (A-P; HE staining. X200 magnification)

action is to directly inhibit the EphB4-Ephrin-B2 interaction to block tumor angiogenesis and therefore induce VEGF expression and deprive tumor cells from vital nutrients such as oxygen. However, by inhibiting angiogenesis, there is a concern that this inhibition may affect wound healing when administered in the neoadjuvant setting, as angiogenesis is a vital component to growth of new tissue.

Materials and Methods: Ten porcines were enrolled into a randomized trial where experimental porcines would receive up to 4 doses of intravenous sEphB4-HSA and then undergo laparoscopic partial nephrectomy, cystotomy, vascular reconstruction, ileotomy, and a complete skin layer excision. Each porcine was then followed post-operative for one to two weeks and then euthanized. Control porcines did not receive sEphB4-HAS but underwent the same study protocol.

Results: Six porcines were randomized to the experimental group and four to the control group. One experimental porcine expired prior to experimentation, and was excluded from analysis. The remaining five experimental porcines were able to receive sEphB4HSA and undergo all surgical procedures successfully without complications. Three experimental porcines were euthanized one week after surgery while two were euthanized two weeks after surgery. During post-operative care, there were not any complications or concerns with any of the experimental swine. Histopathological tissue, radiographic, and hydrostatic pressure analysis did not demonstrate any significant statistical differences between the two cohorts except sEphB4-HSA administration decreases organ inflammation $(\mathrm{p}=0.025)$.

Conclusions: Preliminary preclinical experimentation demonstrates no statistically significant difference is observed when administering sEphB4-HSA prior to laparoscopic urologic surgery in terms of wound healing and tissue recovery.

BRPRS3-21 Capability of Electrical Impedance Spectroscopy as a Sensor for Detecting Iatrogenic Injuries Caused by Veress Needle Insertion: Preliminary In Vivo Study Using Murine Models

J Yun, K Lee, T Kim, J Lee

Department of Urology, Pusan National University Hospital Republic of Korea
Introduction \& Objective: To estimate tumor margin during partial nephrectomy, we developed a needle having electrochemical impedance spectroscopy (EIS) sensor on the tip of a needle which we named "EoN (EIS-on-a needle)". In this study, the impedances of renal parenchyma in accordance with the distance from the tumor were measured by using EoN to evaluate the capability of the device in detecting the tumor margin.

Materials and Methods: Microelectromechanical-system technology was applied to fabricate EoN, which is a 22-gauge needle with interdigitated electrodes on its tip. For the experiment, 10 renal specimen consisted of tumor and its surgical margin resected by partial nephrectomy were used. EoN was inserted into the surgical margin of the specimens and the impedance was measured while the device was accessing toward the tumor at the frequency range from $0.1 \mathrm{kHz}$ to $1 \mathrm{MHz}$. The areas within the specimens where the impedance was measured were categorized into five sections according to the distance from the tumor: 1) surgical margin at $>6 \mathrm{~mm}$ and $\leq 8 \mathrm{~mm}$ from the tumor as section I; 2) between $>4 \mathrm{~mm}$ and $\leq 6 \mathrm{~mm}$ as section II; 3 ) between $>2 \mathrm{~mm}$ and $\leq 4 \mathrm{~mm}$ as section III; 4) between $>0 \mathrm{~mm}$ and $\leq 2 \mathrm{~mm}$ as section IV; and 5) within the tumor as section V. The magnitude and phase angle of impedance between each sections were compared by using repeated-measures analysis of variance at each single frequency.

Results: The mean magnitude and phase angle of the impedances were proved to have a statistically significant variation $(\mathrm{p}<0.05)$ in accordance with the distance from the tumor at the frequency from $10.08 \mathrm{kHz}$ to $1 \mathrm{MHz}$ and at $39.89 \mathrm{kHz}$, respectively. At these frequencies, the mean magnitude and phase angle of section $\mathrm{V}$ was significantly different $(\mathrm{p}<0.05)$ to that of at least one other section of the specimen. However, the mean

Figure 1. (a) Mean magnitude and (b) mean phase of impedance measured in intra-abdominal cavity, liver,
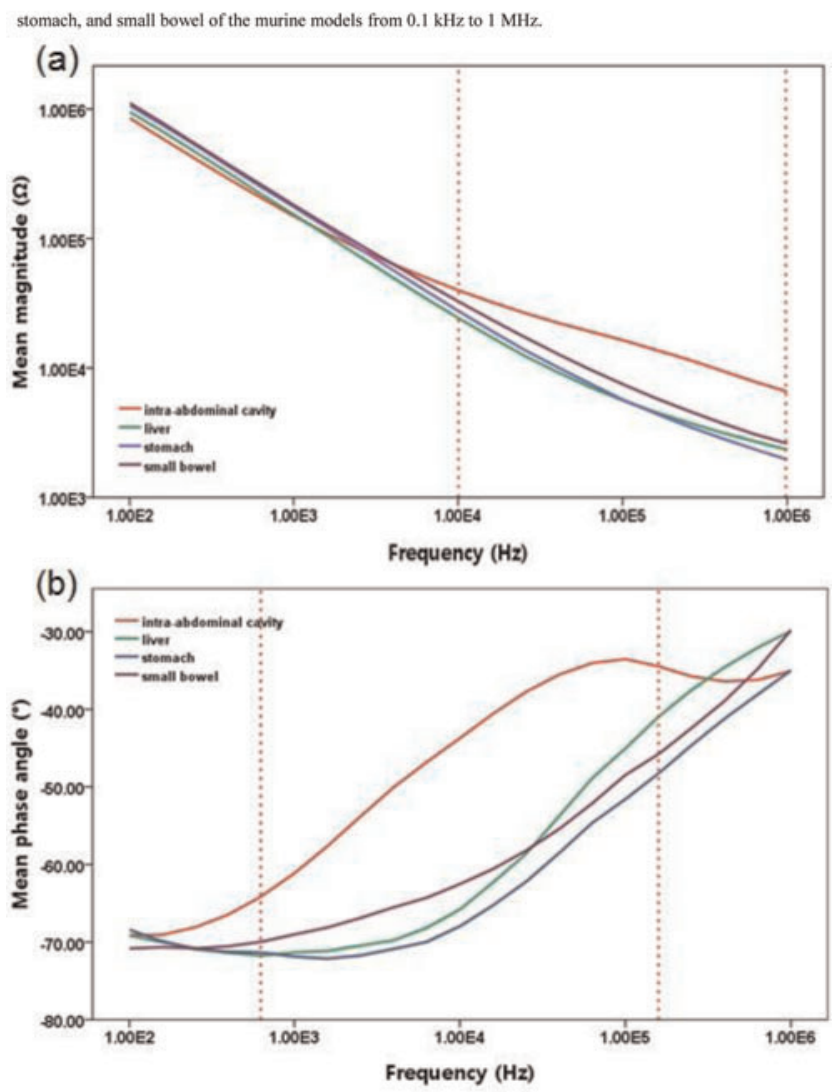
impedance differences between each respective sections other than section $\mathrm{V}$ did not show any statistical significance.

Conclusions: The present thesis has proved the capability of EoN in detecting the tumor when EoN was inserted from the normal renal parenchyma. The depth of tumor margin beneath the renal surface is assumed to be measurable by estimating the inserted length of EoN which has reached an area showing significant change of impedance values at specific frequencies.

BRPRS3-22 Preclinical ex-vivo experiment of photodynamic diagnosis using 5-aminolevulinic acid for upper urinary tract urothelial cancer

R Tanimoto, K Wada, M Ishizuka, K Murakami, T Ishii, Y Mitsui, T Sadahira, Y Kobayashi, M Araki,

$\mathrm{T}$ Watanabe, Y Nasu

Department of Urology, Okayama University Graduate School of Medicine, Dentistry and Pharmaceutical Sciences

Japan

Introduction \& Objective: Protoporphyrin IX (PpIX), one of metabolites from 5-Aminolevulinic acid (5-ALA), is specifically accumulated excessively in cancer cell. PpIX has the nature of photoactivity and emits red fluorescence (600$740 \mathrm{~nm})$ by blue light $(375-445 \mathrm{~nm})$ irradiation. Furthermore, the accumulation and excitation of PpIX produces reactive oxygen species in cancer cell and induces apoptosis. In various types of cancers including urothelial cancer of bladder, 5-ALA has been applied to photodynamic diagnosis and therapy. To evaluate the photodynamic diagnosis using 5-ALA (ALAPDD) for upper urinary tract urothelial cancer (UTUC), we examined the applicability of the flexible ureteroscope using ex-vivo model.

Materials and Methods: We examined the connectivity between the flexible ureteroscope, URF-P6 (Olympus, Tokyo, Japan) and the bicolor medical LED light source, Aladuck LS-DLED (SBI Pharmaceutical Co. Ltd, Tokyo, Japan) by which blue light mode and white light mode can be switched. The light power is measured at $15 \mathrm{~mm}$ far from the receiver of power meter. The signal intensity of red color was monitored at the tip of cotton swab soaked with the different concentration of PpIX $(0$, 1,3 , and $10 \mu \mathrm{M}$ ) under white light and blue light. Similarly, it

Figure 1. The signal intensity of red color under white or blue light

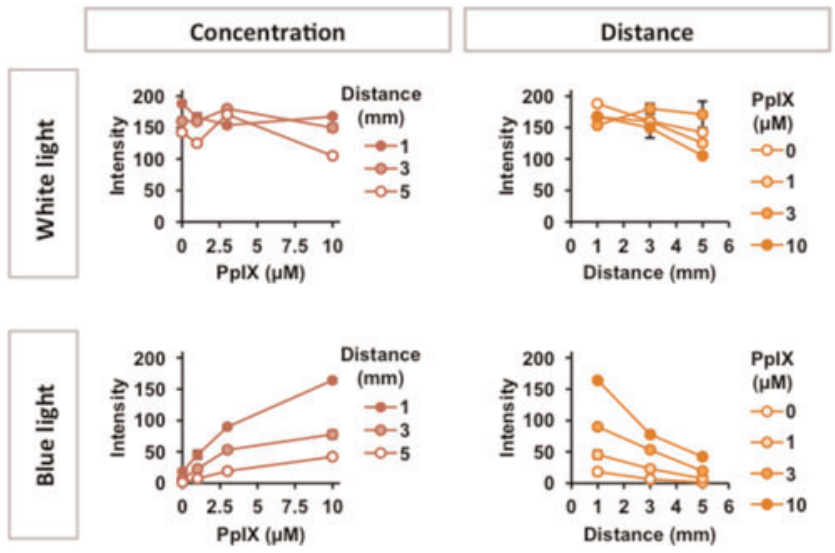

was monitored at different distance between the target and the tip of the ureteroscope $(1,3,5 \mathrm{~mm})$.

Results: The flexible ureteroscope, URF-P6 was connectable and applicable with Aladuck. The light power was $4.7 \mathrm{~mW}$ in blue light mode and $1 \mathrm{~mW}$ in white light mode. The signal intensity of red color increased in dose dependent manner under blue light while no difference was identified under white light. Similarly, the signal intensity was inversely correlated to the distance under blue light but not under white light. (Figure 1.)

Conclusions: Flexible ureteroscope was applicable for ALAPDD. 5-ALA is expected as a novel diagnostic tool for UTUC.

BRPRS3-23 Automated and dynamic classification of bladder cancer using deep learning on real-time confocal laser endomicroscopy images

TC Chang, D Yi, D Rubin, JC Liao

Department of Urology, Stanford University United States

Introduction \& Objective: Advancements in artificial intelligence now enable machines to surpass human capability in performing tasks such as classifying images. These innovations can be applied to medical images to create next generation tools that augment current clinical practice. Confocal laser endomicroscopy (CLE) is an intraoperative optical imaging system that provides high resolution imaging similar to histology in real-time but requires interpretation of the images by the clinician. Our aim was to create an automated system utilizing deep learning that provides real-time diagnosis of CLE images.

Materials and Methods: A deep learning algorithm (convolutional neural network) was trained using a bladder CLE dataset from 81 subjects with IRB approval undergoing cystoscopy/ TURBT. The diagnoses of 458 CLE videos were co-registered with clinical pathology reports to establish the ground truth. The algorithm received the 170,712 CLE images that were individually captured from the videos and their corresponding diagnoses as its only inputs.

Results: Overall accuracy in the prediction of malignant versus benign was $87 \%$ (sensitivity $79 \%$ and specificity $90 \%$ ). The algorithm was capable of providing frame-by-frame diagnoses. Figure 1 depicts screen captures of streaming CLE video, illustrating snapshots of the dynamic diagnostic feedback.

Conclusions: We developed an automated system for real-time, dynamic diagnosis of CLE images. Our deep learning machine achieved high accuracy, sensitivity and specificity in detecting bladder cancer. Every frame is classified in real-time, allowing for live feedback of the streaming CLE images.

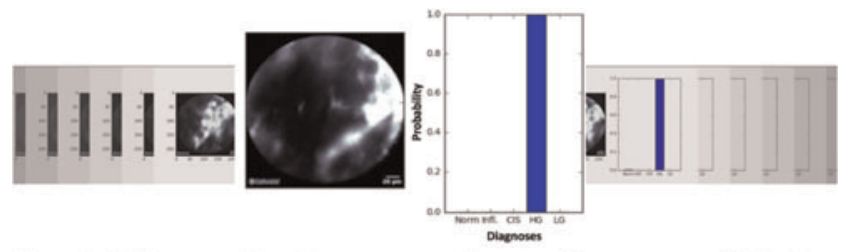

Figure 1: CLE images with real-time, automated diagnoses. Screen capture of CLE video with accompanying histograms providing frame-by-frame prediction of diagnoses. CLE of bladder cancer with $100 \%$ prediction of high grade cancer for that frame. Norm $=$ normal, infl = inflammation, CIS = carcinoma-in-situ, HG = high grade cancer, LG = low grade cancer 
BRPRS3-24 Assessment about heat injury of small intestine induced by surgical sealing devices in animal model

T Suzuki, T Minagawa, T Ogawa, T Uehara, R Hattori, O Ishizuka

Shinshu University School of Medicine Japan

Introduction \& Objective: In recent years, sealing devices become an indispensable tool for laparoscopic surgery, which greatly contributes to control of bleeding and shortening of operation time. Due to the nature of the machine, temperature rise of the tip and thermal damage of the surrounding tissue are inevitable, but there are few reports that the temperature rise of each device is measured directly and the thermal damage of the contacted tissue is actually evaluated. In this report, we evaluated the temperature rise of the tip of the ENSEAL TRIO / Round / G2 Articulating, HARMONIC ACE + (ETHICON), LigaSure V / Blunt Tip / Maryland Jaw (COVIDIEN), BiClamp (ERBE), THUNDERBEAT (OLYMPUS) and the thermal damage of the tissue contacted with each devices.

Materials and Methods: 1) When sealing the porcine small intestine intracorporeally, a thermometer was directly applied to the tip and measured from the start of the operation until the chip temperature dropped to $50^{\circ} \mathrm{C}$. or less. 2) When sealing porcine small intestine extracorporeally, the lower jaw of the tip was pressed against another small intestinal serous membrane. Pathological specimens were prepared in the pressed porcine small intestine and the depth of thermal damage was evaluated.

Results: 1) The highest temperature of the chip was particularly high with LigaSure V and BiClamp, but the other devices also rose above $60^{\circ} \mathrm{C}$, which is thought to cause protein denaturation of tissues. The cooling time to $50^{\circ} \mathrm{C}$ or less was particularly short at ENSEAL TRIO of about 20 seconds, but it took a long time especially around 1 minute at THUNDERBEAT. 2) In the

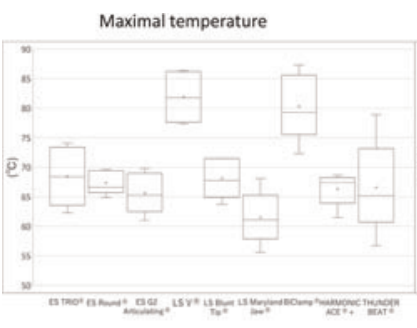

Type of devised

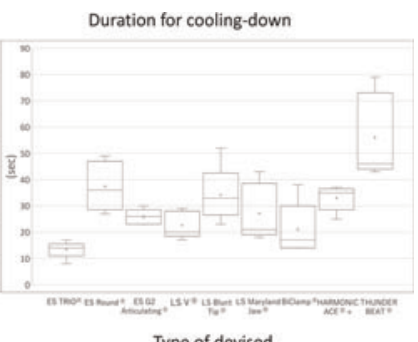

Type of devised

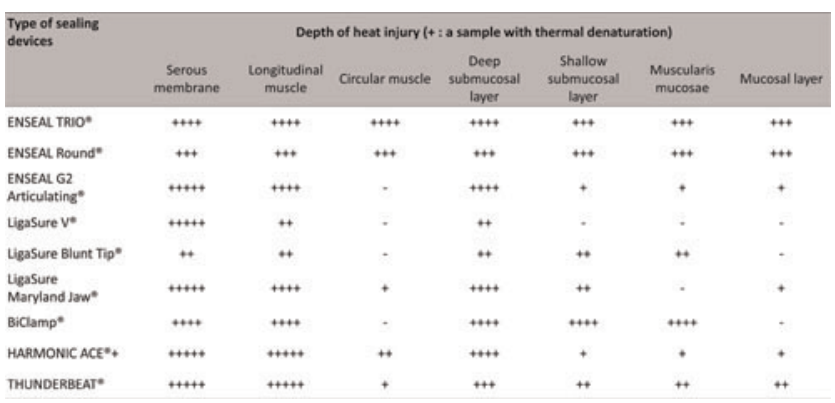

pathological specimens evaluating the degree of depth of thermal damage, thermal damage was extended to the vicinity of the mucosa of the intestinal lumen in any of the devices, although there were some differences.

Conclusions: While sealing devices are useful in laparoscopic surgery, attention should be paid to thermal damage to the contacted tissues. Also, because there are differences in temperature rise and change depending on the instrument, it was thought that should be used after understanding its characteristics.

\section{BRPS4: BENCH TO BEDSIDE: THE SCIENCE OF STONES II}

BRPRS4-1 Holmium-YAG laser: Impact of pulse energy and frequency on local fluid temperature

\author{
M Sourial, J Ebel, N François, G Box, B Knudsen
}

The Ohio State University Wexner Medical Center United States

Introduction \& Objective: Protein denaturation depends on several factors, one of which is temperature. It has been previously shown that temperatures of as low as $60^{\circ} \mathrm{C}$ can cause denaturation. Optical fibers transmit energy to fragment stones, however the temperature rise of its surrounding has never been studied. Understanding the heat impact, especially with the increased use of high powered laser, is an important safety consideration. The objective of this preliminary study is to determine the time it takes from body temperature $\left(37^{\circ} \mathrm{C}\right)$ to $60^{\circ} \mathrm{C}$ at various laser power settings.

Materials and Methods: Four milliliters of normal saline were placed in a glass test tube, corresponding to the average volume of a dilated calyx. A Flexiva TracTip 200 optical fiber was submerged in the saline alongside a NTC-type thermistor to record temperature. A Lumenis VersaPulse Powersuite 100W laser was activated at $0.2-1.5 \mathrm{~J}$ pulse energies, $6-50 \mathrm{~Hz}$ frequencies, and $2-22.5 \mathrm{~W}$ power. Temperature readings were recorded once per second from 37 until $60^{\circ} \mathrm{C}$, and the time was recorded. Heating rate was calculated. This procedure was repeated three times for each setting.

Results: Average time from 37 to $60^{\circ} \mathrm{C}$ for settings (1) $0.2 \mathrm{~J} /$ $10 \mathrm{~Hz}$, (2) $0.6 \mathrm{~J} / 10 \mathrm{~Hz}$, (3) $1 \mathrm{~J} / 10 \mathrm{~Hz}$, and (4) $1.5 \mathrm{~J} / 10 \mathrm{~Hz}$ was $355,95.7,58$, and 43.3 seconds respectively. Time to $60^{\circ} \mathrm{C}$ decreased as frequency increased for a given pulse energy. Average heating rate increased proportionally to power from 0.07 $0.76^{\circ} \mathrm{C} /$ second. Continuing to apply energy resulted in temperatures reaching $100^{\circ} \mathrm{C}$ at all settings.

Conclusions: Use of optical fibers, and particularly at higher power settings, can cause a rapid and substantial increase in the temperature of its surrounding fluid. This could have local tissue effects and some caution with higher pulse energy and frequency should be employed. Further studies incorporating irrigation and live tissue models may aid to further define the risks. 
BRPRS4-2 Save time, save Nephrone.... Emergency drainage for Urosepsis with upper tract calculi

HG Thummar, M Dabhi, A Patel, N T, S D

Sterling

India

Introduction \& Objective: Being in stone belt region and relatively poor socioeconomic status, delayed treatment for stone is very common leading to urosepsis and obstructive uropathy. We examined the characteristics of patients with urosepsis associated with upper urinary tract calculi requiring emergency drainage.

Materials and Methods: We studied 240 patients were admitted to our urological department a total of 273 times for treatment of upper urinary tract calculi, of whom 153 required a total of 159 emergency drainage procedures for urosepsis. We summarized the characteristics of these patients and events, and determined risk factors for emergency drainage using.

Results: In 34 events intensive management, such as the use of vasopressors and anticoagulants, was performed. Transient thrombocytopenia less than $100,000 / \mathrm{mm} 3$ occurred in 28 events. Hyperbilirubinemia occurred in 6 events without prior antibiotic therapy. One patient died of urosepsis. Patients with calculi who underwent emergency drainage required a shorter hospital stay than those without emergency drainage (25.2 vs 14.8 days, $\mathrm{p}$

Conclusions: Our findings suggest that early drainage in urosepsis improves outcome and the frequency of emergency drainage in elderly patients with poor performance status has increased in recent years, at least in our rural area. Preventing calculus formation and urinary tract infection in individuals with poor performance status will be of considerable importance in the future.

BRPRS4-3 Assessing irrigation flows influence on clearance of renal calculi fragments during percutaneous nephrolithotomy: a hydrodynamic computerized and practical model study

Y Barghouthy, S Shlain, G Aviram, A Liberzon,

A Paster, M Sofer

Tel-Aviv Sourasky Medical Center, Sackler School of Medicine, Tel-Aviv University

Israel

Introduction \& Objective: The irrigation flow used in PCNL creates a clear endoscopic view and contributes to flushing out residual fragments, as shown by the vacuum cleaning effect recently described in a model of a single calyx. However, that model does not reflect the hydrodynamic processes within the entire intrarenal collecting system. This study aimed to further analyze these processes using computerized models (CM) for prediction and a PCNL practical model (PM) to validate the calculations.

Materials and Methods: COMSOL Multiphysics ${ }^{\circledR}$ software (Palo Alto, CA, USA) was used to simulate the irrigation flows and the particles' floating and movements in a two-dimensional kidney model based on a kidney computerized tomography acquisition which was graphically translated by computer-aided design software. A similarly shaped transparent model with solid particles was constructed, and PCNL was simulated using a 24FR nephroscope through a 30 FR working sheath, while the movements of the particles were monitored by a high-speed recording digital camera (figure). Particle movement of both models was compared at various flow velocities and nephroscopic positions.

Results: Results for various velocities at $90^{\circ}$ and various angles at a velocity of $1 \mathrm{~m} / \mathrm{s}$ are reported here. The numerical model predicted a significantly increased particle clearance with high-velocity irrigation $(0.25$ vs $1 \mathrm{~m} / \mathrm{s}, 12 \%$ vs $70 \%$, respectively: $\mathrm{p}<0.0001)$, as did the perpendicular positioning of the instrument $\left(45^{\circ}\right.$ vs $90^{\circ}, 1 \%$ vs $\left.70 \%: \mathrm{p}<0.0001\right)$. These results were validated in the practical model (table). This process occurred only in the directly irrigated calyx when the flow and subsequently the particle movements in the other calices are null.

Conclusions: Floating and clearance of particles are produced only when irrigation is perpendicularly directed to the targeted calyx. The effectiveness of the clearance is enhanced at higher flow velocities. There is no effective flow in calyces that are not directly irrigated. The validation of the numerical calculated and predicted model in the practical model confirms that computerized software can be utilized for advanced renal hydrodynamic research and possibly reduce the number of studies performed in animals and humans.

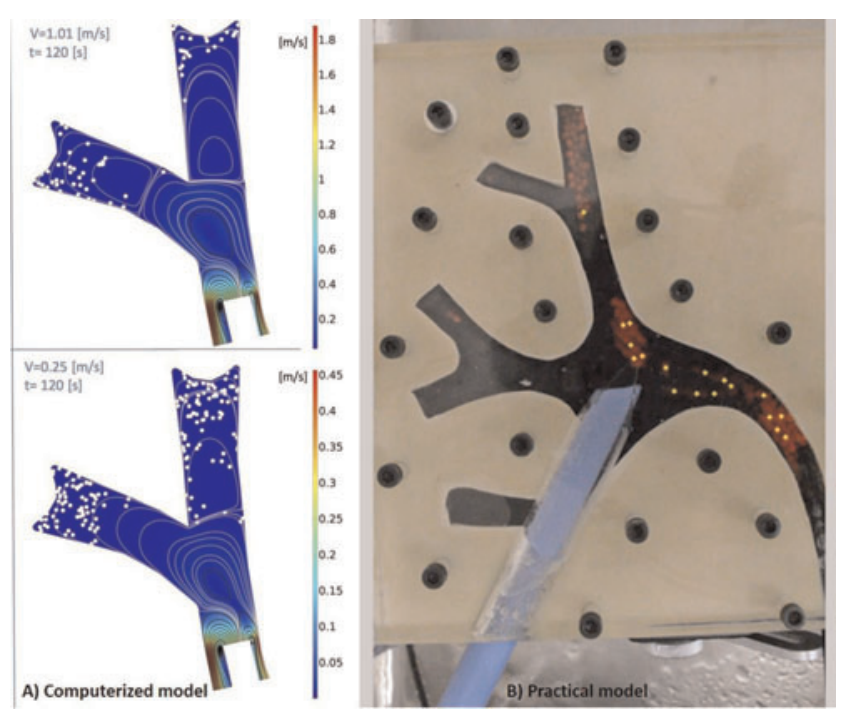

\begin{tabular}{|l|l|l|l|}
\hline Velocity (m/s) at $90^{\circ}$ & $\begin{array}{l}\text { CM Clearance } \\
\text { at } 120 \mathrm{sec}(\%)\end{array}$ & $\begin{array}{l}\text { PM Clearance } \\
\text { at } 120 \mathrm{sec}(\%)\end{array}$ & $\begin{array}{l}\text { P value [OR(90\% } \\
\text { confidence interval)] }\end{array}$ \\
\hline 0.25 & 12 & 10 & $0.8[0.8(0.33-1.98)]$ \\
\hline 0.50 & 41 & 44 & $0.8[1.1(0.83-1.33)]$ \\
\hline 0.75 & 64 & 59 & $0.5[0.8(0.45-1.43)]$ \\
\hline 1 & 70 & 72 & $0.9[1.1(0.09-1.65)]$ \\
\hline $\begin{array}{l}\text { Instrument angle }\left({ }^{\circ}\right) \text { at } 1 \\
\mathrm{~m} / \mathrm{s}\end{array}$ & & & \\
\hline 45 & 1 & 5 & $0.2[5.2(0.59-45)]$ \\
\hline 60 & 17 & 12 & $0.42[0.66(0.29-1.4)]$ \\
\hline 80 & 19 & 30 & $0.09[1.8(0.94-3.5)]$ \\
\hline 90 & 70 & 80 & $0.14[1.7(0.89-3.2)]$ \\
\hline & & & \\
\hline & & 30 & \\
\hline
\end{tabular}


BRPRS4-4 Can Hounsfield unit (HU) measurement on non-contrast computed tomography scan (NCCT) predict urinary stone composition? A validation of 2 different techniques.

Z Choo, A Yuwono, M Lim, Z Liu, C Tan, Y Tan, Y Lee

Urology Department TTSH

Singapore

Introduction \& Objective: Early knowledge of urinary stone composition allows urologists to choose appropriate therapeutic modalities. This study aims to determine the accuracy of predicting urinary stone composition from NCCT by validating 2 methods of evaluating region of interest (ROI) measurement of $\mathrm{HU}$.

Materials and Methods: Our institution's stone analysis database was retrospectively reviewed. Selected patients had at least one NCCT performed prior to stone composition analysis between April 2013 and September 2015. The stones were grouped to calcium oxalate, UA and mixed stones. Parameters analyzed included stone size ie: largest measured diameter in the axial view, stone depth ie: largest coronal view diameter and stone volume ie stone size $\times$ perpendicular diameter $\times$ depth $\times \prod \times 0.167$. Absolute core HU was measured by 2 methods. The 1 st method used ROI encompassing the maximum size of the stone both in the axial and coronal view before a mean was calculated. HU density was obtained by division of core HU by stone size. The 2 nd method involves ROI of $0.5 \mathrm{~mm}^{2}$ at the intersection of the largest transverse and its perpendicular diameter in the axial view. Other analyzed parameters include periphery $\mathrm{HU}$, absolute and relative HU difference as well as HU density. Study was conducted by a urologist and radiologist trainee, both blinded to stone composition. Measurements were all done in same abdominal window and magnification. Data was analyzed by SPSS software.

Results: 67 patients were selected; 17 (25.4\%) calcium oxalate, 6 (9\%) uric acid (UA), and $44(65.7 \%)$ mixed stones. Mean stone size and volume were compared between all 3 groups, respectively, and showed no significant difference [(Stone size: $22.8 \pm 10.2 \mathrm{~mm}$ vs $21.1 \pm 9.7 \mathrm{~mm}$ vs $18.8 \pm 10.3 \mathrm{~mm}$, respectively $\mathrm{p}=0.373$ ), (Stone volume: $5006.4 \pm 6355.6 \mathrm{~mm}^{3}$ vs $3306.4 \pm 4452.9 \mathrm{~mm}^{3}$ vs $6401.9 \pm$ $8681.4 \mathrm{~mm}^{3}$; respectively, $\left.\left.\mathrm{p}=0.608\right)\right]$. For 1 st method, core HU of both calcium oxalate and mixed were higher than UA stones $(1186.1 \pm 181.1$ vs $541.1 \pm 150.9$ and $1098.7 \pm 291.7$ vs $541.1 \pm$ 150.9 ; respectively $\mathrm{p}<0.01$ ). For 2 nd method core $\mathrm{HU}$ of both calcium oxalate and mixed were higher than UA stones $(1164.4 \pm 274.3$ vs $516.2 \pm 75$ and $1055.2 \pm 323.3$ vs $516.2 \pm 75$; respectively $\mathrm{p}<0.01)$. Both method's data did not show any significant difference.

Conclusions: There is a statistical difference in core HU between both calcium oxalate and mixed stones to uric acid stones respectively, for both methods. No other parameters analyzed in this study could differentiate mixed and calcium oxalate stones. Both techniques are acceptable in evaluating ROI of HU to differentiate UA stones.

BRPRS4-5 Enhanced high rate fragmentation of model urinary stones using acoustic bubble coalescence with a commercial shockwave lithotripsy system

\section{TL Hall, WW Roberts}

Department of Biomedical Engineering, University of Michigan United States

Introduction \& Objective: Shockwave lithotripsy (SWL) at ultra low rate (i.e. $<30$ shocks/min) has been shown in the laboratory to

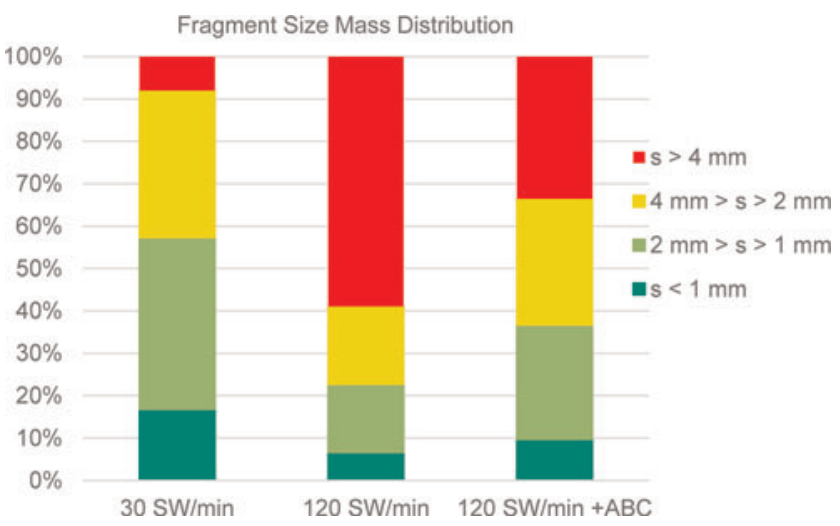

fragment stones more efficiently than high rate $(120 \mathrm{SW} / \mathrm{min})$. This is thought to be because low rate affords sufficient time for cavitation bubbles to dissolve between shocks mitigating shielding of bubbles on subsequent shockwaves. Ultra low rates have not been adopted clinically because of the impractically long procedure times required. We have previously demonstrated with a laboratory electrohydraulic lithotripter unfocused low amplitude acoustic bursts can stimulate rapid coalescence of cavitation bubbles during SWL within 10s of milliseconds enhancing fragmentation of model stones. The objective of this study was to determine if this acoustic bubble coalescence method (termed ABC) could also enhance model stone fragmentation for a commercial SWL system.

Materials and Methods: Cylindrical $1 \mathrm{~cm}$ model stones (Begostone plaster, water, albumen mix) were placed in a vinyl finger cot containing about $5 \mathrm{ml}$ DI water degassed to $80 \%$ oxygen saturation. This was placed at the focus of a Dornier Compact Delta lithotripter in an 801 tank of highly degassed $(<15 \%)$ DI water. Each stone was exposed to 200 shocks at power setting 3 following one of three protocols: $30 \mathrm{SW} / \mathrm{min}(\mathrm{N}=10)$, $120 \mathrm{SW} / \mathrm{min}(\mathrm{N}=15), 120 \mathrm{SW} / \mathrm{min}$ with $\mathrm{ABC}(\mathrm{N}=15)$. ABC consisted of 1600 low amplitude ultrasound pulses lasting $16 \mathrm{~ms}$ applied $2 \mathrm{~ms}$ after each shockwave from an annular array of eight $500 \mathrm{kHz}$ transducer modules around the lithotripter head. The peak amplitude was $1 \mathrm{MPa}$. Following exposure, the contents of each finger cot was sieved through screens of $4 \mathrm{~mm}, 2 \mathrm{~mm}$, and $1 \mathrm{~mm}$ and then allowed to dry for $36-48 \mathrm{hrs}$ prior to weighing.

Results: Stones treated at $30 \mathrm{SW} / \mathrm{min}, 120 \mathrm{SW} / \mathrm{min}$, and $120 \mathrm{SW} /$ min $+\mathrm{ABC}$ resulted in averages of $57 \%, 23 \%$, and $37 \%$ of the fragment mass being less than $2 \mathrm{~mm} .30 \mathrm{SW} / \mathrm{min}$ was significantly better than $120 \mathrm{SW} / \mathrm{min}(\mathrm{p}$-value $=2.8 \mathrm{E}-5)$ and $120 \mathrm{SW} / \mathrm{min}+\mathrm{ABC}$ was significantly better than $120 \mathrm{SW} / \mathrm{min}$ alone $(\mathrm{p}$-value $=0.026)$. Other fragment sizes scaled similarly as shown in the figure.

Conclusions: Bubble coalescence can enhance model stone fragmentation for a clinical lithotripter operating at high shockwave rates. Clinical use of this technique may improve outcomes for SWL procedures.

BRPRS4-6 Differences of metabolic evaluation in 24-hour urine between pre- and postmenopausal non-stone former women

Z Mai, G Zeng

Department of Urology, Minimally Invasive Surgery Center, the First Affiliated Hospital of Guangzhou Medical University. Guangzhou Institute of Urology. Guangdong Key Laboratory of Urology

China (People's Republic) 
Introduction \& Objective: To estimate the differences in 24hour urine compositions associated with urolithiasis between pre- and postmenopausal females who were not stone formers. Materials and Methods: The 24-hour urine samples of female volunteers without urolithiasis were collected from May 2013 to July 2014 during an epidemiology study of urolithiasis among adults aged 18 and older in China. Subjects were excluded from this study if they met the following criteria: incomplete urine samples(urine creatinine $<600 \mu \mathrm{g} / 24 \mathrm{~h}$ ), serum creatinine $>133 \mu \mathrm{mol} / \mathrm{L}$, had urinary stone currently and previously, urinary tract infection, gout, hyperthyroidism, malignancy, had a history of enterectomy, had taken medications of thiazide, allopurinol, vitamin supplements, potassium citrate or calcium supplements during the past two weeks. The compositions associated with urinary stone in 24-hour urine were measured and compared between pre-and postmenopausal women.

Results: A total of 603 female participants provided their 24hour samples to analyze. And 354 women with an average age of $52.5 \pm 14.03$ (19-84) years met our criteria, included 160 premenopausal women and 194 postmenopausal women. Compared to premenopausal women, postmenopausal women had a lower secretion of citrate $(p=0.043)$, magnesium $(p=0.001)$, and creatinine $(p=0.001)$ in 24 -hour urine. Multivariate linear regression analysis showed that the status of menopause was associated with the differences of magnesium $(p=0.003)$ and creatinine $(p=0.002)$ secretion, but was not associated with difference of citrate $(\mathrm{p}=0.402)$ secretion.

Conclusions: Postmenopausal females had a significant lower secretion of magnesium in 24-hour urine than premenopausal females. It may be associated with an increased risk of urolithiasis after menopause among women.

BRPRS4-7 Arbutin as a novel prevention and therapy for calcium oxalate nephrolithiasis

T Dayarathna, S Ali, AN Ali, P Spagnuolo, H Razvi, HS Leong

Mayo Clinic

United States

Introduction \& Objective: Calcium oxalate calculi are the most prevalent kidney stone by composition, accounting for approximately $80 \%$ of all calculi. Through the adaptation of the Drosophila melanogaster model of calcium oxalate nephrolithiasis as a drug screening tool, the therapeutic potential of the the plant based glycoside Arbutin was identified. Here, we further investigate the efficacy of Arbutin as a next generation therapy for calcium oxalate nephrolithiasis.

Materials and Methods: To characterize the interaction of Arbutin with calcium and oxalate ions, isothermal titration calorimetric analysis (ITC) was performed by titrating $25 \mathrm{uM}$ Arbutin onto $2.5 \mathrm{mM}$ solutions of calcium chloride and sodium oxalate respectively. Observations were confirmedfurther investigated by matrix assisted laser deposition/ionization (MALDI). Polarized light and atomic force microscopy (AFM) of in vitro grownsodium oxalate crystals in the presence and absence of Arbutin was conducted to assess the action of Arbutin on crystal growth. The potential effects of Arbutin on human calculi was assessed by incubating $3 \mathrm{mM}$ Arbutin solution with pulverized human calcium oxalate calculi against untreated controls followed by scanning electron microscopy (SEM). Cytoxic effects of Arbutin Human cell cytotoxicity studieson human cell were performed onutilizing ity studies utilizing HEK-293 and FHS174INT cells cells were performed utilizingvia athean Lactate Dehydrogenase (LDH) cytotoxicity assay.

Results: ITC and MALDI analysis revealed revealed that Arbutin interacts with both -calcium and Arbutin-oxalate ions while, with the Arbutin-calcium complex is formation incomposed ofarranged in a 4:1 stoichiometry. AFM and polarized light microscopic images revealed incisors and decaycays of oxalate crystals in the presence of Arbutin. Following incubation with Arbutin, AFM and SEM imaging revealed crystal surface aberrations with a highly active crystal topography. No significant cytotoxic effects of Arbutin on human kidney and intestinal cells was noted.

Conclusions: Arbutin inhibits the growth of calcium oxalate crystals and promotes degradation of formed crystals with no cytotoxic effects noted in vitro.. Whilst further characterization in a mammalian system is warranted, the discovery of this novel anti-lithogenic compound via $D$. melanogaster brings greater recognition to this platform where metabolic features are primary outcomes.

\section{BRPRS4-8 Vitamin D, Bone Turnover Markers and Bone Mineral Density in Recurrent Calcium Stone Formers}

U Dhakad, B Singh, K Kaura, S Sankhwar, S Das

\section{King George's Medical University}

India

Introduction \& Objective: The aim of this study was to examine the status and interrelationship of vitamin D, bone mineral density, bone turnover markers in recurrent calcium stone formers.

Table 1. Parameters in recurrent calcium stone formers (group A) vs, healthy controls (group B) :

\begin{tabular}{|c|c|c|c|}
\hline Parameters & $\begin{array}{l}\text { Group A/ Cases } \\
(\mathbf{n}=\mathbf{5 5 )} \\
(\text { Mean } \pm \text { SD); Median }\end{array}$ & $\begin{array}{l}\text { Group B/ Controls } \\
(n=44) \\
(M e a n \pm S D) ; \text { Median }\end{array}$ & p-value \\
\hline Age (years) & $38.62 \pm 10.07 ; 38$ & $38.23 \pm 9.99 ; 40$ & 0.953 \\
\hline Male/Female, n (\%) & $42(76.36) / 13(23.64)$ & $33(75) / 11(25)$ & 0.876 \\
\hline Smokers/Non-smokers,n (\%) & $40(72.72) / 15(27.27)$ & $30(68.18) / 14(31.82)$ & 0.66 \\
\hline S. Calcium (mg/dl) & $9.62 \pm 1.04 ; 9.5$ & $9.43 \pm 0.63 ; 9.46$ & $0.027^{*}$ \\
\hline S. Phosphorous (mg/dl) & $5.19 \pm 1.97 ; 4.9$ & $6.36 \pm 4.30 ; 4.25$ & 0.632 \\
\hline S. Uric acid (mg/dl) & $5.29 \pm 1.55 ; 5.2$ & $5.27 \pm 2.10 ; 5.13$ & 0.149 \\
\hline S. Sodium (meq/l) & $141.58 \pm 2.24 ; 139.2$ & $141.81 \pm 2.07 ; 139.8$ & 0.59 \\
\hline S. Potassium (meq/l) & $4.52 \pm 0.41 ; 4.12$ & $4.45 \pm 0.42 ; 4.21$ & 0.40 \\
\hline Urine $\mathrm{pH}$ & $5.93 \pm 0.69$ & $5.97 \pm 0.71$ & 0.77 \\
\hline \multicolumn{4}{|l|}{24 Urine (mg/24hr) } \\
\hline Calcium & $192.95 \pm 93.87 ; 190$ & $122.93 \pm 72.82 ; 106.5$ & $0.001^{*}$ \\
\hline Phosphorous & $402.66 \pm 209.29 ; 397$ & $406.48 \pm 215.24 ; 390.5$ & 0.882 \\
\hline Uric acid & $485.60 \pm 306.48 ; 480$ & $420.38 \pm 262.12 ; 418$ & 0.151 \\
\hline S. 25-OH vitamin D $(\mathrm{ng} / \mathrm{ml})$ & $18.20 \pm 6.13 ; 18.8$ & $21.79 \pm 6.20 ; 19.65$ & $0.042^{*}$ \\
\hline S. ALP (IU/L) & $235.15 \pm 58.22 ; 235$ & $245.26 \pm 89.44 ; 230.75$ & $0.046^{*}$ \\
\hline S. B-CTx (crosslaps) $(\mathrm{pg} / \mathrm{ml}$ ) & $3700.86 \pm 1299.01 ; 3692$ & $3583.53 \pm 1203.51 ; 3574.41$ & $0.039^{*}$ \\
\hline \multicolumn{4}{|l|}{ BMD $\left(\mathrm{g} / \mathrm{cm}^{2}\right)$} \\
\hline AP spine & $1.047 \pm 0.157 ; 1.066$ & $1.03 \pm 0.143 ; 1.03$ & 0.590 \\
\hline BMD Right Femur Neck & $0.882 \pm 0.137 ; 0.89$ & $0.919 \pm 0.12 ; 0.919$ & 0.224 \\
\hline BMD Left Femur Neck & $0.859 \pm 0.145 ; 0.853$ & $0.860 \pm 0.254 ; 0.88$ & 0.288 \\
\hline BMD Femur Right & $0.941 \pm 0.153 ; 0.942$ & $0.963 \pm 0.113 ; 0.965$ & 0.844 \\
\hline BMD Femur Left & $0.924 \pm 0.154 ; 0.924$ & $0.964 \pm 0.117 ; 0.954$ & 0.159 \\
\hline BMD Left Forearm & $0.810 \pm 0.118 ; 0.81$ & $0.866 \pm 0.093 ; 0.87$ & $0.030^{*}$ \\
\hline \multicolumn{4}{|l|}{ T-score : } \\
\hline AP spine & $-0.879 \pm 0.897$ & $-1.164 \pm 0.938$ & 0.242 \\
\hline Right Femur Neck & $-1.000 \pm 0.771$ & $-0.948 \pm 1.050$ & 0.827 \\
\hline Left Femur Neck & $-1.232 \pm 0.956$ & $-1.232 \pm 1.101$ & 0.999 \\
\hline Right Femur & $-0.814 \pm 1.015$ & $-0.660 \pm 0.964$ & 0.598 \\
\hline Left Femur & $-0.817 \pm 0.923$ & $-0.784 \pm 1.033$ & 0.896 \\
\hline Left Forearm & $-1.044 \pm 0.867$ & $-1.084 \pm 0.961$ & 0.868 \\
\hline
\end{tabular}


Materials and Methods: This prospective case control study was conducted between November 2015 and October 2016 at rheumatology and urology departments of a tertiary care teaching institution. A total of 99 adult subjects were recruited, including 55 patients of recurrent renal calcium calculi (Group A) and 44 healthy controls (Group B, without any history or evidence or intervention for urolithiasis). These subjects underwent metabolic evaluation including serum creatinine, calcium, phosphorous, sodium, potassium, uric acid, 25-OH-Vitamin D. Bone turnover markers studied were serum alkaline phosphatase and serum $\beta$-crosslaps levels. 24 hour urine tests included calcium, uric acid, phosphorous. Bone mineral density (BMD) was measured at multiple sites in body. Parameters between two groups were analyzed and compared. SPSS 16.0 statistics program was used for analysis and $\mathrm{p}$ value $\leq 0.05$ was considered statistically significant.

Results: Table1. Mean age and sex ratio were similar in group A and group B. Mean serum 25-OH vitamin D level, serum alkaline phosphatase and BMD at left forearm were significantly (p values $0.042,0.046$ and 0.030 respectively) lower whereas mean serum beta-crosslaps level, serum calcium and 24 hours urine calcium were significantly ( $\mathrm{p}$ values $0.039,0.027$ and 0.001 respectively) higher in recurrent stone formers as compared to controls. On multivariate analysis 24 hour urine calcium, 24 hour urine phosphorous, 25-OH Vitamin D and $\mathrm{BMD}$ right femur neck were found to be significant ( $\mathrm{p}$ values $0.006,0.019,0.029$ and 0.032 respectively) risk factors associated with recurrent calcium stone formation.

Conclusions: Idiopathic recurrent calcium stone formation may result from complex interplay between low serum vitamin D, higher normal serum calcium level and idiopathic hypercalciuria, with attendant lower serum alkaline phospahatase and higher serum beta-crosslaps levels.

BRPRS4-9 Metabolic characteristics and risks associated with stone recurrence in young adult stone patients

S Seo, H Kang, H Jang, W Kim, Y Kim, S Yun, W Kim, S Lee CHUNGBUK NATIONAL UNIVERSITY HOSPITAL Republic of Korea

Introduction \& Objective: The aim of the present study was to assess the metabolic characteristics and risk of stone recurrence in young adult stone patients.

Materials and Methods: The medical records of 1,532 patients presenting with renal or ureteric stones at our stone clinic between 1994 and 2015 were retrospectively reviewed. Patients were grouped according to age at first presentation and measurements of clinicometabolic characteristics and risks of stone recurrence were compared.

Results: Early onset stone patients were more likely to be male and have the smallest stone burden. Overall, excretion of urinary stone-forming substances was highest in the intermediate onset group, followed by the early and late onset groups. Importantly, excretion of urinary citrate was lowest in the early onset group. Adjustment for sex and body mass index revealed that younger age at first stone presentation was a significant risk factor for hypocitraturia (odds ratio, 1.763; 95\% confidence interval (CI), 1.305-2.382; $\mathrm{P}<0.001$ ). Kaplan-Meier analyses identified a significant difference between the three age groups in terms of stone recurrence ( $\log$ rank test, $\mathrm{P}<0.001)$. Multivariate Cox regression analyses revealed that younger age at first stone presentation was an independent risk factor for stone recurrence (Hazard ratio, 1.988; 95\% CI, 1.342-2.946; $\mathrm{P}=0.001)$.

Conclusions: Younger age at first stone presentation was a significant risk factor for hypocitraturia and an independent risk factor for stone recurrence. Metabolic evaluation and potassium citrate therapy should be considered for early onset stone patients to prevent recurrence.

BRPRS4-10 Staghorn Stone etiology paradigm Shift : Infective to metabolic !!!

HG Thummar, N Zinto, S D, N T, J Vyas

Sterling

India

Introduction \& Objective: AUA urolithiasis guidelines mentions that calcium oxalate or calcium phosphate stones as unlikely causes of staghorn calculi. Historically staghorn calculi have been thought of as infectious stones, made up primarily of magnesium ammonium phosphate or (struvite) stones. The American Urological Association (AUA) guidelines for management of staghorn calculi continue to state calcium oxalate or calcium phosphate stones as unlikely causes of staghorn calculi. We reviewed our stone data and incidence of infectious and metabolic composition in large stag horn calculi.

Materials and Methods: We reviewed retrospectively for patients who underwent percutaneous nephrolithotomy (PCNL) for complete staghorn calculus from 2007 to 2016. Primary outcome of the study was to identify stone composition in infectious and non-infectious cases. Stone analysis and charts were reviewed for demographics, surgical complications, preoperative urine culture results and outcomes compared to stone type.

Results: 1840 PCNLs were completed at our institution between 2010-2015 for stones $>2 \mathrm{~cm}$. 526 patients (610 kidneys) had large staghorn calculus that met our size criteria. Overall 99 $169(31.9 \%)$ of patients were found to have infection stones, either struvite or carbonate. $211(68,1 \%)$ stones were composed of metabolic based stones without any infectious composition. The primary compositions in the metabolic stone group were calcium oxalate $(48 \%)$, Uric Acid $(21 \%)$, calcium phosphate $(19 \%)$, and mixed (12\%). In patients with purely metabolic stones, $65 \%$ of patients with primarily calcium phosphate hydroxyapatite had positive pre-op urine cultures, while only $12.1 \%$ of patients with primary calcium oxalate stones had positive pre-op urine cultures. Preoperative urine cultures revealed Proteus present ( $4.6 \%$ vs $43.7 \%$ ) for non-infectious and infectious stones. Infectious stones were have more complication as metabolic stones $(\mathrm{p}=0.0179)$. E. Coli was present in preoperative urine cultures (14.8\% vs $3.3 \%$ ) for non-infectious and infectious stones. Proteus was the most common bacteria in infectious stones, while E. Coli was most common with metabolic stones.

Conclusions: This study shows that noninfectious staghorn stone is more common than infection staghorn.. Calcium oxalate was the most common stone composition for staghorn calculi differing from historical reports of staghorn calculi being primarily infectious. Patients with calcium phosphate stones also had a high rate of positive urine cultures. More research is needed on the cause of this paradigm shift. 
BRPRS4-11 Urine and serum fetuin-A levels in patients with urolithiasis

R Arora, N Abrol, B Antonisamy, C Beri, S Kumar,

N Kekre, A Devasia

Medanta The Medicity

India

Introduction \& Objective: Fetuin-A is a glycoprotein secreted by liver and has been shown to inhibit extra osseous mineralization. Urolithiasis may a manifestation in urinary tract due to Fetuin deficiency in urine. The objective of this study was to compare the 24 hour urine and serum fetuin-A levels of patients with and without urolithiasis.

Materials and Methods: Serum and 24 hour urine fetuin-A levels were measured in 41 patients with bilateral, multiple or recurrent urinary tract calculi (Group A) and 41 matched controls with no calculi (Group B). Fetuin levels were measured by enzyme linked immunosorbent assay (ELISA). Serum and urine fetuin-A levels in the two groups were compared.

Results: The median (range) 24 hour urine fetuin-A value in Group A was 11.9 (1.12-221) mg /day and in Group B was 37.7 (1.28-125) $\mathrm{mg} /$ day. This difference was statistically significant (Man-Whitney test, $\mathrm{P}=0.017$ ). The median (range) serum fetuin-A in Group A was 0.67(0.05-2.68) gm/L and in Group B it was $0.99(0.01-5.5) \mathrm{gm} / \mathrm{L}$. The difference between serum values in the two arms was not statistically significant (ManWhitney test, $\mathrm{p}=0.18$ ) but serum creatinine adjusted mean $\log$ serum fetuin and urine fetuin were significantly different in the two arms $(p=0.003)$. The mean \pm SD (range) serum creatinine in Group A was $0.98 \pm 0.25(0.56-1.58) \mathrm{mg} \%$ and in Group B it was $0.83 \pm 0.16(0.58-1.18) \mathrm{mg} \%$, (two sample $\mathrm{t}$ test, $\mathrm{p}=0.003$ ).

Conclusions: Patients with urolithiasis have lower urine fetuinA and creatinine adjusted serum fetuin -A levels.

BRPRS4-12 Establishment of reference values for stone risk factors in 24-hour urine among healthy adult Han population across China

Z Mai, G Zeng

Department of Urology, Minimally Invasive Surgery Center, the First Affiliated Hospital of Guangzhou Medical University. Guangzhou Institute of Urology. Guangdong Key Laboratory of Urology

China (People's Republic)

Introduction \& Objective: To establish the reference intervals of stones risk factors in 24-hour urine for healthy Chinese Han population.

Materials and Methods: From May 2013 to July 2014, we collected 24-hour urine samples of Han adult volunteers during a cross-sectional study of urolithiasis across China. Volunteers were excluded if they had urinary stone, hypertension, diabetes mellitus, gout, hyperparathyroidism, had a history of enterectomy, with serum creatinine $>133 \mathrm{umol} / \mathrm{L}$ and incomplete urine collections. All 24-hour urine samples were analyzed in our hospital, and the standard protocol included volume, $\mathrm{pH}$ value, oxalate, citrate, sodium, potassium, chloride, calcium, phosphate, creatinine, uric acid, magnesium, the ion activity products of calcium oxalate $\left(\mathrm{AP}(\mathrm{CaOx})\right.$ index $\left._{\mathrm{s}}\right)$ and calcium phosphate $\left(\mathrm{AP}(\mathrm{CaP})\right.$ index $\left._{\mathrm{s}}\right)$. We calculated the reference ranges according
Table 1. Medians and reference intervals for 24-hour urine stone risk among healthy adult Han population in China: compared to reference ranges of European Associated of Urology(EAU) guideline

\begin{tabular}{|c|c|c|c|}
\hline Characteristic & $\begin{array}{l}\text { Median (reference } \\
\text { intervals), present } \\
\text { study }\end{array}$ & $\begin{array}{l}\text { Reference ranges } \\
\text { and limits for } \\
\text { medical attention } \\
\text { of EAU guideline }\end{array}$ & $\begin{array}{l}\text { Abnormal rate } \\
\text { when compared } \\
\text { to EAU } \\
\text { guideline, } \%(n / N)\end{array}$ \\
\hline \multicolumn{4}{|l|}{$\begin{array}{l}\text { Creatinine, } \\
\mathrm{mmol} / 24 \mathrm{~h}^{\mathrm{a}}\end{array}$} \\
\hline Men & $11.28(7.20,21.79)$ & $13-18$ & $74.24(98 / 132)$ \\
\hline Women & $8.10(5.52,13.30)$ & $7-13$ & $22.76(28 / 123)$ \\
\hline \multicolumn{4}{|l|}{$\begin{array}{l}\text { Calcium, } \\
\mathrm{mmol} / 24 \mathrm{~h}^{\mathrm{b}}\end{array}$} \\
\hline Overall & $3.85(\leqslant 8.25)$ & $>5.0$ & $26.27(67 / 255)$ \\
\hline Men & $4.09(\leqslant 9.00)$ & & \\
\hline Women & $3.62(\leqslant 7.51)$ & & \\
\hline \multicolumn{4}{|c|}{ Oxalate, $\mathrm{mmol} / 24 \mathrm{~h}^{\mathrm{b}}$} \\
\hline Overall & $0.21(\leqslant 0.59)$ & $>0.5$ & $7.06(18 / 255)$ \\
\hline Men & $0.22(\leqslant 0.58)$ & & \\
\hline Women & $0.21(\leqslant 0.59)$ & & \\
\hline \multicolumn{4}{|c|}{ Citrate, $\mathrm{mmol} / 24 \mathrm{~h}^{\mathrm{c}}$} \\
\hline Overall & $1.89(\geqslant 0.21)$ & $<2.5$ & $68.24(174 / 255)$ \\
\hline Men & $1.50(\geqslant 0.21)$ & & \\
\hline Women & $2.31(\geqslant 0.25)$ & & \\
\hline \multicolumn{4}{|l|}{$\begin{array}{l}\text { Uric acid, } \\
\mathrm{mmol} / 24 \mathrm{~h}^{\mathrm{b}}\end{array}$} \\
\hline Overall & $3.01(\leqslant 6.35)$ & & \\
\hline Men & $3.18(\leqslant 5.76)$ & $>5.0$ & $8.33(11 / 132)$ \\
\hline Women & $2.84(\leqslant 5.41)$ & $>4.0$ & $8.13(10 / 123)$ \\
\hline \multicolumn{4}{|c|}{ Magnesium, $\mathrm{mmol} / 24 \mathrm{~h}^{\mathrm{c}}$} \\
\hline Overall & $3.32(\geqslant 1.14)$ & $<3.0$ & $45.88(117 / 255)$ \\
\hline Men & $2.98(\geqslant 1.41)$ & & \\
\hline Women & $3.54(\geqslant 0.90)$ & & \\
\hline \multicolumn{4}{|c|}{ Phosphorus, $\mathrm{mmol} / 24 \mathrm{~h}^{\mathrm{b}}$} \\
\hline Overall & $15.56(\leqslant 31.24)$ & $>35$ & $0.08(2 / 254)$ \\
\hline Men & $16.43(\leqslant 31.60)$ & & \\
\hline Women & $14.46(\leqslant 31.24)$ & & \\
\hline
\end{tabular}

to the Clinical and Laboratory Standards Institute (CLSI) 2008 guidelines. And we compared these reference intervals with EAU guideline 2015.

Results: A total of 1057 volunteers provided their 24-hour urine samples to analyze, and 802 cases were excluded based on these criteria. A total of 255 subjects with an average age of $52.39 \pm 15.15$ (19-89) years old met our criteria and were eligible in the final analysis, included 132 men and 123women.

The median and reference intervals for creatinine, calcium, oxalate, citrate, uric acid, magnesium, and phosphorus of the present study population were showed in Table 1, with comparisons to reference ranges and limits for medical attention of EAU guideline 2015. The overall abnormal rate of calcium, oxalate, citrate, magnesium, and phosphorus in 24-hour urine was $26.27 \%$, $7.06 \%, 68.24 \%, 45.88 \%$, and $0.08 \%$ respectively when compared with EAU guideline 2015. And the abnormal rate of creatinine was $73.48 \%$ in men and $22.76 \%$ in women, while the abnormal rate of uric acid was $8.33 \%$ in men and $8.13 \%$ in women.

The $95 \%$ reference intervals of $\mathrm{pH}$ values, chloride, sodium, potassium, volume, AP $(\mathrm{CaOx}) \mathrm{Index}_{\mathrm{s}}$, and $\mathrm{AP}(\mathrm{CaP}) \mathrm{Index}_{\mathrm{s}}$ were showed in Table 2.

Conclusions: Healthy adult Han population in China has lower secretion of creatinine, calcium, citrate, and magnesium when 
Table 2. Medians and $95 \%$ reference intervals for 24-hour urine stone risk among healthy adult Han population in China

\begin{tabular}{|c|c|}
\hline Characteristic & $\begin{array}{l}\text { Median (reference intervals), present } \\
\text { study }\end{array}$ \\
\hline \multicolumn{2}{|l|}{$\mathrm{pH}^{\mathrm{a}}$} \\
\hline Overall & $6.22(5.24,7.63)$ \\
\hline Men & $6.18(5.23,7.23)$ \\
\hline Women & $6.29(5.32,8.16)$ \\
\hline \multicolumn{2}{|c|}{ Chloride, $\mathrm{mmol} / 24 \mathrm{~h}^{\mathrm{a}}$} \\
\hline Overall & $153.10(70.0,353.60)$ \\
\hline Men & $141.00(55.20,304.80)$ \\
\hline Women & $162.80(74.40,962.52)$ \\
\hline \multicolumn{2}{|c|}{ Sodium, $\mathrm{mmol} / 24 \mathrm{~h}^{\mathrm{a}}$} \\
\hline Overall & $157.36(69.81,396.80)$ \\
\hline Men & $144.47(67.31,289.49)$ \\
\hline Women & $166.53(72.00,396.84)$ \\
\hline \multicolumn{2}{|l|}{$\begin{array}{l}\text { Potassium, } \\
\mathrm{mmol} / 24 \mathrm{~h}^{\mathrm{a}}\end{array}$} \\
\hline Overall & $40.20(15.40,90.09)$ \\
\hline Men & $33.93(15.23,91.84)$ \\
\hline Women & $37.50(19.42,82.40)$ \\
\hline \multicolumn{2}{|c|}{ Volume, $\mathrm{ml} / 24 \mathrm{~h}^{\mathrm{a}}$} \\
\hline Overall & $1330.00(600.00,2840.00)$ \\
\hline Men & $1330.00(630.00,3140.00)$ \\
\hline Women & $1310.00(600.00,2660.00)$ \\
\hline \multicolumn{2}{|c|}{$\mathrm{AP}(\mathrm{CaOx})$ Index ${ }_{s}{ }^{2}$} \\
\hline Overall & $0.64(0.09,2.11)$ \\
\hline Men & $0.72(0.15,2.44)$ \\
\hline Women & $0.57(0.09,2.11)$ \\
\hline \multicolumn{2}{|c|}{$\mathrm{AP}(\mathrm{CaP})$ Index ${ }^{\mathrm{a}}$} \\
\hline Overall & $21.81(4.94,75.69)$ \\
\hline Men & $24.54(4.58,84.05)$ \\
\hline Women & $17.61(5.02,53.67)$ \\
\hline
\end{tabular}

${ }^{\text {a }}$ Presented with $95 \%$ reference intervals. compared with EAU guideline 2015. And they should have their own reference intervals of stone risk factors in 24-hour urine.

BRPRS4-13 Prevalence of pediatric urolithiasis in Kashgar area of Xinjiang Province in China: an ultrasonography based cross-sectional study

\section{Z Mai, G Zeng}

Department of Urology, Minimally Invasive Surgery Center, the First Affiliated Hospital of Guangzhou Medical University. Guangzhou Institute of Urology. Guangdong Key Laboratory of Urology

China (People's Republic)

Introduction \& Objective: The aim of this study was to investigate the prevalence and associated factors of pediatric urolithiasis in Kashgar area of Xinjiang Province in China.

Materials and Methods: A cross-sectional survey was conducted among children (0-14years) in Kashi area from July to December 2016. Children were selected by a two-stage random clustered sampling method. Participants underwent urinary tract ultrasonographic examinations, provided blood and urine samples to analyze. Their parents or guardians were asked to fill out questionnaires. Children those who were found with any abnormalities of their urinary tract by ulrtasonography would undergo a low-dose computed tomography (CT) to confirm the abnormalities. Prevalence was defined as the proportion of children with urinary stone and binary logistic regression was used to estimate the associated factors. This study has registered on the Clinical Trials.gov (NCT03003312).

Results: A total of 5605 children ( $52.2 \%$ boy) with an average age of $77.4 \pm 63.6$ (1-177) months were selected and invited to participate in the study. And 4849 (51.3\% boy) children completed the investigation, with a response rate of $86.5 \%$. The overall prevalence of urinary stone was $1.8 \%$ (95\% confidence interval (CI):1.5, 2.2). The prevalence of urinary stone was significant higher among children who had a family history of urolithiasis (6.8\% vs $1.6 \%, \mathrm{P}<0.001)$, but it was similar between boys and girls $(1.8 \%$ vs $1.9 \%, \mathrm{P}=0.721)$, between rural areas and urban areas $(1.8 \%$ vs $1.9 \%, \mathrm{P}=0.784)$, and it was decreased with age $(\mathrm{P}<0.001)$. Binary logistic regression analysis showed that without breast feeding, urinary tract infection, had a family history of urinary stone, and excessive sweating were all statistical significantly associated with increased risk of urinary stone.

Conclusions: Urinary stone is a common disease among children in Kashgar area of China and some feeding practice was associated with increased risks of stone formation.

BRPRS4-14 The effect of Aged Vinegar on stone risk factors in 24-hour urine: a pilot randomized controlled trial

Z Mai, G Zeng

Department of Urology, Minimally Invasive Surgery Center, the First Affiliated Hospital of Guangzhou Medical University. Guangzhou Institute of Urology. Guangdong Key Laboratory of Urology

China (People's Republic)

Introduction \& Objective: The aim of this study was to investigate the effect of Aged Vinegar on stone risk factors in 24hour urine.

Materials and Methods: We recruited young male healthy volunteers (aged 18-35 years) to detect the change of 24-hour urine risk factors associated with urinary stone pre- and after consuming Aged Vinegar. The exclusion criteria included: had urinary stone, serum creatinine $>133 \mu \mathrm{mol} / \mathrm{L}$, hematuria, urinary tract infection and urinary diversion. Participants were assigned to Aged Vinegar group or control group by a simple random sampling technique. Participants in Aged Vinegar group drank $15 \mathrm{ml}$ vinegar (Ninghuafu, Sanxi, China) at noon and evening respectively for a period of four weeks, while control group drank $15 \mathrm{ml}$ distilled water. All participants drank vinegar or distilled water under the surveillance of research clinicians. Participants, research clinicians, and trail personnel were not masked to trail assignment. The primary outcome was to compare the $\mathrm{Ph}$ value, volume, creatinine, oxalate, citrate, phosphate, uric acid, calcium, magnesium, potassium, sodium, chloride, the saturability of calcium oxalate () and calcium phosphate () in 24-hour urine between Aged Vinegar group and control group. These 24-hour urine samples were collected before trail, and on the 7th, 14th, 21th, and 28th day after trail beginning. This trial has registered on the Clinical Trials.gov (NCT02649140). 
Results: Between Dec 14, 2015, and Mar 28, 2016, we recruited 53 volunteers, and randomly assigned 50 participants (three were excluded because of ineligibility), including 26 in Aged Vinegar group and 24 in control group. All participants were included in the primary analysis. The analysis of 24-hour urine showed that Aged Vinegar consumption could increase urine volume $(\mathrm{P}=0.020)$, decrease the secretion of calcium $(\mathrm{P}=0.037)$, and the saturability of calcium phosphate() $\mathrm{P}=0.026)$. The differences of $\mathrm{Ph}$ value $(\mathrm{P}=0.233)$, creatinine $(\mathrm{P}=0.072)$, chloride $(\mathrm{P}=0.372)$, potassium $(\mathrm{P}=0.494)$, sodium $(\mathrm{P}=0.374)$, phosphate $(\mathrm{P}=0.547)$, magnesium $(\mathrm{P}=0.432)$, uric acid $(\mathrm{P}=0.081)$, oxalate $(\mathrm{P}=0.079)$, citrate $(\mathrm{P}=0.549)$, and the saturability of calcium oxalate ()$(\mathrm{P}=0.591)$ were not statistical significant between these two groups.

Conclusions: Aged Vinegar could increase urine volume, decrease calcium secretion, and the saturability of calcium phosphate in 24-hour urine.

\section{BRPRS4-15 Percutaneous Treatment of Bladder Stones in Children: 10 Years Experience, Is Blind Access Safe?}

H Ahmadnia, A Kamalati, M Younesi,

A akhavan Rezayat, M Imani

Masshad University of Medical Sciences, Mashhad Iran

Introduction \& Objective: Bladder stones are more common found in children from developing countries. Open Cystolithotomy or transurethral cystolithalopaxy are the traditional treatment but a percutaneous approach ha been advocated. We present our experience with percutaneous cystolithotomy in children with bladder stones without any ultrasonic or fluoroscopic guidance.

Materials and Methods: From April 2001 to October2011, A total of 147 children(135boys and 12girls) with a mean(range)age of 4.07(1-12.5)years underwent Percutaneous cystolithotripsy (PCCL). The mean(range)stone diameter was $2.74(0.8-5) \mathrm{cm} .138$ children $(94 \%)$ had a solitary stone while nine $(6 \%)$ had more than one stone. The main component of the stones were calcium oxalate in 70 patients $(48.6 \%)$.

Results: All children were stone-free after one PCCL; no recurrent stones developed. The mean (range)PCCL procedure time was 29.6(12 to 48)min and intraoperative blood loss was scant. Perioperative complications were few. The mean(range)hospital stay was1.2(1- 3)days.

Conclusions: Blind access PCCL(without any ultrasonic or fluoroscopic guidance)is a facile and safe approach for removing stones in the pediatric bladder stones. Advanta es include the lack of ionizing radiation, no need for opacification by iodine contrast media and low relative cost. We recommend this minimally invasive technique for management of large bladder stones (larger than $1 \mathrm{~cm}$ )in children. To our knowledge, this is the largest single center series reported on percutaneous cystolithotripsy of endemic bladder stones in children.

BRPRS4-16 Differential gene expression profile between papilla, medulla, and cortex leads to plaque and crystal formation

K Taguchi, DT Tzou, M Usawachintachit, BA Sherer, T Yasui, S Ho, ML Stoller, T Chi

UCSF

United States
Introduction \& Objective: Advanced endoscopy facilitates observation of plaques in the renal papilla. While these are thought to be important for lithogenesis, understanding the mechanism by which stones form along the nephron is challenging given the challenge of obtaining deep renal samples from the papilla tip. Understanding both histologic and genomic features of tissue within the kidney is important to elucidate the causes of crystal formation. To analyze gene expression profile among different renal locations, we performed RNA-sequence analysis of needle core biopsy specimens from a stone-forming kidney.

Materials and Methods: Specimens were obtained from a recurrent kidney stone patient who underwent laparoscopic nephrectomy of a stone-forming kidney with normal architecture. Four needle core specimens were taken from each papilla tip. Each specimen was between 2.5 and $3.0 \mathrm{~cm}$ length, and divided into 3 equal length parts representing the papilla, medulla, and cortex. After RNA extraction, samples were analyzed by single-end 50bp RNA-sequence with Illumina HiSeq ${ }^{\circledR} 4000$. Data analysis was performed by DESeq $2^{\mathrm{TM}}$ for differential expression comparison and IPA ${ }^{\circledR}$ for causal network analysis.

Results: Mineralization was observed in the papilla region by 3D-CT imaging and von kossa stain of paraffin embedded samples. 5871, 436, and 356 differentially expressed genes were found in the papilla vs cortex, papilla vs medulla, and medulla vs cortex, respectively. Cluster analysis demonstrated that different patterns of gene expression between the papilla, medulla, and cortex (Figure 1). Causal network analysis showed differentially expressed genes in the papilla demonstrated decreased function of molecule, carboxylic acid, and lipid transport genes as well as increased function of movement and urination disorder, hydronephrosis, polyuria associated genes. Moreover, within 35 genes known to be related to urolithiasis, gene expression of ALPL, AQP1, ATP6V0A4, ATP6V1B1, CASR, CLCN5, CLDN16, KCNJ1 SLC12A1, and UMOD were downregulated by more than $50 \%$ whereas MGP was two-fold upregulated in in the papilla compared to the medulla and cortex.

Conclusions: We demonstrated differential gene expression profiles among renal locations. The papilla showed impairment of genes associated with urolithiasis, indicating their local dysfunction may lead to calcification and plaque formation.

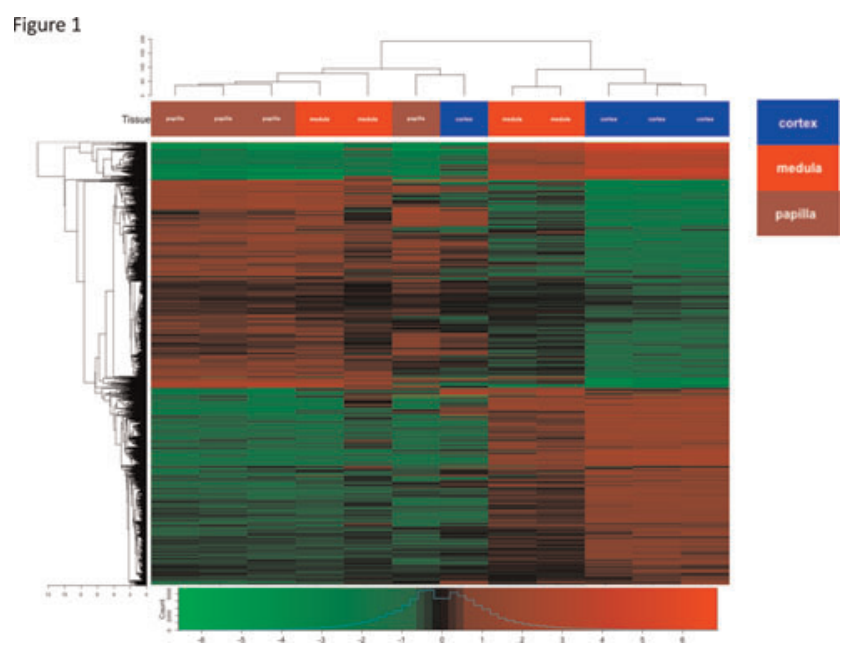


BRPRS4-17 Initial Discovery of Spontaneous Randall's Plaque Stone Overgrowth in an Animal Model

MS Borofsky, JC Williams, JP Lulich

University of Minnesota

United States

Introduction \& Objective: Stone formation in existing animal models used to study stone pathogenesis is frequently an artificially induced process that occurs in response to chemical exposure and dietary manipulation. An animal model that forms stones naturally and via similar mechanisms as humans would be preferable but has not previously been identified. Cats are known to form stones spontaneously though the mechanism by which this occurs has yet to be formally desfribed. Herein, we provide initial evidence of spontaneous stone formation in the feline via a mechanism similar to if not identical to that of human Randall's Plaque (RP) overgrowth.

Materials and Methods: Whole kidney specimens from deceased cats at a single university veterinary center underwent radiographic imaging to identify nephroliths. Stone bearing kidneys were subsequently examined with micro CT. Stone and tissue microdisection and examination was later performed on the largest identified stone via electron dispersion spectroscopy (EDS) and scanning electron microscopy (SEM).

Results: Twenty feline kidneys had evidence of intrarenal stones on high resolution X-ray (Figure 1). The kidney with the largest
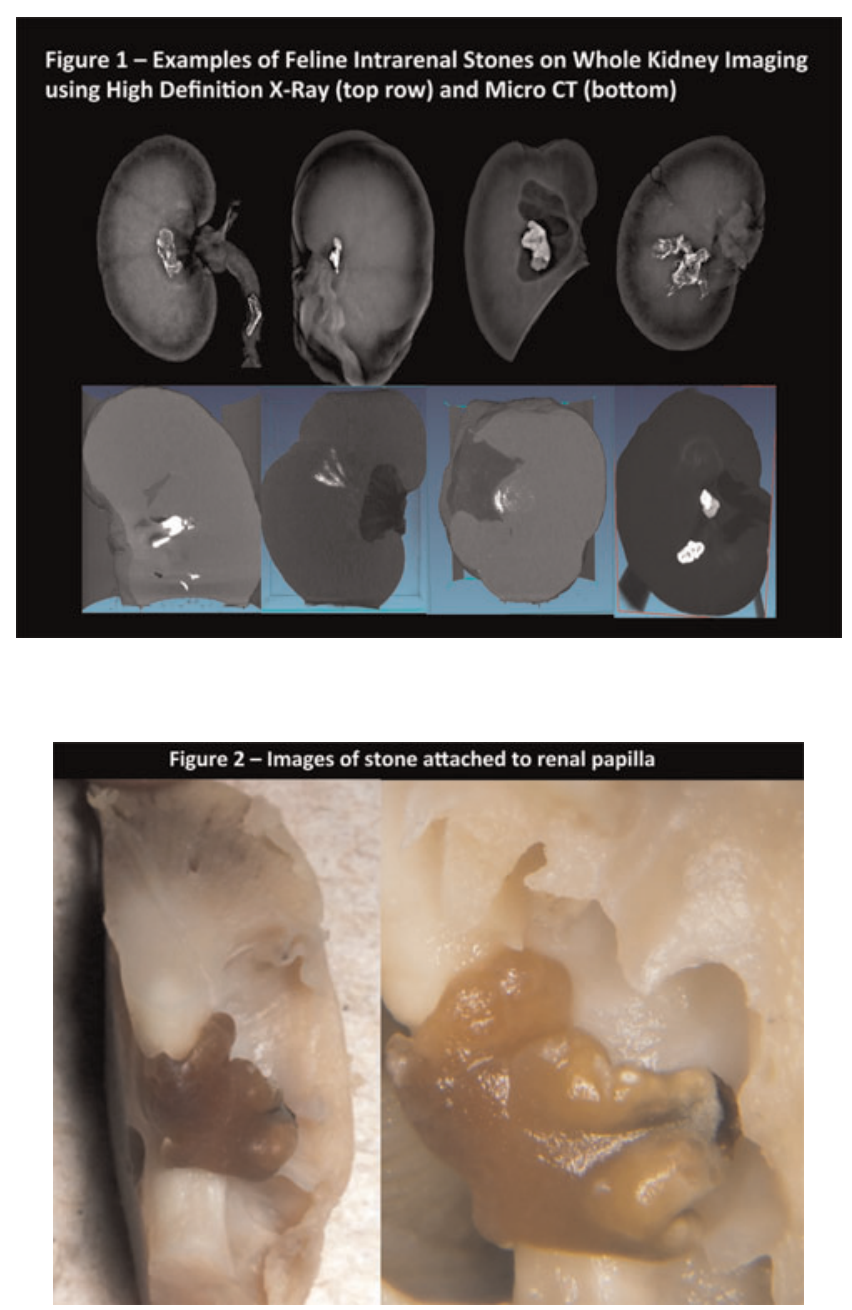

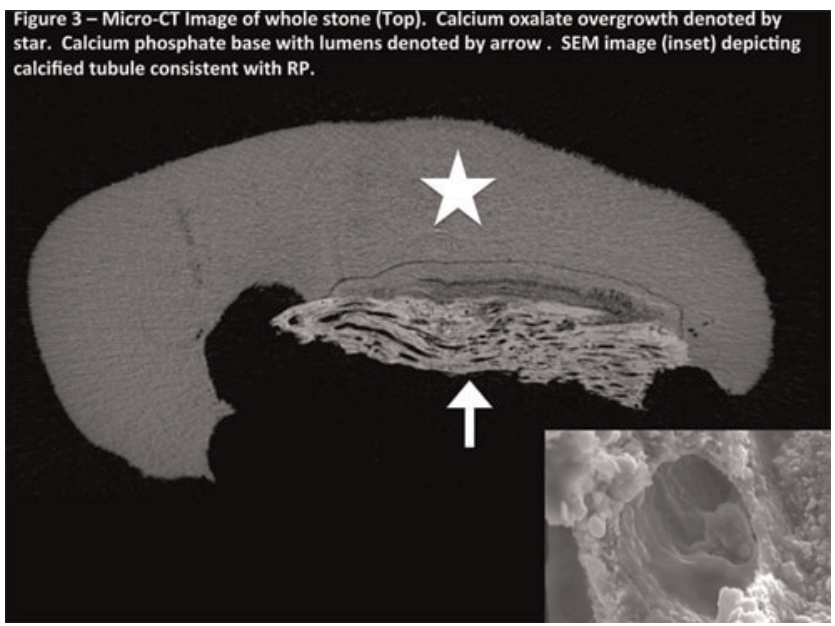

stone was dissected to further analyze the stone/tissue interface. It came from a deceased 15 year-old Bermese male cat diagnosed with stones during the last year of life but without prior history of renal insufficiency. The stone was $9 \times 4.5 \mathrm{~mm}$ and was attached to the papilla (Figure 2). Stone micro CT demonstrated calcium oxalate overgrowing a region of calcium phosphate closest to the papilla. Microstructure of the apatite region on micro CT and SEM showed lumens consistent with calcified renal tubules pathognomonic for RP (Figure 3). Mineral composition was confirmed with EDS. Micro-CT of the tissue beneath the stone showed calcium deposition along the tubules extending deep within the tissue.

Conclusions: Spontaneous stone formation in cats can occur via a RP mechanism indistinguishable from humans in terms of stone composition and microstructure. These findings are the first to describe naturally occurring, spontaneous RP stone overgrowth in a non-human subject and support further investigation of cats as a model to study stone formation.

BRPRS4-18 Canine Kidney Stones As A Comparable Human Stone Model in Lithotripsy Experimentation

JS Ahn, A Randad, W Kreider, MR Bailey, AD Maxwell, JP Lulich, E Furrow, MS Borofsky, MD Sorensen, JD Harper

Department of Urology, University of Washington Medical Center

United States

Introduction \& Objective: Human kidney stones used for in vitro lithotripsy experiments are typically obtained by passage or percutaneous renal access; however they are limited in size and may be subject to manipulation or lithotripsy prior to extraction, thus compromising them before experiments. Artificial stone models also have known limitations. Urinary stones are well documented and researched in canines, with the majority of stones removed intact transurethrally or via open lithotomy, contrary to human stones. We hypothesize that intact canine urinary stones are a suitable alternative human stone model for lithotripsy testing, and that intact canine stones are harder to break than their variably intact human counterparts.

Materials and Methods: We compared pairs of canine and human stones of similar composition and weight, and examined percent stone weight fragmented per minute. Stones were 

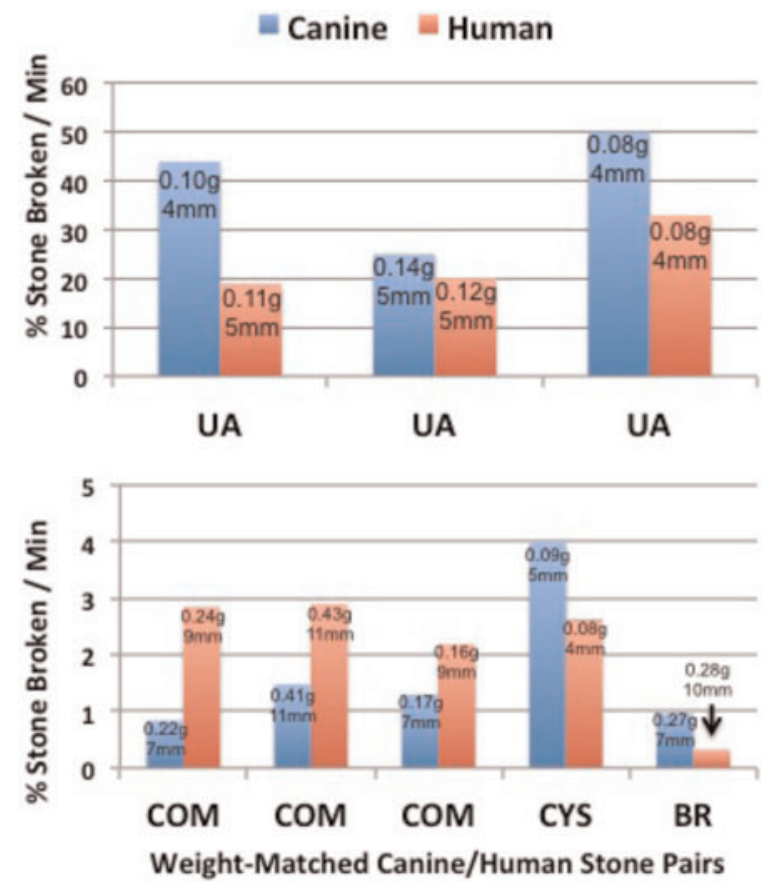

treated with a $335 \mathrm{kHz}$ BWL transducer in a water bath for 45 min or until completely fragmented, defined as no fragments over $2 \mathrm{~mm}$ remaining. The weights of the remaining stone and fragments over $2 \mathrm{~mm}$ after treatment were compared to their associated pre-treatment stone weight. Intact canine calcium oxalate monohydrate (COM), ammonium acid urate (AAU), cystine (CYS), and brushite (BR) stones were obtained through the Minnesota Veterinary Urolith Center. Due to the rarity of AAU stones in humans, we compared canine AAU stones to human uric acid (UA) stones. Stones measured between 4 to $11 \mathrm{~mm}$.

Results: Both human and canine stones produced similar sized fragments during BWL, almost all under $2 \mathrm{~mm}$. Canine AAU and COM stones broke at an average rate of 1.7 (range 1.3-2.3) and 0.5 (range 0.3-0.6) times that of corresponding weight-matched human stones, respectively (3 pairs per stone type)(See Figure). Single pairs of CYS and BR canine stones broke more readily, than corresponding human stones. Species aside, all urate-based stones $(4-5 \mathrm{~mm})$ broke at a range of $19-44 \%$ per minute, while all other stone types $(4-11 \mathrm{~mm})$ broke at $0.3-4 \%$ per minute.

Conclusions: Preliminary results suggest that intact canine stones can have a similar relative response to lithotripsy when compared to human stones of similar type and weight. Further study is warranted to establish a statistical comparison between the 2 groups.

BRPRS4-19 Local and sustainable delivery of lidocaine/ ketorolac to the urethra in an animal model via an analgesic-eluting stent coated with biodegradable nanofibers

Y Lin, K Liu, T Tsai, T Hwang, S Liu

Shin Kong WHS Hospital

Taiwan (Republic of China)

Introduction \& Objective: Pain induced by the stent deployment has been one of the major urinal discomforts. This study developed a novel analgesics-eluting stent coated with electro- spun nanofibers for the sustained release of lidocaine and ketorolac in the ureteral space.

Materials and Methods: Electrospun nanofibers prepared using polylactide-polyglycolide (PLGA) copolymer, lidocaine hydrochloride and ketorolac tris salt dissolved in 1,1,1,3,3,3hexafluoro-2-propanol were used to coat the surface of a double-J stent. The in vitro patterns of drug release were determined by placing the stent in phosphate-buffered saline. In vivo studies were performed using rabbits deployed with the analgesics-
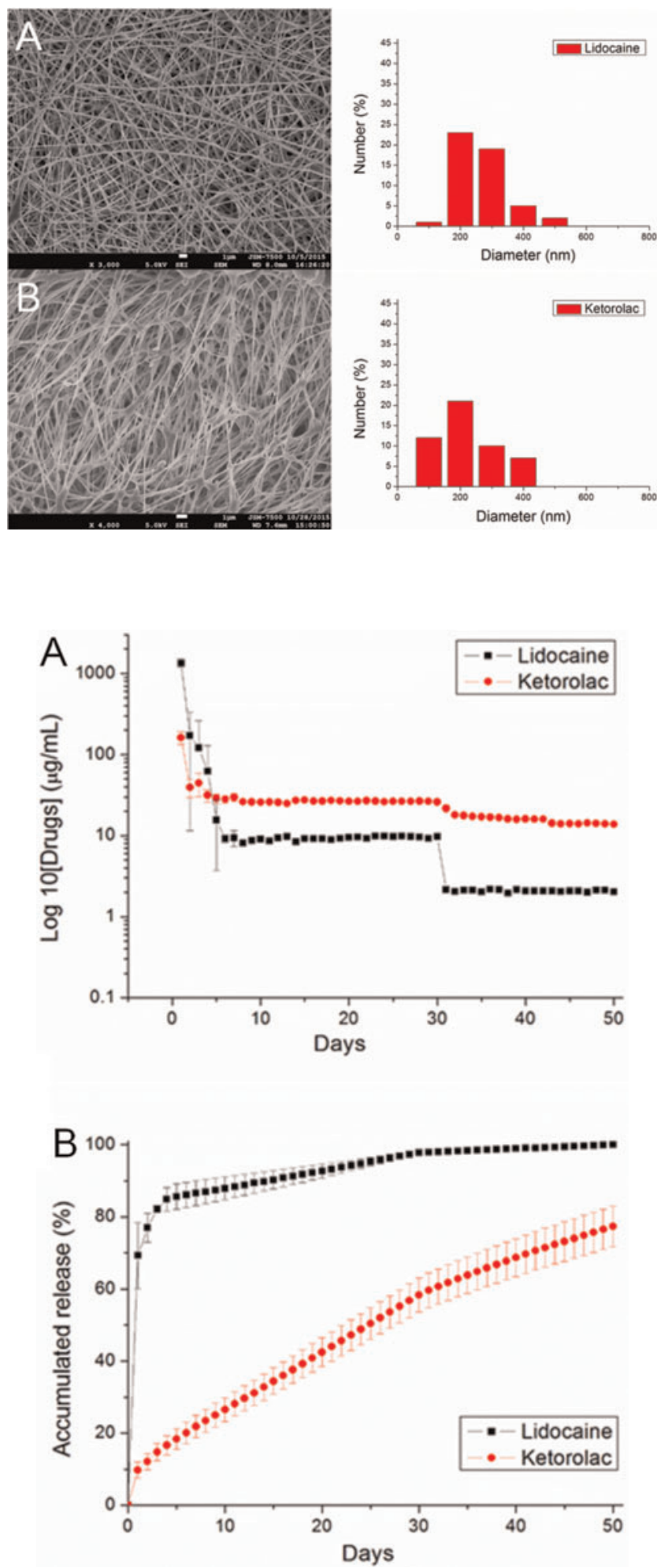
eluting stents. Lidocaine and ketorolac concentrations in the ureteral tissue and in serum and pleural fluid were measured by employing a high performance liquid chromatography (HPLC). Results: The experimental results suggested that the analgesicseluting stents can elute effective levels of lidocaine and ketorolac for more than 50 days. The drug concentration is much greater in the urethra tissue (local) than in serum (systemic). Furthermore, histological analysis did not any noticeable inflammation in the tissue surrounding the stents.

Conclusions: Dual drug-eluting ureteral stent offers a long-term steady release of analgescs in this expeirment. The strategy enhance the future possiblty clinical use of drug-eluting stents.

\section{BRPRS4-20 Evaluation of in vitro burst wave lithotripsy} exposure conditions

C Hunter, JS Ahn, W Kreider, AD Maxwell, BW Cunitz, Y Wang, B Dunmire, MR Bailey, JD Harper, MD Sorensen

Center for Industrial and Medical Ultrasound, Applied Physics Laboratory, University of Washington

United States

Introduction \& Objective: Burst wave lithotripsy (BWL) is a new non-invasive technology for disintegrating kidney stones using bursts of sub-megahertz ultrasound. In vitro studies suggest BWL can deliver acoustic energy to fragment stones at a faster rate without causing cavitation compared to shock wave lithotripsy (SWL). However, B-mode ultrasound observations of porcine kidneys during BWL exposures suggest large, sustained cavitation clouds can occur above certain pressure amplitudes. Such cavitation has been correlated with renal parenchymal injury if allowed to persist and would likely shield the stone to hinder further effective lithotripsy. This work focuses on mimicking conditions that generate sustained bubble clouds in vitro as part of a BWL test bed. Materials and Methods: BWL exposures were delivered to a kidney phantom (polyvinyl alcohol, PVA) with a water-filled collecting system (see figure). BWL exposures at $335 \mathrm{kHz}$ comprised 2,000 pulses delivered at a repetition rate of $40 \mathrm{~Hz}$ and a duty factor of $0.2 \%$. Variable conditions were tested to explore the impact on cavitation of the dissolved gas concentration in water, the confined water volume, and the presence/ absence of a stone. For each test condition, BWL exposures were delivered over a range of focal pressure amplitudes with 10 observations recorded at each. Every observation included high-speed photography and B-mode ultrasound images and was interpreted as above or below the threshold based on detection of sustained bubble activity.

Results: Observations were compiled to yield a cavitation probability, at each amplitude, with the threshold defined as the

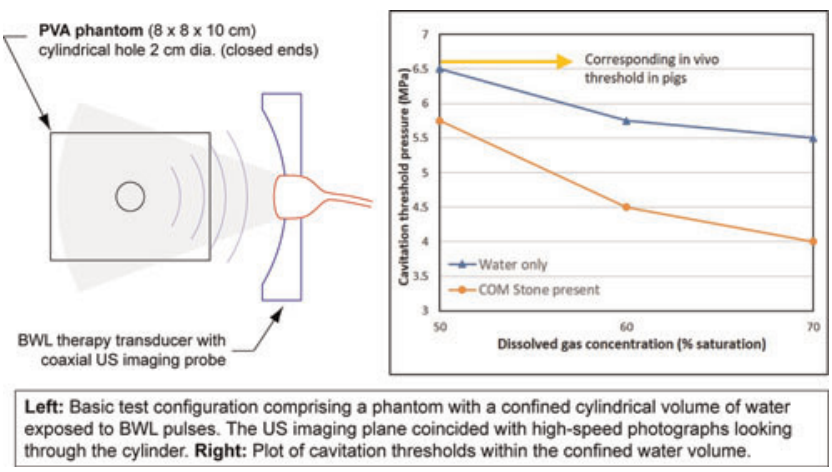

lowest amplitude with non-zero probability. Threshold behavior for generating sustained cavitation clouds within the phantom collecting system mimicked B-mode observations from in vivo pig kidneys without stones. Thresholds decreased with elevated concentrations of dissolved gas and the presence of a human calcium oxalate stone (see figure). Although volumetric confinement was required to generate sustained cavitation clouds, variation of this volume from 10-25 mL did not significantly alter thresholds.

Conclusions: An in vitro test configuration with a volumetrically confined liquid space and controlled gas concentration can simulate cavitation behaviors during BWL exposures. Results suggest that a $50 \%$ dissolved gas concentration most closely represents in vivo observations for the tested exposures.

BRPRS4-21 The Impact of Forces Applied during Ureteral Access Sheath Deployment on Ureteral Injury in a Porcine Model

KS Kaler, RM Patel, R Yoon, DJ Lama, C Hwang, Z Okhunov, M Klopfer, J Landman, RV Clayman

Department of Urology, University of California, Irvine United States

Introduction \& Objective: Widespread use of the ureteral access sheath (UAS) during ureteroscopy has been slowed by concerns over possible ureteral injury during its passage. In this pilot porcine study, using a novel device developed at UC Irvine, we evaluated the force threshold which would induce ureteral injury.

Materials and Methods: With IACUC approval, we measured UAS deployment force using a novel Ureteral Access Sheath Force Sensor (UC Irvine Force Sensor) in a female Yorkshire pig (Figure 1). Under fluoroscopic control, force was continuously measured from the time the UAS contacted the urethral meatus until the tip of the UAS had reached the renal pelvis. Ureteral dilators $(6-9 \mathrm{~F})$, UAS and its obturators $(9.5 \mathrm{~F}, 10 \mathrm{~F}, 11 \mathrm{~F}, 11.5 \mathrm{~F}$, $12 \mathrm{~F}, 13 \mathrm{~F}, 14 \mathrm{~F}, 15 \mathrm{~F}, 16 \mathrm{~F}$ ) sequentially passed twice into both ureters. Ureteroscopic evaluation was initiated after the $9.5 \mathrm{~F}$ UAS obturator was passed.

Results: No ureteral injury occurred at $\leq 4$ Newtons (N). Increasing UAS size resulted in greater force over-time (Figure 2) and larger peak forces (Figure 3). First ureteral injury occurred at

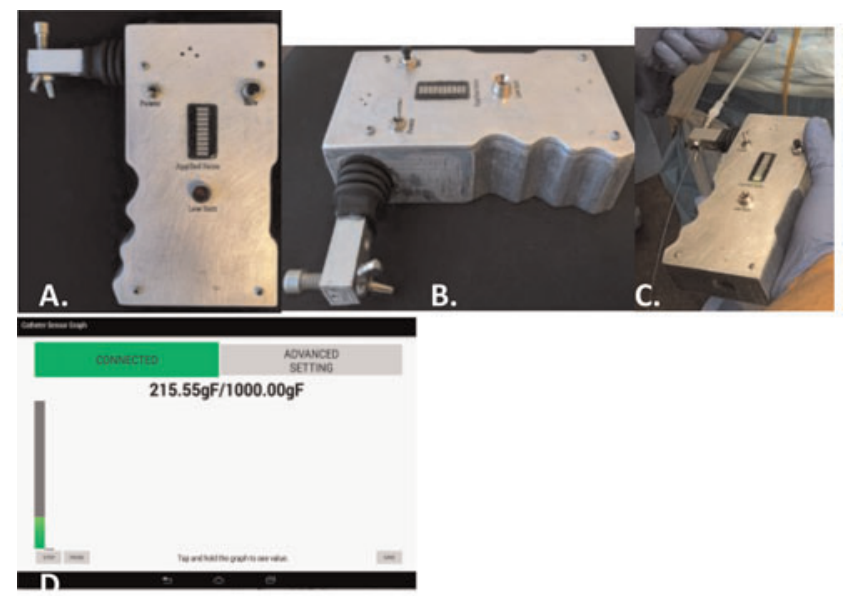

Figure 1: Ureteral Access Sheath Force Sensing Device and Screenshot of Software. A, Top view. B, Side view. C, Device in use. D, Screenshot of real-time reading from force sensor. 
Figure 2. Force Measurements during Ureteral Access Sheath Deployment (Right ureter)

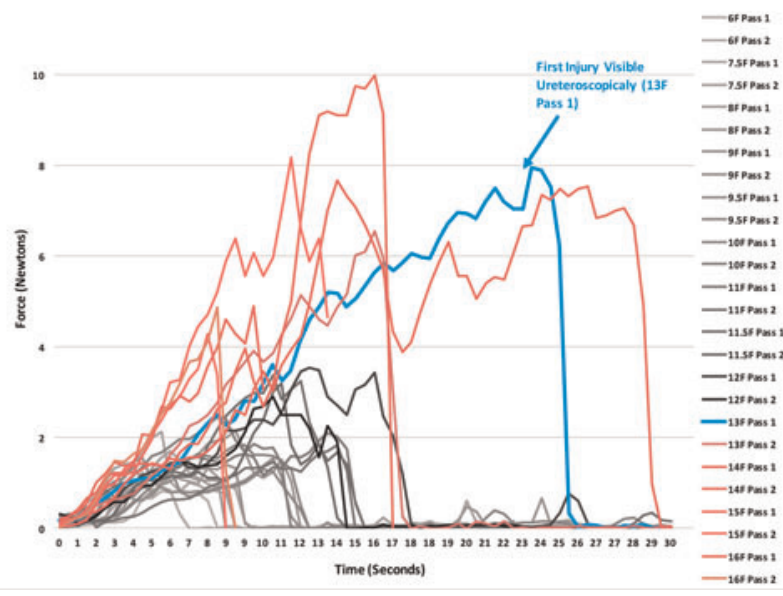

Figure 3. Peak Force Measured During Ureteral Access Sheath Deployment in the Porcine Model for Left and Right Ureter

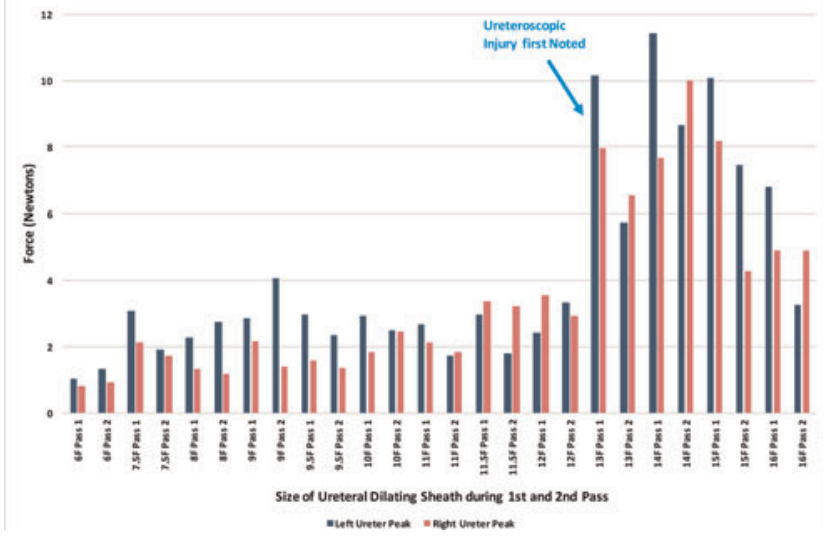

Figure 4. A. Normal Right Proximal Ureter. B. Initial Medial Wall Injury in Proximal Right Ureter and with $13 F$ UAS at peak pressure of $8 \mathrm{~N}$. C. Additional Lateral Wall Injury in the same Proximal Right Ureter with 14F UAS.

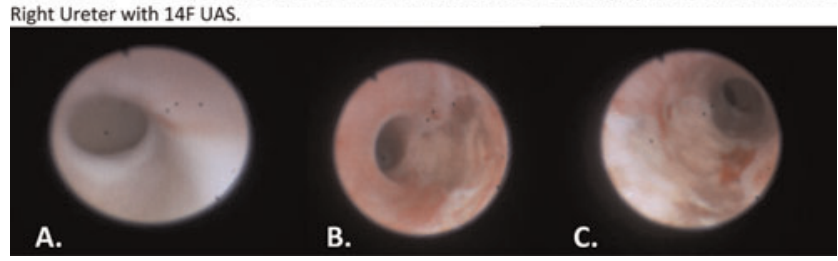

$8 \mathrm{~N}$ (right ureter) and $10 \mathrm{~N}$ (left ureter). Figure 4 shows a normal right ureter before and after deployment of a $13 \mathrm{~F}$ UAS with a peak force of $8 \mathrm{~N}$.

Conclusions: The UC Irvine Force Sensor can reliably measure force while deploying a UAS. Initial ureteral injury occurred at forces $\geq 8 \mathrm{~N}$.
BRPRS4-22 A Multi-centre cohort study evaluating the role of Inflammatory Markers In patient's presenting with acute ureteric Colic (MIMIC).

N Nkwam, T Shah, A O'Keefe, C Gao, T Manning, A Peacocke, S Cashman, A Nambiar, B Lamb, M Cumberbatch, R Pickard, P Erotocritou, D Smith, V Kasivisvanathan

University Hospitals of Leicester NHS Trust, Leicester, United Kingdom

United Kingdom

Introduction \& Objective: There is conflicting evidence on the role of raised inflammatory markers in acute ureteric colic and spontaneous stone passage. Of particular interest are patients discharged with conservative management and whether White Cell Count (WBC) on admission can predict stone passage. If so, it could be used to guide management in these patients. MIMIC aims to assess whether WBC at presentation of acute ureteric colic is associated with likelihood of spontaneous stone passage.

Materials and Methods: Design: Multi-centre cohort study in 71 centres disseminated via the UK British Urology Researchers in Surgical Training (BURST).

Primary Outcome: Spontaneous stone passage (SSP).

Inclusion criteria: Acute renal colic with CT-KUB confirmed ureteric stone.

Follow up: 6 months.

Statistical Analysis: A multivariate logistic regression was performed including: WBC, Neutrophils, CRP, Creatinine, Stone size, Stone position, Hydronephrosis, NSAID use, Medically Expulsive Therapy (MET) use, Antibiotic use.

Results: Data were collected from $\mathbf{4 , 1 8 1}$ patients. $75 \%(\mathrm{n}=3127)$ were discharged with conservative management. $80 \%(n=2516)$ had a confirmed outcome and were included in the multivariate analysis. Overall SSP rate for this cohort was $74 \%(n=1863)$.

WBC was not significantly associated with SSP on either the univariate or multivariate analysis (adjusted OR 0.99 [95\% CI 0.99-1.00], $\mathbf{p}=\mathbf{0 . 5 2 7}$ ).

A number of factors were found to be significant on univariate analysis but after adjusting for key confounding variables in multivariate analysis the strongest predictors of SSP were stone size (OR 0.57 [95\% CI 0.53-0.61], $\mathrm{p}=0.00001$ ) and position (OR 3.31 [95\% CI 2.60-4.22], $\mathrm{p}=0.00001$ ). For every increase in stone size by $1 \mathrm{~mm}$, the odds of SSP decreased by $43 \%$. Stone clearance rate for stones measuring $0-5 \mathrm{~mm}$ was $84 \%$ compared to $42 \%$ for stones measuring $6 \mathrm{~mm}$ or greater. Compared to proximal ureteric stones, distal ureteric stones had a three times greater odds of SSP. Stone clearance rate was $51 \%$ for proximal ureteric stones, $69 \%$ for mid ureteric stones and $83 \%$ for lower/ distal ureteric stones.

Conclusions: To our knowledge MIMIC is the largest contemporary cohort assessing outcomes from acute ureteric colic. Our data shows that patients with acute ureteric colic who are suitable for initial conservative management, WBC alone should not be used to influence decisions on whether to discharge or perform intervention. However, stone size and position should inform clinical decisions. This data will be used to develop a risk calculator for the prediction of spontaneous stone passage. 


\section{MPS1: PCNL I TECHNIQUES}

MP1-1 Super-mini percutaneous nephrolithotomy (SMP) versus retrograde intrarenal surgery for the treatment of 1 to $2 \mathrm{~cm}$ lower pole renal calculi: a multinational multicenter randomized controlled trial

G Zeng, T Zhang, J Fan, W Zhang, S Yang, K Xiao, X Li, H Li, $\mathrm{X} \mathrm{He}, \mathrm{C} \mathrm{Xu}, \mathrm{K}$ Sarica, MS Agrawal

Department of Urology, Minimally Invasive Surgery Center, the First Affiliated Hospital of Guangzhou Medical University. Guangzhou Institute of Urology. Guangdong Key Laboratory of Urology

China (People's Republic)

Introduction \& Objective: The treatment of Lower pole (LP) calculi is controversial, especially $1-2 \mathrm{~cm}$ stones, with competing interventions possessing advantages and disadvantages. A multinational multicenter prospective randomized comparison of super-mini percutaneous nephrolithotomy (SMP) and retrograde intrarenal surgery (RIRS) for the treatment of 1 to $2 \mathrm{~cm}$ LP renal calculi was done to evaluate the safety and efficacy of these procedures.

Materials and Methods: 153 patients with 1 to $2 \mathrm{~cm} \mathrm{LP} \mathrm{renal}$ calculi who underwent treatment between August 2015 and January 2017 were included in this study at 9 Asian centers and 1 European center. These patients were randomized to SMP and RIRS groups. One-step stone-free rate (SFR) and SFR at 1-month postoperatively were the primary outcomes. The secondary outcomes included blood loss, operation duration, pain visual analogue score (VAS) score at $24 \mathrm{~h}$ postoperatively, auxiliary procedures, complications and hospital stay. The study was registered at http://clinicaltrials.gov /(NCT02519634).

Results: The SFR was significantly higher in the SMP group than in the RIRS group (one-step SFR 94.8\% and 75.0\%, P=0.001; overall SFR at 1-month postoperatively $97.4 \%$ and $84.2 \%$, $\mathrm{P}=0.005$ ). The auxiliary procedure rate was lower in the SMP group than in the RIRS group ( $5.2 \%$ vs $25.0 \%, \mathrm{p}=0.001)$. RIRS is superior to SMP in terms of lower haemoglobin drop (10.2 vs $4.3 \mathrm{~g} / \mathrm{L}, \mathrm{P}<0.001)$ and less postoperative pain $(2.0$ vs 1.3 , $\mathrm{P}=0.008)$. There was no significantly difference in the operating time and hospital stay. The overall complication rates were similar in the two group studied.

Conclusions: SMP and RIRS are safe and feasible surgical options in the treatment of 1 to $2 \mathrm{~cm}$ LP renal calculi. SMP provides overall significantly higher SFR and lower auxiliary rate compared with RIRS, with no differences in surgical time and hospital stay, whereas RIRS is superior in terms of lower haemoglobin drop and less postoperative pain.

MP1-2 Clinical effect of prone mini-percutaneous nephrolithotomy with two-step precise puncture method by ultrasound guidance under total paravertebral block anesthesia

H Yang, X Yu, S Wang

China (People's Republic)

Introduction \& Objective: To investigate the the efficiency and safety of mini-percutaneous nephrolithotomy (PCNL) for kidney stones in the prone position with two-step precise puncture method by ultrasound guidance under total paravertebral block (PVB) anesthesia.

Materials and Methods: From December 2015 to March 2016, 45 cases of patients with renal stones underwent prone PCNL after successful PVB. All the precise punctures were achieved under ultrasonographic guidance. The puncture needles first addressed the perinephric fat tissue and confirmed the appropriate insertion direction from kidney surface to target renal calyx, then punctured into renal calyx through renal calyx fornix. The accesses were dilated to F18-F20 gradually, and the stones were fragmented by Holmium laser and the debrises were removed by water vortex. All patients received conventional abdominal plain film and 3dimensional reconstruction of non-contrast abdominal computed tomography before operation. All procedures were performed in prone position, and the intraoperative complications as the postoperative outcome were reviewed. Postoperative complications, recovery, stone free rate, dosage of pain relievers were evaluated. Results: The mean operation time was 59 (25-150) min, no patients complained pain during operation. Time to first postoperative analgesic requirement was $160(60-630) \mathrm{min}$, and the mean dosage of sodium dichlorophenolate was 150 (50-425) mg. Early ambulation could be achieved in 1 hour after operations. The complete stone clearance rate was $93.1 \%$ according to postoperative plain abdominal radiograph. No patients needed blood transfusion and no patients suffered severe complications. Mean time of hospital stay was 5.5 (4-9) days.

Conclusions: The prone PCNL of two-step precise puncture method by ultrasound guidance under paravertebral block anesthesia totally was a safe and efficient approach for renal stones, resulting in quick recovery, high stone free rate and few severe complications.

MP1-3 Prospective comparative study of the efficacy and safety of new-generation versus early-generation system for super-mini-percutaneous nephrolithotomy: a revolutionary approach to improving the irrigation and stone removal

G Zeng, W Zhu, Y Liu

Department of Urology, Minimally Invasive Surgery Center, the First Affiliated Hospital of Guangzhou Medical University. Guangzhou Institute of Urology. Guangdong Key Laboratory of Urology

China (People's Republic)

Introduction \& Objective: The study sought to compare the procedural and clinical results of super-mini-percutaneous nephrolithotomy (SMP) with the use of the first- and new- generation devices.

Materials and Methods: A prospective, comparative cohort study was performed from February 2013 to January 2017. A total of 156 consecutive patients undergoing SMP were enrolled in the study. Of 156 patients, the first 85 consecutive patients underwent SMP with the first-generation system, and the other 71 consecutive patients were treated with the new-generation SMP system. The first-generation SMP system consists of a $7 \mathrm{~F}$ miniaturized nephroscope and a modified $10-14 \mathrm{~F}$ plastic sheath with a suctionevacuation function. The new-generation SMP system consists of a 8 F miniaturized nephroscope and a newly designed irrigationsuction sheath. The primary outcome of the present study was the 
operating time, which was calculated from the starting of percutaneous puncture to the wound closure. Secondary outcomes were the stone-free rate (SFR), blood loss (haemoglobin decrease), hospital stay, and postoperative complications.

Results: The two groups had comparable demographic data such as age, BMI, stone size, stone location, comorbidities, grade of hydronephrosis, and positive preoperative urine culture. The new-generation SMP had a shorter operation time (39.3 vs. $50.5 \mathrm{~min}, \mathrm{p}=0.016$ ), and shorter postoperative hospitalization time (2.1 vs. 3.0 days, $\mathrm{p}<0.001)$ than the first-generation SMP. No significant difference existed between the two groups for SFR, haemoglobin decrease, and tubeless rate. The overall operative complication rates using the Clavien-Dindo grading system were similar between the groups.

Conclusions: The clinical outcomes of SMP with the newgeneration system were favorable. The new-generation SMP system was associated with shorter operation time and postoperative hospitalization time than the first-generation system.

MP1-4 Application of super-mini percutaneous nephrolithotomy (SMP) in the treatment of upper urinary tract calculi

Z Guoxi, Z Xiaofeng, X Rihai, Y Yuanhu, W Gengqing, W Xiaoning

Department of Urology, First Affiliated Hospital of Gannan Medical University

China (People's Republic)

Introduction \& Objective: To introduce our experience in super-mini percutaneous nephrolithotomy (SMP) for the treatment of upper urinary tract calculi, and evaluate its safety and efficacy. Materials and Methods: A total of 39 cases with upper urinary tract calculi underwent SMP. Of these cases, 19 were boys and 20 girls, aged from 24 year to 76 years (mean, 49.3 years). The calculi were found on left side in 18 cases, on right side in 16 and on both side in 5 . The largest size of the calculus was from 0.8 to $2.5 \mathrm{~cm}$ (mean, $2.1 \mathrm{~cm}$ ). Seventeen cases had renal calculi, 18 had proximal ureter calculi and 4 also had renal and proximal calculi. Under general anaesthesia, a 5-F ureteric catheter was first inserted into the collecting system in a retrograde fashion in the lithotomy position. The patient was then turned prone and the desired calyx was punctured under ultrosound guidance. $\mathrm{Ne}$ phrostomy tract dilatation was carried out using fascial dilators of $8-14 \mathrm{~F}$. A 12 or $14 \mathrm{~F}$ sheath was then placed. The stone was visualised and lithotripsy was performed using holmium-yttrium aluminium garnet (YAG) laser. At the end of procedure, a double $\mathrm{J}$ stent was placed. Indications for nephrostomy tube placement included significant residual stone fragments, which would require a second-look procedure and significant pyelocalyceal blood clots or bleeding after the lithotripsy.

Results: SMP was completed successfully in 38 of 39 patients. One patient required conversion to larger nephrostomy tracts for bleeding. The mean operative time was $57.8 \mathrm{~min}$. The initial stone-free rate (SFR) was $92.3 \%$. The SFR at the 3-month follow-up was $94.7 \%$. Two patients required auxiliary procedures for residual stones. Complications occurred in $13.2 \%$ of the patients, all of which were Clavien grade $\leq$ II except one patient who underwent super selective embolization of renal artery for blood and no transfusions were needed. In all, $87.2 \%$ of the patients did not require nephrostomy tubes placed. The mean hospital stay was 3.6 days.

Conclusions: SMP is a safe and effective treatment for renal stones of $<2.5 \mathrm{~cm}$ with less trauma, faster recovery and shorter hospital stay.
MP1-5 A Prospective Randomized Controlled Study of Instantly Phase-II Tubeless Percutaneous nephrolithotomy

L Folin, Z Xiaofeng, X Rihai, Y Yuanhu, W Gengqing, W Xiaoning

Department of Urology, First Affiliated Hospital of Gannan Medical University

China (People's Republic)

Introduction \& Objective: We aimed to evaluate the feasibility, safety and efficacy of instantly phase-II tubeless PCNL and to investigate the inclusion criteria, experience for instantly phaseII tubeless PCNL.

Materials and Methods: A prospective study was conducted on 390 patients with upper urinary calculi. The patients were randomly divided into three groups according to the random number table. Three groups were instantly phase-II tubeless PCNL (130 cases, group A), tubeless PCNL (130 cases, Group B) and standard PCNL (130 cases, group C). All patients were excluded from systemic hemorrhagic disease and stones with empyema. The inclusion criteria of group A were including stone diameter generally $<3 \mathrm{~cm}$, only a single percutaneous renal access, distal renal calculi collection system without obstruction, percutaneous renal access established smoothly, collection system with no active bleeding and perforation, no residual stones or clinically insignificant residual stone (CIRF residual stones $<4 \mathrm{~mm}$ ), no delayed second-look nephroscopy, no significant urinary tract infection, renal empyema, no adjacent organs damage and the surgical time less than 60 minutes. In group A, the NT was removed during the first 12 to 24 hours after PCNL stone extraction with clear urine, no urinary extravasation, no active bleeding and no delayed second-look nephroscopy to clear visible residual stones. The age, sex, stone size, operation time, mean blood loss, stone clearance rate, postoperative visual analogue pain scores (VAS), analgesic requirement, hospital stay and complication rate of all the groups were compared respectively.

Results: All the PCNL procedures of three groups were performed successfully. The difference in the age, stones size, operation time, stone free rate, mean blood loss and complications among the three groups were not statistically significant $(\mathrm{P}>0.05)$. While the VAS, analgesia requirement and hospital stay of instantly phase-II tubeless PCNL and tubeless PCNL were all lower than the standard PCNL, the differences were statistically significant $(\mathrm{P}<0.05)$. No complications were observed in all instantly phase-II tubeless PCNL patients with secondary hemorrhage after NT removal. All the patients were followed up for 1 to 24 months, no complications such as perirenal infection, perinatal urinary cyst and perirenal hematoma were observed.

Conclusions: Instantly phase-II tubeless PCNL appeared to be feasible, safe, and effective for the indication patients. Compared with the standard PCNL, it showed less pain, shorter hospital stay. While compared with the tubeless PCNL, the secondary hemorrhage risk could also be avoided commendably.

MP1-6 The role of super-mini percutaneous nephrolithotomy (SMP) in the treatment of symptomatic lower pole renal stones (LPSs) after the failure of shockwave lithotripsy (SWL) or retrograde intrarenal surgery (RIRS)

G Zeng, J Fan

Department of Urology, Minimally Invasive Surgery Center, the First Affiliated Hospital of Guangzhou Medical University. Guangzhou Institute of Urology. Guangdong Key Laboratory of Urology

China (People's Republic) 
Introduction \& Objective: To assess the safety and efficacy of super-mini percutaneous nephrolithotomy (SMP) in the treatment of symptomatic lower pole renal stones (LPSs) after the failure of shockwave lithotripsy (SWL) or retrograde intrarenal surgery (RIRS).

Materials and Methods: We retrospectively evaluated 44 patients with symptomatic LPSs who previously failed SWL or RIRS treatment and consequently underwent SMP between October 2014 and June 2016. The percutaneous renal access was performed $12-14 \mathrm{~F}$ in size with C-arm fluoroscopy or ultrasonographic guidance. Stone disintegration was performed using either Holmium laser or pneumatic lithotripter. Perioperative parameters along with operative data were assessed in detail.

Results: A total of 44 patients (mean age $49.1 \pm 13.7$ years) with LPSs were included in the study. Mean stone size was $18.4 \pm$ $6.0 \mathrm{~mm}$ (range 9-29), mean operative time was 63.9 $\pm 32.7 \mathrm{~min}-$ utes (range 14-145) and mean hospital stay was $2.8 \pm 1.2$ days (range 1-5). The hemoglobin drop was $12.4 \pm 8.8 \mathrm{~g} / \mathrm{L}$ (range 031 ), no patients required blood transfusion. Complete stone-free status (SFR) was achieved in $40(90.9 \%)$ patients. Clinically insignificant residual fragments (CIRF) were observed in three $(6.8 \%)$ patients and only one $(2.3 \%)$ patient had a $6 \mathrm{~mm}$ residual calculus. A total of three minor complications (urinary tract infection, hemorrhage resolved by hemostatics and renal colic requiring analgesics) were observed postoperatively.

Conclusions: For symptomatic LPSs after the failure of SWL or RIRS, SMP is a safe and efficient auxiliary option and even might be an alternative to SWL or RIRS, while further considering the stone free rates and stone-related events.

MP1-7 Prospective comparative study of Super-mini Percutaneous nephrolithotomy and Miniprec for the treatment of stone lager than $2 \mathrm{~cm}$.

Y Liu, W Zhu, Y Liu, W Wu, W Zhong, J Fan, Y Lan, X Li, G Zeng

Department of Urology, Minimally Invasive Surgery Center, the First Affiliated Hospital of Guangzhou Medical University China (People's Republic)

Introduction \& Objective: The aim of this study was to compare the efficacy and safety of Super-mini percutaneous nephrolithotomy (SMP, F12-F14) and mini-percutaneous nephrolithotomy (Miniperc, F18) in the treatment of renal stones of $2-4 \mathrm{~cm}$ in size.

Materials and Methods: We prospectively compared the outcomes of SMP and Miniperc in our center, consecutive cohort study. Feom July 2014 to January 2017, 336 patients with renal stone larger than $2 \mathrm{~cm}$ were underwent either SMP or miniperc treatment, depending on paitnets' own decision. Demographic data, stone criteria, operative technique, complications, blood transfusion, hemoglobin decrease, stone-free rate and length of hospital stay were compared between the two groups.

Results: 79 patients and 257 patients were subjected to SMP and Miniprec treatment, respectively. Stone burden was comparable for both groups $(3.0 \pm 1.0$ vs $3.1 \pm 0.8, \mathrm{p}<0.05)$. Mean operation time was not significant difference between two groups, while the hospital stay of SMP was much shorter than Miniperc $(2.6 \pm 1.4$ vs $5.2 \pm 2.1, \mathrm{p}<0.001)$. Stone-free rate in postoperative 1 day was similar between two groups $(86.1 \%$ vs $81.7 \%$, $\mathrm{p}=0.923)$, while a higher clearance rate was achieved by SMP at 1 month follow up $(93.7 \%$ vs $85.0 \%, \mathrm{p}<0.05)$. In addition, there was an advantage of SMP over Miniperc in terms of a significantly higher tubeless rate $(82.3 \%$ vs $4.7 \%, \mathrm{p}<0.001)$. No difference was observed between two groups in terms of com- plications $(p=0.526)$. Three patients in the Miniperc group required blood transfusions, compared to none in the SMP group. Conclusions: In the treatment of large size renal stone, SMP is associated with a better stone clearance without increasing the complications. Meanwhile, SMP appears to have significant advantage in terms of higher tubeless procedure and reduced hospital stay.

MP1-8 A comparison of fluoroscopy time for two different techniques of percutaneous nephrolithotomy tract dilation: one-shot vs metal telescopic dilation

I Sedano-Portillo, G Ochoa-Leon, A Gonzalez-Ojeda

IMSS

Mexico

Introduction \& Objective: Percutaneous nephrolithotomy is considered first line treatment for large renal stones. The creation of a nephrostomy tract is a fundamental step of percutaneous nephrolithotripsy. Dilation of the tract may be achieved by many different techniques. The aim of this study was to assess if oneshot dilation could reduce fluoroscopy time compared with metal telescopic dilation.

Materials and Methods: A single blind controlled clinical trial was conducted. Between May 2013 and October 2015, 59 patients who underwent percutaneous nephrolitotomy were included and they were divided into two groups according to the type of tract dilation: group1 (30 patients), one-shot dilation; group 2 (29 patients) metal telescopic dilation. With the patient in lithotomy position, a UPJ Occlusion Balloon Catheter was placed; the patient was then changed to prone position and a renal puncture was performed in the calyx of choice. For one-shot dilation, the central Alken dilator was advanced over the guidewire followed by directly inserting the 30-F Amplatz dilator and then the access sheath

Table 1

Preoperatives variables of Patients

\begin{tabular}{|c|c|c|c|}
\hline Variables & $\begin{array}{c}\text { One-shot } \\
\text { (Group 1) } \\
(n=30)\end{array}$ & $\begin{array}{c}\text { Sequential } \\
\text { (Group.2) } \\
(n=29)\end{array}$ & $\mathrm{p}$-Value \\
\hline \multicolumn{4}{|l|}{ Gender } \\
\hline Female & 15 & 14 & $0.89^{*}$ \\
\hline Male & 15 & 15 & \\
\hline Age (year) & $49.73 \pm 14.39$ & $45.44 \pm 14.47$ & $0.31^{* *}$ \\
\hline \multicolumn{4}{|l|}{$\begin{array}{l}\text { History of open stone } \\
\text { surgery }\end{array}$} \\
\hline Yes & 5 & 6 & $0.16^{*}$ \\
\hline No & 25 & 23 & \\
\hline \multicolumn{4}{|l|}{ Side } \\
\hline Left & 14 & 16 & $0.51^{*}$ \\
\hline Right & 16 & 13 & \\
\hline Horseshoe Kidney & 2 & 3 & $0.48^{\cdots \cdots}$ \\
\hline Stone Burden $(\mathrm{cm})$ & $2.23 \pm 1.15$ & $2.52 \pm 1.14$ & $0.34^{* *}$ \\
\hline \multicolumn{4}{|l|}{ Stone location } \\
\hline Upper calyx & 3 & 2 & \\
\hline Middle calyx & 15 & 14 & $0.90^{*}$ \\
\hline Lower calyx & 8 & 10 & \\
\hline Staghorn calculli & 4 & 3 & \\
\hline
\end{tabular}


Table 2 Decrease of $\mathrm{Hb}$ and $\mathrm{X}$-ray exposure

\begin{tabular}{|c|c|c|c|}
\hline Variable & $\begin{array}{l}\text { One-shot } \\
(\mathbf{n}=\mathbf{3 0})\end{array}$ & $\begin{array}{c}\text { Sequential } \\
(n=29)\end{array}$ & $\mathrm{p}$-Value \\
\hline Difference $\mathrm{Hb}$ pre/post (g/dl) & $0.81 \pm 0.78$ & $2.03 \pm 1.04$ & $<0.001^{\star * *}$ \\
\hline $\begin{array}{l}\text { Difference of serum } \\
\text { cretinine }(\mathrm{mg} / \mathrm{dl})\end{array}$ & $-0.055 \pm 0.071$ & $+0.19 \pm 0.14$ & $<0.001^{* *}$ \\
\hline X-ray exposure (sec) & $69.60 \pm 21.38$ & $100.62 \pm 23.54$ & $<0.001^{\star *}$ \\
\hline
\end{tabular}

under fluoroscopy. In the metal telescopic dilation group, the Alken guide was placed followed by step-wise dilation from $10 \mathrm{~F}$ to $28 \mathrm{~F}$ using metal telescopic dilators. Patients with complex or uncomplicated stones with negative urine culture, prothrombin activity $>80 \%$ and stable renal function were included. Patients with urosepsis, impaired respiratory function and uncontrolled heart failure and renal failure were excluded. X-ray exposure time, blood loss, medical and surgical complications were evaluated.

Results: Baseline characteristics were similar in both groups. Table 1

Group 1 had a smaller postoperative hemoglobin decline than group 2 (0.81 vs. $2.03 \mathrm{~g} / \mathrm{dL} ; \mathrm{p}<0.001)$. Only one patient in each group required transfusion. Group 1 had at $31 \%$ shorter fluoroscopy time than group 2 (69.6 vs. $100.62 \mathrm{~s} ; \mathrm{p}<0.001)$. There was no significant difference in complications or length of hospital stay between the groups. Table 2

Conclusions: The one-shot technique resulted in a marked reduction in fluoroscopy time and bleeding rate without any increase in complications. This technique may benefit both the patient and healthcare personnel, allowing for safer and more precise tract dilation than traditional methods.

MP1-9 The Safety and Efficancy of Minimally Invasive Percutaneous Nephrolithotomy (MPCNL) in Treating Upper Ureteral Calculi of Elderly Patients

$\mathrm{S} \mathrm{Li}, \mathrm{L} \mathrm{He}$

China (People's Republic)

Introduction \& Objective: To evaluate the safety and efficacy of percutaneous nephrolithotomy (PCNL) in treating upper ureteral calculi of elderly patients.

Materials and Methods: We retrospectively analyzed 143 elderly patients with upper ureteral calculi [108 elderly patients underwent ureteroscopic lithotripsy (URL), 35 elderly patients underwent minimally invasive percutaneous nephrolithotomy (MPCNL)] from January 2008 to January 2011 in our department. Results: The stones diameters were $2.0 \pm 0.5 \mathrm{~cm}$ in the URL group, compared with $1.8 \pm 0.6 \mathrm{~cm}$ in the MPCNL group $(\mathrm{P}>0.05)$. The amount of concurrent diseases in the URL group was 1.2, compared with 1.5 in the MPCNL group $(\mathrm{P}>0.05)$. The mean operative time was $48.3 \pm 11.4 \mathrm{~min}$ in the URL group, compared with 59.4 $\pm 14.2 \mathrm{~min}$ in the MPCNL group $(\mathrm{P}>0.05)$. The complication rates were almost the same (11.2\% vs $10.3 \%$, $\mathrm{P}>0.05)$. The postoperative hospitalization time in URL group was significantly higher than that in group MPCNL [(5.8 \pm 2.3$)$ $\mathrm{d}$ vs $(3.6 \pm 2.1) \mathrm{d}, \mathrm{P}<0.05]$. The stone free rate in URL group was $67 \%$, compared with $97 \%$ in the MPCNL group. There was significant difference between the two groups $(\mathrm{P}<0.05)$.

Conclusions: MPCNL can be the first opinion in treating upper ureteral calculi of elderly patients with high stone free rate.
MP1-10 Mini-Percutaneous Nephrolithotomy: Initial Experience

S Moazami, L Cheuck, J Stern

Montefiore Department of Urology

United States

Introduction \& Objective: Percutaneous Nephrolithotomy (PCNL) is the standard of care for renal calculi $>2.0 \mathrm{~cm}$, with ureteroscopy and ESWL considered a first line option for smaller stones. PCNL is a balance between excellent stone free rates and increased invasiveness. Mini-PCNL was created as a way to decrease the burden of intraoperative blood loss, pain, and hospital stay associated with standard PCNL. While small series show comparable stone free rate to standard PCNL, the role of mini-PCNL in kidney stone surgery has yet to be defined. Herein, we present our early experience with mini-PCNL using the 12 French Storz Miniperc system.

Materials and Methods: We prospectively followed patients who underwent a mini-percutaneous nephrolithotomy at Montefiore Medical Center from June 2015 to January 2017. Variables including stone size, complications, post-operative drain management, and length of stay. All patients received a preoperative CT scan. Post-operative imaging to assess residual stone burden was done with either CT scan or ultrasound. Clinically significant stone fragments were defined as $>3 \mathrm{~mm}$.

Results: 22 patients were included in our analysis ( $41 \%$ male, $59 \%$ female). The average stone size was 14 millimeters $(\mathrm{mm})$ (7$30 \mathrm{~mm}$ ). There were no intraoperative or postoperative complications, no post-operative emergency visits, and no patients required a blood transfusion. $10(45 \%)$ cases were done totally tubeless, 11 $(50 \%)$ were tubeless (stent only), and one patient required a nephrostomy tube. All stents were left with a tether, and average stent duration was 4.7 days. 15 patients $(68 \%)$ were discharged the same day. Average length of stay was 0.5 days. 19/22 (86\%) patients were stone free at 4-6 week post-operative ultrasound.

Conclusions: Mini-percutaneous nephrolithotomy is a safe approach for renal and proximal ureteral calculi $>1 \mathrm{~cm}$ in size. Both decreased hospital length of stay and need for post-operative tube insertion, make it an attractive alternative to standard PCNL and ureteroscopy.

MP1-11 Supine Percutaneous Nephrolithotomy is safe and achieves higher stone free rates when combined with retrograde intrarenal surgery (Endoscopic Combined Intra Renal Surgery)

D Yong, S Koh, Y Tan

National Healthcare Group - Tan Tock Seng Hospital Singapore

Introduction \& Objective: Percutaneous Nephrolithotomy (PCNL) has traditionally been done in the prone position. However, with promising results on safety and efficacy of the modified supine technique first described by Valdivia Uria et al, PCNL in the supine position has gained increasing popularity over recent years. Not only is there less cardiovascular and respiratory changes, it also provides the option of combined retrograde intrarenal surgery. The objective of this study was to retrospectively compare Supine PCNL and Endoscopic Combined Intra Renal Surgery (ECIRS) to Prone PCNL in terms of stone free rates and other surgical outcomes.

Materials and Methods: In total, 107 PCNLs (16 ECIRs, 34 supine PCNL only and 57 prone) were performed by a single 
surgeon from Jan 2014 to Sep 2016. Surgical time, estimated blood loss, length of hospital stay, stone free rate $(4 \mathrm{~mm}$ and absolute) and complications were recorded. Comparison was done between the supine and prone group. Further analysis of the ECIRS subgroup was also performed and compared to the latest 16 prone PCNL patients with matching for Guy's stone score. Results: The stone free rate $(4 \mathrm{~mm})$ for patients who underwent ECIRS was significantly higher compared to those who underwent conventional prone PCNL ( $81.3 \%$ vs $43.8 \%, \mathrm{p}=0.028$ ). Although the stone-free rates ( $4 \mathrm{~mm}$ and absolute) were both higher for patients who underwent supine PCNL as compared to prone PCNL (SFR: $85 \%$ vs $68.4 \%, \mathrm{p}=0.063$; aSFR: $55 \%$ vs $45.6 \%, \mathrm{p}=0.36$ ), this did not reach statistical significance. There was no statistical difference in the mean operative time, estimated blood loss and median length of hospital stay when comparison was made between patients who underwent ECIRS or supine PCNL to prone PCNL. No major complications (Clavien $>2$ ) were encountered in all cases. Conclusions: ECIRS allows for better stone free rates due to the advantage of combining Retrograde Intra Renal Surgery to access stone fragments at challenging locations. With greater familiarity, surgical time for supine PCNL and ECIRS should also improve and be comparable or superior to prone PCNL. This study demonstrates that supine PCNL and ECIRS are safe and efficacious methods of managing complex renal calculi and are useful additions to the armamentarium of an endourologist.

\section{MP1-12 Which Way is Best for Stone Fragments and Dust} Extraction During Percutaneous Nephrolithotomy

\section{B Kati, E Pelit, I Yagmur, Y Akin, H Ciftci, E Yeni}

Harran University, Faculty of Meicine

Turkey

Introduction \& Objective: Percutaneus nephrolithotomy (PCNL) is a commonly used type of minimally invasive treatment in kidney stones surgeries. Surgical success is assessed according to residual stone status after surgery. The purpose of this study is to compare the two methods's success and practicality that are applied after the fracture of the stone in the patients who applied PCNL and which enable the removal of the residual stones.

Materials and Methods: Among 102 patients who underwent a single-session of PCNL at our university urology department between June 2015 and November 2016 were evaluated. Previously identified irrigation method (picture 1) and our aspiration method which desribed used in post-operative patients divided

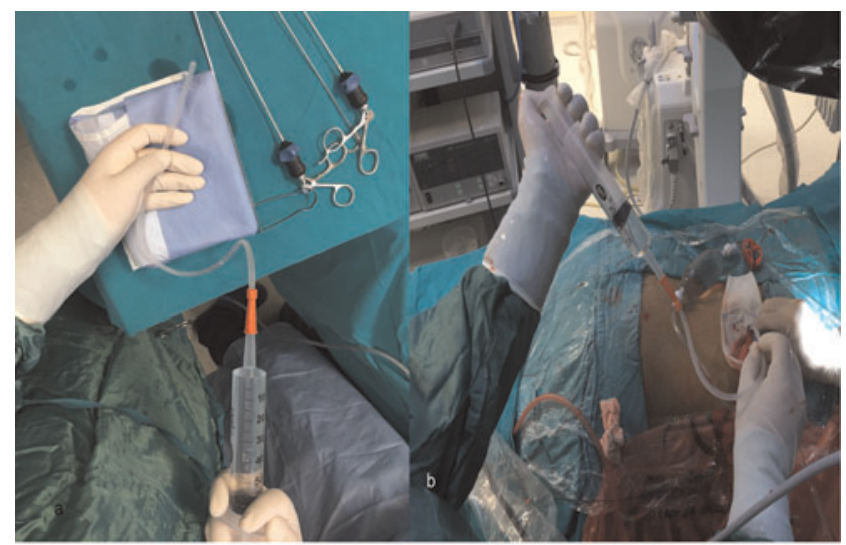

Figure 1a- Preparation of irrigation system 1b- Application of pressurized normal saline with NGT through the sheet.

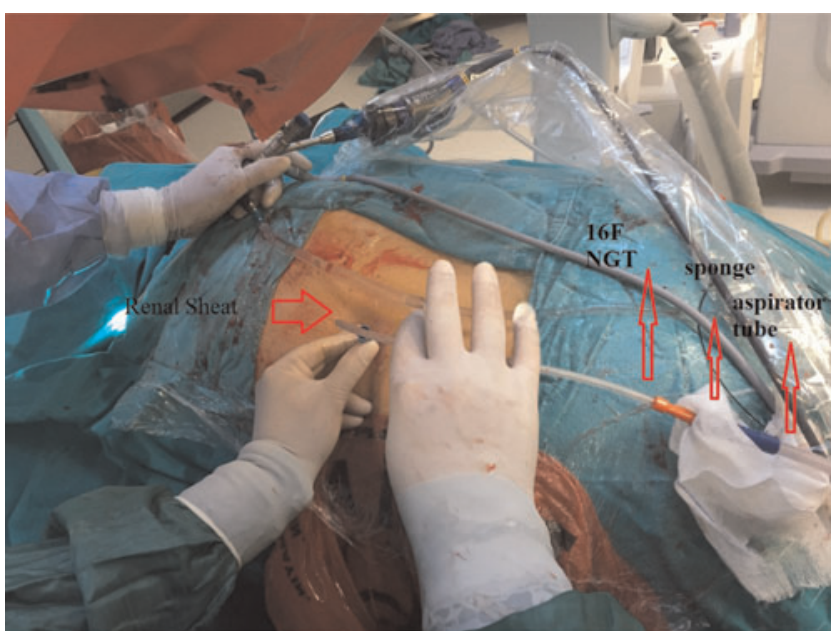

Figure 2- Application of the NGT which connected to the aspirator tip through the renal sheet.

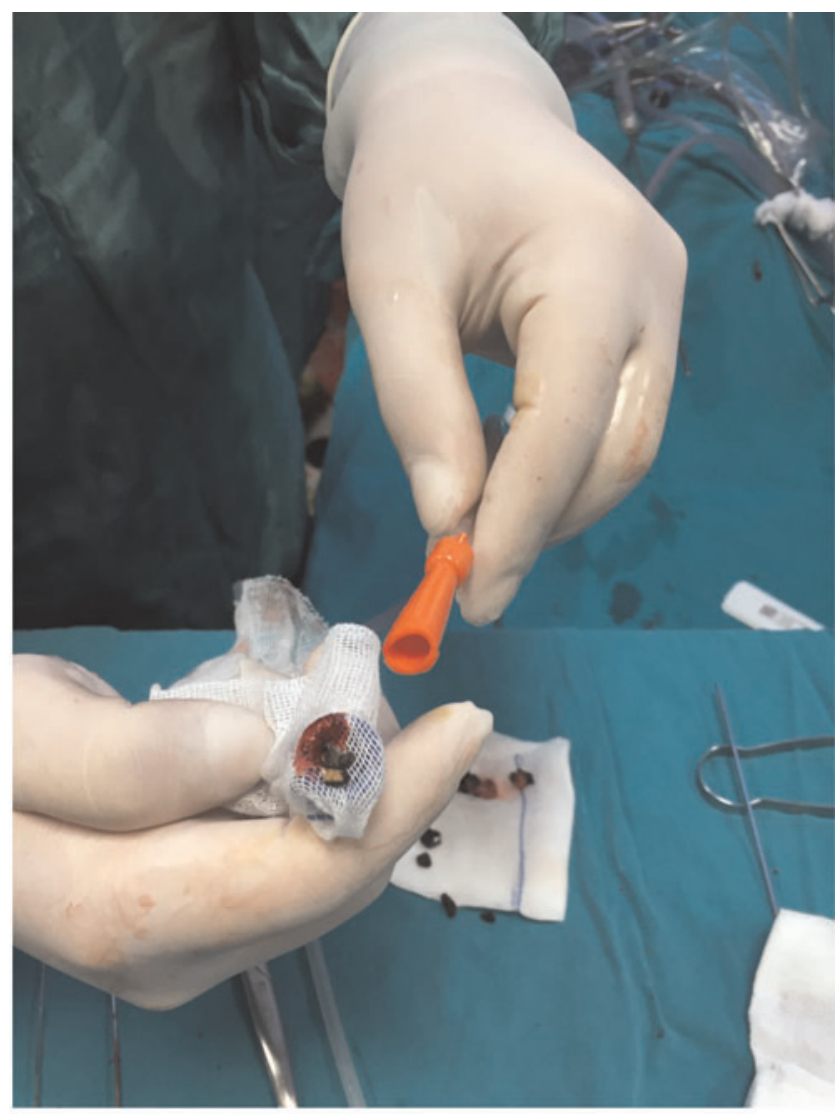

Figure 3- Residual stone fragments in the sponges between the aspirator and the NGT

into two groups of residual fragments was assessed by computer tomoghrapy. We applied our own suction technique in which a $16 \mathrm{~F}$ nasogastric tube (NGT) was combined with a sponge at the head of the aspirator arm. (Figure 2-3) The cut end of the NGT (cut nearly $1 \mathrm{~cm}$ for a full open entrance) was sent through a renal sheath. Then, with negative pressure created by the aspirator, it pulled on the stone fragments or dust and collected them into a sponge at the tip. Saline was sent through the renal sheath with a nephroscope during aspiration. Sometimes stone fragments became stuck in the NGT; however, these were pushed out with a pneumatic lithotripter stick. Suction was performed by gently 
touching the NGT in the renal sheath towards the end. The results were evaluated statistically. A p value less than 0.05 was considered statistically significant.

Results: The age and gender distribution of patients in the irrigation and aspiration groups did not differ significantly ( $p>0.05)$. In irrigation and aspiration groups, stone size did not differ significantly $(p>0.05)$. The amount of residue stones and dust remaining in the irrigation group was significantly higher $(\mathrm{p}<0.05)$ than the aspiration group.

Conclusions: Although many methods have been tried before, we think that the aspiration method we have described is a cheaper, more effective and feasible option.

MP1-13 Minimally Endoscopic Combined Intrarenal Surgery for Staghorn Calculi in Breaststroke Position

D Lai, Y He, X Li, M Chen

Fifth affiliated Hospital, Guangzhou Medical University China (People's Republic)

Introduction \& Objective: to evaluate the clinical value of performing minimally endoscopic combined intrarenal surgery(MECIS) in breaststroke position in managing the staghorn calculi.

Materials and Methods: retrospectively analyzed 30 patients with staghorn calculi, who underwent MECIS under breaststroke position between May to Aug 2016 in our center. The mean age was $42.4 \pm 10.9$ year. There were 10 males and 20 females. The BMI was $23.1 \sim 30.1 \mathrm{~kg} / \mathrm{m}^{2}$. The diameter of stone was $3.4 \sim 5.4 \mathrm{~cm}$.

Results: all procedures were performed successfully with a $20 \mathrm{~F}$ single tract and the patient in the prone split-leg position. Mean operative duration was $116 \pm 9.83 \mathrm{~min}$. The initial stone-free rate was $83.3 \%$, and the final stone-free rate was $87 \%$. Five patients required ancillary treatments: $1 \mathrm{SWL}, 2 \mathrm{MPCNL}$ and 2 clinical observation. One patient required blood transfusion, three patients had postoperative fever, but none had severe complications.

Conclusions: MECIS in breaststroke position is a viable approach for staghorn calculi with high SRF and low complication. With this position, doctors who performed MPCNL are familiar and who performed flexible ureteroscope are suitable.
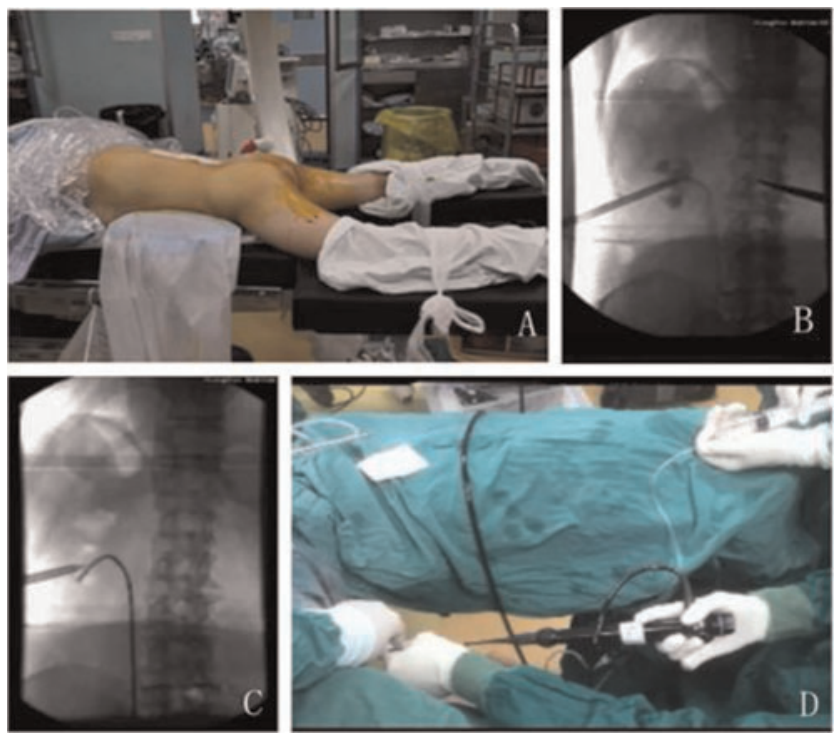

MP1-14 Supracostal access tubeless percutaneous nephrolithotomy: Minimizing complications

M Sourial, N François, G Box, B Knudsen

The Ohio State University Wexner Medical Center United States

Introduction \& Objective: Supracostal access in percutaneous nephrolithotomy (PCNL) may be avoided due to concern for thoracic complications. The objective of the study is to report the safety and efficacy of supracostal access utilizing a tubeless (stent only) PCNL technique.

Materials and Methods: From July 2010 to Oct 2016, 70 patients (76 renal units) underwent a supracostal access tubeless PCNL. The study is a retrospective review of their perioperative and postoperative outcomes. All patients underwent a non-contrast CT prior to the surgery. No nephrostomy tubes were left and all patients had a 7F ureteral stent and Foley catheter placed. The nephrostomy sheath was removed with the patient held in expiration, similar to a chest tube, and the incision closed.

Results: Median (IQR) patient age was $62(48.3$ - 67) years. Median (IQR) BMI and ASA score was 32.9 (27.7 - 39.1) kg/m2 and 3, respectively. Median stone size was $20 \times 21 \mathrm{~mm}$, and 14 $(18 \%)$ patients had complete staghorn stones. The upper calyx was the site of access in $50(66 \%)$ cases. The access was above the $12^{\text {th }}$ and $11^{\text {th }}$ rib in $57(75 \%)$ and $12(16 \%)$ cases, respectively. Median (IQR) length of hospital stay was 30 (28-32) hours. Postoperatively, $48(67 \%)$ patients had no residual fragments $(<2 \mathrm{~mm})$ on postoperative imaging. $8(11 \%)$ patients underwent an ancillary procedure to clear residual stones (7 URS and 1 ESWL), with an additional 7 patients becoming stone-free after this procedure $(76 \%)$. Thoracic complications occurred in 2 (2.6\%) patients: 1 small pneumothorax that resolved with conservative management, and 1 symptomatic ipsilateral pleural effusion requiring thoracocentesis. Other complications occurred in 9 patients $(11.8 \%)$ which included bleeding requiring transfusion (1), fever (4), urinary retention (2), and syncope (2).

Conclusions: Compared to historical controls, our approach to upper tract PCNL utilizing a nephrostomy tube free approach resulted in an overall low thoracic complication rate and facilitated hospital discharge.

MP1-15 A novel method to re-position suboptimally preopertively placed nephrostomy tubes for PCNL without renal re-puncture

S Dekalo, Y Barghouthy, J Ben-Chaim, S Conti,

A Greenstein, M Sofer

Tel-Aviv Sourasky Medical Center, Sackler School of Medicine, Tel-Aviv University

Israel

Introduction \& Objective: Nephrostomy tubes (NT) inserted in emergency settings by interventional radiologists are frequently unsuitable for subsequent percutaneous nephrolithotomy because of long retroperitoneal tracts, acute renal entry angle or direct pelvic location. We report a novel method of adjusting those NTs to be used as PCNL tracts and avoiding renal repuncture.

Materials and Methods: A retrospective search of 981 consecutive PCNLs performed between 2002 and 2017 identified 23 (2\%) patients (average age 67 years, average stone diameter $34 \mathrm{~mm}$ ) with preoperatively inserted NTs. The NTs that were unsuitable for PCNL were adjusted using a novel approach in 

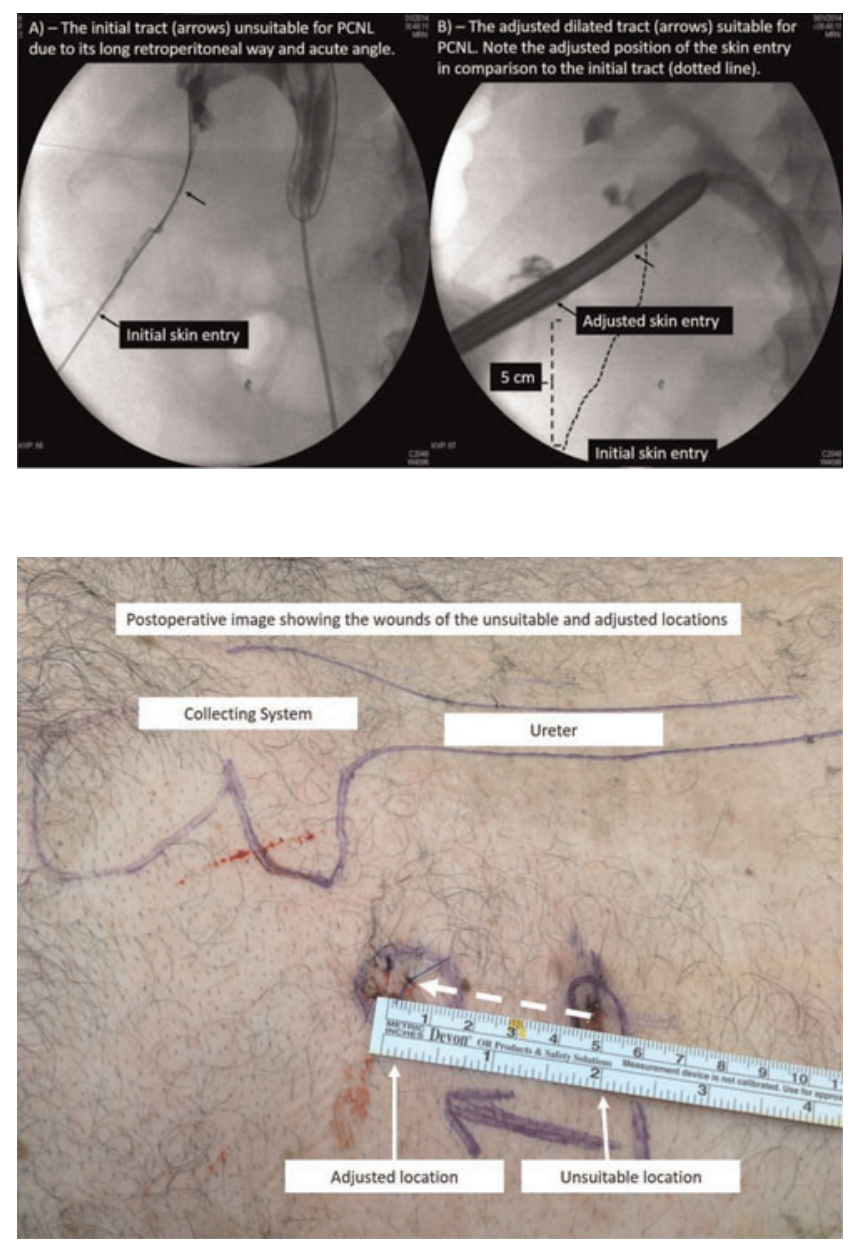

which a 5-mm incision was made at the ideal puncture location (IPL) as signed under fluoroscopic guidance. The pre-inserted NT was removed after passing a guidewire (GW) into the kidney. A Lahey dissector clamp was introduced through the IPL to bluntly dissect a retroperitoneal tunnel and reach the entry wound of the removed NT. The distal tip of the GW was grasped using the clamp and pulled out through the IPL, while its proximal segment was maintained in the kidney. The newly positioned GW was used for PCNL tract preparation without re-puncturing the kidney (figure).

Results: The preoperatively inserted NTs were located in the mid-calyx, lower calyx and renal pelvis in $6(26 \%), 13(57 \%)$ and $4(17 \%)$ cases, respectively. The NT was suitable for PCNL in 5 (22\%) cases, a new renal access was performed in $3(13 \%)$ and the novel adjustment approach was used in 15 (65\%). Staghorn stones were encountered in five cases. The novel NT adjustment was successful in all cases. The place of entry was moved an average of $6 \mathrm{~cm}$ (range 4-7) shortening and aligning the tract axis with the calyx axis. The angle between the tract axis and the calyx axis was reduced in average by $65^{\circ}$. The procedure was done in an average time of 4 minutes. There were no complications related to repositioning.

Conclusions: The novel method of adjusting preoperatively inserted NTs for PCNL by re-positioning their original entry location to the IPL offers the possibility of avoiding kidney re-puncture. It is feasible, safe, and easy to implement, and it spares potential morbidity related to additional puncturing of the kidney.
MP1-16 Single-session Bilateral Tubeless Percutaneous Nephrolithotomy in Supine and Prone Positions: A Comparison Study

\author{
Y Barghouthy, S Dekalo, S Proietti, J Ben-Chaim, I Mintz, \\ G Giusti, M Sofer
}

Tel-Aviv Sourasky Medical Center, Sackler School of Medicine, Tel-Aviv University

Israel

Introduction \& Objective: Bilateral large and complex renal calculi represent one of the most challenging urological pathologies. Affected patients are exposed to renal functional deterioration, recurrent infections, and repeated endourologic interventions that are usually performed under general anesthesia and require long periods of hospital stay. The aim of this study was to assess the outcome of same-session bilateral tubeless percutaneous nephrolithotomy (BPCNL) by supine and prone positions and to compare them to unilateral tubeless PCNL (UPCNL).

Materials and Methods: Consecutive PCNL patients treated at two institutions between 2006-2016 were analyzed. Tubeless BPCNL was performed when indicated. One institution treated all the patients in the supine position and the other initially operated in the prone position and then in the supine position. We identified the following comparison groups: 58 BPCNLs, of them 30 supine (SBPCNL) and 28 prone (PBPCNL), and 1395 UPCNLs. Statistical analysis was conducted.

Results: Patients demographics and baseline data were similar for all groups ( $p>0.05$ ). Comparison of SBPCNLs to PBPCNLs revealed a trend toward longer operative time $(124 \pm 38$ vs $105 \pm 36$ minutes, respectively; $\mathrm{p}=0.49$ ) and a significantly longer hospital stay (3.6 \pm 1.9 vs $2.4 \pm 1.3$ days, respectively; $\mathrm{p}=0.019$ ) for SBPCNLs. Seven planned BPCNLs were converted to UPCNL, resulting in a BPCNL success rate of 58/63 (92\%). In comparison to UPCNL patients, BPCNL patients had a significantly increased mean postoperative creatinine level $(0.74 \pm 0.3$ vs $-0.04 \pm 0.8 \mathrm{~g} / \mathrm{dl} ; \mathrm{p}=0.07 \mathrm{E}$ 7 ), a more pronounced decreased mean postoperative hemoglobin level ( $2 \pm 1.1 \mathrm{vs} 1.4 \pm 1.7 \mathrm{mg} / \mathrm{dl} ; \mathrm{p}=0.026)$, a higher blood transfusion rate $(9 \%$ vs $2 \% ; \mathrm{p}=0.023)$, and a longer hospital stay $(3 \pm 1.7$ vs $1.6 \pm 1.7$ days; $\left.p=0.001^{\mathrm{E}-4}\right)$. The stone-free and overall complication rates were similar for both groups. However, analysis of the distribution of complications by Clavien grade identified a trend toward more complications that were greater than grade II in the UPCNL group compared to the BPCNL group (50\% vs $20 \%$, respectively). This difference did not reach a level of significance $(\mathrm{p}=0.09)$.

Conclusions: BPCNL can be routinely offered to patients presenting with a bilateral indication. Patients should be informed that BPCNL is associated with a higher blood transfusion rate and a longer hospital stay, but that it may spare them from repeat anesthesia and hospitalization. SBPCNL takes longer to perform than PBCNL, but without clinical ramifications.

MP1-17 Ultrasound guided paravertebral nerve block anesthesia of percutaneous nephrolithotomy: a Prospective Randomized, non-blind parallel controlled study

J Hu, X Yu, S Wang

China (People's Republic)

Introduction \& Objective: To assess the clinical safety and feasibility for ultrasound guided paravertebral block anesthesia of percutaneous nephrolithotomy.

Materials and Methods: Between December 2015 to June 2016, 180 patients with renal or ureteral calculi were enrolled and 
evaluated with ultrasonography and CT scan. Of all the 180 patients, 108 males and 82 females. Their mean age was 39.2 years (23-71years). The clinical characteristics of the patients in each group, such as age, gender, BMI index, ASA sataus, mean arterial pressure and disease type had no significant differences $(\mathrm{P}>0.05)$. These patients were randomly enrolled into group general anesthesia ( $\mathrm{G}$ group), group combined spinal epidural anesthesia $(\mathrm{C}$ group) and group paravertebral nerve block anesthesia (P group) according to the random number and underwent percutaneous nephrolithotomy. G group: 35 males and 25 females. Their mean age was $40.1 \pm 11.8$ years and BMI was $25.1 \pm 3.8 \mathrm{~kg} / \mathrm{m} 2$; Renal calculi 52 cases, ureteral calculi 8 cases, Average maximum stone diameter was $2.6 \pm 0.8 \mathrm{~cm}$. C group: 38 males and 22 females. Their mean age was $39.7 \pm 12.4$ years and BMI was $24.6 \pm 4.1 \mathrm{~kg} /$ m2; Renal calculi 54 cases, ureteral calculi 6 cases, Average maximum stone diameter was $2.4 \pm 0.8 \mathrm{~cm}$. P group: 35 males and 25 females. Their mean age was $38.9 \pm 12.7$ years and BMI was $25.4 \pm 4.0 \mathrm{~kg} / \mathrm{m} 2$; Renal calculi 51 cases, ureteral calculi 9 cases, Average maximum stone diameter was $2.5 \pm 0.7 \mathrm{~cm}$. Periprocedural Vital signs, complications, postoperative the eating time, hospitalized day and expense in these three groups were evaluated. Results: Major intraoperative or postoperative complications did not occur in all of the patients. Mean arterial pressure decreased during preoperative changing positions was observed in group $\mathrm{G}$ (mean decreased $8.8 \mathrm{mmHg}$ )and group $\mathrm{C}($ mean decreased $1.9 \mathrm{mmHg}$ ), with significant difference in intra-group $(\mathrm{P}<0.05)$. Postoperative nausea and vomiting was observed in 8 and 2 patients of group $\mathrm{G}$ and group $\mathrm{P}$, respectively $(\mathrm{P}<0.05)$. Postoperative pain was observed in 2 and 7 patients of group $C$ and group $\mathrm{P}$, respectively $(\mathrm{P}>0.05)$. In addition, group $\mathrm{P}$ had with the early eating time post operation $(6.43 \pm 2.39 \mathrm{~h})$, shorter hospitalized day $(4.5 \pm 1.1 \mathrm{~d})$ and lower hospitalized expense $(2.87 \pm 0.32 \mathrm{w})$ compared with other groups $(\mathrm{P}<0.05)$.

Conclusions: Ultrasound guided paravertebral block can provide safe and reliable surgical anesthesia for percutaneous nephrolithotomy, which can accelerate the patients' recovery and is worth widely clinical application and spread.

MP1-18 Comparative analysis of contact holmium laser and ultrasonic lithotripsy effectiveness in the case of minimally invasive percutaneous nephrolithotomy.

D Merinov, A Artemov, S Gurbanov, E Valery, L Arustamov, O Merinova, D Dolotkazin

NII Urology

Russia

Introduction \& Objective: Minimizing of percutaneous access has a number of clinical advantages in terms of reducing the hemorrhagic complications risks, severity of the pain syndrome, possibility of a non-exaggerated termination of an intervention, and a reductionof hospital staying duration. At the same time, decreasing of instrument's working channel diameter influences on the choice of stone contact disintegration method. It was studied the efficiency of the two most popular modern methods, which are used in clinical practice: holmium laser and ultrasonic contact lithotripsy.

Materials and Methods: Percutaneous nephrolithotripsy with usage of mininefroscope (mPNL) $10 \mathrm{Ch}$ and the diameter of tube 16 $\mathrm{Ch}$ from the mono-access were perform on 118 patients with different localized single kidney stones up to $3 \mathrm{~cm}$ in size. For the disintegration of concrements there where use: a holmium laser $(\mathrm{H})$ with a fiber diameter of $600 \mathrm{~nm}$, energy for lithotripsy of $1.8 \mathrm{~J}$, frequency of $12 \mathrm{~Hz}$; Contact ultrasonic lithotriptor Lithoclast Master (US) with probe diameter of $4.8 \mathrm{Ch}$ and lapaxia of fragments. The following aspects were evaluated: the effectiveness in the absence of clinically significant residual fragments immediately after the operation and the time (T) spent to lithotripsy and evacuation of the fragments. In cases of complete removal of the stone and absence of bleeding, operation was finished without nephrostomy.

Results: Holmium mPNL was performed in 63 (53.3\%) cases, 55 $(46.7 \%)$ of the interventions were on patients with ultrasonic lithotripsy. The average size of the stone in the group HmPNL was $2.4(1.3-3) \mathrm{cm}, \mathrm{T} 39.1$ (24-52) min, the efficiency was $90.4 \%$. In the group of USmPNL average stone size was 2.6 (1.2$3.0) \mathrm{cm}$, T $31.3(18-49) \mathrm{min}(\mathrm{p}<0.05)$, the efficiency $-94.5 \%$. Lapaxia, effective fragmentation determined a smaller average duration of intervention by ultrasound in comparison with holmium lithotripsy, which requires, in a significant part of cases, additional time for extraction and landering of fragments.

Conclusions: Usage of ultrasonic lithotripsy with simultaneous lapaxia in mPNL is the most effective method and can be considered as a basic approach for the removal of concrements with small diameters of the working channel instrument.

MP1-19 Use of various entrance and exit strategies to facilitate percutaneous nephrolithotomy as an ambulatory procedure

\section{T Zhou, K Mahagaokar, J Stern}

Montefiore Medical Center

United States

Introduction \& Objective: Percutaneous nephrolithotomy (PCNL) is standard of care for removal of large renal calculi. The procedure traditionally necessitated a post-operative nephrostomy tube but this has been recognized as a significant cause of patient discomfort. Modifications such as tubeless technique with a double-J stent have been developed to decrease morbidity. Revisions to the PCNL "exit" have also allowed for consideration of PCNL as an outpatient ambulatory case. We report on our experience with ambulatory PCNL and to report how our exit strategy is related to same day discharge.

Materials and Methods: PCNL and mini-PCNL were performed on a total of 392 patients from January 2012 to December 2016. Exit strategies were chosen in the following manner. Tubeless technique with double-J stent was selected if there appeared to be ureteral edema or stricture along the ureter, particularly in the distal portion. A modified double $\mathrm{J}$ stent was fashioned into a ureteropelvic junction (UPJ) stent by excising one curl with the other placed in the renal pelvis with extraction string externalized. This was used if UPJ or proximal ureteral edema or trauma was seen. A totally tubeless technique was selected if there did not appear to be any narrowing or edema along the course of the ureter or at the ureteropelvic junction. Criteria for same-day discharge included adequate pain control with oral analgesics and no evidence of anemia, acute kidney injury, or electrolyte abnormalities.

Results: $44(11 \%)$ of our patients were discharged same-day. Of these, 10 (23\%) underwent mini-PCNL (12Fr) and 34 (77\%) standard PCNL. Mean stone diameter was $1.75 \pm 0.72 \mathrm{~cm} .19(43 \%)$ were totally tubeless, 12 (27\%) had a UPJ stent, and $13(30 \%)$ were tubeless with a double-J stent. Patients stayed an average of $286 \pm 101 \mathrm{~min}$ in the recovery room. There were two Clavien Grade I complications: one patient experienced a vasovagal response following removal of her UPJ stent, and another patient had stent discomfort from a double-J stent managed with analgesics and antiemetics. No patients required re-admission to the hospital.

Conclusions: Appropriate selection of PCNL and mini-PCNL as entrance strategies, as well as judicious use of exit strategies based on 
intra-operative findings may facilitate same-day discharge following PCNL with minimal complications. Further studies are needed to determine optimal candidate selection for ambulatory PCNL, choice of entrance and exit strategies, and to perform a cost-analysis.

MP1-20 Ureteropelvic junction stent as an exit strategy for percutaneous nephrolithotomy: an updated series

T Zhou, J Stern

Montefiore Medical Center

United States

Introduction \& Objective: In standard percutaneous nephrolithotomy (PCNL) a nephrostomy tube is left in situ to divert urine, tamponade the tract and maintain access for second-look nephroscopy if necessary. Tubeless technique in which no nephrostomy tube is placed has been shown to be a reasonable alternative to reduce post-op pain and hospital stay. However, ureteral stent placement is still required causing significant quality of life issues and often requiring cystoscopic removal. We update our series using a novel exit strategy, the ureteropelvic junction (UPJ) stent, in patients appropriate for tubeless PCNL.

Materials and Methods: We retrospective reviewed 50 patients undergoing PCNL with UPJ stent chosen as an exit strategy. Inclusion criteria included evidence of proximal ureteral narrowing or edema, no significant bleeding, and nephrostogram or ureteroscopic evidence of distal ureteral patency. At the conclusion of the case a UPJ stent was inserted: the tapered end of a double-J ureteral stent was cut and removed, with a string left attached to the remaining curl. The stent was inserted anterograde under fluoroscopic guidance over wire with its cut end in the mid ureter and proximal end in renal pelvis. The extraction string was brought through the tract and patient instructed to pull it several days later. Primary study endpoints included successful drainage, complications, and emergency department visits.

Results: 49 of 50 patients were successfully drained by a UPJ stent. There were four Clavien grade III complications: one patient required exchange of her UPJ stent with a double-J stent due to distal ureteral clot and three patients required ureteroscopic retrieval of retained stents secondary to missed follow-up appointments. 46 of 50 of the patients tolerated their stent well and self-removed at an average of 4.6 days without any reports of difficulty removing the stent by extraction string. Mean operating time was $141 \pm 17$ min, estimated blood loss $51 \pm 12 \mathrm{cc}$, and mean stone size was $1.98 \pm 0.23 \mathrm{~cm}$ in longest dimension. Patients were hospitalized an average of $1.6 \pm 0.5$ days, including $15(30 \%)$ same day discharges. Conclusions: PCNL with UPJ stent is a safe and effective exit strategy for PCNL that can be offered to select patients with proximal ureteral edema to avoid lower urinary tract symptoms from a distal curl and discomfort from a large-bore nephrostomy tube. Further studies will use a standardized stent symptom questionnaire to validate improvement in stent symptoms over standard double-J stent in tubeless PCNL.

MP1-21 Medically Facilitated Endoscopic Surgery: The Impact of One Week of Pre-operative Tamsulosin on Deployment of 16-French Ureteral Access Sheaths and Stone Free Rates

KS Kaler, S Safiullah, DJ Lama, RM Patel, Y Ko, Z Okhunov, J Landman, RV Clayman

Department of Urology, University of California, Irvine United States

Introduction \& Objective: Medical expulsive therapy is based on pharmacologic ureteral relaxation. We hypothesized this ap- proach might facilitate the deployment of the larger 16 French $(\mathrm{F})$ ureteral access sheath (UAS). We retrospectively analyzed our experience with UAS deployment during endoscopic procedures in patients intentionally pre-treated for 1-week with oral tamsulosin. Materials and Methods: Seventy one patients with non-stented ureters met inclusion criteria between January 2015 and September 2016. Demographic data, tamsulosin usage, ureteral access sheath (UAS) size, deployment failure, ureteral injuries, stone free rates (SFR) (i.e. no stones noted on follow-up CT or $\leq 4 \mathrm{~mm}$ fragments), and complications were collated. Univariate and multivariate analysis was conducted to assess the impact of tamsulosin on UAS size deployment.

Results: There was no statistical difference between the tamsulosin $(n=40)$ group and non-tamsulosin $(n=31)$ group, in regard to demographic data. The tamsulosin group had a significantly higher percentage of $16 \mathrm{~F}$ UAS deployment, $88 \%$ vs. $36 \%(\mathrm{p}<0.001)$, and no significant difference in ureteral injuries $(\mathrm{p}=0.50)$. Univariate and multivariate analysis (Table 2) revealed that tamsulosin pre-treatment statistically significantly increased the odds (15.7 and 33.5, respectively) for successful passage of a 16F UAS. Despite a larger stone volume and higher stone ablation rate in PCNL patients with $16 \mathrm{~F}$ UAS $\left(24075 \mathrm{~mm}^{3}\right.$, $\left.87 \mathrm{~mm}^{3} / \mathrm{min}\right)$ vs. $14 \mathrm{~F}$ UAS $\left(14467 \mathrm{~mm}^{3}, 69 \mathrm{~mm}^{3} / \mathrm{min}\right)$ there was no significant difference in computed tomography (CT) scan) completely stone free rates $(29 \% \%$ vs. $14 \% \% ; \mathrm{p}=0.655)$ or remnant fragments $\leq 4 \mathrm{~mm}(37 \%$ vs. $29 \% \mathrm{p}=1.00)$ (Table 3$)$.

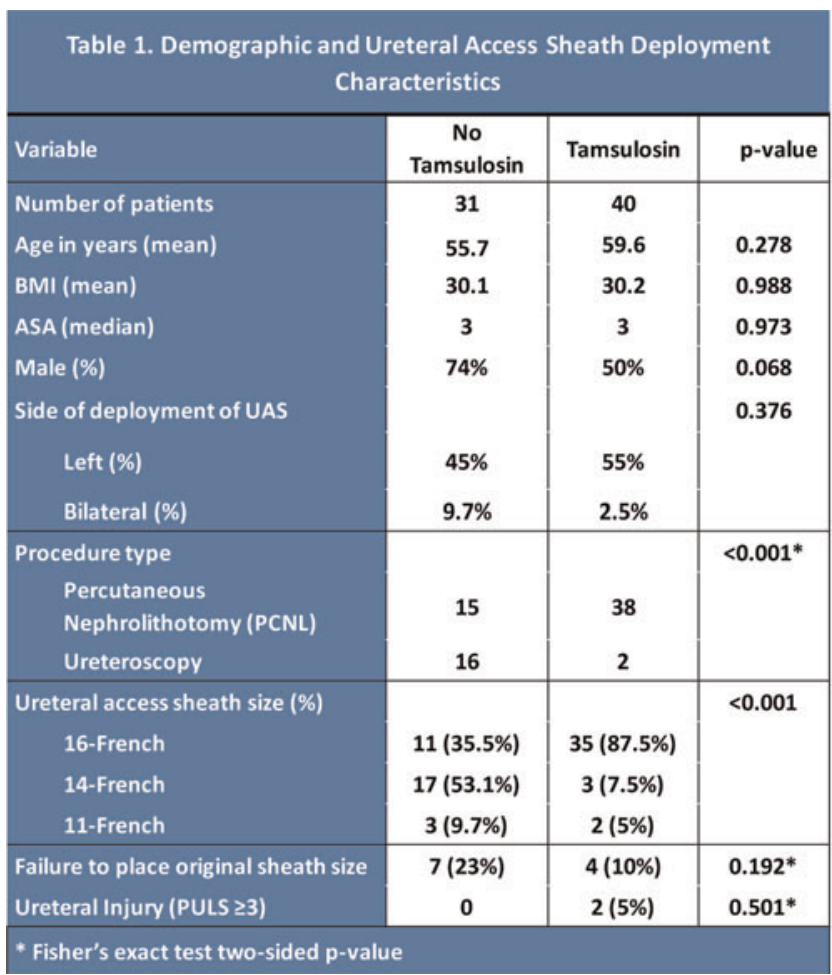

Table 2. Univariate and Multivariate Analysis of Tamsulosin on $16 \mathrm{~F}$ vs. 14F Ureteral Access Sheath Deployment

\begin{tabular}{|c|c|c|c|c|c|c|c|c|}
\hline & \multicolumn{4}{|c|}{ Univariate } & \multicolumn{4}{|c|}{ Multivariate } \\
\hline & \multirow{2}{*}{$\begin{array}{l}\text { Odds } \\
\text { ratio }\end{array}$} & \multicolumn{2}{|c|}{$\begin{array}{c}95 \% \mathrm{Cl} \text { for } \\
\text { OR }\end{array}$} & \multirow{2}{*}{$\begin{array}{c}\mathrm{p} \text { - } \\
\text { value }\end{array}$} & \multirow{2}{*}{$\begin{array}{l}\text { Odds } \\
\text { ratio }\end{array}$} & \multicolumn{2}{|c|}{$95 \% \mathrm{Cl}$ for OR } & \multirow{2}{*}{$\begin{array}{c}\mathbf{p}- \\
\text { value }\end{array}$} \\
\hline & & Lower & Upper & & & Lower & Upper & \\
\hline Surgeon & 1.829 & 0.642 & 5.212 & 0.258 & 0.988 & 0.233 & 4.185 & 0.987 \\
\hline Age (<60 years) & 0.500 & 0.175 & 1.425 & 0.194 & 0.779 & 0.201 & 3.022 & 0.718 \\
\hline Sex (Male) & 1.372 & 0.488 & 3.856 & 0.548 & 3.835 & 0.699 & 21.057 & 0.122 \\
\hline BMI $(<30 \mathrm{~kg} / \mathrm{m} 2)$ & 1.499 & 0.538 & 4.179 & 0.438 & 1.537 & 0.374 & 6.310 & 0.551 \\
\hline (Left) & 0.546 & 0.191 & 1.557 & 0.258 & 0.374 & 0.090 & 1.543 & 0.174 \\
\hline Tamsulosin used & 15.749 & 4.329 & 57.295 & $<.001$ & 33.491 & 5.765 & 194.551 & $<.001$ \\
\hline
\end{tabular}




\begin{tabular}{|c|c|c|c|}
\hline \multirow[b]{2}{*}{ Variables } & \multicolumn{2}{|c|}{ UAS SIZE } & \multirow[t]{2}{*}{ P-value* } \\
\hline & $14 \mathrm{~F}(\mathrm{~N}=7)$ & $16 F(N=38)$ & \\
\hline Mean Preoperative Stone Volume $\left(\mathrm{mm}^{3}\right)$ & 14466.7 & 24075.3 & 0.569 \\
\hline Mean Preoperative Hounsfield Units & 802.9 & 849.7 & 0.688 \\
\hline $\begin{array}{l}\text { Mean Absolute (\%) Stone Volume } \\
\text { Reduction }\left(\mathrm{mm}^{3}\right)\end{array}$ & $\begin{array}{c}13780.8 \\
(86.7)\end{array}$ & $\begin{array}{c}23527.2 \\
(91.9)\end{array}$ & 0.556 \\
\hline $\begin{array}{l}\text { Mean Preoperative Cumulative Stone } \\
\text { Diameter }(\mathrm{mm})\end{array}$ & 58.3 & 58 & 0.988 \\
\hline Mean Absolute (\%) CSD Reduction (mm) & $42.7(66)$ & $45.4(77.5)$ & 0.824 \\
\hline Complete Stone-Free Rate (No residual) & $14.20 \%$ & $28.90 \%$ & 0.655 \\
\hline Stone Free Rate $(<4 \mathrm{~mm})$ & $28.50 \%$ & $36.80 \%$ & 1.000 \\
\hline Mean Case Time (minutes) & 166.4 & 206.2 & 0.359 \\
\hline $\begin{array}{l}\text { Mean Stone Ablation Rate for PCNL } \\
\left(\mathrm{mm}^{3} / \mathrm{min}\right)\end{array}$ & 69 & 87 & 0.72 \\
\hline
\end{tabular}

Conclusions: In this retrospective study, one week of pre-operative tamsulosin was associated with an increase in the deployment of a $16 \mathrm{~F}$ UAS. The larger UAS appears to allow for more efficient stone removal.

MP1-22 The optical needle puncture followed by percutaneous nephrolithotomy using high definition image guide system

K Wada, R Tanimoto, M Araki, K Sasaki, T Sadahira, T Iwata, A Takamoto, Y Kobayashi, T Watanabe, Y Nasu, H Kumon

Department of Urology, Okayama University Graduate School of Medicine, Dentistry and Pharmaceutical Sciences Japan

Introduction \& Objective: Percutaneous nephrolithotomy (PCNL) has been a standard procedure for large nephrolithiasis, especially for staghorn calculi. To perform PCNL safely and effectively, the most important step is to make optimal percutaneous access. We have originally developed an optical puncture needle using high definition image guide (HDIG) system and applied it to making percutaneous access for PCNL. In this study we examined the efficacy and safety of the optical needle puncture followed by PCNL using our originally developed HDIG system.

Materials and Methods: As part of a collaborative research with Takei Medical \& Optical Co. Ltd. (Tokyo, Japan) and Sumita Optical Glass Inc. (Saitama, Japan), ultrathin fiberscope via HDIG system (HDIG scope) and its accessary devices have been developed. After evaluating safety, optical quality and operation performance in an animal study using adult swine, a clinical study on the application of the optical puncture needle for PCNL, in patients with nephrolithiasis, was approved by our ethics committee and carried out at Okayama University Hospital. A $20 \mathrm{G}$ puncture needle equipped with the $0.65 \mathrm{~mm}$ HDIG scope was adopted for making percutaneous access followed by PCNL through 4.8, 10, 20 or 26 Fr sheath.

Results: Between January 2014 and April 2017, 15 patients underwent percutaneous renal access using the optical puncture needle for PCNL. All patients have large volume nephrolithiasis with median stone size $2.5(1.2-4.5) \mathrm{cm}$ including staghorn calculi in 5 cases. Nine patients did not have hydronephrosis. Three patients were performed in modified Valdivia position, and others in prone position. In all cases, optimal percutaneous renal access was created without any complications related with needle puncture. The tract was dilated accordingly. PCNL was completed through $4.8 \mathrm{Fr}$ sheath in 4 cases, 10 Fr sheath in 1 case, 18 Fr sheath in 4 cases, 24 Fr sheath in 6 cases, respectively. Holmium-YAG laser or ultrasonic lithotripter was utilized for nephrolithotomy. Median (range) operative time was 165 (116-315) minutes. Median estimated blood loss including urine was $10(5-500) \mathrm{mL}$. Stone-free rate was $93.3 \%$ (14/15). Clinically insignificant residual stone was observed in 1 case. PCNL-related complications included pyelonephritis in 1 case and bleeding required blood transfusion in 1 case.

Conclusions: The optical needle puncture using HDIG system enabled safe and effective placement of percutaneous renal access. It can convert to PCNL seamlessly with minimum tract size of $4.8 \mathrm{Fr}$.

MP1-23 Papillary versus non papillary puncture in percutaneous nephrolithotomy:A prospective randomized trial

P Kallidonis, I Kyriazis, D Kotsiris, P Ntasiotis,

V Panagopoulos, A Koutava, W Kamal, E Liatsikos

Department of Urology, University of Patras

Greece

Introduction \& Objective: Literature suggests that the percutanoues punctures must be performed at the papilla of the renal calyx and a puncture at the infundibulum or the direction of the pelvis is not advisable due to increased hemorrhagic risk. A prospective randomized study was conducted to investigate the safety in terms of blood loss of the infundibular approach during PCNL.

Materials and Methods: Patients with renal stones with accumulative size of at least $2 \mathrm{~cm}$ were randomly assigned to one of two parallel groups to undergo PCNL with either papillary (Group 1) or infundibular (Group 2) renal access. The primary outcome measures were the hemoglobin drop on $1^{\text {st }}$ postoperative day and the need for transfusion during the first postoperative month. Secondary endpoints included the operative and fluoroscopy time, number of accesses performed, overall complication rate, hospitalization time, complications up to 3 months.

Results: Totally, 27 and 28 patients were enrolled in Group 1 and 2 , respectively. Patient age, BMI and stone size were similar among the groups $(\mathrm{p}=0.672,0.256$ and 0.889 , respectively). Hemoglobin drop and transfusion rate were similar among the groups $(\mathrm{p}=0.916, \mathrm{p}=1.0$, repscetively). Operative time was higher in the case of Group $1(\mathrm{p}=0.027)$. Hospitalization time was not significantly different in the study groups $(p=0.724)$.

Conclusions: The infundibular approach for PCNL to the posterior middle renal calyces is not associated with higher blood loss or transfusion rate in comparison to the respective approach to the fornix of the papilla when the currently described technique is performed.

MP1-24 Multi-Institutional Evaluation of Mini-Percutaneous Nephrolithotomy: An Evaluation of Outcomes and Stone Free Rates for Stones up to $3 \mathrm{~cm}$

D Brooks, M Patel, S Moazami, K Basham, RB Pickens, J Stern, J Gutierrez

Wake Forest Baptist Hospital

United States 
Introduction \& Objective: Percutaneous nephrolithotomy (PCNL) is the surgical standard for treating large or complex renal stones. Since its inception, the technique of PCNL has undergone many modifications. Recently smaller accesses less than 20 French has been developed to help minimize bleeding and postoperative pain while attempting to maintain a high stone free rate. In this multi-institutional study of mini-percutaneous nephrolithotomy (mPCNL) using a 12Fr nephroscope we evaluate perioperative outcomes as well as stone free rates to help determine the role of $\mathrm{mPCNL}$ in the management of nephrolithiasis. Materials and Methods: A retrospective review of all patients who underwent mPCNL at three institutions in the United States was performed. Demographic variables (age, BMI, comorbidities), procedural variables (stone size, stone location, stone composition) and post operative variables (renal drainage, complications, hospital stay, stone free rate) were evaluated.

Results: Data from 114 patients were available for analysis. Mean age was 51.4 years (4-85). Mean body mass index was $29.1 \mathrm{~kg} / \mathrm{m} 2(12.7$ - 48.7). Hypertension, hyperlipidemia, diabetes mellitus, and chronic kidney disease was present in $45.6 \%$,
$32.4 \%, 23.6 \%$ and $14 \%$ of patients respectively. The mean stone size was $16.9 \mathrm{~mm}$ (7-30). Holmium laser or ultrasonic lithotripsy was used to fragment stone in $81.6 \%$ and $18.4 \%$ of cases respectively. Primary stone composition was calcium oxalate monohydrate, calcium phosphate, uric acid, struvite and calcium oxalate dehydrate in $51.2 \%, 28.4 \%, 11.3 \%, 5.6 \%$ and $3.4 \%$ respectively. Postoperative renal drainage was accomplished by nephrostomy tube, stent, both and without any drainage in $61.4 \%, 27.8 \%, 9.6 \%$ and $23.6 \%$ of cases respectively. Overall complication rate was $5.3 \%$ with a $1.75 \%$ rate of serious (Clavien III or higher) complication. There were no blood transfusions in this series. Mean hospital stay was 1.1 days. Stone free rate was $78.3 \%$ with CT, ultrasound and KUB being used to evaluate outcomes in $59 \%, 36 \%$ and $4.5 \%$ respectively.

Conclusions: mPCNL is a safe and feasible option for management of renal calculi up to $3 \mathrm{~cm}$. $\mathrm{mPNCL}$ has a reasonable stone free rate with low complication rate compared to data reported from convention PCNL assess with no evidence of transfusion in this series. Further studies with more patients will help solidify this approach in the management of nephrolithiasis.

\section{MPS2: SHOCK WAVE LITHORIPSY}

\begin{abstract}
K Manoharan, N Sharma, P Kumar
Milton Keynes Hospital

United Kingdom
\end{abstract}

MP2-1 Comparing the success of extracorporeal shock wave lithotripsy in the treatment ureteric calculi between the EDAP TMS Sonolith and the Storz Modulith SLX

Introduction \& Objective: To compare the effectiveness of a new lithotripter, EDAP TMS Sonolith, with the old lithotripter, Storz Modulith SLX, at a district general hospital using a retrospective data analysis.

Materials and Methods: Retrospective data analysis on patients with a solitary ureteric calculi treated with the Storz modulith SLX lithotripter, between September 2007 and March 2010, and the EDAP TMS Sonolith, between July 2013 and March 2015, were identified. Patients treated with both machines were investigated for 3 parameters including calculus location, stone diameter and the presence of a JJ stent. These parameters were correlated to treatment success. Success was defined as complete stone clearance on post-treatment imaging.

\begin{tabular}{|c|c|c|c|c|c|c|}
\hline & \multicolumn{3}{|c|}{ Storz Modulith SLX } & \multicolumn{3}{|c|}{ EDAP TMS Sonolith } \\
\hline & $\begin{array}{l}\text { Successful } \\
(\%)\end{array}$ & Failed (\%) & Total & Successful (\%) & Failed (\%) & Total \\
\hline Total & $66(76.7 \%)$ & $20(23.3 \%)$ & 86 & $41(56.9 \%)$ & $32(44.4 \%)$ & 72 \\
\hline Male & $51(77.3 \%)$ & $15(22.7 \%)$ & 66 & $37(60.7 \%)$ & $27(44.3 \%)$ & 61 \\
\hline Female & $15(75.0 \%)$ & $5(25.0 \%)$ & 20 & $4(44.4 \%)$ & $5(55.6 \%)$ & 9 \\
\hline Average Age & 43.5 & 43.3 & 43.5 & 50.6 & 50.1 & 50.4 \\
\hline $\begin{array}{l}\text { Average Stone } \\
\text { Size }\end{array}$ & 7.8 & 8.5 & 7.9 & 7.4 & 7.5 & 7.5 \\
\hline JJ Stent present & $7(58.3 \%)$ & $5(41.7 \%)$ & 12 & $2(40 \%)$ & $3(60 \%)$ & 5 \\
\hline $\begin{array}{l}\text { No JJ Stent } \\
\text { present }\end{array}$ & $59(79.7 \%)$ & $15(20.3 \%)$ & 74 & $36(52.9 \%)$ & $29(42.6 \%)$ & 68 \\
\hline Stone site & & & & & & \\
\hline PUJ & $15(83.3 \%)$ & $3(16.7 \%)$ & 18 & $9(60.0 \%)$ & $6(40.0 \%)$ & 15 \\
\hline UU & $43(78.2 \%)$ & $12(21.8 \%)$ & 55 & $20(60.6 \%)$ & $13(39.4 \%)$ & 33 \\
\hline LU & $8(61.5 \%)$ & $5(38.5 \%)$ & 13 & $12(48.0 \%)$ & $13(52.0 \%)$ & 25 \\
\hline $\begin{array}{l}\text { Average number of } \\
\text { treatments required } \\
\text { for success }\end{array}$ & & 1.20 & & & 1.54 & \\
\hline
\end{tabular}

Results: There were 95 patients in the Storz Modulith SLX cohort who met inclusion for the study and they in total received 108 treatments. There were 74 patients in the EDAP TMS sonolith cohort who met inclusion for the study and they in total received 129 treatments. The overall success rate for the Storz was $76.7 \%$ compared to $56.9 \%$ for the EDAP TMS. The Storz required fewer treatments of average (1.2) compared to the EDAP TMS (1.54). Conclusions: We found that the Storz Modulith SLX lithotripter was significantly more effective at clearing ureteric stones compared to the EDAP Sonolith lithotripter $(p=0.006)$. It is difficult to interpret the significance of JJ stents in both cohorts due to the relatively small study samples. The results were communicated to the company who have since found several issues with the EDAP machine and rectified the problems and follow up data collection is in progress.

MP2-2 Does Mild Hydronephrosis Induced by full-Bladder Improve Outcomes in Patient Undergoing Shock Wave Lithotripsy for Lower Calyceal Stones?

\author{
E Arda, i Hazar, B Cakiroglu, O Sinanoglu \\ Trakya University \\ Turkey
}

Introduction \& Objective: To compare the outcomes, session and shock wave numbers in patients undergoing standard procedure shock wave lithotripsy (SWL) and patients undergoing SWL with mild hydronephrosis induced by full-bladder following oral hydration before SWL procedure for lower calyceal stones. Materials and Methods: Between january 2014- 2016 a total of 371 patients who underwent SWL, for lower pole calyceal stones $\leq 2 \mathrm{~cm}$, were included into the study. 127 patients were treated in the supine position (Group A), 123 in the prone position (Group B) and 121 in the prone position with full bladder and mild hydronephrosis checked by ultrasound before 
procedure (Group C). There were 286 men and 85 women with a mean age of 36 years and standard deviation (SD) of 11 years. In three groups ultrasound guided Storz Modulite FxSWL device was used.

Results: The median (IQR) number of shocks within the group A, group B and group C were 7600 (3855), 6500 (4300) and 6700 (4915) respectively. Significant difference was found in number of shock waves among 3 groups $(\mathrm{p}<0.01)$. After one session, stone expulsion rate of $13 \%$ (17 / 127) was observed session in group A, 28\% (34/ 123) was observed in group B and 40\% (48/ 121 patients) was observed in group $\mathrm{C}$. The difference between groups was found significant $(\mathrm{p}<0.01)$.

Conclusions: The present study suggests that mild hydronephrotic status induced by full-bladder before SWL can lower cost and patient discomfort by decrease in number ofsessionsand increase in stone clearance.

MP2-3 A prospective randomized study to investigate the effect of power ramping on treatment outcome in extracorporeal shockwave lithotripsy of renal calculi

AC Ng, J Yam, C Yee, W Chu, K Wong, S Leung, B Lau, J Teoh

SH Ho Urology Centre, The Chinese University of Hong Kong Hong Kong

Introduction \& Objective: To assess the effects of voltage escalation in patients receiving SWL for renal stones, particularly treatment success, degree of renal injury, degree of pain experienced and analgesic demand.

Materials and Methods: During Feb 2016 and Dec, 2016 patients with renal stones were prospectively randomised to receive shockwave delivered at either power ramping (Group-1) or constant voltage (Group-2) in a single institute using Modulith SLX-F2. Primary outcome was successful treatment at 12-weeks after one session of lithotripsy, as defined as stone free or stone fragment less than $4 \mathrm{~mm}$ on computerized tomography. Secondary outcome measures included degree of renal injury as access by post-treatment imaging, patients' pain scores, analgesics consumed during treatment.

Results: There were 100 patients recruited during the study period and completed 12-week follow-up. The mean stone size for Group1 and -2 were $8.59 \mathrm{~mm}$ and $8.62 \mathrm{~mm}$ respectively $(p=0.955)$. There was no difference between the other baseline parameters of the two groups. Group-1 has received lower energy shockwaves $(p \mathrm{pp}=0.55)$. The number of patients develop haematoma after SWL for Group-1 \& Group-2 were 18 and 17 respectively $(p=0.98)$. There was no between-group difference in pain scores, analgesic consumption during SWL or immediate complication right after SWL, while Group-1 showed a strong trend to reduce unplanned hospital admission due to SWL treatment. $(p=0.09)$

Conclusions: The power ramping protocol did not show a better treatment outcome, but it has reduced the number of hospital visits due to SWL compare to fixed voltage protocol.

MP2-4 Extracorporeal shock wave lithotripsy in pediatric urolithiasis: A single-center 100 cases experience

Y Tang, P Han

West China Hospital of Sichuan University

China (People's Republic)

Introduction \& Objective: Extracorporeal shock wave lithotripsy (ESWL) is widely used worldwide to treat urinary stone.
But to treat renal and ureteral stone disease in children (under the age of 14 years) are technically challenging. In the minimal invasive era, the ureterscope and Percutaneous nephrolithotomy (PCNL) are developed rapidly. In this study, we assessed the impact of Extracorporeal shock wave lithotripsy (ESWL) on the management of pediatric urolithiasis by analyzing our local practice over the past 100 cases, and determining the role of ESWL in pediatric urolithiasis at the minimal invasive era.

Materials and Methods: We retrospectively reviewed the record of the last 100 children who underwent the ESWL treatment for urolithiasis between Feb 2009 and Dec 2016. The patients' age, gender, stone size, stone location and number, complications, stone-free status, and postoperative complications were recorded. SPSS 22.0 software was used.

Results: The mean age of the children was 5.5 years (range 7 months-14 years). There were 68 renal and 32 ureteral stones. ESWL was performed by using Dornier Compact Delta II lithotripters. $88 \%$ of patients were stone-free at 3 months. Further ESWL treatment was needed in $4 \%$ of the cases (one session, $n=88$; two sessions, $n=4$;). Steinstrasse occurred in 3 patients but 2 of them cleared completely during the follow-up period. No Urinary tract infection was detected because of the strict anti-infection preoperative and no subcapsule hematoma was occurred. It was found that the stone-free rate is higher in diameters $<10 \mathrm{~mm}$ than $\geq 10 \mathrm{~mm}$.

Conclusions: ESWL has favorable results with low rates of complication and stone-free rates in selected pediatric patients. The majority of stones in children can be managed using ESWL. The ESWL techniques allow us to treat stones at an earlier age at very effective and safe ways and it has its own role in minimal invasive era.

\section{MP2-5 Tubeless versus Totally Tubeless Nephrolithotripsy -} A Randomized Study

\section{P Gyawali, S Chapagain, PR Chalise}

TU Teaching Hospital, Maharajgunj

Nepal

Introduction \& Objective: Introduction: Percutaneous nephrotripsy (PCNL) is standard method to remove the kidney stones more than $15 \mathrm{~mm}$ size. Though it is minimal invasive procedure, post operative bleeding, pain and urinoma are main cumbersome complications related with PCNL. Aim of this study is to find out the procedure which has less complications after the surgery. Objectives: To report outcomes of Tubeless and Totally Tubeless percutaneous nephrolithotripsy in a group of randomized patients.

Materials and Methods: From Aug 2013 to Feb 2015 total 61 patients with renal stones between 10 to $30 \mathrm{~mm}$ in size were randomized for tubeless (group-I) and totally tubeless group (group-II). Single tract percutaneous nephrolithotripsy (PCNL) was performed by a consultant urologist using 30 Fr Amplatz sheath and pneumatic lithotripter. Incidence of hemorrhage, urinoma, fever, use of analgesics and hospital stay were studied in post operative periods

Results: Mean age of patients in group -I and group - II was $34.5 \pm 11.5$ and 35.4 years respectively. Change in hemoglobin level in post operative period in both groups was not significant (p 0.53). None of our patient had developed urinoma. Use of Pethidine in group - I and group - II was $130.65 \pm 54.2 \mathrm{mg}$ and $95.16 \pm 45.37 \mathrm{mg}$ (p 0.007) respectively. Hospital stay in both groups was $3.45 \pm 1.3$ and $2.58 \pm 0.7$ days (p 0.003) respectively Conclusions: In selected group of patients totally tubeless PCNL is safe and has less morbidity than tubeless PCNL 
MP2-6 Does ESWL cause more injury to the kidneys than PNL for moderate size $(1.5-2 \mathrm{~cm})$ stones?

U Mete, A Mittal, B Mittal

PGIMER, Chandigarh

India

Introduction \& Objective: To determine and compare the functional outcome of the stone bearing kidney as assessed by differential renal function (DRF) following percutaneous nephrolithotomy( PNL) and extracorporeal shock wave lithotripsy (ESWL) for solitary moderate size renal stone.

Materials and Methods: A total of sixty patients with unilateral moderate size $(1.5-2 \mathrm{~cm})$ renal stone were randomized into two groups, Group I for PNL and Group II for ESWL using computer generated model method. Differential renal function was measured using EC(Ethylenedicysteine) scan before and at 3 months after completion of the procedure. Xray KUB at 3 weeks following PNL and 3 months following first session of ESWL was done to evaluate the stone free status. Stone clearance, procedure related morbidity (like sepsis, haemorrhage requiring blood transfusion, pain medication) and need for auxillary procedure for management of residual stones were also noted.

Results: Average stone size was $19.40+/-5.5$ in PNL group and $15.01+/-3.24$ in ESWL group. Only one patient in the PNL group $(3.3 \%)$ had very small residual. $96.7 \%$ of patients had single tract with tract size upto 24 Fr. In ESWL group 3 (10\%) had residual calculus, for which auxillary procedures were performed. Mean pre and post procedure differential function in patients of PNL were 42.46 and 42.16 which was not statistically significant. Similarly mean pre and post procedure differential function in patients of ESWL group were 44.20 and 42.83 with a mean change of -1.66 which was also not statistically significant. In PNL group differential renal function deteriorated in one patient $(3.3 \%)$, improved in two $(6.7 \%)$ and remained stable in $27(90 \%)$ while in ESWL group differential function deteriorated in 6 patients $(20 \%)$, improved in $3(10 \%)$ and remained stable in $21(70 \%)$ patients. When both PNL and ESWL groups were compared, a trend towards deterioration of DRF was seen in ESWL group ( $\mathrm{p}$ value 0.059). None of the factors in PNL group like number of tracts, tract dilatation, stone size and stone location had affected differential renal function post procedure. Similarly in ESWL group, increase in number of sessions had shown significant deterioration in differential renal function following procedure.(p value 0.044 )

Conclusions: PNL is more efficacious over ESWL in terms of stone free status, with equivalent morbidities. However multiple sessions of ESWL may lead to short term mild deterioration of function of the stone bearing renal unit. We believe PNL should be offered to the patients if surgeon has achieved reasonable proficiency.

MP2-7 Emergency extracorporeal shock wave lithotripsy for upper ureteric stones with or without pre-stenting: A randomized clinical trial

T El-Ghazaly, S Vita, S Hussain, A Al-Obaidy, A Al-Naimi

Hamad Medical Corporation - Teaching Hospital of Weill Cornell Medical College in Qatar

Qatar

Introduction \& Objective: Extracorpreal Shock Wave Lithotripsy (ESWL) is an attractive non-invasive therapeutic modality for urolithiasis typically reserved for elective cases in a controlled setting. Proceeding directly to ESWL without pre-stenting in patients presenting to the emergency room with acute renal colic secondary to upper ureteric calculi can spare patients multiple anesthesia-requiring procedures. In this study, we aim to compare upper ureteric stone clearance with and without prestenting in patients undergoing ESWL within 48 hours of their initial presentation.

Materials and Methods: Between July 2012 and July 2015, 124 patients who had presented to emergency with renal colic secondary to upper ureteric calculi were recruited for this study. Criteria for exclusion included patients with abnormally elevated renal parameters, signs of a concomitant infectious process (fever, leukocytosis, or a positive urine dipstick), pain poorly responding to analgesia, radiolucent stones, or stones smaller than 4-mm or larger than $15-\mathrm{mm}$ in size. 72 patients had been randomly assigned to undergo ESWL directly without pre-stenting (Group A), while 52 patients were assigned for pre-stenting (Group B), with their data and outcomes prospectively collected. Mean patient BMI in both groups was 26.1 and $26.7 \mathrm{~kg} / \mathrm{m} 2$ $(\mathrm{p}=0.49)$, mean skin-to-stone distance was 11 and $10.1 \mathrm{~cm}$ $(p=0.03)$, mean stone size was 7.3 and $7.8 \mathrm{~mm}(p=0.114)$, and mean stone density was 902 and 1078 Hounsfield units $(\mathrm{p}=0.005)$ respectively.

Results: All 124 patients had undergone their first session of ESWL within 48 hours of their initial presentation. 8 patients were lost to follow up in Group A, while one patient was lost to follow up in Group B. Four patients' stones in Group B had migrated to the kidney with stenting and were excluded from the study. Stone clearance in both groups was $61 \%$ and $44 \%$ $(\mathrm{p}=0.068)$ after one session, $91 \%$ vs $59 \%(\mathrm{p}=<0.001)$ by the second session, and $95 \%$ and $73 \%(\mathrm{p}=0.001)$ by the third and final session. No patients in Group A crossed over to Group B or required stenting at any point before their last session.

Conclusions: Emergency ESWL for upper ureteric calculi offers excellent stone clearance outcomes for properly selected patients with an acute presentation of renal colic that has subsided. Proceeding directly for ESWL without pre-stenting was associated with significantly enhanced stone clearance while sparing the patient multiple invasive interventions and their potential morbidity.

MP2-8 Comparison of Fixed and Ramping Voltage Extracorporeal Shockwave Lithotripsy with Acute Renal Injury Biomarkers: Prospective Randomized Controlled Clinical Study

A Atar, K Seker, A Kural, R Turkay, M Yenice, V Tugcu

University of Health Sciences, Bakirkoy Dr. Sadi Konuk Training and Research Hospital, Department of Urology Turkey

Introduction \& Objective: Extracorporeal shock wave lithotripsy (ESWL) is a non- invasive and effective method for the treatment of kidney stones. With it, an optimal treatment protocol has not been established yet which minimizes tissue damage while achieving effective stone breakdown. This study compares ESWLinduced renal injury in different ESWL treatment strategies by measuring urinary tissue inhibitor of metalloproteinases2 (TIMP-2) and insulin-like growth factor-binding protein 7 (IGFBP7) excretion.

Materials and Methods: This study was a prospective, randomized controlled trial in 88 patients between April 2016 and June 2016. Patients were randomized into two groups according 
TABLE LEGENDS

Table 1. Patient and Stone Characteristics

\begin{tabular}{|c|c|c|c|}
\hline & Fixed Group & Ramping Group & p value \\
\hline Patients, $n$ & 44 & 44 & \\
\hline Median Age, yr (IQR) & $47(33-55)$ & $43(34-47)$ & $0.20^{1}$ \\
\hline Gender, $n(\%)$ & & & $0.83^{2}$ \\
\hline Male & $21(48 \%)$ & $19(43 \%)$ & \\
\hline Female & $2352 \%)$ & $25(57 \%)$ & \\
\hline Comorbidities, $n(\%)$ & & & $0.30^{2}$ \\
\hline No & $32(73 \%)$ & 37 (84\%) & \\
\hline Yes & $12(27 \%)$ & $7(16 \%)$ & \\
\hline $\begin{array}{l}\text { Median Body Mass } \\
\text { Index, } \mathrm{kg} / \mathrm{m} 2 \text { (IQR) }\end{array}$ & $25(23-27)$ & $25(24-26)$ & $0.52^{1}$ \\
\hline Stone Side, $n(\%)$ & & & $0.83^{2}$ \\
\hline Left & $24(55 \%)$ & $22(50 \%)$ & \\
\hline Right & $20(45 \%)$ & $22(50 \%)$ & \\
\hline Stone Location, $n(\%)$ & & & $0.41^{3}$ \\
\hline Pelvis & $24(55 \%)$ & $19(43 \%)$ & \\
\hline Upper & $7(16 \%)$ & $6(14 \%)$ & \\
\hline Middle & $8(18 \%)$ & $15(34 \%)$ & \\
\hline Lower pol & $5(11 \%)$ & $4(9 \%)$ & \\
\hline Number of Stone, $n(\%)$ & & & $0.23^{2}$ \\
\hline Single & $40(91 \%)$ & $35(80 \%)$ & \\
\hline Multiple & $4(9 \%)$ & $9(20 \%)$ & \\
\hline Multiple Stone, $n(\%)$ & & & - \\
\hline Lower pol & $3(7 \%)$ & $4(9 \%)$ & \\
\hline Non Lower pol & $1(2 \%)$ & $2(5 \%)$ & \\
\hline Stone Diameter, $\mathrm{n}(\%)$ & & & $0.45^{2}$ \\
\hline$<5 \mathrm{~mm}$ & $1(\% 2)$ & $4(\% 9)$ & \\
\hline $5-10 \mathrm{~mm}$ & $23(\% 52)$ & $19(\% 43)$ & \\
\hline $10-20 \mathrm{~mm}$ & $18(\% 41)$ & $20(\% 46)$ & \\
\hline $20 \mathrm{~mm}$ & $2(\% 5)$ & $1(\% 2)$ & \\
\hline
\end{tabular}

${ }^{1}$ Mann-Whitney $U$ test was applied.

${ }^{2}$ Fisher's Exact test was applied.

${ }^{3}$ Pearson Chi-Square test was applied.

-A test was not performed because the number of observations was insufficient.

to ESWL treatment strategies. In the Ramping (escalating voltage) group and the Fixed (standard voltage) group. Urinary beta2 microglobulin was measured before the procedure, 1 week after the procedure, and urinary TIMP-2 and ILGFBP7 before the ESWL and 2 hours after the ESWL for each patient. In the study primary (comparison of biochemical markers) and secondary (Stone-free rate and affecting factors) endpoints were determined.

Results: There was no a statistically significant difference between the groups in terms of demographic and stone features. There was a statistically significant difference between the baseline and 1 week after creatinine, e - GFR, according to the ramping group in the fixed group $(\mathrm{p}<0.0001)$. Statistically, there was a significant difference between baseline and two hours after TIMP- $2 \times$ IGFBP7 / 1000 concentration value in the Fixed and Ramping group. Considering the 0.3 cut-off value between baseline and two hours after TIMP-2 $\times$ IGFBP7 / 1000 concentration values in constant and ramping group, the increase seen in the fixed group was more. Stone disintegration, stone-free rate, and rates of secondary interventions did not significantly differ between the groups.
Table 2. Comparison of biochemical markers before and after ESWL in Fixed and Ramping groups

\begin{tabular}{|c|c|c|c|}
\hline & Baseline & 1 Week After & $p$ value \\
\hline \multicolumn{4}{|c|}{ Creatinine. median (IQR) } \\
\hline Fixed & $0.77(0.67-0.91)$ & $0.81(0.72-0.98)$ & $<0.0001^{1}$ \\
\hline Ramping & $0.76(0.66-0.87)$ & $0.81(0.65-0.92)$ & $0.081^{1}$ \\
\hline \multicolumn{4}{|c|}{ Urea. median (IQR) } \\
\hline Fixed & $28(22-35)$ & $29.5(24-35)$ & $0.455^{1}$ \\
\hline Ramping & $26(22-28)$ & $25(20-30)$ & $0.781^{1}$ \\
\hline \multicolumn{4}{|c|}{ E-GFR. median (IQR) } \\
\hline Fixed & $102(94-114)$ & $99(83-112)$ & $<0.0001^{1}$ \\
\hline Ramping & $109(101-117)$ & $107(93-115)$ & $0.125^{1}$ \\
\hline \multicolumn{4}{|c|}{ B2 microglobulin, median (IQR) } \\
\hline \multicolumn{4}{|l|}{ Fixed } \\
\hline$<0.21$ & $43(98 \%)$ & $41(93 \%)$ & $0.625^{2}$ \\
\hline$>=0.21$ & $1(2 \%)$ & $3(7 \%)$ & \\
\hline \multicolumn{4}{|l|}{ Ramping } \\
\hline$<0.21$ & $42(95 \%$ & $43(98 \%)$ & $1.000^{2}$ \\
\hline$>=0.21$ & $2(5 \%)$ & $1(2 \%)$ & \\
\hline \multicolumn{4}{|c|}{ Albumin. median (IQR) } \\
\hline Fixed & $4(0.83-8.8)$ & $2.3(0.9-10.6)$ & $0.414^{1}$ \\
\hline \multirow[t]{2}{*}{ Ramping } & $2.12(0.73-10)$ & $2.53(0.63-6.80)$ & $0.074^{1}$ \\
\hline & Baseline & 2 Hours After & \\
\hline \multicolumn{4}{|c|}{ IGFBP7 concentration. median (IQR) } \\
\hline Fixed & $2.87(1.7-4.1)$ & $4.25(3.1-6.1)$ & $<0.0001^{1}$ \\
\hline Ramping & $2.91(1.4-5.4)$ & $4.33(3.2-6)$ & $<0.0001^{1}$ \\
\hline \multicolumn{4}{|c|}{ TIMP-2 concentration. median (IQR) } \\
\hline Fixed & $62.8(28-87)$ & $72.5(37-100)$ & $0.049^{1}$ \\
\hline Ramping & $90(38-113)$ & $90(49-126)$ & $0.020^{1}$ \\
\hline \multicolumn{4}{|c|}{ TIMP-2 X IGFBP7 / 1000. median (IQR) } \\
\hline Fixed & $0.15(0.08-0.18)$ & $0.32(0.28-0.50)$ & $0.001^{1}$ \\
\hline Ramping & $0.27(0.06-0.47)$ & $0.42(0.15-0.70)$ & $0.000^{1}$ \\
\hline
\end{tabular}

${ }^{2}$ McNemar test was applied.

Tablo 3. Secondary outcome results.

\begin{tabular}{|c|c|c|c|c|}
\hline & \multicolumn{2}{|c|}{ Fixed Group } & Ramping Group & p value \\
\hline \multicolumn{4}{|l|}{ Complication, n (\%) } & $1,00^{1}$ \\
\hline No & \multicolumn{2}{|c|}{$38(86 \%)$} & $39(89 \%)$ & \\
\hline Yes & \multicolumn{2}{|c|}{$6(14 \%)$} & $5(11 \%)$ & \\
\hline \multicolumn{4}{|c|}{ Complication Clavien -Dindo Classification } & - \\
\hline Grade1 & & $4(9 \%)$ & $4(9 \%)$ & \\
\hline Grade2 & & $1(2 \%)$ & $1(2 \%)$ & \\
\hline Grade3a & & $1(2 \%)$ & $0(\% 0)$ & \\
\hline \multicolumn{4}{|l|}{ Stone Density } & $0.52^{2}$ \\
\hline $1000 \mathrm{HU}<$ & & $27(61 \%)$ & $23(52 \%)$ & \\
\hline \multicolumn{2}{|l|}{$1000 \mathrm{HU}>$} & $17(39 \%)$ & $21(48 \%)$ & \\
\hline $\begin{array}{l}\text { Median Stone - } \\
\text { Distance, mm (IQR) }\end{array}$ & \multicolumn{2}{|c|}{ Distance, mm (IQR) } & $8,8(7,6-9,7)$ & $0.58^{3}$ \\
\hline \multicolumn{4}{|l|}{ Stone disintegration } & $0.68^{2}$ \\
\hline \multicolumn{2}{|c|}{ Stone Free,no fragments } & $12(27 \%)$ & $12(27 \%)$ & \\
\hline \multicolumn{2}{|l|}{ Fragments $<2 \mathrm{~mm}$} & $8(18 \%)$ & $8(18 \%)$ & \\
\hline \multicolumn{2}{|c|}{ Fragments btw 2- $5 \mathrm{~mm}$} & $10(23 \%)$ & $6(14 \%)$ & \\
\hline \multicolumn{2}{|l|}{ Fragments 5 > } & $14(32 \%)$ & $18(41 \%)$ & \\
\hline \multicolumn{3}{|l|}{ Secondary Intervention } & & $0.49^{2}$ \\
\hline \multicolumn{2}{|l|}{ No } & $36(82 \%)$ & $37(84 \%)$ & \\
\hline \multicolumn{2}{|l|}{ ESWL } & $1(2 \%)$ & $0(\% 0)$ & \\
\hline \multicolumn{2}{|l|}{ URS } & $6(14 \%)$ & 4 (9\%) & \\
\hline \multicolumn{2}{|l|}{ PCNL } & $1(2 \%)$ & $3(7 \%)$ & \\
\hline \multicolumn{5}{|c|}{ 'Fisher's Exact test was applied. } \\
\hline \multicolumn{5}{|c|}{ 2Pearson Chi-Square test was applied. } \\
\hline \multicolumn{5}{|c|}{${ }^{3}$ Mann Whitney-U test was applied. } \\
\hline
\end{tabular}


Conclusions: TIMP-2 and IGFBP7 / 1000 concentration value is a non invasive biomarker that may be used to show renal damage because of early renal damage linked to ESWL. This prospective, randomized study shows that treatment of ESWL at the ramping voltage should be preferred in the prevention of early renal injury.

MP2-9 Lower rate with a Storz lithotripter results in improved stone fragmentation both in vitro and in a clinical setting

\section{K Schmitz, M Spencer, R Cleveland, B Turney \\ Urology Department, Oxford University Hospitals United Kingdom}

Introduction \& Objective: SWL is one of the main modalities used to treat kidney and ureteric stones in the UK. Previous reports have suggested that firing at a lower rate improved fragmentation. The aim of this project was to test the effect of rate on a modern electromagnetic lithotripter both in vitro and with clinical outcomes.

Materials and Methods: In vitro experiments used artificial stones, made of gypsum cement, placed in a water bath mounted on a Storz Modulith SLX-F2 lithotripter. Stones were treated with 300 shocks at an energy level of 6 and a frequency of either 1.5 or $2 \mathrm{~Hz}$. Residual fragments were passed through a $2 \mathrm{~mm}$ sieve. Fragments $>2 \mathrm{~mm}$ were weighed.

Clinical outcomes were evaluated for 528 patients treated between January 2013 and December 2016; with patients selected that were treated with lithotripsy at either 3000 shocks at $1.5 \mathrm{~Hz}(\mathrm{~N}=125)$ or 4000 shocks at $2 \mathrm{~Hz}(\mathrm{~N}=403)$ and also had complete follow up. Note that the reduced number of SWs at $1.5 \mathrm{~Hz}$ meant that the wall clock time of the treatments was the same. Patients were matched by gender, age, stone site / size and number of treatment sessions. Treatment success was defined as complete stone clearance or remaining of clinically insignificant residual fragments $<4 \mathrm{~mm}$ (CIRF).

Results: For in vitro experiments, stones treated at $1.5 \mathrm{~Hz}$ the remaining fragments had a mean mass of $97.9 \mathrm{~g}(\mathrm{~N}=10, \mathrm{SD}=32.7$, range $39.3-131.9 \mathrm{~g})$ compared to $156.9 \mathrm{~g}(\mathrm{~N}=10, \mathrm{SD}=45.2$, range $76.4-228.7 \mathrm{~g}$ ) at $2 \mathrm{~Hz}$. The remaining mass was significantly less at 1.5 than at $2 . \mathrm{Hz}(\mathrm{p}=0.004)$ indicating that shocks fired at a slower rate fragmented stones more effectively. For the clinical data after the matching process, 64 pairs of patients were identified. In these, overall treatment success at $2 \mathrm{~Hz}$ was $67.2 \%$ (50\% stone free, $17.2 \%$ CIRF) and $73.4 \%$ at $1.5 \mathrm{~Hz}$ (46.9\% stone free, $26.6 \%$ CIRF) with $\mathrm{p}=0.439$. Overall treatment success for all 403 patients at $2 \mathrm{~Hz}$ was $68 \%$, (40\% stone free, $28 \%$ CIRF) and for 125 patients at $1.5 \mathrm{~Hz}$ was $62.4 \%$ (38.4\% stone free, $24 \% \mathrm{CIRF}$ ) and were not statistically different $\mathrm{p}=0.247$.

Conclusions: In vitro data, using a modern lithotripter, demonstrates that $1.5 \mathrm{~Hz}$ is significantly more effective than $2 \mathrm{~Hz}$ at fragmenting stones and is consistent with data from older lithotripters. These results support the clinical outcomes in which patients in the $2 \mathrm{~Hz}$ group were treated with 1000 shocks more than in the $1.5 \mathrm{~Hz}$ group yet there was no significant difference regarding overall treatment success, both in the matched pair data and for all patients. Other studies suggest that lower rate and fewer number of shocks results in reduced side-effects. Therefore we conclude that it is preferable to carry out lithotripsy at $1.5 \mathrm{~Hz}$ with the Storz modulith as equivalent fragmentation results from fewer shocks.
MP2-10 External validation of Triple D score and its modification to Triple D-Sscore for better prediction of stone free rates after extracorporeal shock wave lithotripsy.

N Iqbal, M Imran Jamil, A Ur-Rehman, S Akhter

$\mathrm{SIH}$

Pakistan

Introduction \& Objective: Here we wanted to externally validate three D score in our center using third generation Electromagnetic lithotripter. Secondly we wanted to apply it on relatively larger stone volume as compared to the original study. Third point was to see the role of lower pole stone location as well in combination with three D scoring.

Materials and Methods: It was a retrospective study that included 150 adult patients having mean age of $38.1 \pm 15$ years. Out of them $120(80 \%)$ were males and 30(20\%) females. Exclusion criteria included age less than 18 years, coagulation disorders, skeletal deformity, positive urine cultures and abnormal renal anatomy. All of thepatients had CT scan films which were assessed for Stone volume (SV), skin-to-stone distance (SSD), and stone density. Stone free rates and complications were assessed for each category of the score to see their correlation. Stone fragment less than $4 \mathrm{~mm}$ was considered as stone free status for a patient. Patients were followed for 3 months. Triple D scores were calculated for each patient according to the original study. We also made a modification of three D score later on to see its correlation with the stone free rates. In this modification nonlower pole stones were given extra 1 point while lower pole stones were given zero point for the location factor added to the new modification of three D score called as three D-S score.

Results: The mean stone volume was $337 \pm 170 \mathrm{~mm}^{3}$, mean skin to stone distance of $9.74 \pm 2.7 \mathrm{~cm}$ and mean stone density of $890 \pm 540$. According to three D scoring stone free rates were $38 \%, 56 \%, 53 \%$ and $93 \%$ for $0,1,2$ and 3 score respectively(p $0.001)$. There is one limitation according to the three D score that they did not take into account the role of lower pole that results in relatively lower stone free rates according to studies. So we did a modification and added S(site) to three D score in which we gave extra point for non-lower pole site while zero point for the lower pole location in three D-S score. Stone free rates were $36 \%, 47 \%$, $54 \%$ and $99 \%$ for $0,1,2,3$ and 4 score respectively (0.004). Both three D and three D-S scores were not able to predict significant differences regarding complications of SWL.

Conclusions: Three D score may be a better tool for predicting stone free rates after SWL but lower pole stone especially if it is relatively larger in volume may alter the results of three D score, so modified three D-S score more accurate for predicting success in both lower and non-lower pole stones respectively. Further studies may be needed to confirm findings of this study.

MP2-11 Clinical Efficacy and safety of anterograde ureterography for radiolucent ureteral stone localization in extracoporeal shock wave lithotripsy.

J You, H Kim, Y Shin, Y Kim

Department of Urology, Chonbuk National University Medical School, Jeonju, Korea

Republic of Korea

Introduction \& Objective: Percutaneous nephrostomy (PCN) or $\mathrm{JJ}$ stent is needed in patients with urinary tract stones with infection or renal failure. When performing extracorporeal shock wave 
lithotripsy (ESWL), localization of the radiolucent ureteral stone is not easy. We report our experience that anterograde ureterography using a PCN for radiolucent ureteral stone localization in ESWL.

Materials and Methods: Between January 2012 and December 2016, a total of 88 patients underwent ESWL with anterograde ureterography for radiolucent ureteral stone management. A database was kept prospectively for all patients. All patients underwent ESWL after urinary tract infection or acute renal failure was resolved. Ultravist ${ }^{\circledR} 370 \mathrm{mgI} / \mathrm{mL}$ contrast medium was diluted 1:1 with saline. About 5 to $8 \mathrm{cc}$ of this solution was slowly injected through the PCN tract, confirming the renal pelvis and renal calyx. When the ureter was not well visualized or filling defect was not seen, additional contrast agent was injected, paying attention to the patient's pain, contrast leakage, and contrast reflux. The filling defect area was aligned to the F2 zone and then lithotripsy was done in the same manner as a conventional ESWL. Three days to two weeks later, anterograde ureterography was performed through the PCN tract and additional ESWL was performed if a filling defect was present. Stone free was defined as the absence of filling defects on antegrade ureterography or absence of residual stones on CT scan.

Results: The mean age of the patients was $64.45 \pm 11.96$ years, 62 men and 26 women. Of the 88 patients, 50 underwent $\mathrm{PCN}$ for acute renal failure, 38 others had PCN for urinary tract infection. The mean stone size was $9.44 \pm 4.19 \mathrm{~mm}$. The locations of ureteral stones were 47,23 , and 18 in the upper, middle, and lower, respectively. The mean number of ESWL received until the stone was discharged was $1.52 \pm 0.83$ times. The success rate of ESWL was $96 \%$. There was no recurrence of urinary tract infection or acute renal failure.

Conclusions: When performing ESWL for radiolucent ureteral stone, anterograde ureterography using a PCN tract is useful and safe.

MP2-12 Effects of shock wave lithotripsy, for the treatment of kidney stones, on routine blood tests, Neutrophil Gelatinase Associated Lipocalin (NGAL) and Interleukin-18 (IL-18).

\section{Shergill, N Jones, SF Hughes, P Ella-Tongwiss}

Introduction \& Objective: The number of patients undergoing shock wave lithotripsy (SWL) for kidney stones is increasing annually, and as such the development of post-operative complications, such as haematuria and acute kidney injury (AKI) following SWL, is likely to increase. Investigating the response following SWL has not been widely researched. The aim of the study was to evaluate changes in routine blood tests and novel biomarkers (NGAL and IL18) following SWL for the treatment of kidney stones.

Materials and Methods: Twelve patients undergoing SWL for solitary unilateral kidney stones were recruited. Frompatients $(8$ males and 4 females) aged between 31-72 years (median 43 years), venousblood samples were collected pre-operatively (baseline), at 30,120 and 240 minutes post-operatively. Various haematological and biochemical parameters were measured employing a Sysmex XE-5000automated cell counter and a Beckman-Coulter-AU5800 analyser respectively. NGAL and IL-18 concentrations were determined using commercially available ELISA kits.

Results: Significant $(\mathrm{p} \leq 0.05)$ changes were observed in haematological and biochemical parameters (total white cell count, neutrophils, monocytes and sodium) following SWL. NGAL and IL-18 concentrations were determined using commercially available ELISA kits. NGAL concentration significantly increased following SWL, with values peaking at 30 minutes posttreatment $(\mathrm{p}=0.033)$. Although IL-18 concentration increased following SWL, these changes were not significant $(p=0.116)$.
Conclusions: Changes to NGAL and IL-18, and routine blood test were seen at short time periods following SWL, throughout this pilot study. Analysis of such markers, in larger cohorts, may potentially provide valuable data to identify or predict those patients at risk of post-operative complications.

MP2-13 Long-term Risk of New-Onset Hypertension and Diabetes after Treatment of Shock Wave Lithotripsy: Evidence from a National Representative Cohort in Taiwan

\section{Tsai, S Huang}

Far Eastern Memorial Hospital

Taiwan (Republic of China)

Introduction \& Objective: The aim of this study is to evaluate how shock wave lithotripsy (SWL) associates with new-onset hypertension (nHTN) or new-onset diabetes (nDM).

Materials and Methods: The longitudinal health insurance database 2000 (LHID-2000), a data subset randomly sampled from Taiwan national health research Insurance database (NHIRD) between 1996 to 2013, was used for analysis. LHID-2000 contains 1,000,000 beneficiaries randomly selected from the approximately 23.75 million people recorded in the year 2000 registry for beneficiaries of the NHIRD. We applied inclusion and exclusion criteria to establish the study cohort A and B as illustrated in Figure 1. Cox-proportional model was applied for evaluating the associations. Time-varying cox models were further applied to evaluate how SWL treatment session numbers associates with $\mathrm{nHTN}$ or $\mathrm{nDM}$.

Results: The LHID was used to identify 20,789 patients aged between 18 to 65 years and whose first stone surgical treatment time was performed between Jan 1999 to Dec 2011. After a median follow-up of 82.6 and 74.9 months, 2,028 and 688 patients developed hypertension in SWL group and ureteroscopic lithotripsy (URSL) group, respectively. SWL group patients had a higher probability of nHTN compared with URSL group with a hazard ratio(HR) of 1.20 (95\% CI 1.10-1.31) after adjusting for covariates. The risk increased as SWL treatment session numbers increased with a HR of 1.10 (95\% CI 1.00-1.20), 1.30 (95\% CI 1.15-1.48), 1.55 (95\% CI 1.31-1.85), 1.70 (95\% CI 1.32-2.19), and $2.00(95 \%$ CI 1.63-2.45) in one, two, three, four and more than five SWL sessions, respectively. In contrast, the risk of $\mathrm{nDM}$ was similar in SWL group compared with URSL group $(\mathrm{HR}=0.95, \mathrm{p}=0.35)$. Besides, the HR do not increase as SWL sessions increased.

Conclusions: Our study showed that SWL increased nHTN in the long term follow-up. Therefore, alternative treatment for urolithiasis such as endoscopic surgery or medical expulsion therapy, instead of SWL, is suggested to avoid the risk of nHTN. Also, avoiding multiple sessions of SWL is crucial to prevent nHTN risk.

MP2-14 Emergency Extracorporeal Shockwave Lithotripsy (ESWL) for acute ureteric colic: Outcomes from a large UK tertiary centre

I Jubber, S Hori, S Elwakil, J Collie, M Wynn, S Isaacs, S Shah, O Wiseman, S Al-Hayek

Cambridge University Hospital NHS Foundation Trust, Addenbrookes Hospital

United Kingdom

Introduction \& Objective: ESWL can be used for renal or ureteric stones as it doesn't require general anaesthetic and believed to have lower morbidity compared to surgery. However, little data is published on the outcome of this intervention in the 
treatment of acute ureteric colic. The purpose of this study is to evaluate the outcome of emergency ESWL at a large UK tertiary referral centre.

Materials and Methods: Prospectively collected data of emergency ESWL cases over 5 years (2012-2016) was included in the study. Data collected included patient factors (age and sex), stone characteristics (location, dimension and density), significant adverse events. Outcomes of treatment were collected including number of ESWL sessions required and whether further interventions were needed. The data was analysed using a multinomial logistic regression model that accounted for the effect of the potential confounding predictor variables on the outcome of treatment.

Results: A total of 63 patients had emergency ESWL with complete data over the 5-year period of this study. 71\% (45/63) were for upper ureteric, 3\% (2/63) mid-ureteric and 25\% (16/63) for lower ureteric stones. Of these, $79 \%(50 / 63)$ were male and $21 \%(13 / 63)$ were female. The median (+/- standard deviation) age of our cohort was 49 (+/-33) with a mean stone density of 946 (+/-475) Hounsfield Units, mean stone diameter of $7 \mathrm{~mm}(+/-3)$ and mean stone to skin distance of $120 \mathrm{~mm}(+/-50) .71 \%$ of patients $(45 / 63)$ became stone free from ESWL procedure alone. Of these, the majority $(82 \%, 37 / 45)$ required only one session of ESWL although a small minority required 2-3 treatments to become stone free $(18 \%, 8 / 45)$. Of the 45 patients who were stone free from ESWL alone, 16\% (7/ 45) patients required readmission due to pain. The patients who failed ESWL $(29 \%, 18 / 63)$ went on to have surgery. Of these, $17 \%$ (3/18) had a stent insertion as emergency and 83\% (15/18) had ureteroscopy (URS). One patient developed infection post ESWL and required an emergency ureteric stent insertion. No statistically significant correlation was seen between ESWL success or number of ESWL sessions received and the following variables: age, sex, skin to stone distance, stone density, stone size and stone location (all $\mathrm{p}>0.05$ ) though the number was small.

Conclusions: Emergency ESWL is a valid alternative to a URS or stent insertion in acute ureteric colic requiring intervention. However patients need to be carefully counselled on the advantages and disadvantages of all the available options. More studies comparing outcomes between these emergency procedures are needed.

MP2-15 Renal injury protection agents in patients undergoing extracorporeal shockwave lithotripsy (ESWL): A Systematic Review

I Jubber, S Hori, S Al-Hayek

Cambridge University Hospital NHS Foundation Trust, Addenbrookes Hospital

United Kingdom

Introduction \& Objective: Shock wave lithotripsy induced renal damage can occur as a result of multiple mechanisms, including direct mechanical effects, regional vasoconstriction, cavitation bubbles, generation of free radicals through ischaemia reperfusion injury and impairment of the antioxidant defence potential. The aim of this systematic review was to evaluate the efficacy and safety profile of renoprotective agents in ESWL.

Materials and Methods: We performed a literature search of the PubMed database in April 2017 with an English language restriction according to the Preferred Reporting Items for Systematic Reviews and Meta-analyses statement (PRISMA). We developed a search strategy using combinations of search terms for variations of "renal injury and ESWL". Inclusion criteria were randomised controlled trials (RCTs) and controlled studies that investigated the effect of possible renoprotective agents in ESWL available in full text article form.

Results: 7 studies (RCTs) were included. The studies evaluated the renoprotective effects of the following agents: coenzyme Q10, Nature made $\mathrm{R}$ antioxidant, traditional Chinese herbs, mannitol, vitamin $C$, vitamin $E$, a combination of selenium with vitamin $A, C$ and $\mathrm{E}$, verapamil, losartan and nifedipine. The studies all used surrogate markers of renal injury such as: Glomerular filtration rate (GFR), urinary albumin and albumin/creatinine ratio (ACR), $\beta 2$ microglobulin $(\beta 2-\mathrm{MG})$, urinary neutrophil gelatinase-associated lipocalin (uNGAL), urinary N-acetyl-D-glucosaminidase (NAG), urinary $\gamma$-glutamyltransferase $(\gamma \mathrm{GT})$, high sensitivity $\mathrm{C}$-reactive protein (hsCRP) and renal resistive index (RRI).

Statistically significant improvements $(\mathrm{p}<0.05)$ in renal injury parameters were observed for losartan (for urinary albumin at 1 week), coenzyme Q10 (for GFR, ACR and serum $\beta 2-\mathrm{MG}$ at 1 week), Chinese herbs (for urinary NAG at 24 hours, for urinary $\gamma \mathrm{GT}$ at 24 and 72 hours and for urinary $\beta 2-\mathrm{MG}$ immediately after ESWL), mannitol (for urinary $\beta 2-\mathrm{MG}$ immediately after ESWL and for renal RRI immediately, 3 and 7 days after ESWL), and vitamins $\mathrm{C}$ and $\mathrm{E}$ (for hs-CRP on days 2, 7 and 28).

With regards to adverse events, one study reported 1 case of macroscopic haematuria with selenium ACE.

Conclusions: Whilst the evidence shows that there are renoprotective agents which can be effective and relatively safe at protecting patients from renal injury from ESWL on a biochemical level, the clinical relevance of this to clinical practice needs further evaluation in large high quality studies.

\section{MP2-16 Withdrawn}

\section{MP2-17 Shock Wave Lithotripsy for Large Renal Calculi: Is Prophylactic Stenting Beneficial?}

T Kroczak, D Ghiculete, R D’A. Honey, M Ordon, JY Lee, K Pace

\section{St. Michael's Hospital - University of Toronto}

Canada

Introduction \& Objective: For selected patients, shockwave lithotripsy (SWL) is a safe, effective and minimally invasive treatment option for certain upper tract urinary calculi. Complications are generally infrequent following SWL, however fragments may obstruct the ureter and lead to serious obstructive complications, particularly after treating larger stones. It is generally recommended that a ureteric stent be inserted prior to performing SWL for large renal calculi in order to prevent post procedure complications. We set out to determine if ureteral stenting prior to SWL for large renal calculi affects treatment success and complication rates.

Materials and Methods: A matched retrospective cohort study was performed comparing stented and non-stented patients undergoing SWL for solitary large renal calculi at our center from January 2010 to April 2017. Patients were matched according to stone size. Large calculi were defined as those with an area of equal to or greater than $200 \mathrm{~mm}^{2}$ and at least one diameter greater than $15 \mathrm{~mm}$. We compared procedure outcomes and complications at 2 weeks and 3 months between patients with and without a pre-SWL stent. Results: One hundred and sixty-eight patients (84 stented, 84 non-stented matched controls) were identified. There were no demographic differences between stented patients and matched controls; female gender ( $42 \%$ vs $38 \%$ ), mean age ( 57.4 vs $55.3 \mathrm{yrs}$ ), and mean stone area $\left(261.1 \mathrm{~mm}^{2}\right.$ vs $\left.260.8 \mathrm{~mm}^{2}\right)$. No significant differences were noted in patients undergoing SWL with or without 
a stent with respect to presentation to the emergency department post SWL treatment $(13.1 \%$ vs $21.4 \% \mathrm{p}=0.153)$ and rate of Steinstrasse $(10.7 \%$ vs $14.3 \% \mathrm{p}=0.484)$. Ten patients who were not pre-stented $(11.9 \%)$ eventually required ureteric stenting after SWL. After two weeks, single-treatment SWL success rates were similar for patients without a stent compared to those with a stent $(17.9 \%$ vs $19.1 \% \mathrm{p}=0.842)$. No difference in success rate was demonstrated after three or less treatments for patients in both groups $(78.6 \%$ stent vs. $70.2 \%$ no stent $\mathrm{p}=0.216)$.

Conclusions: In selected patients, pre-SWL stenting for large, solitary renal calculi may not improve stone-free rates nor reduce Steinstrasse rates. For patients initially receiving SWL without a stent, few patients required ureteric stenting after SWL due to complications.

\section{MP2-18 The Effect of Stone Dust and Collecting System Flow On Shockwave Lithotripsy}

JS Ahn, A Randad, W Kreider, MR Bailey, AD Maxwell, MD Sorensen, JD Harper

Department of Urology, University of Washington Medical Center

United States

Introduction \& Objective: During shock wave lithotripsy (SWL) stones emit dust which if not displaced, can form a cloud around the stone and potentially interfere with shock wave transmission. Prior in vitro studies show that stone confinement can reduce lithotripsy efficacy; however, it is unclear if stone dust specifically contributes to this effect. We hypothesized that stone dust and stone confinement diminish lithotripsy efficacy and that removing the dust will improve lithotripsy. Our second hypothesis was that active dust displacement by simultaneous continuous irrigation can improve lithotripsy.

Materials and Methods: Using a SWL water bath setup, we broke two types of artificial stones in an open-top ballistic gel cup (Figure 1): Begostone Plus, which produces dust during fragmentation, and crystalline calcite, which does not produce dust. Stones of similar weight and shape were used. To simulate confinement, the open-top gel cup was covered with a Parafilm ${ }^{\circledR}$ membrane. We treated both types of stones for 5 minutes in confined or unconfined states. We assessed stone breakage by comparing the stone's original weight with that of the residual stone and fragments greater than $2 \mathrm{~mm}$.

For our second hypothesis, Begostones placed inside a calyx of an anatomically accurate gel kidney were treated with SWL with or without water irrigation. Irrigation via a continuous infusion pump and 18 gauge calyceal needle access was administered at different rates (Figure 1). Irrigation rates approximated those of known physiologic and loop diuretic-stimulated urine production. We conducted 2-4 trials for each exposure condition.

Results: Unconfined Begostones broke 3.5 times better than those not confined $(\mathrm{p}=0.001)$. There was no significant difference in

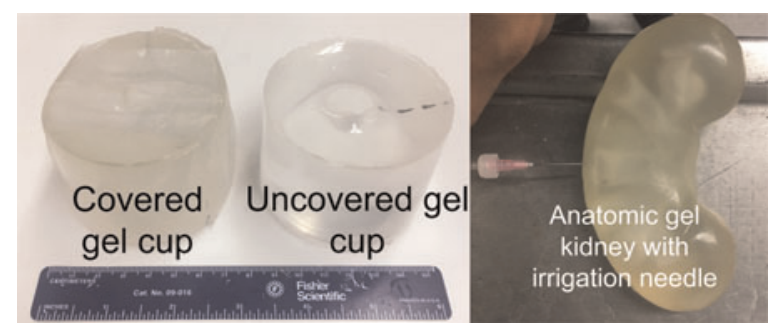

calcite stone breakage between the confined and unconfined groups $(\mathrm{p}=0.12)$. At irrigation rates of 35 and $70 \mathrm{ml} / \mathrm{hr}$, there were no differences between stones treated with versus without irrigation $(\mathrm{p}=0.41$ and 0.38 respectively). At $420 \mathrm{ml} / \mathrm{hr}$, stones broke approximately $35 \%$ better with irrigation than without irrigation $(\mathrm{p}=0.006)$.

Conclusions: Stone dust clouds confined around a stone diminish SWL efficacy. Confinement does not affect lithotripsy in stones that do not produce dust. Collecting system irrigation at loop diuretic-induced rates of urine flow improves lithotripsy despite confinement, but not at physiologic flow rates.

MP2-19 The efficacy and safety of and satisfaction with the transgluteal approach to extracorporeal shockwave lithotripsy via the supine position to treat distal ureter stones: A prospective, randomized, and multicenter study

M Choo, J Han, S Lee

Hallym University Dongtan Sacred Heart Hospital Republic of Korea

Introduction \& Objective: To compared the outcomes of ESWL to treat distal ureter stones with regard to the conventional prone and supine positions using the transgluteal approach through the greater sciatic foramen.

Materials and Methods: A prospective, randomized, singleblind, and multicenter study was conducted between October 2014 and July 2015. The inclusion criteria were radio-opaque distal ureter stones with a maximum diameter of $0.5-2 \mathrm{~cm}$ as measured on a computed tomography (CT) scan. The included 160 patients were treated using the PiezoLith 3000 . The patients were randomly assigned to 2 groups: the prone group $(n=80$; treated in the conventional prone position) and the transgluteal group $(n=80$; treated in the supine position using a transgluteal approach). In the latter group, the focused shock wave was transmitted through the greater sciatic foramen with the head positioned at a $40^{\circ}$ angle to the vertical. Treatment outcomes were assessed using KUB at 2 weeks after treatment. "Stonefree" was defined as the complete clearance of stone fragments. Overall satisfaction was self-reported using a 0-to-5 Likert scale. Results: The mean patient age was $43.2 \pm 11.9$ years, and the mean stone size was $5.16 \pm 1.87 \mathrm{~mm}$. No differences were found in the baseline characteristics of either the patients or the stones. The overall efficacy was $66.9 \%$. The stone-free rate was significantly higher in the transgluteal group $(72.6 \%)$ than in the prone group (54.7\%; odds ratio $2.413,95 \%$ CIs $1.010-5.761, \mathrm{P}=0.023$ ). No serious adverse events were observed due to treatment in either group. The satisfaction score of the transgluteal group was

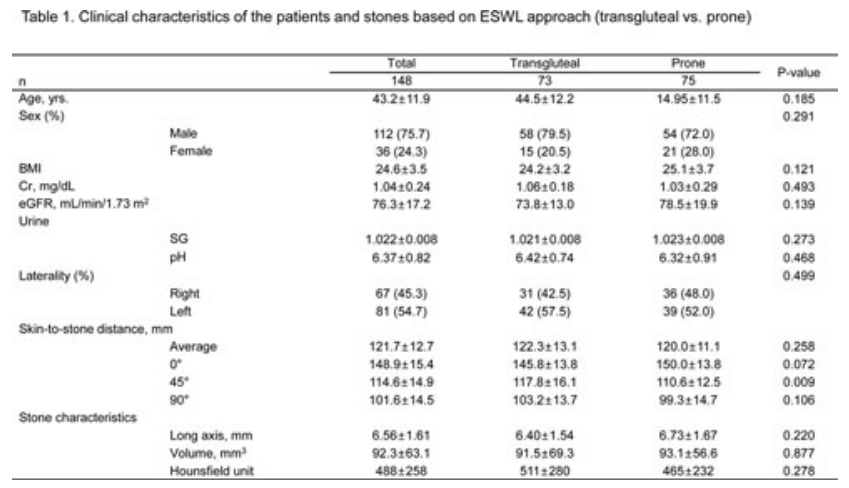




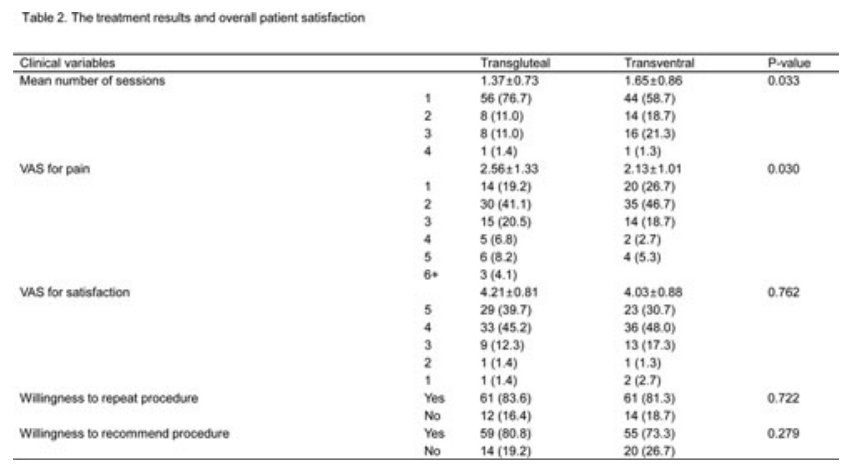

$4.21 \pm 0.81$, and $83.6 \%$ were willing to repeat the same procedure if necessary.

Conclusions: ESWL using the transgluteal approach via the supine position through the greater sciatic foramen was more effective than via the conventional prone position. Furthermore, this approach provided a comparably safe and satisfactory procedure.

MP2-20 The direction of Shockwave lithotripsy (SWL): Publication trends (PubMed) over the last 16 years (20002015).

A Pietropaolo, B Somani

University Hospital Southampton NHS Foundation Trust United Kingdom

Introduction \& Objective: Shockwave lithotripsy (SWL) technology has been continually evolving over the last 3 decades with more procedures now being preformed as a minimally invasive day case procedure. With lessons learnt over this time, the boundaries of SWL are being pushed with more complex stones being treated. We wanted to see the publication trends for SWL as reported on PubMed over the last 16-years.

Materials and Methods: All published papers on 'Urolithiasis', 'kidney stones', 'renal stones', 'ureteric stones', 'shockwave lithotripsy', 'extracorporeal shockwave lithotripsy', 'SWL', 'ESWL', and 'lithotripsy' were searched on PubMed over the last 16-years from 2000-2015. There were no language restrictions and all non-English language papers with published English abstracts were also included in our review. While review articles were included, case reports, laboratory and animal studies, and those papers that did not have a published abstract were excluded from our analysis. Data was divided into two 8-year periods, period-1 (2000-2007) and period-2 (2008-2015).

Results: A total of 887 studies on SWL were published on PubMed 16 year period [English language - $729(82 \%)$ and NonEnglish language - $158(18 \%)]$. The rates of SWL, despite some fluctuations, seem to have steadily decreased over the study period $(\mathrm{p}=0.2)$, although there was a clear fall in the number of Non-English articles published over time (Figure). When comparing the two time periods, there were a total of 486 articles published in period- 1 , which had decreased by almost $20 \%$ to 401 articles in period-2 $(p=0.18)$. The number of English/NonEnglish language articles in period-1 and period-2 were 384/102 and 345/56 articles respectively, suggesting a drop of 10\% and 45\% for English and non-English articles during period-2.

Conclusions: Published papers on SWL have fallen over the last 8years (both in English and regional languages), highlighting a potential stagnation in this procedure or a threat/take over from

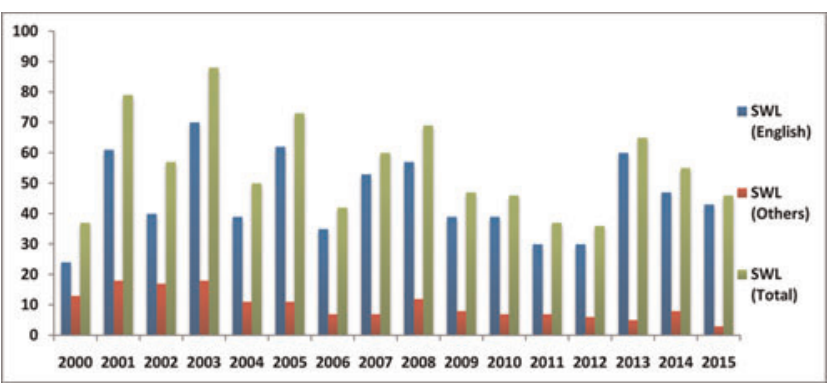

other procedures such as ureteroscopy and minimally invasive percutaneous stone removal techniques.

MP2-21 Shock wave lithotripsy is more effective for residual fragments after percutaneous nephrolithotomy than for primary stones of the same size: A matched pair cohort study

A Aminsharifi, D Irani, H Amirzargar

Advanced Urological Oncology, Duke University Medical Center, Durham, NC

United States

Introduction \& Objective: Shock wave lithotripsy (SWL) is popular for the management of post-percutaneous nephrolithotomy (PCNL) residual fragments. We designed a matched pair study to compare the outcome of SWL on post-PCNL fragments versus primary stones of the same size. We hypothesized that previous percutaneous lithotripsy for the primary stone may affect the outcome of SWL on residual stones because of the disintegration effect of the previous intracorporeal energy on stone fragments.

Materials and Methods: Patients with a single $5-15 \mathrm{~mm}$ fragment confirmed by follow-up computerized tomography 3 months after PCNL were enrolled (study group $n=59$ ). Exclusion criteria were solitary kidney or renal anomalies, serum creatinine level $>2 \mathrm{mg} / \mathrm{dL}$, uncontrolled hypertension, pregnancy, persistent urinary tract infection after adequate antibiotic therapy, irreversible coagulopathy, ureteral obstruction, morbid obesity and skeletal malformations. The control group $(n=67)$ consisted of all adult patients with a single $5-15 \mathrm{~mm}$ renal stone who met the above exclusion criteria and underwent SWL. Demographic data, stone characteristics and 3-month post-SWL outcome were compared between the two groups.

Results: Both groups were similar in age, gender distribution, largest stone diameter and volume. Regardless of stone location, the success rate of SWL (no identifiable stone after 3 months) was significantly higher in the study group $(81.4 \%$ versus $59.7 \%$; $\mathrm{P}=0.008$; OR: $2.95,95 \% \mathrm{CI}$ : 1.301-6.66). Mean stone density in Hounsfield units (HU) was significantly lower in patients with successful SWL $(790.7 \pm 336.46$ versus $945.7 \pm 316.8 ; \mathrm{P}=0.005$, 95\% CI: $-313.71--56.24)$. Receiver operating characteristic curve analysis determined a cutoff point of $750 \mathrm{HU}$ : the success rate was significantly lower in patients with a stone $\mathrm{HU} \geq 750$ (OR: 3.488, 95\% CI: 1.388-8.766). This HU cut off value had no effect on the outcome of SWL in patients with post PCNL RF $(\mathrm{P}=0.14)$. On the other hand, the outcome of SWL was significantly more favorable in control group when $\mathrm{HU}<750(\mathrm{P}=0.02)$. Conclusions: The success rate of SWL was 2.95-fold higher for post-PCNL RFs than in a stone burden-matched control group. The likelihood of stone clearance after SWL was 3.488fold greater when the HU was less than 750. This effect of HU was more prominent in patients receiving SWL for their primary 


\begin{tabular}{|c|c|c|c|}
\hline & $\begin{array}{l}\text { Study group ( } \mathrm{n}=59 \text { ) } \\
\text { Post-PCNL fragenents }\end{array}$ & $\begin{array}{l}\text { Matched control group }(\mathrm{n}=67) \\
\text { Primary stones }\end{array}$ & $\begin{array}{ll}\text { P.Value } \\
\text { P. }\end{array}$ \\
\hline $\begin{array}{l}\text { Age (years) } \\
\text { Gender }\end{array}$ & $46.45 \pm 14.94$ & $45.91 \pm 14.25$ & 0.83 \\
\hline Male & $39(66.1 \%)$ & $42(62.7 \%)$ & \\
\hline Female & $20(33.9 \%)$ & $25(37.3 \%)$ & 0.12 \\
\hline Side of stone & & & \\
\hline Right & $28(47.5 \%)$ & $36(53.7 \%)$ & \\
\hline Left & $31(52.5 \%)$ & $31(46.3 \%)$ & 0.75 \\
\hline Stone size (Max diameter) (mm) & $10.2 \pm 3.14$ & $9.3 \pm 5.52$ & 0.06 \\
\hline Stone volume ( $\left(\mathrm{mm}^{5}\right)$ & $532.8 \pm 413.3$ & $491.4 \pm 246.2$ & 0.06 \\
\hline \multicolumn{4}{|l|}{ Location of stone } \\
\hline Lower calyx & $32(54.2 \%)$ & $20(29.9 \%)$ & \multirow{5}{*}{$<0.001$} \\
\hline Mid calyx & $9(15.3 \%)$ & $6(9 \%)$ & \\
\hline Upper calyx & $6(10.2 \%)$ & $3(4.496)$ & \\
\hline Renal pelvis & $12(20.3 \%)$ & $28(41.8 \%)$ & \\
\hline Ureteropelvicjunction & $0(0 \%)$ & $10(14.9 \%)$ & \\
\hline Stonedensity (HU) & $738.39 \pm 325.29$ & $942.42 \pm 327.01$ & 0.001 \\
\hline
\end{tabular}

PCNL: Percutaneous nephrolithotomy, HU: Hounsfield units

Table 2. Stone-free rate (SFR) after shock wave lithotripsy in the two groups.

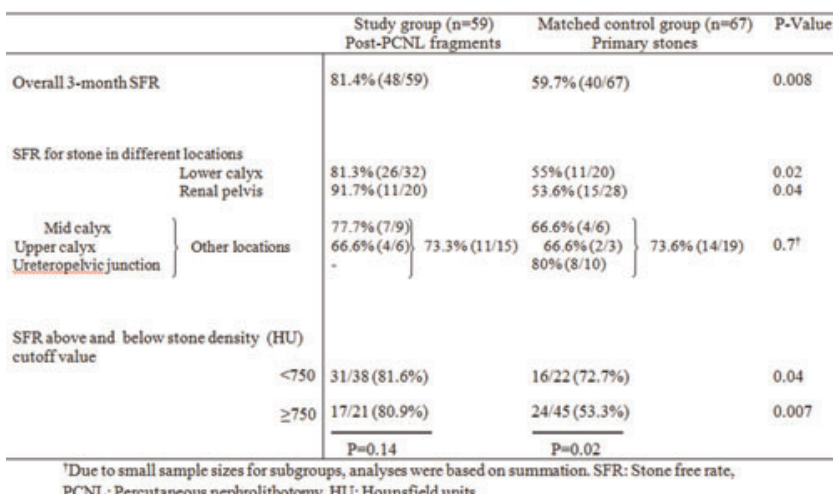

PCNL: Pereutaneous nephrolithotomy, HU: Hounsfield units

stones while SWL was evenly effective on post PCNL RFs with different HUs.

MP2-22 Extracoporeal shock wave lithotripsy treatment for radiolucent ureteral stone with the help of retrograde ureterography using a flexible cystoscopy

J You, H Kim, Y Shin, Y Kim

Department of Urology, Chonbuk National University Medical School, Jeonju, Korea

Republic of Korea

Introduction \& Objective: Localization of the radiolucent ureteral stone during ESWL is difficult. Intravenous contrast medium (IV-CM) administration may overcome this problem. However, IV-CM has several side effects. Patients at risk for adverse reaction to contrast include those with taking Metformin, previous adverse reactions, history of asthma, heart failure, renal insufficiency, and dehydration. We report our experience that ESWL with retrograde ureterography (RGU) for radiolucent ureteral stone localization in patients who have risk factor for IV-CM adverse effect.

Materials and Methods: Between October 2016 and March 2017, a total of 16 patients underwent ESWL with RGU for radiolucent ureteral stone management. All patients had one or more risk factors for IV-CM adverse effect as stated above. All patients were treated on an outpatient basis under analgesia and light sedation if required. The patient prepared the genitalia in an aseptic manner with the supine position on the lithotripsy table.
A ureteral catheter was inserted into the ureteral orifice using 17Fr 'cystoscopy and RGU was performed by injecting diluted contrast media. Contrast medium was injected with as little pressure as possible to prevent the stone upward migration. The filling defect area was aligned to the F2 zone and then lithotripsy was done in the same manner as a conventional ESWL. One to two weeks later, CT scan was performed and additional ESWL was performed if a stone was present. Stone free was defined as the absence of residual stones on CT scan.

Results: The mean age of the patients was $64.8 \pm 11.2$ years, 11 men and 5 women. There were 10 patients had taken Metformin, 8 with renal failure, 4 with contrast allergy, 3 with asthma, and 2 with heart failure. The mean stone size was $8.6 \pm 2.5 \mathrm{~mm}$. The mean number of ESWL received until the stone was discharged was $1.3 \pm 0.6$ times. The success rate was $88 \%$. There was no occurrence of serious adverse effects.

Conclusions: When performing ESWL for radiolucent ureteral stone, RGU using a flexible cystoscopy is useful and safe. We recommend using this method in patients who are expected to have side effects of IV-CM.

MP2-23 Mobile EWSL Treatment For 1-2cm Renal Stones Results and Complications

H Rooney, L Mokool, R Marri, SK Nalagatla

Monklands Hospital, Scotland

United Kingdom

Introduction \& Objective: ESWL is a well established treatments for renal stones $<2 \mathrm{cms}$ [EAU Guidelines]. There is high variability in the global literature for successful treatment of renal stones larger than $1 \mathrm{~cm}$ with mobile SWL. We analysed our results of mobile SWL using a 4th Generation Richard Wolf Piezolith 3000 for $1-2 \mathrm{~cm}$ stones in a District General Hospital setting.

Materials and Methods: We analysed the results of all patients with a renal stone size of 1 to $2 \mathrm{~cm}$ undergoing treatment with mobile SWL as the sole treatment modality between the period of April 2010 to April 2017. Specific information related to the patient's BMI, stone density on CT scan, stone location, along with relevant medical history was documented on the referral form. SWL was performed using a mobile 4th generation Richard Wolf Piezolith 3000 on an outpatient basis. Oral Diclofenac and co-codamol analgesia was used. A maximum of ten patients underwent treatment during these sessions which were held once per month. These sessions were closely supervised by the same lead consultant (SKN). The outcomes were assessed by the same consultant 2 weeks post procedure with $\times$ ray KUB, and the need for further treatment was determined. Patients were advised regarding the possibility of ureteric obstruction and stent insertion post-procedure. Treatment was defined as successful when the residual fragments were of less than $4 \mathrm{~mm}$ in size.

Data was also collected on patients needing any adjuvant treatment.

Results: A total of of 89 patients had Mobile ESWL treatment for $1-2 \mathrm{~cm}$ renal stones. Mean age- 56.7yrs [range16-83 yrs]. 62 were males \& 30 were females. Mean stone size was $12.41 \mathrm{~mm}$. Mean stone density was 950HU [range 431 - 1400HU]. Majority of the stones were in the renal pelvis \& lower calyx [34+33]. 43 patients [58\%]were stone free. 11 patients[15\%] had CIRS. Ten patients needed rigid URS and laser stone fragmentation for stone fragments in the ureter post ESWL. Another 19 patients needed flexi URS \& LASER for stone fragments in the kidney \& 1 had PCNL. 5 patients failed to attend for followup. None of the patients had sepsis or bleeding complications. 
Conclusions: Mobile ESWL is a safe \& effective treatment option for $1-2 \mathrm{~cm}$ renal stones. Patients should be counselled regarding risks of incomplete clearence, need for more than 1 session \& oother djuvant tretments. Results can be further improved by patient selection on the basis of stone location, density and body habitus.

MP2-24 How to accelerate the upper urinary stone discharge after Extracorporeal Shockwave Lithotripsy (ESWL) : a Prospective Multi-center Randomized Controlled Trial about External Physical Vibration Lithecbole (EPVL)

G Zeng, W Wu, Z Yang, F Tang, C Xu, Y Wang, X Gu, X Chen, $\mathrm{R}$ Wang, J Yan, X Wang, W Gao, C Hou, G Alberto, Z Ye

Department of Urology, Minimally Invasive Surgery Center, the First Affiliated Hospital of Guangzhou Medical University. Guangzhou Institute of Urology. Guangdong Key Laboratory of Urology

China (People's Republic)
Introduction \& Objective: To asset the efficacy and safety of EPVL plus ESWL compared with ESWL alone for the treatment of simple upper urinary stones $(<15 \mathrm{~mm})$.

Materials and Methods: All patients with upper urinary stones (immediate EPVL after ESWL while in control group ESWL alone was offered. All patients were reexamined at 1,2 and 4 weeks after ESWL. Stone size, stone location, stone free rate (SFR) and complication rate were compared.

Results: 56 males and 20 females in treatment group were compared to 52 male and 25 females in control group $(p=0.404)$. Median ages were $42.9 \pm 1.5$ years in treatment group and $42.7 \pm 1.3$ years in control group $(\mathrm{p}=0.943)$. Median stone size was $10.0 \pm 0.4 \mathrm{~mm}$ ( 3 to $15 \mathrm{~mm}$ ) in treatment group and $10.4 \pm 0.4 \mathrm{~mm} \mathrm{(4}$ to $15 \mathrm{~mm})$ in control group $(\mathrm{p}=0.622)$. The stone clearance rate in treatment and control group at 1 week after ESWL was 51.3\%(39/ $76)$ and $45.4 \%(35 / 77)(\mathrm{p}>0.05)$, at 2 week was $81.6 \%(62 / 76)$ and $64.9 \%(50 / 77)(\mathrm{p}$

Conclusions: EPVL is a noninvasive, effective and safe adjunctive treatment which increases and accelerates upper urinary stones discharge after ESWL treatment.

\section{MPS3: URETEROSCOPY I}

MP3-1 "Stone-less" or "negative" ureteroscopy - a part of endourologic routine or avoidable source of frustration?

IM Sabler, A Isid, A Lorber, A Latke, G Hidas, E Landau, V Yutkin, D Pode, O Gofrit, M Duvdevani

Hadassah Hebrew University Medical Center, Jerusalem Israel

Introduction \& Objective: A "negative" ureteroscopy is a procedure performed with the intent of removing a kidney or ureteral stone, but in which no stone noted. It may occur when the stone is found to have already been passed or located outside of the collecting system. Not finding a stone at ureterorenoscopy causes frustration to endourologist, patient and his/her family. At our institution, day before endourologic procedure, imaging is done for all patients to ensure stone presence and update its location. Exception are cases in which the patient is clearly symptomatic before the surgery or radiation exposure considerations preclude additional imaging. The aim of the study was to outline risk factors for unnecessary procedures.

Materials and Methods: We retrospectively searched for ureterorenoscopies in which the stone was not found in our prospectively collected and maintained uretrorenoscopic database. Secondary procedures and parenchymal stones were excluded. In every case where presumptive ureteral stone was not found,

\begin{tabular}{|c|c|c|c|c|}
\hline \multicolumn{2}{|c|}{ Parameter } & URS & $\begin{array}{c}\text { Negative } \\
\text { URS }\end{array}$ & $\overline{p-v a l u e}$ \\
\hline \multicolumn{2}{|c|}{ Number of patients } & 560 & $16(2.8 \%)$ & \\
\hline \multicolumn{2}{|l|}{ Mean age } & $52 \pm 15$ & $52 \pm 15$ & \\
\hline \multirow{2}{*}{ Gender } & Female & $144(26 \%)$ & $8(50 \%)$ & \multirow{2}{*}{$p=0.03$} \\
\hline & Male & $416(74 \%)$ & $8(50 \%)$ & \\
\hline \multicolumn{2}{|c|}{ Stone density (HU) } & 995 & 709 & $\mathrm{p}=0.001$ \\
\hline \multicolumn{2}{|c|}{ Radiopaque stones (\%) } & 98.8 & 1.2 & \multirow{2}{*}{$p<<0.05$} \\
\hline \multicolumn{2}{|c|}{ Radiolucent stones (\%) } & 92.2 & 7.8 & \\
\hline \multirow[t]{4}{*}{ Location (\%) } & Kidney & $185(33 \%)$ & $1(1 \%)$ & \multirow[t]{4}{*}{$\mathrm{p}=0.088$} \\
\hline & Upper ureter & $129(23 \%)$ & $5(60 \%)$ & \\
\hline & \begin{tabular}{|l|} 
Mid ureter \\
\end{tabular} & $58(10 \%)$ & 0 & \\
\hline & \begin{tabular}{|l|} 
Lower ureter \\
\end{tabular} & $188(34 \%)$ & $10(60 \%)$ & \\
\hline \multicolumn{2}{|c|}{ Stone burden $\left(\mathrm{mm}^{3}\right)$} & 126 & 289 & $p<<0.05$ \\
\hline
\end{tabular}

formal nephroscopy and whole collecting system revision was completed. Negative procedures - negative group, were compared to the therapeutic ones - therapeutic group in terms of patient and stone characteristics.

Results: From 576 ureterorenoscopies, detected 16 (2.8\%) negative procedures. Mean age was 52 years in both groups. In negative group were: significantly more women $(\mathrm{p}=0.03)$, less dense and radiolucent stones $(\mathrm{p}=0.001$ and $\mathrm{p}<<0.05$, respectivelly) and lower stone volume $(\mathrm{p}<<0.05)$. More negative ureteroscopies were done for distal stone location, but without statistical significance $(\mathrm{p}=0.088)$. BMI, laterality, preoperative obstruction severity, stent or nephrostomy drainage and complications did not differ significantly between the groups.

Conclusions: $\mathbf{2 . 8 \%}$ useless procedures with same complication profile as therapeutic ones. Risk factors include female gender, radiolucent and small stones. Relatively more distal stones were among negative procedures. Development of clear preoperative imaging protocol may decrease number of useless invasive procedures.

\section{MP3-2 SWL vs. URS: Between indications and doctor's} whim

\section{Malkhasyan, I Semanyakin, V Ivanov, D Pushkar}

Russia

Introduction \& Objective: The aim of the study was to evaluate the factors influencing the treatment options of urinary stones. Materials and Methods: We requested data of 300 patients treated for urolithiasis in Moscow region (September 2013 august 2014). The inclusion criteria were solitary, radiopaque non recurrent renal stones $<20 \mathrm{~mm}$, and solitary, radiopaque non recurrent ureteral stones $<12 \mathrm{~mm}$. The exclusion criteria were repeated treatment sessions and emergency cases. We received data on 248 patients. 135 patients were available for follow up 
and consented to take part in the study. Confidence intervals for sample ratio were used for statistical analysis.

Results: Out of 135 patients, $82(61 \%)$ were treated for renal calculi and, 53 (39\%) for ureteral calculi. Median size of ureteral calculi was $9 \mathrm{~mm}(4-12 \mathrm{~m})$, and $14 \mathrm{~mm}(6-20 \mathrm{~mm})$ for renal calculi. 51 patients $(62 \%)$ out of 82 with renal calculi, with median stone size $12 \mathrm{~mm}$, underwent PCNL, and $31(38 \%)$, with the median stone size $10 \mathrm{~mm}$, underwent (SWL). 35 (66\%) out of 53 with renal calculi, with the median stone size $8 \mathrm{~mm}$, underwent URS, and 18 (24\%), with the median stone size $8 \mathrm{~mm}$, underwent (SWL). So $86(64 \%)$ CI $(55.9-72.1)$ of all patients underwent endourological interventions and 49 (36\%) CI (27.9 44.1) (SWL). 18 (21\%) CI $(9.88-26.12)$ out of 86 revealed they have chosen ureteroscopy due to non-availability of SWL, 21 $(25 \%)$ CI $(12.39$ - 29.61) stated that doctor influenced their decision. The remaining 47 (54\%) CI (36.45 - 57.55) patients believed ureteroscopy was their own deliberate choice. Only 8 (16\%) CI (5.14-26.6) patients in SWL group thought that their doctor (has) influenced their decision and 41 (84\%) CI (73.74 94.26) believed SWL was their own deliberate choice. 47 (35\%) patients declared, they were not comprehensively informed on alternative methods of treatment.

Conclusions: Our study showed that $35 \%$ patients treated for renal and ureteral calculi did not receive comprehensive information on alternative methods of treatment. Majority of patients with similar stones size and localization underwent ureteroscopy $86(64 \%)$ vs 49 (36\%). One of the most crucial factors determining treatment options is non-availability of (SWLT) SWL. Urologists inclined to persuade patients for more invasive treatment $21(25 \%)$ vs $8(16 \%)$. The weak point of our study was inability to (asses) assess influence of stone density and stone composition on treatment modalities.

MP3-3 Ureteral stricture formation after removal of proximal ureteral stone: retroperitoneal laparoscopic ureterolithotomy versus ureteroscopy with holmium: YAG laser lithotripsy

C Li, H Hu, L Xu

China (People's Republic)

Introduction \& Objective: To compare the risk of postoperative ureteral stricture formation following retroperitoneal laparoscopic ureterolithotomy (RPLU) and ureteroscopy with Holmium: YAG laser lithotripsy (URSL) in patients with proximal ureteral stones.

Materials and Methods: We retrospectively reviewed the medical records of patients who underwent RPLU or URSL for proximal ureteral stones between April 2011 and May 2015. Patients were allocated into URSL group or RPLU group and the outcomes were compared.

Results: A total of 201 patients who underwent 209 procedures including 159 URSL and 50 RPLU with a median follow-up of 30 months were included. No significant difference was observed among the two groups in most baseline parameters, while the stone size was significantly larger in the RPLU group (11.37 \pm 2.97 vs $14.04 \pm 4.38 \mathrm{~mm}, \mathrm{p}=0.000)$. Patients in RPLU group had markedly longer operative time $(\mathrm{p}=0.000)$ and longer postoperative hospital stay $(\mathrm{p}=0.000)$. The initial and one-month stone-free rates were significantly higher in the RPLU group $(78.6 \%$ vs $100 \%, \mathrm{p}=0.000$ and $82.4 \%$ vs $100 \%, \mathrm{p}=0.001$, respectively). Patients in the RPLU had a higher complication rate $(18.0 \%$ vs $9.4 \%, \mathrm{p}=0.098)$ and lower ureteral stricture rate $(2.5 \%$ vs $2.0 \%, \mathrm{p}=1.000)$, while the difference was not significant. Further logistic regression model
Table 3. Results of multiple logistic regression analysis to determine factors

associated with postoperative complications and ureteral stricture.

\begin{tabular}{cllccc}
\hline \multicolumn{1}{c}{ Items } & Variables & \multicolumn{1}{c}{ Values } & OR & $95 \% \mathrm{Cl}$ & $\mathrm{p}$ value \\
\hline Complications & Procedure & $\mathrm{RPLU}=0 ;$ URSL=1 & 0.28 & $0.09-0.89$ & 0.032 \\
& Sex & Male=0; female=1 & 3.47 & $1.14-10.53$ & 0.028 \\
Ureteral stricture & Procedure & RPLU=0; URSL=1 & 0.90 & $0.07-11.91$ & 0.935 \\
\hline
\end{tabular}

$\mathrm{Cl}$ : confidence interval; OR: odd ratio; RPLU: retroperitoneal laparoscopic

ureterolithotomy; URSL: ureteroscopy with holmium: YAG laser lithotripsy.

$P<0.05$ was considered as statistically significant.

identified RPLU and female sex as independent risk factors for postoperative complication (Odds Ratio[OR] $=3.57, \mathrm{p}=0.032$ and $\mathrm{OR}=3.47, \mathrm{p}=0.028$, respectively), however, URSL was not a independent risk factor for the formation of postoperative ureteral stricture after adjusting confounding variables $(\mathrm{OR}=0.90$, $\mathrm{p}=0.935$ ).

Conclusions: RPLU and URSL have similar postoperative ureteral stricture formation risks. RPLU can provide significantly higher stone clearance rate, but relates with more postoperative complications.

\section{MP3-4 Ureteroscopy in patients with ureteral duplication}

N Chertack, RK Jain, S Sivalingam, MJ Noble, M Monga

Case Western Reserve University

United States

Introduction \& Objective: Ureteral duplication can present challenges in planning and executing management of urolithiasis. We analyzed outcomes of ureteroscopic (URS) treatment of urolithiasis in patients with ureteral duplication.

Materials and Methods: Patients with ureteral duplication who underwent URS were identified in a prospectively collected stone registry at a high volume, tertiary care center. Preoperative, intraoperative, and postoperative data were collected retrospectively. A 1:1 control cohort of patients without duplication was identified, primarily matched by stone location and size, as well as age, BMI and gender. Clinical data and outcomes were compared between duplication and control groups, between partial and complete duplication groups and between patients in whom duplication was identified intraoperatively versus known previously.

Results: We identified 86 patients with ureteral duplication who underwent URS and matching controls. There was no difference in age, gender distribution, BMI, comorbidities and likelihood of presenting with infection. Duplication patients were more likely to have a history of stone disease $(85 \%$ vs $59 \%, \mathrm{p}<0.001)$ and more likely to require additional procedures (13\% vs $3 \%$, $\mathrm{p}=0.026)$. OR time and stone-free rates were not significantly different ( $\mathrm{p}=0.2,0.72$, respectively).

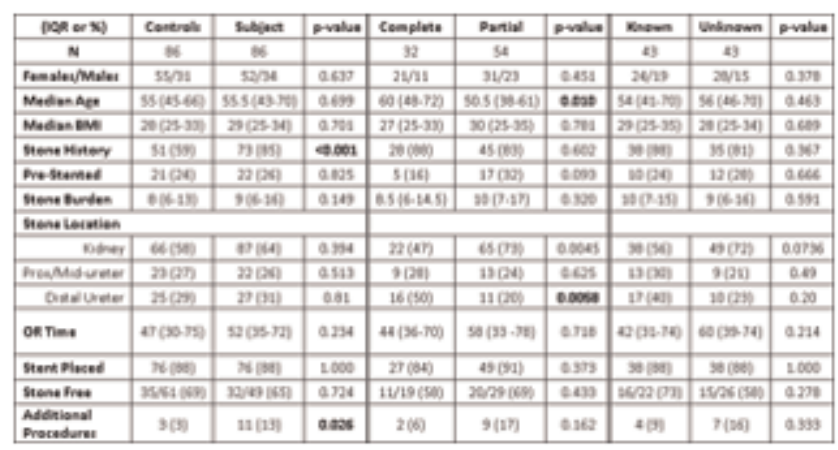


Patients with complete duplication were more likely to have distal ureteral stones (50\% VS 20\%, $\mathrm{P}=0.004)$.

There were no differences in preoperative, operative and postoperative characteristics when comparing patients in whom ureteral duplication was known preoperatively versus identified intraoperatively. There was no difference in complication rate in any of the three comparisons.

Conclusions: Patients with duplicated ureter undergoing ureteroscopy for urolithiasis should be counseled that this anatomic variation does not affect stone-free rate or risk of complications. However, patients may need an additional procedure. Preoperative knowledge of ureteral duplication does not impact outcome of stone treatment.

\section{MP3-5 Indications for Stent Omission after Ureteroscopic} Lithotripsy Defined: An Initial Analysis

PE Bower, J Pereira, S Thavaseelan, G Pareek

\section{Brown University}

United States

Introduction \& Objective: Stent placement after ureteroscopic lithotripsy (URSL) is associated with increased morbidity from pain and lower urinary tract symptoms. Although stent omission (SO) has been proposed to reduce morbidity and visits, stent placement remains nearly ubiquitous and well defined indications for SO remain elusive. We propose evidence based indications for SO and report on our initial experience.

Materials and Methods: Indications for SO were defined as per the attached figure and distributed 11/3/2016. Data from URSL procedures performed by fellowship trained endourologists (GP, ST) was retrospectively reviewed for procedures performed from 11/3/2016-3/24/2017. Patients treated bilaterally, with percutaneous access, or carrying viable pregnancy were excluded. For patients eligible for SO, stone burden, procedure time, number of visits prior to routine follow up imaging, unplanned follow up visits, and whether stent was placed or omitted was recorded. Mean values were compared using student's t-test, and frequency was compared using $\chi^{2}$ test.

Results: Data was gathered on 103 consecutive URSL procedures performed during the study period. 10 cases were excluded. Of the 93 remaining procedures, $35(37.6 \%)$ were eligible for SO, and SO was performed in 19 (20\%) of cases reflecting a 54\% compliance. Patients who had SO had shorter procedure times (19.9 min vs $32.1 \mathrm{~min}, \mathrm{p}<0.01)$ and fewer follow up visits $(0.05$ visits vs 1.0 visits, $\mathrm{p}<0.001$ ) when compared to patients who were eligible for SO but had a stent placed. There was no significant difference in stone burden $(6.8 \mathrm{~mm}$ vs $9.5 \mathrm{~mm}, \mathrm{p}=0.956)$ or unplanned return

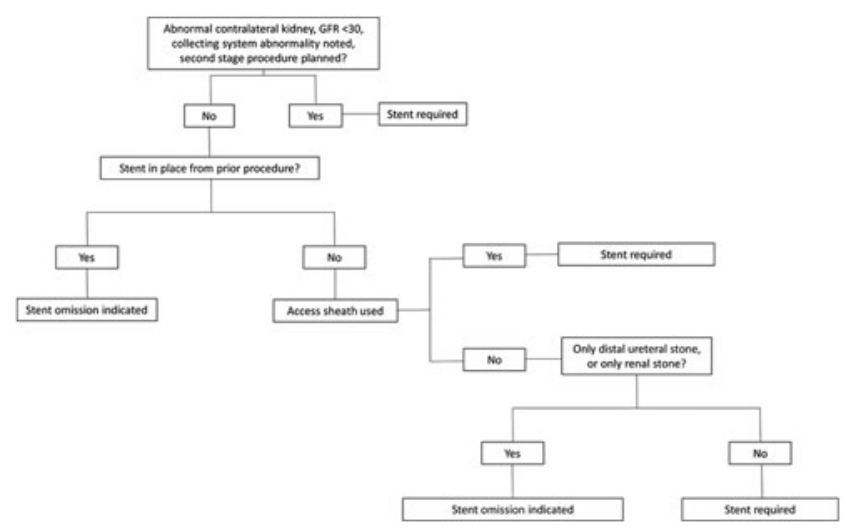

visits $(5.3 \%$ vs $12.5 \%, \mathrm{p}=0.45)$. Patient who had SO performed had a stone burden range of $2-14 \mathrm{~mm}$. The single follow up visit in the SO group was for fatigue and abdominal pain 15 days after URSL. CT was negative for retained fragments or hydronephrosis. Surgeon 1 performed SO in 14/20 eligible cases (70\% compliance). Stratifying results for surgeon 1, patients who had SO had decreased stone burden $(7.7 \mathrm{~mm}$ vs $12.0 \mathrm{~mm}, \mathrm{p}<0.01)$, shorter procedure times (19.2 $\mathrm{min}$ vs $27.3 \mathrm{~min}, \mathrm{p}=0.05)$, and fewer follow up visits $(0.07$ visits vs 1.0 visits, $\mathrm{p}<0.01)$. There was no significant difference in unplanned return visits $(7.1 \%$ vs $0 \%, \mathrm{p}=0.45)$. Conclusions: We provide indications for the safe performance of SO. SO is possible in a significant portion of URSL procedures, and SO decreases the number of postoperative visits after URSL.

\section{MP3-6 CT Imaging: A Predictor of Impacted Ureteral Stones?}

E Parkhomenko, T Tran, JN Thai, K Blum, S Purnell, M Gupta

ICAHN SCHOOL OF MEDICINE AT MOUNT SINAI United States

Introduction \& Objective: A stone is considered impacted if it is not passable with a guide wire or contrast. These stones are more difficult to treat and have a higher morbidity with ureteral stricture rates as high as $24 \%$. Factors predicting stone impaction have not been clearly identified. We sought to evaluate if pre-operative CT findings can predict the presence of an impacted stone.

Materials and Methods: From our prospectively maintained database of 1151 kidney stone formers between 01/2014 - 02/2017, we identified 47 patients with impacted stones (IS) and compared them to 44 who had non-impacted stones (NIS). All patients were treated with ureteroscopic laser lithotripsy by a single surgeon. We excluded patients who had prior stents or surgery for their stone. Computed tomography (CT) was reviewed to calculate stone size, stone volume, degree of hydronephrosis (0-3) and Hounsfield units (HU) of the stone as well as distal and proximal to the stone. Demographic data, CT imaging, labs, and intraoperative factors were used for comparison between groups.

Results: There were no differences in age, gender or BMI between IS and NIS. IS patients had a greater stone size, volume, HU under the stone, HU under/above ratio and degree of hydronephrosis compared to NIS patients. No differences in pre- or post-operative creatinine, stone density or HU above the stone was noted between the two groups. Patients above the cut-off value of $27 \mathrm{HU}$ for the ureter distal to the stone were noted to have impacted stones with a sensitivity of $85 \%$, specificity of $85 \%$, positive predictive value of $85 \%$ and negative predictive

Table 1: Patient and Stone characteristics

\begin{tabular}{|cccc|}
\hline Age & Control & Impacted & $\mathrm{p}$ \\
\hline BMI & 29.0 & 56 & 0.38 \\
\hline Stone size & 7.01 & 8.48 & $<0.05$ \\
\hline Stone Volume & 1061 & 1948 & $<0.05$ \\
\hline Stone HU & 855 & 820 & 0.63 \\
\hline HU under stone & 19.3 & 34.9 & $<0.05$ \\
\hline HU above stone & 14.0 & 11.4 & 0.14 \\
\hline HU under/above & $\mathbf{2 . 1 9}$ & 4.97 & $<0.05$ \\
\hline $\begin{array}{c}\text { Level of } \\
\text { Hydronephrosis }\end{array}$ & 1.22 & 1.66 & $<0.05$ \\
\hline
\end{tabular}


value of $84 \%$. Using a logistic regression model and adjusting for the degree of hydronephrosis and stone size, patients with higher HU under the stone have a higher likelihood of being impacted $(\mathrm{OR}=1.17 ; 95 \%=1.1-1.25 ; \mathrm{p}<0.001)$.

Conclusions: Impacted stones are associated with greater ureteral density distal to the stone, higher stone volumes and greater degrees of hydronephrosis on pre-operative CT. These criteria may help predict which patients are more likely to have impacted stones.

\section{MP3-7 Withdrawn}

MP3-8 Managing Lower calyceal renal stones, flexible ureteroscopy vs ESWL. Is it doctor or patient choice?

A Hammady, M Elbadry

Minia University

Egypt

Introduction \& Objective: With advances of endourologic and laser technology, flexible ureterorenoscopy (FURS) and laser lithotripsy are considered the second line therapy in ESWL-resistant lower calyceal stones. This study aimed to assess patient choice, safety, efficacy and outcome of FURS and holmium: YAG laser comparing its results to ESWL in lower calyceal stones.

Materials and Methods: A prospective randomized study was done from April 2015 to August 2016. It included patients with radiopaque unilateral, single or multiple, LC $\leq 20 \mathrm{~mm}$. Patients were divided into 2 groups according to patient choice. In Group I, patients underwent FURS and laser using $220 \mu \mathrm{m}$ laser fibers. In Group II, patients underwent ESWL. Patients were followed for 3 months by KUB to assess stone-free status defined as no fragments or fragment $\leq 4 \mathrm{~mm}$. stone-free status and complications were compared in both groups.

Results: 114 patients were included in the study. In Group I $(\mathrm{N}=54)$, mean age was 34.2 years and mean stone size was $12.5 \mathrm{~mm}$. 41 patients $(75.9 \%)$ had single stone and 13 patients (24\%) had multiple stones. Stone free status was achieved in 53 patients(98.1\%). Complication rate was $16.7 \%$. In Group II $(\mathrm{N}=60)$, mean age was 35.5 years and mean stone size was $13.3 \mathrm{~mm} .48$ patients $(80 \%)$ had single stone and 12 patients $(20 \%)$ had multiple stones. Stone-free status was achieved in 47 patients $(78.3 \%)$. Complication rate was $23.3 \%$.

Conclusions: Both FURS with LL and ESWL are considered safe in treating LC stones less than $20 \mathrm{~mm}$ with minimal complication rates. However, FURS with LL achieved significantly better stone-free rates. Stone size could predict stone-free status in ESWL

MP3-9 Do Stone Characteristics Influence Avicenna Roboflex Robotic Assisted Retrograde Intra-Renal Surgery Outcomes?

A Patel, N Tokatli, A Kabakci, R Saglam

Spire Roding

United Kingdom

Introduction \& Objective: To study Robotic Assisted Retrograde Intra-Renal Surgery (RA-RIRS) laser stone disintegration outcomes analyzed by stone features.

Materials and Methods: Consecutive upper urinary tract stone patients with stone volume $5000 \mathrm{~mm}^{3}$ undergoing RA-RIRS laser

Table-1
\begin{tabular}{|l|c|c|c|c|c|}
\hline $\mathrm{N}=$ & 6 & 21 & 13 & 16 & 6 \\
\hline Volume Range $\left(\mathrm{mm}^{3}\right)$ & $0-1000$ & $1001-2000$ & $2001-3000$ & $3001-4000$ & $4001-5000$ \\
\hline Mean Volume $\left(\mathrm{mm}^{3}\right)$ & 839.5 & 1603.8 & 2510.3 & 3396.3 & 4187 \\
\hline Mean Laser Fragmentation Time (min) & 26.3 & 50.7 & 59.6 & 65.4 & 85.3 \\
\hline Mean Laser FE (mm'/min) & 31.9 & 31.6 & 42.1 & 51.9 & 49.1 \\
\hline
\end{tabular}

Table-2
\begin{tabular}{|c|c|c|c|c|c|c|c|}
\hline & n & Mean HU & $\begin{array}{c}\text { Mean HU } \\
\text { Density } \\
\left(\mathrm{HU} / \mathrm{mm}^{3}\right)\end{array}$ & $\begin{array}{c}\text { Mean Laser } \\
\text { Fragmentation } \\
\text { Time }(\min )\end{array}$ & $\begin{array}{c}\text { Mean Laser FE } \\
\left(\mathrm{mm}^{3} / \mathrm{min}\right)\end{array}$ & SFR & $\begin{array}{c}\text { Insignificant } \\
\text { residual < } 3 \mathrm{~mm}\end{array}$ \\
\hline HU $<900$ & 32 & $725.3^{* *}$ & 0.338 & 54.6 & 49.4 & $69 \%$ & $28 \% \%^{*}$ \\
\hline HU $\geq 900$ & 30 & $1144^{* *}$ & 0.557 & 61.3 & 35.6 & $63 \%$ & $37 \%$ \\
\hline
\end{tabular}

*3\% significant residual, **28\% Calcium oxalate dihydrate $25 \%$ each Calcium oxalate monohydrate (CaOMh), and mixed mono-dihydrate , $9 \%$ Uric Acid, $6 \%$ Ca Phosphate infection stones, $7 \%$ (others), $* * 90 \%$ CaOMh

stone dusting +/- popcorning under general anesthesia with DJ stent placement, were analysed. Stone free rate (SFR) was defined as no residual, insignificant or significant residual, on 3 month NCCT follow-up. Stone density cut-off was $>$ or $<900$ Hounsfield units (HU).

Results: There were 15 females, 47 males, 4 had hydronephrosis, and 15 were pre-stented. 39 were either renal pelvic or lower calyceal in location. Mean (range) data were: age 39 years (19$62)$; stone diameter $24.9 \mathrm{~mm}$ (20-42), volume $2435 \mathrm{~mm}^{3}$ (6794400), Hounsfield units 928 (537-1480) and fragmentation time $58 \mathrm{~min}$. (15-118). Fragmentation efficiency (FE) was $31.9 \mathrm{~mm}^{3} /$ min for the smallest stones, peaking at $51.9 \mathrm{~mm}^{3} / \mathrm{min}$ for stones with mean volume $3396 \mathrm{~mm}^{3}$ (Table-1). SFR was $98 \%$ (insignificant residual $28 \%$ if $\mathrm{HU}>900$ ). Stone density features related to FE are shown in Table-2.

Conclusions: Larger $\left(>3000 \mathrm{~mm}^{3}\right)$ softer stones $(<900 \mathrm{HU})$ had optimal FE, the latter having higher 3 month complete SFR rates. The higher FE for larger volume stones may be related to lower stone mobility in the collecting system. RA-RIRS potentially allows comfortable, safe, efficient, and successful single procedure minimally invasive fragmentation and stone clearance of large, higher volume softer upper tract stones, with treatment times $<90$ minutes for the largest volume stones.

MP3-10 Defining the rate of encountering unacceptable reusable flexible ureteroscopes for immediate clinical use: 'Bad out of the Box.'

SG Hubosky, KA Healy, DH Bagley

Thomas Jefferson University Hospital United States

Introduction \& Objective: Single-use flexible ureteroscopes offer the advantage of being consistently functional and perfect for immediate clinical use right "out of the box." Cost is the barrier to widespread acceptance of these instruments. Economic models have been put forth which compare the expense of acquiring and maintaining reusable flexible ureteroscopes to that of using single-use flexible ureteroscopes. However, one poorly defined variable in these models is the frequency of encountering an unsuitable reusable flexible ureteroscope at the beginning of a case. We sought to define this in a consecutive series of patients undergoing flexible ureteroscopy.

Materials and Methods: Prospective analysis of all consecutive cases requiring flexible ureteroscopy over three months was undertaken. A combination of fiberoptic and digital flexible ureteroscopes comprised the available inventory. Per protocol, these instruments were grossly cleaned in the endourology suite 
after use and sent to central processing for final cleaning, sterilization (STERRAD®) and packaging. Repairs were managed by a third party repair service when needed. Ureteroscopes were defined as acceptable if they provided reasonable visualization, deflection, an open working channel that would accept passage of instruments and no evidence of gross contamination or overt damage or deformity.

Results: Of 103 consecutive cases, a total of 119 reusable flexible ureteroscopes were unwrapped and $93(90 \%)$ cases were initiated with the first instrument opened. In 10 (9.7\%) cases, the initial ureteroscope opened was unacceptable for use and required opening an additional ureteroscope(s). In 7 cases at least 2 instruments were opened, 3,4 and 5 instruments needed to be opened in 1 case each. One case had to be rescheduled after 4 consecutive instruments were opened and all were unsuitable. Of 17 unfit instruments, 19 problems were noted and included broken deflection (4), dried cleaning solution on the instrument tip (4), inability to pass a laser fiber through the working channel (5), digital camera dislodged from distal bending rubber (2), crushed proximal shaft (1), digital image failure (1), lens trouble causing optical failure (1) and a missing sterilization cap (1). Considering all 119 instruments opened, 17 (14\%) were unsuitable for immediate use.

Conclusions: In up to $14 \%$ of cases, the initially opened reusable flexible ureteroscope is not fit for initiation of the procedure. This rate may vary among institutions depending on repair, processing and nursing practices but represents one area where single use devices can fill an essential and immediate role.

MP3-11 Is endoscopic evaluation at the end of retrograde intra-renal surgery a reliable predictor of post-operative significant residual fragments?

E Alessandria, A Bosio, E Dalmasso, D Peretti, F Vitiello, A Bisconti, P Destefanis, P Gontero

Città della Salute e della Scienza di Torino Italy

Introduction \& Objective: Post-operative imaging used to assess retrograde intra-renal surgery (RIRS) results affects stonefree rates (SFR). Imaging after RIRS may be unnecessary if surgeon\&'s endoscopic evaluation (EE) at the end of RIRS proved to be reliable.

The objective of our study was to assess the reliability of surgeon\&'s EE at the end of RIRS.

Materials and Methods: We made a retrospective analysis on all consecutive RIRS performed for renal stones from January 2009 to August 2016 and included in a prospectively maintained database. RIRS were performed with fiber optics instruments in one-day surgery under spinal anesthesia. A ureteral access sheath was used, laser lithotripsy was performed and significant fragments extracted. Multiple stones, stones $>2 \mathrm{~cm}$ and staged procedures were included. Surgeons recorded their EE particularly about the presence of significant residual fragments (SRF). Residual fragments were considered clinically insignificant (CIRF) if $\leq 4 \mathrm{~mm}$. Primary endpoint of our study was the ability of EE made by an expert surgeon to exclude the presence of SRF at US at 2-3 weeks. Chi-square and Fisher tests were used for statistical analysis.

Results: 294 RIRS were included. EE was available in 281 cases and US in 211. Mean stone size was $12.31 \mathrm{~mm} \pm 4.87 \mathrm{~mm}$ (SD). In 68 cases $(23.13 \%)$ stones were multiple. Post-operative US outcomes significantly differed from urologist\&'s EE in term of SRF, CIRF and SFR $(\mathrm{p}<0.0001)$, independently from the
Table 1. Concordance of EE and US outcomes in relation with the number of RIRS performed per year by the surgeon

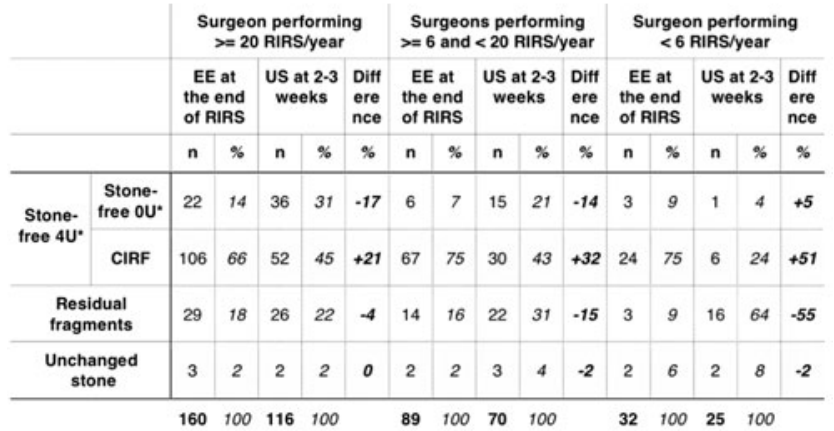

* according to BK Somani, M Desai, O Traxer, S Lahme on Urolithiasis 2014:42:95.

number of RIRS performed per year by the surgeon $(\mathrm{p}<0.001)$. $14 \%$ more patients were found stone-free (SFR 0U) at US compared to EE, $28 \%$ less were found to have CIRF and $14 \%$ more to have SRF. Table 1 shows how the concordance of EE and US outcomes varied in relation with surgeon\&'s expertise. In particular the difference in terms of SRF and CIRF decreased when EE was made by a surgeon performing $\geq 20 \mathrm{RIRS} /$ year. The absence of SRF assessed at EE by a surgeon performing $\geq 20$ RIRS/year was confirmed at US in $92 \%$ of cases. The probability to diagnose at US SRF not assessed at EE increased to $36 \%$ when EE was made by surgeons performing $<20 \mathrm{RIRS} /$ year $(\mathrm{OR}=6.4)$. Conclusions: Post-operative US outcomes significantly differed from urologist\&'s EE. EE underestimated SFR and overestimated CIRF. SRF were underestimated at EE, but an expert surgeon reliably predicted the absence of SRF.

MP3-12 Effect of Dusting and Fragmentation Settings on Stone Models Using an Automated 3-D Gantry System

AH Aldoukhi, TL Hall, WW Roberts, KR Ghani

Department of Urology, University of Michigan United States

Introduction \& Objective: Holmium laser systems are now able to offer fragmentation (Low Frequency-High Pulse Energy) and dusting (High Frequency-Low Pulse Energy) settings for lithotripsy. Most in vitro studies in this field have explored fragmentation using rudimentary motion systems. We assessed the effect of different laser settings on fragmentation utilizing an automated 3-D gantry system for moving the laser fiber using two widely available stone models.

Materials and Methods: We tested two distinct stone models: BegoStone (15:3 powder to water ratio) and Ultracal 30 (100:38 powder to water ratio). Stones were $30(\mathrm{l}) \times 30(\mathrm{w}) \times 3(\mathrm{~h}) \mathrm{mm}$ in size and 3 stones from each stone model were used for each experiment. A 60-Watt system (VersaPulse, PowerSuite, Lumenis, CA) and $200 \mu \mathrm{m}$ fiber (Flexiva 200, Boston Scientific, MA) were used. The laser fiber was stripped and cleaved prior to each experiment. The laser fiber was positioned with a fiber chuck that was connected to a customized 3-D positioner (Velmex, NY). For each experiment, the positioner was programed using Matlab (MathWorks, MA) to advance the fiber at a speed of $1 \mathrm{~mm} / \mathrm{s}$ to make 10 connected lines $20 \mathrm{~mm}$ long with $2 \mathrm{~mm}$ spaces in between. Laser settings assessed were either $8 \mathrm{~W}: 0.2 \mathrm{~J} \times 40 \mathrm{~Hz}, 0.4 \mathrm{~J} \times 20 \mathrm{~Hz}$, $1.0 \mathrm{~J} \times 8 \mathrm{~Hz}$; or $16 \mathrm{~W}: 0.4 \mathrm{~J} \times 40 \mathrm{~Hz}, 0.8 \mathrm{~J} \times 20 \mathrm{~Hz}$, and $1.6 \mathrm{~J} \times 10 \mathrm{~Hz}$. To determine stone fragmentation, the difference in stone weight 


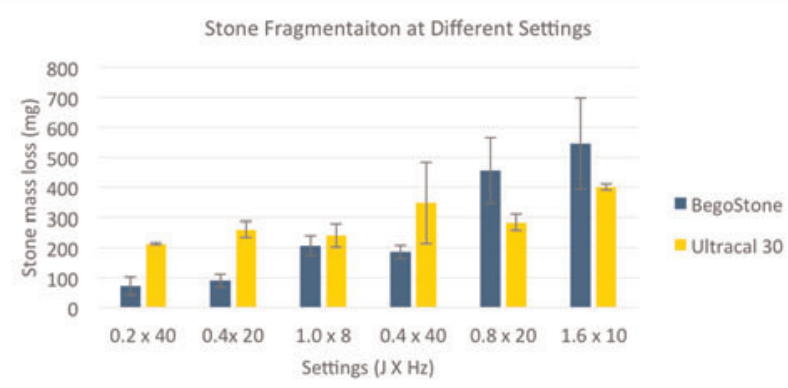

Figure 1: Results of stone fragmentation at different laser settings using 2 stone models

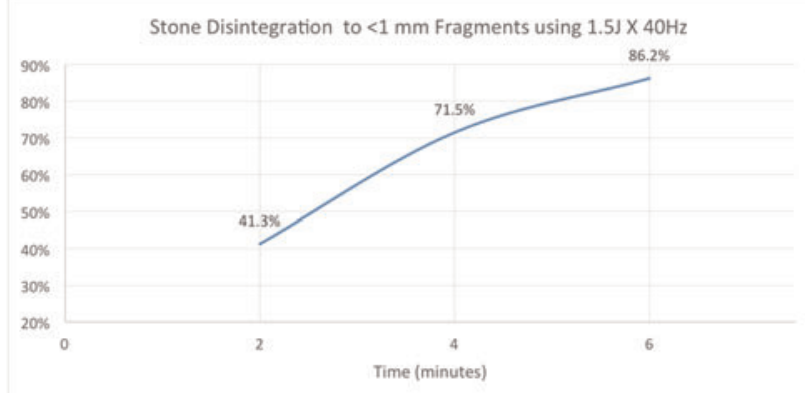

Figure 1: Effect of time on stone disintegration to $<1 \mathrm{~mm}$ (dusting effect) using $1.5 \mathrm{~J} \mathrm{X} 40 \mathrm{~Hz}$ before and after each experiment was recorded. We used microsieves $(1$ and $0.5 \mathrm{~mm})$ to determine sub-millimeter stone fragmentation (dusting) effect.

Results: Stone fragmentation at different laser settings for the two stone models is shown in Figure 1. For BegoStone stones, fragmentation increased when the pulse energy was increased. In contrast, Ultracal stones did not show a similar trend. Regardless of the laser setting, none of the stone models produced fragments $>0.5 \mathrm{~mm}$ in size.

Conclusions: Fragmentation with the BegoStone stone model was more consistent than when using Ultracal; however, the response to laser lithotripsy did not mimic human stone response for dusting settings and fragmentation. Our findings suggest the need for validating the currently used stone models to ensure they replicate clinical findings in the era of high frequency laser lithotripsy.

MP3-13 Non-Contact "Pop-corn" Holmium Laser Lithotripsy: What is the ideal duration for dusting effect?

\section{AH Aldoukhi, TL Hall, WW Roberts, KR Ghani \\ Department of Urology, University of Michigan United States}

Introduction \& Objective: Non-contact high-frequency holmium laser lithotripsy (pop-corn effect) is performed when the laser fiber is activated away from the stone surface, resulting in a whirlpool like effect that breaks stones into fragments. A prior study demonstrated settings of $1.5 \mathrm{~J}$ and $40 \mathrm{~Hz}$ resulted in the best stone disintegration. The aim of this study was to assess the optimal time needed to achieve stone disintegration and the impact on fiber burnback.

Materials and Methods: A 60-W laser (VersaPulse PowerSuite, Lumenis, CA) and a $200 \mu \mathrm{m}$ fiber (Flexiva 200, Boston Scientific, MA) were used. Disc shaped BegoStone (15:3 powder to water ratio / size $5 \mathrm{~mm} \times 2-3 \mathrm{~mm}$; weight $0.3 \mathrm{~g}$ ) were prepared and placed in a glass vial (inner diameter $14 \mathrm{~mm} /$ length $60 \mathrm{~mm}$ ), to simulate a caliceal model. Flow was introduced using a $4 \mathrm{Fr}$ catheter, connected to a saline bag for irrigation $(150 \mathrm{mmHg})$. The laser fiber was positioned $2 \mathrm{~mm}$ away from the highest stone and held in place using a fiber chuck attached to a 3-D positional gantry system. We assessed $1.5 \mathrm{~J} \times 40 \mathrm{~Hz}$ for a duration of 2, 4, and 6 minutes; the experiment was paused at each minute to readjust the fiber. Stone fragments were sieved with a $0.5 \mathrm{~mm}$ sieve to determine dusting result (sub-millimeter fragmentation) and loss in stone mass. Fiber burnback was assessed by measuring fiber tip length before and after each experiment using a digital caliber (Fisher Scientific, NH).
Results: A total of 9 experiments were conducted. At $1.5 \mathrm{~J} \times 40 \mathrm{~Hz}$ setting, the mean percentage loss in stone mass resulting in a sub-millimeter dusting effect was $41.3 \%$ (SD 10.6), $71.5 \%$ (SD 9.0), and 86.2\% (SD 6.6) at 2, 4, and 6 minutes respectively. Stone fragmentation was significantly different when non-contact lithotripsy was performed for 4 vs 2 minutes $(P<0.05)$; however, fragmentation between 4 and 6 minutes was not significantly different $(P=0.08)$ (Figure 1$)$. The mean loss in fiber length (burnback) was 2.1 (SD 0.7), 3.2 (SD 0.8), and 4.0 (SD 0.9) $\mathrm{mm}$ at 2, 4, and 6 minutes respectively; 2 vs 6 minutes, $P=0.054$.

Conclusions: We found an increase in both stone disintegration and fiber burnback with increasing non-contact lithotripsy time. When using $1.5 \mathrm{~J} \times 40 \mathrm{~Hz}$, at least 4 minutes is needed to achieve $>50 \%$ sub-millimeter (dusting) results for stone fragmentation.

\section{MP3-14 Heat Generation During Holmium Laser Litho- tripsy}

AH Aldoukhi, TL Hall, KR Ghani, WW Roberts

Department of Urology, University of Michigan United States

Introduction \& Objective: With increasing interest in high frequency laser lithotripsy techniques, and the advent of higher power multi-cavity laser systems, the effect of high-watt laser settings on heat production within the collecting system is relatively unknown. The aim of this study was to investigate the effect of irrigant flow rate on temperature changes during laser lithotripsy in a simulated laboratory model.

Materials and Methods: The experimental system consisted of a glass test tube (inner diameter $10 \mathrm{~mm} /$ length $75 \mathrm{~mm}$ ) filled with deionized water. Real time temperature was recorded using a thermocouple (Physitemp, NJ) positioned $5 \mathrm{~mm}$ from the bottom of the tube. A $200 \mu \mathrm{m}$ laser fiber (Flexiva, Boston Scientific, MA) was located within the test tube with tip $10 \mathrm{~mm}$ above the bottom. A 4.1 Fr catheter was placed $14 \mathrm{~mm}$ from the bottom of the tube to provide irrigant flow from a pressure bag of room temperature deionized water calibrated to deliver irrigant flow rates of 15 and $39 \mathrm{~mL} / \mathrm{min}$. The test tube was placed in a water bath maintained at $37^{\circ} \mathrm{C}$. During each experiment, temperature was monitored continuously, irrigant flow was started at 20 seconds and laser energy was delivered from a 60-watt holmium laser (VersaPulse PowerSuit, Lumenis, CA) starting at 40 seconds until $100 \mathrm{sec}-$ onds. Irrigant flow was stopped at 120 seconds. The following settings were explored: $0.2 \mathrm{~J} \times 40 \mathrm{~Hz}(8 \mathrm{~W}), 0.5 \mathrm{~J} \times 20 \mathrm{~Hz}(10 \mathrm{~W})$, $1.0 \mathrm{~J} \times 10 \mathrm{~Hz}(10 \mathrm{~W}), 0.5 \mathrm{~J} \times 40 \mathrm{~Hz}(20 \mathrm{~W}), 1.5 \mathrm{~J} \times 20 \mathrm{~Hz}(30 \mathrm{~W})$, $1.0 \mathrm{~J} \times 40 \mathrm{~Hz}(40 \mathrm{~W})$, and $1.5 \mathrm{~J} \times 40 \mathrm{~Hz}(60 \mathrm{~W})$. The fiber was 


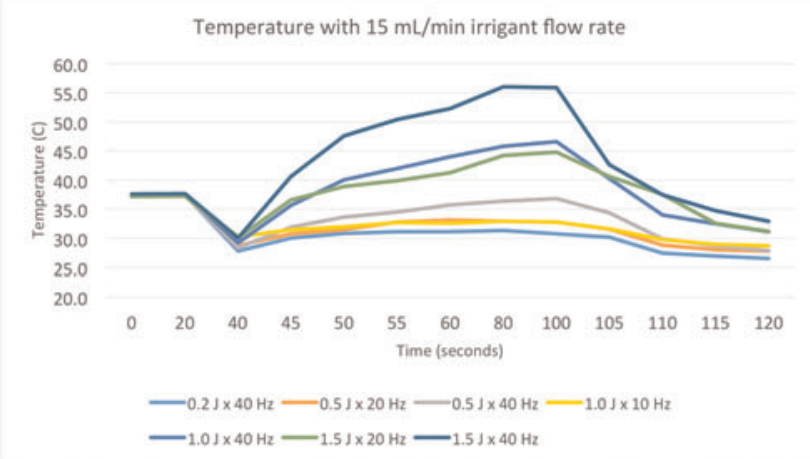

Figure 1: change in temperature at each setting during the experiments at flow rate of $15 \mathrm{~mL}$ min. Each experiment was conducted as follow: the flow was started at 20 second, the laser was started at 40 seconds and stopped at 100 seconds, and the flow was continued until 120 seconds.

stripped and cleaved prior to each experiment. Each experiment was repeated 3 times.

Results: Temperature curves are demonstrated in Figure 1. At $15 \mathrm{~mL} / \mathrm{min}$ flow rate, maximal temperature up to $55.9^{\circ} \mathrm{C}$ was recorded when using settings with power output of $60 \mathrm{~W}$. Temperature did not increase above $38^{\circ} \mathrm{C}$ for any settings with $39 \mathrm{ml} /$ min irrigant flow.

Conclusions: Higher watt laser settings can induce temperature rise of calyceal fluid up to $56^{\circ} \mathrm{C}$ with $15 \mathrm{~mL} / \mathrm{min}$ irrigant flow. Further studies are needed to investigate the heat-sink effects of renal blood flow and tissue perfusion on calyceal temperature rise, as well as the effect of these changes on ureteroscope longevity during laser lithotripsy.

\section{MP3-15 Laser Fiber Burnback: The role of Pulse Width on Tip Degradation}

\section{AH Aldoukhi, TL Hall, WW Roberts, KR Ghani \\ Department of Urology, University of Michigan United States}

Introduction \& Objective: Laser fiber tip degradation (burnback) during ureteroscopic holmium lithotripsy is known to be affected by pulse energy; higher pulse energies lead to greater tip degradation. Holmium lasers now provide the option to alter the pulse width (PW) to short or long PW modes. In this study, we investigated the effect of PW on fiber tip degradation.

Materials and Methods: We used a 120-Watt system (Lumenis Pulse 120, Lumenis, CA) and $200 \mu \mathrm{m}$ fiber (Flexiva 200, Boston Scientific, MA) for this study. Prior to each experiment, the laser fiber was stripped and cleaved. The following settings were used for non-contact laser lithotripsy: $1.0 \mathrm{~J} \times 20 \mathrm{~Hz}$ (pop-corn), $1.0 \mathrm{~J} \times 40 \mathrm{~Hz}$ (high frequency pop-corn), and $0.5 \mathrm{~J} \times 70$ (high frequency pop-dusting) utilizing both short and long $\mathrm{PW}$ for a total duration of 4 minutes. Cuboid Begostone (15:3 powder to water ratio / size $3.0 \times 3.0 \times 1.3 \mathrm{~mm}$; weight $0.19 \mathrm{~g})$ were placed in a glass test tube (inner diameter $10 \mathrm{~mm} /$ length $75 \mathrm{~mm}$ ), to simulate a caliceal model. Flow was introduced using a $4.1 \mathrm{Fr}$ ureteral catheter, connected to a saline bag placed at $100 \mathrm{~cm}$ (flow rate $15 \mathrm{~mL} / \mathrm{min}$ ) with the laser fiber positioned $5 \mathrm{~mm}$ away from the bottom of the tube. The fiber tip length was measured before and after each experiment using a digital caliber (Fisher Scientific, NH). Images of the fiber tip were taken using an upright microscope (Nikon, Japan). Each experiment was repeated 5 times.

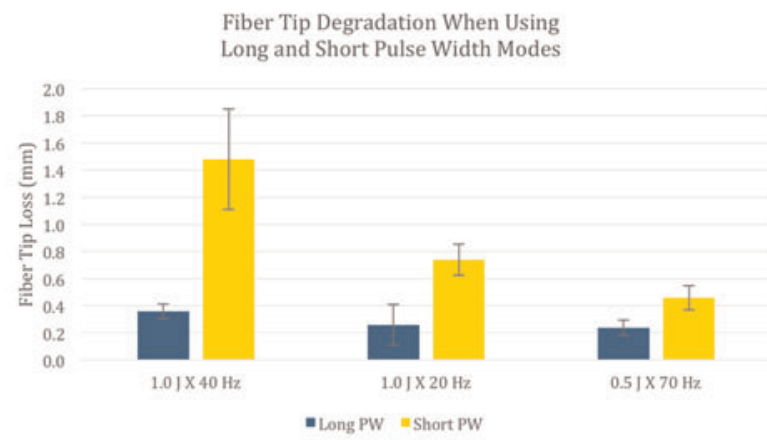

Figure 1: Laser fiber tip degradation during non-contact laser lithotripsy utilizing pop-corning $(1 \mathrm{~J}, 20 \mathrm{~Hz} ; 1 \mathrm{~J}, 40 \mathrm{~Hz})$ and pop-dusting $(0.5 \mathrm{~J}, 70 \mathrm{~Hz})$ settings

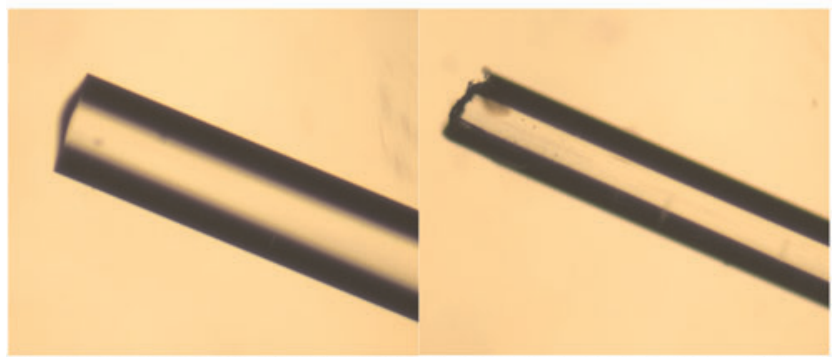

Figure 2: Microscopic image of a cleaved fiber prior to testing at $1.0 \mathrm{~J} \mathrm{X} 40 \mathrm{~Hz}$ utilizing a short PW (Left) and the same fiber after the experiment (right).

Results: Fiber tip degradation was greatest when using short PW compared to long PW at each setting $(P<0.01)$, with the highest degradation for $1.0 \mathrm{~J} \times 40 \mathrm{~Hz}$ with short PW. Figure 1 presents fiber tip degradation at each setting and figure 2 demonstrates the fiber tip before and after experiments conducted utilizing $1.0 \mathrm{~J} \times 40 \mathrm{~Hz}$ with short PW.

Conclusions: Long PW was found to result in less fiber tip degradation for all settings tested, and utilizing long PW can improve the longevity of the laser fiber. Clinical studies are needed to investigate the effect of these settings on power output during holmium laser lithotripsy.

\section{MP3-16 Non-Contact Holmium Laser Lithotripsy: Which settings give the best Dusting effect?}

AH Aldoukhi, TL Hall, WW Roberts, KR Ghani

Department of Urology, University of Michigan United States

Introduction \& Objective: Non-contact high-frequency holmium laser lithotripsy (pop-corn effect) during ureteroscopy (URS) is performed when the laser fiber is activated away from the stone surface in a calyx, resulting in a whirlpool like effect and stone fragmentation. A prior study demonstrated that settings of $1.5 \mathrm{~J}, 40 \mathrm{~Hz}$ resulted in the best stone disintegration for fragment sizes $<2 \mathrm{~mm}$. The aim of our study was to assess submillimeter fragment (dusting effect) size distribution and loss in stone mass utilizing traditional "pop-corning" and high frequency "pop-dusting" settings.

Materials and Methods: Begostone (15:3 powder to water ratio / size $3.0 \times 3.0 \times 1.3 \mathrm{~mm}$; weight $0.19 \mathrm{~g}$ ) were placed in a test tube 


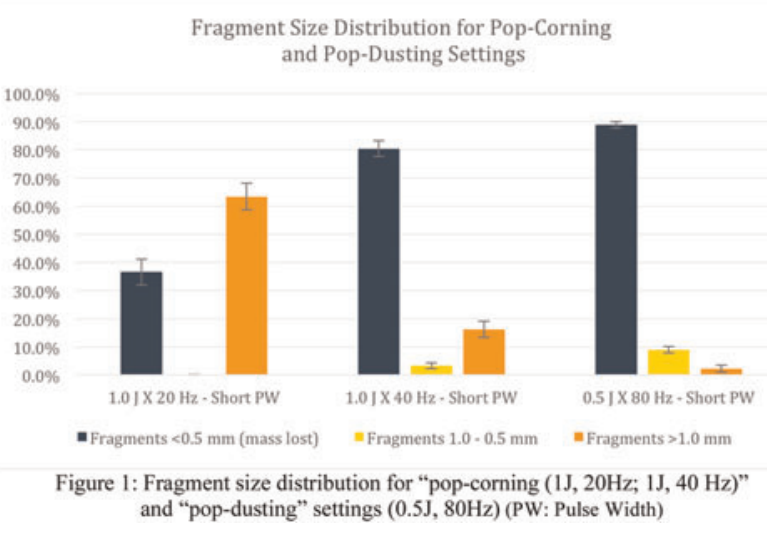

(inner diameter $10 \mathrm{~mm} /$ length $75 \mathrm{~mm}$ ), to simulate a caliceal model. Flow was introduced using a 4.1 Fr catheter connected to a saline bag placed at $100 \mathrm{~cm}$ to provide flow rate of $15 \mathrm{~mL} / \mathrm{min}$. The study was performed utilizing a 120-W laser (Lumenis Pulse 120, Lumenis, CA) and a $200 \mu \mathrm{m}$ fiber (Flexiva 200, Boston Scientific, MA). The laser fiber was positioned $5 \mathrm{~mm}$ away from the bottom of the tube. Laser settings of $1.0 \mathrm{~J} \times 20 \mathrm{~Hz}$ (traditional pop-corn), $1.0 \mathrm{~J} \times 40 \mathrm{~Hz}$ (high-frequency pop-corn), and $0.5 \mathrm{~J} \times 80 \mathrm{~Hz}$ (popdusting) were used utilizing short pulse width mode for a total duration of 4 minutes; experiments were paused every 30 seconds to readjust the fiber tip. Stone fragments were sieved with 1.0 and $0.5 \mathrm{~mm}$ sieves to determine sub-millimeter fragment distribution and loss in stone mass. Each experiment was repeated 5 times.

Results: Loss in stone mass was $36.6 \%$ (SD 4.6\%), 80.4\% (SD $2.8 \%$ ), and $88.9 \%$ (SD $1.1 \%$ ) for $1.0 \mathrm{~J} \times 20 \mathrm{~Hz}, 1.0 \mathrm{~J} \times 40 \mathrm{~Hz}$, and $0.5 \mathrm{~J} \times 80 \mathrm{~Hz}$, respectively. Figure 1 demonstrates the fragment size distribution for each setting. Pop-dusting settings $(0.5 \mathrm{~J} \times 80 \mathrm{~Hz})$ resulted in greater sub-millimeter fragments compared to the other settings $(P<0.001)$.

Conclusions: Compared to traditional pop-corning settings, utilizing low-pulse energy and ultra-high frequency (pop-dusting) during non-contact lithotripsy resulted in the greatest loss in stone mass and the highest amount of sub-millimeter fragments. Utilizing these settings during URS may result in a better dusting effect for optimal spontaneous passage of fragments. Further clinical studies are needed to confirm these findings.

MP3-17 The impact of high-frequency positive-pressure ventilation (HFPPV) on operation time in retrograde intrarenal surgery for stones $>1 \mathrm{~cm}$

M Popiolek, D Al-Rammahi, R Swartz

Department of Urology, Orebro University Hospital Sweden

Introduction \& Objective: Respiration-induced kidney movements may affect the outcomes in retrograde intrarenal surgery (RIRS). However, the effects on operation time and stone-free rates are not well studied. According to an earlier report, HFPPV reduces the respiratory kidney movements and may therefore facilitate lithotripsy. The aim of this study is to the report preliminary results after RIRS with HFPPV and investigate whether it can shorten the operation times and improve stone-free rates (SFR).

Materials and Methods: All consecutive patients $(n=69)$ operated during 2016 with flexibel ureteroscopy and laser lithotripsy at one center for renal or proximal larger than $10 \mathrm{~mm}$ were included. The data was retrieved from a local register and medical records were additionally reviewed with regard to patient and surgery related variables.

Results: Of all, 18 were operated under HFPPV (group A) and 51 received conventional anesthesia (group B). The groups did not differ significantly regarding age, gender and BMI. The median stone size and volume were $17 \mathrm{~mm}, 693 \mathrm{~mm}^{3}$ and $15 \mathrm{~mm}, 400 \mathrm{~mm}^{3}$ in groups A and $B$, respectively $(\mathrm{p}=0.32)$. Ureteral access sheath was utilized in all cases in both groups. Median operation time was 62 minutes (range: $31-139$ ) in group A compared to 73 minutes (range: $33-130)$ in group B $(\mathrm{p}=0.089)$. SFR was $78 \%(14 / 18)$ and $67 \%(34 / 51)$ in groups $\mathrm{A}$ and $\mathrm{B}$, respectively $(\mathrm{p}=0.55)$. Hospitalization length and postoperative infectious complications did not differ significantly between the groups.

Conclusions: HFPPV facilitates a precise and efficient lithotripsy due to reduction of respiration-induced kidney movements, which may result in shortened operation time and improved SFR. Further evaluation with prospective setting is in progress.

MP3-18 The impact of stone density on outcome of ureteronephroscopy

A Shvero, N Kleinmann, Y Abu-Ghanem, A Eisner, D Zilberman, J Ramon, H Winkler

Department of Urology, Sheba Medical Center, Tel Hashomer Israel

Introduction \& Objective: Stone density, measured by Hounsfield units (HU) on non-contrast CT (NCCT), has been linked to success rates of ESWL and PCNL. This study aims to examine the effect of stone density on surgical outcome and complication rate in patients undergoing ureteronephroscopy (RIRS) and laser lithotripsy for kidney stones.

Materials and Methods: Between December 2013 and December 2015, 1327 endourologic procedures were performed at our institution. We reviewed consecutive ureteroscopies in cases with unilateral kidney stones, no ureteral stones, and an available NCCT scan. We gathered demographic data, extent of stone disease (stone burden calculated by summation of stone sizes, weighted stone density for each patient according to burden), surgery reports (complications, duration), post-operative complications, and shortterm clinical follow-up (urinary tract infections, pain, refers to emergency room, repeat procedures, stone-free rate). Univariate and multivariate analysis were performed to assess the relation between stone density and the above parameters.

Results: Study cohort included 177 patients, 118 men, with a median age of 55 years (range 18-84). Average number of stones was 1.6. Median stone burden was $17 \mathrm{~mm}$ (5-67). Median weighted stone density was $1000 \mathrm{HU}$ (246-1720). Average follow-up time after surgery was 115 days. We found no relation between stone density and: stone free rate $(p=0.986)$, intra-operative complications $(\mathrm{p}=0.223)$, post-operative complications $(\mathrm{p}=0.103)$, refers to ER $(\mathrm{p}=0.790)$, UTIs $(\mathrm{p}=0.789)$, and the

Table 1: Surgery duration divided by HU groups

\begin{tabular}{|c|c|c|c|}
\hline & $\mathrm{HU}<1300$ & $1300<\mathrm{HU}$ & $\mathrm{p}$-value \\
\hline $\mathrm{N}$ & 138 & 39 & 0.410 \\
\hline $\begin{array}{c}\text { Average stone } \\
\text { mass (mm) }\end{array}$ & 17.3 & 19.1 & 0.008 \\
\hline $\begin{array}{c}\text { Average surgery } \\
\text { duration ( } \pm \text { standard } \\
\text { deviation) in } \\
\text { minutes }\end{array}$ & $48.3( \pm 33.6)$ & $60.6( \pm 24.4)$ & \\
\hline
\end{tabular}


need for repeat procedures $(\mathrm{p}=0.252)$. Ureteroscopies for stones denser than $1300 \mathrm{HU}$ were $25 \%$ longer than for stones with a density below $1300 \mathrm{HU}$ (table 1).

Conclusions: Stone density was not found to be related to surgery complications, stone free rate, post-operative complications, or the need for further procedures. However, stone density was found to be associated with duration of the procedure. This parameter of the stone should be considered when choosing the surgical approach.

MP3-19 Pelvicalyceal Anatomy Types Impact On The Success Of Procedure In Patients Who Performed Flexible Ureteroscopy

\section{S Kirecci, M Ilgi, C Kutsal, C Yesildal, H Demirel}

şişli hamidiye etfal eğitim araştırma hastahanesi Turkey

Introduction \& Objective: Endourological procedures have highly developed for twenty years, Lastly; flexible urs settled as a powerful treatment modality. This study identifies the using with a certain anatomic renal classification can predict the success rate.

Materials and Methods: Evaluating the data of the 130 patients who was performed flexible urs in our centre between December 2012 with September 2016 as a retrospectively. Two of them were excluded because of the have a horseshoe kidney malformation. Patients are categorised according to pelvicalyceal system anatomy which was used most popular classifications system as called Sampaio. The success rate was defined as the absence of stone fragments or clinical insignificant residual fragments $<4 \mathrm{~mm}$ at the end of the first month by KUB or CT. Qualitative data are analysed with a chi-square $\left(\mathrm{x}^{2}\right)$ test and Quantitative variables were analysed with Nonparametric Kruskal-Wallis test in SPSS 21 IBM software. Results: The mean patient age was 43.1 years and $62.3 \%$ were men. The overall stone-free rate was $\% 71.5$ and mean stone volume was $344.8 \pm 235.3 \mathrm{~mm} 3$, mean operative time was $76.3 \pm 23.8 \mathrm{~min}$, average fluoroscopy time was $13 \pm 18 \mathrm{sec}$ and hospitalisation time was a $32.4 \pm 23.8$ hour. In our study, grouped to the Sampaio A1: $55(\% 42.3)$ is a common type, A2: 24 (\%18.5), B1:31 (\%23.8), B2: 18(\%13.8). Stone-free rates were significantly lower in A2(\%47.8). Other evaluated parameters were not significantly differences between the groups. Sampaio groups' fluoroscopy and operative time statistically similar, however; Sampaio B type kidney have a broad pelvis and simple calyceal body, therefore; operative and fluoroscopy time relatively short and stone-free status were high.

Conclusions: Anatomical details affect the stone-free rates of operation, Preoperative evaluation can help to increase procedure success by using the definitive predictive classification systems. According to the anatomic calyceal body; should manage your approach and enlighten to patients about an additional session requirement and success rate of the operation.

MP3-20 A critical evaluation of Intra-renal pressures based on ureteral access sheath size and irrigation flow rate for safe and effective Robotic Assisted Retrograde Intrarenal Surgery

N Tokatli, K Sarica, A Kabakci, R Saglam, A Patel

Health Sciences University, Kartal Lutfi Kirdar Training and Research Hospital

Turkey
Introduction \& Objective: The intra-renal pressure levels are important for patient safety during Retrograde Intra-renal Surgery (RIRS). Especially, it is very critical where there is no fluid return thru out of ureteral access sheath (UAS). The aim of the study to evaluate effects of varying UAS sizes $(9.5 / 11.5 \mathrm{~F}$ $12 / 14 \mathrm{~F}$ ) and changing irrigation flow rates modulated by custom designed pump system, on intra-renal pressure levels during Robotic Assisted RIRS (RA-RIRS).

Materials and Methods: Possible effects of changing irrigation flow rates on the intra-renal pressure values during RA-RIRS were evaluated by using a customized pump system adjusted for stepwise flow rate increases between $5-60 \mathrm{ml} / \mathrm{min}$. Two different flexible uretero-renoscopes (Flex-X2 and Flex-XC, Karl Storz $\mathrm{GmbH}$, Germany) were used to measure the flow rates through each instrument working channel (free and $270 \mu \mathrm{m}$ laser fiber inserted) for a set irrigant heights and different flow rates levels of customized pump. Following the assessment of baseline flow rates with constant flow, the study was repeated by using a custom designed pump system (ELMED, Turkey). Intrarenal pressures during RA-RIRS were measured percutaneously by using urodynamic system (MMS, Netherland) thru 5 different types of access sheaths $(9.5 / 11.5 \mathrm{~F}$ Cook, 10/12F Rocamed, 10.7/12.7F Cook, 11/13F and 12/14F Boston Scientific) positioned in the proximal ureter of the Minnesota University kidney model. The validation of the pressure studies of the Minnesota University kidney model has been evaluated with the measurements of pressure thru nephrostomy tube during PCNL / RIRS combination treatment of a kidney stone.

Results: Smaller sized UAS (9.5/11.5F) limited irrigant efflux along the outer side and caused increased intrarenal pressures in a short time even without a pump system. For larger UAS sizes, intrarenal pressure levels continued to remain $<30 \mathrm{cmH} 2 \mathrm{O}$ even with irrigation rates of up to $20 \mathrm{ml} / \mathrm{min}$. Finally, when $11 / 13 \mathrm{~F}$ and $12 / 14 \mathrm{~F}$ UAS was used intrarenal pressures were $<30 \mathrm{cmH} 2 \mathrm{O}$ despite irrigation flow rates up to $60 \mathrm{ml} / \mathrm{min}$.

Conclusions: Use of an appropriate sized UAS during RA-RIRS is of paramount importance. Careful irrigant flow rate adjustment with precisely controlled intrarenal pressure values, preferably using a specially designed pump system, can enable surgeons to perform RA-RIRS safely under clear vision with larger UAS sizes. This approach may increase stone free rates and reduce pressure build-up plus infection-related complication risk, particularly in cases with large and complex stones requiring longer operating times.

\section{MP3-21 Endoscopic Assessment Of Renal Papillae: Im- plications Of Compound Versus Simple Papillae Status On Stone Analysis}

BB Anderson, CU Nottingham, MA Adamsky, E Worcester, F Coe, GS Gerber

IU Health School of Medicine

United States

Introduction \& Objective: Digital ureteroscopy allows detailed visualization of renal papillae. The purpose of this study is to determine if the presence of compound papillae is associated with a specific stone type or stone recurrence.

Materials and Methods: This is an IRB-approved, single surgeon, single center prospective study of patients undergoing endoscopic stone removal from 2013 to 2017. Demographics and baseline characteristics were collected. Ureteroscopy was performed with a flexible, digital ureteroscope, and images of papillae were recorded systematically. Operative videos of 


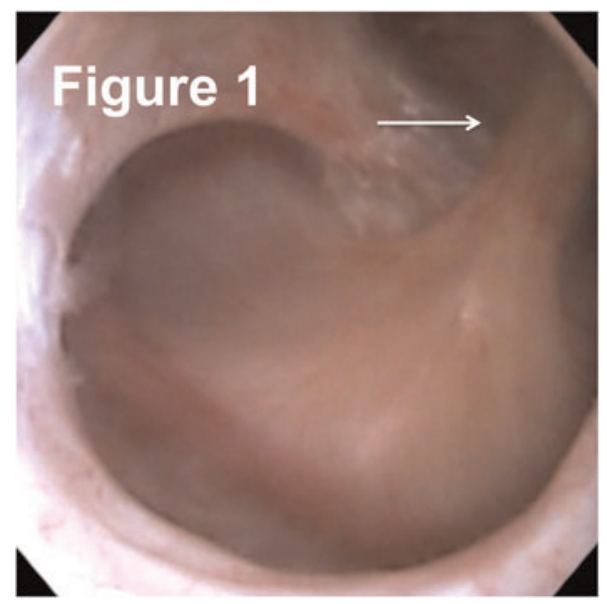

individual papillae were analyzed for simple versus compound papillary morphology. Papillae were determined to be compound when two or more distinct papillae were connected by a tissue bridge (Figure 1). Stone type and stone recurrence rates in patients with compound papillae were compared to those with only simple papillae.

Results: Subjects to date include 118 patients who had adequate papillae videos, of which 60 had at least one compound papilla. Between compound and simple papillae groups, there were no significant differences in age, race, sex, BMI, Charlson Comorbidity Index, prior stone history, or family history of stones. Preoperatively both groups had similar serum creatinine and urine $\mathrm{pH}$, but patients with compound papillae had a smaller median stone size ( $7 \mathrm{vs} 9 \mathrm{~mm}, \mathrm{p}=0.02)$. On postoperative stone analysis, patients with compound papillae more commonly had stones associated with acidic urine (calcium oxalate and uric acid; $71 \%$ vs $53 \%, \mathrm{p}=0.05$ ).

Conclusions: In this study we observed a higher rate of acidic urine associated stones in patients with compound papillae. Further studies into the physiology of stone formation in compound papillae may allow clinicians to guide patients about prognosis and stone prevention when this phenomenon is observed endoscopically.

MP3-22 Reusable vs. disposable flexible ureterorenoscopy (fURS): How to strike a balance? Case-based Cost-Benefit Analysis

T Ozimek, MH Schneider, MC Hupe, JR Wiessmeyer,

PL Chlosta, J Cordes, AS Merseburger, MW Kramer

Department of Urology University Hospital Schleswig-Holstein (Campus Lübeck)

Germany

Introduction \& Objective: Increasing number of fURS procedures, fragility of devices together with growing costs of its maintenance and repair are a substantial burden for urological departments. The already present on the market disposable single-use fURS devices offer many advantages compared to reusable fURS. LithoVue ${ }^{\mathrm{TM}}$ is the model with the highest evidence of clinical utility. In our study the economic aspects of reusable fURS application were assessed and compared with potential costs and benefits of single-use fURS (LithoVue ${ }^{\mathrm{TM}}$ ).

We attempted to establish indications for single-use fURS based on potential risk factors of reusable fURS damage.
Table 1. Damaged fURS - Preoperative Characteristics

\begin{tabular}{|c|c|}
\hline Number of Patients & 32 \\
\hline Male/Female & $21 / 11$ \\
\hline Age & $56(\mathrm{SD} \pm 18)$ \\
\hline \multicolumn{2}{|l|}{ Affected side } \\
\hline Left & $21(65.63 \%)$ \\
\hline Right & $11(34.37 \%)$ \\
\hline DJ Prestenting & $26 / 32(81.25 \%)$ \\
\hline \multicolumn{2}{|l|}{ fURS Indication } \\
\hline Diagnostic & $9 / 32(28.13 \%)$ \\
\hline Kidney stone & $23 / 32(71.87 \%)$ \\
\hline Recurrent Urolithiasis Patients & $14 / 23(60.87 \%)$ \\
\hline \multicolumn{2}{|l|}{ Recent Urolithiasis Procedures } \\
\hline SWL & $5 / 23(21.74 \%)$ \\
\hline URS & $5 / 23(21.74 \%)$ \\
\hline PNL & $2 / 23(8.69 \%)$ \\
\hline \multicolumn{2}{|l|}{ Stone characteristics } \\
\hline Single stone & $9 / 23(39.13 \%)$ \\
\hline Multiple stones & $14 / 23(60.87 \%)$ \\
\hline Stone diameter & $8(\mathrm{SD} \pm 6.67)$ \\
\hline$<10 \mathrm{~mm}$ & $12 / 23(52.17 \%)$ \\
\hline $10-20 \mathrm{~mm}$ & $8 / 23(34.78 \%)$ \\
\hline$>20 \mathrm{~mm}$ & $3 / 23(13.04 \%)$ \\
\hline Stone density (HU) & $950(\mathrm{SD} \pm 292)$ \\
\hline \multicolumn{2}{|l|}{ Stone location } \\
\hline Lower pole & $20 / 23(86.96 \%)$ \\
\hline Upper pole & $1 / 23(4.35 \%)$ \\
\hline Renal Pelvis & $2 / 23(8.69 \%)$ \\
\hline Concomitant ureteral Stone & $7 / 23(30.43 \%)$ \\
\hline
\end{tabular}

Materials and Methods: Single-center retrospective cost analysis based on actual costs of reusable fURS program compared with potential costs of LithoVue ${ }^{\mathrm{TM}}$ based on the assumed price offered by the manufacturer. Consecutive case analysis of damaged fURS to determinate potential risk factors of fURS damage.

Results: Study group consisted of 423 reusable fURS procedures conducted between January 2013 and December 2016. In this period, $102(24.11 \%)$ diagnostic fURS and 321

Table 2. Damaged fURS - Intraoperative Characteristics.

\begin{tabular}{ll}
\hline $\begin{array}{l}\text { OR Time } \\
\text { Laser (Ho:YAG) Application }\end{array}$ & $75 \mathrm{~min}(\mathrm{SD} \pm 32 \mathrm{~min})$ \\
$\quad$ via fURS & $16 / 32(50 \%)$ \\
Total Laser Energy $(\mathrm{kJ})$ & \\
$\quad$ via fURS & $1,38(\mathrm{SD} \pm 2.17)$ \\
fURS Insertion & \\
$\quad$ besides safety guidewire & $6 / 32(18.75 \%)$ \\
$\quad$ over the guidewire & $18 / 32(56.25 \%)$ \\
$\quad$ ureteral access sheath & $8 / 32(25 \%)$ \\
Lower Pole Inspection & $31 / 32(96.88 \%)$ \\
Infundibulopelvic Angle (retrograde Pyelography) & \\
$\quad \leq 50^{\circ}$ & $18 / 32(56.25 \%)$ \\
$\quad>50^{\circ}$ & $10 / 32(31.25 \%)$ \\
$\quad$ no data & $4 / 32(12.5 \%)$ \\
Stone-free rate & $10 / 23(43.48 \%)$ \\
Repair reports & \\
Leakiness & $18 / 32(56.25 \%)$ \\
Tip break-off & $7 / 32(21.86 \%)$ \\
& $7 / 32(21.86 \%)$ \\
\hline &
\end{tabular}


(75.89\%) fURS for kidney stone therapy were performed. In 32 out of $423(7.57 \%)$ fURS cases devices were deemed defective. Nine fURS were used for diagnostic procedures $(9 / 102 ; 8.82 \%), 7$ for fURS with stone removal (7/148; $4.73 \%), 16$ for fURS with stone removal and laser (Ho:YAG) application $(16 / 173 ; 9.25 \%)$. Average cost per reusable fURS procedure was $503.26 €$.

Conclusions: Disposable fURS is a more expensive option for high volume centers. Based on our case analysis, larger multiple lower pole stones in recurrent stone formers treated with laser disintegration, as well as steep infundibulopelvic angle (IPA $\leq 50^{\circ}$ ) seem to be main risk factors for fURS damage. For these cases, disposable fURS may be a cost-effective alternative, however a prospective comparison of economic outcomes between disposable and reusable fURS, together with confirmation of damage risk factors is needed.

MP3-23 Effect of Ureteral Access Sheath Use on Postoperative Visual Analogue Scale and Verbal Rating Scale Levels During Retrograde Intrarenal Surgery

O Kilic, M Akand, E Altintas, G Yildiz, S Goktas

Selcuk University, School of Medicine, Department of Urology Turkey

Introduction \& Objective: Ureteral access sheath (UAS) has been used to lower intrapelvic pressure as well as to decrease morbidity for repeating entries during retrograde intrarenal surgery (RIRS). It has been reported that use of UAS also decreases the postoperative pain level of the patients. In this study, we aimed to compare the pain level of the patients who underwent RIRS either with or without UAS.

Materials and Methods: Eighty patients who were planned to undergo RIRS by the same surgeon were enrolled in this study, where they were randomized either to Group 1 (with UAS) or Group 2 (without UAS) in a 1:1 ratio. Postoperative pain levels were measured during previously determined time scales by another single physician blinded to use of UAS by using visual analogue scale (VAS) and verbal rating scale (VRS). Demographic data as well as operation duration, number of laser shoots and stone burden were also collected.

Results: Demographic data are shown in Table 1. Operation duration and number of shoots were $38 \mathrm{~min}$ and 6001 versus $36 \mathrm{~min}$ and 5232 in Group 1 and 2, respectively. Stone burden was 948.9 and $871.6 \mathrm{~mm}^{2}$ in Group 1 and 2, respectively. No significant difference was observed among demographic and

Table 1: Demographic and operative data.

\begin{tabular}{|l|c|c|}
\hline & Group 1 (with UAS) & Group 2 (without UAS) \\
\hline Age & $48 \pm 12.3$ & $45.5 \pm 15.58$ \\
\hline Sex (Female/Male) & $14 / 26$ & $16 / 24$ \\
\hline BMI (kg/m ${ }^{2}$ ) & $28.3 \pm 4.8$ & $28.1 \pm 4.9$ \\
\hline Stone burden (mm ${ }^{2}$ ) & $948.9 \pm 1900$ & $871.6 \pm 1418.8$ \\
\hline $\begin{array}{l}\text { Operation duration } \\
\text { (min) }\end{array}$ & $38.4 \pm 25.6$ & $36.0 \pm 22.4$ \\
\hline Number of shoots & $6001 \pm 7183.5$ & $5232 \pm 4551.1$ \\
\hline
\end{tabular}

Table 2: Postoperative VAS and VRS data.

\begin{tabular}{|l|c|c|c|c|c|c|c|c|c|c|}
\hline & \multicolumn{9}{|c|}{ VAS } & \multicolumn{5}{c|}{ VRS } \\
\cline { 2 - 11 } & $\begin{array}{c}0 \\
\mathrm{~min}\end{array}$ & $\begin{array}{c}10 \\
\mathrm{~min}\end{array}$ & $\begin{array}{c}30 \\
\mathrm{~min}\end{array}$ & $\begin{array}{c}60 \\
\mathrm{~min}\end{array}$ & $\begin{array}{c}120 \\
\mathrm{~min}\end{array}$ & $\begin{array}{c}0 \\
\mathrm{~min}\end{array}$ & $\begin{array}{c}10 \\
\mathrm{~min}\end{array}$ & $\begin{array}{c}30 \\
\mathrm{~min}\end{array}$ & $\begin{array}{c}60 \\
\mathrm{~min}\end{array}$ & $\begin{array}{c}120 \\
\mathrm{~min}\end{array}$ \\
\hline Group 1(with UAS) & 3.60 & 3.38 & 3.20 & 3.13 & 2.23 & 0.93 & 0.93 & 0.83 & 0.83 & 0.55 \\
\hline $\begin{array}{l}\text { Group 2 (without } \\
\text { UAS) }\end{array}$ & 3.60 & 3.53 & 3.85 & 3.82 & 2.80 & 0.98 & 0.92 & 1.08 & 1.00 & 0.60 \\
\hline
\end{tabular}

$p$ values: VASO $p<0.730$ VAS10 $p<0.597$ VAS30 $p<0.287$ VAS60 $p<0.269$ VAS120 $p<0.704$

$p$ values: VRSO $p<0.617$ VRS10 $p<0.83$ VRS30 $p<0.238$ VRS60 $p<0.441$ VRS120 $p<0.828$

operative data. There was no difference for postoperative VAS and VRS levels between two groups. No difference was observed for mean VAS and VRS values among defined time scales (Table 2). There was only a negative correlation between age and VAS $0 \mathrm{~min}$, VAS $10 \mathrm{~min}$ and VAS $60 \mathrm{~min}(\mathrm{p}<0.044, \mathrm{p}<0.044$ and $\mathrm{p}<0.008$, respectively).

Conclusions: Although a difference for pain levels was expected due to lower intrarenal pressure in Group 1, no statistical significance was observed among two groups. This may be attributed to mucosal injury and edema due to UAS use. This study shows that use of UAS doe not make a difference in postoperative pain levels.

MP3-24 Limitations of the Lithovue single use digital flexible ureteroscope

S Bazargani, S Ghodoussipour, E Thompson, A Shah, A Mitra, MD Dunn

\section{UCLA Department of Urology}

United States

Introduction \& Objective: A new disposable flexible digital ureteroscope (Lithovue, Boston Scientific, Marlborough, Mass) was recently released as an alternative to non-disposable digital scopes. This series reviews our initial experience with this new device with a focus on identifying its limitations.

Materials and Methods: We retrospectively reviewed our first 75 cases with the 7.7Fr disposable flexible digital ureteroscope and identified any problems, difficulties, or complications related to the scope itself.

Results: Of the 75 patients, 47 were female 28 were male; 39 cases were left sided, 30 were right sided, and 6 were bilateral. The reasons for ureteroscopy include stone disease in 60 patients, ureteral stricture disease in 5 patients, and upper tract transitional cell carcinoma (TCC) in 10 patients. There was a mechanical failure in 3 patients; one was intraoperative and the remaining were before use. A replacement scope was used for each of these cases. There was difficulty in getting up the ureter (ureterovesical junction) in 3 patients; one due to distal ureteral narrowing requiring balloon dilation, the other 2 due to proximal ureter narrowing requiring stenting. Passage of the scope was relatively effortless in the remaining retrograde URS patients. Two patients underwent antegrade ureteroscopy through an established nephrostomy tract to treat ureteroenteric anastomotic strictures. There was mild interference in the video system during laser lithotripsy of hard stones (calcium oxalate monohydrate but did not prevent treatment. But the system was incompatible with the use of electrocautery. During fulguration of upper tract TCC, the system continually shut down during the 
use of a 3Fr electrode. Another difficulty with visualization occurred during antegrade ureteroscopy for 2 ureteroileal anastomotic strictures. Due to problems with distant focusing, there was difficulty identifying the true lumen past the stricture, which lead to inaccurate incision of the soft tissue resulting in extravasation in both cases.
Conclusions: This new disposable ureteroscope works well for routine ureteroscopy with laser lithotripsy but should not be used with electrocautery and avoided for antegrade incision of ureteroenteric anastomotic strictures. Its strength is in near focusing rather than distant focusing which is fine for stones but not for the latter situation.

\section{MPS4: STONES: CLINICAL OUTCOMES AND INFECTIONS}

\author{
MP4-1 Routine Urine Culture May Not Be Necessary \\ Prior to Ureteroscopy
}

SK Bechis, S Patel, A Alagh, RL Sur, DG Kronenberg

UC San Diego School of Medicine

United States

Introduction \& Objective: The overuse of preoperative tests such as urine culture (UCx) contributes to increased costs on the health care system and may not improve outcomes after surgery. The aim of this study was to determine the value of pre-operative urine culture versus urinalysis for ureteroscopic surgery.

Materials and Methods: We performed a retrospective review of 64 consecutive ureteroscopic procedures for stone disease or upper tract evaluation performed by a single surgeon from January to October 2016. In our practice, preoperative urinalysis and UCx is sent for each patient. Perioperative antibiotics are given according to the AUA Best Practice statement. Inclusion criteria were subjects undergoing initial treatment with ureteroscopic lithotripsy or diagnostic ureteroscopy who had a urinalysis within 30 days of surgery. Subjects with a previously placed ureteral stent, no urinalysis data, recurrent urinary tract infections (UTIs), or undergoing staged procedures were excluded. All subjects returned within 4 weeks for post-operative followup and the primary outcome - rate of infectious complications - for both negative and abnormal urinalysis was determined.

Results: Forty-three (43) subjects met inclusion criteria, with 24 subjects being male $(56 \%)$. Operative side was left in 17 cases, right in 19 cases, and bilateral in 7 cases. The average operative time was 103 minutes, and average time to follow up was 19 days. Of the 27 stones sent for analysis, stone composition was pure calcium oxalate in 16 subjects $(59 \%)$, mixed calcium oxalateapatite in $10(37 \%)$, and pure calcium phosphate in $1(4 \%)$. Overall, of the 41 subjects with negative preoperative urinalysis (nitrite negative), none had $>100,000$ colonies $/ \mathrm{mL}$ on preoperative culture and 3 had asymptomatic bacteruria $(<100,000$ colonies $/ \mathrm{mL})$. None of these 3 asymptomatic bacteruria patients developed postoperative UTI. Two of 41 subjects (5\%) developed a postoperative UTI with positive postoperative urine culture. Three subjects who complained of dysuria and stent pain had postoperative urine cultures sent which were negative. Two subjects had nitrite positive urinalysis preoperatively, and both had positive preoperative urine cultures which were treated with antibiotics.

Conclusions: Our study demonstrated that preoperative urine culture may be unnecessary for uncomplicated subjects with a negative urinalysis (nitrite negative) undergoing ureteroscopy. If further studies validate these findings, there may be a role for omitting pre-operative urine cultures in low risk patients-thus offering potential for savings to the health care system. Defining low risk patients will be necessary as well.
MP4-2 Risk Factors of Readmission for Infection following pneumatic transurethral lithotripsy for Ureteral Stones

S Rabani, s Rabani

Yasuj Unhversity of Medical Sciences Iran

Introduction \& Objective: Urinary tract infection and even urosepsis after ureteroscopy (URS) for ureteral stone lithotripsy is a clinically significant event that may lead to serious morbidity and mortality and necessitating rehospitalization. We have evaluated the risk factors associated with infection after URS for pneumatic lithotripsy in ureteral stones leading to unplanned hospital return

Materials and Methods: We have retrospectively reviewed the records of all adult patients who underwent transurethral lithotripsy for ureteral stones by pneumatic lithotriptor 8-9.8 F, in a single center and by a single surgeon, from October 2009 to November 2016. Size, location, opacity of stones, duration of operation, antibiotics prescription before operation, history of previous operation on the ipsilateral ureter, irrigation fluid height, preoperative related comorbidities such as urine culture, diabetes mellitus, duration of hospital stay before operation, degree of hydronephrosis, and patient demographics were collected data. Inclusion criteria were all adult patients with ureteral stones who needed intervention for transurethral lithotripsy without anesthetic contraindication and could tolerate lithotomy position. Data analyses were performed to determine the risk factors of rehospitalization

Results: 1086 patients were enrolled in this study. Male to female ratio was about 1.83 (703 male and 383 female patients), average age was $41.6(+$ + 20.7) years. Overall 321 patients returned to the hospital, within these groups of the patients, 67 patients were admitted again for infections only as a complication of endoscopic intervention $(6.16 \%)$. Two diabetic patients with large proximal ureteral stones developed fungal infection and retroperitoneal fungal abscess formation with long term hospitalization in their return admissions. 31 patients underwent second time transurethral lithotripsy after control of infection processes and the remainders were managed conservatively.

Conclusions: Stone size, stone location, duration of operation, and the height of irrigation fluid were associated with greater likelihood of developing urinary tract infection especially when these factors were accompanied with residual obstructing stones. When the ureteroscope hasn't a good visual field due to poor calibration, the time of operation will be increased and we have to use a higher pressure of irrigation fluid for a better vision and we think that the higher pressure may cause the major role in development of urinary tract infection during the procedure of URS for ureteral stones. In our study the most common cause of readmission after URS for ureteral stone was pain due to residual obstructing stones. 
MP4-3 Treatment of Non-Obstructive Urolithiasis is Effective in Treatment of Recurrent Urinary Tract Infections

DK Agarwal, FJ Maldonado, ME Westerman, ME Rivera, AE Krambeck, JJ Knoedler

Department of Urology, Mayo Clinic, Rochester, MN United States

Introduction \& Objective: Patients with recurrent UTIs and non-obstructing urologic calculi provide a clinical quandary. There is growing evidence that removing these stones may be beneficial, but further studies are required to validate this. We sought to determine if removing non-obstructive stones was effective in treatment of recurrent UTI.

Materials and Methods: A retrospective review was performed of patients undergoing elective management of non-obstructive, non-struvite upper tract urinary calculi with recurrent UTI from 2009-2016. UTI within 30 days of the operation were considered to be post-operative complications and not recurrence. Beyond 30 days, $>3$ UTI in a year interval was considered recurrent.

Results: 46 patients met inclusion criteria. 42 (91.3\%) were female. Median age was 63.7 years (IQR 49.1-73.4). Median stone size (or largest if multiple) was $14 \mathrm{~mm}$ (IQR 9-20). 32 (70\%) had multiple stones and 13 (28\%) were bilateral. 20 (43.5\%) underwent ureteroscopy only, $26(56.5 \%)$ underwent PCNL +/- URS. None underwent ESWL. Stone culture was available for $35(76 \%)$ patients and $19(54.2 \%)$ had a positive culture. Notably, $31.5 \%$ of stone cultures were not concordant with preoperative urine culture. $18(39 \%)$ had residual stone. Twenty-three $(50 \%)$ patients had a UTI $>30$ days after treatment, but only five $(10.9 \%)$ had recurrent UTI after treatment at a median follow up of 2.9 years (IQR 2.-4.3). Of the five, one had recurrent UTIs $>1$ year after treatment. $18(39 \%)$ had residual stone. The presence of residual stone was a risk factor for recurrent UTI after treatment ( $p$ Conclusions: Stone removal for patients with recurrent UTIs has a high rate of success $(89.1 \%)$ in treatment of recurrent UTIs. Residual stone after treatment increases the risk of recurrent UTIs and should be addressed to lower UTI future UTI risk.

MP4-4 Early Discharge Following Decompression for Sepsis and an Obstructing Stone? A Multi-Institutional Study to Identify Predictors of Antibiotic Sensitivity

T Tran, M Cancian, E Parkhomenko, G Pareek, M Gupta

Icahn School of Medicine at Mount Sinai United States

Introduction \& Objective: Patients presenting with sepsis and an obstructing stone undergo urgent urinary tract decompression. Following this, patients are hospitalized for hemodynamic support and broad spectrum antibiotics. Urine culture results are used to tailor outpatient antibiotic therapy. At times patients achieve early clinical stability but remain hospitalized while awaiting antibiotic sensitivities. We sought to identify predictors of antibiotic resistance that may allow clinicians to select candidates for discharge on empiric oral antibiotics prior to culture results being available. Materials and Methods: All patients that underwent emergent urinary tract decompression for sepsis and an obstructing ureteral stone over the last 2 years at the two above institutions were included. Clinical factors, including urine culture sensitivities and patient demographics were recorded. Student's t-test and the chi-squared test were used to identify statistical difference.

Results: 134 patients were identified that met inclusion criteria. Eighty-four patients $(62.7 \%)$ had urine cultures with antibiotic

\begin{tabular}{|c|c|c|c|}
\hline & Sensitive $(n=50)$ & Resistant $(n=84)$ & p value \\
\hline Age & 59.9 years & 58.9 years & 0.763 \\
\hline Gender & $22.0 \%$ male & $27.1 \%$ male & 0.516 \\
\hline BMI & $31.2 \mathrm{~kg} / \mathrm{m}^{2}$ & $31.8 \mathrm{~kg} / \mathrm{m}^{2}$ & 0.874 \\
\hline Diabetes & $22.0 \%$ & $22.4 \%$ & 0.962 \\
\hline Paraplegia & $2.0 \%$ & $4.7 \%$ & 0.425 \\
\hline Stone Size & $6.7 \mathrm{~mm}$ & $7.6 \mathrm{~mm}$ & 0.249 \\
\hline WBC, serum & 15.0 & 15.9 & 0.403 \\
\hline Temperature & $99.3^{\circ} \mathrm{F}$ & $100.4^{\circ} \mathrm{F}$ & 0.489 \\
\hline Systolic Blood Pressure & $113 \mathrm{mmHg}$ & $113 \mathrm{mmHg}$ & 0.782 \\
\hline Heart Rate & $107 \mathrm{bpm}$ & $109 \mathrm{bpm}$ & 0.498 \\
\hline $\begin{array}{c}\text { Previous Urologic } \\
\text { Surgery }\end{array}$ & $22.0 \%$ & $44.7 \%$ & 0.008 \\
\hline Previous Ureteroscopy & $8.0 \%$ & $38.9 \%$ & 0.0002 \\
\hline Previous PCNL & $4.0 \%$ & $7.1 \%$ & 0.471 \\
\hline ICU admission (postop) & $12.0 \%$ & $27.1 \%$ & 0.039 \\
\hline Length of stay, mean & 3.4 days & 5.4 days & 0.026 \\
\hline Positive Blood Culture & $24.0 \%$ & $48.2 \%$ & 0.005 \\
\hline
\end{tabular}

\begin{tabular}{|c|c|c|c|}
\hline Antibiotic & $\begin{array}{l}\text { Providence, Rhode } \\
\text { Island }\end{array}$ & New York, New York & p value \\
\hline Ampicillin / sulbactam & $12.3 \%$ & $60.0 \%$ & 0.0002 \\
\hline Axtreonam & $7.7 \%$ & $10.0 \%$ & 0.746 \\
\hline Cephalexin & $13.8 \%$ & $30.0 \%$ & 0.212 \\
\hline Ciprofloxacin & $35.4 \%$ & $45.0 \%$ & 0.619 \\
\hline Gentamicin & $16.9 \%$ & $30.0 \%$ & 0.330 \\
\hline Levofloxacin & $29.2 \%$ & $45.0 \%$ & 0.302 \\
\hline Meropenem & $1.5 \%$ & $5.0 \%$ & 0.378 \\
\hline $\begin{array}{l}\text { Piperacillin / } \\
\text { tazobactam }\end{array}$ & $3.1 \%$ & $20.0 \%$ & 0.009 \\
\hline $\begin{array}{c}\text { Trimethoprim / } \\
\text { sulfamethoxazole }\end{array}$ & $29.5 \%$ & $45.0 \%$ & 0.302 \\
\hline
\end{tabular}

resistance. Comparison was made between patients with pansensitive and resistant urine cultures. Patients with resistant cultures were more likely to have had previous urologic surgery $(44.7 \%$ vs. $22.0 \%, p=0.008)$ - the most notable difference was in patients that had had previous ureteroscopy $(38.9 \%$ vs. $8.0 \%$, $\mathrm{p}=0.0002$ ). Those with resistant cultures were more likely to require postoperative ICU-level care $(27.1 \%$ vs. $12.0 \%, \mathrm{p}=0.039)$, have bacteremia $(48.2 \%$ vs. $24.0 \%, \mathrm{p}=0.005)$ and a longer length of stay ( 5.4 vs. 3.4 days, $p=0.026)$. Resistance patterns were noted to be similar between the two institutions.

Conclusions: Patients that have had previous urologic surgery, especially ureteroscopy, appear to be poor candidates for early discharge on empiric antibiotics prior to the completion of urine culture results due to a higher likelihood of having antibiotic resistance. These results were noted to be consistent at both institutions participating in this study.

MP4-5 Impact of the timing of ureteral stent placement on outcomes in patients with obstructing ureteral calculi and presumed infection

C Faw, J Hollingsworth, SN Ambani, KR Ghani, WW Roberts, CA Dauw

University of Michigan

United States

Introduction \& Objective: Acute renal colic due to an obstructing ureteral calculus is a common cause of emergency 
Table. Impact of stent placement before and after 10 hours in those with SIRS criteria and obstructing ureteral stones

\begin{tabular}{|c|c|c|c|}
\hline & $\begin{array}{c}\text { Under 10 Hr } \\
\mathbf{N = 2 1}\end{array}$ & $\begin{array}{c}\text { Over 10 Hr } \\
\mathbf{N}=\mathbf{2 7}\end{array}$ & P-value \\
\hline Age & 49.01 & 51.78 & 0.62 \\
\hline Gender & 0.38 & 0.41 & 0.85 \\
\hline BMI & 30.63 & 30.36 & 0.90 \\
\hline American Society of Anesthesiology Score & 2.38 & 2.44 & 0.73 \\
\hline Stone Size (mm) & 5.48 & 6.04 & 0.48 \\
\hline Positive Urine Culture & 0.62 & 0.56 & 0.65 \\
\hline Admission Temperature $\left({ }^{\circ} \mathrm{C}\right)$ & 37.51 & 36.99 & 0.08 \\
\hline Admission Heart Rate & 100.57 & 87.67 & 0.02 \\
\hline Admission Respiratory Rate & 19.05 & 20.26 & 0.24 \\
\hline Admission WBC & 17.25 & 13.89 & 0.14 \\
\hline Time to Urology Consult (hours) & 4.93 & 9.67 & 0.01 \\
\hline ICU Admission & 0.14 & 0.11 & 0.74 \\
\hline Stepdown Care & 0.76 & 0.93 & 0.11 \\
\hline Hospital Duration (hours) & 45.71 & 82.41 & 0.04 \\
\hline
\end{tabular}

department (ED) visits. In many cases, pain can be managed and patients discharged without need for intervention. However, in the case of obstruction due to a ureteral stone and concern for infection, renal decompression often via stent placement, is vital to avoid patient morbidity and mortality. While it seems selfevident that rapid decompression in this scenario is optimal, there is little data on this subject. We thus sought to understand how time to stent placement impacts outcomes in patients with obstructing ureteral stones and concern for infection.

Materials and Methods: Using a prospective urology consult database (2011-2016), we identified patients who presented to the ED with an obstructing ureteral stone, met two or more SIRS criteria (temperature $(\mathrm{T})>38 \mathrm{C}$ or 90 , respiratory rate $(\mathrm{RR})>20$, white blood cell count $(\mathrm{WBC})>12$ or $<4$ ) and underwent ureteral stent placement. We compared outcomes in those who received a stent within 10 hours to those who did not. The primary outcome of interest was the impact of stent timing on intensity of subsequent care (need for intensive care (ICU) or stepdown unit admission) as well as overall length of stay (LOS). Statistical analysis was performed using chi-square and t-testing where appropriate.

Results: For patients that met study criteria $(\mathrm{N}=48)$, mean $\mathrm{T}, \mathrm{HR}$, $\mathrm{RR}$, and WBC on ED admission were 37.2, 93.3, 19.7, and 15.4, respectively. Overall $58.3 \%$ had a positive urine culture with the majority being E. coli (50.0\%), Staphylococcus (14.3\%), and Klebsiella (14.3\%). There was no difference between groups with respect to age, gender, BMI, stone size, presence of positive urine culture, or comorbidity (Table). Those who were stented within 10 hours more commonly had higher heart rate at admission and a more expeditious urology consult. While need for ICU or stepdown care did not differ between groups, those stented within 10 hours had a significantly shorter LOS (45.7 vs. 82.4 hours, $p=0.04$ ).

Conclusions: In patients with an obstructing ureteral calculus and concern for infection, there is a beneficial effect to timelier stent placement in the form of decreased overall LOS. This information is important to both patients with stone disease as well as payors.

\section{MP4-6 Withdrawn}

MP4-7 Stone culture: Is it essential in the urolithiasis endoscopial approach?

A Rivero, C Manso, B de la Cruz, JM Hernández, JM Adot, J Montero, E Gutiérrez

Spain

Introduction \& Objective: Preoperative urine culture is a security tool, recommended by main urolithiasis guidelines. However, its yield is discussed currrently and experts prefer intraoperative culture.
Our review analyzes microbiology data in stones of patients who had endsoscopic lithotripsy in our institution from the last twelve months. All of them had negative preoperative urine culture.

Materials and Methods: We planned an observational prospective study. We performed a microbiological analysis in a preoperative urine culture, an intraoperative urine culture and a stone culture (cortex and core). All patients with endoscopic lithotripsy were included. We only choose patients with a negative preoperative urine culture.

Results: A $23.6 \%$ of them, had a positive intraoperative urine culture and a $25 \%$ of them, had a positive stone culture. There were differences in a $31 \%$ of those cultures (intraoperative vs stone). We found higher yield rates in cortex/core 12.8:1.

Conclusions: In our expirence, intraoperative urine control is an usefull tool and it can help us to choose the correct antibiotic therapy. Our cortex's data have more rentability.

MP4-8 Risk and Protective Factors for Recurrent Urinary Tract Infection Following Percutaneous Nephrolithotomy

\section{Au, C Tsai, P Ku, S Chung, C Chang}

Far Eastern Memorial Hospital

Taiwan (Republic of China)

Introduction \& Objective: Pre-existed urinary tract infection (UTI) is often associated with large nephrolithiasis. This is the first study to evaluate the predictors of recurrent UTIs in patients status post percutaneous nephrolithotomy (PCNL).

Materials and Methods: We retrospectively analyzed the medical records of patients with renal stones and pre-operative UTIs who underwent PCNL in one tertiary hospital from January 2010 to June 2015. Patient's demographics as well as stone composition, infected organism and post-operative recurrent UTIs within 12 months were reviewed. Fisher's exact test and logistic regression were applied for univariate analysis and multivariate analysis respectively in order to determine the predictors of post-operative UTIs.

Results: A total of 201 patients with pre-existed UTIs and large renal stones who had undergone PCNL were recruited. 118 $(59 \%)$ patients were infection free while $83(41 \%)$ patients experienced recurrent UTIs post-operatively. Escherichia coli, Proteus mirabilis and Streptococcus species were the most frequently isolated bacterial organisms pre-operatively in both groups. Using the univariate analysis, significant predictors associated with recurrent UTIs were diabetes mellitus $(\mathrm{OR}=2.109$, 95\% CI:1.13-3.93, $\mathrm{p}=0.019)$ and stone free $(\mathrm{OR}=0.482,95 \%$ CI:0.269-0.865, $p=0.014)$. In multivariate logistic regression analysis, stone free $(\mathrm{OR}=0.39,95 \%$ CI $0.20-0.76, p=0.005)$, diabetes mellitus ( $\mathrm{OR}=2.44,95 \% \mathrm{CI}: 1.21-4.94, p=0.005)$, age $\geq 65(\mathrm{OR}=2.39,95 \% \mathrm{CI}: 1.12-5.12, p=0.025)$, female gender $(\mathrm{OR}=2.65,95 \% \mathrm{CI}: 1.21-5.78, p=0.015)$ were significant predictors for recurrent UTIs. However, hypertension and BMI $\geq 27$ were not statistically significant predictors.

Conclusions: Stone free was a strong protective factor while diabetes mellitus, age $\geq 65$ and female gender were significant risk factors for post-PCNL recurrent UTI.

MP4-9 Predictive Value of Preoperative Inflammatory Response Biomarkers for Metabolic Syndrome and postPCNL systemic inflammatory response syndrome (SIRS) in Patients with Kidney Stones

K Tang, H Liu, K Jiang, H Xu, Z Ye

China (People's Republic) 
Introduction \& Objective: This study aimed to evaluate the diagnostic value of the inflammatory response biomarkers for the early detection of metabolic syndrome (MetS) and their predictive value for post-PCNL systemic inflammatory response syndrome (SIRS) in patients with kidney stones.

Materials and Methods: We retrospectively enrolled hospital records of 513 patients with kidney stones and 204 age and sexmatched healthy controls. Preoperative neutrophil to lymphocyte ratio (NLR), derived neutrophil to lymphocyte ratio (dNLR), lymphocyte to monocyte ratio (LMR) and platelet to lymphocyte ratio (PLR) were analyzed. The diagnostic and predictive values of NLR, dNLR, LMR and PLR were estimated using the sensitivity, specificity, the receiver operating characteristic (ROC), area under curve (AUC), and Cox regression analysis.

Results: Across our cohort 181 of 513 patients with kidney stones had post-PCNL sirs and 83 patients accompanied with MetS. While the NLR, dNLR, LMR and PLR values in patients with kidney stones were significantly higher than those observed in controls $(\mathrm{p}<0.001)$, they had limited diagnostic value based on the ROC area under the curve (AUC) curve analyses values of 0.648 , $0.625,0.662,0.53$, respectively. Among patients with kidney stones, the NLR, dNLR, LMR and PLR were significantly higher in patients with than without MetS and post-PCNL SIRS $(\mathrm{p}<0.001)$. ROC curve analysis indicated that NLR, dNLR, LMR and PLR for predicting patients with kidney stones and MetS, displayed AUC of $0.730,0.717,0.627$ and 0.606 , respectively $(\mathrm{P}<0.01)$. In addition, the ROC curves, using post-PCNL SIRS as the end-point for NLR, dNLR, LMR and PLR with AUC of 0.831, 0.813, 0.723 and 0.685, respectively $(\mathrm{P}<0.001)$. Multivariate analysis revealed that NLR represents an independent factor for predicting MetS $(\mathrm{HR}=0.248$, $95 \% \mathrm{CI}=0.100 \hat{\mathrm{E}}$ " $0.611, \quad \mathrm{P}=0.002)$ and post-PCNL SIRS $(\mathrm{HR}=7.516,95 \% \mathrm{CI}=1.051 \hat{a} €$ "53.761, $\mathrm{P}=0.045)$, respectively. Conclusions: Preoperative NLR is a simple, effective, and a practical predictor of MetS and post-PCNL SIRS in patients with kidney stones. It has potential value in public health practice for management of patients with kidney stone and treatment intervention following PCNL.

MP4-10 Routine Complete Blood Count Has Limited Value Post Percutaneous Nephrolithotomy in Identifying Hemorrhagic or Infectious Complications

T Kroczak, M Ordon, K Pace, R D’A. Honey, JY Lee

St. Michael's Hospital - University of Toronto Canada

Introduction \& Objective: Percutaneous nephrolithotomy (PCNL) is a minimally invasive procedure with low complication rates reported by high volume centers; hemorrhage and sepsis rates are typically $<5 \%$. Yet post-operative bloodwork including complete blood count (CBC) is routinely performed in the early postoperative period at most centers. We set out to determine how effective routine post-operative $\mathrm{CBC}$ was in identifying complications after PCNL, as it may represent a low-value care practice. Materials and Methods: A retrospective chart review was performed of all PCNL procedures at our center from Jan 2014 to Dec 2015. PCNL cases performed on renal transplant patients, strictures or urothelial tumours were excluded from the analysis. Patient demographic and stone characteristics were collected for analysis along with post-operative outcome data.

Results: Two hundred and forty eight patients (134 female \& 114 males) underwent PCNL for urolithiasis. Mean age was 55.9 years, mean ASA score 2.5, and mean length of stay in hospital was 1.59 days (+/-3.6). Most patients $(208,83.9 \%)$ had neither ureteric stent nor percutaneous tube prior to PCNL. Post-operatively, five (2\%) patients required extended CBC monitoring and $2(0.8 \%)$ patients required transfusion, one of whom required embolization $(0.4 \%)$. All patients that required transfusion demonstrated abnormal vital signs (tachycardia or hypotension). Of the five patients that required only extended $\mathrm{CBC}$ monitoring, one had abnormal vital signs and four had normal vital signs. Mean hospital length of stay for patients undergoing $\mathrm{CBC}$ monitoring was $2.2(+/-1.6)$ days compared to $1.54(+/-3.7)$ days for those with a normal postoperative course. When comparing patients with no sign of postoperative hemorrhage to those requiring monitoring or intervention, pre-op anticoagulation or antiplatelet therapy was associated with a higher risk of complication (1.0 vs $1.13, \mathrm{p}<0.001)$. Only $9(3.6 \%)$ patients developed SIRS or sepsis post-PCNL. All of these patients developed abnormal vital signs post-PCNL (fever, tachycardia, or hypotension). Patients that developed either SIRS or sepsis were more likely to have a positive pre-operative urine culture $(\mathrm{p}=0.015)$.

Conclusions: Routine post-operative CBC after PCNL does not improve identification of hemorrhage or infection. Abnormal vital signs alone identified all patients that required transfusion or embolization after PCNL and the development of SIRS or sepsis. Length of stay for patients undergoing CBC monitoring in the setting of normal vital signs, was a full day longer and resulted in no clinical benefit. Post-operative $\mathrm{CBC}$ might be beneficial in anti-coagulated patients.

MP4-11 Preoperative urine culture analysis in 284 consecutive patients undergoing percutaneous nephrolithotomy: are current antibiotic prophylaxis recommendations adequate?

A Garza-Gangemi, J Dominguez-Rodriguez,

J Cruz-Ruíz, C Mendez-Probst

Instituto Nacional de Ciencias Médicas y Nutrición Salvador Zubirán

Mexico

Introduction \& Objective: Urinary tract infections are one of the most common complications following percutaneous nephrolithotomy (PCNL) occurring in up to $39 \%$ of patients. Antibiotic prophylaxis is indicated for every patient and recommendations are vastly empiric or based on older epidemiological data. The increasing prevalence of multidrug resistant (MDR) bacteria worldwide is alarming and of particular concern is the complexity associated with antibiotic prophylaxis in these patients. The aim of this study was to investigate the microbiology and prevalence of MDR bacteria in our patient population.

Materials and Methods: Preoperative urine cultures were collected from 284 consecutive patients undergoing PCNL in a tertiary care center from 2010 to 2017. Patient demographics, comorbidities, stone parameters, the presence of hydronephrosis on imaging studies, and preoperative stent use were recorded for every patient. Single or multiple culture growth was registered. The frequency of isolated bacteria along with their antibiotic resistance pattern was described.

Results: The mean age was $49.4 \pm 13.4$ years and $61.3 \%$ of the patients were female. Staghorn calculi were observed in $45.5 \%$ of the patients and hydronephrosis was present in $42.7 \%$. Preoperative stent use was documented in $24.3 \%$ of the patients. One hundred and sixty three $(57.4 \%)$ patients had negative cultures, $107(37.7 \%)$ had a single microorganism growth and $14(5 \%)$ reported multiple isolations. Escherichia coli was isolated in 79 patients $(57 \%)$ of the cultures and $38(53.2 \%)$ were extended spectrum beta lactamases (ESBL) producing organisms. Other 


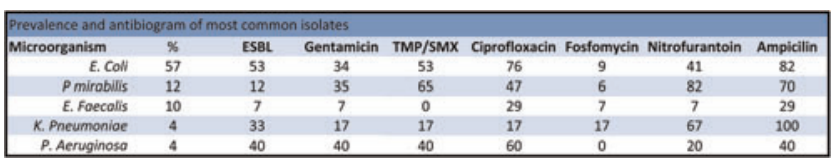

common isolated bacteria were $P$. mirabilis $(12.3 \%)$, E. faecalis (10.1\%), K. pneumonae (4.3\%) and P. aeuroginosa (3.6\%).

Conclusions: More than $40 \%$ of preoperative urine cultures are positive and the most frequent isolated bacteria is $E$. coli. The prevalence of ESBL producing bacteria in our population is concerning and these results suggest that current empiric antibiotic practice may be inadequate in our population.

\section{MP4-12 Prediction on infectious complications after per-} cutaneous nephreolithotomy

B Guliev, E Stetchik, A Zaikin

North-West medical university by Mechnikov Russia

Introduction \& Objective: Percutaneous nephrolithotripsy (PCNL) is characterized by such complications as bleeding, exacerbation of chronic pyelonephritis, trauma of colon, and pleural cavity. Infectious complications can be associated with technical issues of PCNL (constant irrigation, increasing of pelvis pressure). However, infection is not always observed after PCNL. The aim of study is to determine preoperative factors, which can be predictors of infectious complications of PCNL.

Materials and Methods: $96(7,1 \%)$ of 1358 patients, treated with PCNL, were included in our study. Urine culture from bladder before PCNL and from pelvis after renal cavitary system punction were performed, as well as microbiological analysis of stone. We considered such demographic characteristics of patients as age, sex, body mass index, previous endoscopic and open operations on kidneys, hydronephrosis stage, size and localization of stone, number of percutaneous accesses and residual fragments, intraoperative complications. Patients with nephrostomy tube before PCNL, previous operations in upper urinary tract, stones or tumors in bladder were excluded. Patients with diabetes mellitus were also excluded. Patients were divided in two groups: the first one - without fever after PCNL; the second one - with fever $\left(>38^{\circ} \mathrm{C}\right)$. We analyzed connection of patient's characteristics with postoperative fever. Student's t-test was used for comparison of quantitative data in research groups with normal distribution.

Results: We discovered that frequency of infection complications after PCNL didn't depend on patient's sex and age, stone size and body mass index. There were no significant differences in operation time in both groups $(58,4 \pm 28,6 \mathrm{~min}$ - I group, $62,4 \pm 30,6 \mathrm{~min}$ - II group). The number of complications in II group was high $(36,4 \%$ vs $18,8 \%)$, but there was no significant difference as well. Urine culture from bladder, pelvis and microbiological analysis of stone fragment were positive in $24(25,0 \%), 18(18,7 \%), 16$ $(16,7 \%)$ patients respectively. Postoperative fever was observed in $11(11,5 \%)$ of 96 patients. In patients with fever only in $45,4 \%$ bacteria growth from cultures of bladder and pelvis was identified, and fever was observed in $63,6 \%$ of patients with positive urinary culture from pelvis and stone. In patients with positive urine culture fever was absent in $19(22,4 \%), 11(12,9 \%)$ and $9(10,6)$ cases respectively.

Conclusions: Thus, probability of fever in cases with positive urinary culture from pelvis and stone is significantly higher than in cases with positive culture from urinary bladder.
MP4-13 The clinical yield of Stone culture in patients undergoing percutaneous nephrolithotripsy

A Nevo, R Holland, R Gilad, D Lifshitz

UPMC

United States

Introduction \& Objective: Stone colonization is a wellrecognized risk factor for sepsis following percutaneous nephrolithotripsy (PCNL).

Several studies have suggested that SC should be obtained routinely during PCNL, however, the clinical yield of this practice has not been studied.

We aimed to determine the incidence at which sepsis was caused by stone pathogens, and to assess the probability of SC to modify the antibiotic treatment in patients with post-operative sepsis.

Materials and Methods: A prospectively collected Database of patients undergoing PCNL between 2005 and 2017 was queried for patients in whom UC and SC were obtained.

Sepsis was defined as SIRS with positive blood or UC, obtained during sepsis (CDS).

To study the incidence at which sepsis was caused by urine and stone pathogens, the concordance between UC, SC, and CDS was examined.

The probability of SC to modify the antibiotic treatment was assessed by identifying highly resistant pathogen in SC.

Regression analysis was done to identify risk factors for stone colonization by these pathogens.

Results: among 512 patients who met the inclusion criteria, UC and SC were positive in 137 (26.7\%) and 117 (22.8\%) patients, respectively. in $88(17 \%)$ patients, $\mathrm{UC}$ did not predict SC results.

SIRS and Sepsis occurred in $50(9.8 \%)$ and $17(3 \%)$ patients, respectively.

Among patients with sepsis, SC predicted CDS results in 7 $(41 \%)$ patients. In 3 of which, UC was different from CDS while SC was identical, and in $2(11.7 \%)$ of these patients, different treatment was required because of resistant pathogens.

Overall, highly resistant pathogens were isolated from 25 (4.9\%) SC: carbapenem-only susceptible gram-negative bacteria in 12 patients, and candida and Vancomycin-only susceptible gram-positive bacteria in 6 patients each.

Univariable and multivariable regression analyses of risk factors for colonization with resistant pathogens are presented in the table.

\section{Conclusions:}

- $41 \%$ of sepsis events were caused by stone pathogens, however, only in $2(11.7 \%)$ patients, the culprit was not isolated from $\mathrm{UC}$, representing $0.4 \%$ of the cohort.

\begin{tabular}{|c|c|c|c|c|}
\hline variable & $\begin{array}{l}\text { resistant } \\
\text { pathogen } \\
(25)\end{array}$ & $\begin{array}{l}\text { others } \\
(487)\end{array}$ & univariable $\mathrm{Pv}$ & $\begin{array}{l}\text { Multivariable } \\
\text { Pv }\end{array}$ \\
\hline $\begin{array}{l}\text { Prior kidney drainage (\%) } \\
\text { nephrostomy tube (\%) } \\
\text { JJ stent (\%) }\end{array}$ & $\begin{array}{c}10(40) \\
10(40) \\
0(0)\end{array}$ & $\begin{array}{l}122(25) \\
71(14.6) \\
51(10.5)\end{array}$ & $\begin{array}{c}0.005 \\
0.001 \\
0.9\end{array}$ & 0.01 \\
\hline $\begin{array}{l}\text { Median Chsrlson comorbidity } \\
\text { index }\end{array}$ & 1 & 3.5 & 0.003 & 0.03 \\
\hline $\begin{array}{l}\text { Stone composition \% } \\
\text { CAOX } \\
\text { Uric acid } \\
\text { Calcium Phosphahte } \\
\text { Struvite } \\
\text { Cystine } \\
\end{array}$ & $\begin{array}{c}21.4 \\
35.7 \\
7.1 \\
28.4 \\
7.1\end{array}$ & $\begin{array}{c}64.4 \\
18.8 \\
7 \\
5.8 \\
3.2\end{array}$ & 0.002 & 0.025 \\
\hline $\begin{array}{l}\text { Median number of } \\
\text { hospitalizations during the } \\
\text { previous year }\end{array}$ & 2 & 0 & $<0.001$ & 0.23 \\
\hline Prior UTI & $14(56)$ & $118(24.2)$ & $<0.001$ & 0.22 \\
\hline Median age (q1, 3 3) & $53(43,65)$ & $65(52,75)$ & 0.07 & \\
\hline Female gender $(\%)$ & $12(48)$ & $177(36)$ & 0.12 & \\
\hline Diabetes Melitus (\%) & $8(32)$ & $115(23.6)$ & 0.22 & \\
\hline Indwelling catheter (\%) & $1(4)$ & $22(4.5)$ & 0.73 & \\
\hline
\end{tabular}


- Overall, a highly resistant pathogen which may necessitate treatment change were found in $4.9 \%$ of the cohort.

- SC should be obtained selectively from paitents who have increased risk for colonization with resistant pathogens

MP4-14 Gentamicin and Ciprofloxacin resistance in PCNL Stone Cultures

N Smyth, A McPhee, G Jones

Glasgow Royal Infirmary

United Kingdom

Introduction \& Objective: PCNL carries a significant risk of sepsis. Peri-operative prophylactic antibiotics are important in reducing this risk. Conflicting evidence exists as to whether extended post operative antibiotic prophylaxis for patients undergoing PCNL impacts on sepsis rates. Concerns regarding likely increase in antibiotic resistance have also been raised. We aim to assess the rates of bacterial resistance to Gentamicin and ciprofloxacin - commonly used prophylactic antibiotics in patients undergoing PCNL and to assess whether the use of extended post operative prohylaxis has impacted on these rates over time.

Materials and Methods: Retrospective review of intraoperative stone cultures from PCNLS undertaken between September 2012 and November 2016 at a single centre where the standard prophylactic regime includes single dose Gentamicin +3.5 days Ciprofloxacin to assess antibiotic resistance rates. Resistance rates were compared for those undertaken in the first two years of data collection with those undertaken in the latter two years.

Results: 182 PCNLs were performed from September 2012 to November 2016, of which 94 had positive stone cultures. 91 PCNLs were undertaken between sept 2012 and September 2014 (24months), of which 46 (50.5\%) had positive stone cultures. A further 91 PCNLs were undertaken between September 2014 and November 2016 (26months) of which 48 (52.7\%) had positive stone cultures. Of those with positive stone cultures, the rate of ciprofloxacin resistance in the earlier group was $21.7 \%(n=10)$ the rate of gentamicin resistance was $17.4 \%(n=8), 10.9 \%(n=5)$ were resistant to both antibiotics. The second group $16.7 \%(\mathrm{n}=8)$ of positive stone cultures showed resistance to ciprofloxacin; $6.2 \%(n=3)$ gentamicin resistance and $6.2 \%(n=3)$ were resistant to both agents. There was no change to technique or antibiotic prophylactic regime over this time period to explain this. The overall resistance rates observed in this population of positive cultures were $19.1 \%$ for Ciprofloxacin and $11.7 \%$ which are comparable with figures published in 2014 in the UK 5year antimicrobial resistance report $(18.8 \%, 10.5 \%)$.

Conclusions: Gentamicin and ciprofloxacin resistance rates have not changed and in fact decreased over time in patients undergoing PCNL despite the same prophylaxis regime. This does not support concerns relating to increasing resistance caused by extended prophylaxis regimes. Evidence remains controversial regarding the ideal prophylactic regime for PCNL.

MP4-15 What's growing in our renal stones? - Not Just the Usual Suspects

N Smyth, A McPhee, G Jones

Glasgow Royal Infirmary

United Kingdom

Introduction \& Objective: Infection and sepsis are significant risks associated with PCNL. Positive intra-operative stone cul-
Summary of organisms cultured on stone fragments collected at PCNL

\begin{tabular}{|l|l|}
\hline Proteus & $23(24.4 \%)$ \\
\hline $\begin{array}{l}\text { Staphylococci (incl. staph epidermis, staph. } \\
\text { aureus, staph. capitis, staph. simulans) }\end{array}$ & $22(24.4 \%)$ \\
\hline E.Coli & $20(21.2 \%)$ \\
\hline Enterococcus & $17(18.1 \%)$ \\
\hline $\begin{array}{l}\text { Streptococci (incl.Group B Strep, strep } \\
\text { mileri, strep oralis) }\end{array}$ & $5(5.3 \%)$ \\
\hline Pseudomonas & $5(5.3 \%)$ \\
\hline Candida & $5(5.3 \%)$ \\
\hline Serratia Marcescens & $3(3.2 \%)$ \\
\hline Klebsiella & $2(2.1 \%)$ \\
\hline "gram positive cocci" & $2(2.1 \%)$ \\
\hline "mixed anaerobes" & $2(2.1 \%)$ \\
\hline Morganella morganii & $1(1 \%)$ \\
\hline Providencia Stuartii & $1(1 \%)$ \\
\hline Micrococcus luteus & $1(1 \%)$ \\
\hline
\end{tabular}

tures are known to increase the risk of post operative sepsis. Knowing what organisms colonise bacteria can help guide both prophylactic and treatment antibiotic choice. We aim to investigate the specific organisms which colonise large renal stones treated with PCNL.

Materials and Methods: A retrospective study of all PCNLS undertaken between September 2012 and November 2016 to determine the rate of positive intraoperative stone culture and assess which organisms were most likely to colonise such stones. Results: 182 PCNLs were undertaken on 164 patients. 169 stone fragments were sent for microscopy and culture. The rate of positive culture was $55.6 \%(\mathrm{n}=94)$. The most common organism detected was Proteus $24.4 \%(n=23)$ followed by Staphylococci $24.4 \%(n=22)$ then E.Coli $(21.2 \%)$ and Enterococcus $(18.1 \%)$. 23 stones grew more than 1 organism and a single stone grew 3 different bacteria as well as candida. The table below documents all organisms grown in our stone population.

Conclusions: A wide variety of organisms were grown in intraoperative stone cultures including gram negative and positive bacteria and fungal species. Stones are often colonised with more than one agent. Surprisingly high rates of streptococcal and staphylococcal agents were found which supports the use of broad spectrum antibiotic prophylaxis in stone surgery. Intraoperative stone culture should form an important part of the assessment of such patients in order to guide antibiotic treatment for those who develop subsequent infection and also to help determine the best prophylaxis for future procedures.

MP4-16 Is pre-operative automated cytometry adequate for the screening of pre-operative urine cultures before PCNL?

N Smyth, A McPhee, G Jones

Glasgow Royal Infirmary

United Kingdom

Introduction \& Objective: Percutaneous nephrolithotomy carries a significant risk of sepsis. Pre-operative urine culture forms and important part of standard work up to assess for active infection and guide antibiotic prophylaxis. We aim to assess the correlation between pre-operative urine cultures and interoperative stone culture results in patients undergoing PCNL at a 
single trust where pre-operative urine samples are assessed by plating for bacterial growth only if initial automated cytometry results are positive.

Materials and Methods: Retrospective analysis of all PCNLs undertaken by a single surgeon at one hospital trust between September 2012 and November 2016. As standard practice preoperative urine samples underwent initial cytometry followed by plating for culture and sensitivities in the case of positive cytometry. Stone fragments obtained at PCNL were sent routinely for microscopy and culture.

Results: 182 PCNLs were undertaken on 164 patients. Of these, 169 procedures investigated had both pre-operative urine samples and intra-operative stone cultures available. 55 (32.5\%) pre-op cultures were positive - of which $23(13.6 \%)$ were reported as "heavy mixed growth" - mostly csu/neph/urostomy. 94 (55.6\%) stones were positive for bacterial culture, of these $48(50.5 \%)$ were associated with negative pre-op cultures. Just one positive preoperative urine culture was associated with a negative stone culture. Conclusions: Our results reveal a high $(50.5 \%)$ false negative rate of pre-operative urine cultures assessed in this method. This rate is significantly higher than previous studies which involved preoperative urine samples that were plated for culture as a routine. These results highlight the importance of routinely sending intraoperative stone cultures to better guide antibiotic treatment in those who develop post operative sepsis. Our results also suggest that using automated cytometry as an initial routine investigation for pre-operative urine may miss potential bacterial growth associated with renal stones and that such a technique for pre-operative urine analysis may not be adequate for the optimum prediction of bacterial colonisation in patients undergoing stone surgery.

MP4-17 Urinary tract infections in patients treated with percutaneous nephrolitotomy and their risk factors

E Hernández Méndez, C Martínez Arroyo, J Herrera Muñoz, I Calvo Vázquez, P Cortés Raygoza, M Ortega González, M Cantellano Orozco, G Fernández Noyola, J Morales Montor, C Pacheco Gahbler

Hospital General Dr. Manuel Gea González Mexico

Introduction \& Objective: Infectious perioperative complications rates after percutaneous nephrolitotomy (PCNL) have been reported between $0.5-10 \%$. Positive urinary cultures are found in $15-18 \%$ of patients after PCNL. Gram negative bacilli are the most isolated microorganisms. PCNL is categorized as a cleancontaminated or contaminated surgery. Postoperative (PO) bacteriuria usually occurs in stone patients with preoperative sterile urine without prophylactic antibiotic administration.

Evaluate the association between preoperative and transoperative risk factors and $\mathrm{PO}$ urinary tract infections in patients treated with PCNL.

Materials and Methods: A retrospective study was conducted in patients treated in the Hospital General "Dr. Manuel Gea González”' (Mexico City) since January 2015 to March 2017. All patients were clinically examined prior to PCNL and after the treatment focused on symptoms of infectious complications.

Results: 80 PCNL were performed in a total of 74 patients, 39 $(52.7 \%)$ women and $35(47.3 \%)$ men. 3 patients underwent more than 1 PCNL. Preoperative (PreO) bacteriuria was more common in women $10(25.6 \%)$ than in men $8(22.8 \%) .28(37.8 \%)$ patients were diagnosed with PO infectious complications, $22(78.5 \%)$ had PO fever, 6 (21.4\%) sepsis and 4 (1.4\%) developed renal abscesses that were treated with percutaneous drainage. ESBL

\begin{tabular}{|c|c|c|c|}
\hline Patient characteristic & $\mathrm{n}=\mathbf{2 8}$ & OR (CI 95\%) & p value \\
\hline Male sex & $17(60.7 \%)$ & $2.1(1.3-2.3)$ & 0.03 \\
\hline Diabetes mellitus & $22(78.5 \%)$ & $3.2(1.4-5.2)$ & 0.02 \\
\hline Previous PCNL & $3(10.7 \%)$ & $1.8(0.90-3.6)$ & 0.09 \\
\hline Body mass index $>30$ & $16(57.1 \%)$ & $2.5(2.0-6.4)$ & 0.02 \\
\hline Stone size > $2.5 \mathrm{~cm}$ & $10(35.7 \%)$ & $1.9(1.2-3.6)$ & 0.02 \\
\hline Staghorn calculi & $8(28.5 \%)$ & $3.4(2.9-5.6)$ & 0.01 \\
\hline Surgery time $>90$ min & $16(57.1 \%)$ & $2.8(0.9-3.6)$ & 0.08 \\
\hline$>1$ nephrostomy tracts & $16(57.1 \%)$ & $1.1(0.9-1.6)$ & 0.15 \\
\hline $\begin{array}{c}\text { Pre0 positive urine } \\
\text { culture }\end{array}$ & $12(42.8 \%)$ & $1.3(0.8-1.2)$ & 0.10 \\
\hline $\begin{array}{c}\text { Pre0 isolated ESBL E. } \\
\text { Coli }\end{array}$ & $8(28.5 \%)$ & $1.7(1.3-2.7)$ & 0.05 \\
\hline
\end{tabular}

Escherichia Coli (E. Coli) was isolated in 14 (18.9\%) patients and was responsible of $50 \%$ of infectious complications. Multivariate analysis of risk factors associated with PO urinary tract infections (UTI) is shown in table 1 .

Conclusions: UTI are common complications following PCNL and $50 \%$ are caused by ESBL E. Coli. Prophylactic antibiotics are important for prevention especially in patients with negative preoperative urine cultures. Male sex, chronic disease, calculi size, staghorn form and obesity are preoperative factors that are strongly associated with PO infectious complications.

MP4-18 Prospective Randomized Trial of Antibiotic Prophylaxis Duration for Percutaneous Nephrolithotomy in Low-Risk Patients: Preliminary Results

P Samson, G Gaunay, S Derisavifard, D Leavitt, B Morganstern, V Patel, M Elmasri, AD Smith, DM Hoenig, Z Okeke

Smith Institute for Urology United States

Introduction \& Objective: The American Urologic Association (AUA) recommends 24 hours or less of perioperative antibiotics for percutaneous renal surgery; however, duration of antibiotic therapy varies based on individual practice patterns. Only recently have small, randomized studies been published to support the use of 24 hours of antibiotics in low risk patients undergoing percutaneous nephrolithotomy (PCNL). We compared the efficacy of 24-hour antibiotic coverage versus short-course protocol of antibiotic prophylaxis for PCNL.

Materials and Methods: Low risk patients with a sterile preoperative urine culture undergoing PCNL were randomized to either antibiotics for 24 hours $(24 \mathrm{H})$ or until external urinary catheters were removed (CR). Per AUA recommendations, patients were given a $1^{\text {st }}$ generation cephalosporin, or ciprofloxacin in cases of penicillin allergy. Exclusion criteria included age 1 week, plan for multi-stage procedure, immunosuppression, pregnancy, multiple antibiotic allergies, and patients who are breastfeeding. Descriptive and Fisher's exact test were used to compare infectionrelated events and complication rates within 30 days of the procedure between groups.

Results: Sixty-one patients have been randomized to either $24 \mathrm{H}$ $(n=31)$ or CR $(n=30)$. Mean duration of antibiotic administration was 24.0 hours and 42.4 hours in the $24 \mathrm{H}$ and CR groups, respectively. Mean age was 55 years and 62 years in the $24 \mathrm{H}$ and CR groups $(p=0.037)$, respectively. Other parameters in demographics, comorbidities, and surgical parameters (including stone size, operative time, number of punctures, dilations, and types of tube) were similar between groups. There have been no differences in febrile episodes $(9.7 \%$ vs $10 \%, \mathrm{p}=0.967)$ or rates of systemic 
inflammatory response syndrome (SIRS) (10.5\% vs $9.5 \%$, $\mathrm{p}=0.393$ ) between the $24 \mathrm{H}$ and $\mathrm{CR}$ groups. Only one patient in each arm had evidence of bacteremia. One patient in the $24 \mathrm{H}$ group had a post-operative urinary tract infection compared to two patients in the CR group. Overall complication rates were similar between the two groups.

Conclusions: In the early stages of our study, a 24-hour protocol for antibiotic prophylaxis is not associated with increased risk of infection-related events compared to giving antibiotics until external catheters are removed in patients with low infectious risk undergoing PCNL.

MP4-19 Risk factor analysis of systemic inflammatory response syndrome (SIRS) after retrograde intrarenal surgery in patients with kidney stone

K Tang, H Liu, H Xu, Z Ye

China (People's Republic)

Introduction \& Objective: The aim of this study was to analyze the pre- and intraoperative risk factors that affect the development of postoperative systemic inflammatory response syndrome (SIRS) after retrograde intrarenal surgery (RIRS) in patients with kidney stone.

Materials and Methods: A total of 325 adult patients with the complete data who underwent RIRS for renal stones were included in the recent study. Patients' data including past renal surgery, nephrostomy insertion and recurrent urinary infection were obtained. Preoperative urine culture, renal pelvic urine culture and stone culture were obtained from all patients. The intraoperative data were prospectively noted. All patients were followed up postoperatively for signs of SIRS and sepsis.

Results: The SIRS was significantly correlated with the patients' gender $(\mathrm{P}<0.001)$, diabetics comorbidities $(\mathrm{P}<0.001)$, blood glucose level $(\mathrm{P}=0.02)$, operative time $(\mathrm{P}<0.001)$, staghorn stones $(\mathrm{P}<0.001)$, hydronephrosis $(\mathrm{P}=0.04)$, and preoperational urinary tract infection $(\mathrm{P}=0.01)$. There was no significant correlation between the SIRS and the patients' age, body mass index, preoperative hemoglobin level, preoperative serum creatinine, and transfusion. By multivariate logistic regression analysis, presence of females, diabetics, infection stone, stone burden, recurrent urinary tract infection and prolonged lithotripsy time were associated with both SIRS and sepsis development.

Conclusions: Presence of females, diabetics, infection stone, large stone size, recurrent urinary tract infection and prolonged lithotripsy time can be identified as independent predictors for the development of SIRS and sepsis following RIRS.

MP4-20 Tailored optimal perioperative antimicrobial prophylaxis in retrograde intrarenal surgery: evidence from a prospective randomized trail

G Zeng, J Fan, Z Zhao

Department of Urology, Minimally Invasive Surgery Center, the First Affiliated Hospital of Guangzhou Medical University. Guangzhou Institute of Urology. Guangdong Key Laboratory of Urology

China (People's Republic)

Introduction \& Objective: To evaluate the incidence of infectious complications after zero vs. single vs. two doses of antibiotic prophylaxis (ABP) in patients undergoing retrograde intrarenal surgery (RIRS), as the appropriate prophylaxis is unclear for this procedure.

Materials and Methods: A prospective randomized trial was conducted between August 2014 and November 2016. 300 consecutive patients with preoperative sterile urine undergoing RIRS were randomized into three groups and received ciprofloxacin-based different ABP regimes (group 1, zero-dose; group 2, single dose, 30 min before the start of surgery; group 3 , two doses, first dose at $30 \mathrm{~min}$ before the start of surgery and additional dose within 6 hours postoperatively). The primary endpoint was the systemic inflammatory response syndrome (SIRS).

Results: A total of 265 patients (group 1: 88, group 2: 88, group 3:89) were eligible for final analysis. Baseline characteristics were similar between three groups. SIRS was recorded in 8 $(9.1 \%), 3(3.4 \%)$ and $1(1.2 \%)$ patients in group 1, 2, 3, respectively. In subgroup analysis, SIRS occurred respectively in $5.0 \%$, $5.1 \%$ and $0 \%$ of patients with stones $\leq 200 \mathrm{~mm}^{2}(\mathrm{P}=0.257)$; for stones $>200 \mathrm{~mm}^{2}$, low rates of SIRS were still observed in group $2(0 \%)$ and group $3(2.7 \%)$, however, $17.9 \%$ were developed in group $1(\mathrm{P}=0.011)$. No urosepsis were developed in any groups. Conclusions: RIRS without antibiotic prophylaxis is safe for low-risk patients with stones $\leq 200 \mathrm{~mm}^{2}$, and prophylactic antibiotics are not recommended for those patients. However, single dose prophylaxis seems necessary and sufficient for patients with stones $>200 \mathrm{~mm}^{2}$.

MP4-21 The role of various novel inflammatory biomarkers, following Flexible Ureterorenoscopy, for the treatment of kidney stones.

SF Hughes, AJ Moyes, I Shergill

Betsi Cadwaladr University Health Board (BCUHB)

United Kingdom

Introduction \& Objective: With increasing use of flexible ureterorenoscopy worldwide, the development of postoperative complications, such as infection, is likely to increase. Investigating the inflammatory response following flexible ureterorenoscopy has not been widely researched. The main purpose of this pilot study was to test the hypothesis that flexible ureterorenoscopy results in changes to novel inflammatory markers.

Materials and Methods: 40 consecutive patients aged between 27-87 years (median 49 years) undergoing Flexible Ureterorenoscopy for the treatment of kidney stones were recruited (26 males, 14 females). Blood samples were collected from each patient at four time points: baseline (preoperatively) followed by 30 minutes, 120 minutes and 240 minutes post-operatively. Specific inflammatory markers, IL10, MCP-1, IL-6, VEGF and MIP-1a were measured using a Bio-Rad Bio-Plex 100 analyser.

Results: Following Flexible Ureterorenoscopy, there was an increase in VGEF $(p=0.01)$, and IL-6 $(p=0.07)$ concentrations. There were no significant changes to IL-10 $(p=0.615)$ and MCP$1(\mathrm{p}=0.859)$, although there was a trend of decreasing MIP-1a concentration $(\mathrm{p}=0.357)$.

Conclusions: Changes to inflammatory markers such as VEGF, IL-6, MIP-1a, were observed at short time periods following flexible ureterorenoscopy. This response may represent a more relevant assessment of the true extent of inflammation. Analysis of such markers, in larger cohorts, may potentially provide valuable data to identify or predict those patients at risk of postoperative complications, such as infection. 
MP4-22 Prophylactic Antibiotic Utilization Patterns and Postoperative Infectious Complications Following Percutaneous Nephrolithotomy

SW Reese, TR McClintock, RE Krasnow, M Mossanen, SL Chang, R Korets

Brigham and Women's Hospital United States

Introduction \& Objective: Percutaneous nephrolithotomy (PCNL) has become the gold standard for treatment of staghorn or large renal calculi. While highly effective, the risk of complications for PCNL is not insignificant, with Clavien 2 or higher complications having been reported in approximately $9 \%$ of cases. Infection is a major cause of morbidity and added cost, with sepsis accounting for the most common cause of postoperative mortality. Though the AUA Best practice statement recommends $<24$ hours antibiotic prophylaxis, utilization of regimens remains highly heterogeneous and there are little data regarding how various antibiotic choices affect subsequent incidence of postoperative infectious complications.

Materials and Methods: We utilized the Premier Hospital Database, a national discharge database to identify patients who had undergone PCNL from 2006-2015. Patients with pre-existing diagnosis of urinary tract infection or sepsis were excluded. We examined patient, surgeon, and hospital characteristics and then characterized all associated data regarding inpatient intravenous and oral antibiotics administered from 1 day prior to the index surgery through time of discharge. Trends in antibiotic utilization were assessed via the chi-square test for linear trend. Binary outcomes were evaluated using multivariable logistic regression. All statistical analysis was performed using SAS (North Carolina, USA).

Results: Between 2006 and 2015 we identified 107,778 patients undergoing PCNL that met our inclusion and exclusion criteria. There were 599 unique antibiotic regimens. First generation cephalosporins were the most commonly prescribed antibiotics $(47.1 \%)$; the overall rate of sepsis was $1.9 \%$. Combination antibiotic regimens were associated with higher rate of any infection (1.44 [95\% CI 1.33-1.55]), sepsis (1.63 [1.42-1.87]), and UTI (1.34 [1.22-1.47]). Longer duration antibiotic use $(>24 \mathrm{~h})$ was associated with higher rates of UTI (1.15 [1.08-1.24]), though not with any infection (1.03 [0.97-1.09]) or sepsis (1.08 [0.97-1.20]). Conclusions: There is a high degree of heterogeneity in utilization of antibiotic regimens in PCNL, with choice of antibiotic class, combination, and duration yielding significant associations with subsequent clinical outcomes. Broad spectrum regimens resulted in significant increase in infectious risk with respect to any infection, sepsis, and UTI. Longer duration coverage was associated with higher rate of UTI. These data may be utilized to inform selection of antibiotics for PCNL, which often require case-by-case tailoring with respect to prior cultures and risk stratification for postoperative infection.

MP4-23 Stone Microbiology and Antibiotic Susceptibility based on Renal Calculi culture

R Galeana Ruiz, R Romero Aguilar, F Gonzalez Gonzalez, E Mendoza Villanueva, SA Victor, R Carvajal Garcia

ISSSTE

Mexico

Introduction \& Objective: Percutaneous nephrolitotomy is the gold standard for renal calculi. The main complication is urinary tract infection which can lead to severe sepsis. Better manage- ment and prognosis can be ameliorated by a simple urinary and stone culture during surgery.

Materials and Methods: 76 Patients who undergone percutaneous nephrolitotomy during 1 year where followed, 59 had eligibility criteria. Gender, urine culture before intervention, renal stones culture and susceptibility and antibiotic resistance was recorded.

Results: Mean age was 52.3 years, $60 \%$ female and $40 \%$ male. $47 \%$ of urine cultures where positive for bacteria, in which $55 \%$ was E. coli, $11 \%$ Pseudomona Aeruginosa, 11\% Proteus. Renal stones cultures $51 \%$ where positive for bacteria, E.coli $51 \%$ in which $30 \%$ ESBL, 23\% Pseudomona, 7\% Enterococus/Burkholderia. Our finding was that sensibility to antibiotics was reduced in cases where urine and renal calculi culture was positive for bacteria

Conclusions: Antibiotic resistance is increased in patients with both: urine and renal stone calculi cultures positive for bacteria. In our clinical practice, urinary tract infections, severe sepsis and antibiotic resistance can be predicted by having a simple urine and renal stone culture during surgery to prevent complications.

MP4-24 Emergent double J stenting: It's role in urosepsis with non-dilated pelvicalyceal systems

\section{E Fernandes, A Thakarkar}

Vision Hospitals, Healthway Hospitals

India

Introduction \& Objective: Urosepsis associated with Pyonephrosis(PN) has a poor outcome if decompression of pelvi calyceal system(PCS) is not done on time. Usually there is radiological evidence of an obstructed PCS with PN. However, PN could well be present in a nondialted PCS especially in patients(pts) with concomitant acute renal failure(ARF). We present a series of 4 such pts who presented with urosepsis and ARF. Based solely on clinical and biochemical parameters, these patient underwent Double J(DJ)stenting to decompress the non dilated PCS's with PN.

Materials and Methods: Of the 4 pts(2-males, 2-females), aged 50-75yrs. All pts had fever with decreased urine output. 2 pts had anuria. All had tachycardia, tachypnea and hypotension. Leucocytosis, Neutrophilia, deranged RFT's(Serum Creatinine $>7 \mathrm{mg} \%$ ), deranged coagulation profile and pyuria was seen in all the pts. 2 pts had severe fluid overload and required urgent Hemodialysis. 1 pt was intubated and rest of them required intermittent Non Invasive ventilation. Antibiotic and Ionotropic support was started for all pts. High Procalcitonin levels was the hallmark in all pts. All the radiological test( Ultrasound, CT scan)did not show any evidencence of PCS obstruction or pyonephrosis. With ongoing supportive treatments pts underwent bilateral DJ stentings under local anesthesia with minimal sedation. Purulent efflux was seen in all the pts, either unilateral or bilateral. All had positive bacterial culture while two had a mixed fungal infection, which was appropriately treated Results: After stenting, within 2 days pts showed signs of resolving sepsis. PCT level declined, Urine output picked up and the creatinine levels reduced in a week to 10 days time.Pts were dichareged on oral antibiotics. Ultrasound was done at 6weeks. At 8 weeks DJ Stent removal and bilateral check Ureterorenocopy( URS) and flexible URS was done. Only one pt had necrosed papillae which were removed.

Conclusions: Urosepsis associated with PN is a life threatening condition. With radiological evidence of PCS dilatation, PCN is the decompression method of choice. In the absence of PCS dilatation, the diagnosis of PN itself, is difficult and should be strongly suspected. Dilatation may not be evident due to the associated ARF. Retrograde bilateral DJ stenting under LA and sedation is the best option to decompress these systems. At follow up bilateral URS is recommended. 


\section{MPS5: EDUCATION AND SIMULATION I}

\begin{abstract}
MP5-1 Skills Acquisition Trend and Outcomes in the transition from Laparoscopic to Robot Assisted Radical Cystectomy
\end{abstract}

T Yeong, SS Kommu, P Rouse, P Macneal, A Samateh, J Jaun, SH Garnett, PD Rimington, (S Academic Research Group

East Sussex Healthcare NHS Trust

United Kingdom

Introduction \& Objective: Current exponential uptake of robotic surgery has ushered concern about the impact of skill acquisition on outcomes during the transition to the robotic platform in surgical extirpation and reconstruction. Herein, we aim to decipher the outcomes during the transition phase form Laparoscopic Radical Cystectomy (LRC) and Robot-Assisted Radical Cystectomy (RARC) for Bladder Cancer (BC) using the template of a single experienced minimally invasive urological surgeon.

Materials and Methods: The final 40 LRCs were compared with the first 40 RARCs during the transition period. The 80 patients underwent their respective procedures between October 2013 and October 2016 at a tertiary referral centre, uro-oncological centre. Data was retrospectively evaluated from a prospectively maintained database. We compared the different parameters such as cystectomy time, length of total hospital stay, lymph node dissection, resection margins, in-patient complications and 30-day readmission. Data were analysed using SPSS Statistical Analysis Software.

Results: The median age for all patients is 70yrs $(36-84)$, with median age of $71 \mathrm{yrs}(36-84)$ for RARCs and $70(51-82)$ for LRCs. Of total 80 patients, 62 (77.5\%) were male and $18(22.5 \%)$ were female. There were no significant differences in the rate of in-patient complications $(\mathrm{p}>1.00)$ and 30-day re-admission ( $p>0.05)$ between those who had RARCs and LRCs.

Conclusions: The transition from laparoscopic to robotic platform was not found to impact on cystectomy time, total length of hospital stay, lymph node dissection, resection margins, in-patient complications and 30-day re-admission rates. Current findings indicate the transition is safe.

MP5-2 Upper body position analysis using optical motion capture during laparoscopic suturing maneuvers

K Takayasu, K Yoshida, T Mishima, M Watanabe, T Matsuda, H Kinoshita

Kansai Medical University

Japan

Introduction \& Objective: This study aimed to analyze the differences in posture patterns during laparoscopic surgery among medical students/surgeons with three skill levels using an optical motion capture system. Understanding the differences in surgical posture patterns depending on skill level might be an important factor in efficiently alleviating fatigue and acquiring skills.

Materials and Methods: Thirty participants were stratified into novice, intermediate, and experienced groups. The participants' upper body motions during a suturing task were captured, including the average angle and angle variability (axilla, elbow, wrist) to assess basic upper body positioning; joint fixation and hand

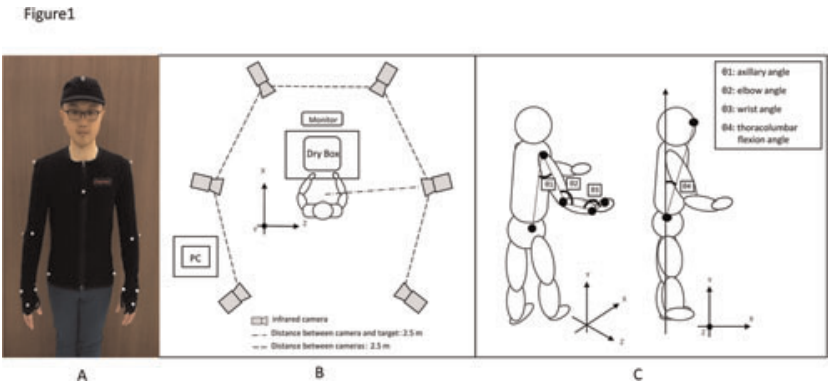

Figure2a: Average of joint angle (right side) for experts, intermediates and novices $\theta 1$ : axilla, $\theta 2$ : elbow, $\theta 3$ : wrist, $\theta 4$ Thoracolumbar flexion

*Post hoc test, Steel-Dwass test

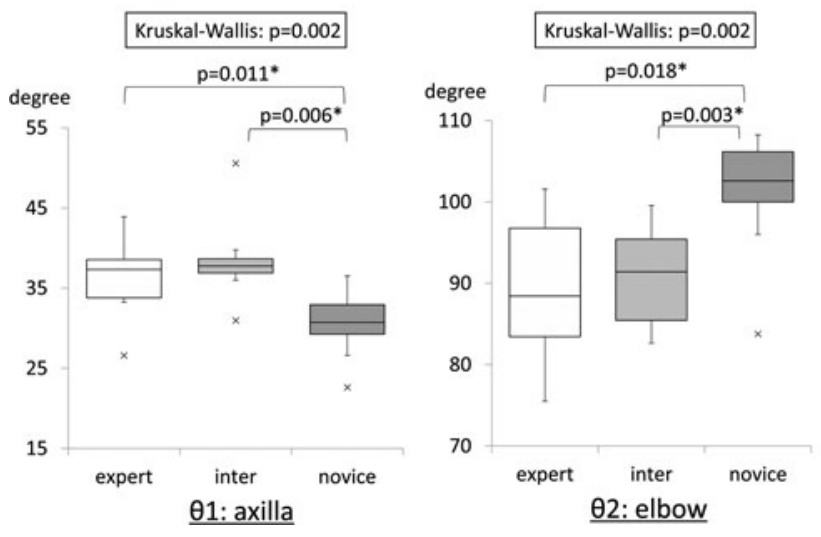

Figure2b: Average of joint angle (right side) for experts, intermediates and novices $\theta 1$ : axilla, $\theta 2$ : elbow, $\theta 3$ : wrist, $\theta 4$ Thoracolumbar flexion

*Post hoc test, Steel-Dwass test
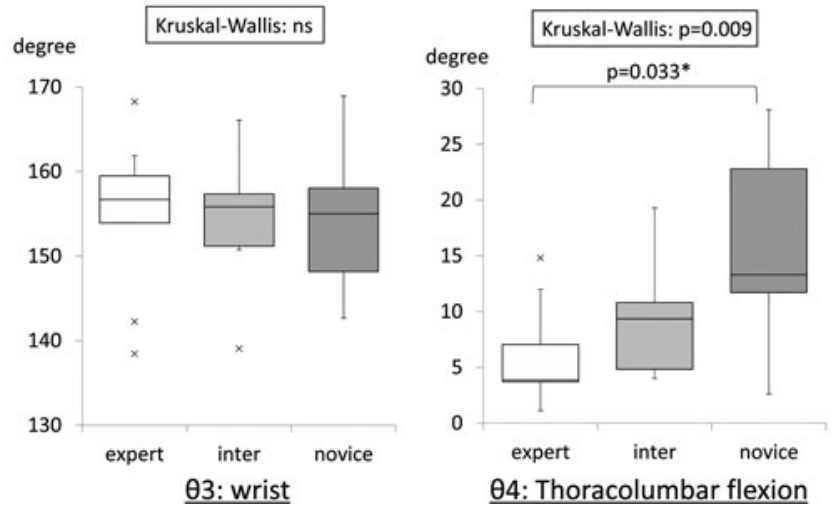

movement to assess efficiency; trunk (chest, head) movements to assess trunk stability; and the thoracolumbar flexion angle.

Results: The experienced group posture versus novice group posture showed that in the experienced group (1) the arms were more loosely held at their sides, and their elbows were bent; (2) there was a greater change in armpit angle and more fluid posture; (3) hand movements were faster and the side-to-side movements were larger; (4) the head and chest were more stable, 
with no stooping. Intermediate group posture versus that of the experienced group showed that the range of movement of the head longitudinally was 1.5 times greater for the intermediate group, their forward leaning tended to be greater, and their hand movement was slower.

Conclusions: Posture patterns of three groups of surgeons with different technical levels during a laparoscopic suturing task revealed differences in the operating limb positions according to the level of experience. These results may provide new insights about efficient acquisition of technical skill and reduced physical stress during laparoscopic surgery.

MP5-3 The validation of a novel Robot-Assisted Radical Prostatectomy virtual reality module

P Harrison, N Raison, K Ahmed, W Watkinson

Kings College London

United Kingdom

Introduction \& Objective: Basic virtual reality (VR) simulation is an effective method of robotic surgical skills training. Novel VR full procedural training tools require rigorous assessment prior to incorporation into surgical training programmes. We have performed the first validation of full procedural VR training module and analysis of novice learning curves.

Materials and Methods: 13 novice, 24 intermediate and 8 expert surgeons completed the validation study. Bladder neck dissection (BND) and urethrovesical anastomosis (UVA) tasks were completed as part of the prostatectomy training module.
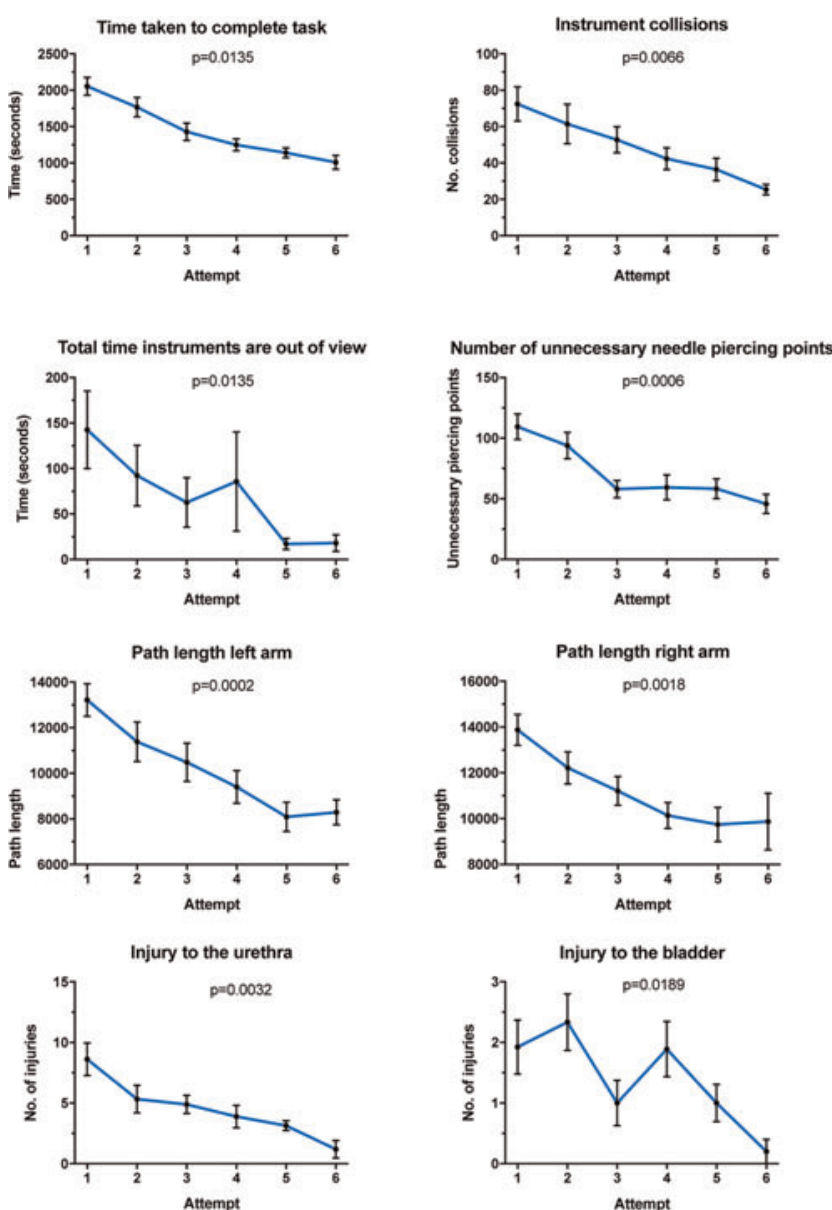

Surgeons completed feedback questionnaires assessing the realism, educational impact, acceptability and feasibility of the VR prostatectomy module. All novice surgeons completed a 5.5hour training programme.

Results: Realism was scored highly for BDN (mean 3.4/5) and UVA (3.74/5) task as was importance of BDN (4.32/5) and UVA (4.6/5) for training. It was rated a feasible (3.95/5) and acceptable $(4 / 5)$ tool for training. Experts performed significantly better than novice group in 6 metrics in the UVA including time $(\mathrm{p}=0.0005)$, distance by camera $(p=0.0010)$ and instrument collisions $(\mathrm{p}=0.0033)$ as well as task specific metrics such as number of unnecessary needle piercing points $(\mathrm{p}=0.0463)$. In novice surgeons, a significant improvement in performance after training was seen for BND in time $(\mathrm{p}<.0001)$, instrument collisions $(p=0.0013)$, total time instruments are out of view $(p=0.0251)$, number of movements of left instrument $(\mathrm{p}=0.0003)$ and right instrument $(\mathrm{p}=0.0002)$. In UVA, a significant improvement was seen in many metrics including time $(p=0.0135)$, instrument collisions $(p=0.0066)$ and task specific metrics such as injury to the urethra $(\mathrm{p}=0.0032)$ and bladder $(\mathrm{p}=0.0189)$ (Figure 1).

Conclusions: Surgeons found this full procedural VR training module to be realistic as well as a feasible and acceptable component for a robotic surgical training programme. Construct validity was proven between expert and novice surgeons. Novice surgeons have shown a significant learning curve over 5.5 hours of training, suggesting this module could be used in a surgical curriculum for acquisition of technical skills. Further implementation of this module into the curriculum and continued analysis would be beneficial to gauge how this module can be fully utilised.

MP5-4 Construct validity of laser fragmentation as a marker of expertise in ureteroscopy

J Hendry, L Kerr, C McIlhenny

NHS Forth Valley

United Kingdom

Introduction \& Objective: In learning ureteroscopy and laser fragmentation there is a learning curve for trainees from supervised practice to then gain independent competence. Workplace based assessments are used in the UK and give a global judgement of competency however at present there is no marker of expertise. We sought to evaluate the construct validity of laser fragmentation time in ureteroscopy between expert and trainee urologist.

Materials and Methods: Prospective stone database was recorded for all patients undergoing elective ureteroscopy for renal or ureteric calculi. Patient age, sex, comorbidity, BMI preoperative MSSU, ASA and pre operative stent were recorded for all patients. Stone burden was calculated on preoperative CT KUB using cubic volume of each stone and multiplying by Hounsefield unit to give $\mathrm{HUcm}^{3}$. Where there were multiple stones the volumes were summed and a mean density from all the stones used to calculate total density volume. The operative time was recorded from start of procedure to end of surgery and fragmentation time as laser active time. Cases where trainee and expert did parts of the procedure jointly were excluded. Post operative complications, stone free status and length of stay were recorded.

Results: Forty seven patients undergoing ureteroscopy and laser stone fragmentation with one operating surgeon between September 2016 and March 2017 were included. Procedures were performed by an expert endourologist $(\mathrm{N}=27)$ or one of two junior trainees under direct supervision $(\mathrm{N}=20)$. The mean $\mathrm{HUCm}^{3}$ varied between expert and trainee cases significantly 
$\left(1075.5 \mathrm{HUcm}^{3}\right.$ vs. $299.7 \mathrm{HUcm}^{3}$, ANOVA p $\left.=0.019\right)$. There was no significant variation in overall operative time or fragmentation time totals (46.1 vs 43.9 and 18.8 vs 16.2 ) however when looking at fragmentation volume / minute there is marked difference between expert and trainee (54.7 vs 20.73, ANOVA $p=0.017$.) When looking at laser energy there is no significant difference in laser energy used per unit volume of stone between grade of surgeon (196.6 vs 218.4, ANOVA $\mathrm{p}=0.75$.)

Conclusions: Overall laser fragmentation time, laser energy and operation time cannot be used as a differentiating factor of expertise due to varied stone burden and case complexity. However fragmentation volume per minute, when accounting for stone size and density, demonstrates construct validity and should be utilised as a marker of competence.

MP5-5 Evaluation of a 3D Virtual Reality Model for Surgical Planning and Education

S Safiullah, DJ Lama, RM Patel, RC James, A Aljadaan, M Laughery, RV Clayman, J Landman

Department of Urology, University of California, Irvine, Orange, CA

United States

Introduction \& Objective: Preoperative imaging is the cornerstone of well-planned, safe surgery. Advancements in threedimensional (3D) imaging technology have allowed for improved understanding of patient anatomy. Virtual reality (VR) technology has similarly improved to the extent that it may become an adjunctive tool in surgical planning and education. We report our initial experience of developing VR models with subsequent use in a VR environment using commercially-available technology.

Materials and Methods: Axial CT scans of 5 patients with a small renal mass (SRM) were segmented via 3D Slicer (www .slicer.org) and converted into 3D Object files (.obj). These were imported to a VR software (Bosc, Pear Medical, Seattle, WA), enabling interaction with the VR kidney models through a headmounted display (HMD). The CT scans and corresponding VR models were reviewed in randomized order by 3 medical students (DL and two non-authors), who then completed a questionnaire $(0-10)$ after studying each CT scan and VR model to assess their understanding of the SRM and its relationship to other structures (e.g. vasculature, hilum, ureter).

Results: While wearing the HMD, participants could manipulate the VR kidneys and associated structures in VR using their own hands as input. In addition, the VR kidneys could be cleanly

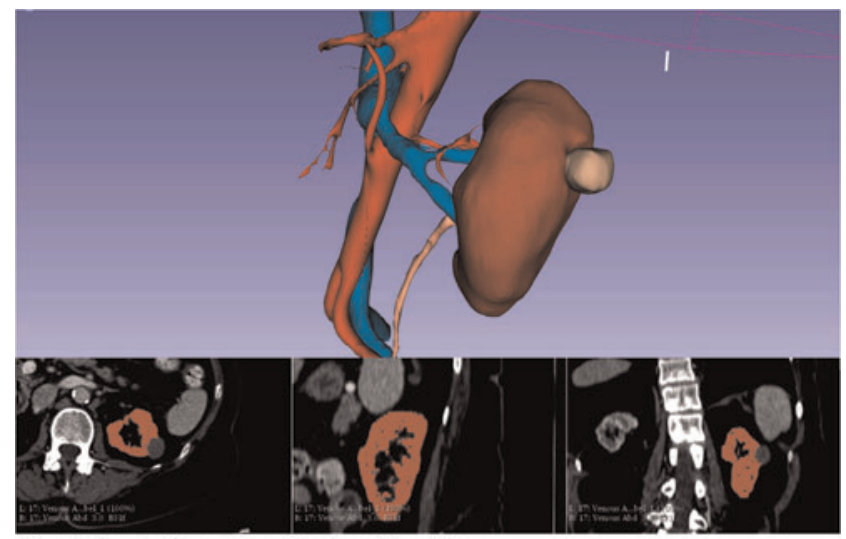

Figure 1: Example of image segmentation for model rendering

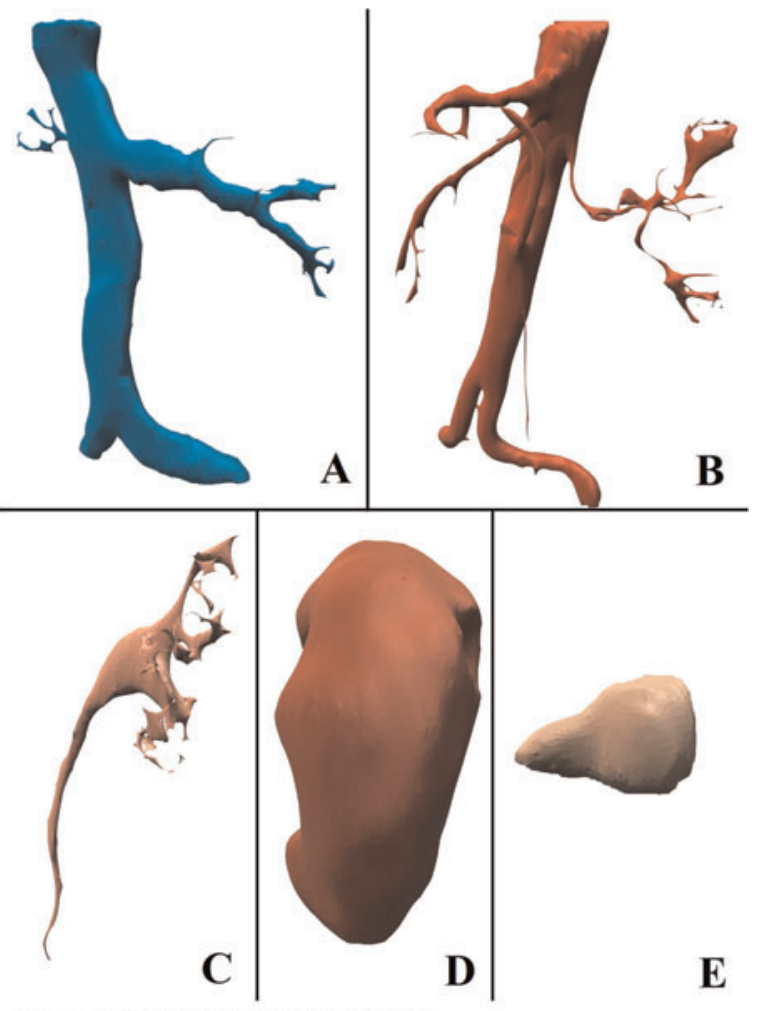

Figure 2: Rendered structures;

A) Venous; B) Arterial; C) Collecting System;

D) Kidney; E) Mass

\begin{tabular}{|l|l|l|l|}
\hline Questions & $\begin{array}{l}\text { CT Score } \\
\text { (N=15) } \\
\text { (range) }\end{array}$ & $\begin{array}{l}\text { VR Score } \\
\text { (N=15) } \\
\text { (range) }\end{array}$ & p-value \\
\hline $\begin{array}{l}\text { How well do you understand the } \\
\text { relationship between the renal } \\
\text { mass and the following structures? }\end{array}$ & & & \\
\hline Arterial vasculature & $4(0-7)$ & $9(7-10)$ & 0.0001 \\
\hline Venous vasculature & $3(0-9)$ & $9(6-10)$ & 0.0002 \\
\hline Hilar anatomy & $5(0-9)$ & $10(5-10)$ & 0.0002 \\
\hline Intrarenal collecting system & $5(0-8)$ & $9.5(8-10)$ & 0.0001 \\
\hline \multicolumn{1}{|c|}{ Ureter } & $7(0-9)$ & $10(8-10)$ & 0.0001 \\
\hline $\begin{array}{l}\text { How well do you understand the } \\
\text { tumor location? }\end{array}$ & $6(0-8)$ & $10(9-10)$ & $<0.0001$ \\
\hline
\end{tabular}

separated from the renal vasculature and the collecting system; all VR scores were significantly higher than CT scores (Table 1). Conclusions: VR technology can be used to import CT imaging data to create a patient specific virtual 3D kidney model which in turn can be viewed and manipulated. Use of VR technology improved the viewer's ability to understand the relationship of the SRM to the underlying renal structures.

MP5-6 Assimilation of Skills: A Survey Among Percutaneous Nephrolithotomy Course Participants

RM Patel, A Walia, KS Kaler, Z Okhunov, J Landman, RV Clayman

Department of Urology, University of California, Irvine, Orange, CA

United States 
Introduction \& Objective: We sought to determine the effect on clinical practice of training received in percutaneous nephrolithotomy (PCNL) at three AUA (American Urological Association) PCNL courses given in 2015.

Materials and Methods: Participants of the 3 AUA sponsored PCNL courses in 2015 were invited to participate in a 17-question web-based survey that was distributed via SurveyMonkey ${ }^{\circledR}$ from November 2016 to January 2017.

\begin{tabular}{|c|c|}
\hline Course location & Total (\%) \\
\hline Smith Institute of Urology, New Hyde Park, NY & $25 \%$ \\
\hline University of California, Irvine, CA & $32 \%$ \\
\hline University of Minnesota, Minneapolis, MN & $43 \%$ \\
\hline \multicolumn{2}{|l|}{ Age } \\
\hline$<30$ & $0 \%$ \\
\hline $30-50$ & $45 \%$ \\
\hline $51-70$ & $52 \%$ \\
\hline$>70$ & $2 \%$ \\
\hline \multicolumn{2}{|l|}{ Years since residency completion } \\
\hline $0-5$ years & $21 \%$ \\
\hline $6-10$ years & $26 \%$ \\
\hline $11-20$ years & $16 \%$ \\
\hline+21 years & $37 \%$ \\
\hline \multicolumn{2}{|l|}{ Fellowship trained in Endourology } \\
\hline Yes & $28 \%$ \\
\hline No & $72 \%$ \\
\hline \multicolumn{2}{|l|}{ Practice type } \\
\hline Solo & $23 \%$ \\
\hline Group & $51 \%$ \\
\hline Academic & $19 \%$ \\
\hline Other & $7 \%$ \\
\hline \multicolumn{2}{|l|}{$\begin{array}{l}\text { Were you performing your own access for PCNL } \\
\text { prior to the course? }\end{array}$} \\
\hline Yes & $42 \%$ \\
\hline No & $58 \%$ \\
\hline \multicolumn{2}{|l|}{$\begin{array}{l}\text { Were you performing PCNL after radiology obtained } \\
\text { access prior to the course? }\end{array}$} \\
\hline Yes & $60 \%$ \\
\hline No & $40 \%$ \\
\hline \multicolumn{2}{|l|}{$\begin{array}{l}\text { Have you performed your own access for a PCNL } \\
\text { since your participation in the PCNL course? }\end{array}$} \\
\hline Yes & $67 \%$ \\
\hline No & $33 \%$ \\
\hline \multicolumn{2}{|l|}{$\begin{array}{l}\text { If you are obtaining your own access, what } \\
\text { technique do you use? }\end{array}$} \\
\hline Ultrasound guided & $7 \%$ \\
\hline Fluoroscopy guided & $56 \%$ \\
\hline Ultrasound and fluoroscopy guided & $24 \%$ \\
\hline Fluoroscopy guided endoscopically assisted & $12 \%$ \\
\hline \multicolumn{2}{|l|}{$\begin{array}{l}\text { How many PCNLs have you performed in the last } \\
\text { year? }\end{array}$} \\
\hline 0 & $7 \%$ \\
\hline $1-5$ & $35 \%$ \\
\hline $6-10$ & $28 \%$ \\
\hline $11-15$ & $14 \%$ \\
\hline $16+$ & $16 \%$ \\
\hline
\end{tabular}

Table 1: Demographics of survey respondents and responses on practice patterns

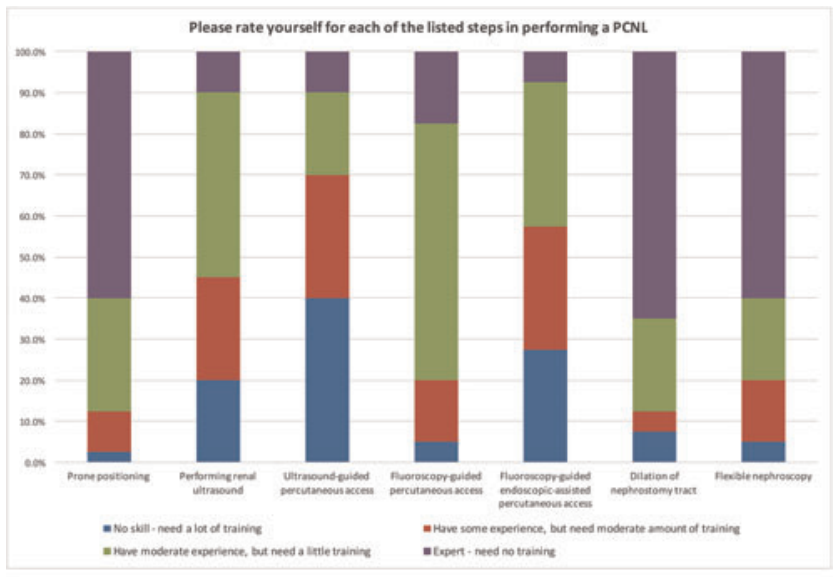

Figure 1: Self-assessment of performing PCNL

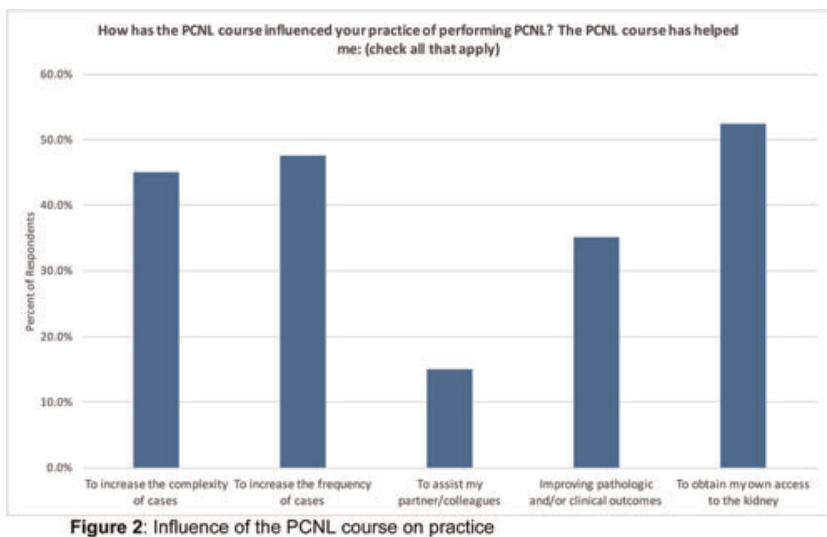

Results: A total of 89 participants attended the three AUA PCNL courses offered in 2015 and 44 (49.4\%) individuals participated in the survey. Demographics of participants are presented in Table 1. Age, fellowship training in Endourology, and type of clinical practice were found to have no significant correlation with the influence of the PCNL course on practice. There was a $25 \%$ increase in the number of participants who obtained their own access for PCNL following participation in a course and the preferred method of obtaining access was fluoroscopy guided (Table 1). After completing the course and returning to practice, attendees were asked to rate their skills in the various steps of PCNL; responses are summarized in Figure 1. No significant difference was observed between AUA PCNL course location and technique used for obtaining access, self-rated skills, or recommending the course to a colleague. Changes in practice patterns after course completion is summarized in Figure 2 with $90 \%$ of respondents stating they either "agree" or "strongly agree" to recommend an AUA PCNL course to a colleague.

Conclusions: One year following completion of an AUA sponsored PCNL course, $25 \%$ of participants reported a successful assimilation of skills independent of course location. Long-term follow-up is necessary to evaluate for lasting effects of the training received.

MP5-7 Prostate Dynamic Anastomotic Template On Robot (Pro-DATOR ${ }^{\mathrm{TM}}$ ) - A Natural Simulation Platform for Optimal Skills Acquisition in Vesicourethral Anastomosis prior to Robotic Prostatectomy

SS Kommu, L Samateh, RA Persad, P Rouse, SH Garnett, PD Rimington, (S Academic Research Group

Eastbourne District General Hospital: East Sussex Healthcare NHS Trust

United Kingdom

Introduction \& Objective: The utility of the robotic platform as a tool for surgical extirpation and reconstruction in urology has ushered a recent near exponential increase in uptake. A pivotal part of training is currently ascribed to skills acquisition using Virtual Reality Robotic Simulators as part of fellowship training programmes. Robot Assisted Radical Prostatectomy (RARP) demands skill with little margin for error.Vesicourethral anastomosis (VUA) remains one of the most complex steps in performing RARP. Herein, we describe a Natural Simulation Platform (NSP) and decipher its face, content, and construct validity. 
Materials and Methods: The NSP consisted of a purpose built model with optical projection and layered tissue matrix that has striking resemblance to the live human case. The platform was built such that the da Vinci Surgical Systems robot could be docked [Prostate Dynamic Anastomotic Template on Robot (ProDATOR $\left.^{\mathrm{TM}}\right)$ ]. An independent invigilator measured the working platform to ensure accurate representation of the cave of retzius and surrounding anatomical landmarks including endopelvic fascia. The working platform used optical projections such that depth and working surface mimicked an urethra and bladder neck. A robotic Needle Holder ${ }^{\circledR}$, Prograsps ${ }^{\circledR}$ and Marylands $\AA$ were deployed for continuous VUA. A barbed suture was used. Each case was recorded. Trainees and consultant surgeons participated. We divided subjects into 2 groups, those with experience and novices (trainees). Each subject performed several simulated VUA using ProDATOR $^{\mathrm{TM}}$ )].Video recordings of the console view and forearm ergonomics and direct instrument collisions were tallied. A scoring algorithm recorded the data from each performance.

Results: The novice group consisted of 4 residents. The experienced surgeons $(n=4)$ had each previously performed at least 20 robotic surgeries. The experienced group outperformed the novice group in most variables, including task time, total score, total economy of motion and number of instrument collisions with striking resemblance to the live human case.

Conclusions: The Natural Simulation Platform (NSP) Prostate Dynamic Anastomotic Template on Robot (Pro-DATOR ${ }^{\mathrm{TM}}$ ) represents one of the most advanced tool to permit optimal skills acquisition for VUA and provided excellent face, content, and construct validity. The NSP represents the next step in the current paradigm of preparing for the live human case and acts as a more advanced platform than virtual reality. Large scale studies are indicated to further develop similar tools for advanced skills acquisition.

MP5-8 Simulator Based Development of "Trans-Urethral Resection of Prostate" Skills and implementation of "Global Rating Scale" Evaluation System in Urology Postgraduate training.

K Soomro, Z Rajpar, I Memon, S Memon

Liaquat University of Medical and Health Sciences (LUMHS) Jamshoro/ Hyderabad

Pakistan

Introduction \& Objective: After the introduction of simulators in urology, education in urology has evolved significantly. Specially in endourology, development of Transurethral Resection of Prostate (TURP) skills are shifted from "direct on patient" to simulator based learning. we evaluated the development of TURP procedure skills based on virtual reality simulator (VirtaMed | UroSim ${ }^{\mathrm{TM}}$ ) and assessment of skills based on Global Rating Scale (GRS) evaluation system.

Materials and Methods: Of 17 participants, 4 were Urology faculty and 13 postgraduate urology trainees. All participants performed TURP basic procedural skills on simulator before attempting TURP full procedure (Easy 1 mode). Five attempts of real time TURP full procedure were performed by all candidates. Participants were allowed to resect maximum possible prostate tissue (goal $=\geq 85 \%)$. Skills were assessed in terms of Resection, Bleeding control and safety. Data was obtained from Simulator based scores and objective assessment based on GRS scores. We analyzed the data from the first and fifth attempt of all participants, and differences were compared.
Results: In faculty, there was no significant deference in Resection score $(112 \pm 8.6 / 120: p=0.18)$, Bleeding control score $(70 / 70)$, Safety score $(70 \pm 14.14 / 80: \mathrm{p}=0.2)$ and total score $(257 \pm 36.8 /$ $300: p=0.1)$, whereas in residents there was statistically significant deference in Resection score (86.69 $\pm 9.95 / 119 \pm 0.5: \mathrm{p}=<0.001)$, Bleeding control score $(57 \pm 10.2 / 69 \pm 0.7: \mathrm{p}=0.001)$, Safety Score $(48.46 \pm 14.0 / 77.69 \pm 4.3: \mathrm{p}=<0.001)$ and total score $(196 \pm 39.0 /$ $298 \pm 3.65: \mathrm{p}=<0.001$ ). In comparing $1^{\text {st }}$ attempts (Faculty vs Residents), statistically significant difference in resection score $(112 \pm 8.6 / 86.69 \pm 9.95: \mathrm{p}=<0.000)$, bleeding control score $(70 /$ $57 \pm 10.2: \mathrm{p}=0.001), \quad$ safety score $(70 \pm 14.14 / 48.46 \pm 14.0: \mathrm{p}=$ $0.01)$ and total score $(257 \pm 36.81 / 196 \pm 39.0: \mathrm{p}=0.01)$. Trainee's achieved comparable skill's scores vs faculty at the end of study (Total Scores. 298.0 $\pm 3.6 / 300: p=0.3$ ). GRS scores of trainees also significantly improved $\left(1^{\text {st }} 12.0 \pm 2.97 / 5^{\text {th }} 19.15 \pm 3.10: p=<0.001\right)$. Conclusions: Simulation based TURP training significantly improve operative skills in terms of Resection, Bleeding Control and Safety. GRS is effective tool in assessment of operative skills and may be used for evaluation of urology residents periodically.

MP5-9 Analysis of the surgeon's posture during robotic simulator tasks using motion capture system

K Yoshida, K Takayasu, T Matsuda

Kansai Medical University

Japan

Introduction \& Objective: Surgeons are sometimes forced to maintain uncomfortable joint positions during robotic surgery due to the high degree of instrument maneuverability. This study aimed to use an optical motion capture system to analyze the differences in posture patterns during robotic simulator tasks between surgeons at two skill levels.
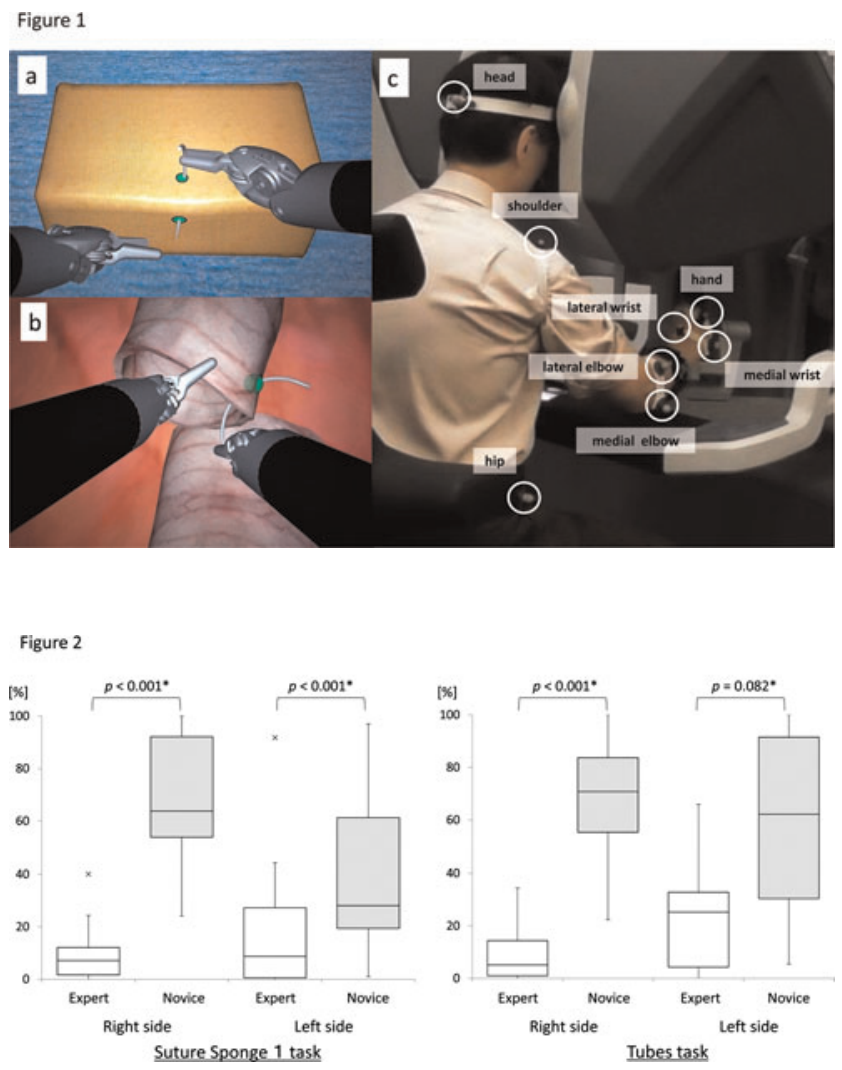
Materials and Methods: Ten experienced and ten novice surgeons performed two tasks in a da Vinci Skills Simulator (Figure 1a and 1b): Suture Sponge 1 (SP) and Tubes (TU). The participants' upper body motion during each task was captured, including the joint angles (axilla, elbow, and wrist: Figure 1c), the percentage of time when the wrist height was lower than the elbow height (PTW), and the height of the elbow and wrist relative to the armrest.

Results: The novice group exhibited significantly more excessive stretch in both elbow angles and extension $\left(>50^{\circ}\right)$ in both wrist angles than the experienced group. The novice group had significantly lower PTW than the experienced group on the right side in both tasks (both $\mathrm{p}<0.001)$, and on the left side in $\mathrm{SP}(\mathrm{p}<0.001)$. Compared with the experienced group, the novice group had a significantly higher elbow relative to the armrest on the right side (SP, TU: $p<0.05$, Figure 2), and a significantly lower wrist relative to the armrest on the right side (SP, TU: $\mathrm{p}<0.05$ ).

Conclusions: An optical motion capture system can detect the differences in posture patterns in the positional relationship between the elbow and wrist and the joint angles of the upper limb between two groups of surgeons at different skill levels during robotic simulator tasks.

MP5-10 The Training Concept of Whole Procedure Equivalent in Robot Assisted Radical Prostatectomy - Results of a single surgeon in a Fellowship Training Programme

P Rouse, SS Kommu, A Samateh, P Sturch, SH Garnett, PD Rimington, (S Academic Research Group

Eastbourne District General Hospital: East Sussex Healthcare NHS Trust

United Kingdom

Introduction \& Objective: We report on our experience with a modular, non-sequential training concept of Whole Procedure Equivalent (WPE) and its application in a Pelvic Uro-oncology Fellowship Training Programme for Robot Assisted Radical Prostatectomy (RARP).

Materials and Methods: RARP was broken down into standardized steps: A Preprocudural Prostate Mapping Chart (PPMC), Patient Positioning, Port Placement, Anterior Dissection, Dorsal Vein Complex Suturing, Bladder Dissection, Posterior Dissection, Dissection of Pedicles, Posterior Reconstruction and Vesicourethral Anastomosis. The PPMC is a novel platform with 3-D toggle capabilities which involves a detailed summary of the patient including correlation of MRI and biopsy findings coupled with nomograms including those described by Partin and Briganti. These steps can be learnt/performed in a non-sequential manner. A colour code of performance was used: [White when the trainee was in an assistant role. Red when a step was being taught such that the fellow was under active or pro-active supervision. Orange when a step was completed with passive supervision (NO INPUT needed by the supervising trainer but trainer present). Blue when a step could be completed independently but noted to require minimal adjustment upon review of video recording. Green when a step could be completed independently but noted to require no adjustment upon review of video recording]. A simple formula was used to assess how many times the trainee had completed the individual steps.

Results: A total of 100 cases were analyzed. The fellow did not impact on total operative time (mean 135 minutes), estimated blood loss (median $<50 \mathrm{mls}$ ), complication rate (One Clavien grade $\geq 2$ complications) nor hospital stay (median 28 hours). After 100 procedures, the trainee has performed a large number of steps and has competently performed 20 procedures with passive supervision, including 8 moderate-difficult cases in- cluding presence of median lobes. Following this the fellow embarked on independent cases.

Conclusions: The current modular non-sequential platform of skill acquisition and delivery can potentially be used effectively and with no impact on patient outcomes in fellowship training programmes for RARP. This is especially important to note within the constraints of service provision in most tertiary referral centers.

MP5-11 Predictors of trainees' robotic anastomosis competence evaluation (RACE) scores of a simulated da Vinci robot-assisted urethrovesical anastomosis on a novel 3Dprinted bladder model

NC Wong, J Hoogenes, U Blankstein, K Kim, Y Guo, B Shayegan, ED Matsumoto

McMaster University

Canada

Introduction \& Objective: Surgical simulation is becoming more accessible and popular, yet no standardized curricula or evaluation metrics for simulator training exist. We evaluated the predictability of trainees' scores on the Robotic Anastomosis Competence Evaluation (RACE) (Raza et al., 2015) after performance of a robot-assisted urethrovesical anastomosis (UVA) on a novel 3D-printed bladder model.

Materials and Methods: We developed a unique robotic training curriculum to mimic the skills required to complete a UVA. Medical students, residents, and fellows were randomized to training on either the dV-Trainer or the da Vinci Surgical Skills Simulator (dVSSS). Participants then used the da Vinci robot to complete a UVA on the bladder model affixed within a silicone torso. The simulator composite scores for each of the three progressively difficult tasks were uploaded, and three blinded expert robotic surgeons independently evaluated endoscopic videos of each UVA performance using RACE and the Global Evaluative Assessment of Robotic Skills (GEARS, Aghazadeh et al., 2015). Results: 39 participants ( 10 medical students, 26 residents, 3 fellows) completed the study. Mean age was $26.7 \pm 0.9,26$ were male. For the UVA task, mean GEARS and RACE scores (both out of a total score of 30 across 6 domains) were $20.6 \pm 0.9$ and $21.2 \pm 1.0$, respectively. On univariate analysis, higher level of training, previous robotic simulation experience, previous laparoscopic and/or robotic experience, dVSSS curricula, higher cumulative simulator scores, and high GEARS scores were all positively associated with RACE scores. On multivariate regression analysis, GEARS scores were shown to be an independent predictor for RACE scores.

Conclusions: This study demonstrated that the GEARS scores were an independent predictor of positive performance of a simulated UVA on a 3D-printed bladder model based on RACE scores. Further research is required to determine additional significant predictors of trainees' success in performing a UVA in a simulated setting.

MP5-12 Development and validation of a 3D-printed bladder model for laparoscopic simulated urethrovesical anastomosis training for radical prostatectomy

Y Guo, J Hoogenes, NC Wong, K Kim,

B Shayegan, ED Matsumoto

McMaster University

Canada 
Introduction \& Objective: Minimally invasive urological surgeries such as laparoscopic prostatectomy are associated with steep learning curves. With competency-based training on the horizon, and the recent reduction of resident work hours, surgical educators have shifted some training outside of the OR to surgical skills labs. To reduce learning curves and improve education at our centre, we developed a 3D-printed bladder model for simulated urethrovesical anastomosis (UVA) training. Our objective was to validate this model for laparoscopic training.

Materials and Methods: After several iterations, the final model was produced using a unique polymer, and the dimensions mimic the anatomical structures of a human bladder and urethra. To validate this model for its use in simulation, we surveyed urology residents, fellows, and staff after they completed a laparoscopic training course where they were tasked to complete a UVA after receiving didactic training. Laparoscopic video trainers were used and the model was affixed inside the simulated torso (Figure). Each participant had their own model. All participants completed an exit questionnaire that was used to validate the model using 5-point Likert scales within 4 domains.

Results: Urology residents (junior and senior), fellows, and staff from 7 urology programs completed the course $(\mathrm{N}=24)$. Mean age was $29.8(\mathrm{SD}=4.6)$, and 21 were male. $87 \%$ had basic and $62 \%$ had advanced simulation training prior to the course. For face validity, participants scored an overall mean of 3.6 on the anatomical realism domain and rated overall task-based usefulness a mean of 3.8, with the UVA task a 4.1, and suturing, knot tying, and cutting a 4.4. For content validity, participants rated overall usefulness as a training tool a 4.3, with improving operative technique scoring 4.4. Overall reaction scored a mean of 4.2 over 6 domains. A mean of 4.1 was found for "skills learned on model will transfer to the OR", and the majority thought that the model should be incorporated into the training curriculum (4.3).

Conclusions: Participants' scores established that this low-cost model (\$14USD/model) has face and content validity for laparoscopic UVA training within this sample. Research is currently undway to establish the model's contruct validity.

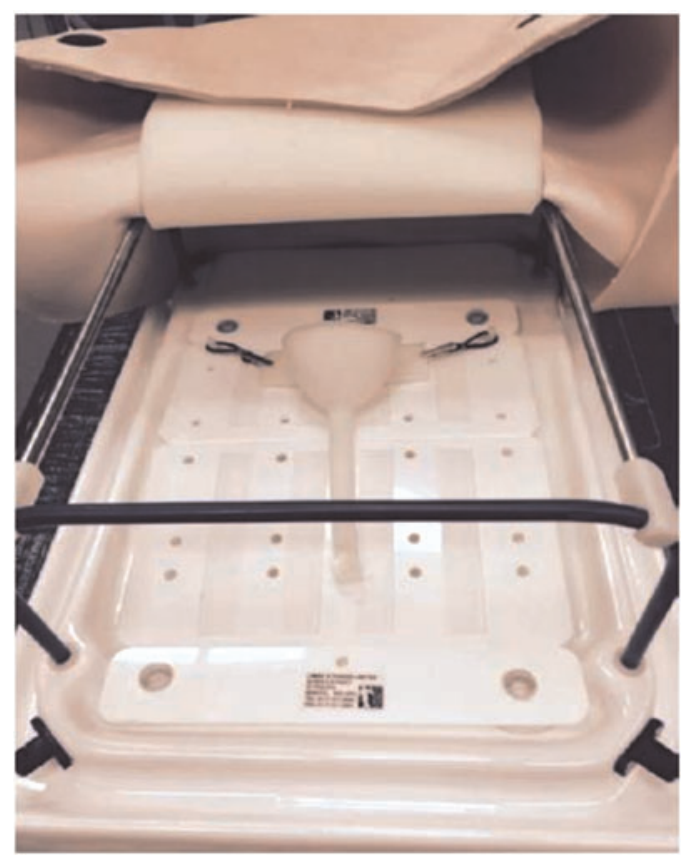

MP5-13 Skill Acquisition Trend (SATrend) - A Novel Approach for Redefining "The Learning Curve" in Minimally Invasive Urological Surgery

SS Kommu, SH Garnett, PD Rimington, (S Academic Research Group

Eastbourne District General Hospital: East Sussex Healthcare NHS Trust

United Kingdom

Introduction \& Objective: In any minimally invasive urological training programme, skill acquisition and delivery mandates a modus upon which transferrable and learned tasks can be gauged and compared. The concept of the "learning curve" is currently used extensively to describe the patterns of skill acquisition. The traditional description of the learning curve includes "steep", "narrow", "flat" etc. These descriptions have inherent limitations. The current and evolving patterns of skill acquisition and delivery as it pertains to laparoscopic and robotic surgery may be better defined with a more robust and quantifiable means of describing the traditional learning curve. Herein, we aim to describe and test a novel means of defining skill acquisition using the concept of Skill Acquisition Trend (SATrend).

Materials and Methods: It is recognized that during any task performance, there is (1) an End Point and (2) Error Pattern. SATrend was developed to include patterns of error on the same graph such that a dual pattern of end point achievement and error/penalty would be described in one graphic format. One task was defined as complex (triple knot tie) and the other simple (picking up 5 buttons and placing into a container). Four teenagers who had no concept of laparoscopy were recruited for the study. Metrics were then applied to each task and included time to completion of task, redundant movements and errors. Each task was performed 50 times by each participant. Five End Point Achievement Patterns (EPAP) and 2 Error Occurrence Patterns (EOP) were noted. The observed patterns were applied to the advanced platform of ex-vivo vesicourethral anastomosis (VUA) and in the live human case of robot assisted radical prostatectomy (RARP).

Results: EPAP had two distinct phases and 5 distinct patterns. EPAP Phase 1 involved the first 10 cases in which the skill acquisition was noted to be erratic. Phase 2 consisted a predictable linear pattern in a positive trend. EOP consisted of 2 phases and 2 patterns independent of number of cases. EOC Phase 1 consisted of and erratic phase but remained erratic despite number of attempts in some candidates. EOC Phase 2 consisted of rapid decrease in error occurrence in remaining candidates. When applied to the live human case (VUA in RARP), the patterns were noted on video analysis [Table 1].

Conclusions: Skill Acquisition Trend (SATrend) is an advanced platform for analysis, description and standardisation of the traditional "Learning Curve" in minimally invasive surgery. The current description could potentially permit early identification of those candidates who are likely to require further targeted training.

WCE 2017 Kommu et al SATrend

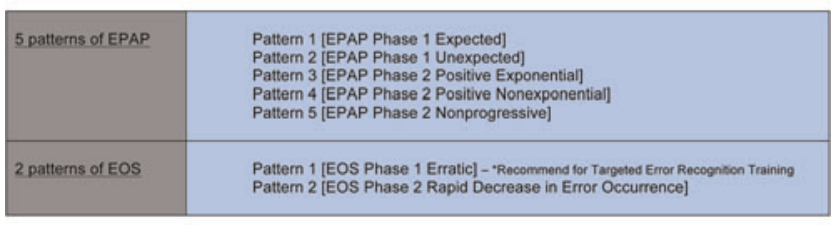

TABLE 1. SATrend Patterns 
MP5-14 Comparison of 3D printed prostate models with standard radiological information to aid understanding of the precise location of prostate cancer: a construct validation study.

J Ebbing, F Jäderling, J Collins, O Akre, S Carlsson, J Höijer, M Olsson, P Wiklund

University Hospital Basel, Urological University Clinic BaselLiestal

Switzerland

Introduction \& Objective: To investigate the reliability to impart the exact location of prostate cancer in the prostate to healthcare professionals of different expertise by written magnetic resonance imaging (MRI) reports and MRI presentations in multidisciplinary team meetings (MDT), as current standard methods, compared to 3D printed prostate models as a new technology to describe the location of prostate cancer (PCA) lesion.

Materials and Methods: We used a 3x3 Latin square array with three different PCA cases to assess the three information tools for the orientation of PCA locations: (A) Written MRI report, (B) 3D printed model, and (C) MRI presentation in MDT. Construct validation was performed using two healthcare groups with different levels of expertise: (1) Expert urologists in PCA, (2) Medical students. After each information tool the study participants plotted the tumour location in a prostate template. A scoring system was used to evaluate the drawings for the accuracy of plotting tumour position with a maximum of 30 points per case available. Data are shown as median scores with interquartile range. Median regression was used to identify differences between the groups and information tools.

Results: A total of 162 drawings were evaluated. Within the expert group no significant difference was seen in the overall scoring results between the information tools $(\mathrm{p}=0.34)$. Medical students performed significantly worse with MDT information $(\mathrm{p}=0.03)$. Experts performed better in all three information tools compared to students, resulting in a significantly $25 \%$ higher overall total score in the expert group (25.0 [22.3-26.7] vs. 20.0 [15.0-24.0], $\mathrm{p}<0.001)$. The difference was largest after MDT information with experts showing a $49 \%$ better scoring $(\mathrm{p}<0.001)$ and second largest with the 3D printed models showing a $17 \%$ better scoring of the experts $(p=0.07)$. No statistically significant difference was shown in the written MRI report scoring results between experts and students (Table 1). Quality and rates of major mistakes and major complete accuracy in reproduction of the exact tumour location were distributed differently between the three tools in each group.

Conclusions: Overall construct validation of the information tools was achieved with experts showing more accurate plotting of prostate cancer location than medical students who had more difficulty with understanding the exact tumour location, especially in the MDT setting. However, both written reports and 3D printer models orientated medical students better compared to

\begin{tabular}{|c|c|c|c|c|c|}
\hline & $\begin{array}{l}\text { Intromation tool A } \\
\text { (Writhen MRI Irepoch) }\end{array}$ & 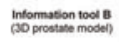 & 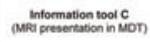 & $\begin{array}{l}\text { Orearal total } \\
\text { score }\end{array}$ & peratuen \\
\hline $\begin{array}{l}\text { Group } 1 \\
\text { Expent urologists }\end{array}$ & $\begin{array}{c}240 \\
(220250) \\
00016\end{array}$ & $\begin{array}{c}250 \\
(20.0267) \\
83.3 \%\end{array}$ & $\begin{array}{l}2533 \\
(237-27.0) \\
24.3 \%\end{array}$ & $\begin{array}{c}250 \\
223.2877 \\
83.3 \%\end{array}$ & 0.34 \\
\hline $\begin{array}{l}\text { Group } 2 \\
\text { Modicat masterts }\end{array}$ & $\begin{array}{c}200 \\
1702247 \\
7336\end{array}$ & $\begin{array}{c}213 \\
173223 n \\
7100\end{array}$ & $\begin{array}{c}170 \\
(12321.7) \\
\text { se.6\% }\end{array}$ & $\begin{array}{c}200 \\
(150240) \\
66,7 \times\end{array}$ & 0.093 \\
\hline presure & 025 & 0.07 & $\infty 001$ & $\$ 001$ & \\
\hline
\end{tabular}

MRI presentations in MDT. Indicating that the 3D printed models might be easier to understand than the current gold standard MDT conferences. Therefore, 3D models may have a role in orientating less experienced individuals, such as surgical trainees.

MP5-15 A novel 3D-printed bladder model for training robot-assisted urethrovesical anastomosis

J Hoogenes, NC Wong, Y Guo, K Kim, B Shayegan, ED Matsumoto

\section{McMaster University}

Canada

Introduction \& Objective: Robot-assisted radical prostatectomy (RARP) training is often conducted via intraoperative mentoring and proctoring, which is time consuming, expensive, and can put patients at risk. Simulation offers an alternative method of training, especially for specific tasks within a larger procedure, where the trainee can practice in a safe and accessible environment. The urethrovesical anastomosis (UVA) is one of the most challenging steps in RARP and has a steep learning curve. We developed and validated a 3D-printed bladder model for simulated robotic UVA training.

Materials and Methods: We developed a 3D-printed bladder model using computer-aided design software with rapid prototyping using a Lulzbot Taz $43 \mathrm{D}$ printer. The model is composed of a unique polymer with characteristics similar to the human bladder and urethra with regards to size and handling for incising and cutting, needle penetration, and suture pull-through. Medical students and residents performed a series of UVA-related tasks on a robotic simulator, and then performed a UVA on the bladder model, which was affixed within a silicone torso and docked to the da Vinci Si robot. Each session was videotaped via the endoscopic camera. Participants completed an exit survey and each video, including the end product, was reviewed by three blinded expert raters using the GEARS and RACE global rating scales (Figure).

Results: Participants, who were part of a larger study, included residents (11 junior and 7 senior) who had at least some robotic surgical experience. Using a 5-point Likert scale, participants agreed that the bladder model was anatomically realistic (3.2)
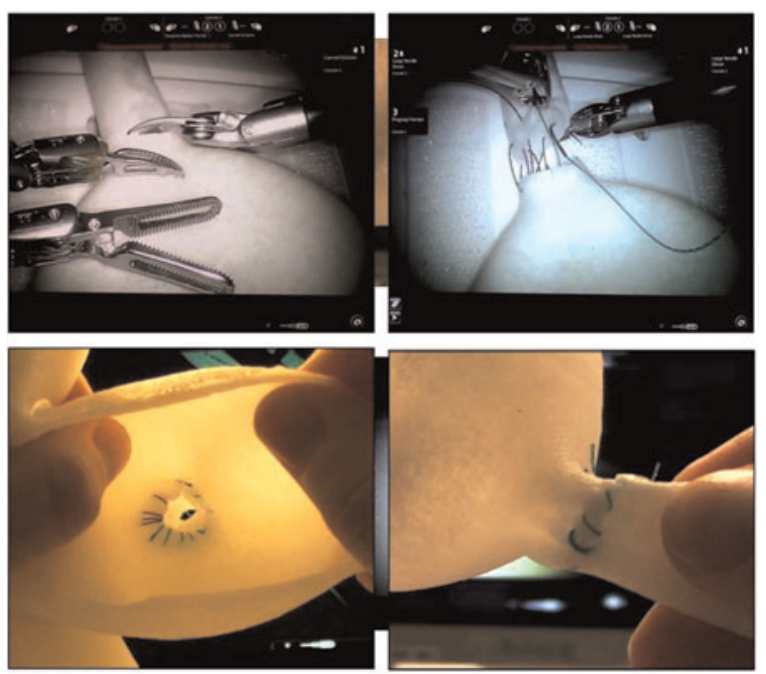
and that it increased their confidence for performing an in vivo UVA (3.6). The GEARS and RACE scores showed high interrater reliability with an intraclass correlation coefficient of 0.845 [95\% CI $=0.706-0.924$ ] and 0.864 [95\% CI $=0.742-0.933$ ], respectively, among raters.

Conclusions: Our 3D-printed UVA model shows preliminary face, content, and construct validity; however, further research must be completed with a larger sample with multiple levels of training.

MP5-16 Non-Technical Skills for Urological Surgeons (NoTSUS): Development and Validation of a Scenario-based Ureteroscopy Curriculum

A Aydin, K Ahmed, O Brunckhorst, N Raison, F Dar, H Aya, C Lovegrove, M Iqbal, A Al-Jabir, T Abe, J Brewin,

C McIlhenny, S Jain, M Shabbir, S Khan, P Dasgupta

MRC Centre for Transplantation, King's College London United Kingdom

Introduction \& Objective: Considerable attention has been given to the development of simulation-based technical skills training. Although several studies have illustrated the importance of non-technical skills training, this area is often overlooked in many surgical specialties. The aim of this study is to develop and validate an ureterorenoscopy procedure-specific non-technical skills curriculum for urological surgeons.

Materials and Methods: A Delphi technique was employed to consult experts in urolithiasis and non-technical skills training and determine the contents and scenarios for the curriculum. Senior specialists and junior urology residents from around the United Kingdom and Europe were invited to participate in a one-day training session. Participants were divided into four groups, with up to four in each group. Four independent scenarios were conducted within the previously validated Full Immersion Simulation envi- ronment (Imperial College, London, UK). Two faculty members, with expertise in urolithiasis and non-technical skills training, supervised participants. Faculty and the remaining participants in each group observed the sessions through a video-link. Each candidate was given individual feedback and assessed using a modified Non-technical Skills for Surgeons (NOTSS), entitled Nontechnical Skills for Urological Surgeons (NOTSUS), rating scale for ureterorenoscopy. Sessions were concluded with an evaluation questionnaire.

Results: A significant majority stated that they felt they were in the real operating room (mean: $4.3 / 5$ ), behaved as they did in real operations (mean: 4.4/5) and that the team interactions were realistic (mean: 4.6/5). Participants rated the day to be very useful (mean: 4.6/5), stated that it significantly improved their technical (mean: 3.75/5) and non-technical skills (mean: 4.5/5) and added that this form of simulation adds value to simulationbased training (mean: 4.7/5). It was also felt that the session provided them with transferrable skills, which can be taken into the operating room (mean: 4.7/5). A One-way ANOVA test revealed significant improvement in non-technical skills with consecutive scenarios $(\mathrm{p}=0.0037)$ in each group.

Conclusions: The non-technical skills curriculum for ureterorenoscopy revealed to be useful for urological residents and specialists. Participants rated the programme highly in all parameters, demonstrating educational value and acceptability. The participants will be followed in their individual institutions for transfer validity.
MP5-17 Validation Measures of a Partial Nephrectomy Training Model

SM Monda, JR Weese, BG Anderson, R Venkatesh, B Cheng, RS Figenshau

Washington University in St. Louis and Saint Louis University United States

Introduction \& Objective: More partial nephrectomies are performed every year as a surgical treatment for kidney cancer. However, this procedure remains technically challenging and a significant learning curve exists. Warm ischemia time, one important prognostic indicator and measure of surgeon performance, has been shown to plateau only after a surgeon has performed 30 cases. Currently, no established practice model exists for partial nephrectomy training. We created silicone renal tumor models using $3 \mathrm{D}$ printed molds of a patient's kidney with a mass. Here we present the results of the face, content, and construct validity testing we performed on these models.

Materials and Methods: We recruited four medical students, fourteen urology residents, three endourology fellows, and three attending surgeons to perform simulated partial nephrectomies on silicone renal tumor models. Four trials each were performed with a da Vinci surgical robot on two different days. Operation specific metrics - renal artery clamp time and surgical margins - were recorded for each trial. Validated measures of self-assessed operative demand (NASA TLX) and blinded reviewer-assessed surgical performance (GEARS) were also recorded across trials. Model face validity was evaluated via survey by participating fellows and attendings on a 0-100 slider bar scale anchored at unrealistic and realistic.

Results: Renal artery clamp time results and face validity results are shown in Figures 1 and 2 respectively. Surgeon training level and trial number were both found to be predictive of clamp time on linear regression $(\mathrm{p}<0.005)$. Logistic regression also demonstrated a reduction in positive margin rates across trial number $(p<0.05)$. Results of reviewer-assessed surgical performance is currently being collected from video footage.

Conclusions: Face validity measures indicate this model adequately represents reality. Construct validity was demonstrated both within and between groups; we observed improved surgical performance over the four trials as well as better performance in urologists of higher training levels. This model may have potential for broader application and integration into minimally invasive surgery training programs. Future work will establish

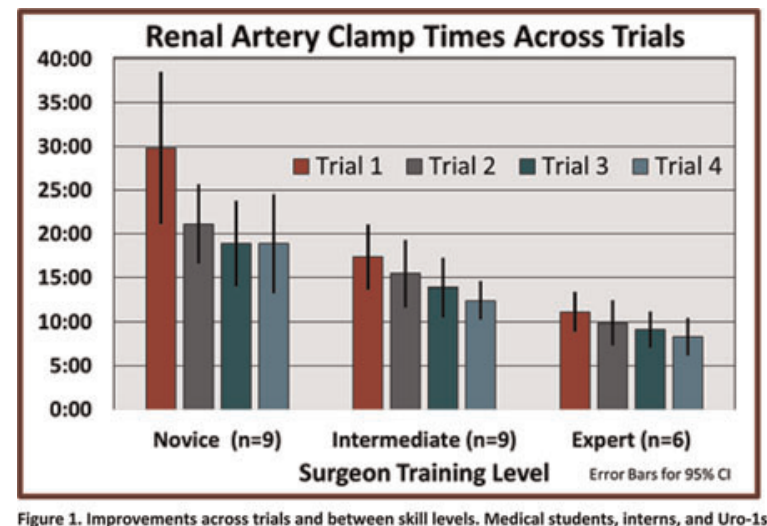

were novice, Uro-2s through Uro-4s were intermediate, and attendings and fellows were expert 


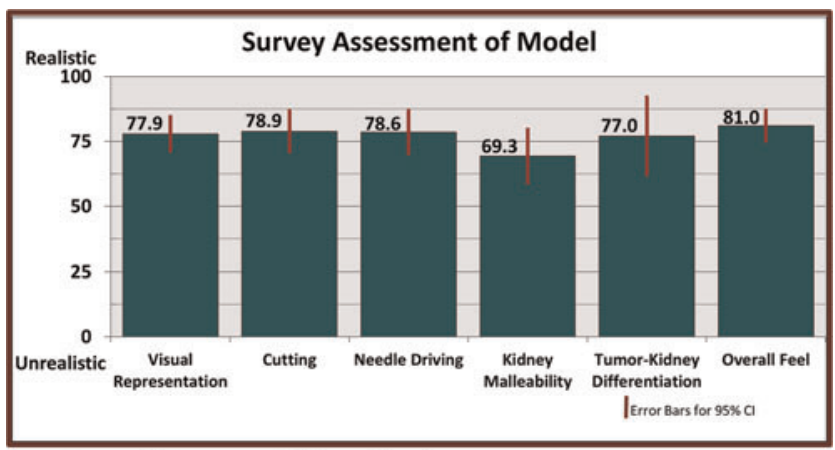

Figure 2. Face validity survey results for model realism.

training tumors of different anatomical difficulty, and test the correlation between model performance and operating room performance.

MP5-18 The development of novel poly vinyl alcohol training model for robotic partial nephrectomy

S Isotani, Y Noma, M Kurosawa, T China, T Ieda, K Kitamura, M Nagata, Y Wakumoto, S Muto, S Horie

Juntendo University, Graduate School of Medicine Japan

Introduction \& Objective: The robot partial nephrectomy (RPN) is rapidly evolving minimum invasive surgery for nephron sparing surgery. The trend towards RPN leads to the requirement for training tools specific for robotic techniques such as tumor resection with avoiding trauma of renal parenchyma, and renorrhaphy suturing. We developed the inanimate poly vinyl alcohol (PVA) model having a texture similar to actual renal parenchyma and tumor for physical learning experience in RPN training. The face and construct validity by learning performance was examined.

Materials and Methods: The RPN training models made by PVA having $4 \mathrm{~cm}$ diameter tumor was prepared. To reproduce the kidney and tumor, 3D printed data of the actual patient was extracted. A single surgeon had not experienced RPN performed 20 surgical RPN training procedures using the da Vinci Si robotic system. The RPN training this surgery were (1) marking of surgical margin by ultrasound, (2) tumor resection and vascular

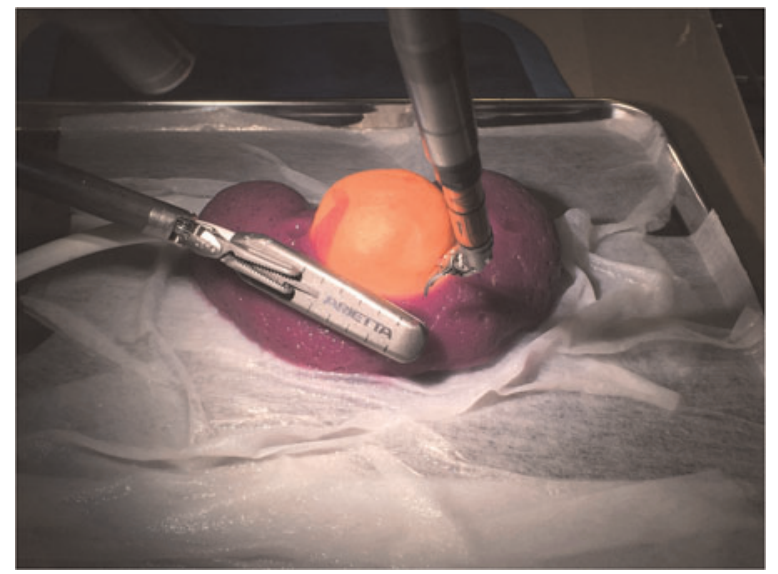

control of the intrarenal arterial branch, (3) deep renorrhaphy and parenchymal renorrhaphy by continuous suturing with sliding clip method. The face validity and construct validity was examined by the time of each procedure, presence or absence of exposure of tumor.

Results: The reproducibility of the tumor and renal parenchyma was good, and surgical training was perfumed successfully in all cases. The both of ultrasonic probes and energy devices could be performed to identify tumors and marking of surgical margin. The duration of tumor resection and renorrhaphy was reduced from 37 to 10 and 35 to 15 minutes respectively. Clear learning curves were observed presenting the construct validity of the model. In the early cases, the exposure of the tumor (positive margin) was observed in 3 cases, and the lesion tears in the renal parenchyma were observed in 2 cases, but these were not recognized in later cases.

Conclusions: The novel training method using PVA model for RPN. was examined. Our method demonstrated face and construct validity. This training model can contribute to improve in the early stage education of RPN.

\section{MP5-19 Construction and Assessment of An Innovative Indigenious Ultrasound Guided Puncture Simulator}

AV Rawandale-patil, LG Patni, G Ladumor

Institute of Urology, Dhule, MS

India

Introduction \& Objective: Percutaneous nephrolithotomy (PCNL) has a significant learning curve. Ultrasound guided puncture has its definite indications. Ultrasound guided simulators for renal access is still not widely available. We describe and validate our own, portable, active mannequin type of ultrasound guided renal access simulator.

Materials and Methods: Study of the retroperitoneal anatomy of the renal region was conducted using CT sections and standard anatomy literature. The simulator was constructed with two components: a plastic mannequin and an insert. The insert was created using a plastic container. Green \& red colored water filled tubes (representing the major vessels and the bowel); a silicon kidney replica, aluminium ribs and special mixture of wax were used to reconstruct the retroperitoneal anatomy. The simulator allowed ultrasound guided puncture, saline aspiration for confirmation and wire parking into the kidney/ureter. Internal high definition camera placed in the mannequin provided visual feedback at end of the task. Evaluation was done using a three step test, global rating system (GRS) score and trainee feedback

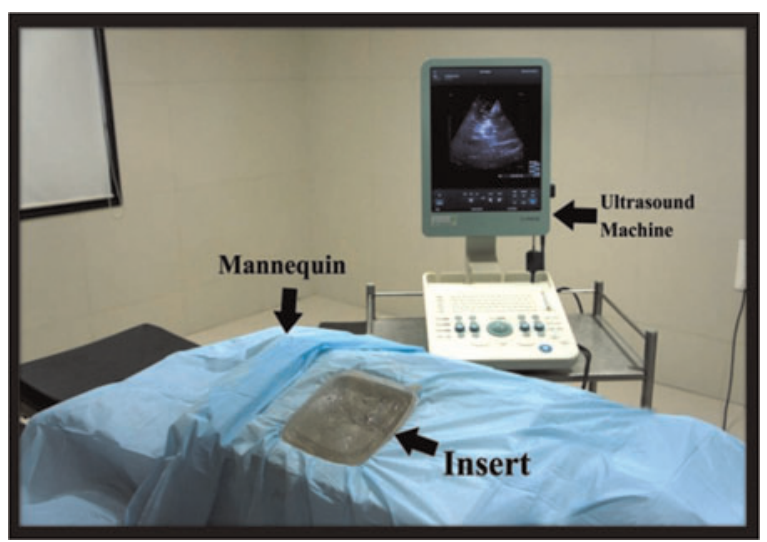




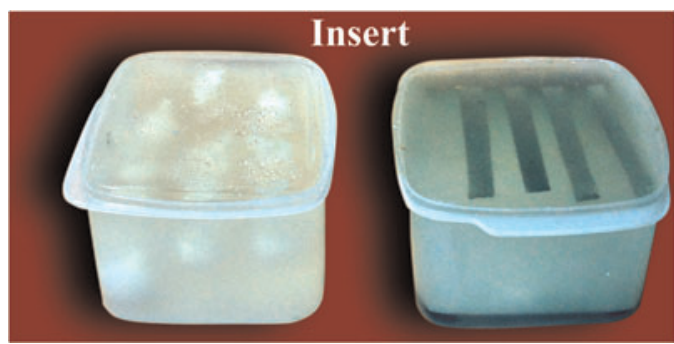

was analysed using Spearman rank order correlations and paired t test.

Results: A total of 16 urology trainees and 2 experts evaluated the simulator. Face and content validity was satisfactory. All subjects demonstrated statistically significant betterment (Spearman rank order correlations) in their GRS scores, total time, fluoroscopic time and attempted needle punctures. Subjective simulator assessment indicated a high degree of satisfaction on effectiveness. Conclusions: Our portable ultrasound guided PCNL simulator is portable, uses the usual initial puncture needle with any access technique. The end of task confirmatory saline aspiration and inspectory confirmation of the puncture facilitates faster learning. It allows evaluation and supervised, repetitive tailored learning in a controlled, low stress environment. It has low initial and maintenance cost \& is recyclable. Multicentre and larger cohort construct validity assessments are further needed for integration into training programs.

MP5-20 Comparing the K-Box and Advanced Scope Trainer for Flexible Ureterorenoscopy Training: A Randomised Controlled Trial

F Nawab, A Aydin, B Smith, T Abe, S Khan,

P Dasgupta, K Ahmed

Kings College London

United Kingdom

Introduction \& Objective: Flexible ureterorenoscopy is an highly utilised procedure in urolithiasis treatment. It is however, technically difficult with training often limited by high cost and exposure. The study aimst to investigate the difference in acquiring technical skills in two bench models; Kidney box (Kbox) and Advanced Scope Trainer (AST), for use in flexible ureterorenoscopy training.

Materials and Methods: This randomised controlled trial recruited 30 novices (medical students), with an average age of 21 (range 18-27), with no prior experience in performing ureteroscopy. Participants were randomised into 2 cohorts using a blocked randomisation process. Each cohort received a didactic 30-minute lecture and tutorial followed by a baseline assessment on the Endoscopic Urinary Tract Model (SimPortal, Minnesota, USA) followed by four subsequent training sessions on either the K-Box or AST, in which 2 standardised tasks were undertaken, concluding with a final assessment on the Endoscopic Urinary Tract model.

Results: There was an overall reduction of $55.8 \%$ in average total time taken from session $1-4$ for the AST participants, i.e. From 919.3 seconds to 405.9 seconds $(\mathrm{p}<0.0001)$ compared to $52 \%$ in the K-Box arm, with a reduction from 1000 seconds to 480.6 seconds in session $(\mathrm{p}<0.0001)$. For the K-box total (task 1 and task 2 combined) average task specific checklist scores increased from the baseline (BA) to final assessments (FA) from
20.80 to 31.87 , i.e. a $53.7 \%$ increase $(\mathrm{p}<0.0001)$. For the AST, the total average checklist score also increased from 20.60 in the BA to 31.67 in the FA, thus a $53.7 \%$ increase $(p<0.0001)$. When analysing the time taken in the context of the baseline and final assessment tasks, the results show that both the K-Box and the AST lead to a reduction in the time taken to complete the task from BA to FA. For the K-box participants the average time change from BA to FA was 330.6 seconds, i.e. $52 \%(\mathrm{p}<0.0001)$, and for the Advanced Scope Trainer, there was an average reduction of 338.4 seconds, which also is a $52 \%$ reduction in time $(\mathrm{p}=0.0002)$.

Conclusions: The bench models evaluated, K-Box and Advanced Scope Trainer, were both effective in terms of acquisition of technical skills relating to flexible ureterorenoscopy, with no significant difference found between each model.

\section{MP5-21 Withdrawn}

MP5-22 Construction And Assessment Of An Innovative Virtual (Radiation Free) Fluoroscopy Optical Light Based Percutaneous Nephrolithotomy Simulator: An Indigenous Approach To Training

AV Rawandale-patil, LG Patni, G Ladumor

Institute of Urology, Dhule, MS

India

Introduction \& Objective: Percutaneous renal access has a steep learning curve. Virtual reality simulators have prohibitive pitfalls. We describe our innovative virtual fluoroscopy percutaneous nephrolithotomy (PCNL) simulator.

Materials and Methods: The virtual fluoroscopy simulator was conceived, designed, engineered, and patented by Tejnaksh innovations Inc. 2012 (Figure 1). It has been constructed using a CAD designed stand alone fiber box torso. The box replicates the patient's body shape and has window on the top which is fitted with a translucent interface (paper / thin white cloth) using magnetic strips. An isocentric C-arm was constructed. The lower tip of the $\mathrm{C}$-arm is fitted with a 12 volt light source emitting visible light and the upper tip with a high definition camera. A metallic kidney pelvi-calyceal system replica is suspended below the translucent interface screen at the point of isocentricity. The lamp is turned on and off by a footswitch which casts a shadow on the translucent screen captured by the camera. Evaluation

\begin{tabular}{|c|c|c|c|c|c|c|c|c|}
\hline \multicolumn{7}{|c|}{ Objective Measurements: Spearman correlation } \\
\hline & \multicolumn{2}{|c|}{ Pretest } & p value & \multicolumn{2}{|c|}{ Post test } & p value & Controls (experts) \\
\hline & Group1 & Group 2 & & Group1 & Group 2 & & \\
\hline Time per attempt (secs) & 310.41 & 317.8 & $<0.005$ & 211.35 & 157.17 & $<0.005$ & 90 \\
\hline Fluoroscopy time per attemptssocs) & 107 & 105 & $<0.005$ & 85 & 57 & $<0.005$ & 20 \\
\hline No. of failed attempts/ success & 5.32 & 5.31 & $<0.005$ & 4.32 & 2.27 & $<0.005$ & 1.5 \\
\hline Total time (secs) 3 attempts & 931.23 & 953.40 & $<0.005$ & 634.05 & 471.51 & $<0.005$ & 270 \\
\hline \multicolumn{7}{|c|}{ GRS (Range 1.5) } & & \\
\hline Identify anatomy & 3.14 & 3.18 & $<0.005$ & 3.54 & 4.16 & $<0.005$ & 4.96 \\
\hline Procedure planning & 3.28 & 3.23 & $<0.005$ & 3.58 & 4.87 & $<0.005$ & 4.87 \\
\hline Instrument use & 3.27 & 3.75 & $<0.005$ & 3.87 & 4.64 & $<0.005$ & 4.93 \\
\hline Ability to perform task & 2.40 & 2.79 & $<0.005$ & 2.95 & 4.13 & $<0.005$ & 4.90 \\
\hline Overall performance & 2.50 & 3.06 & $<0.005$ & 3.10 & 4.74 & $<0.005$ & 4.97 \\
\hline
\end{tabular}




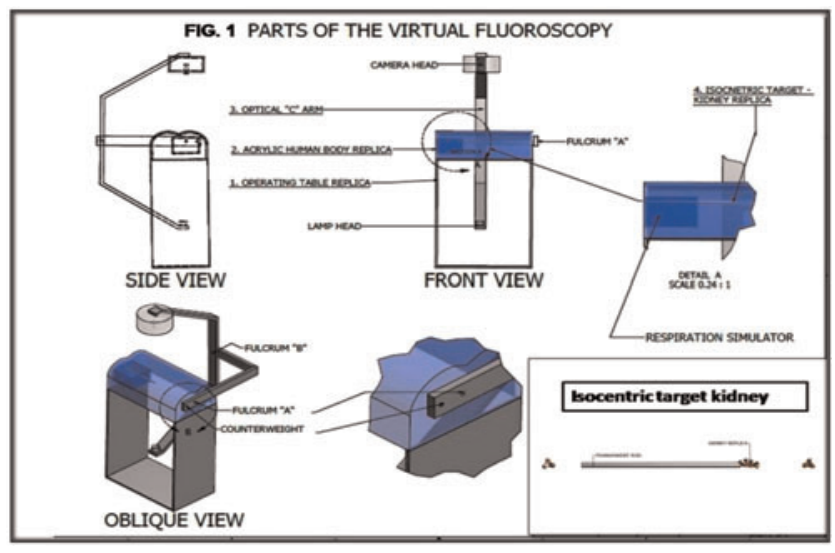

using a 3 steps test, global rating system scoring (GRS) performance score and trainee feedback form, was done.

Results: A total of 10 urology trainees and 2 experts evaluated the simulator. Face and content validity demonstrated a satisfactory resemblance of the virtual fluoroscopy image to the image of the actual fluoroscopy machine. Trainees demonstrated statistically significant improvement in GRS scores, total time, fluoroscopic time and attempted needle punctures after training. Conclusions: Our innovative portable virtual fluoroscopy PCNL simulator uses visible light to reproduce fluoroscopy images. The usual puncture needle with any access technique can be used. It replicates repetitive respiratory movements. The alarm allows trainee evaluation and supervised, repetitive tailored learning in a controlled, low stress environment, It has low initial and maintainance cost and serves as a satisfactory initial puncture practice station. Such virtual fluoroscopy simulators would open up newer avenues in PCNL simulation.

MP5-23 Hands on wet lab and live surgery training in PCNL: any impact to surgical skills of attending surgeons?

I Kyriazis, P Kallidonis, P Ntasiotis, D Kotsiris,

V Panagopoulos, T Vrettos, E Liatsikos

Department of Urology, University of Patras

Greece

Introduction \& Objective: The clinical value and efficiency of hands-on training courses in percutaneous nephrolithotomy (PCNL) remains undocumented. During the last 5 years, a two day international intensive hands-on training course in fluoroscopic guided prone PCNL is taking place in our department on a monthly basis. Course includes wet lab training in the porcine model and live surgery training. In this work we report the outcomes of a survey send to course participants questioning the impact of the course to their clinical practice.

Materials and Methods: A survey consisted of 26 questions was distributed online to a total of 91 trainees that had completed the course. Comparison of pre and post course surgical practices was performed using the "N-1" Chi-squared test.

Results: A total of 64 trainees responded to our online survey with $55.6 \%$ and $41.3 \%$ reporting a modest or major impact to their practice accordingly. Notable changes in puncture and dilation methods were noted while a uniform reduction in puncture and operative times was documented. $79.4 \%$ responded that the course increased the safety of their procedure, $73 \%$ that it reduced operating times, $39.7 \%$ that increased their stone free rates, $23.8 \%$ that reduced their complications and $23.8 \%$ that changed their instrumentation they were using in the past. Subgroup analysis including only well experienced surgeons revealed a similar impact to their practice.

Conclusions: In the proper setting, an intensive hands on PCNL course can have a significant impact to attending physicians and participation to such events even for experienced surgeons should be encouraged.

MP5-24 Preoperative simulation of PCNL using three-dimensional printed model

\section{R Takazawa, S Kitayama, S Yoshida, T Tsujii}

Tokyo Metropolitan Ohtsuka Hospital Japan

Introduction \& Objective: Although percutaneous nephrolithotomy (PCNL) has been recommended as the first-line treatment for renal stones larger than $2 \mathrm{~cm}$, its major complication rate is not negligible. One of the most important procedure in

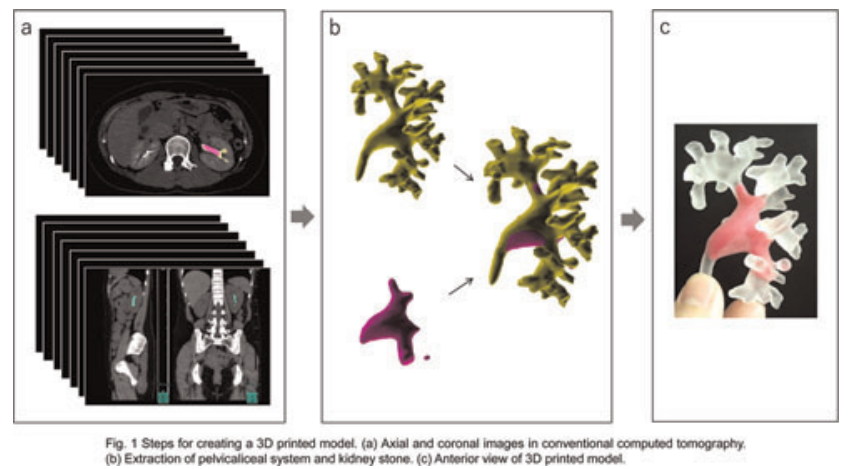

Table 1. Clinical characteristics of patients and kidney stones

\begin{tabular}{|c|c|}
\hline Variables & Values \\
\hline \multicolumn{2}{|l|}{ Patient factors } \\
\hline Age (years) & $65.5(24-78)$ \\
\hline \multicolumn{2}{|l|}{ Sex } \\
\hline Male & $8(57 \%)$ \\
\hline Female & $6(43 \%)$ \\
\hline BMI $\left(\mathrm{kg} / \mathrm{m}^{2}\right)$ & $23.8(17.5-29.1)$ \\
\hline $\mathrm{Hb}(\mathrm{g} / \mathrm{dL})$ & $14.2(10.2-15.9)$ \\
\hline Het $(\%)$ & $42.8(30.3-48)$ \\
\hline eGFR $\left(\mathrm{mL} / \mathrm{min} / 1.73 \mathrm{~m}^{2}\right)$ & $65.8(39.9-97.2)$ \\
\hline History of pyelonephritis & $3(21 \%)$ \\
\hline Positive preoperative bladder urine culture & $4(29 \%)$ \\
\hline \multicolumn{2}{|l|}{ Stone factors } \\
\hline \multicolumn{2}{|l|}{ Side } \\
\hline Right & $8(57 \%)$ \\
\hline Left & $6(43 \%)$ \\
\hline Staghorn calculi & $6(43 \%)$ \\
\hline \multicolumn{2}{|l|}{ CT density } \\
\hline Mean $(H U)$ & $1060(559-1391)$ \\
\hline Highest (HU) & $1523(1261-1758)$ \\
\hline \multicolumn{2}{|l|}{ Size } \\
\hline Diameter $(\mathrm{mm})$ & $34.5(23-84)$ \\
\hline Circumference $(\mathrm{mm})$ & $97.5(65-311)$ \\
\hline Area $\left(\mathrm{mm}^{2}\right)$ & $468.5(270-2173)$ \\
\hline Volume $\left(\mathrm{mm}^{3}\right)$ & $9.2 \times 10^{3}\left(4.7-48.0 \times 10^{3}\right)$ \\
\hline
\end{tabular}

Categorical variables are shown as number of patients (\%). Continuous variables are shown as median (full range). BMI=body mass index, $\mathrm{Hb}=$ hemoglobin, Hct=hematocrit, eGFR=estimated glomerular filtration rate. 
Table 2. Surgical outcomes

\begin{tabular}{|c|c|}
\hline Variables & Value \\
\hline Total operation time (min) & $133(88-184)$ \\
\hline \multicolumn{2}{|l|}{ Times of puncture } \\
\hline 1 & $11(79 \%)$ \\
\hline$\geq 2$ & $3(21 \%)$ \\
\hline $\mathrm{Hb}$ change $(\mathrm{g} / \mathrm{dL})$ & $-0.65(-0.5-2.7)$ \\
\hline Het change (\%) & $-1.25(-8.2-4)$ \\
\hline eGFR change $\left(\mathrm{mL} / \mathrm{min} / 1.73 \mathrm{~m}^{2}\right)$ & $3.3(-12.1-13.2)$ \\
\hline Stone clearance by a single PNL session & $9(64 \%)$ \\
\hline Additional treatment & $5(36 \%)$ \\
\hline \multicolumn{2}{|l|}{ PNL } \\
\hline Same tract & 4 \\
\hline Another tract & 0 \\
\hline URS & 1 \\
\hline Overall stone clearance & $12(86 \%)$ \\
\hline Overall complications encountered & $3(21 \%)$ \\
\hline \multicolumn{2}{|l|}{ Clavien grade } \\
\hline 0 & $11(79 \%)$ \\
\hline I (Renal pelvic perforation) & $1(7 \%)$ \\
\hline II (Postoperative fever $>38 \mathrm{C}$ managed with antibiotics) & $1(7 \%)$ \\
\hline IIla (Myocardial infarction required catheter intervention) & $1(7 \%)$ \\
\hline
\end{tabular}

PCNL is to create a tract in the appropriate renal calyx. To achieve safe PCNL, we prepared a three-dimensional (3D) printed model reproducing renal pelvicaliceal system and stones in full scale and performed a preoperative simulation.

Materials and Methods: Based on the contrast CT image, a 3D model was created using an inkjet 3D printer. The shape of the renal pelvicaliceal system was reproduced with a semi-transparent material (TangoPlus FLX $930^{\circledR}$ ) and the stone was reproduced with a red hard material (Vero Magenda ${ }^{\circledR}$ ). (Figure 1) Preoperatively, we examined the selection of the calyx to be punctured. Especially in the case of staghorn stone, we also examined the position which rigid nephroscope can reach.

Results: We prepared a total of 14 cases and verified its usefulness. In 9 cases, stone-free was achieved with the initial PCNL, the remaining 5 cases required additional treatment (4 cases of PCNL, 1 case of ureteroscopy). Perioperative complications included one case of a fever of 38 degrees or more requiring prolongation of antibiotic administration, and one case of myocardial infarction that developed 48 hours later. There was no bleeding that required blood transfusion. (Table $1 \&$ 2)

Conclusions: The preoperative simulation using a 3D printed model can greatly contribute to improvement of treatment outcome and reduction of perioperative complications by enabling stable operation.

\section{MPS6: PCNL II TECHNIQUES AND COMPLICATIONS}

\author{
MP6-1 Comparison of the Effect of Tolterodine and Ga- \\ bapentin With Placebo in Reducing Catheter Related \\ Bladder Discomfort After Percutaneous Nephrolithotomy: \\ A Randomised Clinical Trial. \\ R Maghsoudi, M Etemadian, S Farhadi Niaki, A Kashi \\ Iran University of Medical Science \\ Iran
}

Introduction \& Objective: To compare the influence of tolterodine, gabapentin with placebo in reducing catheter related bladder discomfort (CRBD) after percutaneous nephrolithotomy (PCNL) opetations. To our best knowledge this is the first trial to compare tolterodine with gabapentin in reducing CRBD.

Materials and Methods: The design of the study was a double blind (evaluator and patient) parallel group randomized clinical trial. After obtaining informed consent from PCNL candidates, patients were divided into 3 treatment groups by balanced blocked randomization. Visual analogue pain scales (VAS) were used to document bladder discomfort after the operations. Usage of pain medications was documented after the operation in treatment groups. The primary endpoint of interest was the VAS scores after the operation. Secondary endpoints included the amount of narcotic and non-narcotic pain medications.

Results: The mean \pm SD of paracetamol injections in the tolterodine, gabapentin and placebo groups were $1.82 \pm 0.85,1.78 \pm 0.71$, and $3.56 \pm 0.75(\mathrm{p}<0.001)$. The mean \pm SD of narcotic udage in the three groups of tolterodine, gabapentin and placebo were $0.42 \pm 0.54$, $0.68 \pm 062$, and $2.36 \pm 0.64$ respectively $(\mathrm{p}<0.001)$. Difference in VAS scores in gabapentin and tolterodine groups versus placebo was statistically significant $(\mathrm{p}<0.001)$. In patients with a history of ureteral DJ catheter insertion, there was a statistically significant trend for VAS scores difference between placebo and intervention groups. The usage amounts of paracetamol and VAS scores between tolterodine and gabapentin groups were not statistically significant. Conclusions: The results of this study indicate the beneficial effects of tolterodine and gabapentin in alleviating CRBD in patients after PCNL operations. The use of these medications will also reduce the necessity for injecting narcotic and nonnarcotic pain medication. We recommend the routine usage of these medication after operations in which catheters are kept after the operation.

MP6-2 Bilateral percutaneous nephrolithotomy on the same procedure: A retrospective analysis of its safety and efficiency

J Pelletier, I fugaru, F Soucy, B Laroche, J Cloutier

université Laval

Canada

Introduction \& Objective: Percutaneous nephrolithotomy (PCNL) has been practiced for many years and has become the gold standard for more complex nephrolithiasis. When it comes to patients with bilateral large kidney stone burden, there is no consensus yet as to proceed to a synchronous PCNL (sBPCNL) or do a staged surgery. To assess the safety and effectiveness of sBPCNL in patients with bilateral renal calculi, we performed a review of the operative and postoperative outcomes of sBPCNL. Our second endpoint was to evaluate the validity of the nephrolithometry nomogram (CROES) with sBPCNL.

Materials and Methods: Using hospital coding data, we identified 802 patients who underwent a PCNL between January 2006 and June 2016. 25 of them underwent a sBPCNL. We compared the outcomes of this group with a selected cohort of 9 patients 
who were initially scheduled for sBPCNL but underwent an unilateral PCNL and patients who had a staged PCNL during within one week. The Charlson Comorbidity Index (CCI) was used to compare comorbidities. The stone characteristics were analysed using the CROES nephrolithometry scoring system to predict the stone free rate (SFR) for each renal unit. Postoperative outcomes were evaluated with the modified Clavien Dindo score. The SFR was analysed with an X-ray or a computed tomography. Treatment success was defined as residual fragments less than $4 \mathrm{~mm}$.

Results: The median CCI was 3 (0-9) for the sBPCNL and 5 (18) for the control group. The control group had slightly higher stone burden with a mean of $412,2 \mathrm{~mm}^{2}$ and the sBPCNL group with a mean of $397.6 \mathrm{~mm}^{2}$. Mean operating time was $178 \mathrm{~min}$ (65-380) for the sBPCNL and $115 \mathrm{~min}(60-173)$ for the control group. Fifteen $(60 \%)$ patients in the sBPCNL group had no post-operative complications (Clavien 0) compared to $22 \%$ in the control group. There was no Clavien score higher than 2 for the sBPCNL. In the control group, 2 patients scored 1 (22\%), 4 had a scored $2(44 \%)$ and one patient required intensive care (Clavien 4a). The SFR for the first side operated was achieved in 19 patients $(73.1 \%)$ who underwent a sBPCNL and $5(55.6 \%)$ patients for the staged procedure. The SFR for the second side operated was achieved in 16 patients $(61.5 \%)$ for the sBPCNL compared to $3(33.3 \%)$ for the staged procedure. Overall, the first side operated had a better SFR than the second side in only $28,6 \%$. CROES scores in both group overestimated the SFR by over $10 \%$.

Conclusions: From our data, sBPCNL manipulation is feasible and safe. It can be offered to selected patients with medium-sized bilateral renal stones in high-volume centers by experienced surgeons. The CROES nephrolithometry scoring system seems to overestimate our SFR.

MP6-3 Percutaneous Nephrolithotomy for Renal Calculi up to $15 \mathrm{~mm}$ : What should the ideal tract size be?

RA Kukreja

Urocare

India

Introduction \& Objective: Reducing the Percutaneous Nephrolithotomy (PCNL) access tract size has been found to decrease the morbidity associated with the procedure. The modification in technique required is to fragment the stone into smaller particles and remove them using the vacuum cleaner effect. This prospective study compares the efficacy and morbidity of reducing the tract size from the standard $24 \mathrm{Fr}$ to $16.5 \mathrm{Fr}$ and $12 \mathrm{Fr}$ for stones sized from 10$15 \mathrm{~mm}$.

Materials and Methods: 125 patients were enrolled in this prospective study and distributed into 3 groups based on the tract size used to clear the stone (Group A: 11/12Fr, N=40; Group B: 16.5/ $18 \mathrm{Fr}, \mathrm{N}=45$ and Group C: 22/24Fr, $\mathrm{N}=40$ ). The equipment used comprised of: 11/12Fr MiniPNL XS sheath with 7.5 Fr nephroscope (Karl Storz) for group A, the 16.5/18 Fr MiniPNL sheath with 12Fr nephroscope (Karl Storz) for group B and 22/24 Fr Amplatz sheath with 20.5 Fr Nephroscope (Richard Wolf, Germany). Critical factors assessed were procedure time, fluoroscopy time, blood loss, pain score, stone clearance status and complications

Results: All the 3 groups were comparable with respect to age, BMI, creatinine and stone size. The blood loss (assessed by
Hemoglobin and PCV drop) was comparable for group A and B $(Z=1.308, p>0.05)$, but significantly less for both groups $A$ and $B$ as compared to group $C(Z=3.298, p<0.01$ for group $B$ vs $C ; Z=4.53, p<0.01$ for groups $A$ vs $C)$. Groups $B$ and $C$ were comparable with regards to the pain score $(Z=1.012$ at 6 hours and $\mathrm{Z}=0.179$ at 24 hours, $\mathrm{p}>0.05)$. However the pain was significantly less in group A (A vs B: $Z=2.102$ at 6 hours and $Z=2.045$ at 24 hours, $p<0.05$; $A$ vs $C: Z=2.83$ at 6 hours and $\mathrm{Z}=1.88$ at 24 hours, $\mathrm{p}<0.05$ ). All the patients in group A underwent a tubeless procedure whereas nephrostomy was placed in only 2 patients in group B and 7 patients in group C. There was no significant difference in the procedure time or fluoroscopy time amongst all the 3 groups. Only 2 patients (one in group A and one in group C) had a residual fragment greater than $3 \mathrm{~mm}$.

Conclusions: The 12Fr tract offers the least morbidity in terms of blood loss, pain score and nephrostomy tube requirement and maintains comparable stone clearance at par with the larger tracts. The 12Fr tract should be the standard size used for PCNL for stones up to $15 \mathrm{~mm}$ size.

\section{MP6-4 Supine Percutaneous Lithotomy for Partial and Complete Staghorn Renal Stones}

\section{Mokool, Z Kratiras, M Alsawi, S Yallappa, SK Nalagatla}

Monklands District General Hospital, Scotland

United Kingdom

Introduction \& Objective: Percutaneous lithotomy is the treatment of choice for staghorn stones. The majority of these procedures are done in the prone position. Supine percutaneous nephrolithotomy (SPCNL) is gradually gaining popularity due to anaesthetic and surgical advantages over prone PCNL (PPCNL).

Our study aims to evaluate the efficacy and safety of SPCNL in the management of partial and complete staghorn renal calculi.

Materials and Methods: Patients undergoing SPCNL between 2013 and 2017. Our inclusion criteria were all patients undergoing SPCNL for partial or complete staghorn stones with complete data available. Key variables collected were: patient demographics, stone size, radiodensity (HU), puncture site, complete or incomplete clearance, additional procedures required, complications and post-operative hospital stay.

Results: A total of 14 patients underwent SPCNL for partial (3) or complete (11) staghorn calculi during this period. Male to female ratio was $2: 1$. The average stone size was $40 \mathrm{~mm}$ and average radiodensity $1046 \mathrm{HU}$. Access was gained into mid pole calyx (3), lower pole calyx (10) and 1 patient had a double puncture (middle and lower calyx). In $28.5 \%$ of the cases (4/14) complete stone clearance was achieved. One patient failed to attend for follow up. $71.5 \%$ of the patients needed additional procedure to clear residual stones which included further PCNL, flexible ureteroscopy and laser and shockwave lithotripsy. 2 of the patients (14\%) developed post operative sepsis and 1 patient $(7.1 \%)$ required a transfusion. There were no other complications. The average post procedure hospital stay was 4 days (range 2-10).

Conclusions: SPCNL monotherapy is safe in clearing both partial and complete staghorn calculi. However, SPCNL monotherapy is not very effective in completetly clearing staghorn stones. A combination of percutaneous lithotomy and other adjunct procedures is required to achieve complete stone clearance. 
MP6-5 Tubeless PCNL as daycare procedure: is it safe?

A Rehman, W Rahim, I Khan, D Shohab, I Jamil, G Nawaz, S Muhammad, S Akhter

Pakistan Kidney Institute, Islamabad

Pakistan

Introduction \& Objective: Percutneous nephrolithotomy (PCNL) has now become the standard minimal invasive option for renal stones. And various forms of PCNL have been introduced to decrease the morbidity and hospital stay of the patients.

The objective of our study was to assess the outcomes and safety of tubeless PCNL as a day care procedure.

Materials and Methods: It is a retrospective analysis of 69 patients who underwent PCNL as daycare procedure in Dept of Urology, Pakistan Kidney Institute from August 2015 - July 2016.

Data regarding patient age, gender distribution, stone size, stone location, stone clearance, mean operative time, mean hospital stay and post operative complications were compiled by chart review.

Results: Total of 69 patients with mean age of 38.18 years \pm 13.86 years were included of which $15(21.4 \%)$ were females and $54(77.1 \%)$ were males. The mean post operative stay in hospital was $21.55 \pm 3.90$ hours and complete stone clearance was achieved in $71 \%$ of cases.

Mean stone size was $3.07 \pm 0.94 \mathrm{~cm}$. Whereas mean operating time was $136.59 \pm 30.69 \mathrm{~min}$ and a range from $75-190 \mathrm{~min}$.

Ancillary procedures like ESWL done in 20 patients while two patients need URS for residual ureteric stone.

Post PCNL febrile UTI was the major complication in 5 patient which resolved after injectable antibiotics and Double J stent removal.

Conclusions: Tubeless PCNL is a well known rising technique which can be safely done in patients as a day care procedure with acceptable stone clearance, minimal post operative complications and a less hospital stay.

MP6-6 Analysis of factors affecting Radiation Exposure during Percutaneous Nephrolithotomy procedures

S Balaji, A Singh, A Ganpule, R Sabnis, M Desai

Muljibhai Patel Urological Hospital

India

Introduction \& Objective: Percutaneous Nephrolithotomy (PCNL) is single most common procedure performed for large stone burden and is associated with potential Radiation Exposure (RE). The objective of this study is to measure and analyze various factors affecting RE to surgeons during PCNL.

Materials and Methods: Total of 212 patients who underwent PCNL during study period were analyzed. Informed consent was obtained from enrolled patients. Surgico 60DHFTM, digitalised mobile surgical Carm image intensifier system was used in all cases. RE was determined using pen dosimeter worn outside lead apron of surgeon

Results: We utilized Multivariate regression analysis to identify various parameters which affect $\mathrm{RE}(\mathrm{mSv})$. The parameters analyzed were age, number and size of stones, BMI of patients, Hounsfield unit of stone(HU), degree of hydronephrosis, number of tracts, mode of access to PCS, sheath size( $\mathrm{Fr})$, operative time(minutes), placement of DJ/ureteric catheter/PCN and $\mathrm{kV}$ used for exposure. Significant statistical relationship was seen between stone volume $(\beta=0.356, \mathrm{p}$
Conclusions: Mean RE per procedure was $0.21 \pm 0.11 \mathrm{mSv}$. Increasing size and low HU of stone, increasing number of tracts, fluoroscopic access to PCS, increasing sheath size and $\mathrm{kV}$ are found to increase radiation exposure. Although the exposure levels are within safety limits, serial monitoring and constant vigilance are mandatory to sensitize surgeons

\section{MP6-7 Withdrawn}

MP6-8 Percutaneous Nephrolithotomy Puncture and Tract Dilation: Evidence on the Safety of Approaches to the Infundibulum of the Calyx.

P Kallidonis, I Kyriazis, P Ntasiotis, D Kotsiris, C Kalogeropoulou, D Apostolopoulos, P Kitrou, E Liatsikos

Department of Urology, University of Patras

Greece

Introduction \& Objective: To investigate the anatomical relations of the papillary, infundibular and pelvic approach to percutaneous nephrolithotomy (PCNL) and evaluate the amount of vascularization at the respective sites.

Materials and Methods: ${ }^{99 \mathrm{~m}} \mathrm{Tc}$-dimercaptosuccinic acid SPECT/CT renal scintigraphies or Computerized tomographies perfusion (CTP) were performed in 40 patients (prone $n=20$ or supine position $n=20)$. The angle of approach (AoA) for access tracts to the mid-calyceal papilla, infundibulum and pelvis as well as the respective regions of interest (ROIs) were designed. The AoA and the blood supply related to the ROIs were compared

Results: The design of access tracts aiming to the renal pelvis, papilla and infundibulum of the renal calyx was impossible for the non-dilated collecting systems since all these tracts were in close vicinity. In both SPECT/CT and CTP, there was no statistical difference between the AoA for infundibular or pelvic access in comparison to the papillary puncture in either prone or supine position regardless of the degree of dilation of the system. The comparison of the measurements in the ROIs showed that there was no difference in blood supply between the infundibular or pelvic access in comparison to the papillary approach in both positions regardless to the degree of collecting system dilation. Conclusions: The SPECT/CT and CTP showed that punctures to the renal papilla, infundibulum and pelvis of the middle calyces of the kidney have similar angles of approach. The sites of dilation of the respective approaches are not related with significant differences in terms of vascularization of the renal parenchyma.

MP6-9 Inferior renal displacement using a Ureteric Balloon Catheter- A Novel Renal Displacement Technique and A Systematic Review of the Literature

M Alsawi, L Mokool, S Yallappa, T Amer, SK Nalagatla

Monklands Hospital, Scotland

United Kingdom

Introduction \& Objective: Optimal planning and execution of percutaneous access into the renal collecting system is crucial to the success of PCNL. In various situations, the literature reports that percutaneous access has been achieved through a supracostal approach. This technique provides direct access to most of the intrarenal collecting system; however, it is associated with a significant risk of pleural and intrathoracic iatrogenic injuries. 
There has naturally been reluctance in using the supracostal approach during percutaneous renal surgery for the reasons described above. Newer techniques to replace supracostal approach have been suggested, notably renal displacement methods. There are currently no guidelines or systematic review of renal displacement techniques. We therefore carried out a systematic review to distil the literature in this domain and to assess the viability of this approach. We also present our own institution's novel inferior renal displacement technique using a ureteric balloon catheter (UBC) and we compare it to other techniques. Materials and Methods: We performed a systematic review according to the Cochrane diagnostic accuracy reviews guidelines. All papers were extracted independently. Studies reporting on the treatment of patients undergoing PCNL in supine or prone positions who required any form of displacement of the kidney were included. We compared the following variables 1) Technical aspects of displacement method 2) Outcome measures for extraction 3) Period 4) Journal and location of the study 5) Study population demographics 6) Stone free rates and 7) Complications.

Results: The literature search yielded 6 possible studies with 3 excluded after abstract review. All the included studies were published between 1981 and 2016 with the total number of participants being 35 . The studies were conducted in 3 different countries (USA, UK and Morocco). Our own institutions data was then collected retrospectively and included 10 patients undergoing PCNL using our novel UBC technique. We achieved successful percutaneous access during PCNL in 9/10 cases (Supine PCNL $=2$, Prone PCNL $=7$ ). There were no complications encountered in all 10 cases.

Conclusions: Our UBC approach is a novel renal displacement technique that provides a safe alternative to the renal supracostal approach in percutaneous renal surgery. Compared to other techniques, it is a simpler technique that achieves comparable stone clearance rates as it allows ready access to the desired calyx. It is less traumatic as is stabilizes the kidney during respiration and also the risk of intrathoracic complications avoided.

MP6-10 Post-Operative Pain Management after Percutaneous Nephrolithotomy: A Randomized Double-Blind Controlled Study Assessing Electro-Acupuncture

E Parkhomenko, T Tran, R Chugh, JN Thai, K Blum, J Capodice, S Purnell, M Gupta

\section{ICAHN SCHOOL OF MEDICINE AT MOUNT SINAI} United States

Introduction \& Objective: Percutaneous nephrolithotomy (PCNL) is the gold standard procedure for large renal calculi, but post-operative pain remains a major concern. More recently, acupuncture has been described as an adjunct to reduce pain following abdominal, spinal, and orthopedic surgeries. Among the benefits of acupuncture are its ease of performance, non-invasiveness, and lack of significant side effects. In comparison to traditional acupuncture, electro-acupuncture has shown enhanced efficacy, possibly due to central endorphin release. We sought to investigate the effects of electro-acupuncture on patients undergoing PCNL.

Materials and Methods: A double blind, randomized, sham controlled study design was used. Fifty-one patients undergoing PCNL by a single surgeon were randomized to one of three groups: true electro-acupuncture (EA, $\mathrm{n}=17)$, sham electro-acupuncture (Sham, $\mathrm{n}=17$ ), and no acupuncture (Control, $\mathrm{n}=17$ ). Acupuncture was performed by a licensed acupuncturist $1 \mathrm{hr}$ prior to surgery. All study personnel, except the acupuncturist were blinded to the intervention. PCNL was performed according to

\begin{tabular}{|c|c|c|c|c|c|c|}
\hline Flank VAS Score & $1 \mathrm{hr}$ & $2 \mathrm{hr}$ & $3 \mathrm{hr}$ & $4 \mathrm{hr}$ & $8 \mathrm{hr}$ & $24 \mathrm{hr}$ \\
\hline Acupuncture & 3.35 & $3.06^{*}$ & 2.82 & 4.35 & $3.24^{*}$ & $1.53^{*}$ \\
\hline Sham & 4.84 & 4.11 & 3.74 & 4.05 & 3.53 & $3.11 t$ \\
\hline Control & 4.65 & 5.18 & 4.24 & 5.06 & 5.18 & 4 \\
\hline \multicolumn{7}{|c|}{ Abdominal VAS Score } \\
\hline Acupuncture & 0.47 & 0.29 & 0.47 & 0.82 & 0.29 & $0.18^{*}$ \\
\hline Sham & 0.47 & 0.68 & 0.84 & 1.26 & 0.47 & 0.89 \\
\hline Control & 1.76 & 1.47 & 1.65 & 1.41 & 1.29 & 1.12 \\
\hline \multicolumn{7}{|c|}{ Cumulative Morphine Equivalents } \\
\hline Acupuncture & $0.99 *$ & $1.76^{*}$ & 2.73* & 4.28* & 9.5 & 25.91 \\
\hline Sham & 1.26 & 3.70 & 4.25 & 5.65 & 13.85 & 31.95 \\
\hline Control & 3.62 & 5.64 & 7.07 & 9.98 & 14.42 & 27.5 \\
\hline
\end{tabular}

*True vs. control $p<0.05,+$ True vs. sham $p<0.05$

standard protocol and without intra-operative nerve block or local anesthetic. Pain scores (visual analog scale), narcotic use (morphine equivalents), and side effects were recorded at set intervals post-operatively.

Results: Mean VAS scores for flank and abdomen in the EA group were lower at all time periods compared to sham and control groups. In fact, 2 patients in the EA group did not require any post-operative narcotics. Mean cumulative opioid usage was lower in the EA group immediately post-operatively compared to both sham and control groups (Table 1). No differences between groups were found for nausea and vomiting. No adverse effects of EA were noted.

Conclusions: Electro-acupuncture significantly reduces acute post-operative pain and narcotic usage without any adverse effects. This promising adjunct for post-operative pain control warrants further validation.

MP6-11 Randomized clinical trial of standard minipercutaneous nephrolithotomy versus standard percutaneous nephrolithotomy

R Haghighi

Iran

Introduction \& Objective: One of the most important difference of various percutaneous nephrolithoto mytechniques is the size of renal access that leads to the broad spectrum of complications and out-comes. The ultra mini- PCNL is a technique using ureteroscope and 14Fr metal sheath, whichcould exert less negative effects. So this study assessed standard mini percutaneous nephrolithotomy versus standard percutaneous nephrolithotomy.

Materials and Methods: This study is a Clinical randomized trial that was conducted in 2016. Seventy patients suffered from renal or upper ureteral stones between 10-20 millimeters in diameters who candidate for PCNL were categorised into two equal groups. The procedure was min-imally invasive percutaneous nephrolithotomy using 8.9 - 9 Fr ureteroscope through 14-Fr metal sheath. The ureteral stones were fragmented by pneumatic lithotripsy. Furthermore, we assessed visual analog scale to determine the postoperative pain in both groups. Data were analyzed using spss version 13 and statistical analysis.

Results: Patients underwent a standard percutaneous nephrolithotomy in control group, while patients in case group underwent Ultra mini-PCNL procedure for their renal or upper ureteral stones. We found statistically significant differences in postoperative hemoglobin values, hemoglobin drop, transfusion 
rate, duration of hospitalization and postoperative pain (VAS score) between two groups ( $\mathrm{P}$ value $<0.05$ ). There were no significant difference regarding operation time, need to auxiliary procedure and stone-free rate.

Conclusions: A minimally invasive percutaneous nephrolithotomy method using 8.9-9 Fr ureteroscope can play an important role in the treatment of renal and upper ureteral stones less than 20 millimeters in diameters with less blood loss, hospitalization stay, need of transfusion and postoperative pain.

MP6-12 Super-mini percutaneous nephrolithotomy (SMP) for renal stone $<25 \mathrm{~mm}$ in pediatric patients: Could it be an alternative to shockwave lithotripsy (SWL) ?

K Sarica, B Eryildirim, A Tuerxun, A Batuer, K Erdem, A Buz, G Zeng

Health Sciences University, Kartal Lutfi Kirdar Training and Research Hospital

Turkey

Introduction \& Objective: To evaluate the efficacy of two different techniques (shock wave lithotripsy (SWL) vs super-mini percutaneous nephrolithotomy (SMP) in terms of success as well as complication rates in pediatric renal stones sizing $<25 \mathrm{~mm}$.

Materials and Methods: A total of 219 children (aging between 1-17 years) undergoing two different treatment modalities (SWL vs SMP) for kidney stones $<25 \mathrm{~mm}$ with were included. Depending on the type of the procedure applied, children were divided into two different groups; (Group 1, $\mathrm{n}=108$ ) children treated with SWL and (Group 2, n=111) children treated with SMP. All treatment related parameters (stone free rates, number of sessions, treatment duration, hospitalization, presence of the residual fragments, complications as well as the need for additional interventions) were noted and evaluated between two groups in a comparative manner.

Results: Evaluation of our data have clearly demonstrated that the percentage of residual fragments after SWL was significantly higher in cases undergoing SWL procedure when compared with SMP. Although SWL required several sessions under general anesthesia in a certain per cent of the cases (54.6\%), SMP was successful in one session in all of the cases. Last but not least, in addition to the similar minor complication rates observed in both group of cases, no major complication observed in any case and no case in both groups again required blood transfusion after these two procedures with no significant drop rates in $\mathrm{Hb}$ levels. Conclusions: Although SWL is still the preferred treatment modality for the majority of kidney stones in children with its safe and non-invasive nature, SMP modality may be applied as a valuable alternative in this specific patient population with its excellent stone free rates obtained in a single session and acceptable complication rates in the minimal invasive management of stones $<25 \mathrm{~mm}$.

MP6-13 Flank suspended supine position versus standard supine and prone position in percutaneous nephrolithotomy

M Mahmoud, A Abdel Aal, M Abdel Azim,

Sa El Hamshary, E El-Barky

Benha Faculty of Medicine, Benha University

Egypt

Introduction \& Objective: Percutaneous nephrolithotmy (PCNL) has become the choice of modality for treatment of large
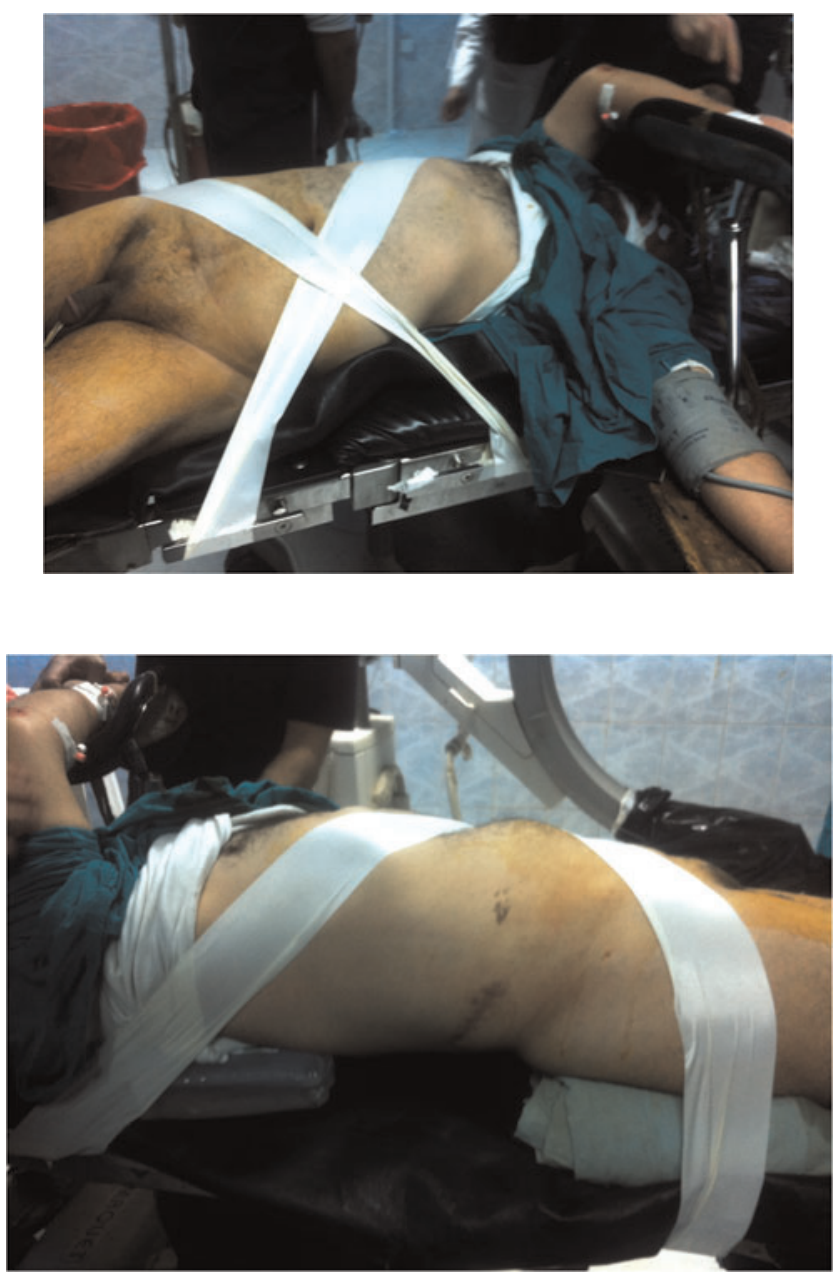

and complicated renal calculi. Prone position remains the standard method for positioning patients for PCNL and has several advantages including wide operation field, and a large room for instrument maneuvering. A new positioning called the flank suspended supine position (FSSP) is proposed to avoid most of the inherit draw backs of supine and prone position.

\section{Results and Graphs}

Table (1): Sex of the patients in the 3 groups

\begin{tabular}{|c|c|c|c|c|c|}
\hline & $\begin{array}{l}\text { Group A } \\
\text { FSSP } \\
\mathrm{N} \quad(\%)\end{array}$ & $\begin{array}{l}\text { Group B } \\
\text { supine } \\
\mathrm{N} \quad(\%)\end{array}$ & $\begin{array}{l}\text { Group C } \\
\text { prone } \\
\mathrm{N} \quad(\%)\end{array}$ & $\mathrm{X}^{2}$ test & $\begin{array}{l}\mathrm{P} \\
\text { value }\end{array}$ \\
\hline Sex & & & & & \\
\hline Male & $11(55 \%)$ & $12(60 \%)$ & $15(75 \%)$ & 1.87 & 0.393 \\
\hline Female & $9 \quad(45 \%)$ & $8 \quad(40 \%)$ & $5 \quad(25 \%)$ & & \\
\hline
\end{tabular}

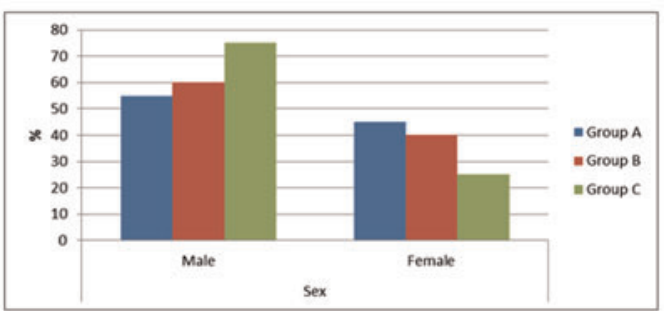

Fig. (1): Sex of the patients in the 3 groups 
Table (2): Showing the age of the patients in the 3 groups

\begin{tabular}{|c|c|c|c|c|c|}
\hline & $\begin{array}{l}\text { Group A } \\
\text { FSSP } \\
\mathrm{N}\end{array}$ & $\begin{array}{l}\text { Group B } \\
\text { supine } \\
\mathrm{N}\end{array}$ & $\begin{array}{l}\text { Group C } \\
\text { prone } \\
\mathrm{N}\end{array}$ & $\mathrm{X}^{2}$ test & $\begin{array}{l}\mathrm{P} \\
\text { value }\end{array}$ \\
\hline $\begin{array}{l}\text { Age } / y \\
\text { Mean } \pm \text { SD }\end{array}$ & $39.05 \pm 14.93$ & $42.35 \pm 14.31$ & $41.15 \pm 15.18$ & $\mathrm{Stt}=0.254$ & 0.667 \\
\hline
\end{tabular}

Table (3): Showing the BMI in the 3 groups

\begin{tabular}{||l|l|l|l|l|l|}
\hline & Group A & Group B & Group C & $\mathrm{X}^{2}$ test & $\mathrm{P}$ \\
& FSSP & supine & prone \\
$\mathrm{N}$ & $\mathrm{N}$ & & value \\
\hline BMI & $28.81 \pm 6.16$ & $25.68 \pm 6.74$ & $27.94 \pm 6.65$ & $\mathrm{Stt}=1.23$ & 0.30 \\
Mean $\pm \mathrm{SD}$ & & & & & \\
\hline
\end{tabular}

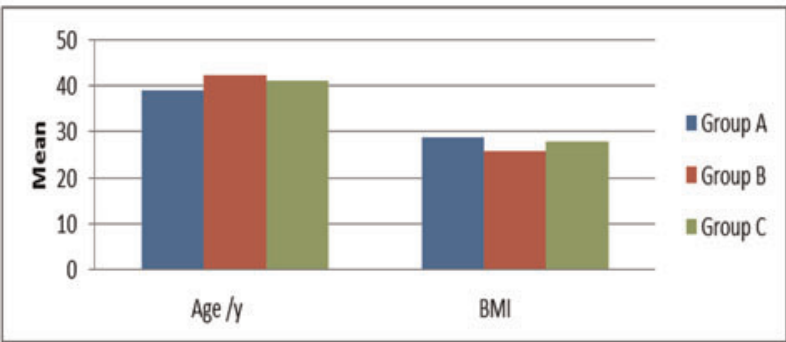

Fig. (2): showing the age and the BMI in the 3 groups

Table (4): comparing the 3 groups according to the side of the stone

\begin{tabular}{|l|l|l|l|l|l|}
\hline & $\begin{array}{l}\text { Group A } \\
\text { FSSP } \\
\mathrm{N}(\%)\end{array}$ & $\begin{array}{l}\text { Group B } \\
\text { Supine } \\
\mathrm{N}(\%)\end{array}$ & $\begin{array}{l}\text { Group C } \\
\text { Prone } \\
\mathrm{N}(\%)\end{array}$ & $\mathrm{X}^{2}$ test & $\begin{array}{l}\mathrm{P} \\
\text { value }\end{array}$ \\
\hline $\begin{array}{l}\text { Side } \\
\mathrm{Rt} \\
\mathrm{Lt}\end{array}$ & $\begin{array}{l}13(65 \%) \\
7(35 \%)\end{array}$ & $\begin{array}{l}14(70 \%) \\
6(30 \%)\end{array}$ & $\begin{array}{l}9(45 \%) \\
11(55 \%)\end{array}$ & 2.92 & 0.233 \\
\hline
\end{tabular}

Table (5): comparing the 3 groups according to the number of the stones

\begin{tabular}{|l|l|l|l|l|l|}
\hline & $\begin{array}{l}\text { Group A } \\
\text { FSSP } \\
\text { N(\%) }\end{array}$ & $\begin{array}{l}\text { Group B } \\
\text { Supine } \\
\text { N(\%) }\end{array}$ & $\begin{array}{l}\text { Group C } \\
\text { Prone } \\
\text { N(\%) }\end{array}$ & $X^{2}$ test & $\begin{array}{l}\text { P } \\
\text { value }\end{array}$ \\
\hline $\begin{array}{l}\text { Number } \\
\text { Single } \\
\text { Multiple }\end{array}$ & $\begin{array}{l}11(55 \%) \\
9(45 \%)\end{array}$ & $\begin{array}{l}13(65 \%) \\
7(35 \%)\end{array}$ & $\begin{array}{l}16(80 \%) \\
4(20 \%)\end{array}$ & 2.85 & 0.241 \\
\hline
\end{tabular}

Table (6): comparing the 3 groups according to the size of the stone

\begin{tabular}{|l|l|l|l|l|l|}
\hline & $\begin{array}{l}\text { Group A } \\
\text { FSSP }\end{array}$ & $\begin{array}{l}\text { Group B } \\
\text { Supine }\end{array}$ & $\begin{array}{l}\text { Group C } \\
\text { Prone }\end{array}$ & St t test & $\begin{array}{l}\text { P } \\
\text { value }\end{array}$ \\
\hline $\begin{array}{l}\text { Size } / \mathrm{cm} \\
\text { Mean } \pm \text { SD }\end{array}$ & $2.82 \pm 0.91$ & $2.71 \pm 0.79$ & $2.57 \pm 0.59$ & 0.526 & 0.594 \\
\hline
\end{tabular}

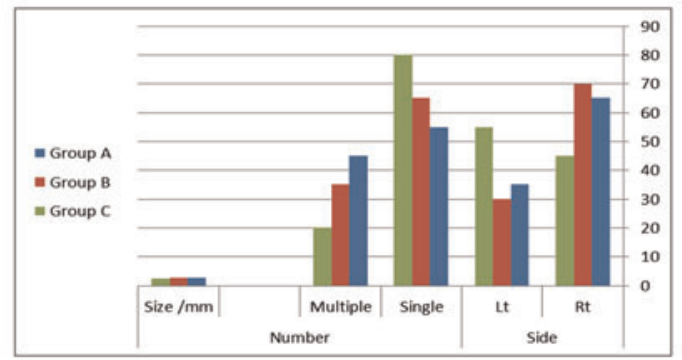

Fig (3): showing the side, the number, and the size in the $\mathbf{3}$ groups
Table (7): comparing the 3 groups according to the site of the stone

\begin{tabular}{|l|l|l|l|l|l|}
\hline & $\begin{array}{l}\text { Group A } \\
\text { FSSP } \\
\text { N(\%) }\end{array}$ & $\begin{array}{l}\text { Group B } \\
\text { Supine } \\
\text { N(\%) }\end{array}$ & $\begin{array}{l}\text { Group C } \\
\text { Prone } \\
\text { N(\%) }\end{array}$ & FET & $\begin{array}{l}\text { P } \\
\text { value }\end{array}$ \\
\hline Site of stone: & & & & & \\
Upper & $3(15 \%)$ & $0(0 \%)$ & $1(5.3 \%)$ & 7.63 & 0.447 \\
Middle & $4(20 \%)$ & $6(30 \%)$ & $7(36.8 \%)$ & & \\
Lower & $8(40 \%)$ & $9(45 \%)$ & $6(31.6 \%)$ & & \\
Pelvis & $5(25 \%)$ & $5(25 \%)$ & $3(15.8 \%)$ & & \\
Multi-site & $0(0 . \%)$ & $0(0 \%)$ & $2(10.5 \%)$ & & \\
\hline
\end{tabular}

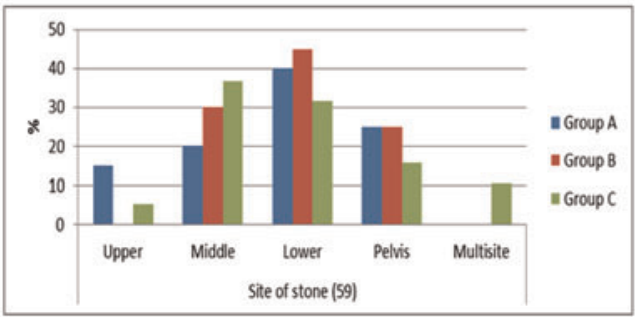

Fig. (4): showing the site of the stones

Table (8): showing the puncture site:

\begin{tabular}{|l|l|l|l|l|l|}
\hline & $\begin{array}{l}\text { Group A } \\
\text { FSSP } \\
\mathrm{N}(\%)\end{array}$ & $\begin{array}{l}\text { Group B } \\
\text { Supine } \\
\mathrm{N}(\%)\end{array}$ & $\begin{array}{l}\text { Group C } \\
\text { Prone } \\
\mathrm{N}(\%)\end{array}$ & FET & $\begin{array}{l}\text { P } \\
\text { value }\end{array}$ \\
\hline $\begin{array}{l}\text { Puncture site: } \\
\text { Middle }\end{array}$ & $\begin{array}{l}4(20 \%) \\
\text { Lower }\end{array}$ & $\begin{array}{l}6(30 \%) \\
16(80 \%)\end{array}$ & $\begin{array}{l}4(20 \%) \\
14(70 \%)\end{array}$ & 0.769 & 0.799 \\
\hline
\end{tabular}

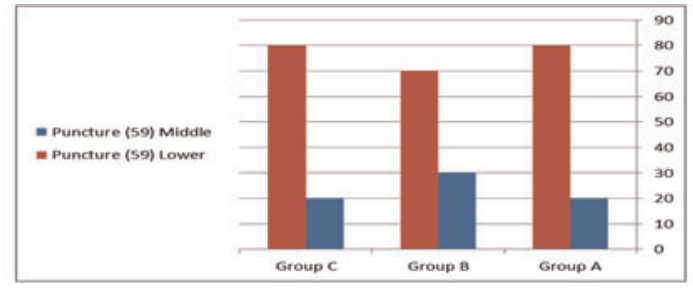

Fig. (5): comparing the puncture site between the 3 groups

Table (9): showing operative time in the 3 groups

\begin{tabular}{|l|l|l|l|l|l|}
\hline & Group A & Group B & Group C & St t test & $\begin{array}{l}\text { P } \\
\text { value }\end{array}$ \\
\hline $\begin{array}{l}\text { Operative time } \\
(\text { min }) \\
\text { Mean } \pm \mathrm{SD}\end{array}$ & $61.65 \pm 15.14$ & $75.95 \pm 18.11$ & $78.4 \pm 9.7$ & 7.54 & $0.01^{* *}$ \\
\hline
\end{tabular}

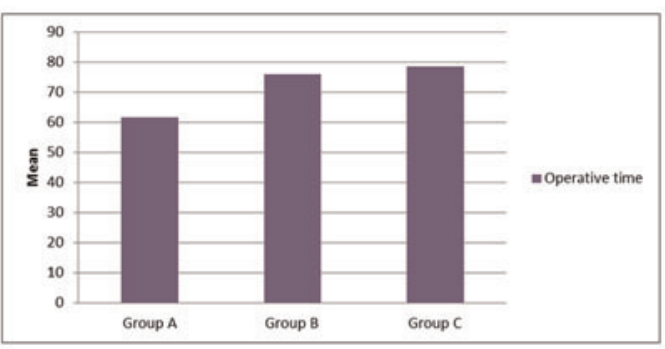

Fig. (6): showing the operative time in the 3 groups. 
Table (10) showing the intraoperative morbidity in the 3 groups

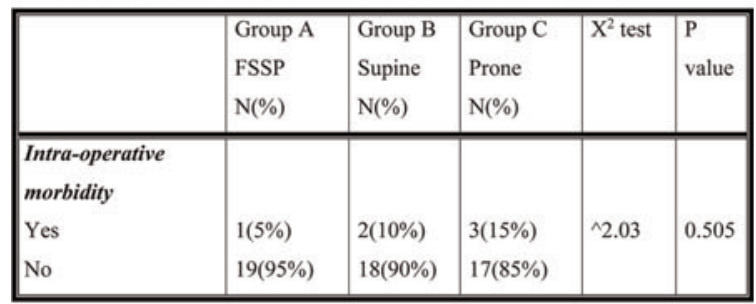

Table (11) showing the intraoperative blood loss which needed blood transfusion in the 3 groups:

\begin{tabular}{|l|l|l|l|l|l|}
\hline & $\begin{array}{l}\text { Group A } \\
\text { FSSP } \\
\mathrm{N}(\%)\end{array}$ & $\begin{array}{l}\text { Group B } \\
\text { Supine } \\
\mathrm{N}(\%)\end{array}$ & $\begin{array}{l}\text { Group C } \\
\text { Prone } \\
\mathrm{N}(\%)\end{array}$ & FET & $\begin{array}{l}\mathrm{P} \\
\text { value }\end{array}$ \\
\hline Blood loss & $1(10 \%)$ & $2(15 \%)$ & $2(15 \%)$ & 0.424 & 1.0 \\
Present & $19(90 \%)$ & $18(85 \%)$ & $18(85 \%)$ & & \\
\hline Absent & & & & \\
\hline
\end{tabular}

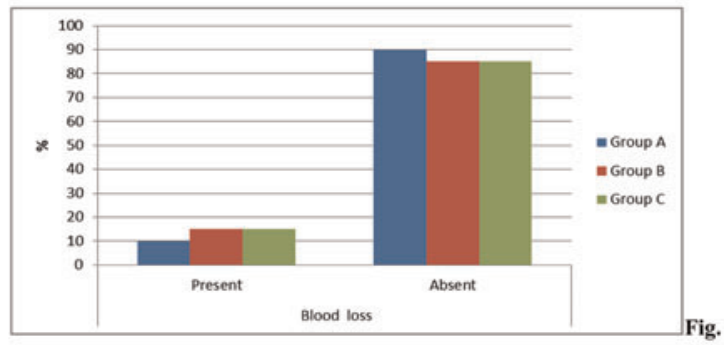

(7): showing the blood loss which needed blood transfusion in the $\mathbf{3}$ groups

Table (12): showing the postoperative morbidity in the 3 groups

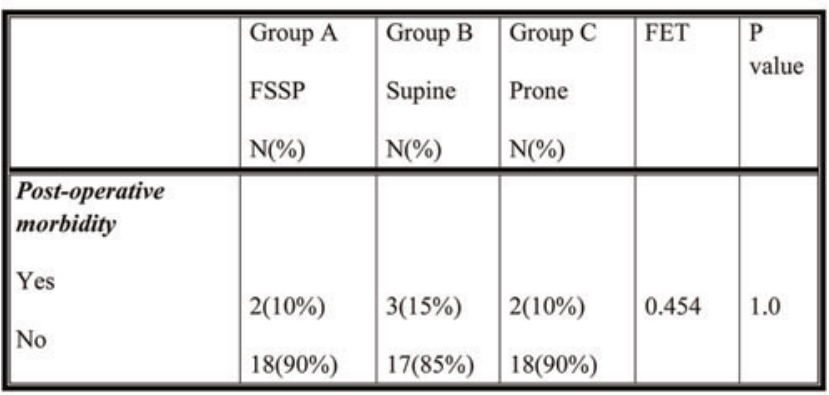

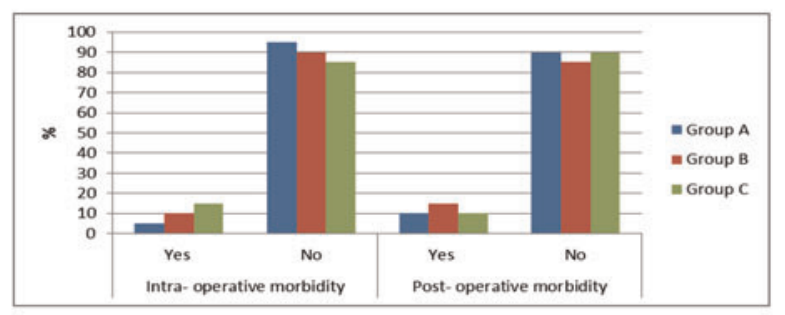

Fig. (8): showing comparison between the 3 groups regarding the intraoperative \& postoperative morbidity

Objective: is to compare the outcome of PCNL in flank suspended supine position, standard prone and standard supine position.

Materials and Methods: This study was conducted on $60 \mathrm{pa}$ tients with renal stone more than $1.5 \mathrm{~cm}$ from March 2013 to
Table (13): showing comparison between the studied groups according to the patients who needed re-treatment in the form of second look PCNL

\begin{tabular}{|c|c|c|c|c|c|}
\hline & $\begin{array}{l}\text { Group A } \\
\text { FSSP } \\
\mathrm{N}(\%)\end{array}$ & $\begin{array}{l}\text { Group B } \\
\text { Supine } \\
\mathrm{N}(\%)\end{array}$ & $\begin{array}{l}\text { Group C } \\
\text { Prone } \\
\mathrm{N}(\%)\end{array}$ & $\mathrm{X}^{2}$ test & $\begin{array}{l}\mathrm{P} \\
\text { value }\end{array}$ \\
\hline $\begin{array}{l}\text { Re ttt with pcnl } \\
\text { yes } \\
\text { No }\end{array}$ & $\begin{array}{l}1(5 \%) \\
19(95 \%)\end{array}$ & $\begin{array}{l}2(10 \%) \\
18(90 \%)\end{array}$ & $\begin{array}{l}1(5 \%) \\
19(95 \%)\end{array}$ & ${ }^{\wedge} 0.0$ & 1.0 \\
\hline
\end{tabular}

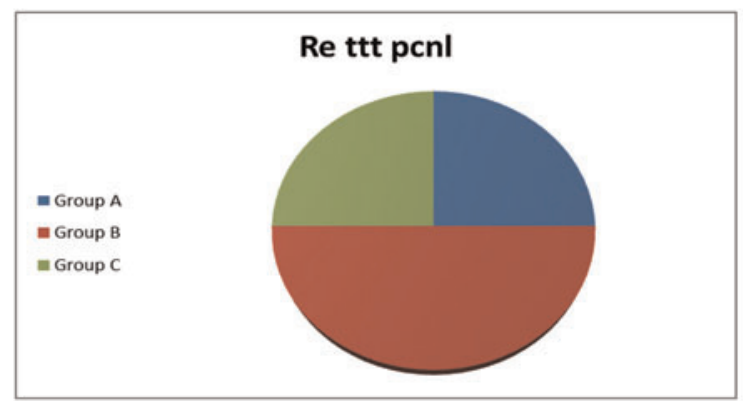

Fig. (9): showing the comparison between the 3 groups

Table (14): comparing the 3 groups according to the needed auxiliary treatment in the form of ureteroscopy and ESWL

\begin{tabular}{|c|c|c|c|c|c|}
\hline & $\begin{array}{l}\text { Group A } \\
\text { FSSP } \\
\mathrm{N}(\%)\end{array}$ & $\begin{array}{l}\text { Group B } \\
\text { Supine } \\
\mathrm{N}(\%)\end{array}$ & $\begin{array}{l}\text { Group C } \\
\text { Prone } \\
\mathrm{N}(\%)\end{array}$ & $\mathrm{X}^{2}$ test & $\begin{array}{l}\mathrm{P} \\
\text { value }\end{array}$ \\
\hline Auxillary ttt & & & & & \\
\hline Eswl & $1(5 \%)$ & $2(10 \%)$ & $2(10 \%)$ & ^ 0.0 & 1.0 \\
\hline UrS & . & . & $1(5 \%)$ & & \\
\hline
\end{tabular}

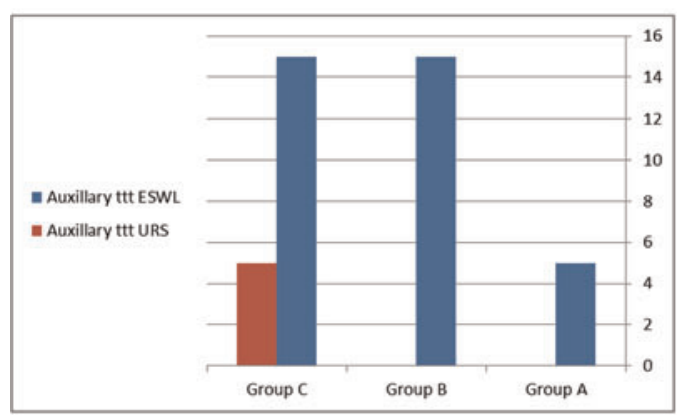

Fig. (10): comparing the 3 groups according to the need of auxiliary treatment

October 2016 in Urology Department, Benha University Hospital, and were divided randomly into 3 groups: group $(\boldsymbol{A}): 20$ patients were treated by PCNL in flank suspended supine position (FSSP), group (B): 20 patients were treated by PCNL in standard supine position and group $(\boldsymbol{C}): 20$ patients were treated by PCNL in the prone position. Exclusion creiteria were renal anomalies and bleeding diathesis. Informed written consent were taken from all patients after institutional review board approval. 
Table (15): Comparing the stone free rate between the 3 groups

\begin{tabular}{|l|l|l|l|l|l|}
\hline & Group A & Group B & Group C & $\mathrm{X}^{2}$ test & $\begin{array}{l}\mathrm{P} \\
\text { value }\end{array}$ \\
& FSSP & Supine & Prone & & \\
\hline Stone free rate & $\mathrm{N}(\%)$ & $\mathrm{N}(\%)$ & $\mathrm{N}(\%)$ & & \\
Residual & $1(5 \%)$ & $4(20 \%)$ & $3(15 \%)$ & $\wedge 2.03$ & 0.505 \\
No & $19(95 \%)$ & $16(80 \%)$ & $17(85 \%)$ & & \\
\hline
\end{tabular}

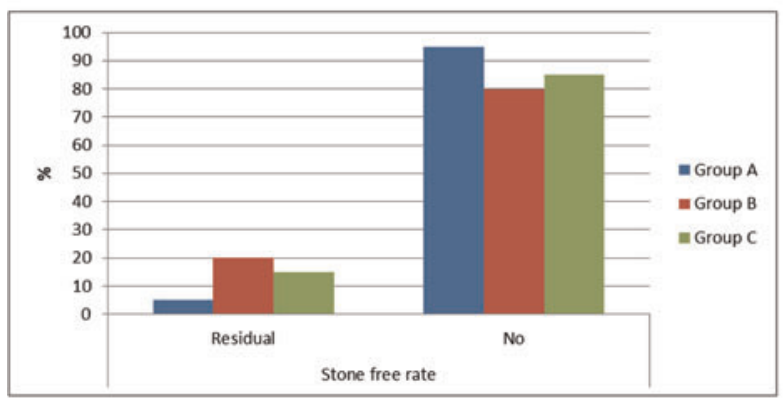

Fig. (11): comparing the 3 groups according to the stone free rate

Results: Total number was 60 patients divided into 3 groups A,B, and $\mathbf{C} 20$ patients in each group. The mean body mass index(BMI) by $\mathbf{K g} / \mathbf{s q} . \mathbf{m}$ in group $\mathbf{A}$ was $(25.81 \pm 6.16)$, in group $\mathbf{B}$ was $(27.68 \pm 6.74)$, and in group $\mathbf{C}$ the mean BMI was $(30.94 \pm 6.65)$. According to the stone multiplicity in group $\mathbf{A}$ there were $\mathbf{1 1}$ cases of single renal stones $(55 \%)$ and $9(45 \%)$ cases of multiple renal stones, in group B there were $\mathbf{1 3}$ single renal stones (65\%) and $\mathbf{7}$ cases $(35 \%)$ of multiple renal stones as in group $\mathbf{C}$ there were $\mathbf{1 5}$

\section{Data management}

The clinical data were recorded on a report form. These data were tabulated and analyzed using the computer program SPSS (Statistical package for social science) version 16 to obtain:

\section{Descriptive data}

Descriptive statistics were calculated for the data in the form of:

1. Mean and standard deviation $( \pm S D)$ for quantitative data.

2. Frequency and distribution for qualitative data.

\section{Analytical statistics}

In the statistical comparison between the different groups, the significance of difference was tested using one of the following tests:-

1- ANOVA test (F value):-Used to compare mean of more than two groups of quantitative data.

2- Inter-group comparison of categorical data was performed by using chi square test ( $X^{2}$-value) and fisher exact test (FET).

$$
x^{2}=\frac{\sum(\text { observed }-\operatorname{expected})^{2}}{\text { Expected }}
$$

Expected $=\frac{\text { col.total } x \text { row total }}{\text { Grand total }}$

A $P$ value $<0.05$ was considered statistically significant (S) while $>0.05$ statistically insignificant $\mathrm{P}$ value $<0.01$ was considered highly significant (HS) in all analyses.
(80\%) single renal stones and $5(20 \%)$ multiple renal stones. Intraoperative morbidity occurred in one patient (5\%) for group A, two patients (10\%) for group B and three patients (15\%) for group C. The mean operative time were $61.65 \pm 15.14$ in group $\mathrm{A}$, $75.95 \pm 18.11$ in group B and 78.4 \pm 9.7 in group C. with significant $\mathrm{P}$ value (0.001). Postoperative complications occurred in two patient (10\%) for group A, three patients (15\%) for group B and two patients (10\%) for group C.

Conclusions: PCNL in the FSSP position is safe, effective and suitable for the patients especially morbidly obese patients, it has several advantages, like less operative time, ability to increase the space between the last rib and the iliac crest increasing the maneuvering space for the instruments and better control of airway during the procedure. However, the supine position, and the FSSP position is not a substitute for the prone position for PCNL. We need more prospective randomized studies in this field to draw an affirmative conclusion.

MP6-14 Complications of upper-pole calyceal puncture in patients having supine percutaneous nephrolithotomy

L Mokool, S Yallappa, Z Kratiras, SK Nalagatla

Monklands District General Hospital, Scotland United Kingdom

Introduction \& Objective: Upper-pole puncture is mainly used for access in cases with upper calyceal stones or large and complex stone burden. However, this poses an increased risk of both thoracic and non-thoracic complications.

Our study aims to evaluate the efficacy and safety of upper pole puncture in supine percutaneous nephrolithotomy (SPCNL). We also include our experience with the ureteric balloon catheter (UBC) displacement technique in cases with difficult to access supracostal calyces.

Materials and Methods: A retrospective analysis of patients undergoing SPCNL between 2013 and 2017 was carried out. The inclusion criterion was all patients who had upper pole calyceal puncture for access with complete data. Key variables collected were: patient demographics, stone size, radiodensity (HU), complete or incomplete clearance, additional procedures required, complications and post-operative hospital stay.

Results: Eight patients had upper-pole puncture for access during SPCNL. The stone positions were as follows: 1 complete staghorn, 2 upper calyceal, 2 pelvic and 3 pelvic stones with smaller stones in the upper and lower poles. The average stone size was $23.6 \mathrm{~mm}$ and average radiodensity $944 \mathrm{HU}$. Complete stone clearance was achieved in 5 cases $(62.5 \%)$. The rest required additional flexible ureteroscopy and laser fragmentation. The UBC technique (inferior renal displacement) was used in 3 cases to access upper-pole calyces. Two patients $(25 \%)$ became septic post operatively and two required a blood transfusion. There were no pleural or collateral organ injuries. Conclusions: Upper-pole puncture is effective and safe and coupled with the UBC displacement technique allows access to supracostal calyces and minimises the risks of thoracic complications.

MP6-15 Risk of complications with endoscopic renal papillary biopsy

ME Rivera, J Lingeman, N York, JC Williams, AE Krambeck

Department of Urology, Mayo Clinic (Rochester, MN)

United States 
Introduction \& Objective: The evidence concerning the safety of papillary biopsy in the study of renal stone formation is limited. Herein, we discuss our experience with endoscopic papillary biopsy.

Materials and Methods: An institutional review board approved, prospectively maintained database of patients undergoing percutaneous nephrolithotomy was utilized. Following stone burden clearance study patients underwent flexible nephroscopy to assess the papillary architecture. Biopsies of the papillary tip were taken using a 5 Fr biopsy forcep from representative papillae. Identified patients who underwent papillary biopsy were compared to a non-biopsy cohort and evaluated for post-operative complications.

Results: A total of 1448 patients were identified and, of those, 190 underwent papillary biopsy. Comorbidities were similar between the two groups; however, patient's who underwent papillary biopsy were younger (mean 49.5 versus 53.1 years, $\mathrm{p}<0.005)$ and were less likely to have a pre-operative diagnosis of hypertension $(\mathrm{p}<0.01)$. There were no differences in transfusion or complication rates between patients who underwent biopsy to those who did not (1.6\% versus $3.7 \%, \mathrm{p}=0.19$ and $4.7 \%$ versus $6.5 \%, p=0.4$, respectively). Likewise, there were no significant differences in pre-, post-operative or change in renal function (mean (SD) Creatinine $1.10(0.68)$ versus $1.02(0.38)$ $\mathrm{mg} / \mathrm{dl}, 1.27(0.57)$ versus $1.26(0.83) \mathrm{mg} / \mathrm{dl}, 0.28(0.77)$ versus $0.20(0.55) \mathrm{mg} / \mathrm{dl} \mathrm{p}=0.13,0.97$, and 0.08 respectively).

Conclusions: Patients who underwent papillary biopsy were younger, there were no differences in change in renal function, risk for a transfusion or likelihood of experiencing a complication. Renal papillary biopsy remains a safe modality in the study of stone pathogenesis.

MP6-16 Characterization of Thoracic Complications associated with Percutaneous Nephrolithotomy

A Serrano, C Trujillo, A Bravo-Balado, M Echeverry, T Higuera, C Barco, A Díaz, D Robledo, J Caicedo, J Cataño, M Plata

Hospital Universitario Fundación Santa Fe de Bogotá and Universidad de los Andes School of Medicine, Bogotá D.C., Colombia

Colombia

Introduction \& Objective: Percutaneous nephrolithotomy (PCNL) is associated with a variety of complications. Thoracic events have been reported and may result in high morbidity. However, there is scarce evidence regarding the risk factors associated with these entities. We aim to describe the most common thoracic complications, their clinical presentation and

Table 1. Baseline characteristics

\begin{tabular}{lr} 
& \\
\cline { 2 - 2 } Diabetes Mellitus & $n(\%)$ \\
High blood pressure & $20(14.9)$ \\
Previous history of renal stone & $50(37.3)$ \\
Chronic kidney disease & $94(70.1)$ \\
Scoliosis of the spine & $8(5.9)$ \\
Horseshoe kidney & $2(1.5)$ \\
Monorenal & $6(4.4)$ \\
Pyeloureteral junction stenosis & $5(3.7)$ \\
Ureteral duplication & $7(5.1)$ \\
Previous PCNL & $3(2.2)$ \\
$\mathrm{n}=136$ & $27(19.9)$ \\
PCNL: percutaneous nephrolithotomy &
\end{tabular}

Table 2. Thoracic complications

\begin{tabular}{lr}
\hline & $n(\%)$ \\
\cline { 2 - 2 } $\begin{array}{l}\text { Hydrothorax managed by intercostal draining under local } \\
\text { anaesthesia }\end{array}$ & $1(0.7)$ \\
Pleural effusion managed by watchful waiting & $4(2.8)$ \\
Hemothorax managed by intercostal draining under local & \\
anaesthesia & $2(1.4)$ \\
Hyposaturation managed by oxygen & $3(2.2)$ \\
Atelectasis managed by oxygen & $2(1.4)$ \\
Pulmonary embolism managed by IVC filter & $1(0.7)$ \\
Postoperative pneumonia managed by antibiotics & $2(1.5)$ \\
Clavien-Dindo & \\
$\quad$ I & $2(1.4)$ \\
II & $8(5.9)$ \\
IIIb & $2(1.4)$ \\
$\quad$ IVa & $3(2.2)$ \\
Time of presentation and diagnosis & \\
Immediate ( $\leq 24$ hours) & $8(50)$ \\
$\quad$ Mediate $(>24$ hours -1 week) & $7(43.8)$ \\
Late (>1 week, $<1$ month) & $1(6.2)$ \\
\hline $\mathrm{n}=136$ & \\
IVC $=$ inferior vena cava &
\end{tabular}

severity, as well as associated factors and management of patients undergoing the aforementioned procedure.

Materials and Methods: We conducted an observational retrospective study between 2010 and 2017 in a referral center. Data was obtained from medical records of patients aged $\geq 18$ years who underwent prone PCNL for the treatment of kidney stones. Descriptive and inferential statistics were employed.

Results: A total of 136 patients were included, out of which $56.6 \%$ were males and $43.4 \%$ were females. Baseline characteristics are summarized in Table 1. Median age was 46 years old (range 22-82, IQR 21.5) and median BMI was 26.1 (range 18-41, IQR 5). Complete and partial staghorn calculi were found in $22.6 \%$ and $25.4 \%$ of cases, respectively. Regarding renal pole access, $69.9 \%$ were upper pole, $15.4 \%$ lower pole, $5.1 \%$ mid pole and the rest had multiple access tracts. The overall rate of complications was $26.5 \%(n=36)$, out of which $41.7 \%(n=15)$ were of pulmonary origin (Table 2 ). The main presenting symptoms were: hyposaturation $(n=9)$, pleuritic pain $(n=2)$, fever $(n=2)$ and dyspnea $(n=2)$. A univariate logistic regression analysis found that a complete staghorn calculi $(\mathrm{OR}=8.4 \mathrm{CI} 95 \%$ 2.6-27.6, $\mathrm{p}<0.0001)$ and the upper pole access $(\mathrm{OR}=7.7 \mathrm{CI} 95 \% 1.0-60.7, \mathrm{p}=0.05)$ were associated with thoracic complications.

Conclusions: The frequency of thoracic complications in PCNL in our study is similar to other reports in the literature. We believe urologists who treat complex stones or prefer an upper pole access must consider these factors before performing PCNL. In addition, they must be familiar with the clinical presentation and management of thoracic complications since it might reduce the morbidity associated with this procedure.

MP6-17 Predictive Factors for Complications assoicated with Percutaneous Nephrolithotomy

K Lee, S Choi, T Kim, T Oh, C Yoon, W LEE, J Lee

Department of Urology, Pusan National University Hospital, Republic of Korea

Introduction \& Objective: Although percutaneous nephrolithotomy (PNL) is widely used as a noninvasive surgical method for the removal of renal stones larger than $2 \mathrm{~cm}$, it may cause a variety of perioperative or postoperative complications 
Table 1. Correlation of patients' characteristics and complications. All data represent p-values of univariable and multivariable analyses.

\begin{tabular}{|c|c|c|c|c|c|c|}
\hline & \multicolumn{2}{|c|}{ Residual stone } & \multicolumn{2}{|c|}{ Massive hemorrhage } & \multicolumn{2}{|c|}{ Infection } \\
\hline & Univariable & Multivariable & Univariable & Multivariable & Univariable & Multivariable \\
\hline Gender & 0.556 & & 0.326 & & 0.683 & \\
\hline Age & 0.576 & & 0.980 & & 0.987 & \\
\hline BMI & 0.416 & & 0.272 & & 0.151 & \\
\hline \multicolumn{7}{|l|}{$\begin{array}{l}\text { Underlying } \\
\text { disease }\end{array}$} \\
\hline Hypertension & 0.705 & & 0.028 & 0.041 & 0.436 & \\
\hline DM & 0.733 & & 0.093 & & 0.049 & 0.075 \\
\hline CVA & 0.574 & & 0.454 & & 0.648 & \\
\hline Hospitalization & 0.040 & 0.627 & 0.016 & 0.698 & 0.000 & 0.127 \\
\hline \multicolumn{7}{|l|}{ Stone position } \\
\hline $\begin{array}{l}\text { Complete } \\
\text { staghorn }\end{array}$ & 0.071 & & 0.005 & 0.819 & 0.179 & \\
\hline $\begin{array}{l}\text { Partial } \\
\text { staghom }\end{array}$ & 0.177 & & 0.141 & & 0.595 & \\
\hline Renal pelvis & 0.097 & & 0.003 & 0.341 & 0.161 & \\
\hline Renal calyx & 0.144 & & 0.088 & & 0.476 & \\
\hline $\begin{array}{l}\text { Multiple } \\
\text { puncture }\end{array}$ & 0.422 & & 0.934 & & 0.004 & 0.002 \\
\hline Operation time & 0.007 & 0.080 & 0.003 & 0.102 & 0.148 & \\
\hline Prior ESWL & 0.921 & & 0.520 & & 0.399 & \\
\hline Balloon dilation & 0.487 & & 0.758 & & 0.022 & 0.082 \\
\hline \multicolumn{7}{|l|}{$\begin{array}{c}\text { Stone } \\
\text { composition }\end{array}$} \\
\hline $\begin{array}{l}\text { Calcium } \\
\text { oxalate }\end{array}$ & 0.310 & & 0.013 & 0.772 & 0.801 & \\
\hline $\begin{array}{c}\text { Calcium } \\
\text { phosphate }\end{array}$ & 0.855 & & 0.001 & 0.152 & 0.143 & \\
\hline $\begin{array}{l}\text { Calcium } \\
\text { carbonate }\end{array}$ & 0.693 & & 0.097 & & 0.748 & \\
\hline Uric acid & 0.144 & & 0.323 & & 0.079 & \\
\hline Struvite & 0.002 & 0.032 & 0.000 & 0.084 & 0.001 & 0.009 \\
\hline
\end{tabular}

which occasionally may be life threatening. The purpose of this study is to investigate the predictive factors of major complications in PNL.

Materials and Methods: Medical records of 105 patients who underwent PNL for renal and upper ureteral stones from September 2009 to July 2012 were retrospectively reviewed and analyzed. Correlation between the major complications of PNL (residual stones, massive hemorrhage, infection, and adjacent organ injury) and variables such as age, sex, body mass index, size and type of stone, operation time, dilatation method and number of renal puncture, underlying disease, and history of urinary stones was evaluated and compared by using univariable and multivariable analysis. Stones $\geq 4 \mathrm{~mm}$ in the postoperative imaging studies (CT or X-ray) or those $\geq 2 \mathrm{~mm}$ which occur stone-related event were determined as the residual stones. Massive hemorrhage was determined to be the cases requiring blood transfusion. Infection was defined when the postoperative body temperature exceeded $38.3^{\circ} \mathrm{C}$ or maintained at $38^{\circ} \mathrm{C}$ over 1 hour. The presence of adjacent organ injuries were evaluated in the cases suspicious of infection.

Results: Among the 14 cases $(13.3 \%)$ showing residual stones, extracorporeal shock lithotripsy and second PNL was performed in $10(9.5 \%)$ and 4 cases $(3.8 \%)$, respectively. Blood transfusion was given in 27 cases $(25.7 \%)$ and $6(5.7 \%)$ of these cases underwent arterial embolization due to pseudoaneurysm. Infection was occurred in 9 cases $(8.6 \%)$ which were successfully controlled by antibiotic treatment without exacerbation to sepsis. Adjacent organ injury was found in one case and was managed by chest tube insertion. According to the multivariable analysis, struvite composition, hypertension, and multiple puncture and struvite composition were the predictive factors of residual residual stones, massive hemorrhage, and infections, respectively (Table 1).

Conclusions: PNL is a safe and successful treatment modality and its predictive factors regarding major complications can be useful in patient care and management.

MP6-18 Hydrothorax following supracostal PCNL is influenced by the type of post procedural drainage: A Prospective Randomized Study

H Goldberg, A Nevo, Y Shtabholtz, MA Lubin, J Baniel, Y Ehrlich, D Lifshitz

Endourology unit, Rabin medical center, and Sackler Faculty of Medicine, Tel Aviv University, Tel Aviv, Israel Israel

Introduction \& Objective: Supracostal upper pole access is often the most effective approach for staghorn calculi, upper ureteral calculi, and complex lower pole stones. However, it is associated with a higher rate of pulmonary complications. We routinely place a double-j stent following an upper pole access to prevent and treat a potential nephro-pleural fistula. A nephrostomy tube is placed at the surgeon's discretion. We hypothesized that a nephrostomy tube may increase the rate of hydrothorax and designed the current study to test this hypothesis. Materials and Methods: Patients planned for PCNL with an upper pole access based on the preoperative CT were prospectively randomized into two groups (NCT02036398) according to the post procedural drainage: group 1 with double $\mathrm{j}$ stent and a nephrostomy tube (12Fr Folly catheter) and group 2 with a double j stent only ("tubeless"). Chest X-ray was performed immediately following surgery and the following day. Patient's data and perioperative data were compered between the groups and analyzed.

Results: Overall 74 patients were recruited thus far. Table 1 demonstrates no significant differences in demographic, and preoperative data between the groups. Table 2 shows all relevant

\begin{tabular}{|c|c|c|c|}
\hline & $\begin{array}{c}\text { Group 1 } \\
\text { (Nephrostomy + DJ stent) }\end{array}$ & $\begin{array}{c}\text { Group 2 } \\
\text { (Only DJ stent) }\end{array}$ & $\begin{array}{c}p \\
\text { Value }\end{array}$ \\
\hline Number of patients & 37 & 37 & - \\
\hline Mean age (SD) & 57.2 & 55.4 & 0.61 \\
\hline \multicolumn{4}{|l|}{ Gender, $n$} \\
\hline Male & 23 & 24 & 0.77 \\
\hline Females & 14 & 13 & \\
\hline $\begin{array}{l}\text { Mean Age adjusted Charlson score } \\
\text { (SD) }\end{array}$ & $2.3(2.2)$ & $2.1(2)$ & 0.64 \\
\hline Mean BMI (SD) & $31.6(8)$ & $30.2(5.7)$ & 0.4 \\
\hline \multicolumn{4}{|l|}{ Laterality, $n$} \\
\hline Right & 17 & 20 & 0.29 \\
\hline Left & 20 & 17 & \\
\hline Mean number of stones (SD) & $3.3(1.8)$ & $2.8(1.7)$ & 0.26 \\
\hline Mean largest stone size, $\mathrm{cm}(S D)$ & $24.2(10.1)$ & $23(8.6)$ & 0.6 \\
\hline \multicolumn{4}{|l|}{ Largest stone location, \% } \\
\hline Upper pole & $3(11.1 \%)$ & $4(10.5 \%)$ & \\
\hline Mid pole & 0 & $1(2.7 \%)$ & \\
\hline Real pelvis & $7(25.9 \%)$ & $14(36.8 \%)$ & 0.3 \\
\hline Lower pole & $6(22.3 \%)$ & $3(7.9 \%)$ & \\
\hline Complete Staghorn & $10(37 \%)$ & $12(31.6 \%)$ & \\
\hline Partial staghorn & $1(3.7 \%)$ & $4(10.5 \%)$ & \\
\hline
\end{tabular}




\begin{tabular}{|c|c|c|c|}
\hline & $\begin{array}{c}\text { Group 1 } \\
\text { (Nephrostomy + DJ } \\
\text { stent) }\end{array}$ & \begin{tabular}{|c|} 
Group 2 \\
(Only DJ stent)
\end{tabular} & $\begin{array}{c}p \\
\text { Value }\end{array}$ \\
\hline Mean Operative time, $\min (S D)$ & $116(38.8)$ & $105.1(28.4)$ & 0.2 \\
\hline $\begin{array}{l}\text { Mean Duration of Nephrostomy tube, days } \\
\text { (SD) }\end{array}$ & $2(1.45)$ & 0 & $\cdot$ \\
\hline Mean Duration of catheter, days (SD) & $2.8(1.5)$ & $2.5(0.9)$ & 0.27 \\
\hline Mean Duration of DJ stent, days (SD) & $29(18.7)$ & $35.5(63.7)$ & 0.64 \\
\hline Stone free rate, \% & $84 \%$ & $89.5 \%$ & 0.52 \\
\hline Percentage of hydrothorax\% & $35.1 \%$ & $18.9 \%$ & 0.05 \\
\hline \multicolumn{4}{|l|}{ Hydrothorax treatment, $n$ (\%) } \\
\hline Conservative treatment & $10(77 \%)$ & $5(71.4 \%)$ & \\
\hline Pleural aspiration & 0 & $1(14.3 \%)$ & 0.4 \\
\hline Chest Tube insertion & $1(8 \%)$ & $1(14.3 \%)$ & \\
\hline Removal of Nephrostomy Tube & $2(15 \%)$ & & \\
\hline \multicolumn{4}{|l|}{ Additional complications, \% } \\
\hline $\begin{array}{l}\text { Significant loss of blood in surgery } \\
\text { (drop in >2 units of Hemoglobin) }\end{array}$ & $7.4 \%$ & $5.3 \%$ & 0.72 \\
\hline Fever & $7.4 \%$ & $5.3 \%$ & 0.72 \\
\hline Urosepsis & $3.7 \%$ & $5.3 \%$ & 0.77 \\
\hline
\end{tabular}

operative, postoperative and complications data, with similar operative time and stone free rate. The rate of hydrothorax was significantly lower in the "tubeless" patients (18.9\% vs. $35.1 \%$, $\mathrm{p}=0.05)$. Of the entire cohort of 74 patients, three patients $(4 \%)$ required active intervention for hydrothorax: one patient underwent aspiration (group 2) and two patients, one from each group, received a chest tube. Univariate and multivariate logistic regression analysis demonstrate that the only predictor for hydrothorax in the "tubeless" patients (group 2) was decreased catheter duration (HR 0.141, 95\% CI: 0.021-0.957, p=0.045). Variables in the model included age, gender, BMI, largest stone size, number of stones, laterality, puncture site (predominantly supra 12), operative time, and duration of catheter. No predictors for hydrothorax were found in group 1.

Conclusions: Placement of a nephrostomy tube in addition to a double-j stent following an upper pole access increases the rate of hydrothorax. A longer catheter dwelling time may decrease the rate of hydrothorax.

MP6-19 A Minimally Invasive Angioembolization Technique to Treat Delayed Hemorrhage After percutaneous Nephrolithotomy

R Maghsoudi, M Etemadian, A Kashi, M Banaei

Iran University of Medical Science

Iran

Introduction \& Objective: To present the results of our new method of angioembolization for delayed refractory bleeding after percutaneous nephrolithotomy.

Materials and Methods: The records of all patients who underwent PCNL in our referral center from 2010 to 2016 were investigated for the treatment of refractory postoperative bleeding by angioembolization. In our new technique, we use crushed cardiac stents to embolize pseudoaneurysm or arteriovenous fistula.

Results: 14 cases were treated by angioembolization. Preoperative Color Doppler ultrasonography had revealed pseudoaneurysm in 6, arteriovenous fistula in 7 and both in 1 patient. 3 patients were dealt with by the standard protocol incorporating two coils with satisfactory results in one session. Eleven patients were treated with angioembolization using crushed cardiac stents. 9 patients were successfully treated in the first session using our new technique. In two patients, a second angioembolization session was needed with our new method to control bleeding. In 8 patients, 2 crushed stents were used to control bleeding under angiographic monitoring. In 3 patients, 1 coil was enough for bleeding cessation. The cost of standard angioembolization coils is several times higher than the used cardiac stents in this report.

Conclusions: Our new technique was able to control refractory postoperative bleeding in PCNL in most patients with considerably lower cost in comparison with the standard protocol.

MP6-20 Cessation of Tract Hemorrhage using the Dual Catheter Hemostatic Sandwich Technique Following Percutaneous Nephrolithotomy

B Mattison, M Keheila, I Kelly, S Abourbih, J Cheng, M Pierce, H Wagner, DD Baldwin

Loma Linda University

United States

Introduction \& Objective: During percutaneous nephrolithotomy (PCNL), damaged vessels within the percutaneous tract can lead to persistent bleeding. We have previously described a technique to control percutaneous tract hemorrhage using a dual-nephrostomy gelatin matrix hemostatic sealant (GMHS) sandwich. The purpose of this study is to review the safety, efficacy, and clinical outcomes of this technique in a series of patients.

Materials and Methods: A single institution, retrospective review of 304 PCNL procedures was performed. The clinical and perioperative characteristics of patients receiving the dual catheter hemostatic sandwich technique either with or without GMHS were reviewed. In the hemostatic sandwich technique, a 22 Fr Council-tip catheter is placed through the nephrostomy tract at completion of PCNL and the balloon is inflated just inside the nephrostomy tract. Then a $16 \mathrm{Fr}$ Council-tip catheter is inserted so the balloon is positioned just below the skin. GMHS is injected between the two catheters and then the second balloon is inflated. This technique was performed only in cases with moderate or severe persistent tract bleeding failing conservative measures including manual compression (10 minutes). For cases of moderate tract bleeding, GMHS was omitted in select patients to minimize cost.

Results: Of 304 PCNLs, 27 patients received the dual catheter hemostatic technique ( 24 with and 3 without GMHS). The mean operative time was 197 minutes, mean EBL was $254 \mathrm{cc}$. Transfusion of two units of blood was required in each of $2(7.4 \%)$ patients. For patients who received the dual catheter hemostatic technique the hemoglobin stabilized at an average of 2.14 days and there was an insignificant change in serum creatinine when comparing preoperative and postoperative levels. There were no complications related to the application of GMHS.

Conclusions: The dual catheter hemostatic sandwich technique is a safe and effective way to control tract bleeding following PCNL and may reduce the risk of delayed hemorrhage. There were no cases of delayed bleeding, allergic reactions, ureteral obstruction or any other complications related to the technique. The dual catheter hemostatic technique should be considered in cases of moderate or significant persistent tract bleeding. 
MP6-21 Effect of pelvicalyceal anatomy on perioperative bleeding during percutaneous nephrolithotomy

SK Devana, R Patel, RS Mavuduru, A Lal, GS Bora, SK Singh, AK Mandal

Post Graduate Institute of Medical Educatio \& Research, Chandigarh, India

India

Introduction \& Objective: Perioperative bleeding is a significant morbidity during percutaneous nephrolithotomy (PNL). The impact of pelvicalyceal (PC) anatomy on perioperative bleeding during PNL is not well established.

Materials and Methods: This was a prospective study done on patients with renal stone disease undergoing PNL fromJuly 2015 to December 2016. Patients with serum creatinine $\geq 1.5 \mathrm{mg} / \mathrm{dl}$, history of contrast allergy and anomalous kidney were excluded. Preoperatively all patients underwent CT pyelography using 128-slice multidetector CT (Siemen Somatom definition flash) with a $16 \times 1.25-\mathrm{mm}$ configuration. PNL was performed in prone position using rigid nephroscope. The access tract was dilated till 24 Fr using Alkens dilators. PC anatomical variables on CT pyelography such as degree of hydronephrosis, type of PC anatomy (Sampaio classification), IL (infundibular length) and IW (infundibular width) of the punctured calyx, infundibulo pelvic angle and upper lower calyceal angle were recorded. Fall

\begin{tabular}{|c|c|c|c|c|}
\hline \multicolumn{2}{|l|}{ Variable } & $\mathrm{Hb}$ fall $<1.25 \mathrm{gm} / \mathrm{dl}(\mathrm{n}=29)$ & $\mathrm{Hb}$ fall $>1.25 \mathrm{gm} / \mathrm{dl}(\mathrm{n}=29)$ & $P$ valuc \\
\hline \multicolumn{2}{|l|}{ Mean age(yrs) } & $44,4 \pm 12.9$ & $40.1 \pm 12.8$ & 0.208 \\
\hline \multicolumn{5}{|l|}{$\operatorname{Sex}(n)$} \\
\hline \multicolumn{2}{|l|}{ Male } & $18(52.9 \%)$ & $16(47.1 \%)$ & 0.395 \\
\hline \multicolumn{2}{|l|}{ Female } & $11(45.8 \%)$ & $13(54.2 \%)$ & \\
\hline \multicolumn{2}{|l|}{ Mean BMI $\left(\mathrm{kg} / \mathrm{m}^{2}\right)$} & $23.7 \pm 2.9$ & $24.4 \pm 3.6$ & 0.436 \\
\hline \multicolumn{2}{|l|}{$\mathrm{H} / \mathrm{o}$ previous surgery } & $8(57.1 \%)$ & $6(42.9 \%)$ & 0.380 \\
\hline \multicolumn{2}{|l|}{ Mean stone burden( $\left(\mathrm{cm}^{2}\right)$} & $2.94 \pm 3.6$ & $3.76 \pm 2.8$ & 0.342 \\
\hline \multicolumn{5}{|l|}{ Tracts needed } \\
\hline \multicolumn{2}{|l|}{ Single } & $24(50 \%)$ & $24(50 \%)$ & 1.000 \\
\hline \multicolumn{2}{|l|}{ Multiple } & $5(50 \%)$ & $5(50 \%)$ & \\
\hline \multicolumn{2}{|l|}{ Stone clearance } & $27(93.1 \%)$ & $21(72.4 \%)$ & 0.079 \\
\hline \multirow[t]{2}{*}{ Degree of hydronephrosis } & Grade UIII & $23(50 \%)$ & $23(50 \%)$ & \\
\hline & Grade III//V & $6(50 \%)$ & $6(50 \%)$ & 1.0 \\
\hline Type of PCS & Al & $16(57.1 \%)$ & $12(42.9 \%)$ & \\
\hline \multirow{3}{*}{ (Sampaio classification) } & $\mathrm{A} 2$ & $3(33.3 \%)$ & $6(66.7 \%)$ & 0.224 \\
\hline & B1 & $6(37.5 \%)$ & $10(62.5 \%)$ & \\
\hline & B2 & $4(80 \%)$ & $1(20 \%)$ & \\
\hline \multicolumn{2}{|l|}{$\mathrm{IL}(\mathrm{cm})$} & $1.90 \pm 0.25$ & $2.04 \pm 0.25$ & 0.036 \\
\hline \multicolumn{2}{|l|}{$\mathrm{IW}(\mathrm{cm})$} & $0.83 \pm 0.13$ & $0.87 \pm 0.17$ & 0.342 \\
\hline \multicolumn{2}{|l|}{$\mathrm{ULA}^{\circ}$} & $116.48 \pm 11.67$ & $119.34 \pm 15.19$ & 0.425 \\
\hline \multicolumn{2}{|l|}{$\mathrm{IPA}^{\circ}$} & $66.66 \pm 12.36$ & $62.38 \pm 10.42$ & 0.160 \\
\hline \multicolumn{2}{|c|}{ Duration of nephrostomy placement } & $2.03 \pm 1.47$ & $4.17 \pm 2.50$ & 0.001 \\
\hline \multicolumn{2}{|l|}{ Hospital stay } & $4.17 \pm 1.77$ & $5.52 \pm 2.33$ & 0.003 \\
\hline
\end{tabular}

in hemoglobin from baseline was estimated on post operative day 1 .

Results: Total 108 patients underwent PNL during the study period, 58 patients were eligible for evaluation according to the study protocol. The median fall in hemoglobin $(\mathrm{Hb})$ was $1.25 \mathrm{gm} /$ dl. Patients were divided into two groups based on $\mathrm{Hb}$ fall. Both the groups ( $\mathrm{Hb}$ fall $<1.25$ or $>1.25 \mathrm{gm} / \mathrm{dl}$ ) were comparable for their demographic profile, number of tracts used, stone burden and stone free rate. Only IL of the punctured calyx was significantly longer in group with fall in hemoglobin $>1.25 \mathrm{gm} / \mathrm{dl}$ $(2.04 \pm 0.25 \mathrm{cms}$ vs $1.90 \pm 0.25 \mathrm{cms}$; p value 0.036 ; Table). On ROC infundibular length of $\geq 1.95 \mathrm{cms}$ had a sensitivity of $62.1 \%$ and specificity $55.2 \%$ in predicting fall in $\mathrm{Hb}>1.25 \mathrm{gm} / \mathrm{dl}$ following PNL.

Conclusions: Lengthy infundibulum of the punctured calyx increases the risk of perioperative bleeding in PNL. This may lead to delay in nephrostomy removal further leading to prolonged hospital stay.

MP6-22 Tubeless Mini Percutaneous Nephrolithotomy: A Single Institution Experience with Outpatient Management of Renal Calculi

S Fisher, M Jennings, CA Winter, K Basham, RB Pickens, WM White, B Waters

University of Tennessee Medical Center Knoxville United States

Introduction \& Objective: Percutaneous nephrolithotomy (PCNL) for the treatment of renal calculi has traditionally been an inpatient procedure often requiring a multi-day hospitalization. Large-bore nephrostomy tubes and ureteral stents have also been traditionally placed for control of bleeding and urinary drainage. Currently, more advanced technology as well as smaller operating nephroscopes and lithotripters have made PCNL a less morbid procedure with the possibility of being done on an outpatient basis. Our aim is to show that mini-PCNL can be safely performed in a tubeless fashion on an outpatient basis.

Materials and Methods: We performed a retrospective chart review of patients that underwent mini-PCNL at our institution by a single fellowship-trained endourologist. Only those patients who were discharged home the same day without nephrostomy tubes or ureteral stents were included. Cases were performed using mini-nephroscopes with an outer diameter of 15 to $17.5-\mathrm{Fr}$.

Results: There were 15 patients included for analysis. Average age was 51.3 years. The group was $53 \%$ female and $47 \%$ male. Mean BMI and ASA score were 29.31 and 2.4, respectively. Total operative time averaged 55.6 minutes [range $27-106$ ]. Mean estimated blood loss was $12.87-\mathrm{mL}$ [range 3-30-mL]. Stone size ranged from $1.3-\mathrm{cm}$ to $3-\mathrm{cm}$. Left and right sided stones were split evenly. There were multiple stones in $47 \%$ of patients. Primary stone location varied, but the majority were in the renal pelvis or the lower pole. Renal access was obtained in a middle or interpolar calyx $53 \%$ of the time, and $47 \%$ in the lower pole. Laser or ultrasonic lithotripsy was utilized. FLOSEAL was administered in the tract, and no stents or nephrostomy tubes were left. All patients were discharged home. No patients were readmitted or had unplanned ER visits so far. All patients with follow-up were stone free on KUB and RUS imaging. 
Conclusions: Mini-PCNL using operating nephroscopes up to 17.5-Fr can be safely performed on an outpatient basis in a tubeless fashion without nephrostomy tubes or ureteral stents. Some of our patients went home with Foley catheters that were removed the following morning. With the advent of improved optics and smaller ultrasonic lithotripters, mini-PCNL is a worthwhile option for patients with renal calculi that can be accomplished safely in a cost-saving outpatient basis, all while rendering patients stone free with one procedure. With changes in reimbursement for hospitals and physicians in the future, outpatient mini-PCNL could serve as a sound option for those with moderate to large renal stones that wish to be rendered stone free in one operation and avoid ureteral stents.

MP6-23 Effect of nephrostomy tube calibre (12F vs 24F) on the outcome of PCNL-A prospective randomized clinical study.

S Tiwari, S Gupta, I Singh

University College of Medical Sciences (University of Delhi) \& GTBH.

India

Introduction \& Objective: Percutaneous nephrolithotomy(PCNL) is a standard procedure with the placement of a percutaneous nephrostomy (PCN) tube at the termination of PCNL being integral for providing unimpeded post op drainage. In addition a PCN tube also helps in tamponading the PCN tract to control bleedingand in providean access to the pelvicalyceal system should a second look nephroscopy be deemed necessary. Most urologists prefer a PCN tube that is $2 \mathrm{~F}$ to $3 \mathrm{~F}$ lower than the calibre of the PCN tract. Some patients develop complications with pain and prolonged urinary leak at PCN site which may prolong the hospital stay. Placing a JJ stent in place of PCN tube (tubeless PCNL) may circumvent the problem of PCN tube however this may be feasible only in select patients. A narrow calibre PCN tube could be associated with a superior patient outcomes in terms of pain and complications versus a wider calibre $24 \mathrm{~F} \mathrm{PCN}$ tube; this formed the rationale for the current study. This study aimed to evaluate and compare the clinical outcome (complications/morbidity) after using $12 \mathrm{f}$ Vs 24f calibre PCN tube in patients undergoing standard PCNL. Materials and Methods: Design, setting and participants included a randomized parallel group of selected patients in whom PCNL was indicated as per protocol between November 2014 and April 2016 in the outpatient department of our tertiary care teaching Medical College Hospital. The protocol was approved by local Institutional ethics Committee for Human Research and is submitted/registered with CTRI/2016/11/007443. Eligible patients undergoing PCNL were randomised to receive either (12fr) or (24 fr) PCN tube. Outcome measures were compared in both groups till time to discharge.

Results: The12Fnephrostomy group showed significantly less postoperative pain and analgesic requirement as compared to 24 F nephrostomy group $(\mathrm{p}<0.001)$. There was no significant difference in duration of hematuria, PCN site leak, PCN indwelling time and hospital stay between two groups. Table-1 shows a summary of salient baseline parameters and intragroup comparatives of the two groups.

Conclusions: A narrow caliber nephrostomy(12 fr) was associated with significantly lower op morbidity and complications than a wider caliber nephrostomy ( $24 \mathrm{fr}$ ) in terms of post op pain

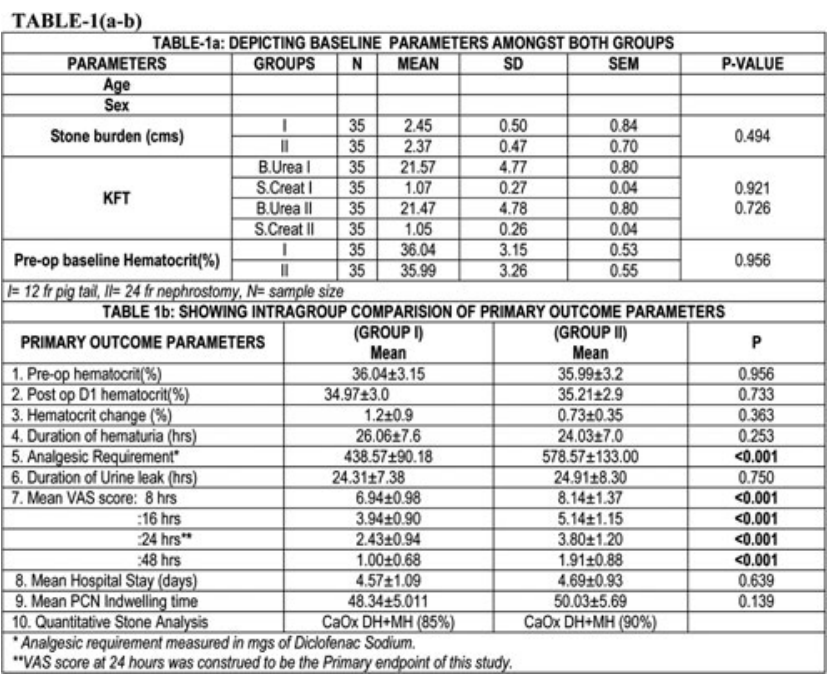

and analgesic requirement. While the safety, efficacy and feasibility of using narrow calibre PCN tubes could be demonstrated in this study, larger randomised controlled studies are still necessary to better document the precise benefit if any of using lower calibre nephrostomy tubes before this could be advocated routinely in most uncomplicated PCNL cases.

MP6-24 Modified supine percutaneous nephrolithotomy with combined fluoroscopy \& ultrasound-guided renal puncture: Initial single-surgeon experience at a UK teaching hospital.

\section{N Nkwam, M Khan}

University Hospials of Leicester NHS Trust, Leicester, United Kingdom

United Kingdom

Introduction \& Objective: To report the outcomes of our initial experience of performing percutaneous nephrolithotomy (PCNL) in the supine position using ultrasound (US) guidance in addition to fluoroscopy for renal access.

Materials and Methods: The first 34 consecutive modified supine PCNL cases performed by a single Consultant Urologist inserting their own percutaneous tracts using US-guidance and fluoroscopy were evaluated. Operative data was collected prospectively over a 10-month period including any complications. Stone-free rate (SFR) was evaluated 3 months post-operatively with a plain abdominal film or CT scan.

Results: Renal access was achieved in all 34 cases. Mean age was 57 years (19-82) with 14 males and 20 females. Mean stone size $37.2 \mathrm{~mm}(20-72 \mathrm{~mm})$. Overall SFR was $85.3 \%$ at a mean follow up of 11.2 months. There was one Clavien Grade I, and three Clavien Grade II complications of post-op fever requiring treatment with intravenous antibiotics. No patients developed urosepsis, required blood transfusion or sustained any visceral injury. Median in-patient stay was 1 night (1-4).

Conclusions: Modified supine PCNL with combined fluoroscopy and US-guided renal access has a low incidence of complications allowing for short hospital stay. 


\section{MPS7: STENTS}

MP7-1 The American Stent Epidemic: An International Survey of Ureteral Stenting Practices Following Routine Ureteroscopy

J Pereira, PE Bower, G Pareek, S Thavaseelan

Minimally Invasive Urology Institute, Alpert Medical School at Brown University

United States

Introduction \& Objective: Ureteral stent placement after routine ureteroscopy (R-URS) results in added patient morbidity and excess cost of treatment. Stent omission after R-URS is safe and accepted by current guidelines. In a value based healthcare model, the added morbidity and cost of routine stent placement may be scrutinized. We seek to describe ureteral stenting practices and cost awareness both domestic and abroad.

Materials and Methods: A survey was sent to members of the Endourological Society using an international e-mail listserv. Respondents were grouped by practice location (domestic/abroad). Practice patterns and attitudes were evaluated using logistic regression, adjusted for respondent features including fellowship training, years in practice, nephrolithiasis volume, and practice setting.

Results: 219 completed responses of 2000 were received. $39 \%$ of respondents practice in the United States (US), 60\% completed an endourology fellowship and median years in practice was 14 (IQR $8,23)$. Those abroad more frequently held non-academic hospital employed positions, compared to those in the US $(p<0.01)$. No other significant differences in characteristics were noted.

$56 \%$ and $72 \%$ of respondents reported insertion of a ureteral stent after R-URS more than $75 \%$ of the time for ureteral and renal stones, respectively. Stent insertion more than $75 \%$ of the time was more frequent among US participants for both ureteral and renal stones $(\mathrm{p}<0.01 \mathrm{p}=0.03$, respectively). Overall, $88 \%$ reported knowledge of ureteral stent cost, while $80 \%$ reported knowledge of ureteral access sheath cost. Cost knowledge rates were lower among US participants (table1).

On multivariable analysis, US respondents were more likely to place ureteral stents after R-URS for ureteral or renal stones more than $75 \%$ of the time when compared to those abroad (OR 4.64 $\mathrm{p}<0.01$; OR $2.75 \mathrm{p}<0.01$, respectively). Domestic participants were also less likely to report knowledge of ureteral stent (OR 0.29 $p=0.01)$ and access sheath cost $(O R 0.45 p=0.04)$. Knowing stent cost was associated with decreased likelihood of ureteral stenting more than $75 \%$ of the time after R-URS for a ureteral stone, though not statistically significant $(\mathrm{OR} 0.61 \mathrm{p}=0.35)$

Table 1. Ureteral stent utilization following routine ureteroscopy and cost knowledge, by practice location.

\begin{tabular}{|c|c|c|c|c|c|}
\hline & & $\begin{array}{l}\text { Overall } \\
\mathrm{N}=219\end{array}$ & $\begin{array}{l}\text { USA } \\
\mathrm{N}=78 \\
\end{array}$ & $\begin{array}{l}\text { Abroad } \\
\mathrm{N}=141\end{array}$ & p-value \\
\hline \multirow{4}{*}{$\begin{array}{c}\% \text { of cases stented } \\
\text { after R-URS* for } \\
\text { a ureteral stone, } \\
\%(n)\end{array}$} & $<25 \%$ & $15.1 \%(33)$ & $3.9 \%(3)$ & $21.3 \%(30)$ & \multirow{4}{*}{$<0.01^{2}$} \\
\hline & $25-50 \%$ & $9.6 \%(21)$ & $6.4 \%(5)$ & $11.4 \%(16)$ & \\
\hline & $51-75 \%$ & $19.2 \%(42)$ & $11.4 \%(16)$ & $23.4 \%(33)$ & \\
\hline & $>75 \%$ & $56.2 \%$ (123) & $78.2 \%(61)$ & $44.0 \%(62)$ & \\
\hline \multirow{4}{*}{$\begin{array}{c}\% \text { of cases stented } \\
\text { after R-URS* for } \\
\text { a renal stone, } \\
\%(n)\end{array}$} & $<25 \%$ & $6.9 \%(15)$ & $1.3 \%(1)$ & $10.1 \%(14)$ & \multirow{4}{*}{$0.03^{\mathrm{a}}$} \\
\hline & $25-50 \%$ & $6.9 \%(15$ & $6.4 \%(5)$ & $7.2 \%(10)$ & \\
\hline & $51-75 \%$ & $14.3 \%(31)$ & $10.3 \%(8)$ & $16.6 \%(23)$ & \\
\hline & $>75 \%$ & $71.9 \%(156)$ & $82.1 \%(64)$ & $66.2 \%(92)$ & \\
\hline \multirow{2}{*}{$\begin{array}{c}\text { Cost Knowledge, } \\
\%(\mathrm{n})\end{array}$} & Stent & 88.1 (193) & $79.5 \%(62)$ & $92.9 \%(131)$ & $<0.01^{b}$ \\
\hline & Access Sheath & $80.4 \%(176)$ & $71.8 \%(56)$ & $85.1 \%(120)$ & $0.02^{\mathrm{b}}$ \\
\hline
\end{tabular}

-R-URS= Routine ureteroscopy 'Fischer's Exact Test of Ind
Conclusions: Ureteral stenting after R-URS is over utilized in the US compared to abroad. This phenomenon is multifactorial in nature, but increased education related to cost may reduce utilization rates.

MP7-2 Office-based ureteric stent removal is achievable, cheaper and associated with reduced stent dwell time, stentrelated symptoms and complications, compared with traditional removal methods.

E Baston, A Palmer, S Wellum, Z Bredow, G Storey, D Van Dellen, B Grey

Central Manchester University Hospitals NHS Foundation Trust United Kingdom

Introduction \& Objective: Ureteric stent placement after stone procedures is commonplace as is stent morbidity, which is experienced by $80 \%$ of patients. Demand for diagnostic pathways in endoscopy suites results in stent removal often not receiving the required priority. As well as using stents with extraction strings, the introduction of a portable single use flexible cystoscope for ureteric stent removal (IsirisTM), provides flexibility to negotiate these clinical pressures, relocating stent removal to the clinic. This study aimed to determine whether such flexibility reduced stent dwell time with the assumption this would improve patient experience and decrease associated complications

Materials and Methods: A retrospective review of ureteric stents placed by a single surgeon during stone procedures was undertaken. Data collection included; patient demographics; stent dwell times; the number of emergency department (ED) attendances and hospital readmissions; cancellation of procedures; the number of urinary tract infections and the clinical course, post stent removal. These data were compared with the method of stent removal employed.

Results: In total, 162 stents were removed (113 Standard, 34 IsirisTM, 15 via strings). There was a reduction in excess dwell time in both IsirisTM (median 1 day, mean 1.37 days, $p=0.0009$ ) and Strings Groups (median 0.96 days, mean 0.96 days, $\mathrm{p}=0.022$ ) compared with the Standard Group (median 8 days, mean 15.34 days). ED attendances were reduced by $33.5 \%$ in the IsirisTM Group compared with the Standard Group, there were no ED attendances in the Strings group. Readmissions were reduced in the IsirisTM group by $22 \%$. Reductions in length of stay, urine infections and cancellation on the day of procedures were also observed. Conclusions: The clinical flexibility provided by IsirisTM and 'stents on strings' has objectively improved patient experience and is associated with a reduction in complications. Such service improvement increases quality further by increasing diagnostic capacity and cost efficacy.

MP7-3 Efficacy and cost comparisons of metallic versus polymer stents in the management ketamine induced ureteric strictures

D Yong, A Ismail, G Lee

Nine Wells Hopsital

United Kingdom 
Introduction \& Objective: The exposure of recreational substances such as ketamine is becoming more prevalent and associated with severe urinary symptoms. The inflammatory changes and contraction of the bladder is also associated with ureteric strictures and obstructions. The reconstructive management of such ureteric strictures may not be possible due to ongoing changes with continuing substance abuse. In the acute settling, the use of polymer stent plays the main role in relieving the obstruction or infection. The repeated replacement of stents is a viable option, however the metallic stents allows longer indwelling intervals and resilient to further obstructions. We compare our experience of Resonance and polymer stents efficacy and cost, in the long-term management of ketamineinduced ureteric obstructions.

Materials and Methods: Retrospective analysis of four patients with Polymer and Resonance stent insertions between 2009 and 2016 for ketamine induced ureteric strictures. Patients demographic, stent patency, complications and costs were recorded. Patients were also followed-up after the removal of the prosthesis to ensure obstruction relief.

Results: Six patients were identified with the insertions of three pairs of Polymer and Resonance stents each, due to bilateral ketamine induced ureteric obstruction. The mean age was 30 years (Range 25-41). The patients had interval of ketamine exposure ranging from two to twelve years. The stents were all inserted retrograde manner, one month following the stabilization of renal functions with initial nephrostomy insertion and ante-grade stenting. Upon cessation of ketamine abuse for more than one year with stabilization of renal functions, the stents were removed with continuing close monitoring. The patients were rendered stent free at mean interval of 16 months (Range: 15-18 months), with renal function stabilized up to two-year follow-up (Range 6-24 months). The average cost for the polymer stent intervention was USD12K, versus USD4.3K in the Resonance arm.

Conclusions: The Resonance metallic ureteral stent is an effective alternative to polymer-based stents for patients with ketamine induced upper urinary tract obstruction. The potential advantages of metallic ureteral stents include greater tensile strength allowing increased dwell times, while patients undergoing rehabilitation. Our results demonstrated good stent efficacy with one third of total costs in the conventional polymer method. The functional preservations of kidneys persisted up to two years beyond the stenting. Stringent monitoring of renal functions and complications are warranted to prevent further occlusions.

\section{MP7-4 Withdrawn}

MP7-5 Experimental Study on the Effectiveness of an Anti-Reflux Ureteral Stent

N Liu, Z Liu, J Yang

China (People's Republic)

Introduction \& Objective: A double-J stent design bypasses the ureterovesical junction, enables bladder pressure reflection to the renal pelvis and causes vesicoureteral reflux (VUR). To solve this problem, we developed a new double $\mathrm{J}$ stents with anti-reflux device. To investigate the drainage potency and anti-reflux

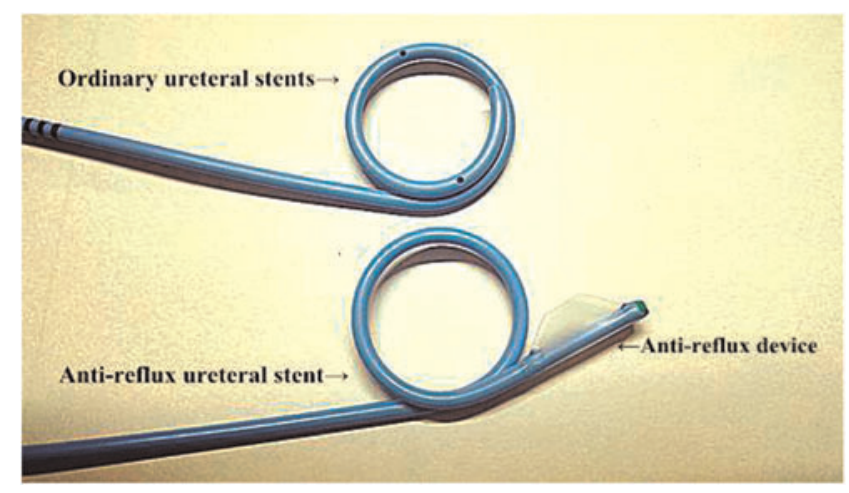

properties of a novel anti-reflux ureteral stent in animal models, with the aim to increase feasibility of clinical application.

Materials and Methods: Bilateral ureters of 6 pigs were randomly divided into group A and group B. Ordinary ureteral stents were placed in ureters of animals in group A, whereas novel antireflux ureteral stents were placed in ureters of animals in group B. Ureters without a stent placement were placed into group C. Drainage potency and anti-reflux properties of the animals in the three groups were examined, and the data was compared and analyzed.

Results: For the detection of drainage potency and anti-reflux property animals underwent surgery, and saline was injected into the pelvis fistula under pressure of $30 \mathrm{cmH}_{2} \mathrm{O}$ and $50 \mathrm{cmH}_{2} \mathrm{O}$. The difference in drainage potency of stents in animals in group A and group B was not statistically significant $(\mathrm{P}>0.05)$, however it was superior to that of animals in group $\mathrm{C}(\mathrm{P}<0.05)$. No stent that was placed in animals in group A showed anti-reflux properties at the time of urination $(0 \%, 0 / 6)$, whereas all stents placed in animals in group $\mathrm{C}$ displayed anti-reflux property $(100 \%, 6 / 6)$. The average ureteropelvic pressures in groups A, B, and $\mathrm{C}$ were $16.67 \pm 1.63 \mathrm{mmHg}, \quad 8.17 \pm 0.75 \mathrm{mmHg}$, and $7.08 \pm 0.90 \mathrm{mmHg}$, respectively, and differences between groups were statistically significant $(\mathrm{P}<0.05)$.

Conclusions: We found that the anti-reflux ureteral stent displays better anti-reflux properties compared to a traditional ureteral stent. In addition, the drainage potency between antireflux ureteral stents and ordinary ureteral stents was similar. However, the anti-reflux stent has certain limitations, which should be improved through clinical studies. To reduce postplacement complications in patients, all product properties should be perfected before application in the clinic. The antireflux device of the ureteral stent is designed to target internal reflux. We believe that the VUR that occurs after stent placement is mainly caused by internal reflux.

MP7-6 A prospective observational study about stentrelated symptoms after ureteroscopy assessed through a validated questionnaire.

E Alessandria, A Bosio, E Dalmasso, D Peretti, A Bisconti, P Destefanis, $\mathrm{P}$ Gontero

Città della Salute e della Scienza di Torino Italy

Introduction \& Objective: Double J ureteral stents can cause major discomfort. Nevertheless, they are still often used at the 
end of ureteroscopy. The Ureteric Stent Symptoms Questionnaire (USSQ) is a specific validated tool to evaluate the impact of ureteral stents on different aspects of life.

The objective of our study was to evaluate how patients tolerate ureteral stent after ureteroscopy using the USSQ.

Materials and Methods: A prospective single-institutional observational study was organized. Since January 2010 to October 2015 , after a semirigid or flexible ureteroscopy with double $\mathrm{J}$ positioning, patients were asked to complete the validated Italian version of the USSQ. The USSQ consists of 6 sections exploring urinary symptoms, body pain, general health, work performance, sexual matters, additional problems and general satisfaction.

Endpoint of the study was to assess the true extent of stent-related symptoms in the population and their impact on quality of life.

Results: 232 patients completed the USSQ. 59\% experienced a daily urinary frequency $\geq 1$ hour. $90 \%$ had $\geq 1$ micturition during the night. $87 \%$ complained of urgency, $37 \%$ of urge incontinence. $82 \%$ experienced burning at voiding and $81 \%$ sensation of incomplete bladder emptying. $69 \%$ had episodes of macroscopic hematuria. Urinary symptoms represented a problem for $88 \% .79 \%$ would feel unhappy if they had to spend the rest of their life with their urinary symptoms. The stent was cause of pain or discomfort in $83 \%$. Median Visual Analogue Scale (VAS) score was 5 (range $0-10)$. Pain was felt in the kidney back area by $31 \%$, in the kidney front/side area by $26 \%$ and in the bladder area by $31 \% .73 \%$ complained of pain during physical activities. Pain caused sleep interruption in $44 \%$, occurred at voiding in $77 \%$ and interfered with everyday life in $77 \%$. 47\% used painkillers. General health, working and sexual activity were also negatively influenced by the stent: patients needed bed rest for a median of 2 days and reduced their daily activities for a median of 5 half days, $45 \%$ reduced their usual working hours and $46 \%$ stopped sexual life after stent insertion. $62 \%$ of patients would be unsatisfied (52\% unhappy or terrible) if further ureteral stenting would be proposed in future.

Conclusions: Double J ureteral stents after ureteroscopy heavily affect all USSQ domains and patients $\&^{\prime}$ quality of life. Pain, urinary and sexual symptoms adversely influence daily life and working activity in the majority of patients. Stents should be placed only when absolutely needed and should be left in place as short as possible.

MP7-7 A multi-centre analysis of antibiotic susceptibility of urinary tract isolates in patients with ureteric stents and associated UTI.

A McPhee, N Smyth, M Hinckley, W Ho, D Clark, B Jones, L Cottom

Glasgow Royal Infirmary

United Kingdom

Introduction \& Objective: Indwelling ureteric stents can act as a nidus for infection and as such are associated with an increased incidence of urinary tract infection (UTI). In a previous study at a single centre we demonstrated a significant difference in the bacterial isolates in patients with ureteric stent associated UTI compared to the epidemiological data collected by Health Protection Scotland (HPS). Furthermore a significant difference in the antibiotic susceptibility of comparable urinary tract isolates was noted. This study aims to expand upon the last by reviewing patients across all seven hospitals within the NHS Greater Glasgow \& Clyde (NHS GG\&C) health board.

Materials and Methods: We performed a retrospective analysis of a 12 month period across all hospitals in our health board.
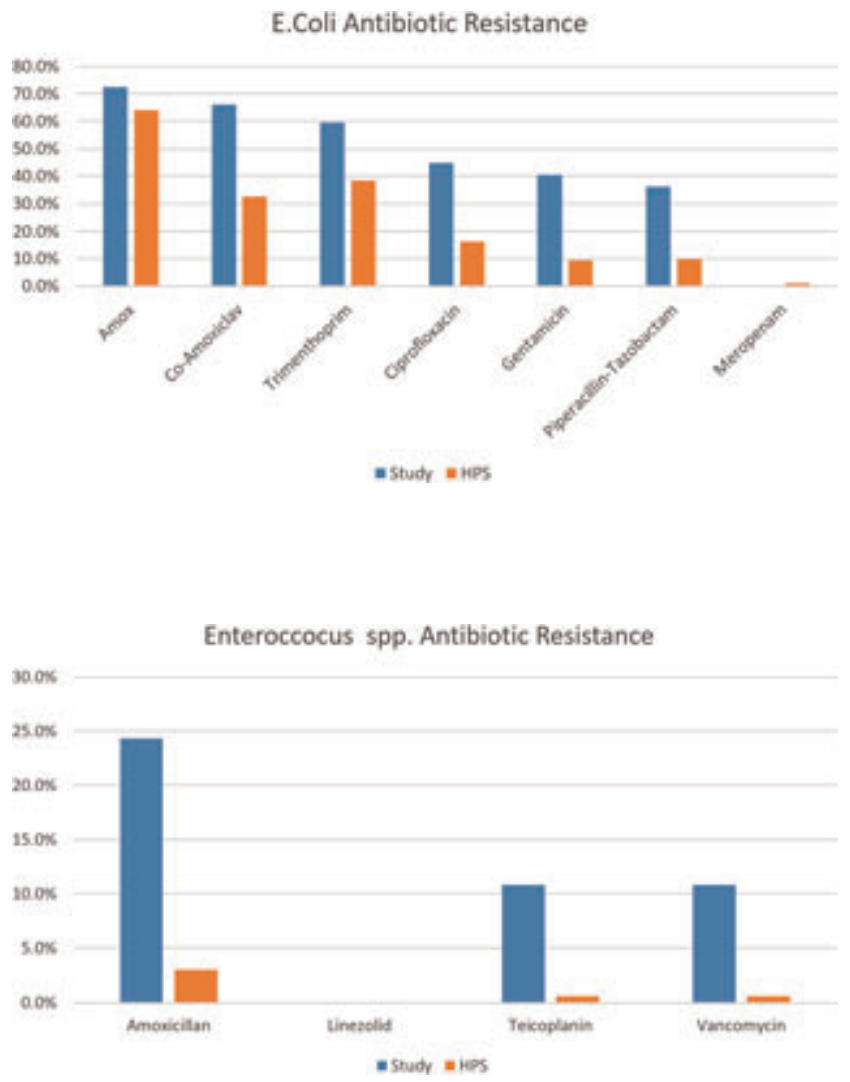

Patients were identified using our electronic records system (Opera/Clinical Portal). Patients with a microbiologically proven UTI associated with a ureteric stent were included, renal transplant patients were excluded.

Results: 647 patients were identified with an indwelling stent. Of these $129(19.9 \%)$ had positive bacteruria. A statistically significant difference in the distribution of urinary tract isolates in our study population was demonstrated when compared to epidemiological data from HPS. Furthermore the Antimicrobial susceptibility of urinary tract isolates in our population was also compared to HPS resistance data. A statistically significant increase in resistance towards several antibiotics was demonstrated in Escherichia coli \& Enterococcus spp.

Conclusions: Our study shows that urinary isolates associated with ureteric stents were more resistant to antibiotics used as empirical therapy in NHS GG\&C and as peri-operative prophylaxis. We believe empirical antibiotic guidance for such patients should be re-evaluated This study also raises questions regarding the high level of resistance demonstrated. Does this population represent the tip of the spear of antimicrobial resistance and are worthy of ongoing surveillance. Or does the process of having an indwelling stent encourage resistance through biofilm development or some as yet unknown mechanism.

\section{MP7-8 Parallel and Intraluminal Stenting}

M Eshghi, A Fathollahi, M Ferretti, SA Fullerton, JH Hillelsohn, NH Patel

New York Medical College

United States 
Introduction \& Objective: In the majority of cases after ureteroscopy double pigtail stent is placed under fluoroscopic control to confirm proper positioning. In certain cases such as pregnancy the use of fluoroscopy is not advised. We described the technique of stent placement after rigid or flexible ureteroscopy without the need for fluoroscopic control.

Materials and Methods: In the earlier years of ureteroscopy rigid large diameter ureteroscope with 10-12 Fr size were used at the completion of the procedure we preformed intraluminal stenting. A 4-5 Fr stent was introduced to the lumen of ureteroscope over a wire with the tip of scope at the level UPJ. Once the proximal end of the stent entered and was positioned in the renal pelvis. The rigid ureteroscope would be withdrawn with the stent held in place with a 5 Fr catheter to serve as a pusher. Once the tip of the ureteroscope is placed at the bladder neck the wire is removed and the stent's distal end would curl in the bladder. Even more modern semi rigid ureteroscopes of 9-10 Fr size have a 5 Fr lumen which still allows for this maneuver to be preformed. We use parallel stenting technique in the last 2 decades with the use of flexible ureteroscope. In this technique while the tip of the flexible ureteroscope is at the UPJ we advance a 4.8 Fr stent parallel to the stent while observing the entry into the renal pelvis. The ureteroscope is withdrawn while the stent is held in place with an open ended catheter or a regular pusher in female patients. The ureteroscope is withdrawn and the distal end positioning is the same as with rigid ureteroscopy. The slim design of the modern digital flexible ureteroscope allows for this maneuver to be done very easily which is of great value during ureteroscopy cases in pregnancy. In other situations it eliminates several steps of changing scopes and reduces operating room time.

Results: These 2 techniques with their modifications over a period of 30 years have been used in at least over 500 cases and procedures were reported as stent placement under fluoroscopic control in OR documents and not specifically identified as a separate technique. In rare cases parallel technique (less than $5 \%$ ) the parallel techniques failed due to inadequate lumen in the ureter. In 3 cases the proximal end migrated distally which was noted at the time and was adjusted. No complications occurred as a result of these maneuvers. The obvious limitations of this technique is that it cannot be used for large stents intraluminally and in some cases in the parallel technique.

Conclusions: We will demonstrate the technique of intraluminal and parallel stenting to decrease fluoroscopic and operating time without any compromise, it is less traumatic at the same time it does not require any additional instruments.

\section{MP7-9 Ureteric Stenting: Analysis Of Antegrade \& Ret-} rograde Procedures And Timeliness Of Removal

P Parameshwaran, K Stephenson, N Sheikh

Basildon \& Thurrock University Hospital, UK United Kingdom

Introduction \& Objective: In the UK, approximately 12,000 patients are admitted yearly due to nephrolithiasis, with stones having a prevalence of $2-3 \%$ across the population. Though the typical presentation is one of acute pain, ureter obstruction compounded by urosepsis is the true emergency and ureteric stenting the forerunner as the therapy of choice.

The objective was to test the correlation between ureteric stent options and demographic status and anatomical location, and calculate the mean time to stent removal.
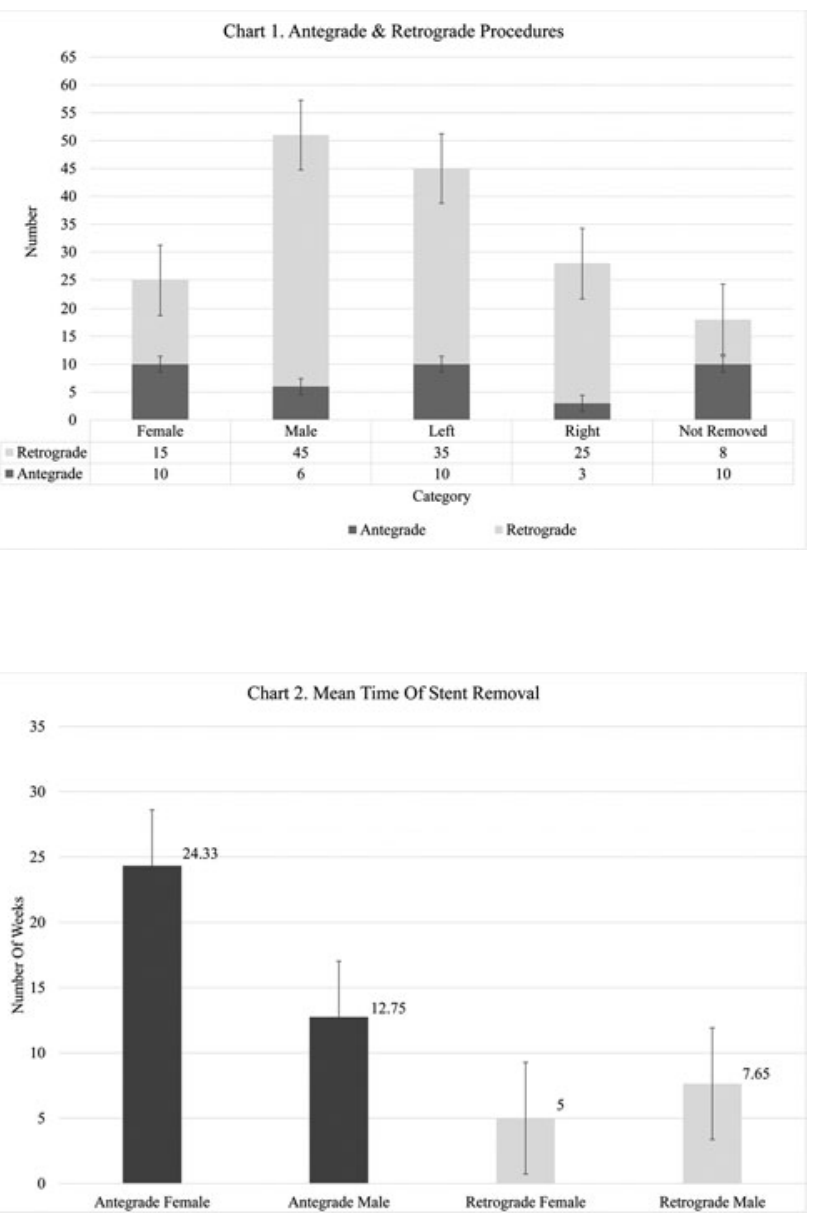

Materials and Methods: 76 patients were enrolled into this retrospective study at Basildon \& Thurrock University Hospital during the year 2016. Data was obtained from electronic medical records and Ormis software.

Results: A ratio of 2.04 males:females with mean ages of 53.24 $( \pm 14.09)$ and $54.08( \pm 18.79)$ respectively was in keeping with literature. Females presented younger with a modal age of 33 compared to 47 in males, and had a lower value for the range of age presentation at 18 versus 23 in males.

$40 \%$ of females had radiological therapy by antegrade procedures compared to $13.3 \%$ in males. A predominance of urethral tract obstruction to develop on the left in both males (31/49 cases) and in females (14/24 cases) was seen (chart 1).

Chi-Square calculations were performed. P (0.04558) was significant $(<0.5)$ when testing antegrade and retrograde procedures to gender. No further statistical significance was obtained for procedure and ureter position, or gender and ureter position.

Conclusions: In this study, antegrade stenting was more commonly performed in females, but retrograde stenting was more appropriate overall. The mean time to for stent removal is 12.43 weeks. 18 patients are still waiting for their stent to be removed, $62.5 \%$ of patients with antegrade stents. The mean time taken for stent removal exceeds the suggested literature value of 6 weeks, particularly for antegrade stents (chart 2).

This remains an area of development and should be targeted to each individual and clinical presentation. A potential for future consideration is the implmentation of a stent registry to ensure timely removal. 
MP7-10 Comparison of polymer and Metallic ureteral stent in the management of benign and malignant ureteric obstructions: Six years experience

A Ismail, N Chia, G Lee

University Malaya Kuala Lumpur

Malaysia

Introduction \& Objective: The reconstructive management of ureteric strictures, both of benign and malignant natures may not be desirable or possible due to various risk factors. The repeated utilization of the polymer stents has been recognized to be the standard of care, but the emergence of the metallic ureteral may play a role to reduce the interval of stent change and increase radial strength. We compare our six-year experience of Resonance stents with the polymer prosthesis, in the management of ureteric obstructions, assessing the patency rates, adversity and cost in the treatment of both malignant and benign strictures.

Materials and Methods: Retrospective analysis of patient with Resonance stent insertions between 2009 and 2016 were carried out. Patients demographic, etiology of occlusion, stent patency and complications were recorded. The necessity for stent change was identified by deterioration of renal function patients with bilateral stents.

Results: Forty-four Resonance stents were inserted in 24 patients, of whom 14 had bilateral stents, ten unilateral and six reinsertions (all bilateral). The mean age was 60 years (Range 38-79). Fourteen men and 10 women were treated, with 28 stents in malignant obstructions and 16 benign causes. The mean follow-up was 38 months ( 3 to 70 months). The longest functioning stent was 55 months. The stent patency for both malignant and non-malignant causes was $75 \%$. Half of the patients with malignancy were deceased with functioning stent. The mean interval of change due to obstruction was 11 months (7 to 23 months). The non-malignant group had patency interval of 18 months ( 3 to 70 months) and mean interval of change is 12 months in 3 patients. The polymer stents were inserted in 18 patients with strictures, of whom 10 were for palliative malignancy. The mean interval of change was 4 months in both malignant and benign strictures, with monitoring up to 36 months. Equal number of patients in each arm had recurrent urinary tract infections (6 patients), sepsis ( 2 patients) and one metallic stent migration noted. The mean total costs of polymer intervention were USD36K, compared to USD5K in Resonance arm.

Conclusions: The Resonance metallic ureteral stent is an effective alternative to polymer-based stents for select patients with upper urinary tract obstruction. The potential advantages of metallic ureteral stents include greater tensile strength allowing increased dwell times. Our results demonstrated good stent efficacy in both malignant and benign ureteric obstructions. Stringent monitoring of renal functions is warranted to prevent stent occlusions.

MP7-11 Risk factors for re-infection in patients drained with DJ stent for ureterolithiasis and sepsis

E Kord, G Goltsman, S Hirsh, Y Siegel, A Zisman

Assaf harofe medical center

Israel

Introduction \& Objective: Currently, there are no guidelines defining the optimal time frame for definitive stone treatment in stented patients after an event of urosepsis with ureteral stone and following resolution of infection. Objective: to evaluate risk factors for re-infection and re-admission in this sub-set of patients.

Materials and Methods: The records of 176 patients admitted between 2009-2016 due to urosepsis and ureterolithiasis and were primarily drained with a DJ stent, were retrospectively reviewed. A comparison was carried out between 129 patients with no re-infection and 47 patients $(27 \%)$ re-admitted prior to definitive stone treatment due to re-infection in the presence of a ureteral DJ stent. Univariate and multivariate analysis were performed.

Results: Re-infection rate that required admission was $26.7 \%$ (47/176). After comparing the two groups we had found that the presence of hypertension $(p=0.03)$, CKD $(p=0.05)$, A higher peak glucose level $(\mathrm{p}=0.008)$ and a higher peak creatinine level $(\mathrm{p}=0.001)$ during initial hospitalization were found to be statistically significant risk factors for recurrent infection. Patients with re-infection were more likely to be diagnosed later in their initial presentation with sepsis $(\mathrm{p}=0.01)$. In multivariate analysis, we found that during initial hospitalization, every $1 \mathrm{mg} / \mathrm{dl}$ increase in peak creatinine level above $1.2 \mathrm{mg} / \mathrm{dL}$ and an increase in $10 \mathrm{mg} / \mathrm{dL}$ peak glucose level above $140 \mathrm{mg} / \mathrm{dL}$ conveys an OR of $1.41(\mathrm{p}=0.002)$ and $2.05(\mathrm{p}=0.05)$ for recurrent infection respectively. $37.5 \%$ of patients re-admitted for re-infection do so within 3 weeks following the primary septic episode. Mortality: one case $(0.8 \%)$ during the primary septic episode and three cases $(6.3 \%)$ during re-admission.

Conclusions: Patients who suffer from hypertension and chronic kidney disease or present a higher glucose and creatinine peak values during initial hospitalization are at risk for re-infection after drainage with DJ stent due to urosepsis and ureterolithiasis. Recurrent infection requiring re-admission is most probable during the first 3 weeks after discharge possibly due to an inability to eradicate urinary bacteria in the presence of a ureteral stent. During the period between drainage and definite stone treatment, an episode of re-infection is associated with an increased risk for mortality. Early definitive stone treatment following primary sepsis and drainage may eliminate re-infection rate.

\section{MP7-12 Forgotten Double J Catheter Management}

AM Cardenas Ortiz, LF Takano Araujo, PR Kawano, HA Yamamoto, R Guerra da Silva, P Pajolli, OE Hidetoshi Fugita

UNESP

Colombia

Introduction \& Objective: Xanthogranulomatous pyelonephritis (XGPN) is considered a relative contraindication to laparoscopic nephrectomy (LN) because of the potential surgical difficulty. The objective of this stydy is to identify preoperative predictors of surgical difficulty or conversion from laparoscopic to open approach in XGPN based on clinical, laboratory and tomography $(\mathrm{CT})$ parameters

Materials and Methods: 320 patients from 2006 to 2015 of that underwent transperitoneal laparoscopic nephrectomy for benign conditions, among which $23(7.18 \%)$ had a confirmative histopathological diagnosis of XGPN were further analyzed. Data regarding age, sex, operative time, bleeding, length of hospital stay, radiological exams and complications were evaluated.

Results: Three hundred and twenty patients underwent transperitoneal laparoscopic nephrectomy for benign conditions 
between 2006 and 2016, among whom 23 (7.18\%) had a confirmatory histopathological diagnosis of XGPN. The mean age was $46 \pm 17,6$ years. Mean BMI was $25 \pm 6,38$. Five cases $(21.7 \%)$ had open conversion. Operative time was longer in cases of staghorn stone and open conversion ( $\mathrm{p}$ : <0,0001). Bleeding rate was longer in the presence of staghorn stone (p: 0,006) in patients with conversion to open approach (p: <0.0001) and in patients that developed any complication (p: 0,03). There was higher hospital length stay between the patients that had a conversion of approach (p: <0,001).

Conclusions: The laparoscopic treatment of XGPN remains challenging and controversial, due to the technical difficulties posed by the inherent inflammatory process. In our series the bleeding rate is the factor that predict open conversion, higher operative time, higher probability of complication and it is in relation with the presence of staghorn stone. Higher grade complications will be less if novice surgeons are accompanied by advanced laparoscopic surgeons while obtaining the surgical proficiency curve.

MP7-13 Two weeks too long: Optimal duration for ureteric pre-stenting and its physiological effects on the ureter in a porcine model.

K Lim, Y Lim, L Ng, A Sim

Singapore General Hospital

Singapore

Introduction \& Objective: Introduction: Ureteral stents are an integrate part of any Urological practice. Pre-stenting of the ureter is commonly performed to allow for passive dilatation and better access to the urinary system during subsequent procedures. EAU guidelines suggest a 1-2 weeks duration for pre-stenting. There is minimal literature on the incidence of pre-stenting and there is no consensus on the duration required to achieve ureteral dilatation. Objective: Using a porcine model, our primary objective is to investigate the temporal rate of ureteral dilatation, which in turn informs the minimal duration required for prestenting to achieve significant ureteric dilatation over 2 weeks. Our secondary objective is to measure the ureteral compliance of the stented and the un-stented ureters.

Materials and Methods: Methods: A total of three female pigs between $40-50 \mathrm{kgs}$ were used. Intravenous pyelograms (IVP) were performed and ureteral measurement taken on day $0,5,7$, and $14.100 \mathrm{ml}(2 \mathrm{ml} / \mathrm{kg})$ of intravenous contrast was infused on each day. An abdominal binder was applied during infusion to compress the distal ureters and enhance contrast collection in the upper urinary tracts as per human protocol. 15 mins after infusion, the abdominal binder was released and serial IVP obtained. Unilateral stenting with $4.8 \mathrm{Fr} \times 26 \mathrm{~cm}$ stent on day 0,5 and 7 were performed. Stents were removed prior to IVPs at the following session and replaced thereafter. A blinded reader measured the maximal ureteral widths; a minimal of 3 readings at 4 levels was obtained bilaterally for each pig. On day 14, bilateral nephroureterectomy were performed and ureteral compliance was measured in the stent and un-stented ureter.

Results: There were significant amount of ureteric dilatation between day 0 and day 5 for all 3 pigs $\left(\mathrm{p}_{1}=0.001, \mathrm{p}_{2}=3=0.01\right)$. While ureteral dilatation continued up to day 14 , the rate of ureteral dilatation appears to plateau after day $5\left(\mathrm{p}_{1}=0.416\right.$, $\mathrm{p}_{2}=0.344$ and $\left.\mathrm{p}_{3}=0.774\right)$. Ureteric compliance in the stented ureter is better compared to a non-stent ureter $\left(P_{1}=0.65\right.$ vs. 0.13 ,
$P_{2}=0.8$ vs. $0.04, P_{3}=0.62$ vs. 0.2 ). An unexpected observation was the ureteric dilatation and increased tortuosity in the unstented ureter in 2 of the 3 pigs $\left(\mathrm{p}_{1}=0.152, \mathrm{p}_{2}=0.007\right.$ and $\mathrm{p}_{3}=0.018$ ).

Conclusions: Our results suggest optimal pre-stenting may be achieved in 5 days in a porcine model. It can potentially form the basis to start randomised human trials. Unilateral ureteric dilatation triggers a systemic response. A systemic vector may be responsible for ureteric dilatation.

\section{MP7-14 Retained Stents: who is at risk?}

RK Jain, H Chaparala, M Omar, S Sivalingam, MJ Noble, M Monga

Cleveland Clinic Foundation

United States

Introduction \& Objective: Encrustation of retained ureteral stents can lead to significant morbidity. We examined the treatment of patients with retained stents.

Materials and Methods: Patients with retained stents were identified in a prospectively collected stone registry at a high volume, tertiary care center. The electronic medical record was queried using a relational database management program to parse operative notes for the terms "retained" and "encrusted." The generated list was manually validated and data were collected and analyzed retrospectively.

We collected demographics, medical history, insurance type, sugical and posoperative data. Preoperative degree of encrustation was graded using FECal system (1-2: upper OR lower coil WITHOUT body involvement, 3: upper OR lower WITH body,

\begin{tabular}{|l|c|c|c|}
\hline & Control & Retained Stent & p-value \\
\hline $\mathrm{N}$ & 4962 & 66 & \\
\hline Age & $53.2 \pm 15.5$ & $50 \pm 18$ & 0.11 \\
\hline Sex (male) & $2776(57.17 \%)$ & $39(57.35 \%)$ & 1 \\
\hline BMI & $30.1 \pm 7.4$ & $29.7 \pm 8.7$ & 0.6 \\
\hline INSURANCE & & & $<0.001$ \\
\hline Private & $2538(51.86 \%)$ & $14(20.59 \%)$ & \\
\hline Medicare & $153931.45 \%)$ & $18(26.47 \%)$ & \\
\hline Medicaid & $318(6.5 \%)$ & $13(19.12 \%)$ & \\
\hline Self-Pay/None & $499(10.2 \%)$ & $23(33.82 \%)$ & \\
\hline COMPOSInON & & 51 & $<0.001$ \\
\hline COM & $3096(63.99)$ & $13(25.49 \%)$ & \\
\hline CAP & $783(16.18 \%)$ & $23(45.1 \%)$ & \\
\hline COD & $391(8.08 \%$ & 0 & \\
\hline UA & $441(9.12 \%)$ & $8(15.69 \%)$ & \\
\hline Struvite & $85(1.76 \%)$ & $3(5.88 \%)$ & \\
\hline Cystine & $3(5.88 \%)$ & $27(0.56 \%)$ & \\
\hline other & $1(1.96 \%)$ & $15(0.31 \%)$ & \\
\hline
\end{tabular}




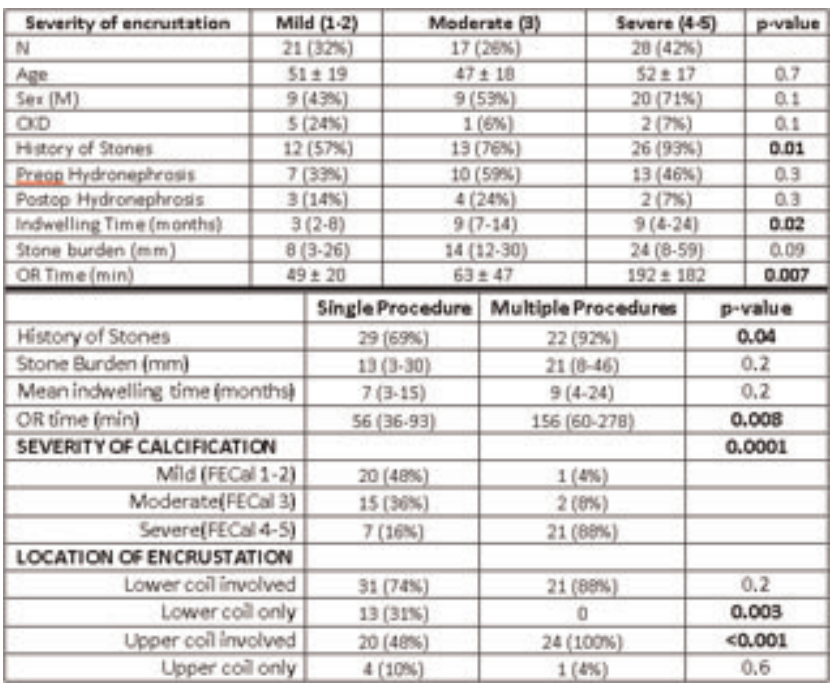

4: upper AND lower WITHOUT body, 5: upper AND lower WITH body).

A control group representative of patients undergoing ureteroscopy for urolithiasis was identified for comparison of demographic and insurance data.

Results: Sixty six patients with retained, encrusted stent were identified. Indication for stent insertion was most commonly obstructing stone $(53 \%)$, post-URS (15\%) and ESWL (11\%). The control group consisted of 4962 patients. There were no differences in age, BMI or gender distribution. The encrusted stent group were more likely to have Medicaid or no insurance $(\mathrm{p}<0.001)$. Longer indwelling time (mild: 3 mos, moderate/severe: $9 \mathrm{mos}, \mathrm{p}=0.02$ ) and prior history of stones predicted higher degree of encrustation, i.e. higher FECal grade (mild: 57\%, moderate: $76 \%$, severe: $93 \%, \mathrm{p}=0.01$ ). Patients with prior history of stones, higher grade encrustation and upper coil encrustation were more likely to require $>1$ procedure for stent clearance $(\mathrm{p}=0.04, \mathrm{p}=0.0001, \mathrm{p}<0.001$, respectively).

Conclusions: Encrustation of retained stents is a preventable phenomenon which can lead to significant morbidity. Indwelling time and history of stone disease are predictive of degree of encrustation. Upper coil encrustation increases complexity of management, more often requiring multiple procedures.

MP7-15 Ureteral Stent Placement in Clinic Using Nitrous
Oxide Analgesia: One Institution's Experience

GL Machen, PA Milburn, PS Lowry, MM El Tayeb, L Tsai

Texas A\&M College of Medicine/Baylor Scott and White United States

Introduction \& Objective: Given advances in technology, there is an increasing interest in performing endourologic procedures in clinic. In particular, stent placements have traditionally been completed in the operating room. At our institution, we utilize a nitrous oxide analgesia system which we believe allows us to perform multiple procedures safely in the clinic, including stent placements and exchanges. We attempt to briefly describe our experience below.
Materials and Methods: After IRB approval was obtained, a retrospective chart review was undertaken utilizing the CPT code 52332 to identify stent placements and exchanges. Stent placements done in the operating room were excluded. In each instance, either a 15.5 Fr flexible or 22 Fr rigid cystoscope were used. All patients received 1\% lidocaine jelly for topical anesthesia, and the vast majority were given a single dose of a periprocedural oral antibiotic. Standard fluoroscopy was utilized; retrograde pyelograms were performed at the discretion of the surgeon to aid in stent placement. Further, nitrous oxide analgesia was administered in select cases.

Results: A total of 565 stent placements were performed from $2 / 1 / 2014$ to $2 / 1 / 2016$. $59 \%$ of the patients were female, and the average age was $56.4 .76 \%$ of the procedures were primary stent placements, while $24 \%$ were exchanges. In $9.6 \%$ of the procedures, bilateral renal units were stented. The most common indication was an obstructing ureteral stone (61.4\%). Of note, pelvic malignancies causing ureteral obstruction were the indication in $9.4 \%$. The average procedure time was $12.7 \mathrm{~min}$ (SD 8.0). An average of $1.7 \mathrm{~min}$ of fluoro were used (SD 1.4). Nitrous was utilized in $77 \%$ of the procedures for an average of 11.8 minutes (SD 7.6). Average pre-procedure Aldrete scores were 9.95 (SD 0.32), while post-procedure was 9.98 (SD 0.18). A total of 50 complications occurred, the most common of which was UTI $(72 \%)$. Only 17 (3\%) had to be admitted post-procedure, most commonly for pyuria. Only 6 stent placements failed, including 1 aborted due to patient intolerance. Of the failures, 3 required PCN placement, while 3 had stents placed in the OR. None had any long term sequelae.

Conclusions: To our knowledge, this is the largest series of clinic endourologic procedures. Office stent placements and exchanges utilizing nitrous oxide seem to be a safe and tolerable alternative to placement in the OR given the low failure rate and lack of long term complications even in complicated stent placements. Additional benefits include avoidance of a general anesthetic and increased convenience for both the patients and surgeons.

MP7-16 Impact of loop-tail ureteral stents on ureteral stent-related symptoms immediately after ureteroscopic lithotripsy: Comparison with pigtail ureteral stents

M Taguchi, T Inoue, K Muguruma, T Murota, H Kinoshita, T Matsuda

Department of Urology and Andrology, Kansai Medical University

Japan

Introduction \& Objective: To evaluate urination-related quality of life (QOL) in patients with an indwelling ureteral stent immediately after ureteroscopic lithotripsy (URSL) for upper urinary calculi. We compared the effects of loop-tail and pigtail ureteral stents on urination-related QOL.

Materials and Methods: Of 135 patients who underwent URSL between May 2014 and March 2015 at our hospital, we retrospectively analyzed the records of 70 patients (42 men, 28 women; median age, 63 years) in whom the stent tail was positioned inside the bladder without crossing the midline and who completed the Core Lower Urinary Tract Symptoms Score questionnaire (CLSS) pre- and postoperatively.

Results: There were significant differences in incomplete emptying $(\mathrm{p}=0.048)$ and bladder pain $(\mathrm{p}=0.041)$ between patients 
with loop-tail versus pigtail ureteral stents after URSL. In the multivariate analysis, stent type had a stronger association with incomplete emptying $(\mathrm{p}=0.022)$ and bladder pain $(\mathrm{p}=0.018)$ than age, gender, BMI, stent side, operation time, diameter of ureteral access sheath and stent type.

Conclusions: Patients with loop-tail ureteral stents had better urination-related QOL in the immediate post-URSL stage than patients with pigtail stents.

MP7-17 Long-term outcomes of double-layered polytetrafluoroethylene membrane-covered self-expandable segmental metallic stents (Uventa) in patients with chronic ureteral obstructions: Is it really safe?

D Kim, M Kim, B Hong, H Park

Seoul Asan medical center

Republic of Korea

Introduction \& Objective: To evaluate the long-term clinical efficacy and safety of double-layered polytetrafluoroethylene membrane-covered self-expandable segmental metallic stents (Uventa) in patients with chronic ureteral obstruction.

Materials and Methods: In a retrospective study, a total of 50 ureter units (44 patients) with chronic obstructions were included from July 2010 to May 2015. Indications for Uventa placement were primary stenting for malignant ureteral obstruction, failed conventional polymeric Double-J stent (PS) or percutaneous nephrostomy (PCN) technique, with comorbidities or fears limiting PS/PCN changes, or with irritation or pain due to PS/PCN. Patients underwent Uventa stent placement using the antegrade or retrograde approach.

Results: There were no immediate procedure-related complications, and all stents were placed in the proper sites. During the median follow-up of 30.9 (IQR, 8.1-49.0) months, the primary (no obstruction and no additional intervention) and overall success (no obstruction and no additional intervention except supplementary Uventa) were $30.0 \%$ and $34.0 \%$, respectively. Moreover, 14 of 50 ureter units $(28.0 \%)$ experienced major complications ( $\geq$ Clavien-Dindo class IIIb), such as ureteroarterial fistula (3 cases, $6.0 \%$ ), ureteroenteric fistula $(3,6.0 \%)$, ureterovaginal fistula (one, $2.0 \%$ ), ureter perforation (one, $2.0 \%$ ), uncontrollable bleeding (one, $2.0 \%$ ), and complete obstruction (5, $10.0 \%)$. On univariate analysis, major complications were associated with female $(\mathrm{OR}=6.000)$, cervical cancer $(\mathrm{OR}=4.667)$, ureteral stricture length $(\geq 6.0 \mathrm{~cm}, \mathrm{OR}=4.583)$, and placement duration ( $\geq 24.0$ months, $\mathrm{OR}=20.429$; all $\mathrm{p}<0.05)$.

Conclusions: In long-term follow-up, the Uventa stent demonstrated poor treatment outcomes with frequent major complications in patients with chronic ureteral obstructions.

MP7-18 A swellable drug-eluting ureteral stent for the treatment of upper tract urothelial carcinoma - a proof of concept in-vivo porcine study

K Chen, W Lim, G Xiong, Y Huang, S Venkatraman, T Chong

Department of Urology, Singapore General Hospital,

Singapore

Singapore

Introduction \& Objective: Upper tract urothelial carcinoma unlike primary bladder urothelial carcinoma is relatively un- common but more lethal. Multiple agents have been investigated as adjuvant local therapy. However the challenge in the ureter remains a mechanical one. Achieving adequate contact and dwell time of topical therapy in a tubular structure with constant urine flow is crucial. We investigate the feasibility of a swellable drugeluting stent for delivery of mitomycin $\mathrm{C}(\mathrm{MMC})$ in the porcine ureter.

Materials and Methods: The delivery platform comprises of a conventional double $\mathrm{J}$ ureteral stent with a bilayer coating. The $1^{\text {st }}$ layer consists of a polymer coating dispersed with MMC, and the $2^{\text {nd }}$, a hydrogel that swells upon contact with urine to effect close apposition with ureteric mucosa facilitating lateral transfer of drug. Drug release kinetics was demonstrated by our group previously in ex-vivo bench tests.

The MMC coated (400ug) 6 Fr stents were inserted unilaterally in 3 female pigs, with the MMC segment positioned in mid ureter, to study the in-vivo pharmacokinetics of MMC in porcine ureteric tissue at 3 time points (post insertion Day 1, 2 and 8). Uncoated 6 Fr stents without MMC were inserted in contralateral ureter as reference. In a control pig resembling current clinical practice, uncoated $6 \mathrm{Fr}$ stents were inserted bilaterally and intravesical MMC (40 mg / $40 \mathrm{mls}$ saline) was instilled on day 8 for 1 hour before sacrifice. Intravenous urograms were performed and serum creatinine measured in all pigs prior to sacrifice. MMC levels were measured at the proximal, mid and distal ureter; and periureteric fat (mid ureter) by LCMS.

Results: At day 1, only the mid ureter of the test side, corresponding to position of MMC coated segment of stent, had significantly higher levels of MMC detected $(0.204 \pm 0.093 \mathrm{ng} / \mathrm{mg}$ of tissue) compared to the proximal and distal ureters $(0.003 \pm 0.001 \mathrm{ng} / \mathrm{mg}$ and $0.002 \pm 0.001 \mathrm{ng} / \mathrm{mg})$. MMC levels were greatly reduced by day 2 in the mid ureter $(0.070 \pm 0.106 \mathrm{ng} / \mathrm{mg})$. No MMC was detected on day 8. Serum and periureteric fat MMC levels on Day 1, 2 and 8 were negligible.

At day 1 , no hydronephrosis was seen despite a fully expanded hydrogel. On day 2 , hydronephrosis was seen although serum creatinine was normal. There were no technical issues with stent insertion or removal (ex vivo).

In the control pig with intravesical MMC, insignificant amounts of MMC were detected throughout the ureters.

Conclusions: We have developed a swellable drug-eluting stent prototype that exhibits targeted drug release in the porcine ureter within 48 hours of stent insertion. This proof of concept will form the basis of targeted delivery of MMC in the treatment of upper tract urothelial carcinoma.

MP7-19 Uventa, a new covered metallic ureteric stent: initial experience in a United Kingdom centre.

A Pai, S Janardanan, P James, H Ni Raghallaigh, R Kulkarni

St. Peter's Hospital

United Kingdom

Introduction \& Objective: Patients presenting with malignant ureteric obstruction have a limited life span. Metallic stents are superior to conventional JJ stents due to their longer patency and reduced need for change. We present our initial experience with a new covered metallic stent - Uventa, in this group of patients. Materials and Methods: An ethical committee approved clinical trial has been set up for the evaluation of this stent. Ten patients with malignant ureteric obstruction with an anticipated life span of 6 months or more are eligible. Ease of insertion, 
technical aspects, decompression of upper tract obstruction, durability \& complications were evaluated. Requirement of ancillary procedures, migration, encrustation and obstruction were recorded prospectively.

Results: 10 insertions were performed in 7 patients. Median patient age was 68.0 years (39-85 years). One stent was misplaced. A JJ stent was placed through the uventa stent. In 6 patients the stent remained patent. 3 patients have died due to tumour progression. The stent was patent in all these patients. The duration of patency was 10, 12 and 12 months. Mean follow up was 8.8 months (range 2-18 months). Renal function improved in all patients. No stent related symptoms were reported. There were no migrations or stent related symptoms.

Conclusions: Our initial experience is satisfactory. Repositioning the stent can be difficult. Long term follow up is required for full evaluation.

\section{MP7-20 The ALLIUM ${ }^{\circledR}$ ureteral stent in the manage- ment of ureteral disorders}

F De Marco, C Corsi, L Grillenzoni, S Di Nicola, A Alfarone, M Pozza, U Parente, P Vicini, C Leonardo, G Ricciuti

INI - Urology Unit

Italy

Introduction \& Objective: Ureteral stenosis is severe, difficult to treat and significantly affects the quality of life of patients. Usually the definitive resolution is surgical of reconstructive nature or ureteral stent placement. We evaluated the use of new expandable ureteral stent (ALLIUM $®$ ) as alternative to standard indwelling ureteral stent or reconstructive surgery

Materials and Methods: From September 2013 to December 2016, 82 patients were enrolled in the study and underwent to endoscopic positioning of the urinary tract Allium ${ }^{\circledR}$. Of the 82 patients treated, 31 had ureteral stenosis post-ureterorenoscopy, in these patients an ureteral balloon dilatation was always performed, 36 patients had stenosis as a complication of oncological disorders, 2 stenosis in patients after ureteral re-implantation, 5 stenosis in neo bladder ureteral anastomosis, 5 patients with stenosis post pieloplasty. 3 patients with urinary fistula.

Results: Operating times were short (maximum 60 minutes), postoperative hospitalization was $<48$ hours in all cases except a case. There have been no cases with post-operative fever, no cases of stent migration. All patients with stenosis post-ureterorenoscopy removed the Allium ${ }^{\circledR}$ after 6 months, 23 of 31 had no hydronephrosis at 6 months follow-up, the other patients were submitted to reconstructive surgery. Patients with urinary fistula removed the Allium ${ }^{\circledR}$ stent after 6 months and in all 3 patients the fistula was resolved. Patients with neobladder ureteral anastomosis had no hydronephrosis at 6 and 12 months, in these cases the Allium ${ }^{\circledR}$ stent was not removed. In patients with stenosis post Pieloplasty, the Allium ${ }^{\circledR}$ stent was removed after 6 monts, in 2 cases other surgical procedure was required because of persistent hydronephrosis. In patient with stenosis due to oncological disorders, the Allium ${ }^{\circledR}$ stent was not removed, all patients that are still living had no significant hydronephrosis and did not have discomfort or other complications. All patients with stenosis after re-implantation and stenosis in neobladder anastomosis, did not remove Allium ${ }^{\circledR}$ and have complete resolution of hydronephrosis

Conclusions: Allium ${ }^{\circledR}$ ureteral can be considered a device that require a short learning curve, minimal post-operative compli- cations, lower negative impact on the quality of life of patients, and is effective in treating ureteral obstructions. However, we need more cases to understand if this device can be considered as first line treatment of ureteral stenosis

MP7-21 Retrograde pyelography predicts retrograde ureteral stenting failure and reduces unnecessary stenting trials in patients with advanced non-urological malignant ureteral obstruction

H Seo, J Chung, J Joung, Y Suh, J Kim, K Lee

Department of Urology, Center for the Prostate Cancer, Research Institute and Hospital of National Cancer Center Republic of Korea

Introduction \& Objective: Many previous studies have suggested baseline characteristics such as serum creatinine level, degree of hydronephrosis, male sex, level of obstruction, and presence of bladder invasion as predictive factors for retrograde ureteral stenting (RUS) failure in patients with malignant ureteral obstruction (MUO). The present study also aimed to evaluate other predictive factors, especially the clinical significance of intraoperative retrograde pyelographic (RGP) findings, for prediction of RUS failure and prevention of unnecessary and ineffective RUS trials in the management of patients with nonurological MUO from a single institute.

Materials and Methods: Between 2005 and 2014, medical records of 284 MUO patients with 712 RUS trials including 63 $(22.2 \%)$ having bilateral MUO were retrospectively reviewed. RUS failure was defined as the inability to place ureteral stents by

\begin{tabular}{|c|c|c|c|}
\hline Variables & Stent $(\mathrm{N}=244)$ & $\mathrm{PCN}(\mathrm{N}=40)$ & $\mathrm{p}$-value \\
\hline Age (years) & $54.1 \pm 13.1$ & $53 \pm 13.8$ & 0.622 \\
\hline Sex, Male/Female & $76 / 166(31.4 / 68.6)$ & $25 / 15(62.5 / 37.5)$ & 0.468 \\
\hline Body mass index $\left(\mathrm{kg} / \mathrm{cm}^{2}\right)$ & & & 0.901 \\
\hline Underweight & $21(8.6)$ & $2(5.0)$ & \\
\hline Normal & $217(88.9)$ & $37(92.5)$ & \\
\hline Obese & $6(2.5)$ & $1(2.5)$ & \\
\hline Patient's ECOG 0/1/2 & $205 / 35 / 4(84 / 14.4 / 1.6)$ & $33 / 7 / 0(82.5 / 17.5 / 0)$ & 0.801 \\
\hline Pre-stent Treatment & & & 0.974 \\
\hline Surgery & $35(14.5)$ & $5(12.5)$ & \\
\hline Radiotherapy & $28(11.6)$ & $5(12.5)$ & \\
\hline Chemotherapy & $155(64.3)$ & $27(67.5)$ & \\
\hline None or follow-up & $23(9.5)$ & $3(1.1)$ & \\
\hline Hydronephrosis, Bil/Unilateral & $50 / 194(20.4 / 79.6)$ & $13 / 27(32.5 / 67.5)$ & 0.049 \\
\hline Degree $1 / 2 / 3 / 4$ & $\begin{array}{l}20 / 80 / 87 / 18 \\
(9.8 / 39 / 42.4 / 8.8)\end{array}$ & $\begin{array}{l}2 / 11 / 11 / 8 \\
(6.3 / 34.4 / 34.4 / 25)\end{array}$ & 0.086 \\
\hline Primary cancer & & & 0.524 \\
\hline Gynecologic cancer & $55(22.5)$ & $9(22.5)$ & \\
\hline Lung cancer & $8(3.3)$ & $2(58.0)$ & \\
\hline Head and neck cancer & $1(0.4)$ & 0 & \\
\hline Osteologic cancer & $1(0.4)$ & $1(2.5)$ & \\
\hline $\begin{array}{l}\text { Usteologic cancer } \\
\text { Breast cancer }\end{array}$ & $1(0.4)$ & $4(10.0)$ & \\
\hline $\begin{array}{l}\text { Breast cancer } \\
\text { Colocrectal cancer }\end{array}$ & $11(4.5)$ & $8(20.0)$ & \\
\hline $\begin{array}{l}\text { Colocrectal cancer } \\
\text { Hepatobiliary cancer }\end{array}$ & $62(25.5)$ & $2(5.0)$ & \\
\hline & $6(2.5)$ & $14(35.0)$ & \\
\hline Stomach cancer & $91(37.4)$ & 0 & \\
\hline Hematologic cancer & $5(2.1)$ & 0 & \\
\hline Others & $3(1.2)$ & & \\
\hline $\begin{array}{l}\text { Baseline sCreatinine level } \\
\text { (mg/dL) }\end{array}$ & $1.3 \pm 0.8$ & $1.5 \pm 1.1$ & 0.062 \\
\hline sCr category $(\mathrm{mg} / \mathrm{dL})<1.5$ & $170(70.5)$ & $28(70.0)$ & 0.097 \\
\hline $1.5-2.5$ & $61(25.3)$ & $7(17.5)$ & \\
\hline$>2.5$ & $10(4.1)$ & $5(12.5)$ & \\
\hline \multicolumn{4}{|l|}{ Retrograde pyelography findings } \\
\hline $\begin{array}{l}\text { Severity of hydronephrosis } \\
\text { mild/moderate/severe }\end{array}$ & $\begin{array}{l}29 / 65 / 115 \\
(13.9 / 31.1 / 55)\end{array}$ & $\begin{array}{l}3 / 11 / 19 \\
(9,1 / 33,3 / 57.6)\end{array}$ & 0.807 \\
\hline $\begin{array}{l}\text { Obstruction level } \\
\text { None/distal/mid/proximal/multi } \\
\text { ple }\end{array}$ & $\begin{array}{l}2 / 103 / 67 / 45 / 27 \\
\text { (0.8/42.2/27.5/18.4/11. } \\
\text { 1) }\end{array}$ & $\begin{array}{l}0 / 18 / 8 / 8 / 6 \\
(0 / 45 / 20 / 20 / 15)\end{array}$ & 0.514 \\
\hline $\begin{array}{l}\text { Ureteral kinking } \\
\text { none/Z-shape/ pigtail shape }\end{array}$ & $\begin{array}{l}114 / 84 / 18 \\
(52.8 / 38.9 / 8.3)\end{array}$ & $\begin{array}{l}17 / 11 / 4 \\
(53.1 / 34.4 / 12.5)\end{array}$ & 0.658 \\
\hline Irreversibility of ureteral kinking & 31 (14.3) & $10(31.3)$ & 0.022 \\
\hline Abnormal ureteral lateralization & $18(8.2)$ & $2(6.3)$ & 1.0 \\
\hline Bladder invasion & $24(10.8)$ & $13(41.9)$ & $<0.001$ \\
\hline Stent size $(\mathrm{Fr})$ & $6.8 \pm 4.0$ & $6.5 \pm 0.5$ & 0.679 \\
\hline
\end{tabular}




\begin{tabular}{llcc}
\hline Times of Stenting & $2.5 \pm 2.6$ & $2.7 \pm 2.6$ & 0.668 \\
Stent duration (mean, months) & $8.6 \pm 12.5$ & $8.5 \pm 11.1$ & 0.028 \\
Survival & $37(15.2)$ & $4(10.0)$ & 0.475 \\
Overall Survival & $17.9 \pm 23.2$ & $11.4 \pm 12.9$ & 0.013 \\
I time (median, months) & & & \\
\hline
\end{tabular}

cystoscopy, recurrent stent obstruction within one month, or non-relief of azotemia within one week from prior RUS. Clinicopathological parameters and retrograde pyelographic findings (RGP) were analyzed to investigate the predictive factors for the RUS failure and conversion to percutaneous nephrostomy in multivariate analysis with a statistical significance of $\mathrm{p}<0.05$.

Results: The RUS failure was detected in $14.1 \%$. The mean number of RUS placements and indwelling duration of the ureteral stents were $2.5 \pm 2.6$ times and 8.6 \pm 4.0 months, respectively. Multivariate analyses identified chemotherapy, baseline serum creatinine level $>2.5 \mathrm{mg} / \mathrm{dL}$, normal body mass index, male sex, age, stent caliber, and several specific RGP findings as significant predictive factors for RUS failure $(\mathrm{p}<0.05)$. The significant RGP findings included grade 1-2 unilateral hydronephrosis, reversible Z-shaped ureteral kinking, irreversible ureteral kinking, presence of bladder invasion, and lateralized ureteral direction $(\mathrm{p}<0.05)$.

Conclusions: This study identified the importance of RGP findings and other significant predictive factors for preventing RUS failure and unnecessary and ineffective RUS trials.

\section{MP7-22 Withdrawn}

MP7-23 The rendezvous technique of Ureteric stenting: when, how and why?

S Janardanan, P James, A Pai, R Kulkarni

St. Peter's Hospital

United Kingdom

Introduction \& Objective: Conventional techniques of stenting may fail in complex ureteric strictures. A rendezvous technique combines the benefits of both ante-grade as well as retrograde approach. We present our outcomes of a protocol driven policy of primary rendezvous technique and points of technique.

Materials and Methods: We retrospectively analysed 31 patients who underwent rendezvous stenting in our institution between November 2011 to November 2016. This was considered if the ureteric obstruction was complete, distal, involving the ureteric orifice, in an ileal conduit or the stricture was longer than $1.5 \mathrm{~cm}$. It was also chosen if the strictures were multiple. The strictures were located in distal ureter in 27 patients, middle third in 2 and upper ureter in 2 . In 16 patients ureteric obstruction was caused by recurrent benign pathology such as radiation fibrosis, stone impaction, failed pyeloplasty and retro-peritoneal haematoma. In 15 it was caused by malignancy of colonic, prostate and bladder origin. In 25 patients, this was a primary procedure, in 4 after failed retrograde and in 2 failed ante-grade stenting. All patients had a nephrostomy inserted at least 24 hours before. General anaesthesia was preferred. Peri-operative antibiotics were used according to the institutional guidelines. The use of a Terumo guide wire, biliary manipulating curved tip catheter and ureterosope ensured a successful placement of the stent in all cases. 12 patients had tumour resection at the ureteric orifice.

Results: Stents were inserted in all patients. There were no failures. The median length of stay was 1 day $(1-16)$. There was no procedure related morbidity or mortality.

Conclusions: The rendezvous technique has a higher success rate than ante-grade or retrograde stenting. It is more comfortable for the patient, reduces the need for multiple procedures and shortens the length of stay and cost.

MP7-24 Malignant ureteral obstruction - Balloon dilatation and decompression using Tandem ureteral stents

A Shvero, N Kleinmann, M Laufer, ZA Dotan, D Zilberman, J Ramon, H Winkler

Department of Urology, Sheba Medical Center, Tel Hashomer, Israel

Israel

Introduction \& Objective: In cases of extraluminal malignant ureteral obstruction (MUO), it is essential to decompress the kidney in order to preserve kidney functionality. In most cases, ureteral stents are not durable to extraluminal pressure, and therefore, in many cases, percutaneous drainage is necessary. Percutaneous drainage has significant quality of life implications. In an artificial ureteral model, we found that decompression of an obstructed ureter using tandem stents - is more durable to extraluminal pressure than a single stent. In this study, we report our clinical experience with patients suffering from MUO who were treated with balloon dilation and insertion of tandem ureteral stents (TUS).

Materials and Methods: A retrospective review was performed of all patients undergoing TUS insertion for extraluminal MUO at our institution from June 2014 to May 2017. First, we performed a retrograde pyelography to characterize the obstructed segment in detail. Then we dilated the obstructed segment using a balloon dilator. Finally, two ureteral stents were inserted. Patients' courses were reviewed for clinical follow-up, imaging tests and serum creatinine levels.

Results: Our cohort included 59 patients (15 men and 44 women), and 83 renal units (44 right, 39 left). Median age was 60 years (range 27-85). 24 patients had bilateral obstruction. Median length of the obstructed segment was $4.5 \mathrm{~cm}$ (range 0.5 $10 \mathrm{~cm})$. Length of stay after the procedure was 1 day. Median follow-up time was 27 weeks (1-132). In 13 cases (15.6\%) there was failure in draining the kidney: in 5 cases due to UTI, and in 8 cases due to a rise in serum creatinine levels. Furthermore, in one case, the stents were taken out due to severe LUTS. Post-procedural serum creatinine levels were 0.46 lower than pre-procedural.

Conclusions: Using TUS in cases of extraluminal MUO is a proper solution in this patient group, with an improvement in quality of life. 


\section{MPS8: URETEROSCOPY II}

MP8-1 Placement of ureteral access sheath without fluoroscopic guidance: 122 cases in a single center

S Wang, X Zhang, B Xiao, W Hu, S Chen, J Li

Department of Urology, Beijing Tsinghua Changgung Hospital China (People's Republic)

Introduction \& Objective: Retrograde flexible ureteroscopy (RIRS) is widely performed in treatment of renal and upper ureter stones. In this procedure, a ureteral access sheath (UAS) is often needed to be placed. This process is generally completed under fluoroscopy for safety. In our practice, we finished 122 cases of flexible ureteroscopy without fluoroscopy guidance to see if a UAS could be safely placed without fluoroscopy.

Materials and Methods: A retrospective analysis of patients who underwent RIRS for renal and upper ureter calculus between December 2014 and December 2016 was performed. All patients received general anesthesia with standard lithotomy position. Ureteroscopy $(9.5 \mathrm{~F})$ was always performed initially before RIRS to evaluate the ureter. A wire was placed to guide the entrance of UAS, which was placed under direct vision of rigid ureteroscope. At the end of lithotripsy and removal of UAS, we used rigid ureteroscopy to check the entire length of the ureter to evaluate possible injuries. A 6Fr double $\mathrm{J}$ stent would be left for 3-4 weeks. Patients were evaluated for age, gender, size of the stone, placement of stent preoperative or not, duration of operation, postoperative hemoglobin. Complication rates were assessed according to the Post Ureteroscopic Lesion Scale and Modified Clavien Classification System.

Results: 122 patients ( 81 male, 41 female) with a mean age of 46.2 years (range 19-77 years) were included in the study, among which, 29 (21 male and 8 female) had a double J stent placed 1-2 weeks before RIRS. Mean stone size was $19 \mathrm{~mm}$ (range 8$25 \mathrm{~mm})$. UAS placement was successful in 111 patients including all those who had a double $\mathbf{J}$ stent placed preoperatively and 82 patients who hadn't received stent placement preoperative. Success rate of UAS placement was $90.98 \%$. PULS grade 1 lesions occurred in 7 patients while grade 2 lesions occurred in 3 patients. No grade 3-5 lesions were observed. Mean duration of operation was $115 \mathrm{~min}$ (range $58-137 \mathrm{~min}$ ). Mean temperature and hemoglobin on the 1 st postoperative day were $37.2^{\circ} \mathrm{C}$ (range $36.5-38.8^{\circ} \mathrm{C}$ ) and $136 \mathrm{~g} / \mathrm{L}$ (range $110-157 \mathrm{~g} / \mathrm{L}$ ), respectively.

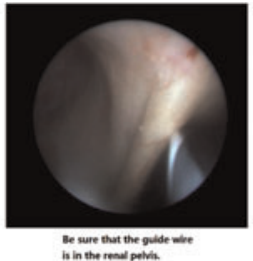

Ge sure that the gidde wite
is in the rend petich.

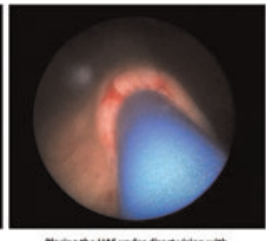

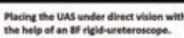

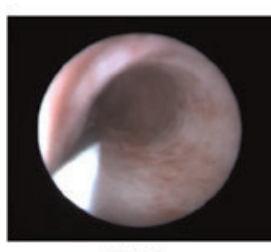

PULS 0

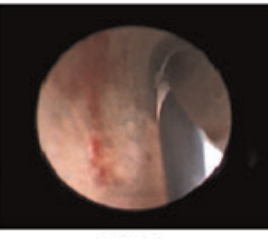

PULS 1

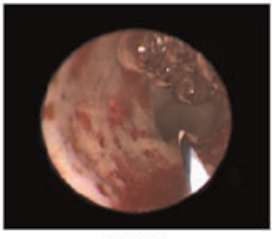

PUIS 2
Clavien I complications were noted in 30 cases (transient hematuria in 16 patients, fever in 14 patients). No major complications (Clavien II-V) were noted. During the 3-month follow-up after removal of the stent, there was no ureteral stricture.

Conclusions: Placement of UAS without fluoroscopy confirmed to be feasible and safe. It would reduce radiation exposure to the doctors and patients while not increase the risk of infection, bleeding and ureteral stricture. If resistance were encountered when entering the UAS, a direct monitor with 8Fr ureteroscope or a dilation with the inner core of the sheath could be helpful.

MP8-2 Accuracy of Predicting Stone Composition During Endoscopic Evaluation

T Marien, A Darves-Bornoz, J Robles, N Miller

Vanderbilt University Medical Center

United States

Introduction \& Objective: Knowledge of kidney stone composition is essential for the medical management of nephrolithiasis. The aim of this study is to assess the accuracy of stone composition prediction based on endoscopic evaluation.

Materials and Methods: A retrospective chart review was performed on all patients who underwent percutaneous nephrolithotomy or ureteroscopy for nephrolithiasis between October 2014 and September 2015 by an experienced Endourologist. Operative reports, stone analyses (SA), and medical history were reviewed. Known cystine stone formers were excluded. A stone component was considered predominant when it comprised greater than $50 \%$ of the specimen.

Results: 146 patients had endoscopic stone composition prediction and SA results available for review. Accuracy was high at

Table 1: Accuracy of Endoscopic Stone Composition Assessment

\begin{tabular}{|l|l|l|l|}
\hline Predominant Stone Composition & Sensitivity & Specificity & Accuracy \\
\hline Calcium (132) & $93 \%$ & $78 \%$ & $92 \%$ \\
\hline Uric acid (11) & $72 \%$ & $100 \%$ & $98 \%$ \\
\hline Infectious (3) & $100 \%$ & $94 \%$ & $94 \%$ \\
\hline
\end{tabular}

Table 2: Comparison of Correct and Incorrect Endoscopic Stone Composition Assessments

\begin{tabular}{|c|c|c|c|}
\hline & Correct $(n=134)$ & Incorrect $(n=12)$ & $P$ value \\
\hline Age, years old, mean (range) & $53(19-80)$ & $62(26-82)$ & 0.040 \\
\hline Female gender, no. (\%) & $69(51 \%)$ & $3(25 \%)$ & 0.079 \\
\hline $\mathrm{BMI}, \mathrm{kg} / \mathrm{m}^{2}$, mean (range) & $31(17-52)$ & $26(17-35)$ & 0.045 \\
\hline \multicolumn{4}{|l|}{ Laterality } \\
\hline Left & $63(47 \%)$ & $6(50 \%)$ & 0.79 \\
\hline Right & $65(48 \%)$ & $5(42 \%)$ & \\
\hline Bilateral & $6(5 \%)$ & $1(8 \%)$ & \\
\hline \multicolumn{4}{|l|}{ Procedure } \\
\hline Ureteroscopy & $114(85 \%)$ & $7(58 \%)$ & 0.017 \\
\hline Percutaneous nephrolithotomy & 19 (14\%) & $4(33 \%)$ & \\
\hline Both & $1(1 \%)$ & $1(8 \%)$ & \\
\hline \multicolumn{4}{|c|}{ Predominant stone composition, no. (\%) } \\
\hline Calcium oxalate (101) & $98(73 \%)$ & $3(25 \%)$ & 0.002 \\
\hline Calcium phosphate (32) & $26(19 \%)$ & $6(50 \%)$ & \\
\hline Uric acid (10) & $8(6 \%)$ & $3(25 \%)$ & \\
\hline Infectious (3) & $3(2 \%)$ & $0(0 \%)$ & \\
\hline Diabetes mellitus & $7(5 \%)$ & $3(25 \%)$ & 0.93 \\
\hline Urinary tract infections & $11(8 \%)$ & $7(50 \%)$ & $<0.0001$ \\
\hline Bowel segment in urinary tract" & $2(1 \%)$ & $6(58 \%)$ & $<0.0001$ \\
\hline Gout & $6(4 \%)$ & $0(0 \%)$ & 0.45 \\
\hline
\end{tabular}

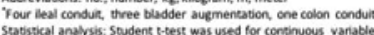


92-98\% (Table 1). The specificity for uric acid (UA) endoscopic prediction was $100 \%$ and the sensitivity for infectious stones was $100 \%$. Three predominantly UA stones were mixed with $22-24 \%$ calcium oxalate and were predicted to be calcium. Eight of the nine calcium stones predicted to be infectious contained calcium phosphate (CAP). These nine patients all had a history of urinary tract infections (UTIs) (7) and/or a bowel augment or diversion (6). On univariate analysis, age, BMI, procedure, stone composition, UTIs and augment/diversion were associated with incorrect assessment (Table 2). On multivariate analysis, age, and augment/diversion remained independent predictors $(\mathrm{p}<0.01)$.

Conclusions: On gross examination, there are characteristics of kidney stones that help predict stone composition with high accuracy. Incorrect prediction most commonly occurred when CAP stones were mistaken for infectious stone in patients with risk factors. Intraoperative description and prediction of stone composition is recommended as can be beneficial in instituting early alkalinization therapy for UA stones, managing infectious stones with stone culture and antibiotics, and can be helpful when the SA does not fit the clinical picture or the specimen is lost.

MP8-3 Fluid Dynamic Analysis of Manual Pressure Infusor Irrigation

DJ Lama, RM Patel, S Safiullah, J Landman, RV Clayman

Department of Urology, University of California, Irvine,

Orange, CA

United States

Introduction \& Objective: The manual hand-pump infuser device is a common method of compressing fluid to generate irrigation for endoscopic urologic surgery. To our knowledge, the flow rate and accuracy of the device's manometer has not been investigated. Herein, we performed an in vitro analysis of the handpump infuser during irrigation through a flexible ureteroscope with and without various sized instrumentation in the working channel. Materials and Methods: The Flex-X2 ureteroscope (Karl Storz Inc., Germany) was attached to a 3-liter bag of saline using standard irrigation tubing. The saline was placed into a handpump infuser (Ethox Infu-surg®, SunMed LLC., Grand Rapids, MI) and suspended from an intravenous pole. A three-way valve was attached with one valve to the irrigant, one valve to a digital manometer (DM8252, General Tools and Instruments LLC., Secaucus, NJ), and the third valve connected to the ureteroscope. Two trials were completed; flow and pressure measurements were performed at three hand-pump infuser settings (250, 200, and $150 \mathrm{mmHg}$ ) as indicated by the device's manometer. Once the device was set at the desired pressure, it was not reinflated over the course of the trial. Data were collected at set time intervals; the working channel flow was studied when it was open, or when occupied with a $200 \mathrm{~nm}$ holmium laser fiber (Cook Medical Inc., Bloomington, IN), a 1.7F Ngage nitinol stone basket (Cook Medical Inc. Bloomington, IN), and a $2.67 \mathrm{~F}$ guidewire (Amplatz Super Stiff, Boston Scientific Co., Malborough, MA). The digital manometer was tared before measuring the inflow pressure at each time interval.

Results: The hand-pump infuser's manometer underestimated the pressure of irrigant delivered to the ureteroscope by 45 to $50 \mathrm{mmHg}$ (Table 1). Flow through the ureteroscope decreased as the diameter of the instrumentation increased (Figure 1). Using the formula for exponential decay, $\mathrm{Ve}=\mathrm{Voe}^{\wedge}$-rt, where $\mathrm{Ve}=$ volume expected, $\mathrm{Vo}=$ volume observed, $\mathrm{r}=$ rate constant for $250 \mathrm{mmHg}$, and $\mathrm{t}=$ time the observed depletion of irrigant
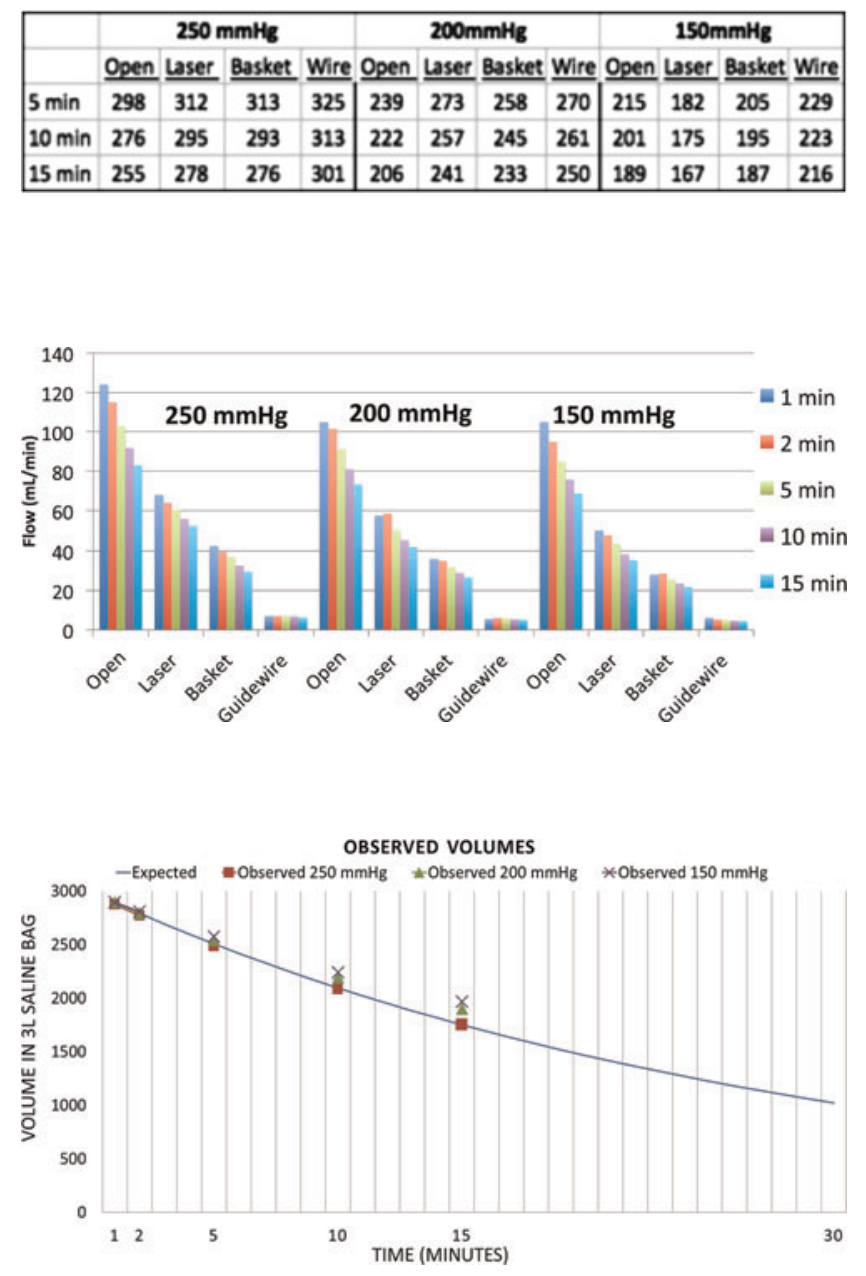

volume for an open-channel was similar to the expected exponential decay $(\mathrm{p}=0.678)$ (Figure 2$)$.

Conclusions: The pressure measured at the point of inflow to the ureteroscope is commonly $40 \mathrm{mmHg}$ or more greater than the pressure displayed on the hand-pump infuser's manometer. With an unoccupied working channel, irrigation volume falls exponentially, and thus an assistant is needed to frequently maintain the desired infuser pressure.

MP8-4 Moses Technology from Stone Simulator to Early Clinical Experience

A Ibrahim, S Badaan, S Andonian, M Elhilali

McGill University Health Centre

Canada

Introduction \& Objective: Holmium laser is the most frequently used energy for ureteroscopic lithotripsy but still has some limitations such as stone migration during lithotripsy. Lumenis has recently developed a new Holmium laser technology called "Moses", which was associated with reduced stone retropulsion in in vitro and animal studies. However, there are no simulator nor clinical studies evaluating the effectiveness of this new Moses technology in minimizing stone retropulsion during laser lithotripsy. Therefore, our objectives were to compare the 
fragmentation efficiency of the new Moses mode with the conventional Regular mode using a stone simulator model. In addition, we compared both modes in a pilot clinical study in terms of stone retropulsion and peri-operative complications.

Materials and Methods: The Lumenis ${ }^{\circledR}$ Pulse $^{\mathrm{TM}} \mathrm{P} 120 \mathrm{H}$ Holmium laser system together with Moses D/F/L fibers were used to compare Regular mode with Moses mode in a stone simulator model using flexible ureteroscopy with pre-prepared artificial stones (plaster of paris). Efficiency of laser lithotripsy was measured by procedural time, and lasing-pausing ratio. The two modes were then compared in a pilot clinical study, where the degree of stone retropulsion was graded subjectively on a Likert scale from zero-no retropulsion to 3-maximum retropulsion.

Results: With the stone simulator model, when compared with the Regular mode, the Moses mode was associated with significantly shorter procedural time (13.9 vs. $9.1 \mathrm{~min})$ for fragmentation and (9.3 vs. $7.1 \mathrm{~min}$ ) for dusting (average $35 \%$ reduction for fragmentation and $23 \%$ for dusting settings, $\mathrm{p} \leq 0.01$ ) and less pausing time during each procedure, $\mathrm{p} \leq 0.01$. A significant reduction in stone retropulsion was noticed when using Moses mode $(\mathrm{p}<0.01)$. However, There were no significant differences between both modes in total lasing time and total energy required to fragment stones $(\mathrm{p}>0.05)$. Twenty-two patients were included in the pilot clinical study. When compared with the Regular mode, Moses mode was associated with significantly less retropulsion $(p=0.002)$. Subjectively, two grades differences were observed between both modes. No intra-operative complications were detected. The stone-free rate after one month was $90.9 \%$.

Conclusions: Using the stone simulator setup, the Moses technology was associated with more efficient laser lithotripsy (shorter procedural time and less pausing time) due to significantly reduced stone retropulsion. The initial clinical experience demonstrated the safety of this new technology.

\section{MP8-5 Evaluating the Ergonomics of Flexible Uretero- scopy}

WW Ludwig, G Lee, J Ziemba, BR Matlaga

Brady Urological Institute, Johns Hopkins School of Medicine United States

Introduction \& Objective: To date, the ergonomics of flexible ureteroscopy (URS) have not been well described. We performed a study to define the biomechanical stresses on the urologist performing URS, and also to investigate the effect of the type of ureteroscope on these parameters.

Materials and Methods: Electromyography (EMG) was used to quantify the activation level of the muscle groups used in URS. Surface EMG electrodes (Delsys, Boston, MA) were placed on the right and left thenar eminence, flexor carpi ulnaris (FCU), extensor carpi ulnaris (ECU), biceps, triceps and deltoid. Three endoscopes were studied: single-use digital (Boston Scientific LithoVue); reusable digital (Karl Storz Flex- $X^{\mathrm{c}}$ ); reusable fiberoptic (Karl Storz Flex- $\mathrm{X}^{2}$ ). Each ureteroscope was used to perform a set sequence of tasks in a training model. The maximum voluntary contraction levels for each muscle were recorded; data were collected at $1,000 \mathrm{~Hz}$ to normalize activation levels between muscle groups. EMG recordings were full-wave rectified and the time integral of data was used to calculate cumulative muscular workload (CMW). CMW was adjusted by performance time to calculate average muscular work per second.

Results: The URS CMW was greatest for the ECU, followed in descending order by the right and left thenar eminence, FCU,

Table 1. Average CMW.
\begin{tabular}{|c|c|c|c|c|c|c|c|}
\hline & $\begin{array}{c}\text { Right } \\
\text { Thenar }\end{array}$ & FCU & ECU & Biceps & Triceps & Deltoid & $\begin{array}{c}\text { Left } \\
\text { Thenar }\end{array}$ \\
\hline LithoVue & 227.0 & 72.6 & 305.7 & 20.6 & 12.8 & 20.6 & 133.9 \\
\hline Flex-X & 175.5 & 66.9 & 285.5 & 25.6 & 12.1 & 24.7 & 134.3 \\
\hline Flex-X $\mathbf{X}^{\mathbf{2}}$ & 371.3 & 113.0 & 492.8 & 60.0 & 17.2 & 49.5 & 196.7 \\
\hline
\end{tabular}

Table 2: Average Muscular Work per Second.
\begin{tabular}{|c|c|c|c|c|c|c|c|}
\hline & $\begin{array}{c}\text { Right } \\
\text { Thenar }\end{array}$ & FCU & ECU & Biceps & Triceps & Deltoid & $\begin{array}{c}\text { Left } \\
\text { Thenar }\end{array}$ \\
\hline LithoVue & 3.0 & 0.9 & 3.9 & 0.2 & 0.2 & 0.3 & 1.7 \\
\hline Flex- $\mathrm{X}^{\mathrm{c}}$ & 2.4 & 0.9 & 3.8 & 0.4 & 0.2 & 0.4 & 1.8 \\
\hline Flex- $\mathrm{X}^{2}$ & 3.6 & 1.1 & 4.7 & 0.6 & 0.2 & 0.5 & 1.9 \\
\hline
\end{tabular}

biceps, deltoid and triceps. Both LithoVue and Flex- $\mathrm{X}^{\mathrm{c}}$ had lower CMWs for every muscle group when compared to the Flex- $\mathrm{X}^{2}(\mathrm{p}=\mathrm{c}$ ureteroscopes had similar average muscular work per second and both were lower than the Flex- $\mathrm{X}^{2}$ for the right thenar, ECU, biceps and deltoid activation $(\mathrm{p}=<0.05)$ (Table 2). Conclusions: This study provides the first description of EMGmeasured ergonomics of URS. The LithoVue and Flex- $X^{\mathrm{c}}$ ureteroscopes have similar profiles, and both have significantly better ergonomic metrics than the Flex- $\mathrm{X}^{2}$.

MP8-6 Crowd Sourcing Evaluation of Ureteroscopic Videos using the Post-Ureteroscopic Lesion Scale (PULS) to Assess Ureteral Injury Following Ureteral Access Sheath Deployment

KS Kaler, K Bettir, S Safiullah, DJ Lama, R Yoon, J Landman, RV Clayman

Department of Urology, University of California, Irvine United States

Introduction \& Objective: We hypothesized that crowdsourcing assessment could be applied to the PULS Scale for ureteral injury.

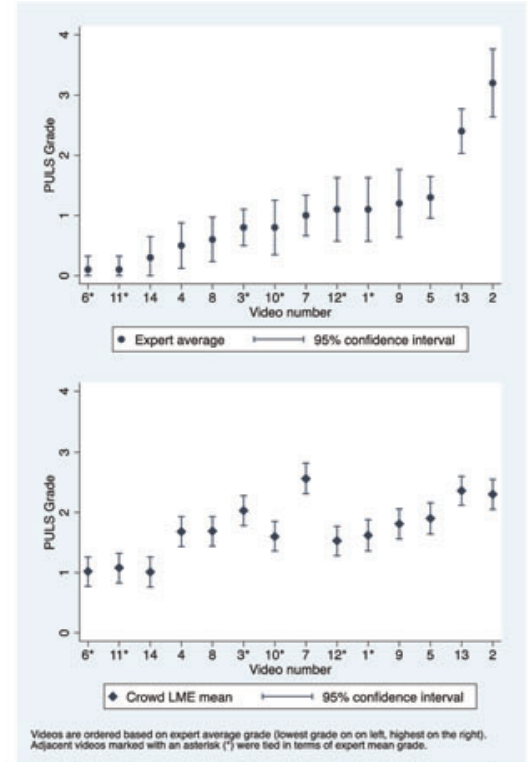

Figure 1. Comparison of Expert and Crowd Mean Scores for Each Video Arranged According to Highest Expert PULS Score to the Right (Video 2). 
Materials and Methods: Fourteen ureteroscopic videos from a single institution were evaluated by 10 experts and were then subjected to crowdsourcing assessment. Table 1 shows relevant clinical information. Table 2 shows review scores by experts for each video. After crowd reviewers underwent appropriate training, the CSATSÒ (CSATS, Inc.) platform was used to obtain 2,100 crowd reviews of the ureteroscopic tapes; this was completed in 49 hours. The mean PULS scores were determined using a linear mixed-effects (LME) model. The intraclass correlation coefficient (ICC) was also calculated as a measure of the agreement among

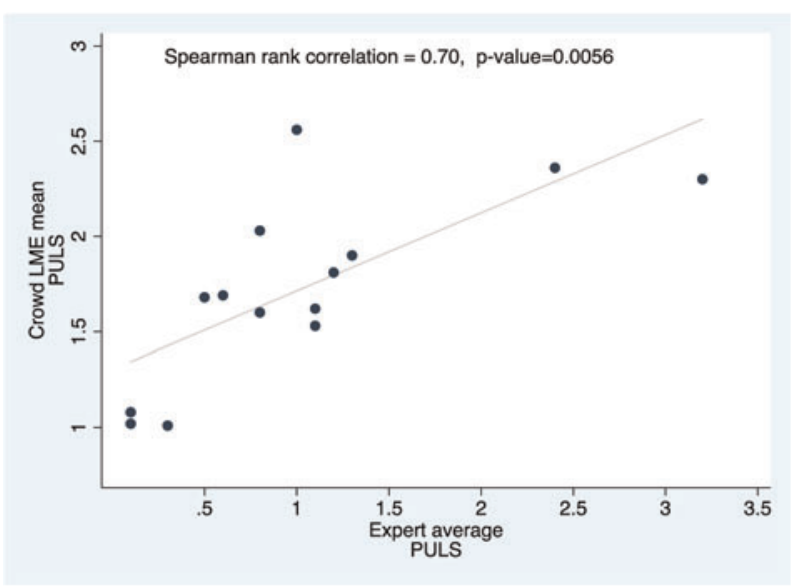

Figure 2. Spearman Rank Correlation with Crowd and Expert PULS Scores showing a Moderately Strong Correlation.

Table 1. Relevant Clinical Information during Ureteral Access sheath Deployment

\begin{tabular}{|c|c|c|c|c|c|c|c|c|}
\hline $\begin{array}{c}\text { Video } \\
\text { Number }\end{array}$ & Age & $\begin{array}{c}\text { Pre- } \\
\text { Procedure } \\
\text { Stent } \\
\text { (French) }\end{array}$ & $\begin{array}{c}\text { Post- } \\
\text { procedure } \\
\text { Stent } \\
\text { French) }\end{array}$ & $\begin{array}{c}\text { One Week } \\
\text { Preoperative } \\
\text { Tamsulosin } \\
\text { (Yes/No) }\end{array}$ & $\begin{array}{c}\text { UAS Size } \\
\text { (French) }\end{array}$ & $\begin{array}{c}\text { UAS } \\
\text { length } \\
\text { (cm) }\end{array}$ & $\begin{array}{c}\text { Injury } \\
\text { (Yes } \\
\text { (No) }\end{array}$ & $\begin{array}{c}\text { Location } \\
\text { of Injury }\end{array}$ \\
\hline $\mathbf{1}$ & 32 & $7 / 14$ & None & Yes & None & None & No & \\
\hline $\mathbf{2}$ & 32 & None & $7 / 14$ & Yes & 16 & 35 & Yes & Mid \\
\hline $\mathbf{3}$ & $\mathbf{7 2}$ & None & 6 & Yes & 16 & 55 & No & \\
\hline $\mathbf{4}$ & 69 & None & $7 / 10$ & Yes & 16 & 55 & Yes & Proximal \\
\hline $\mathbf{5}$ & 56 & None & 6 & Yes & 16 & 35 & No & \\
\hline $\mathbf{6}$ & 63 & 6 & 6 & Yes & 16 & 55 & No & \\
\hline $\mathbf{7}$ & 91 & None & $7 / 14$ & Yes & 11 & 55 & No & \\
\hline $\mathbf{8}$ & 40 & None & 6 & Yes & 11 & 35 & No & \\
\hline $\mathbf{9}$ & 72 & None & 6 & Yes & 16 & 35 & No & \\
\hline $\mathbf{1 0}$ & 72 & None & 6 & Yes & 16 & 45 & No & \\
\hline $\mathbf{1 1}$ & 53 & None & 6 & Yes & 16 & 55 & No & \\
\hline $\mathbf{1 2}$ & 38 & None & 6 & Yes & 16 & 35 & No & \\
\hline $\mathbf{1 3}$ & 61 & None & 6 & Yes & 16 & 35 & Yes & Proximal \\
\hline $\mathbf{1 4}$ & 52 & None & 6 & No & 16 & 35 & No & \\
\hline
\end{tabular}

\begin{tabular}{|c|c|c|c|c|c|c|c|c|c|c|c|c|c|c|}
\hline \multirow{2}{*}{ Video } & \multicolumn{10}{|c|}{ Reviewer } & \multirow{2}{*}{$\begin{array}{l}\text { Mean } \\
\text { score }\end{array}$} & \multirow{2}{*}{$\begin{array}{c}\text { Median } \\
\text { score }\end{array}$} & \multirow{2}{*}{$\begin{array}{l}\text { Range } \\
\text { (Min) }\end{array}$} & \multirow{2}{*}{$\begin{array}{l}\text { Range } \\
\text { (Max) }\end{array}$} \\
\hline & A & B & c & D & $E$ & $F$ & G & $\mathrm{H}$ & 1 & 1 & & & & \\
\hline 1 & 2 & 1 & 1 & 0 & 2 & 0 & 1 & 1 & 2 & 1 & 1.1 & 1 & 0 & 2 \\
\hline 2 & 3 & 4 & 3 & 3 & 5 & 3 & 3 & 2 & 3 & 3 & 3.2 & 3 & 2 & 5 \\
\hline 3 & 1 & 1 & 1 & 1 & 1 & 1 & 1 & 0 & 1 & 0 & 0.8 & 1 & 0 & 1 \\
\hline 4 & 0 & 1 & 0 & 0 & 1 & 0 & 1 & 1 & 0 & 1 & 0.5 & 0.5 & 0 & 1 \\
\hline 5 & 1 & 1 & 1 & 1 & 2 & 1 & 1 & 2 & 2 & 1 & 1.3 & 1 & 1 & 2 \\
\hline 6 & 0 & 0 & 0 & 0 & 0 & 0 & 0 & 0 & 1 & 0 & 0.1 & 0 & 0 & 1 \\
\hline 7 & 1 & 1 & 1 & 0 & 2 & 1 & 1 & 1 & 1 & 1 & 1.0 & 1 & 0 & 2 \\
\hline 8 & 0 & 1 & 0 & 0 & 1 & 1 & 0 & 1 & 1 & 1 & 0.6 & 1 & 0 & 1 \\
\hline 9 & 1 & 1 & 1 & 1 & 1 & 1 & 0 & 1 & 3 & 2 & 1.2 & 1 & 0 & 3 \\
\hline 10 & 0 & 1 & 0 & 1 & 2 & 0 & 1 & 1 & 1 & 1 & 0.8 & 1 & 0 & 2 \\
\hline 11 & 0 & 0 & 0 & 0 & 1 & 0 & 0 & 0 & 0 & 0 & 0.1 & 0 & 0 & 1 \\
\hline 12 & 1 & 2 & 1 & 0 & 2 & 1 & 0 & 1 & 2 & 1 & 1.1 & 1 & 0 & 2 \\
\hline 13 & 3 & 2 & 2 & 2 & 3 & 2 & 2 & 2 & 3 & 3 & 2.4 & 2 & 2 & 3 \\
\hline 14 & 0 & 1 & 0 & 0 & 1 & 0 & 0 & 0 & 1 & 0 & 0.3 & 0 & 0 & 1 \\
\hline $\begin{array}{c}\text { Mean } \\
\text { response }\end{array}$ & 0.9 & 1.2 & 0.8 & 0.6 & 1.7 & 0.8 & 0.8 & 0.9 & 1.5 & 1.1 & 1.0 & 1 & & \\
\hline
\end{tabular}

experts. Spearman's rank correlation (rho) was used to quantify the strength of the relationship between the crowd LME mean and the experts $((>0.8)$ very strong agreement, $(0.6$ to 0.8$)$ moderately strong agreement, $(0.3$ to 0.5$)$ fair agreement, and (

Results: The ICC for the ten experts was 0.68 (95\% confidence interval 0.49 to 0.86 ). Figure 1 shows expert averages, crowd LME means and 95\% confidence intervals. Figure 2 shows the correlation between the crowd LME means and expert averages across all videos; this was $0.70(\mathrm{p}=0.0056)$. When the expert mean PULS was $<1$, the crowd scored those videos at 1 to 2 . The highest scored video by the experts was 3.2; the crowd scored the same video much lower, at 2.25.

Conclusions: There is a moderately strong correlation between crowd and expert PULS rating. Refinement of the training, and exposure to the nuances of ureteral injuries particularly for PULS 3 (i.e. urothelial separation) is needed.

MP8-7 Reducing the Narcotic Burden: Comparison of Postoperative Outcomes of Opioids vs Non-opioids for PostUreteroscopy Pain Control

T Cisu, D Sobel, K Sternberg

University of Vermont College of Medicine United States

Introduction \& Objective: Despite playing an important role in the treatment of nephrolithiasis, ureteral stents remain a source of significant morbidity for urologic patients. With growing concern regarding narcotic medication prescriptions for the management of postoperative pain, the urologic community should assess current opioid prescription patterns. In this study, we evaluate the efficacy of not prescribing an opioid at discharge compared to standard opioid protocols postoperatively.

Materials and Methods: Charts of patients who underwent ureteroscopy with stent placement over a five-month period from November 2016 to March 2017 were reviewed retrospectively. Whenever possible, efforts were made to substitute the opioid on discharge for either no prescription or diclofenac, a non-steroidal anti-inflammatory drug (NSAID). Patients with a history of renal dysfunction or prior opioid tolerance were not offered diclofenac. Efficacy of the medication was determined by measuring the frequency of adverse events postoperatively including visits to the emergency room for stent symptoms, stent symptom related clinic telephone calls, early stent removal, and requests for prescription refills for pain medication.

Results: 75 patients underwent ureteroscopy with stent placement: 30 were given an opioid prescription (40.0\%), and 35 were discharged without opioids (46.7\%). Of those without an opioid, 33 received diclofenac and 2 patients received no pain medication. 10 patients were excluded from analysis due to the presence of a pain management plan or active use of opioids. A similar percentage of patients receiving opioids and non-opioids had postoperative visits to the emergency room for genitourinaryrelated concerns (3 patients receiving opioids (10.0\%) and 6 patients without opioids $(17.1 \%) ; \mathrm{p}=0.4120)$. Patients in the non-opioid group made significantly fewer number of telephone calls compared to patients given opioids (18 patients receiving opioids (60.0\%) and 10 patients without opioids (28.6\%); $\mathrm{p}=0.0114$ ). Similarly, patients not given an opioid at discharge requested fewer pain medication refills (7 patients receiving opioids (23.3\%) and 1 patient without opioids $(2.86 \%)$; $\mathrm{p}=0.0130$ ). 
Conclusions: This study demonstrates the feasibility of avoiding the use of opioids for appropriately selected patients undergoing ureteroscopy with stent placement. Prospective studies are needed to define which patients are suitable for this approach to help address the increasing opioid burden in our patient population.

MP8-8 A pilot study evaluating changes to haematological and biochemical tests after Flexible Ureterorenoscopy for the treatment of kidney stones.

SF Hughes, AJ Moyes, I Shergill

Betsi Cadwaladr University Health Board (BCUHB)

United Kingdom

Introduction \& Objective: Currently there is limited research documenting the changes in blood parameters, following Flexible Ureterorenoscopy for the treatment of kidney stones. The aims of this pilot study was to determine whether there were any changes in haematology and biochemistry parameters, following Flexible Ureterorenoscopy for the treatment of kidney stones.

Materials and Methods: 40 consecutive patients aged between 27-87 years (median 49 years) undergoing Flexible Ureterorenoscopy for the treatment of kidney stones were recruited (26 males, 14 females). Blood samples were collected from each patient at four time points: baseline (pre-operatively) followed by 30 minutes, 120 minutes and 240 minutes post-operatively. All haematological, haemostatic and biochemical testing were undertaken via the Sysmex XE-5000, Sysmex CS2100, Beckman Coulter AU5800 and AU680 analysers.

Results: There was a significant decrease observed following Flexible Ureterorenoscopy in the following parameters: lymphocytes $(p=0.007)$, eosinophils $(p=0.001)$, basophils $(p=0.001)$, haemoglobin $(p=0.002)$, red blood cells $(p=0.001)$, platelet count $(\mathrm{p}=0.001)$, fibrinogen concentration $(\mathrm{p}=0.001)$, von Willebrand factor $(\mathrm{p}=0.046), \mathrm{C}$ reactive protein $(\mathrm{p}=0.01)$, total protein $(p=0.001)$, albumin $(p=0.001)$, globulin $(p=0.001)$ and alkaline phosphatase $(p=0.001)$. In addition, there was a significant increase observed in the following parameters: white blood cells $(\mathrm{p}=0.001)$, neutrophils $(p=0.001)$, activated partial thromboplastin time $(p=0.001)$, total bilirubin $(p=0.012)$, creatinine $(p=0.008)$, sodium $(\mathrm{p}=0.002)$ and potassium $(\mathrm{p}=0.001)$. Limiting factors for this study were the sample size, and restriction on the recruitment time points. Conclusions: Significant changes were noted in haematology and biochemistry parameters occur following Flexible Ureterorenoscopy. Some of the data presented in this study may represent the 'normal' post-operative response following FURS, as no major complications occurred, in the majority of our patients. This data on the 'normal response' will need to be validated and may ultimately aid clinicians in distinguishing patients at risk of complications, if reproduced in larger multi-centre studies.

MP8-9 A prospective, observational study on the feasibility of a single-session endoscopic combined intrarenal surgery for ipsilateral renal stones and retrograde intrarenal surgery for contralateral renal stones: initial experiences

J Park, K Ko, W Bang, M Choo, S Lee, D Lee, H Kim, K Koo, S Yoo, M Cho, H Jeong, S Cho, T Choi, K Yoo

Kyung hee university hospital at Gangdong Republic of Korea

Introduction \& Objective: To analyze the feasibility of performing endoscopic combined intrarenal surgery (ECIRS) of the
Table 1 Patient and stone characteristics

\begin{tabular}{|c|c|c|}
\hline Variables & Value & $P$ \\
\hline \multicolumn{3}{|l|}{ Patient characteristics } \\
\hline No & 26 & \\
\hline Mean age (years) & $60.6 \pm 12.1(38-82)$ & \\
\hline Male: female (\%) & $15(57.7 \%): 11(42.3 \%)$ & \\
\hline Mean body mass index $\left(\mathrm{kg} / \mathrm{m}^{2}\right)$ & $26.4 \pm 5.4(18.4-46.2)$ & \\
\hline \multicolumn{3}{|l|}{ Stone characteristics } \\
\hline Presence of hydronephrosis without obstruction & $13(50 \%)$ & \\
\hline PCNL side & $7(26.9 \%)$ & 0.749 \\
\hline RIRS side & $6(23.1)$ & \\
\hline Mean infundibulopelvic angle ( $\left(^{\circ}\right)$ & $57.5 \pm 16.4(26.8-96.0)$ & \\
\hline PCNL side & $57.4 \pm 16.0(33.0-96.0)$ & 0.847 \\
\hline RIRS side & $58.3 \pm 17.0(26.8-92.7)$ & \\
\hline Mean Hounsfield unit & $857.4 \pm 304.2(333.0-1394.0)$ & \\
\hline PCNL side & $868.13 \pm 295.2(340.0-1394.0)$ & 0.802 \\
\hline RIRS side & $846.6 \pm 318.4(333.0-1321.0)$ & \\
\hline \multicolumn{3}{|l|}{ S-ReSC scores: 1-2/3-4/25 } \\
\hline PCNL side & $3 / 16 / 7$ & $<0.001$ \\
\hline RIRS side & $18 / 3 / 5$ & \\
\hline Mean maximal stone size $(\mathrm{mm})$ & $26.5 \pm 15.6(4.0-82.0)$ & \\
\hline PCNL side & $36.0 \pm 16.0(15-82)$ & $<0.001$ \\
\hline RIRS side & $17.1 \pm 7.6(4-35)$ & \\
\hline Mean total stone volume $\left(\mathrm{mm}^{3}\right)$ & $15003.8 \pm 12332.2(2482.9-45206.0)$ & \\
\hline PCNL side & $12709.3 \pm 11132.6(1082.6-37596.4)$ & $<0.001$ \\
\hline RIRS side & $2294.5 \pm 2316.9(33.5-8402.0)$ & \\
\hline Mean number of stones & $6.2 \pm 4.8(2-18)$ & \\
\hline PCNL side & $3.4 \pm 2.7(1-10)$ & 0.362 \\
\hline RIRS side & $2.7 \pm 2.7(1-10)$ & \\
\hline Staghorn (PCNL/RIRS) & $11(42.3 \%) / 6(23.1 \%)$ & 0.139 \\
\hline \multicolumn{3}{|l|}{ Main stone composition } \\
\hline Calcium oxalate monohydrate & 15 & \\
\hline Uric acid & 9 & \\
\hline Carbonate apatite & 1 & \\
\hline Others & 1 & \\
\hline Grade III-V & 0 & \\
\hline
\end{tabular}

PCNL percutaneous nephrolithotomy, RIRS retrograde intrarenal surgery, S-ReSC Seoul National University Renal stone Complexity

\begin{tabular}{|c|c|c|}
\hline Variables & Value & $P$ \\
\hline Mean operative time (min) & $188.0 \pm 88.4(40-420)$ & \\
\hline PCNL side & $112.9 \pm 76.7(20-335)$ & 0.032 \\
\hline RIRS side & $75.1 \pm 41.3(15-170)$ & \\
\hline Mean hospital day (d) & $2.1 \pm 1.8(1-8)$ & \\
\hline Transfusion (\%) & $1(3.8 \%)$ & \\
\hline Stone-free status & $19(73.1 \%)$ & \\
\hline PCNL side & $20(76.9 \%)$ & 0.248 \\
\hline RIRS side & $24(92.3 \%)$ & \\
\hline \multicolumn{3}{|l|}{ Complications } \\
\hline Grade I & 0 & \\
\hline Grade II & $2(7.7 \%)$ & \\
\hline Fever more than 38.0 with antibiotics & 1 & \\
\hline Bleeding without embolization & 1 & \\
\hline Grade III-V & 0 & \\
\hline
\end{tabular}




\begin{tabular}{|c|c|c|c|}
\hline Variables & No residual stone & Residual stone & $P$ \\
\hline Number of procedures (n, \%) & $19(73.1 \%)$ & $7(26.9 \%)$ & \\
\hline Male:female (\%) & $10(52.6 \%): 9(47.4 \%)$ & $5(71.4 \%): 2(28.6 \%)$ & 0.390 \\
\hline Mean body mass index $\left(\mathrm{kg} / \mathrm{m}^{2}\right)$ & $25.7 \pm 4.1$ & $28.3 \pm 8.4$ & 0.280 \\
\hline Mean operation time ( $\mathrm{min}$ ) & $187.0 \pm 91.8$ & $190.7 \pm 85.1$ & 0.926 \\
\hline Number of stones & $5.1 \pm 4.3$ & $9.1 \pm 5.3$ & 0.053 \\
\hline Total stone volume (mm³) & $15348.7 \pm 11913.4$ & $14067.5 \pm 14369.9$ & 0.820 \\
\hline \multicolumn{4}{|l|}{ PCNL side } \\
\hline Mean Hounsfield unit & $822.0 \pm 328.3$ & $993.3 \pm 121.8$ & 0.064 \\
\hline S-ReSC scores: 1-2/3-4/25 & $3 / 11 / 5$ & $0 / 5 / 2$ & 0.531 \\
\hline Number of stones & $2.7 \pm 2.4$ & $5.3 \pm 2.9$ & 0.032 \\
\hline Maximal diameter of stone $(\mathrm{mm})$ & $37.4 \pm 17.8$ & $32.1 \pm 9.1$ & 0.466 \\
\hline Total stone volume $\left(\mathrm{mm}^{3}\right)$ & $13256.9 \pm 11000.6$ & $11223.1 \pm 12240.2$ & 0.688 \\
\hline Mean operation time $(\mathrm{min})$ & $117.6 \pm 83.1$ & $100.0 \pm 59.2$ & 0.614 \\
\hline Fragmentation efficacy & $138.5 \pm 125.2$ & $112.6 \pm 72.0$ & 0.613 \\
\hline \multicolumn{4}{|l|}{ RIRS side } \\
\hline Mean Hounsfield unit & $794.6 \pm 341.4$ & $987.9 \pm 202.3$ & 0.175 \\
\hline S-ReSC scores: $1-2 / 3-4 / 25$ & $13 / 3 / 3$ & $5 / 0 / 2$ & 0.462 \\
\hline Number of stones & $2.3 \pm 2.6$ & $3.9 \pm 2.7$ & 0.201 \\
\hline Maximal diameter of stone $(\mathrm{mm})$ & $17.4 \pm 8.2$ & $16.1 \pm 6.0$ & 0.695 \\
\hline Total stone volume $\left(\mathrm{mm}^{3}\right)$ & $2091.9 \pm 2072.7$ & $2844.5 \pm 2999.1$ & 0.474 \\
\hline Mean operation time (min) & $69.4 \pm 37.8$ & $90.7 \pm 49.3$ & 0.250 \\
\hline Fragmentation efficacy & $29.1 \pm 29.4$ & $26.0 \pm 14.7$ & 0.797 \\
\hline
\end{tabular}

supine miniaturized percutaneous nephrolithotomy (miniPCNL) and retrograde intrarenal surgery (RIRS) for patients with bilateral stones in a single-session through this prospective, observational study.

Materials and Methods: The records of consecutive patients who underwent ECIRS of supine mini-PCNL using a $15 \mathrm{Fr}$ miniaturized nephroscope and RIRS in the ipsilateral side and RIRS in the contralateral side were prospectively reviewed. After the mini-PCNL procedure, flexible ureterorenoscopes were inserted into the PCNL side and the active removal of the remnant stones was performed simultaneously. Subsequently, RIRS procedures were performed on the contralateral sides.

Results: Overall, 26 patients were included in the analysis. The stone-free rate of the initial ECIRS side was $76.9 \%(20 / 26)$ and the stone-free rate of the contralateral RIRS side was $92.3 \%$ (24/26). Complications occurred in two patients (postoperative bleeding and urinary tract infection); however, these conditions were completely resolved with appropriate medications within two weeks postoperatively. Univariate logistic regression analyses showed that accumulation of surgical experience $(\mathrm{OR}=117.3$, $P=0.046$ ) was a significant predictors for stone-free status.

Conclusions: Performing ECIRS for ipsilateral renal stones and contralateral RIRS for patients with bilateral stones in a single session is feasible.

MP8-10 Published (PubMed) trends and bibliometric analysis of Ureteroscopy (URS) for stone disease over a 16year period (2000-2015)

A Pietropaolo, R Geraghty, B Somani

University Hospital Southampton NHS Foundation Trust United Kingdom

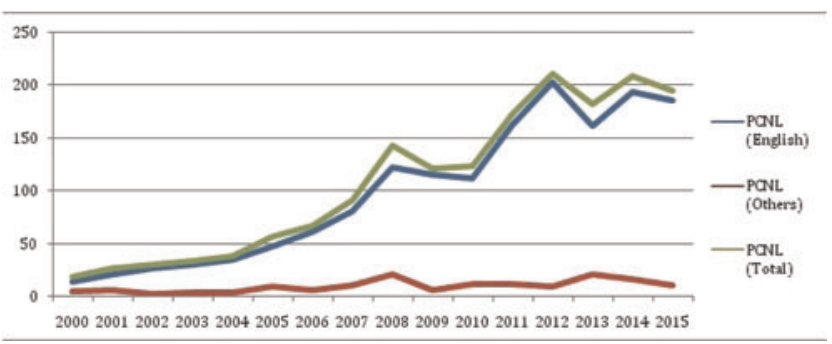

Introduction \& Objective: Ureteroscopy (URS) is increasingly used for treatment of urolithiasis. With widening indications for its use in high risk and complex stone disease, there has also been a proportionate increase in the yearly publications for URS. We wanted to see the publication trends for URS as reported on PubMed over the last 16-years

Materials and Methods: All published papers on 'Urolithiasis', 'ureteroscopy', 'URS', 'kidney stones', 'renal stones', 'ureteric stones', 'retrograde intrarenal stone surgery' and 'RIRS' were searched on PubMed over the last 16-years from 2000-2015. There were no language restrictions and all non-English language papers with published English abstracts were also included in our review. While review articles were included, case reports and those papers that did not have a published abstract were excluded from our analysis. Similarly animal and laboratory studies were also excluded from our analysis. Data was divided into two 8-year periods, period-1 (2000-2007) and period-2 (2008-2015)

Results: A total of 1200 URS papers were published on PubMed over these 16 years, of which majority $(1084,90 \%)$ were in English. Of the non-English articles $(116,10 \%)$ majority were in French $(n=38)$ and Spanish $(n=26)$. There was a linear increase in the rates of URS over the study period (Figure 1) for English language articles from 24 articles in 2000 to 176 articles in 2015 $(\mathrm{p}<0.001)$. When comparing the two time periods, there were a total of 323 articles published in period-1, which had increased by $x 2.7$ times $(171 \%$ rise) to 877 articles in period $-2(p=0.001)$. The number of English/Non-English language articles in period1 and period-2 were 280/43 and 804/73 articles respectively.

Conclusions: Published papers on ureteroscopy have risen over the last 2 decades (both in English and regional languages), highlighting a growing popularity of this minimally invasive procedure for stone disease.

MP8-11 In Vitro Comparison of Stone Fragmentation When Using Various Settings with Modern Variable Pulse Holmium Lasers

J Bell, KL Penniston, SY Nakada

University of Kentucky College of Medicine United States

Introduction \& Objective: There is limited data regarding optimal laser and energy settings during stone fragmentation. We assessed effects on fragmentation using a variety of energy and frequency settings with two variable pulse laser systems.

Materials and Methods: Artificial stones were created using BegoStone Plus (Streeper et al 2016). A clear PVC tube was closed at one end with a removable plug to create the in vitro 
FIGURE 1 - Graph showing the mean treatment time and watts for the calyceal studies.

Markers represent the mean treatment time with standard deviation shown by error bars. The $120 \mathrm{H}$ laser was tested using the following settings: $0.2 \mathrm{~J} \times 40 \mathrm{~Hz}, 0.2 \mathrm{~J} \times 70 \mathrm{~Hz}, 0.6 \mathrm{~J} \times 10 \mathrm{~Hz}, 0.6 \mathrm{~J} \times 40 \mathrm{~Hz}, 0.8 \mathrm{~J} \times 8 \mathrm{~Hz}, 1.2 \mathrm{~J} \times 10 \mathrm{~Hz}, 1.2 \mathrm{~J} \times 25 \mathrm{~Hz}$ $1.5 \mathrm{~J} \times 10 \mathrm{~Hz}$, and $1.5 \mathrm{~J} \times 25 \mathrm{~Hz}$. The $\mathrm{H}-30$ device was tested using the following settings: $0.5 \mathrm{~J} \times 20 \mathrm{~Hz}, 0.8 \mathrm{~J} \times 8 \mathrm{~Hz}$, $1.0 \mathrm{~J} \times 15 \mathrm{~Hz}, 1.5 \mathrm{~J} \times 10 \mathrm{~Hz}$, and $2.03 \times 5 \mathrm{~Hz}$

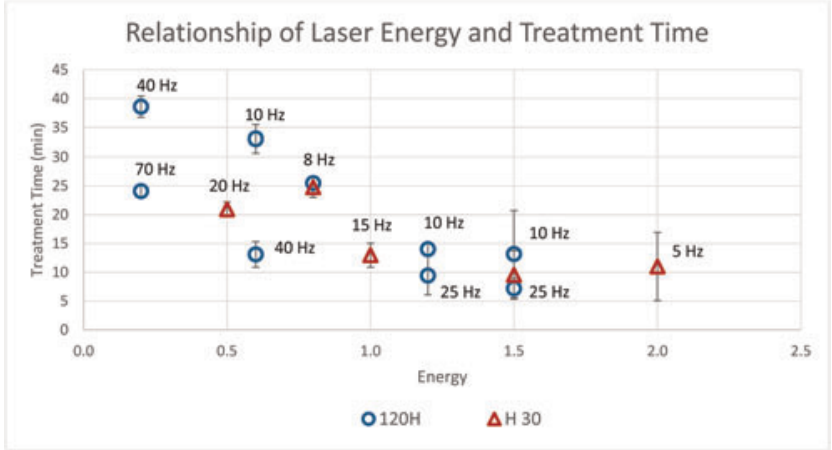

FIGURE 2 - Graph showing the relationship of the pulse width on retropulsion distance.

The markers shown represent the mean retropulsion distance. Error bars represent the standard deviation for each study arm. The $\mathrm{H}-30$ laser offers long and short pulse widths, whereas the $120 \mathrm{H}$ laser offers long, medium and short pulse widths.

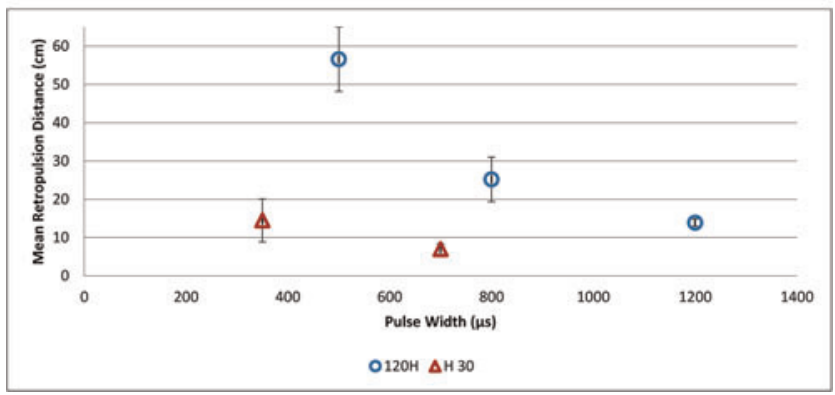

ureteral and calyceal environments. The Lumenis Pulse $120 \mathrm{H}$ and the Cook Rhapsody H-30 holmium lasers were studied. The in vitro calyceal studies measured the time required to fragment stones to $<2 \mathrm{~mm}$ fragments $(\mathrm{n}=56)$. In vitro ureteral studies measured retropulsion effects after 5 minutes of laser time $(\mathrm{n}=15)$.

Results: Complete treatment of stone phantoms in the calyceal model with the $120 \mathrm{H}$ required $10.9 \mathrm{~min}$ at $\geq 1 \mathrm{~J}$ vs $26.9 \mathrm{~min}$ at $<1 \mathrm{~J}$ $(\mathrm{P}<0.001)$ compared to $11.2 \mathrm{~min}$ at $\geq 1 \mathrm{~J}$ vs $22.8 \mathrm{~min}$ at $<1 \mathrm{~J}$ $(\mathrm{P}<0.001)$ using the $\mathrm{H}-30$. Treatment times were similar between the $120 \mathrm{H}$ and $\mathrm{H}-30$ laser systems 25.5 vs $24.8 \mathrm{~min}$ $(\mathrm{P}=0.861)$ using $0.8 \mathrm{~J} \times 8 \mathrm{~Hz}$ and 13.2 vs $9.5 \mathrm{~min}(\mathrm{P}=0.061)$ using $1.5 \mathrm{~J}$ and $10 \mathrm{~Hz}$.

Retropulsion distances using the $120 \mathrm{H}$ were $13.9 \mathrm{~cm}$ using long pulse, $25.2 \mathrm{~cm}$ using medium pulse, and $56.6 \mathrm{~cm}$ using short pulse $(\mathrm{P}<0.02)$. Retropulsion distances using the $\mathrm{H}-30$ laser were $7 \mathrm{~cm}$ using long pulse and $14.5 \mathrm{~cm}$ using short pulse which differed from the $120 \mathrm{H}(\mathrm{P}<0.001)$.

Conclusions: Laser fragmentation was faster with either laser using higher energy settings. Treatment times using the $120 \mathrm{H}$ and the H-30 lasers were equivalent. Retropulsion distances were less with both lasers when longer pulse widths were used. Retropulsion distance was less overal with the H-30 laser.
MP8-12 Adverse Events Related to Holmium Laser Lithotripsy at the time of Ureteroscopy: Looking under the hood of the FDA

T Osumah, AH Aldoukhi, J Hollingsworth, WW Roberts, KR Ghani

Department of Urology, University of Michigan United States

Introduction \& Objective: Ureteroscopy (URS) and holmium laser lithotripsy is the most common treatment modality for upper urinary tract stones in North America. Despite its popularity, studies reporting adverse events (AEs) from its use are limited. We investigated its safety by assessing a medical device reporting database to determine the prevalence, type and severity of associated AEs

Materials and Methods: We assessed the United States Food and Drug Administration (FDA) Manufacturer and User Facility Device Experience (MAUDE) database, a prospectively maintained reporting system of AEs associated with medical devices. Using the term "holmium laser", we abstracted all reported AEs related to URS over the last 10 years. Events were classified as machine/system or laser fiber related. The severity of injuries from AEs were stratified using the Common Terminology Criteria for Adverse Events (CTCAE v4.0)

Results: From 2007 to 2017, 499 reports were noted, with a significant increase in AEs over this time (Figure 1; $r=0.95$, $\mathrm{p}<0.0001)$. There were 483 fiber-related $(96.8 \%)$ events, 16 $(3.2 \%)$ system-related and $2(0.4 \%)$ sterility events (hair noted in sterile packaging). System-related AEs included generator failure and overheating. The majority of fiber-related AEs were fiber shaft fractures $(47.1 \%)$, followed by fiber tip fractures $(28.1 \%)$ and fiber failures (21.2\%). Most fiber-related events $(73.9 \%)$ occurred with small laser fibers $(<273 \mu \mathrm{m})$. Of the reported AEs, $97(19.4 \%)$ resulted in an injury to the patient, while $31(6.2 \%)$ resulted in an injury to the surgeon or operating room (OR) personnel. Patient injuries were Grade 1 (asymptomatic, no intervention indicated) in $42.3 \%$, Grade 2 (moderate, intervention indicated) in $49.5 \%$ and Grade 3 (severe/not life-threatening) in $5.2 \%$. There was a significant increase in patient injury reports over this period. Most patient injuries (51.5\%) were from fiber tip fractures occurring during lithotripsy, with the fiber tips requiring retrieval or remained indwelling. Other injuries were burns resulting in skin damage (38\%). Injuries to OR personnel were all laser burns to the body from fractured fibers $(67.7 \%)$, overheated connectors $(19.4 \%)$, or energy pulses to the fingers $(25.8 \%)$

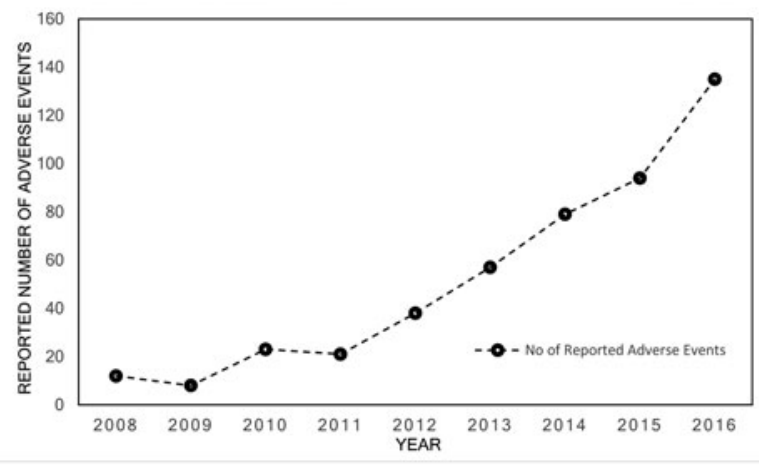

Figure 1. Adverse events related to URS and holmium laser lithotripsy reported to the FDA MAUDE database from 2008 - 2016 (2007 and 2017 were years with incomplete data and excluded). 
Conclusions: AE reports to the FDA related to URS and holmium laser lithotripsy have increased steadily over the last decade. Consequently, a rise in patient injuries has followed. Whether this reflects a true increase in incidence is uncertain as the use of URS has also increased over this period. Improved design, surveillance and manufacturer-user training may help reduce the frequency of these AEs.

MP8-13 Cost-Effective Semi-Rigid Ureteroscopy With Laser Dusting Is Effective For The Treatment Of Sizable Proximal Ureteral Calculi

AH Gabr, ER Tawfiek

Minia University, Egypt

Egypt

Introduction \& Objective: To evaluate the effectiveness of the low-cost and readily available semi-rigid ureteroscope in the management of proximal ureteric stones up to $2.5 \mathrm{~cm}$.

Materials and Methods: Patients and methods: The data of 271 patients who had ureteroscopy for proximal ureteral stones up to $2.5 \mathrm{~cm}$ diameter were prospectively analyzed. Patient demographics, size and density of stones, stone clearance, the need to use flexible ureteroscopy or other treatment modalities, and complications of the procedure were evaluated. For sake of cost reduction, we limited our instrumentation to the $6 \mathrm{~F}$ Semi-rigid ureteroscope, with the flexible urteroscope available in case of failure reaching the stone. Laser dusting, utilizing Holmium:YAG laser, with setting sof $0.2 \mathrm{Jx} 60 \mathrm{~Hz}$ were used. Success rate was defined as stone clearance on radiography at 1 month postoperatively.

Results: The mean age of patients (163 males and 108 females) was $37 \pm 7.1$ years old. Mean stone size was $16.3 \pm 4.1 \mathrm{~mm}$. Using Semi-rigid ureteroscope stone clearance was achieved in 265 $(97.8 \%)$ patients, with a remaining $6(2.2 \%)$ patients in whom flexible ureteroscopy was used due to stone migration (2 patients) or failure to proceed with the semi-rigid ureteroscope through the ureter. Post-operative ureteral stents were placed in $89.3 \%$ of patints, of which 46.4 were with extraction strings. There were no recodered intra- or post-operaive complications. Conclusions: Our study demonstrates that Semi-rigid ureteroscopy, with laser dusting, is a cost-effective and safe option for the treatment of sizable proximal ureteral stone, up to $2.5 \mathrm{~cm}$, with a very high stone-free rate.

MP8-14 Primary Retrograde Intrarenal Surgery versus Excorporeal Shockwave Lithotripsy in High Risk Renal Stones

\section{Ab. Rashid, A Wahi, R Hassan, H Ghazali}

International Islamic University Malaysia

Malaysia

Introduction \& Objective: Incidence of urolithiasis is high in our country. Treatment modalities vary depending to the size and location of stone and patient's fitness for invasive treatment. Identifying the most suitable treatment modalities according to patient's clinical characteristic is important to get optimum stone clearance with least complication and better cost-benefit.
General Objectives

1. To prove that primary RIRS is more effective than ESWL in treatment of renal stone with suspected failure of ESWL

Specific objectives

1. To show that RIRS has better stone clearance in treatment of renal stone with suspected failure of ESWL

2. To show that RIRS is more cost effective in treatment of renal stone with suspected failure of ESWL

3. To identify factors that favors RIRS than ESWL in treatment of renal stone with suspected failure of ESWL

Materials and Methods: This is a randomized controlled trial done for a period of two years from from August 2014 to July 2016 in Hospital Tengku Ampuan Afzan Kuantan, Pahang, MalaysIa.

Inclusion criteria

1. Lower pole stone

2. Stone size of $1.5-2 \mathrm{~cm}$

3. Obesity (BMI $>30 \mathrm{~kg} / \mathrm{m} 2)$

Patients were randomized into two groups, primary RIRS and ESWL group Patients were stented at least 3 weeks prior to RIRS. Retrograde intrarenal surgery was performed using a flexible ureterorenoscope. Stones were fragmented using holmium laser and retrieved using either basket or forceps. ESWL will be given for the maximum of 3 sessions. ESWL will be stopped once the stone has been cleared or the maximum 1500kj has been given. Patients were followed up with KUB x-ray and ultrasonography of the kidney 3 weeks after the surgery/procedure. The duration of follow up is at least 12 weeks ( 3 sessions of ESWL 3 weeks apart and follow up after 3 weeks). Success is defined as stone less than $4 \mathrm{~mm}$ in size measured by ultrasonography.

Results: 78 patients were enrolled in the study. Stone clearance rate in primary RIRS group is higher $(80.6 \%)$ compared to ESWL group $(36.8 \%)$ and it is statistically significant $(\mathrm{p}<0.001)$. There was no significant association of stone clearance with stone size, location and body mass index (BMI) in between these 2 groups. The complication rate is $19.4 \%$ in primary RIRS group and $18.4 \%$ in ESWL group.

Conclusions: Primary retrograde intrarenal surgery (RIRS) has significantly higher success rate of stone clearance in comparison with extracorporeal shockwave lithotripsy (ESWL). There is no independent factors in this study that significantly that favours primary RIRS. ESWL in short-term serve as more cost-effective modality compared to primary RIRS but failure of ESWL will eventually cause higher cost in the treatment.

MP8-15 One surgeon active stone retrieval procedure in flexible ureteroscopy for urinary calculi

S Okada, S Hamamoto, T Inoue, S Minagawa, H Morikawa, T Matsuda, H Miura

Department of Urology, Gyotoku General Hospital

Japan

Introduction \& Objective: Flexible ureteroscopy (f-URS) is now challenging. In the course of f-URS, stone retrieval with basket forceps is one of the most stressful procedures. Worldwide, in a stone retrieval, it seems to be common practice for $\mathrm{f}$ URS to be conducted by the operator, basket forceps to be manipulated by an assistant (two-surgeon stone retrieval procedure). However, the outcomes of this procedure may be influenced by several factors, such as the skills of the assistant and combination of two surgeons. We established a new stone retrieval procedure, that is, the operator simultaneously conducts 
f-URS and manipulates basket forceps (one-surgeon stone retrieval procedure). We evaluated the efficacy of this procedure and its superiority to the two-surgeon stone retrieval procedure. Materials and Methods: We evaluated whether non-experienced urologist could master this procedure and differences between the one and two surgeon procedures by comparing the results of a task performed using each procedure in an off-site simulator for f-URS. The task consisted of retrieving three selected renal calyx stones and was performed by 7 operators.

Results: The mean task completion time of the non-experienced urologists with the one-surgeon procedure after training was significantly shorter at 203 seconds than that before training at 599 seconds $(p=0.035)$. No difference was found in the mean task completion time between the two procedures after training. However, the mean (SD) number of times when the basket was opened and closed in the two-surgeon procedure was 10.9 (6.6) times, which was significantly more than that in the one-surgeon procedure of $5.1(1.8)$ times $(\mathrm{p}=0.017)$. Furthermore, retrieval failure and retrieval mistake (retrieving stone that is not the target) were often found in the two-surgeon procedure.

Conclusions: The one-surgeon stone retrieval procedure is an effective and acceptable technique for any urologist. The twosurgeon procedure retrieves stone using basket forceps opened widely (forceps-lead procedure), while one-surgeon procedure retrieves stone by URS leading process, in which URS leads the minimally opened basket forceps to the stone (URS-lead procedure). URS-lead procedure could decrease stone retrieval-related events, such as mucosal bleeding and scratches. Onesurgeon procedure is an effective and safe technique considering the URS-lead maneuver and less affected by the assistant's skills.

\section{MP8-16 Withdrawn}

\section{MP8-17 Withdrawn}

MP8-18 Micro-costing analysis demonstrates comparable costs for Lithovue versus reusable flexible ureteroscope use

K Taguchi, M Usawachintachit, DT Tzou, D Isaacson, BA Sherer, I Metzler, ML Stoller, T Chi

UCSF

United States

Introduction \& Objective: Reusable ureteroscope durability and need for repair are significant sources of expense and inefficiency for patients and urologists. Utilization of LithoVue ${ }^{\mathrm{TM}}$ (Boston Scientific), a disposable flexible digital ureteroscope, may address some of these concerns. To identify its economic impact on clinical care, we performed a micro-cost comparison between flexible reusable fiberoptic ureteroscopes (URF-P6 ${ }^{\mathrm{TM}}$ : Olympus) and LithoVue ${ }^{\mathrm{TM}}$.

Materials and Methods: For this prospective, single-center micro-costing study, all consecutive ureteroscopies performed during one week each in July and August 2016 utilized either URF-P6 ${ }^{\mathrm{TM}}$ or LithoVue ${ }^{\mathrm{TM}}$ ureteroscopes respectively. Workflow data were collected, including intraoperative events, post-operative reprocessing cycle timing, consumables usage, and scope cost data. Results: Intraoperative data analysis showed mean total operating room time for URF-P6 ${ }^{\mathrm{TM}}$ and LithoVue ${ }^{\mathrm{TM}}$ cases were $93.4 \pm 32.3$ and $73.6 \pm 17.4$ minutes, respectively $(p=0.093)$. Mean cost of operating room usage per case was calculated at $\$ 1,618.72 \pm 441.39$ for URF-P6 ${ }^{\mathrm{TM}}$ and $\$ 1,348.64 \pm 237.40$ for

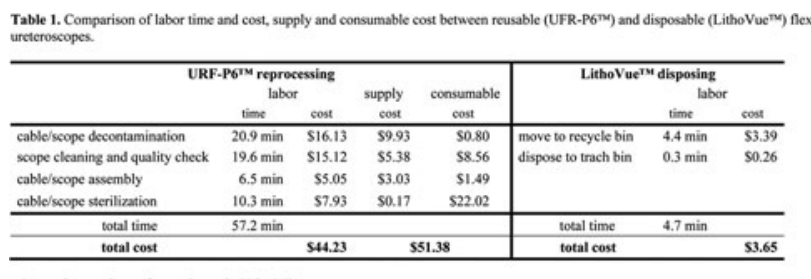

min. - minutes. Costs (S) are shown in U.S. dollars.

Figure 1
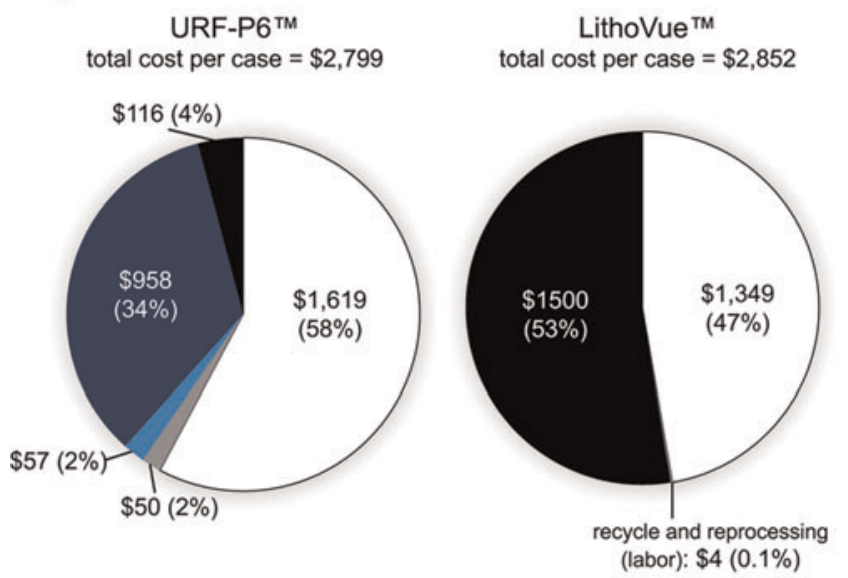

$\square$ operative room
$=$ recycle and reprocessing (labor)
= recycle and reprocessing (consumables)
= scope repair
- scope acquisition

LithoVue $^{\mathrm{TM}}$ based on institutional cost rates exclusive of disposables. Postoperative data analysis revealed costs of $\$ 107.27$ for labor and consumables during reprocessing for URF-P6 ${ }^{\mathrm{TM}}$ ${ }^{\mathrm{TM}}$ cases. (Table 1) The costs of scope repair and capital acquisition for each URF-P6 ${ }^{\mathrm{TM}}$ case were $\$ 957.71$ and $\$ 116.02$, respectively. The total ureteroscope cost per case for URF-P6 ${ }^{\mathrm{TM}}$ and LithoVue ${ }^{\mathrm{TM}}$ were $\$ 2,799.72$ and $\$ 2,852.29$, respectively. (Figure 1)

Conclusions: Micro-cost analysis revealed that the cost of LithoVue ${ }^{\mathrm{TM}}$ acquisition is higher per case compared to reusable fiberoptic ureteroscopes, but savings are realized in labor, consumables, and repair. When accounting for these factors, the total cost per case utilizing these two scopes were comparable.

MP8-19 Prospective Evaluation of Stone Free Rates by Computed Tomography After Aggressive Ureteroscopy

N Canvasser, I Sorokin, A Lay, E Kolitz, J Antonelli, M Pearle

Department of Urology, UC Davis Medical Center

United States

Introduction \& Objective: Previous retrospective and prospective studies have shown surprisingly low stone free rates 


\begin{tabular}{|r|c|c|c|}
\hline \multicolumn{1}{|c|}{ Subgroup } & $\mathrm{n}$ & SF & $\%$ \\
\hline Renal and Ureteral Stones & & & \\
\hline All stones & 169 & 88 & $52 \%$ \\
\hline $\begin{array}{r}\text { All stones, } \\
\text { exclude } \leq 2 \mathrm{~mm} \text { RF }\end{array}$ & 169 & 118 & $70 \%$ \\
\hline $\begin{array}{r}\text { Solitary stone, } \\
\leq 10 \mathrm{~mm}\end{array}$ & 37 & 27 & $73 \%$ \\
\hline Solitary stone & 20 & 13 & $65 \%$ \\
\hline $1-3$ stones & 35 & 23 & $66 \%$ \\
\hline All stones & 40 & 24 & $60 \%$ \\
\hline Renal Stones, s10mm aggregate & & & \\
\hline Solitary stone & 6 & 2 & $33 \%$ \\
\hline $1-3$ stones & 23 & 9 & $39 \%$ \\
\hline All stones & 33 & 10 & $30 \%$ \\
\hline All stones & 24 & 21 & $88 \%$ \\
\hline Ureteral Stones, $\leq 10 \mathrm{~mm}$ aggregate & & & \\
\hline
\end{tabular}

Table 1. Stone free (SF) rates for subgroups. RF- residual fragment

(SFR) (approximately 50\%) after ureteroscopy (URS) and laser lithotripsy using stringent computed tomography (CT) criteria. Our goal was to determine SFR by CT after URS and aggressive fragment retrieval, quantified by the number of ureteroscope passes through a ureteral access sheath (UAS).

Materials and Methods: We prospectively evaluated patients undergoing URS for kidney stones at our institution from December 2015 to February 2017. UASs were used in all patients except those with distal ureteral stones. Every attempt was made to extract all stone fragments, regardless of size, after fragmentation. Patient demographics, stone and operative details, and the number of ureteroscope passes for stone extraction were recorded. CT was obtained post-operatively in all patients at 6-8 weeks. Patients rendered stone free (SF) were compared to those with residual fragments (RF) using the student's T-test and chi-squared test, and binary logistic regression identified factors associated with SF status. Statistical significance was set at $\mathrm{p}<0.05$.

Results: In total, 132 patients (169 renal units, RU) underwent URS with completed radiographic follow up. Median number of stones per renal unit was 2.0 (IQR 1.0-4.5), aggregate stone size was $13 \mathrm{~mm}$ (IQR 7-19 mm), number of UAS passes was 33.5 (IQR 17-60, range 1-230), and mean OR time was $91 \pm 43 \mathrm{~min}-$ utes. Overall SFR was $52 \%$. Subgroup analysis is shown in table 1. Among RUs with RFs, mean number of RF was $2.1 \pm 1.4$ with median RF size of $3 \mathrm{~mm}$ (IQR 2-5 mm). On univariate analysis, SF cohort had fewer stones $(2.0$ vs. $3.0, \mathrm{p}<0.001)$ and smaller aggregate stone size (10 vs. $17 \mathrm{~mm}, \mathrm{p}<0.001)$ and a larger proportion of ureter only stones $(30.7 \%$ vs. $6.2 \%, \mathrm{p}<0.001)$, solitary stones $(36 \%$ vs. $20 \%, \mathrm{p}=0.02)$ and pre-stented RUs $(17 \%$ vs. $7 \%$, $\mathrm{p}=0.047)$, compared to RF cohort. There was no significant difference in OR time between groups ( 87 vs. 96 minutes, $p=0.2$, respectively). Binary logistic regression revealed that increased stone number (OR 0.77, 95\%CI 0.63-0.93, $\mathrm{p}=0.008)$ and ureter only stones (OR 4.35, 95\%CI $1.34-14.1, \mathrm{p}=0.02)$ were significant predictors of SF status.

Conclusions: SFRs by CT after URS are disappointingly low despite aggressive manual fragment retrieval. However, acceptable SFRs are achieved for ureteral stones and renal stones $\leq 10 \mathrm{~mm}$.

MP8-20 Withdrawn
MP8-21 Pediatric Ureteropyeloscopy In Supine AbductedThigh Position

M Hosseini, A Aminsharifi, A Eslahi, R Haghpanah

shiraz nephro-urology research center, shiraz university of medical sciences, shiraz, iran

Iran

Introduction \& Objective: Ureteroscopy in pediatric age group is more challenging than adults. Lithotomy position on standard operating tables is difficult and placement of the child in this position may be traumatic to the hip joint. We have done ureteroscopy in children in supine position with only abducted thigh.

Materials and Methods: Between March 2013 to April 2017, 117 ureteroscopy were done in 72 boys and 45 girls.

Mean age was 4.5 years (10 months to 15 years), and URS mostly done for stone management, In supine position with abducted-thigh. We use an arm-board that attached to the side of the table perpendicularly, and placed the patient in position which one leg rested on the arm-board and another one on the edge of the table and secured them. The procedure was done in ergonomic status and no need for lithotomy

Results: All procedures were done successfully. No any complication was seen related to utreteroscopy or position.

Conclusions: Ureteroscopy and lithotripsy in supine abductedthigh position in children seems safe and good alternative to lithotomy.

MP8-22 Does ureteral access sheath usage result in permanent damage in the ureter?

A Gücük, G Söyler, U Üyetürk, B Yılmaz, Nalbant, S Gücük, A Kükner, A Çetinkaya

Abant Izzet Baysal Univercity Medical Faculty Turkey

Introduction \& Objective: Evaluation of clinical stenosis or precursor histological changes that ureteral access sheaths (UAS) commonly used in ureteroscopic surgeries may cause in the long term in ureter with the rabbit model

Materials and Methods: In this study, 72 New Zealand white male rabbits (weight, $3.0-3.5 \mathrm{~kg}$ ) were used. The animals were divided into 9 groups according to the catheter diameter and duration as shown in table 1 (Table 1). According to their groups, only the right side ureters of the rabbits were endoscopically fitted with $2 \mathrm{~F}$ and $3 \mathrm{~F}$ ureter catheters to correspond to UAS in humans, and the catheters were left in place and withdrawn after a specified period of time. To determine the long-term effects of

Table 1: Groups according to catheter size and duration

\begin{tabular}{llll}
\hline & 2F & 3F & $\begin{array}{l}\text { Cystoscopy } \\
\text { alone }\end{array}$ \\
\hline 1 hour & Group 1 & Group 5 & \\
\hline 2 hour & Group 2 & Group 6 & \\
\hline 3 hour & Group 3 & Group 7 & \\
\hline 4 hour & Group 4 & Group 8 & \\
$\begin{array}{l}\text { Cystoscopy } \\
\text { alone }\end{array}$ & & & $\begin{array}{l}\text { Group } \\
\text { (Control) }\end{array}$ \\
\hline
\end{tabular}


access sheaths, rabbits were fed for 1 month and then sacrificed. At the end of the procedure, all the ureters were excised with open surgical procedure and evaluated macroscopically, microscopically and histologically. The ureters were separated from the upper, middle and lower parts and ureter diameters were measured from 4 different points. FGF-2 (+) labeled fibroblasts were counted in connective tissue as stenosis precursors. Ureter diameters and fibroblast counts were compared between groups. Results: Macroscopically or microscopically, no stenosis or its indirect indicator proximal dilatation was found in any group. During the comparisons where the mean of the four measurements made at three different levels were taken, the diameters of groups 4, 6 and 8 were significantly lower than the control group $(\mathrm{p}<0.05)$ when superior ureteral compartments were compared. There was no significant difference in the comparison of mid-ureter sections. When lower ureter diameters were compared, ureter diameter of group 8 was significantly lower than groups 1, 2, 3 and the control group. When the groups were compared in terms of their FGF values, there was a significant difference in FGF-2 counts at all three ureter levels $(\mathrm{p}<0.05)$. In the upper level, these differences were higher in group 8 than in groups 1, 2, 3 and the control group. In the middle and lower levels, groups 5, 6, 7 and group 8 were higher than the control group.

Conclusions: Although it does not lead to clinical stenosis, the use of UAS may lead to histological changes that are the precursor of stenosis, as its diameter and duration increase. For this reason, diameter and time should be carefully considered during use.

MP8-23 Early experience of retrograde intra-renal surgery in Mongolia

D Batbold, A Sankjaajamts, T Zev, M Munkhbaatar, S Avirmed, J Jodov, J Bayarlakh

Intermed hospital of Mongolia Mongolia

Introduction \& Objective: The management of renal stone is main problem and there is no golden standard technique in Mongolia. Retrograde intra-renal surgery (RIRS) has been used to remove stones using YAG laser or standard technique throughout the world. However the only one hospital performs RIRS in our country. Therefore, we are presenting our small experience of that surgery.

Materials and Methods: A Total 45 patients were included in this retrospective study. All patients are underwent retrograde intra-renal surgery by using flexible endoscopy with YAG laser, at the Ulaanbaatar Songdo hospital, between 2014Jan-2016Dec. The surgical charts of all patients reviewed. Gender, operation time, body mass index, hospital stay, stone size, site of stone, stone free rate and complication were analyzed.

Results: During study period 45 patient were underwent RIRS for renal stone. Stones were successfully accessed in all of the cases by the RIRS with YAG laser. There were 7 patients bilateral, 18 patients with right sided, 20 patients with left sided stone. Mean operation time was $151 \pm 54.2 \mathrm{~min}$, body mass index $28.1 \pm 4.3$, stone size $1.3 \pm 0.5 \mathrm{~cm}$, stone free rate $95.5 \%$, hospital stay $5.1 \pm 2.2$ day. The complication rate was $4.4 \%$, which consisted of 2 cases with ureteral perforation.

Conclusions: Retrograde intra-renal surgery by using laser is feasible and safe in renal stone and more benefits for patients. Therefore we believe that patient with renal stone should be treated RIRS by using laser. Nevertheless, more evidence and studies are needed to ascertain the risks and benefits of this technique. This is the first reported data in Mongolia to our knowledge

MP8-24 Utilization of pressurized vs non-pressurized irrigation during ureteroscopy in the absence of ureteral access sheath: A comparative retrospective study

K Doersch, A El Mekresh, GL Machen, PA Milburn, K Hart, MM El Tayeb, L Tsai

Texas A\&M College of Medicine/Baylor Scott and White United States

Introduction \& Objective: Ureteroscopy (URS) is a common urologic procedure for removing upper urinary tract stones and evaluating other abnormalities of the urinary tract. Pressurized irrigation is frequently used to aid visualization by increasing the flow through the working channel. However, this can lead to postoperative renal colic, intrarenal reflux, rupture of the fornix, and, more seriously, sepsis, especially in the absence of ureteral access sheath (UAS). Here within, we evaluate the safety of pressurized irrigation during ureteroscopy in the absence of UAS.

Materials and Methods: After IRB approval, a retrospective chart review was performed comparing patients in whom pressurized irrigation was used during URS in the absence of UAS to those in whom pressure was not used from February 2014 to September 2016. Pressurized irrigation was performed utilizing automated external compression to the irrigation bag with maximum pressure set at $150 \mathrm{~mm} \mathrm{Hg}$; in the other cohort, hand irrigation was performed using $60 \mathrm{ml}$ syringe and IV extension tubing. Statistics were performed in Prism (Graph Pad) and included means, standard deviations, chi squared tests, and student's t-tests.

Results: Group (A) consisted of 206 patients in which pressurized irrigation was used. Group (B) consisted of 24 patients in which hand irrigation was used during their URS. In group (A), $54.9 \%$ were male vs in group (B), 37.5\%. Group (B) were younger on average, with a mean age of $44 \pm 19.0$, compared with group (A) whose mean age was $53.7 \pm 18.9$ ( $\mathrm{p}>0.9999)$. Emergency Department (ED) return rate was $29(14.1 \%)$ vs $6(25 \%)$ in groups $(\mathrm{A})$ and $(\mathrm{B})$, respectively $(\mathrm{P}=0.2235)$. Most complications in both cohorts were UTI and pain-associated complications. Urinary tract infection was encountered in 4 vs 0 patients in groups (A) and (B), respectively ( $p>0.9999)$. No occurrences of sepsis in either group. There was no calyceal rupture or intraoperative extravasation in either cohort.

Conclusions: Pressurized irrigation in the absence of UAS during URS appears to be safe. There was no significant difference in procedure times and, although there were more complications and ED visits in the hand irrigation group, many of these were experienced by a single patient who experienced a significant number of complications. 


\section{MPS9: NON-SURGICAL \& MEDICAL MANAGEMENT OF STONES I}

MP9-1 Medical dissolution therapy for the treatment of uric acid nephrolithiasis

C Gridley, M Sourial, B Knudsen

The Ohio State University Wexner Medical Center United States

Introduction \& Objective: Uric acid (UA) nephrolithiasis represents $10 \%$ of kidney stones in the United States with low urine $\mathrm{pH}$ and high saturation of UA the main risk factors for stone development. Dissolution therapy for UA kidney stones via urinary alkalization is an established strategy for treatment. We present our experience in treating UA nephrolithiasis with medical dissolution therapy.

Materials and Methods: A retrospective review was performed of UA stone patients treated between July 2007 and July 2016 with medical dissolution therapy. Patients were identified using ICD- 9 codes. Patients were treated with potassium citrate and/or allopurinol. Serial imaging and urine $\mathrm{pH}$ were obtained at follow up. Demographics, aggregate stone size, time to stone clearance, urine $\mathrm{pH}$ (office dip), and complications were recorded.

Results: Twenty-three patients were identified that underwent medical dissolution therapy for UA nephrolithiasis: thirteen men and ten women. Median age at presentation was 56 years. Median BMI was 45. Median urine $\mathrm{pH}$ was 5.5. Median stone burden at time of presentation measured $30 \mathrm{~mm}$. Urine $\mathrm{pH}$ in the office was raised to a median of 6.5 . Three patients did not tolerate treatment and discontinued dissolution therapy. Fourteen patients $(70 \%)$ showed complete resolution of nephrolithiasis while six patients $(30 \%)$ showed partial reduction. Median time to complete stone clearance was 3 months. Patients with partial response showed a reduction in stone burden of $65.6 \%$

Conclusions: Medical dissolution therapy is a viable, non-invasive option for UA nephrolithiasis.

MP9-2 Do patients with non-alcoholic fatty liver disease have predictable 24 -hour urine abnormalities?

E Ghiraldi, A Lee, C Tong, J Friedlander

Albert Einstein Medical Center

United States

Introduction \& Objective: Patients with non-alcoholic fatty liver disease (NAFLD) have predictable hepatic dysfunction with metabolic consequences. NAFLD has already been linked to diabetes mellitus (DM) and metabolic syndrome, and new population-based research suggests NAFLD is also associated with nephrolithiasis. Herein we sought to identify urinary abnormalities in stone-forming patients with NAFLD and compare this to patients without NAFLD.

Materials and Methods: A retrospective chart review was performed of patients treated by a single surgeon for nephrolithiasis between August 2014 and September 2016. Patients who submitted 24-hour urine samples were divided into two groups, those who did and did not have NAFLD. Analyses of metabolic abnormalities related to patients with and without
NAFLD were performed using univariate analysis and multivariate logistic regression.

Results: Ninety-four patients met inclusion criteria, 32.9\% (31/ 94) of whom had a history of NAFLD. Among patients with NAFLD, mean age was 54 (26-83 years), 54\% (17/31) were female, and $33 \%(10 / 30)$ were African American. Of NAFLD patients, $62.7 \%(32 / 51)$ had calcium oxalate stones, $11.8 \%(6 / 51)$ uric acid stones, $2.0 \%$ (1/51) calcium phosphate stones. On univariate analysis patients with NAFLD had more acidic urine ( $\mathrm{pH} 40 \mathrm{mg} /$ day: $53.3 \%$ vs $26.9 \%$; $\mathrm{p}=0.013$ ), and higher supersaturation of uric acid ( $>1: 58.6 \%$ vs $35.5 \%$; $\mathrm{p}=0.038$ ). On multivariate adjusted analysis, elevated urinary oxalate (OR 9.777, 95\% CI 2.345-40.768, $\mathrm{p}=0.0018)$ and supersaturation of uric acid (OR 2.912, 95\% CI 1.025-8.271, p=0.0448) were found to be associated with NAFLD.

Conclusions: Stone-forming patients with NAFLD are at risk for nephrolithiasis. Our results demonstrate these patients have elevated urinary oxalate and higher supersaturation of uric acid. These abnormalities must be addressed to reduce stone formation in this population.

MP9-3 A Prospective Randomized Controlled Trial Comparing the Efficacy of Low-oxalate Diet versus Vitamin B6 and Magnesium Supplementation versus Both in Idiopathic Hyperoxaluria

JN Thai, T Tran, E Parkhomenko, K Blum, M Gupta, S Purnell

Department of Urology, Ichan School of Medicine at

Mount Sinai

United States

Introduction \& Objective: Urinary oxalate is implicated in the formation of calcium oxalate $(\mathrm{CaOx})$ stones. Evidence shows a

\begin{tabular}{|l|c|c|c|}
\hline & $\begin{array}{c}\text { Diet Only } \\
(\mathbf{N}=17)\end{array}$ & $\begin{array}{c}\text { Supplements Only } \\
(\mathrm{N}=17)\end{array}$ & $\begin{array}{c}\text { Combination } \\
(\mathrm{N}=\mathbf{1 7})\end{array}$ \\
\hline Mean Age (range), years & $53.4(25-78)$ & $56.1(20-80)$ & $53.4(23-78)$ \\
\hline Male:Female & $15: 2$ & $12: 5$ & $15: 2$ \\
\hline Mean starting 0x24 & 51.1 & 52.1 & 52.9 \\
\hline Range Starting 0x24 & $40-74$ & $40-65$ & $41-79$ \\
\hline Mean ending 0x24 & $41.1(\mathrm{~N}=7)$ & $51.1(\mathrm{~N}=12)$ & $54.3(\mathrm{~N}=4)$ \\
\hline Range Ending 0x24 & $31-50$ & $25-88$ & $34-74$ \\
\hline Study Completion rate & $41.2 \%(7 / 17)$ & $70.6 \%(12 / 17)$ & $23.5 \%(4 / 17)$ \\
\hline Non-compliance rate & $11.8 \%(2 / 17)$ & $5.9 \%(1 / 17)$ & $17.6 \%(3 / 17)$ \\
\hline Lost to follow up rate & $17.6 \%(3 / 17)$ & $11.8 \%(2 / 17)$ & $35.3 \%(6 / 17)$ \\
\hline In Progress & $29.4 \%(5 / 17)$ & $5.9 \%(1 / 17)$ & $23.5 \%(4 / 17)$ \\
\hline $\begin{array}{l}\text { Number of Patients with } \\
\text { Decrease in Ox24/Total } \\
\text { Completed Study (\%) }\end{array}$ & $5 / 7(71.4 \%)$ & $6 / 12(50 \%)$ & $2 / 4(50 \%)$ \\
\hline $\begin{array}{l}\text { Mean Absolute Decrease in } \\
\text { Ox24/3-months }\end{array}$ & 16.6 & 13.7 & 12.0 \\
\hline Range Absolute Decrease & $6-33$ & $1-24$ & $10-14$ \\
\hline & & & \\
\hline
\end{tabular}


diet low in oxalate-rich foods can prevent $\mathrm{CaOx}$ kidney stones. Recent research found that supplementation with vitamin B6 (VB6) and magnesium (Mg) targets the pathways of oxalate production and absorption, respectively, to reduce urinary excretion of oxalate. Urologists who treat $\mathrm{CaOx}$ stones often prescribe dietary changes, vitamin supplementation, or both. However, no studies to date have compared the efficacy of these treatments. Our study is the first prospective randomized controlled trial to quantify the effects of dietary changes versus vitamin supplementation versus a combination of both treatments for the prevention of $\mathrm{CaOx}$ stones.

Materials and Methods: Patients were recruited from a urology clinic at a major tertiary referral center. Patients were eligible for enrollment if they made $\mathrm{CaOx}$ kidney stones and had newly diagnosed idiopathic hyperoxaluria (urine oxalate levels $\geq 40 \mathrm{mg}$ / day). Once enrolled, patients were randomized into 1 of 3 treatment groups: low-oxalate diet (D), supplementation (S) with a combination pill of $25 \mathrm{mg}$ vitamin B6 and $400 \mathrm{mg}$ Magnesium, or both (DS). Patients were asked to adhere to their prescribed treatment regimen for 3 months and then had a repeat 24-hour urine. End data were evaluated as an absolute decrease in Ox24. Results: Mean age is 54.3 years. Male to female ratio is 42 to 9 . 17 patients are enrolled in the D group, 17 in the $S$ group, and 17 in the DS group. The $\mathrm{S}$ patients had the highest study completion rate at $71 \%(12 / 17)$. Non-compliance and lost to follow up rates were highest in the DS at $18 \%(3 / 17)$ and $35 \%(6 / 17)$, respectively. In the D group, 71\% (5/7) had lower Ox24 after the 3month period. On average the $\mathrm{D}$ had the highest absolute decrease in Ox24. Table 1.

Conclusions: This is the first prospective randomized controlled study comparing the efficacy of a low-oxalate diet versus supplementation versus a combination of both for the treatment of idiopathic hyperoxaluria. We found that patients are more likely to adhere to a treatment plan when prescribed supplements only. However, those who are prescribed a low-oxalate diet, when compliant, benefitted the most with the greatest overall reduction in urinary oxalate levels.

MP9-4 Does saturation index predict stone activity in patients with calcium oxalate nephrolithiasis?

N Canvasser, I Sorokin, A Lay, E Kolitz, X Li, B Adams-Huet, J Poindexter, N Maalouf, O Moe, K Sakhaee, J Antonelli, M Pearle

Department of Urology, UC Davis Medical Center United States

Introduction \& Objective: Supersaturation, the driving force for crystallization, should predict stone formation, and estimates of supersaturation could constitute a useful surrogate for stone recurrence. EQUIL 2 has been the gold standard for estimating urinary saturation of stone-forming salts (relative saturation ratio, RSR). The Joint Expert Speciation System (JESS) is an alternative program that takes into account soluble complexes not considered by EQUIL 2 and is thought to provide a more accurate estimate of supersaturation (saturation index, SI). Our goal was to determine if SI calcium oxalate $(\mathrm{CaOx})$ correlates with stone activity in $\mathrm{CaOx}$ stone formers.

Materials and Methods: We reviewed patient charts from 2005 - 2016 from our stone clinic and identified stone formers with $>50 \% \mathrm{CaOx}$ stone composition, and baseline and at least one follow-up 24-hour urine collection after the initiation of drug and/or dietary therapy. Patients with renal tubular acidosis, nephrocalcinosis, or anatomic abnormalities were excluded. $\mathrm{Pa}-$ tient demographics, follow-up imaging, 24-hour urine studies, and all surgeries were recorded in a timeline for each patient. SI was calculated using JESS. Stone recurrence was defined as a new stone or $>2 \mathrm{~mm}$ stone growth on imaging, or treatment/ passage of a new stone. For patients without stone recurrence (NR), we compared initial SI CaOx to the mean of the last two SI $\mathrm{CaOx}$ during follow-up. For patients who recurred $(\mathrm{R})$, we compared initial $\mathrm{SI} \mathrm{CaOx}$ to the last $\mathrm{SI} \mathrm{CaOx}$ prior to recurrence. Statistical analysis was performed using SAS, and significance was set at $\mathrm{p}<0.05$.

Results: In total, $118 \mathrm{R}(36 \%)$ and 208 NR (64\%) met inclusion criteria, with a median follow up of 21.7 and 22.0 months, respectively $(\mathrm{p}>0.05)$. We found no significant difference between $\mathrm{R}$ and NR with respect to initial SI (6.5 vs. 6.7, respectively, $\mathrm{p}=0.66)$, follow-up SI (5.3 vs. $5.7, \mathrm{p}=0.33)$, or $\Delta \mathrm{SI}(-1.2$ vs. $1.0, \mathrm{p}=0.71)$. However, both groups independently showed significant improvement from initial to follow up SI values $(\mathrm{p}<0.001$ and $\mathrm{p}=0.01$, respectively). Receiver operating characteristic analysis for prediction of stone recurrence demonstrated area under the curve for SI $\mathrm{CaOx}$ of 0.49 , which was similar to RSR $\mathrm{CaOx}$ (0.49), urine total volume (0.50), urine calcium (0.51), urine citrate (0.52), and urine oxalate $(0.52)$.

Conclusions: Absolute SI is no better a predictor of stone recurrence than RSR or other urinary parameters (total volume, urine calcium, citrate or oxalate). Although $\Delta$ SI reflects the impact of treatment on urinary stone risk, it does not predict actual efficacy in preventing recurrence. Therefore, SI may not be a good surrogate for stone recurrence.

\section{MP9-5 Feasibility and Safety of Individual Renal Unit Urine Sampling for Metabolic Analysis}

JB Ziemba, R Gurnani, MA Gorin, WW Ludwig, BR Matlaga, JR Asplin

Perelman School of Medicine at the University of Pennsylvania United States

Introduction \& Objective: Preventing kidney stone recurrence relies on detecting and modifying urine chemistry abnormalities. The assumption is that an abnormality is due to a metabolic defect present in both kidneys. However, we hypothesize that clinically significant unilateral defects exist that are not detectable in pooled bladder urine. To identify these patients, we have developed a protocol to sample urine from each individual renal unit.

Materials and Methods: In this prospective pilot study, adults undergoing retrograde upper urinary tract surgery were eligible for inclusion. Excluded were patients with a solitary kidney, suspected urothelial malignancy, or urinary tract infection. Following informed consent, all patients proceeded to the operating room. After induction of anesthesia, cystoscopy with ureteral catheterization (open-ended 5-french) was performed up to $25 \mathrm{~cm}$ in each ureter. Urine was collected via gravity drainage for 10 minutes and urine samples with adequate volume were analyzed for chemistry concentrations (Litholink, Chicago, IL).

Results: To date, a total of 25 patients were screened for eligibility with 12 excluded and 3 declining participation. The remaining 10 patients were consented, but 1 had a complication with anesthesia precluding sample collection, resulting in a total of 9 participants (18 renal units). All patients successfully 
Table 1: Urine Sample Characteristics

\begin{tabular}{|l|l|l|l|l|}
\hline \multirow{2}{*}{ Patient } & \multicolumn{2}{|c|}{ Urine Volume (cc) } & \multicolumn{2}{c|}{ Urine Creatinine (mg/dL) } \\
\cline { 2 - 5 } & Right & Left & Right & Left \\
\hline 1 & 0.50 & 1.50 & 80.44 & 42.86 \\
\hline 2 & 0 & 1.50 & 0 & 85.04 \\
\hline 3 & 0 & 17.00 & 0 & 53.84 \\
\hline 4 & 0 & 7.50 & 0 & 105.53 \\
\hline 5 & 0.15 & 0.25 & 122.45 & 246.28 \\
\hline 6 & 20.00 & 20.00 & 15.16 & 11.56 \\
\hline 7 & 1.90 & 0 & 144.39 & 0 \\
\hline 8 & 4.00 & 4.20 & 83.47 & 81.58 \\
\hline 9 & 0 & 17.00 & 0 & 26.92 \\
\hline Median (IQR) & $1.9(0.5-4.00)$ & $5.85(1.5-17.00)$ & $83.47(80.44-122.45)$ & $67.71(38.88-90.16)$ \\
\hline
\end{tabular}

Table 2: Individual Renal Unit Urinary Chemistry Profiles

\begin{tabular}{|c|c|c|c|c|c|c|c|c|c|c|c|c|}
\hline Patient & Side & pH & UA & Amm & Ca & $\mathrm{Na}$ & K & Phos & $\mathrm{Mg}$ & UUN & Cit & $0 x$ \\
\hline \multirow[t]{2}{*}{1} & \begin{tabular}{|l|} 
Right \\
\end{tabular} & & & 0.30 & 0.04 & 1.13 & 0.94 & 0.65 & 0.04 & 2.70 & 0.03 & 0.29 \\
\hline & Left & & & 0.33 & 0.04 & 1.21 & 1.00 & 0.66 & 0.04 & 3.05 & 0.01 & 0.31 \\
\hline 2 & Left & & & 0.56 & 0.23 & 2.06 & 3.05 & 0.55 & 0.05 & 4.79 & 0.41 & 0.47 \\
\hline 3 & Left & 5.53 & 0.22 & 0.05 & 0.00 & 1.32 & 2.11 & 0.30 & 0.03 & 5.51 & 0.23 & 0.42 \\
\hline 4 & Left & 4.89 & 0.25 & 0.11 & 0.05 & 0.51 & 2.12 & 0.23 & 0.03 & 5.03 & 0.03 & 0.22 \\
\hline \multirow[t]{2}{*}{5} & Right & & & 0.19 & 0.06 & 2.44 & 1.71 & 0.29 & 0.03 & 1.77 & & \\
\hline & Left & & & & 0.05 & & & & & & & \\
\hline \multirow[t]{2}{*}{6} & Right & 7.20 & 0.58 & 0.13 & 0.12 & 6.68 & 4.05 & 0.90 & 0.08 & 8.57 & 0.24 & 0.07 \\
\hline & Left & 6.16 & 0.42 & 0.32 & 0.02 & 5.27 & 2.97 & 0.50 & 0.07 & 8.51 & 0.27 & 0.34 \\
\hline 7 & Right & & & 0.11 & 0.08 & 1.58 & 0.92 & 0.14 & 0.04 & 2.78 & 0.21 & 0.14 \\
\hline \multirow[t]{2}{*}{8} & Right & & & 0.10 & 0.10 & 1.07 & 3.66 & 0.41 & 0.05 & 9.63 & 0.35 & 0.14 \\
\hline & Left & & & 0.13 & 0.11 & 1.03 & \begin{tabular}{|l|}
3.28 \\
\end{tabular} & 0.40 & 0.05 & 9.44 & 0.30 & 0.15 \\
\hline 9 & Left & 7.04 & 0.36 & 0.23 & 0.02 & 1.37 & 2.55 & 0.19 & 0.01 & 5.02 & 0.31 & 0.16 \\
\hline \multicolumn{13}{|c|}{$\begin{array}{l}\text { Values are expr } \\
\text { UA: Uric Acid }\end{array}$} \\
\hline \multicolumn{13}{|c|}{$\begin{array}{l}\text { UA: Uric Acid } \\
\text { Amm: Ammonia }\end{array}$} \\
\hline \multicolumn{13}{|c|}{ UUN: Urea Nitroger } \\
\hline \multirow{2}{*}{\multicolumn{13}{|c|}{ Cit: Citrate }} \\
\hline & & & & & & & & & & & & \\
\hline
\end{tabular}

Ox: Oxalate

underwent catheterization of each renal unit. Only 4 (44\%) patients had urine output from both kidneys (Table 1). Table 2 shows the individual renal unit urinary chemistry profiles. One patient (11\%; patient \#6) demonstrated a significant difference in $\mathrm{pH}$, ammonia, and calcium between renal units. No patient experienced an adverse event related to ureteral catheterization.

Conclusions: Individual renal unit urine sampling is feasible and safe. Urine volumes captured are variable, but differences in chemistry concentration are still detectable. Data from additional patients will allow us to determine if these differences are clinically significant.

\section{MP9-6 Mineral water in the prevention of renal stones}

P Sundaram, T Chong

Singapore

Introduction \& Objective: The effect of increased fluid intake in the prevention of urinary stones is well accepted. Due to their increased magnesium, calcium and bicarbonate content, mineral water may have additional protection against calcium oxalate stone formation. Therefore mineral water intake could be more acceptable than dietary restrictions which patients find difficult to adhere to. The aim of this pilot study is to assess whether the intake of mineral water in healthy subjects and stone formers will alter $24 \mathrm{hr}$ urinary composition.

Materials and Methods: A total of 10 healthy volunteers and 10 hyperoxaluric stone formers were recruited. While on their usual diet, 24hr urine collection was obtained. During the water intake phase, they were to consume at least $1.25 \mathrm{~L}$ of mineral water each day, preferably at meal times in addition to their usual fluid intake. Following this, the stone formers underwent an additional washout phase where they would revert to their usual diet. At the end of each phase, a $24 \mathrm{hr}$ urine collection was performed. The urine samples were assessed for urinary concentrations of calcium, magnesium, oxalate and citrate. The relative supersaturation of calcium oxalate was calculated using Tiselius risk index. Results: In the healthy volunteer study, there was a decrease in urinary oxalate excretion from $0.36 \mathrm{mmol} /$ day to $0.26 \mathrm{mmol} /$ day. Urinary magnesium showed a significant increase from $3.1 \mathrm{mmol} /$ day to $4.4 \mathrm{mmol} /$ day while urinary citrate showed a slight increase from $2.7 \mathrm{mmol} /$ day to $2.8 \mathrm{mmol} /$ day. The relative supersaturation showed a significant decrease from 1.13 to 0.63 . The stone formers study showed significant decrease in urinary oxalate from $0.53 \mathrm{mmol} /$ day to $0.29 \mathrm{mmol} /$ day $(\mathrm{p}=0.009)$ but later increased to $0.43 \mathrm{mmol} / \mathrm{day}(\mathrm{p}=0.026)$ when the mineral water was held off. Urinary magnesium significantly increased from $3.01 \mathrm{mmol} /$ day to $4.92 \mathrm{mmol} /$ day $(\mathrm{p}=0.008)$ with the intake of mineral water but decreased to $3.29 \mathrm{mmol} /$ day $(\mathrm{p}=0.078)$. Likewise citrate levels significantly increased from $2.27 \mathrm{mmol} /$ day to $4.88 \mathrm{mmol} /$ day $(\mathrm{p}=0.010)$ then decreased to $2.94 \mathrm{mmol} /$ day $(p=0.053)$ after the washout phase. The Tiselius risk index decreased from 1.04 to $0.51(\mathrm{p}=0.052)$ but significantly increased to $1.26(\mathrm{p}=0.013)$ while the urinary $\mathrm{pH}$ increased from 5 to 7 then decreased to 6 after the washout phase.

Conclusions: Mineral water significantly reduces urinary oxalate, increases urinary magnesium and citrate excretion. These changes reduce the supersaturation of calcium oxalate. After the washout phase, all the changes reversed, specifically the risk index increased. Moving forward, we aim to study the effects of mineral water intake in a prospective randomized controlled trial with a larger sample size.

MP9-7 Association between serum 25-OH-vitamin D and 24-hour urine calcium in urolithiasis patients

S Tavasoli, M Taheri, F Bagheri Amiri, M Parvin, A Basiri

Urology and Nephrology Research Center (UNRC) Iran

Introduction \& Objective: Hypercalciuria is an important and common urinary metabolic abnormality among stone formers. Although vitamin D deficiency (VDD) has been known as a risk factor for many chronic diseases, there is a traditional concern for the treatment of VDD, due to the probable relationship between serum vitamin $\mathrm{D}$ and urine calcium. The aim of current study was to evaluate the relationship between 24-hour urine calcium (UCa) and serum 25-OH-vitamin D [25(OH)D] in urolithiasis patients.

Materials and Methods: This retrospective cross-sectional study was performed based on medical reports of urolithiasis patients referred to stone prevention clinic in Labbafinejad Hospital, Tehran, Iran, from 2010 to 2017. Primary variables included UCa and 25(OH)D. Age, gender, body mass index, serum parathyroid (PTH), 24-hour urine urea (UUr), 24-hour urine creatinine (UCr), and 24-hour urine sodium (UNa) were considered as covariates.

All statistical analyses were performed using SPSS software, version 23. UCa were categorized to normal $(<200 \mathrm{mg} / 24 \mathrm{~h})$ and hypercalciuria $(\geq 200 \mathrm{mg} / 24 \mathrm{~h}) .25(\mathrm{OH}) \mathrm{D}$ were categorized to $\geq 30 \mathrm{ng} / \mathrm{mL}, 20-30 \mathrm{ng} / \mathrm{mL}$ and $<20 \mathrm{ng} / \mathrm{mL}$. The relationship between UCa and $25(\mathrm{OH}) \mathrm{D}$ or other variables was assessed by chi- 
square test and univariate logistic regression. Backward LR logistic regression method was used in multivariate analyses.

Results: Two hundred and forty-nine patients were included in the study. Mean UCa was $199.55 \mathrm{mg} / 24 \mathrm{~h}$ (SD: 102.80 , range $25-$ 525 ) and mean $25(\mathrm{OH}) \mathrm{D}$ was $25.0 \mathrm{ng} / \mathrm{mL}$ (SD: 16.73 , range 294). One hundred and ten (44.2\%) patients had hypercalciuria. Distribution of $25(\mathrm{OH}) \mathrm{D}$ were as follows: $\geq 30 \mathrm{ng} / \mathrm{mL}$ : 69 (27.7\%), $20-30 \mathrm{ng} / \mathrm{mL}: 69$ (27.7\%), and $<20 \mathrm{ng} / \mathrm{mL}: 111$ (44.6\%). There was no correlation between $\mathrm{UCa}$ and $25(\mathrm{OH}) \mathrm{D}$ in univariate analyses, although age, UUr, UNa, and PTH were significantly correlated to UCa. In multivariate analyses, $25(\mathrm{OH}) \mathrm{D} 20-30 \mathrm{ng} / \mathrm{mL}$ did not increase the risk of hypercalciuria significantly (OR: 1.37, 95\% CI: 0.70-2.67). However, values $\geq 30 \mathrm{ng} / \mathrm{mL}$, compared to $<20 \mathrm{ng} / \mathrm{mL}$, increased the risk significantly (OR: $2.37,95 \%$ CI: $1.20-4.66$ ). Age $\geq 49$ years decreased the risk of hypercalciuria (OR: $0.45,95 \% \mathrm{CI}: 0.25-0.80)$ compared to age $<49$. UUr $\geq 24 \mathrm{gr} / 24 \mathrm{~h}$ increased the risk of hypercalciuria compared to values $<24 \mathrm{gr} / 24 \mathrm{~h}(\mathrm{p}=0.001)$.

Conclusions: Our study showed that although $25(\mathrm{OH}) \mathrm{D} \geq 30 \mathrm{ng} /$ $\mathrm{mL}$ increased the risk of hypercalciuria, serum range of 20-30 ng/ $\mathrm{mL}$ did not change the risk, compared to values $<20 \mathrm{ng} / \mathrm{mL}$. These results could have implication in the goal of VDD treatment in Iranian patients with urolithiasis.

MP9-8 Association between 24-Hour urine metabolic abnormalities and stone recurrence in patients with urolithiasis

M Taheri, S Tavasoli, F Taheri, F Bagheri Amiri, A Basiri

Urology and Nephrology Research Center (UNRC) Iran

Introduction \& Objective: To evaluate the prevalence of 24hour urine metabolic abnormalities (UMAs) in patients with urolithiasis and the relationship between them to stone recurrence.

Materials and Methods: A retrospective review was performed on medical reports of patients referred to our tertiary metabolic stone clinic in Labbafinejad Medical Center, Tehran, Iran. Based on stone recurrence status, stone formers $(\mathrm{SF})$ were classified into three groups; Group 1: First time SF, Group 2: Recurrent SF with history of recurrent event in any time before the last two years and, Group 3: Recurrent SF with the history of stone event during the last two years and before. Age, gender, body mass index (BMI) was considered as co-variates. we excluded all medical diseases or medications that may affect urine chemistries. Statistical analyses were performed using SPSS software, version 23. Chi square test was used for assessing the relation between UMAs and groups and, univariate logistic regression for the effect of UMAs on recurrent events.

Results: 780 patients were included in the study. 232 were women and 548 were men, the mean age was 47.31 (SD: 11.83, range: $18-78$ ), and the mean BMI was 28.53 (SD: 4.58, range: 1645). The prevalence of UMAs in Group 1, 2 and 3 SF are shown

Table 1. prevalence of UMAs in our patients.
\begin{tabular}{|l|l|l|l|l|}
\hline & Group 1 (n=57) & Group 2 (n=314) & Group 3 (n=409) & P value \\
\hline Low Urine volume & $63.3 \%$ & $77.1 \%$ & $76.5 \%$ & 0.07 \\
\hline Hyperoxaluria & $33.3 \%$ & $36.9 \%$ & $41.2 \%$ & 0.34 \\
\hline Hypercalciuria & $38.6 \%$ & $56.5 \%$ & $42.5 \%$ & 0.001 \\
\hline Hyperuricosuria & $15.8 \%$ & $17.9 \%$ & $24.9 \%$ & 0.04 \\
\hline Hypocitraturia & $36.8 \%$ & $35.8 \%$ & $43.9 \%$ & 0.08 \\
\hline Hypomagnesuria & $66.0 \%$ & $58.3 \%$ & $60.6 \%$ & 0.55 \\
\hline
\end{tabular}

Table 2. The frequency of simultaneous urine metabolic abnormalities (UMAs).

\begin{tabular}{|l|r|r|r|r|}
\hline & Group 1 $(n=57)$ & Group 2 ( $n=314)$ & Group 3 ( $=409)$ & Total \\
\hline No UMA & 1 & 4 & 9 & 14 \\
& $1.8 \%$ & $1.3 \%$ & $2.2 \%$ & $1.8 \%$ \\
\hline One UMA & 10 & 24 & 31 & 65 \\
& $17.5 \%$ & $7.6 \%$ & $7.6 \%$ & $8.3 \%$ \\
\hline Two UMAs & 17 & 85 & 108 & 210 \\
& $29.8 \%$ & $27.1 \%$ & $26.4 \%$ & $26.9 \%$ \\
\hline Three UMAs & 19 & 130 & 144 & 293 \\
& $33.3 \%$ & $41.4 \%$ & $35.2 \%$ & $37.6 \%$ \\
\hline Four UMAs & 9 & 62 & 94 & 165 \\
& $15.8 \%$ & $19.7 \%$ & $23.0 \%$ & $21.2 \%$ \\
\hline Five UMAs & 1 & 8 & 19 & 28 \\
& $1.8 \%$ & $2.5 \%$ & $4.6 \%$ & $3.6 \%$ \\
\hline Six UMAs & 0 & 1 & 4 & 5 \\
& $0.0 \%$ & $0.3 \%$ & $1.0 \%$ & $0.6 \%$ \\
\hline
\end{tabular}

in Table 1. There were no significant differences between prevalence of simultaneous UMA in three groups $(p=0.22)$ and, the presence of 3 simultaneous UMAs $(37.6 \%)$ was the most frequent that followed by $2(26.9 \%)$ and $4(21.2 \%)$, respectively (Table 2). In comparison between First SF and Recurrent SF groups, we found that the presence of 3 (OR: 2.62, 95\% CI: $1.16-$ 5.95 ) or $\geq 4$ simultaneous UMAs (OR: 3.42 , 95\% CI: $1.35-8.63$ ) versus one UMA, can increase the chance of recurrence event. Conclusions: This study reveals that the presence of three or more simultaneous UMAs, is associated with increase in stone recurrence events and appropriate medical management can be preventive against recurrent stone formation.

MP9-9 Dietary Assessment and 24 Hour Urine Collections in the Management of Hypercalciuria: Are Both Needed?

J Bell, M Ehlers, A Schweitzer, SY Nakada, KL Penniston

University of Kentucky College of Medicine

United States

Introduction \& Objective: The management of hypercalciuria may include dietary and/or medical therapy. Dietary advice is often based on 24 hour urine results alone, without considering whether there is an influence of diet on urinary calcium excretion. We studied the 24 hour urine results and dietary habits of patients with a history of stones.

Materials and Methods: Dietary questionnaires designed to assess habitual intake are completed by all our patients. These were matched with the 24-hour urine results of those who presented to our metabolic stone clinic between May 2016 and April 2017. All dietary questionnaires were reviewed by a registered dietician; nutrient intake was quantified. Suspected over- and under-collections for urine were eliminated from the dataset.

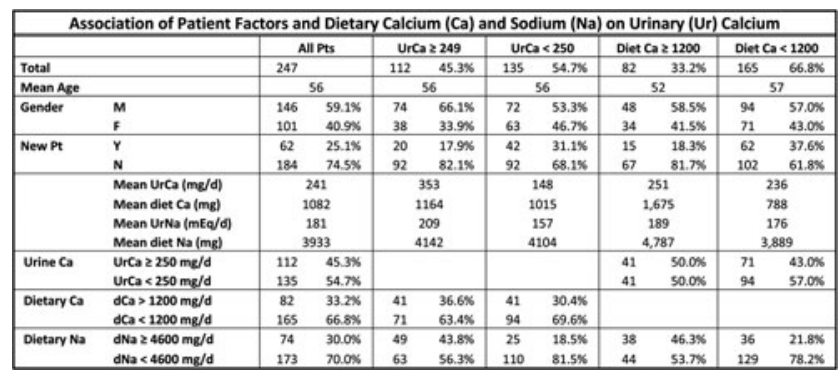


Patients with urine collections $>90$ days from dietary data were not included.

Results: Our series included 247 patients, 59\% male; mean age $56 \pm 14$ years. Mean calcium intake was $1,082 \mathrm{mg} / \mathrm{d}$ (range, 200$5,675 \mathrm{mg} /$ day). Mean urinary calcium excretion was $241 \mathrm{mg} / \mathrm{d}$ (range, 9-691 mg/d). Calcium intake $\geq 1,200 \mathrm{mg} /$ day was identified in $33 \%$. Hypercalciuria ( $\geq 250 \mathrm{mg} / \mathrm{d}$ ) was observed in $45 \%$. Of these, $50 \%$ reported consuming $>1,200 \mathrm{mg} / \mathrm{d}$ of calcium. There was no difference in either sodium or calcium intake for patients with hypercalciuria vs those with normal urine calcium (4,142 vs 4,104 mg/d, P=0.87; and 1,164 vs 1,015 mg/d, P = 0.57 respectively).

Conclusions: The majority of patients $(67 \%)$ reported dietary calcium intake below the RDA $(1,200 \mathrm{mg} / \mathrm{d})$. Of patients with hypercalciuria, half reported suboptimal calcium intake. Additionally, $56 \%$ of these did not have a high sodium intake $(>4,600 \mathrm{mg} / \mathrm{d})$. Thus, neither calcium nor sodium restriction would be likely to address the hypercalciuria of these patients. Management of patients with hypercalciuria may benefit from a dietary assessment in order to rule in or out dietary contributors and avoid unnecessary dietary recommendations or restrictions.

MP9-10 Association of subcutaneous adipose tissue with nephrolithiasis as measured by computed tomography

HS Alakrash, B Alharbi, J Hoogenes, K Kim, O Aljarallah, A Kapoor, B Shayegan, ED Matsumoto

McMaster University

Canada

Introduction \& Objective: Both obesity and metabolic syndrome are considered to be risk factors for nephrolithiasis. Studies have shown their association with various urinary biochemical abnormalities that increase the propensity for kidney stone formation. We have previously shown that increased visceral adipose tissue (VAT, $\mathrm{cm}^{2}$ ) is significantly associated with metabolic syndrome, diabetes, hypertension, and hyperlipidemia, which are risk factors for stone development. Subcutaneous adipose tissue (SAT, $\mathrm{cm} 2$ ) may also be a more accurate measure of obesity than body mass index (BMI), and is strongly associated with insulin resistance, a prerequisite for the formation of kidney stones in obese patients. Our objective was to determine the association between SAT and metabolic parameters in obese pre-bariatric patients with history of urolithiasis and computed tomography (CT) of abdomen and pelvis.

Materials and Methods: We retrospectively reviewed the records of 200 obese patients who had CT abdomen and pelvis as pre-bariatric surgery workup. SAT was measured using CTbased fat delineation program AnalyzePro®, where axial slices were obtained at the level of the umbilicus (L4 and L5 [SAT1]) and at the L1-L2 level (SAT2). We analyzed the association of SAT1 and SAT2 measurements on comorbidities, urolithiasis risk factors, stone composition, urine metabolites, and metabolic syndrome.

Results: The mean age was $51.2( \pm 11.8)$ and $118(59 \%)$ were female. Based on BMI, $75.5 \%$ of the sample were obese (more females were obese, $p=0.001$ ), while with SAT1 calculation $93.5 \%$ were obese, and with SAT2 $71.1 \%$ were obese. Stone composition showed $59.9 \%$ mixed, $11.9 \%$ calcium oxalate, $8 \%$ uric acid, and the remaining were $\mathrm{p}=0.01$ ), while SAT2 was significant for diabetes $(p=0.018)$, hypertension $(p=0.006)$, and metabolic syndrome $(p=0.02)$. At both SAT1 and 2 levels, 24-hour urinalysis was significant for high levels of urinary sodium, urate, phosphate, and citrate (all $p$

Conclusions: Increased SAT was significantly associated with hypertension, diabetes, and metabolic syndrome, which are risk factors for nephrolithiasis. Obesity at both SAT levels showed significantly high levels of urinary sodium, urate, phosphate, and citrate. Our findings may support an easily reproducible marker of metabolic syndrome that can be used to stratify risk for development of nephrolithiasis and serve as a target for patient treatment, management, and counseling.

MP9-11 Relationship of Sodium Intake to Urinary Sodium Excretion in Stone Formers: Which Foods Contribute the Most?

J Bell, M Ehlers, A Schweitzer, SY Nakada, KL Penniston

University of Kentucky College of Medicine

United States

Introduction \& Objective: The ability of dietary salt (sodium chloride) to enhance urinary calcium excretion is well-known. Identifying chronic high salt intake from occasional high intake using 24 hour urine collections alone is problematic as the 24 hour collection reflects a limited time frame and does not always represent chronic dietary habits. Moreover, as added salt is known to contribute only a small percentage to intake and to urinary sodium $(\mathrm{Na})$ excretion, patients claiming to "never use the salt shaker" often question findings of hypernatriuria. We assessed dietary sources of $\mathrm{Na}$ in patients with a history of stones. Materials and Methods: Dietary questionnaires to assess habitual intake are completed by all our patients. These were matched with the 24-hour urine results of those presenting to our metabolic stone clinic from 5/2016-4/2017. Dietary questionnaires were reviewed by a registered dietician. Nutrient intake was quantified; food categories reflecting $\mathrm{Na}$ intake were identified. Patients with suspected over- and under-collections for urine were not included nor were those with urine collections $>90$ days from dietary data.

Results: Of 247 patients (mean age $56 \pm 14$ years), 59\% were male. Mean Na intake was $181 \mathrm{mEq} / \mathrm{d}$ (range, 71-524 mEq).* Na intake $\geq 200 \mathrm{mEq} / \mathrm{d}$ was identified in $30 \%$. Mean urinary $\mathrm{Na}$ excretion was $183 \mathrm{mEq} / \mathrm{d}$ (range, $37-551 \mathrm{mEq}$ ). Urinary $\mathrm{Na}$ excretion $\geq 200 \mathrm{mEq} / \mathrm{d}$ was observed in $35 \%$. The most common

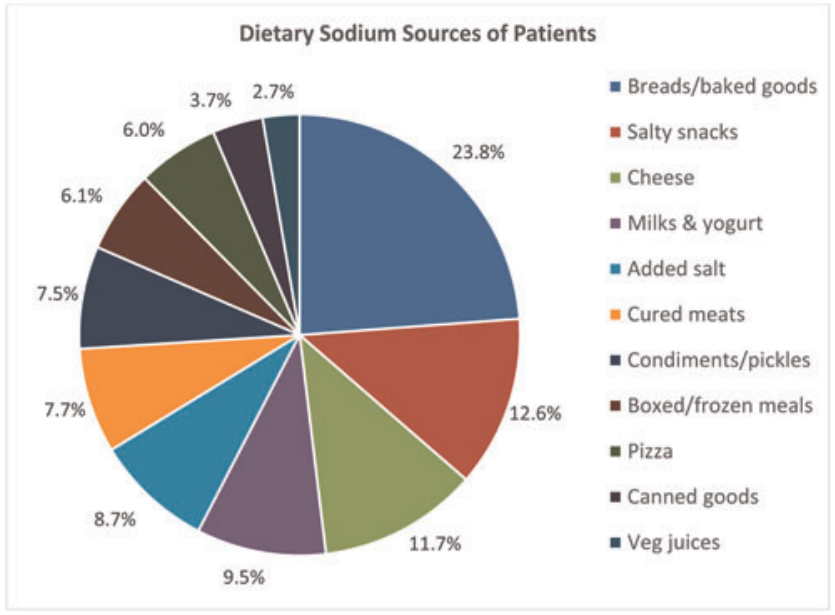


food source of $\mathrm{Na}$ was breads and baked goods (24\%), followed by salty snacks $(13 \%)$, cheese $(12 \%)$, and then milks (both dairy and non-dairy) and yogurts (10\%). There was no difference in food salt sources between those with higher vs lower urinary sodium excretion. The percentage of total $\mathrm{Na}$ intake for all food categories is shown (figure).*mEq sodium is converted to $\mathrm{mg}$ by multiplying by 23 , thus $4,600 \mathrm{mg} / \mathrm{d}$ is equivalent to $200 \mathrm{mEq} / \mathrm{d}$ Conclusions: One-third of patients had high urinary Na excretion $(\geq 200 \mathrm{mEq} / \mathrm{d})$; about the same number $(30 \%)$ reported intake $\geq 200 \mathrm{mEq} / \mathrm{d}$. The other two-thirds of patients had either higher or lower $\mathrm{Na}$ excretion than reported intake. Dietary assessment may help to distinguish the occasional intake of high dietary salt, which may manifest in a single 24 hour collection as hypernatriuria, from chronic high salt intake, which could falsely manifest as urinary $\mathrm{Na}$ under the risk cutoff. Moreover, dietary assessment can identify sources of salt intake and lead to more targeted nutrition counseling.

\section{MP9-12 Pharmaceutical Burden and Medication Com- pliance in Patients with Cystinuria}

BA Sherer, D Isaacson, Z Kornberg, M Usawachintachit, DT Tzou, K Taguchi, S Wiener, T Chi, ML Stoller

UCSF

United States

Introduction \& Objective: Cystinuria is a rare orphan disease marked by chronic, recurrent, debilitating episodes of cystine urolithiasis, often requiring repetitive procedural intervention. Patients face a rigorous, life-long pharmaceutical burden, often ingesting dozens of pills per day, many of which have known side effects. The objective of this study is to determine pharmaceutical burden and medication compliance in patients with cystinuria.

Materials and Methods: Under an institutional review board approved protocol, cystinuric patients from an established institutional database $(n=42)$ were asked to complete a brief standardized telephone survey evaluating six domains of medical therapy: 1) current and historical medication/supplement use, 2) daily medication dosing, 3) medication compliance, 4) barriers to medication continuation, 5) side effects, and 6) goals/desires for alternative therapies. When available, 24 hour urine studies were retrospectively reviewed to determine interval changes in quantitative urinary cystine, urine $\mathrm{pH}$, and urinary citrate during therapy.

Results: Of patients completing the survey $(n=22), 95 \%$ had currently or previously taken at least one medication for cystinuria including potassium citrate, tiopronin, captopril, penicillimine, and/or a supplement. Most (77\%) patients had taken combination therapy regimens, most commonly tiopronin + potassium citrate $(n=13)$. For patients currently on medication $(\mathrm{n}=19)$, the average tablets/doses ingested per day was is 11.4 (range 4 to 23). Overall, daily pharmaceutical burden for each patient is depicted in Figure 1. Despite good compliance (77\% reported taking at least $75 \%$ of their scheduled doses), only $33 \%$ of treated patients were convinced that their medication regimens had helped to decrease stone episodes. Side effects were commonly attributed to tiopronin and most often included malodorous urine $(n=7)$ and nausea/GI upset $(n=4)$. Despite impressive medication compliance; at a mean interval of 7.7 years between initial and current 24 hour urine studies, there were only marginal improvements in average quantitative urinary cystine $(988 \mathrm{mg}$ to 949mg), urine $\mathrm{pH}$ ( $\mathrm{pH} 6.4$ to $\mathrm{pH} 7.1$ ), and urinary citrate $(577 \mathrm{mg}$ / d to $605 \mathrm{mg} / \mathrm{d}$ ).

Conclusions: Cystinuric patients face a substantial pharmaceutical burden and display heroic individual efforts in attempted stone prevention, often ingesting more than a dozen tablets per day. Despite their best efforts, many do not feel that these exhaustive regimens are successful. Cystinurics express a strong desire for pharmaceutical options with better efficacy, less dosing frequency, and fewer side effects.

MP9-13 Long-term treatment of vitamin D insufficiency or deficiency in postmenopausal women with or without hypercalciuria does not increase urinary calcium excretion

KL Penniston, RA Jhagroo, AC Staples, SY Nakada, KE Hansen

University of Wisconsin School of Medicine and Public Health, Department of Urology

United States

Introduction \& Objective: Vitamin D (VD) repletion in calcium $(\mathrm{Ca})$ stone formers is sometimes questioned, especially if long-term, if higher doses to achieve adequate VD status are required, and/or in those with hypercalciuria ( $>250 \mathrm{mg} / \mathrm{d})$. We assessed the effect of $1 \mathrm{y}$ VD repletion on urinary $\mathrm{Ca}$ excretion. Materials and Methods: The study was randomized, doubleblind, and placebo-controlled. With IRB approval, postmenopausal women with low VD status [serum $25(\mathrm{OH}) \mathrm{D}<30 \mathrm{ng} / \mathrm{mL}$; $\mathrm{n}=90 ; 61 \pm 6 \mathrm{y} ;$ BMI $30.8 \pm 6.8]$ were randomized to placebo, low-dose (LD) VD (800 IU/d), or high-dose (HD) VD (50,000 IU for $15 \mathrm{~d}$ followed by 50,000 IU twice/month) for $1 \mathrm{y}$. Prefilled 31-day pill boxes were dispensed; remaining capsules were counted at study visits $(60,120, \& 365 \mathrm{~d})$, at which time participants reported to our clinical research unit for 24-h urine collections. Multiple-day weighed diet records prior to each study visit were completed by all to establish individuals' usual nutritional intake. Dietary intake was reproduced for each participant during the 24-h study visits.

Results: Median baseline urinary Ca excretion was $209 \mathrm{mg} / \mathrm{d}$. Compliance with treatment was estimated at approximately $100 \%$ across all arms. Overall, urinary Ca excretion was unchanged from baseline to $1 \mathrm{y}(228 \pm 70$ to $219 \pm 92 \mathrm{mg} / \mathrm{d}$; $\mathrm{P}=0.25$, paired $\mathrm{t}$-test). Only women in the HD group achieved $25(\mathrm{OH}) \mathrm{D} \geq 30 \mathrm{ng} / \mathrm{mL}$ ( $21 \pm 3$ to $56 \pm 12 \mathrm{ng} / \mathrm{mL} ; \mathrm{P}<0.001)$. Despite the significant rise in VD status in this group, urinary $\mathrm{Ca}$ excretion did not increase $(256 \pm 86$ to $250 \pm 131 \mathrm{mg} / \mathrm{d}$; $\mathrm{P}=0.74)$. Nor did urinary $\mathrm{Ca}$ excretion change in the other treatment groups ( $\mathrm{P} \geq 0.27$ for within-group pairwise comparisons from

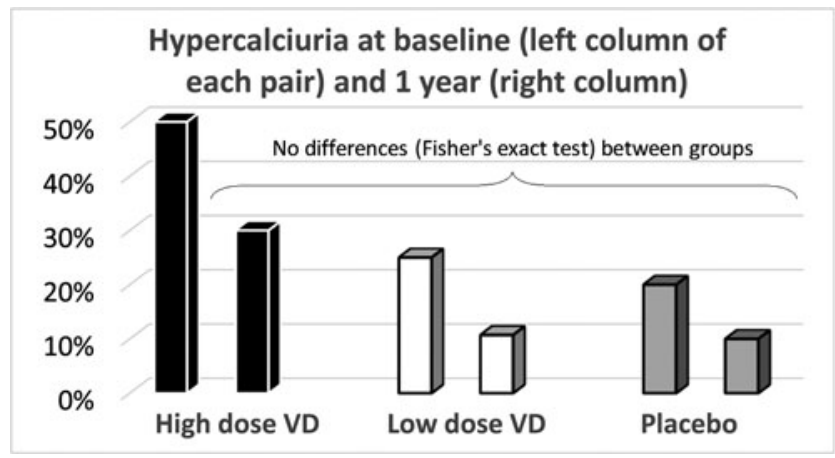


baseline to $1 \mathrm{y})$. The incidence of new-onset hypercalciuria was not affected by VD treatment $(\mathrm{P} \geq 0.07)$ and was $7 \%, 19 \%$, and $12 \%$ for HD, LD, and placebo groups. Among women across all groups with hypercalciuria at baseline $(n=28)$, Ca excretion did not change ( $312 \pm 60$ to $273 \pm 130 \mathrm{mg} / \mathrm{d} ; \mathrm{P}=0.07)$, but tended to be lower at $1 \mathrm{y}$. Among these, hypercalciuria was resolved in $40 \%, 57 \%$, and $50 \%$ in $\mathrm{HD}, \mathrm{LD}$, and placebo groups, respectively. VD treatment did not affect resolution of hypercalciuria ( $\mathrm{P} \geq 0.20$, figure).

Conclusions: Despite a significant rise in VD status, VD repletion at 50,000 IU twice monthly for $1 \mathrm{y}$, following a loading dose, did not increase urinary $\mathrm{Ca}$ excretion in postmenopausal women with or without hypercalciuria. Evidence suggests the risk for hypercalciuria is low following VD repletion in patients with low VD status.

MP9-14 Correlation between cystine concentration and cystine capacity in cystinuric patients

N Canvasser, I Sorokin, J Friedlander, D Mollengarden, J Antonelli, M Pearle

Department of Urology, UC Davis Medical Center United States

Introduction \& Objective: The therapeutic goal in patients with cystinuria has traditionally been a cystine concentration $<250 \mathrm{mg} / \mathrm{L}$. However, this endpoint fails to take into account other factors that influence cystine stone formation, such as $\mathrm{pH}$. A proprietary assay from Litholink Corporation (Chicago, IL) measures "cystine capacity," which reflects the change in a solid phase of cystine incubated in patient urine, for which the recommended goal is $>150 \mathrm{mg} / \mathrm{L}$, although this cutpoint was empirically, not clinically, derived. Our goal was to analyze urine collections from cystine stone formers to determine correlation between cystine concentration and capacity.

Materials and Methods: With IRB approval, we prospectively followed cystine stone patients in our clinic from 2005-2016. 24hour urine studies obtained at baseline and during followup were sent to Litholink ${ }^{\mathrm{TM}}$ for analysis of cystine excretion, capacity and other factors. Urine collections were categorized according to a cystine concentration cutpoint of $250 \mathrm{mg} / \mathrm{L}$ as 'favorable' or 'unfavorable'. Binary analysis and Pearson correlation was performed between cystine concentration and capacity. Statistical significance was set at $\mathrm{p}<0.05$.

Results: A total of 43 patients collected 219 24-hour urines analyzed for cystine capacity and excretion. Mean values among all urine collections were: $\mathrm{pH} 6.98 \pm 0.36$, total volume $3.6 \pm 1.3 \mathrm{~L} / \mathrm{d}$, cystine concentration $286 \pm 147 \mathrm{mg} / \mathrm{L}$ and capacity $22 \pm 116 \mathrm{mg} / \mathrm{L}$. Among all urine collections, $105 \mathrm{had}$ favorable cystine concentration $(150 \mathrm{mg} / \mathrm{L}), 14$ had favorable capacity

Table 1. Discordance between cystine concentration and cystine capacity at various capacity cutpoints among 105 24-hour urine analyses with cystine concentration $<250 \mathrm{mg} / \mathrm{L}$.

\begin{tabular}{|c|c|}
\hline $\begin{array}{c}\text { Cystine capacity } \\
\text { (mg/L) }\end{array}$ & $\begin{array}{c}\text { No. of urines with } \\
\text { favorable cystine } \\
\text { concentration and } \\
\text { unfavorable capacity }\end{array}$ \\
\hline$<150$ & $93(89 \%)$ \\
\hline$<140$ & $92(88 \%)$ \\
\hline$<130$ & $86(82 \%)$ \\
\hline$<120$ & $79(75 \%)$ \\
\hline$<110$ & $73(70 \%)$ \\
\hline$<100$ & $67(64 \%)$ \\
\hline$<90$ & $62(59 \%)$ \\
\hline
\end{tabular}

( $>150 \mathrm{mg} / \mathrm{L})$, but only $12(11 \%)$ had both. Although there was strong correlation between capacity and concentration $(r=-0.9$, $\mathrm{p}<0.001$ ), there was significant discordance between the 2 parameters, depending on the capacity cutpoint used: even at a cutpoint of $90 \mathrm{mg} / \mathrm{L}$, only $41 \%$ of patients had both parameters favorable. Table 1 shows the discordance between favorable cystine concentration and favorable cystine capacity at varying capacity cutpoints.

Conclusions: Despite near perfect inverse correlation between cystine concentration and capacity, many 24-hour urines with therapeutic cystine concentrations have unfavorable cystine capacity, even at lower than recommended therapeutic cystine capacity. Further work is needed to determine the ideal measure and therapeutic goal for patients with cystinuria.

MP9-15 Effect of Increased Fluid Intake on 24-hour Urinary pH in Stone Formers with Low Urine Volume

D Wollin, B Winship, W Tom, LG Davis, E Cone, C Scales, M Ferrandino, M Lipkin, GM Preminger

Duke University Medical Center

United States

Introduction \& Objective: Urolithiasis is a common condition affecting 1 in 11 people in the United States. Through meticulous medical and dietary therapy, the risk of subsequent stone episodes can be reduced by more than half. While urinary alkalinization is the cornerstone of pharmacologic management, little is known about the effect of improved fluid intake on urinary $\mathrm{pH}$. Our aim was to assess the change in urinary $\mathrm{pH}$ associated with conservative dietary management, focusing specifically on an increase in fluid intake and 24-hour urine volume in the absence of directed alkalinizing therapy.

Materials and Methods: We queried a retrospectively-maintained database of demographic data and 24-hour urine analyses of 2,197 kidney stone formers from 2000 to 2015. Inclusion criteria included patients over age 17, multiple 24-hour urine collections, and a baseline low urine $(<2 \mathrm{~L} / 24 \mathrm{~h})$ and low urine $\mathrm{pH}$ $(<6.0)$. Patients who were treated with thiazide diuretics or alkalinizing agents were excluded to ensure that all effects were due to dietary modifications alone. Patients with an increased 24hour urine volume on a subsequent collection were then analyzed to evaluate a change in 24-hour urinary $\mathrm{pH}$. The urinary $\mathrm{pH}$ means from pre- and post-intervention were compared using Student's T-test, with univariate and multivariate analyses used to construct a model to predict follow-up results.

Results: Using the inclusion and exclusion criteria, 163 patients were identified (94 male, 69 female). The mean 24-hour urine volume, $\mathrm{pH}$, citrate, and urea nitrogen significantly increased, while ammonium and sulfate remained stable (Table 1). Multivariate analysis produced a best-fit model utilizing initial urine volume, $\mathrm{pH}$, ammonium and change in urine volume, citrate, and ammonium. The model demonstrated a correlation between an

\begin{tabular}{|r|c|c|c|}
\hline Table 1: Pre-intervention and Post-intervention 24-hour urine values & \\
\hline & Pre-intervention mean (SD) & Post-intervention mean (SD) & p \\
\hline Vol (L) & $1.31(0.37)$ & $2.12(0.82)$ & $<0.001$ \\
\hline pH & $5.65(0.21)$ & $6.11(0.51)$ & $<0.001$ \\
\hline Citrate (mg/day) & $508.08(274.33)$ & $676.23(383.26)$ & $<0.001$ \\
\hline Ammonium (mmolday) & $35.50(15.76)$ & $34.53(18.96)$ & 0.616 \\
\hline Sulfate (mEq/day) & $36.11(13.87)$ & $38.80(14.22)$ & 0.098 \\
\hline Urea Nitrogen (g/day) & $9.89(3.44)$ & $11.07(3.83)$ & 0.004 \\
\hline
\end{tabular}




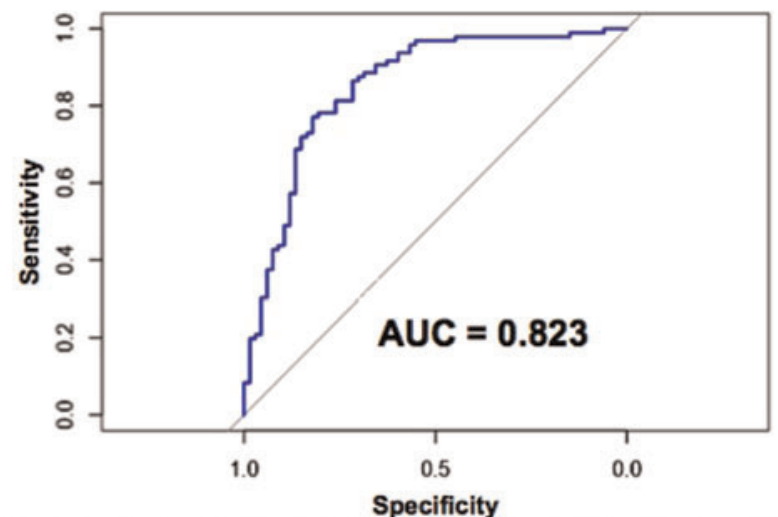

Figure 1: Receiver Operating Characteristic Curve of Multivariate Model Predicting Favorable PostIntervention Urine $\mathrm{pH}$

increase in urine volume and increase in urine $\mathrm{pH}$ in this population with an AUC of 0.823 (Figure 1).

Conclusions: These data show that conservative dietary recommendations, and specifically a recommendation for increased fluid intake without directed alkalinizing therapy, is associated with a significant improvement in urinary $\mathrm{pH}$ in certain patients. Given the ability for this model to output a probability of favorable $\mathrm{pH}$ results based on initial urinary parameters, it could potentially be used to assist in predicting response to dietary recommendations in patients.

\section{MP9-16 Withdrawn}

MP9-17 Do Metabolic Factors Influence the Formation and Recurrence of Bladder Calculi?

JN Thai, T Tran, E Parkhomenko, K Blum, M Gupta, S Purnell

Department of Urology, Ichan School of Medicine at Mount Sinai

United States

Introduction \& Objective: Metabolic abnormalities are associated with an increased risk for renal calculi. However, no studies to date have investigated whether metabolic factors affect the formation or recurrence of adults with bladder calculi. We aim to characterize patients with primary bladder stones, with and without kidney stones, based on their clinical factors, 24-hour urine parameters, and stone composition.

Materials and Methods: We retrospectively reviewed medical records of patients referred to a large-volume urology clinic for primary bladder stones and classified them based on their history of kidney stones. We investigated relationships between metabolic factors, clinical characteristics, and stone composition. We used descriptive statistics, multivariate analysis, and one-way ANOVA to look at associations between these parameters.

Results: Final analysis included 58 men. 16 men were classified as forming only bladder stones. $72 \%(42 / 58)$ of these men had a history of concurrent kidney stones. Bladder stone only formers were older ( 70 years versus 63 years), heavier (BMI of 26.5 versus 29.6) and had greater total stone burden $(3.4 \mathrm{~cm}$ versus $1.9 \mathrm{~cm})$, higher bladder stone recurrence rates (38\% versus $24 \%$ ), and significantly lower urine $\mathrm{pH}(\mathrm{p}=0.01)$ compared to the concomitant kidney stone formers. Bladder stone only formers had a higher

\begin{tabular}{|c|c|c|c|}
\hline & $\begin{array}{c}\text { Bladder Stones } \\
\text { Only ( } \mathrm{N}=16)\end{array}$ & $\begin{array}{c}\text { Concomitant } \\
\text { Kidney Stones } \\
(\mathrm{N}=42)\end{array}$ & $p$-value \\
\hline Mean Age, years & 70.2 & 62.8 & 0.06 \\
\hline Mean BMI & 26.5 & 29.6 & 0.03 \\
\hline Mean Stone Burden, cm & 3.4 & 1.9 & 0.04 \\
\hline Mean Serum UA & 5.6 & 5.9 & 0.34 \\
\hline $\begin{array}{l}\text { Mean Number of Metabolic Abnormalities } \\
\text { in 24-Hour Urine }\end{array}$ & 5.3 & 4.8 & 0.56 \\
\hline Metabolic Syndrome, N (\%) & $2(12.5)$ & $14(33.3)$ & 0.12 \\
\hline Mean Post-void Residual, ml & 67.8 & 99.5 & 0.53 \\
\hline Mean Max Flow Rate, $\mathrm{ml} / \mathrm{sec}$ & 7.8 & 7.9 & 0.17 \\
\hline Prior history of BPH, N (\%) & $11(68.8 \%)$ & $25(59.5 \%)$ & 0.84 \\
\hline \multicolumn{4}{|l|}{ Mean Metabolic Parameter Levels } \\
\hline Vol24 & 1.66 & 1.96 & 0.33 \\
\hline SSCaOx & 8.59 & 6.44 & 0.10 \\
\hline $\mathrm{Ca} 24$ & 200.50 & 195.69 & 0.91 \\
\hline $0 \times 24$ & 43.40 & 41.00 & 0.66 \\
\hline Cit24 & 731.30 & 608.28 & 0.31 \\
\hline SSCaP & 0.56 & 1.08 & 0.09 \\
\hline $\mathrm{pH}$ & 5.44 & 6.16 & $0.01^{*}$ \\
\hline SSUA & 1.85 & 0.99 & $0.02^{*}$ \\
\hline UA24 & 0.62 & 0.63 & 0.79 \\
\hline $\mathrm{Na} 24$ & 142.90 & 169.07 & 0.24 \\
\hline K24 & 70.80 & 72.17 & 0.87 \\
\hline Mg24 & 96.20 & 102.52 & 0.66 \\
\hline P24 & 0.89 & 0.93 & 0.67 \\
\hline Nh424 & 38.90 & 36.24 & 0.57 \\
\hline $\mathrm{Cl} 24$ & 157.90 & 175.45 & 0.42 \\
\hline Sul24 & 49.70 & 42.10 & 0.14 \\
\hline UUN24 & 12.64 & 11.70 & 0.44 \\
\hline
\end{tabular}

* Denotes statistical significance

incidence of uric acid (UA) composition compared to their counterparts (56\% versus $24 \%$ ). Patients who formed only bladder stones were also more likely to have BPH (69\% versus $60 \%)$.

Conclusions: Primary bladder stone formers had surprisingly high rates of kidney stone formation and higher incidence of recurrence of bladder stones, specifically. Outlet obstruction secondary to BPH may be the cause of bladder stones. These patients were also found with multiple metabolic disturbances contributing to the formation and recurrence of stones. The use of metabolic profiles could be helpful in offering appropriate treatments. Both groups, regardless of whether they made only bladder stones or both bladder and kidney stones, were metabolically similar, suggesting that aggressive treatment whether due to bladder or kidney stones, may be warranted in patients with multiple metabolic abnormalities and recurrence of stones.

MP9-18 Stone composition and lithogenic risk factors in patients with ureteropelvic junction and renal calculi

M Shahait, A Maganty, MJ Semins, T Tarin, S Jackman, T Averch, MC Ost

UPMC

United States

Introduction \& Objective: Concomitant lithiasis in patients with ureteropelvic junction obstruction (UPJO) is not uncommon. The mechanism of stone formation in this subgroup of patients is an issue of debate amongst the urological community. We sought to assess the stone composition and associated urine milieu in this subset of patient.

Materials and Methods: We reviewed the medical records of all patients who presented with simultaneous stone and UPJO 
between 2005-2016. We included patients with preoperative radiological studies and/or reports that demonstrate ureteropelvic junction obstruction and a concurrent renal calculus. The stone type was defined by the predominant stone component, represented by at least $60 \%$ of all components.

Results: We identified 42 patients with UPJO and renal calculi. The male to female ratio is $1: 1.1$. The chemical composition of stone was calcium oxalate in $64.3 \%$ of the cases, Calcium phosphate in $28.7 \%$, and uric acid in $7.0 \%$. Of those, 12 patients completed comprehensive metabolic workup. Metabolic abnormality was observed in $75 \%$ of the cases. The percentage of patients with hypercalciuria, hypocitraturia, low urine $\mathrm{PH}$, and hyperoxaluria was $41.6 \%, 41.6 \%, 33.3 \%$, and $25 \%$, respectively. Conclusions: A substantial number of UPJO patients who present with concomitant stone have metabolic abnormalities. Early identification of these abnormalities may direct the medical treatment to decrease stone recurrence rate.

MP9-19 Growing older, growing stones? Increasing health care use by older adults with stones

B Winship, T Hobbs, D Wollin, M Lipkin, K Schmader, GM Preminger, C Scales

United States

Introduction \& Objective: Urinary stone disease (USD) has long afflicted the working age population. Little is known regarding changing patterns of health care utilization in the face of rapidly aging populations in the United States (US). The objective of our research was to examine trends in health care utilization for USD by age, with a particular focus on older adults. Materials and Methods: We used data from the Nationwide Inpatient Sample (NIS) and the Nationwide Emergency Department Sample (NEDS) to estimate US trends for inpatient and emergency department care utilization from 2006 to 2014. Older adults were defined as individuals aged at least 65 years. Encounters for USD were identified using diagnostic codes and established algorithms. We estimated age- and sex- specific utilization rates for emergency department (ED) visits and inpatient discharges. Estimates were compared using the Z-test.

Results: The age-adjusted rate of ED visits grew by $19.3 \%$ in the US from 2006 to 2014 ( $\mathrm{p}<0.001$ ). Utilization rates demonstrated the greatest increase among older adults (Figure). Among females aged at least 65 years, ED utilization grew by over $50 \%$,

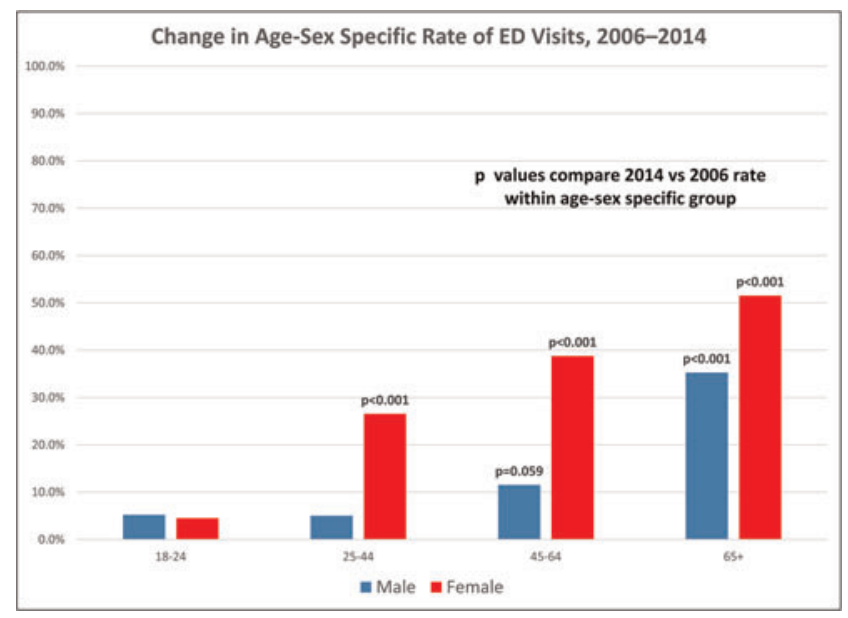

from 327 to 496 per $100,000(\mathrm{p}<0.001)$. Among similarly aged males, utilization grew by $35 \%$, from 668 to 904 per 100,000 $(\mathrm{p}<0.001)$. Utilization of inpatient care was greatest among older adults. While the age-adjusted use of inpatient care grew modestly $(7.8 \%, \mathrm{p}<0.001)$, large increases in inpatient care were noted among older adults: females, $32 \%$ increase $(\mathrm{p}<0.001)$ and males, $23 \%$ increase $(\mathrm{p}<0.001)$. These age- and sex- specific rate changes suggest that increasing utilization is NOT due to an aging population.

Conclusions: Health care utilization for USD is growing rapidly among older adults, and faster than any other age group. These trends do not appear to reflect changing population demographics, but rather intrinsic changes in the burden of urinary stone disease. Further investigation is required to identify potential mechanisms for the rising burden of USD among older adults.

MP9-20 Virtual Stone Clinic - The Future of Stone Management in the United Kingdom?

T Smith, O Blach, K Guest, A Symes

Maidstone and Tunbridge Wells NHS Trust

United Kingdom

Introduction \& Objective: United Kingdom outpatient waiting lists are increasing. In January 2016, 976 patients were awaiting a Brighton Urology appointment, with routine stone-clinic referrals seen after 6 months. Patient experience was poor with high DNA rates. A virtual stone clinic (VSC) was created to improve the service.

Materials and Methods: VSC set up was based on the awardwinning virtual fracture-clinic. A consultant-led once-weekly VSC was supported by a MDT (stone-registrar, ESWL radiographer and nurse). Referrals were triaged direct from source. A target of 20-30 patients per week was set and a tariff of $£ 64$ agreed. We assessed outcomes following the first 5 months of the service.

Results: 468 patients were seen. 91(24.3\%) were discharged without further investigation. Of the $377(60.7 \%$ ) who required follow up, $283(75 \%)$ were brought back to the VSC, and $94(25 \%)$ were invited to an outpatient appointment (to discuss invasive treatment or for metabolic evaluation). $78.8 \%$ were discharged following second virtual clinic review. Income from the 1hour-long weekly VSC was $£ 29,952$, vs. $£ 51,976$ from 4hour-long comparable outpatient clinics. The projected income had the VSC run for 4hours was $£ 119,808$. The 6 month waiting list was cleared within 2 months. All new referrals were reviewed by a Consultant within 1 week. Feedback from patients was good with 1 complaint $(0.002 \%)$ and 20 DNA's(4.3\%).

Conclusions: The VSC is cost effective and has clear benefits with regards to reducing waiting lists and improving patient care. VSCs avoid time-consuming telephone follow-up clinics and free-up outpatient appointments for other activity or complex stone patients. Early experience suggests patients enjoy the service.

MP9-21 Are Patients with Pure Uric Acid Stones Metabolically and Clinically Different than those with Mixed Uric Acid Stones?

JN Thai, T Tran, E Parkhomenko, K Blum, M Gupta, S Purnell

Department of Urology, Ichan School of Medicine at Mount Sinai

United States 


\begin{tabular}{|c|c|c|c|}
\hline & \multicolumn{2}{|c|}{ GROUPS } & \multirow[b]{2}{*}{ p-value } \\
\hline & $\begin{array}{l}\text { Pure UA } \\
(\mathrm{N}=26)\end{array}$ & $\begin{array}{l}\text { Mixed UA } \\
(\mathrm{N}=66)\end{array}$ & \\
\hline \multicolumn{4}{|l|}{$\begin{array}{l}\text { Demographics and Clinical } \\
\text { Characteristics }\end{array}$} \\
\hline Mean age, years & 64.5 & 61.9 & 0.29 \\
\hline Female, $\%$ & 15.4 & 30.3 & 0.23 \\
\hline Mean BMI & 30.5 & 29.4 & 0.43 \\
\hline Mean stone burden, $\mathrm{cm}$ & 2.2 & 1.7 & 0.25 \\
\hline BPH history, N (\%) & $4(15.4)$ & $12(18.2)$ & 0.75 \\
\hline Prior history of stones, $\mathrm{N}(\%)$ & $20(76.9)$ & $47(71.2)$ & 0.74 \\
\hline Serum UA & 6.3 & 5.8 & 0.19 \\
\hline \multicolumn{4}{|l|}{$\begin{array}{l}\text { Mean Metabolic Parameter Levels in } \\
\text { 24-hour Urine }\end{array}$} \\
\hline Vol24 & 1.9 & 1.9 & 0.52 \\
\hline SSCaOx & 4.3 & 5.4 & 0.16 \\
\hline $\mathrm{Ca} 24$ & 122.9 & 140.3 & 0.41 \\
\hline $0 \times 24$ & 38.8 & 41.1 & 0.49 \\
\hline Cit24 & 500.4 & 601.8 & 0.19 \\
\hline SSCaP & 0.3 & 0.50 & 0.15 \\
\hline $\mathrm{pH}$ & 5.4 & 5.7 & $0.03^{*}$ \\
\hline SSUA & 1.5 & 1.4 & 0.57 \\
\hline UA24 & 0.6 & 0.6 & 0.33 \\
\hline $\mathrm{Na} 24$ & 175.2 & 168.2 & 0.71 \\
\hline K24 & 74.8 & 78.5 & 0.67 \\
\hline Mg24 & 85.6 & 99.7 & 0.13 \\
\hline P24 & 1.0 & 0.9 & 0.29 \\
\hline Nh424 & 38.9 & 29.7 & $0.02^{*}$ \\
\hline $\mathrm{Cl} 24$ & 187.7 & 179.8 & 0.67 \\
\hline Sul24 & 53.2 & 43.7 & 0.05 \\
\hline UUN24 & 14.5 & 12.2 & 0.08 \\
\hline
\end{tabular}

Introduction \& Objective: It is known that uric acid (UA) can serve as a nidus for calcium oxalate stone deposition through heterogeneous nucleation and epitaxy. Traditionally, pure UA stones are felt to form in the setting of supersaturation, whereas calcium oxalate stones form at the tubular level as a result of tissue damage and/or metabolic abnormalities. Few studies have investigated differences in metabolic profiles between pure UA stone formers and those with mixed UA stones. We aimed to better understand the differences between these patient populations by evaluating and comparing their metabolic and clinical factors.

Materials and Methods: We queried our Institutional Review Board-approved endourology database to identify those with UA stones. Patients with pure and mixed UA stones were compared using descriptive statistics, multivariate analysis, and one-way ANOVA to look at associations between metabolic and clinical factors. Parameters evaluated included demographics (i.e., age, BMI, sex), total stone burden, serum UA, stone recurrence, past medical history (i.e., diabetes, metabolic syndrome, BPH), and 24-hour urine parameters.

Results: 92 patients with uric acid stones were identified. Mean age was 62.4 years and $26.1 \%$ were female. $26(28.2 \%)$ patients made pure UA stones while 66 (71.8\%) made mixed UA stones. Pure UA stone formers were older and heavier, had higher total stone burden, higher serum UA, increased incidence of stone recurrence and were predominantly male $(77.0 \%)$. They also had a higher incidence of diabetes $(42.3 \%$ versus $25.8 \%)$ and metabolic syndrome $(23.1 \%$ versus $12.1 \%)$. When comparing metabolic profiles, pure UA stone formers had significantly lower urine $\mathrm{pH}(5.43 \pm 0.39$ versus $5.72 \pm 0.58 ; \mathrm{p}=0.03)$, higher urine ammonium levels (38.86 \pm 16.87 versus $29.71 \pm 14.02 ; \mathrm{p}=0.02)$, and higher Sul24 (53.23 \pm 21.75 versus $43.67 \pm 18.66 ; \mathrm{p}=0.05)$. On average, patients who form pure UA stones had more metabolic abnormalities on 24-hour urine than those with mixed UA stones (7 versus 6).
Conclusions: Overall, patients who form pure UA stones were metabolically and clinically different than those who form mixed UA stones. Pure UA stone formers were more likely to have metabolic syndrome as well as metabolic abnormalities in their urine. They were also found to have higher protein intake and higher acid secretion in their urine, which warrants consideration for dietary-based treatments in these types of stone formers.

MP9-22 Impact of metabolic risk factors on stone recurrence in patients submitted to nephrectomy due to struvite stones

A Danilovic, IA Wei, T Ferreira, S Reis, A Brito, F Vicentini, F Torricelli, G Marchini, C Batagello, E Mazzucchi, W Nahas, M Srougi

Hospital das Clinicas da Faculdade de Medicina da Universidade de Sao Paulo

Brazil

Introduction \& Objective: Despite struvite stones are caused by urea splitting bacteria, patients submitted to nephrectomy due to struvite stones may have metabolic risk factors for stones. Our aim was to evaluate the frequency of metabolic risk factors in

\begin{tabular}{|c|c|c|c|c|}
\hline \multirow{2}{*}{\multicolumn{2}{|c|}{ Variable }} & \multicolumn{2}{|c|}{ Stone Composition } & \multirow[b]{2}{*}{$\begin{array}{c}\mathrm{p}- \\
\text { value }\end{array}$} \\
\hline & & $\begin{array}{c}\text { Struvite } \\
(37.9 \% 36 / 95)\end{array}$ & $\begin{array}{c}\text { Calcium } \\
(62.1 \% 59 / 95)\end{array}$ & \\
\hline \multicolumn{2}{|c|}{ Female (\%) } & 46.6 & 53.4 & 0.08 \\
\hline \multicolumn{2}{|c|}{ Age $(y$, mean $\pm S D)$} & $48.1 \pm 14.0$ & $48.5 \pm 13.4$ & 0.976 \\
\hline \multicolumn{2}{|c|}{$\mathrm{BMI}\left(\mathrm{Kg} / \mathrm{m}^{2}\right.$, mean $\left.\pm \mathrm{SD}\right)$} & $25.6 \pm 5.4$ & $27.2 \pm 5.2$ & 0.863 \\
\hline \multirow{8}{*}{ Charlson } & 0 & $69.4 \%$ & $59.3 \%$ & \multirow{8}{*}{0.857} \\
\hline & 1 & $2.8 \%$ & $10.2 \%$ & \\
\hline & 2 & $13.9 \%$ & $10.2 \%$ & \\
\hline & 3 & $2.8 \%$ & $5.1 \%$ & \\
\hline & 4 & $5.6 \%$ & $6.8 \%$ & \\
\hline & 5 & $2.8 \%$ & $3.4 \%$ & \\
\hline & 6 & $2.8 \%$ & $3.4 \%$ & \\
\hline & 9 & $0 \%$ & $1.7 \%$ & \\
\hline \multirow{4}{*}{ ASA } & 1 & $41.7 \%$ & $23.7 \%$ & \multirow{4}{*}{0.080} \\
\hline & 2 & $38.9 \%$ & $66.1 \%$ & \\
\hline & 3 & $16.7 \%$ & $8.5 \%$ & \\
\hline & 4 & $2.8 \%$ & $1.7 \%$ & \\
\hline \multirow{3}{*}{ MDRD } & 1 & $13.9 \%$ & $13.6 \%$ & \multirow{3}{*}{0.811} \\
\hline & 2 & $55.6 \%$ & $49.2 \%$ & \\
\hline & 3 & $30.6 \%$ & $37.3 \%$ & \\
\hline \multicolumn{2}{|c|}{$\begin{array}{l}\text { Contralateral kidney stone } \\
\text { before nephrectomy }\end{array}$} & $22.2 \%$ & $33.9 \%$ & 0.255 \\
\hline \multicolumn{2}{|c|}{ Follow-up (mo., mean \pm SD) } & $66.3 \pm 33.2$ & $58.2 \pm 25.4$ & 0.07 \\
\hline \multicolumn{5}{|c|}{ 24-hour urinalysis } \\
\hline \multicolumn{2}{|c|}{$\begin{array}{l}\text { Urinary Volume } \\
(\mathrm{mL}, \text { mean } \pm S D)\end{array}$} & $1810 \pm 527$ & $1864 \pm 607$ & 0.186 \\
\hline Hypocitraturia & Yes & $61.1 \%$ & $66.1 \%$ & 0.663 \\
\hline
\end{tabular}




\begin{tabular}{|c|c|c|c|c|}
\hline & No & $38.9 \%$ & $33.9 \%$ & \\
\hline \multirow{2}{*}{ Hypercalciuria } & Yes & \multirow{2}{*}{$\begin{array}{l}5.6 \% \\
94.4 \%\end{array}$} & \multirow{2}{*}{$\begin{array}{l}23.7 \% \\
76.3 \%\end{array}$} & \multirow{2}{*}{0.025} \\
\hline & No & & & \\
\hline \multirow{2}{*}{ Hypernatriuria } & Yes & \multirow{2}{*}{$\begin{array}{l}13.9 \% \\
86.1 \%\end{array}$} & \multirow{2}{*}{$\begin{array}{l}11.9 \% \\
88.1 \%\end{array}$} & \multirow{2}{*}{0.761} \\
\hline & No & & & \\
\hline \multirow{2}{*}{ Hyperuricosuria } & Yes & \multirow{2}{*}{$\begin{array}{c}0 \% \\
100 \%\end{array}$} & \multirow{2}{*}{$\begin{array}{l}3.4 \% \\
96.6 \%\end{array}$} & \multirow{2}{*}{0.524} \\
\hline & No & & & \\
\hline \multirow{2}{*}{ Hyperuricemia } & Yes & \multirow{2}{*}{$\begin{array}{l}36.1 \% \\
63.9 \%\end{array}$} & \multirow{2}{*}{$\begin{array}{l}25.4 \% \\
74.6 \%\end{array}$} & \multirow{2}{*}{0.354} \\
\hline & No & & & \\
\hline \multirow{2}{*}{ Hypercalcemia } & Yes & \multirow{2}{*}{$\begin{array}{c}2.8 \% \\
97.2 \%\end{array}$} & \multirow{2}{*}{$\begin{array}{l}13.2 \% \\
86.2 \%\end{array}$} & \multirow{2}{*}{0.146} \\
\hline & No & & & \\
\hline \multirow{2}{*}{$\begin{array}{l}\text { Renal Tubular } \\
\text { Acidosis }\end{array}$} & Yes & \multirow{2}{*}{$\begin{array}{c}0 \% \\
100 \%\end{array}$} & \multirow{2}{*}{$\begin{array}{l}3.4 \% \\
96.6 \%\end{array}$} & \multirow{2}{*}{0.524} \\
\hline & No & & & \\
\hline \multirow{2}{*}{$\begin{array}{c}\text { Primary } \\
\text { Hyperparathyroidism }\end{array}$} & Yes & \multirow{2}{*}{$\begin{array}{c}0 \% \\
100 \%\end{array}$} & \multirow{2}{*}{$\begin{array}{l}16.9 \% \\
83.1 \%\end{array}$} & \multirow{2}{*}{0.012} \\
\hline & No & & & \\
\hline
\end{tabular}

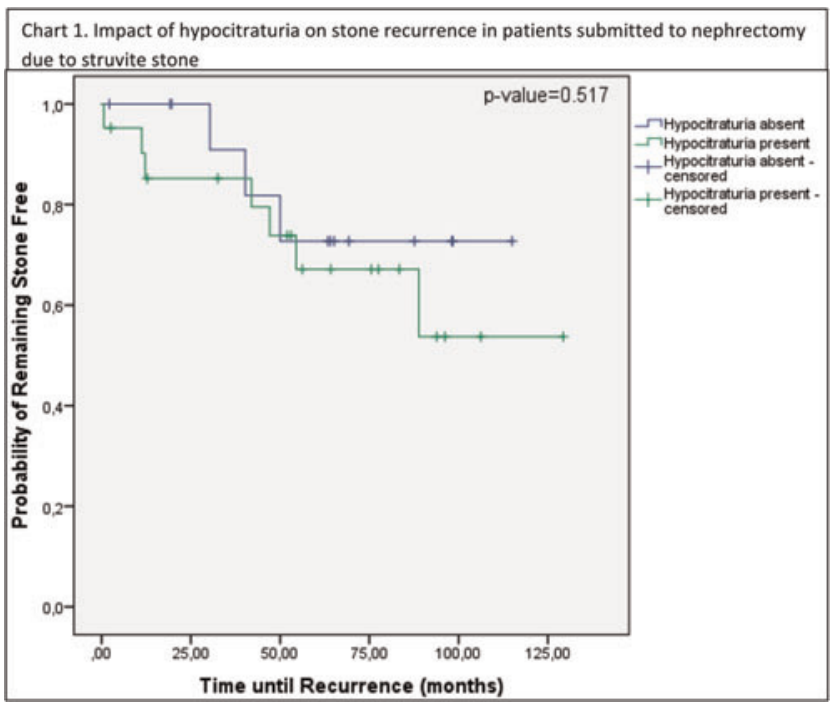

patients submitted to nephrectomy as a result of struvite stones and its impact on stone recurrence in the remaining kidney.

Materials and Methods: Electronic charts of patients submitted to nephrectomy due to urolithiasis between 2006 and 2013 at our Institution were studied. Inclusion criteria was one 24-hour urinalysis with urine creatinine $>800 \mathrm{mg} / 24 \mathrm{~h}$ for men and $>600 \mathrm{mg} /$ $24 \mathrm{~h}$ for women obtained after nephrectomy. Patients with chronic kidney disease stage 4 or 5, current urinary infection or use of thiazide, citrate or allopurinol were excluded. Metabolic abnormalities were treated accordingly during follow-up. Stone recurrence was evaluated by annual ultrasound confirmed by computed tomography when positive for new stone formation or stone growth.

Results: Comparative analysis between groups of patients with struvite and calcium stones was summarized on table 1 . Overall recurrence rate was $39.0 \%$ in calcium group and $30.6 \%$ in struvite group ( $p$-value $=0.509$ ). Recurrence rate was not significantly affected by hypocitraturia in calcium stone formers with a median occurrence of 64.6 months \pm 3.2 vs. 83.1 months
$[$ IC95\% $=58.199-70.934](\mathrm{p}$-value $=0.123)$ and in patients with struvite stones with a mean occurrence of 91.3 months \pm 11.4 vs. 94.6 months \pm 10.1 (p-value $=0.517)($ Chart 1$)$. Hypercalciuria and primary hyperparathyroidism were independently associated with a higher recurrence rate in calcium stone formers with a median occurrence of 45.2 months \pm 4.5 [IC95\% $=36.266$ 54.067 ] vs. 88.1 months \pm 15.2 [IC95\% $=58.373-117.893$ ] (pvalue $=0.03)$ and 39.3 months $\pm 8.1[$ IC95\% $=23.392-55.208]$ vs. 83.1 months \pm 14.0 [IC95\% $=55.663$ - 110.603] (p-value $=0.038$ ), respectively.

Conclusions: Patients with struvite stones should have a 24-hour urinalysis after stone eradication since they have a high frequency of hypocitraturia, comparable to calcium stone formers. Patients treated to correct hypocitraturia have a stone recurrence rate similar to patients with no hypocitraturia.

MP9-23 Non Compliance in Recurrent Nephrolithiasis Undergoing Metabolic Evaluation

E Kwenda, Z Chen, BK Carew, VG Bird, M Prosperi, VY Bird

UF College of Medicine

United States

Introduction \& Objective: Introduction: Kidney stone formation poses a high cost to our health care system, with $50 \%$ recurrence within five years. Kidney stone recurrence may be reduced with follow up and patient education. We aim to identify recurrent-kidney stone formers(R-KSF) who did not follow up to complete metabolic evaluation and patient specific kidney stone prevention education.

Materials and Methods: Methods: A retrospective study, $\mathrm{N}=1004 \mathrm{KSF}$ identified 606 patients as RKSF. Patients were stratified in three groups: private insurance, Medicare and Medicaid, and no insurance. Univariate analysis was performed for all subjects and stratified univariate analyses were performed by gender and age group among uninsured and noninsured individuals.

Results: Private insured and Medicaid/Medicare patients had no significant differences in follow up and work up. Medicare and Medicaid patients were slightly less likely to follow up when compared to insured individuals OR: $0.91,95 \%$ CI: $0.57,1.45$. Age played the largest role in compliance; individuals under 60 were more likely to undergo assestments in the insured group, OR: $1.94(85 \%$ CI 1.39-2.70) when compared to those over 60 . Noninsured individuals were more likely not to comply with follow up(p<0.001)OR: 0.21 ; 95\% CI $0.11,0.50$. In the noninsured group, gender was the biggest predictor of follow-up and compliance, and showed significant gender disparity, with noninsured males 4.11 times(95\% CI: $1.07,15.78)$ more likely than females to follow up.

Conclusions: Decreased compliance in the insured group was associated with age over 60 . The uninsured RKSF have the poorest compliance, specially uninsured women. Measures to identify this population should be in place to prevent further recurrent episodes in an already uninsured and impoverished population.

MP9-24 Quality of life and satisfaction in a virtual stone clinic: a pilot study

J Hendry, L Kerr, C McIlhenny

NHS Forth Valley

United Kingdom 
Introduction \& Objective: Virtual clinics have been used in several specialities in order to decrease face to face clinic appointments (FTF), saving patient and clinician time. It is unknown if this model is acceptable with recurrent stone disease who attend for annual surveillance. We sought to assess recurrent stone clinic attenders' quality of life and satisfaction with being followed up in a novel virtual stone clinic. In addition we analysed the financial impact of traditional (FTF) versus a virtual stone clinic

Materials and Methods: Patients due annual stone clinic review were allocated to the pilot virtual stone clinic. A Symptom questionnaire and two validated quality of life questionnaires (EQ -5D, general QOL) and (WIS-QOL, stone specific QOL) were posted to patients. They were given a 2-week window to attend for XRKUB. Subsequently they received a letter from the consultant urologist following review of symptom scores and imaging with an outcome of further virtual stone clinic, advice for intervention and FTF clinic appointment, or discharge.

Results: The initial forty six patients were included in this pilot. There were 31 male and 15 female patients with mean age of 60.9 years. Twelve were deemed stone free, thirty had unchanged stone burden and four had increasing stone size based on XRKUB. Ten patient patients complained of pain symptoms.
$85 \%$ of patients found the flexibility of XRKUB timing as very or extremely helpful and 59\% were able to avoid time off work with the new clinic model. $96 \%$ of patients would be happy to have ongoing follow up using this clinic format. Costing analysis showed a decrease in direct costs per patient to $£ 7.92$ in the virtual clinic compared to $£ 24.74$ in the FTF clinic. Generic and disease specific QOL scores of EQ5D and WISQOL were only found to be weakly correlated (Pearsons $r=0.36$ ) in contrast to original validating studies. However, WISQOL scores varied significantly between symptomatic and non symptomatic patients (79.2 vs 87.6, p=0.03, ttest) whilst the EQ5D failed to differentiate between groups ( 0.80 vs $83.8, p=0.13$ ttest). There was no difference in satisfaction scores or generic or disease specific QOL scores in those with stones versus no stone burden. Conclusions: The virtual clinic provides time and financial benefits and is widely acceptable for patients. Stone free patients and those with stones had similar quality of life scores and satisfaction scores for the virtual clinic suggesting this model would be acceptable across a heterogeneous population. In this cohort the WISQOL, a relatively new stone specific QOL questionnaire is able to detect patient perspectives in symptomatic versus non symptomatic patients where the generic EQ5D tool cannot.

\section{MPS10: EPIDEMIOLOGY, HEALTH POLICY, SOCIOECONOMICS \& OUTCOMES}

MP10-1 Pre-Operative Nomograms for Predicting Renal Function at 2 Years after Donor Nephrectomy

C Shum, CD Bahler, CP Sundaram

Khoo Teck Puat Hospital

Singapore

Introduction \& Objective: The life-long loss of nephron mass among donors may result in post-operative renal insufficiency. Being able to predict post-operative renal function prior to donor nephrectomy (DN) would help with counseling patients and managing expectations. We used our institutional database to

\begin{tabular}{|c|c|c|c|c|}
\hline & $\begin{array}{c}\text { Included cases } \\
(n=262)\end{array}$ & $\begin{array}{c}\text { Excluded } \\
\text { cases }(n=253)\end{array}$ & p-value \\
\hline \multicolumn{2}{|l|}{ Age (year) } & $41.5 \pm 11.2$ & $38.0 \pm 10.4$ & $<0.001$ \\
\hline \multicolumn{2}{|l|}{ Male gender (n, \%) } & $89(34.0 \%)$ & $112(44.3 \%)$ & 0.017 \\
\hline \multicolumn{2}{|l|}{ African American race $(n, \%)$} & $14(5.3 \%)$ & $18(7.1 \%)$ & 0.405 \\
\hline \multicolumn{2}{|l|}{ Body mass index $\left(\mathrm{kg} / \mathrm{m}^{2}\right)$} & $26.4 \pm 3.8$ & $26.9 \pm 4.1$ & 0.160 \\
\hline \multicolumn{2}{|l|}{ Left-sided DN (n, \%) } & $162(61.8 \%)$ & $159(62.8 \%)$ & 0.812 \\
\hline \multicolumn{2}{|l|}{ Volume of contralateral kidney ( $\mathrm{ml}$ ) } & $190.3 \pm 42.1$ & $190.8 \pm 40.3$ & 0.961 \\
\hline \multicolumn{2}{|c|}{ Pre-operative creatinine level (mg/dL) } & $0.83 \pm 0.16$ & $0.87 \pm 0.18$ & 0.003 \\
\hline \multicolumn{2}{|c|}{ Pre-operative eGFR by MDRD formula ( $\left.\mathrm{m} / \mathrm{min} / 1.73 \mathrm{~m}^{2}\right)$} & $94.5 \pm 18.0$ & $93.9 \pm 18.0$ & 0.732 \\
\hline \multicolumn{2}{|c|}{ Pre-operative eGFR by CKD-EPI formula ( $\left.\mathrm{m} / \mathrm{min} / 1.73 \mathrm{~m}^{2}\right)$} & $98.4 \pm 16.1$ & $98.6 \pm 16.7$ & 0.885 \\
\hline \multicolumn{5}{|c|}{ Post-operative eGFRs (endpoints) } \\
\hline \multicolumn{2}{|c|}{$\begin{array}{l}\text { eGFR by MDRD formula at } 2 \text { years after DN } \\
\left(\mathrm{ml} / \mathrm{min} / 1.73 \mathrm{~m}^{2}\right)\end{array}$} & $62.0 \pm 13.7$ & & \\
\hline \multirow{5}{*}{$\begin{array}{l}\text { eGFR by MDRD formula at } 2 \\
\text { years after DN }(n, \%)\end{array}$} & $290 \mathrm{~m} / \mathrm{min} / 1.73 \mathrm{~m}^{2}$ & $10(3.8 \%)$ & & \\
\hline & 60 to $<90 \mathrm{ml} / \mathrm{min} / 1.73 \mathrm{~m}^{2}$ & $119(45.4 \%)$ & & \\
\hline & 30 to $<60 \mathrm{ml} / \mathrm{min} / 1.73 \mathrm{~m}^{2}$ & $132(50.4 \%)$ & & \\
\hline & 15 to $<30 \mathrm{ml} / \mathrm{min} / 1.73 \mathrm{~m}^{2}$ & $1(0.4 \%)$ & & \\
\hline & $<15 \mathrm{ml} / \mathrm{min} / 1.73 \mathrm{~m}^{2}$ & 0 & & \\
\hline \multicolumn{2}{|c|}{$\begin{array}{l}\text { eGFR by CKD-EPI formula at } 2 \text { years after DN } \\
\left(\mathrm{mU} / \mathrm{min} / 1.73 \mathrm{~m}^{2}\right)\end{array}$} & $65.5 \pm 15.5$ & & \\
\hline \multirow{5}{*}{$\begin{array}{l}\text { eGFR by CKD-EPI formula at } 2 \\
\text { years after } D N(n, \%)\end{array}$} & $290 \mathrm{mV} / \mathrm{min} / 1.73 \mathrm{~m}^{2}$ & $17(6.5 \%)$ & & \\
\hline & 60 to $<90 \mathrm{ml} / \mathrm{min} / 1.73 \mathrm{~m}^{2}$ & $137(52.3 \%)$ & & \\
\hline & 30 to $<60 \mathrm{ml} / \mathrm{min} / 1.73 \mathrm{~m}^{2}$ & $107(40.8 \%)$ & & \\
\hline & 15 to $<30 \mathrm{ml} / \mathrm{min} / 1.73 \mathrm{~m}^{2}$ & $1(0.4 \%)$ & & \\
\hline & $<15 \mathrm{~m} / / \mathrm{min} / 1.73 \mathrm{~m}^{2}$ & 0 & & \\
\hline
\end{tabular}

\begin{tabular}{|c|c|c|c|}
\hline \multicolumn{4}{|c|}{ Endpoint: eGFR by MDRD formula at 2 years after DN } \\
\hline \multirow{2}{*}{\multicolumn{2}{|c|}{ Parametric covariates }} & Pearson correlation & $p$-value \\
\hline & & -0.538 & $<0.001$ \\
\hline \multicolumn{2}{|l|}{ Body mass index } & -0.126 & 0.044 \\
\hline \multicolumn{2}{|c|}{ Volume of contralateral kidney } & 0.200 & 0.003 \\
\hline \multicolumn{2}{|c|}{ Pre-operative creatinine level } & -0.332 & $<0.001$ \\
\hline \multicolumn{2}{|c|}{ Binary covariates } & Mean eGFRMDRD2year & $p$-value \\
\hline \multirow{2}{*}{ Gender } & Male & $63.5 \pm 12.7$ & \multirow{2}{*}{0.205} \\
\hline & Female & $61.2 \pm 14.2$ & \\
\hline \multirow{2}{*}{ African American race } & No & $61.8 \pm 13,7$ & \multirow{2}{*}{0.309} \\
\hline & Yes & $65.6 \pm 13.6$ & \\
\hline \multirow{2}{*}{ Side of DN } & Left & $62.6 \pm 14.4$ & \multirow{2}{*}{0.393} \\
\hline & Right & $61.1 \pm 14.5$ & \\
\hline \multicolumn{4}{|c|}{ Endpoint: eGFR by CKD-EPI formula at 2 years after DN } \\
\hline \multicolumn{2}{|c|}{ Parametric covariates } & Pearson correlation & $\rho$-value \\
\hline \multicolumn{2}{|c|}{ 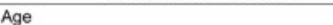 } & -0.593 & $<0.001$ \\
\hline \multicolumn{2}{|l|}{ Body mass index } & -0.138 & 0.027 \\
\hline \multicolumn{2}{|c|}{ Volume of contralateral kidney } & 0.182 & 0.007 \\
\hline \multicolumn{2}{|c|}{ Pre-operative creatinine level } & -0.355 & $<0.001$ \\
\hline \multicolumn{2}{|c|}{ Binary covariates } & Mean eGFRCKDEPI2year & $p$-value \\
\hline \multirow{2}{*}{ Gender } & Male & $66.1 \pm 14.5$ & \multirow{2}{*}{0.622} \\
\hline & Female & $65.1 \pm 16.1$ & \\
\hline \multirow{2}{*}{ African American race } & No & $65.5 \pm 15.6$ & \multirow{2}{*}{0.931} \\
\hline & Yes & $65.8 \pm 15.0$ & \\
\hline \multirow{2}{*}{ Side of DN } & Left & $66.3 \pm 16.2$ & \multirow{2}{*}{0.281} \\
\hline & Right & $64.2 \pm 14.4$ & \\
\hline
\end{tabular}

\begin{tabular}{|l|c|c|c|c|}
\hline \multicolumn{5}{|c|}{ Endpoint: eGFR by MDRD formula at $\mathbf{2}$ years after DN } \\
\hline \multicolumn{1}{|c|}{ Pre-operative covariates } & $\boldsymbol{B}$ & $\mathbf{9 5 \%} \mathrm{Cl}$ for $\boldsymbol{B}$ & $\boldsymbol{\beta}$ & $\boldsymbol{p}$-value \\
\hline Age & -0.548 & $-0.663--0.434$ & -0.466 & $<0.001$ \\
\hline Gender & -9.760 & $-13.662--5.858$ & -0.351 & $<0.001$ \\
\hline African American race & 7.194 & $1.992-12.396$ & 0.132 & 0.007 \\
\hline Body mass index & -0.338 & $-0.687-0.012$ & -0.101 & 0.058 \\
\hline Volume of contralateral kidney & 0.014 & $-0.022-0.051$ & 0.046 & 0.441 \\
\hline Pre-operative creatinine level & -47.181 & $-57.733--36.629$ & -0.564 & $<0.001$ \\
\hline \multicolumn{4}{|r|}{ Endpoint: eGFR by CKD-EPI formula at 2 years after DN } \\
\hline \multicolumn{1}{|c|}{ Pre-operative covariates } & $\boldsymbol{B}$ & $\mathbf{9 5 \%}$ Cl for $\boldsymbol{B}$ & $\boldsymbol{\beta}$ & $\boldsymbol{p}$-value \\
\hline Age & -0.714 & $-0.840--0.589$ & -0.529 & $<0.001$ \\
\hline Gender & -8.796 & $-13.078--4.513$ & -0.276 & $<0.001$ \\
\hline African American race & 4.165 & $-1.544-9.875$ & 0.067 & 0.152 \\
\hline Body mass index & -0.353 & $-0.737-0.030$ & -0.092 & 0.071 \\
\hline Volume of contralateral kidney & 0.018 & $-0.022-0.058$ & 0.051 & 0.380 \\
\hline Pre-operative creatinine level & -51.958 & $-63.539--40.378$ & -0.542 & $<0.001$ \\
\hline
\end{tabular}



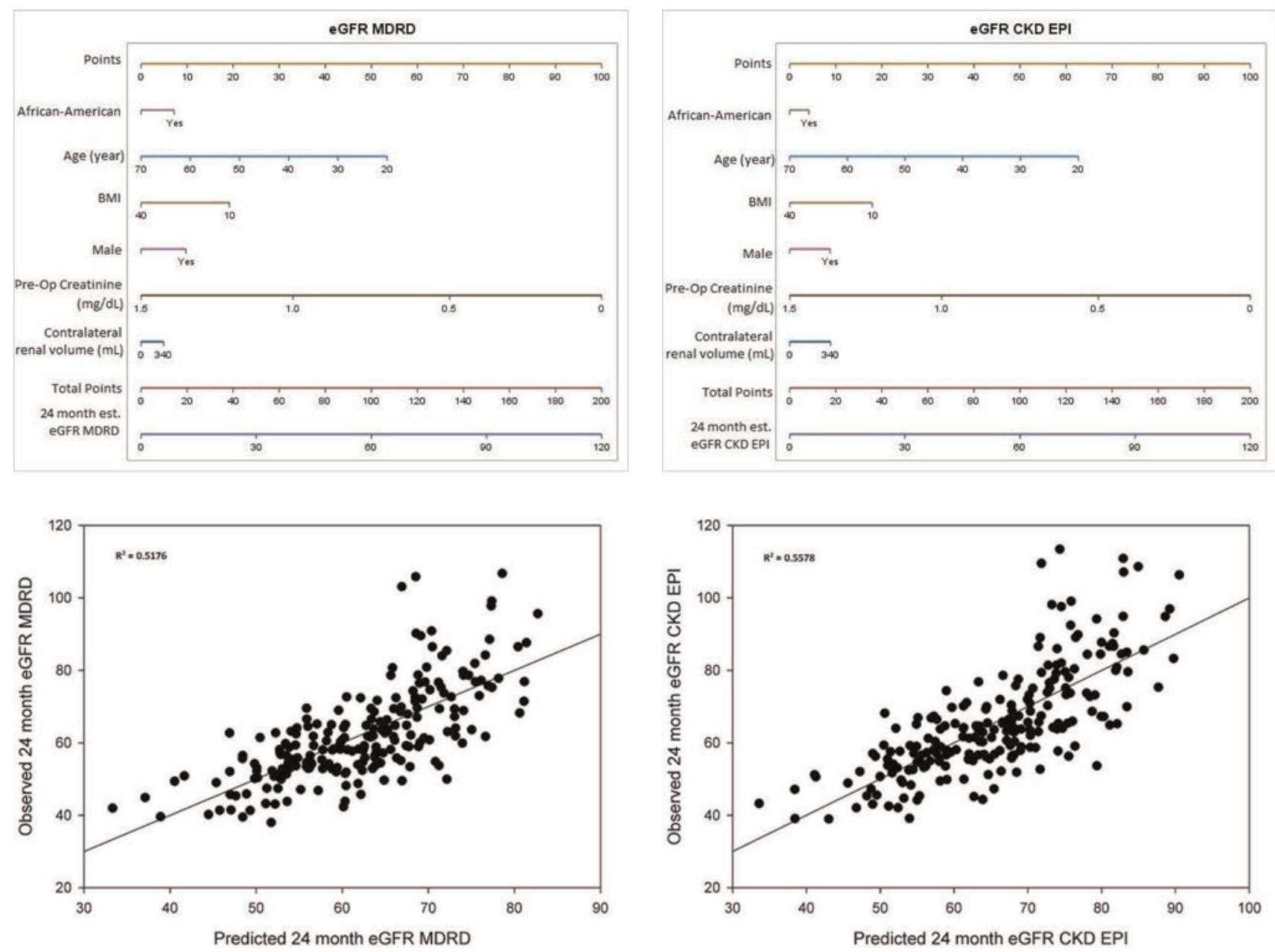

develop nomograms that would predict estimated glomerular filtration rates (eGFRs) at 2 years after DN.

Materials and Methods: Pre-operative covariates included age, gender, race, body mass index (BMI), side of DN, contralateral renal volume and pre-operative serum creatinine level. We used serum creatinine level at 2 years after DN to calculate eGFRs using the MDRD and the CKD-EPI formulae, to be defined as endpoints (eGFRMDRD2year and eGFRCKDEPI2year). Patients with missing data that prevented calculation of eGFRs at 2 years were excluded. We identified associations between preoperative covariates and endpoints eGFRMDRD2year and eGFRCKDEPI2year. Based on such univariate analyses and literature review, we selected pre-operative covariates of interest and included them in two multivariate linear regression models. Two nomograms were constructed for the endpoints, and were internally validated by bootstrapping.

Results: Table 1 shows the baseline characteristics of the included and excluded cases. The mean eGFR dropped from $95 \mathrm{ml} / \mathrm{min} / 1.73 \mathrm{~m} 2$ pre-operatively to $65 \mathrm{ml} / \mathrm{min} / 1.73 \mathrm{~m} 2$ at 2 years after DN, with 40 to $50 \%$ of donor developing stage 3 CKD. Table 2 shows the initial univariate analysis. Age, BMI, contralateral renal volume and pre-operative serum creatinine level had significant associations with eGFRs at 2 years. Table 3 shows the multivariate linear regression models. Age, gender and pre-operative serum creatinine level had significant associations with eGFRs at 2 years. African American

race was a significant factor for eGFR at 2 years by the MDRD formula. Figures 1 and 2 are the nomograms to predict endpoints eGFRMDRD2year and eGFRCKDEPI2year, with the associated scatter plots of predicted against observed values. Internal validated $\mathrm{R} 2$ values were 0.52 and 0.56 respectively. Conclusions: We constructed and internally validated two nomograms to predict renal function at 2 years after DN, defined by two widely used formulae for eGFRs.

MP10-2 Stone Size at Initial Presentation at a Tertiary Care Center: Demographics

MJ Wardenburg, J Brown, R Ruchi, X Wen, VG Bird, VY Bird

Department of Urology, University of Florida, Gainesville,

Florida

United States

Introduction \& Objective: Urinary lithiasis will affect 1 in 11 people in the United States. These recent findings represent a marked increase in stone disease compared with the National Health and Nutrition Examination Survey III cohort in 1994, particularly in African-American, non-Hispanic minorities, and Hispanic individuals. However, few studies have elucidated the specific demographics of patients with significant stone disease 
requiring surgical treatment, particularly in the elderly. We believe this to be the largest study of its kind in patients $>60$ years of age.

Materials and Methods: Retrospective review of patients undergoing surgical treatment for urinary lithiasis at a tertiary care center was performed. Procedures included SWL, URS, and PCNL. We recorded demographics including ethnicity, age, BMI, ASA, and stone burden $(<2,2-4$ and $>4 \mathrm{~cm}$ cumulative stone burden in longest dimension) at presentation. Continuous variables were presented as mean $\pm \mathrm{SD}$ and compared using an independent t-test. Categorical variables were examined using chi-squared or fisher exact test.

Results: Out of 1004 patients identified, $60 \%, 25 \%$ and $15 \%$ presented with stone burden $<2 \mathrm{~cm}, 2-4 \mathrm{~cm}$ and $>4 \mathrm{~cm}$ respectively. Female gender was $48.2 \%$. There was no significant difference between genders and stone size at presentation $(p=0.92)$. Mean ages at presentation were $49.3 \pm 7.8,55.8 \pm 14.7$ and $57.2 \pm 13.9$ for stone sizes $<2,2-4$ and $>4 \mathrm{~cm}$ respectively $(\mathrm{p}<0.001)$. Mean BMI was $29.6 \pm 7.8,31.1 \pm 9.4$ and $31.3 \pm 7.6$ for stones $<2,2-4$ and $>4 \mathrm{~cm}$ respectively $(\mathrm{p}<0.01)$. Mean ASA scores were $2.4 \pm 0.7,2.6 \pm 0.6$ and $2.7 \pm 0.5$ for stones $<2,2-4$ and $>4 \mathrm{~cm}$ respectively $(\mathrm{p}<0.001)$. Of the entire group, $15 \%$ were minorities (58\% African American, 35\% Hispanic, and 7\% other ethnicity). When comparing all minority groups with Caucasians, $52 \%$ vs $62 \%, 32 \%$ vs $24 \%$ and $16 \%$ vs $14 \%$ presented with stones $<2,2-4$ and $>4 \mathrm{~cm}$ respectively $(\mathrm{p}=0.0477)$. Analysis of patients by age decade at stone presentation revealed that $11 \%, 14 \%, 17 \%, 23 \%, 21 \%, 10 \%$ and $2 \%$ presented at age $21-30,31-40,41-50,51-60,61-70,71-80$ and $>80$ years of age, respectively. Stone frequency increased per decade of age and peaked in the fifth decade. However, stone size $2-4 \mathrm{~cm}$ was more common in the sixth decade of age.

Conclusions: Our results demonstrate that at time of presentation to a tertiary care center, higher age, BMI, ASA score, and minority status are significantly associated with larger stones. Further investigation is needed to discern what other factors may be associated with these disparities.

MP10-3 Post-Acute Care as a Driver of Episode Cost Variation for Ambulatory Stone Surgery

J Hollingsworth, J San Juan, H Hou, J Dupree, KR Ghani

University of Michigan

United States

Introduction \& Objective: Because many post=acute care (PAC) services, including emergency department (ED) visits, after ambulatory stone surgery are potentially avoidable, they are coming under payer scrutiny. In this context, we analyzed claims data to describe variation in total episode costs for ambulatory stone surgery across a diverse set of hospitals, examining PAC as a driver of this variation.

Materials and Methods: We used Medicare and private insurer claims to identify patients who underwent ambulatory stone surgery (ureteroscopy or shockwave lithotripsy) at hospitals in Michigan (2012-2015) from the Michigan Value Collaborative. We defined surgical episodes that extended from the surgery date through 30 days post-discharge and totaled costs for all relevant services during this window. We then categorized component payments to the hospital for the index surgery, as well as those for professional services, subsequent hospitalizations, and PAC. Finally, after aggregating across episodes within a year by hos-

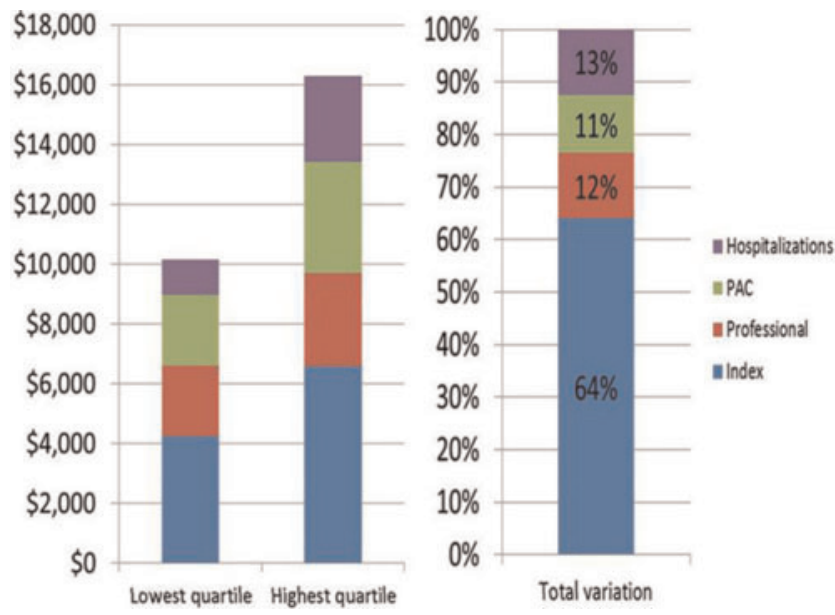

pital, we placed hospitals into quartiles based on their mean total costs and compared component payments at high- versus lowcost hospitals

Results: In total, we identified 7,807 patients who underwent ambulatory stone surgery at 69 hospitals in Michigan. The mean total cost for hospitals was $\$ 9,538$ ( $\$ 13,044$ and $\$ 9,037$ for episodes associated with and without an ED visit after surgery, respectively) and ranged from $\$ 7,317$ to $\$ 11,914$ across hospital quartiles $(62.8 \%$ difference, $\mathrm{P}<.001)$. Payments were higher for hospitals in the highest cost quartile across all payment components (Figure). The index surgery payments contributed the most $(64 \%)$ to the variation in total costs between high- and low-cost hospitals (Figure), followed by those for subsequent hospitalizations (13\%), professional payments (12\%), and PAC payments $(11 \%)$.

Conclusions: We observed significant variation in total episode costs for ambulatory stone surgery, driven, in part, by payments for PAC. As such, efforts to reduce the use of PAC services, including ED visits, after ambulatory stone surgery is likely to improve cost-efficiency.

MP10-4 The "Acute" Stone Clinic Effect: Improving Healthcare Delivery by Reorganizing Clinical Resources

MA Asmus, S De, T Schuler, D Bochinski, T Wollin

University of Alberta

Canada

Introduction \& Objective: To determine the time to specialist urologic consultation and definitive management after establishing a subspecialist administered Acute Stone Clinic (ASC) for adults with symptomatic upper tract stones in a publicallyfunded, universal healthcare system.

Materials and Methods: We retrospectively reviewed 337 adult referrals for stone management. Three distinct 9 week periods were assessed. Group 1 was seen/treated by their individual urologist prior to inception of a general urology Emergency Clinic (pre-EC). Group 2 were seen in a pooled Emergency Clinic (EC), and Group 3 was the ASC.

Results: 337 patients (75-pre-EC, 91-EC, 171-ASC) were reviewed. Mean time to consultation for pre-EC, EC and ASC 
cohorts was 29,7 and 7 days respectively $(\mathrm{p}<0.05)$, while loss to follow up decreased from $13 \%$ to $5 \%(\mathrm{p}<0.05)$. On average, the number of patients seen per week increased from 9 to 20. Mean time to stone surgery from date of referral was $75 \mathrm{~d}$ pre-EC, $43 \mathrm{~d}$ $\mathrm{EC}$, and $25 \mathrm{~d}$ ASC $(\mathrm{p}<0.05)$. The percentage of patients undergoing surgery was between $59-63 \%$ per cohort, however, the number of patients increased from 5 to 11 /week.

Conclusions: By reorganizing clinical resources, a dedicated acute stone clinic was able to increase patient capacity, while reducing time to consultation and surgery.

MP10-5 Modeling operating room costs for treatment of ureteral calculi using time-driven activity-based costing

TR McClintock, DF Friedlander, AY Feng, S Ganeshan, MA Shah, SL Chang, TW Feeley, AM Bader, GE Haleblian

Brigham and Women's Hospital and Harvard Medical School United States

Introduction \& Objective: Urolithiasis ranks as the most costly nonmalignant urologic condition in the United States, accounting for more than $\$ 10$ billion in annual aggregate medical expenditures. Given the subjectivity that exists in
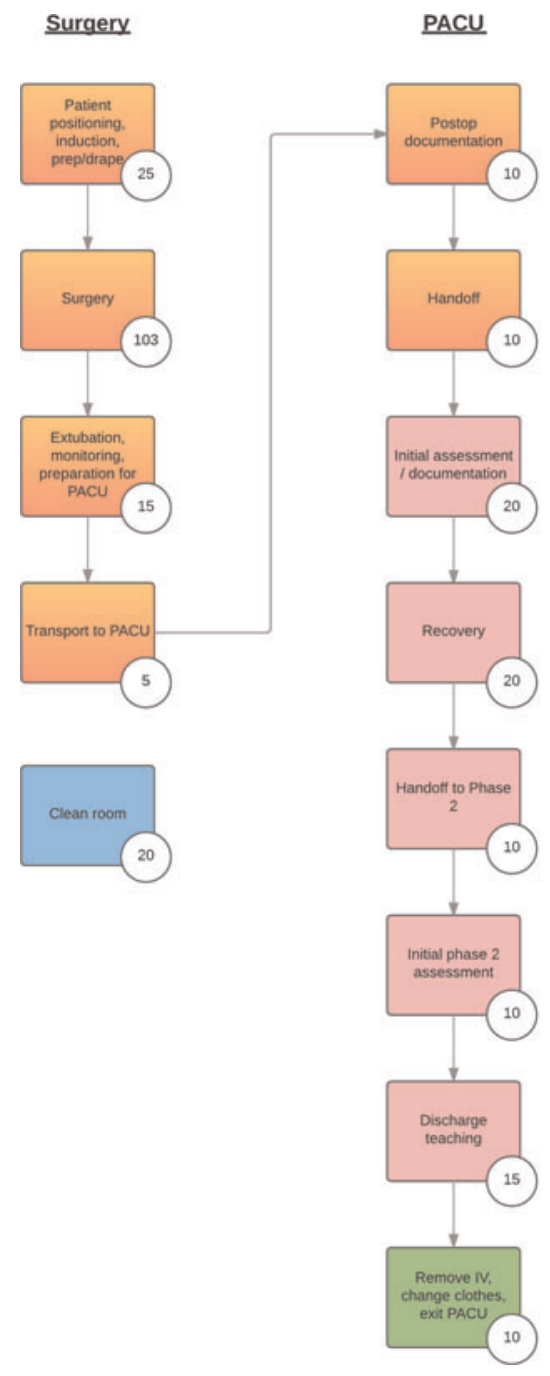

choosing the timing and approach of procedural intervention, there exists a significant opportunity for optimizing costs within each given episode of care. Cost optimization based on conventional accounting measures such as billing or reimbursement data, however, can be cumbersome and wildly inaccurate. A more effective and precise approach is time-driven activitybased costing (TDABC), wherein all specific processes related to a patient's episode of care are identified at a highly granular level; within each process, all related resource and personnel utilization costs are then estimated. Here, we have utilized TDABC to model operating room costs of both shockwave lithotripsy (SWL) and ureteroscopic lithotripsy (URS) for the treatment of ureteral calculi.

Materials and Methods: We defined all phases of care that comprise the full care cycle for any patient who presents to our institution with a ureteral stone. Here, we focus only on the phases of care relevant to the day of definitive stone procedure. Based on interviews with clinical personnel and observation of clinical processes, detailed process maps were created to illustrate the flow of patients through each respective phase. For every step, times were estimated and relevant personnel were defined. We then determined the cost for each personnel member to be involved with his or her respective steps in the care process by calculating the capacity cost of all participants. Major equipment costs (including yearly depreciation and maintenance), as well as costs of space, utilities, and consumables were obtained.

Results: Utilizing TDABC methodology, the true OR costs of intervention for ureteral stones were determined to be $\$ 3739$ for SWL and \$3472 for URS.

Conclusions: TDABC has allowed for delineation of true OR costs in treatment of ureteral stones. This is a novel approach to cost accounting for these procedures, and we believe our models represent the most accurate estimation of OR costs for ureteral stone treatment to date. Future work to apply TDABC to additional phases of care relevant to stone management will further elucidate costs for the full cycle of care and be of high relevance in the context of alternative payment modalities and efforts to improve value in care delivery.

MP10-6 Improving value in urologic surgery episodes of care through time-driven activity-based costing

TR McClintock, DF Friedlander, S Ganeshan, MA Shah, SL Chang, TW Feeley, GE Haleblian

Brigham and Women's Hospital and Harvard Medical School United States

Introduction \& Objective: Bundled payments and other valuebased reimbursement approaches have begun to be introduced as a mechanism to contain or reduce health care costs. Key to valuebased restructuring is a firm understanding of how much it costs to deliver patient care and how those costs can be weighed against corresponding outcomes. This exposes the dire need for more accurate measurement of true costs, as historical costing systems within health care often have little association with actual resource utilization. Time-driven activity-based costing (TDABC) is an approach to cost accounting that provides this requisite accuracy through clearly defining the time and per-unit resource cost necessary to provide care. Such clarity is highly relevant for such cost intensive encounters as surgical care, where awareness of resource utilization within an full episode of 
care can serve as a crucial driver of cost reduction, improved efficiency, and higher value care.

Materials and Methods: TDABC is a bottom up approach that relies on creating process maps of patient care to clearly define each specific step within all relevant phases of care. Maps are created through observation and face-to-face iterative discussion with the individuals most aware of the care process. For each step, it is necessary to identify the amount of time and personnel required. Personnel costs are determined by first defining the time capacity for each individual involved in the care process, which is 365 days minus time for weekends, vacation, research, and other non-clinical time. Dividing salary by time capacity yields a capacity cost rate, signifying the cost per minute of assigning a particular individual to any task. Space costs are determined by dividing depreciation-adjusted construction costs by the associated facility size; these are assigned to each step. Fixed and consumable equipment costs are also added to the overall sum.

Results: TDABC has been utilized to determine true costs of surgical episodes of care, including to a limited degree within urology. In our institution, TDABC has been successfully applied to surgical episodes of care and stands to serve as the basis for improvements in care delivery.

Conclusions: TDABC is a feasible and effective approach to cost accounting for episodes of surgical care within urology. More widespread implementation of this approach and formal integration of these principles into hospital accounting systems would yield more accurate cost data, driving down expenses and enabling healthcare entities to more capably compete in the emerging landscape of bundled reimbursement.

MP10-7 Comparing the Utilization and Outcomes of Extracorporeal Shock Wave Lithotripsy between Inpatient and Outpatient Settings in New York State, 1990-2012

A Yang, KH Bilal, M Finkelstein, MA Palese

Icahn School of Medicine at Mount Sinai United States

Introduction \& Objective: This study investigates the trends and disparities in the utilization and outcomes of extracorporeal shock wave lithotripsy (ESWL) in the management of urolithiasis between outpatient and inpatient settings.

Materials and Methods: The New York Statewide Planning and Research Cooperative System database was used to identify re-

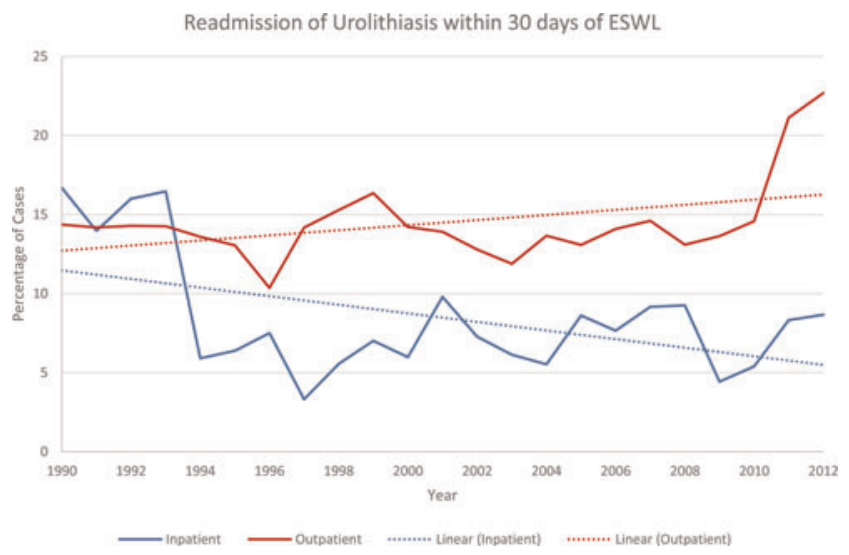

cords of patients diagnosed with urolithiasis between 1990 and 2012. Patients who underwent ESWL were selected using ICD9 and CPT codes. Records were stratified according to the setting. Statistical analysis was performed using the chi-square test. Binary logistic regression was used to assess short-term outcomes while adjusting for patient age, sex, race, Charlson comorbidity index and procedure year.

Results: From 1990 to 2012, cases of urolithiasis in New York State increased from 22,340 to 100,679. In the outpatient setting, the proportion of cases treated using ESWL decreased from $42.8 \%$ to $8.58 \%(\mathrm{p}<0.05)$. Inpatient cases decreased from $13.15 \%$ to $1.73 \%(\mathrm{p}<0.05)$. Compared to inpatient, outpatient ESWL cases had significantly higher readmission rates within 30 days $(\mathrm{OR}=1.8, \mathrm{p}<0.05)$ and 90 days $(\mathrm{OR}=2.4, \mathrm{p}<0.05)$. Outpatient readmission rates within 30 days also increased from 1990 to $2012(\mathrm{OR}=1.8, \mathrm{p}<0.05)$, while inpatient readmission rates decreased in the same period $(O R=0.5, p<0.05)$. This trend was also true for readmission within 90 days $(\mathrm{p}<0.05)$. Outpatient cases were more likely to undergo a second ESWL within 30/90 days $(\mathrm{OR}=4.4, \mathrm{p}<0.05) \quad(\mathrm{OR}=5.9, \mathrm{p}<0.05)$. Complications within 30/90 days did not differ.

Conclusions: From 1990 to 2012, ESWL became less common for the treatment of urolithiasis in both inpatient and outpatient settings, suggesting ESWL being increasingly performed in office settings and the increased use of endoscopic approaches. Between these two settings, there are disparities in ESWL's success rate, which have continued to grow since 1990. Further studies of these differences is needed.

MP10-8 Nephrectomy for calculous disease: analysis of United States trends and outcomes from 2001 to 2014

V Dombrovskiy, E Olweny

Rutgers-Robert Wood Johnson Medical School United States

Introduction \& Objective: Nephrectomy plays a role in the management of complex nephrolithiasis associated with loss of kidney function, and by some estimates is performed in $0.5-1 \%$ of patients hospitalized with upper urinary tract calculi (UUTC). We sought to describe nationwide trends and outcomes for adult patients undergoing nephrectomy $(\mathrm{Nx})$ for the management of UUTC in the United States between 2001 and 2014. 
Materials and Methods: Adults $(\geq 18 \mathrm{yr})$ hospitalized with a principal diagnosis of kidney or ureteral stones, and who underwent surgical interventions for UUTC including Nx, were selected from the Nationwide/National Inpatient Sample (NIS) database for 2001-2014, using the appropriate ICD-9-CM diagnosis and procedure codes. Trends over time in use of nephrectomy, as well as patient and hospital characteristics, hospital length of stay (LOS), cost and complications were analyzed. Chisquare and Cochran-Armitage trend tests, logistic regression analysis and Wilcoxon rank sum test were used in statistical analysis as appropriate.

Results: An estimated $9232 \mathrm{Nx}$ procedures were performed over the study period, $20 \%$ of which were emergent procedures. $71 \%$ of the patients were female and $48.4 \%$ were white. The majority of procedures $(63.3 \%)$ were performed in the Midwest and the South, and in urban teaching hospitals $(58.1 \%)$. Proportion of $\mathrm{Nx}$ as a fraction of all procedures decreased significantly over time, from $0.71 \%$ in 2001 to $0.59 \%$ in 2014 ( p<0.0001). Complications of any kind occurred in $38.3 \%$, with a significantly lower rate in urban teaching (35\%) vs. rural $(45.9 \%)$ and urban non-teaching hospitals $(42.2 \%)$ respectively $(\mathrm{p}<0.0001$ for all). Median LOS decreased over time from 5 to 4 days $(\mathrm{P}<0.0001)$ whereas median cost increased from $\$ 11,097$ to $\$ 13,237(\mathrm{P}=0.02)$.

Conclusions: Nephrectomy for calculous disease is infrequently performed in the United States and its contemporary utilization has significantly decreased over time. Associated complication rates are moderately high, particularly within non-teaching hospitals, reflecting the technical difficulty of these cases, and highlighting a possible benefit of regionalization of care for these challenging cases.

MP10-9 Unplanned readmission rates and postoperative complications after day-case surgery in Urology: Retrospective study over 1 year

F Colomb, I Bentellis, P Regnier, P Treacy, L Vignot, R Haider, Y Bodokh, B Tibi, P Romain, J Fallot, J Amiel, D Chevallier, M Durand

Departement of Urology, Andrology and Renal transplantation, Hospital Pasteur II, CHU Nice

France

Introduction \& Objective: The development of ambulatory surgery in France is booming, promoted by current health policy changes in reimbursements and technological advances. Few studies in the literature refer to the rate of readmission to emergency departments for postoperative complications [1]. We retrospectively analyzed the rate of unplanned readmission after ambulatory surgery in our center in 2016.

[1]Int. braz j urol. vol.33 no.3 Rio de Janeiro May/June 2007

Adverse events and readmissions after day-case urological surgery

Alvaro Paez; Enrique Redondo'; Ana Linares'; Emilio Rios ${ }^{\mathrm{I}}$; Jorge Vallejo'; Margarita Sanchez-Castilla ${ }^{\mathrm{I}}$

Materials and Methods: We retrospectively studied the 295 patients validated for ambulatory surgery by the Urologist in our center over a period of one year (2016).

The usual Urological acts with low and moderate hemorrhagic risk were proposed for this mode of management to the anesthetist before being programmed. 7 typologies of act were constituted.
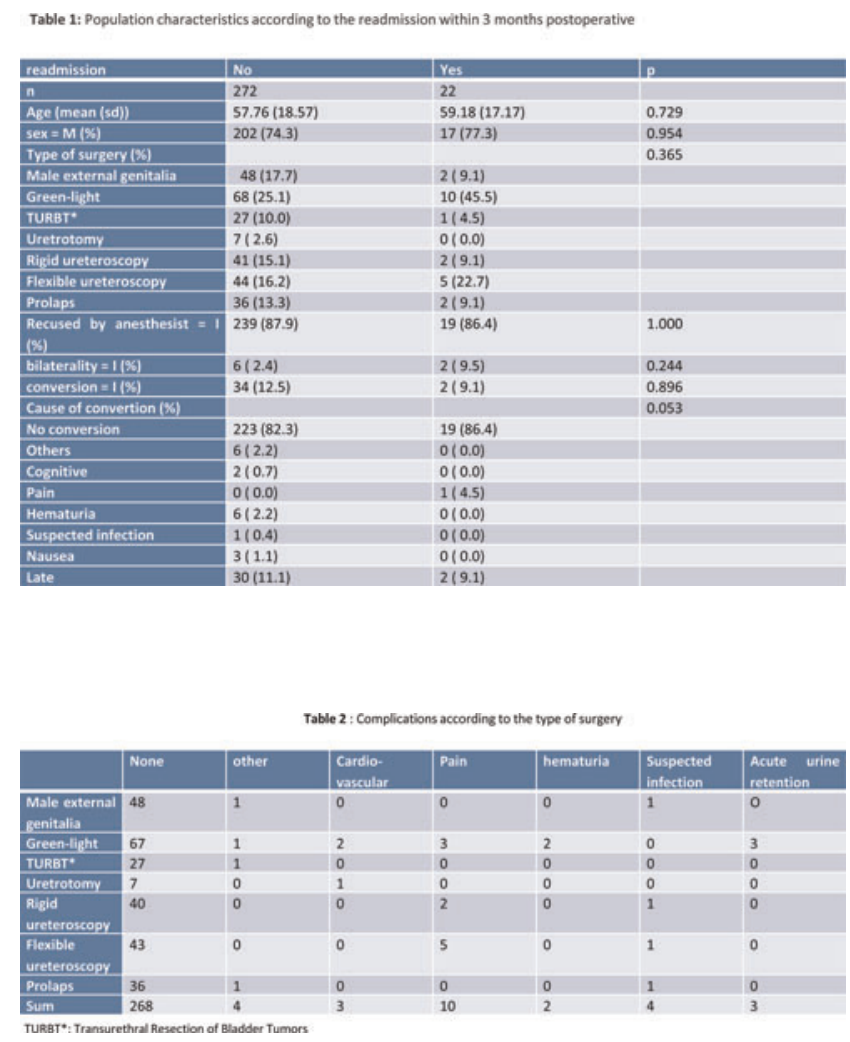

GreenLight laser prostate resection techniques and holmium lasertritis for ureteroscopy were feasible.

The primary outcome measure was the unplanned readmission rate defined as any consultation to emergencies within 3 months after the act. Medical data included age, gender, medical validation for ambulatory, conversion to regular hospitalization, readmissions and complications within 3 months.

Results: 295 patients were analyzed, the mean age was 61 years (range 5-95). 20\% were aged $>75$ year old. Discharge schedule was not completed in $12.2 \%$, essentially due to a delayed outpatient $(72.2 \%)$. The readmission rate was $1.4 \%$ within 24 hours and $7.8 \%$ within 3 months (Table 1). Urological complications were the most frequent (Table 2) $73 \%$ with an average delay of $10 \mathrm{~J}(0-85)$ On the basis of the multivariate analysis, none of the qualitative and quantitative factors tested were related to readmissions and complications.

Conclusions: The rate of readmission and complications at 3 months after ambulatory urologic surgery from a standard recruitment is low.

The results of this series highlight prospects for progression to decrease conversions and the major complication, which is pain.

Larger, multicenter studies are needed to confirm these preliminary results.

MP10-10 Trends and Sociodemographic Disparities in the Surgical Management of Benign Prostatic Hyperplasia, 1997-2012

A Yang, M Finkelstein, KH Bilal, MA Palese

Icahn School of Medicine at Mount Sinai

United States 

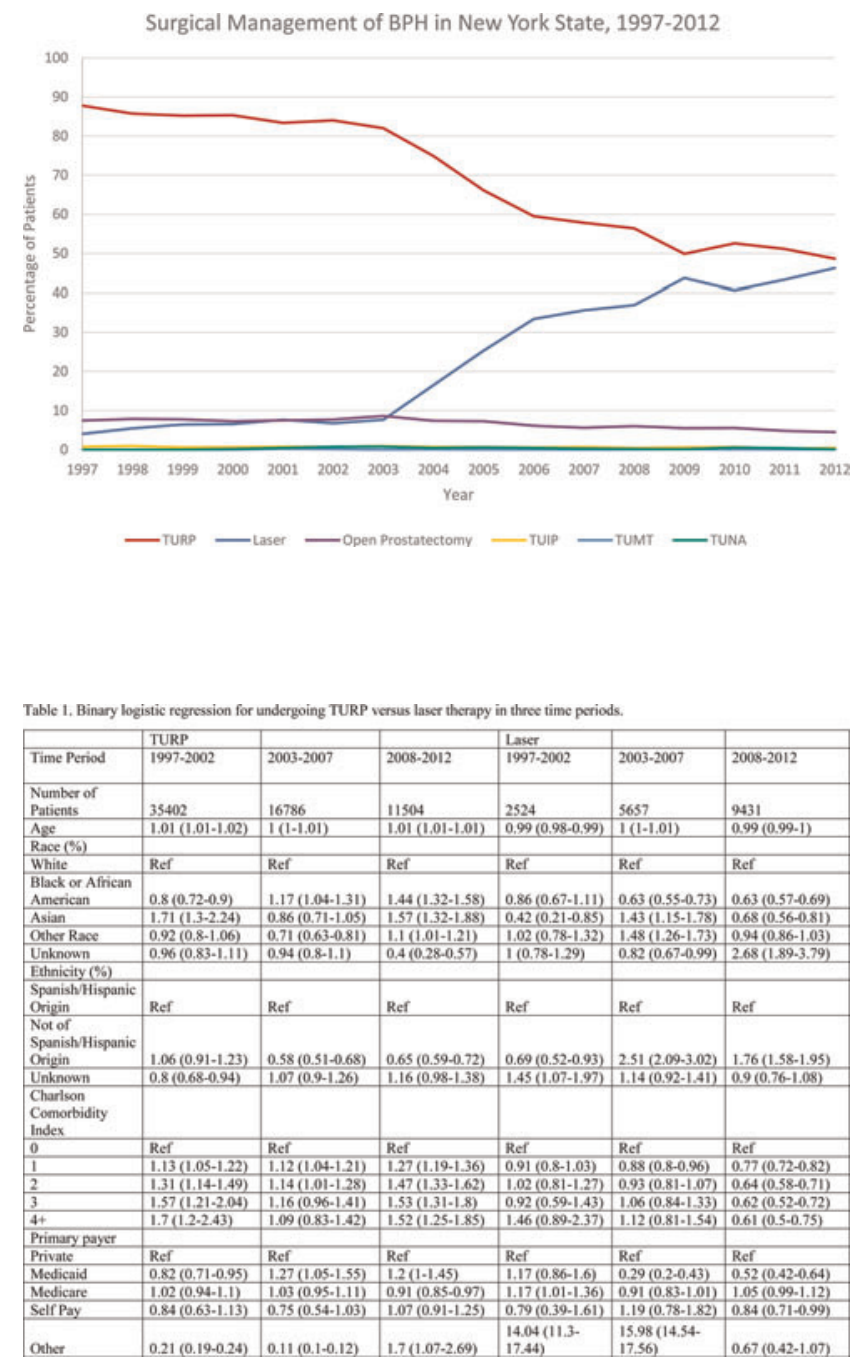

Introduction \& Objective: There are few studies assessing the effect of changing preferences in the surgical treatment for benign prostatic hyperplasia $(\mathrm{BPH})$ on existing disparities. We examined the trends in surgical procedures for $\mathrm{BPH}$ and analyzed the sociodemographic disparities of the two most common procedures, transurethral resection of the prostate (TURP) and laser therapy.

Materials and Methods: Records of patients who had BPH, excluding a history of prostate cancer, were extracted from the Statewide Planning and Research Cooperative System (SPARCS) database. Patients with surgical management of BPH were identified using ICD9 and CPT codes. The chi-square test was used to compare categorical variables. Binary logistic regression was used to assess predictors of undergoing TURP or laser therapy in three time periods (1997-2002, 2003-2007, 20082012).

Results: Of 88,254 patients who underwent surgical management of BPH, TURP accounted for $72 \%$ of the patients and laser surgery was performed on 20\% of patients. From 1997 to 2012, the proportion of patients receiving TURP decreased from $88 \%$ to $49 \%$ ( $\mathrm{p}<0.01)$, while laser surgery cases increased from $4 \%$ to $46 \%$ ( $\mathrm{p}<0.01$ ). Patients undergoing TURP in 1997-2002 were more likely to be Caucasian than African-American (OR $0.8 \mathrm{CI}$ 0.72-0.90), and have private insurance than Medicaid (OR 0.82
CI 0.71-0.95). In 2008-2012, these trends reversed ( $<<0.05)$. For laser surgery, patients in 2008-2012 were less likely to be Black (OR 0.63 CI 0.57-0.69), Hispanic (OR 1.76 CI 1.58-1.95), have comorbidities or have Medicaid (OR 0.52 CI 0.42-0.64). These disparities did not exist in 1997-2002.

Conclusions: The surgical treatment for BPH has shifted from TURP to laser surgery from 1997 to 2012. Sociodemographic disparities in 1990 associated with TURP were largely replaced by differences in access to laser therapy by 2012, suggesting these disparities have shifted as well and still remain in the surgical management of $\mathrm{BPH}$.

MP10-11 Ethnicity and family history impact age of onset and number of episodes for urolithiasis: a prospective cohort study from ReSKU

K Taguchi, DT Tzou, M Usawachintachit, BA Sherer, ML Stoller, T Chi

\section{UCSF \\ United States}

Introduction \& Objective: Family history is thought to increase relative risk for urolithiasis. Considering genetic risks in the context of ethnicity is a novel approach that would contextualize the relationship between patient background and clinical presentation. Our objective was to evaluate the relationship between patient family history, ethnicity and clinical outcomes for urolithiasis.

Materials and Methods: This was a prospective cohort study utilizing ReSKU ${ }^{\mathrm{TM}}$, the Registry for Stones of the Kidney and Ureter. Nephrolithiasis patient demographic, presenting symptoms, family history and treatment history were obtained at first clinic visit. Patients were separated into three major ethnicity groups (White, Asian, Hispanic/Latino). Detailed family history was collected for three generations.

Table 1.

\begin{tabular}{|c|c|c|c|}
\hline & Family history (-) & Family history (+) & p value \\
\hline & $n=365$ & $n=191$ & \\
\hline age of first clinic visit, mean(SD) & $54.25(16.33)$ & $51.21(16.29)$ & 0.037 \\
\hline body mass index $\left(\mathrm{kg} / \mathrm{m}^{2}\right)$, mean(SD) & $28.33(7.80)$ & $28.05(7.75)$ & 0.688 \\
\hline female gender, $n(\%)$ & $167(45.8)$ & $102(53.4)$ & 0.090 \\
\hline symptomatic presentation, $n(\%)$ & $335(92.3)$ & $181(94.8)$ & 0.295 \\
\hline number of previous stone episodes, $n(\%)$ & & & $<0.001$ \\
\hline 0 & $187(55.7)$ & $72(40.0)$ & \\
\hline 1 & $65(19.3)$ & $30(16.7)$ & \\
\hline 2 & $15(4.5)$ & $15(8.3)$ & \\
\hline 3 & $13(3.9)$ & $12(6.7)$ & \\
\hline 4 & $10(3.0)$ & $6(3.3)$ & \\
\hline 5 & $12(3.6)$ & $4(2.2)$ & \\
\hline$>6$ & $34(10.1)$ & $41(22.8)$ & \\
\hline previous procedures for stone, $n(\%)$ & $97(26.6)$ & $69(36.1)$ & 0.025 \\
\hline age of onset for first stone episode, $n(\%)$ & & & 0.007 \\
\hline no previous stone & $183(55.5)$ & $72(39.6)$ & \\
\hline since age $<10$ & $3(0.9)$ & $2(1.1)$ & \\
\hline since age $11-20$ & $22(6.7)$ & $21(11.5)$ & \\
\hline since age $21-30$ & $28(8.5)$ & $20(11.0)$ & \\
\hline since age $31-40$ & $23(7.0)$ & $22(12.1)$ & \\
\hline since age $41-50$ & $32(9.7)$ & $23(12.6)$ & \\
\hline since age $51-60$ & $27(8.2)$ & $9(4.9)$ & \\
\hline since age $>60$ & $12(3.6)$ & $13(7.1)$ & \\
\hline bilateral stone, $n(\%)$ & $294(80.5)$ & $157(82.2)$ & 0.732 \\
\hline total stone burden $(\mathrm{mm})$, median[IOR] & $14.00[7.00,34.00]$ & $13.00[8.00,28.00]$ & 0.941 \\
\hline
\end{tabular}


Results: A total of 556 patients were enrolled during the study period, of which 191 (34.4\%) had a family member with urolithiasis. Within these 191 patients, $15.2 \%$ had both $1^{\text {st }}$ and $2^{\text {nd }}$ degree of relatives with stones, $42.2 \%$ had paternal and $31.9 \%$ had maternal positive family history. White, Asian, and Hispanic/Latino ethnicity encompassed $75.1 \%, 12.7 \%$, and $7.9 \%$ of patients respectively. Positive family history was associated with younger age of first clinic visit $(p=0.037)$, larger number of previous stone episode $(\mathrm{p}<0.001)$, history of previous procedure for stone $(p=0.025)$, and younger age of onset for first stone episode $(\mathrm{p}=0.007)$. (Figure 1$)$ White ethnicity was associated with larger number of previous stone episodes and younger age of onset for first stone episode ( $\mathrm{p}=0.025$ and 0.008 , respectively). Having $1^{\text {st }}$ and $2^{\text {nd }}$ degree relatives, paternal family history, and maternal family history were associated with younger age of onset for first stone episode $(\mathrm{p}=0.002,0.048$, and 0.01 , respectively). Multivariate regression analysis revealed that Asian ethnicity demonstrated a 0.11 odds ratio $(p=0.046)$ for younger onset age for first stone episode compared to 5.70 for having $1^{\text {st }}$ and $2^{\text {nd }}$ degree relatives with stones $(\mathrm{p}=0.018)$.

Conclusions: Our results demonstrate that family history of urolithiasis was associated with age of onset and number of stone episodes. Moreover, the type of relative and patient ethnicity may impact clinical presentation.

MP10-12 30 Day Unplanned Readmission After Minimally Invasive Urological Procedure at a Tertiary Care Academic Center.

AE Ward, SS Parikh, NH Patel, JH Hillelsohn, SA Fullerton, JL Phillips, MS Choudhury, M Eshghi

New York Medical College

United States

Introduction \& Objective: Readmission within 30 days of discharge following an in-patient admission is increasingly used as a quality metric and has implications on potential reimbursement models. Currently, reported 30 day unplanned readmission rates to urology services are largely based on pooled national databases from a wide variety of patient populations, demographics, case mix indices, and healthcare settings. This study was to determine a 30 day unplanned readmission rate to the urology service of a tertiary care academic university hospital with a high average case mix index (CMI) of 1.5 over 6 years, and to assess the risk factors associated with readmission.

Materials and Methods: We reviewed all 30 day unplanned readmissions for 10 minimally invasive index procedures from our adult urology service between January 2010 and December 2015. Each case was analyzed for the cause of readmission, the patient demographics, and other contributing factors that led to readmission. Non-parametric univariate and regression analysis was completed on the aggregate with an alpha set at 0.05. Planned multi-stage procedures were excluded.

Results: We found that our 30 day unplanned readmission rate following minimally invasive urologic and endourologic procedures was stable over a 6 year period with an overall rate of $2.2 \%$. Main factors that were related to readmission were patient age, shorter initial length of stay, and inadequate medical prevention. The main cause of readmission was UTI $(20.0 \%)$, followed by pain $(17.0 \%)$ and hematuria $(15 \%)$.

Conclusions: Our data reflects a 30 day unplanned readmission rate for 10 minimally invasive urologic and endourologic index

\begin{tabular}{|c|c|c|c|c|}
\hline \multirow[b]{2}{*}{ Procedure } & \multicolumn{2}{|c|}{$\begin{array}{l}\text { Minimally Invasive and } \\
\text { Endourological Procedures }\end{array}$} & \multicolumn{2}{|c|}{ Comparative Open Procedures } \\
\hline & Total & $\begin{array}{l}\text { Unplanned } \\
\text { Readmissions (\%) }\end{array}$ & Totals & $\begin{array}{c}\text { Unplanned } \\
\text { Readmissions (\%) }\end{array}$ \\
\hline TURBT & 302 & $5(1.7 \%)$ & - & - \\
\hline TURP & 186 & $6(3.2 \%)$ & - & - \\
\hline Lap Prostatectomy & 104 & $4(3.8 \%)$ & 30 & $1(3.33 \%)$ \\
\hline Lap Partial Nephrectomy & 87 & $3(3.4 \%)$ & 32 & $4(12.5 \%)$ \\
\hline Lap Radical Nephrectomy & 71 & $2(2.8 \%)$ & 52 & $1(1.9 \%)$ \\
\hline Lap Cystectomy & 12 & $1(8.3 \%)$ & 25 & $7(28.0 \%)$ \\
\hline Ureteroscopy & 816 & $11(1.3 \%)$ & $\cdot$ & $\cdot$ \\
\hline PCNL & 206 & $6(2.9 \%)$ & $\cdot$ & - \\
\hline Adrenalectomy & 21 & $1(4.8 \%)$ & 1 & $1(100 \%)$ \\
\hline Nephroureterectomy & 33 & $1(3.3 \%)$ & - & - \\
\hline OVERALL & 1836 & $40(2.2 \%)$ & - & - \\
\hline
\end{tabular}

procedures at a major academic urology service with a complex patient population. Compared to a cohort of similar patients, laparoscopic procedures generally had a similar or more favorable outcome compared to the same open procedure. Planned follow up with urologic evaluation as an outpatient within 1 week after elective discharge, as well as improved pain management may improve readmission rates.

MP10-13 Prevalence of Kidney Stones Changing in Male to Female Ratio: National Health and Nutrition Examination Survey (NHANES) 2007-2014

\section{Z Chen, M Prosperi, VY Bird}

UF Urology

United States

Introduction \& Objective: Purpose: To estimate the latest prevalence of kidney stones in the US using data from the most recent National Health and Nutrition Examination Survey (NHANES), and describe the trends in kidney stone prevalence in different genders included in the most recent reported NHANES data.

Materials and Methods: A cross-sectional analysis of response to the 2007-2014 NHANES was performed. The data contains 4 distinct survey circles (2007-08, 09-10, 11-12 and 13-14). Kidney stone history were assessed by question "Have you ever had kidney stones?". The percent prevalence of self-reported kidney stone history in each circle were calculated using weights and design factors reported by NHANES. The trends of kidney stone prevalence in males and females at different age groups were compared. All statistical analysis was performed using SAS ver.9.4.

Results: The overall age-adjusted prevalence in 2013-14 was $10.3 \%$, a slight increase than the prevalence in 2007-08 (9.4\%). The prevalence in males $(11.4 \%)$ was higher than in females $(9.5 \%)$. However, when comparing the prevalence by each survey circle, a steady increase was observed in females $(6.6 \%$, $7.7 \%, 8.7 \%$ and $9.5 \%$ in each cycle respectively, $\mathrm{p}<0.05)$, while in males, a slight decrease was observed $(12.7 \%, 10.5 \%, 9.1 \%$ and $11.4 \%$ in each circle respectively). When looking at the prevalence at different age groups, a drastic increase in younger (20-39 years old) female was observed, the prevalence almost doubled $(\mathrm{p}<0.05)$ in $2013-14(7.5 \%)$ comparing to $2007-08$ 
(3.9\%), similar trend also presented in mid-aged females (40-59 years old), the prevalence increased from $7.4 \%$ to $10.8 \%$. Prevalence in males and older females were relatively stable.

Conclusions: The prevalence of kidney stones according to the most recent NHANES data, demonstrates increasing trends, especially in younger females. Future research should focus on examining risk factors that may associated with the gender difference in kidney stone formers.

MP10-14 Kidney Stones Are Positively Associated With Sleep Disorders: National Health and Nutrition Examination Survey (NHANES) 2011-2014

\section{Z Chen, M Prosperi, VY Bird}

UF Urology

United States

Introduction \& Objective: Background: Sleep disorders, such as sleep apnea, could elevate urinary uric acid excretion and decrease testosterone level, thus affecting normal renal physiology. Recent studies suggest that sleep disorders may be linked to kidney stone formation, but few population-based studies specifically examine this association. Objectives: This analysis utilizes 2011-2014 National Health and Nutrition Examination Survey (NHANES) to examine the association between history of kidney stones and sleep disorders. We also examined the trends of demographic characteristics in people with history of kidney stones.

Materials and Methods: Methods: Participants who responded to the question "Have you ever had kidney stones?" were included. Univariate and multivariate analysis were conducted to identify association between history of kidney stones and sleep disorder, as well as demographic variables including age, gender, race and BMI. History of diabetes and gout were included in the multivariate model to adjust potential confounders.

Results: A total of 11,306 participants responded to the question regarding kidney stones history, and among them, 8.78\% (993) respondents reported history of kidney stones. Of all participants who had kidney stones, $51.36 \%$ were male. The rate of kidney stones was highest $(22.66 \%)$ in age group 60-69. The rate of obesity $(45.82 \%)$ and sleep disorder $(14.43 \%)$ were significantly higher in those who had kidney stones history comparing to those who never had kidney stones (8.66\% and $3589 \%$ respectively, both p-value $<0.0001)$. After controlling for other risk factors, obesity (OR: $2.26,95 \%$ CI $(1.10,4.66)$, non-Hispanic white (OR: $1.32,95 \%$ CI $(1.05,1.66)$ and sleep disorder (OR: 1.29, 95\% $(1.04,1.59))$ were statistically significantly associated with higher risk of history of kidney stones. Contradictory to previous reports, gender was not associated with history of kidney stones (OR: $0.82,95 \%$ CI $(0.76,1.01)$ ).

Conclusions: Our analysis demonstrated that history of kidney stones is positively associated with sleep disorder, as well as race and BMI category. However, due to the cross-sectional nature of NHANES, future longitudinal studies may help understand the mechanisms in these associations.

MP10-15 A retrospective study using the Korea National Database for prognosis of prostate cancer in patients with end-stage renal disease (2003-2010)

H Seo, S Kim, J Joung, Y Kim, E Lee, K Lee, J Hong
Department of Urology, Center for the Prostate Cancer, Research Institute and Hospital of National Cancer Center Republic of Korea

Introduction \& Objective: The aims of the present study were to evaluate the prevalence and prognosis of prostate cancer-endstage renal disease (PC-ESRD); and to determine the risk factors of overall survival (OS) and PC-specific survival (CSS), and differences in PC-related clinical therapeutic patterns between patients with and without PC-ESRD.

Materials and Methods: Using the nationwide Korean Health Insurance System Database and Korean Central Cancer Registry data based on the International Classification of Disease (ICD), Tenth Edition diagnosis code of PC, 38925 patients with PC between 2002 and 2011 who completed follow-up until 2012 and had available survival data were selected. The Surveillance, Epidemiology, and End Results (SEER) staging classification was used to stage PC. Diagnostic ICD codes for diabetes, renal failure, dialysis, and kidney transplantation were used to select patients with ESRD before PC was diagnosed. The prevalence and prognostic risk factors of OS were statistically analyzed.

Results: In this study, 3945 (10.1\%) patients with ESRD were diagnosed as having PC, which included $3.9 \%$ on dialysis $(\mathrm{N}=152)$ and $0.2 \%$ with kidney transplantation $(\mathrm{N}=10)(\mathrm{D}-\mathrm{TPL}$ group), and of those, $3783(95.9 \%)$ had neither dialysis nor transplantation (non-D-TPL ESRD group). Overall, 9406 (24.2\%) patients died, including 697 (7.4\%) PC-specific deaths. Compared to the non-D-TPL group, the D-TPL group had a significantly higher rate of advanced stages of PC, history of hormonal therapy for $\mathrm{PC}$, higher death rate, and lower rate of surgical therapy $(\mathrm{p}<0.05)$. The median respective OS/PC-specific survival time/5-year survival rate for the non-ESRD (37.4/ $21.1 / 80.1 \%$, non-D-TPL ESRD (30.5/17.8/64.5\%), dialysis ESRD (8.9/11.4/59.1\%), and transplantation ESRD (36.9/not applicable/100\%) groups were significantly different $(\mathrm{p}<0.001)$. Results of multivariate analysis showed that the presence of ESRD (hazard ratio [HR] 1.614), age (HR 1.296-4.385), body mass index (underweight, HR 1.428; overweight and obese, HR 0.774 and 0.778, respectively), SEER stage (HR 1.277-5.052), no treatment within 6 months after diagnosis (HR 2.321), no surgery (HR 1.436), no chemotherapy (HR 0.750), no radiotherapy (HR 0.750), no hormonal therapy (HR 0.464), non-adenocarcinoma pathology (HR 1.363), and CGI grade 2 (HR $0.600-0.708)$ were significant risk factors of OS ( $p<0.001$, all). Conclusions: The nationwide prevalence of $\mathrm{PC}$ in patients with ESRD was $10.1 \%$, and the presence of ESRD, including those with ESRD with and without dialysis and kidney transplantation, was a significant survival factor along with other significant clinicopathological factors.

MP10-16 Worldwide trends in the role of warmer climate on the incidence of renal colic and kidney stone disease (KSD): Evidence from a systematic review of literature

R Geraghty, S Proietti, M Archer, A Pietropaolo, BK Somani

University Hospital Southampton NHS Foundation Trust United Kingdom

Introduction \& Objective: Several studies have examined the link between temperature or season and urolithiasis. These studies have demonstrated that higher temperatures or the summer months have a demonstrably higher rate of presentations 


\begin{tabular}{|c|c|c|c|c|c|c|c|}
\hline & Study & $\begin{array}{c}\text { Data collected } \\
\text { from }\end{array}$ & $\begin{array}{l}\text { Study } \\
\text { Period } \\
\text { (Years) }\end{array}$ & $M: F(n)$ & $\mathrm{R}^{2}$ & $\beta$ & $\mathbf{P}$ \\
\hline \multirow{3}{*}{$\begin{array}{l}\text { Proven } \\
\text { Stones }\end{array}$} & \begin{tabular}{|l|l|} 
Archer et al. 2016 \\
\end{tabular} & 1 Hospital & 3 & $925^{\circ}$ & 0.24 & 0.49 & 0.002 \\
\hline & Lee et al. 2016 & 4 Hospitals & 8 & 9712:4806 & 0.74 & 0.86 & $<0.001$ \\
\hline & Sirohi et al. 2014 & 1 Hospital & 5 & 2290:1355 & 0.74 & 0.86 & $<0.001$ \\
\hline \multirow{9}{*}{$\begin{array}{l}\text { Renal } \\
\text { Colic }\end{array}$} & Al-Hadramy et al. 1997 & 1 Hospital & 3 & $\begin{array}{c}1342 \text { (M } \\
\text { only) }\end{array}$ & 0.89 & 0.94 & $<0.001$ \\
\hline & $\begin{array}{l}\text { Cepeda Delgado et al. } \\
2015\end{array}$ & 1 Hospital & 9 & $5187: 4143$ & 0.46 & 0.68 & 0.016 \\
\hline & Cervellin et al. 2012 & 1 Hospital & 9 & $7580: 3222$ & 0.84 & 0.92 & $<0.001$ \\
\hline & Chen et al. 2008 & National Data & 4 & $\begin{array}{c}206,978: \\
63,324\end{array}$ & 0.78 & 0.88 & $<0.001$ \\
\hline & Condemi et al. 2014 & 1 Hospital & 3 & $2270: 1781$ & 0.51 & 0.72 & 0.009 \\
\hline & Fukuhara et al. 2016 & 1 Hospital & 8 & $346: 145$ & 0.54 & 0.74 & 0.006 \\
\hline & Lo et al. 2009 & $\begin{array}{l}\text { Regional Data } \\
\text { (Auckland) }\end{array}$ & 9 & 5385:2275 & 0.74 & 0.86 & $<0.001$ \\
\hline & Lujan et al. 2011 & 1 Hospital & 1 & $868: 619$ & 0.13 & 0.36 & 0.254 \\
\hline & Park et al, 2015 & National Data & 4 & $1,702,913^{\circ}$ & 0.65 & 0.81 & 0.002 \\
\hline ESWL & Lin et al. 2014 & 1 Hospital & 5 & 4685:1931 & 0.70 & 0.84 & 0.001 \\
\hline
\end{tabular}

with urolithiasis. However, no systematic review has been performed to examine this effect.

Materials and Methods: A systematic review was conducted using studies identified by a literature search between January 1990 and June 2016. All English language articles reporting on monthly admissions for urolithiasis with mean temperature for each month. Two reviewers independently extracted the data from each study. Data was analyzed using SPSS version 24.

Results: A total of 13 studies were included for analysis from a literature search yielding 59 studies. The studies took place in UK, South Korea, USA, Saudi Arabia, Spain, Italy, Taiwan, Japan and New Zealand. The study periods covered a mean of 5.5 years (range: 1-9). 3 studies demonstrated data on proven stones, one study used data from monthly ESWL and the remainder demonstrated data on presentation to emergency departments for renal colic. 2 of the studies demonstrated national data, 1 study used regional data, 1 study used data from four hospitals and the remaining studies used data from single hospitals. Given the heterogeneity of the data, studies were examined individually. All apart from one study demonstrated a significant association between temperature and the observed parameter for stone presentation.

Conclusions: There is a statistically significant linear association between temperature and urolithiasis presentation across multiple studies.

MP10-17 Trends of Ambulatory Urological Surgery in the State of Florida: 2010-2014

HD Patel, BR Matlaga, JB Ziemba

Brady Urological Institute, Johns Hopkins School of Medicine United States

Introduction \& Objective: Although it is estimated that more than half of all urological procedures are performed in the ambulatory setting, there remains little data on the actual volume, variety, and cost of these procedures. To address this area of need, we performed an analysis of an all-payer ambulatory surgery database from the State of Florida over a 5-year period.

Materials and Methods: The Healthcare Cost and Utilization Project State Ambulatory Surgery and Services Databases (SASD) contain uniform encounter-level data inclusive of all payers. Florida state files from 2010-2014 (most recent year available) were obtained. Data extracted included patient demographics, facility type (freestanding/non-freestanding), surgeon identification, procedure number and type (CPT code) with

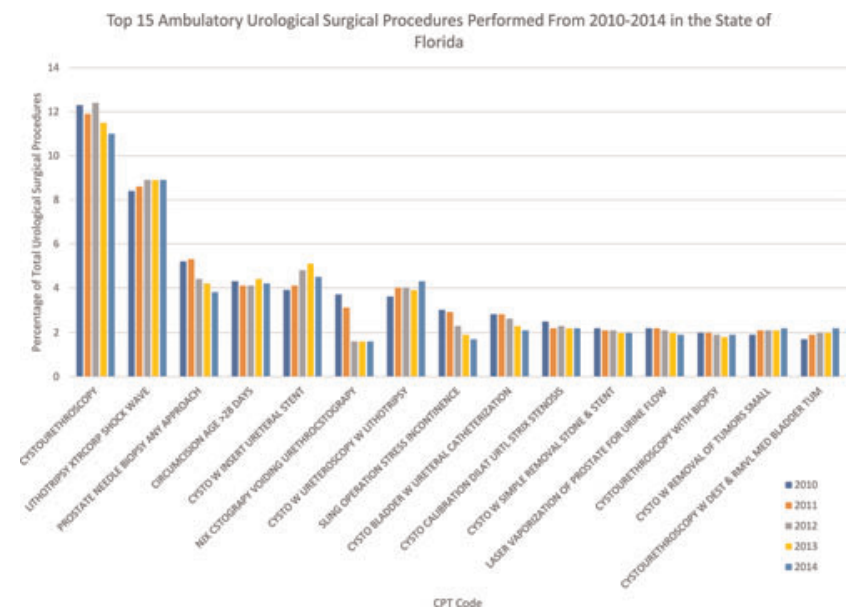

associated diagnosis (ICD-9), and total charge. Total charges were compared across study years.

Results: A total of 557,176 patients underwent 862,462 urological procedures over the 5-year period. This accounted for nearly $6 \%$ (range: $5.69-5.96 \%$ ) of all ambulatory surgical procedures performed in the state. The mean patient age was $57.32 \pm 22.43$ years with $63.1 \%$ male, $72.9 \%$ Caucasian, $50.3 \%$ lived in a large metropolitan area, and the median number of comorbid chronic conditions was 1 (IQR: 0-2). The majority of the procedures $(65.3 \%)$ were performed in a non-freestanding facility by 11,009 different surgeons. The top 20 surgeons accounted for $10.2 \%$ of all cases. The median number of CPT codes and diagnoses per record were 2 (IQR: 1-8) and 2 (IQR: 1-5), respectively. The top 15 most frequently performed primary procedures in each year are outlined in Figure 1. Medicare (45.5\%) and private insurance $(39.5 \%)$ accounted for the majority of payers with the mean charge per case of $\$ 14,995.17 \pm \$ 16,569.81$. The mean charge per case increased each year over the 5-year study period (2010: $\$ 11,233.05$ vs 2014: $\$ 18,359.87$; one-way ANOVA $\mathrm{p}<0.001)$.

Conclusions: Urological surgery represented nearly $6 \%$ of all ambulatory surgery cases in the State of Florida from 2010-2014. The most common procedure performed was cystourethroscopy followed by shockwave lithotripsy. The mean charge per case increased each year from 2010-2014.

MP10-18 Predicting Adverse Outcomes in Laparoscopic Nephrectomies Utilizing a Modified Frailty Index

I Levy, M Finkelstein, MA Palese

Icahn School of Medicine at Mount Sinai United States

Introduction \& Objective: The prevalence of frailty in geriatric surgical patients is higher compared to non-operative counterparts. This study aims to determine the effect of frailty on patient outcomes including Clavien-Dindo (CD) 4 and 5 complications and 30-day mortality for laparoscopic nephrectomy patients in comparison to other predictive indices using the modified frailty index $(\mathrm{mFI})$.

Materials and Methods: Patients undergoing laparoscopic nephrectomy from 2008 to 2014 were queried using the ACSNSQIP database. The mFI was developed using the Canadian 


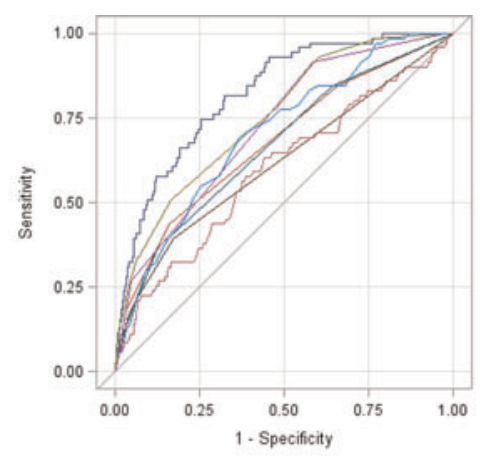

\begin{tabular}{|c|l|l|}
\hline \multicolumn{3}{|c|}{ 30-Day Mortality ROC Curve (Area) } \\
\hline Model & & 0.8221 \\
\hline mFI & & 0.6759 \\
\hline CSHA-FI & & 0.6677 \\
\hline CCI & & 0.6168 \\
\hline ASA & & 0.7225 \\
\hline ASA mFI & & 0.7516 \\
\hline Age & & 0.7043 \\
\hline Total Operative Time & & 0.5965 \\
\hline
\end{tabular}

Study of Health and Aging Frailty Index (CSHA-FI) as a model. The $\mathrm{mFI}$ was compared with other associative indices such as the American Society of Anesthesiology (ASA) classification and the Charlson Comorbidity Index (CCI). Multivariable regression adjusting for patient demographics of age, sex, ethnicity, smoking status, and additional lymph node dissection to predict CD 4 and 5 complications and a receiver operator characteristic (ROC) curve used to predict 30-day mortality were analyzed.

Results: 16,645 patients were identified. $5677(34.1 \%)$ had a $\mathrm{mFI}$ of $0,8228(49.4 \%)$ had a $\mathrm{mFI}$ of $1,2182(13.1 \%)$ had a $\mathrm{mFI}$ of 2 , and $558(3.4 \%)$ had a $\mathrm{mFI} \geq 3$. Patients with increasing frailty were older, had a greater percentage of males, non-whites, and smokers, and scored higher on ASA and CCI indices $(\mathrm{P}<0.0005)$. Patients with increasing frailty also experienced increased CD 4 or 5 complications and 30-day mortality $(\mathrm{P}<0.0005)$. Laparoscopic nephrectomy patients with $\mathrm{mFI} \geq 3$ had the highest adjusted odds for CD 4 or 5 complications $(\mathrm{OR}=2.772$, CI: $1.616-4.755, \mathrm{P}<0.0005)$ in comparison with non-frail laparoscopic nephrectomy patients. These odds were higher than the CSHA-FI, ASA, CCI, and stratified patient age. Additionally, a variable combining $\mathrm{mFI}$ and ASA had fair sensitivity and specificity for predicting 30-day mortality in laparoscopic nephrectomy patients $(\mathrm{C}$-statistic $=0.7516, \mathrm{P}<0.0005)$ which was better than ASA, mFI, CCI, or patient age individually (Figure 1).

Conclusions: Increasing $\mathrm{mFI}$ scores are associated with worsening outcomes for patients undergoing laparoscopic nephrectomy and are more predictive of CD 4 and 5 complications than other indices. A combined $\mathrm{mFI}$ and ASA variable can be used to predict 30-day mortality for laparoscopic nephrectomy patients better than $\mathrm{mFI}, \mathrm{ASA}$, or patient age alone.

MP10-19 Urologic Cancer Disparities among Hispanic Americans and Native Americans in Four U.S.-Mexico Border States

A Bergersen, K Batai, J Michalak, M Craig, E Price, BR Lee

University of Arizona College of Medicine, Department of Surgery, Division of Urology

United States

\begin{tabular}{|c|c|c|c|c|c|}
\hline & $\begin{array}{c}\mathrm{NA} \\
\text { Rate }(95 \% \mathrm{C} . \mathrm{L}) \\
\end{array}$ & $\begin{array}{c}\text { HA } \\
\text { Rate }(95 \% \text { C.1.) }\end{array}$ & $\begin{array}{c}\text { EA } \\
\text { Rate }(95 \% \text { C. } . .)\end{array}$ & $\begin{array}{c}\text { NAEA } \\
\text { Ratio }\end{array}$ & $\begin{array}{c}\text { HAVEA } \\
\text { Ratio }\end{array}$ \\
\hline \multicolumn{6}{|l|}{ Prostate } \\
\hline Arizona & $69.2(61.2-78.1)$ & $69.4(65.9 .73 .2)$ & $84.9(83.3-86.4)$ & $0.82^{\circ}$ & $0.82^{*}$ \\
\hline Califomia & $50.0(43.3-57.7)$ & $104.5(102.8-106.1)$ & $119.1(118.1-120.0)$ & $0.42^{*}$ & $0.88^{\circ}$ \\
\hline New Mexico & 67.5 (57.9.78.5) & $90.2(85.8-94.8)$ & $106.8(103.3-110.5)$ & $0.63^{*}$ & $0.84^{*}$ \\
\hline Texas & $51.9(22.5-110.6)$ & $85.6(83.8-87.4)$ & $107.4(106.3-108.5)$ & 0.48 & $0.80^{*}$ \\
\hline U.S. & $85.1(81.4-89.0)$ & $104.9(104.0-105.8)$ & $114.8(114.5-115.0)$ & $0.74^{*}$ & $0.91^{\circ}$ \\
\hline \multicolumn{6}{|l|}{ Kidney } \\
\hline Arizona & $24.9(22.1-28.0)$ & $19.1(18.0-20.4)$ & $14.9(14.4-15.3)$ & $1.67^{*}$ & $1.28^{\circ}$ \\
\hline Califomia & $11.7(9.6-14.2)$ & $15.7(15.3-16.1)$ & $14.8(14.6-15.1)$ & $0.79^{\circ}$ & $1.06^{\circ}$ \\
\hline New Mexico & $23.1(19.8-26.9)$ & $15.6(14.4-16.9)$ & $11.6(10.7-12.5)$ & $1.99^{*}$ & $1.34^{*}$ \\
\hline Texas & & $20.3(19.8-20.8)$ & $17.8(17.4-18.1)$ & & $1.14^{\circ}$ \\
\hline U.S. & $23.3(22.1-24.5)$ & $15.9(15.7-16.1)$ & $16.3(16.2-16.3)$ & $1.43^{\circ}$ & $0.98^{\circ}$ \\
\hline \multicolumn{6}{|l|}{ Bladder } \\
\hline Arizona & $4.9(3.6-6.6)$ & $9.3(8.4-10.3)$ & $21.2(20.6-21.7)$ & $0.23^{*}$ & $0.44^{*}$ \\
\hline California & $7.3(5.5-9.6)$ & $10.3(10.0-10.7)$ & $23.0(22.7-23.3)$ & $0.32^{*}$ & $0.45^{\circ}$ \\
\hline New Mexico & $3.4(2.1-5.2)$ & $9.6(8.6-10,7)$ & $18.2(17.2-19.3)$ & $0.19^{*}$ & $0.53^{\circ}$ \\
\hline Texas & & $8.8(8.4-9.2)$ & $19.2(18.9-19.5)$ & & $0.46^{*}$ \\
\hline us & $11.6(10.6-12.6)$ & $11.4(11.2-11.6)$ & $23.0(23.0-22.6)$ & $0.50^{*}$ & $0.50^{\circ}$ \\
\hline
\end{tabular}

Introduction \& Objective: Overall cancer incidence and mortality rates among Hispanic Americans (HAs), Native Americans (NAs), and Alaskan Natives are lower compared to European Americans (EAs) of non-Hispanic origin. However, NAs and HAs are very heterogeneous groups. Cancer incidence and mortality rate vary among HA subgroups and regionally among NAs. The objective of this study is to assess the extent of urologic cancer disparities among HAs and NAs compared to EAs in four U.S.-Mexico border states: Arizona (AZ), California (CA), New Mexico (NM), and Texas (TX).

Materials and Methods: We obtained prostate $(\mathrm{CaP})$, kidney $(\mathrm{CaK})$, and bladder cancer $(\mathrm{CaB})$ incidence rates (age-adjusted incidence rates per 100,000 using 2000 US standard population) between 2009 and 2013 from the North American Association of Central Cancer Registries.

Results: $\mathrm{CaP}$ was more common than $\mathrm{CaK}$ and $\mathrm{CaB}$ in all three race/ethnicity groups. $\mathrm{CaP}$ incidence rate was higher in $\mathrm{EAs}$ than NAs/HAs, but HAs and NAs were more likely to be diagnosed with metastatic $\mathrm{CaP}$ than EAs. The proportion of NA men diagnosed with metastatic CaP was $17 \%$ and $19 \%$ in AZ and NM respectively, while only $5 \%$ of EA men were diagnosed with metastatic $\mathrm{CaP}$. $\mathrm{CaK}$ was more common than $\mathrm{CaB}$ in NAs and HAs. HAs had significantly higher CaK incidence rate than EAs in all border states. $\mathrm{CaK}$ incidence rate in NAs was significantly higher than EAs in $\mathrm{AZ}$ and NM, but lower in CA. In EAs, CaB was more common than $\mathrm{CaK}$, and $\mathrm{CaB}$ incidence rate was significantly higher than in NAs and HAs. Incidence rates were compared between races/ethnicities and further stratified by age group (

Conclusions: Although HAs and NAs showed lower CaP incidence rates among the younger age group, HAs and NAs showed higher incidence rates for metastatic $\mathrm{CaP}$, possibly due to differences in screening participation compared to EAs. NAs and HAs in border states were also found to have a heavier burden of $\mathrm{CaK}$ than EAs. These findings indicate a need for greater understanding and exploration of the underlying causes of these disparities.

\section{MP10-20 Understanding the Time Course of Nephro- lithiasis Management}

RL Sur, SK Bechis, RM Shapiro, DG Kronenberg

UC San Diego School of Medicine

United States 
Introduction \& Objective: The rising incidence and cost of managing kidney stones places a major burden on the United States healthcare system. Given the calls for more cost-effective, value-based healthcare, there is a growing need for timely access to care. We sought to assess the time course of stone disease treatment from onset of symptoms to stone passage or definitive treatment to better characterize the current state of management. Materials and Methods: We conducted a retrospective study of patients treated for nephrolithiasis at a single, tertiary, academic center. Using the Electronic Medical Record, patients were selected who had symptomatic renal or ureteral stones, subsequent urology evaluation, and documented resolution of their stone(s) by stone passage or procedure. Primary outcomes were the time measured in days from initial presentation at the Emergency Department (ED) to procedure or stone passage. Secondary outcomes included time in days to confirmatory imaging, time in days to urology evaluation, and delays to procedure scheduling $>14$ days, designated "delay in treatment time." Descriptive statistical analyses were performed.

Results: 62 patients ( $40.3 \%$ female) between 08/2016-02/2017 met selection criteria. 48 patients $(77.4 \%)$ had ureteral stones, 11 $(17.7 \%)$ had renal stones, $3(4.9 \%)$ had both. Most patients were treated with ureteroscopic laser lithotripsy (56.5\%). Mean time from onset of symptoms to ED presentation was 2.0 days. Mean time from ED to clinic visit was 16.3 days. Mean time from clinic to procedure/stone passage and to confirmatory imaging was 33.3 and 36.4 days, respectively. Mean time from initial presentation to procedure/stone passage was 48.9 days. 38 patients had documented causes for delay in treatment. Of this cohort, 18 $(51.4 \%)$ were due to provider availability, $6(15.8 \%)$ due to contraindications to surgery, $3(7.9 \%)$ lost to follow up, and 2 $(5.3 \%)$ had positive urine cultures.

Conclusions: For patients with symptomatic nephrolithiasis at our institution, the mean time between initial presentation to resolution via procedure or recorded stone passage was about 7 weeks. However, delays in treatment occurred in $>50 \%$ of the cohort with provider availability as primary etiology. Obstacles contributing to provider availability such as payer/insurance status and geographic distance travelled might be better identified in a prospectively designed study.

MP10-21 Care Pathway Variation and Surgical Cost Measurement for Percutaneous Nephrolithotomy with Time-Driven Activity-Based Costing

I Metzler, D Issacson, M Usawachintachit, M Hudnall, DT Tzou, K Taguchi, T Chi

University of California San Francisco School of Medicine United States

Introduction \& Objective: The majority of health care cost data are based on charges or reimbursements, but these approaches fail to accurately represent the money hospitals actually spend to provide services. Time-driven activity-based costing (TDABC) provides a methodology to better understand true costs of providing a health care service and the variations in cost based on variance in timing. We applied TDABC analysis to a percutaneous nephrolithotomy (PCNL) care pathway.

Materials and Methods: The care pathway for PCNL was defined as the time from patient arrival to the preoperative area to discharge from post anesthesia care unit (PACU) to the hospital floor. Process maps were created with perioperative stakeholders to define the activities involved in PCNL. Stop-watch timing was

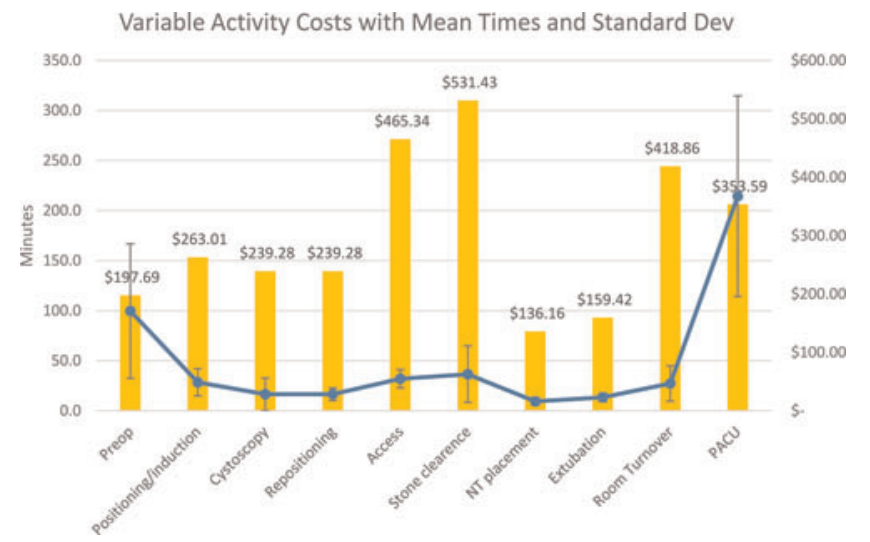

performed for eleven PCNL cases. The cost rate for attending urologists was calculated using publicly available salaries and estimated capacity, additional cost rates were estimated using a ratio of the averaged salaries for each staff position.

Results: The activities demonstrating the greatest time variance were PACU recovery, preoperative holding, and stone clearance $(214 \pm 100.3,99 \pm 67.2$ and $36 \pm 28.2$ minutes respectively). The activities with the least variation were anesthesia extubation, nephrostomy tube placement and patient repositioning (13 \pm 3.9 , $9 \pm 4.3,16 \pm 6.3$ minutes respectively). Total cost including disposables and overhead for the average PCNL was $\$ 5319$. Preoperative care accounted for $\$ 470(7 \%)$, intraoperative care accounted for $\$ 4351(84 \%)$ and post-operative care accounted for $\$ 353(9 \%)$ of the total. Thirty-seven percent of cost was attributable to disposables. Theoretical modeling with an attending performing all perioperative activities (rather than a resident) increased the human resource cost by $31 \%$. Removing the highest time outliers from each activity reduced the cost by $21 \%$.

Conclusions: Although utilizing the operative room is the most cost intensive activity for PCNL, variation exists during different phases of care. Opportunities for standardization to reduce cost is greatest in the pre- and post-operative areas. The TDABC methodology allows for estimation of true costs for PCNL and modeling of care pathway variation in order to understand health care costs and target areas for increased care value.

MP10-22 Defining the Costs of Reusable Flexible Ureteroscope Reprocessing Using Time-Driven Activity-Based Costing (TDABC)

D Isaacson, I Metzler, T Ahmad, S Rosenberg-Wohl, K Taguchi, M Usawachintachit, DT Tzou, BA Sherer, ML Stoller, T Chi

University of California San Francisco School of Medicine United States

Introduction \& Objective: Ureterorenoscopy with reusable fiber-optic or digital flexible ureteroscopes requires an infrastructure and dedicated technical staff to properly and promptly reprocess scopes in between cases. Optimal ureteroscope reprocessing balances thorough disinfection and avoidance of damage to fragile components while minimizing personnel-time invested, reducing use of disposable supplies and ensuring that scopes are ready for subsequent cases without causing expensive delays. This work aims to characterize the resources consumed in 


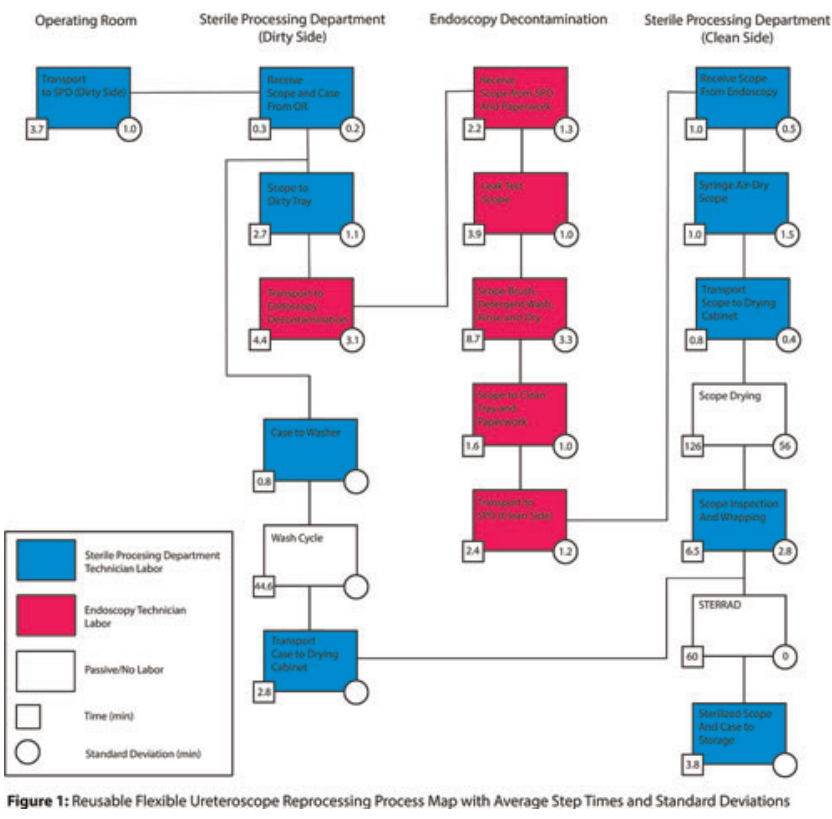

the reprocessing of reusable flexible ureteroscopes and to identify high-variance steps for potential cost-reductive improvements through applying time-driven activity-based costing (TDABC).

Materials and Methods: As part of a prospective time-motion study conducted at UCSF between July 2016 - April 2017, we followed reusable flexible ureteroscopes (URF-P6, Olympus, Tokyo, Japan) through reprocessing following the conclusion of ureteroscopy cases. We observed and timed individual steps in reprocessing to generate an overall process map. Steps requiring technician labor vs. passive and automated steps were noted. The variance for each step was calculated across all observations of that step. We measured disposables used and calculated their cost. Sterile processing department (SPD) and endoscopy personnel costs were estimated based on San Francisco averages for salary plus benefits.

Results: The overall process map was defined based on observation of ten flexible ureteroscopes and is detailed in Figure 1 Reprocessing averaged $229 \pm 74$ minutes, of which 47.7 minutes required labor by SPD/endoscopy personnel (\$36.88). Disposables averaged $\$ 45.35$ per scope reprocessed. Labor-dependent steps and automated steps (wash cycle, STERRAD) demonstrated low variance. The highest-variance step was scope drying at $126 \pm 56$ minutes, a value noted to be highly influenced by the time the SPD technician spent flushing the scope working channel with air and the position of the scope in the drying cabinet.

Conclusions: Flexible reusable ureteroscope reprocessing at UCSF requires $3.8 \pm 1.2$ hours and costs an average of $\$ 82.23$ per scope. Most reprocessing steps are performed efficiently and consistently, however scope drying time varies widely with preparation technique. Streamlined scope drying protocols may help speed reprocessing and prevent delays in availability.

MP10-23 Impact of race/ethnicity and socioeconomic status on stone characteristics: results from ReSKU - the Registry for Stones of the Kidney and Ureter

M Usawachintachit, DT Tzou, K Taguchi, BA Sherer, BD Duty, JD Harper, MD Sorensen, RL Sur, RM Sweet, ML Stoller, T Chi
University of California San Francisco School of Medicine United States

Introduction \& Objective: Socioeconomic status (SES) reflects a combination of education, income, and occupation for individuals. The relationship between SES and kidney stones remains unknown. This study was aimed to examine the association between SES and urinary stone presentation.

Materials and Methods: ReSKU - the Registry for Stones of the Kidney and Ureter - is an ongoing prospectively maintained, automated urinary stone registry centered at the University of California, San Francisco. From November 2015 to October 2016, all nephrolithiasis patients who presented with a urinary stone episode were enrolled. Patient demographics, clinical presentation, and stone characteristics are automatically extracted from the electronic health record and stored in a secure data warehouse. Gross household income for patient postal address was obtained from U.S. Census Bureau data. Patient factors were correlated to stone characteristics using univariate and multivariate models.

Results: 411 new stone patients were enrolled into ReSKU. The most common race/ethnicity was non-Hispanic White $(71.8 \%)$, following by Asian (17.8\%) and Hispanic White (10.8\%). Most patients reported their highest education level as "some college or college degree" (48.2\%), following by "high school or less" $(27.6 \%)$ and "graduate school" $(24.2 \%)$ and their mean annual income was $\$ 77,944.4 \pm 34,841.4$. Staghorn stone was present in $10.4 \%$ of patients. The overall mean total stone burden at presentation was $19.8 \pm 25.5 \mathrm{~mm}$.

No association existed between race/ethnicity and the presence of staghorn stone $(\mathrm{p}=0.47)$, or stone burden $(\mathrm{p}=0.29)$. Education level was significantly associated with the presence of staghorn stone $(p=0.47)$, or stone burden $(p=0.29)$. Education level was significantly associated with the presence of staghorn stone $(\mathrm{p}<0.01)$. Staghorn stones presented in $20.9 \%$ of patients with high school level or less. Similarly, mean stone burden was significantly higher in patients with high school level of education $(\mathrm{p}<0.01)$. Patients with the lowest income quartile presented with staghorn stones five times more often than the highest quartile $(16.8 \%$ versus $3 \%, \mathrm{p}<0.01)$, and income status was inversely correlated to total stone burden $(\mathrm{p}<0.01)$. Multivariate analysis demonstrated a strong correlation between education level and the presence of staghorn stone and total stone burden.

Conclusions: Lower education level and annual household income were strongly associated with higher total stone burden and the presence of staghorn stones, independent of race/ethnicity. Data collection in ReSKU is continuously ongoing to validate these findings.

MP10-24 Feasibility of Performing MRI Prostate before Prostate Biopsy in a District General Hospital in the UK

Y Phan, A Loh, A Anandakumar, S Umranikar, N Lynn

Royal Shrewsbury Hospital

United Kingdom

Introduction \& Objective: Men with abnormal digital rectal examination or raised PSA usually undergo transrectal ultrasound (TRUS) prostate biopsies. NICE guidelines do not recommend routine MRI prostate before prostate biopsy unless they have a previous negative prostate biopsy. However, all men with positive prostate biopsies will have MRI prostates. The recent 
publication of PROMIS (Prostate MR Imaging Study) trial suggests that MRI prostate can reduce unnecessary biopsies by a quarter and can improve detection of clinically significant cancer. In light of this, we would like to determine if performing MRI prostate before biopsy is likely to increase workload in our radiology department in a district general hospital in the UK.

Materials and Methods: Patients who underwent TRUS prostate biopsy between 3 Dec 2015 to 28 April 2016 were identified. Their data were analysed retrospectively. 1 year follow-up was chosen to see how many patients would have had MRIs.

Results: 173 patients were listed for prostate biopsies but only 158 patients had biopsies with an average age of 69.8 years old (range: 49-88 years old) and an average PSA of 48.1ug/l (range: 0.5-3283.1ug/l). 57 patients had a negative prostate biopsy during this period. $30 / 57$ patients did not have a MRI at all; 12/57 patients had a MRI after biopsy; $1 / 57$ patient had a MRI as an acute setting after biopsy to look for abscesses; and 14/57 patients had a MRI before biopsy.

Conclusions: In our study, 30/158 (19.0\%) did not have any MRI prostate in 1 year after their first prostate biopsy. However, it is possible that this group of patients will have a MRI prostate in the second year or later. If we were to perform a MRI prostate before TRUS prostate biopsy for all patients, it would increase $19.0 \%$ workload for our radiology department.

\section{MPS11: URETEROSCOPY III}

\section{MP11-1 Withdrawn}

MP11-2 Bilateral simultaneous ureteroscopic (BS-URS) approach in the management of Bilateral Urolithiasis is a safe and effective strategy in the contemporary era - Evidence from a Systematic Review

\section{R Geraghty, BP Rai, P Jones, A Pietropaolo, BK Somani \\ University Hospital Southampton NHS Foundation Trust} United Kingdom

Introduction \& Objective: Ureteroscopic treatment of urolithiasis has become safer and more effective in the contemporary era. This has opened the possibility of simultaneous ureteroscopy for bilateral stones, thus avoiding the need for further procedures. This review evaluates the current evidence base for bilateral simultaneous ureteroscopy (BS-URS).

Materials and Methods: A systematic review was conducted using studies identified by a literature search between January 1990 and June 2016. All English language articles reporting on outcomes of BS-URS for urolithiasis were included. Two reviewers independently extracted the data from each study. Data

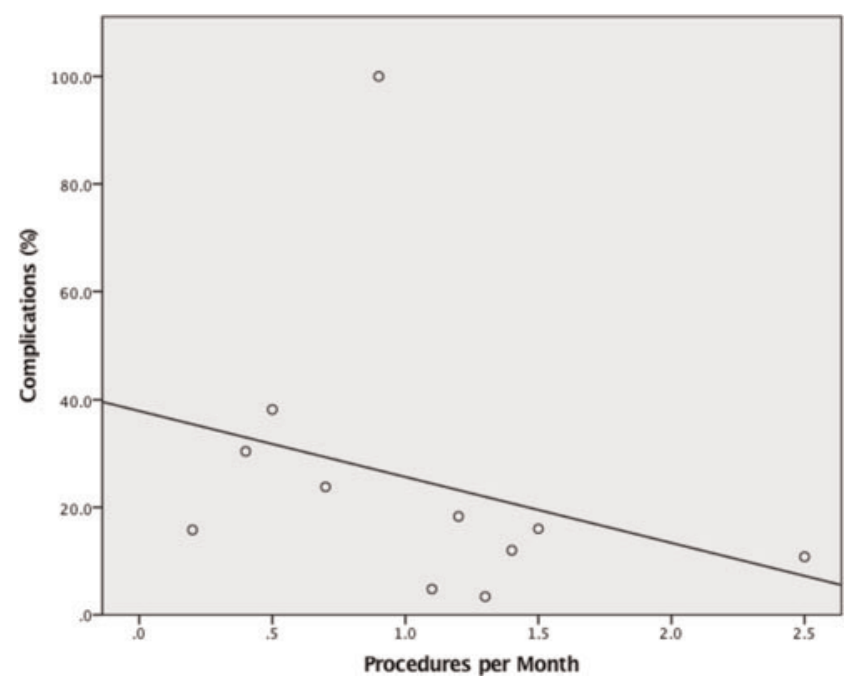

was split into two periods: period 1: 2003-2012 and period 2: 2013-2016, and analyzed using SPSS version 24.

Results: A total of 11 studies (491 patients) were identified from a literature search of 148 studies with mean age of 45 years and a male: female ratio of 2:1. Mean operative time was 69 minutes $(\mathrm{SD}= \pm 15)$. The initial and final stone free rate (SFR) was $87 \%$ and 93\% respectively. Post-operative stents were placed in $89 \%$ of patients with a mean hospital stay of 1.6 days $(S D= \pm 0.5)$. Overall there was a significant negative association between case volume (procedures per month) and complication rate $(\mathrm{p}=0.045)$. Mean hospital stay was significantly longer in period 1 (1.9 days, $\mathrm{SD}= \pm 0.5)$ than period $2(1.3$ days, $\mathrm{SD}= \pm 0.3)$ and complications were also significantly higher in the period $1(47 \%)$ compared to period $2(12 \%)(\mathrm{p}<0.001)$. There were 6 studies examining holmium laser (HL) lithotripsy and 3 examining pneumatic lithotripsy (PL). There were significantly more complications after PL than HL $(54.9 \%$ vs. $16.7 \%, \mathrm{p}=0.007)$ and a trend towards longer hospital stay $(1.98$ vs. $1.21, \mathrm{p}=0.041)$. However, there was no difference in SFR between these two fragmentation modalities.

Conclusions: Simultaneousbilateral ureteroscopic treatment of urolithiasis is safe and effective in the contemporary era. Safety is increased in centers with increased number of procedures performed and with the use of laser lithotripsy.

MP11-3 The role of preoperative ureteral stenting in retrograde intrarenal surgery.

S Yoo, H Yuk, S Lee, H Kim, C Jeong

Seoul National University Hospital Republic of Korea

Introduction \& Objective: During retrograde intrarenal surgery(RIRS), Placing the stent before surgery can theoretically expand the ureter to improve access and removal of stones. The purpose of this study was to investigate the effect of preoperative ureteral stenting on access and operation.

Materials and Methods: We retrospectively analyzed RIRS cases from October 2013 to June 2016. The patients were divided into two groups according to the preoperative ureteral stent insertion. The character of stone(size, number, density, location) and access sheath success rate, balloon dilatation rate, perioperative complication, operative time, Hospitalization time, Period with stent, postoperative UTI rate, stone free rate and additional treatment rate were analyzed. 
Table1. patient's characteristics

\begin{tabular}{|c|c|c|c|}
\hline Variables & Non-stented group $(n=49)$ & Prestented group $(n=73)$ & $p$-value \\
\hline Mean Age & $56.7 \pm 14.0$ & $54.6 \pm 14.6$ & 0.440 \\
\hline Mean BMI & $23.3 \pm 8.7$ & $24.4 \pm 2.9$ & 0.413 \\
\hline \multicolumn{4}{|l|}{ Gender } \\
\hline Male & $30(61.2 \%)$ & $41(56.2 \%)$ & \multirow[t]{2}{*}{0.579} \\
\hline Female & $19(38.7 \%)$ & $32(43.8 \%)$ & \\
\hline DM & $11(22.4 \%)$ & $17(23.3 \%)$ & 0.914 \\
\hline HTN & $15(30.6 \%)$ & $30(41.1 \%)$ & 0.239 \\
\hline CVA & $1(2.0 \%)$ & $4(5.5 \%)$ & 0.348 \\
\hline CVD & $5(10.2 \%)$ & $6(8.2 \%)$ & 0.707 \\
\hline Preoperative $\mathrm{Hb}$ & $13.5 \pm 1.9$ & $12.5 \pm 3.9$ & 0.570 \\
\hline Preoperative $\mathrm{Cr}$ & $0.97 \pm 0.29$ & $0.97 \pm 0.62$ & 0.966 \\
\hline Preoperative GFR & $68.18 \pm 32.87$ & $79.01+25.12$ & 0.042 \\
\hline \multicolumn{4}{|l|}{$\begin{array}{l}\text { Modified S-ReSC } \\
\text { score }\end{array}$} \\
\hline \multicolumn{4}{|l|}{ Laterality } \\
\hline Right & $24(49.0 \%)$ & $32(43.8 \%)$ & \multirow[t]{2}{*}{0.461} \\
\hline Left & $25(51.0 \%)$ & $41(56.2 \%)$ & \\
\hline Density of stones(HU) & $837.9 \pm 399.6$ & $915.1 \pm 350.7$ & 0.262 \\
\hline Stone max diameter & $14.47 \pm 8.43$ & $15.27 \pm 10.85$ & 0.662 \\
\hline Number of stone & $1.90 \pm 1.39$ & $2.33 \pm 2.35$ & 0.206 \\
\hline 1 & $28(57.1 \%)$ & $35(47.9 \%)$ & \multirow[t]{3}{*}{ NS } \\
\hline 2 & $9(18.4 \%)$ & $17(23.3 \%)$ & \\
\hline 23 & $12(24.5 \%)$ & $21(28.8 \%)$ & \\
\hline
\end{tabular}

Table2. Perioperative outcomes

\begin{tabular}{|l|l|l|l|l|}
\hline Variables & $\begin{array}{l}\text { Non-stented group } \\
(\mathrm{n}=49)\end{array}$ & $\begin{array}{l}\text { Prestented group } \\
(\mathrm{n}=73)\end{array}$ & $\mathrm{p}$-value & OR \\
\hline Intraop. complication & $0(0 \%)$ & $3(4.1 \%)$ & 0.151 & 0.588 \\
\hline Intraop. transfusion & $0(0 \%)$ & $1(1.4 \%)$ & 0.411 & 0.595 \\
\hline $\begin{array}{l}\text { Assess sheath } \\
\text { successful insertion }\end{array}$ & $43(87.8 \%)$ & $71(97.3)$ & 0.038 & 4.953 \\
\hline Balloon dilatation & $5(10.2 \%)$ & $2(2.7 \%)$ & 0.082 & 0.248 \\
\hline Postop. UTI & $3(6.1 \%)$ & $6(8.2 \%)$ & 0.664 & 1.373 \\
\hline Stone free rate Omm & $34(69.4 \%)$ & $53(72.6 \%)$ & 0.700 & 1.134 \\
\hline Stone free rate <4mm & $43(87.8 \%)$ & $65(89.0 \%)$ & 0.827 & 1.169 \\
\hline Additional Treatment & $4(8.2 \%)$ & $6(8.2 \%)$ & 0.991 & 1.007 \\
\hline Operative Time (min) & $68.8 \pm 40.8$ & $65.2 \pm 46.4$ & 0.664 & $\mathrm{NS}$ \\
\hline Hospitalization period(day) & $1.3 \pm 0.8$ & $1.2 \pm 0.8$ & 0.678 & $\mathrm{NS}$ \\
\hline $\begin{array}{l}\text { Postoperative Period with } \\
\text { stent (day) }\end{array}$ & $14.0 \pm 8.2$ & $15.8 \pm 15.8$ & 0.471 & $\mathrm{NS}$ \\
\hline
\end{tabular}

Table3. Operation times classified by stone size

\begin{tabular}{|l|l|l|l|l|l|}
\hline \multirow{2}{*}{$\begin{array}{l}\text { Size of } \\
\text { Stone }(\mathrm{cm})\end{array}$} & \multicolumn{2}{l|}{ Non-stented } & \multicolumn{2}{l|}{ Prestented } & \multirow{2}{*}{-value } \\
\cline { 2 - 5 } & $\mathrm{n}$ & Operative time $(\mathrm{min})$ & $\mathrm{n}$ & Operative time(min) & \\
\hline$<1 \mathrm{~cm}$ & 11 & $48.6 \pm 24.5$ & 16 & $40.3 \pm 23.3$ & 0.019 \\
\hline $1-2 \mathrm{~cm}$ & 32 & $67.6 \pm 34.2$ & 48 & $64.2 \pm 48.4$ & 0.777 \\
\hline$\geq 2 \mathrm{~cm}$ & 6 & $128.5 \pm 46.9$ & 9 & $93.9 \pm 52.2$ & 0.214 \\
\hline
\end{tabular}


Introduction \& Objective: The target of our study defines to clinical features which affect the stone-free rates(SFR) of the procedures.

Materials and Methods: As a retrospectively; 164 flexible ureteroscopy procedures for kidney stone treatment was regarded between December 2012 with October 2016 at the single centre. Stone-Free status was controlled with the KUB or CT at the first month of the procedures. The success rate was confirmed as the absence of stone fragments or clinical insignificant residual fragments $<4 \mathrm{~mm}$. Clinical parameters related to the patient, stone and operation were recorded. Multivariate Logistic Regression analysis with forwarding selection was used to determine independent factors

Results: The mean total stone volume(TSV) was $364.6 \mathrm{~mm}^{3}$, Hounsfield Unit was 1099.3, Operative time was 75.4 min and the overall stone-free rate was 120 (\%73.2). Stone size, Hounsfield Unit, Fluoroscopy time, Operative time and Stone count were found significant factors to the stone-free status of patients. Additionally; reduced multivariate analyses showed that Stone volume $(\mathrm{p}<0.001)$, Operative time (p:0.002), Hounsfield Unit $(\mathrm{p}=0.004)$ are the three independent predictors of the success rate of procedures. High stone volume, longer operative time and stiffer stone have led to decreased success rate of Furs.

Conclusions: Our data indicate that Preoperative findings such as Stone Volume, Hounsfield Unit and Operative time affect stone clearance rate. Amongst the main factors in Furs, Total stone volume has the strongest impact on the SFR. Best results were obtained for Small, Soft and Short time fragmented (3s Rules) Stones.

MP11-7 Sequential balloon dilation Vs direct access sheath insertion pre flexible ureteroscopy in non pre-stented patients: Retrospective comparative study

AM Elmekresh, DM Masten, GL Machen, PS Lowry, MM El Tayeb, L Tsai

Texas A\&M College of Medicine/Baylor Scott and White United States

Introduction \& Objective: To evaluate the efficacy and the primary outcome associated with sequential ureteral balloon dilation (SBD) prior to ureteral access sheath (UAS) insertion in non-pre-stented patients.

Materials and Methods: After IRB approval, a retrospective review of non pre stented patients undergoing ureteroscopy for kidney, and proximal ureteral stones between February 2014 and September 2016 was performed. Cook Flexor 12/ 14 FR UAS was used in all cases. In our institution, one surgeon sequentially dilates the entire ureter using the 12 $\mathrm{FR} / 4 \mathrm{~cm}$ cook ureteral balloon prior to UAS insertion; this technique is only used in non-pre-prestented ureter. Evaluation of the primary outcome of using SBD vs direct UAS insertion use in the terms success of UAS insertion, duration of the procedure, intra/post-operative complications, ER return rate and stone free rate (SFR) at 6 weeks. Statistics were performed using SPSS and included means with standard deviations, chi squared tests, and independent t-tests when appropriate.

Results: A total of 124 patients were identified, 63 patients in group (A) who had SBD versus 61 patients in group (B) who had direct UAS insertion. Patients' demographics are combarable with no statistifical significance between both cohorts. Mean procedure time was 58.1 vs 63.9 mins in group (A) and (B), respectively, $(\mathrm{P}=0.321)$. UAS insertion was successfully inserted in $100 \%$ in group (A) vs $81 \%$ in group $(B),(p=0.01)$. Tear of the ureter was encountered in 4 patients $(6.3 \%)$ in group (A), $(\mathrm{p}=0.039)$. Post-operative complications in terms of (hematuria, pyelonephritis, and flank pain) were encountered among $9 \mathrm{~Pa}-$ tients (14.7\%) vs 2 (3.17) \% in groups (A) and (B), respectively, $(\mathrm{p}=0.023)$. The ER return was encountered in 14 patients $(22.9 \%)$ vs $9(14.2 \%)$ in groups (A) and (B), respectively, $(\mathrm{P}=0.619)$. The SFR was $96.7 \%$ vs $96.8 \%$ in groups $(\mathrm{A})$ and $(\mathrm{B})$, respectively, $(\mathrm{P}=0.974)$. Utilization of the UAS and postoperative complication rate were statistical significant among patients who underwent $\operatorname{SBD}(\mathrm{p}=0.01 \& \mathrm{p}=0.023$, respectively). While the procedure time, ER return rate and the SFR were of no statistical significance.

Conclusions: Sequential balloon dilation followed by UAS and Flexible ureteroscopy for stone removal was associated with more success in UAS insertion. However, it was associated with higher rate of intra/post-operative complications. There was no difference in SFR between both cohorts. Therefore, SBD of the ureter has no superiority.

MP11-8 Ureterorenoscopy and Laser Lithotripsy for Upper Tract Stones Using " Stone Dusting" Technique

MA Hussein, I Saad, A Abdalla, S Sadek

Egyptian military medical services

Egypt

Egypt

Introduction \& Objective: Laser settings during ureterorenoscopy (URS) and laser lithotripsy (LL) have recently been

\begin{tabular}{|c|c|}
\hline \multicolumn{2}{|c|}{ Patients $n=60$} \\
\hline Mean Age in Years \pm SD (range) & $50.5 \pm 13.9(22-79)$ \\
\hline $\begin{array}{l}\text { Gender } \\
\text { - Male }(\%) \\
\text { - Female }(\%)\end{array}$ & $\begin{array}{l}20(33) \\
40(67)\end{array}$ \\
\hline Mean $\mathrm{BMI}$ in $\mathrm{kg} / \mathrm{m}^{2}$ (range) & $28.2 \pm 3.8(20-35)$ \\
\hline \begin{tabular}{|l} 
Stone Site \\
- Ureter (\%) \\
- Pelvis (\%) \\
- Calyx (\%) \\
- Pelvicalyceal (\%)
\end{tabular} & $\begin{array}{l}43(71.7) \\
10(16.7) \\
2(3.3) \\
5(8.3)\end{array}$ \\
\hline $\begin{array}{l}\text { Stone Multiplicity } \\
\text { - Single }(\%) \\
\text { - Multiple (\%) }\end{array}$ & $\begin{array}{l}50(83.3) \\
10(16.7)\end{array}$ \\
\hline $\begin{array}{l}\text { Mean Stone Size in } \mathrm{cm} \pm \mathrm{SD} \\
\text { (range) }\end{array}$ & $1.6 \pm 0.55(0.5-3)$ \\
\hline Mean HFU \pm SD (range & $794.2 \pm 296.6(290-1800)$ \\
\hline $\begin{array}{l}\text { Mean operative time in } \min \pm \mathrm{SD} \\
\text { (range) }\end{array}$ & $58.5 \pm 16.2(30-100)$ \\
\hline $\begin{array}{l}\text { Mean total energy delivered in J } \pm \\
\text { SD (range) }\end{array}$ & $6836.2 \pm 3018(1002-19314)$ \\
\hline $\begin{array}{l}\text { Stenting } \\
\text { - DJ (\%) } \\
\text { - Ureteric Catheter (\%) }\end{array}$ & $\begin{array}{l}38(63.3) \\
22(36.7)\end{array}$ \\
\hline $\begin{array}{l}\text { Stone Free } \\
\text { - Yes (\%) } \\
\text { - No (\%) }\end{array}$ & $\begin{array}{l}55(91.7) \\
5(8.3)\end{array}$ \\
\hline $\begin{array}{c}\text { Stone Migration } \\
\text { - Yes (\%) } \\
\text { - No }(\%)\end{array}$ & $\begin{array}{l}58(96.7) \\
2(3.3)\end{array}$ \\
\hline $\begin{array}{r}\text { Complications } \\
\text { - Yes (\%) } \\
\text { - No (\%) } \\
\end{array}$ & $\begin{array}{l}11(18.3) \\
49(81.7)\end{array}$ \\
\hline
\end{tabular}


increasingly studied aiming at enhancing stone fragmentation while minimizing complications. Studies have reported that low energy-high frequency (LEHF) settings of LL may produce smaller stone fragments i.e. stone dusting with less retroplusion. We aim to evaluate the safety, efficacy and complications of URS with Holmium:YAG LL using LEHF “dusting" settings for upper urinary tract (UUT) stones.

Materials and Methods: This was a prospective study conducted at Maadi Military and Cairo University Hospitals from April 2015 to December 2015. It included patients with single or multiple UUT (mid and upper ureter, pelvic, and calyceal) radioluscent or radio-opaque stones $<3 \mathrm{~cm}$ in maximum diameter. Patients with BMI $>35$ were excluded. URS was done using semirigid or flexible ureteroscopes depending on stone site. LL was set to low energy $(0.5 \mathrm{~J})$ and high frequency $(20 \mathrm{~Hz})$ aiming at stone dusting. No stone stabilization devices were used. Stone free (SF) was defined as having no fragments or fragments $\leq 2 \mathrm{~mm}$ on non-contrast $\mathrm{CT}$ at 4 weeks. Perioperative parameters, SF rate (SFR) and complications were analyzed.

Results: Our study included 60 patients. Table 1 shows perioperative parameters. On analysis patients who were SF had a significantly lower BMI and smaller stone size $(27.8 \mathrm{~kg} / \mathrm{m} 2$ vs. $32.5 \mathrm{~kg} / \mathrm{m} 2$ and $1.5 \mathrm{~cm}$ vs. $2.3 \mathrm{~cm}$, respectively; p0.05). Additionally, 96\% (48/50) of patients with single stones were SF vs. $70 \%(7 / 10)$ of patients with multiple stones $(p<0.05)$. Operative time correlated with age, stone number, size and site, and HFU $(\mathrm{p}<0.05)$. The need for DJ stenting correlated with stone size and operative time $(1.77 \mathrm{~cm}$ vs. $1.17 \mathrm{~cm}$ and $62.7 \mathrm{~min}$ vs. $51.2 \mathrm{~min}$, respectively; $\mathrm{p}<0.05)$ but not stone site. The occurrence of complications did not correlate with age, BMI, stone size and site, or operative time. Total energy delivered correlated with stone size and HFU $(\mathrm{p}<0.05)$.

Conclusions: URS with LL using LEHF "stone dusting" setting is safe and effective in management of UUT stones. It achieves a SFR of $>90 \%$ with an acceptable complication rate and minimal stone migration. Further comparative studies are needed to compare stone dusting to other fragmentation techniques, namely basketing.

MP11-9 Endoscopic recognition of Kidney Stones: The new challenge for urologist?

V Estrade, I Jour, M Daudon, O Traxer

\section{CH ANGOULEME/ FRANCE}

France

Introduction \& Objective: To assist the urologists in recognizing different types of kidney stones intra-operatively through their morphological classification prior to their fragmentation by laser photo-vaporisation. Aim of this work is to also validate a sheet of endoscopic morphological classification of kidney stones in parallel to their corresponding microscopic analysis.

Materials and Methods: Between June 2015 and December 2016, 250 kidney stones were visually identified through a combined approach by a urologist and a biologist, the former providing intra-operative imaging of the stones' surface while the latter performing microscopic analysis of stone sections. All the stones were treated with Laser ureterorenoscopy.

Results: Validation of a sheet for the morphological endoscopic recognition (Annexes 1 to 3) of pure (Ia Ib Id Ie / IIa IIb / IIIb / IVA1 IVa2 IVb IVc IVd / Va) and mixed stones (IIIa / Ia / (IIbIVa) c / (IIb / IVa)i along with crystalline conversion (IIb / Ia Conclusions: With the help of this intraoperative endoscopic sheet, urologists can now immediately recognize the type of
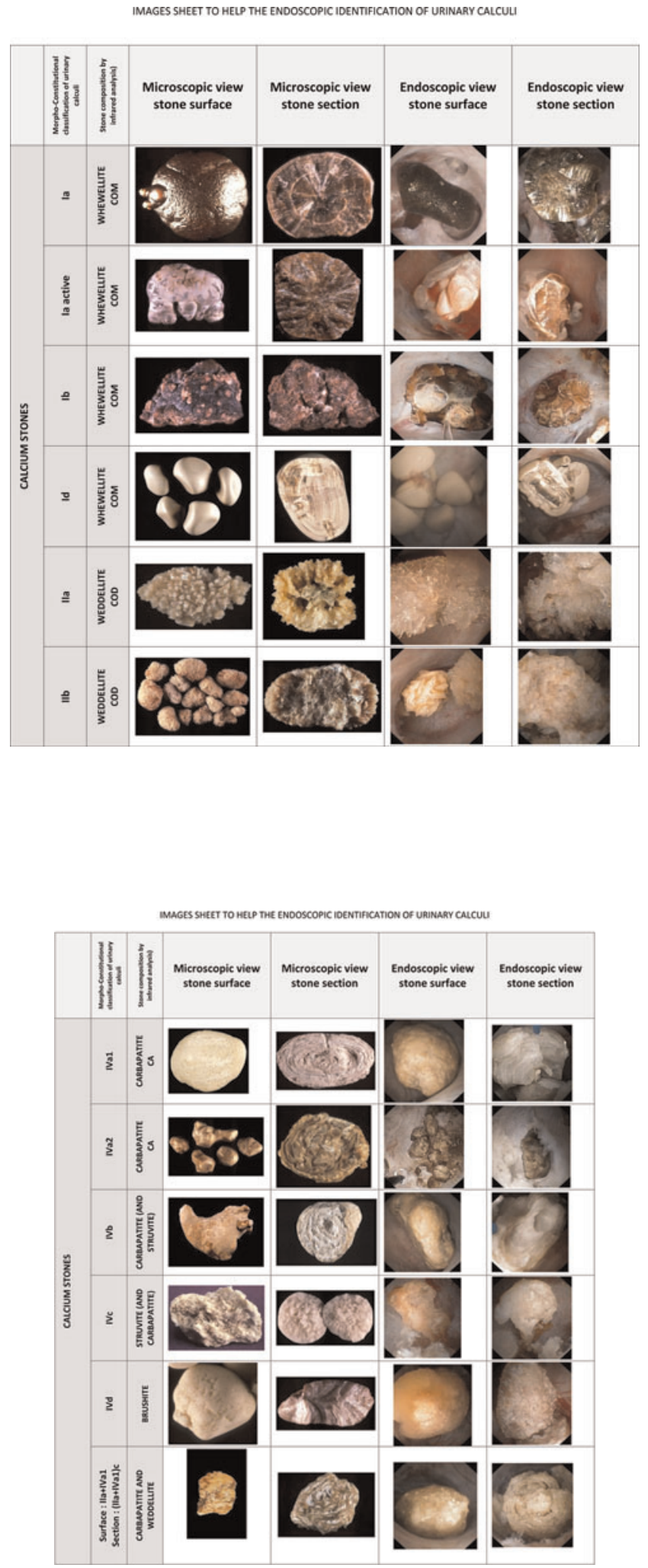

kidney stones during ureterorenoscopy prior to stone vaporisation. This intraoperative inspection must be complemented by fragments and/or powder analysis by the biologist, who would systematically conduct an additional infrared spectrophotometric analysis. 
IMAGES SHEET TO HELP THE ENDOSCOPIC IDENTIFICATION OF URINARY CALCUL

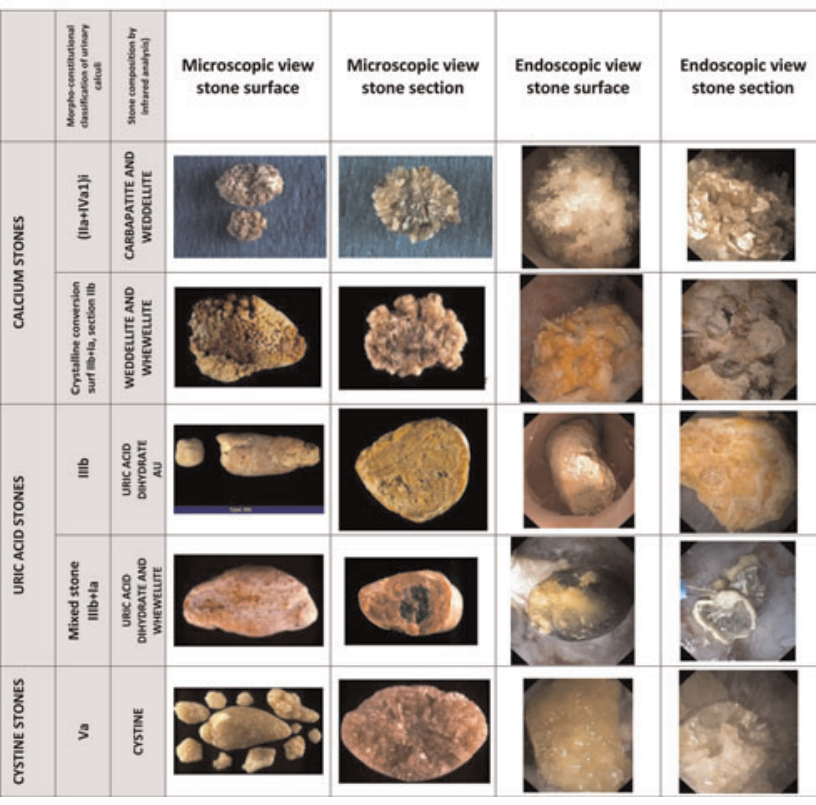

MP11-10 Urolithiasis after bariatric surgery: a five-year follow-up study

A Ostrowski, R Williams, D Thiel, R Pak

Mayo Clinic Florida

United States

Introduction \& Objective: Current bariatric surgery trends, including Roux-en-Y gastric bypass (RYGB), laparoscopic gastric banding (LGB), and laparoscopic sleeve gastrectomy (LSG) have elicited discussion as to their effects on urolithiasis development. A previous study at our center prospectively evaluated 47 consecutive patients receiving bariatric surgery in our comprehensive care bariatric practice, demonstrating altered select urinary parameters at 1-year follow-up, like oxalate and citrate, that are associated with increased risk for urolithiasis. We seek to provide a 5-year follow-up evaluation for incidence of urolithiasis formation in this patient cohort.

Materials and Methods: Our patient population includes all patients previously enrolled in a prospective study conducted at Mayo Clinic Jacksonville to evaluate 24-hour urine profiles following bariatric surgery in a modern comprehensive care bariatric clinic. A chart review was conducted on these patients to evaluate for new diagnosis of urolithiasis. No exclusion criteria were used. Results: A total of 4 out of 47 patients $(8.5 \%)$ were newly diagnosed with urolithiasis events after their bariatric surgery. Of these 4, 3 patients had shown decreases in their 24-hour urine citrate levels (one patient did not have follow-up for 24-urine citrate), and 2 had definitive increase in their urinary oxalate, with the other 2 the same or only slightly lower. Of those patients with new urolithiasis, 2 had RYGB, 1 had LGB, and 1 had a LSG. Of the 47 patients in this cohort, $6(12.8 \%)$ had developed microscopic hematuria during their follow-up period, of which 2 were confirmed to have new urolithiasis.

Conclusions: This 5-year follow-up study suggests that bariatric surgery is associated with an increased risk of urolithiasis, especially when 24-hr urine citrate and oxalate levels are altered in a fashion associated with urolithiasis risk. Between the types of surgical procedures observed, there does not appear to be any difference in risk of urolithiasis formation.

\section{MP11-11 Ureteral Access Sheath Use and Incidence of Hydronephrosis}

BJ Otto, R Terry, JM Shields, VG Bird

\section{SUNY Downstate}

United States

Introduction \& Objective: Ureteral access sheaths (UAS) are commonly used during retrograde ureteroscopic treatment of nephrolithiasis. UAS facilitate multiple ureteroscope passages, stone extraction, and irrigation/flow. Reports describe a spectrum of ureteral injuries; the long-term consequences of which are unknown. Potential risk of ureteral injury and stricture formation from UAS has been debated. We reviewed our UAS experience, with long-term follow-up, to identify risks and predictors of postoperative hydronephrosis.

Materials and Methods: 527 consecutive flexible ureteroscopic lithotripsy procedures, with use of a UAS, between September 2010 and March 2016 at a tertiary care center were reviewed. A single surgeon performed all ureteroscopic lithotripsies utilizing $12 / 14 \mathrm{Fr}$ UAS. We evaluated patient, stone, and operative factors, and post-operative imaging after stent/nephrostomy tube removal. Results: UAS were successfully placed in 502(95.3\%) procedures (Table 1). Mean aggregate stone size was $15.4 \mathrm{~mm} .272(51.6 \%)$ patients had pre-operative stenting. 38(7.2\%) patients required

Table 1

Demographics, OR, Stone, Ureteral Access Sheath, and Follow-up Details

\begin{tabular}{|c|c|c|}
\hline \multicolumn{2}{|c|}{ Demographics } & Value (\%) \\
\hline \multicolumn{2}{|l|}{ Total number of patients } & 527 \\
\hline \multicolumn{2}{|l|}{ Male } & $277(52.6)$ \\
\hline \multicolumn{2}{|l|}{ Caucasian } & $463(87.9)$ \\
\hline \multicolumn{2}{|l|}{ Age, mean $\pm S D$ (years) } & $52.4 \pm 15.6$ \\
\hline \multicolumn{2}{|l|}{ BMI, mean $\pm S D\left(k g / m^{\wedge} 2\right)$} & $31 \pm 9.3$ \\
\hline \multicolumn{2}{|l|}{ ASA Score, mean \pm SD } & $2.5 \pm 0.7$ \\
\hline \multicolumn{3}{|c|}{ Stone History and Characteristics } \\
\hline \multicolumn{2}{|c|}{ Patients with history of nephrolithiasis } & $383(72.7)$ \\
\hline \multicolumn{2}{|c|}{ Patients with prior same-sided stone procedure } & $312(59.2)$ \\
\hline \multicolumn{2}{|c|}{ Stone Size, mean $\pm S D(\mathrm{~mm})$} & $15.4 \pm 9.8$ \\
\hline \multicolumn{2}{|l|}{ Patients with multiple stones } & $263(49.9)$ \\
\hline \multicolumn{3}{|l|}{ Stone Location } \\
\hline \multicolumn{2}{|l|}{ Ureter Only } & $110(20.9)$ \\
\hline \multicolumn{2}{|l|}{ Ureter and Kidney } & $80(15.2)$ \\
\hline \multicolumn{2}{|l|}{ Kidney Only } & $335(63.6)$ \\
\hline \multicolumn{2}{|c|}{$\begin{array}{l}\text { Patients with pre-operative stent } \\
\text { OR Details }\end{array}$} & $272(51.6)$ \\
\hline OR Details & & \\
\hline \multicolumn{2}{|c|}{ Operative Time, mean $\pm \mathrm{SD}$ (minutes) } & $69.6 \pm 35.9$ \\
\hline \multicolumn{3}{|c|}{ Ureteral Access Sheaths Deployment } \\
\hline \multicolumn{2}{|l|}{ Successful deployment } & $502(95.3)$ \\
\hline \multicolumn{2}{|c|}{ Partial deployment (past level of iliac vessels) } & $15(3.0)$ \\
\hline \multirow{2}{*}{$\begin{array}{l}\text { Failed } \\
\text { deployment }\end{array}$} & Unable to pass ureteral orifice & $8(1.6)$ \\
\hline & \begin{tabular}{|l|} 
Unable to pass level of iliac vessels \\
\end{tabular} & $17(3.4)$ \\
\hline \multicolumn{3}{|l|}{ Post-operative Details } \\
\hline Patients needing additiona & procedures & $73(13.9)$ \\
\hline Time to first imaging, mear & $\pm S D$ (days) & $140.8(207.9)$ \\
\hline Total imaging follow-up, m & an $\pm S D$ (days) & $497.5(475.9)$ \\
\hline Follow-up & & \\
\hline Range (days) & & $12-1958$ \\
\hline Median (days) & & 328 \\
\hline Patients with $\leq 1$ year follo & -up & $285(54.1)$ \\
\hline Patients with $1-2$ years foll & w-up & $101(19.2)$ \\
\hline Patients with 2-3 years foll & w-up & $68(12.9)$ \\
\hline Patients with $3-4$ years foll & w-up & $52(9.9)$ \\
\hline Patients with $4-5$ years foll & w-up & $21(4.0)$ \\
\hline Post-operative persisten & Hydronephrosis & \\
\hline Patients with post-operativ & persistent hydronephrosis & $22(4.2)$ \\
\hline & Mild & $16(72.7)$ \\
\hline Degree of hydronephrosis & \begin{tabular}{|l|} 
Moderate \\
\end{tabular} & $5(22.7)$ \\
\hline & Severe & $1(4.6)$ \\
\hline Symptomatic & & $3(13.6)$ \\
\hline Resolved & & $13(59.1)$ \\
\hline Persistent hydronephrosis & vithout obstruction & $3(13.6)$ \\
\hline Persistent hydronephrosis & vith obstruction & $6(37.5)$ \\
\hline Time to resolution, mean \pm & $\mathrm{D}$ (days) & $267 \pm 159$ \\
\hline
\end{tabular}




\begin{tabular}{|c|c|c|c|c|}
\hline . & & $\begin{array}{l}\text { Hydronephrosis } \\
(n=505)\end{array}$ & $\begin{array}{l}\text { No Hydronephrosis } \\
(n=22)\end{array}$ & $\begin{array}{l}\mathrm{p}- \\
\text { value }\end{array}$ \\
\hline \multicolumn{5}{|l|}{ Clinical/Demographic } \\
\hline \multicolumn{2}{|l|}{ Age (mean, SD) } & $52(15.7)$ & $50(13.8)$ & 0.527 \\
\hline \multirow[t]{2}{*}{ Gender (No., \%) } & Female & $239(95.6)$ & $11(4.4)$ & \multirow[t]{2}{*}{0.806} \\
\hline & Male & $266(96.0)$ & $11(4.0)$ & \\
\hline \multirow[t]{2}{*}{ Race (No., \%) } & Caucasian & $446(96.3)$ & $17(3.7)$ & \multirow[t]{2}{*}{0.105} \\
\hline & Non-caucasian & $57(91.9)$ & $5(8.1)$ & \\
\hline \multicolumn{2}{|l|}{ BMI (mean, SD) } & $31(9.2)$ & $31.2(11.4)$ & 0934 \\
\hline \multirow{2}{*}{$\begin{array}{l}\text { ASA score group } \\
\text { (No., \%) }\end{array}$} & 1 or 2 & $222(95.7)$ & $10(4.3)$ & \multirow[t]{2}{*}{\begin{tabular}{|l|l|}
0.89 \\
0.89
\end{tabular}} \\
\hline & 3 or 4 & $283(95.9)$ & $12(4.1)$ & \\
\hline \multirow{2}{*}{$\begin{array}{l}\text { History of ipsilateral } \\
\text { stone (No., \%) }\end{array}$} & No & $138(95.8)$ & $6(4.2)$ & \multirow[t]{2}{*}{0.996} \\
\hline & Yes & $138(95.8)$ & $16(4.2)$ & \\
\hline \multirow{2}{*}{$\begin{array}{l}\text { History of ipsilateral } \\
\text { stone procedure }\end{array}$} & No & $201(96.6)$ & $7(3.4)$ & \multirow[t]{2}{*}{0.423} \\
\hline & Yes & $297(95.2)$ & $15(4.8)$ & \\
\hline \multicolumn{5}{|l|}{ Stone/Operative Details } \\
\hline \multicolumn{2}{|l|}{ Stone size (mean, SD) } & $15.4(9.8)$ & $15(10.5)$ & 0.851 \\
\hline \multirow[t]{2}{*}{ Stone number } & Single & $253(96.2)$ & $10(3.8)$ & \multirow[t]{2}{*}{0.831} \\
\hline & Multiple & $252(95.8)$ & $11(4.2)$ & \\
\hline \multirow[t]{3}{*}{ Stone location } & Kidney & $320(95.5)$ & $15(4.5)$ & \multirow[t]{3}{*}{0.574} \\
\hline & $\begin{array}{l}\text { Kidney and } \\
\text { ureter }\end{array}$ & $77(97.5)$ & $2(2.5)$ & \\
\hline & Ureter & $107(97.3)$ & $3(2.7)$ & \\
\hline \multirow[t]{2}{*}{ Pre-operative stent } & No & $243(96.4)$ & $9(3.6)$ & \multirow[t]{2}{*}{0.496} \\
\hline & Yes & $260(95.2)$ & $13(4.8)$ & \\
\hline \multirow{2}{*}{$\begin{array}{l}\text { Successful UAS } \\
\text { placement }\end{array}$} & No & $23(92.0)$ & $2(8.0)$ & \multirow[t]{2}{*}{0.327} \\
\hline & Yes & $482(96.0)$ & $20(4.0)$ & \\
\hline \multicolumn{2}{|c|}{ Operative time (mean, SD) } & $70(35.9)$ & $557(33.2)$ & 0.202 \\
\hline
\end{tabular}

additional procedures for residual stone. Post-operatively, imaging revealed $22(4.2 \%)$ patients with persistent hydronephrosis: mild$16(72.7 \%)$, moderate-5(22.7\%), severe-1(4.6\%); of this group, hydronephrosis resolved without intervention in $13(59.1 \%)$ patients. Mean resolution time of hydronephrosis on interval imaging was 267days. Post-operative functional imaging revealed $3(13.6 \%)$ patients with persistent hydronephrosis without obstruction; 6(37.5\%) patients had persistent hydronephrosis with evidence of obstruction as well as evidence of ipsilateral obstruction on pre-operative imaging. Additional analysis found no factors associated with postoperative hydronephrosis (Table 2).

Conclusions: UAS use during ureteroscopic lithotripsy was successful in most patients. Prevalence of post-operative hydronephrosis is low, with the majority of hydronephrosis resolving without intervention. Use of $12 / 14 \mathrm{Fr}$ UAS is not associated with ipsilateral ureteral obstruction.

MP11-12 Analysis of ureter stone in ureteroscopic lithotripsy: A single institution experience.

T Oh, J Lee, S Park, I Seo

Republic of Korea

Introduction \& Objective: The aim of this study was to compare the characteristics of previous ureteral stones in patients who underwent ureteroscopic lithotripsy (URSL).

Materials and Methods: From January 2010 to December 2014, patients who underwent URSL after diagnosing ureteral stones at our hospital were enrolled. Patients who did not have follow-up, or who did not have any evidence of stones free were examined through general urinalysis, plain radiography (KUB, DIP), and computed tomography were excluded. Patients with preoperative renal stones, and who underwent flexible ureterosopic lithotripsy were excluded. A total of 450 patients were retrospectively studied. Stone free was defined as the absence of any residual disease, or a remnant of less than $3 \mathrm{~mm}$ without any symptoms. Results: The mean age of the patients was $54.9 \pm 14.6$ years and the mean stone size was $7.63 \pm 4.3 \mathrm{~mm}$. The male to female ratio was $275(61.1 \%)$ to $175(38.9 \%)$. The stone composition was calcium oxalate monohydrate $(201,44.7 \%)$, calcium oxalate
Table 1. Comparison of uric acid and Non-uric acid stone

\begin{tabular}{lccc}
\hline & Uric acid (112) & Non-uric acid (338) & P-value \\
\hline Sex (Male:Female) & $86: 26$ & $188: 150$ & 0.000 \\
Laterality (Rt:Lt.) & $51: 61$ & $163: 175$ & 0.341 \\
Size (maximal diameter, cm) & $8.21 \pm 4.22$ & $7.45 \pm 4.35$ & 0.283 \\
Hounsfield unit (HU) & $495.96 \pm 247.44$ & $870.89 \pm 500.32$ & 0.000 \\
URSL success rate (\%) & $101(90.2)$ & $319(94.4)$ & 0.097 \\
Recurrence rate (\%) & $32(28.6)$ & $55(16.3)$ & 0.004 \\
Mean durations until recurrence & $18.9 \pm 13.0$ & $25.8 \pm 15.6$ & 0.031 \\
(month) & & & \\
\hline
\end{tabular}

URSL, ureteroscopic lithotripsy

mono / dihydrate $(73,16.2 \%)$, calcium phosphate $(15,3.3 \%)$, uric acid $(112,24.9 \%)$, carbonite apatite $(38,8.4 \%)$, and the proportion of uric acid is higher than in the past. The stone free rate of URSL was $93.3 \%$ (420/450). The recurrence rate was $19.3 \%$ (87/ $450)$ and the mean recurrence time was $23.3 \pm 14.9$ months. As a result of the comparison between uric acid and non-uric acid stone, the incidence in men was significantly higher in uric acid group $(p=0.000)$. There was a statistically significant difference in the recurrence rate, duration of recurrence, and Hounsfield unit $(\mathrm{p}<0.05)$ between uric acid and non-uric acid stone.

Conclusions: The high success rate results support URSL as the effective treatment for ureter stone. In the case of uric acid stone, the recurrence rate was higher than in the past. Similar to previous studies, the recurrence rate was high and the recurrence period was short in the uric acid stone. Active prophylactic treatment for uric acid stone patients will be necessary.

\section{MP11-13 Analysis of surgical complications of retrograde} intrarenal surgery

B Kim, Y Lee, J Chung, Y Ha, S Choi, J Lee, H Kim, T Kim, E Yoo, T Kwon, S Chung

Kyungpook National University School of Medicine Republic of Korea

Introduction \& Objective: Retrograde intrarenal surgery (RIRS) is being used widely as the first operative management for renal calculi along with extracorporeal shock wave lithotripsy (ESWL) and percutaneous nephrolithotomy (PCNL). The removal rate of renal calculi of RIRS is higher than that of ESWL and invasiveness of RIRS is lesser than that of PCNL. Although RIRS is getting popularity, there are few studies about surgical complications of RIRS. Therefore, we tried to analyze the surgical complications of RIRS in our center.

Materials and Methods: We identified 312 patients underwent RIRS from January 2010 to May 2015. The sort of surgical complications, the incidences rate of complication, the severity of each complication and the risk factors were verified retrospectively with logistic regression analysis. The severity was based on modified Clavien classification. Febrile urinary tract infection (UTI) was defined as the body temperature $>38^{\circ} \mathrm{C}$ with pyuria or bacteriuria within a month after the operation. Gross hematuria and flank pain were defined as surgical complication when these symptoms persist over than 2 weeks after the operation. The success of the operation was defined as complete disappearance of stone or presence of asymptomatic small fragments lesser than $4 \mathrm{~mm}$ in non-contrast computed tomography (CT) at post-operative 1 month.

Results: Of 312 cases, ureter stone was accompanied in 129 cases (41.3\%) and bilateral renal calculi was in 64 cases $(20.5 \%)$. The mean size of stones was $11.0 \mathrm{~mm}$, and mean operation time was 81.5 minutes. Overall success rate was $64.0 \%$ (200/312). Incidence rate of complications was $30.7 \%$ (96/312). The febrile UTI was developed in 54 cases $(17.3 \%)$, as the most common complication. 
Persistent flank pain and gross hematuria was observed in 38 cases (12.2\%) and 27 cases (8.7\%), respectively. Perirenal hematoma and hydronephrosis after ureteral stent removal was observed in 5 cases $(1.6 \%)$ and 18 cases $(5.8 \%)$, respectively. None of ureteral injury or ureteral stenosis was observed. According to modified Clavien classification, 47 (15.1\%), 29 (9.3\%), and 20 cases $(6.4 \%)$ were classified into grade I, II, and III, respectively, and there was no case of in grade IV or higher. In logistic regression analysis, any statistically significant risk factor was not identified.

Conclusions: Various surgical complications after RIRS can be developed such as febrile UTI, perinephric hematoma, hydronephrosis, persistent flank pain and gross hematuria. However, most cases were minor complications, and serious complications were rarely observed. As the operative management for renal calculi, RIRS can be considered to be effective and safe.

MP11-14 Management of nephrolithiasis with a large ureteral access sheath is not associated with increased risk of surgical complications and stricture formation

A Khambati, B Jordan, S Yoo, K Perry, R Nadler

Northwestern University

United States

Introduction \& Objective: Ureteral access sheaths (UAS) are commonly used in the management of nephrolithiasis. There is limited data on complications associated with UAS, especially with the large 14/16 F sheath. We evaluated and compared surgical complications and outcomes associated with UAS use between the smaller (10-14 F) and the large (14/16 F) UAS sheaths. Materials and Methods: We identified 355 patients who underwent ureteroscopy for proximal or renal stones with a UAS between 2010 and 2015 at our institution. All patients had an initial 3 month follow-up CT scan. Patient demographic and clinical information including JJ stent status, surgical complications, hospital admission and 90-day readmission rates, and post-operative hydronephrosis and strictures were assessed.

Results: In total, 335 patients were included, 170 men (50.7\%) and $165(49.3 \%)$ women. Median age was 55 . The stones were larger in the $14 / 16$ UAS group ( 10 vs $12 \mathrm{~mm}, \mathrm{p}<0.001)$. There was no significant difference in the rate of intraoperative $(3.8 \%$ vs $2.8 \%$ $\mathrm{p}=0.24)$ and post-operative $(6.3 \%$ vs $3.9 \%, \mathrm{p}=0.84)$ complications, hospital admission, readmission and stone-free rates between the $10-14 \mathrm{~F}$ and 14/16 UAS groups. In total, 3 (0.8\%) strictures

Table 1 Univariate analysis of surgical outcomes and complications based on UAS size

\begin{tabular}{|l|l|l|l|}
\hline Parameter & $10-14$ UAS & $14 / 16$ UAS & p-value \\
\hline Number of patients & 157 & 178 & \\
\hline Post-operative stent & $151(96.1)$ & $173(97.1)$ & 0.89 \\
\hline Overall SFR & $125(79.6)$ & $135(75.8)$ & 0.40 \\
\hline SFR by size & $67(91.7)$ & & \\
\hline$<10 \mathrm{~mm}$ & $67(70.1)$ & $54(88.5)$ & 0.52 \\
\hline $10-20 \mathrm{~mm}$ & $4(57.1)$ & $54(72.9)$ & 0.69 \\
\hline$>20 \mathrm{~mm}$ & $23(14.6)$ & $27(62.7)$ & 0.77 \\
\hline Post-operative admission & $6(3.8)$ & $30(16.8)$ & 0.58 \\
\hline Intraoperative complication & $151(96.1)$ & $5(2.8)$ & 0.24 \\
\hline Uneventful & $1(0.6)$ & $173(97.1)$ & \\
\hline Bleeding & $2(1.3)$ & $4(2.2)$ & \\
\hline Perforation & $1(0.6)$ & $1(0.5)$ & \\
\hline Avulsion & $2(1.27)$ & 0 & \\
\hline Submucosal wire & $10(6.3)$ & 0 & \\
\hline Early Post-operative complication & $5(3.2)$ & $7(3.9)$ & 0.84 \\
\hline UTI/pyelonephritis & $1(0.6)$ & $3(1.7)$ & \\
\hline Sepsis & $1(0.6)$ & $1(0.5)$ & \\
\hline Stent malfunction & $2(1.3)$ & $1(0.5)$ & \\
\hline Steinstrasse/colic & $1(0.6)$ & $2(1.1)$ & \\
\hline Renal abscess & $15(9.5)$ & 0 & \\
\hline Hospital readmission (within 90 days) & & $11(6.1)$ & 0.24 \\
\hline Late post-operative outcome & $6(3.8)$ & & \\
\hline Benign hydronephrosis & $2(1.3)$ & $9(5.0)$ & 0.58 \\
\hline Ureteral stricture & & $1(0.5)$ & 0.60 \\
\hline \multicolumn{1}{|c|}{ SFR Stone free rate } & & \\
\hline
\end{tabular}

were diagnosed, with only 1 in the 14/16 UAS group. No difference was detected in total complications based on sheath size on logistic regression analysis. Mean follow-up was 21.7 months.

Conclusions: The risk of surgical complications and stricture formation associated with UAS is low. The use of a 14/16 sheath may allow treatment of large renal stones safely without an increased risk of complications.

\section{MP11-15 Return for Unplanned Care after Ureteroscopy -} Does the Type of Pain Medication Matter?

PA Milburn, A El Mekresh, GL Machen, MM El Tayeb, L Tsai

Texas A\&M College of Medicine/Baylor Scott and White United States

Introduction \& Objective: Ureteroscopy causes discomfort for patients postoperatively. This manifests as flank or bladder pain, and most postoperative medication regiments include a centrally acting opioid medication. Data on the most appropriate pain medication after ureteroscopy is limited. There is increasing scrutiny on narcotic prescriptions in America to reduce abuse, and narcotic use is associated with many undesirable and dangerous side effects. For this reason, we sought to evaluate whether different strengths of postoperative opioid medications impacted the rate of patient return to the emergency room (ER), unplanned clinic visits for pain, or overall 3-month readmission rate.

Materials and Methods: After IRB approval, retrospective chart review was performed for patients who underwent outpatient ureteroscopy for purposes of renal or ureteral stone removal from February 1, 2014 through March 31, 2016. The pain medication that was prescribed and the patient's postoperative course was evaluated for the first 3 months after surgery. Both ER visits and clinic calls/visits were analyzed. Clinic and ER contacts were excluded if they did not pertain to urinary or flank pain. Pain medications were classified into 4 categories based on their DEA drug schedule: Schedule II, III, IV, and over the counter (OTC) medications. A Chi-square test was used for analysis.

Results: 475 patients were identified with complete charts and follow up. The results of our data are summarized in the table below. 199 patients received schedule II medicines, 165 received schedule III medications, 72 received schedule IV medications, and 39 received OTC medications.. Overall 138 (29\%) of the patients had unplanned visits for care with 59 calls or visits to the clinic, and 79 visits to the emergency room for pain. The rates of return to ER, clinic, or re-admission within 3 months were not significantly different from each other.

Conclusions: The strength of pain medication prescribed after ureteroscopy had no bearing on whether the patient would contact the urologist due to pain. Specifically, medications such as hydrocodone and oxycodone, which have a higher abuse potential, did not prevent out of control pain in the postoperative period better than safer alternatives. Re-admission rate within 3 months was not impacted by postoperative pain medication either. This information should be considered to improve prescription narcotic stewardship in the world of increasing oversight, overdose, and abuse.

\begin{tabular}{|l|l|l|l|l|}
\hline & $\begin{array}{l}\text { Total } \\
\text { Number }\end{array}$ & Returned to ER & $\begin{array}{l}\text { Contacted } \\
\text { Clinic }\end{array}$ & $\begin{array}{l}\text { Re-Admission } \\
\text { within 3 Months }\end{array}$ \\
\hline Schedule II (hydrocodone) & 199 & $29(25.6 \%)$ & $22(11.1 \%)$ & $17(15.4 \%)$ \\
\hline Schedule III (codeine) & 165 & $25(14.6 \%)$ & $17(10.3 \%)$ & $12(8.5 \%)$ \\
\hline Schedule IV (tramadol) & 72 & $15(15.2 \%)$ & $12(16.7 \%)$ & $4(7.3 \%)$ \\
\hline OTC & 39 & $10(20.8 \%)$ & $8(20.5 \%)$ & $6(5.6 \%)$ \\
\hline p value & & 0.254 & 0.207 & 0.313 \\
\hline
\end{tabular}


MP11-16 The feasibility of Poly FURS combined with ultrasound guided all seeing needle Micropen to treatment lower calyceal stones over $\mathbf{2} \mathrm{cm}$ in size with Infundibular pelvic angle less than 60 degree

\section{Y Wenzeng}

China (People's Republic)

Introduction \& Objective: Objectives: To assess the efficacy of Poly FURS combined with ultrasound guided all seeing needle Micro-pcn to treatment lower calyceal stones over $2 \mathrm{~cm}$ in size with Infundibular pelvic angle (IPA) less than 60 degree

Materials and Methods: Between January 2016 and January 2017,63 cases with lower pole kidney stones were enrolled in this study. Infundibulopelvic angle (IPA) were less than 60 degree, 37 cases of multiple stones, single stone in 26 cases, the patients were under retrograde Poly FURS (male $70 \mathrm{~cm}$ type, female $42 \mathrm{~cm}$ type) combined with ultrasound guided all seeing needle Micropcn Lithotripsy, and retrospectively analysis the clinical data of theses patients, including the operation time, the amount of bleeding, stone free rate(SFR), complications, to assess the safety and effectiveness of the approach ize:12.0pt;line-height:150\%; font-family: "Times New Roman", "serif", > less than 60 degree Results: The operation time were $45 \pm 13 \min (30 \sim 60 \mathrm{~min})$, FURS lithotripsy time were $27.5 \pm 7.5 \mathrm{~min}(20 \sim 35 \mathrm{~min})$, all seeing needle puncture time were $4 \pm 1 \mathrm{~min}(3 \sim 5 \mathrm{~min})$, Micro-pcn lithotripsy time were $10 \pm 2 \min (8 \sim 12 \mathrm{~min})$. the main role of FURS lithotripsy in 48 cases, and the Micro-pcn lithotripsy in 15 cases, postoperative complications of fever in 3 cases, pain in 2 cases; no blood transfusion patients; 2 cases changed the operation into percutaneous nephrolithotomy due to a breezing vision. Postoperative hospital stay 2 to 3 days, postoperative stone resection in 3 cases, 2 weeks after ESWL, stones removed $95.1 \%$

Conclusions: Retrograde Poly FURS combined with ultrasound guided all seeing needle Micropcn to treatment lower calyceal stones over $2 \mathrm{~cm}$ in size with Infundibular pelvic angle less than 60 degree is safe, effective and feasible. pt;line-height:150\%;font-family: "Times New Roman", "serif"'> $>20 \sim 35 \mathrm{~min}$ ), all seeing needle puncture time were $4 \pm 1 \min (3 \sim 5 \mathrm{~min})$, Micro-pcn lithotripsy time were $10 \pm 2 \min (8 \sim 12 \mathrm{~min})$. the main role of FURS lithotripsy in 48 cases, and the Micro-pen lithotripsy in 15 cases, postoperative complications of fever in 3 cases, pain in 2 cases; no blood transfusion patients; 2 cases changed the operation into percutaneous nephrolithotomy due to a breezing vision. Postoperative hospital stay 2 to 3 days, postoperative stone resection in 3 cases, 2 weeks after ESWL, stones removed 95.1\%

MP11-17 Flexible ureteroscopy with holmium laser lithotripsy completely fluoroless for treating kidney stones: feasibility, outcomes and complications. "Our experience".

BO Manzo, E Lozada, J Sanchez, G Manzo, H Sanchez, R Maldonado, M Vanzzini

Endourology Department at Hospital Regional de Alta Especialidad del Bajío

Mexico

Introduction \& Objective:

Introduction

In recent years urinary stones incidence has markedly increased in the United States and around the world, this increase has caused that the number of flexible ureteroscopies performed the period between 1994 to 2004 have had an $83 \%$ increase. This increase in flexible ureteroscopy for urinary stones treatment has been facili-

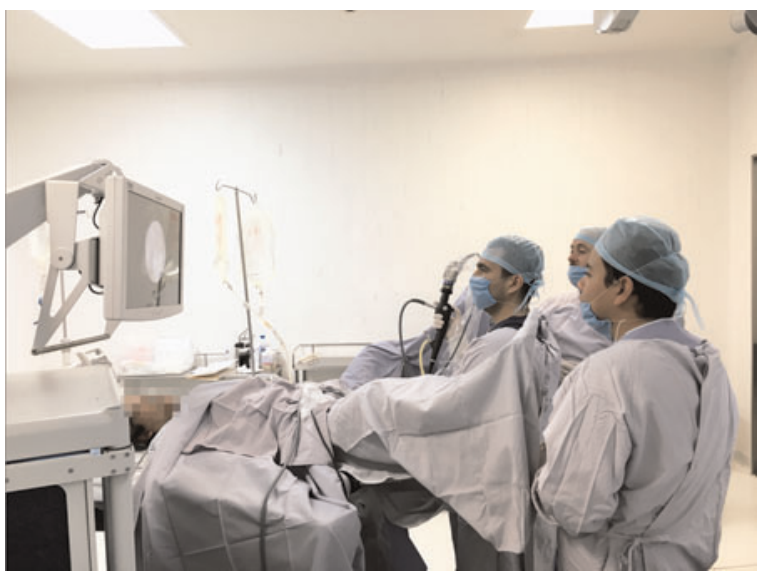

tated by improvements in new flexible ureteroscopes that allow easy access to the urinary tract with excellent optical vision ${ }^{1,2}$. Objetive:

To present feasibility, safety, outcomes and complications of flexible ureteroscopy for kidney stones treatment without fluoroscopy and compare them to conventional ureteroscopy withfluoroscopy use.

Materials and Methods: All patients treated for kidney stones by flexible ureteroscopy with Holmium laser lithotripsy from February 2014 to February 2015 at our endourology department were retrospectively evaluated. Patients demographic characteristics, stone features (size, number and location), surgical variables (fluoroscopy use, operative and fluoroscopy time), complications and success rate (by means of stone-free rate) were included in the analysis.

Results: Over a 12 months period 216 patients were diagnosed and treated for kidney stones by flexible ureteroscopy with Holmium laser lithotripsy were evaluated. A total of 100 patients met inclusion criteria and were included in our statistical analysis; 33 flexible ureteroscopies were performed under fluoroscopy (group1)

\begin{tabular}{|c|c|c|c|c|c|}
\hline & & DJS & UC & Tubeless & \multirow{2}{*}{ P Value } \\
\hline \multicolumn{2}{|l|}{ Number of patients } & 106 & 214 & 196 & \\
\hline \multicolumn{2}{|l|}{ Stone volume $\left(\mathrm{mm}^{2}\right)$} & 696.78 & 292.09 & 210.03 & $p<<0.005$ \\
\hline \multicolumn{2}{|l|}{ Hounsfield units (HU) } & \begin{tabular}{|l|}
1047.82 \\
\end{tabular} & 1005.56 & 907.49 & $p<<0.005$ \\
\hline \multicolumn{2}{|l|}{ Billateral tratment } & 2 & 3 & 2 & $p=0.822$ \\
\hline \multirow[t]{3}{*}{ Stone location } & Kidney & $43(28.9 \%)$ & $47(31.5 \%)$ & \begin{tabular}{|l|l|}
$59(39.6 \%)$ \\
\end{tabular} & \multirow[t]{3}{*}{$p=0.001$} \\
\hline & Ureter & $52(15.5 \%)$ & $158(47 \%)$ & $126(37.5 \%)$ & \\
\hline & Both & $11(34.4 \%)$ & $10(31.3 \%)$ & $11(34.4 \%)$ & \\
\hline \multirow[t]{3}{*}{ Ureteroscope used } & Semi-rigid & $42(14.5 \%)$ & $136(47.1 \%)$ & $111(38.4 \%)$ & \multirow[t]{3}{*}{$p=0.003$} \\
\hline & Flexible & $59(28.2 \%)$ & $73(34.9 \%)$ & $77(36.8 \%)$ & \\
\hline & Both & $5(26.3 \%)$ & $6(31.6 \%)$ & $8(42.1 \%)$ & \\
\hline \multirow{5}{*}{$\begin{array}{l}\text { Hydronephrosis } \\
\text { grade }\end{array}$} & 0 & $20(17.1)$ & $42(35.9 \%)$ & $55(47 \%)$ & \multirow{5}{*}{$p<<0.005$} \\
\hline & 1 & $36(23.5 \%)$ & $56(36.6 \%)$ & $61(39.9 \%)$ & \\
\hline & 2 & $17(11.3 \%)$ & $77(51.3 \%)$ & $56(37.3 \%)$ & \\
\hline & 3 & $27(30.7 \%)$ & $37(42 \%)$ & $24(27.3 \%)$ & \\
\hline & 4 & $6(66.7 \%)$ & $3(33.3 \%)$ & 0 & \\
\hline \multirow[t]{2}{*}{ Prior stent } & Yes & $23(16.2 \%)$ & $62(43.7 \%)$ & $57(40.1 \%)$ & \multirow[t]{2}{*}{$0=0.202$} \\
\hline & No & $82(21.9 \%)$ & $153(40.9 \%)$ & $139(37.2 \%)$ & \\
\hline \multirow[t]{2}{*}{ Complications } & Yes & $5(19.2 \%)$ & $14(53.8 \%)$ & $7(26.9 \%)$ & \multirow[t]{2}{*}{$p=0.390$} \\
\hline & No & $101(20.6 \%)$ & $201(40.9 \%)$ & $189(38.5 \%)$ & \\
\hline \multirow[t]{2}{*}{ Acute renal failure } & Yes & $1(7.7 \%)$ & $7(53.8 \%)$ & $5(38.5 \%)$ & \multirow[t]{2}{*}{$p=0.461$} \\
\hline & No & $105(20.8 \%)$ & $208(41.3 \%)$ & $191(37.9 \%)$ & \\
\hline \multirow[t]{2}{*}{ Fever } & Yes & $5(29.4 \%)$ & $8(47.1 \%)$ & $4(23.5 \%)$ & \multirow[t]{2}{*}{$p=0.401$} \\
\hline & No & $101(20.2 \%)$ & $207(41.4 \%)$ & $192(38.4 \%)$ & \\
\hline \multirow[t]{2}{*}{ Stone street } & Yes & 0 & 0 & $2(0.8 \%)$ & \multirow[t]{2}{*}{$p=0.193$} \\
\hline & No & $106(20.6 \%)$ & $215(41.7 \%)$ & $194(37.7 \%)$ & \\
\hline
\end{tabular}

Table 2: Postoperative VAS and analgesia consumption

\begin{tabular}{|l|l|c|c|c|c|}
\hline \multicolumn{2}{|c|}{} & DJS & UC & Tubeless & $p$ Value \\
\hline POD 0 & Mean VAS & $2.47 \pm 2.96$ & $2.65 \pm 3.20$ & $2.5 \pm 3.42$ & $p=0.563$ \\
\cline { 2 - 6 } & Mean \# of pain killer required & $0.98 \pm 1.26$ & $1.05 \pm 1.48$ & $0.88 \pm 1.36$ & $p=0.444$ \\
\hline \multirow{2}{*}{ POD 1 } & Mean VAS & $1.08 \pm 2.07$ & $1.63 \pm 2.69$ & $1.56 \pm 2.73$ & $p=0.186$ \\
\cline { 2 - 6 } & Mean \# of pain killer required & $0.49 \pm 1.12$ & $0.67 \pm 1.34$ & $0.69 \pm 1.58$ & $p=0.461$ \\
\hline \multirow{2}{*}{ POD 2 } & Mean VAS & $0.36 \pm 1.65$ & $0.40 \pm 1.66$ & $0.44 \pm 1.82$ & $p=0.92$ \\
\cline { 2 - 6 } & Mean \# of pain killer required & $0.13 \pm 0.46$ & $0.17 \pm 0.76$ & $0.22 \pm 0.98$ & $p=0.672$ \\
\hline
\end{tabular}




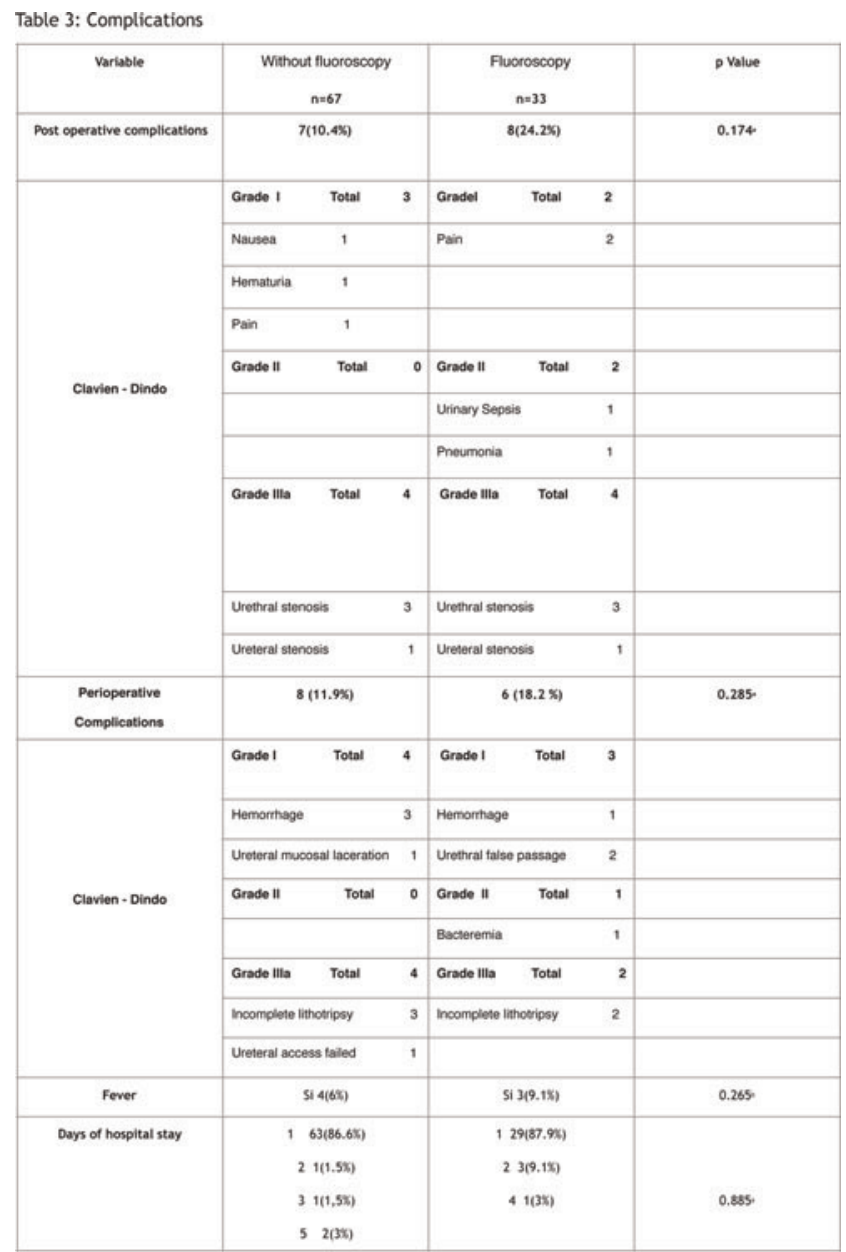

"Comparison between groups was performed using the $x^{2}$ test and results are reported as frequency and percentage.

-Comparison between groups was performed using Fisher's exact test. results are reported as frequency and percentage

and 67 were performed without it (group 2). Mean operating time was $94.33(+/-37.2)$ and $98.29(+/-49.4)$ minutes respectively. Mean stone volume was $78.5 \mathrm{~mm}^{2}\left(50-137.4 \mathrm{~mm}^{2}\right)$ in group 1 and $82.4 \mathrm{~mm}^{2}\left(50-166.4 \mathrm{~mm}^{2}\right)$ in group 2. Stone-free rate was $63.6 \%$ and $64.2 \%$ respectively. Perioperative complications were 6 $(18.2 \%)$ and $8(11.9 \%)$ respectively and postoperative complications were $8(24.2 \%)$ and $7(10.4 \%)$.

Conclusions: Flexible ureteroscopy with holmium laser lithotripsy completely fluoroless is feasible and safe for kidney stones treatment in majority of patients, thus reducing risk of radiation exposure to patients and medical staff while maintaining surgical success rate by means of stone free status. Even though it is necessary randomized controlled studies to further evaluate complete fluoroless ureteroscopy and confirm our results.

MP11-18 Does retrograde treatment of upper urinary tract stones necessitate postoperative upper urinary tract drainage? Conclusions from over 500 single center consecutive cases

IM Sabler, A Isid, A Lorber, A Latke, D Shurka, T Harhel, S Sfoungaristos, V Yutkin, G Hidas, E Landau, D Pode, O Gofrit, M Duvdevani

Hadassah Hebrew University Medical Center, Jerusalem, Israel Israel
Table 1: Demographic data and stone features.

\begin{tabular}{|c|c|c|c|}
\hline Variable & $\begin{array}{c}\text { without fuccoscopy } \\
\qquad n=67\end{array}$ & $\begin{array}{l}\text { muroscopy } \\
n=33\end{array}$ & p \\
\hline Gender & $\begin{array}{l}\text { Female 33(49.38) } \\
\text { Mole 34(50.7x) }\end{array}$ & $\begin{array}{l}\text { Female } 8(24.28) \\
\text { Male 25(75.8x) }\end{array}$ & 0.017 \\
\hline Age & $49.16+12.58$ & $48.75+14.56$ & 0.367 . \\
\hline Stone number & $\begin{array}{ll}1 & 40(59.75) \\
2 & 15(22.45) \\
3 & 6(95) \\
4 & 0(05) \\
5 & 2(35) \\
6 & 1(1.5)\end{array}$ & $\begin{array}{ll}1 & 18(54.5 x) \\
2 & 7(21.2 x) \\
3 & 4(12.15) \\
4 & 2(6.1 \times) \\
5 & 1(3 x) \\
6 & 0(0 x)\end{array}$ & 0.543 \\
\hline Maximum stone diameter & 10(8-19,5) & $13(8.5-16)$ & 0.671 \\
\hline Stone volumen & $82.4(50-166.4)$ & $78.5(50-137.4)$ & 0.885 \\
\hline Previous PNL & 18 (26.88) & 10 (30.38) & 0.719 \\
\hline Previous SWL & $18(26.95)$ & $10(30.35)$ & 0.814 \\
\hline Doble J catheter & 31 (41.68) & $12(36.45)$ & 0.470 \\
\hline
\end{tabular}

- Comparison between groups was performed using the $x^{2}$ test and results are reported as frequency and percentage.

b Results are reported as mean and standard deviation. For comparison between independent groups student " $\mathrm{t}$ " test was used.

'Comparison between groups was performed using Fisher's exact test. results are reported as frequency and percentage

¿ Comparison between groups was performed using Withney's U Mann test. Results are reported as median and interquartile range (25-75).

Table 2: Intraoperative variables

\begin{tabular}{|l|c|c|c|}
\hline Variable & Without fluoroscopy & Fluoroscopy & p value \\
\hline Fluoroscopy time & & $8(3.5-11.5)$ seconds & \\
\hline Operative time & $98.29(+/-49.4)$ & $94.33(++-37.2)$ & 0.888 \\
\hline Infundibular stenosis & $19(28.4 \%)$ & $13(29.4 \%)$ & 0.266. \\
\hline Hydronephrosis & $14(20.9 \%)$ & $8(24.2 \%)$ & 0.435 \\
\hline Ureteral stenosis & $2(3 \%)$ & 0 & 0.316 \\
\hline $\begin{array}{l}\text { Uretero-pelvic junction } \\
\text { stenosis }\end{array}$ & $7(10.4 \%)$ & 0 & 0.054 \\
\hline Ureteroscope damage & $4(6 \%)$ & $1(3 \%)$ & 0.526 \\
\hline
\end{tabular}

- Comparison between groups was performed using the $x^{2}$ test and results are reported as frequency and percentage.

b Results are reported as mean and standard deviation. For comparison between independent groups student " $t$ " test was used.

c Comparison between groups was performed using Fisher's exact test. results are reported as frequency and percentage

Diagram 1: Mean postoperative VAS, severe

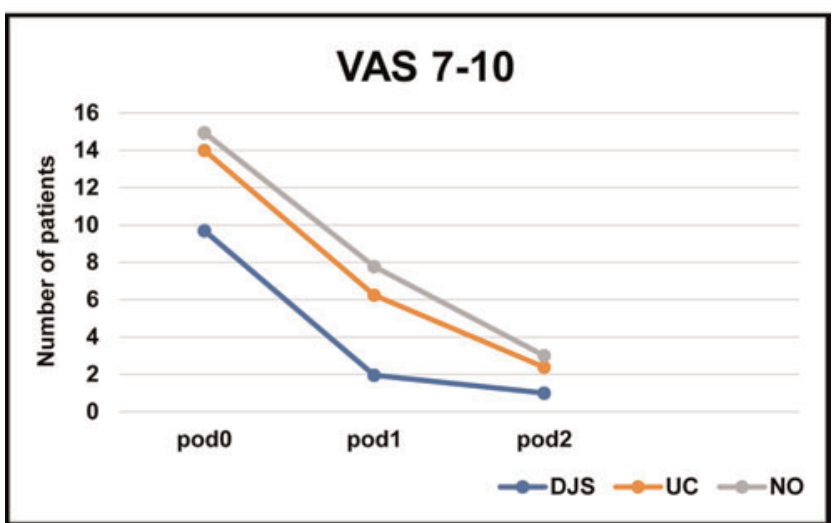


Introduction \& Objective: Stent (DJS) drainage following ureteroscopy is recommended in several scenarios and in some selected situations tubeless (TL) approach is possible. In other cases ureteral catheter (UC) attached to urethral catheter is left for 24-48 hrs. We evaluated these three subgroups, and compared postoperative complications and pain

Materials and Methods: Prospectively collected database of 516 consecutive patients that underwent ureterorenoscopy between 2010 and 2016. The cohort was divided according to postoperative drainage as: TL, UC and DJS. The data consisted of demographic parameters, stone location, number and burden, hydronephrosis grade, postoperative complications (fever, acute renal failure, obstruction of the upper urinary tract by stone street), pain had been assessed by Visual Analog Scale (VAS) and analgesic used. Data was evaluated using Chi Square or spearman's tests and Pearson analysis

Results: There were 196 (38\%) TL, 214 (41\%) UC and 106 (21\%) DJS cases. Patients that were drained with DJS had significantly higher stone volume, higher stone density and more severe obstruction (table 1). Postoperative complications (table 1) and mean VAS or analgesic use (table 2) were not different between the groups. The trend was for highest VAS in TL and UC groups at POD 0 and 1 (diagram 1)

Conclusions: Tubeless approach is safe in carefully selected cases. UC drainage may be omitted and, in borderline cases, DJS should be considered. At immediate postoperative process DJS group "doing better" in terms of symptoms. Tubeless procedures may facilitate outpatient approach to upper urinary tract stones treatment with a better patient satisfaction

\section{MP11-19 Withdrawn}

\section{MP11-20 Upper Tract Imaging after Ureteroscopy: Risk} Factors for Hydronephrosis

N François, M Sourial, J Cooper, H Miyagi, G Box, B Knudsen

The Ohio State University Wexner Medical Center United States

Introduction \& Objective: Routine upper tract imaging following ureteroscopy (URS) remains controversial with some recommending imaging in all patients and others suggesting a more selective approach. The concern for silent obstruction related to the stone and intervention drives the decision to obtain routine post-operative upper tract imaging after ureteroscopy. In addition, the use of a ureteral access sheath (UAS), which can cause ureteral tears, may further drive the decision to image after ureteroscopy. We retrospectively reviewed the incidence of hydronephrosis in a consecutive series of patients that underwent routine upper tract imaging after ureteroscopy.

Materials and Methods: Retrospective chart review of patients who underwent URS for nephroureterolithiasis by two urologists between 1/2012-9/2016. Stone size and location, type of ureteroscope, UAS, laser fiber, and stone basket use, and the use of a ureteral stent pre- and post-operatively were extracted from patient charts. Follow-up renal ultrasound and KUB plain xray findings approximately eight weeks after surgery were recorded. Results: Six hundred and fifty-nine patients underwent 824 URS. Four hundred and ninety cases utilized a UAS (92.1\%). Post-operative hydronephrosis was noted following 42 cases $(5.1 \%)$. Eleven of these patients $(26.2 \%)$ reported associated flank pain, and five (11.9\%) underwent secondary URS procedures. On follow-up imaging at eight weeks, 20 patients $(47.6 \%)$ demonstrated resolution

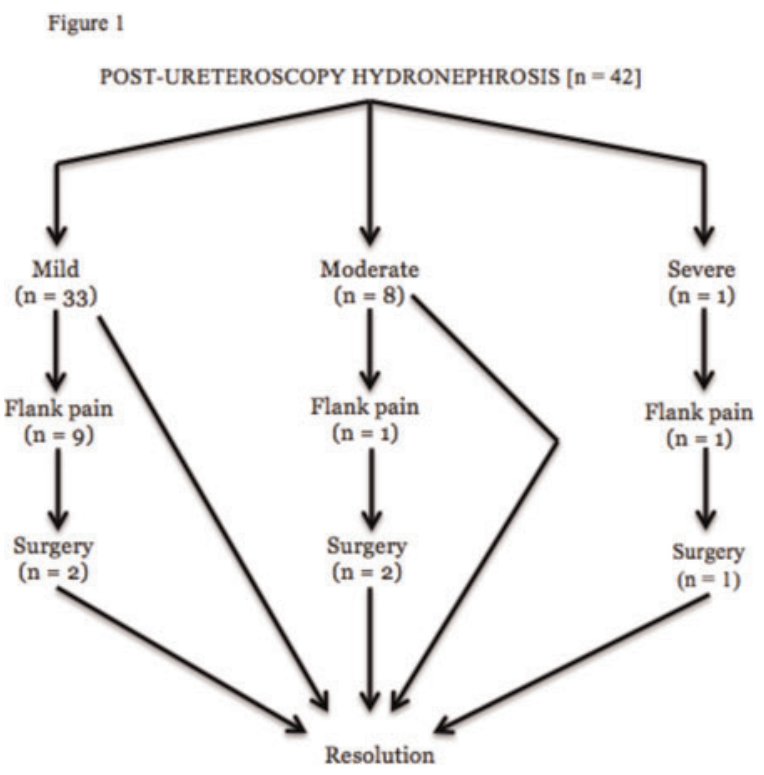

Table 1: Baseline patient characteristics

\begin{tabular}{|c|c|}
\hline CHARACTERISTIC & MEAN $( \pm$ SD) or $\mathrm{n}(\%)$ \\
\hline Age at operation (years) & $48.6( \pm 15.0)$ \\
\hline Male gender $(\%)$ & $320(48.6 \%)$ \\
\hline BMI $(\mathrm{kg} / \mathrm{m})$ & $31.8( \pm 9.1)$ \\
\hline
\end{tabular}

Table 2: Procedural characteristics

\begin{tabular}{|c|c|}
\hline CHARACTERISTIC & MEAN $( \pm \mathrm{SD})$ or $\mathrm{n}(\%)$ \\
\hline Operative time (minutes) & $40.4( \pm 22.9)$ \\
\hline Stone size (mm) & $8.4( \pm 4.9)$ \\
\hline Multiple ipsilateral stones & $132(20.0 \%)$ \\
\hline Pre-operative stent & $148(22.5 \%)$ \\
\hline Ureteral access sheath use & $490(92.1 \%)$ \\
\hline Laser use & $547(83.0 \%)$ \\
\hline Basket extraction & $492(74.7 \%)$ \\
\hline Post-operative stent & $548(83.2 \%)$ \\
\hline Mean stent duration (days) & $10.3( \pm 20.9)$ \\
\hline
\end{tabular}

of hydronephrosis, including one ureteral stricture $(0.12 \%)$ treated with balloon-dilation. Hydronephrosis was not statistically significantly more common with UAS use, laser, stone-basketing.

Conclusions: We detected $0.12 \%$ of silent obstruction, however the $5.1 \%$ observed incidence of hydronephrosis on follow-up imaging at eight weeks suggests routine follow-up upper tract imaging after URS remains valuable. 
MP11-21 Preliminary evaluation of High Frequency Jet Ventilation in RIRS from a tertiary center

MN Hasan, M Brehmer, P Harbut

Karolinska Institute

Sweden

Introduction \& Objective: RIRS with the useage of laser in treating upper urinary tract conditions minimally invasive has been increased at the last years Renal movements caused by respiration has always made retrograde intrarenal surgery (RIRS) challenging because of the effort to treat a moving target. To date there is no published data about the usage of high frequency jet ventilation (HFJV) in RIRS. HFJV is a high frequency ventilation mode, widely used in ENT. Within urology this technique has been previously reported during ESWL to reduce renal movement from $32 \mathrm{~mm}$ to $10 \mathrm{~mm}$ which resulted in reducing stone movement and time and shock energy requirements, therefore decreasing exposure to X-rays and perinephric trauma. The method is not adapted widely because of the need of a special ventilator equipment as well as training and experience in this field. We are evaluating HFJV ventilatory protocol in the treatment of renal stones especially larg stones

Materials and Methods: HFJV is accessible in our institution and we could even use it in RIRS of large renal stones to demonstrate its feasibility, safety and may be reducing the operating time and improving the outcomes of the surgery. We are evaluating the first 15 patients when HFJV were used in order to reduce renal movements during RIRS, under general, total intravenous anesthesia RIRS for renal stones between 7-24 mm in size done on 15 patients at the age of 23-83 years All the patients had an access sheath 10/12 Fr, endoflow pump, digital flexible ureteroscope and laser with two settings of $6 \mathrm{hz}-1,2 \mathrm{~J}$ and $10 \mathrm{hz}-0,5 \mathrm{~J}$ used for stone disintegration. 12 of 15 get a DJ stent postoperatively

Results: 1 of 15 patients developed UTI 3 days postoperatively and was admitted for intravenous treatment. 1 patient admitted because of stent migration for stent extraction and 1 patient admitted after stent extraction because of pain. The total operative time(Laser) was between 20-90 min No complications reported Conclusions: Renal movements during RIRS are challenging especially in cases where precise maneuvers during treatment are required. This preliminary report reveal HFJV as a safe, feasible method that can be used in RIRS to significantly facilitate the procedure allowing more precise maneuvers, may minimize the operating time and the risk for subsequent UTI.

MP11-22 Revisiting the $15 \mathrm{~mm}$ renal stone size threshold in the modern RIRS era

H Goldberg, D Golomb, Y Shtabholtz, S Tapiero, G Creiderman, A Shariv, J Baniel, Y Ehrlich, D Lifshitz

Endourology unit, Rabin medical center, and Sackler Faculty of Medicine, Tel Aviv University, Tel Aviv, Israel Israel

Introduction \& Objective: Retrograde renal surgery (RIRS) has been steadily gaining popularity and is now often offered as a first line treatment option for larger renal stones (RSs). The study objective was to investigate if the effectiveness of RIRS remains the same over the $1-2 \mathrm{~cm}$ renal stone size range in comparison to smaller stones.

Materials and Methods: From our data base of 2750 ureteroscopies we identified 635 consecutive patients who underwent RIRS for RSs between 2004 and 2014. Patients were divided to three stone size groups: $<10 \mathrm{~mm}, 10-15 \mathrm{~mm}, 15-20 \mathrm{~mm}$. Preoperative,
Table 1. Odds Ratios determining significant difference in stone free rate for different stone sizes:

\begin{tabular}{|c|c|c|c|c|c|c|}
\hline Group & SFR* & $\begin{array}{c}\text { Failure } \\
\text { rate }\end{array}$ & $\begin{array}{c}\text { Odds } \\
\text { (Success/Failure) }\end{array}$ & $\begin{array}{c}\text { Odds } \\
\text { Ratio* }\end{array}$ & $\begin{array}{c}\mathbf{9 7 . 5 \%} \text { Wald Confidence } \\
\text { Interval }\end{array}$ \\
\hline $\mathbf{1}(<\mathbf{1 0} \mathbf{m m})$ & $94.1 \%$ & $5.9 \%$ & 15.9 & 1 & & \\
\hline $\mathbf{2 ( 1 0 - 1 5} \mathrm{mm})$ & $90.1 \%$ & $9.9 \%$ & 9.1 & 0.572 & 0.27 & 1.206 \\
\hline $\mathbf{3 ( > 1 5} \mathrm{mm})$ & $85 \%$ & $15 \%$ & 5.7 & 0.358 & 0.153 & 0.914 \\
\hline
\end{tabular}

*With group 1 serving as reference

Table 2. Multivariate analysis for predicting stone-free rate in RIRS for stones $>=15 \mathrm{~mm}$

\begin{tabular}{|c|c|c|c|c|c|}
\hline Variable & B & SE & OR & $95 \% \mathrm{Cl}$ & $P$ value \\
\hline Age & -0.92 & 0.45 & 0.91 & $0.83-0.99$ & 0.041 \\
\hline Age adjusted Charlson score & 0.45 & 0.29 & 1.57 & $0.9-2.76$ & 0.121 \\
\hline $\begin{array}{l}\text { Gender } \\
\text { Male (reference) } \\
\text { Female }\end{array}$ & -0.16 & 0.675 & 0.85 & $0.23-3.19$ & 0.81 \\
\hline $\begin{array}{l}\text { Ipsilateral previous procedures } \\
\text { Done (reference) } \\
\text { Not done }\end{array}$ & -0.37 & 0.7 & 0.69 & $0.18-2.7$ & 0.6 \\
\hline $\begin{array}{l}\text { Preoperative DJ stent insertion } \\
\text { Inserted (reference) } \\
\text { Not inserted }\end{array}$ & 0.93 & 0.85 & 2.54 & $0.48-13.4$ & 0.27 \\
\hline $\begin{array}{l}\text { Ureteral stone treated } \\
\text { Treated (reference) } \\
\text { Not treated }\end{array}$ & -1.5 & 0.7 & 0.22 & $0.57-0.88$ & 0.032 \\
\hline $\begin{array}{l}\text { Lower pole renal stone } \\
\text { Lower pole stone (reference) } \\
\text { All other locations }\end{array}$ & 0.36 & 0.85 & 1.4 & $0.3-6.66$ & 0.65 \\
\hline $\begin{array}{l}\text { Renal stone }>=20 \mathrm{~mm} \\
>=20 \mathrm{~mm} \text { (reference) } \\
<20 \mathrm{~mm}\end{array}$ & 0.67 & 0.74 & 1.95 & $0.46-8.35$ & 0.37 \\
\hline Operative time & 0.02 & 0.014 & 1.02 & $0.99-1.05$ & 0.15 \\
\hline Number of complications & -2.39 & 0.92 & 0.91 & $0.015-0.56$ & 0.01 \\
\hline $\begin{array}{l}\text { Renal stone composed of calciur } \\
\text { oxalate ( } \mathrm{CaOx} \text { ) } \\
\text { Composed of } \mathrm{CaOx} \text { (reference) } \\
\text { Not composed of } \mathrm{CaOx}\end{array}$ & -0.951 & 1.08 & 0.39 & $0.047-3.19$ & 0.38 \\
\hline
\end{tabular}

operative, stone free rate (SFR) and follow-up data were analyzed and compared.

Results: The SFR for the three groups was $94.1 \%, 90.1$ and $85 \%$, respectively. Logistic regression analysis showed that the odds ratio (OR) for SFR of patients with RS $>15 \mathrm{~mm}$ (with respect to patients with RS15 mm (Table 1). The efficiency quotient calculated for stones larger and smaller than $15 \mathrm{~mm}$ was $83.9 \%$ vs. $91.8 \%$, respectively (p $15 \mathrm{~mm}(73.6 \pm 29.9$ vs. $53 \pm 19.4 \mathrm{~min}, \mathrm{p}+2$ days vs. $1.8 \pm 1.8$ days, $\mathrm{p}=0.031$, respectively). Moreover, the complication rate was almost two times higher $(10 \%$ vs $5.4 \%, \mathrm{p}=0.08)$. Older age, concomitant ureteral stones and number of complications were independent predictors of failure in the large stone group (Table 2).

Conclusions: While the overall SFR following RIRS for RSs up to $2 \mathrm{~cm}$ is generally high, the SFR of stones $\geq 15 \mathrm{~mm}$ is significantly lower, with longer operating time and hospital stay, and a higher complication rate.

MP11-23 Flexible ureteroscopy in patients with nephrolithiasis associated with autosomal dominant polycystic kidney disease (ADPKD)

P Geavlete, D Georgescu, R Multescu, C Ene, V Iordache, G Balan, B Geavlete

Department of Urology,"Saint John" Emergency Clinical Hospital, Bucharest, România;

Romania

Introduction \& Objective: Nephrolithiasis associated with autosomal dominant polycystic disease impose specific management 
problems. The aim of this study was to evaluate the safety and efficacy of flexible ureteroscopy and holmium laser lithotripsy for the management of these cases.

Materials and Methods: Between January 2007 - January 2017 in "Saint John" Emergency Clinical Hospital, flexible ureteroscopy with laser lithotripsy was performed in 11 cases with nephrolitiasis associated with ADKPD. We retrospectively reviewed the efficacity and the safety of these procedures.

Results: The mean age of the patients was 61 years (between 52 and 71 years). Seven patients underwent chronic dialisis. Stone location was pyelic în 5 cases, inefrior calyx in one case and multiple in 3 cases. One patient presented bilateral lithiasis. Stone sizes were between $1 \mathrm{~cm}$ and $2.4 \mathrm{~cm}$. Stone free rate after one procedure was $72.7 \%$ and $90.9 \%$ after the second ones. The complication rate was $27.2 \%$, (3 cases) being represented by fever in one case, persistent hematuria in one case and renal colic in another one.

Conclusions: Flexible ureteroscopy with holmium laser lithotripsy is a safe and effective method for the treatment of patients with ADPKD and associated nephrolithiasis.

MP11-24 Comparison of Two Novel Single-Use Flexible Ureteroscopes to Currently Existing Reusable and SingleUse Flexible Ureteroscopes

D Wollin, B Winship, R Jiang, W Tom, D Radvak, C Scales, M Ferrandino, W Simmons, GM Preminger, M Lipkin

United States

Introduction \& Objective: Due to the high cost and limited durability associated with reusable ureteroscopes, single-use ureteroscopes have been gaining popularity in recent years. We aimed to directly compare two new single-use ureteroscopes - the YouCare fiberoptic flexible ureteroscope (YC-FR-A) and NeoFlex digital flexible ureteroscope - to contemporary reusable/ single-use flexible ureteroscopes in regards to optics, resolution, deflection, and irrigation flow.

Materials and Methods: Five flexible ureteroscopes, YC-FR-A (YouCare Tech, China), NeoFlex (NeoScope Inc, USA), LithoVue (Boston Scientific, USA), Flex-Xc (Karl Storz, Germany),

\begin{tabular}{|l|c|c|c|c|c|}
\hline & YC-FR-A & Neoflex & LithoVue & Flex-XC & Cobra \\
\hline Image Plattorm & Fiberoptic & Digital & Digital & Digital & Fiberoptic \\
\hline Working Channel & $4.3 \mathrm{~F}$ & $3.5 \mathrm{~F}$ & $3.6 \mathrm{~F}$ & $3.6 \mathrm{~F}$ & Dual $3.3 \mathrm{~F}$ \\
\hline Resolution at 10mm (lines/mm) & 5.04 & 10.1 & 7.13 & 8.00 & 4.00 \\
\hline Image Distortion & $4.3 \%$ & $14 \%$ & $3.6 \%$ & $22.6 \%$ & $16.7 \%$ \\
\hline Maximum Deflection & $349^{\circ}$ & $264^{\circ}$ & $276^{\circ}$ & $263^{\circ}$ & $253^{\circ}$ \\
\hline Maximum Flow Rate (mL/min) & 59.0 & 40.0 & 40.3 & 38.4 & 28.8 \\
\hline
\end{tabular}

Table 1: Optical and functional characteristics of the tested ureteroscopes

and Cobra (Richard Wolf, Germany), were assessed in vitro for image resolution, distortion, color representation, and grayscale imaging. Ureteroscope deflection was tested with an empty channel followed by placement of a $200 \mu \mathrm{m}$ laser fiber and a $1.9 \mathrm{~F}$ wire basket. Irrigation flow was measured using normal saline at a height of $100 \mathrm{~cm}$ through an empty channel, channel with $200 \mu \mathrm{m}$ laser fiber, and channel with $1.9 \mathrm{~F}$ basket.

Results: The optical and functional characteristics of the five ureteroscopes are shown in Table 1. The YC-FR-A showed a resolution of 5.04 lines $/ \mathrm{mm}$ and $4.3 \%$ image distortion compared to the NeoFlex resolution of 10.1 lines $/ \mathrm{mm}$ and $14 \%$ image distortion. No substantial difference was demonstrated in color reproducibility or in the discernment of gray-scales between ureteroscopes. The YC-FR-A had an impressive one-way deflection of 349 degrees at baseline but lacks two-way deflection capability, while the NeoFlex deflection was consistent with current dual-deflection ureteroscopes. With an empty channel, the YC-FR-A showed a maximum flow rate of $59 \mathrm{~mL} / \mathrm{min}$, which is the highest flow rate among the tested ureteroscopes, while the NeoFlex had a similar irrigation capability with the other devices. Conclusions: The YC-FR-A fiberoptic ureteroscope has comparable resolution to the Cobra fiberoptic ureteroscope but cannot match the digital ureteroscopes tested. Although the one-way deflection and ergonomics of the YC-FR-A are not intuitive, this scope can be a viable alternative to the current reusable/single-use flexible ureteroscopes on the market. Newer single-use ureteroscopes, such as the NeoFlex, appear comparable to current reusable ureteroscopes with regard to imaging and functional capability.

\section{MPS12: STONES: INSTRUMENTATION}

MP12-1 Surgeon Assessment of the Dakota Stone Retrieval Device

SK Bechis, JE Abbott, RL Sur, DG Kronenberg

UC San Diego School of Medicine

United States

Introduction \& Objective: The Dakota ${ }^{\mathrm{TM}}$ Nitinol Stone Retrieval Device (Boston Scientific, Boston, MA) is a newly released hybrid grasper basket designed to capture, reposition and extract a wider variety of renal stones, including small stone fragments under $2 \mathrm{~mm}$. Its superior durability is reported to facilitate more complete stone removal in a greater number of patients. We conducted a survey to solicit early feedback from urologists performing ureteroscopy and stone extraction with the Dakota device.

Materials and Methods: A questionnaire assessing various aspects of the Dakota device including general grasping ability,

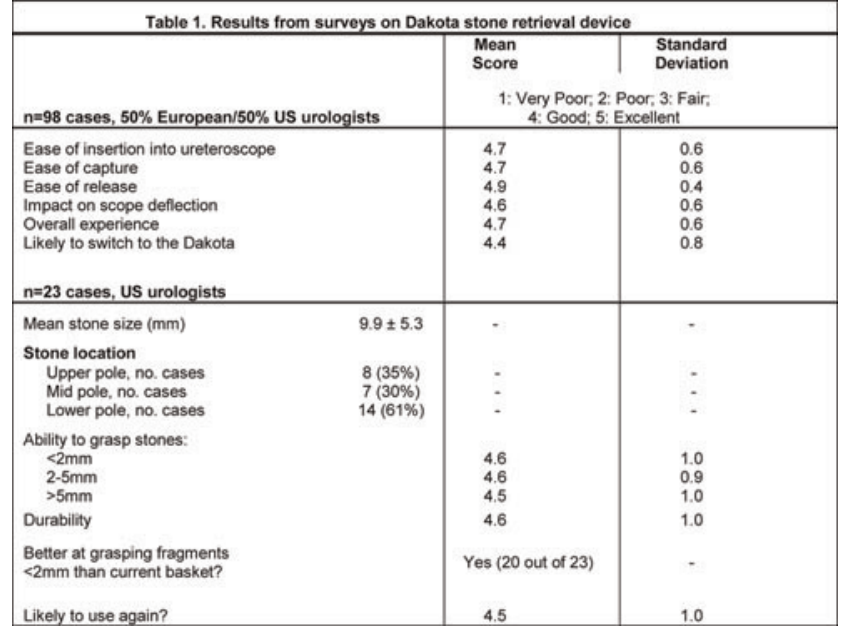


ease of insertion into the ureteroscope, and overall experience was distributed to urologists in Europe and the US performing ureteroscopic lithotropsy with the Dakota device. A supplemental survey was distributed to US surgeons assessing ability to capture, manipulate and retrieve stones of varying sizes $(5 \mathrm{~mm})$, durability, surgeon experience with grasping stone fragments

Results: Surveys were answered for 98 cases by urologists in the US and Europe. US surgeons accounted for $50 \%$ of all respondents (48). Performance scores are shown in Table 1. Supplemental questionnaires were answered for 23 cases by US urologists. Mean stone size was $9.9 \pm 5.3 \mathrm{~mm}$. Twenty out of 23 (87\%) surgeons rated the Dakota as better at grasping stone fragments

Conclusions: As determined by an initial survey at the time of surgery, the Dakota Stone Retrieval Device offers an improved ability to grasp small stone fragments, particularly $<2 \mathrm{~mm}$ and lower pole stones, with improved durability. It should be considered as a valuable addition to the stone surgeon's ureteroscopic arsenal.

MP12-2 Holmium Laser Lithotripsy with Moses Technology - Initial Evaluation

A Symes

Brighton and Sussex University Hospitals NHS Trust United Kingdom

Introduction \& Objective: Moses $^{\mathrm{TM}}$ is a new technology designed to deliver laser energy effectively and reduce stone retropulsion during Holmium laser lithotripsy thus potentially enabling less stone migration and increased surgical efficiency. This study provides the first European clinical evaluation of the technology.

Materials and Methods: 17 patients were included in this evaluation of the Lumenis pulse $120 \mathrm{H}$ Holmium laser, with

\begin{tabular}{|l|l|l|l|}
\hline Stone location & Kidney & ureter & both \\
\hline No of cases & 10 & 6 & 1 \\
\hline no. of stones & 1 & 2 & $>3$ \\
\hline No of cases & 11 & 1 & 4 \\
\hline Aver stone volume & $12.05 \mathrm{~mm}(6-30)$ & \multicolumn{2}{l|}{} \\
\hline Aver HU & \multicolumn{2}{|l|}{$95.7(650-1400)$} & \\
\hline Patient age (y) & \multicolumn{4}{|l|}{$54.53(35-76)$} & \\
\hline patient gender & male & female & recurrence \\
\hline & $58.8 \%$ & $41.2 \%$ & 6 \\
\hline patient history & newly & failed ESWL & 2 \\
\hline No of cases & 9 & 2 &
\end{tabular}

\begin{tabular}{|c|c|c|c|}
\hline & Aver & $\max$ & $\min$ \\
\hline Average lasing time (min) per case & 8.36 & 33.14 & 0.59 \\
\hline Average procedure time (min) per case & 40.3 & 150 & 12.33 \\
\hline Average energy $(\mathrm{Kj})$ per case & 5.9 & 35.98 & 0.57 \\
\hline complications & no & yes & \\
\hline No. of cases & 17 & 0 & \\
\hline$\%$ of cases & $100 \%$ & & \\
\hline damage to scope & no & yes & \\
\hline No. of cases & 17 & 0 & \\
\hline
\end{tabular}

\begin{tabular}{|l|l|l|l|l|l|}
\hline Subjective ranking (no. of cases) & 0 & 1 & 2 & 3 & N/A \\
\hline stone retropulsion rating (Moses mode) & 11 & 6 & - & - & \\
\hline $\begin{array}{l}\text { Fiber flexibility limitation (Moses 200 } \\
\text { D/F/L) }\end{array}$ & 12 & 1 & - & - & 4 \\
\hline $\begin{array}{l}\text { Fiber durability } \\
\text { (reduction in energy transmission) }\end{array}$ & 16 & - & - & - & 1 \\
\hline
\end{tabular}

Moses $^{\mathrm{TM}}$ technology. Moses ${ }^{\mathrm{TM}} \mathrm{D} / \mathrm{F} / \mathrm{L}$ fibres (Moses $200 \mathrm{D} / \mathrm{F} / \mathrm{L}$ $(n=13)$ and Moses $365 \mathrm{D} / \mathrm{F} / \mathrm{L}(\mathrm{n}=4)$ were used through differing rigid and flexible ureteroscopes. In most cases (>80\%) settings suitable for stone 'dusting' were utilized $(0.2 \mathrm{~J} / 50 \mathrm{~Hz})$. In addition, stone fragmentation parameters $(0.5-0.8 \mathrm{~J} / 10 \mathrm{~Hz})$ were also set. Moses ${ }^{\mathrm{TM}} \mathrm{A}$ ("Contact") was tested in all cases, and Moses ${ }^{\mathrm{TM}}$ B ("Distance") was also evaluated in 4 cases. A comparison was made intraoperatively with regular mode following physician subjective view and ranking.

Results: Comparison with 'regular mode' within each case demonstrated less retropulsion with Moses $^{\mathrm{TM}}$ settings, which gave controlled 'dusting' of stones. All cases were rendered stone free without any complication. $30 \%$ of patients required post-operative stenting (all removed at day 3 on a string). The $\mathrm{D} / \mathrm{F} / \mathrm{L}$ fibres performed very well with no visible degradation at the end of the procedure or inhibition of flexibility of scopes.

Conclusions: Moses ${ }^{\mathrm{TM}}$ technology allowed for effective and efficient stone clearance during Holmium laser lithotripsy. Moses $^{\mathrm{TM}}$ A (Contact) settings provided excellent controlled stone dusting, which presented a real improvement compared to existing settings. Moses ${ }^{\mathrm{TM}} \mathrm{B}$ (Distance) or regular fragmentation settings were used at the end of procedures for 'popcorning' effect, with good results.

\section{MP12-3 Optimizing Configuration of Thulium (Tm) Fiber Laser for Lithotripsy}

P Glybochko, G Altshuler, A Vinarov, L Rapoport, D Enikeev, N Sorokin, A Dymov, V Vinnichenko, A Kovalenko, I Yaroslavsky

I.M. Sechenov First Moscow State Medcal University Clinic of Urology

Russia

Introduction \& Objective: Tm fiber lasers have demonstrated promise as energy sources for transurethral lithotripsy. However, specific configuration and parameter set optimal for the application remain a subject of discussion. This work had two objectives: 1) Clinical evaluation of a modulated $120 \mathrm{~W}$ Continuous-Wave (CW) Tm laser; 2) In vitro characterization and optimization of a modified super-pulsed (500 W peak power) Tm laser, using a Holmium (Ho) laser system as benchmark.

Materials and Methods: 1) In clinical setting, 20 patients undergoing BPH surgery have been also treated for urolithiasis with a clinical $120 \mathrm{~W}$ Tm system, operated in pulse mode $(1.3 \mathrm{~ms}$ pulsewidth, $1.5 \mathrm{~J}$ pulse energy). Total of 3 cases of kidney stones and 20 cases of bladder stones have been treated. 2) In laboratory setting, a prototype super-pulsed Tm system capable of operation with up to 50 Waverage power was compared to a top-of-the-line Ho system (up to $120 \mathrm{~W}$ average power and $80 \mathrm{~Hz}$ rep rate), with settings matched in terms of average power and/or pulse energy. The ablation rates for human $\mathrm{COM}, \mathrm{COM} / \mathrm{CaP}$ and uric acid stones, magnitude of the retropulsion effect (after a single pulse) for Bego phantoms, as well as temperature rise at water outlet vs inlet, and maximum temperature rise in the cuvette during treatment were evaluated. 
Results: 1) Clinically, $16(80 \%)$ cases of bladder stones have been successfully resolved. Analysis of the outcomes revealed that hard stones (COM composition) were resistive to the treatment. It was hypothesized that the situation could be rectified through increase of the peak power of the system. 2) In vitro, the ablation rates measured with Tm system were about 1.9 times (forfragments $<3 \mathrm{~mm}$ ) and 3.9 times (for fragments $<1 \mathrm{~mm}$ ) higher than those of Ho system, at equivalent settings. The temperature rise in the cuvette was nearly identical for the two lasers. The average retropulsion distance after a single pulse was substantially shorter with the Tm laser.

Conclusions: 1) $120 \mathrm{~W} \mathrm{CW} \mathrm{Tm} \mathrm{fiber} \mathrm{laser} \mathrm{system} \mathrm{is} \mathrm{adequate} \mathrm{for}$ treatment of bladder stones with density $<1200 \mathrm{HU}$, but sub-optimal for treatment of harder stones. 2) Super-pulsed $500 \mathrm{~W}$ peak power Tm system is effective on all types of stones investigated and demonstrates a significantly higher ablation rate than $\mathrm{Ho}$ laser, while maintaining equivalent thermal regime and reduced retropulsion.

MP12-4 Percutaneous nephrolithotomy assisted by a novel negative pressure sheath

D Lai, X Li, Y He, M Sheng

Fifth affiliated Hospital, Guangzhou Medical University China (People's Republic)

Introduction \& Objective: to explore the clinical outcome of using a novel negative pressure sheath in minimally invasive percutaneous nephrolithotomy (MPCNL).

Materials and Methods: From March to October 2016, 50 patients who underwent MPCNL combined with negative pressure sheath in the Fifth Affiliated Hospital of Guangzhou Medical University (32 patients) and Jinshazhou Hospital of Guangzhou University of Traditional Chinese Medicine (18 patients) was retrospectively analyzed. The operative time, stone composition, stone clearance rate, preoperative and postoperative hemoglobin level and creatinine were recored. Complications such as postoperative fever, hemorrhage, sepsis were analyzed.

Results: All operations were performed successfully. The mean diameter of the calculi was $3.5 \mathrm{~cm}(5.5-7.0 \mathrm{~cm})$. The mean operation time was $32 \mathrm{~min}$ (25-45 min). Postoperative hospital stay was 5 to 9 days. The stone clearance rate was $94 \%(47 / 50)$. Significant hemorrhage was not found after operation and no cases underwent superselective renal artery embolization. 3 cases $(6 \%)$ had postoperative fever.
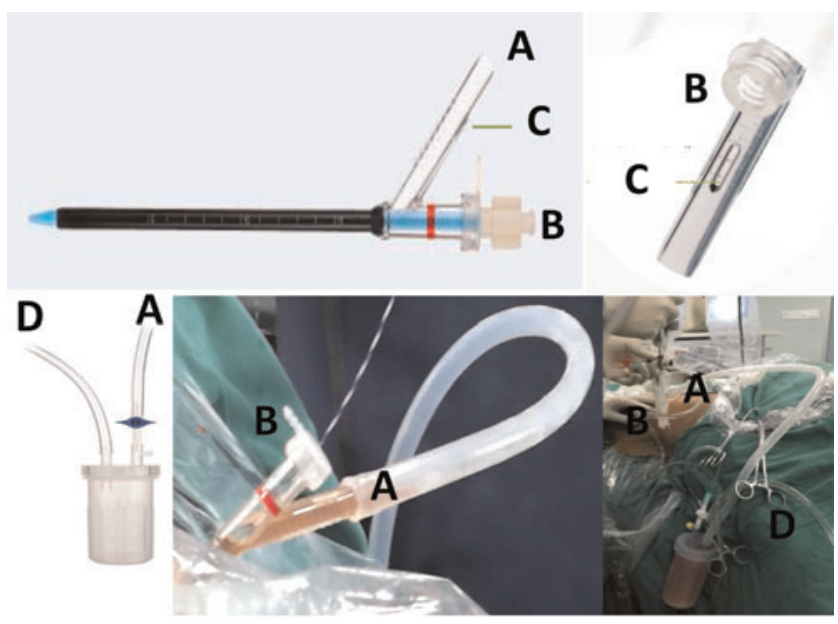

Conclusions: Using negative pressure sheath in MPCNL can significantly shorten the operative time and reduce the rate of complications.

MP12-5 Standardized Reporting of Device-Related Adverse Events During Percutaneous Nephrolithotomy: Review of the Manufacturer and User Facility Device Experience (MAUDE) Database

NH Patel, A Schulman, N Uppaluri, J Bloom, JL Phillips, S Konno, MS Choudhury, M Eshghi

New York Medical College

United States

Introduction \& Objective: Percutaneous nephrolithotomy (PCNL) is a standard approach for the access to and treatment of large stones from the upper urinary tract. PCNL is a complex, multistep surgery requiring multiple classes of devices. Malfunctions of devices are important to best address issues of patient safety and surgical quality which has not yet been standardized. Herein we describe the first report of device-related adverse events during percutaneous nephrolithotomy (PCNL) in the MAUDE database using a recently developed standardized classification system.

Materials and Methods: The MAUDE database was queried for "percutaneous nephrolithotomy" from 2006 to 2016. The circumstances and patient complications associated with various classes of devices used during PCNL were identified. We then utilized a novel, validated MAUDE classification reporting system to categorize clinical events. Logistic regression analysis was performed to identify associations between device classes and adverse patient events.

Results: 218 unique device-related events were reported. The most common classes included: lithotripter 53 (24.3\%), wires 43 (19.7\%), balloon dilators 30 (13.8\%) and occlusion balloons 28 (12.8\%). Reported patient complications included need for a second procedure $12(28.6 \%)$, bleeding $8(19.0 \%)$, retained fragments $7(16.7 \%)$, prolonged procedure $4(9.5 \%)$, ureteral injury $2(4.8 \%)$ and conversion to an open procedure $3(7.1 \%)$. Using the novel MAUDE classification system, $176(81 \%)$ were Level I (mild), 26 (12\%) were Level II (moderate), 15 (7\%) were Level III (severe) and 1 (0.5\%) was Level IV (life threatening). On univariate analysis, balloon dilators had the highest risk of Level II-IV complications compared to the other device classes [OR: 4.33, CI: 1.978-9.493, $\mathrm{p}=0.0002]$. The device was evaluated by the manufacturer in $93(42.7 \%)$ cases, with $54.8 \%$ of reviewed cases listing the source of malfunction as misuse by the operator.

Conclusions: PCNL is subject to a wide range of device-related adverse events that in some cases results in significant patient morbidity. The novel MAUDE classification system is

\begin{tabular}{|c|c|c|c|c|c|c|}
\hline & Overall & Lithotripters & Wires & $\begin{array}{c}\text { Balloon } \\
\text { Dilators }\end{array}$ & $\begin{array}{c}\text { Occlusion } \\
\text { Balloons }\end{array}$ & $\begin{array}{c}\text { Nephrostomy } \\
\text { Tubes }\end{array}$ \\
\hline Level I & 176 & 51 & 31 & 20 & 24 & 9 \\
& $(80.7 \%)$ & $(96.2 \%)$ & $(72.1 \%)$ & $(57.1 \%)$ & $(85.7 \%)$ & $(81.8 \%)$ \\
\hline Level II & 26 & 1 & 7 & 13 & 3 & 0 \\
& $(11.9 \%)$ & $(1.9 \%)$ & $(16.3 \%)$ & $(37.1 \%)$ & $(10.7 \%)$ & $(0 \%)$ \\
\hline Level III & 15 & 1 & 5 & 2 & 1 & 2 \\
& $(6.9 \%)$ & $(1.9 \%)$ & $(11.6 \%)$ & $(5.7 \%)$ & $(3.6 \%)$ & $(18.2 \%)$ \\
\hline Level IV & 1 & 0 & 0 & 0 & 0 & 0 \\
& $(0.5 \%)$ & $(0 \%)$ & $(0 \%)$ & $(0 \%)$ & $(0 \%)$ & $(0 \%)$ \\
\hline
\end{tabular}

Table 1- MAUDE Classification System highlighting most common types of device complications 
useful for more standardized, clinically-relevant reporting of events.

MP12-6 A novel approach: simultaneous catch and blast Ureteroscopy lithotripsy with in situ dormia basket Vs standard stone fragmentation

HG Thummar, K Thummar, N Zinto, J Vyas, N T, P Suthar

sterling

India

Introduction \& Objective: Ureteroscopy is the gold standard surgical treatment option for impassable ureteral stone with minimal morbidity and good stone clearance. Stone fragment migration is worrisome problem during URS, especially using pneumatic lithotripsy. We compared our results of uretereoscopy in situ dormia basket stone fragmentation Vs standard fragmentation.

Materials and Methods: We divided randomly 30 patients of ureteroscopy in each group i.e. group A in situ dormia; group B control. We used 6fr ureteroscope which has dual channel. We studied various parameters prospectively like stone fragmentation time, stone migration rate, operative time, stone clearance rate, fluoroscopy time, need for stent placement and emergency room visit, cost.

Results: Stone fragmentation time, stone migration rate, operative time, fluoroscopy time, need for stent placement, and emergency room visit was significantly less in group A. While Stone clearance rate was significantly higher in group A compare to group B.

Conclusions: Stone fragmentation with simultaneous and in situ dormia basket gives better stone free rates and less stone migration, reduces operative time compare to standard stone fragmentation during urederoscopy.

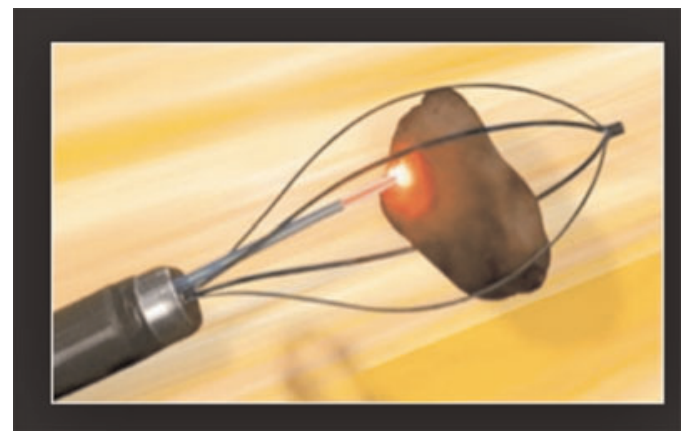

MP12-7 Computed tomography Hounsfield parameters for discrimination of predominantly composed calcium oxalate mono- and di- hydrate stones: preliminary results

A Gallioli, M Fontana, E De Lorenzis, L Boeri, S Zanetti, F Palmisano, M Talso, F Longo, P Dell'Orto, M Ferruti, E Montanari

Department of Urology, Fondazione IRCCS Ca' Granda Ospedale Maggiore Policlinico, Università degli Studi di Milano

Italy
Table 1. Type of surgery and stone characteristics at CT of the 2 groups.

\begin{tabular}{|c|c|c|c|}
\hline & $\mathrm{CaO} \times \mathrm{MH}$ & $\mathrm{CaO} \times \mathrm{DH}$ & P-value \\
\hline $\begin{array}{l}\text { Surgery } \\
\text { Ureteroscopy } \\
\text { RIRS } \\
\text { PCNL }\end{array}$ & $\begin{array}{l}20(38 \%) \\
20(38 \%) \\
12(26 \%)\end{array}$ & $\begin{array}{c}2(12 \%) \\
10(59 \%) \\
5(29 \%)\end{array}$ & 0,12 \\
\hline Stone Diameter & $10,85 \pm 5,75$ & $12,60 \pm 7,24$ & 0,31 \\
\hline Stone Area & $76,08 \pm 77,52$ & $85,00 \pm 76,65$ & 0,68 \\
\hline Stone Volume & $844,1 \pm 1399,0$ & $967,8 \pm 1525,0$ & 0,76 \\
\hline HUC & $1232,0 \pm 268,2$ & $1036,0 \pm 411,8$ & 0,03 \\
\hline HUP & $201,1 \pm 137,4$ & $179,6 \pm 40,7$ & 0,53 \\
\hline$\Delta \mathrm{HU}$ & $1027,0 \pm 291,4$ & $856,5 \pm 401,9$ & 0,06 \\
\hline HUD & $97,1 \pm 39,2$ & $72,6 \pm 33,8$ & 0,02 \\
\hline HUM & $894,6 \pm 232,3$ & $770,1 \pm 276,8$ & 0,07 \\
\hline
\end{tabular}

Introduction \& Objective: To evaluate the predictive value of Hounsfield (HU) parameters at pre-operative Computed Tomography (CT) for the discrimination between calcium oxalate mono- $(\mathrm{CaOxMH})$ and di- hydrate $(\mathrm{CaOxDH})$ stones.

Materials and Methods: We retrospectively analyzed 69 patients who underwent percutaneous or endoscopic lithotripsy from November 2010 to March 2017. Inclusion criteria were: I-II) the availability of a pre-operative CT-scan and the stone biochemical analysis, III) a maximum stone diameter $>4 \mathrm{~mm}$, IV) a $\mathrm{CaOx}$ stone component $>50 \%$. We divided the cohort in 2 groups: group $\mathrm{A}(\mathrm{CaOxMH})$ and group $\mathrm{B}(\mathrm{CaOxDH})$, including 52 and 17 patients respectively. All images were reviewed by a single urologist. Stone volume, HU mean value (HUM), core (HUC) and periphery (HUP) HU and their absolute difference $(\Delta \mathrm{HU})$ were evaluated at abdomen windows. HU density (HUD) was defined as the ratio between mean HU and the stone's largest diameter. Demographics and clinical data were included. Receiver Operating Characteristic (ROC) curves were calculated to test the predictive power of these parameters to differentiate $\mathrm{CaOxMH}$ from $\mathrm{CaOxDH}$. Cut-offs was defined as Youden J Index.

Results: The mean age was 57 years in both groups. Group A was composed by 35 men and 17 women, group B by 11 men and 6 women $(p=0,99)$.

Table 1 reports type of surgery and stone characteristics of the 2 groups. $\mathrm{CaOxMH}$ stones resulted hyperdense than $\mathrm{CaOxDH}$ in terms of HUD (mean \pm SD 97,1 $\pm 39,2$ vs $72,6 \pm 33,8 ; \mathrm{p}=0,02)$ and HUC (mean \pm SD $1232 \pm 268,2$ vs $1036 \pm 411,8 ; p=0,03)$. The other HU variables could not discriminate the 2 groups. HUD best differentiated the two groups (cut-off $85 \mathrm{HU} / \mathrm{mm}$; specificity $62 \%$, sensitivity $65 \%$; likelihood ratio 1,68). The AUC of HUD was 0,67 . In case of HUD $<85 \mathrm{HU} / \mathrm{mm}$ the probability of $\mathrm{CaOxDH}$ was 2,7 folds higher.

Conclusions: The current study evaluates the clinical applications of $\mathrm{HU}$ parameters in the characterization between $\mathrm{CaOxMH}$ and $\mathrm{CaOxDH}$ calculi. At present, there is no proven method to differentiate calcium oxalate stone types prior to endosurgery or SWL. We found that HUD is the best $\mathrm{HU}$ variable to distinguish the composition of calcium oxalate calculi. These findings can help the clinician to select the best candidates to SWL and could potentially drive the patient alimentary behaviour since $\mathrm{CaOxMH}$ and $\mathrm{CaOxDH}$ are generally due to hyperoxaluria and hypercalciuria, respectively. 
MP12-8 In patients with obstructing ureteral calculi, the severity of perinephric stranding is predictive of creatinine elevation

D Papagiannopoulos, J Ebersole, M Farrell, G White, LA Deane

University of California San Diego

United States

Introduction \& Objective: Patients who present to the emergency room with obstructing ureterolithiasis often have an elevated creatinine, which may lead to early intervention. Pyelovenous and pyelolymphatic backflow from acute ureteral obstruction, evident radiographically as perinephric stranding, may result in an artificially elevated serum creatinine. We explored a possible relationship between degree of perinephric stranding and changes to serum creatinine in those presenting with acute ureterolithiasis. Secondarily, we investigated potential associations between perinephric stranding and stone location, degree of hydronephrosis, and the presence of concomitant urinary tract infection.

Materials and Methods: After obtaining IRB approval, the institution's radiology dictation system (Fluency Discovery, M Modal) was queried for non-contrast CT abdomen/pelvis studies mentioning "ureteral stone" over a 10-month period (July-April 2016) from the Rush University Medical Center Emergency Room. A single radiologist, who was blinded to clinical data, reviewed all CT scans and coded stone size, location, and degree of perinephric fat stranding. For patients who met inclusion criteria, a retrospective chart review of pertinent clinical data was performed. Results: Of the 148 patients who met inclusion criteria, the mean patient age was 46 years (SD 14.6) and $55.7 \%(n=83)$ were male. Within this cohort, $32.4 \%(n=48)$ were admitted for pain $(n=23$, $47.9 \%)$, infection $(n=11,22.9 \%)$, stone size $(n=7,14.6 \%)$, acute kidney injury $(n=6,12.5 \%)$, or anemia $(n=1,2.1 \%)$. Ultimately, $29.7 \%(n=44)$ of patients required surgical intervention: primary ureteroscopy in $43.2 \% \quad(n=19)$ and staged ureteroscopy in $56.8 \%(n=25)$ of cases. Of the 100 patients with a baseline serum creatinine on file, those with moderate-severe perinephric stranding had greater odds of an elevated creatinine from baseline when compared to those with absent or mild stranding (OR 3.22, p=0.014). Degree of perinephric stranding was not associated with stone location, degree of hydronephrosis, or urine culture results $(\mathrm{p}>0.05)$.

Conclusions: In the setting of acute obstructive ureterolithiasis, patients with moderate-severe perinephric stranding had greater odds of a creatinine elevation from baseline. This elevation in creatinine, in the setting of obstructing ureteral calculi, may be a consequence of pyelovenous and/or pyelolymphatic backflow. Conversely, perinephric stranding was not associated with the presence of infection.

MP12-9 Is Limited Non-Contrast Pelvic CT really indicated for asymptomatic patients with small distal ureteric calculi?

N Hicks, W Finch, SO Irving, N Burgess

Norfolk and Norwich University Hospital United Kingdom

Introduction \& Objective: Our objective was to investigate whether patients with small asymptomatic distal ureteric calculi require follow-up imaging to confirm stone passage.
Materials and Methods: Patients were identified from a prospective database of all new cases of urolithiasis presenting in a UK teaching hospital over an 18 month period from January 2015 to June 2016. Those who had a computer-tomography (CT) confirmed distal ureteric calculus of $5 \mathrm{~mm}$ or less were included. Results: 14 cases were added to our database over the study period. Of which 192 patients met study inclusion criteria. 167 patients were managed conservatively. Of these 138 attended for follow-up over a mean time period of 5.38 weeks $(\mathrm{SD}=3.46)$. The remaining 29 patients were either re-admitted, were from out of area or did not attend follow-up. At follow-up, 107 patients did not have evidence of stone passage. 20 of these patients were symptomatic and a majority of 18 had a follow-up CT scan. Persistent calculus was demonstrated on three. The remaining two patients had radiographs; neither showed a calculus Of the 87 patients who were asymptomatic at follow-up 53 underwent a limited non-contrast pelvic CT to determine if their calculus remained in situ. Four $(7.5 \%)$ had not passed the calculus. The mean time between initial and follow-up CT was 10.66 weeks $(\mathrm{SD}=7.35)$. In 23 patients the decision was made mutually between patient and urologist for no further imaging. None have represented. Two patients did not attend for their follow-up CT scan. Of the remaining patients six had ultrasound and three had radiograph follow up. Persistent calculus was seen on one radiograph.

Conclusions: In this limited study, although the numbers are small, the detection rate for persistent small distal ureteric calculus in asymptomatic patients of $7.5 \%$ is important when considering follow-up in these cases. When the drawbacks of repeated CT imaging are weighed against the risk of undetected calculi (such as atrophic kidney) it would appear from these results we are still reliant of radiological follow-up

MP12-10 What Doses Are Our Patients Receiving? An audit examining the radiation dose associated with CT KUB, CT IVU and intra operative fluoroscopy during ureteroscopy and PCNL

M Sahu, P Patel, N Lobo, J Makanjuola, I Honey, D Gallagher, D Phillips, G Rottenberg, S Willis, J Glass, K Thomas, M Bultitude

Guy's and St Thomas' NHS Foundation Trust

United Kingdom

Introduction \& Objective: All urologists are familiar with standard textbook reference doses for CT scans and quote these to our patients. However, what doses are patients actually receiving in reality and do they conform to radiation guidelines? The typical dose length product (DLP) for CT KUBs and CT IVUs in the UK is $355 \mathrm{mGy}-\mathrm{cm}$ and $960 \mathrm{mGy}-\mathrm{cm}$ respectively (Public Health England,2011). We audited our practice as well as analysing doses received during stone procedures.

Materials and Methods: For CT data, patients were retrospectively identified from the Computerised Radiology Information System (CRIS ${ }^{\circledR}$ ). All CT KUBs between September 2014 and April 2015 were analysed. A smaller cohort of CT IVU patients were analysed between November 2015 and December 2015. Data on consecutive patients undergoing ureteroscopy and PCNL was collected prospectively. Estimated radiation doses were calculated.

Results: 669, 70, 67 and 24 patients underwent CT KUB, CT IVU, ureteroscopy and PCNL respectively. The median DLP was 
176.0mGy-cm and $639 \mathrm{mGy}-\mathrm{cm}$ for CT KUB and CT IVU respectively. This corresponds to an estimated radiation dose of $2.3 \mathrm{mSv}$ and $8.3 \mathrm{mSv}$. For ureteroscopy the dose-area product (DAP) was $158 \mu \mathrm{Gym}^{2}$ giving an effective dose of $0.2 \mathrm{mSv}-$ although this falls to $0.09 \mathrm{mSv}$ for patients with BMA

Conclusions: Median doses of radiation from CT KUB and CT IVU at our institution compare favourably with average doses reported nationally. We are now able to give informed local data to our patients to reassure them regarding radiation safety for diagnostic procedures and therapeutic interventions

MP12-11 Furs Versus PNL; Total Stone Volume Measurement By Non-Contrast CT (NCCT) Could Be Defined Precise Cut-off Value For The Management Of Procedures

\author{
M Ilgi, S Kirecci, C Kutsal, A Dalkilic
}

şişli hamidiye etfal eğitim araştırma hastahanesi

Turkey

Introduction \& Objective: Guidelines suggest that Furs or SWL for smaller than $2 \mathrm{~cm}$ kidney stone and PNL for bigger than $2 \mathrm{~cm}$. However; Calculi are asymmetric spherical bodies correlate with volume, to determine optimal patient group who proper for flexible ureteroscopy treatment for kidney stones, and the precise cut-off value to decide either Pnl or Furs as a total stone volume.

Materials and Methods: We have analysed retrospective cohort study of the 164 Furs procedures for renal stone retrieval between December 2012 with October 2016 at the single centre. Stone Free Rate of the procedure was controlled with KUB or CT at the end of the first month. The success rate was determined as the absence of stone fragments or clinical insignificant residual fragments $<4 \mathrm{~mm}$. Demographic features, Clinical and perioperative findings were collected. Area Under Curve (AUC) was used to define a relation between Total Stone Volume (TSV) and Stone Free Rate(SFR), and a Roc Curve was plotted to determine an absolute Cut-off value.

Results: The mean total stone volume(TSV) was $364.6 \mathrm{~mm}^{3}$, and the overall stone-free rate was 124 (\%75.6). The area under the

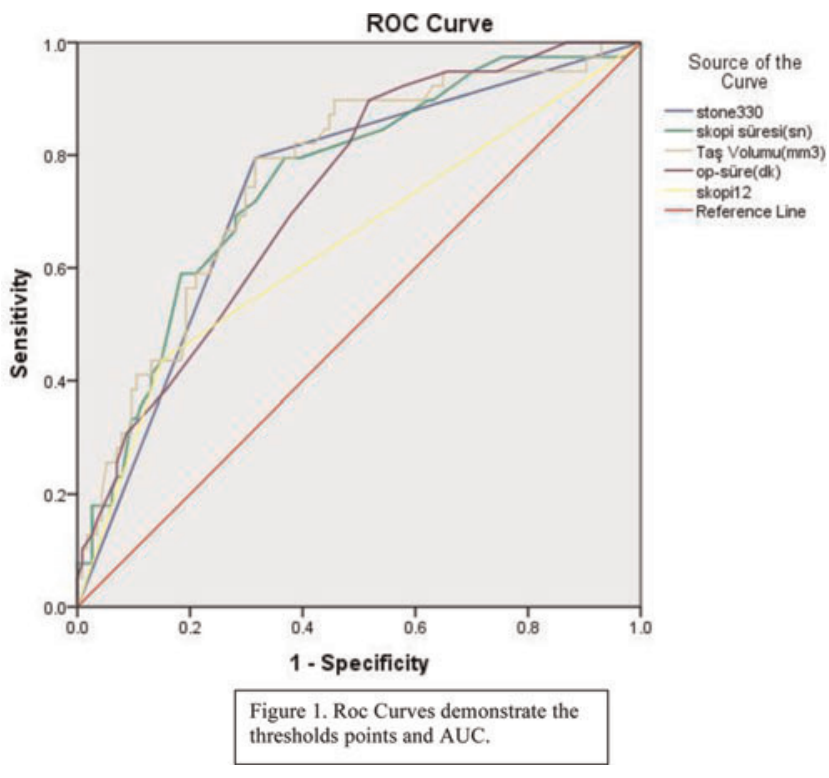

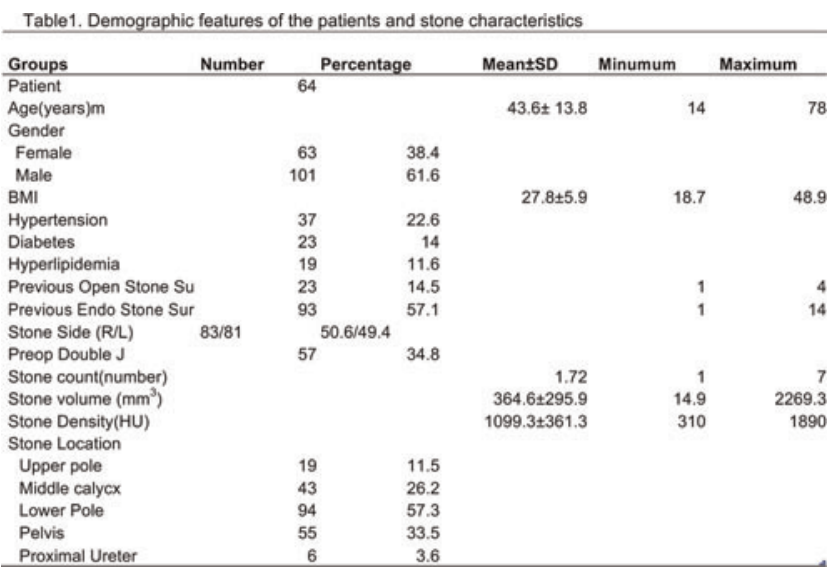

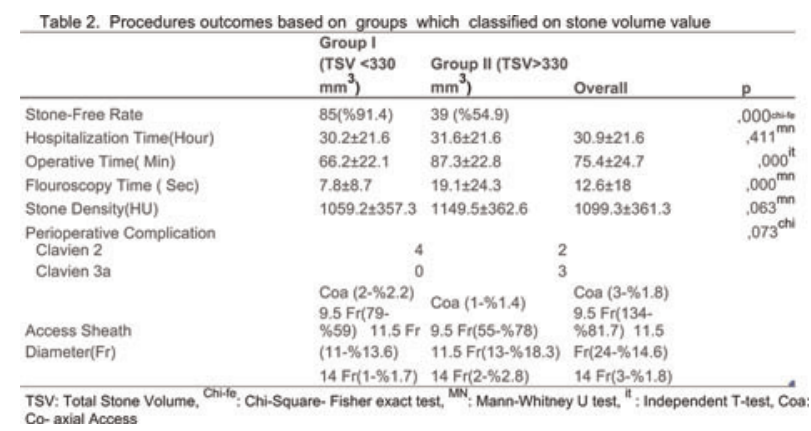

curve for the total stone volume was 0.743 . We identified that $330 \mathrm{~mm}^{3}$ showed the highest sensitivity and specificity to predict the stone-free status, therefore; this point was set a cutoff value for total stone volume. Patient count who has TSV smaller than $330 \mathrm{~mm}^{3}$ was 93 (\%56.7). The rest of patients (\%43.3) have over stone volume lower SFR and PNL or SWL can be recommended to increase the success rate of the procedure.

Conclusions: The Total Stone volume is the strongest impact on the SFR.TSV smaller than $330 \mathrm{~mm} 3$ Furs should be kept in mind firstly if it has a higher volume PNL can be an option at the Management of kidney stone patients treatment.

\section{MP12-12 Imaging Patterns After Percutaneous Nephro- lithotomy}

JS Ahn, S Holt, P May, MD Sorensen, JD Harper

Department of Urology, University of Washington Medical Center

United States

Introduction \& Objective: There are various imaging practices following PCNL. Imaging after ureteral instrumentation for nephrolithiasis is suggested by the American Urologic Association (AUA) to ensure stone fragment clearance, resolution of hydronephrosis, and rule out ureteral stricture formation. No prior studies to our knowledge have examined post-PCNL imaging patterns. We sought to characterize imaging utilization patterns following PCNL. 
Materials and Methods: Using Marketscan ${ }^{\circledR}$, a private employer-based insurance database, we identified patients 17-64 years old undergoing PCNL between 2007-2014. A minimum of 12 months of enrollment was required. We identified cumulative post-operative imaging modalities at 3, 6 and 12 months by CPT and ICD-9 codes, including computed tomography (CT), renal ultrasound (RUS), abdominal X-ray (KUB), and intravenous pyelogram (IVP). RUS or KUB imaging on the same day of the procedure was excluded to avoid inclusion of intra-operative imaging. Utilization patterns by demographic factors were assessed using chi-squared test.

Results: A total of 6,495 met inclusion criteria. 29\%,21\%, and $15 \%$ of patients had no imaging by 3,6 , and 12 months, respectively after their procedures. For patients that received postoperative imaging, the most common modality within 3 months was KUB (46\%), followed by CT (34\%), RUS (19\%), then IVP (4\%). This distribution remained relatively constant at 12 months. Of the $71 \% \%$ with imaging within 3 months, roughly half $(54 \%)$ were imaged within 3 days, most commonly CT $(61 \%)$, then KUB (37\%). At 3, 6, and 12 months, approximately $67 \%, 56 \%$, and $51 \%$, respectively, of PCNL patients lacked any anatomic imaging (RUS, CT, or IVP) that could identify obstruction. Over the 7-year study period, US increased by approximately $13 \%$, while CT and KUB rates remained relatively constant. Younger age and North Central region location were independently associated with higher rates of CT imaging. Living within a metropolitan statistical area or North East region was independently associated with higher rates of RUS.

Conclusions: National imaging patterns in adult insured patients undergoing PCNL are variable and differ by type and timing of imaging, patient age, and geographic location. Despite AUA recommendations to periodically obtain follow-up imaging in nephrolithiasis patients, nearly $30 \%$ of PCNL patients are not imaged within 3 months and $67 \%$ do not have anatomic imaging during that timeframe. $\mathrm{CT}$ is the most common postoperative imaging within 3 days.

\author{
MP12-13 Stone management in patients with ureter- \\ opelvic junction obstruction is possible not only by lithot- \\ omy during laparoscopic pyeloplasty but also by secondary \\ lithotripsy. \\ M Nishi, K Matsumoto, D Ishii, T Hirayama, \\ T Fujita, M Iwamura \\ Department of Urology, Kitasato University School of \\ Medicine \\ Japan
}

Introduction \& Objective: Laparoscopic pyeloplasty (LPP) is widely used to treat ureteropelvic junction obstruction (UPJO). Some patients with UPJO have kidney stones in the dilated renal cavity, and these stones can be removed during LPP. However, little is known about the outcomes of primary lithotomy during LPP and secondary lithotripsy after LPP. In the present study, we retrospectively reviewed the clinical features of patients with both UPJO and kidney stones who underwent LPP, and we analyzed the stone treatment outcomes from primary intraoperative lithotomy and secondary postoperative lithotripsy.

Materials and Methods: Between October 1998 and December 2015, 222 patients with 231 kidneys underwent LPP at our institution. In these patients, 18 patients with 20 kidneys had a stone at the time of LPP. We compared the UPJO patients with and without kidney stones in terms of the clinical features, including age, sex, laterality, BMI, configuration of hydronephrosis, renogram findings, and presence of crossing vessels. In 17 patients, 18 kidneys had clinically significant stones with a diameter over $3 \mathrm{~mm}$ located in the renal pelvis. We analyzed the outcomes for these stones treated with LPP followed by lithotripsy for the residual stones.

Results: Operative time were longer and crossing vessels were rarely seen in patients with stones than in those without stones $(\mathrm{p}=0.04$, respectively). Of 18 kidneys with clinically significant stones, 7 kidneys became stone free with lithotomy during LPP alone (38.8\%). Ten of 11 kidneys with residual stones were treated with secondary procedures ( 9 with shockwave lithotripsy [SWL], 1 with transurethral ureterolithotripsy [TUL]). Eight of 10 kidneys with residual stones became stone free; however, 1 kidney with residual stone did not receive further treatment because patient declined it. Finally, 15 of 18 kidneys $(83.3 \%)$ became stone free after primary and secondary procedures, with a median postoperative period of 30 months (range: 3-98). Treatment failed in 2 kidneys. After secondary procedures, both had both dilated calices; 1 was treated with SWL before LPP, leading to many tiny stones in the lower dilated calix.

Conclusions: Stone management for patients with UPJO can be done not only by lithotomy during LPP but also by secondary lithotripsy after LPP. UPJO patients with stones, especially those with dilated calices, should not be treated by lithotripsy prior to pyeloplasty.

MP12-14 Comparison of three different endoscopic techniques in treatment of bladder stones

J Choi, Y Ko, P Song, K Moon, H Jung, J Kim, Y Ha

Department of Urology, College of Medicine, Yeungnam University

Republic of Korea

Introduction \& Objective: The aim of this study was to compare three endoscopic techniques used in the treatment of bladder stones: transurethral use of cystoscope with pneumatic lithoclast or nephroscope with pneumatic lithoclast and nephroscope with ultrasonic lithoclast.

Materials and Methods: From January 2013 to May 2016, 107 patients of bladder stone underwent endoscopic treatment. The patients were classified three groups according to different endoscopic technique and energy modalities: group 1 (transurethral removal using cystoscope with pneumatic lithoclast), group 2 (transurethral removal using nephroscope with pneumatic lithoclast) and group 3 (transurethral removal using nephroscope with ultrasonic lithoclast). Baseline and

Table 1. Comparison of all the three groups for various variables

\begin{tabular}{lcccc}
\hline & Group 1 & Group 2 & Group 3 & P-value \\
\hline No of patients & 65 & 21 & 21 & \\
Male : female & $57: 8$ & $19: 2$ & $18: 3$ & 0.726 \\
Age (years) & $66.7 \pm 13.5$ & $67.9 \pm 13.3$ & $63.5 \pm 13.7$ & 0.622 \\
Stone burden (mm3) & $5238.2 \pm 4818.4$ & $4656.8 \pm 2252.1$ & $6350.7 \pm 5534.1$ & 0.472 \\
Operation time (mins) & $71.3 \pm 46.6$ & $33.0 \pm 13.7$ & $24.6 \pm 8.0$ & $<0.001$ \\
Mean urethral entries & $2.1 \pm 1.7$ & $1.03 \pm 0.12$ & $1.05 \pm 0.08$ & 0.081 \\
Hospital stay (days) & $22 \pm 0.9$ & $2.3 \pm 0.4$ & $2.1 \pm 0.3$ & 0.594 \\
\hline
\end{tabular}


perioperative data were retrospectively compared between three groups.

Results: No statistical significance was found in all the groups regarding the age, male to female ratio, stone size, [Table 1]. Statistical significant difference was observed in operating time: group 1 (71.3 \pm 46.6 mins), group $2(33.0 \pm 13.7$ mins $)$, and group 3 (29.6 \pm 8.0 mins). Complete clearance achieved in all the patients. Group 1 had more number of urethral entries as compared to other two groups in consideration. There was no significant complication in all cases.

Conclusions: Removal of bladder stones by the using a nephroscope have more advantage in operation time than using cystoscopy. Ultrasonic lithoclast is safe and efficacious energy modality, and also have advantage in operation time compared with pneumatic lithoclast.

MP12-15 Impact of preoperative $\alpha$-adrenergic antagonists on ureteral access sheath insertion force and the upper limit of force to avoid ureteral mucosal injury: A randomizedcontrolled study

K Koo, J Yoon, Y Ha, B Chung, D Lee

Yonsei University College of Medicine

Republic of Korea

Introduction \& Objective: Primary access of the ureteral access sheath (UAS) is not always possible and often excessive force is exerted, thereby increasing the risk of ureteral injury. A randomized controlled trial was performed to investigate the efficacy of preoperative alpha-blockade on reducing UAS insertion forces (UASIF) and to appreciate the upper limit of UASIF to avoid ureteral injury.

Materials and Methods: From December 2015 to October 2016, 88 patients with ureteropelvic junction or renal pelvis stones planned for retrograde intrarenal surgery (RIRS) were prospectively enrolled. Patients were randomly assigned to a control group $(n=37)$ or to an experimental group who received alpha-blockade with tamsulosin $0.4 \mathrm{mg}$ q.d. for seven days prior to RIRS $(n=39)$. Pre-stented patients were excluded from randomization $(n=12)$. A homemade UASIF gauge was adapted to measure the maximal UASIF at the ureterovesical junction (UVJ) and the proximal ureter. The degree of mucosal injury was recorded.

Results: UASIF of the alpha-blockade group was significantly lower than controls at the UVJ $(260.1 \pm 180.2 \mathrm{~g}$ vs. $524.2 \pm 237.5 \mathrm{~g}$; $\mathrm{p}=0.017)$, however, not at the proximal ureter $(367.2 \pm 175.2 \mathrm{~g}$ vs. $647.7 \pm 294.3 \mathrm{~g} ; \mathrm{p}=0.054)$. The alpha-blockade group exhibited comparable UASIF with the pre-stented group at the UVJ

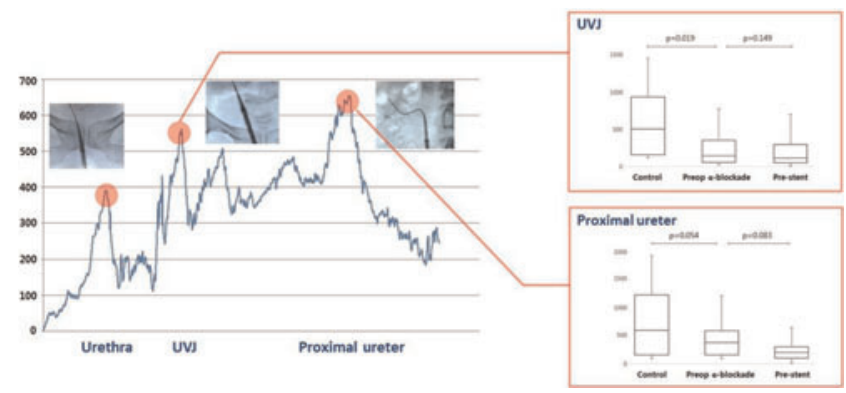

$(260.1 \pm 180.2 \mathrm{~g}$ vs. $99.8 \pm 19.9 \mathrm{~g} ; \mathrm{p}=0.149)$ and the proximal ureter $(367.2 \pm 175.2 \mathrm{~g}$ vs. $131.4 \pm 75.2 \mathrm{~g} ; \mathrm{p}=0.081)$. The rate of mucosal injury was lower in the alpha-blockade group compared to controls $(p=0.028)$. Mucosal injury ( $\geq$ grade 2$)$ did not occur in cases with UASIF

Conclusions: Preoperative alpha-blockade mimics the effect of pre-stenting and reduces maximum UASIF and consequent risk of ureteral injury. If the UASIF exceeds $600 \mathrm{~g}$, the procedure could be terminated with stent placement and followed later by pre-stented RIRS.

MP12-16 Effects of Laser Settings and Irrigation Rates on Ureteral Temperature During Laser Lithotripsy, an In Vitro Model

D Wollin, B Winship, W Tom, C Scales, M Ferrandino, W Simmons, GM Preminger, M Lipkin

Duke University Medical Center

United States

Introduction \& Objective: Ureteroscopic laser lithotripsy requires constant irrigation for visualization within the upper urinary tract while also allowing for temperature stabilization. Despite this need, there is little data on the effect of different irrigation and laser settings on the ureteral wall and surrounding tissues. This becomes especially important given the fact that thermal cytotoxicity has been shown at temperatures greater than $41^{\circ} \mathrm{C}$. Our aim was to evaluate the effect of laser settings and irrigation flow on ureteral temperature in an in vitro setting.

Materials and Methods: An in vitro setup was developed to mimic ureteroscopic laser lithotripsy allowing for clinically relevant irrigation flow and near-continuous temperature monitoring. The probe tip of a multilogger thermometer (OMEGA Engineering, Stamford, CT) was positioned $1 \mathrm{~mm}$ from the tip of the laser fiber, which was fired for 60 seconds at various settings $(0.2 \mathrm{~J} / 50 \mathrm{~Hz}, 0.6 \mathrm{~J} / 6 \mathrm{~Hz}, 0.8 \mathrm{~J} / 8 \mathrm{~Hz}, 1 \mathrm{~J} / 10 \mathrm{~Hz}, 1 \mathrm{~J} / 20 \mathrm{~Hz})$ within a tubing system that allowed for specified flow rates $(100 \mathrm{cc} / \mathrm{min}$, $50 \mathrm{cc} / \mathrm{min}, 0 \mathrm{cc} / \mathrm{min})$. Temperatures were logged at 5 second intervals, along with the overall maximum temperature for each trial. Each laser/flow setting was repeated in duplicate. Maximum temperature values for each trial were compared using ANOVA across irrigation settings.

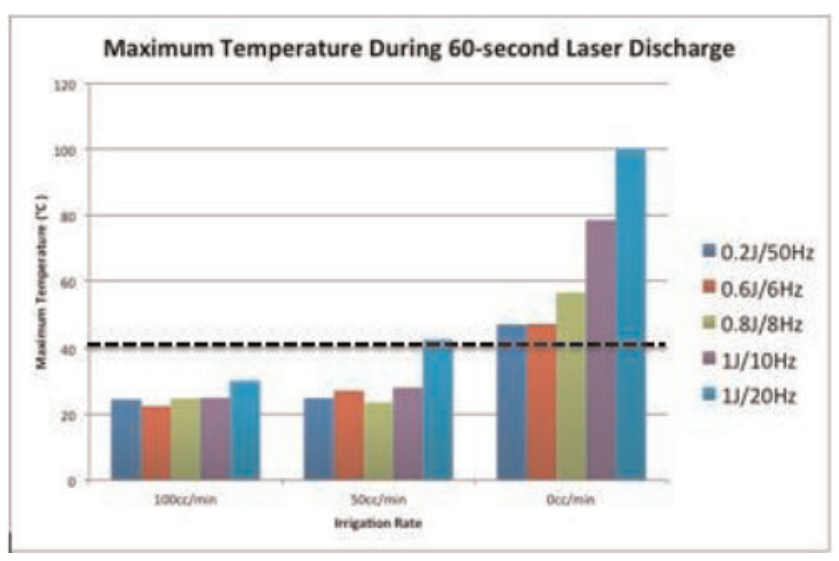


Results: At an irrigation rate of $100 \mathrm{cc} / \mathrm{min}$, only the $1 \mathrm{~J} / 20 \mathrm{~Hz}$ laser setting produced a significantly higher maximum temperature $(\mathrm{p}<0.01)$, although this was not clinically significant at a maximum of $30.7^{\circ} \mathrm{C}$. At a lower irrigation rate of $50 \mathrm{cc} / \mathrm{min}$, the $1 \mathrm{~J} / 20 \mathrm{~Hz}$ setting was again the only significantly higher maximum temperature $(\mathrm{p}<0.03)$, although this temperature crossed the toxic threshold at a maximum of $43.4^{\circ} \mathrm{C}$. With no flow, all maximum temperatures reached over $41^{\circ} \mathrm{C}$, with $0.8 \mathrm{~J} / 8 \mathrm{~Hz}$, $1 \mathrm{~J} / 10 \mathrm{~Hz}$, and $1 \mathrm{~J} / 20 \mathrm{~Hz}$ each statistically higher than the lowerenergy settings $(p<0.05)$. Maximum temperature at $1 \mathrm{~J} / 20 \mathrm{~Hz}$ with no irrigation was over $100^{\circ} \mathrm{C}$, which produced boiling of the fluid column.

Conclusions: Despite increasing laser settings, adequate irrigation is able to maintain a relatively stable temperature within an in vitro ureteral system. As irrigation rates decrease, even lower power laser settings produce a statistically and clinically significant increase in maximum temperature, potentially causing tissue injury. Animal models may help to further quantify this damage and assess optimal laser and flow settings for ureteroscopic lithotripsy.

MP12-17 Vaporizing effect of popcorn technique for laser lithotripsy; comparing the different setting of high energy in calyceal model.

K Jongjitaree, E Chotikawanich

Siriraj hospital, Mahidol university, Thailand Thailand

Introduction \& Objective: To assess the effectiveness of laser lithotripsy in each setting of Ho-YAG laser along a wide range of energies, frequencies and power. Outcomes of this study were the amount of remained stone fragment after laser lithotripsy, time to stone disintrigation and fiber laser degenaration.

Materials and Methods: Two different types of phantom stones were made, soft stone, which mimic to uric acid stone, and hard stone, which mimic to calcium oxalate monohydrate stone. Both types of stone were made into round shape, $10 \mathrm{~mm}$ in diameter. All phantom stones were fired with multiple energy setting of Ho:YAG laser, using a $200 \mu \mathrm{m}$ diameter laser fiber with noncontact technique (popcorn). The lithotripsy setting was devided in to 5 settings, $1 \mathrm{Jx} 20 \mathrm{~Hz}, 2 \mathrm{Jx} 10 \mathrm{~Hz}, 1.5 \mathrm{Jx} 20 \mathrm{~Hz}, 3 \mathrm{Jx} 10 \mathrm{~Hz}$, $2 \mathrm{~J} \times 20 \mathrm{~Hz}$. The lithotripsy was conducted in calyceal model which had $2 \mathrm{~mm}$ filter. All stone vanishing from artificial calyx was the end point of the experiment. All fragments that pass through the filter of each setting were dried and weight to compare residual fragments. Vaporizing effect was calculated and compared among the settings.

Results: In our study, the setting that had the greatest disintegration efficiency in term of time for hard stone were $3 \mathrm{Jx} 10 \mathrm{~Hz}$ and $2 \mathrm{Jx} 20 \mathrm{~Hz}$ at mean time $9 \mathrm{~min} 55 \mathrm{sec}$ and $8 \mathrm{~min} 51 \mathrm{sec}$, respectively. However there was no different upon setting for soft stone. The setting that had the greastest amount of remained stone fragmentation was $1 \mathrm{Jx} 20 \mathrm{~Hz}$. It caused residual fragments in hard stone $19.02 \%$ while greater than the best setting, $2 \mathrm{~J} \times 20 \mathrm{~Hz}, 11.2 \%$ (p-valve $=0.006$ ). Fiber tip degeneration was longer in hard stone comparing with soft stone (p-value

Conclusions: The fastest settings with maximum vaporizing effect for hard stone were $2 \mathrm{Jx} 20 \mathrm{~Hz}$ and $3 \mathrm{Jx} 10 \mathrm{~Hz}$. Fiber tip degenaration was responsible to stone hardness not setting of the laser.
MP12-18 How Do You Like Your Popcorn? An Evaluation of Laser Settings and Location in the Efficiency of the Popcorn Effect

D Wollin, B Winship, R Jiang, W Tom, D Radvak, C Scales, M Ferrandino, W Simmons, GM Preminger, M Lipkin

Duke University Medical Center

United States

Introduction \& Objective: There are many techniques for laser lithotripsy of urinary stones. The "popcorn" method involves placing a laser fiber in the center of a collection of stones and firing continuously, allowing fragments to further dust into smaller particles. Our aim was to examine different locations and laser settings on the efficiency of this lithotripsy method.

Materials and Methods: Pre-fragmented BegoStone phantoms were created between $2-4 \mathrm{~mm}$ in size to mimic typical popcorning conditions. A $0.5 \mathrm{~g}$ collection of fragments was placed into two 3D-printed models (a $2 \mathrm{~cm}$ spherical calyx model and $4 \times 2 \mathrm{~cm}$ ellipsoid pelvis model, Figure 1) and a $200 \mu$ laser fiber

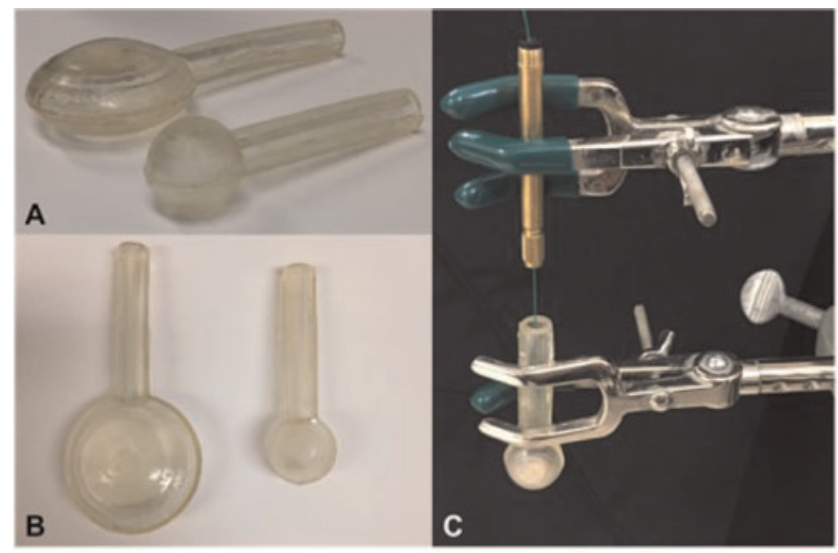

Figure 1: 3D-printed calyceal and pelvis models (A, B) and experimental setup (C)

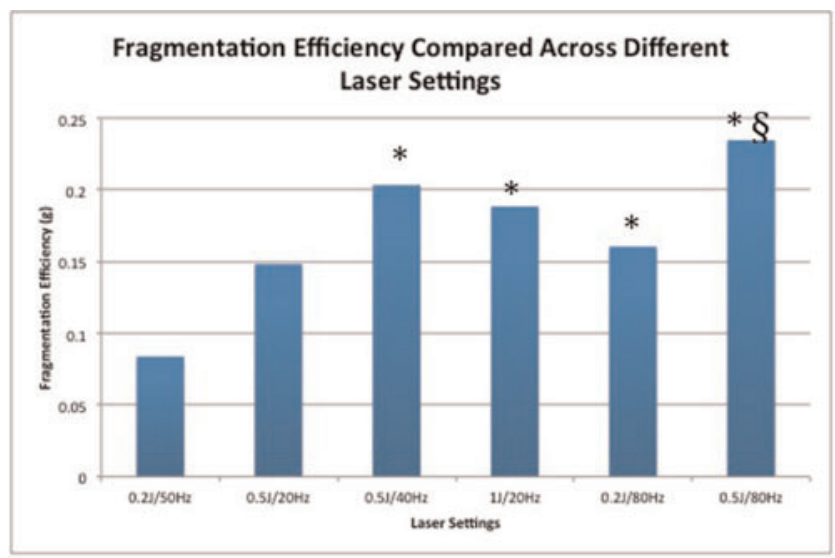

Figure 2: Fragmentation efficiency (as calculated by mass lost as sub-2 $\mathrm{mm}$ particles) across different lase settings ( $(*$, significantly different from $0.2 \mathrm{~J} / 5 \mathrm{OHz}$; 5 , significantly different from $0.5 \mathrm{~J} / 2 \mathrm{Hzz}$ ) 
was positioned at the top of the stones. The laser was fired for 2 minutes with constant irrigation, with 5 trials performed at each setting: $0.2 \mathrm{~J} / 50 \mathrm{~Hz}, 0.5 \mathrm{~J} / 20 \mathrm{~Hz}, 0.5 \mathrm{~J} / 40 \mathrm{~Hz}, 1 \mathrm{~J} / 20 \mathrm{~Hz}, 0.2 \mathrm{~J} /$ $80 \mathrm{~Hz}, 0.5 \mathrm{~J} / 80 \mathrm{~Hz}$. The fragmentation efficiency was determined by calculating the mass of stones reduced to sub- $2 \mathrm{~mm}$ particles after $48 \mathrm{~h}$ of drying. Statistical analysis was performed with ANOVA and Student's T-test. Additionally, high-speed photography was used to examine the mechanism of the popcorn effect.

Results: The trials within the calyx model were significantly more efficient compared to the pelvis model $(0.19 \mathrm{~g}$ vs $0.15 \mathrm{~g}, \mathrm{p}=0.01)$. When comparing laser settings, there was a difference between groups by one-way ANOVA $\left(\mathrm{F}[5,54]=8.503, \mathrm{p}=5.47 \times 10^{-6}\right)$. Post hoc tests showed that $0.5 \mathrm{~J} / 80 \mathrm{~Hz}$ was significantly more efficient than the low-power settings $0.2 \mathrm{~J} / 50 \mathrm{~Hz}$ and $0.5 \mathrm{~J} / 20 \mathrm{~Hz}$ $(\mathrm{p}<0.05)$. Additionally, $0.2 \mathrm{~J} / 50 \mathrm{~Hz}$ was significantly less efficient than $0.5 \mathrm{~J} / 40 \mathrm{~Hz}, 1 \mathrm{~J} / 20 \mathrm{~Hz}$, and $0.2 \mathrm{~J} / 80 \mathrm{~Hz}$ as well (Figure 2). Highspeed imaging shows the majority of fragmentation is due to intermittent stone contact with the laser as opposed to stone-stone interaction.

Conclusions: The popcorn effect is most efficient in a smaller space as in the calyx model and as such we recommend displacement of stones into a calyx for popcorning. No statistical difference was seen between high-power settings, although $0.5 \mathrm{~J} /$ $40 \mathrm{~Hz}$ and $0.5 \mathrm{~J} / 80 \mathrm{~Hz}$ performed best, suggesting that a moderate energy popcorning method with at least $0.5 \mathrm{~J}$ per pulse is the most efficient setting.

MP12-19 Digital flexible ureteroscopy: Olympus vs Storz technology differences

P Geavlete, D Georgescu, M Jecu, R Multescu, B Geavlete

Department of Urology,'Saint John" Emergency Clinical Hospital, Bucharest, România;

Romania

Introduction \& Objective: In modern urological practice, flexible ureteroscopy became a routine procedure in many centers. The aim of this study was the comparison of feasibility a two of the new instruments.

Materials and Methods: We evaluated, in our clinic, 40 flexible ureteroscopy used for minimally-invasive treatment of pyelocaliceal lithiasis. In 20 cases we used Flex-Xc endoscope whereas in others patients it was utilized Olympus URF-V2 ureteroscope. The procedures were performed by two surgeons who used alternatively these technologies. At the end of the interventions, the surgeons have noted the performance of the two instruments with a score between 1 to 5. They were analyzed maneuverability, visibility, irrigation flow and maximum deflection.

Results: Both models have demonstrated good handling, with a slight advantage for Flex-Xc. Regarding visibility, no significant differences were recorded, however URF-V2 was being designated slightly higher. Regarding loss of deflection and irrigation flow during use of auxiliary tools results were similar. However, it has been found that the average loss of deflection was higher at Flex-Xc in comparison with URF-V2. Finally, the assessments of the two surgeons were significantly close: 4.75 for URF-V2 and 4.65 for Flex-Xc respectively.
Conclusions: The latest models of flexible ureteroscopes have proved to be effective instruments for upper urinary tract endoscopic interventions. Huge technological advances of recent years gives us confidence of improved new tools that meet the highest standards.

MP12-20 Dual Usage of a Stone Basket: Stone Capture and Retropulsion Prevention.

T Kroczak, D Ghiculete, R Sowerby, K Pace, R D’ A. Honey

St. Michael's Hospital - University of Toronto

Canada

Introduction \& Objective: Stone migration during ureteroscopy (URS) for proximal ureteric calculi is a constant challenge. Several retropulsion prevention devices have been developed in order to optimize URS outcomes. Our technique involves capturing the stone within a four wire Nitinol stone basket and then preforming laser lithotripsy to dust the stone while it is engaged in the basket. The dusted fragments wash out with the irrigation fluid and once small enough the remaining stone is removed intact.

Materials and Methods: A retrospective chart review was preformed of all proximal URS procedures preformed with semi rigid URS for a solitary calculus (2000-2016). Patient characteristics, procedure time, stone size and composition, retropulsion rate, use of flexible URS, stone free rate, and complications were collected. We compared our new technique introduced in 2010 to URS control procedures that did not use retropulsion prevention techniques or devices. Chi squared analysis and ANOVA were used for statistical analysis.

Results: One hundred and fifty two patients (97 males \& 55 females) underwent URS for proximal ureteric calculi. Mean stone diameter was $9.3 \pm 3.4 \mathrm{~mm}$ with similar impaction rate between both groups $(44.3 \%$ vs. $42.5 \%$ control, $\mathrm{p}=0.587)$. The mean surgical procedure time was $53.3 \mathrm{~min} \pm 17.9$ for the new technique and $65.2 \mathrm{~min} \pm 29.2$ for the control group $(\mathrm{p}=0.005)$. Compared to the new technique, the control group had a higher rate of retropulsion ( $16.5 \%$ vs. $34.3 \%$, respectively, $\mathrm{p}=0.011$ ) and required flexible URS more often to exclude or remove residual fragments $(24.1 \%$ vs. $59.1 \%$, respectively, $\mathrm{p}=0.001)$. Using the new technique stone free rates were higher (78.2\% vs. $68.5 \%, p=0.177)$ and there was a lower likelihood of leaving residual fragments both $<3 \mathrm{~mm}$ and $\geq 3 \mathrm{~mm}(\mathrm{p}=0.002)$. Rate of ureteric injury and secondary procedures were similar between both techniques.

Conclusions: Our novel technique results in shorter operative time, lower retropulsion rates and decreases post-operative residual stone fragments.

MP12-21 A Multicenter, Prospective, Observational Study to Investigate the Feasibility of the Disposable Flexible Ureterorenoscopy (Lithovue ${ }^{\circledR}$ ) in Patients with Renal Stones

S Cho, J Lee, D Shin, I Seo, H Park

SMG-SNU Boramae Medical Center

Republic of Korea

Introduction \& Objective: The aim of this study is to investigate the feasibility of the disposable Flexible Ureterorenoscopy 


\begin{tabular}{|c|c|}
\hline Variables & Variables \\
\hline \multicolumn{2}{|l|}{ Patient characteristics } \\
\hline No & 62 \\
\hline Age (years) $( \pm \mathrm{SD})$ & $57.3( \pm 13.9)$ \\
\hline Male : female (\%) & $38(61.3 \%): 24(38.7 \%)$ \\
\hline Body mass index $\left(\mathrm{kg} / \mathrm{m}^{2}\right)( \pm \mathrm{SD})$ & $26.0( \pm 4.6)$ \\
\hline \multicolumn{2}{|l|}{ Creatinine (mg/dl) } \\
\hline Preoperative $( \pm S D)$ & $0.9( \pm 0.3)$ \\
\hline Change within 1 months postoperative ( $\pm \mathrm{SD}$ ) & $0.9( \pm 0.3)$ \\
\hline \multicolumn{2}{|l|}{ Estimated GFR (mL/min/1.73 m²) } \\
\hline Preoperative $( \pm S D)$ & $83.3( \pm 20.4)$ \\
\hline Change within 1 months postoperative ( $\pm \mathrm{SD}$ ) & $-1.0( \pm 1.5)$ \\
\hline \multicolumn{2}{|l|}{ Hemoglobin (mg/dl) } \\
\hline Preoperative ( \pm SD) & $13.7( \pm 1.8)$ \\
\hline Immediate postoperative minus preoperative $( \pm S D)$ & $-0.9( \pm 1.5)$ \\
\hline Diabetes/ hypertension & $13(21.0 \%) / 26(41.9 \%)$ \\
\hline \multicolumn{2}{|l|}{ Stone characteristics } \\
\hline Previous ESWL history & $13(21.0 \%)$ \\
\hline Previous URS history & $3(4.8 \%)$ \\
\hline Presence of hydronephrosis without obstruction & $27(43.5 \%)$ \\
\hline Infundibulo-pelvic angle $($ IPA $)\left({ }^{\circ}\right)( \pm S D)$ & $53.1( \pm 18.2)$ \\
\hline Houns-field unit $( \pm S D)$ & $761.9( \pm 348.6)$ \\
\hline Diverticular stone & $1(1.6 \%)$ \\
\hline Stone laterality: Right / Left & $29(46.8 \%) / 33(53.2 \%)$ \\
\hline Radiopaque/radiolucent & $51(82.3 \%) / 11(17.7 \%)$ \\
\hline S-ReSC scores: $1-2 / 3-4 / \geq 5$ & $49(79.0 \%) / 9(14.5 \%) / 4(6.4 \%)$ \\
\hline Maximal stone size $(\mathrm{mm})( \pm \mathrm{SD})$ & $15.4( \pm 5.4)$ \\
\hline Total stone volume $\left(\mathrm{mm}^{3}\right)( \pm \mathrm{SD})$ & $1376.2( \pm 1433.2)$ \\
\hline Number of stones ( $\pm \mathrm{SD}$ ) & $2.0( \pm 16)$ \\
\hline \multicolumn{2}{|l|}{ Main stone composition } \\
\hline Calcium oxalate monohydrate & $48(77.4 \%)$ \\
\hline Uric acid & $11(17.7 \%)$ \\
\hline Carbonate apatite & $3(4.8 \%)$ \\
\hline
\end{tabular}

(Lithovue ${ }^{\circledR}$ ) in patients with renal stones in a prospective, observational study.

Materials and Methods: Consecutive patients who underwent planned unilateral ureterorenoscopic stone surgery by Lithovue ${ }^{\circledR}$ were included. Sixty-two patients were enrolled considering $20 \%$ drop-out rate. To evaluate the feasibility of Lithovue ${ }$, surgeons' perspectives on the maneuverability, general perception about visibility of the monitor and the irrigation systems were evaluated: 1 (very good), 2 (good), 3 (not different), 4 (poor), and 5 (very poor).

Results: The mean age was $57.3 \pm 13.9$ years and the stone size was $15.4 \pm 5.4 \mathrm{~mm}$. Clinical success of the overall stone free rate is $82.3 \%$. Complication occurred in 4 cases of the Clavien classification grade I in a single case (hematuria and pain) and II in 3 cases (fever). The comparative parameters of (1) maneuverability, (2) perception score of the monitor system and (3) perception score of the irrigation channel were 2.5 (good to no difference), 2.5, and 3.0 (no difference), respectively. Maximal deflection angle of 270 degrees were preserved in 53 cases
$(85.5 \%)$. The impaired deflection angles at the end of surgery were 260 degrees ( 1 case), 240 degrees ( 3 cases), 230 degrees (1 case), and 180 cases ( 4 cases).

Conclusions: Lithovue ${ }^{\circledR}$ was feasible for surgical removal of renal stones. The strong points were maneuverability and the monitor system. Poor judgment was predominantly related to the irrigation system.

MP12-22 Laser versus pneumatic lithotripsy with semi rigid ureteroscope. A comparative randomized study.

S Rabani, s rabani

Yasuj Unhversity of Medical Sciences Iran

Introduction \& Objective: Ureteral stones are among the most common disorders in urologic field. Miniaturization of endoscopic devices in urology and extracorporeal shock wave lithotripsy (SWL) has revolutionized management of ureteral stones. The aim of this study was to compare the efficacy and results of Laser versus pneumatic lithotripsy with semi rigid ureteroscope in a randomized prospective clinical trial.

Materials and Methods: During a prospective randomized clinical trial between February 2015 and September2016, 117 adult patients underwent TUL. Ureteroscope 8-9.8 f (wolf. Germany) was used for stone access. Exclusion criteria were: uncorrected coagulopathy, pregnancy, deformity and inability to have lithotomy position, and inability to access the stone during URS. The patients were randomized in 2 groups: In group one 58 patients with ureteral stones underwent ureteroscopy (URS) and stone fragmentation was done by holmium: yttrium-aluminumgarnet (Ho:YAG) laser lithotripsy (LL) and in group 2, 59 patients underwent pneumatic lithotripsy (PL) by using the same ureteroscope.

Results: Mean age was 41.77 years and 41.1years in group one and 2 respectively ( $\mathrm{p}$ value: 0.79 ), mean stone size was $9.29 \mathrm{~mm}$ and $9.77 \mathrm{~mm}$ in group one and 2 respectively ( $\mathrm{p}$ value: 0.45 ), male to female ratio was 36 to 22 and 40 to 19 in group one and 2 respectively ( $\mathrm{p}$ value: 0.56 ). The success rate for stone clearance was $79.31 \%$ and $77.96 \%$ in group one and 2 respectively ( $\mathrm{p}$ value 0.52 ). Complications were shown in table one for both groups. Duration for operations was shown in table 2 for both groups.

Conclusions: In this study, we evaluated the results of the two technique in patients with ureteral stones. According to the results of this study no significant difference was seen between demographic characters and size of the stones in the two groups. In both techniques acceptable results were achieved, but in pneumatic group, the duration of operatin was shorter and the cost was less thanLL, that may be a result of more experience in working with pneumatic lithotripsy in our center. Ureteroscopic interventions can cause complications such as ureteral avulsion, access problem, stone migration, urosepsis, and finally ureteral stricture. There was no major complication in our study and also no statistical significant differences ( $\mathrm{p}$ value: 0.7 ), between the two groups, but upward migration of the stone fragments was more common in pneumatic group, albeit with no significant statistical outcome ( $p$ value: 0.7 ). Finally, this study shows that pneumatic lithotripsy may have advantages of the same efficacy, but is less expensive, and may have a shorter time of operation in some centers. 


\section{MPS13: PCNL III OUTCOMES}

MP13-1 Short and long-term Quality of life (QoL) in patients with percutaneous nephrostomy (PCN), and comparision to other forms of urinary drainage: Systematic review of literature

F New, B Somani

Royal Bournemouth Hospital

United Kingdom

Introduction \& Objective: To review the quality of life (QoL) in patients undergoing percutaneous nephrostomy (PCN) compared directly to other forms of urinary drainge in benign and malignant disease.

Materials and Methods: A review of literature from inception to Apil 2017 for all prospective English language articles comparing QoL in patients with benign or malignant ureteric obstruction undergoing PCN compared to other methods of urinary drainage. Articles which did not have QOL as their primary outcome were excluded, and articles that did not compare PCN to other forms of ureteral drainage were excluded.

Results: Five studies (169 patients) were included. Three compared PCN to JJ stents in benign disease, of these three all showed similar QOL in both groups, but all favoured the ne-

Table 1: Details of all studies reporting on long-term QOL (Quality of life)

\begin{tabular}{|l|l|l|l|l|l|l|l|}
\hline Author & $\begin{array}{l}\text { Year } \\
\text { Published }\end{array}$ & Journal & $\begin{array}{l}\text { Review } \\
\text { period }\end{array}$ & M:F & $\begin{array}{l}\text { Mean } \\
\text { age } \\
\text { (years }\end{array}$ & $\begin{array}{l}\text { Patient } \\
\text { number }\end{array}$ & $\begin{array}{l}\text { QOL tool } \\
\text { used }\end{array}$ \\
\hline $\begin{array}{l}\text { Desgrandchamps } \\
\text { F, }\end{array}$ & 2007 & $\begin{array}{l}\text { J } \\
\text { Endourology }\end{array}$ & $\begin{array}{l}2006- \\
2007\end{array}$ & $9: 10$ & 65 & 19 & $\begin{array}{l}\text { EORTC } \\
\text { QLC-30 }\end{array}$ \\
\hline Monsky, W & 2013 & $\begin{array}{l}\text { Cardiovasc } \\
\text { Intervent } \\
\text { Radiol }\end{array}$ & $\begin{array}{l}2012- \\
2013\end{array}$ & $19: 26$ & & 46 & FACT BL \\
\hline Joshi, H B & 2001 & $\begin{array}{l}\text { J } \\
\text { Endourology }\end{array}$ & $\begin{array}{l}1999- \\
2000\end{array}$ & $22: 12$ & 56 & 34 & $\begin{array}{l}\text { EuroQOL } \\
\text { EQ-5D }\end{array}$ \\
\hline Phillip T & 2016 & $\begin{array}{l}\text { J } \\
\text { Endourology }\end{array}$ & $\begin{array}{l}2015- \\
2016\end{array}$ & $16: 13$ & 56 & 30 & $\begin{array}{l}\text { Wisconsin } \\
\text { stone } \\
\text { QOL }\end{array}$ \\
\hline Mokhmalji, H & 2001 & $\begin{array}{l}\text { J } \\
\text { Endourology }\end{array}$ & $\begin{array}{llll}1996- \\
1998\end{array}$ & $21: 19$ & 52 & 40 & $\begin{array}{l}\text { German } \\
\text { WHO } \\
\text { HRQOL } \\
\text { index and } \\
\text { Institute } \\
\text { of mental } \\
\text { health } \\
\text { Mannheim }\end{array}$ \\
\hline
\end{tabular}

Table 2: Details of the type of intervention and the disease itself.

\begin{tabular}{|l|l|l|l|l|}
\hline Author & $\begin{array}{l}\text { Nephrostomy } \\
\text { only (no. pts) }\end{array}$ & $\begin{array}{l}\text { Neph vs stent } \\
\text { (no. pts) }\end{array}$ & $\begin{array}{l}\text { Neph vs extra } \\
\text { anatomical } \\
\text { stent (no. pts) }\end{array}$ & $\begin{array}{l}\text { Benign or } \\
\text { malignant } \\
\text { disease }\end{array}$ \\
\hline $\begin{array}{l}\text { Desgrandchamps } \\
\text { F, }\end{array}$ & 0 & 0 & 19 & Malignant \\
\hline Monsky, W & 15 & 15 & 15 & Malignant \\
\hline Joshi, HB & 13 & 21 & 0 & $\begin{array}{l}\text { Benign } \\
\text { (stone } \\
\text { obstruction) }\end{array}$ \\
\hline Phillip T & 0 & 30 & 0 & $\begin{array}{l}\text { Benign } \\
\text { (post } \\
\text { PCNL) }\end{array}$ \\
\hline Mokhmalji, H & 0 & 40 & 0 & $\begin{array}{l}\text { Benign ( } \\
\text { obstructing } \\
\text { stones) }\end{array}$ \\
\hline
\end{tabular}

phrostomy group due to increased urinary symptoms in the JJ stent group. Two of the studies compared QOL in malignant diease, comparing PCN to extra-anatomical stents and internal JJ stents. One of the studies showed that the extra-anatomical stent had better QOL than the PCN group, however the other study demonstrated that the PCN group had a better QOL than the JJ stent group due to urinary symtoms.

Conclusions: In patients with malignant or benign obstruction undergoing $\mathrm{PCN}$ or other forms of urinary drainge, QOL is slightly improved in PCN, due the decreased urinary symptoms. However the overall QOL was shown to be equal in all groups and the treatment choice should be made on an individual bases.

MP13-2 Outcomes of Retrogade Intrarenal Surgery in Shockwave Lithotripsy Failed Intermediate Size Lower Calyceal Calculi

B Singh, K Kaura, S Sankhwar, A Goel

King George's Medical university India

Table 1. Patient Characteristics and Outeomes of RIRS in Shockwave Failed (SWF) vs. Treatment Native (TN) Calculi Groups

\begin{tabular}{|c|c|c|c|}
\hline Variable & $\begin{array}{c}\text { Group A, SWF } \\
\underset{(\mathrm{n}=32)}{ }\end{array}$ & $\begin{array}{l}\text { Group B, TN } \\
(n=32)\end{array}$ & P value \\
\hline Age (years) (Mean \pm SD & $50.40 \pm 9.80$ & $47.65 \pm 5.20$ & 0.235 \\
\hline Sex (Men/Women) (Mean \pm SD & $24 / 08$ & $22 / 10$ & 0.781 \\
\hline Body mass index $\left(\mathrm{kg} / \mathrm{m}^{2}\right)($ Mean \pm SD & $27.31 \pm 5.90$ & $26.40 \pm 6.30$ & 0.553 \\
\hline Stone size $(\mathrm{mm})($ Mean \pm SD & $13.80 \pm 2.30$ & $14.85 \pm 3.01$ & 0.122 \\
\hline Serum creatinine (mg/dL) (Mean \pm SD & $1.10 \pm 0.31$ & $1.10 \pm 0.21$ & 1.0 \\
\hline Co-Morbidities, n (\%) & $14(43.75)$ & $08(25)$ & 0.187 \\
\hline Mean operative time ( $\min$.) (Mean \pm SD & $84.70 \pm 18.03$ & $75.91 \pm 19.80$ & 0.0428 \\
\hline Mean hospital stay (hours) (Mean \pm SD & $68 \pm 15.30$ & $58 \pm 12.30$ & 0.005 \\
\hline Overall response rate, $\mathrm{n}(\%)$ & $25(78.13)$ & $27(84.40)$ & 0.75 \\
\hline Complete clearance, $\mathrm{n}(\%)$ & $23(71.80)$ & $26(81.30)$ & 0.556 \\
\hline Insignificant residual fragments, n (\%) & $2(6.31)$ & $1(3.10)$ & 1.00 \\
\hline $\begin{array}{l}\text { Significant residual calculi, } \mathrm{n}(\%) \\
\text { - Difficult access } \\
\begin{array}{l}\text { Stone fragmentation/ manipulation } \\
\text { difficulties }\end{array} \\
\text { - Edema/oozing }\end{array}$ & $\begin{array}{l}7(21.87) \\
04(57.14) \\
02(28.57) \\
01(14.28)\end{array}$ & $\begin{array}{l}5(15.60) \\
03(60) \\
02(40) \\
-\end{array}$ & $\begin{array}{l}0.770 \\
1.00 \\
1.00 \\
-\end{array}$ \\
\hline \multicolumn{4}{|l|}{ Auxiliary procedure, $\mathrm{n}(\%)$} \\
\hline SWL & $01(3.10)$ & $03(9.41)$ & 0.612 \\
\hline Redo RIRS & $03(9.40)$ & $01(3.10)$ & 0.612 \\
\hline Patient unwilling for further treatment & $03(9.40)$ & $01(3.10)$ & 0.60 \\
\hline Complications, n(\%) & $13(40.6)$ & $07(20)$ & 0.176 \\
\hline Clavien grade I & $9(28.10)$ & $5(15.61)$ & 0.364 \\
\hline Colic & $2(6.30)$ & $1(3.10)$ & 1.000 \\
\hline Ileus & $5(15.60)$ & $3(9.40)$ & 0.707 \\
\hline Transient hematuria & $2(6.31)$ & $1(3.10)$ & 1.000 \\
\hline \multicolumn{4}{|l|}{ Clavien grade II, $\mathrm{n}(\%)$} \\
\hline Sepsis & $3(9.40)$ & $1(3.10)$ & 0.612 \\
\hline $\begin{array}{l}\text { Clavien grade III, } \mathrm{n}(\%) \\
\text { Ureteric perforation/injury }\end{array}$ & $1(3.11)$ & $1(5.70)$ & 1.000 \\
\hline
\end{tabular}


Introduction \& Objective: For intermediate size $(1-2 \mathrm{~cm})$ lower calyceal (LC) calculi, retrograde intrarenal surgery (RIRS) is shown to have better outcomes than shockwave lithotripsy (SWL). However, SWL being less invasive is still preferred by many patients and physicians in this situation. Failures of SWL can be treated by RIRS or PCNL. As literature is sparse regarding outcomes of RIRS in SWL resistant inferior calyceal calculi, present study aims to find efficacy and safety of RIRS, in SWL failures with residual intermediate size calculi.

Materials and Methods: In this prospective case-control study at a tertiary teaching institution, 64 adult patients ( $>18$ years of age) with $1-2 \mathrm{~cm}$ size, radiopaque LC calculi were recruited between June 2012 and Dec 2016. Of these, 32 (group A) were SWL failed (non fragmentation or non expulsion after a maximum of 3 sessions; 3000-4000 shocks, 25 KV @60-90 Hz) and 32 (group B) were treatment naïve (TN) controls, matched for age, BMI and socio cultural status. RIRS was done under combined epidural and spinal anaesthesia using 7.5 Fr flexible ureterorenoscope, access sheath and Ho:YAG laser with $272 \mu$ fibre at a setting of 8-12 watt/5-12 Hz. Relocation of stones/fragments was done to a more favourable location by basketing for better visualization. Larger stone fragments were removed using dormia basket and finer stone dust was left in situ. Patients were assessed at 2 weeks (at the time of DJ stent removal) and 1 month, clinically and by ultrasonography and X-ray KUB region. Postoperative complications (using Clavien-Dindo classification) and outcomes (overall response rate; no or $<4 \mathrm{~mm}$ (insignificant) residual fragments, complete clearance; no residual fragments, failure $>4 \mathrm{~mm}$ residual fragments) were recorded. Chi-square/ fisher exact test and unpaired t-test were applied to analyse the data using SPSS ver.16. P value $<0.05$ was considered statistically significant.

Results: Table 1. shows that in SWL resistant group, mean operative time and hospital stay were significantly $(\mathrm{p}=0.042$ and $p=0.005$ respectively) higher. Demographic parameters, success rate and complications of RIRS were similar in SWL resistant and treatment naïve groups.

Conclusions: RIRS is an effective treatment modality for SWL failed intermediate size inferior calyceal calculi with success and complication rate equal to that in treatment naïve patients.

MP13-3 Natural history, complications, and re-intervention rates of residual stone fragments following percutaneous nephrolithotomy

AS Emmott, H Brotherhood, RF Paterson, D Lange, BH Chew

Faculty of Medicine, University of British Columbia Canada

Introduction \& Objective: The management of residual fragments (RFs) that persist after percutaneous nephrolithotomy (PCNL) is under discussion. RFs have the potential to grow and/ or cause recurrence of symptoms, urinary tract infection and ureteral obstruction. The aim of this study was to retrospectively follow patients with RFs after PCNL to identify predictors of stone-related events (re-interventions and complications) and identify the stone-free rate after PCNL at our institution.

Materials and Methods: Data was retrospectively collected from patients who underwent PCNL from 2008 to 2013 at Vancouver General Hospital. Patients with RFs of any size following PCNL on post-operative day 1 CT-KUB were included and patients with planned secondary interventions were ex-

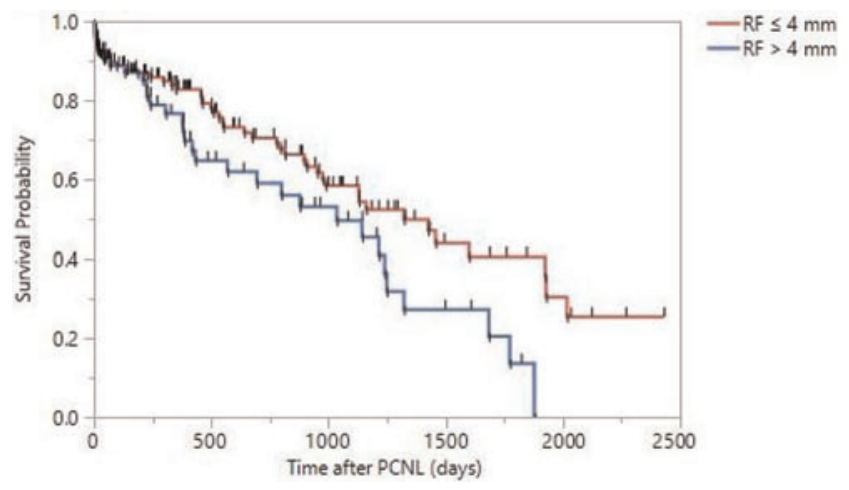

cluded. Subgroup analysis was performed on subjects with CT, KUB X-ray or US within 2 years after PCNL to determine RF growth or passage.

Results: Of 658 patients who received a post-operative CT-KUB on day one, 299 patients (45\%) had RFs $1 \mathrm{~mm}$ or larger. 263 patients met study criteria and were included. The size of RFs, using a 4mm cutoff, did not predict the passage of RFs $(\mathrm{P}=0.173)$ or growth of RFs $(\mathrm{P}=0.572)$. On multivariate analysis, previous history of renal stones and size of RF were predictive for stone-related events $(\mathrm{P}=0.002$ and 0.027, respectively). Kaplan-Meier analysis identified RFs $>4 \mathrm{~mm}$ having a shorter survival time before occurrence of stone-related events (Figure 1, $\mathrm{P}=0.044$ ).

Conclusions: The true stone-free rate was $55 \%$ following PCNL. However, $82.5 \%$ were stone free or had RFs $4 \mathrm{~mm}$ or less, which correlates with previous studies. Larger RFs had higher rates of stone-related events. The growth and spontaneous passage of RFs was independent of RF size, emphasizing the importance of obtaining a stone-free status following PCNL.

MP13-4 Contemporary Trends In Utilization And Outcomes Of Percutaneous Nephrolithotomy In The United States From 2003 To 2014

S Harmouch, J Leow, C Meyer, Y Wang, SL Chang, B Chung, Q Trinh, R Korets, N Bhojani

Division of Urology, Université de Montréal

Canada

Introduction \& Objective: Contemporary data has demonstrated that flexible ureteroscopy (URS) is increasingly challenging percutaneous nephrolithotomy (PCNL) in the treatment for medium to large sized kidney stones. However, limited updated data on contemporary utilization rates and outcomes of PCNL exists. In this study, we aimed to characterize national utilization trends, perioperative outcomes and costs using a contemporary population-based cohort for symptomatic patients with a total renal stone burden $>20 \mathrm{~mm}$.

Materials and Methods: Using the Premier Healthcare Database, we identified 225,321 patients diagnosed with kidney/ ureter calculus who underwent PCNL at 447 different hospitals across the United States from 2003 to 2014 (Table 1). Outcomes included 90-day postoperative complications (as classified by the Clavien-Dindo system), prolonged hospital length of stay, operating room time, blood transfusions and direct hospital costs. Temporal trends were quantified by estimated annual percent 


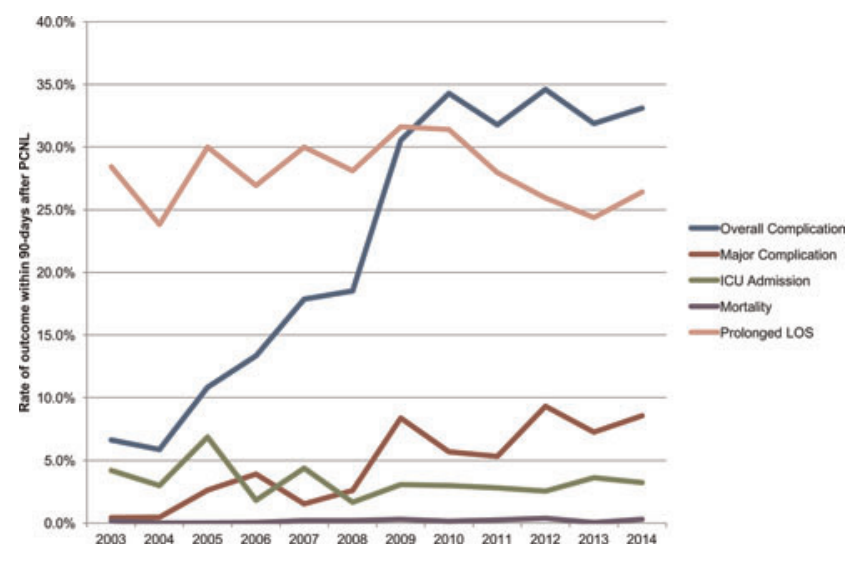

change (EAPC) using least squares linear regression analysis. Multivariable logistic regression was performed to identify predictors of outcomes.

Results: PCNL utilization rates initially increased from $6.7 \%$ (2003) to $8.9 \%(2008)(\mathrm{EAPC}+5.60 \%, \mathrm{p}=0.02)$, before plateauing at $9.0 \%(2008-2011)$, then declining to $7.2 \%$ in 2014 (EAPC $-4.37 \%, p=0.02$ ). Overall (Clavien $\geq 1$ ) and major complication (Clavien $\geq 3$ ) rates rose significantly (EAPC: $+12.2 \%$ and $+16.4 \%$ respectively, both $\mathrm{p}<0.001$ ) (Figure 1 ). Overall/major complication and blood transfusion rates were $23.1 \% / 4.8 \%$ and $3.3 \%$ respectively. Significant predictors of overall complications include higher Charlson comorbidity in$\operatorname{dex}(\mathrm{CCI})(\mathrm{CCI} \geq 2$ : OR 2.08, p < 0.001) and more recent year of surgery (2007-2010: OR 3.20, 2011-2014: OR 4.39, both $\mathrm{p}<0.001)$. Higher surgeon volume was significantly associated with decreased overall $(\mathrm{OR} 0.992, \mathrm{p}<0.001)$ and major $(\mathrm{OR}$ 0.991, $\mathrm{p}=0.01$ ) complications.

Conclusions: Contemporary decrease in utilization of PCNL may be due to an increase in the use of flexible URS. Possibly, increasing complex cases being managed with PCNL may contribute to the rise in complication rates. Numerous patient, hospital and surgical characteristics affect complication rates and may have implications on referral patterns and patient selection.

MP13-5 Perioperative aspirin use during percutaneous nephrolithotomy (PCNL): our single center experience.

J Ebel, M Sourial, B Knudsen

The Ohio State University Wexner Medical Center United States

Introduction \& Objective: The rates of heart disease and nephrolithiasis continue to increase in the United States. As more patients on aspirin for secondary prevention present with large kidney stones, urologists are increasingly faced with the decision to proceed with the less effective but lower risk ureteroscopy or the higher risk but more effective percutaneous nephrolithotomy (PCNL). Leavitt, Smith, and Okeke recently published their experience performing PCNLs on seventeen patients continued on aspirin perioperatively and concluded that the procedure was safe. We wish to add our center's experience to this body of knowledge.

Materials and Methods: We retrospectively reviewed our single center, single surgeon, experience of 199 PCNLs performed between January 2013 and September 2016. Hemoglobin preand post-operatively was recorded as were the relevant related variables of age, sex, BMI, operative duration, skin-to-stone distance, stone size, aspirin dose, aspirin indication, number of blood transfusions, and Clavien-Dindo complication classification. Correlations between hemoglobin and explanatory variables were then explored with linear regression and the Wilcoxon rank-sum test.

Results: Of the 199 PCNLs performed at our institution in the range of our study, 27 procedures on 23 patients were conducted without discontinuing aspirin perioperatively. Coronary artery disease was the most common indication for aspirin use. In 24 cases, the patient was on $81 \mathrm{mg}$ of aspirin perioperatively with the remaining three patients on $325 \mathrm{mg}$ of aspirin daily. The average patient experienced a $1.3 \mathrm{~g} / \mathrm{dl}$ drop in hemoglobin perioperatively. The largest drop was $4.1 \mathrm{~g} / \mathrm{dl}$, but the lowest postoperative hemoglobin was $8.2 \mathrm{~g} / \mathrm{dl}$. No significant associations were found between hemoglobin decline and age, sex, BMI, operative duration, skin-to-stone distance, or stone size. There were no Clavien-Dindo grade III or higher complications in the course of our review, and no patients required a blood transfusion or embolization. In one case, a patient required a three day inpatient stay following stent removal for continuous bladder irrigation after developing gross hematuria but this resolved spontaneously.

Conclusions: In our single center experience, PCNLs performed on patients taking aspirin perioperatively were not associated with the need for blood transfusion nor the occurrence of highgrade complications. It is our experience that this practice is safe when performed by expert hands.

MP13-6 Should MiniPercutaneous Nephrolithotomy (MiniPNL) be the ideal tract for Medium sized Renal Calculi $(16-30 \mathrm{~mm})$ ?

\section{RA Kukreja}

Urocare

India

Introduction \& Objective: Reducing the Percutaneous Nephrolithotomy (PCNL) access tract size has been found to decrease the morbidity associated with the procedure. Prolonged procedure time is a concern. The modification in technique required is to fragment the stone into smaller particles and remove them using the vacuum cleaner effect.

This prospective study compares the efficacy and morbidity of reducing the tract size from the standard $24 \mathrm{Fr}$ to $16.5 \mathrm{Fr}$ for stones sized from 16-30mm.

Materials and Methods: 104 patients were enrolled in this prospective study and distributed into 2 groups based on the tract size used to clear the stone (Group A: $16.5 / 18 \mathrm{Fr}, \mathrm{N}=51$ and Group B: 22/24Fr, $N=53$ ). The equipment used comprised of: 16.5/18 Fr MiniPNL sheath with 12Fr nephroscope (Karl Storz) for group A and 22/24 Fr Amplatz sheath (Cook) with $20.5 \mathrm{Fr}$ Nephroscope (Richard Wolf, Germany) for group B. Critical factors assessed were procedure time, fluoroscopy time, blood loss, pain score, stone clearance status and complications.

Results: Both the groups were comparable with respect to age, BMI, creatinine and stone size. The blood loss (assessed by Hemoglobin and PCV drop) was significantly less for group A $(\mathrm{Z}=4.005, \mathrm{p}<0.001)$. Both the groups were comparable with regards to the pain score $(Z=1.446 ; p>0.05)$. Nephrostomy was 
placed in 3 patients in group A and 13 patients in group B. There was no significant difference in the procedure time amongst the 2 groups. A total of 6 patients (3 in each group) had residual fragments greater than $3 \mathrm{~mm}$.

Conclusions: The 16.5Fr MiniPNL tract offers lower morbidity in terms of blood loss and nephrostomy tube requirement and maintains comparable stone clearance as compared to larger tract size. It should be the standard size used for PCNL for medium sized renal stones.

MP13-7 Continuing Aspirin Does Not Increase Blood Loss From Percutaneous Nephrolithotomy

T Tran, E Parkhomenko, JN Thai, K Blum, M Gupta

Icahn School of Medicine at Mount Sinai United States

Introduction \& Objective: Patients with vascular or cardiac disease may benefit from continuing antiplatelet therapy during surgery. Percutaneous nephrolithotomy (PCNL) is a transparenchymal procedure that can be associated with significant bleeding. Recent studies have demonstrated safety with continuing aspirin during PCNL. However, these studies were notable for patients having large postoperative nephrostomy tubes that may have provided additional hemostatic control. Here, we evaluated our experience with continuing aspirin in patients undergoing PCNL.

Materials and Methods: All patients that underwent PCNL over the last 3 years were evaluated. Hematocrit levels were recorded preoperatively, one hour after surgery and on postoperative day 1. Clinical and demographic parameters were recorded. Statistical difference was assessed using Student's t-test and chisquare test.

Results: 218 patients underwent PCNL during this time period. 20 patients continued aspirin through surgery and an additional 20 stopped aspirin one week prior to their procedure. No difference in mean hematocrit decrease was noted between patients that never took aspirin, those that stopped and those that continued on postoperative day 0 ( $-2.40 \%$ vs. $-2.62 \%$ vs. $-2.38 \%$, respectively, $\mathrm{p}=0.86)$ or postoperative day $1(-2.37 \%$ vs $-2.94 \%$ vs. $-2.11 \%, \mathrm{p}=0.34)$. No significant difference in preoperative platelet count, operating room time, estimated blood loss, and postoperative nephrostomy tube size (16 French) was noted between the groups.

Conclusions: Continuing aspirin through PCNL does not appear to lead to increased bleeding.

MP13-8 Is PCNL changing in the UK - Analysis of 9500 cases from the BAUS PCNL Registry

W Finch, JN Armitage, J Withington, SO Irving, S Fowler, $\mathrm{N}$ Burgess, O Wiseman

Cambridge University Hospital NHS Foundation Trust, Addenbrookes Hospital.

United Kingdom

Introduction \& Objective: PCNL indications, practices and techniques are evolving. BAUS developed an online data registry in January 2010 that now includes $>9500$ procedures. We evaluate outcomes and practices in PCNL and compare with previous

\begin{tabular}{|l|l|l|l|l|l|l|l|}
\hline Table 1 & \multicolumn{3}{|l|}{ Ik Analysis } & \multicolumn{3}{|l|}{$5 \mathrm{k}$ Analysis } & \multicolumn{2}{l|}{ 9k Analysis } & \\
\hline & $\mathrm{N}$ & $\%$ & $\mathrm{~N}$ & $\%$ & $\mathrm{~N}$ & $\%$ & Chi square test \\
\hline Visceral Injury & 4 & 0.3 & 17 & 0.3 & 22 & 0.2 & $\mathrm{Ns}$ \\
\hline Transfusion & 24 & 2.5 & 117 & 2.4 & 199 & 2.2 & $\mathrm{Ns}$ \\
\hline Post op fever & 155 & 16 & 551 & 12.1 & 916 & 10.7 & $\mathrm{p}=0.02$ \\
\hline Sepsis & 25 & 2.4 & 128 & 2.8 & 215 & 2.5 & $\mathrm{Ns}$ \\
\hline Clavien 3-5 & 17 & 1.4 & 84 & 1.6 & 229 & 2.4 & $\mathrm{p}=0.002$ \\
\hline
\end{tabular}

analyses of the registry at 1000 and 5000 procedures to highlight significant changes in PCNL practice in the UK.

Materials and Methods: The BAUS PCNL registry was analysed between 2010 and 2017. We evaluated patient position, access and tract dilatation methods, grade of operator and fragmentation device used. Post-operative drainage, stone free rate at day 1 and complications were also analysed.

Results: Overall 9536 procedures were available for analysis and compared with the previous analyses of 1028 cases in 2011 ( $1 \mathrm{k}$ analysis) and 5191 cases in 2015 (5k analysis). Submission of cases has stabilised at $\sim 2,200$ cases per year for the last 3 years. The majority of PCNL is still prone, but supine positioning continues to significantly increase when compared with $5 \mathrm{~K}$ analysis $(22.4 \%$ vs. $16.2, \mathrm{p}=0.0001)$. Percutaneous access by interventional radiologist showed a small but significant decrease compared with $5 \mathrm{~K}$ analysis $(63.3 \%$ vs. $66.3 \%$, $\mathrm{p}=0.0004$ ), but not significantly different from $1 \mathrm{~K}$ analysis. No further significant changes in tract dilatation methods have been seen with balloon dilatation the most used technique (64.3\%). Significantly more Consultants perform PCNL themselves rather than their trainees $(96.5 \%$ vs. $84.4 \%(5 \mathrm{~K})$ vs. $79.0 \%(1 \mathrm{k})$, $\mathrm{p}=0.0001)$. Laser fragmentation has significantly increased in usage compared with $5 \mathrm{k}$ and $1 \mathrm{k}$ analyses $(9.4 \%$ vs. $7.0 \%$ vs. $5.8 \%, \mathrm{p}=0.0001$ ), with similar usage of ultrasound, lithoclast and lift out. Sub-analysis of 4490 cases showed $25.8 \%$ of cases used multiple stone fragmentation modalities. Nephrostomy tube drainage post operatively appears to have significantly reduced (72.6\% vs. $75.6 \%(5 \mathrm{k}), \mathrm{p}=0.0001)$. Intraoperatively $78.5 \%$ of patients were believed to be stone free, which was confirmed in $69.1 \%$ with postoperative imaging, similar to the previous analyses. Complication rates are shown in Table 1.

Conclusions: The BAUS PCNL registry remains a unique resource for UK surgeons, enabling individuals to benchmark their practice against national outcome data and counsel their patients as to possible outcomes. PCNL practices continue to evolve in the UK. Continued contribution of data and subsequent careful analysis of the registry allows us a better understanding of PCNL in the UK.

MP13-9 How can we measure successful outcome for PCNL? Further planned treatment vs. traditional measurements of stone free rate: Analysis of a national registry.

W Finch, JN Armitage, J Withington, SO Irving, S Fowler, $\mathrm{N}$ Burgess, O Wiseman

Cambridge University Hospital NHS Foundation Trust, Addenbrookes Hospital.

United Kingdom

Introduction \& Objective: PCNL is a complex Endourology procedure, and encompasses a spectrum of stone disease and techniques. Traditionally stone free rate is a marker of treatment 
success. Increasing variation of imaging modalities used and timing of imaging results in heterogeneous methodology for the documentation of stone free rate within a national registry. Intraoperatively, can "further planned stone treatment" be used as a surrogate marker of stone free rate and treatment success?

Materials and Methods: 9084 cases from the BAUS PCNL registry record stone free rate (SFR) intraoperatively. These cases were analysed for SFR records intra-operatively, post-op day 1 and at 3 months follow-up (FU). SFR records were compared with intention for "further planned stone treatment". Subgroup analysis was performed for variation with stone size and stone complexity measured by Guys stone score (GSS).

Results: Of the 9084 cases recording intraoperative SFR, 78.4\% had records for SFR on post-op day $1,37.3 \%$ at 3 months FU and $60.6 \%$ recorded intention for "further planned stone treatment", at the time of surgery. X-ray KUB was most the popular imaging modality used $(67.9 \%)$ followed by CT and USS $(16.1 \%$ and $6.1 \%$ respectively). Using intention for "further planned stone treatment" as a marker of success compared to complete clearance on fluoroscopy intraoperatively had a sensitivity of $92.7 \%$ (95\% CI 91.81-93.53) and specificity of $72.6 \%$ (95\% CI 70.4874.67). When compared to clearance on post-op day 1 imaging the sensitivity was $97.6 \%$ (95\% CI 96.91-98.23) and specificity $63.08 \%$ (95\% CI 61.02-65.10). Subgroup analysis of stone diameter and GSS revealed no statistically significant differences in treatment success when comparing stone free rates with intention for "further planned stone treatment".

Conclusions: Treatment success when measured by stone free rate demonstrates variable reporting of rates and heterogeneous imaging modalities within our national registry. Using intention for "further planned stone treatment", does show correlation with reported SFR intraoperatively and post-op day 1 . Accurate recording of SFR to measure treatment success within a national registry requires enhanced submission of FU data and a consistent approach to the timing and imaging modality used to measure this. Identify patients who have received further stone treatment within an agreed period would be a further useful measure of treatment success.

MP13-10 Effect of open surgery and shock wave lithotripsy (swl) on percutaneous nephrolithotomy in elderly patients

I Aridogan, V Izol, A Borekoglu, N Akdogan, I Sukur, M Deger

University of Cukurova

Turkey

Turkey

Introduction \& Objective: Efficiency of percutaneous nephrolithotomy (PCNL) operation which is safely applicable to almost every age group was evaluated in the population aged over 65 undergone an open kidney stone surgery or Shock Wave Lithotripsy (SWL).

Materials and Methods: Data related to 233 patients aged over 65 who have undergone a PCNL operation in our clinic, was retrospectively analyzed. Operation, scopy, nephrostomy withdrawal period, hospitalization time and blood transfusion rates were evaluated. Effects of the open surgery or shock wave lithotripsy (SWL) previously carried out on parameters were examined.

Results: Out of those who have undergone a PCNL operation between July 1997 and October 2012, 233 patients were aged over 65.32 of 233 patients have previously experienced an open kidney surgery operation in their past. The patients who have undergone an open surgery before had a mean operative time of $76.2 \pm 33.6 \mathrm{~min}$, a scopy time of $10.8 \pm 6.8 \mathrm{~min}$, a nephrostomy withdrawal period of 2,3 $\pm 1,4$ days and a hospitalization time of $4.0 \pm 1.9$ days. The patients who have not undergone any open surgery in their past had a mean operative time of $78,7 \pm 49,5 \mathrm{~min}$, a scopy time of $10,3 \pm 6,9 \mathrm{~min}$, a nephrostomy withdrawal period of $2,3 \pm 1,9$ days and a hospitalization time of $3,8 \pm 2,5$ days. A total of 28 patients had a blood transfusion and 6 of them had undergone an open surgery before. The patients who have undergone a SWL in their past had a mean operative time of 75,8 $\pm 33,5 \mathrm{~min}$, a scopy time of $8,72 \pm 3,5 \mathrm{~min}$, a nephrostomy withdrawal period of 2,6 $2 \pm 2,9$ days and a hospitalization time of 4,2 $\pm 3,3$ days. The patients who have not undergone any SWL in their past had a mean operative time of 78,6 $\pm 48,6 \mathrm{~min}$, a scopy time of $10,5 \pm 7,1 \mathrm{~min}$, a nephrostomy withdrawal period of 2,2 $\pm 1,7$ days and a hospitalization time of 3,8 $\pm 2,3$ days. Statistically there was no difference between the patients with or without SWL experience in their past $(\mathrm{p}>0.05)$.

Conclusions: PCNL can be safely applied with higher stone freeness rates and lower complication rates to the patient group aged over 65 where systemic disorders are increased and body reserves are decreased.

MP13-11 Comparison of percutaneous nephrolithotomy results in different age groups

V Izol, I Aridogan, A Borekoglu, N Akdogan, O Yilmaz, F Ok

University of Cukurova

Turkey

Turkey

Introduction \& Objective: Percutaneous nephrolithotomy (PCNL) is a minimally invasive surgical method for the treatment of kidney stones. We have examined the results of this method applicable to almost every age group in the population aged 65 and older in order to assess the efficiency on different age group.

Materials and Methods: In this study, efficiency of the PNL operations introduced to 233 patients aged 65 years and over, who have undergone a PNL surgery in our clinic was evaluated. Patients' data which is retrospectively documented has been analyzed. Operative time, scopy time, nephrostomy withdrawal period, hospitalization time and blood transfusion rates were evaluated.

Results: 1046 patients included in the study have undergone an PNL operation between July 1997 and October 2012, of whom 233 patients were aged 65 years and over, and 813 patients were aged between 50-65 years. Findings about the patients are shown in Table 1. Fever was detected in 71 of the 813 patients $(8.7 \%)$ in the control group, for the elder group it was 22 out of

\begin{tabular}{|lccc|}
\hline Table 1 & $>65$ years & $50-64$ years & $\mathrm{p}$ Volue \\
\hline Age (years) & $69,66 \pm 4,62$ & $55,75 \pm 4,14$ & \\
\hline Stone size $\left(\mathrm{mm}^{2}\right)$ & $611,18 \pm$ & $611,41 \pm 561,740$ & $>0,05$ \\
& 607,497 & & \\
\hline Operative time (min) & $78,43 \pm 47,59$ & $76,64 \pm 45,37$ & $>0,05$ \\
\hline Amount of Perioperative Bleeding (ml) & $665(300-1500)$ & $510(200-1500)$ & $>0,05$ \\
\hline Perioperative Blood Transfusion Rate & $\% 12$ & $\% 11,8$ & $>0,05$ \\
\hline Scopy time (min) & $10,43 \pm 6,91$ & $11.7 \pm 7.6$ & $<0,05$ \\
\hline Nephrostomy withdrawal period (days) & $2.3 \pm 1.8$ & $3.5 \pm 2.2$ & $>0,05$ \\
\hline Hospitalization Time (days) & $3,87 \pm 2,46$ & $4,27 \pm 3,73$ & $>0,05$ \\
\hline
\end{tabular}


233 patients (\%9.4) who were suffering from fever, and there was statistically no difference between them.

Conclusions: Stone freeness rates, blood transfusion rates and many perioperative data of PNL operation carried out on elderly patients are similar to those of non-elderly patients after the completion of learning curve. Even though comorbidity is increased and body reserves are decreased in the patients over 65 years, PNL is applied to the patients of such age group.

MP13-12 Role of minimally invasive PCNL techniques: Micro and Ultra-mini PCNL $(<15 \mathrm{Fr})$ in the paediatric population - A Systematic Review

P Jones, A Pietropaolo, OM Aboumarzouk, B Somani

University Hospital Southampton NHS Foundation Trust United Kingdom

Introduction \& Objective: Management of paediatric stone disease is challenging, with standard PCNL having a good stone free rate (SFR) but with an associated high complication rates. Miniaturisation of this technique has led to the rise of minimally invasive PCNL techniques such as micro (

Materials and Methods: A Cochrane style search was performed and the following bibliographic databases were accessed: Pubmed, Science direct, Scopus and Web of Science. This was carried out in accordance with the Preferred Reporting Items for Systematic reviews and Meta-Analyses (PRISMA) guidelines

Results: A total of 14 studies (456 patients) including 8 on micro-PCNL (m-PCNL, $\mathrm{n}=233$ ) and 6 on ultra-mini PCNL (UMP, $\mathrm{n}=223$ ) were included. Mean stone size ranged from 12$16.5 \mathrm{~mm}$ (m-PCNL) and 12-41 mm (UMP), the overall SFR ranged from $80 \%-100 \%$ (m-PCNL) and $85 \%-100 \%$ (UMP). The overall complication rates for all studies were $11.2 \%$, which was slightly higher for UMP (13.9\%). Post-operative renal colic or fragment obstruction was only seen in m-PCNL, but there was a statistically significant rate of extravasation or renal pelvicalyceal perforation and haematuria for UMP compared to $\mathrm{m}$ PCNL $(\mathrm{p}<0.05)$.

Conclusions: Miniaturised PCNL techniques can deliver high stone free rates with a small risk of Clavien I/II complications. The size of tract seems to influence the nature of complications, with higher haematuria and renal extravasation with increasing tract size.

MP13-13 The Role of Frailty Assessment in the Prediction of Post-operative Complications for Percutaneous Renal Surgery

V Vasudevan, P Samson, G Gaunay, K Ren, Z Okeke, AD Smith, DM Hoenig

Smith Institute for Urology

United States

Introduction \& Objective: Surgical complications are difficult to predict, despite the existing assessment tools used by physicians. Frailty, as a phenotype, has shown great promise in the estimation of postoperative risk amongst the elderly and infirm. We sought to evaluate the use of a frailty index as a predictive tool on patients undergoing percutaneous renal surgery at a high volume institution.
Materials and Methods: Frailty was prospectively analyzed prior to surgery in all consenting patients using the Hopkins Frailty Index, consisting of 5 components yielding an additive score. Patients were categorized as not frail, intermediate, or severely frail, depending on overall score. Primary outcomes assessed were complications during admission and the 30-day complication rate. Secondary outcomes included overall hospital length of stay (LOS) and discharge location.

Results: 78 patients were included in the analysis. In total, 58 (74\%), $13(17 \%)$, and 7 (9\%) patients were in the not frail, intermediate, and severely frail categories. Overall, intermediate or frail patients were more likely to have underlying chronic kidney disease $(\mathrm{p}<0.05)$, anemia $(\mathrm{p}<0.01)$, or prior abdominal surgery $(\mathrm{p}<0.04)$. Intraoperatively, intermediate or frail patients were not more likely to experience higher blood loss or to have a higher stone burden. Of the five frailty metrics, grip strength alone was predictive of post-operative complications $(\mathrm{p}<0.01)$. When compared with the American Society of Anesthesiologists (ASA) score and Charlson comorbidity index, frailty class alone was predictive of the incidence of complications during admission $(\mathrm{p}<0.03)$, but not 30-day post-operative complications $(p=0.33)$. Extended length of stay $(p<0.001)$, along with specific complications like fever $(\mathrm{p}<0.0063)$, sepsis $(\mathrm{p}<0.0001)$, bacteremia $(\mathrm{p}<0.0004)$, hemorrhage requiring embolization $(\mathrm{p}<0.03)$, and hemodynamic instability requiring intensive care (ICU) admission $(\mathrm{p}<0.0008)$ were more common with in individuals with higher frailty scores. Frailer individuals tended to require higher level of care upon hospital discharge $(\mathrm{p}<0.0001)$. Conclusions: Frailty assessment appears useful in stratifying those at risk for extended hospitalization and those unable to return home without assistance following percutaneous renal surgery. Risks of sepsis, bacteremia, and post-operative hemorrhage may be higher in frail individuals. Incorporated into the preoperative assessment, the frailty phenotype may give further insight into treatment decisions and the prediction of postoperative outcomes and may represent a modifiable marker for patient optimization prior to surgery.

MP13-14 Does percutaneous nephrolitotomy affect renal function in the first year after surgery?

E Hernández Méndez, C Martínez Arroyo, J Herrera Muñoz, I Calvo Vázquez, P Cortés Raygoza, M Ortega González, M Cantellano Orozco, G Fernández Noyola, J Morales Montor, C Pacheco Gahbler

Hospital General Dr. Manuel Gea González Mexico

Introduction \& Objective: Renal failure occurs in $2-15 \%$ of patients with urolithiasis and it is associated with multifactorial causes that enclose chronic and metabolic disease, hydronephrosis, repeated stone surgeries, environmental and genetic factors. Many studies have associated stone disease with varying degrees of impaired kidney failure. However, there are only a few reports of the impact of surgery as percutaneous nephrolitotomy (PCNL) in terms of renal function.

Evaluate the effect of PCNL in renal function during the first year after surgery.

Materials and Methods: We performed a retrospective study in patients treated in the Hospital General "Dr. Manuel Gea González" (Mexico City). We reviewed their clinical records since January 2015 to March 2017. All patients were clinically 
Comparison of GFR during the first year after PCNL

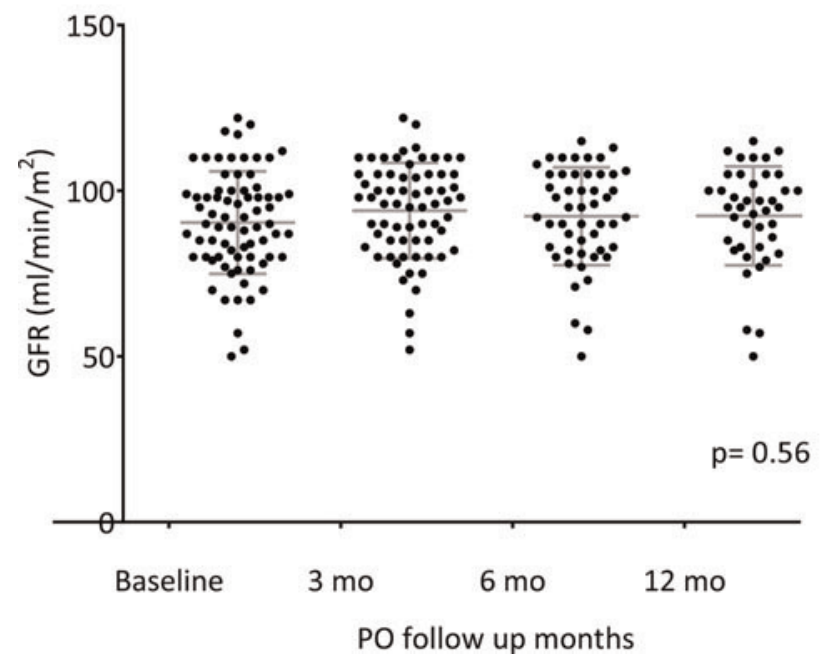

examined prior to percutaneous nephrolitotomy and renal function was evaluated at 3, 6 and 12 postoperative (PO) months using the Chronic Kidney Disease Epidemiology Collaboration (CKDEPI) equation to calculate glomerular filtration rate (GFR).

Results: 80 PCNL were performed in a total of 74 patients, 39 $(52.7 \%)$ women and $35(47.3 \%)$ men. 3 patients underwent more than 1 PCNL and only 44 patients completed 1st-year follow up. Mean baseline GFR $\left(\mathrm{ml} / \mathrm{min} / \mathrm{m}^{2}\right)$ was $92 \pm 15.2$ and at 3,6 and 12 PO months $96 \pm 14.4,93.7 \pm 14.7$ and $95 \pm 14.9$ respectively. Nevertheless there was not significant difference comparing these results (Figure 1). Men had a higher improvement of their 1st-year PO GFR with $8 \pm 5.2 \mathrm{ml} / \mathrm{min} / \mathrm{m}^{2}$ compared to women with $5 \pm 2.3$ (p 0.06). Chronic and metabolic disease, stone size, body mass index and more than 1 PCNL did not affect renal function after 1styear follow up in this population.

Conclusions: PCNL is a safe procedure and does not affect GFR during the first year after surgery. In fact, we observed a slightly improvement in PO renal function that was most evident in men. A cohort study that includes a bigger population is necessary in order to confirm our findings.

MP13-15 Stone morphometry: tract (s) and procedure (s) predictor for percutaneous nephrolitotomy

J Sedano Basilio, C Martínez Arroyo, U Sánchez Aquino, E Hernández Méndez, M Cantellano Orozco, G Fernández Noyola, J Morales Montor, C Pacheco Gahbler

Hospital General Dr. Manuel Gea González Mexico

Introduction \& Objective: Percutaneous nephrolithotomy (PCNL) is the standard treatment for renal stones $>2 \mathrm{~cm}$. There is no precise consensus for the term "staghorn calculi", "partial" or "complete", designations do not imply any volume judgment. The stone free rate after monotherapy, has been found dependent on stone burden. More important than stone burden is the term "staghorn morphometry", which is defined as the stone volumetric burden distribution in the collecting system.

Determine the "stone morphometry" by preoperative computed tomography (CT) study in Mexican population.

Materials and Methods: A restrospective study was conducted in the Hospital General “Dr. Manuel Gea González” since 2012

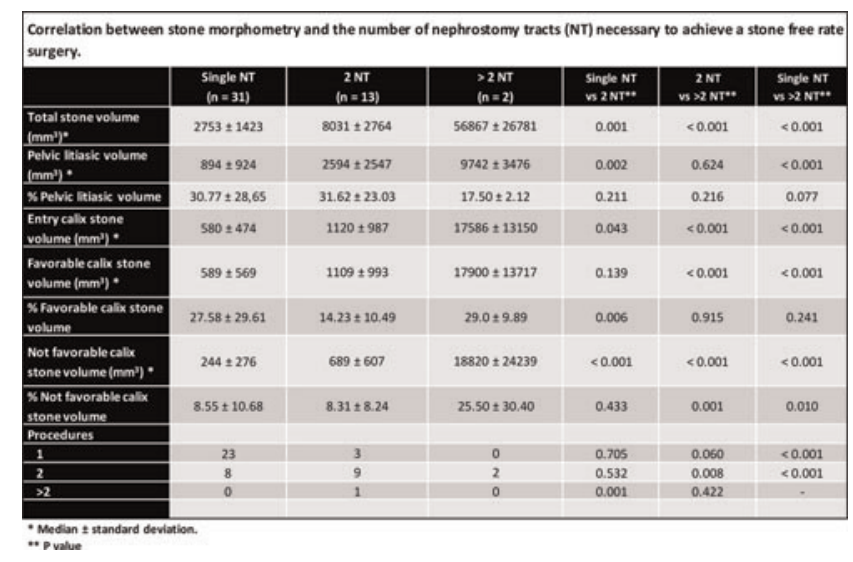

to 2016 in patients with dianosis of renal lithialsis. All patients had a CT scan previous to PCNL. Using OsiriX Software v.7.5.1, we performed measurement of the total stone volume (TSV): volume calculated with the reconstruction of the entire image of the stone, entry calix stone volume (ECSV), and favorable calix stone volume (FCSV). Measurements were compared in terms of the number of nephrostomy tracts.

Results: 46 patients were include in this study. A TSV of $2753 \pm 1423 \mathrm{~mm}^{3}$ versus $56867 \pm 26781 \mathrm{~mm}^{3}(\mathrm{p}<0.001)$, ECSV of $580 \pm 474 \mathrm{~mm}^{3}$ compared to $17586 \pm 13150 \mathrm{~mm}^{3}(\mathrm{p}<0.001)$ and FCSV of $589 \pm 569 \mathrm{~mm}^{3}$ in comparison with $17900 \pm 13717 \mathrm{~mm}^{3}$ $(\mathrm{p}<0.001)$, were found in patients with a single tract and those with $\geq 2$ tracts respectively. TSV $>5000 \mathrm{~mm}^{3}$ has 8 times greater risk for $\geq 2$ procedures / tracts necessaries to obtain a stone free surgery result (CI 95: 2.05-33). These volumetric characteristics could be considered to regulate behavior in treatment by PCNL (Table 1).

Conclusions: Stone morphometry provides the volumetric stone distribution to be treated, and significantly influences to determine the number of tracts / procedures necessary to achieve a free stone state in patients with staghorn calculi who underwent PCNL in our population.

MP13-16 Costs Variations For Percutaneous Nephrolithotomy In The United States From 2003 To 2015: A Contemporary Analysis Of An All-Payer Discharge Database

M Meskawi, J Leow, SL Chang, Q Trinh, R Korets, N Bhojani, A Valiquette, B Chang

University of Montreal Health Center (CHUM)

Canada

Introduction \& Objective: Percutaneous nephrolithotomy is the first-line treatment for symptomatic patients with a total renal stone burden larger than $2 \mathrm{~cm}$. Our objective was to evaluate population-based costs variations and predictors of outlier costs for percutaneous nephrolithotomy in the United States.

Materials and Methods: Using the Premier Healthcare Database, we identified all patients diagnosed with kidney/ureter calculus who underwent percutaneous nephrolithotomy from 2003 to 2015 . We evaluated 90-day direct hospital costs, defining high- and low-cost surgery as those $>90$ th and 10th percentile, respectively. We constructed a multilevel hierarchical regression model and calculated the pseudo- $\mathrm{R}^{2}$ of each variable, which translates to the percentage variability contributed by that variable on 90-day direct hospital costs. 
Table 1. Multivariable Logistic Regression For Patient-, Sugical- And Hospital-Level Predictors Of High Cost $\left(>90^{\text {th }}\right.$ Percentile) Percutaneous Nephrolithotomy

\begin{tabular}{|c|c|c|c|c|}
\hline \multirow{2}{*}{$\begin{array}{l}\text { Patient characteristics } \\
\text { Age }\end{array}$} & \multirow{2}{*}{$\begin{array}{c}\text { Odds Ratio } \\
0.99\end{array}$} & \multicolumn{2}{|c|}{$\begin{array}{l}95 \% \text { Confidence } \\
\text { Intervals }\end{array}$} & \multirow{2}{*}{$\frac{\text { P value }}{0.09}$} \\
\hline & & 0.98 & 1.002 & \\
\hline \multicolumn{5}{|l|}{ Gender } \\
\hline Female vs. male & 0.95 & 0.72 & 1.25 & 0.7 \\
\hline \multicolumn{5}{|l|}{ Race } \\
\hline Non-White vs. white & 0.96 & 0.67 & 1.36 & 0.8 \\
\hline \multicolumn{5}{|l|}{ Marital status } \\
\hline Non-married vs. married & 1.09 & 0.78 & 1.53 & 0.6 \\
\hline \multicolumn{5}{|l|}{ Insurance status } \\
\hline Medicaid vs. medicare & 0.96 & 0.52 & 1.79 & 0.9 \\
\hline Private vs. medicare & 0.52 & 0.34 & 0.80 & $<0.001$ \\
\hline Other vs. medicare & 0.41 & 0.20 & 0.83 & 0.01 \\
\hline \multicolumn{5}{|l|}{ Charlson comorbidity score } \\
\hline 1 vs. 0 & 1.19 & 0.88 & 1.61 & 0.3 \\
\hline$\geq 2$ vs. 0 & 1.81 & 1.18 & 2.79 & 0.01 \\
\hline \multicolumn{5}{|l|}{ Hospital characteristics } \\
\hline \multicolumn{5}{|l|}{ Hospital teaching status } \\
\hline Teaching vs. Non-teaching & 1.39 & 0.86 & 2.24 & 0.2 \\
\hline \multicolumn{5}{|l|}{ Hospital bed size } \\
\hline $300-499$ vs. $<300$ beds & 1.04 & 0.58 & 1.89 & 0.9 \\
\hline 2500 vs. $<300$ beds & 1.48 & 0.73 & 3.00 & 0.3 \\
\hline \multicolumn{5}{|l|}{ Hospital location } \\
\hline Urban vs. rural & 0.75 & 0.24 & 2.36 & 0.6 \\
\hline \multicolumn{5}{|l|}{ Hospital region } \\
\hline Northeast vs. Midwest & 2.04 & 1.09 & 3.81 & 0.03 \\
\hline South vs. Midwest & 0.79 & 0.39 & 1.59 & 0.5 \\
\hline West vs. Midwest & 1.25 & 0.58 & 2.73 & 0.6 \\
\hline \multicolumn{5}{|l|}{ Hospital volume } \\
\hline$>75$ th $(>19 / y r)$ vs. $\leq 75$ th $(\leq 19 / y r)$ & 0.98 & 0.54 & 1.77 & 0.9 \\
\hline \multicolumn{5}{|l|}{ Surgical characteristics } \\
\hline \multicolumn{5}{|l|}{ Surgeon volume } \\
\hline$>75$ th $(>6 / y r)$ vs. $\leq 75$ th $(\leq 6 / y r)$ & 1.33 & 0.89 & 1.99 & 0.2 \\
\hline \multicolumn{5}{|l|}{ Year of surgery } \\
\hline $2007-2010$ vs. $2003-2006$ & 1.40 & 0.86 & 2.28 & 0.2 \\
\hline $2011-2015$ vs. $2003-2006$ & 1.09 & 0.61 & 1.96 & 0.8 \\
\hline
\end{tabular}

Table 2. Multivariable Logistic Regression For Patient-, Sugical- And Hospital-Level Predictors Of Low Cost $\left(<10^{\text {th }}\right.$ Percentile) Percutaneous Nephrolithotomy

\begin{tabular}{|c|c|c|c|c|}
\hline \multirow{2}{*}{$\begin{array}{l}\text { Patient characteristics } \\
\text { Age }\end{array}$} & \multirow{2}{*}{$\begin{array}{c}\text { Odds Ratio } \\
1.003\end{array}$} & \multicolumn{2}{|c|}{$\begin{array}{l}\mathbf{9 5 \%} \text { Confidence } \\
\text { Intervals }\end{array}$} & \multirow{2}{*}{$\begin{array}{c}\text { p value } \\
0.7\end{array}$} \\
\hline & & 0.99 & 1.02 & \\
\hline \multicolumn{5}{|l|}{ Gender } \\
\hline Female vs. male & 0.99 & 0.73 & 1.34 & 1 \\
\hline \multicolumn{5}{|l|}{ Race } \\
\hline Non-White vs. white & 0.77 & 0.48 & 1.24 & 0.3 \\
\hline \multicolumn{5}{|l|}{ Marital status } \\
\hline Non-married vs. married & 0.85 & 0.57 & 1.26 & 0.4 \\
\hline \multicolumn{5}{|l|}{ Insurance status } \\
\hline Medicaid vs. medicare & 1.39 & 0.68 & 2.84 & 0,4 \\
\hline Private vs. medicare & 1.33 & 0.80 & 2.22 & 0.3 \\
\hline Other vs. medicare & 1.00 & 0.60 & 1.69 & 1 \\
\hline \multicolumn{5}{|l|}{ Charlson comorbidity score } \\
\hline 1 vs. 0 & 0.85 & 0.63 & 1.15 & 0.01 \\
\hline$\geq 2$ vs. 0 & 0.69 & 0.43 & 1.09 & $<0.001$ \\
\hline \multicolumn{5}{|l|}{ Hospital characteristics } \\
\hline \multicolumn{5}{|l|}{ Hospital teaching status } \\
\hline Teaching vs. Non-teaching & 0.33 & 0.17 & 0.63 & $<0.001$ \\
\hline \multicolumn{5}{|l|}{ Hospital bed size } \\
\hline $300-499$ vs. $<300$ beds & 1.35 & 1.15 & 1.60 & $<0.001$ \\
\hline$\geq 500$ vs. $<300$ beds & 0.61 & 0.49 & 0.75 & $<0.001$ \\
\hline \multicolumn{5}{|l|}{ Hospital location } \\
\hline Urban vs. rural & 2.77 & 1.27 & 6.03 & 0.01 \\
\hline \multicolumn{5}{|l|}{ Hospital region } \\
\hline Northeast vs. Midwest & 1.54 & 0.53 & 4.44 & 0.4 \\
\hline South vs. Midwest & 2.06 & 0.95 & 4.47 & 0.1 \\
\hline West vs. Midwest & 1.11 & 0.50 & 2.50 & 0.8 \\
\hline \multicolumn{5}{|l|}{ Hospital volume } \\
\hline$>75$ th $(>19 / y r)$ vs. $\leq 75$ th $(\leq 19 / y r)$ & 0.62 & 0.30 & 1.30 & 0.2 \\
\hline \multicolumn{5}{|l|}{ Surgical characteristics } \\
\hline \multicolumn{5}{|l|}{ Surgeon volume } \\
\hline$>75$ th $(>6 / y r)$ vs. $\leq 75$ th $(\leq 6 / y r)$ & 1.74 & 0.88 & 3.41 & 0.1 \\
\hline \multicolumn{5}{|l|}{ Year of surgery } \\
\hline $2007-2010$ vs. $2003-2006$ & 0.82 & 0.38 & 1.75 & 0.6 \\
\hline $2011-2015$ vs. $2003-2006$ & 0.88 & 0.39 & 1.97 & 0.8 \\
\hline
\end{tabular}

Results: A total of 114,581 patients underwent percutaneous nephrolithotomy during the 12-year study period. Mean cost in the low-cost group was \$5,787 (95\% CI: \$5716-\$5856) vs. $\$ 38,590$ (95\% CI: $\$ 37,357-\$ 39,923)$ in the high-cost group. Cost variations were substantially impacted by patient $(63.7 \%)$ and surgical $(18.5 \%)$ characteristics and less so by hospital characteristics $(3.9 \%)$. Significant predictors of high costs included more comorbidities ( $\geq 2$ vs. 0 : OR 1.81, $\mathrm{p}=0.01$ ) and hospital region (Northeast vs. Midwest: OR 2.04, $\mathrm{p}=0.03$ ). Predictors of low cost were hospital bed size of $300-499$ beds OR 1.35, $\mathrm{p}<0.01$ ) and urban hospitals (OR 2.77, $\mathrm{p}=0.01$ ) Factors less likely to be associated with low cost PCNL were more comorbidities (CCI $\geq 2$ : OR 0.69, p $<0.0001$ ), larger hospitals (OR $0.61, \mathrm{p}=0.01)$ and teaching hospitals $(\mathrm{OR} 0.33, \mathrm{p}<0.0001)$.

Conclusions: Our contemporary analysis demonstrates that patient and surgical characteristics had a significant effect on costs associated with percutaneous nephrolithotomy. Poor comorbidity status contributed to high costs highlighting the importance of patient selection.

MP13-17 Is there any difference in outcomes among variations of position in percutaneous nephrolithotomy?

PA Melo, F Vicentini, C Murta, J Claro, R Perrella, M Hisano

Hospital Brigadeiro

Brazil

Introduction \& Objective: Percutaneous nephrolithotomy (PCNL) is the standard surgical procedure for large renal calculi. The best patient positioning on the surgical table is a controversial issue. Our aim is to compare the outcomes of PCNL performed in the classic prone position and in some variations of supine position.

Materials and Methods: We made a retrospective analysis of patients submitted to PCNL from June 2011 to October 2016 in the prone position (PRON) and in three variations of supine position: complete supine (COMPSUP), original Valdivia supine position (VALD), and Galdakao-modified supine Valdivia (GALD). All the patients had a complete pre-operative evaluation, including a non-contrast-enhanced abdominal computed tomography (CT). Success was defined as the absence of fragments larger than $4 \mathrm{~mm}$ on the first post operative day (POD) CT. Complications were classified according to Clavien Score. The significance level adopted was $\mathrm{p}<0.05$.

Results: 393 PCNLs were performed: 100 in COMPSUP, 94 in VALD, 100 in GALD, and 100 in PRON. The analyzed groups

\begin{tabular}{|c|c|c|c|c|c|c|}
\hline & COMPSUP & VALD & GALD & PRON & TOTAL & p value \\
\hline Number of PCNLs, $\mathrm{n}$ & 100 & 94 & 100 & 99 & 393 & \\
\hline $\begin{array}{l}\text { Number of puncture tracts, } \\
\text { mean (SD) }\end{array}$ & $1.39(0.75)$ & $1.38(0.70)$ & $1.25(0.50)$ & $1.20(0.45)$ & $1.31(0.61)$ & 0.073 \\
\hline $\begin{array}{l}\text { Operative time (min), mean } \\
\text { (SD) }\end{array}$ & $90.50(44.36)$ & $111.02(40.87)$ & $120.85(49.49)$ & $123.48(45.14)$ & $111.44(46.84)$ & $<0.001$ \\
\hline $\begin{array}{l}\text { Fluoroscopy time (min), mean } \\
\text { (SD) }\end{array}$ & $12.12(7.50)$ & $15.60(8.07)$ & $14.23(9.47)$ & $15.08(8.08)$ & $14.23(8.39)$ & 0.020 \\
\hline Success rate, \% & $58.0 \%$ & $48.9 \%$ & $49.0 \%$ & $47.5 \%$ & $50.9 \%$ & 0.428 \\
\hline Stone-free rate, \% & $43.0 \%$ & $34.0 \%$ & $35.0 \%$ & $37,4 \%$ & $37,4 \%$ & 0.565 \\
\hline Blood transfiusion rate, \% & $8.0 \%$ & $2.1 \%$ & $3.0 \%$ & $8.1 \%$ & $5.3 \%$ & 0.118 \\
\hline $\begin{array}{l}\text { Drop in hemoglobin level } \\
\text { (gal), mean (SD) }\end{array}$ & $2.22(1.46)$ & $1.91(1.28)$ & $1.97(1.22)$ & $2.34(1.39)$ & $2.11(1.35)$ & 0.092 \\
\hline Complications, n & & & & & & \\
\hline Clanien I & 3 & 1 & 0 & 8 & 12 & 0.001 \\
\hline Clavien II & 7 & s & i & 6 & 19 & \\
\hline Clavien III & 6 & 5 & 7 & 6 & 24 & \\
\hline Clavien $N$ & 4 & 0 & i & 3 & & \\
\hline Clavien $V$ & 0 & 0 & 0 & 0 & 0 & \\
\hline Total, \% & $20.0 \%$ & $11.7 \%$ & $9.0 \%$ & $23.2 \%$ & $16.0 \%$ & 0.019 \\
\hline $\begin{array}{l}\text { Major complications (Clavien } \\
\text { higher of equals to III), \% }\end{array}$ & $10.0 \%$ & $53 \%$ & $8.0 \%$ & $9.1 \%$ & $8.1 \%$ & 0.663 \\
\hline $\begin{array}{l}\text { Hospital stay (hours), mean } \\
\text { (SD) }\end{array}$ & $33.12(49.38)$ & $53.66(36.68)$ & $54.30(32.99)$ & $67.92(58.75)$ & $37.23(45.90)$ & 0.070 \\
\hline Tubeless rate, \% & $31.3 \%$ & $21.3 \%$ & $14.0 \%$ & $10.1 \%$ & $19.1 \%$ & 0.001 \\
\hline
\end{tabular}


were homogeneous regarding age, gender, BMI, stone size and Guy's Score. The overall immediate success rate was $50.9 \%$ and it was similar among groups $(\mathrm{p}=0.428)$. There was also no difference among groups regarding to the number of punctures $(p=0.073)$, stone-free rate $(p=0.565)$, blood transfusion $(p=0.118)$, drop in hemoglobin level $(p=0.092)$, length of hospital stay $(\mathrm{p}=0.070)$, and severe complications (Clavien $\geq 3$ ) $(p=0.663)$. There was statistically significant difference in relation to operative time $(\mathrm{p}<0.001)$, fluoroscopy time $(\mathrm{p}=0.020)$, and tubeless rate $(\mathrm{p}=0.001)$. In post hoc analysis, we observed that COMPSUP had a lower operative time than all the other positions. COMPSUP had a lower fluoroscopy time than VALD. Regarding tubeless rate, COMPSUP had a higher rate and PRON had a lower rate than the other positions.

Conclusions: Patient positioning in PCNL does not seem to impact the success rate and severe complications rate. However, COMPSUP appears to be associated with a lower surgical time than the other studied positions.

MP13-18 Ambulatory Percutaneous Nephrolithotomy: 2 year 150 case experience

JE Abbott, J Davalos

University of Maryland Baltimore Washington Medical Center United States

Introduction \& Objective: Percutaneous Nephrolithotomy (PNL) is a procedure that has traditionally been performed in an inpatient setting with at least an overnight stay. Many surgical procedures have evolved over time from an inpatient setting to an ambulatory surgery center (ASC) setting. Feasibility of Ambulatory PNL (aPNL) was shown in our initial pilot series of 25 cases. ${ }^{1}$ This 150 case series is reviewed to further evaluate outcomes with a more robust data set.

Materials and Methods: We present a series of our initial 150 patients who underwent PNL in an ASC from April 2015 to March 2017. Each aPNL was performed by one of two surgeons with the same operative team. The surgeon and operative team had extensive experience with PNLs performed in a hospital setting. All procedures were performed with the operative surgeon obtaining renal access and all procedures were performed tubeless (ureteral stent without a nephrostomy tube). All patients also had hemostatic plugs placed into the access tract with a local intercostal block performed to aid with pain control. ${ }^{2}$ All cases were reviewed and demographic date and case details were analyzed.

Results: Of the 150 aPNL reviewed (Table 1), there were 77 women, 78 left side, mean age 55 (21-84), mean BMI 31 (17-49), and mean stone burden $29 \mathrm{~mm}$ (10-110). Stone free rate was $97 \%$. Seven patients had complications. One Clavien 4a pulmonary embolism (PE) required a 7 day hospital admission. One Clavien 2 sepsis requiring 4 days of hospitalization and IV antibiotics. One Clavien $3 \mathrm{~A}$ for wound infection and urinoma requiring nephrostomy tube placement. Four Clavien 1 presentations (two for pain, one for nausea, one for observation due to concern for bleeding) of which three required overnight hospital admission.

Conclusions: This consecutive 150 case series further demonstrates the safety of aPNL. While seven patients experienced complications; none of the outcomes of the adverse events were affected by the site of service. The patients experienced complications not uncommon to many types of procedures (postoperative pain and nausea) that were managed with an ER visit or short hospital stay. The PE was managed in a routine manner. With an experienced surgeon, well trained operative team and with modifications to the procedure focusing on post-operative pain control, PNL can be safely and effectively performed in an ASC. Ongoing collaborative data collection is needed to continue to evaluate the safety of aPNL. 1. Davalos JG, Abbott JE. Ambulatory PCNL: Initial Case Series. J Urology. April 2016. Vol. 195 (4), Supplement 1: MP51-20, e688-e689.

\section{MP13-19 Withdrawn}

MP13-20 Percutaneous Nephrolithotomy (PCNL): 15 Years Experience with Over 15,000 cases

M Hosseini, A Aminsharifi, D Irani, A Eslahi, R Haghpanah, A Yousefi, R Inaloo

shiraz nephro-urology research center, shiraz university of medical sciences, shiraz, iran

Iran

Introduction \& Objective: Currently, PCNL is the modality of choice for large, complex renal stones, however, such as open surgery has complication. We investigated the results and complications of PCNL in our high volume, training, referral center. Materials and Methods: Between Sept. 2002 and Feb., 2017, a total of 15,217 PCNL procedures were done in our center;8604 men and 6613 women, Mean age 38.4 year (18-82), Mean stone size $23.5 \mathrm{~mm}$ (17-71).PCNL was done by experienced, training urologist (Fellowships), and residents. Procedure was done in standard, No Nephrostomy and totally tubeless methods. Floroscopy and ultrasound were used for access. General, epidural, and spinal were the methods of anesthesia. We recorded the results and complications of our patients annually.

Results: Early stone-free rate was $87.6 \%$ and after 3 weeks with ancillary procedures (URS, SWL) 92.5\%. Complications according to Clavein classification: -Grade I (764): Fever $>38.3 \mathrm{C}$ 241, Bleeding 275, Hyponatremia (PCNL Sx.) 18, PCS perforation 159, Pneumo/hydro/hemothorax 47, Renal failure 24; Grade II (344): Transfusion 249, UTI 31, ileus 48, pneumonia 16; -Grade III (589): Access failure 98, Clot retention 39, late hematuria 114, angioembolization 51, Conversion to open 29, Visceral injury 15, Perinephric collection 33, Chest tube 17, Scapular fracture 1, Re-PCNL 240; -Grade IV (163): Renal failure requiring dialysis (18), Kidney exploration and repair (18), Heart failure (25), Arrhythmia requiring ICU (31), Sepsis (11), Myocardial infarction (55); -Grade V (22): sepsis 6, MI 12, Bleeding 2, pulm. emboli 1, unknown 1. Although some of the minor and major complications were managed by referring physicians which did not report to us.

Conclusions: PCNL seems the best treatment option for large renal stones and is a less invasive method. we should consider minor and major complications such as open surgery and manage them properly.

MP13-21 The Clinical Safety and Efficacy of Upper Pole Access Percutaneous Nephrolithotomy (uPPCNL) for Inferior Pole Stones

D Hurtado, JA Abraham

National Kidney and Transplant Institute Philippines

Introduction \& Objective: Traditionally, percutaneous nephrolithotomy (PCNL) for lower pole stones are directly removed through an inferior polar access. We preferentially treated 
inferior pole calculi with an upper polar access and evaluated our clinical outcomes.

Materials and Methods: Between January 2010 and April 2016, 32 patients with inferior calyceal stones were treated uPPCNL. All stones were diagnosed using an unenhanced CT scan. The efficacy (stone-free rate) was determined by comparing the preoperative and postoperative imaging. Clinical safety was assessed based on intraoperative parameters pertaining to operative time, blood loss, urinary extravasation, calyceal injury, pelvic perforation and other untoward events. These complications were summarized using the Clavien-Dindo grading system.

Results: The male to female ratio is 1.1:1. All stones included in the study were pure inferior calyceal in location. The average stone size was $1.65 \pm 0.84 \mathrm{~cm}$ (Range:0.6-4.4) with a mean durility of $936 \pm 298 \mathrm{HU}$ (Range:350-1500). Stone-free rate was $96.8 \%$ (31/32) after a single session of PCNL. The mean operative time was $97 \pm 43$ minutes (Range:40-230). According to the Clavien-Dindo Classification, 26 (81.3\%) had no complication, 5 $(15.6 \%)$ had Grade 1 (fever), and 1 (3.1\%) had Grade 2.

Conclusions: uPPCNL is effective and safe for patients with inferior calyceal stones and confers the following advantages (1) shorter skin-to-calyceal distance (2) straight line to the UPJ and inferior pole (3) a panoramic view of the collecting system (4) less stone migration (5) minimal torque of the nephroscope This minimally invasive procedure achieves a high stone clearance rate with acceptably low complication rates.

MP13-22 PCNL in kidney with borderline renal function: A single institution experience

M Shahait, O Ayyash, S Jackman, T Averch

UPMC

United States

Introduction \& Objective: Prior to the treatment of staghorn calculi, the American Urologic Associa!on recommends urologists obtain a functional imaging study if clinically significant loss of renal function in the involved kidney is suspected. Furthermore, it endorses nephrectomy for kidneys with poor function as they may act as source of infection and pain. We sought to investigate the feasibility of percutaneous nephrolithotomy (PCNL) in kidneys with borderline renal function, particularly with regards to post-operative complications.

Materials and Methods: We performed a retrospective review of patients with borderline kidney function (split func!on $<35 \%$ ) who underwent PCNL between 2003-2015. Inclusion criteria necessitated work-up with func!onal imaging (DMSA scan). PCNL was offered as a treatment op!on for patients who refused nephrectomy or observation

Results: Twenty seven pa!ents with borderline kidney function underwent PCNL during the study period. The median age of the pa!ents was 53 (IQR 40-61); male to female ratio was 1:1.4. The percentage of patients in our cohort with a split function of 10-19, 2029 , and $30-35$ was $26 \%, 44.4 \%$, and $29.6 \%$, respectively. In $26 \%$ of the cases, interventional radiology inserted a nephrostomy tube preoperatively. A second look procedure was performed in $52 \%$ of cases. Seventy eight percent of the cases were stone free after immediate post-op imaging; three out of the 6 cases with residual fragments underwent retrograde ureteroscopy/laser lithotripsy. One case developed immediate post-op bleeding that was treated conservatively. The median creatinine at 1 month post-op follow-up was 0.9

Conclusions: For staghorn calculi in kidneys with borderline func!on, PCNL is a feasible treatment op!on with minimalrisk of complica!ons including bleeding and repeat procedures. Future prospective studies should assessthe long term benefit of salvaging borderline functional kidneys in patients with nephrolithiasis

\section{MP13-23 Outpatient PCNL Outcomes: The UC San Diego} Experience

\section{DS Han, SK Bechis, JE Abbott, RL Sur}

UC San Diego School of Medicine, Department of Urology United States

Introduction \& Objective: Percutaneous nephrolithotomy (PCNL) is traditionally performed with an overnight stay due to concern for postoperative infection or hemorrhage. Outpatient PCNL has been described for highly selected patients. We present a series of outpatient PCNL in a tertiary care kidney stone center. Materials and Methods: In July 2015 we began attempting outpatient PCNL and by February 2016 it became standard algorithm at our institution. In total 54 patients underwent planned outpatient PCNL from July 2015 to October 2016. Each was performed at a tertiary care facility by a single surgeon who obtained renal access intraoperatively. Patients had thrombin soaked gel foam plugs placed into the access tract and local marcaine intercostal blocks. Demographic/perioperative data, comorbidity classification (ASA Score and Charlson Index) and outcomes were analyzed with descriptive statistics.

Results: 55 PCNLs (one bilateral) were performed: $45 \%$ males, mean age 55 years (20-85), BMI 27.9 (16-50), and stone burden $25.4 \mathrm{~mm}$ (9-54). Two were complete staghorn calculi, 11 had stone burden $>30 \mathrm{~mm}$, and 4 cases involved encrusted stents. One patient had PCNL performed on a transplant kidney. 21 patients had an ASA $>=3$ and 18 had a Charlson Comorbidity Index $>=4$. Mean OR time was $180+/-56$ minutes. 48 of 55 cases were tubeless with internalized ureteral stents placed. Clinical stone-free rate was $87 \%$ (48/55). Mean postoperative hematocrit and creatinine changes were $-1.3 \mathrm{~g} / \mathrm{dL}$ and $+0.03 \mathrm{mg} / \mathrm{dL}$, respectively. $72 \%$ (39) patients were discharged home that same day while $28 \%$ (15) were observed overnight and discharged the day after surgery: 5 for social reasons, 7 for nausea/pain control, 2 for slow recovery from anesthesia, and 1 for persistent drainage from access site requiring nephrostomy tube exchange. One staghorn patient stayed overnight for slow recovery without post op complications and the other was sent home same day but readmitted for pyelonephritis. The 30 day complication rate was $20 \%(11 / 55) .9$ patients had Clavien grades I-II (7 UTIs requiring treatment, 1 wound infection, 1 gross hematuria without need for transfusion). One patient had a post-op urinoma after stent removal requiring drain placement (Clavien IIIb) while another had a misplaced stent requiring surgical removal. 13\% (7) of patients required hospital readmission.

Conclusions: Outpatient PCNL-even in those with higher comorbidity status-can be safely performed on most patients with acceptably low major complication rates. A subset such as those with complete staghorn calculi may require overnight observation.

\section{MP13-24 Ambulatory Percutaneous Nephrolithotomy: A Cost-Reducing Innovation}

D Beiko, K Pace, S Andonian

Queen's University

Canada

Introduction \& Objective: Globally, growth in healthcare expenditures is becoming unsustainable. In 2016, Canada's healthcare expenditure was estimated at $\$ 228$ billion $(\$ 6,299$ per 
person) or $11 \%$ of gross domestic product. The shift from inpatient to outpatient surgical care-largely led by urology-has helped reduce healthcare costs. Ambulatory percutaneous nephrolithotomy (aPCNL) is a relatively new innovation that offers potential cost savings and has been shown to be safe and effective in properly selected patients. Our objective was to quantify potential cost savings of aPCNL in Canada.

Materials and Methods: A review of the databases and reports of the Canadian Institute for Health Information (CIHI, an independent, not-for-profit organization that provides essential information on Canadian healthcare systems) was performed to determine multiple estimated data points including: number of PCNL cases performed annually; average total cost of a single PCNL operation; average length of stay for PCNL in Canada; average daily cost of an overnight stay in a standard hospital bed. The formula used was as follows: cost of aPCNL $=$ total cost of standard PCNL - cost of postoperative hospital stay. These calculations were based on the following assumptions: cost of an average postoperative hospital stay provides an estimate of the cost savings of aPCNL; patients were not admitted to the intensive care unit postoperatively; aPCNL has the same ER visit and readmission rates as standard PCNL.

Results: An estimated 6,000 adult PCNL cases occur annually in Canada. The average length of stay for PCNL in an adult is 5.1 days and the average estimated daily cost of a standard hospital bed is \$2000 per day. Excluding hospital stay, CIHI's estimated average cost of PCNL is $\$ 9,212$ - the estimated cost of aPCNL. The total cost of standard PCNL is calculated at $\$ 19,412$. The resulting cost savings of aPCNL is approximately $\$ 10,200$ per PCNL case in Canada.

Conclusions: With potential savings of over $\$ 10,000$ per PCNL case in Canada, aPCNL represents an innovative surgical approach that could reduce costs across global healthcare systems. Understandably, not all patients are suitable for same-day discharge following PCNL but if even 5\% of PCNL cases could be performed on an outpatient basis, an estimated cost savings greater than $\$ 3 \mathrm{M}$ per year (300 cases $\times \$ 10,200 /$ case) could be realized in Canada alone.

\section{MPS14: PEDIATRIC ENDOUROLOGY, LAPAROSCOPY AND ROBOTICS}

MP14-1 Unique Combined Endoscopic Approach to Large Staghorn Calculus in a 2-Year-Old

N Jung, D Herz

University of TN College of Medicine Chattanooga/Erlanger Health System

United States

Introduction \& Objective: A 2 year old neurodevastated tracheostomy dependent girl presented with Proteus urosepsis. Past medical is history of seizure disorder treated with Topomax. No prior genitourinary surgery or UTI. 24-hour urine cysteine normal. CT scan of the abdomen and pelvis demonstrated left $5 \times 3 \times 2 \mathrm{~cm}$ staghorn calculus. (Figure 1)

Materials and Methods: Despite attempts at upper/lower pole percutaneous access, only a 6Fr nephrostomy tube could be placed into the left mid-pole calyx. Anterograde nephrostogram revealed

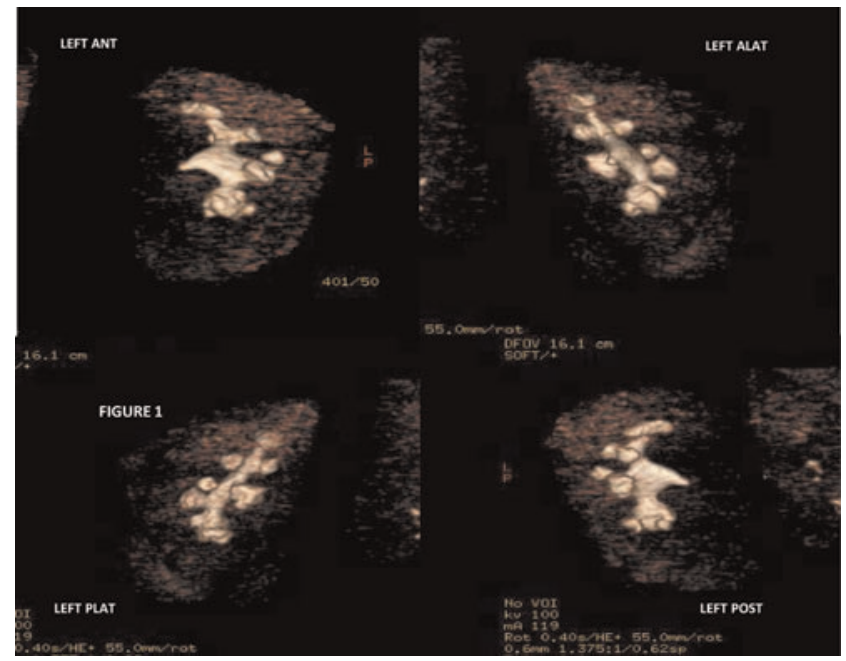

no hydronephrosis, however there was ureteral dilation down to the bladder level. Due to suboptimal percutaneous access, initial ureteroscopic management was chosen. Second look ureterscopy was performed after 2 weeks of nephroureteral drainage. Completion mini-PCNL was performed at 3 weeks. Stone fragment chemical analysis and 24-hour urine analysis were obtained. Repeat CT scan and nephrostomy removal were performed at 4 weeks.

Results: Initial ureteroscopic laser lithotripsy cleared $\sim 80 \%$ of initial stone burden. Stone composition was $25 \%$ calcium phosphate and $75 \%$ magnesium ammonium phosphate. Second look ureteroscopy and CT scan revealed $8 \mathrm{~mm}$ lower and $5 \mathrm{~mm}$ upper pole stone fragments. Completion mini-PCNL performed through the established nephrostomy tract cleared remaining fragments. There were no complications, unplanned operations, or post-op infections or organ failure.

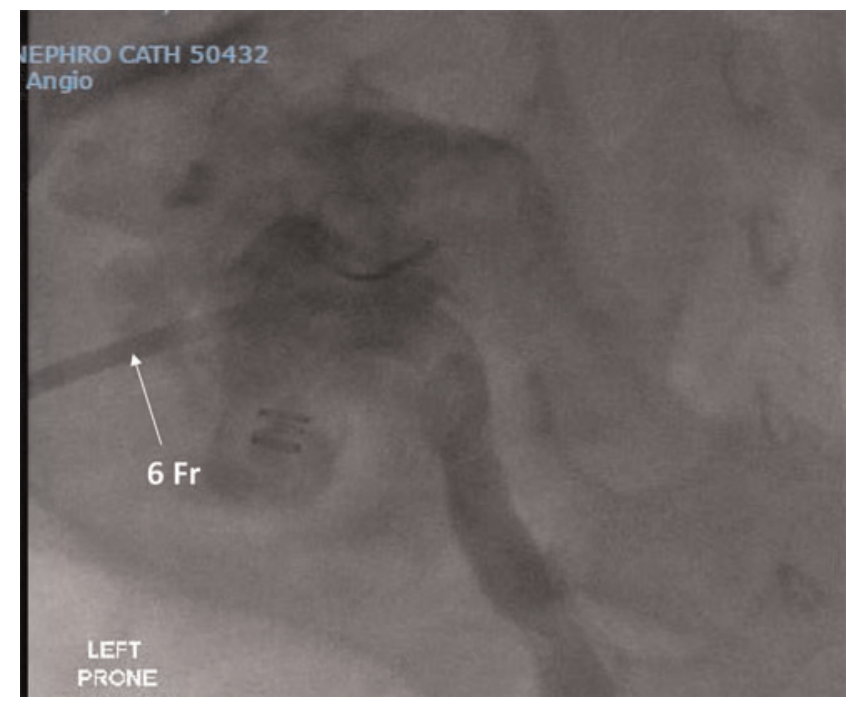


Conclusions: PCNL offers superior stone clearance rates for large renal calculi. However, these outcomes are predicated on having 24-30 Fr kidney access. Given the absence of hydronephrosis, significant discrepancy between caliceal and infundibular caliber and standard PCNL sheath, and presence of ureteral dilation (Figure 2), we chose initial ureteroscopic laser lithotripsy. Completion min-PCNL was only required for small fragments ureteroscopically inaccessible. This case illustrates a safe unconventional approach to an uncommon surgically complex problem.

MP14-2 Combined use of flexible ureteroscopic lithotripsy with micro-percutaneous nephrolithotomy in pediatric multiple kidney stones

W Wang, J Li

Beijing Friendship Hospital, Capital Medical University China (People's Republic)

Introduction \& Objective: To investigatethe clinical value of the treatment of pediatric multiple kidney stones using flexible ureteroscopic lithotripsy (FUL) combined with micro-percutaneous nephrolithotomy (microperc).

\begin{tabular}{|c|c|}
\hline Demographics & Value \\
\hline \multicolumn{2}{|l|}{ Gender } \\
\hline Male & $10(66.7 \%)$ \\
\hline Female & $5(33.3 \%)$ \\
\hline \multicolumn{2}{|l|}{$\operatorname{Age}(y)$} \\
\hline Range & $1-8$ \\
\hline Mean & $3.8 \pm 1.6$ \\
\hline Patients aged $\leqslant 3 y$ & $8(53.3 \%)$ \\
\hline Patients aged $>3 y$ & $7(46.7 \%)$ \\
\hline \multicolumn{2}{|l|}{ Symptoms } \\
\hline Hematuria & $6(40.0 \%)$ \\
\hline Urinary tract infection & $5(33.3 \%)$ \\
\hline Abdominal pain & $3(20.0 \%)$ \\
\hline Fever & $1(6.67 \%)$ \\
\hline \multicolumn{2}{|l|}{ No.laterality(n) } \\
\hline Right & $8(53.3 \%)$ \\
\hline Left & $7(46.7 \%)$ \\
\hline \multicolumn{2}{|l|}{ Stones(n) } \\
\hline Multiple & $11(73.3 \%)$ \\
\hline Single & $4(26.7 \%)$ \\
\hline \multicolumn{2}{|l|}{ Stone burden $(\mathrm{cm})$} \\
\hline Range & $1.5-5.0$ \\
\hline Mean & $3.4 \pm 0.9$ \\
\hline \multicolumn{2}{|l|}{ Hydronephrosis } \\
\hline Yes & $9(60.0 \%)$ \\
\hline No & $6(40.0 \%)$ \\
\hline \multicolumn{2}{|l|}{ Urine test } \\
\hline High white blood cell & $10(66.7 \%)$ \\
\hline Normal white blood cell & $5(33.3 \%)$ \\
\hline \multicolumn{2}{|l|}{ Urine culture } \\
\hline Positive & $2(13.3 \%)$ \\
\hline Negative & $11(73.3 \%)$ \\
\hline Contaminated & $2(13.3 \%)$ \\
\hline \multicolumn{2}{|l|}{ Urine nitrite } \\
\hline Positive & $2(13.3 \%)$ \\
\hline Negative & $13(86.7 \%)$ \\
\hline
\end{tabular}

Table 2.Intraoperative and postoperative data

\begin{tabular}{ll}
\hline Variable & Value (\%) \\
\hline $\begin{array}{l}\text { Passive dilatation } \\
\text { Yes }\end{array}$ & $15(100 \%)$ \\
No & $0(0 \%)$ \\
Stone clearance & \\
Complete & $13(86.7 \%)$ \\
Partial & $2(13.3 \%)$ \\
Postoperative complications(Clavien classification) & \\
$\quad$ Grade I & $6(40.0 \%)$ \\
$\quad$ Hematuria & $6(40.0 \%)$ \\
Grade II & $1(6.7 \%)$ \\
$\quad$ Low fever(<38.5 c) & $1(6.7 \%)$ \\
Grade IIIb & $1(6.7 \%)$ \\
$\quad$ Steinstrasse with high fever( $\left.>38.5^{\circ} \mathrm{c}\right)$ & $1(6.7 \%)$ \\
Stone composition & \\
Mixed(calcium oxalate morlohydrate and dehydrate) & $5(33.3 \%)$ \\
L-cystine & $4(26.7 \%)$ \\
Calcium oxalate morlohydrate & $4(26.7 \%)$ \\
Struvite & $2(13.3 \%)$ \\
\hline
\end{tabular}

Materials and Methods: In total, 15 pediatric patients with multiple renal calculi between July 2015 to September 2016 were selected for FUL combined with microperc, including 10 boys and 5 girls; patients' mean age was 3.8 years (range 1-8 years); 7 cases involved the left side, and 8 cases involved the right side; and the mean stone diameter was $3.4 \mathrm{~cm}$ (range $1.5-5 \mathrm{~cm}$ ). All the patients had retained 4.7Fr double $\mathrm{J}$ stent for passive dilatation of the ureter 14 days prior to surgery. FUL was first performed in the lithotomy position to fragment the stones which were located in the renal pelvis, upper and mid renal calyx. Patients were then moved to a prone position, and microperc was performed. Percutaneous renal access to the lower calyx was achieved using a $4.8 \mathrm{~F}$ "all seeing needle" (polyDiagnost, Pfaffenhofen, Germany) with ultrasound guidance, and stone fragmentation was performed with a 200-um holmium laser in different setting to achieve dusting of fragments of $1-2 \mathrm{~mm}$ according to stone disintegration. "Flushing fluid" irrigation was used during FUL (pump irrigation) and microperc (manual irrigation).

Results: All the 15 pediatric patients with multiple kidney stones underwent combined FUL and microperc successfully. The onesession surgical stone free rate (SFR) was $86.7 \%$ (13/15). The mean surgical time was $80 \mathrm{~min}$ (range $50-180 \mathrm{~min}$ ), and the mean amount of flushing fluid used was 4,228 ml (range 120-12,000 ml). According to the modified Clavien classification, Grade 1, Grade 2, Grade $3 \mathrm{~b}$ postoperative complications occurred in 6,1 , and 1 patient respectively. The mean hemoglobin drop was $0.4 \mathrm{~g} / \mathrm{dl}(0.1-$ $0.8 \mathrm{~g} / \mathrm{dl})$, no patients needed transfusion. The average hospital stay was 3 days (range, 2-5 days).

Conclusions: Combined FUL and microperc is a safe, effective, minimally invasive operation to remove multiple renal calculi in children in selected cases.

MP14-3 Staged ureteroscopic management of large bilateral renal calculi in prepubescent patients

EM Sebesta, AC Small, MJ Lipsky, GW Moran, OD Shah, P Casale

NewYork-Presbyterian Hospital / Columbia University Medical Center

United States

Introduction \& Objective: The management of large renal calculi in children is controversial. The 2016 AUA surgical stone 
FIGURE: Treatment schema for staged ureteroscopic management of large $(>20 \mathrm{~mm})$ bilateral renal calculi in prepubscent patients

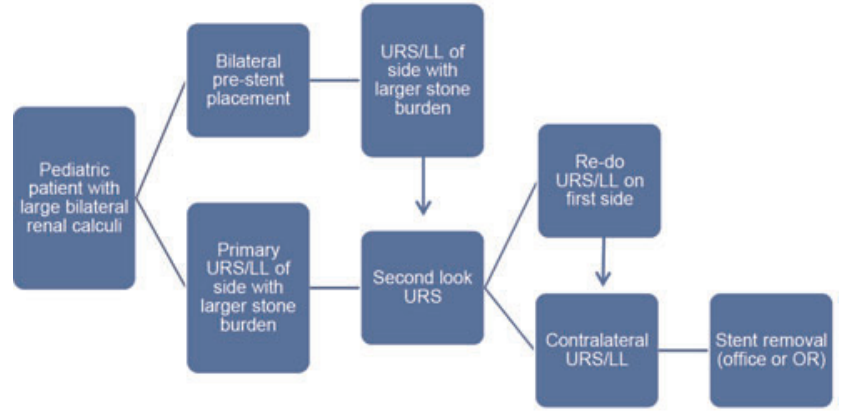

Mean $3.4 \pm 0.5$ procedures $(n=17)$

guidelines state that percutaneous nephrolithotomy (PCNL) and shockwave lithotripsy are acceptable options for pediatric patients with renal stone burden $>20 \mathrm{~mm}$, without mention of ureteroscopy (URS). However, a randomized trial of PCNL vs URS for pediatric stones $>20 \mathrm{~mm}$ showed fewer complications and shorter hospital stays with URS than PCNL, albeit with lower stone free rates (Saad 2015). We present our experience with staged URS for large $(>20 \mathrm{~mm}$ ) bilateral renal calculi.

Materials and Methods: We retrospectively reviewed prepubescent patients $\leq 12$ years old with bilateral stones $>20 \mathrm{~mm}$ on either or both sides, from 2011 to 2016. Patients were managed with staged URS and laser lithotripsy (LL) (FIGURE). All procedures utilized an access sheath (12 or 9.5 Fr), Storz Flex-X2 flexible ureteroscope (7.5 Fr), and holmium laser. Baseline characteristics, surgical technique and outcomes were evaluated. Results: 17 patients (34 renal units) with mean age $7.2 \pm 2.7$ years underwent staged URS for bilateral renal calculi. $65 \%$ were girls. Underlying diagnoses included ketogenic diet $(41 \%)$, cystinuria $(35 \%)$, neurogenic bladder with UTI (12\%) and topiramate usage (12\%). Mean stone size was $3.1 \pm 0.9 \mathrm{~cm}$ (range 1.5 to $4.5 \mathrm{~cm}$ ) with $59 \%$ staghorn calculi. Patients underwent mean $3.4 \pm 0.5$ procedures with an operating time of $177 \pm 58$ minutes. Patients underwent primary URS/LL (24\%) or were pre-stented bilaterally $(76 \%)$ then returned for URS/LL of the side with larger stone burden. They next underwent second-look URS on the side of the first procedure, and if stone free, URS/LL of the contralateral side was performed. If the first side was not stone free, further URS/LL was performed, and patients returned for a fourth procedure for contralateral URS/LL. Bilateral simultaneous URS/LL was not performed. Complications occurred in $12 \%$ ( 1 febrile UTI after stent removal and 1 calyceal rupture requiring stent). Patients underwent follow up renal ultrasound at 4-6 weeks and all were stone free.

Conclusions: Management of large bilateral calculi is feasible, safe and effective with a ureteroscopic approach. This may be preferable to PCNL for some patients, especially those who may require future stone procedures. Preoperative counseling and careful staged planning are required for successful outcomes.

MP14-4 A contemporary update of paediatric urinary stone composition in the United Kingdom \& the Republic of Ireland.

PT Grice, N Nkwam, D Bodiwala, N Armugam, N Mamooowala, M Wells, V Lee, G Ball, P Gupta, P Patel

University Hospitals of Leicester NHS Trust, Leicester, United Kingdom

United Kingdom
Introduction \& Objective: Urolithiasis is a recurrent condition with up to $50 \%$ of patients experiencing repeated episodes within 10 years. Performing stone analysis for the comprehensive evaluation of children with urinary tract calculi can go a long way to reducing morbidity and recurrence.

Materials and Methods: We retrospectively reviewed stone specimens from paediatric patients submitted to the chemical pathology department of a tertiary UK teaching hospital between 2010 and 2015. Samples were received from England, Scotland, and the Republic of Ireland. All specimens were analysed using infrared spectroscopy.

Results: A total of 220 samples were reviewed -143 boys and 77 girls; median age of 9 years (0-17 years). Most specimens came from Yorkshire (49.5\%), East Midlands (21.8\%), and the Republic of Ireland (18.2\%). The most prevalent stone components in males compared to females were calcium phosphate (37.1\% cf $41.6 \%)$, calcium oxalate $(31.5 \%$ cf $35.5 \%)$, struvite $(11.2 \%$ cf $14.3 \%)$ and cystine $(10.5 \%$ cf $1.3 \%, \mathrm{p}=0.01)$, respectively. Cystine was the only crystal to demonstrate statistical significance between the sexes.

Conclusions: This is the largest pediatric stone analysis database in Europe. Calcium oxalate crystals appears to be the most prevalent in this age group in much of the published literature worldwide. Interestingly, the most prevalent crystal in this analysis was calcium phosphate. It is unclear if this is due to consumed local water content or variations in dietary and lifestyle factors. This study provides valuable contemporary insight into the composition of urinary tract calculi in our paediatric population and can help to address risk factors accordingly.

MP14-5 Mini-percutaneous cystolithotripsy (PCCL) in pre-school children

\section{MS Agrawal, D Mishra}

Global Rainbow Healthcare, Agra, India India

Introduction \& Objective: The greatest limiting factor in endourological procedures for vesical calculi in small children is the narrow urethral calibre, not only related to instrumentation but also for clearance of stone fragments. A percutaneous suprapubic approach circumvents the problem of urethral calibre in these situations. We present our experience of mini-percutaneous cystolithotripsy in pre-school children.

Materials and Methods: This study was conducted from January 2011 till December 2016. Data was collected prospectively. All patients underwent suprapubic cystolithotripsy using mini nephroscope (Karl Storz, 12 F) with 15 Fr access sheath. Vesical puncture was done after filling the bladder and under Ultrasonic guidance. Holmium Laser with power setting of 2040 watts was used for fragmentation of stone. No suture or suprapubic catheter was left in. All children were discharged on next day.

Results: We had 16 patients with mean age of 2.4 years (1-5), 13 were male and 3 were female. Mean stone size was $1.86 \pm 0.82 \mathrm{~cm}$ (1 - $3.6 \mathrm{~cm})$. Mean operative duration was 33.5 minutes (21-51). No suprapubic catheter was left in any patient. All fragments were cleared on table and all children were stone-free on post-op imaging. No complications were detected. At follow-up ranging from six months to six years, there was no recurrence of stones. Conclusions: Suprapubic cystolithotripsy with mini-perc is a feasible safe and effective minimally invasive treatment option for bladder stones in children, with good success rate. 
MP14-6 Pre-Operative Tamsulosin and Ureteral Orifice Navigation in Pediatric Patients: Is there any benefit?

C Morley, O AL-Omar

United States

Introduction \& Objective: Balloon dilation of the ureteral orifice (UO) is not recommended in pediatric patients. Ureteral stents (US) are placed for passive dilation resulting in another procedure. We aim to evaluate whether pre-operative tamsulosin increases the rate of ureteral navigation for ureteroscopy (URS). Materials and Methods: We retrospectively reviewed all pediatric patients who underwent URS at our institution from January 2013 to March 2016. All cases were performed by a single surgeon using a standard approach with semi-rigid ureteroscope (Wolf 4.5 Fr) for distal and mid ureteral stones and flexible ureteroscope (Storz 7.5 Fr) with or without a ureteral access sheath (Cook 9.5 Fr) for proximal ureteral and kidney stones. Patients were separated into two groups: those who took tamsulosin $0.4 \mathrm{mg}$ daily $\geq 48$ hours pre-operatively and those who did not take tamsulosin pre-operatively. The student $\mathrm{T}$ test, $\mathrm{Z}$ test, and chi square test were used for statistical analysis.

Results: A total of 68 patients underwent URS with 32 taking pre-operative tamsulosin, 19 without tamsulosin, and 17 patients were excluded. There was no significant difference between the groups with consideration to age and weight of the patients and size or location of the stones. We were able to navigate the ureter in 27 of 32 patients (84.4\%) who took tamsulosin and 10 of 19 patients $(52.6 \%)$ who did not take tamsulosin $(p=0.014)$. We were able to navigate the ureter in 12 of 14 patients $(85.7 \%)$ in the tamsulosin group and 1 of 4 patients $(25.0 \%)$ in the no tamsulosin group for mid and distal stones $(p=0.016)$. For proximal ureteral and renal stones, we were able to navigate the ureter in 15 of 18 patients $(85.7 \%)$ in the tamsulosin group and 9 of 15 patients $(60.0 \%)$ in the no tamsulosin group $(\mathrm{p}=0.133)$. We did not observe any adverse effect from tamsulosin.

Conclusions: Pre-operative tamsulosin did significantly increase the success rate of ureteral navigation for URS, particularly during semi-rigid URS for distal/mid ureteral stones.

MP14-7 Post-Surgical Imaging Patterns in Pediatric Nephrolithiasis: Opportunities for Improvement

J Ellison, P Merguerian, B Fu, S Holt, T Lendvay, J Gore, M Shnorhavorian

University of Washington

United States

Introduction \& Objective: One-third of children with nephrolithiasis will require surgical intervention. Post-surgical

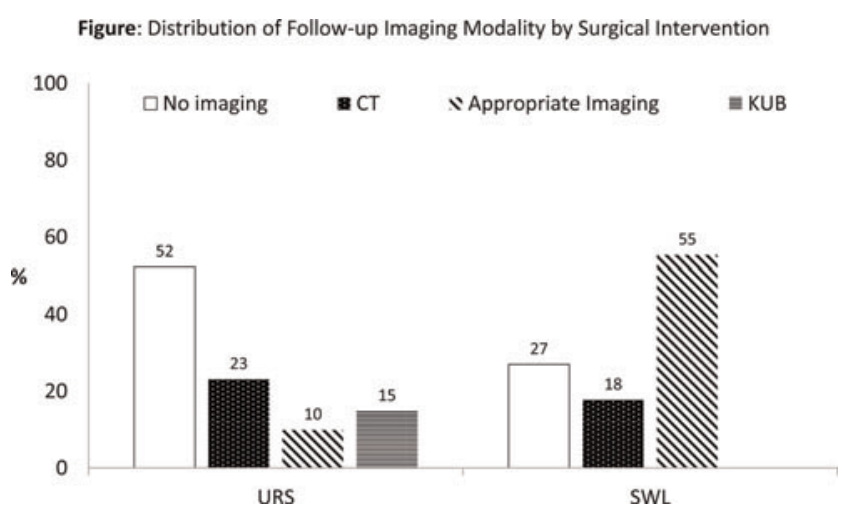

\begin{tabular}{|c|c|c|c|c|}
\hline \multicolumn{5}{|c|}{ Table: Odds of Appropriate Follow-up Imaging for URS and SWL } \\
\hline & \multicolumn{2}{|c|}{ URS } & \multicolumn{2}{|c|}{ SWL } \\
\hline Demographic & OR & CI & OR & CI \\
\hline Age (years) & 1.09 & $1.05-1.14$ & 0.97 & $0.94-1.01$ \\
\hline \multicolumn{5}{|l|}{ Gender } \\
\hline Male & 1.00 (ref) & & 1.00 (ref) & \\
\hline Female & 1.27 & $1.00-1.61$ & 1.45 & $1.18-1.78$ \\
\hline \multicolumn{5}{|l|}{ Comorbidity } \\
\hline $\begin{array}{l}\text { Presence of Chronic } \\
\text { Complex Condition }\end{array}$ & 1.23 & $0.90-1.70$ & 1.13 & $0.81-1.57$ \\
\hline \multicolumn{5}{|l|}{ Region } \\
\hline Northeast & 1.00 (ref) & & 1.00 (ref) & \\
\hline North Central & 0.93 & $0.63-1.37$ & 1.43 & $0.99-2.05$ \\
\hline South & 0.97 & $0.68-1.40$ & 1.49 & $1.08-2.06$ \\
\hline West & 1.08 & $0.67-1.71$ & 1.00 & $0.66-1.52$ \\
\hline Unknown & 1.61 & $0.86-3.01$ & 1.18 & $0.53-2.59$ \\
\hline \multicolumn{5}{|l|}{ Stone Location } \\
\hline $\begin{array}{l}\text { Ureter } \\
\text { Kidney }\end{array}$ & $\begin{array}{c}1.03 \\
1.00 \text { (ref) }\end{array}$ & $0.79-1.36$ & $\begin{array}{c}1.05 \\
1.00 \text { (ref) }\end{array}$ & $0.85-1.31$ \\
\hline \multicolumn{5}{|l|}{ Visit Complexity } \\
\hline ED visit & 8.85 & $6.23-12.56$ & 5.45 & $1.67-17.86$ \\
\hline Repeat Intervention & 2.09 & $1.66-2.63$ & 3.10 & 2.33-4.12 \\
\hline
\end{tabular}

imaging can define treatment success and assess for complications. We explored nationwide imaging patterns in children following surgical intervention for nephrolithiasis, hypothesizing that surgery type and repeat intervention would impact choice of post-surgical imaging.

Materials and Methods: Claims from MarketScan (20072013), an employer-based dataset of privately insured patients, were used to assess all children 1-18 undergoing surgical intervention with shock wave lithotripsy (SWL) or ureteroscopy (URS) for nephrolithiasis, excluding patients undergoing surgical treatment for nephrolithiasis within 6 months. Patient demographics including age, gender, and region of care were collected, as were return emergency department (ED) visits and repeat interventions. Primary outcome was imaging within 90 days of surgery. Appropriate imaging was defined as either abdominal x-ray (KUB) or ultrasound (US) for SWL or US alone for URS. Using logistic regression, odds for appropriate imaging in follow-up were calculated.

Results: A total of 4,251 children were evaluated including 2604 (61.3\%) undergoing URS and 1,647 (38.7\%) undergoing SWL. Median age was 16 (range 1-18) and the majority of patients were female $(2710,63.7 \%)$. Overall, 1169 (27.5\%) children had appropriate post-surgical imaging. Distribution of post-surgical imaging by procedure is shown in the Figure. As compared to URS, children undergoing SWL had higher rates of appropriate post-surgical imaging than those undergoing URS $(\mathrm{p}<0.0001)$. Odds of appropriate follow-up imaging by surgical modality are shown in the Table.

Conclusions: A low proportion of children with nephrolithiasis receive post-surgical imaging. Choice of follow-up imaging may impact assessment of surgical success. The lack of any follow-up imaging, especially following URS, is concerning given the risk of unrecognized silent obstruction. Further work to standardize postsurgical imaging patterns for pediatric nephrolithiasis is needed.

MP14-8 Renal Papillary Mapping and Quantification of Randall's Plaque in Pediatric Calcium Oxalate Stone Formers

\author{
A Darves-Bornoz, J Thomas, T Marien, N Miller \\ Vanderbilt University Medical Center \\ United States
}

Introduction \& Objective: Randall's plaque (RP) with attached stones is recognized as a primary mechanism for stone formation 


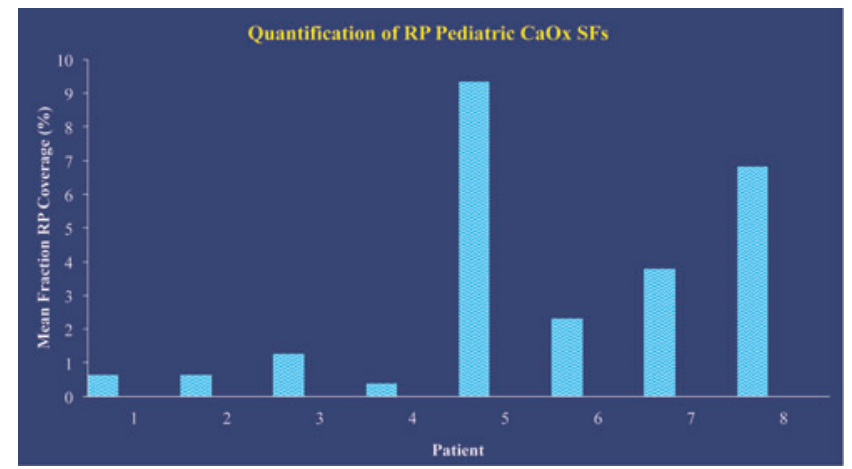

\begin{tabular}{|c|c|c|c|c|c|c|c|c|c|c|c|}
\hline \multirow{2}{*}{\multicolumn{2}{|c|}{ Age }} & \multirow{2}{*}{$\begin{array}{l}\text { Stone } \\
\text { Burden } \\
\text { (mm) }\end{array}$} & \multirow{2}{*}{$\begin{array}{l}\text { Prior } \\
\text { Stone } \\
\text { Events }\end{array}$} & \multirow{2}{*}{$\begin{array}{c}\text { nof } \\
\text { Papilis } \\
\text { Mapped }\end{array}$} & \multicolumn{4}{|c|}{ Quantification of RP in papilla (n of papilla) } & \multirow{2}{*}{$\begin{array}{c}\text { Xof } \\
\text { Papilla } \\
\text { with } \\
\text { Plaque }\end{array}$} & \multirow[t]{2}{*}{$\begin{array}{l}\text { Attached } \\
\text { Stone }\end{array}$} & \multirow[t]{2}{*}{$\begin{array}{c}\text { Stone } \\
\text { Composition }\end{array}$} \\
\hline & & & & & None & Mild & Moderate & Severe & & & \\
\hline 1 & 13 & 5 & 0 & 10 & 6 & 4 & 0 & 0 & 40 & 0 & $100 \% c 00$ \\
\hline 2 & 17 & 8.5 & 1 & 9 & 0 & 9 & 0 & 0 & 100 & 0 & $100 \% \mathrm{com}$ \\
\hline 3 & 12 & 20 & 0 & 6 & 0 & 6 & 0 & 0 & 100 & 0 & $\begin{array}{l}34 \times \text { COM; } \\
66 \times C O D\end{array}$ \\
\hline 4 & 9 & 6 & 1 & 7 & 0 & 7 & 0 & 0 & 100 & 0 & \$100COD \\
\hline 5 & 17 & 9 & 3 & 9 & 0 & 0 & 6 & 3 & 100 & 0 & $\begin{array}{l}84 \times C O M \text {; } \\
16 \% H A\end{array}$ \\
\hline 6 & 11 & 5 & 0 & 16 & 2 & 11 & 3 & $\overline{0}$ & 87.5 & 0 & $100 \% \mathrm{COD}$ \\
\hline 7 & 17 & 10 & 0 & 4 & 0 & 1 & 3 & 0 & 100 & 0 & $100 \% C 0 D$ \\
\hline 8 & 9 & 4 & $\overline{0}$ & 11 & 0 & 5 & 3 & 3 & 100 & 1 & $\begin{array}{l}75 \% C 0 D, \\
25 \% H A\end{array}$ \\
\hline
\end{tabular}

in adult calcium oxalate stone formers ( $\mathrm{CaOx} \mathrm{SF})$. The role of RP in pediatric stone pathogenesis is unknown with no reported studies to date. The purpose of this study is to investigate renal papillary abnormalities and quantify RP in pediatric $\mathrm{CaOx}$ SF.

Materials and Methods: 8 pediatric $\mathrm{CaOx}$ SF underwent ureteroscopy for symptomatic urolithiasis. The collecting system was mapped using a digital ureteroscope. Video for each patient was then reviewed using a retrograde pyelogram to confirm the location of each papilla. A single investigator (NLM) reviewed the video to quantify RP and other papillary abnormalities such as pitting and Bellini duct plugs. Each papilla was graded as having mild $(<10 \%)$, moderate $(10-50 \%)$, or severe $(>50 \%)$ amount of RP. Using computer software, a single investigator calculated the amount (\%) of papillary surface area covered in RP. Patient history and available 24 hour urine data was analyzed.

Results: An average of 9 papillae were mapped per patient. RP was present in $100 \%$ of patients and in $88.8 \%(64 / 72)$ of all papillae examined. When present, RP was uniformly distributed throughout the kidney without preferential distribution to a region or pole. The amount of RP on the papillae was graded as mild in $60 \%$, moderate in $20.8 \%$, and severe in $8.3 \%$ (Table 1). Other papillary abnormalities were rare in pediatric SF with Bellini duct plugging in $9.7 \%$ and pitting in $15.2 \%$ papillae. No correlation was found between the amount of plaque and age at first stone or number of prior stones $(\mathrm{p}=0.84)$. Attached stones were rare (1/8 patients). Mean RP surface area per papillum ranged from 0.39 to $6.82 \%$ (Figure 1 ). The two patients with severe RP had a small amount of calcium phosphate in their stone analysis.

Conclusions: RP is common in pediatric $\mathrm{CaOx} \mathrm{SF}$, while pitting and Bellini duct plugging are not. Compared to adult $\mathrm{CaOx} \mathrm{SF}$ where up to $75 \%$ of stones are found attached to RP, attached stones were rare. The significance of these findings in pediatric stone pathogenesis remains uncertain in this early report, however ongoing research to include correlation with $24 \mathrm{hr}$ urine data and stone recurrence is currently underway.

MP14-9 Outcomes of flexible ureteroscopic lithotripsy for treatment of kidney stones in infants

W Wang, J Li

Beijing Friendship Hospital, Capital Medical University China (People's Republic)

Introduction \& Objective: We evaluated the outcome of flexible ureteroscopic lithotripsy for treatment of kidney stones in infants.

Materials and Methods: One hundred and seventy-five infants with kidney stones were included in this study: 105 males and 70 females. The median age was 16 months (range, 3-36 months). Sixty-five patients had left-side calculi, 68 of them had right-side calculi, and 42 had bilateral calculi. The median diameter of the calculi was $1.8 \mathrm{~cm}$ (range, $1.0-3.2 \mathrm{~cm}$ ). Retrograde intrarenal surgery was performed by an $8 \mathrm{Fr} / 30 \mathrm{~cm}$ flexible ureterorenoscope $\left(\right.$ POLY $\left.{ }^{\circledR}\right)$ combined with a holmium laser. CT scanning or radiography of the kidneys, ureters, and bladder (KUB) region was performed one month after the operation to confirm the clearance of calculi.

Results: All the 175 infants with calculi in 217 sides underwent flexible ureteroscopic lithotripsy. The median operation time was 32 min (range, 10-90mins). The median amount of flushing fluid was $550 \mathrm{~mL}$ (range, $100-1400 \mathrm{~mL}$ ). The stone-free rate after a single session treatment was 92.2\% (200/217), within which 15 infants underwent simultaneous bilateral flexible ureteroscopy lithotripsy. Catheters were retained for 24-48 hours after the operation. Continuous high fever due to reflux $\left(39-40{ }^{\circ} \mathrm{C}, 3-\right.$ 5 days) was present in seven cases. Flushing fluid extravasation was found in two infants. Some patients with minor complications, such as mild hematuria, irritation symptoms, and low fever, recovered without treatment. The duration of hospitalization time after the operation was approximately 1-7 days.

Conclusions: Flexible ureteroscopic lithotripsy is a safe, highly efficient, minimally invasive, and reproducible operation for removal of renal calculi in infants. This technique is a convenient method for postoperative management of patients that enhances their rapid recovery.

\section{MP14-10 Subspecialty Practice Patterns in the Treatment of Pediatric Urolithiasis}

R Davis, NJ Farber, A Kaplan, R Patel, R Steckler, S Elsamra

Rutgers Robert Wood Johnson Medical School

United States

Introduction \& Objective: Recent epidemiological data suggests that the prevalence of pediatric urolithiasis is rapidly increasing. The trends in practice patterns for the surgical treatment of pediatric urolithiasis have not been well described, as both pediatric urologists and endourologists participate in the care of this condition. Our study compared endourology versus pediatric urology exposure to pediatric stone cases during fellowship training, current comfortability in treating pediatric stone cases, and access to pediatric surgical equipment. 


\begin{tabular}{|c|c|c|c|c|c|c|c|c|c|}
\hline \multicolumn{10}{|c|}{ Comfortable or Very Comfortable with Surgical Modality } \\
\hline & \multicolumn{3}{|c|}{ URS } & \multicolumn{3}{|c|}{ ESWL } & \multicolumn{3}{|c|}{ PCNL } \\
\hline & Ped (\%) & Endo (\%) & p value & Ped (\%) & Endo (\%) & p value & Ped (\%) & Endo (\%) & p value \\
\hline $\begin{array}{c}\text { Infant } \\
\text { (0-12 mos) }\end{array}$ & 45 & 30 & 0.29 & 39 & 24 & 0.27 & 48 & 22 & 0.053 \\
\hline \begin{tabular}{c|c} 
Toddler \\
(12 $\mathrm{mos}-4$ yrs)
\end{tabular} & 68 & 40 & 0.04 & 65 & 36 & 0.04 & 60 & 36 & 0.08 \\
\hline $\begin{array}{c}\text { School } \\
(5-12 y r s)\end{array}$ & 91 & 68 & 0.04 & 82 & 64 & 0.11 & 91 & 64 & 0.02 \\
\hline $\begin{array}{l}\text { Adolescent } \\
\text { (13-18 yrs) }\end{array}$ & 96 & 91 & 1 & 87 & 79 & 0.53 & 87 & 90 & 0.7 \\
\hline \multicolumn{10}{|c|}{ Would not perform or Uncomfortable with Surgical Modality } \\
\hline & \multicolumn{3}{|c|}{ URS } & \multicolumn{3}{|c|}{ ESWL } & \multicolumn{3}{|c|}{ PCNL } \\
\hline & $\operatorname{Ped}(\%)$ & Endo (\%) & p value & Ped (\%) & Endo (\%) & p value & Ped (\%) & Endo (\%) & p value \\
\hline $\begin{array}{c}\text { Infant } \\
(0-12 \text { mos })\end{array}$ & 50 & 55 & 0.8 & 35 & 57 & 0.13 & 43 & 67 & 0.04 \\
\hline $\begin{array}{c}\text { Toddler } \\
\text { (12 } \mathrm{mos}-4 \mathrm{yrs})\end{array}$ & 14 & 43 & 0.03 & 21 & 45 & 0.07 & 21 & 45 & 0.07 \\
\hline $\begin{array}{c}\text { School } \\
(5-12 y r s)\end{array}$ & 5 & 20 & 0.15 & 17 & 28 & 0.39 & 4 & 26 & 0.03 \\
\hline $\begin{array}{l}\text { Adolescent } \\
\text { (13-18 yrs) }\end{array}$ & 5 & 4 & 1 & 9 & 10 & 1 & 4 & 6 & 1 \\
\hline
\end{tabular}

Materials and Methods: A survey was designed and distributed to all pediatric urology fellowship programs and the Endourological Society. Inclusion criteria included completion of a fellowship in either pediatric urology or endourology, exclusion criteria included completion of both fellowships. Pediatric age group was stratified into $<12$ months old, 12 months to 4 years, 5 years to 12 years, and 13 years to 18 years. Exposure to and comfortability performing extracorporeal shockwave lithotripsy (ESWL), ureteroscopy (URS) and percutaneous nephrolithotomy (PCNL) was assessed for each age group. Exposure was assessed as a yes or no answer while comfortability was expressed on a scale from 1 to 5 with 1 being "would not do" to 5 being "very comfortable" with each procedure.

Results: 88 urologists responded to the survey; 9 surveys were excluded. 72 surveys met inclusion criteria, with $23(31.9 \%)$ surveys completed by pediatric urologists and $49(68.1 \%)$ surveys by endourologists. During fellowship training, pediatric urologists had significantly more exposure to all surgical modalities in multiple age groups compared to endourologists and were significantly more comfortable performing surgical procedures in certain age groups (Table 1). There were no differences in the availability of pediatric surgical equipment between pediatric urologists and endourologists.

Conclusions: Pediatric urologists, compared to endourologists, have significantly more exposure during fellowship training and are more comfortable performing surgical treatment for urolithiasis across most pediatric age groups. This survey demonstrates an educational opportunity for endourology fellowships, as they may benefit from greater exposure to pediatric patients with stones.

MP14-11 Trends of intervention for paediatric stone disease over the last two decades

\section{A Pietropaolo, B Somani}

University Hospital Southampton NHS Foundation Trust United Kingdom

Introduction \& Objective: There has been a gradual rise of kidney stone disease (KSD) and related interventions in the paediatric age group. We wanted to see the publication trends for interventions for paediatric KSD as reported on PubMed over the last 16 years.

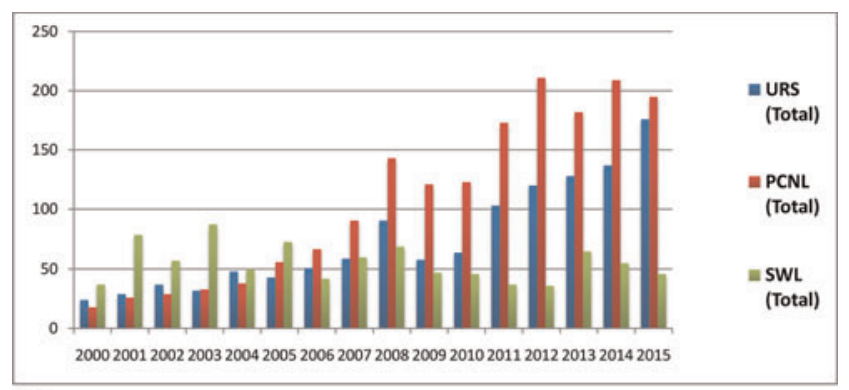

Fig1

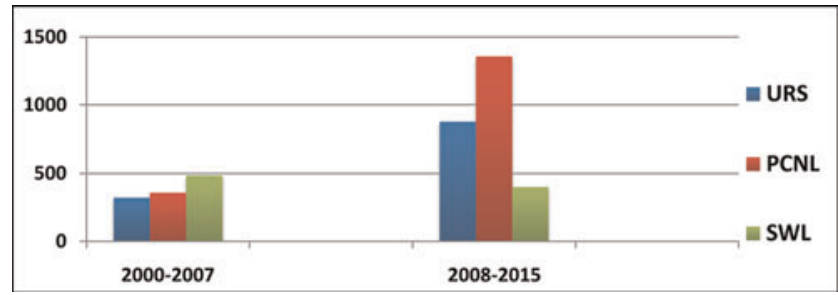

Materials and Methods: All published papers on 'Urolithiasis', 'kidney stones', 'renal stones', 'ureteric stones', 'percutaneous nephrolithotomy', 'percutaneous stone surgery', 'PCNL' and 'PNL', 'ureteroscopy', 'URS', 'shockwave lithotripsy', 'extracorporeal shockwave lithotripsy', 'SWL', 'ESWL', 'lithotripsy', 'open stone surgery', 'laparoscopic surgery', 'robotic surgery', 'pyelolithotomy', 'paediatrics' and 'kids' were searched on PubMed over the last 16-years from 2000-2015. Data was included for ureteroscopy (URS), percutaneous stone surgery (PCNL), shockwave lithotripsy (SWL), open stone surgery and laparoscopic stone surgery. We restricted our search to English language articles with published abstracts. While review articles were included, case reports, laboratory and animal studies, those papers that did not have a published abstract and all adult studies were excluded from our analysis. Data was divided into two 8year periods, period-1 (2000-2007) and period-2 (2008-2015).

Results: During the last 16-years, a total of 339 papers have been published on paediatric stone disease on PubMed. This includes URS ( $\mathrm{n}=95)$, PCNL $(\mathrm{n}=97)$, SWL $(\mathrm{n}=102)$, open stone surgery $(n=34)$ and laparoscopic stone surgery $(n=11)$ (Fig 1). When comparing the two time periods, 92 published papers for all interventions in period- 1 and this had risen almost three-fold $(+270 \%)$ to 247 papers in period-2 (Fig 2).

Conclusions: Published papers on intervention for KSD in paediatric age group has risen over the last 8 years. While URS, SWL, open surgery and laparoscopic surgery have all doubled, PCNL has risen 5-fold reflecting an increase in the new minimally invasive PCNL techniques.

MP14-12 Emergency Department visits, readmissions, and complications after ureteroscopy for pediatric urolithiasis

RN Prasad, AC Strine, CW Concodora, ME Schulte,

BA VanderBrink, PH Noh, E Minevich, PP Reddy, WR DeFoor

Division of Pediatric Urology, Cincinnati Children's Hospital

Medical Center

United States 


\begin{tabular}{|c|c|}
\hline \multicolumn{2}{|c|}{ Demographics } \\
\hline \multicolumn{2}{|l|}{$\begin{array}{l}\text { Median age at surgery in years (IQR) } \\
\text { Gender }(\%)\end{array}$} \\
\hline $\begin{array}{l}\text { Gender }(\%) \\
\text { Male } \\
\text { Female }\end{array}$ & $49(30.2)$ \\
\hline \multicolumn{2}{|l|}{ Race (\%) } \\
\hline Caucasian & $151(93,2)$ \\
\hline African American & $4(2.5)$ \\
\hline Hispanic & 3(1.9) \\
\hline Other & $4(2.5)$ \\
\hline \multicolumn{2}{|l|}{ Primary insurance (\%) } \\
\hline Private & $110(67.9)$ \\
\hline Public & $52(32.1)$ \\
\hline Median body mass index (IOR) & $19.9(17.1-25.1)$ \\
\hline \multicolumn{2}{|c|}{ Preoperative } \\
\hline Congenital anomalies of kidney and urinary tract $(\%)$ & $17(10.5)$ \\
\hline Neurogenic bladder (\%) & $7(4.3)$ \\
\hline Bowel and bladder dysfunction (\%) & $5(3.1)$ \\
\hline Personal history of urolithiasis (\%) & $98(60.5)$ \\
\hline Family history of urolithiasis (\%) & $78(48.1)$ \\
\hline Failure of medical expulsive therapy $(\%)$ & $96(59.3)$ \\
\hline Treatment of urinary tract infection in last 30 days (\%) & $11(6.8)$ \\
\hline Endoscopic procedure in last 30 days (\%) & $77(47.5)$ \\
\hline Extracorporeal shock wave lithotripsy in last 30 days $(\%)$ & $5(3.1)$ \\
\hline Preoperative stenting (\%) & $86(53.1)$ \\
\hline Median number of stones (IQR) & $2.0(1.0-3.0)$ \\
\hline Median size of largest stone in mm (IQR) & $5.0(4.0-7.0)$ \\
\hline \multicolumn{2}{|l|}{ Location of stones $(\%)$} \\
\hline Distal ureter & $48(29.6)$ \\
\hline Middle ureter & $6(3.7)$ \\
\hline Proximal ureter & $8(4.9)$ \\
\hline Kidney & $72(44.4)$ \\
\hline Ureter and kidney & $28(17.3)$ \\
\hline \multicolumn{2}{|c|}{ Intraoperative } \\
\hline Median ASA classification (IQR) & $2.0(2.0-2.0)$ \\
\hline Positive intraoperative urine culture (\%) & $6(3.7)$ \\
\hline Bilateral procedure $(\%)$ & $10(6.2)$ \\
\hline \multicolumn{2}{|l|}{ Type of ureteroscopy (\%) } \\
\hline Semi-rigid & $40(24.7)$ \\
\hline Flexible & $98(60.5)$ \\
\hline Both & $24(14.8)$ \\
\hline Ureteral dilation (\%) & $37(22.8)$ \\
\hline Use of ureteral access sheath (\%) & $23(14.2)$ \\
\hline Postoperative stenting (\%) & $80(49.4)$ \\
\hline Median operative time in minutes (lQR) & $33.0(24.3-54.0)$ \\
\hline \multicolumn{2}{|l|}{ Median operative time in minutes $(\mathrm{IQR})$} \\
\hline Same day surgery $(\%)$ & $123(75.9)$ \\
\hline Narcotic use (\%) & $104(64.2)$ \\
\hline a-blocker use with stent (\%) & $39(48.8)$ \\
\hline Anticholinergic use with stent (\%) & $51(63.8)$ \\
\hline Antibiotic use (\%) & $114(70.4)$ \\
\hline
\end{tabular}

Introduction \& Objective: The management of pediatric urolithiasis has evolved, with ureteroscopy (URS) becoming a firstline surgical option for a majority of upper-tract stones. A growing body of evidence has focused on the identification of risk factors leading to unplanned care and complications after URS to reduce the cost of care in adults. However, a paucity of evidence is available in the pediatric population. We sought to determine the rates of Emergency Department (ED) visits, readmissions, and complications after URS and to identify their predictors in a pediatric population.

Materials and Methods: A retrospective review was performed for patients under 21 years of age who underwent URS for uppertract stones at our institution between 2011-2015. Demographic and perioperative data were collected. A univariate analysis was performed to identify all variables associated with ED visits, readmissions, and complications after URS. A multivariate logistic regression model was fitted for variables with a p-value $\leq 0.05$ on univariate analysis and clinically relevant variables (age, gender, insurance, American Society of Anesthesiologists classification, size of largest stone, and postoperative stenting). Results: A total of 162 URS cases were identified. Demographic and perioperative data are provided in Table 1. The rates of 30day ED visits, readmissions, and complications after URS were $9.9 \%, 6.2 \%$, and $4.3 \%$, respectively. Pain alone was the most common complaint for $43.8 \%$ and $40.0 \%$ of patients who presented to ED and were readmitted, respectively. On multivariate analysis, the development of a complication was independently predictive of ED visits (OR 29.5, $\mathrm{p}=0.002$ ) and readmissions $(\mathrm{OR} 38.6, \mathrm{p}=0.0006)$ after URS. A positive intraoperative urine culture was also independently predictive of complications (OR 25.3, $\mathrm{p}=0.01)$ after URS.
Conclusions: A majority of ED visits and readmissions after URS were due to pain. Both ED visits and readmissions may be reduced by the avoidance of complications. A preoperative urine culture should also be considered to ensure the sterility of urine before URS.

MP14-13 Radiologic evaluation of children prior to SWL: to what extent they are exposed to radiation?

B Eryildirim, O Turkoglu, C Goktas, O Kavukoglu, K Sarica

Health Sciences University, Kartal Lutfi Kirdar Training and Research Hospital

Turkey

Introduction \& Objective: To evaluate the average radiation exposure in children with renal stones before SWL treatment.

Materials and Methods: Mean radiation exposure values were evaluated in 110 children before SWL treatment. While some children referred to the emergency department (ED) with colic pain, remaining cases referred to outpatient department (OD). Although low-dose NCCT was performed in ED; KUB and abdominal sonography were first performed in other cases referring to OD where CT has been applied if needed. Type of imaging modality used and the mean radiation exposure were evaluated and comparatively evaluated with respect to the department referred, patient as well as stone related parameters.

Results: 49 children referred to ED and 61children referred to OD. Mean stone size was $7.24 \pm 0.29 \mathrm{~mm}$. 62 cases had opaque stones. Mean radiation exposure values were higher in children referring to ED than the other cases. However, there was no significant difference between two groups regarding the mean number of KUB, IVU and sonographic evaluation perforemed prior to SWL management. There was a significant correlation between the mean radiation exposure and the stone size as well degree of hydonephrosis in a positive manner. Although a significant correlation was present between the mean radiation exposure and stone opacity in a negative manner; there was no correlation with respect to the other related parameters.

Conclusions: Unnecessary use of X-ray based imaging modalities in children could be effectively avoided by using KUB and US combination beginning from the diagnostic phase of stone disease.

MP14-14 Outcomes of percutaneous nephrolithotomy (PCNL) in pediatric patients with spina bifida and/or nonambulatory status: Are all PCNL patients created equally?

J Van Batavia, T Kawal, D Chu, A Shukla, A Srinivasan

Children's Hospital of Philadelphia

United States

Introduction \& Objective: Percutaneous nephrolithotomy (PCNL) utilization for pediatric stones has risen due to technological advances in optic system quality and development of smaller instruments. PCNL is usually reserved for patients with larger stone burdens and staghorn calculi which may be more likely to occur in children with spina bifida (SB) and/or those who are non-ambulatory (NA). The outcomes of PCNL in these children may differ from the general pediatric population although this has not been addressed by any study to date. We 
hypothesized that SB/NA patients would have higher complication rates, longer length of stays (LOS), and worse outcomes in terms of stone-free rate.

Materials and Methods: We retrospectively reviewed our IRB approved pediatric stone database for all PCNL procedures. Baseline characteristics, operative findings and outcomes of interest were assessed for all patients. Patients with SB or NA status were compared to children without the conditions. Baseline characteristics between groups were compared with Fisher's exact test and Mann-Whitney U test. Multivariate linear and logistic regression were used to assess continuous outcome variables and binary outcome variables, respectively.

Results: 39 PCNL procedures in 33 patients (median age 13.0 yrs, IQR $=5.1-17.6 ; 19 \mathrm{~F}, 14 \mathrm{M}$ ) were identified. In total, 12 children had SB and of this group 9 had neurogenic bladder and 11 were NA. Six other children were NA due to other causes for a total of 17 non-ambulatory children. There were no baseline differences between the SB/NA children and those without either condition, except for tract length for access which was significantly longer in the SB/NA group. Neurogenic bladder by itself was not an independent risk factor for any outcome while SB diagnosis and NA status were both independently (and when grouped together) significantly associated with increased LOS (2.6 days longer, 95\% CI 0.3-4.9), less estimated blood loss, increased stone-free rate (OR 19.1, 95\% CI 1.4-253.1), and decreased need for future stone surgery (OR $0.17,95 \% \mathrm{CI} 0.03-0.89$ ). SB/NA status was not associated with complication rate or transfusion rate in multivariate analysis controlling for age, gender, history of UTI, number of stones, and total stone burden.

Conclusions: PCNL can safely be performed in children with SB and those who are non-ambulatory. While LOS is longer in these patients, stone free rate is higher without any increased risk in complication or transfusion rate. Future studies are needed to elicit the underlying causes for these differences.

\section{MP14-15 Percutaneous Nephrolithotomy(PCNL) in Chil-} dren under Age 15

M Hosseini, A Eslahi, A Derakhshan, M Basiratnia

shiraz nephro-urology research center, shiraz university of medical sciences, shiraz, iran

Iran

Introduction \& Objective: Nowdays, Renal calculi is a common problem in children. ESWL is the first choice in this age group, but sometimes other interventions may be indicated. In such cases PCNL seems the less invasive and more safe option than open surgery. We evaluated the results and complications of the pediatric PCNL in our referral training center.

Materials and Methods: Between September 2002 and November 2016, a total of 179 children, who had failure of and/or their parents refuse SWL underwent PCNL. The procedure was done under general anesthsia, in prone position, with ureteral cathter 3 or $4 \mathrm{~F}$, diluted contrast injection and fluoroscopic or ultrasonographic guided nephrostomy by Chiba needle 18G. Tract dilation performed with Alken telescopic dilators. Nephroscopy were done with adult size 24 Fr. Nephroscope in 89 , pediatric in 51 and semirigid ureteroscpe in 39 cases. Lithotripsy was done with pneumatic lithoclast and Saline solution used as irrigation. Nephrostomy tube was inserted in 32 with ureteral stent, tubeless(No nephrostomy) in 121 and totally tubeless in 33. Ureteral stent and Foley catheter removed 12-24 hours after operation.
Results: Of total 179 patients, 104 were boys, 75 girls, with mean age 9.2 years(9months-15years) with renal stone $=>20 \mathrm{~mm}$. Mean operation time was $70 \mathrm{~min}(40-105)$ and radiation 0.5 $\min (0.2-1.9)$. Five patients had residual fragment less than $5 \mathrm{~mm}$, passed spontaneously in 2 weeks after operation, 7 underwent second look nephroscopy, and 2 ureteroscopy for migrated stone fragments to distal ureter. Postoperatively, 21 patients developed low grade fever, 2 sepsis, 8 transfusion, and 4 raising of normal creatinine which improved with conservative management.

Conclusions: PCNL seems a good option for treatment of the nephrolithiasis in children, however, it needs experienced hands, suitable equipments and meticolous procedure.

MP14-16 Percutaneous Nephrolithotomy in Pediatric Patients with Solitary Kidney

I Aridogan, V Izol, N Satar, N Akdogan, F Ok, B Kizilgok

University of Cukurova

Turkey

Introduction \& Objective: In this study, the safety and efficacy of percutaneous nephrolithotomy (PCNL) was assesed on the treatment of solitary kidneys with stone disease in children.

Materials and Methods: Between September 1997- May 2017, PCNL was performed on 26 pediatric patients who had solitary kidney with stone disease. Twelve patients had non-functioning kidney, seven patients had agenesia and seven patients nephrectomy in the past. Mean age was 9.1 years (1.5-18 years), $16(61.5 \%)$ patients were male and $10(38.5 \%)$ patients were female. Ten $(38.5 \%)$ patients had previous open renal surgery. Initially open tip ureter catheter was inserted under flouroscopy in lithotomy position. Following percutaneous needle access to an appropriate renal calyx, tract was dilated by the help of amplatz dilators and a renax tube with a minimum required diameter is inserted. Stones were visualized with a rigid nephroscope or a ureteroscope and extracted after pneumatic, ultrasonic or holmium laser lithotripsy when necessary.

Results: 23 (88.4\%) patients were rendered stone-free. 6 patients required blood transfusions. seven patients had fever at postoperative days. There was no impairment in renal functions after the surgery.

Conclusions: PCNL is postulated as a gold standard for the treatment of kidney stones and it is also effective and reliable in the treatment of solitary kidneys with stone disease.

\section{MP14-17 Laparoendoscopic Reduced Port Pyeloplasty for} Children and Young Patients.

K Takei, M Yamasaki, K Iwasaki, M Hanada, Y Akita, K Hirai, T Ando, T Shin, T Nomura, F Sato, T Terachi, H Mimata

Japan

Introduction \& Objective: Laparoendoscopic pyeloplasty is known to be comparable in both performance and safety to laparotomy, as well as less invasive. To obtain even less invasiveness, laparoendoscopic single-site (LESS) surgery or reduced port surgery (RPS), which uses small diameter forceps and utilizes a smaller number of ports, has been recently performed in various diseases. In this report, we will discuss the experience of laparoendoscopic reduced port pyeloplasty. 


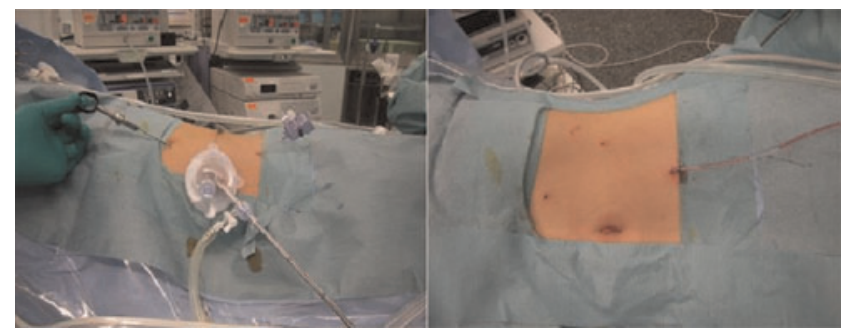

Materials and Methods: From May 2011 to February 2017, we performed 6 cases of laparoendoscopic reduced port pyeloplasty for children and young patients (under 35 years old) with ureteropelvic junction obstruction (UPJO) at the Oita University Hospital. Briefly describing the surgical method, an access platform (GelPOINT, SILS Port, or EZ Access) was placed through a $2-3 \mathrm{~cm}$ umbilical incision, and one or two additional trocars (3- or 5-mm) or BJ-needle were inserted at the location of the xiphoid process or the rib bow. The affected side was right in all cases. The Anderson-Hynes method was performed in 5 cases, and only dissection was performed in 1 case. Surgical outcomes, perioperative complications, postoperative pain and cosmesis were analyzed retrospectively.

Results: The mean operation time was $362.2 \pm 113.6 \mathrm{~min}$. In all cases, the estimated blood loss was minimal, and no perioperative complications were observed. The mean number of additional ports was $2.7 \pm 0.81$, the mean number of use of analgesia was $3.5 \pm 2.4$ times, and the postoperative hospital stay was $7.2 \pm 0.98$ days on average duration. Surgical scarring was minimal and cosmesis was good. All cases resulted in improvement of the symptom with UPJO after surgery.

Conclusions: Although there is a little extension of the operating time, laparoendoscopic reduced port pyeloplasty is a safe and feasible procedure with good cosmesis. RPS is well tolerated and is a good option for urological surgery for children and young patients.

MP14-18 Outpatient robotic extravesical ureteral reimplantation: short-term outcome assessment of safety

EJ Fichtenbaum, AC Strine, CW Concodora,

ME Schulte, PH Noh

Division of Pediatric Urology, Cincinnati Children's Hospital Medical Center

United States

Introduction \& Objective: Robotic extravesical ureteral reimplantation has been established as a viable option for surgical management of congenital vesicoureteral reflux. Typically, this procedure is associated with a hospital stay for routine postoperative care. In an effort to reduce the healthcare burden of hospitalization and further utilize the benefits of minimally invasive surgery, robotic surgery was performed as an outpatient procedure. The aim of the study was to assess the safety of performing pediatric outpatient robotic extravesical ureteral reimplantation.

Materials and Methods: A retrospective review was performed from June 2012 to March 2017 of patients who underwent outpatient robotic extravesical ureteral reimplantation. No regional blocks were utilized. All procedures were unilateral. One camera port and 2 instrument ports were utilized. No assistant ports were used. Patients were discharged from the recovery room, without an extended stay, as a scheduled outpatient procedure. Postoperative outcomes were assessed during the 30 days after surgery, specifically focusing on emergency room (ER) visits, hospital readmission, and Clavien-Dindo (CD) grade I-V complications.

Results: A total of 14 patients were identified (1 male, 13 females). Median age (months) and weight (kg) were 90 (range 43210) and 27 (range 13-97), respectively. Median robotic console time (minutes) was 143 (range 84-257). No patient required inpatient care for pain management. Two (14\%) patients required unplanned antibiotics postoperatively (CD grade II) for management of simple bacterial cystitis and pneumonia. The patient with the pneumonia was diagnosed during a subsequent ER visit. No CD grade III or higher complications or hospital readmissions were observed for any patient.

Conclusions: Pediatric robotic extravesical ureteral reimplantation is safe as an outpatient procedure. Further evaluation is warranted to assess long-term outcomes of these procedures on a larger scale.

MP14-19 Does side matter? The impact of laterality on pediatric laparoscopic pyeloplasty

D Bos, J Moore, M Rickard, A Lorenzo, R Romao,

D D'Souza, L Braga

McMaster University

Canada

Introduction \& Objective: Anatomically, surgical access to the ureteropelvic junction may differ on the right vs. the left side.

\begin{tabular}{|c|c|c|c|}
\hline & $\begin{array}{c}\text { Right } \\
\mathrm{n}=35(\%)\end{array}$ & $\begin{array}{c}\text { Left } \\
\mathrm{n}=70(\%)\end{array}$ & p value \\
\hline Age (months) & $101.1 \pm 92.3$ & $102.9 \pm 57.5$ & 0.90 \\
\hline Gender (males) & $18(51)$ & $47(67)$ & 0.12 \\
\hline Baseline DRF (\%) & $46.3 \pm 8.4$ & $45.9 \pm 12.5$ & 0.90 \\
\hline Baseline APD (mm) & $28.1 \pm 13.9$ & $28.1+11.7$ & 0.65 \\
\hline $\begin{array}{c}\text { Baseline SFU Grade } \\
-\quad 2 \\
=-3 \\
-\quad 4\end{array}$ & $\begin{array}{c}1(3) \\
16(47) \\
17(50)\end{array}$ & $\begin{array}{c}1(1) \\
40(57) \\
28(42)\end{array}$ & 0.53 \\
\hline $\begin{array}{l}\text { Symptomatic Presentation } \\
\begin{array}{l}\text { - UTI } \\
\text { - Sepsis } \\
\text { - Abdominal pain } \\
\text { - Trauma } \\
\text { - Incidental }\end{array}\end{array}$ & $\begin{array}{l}26(74) \\
4(15) \\
2(8) \\
14(54) \\
0(0) \\
6(23)\end{array}$ & $\begin{array}{l}60(86) \\
9(15) \\
1(2) \\
42(70) \\
1(2) \\
7(11)\end{array}$ & 0.41 \\
\hline $\begin{aligned} & \text { Etiology of UPJO } \\
&- \text { Classic } \\
&- \text { Long segment } \\
&- \text { Kink } \\
&- \text { Polyps } \\
&- \text { Crossing vessel } \\
&- \text { High insertion }\end{aligned}$ & $\begin{array}{c}12(42) \\
1(3) \\
8(28) \\
1(3) \\
3(10) \\
4(14)\end{array}$ & $\begin{array}{c}12(21) \\
1(2) \\
11(19) \\
4(7) \\
24(42) \\
5(9)\end{array}$ & 0.11 \\
\hline Surgical time (mins) & $272.8 \pm 86.3$ & $220+54.9$ & $<0.05$ \\
\hline Retrograde pyelogram & $25(71)$ & $45(64)$ & 0.40 \\
\hline Transmesenteric approach & $6(17)$ & $53(76)$ & $<0.05$ \\
\hline Postoperative complications (\%) & 2.67 & 5.27 & 0.40 \\
\hline Postoperative $\mathrm{T} 1 / 2$ time (mins) & $11.3 \pm 10.3$ & $13.9 \pm 13.5$ & 0.49 \\
\hline Postoperative DRF (\%) & 45.5 & 40.64 & 0.64 \\
\hline Improvement in APD (\%) & 49.9 & 56.6 & 0.23 \\
\hline LOS (days) & $2.6 \pm 0.9$ & $2.8+2$ & 0.49 \\
\hline
\end{tabular}

APD: renal pelvis anteriorposterior diameter; DRF: differential renal function; $S F U$ : society of fetal urology; UPJO: ureteropelvic junction obstruction; LOS: length of stay 
Herein we evaluate whether laterality impacts laparoscopic pyeloplasty perioperative and postoperative outcomes in pediatric patients.

Materials and Methods: Our prospectively collected, multicenter pediatric pyeloplasty database (2008-16) was reviewed $(\mathrm{n}=175)$. We included all patients who underwent a laparoscopic approach $(n=105)$ and divided them into 2 cohorts based on the laterality of their pyeloplasty (right vs. left). We collected data on baseline patient characteristics, surgical approach, operative time, postoperative complication rate, length of stay, and pre and postoperative renal function. Data was analyzed between groups with independent t-test and Chi-square for continuous and dichotomous variables respectively.

Results: Of 105 patients included in the analysis, 65 were male $(62 \%)$, with a mean age of $8 \pm 5$ years and 70 were left sided $(67 \%)$. Patient baseline characteristics were similar for both groups including surgical indications, hydronephrosis grade, and renal function (Table 1). Laterality of procedure was associated with prolonged operative time in patients on the right side ( $273 \pm 86$ min.), compared to the left $(220 \pm 55$ min.; $p<0.05)$. A transmesenteric approach was more common in the left-sided cohort $(77 \%$ vs. $18 \%, \mathrm{p}<0.05)$, while the majority of right-sided cases $(82 \%)$ required colon mobilization. Despite this, laterality was not associated with a difference in postoperative complications, length of hospital stay, or postoperative renal function.

Conclusions: We found that laterality of the procedure was associated with prolonged operative time in patients who had a right-sided pyeloplasty, highlighting the technical challenge of a right-sided approach due to anatomical challenges despite the advantages of laparoscopic visualization.

MP14-20 The clinical application of laparoscopy combined flexible ureteroscope in the treatment of hydronephrosis associated with upper urinary tract calculi

S Jie, Z Liang

shanghai children's medical center

China (People's Republic)

Introduction \& Objective: To discuss the feasibility and safety of the laparoscopy combined flexible ureteroscope in the treatment of hydronephrosis with calculi.

Materials and Methods: A retrospective analysis of 5 patients with hydronephrosis and upper urinary tract stones treated by laparoscopy combined with flexible ureteroscope. Lower ureter was pull out of abdominal wall in the operation of laparoscopic ureteral reimplantation, then flexible ureteroscope was performed through the pulled out ureter to handle the calculi.

Results: all operations were successful completed without major surgical complications. The hydronephrosis was significantly improved after removal of the DJ tube three month later, and there were no significant residual stones.

Conclusions: It is feasible to treat hydronephrosis associated with upper urinary tract calculi by laparoscopy combined flexible ureteroscope.

MP14-21 Durability of pediatric robotic pyeloplasty: Should we follow long-term?

C Rowe, M Shapiro, J Ellison, T Lendvay

University of Washington

United States

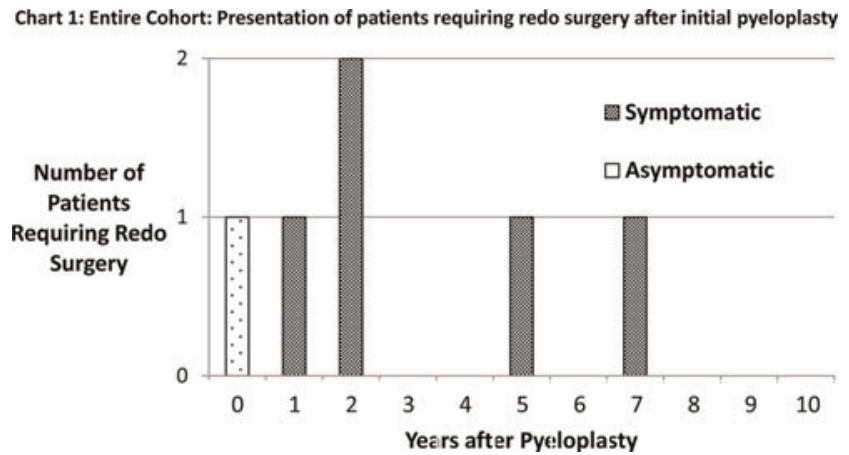

Introduction \& Objective: Robot-assisted laparoscopic pyeloplasty is an established treatment for ureteropelvic junction (UPJ) obstruction. The literature in children is limited to shortterm follow-up and the benefit of long-term imaging is unclear. We report our long-term outcomes after robotic pyeloplasty over a 10-year experience.

Materials and Methods: Patients who underwent robotic pyeloplasty at our institution since 2006 with available post-operative imaging were included. Failures were defined as patients requiring a redo surgery due to concern for obstruction. Clinical length of follow-up was calculated to the most recent check in clinic or by phone. Our long-term cohort was defined as any patient with more than 5 years clinical follow-up.

Results: 169 patients underwent robotic pyeloplasty between 2006 and 2016. Of these, 146 had available post-operative imaging and 36 had long-term follow up with median clinical follow up 6.9 years (range $5.2-10.1$ ). Most patients in the long-term cohort were boys (72\%) and one patient $(2.8 \%)$ had prior surgery on the UPJ. Median age at time of surgery was 83.5 months (4 215). One surgeon performed $94 \%$ of the operations, using the dismembered pyeloplasty technique for most (80\%) with nearly all having an intra-operative stent $(97 \%)$. Median operative time for the long-term cohort was 210 minutes $(128-388)$ and median length of stay was 2 days $(1-8)$ with $44 \%$ of patients discharged POD1. Within the long-term cohort, five (14\%) patients experienced post-operative complications. Overall, $92 \%$ of pyeloplasties followed long-term were successful.

Three patients required a redo operation in the long-term cohort. All presented with flank pain including two followed annually with reassuring imaging. The failure rate was similar between the long-term and the overall cohort (six failures) with only one presenting asymptomatically with worsening immediately post-operative imaging.

Conclusions: Robot-assisted laparoscopic pyeloplasty is safe and durable in pediatric patients but failures can present years after the initial surgery. With only one patient in the entire cohort presenting with asymptomatic failure, it remains to be seen whether long-term imaging is required to follow these patients. Further study and multi-institutional collaborations will be required to truly understand the comparative effectiveness of robotic pyeloplasty.

MP14-22 Establishment of a Pediatric Urology Robotic Program: Ancilllary and Technical Results in Robotic Assisted Pyeloplasty

AC Keenan, TG Leffler, RR Su, SY Nakada

University of Wisconsin-Madison

United States 

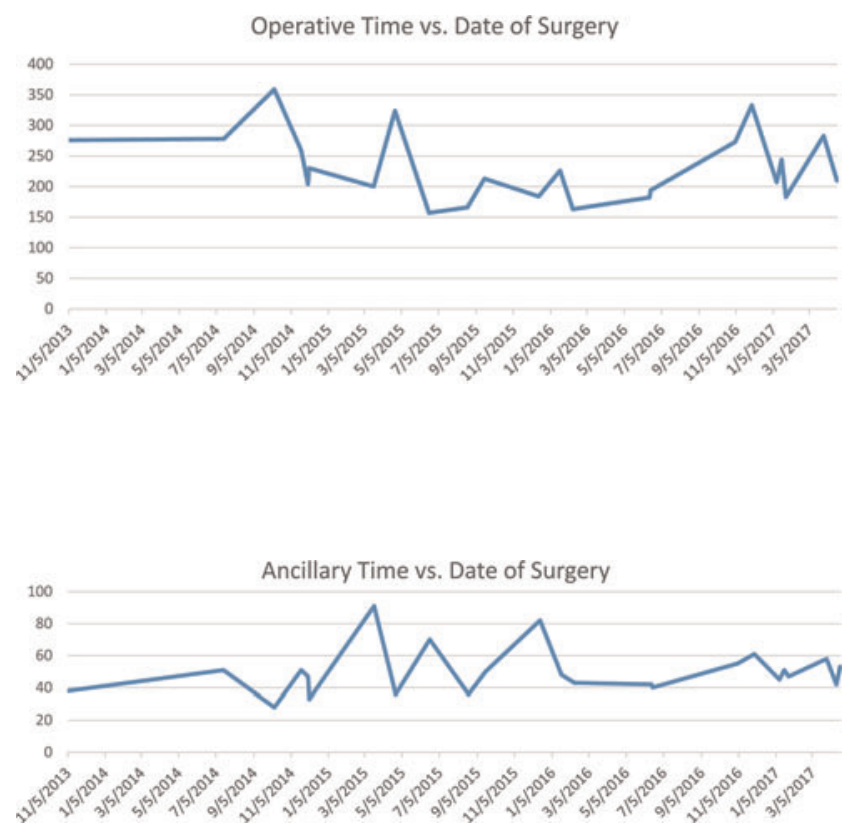

Introduction \& Objective: We examined the technical surgical data and clinical outcomes of patients undergoing robotic-assisted laparoscopic pyeloplasty in a new robotic surgery program. We hypothesize that the early outcomes of this new program would reflect published literature, and that operative times will decrease over time and will be dominated by ancillary (non-operative) time related to the use of robotic technology.

Materials and Methods: This study retrospectively reviewed the UW-Health Healthlink database for patients that underwent robotic pyeloplasty performed from October 2013 to January 2017. Data collected included: demographics, surgery date, UPJ side, grade of hydronephrosis pre- and post-op, differential function, $\mathrm{T} 1 / 2$, presence of contralateral hydronephrosis, and operative and ancillary OR time.

Results: Twenty-four patients were assessed (17 male; 7 female). Mean age was $7.2 \pm 5.6$ years. UPJ presented on the left side in 16 patients, 8 on the right $(67 \% ; 33 \%)$. Mean operative time was $232.4 \pm 55.4$ minutes and remained relatively stable throughout the study period. Mean ancillary time was $49.9 \pm 14.7$ minutes and decreased over the study period. Hydronephrosis grading was available for 8 patients, $62.5 \%$ of whom experienced a grading decrease. Two patients underwent a second revision pyeloplasty.

Conclusions: Our results indicate that operative times remained relatively stable, and overall OR times did not significantly decrease, reflecting a stable portion of the learning curve for operative support teams adopting new technology. This may be because the first 21 cases were performed in the adult hospital OR, before transitioning the technology to the children's hospital. We demonstrate the feasibility and clinical safety of establishing a new pediatric robotic surgical program. Early focus on robotic training for operative support staff may minimize the increased ancillary OR time seen in the initial phases of establishing a new program.
MP14-23 Paediatric Needleoscopic Pyeloplasty-Our initial experience in Advanced Centre of Kidney and Urology

M Naser, M Hossain, M Isam

Shaheed Suhrawardy Medical College Hospital Bangladesh

Introduction \& Objective: Introduction: Laparoscopic dismembered pyeloplasty for correction of ureteropelvic junction obstruction (UPJO) in the pediatric population is comparable to open dismembered pyeloplasty in success rates. Experience with this procedure however remains limited in Bangladesh. Objectives: To report our initial experience with needlescopic pyeloplasty (NP) in children with pelvi-ureteric junction (PUJ) obstruction, and to evaluate the results for these patients.

Materials and Methods: Methods: Between February 2016 to April 2016, we have done sixteen consecutive needlescopic pyeloplasty for pelviureteric junction obstruction, confirmed by sonography and intravenous urography and isotope renogram. Ten boys and 6 girls, with the age range of two and half months to eight years, underwent this procedure. Four needed prior percutaneous nephrostomy. Seven were on the right side and nine on the left side. $3 \mathrm{~mm}$ ports and $2.77 \mathrm{~mm}$ telescope were used in all the procedure. The patient was placed in a lateral position with three ports in left side and four on the right side. The PUJ was resected and the anastomosis made using absorbable sutures. A JJ stent was inserted by needlescopy in the all patients. Follow-up included clinical and ultrasound assessment, and isotope renography at 6 months.

Results: NP was possible in all the patients. The mean operative time was $145 \mathrm{~min}$ (range $70-270 \mathrm{~min}$ ), and mean hospital stay was 4.2 (3-8) days. No major complications were seen in the perioperative period. All patients were asymptomatic after removal of the stent. USG shows reduction of the degree of hydronephrosis in all patients, and had also improved PUJ drainage on isotope renography.

Conclusions: NP is effective and safe in children with minimal morbidity and gives excellent short-term results. The feasibility is also good in patients younger than 1 year. The transabdominal approach revealed good exposition without disadvantage to the patient. However, the operative time remains longer than open pyeloplasty.
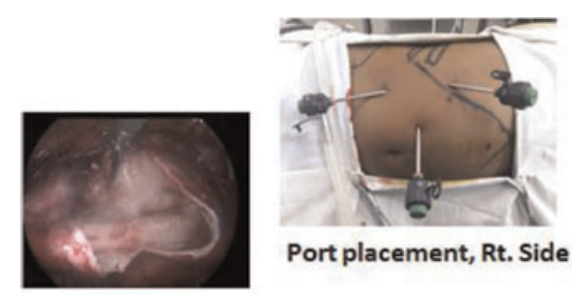

Port placement, Rt. Side

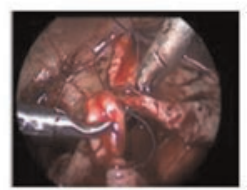

Trans section of the Pelvis Spatulated Anastomosis
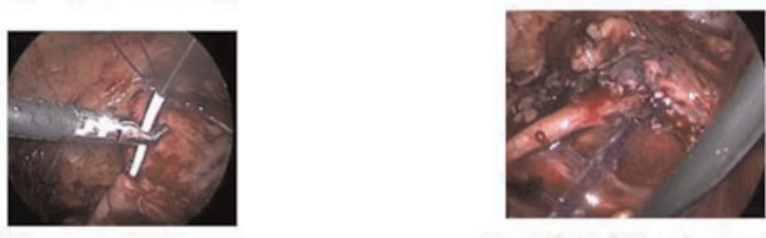

Insertion of J-J stent 


\section{MPS15: PROSTATE CANCER I}

MP15-1 Prostate cancer distribution and pathological stage in patients diagnosed by transrectal systemic random biopsy

$\mathrm{K} \mathrm{Wu}$

Department of Urology, College of Medicine and Hospital, National Cheng Kung University

Taiwan (Republic of China)

Introduction \& Objective: Prostate cancer is one of the most commonly diagnosed cancers in men. The diagnosis is established by histopathologic examination of extended transrectal ultrasound (TRUS) biopsy. However, little research has been done with regard to anatomic distribution of prostate cancer. Therefore, the aim of this study is to investigate the anatomic distribution of prostate cancer in patients diagnosed by TRUS guided 10-core systemic random biopsy. In addition, our study was also attempt to establish a predictive nomogram to predict the pathological stage in patients with prostate cancer via anatomic distribution of prostate cancer. Materials and Methods: From September 2005 to July 2014, 545 men received TRUS-guided 10-core systemic random biopsy at our hospital because of serum prostate specific antigen (PSA) over $4.0 \mathrm{ng} / \mathrm{ml}$ and/or abnormal digital rectal examination. All of them received PSA, digital rectal examination (DRE), PSA density, and transitional zone index (TZ index). Each core was reported separately, including malignancy or benignity, Gleason score, and the proportion of malignancy. If patient be treated with radical prostatectomy, the association between anatomic distribution of prostate cancer and pathological stage were analyzed.

Results: Among 545 patients, 152 men (27.9\%) were diagnosed with prostate cancer, including 64 of $370(17.3 \%)$ men with normal DRE and 88 of $175(50.3 \%)$ men with abnormal DRE. Prostate cancer in men with abnormal DRE exhibited significantly older age (71.0 vs. 67.9, $p=0.008)$, higher serum PSA (25.5 vs. 15.7, $p=0.003)$, higher PSA density ( 0.71 vs. $0.41, p=0.002)$, higher percent of cores positive $(p=0.022)$, and higher Gleason score $(p=0.0006)$ than men with normal DRE. The most detection site of prostate cancer in men with abnormal DRE was left basal parasagittal $(59.1 \%)$, following by left mid-lateral $(55.7 \%)$. The most detection site of prostate cancer in men with normal DRE was left middle lateral (46.8\%), following by left apex (45.3\%). Nomograms were developed for the predicted probability of pathological stage. Conclusions: In our study, men with abnormal DRE has higher malignancy rate than men with normal DRE but elevated PSA $(50 \%$ vs. $16 \%$ ). Prostate cancer in men with normal DRE exhibited younger age, lower serum PSA, lower PSA density, less positive cores, and less Gleason score than men with abnormal DRE. The cancer foci detected by biopsies of the prostate are equally distributed even subgroup analysis depending on location of positive cores or prostate size. TRUS guided systemic random biopsy might help clinicians to predict pathological stage and determine the treatment policy.

MP15-2 The Clinical Outcome of Prostate Ductal Adenocarcinoma: a matched cohort comparative study

NS Khan, Y Kiberu, O El Hage, M Suleman, A Chandra, S Morris, P Dasgupta

MRC centre for Transplantation, King's College London, London, UK

United Kingdom
Introduction \& Objective: Prostate Ductal Adenocarcinoma (PDA) is a rare variant of prostate cancer (PCa). There are conflicting reports regarding the oncological outcome and prognosis of PDA. To date there is no consensus on what is the best treatment option.

Materials and Methods: All patients who were diagnosed with PDA in a single institution during 2005-2015 period were included. Treatment included radical prostatectomy, radiotherapy or hormone manipulation. Patients who had Gleason 8 prostate acinar adenocarcinoma (PAA) served as control groups.

Results: Of 7638 patients who were diagnosed with PCa, 139 $(1.8 \%)$ had PDA and 811 had PAA. PSA at presentation was lower for PDA. 317 (224-PAA, 93-PDA) patients were treated with radical prostatectomy. 313 (295-PAA, 18-PDA), had radical radiotherapy. 320 (292-PAA, 28-PDA) had hormone treatment. In the surgery group positive margin were $52 \%$ (PDA) vs $54 \%$ (PAA), $\mathrm{p}=0.14$. At follow up 59\% (PDA) vs 47\% (PAA) had biochemical recurrence, and $12 \%(\mathrm{PDA})$ vs $5.3 \%(\mathrm{PAA}), \mathrm{p}=0.09$ developed distant metastasis and $4.5 \%$ (PDA) vs $5.3 \%$ (PAA) have died. In the radiotherapy group 16\%(PDA) vs 22\% (PAA) had biochemical recurrence, $16 \%(\mathrm{PDA})$ vs $9 \%(\mathrm{PAA}), \mathrm{p}=0.01$ developed distant metastasis and $17 \%(\mathrm{PDA}) \mathrm{vs} 18 \%(\mathrm{PAA}), \mathrm{p}=0.76$ have died. In the hormone group $71 \%$ (PDA) vs $47 \%$ (PAA) developed metastasis at 28 months and $71 \%$ (PDA) vs $45 \%$ (PAA) have died. The mean follow up for all groups is 60 months.

Conclusions: PDA patients had higher risk of metastasis compared to the acinar group regardless of the treatment offered. This may indicate a more aggressive phenotype in PDA patients compared to classical acinar type. An early adjuvant treatment may be required in prostate ductal cancer.

MP15-3 Early outcomes of focal brachytherapy for localized prostate cancer: comparison with whole gland brachytherapy.

V Srougi, E Barret, I Nunes-Silva, F Rozet, M Galiano, R Sanchez-Salas, J Cosset, X Cathelineau

Institut Montsouris

France

Introduction \& Objective: Focal brachytherapy (FBT) emerged as a reasonable option to treat patients with favorable prostate cancer (PCa). Purpose: To evaluate the early functional and oncological outcomes of FBT in the treatment of localized PCa.

Materials and Methods: A retrospective review was performed at our prospective collected PCa database, searching for patients treated with FBT between 2010 and 2015. A cohort of 100 individuals treated with whole gland brachytherapy (WBT) in the same period was assessed in order to perform a comparison. IPSS, ICS and IIEF5 questionnaires were used to evaluate urinary symptoms, continence and sexual function, respectively, at 6, 12 and 24 months. All patients in the FBT group had a control prostate biopsy; PCa recurrence was defined as positive biopsy at the same treatment site. In the WBT group, PCa recurrence was defined by PSA increase $>$ nadir +2 .

Results: Forty patients were included in the FBT group with complete oncological follow-up. Twenty-seven patients had completed questionnaires with 24 months of follow-up (included 
Table 1 - Demographic characteristics: FBT vs. WBT.

\begin{tabular}{|l|c|c|c|}
\hline & FBT & WBT & $p$ \\
\hline Median age (years) & $61(50-79)$ & $65(46-76)$ & 0.05 \\
\hline Mean PSA & $7.2 \pm 2.5$ & $6.8 \pm 2.5$ & 0.47 \\
\hline Clinical stage & & & \\
\hline T1c (\%) & $34(85)$ & & \\
\hline T2a (\%) & $6(15)$ & & \\
\hline Gleason score & & & $0.78^{*}$ \\
\hline s6 (\%) & $75(32)$ & $82(82)$ & \\
\hline$=7(\%)$ & $25(8)$ & $18(18)$ & \\
\hline Mean prostate volume (ml) & $41.8 \pm 15$ & $34.4 \pm 8$ & $<0.001$ \\
\hline Mean BT volume (ml) & $12.7 \pm 4$ & $34.1 \pm 7$ & $<0.001$ \\
\hline Median \% of irradiated vol (ml) & $32 \%(17-50)$ & & \\
\hline Median of implanted seeds & $36(23-57)$ & $63(44-90)$ & $<0.001$ \\
\hline Median D90 & $183(172-190)$ & $180(171-190)$ & $<0.001$ \\
\hline Median V100 & $99.7 \%(98-100)$ & $99.8 \%(98-100)$ & 0.15 \\
\hline
\end{tabular}

in the functional assessment). Initial presentation and treatment characteristics are exhibited on table 1. Median follow-up for FBT and WBT groups were 34 months (range: 10-68 months) and 24 months (range: 24-36 months), respectively. There was no difference in recurrence rates between-groups (FBT $7.5 \%$ vs. WBT $3 \%, p=0.35$ ) (fig. 1). WBT group had significant worsening of urinary symptoms when compared to FBT group at 6 months $(p=0.003)$, but not with 12 and 24 months. Urinary continence and sexual function was similar between-groups.

Conclusions: FBT has satisfactory oncological outcomes and less urinary symptoms at early follow-up when compared to WBT. Urinary continence and sexual functional is the same as WBT.

\section{MP15-4 Tumor Characteristics and Survival Outcomes of Prostate Cancer Diagnosed on Repeat Biopsies}

\section{Tseng, C Chen, I Chiang}

National Taiwan University Hospital

Taiwan (Republic of China)

Introduction \& Objective: We aimed to evaluate the tumor presentation and survival outcomes of patients with prostate

Figure 1. Kaplan-Meier survival curves of (A) all-cause mortality and (B) prostate cancerspecific mortality in 327 prostate cancer stratified by numbers of biopsy session.
A

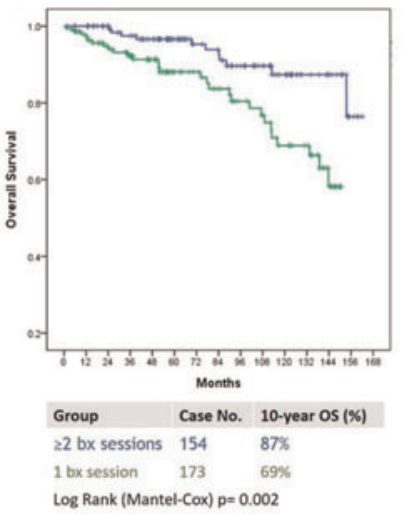

B

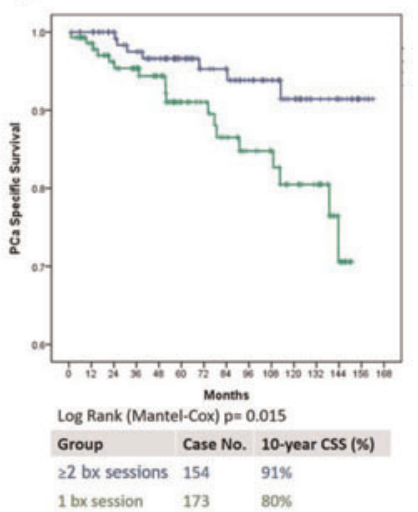

22 bx sessions $154-91 \%$
Figure 2. Kaplan-Meier survival curves of all-cause mortality (A) and prostate cancer-specific mortality (B) in age $\leq 70$ years old group (1) and age $>70$ years old group (2). Both groups were stratified by numbers of biopsy session.

A1
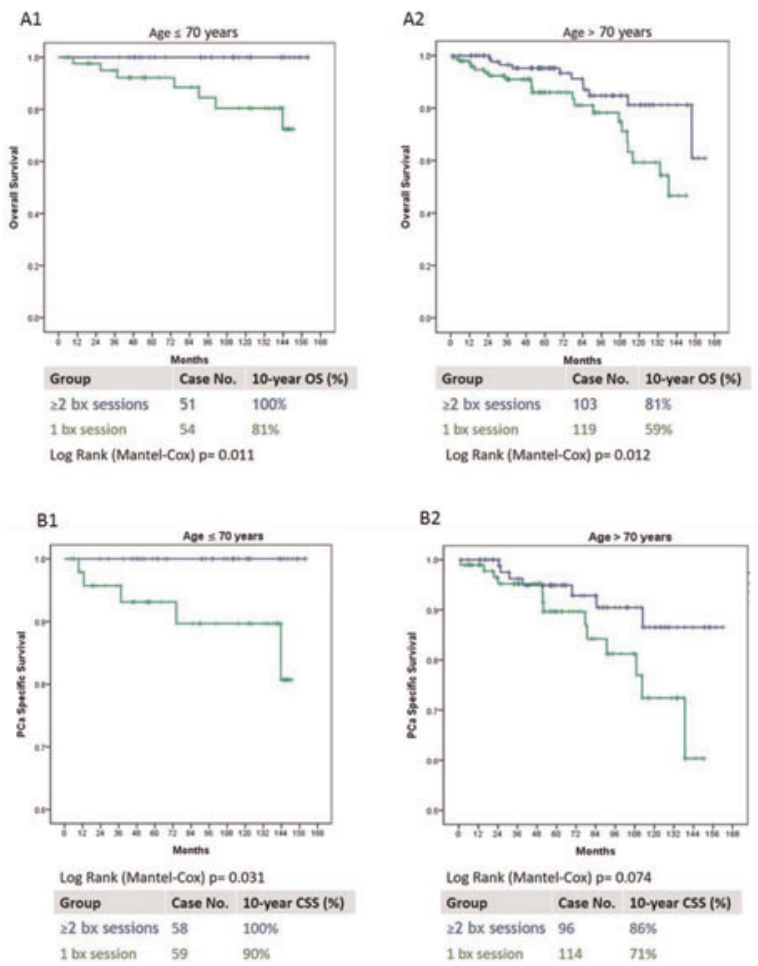

Figure 3. Kaplan-Meier survival curves of all-cause mortality (A) and prostate cancer-specific mortality (B) in different PSA level groups as following: PSA $<10 \mathrm{ng} / \mathrm{ml}$ (1), PSA $10-20 \mathrm{ng} / \mathrm{ml}$ (2) and PSA $>20 \mathrm{ng} / \mathrm{ml}$ (3). Each group was stratified by numbers of biopsy session.
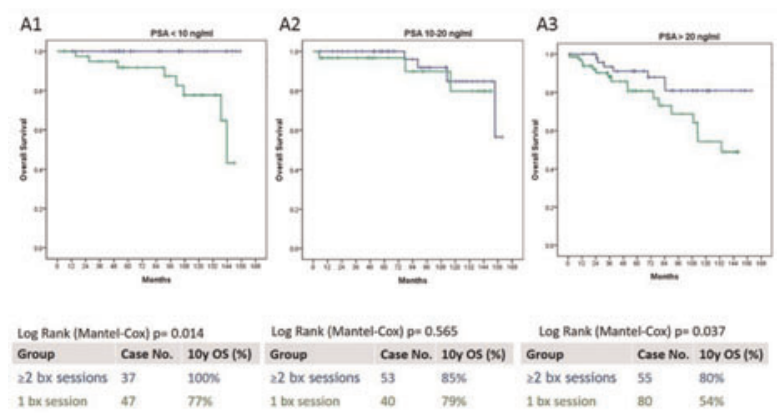
Case No. 10 y OS $(x)$ Group Case No. 10y OS (x)

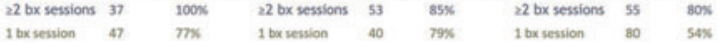
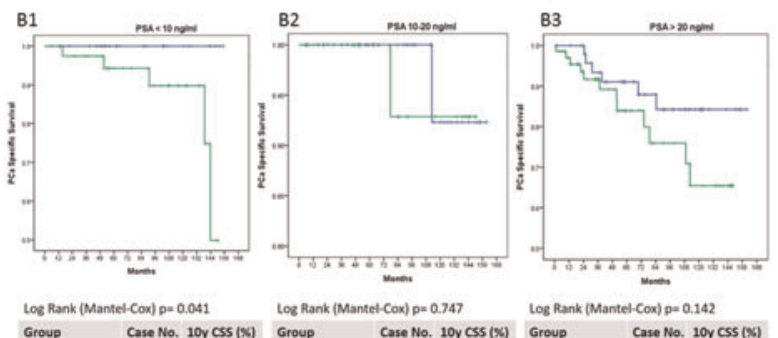

22 bx sessions $37 \quad 1000$ Log Rank (Mantel-Cox) $p=0.747$ Log Rank (Mantel-Cox) p= 0.142

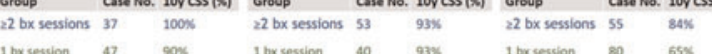


Table 1. Clinical characteristics of prostate cancer stratified by numbers of prostate

biopsies

\begin{tabular}{|c|c|c|c|}
\hline & \multicolumn{2}{|c|}{ No. of biopsies } & \multirow[b]{2}{*}{ PValue } \\
\hline & 1 & 2 or greater & \\
\hline No. of patients & $\mathrm{N}=173$ & $\mathrm{~N}=154$ & \\
\hline \multicolumn{4}{|l|}{ Age, Years } \\
\hline Median Age (IQR) & $73(68-79)$ & $73.5(67.8-79)$ & 0.725 \\
\hline Range & $44-92$ & $49-97$ & \\
\hline \multicolumn{4}{|l|}{ Prostate-specific antigen $(\mathrm{ng} / \mathrm{ml})$} \\
\hline Median PSA (IQR) & $17.4(9.2-79.3)$ & $16.8(9.9-27.0)$ & 0.332 \\
\hline Range & $3.99-2052$ & $2.14-6232$ & \\
\hline PSA range & & & 0.048 \\
\hline PSA 10 & 48 & 39 & \\
\hline PSA $>10-20$ & 40 & 55 & \\
\hline PSA $>20-50$ & 30 & 40 & \\
\hline PSA $>50$ & 49 & 17 & \\
\hline clinical stage T category (\%) & & & 0.014 \\
\hline cT1a-b & $0(0 \%)$ & $23(14.9 \%)$ & \\
\hline cT1c & $107(61.8 \%)$ & $96(62.3 \%)$ & \\
\hline cT2a & $23(13.3 \%)$ & $15(9.7 \%)$ & \\
\hline $\mathrm{cT} 2 \mathrm{~b}$ & $9(5.2 \%)$ & $6(3.9 \%)$ & \\
\hline cT2c & $10(5.8 \%)$ & $5(3.2 \%)$ & \\
\hline ст3а & $18(10.4 \%)$ & $7(4.5 \%)$ & \\
\hline cT3b & $6(3.5 \%)$ & $2(1.3 \%)$ & \\
\hline clinical stage $\mathrm{N}$ category (\%) & & & $<0.001$ \\
\hline NO & $132(76.3 \%)$ & $137(89.0 \%)$ & \\
\hline N1 & $3(1.7 \%)$ & $9(5.8 \%)$ & \\
\hline $\mathrm{Nx}$ & $38(22.0 \%)$ & $8(5.2 \%)$ & \\
\hline clinical stage $\mathrm{M}$ category (\%) & & & 0.001 \\
\hline MO & $145(83.8 \%)$ & $145(94.2 \%)$ & \\
\hline M1 & $28(16.2 \%)$ & $9(5.8 \%)$ & \\
\hline No. GS (\%) & & & 0.047 \\
\hline $5 \sim 6$ & $60(34.6 \%)$ & $70(45.5 \%)$ & \\
\hline $3+4=7$ & $38(22.0 \%)$ & $42(27.3 \%)$ & \\
\hline $4+3=7$ & $32(18.5 \%)$ & $19(12.3 \%)$ & \\
\hline $8 \sim 10$ & $43(24.9 \%)$ & $23(14.9 \%)$ & \\
\hline \multicolumn{4}{|l|}{ Biopsy cores (IQR) } \\
\hline Median No. taken & $12(6-12)$ & $12(12-12)$ & 0.012 \\
\hline Median No. positive & $4(2-6)$ & $3(2-4)$ & $<0.001$ \\
\hline Median \% Positive / Total cores & $46.7(20.0-83.3)$ & $17.8(8.3-33.3)$ & $<0.001$ \\
\hline Max \% involved per core (IQR) & $60(30-80)$ & $30(10-70)$ & $<0.001$ \\
\hline Bilateral disease No. (\%) & & & $<0.001$ \\
\hline yes & $99(57.2 \%)$ & $40(26.0 \%)$ & \\
\hline no & $72(41.6 \%)$ & $108(70.1 \%)$ & \\
\hline
\end{tabular}

cancer diagnosed on either initial prostate biopsy or repeat biopsy sessions.

Materials and Methods: We retrospectively reviewed 1654 patients who had undergone prostate biopsy in National Taiwan University Hospital (NTUH) from 2002 to 2005. We identified 620 patients with prostate cancer and divided them into two groups: 1) men diagnosed as prostate cancer on initial prostate biopsy and 2) on repeat prostate biopsies. The primary outcomes were overall survival and prostate cancer-specific survival. The relative risks were analyzed by univariate and multivariate Cox proportional hazards.

Results: This cohort consisted of 620 patients with prostate cancer, 466 (75.2\%) of whom were diagnosed at first biopsy, 96 $(15.5 \%)$ at second biopsy and $58(9.3 \%)$ at third or more biopsy sessions. The median age was 73 years old (IQR 68-79). Median initial PSA at biopsy was $16.8 \mathrm{ng} / \mathrm{ml}$ (IQR 9.6-42.7). Overall survival $(\mathrm{p}=0.002)$ and prostate cancer-specific survival $(\mathrm{p}=0.015)$ were both superior in the group of repeat biopsy than those diagnosed on initial prostate biopsy. The 10-year overall survival were $87 \%$ versus $69 \%$ in repeat biopsies set and ma- lignant initial biopsy set. Patients diagnosed on repeat biopsy with serum PSA $<10 \mathrm{ng} / \mathrm{ml}$ had a superior 10 -year overall survival rate of $100 \%$ than initial positive biopsy set $(\mathrm{p}=0.014)$.

Conclusions: Prostate cancer patients diagnosed on repeat biopsies would have more favorable tumor characteristics, superior overall survival and prostate cancer-specific survival. Patients with most outstanding survival outcomes were those who had low serum PSA, younger age and diagnosis at repeat biopsy.

MP15-5 Focal Therapy of Prostate Cancer May Lead to Undertreatment of Prostate Cancer: A Study on Laterality Agreement Between Clinial and Pathological Prostate Cancer

A Chow, K Gallo, D Papagiannopoulos, M Montour, C Coogan, K Latchamsetty

Rush University Medical Center

United States

Introduction \& Objective: Focal therapy has emerged as an alternative treatment to radical prostatectomy (RP). However, the decision to perform focal therapy assumes that the disease can be accurately localized on preoperative biopsy. We sought to compare the laterality of disease on transrectal ultrasound guided biopsy (TB) and MRI fusion biopsy (MFB) to final pathology. Materials and Methods: We performed a retrospective review of the UroPartners database of all patients who underwent RP from January 2015 to October 2016. Pathology reports from biopsy and prostatectomy specimens were reviewed to determine disease laterality.

Results: 392 men underwent RP, of which 347 were diagnosed by TB and 45 by MFB. Laterality was concordant in $212 / 347$ $(61 \%)$ in the TB group and in $31 / 45(69 \%)$ in the MFB group $(\mathrm{p}=0.33)$. Unilateral disease was found in $159 / 347(45.8 \%)$ of TB patients and 13/45 (28.9\%) MFB patients. In these cohorts, $117 / 159(73.5 \%)$ and $11 / 13(84.6 \%)$ were found to have bilateral disease on final pathology $(\mathrm{p}=0.5) .11 / 13(84.6 \%)$ patients who underwent MFB had a positive region of interest (ROI), of which $10(76.9 \%)$ had additional ipsilateral disease detected with systematic biopsy.

Conclusions: There was no significant difference in laterality agreement when comparing TB with MFB. The oncologic control of focal prostate therapy should be investigated further given the significant proportion of bilateral disease that was not apparent at time of biopsy.

MP15-6 Is PSA Density Still Relevant? Incidence and Predictive Factors of Prostate Cancer Upgrading

A Chow, K Gallo, D Papagiannopoulos, M Montour,

C Coogan, K Latchamsetty

Rush University Medical Center

United States

Introduction \& Objective: Gleason grade groups (GG) are used to guide treatment strategies and to predict oncologic outcomes. We sought to compare the concordance rate of the highest GG detected on TRUS biopsy (TB) or MRI fusion biopsy (MFB) with final pathology. Furthermore, we aimed to identify factors that may predict accurate GG of final pathology. 
Materials and Methods: We performed a retrospective review of patients in the UroPartners database who underwent radical prostatectomy (RP) from January 2015 to October 2016. We reviewed clinical and pathological GG, pre-biopsy PSA, prostate volume, and BMI. Univariate and multivariate analyses were performed to identify factors that influence upgrading of disease Results: 411 men underwent RP of which 364 (88.6\%) were diagnosed by TB and 47 (11.4\%) by MFB. For TB and MFB, GG concordance was $53 \%$ and $59 \%(\mathrm{p}=0.44)$, upgrading was $24 \%$ and $21 \%(\mathrm{p}=0.85)$, and downgrading was $23 \%$ and $19 \%$ $(\mathrm{p}=0.67)$, respectively. PSA density (OR 5.00, $\mathrm{p}=0.002)$, prostate volume (OR 0.99, $\mathrm{p}=0.048)$, and pre-biopsy PSA (ORÂ $1.03, \mathrm{p}=0.021$ ) were predictive of upgrading. Time from biopsy to RP and number of positive cores were not associated with upgrading.

Conclusions: Our concordance and upgrading rates were similar between imaging modalities. PSA density was significantly more predictive of disease upgrading than prostate volume or prebiopsy PSA individually. PSA density can be an insightful tool for patient counseling to guide treatment decisions and expectations prior to prostatectomy.

MP15-7 The prognostic impact of downgrading and upgrading from biopsy to radical prostatectomy in a contemporary grading system for prostate cancer

D Koh, W Jang, J Park, J Lee, Y Kim, I Cho, Y Choi

Republic of Korea

Introduction \& Objective: Recently, a new prostate cancer (PC) grading system has been introduced, where Gleason score (GS) 7(3+4) and GS 7(4+3) are categorized into grade group (GG) 2 and 3, respectively. However, whether downgrading and upgrading from needle biopsy (NB) to radical prostatectomy (RP) affects oncologic outcomes is currently unknown. Here, we investigated the prognostic impact of downgrading and upgrading from biopsy to radical prostatectomy in the new classification.

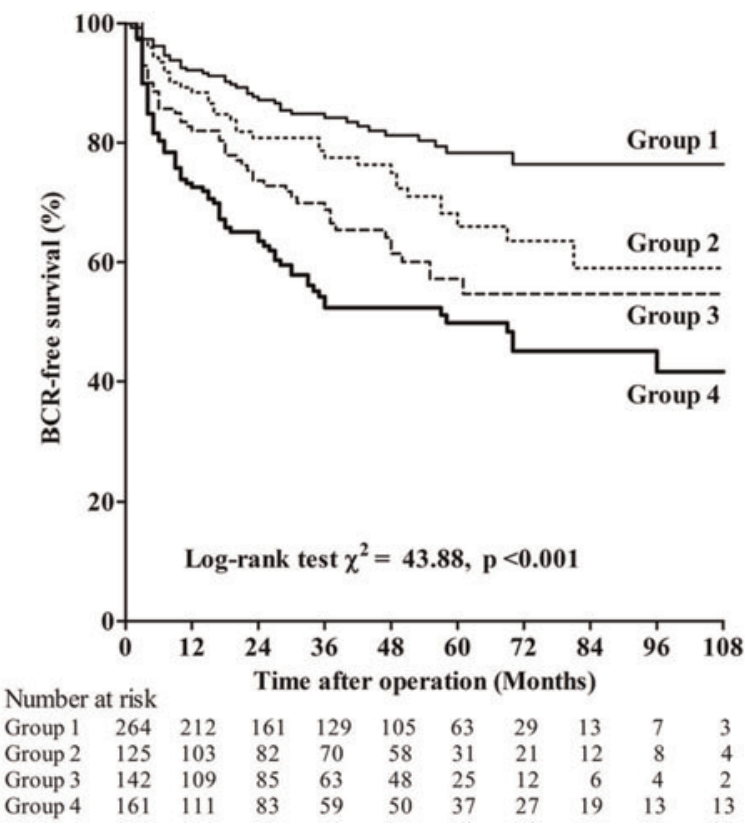

Table 1. Baseline clinical and pathological characteristics

\begin{tabular}{|c|c|c|c|c|c|}
\hline Variable & $\begin{array}{l}\text { Group 1* } \\
n=264\end{array}$ & $\begin{array}{l}\text { Group 2 } \\
n=125\end{array}$ & $\begin{array}{l}\text { Group } 3 \\
n=142\end{array}$ & $\begin{array}{l}\text { Group } 4 \\
n=161\end{array}$ & $\mathrm{p}$ value \\
\hline Age, years & & & & & 0.652 \\
\hline Median & 66 & 65 & 67 & 67 & \\
\hline IQR & $61-71$ & $62-71$ & $62-72$ & $62-71$ & \\
\hline Year of surgery & & & & & $<0.001$ \\
\hline Median & 2010 & 2009 & 2010 & 2009 & \\
\hline IQR & 2009-2012 & $2008-2010$ & $2008-2011$ & $2006-2010$ & \\
\hline $\mathrm{PSA}, \mathrm{ng} / \mathrm{ml}$ & & & & & $<0.001$ \\
\hline Median & 7.5 & 8.7 & 10.7 & 9.3 & \\
\hline IQR & $5.2-12.2$ & $5.6-13.3$ & $7.0-16.2$ & $6.4-15.9$ & \\
\hline Tumor volume, $\mathrm{cm}^{3}$ & & & & & 0.010 \\
\hline$<1$ & $78(29.5)$ & $39(31.2)$ & $31(21.8)$ & $38(23.6)$ & \\
\hline $1-5$ & $159(60.3)$ & $76(60.8)$ & $88(62.0)$ & $96(59.6)$ & \\
\hline$\geq 5$ & $27(10.2)$ & $10(8.0)$ & $23(16.2)$ & $27(16.8)$ & \\
\hline Pathological T stage & & & & & $<0.001$ \\
\hline $\mathrm{T} 2$ & $116(43.9)$ & $48(38.4)$ & $42(29.6)$ & $42(26.1)$ & \\
\hline T3a & $135(51.2)$ & $71(56.8)$ & $84(59.1)$ & $93(57.8)$ & \\
\hline T3b & $13(4.9)$ & $6(4.8)$ & $16(11.3)$ & $26(16.1)$ & \\
\hline Surgical margin & & & & & 0.006 \\
\hline Negative & $131(49.6)$ & $66(52.8)$ & $62(43.7)$ & $59(36.6)$ & \\
\hline Positive & $133(50.4)$ & $59(47.2)$ & $80(56.3)$ & $102(63.4)$ & \\
\hline $\begin{array}{l}\mathrm{NB}=\text { needle biopsy; } \\
\text { antigen; } \mathrm{RP}=\text { radical } \\
{ }^{*} \mathrm{Group} 1: \mathrm{NB} \text { and RP } \\
\text { Group 2: NB GG3 t } \\
\text { Group 3: NB GG2 ts } \\
\text { Group 4: NB and RP }\end{array}$ & $\begin{array}{l}\mathrm{G}=\text { grade gro } \\
\text { rostatectomy } \\
\text { GG2 } \\
\text { RP GG2 (dow } \\
\text { RP GG3 (upgr } \\
\text { GG3 }\end{array}$ & $\begin{array}{l}\mathrm{IQR}=\text { inter } \\
\text { rade) } \\
\text { e) }\end{array}$ & rtile range: & $\mathrm{A}=$ prostate & ecific \\
\hline
\end{tabular}

Table 2. Univariate and multivariate analysis of factors associated with biochemical recurrence following RP

\begin{tabular}{|c|c|c|c|c|}
\hline \multirow[t]{2}{*}{ Variable } & \multicolumn{2}{|l|}{ Univariate } & \multicolumn{2}{|l|}{ Multivariate } \\
\hline & $\mathrm{HR}(95 \% \mathrm{Cl})$ & $\mathrm{p}$ value & HR $(95 \% \mathrm{Cl})$ & p value \\
\hline Age, years & $1.006(0.986-1.026)$ & 0.579 & & \\
\hline Year of surgery & $0.928(0.889-0.969)$ & $<0.001$ & $0.968(0.922-1.017)$ & 0.968 \\
\hline $\mathrm{PSA}, \mathrm{ng} / \mathrm{ml}$ & $1.008(1.005-1.011)$ & $<0.001$ & $1.006(1.003-1.010)$ & $<0.001$ \\
\hline \multicolumn{5}{|c|}{ Tumor volume, $\mathrm{cm}^{3}$} \\
\hline$<1$ & $1(\operatorname{Ref})$ & & 1 (Ref) & \\
\hline $1-5$ & $2.158(1.438-3.237)$ & $<0.001$ & $1.708(1.128-2.587)$ & 0.012 \\
\hline$\geq 5$ & $5.575(3.530-8.806)$ & $<0.001$ & $3.177(1.897-5.321)$ & $<0.001$ \\
\hline \multicolumn{5}{|c|}{ Pathological T stage } \\
\hline $\mathrm{T} 2$ & 1 (Ref) & & 1 (Ref) & \\
\hline T3a & $1.860(1.321-2.618)$ & $<0.001$ & $1.378(0.963-1.972)$ & 0.079 \\
\hline $\mathrm{T} 3 \mathrm{~b}$ & $4.491(2.905-6.941)$ & $<0.001$ & $1.846(1.122-3.037)$ & 0.016 \\
\hline \multicolumn{5}{|l|}{ Surgical margin } \\
\hline Negative & 1 (Ref) & & 1 (Ref) & \\
\hline Positive & $2.616(1.922-3.560)$ & $<0.001$ & $1.762(1.268-2.448)$ & $<0.001$ \\
\hline \multicolumn{5}{|l|}{ Group* } \\
\hline Group 1 & 1 (Ref) & & 1 (Ref) & \\
\hline Group 2 & $1.621(1.037-2.534)$ & 0.034 & $1.675(1.064-2.638)$ & 0.026 \\
\hline Group 3 & $2.308(1.535-3.470)$ & $<0.001$ & $1.908(1.261-2.889)$ & 0.002 \\
\hline Group 4 & $3.250(2.234-4.728)$ & $<0.001$ & $2.699(1.845-3.950)$ & $<0.001$ \\
\hline $\begin{array}{l}\text { NB = needle biop } \\
\text { prostate specific } \\
{ }^{*} \text { Group 1: NB an } \\
\text { Group 2: NB GC } \\
\text { Group 3: NB GC } \\
\text { Group 4: NB an }\end{array}$ & $\begin{array}{l}\text { = grade group; } \mathrm{HR}= \\
\text {; } \mathrm{RP}=\text { radical prostated } \\
\mathrm{G} 2 \\
\mathrm{P} \mathrm{GG} \text { (downgrade) } \\
\text { P GG3 (upgrade) } \\
\mathrm{G} 3\end{array}$ & $\begin{array}{l}\text { zard ratio } \\
\text { my }\end{array}$ & $\mathrm{QR}=$ interquartile rang & ;PSA $=$ \\
\hline
\end{tabular}

Materials and Methods: We retrospectively reviewed the medical records of 3253 patients with localized (pT2-3N0M0) PC who underwent RP at our institution between 1995 and 2014. We identified 739 patients with GS 7 PC on both NB and RP specimens. After exclusion of patients who had received neoadjuvant or adjuvant treatment (i.e., radiation, androgen deprivation therapy, or both) and those with incomplete pathological or follow-up data, 692 men were included in the final 
analysis. We analyzed data using Kaplan-Meier methods with log-rank tests and multivariate Cox regression models.

Results: Of the 692 patients enrolled in this study, 264 (38.1\%), $125(18.1 \%), 142(20.5 \%)$, and $161(23.3 \%)$ patients were classified as group 1 (NB and RP GG2), group 2 (NB GG3 downgraded to RP GG 2), group 3 (NB GG2 upgraded to RP GG3), and group 4 (NB and RP GG3), respectively. Kaplan-Meier curves showed significant differences in biochemical recurrence $(\mathrm{BCR})$-free survival across the groups (Log-rank test, $\mathrm{p}<0.001$ ). In multivariate Cox regression analyses, these groups were significantly associated with BCR (group 2: HR 1.675, $\mathrm{p}=0.026$; group 3: HR 1.908, p =0.002; and group 4: HR 2.699, $\mathrm{p}<0.001$ ). Conclusions: Downgrading and upgrading from NB to RP was an independent predictor of BCR, and could be due to the amount of Gleason pattern 4.

MP15-8 Robotic, Laparoscopic, and Open Approaches in Radical Prostatectomy Effect on Mortality, Readmission, and Length of Stay.

M Finkelstein, KH Bilal, MA Palese

Icahn School of Medicine at Mount Sinai United States

Introduction \& Objective: The effect of hospital volume on certain procedural outcomes has shown fewer deaths, shorter hospital stays, fewer complications, decreased transfusions, and earlier discharges. Concurrent with changes in the volume of radical prostatectomies (RP) there has been a simultaneous trend toward centralization of complex cancer procedures at high volume centers with high volume surgeons. One explanation for this phenomenon is the advent of robotic-assisted laparoscopic surgical technology, which encourages centralization through the capital investments required. In this study, robotic assisted laparoscopic radical (RALP), standard laparoscopic (LRP), and open (ORP) approaches for RP were reviewed for readmission, 1-year mortality, and length of stay and then analyzed in the context of patient and physicianlevel variables as well as physician and hospital volume.

Materials and Methods: The New York Statewide Planning and Research Cooperative System was queried over the period from 2009 to 2015 for RP (ICD-9 60.5). The American Medical Association Masterfile was used to supplement each procedure with information on an identified urologist and the American Hospital Association Dataviewer was used to obtained information on the associated facility. High volume urologists and high volume hospitals were identified such that each accounted for $25 \%$ of all cases. A stay was considered long if it lasted more than 5 days. Multivariate analysis was performed to calculate adjusted odds ratios, taking into account age, ethnicity, race, insurance, comorbidities, surgeon time from medical school graduation, hospital teaching affiliation, hospital setting, and procedure year. Results: RALP was associated with decreased 1-year mortality (OR 0.548, 95\% CI 0.348-0.865) and decreased prolonged length of stay (OR 0.400, 95\% CI 0.349-0.458) over the open approach. There was no statistically significant difference in readmission rate with RALP. LRP was found to be associated with decreased prolonged length of stay (OR $0.307,95 \%$ CI $0.150-0.556$ ) over ORP, but no association was found with readmission or 1-year mortality. Urologist volume was associated with a $0.5 \%$ decrease in mortality for every increase in hospital volume of 10 cases per year (OR 0.995, 95\% CI 0.992-0.998), and a $2.8 \%$ decrease in long stay (OR 0.972, 95\% CI 0.968-0.976).
Conclusions: The robotic approach was found to be beneficial for lowering 1-year mortality and lowering the incidence of long stays, but no significant difference in readmission was found over the open approach. The laparoscopic approach was found to be beneficial for lowering long stay, but no difference was found for readmission or 1-year mortality.

\section{MP15-9 Withdrawn}

\section{MP15-10 Withdrawn}

MP15-11 Lateral Bladder Neck Dissection Technique and Triple-layer Posterior Bladder Wall Reconstruction Lead to Early Removal of Foley Catheter and Improved Urinary Continence After Robotic-assisted Radical Prostatectomy

G Sung, S Kim

Dong-A University Hospital

Republic of Korea

Introduction \& Objective: A variety of surgical techniques have been employed in an attempt to improve early return of continence after robotic-assisted radical prostatectomy (RARP). This study was underwent to evaluate the influence of lateral bladder neck dissection technique (LBND) and triple-layer posterior bladder wall reconstruction (TPWR) on the early removal of Foley catheter and urinary continence after RARP.

Materials and Methods: Total of 485 patients with localized prostate cancer underwent RALP, with 187 men undergoing standard bladder neck dissection with Ven velthoven continuous suturing (group 1) and 298 men undergoing LBND with TPWR and Ven velthoven continuous suturing (group 2). Pre- and postoperative urinary function and continence recovery were evaluated and compared between the two groups.

Results: The overall positive margin rate was lower in group 2 with $11.2 \%$ and $15.6 \%$ for group $1(\mathrm{p}=0.045)$. Early removal of Foley catheter less than at POD $7^{\text {th }}$ was seen in $73.8 \%$ in group 2 whereas only $16.2 \%$ was seen in group 1 . Postoperative continence was evaluated by EPIC short form questionnaire. At POD 1 and 6 months, the mean urinary function scores were higher in group 2 compared to group 1 with statistical significance $(\mathrm{p}=0.031$ at 1 month, $\mathrm{p}=0.043$ at 6 months). Group 2 showed significantly higher continence rates at 1 and 6 months postRALP than group 1 . In group 2 , the recovery of continence approached to $87.2 \%$ at postoperative 6 months showing early return of continence.

Conclusions: Early removal of Foley catheter with improved postoperative continence and urinary functions after RALP can be achieved by lateral bladder neck dissection technique and triple-layer posterior bladder wall reconstruction with Ven velthoven continous suturing.

MP15-12 Retzius-sparing radical prostatectomy: a reversed evolution from robotic-assisted to laparoscopic methodology

\section{J Hu, K Chiu}

Division of Urology, Department of Surgery, Taichung

Veterans General Hospital, Taichung, Taiwan

Taiwan (Republic of China) 
Introduction \& Objective: The developement of robotic-assisted Retzius-sparing radical prostatectomy is based on the idea of avoiding the destruction of the anatomic structures around the prostate (ie, Santorini plexus, puboprostatic ligaments, endopelvic fascia, veil of Aphrodite) by passing through a Douglas incision. The theoretical benefits of anatomic preservation might achieve better functional outcomes. We modified the technique of Retziussparing radical prostatectomy with a reversed evolution from robotic-assisted to laparoscopic methodology.

Materials and Methods: From May 2015 to March 2017, consecutive patients with prostate cancer underwent Retziussparing laparoscopic radical prostatectomy (RS-LRP) at Taichung Veterans General hospital. The general characters of patients, peri-operative parameters, functional and oncologic outcomes were collected prospectively. The surgical techniques including trocar positions and detailed operative steps had been described in our prior video (Videourology. January 2017, 31. doi: 10.1089/vid.2016.0044). Cystography was routinely performed on post-operative day 3 and the Foley catheter would be removed on post-operative day 4 if no leakage on cystography. Continence was defined as no need for any pad in daily life (including protective pad).

Results: Amoung total 15 patients received RS-LRP, the median age (interquartile range) was 66( 62.25-68.75) years old and the initial prostate specific antigen was $15.6(9.59-26.55) \mathrm{ng} / \mathrm{dl}$. The operative time was $200(182.5-237.5)$ minutes while the period of urethrovesical anastomosis was 50 (40-58.75)minutes. The amount of blood loss was $100(100-200) \mathrm{ml}$. The specimen volume was 30 (25-50) gm and the tumor percentages in specimen was 20 (11.25-28.75) \%. Three patients received bilateral neurovascular bundles (NVB) preserving and six patients received unilateral NVB preserving. The other six patients received prostatectomy without NVB preserving. The catheterization period was 4 (4$4.75)$ days. Ten patients $(66.67 \%)$ got immediate continence at the first day of Foley catheter removal. The other five patients had mild post-operative anastomotic leakage and initial mild stress urine incontinence but all of them got recovery to no need for pad within postoperative 2 months.

Conclusions: At the very first time we found the RS-LRP is a feasible option for localized prostate cancer. It resulted in better functional outcome especially in early even immediate continence and with less financial cost for patients in Taiwan's national health system. Further studies are needed to define the benefits of this surgical methodology on the pentafecta of prostate cancer.

MP15-13 Midterm outcomes of four-port extraperitoneal laparoscopic radical prostatectomy for high-risk prostate cancer within Asian population

$\mathrm{RC} \mathrm{Wu}, \mathrm{V}$ Lin

E-Da hospital

Taiwan (Republic of China)

Introduction \& Objective: At diagnosis Asian patients tend to have higher stage prostate cancer compared to other races. This article aims to investigate the outcomes using four-port extraperitoneal laparoscopic radical prostatectomy (EPLRP) as the first step in the multimodality treatment strategy for Asian patients with high-risk prostate cancer (HRPC).

Materials and Methods: A cohort of 202 patients underwent EPLRP between January 2006 to January 2016, of which 122 $(60.3 \%)$ had HRPC as defined by D'Amico classification. The median age was 68 years ( 48 - 82), PSA level of $17.8 \mathrm{ng} / \mathrm{mL}$ (3.3 - 191.1) and the biopsy Gleason sum of 7 (6-10). All patients underwent pelvic lymphadenectomy and some of them underwent neurovascular bundle preservation according to their risk category.

Results: The perioperative outcomes included a median operative time of 185 minutes $(65-380)$, total blood loss of $150 \mathrm{ml}$ (30 - 500) and 10 days $(6-25)$ postoperative hospitalization. No patient was converted to open surgery. The median specimen weight was $42 \mathrm{~g}(19-124)$, lymph node yield was $10(0-35)$ with $11.5 \%$ positivity and a positive surgical margin rate of $28.7 \%$. The median follow-up period was 37 months $(6-129)$. $96.7 \%$ patients achieved continence and $53.8 \%$ of the 39 potent patients prior to surgery maintained their potency at one year after EPLRP. The 5-year cancer-specific, overall and biochemical recurrence free survival rate was $98.8 \%, 92.2 \%$ and $68.7 \%$, respectively.

Conclusions: Four-port extraperitoneal laparoscopic radical prostatectomy can provide low morbidity, fair functional and oncological outcomes as the first step of the multimodality treatment strategy for high-risk prostate cancer within Asians.

MP15-14 Extended Thromboprophylaxis May Be Associated with Increased Symptomatic Lymphocele Formation Following Robotic Prostatectomy with Pelvic Lymph Node Dissection

J Pereira, H Shillan, C Tucci, B Gershman, G Pareek, D Golijanin, J Renzulli

Minimally Invasive Urology Institute, Alpert Medical School at Brown University

United States

Introduction \& Objective: Utilization of pelvic lymph node dissection (PLND) at the time of robotic prostatectomy (RP) has increased in light of the growing evidence of its therapeutic benefit. However, PLND causes added patient morbidity including increased rates of thrombemobolic events (TEE). Extended thromboembolic prophylaxis (EP) at the time of PLND has been shown to reduce rates of TEE following radical cystectomy. Extrapolating from this data, EP was administered to patients undergoing RP with PLND at out institution. In this study, we evaluated the association of EP with symptomatic TEE and lymphocele formation in this population.

Materials and Methods: We identified 133 patients without history of prior TEE from a prospectively maintained institutional database of patients who underwent RP with PLND from 1/2014 through 7/2016. Baseline clinicopathologic features were recorded, including type and duration of pharmacologic thromboprophylaxis. SP was defined as the administration of pharmacologic TEE prohylaxis during the index hospitalization, while EP was defined as pharmacologic TEE prophylaxis with 40mg enoxaparin for 30 days postoperatively. The primary endpoint was symptomatic TEE or lymphocele requiring therapy. The associations of clinicopathologic features with VTE were evaluated using logistic regression.

Results: A total of 133 patients underwent RP with LND. Of these, $98(74 \%)$ received SP, while 35 (26\%) received EP. Median follow up time was 284 (IQR 103,496) days. There were no statistically significant differences in baseline clinicopathologic features across SP and EP groups. The overall VTE rate was $7.5 \%$, with TEE rates of $8.6 \%$ and $7.1 \%$ in the EP and SP groups, respectively. EP was not statistically significantly associated 
with VTE (univariable OR $1.21 \mathrm{p}=0.78$ ). The overall lymphocele rate was $8.3 \%$. EP was associated with an increased rate of lymphocele formation compared to SP (14.3\% versus $6.1 \%)$, although the difference did not reach statistical significance (univariable OR $2.55, \mathrm{p}=0.14$ ) on univariate analysis.

Conclusions: In this contemporary cohort of patients undergoing RP with PLND, rates of VTE were not statistically significantly different between SP and EP. EP was associated with an increased incidence of lymphocele formation, though this difference did not reach statistical significance. However, given the clinical significance of the increased rate of symptomatic lymphocele formation and the morbidity associated with their treatment, we have halted this practice at our institution.

MP15-15 Analysis of extended lymph node dissection during robot-assisted radical prostatectomy: External validation of preoperative nomograms.

K Kiyoshima, J Inokuchi, K Imada, T Dejima, E Kashiwagi, A Takeuchi, M Shiota, K Tatsugami, M Eto

Department of Urology, Graduate School of Medical Sciences, Kyushu University

Japan

Introduction \& Objective: To review the quality of our extended pelvic lymph node dissection (ePLND) and to evaluate the accuracy of preoperative nomograms predicting lymph node involvement (LNI) in patients who underwent ePLND during robot-assisted radical prostatectomy (RARP).

Materials and Methods: The study included 133 patients who underwent ePLND during RARP between November 2011 and August 2016. We performed ePLND in patients with T3 disease, D'Amico high-risk disease and in those where the risk of LNI was higher than $5 \%$ as estimated by the Japan PC Table. Preoperative and postoperative patients' data were collected retrospectively. We carried out ROC analyses for external validation of preoperative LNI-predicting nomograms.

Results: The median number of lymph nodes removed was 19 . LNI found in 27 of the 133 patients $(20.3 \%)$. In the 27 patients with LNI, a total 51 positive nodes were detected, $23.9 \%$ of which were in the obturator region, $16.7 \%$ in the external iliac region and $60.0 \%$ in the internal iliac region. In the external validation of LNI prediction by preoperative nomograms using ROC analyses, AUC of the Briganti nomogram was 0.699 whereas those of the Japan PC Table and MSKCC were 0.606 and 0.690 , respectively.

Conclusions: The number of lymph nodes removed and lymph node positivity by ePLND during RARP in our institution were comparable to previous reports based on an open radical prostatectomy series. In this study, the Japan PC Table was inferior to the Briganti nomogram for prediction of LNI, and therefore the development of an updated nomograms for Japanese patients is required.

MP15-16 Clinical outcomes of robot-assisted radical prostatectomy (RARP) with retroperitoneal approach

K Takahara, A Kawai, M Ito, M Hikichi, K Fukaya, M Ichino, N Fukami, H Sasaki, M Kusaka, R Shiroki

Department of Urology, Fujita Health University School of Medicine, Japan

Japan
Introduction \& Objective: Robot-assisted radical prostatectomy (RARP) has become a widely adopted procedure to treat localized prostate cancer (PCa). However, it is sometimes difficult to perform with usual intraperitoneal approach for patients those who have prior abdominal operations. Herein, we performed RARP with retroperitoneal approach for PCa cases with prior abdominal operations to evaluate feasibility and clinical outcomes.

Materials and Methods: In all, 792 PCa patients underwent RARP from August 2009 to March 2017; 15 patients who were performed with retroperitoneal approach comprised the study cohort. The trocar was placed below the navel, and retroperitoneal space was dilated using balloon dilator. Every port was placed with approximately $3 \mathrm{~cm}$ caudal than transperitoneal position. We maintained at least $6 \mathrm{~cm}$ distance between each port, and the patient was placed in a 10 degrees head- down (Trendelenburg) position.

Results: The mean age of the patients was 67.7 years (57 75), and the mean preoperative serum PSA level was $5.9 \mathrm{ng} / \mathrm{ml}(4.3 \sim 10.7)$. Clinical stage was cT2a in 5 patients, cT2b in 4, and cT2c in 6. The D'Amico Risk classification was low risk in one patient, intermediate risk in 8 , and high risk in 6. The details of abdominal operations in 15 patients were 3 cases for nephrectomy, 2 for panperitonitis, 2 for appendectomy, and 9 for others, including overlap. The mean operation time and console time were 173 minutes $(124 \sim 279)$ and 116 minutes (81 219), respectively. The mean estimated intraoperative blood loss was $227 \mathrm{~mL}(50 \sim 600)$, and there were no cases that required a conversion to open surgery or intra-operative blood transfusions. The mean postoperative catheterization time and postoperative hospital length of stay were 7.2 days $(6 \sim 13)$ and 10.7 days $(8 \sim 17)$, respectively. Post-operative complications included one patient with total retention of urine (Clavien II), one with lymphocele (Clavien I), and one with anastomotic leak (Clavien I). In 777 patients who underwent RARP with intraperitoneal approach during the same period, the mean operation time and console time were 169 minutes $(80 \sim 570)$ and 130 minutes $(53 \sim 515)$, respectively, and the mean estimated intraoperative blood loss was $171 \mathrm{~mL}(10 \sim 1000)$. There were no significant differences of these three factors between them although total number of patients was different.

Conclusions: We proposed RARP could be safely performed with retroperitoneal approach for PCa patients who had prior abdominal operations.

MP15-17 Effects of Previous Hernia Repair on Robotic Assisted Laparoscopic Radical Prostatectomy; A MatchedPair Analyse Study

AH Al-Shareef Al-Shaibani, Y Akin, T Almouhissen, J Rassweiler, M SaadAlshehri, A SerdarGözen, D Teber

Urology department, Al-Sadar teaching hospital, Al-Najaf, IRAQ

Iraq

Introduction \& Objective: To evaluate results of robotic assisted laparoscopic radical prostatectomy (RALP) series considering patients with previous laparoscopic inguinal hernia repair (LIHR).

Materials and Methods: Between, March 2008 and August 2014, our database were investigated and data of 987 RALP patients were evaluated. Age, prostate specific antigen (PSA), 
body mass index, American Society of Anaesthesiologists score, serum haemoglobin level, and Gleason scorewere considered as criteria of matched pair analyses. Thirty two patients underwent RARP, who had previous LIHR using mesh, consisted of Group 1 and 32 patients without LIHR consisted of Group 2. Additionally, preoperative and operative data were recorded. Complications were evaluated according to Clavien-Dindo classification. Statistical analyses performed and statistical significant $\mathrm{p}$ was accepted as $\mathrm{P} \leq 0.05$.

Results: Mean follow-up was $20.3 \pm 3.2$ months. Preoperative parameters were similar between groups. There were significant more peritoneum opening, time of anastomosis, trocar placement, preparing retzius space, pelvic lymph node dissection (PLND)and operation were observed in Group 1 than Group 2 (respectively; $\mathrm{P}=0.01, \mathrm{P}=0.05, \mathrm{P}=0.004, \mathrm{P}=0.001, \mathrm{P}=0.01$, $\mathrm{P}=0.002$ ). Mean estimated blood loss and time for endopelvic dissection were comparable between groups. Additionally, there was no open conversion, bladder and pelvic vessel injury. The most common complication was postoperative fever (Clavien 1). Conclusions: PLND can be performed safely during extraperitoneal RALP in patients with previous LIHR. Steps of operation including PLND can be take longer time in patients with LIHR.

MP15-18 Comparison of two templates of lymphadenectomy in patients affected by high risk prostate cancer

R Sanseverino, G Napodano, U Di Mauro, O Intilla, G Molisso, T Realfonso

Dept. of Urology Umberto I Hospital Italy

Introduction \& Objective: High risk prostate cancer treatment considers an extended lymphadenectomy. We have compared two templates of pelvic lymphadenectomy in high risk patients undergone an extraperitoneal or transperitoneal laparoscopic radical prostatectomy.

Materials and Methods: Two consecutive series of patients affected by high risk prostate cancer underwent laparoscopic radical prostatectomy. In group 1 (116 pts), the procedure was realized by a preperitoneal access with an extended lymphadenectomy including external iliac and obturator nodes; in group 2 (35 pts), access was transperitoneal with a broader lymphade-

\begin{tabular}{|c|c|c|c|c|c|}
\hline & & All pts & Group 1 & Group 2 & $\mathrm{p}$ value \\
\hline Age & $y$ & $66.8 \pm 5.5$ & $67.0 \pm 5.6$ & $66.0 \pm 4.9$ & 0.33 \\
\hline PSA & $\mathrm{ng} / \mathrm{ml}$ & $11.8 \pm 8.1$ & $10.9 \pm 6.2$ & $15.0 \pm 12.0$ & 0.06 \\
\hline PSAD & $\mathrm{Ng} / \mathrm{ml} / \mathrm{cc}$ & $0.25 \pm 0.20$ & $0.22 \pm 0.16$ & $0.32 \pm 0.28$ & 0.07 \\
\hline BMI & $\mathrm{n}$ & $27.1 \pm 3.4$ & $27.1 \pm 3.4$ & $27.2 \pm 3.5$ & 0.85 \\
\hline ASA & $\begin{array}{l}\text { I } \\
\text { II } \\
\text { III } \\
\text { IV (\%) }\end{array}$ & $\begin{array}{l}2.9 \\
36.3 \\
57.8 \\
2.9\end{array}$ & $\begin{array}{l}2.5 \\
29.1 \\
65.8 \\
2.5\end{array}$ & $\begin{array}{l}5.1 \\
57.7 \\
31.2 \\
6.0\end{array}$ & NS \\
\hline C Stage & $\begin{array}{l}\text { T1c } \\
\text { T2 } \\
\text { T3 }\end{array}$ & $\begin{array}{l}43.7 \\
51.0 \\
5.3\end{array}$ & $\begin{array}{l}48.3 \\
48.3 \\
3.4\end{array}$ & $\begin{array}{l}28.6 \\
60.0 \\
11.4\end{array}$ & $\begin{array}{l}0.84 \\
0.02\end{array}$ \\
\hline Bx Gleason & $\begin{array}{l}3+3 \\
3+4 \\
4+3 \\
>7\end{array}$ & $\begin{array}{l}6.6 \\
4.6 \\
49.7 \\
39.1\end{array}$ & $\begin{array}{l}8.6 \\
3.4 \\
58.6 \\
29.4\end{array}$ & $\begin{array}{l}0 \\
8.6 \\
20.0 \\
71.4\end{array}$ & 0.004 \\
\hline
\end{tabular}

\begin{tabular}{|c|c|c|c|c|c|}
\hline & & Total & Group 1 & Group 2 & p value \\
\hline Operative time & $\min$. & 248.358 .7 & 241.759 .6 & 271.149 .71 .8 & 0.01 \\
\hline Prostate volume & gr. & 52.420 .1 & 52.720 .6 & $51.3 \quad 18.8$ & 0.72 \\
\hline Path. Stage & $\begin{array}{l}\text { pT2 } \\
\text { pT3a } \\
\text { pT3b } \\
\text { pT4 }\end{array}$ & $\begin{array}{l}61.6 \\
23.9 \\
13.2 \\
1.3\end{array}$ & $\begin{array}{l}66.4 \\
23.3 \\
9.4 \\
0.9\end{array}$ & $\begin{array}{l}45.7 \\
25.7 \\
25.7 \\
2.9\end{array}$ & 0.01 \\
\hline Pathol. Gleason & $\begin{array}{l}3+3 \\
3+4 \\
4+3 \\
>7\end{array}$ & $\begin{array}{l}3.3 \\
17.9 \\
44.4 \\
34.4\end{array}$ & $\begin{array}{l}4.3 \\
20.6 \\
43.1 \\
32.0\end{array}$ & $\begin{array}{l}0 \\
8.6 \\
48.5 \\
42.9\end{array}$ & 0.14 \\
\hline Positive SM & $\%$ & 21.8 & 18.1 & 34.3 & 0.06 \\
\hline Complications & $\%$ & 21.2 & 25.0 & 8.6 & 0.04 \\
\hline Lymphocele & $\%$ & 4.0 & 5.2 & 0 & 0.21 \\
\hline $\begin{array}{l}\text { Number nodes } \\
\text { removed }\end{array}$ & $\mathbf{n}$ & 20.411 .0 & 16.68 .3 & 33.39 .4 & $<0.001$ \\
\hline Positive nodes & $\%$ & 6.6 & 1.7 & 28.0 & $<0.001$ \\
\hline
\end{tabular}

nectomy consisting of common iliac, external iliac, hypogastric and obturator nodes. We have compared perioperative outcomes in terms of number of nodes removed, positive nodes, complications in the two groups of patients. Statistical analysis has been realized using SPSS 24

Results: Data on 151 patients were analyzed. Baseline characteristics are reported in table 1. Preoperative data were balanced between two groups of patients except for biopsy Gleason score. Postoperative outcomes are listed in table 2: Group 2 patients presented worse pathological stage, longer operative time, more nodes removed (mean 33.3 vs $16.6, \mathrm{p}<0.001$ ) and more positive pathological nodes $(22.9$ vs $1.7 \%, \mathrm{p}<0.001)$. Moreover, a wider lymphadenectomy template was not associated to greater risk of complications or lymphocele.

Conclusions: In our retrospective analysis, a transperitoneal laparoscopic radical prostatectomy with an extended lymphadenectomy template including obturator, external iliac, common iliac and hypogastric nodes allows to remove a greater number of nodes, to obtain a more positive nodes without increasing risk of complications.

MP15-19 Significance of erection hardness score as a diagnostic tool to assess erectile function recovery in Japanese men after robot-assisted radical prostatectomy.

Y Okamura, J Furukawa, K Shigemura, K Harada, $\mathrm{N}$ Hinata, T Isihimura, M Fujisawa

Division of Urology, Kobe University Graduate School of Medicine

Japan

Introduction \& Objective: The objective of this study was to characterize time-dependent recovery of erectile function in Japanese patients following robot-assisted radical prostatectomy (RARP) using the erection hardness score (EHS).

Materials and Methods: This study prospectively included 170 Japanese patients with localized prostate cancer (PC) undergoing RARP without neoadjuvant hormonal therapy. The erectile function of each patient was assessed based on the International Index of Erectile Function-5 (IIEF-5) and EHS at the baseline and on every visit to an outpatient clinic after RARP. In this series, potency was defined as the ability to have an erection sufficient for intercourse, corresponding to EHS $\geq 3$, while patients with EHS $\geq 2$ were regarded as those with erectile function. Results: Of these 170 patients, 20 and 75 underwent bilateral and unilateral nerve-sparing procedures, respectively; however, nonnerve-sparing procedures were performed in the remaining 75. A proportional increase in the IIEF-5 score according to EHS was 
noted at 24 months after RARP. At 6, 12 and 24 months after RARP, the recovery rates of erectile function were 11.9, 21.7 and $35.8 \%$, respectively, while those of potency were 3.8, 9.8 and $13.7 \%$, respectively. Of several factors examined, the age, preoperative IIEF-5 score and nerve-sparing procedure were identified as independent predictors of erectile function recovery.

Conclusions: These findings suggest that favorable erectile function recovery could not be achieved in Japanese PC patients even after the introduction of RARP; therefore, it might be preferable for such a cohort to use EHS rather than IIEF-5 as an assessment tool for the postoperative recovery of erectile function.

MP15-20 Cytoreductive Laparoscopic Radical Prostatectomy for Men with Oligo-Metastatic Prostate Cancer

S Liu, J Wang, T Li, B Hong, K Gong

Department of Urology, Peking University First Hospital China (People's Republic)

Introduction \& Objective: To present our preliminary surgical experience with cytoreductive laparoscopic radical prostatectomy for patients with oligometastatic prostate cancer.

Materials and Methods: 5 selective cases with oligometastatic prostate cancer diagnosed by bone scan and biopsy of prostate underwent cytoreductive laparoscopic radical prostatectomy. The operating time, estimated blood loss and perioperative complication were recorded and evaluated. Follow up studies were performed with an evaluation for postoperative PSA level and the status of the urinary voiding.

Results: The mean age was 58.8 years (range 55-61 years), initial PSA level was $85.37 \mathrm{ng} / \mathrm{ml}$ (range $8.56-280.0 \mathrm{ng} / \mathrm{ml}$ ), biopsy Gleason score was 7 (range 6-9), Preoperative clinical stage 1 case T4N1M1,1 case T3N1M1,2 cases T3N0M1,1 case T2N0M1. All the operations were successfully performed. The total operative time range was $110-300 \mathrm{~min}$ with mean time of $240 \mathrm{~min}$. The blood loss was $85-340 \mathrm{ml}$ with mean $120 \mathrm{ml}$ and no blood transfusion was required. The catheter was removed after a mean (range) of $14(9-16)$ days. No intra-operative complications occurred. 4 patients had positive surgical margins. The mean (range) hospital stay was $15(10-24)$ days after surgery. All the cases were continent after removal of the catheter. No cases demonstrated vesico-urethral stricture. All 5 patients had decreased PSA after operation 6 weeks.

Conclusions: Cytoreductive laparoscopic radical prostatectomy for patients with oligometastatic prostate cancer could be safe, effective, and appropriate. Cytoreductive laparoscopic radical prostatectomy might be a treatment option in the multimodal management of oligo-metastatic prostate cancer.

MP15-21 Surgeon Technical Performance Impacts Patient Outcomes in Robotic-Assisted Radical Prostatectomy

M Goldenberg, L Goldenberg, T Grantcharov

University of Toronto

Canada

Introduction \& Objective: Introduction: There is limited, yet compelling evidence supporting the role of surgeon technical performance in influencing patient outcomes. To date, this con-
Table-3: Binary Logistic Regression Models

\begin{tabular}{ccccc} 
& OR & $95 \% \mathrm{Cl}$ & $\mathrm{p}$-value \\
\hline Total GEARS & .55 & .33 & .91 & $\mathbf{p}=. \mathbf{0 2}$ \\
\hline Age & 1.09 & .98 & 1.23 & \\
\hline BMI & .98 & .77 & 1.26 & \\
\hline Prostate Weight & 1.00 & .97 & 1.04 & \\
\hline Bladder Neck GEARS & & & & \\
\hline Age & .69 & .51 & .94 & $\mathbf{p}=.01$ \\
\hline BMI & 1.11 & .99 & 1.24 & \\
\hline Prostate Weight & .98 & .79 & 1.31 & \\
\hline & 1.00 & .97 & 1.04 & \\
\hline Urethrovesical Anastomosis GEARS & .70 & .51 & .94 & $\mathbf{p}=.03$ \\
\hline Age & 1.12 & .97 & 1.20 & \\
\hline BMI & .98 & .78 & 1.04 & \\
\hline Prostate Weight & 1.00 & .97 & 1.04 & \\
\hline
\end{tabular}

GEARS = Global Evaluative Assessment of Robotic Skil $\mathrm{OR}=$ Odds Ratio, $\mathrm{Cl}=$ Confidence Interval, $\mathrm{BMI}=$ Body mass index

cept has been underexplored in urologic surgery. We hypothesized that a surgeon's technical performance plays a role in predicting an early return to continence after robotic-assisted radical prostatectomy (RARP).

Materials and Methods: Methods: We conducted a retrospective, matched case-control analysis of prospectively collected unedited RARP endoscopic videos performed by a single surgeon. A blinded observer with expertise in intraoperative video analysis evaluated clinically relevant steps of RARP using the Global Evaluative Assessment of Robotic Skill (GEARS) and the Generic Error Rating Tool (GERT). The primary outcome was continence status at 3 months post-operatively, defined as patient use of less than or equal to a single precautionary pad. Mann-Whitney $U$ tests examined differences in predictor variables between cases and controls, and multivariate analysis was conducted using binary logistic regression models.

Results: 24 incontinent patients were matched for age, BMI, preoperative International Prostate Symptoms Score, use of posterior/anterior hitch, prostate weight, and learning curve position. No statistically significant difference in errors between groups was observed using the GERT. On multivariate analysis, overall case GEARS score was independently predictive of three-month continence status $(O R=.55,95 \%$ CI .33-.91), as were urethrovesical anastomosis $(O R=.70,95 \% \mathrm{CI} .50-.97)$ and bladder neck GEARS scores $(O R=.69,95 \%$ CI .51-.94).

Conclusions: Our study generates the hypothesis that there may be a link between surgeon technical performance and functional outcomes in RARP. This relationship may have implications for the accreditation and training of future urologists, and warrants further investigation.

MP15-22 Utility of preoperative mpMRI for planning side-specific nerve sparing in patients undergoing robotic prostatectomy

MB Schaefer, J Piotrowski, P Langenstroer, W See, K Jacobsohn

Medical College of Wisconsin United States

Introduction \& Objective: Multi-parametric magnetic resonance imaging (mpMRI) is increasingly used in the diagnosis 
and staging of prostate cancer $(\mathrm{CaP})$. The utility of mpMRI for defining side specific extracapsular extension (ssECE) and planning side-specific nerve sparing (ssNS) is not well defined. This study looks at the utility of preoperative mpMRI to predict ssECE and therefore alter the degree of ssNS in patients undergoing robotic-assisted laparoscopic radical prostatectomy (RARP).

Materials and Methods: We retrospectively reviewed all patients at a single institution with a preoperative mpMRI who underwent radical prostatectomy between June 2014-September 2016. A validated side-specific nomogram (N) using clinical data was used to determine predicted ssECE and plan grade of NS. Preoperative MRI was used to further refine surgical planning. Grade of MRI-influenced NS was determined by absence, presence or equivocal finding of ssECE. Parametric and non-parametric statistical tests detected differences in outcomes between $\mathrm{N}$ and MRI predictions. A linear regression model produced receiver-operating characteristic curves (ROC) and assessed prediction power of MRI vs. data utilized by N.

Results: 183 patients with a total of 364 specific sides were analyzed. MRI alone performed similar to $\mathrm{N}$ alone in predicting ssECE (Fig 1). The addition of MRI to N provided the most accurate preoperative prediction of $\operatorname{ssECE} \quad(\mathrm{AUC}=0.87$, $\mathrm{p}=0.004, \mathrm{p}=0.028$ ). In sides with low, intermediate and high risk of ECE by nomogram alone, MRI $+\mathrm{N}$ widened the grade of NS in 30,0 , and 0 patients $(17.3,0$ and $0 \%)$, and narrowed the grade of NS in 0,86 , and $16,(0,53.1$, and $55.2 \%) .18 \%$ of sides predicted by $\mathrm{N}$ to have low risk ECE had possible/very likely ECE on MRI. Final pathology was positive for ECE in $20 \%$ of
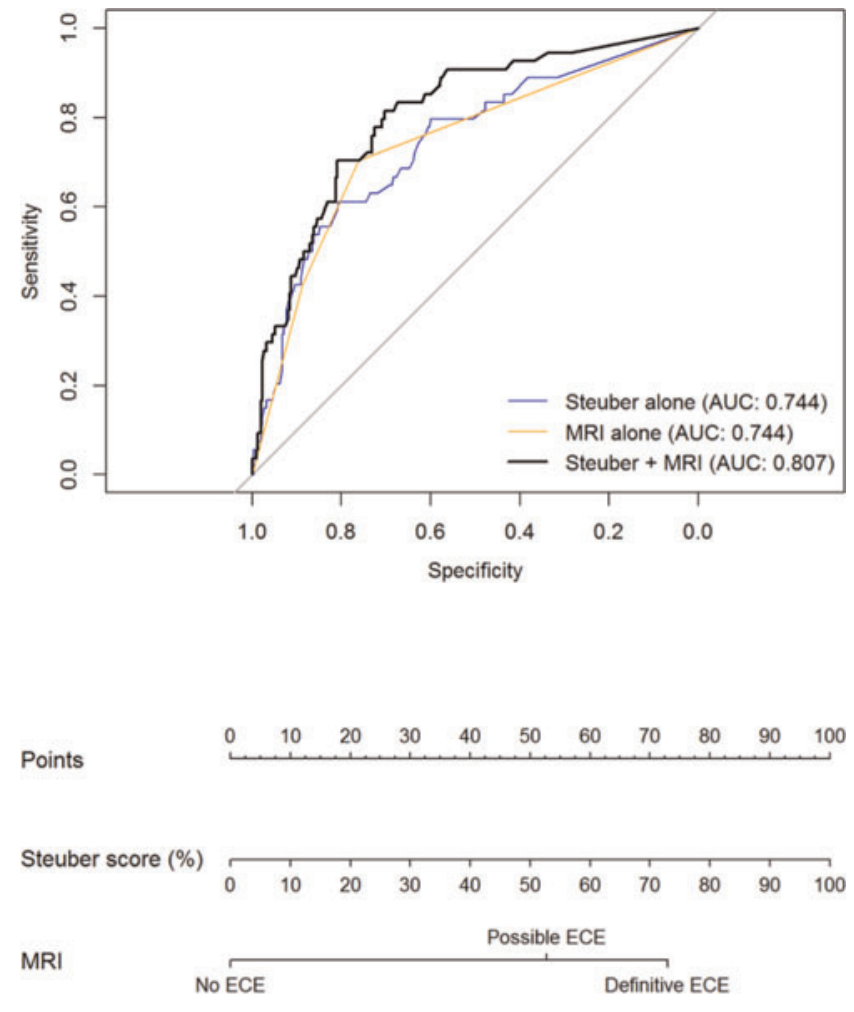

Total Points

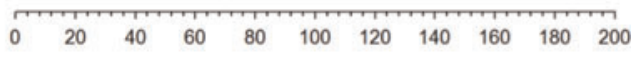

Prob. of positive ECE

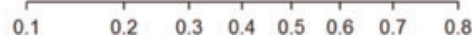

these patients. Overall, MRI altered side specific NS operative plans in $50 \%$ of sides compared with clinical and biopsy data alone. A nomogram incorporating $\mathrm{N}$ and MRI results was generated (Fig 2).

Conclusions: Prediction of ssECE by mpMRI is similar to the previously validated nomogram. Combining mpMRI and $\mathrm{N}$ produces a more sensitive, and specific prediction of ssECE and allows a more accurate prospective NS plan.

MP15-23 Narrow Bony Pelvic Dimensions are Associated with Symptomatic Lymphocele

A Hamada, W Devan, I Tuerk

LoUIS a Johnson VAMC

United States

Introduction \& Objective: Objectives are to evaluate risk factors for development of symptomatic lymphocele (SL) in men who underwent robotic assisted radical prostatectomy (RARP) with pelvic lymph node dissection (PLND), specifically but not limited to the role of bony pelvic dimensions.

Materials and Methods: This study was a single-center, IRBapproved, retrospective, case series at a high-volume cancer center between November 2008 and October 2014. Data included 1,196 patients with PC who underwent RARP with PLND based upon $\geq 2 \%$ MSKCC nomograms risk of lymph node invasion. Of these, 865 patients underwent RARP with PLND. Men with SL and asymptomatic (ASL) and those with no lymphocele (NL) were compared for the perioperative, demographic, clinical presentation and treatment pathways data. Furthermore, Pelvimetry data was obtained in these patients by using anteroposterior, transverse, and sagittal CT scans to measure anteroposterior (AP), oblique (O), trans-ischial (TIS), transfemoral diameters $(\mathrm{T})$, pubic to acetabulum dimension (PTAD) and acetabular width (AW).

Results: After mean follow up of 14 weeks, SL with mean size 7. $94 \mathrm{~cm}$ was seen in 62 of them (7. 2\%) confirmed on CT radiographs, whereas, asymptomatic lymphocele (ASL) with mean size $4.03 \mathrm{~cm}$ was seen in 129 patients (14.9\%). Pelvimetry data was available for 816 patients and showed significant differences in AP and $\mathrm{T}$ diameters as well as PTAD and PTAD/AW ratios among these three groups. Men with SL had significantly shorter AP diameters and larger lymphocele sizes compared with men with ASL. Cox regression and backward stepwise regression showed smaller PTAD [odds ratio (OR) 2. 53, 95\% CI (1. 75-3.

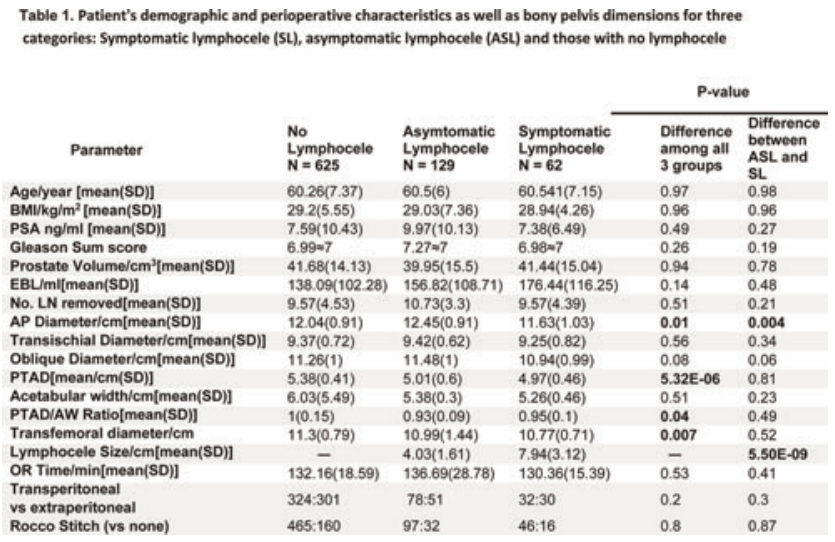


68) and Rocco stitch (OR 1. 88, 95\% CL (1. 23-2. 90)) were significantly associated with SL. Comparing men with SL to ASL, smaller AP diameter (OR 1. 13, 95\% CI (1.04-1. 22)) was significantly associated with SL. Stratified analysis by trans and extraperitoneal approach yielded similar results to overall analysis.

Conclusions: SL are more likely to develop after PLND during RALP in men with narrower pelvic dimensions. This may reflect smaller pelvic peritoneal absorptive surface area or earlier compression of adjacent structures resulting in generation of clinical symptoms.

MP15-24 The Ben Stiller effect: The online and social media impact of the Ben Stiller essay and the ProtecT Study on public awareness of prostate cancer

T Cisu, K Sternberg

University of Vermont College of Medicine United States

Introduction \& Objective: Newly published medical research and celebrity medical announcements can have a profound effect on raising public awareness concerning a specific disease. The recent release of the ProtecT Study in September 2016 and the publication of actor-comedian Ben Stiller's account with prostate cancer three weeks later have both garnered much attention in news media outlets and on social media. Our objective was to examine trends in online search queries and Twitter posts as a proxy for public awareness of prostate cancer as a result of these two major events.

Materials and Methods: Google Trends and Twitter were used to identify the online and social media impact of the ProtecT Study publication on September 14-15 and the Stiller essay on October 4-5. Using \#prostatecancer, Symplur was used to quantify Twitter activity including total number of posts ("tweets"), views ("impressions"), and rate of posting per hour. Similarly, Google Trends, a publicly-available data acquisition tool for quantification of search engine queries, was used to investigate the keyword "prostate cancer" over the same time period. Weekly relative search volume (RSV) means and distribution were tested using one-way analysis of variance.

Results: The baseline average Twitter activity for August 2016 was 804 tweets/day, 11 tweets/hour, and 4.7 mil impressions. The

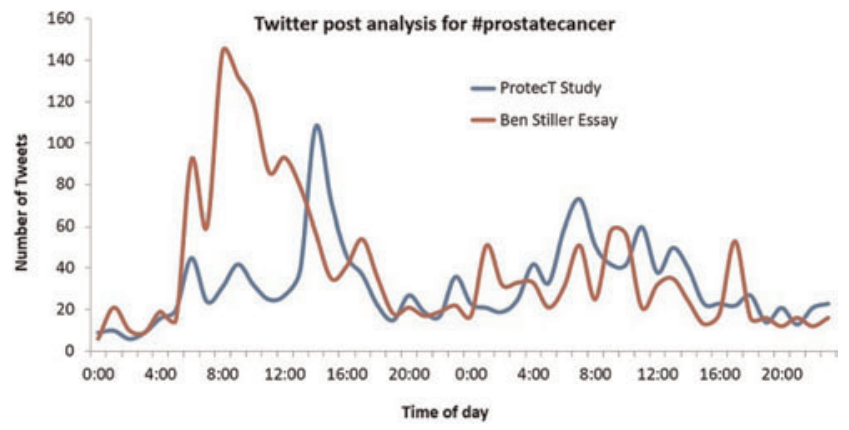

Google Trends Relative Search Volume for keyword "Prostate Cancer"

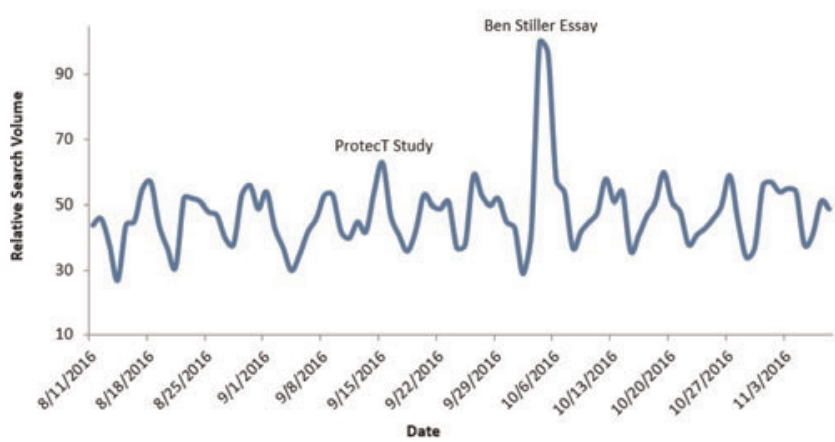

ProtecT Study resulted in 1,540 tweets/day (85\% increase), 32 tweets/hour, and 9.8mil impressions (107\% increase). The Ben Stiller essay resulted in 1,896 tweets/day (128\% increase), 40 tweets/hour, and 31.1mil impressions (557\% increase). The mean baseline RSV for Google in August 2016 was 43.7 $[\mathrm{SD}=7.7]$, compared to $55.5[\mathrm{SD}=12.0]$ for the ProtecT Study and $94.5[\mathrm{SD}=7.8]$ for the Ben Stiller essay.

Conclusions: Acknowledging the impact of celebrity outreach on medical topics is important for medical providers. The role of physicians should include helping patients understand the content and accuracy of information available online and on social media.

\section{MPS16: STONES: EPIDEMIOLOGY \& CLINICAL OUTCOMES}

MP16-1 Holmium Laser Versus Pneumatic Lithotripsy for Bladder Calculi: Which is Faster?

P Budny, M Lacey, M Avallone, R O'Connor, C Davis

Medical College of Wisconsin

United States

Introduction \& Objective: Endoscopic management of bladder calculi can be performed with either holmium laser or pneumatic lithotripsy. There is a paucity of data regarding which modality is more time efficient in treating bladder calculi. The aim of our study is to determine which lithotriptor has greater lithotripsy efficiency. Materials and Methods: Retrospective review was conducted for patients undergoing cystolithalopaxy from 2011 to 2016. Stone size and density were measured from pre-operative CT imaging and operative data was extracted from the electronic medical record. Statistical analysis was performed with student- $t$ test and analysis of covariance.

Results: 13 patients underwent pneumatic lithotripsy utilizing the Cook Stone Breaker ${ }^{\circledR}$ and 26 patients underwent laser 


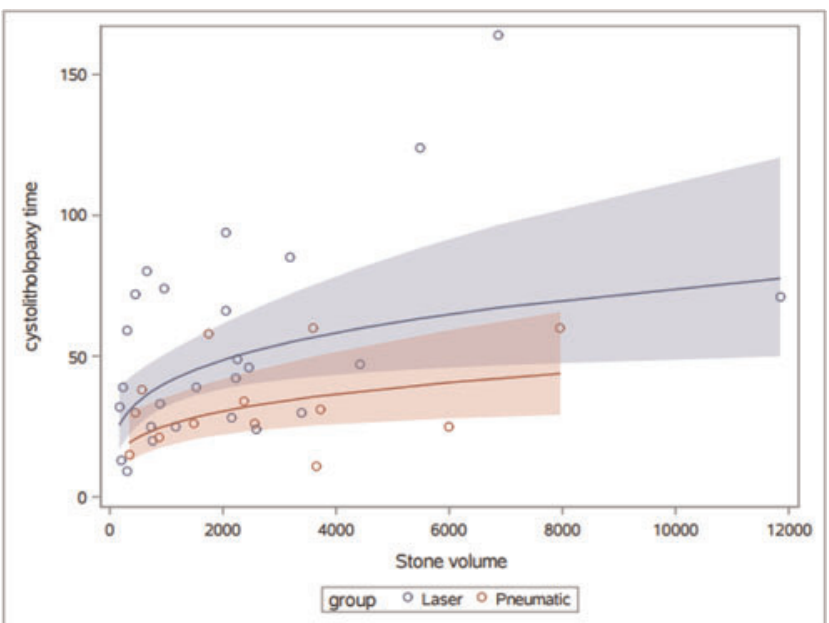

\begin{tabular}{|l|l|l|}
\hline Stone Size $\left(\mathrm{mm}^{3}\right)$ & $\begin{array}{l}\text { Laser procedure time } \\
(\mathrm{min})\end{array}$ & $\begin{array}{l}\text { Pneumatic procedure } \\
\text { time }(\mathrm{min})\end{array}$ \\
\hline 1000 & 40 & 25 \\
\hline 2000 & 44 & 28 \\
\hline 5000 & & \\
\hline & 61 & 38 \\
\hline
\end{tabular}

lithotripsy with a $100 \mathrm{~W}$ Holmium laser. By t-test, there is no significant difference between the laser and pneumatic groups in cumulative stone volume, stone density, or procedure time. When controlling for stone volume on a per-procedure basis with analysis of covariance, laser lithotripsy procedures are 1.6 times longer compared to pneumatic lithotripsy procedures $(\mathrm{P}=0.0212$, 95\% confidence interval 1.08, 2.37).

Conclusions: Pneumatic and holmium laser are effective to treat bladder calculi, however, pneumatic lithotripsy is more time efficient.

MP16-2 A coast-to-coast descriptive analysis of discharge summaries for renal colic from emergency departments in the United States

T Cisu, A Pham, K Sternberg

University of Vermont College of Medicine United States

Introduction \& Objective: Renal colic is the third most common reason for an emergency room visit. Prior studies have noted the wide variation in practice patterns for the management of nephrolithiasis in emergency departments (EDs) nationwide. This

\begin{tabular}{|l|l|l|l|}
\cline { 2 - 4 } & East Coast & West Coast & Combined \\
\hline Etiology & $30 / 34(88.2)$ & $12 / 12(100)$ & $42 / 46(91.3)$ \\
\hline Common Symptoms & $25 / 34(73.5)$ & $7 / 12(58.3)$ & $32 / 46(69.6)$ \\
\hline Expected Course & $10 / 34(29.4)$ & $2 / 12(16.7)$ & $12 / 46(26.1)$ \\
\hline Mentions Imaging & $15 / 34(44.1)$ & $5 / 12(41.7)$ & $20 / 46(43.5)$ \\
\hline CT scan & $11 / 15(73.3)$ & $3 / 5(60.0)$ & $14 / 20(70.0)$ \\
\hline X-ray & $13 / 15(86.7)$ & $5 / 5(100)$ & $18 / 20(90.0)$ \\
\hline Return to ED & $28 / 34(82.4)$ & $9 / 12(75.0)$ & $37 / 46(80.4)$ \\
\hline Return to PCP & $15 / 34(44.1)$ & $5 / 12(41.7)$ & $20 / 46(43.5)$ \\
\hline Follow up & $24 / 34(70.6)$ & $10 / 12(83.3)$ & $34 / 46(73.9)$ \\
\hline Urologist & $7 / 24(29.2)$ & $0 / 10(0)$ & $7 / 34(20.6)$ \\
\hline Fluids & $33 / 34(97.1)$ & $10 / 12(83.3)$ & $43 / 46(93.5)$ \\
\hline Strain Urine & $32 / 34(94.1)$ & $10 / 12(83.3)$ & $42 / 46(91.3)$ \\
\hline NSAIDs & $10 / 34(29.4)$ & $4 / 12(33.3)$ & $14 / 46(30.4)$ \\
\hline Exercise & $9 / 34(26.5)$ & $3 / 12(25)$ & $12 / 46(26.1)$ \\
\hline Dietary & $15 / 34(44.1)$ & $5 / 12(41.7)$ & $20 / 46(43.5)$ \\
\hline
\end{tabular}

study evaluates the contents of generic ED discharge summaries obtained from hospitals in the United States.

Materials and Methods: Discharge summaries for patients presenting to the ED with renal colic were obtained from hospitals in the United States from October 2016 to April 2017, primarily in New England and the West Coast. These were acquired by calling medical records offices or emergency departments directly. Discharge instructions for "renal colic" or "kidney stone pain" were requested. Hospitals using the same discharge instruction set or template were each included in the evaluation and analysis as the goal was to identify and quantify the types of instructions being given to this patient population. Information regarding etiology and symptoms, diagnosis, prevention, treatment, and follow-up care was collected from each discharge summary. Results: A total of 134 EDs were contacted, of which 46 provided discharge summaries for renal colic. 42 mentioned the etiology of stone pain $(91.3 \%)$ and 32 discussed common symptoms of kidney stones $(69.6 \%)$. Less than one-third $(26.1 \%)$ described the expected course of kidney stones. About one-half (43.5\%) mentioned imaging that was or should be done in management of renal colic. Nearly all summaries advised drinking plenty of fluids $(93.5 \%)$, straining the urine for future stone analysis $(91.3 \%)$ and $30.4 \%$ advised taking NSAIDs for pain relief. There were varied recommendations regarding kidney stone prevention including increasing exercise $(26.1 \%)$, and eating certain foods $(43.5 \%)$. Specific dietary information included recommendations to increase intake of phytate-containing foods (wheat, rice, rye, barley, beans), calcium-rich foods, and citrate-rich foods, while reducing intake of high-oxalate foods (spinach, rhubarb, peanuts, cashews, grapefruit), high-protein foods (animal meats), caffeinated foods and drinks, sodium, and vitamin C.

Conclusions: ED discharge instructions for patients with diagnosed or suspected urolithiasis vary both by hospital and region. Most provide information regarding symptoms, diagnosis, reasons to return to the ED, staying hydrated, and straining urine. This study highlights the need for a standardized set of educational instructions for this patient population.

\section{MP16-3 The Effect of Distance on Health-Related Quality of Life for Patients with Urolithiasis}

GL Narang, L Elizabeth Wiener, KL Penniston, J Antonelli, T Averch, S Sivalingam, BH Chew, VG Bird, VM Pais, RL Sur, T Chi, SY Nakada, GG Koch, DP Viprakasit

University of North Carolina School of Medicine United States 
Introduction \& Objective: Urolithiasis carries significant impact on health-related quality of life (HRQoL). Kidney stones are associated with higher levels of stress, depression, and anxiety. Treatment is often necessary and reasonable access to care may be an unexplored barrier. Travel distance has been shown to have direct effects on HRQoL for a variety of illnesses. The relationship between distance to care and HRQoL in the context of stones has not yet been investigated. We hypothesize that increased distance to stone treatment centers is associated with decreased HRQoL.

Materials and Methods: Patients with a history of stones were enrolled at 10 tertiary centers as part of the QOL Stone Consortium of North America. HRQoL data were obtained using the Wisconsin Stone Quality of Life Questionnaire (WISQOL). Geodesic distance between patient home ZIP code and their stone center ZIP code was computed using national ZIP code data. Spearman's correlations were calculated between total WISQOL score and distance to stone centers. To assess the effect of distance on HRQoL more extensively, linear models with demographics, a composite variable for stones and/or symptoms, distance, and the distance $\times$ composite variable were fit to the data.

Results: A total of 1512 patients were enrolled, including 53\% male, $80 \%$ non-Latino white and mean age 53 years. Mean distance to stone centers was 40 miles (range 0-2,346), with 73\% reporting current stones and $45 \%$ symptoms. WISQOL scores and distance were statistically significantly negatively correlated for patients who have stones, symptoms, and both $(\mathrm{p}<0.0001$, $p=0.0002, p=0.0005)$. Linear modeling revealed decreased WISQOL scores for those with stones and/or symptoms with larger distance $(p=0.0009)$, with symptoms contributing the greatest effect, even after taking other demographic factors into account. At a distance of 100 miles, WISQOL scores decreased by 8,25 , and 38 points for a patient with only stones, only symptoms, and both stones and symptoms, respectively, when compared to an identical patient without stones or symptoms.

Conclusions: Distance to stone centers has a negative effect on HRQoL in the presence of stones and/or symptoms, with incremental decline in HRQoL as distance increases. The presence of current symptoms has the largest contribution to detriments in HRQoL with increasing distance. Difficulties with access to care, delayed time to treatment, and inadequate local treatment may be contributing factors for worse HRQoL at increased distance. Further study is required to fully understand the relationship between distance and HRQoL for patients with urolithiasis.

MP16-4 Establishing the Cambridge Renal Stone Patient reported outcome measures reliability.

M Ragab, N Baldin, J Collie, M Tran, S Al-Hayek, JN Armitage, O Wiseman

Cambridge University Hospital Nhs Foundation Trust, Addenbrookes Hospital.

United Kingdom

Introduction \& Objective: Surgeon-reported outcomes have been the main measures in reporting surgical results. An emerging paradigm to assess patients' experience from the qualitative aspect has increased the interest in Patient Reported Outcome Measures (PROMs). The primary stages of generating and selecting items for the Cambridge Renal Stone PROM (CRESP) via focus groups and semi-structured interviews were presented previously. Our aim is establishing the test-retest reliability and internal consistency of the developed kidney stone disease-specific PROM.
Materials and Methods: In a pilot study, patients with radiologically proven renal calculi presenting to our stone clinic were invited to participate in test-retest of our developed renal stone disease-specific PROM along with demographic and clinical data. Results: Analysis was performed to determine internal consistency within domains using Cronbach's alpha with a mean of 0.91 (range 0.89 to 0.93 ) and Cronbach's alpha between domains was 0.93. Average inter-item Pearson's and Spearman's correlation within domains was performed, with Pearson's correlation mean of 0.78 (range 0.73 to 0.86 ) and Spearman's correlation mean of 0.73 (range 0.66 to 0.76 ). Test-retest Pearson's correlation mean was 0.84 (range 0.51 to 0.95 ). Bland-Altman analysis was conducted for all domains, the overall mean of differences is 0.10 and $95 \%$ limits of agreement were between $-1.05 \& 1.25$.

Conclusions: After excluding redundant questions, test-retest reliability, internal consistency within domains and average inter-item correlation within domains were high. We will now evaluate renal calculi therapy prospectively using CRESP, in addition to establishing the construct validity together with the EQ-5D-5L and criterion validity.

MP16-5 Urolithiasis in the Elderly: Protocol driven care and outcomes

A Pai, S Janardanan, P James, H Ni Raghallaigh, R Kulkarni

St. Peter's Hospital

United Kingdom

Introduction \& Objective: There is an increasing incidence of urolithiasis in the elderly. However this cohort represents diagnostic and management challenges. We evaluate our institution's experience.

Materials and Methods: We retrospectively analysed all patients over the age of 80 who required intervention for calculi between August 2010 and March 2017. High risk assessment with NSQIP frailty index was used in all patients before intervention was considered.

Results: There were 57 patients aged 80 and above.

Patients had a median NSQIP score of 3; indicating a high degree of frailty.

Median stone size was $11.8 \mathrm{~mm}$. 17 patients presented with renal colic. 16 presented with urosepsis. Of those patients who presented with an infected, obstructed urinary system, 31\% required intensive care admission.

Fifty patients had ureterorenoscopy. Three patients had PCNL. One patient had lithotripsy. Three patients were managed with stent alone. Stone free rates were $90 \%$.

Median length of stay after the definitive stone procedure was 1 day. After definitive stone procedure, one patient required high dependency unit admission due to cardiac complications. There were no other complications above Clavien Grade 2.

Conclusions: Elderly patients with urinary calculi present atypically. High risk assessment with frailty index can aid anaesthetic and surgical planning for definitive procedures. These patients tolerate definitive stone management and have good outcomes. Age should therefore not be a bar to definitive treatment of calculi in the elderly.

MP16-6 Renal Forniceal Rupture: Is Conservative Management Safe?

T Nikonow Morgan, J Bandari, M Shahait, A Nevo, T Averch

UPMC

United States 
Introduction \& Objective: Forniceal rupture is a condition of perirenal urinary extravasation often associated with ureteral obstruction. Treatment considerations for this condition have not been standardized, and there is very limited information in the literature regarding clinical practice

Materials and Methods: We retrospectively searched all radiographic records for patients treated at our institution between January 2009 and January 2016 using the terms "forniceal rupture," "fornix rupture," "calyx rupture," or "calyceal rupture," and identified 111 patients. Each patient was followed for two months from presentation. Three patients were excluded for age $<18$ and five were excluded for incomplete records.

Results: Of the 103 patients analyzed, the median age at presentation was 64 years (IQR 52-73) and 56 (54\%) were female. The etiology of forniceal rupture was most commonly urolithiasis $(73 \%)$, with cancer being the next most common cause $(11 \%)$. Most cases $(61 \%)$ were caused by small $(1-5 \mathrm{~mm})$ stones in the distal ureter. Thirty-two patients $(31 \%)$ were treated surgically with ureteral stent placement upfront; 27 of those patients were stone patients and most had some clinical factors making them higher risk. There was only 1 operative complication during the study period. Only 1 patient developed an abscess. Fortythree patients $(42 \%)$ were sent home from the ER. Of the patients who were admitted, the average hospital stay was 3 days (IQR 2$6)$. For the entire cohort, there were $6(6 \%)$ re-admissions in the study period.

Conclusions: Very limited data exists in the literature regarding clinical practice in the treatment of forniceal rupture. There are studies showing favorable outcomes of this condition in institutions with operative intervention rates from $59 \%$ to $99 \%$. Clinical practice at our institution is conservative treatment of forniceal rupture in the absence of infection, kidney failure, or other risk factors with few complications or readmissions.

\section{MP16-7 Evaluation of Ethnic Differences in Kidney Stone} Composition in a United States Cohort

R Patel, K Rhee, K Radadia, E Olweny

Rutgers Robert Wood Johnson Medical School United States

Introduction \& Objective: Epidemiological studies in North America have shown that kidney stone prevalence varies according to race $\&$ ethnic origin. However, data on the variation of kidney stone composition according to race / ethnicity are sparse. We sought to characterize the differences in kidney stone composition according to race / ethnicity in a diverse United States cohort.

Materials and Methods: Adult patients treated for kidney stones at our institution with information on chemical composition from at least one stone sample were identified by database query; analysis was restricted to the first submitted stone. Patients were grouped according to race / ethnic categories recognized by the United States Census Bureau, namely White, Black, Hispanic, Asian, Middle Eastern and Other. Each stone was classified within a single specific group, i.e. calcium oxalate monohydrate (COM) or dihydrate (COD), uric acid (UA), apatite (HA), brushite (BT), struvite (ST), cystine (Cy) or ammonium urate $\left(\mathrm{NH}_{4} \mathrm{U}\right)$. Stones were classified as UA, BT, ST, Cy or $\mathrm{NH}_{4} \mathrm{U}$ based on any presence of those constituents, and as COM, COD or HA based on the majority $(>50 \%)$ constituent. Distributions of stone types within the ethnic categories were
Table 1: Distribution of kidney stone composition according to ethnic origin

\begin{tabular}{|c|c|c|c|c|c|c|c|}
\hline $\begin{array}{c}\text { Stone } \\
\text { Composition }\end{array}$ & $\begin{array}{c}\text { White } \\
\# \\
\%\end{array}$ & $\begin{array}{c}\text { Black } \\
\# \\
\%\end{array}$ & $\begin{array}{c}\text { Hispanic } \\
\# \\
\%\end{array}$ & $\begin{array}{c}\text { Asian } \\
\# \\
\%\end{array}$ & $\begin{array}{c}\text { Middle } \\
\text { Eastern } \\
\# \\
\% \\
\%\end{array}$ & $\begin{array}{c}\text { Other } \\
\# \\
\%\end{array}$ & $\begin{array}{c}\text { Total } \\
\# \\
\%\end{array}$ \\
\hline UA & $\begin{array}{c}28 \\
12.9 \\
\end{array}$ & $\begin{array}{c}7 \\
14.6 \\
\end{array}$ & $\begin{array}{c}8 \\
9.6 \\
\end{array}$ & $\begin{array}{c}2 \\
6.3 \\
\end{array}$ & $\begin{array}{l}0 \\
0 \\
\end{array}$ & $\begin{array}{l}0 \\
0 \\
\end{array}$ & $\begin{array}{c}45 \\
11.1 \\
\end{array}$ \\
\hline COM & $\begin{array}{l}108 \\
49.8 \\
\end{array}$ & $\begin{array}{c}20 \\
41.7 \\
\end{array}$ & $\begin{array}{c}37 \\
44.6 \\
\end{array}$ & $\begin{array}{c}22 \\
68.8 \\
\end{array}$ & $\begin{array}{c}16 \\
88.9 \\
\end{array}$ & $\begin{array}{c}4 \\
66.7 \\
\end{array}$ & $\begin{array}{r}207 \\
51.2 \\
\end{array}$ \\
\hline COD & $\begin{array}{c}13 \\
6 \\
\end{array}$ & $\begin{array}{c}3 \\
6.3 \\
\end{array}$ & $\begin{array}{l}5 \\
6 \\
\end{array}$ & $\begin{array}{c}5 \\
15.6 \\
\end{array}$ & $\stackrel{2}{11.1}$ & $\begin{array}{c}1 \\
16.7 \\
\end{array}$ & $\begin{array}{l}29 \\
7.2 \\
\end{array}$ \\
\hline HA & $\begin{array}{c}43 \\
19.8 \\
\end{array}$ & $\begin{array}{l}12 \\
25 \\
\end{array}$ & $\begin{array}{r}21 \\
25.3 \\
\end{array}$ & $\begin{array}{c}1 \\
3.1 \\
\end{array}$ & $\begin{array}{l}0 \\
0 \\
\end{array}$ & $\begin{array}{l}0 \\
0 \\
\end{array}$ & $\begin{array}{c}77 \\
19.1 \\
\end{array}$ \\
\hline ST & $\begin{array}{l}15 \\
6.9 \\
\end{array}$ & $\begin{array}{c}4 \\
8.3 \\
\end{array}$ & $\begin{array}{l}5 \\
6 \\
\end{array}$ & $\begin{array}{l}0 \\
0 \\
\end{array}$ & $\begin{array}{l}0 \\
0 \\
\end{array}$ & $\begin{array}{l}0 \\
0 \\
\end{array}$ & $\begin{array}{l}24 \\
5.9 \\
\end{array}$ \\
\hline BR & $\begin{array}{c}8 \\
3.7 \\
\end{array}$ & $\begin{array}{c}1 \\
2.1 \\
\end{array}$ & $\begin{array}{c}7 \\
8.4 \\
\end{array}$ & $\begin{array}{c}2 \\
6.3 \\
\end{array}$ & $\begin{array}{c}0 \\
0.0 \\
\end{array}$ & $\begin{array}{c}1 \\
16.7 \\
\end{array}$ & $\begin{array}{l}19 \\
4.7 \\
\end{array}$ \\
\hline $\mathrm{NH}_{4} \mathrm{U}$ & $\begin{array}{l}0 \\
0 \\
\end{array}$ & $\begin{array}{c}1 \\
2.1 \\
\end{array}$ & $\begin{array}{l}0 \\
0 \\
\end{array}$ & $\begin{array}{l}0 \\
0 \\
\end{array}$ & $\begin{array}{l}0 \\
0 \\
\end{array}$ & $\begin{array}{l}0 \\
0 \\
\end{array}$ & $\begin{array}{c}1 \\
0.3 \\
\end{array}$ \\
\hline Cy & $\begin{array}{c}2 \\
0.9 \\
\end{array}$ & $\begin{array}{l}0 \\
0 \\
\end{array}$ & $\begin{array}{l}0 \\
0 \\
\end{array}$ & $\begin{array}{l}0 \\
0 \\
\end{array}$ & $\begin{array}{l}0 \\
0 \\
\end{array}$ & $\begin{array}{l}0 \\
0 \\
\end{array}$ & $\begin{array}{c}2 \\
0.5 \\
\end{array}$ \\
\hline Total & 217 & 48 & 83 & 32 & 18 & 6 & 404 \\
\hline
\end{tabular}

Kidney stone composition based on ethnic origin. $R=0.05$ for comparison of proportions. $U A=$ uric acid stones; $C O M=$ calcium oxalate monohydrate stones; $C O D=$ calcium oxalate dihydrate stones; $H A=$ hydroxyapatite stones; $S T=$ struvite stones; $B R=$ brushite stones; $\mathrm{NH}, \mathrm{U}=$ ammonium urate stones; $\mathrm{Cy}=$ cysteine stones

compared using chi-squared tests. Multinomial logistic regression was used to estimate relative likelihood of occurrence of each stone type (COM referent).

Results: 404 patients were identified, of whom $52 \%$ were male. The distribution of kidney stone composition according to ethnic origin is shown in Table 1. There was a statistically significant difference in the distribution of stone compositions according to race / ethnicity $(\mathrm{p}=0.05)$. Distribution of stone types significantly varied by gender, but only for Whites, Asians and Hispanics. On multivariable regression, HA stones were found to be significantly more likely to occur in younger, female, and White patients.

Conclusions: Kidney stone composition appears to vary according to race / ethnicity, gender and age, among other factors. Further studies elucidating the causal effects of these differences may contribute towards future understanding of the pathogenesis of the disease.

MP16-8 Impact of Day or Hour on Diagnosis, Admission and Treatment Outcomes of Acute Stone Episode in the Emergency Room Setting

C Tong, E Ghiraldi, A Lee, T Li, PC Dreher, J Brandt, J Friedlander

Albert Einstein Medical Center

United States

Introduction \& Objective: It has been found that day of the week can influence and delay a patient receiving urgent decompressive intervention in the setting of acute stone disease and sepsis, a phenomenon known as the weekend effect. Our study aims to compare patients on diagnosis and treatment outcomes who present with acute stone colic to our emergency department (ED) on the weekend to regular weekdays, as well as compare weeknights to regular working hours.

Materials and Methods: A cross-sectional, retrospective, IRBapproved chart review was performed on patients who presented with suspected kidney or ureteral stones to the ED over a 6month period. Patients were divided into cohorts based on when 
they presented to the emergency department: 8PM to 6AM versus $6 \mathrm{AM}$ to $8 \mathrm{PM}$, and Friday at $8 \mathrm{PM}$ to Monday at $6 \mathrm{AM}$ versus Monday at 6AM to Friday at 8PM. We recorded information about imaging used, location and size of stones, need for urologic consultation and treatment outcomes of the patients. The cohorts were then compared using univariate statistical models.

Results: 335 patients met inclusion criteria; 117 patients presented on the weekend, and 151 patients presented between the hours of 6PM and 8AM during the week. US use significantly increased overnight as other imaging modalities (MRI, CT with contrast $)$ decreased $(p=0.0183)$. There was no difference in use of imaging modality on weekends $(p=0.3047)$. The urology service was equally as likely to be called for consultation during the day as they were at nighttime, as well as during the weekday or over the weekend $(p=0.6659$ and $p=0.5073$, respectively). Disposition variables, including admission rates or discharge medications such as narcotics and medical expulsive therapy, also showed no statistical significance.

Conclusions: When examining for the weekend effect at our institution, we found no differences in patient outcomes for the diagnosis and treatment of acute stone episodes at our ED. Unexpectedly there was no difference in the frequency of consults to the urology service across time periods. This could be a result of the 24-hour availability of the urology consult service through our residency program. Further work is necessary to validate these results.

MP16-9 Incidence, Treatment and Risk of Adverse Birth Outcomes for Kidney Stones in Pregnancy- A PopulationBased Study

M Ordon, J Kroft, J Dirk, J Slater, E McArthur, S Dixon, B Welk

St. Michael's Hospital, University of Toronto Canada

Introduction \& Objective: The contemporary incidence of symptomatic nephrolithiasis in pregnancy is unknown and there is conflicting evidence as to the risks for perinatal complications associated with stones. Our objective was to perform a population-based study to determine the incidence of nephrolithiasis in pregnancy, the risk of adverse perinatal/neonatal outcomes and treatment trends for stones in pregnancy.

Materials and Methods: We performed a population-based matched cohort study using Ontario's healthcare administrative databases. All pregnancies in the province from 2004 to 2014 were identified. The study exposure was hospital admission for nephrolithiasis during pregnancy. Each pregnancy with nephrolithiasis was matched to up to 6 pregnancies without nephrolithiasis based on age, region of residence, income quintile, year of cohort entry, prior births and multiple births. The primary outcome was any adverse birth outcome (ABO) defined as preterm birth, low birth weight, and infant death. Secondary outcomes included premature rupture of membranes (PROM), pre-eclampsia and caesarian section $(\mathrm{C} / \mathrm{S})$, as well as the type and frequency of intervention for stones in pregnancy. Logistic regression models, with generalized estimating equations, were utilized to assess any differences in study outcomes across the exposure groups.

Results: Of 1.39 million pregnancies identified there were 2,863 pregnancies with nephrolithiasis $(0.2 \%)$, which were matched with 17,171 pregnancies without nephrolithiasis (Baseline characteristics in Table 1). A pregnancy with nephrolithiasis had a significantly increased risk for $\mathrm{ABO}$ compared with matched
Table 1. Baseline Characteristics for Pregnancies with Nephrolithiasis and Matched Pregnancies without Nephrolithiasis in the Province of Ontario between April 2004 and December 2014.

\begin{tabular}{|c|c|c|c|}
\hline & $\begin{array}{l}\text { Not Exposed } \\
N=17,171\end{array}$ & $\begin{array}{l}\text { Exposed } \\
N=2,863\end{array}$ & St Diff* \\
\hline \multicolumn{4}{|l|}{ Age at index } \\
\hline Mean (SD) & $28.84(2.16)$ & $28.81(5.4)$ & 0.01 \\
\hline Median (IQR) & $29(25-33)$ & $29(25-33)$ & \\
\hline \multicolumn{4}{|l|}{ Income quintile } \\
\hline Quintile 1 (Lowest income) & $24.9 \%$ & $24.9 \%$ & 0 \\
\hline Quintile 2 & $22.0 \%$ & $22.0 \%$ & 0 \\
\hline Quintile 3 & $20.1 \%$ & $20.1 \%$ & 0 \\
\hline Quintile 4 & $19.1 \%$ & $19.1 \%$ & 0 \\
\hline Quintile 5 (Highest Income) & $13.8 \%$ & $13.8 \%$ & 0 \\
\hline \multicolumn{4}{|l|}{ Year of index date } \\
\hline 2004-2006 & $25.1 \%$ & $25.1 \%$ & 0 \\
\hline 2007-2009 & $28.5 \%$ & $28.5 \%$ & 0 \\
\hline $2010-2012$ & $30.0 \%$ & $30.0 \%$ & 0 \\
\hline 2013-2014 & $16.4 \%$ & $16.3 \%$ & 0 \\
\hline \multicolumn{4}{|l|}{ Residential Status } \\
\hline Urban & $82.2 \%$ & $82.2 \%$ & 0 \\
\hline Rural & $17.8 \%$ & $17.8 \%$ & 0 \\
\hline \multicolumn{4}{|l|}{$\begin{array}{l}\text { Number of Prior } \\
\text { Pregnancies }\end{array}$} \\
\hline 0 & $47.1 \%$ & $47.1 \%$ & 0 \\
\hline 1 & $33.6 \%$ & $33.6 \%$ & 0 \\
\hline 2 & $13.0 \%$ & $13.0 \%$ & 0 \\
\hline $3+$ & $6.3 \%$ & $6.3 \%$ & 0 \\
\hline \multicolumn{4}{|l|}{ Multibirth (e.g. twins) } \\
\hline Yes & $1.5 \%$ & $1.5 \%$ & 0 \\
\hline \multicolumn{4}{|l|}{ ADG Score } \\
\hline Mean (SD) & $7.79(1.62)$ & $8.71(4.19)$ & 0.29 \\
\hline Median (IQR) & $8(5-11)$ & $9(6-12)$ & \\
\hline \multicolumn{4}{|l|}{ Comorbidities } \\
\hline History of Kidney Stones & $0.3 \%$ & $6.1 \%$ & 0.33 \\
\hline Hypertension & $2.3 \%$ & $3.5 \%$ & 0.07 \\
\hline Diabetes & $1.6 \%$ & $1.6 \%$ & 0 \\
\hline $\begin{array}{l}\text { Inflammatory Bowel } \\
\text { Disease }\end{array}$ & $0.8 \%$ & $1.5 \%$ & 0.07 \\
\hline Gout & $0.0 \%$ & $0.0 \%$ & 0 \\
\hline
\end{tabular}

*St Diff= standardized differences. This metric describes differences between group means relative to the pooled standard deviation; differences greater than $10 \%$ reflect the potential for meaningful imbalance.

pregnancies without nephrolithiasis $(13.5 \%$ vs. $8.8 \%$ OR 1.62 , $95 \%$ CI 1.43, 1.82, p <0.0001). Pregnancies with nephrolithiasis also had a greater risk fro pre-eclampsia $(\mathrm{OR} 1.42, \mathrm{p}=0.04)$ and C/S (OR 1.39, p<0.0001), but no PROM.

Conclusions: Our study demonstrated an increased risk of $\mathrm{ABO}$ in women with a hospital admission for nephrolithiasis during pregnancy.

MP16-10 Preoperative DJ stent placement vs. direct ureteroscopy: a retrospective comparative study

AF Navetta, TD Durdin, A El Mekresh, GL Machen, MM El Tayeb, L Tsai

Texas A\&M College of Medicine/Baylor Scott and White United States

Introduction \& Objective: Ureteral stenting prior to ureteroscopy (URS) has been credited with improved stone-free rates and reduced operative times; however, the AUA guidelines on the surgical management of urolithiasis advocate against routine prestenting. We chose to compare the perioperative outcomes of patients with and without a ureteral stent at the time of URS at our institution.

Materials and Methods: After IRB Approval, a retrospective review of patients undergoing semi-rigid and/or flexible URS between February 2014 and April 2016 was conducted. Patient demographics and perioperative outcomes were compared based on the presence or absence of a double-J ureteral stent prior to 


\begin{tabular}{|c|c|c|c|}
\hline & Prestent $(n=295)$ & Direct $(n=163)$ & P-value \\
\hline Age & 53.0 & 53.9 & 0.72 \\
\hline Mean ASA Score & 2.53 & 2.35 & 0.58 \\
\hline Indication for surgery & & & 0.16 \\
\hline $\begin{array}{l}\text { Symptomatic } \\
\text { Obstruction }\end{array}$ & 216 & 114 & \\
\hline Renal Stone & 63 & 26 & \\
\hline Other & 12 & 12 & \\
\hline Stone Location & & & 0.002 \\
\hline Distal & 58 & 57 & \\
\hline Mid & 17 & 6 & \\
\hline Proximal & 77 & 25 & \\
\hline Renal & 57 & 39 & \\
\hline Multiple & 37 & 15 & \\
\hline \multicolumn{4}{|l|}{ Mean Stone Size $(\mathrm{mm})$} \\
\hline Right & 8.05 & 6.34 & 0.65 \\
\hline Left & 6.63 & 6.83 & 0.71 \\
\hline $\begin{array}{l}\text { Preop Positive Urine } \\
\text { culture }\end{array}$ & 58 & 16 & 0.006 \\
\hline \multicolumn{4}{|l|}{ Table 2} \\
\hline & Prestent $(n=295)$ & Direct $(n=163)$ & P-value \\
\hline Operative Time (mins) & 71.3 & 72.9 & 0.57 \\
\hline Type of Scope & & & $<0.001$ \\
\hline Flex & 190 & 80 & \\
\hline Semi-rigid & 52 & 58 & \\
\hline Flex/Semi-rigid & 20 & 14 & \\
\hline
\end{tabular}

URS. Nurse calls, return to the emergency department and readmission within 90 days were also compared. Chi-square analysis was used for nominal data while Student's t-test was used for interval data.

Results: 458 patients underwent URS during the study period. 295 patients were prestented (psURS) while 163 went directly for ureteroscopy (dURS). There was no difference in age, mean ASA score, indication for surgery or mean stone size (Table 1). PsURS had more proximal ureteral stones while dURS had more distal stones. PsURS was also more likely to have UTI prior to surgery. Prestenting did not influence operative time and psURS patients were more likely to undergo flexible URS (Table 2). The psURS cohort utilized an access sheath more often $(<0.001)$ and had less ureteral dilation $(\mathrm{p}<0.001)$. There was failure to reach the stone in 3-patients undergoing dURS vs. 0 patients with psURS $(p=0.02)$; however, there was no difference in stone free rates $(p=0.37)$. There was no increased risk of ureteral injury in the dURS cohort $(\mathrm{p}=0.24)$. PsURS and dURS yielded no difference in calls to the nurse $(\mathrm{p}=0.20)$ and return to the ED within 90 days $(p=0.80)$. Readmission within 90 days was more likely after psURS (32 vs 7 readmissions, $\mathrm{p}=0.02$ ).

Conclusions: Presence of a ureteral stent at the time of URS offers no advantage vs. dURS, but is associated with an increased risk of readmission within 90 days.

MP16-11 Correlation of Stress in Kidney Stone Patients with the Wisconsin Stone Quality of Life Questionnaire

CJ Lundeen, BH Chew, T Chi, SY Nakada, KL Penniston

University of British Columbia, Faculty of Medicine, Department of Urologic Sciences

Canada
Introduction \& Objective: Stress may play a role in endocrinologic causes of kidney stones, be a consequence of stones, or both. We sought to determine if stress was correlated with healthrelated quality of life (HRQOL) in our kidney stone patients.

Materials and Methods: With IRB approval at participating sites, a subset of patients from the North American Stone Quality of Life Consortium were administered the validated Perceived Stress Scale (PSS-10) and the Wisconsin Stone Quality of Life questionnaire (WISQOL), a validated stone-specific, patient-reported outcome to assess HRQOL. WISQOL total and domain scores were compared with PSS-10 scores. WISQOL and PSS-10 scores were also compared by patients' stone and symptom statuses.

Results: Patients ( $\mathrm{n}=114 ; \mathrm{M}=56, \mathrm{~F}=58$; age $55 \pm 15 \mathrm{y}$ ) from 3 centers participated; $78 \%$ were white Caucasian, $6 \%$ Hispanic/ Latino, $11 \%$ Asian, and 4\% Black/African American. Most $(90 \%)$ were mixed calcium stone formers. Duration of stone disease varied (mean $10 \mathrm{y}$; median $4.5 \mathrm{y}$ ) as did patients' estimated number of lifetime stone events (mean 4.6; median 2.0). Among patients with active stone symptoms (27\% of sample), total WISQOL and PSS-10 scores were inversely correlated (Pearson correlation coefficient, $-0.235 ; \mathrm{P}=0.014$ ), demonstrating that lower HRQOL was associated with more stress. Domain-level analysis revealed that lower HRQOL scores in domains 1 (emotional impact) and 4 (vitality) were also associated with more stress $(\mathrm{P}=0.011$ and 0.0065 , respectively). While the WISQOL discriminated patients with stones at survey completion from patients without $(\mathrm{P}=0.019$ for difference in total scores), the PSS-10 did not ( $\mathrm{P}=0.73$ for difference in PSS- 10 scores). Similarly, the WISQOL distinguished symptomatic patients from asymptomatic ( $\mathrm{P}<0.0001$ for difference) while the PSS-10 could not $(P=0.46)$.

Conclusions: The inverse relationship between the PSS-10 and WISQOL showed overall that patients with higher levels of stress had lower HRQOL. Patients with stone-related symptoms scored lower on the WISQOL for emotional impact and vitality than asymptomatic patients and had significantly more stress. Despite the association of stress and lower HRQOL in patients with stones and symptoms, stress alone did not explain the HRQOL differences between these groups. This study showed that stone patients with a lower HRQOL had more stress. A future comparison of stress levels in stone patients compared to non-stone forming controls may help us determine if stress has a reactive or causative role in kidney stone disease.

\section{MP16-12 Renal Colic in Pregnancy-From Prenatal to} Postpartum Management

W Chen, S Sivalingam

Case Western Reserve University School of Medicine United States

Introduction \& Objective: Renal colic during pregnancy often presents a clinical dilemma as symptoms can mimic pain due to physiological hydronephrosis from the gravid uterus. The accurate diagnosis of renal colic is challenging as CT based imaging must be avoided in this cohort of patients. The purpose of our study was to evaluate diagnosis and management of renal colic in pregnancy in a tertiary care center.

Materials and Methods: A retrospective review of consecutive pregnant patients presenting with suspected renal colic within our institution from January 2013 to September 2015 was performed. Patients were identified based on ICD-9 codes for flank 
pain or any stone related diagnosis. Demographic data, imaging results, treatment plan and final management up to 1 year postpartum was recorded.

Results: Two hundred thirteen pregnant patients presented with flank pain. The following imaging was performed: renal ultrasound in $95 \%$ of patients, CT in one patient, and KUB x-ray in one patient. Overall, 154 patients $(72 \%)$ were diagnosed with urolithiasis, while 59 patients $(28 \%)$ were given other diagnoses; $54 \%$ had imaging findings indicative of a stone. Most were discharged with analgesics and observation; 12 patients underwent stent placement, 8 underwent nephrostomy tube placement, 2 underwent ureteroscopy, and 16 were prescribed tamsulosin for medical expulsive therapy. Within a year of delivery, 61 patients returned for follow-up with $85 \%$ receiving CT. Renal ultrasound and KUB x-ray were used less frequently for the initial followup. Twenty patients received more than one imaging technique (e.g. an ultrasound and a CT). Forty seven patients were found to have stones of which 20 underwent further treatment. Three patients underwent ESWL, 10 underwent ureteroscopy, and 7 were prescribed tamsulosin.

Conclusions: Renal ultrasound was the imaging modality of choice in pregnant patients presenting with flank pain and most were managed conservatively. It is notable that the majority of patients $(60 \%)$ did not follow up after delivery, while $77 \%$ of those who did return for follow-up were found to have stones, indicating that closer follow-up is warranted in these patients.

MP16-13 Does diabetes mellitus influence the surgical management of urinary tract stones among patients in the United Kingdom?

M Elhadi, J D'Costa, C De Vogel, R Devarajan, U Otite

Sandwell and West Birmingham Hospitals Nhs Trust United Kingdom

Introduction \& Objective: Urolithiasis is one of the most common urological problems in the United Kingdom; with an annual incidence of 1-2 cases per 1000 people in England. 6\% of the adult population in 2013 were diagnosed with diabetes in England. Several studies suggest there is an association of diabetes with stone formation, recurrence, and morbidity. Our study aimed to compare the prevalence of risk factors like metabolic syndrome, urinary tract infections, age, gender and ethnicity among diabetics versus non-diabetics and to assess how diabetes affects the biochemical and surgical outcomes of urolithiasis.

Materials and Methods: Patients who treated surgically for urolithiasis with either ureteroscopy or percutaneous nephrolithotomy over three years were retrospectively analysed. Information was cross-referenced with electronic notes to produce biochemical and surgical data.

Results: Of 182 patients treated, 31 (17\%) type 2 diabetes. The mean age of diabetics was significantly higher than non-diabetics by nearly 12 years ( $\mathrm{p}$-value <0.001). Hypertension, hyperlipidaemia, obesity and UTIs were more prevalent among diabetics (p-value $<0.001,<0.001,0.01,0.009$ respectively). Diabetics were at higher risk of stone recurrence at 1 year $(p$-value $=0.04)$ compared to non-diabetics. Stone recurrence was not significantly different at 3 and 5 years between the two groups. Diabetics had higher urinary oxalate, and nearly statistically significant lower phosphate levels ( $p$-value $=0.007,0.076$ respectively).

Conclusions: Diabetics with urolithiasis were significantly older and associated with metabolic syndrome. UTIs were more prevalent among diabetics which put them at risk of postoperative complications. Diabetics were at higher risk of stone recurrence at 1 year compare to non-diabetics. The biochemical urinary findings can guide the management of recurrent stone formers with diabetes.

MP16-14 AiRSTRiP: An Acute Rapid Stone Treatment Pathway to Reduce Treatment Time

M Sirohi, A Wang, SE Wason, P Muraki,

CF McCammon, J Myers, P Venkataram, JW Lambert, JB Malcolm, MD Fabrizio

Eastern Virginia Medical School

United States

Introduction \& Objective: Patients who present to the emergency room (ER) with acute renal colic can have lengthy wait times to be seen by a urologist and further wait times for definitive therapy. A prior study by our group demonstrated that mean time to definitive stone treatment was up to 47 days. This delay to treatment time reinforced the need to develop measures to decrease costs through improved efficiency. We have developed a quality initiative pathway, entitled "AiRSTRiP," intended to address these issues of delayed time to treatment. The primary objective is to allow for stone passage or surgical treatment within 21 days from initial emergency room (ER) presentation after implementation of the pathway.

Materials and Methods: We reviewed our data from our prior published study and compared it to retrospective and prospective data mined from our database of patients who were operated on from July 2016 to February 2017. Several factors, including patient demographics, disease characteristics, follow up and treatment times, and stone characteristics were examined. Midway through the commencement of the study, we instituted the AirSTRiP pathway in order to assess its efficacy in reducing ER to office and treatment times.

Results: Of a total of 2072 procedures performed, 523 were examined and 103 patients qualified for our study. 64 patients were in our pre-pathway cohort and 39 in our post-pathway cohort. Most patients $(36 \%)$ underwent treatment at the ambulatory surgery center (ASC). There was no significant difference in the mean time of ER to office follow up between the pre- and post-pathway cohorts (pre-pathway 4.3 days vs. post-pathway 4.6 days; $p=0.8$ ) or between our prior data and the pre-pathway ER to treatment times $(p=0.051)$. There was a difference in the ER to treatment times between pre- and post-pathway patients (22.1 vs. 12.7 days; $p$

Conclusions: We have shown, for the first time that access to an ASC and implementation of a pathway can help to diminish time to treatment for stone disease while maintaining quick ER follow up times. This study highlights the importance of a streamlined and systematic approach to healthcare, as providers are increasingly scrutinized to provide patients with more efficient methods of treatment and will soon be judged by patient satisfaction, while still instituting appropriate medical care.

\section{MP16-15 Characterization of Upper Tract Urolithiasis in Patients Following Urinary diversion}

M Shahait, O Ayyash, J Denk, H Taan, S Jackman, MC Ost, T Averch, MJ Semins

UPMC

United States 
Introduction \& Objective: Urinary diversion has been implicated in causing several metabolic derangements that may hasten stone formation. We sought to characterize the stone type and urinary lithogenic risk factors.

Materials and Methods: We queried our institutional stone database (1/2010-12/2016) for urinary diversion patients who had stone related surgery. We included in our final analysis patients who were diagnosed with upper tract stones and had a stone analysis or 24 urine collection. We excluded patients with a stone in the diversion, those with augmentation cystoplasty. There was one patient with struvite stone that was excluded. The stone type was defined by the predominant stone component, represented by at least $60 \%$ of all components.

Results: Twenty-one patients were included in the final analysis (5 female, 16 male). Median age was 65.3 (IQR 59-75.9). Median Body mass index was 28.8 (IQR 22.9-32.8). Sixteen patients had an incontinent diversion, and five patients had a continent urine diversion. All patients had at least one abnormality on 24-hr urine collection study. The percentage of patients in this cohort with high urine $\mathrm{PH}$, hypocitraturia, and Hyperoxaluria was $87 \%$, $75 \%$, and $37.5 \%$, respectively. Calcium Phosphate Calcium oxalate and mixed stone was observed in $41 \% 35 \%$, and $24 \%$ of the analyzed stone.

Conclusions: Patients with urinary diversion harbor significant metabolic derangement, thus they are prone to develop recurrent metabolic stone. The astute urologist should exclude metabolic disorder in the clinical management of patients with urinary diversion.

MP16-16 Factors Predictive of Surgical Intervention in Patients Who Present to the Emergency Department with Renal Colic

P Samson, W Wu, S Derisavifard, S Gurram, S Ahn, K Ren, J Chu, AD Smith, C Hartman

Smith Institute for Urology

United States

Introduction \& Objective: Urolithiasis is a commonly encountered problem in the Emergency Department (ED). Most

\begin{tabular}{|c|c|c|c|}
\hline & $\begin{array}{c}\text { Conservative } \\
(n=328)\end{array}$ & Surgical ( $n=56)$ & p-value \\
\hline \multicolumn{4}{|l|}{ Univariate Analysis } \\
\hline Mean Age [years \pm SD] & $50.2 \pm 14.3$ & $54.9 \pm 14.5$ & 0.0225 \\
\hline Gender [\% female] & 45.4 & 51.8 & 0.3778 \\
\hline Diabetes [\%] & 15.9 & 30.4 & 0.009 \\
\hline Reported fever [\%] & 6.71 & 30.4 & $<0.0001$ \\
\hline Vomiting [\%] & 34.4 & 44.6 & 0.1418 \\
\hline Dysuria [\%] & 17.4 & 17.9 & 0.9304 \\
\hline Fever in the ED* $[\%]$ & 1.83 & 28.6 & $<0.0001$ \\
\hline Leukocytosis** [\%] & 31.1 & 51.8 & 0.0025 \\
\hline Serum creatinine $[\mathrm{mg} / \mathrm{dl}=\mathrm{SD}]$ & $1.15 \pm 0.86$ & $1.54 \pm 0.82$ & $<0.0001$ \\
\hline Positive nitrites [\%] & 1.52 & 17.9 & $<0.0001$ \\
\hline Stone size $[\mathrm{mm}]$ & $4.08 \pm 3.38$ & $7.00 \pm 4.62$ & $<0.0001$ \\
\hline Total time in ED [hours $\pm S D$ ] & $8.47 \pm 5.76$ & $10.9 \pm 6.78$ & 0.0004 \\
\hline Total opioid amount $[\mathrm{mg} \pm \mathrm{SD}]$ & $4.92 \pm 5.51$ & $7.16 \pm 6.29$ & 0.0047 \\
\hline Observation unit [\%] & 8.84 & 8.93 & 1.0 \\
\hline Multivariate model & OR & $95 \% \mathrm{Cl}$ & p-value \\
\hline Reported fever & 4.089 & $1.720-9.722$ & 0.0014 \\
\hline Stone size & 1.165 & $1.061-1.280$ & 0.0014 \\
\hline Leukocytosis & 1.978 & $1.022-3.831$ & 0.043 \\
\hline Total opioid amount & 1.072 & $1.013-1.134$ & 0.0165 \\
\hline \multicolumn{3}{|l|}{ Initial Pain Med Choice } & \multirow{5}{*}{0.0250} \\
\hline None & 1 & Reference & \\
\hline NSAID & 1.520 & $0.172-13.400$ & \\
\hline Opioid & 2.459 & $0.297-20.375$ & \\
\hline & & & \\
\hline
\end{tabular}

patients, however, are discharged from the ED and managed expectantly. Previous studies have examined which factors may contribute to eventual surgical intervention for those who fail conservative therapy; however, no studies have examined what factors warrant early intervention. Our goal was to determine the factors predictive of urgent surgical management in patients presenting to the ED with renal colic.

Materials and Methods: We performed retrospective analysis of patients presenting to the ED between 2014-2015 with renal colic, a ureteral calculus, or a renal calculus as determined by ICD-9 coding. Patients who had radiographic evidence of urolithiasis by computed tomography, and had available serologic testing and urinalysis were included. Demographics, medical history, physical examination, laboratory data, imaging results, and hospital course were examined. Univariate (UVA) analysis using two-sample t-test, Mann-Whitney, chi-square test, or Fisher's exact test, and multivariate (MLA) logistic regression analysis were performed to determine clinical factors associated with operative intervention during the same hospitalization.

Results: 384 patients met inclusion criteria, of which 56 (14.6\%) underwent surgical intervention. Ureteral stenting (US) was the most common procedure $(75 \%)$ followed by ureteroscopy (19.4\%), and percutaneous nephrostomy (PCN) tube placement $(5.36 \%)$. Reported indications for temporary drainage with US or PCN included sepsis, intractable pain, acute kidney injury (AKI), or a combination thereof. Patients underwent ureteroscopy for intractable pain or AKI. Factors associated with operative intervention are presented in Table 1. Stone size, leukocytosis, reported fever, initial pain medication choice, and total opioid dosage given were independent predictors of intervention on MLA. Fever in the ED and presence of nitrites on urinalysis were excluded from MLA because of low frequency of events.

Conclusions: Stone size, leukocytosis, reported fever, and total opioid dosage were associated with need for surgical intervention in patients presenting to the ED with flank pain secondary to an obstructing ureteral stone. Prompt recognition by the ED staff of these factors may allow for earlier surgical intervention.

MP16-17 Managing Renal Colic in the Emergency Room Observation Unit: Predictors of Hospital Admission

P Samson, W Wu, S Gurram, S Derisavifard, D Friedman, K Ren, AD Smith, C Hartman

Smith Institute for Urology

United States

Introduction \& Objective: Emergency department (ED) observation units (OU) help reduce length of stay and unnecessary hospital admissions; however, monitoring patients with renal colic in OUs has not been extensively studied. As such, placement and management of a patient with renal colic in an $\mathrm{OU}$ is based on clinical judgment. We aim to assess if treating renal colic patients in our OU is efficacious and to determine the clinical and radiologic parameters that may predict hospital admission.

Materials and Methods: Patients monitored in the ED OU at our institution between 2014 and 2015 with ICD-9 diagnosis codes for renal colic, ureterolithiasis, or nephrolithiasis were retrospectively reviewed. Inclusion criteria encompassed radiographic evidence of urinary calculi on computed tomography (CT), serum studies, and urinalysis (UA). ED parameters before OU placement involving history, physical exam, laboratory data, 
Table 1: Clinical predictors of admission from ou for renal colic

\begin{tabular}{|c|c|c|c|}
\hline Clinical or radiologic parameter & Discharged $(n=81)$ & Admitted ( $n=14)$ & p-value \\
\hline \multicolumn{4}{|l|}{ Univariate Model } \\
\hline Repeat ER visit [\%] & 9.9 & 2.1 & 0.204 \\
\hline Mean Age [years \pm SD] & $55.6 \pm 9.4$ & $49.3 \pm 13.2$ & 0.161 \\
\hline Gender [\% female] & 43.2 & 28.6 & 0.234 \\
\hline Diabetes [\%] & 19.8 & 14.3 & 0.478 \\
\hline Subjective fever [\%] & 4.9 & 0 & 0.523 \\
\hline Objective fever* $[\%]$ & 1.2 & 14.3 & 0.056 \\
\hline Vomiting [\%] & 44.4 & 42.9 & 0.575 \\
\hline Dysuria $[\%]$ & 17.3 & 14.3 & 0.568 \\
\hline White blood cell $(W B C)>12,000[\%]$ & 39.5 & 50.0 & 0.329 \\
\hline Serum creatinine $>1.5[\mathrm{mg} / \mathrm{dL} \pm \mathrm{SD}]$ & 21.0 & 50.0 & 0.029 \\
\hline Stone size $>5 \mathrm{~mm}[\%]$ & 11.1 & 21.4 & 0.247 \\
\hline Moderate hydronephrosis [\%] & 12.3 & 28.6 & 0.167 \\
\hline Perinephric stranding [\%] & 64.2 & 64.3 & 0.623 \\
\hline Proximal stone location [\%] & 30.9 & 21.4 & 0.347 \\
\hline Time to first pain medication $[\min \pm S D]$ & $155.0 \pm 209.9$ & $111.5 \pm 53.7$ & 0.518 \\
\hline Total ketoralac dosage $[\mathrm{mg} \pm \mathrm{SD}]$ & $26.7 \pm 25.1$ & $24.6 \pm 30.9$ & 0.497 \\
\hline Total opioid dosage $[\mathrm{mg} \pm \mathrm{SD}]$ & $10.6 \pm 7.8$ & $16.8 \pm 12.1$ & 0.052 \\
\hline Total time in ED [hours $\pm S D$ ] & $23.5 \pm 7.5$ & $25.2 \pm 7.6$ & 0.345 \\
\hline Total time in ou [hours $\pm \mathrm{SD}$ ] & $15.4 \pm 6.8$ & $16.2 \pm 7.2$ & 0.834 \\
\hline
\end{tabular}

radiographic findings, and clinical course were assessed. Fisher's exact and Mann-Whitney $\mathrm{U}$ tests were used to determine statistical significance $(\mathrm{p}<0.05)$ for categorical and contunuous variables, respectively.

Results: 95 patients met inclusion criteria, of which 14 (14.8\%) were admitted for 1 or more of the following: pain control (57.2\%), acute kidney injury (AKI) $(21.4 \%)$, oral intolerance $(14.3 \%)$, and fevers $(14 \%)$. A serum creatinine $>1.5 \mathrm{mg} / \mathrm{dl}$ predicted inpatient admission from the OU $(p=0.029)$. Documented fever $>38$ degrees $\mathrm{C}$ and amount of total opiates given trended towards but did not reach statistical significance $(p=0.056 \& 0.052$, respectively). There were no differences in patients discharged versus those admitted from the OU regarding any other clinical or radiologic parameter assessed (Table 1).

Conclusions: The majority of patients monitored in an OU for renal colic are effectively managed and ultimately discharged home. A serum creatinine of $>1.5 \mathrm{mg} / \mathrm{dL}$ was the only factor associated with hospital admission after being managed in the OU. Additionally, an objective fever and the total amount of opiates given may also be associated with admission in a larger sample.

MP16-18 Preferences in Surgical Management in the Setting of Bilateral Stone Disease

ME Rivera, K Heinsimer, J Lingeman, AE Krambeck

Department of Urology, Mayo Clinic (Rochester, MN) United States

Introduction \& Objective: Bilateral stone disease presents a potential challenging situation to the urologist. Conflicting data exists regarding bilateral simultaneous versus staged procedures including ureteroscopy and percutaneous nephrolithotomy (PCNL). A survey of endourologists was performed to assess preferences of management of bilateral stone disease.

Materials and Methods: An online survey was sent to endourologists via the Endourological Society email listserv. Data collected included preference of bilateral percutaneous nephrolothomy (PCNL), bilateral ureteroscopy (URS), and rationale for performing procedures.

Results: A total of 153 urologists completed the survey. Of these, $58(38 \%)$ perform bilateral PCNL under the same an- esthesia, 74 (48\%) perform bilateral URS but not PCNL, and $21(14 \%)$ do not perform bilateral stone procedures under the same anesthetic. Those who do not perform bilateral PCNL, were willing to offer their patients staged PCNLs (88\%), unilateral PCNL and contralateral URS (10\%) and/or unilateral PCNL with observation of the contralateral side (5\%). Reasons for not performing bilateral PCNLs included: rarely perform bilateral renal stone surgery $(35 \%)$, duration of bilateral procedures $(53 \%)$, bilateral renal injury $(48 \%)$, bleeding risk $(34 \%)$, patient discomfort $(23 \%)$, reimbursement $(13 \%)$, burden of bilateral procedure to operating room staff (11\%), and hospital policy $(6 \%)$. Urologists who were willing to do unilateral PCNL and contralateral URS $(n=9)$ felt their method of treatment was safer (56\%), had less risk of acute kidney injury $(78 \%)$, and less blood loss (11\%). Regarding bilateral ureteroscopy, $131(85 \%)$ were willing to perform bilateral URS under the same anesthesia, while $14 \%$ would do staged URS, and $1 \%$ would do unilateral URS and observe the contralateral side. Overall, $97 \%$ of urologist are willing to do bilateral URS compared to only $78 \%$ of urologist who do not do bilateral PCNL. A scenario of a patient with $2.5 \mathrm{~cm}$ stones, amenable to PCNL was given and those who perform bilateral PCNL, chose bilateral PCNL most often (74\%), staged PCNL (12\%), staged URS (5\%), bilateral URS (5\%), and unilateral PCNL with contralateral URS $(4 \%)$. Urologists who do not perform bilateral PCNLs elected to perform staged PCNL (83\%), URS (12\%), unilateral PCNL and contralateral URS (3\%), and bilateral URS (2\%).

Conclusions: A majority of those surveyed do not perform bilateral PCNL but would perform bilateral URS with duration of the procedure and concern for bilateral renal injury representing the most common reasons. Further investigation into whether bilateral procedures do truly increase the risk of complications, including bleeding and bilateral renal injury is warranted.

MP16-19 The Importance of Urologist Estimation of Stone Burden: Results from the Registry for Stones of the Kidney and Ureter (ReSKU)

DT Tzou, D Isaacson, M Usawachintachit, ZJ Wang, K Taguchi, RS Hsi, BA Sherer, ML Stoller, T Chi

UCSF

United States

Introduction \& Objective: A discrepancy often exists between urologists' and radiologists' estimation of a patient's true stone burden. Current guideline treatment recommendations are based

Table 1 Discrepancies in urologic interpretation vs radiologic report of aggregate stone size based on AUA Guidelines size thresholds

\begin{tabular}{|c|c|c|c|}
\hline $\begin{array}{l}\text { Stone-containing renal } \\
\text { units ( } \mathrm{n}=303)\end{array}$ & $\begin{array}{c}\text { Urologic } \\
\text { Interpretation } \\
\leq 10 \mathrm{~mm}\end{array}$ & $\begin{array}{c}\text { Urologic } \\
\text { Interpretation } \\
>10 \mathrm{~mm},<20 \mathrm{~mm}\end{array}$ & $\begin{array}{c}\text { Urologic } \\
\text { Interpretation } \\
\geq 20 \mathrm{~mm}\end{array}$ \\
\hline $\begin{array}{c}\text { Radiologic Report } \\
\leq 10 \mathrm{~mm}\end{array}$ & $\begin{array}{c}137 \\
(45 \%)\end{array}$ & 24 & 11 \\
\hline Radiologic Report & 5 & $(8 \%)$ & $(4 \%)$ \\
$>10 \mathrm{~mm},<20 \mathrm{~mm}$ & $(2 \%)$ & 25 & 13 \\
\hline Radiologic Report & 0 & $(8 \%)$ & $(4 \%)$ \\
$\geq 20 \mathrm{~mm}$ & $(0 \%)$ & $(<1 \%)$ & 39 \\
\hline Radiologic Report & 21 & 5 & $(13 \%)$ \\
unclear & $(7 \%)$ & $(2 \%)$ & $(7 \%)$ \\
\hline
\end{tabular}

Shaded boxes $=$ size discrepancy or unclear report, total $=102(34 \%)$

White boxes $=$ size concordance, total $=201(66 \%)$ 
on stone size and therefore accurate stone measurements are crucial in directing patients to the appropriate treatment. With an increasing trend of basing quality of care on the amount of stone treated versus left behind, an accurate stone burden estimation represents an essential starting point for any determination of operative success. This study aimed to compare the number, size and location of stone burden recorded by urologists and radiologists, and determine what discrepancies exist and how these differences could impact potential stone management.

Materials and Methods: From October 2015 to August 2016, 371 new stone patients were prospectively enrolled into the Registry for Stones of the Kidney and Ureter (ReSKUTM). The treating urologist personally reviewed all computed tomography (CT) imaging, recording key characteristics such as stone number, location, and total stone burden. Stone burden was based on the largest aggregate linear dimension from axial and coronal views. A blinded, retrospective review of the corresponding radiologic reports for these patients was then performed, comparing how often these key attributes were mentioned. A report was categorized as unclear for each characteristic when it failed to specifically mention the number, location or total stone burden.

Results: A total of 219 patients had both CT images and an official report available for review. With regards to stone number, 107/219 (24\%) of reports were considered unclear. For radiology and urology respectively, mean reported single stone size was $8.7 \pm 9.2 \mathrm{~mm}$ vs $10.7 \pm 12.3 \mathrm{~mm}$, while mean reported multiple stone burden was $12.8 \pm 9.9 \mathrm{~mm}$ vs $21.8 \pm 19.2 \mathrm{~mm}$ (both $\mathrm{p}<0.001$ ). Of the 303 right and left renal units that contained stones, $102(34 \%)$ had a description in the radiology report that was either unclear or based on size discrepancy could have resulted in a potential change in surgical management (Table 1).

Conclusions: A statistically significant difference was found in the estimation of stone size between urologists' and radiologists' interpretation of CT scans. To optimize appropriate surgical selection and allow for quality outcomes measurements, it is highly recommended that urologists perform their own imaging interpretation.

\section{MP16-20 Effects of Hounsfield unit value on percutaneous nephrolithotomy outcomes.}

H Moon, W Bae, U Ha, S Hong, J Lee, S Kim, H Cho

Department of urology, Seoul St. Mary's hospital Republic of Korea

Introduction \& Objective: To evaluate the effects of hounsfield unit value on percutaneous nephrolithotomy outcomes and operative parameters.

\begin{tabular}{|c|c|c|c|c|}
\hline \multicolumn{5}{|c|}{ 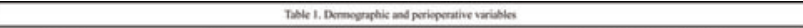 } \\
\hline Vwriatles & Total & 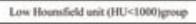 & 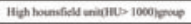 & 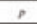 \\
\hline 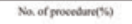 & 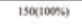 & 92(61.36) & $\operatorname{secos} \times 5)$ & $\cdot$ \\
\hline 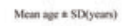 & $54,30=1,23$ & $5432.1261[30.89]$ & 54.23:142Y|1:80] & oss9 \\
\hline \multicolumn{5}{|l|}{$\operatorname{sen}(0,5)$} \\
\hline Male & mosos) & 62 & s & $0.3 \times 5$ \\
\hline \multicolumn{5}{|l|}{$\operatorname{side}(\cos 5)$} \\
\hline Leth & mansong & $4(62 \times 5)$ & 2607.150 & 0.095 \\
\hline Stume dantimm) & 2000.0 .9 & 158.0 .44 & 20100.45 & 0.563 \\
\hline 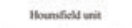 & 887.54 .2138 .39 & Gastazisss [02:-men] & 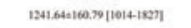 & 0.0000 \\
\hline Mean exprative ingreming & 5658252506 & $3583+22001[32-180]$ & $5781+27 \times 120.167]$ & o.ow \\
\hline 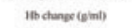 & $21 \mathrm{sel}, 0$ & 21601.53 & 2211.3 & 0.006 \\
\hline Mat change 60 & 6364.42 & 629457 & Gate3.61 & 0.785 \\
\hline 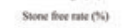 & 152006: & semeng & sases.20 & 0.79 \\
\hline Cenglication rose (s) & 241050 & $14(1,250)$ & 1017250 & a.911 \\
\hline Radio eposity & 13409350 & тая.:.5\%) & 540655 & 0.002 \\
\hline
\end{tabular}

Materials and Methods: The patients evaluated in our institute between April 2014 to December 2016 that had single tract percutaneous nephrolithotomy with single stone(range $1.5 \mathrm{~cm}-3.5 \mathrm{~cm})$ were retrospectively reviewed. The patients were divided into two groups according to their hounsfield unit in a low hounfield group ( $\mathrm{HU}<1000)$ or a high hounsfield unit group (HU >1000), and we evaluated stone free rate, complication rate, operation time, hemoglobin, hematocrit change, and radio opacity.

Results: The study included 96 males (64\%), 54 females (36\%) with a mean \pm SD age of $54.30 \pm 13.23$ years (range $23-78$ ). The overall stone free rate was $88.0 \%$, low HU group $(\mathrm{N}=92)$ was $89.1 \%(\mathrm{n}=82)$, high HU group $(\mathrm{N}=58)$ was $86.2 \%(\mathrm{n}=50)$. The total complication rate was $16 \%(\mathrm{~N}=24)$, low $\mathrm{HU}$ group $(\mathrm{n}=14)$ was $15.2 \%$, high HU group $(n=10)$ was $17.2 \%$. The stone free rate and complication rate did not differ significantly between two groups ( $\mathrm{p}>0.05)$. The operation time was $55.34 \pm 23.7$ minutes, $54.28 \pm 14.2$ minutes, respectively $(\mathrm{p}=0.296)$. Hb \& Hct change was $2.17 \pm 1.52,2.23 \pm 1.25$. Also did not differ significantly between two groups ( $p>0.05)$. On KUB X-ray, $18.4 \%(n=17)$ of low HU group were radiolucent and $98.2 \%(n=57)$ of high $\mathrm{HU}$ group were radio-opaque.

Conclusions: Measuring the Hounsfield unit value seems to have little utility in predicting the surgical outcomes. However, the hounsfield unit value influences the radio opacity in fluoroscopy, so further studies are needed.

\section{MP16-21 Flexible ureteroscopy in anticoagulated patients}

P Geavlete, D Georgescu, C Ene, V Iordache, R Multescu, B Geavlete

Department of Urology,’Saint John” Emergency Clinical Hospital, Bucharest, România;

Romania

Introduction \& Objective: We analyzed the complications of flexible ureteroscopy in patients with long term anticoagulant treatment.

Materials and Methods: Between February 2016 and February 2017 in our clinic were performed 47 flexible ureteroscopy at patients with anticoagulant treatment. The anticoagulant medication included warfarin (Sintrom), enoxaparin (Clexane) and non-vitamin $\mathrm{K}$ antagonists for example rivaroxaban (Xarelto), dabigatran (Pradaxa), apibaxan (Eliquis). The excluded patients used an anticoagulant and an antiplatelet treatment.

Results: From the 47 patients included, $29(61,70 \%)$ had the AC treatment stopped and $18(38,29 \%)$ the AC have been replaced by enoxaparin. The average age of the patients is 71,3 years, and the majority of the patients $(66.6 \%)$ have been subjected to the procedure with stone free rate of $73 \%$. In patients who required a second procedure was preferred keeping enoxaparin bridges and stone free rate was $87,5 \%$. Patients who stopped preoperative anticoagulant had the lowest rate of perioperative bleeding compared to those with Enoxaparin bridge (Clexane) 3,44\% respectively $11,11 \%$.

Conclusions: In conclusions flexible ureteroscopy is a safe treatment in selected cases and with adequate preoperative preparation. $\mathrm{AC}$ shunting may increase the risk of preoperative bleeding.

MP16-22 Withdrawn 
MP16-23 Worldwide Trends of Urinary Stone Disease Treatment over the last two Decades: A Systematic Review

R Geraghty, P Jones, A Pietropaolo, BK Somani

University Hospital Southampton NHS Foundation Trust United Kingdom

Introduction \& Objective: Numerous studies have reported on regional or national trends of stone disease intervention. However, no paper has yet examined the global trend of stone disease treatment. We aimed to complete this examination.
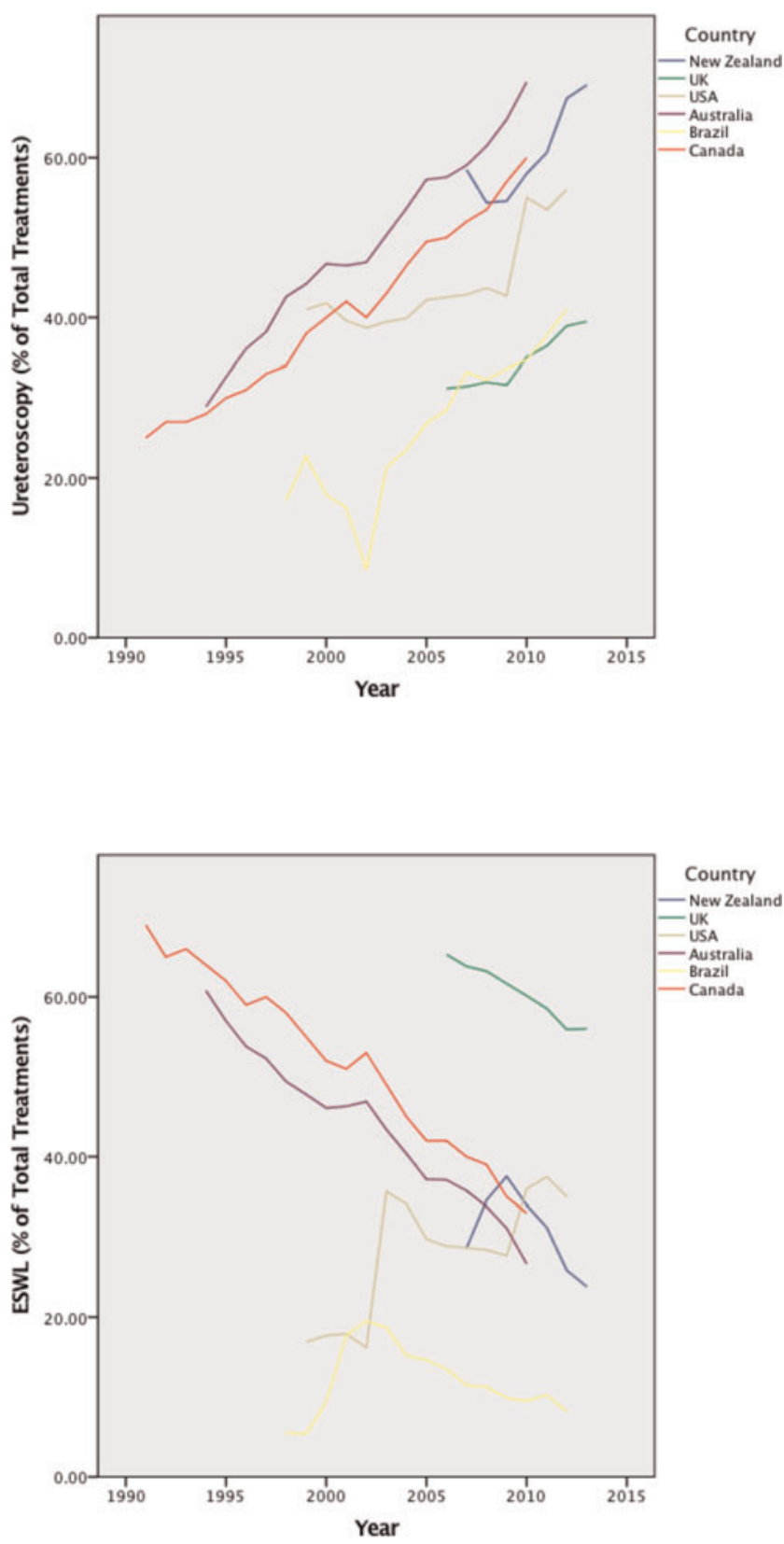

Materials and Methods: A systematic review of papers from 1996 to August 2016 for all English language articles reporting on trends of stone disease intervention was performed by 2 reviewers independently. Authors were contacted in the case of data not being exact. If the author did not reply, data was estimated from graphs. Data was examined using SPSS version 24. Trends were analysed using linear regression.

Results: The systematic review yielded 120 papers, of which 8 were included in the review. 5 papers had numerical data available and a further 3 had only percentage of total treatment. Overall there were 6 countries with available data: UK, USA, New Zealand, Australia, Canada and Brazil. There was significant linear regression between share of total treatments and year for ureteroscopy $\left(b=0.45, R^{2}=0.20, p<0.001\right)$. The remaining modalities had non-significant trends.

Conclusions: The share of total treatment for urolithiasis across the published literature has increased for ureteroscopy and shockwave lithotripsy whereas percutaneous nephrolithotomy and open surgery have remained static from 1994 to 2014.

MP16-24 Clinical outcomes of endoscopic management for $2 \mathrm{~cm}$ or more of large renal calculi

K Tanabe, M Kobayashi, Y Kojima, H Kameoka

hoshi general hospital

Japan

Introduction \& Objective: Objectives: Treatment of renal calculus $\geqq 2 \mathrm{~cm}$ is challenging. According to previous guidelines, patients with large renal calculi $\geqq 2 \mathrm{~cm}$, including staghorn calculi, can undergo percutaneous nephrolithotripsy (PNL) first. Even though it has been reported that endoscopic combined intrarenal surgery (ECIRS) is safe and effective for these patients, there is still a lack of adequate evidence about the feasibility of ECIRS. So we introduced ECIRS, and investigated the clinical outcomes of endoscopic management for the total stone size $\geqq 2 \mathrm{~cm}$ in our department.

Materials and Methods: Patients and Methods: We evaluated a total of 75 patients who underwent ECIRS in 38 cases, TUL in 31 cases, PNL in 5 cases, and 1 case of laparoscopic pyelolithotomy for the total renal stone size $\geqq 2 \mathrm{~cm}$ between March 2012 and November 2016, retrospectively. Basically ECIRS was selected for the maximum diameter $\geqq 30 \mathrm{~mm}$, TUL was selected for the maximum diameter $<20 \mathrm{~mm}$, others were case by case.

Results: We treated 13 cases with complete staghorn, 12 cases with partial staghorn, 33 cases with single, and 17 cases with multiple calculus. The success rate was $61.5 \%, 100 \%, 97 \%$, and $94.1 \%$, respectively. Complications included one blood transfusion, five fevers over $38.5^{\circ} \mathrm{C}$, one renal pelvic injury, two ureteral injuries, but no severe complications occurred. The mean surgical time was 149 minutes. The mean hospital stay was 9.5 days.

Conclusions: The outcomes of our study have demonstrated that ECIRS is a safe and effective treatment modality, especially in the treatment for large renal calculi. 


\section{MPS17: PROSTATE CANCER II}

MP17-1 Pathologic outcomes in patients affected by very low risk and low risk prostate cancer and eligible for active surveillance

R Sanseverino, G Napodano, O Intilla, U Di Mauro, G Molisso, T Realfonso

Dept. of Urology Umberto I Hospital Italy

Introduction \& Objective: Active surveillance (AS) has emerged as a valid option for the conservative management of low risk prostate cancer (PCa). The D'Amico classification is commonly used criterion for identification of low risk patients. However upgrading and upstaging at radical prostatectomy occurred in $20-54 \%$ and $6-26 \%$ of patients, respectively. Therefore

Table1. Baseline characteristics

\begin{tabular}{|l|c|c|c|c|}
\hline & & LR & VLR & P value \\
\hline Age (average) & $\mathrm{y}$ & $65.8 \pm 5.4$ & $66.3 \pm 5.6$ & 0.54 \\
\hline BMI (average) & & $26.6 \pm 2.8$ & $26.8 \pm 2.8$ & 0.75 \\
\hline PSA (ng/ml) & $\mathrm{ng} / \mathrm{ml}$ & $6.4 \pm 1.9$ & $6.2 \pm 2.1$ & 0.48 \\
\hline PSAD & $\mathrm{ng} / \mathrm{ml} / \mathrm{cc}$ & $0.12 \pm 0.05$ & $0.12 \pm 0.06$ & 0.52 \\
\hline Clinical stage & T1c & 88.4 & 93.1 & 0.54 \\
& T2a & 11.6 & 6.9 & \\
\hline Prostate volume & $\mathrm{cc}$ & $57.4 \pm 21.0$ & $58.8 \pm 24.0$ & 0.70 \\
\hline Biopsy positive cores & $\%$ & $27.4 \pm 18.5$ & $15.5 \pm 6.5$ & $<0.001$ \\
& & & &
\end{tabular}

Table 2. Pathological outcomes in LR and VLR patients

\begin{tabular}{|c|c|c|c|c|}
\hline & & LR & VLR & p value \\
\hline Number of removed nodes & $n$ & $12.2 \pm 6.3$ & $11.1 \pm 5.1$ & 0.35 \\
\hline Surgical Margin & $\%$ & 4.9 & 5.3 & 0.93 \\
\hline LAD & $\begin{array}{l}\mathrm{Nx} \\
\text { Obturator } \\
\text { lliac-obturator }\end{array}$ & $\begin{array}{l}21.4 \\
62.1 \\
16.5\end{array}$ & $\begin{array}{l}31.0 \\
56.9 \\
12.1\end{array}$ & 0.36 \\
\hline Pathological Stage & $\begin{array}{l}\text { TO } \\
\text { T2a } \\
\text { T2b } \\
\text { T2c } \\
\text { T3a } \\
\text { T3b }\end{array}$ & $\begin{array}{c}3.0 \\
19.8 \\
6.0 \\
62.2 \\
7.0 \\
2.0\end{array}$ & $\begin{array}{c}5.3 \\
28.0 \\
7.0 \\
57.9 \\
0 \\
1.7\end{array}$ & 0.64 \\
\hline GLEASON PT & $\begin{array}{l}0 \\
3+3 \\
3+4 \\
4+3 \\
4+4 \\
9\end{array}$ & $\begin{array}{c}3.0 \\
35.6 \\
36.6 \\
17.8 \\
5.9 \\
1.0\end{array}$ & $\begin{array}{c}5.3 \\
40.3 \\
31.6 \\
17.5 \\
5.3 \\
0\end{array}$ & 0.95 \\
\hline Unfavorable disease & $\%$ & 28.2 & 22.4 & 0.43 \\
\hline Pts with complication & $\%$ & 10.7 & 8.6 & 0.67 \\
\hline
\end{tabular}

Tab 3. Multivariate analysis for predictive factors of Unfavorable disease in LR and VLR patients

\begin{tabular}{|l|c|c|c|c|c|c|}
\hline & \multicolumn{3}{|c|}{ LR } & \multicolumn{3}{c|}{ VLR } \\
\hline & OR & P value & Cl (95\%) & OR & P value & Cl (95\%) \\
\hline Age & 1.093 & 0.26 & $0.94-1.28$ & 1.13 & 0.31 & $0.89-1.45$ \\
\hline BMI & 0.935 & 0.57 & $0.74-1.18$ & 0.86 & 0.39 & $0.61-1.21$ \\
\hline PSA & 0.905 & 0.60 & $0.62-1.31$ & 0.98 & 0.95 & $0.55-1.73$ \\
\hline PSAD & $\mathbf{5 . 4 1 7}$ & $\mathbf{0 . 0 2}$ & $\mathbf{1 . 2 8 - 2 2 . 8}$ & 11.79 & 0.05 & $0.97-143.3$ \\
\hline \% positive biopsy cores & 0.996 & 0.86 & $0.96-1.04$ & 1.04 & 0.96 & $0.86-1.17$ \\
\hline Clinical Stage & 0.339 & 0.20 & $0.67-1.76$ & 0.29 & 0.45 & $0.01-7.32$ \\
\hline N removed nodes & 0.917 & 0.13 & $0.82-1.03$ & 0.80 & 0.07 & $0.62-1.02$ \\
\hline
\end{tabular}

more restrictive criteria are adopted in several AS protocols. Italian arm (SIURO) of Prostate Cancer Research International Active Surveillance (PRIAS) inclusion criteria are stage cT1c/ T2a, Gleason score.

To evaluate pathologic outcomes in patients affected by very low risk (VLR) and low risk (LR) prostate cancer and eligible for Active Surveillance

Materials and Methods: we conducted a retrospective analysis in patients with low risk prostate cancer who underwent Laparoscopic Radical Prostatectomy (LRP) at our institution from 2005 to 2016. We identified patients with low risk (LR) PCa defined as cT1c-T2a, Gleason score $<7$, PSA $\leq 10 \mathrm{ng} / \mathrm{ml}$ and patients with very low risk (VLR) PCa as defined by Italian PRIAS (cT1c-2a, Gleason score $<7$, PSA $\leq 10 \mathrm{ng} / \mathrm{ml}$, PSAD $\leq 0,20 \mathrm{ng} / \mathrm{ml} / \mathrm{cc}, \leq 2$ positive cores). Complete information on PSA, PSA density (PSAD), clinical stage, Gleason score, percentage of positive cores, number of nodes removed, and pathological outcomes were available. We evaluate GS upgrading (to primary pattern 4 ), non-organ confined disease and unfavorable disease ( $\geq \mathrm{pT} 3, \mathrm{GS} \geq 4+3, \mathrm{pN} 1)$ in LR and VLR patients. Predictive factors of unfavorable disease were analyzed by logistic regression analysis (SPSS 24).

Results: We identified 103 patients with LR Prostate cancer. Of these, 58 patients have VLR cancer according with PRIAS criteria. Baseline characteristic of patients are described in table 1. There were no significant differences between LR and VLR patients. Pathological outcomes revealed upstaging in $9 \%$ and $1.7 \%$, upgrading in $24.7 \%$ and $22.8 \%$ in LR and VLR patients, respectively. Unfavorable disease occurred in $28.2 \%$ and $22.4 \%$ of LR and VLR patients, respectively [table 2]. At multivariate analysis, PSAD was the only predictive factor of unfavorable disease in LR patients [table 3].

Conclusions: In our experience upstaging and upgrading at Laparoscopic radical prostatectomy occurred in $9 \%$ and $25 \%$ of low risk patients and in $2 \%$ and $23 \%$ of very low risk patients. About a quarter of the patients presented unfavorable disease (non organ confined, primary Gleason 4). PSA density was the only predictive factor of unfavorable disease.

MP17-2 Predictive factors of upstaging, upgrading and adverse pathological features in favourable GS $3+4$

R Sanseverino, G Napodano, U Di Mauro, O Intilla, G Molisso, T Realfonso

Dept. of Urology Umberto I Hospital Italy

Introduction \& Objective: Active surveillance (AS) is a valid option for the treatment of low risk prostate cancer. Whether or not AS could be offered also to patients with intermediate risk prostate cancer is a debated issue. Some AS protocols included selected patients (older) with Gleason score $3+4$. In our study we evaluated the risk of upgrading and upstaging and predictive factors of adverse disease in patients with favourable Gleason score $3+4$ and identified predictive factors.

Materials and Methods: From database of our institution, we identified patients undergone a laparoscopic pelvic lymphadenectomy (LAD) and radical prostatectomy; data on age, BMI, 
Table 1. Baseline characteristics

\begin{tabular}{|l|c|c|}
\hline Pts & $\mathrm{n}$ & 82 \\
\hline Age & $\mathrm{y}$ & $65.1 \pm 6.1$ \\
\hline BMI & $\mathrm{n}$ & $27.5 \pm 3.0$ \\
\hline PSA & $\mathrm{ng} / \mathrm{ml}$ & $6.7 \pm 1.9$ \\
\hline Prostate volume & $\mathrm{cc}$ & $55.6 \pm 20.3$ \\
\hline PSAD & $\mathrm{ng} / \mathrm{ml} / \mathrm{cc}$ & $0.13 \pm 0.06$ \\
\hline C Stage (\%) & $\mathrm{Tlc}$ & 36.6 \\
& $\mathrm{~T} 2 \mathrm{a}$ & 63.4 \\
\hline Positive core percentage & $\%$ & $39.3 \pm 24.0$ \\
\hline
\end{tabular}

Table 2. Surgical and pathological outcomes

\begin{tabular}{|l|l|c|}
\hline Pathological Stage & pT2a & 17.2 \\
& pT2b & 7.3 \\
& pT2c & 65.8 \\
& pT3a & 7.3 \\
& pT3b & 2.4 \\
\hline Pathological Gleason & $3+3$ & 4.9 \\
& $3+4$ & 70.7 \\
& $4+3$ & 19.5 \\
& $>7$ & 4.9 \\
\hline Positive SM & $\%$ & 5 \\
\hline LAD template & Obturator (I) & 48.8 \\
& Iliac-obturator (II) & 51.2 \\
\hline $\mathrm{n}^{\circ}$ removed nodes & $\mathrm{n}$ & $20.0 \pm 10.4$ \\
\hline pN1 & $\%$ & 2.4 \\
\hline Adverse pathology & $\%$ & 31.7 \\
\hline
\end{tabular}

Table 3 Predictive factors of upstaging ( $\geq \mathrm{pT} 3)$ : univariate analysis

\begin{tabular}{|l|c|c|c|}
\hline \multicolumn{4}{|c|}{ UPSTAGING } \\
\hline & Yes & No & p value \\
\hline cTlc vs cT2a (\%) & 11.5 vs 6.7 & 88.5 vs 93.3 & 0.61 \\
\hline LAD template: I vs II (\%) & 0 vs 19.1 & 100 vs 80.9 & 0.22 \\
\hline Age (y) & $65.0 \pm 2.3$ & $65.1 \pm 6.4$ & 0.99 \\
\hline BMI (n) & $29.9 \pm 5.5$ & $28.1 \pm 2.8$ & 0.24 \\
\hline PSA (ng/ml) & $7.9 \pm 0.5$ & $6.5 \pm 2.0$ & 0.19 \\
\hline PSAD (ng/ml/cc) & $0.13 \pm 0.02$ & $0.13 \pm 0.06$ & 0.99 \\
\hline Positive core percentage (\%) & $58.3 \pm 31.9$ & $37.2 \pm 22.6$ & 0.09 \\
\hline Prostate volume (cc) & $59.5 \pm 12.4$ & $55.2 \pm 21.1$ & 0.61 \\
\hline $\mathrm{n}^{\circ}$ removed nodes (n) & $25.5 \pm 14.6$ & $19.4 \pm 9.9$ & 0.29 \\
\hline
\end{tabular}

PSA, PSAD, positive cores percentage, clinical stage, Gleason score, lymphadenectomy template, prostate volume, number of removed nodes were available. We correlated these variables with upstaging $(\geq \mathrm{pT} 3)$, upgrading $(\geq \mathrm{GS} 4+3)$ and adverse pathological outcomes (non-organ confined disease or $\geq \mathrm{GS} 4+3$ or pN1) by logistic regression analysis (SPSS 24).
Table 4A Predictive factors of upgrading: univariate analysis

\begin{tabular}{|l|c|c|c|}
\hline \multicolumn{4}{|c|}{ UPGRADING } \\
\hline & Yes & No & p value \\
\hline cTle vs cT2a (\%) & 26.9 vs 20.0 & 73.1 vs 80.0 & 0.62 \\
\hline LAD template: I vs II (\%) & 30.0 vs 19.1 & 70.0 vs 80.9 & 0.60 \\
\hline Age (y) & $66.4 \pm 3.4$ & $64.6 \pm 6.7$ & 0.43 \\
\hline BMI (n) & $28.3 \pm 3.5$ & $27.1 \pm 2.7$ & 0.32 \\
\hline PSA (ng/ml) & $7.9 \pm 1.9$ & $6.3 \pm 1.8$ & 0.02 \\
\hline PSAD (ng/ml/cc) & $0.15 \pm 0.06$ & $0.13 \pm 0.06$ & 0.28 \\
\hline Positive core percentage (\%) & $33.9 \pm 25.2$ & $41.0 \pm 23.7$ & 0.42 \\
\hline Prostate volume (cc) & $56.9 \pm 13.5$ & $55.2 \pm 22.3$ & 0.77 \\
\hline $\mathrm{n}^{\circ}$ removed nodes (n) & $19.0 \pm 11.9$ & $20.4 \pm 10.0$ & 0.72 \\
\hline
\end{tabular}

Table 4B Predictive factors of upgrading: multivariate analysis

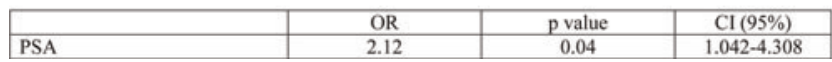

Table 5A Predictive factors of Adverse pathology: univariate analysis

\begin{tabular}{|l|c|c|c|}
\hline \multicolumn{4}{|c|}{ ADVERSE PATHOLOGY } \\
\hline & yes & No & p value \\
\hline cTle vs cT2a (\%) & 38.4 vs 20.0 & 61.5 vs 80.0 & 0.22 \\
\hline LAD template: I vs II (\%) & 30.0 vs 33.3 & 70 vs 66.4 & 0.66 \\
\hline Age (y) & $65.9 \pm 3.2$ & $64.7 \pm 7.1$ & 0.43 \\
\hline BMI (n) & $28.1 \pm 3.4$ & $27.1 \pm 2.8$ & 0.45 \\
\hline PSA (ng/ml) & $7.9 \pm 1.6$ & $6.1 \pm 1.8$ & 0.004 \\
\hline PSAD (ng/ml/cc) & $0.15 \pm 0.06$ & $0.13 \pm 0.06$ & 0.36 \\
\hline Positive core percentage (\%) & $41.5 \pm 29.5$ & $38.2 \pm 21.5$ & 0.51 \\
\hline Prostate volume (cc) & $58.1 \pm 13.1$ & $54.4 \pm 23.0$ & 0.64 \\
\hline $\mathrm{n}^{\circ}$ removed nodes $(\mathrm{n})$ & $21.1 \pm 12.9$ & $19.5 \pm 9.1$ & 0.63 \\
\hline
\end{tabular}

Table 5B. Predictive factors of Adverse pathology: multivariate analysis

\begin{tabular}{|l|c|c|c|}
\hline & OR & p value & CI (95\%) \\
\hline PSA & 2.87 & 0.01 & $1.326-6.200$ \\
\hline
\end{tabular}

Results: Baseline characteristics of the 82 patients with favourable Gleason score $3+4 \mathrm{PCa}$ are reported in table 1 . Surgical and pathological outcomes are reported in table 2. Upstaging to $\geq \mathrm{pT} 3$ occurred in $9.7 \%$ of patients; no variables were associated to upstaging (table 3 ). Upgrading occurred in $24.4 \%$ of patients; PSA was the only factor associated to upgrading [OR 2.12, p 0.04] (tables 4A and 4B). Adverse pathological outcomes (non organ confined disease or primary GS4 or pN1) occurred in $31.7 \%$ of patients; PSA correlated with adverse pathological outcomes [OR 2.87, p 0.01] (tables 5A and 5B). Downgrading occurred in about $5 \%$ of patients.

Conclusions: In patients with favourable Gleason score $3+4$, adverse pathological outcomes, defined as non organ confined disease or Gleason score $4+3$ or Pn1, occurred in $32 \%$ of the patients. PSA was a predictive factor of upgrading and adverse pathological features.

MP17-3 Prostate-specific antigen density as a predictor of Gleason score upgrading

D Bos, J Hoogenes, K Kim, F Farrokhyar, B Shayegan

McMaster University

Canada

Introduction \& Objective: Post-operative Gleason score upgrading has been reported to occur in up to $57 \%$ of prostate 
Table 1. Predictors of Gleason score upgrading following surgical intervention $(n=399)$

\begin{tabular}{lcc}
\hline \multicolumn{1}{c}{ Patient characteristics } & Odds ratio $(95 \% \mathrm{CI})$ & $\mathrm{p}$-value \\
\hline Age & $1.03[0.99-1.08]$ & 0.11 \\
Body mass index & $0.98[0.92-1.05]$ & 0.61 \\
Pre-operative Gleason score & $0.06[0.02-0.17]$ & $<0.001$ \\
Prostate weight & $1.0[0.98-1.01]$ & 0.92 \\
D'Amico risk & $0.45[0.15-1.35]$ & 0.16 \\
PSA density (ratio of PSA to gland volume) & $4.16[0.77-22.4]$ & 0.09 \\
Ratio of positive cores to cores sampled & $2.7[0.96-7.50]$ & 0.059 \\
\hline
\end{tabular}

cancer cases. While prostate biopsy Gleason score remains one of the strongest prognostic indicators for predicting prostate cancer aggressiveness, prostate-specific antigen density (PSAD) has been suggested to also be a significant predictor of pathologic upgrading. Our study aimed to explore the potential association between PSAD and Gleason score upgrading.

Materials and Methods: A prospectively collected database of patients who underwent robot-assisted radical prostatectomy (RARP) at an academic tertiary care center was retrospectively reviewed. Patients were divided into two cohorts based on the presence of post-operative Gleason score upgrading. We collected data on baseline patient demographics, pathological Gleason score, PSAD, and associated preoperative biopsy characteristics. Logistic regression model was applied to determine the predictors of upgrade including the baseline demographics and disease specific variables.

Results: Of the 399 patients included in the analysis, mean age (years) was 63.2 \pm 6.9 , mean pre-operative prostate-specific antigen was $8.8 \mathrm{ng} / \mathrm{mL} \pm 6.9$, and mean gland volume was $37.5 \mathrm{ml}$ \pm 16.6. A total of $118(30 \%)$ patients had Gleason score upgrading post-RARP. Pre-operative Gleason score was an independent predictor of pathological upgrading (odds ratio [OR] $0.06,95 \%$ confidence interval $[\mathrm{CI}] 0.02-0.17, \mathrm{p}<0.001)$. Elevated PSAD and ratio of positive cores were both positively related to pathological upgrading with an OR of 4.16 (CI 0.77$22.4, p=0.09)$ and $2.7(\mathrm{CI} 0.96-7.50, \mathrm{p}=0.06)$, respectively (Table 1).

Conclusions: In this sample of patients undergoing RARP for clinically localized prostate cancer, pre-operative Gleason score and PSAD were both associated with pathological upgrading; however, only the former demonstrated statistical significance.

MP17-4 Comparison of surgical, oncological, and functional outcomes of robot assisted and laparoscopic radical prostatectomy

U Boylu, A Inkaya, A Adiguzel, TU Sen, E Kucuk

Department of Urology, Health Sciences University Umraniye Training \& Research Hospital, Istanbul, Turkey

Turkey

Introduction \& Objective: To compare the oncological and functional outcomes of robot assisted radical prostatectomy (RARP) and laparoscopic radical prostatectomy (LRP).

Materials and Methods: Between 2012 and 2016, we performed 635 RARP and 48 LRP. All operations were performed transperitoneally using the posterior approach. The surgical, oncological, and functional data were collected prospectively in the institutional database. The patients were followed-up every 3
Table 1 Sugical and oncological outcomes of RARP and LRP groups

\begin{tabular}{|c|c|c|c|}
\hline & RARP & LRP & $\mathbf{p}$ \\
\hline Number & 635 & 48 & - \\
\hline Age (SD, years) & $62,3(6.5)$ & $62,3(6.5)$ & 0.225 \\
\hline Operating time $(\mathrm{SD}, \mathrm{min})$ & $206(2.2)$ & $248(24)$ & 0.01 \\
\hline $\begin{array}{l}\text { Estimated blood loss, } \mathrm{ml} \\
\text { (sd) }\end{array}$ & $172(5.8)$ & $183(23)$ & 0.108 \\
\hline Transfusion rate, $(\%)$ & $7 / 635(\% 1.1)$ & $2 / 48(\% 4.1)$ & 0.05 \\
\hline Hospital stay, day (sd) & $3.02(0.6)$ & $3.68(0.29)$ & 0.09 \\
\hline $\begin{array}{l}\text { Uretral catheter time, day } \\
\text { (sd) }\end{array}$ & $9.2(0.11)$ & $11.6(0.97)$ & 0.08 \\
\hline $\begin{array}{l}\text { Early }(<30 \mathrm{~d}) \text { complications } \\
\text { (Clavien score } \geq 3 \text { ) (\%) }\end{array}$ & $11 / 635(\% 1.73)$ & $3 / 48(\% 6.2)$ & 0.005 \\
\hline $\begin{array}{l}\text { Intermediate ( } 31-90 \text { d) } \\
\text { complications (Clavien } \\
\text { score } \geq 3 \text { ) (\%) }\end{array}$ & $2 / 635(\% 0.3)$ & $2 / 48(\% 4.15)$ & 0.001 \\
\hline Overall PSMs, no (\%) & $108 / 635(\% 17)$ & $6 / 48(\% 12.5)$ & . \\
\hline pT2 PSM & $39 / 373(\% 10.4)$ & $2 / 30(\% 6.6)$ & 0,360 \\
\hline PT3 PSM & $69 / 262(\% 26.3)$ & $4 / 18(\% 22.2)$ & 0,549 \\
\hline
\end{tabular}

Table 2 Continence rates in RARP and LRP groups
\begin{tabular}{|l|l|l|l|l|}
\hline Continence (\%) & $1 . \mathrm{mo}$ & $3 . \mathrm{mo}$ & $6 . \mathrm{mo}$ & $12 . \mathrm{mo}$ \\
\hline RARP & $490 / 635(\% 77)$ & $529 / 635(\% 83)$ & $594 / 635(\% 93.5)$ & $612 / 635(\% 96.3)$ \\
\hline LRP & $32 / 48(\% 66)$ & $38 / 48(\% 79)$ & $42 / 48(\% 87)$ & $44 / 48(91)$ \\
\hline P value & 0.31 & 0.81 & 0.40 & 0.41 \\
\hline
\end{tabular}

Table 3 Potency rates in RARP and LRP groups

\begin{tabular}{|l|l|l|l|l|}
\hline Potency & $1 . \mathrm{mo}$ & $3 . \mathrm{mo}$ & $6 . \mathrm{mo}$ & $12 . \mathrm{mo}$ \\
\hline RARP & $182 / 542(\% 33.5)$ & $244 / 542(\% 45)$ & $285 / 542(52.5)$ & $362 / 542(\% 66)$ \\
\hline LRP & $12 / 48(\% 25)$ & $16 / 48(\% 33)$ & $20 / 48(\% 41)$ & $26 / 48(\% 54)$ \\
\hline P değeri & 0.52 & 0.34 & 0.37 & 0.2 \\
\hline
\end{tabular}

months for the first 2 years, every 6 months for the $2^{\text {nd }}$ two years, and every year afterwards.

Results: Estimated blood loss, hospital stay, and urethral catheter removal times were similar in RARP and LRP groups. However, operating time (206 vs. $248 \mathrm{~min}, \mathrm{p}=0.01$ ) and Clavien grade $\geq 3$ complication rates $(1.73 \%$ vs $6.2 \%, p=0.005)$ were in favor of RARP. Biochemical recurrence rates were similar $(7.7 \%$ vs. $7.2 \%$ in $\mathrm{pT} 2[\mathrm{p}=0.61]$ and $18.7 \%$ vs $22 \%$ in $\mathrm{pT} 3[\mathrm{p}=0.85])$ in RARP and LRP groups, respectively. (Table 1) Continence rates were $96 \%$ for RARP and $91 \%$ for LRP $(p=0.41)$. (Table 2$)$ Although not significant, potency rate at 1 year was in favor of RARP (66\% vs. $54 \%, \mathrm{p}=0.2$ ) (Table 3 )

Conclusions: RARP and LRP provides similar oncological and functional outcomes in the treatment of prostate cancer. RARP has favorable results in terms of operating time and complication rates in comparison to LRP

MP17-5 Secondary Procedures follow Open verses Robotic Radical Prostatectomy

E Fram, A Srivastava, J Fleischmann

Montefiore Medical Center

United States

Introduction \& Objective: Each year over 100,000 American men have radical prostatectomies. The most common long term urological issues following this procedure are urinary incontinence and erectile dysfunction. Prior reports have characterized the rates of surgical intervention for post-radical prostatectomy 


\begin{tabular}{|c|c|c|c|c|}
\hline & Total & Robotic & Open & $\mathrm{p}$ \\
\hline $\begin{array}{l}\text { Age: mean (SD) } \\
\text { Age }\end{array}$ & $\begin{array}{c}477 \\
58.9(7.2)\end{array}$ & $\begin{array}{c}334 \\
59.8(7.2)\end{array}$ & $\begin{array}{c}143 \\
57.5(7.1)\end{array}$ & 0.004 \\
\hline Race: n (\%) & & & & 0.039 \\
\hline Black & 170 & 109 & 61 & \\
\hline Caucasian & 69 & 45 & 24 & \\
\hline Hispanic & 132 & 94 & 38 & \\
\hline Other & 17 & 13 & 4 & \\
\hline Unknown & 89 & 74 & 16 & \\
\hline BMI: mean (SD) & $27.8(4.3)$ & $27.6(4.0)$ & $28.2(4.8)$ & 0.134 \\
\hline Overweight: $\mathrm{n}(\%)$ & 192 & 141 & 51 & 0.072 \\
\hline Obese: $n(\%)$ & 71 & 41 & 112 & 0.061 \\
\hline Comorbidities: $\mathrm{n}(\%)$ & & & & \\
\hline BPH & 201 & 158 & 43 & $<0.001$ \\
\hline DM & 100 & 68 & 32 & 0.351 \\
\hline ED & 22 & 17 & 5 & 0.309 \\
\hline HTN & 287 & 195 & 92 & 0.132 \\
\hline
\end{tabular}

laparoscopic radical prostatectomies erectile dysfunction. We sought to compare the rates of secondary procedures between open and robotic radical prostatectomy.

Materials and Methods: A review of all patients aged 40-75 who underwent open or robotic radical prostatectomy between January 2009 and March 2013 at a single institution was performed. Demographic information, including common comorbidities and the presents of benign prostatic hyperplasia and erectile dysfunction prior to the initial procedure were recorded. Secondary procedures for urinary incontinence, including artificial urethral sphincters and male urethral slings, and for erectile dysfunction, including placement of inflatable and malleable penile prostheses were identified, up to March of 2017, providing a minimum 4 years and maximum 8 years follow up. Analysis was performed using IBM SPSS v20.

Results: 477 men met our inclusions criteria, $334(75 \%)$ of whom had a robotic prostatectomy (Table 1). Men who had robotic prostatectomies were slightly older $(p=0.039)$ and were more likely to carry a diagnosis of benign prostatic hyperplasia $(\mathrm{p}<0.001)$. After a robotic radical prostatectomy, $18(5.4 \%)$ eventually underwent a procedure for urinary incontinence, compared to $5(3.5 \%)$ patients who had open radical prostatectomies $(\mathrm{p}=0.26) .27(8.1 \%)$ men who had robotic procedures underwent a second procedure for erectile dysfunction, compared to $8(5.6 \%)$ of those who had an open procedure $(\mathrm{p}=0.23)$. There was no significant difference in the time until procedures, which was between six months and six years.

Conclusions: We observed no significant differences in the rates of secondary procedures for urinary incontinence or erectile dysfunction following open verses robotic radical prostatectomy. There were insufficient numbers of secondary procedures to evaluate what pre-operative risk factors predispose patients to needing secondary procedures.

MP17-6 Impact of Gleason score at the positive surgical margin site on biochemical recurrence following robotassisted radical prostatectomy.

K Kanao, M Saito, M Sugie, H Muramatsu, S Morinaga, K Kajikawa, I Kobayashi, G Nishikawa, Y Kato, M Watanabe, K Nakamura, M Sumitomo

Aichi Medical University Japan
Introduction \& Objective: Recently, a paper reported that a lower Gleason score at the positive surgical margin is independently associated with a decreased risk of early biochemical recurrence after radical prostatectomy. However, it does not reach consensus whether he Gleason score at the margin should be reported in addition to highest Gleason score. In this study we evaluate whether the positive surgical margin Gleason score affects the risk of biochemical recurrence following robot-assisted radical prostatectomy.

Materials and Methods: A total of 307 consecutive patients undergoing robot-assisted radical prostatectomy and pelvic lymph node dissection for localized prostate cancer were included in the study. The specimens with positive surgical margin were reviewed and the Gleason score at the positive surgical margin were compared with the highest Gleason score in the specimens. Kaplan-Meier estimates of recurrence-free survival were calculated by both Gleason scores. Cox proportional hazards models including Gleason score, preoperative prostate specific antigen and pathological stage were used to predict biochemical recurrence and concordance index were compared between Gleason score at the margin and highest Gleason score. Results: Positive margins were identified in 68 (22.1\%) patients. The Gleason score at the positive surgical margin was lower than the highest Gleason score in 10 patients. Kaplan-Meier estimates demonstrated improved freedom from biochemical recurrence among patients with a lower Gleason score at the margin. In multivariate Cox models having a lower grade margin was significantly associated with a decreased risk of biochemical recurrence $(p=0.005)$. The concordance index of the model using Gleason score at the positive surgical margin (0.840) was higher than the model using highest Gleason score in the specimen (0.834).

Conclusions: The Gleason score at the positive surgical margin is better than the highest Gleason score in the specimen for predicting a risk of biochemical recurrence following robot-assisted radical prostatectomy.

MP17-7 A novel technique of of robotic radical prostatectomy: combining the best of anterior and retropubic approach

I Banerjee, A Mallya, S Arakere, T Jindal, A Mandhani, RK Ahlawat, FA Zafar

Fortis escorts kidney and urology institute India

Introduction \& Objective: A major component in trifecta of robot assisted laparoscopic radical prostatectomy(RALP) is continence. Existing studies have shown, the continence at 1 and 3 months after standard anterior approach RALP are as low as $20-25 \%$ and $45-60 \%$ respectively. The Retzius sparing approach improves the rate to $80-90 \%$ at 3 months. We report a new technique of RALP with a combined anterior-posterior approach involving anatomic preservation of continence mechanism.

Materials and Methods: A total of 43 patients of localized carcinoma prostate who underwent RALP in our institute by a single surgeon with at least 6 months follow up were included in the study. Patients with metastatic disease, history of previous radiation, previous TURP and neoadjuvant hormonal therapy were excluded. Data was prospectively collected. Propensity score matching using seven preoperative variables was done, making two groups of standard anterior and combined RALP 


\begin{tabular}{|c|c|c|}
\hline & Standard Anterior & Combined \\
\hline $\begin{array}{l}\text { Continence at } 1 \text { month } \\
\text { Dry } \\
1 \text { security pad } \\
\text { Incontinent }(2 \text { or more } \\
\text { pads) }\end{array}$ & $\begin{array}{l}2(10 \%) \\
6(30 \%) \\
12(60 \%)\end{array}$ & $\begin{array}{l}5(22.7 \%) \\
7(31.8 \%) \\
10(45.5 \%)\end{array}$ \\
\hline $\begin{array}{l}\text { Continence at } 3 \text { months } \\
\text { Dry } \\
1 \text { security pad } \\
\text { Incontinent }\end{array}$ & $\begin{array}{l}4(20 \%) \\
8(40 \%) \\
8(40 \%)\end{array}$ & $\begin{array}{l}9(42.9 \%) \\
8(38.1 \%) \\
4(19 \%)\end{array}$ \\
\hline $\begin{array}{l}\text { Erectile function at } 1 \\
\text { month } \\
\text { Normal morning } \\
\text { tumescence }\end{array}$ & $2 / 13$ & $2 / 15$ \\
\hline $\begin{array}{l}\text { Erectile function at } 3 \\
\text { months } \\
\text { Normal morning } \\
\text { tumescence }\end{array}$ & $4 / 13$ & $6 / 15$ \\
\hline
\end{tabular}

Functional outcome
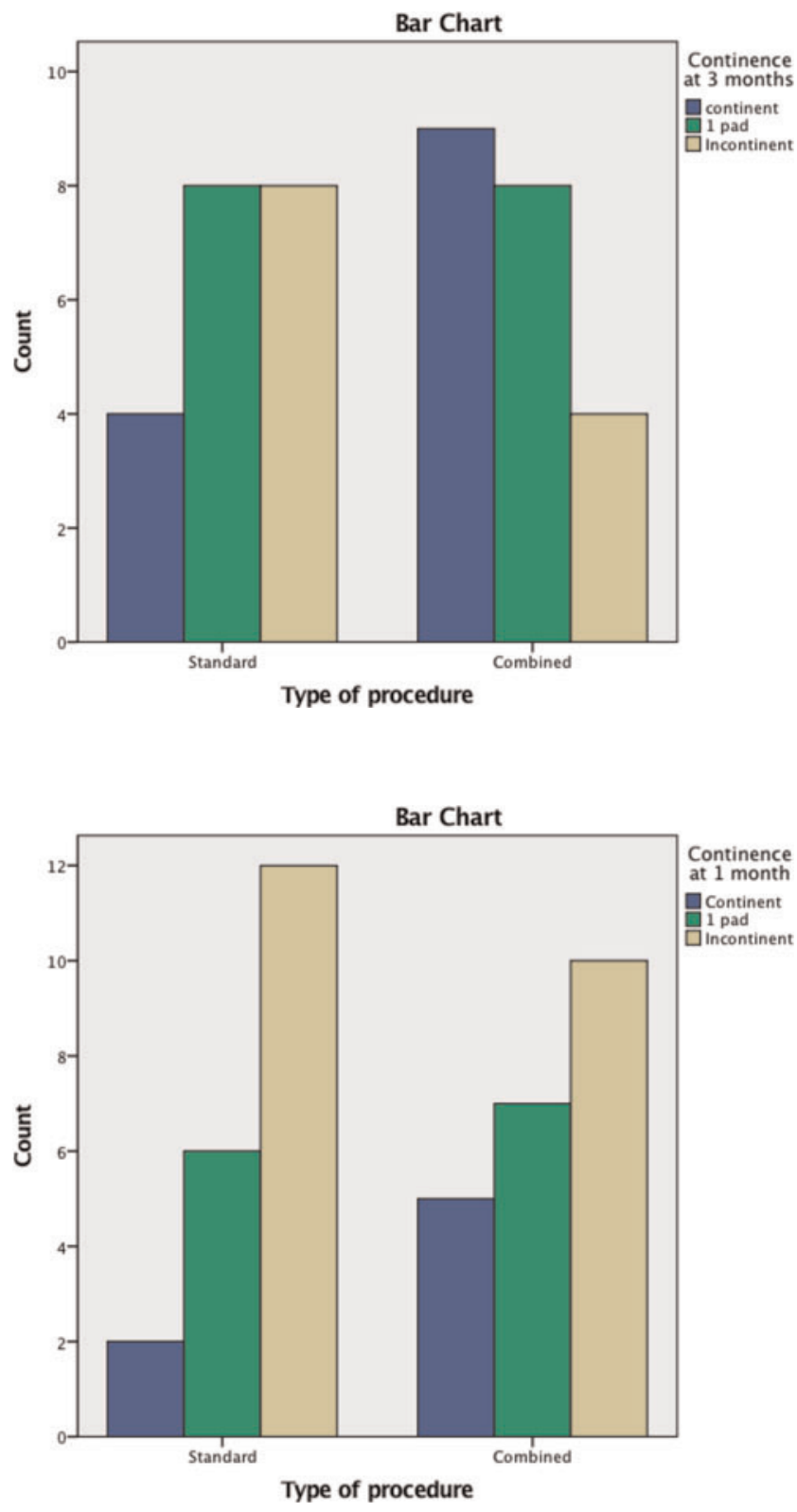

\begin{tabular}{|c|c|c|}
\hline & Standard anterior & Combined \\
\hline $\begin{array}{l}\text { Final staging } \\
\text { pT2a } \\
\text { p2b } \\
\text { pT2c } \\
\text { pT3a } \\
\text { PT3b }\end{array}$ & $\begin{array}{l}1 \\
1 \\
7 \\
6 \\
4\end{array}$ & $\begin{array}{l}2 \\
13 \\
6 \\
1 \\
\end{array}$ \\
\hline $\begin{array}{l}\text { Final specimen Gleason } \\
3+3 \\
3+4 \\
4+3 \\
4+4 \\
4+5 \\
5+4 \\
5+5\end{array}$ & $\begin{array}{l}7 \\
5(\text { Gleason } 7=12) \\
3(\text { GS } 8 \text { n above } 7) \\
1 \\
2 \\
1\end{array}$ & $\begin{array}{l}3 \\
8 \\
4(G S 7=12) \\
4(G S 8 \text { n above } 7) \\
3\end{array}$ \\
\hline Total no of lymph node yield & $25.82 \pm 8.97$ & $22.76 \pm 7.27$ \\
\hline Lymph node positive >2 & 1 & 1 \\
\hline $\begin{array}{l}\text { Margin positive } \\
\text { Multifocal } \\
\text { Unifocal }\end{array}$ & $\begin{array}{l}9 \\
3 \\
6\end{array}$ & $\begin{array}{l}8 \\
2 \\
6\end{array}$ \\
\hline $\begin{array}{l}\text { Site of margin positive } \\
\text { Apex } \\
\text { Bladder neck }\end{array}$ & $\begin{array}{l}\text { 6( pT2c1,3p 3T3b,2 pT3a) } \\
\text { 2(pT3b,pT3a) }\end{array}$ & $\begin{array}{l}1 \text { (pT2c) } \\
1 \text { (рT3a) }\end{array}$ \\
\hline $\begin{array}{l}\text { Post op hormonal therapy } \\
\text { Biochem recurrence } \\
\text { Lymph node positive }\end{array}$ & $\begin{array}{l}2 \\
1 \\
\end{array}$ & $\begin{array}{l}1 \\
1 \\
\end{array}$ \\
\hline
\end{tabular}

Pathological results

comparable. In our technique dorsal venous complex, endopelvic fascia and urethral sphincter was preserved; it also involved both anterior and posterior reconstruction. Patient were followed up at one month, three months, 6 months following radical prostatectomy. Primary outcome measure was continence at 1 and 3 months. Continence was defined as 0 pad or 1 safety pad. All statistical analysis was done using SPPSS v 23(IBM Corp, Armonk, NY, USA).

Results: A total of 22 patients out of of 43 underwent the combined approach and 21 standard anterior approach. There were no significant differences in intra operative blood loss, length of hospital stay, pathological stage, Gleason scores, positive surgical margins, total lymph node yield between the standard anterior and combined RALP groups. Combined approach (135.71 \pm 35.95 minutes) had a shorter console time as compared to the standard anterior approach (162.86 \pm 32.05 minutes. $\mathrm{p}=01)$ Recovery of early continence at $1(55.2 \%$ VS $40 \%$ ) and $3(81 \%$ VS 60\%) months after RALP was better in the combined group than in the standard anterior group

Conclusions: By modifying the standard anterior approach we have achieved results very close to Retzius sparing approach in terms of achieving early continence at 3 months. Our technique is reproducible and technically less demanding than the Retzius sparing approach. A randomised trial comparing the techniques is underway in our institute to shed more light.

MP17-8 The prediction of T3a disease on pre-operative 3T mp-MRI: a correlation study with the pathology of robotic assisted radical prostatectomy

K Lin, E Huang, S Shen, I Huang, C Lin, T Lin, H Chung, S Lu, W Huang, H Wu, Y Chang, A Lin

Taipei Veterans General Hospital

Taiwan (Republic of China)

Introduction \& Objective: Radical prostatectomy is the surgical treatment of choice for localized prostate cancer, which has shown favorable cancer control in patients with organ-confined 


\begin{tabular}{|c|c|c|c|c|}
\hline & & $\begin{array}{l}\text { Group of correct } \\
\text { prediction of EPE } \\
\text { (presence \& } \\
\text { location) } \mathrm{N}=142\end{array}$ & $\begin{array}{l}\text { Group of incorrect } \\
\text { prediction of EPE } \\
\text { (presence \& location) } \\
\mathrm{N}=62\end{array}$ & P value \\
\hline Age(medianะSD,year) & & $66.5 \pm 6.2$ & $65.2 \pm 6.4$ & 0.189 \\
\hline $\mathrm{BMI}\left(\right.$ median $\left.\pm \mathrm{SD}, \mathrm{kg} / \mathrm{m}^{2}\right)$ & & $24.7 \pm 3.0$ & $25.6 \pm 3.1$ & 0.053 \\
\hline $\begin{array}{l}\text { Radiological Prostate } \\
\text { volume(ml) }\end{array}$ & & $44.2+23.2$ & $36.5 \pm 19.1$ & 0.023 \\
\hline $\begin{array}{l}\text { Initial PSA level } \\
\text { (median } 5 \text { SD, ng/ml) }\end{array}$ & & $10.2 \pm 7.7$ & $11.5 \pm 11.0$ & 0.335 \\
\hline Biopsy Gleason score & $\begin{array}{l}<7 \\
\Rightarrow 7 \\
>7\end{array}$ & $\begin{array}{l}73(73.08) \\
42(61.8 \%) \\
27(75.08)\end{array}$ & $\begin{array}{l}27(27.0 \%) \\
26(38.2 \%) \\
9(25.0 \%)\end{array}$ & 0.221 \\
\hline Positive cores of TRUS biopsy & & $3.0 \pm 2.4$ & $3.4 \pm 2.1$ & 0.350 \\
\hline Clinical stage & $\begin{array}{l}\mathrm{T} 1 \\
\mathrm{~T} 2 \\
\mathrm{~T} 3\end{array}$ & $\begin{array}{l}73(79.3 \%) \\
46(63.0 \%) \\
0\end{array}$ & $\begin{array}{l}19(20.7 \%) \\
27(37.0 \%) \\
1(100.0 \%)\end{array}$ & 0.019 \\
\hline D'amico risk group & $\begin{array}{l}\text { Low risk } \\
\text { Intermediate } \\
\text { High risk }\end{array}$ & $\begin{array}{l}55(84.6 \%) \\
60(64.0 \%) \\
32(60.4 \%)\end{array}$ & $\begin{array}{l}10(15.4 \%) \\
31(36.0 \%) \\
27(39.6 \%)\end{array}$ & 0.006 \\
\hline
\end{tabular}

disease. Patients with locally advanced disease may have worse outcome but still can benefit from the surgery. Whether to evaluate the surgical candidates with 3T multiparametric magnetic resonance imaging (mp-MRI) pre-operatively can differentiate patients with localized disease from locally advanced prostate cancer is an interesting issue. Hence, we retrospectively reviewed the patients who received robotic-assisted radical prostatectomy in our hospital to correlate pre-operative $3 \mathrm{~T} \mathrm{mp}$ MRI with the surgical pathology.

Materials and Methods: From 2010 to 2016, 230 patients with prostate cancer received pre-operative $3 \mathrm{~T} \mathrm{mp-MRI} \mathrm{and} \mathrm{robotic}$ assisted radical prostatectomy were identified. The clinical data, PSA, Gleason score, MRI stage, extraprostatic extension location on MRI, pathological stage, and location of EPE were retrospectively reviewed. The correlation of EPE on pre-operative mp-MRI and pathology was performed.

Results: The mean age of the patients was $65.9 \pm 6.2$ years (range 50-87). Pre-operative PSA was $11.1 \pm 9.1 \mathrm{ng} / \mathrm{ml}$ (range 0.4-76.6). D'amico risk group was $28.3 \%$ in low risk, $40.4 \%$ in intermittent risk, and $31.3 \%$ in high risk. Pathological stage was $56.1 \%$ in T2 stage, $36.5 \%$ in T3a stage and $7.4 \%$ in T3b stage. EPE was noticed in $33.5 \%$ patients on $3 \mathrm{~T}$ mp-MRI and $36.5 \%$ on final pathology. The sensitivity and specificity of predicting EPE on 3T mp-MRI were $60.7 \%$, and $82.2 \%$, respectively. The interrater agreement between MRI stage and pathological stage was moderate (weighted Kappa $=0.54$ ). The factors influencing the agreement of MRI stage and pathological stage included positive cores of TRUS biopsy, the radiological size of prostate, and the surgical size of prostate. The accuracy of correct prediction of EPE (presence and location) on 3T mp-MRI was $69.6 \%$. The correlation of the location of EPE on MRI and surgical specimen were better at anterior and lateral aspect of prostate and worse at apex, anterior fibromuscular stroma and bladder neck. The factors influencing the accuracy of correct prediction of EPE (presence and location) on 3T-MRI included higher clinical stage, higher D' amico risk group, and smaller radiological size of the prostate. (Table)

Conclusions: $3 \mathrm{~T} \mathrm{mp-MRI} \mathrm{had} \mathrm{fair} \mathrm{sensitivity,} \mathrm{specificity} \mathrm{and}$ diagnostic accuracy on EPE of prostate cancer. The factors influencing the accuracy of correct prediction of EPE (presence and location) on $3 \mathrm{~T} \mathrm{mp}-\mathrm{MRI}$ included higher clinical stage, higher D'amico risk group, and smaller radiological size of the prostate.
MP17-9 The factors influencing the performance of nerve sparing technique on sides of extraprostatic extension on 3T mp-MRI prior to robotic assisted radical prostatectomy

E Huang, K Lin, S Shen, I Huang, C Lin, T Lin, H Chung, S Lu, W Huang, H Wu, Y Chang, A Lin

Taipei Veterans General Hospital

Taiwan (Republic of China)

Introduction \& Objective: Radical prostatectomy is the surgical treatment of choice for localized prostate cancer, which has shown favorable cancer control in patients with organconfined disease. Multiparametric magnetic resonance imaging (mp-MRI) is a reasonable approach for surgical planning. We retrospectively reviewed the patients who received roboticassisted radical prostatectomy (RARP) in our hospital to evaluate the impact of pre-operative mp-MRI on nerve-sparing technique and surgical margin.

Materials and Methods: From 2010 to 2016, 230 patients with prostate cancer received 3T mp-MRI and robotic assisted radical prostatectomy were identified. The clinical data, PSA, Gleason score, MRI stage, extraprostatic extension(EPE) location on MRI, pathological stage, location of EPE, and positive surgical margin (PSM) status of pathology were retrospectively collected. The associations between EPE location on pre-operative mpMRI and EPE/PSM on pathology were analyzed.

Results: The mean age of the patients was 65.9 \pm 6.2 (range 5087). D'amico risk group was $28.3 \%$ in low risk, $40.4 \%$ in intermittent risk, and $31.3 \%$ in high risk. Pathological stage was $56.1 \%$ in $\mathrm{T} 2$ stage, $36.5 \%$ in $\mathrm{T} 3 \mathrm{a}$ stage and $7.4 \%$ in $\mathrm{T} 3 \mathrm{~b}$ stage. EPE was noticed in $33.5 \%$ patients on $3 \mathrm{~T}$ mp-MRI and $36.5 \%$ on final pathology. The factors influencing the PSM of RARP included small radiological prostate volume, higher Gleason score, higher MRI stage, and higher D' amico risk group. There were 82 sides (right or left side) of prostate suspected to have EPE on preoperative MRI. Nerve-sparing was still performed in $41(50.0 \%)$ of these sides. $9(22.0 \%)$ sides were found to have PSM on the same locations of EPE on mp-MRI. Nerve-sparing was not performed in the other $41(50.0 \%)$ patients. PSM was noted in 12 (29.3\%) sides on the same locations of EPE on mp-MRI. However, there was no significant difference of PSM rate in regards to the performance of nerve-sparing technique or not. $(p=0.448)$ The factors influencing the decision making to perform nerve sparing technique on patients diagnosed as EPE on mp-MRI

\begin{tabular}{|c|c|c|c|c|}
\hline & & $\begin{array}{l}\text { Nerve- } \\
\text { sparing on } \\
\text { locations of } \\
\text { EPE on mp- } \\
\text { MRI }\end{array}$ & $\begin{array}{l}\text { No nerve- } \\
\text { sparing on } \\
\text { locations of } \\
\text { EPE on mp- } \\
\text { MRI }\end{array}$ & $P$ value \\
\hline $\begin{array}{l}\text { Counted numbers of sides of } \\
\text { RALP }\end{array}$ & & 41 & 41 & \\
\hline Age(median \pm SD) & & $65.6 \pm 5.8$ & $68.6 \pm 7.5$ & 0.044 \\
\hline $\mathrm{BMI}($ median $\pm \mathrm{SD})$ & & $25.9 \pm 3.0$ & $24.9 \pm 2.9$ & 0.133 \\
\hline $\begin{array}{l}\text { Radiological Prostate } \\
\text { volume }(\mathrm{ml})\end{array}$ & & $37.3 \pm 21.3$ & $38.5 \pm 16.6$ & 0.781 \\
\hline $\begin{array}{l}\text { Initial PSA level } \\
(\text { median } \pm S D, n g / m l)\end{array}$ & & $13.6 \pm 13.4$ & $14.4 \pm 10.6$ & 0.793 \\
\hline Biopsy Gleason score & $\begin{array}{l}<7 \\
=7 \\
>7\end{array}$ & $\begin{array}{l}21(65.6 \%) \\
15(45.5 \%) \\
5(29.4 \%)\end{array}$ & $\begin{array}{l}11(34.4 \%) \\
18(54.5 \%) \\
12(70.6 \%)\end{array}$ & 0.043 \\
\hline Positive cores of TRUS biopsy & & $3.1 \pm 2.0$ & $4.6 \pm 3.0$ & 0.009 \\
\hline D'amico risk group & $\begin{array}{l}\text { Low risk } \\
\text { Intermediate } \\
\text { High risk }\end{array}$ & $\begin{array}{l}10(71.4 \%) \\
14(48.3 \%) \\
17(43.6 \%)\end{array}$ & $\begin{array}{l}56(28.6 \%) \\
15(51.7 \%) \\
19(56.4 \%)\end{array}$ & 0.197 \\
\hline PSM & & $9(22.0 \%)$ & $12(29.3 \%)$ & 0.448 \\
\hline
\end{tabular}


included younger age, less biopsy Gleason score, and less positive cores of TRUS biopsy. (Table) PSM rate for sides without EPE on pre-op mp-MRI was only $2.2 \%$ which was significantly lower than that of the counterpart.

Conclusions: Whether to perform nerve-sparing technique in RARP or not in patients suspected to have EPE on pre-operative $\mathrm{mp}-\mathrm{MRI}$ showed no difference in the final PSM rate. The factors influencing the decision making to perform nerve-sparing were younger age, less biopsy Gleason score, and less positive cores of TRUS biopsy.

MP17-10 Perioperative, oncologic and functional outcomes of salvage robotic radical prostatectomy after focal therapy failure

I Nunes-Silva, E Barret, V Srougi, P Capogrosso, R Sanchez-Salas, F Rozet, M Galiano, X Cathelineau

Institut Montsouris

France

Introduction \& Objective: Salvage surgery is an option for treating prostate cancer(PCa) after focal therapy(FT) failure.

Our objective was to assess the impact of FT on perioperative, oncologic and functional outcomes in men who underwent salvage robotic-assisted radical prostatectomy(S-RARP) compared to primary RARP(P-RARP).

Materials and Methods: Focal therapy (FT) was performed as an experimental treatment option in patients presenting low- to intermediate risk PCa according to D'Amico criteria, cT1c cT2a, PSA 10 years. FT was defined as target ablation of the index lesion plus around $1 \mathrm{~cm}$ of safety margin within the normal ipsilateral prostatic parenchyma. Prospective data were reviewed for 2775 men who underwent RARP for localized PCa between 2000 and 2016. The S-RARP group included 25 men who underwent S-RARP after FT failure, however 3 men were excluded due to a FU shorter than $1 \mathrm{yr}$, remaining 22 men for analysis. The P-RARP group was defined using matched-pair 1:2 selection of 44 of 2750 P-RARP patients.

The primary and secondary end points were between-group differences in functional outcomes and oncologic data, respectively.

Results: Complication rates were comparable $(p>0.05)$. Pad-free probability (49.5\% vs $62.4 \%$ at 1 year, $p=0.8 ; 73 \%$ vs $76.5 \%$ at 2 years, $p=0.8$ ) was comparable between groups. Recovery of erectile function was significantly lower with S-RARP (mean $3 \pm 2$ vs 9.22 $\pm 6.55, p=0.008$ ), and S-RARP showed significantly lower probability of cumulative biochemical recurrence (BCR)-free survival $(67.6 \%$ vs $95.1 \%$ at 1 year, $p=0.001 ; 56.3 \%$ vs $92.4 \%$ at 2 years, $p=0.001]$. S-RARP presented significantly increased risk of BCR (HR 4.8, 95\% CI 1.67-13.76, $p=0.004$ ).

Conclusions: S-RARP following FT failure is feasible, with acceptable complication rates. However, patients assigned to primary FT should be advised about a poorer prognosis in terms of oncological control and lower erectile recovery rates in case of a future salvage surgery.

MP17-11 Prevention Of Urethral Retraction With Stay Sutures (PURS) During Robot Assisted Radical Prostatectomy Improves Early Urinary Control: A Prospective Cohort Study

OB Argun, MB Tuna, T Doganca, I Tufek, P Mourmoris, C Obek, AR Kural
Acibadem University, Department of Urology, Istanbul, Turkey Turkey

Introduction \& Objective: To evaluate early continence rates with a novel modified vesico-urethral anastomosis technique based on prevention of urethral retraction using anastomosis sutures as stay sutures during robot assisted radical prostatectomy.

Materials and Methods: Between January and June 2016, 60 patients were enrolled and data were collected prospectively. A modified vesico-urethral anastomosis was performed by a single surgeon. This cohort was compared with match paired consecutive 60 patients operated with standard continuous running anastomosis technique. The new technique is based on stabilizing the posterior urethra with 2 anastomosis sutures (used as stay sutures) before transecting the prostate from urethra. Two 3/0 barbed sutures were passed from the posterior aspect of the urethra at 5 o'clock and 7 o'clock positions and then used for vesicourethral anastomosis. The outcomes were prospectively followed and groups compared regarding continence. International Consultation on Incontinence Questionnaire Short Form (ICIQ-SF), previously validated in Turkish, was used to assess incontinence and its impact on the quality of life. It was performed at 3 points in time: Prior to surgery, first week of catheter removal and postoperative first month. Moreover, pad use was evaluated as a more stringent criterion for continence status at first, third, sixth month and first year of surgery. The response to pad test was either a yes or no.

Results: Age was significantly lower in the standard anastomosis cohort $(61 \pm 7.5$ vs. $64 \pm 7.6, \mathrm{p}<0.05)$. Anastomosis was completed at a shorter time in the modified anastomosis group (15.1 min vs. $18.5 \mathrm{~min}, \mathrm{p}=0.05$ ). Based on the ICIQ-SF results at first week and postoperative first month, the severity and bother of incontinence was significantly less in the modified anastomosis group (12.7 vs. 4.1 and 10.1 vs. $2.6, \mathrm{p}<0.001)$. Patients in the modified anastomosis cohort reported significantly better continence rates at both postoperative month 1 (73\% vs. $39 \%$, $\mathrm{p}<0.0001)$ and month 3 (83\% vs. 53\%, p=0.0004). The continence difference between the groups at postoperative sixth month $(\mathrm{p}=0.13)$ and year $1(\mathrm{p}=0.15)$, was statistically insignificant. Four patients in modified anastomosis group (4/60) and 1 in standard anastomosis group (1/60) necessitated temporary urethral recatheterization due to urinary retention.

Conclusions: Modified anastomosis technique achieves superior early continence rates compared to standard technique. Further randomized studies are needed to better evaluate the efficacy and feasibility of this technique.

MP17-12 Prostate Cancer in Men <55: Rates of Functional Outcomes Post Robotic Assisted Radical Prostatectomy

E Hernandez, G Ogaya-Pinies, H Palayapalayam, $\mathrm{T}$ Woodlief, T Rogers, V Patel

Global Robotics Institute

United States

Introduction \& Objective: The recovery of functional outcomes within one year of undergoing a RP are of high importance to most patients, with return of continence and potency being a priority. Age, comorbidities, rehabilitation compliance, pre-op SHIM and degree of nerve spare encompass some of the main impactors of recovery outcomes. 
Materials and Methods: In a retrospective analysis of our IRB approved database between 2008 and 2016, 1411 patients who underwent RARP were $55 \mathrm{y} / \mathrm{o}$ or less. Within this group, 1,087 patients had at least of one year of follow-up. Date of return of continence and potency were self-reported. Return of continence was defined as the use of no pads each day. Return of potency was defined as the ability to achieve an erection and have successful intercourse more than $50 \%$ of the time. We then subdivided these men three ways in order to access both time to and overall recovery of functional outcomes as follows: By Age; By Age and Pre-operative SHIM; and By age, SHIM and degree of

\begin{tabular}{|c|c|c|c|c|c|c|c|c|}
\hline & $\mathrm{N}$ & Age & Pre-Op SHIM & PSA & $\begin{array}{l}\text { "of days } \\
\text { Cont. }\end{array}$ & $\begin{array}{l}\text { I of days } \\
\text { Pot. }\end{array}$ & 8 cont & \% Pot \\
\hline$\leq 45$ & 139 & $43 \pm 0.2$ & $22.65 \pm 0.35$ & $5.7 \pm 0.5$ & $45 \pm 3$ & $122 \pm 16$ & $\begin{array}{c}137 \\
(99 \%)\end{array}$ & $\begin{array}{l}126 \\
(916)\end{array}$ \\
\hline 46550 & 320 & $49 \pm 0.1^{*}$ & $22.13 \pm 0.27$ & $5.9 \pm 0.3$ & $59 \pm 4$ & $119 \pm 11$ & $\begin{array}{c}311 \\
(97 \%)\end{array}$ & $\begin{array}{c}280 \\
(88 \%)\end{array}$ \\
\hline 51555 & 628 & $53 \pm 0.2^{* \star n}$ & $21.51 \pm 0.21^{\circ}$ & $5.2 \pm 0.2$ & $65 \pm 4^{4}$ & $159 \pm 11^{\wedge}$ & $\begin{array}{c}615 \\
(98 \%)\end{array}$ & $\begin{array}{l}514 \\
(82 \%)\end{array}$ \\
\hline
\end{tabular}

\section{By age and SIM:}

(\#=significant versus $\leq 19$ )

\begin{tabular}{|c|c|c|c|c|c|c|c|c|}
\hline $\mathbf{4 5}$ & N & Age & Pre-0p SHIM & PSA & $\begin{array}{c}\text { " of } \\
\text { days } \\
\text { cont. }\end{array}$ & $\begin{array}{c}\text { " of days } \\
\text { Pot. }\end{array}$ & \% Cont & \% Pot \\
\hline$\leq 19$ & 23 & $43 \pm 0.3$ & $14.70 \pm 0.90$ & $8.4 \pm 1.8$ & $54 \pm 13$ & $166 \pm 39$ & $\begin{array}{c}20 \\
(87 \%)\end{array}$ & $\begin{array}{c}17 \\
(74 \%)\end{array}$ \\
\hline 220 & 116 & $43 \pm 0.2$ & $24.23 \pm 0.12 \#$ & $5.2 \pm 0.4 \pi$ & $43 \pm 2$ & $115 \pm 17$ & $\begin{array}{c}115 \\
(99 \%)\end{array}$ & $\begin{array}{c}109 \\
(94 \%)\end{array}$ \\
\hline
\end{tabular}

\begin{tabular}{|c|c|c|c|c|c|c|c|c|}
\hline 46550 & N & Age & Pre-0p SHIM & PSA & $\begin{array}{c}\text { \# of } \\
\text { days } \\
\text { cont. }\end{array}$ & $\begin{array}{c}\text { \# of days } \\
\text { Pot. }\end{array}$ & * cont & \% Pot \\
\hline 519 & 64 & $48 \pm 0.2^{*}$ & $13.95 \pm 0.69$ & $6.0 \pm 0.8$ & $69 \pm 11$ & $158 \pm 37$ & $\begin{array}{c}61 \\
(95 \%)\end{array}$ & $\begin{array}{c}48 \\
(75 \%)\end{array}$ \\
\hline 220 & 256 & $49 \pm 0.1^{*}$ & $24.18 \pm 0.08$ & $5.9 \pm 0.4$ & $56 \pm 4$ & $111 \pm 11$ & $\begin{array}{c}250 \\
(98 \%)\end{array}$ & $\begin{array}{c}232 \\
(91 \%)\end{array}$ \\
\hline
\end{tabular}

\begin{tabular}{|c|c|c|c|c|c|c|c|c|}
\hline 51555 & N & Age & Pre-Op SHIM & PSA & $\begin{array}{c}\text { \# of } \\
\text { days } \\
\text { Cont. }\end{array}$ & $\begin{array}{c}\text { \# of days } \\
\text { Pot. }\end{array}$ & * Cont & \% Pot \\
\hline 519 & 139 & $53 \pm 0.1^{* \wedge}$ & $13.15 \pm 0.48$ & $4.9 \pm 0.3$ & $66 \pm 7$ & $222 \pm 39$ & $\begin{array}{c}138 \\
(99 \%)\end{array}$ & $\begin{array}{c}85 \\
(61 \%)\end{array}$ \\
\hline 220 & 489 & $53 \pm 0.1^{* \wedge}$ & $23.88 \pm 0.07 \|$ & $5.3 \pm 0.2$ & $65 \pm 4$ & $146 \pm 10 \#$ & $\begin{array}{c}477 \\
(98 \%)\end{array}$ & $\begin{array}{c}429 \\
(88 \%)\end{array}$ \\
\hline
\end{tabular}

Pot: ((Old_Low vs Middle_High)(OId_Low versus Young_High))

By Age, SHIM and NS:

\begin{tabular}{|c|c|c|c|c|c|c|c|c|c|}
\hline$\$ 45$ & SHIM & N & Age & Pre-Op SHIM & PSA & I of days Cont. & " of days Pot. & \% Cont & X pot \\
\hline \multirow[t]{2}{*}{ NNS } & $\leq 19$ & 0 & & $\cdot$ &. & $\cdot$ & $\cdot$ & $\because$ & - \\
\hline & 220 & 0 &. & 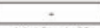 & $\cdot$ & $\cdot$ & $\cdot$ & 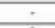 & - \\
\hline \multirow[t]{2}{*}{ PNS } & $\leq 19$ & 10 & $44 \pm 0.3$ & $14.10 \pm 1.69$ & $9.0 \pm 2.1$ & $71 \pm 26$ & $210 \pm 105$ & 9 & $\begin{array}{c}5 \\
\text { (sox) }\end{array}$ \\
\hline & 220 & 30 & $43 \pm 0.3$ & $24.20 \pm 0.25$ & $7.5 \pm 1.3$ & $46 \pm 6$ & $136 \pm 26$ & $\begin{array}{c}30 \\
(100 \mathrm{~s})\end{array}$ & $\begin{array}{c}28 \\
(936)\end{array}$ \\
\hline \multirow[t]{2}{*}{ FNS } & $\leq 19$ & 13 & $43 \pm 0.5$ & $15.15 \pm 0.97$ & $8.1 \pm 2.9$ & $42 \pm 12$ & $149 \pm 38$ & $\begin{array}{c}13 \\
(1000 \mathrm{x})\end{array}$ & $\begin{array}{c}12 \\
(925)\end{array}$ \\
\hline & $\geq 20$ & 86 & $43 \pm 0.3$ & $24.22 \pm 0.15$ & $4.3 \pm 0.2$ & $42 \pm 3$ & $108 \pm 22$ & $\begin{array}{l}85 \\
(9904)\end{array}$ & $\begin{array}{c}81 \\
(94 \times)\end{array}$ \\
\hline
\end{tabular}

\begin{tabular}{|c|c|c|c|c|c|c|c|c|c|}
\hline 46550 & SHIM & $\mathrm{N}$ & Age & Pre-Op SHIM & PSA & \# of days Cont. & $\begin{array}{c}\text { " of days } \\
\text { Pot. }\end{array}$ & \% Cont & \% pot \\
\hline \multirow[t]{2}{*}{ NNS } & $\$ 19$ & 1 & $50 \pm=$ & $18 \pm=$ & $6.0 \pm=$ & 61:- & . & $\begin{array}{c}1 \\
(1000 x)\end{array}$ & 0 \\
\hline & 220 & 2 & $49 \pm 0.0$ & $23.00 \pm 2.00$ & $10.0 \pm 5.0$ & $122 \pm=$ & - & $\begin{array}{c}1 \\
\text { (50:5) }\end{array}$ & 0 \\
\hline \multirow[t]{2}{*}{ PNS } & $\$ 19$ & 25 & $49 \pm 0.3$ & $12.32 \pm 1.28$ & $7.9 \pm 1.8$ & $51 \pm 9$ & $204 \pm 87$ & $\begin{array}{c}23 \\
(92 \times)\end{array}$ & $\begin{array}{c}16 \\
(645)\end{array}$ \\
\hline & 220 & 88 & $49 \pm 0.1$ & $24.08 \pm 0.14$ & $6.2 \pm 0.41$ & $64 \pm 8$ & $77 \pm 24$ & 87 & 77 \\
\hline \multirow[t]{2}{*}{ FNS } & $\leq 19$ & 38 & $480.2 \pm$ & $14.92 \pm 0.78$ & $4.8 \pm 0.7$ & $80 \pm 17$ & $135 \pm 36$ & 37 & $\begin{array}{c}32 \\
(845)\end{array}$ \\
\hline & 220 & 166 & $49 \pm 0.1$ & $24.25 \pm 0.09$ & $5.6 \pm 0.5$ & $52 \pm 4$ & $96 \pm 11$ & $\begin{array}{l}162 \\
(988)\end{array}$ & $\begin{array}{r}155 \\
(93 \times)\end{array}$ \\
\hline
\end{tabular}

\begin{tabular}{|c|c|c|c|c|c|c|c|c|c|}
\hline 51555 & SHIM & N & Age & Pre-Op SHIM & PSA & " of days Cont. & $\begin{array}{l}\text { "of days } \\
\text { Pot. }\end{array}$ & $\%$ cont & \% pot \\
\hline \multirow[t]{2}{*}{ NNS } & $\leq 19$ & 5 & $53 \pm 0.7$ & $3.40 \pm 1.47$ & $8.8 \pm 2.1$ & $88 \pm 21$ & $189 \pm 150$ & $\begin{array}{c}5 \\
(1000)\end{array}$ & $\begin{array}{c}2 \\
(000 x)\end{array}$ \\
\hline & $\geq 20$ & 2 & $53 \pm 0.5$ & $25.00 \pm 0.00$ & $4.0 \pm 1.0$ & $119 \pm 64$ & $462 \pm 269$ & $\begin{array}{c}2 \\
(1005)\end{array}$ & $\begin{array}{c}2 \\
(1000)\end{array}$ \\
\hline \multirow[t]{2}{*}{ PNS } & $\leq 19$ & 61 & $53 \pm 0.2$ & $13.16 \pm 0.71$ & $5.8 \pm 0.4$ & $63 \pm 11$ & $276 \pm 59$ & $\begin{array}{l}60 \\
(988 \times)\end{array}$ & $\begin{array}{c}33 \\
\text { (54\%) }\end{array}$ \\
\hline & 220 & 176 & $53 \pm 0.1$ & $23.80 \pm 0.13$ & $6.3 \pm 0.4$ & $70 \pm 7$ & $199 \pm 25$ & $\begin{array}{l}171 \\
(97 \times)\end{array}$ & $\begin{array}{c}147 \\
(845)\end{array}$ \\
\hline \multirow[t]{2}{*}{ FNS } & $\leq 19$ & 73 & $53 \pm 0.2$ & $13.82 \pm 0.62$ & $4.0 \div 0.3$ & $66 \pm 9$ & $189 \pm 53$ & $\begin{array}{c}73 \\
(1000)\end{array}$ & $\begin{array}{c}50 \\
(68 \mathrm{x})\end{array}$ \\
\hline & 220 & 311 & $53 \pm 0.1$ & $23.92 \approx 0.09$ & $4.8 \pm 0.2$ & $61 \pm 5$ & $116: 9$ & $\begin{array}{l}304 \\
(988)\end{array}$ & $\begin{array}{r}280 \\
(905)\end{array}$ \\
\hline
\end{tabular}

POTENCY:

Old_Low_PNS -> Old_High_FNS

Old_High_PNS $>>$ Old_High_FNS

Old_High_FNS $\rightarrow$ Old_High_FNS nerve spare. Statistical analysis was completed in JMP 12 utilizing a Tukey all pairs test.

Results: When subdivided by age, while there was no significant difference in the percentage of men in each age subgroup in return to continence, there was a significant increase in the number of days to return to continence in Group 3 (65 \pm 4 ) versus Group $1(45 \pm 3)$. While the percentage of men and the number of days to potency in Group $3(82 \% ; 159 \pm 11)$ was significantly higher than Group $1(91 \% ; 122 \pm 16)$. When subdivided by both age and pre-op SHIM, there was no significant difference in the number of days to continence or percentage of its return within one year. The number of days until return to potency was significantly different in Group 3 only between Subgroup a (SHIM

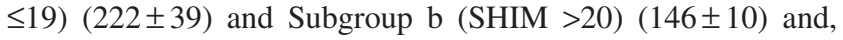
overall Group 3 had both a lower overall percentage of patients with a return to potency as well as a significant increase in the number of days to potency in subgroup a (SHIM $\leq 19)$. When subdivided by all factors (age, pre-op SHIM, and NS), there were no significant differences in the number of days or percentage of patients who achieved continence within one year. While potency in group 3 was mostly affected by both degree of nerve spare and pre-operative SHIM, with the main significant difference being in Group 3b when subdivided into FNS versus PNS. Conclusions: Age, pre-operative SHIM and degree of nerve spare all have a significant effect on the return of continence and potency in men $\leq 55$ yo. Increases in age have a significant impact on recovery of potency. Younger patients with a pre-operative SHIM > 20 who underwent a FNS report the fastest return to both continence and potency.

MP17-13 Artificial intelligence with machine learning: predicting outcomes following robotic prostatectomy

NC Wong, L Patterson, B Shayegan, D Bos

McMaster University

Canada

Introduction \& Objective: Machine learning allows for the analysis and interpretation of "big data" in an automated and adaptive fashion. Predictive algorithms can arm clinicians with knowledge that can provide personalized medicine to patient care. Herein, we trained and compared multiple machine learning algorithms for the prediction of biochemical recurrence following robotic-assisted laparoscopic prostatectomy (RALP).

Materials and Methods: A prospectively collected dataset of 288 patients who underwent RALP was evaluated. We employed multiple supervised machine learning algorithms and used demographic, clinical, imaging, pathological and operative findings in a hypothesis-free manner to build models that could identify patients with biochemical recurrence at 1-year followup. Multiple machine learning algorithms were used to build predictive models including K-Nearest Neighbor, Artificial Neural Networks, Bayesian Networks, Support Vector Machines, Linear Regression, Decision Trees and Random Forest. Results: The mean age of our cohort was $63.4 \pm 6.5$ years with a mean pre-op PSA of 10 . The majority of men had Gleason 7 prostate cancer and the mean prostatic volume was $40 \mathrm{~g}$. During RALP, $65 \%$ of men underwent nerve sparing (unilateral and bilateral), mean estimated blood loss was $180 \pm 119 \mathrm{~mL}$ and mean OR time was $189 \pm 26$ minutes. The average length of stay was $1.0 \pm 0.9$ days. On final pathology, $42 \%$ had extracapsular extension, $15 \%$ had seminal vesicle involvement and $5 \%$ had 
lymph node involvement. Our machine-learned models were able to predict biochemical recurrence with an accuracy score ranged between 0.75 and 0.84 , and area under the curve scores between 0.63 and 0.87 . The support vector machines had the highest accuracy score (0.84) and the artificial neural network model had the highest area under the curve scores $(0.87)$ in our patient cohort.

Conclusions: Machine learning approaches can produce accurate disease predictability. These tools may prove clinically useful for the automated prediction of patients who develop early biochemical recurrence following RALP for which appropriate treatment options can improve patient outcomes and quality of life.

MP17-14 Impact of perineural invasion and tumor volume at prostate biopsy on long-term biochemical recurrence after radical prostatectomy

J Kim, W Ham, S Park, J Ha, S Kang

Severance Hospital

Republic of Korea

Introduction \& Objective: The significance of prostate biopsy perineural invasion (Bx PNI) remains uncertain, and recently, Bx tumor volume has shown to improve the preoperative estimation of the risk for non-organ confined prostate cancer among men with PNI on biopsy. We assessed whether the preoperative prediction model that adds Bx PNI and tumor volume can predict long-term biochemical recurrence (BCR) better than that only based on conventional preoperative variables.

Materials and Methods: Among 4,404 men with radical prostatectomy (RP, 1992-2014), we reviewed 1,512 in whom Bx review was done to verify whether PNI was present or not. Bx tumor volume was based on maximum percent core involvement with cancer. We used multivariate logistic regression analyses to evaluate the relationship of PNI and tumor volume with advanced pathologic features, and Cox regression analyses for BCR for men with complete follow-up.

Results: The group with PNI showed older age, higher prostatespecific antigen (PSA) and Bx Gleason score (GS), worse clinico-pathological stage, and higher rates of positive surgical

Table 1. Multivariate logistic regression analyses of factors predicting ECE and SVI at RP in PC patients verified for PNI status

\begin{tabular}{|c|c|c|}
\hline \multirow[t]{2}{*}{ Variable } & ECE & SVI \\
\hline & OR (95\% Cl), p-value & OR (95\% Cl), p-value \\
\hline Age & $1.02(1.01-1.03),<0.001$ & $1.01(1.00-1.03), 0.044$ \\
\hline PSA & $1.09(1.07-1.10),<0.001$ & $1.08(1.06-1.10),<0.001$ \\
\hline \multicolumn{3}{|l|}{$B \times G S$} \\
\hline$\leq 6$ (reference) & 1 & 1 \\
\hline 7 & $2.15(1.89-2.44),<0.001$ & $3.94(2.82-5.51),<0.001$ \\
\hline$\geq 8$ & $3.47(2.85-4.22),<0.001$ & $8.22(5.66-11.95),<0.001$ \\
\hline \multicolumn{3}{|l|}{ Clinical stage } \\
\hline T1-T2a (reference) & 1 & 1 \\
\hline$T 2 b-T 2 c$ & $2.27(1.84-2.80),<0.001$ & $1.92(1.45-2.54),<0.001$ \\
\hline T3a-T4 & $3.85(1.57-9.46), 0.003$ & $4.02(1.81-8.93), 0.001$ \\
\hline BX PNI & $2.00(1.76-2.28),<0.001$ & $1.63(1.30-2.05),<0.001$ \\
\hline \multicolumn{3}{|l|}{$\operatorname{Max} \%$} \\
\hline$\leq 35.0 \%$ (reference) & 1 & 1 \\
\hline $35.1-60.0 \%$ & $1.40(1.20-1.63),<0.001$ & $1.73(1.21-2.47), 0.003$ \\
\hline $60.1-80.0 \%$ & $2.07(1.75-2.44),<0.001$ & $1.81(1.25-2.63), 0.002$ \\
\hline$>80.0 \%$ & $2.84(2.39-3.38),<0.001$ & $2.98(2.10-4.24),<0.001$ \\
\hline
\end{tabular}

$\mathrm{Cl}=$ confidence interval; $\mathrm{ECE}=$ extracapsular extension; $\mathrm{GS}=\mathrm{Gleason}$ score; $\mathrm{Max} \%=$ maximum percent core involvement with cancer; $\mathrm{OR}=$ odds ratio; $\mathrm{PC}=$ prostate cancer; $\mathrm{PNI}=$ perineural invasion; $\mathrm{PSA}=$ prostate-specific antigen; $\mathrm{RP}=$ radical prostatectomy; $\mathrm{SV}=$ = seminal vesicle invasion
Table 2. Multivariate Cox regression analyses of factors predicting BCR in PC patients verified for PNI status

\begin{tabular}{|c|c|c|}
\hline \multirow[t]{2}{*}{ Variable } & Conventional & Extended \\
\hline & HR ( $95 \% \mathrm{Cl})$, p-value & HR (95\% Cl), p-value \\
\hline Age & $1.00(0.99-1.02), 0.548$ & $1.01(0.99-1.02), 0.408$ \\
\hline PSA & $1.06(1.05-1.07),<0.001$ & $1.05(1.05-1.06),<0.001$ \\
\hline \multicolumn{3}{|l|}{$B \times G S$} \\
\hline$\leq 6$ (reference) & 1 & 1 \\
\hline 7 & $3.73(2.97-4.69),<0.001$ & $3.17(2.51-4.01),<0.001$ \\
\hline$\geq 8$ & $8.29(6.41-10.71),<0.001$ & $6.84(5.23-8.93),<0.001$ \\
\hline \multicolumn{3}{|l|}{ Clinical stage } \\
\hline T1-T2a (reference) & 1 & 1 \\
\hline $\mathrm{T} 2 \mathrm{~b}-\mathrm{T} 2 \mathrm{c}$ & $2.23(1.81-2.76),<0.001$ & $1.80(1.44-2.25),<0.001$ \\
\hline T3a-T4 & $3.36(1.99-5.66),<0.001$ & $2.73(1.62-4.62),<0.001$ \\
\hline BX PNI & & $1.69(1.40-2.03),<0.001$ \\
\hline \multicolumn{3}{|l|}{ Max\% } \\
\hline$\leq 35.0 \%$ (reference) & & 1 \\
\hline $35.1-60.0 \%$ & & $1.09(0.85-1.41),<0.001$ \\
\hline $60.1-80.0 \%$ & & $1.24(0.95-1.62), 0.118$ \\
\hline$>80.0 \%$ & & $1.32(1.02-1.73), 0.038$ \\
\hline
\end{tabular}

$\mathrm{BCR}=$ biochemical recurrence; $\mathrm{Bx}=$ biopsy; $\mathrm{Cl}=$ confidence interval; $\mathrm{GS}=\mathrm{Gleason}$ score; $\mathrm{Max} \%=$ maximum percent core involvement with cancer; $\mathrm{HR}=$ hazard ratio; $\mathrm{PC}=$ prostate cancer; $\mathrm{PNI}=$ perineural invasion; $\mathrm{PSA}=$ prostate-specific antigen

margin and lymph node invasion. Multivariate logistic regression analyses showed that PNI and tumor volume were significant predictors for extracapsular extension and seminal vesicle invasion (Table 1). At a median follow-up of $3 \mathrm{yr}, 542$ men had BCR. BCR curve for group with PNI was lower than that without PNI $(\mathrm{p}<0.001)$. PNI and tumor volume $>80 \%$ were significant predictors for BCR on multivariate analysis (Table 2). Adding $\mathrm{PNI}$ and tumor volume to conventional model improved the fit to the data $(\mathrm{P}<0.001)$, but the c-index only minimally increased from 0.816 to 0.821 .

Conclusions: Prostate Bx PNI and higher tumor volume are associated with advanced pathological features and poorer BCRfree survival after RP, but provide limited additional benefit for risk stratification.

MP17-15 Efficacy of Liposomal Bupivicaine in Robotic Assisted Laparoscopic Prostatectomy: A Prospective Randomized Comparison

I Kelly, S Abourbih, M Pierce, C Bautista, J Lee, B Mattison, J Cheng, H Wagner, M Keheila, B Hu, H Ruckle, DD Baldwin

Loma Linda University Medical Center

United States

Introduction \& Objective: Liposomal bupivacaine (LB) was developed to extend the duration of action for bupivacaine from 8 to 72 hours. It was approved by the FDA for postsurgical analgesia based upon trials for bunionectomy and hemorrhoidectomy. Little data exists on its application after urologic surgery. The purpose of this study was to determine whether LB improves outcomes following robotic assisted laparoscopic prostatectomy (RALP).

Materials and Methods: Forty patients undergoing RALP were prospectively randomized to receive local anesthesia with $30 \mathrm{~mL}$ $\mathrm{LB}$ or $30 \mathrm{~mL} 0.25 \%$ bupivacaine (BP) at the completion of surgery. Primary endpoint was duration of hospital stay, while secondary endpoints were post-operative pain scores (numeric pain score scale) and opioid use in morphine equivalents. 


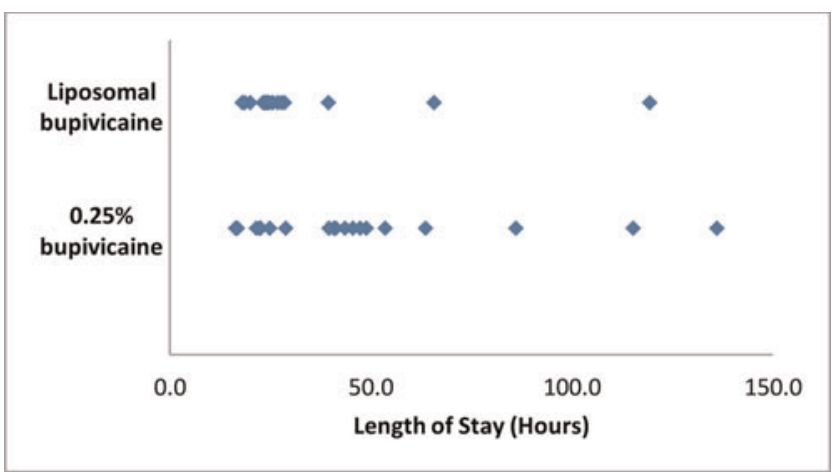

Figure 1: RALP Length of Stay

Patients and personnel administering the pain questionnaires were blinded to the anesthetic received. Independent sample ttest and chi-square tests were performed.

Results: Twenty patients were randomized to each group. LB and BP groups had similar baseline and surgery characteristics, with no adverse local anesthetic complications. Those receiving LB had decreased hospital stay (32.0 hours vs. 46.9 hours, $\mathrm{p}=0.10)$ and postoperative morphine equivalents $(9.2 \mathrm{mg}$ vs. $16.1 \mathrm{mg}, \mathrm{p}=0.11$ ) compared to those receiving $\mathrm{BP}$, but this did not reach significance. However, patients in the LB group were significantly more likely to be discharged on day 1 compared to the BP group $(p=0.01$, Figure 1). Postoperative pain scores on the morning of postoperative day 1 and at discharge were similar for the LB and BP groups (2.0 vs. 2.5, p=0.39, and 2.2 vs. 2.5, $\mathrm{p}=0.56$, respectively).

Conclusions: Patients receiving liposomal bupivicaine after RALP were more likely to be discharged on the first post-operative day, demonstrating that liposomal bupivicaine overcomes barriers to discharge in this population.

MP17-16 Basal Location of Prostatic Cancer on Multiparametric MRI as a New Predictor for Biochemical recurrence after Robotic Radical Prostatectomy

A Mahran, R Abboud, C Buzzy, M Wang, M Glover, I Jaeger, V Gulani, L Ponsky

University Hospitals Cleveland Medical Center United States

Introduction \& Objective: Robot-assisted laparoscopic Radical prostatectomy (RALP) is a common treatment option in patients with high-grade prostate cancer. Ranges for the risk of developing biochemical recurrence (BCR) ten years after radical prostatectomy ranged from $21-37 \%$, depending on the definition of BCR. A variety of predictors for BCR have been reported and on multivariate models it has been shown that PSA and Gleason score are the most significant determinants of outcome. With the increase in utility of MRI as a diagnostic tool, we can use pretreatment imaging as further parameters to consider for developing BCR. Here, our aim is to assess mpMRI parameters along with relevant clinic variables to identify the patients whom are at risk for BCR.

Materials and Methods: Under IRB-approved database, we studied retrospectively a cohort of patients, who had mpMRI of

\begin{tabular}{|l|l|l|l|l|}
\hline \multirow{2}{*}{ Variables } & \multicolumn{4}{|c|}{ Univariate and multivariate cox regression models } \\
\cline { 2 - 6 } & HR $(95 \%$ CI) & P-value & HR $(95 \%$ CI $)$ & P-value \\
\hline Pre-operative PSA & $1.032(1.008-1056)$ & 0.01 & & \\
\hline PSA density & $2.232(1.208-4.124)$ & 0.01 & & \\
\hline $\begin{array}{l}\text { TRUS biopsy Gleason } \\
\text { score } \geq 4+4\end{array}$ & $2.320(1.040-5.180)$ & 0.04 & & \\
\hline $\begin{array}{l}\text { PIRADS 4 or 5 prostatic } \\
\text { mid lesions }\end{array}$ & $3.565(1.332-9.544)$ & 0.011 & & 0.0002 \\
\hline $\begin{array}{l}\text { PIRADS 4 or 5 prostatic } \\
\text { basal lesions }\end{array}$ & $4.972(2.118-11.672)$ & 0.0001 & $5.592(2.288-13.667$ & \\
\hline $\begin{array}{l}\text { PIRADS 4 or 5 } \\
\text { peripheral zone lesions }\end{array}$ & $2.678(0.916-7.830)$ & 0.072 & & \\
\hline $\begin{array}{l}\text { MRI- extracapsular } \\
\text { extension }\end{array}$ & $2.174(0.980-4.823)$ & 0.056 & & \\
\hline $\begin{array}{l}\text { MRI- seminal vesicle } \\
\text { invasion }\end{array}$ & $3.202(1.327-7.727)$ & 0.01 & & \\
\hline
\end{tabular}

the prostate within 11 months prior to have RALP with a median of 3 months. mpMRI was carried out within the University Hospitals of Cleveland between January 2014 and July 2016. 3-T MRI didn't include the use of endorectal coil. We defined biochemical recurrence as patients who had PSA $\geq 0.2 \mathrm{ng} / \mathrm{ml}$ or those who needed secondary treatment after RALP (only 5 out of 25 patients; their lowest detectable PSA was $0.12 \mathrm{ng} / \mathrm{ml})$. Univariate and multivariate Cox regression models with hazard ratios (HR) were assessed at $95 \%$ CI. SPSS 24 was used of statistical analysis. A p-value $<0.05$ was considered statistically significant. Results: A total of 75 patients were included. After a median follow-up of 8 months, biochemical recurrence was found in 25 patients $(33.3 \%)$. On univariate analysis, we identified pre-operative PSA (HR 1.032, $\mathrm{P}=0.01$ ), PSA density (HR 2.232, $\mathrm{P}=0.01$ ), TRUS biopsy final Gleason score (HR 1.580, $\mathrm{P}=0.037$ ), TRUS biopsy Gleason score $\geq 4+4$ (HR 2.32, $\mathrm{P}=0.04$ ) were independent predictors for BCR. From mpMRI, we identified PIRADS 4 or 5 prostatic mid location lesions (HR $3.565, \mathrm{P}=0.011$ ), PIRADS 4 or 5 prostatic basal lesions (HR $4.972, \mathrm{P}=<0.001$ ), PIRADS 4 or 5 peripheral zone lesions (HR 2.678, $\mathrm{P}=0.072$ ), extracapsular extension ( HR 2.174, $\mathrm{P}=<$ 0.056), and seminal vesicle invasion (HR 3.202, $\mathrm{p}=0.01$ ). On multivariate cox regression, we identified PIRADS 4 or 5 lesions located in the basal region of the prostate as the most relevant independent predictor for BCR (HR 5.592, $\mathrm{p}=0.0002$ ).

Conclusions: Patients with clinically significant mpMRI lesions detected in the prostatic base are at high risk of biochemical recurrence after RALP. Future studies are encouraged to validate our model.

MP17-17 Retzius-sparing Robot-assited Laparoscopic Radical Prostatectomy: A Preliminary Result of First 20 cases

H Liu, T Zhou, T Lin, J Huang

Department of Urology, Sun Yat-sen Memorial Hospital China (People's Republic)

Introduction \& Objective: Retzius-sparing robot-assisted laparoscopic radical prostatectomy (RS-RALRP) is an emerging surgical approach for localized prostate cancer with excellent continence outcome. But the new approach may lead to a learning curve, which may affect the pathological and perioperative results. We herein report our preliminary result of first 20 cases.

Materials and Methods: From June 2016 to January 2017, 20 patients with clinically localized prostate cancer underwent RSRALRP. A 3-arm Da Vinci system were set up. Pelvic lymph 


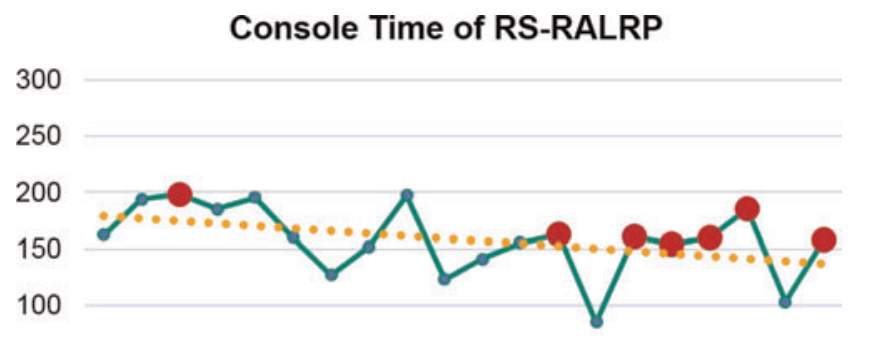

50

0

$\begin{array}{llllllllllllllllllll}1 & 2 & 3 & 4 & 5 & 6 & 7 & 8 & 9 & 10 & 11 & 12 & 13 & 14 & 15 & 16 & 17 & 18 & 19 & 20\end{array}$

node dissection (PLND) was performed before prostatectomy in 7 cases in D'Amico high risk group. The peritoneum was incised posterior to the bladder. With identification of seminal vesicles, the posterior aspect of prostate was dissected. The pedicles of prostate were divided with Hem-o-lok and scissor. The bladder neck was fully dissected and then transected. Anterior aspect of prostate was then dissected to the apex. After division of urethra, the anastomosis was finished with running suture. Post-operative cystogram was scheduled at 7-10 days after surgery. Perioperative and pathology results were collected. The follow-ups were at regular interval.

Results: All operations were succeed without conversion to traditional approach or open surgery. Mean console time was 158 minutes (range 85-198) (Fig 1) with estimated blood loss of 120ml (50-600). In 7 cases with PLND, the mean lymph node (LN) count was 21.7 (8-21). In terms of pathology, 12 patients (60\%) were diagnosed localized disease (T2a:5, T2b:5, T2c:2) and in eight patients (40\%) with locally advanced disease (T3a:4, T3b:4), one had a metastatic LN (T3b). Four patients (20\%) had positive surgical margin. Cystogram showed five patients suffered from anastomotic leakage at 7-10 days after surgery. Follow-up results showed all the patients are free from biochemicalrecurrence. By the end of the first week after catheter removal, 13 patients $(65 \%)$ were continent (less than one pad). By the end of 1-month, 3-month and 6-month, the number was 17/20 (85\%), $17 / 20(85 \%)$ and 6/7 (83\%) respectively.

Conclusions: RS-RALRP is a feasible approach, even for a robot-novice, with acceptable perioperative outcome and pathological outcome and excellent early post-operative continence.

MP17-18 Salvage Robot-Assisted Radical Prostatectomy: 6-year Single Center Experience

MW Mekhail, G Cacciamani, FZ Husain, PW Mekhail, S Chopra, L Medina, C Fay, M Oishi, A Berger, R Sotelo, H Djaladat, M Desai, IS Gill, A Abreu, M Aron

university of southern california

United States

Introduction \& Objective: We report our single-center 6-year experience of salvage robot-assisted radical prostatectomy (sRARP) for recurrent prostate cancer, including functional and perioperative outcomes and rate of biochemical failure.

Materials and Methods: A retrospective chart review was performed of 63 patients who underwent sRARP, between October 2011 and January 2017, at our institution. Primary treatment modality included: external beam radiotherapy in 30 patients (47.6\%); brachytherapy in $10(15.9 \%)$; combined external beam radiotherapy and brachytherapy in $5(7.9 \%)$; HIFU in $3(4.8 \%)$; cryoablation in $11(17.5 \%)$; proton beam therapy in 3 $(4.8 \%)$; and transurethral hyperthermia in $1(1.6 \%)$. The measured endpoints were positive surgical margins (PSM), 30-day postoperative complications (POC), urinary continence (UC) (use of 0 pads), erectile function (EF) (SHIM score $>21$ ), and biochemical failure (BF) (PSA $>0.2 \mathrm{ng} / \mathrm{ml})$. POC were graded using the Clavien-Dindo classification. Continuous variables are shown as mean, standard deviation, and minimum and maximum values (range). Statistical analysis was performed using IBM SPSS Statistics 24.0, with $\mathrm{p}<0.05$ considered statistically significant.

Results: The mean (range) patient age and PSA were 68 years $(52$ - 84) and $10 \mathrm{ng} / \mathrm{mL}(1.8$ - 50), respectively. Pre-sRARP Gleason score was 6 in 11 patients (17.5\%), 7 in $20(32.3 \%)$, and 8 in $17(27 \%)$. Clinical stage was cT1 in 0 patients (0\%), cT2a in $5(7.9 \%)$, cT2b in $1(1.6 \%)$, cT2c in $13(20.6 \%)$, cT3a in 18 $(28.6 \%)$, cT3c in $1(1.6 \%)$, and cT4 in $1(1.6 \%)$. Final pathology showed Gleason 7 in 26 patients (41.3\%), stage pT3a in 18 $(28.6 \%)$, and $\mathrm{pN} 1$ in 17 (27\%). PSM occurred in 20 patients $(31.7 \%)$. Mean (range) operative time was 256 minutes (150 478), estimated blood loss was $124 \mathrm{~mL}(10$ - 450), and length of hospital stay was 2.9 days ( 1 - 14). There was no conversion from robotic to open approach. One intraoperative complication occurred (rectal injury, 1.6\%). Low-grade (I-II) POC occurred in 6 patients $(9.5 \%)$, and high-grade IIIa POC in $5(7.9 \%)$ and IIIb POC in $9(14.3 \%)$. There were no deaths. For those patients whose data was available at last follow-up, 10 of 46 patients were continent $(21.7 \%)$ and 5 of 38 were potent (13.2\%). BF occurred in 7 patients $(11.1 \%)$ at a median (range) of 7.6 months $(1-12)$. Our analysis showed an association between $\mathrm{BF}$ and preoperative PSA, pre-sRARP Gleason score, and final pathology Gleason score.

Conclusions: Our single center experience with sRARP has demonstrated this to be a safe and effective treatment modality for recurrent prostate cancer, with acceptable perioperative and functional outcomes and rate of biochemical failure.

MP17-19 Robot-Assisted Laparoscopic Radical Prostatectomy In Coagulopathic Patients: Diagnosis And Management

A Hsieh, J Stites, G Portman, M Degen, K Basralian, I Sawczuk, R Munver

Hackensack University Medical Center United States

Introduction \& Objective: Based on the prostate's rich blood supply, coagulopathic patients with clinically localized prostate cancer are often encouraged to undergo nonsurgical treatments rather than radical prostatectomy. However the robotic technique, aimed at minimizing blood loss, may make surgery a viable option for this patient population. We examined whether patients with coagulopathies undergoing robot-assisted laparoscopic radical prostatectomy (RALP) are at a higher risk of bleeding events than those without documented abnormal coagulation profiles.

Materials and Methods: A retrospective review was performed of all RALPs performed in patients with postoperative bleeding complications between 2004 and 2017 at a single institution. The study variables included operative (OR) time, estimated blood 
loss (EBL), need for blood transfusion, postoperative hemorrhagic complications, and length of hospital stay (LOS). Data was compared to patients with postoperative bleeding following RALP with normal coagulation profiles.

Results: A total of 1084 consecutive RALPs were reviewed and $14(1.3 \%)$ patients with postoperative bleeding were identified. The cohort included 6 patients with a known coagulopathy and 8 without a documented history of coagulopathy. The mean age was 59.1 years (51-58) OR time $170 \mathrm{~min}(133-225)$, EBL $250 \mathrm{~mL}$ (50-500), and median LOS 4.5 days (2-62) for the known coagulopathic patients, and age 58.6 years (52-65), OR time $152 \mathrm{~min}$ (122-184), EBL $222 \mathrm{~mL}$ (50-700), and LOS 3 days (1-7 days) for the patients without known coagulopathy. Blood transfusion rates were $100 \%$ the coagulopathic group with a mean of $3.2 \mathrm{U}$ RBCs (2-6), and 50\% in the group without known coagulopathy with a mean of $1.7 \mathrm{U}$ RBCs (1-2). Re-exploration was performed in 2 patients (coagulopathic group) and in 1 patient (group without known coagulopathy). Intraoperative findings revealed pelvic/intraperitoneal hematomas without active sources of bleeding. There was no significant difference between the mean age, OR time, and EBL between the groups. Postoperative LOS, blood transfusion rate, and re-exploration rates were statistically higher in the group of patients with known coagulopathies $(\mathrm{p}<0.01)$.

Conclusions: RALP should be performed with caution in patients with known coagulopathies. This study demonstrated no significant difference in preoperative/intraoperative variables, however patients with known coagulopathies had higher rates of postoperative hemorrhagic complications, blood transfusion rates, and LOS. This study further emphasizes the importance of obtaining a thorough history with regard to bleeding tendencies in patients undergoing RALP.

MP17-20 Robot assisted radical prostatectomy in patients with oligometastatic prostate cancer

W Jang, D Koh, J Park, J Lee, Y Kim, I Cho, Y Choi

YUMC

Republic of Korea

Introduction \& Objective: There is growing interest in the role of local treatment in metastatic prostate cancer. However, there is a lack of data for robot assisted radical prostatectomy as therapy in this setting. Thus, we investigated perioperative and oncologic outcomes of robot assisted radical prostatectomy in patients with oligometastatic prostate cancer.

Materials and Methods: We retrospectively evaluated 38 patients with oligometastatic prostate cancer treated with robot assisted radical prostatectomy between 2007 and 2014 at a single high-volume tertiary care center. Oligometastatic disease was defined as five or fewer hot spots on bone scan with or without suspicious pelvic or retroperitoneal nodal involvement at preoperative imaging studies. Perioperative outcomes, radiographic progression-free, cancer-specific, and overall survival were evaluated.

Results: Median follow up duration was 44.5 months. Median patient age at surgery was 65 years. Median PSA at diagnosis was $39.0 \mathrm{ng} / \mathrm{ml}$. Median operation time and console time were 147 and 90 minutes, respectively. Median estimated blood loss was $300 \mathrm{ml}$. Length of hospitalization and catheterization were 5 days and 10 days, respectively. Three patients $(7.9 \%)$ received blood transfusions and 2 patients $(5.3 \%)$ experienced grade 3 postop-

\begin{tabular}{|c|c|}
\hline Variable & $\operatorname{RARP}(n=38)^{*}$ \\
\hline Age, years, median (IQR) & $65(62-69)$ \\
\hline PSA, ng/ml, median (IQR) & $39.0(15.0-84.5)$ \\
\hline \multicolumn{2}{|l|}{ Charlson comorbidity index } \\
\hline 0 & $29(76.3)$ \\
\hline$\geq 1$ & $9(23.7)$ \\
\hline \multicolumn{2}{|l|}{ Biopsy Gleason score } \\
\hline$\leq 8$ & $26(68.4)$ \\
\hline$\geq 9$ & $12(31.6)$ \\
\hline \multicolumn{2}{|l|}{ Clinical T stage } \\
\hline$\leq \mathrm{eT} 2$ & $5(13.2)$ \\
\hline$\geq \mathrm{cT} 3$ & $33(86.8)$ \\
\hline \multicolumn{2}{|l|}{ Clinical N stage } \\
\hline eNo & $21(55.3)$ \\
\hline $\mathrm{eN1}$ & $17(44.7)$ \\
\hline \multicolumn{2}{|l|}{ Neoadjuvant ADT } \\
\hline No & $16(42.1)$ \\
\hline Yes & $22(57.9)$ \\
\hline Variable & Value \\
\hline Operation time, minutes, median (IQR) & 147 (135-186) \\
\hline Console time, minutes, median (IQR) & $90(67-114)$ \\
\hline Estimated blood loss, ml, median (IQR) & $300(200-500)$ \\
\hline Blood transfusion & $3(7.9)$ \\
\hline Hospital stay, days, median (IQR) & $5(4-7)$ \\
\hline Catheter removal, days, median (IQR) & $10(10-12)$ \\
\hline Reoperation & $1(2.6)$ \\
\hline Readmission & $0(0.0)$ \\
\hline \multicolumn{2}{|l|}{ Postoperative complications" } \\
\hline 1 & $3(7.9)$ \\
\hline 2 & 1 (2.6) \\
\hline 3 & $2(5.2)$ \\
\hline \multicolumn{2}{|l|}{ Pathological Gleason score } \\
\hline$\leq 8$ & $13(34.2)$ \\
\hline$\geq 9$ & $25(65.8)$ \\
\hline \multicolumn{2}{|l|}{ Positive surgical margin } \\
\hline No & $8(21.1)$ \\
\hline Yes & $30(78.9)$ \\
\hline \multicolumn{2}{|l|}{ Pathological T stage } \\
\hline pT2 & $1(2.6)$ \\
\hline pT3-4 & $37(97.4)$ \\
\hline \multicolumn{2}{|l|}{ Pathological N stage } \\
\hline $\mathrm{pNx}$ & $3(7.9)$ \\
\hline pNo & $24(63.2)$ \\
\hline pN1 & $11(28.9)$ \\
\hline
\end{tabular}

erative complications. Overall, 37 (97.4\%) and $11(28.9 \%)$ patients had T3-4 disease and lymph node metastasis. Five year radiographic progression-free, cancer-specific, and overall survival rates were $54.5 \%, 78.3$, and $63.8 \%$, respectively.

Conclusions: Our findings support the feasibility and effectiveness of robot assisted radical prostatectomy in a highly selected cohort of prostate cancer patients with bone metastases. 
MP17-21 A Rectal Swab Guided Prophylaxis Program on the Incidence of Infectious Complications Following TransRectal Ultrasound Guided Prostate Biopsy and Fiducial Marker Placement

A Van Hoof, N Ruhotina MD, C Pieczonka MD, B Omarbasha MD, Y Yang MD, S Faisal, DM Albala

Associated Medical Professionals United States

Introduction \& Objective: Trans-Rectal Ultrasound guided prostate biopsy (TRUSBX) and fiducial marker placement (TRUSFM) are noted sources of infectious complications. While data describing the risk of post-TRUSFM infection are lacking, there is an abundance of evidence describing the increasing rate of post-TRUSBX infectious complications. Particularly significant is the rising incidence of more serious infections such as Sepsis and UTIs, which are associated with a high degree of morbidity and cost. Recent evidence links this to a concomitant rise in prevalence of flouroquinolone resistant $(\mathrm{FQR})$ organisms and suggests that use of empirical prophylaxis needs reevaluation. This study aims to make a case for adopting a Rectal Swab (RS) guided prophylaxis by showcasing the effectiveness and feasibility of implementing such a protocol in a large private practice with multiple locations. Additionally, we will be able to better describe the risk of infection associated with TRUSFM.

Materials and Methods: From January 1st, 2011 through May, 30th 2015 we observed the difference in rates of infectious sequelae post-TRUSBX and post-TRUSFM in men who received RS-guided prophylaxis vs empirical prophylaxis with fluoroquinolones per AUA guidelines. RS specimens were collected from patients using a BBL culture swab and plated on selective media containing ciprofloxacin to identify FQR. Standard FQ prophylaxis was prescribed to patients showing FQ sensitivity and patients with cultures positive for $\mathrm{FQR}$ organisms received targeted prophylaxis based on further susceptibility testing.

Results: 5,084 men underwent 1,106 TRUSFM and 5,843 TRUSBX. The prophylactic regimen was prescribed empirically for 2,296 TRUSBXs and 404 TRUSFMs; of these $83(3.61 \%)$ and $21(5.20 \%)$ resulted in infectious complications respectively. A RS-guided prophylactic regimen was used for 3,547 TRUSBXs and 707 TRUSFMs; of these $27(0.76 \%)$ and $7(1.00 \%)$ resulted in infection. 4,248 RS were performed and cultured on 3,294 men. Of these, $472(11.2 \%)$ of the rectal swabs were positive, and 393 men (11.9\%) were found to have at least one FQR organism. Of the FQR organisms identified (96.27\% being E. coli) $83.7 \%$ were multidrug resistant and $37.5 \%$ possessed co-resistances to at least 5 other antimicrobials. Co-resistance rates for specific antimicrobials were as high as $70 \%$ (Ampicillin).

Conclusions: The considerably lower infection rates observed in men receiving RS guided prophylaxis along with the significant prevalence of $F Q R$ displays the advantage of adopting the practice of a rectal swab program. Additionally, the high prevalence of multidrug resistance suggests that alternative methods such as augmented or multi-drug prophylaxis regimens that are commonly empirically prescribed would likely have limited success.

MP17-22 Clinical utility of gene panel associated with increased risk of hereditary prostate cancer: A pilot study

H Palayapalayam Ganapathi, P Patel, G Ogaya-Pinies, E Hernandez, T Rogers, V Patel

Global Robotics Institute at Florida Hospital Celebration Health, University of Central Florida

United States
Introduction \& Objective: Prostate cancer can often have a familial hereditary pattern that can be passed through generations. In addition their progeny are also at increased risk for these cancers. The myRisk Hereditary Cancer TM test from Myriad corp analyzes mutations in 25-gene panel predisposing familial hereditary cancers including prostate, breast, ovary, colorectal and pancreatic cancers. We assessed the clinical utility of myRiskTM test in men underwent robotic-assisted laparoscopic radical prostatectomy.

Materials and Methods: Men with Gleason score $\geq 7$ prostate cancer and one or more significant family history of cancers were offered myRisk TM gene panel test (we captured history of 3 degrees of family members on both maternal and paternal side diagnosed with prostate / breast /ovary / colorectal /pancreatic cancer). Twenty-one patients consented for gene panel testing and samples were collected (18 blood and 3 saliva). Sequencing and large rearrangement analyses of 25 gene panel were performed including BRCA1, BRCA2, CHEK2 and TP53 (These 4 genes are known to be associated with increased risk of prostate cancer).

Results: Total 30 patients had this gene panel analysis. Median patient age was 65 years and mean PSA was $8.7 \mathrm{ng} / \mathrm{ml}$. Positive family history was present for prostate cancer in 16 , breast cancer in 8 and colon cancer in 3 of these patients ( 3 patients had positive family history of both prostate and breast cancers). Four out of these 30 patients $(13 \%)$ reported to have clinically significant mutations for hereditary cancers (2 - BRCA2, 1 - BRCA1 and 1MUTYH). BRCA1 gene mutation is associated with hereditary breast, ovary, pancreatic and prostate cancer (Hereditary Breast and Ovarian Cancer syndrome - HBOC). In addition to these, BRCA 2 predisposes to melanoma. Demographics and pathological parameters were compared between patients with positive versus negative gene panel results.

Conclusions: Locally advanced tumors $(\geq \mathrm{pT} 3 \mathrm{~b})$ or Higher Gleason (9) and/or larger tumor dimension had higher chance of finding hereditary mutations. It is worth considering genetic evaluation in patients with high risk prostate cancer and family history of breast, ovary, pancreatic, colon and prostate cancers. Larger studies needed for confirmation.

MP17-23 Multivariate Analysis of Factors Affecting Biochemical Recurrence After Radical Prostatectomy

JV Balingit, L Sapno, C Evangelista, D Lusaya, J Castillo, D Serrano, J So

St. Luke's Medical Center Philippines

Introduction \& Objective: This study aims to evaluate effects on biochemical recurrence (BCR) of these prognostic factors after radical prostatectomy (RP): patients' clinical $\mathrm{T}$ stage, prognostic grade grouping (PGG) of RP specimen, technique of RP used (Open vs. Robot-assisted Laparoscopic), presence of positive surgical margin (PSM), length of PSM, PGG at PSM, extraprostatic extension (EPE) at PSM, and presence of detectable PSA at 4-6 weeks after RP. It also aims to identify which are independent predictors of risk for BCR.

Materials and Methods: This is a retrospective study. Patients included are those who underwent RP at St Luke's Medical Center from April 2009 to December 2015 with histopathology reports read by a single urologic pathologist and with complete follow-up for at least 1 year. Using Pearson Chi-square and z-test 
with level of significance set at 0.05 , the clinicopathologic variables including: T stage, PGG of RP specimen, length of PSM, PGG at PSM, EPE at PSM, and presence of detectable PSA after RP were assessed to idenify which among these factors were predictive of BCR. Multinomial regression analysis was used to identify which among the variables were independent predictors of risk for BCR.

Results: 165 patients underwent RP from April 2009 to December 2015, of which 72 were eligible for inclusion in the final analysis. Clinical T2 stage was found to be a predictor of BCR with odds ratio of $13.000(\mathrm{p}<0.001)$ compared to stage T1. PGG of final histopathology report of prostatectomy specimen was found to be a predictor of BCR, as those with grade group III, IV and $\mathrm{V}$ had significantly increased risk of BCR with odds ratio of $5.542(\mathrm{p}=0.003)$ compared to those with grade group I and II. Patients with positive margins had increased risk of BCR, with odds ratio of $13.458(\mathrm{p}<0.001)$ compared to those with negative margins. PGG at the PSM was found to be a predictor of BCR, with a grade grouping of III, IV or $\mathrm{V}$ at the positive margin predicting BCR with odds ratio of $20.625(\mathrm{p}=0.008)$ compared to grade grouping of I or II at the margin. Detectable PSA after $\mathrm{RP}$ was found to be a predictor of BCR, with odds ratio of $115.000(\mathrm{p}<0.001)$ compared to undetectable PSA after RP. Technique of RP, measured length of PSM, and EPE at PSM were not found to predict BCR. Furthermore, clinical T stage $(p=0.007)$ and detectable PSA after RP $(p<0.001)$ were found to be independent predictors of $\mathrm{BCR}$ among the risk factors examined.

Conclusions: Of the factors examined, clinical T stage, PGG of RP specimen, presence of PSM, PGG at positive margins, and detectable PSA were found to be significant predictors of BCR. Furthermore, multivariate analysis showed that clinical $\mathrm{T}$ stage and detectable PSA after RP were independent predictors of BCR.

\section{MPS18: IMAGING} MP18-1 Automated Radiographic Stone Measurement: An
Improved Standardization for Stone Volume and Density.

NM Posielski, J Bell, SY Nakada, PJ Pickhardt

University of Wisconsin School of Medicine and Public Health, Department of Urology

United States

Introduction \& Objective: Management of nephrolithiasis is driven by radiographic assessment of stone volume and density, yet, there exists no clear standard of measuring stones. Auto-

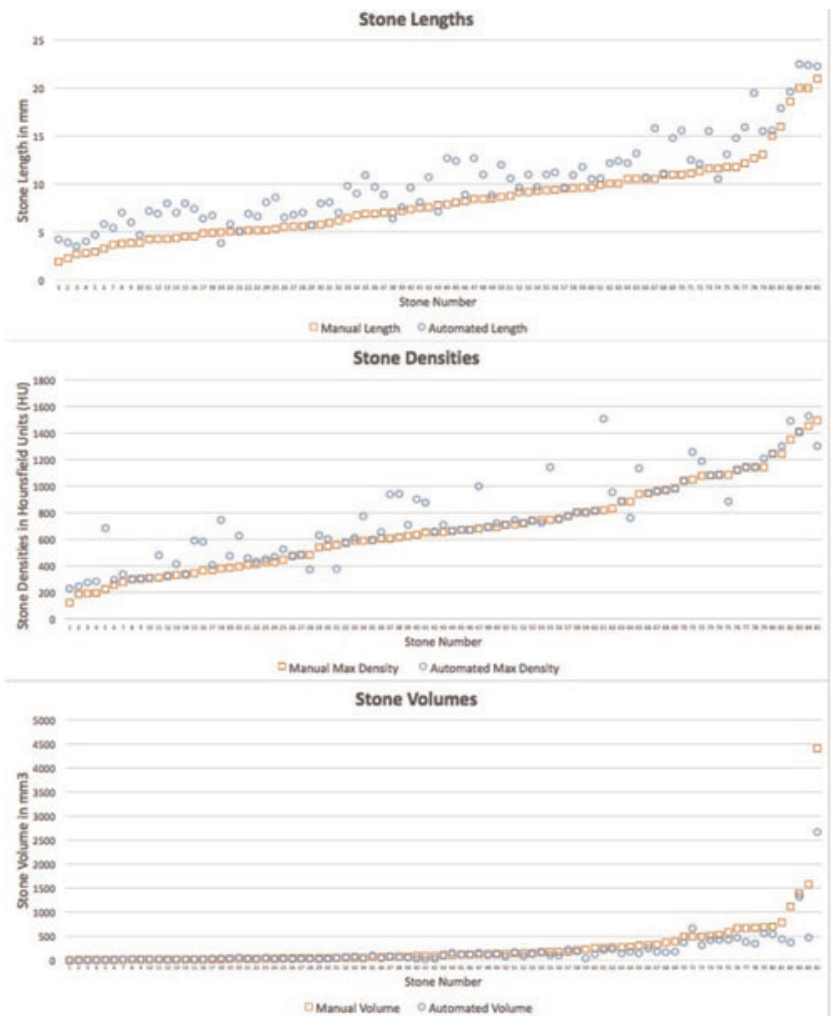

mated stone measurement software provides a comprehensive stone profile including three dimensional volume measurements and eliminates the inter-observer variability seen when measuring stones manually. Our goal was to compare this novel software to conventional manual methods of stone measurement.

Materials and Methods: A single reader (JRB) reviewed kidney and ureteral stones identified on CT scans of patients with known nephrolithiasis. The stones were measured manually to obtain length, width and height. Volume was calculated using the formula for an ellipse, $0.52 \times \mathrm{LxHxW}$. The same stones were then assessed using computer software, which provided automated measurement of stone length, density and volume. Percent difference was calculated between manual measurements and automated software data.

Results: Eighty-five stones were identified on 42 CT scans in 17 patients. Stones were an average of $8 \mathrm{~mm}(1.9-21 \mathrm{~mm})$ in length with a mean maximum density of $451 \mathrm{HU}(21-1492 \mathrm{HU})$ and average stone volume of $182 \mathrm{~mm}^{3}\left(2.8-266 \mathrm{~mm}^{3}\right)$. The automated program closely approximated manual measurements with a mean percent error of $12.4 \%$ for density, $21.6 \%$ for length and $59.2 \%$ for volume.

Conclusions: Comprehensive stone profiling was most concordant measuring stone density, followed by stone length and volume. Automated measurement software is highly reproducible, eliminates inter-observer variability and is capable of measuring stones as small as $1.9 \mathrm{~mm}$ as well as those with complex geometry. This technology offers future promise to improve clinical decision-making in both the surveillance and treatment of renal and ureteral stones.

MP18-2 Feasibility of Automating the Measurement of Kidney Stone Diameter, Volume, and Density on CT

WW Ludwig, P Li, J Ziemba, R Gurnani, S Lim, C Jun, S Kawamato, G Fung, BR Matlaga, D Stoianovici

Brady Urological Institute, Johns Hopkins School of Medicine United States 

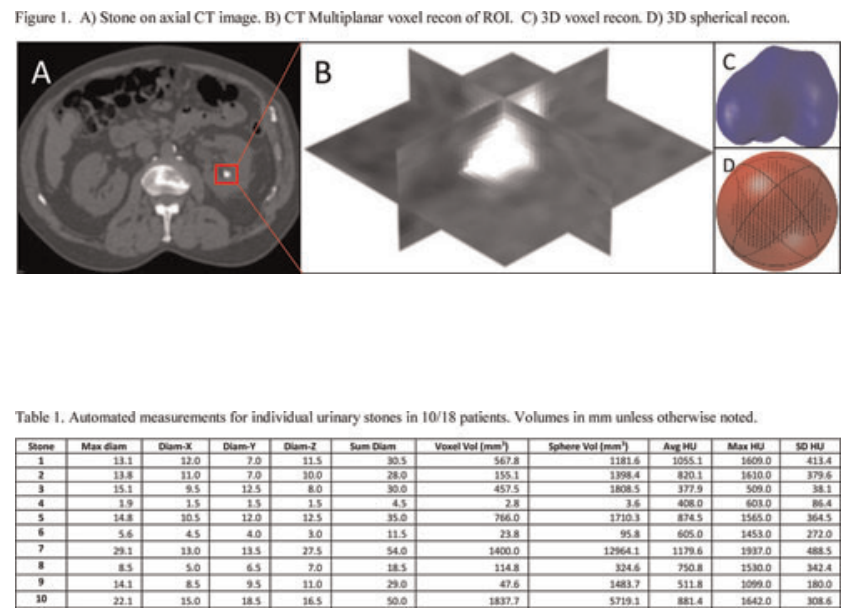

Introduction \& Objective: Several options exist for estimating renal and ureteral stone burden on CT, including volume, surface area, and maximum diameter. No specific measure is accepted as a gold standard for use in research or clinical care, as calculation of these individual parameters is difficult and time consuming. Therefore, we developed an automated tool for calculating relevant urinary stone parameters on CT.

Materials and Methods: An algorithm was developed that identifies stones on CT based on an attenuation threshold within a region of interest (ROI). A threshold of 250 Hounsfield units (HU) was selected to ensure that the stone remains a single object, while eliminating adjacent soft-tissue. For each CT, the images were exported, an ROI was identified by a board-certified radiologist, and the algorithm was applied to this ROI (MATLAB 9.1; Natick, MA) (Figure 1). Stone parameters analyzed included volume, maximum diameter, largest diameter in $\mathrm{x}, \mathrm{y}$ and $\mathrm{z}$ dimensions, cumulative diameter, and HU. Volume was measured by summing all voxels within the stone and this value was correlated (Pearson's) to the calculated volume using the formula for a sphere $\left(4 / 3 \pi r^{3}\right.$, where $r$ is the maximum radius).

Results: As a pilot validation study of the algorithm, a total of 18 consecutive patients with a history of nephrolithiasis who underwent a CT from 9/2016-1/2017 were included in this analysis. Table 1 outlines the calculated parameters for 10 representative stones. The correlation between measured (voxel sum) and calculated (sphere formula) stone volume was 0.807 .

Conclusions: Automated calculation of clinically relevant urinary stone parameters, such as maximum diameter, measured volume, and stone density can easily be obtained and visualized at the point-of-care. Measured and calculated stone volume are moderately correlated, yet differences are likely due to the variability in stone shape. Future investigations will determine how automated stone measurements may aid in clinical outcome prediction.

MP18-3 Role of dual energy CT in the management of urinary uric acid calculi

O Levi, N Idkedek, A Sidi, A Tsivian, E Surin, E Tavdi, R Bass, D Khunovich

Department of Urology, E.Wolfson Medical Center, Holon, Israel

Israel
Introduction \& Objective: Non contrast CT is the imaging of choice for the diagnosis of urinary calculi. Measured density of the calculus in Hounsfield Units (HU), may hint on the stone composition, but remains inefficient for determining treatment. It has been demonstrated that uric acid calculi density will not measure above $700 \mathrm{HU}$. Nowadays, a decision to initiate treatment with urine alkalinization is based mainly on calculi density and absence of radiopaque shadow on abdominal x-ray. However, this strategy may fail because of erroneous identification of chemical composition of the stone. Application of dual energy CT (DECT) enables identification of uric acid calculi. This technology involves two consequent scans of low and high energy. The results are reported in color scheme were red represents uric acid calculus, blue is non-uric acid and red and blue indicates a mixed calculus.

Materials and Methods: To examine the reliability of DECT for identification of the chemical composition of urinary calculi and as a measure of treatment success of uric acid stones. Patients and methods At our institution a patient with a calculus larger than $3 \mathrm{~mm}$ undergoes further evaluation with DECT. From January 2015 to July 2016 four hundred and sixty eight calculi were scanned by DECT in 284 patients. Treatment with urine alkalinization was proposed to patients in whom DECT has indicated uric acid calculi. Efficacy of treatment, defined as disappearance of the calculus was recorded. In cases of spontaneous calculus expulsion or surgical removal the calculus biochemical composition was determined.

Results: Of the 468 calculi scanned with DECT, 398 (85\%) were identified as non-uric acid, $58(12 \%)$ were categorized as uric acid calculi and $12(3 \%)$ were determined as mixed calculi. Thirty three of the uric acid calculi were located in the ureter and 25 in the kidney. The average volume of the calculus was $69 \mathrm{~mm}^{2}$. Density span measured in HU was $130-640$ for uric acid calculi. Among 29 patients with uric acid calculi 13 were treated by urine alkalinization, 5 have undergone, at their own preference, an operation, in 3 the calculus has passed spontaneously while 9 were lost from follow up. All 34 calculi which were treated with urine alkalinization responded to treatment. Thirty three have dissolved completely and one became significantly smaller. All 9 calculi which were subjected to composition analysis were found to be uric acid calculi.

Conclusions: DECT is highly reliable for identification of urinary uric acid calculi. Uric acid calculi, identified by DECT, will respond to urine alkalinization.

MP18-4 CT software-based calculation of stone volume is accurate and reproducible compared to the ellipsoid formula in an in-vitro model

RK Jain, M Omar, H Chaparala, A Kahn, L Kahn, S Sivalingam

Cleveland Clinic Foundation

United States

Introduction \& Objective: Urinary calculus size and volume is typically reported as longest axial diameter (LD) which can be misleading when assessing stone burden, especially for large and irregular stones. Volumetric assessment by CT-based software has been shown to be promising. We compared the accuracy and reproducibility of stone volume estimated by a standard ellipsoid formula (EF) and CT-based algorithm (CT) to true volume (TV) by water displacement in an in-vitro model.

Materials and Methods: Ninety radio-opaque phantom stones were created using clay (size range: $0.5-40 \mathrm{cc}$, density: $814 \mathrm{HU}$ 


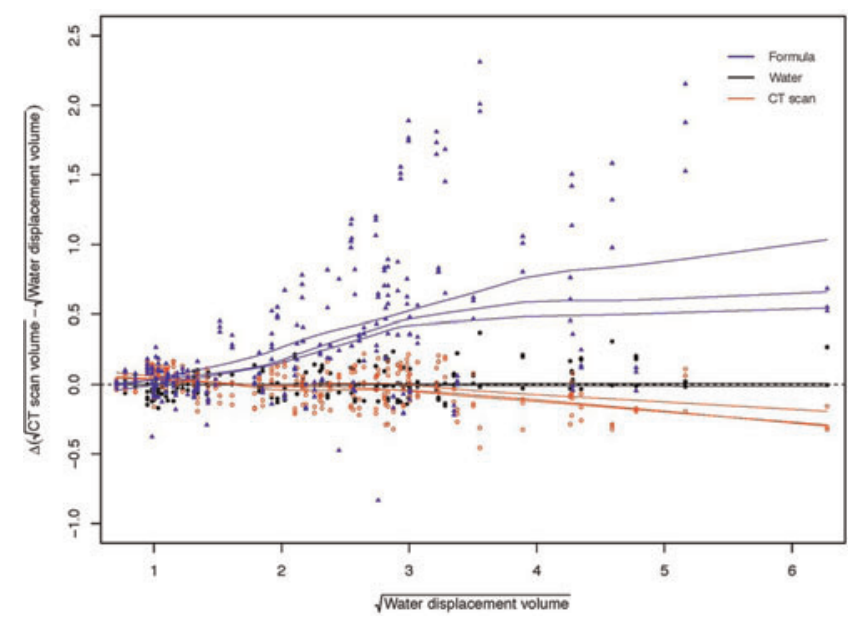

土91) and scanned with CT. For each stone, dimensions for EF estimate were measured using AGFA IMPAX Clinapps software. The region-growing algorithm in the AGFA Volume Viewing 3D software module was used for CT estimate. TV was calculated by water displacement. All measurements were repeated three times, and both inter, and intra-class correlation coefficients (ICC) were calculated. Matched-pair analysis was performed to compare each estimate to TV for the whole group as well as sub-groups based on volume.

Results: The median stone volumes were $4.7 \mathrm{cc}(0.47-36.1 \mathrm{cc})$, $4.75 \mathrm{cc}(0.5-39.3 \mathrm{cc})$, and $5.9 \mathrm{cc}(0.49-47 \mathrm{cc})$, for TV, CT, and EF, respectively. All three groups had a high ICC $(\geq 0.98)$, indicating overall agreement, but there was a stastically significant difference between EF and TV. Overall, there was a significant difference between EF and TV $(p<0.0001)$ but not between CT and TV $(p=0.5)$. The stones were then stratified into 3 groups based on volume. For small stones $(<1.5 \mathrm{cc})$, neither CT nor EF estimates were significantly different to TV (0.05). For intermediate and larger stones $(1.5-6 \mathrm{cc},>6 \mathrm{cc})$, CT estimate was not significantly different from TV $(p>0.05)$ but EF estimate was significantly different $(\mathrm{p}<0.05)$.

Conclusions: CT-based software accurately and consistently estimates the true stone volume across all stone sizes. Compared to ellipsoid formula, CT-based estimates are more accurate and consistent between trials. CT software-based volumetric assessment of stones may have an important role in the clinical management of patients with urinary stones.

\section{MP18-5 Non-Invasive Diagnosis of Renal Cell Carcinoma Subtypes}

\section{A Bergersen, E Price, B Kalb, BR Lee}

University of Arizona College of Medicine, Department of Surgery, Division of Urology

United States

Introduction \& Objective: Renal cell carcinoma (RCC) is the $7^{\text {th }}$ highest incidence cancer in the US, and its incidence has been steadily increasing over the past 20-30 years. Due to the increasing use of cross-sectional imaging, most renal masses are identified incidentally. These lesions tend to be smaller and $\sim 20-30 \%$ of these lesions are benign. Given the potential benign nature of a significant proportion of these masses, accurate diagnosis prior to initiating treatment is becoming more important. It is now recommended to perform biopsy under certain conditions, including when there is suspicion of lymphoma, abscess, or metastases to the kidney, to confirm diagnosis in the setting of bilateral renal masses, and to establish diagnosis prior to initiating treatment for presumed metastatic RCC. However, biopsies are not without complications. For this reason, we evaluated the possible use of MRI to assess tumor characteristics radiographically. The purpose of this study is to demonstrate the ability of MRI to non-invasively differentiate among the RCC subtypes to guide patient counseling and management.

Materials and Methods: We conducted a retrospective chart review of all patients who underwent partial or radical nephrectomy for suspected malignancy at our institution between 2013-2016. Preoperative radiologic diagnoses obtained on MRI were compared with corresponding post-operative final histology on pathological specimen. Preoperative MRI tumor assessment was completed and recorded prior to surgical pathology outcome assessment.

Results: Of the 93 patients identified as receiving either partial or radical nephrectomy, 43 underwent preoperative MRI tumor assessment at our institution. Based on the appearance on T2 post-Gadolinium enhancement, clear cell RCC histology appeared markedly different compared to papillary RCC. Of those patients with histological subtype indicated in their radiology report, $90 \%$ were correctly identified as clear cell type histology, and $80 \%$ were correctly identified as papillary. At least $76 \%$ of subtypes were accurately identified (with the misidentification more likely to occur in rarer RCC).

Conclusions: Prediction of RCC histology by MRI may aid in counseling patients with significant comorbidities and may aid decision making on pre-surgical planning. Further optimization of MRI imaging protocols may hold the promise of even greater accuracy of non-invasive tumor assessment. A prospective study is planned.

MP18-6 Conventional Versus Computer Assisted Stereoscopic Ultrasound Needle Guidance for Renal Access: A Randomized bench-top crossover trial

A Thomas, J Thomas, B Mattison, B West, D Ruckle, M Keheila, S Abourbih, D Baldwin, DD Baldwin, J Cheng

Loma Linda University

United States

Introduction \& Objective: During urologic surgery, ultrasound (US) is an established method for needle guidance, but difficulty in visualizing the needle trajectory may add technical complexity to the procedure. The purpose of this study was to compare conventional ultrasound and a computer assisted stereoscopic needle guidance system for obtaining renal access and mass biopsy.

Materials and Methods: Seventy-one subjects were randomly assigned to target one structure in either a renal access or mass biopsy phantom using computer-assisted stereoscopic or conventional ultrasound guidance in two crossover trials. Recorded outcomes included time for needle adjustment, time to hit the designated target, number of successful trials, number of punctures, and number of course corrections. Participant demographics and subjective rating of the 2 modalities were recorded. Statistical analysis was performed using the student t-test for 
numerical variables and the chi-square test for categorical variables. A P value $<0.05$ was considered significant.

Results: Of the 71 enrolled subjects, 11 were attending physicians, 27 were residents, and 32 were medical students. Overall the computer-assisted stereoscopic system significantly shortened the access time between skin puncture and target contact compared to conventional US (79.4 vs. $51.1 \mathrm{~s} ; \mathrm{p}=0.009)$ respectively. Number of needle course corrections during computer-assisted trials was significantly decreased compared to conventional US $(0.48$ vs. $2.53 ; \mathrm{p}<0.001)$. There was no significant difference in the number of successful punctures between conventional US and computer assisted trials (1.90 vs. $1.71 ; p=0.236)$ respectively. Novice subjects were significantly faster with computer-assisted US needle guidance ( $70 \mathrm{sec}$ vs. $107 \mathrm{sec} ; \mathrm{p}<0.001$ ), while experienced ultra-sonographers trended towards faster overall performance with conventional US needle guidance ( $91 \mathrm{sec}$ vs. $126 \mathrm{sec} ; \mathrm{p}=0.052$ ). Seventy-three percent of subjects preferred the computer-assisted system over conventional ultrasound.

Conclusions: A computer-assisted stereoscopic system may simplify ultrasound-guided procedures by reducing needle access time and number of course corrections. The computer-assisted system was more beneficial with inexperienced subjects but was preferred by $73 \%$ of subjects overall.

MP18-7 Correlation between Confocal laser endomicroscopy (Cellvizio $\left.{ }^{\circledR}\right)$ and histological grading of upper tract urothelial carcinoma: a step forward for a better selection of patients suitable for conservative management.

A Breda, A Territo, F Sanguedolce, G Basile, J Gaya, F Algaba, J Palou, H Villavicencio

Fundació Puigvert, University Autonoma of Barcelona, Spain Spain

Introduction \& Objective: Despite the recent growing interest in the conservative management of upper tract urothelial carcinoma (UTUC), diagnostic process is still lacking of accurate tools, especially for what concerns potential risk of tumours misclassification. Real-time confocal laser endomicroscopy (CLE) provides in vivo microscopic images of tissues using a low-energy laser light source. The aim of the study is to describe our initial experience with CLE for the real-time characterization of UTUC.

Materials and Methods: 16 flexible ureteroscopies [Karl Storz, Tuttlingen, Germany] were performed at our center with CLE for UTUC. Lesions were pre-operatively identified at CT scan. Cellvizio ${ }^{\circledR}$ system was used during fURS to perform CLE on the targeted lesions. Biopsies were then performed. Surgeon's CLE readings (low-grade/high-grade/CIS) were documented in the operation notes. A dedicated genitourinary pathologist -blinded to the surgeon reading- examined all specimens. A third person collected prospectively the CLE readings and the histopathological reports. Cohen's Kappa analysis was performed to test inter-observer agreement.

Results: Mean diameter of tumors at CT scan was $26 \mathrm{~mm}$ (range 5-50 mm). In 10 patients CLE allowed to obtain images with characteristic features compatible with low-grade (LG) UTUC; in 5 patients with high grade (HG) UTUC and in one case CIS. We found correspondence between the CLE images and the final histopathological results in 9/9 cases of LG UTUC (100\%), in 5/6 cases of HG UTUC (83\%) and in 1/1 case of CIS (100\%). Substantial agreement was found at inter-observer agreement $(\mathrm{k}=0.64)$. Neither complications nor limitations related to the use of CLE were recorded.

Conclusions: CLE is a promising new technology in providing a reliable real-time histological characterization of UTUC lesions. Ideal targets might be UTUC patients potentially candidates for conservative management. The diagnostic process is still lacking of accurate tools. In this study we find that CLE, using the Cellvizio ${ }^{\circledR}$ system, seems to help the clinician to have a realtime histological characterization of UTUC lesions. This could better select patient for a conservative treatment.

MP18-8 Gallium labelled-prostate-specific membrane antigen-Positron Emission Tomography/Computed Tomography (GA-PSMA-PET/CT) vs. Choline-PET/CT in Malignant Prostate Cancer - A Systematic Review

M Moghul, AR Rhudd, BP Rai

North Middlesex University Hospital NHS Trust, London

Deanery

United Kingdom

Introduction \& Objective: Recently the role of Prostate-specific membrane antigen-positron emission tomography/computed tomography (PSMA-PET/CT) has become more prominent in helping to diagnose recurrent prostate cancer and malignant prostate cancer. The role of PSMA as a radiotracer appears to be surpassing that of choline tracers in prominent centres. We have evaluated current data regarding the use of PSMA-PET/CT scans compared to Choline PET/CT scans.

Materials and Methods: We performed a review of PubMed/ Medline, Clinical trials.gov and the Cochrane Library in March 2017 according to the Preferred Reporting Items for Systematic Review and Meta-analysis (PRISMA) statement.

Results: 4 studies were included in this review having directly compared 68GA-PSMA-PET/CT and Choline PET/CT scans. Choline tracers used were $11 \mathrm{C}$ in one study and $18 \mathrm{~F}$ in the other studies. Overall 230 men had both 68GA-PSMA PET and choline-PET/CT scans. Overall detection rates for biochemical recurrence were $81.1 \%$ for GA-PSMA-PET and $70.3 \%$ for choline$\mathrm{PET} / \mathrm{CT}$. This indicates that GA-PSMA PET/CT is significantly better than choline-PET/CT for detecting recurrence of prostate cancer $(\mathrm{P}<0.005)$. 68GA-PSMA-PET/CT also showed higher detection rates at lower PSA values. Lesions missed on PSMAPET/CT but captured by choline-PET/CT were found to be negligible (1-2\%).

Conclusions: 68GA-PSMA-PET/CT appears to offer better diagnostic capability than choline-PET/CT, as well as similar if not higher sensitivities and specificities, indicating justification for replacing choline as the radiotracer of choice in prostate cancer and guiding further management decisions.

MP18-9 The diagnostic outcomes of men with a negative Multi-Parametric Magnetic Resonance Imaging (mpMRI) for the diagnosis of Prostate cancer, in low-risk biopsy naive men

AR Rhudd, M Moghul, J McDonald

North Middlesex University Hospital NHS Trust United Kingdom

Introduction \& Objective: The routine use of pre-biopsy multiparametric MRI (mpMRI) for the early diagnosis of prostate cancer $(\mathrm{CaP})$ is controversial. A consensus on the optimal 
management algorithm of a low risk man with a negative mpMRI is unclear. Current guidelines suggest prostate specific antigen (PSA), digital rectal examination (DRE) and shared decisionmaking should be used to determine if a biopsy should be done. mpMRI has the potential to be used as an additional screening tool to select some men to avoid biopsy, potentially lowering the associated harms and over diagnosis. This study evaluated the outcomes of low risk, mpMRI negative men at our institution.

Materials and Methods: Between September 2015 and August 2016, 206 men with a PSA $<10 \mathrm{ug} / \mathrm{L}$ underwent mpMRI for suspected CaP. 22 men were excluded for: previous TURP, previous MRI, prior biopsy or known CaP. 184 men were evaluated for PSA, MRI findings, Prostate Imaging Reporting and Data System (PI-RADS) score, transrectal ultrasound guided (TRUS) biopsy findings and Gleason scores.

Results: $57 / 184$ (31\%) of mpMRIs done were thought to have no evidence of $\mathrm{CaP}$ (PI-RADS <3). 24/57(42\%) of men with a negative MRI went on to have a TRUS biopsy. Of those biopsied, $9 / 24(37.5 \%)$ were positive and 15/24 (62.5\%) were negative for CaP. 6/9 (66.7\%) positive biopsies were Gleason $3+3$ (clinically insignificant $\mathrm{CaP}$ ). $3 / 24$ (12.5\%) of men with a negative MRI had clinically significant $\mathrm{CaP}$, as high as Gleason $4+5$.

Conclusions: Less than half of the men at low risk of prostate cancer (PSA $<10 \mathrm{ug} / \mathrm{L}$ ) that have a negative MRI went on to have a biopsy done. $12.5 \%$ of men with a negative mpMRI will have clinically significant $\mathrm{CaP}$. These results indicate that until the performance of mpMRI is improved and definitive surveillance protocols are devised TRUS biopsy continues to play a role in men with negative mpMRIs.

MP18-10 Accuracy of High-Frequency Endoluminal Ultrasonography for Clinical Staging of Upper Tract Urothelial Carcinoma

JA Farnum, R Vikram, A Rao, D Bedi, C Dinney, SF Matin

MD Anderson Cancer Center

United States

Introduction \& Objective: Staging of upper tract urothelial carcinoma (UTUC) remains a dilemma due to imaging and biopsy limitations leading to understaging. We determine the accuracy of endoluminal ultrasound (ELUS) for clinical staging of UTUC.

Materials and Methods: Patients evaluated for UTUC underwent retrograde pyelography and ureteroscopy. ELUS was performed using mechanical radial scanning at $20 \mathrm{MHz}$ in B-mode with a $5 \mathrm{~F}$ probe. Cine clips were evaluated by 2 radiologists blinded to findings. Results were compared to pathology. Inclusion criteria were patients who underwent NU without pretreatment, or managed endoscopically for cTa-1 disease without recurrence for $>1$ year.

Results: From 9/2008 to 9/2013, 53 patients underwent ELUS without complication. 32 patients met inclusion criteria, and 27 (84.4\%) had conclusive ELUS imaging. ELUS accurately identified 16 of 21 patients with non-MI disease (18 pTa, 2 pT1, 1 CIS) and 1 of 6 patients with at least muscle-invasive (MI) disease (2 pT2, 4 pT3). For pT2 disease, the positive predictive value (PPV) was $16.7 \%$ and the negative predictive value (NPV) was $76.2 \%$. For pT3 disease, the PPV was $0 \%$ and the NPV was $81.8 \%$. Overall accuracy was $51.9 \%$, while for only non-MI disease it was $76.2 \%$.

Conclusions: With current technique and instrumentation, ELUS has poor PPV for stage pT2-3 disease, likely due to poor assessment of invasion of the muscularis. While it may prove useful in select cases to confirm findings of non-MI disease, improvement of current instrumentation is needed before recommending its routine use to confidently confirm T2 disease or higher.

MP18-11 Next-generation ultrasound methods for kidney stone characterization: Feasibility of non-linear beamforming techniques

RS Hsi, J Tierney, S Schlunk, P Karve, R Duddu, B Byram

Vanderbilt University Medical Center

United States

Introduction \& Objective: New ultrasound imaging methods are needed to address the poor sensitivity, specificity, and sizing of kidney stones. Our group has been studying advanced beamforming techniques that address off-axis scattering and multipath scattering in order to enhance B-mode images. These non-linear image formation techniques include short-lag spatial coherence (SLSC) imaging, mid-lag spatial coherence compounding (SLSC Comp), and aperture domain model image reconstruction (ADMIRE). SLSC improves image quality by making images correlated to the spatial phase of the ultrasound wavefronts returning to the transducer, as compared to the traditional image formation techniques that are sensitive to the amplitude of the wavefronts. Mid-lag SLSC Comp (MLSC) is designed specifically for improving ultrasound sensitivity by enhancing coherent scatterers like stones, while suppressing the scattering that originates from soft tissues. ADMIRE is designed to retain characteristic B-Mode features in the process of improving image quality. We also include plane wave synthetic focusing (PWSF), which synthesizes continuous transmit focusing through the field of view. The purpose of this study was to demonstrate in vitro feasibility of these techniques for stone imaging.

Materials and Methods: Human calcium-based kidney stones $(n=12)$ ranging from $0.2-1.8 \mathrm{~cm}$ at the longest axis were rehydrated and de-gassed. A flexible research ultrasound system (Verasonics Vantage 128 system) and linear array transducer (ATL 7-4) were used to image the stones placed on gel phantoms within a water bath at 4 and $8 \mathrm{~cm}$ depths. Stone detection, sizing, and stone shadow contrast were compared among the different beamforming techniques.

Results: All stones were visualized with all techniques at all depths. Compared to traditional B-mode, the posterior stone shadow appeared qualitatively more distinct and of uniform width with ADMIRE. The edges of the stone were most sharp with ADMIRE and SLSC. SLSC Comp technique was able to suppress the gel phantom signal. Compared to true stone size, the sizing error for PWSF, SLSC, ADMIRE and MLSC were $0.57 \pm 0.79 \mathrm{~mm}, 0.69 \pm 1.0 \mathrm{~mm}, 0.48 \pm 2.9 \mathrm{~mm}$, and $0.76 \pm$ $0.71 \mathrm{~mm}$, respectively (ANOVA $\mathrm{p}>0.05$ ). The shadow contrast

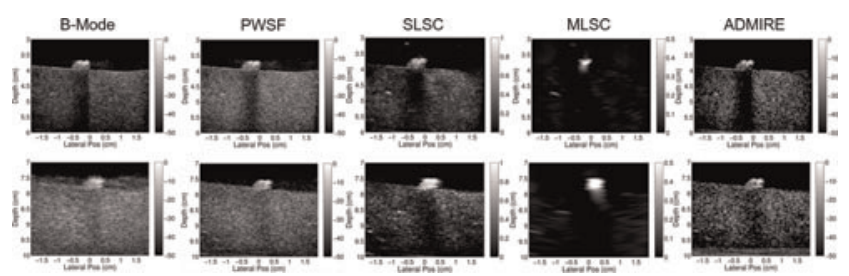


among B-mode, PWSF, SLSC, and ADMIRE were $6.6 \pm 5.0 \mathrm{~dB}$, $7.5 \pm 5.2 \mathrm{~dB}, 13.6 \pm 10.7 \mathrm{~dB}$, and $15.3 \pm 10.1 \mathrm{~dB}$, respectively (ANOVA $\mathrm{p}<0.001$ ).

Conclusions: The SLSC Comp technique appears useful as a stone detection method, whereas PWSF performs best for the sizing task; although, this relationship is not significant here. The stone shadow is enhanced effectively by both SLSC and ADMIRE. Future work is ongoing to evaluate these techniques at greater depths and for embedded stones within kidney models.

MP18-12 Change in nephrometry scoring in small renal masses $(<4 \mathrm{~cm})$ on active surveillance can predict grade of tumours: Tayside Active Surveillance Cohort (TASC) study

W Zhang, C Wei, M Szewczyk-Bieda, C Paterson, G Nabi

United Kingdom

Introduction \& Objective: To determine whether serial nephrometry scores could predict possibility of malignancy and correlate with the grade of cancer on histopathology in patients with small renal masses (SRMs) opting for active surveillance initially.

Materials and Methods: One hundred sixteen patients between January 2000 and December 2016 undergoing partial nephrectomy were analysed using different nephrometry scoring systems. The scores were correlated with histology, grade of cancer and complications of nephron sparing surgery. Measurement of nephrometry scores (RENAL, PADUA, C-Index) in SRMs on serial CT scan images in patients on active surveillance opting for surgery due to change in size or in image characteristics. The scores were correlated with grade, stage, and complications following surgery

Results: Mean age of the cohort $(n=116)$ was $59.75( \pm 12.4)$. Amongst the patients opting for partial nephrectomy, 40 were on active surveillance for at least 12 months (12-60 months) prior to surgery. Nephrometry scores measured on serial CT scan images of these patients showed a significant correlation between increase in score, presence of malignancy and grade of cancer (low vs. high grade). A significantly high increase change in nephromtery scores for solid renal masses was observed compared to cystic. There were higher complications in patients with high nephrometry scores using RENAL and PADUA methods ( $p$ Value 0.02

Conclusions: Increase in nephrometry scoring measurements on serial CT images can accurately predict presence of malignancy and grade of cancer in patients opting for active surveillance and this should be considered as a part of active surveillance protocols.

MP18-13 The significance of bladder filling during computerized tomography in the diagnosis of bladder rupture

$\mathrm{T} \mathrm{Oh}$

Republic of Korea

Introduction \& Objective: Computerized tomography(CT) is the method of choice for establishing patients with abdominal and/or pelvic trauma. However, the sensitivity of CT for detecting bladder rupture has been questioned. We investigated the roles of $\mathrm{CT}$ as the initial evaluation of abdominal and pelvic trauma in diagnosis of bladder rupture.
Materials and Methods: We reviewed the medical records and radiographs of 93 patients with bladder rupture for last 10 years. And among them, all radiographs of 60 patients who underwent both CT and retrograde cystography were evaluated independently by two urologist who had no knowledge of the final diagnosis.

Results: Among 60 patients, all of patients were correctly diagnosed by retrograde cystography, but the CT diagnosis was correct in only 46 patients $(76.7 \%)$, who were 37 patients $(80.4 \%)$ with showed the intraperitoneal rupture and 9 patients $(19.6 \%)$ with extraperitoneal rupture. And of the 14 patients(23.3\%) who were negatively by the CT, all showed the sign id inadequate bladder distention.

Conclusions: We suggest that CT, if properly performed with adequate bladder filling, is as sensitive for detection of bladder injuries as conventional cystography. Especially, in trauma patients with hematuria and suspected other organ injury, CT-cystography with bladder filling may be as accurate as conventonal cystography and obviate the need for an additional cystography.

MP18-14 Posture specific 3D pelvic reconstructions in pelvic organ prolapse derived from open magnetic resonance imaging

MM Abdulaziz, L Stothers, AJ Macnab

$\mathrm{PhD}$ Candidate,

Canada

Introduction \& Objective: Introduction: Gravity impacts pelvic organ location, degree of prolapse, and the integrity of the pelvic floor musculature. Newly available upright open configuration magnetic resonance imaging now allows pelvic structures to be imaged in different positions including when the patient is sitting and upright.

Objective: The objective is to describe a new imaging modality and protocol for image that allows the impact of gravity on POP to be quantified.

Materials and Methods: Methods: Subjects: Volunteer women waiting for POP surgery and controls (asymptomatic, nulliparous) completed a history, physical exam with POPQ staging, and UGDI, CRADI-8 and POPDI questionnaires, and imaging using a $0.5 \mathrm{~T}$ open configuration MRI scanner in the supine,

Figure 1: Orange $\rightarrow$ urethra, purple $\rightarrow$ vagina, green $\rightarrow$ rectum, pink $\rightarrow$ muscles, yellow $\rightarrow$ bladder, red $\rightarrow$ uterus.

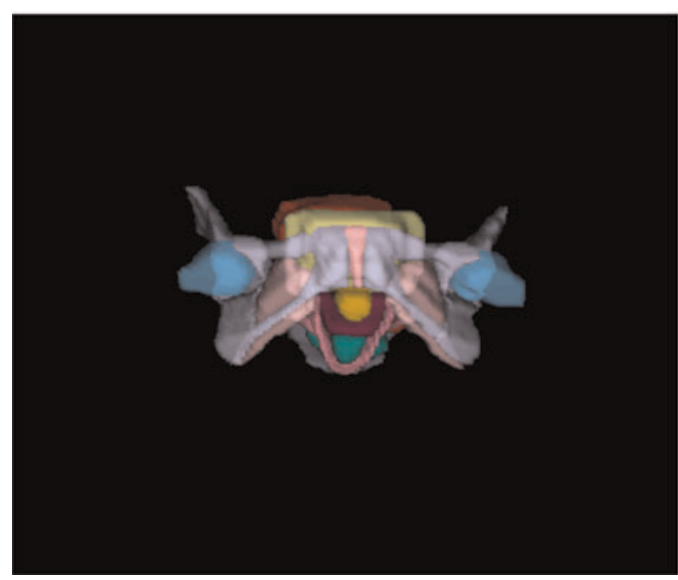


seated and upright position. A T2-weighted sagittal image of the midline structures including the symphysis, urethra, and coccyx was acquired using a protocol iteratively developed (TR/effective TE, 2500/16; echo train length, 32; bandwidth, $32 \mathrm{kHz}$; excitation, one; matrix size, 256 160; field of view, $0.5(24 \mathrm{~cm})$; section thickness, $5 \mathrm{~mm}$, slice gap, 1). Standing included use of intermittent pneumatic compression.

Analysis: Changes in organ position were quantified following overlay of pelvic reference lines generated for each image using mathematical modeling on each image. 2D data extracted from thin-section images were transformed to 3D using Analyze 12.0 (c), multiplane visualization of grayscale images with manual segmentation on up to 50 axial images and a surface-rendering method.

Results: $\mathrm{N}=20$ (10 controls, 10 POP); control age mean 33 years (range 23-56) and POP age mean 53 years (range47-64). Considerable elongation along the PCL and lowest point of the bladder was evident in patients with POP, comparing supine and standing images $(p=0.03)$, but not in the control group $(p=0.07)$. Time for supine, sitting and standing images were 6.30, 2 and 2 minutes respectively.

Conclusions: Open upright MRI is a new technology allowing gravity-influenced quantification of POP through application of pelvic reference line measures and $3 \mathrm{D}$ reconstruction offering new levels of detail for enhanced surgical planning and staging.

\section{MP18-15 Withdrawn}

MP18-16 Contrast-enhanced ultrasound as a radiation free alternative to fluoroscopic nephrostogram for evaluating ureteral patency

T Chi, M Usawachintachit, S Weinstein, MP Kohi, A Taylor, DT Tzou, HC Chang, ML Stoller, J Mongan

University of California San Francisco School of Medicine United States

Introduction \& Objective: Fluoroscopic nephrostogram is commonly used to evaluate ureteral patency after percutaneous nephrolithotomy (PCNL). However, it can incur a significant exposure to ionizing radiation. We have reported feasibility and safety for contrast-enhanced ultrasound (CEUS) with collecting system microbubble contrast injection to obviate the need for radiation exposure. In this study, we compared CEUS to fluoroscopic nephrostogram in evaluating ureteral patency after PCNL.

Materials and Methods: In this prospective cohort non-inferiority study, we enrolled eligible patients with kidney and proximal ureteral stones who underwent PCNL. On postoperative day 1, patients received both CEUS and fluoroscopic nephrostograms within 2 hours of each other to evaluate ureteral patency, the primary outcome for this study. Results from both imaging studies were reviewed in a blinded fashion by two experienced radiologists and compared.

Results: Ninety-two pairs of imaging studies were performed in 82 patients during the study period from September 2015 to October 2016. Five study pairs were excluded due to technical errors preventing imaging interpretation. Females slightly predominated males with a mean age of $50.5 \pm 15.9$ years and a mean body mass index of $29.6 \pm 8.6 \mathrm{~kg} / \mathrm{m}^{2}$. For the remaining 87 sets of studies, $69(79.3 \%)$ demonstrated concordant findings
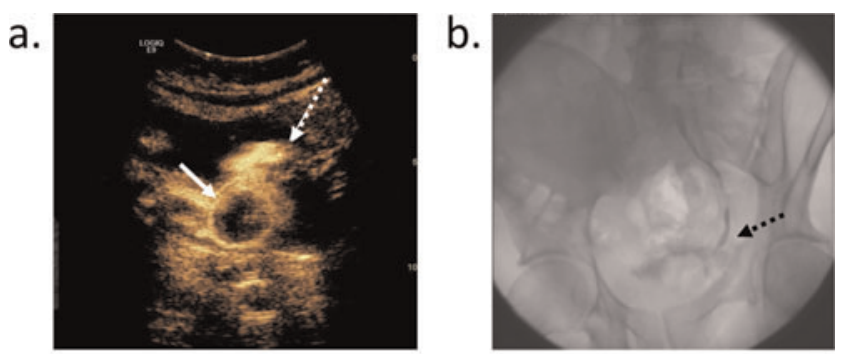

Figure 1. Contrast-enhanced ultrasound and fluoroscopic nephrostogram images from a 60-year old male with a patent collecting system 1 day after PCNL. (a) One minute after injection of ultrasound contrast agent via a preplaced nephrostomy tube, contrastenhanced ultrasound image of the bladder identifies contrast agent within the bladder (white dashed arrow). There is also a Foley balloon seen (white solid arrow). (b) Fluoroscopic nephrostogram confirms ureteral patency and contrast agent entering the bladder (black dashed arrow)

regarding ureteral patency between the two imaging techniques (Figure 1) and $18(20.7 \%)$ were discordant. Fifteen of the 17 patients who demonstrated antegrade urine flow only on CEUS had their nephrostomy tube removed on the same day without experiencing any subsequent adverse events. No adverse events were noted related to ultrasound contrast injection. While CEUS utilized no ionizing radiation, fluoroscopic nephrostograms provided a mean radiation exposure dose of $13.1 \pm 17.5 \mathrm{mGycm}^{2}$. Conclusions: CEUS can be safely performed to evaluate for ureteral patency following PCNL. This imaging technique was mostly concordant with fluoroscopic findings, and it may be a more sensitive modality for predicting clinically significant ureteral patency.

MP18-17 Radiation Exposure from Computed Tomography for Urolithiasis: Results from the Registry for Stones of the Kidney and Ureter (ReSKU)

DT Tzou, M Usawachintachit, D Isaacson, K Taguchi, BA Sherer, ML Stoller, T Chi

\section{UCSF}

United States

Introduction \& Objective: Patients with kidney stones routinely have computed tomography (CT) scans as part of their diagnostic workup. The recommended yearly dose limit for occupational radiation exposure by the International Commission on Radiological Protection is $50 \mathrm{mSv}$, yet how often this limit is exceeded for stone patients remains unknown. Radiation exposure beyond this limit may impart long-term risks of secondary malignancies. This study aimed to identify annual effective radiation doses received by consecutive stone patients referred to a tertiary care stone clinic.

Materials and Methods: From October 2015 to March 2017, newly referred stone patients were prospectively enrolled into the Registry for Stones of the Kidney and Ureter - ReSKUTM at the University of California San Francisco (UCSF) for this cohort study. For each patient, all available CT scans of the abdomen/ pelvis performed prior to their initial visit were reviewed. Measures of radiation exposure such as volume computed tomography dose index $\left(\mathrm{CTDI}_{\mathrm{vol}}\right)$ and dose-length-product (DLP) were recorded. Effective dose (ED) was calculated by multiplying the DLP by a validated conversion factor (0.015) - appropriate for $\mathrm{CT}$ scans of the abdomen and pelvis. Both 
univariate and multivariate regression analysis were performed to determine factors associated with patient ED, and a logistic model was used to dichotomize patients receiving a $>50 \mathrm{mSv} /$ year cumulative ED.

Results: Of 594 patients enrolled into ReSKU ${ }^{\mathrm{TM}}, 458$ patients had at least $1 \mathrm{CT}$ abdomen/pelvis available for review. Including UCSF, CT scans were performed at 135 unique radiologic imaging centers. Only $5.5 \%$ of the $819 \mathrm{CT}$ scans performed met criteria for a low dose CT scan (ED $50 \mathrm{mSv} /$ year (mean $77 \mathrm{mSv}$, range 51$210 \mathrm{mSv}$ ). Univariate logistic analysis demonstrated a statistically significant association of body mass index (BMI) (OR 1.1), number of scans (OR 3.8), performing location (OR 2.6), and multi-phase scans (OR 3.7) with patients receiving a $>50 \mathrm{mSv} / \mathrm{year}$ cumulative ED $(p<0.01)$, respectively. Multivariate linear regression analysis revealed that high BMI, multi-phase scans, and number of scans was associated with higher $\operatorname{ED}(\mathrm{p}<0.01)$.

Conclusions: A subset of kidney stone patients continue to receive dangerous ionizing radiation exposure from CT scans. Statistical analysis indicated that higher effective dose was associated with patients who had higher BMI, multi-phase CT scans, and a higher number of scans performed. All practitioners should be cognizant of the amount of radiation received by kidney stone patients and continued efforts to utilize low-dose CT protocols should be a priority within the medical community.

MP18-18 Radiation Exposure from KUB (kidney-ureterbladder) Radiographs for Nephrolithiasis is Not Trivial

J Kuebker, J Shuman, RS Hsi, S Herrell, N Miller

Vanderbilt University Medical Center

United States

Introduction \& Objective: Given the recurrent nature of nephrolithiasis, patients with this disease are at risk of being exposed to excessive ionizing radiation. As the risk of radiation exposure is thought to be stochastic, urologists will often obtain a KUB as an alternative to computed tomography (CT), albeit with lower sensitivity and specificity. Professional societies' guidance on acceptable KUB dose is .7-.8mSv. Anecdotally, we have noticed increasing numbers of images being acquired in KUB exams and thus sought to investigate effective dose of KUBs using contemporary equipment and techniques.

Materials and Methods: A retrospective review was performed over a 6-month period to identify all stone patients with a visit to a single endourologist. Medical records were reviewed to identify patients undergoing KUB. Patients were included if a CT scan was available for review within 3 years. Age, gender, BMI, and anterior/posterior abdominal diameter on CT were analyzed for association with effective dose. Effective dose was estimated using a Monte Carlo based simulation program. When present, outside KUB films were compared to those obtained at our institution.

Results: 54 patients were identified with a KUB obtained for their clinic visit. Mean \pm SD age was $55 \pm 14$ years. Patients tended to be obese with a mean BMI of $31.6 \pm 6.7 \mathrm{~kg} / \mathrm{m}^{2}$ and abdominal thickness of $28.6 \pm 4.4 \mathrm{~cm} .92 .6 \%(50 / 54)$ of exams contained multiple radiographs (range 1-4). Only $25.9 \%(14 / 54)$ of the exams' effective dose were under the standards set by professional societies (.7-.8mSv). Range of effective dose was .14-6.95 mSv. Mean effective dose was $2.15 \pm 1.67 \mathrm{mSv}$. BMI, abdominal thickness, and image count were all associated with an increase in effective dose $(\mathrm{p}<.01$ for each). Abdominal thickness was associated with $>1$ image being performed $(\mathrm{p}=.0295) .41 \mathrm{KUBs}$ were identified from outside facilities and similar to internal KUBs, $88 \%$ of these exams contained $>1$ image (range 1-4).

Conclusions: KUB in the contemporary setting is associated with a much higher effective dose than is often referenced. This increased dose is associated with both larger patient size and an increase in the number of images acquired. These findings are likely reproducible as increased patient size is widespread and the number of multiple image studies were similar at outside facilities. Potential causes of increased dose include shifts in technique with digital radiography and larger patients requiring more energy and multiple images to complete an exam.

\section{MP18-19 Availability and Utilization of Reduced Dose Computed Tomography for Nephrolithiasis}

\section{J Kuebker, J Robles, N Miller, S Herrell, RS Hsi \\ Vanderbilt University Medical Center \\ United States}

Introduction \& Objective: Reduced dose computed tomography (RDCT) protocols have been developed to combat repeated high levels of radiation exposure kidney stone patients often receive during acute episodes and surveillance. Prior reports show RDCT is greatly underutilized with only $2 \%$ of stone protocol CTs reaching a "low dose" threshold of $<3 \mathrm{mSv}$. The purpose of this study was to determine the availability and utilization of RDCT among endourologists.

Materials and Methods: A survey was designed to investigate the availability and indications for RDCT use among members of the Endourological Society. Demographics of responders were collected along with practice details to determine association with RDCT availability.

Results: Response rate was $7.6 \%$ with 214 of 2822 invitees completing the survey. $44 \%$ of responders were from North America. $76 \%$ of North American and $56 \%$ of international participants were in academic practice. $60 \%$ of North American and $52 \%$ of international responders reported $>50 \%$ of their practice composed of stone disease. Fellowship rates were $67 \%$ in North America and 50\% internationally. RDCT was available for $77 \%$ of North American urologists and $71 \%$ overall. Geographic location, fellowship experience, year of residency completion, and percentage of practice devoted to stones were not associated with availability of RDCT in North America or internationally. Urologists in academic practice were significantly more likely to have RDCT available compared to other practice types $(\mathrm{p}=.028)$. If RDCT was available, 93\% reported utilization, while only $7 \%$ did not. The most common reasons for not utilizing RDCT were reduced image quality (40\%) and concerns of missing other diagnoses $(30 \%)$. The majority of responders utilize RDCT in patients with renal colic (80\%), to confirm stone passage (78\%), and to assess for residual stone burden after percutaneous nephrolithotomy (76\%). Rates were lower for surveillance in stone formers (57\%) and in pregnancy when ultrasound and KUB were inconclusive (43\%) RDCT was unavailable for $22 \%$ of responders and $7 \%$ were unsure. The most common reasons cited for unavailability were unfamiliarity with the technique (39\%), inadequate equipment (22\%), and poor coordination with radiology departments (22\%). All participants without RDCT capability reported they would utilize the technique if available.

Conclusions: RDCT is relatively accessible worldwide, but is more available in academic settings. Greater availability and utilization of RDCT may occur with educational efforts and improved coordination between urologists and the departments that order and perform these exams. 
MP18-20 Identifying the Relationship between Fluoroscopy Pulse Rate and Radiation Absorbed Dose

T Yecies, MJ Semins, M Sheetz, M Duranko, H Taan, M Shahait, R Chaudhry

UPMC

United States

Introduction \& Objective: Techniques in reducing overall radiation dose during fluoroscopy are important for both patients and surgeons. Modern fluoroscopy units have settings that can be modified to lower radiation exposure, including low-dose and pulsed fluoroscopy. Prior studies have demonstrated that reducing pulse rate yields reductions in radiation dose that are less then would be expected if pulse rate and radiation dose had a linear relationship. It is unclear to what extent this finding stems from automated exposure adjustments performed by $\mathrm{C}$-arm devices, changes in surgeon behaviour, or limitations in using proxy measurements such as fluoroscopy time or C-arm reported radiation dose. We aimed to identify the relationship between fluoroscopy pulse rate and radiation absorbed dose in a controlled setting with equivalent exposure behavior.

Materials and Methods: Using a simulated patient model, 60 second fluoroscopy exposures were performed using pulse rates of $30,8,4,2$, and 1 pulse(s) per second (pps). Each experiment was performed in triplicate using both standard and low-dose settings using a GE OEC 9800 plus C-arm. Landauer nanoDo$\mathrm{t}^{\mathrm{TM}}$ OSL dosimeters were used to measure the radiation absorbed dose. The relationship between fluoroscopy pulse rate and radiation absorbed dose was analysed for both standard and low-dose settings. The correlation between radiation absorbed dose and proxy measurements including fluoroscopy time and C-arm reported radiation dose was evaluated.

Results: Fluoroscopy pulse rate and radiation absorbed dose demonstrated a near-perfect linear correlation for both standard $\left(\mathrm{R}^{2}=0.995\right)$ and low-dose $\left(\mathrm{R}^{2}=0.998\right)$ settings. For any given pulse rate, using the low-dose setting reduced radiation absorbed dose by $58 \pm 2.8 \%$. Fluoroscopy time demonstrated a linear relationship with radiation absorbed dose for both standard $\left(\mathrm{R}^{2}=0.996\right)$ and low-dose $\left(\mathrm{R}^{2}=0.990\right)$ settings; however, for any given pulse rate fluoroscopy time did not change with the use of the low-dose setting. C-arm reported radiation dose correlated linearly with radiation absorbed dose $\left(\mathrm{R}^{2}=0.999\right)$ but consistently under-estimated measured values by an average of $49 \pm 3.5 \%$. Using, a combination of $1 \mathrm{pps}$ and low-dose fluoroscopy reduced radiation absorbed dose by an average of $97.7 \pm 0.1 \%$.

Conclusions: With equivalent exposure times, radiation absorbed dose decreases linearly with fluoroscopy pulse rate. While fluoroscopy time correlates with absorbed radiation dose, it does not account for the effect of the low-dose setting. These findings allow for surgeons to make an informed decision when optimizing the trade-off between radiation exposure and image quality.

MP18-21 The application of ImageJ in stone analysis on $X$-Ray images: A new modality for detecting changes in stone mass and density

ME Hassan, J Metcalfe, M Wilson, K Subramonian

Queen Elizabeth Hospital Birmingham

United Kingdom

Introduction \& Objective: Asymptomatic non-obstructing renal calculi are followed up with Xray-KUB which demonstrates changes in stone size and location, without information about changes in stone density or mass. We introduce a new modality to analyze X-ray images using Image J software to quantify the stone mass/density in comparison with CT scan.

Materials and Methods: Image J is an open source image processing software which we used to develop a program to analyse stone mass, which represents an indirect measurement of stone size and density. To validate the analysis, two investigators analyzed the images and their results compared using Student's t test. The stones on X-ray were given a score that was compared with CT Hounsfield units. The data from the ImageJ analysis and CT were compared using student's t test and Pearson $r$ correlation test.

Results: Sixty four images from nine patients who underwent Xray and CT images over the follow up period were analysed. The range of follow up period was between 4 to 7 years with an average of 4.56 years. The results have shown significant correlation between the images analysed by two investigators. This demonstrated that the method of analysis is robust and the correlation coefficient was $(r=0.9205)$. The comparison between ImageJ X-Ray analysis and CT images have shown a significantly $(P<0.0001)$ positive correlation between both results $(\mathrm{r}=0.8108)$.

Conclusions: Stone analysis of plain X-ray KUB using ImageJ could be a useful modality in assessing the change in stone mass/ density over time which may guide stone management. Further analysis and assessment of this method is required to develop a robust software which could be used in the clinic to reduce the frequency of CT scans which will impact on patient safety by reduction of radiation exposure.

MP18-22 Does Intermittent Fluoroscopic Screening During Endourological Procedures Reduce Exposure to Radiation?

L Mokool, T Amer, A Ramsay, SK Nalagatla

Monklands District General Hospital, Scotland United Kingdom

Introduction \& Objective: The use of fluoroscopy during endourological procedures is a common practice. Urologists and other theatre staff are at risk of increased exposure to radition especially in the form of scatter radiation. ALARA (As Low As Possible Reasonably Achievable) is a radiation safety principle for minimising radiation doses by employing all reasonable methods. This is not only a sound safety principle but also a regulatory requiremnet for all radiation safety programs.

The aim of our study is to compare the dose of radiation and exposure time between continuous screening and intermittent flash fluoroscopy during elective endourological procedures.

Materials and Methods: An analysis of patients undergoing both rigid (RURS) and flexible ureteroscopy (FURS) was initially carried out, using intermittent flashes of fluoroscopy to reduce radiation exposure. The key variables collected were: patient demographics, cummulative radiation dose, radiation exposure time. We also recorded the total number of flashes during each procedure and any complications that occured.

For comparison, a retrospective analysis was done of patients undergoing the same procedures when continuous screening was mainly used. The same variables as above were collected.

Results: The intermittent screening group included 32 procedures of which 22 are FURS and 10 RURS. The overall mean dose of radiation used was $5.42 \mathrm{mGy}$ and the average exposure 
time was $15.5 \mathrm{~s}$. The mean radiation dose used for URS was 5.12 mGY and mean exposure time was $10.8 \mathrm{~s}$. The values for FURS were $5.29 \mathrm{mGy}$ and $16.9 \mathrm{~s}$. The continuous screening group comprised 30 procedures, of which 17 were FURS and the rest RURS. The overall mean dose of radiation used was $20.41 \mathrm{mGY}$ and the average exposure time was $39.74 \mathrm{~s}$. The mean radiation used for RURS was $8.74 \mathrm{mGY}$ and exposure time $21 \mathrm{~s}$. The values for FURS were $28.43 \mathrm{mGY}$ and 53s respectively. This translates to a $73.4 \%$ reduction in overall radiation dose and $61 \%$ reduction in overall exposure time. The average dose of radiation per flash was also very low at $0.277 \mathrm{mGy}$ and there were no intraoperative complications.

Conclusions: Although the numbers are small, our study shows that using fluoroscopy intermittently during endourological procedures is safe and significantly reduces exposure to radiation. We plan to explore this futher by recruiting more patients into the study. Additionally, we intend to determine if intermittent flash fluoroscopy can be safely used in the emergency setting.

MP18-23 Diagnostic accuracy of low and ultra-low radiation dose CT (computerised tomography) of the urinary tract for identification of stones in patients with renal colic.

FE Rodger, A Ng, N Santoni, OM Aboumarzouk

United Kingdom

Introduction \& Objective: CTKUB is the investigation of choice for the investigation of acute renal colic however radiation exposure can be a concern in young patients with recurrent presentations. We aim to investigate the diagnostic accuracy of low and ultra-low radiation dose CT of the urinary tract for detection of urinary tract stones in patients with renal colic.

Materials and Methods: A systematic review of the literature from 1995-2017 was carried out. Literature search and data extraction were conducted by two reviewers. The systematic review was performed according to the Cochrane diagnostic accuracy review guidelines. Specificity and Sensitivity values were calculated and summary receiver operating characteristics were calculated for low-dose $(<3.5 \mathrm{mSv})$ and ultra-low dose $(<$ $1.9 \mathrm{mSv})$ CT separately.

Results: A total of 12 studies were included following screening. A total of 1439 patients were included in the review (475 in the

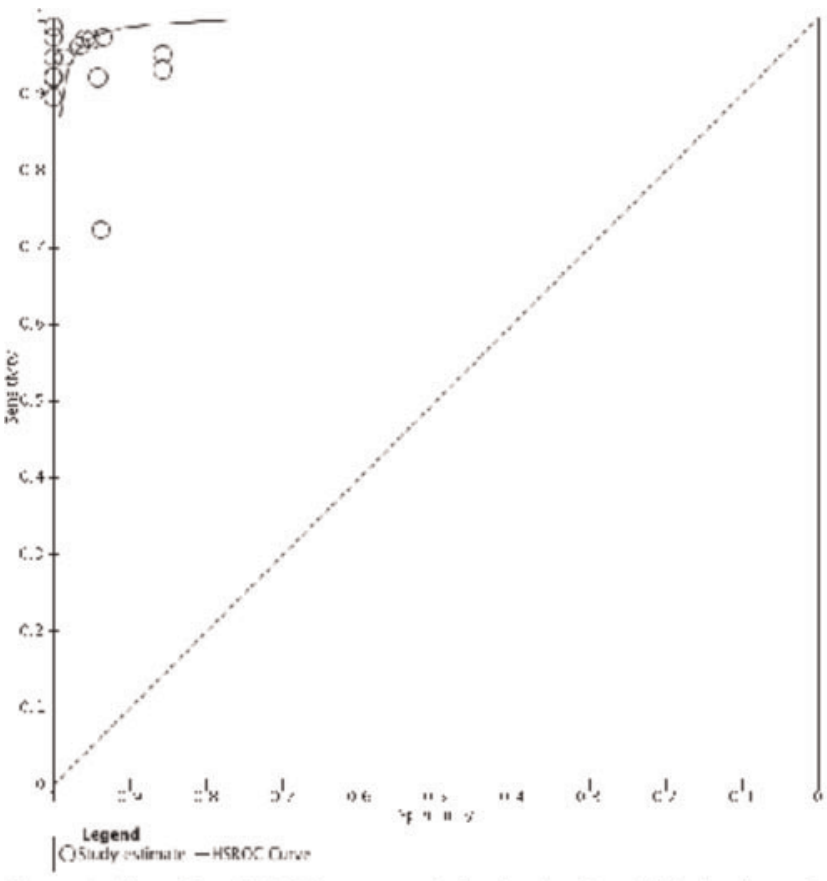

Figure 1. Hierarchical HSROC curve graph for ultra-low dose CT in the diagnosis of urinary tract stones.

low-dose CT group and 964 in the ultra-low dose CT group). Mean effective radiation doses were 2.1-4.5mSv for low-dose and 0.48-1.9 $\mathrm{mSv}$ for ultra-low dose. Using standard dose CTKUB as the reference standard the sensitivity of low-dose CTKUB ranged from $90-98 \%$ and specificity from $88-100 \%$. The sensitivity of ultra-low dose CTKUB ranged from $72-99 \%$ and the specificity from 86 to $100 \%$.

Conclusions: Low and ultra-low dose CTKUB provide effective methods the identification of urinary tract stones. High sensitivity and specificity are maintained despite significant radiation dose reductions compared to standard dose CT. This provides an opportunity to reduce lifetime radiation exposure in patients with urinary tract stones This will be of particular benefit in young patients with recurrent stones disease.

\section{MPS19: MIS ADRENAL SURGERY AND LESS/NOTES}

MP19-1 Impact of surgeon's awareness of radiation safety on fluoroscopy time during flexible ureteroscopy.

B Becker, AJ Gross, C Netsch

Asklepios Klinik Barmbek, Department of Urology Germany

Introduction \& Objective: Fluoroscopic guidance is routinely used during ureterorenoscopy for nephroureterolithiasis. According to the ALARA principles, radiation dose should be kept low to decrease the risk of negative long-term effects for medical personnel. This study evaluates the impact of the doctors' awareness regarding x-ray exposure for pa- tients undergoing flexible ureteroscopy (fURS) for nephroureterolithiasis.

Materials and Methods: Patients with fURS and laserlithotripsy for nephroureterolithiasis were included in this study. Group $1(n=25)$ was analyzed retrospectively regarding patient characteristics, stone characteristics, procedure characteristics, outcome and $\mathrm{x}$-ray exposure. Group $2(\mathrm{n}=25)$ was analyzed with the same parameters prospectively after the surgeons have been instructed to keep fluoroscopic time as low as possible.

Results: There were no differences between the groups with regard to patient characteristics. The mean stone number was 1.88 in group 1 and 1.76 in group 2 . The stone burden was measured as the cumulative, longest diameters in $\mathrm{mm}$ of the stones and did not 
differ between the groups ( $10.6 \mathrm{~mm}$ vs. $11.5 \mathrm{~mm}$ ). There were no differences regarding intraoperative (Satava classification system) and postoperative (Clavien-Score) complications between the groups. One patient in group 2 needed a second operation for complete stone removal. Regarding the time of surgery, the two groups differed significantly $(54 \pm 26.9 \mathrm{~min}$ vs. $77 \pm 33.9 \mathrm{~min})$ ( $\mathrm{p}<0.05)$. The mean fluoroscopy time (36 vs. 29.4 seconds) was significantly shorter in group $2(\mathrm{p}<0.05)$.

Conclusions: Knowledge about radiation exposure leads to a relevant decrease in fluoroscopy time and the use of radiation. However, reduction of fluoroscopic time leads to a prolonged time of surgery.

MP19-2 The advantages of robotic-assisted laparoscopic surgery for huge adrenal tumors inferior vena cava and beside great vessels (Report of 58 Cases)

S Zhong, Z Fang, Z Wu, S Chen, M Zhang, F Gao, X Wang, W He, T Xu, Y Zhu, Q Ding, Z Shen

China (People's Republic)

Introduction \& Objective: To summarize the surgical treatment experience of robotic-assisted laparoscopic surgery for huge adrenal tumors inferior vena cava and beside great vessels, and meanwhile, to analyze surgical techniques and advantages.

Materials and Methods: The data of 58 patients with huge adrenal tumors inferior vena cava and beside great vessels managed by robotic-assisted transperitoneal laparoscopic surgery during January 2012 to December 2016 were retrospectively analyzed, of them 31 were males and 27 were females, with ranging 19-59 years old. The patients were on lateral position. Open sulci paracolici, dissociate the upper polar of the kidney and adrenal gland near the vena cava or aorta to expose the whole adrenal gland and tumor. If the tumor on the left side, we can firstly dissociate left renal vein and control left adrenal central vein. If the tumor on the right side, open the lateral peritoneum along the ascending side ditch and cut off the ligamenta hepatocolicum, subsequently, turn the ascending colon inwards to protect the inferior vena cava, and raise the tumor to control the adrenal central vein. The lesion can be removed completely by using bipolar electrocoagulation or Hem-O-Lok.

Results: Operations were successfully completed. No great vessel injury. The mean operation time was $(62 \pm 15) \mathrm{min}$, the mean blood loss was $60.5 \mathrm{~mL}$, and the mean postoperative hospital stay was $(5.02 \pm 0.63) \mathrm{d}$. The mean tumor diametre was 7.1 $(5.5-12) \mathrm{cm}$. The pathological results showed that of them, 43 were pheochromocytoma, 5 were retroperitoneal paraganglioma, 3 were adrenal adenoma, 3 were adrenocortical carcinoma, and 4 were myelolipoma.

Conclusions: Due to the limitions of conventional laparoscopic operation and large trauma of open surgery, we select roboticassisted laparoscopic surgery for treatment. The key points of manipulation are as follows: dissociating the vessels carefully; the rich surgical experience of assistants. Robotic-assisted laparoscopic surgery is promising to be a standard for huge adrenal tumors inferior vena cava and beside great vessels.

MP19-3 Long term outcome evaluation of laparoendoscopic single site (LESS) adrenalectomy

\section{LO, H Lee}

Kaohsiung Medical University Hospital, Kaohsiung Medical University

Taiwan (Republic of China)

\begin{tabular}{ll} 
Table 1 Patient demographics & \\
\hline & LESS adrenalectomy ( $\mathrm{n}=123)$ \\
\hline $\begin{array}{l}\text { Age (years) } \\
\text { Sex, } \mathrm{n}(\%)\end{array}$ & $50.9 \pm 12.2$ \\
$\quad$ Male & $58(47.2)$ \\
$\quad$ Female & $65(52.8)$ \\
BMI (kg/m ${ }^{2}$ ) & $25.4 \pm 4.4$ \\
Tumor site, $n$ (\%) & \\
Left & $79(64.2)$ \\
$\quad$ Right & $44(35.8)$ \\
Pathologic diagnosis, $\mathrm{n}(\%)$ & \\
$\quad$ Conn's syndrome & $84(68.3)$ \\
Cushing's syndrome & $6(4.9)$ \\
Pheochromocytoma & $4(3.3)$ \\
Non-functioning tumor & $24(19.5)$ \\
Malignant & $1(0.8)$
\end{tabular}

Table 2 Surgical outcomes

\begin{tabular}{ll}
\hline & LESS adrenalectomy $(\mathrm{n}=123)$ \\
\hline Operation time (min) & $114.1 \pm 31.1$ \\
Blood loss (ml) & $65.1 \pm 68.7$ \\
Resumption and oral intake (days) & $0.24 \pm 0.43$ \\
Analgesics after surgery ( $\mathrm{n})$ & $0.35 \pm 0.59$ \\
Length of hospital stay (days) & $5.4 \pm 1.2$ \\
Tumor size $(\mathrm{cm})$ & $4.3 \pm 1.9$ \\
\hline
\end{tabular}

Introduction \& Objective: Laparoendoscopic single site (LESS) adrenalectomy have become a feasible choice for adrenal lesions and is substitute to conventional open adrenalectomy. This study is to describe the characteristics of adrenal tumor after LESS retroperitoneal adrenalectomy in our experience.

Materials and Methods: From January 2010 to August 2016, 123 patients received a LESS retroperitoneal approach in our hospital. We retrospectively review these patients and analyze the characteristics of adrenal tumors. The incision wound is below the 12th rib in the posterior axillary line and is about 2.3 to $3.2 \mathrm{~cm}$ length. A $5 \mathrm{~mm} 300$ rigid laparoscope and other conventional laparoscopic instruments are manipulated via a commercial port (LagiPort). After operation, no drain tube is placed.

Results: Among 123 adrenal tumors receiving operation, Conn's disease is the most common disease (68.3\% Conn's disease, $19.5 \%$ non-functional, $4.9 \%$ Cushing disease, 3.3\% Pheochromocytoma, $0.8 \%$ malignancy, respectively). In our finding, left side adrenal tumors $(64.2 \%)$ are more than right side tumors (35.8\%). Mean operating time was $114.1 \pm 31.1 \mathrm{~min}$ and mean blood loss was $65.1 \pm 68.7 \mathrm{ml}$. Mean hospital stay was $5.4 \pm 1.2$ days. The postoperative course was uneventful without complications.

Conclusions: LESS retroperitoneal adrenalectomy is a safe and satisfied procedure for patients with adrenal tumors. Besides, we discovered the different distribution of adrenal disease between Asian and Western world. Conn's disease takes more than half of adrenal tumors in Taiwanese population.

MP19-4 Robotic-assisted Adrenalectomies: An Initial Experience

S Saad, B Namdarian, M Ravindraanandan,

B Challacombe, A Fernando

Guys Hospital

United Kingdom 
Introduction \& Objective: The role of robot-assisted minimally invasive surgery is developing, with various studies demonstrating safety, feasibility and effectiveness. The laparoscopic approach has been commonplace but the robot offers potential advantages in precision and safety, however, data is still lacking regarding longer term outcomes and efficacy. We aimed to review our procedures over the last 5 years to establish caseload, and assess pathological and surgical outcomes.

Materials and Methods: Retrospective review of tertiary center operative database between January 2012 and December 2016 identified patients who underwent robotic adrenalectomy. Variables considered were patient demographics, operative parameters including operative time, length of stay, blood loss, histopathology and peri-operative complications.

Results: 25 cases were identified in our cohort. 2 had a simultaneous nephrectomy for another pathology and 1 underwent partial adrenalectomy. Median patient age was 63 (Mean 60.7, Range 3585 ). Median operating time was $110 \mathrm{mins}$ (mean 110min). Median blood loss was $55 \mathrm{mls}$ (mean $75 \mathrm{ml}$ ). Pathology is listed below with median lesion size 40mm (mean 42.3mm, range $15-100 \mathrm{~mm}$ ). Pathology Number:

Metastasis 7 Phaeo 6 Adenoma 4 Lymphangioma 1 Pseudocyst 1 Adenomatoid tumour 1 Oncocytoma 1 Adenocarcinoma 1 Median LOS was 4 days (mean 3.8 days). There were no intraoperative complications or transfusions with a single Clavien 1 wound haematoma post-operative complication.

Conclusions: At our centre Robotic Adrenalectomy has been demonstrated as a safe and effective technique. Favourable surgical outcomes and equivalent length of stay were demonstrated compared with the literature for laparoscopic and robotic assisted surgery, whilst allowing for management of complicated endocrine and metastatic lesions.

\section{MP19-5 Transperitoneal Robotic Assistant Laparoscopic} Resection of Large Adrenal Tumors

Z Shen, S Chen, S Zhong, M Zhang

China (People's Republic)

Introduction \& Objective: To summarize our experience in transperitoneal robotic assistant laparoscopic resection of large adrenal tumors.

Materials and Methods: The clinical data of 23 patients with large adrenal tumor exceeding $6 \mathrm{~cm}$ in diameter, which were treated by transperitoneal da Vinci robotic assistant laparoscope from March 2013 to June 2016, were reviewed.

Results: All of the operations were finished successfully with no subsidiary injury and open operation. Tumors were located at left adrenal in 15 cases and right in 8 cases. The average diameter was $8.0 \mathrm{~cm}(6 \mathrm{~cm}$ to $12 \mathrm{~cm})$. The diagnosis of the tumors include 8 cases of pheochromocytoma, 3 cases of cortical adenoma, 2 cases of cortical carcinoma, 2 cases of myelolipoma, 1 case of schwannoma, 1 case of adrenal gland cyst, 1 case of diffuse large B cell lymphoma, 1 case of bronchogenic cyst, 1 case of angioleiomyolipoma, 1 case of mature teratoma, 1 case of adrenal hematoma, and 1case of epithelioid tumor. Average operative time was $78 \mathrm{~min}$ (30-150 min). Estimated blood loss was $215 \mathrm{ml}$ (20-1200ml). 1 case was intraoperative blood transfusion. Average postoperative hospital stay was 6 days (3-11 days). All patients were followed up for 1-40 months except 2 missing follow-up cases. The followed up time ranged from 1-40 months, all 23 patients survive with no tumor recurrence.
Conclusions: Transperitoneal robotic assistant laparoscopic has the advantage of clear image and operative accuracy. Based on our favorable experience, robotic adrenalectomy has become our preferred minimally invasive surgical approach for removing large adrenal tumors for its less stimulating to the tumor and gentle operation.

\section{MP19-6 Withdrawn}

\section{MP19-7 Bilateral Simultaneous Laparoscopic Adrena-} lectomy: A Multicenter Experience

A Tuncel, A Erkan, H Langenhuijsen, T Mikhaylikov, M Arslan, Y Aslan, D Berker, Y Ozgok, A SerdarGözen

University of Health Sciences, Ankara Numune Research and Training Hospital, Dept. of Urology, Ankara-Turkey Turkey

Introduction \& Objective: Minimally invasive techniques play an impostant role in adrenal gland surgery. In this multicenter study, we report our experience in a series of bilateral simultaneous laparoscopic adrenalectomy experience.

Materials and Methods: Between 2012 and 2016, a total of 46 patients underwent simultaneous bilateral laparoscopic adrenalectomy at 6 different centers. Patients were divided into two groups as bilateral transperitoneal laparoscopic adrenalectomy (BTLA) (n:24) and bilateral retroperitoneal laparoscopic

Table: Perioperative and postoperative parameters of the patients.

\begin{tabular}{|c|c|c|c|}
\hline & BTLA (n:24) & BRLA (n:22) & $p$ value \\
\hline Operation time (min) & $\begin{array}{l}169.7 \pm 77.3(70- \\
360)\end{array}$ & $\begin{array}{l}154.1 \pm 55.8(86- \\
282)\end{array}$ & 0.44 \\
\hline Estimated blood loss (ml) & $\begin{array}{l}150.4 \pm 318.5(20- \\
1600)\end{array}$ & $56.3 \pm 93.2(5-400)$ & 0.19 \\
\hline $\begin{array}{l}\text { Length of hospitalization } \\
\text { (days) }\end{array}$ & $6.9 \pm 3.6(2-16)$ & $5.4 \pm 2.9(3-15)$ & 0.13 \\
\hline Intraoperative complications & $1(4.2 \%)$ & $2(9.1 \%)$ & 0.51 \\
\hline Satava classification & & & \\
\hline Grade 1 & - & - & \\
\hline Grade 2 & $1(4.2 \%)$ & $2(9.1 \%)$ & \\
\hline Grade 3 & - & - & \\
\hline $\begin{array}{l}\text { Postoperative complications } \\
\text { Clavien-Dindo }\end{array}$ & $1(4.2 \%)$ & $2(9.1 \%)$ & 0.51 \\
\hline classifications & & & \\
\hline Grade I & - & $1(4.5 \%)$ & \\
\hline Grade 2 & - & $1(4.5 \%)$ & \\
\hline Grade $3 a$ & - & - & \\
\hline Grade $3 b$ & $1(4.2 \%)$ & - & \\
\hline Grade $4 a$ & - & - & \\
\hline Grade $4 b$ & - & - & \\
\hline Grade 5 & - & - & \\
\hline Conversion to open surgery & $1(4.2 \%)$ & - & 0.34 \\
\hline $\begin{array}{l}\text { Conversion to transperitoneal } \\
\text { approach }\end{array}$ & & $5(22.7 \%)$ & \\
\hline
\end{tabular}

BTLA: Bilateral transperitoneal laparoscopic adrenalectomy BRLA: Bilateral retroperitoneal laparoscopic adrenalectomy 
adrenalectomy (BRLA) (n:22). Patients' age, gender, BMI, operative indications, mass size, operation time, blood loss, length of hospitalisation, intraoperative and postoperative complications and pathology reports were analyzed.

Results: Overall, preoperative diagnoses were Cushing's syndrome in 30 patients, $(65.2 \%)$, pheochromocytoma in 10 patients $(21.7 \%), 3$ patients $(6.5 \%)$ operated for bilateral incidentaloma greater than $4 \mathrm{~cm}$, one patient $(2.2 \%)$ operated upon detection of bilateral contrast enhanced adrenal masses, one patient (2.2\%) operated upon diagnosis of ectopic adrenocorticotrophic hormone secretion and one patient $(2.2 \%)$ operated upon bilateral adrenal metastasis of bronchial carcinoma. Perioperative and postoperative parameters are summarized in the Table.

Conclusions: We believe that either transperitoneal or retroperitoneal synchronous laparoscopic bilateral adrenalectomy seems to be feasible, safe and applicable techniques in patients with bilateral adrenal pathology.

\section{MP19-8 Withdrawn}

MP19-9 Initial experience of 243 cases in transperitoneal robotic-assisted laparoscopic surgery for adrenal diseases

Z Shen

China (People's Republic)

Introduction \& Objective: To summarize our experience in robotic assisted laparoscopic surgery for adrenal diseases

Materials and Methods: The clinical data of 243 patients with adrenal tumor treated by robotic assisted laparoscopic surgery from March 2010 to February 2017 were retrospectively reviewed. There were 99 men and 144 women. The mean age was 51.6 years (range, 19 84). Tumors were located at left adrenal in 140 cases, right in 97 cases, and both sides in 6 cases. The average diameter was $3.32 \mathrm{~cm}$ (range, $0.8-12 \mathrm{~cm}$ ). However, there were 41 cases whose tumor diameter were greater than $5 \mathrm{~cm}$.

Results: There were 2 cases of conversion during operation, 1 case converted to open surgery and the other to the traditional laparoscope surgery. The mean operative time was $35 \mathrm{~min}$ (range, 20-130 min). Estimated blood loss was $80 \mathrm{ml}$ (range,20$1200 \mathrm{ml}$ ). Blood transfusion was needed in 6 cases. The mean postoperative hospital stay was $5 \mathrm{~d}$ (range, $3-20 \mathrm{~d}$ ). The pathological diagnosis included 37 cases of pheochromocytoma, 149 cases of cortical adenoma, 3 cases of cortical carcinoma, 5 cases of metastatic tumor, 9 cases of adrenal myelolipoma, 3 cases of adrenal cyst, 2 cases of bronchogenic cyst, 25 cases of adrenal nodular hyperplasia, 2 cases of angiomyolipomas, 1 case of mature teratoma, 1case of diffuse large B-Cell lymphoma, 1 case of angioma, and 4 cases of neurofibromatosis.

Conclusions: Robotic assisted laparoscopic adrenalectomy was safe and effective. Robotic assisted laparoscopic surgery has the advantages over laparoscopic surgery in the treatment of complicated adrenal tumor, such as large adrenal tumors, pheochromocytoma, bilateral adrenal diseases, Cushing's diseases, overweight and obese patients with adrenal diseases.

MP19-10 Comparison of Minimally-Invasive Versus Open Adrenalectomy: An Institutional Review

P Heller, P Fish, A Rawlings, N Pokala, K Murray

University of Missouri

United States

\begin{tabular}{|c|c|c|c|}
\hline & $\begin{array}{c}\text { MIS } \\
\text { (mean) }\end{array}$ & $\begin{array}{c}\text { Open } \\
\text { (mean) }\end{array}$ & p value \\
\hline $\begin{array}{c}\text { Age at time of } \\
\text { surgery (years) }\end{array}$ & 49.9 & 50.5 & 0.8325 \\
\hline BMI & 31.6 & 29.9 & 0.2627 \\
\hline EBL (mL) & 142.6 & 613.4 & $<0.0001$ \\
\hline LOS (days) & 3.46 & 8.37 & $<0.0001$ \\
\hline $\begin{array}{c}\text { Tumor maximum } \\
\text { dimension on preop } \\
\text { imaging (cm) }\end{array}$ & 4.15 & 6.77 & 0.004 \\
\hline $\begin{array}{c}\text { Tumor maximum } \\
\text { dimension on final } \\
\text { pathology (cm) }\end{array}$ & 4.21 & 6.37 & 0.0232 \\
\hline
\end{tabular}

Introduction \& Objective: To compare outcomes of minimally invasive surgical technique (MIS) versus open adrenalectomy over a 12-year period when performed by general surgeons and urologists at a single institution.

Materials and Methods: With Institutional Review Board approval, a retrospective chart review was conducted and 164 patients who underwent adrenalectomy between 2004 and 2016 at the University of Missouri were identified. Key data points were recorded including patient clinical factors, surgical approach, surgeon specialty, blood loss, operative time, clinical and pathological tumor size, final surgical pathology, post-operative complications, readmissions and length of stay. MIS included both robotic and laparoscopic procedures. Student's t-test was performed for variables to compare MIS and open surgical procedures.

Results: Of the 164 patients, 115 (70\%) underwent MIS adrenalectomy and $49(30 \%)$ underwent open adrenalectomy. There was no difference in age or BMI at time of operation between the two groups as shown in Table 1. The maximum measured diameter of adrenal mass on pre-operative imaging was significantly larger in those undergoing open versus MIS procedures $(6.7 \mathrm{~cm}$ vs $4.15 \mathrm{~cm}, \mathrm{p}=0.004)$. This also proved to be true for tumor size on the final pathological specimen. The mean size on pre-operative imaging was very concordant with the final pathological size. As shown in table 1, those patients selected for MIS procedures, had lower EBL $(p<0.0001)$ and length of stay in hospital $(p<0.0001)$ along with smaller masses. While in the operating room, 10 patients $(20.4 \%)$ undergoing open adrenalectomy required blood transfusion of at least 1 unit PRBCs, while none of the MIS patients required intraoperative transfusion. In the 30-day post-operative period, 7 patients $(6.1 \%)$ who underwent open surgery required readmission while none of the MIS patients were readmitted to our institution within 30 days after surgery. Overall final surgical pathology diagnosed $79 \%$ benign masses, $7 \%$ primary malignant tumors and $14 \%$ metastatic lesions of the adrenal gland.

Conclusions: Over a 12-year period at our institution, patients undergoing minimally invasive adrenalectomy had significantly less operative blood loss and shorter hospital stays when compared to patients who underwent open adrenalectomy. A MIS approach to adrenalectomy is safe with decreased blood loss and length of hospitalization in appropriately selected patients with small adrenal masses on pre-operative imaging.

\section{MP19-11 Role of open adrenalectomy in the laparoscopic era}

J Kwok, A Lua, Y Chong

Department of Urology, Tan Tock Seng Hospital Singapore 
Introduction \& Objective: In the era of laparoscopic surgery, the conventional approach to adrenalectomy is that of laparoscopy. However, there is still a role for open adrenalectomy for large tumors and pheochromocytomas. We review our institution's laparoscopic and open adrenalectomy case series.

Materials and Methods: This is a retrospective review of adrenalectomies in our institution from 1 March 2011 to 7 May 2017. A total of 42 cases were reviewed, extracting data including approach used, indication for surgery, estimated blood loss, duration of surgery, length of stay, and complications.

Results: Indications for surgery included 20 for primary hyperaldosteronism, 5 for Cushing's, 9 for likely malignancy, and 8 pheochromocytomas. Of the 42 cases, 4 were open adrenalectomies and 38 laparoscopic adrenalectomies planned as the initial approach. 3 laparoscopic cases were converted to open intraoperatively. For the open cases, 1 was for malignancy and 3 for pheochromocytomas. The mean adrenal nodule size as expected was larger in the open compared to the laparoscopic series $(10 \mathrm{~cm}$ [range $8.7-10.6$ ] vs. $2.29 \mathrm{~cm}$ [range $0.6-6.5$ ], $\mathrm{p}=0.0001)$. Mean estimated blood loss was lower in the laparoscopic group $(140 \mathrm{mls}$ vs. $475 \mathrm{mls}, \mathrm{p}=0.0196)$. There was a longer mean duration of surgery in the open group (250mins vs. 190mins, $p=0.0455$ ), accountable for by the complexity and size of the tumors selected for open surgery. Mean length of stay was shorter in the laparoscopic group (3.4 days vs. 8 days, $\mathrm{p}=0.0001$ ). In analysis of the laparoscopic retroperitoneal versus transperitoneal approaches, operation duration, estimated blood loss and length of stay were not statistically significant. Of the open cases, all incisions used were a thoracolumbar incision, whereas in laparoscopic converted to open cases were of a subcostal incision. The main reasons for conversion to open were that of difficult anatomy with bleeding, dense adhesions, and labile blood pressure in a pheochromocytoma case. Mean tumor size was larger in the laparoscopic converted open cases compared to non-converted cases $(5.16 \mathrm{~cm}$ [range $4.2-6.5]$ vs. $2.71 \mathrm{~cm}$ [range 0.6-6.1], $\mathrm{p}=0.0194)$.

Conclusions: There is still an important role of open adrenalectomy in large functional adrenal nodules or malignant tumors, and on encountering high blood loss or difficult anatomy in the laparoscopic approach. Surgeons should be aware and familiar with the open approach and consider open adrenalectomy as an initial approach in patients where laparoscopy will be very technically challenging. Laparoscopic adrenalectomy has a lower estimated blood loss and decreased length of stay and should still be the first choice of approach in appropriate cases.

MP19-12 Natural Orifice Transluminal Endoscopic surgery for Donor Nephrectomy- outcomes in comparison with the laparoscopic Donor Nephrectomy

KM Ramaswami, H Pothiyedath, P Birud

\section{METROMED INSTITUTE OF ADVANCED UROLOGY AND RENAL TRANSPLANT \\ India}

Introduction \& Objective: Donor nephrectomy is usually done via either an open surgical approach, purely laparoscopic approach, hand assisted or robotic approach. Trans vaginal retrieval has been attempted at few centres and not widely practiced.. The main points of debate concerning the procedure is fear of infectious complications, issues involved in removing large kidneys, nulliparous donors. The use of endocatch bag, vaginal swab culture, proper vaginal preparation, episiotomy incision if nee-
Operative details and receiver outcomes in Laparoscopic donor nephrectomy (LDN) VS Transvaginal natural orifice assisted laparoscopic donor nephrectomy (TVNALDN)

\begin{tabular}{|c|c|c|c|c|c|}
\hline & SLDN & $N=160$ & TVNALDN & $N=40$ & PVALUE \\
\hline & MEAN +/-SD & & MEAN+/-SD & & \\
\hline \multicolumn{6}{|l|}{ TXN } \\
\hline WIT (s) & $\begin{array}{l}176.5+/- \\
61.3\end{array}$ & & $182+/-38.9$ & & 0.193 \\
\hline $\begin{array}{l}\text { DONOR } \\
\text { OT(min) }\end{array}$ & $64.8+/-16.2$ & & $68.7+/-14.9$ & & 0.503 \\
\hline $\begin{array}{l}\text { HOSP.STAY } \\
\text { (days) }\end{array}$ & $2.3+/-0.4$ & & $2.3+/-0.5$ & & 0.973 \\
\hline $\operatorname{VAS}($ at $D / d$ ) & $2+/-0.5$ & & $1.6+/-0.5$ & & 0.973 \\
\hline \multicolumn{6}{|l|}{$\begin{array}{l}\text { CREAT } \\
(\mathrm{mg} / \mathrm{dl})\end{array}$} \\
\hline AT D/d & $1.36+/-0.50$ & & $1.24+/-0.67$ & & 0.852 \\
\hline 6MONTHS & $1.49+/-0.58$ & & $1.40+/-0.67$ & & 0.159 \\
\hline 12MONTHS & $1.35+/-0.41$ & & $1.35+/-0.63$ & & 0.734 \\
\hline
\end{tabular}

TXN - TRANSPLANT

SDLN - STANDARD LAP DONOR NEPHRECTOMY

VAS - VISUAL ANALOGUE SCALE

DONOR \& RECIEVER CHARECTERISTICS - Laparoscopic donor nephrectomy (LDN) VS Transvaginal natural orifice assisted laparoscopic donor nephrectomy (TVNALDN)

\begin{tabular}{|c|c|c|c|c|c|}
\hline & SLDN & $N=160$ & TVNALDN & $\mathrm{N}=40$ & PVALUE \\
\hline & MEAN+/-SD & $n$ & MEAN+/-SD & $n$ & \\
\hline \multicolumn{6}{|l|}{ DONOR } \\
\hline AGE (yrs) & $48.2+/-12.2$ & & $46.9+/-11$ & & 0.559 \\
\hline $\mathrm{BMI}(\mathrm{kg} / \mathrm{m} 2)$ & $30.5+/-5.2$ & & $30.3+/-4.1$ & & 0.998 \\
\hline CREAT(POP) & $0.96+/-0.21$ & & $0.97+/-0.12$ & & 0.079 \\
\hline $\begin{array}{l}\text { OBESE } \\
(>30 \mathrm{BMI})\end{array}$ & & $68(42.5 \%)$ & & $12(30.0 \%)$ & 0.15 \\
\hline Rt NX & & 15 & & 0 & \\
\hline Lt NX & & 145 & & 40 & \\
\hline H/O ABD SX & & $47(29.3 \%)$ & & $9(22.5 \%)$ & 0.39 \\
\hline \multicolumn{6}{|l|}{ RECIEVER } \\
\hline MALE & & $117(73.1 \%)$ & & $31(77.5 \%)$ & 0.57 \\
\hline FEMALE & & $43(26.9 \%)$ & & $9(22.5 \%)$ & \\
\hline AGE(yrs) & $39.1+/-16.2$ & & $36,2+/-15.4$ & & 0.57 \\
\hline
\end{tabular}

SDLN - STANDARD LAP DONOR NEPHRECTOMY

BMI - BODY MASS INDEX

RT NX-RIGHT NEPHRECTOMY

LT NX - LEFT NEPHRECTOMY

ABD SX-ABDOMEN SURGERY

ded makes this procedure much hassle free. We have done 40 cases of transvaginal retrieval of donor kidney recently in selected cases and outcomes is compared with our own series of 160 cases of laparoscopic donor nephrectomy in which kidney has been retrieved through pfannesteil incision.

Materials and Methods: Patient is given modified kidney position with lithotomy. 5 port transperitoneal approach. Hilar dissection followed by division of adrenal vein, gonadal vein and lumbar vein between haemostatic clips. Colpotomy tube was 
placed through the posterior colpotomy and the endo catch bag was introduced. Ureter was divided at the pelvic brim level. Kidney was placed in the sterile pouch and Hilar vessels were clipped with haemolock and divided, retrieved through vagina. In complete laparoscopic donor nephrectomy kidney was retrieved through a pfannesteil incision.

Results: There were no intraoperative and postoperative complications with good graft outcome in both series.

(two ables attached)

Conclusions: Transvaginal retrieval of kidney is the Next generation for kidney donation in selected cases and is the available least minimal invasive method for donor nephrectomy.

MP19-13 Complications of suprapubic-assisted laparoendoscopic single-site surgery (SA-LESS) in urology

Z Xiaofeng, X Gang, Z Guoxi, Y Yuanhu, $X$ Rihai, W Gengqing

Department of Urology, First Affiliated Hospital of Gannan Medical University

China (People's Republic)

Introduction \& Objective: To analyze the complications of urologic suprapubic-assisted laparoendoscopic single-site surgery (SA-LESS), and to explore effective measures for its prevention and management.

Materials and Methods: From July 2010 to April 2015, a total of 573 cases underwent SA-LESS in our center. The median age was 45.4(range 6 to 78) years and the median body mass index was 23.6 (range 15.3 to 32.8 ) $\mathrm{kg} / \mathrm{m} 2$. The various SA-LESS procedures included nephrectomy $(\mathrm{N}=214$; simple 150 , radical 64), partial nephrectomy $(\mathrm{N}=16)$, heminephroureterctomy for duplex kidney $(\mathrm{N}=15)$, nephroureterectomy $(\mathrm{N}=29)$, adrenalectomy $(\mathrm{N}=43)$, renal cyst excision $(\mathrm{N}=34)$, pyelolithotomy or ureterolithotomy $(\mathrm{N}=183)$, pyeloplasty $(\mathrm{N}=15)$ and ureterovesical reimplantation $(\mathrm{N}=24)$. Intraoperative and postoperative complications were graded according to Satava and ClavienDindo grade classifications, respectively. The major complications and their treatments were mainly analyzed.

Results: Among the 573 SA-LESS procedures, there were 65 (11.34\%) complications occurred, including 21 (3.66\%) major complications. The intraoperative major complications included inferior vena cava injury $(\mathrm{N}=3)$, renal vascular injury $(\mathrm{N}=3)$, iliac vein injury $(\mathrm{N}=1)$, pleural injury $(\mathrm{N}=1)$, duodenal injury $(\mathrm{N}=1)$, ileum injury $(\mathrm{N}=1)$, spleen injury $(\mathrm{N}=1)$ and femoral nerve injury $(\mathrm{N}=1)$. The postoperative major complications included secondary hemorrhage $(\mathrm{N}=5)$, urinary leakage $(\mathrm{N}=3)$ and ureteropelvic junction stenosis after pyeloplasty $(\mathrm{N}=1)$. No intraoperative and postoperative deaths occurred.

Conclusions: SA-LESS is safe and feasible in urology, but there are some major complications for consideration.

MP19-14 Application of Cumulative Summation (CUSUM) in learning curve analysis of Suprapubic-assisted laparoendoscopic single-site nephrectomy in one center

Z Xiaofeng, X Hui, Z Guoxi, Y Yuanhu, X Rihai, W Gengqing, W Xiaoning

Department of Urology, First Affiliated Hospital of Gannan Medical University

China (People's Republic)
Introduction \& Objective: To evaluate the learning curve of Suprapubic-assisted laparoendoscopic single-site surgery (SALESS) nephrectomy in our center.

Materials and Methods: From April 2013 to April 2016, 60 patients underwent SA-LESS nephrectomy performed by two associate chief physicians (group B and C, 30 patients in both groups). There were 25 males and 5 females with mean age $48.4 \pm 9.4$ years in group B, and 23 males and 7 females with mean age $52.6 \pm 12.0$ years in group C. Operative time, estimated blood loss, converting to conventional laparoscopy or open surgery, intra and postoperative complications in both groups were recorded, The data from both group $\mathrm{B}$ and $\mathrm{C}$ were compared with those of group A (Standard group with 30 patients who underwent SA-LESS nephrectomy performed by a skillful chief physician, 26 males and 4 females with mean age $50.8 \pm 12.3$ years). All the patients in the three groups were diagnosed with upper urinary tract calculus or ureteral stricture with severe ipsilateral hydronephrosis (no-function kidney) and a normal contralateral kidney. The sum of the Cumulative Summation (CUSUM) was also cumulated. Individual learning curve and total learning curve were established.

Results: All the 30 procedures in group A were successfully completed with an average operative time $123.3 \pm 28.4(85 \sim 175)$ min and an average estimated blood loss $121.7 \pm 43.1(65 \sim 280)$ $\mathrm{ml}$. There were 28 procedures successfully finished in group B and $\mathrm{C}$, respectively. The average operative time was $141.7 \pm 49.4$ (90 260) $\mathrm{min}$ and the average estimated blood loss was $165.2 \pm 81.9(70 \sim 450) \mathrm{ml}$ in group $\mathrm{B}$, and the average operative time was $133.1 \pm 33.7(90 \sim 235) \mathrm{min}$ and the average estimated blood loss was $140.0 \pm 72.3(75 \sim 500) \mathrm{ml}$ in group C. There was 1 case converted to conventional laparoscopy and 1 case converted to open surgery in group B with 4 (13.3\%) complications (Satava I 2 cases, Satava II 1 case, Clavien-Dindo I 1 case) in all. There was 1 case converted to conventional laparoscopy in group C with $4(13.3 \%)$ complications (Satava I 1 case, Satava II 2 cases, Clavien-Dindo II 1 case) in all. Statistical analysis showed that the number corresponding to the slope changed into negative was 15 and 11 for group $\mathrm{B}$ and $\mathrm{C}$, respectively, and 13 was confirmed in the total learning curve.

Conclusions: SA-LESS nephrectomy is feasible and safe for an experienced laparoscopic surgeon, who can master it after he (or she) finished 13 cases of SA-LESS nephrectomy.

MP19-15 A comparison of laparoendoscopic single-site (LESS) surgery versus conventional procedures in laparoscopic donor nephrectomy: a Japanese multi-institutional retrospective study

H Tsuruta, T Inoue, M Miura, M Yanishi, K Harada, F Sato, M Nitta, K Yoshimura, J Hagiuda, K Shinoda, T Kobayashi, K Nakagawa, H Mimata, T Terachi, T Matsuda, T Habuchi

Akita University Graduate School of Medicine Japan

Introduction \& Objective: Laparoendoscopic single-site donor nephrectomy (LESSDN) has been reported to be feasible and effective in terms of cosmesis and invasiveness. However, only few studies conducted by multiple surgeons in multi-institutions have been reported. We compared the clinical data and outcome between LESSDN and conventional laparoscopic donor nephrectomy (LDN) procedures in multi-institutes in Japan.

Materials and Methods: From January 2009 to December 2015, the clinical data of 225 donors who underwent LESSDN and 142 

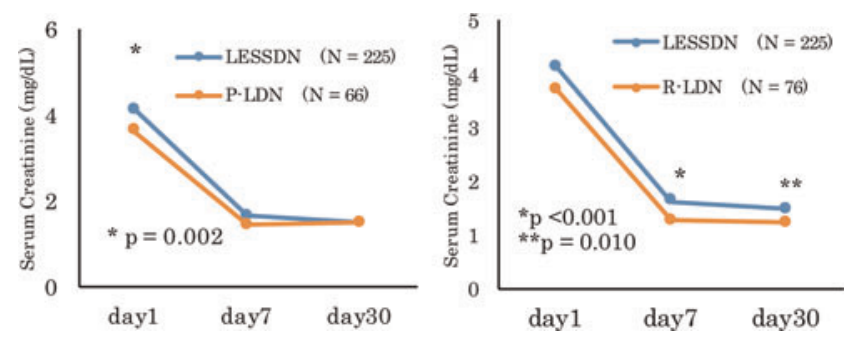

donors who underwent LDN in the same period were collected from 10 institutes. All LESSDNs were performed transperitoneally, while the LDN was transperitoneal in 66 (P-LDN) and retroperitoneal in $76(\mathrm{R}-\mathrm{LDN})$ procedures.

Results: In the LESSDN group, the single site was placed pararectal in $164(72.8 \%)$ and umbilical in $56(24.8 \%)$. Nine patients $(4.0 \%)$ donated the right kidney. Additional $<3 \mathrm{~mm}$ trocars were used in 10 donors $(4.4 \%)$. GelPOINT ${ }^{\circledR}$ was used for the single-site device in 188 cases $(83.5 \%)$. Multiple surgeons comprising 2-8 per institute performed the operations. No significant differences were observed between the three groups in estimated blood loss and warm ischemic time. The operative time was significantly shorter in the LESSDN group than in the R-LDN group but did not significantly differ from that in the PLDN group ( $240 \mathrm{~min}$ vs. $262 \mathrm{~min}$ vs. $252 \mathrm{~min}, \mathrm{p}=0.017$ and $\mathrm{p}=0.056)$. No significant difference was observed between the three groups in the rates of blood transfusion, open conversion, visceral injuries, and postoperative complications of ClavienDindo $\geq$ Grade II. No significant differences were observed between the three groups in the dose of analgesics and the rate of delayed graft function. The postoperative serum creatinine level of recipients was significantly higher in the LESSDN group than in the R-LDN group (1.61 vs. $1.25 \mathrm{mg} / \mathrm{dL}, \mathrm{p}<0.001$, day 7 and 1.45 vs. $1.20 \mathrm{mg} / \mathrm{dL}, \mathrm{p}=0.010$, day 30$)$ and $\mathrm{P}-\mathrm{LDN}$ group (4.10 vs. $3.61 \mathrm{mg} / \mathrm{dL}, \mathrm{p}=0.002$, day 1$)$.

Conclusions: In this multi-institutional study in Japan, LESSDN was less favorable compared to LDN regarding the short-term postoperative graft function. However, there was no difference in outcomes regarding the complication rate, postoperative pain, open conversion, or blood transfusion rate.

MP19-16 Optimization of the key techniques in suprapubicassisted laparoendoscopic single-site nephroureterectomy

Z Xiaofeng, Z Guoxi, W Gengqing, Y Yuanhu, X Rihai, W Xiaoning

Department of Urology, First Affiliated Hospital of Gannan Medical University

China (People's Republic)

Introduction \& Objective: To discuss the optimization of the key techniques in suprapubic-assisted laparoendoscopic singlesite surgery nephroureterectomy (SA-LESS-NU).

Materials and Methods: Nineteen consecutive patients including 13 males and 6 females, with a mean age of 63 yeas (range 18 to 92), were subjected to SA-LESS-NU in our center. There were 15 renal pelvic carcinomas and 4 ureteral carcinomas in this study. The patients were administrated general endotracheal anesthesia and secured on the operating table in lateral decubitus with right side elevated $70^{\circ}$. One 5- and $10-\mathrm{mm}$ trocars were inserted at the medial margin of umbilicus. A 5-mm trocar was inserted into abdominal cavity below the pubic hairline under the direct vision from a 5-mm flexible-tip $0^{\circ}$ laparoscope through the umbilical trocar. The operation was performed using conventional operating apparatus placed in the abdominal trocars, under direct vision achieved by a $5-\mathrm{mm}$ flexible-tip $0^{\circ}$ laparoscope placed through the trocar below the pubic hairline. Firstly, the distal ureter was dissected and blocked by a Hem-Olok. Then, the ureter was isolated completely and radical nephrectomy was performed according to the method of the standard laparoscopy. The distal ureter was isolated, and the uterine artery (for female) and superior vesical arteries were blocked. Finally, the bladder cuff excision was performed and the incision was sutured. The specimen was removed after the incisions below the pubic hairline was enlarged transversely and the rectus abdominis muscle sheath was incised vertically. The intravesical therapy was performed at the end of the operation.

Results: All the procedures were successfully performed except one patient who underwent conversion of standard laparoscopic nephroureterectomy for the injury of vena cava. The median operative time was 150 (range 105 to 280) minutes, and the median estimated blood loss was 140 (range 30 to 600) $\mathrm{ml}$. There was no other major perioperative complication occurred. All the patients resumed ambulation on postoperative day 1. Pelvic drainage tube was removed on postoperative day 2-3. Urethral catheter was removed on postoperative day 6-7 (irrigation of bladder with Pirarubicin before this procedure). The patients were discharged on postoperative day 8-9. The mean follow-up of 11.7 (range 3 to 21) months showed hidden umbilicus scar. The scar below the pubic hairline was not detectable because of the covering of the pubic hairs. There was no incision hernia in umbilicus and below the pubic hairline to occur. There was no recurrence and metastasis occurred.

Conclusions: The optimization of the key techniques in SALESS-NU may reduce the blood loss, shorten the operative time, and improve the operation safety.

MP19-17 The decline of LESS: a survey of the Endourological society to identify shortcomings and guidance for future directions

I Sorokin, BA Johnson, N Canvasser, B Irwin, R Autorino, E Liatsikos, J Cadeddu, A Rane

UT Southwestern

United States

Introduction \& Objective: To analyze the most recent temporal trends in the adoption of urologic laparoendoscopic single-site

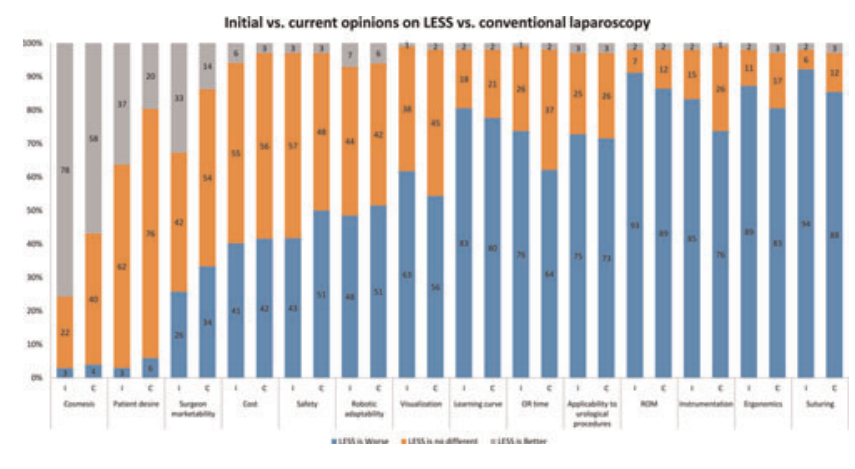




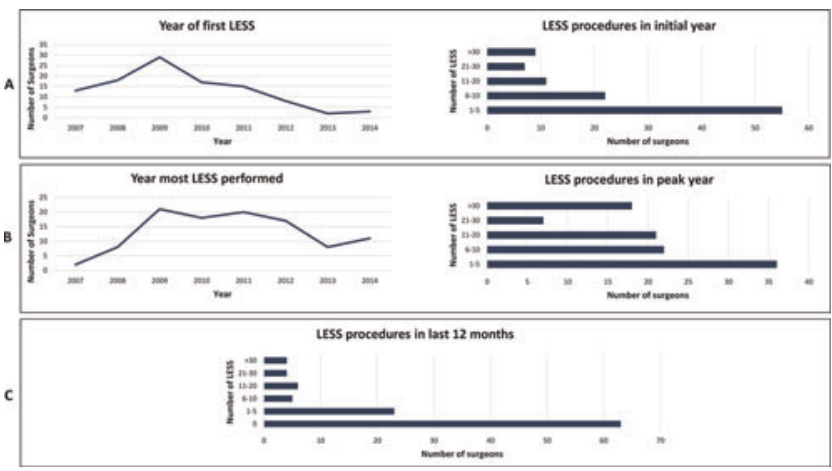

(LESS), to identify the perceived limitations associated with its decline, and to determine factors that might revive the role of LESS in the field of minimally invasive urological surgery.

Materials and Methods: A 15 question survey was created and sent to members of the Endourological Society in September 2016. Only members who performed LESS procedures in practice were asked to respond.

Results: In total, 106 urologists responded to the survey. Most of the respondents were from the United States (35\%) and worked in an academic hospital (84.9\%). Standard LESS was the most popular approach $(78.1 \%)$, while $14.3 \%$ used robotics and $7.6 \%$ used both. 2009 marked the most popular year to perform the initial $(27.6 \%)$ and the majority (20\%) of LESS procedures. The most common LESS procedure was a radical/simple nephrectomy $(51 \%)$ followed by pyeloplasty $(17.3 \%)$. In the past 12 months, $60 \%$ of respondents had performed no LESS procedures. Compared to conventional laparoscopy, respondents only believed cosmesis to be better, however this enthusiasm waned over time. Worsening shifts in enthusiasm for LESS also occurred with patient desire, marketability, cost, safety, and robotic adaptability. The highest rated factor to help LESS regain popularity was a new robotic platform.

Conclusions: The decline of LESS is apparent, with few urologists continuing to perform procedures attributed to multiple factors. The availability of a purpose-built robotic platform and better instrumentation might translate into a renewed future interest of LESS.

MP19-18 Comparison of Robotic Laparo-Endoscopic Single Site (R-LESS) and Multiport Robotic Nephrectomy and Partial Nephrectomy Utilizing the Da Vinci-XI Platform

A Bindayi, Z Hamilton, M Holden, M McDonald,

K Yim, IH Derweesh

Department of Urology, UC San Diego Health, La Jolla, California, USA

United States

Introduction \& Objective: Feasibility of Laparo-endoscopic Single-Site Surgery (LESS) has been demonstrated in a number of procedures. Adoption of this technique has been hampered by the technical challenges associated with the surgical approach. Introduction of DaVinci Xi Robotic Platform, with standardized $8 \mathrm{~mm}$ ports for camera and all instruments, and a smaller arm profile, may reduce some of the challenges associated with robotic adaptation of LESS (RLESS). We performed a matched pair comparison of R-LESS and multiport robotic nephrectomy (RRN) and partial nephrectomy (RPN).

Materials and Methods: Single surgeon nonrandomized prospective comparison of R-LESS and multiport RRN and RPN. All operations were performed utilizing the DaVinci XI platform (Intuitive, Sunnyvale, CA, USA). 20 patients underwent LESS (10 RN/10 PN) and 20 patients underwent MPL (6 RN/14 PN) from 2/2015-2/2016. All cases were performed with via transperitoneal approach. For R-LESS RN and PN, access was obtained by Gel GELPoint (Applied Medical, Santa Marghareta, CA, USA) through which all trocars were inserted. R-LESS-RN and -PN recapitulated all standard steps of Multiport Robotic RRN and RPN.

Results: All 20 R-LESS cases were successfully performed. For R-LESS-PN and multiport RPN: Mean tumor diameter (cm) was 2.5 and $2.8(\mathrm{p}=0.203)$; mean ischemia time (min) was 18.2 and $16.4(\mathrm{p}=0.432)$; operative time $(\mathrm{min})$ was 160 and 149 $(\mathrm{p}=0.109)$. There were no significant differences with respect to preoperative/postoperative estimated Glomerular Filtration Rate $(p=0.653)$. For R-LESS-RN and Multiport RRN: Mean tumor diameter $(\mathrm{cm})$ was 6.8 and $7.3(\mathrm{p}=0.514)$; mean estimated blood loss $(\mathrm{mL})$ was 125.5 and 100.9 , respectively $(\mathrm{p}=0.720)$. For LESS and Multiport Robotic groups: mean hospital stay (days) were 1.7 and $2.3(p=0.107)$; and analgesic use were 4.5 morphine equivalents vs. 11.6 , respectively $(\mathrm{p}=0.036)$. No patients required transfusions, all had negative margins, and all are currently alive and there were no significant differences in complication rates $(\mathrm{p}=0.431)$.

Conclusions: In a matched prospective comparison, R-LESS is comparable to multiport RRN and RPN in terms of perioperative parameters and may confer benefit with respect to analgesic requirement. Randomized evaluation and longer-term assessment and follow up are requisite.

MP19-19 Single Port Laparoscopic Repair of Post Retropubic Radical Prostatectomy Inguinal Hernia Via Previous Operative Scar: Case Series, Initial Experience

J Jo, Y Kim, S Park, H Choi, H Moon, K Kim, S Lee

Hanyang University Hospital

Republic of Korea

Introduction \& Objective: Inguinal hernia is one of the most common complications of radical prostatectomy which needs surgical repair. Surgical repair of inguinal hernia after prostatectomy cannot be avoid the additional scar on abdominal wall. However, in this study we evaluated the safety and feasibility of the inguinal hernia repair via previous prostatectomy wound without additional scar.

Materials and Methods: Total ten patients were enrolled who had developed inguinal hernia as a complication of retropubic radical prostatectomy. The repair of inguinal hernia was conducted transperitoneally using home-made glove single port through $2 \mathrm{~cm}$ incision of the upper part of previous prostatectomy scar. The period between occurrence of inguinal hernia and radical prostatectomy and the operative time were evaluated. The follow-up time, and the complications of inguinal hernia repair were also evaluated.

Results: The mean age of patients was $67.50 \pm 7.5$ and all the patients had no previous history of inguinal hernia. The inguinal 
Fig.1. Mesh application. (A) Inguinal hernia.

(B) Mesh application. (C) Wound closure.

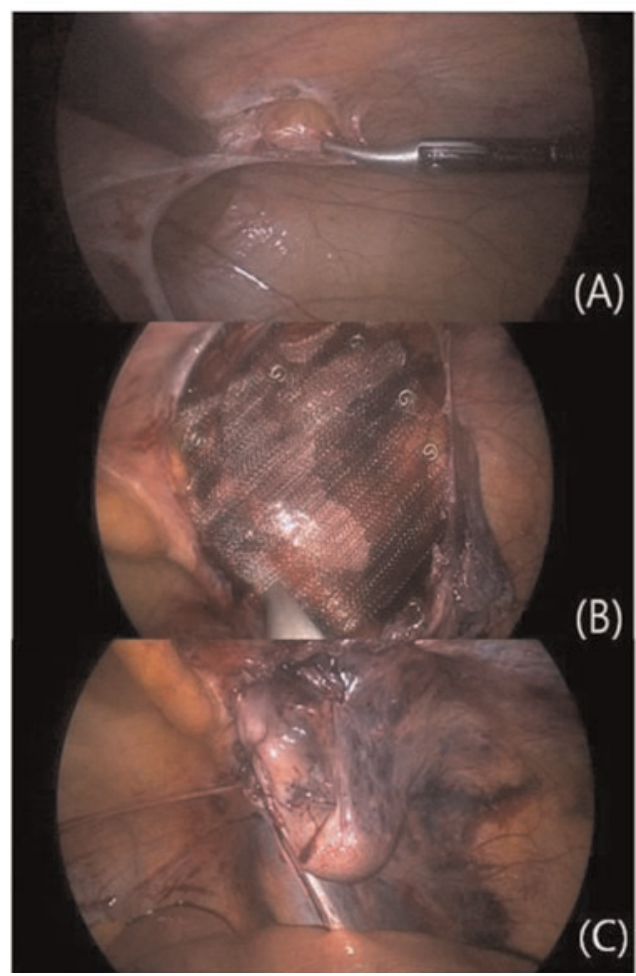

MP19-20 Laparoendoscopic single-site nephrectomy for children with an ectopic ureter associated with a hypoplastic kidney. Two cases report.

S Sejiyama, S Abe, K Takei, N Yamanaka, M Hanada, T Shibuya, M Yamasaki, T Shin, K Mori, T Ando, T Nomura, F Sato, H Mimata

Japan

Introduction \& Objective: To present our experience of laparoendoscopic single-site (LESS) nephrectomy for children with a single-system ectopic ureter associated with an ipsilateral hypoplastic kidney.

Materials and Methods: Retroperitoneal LESS nephrectomy was performed in two 7-year-old girls with a chief complaint of A $3 \mathrm{~cm}$ flank incision was made, and then lumber muscle splittechnique was used to reach retroperitoneal space. A single access platform (GelPOINT Mini $\left.{ }^{\circledR}\right)$ with three attached-ports was placed in the incision, and a $5 \mathrm{~mm}$ flexible endoscope was used. One case required one additional $3 \mathrm{~mm}$ port.

Results: The operative time was 189 and 103 minutes, respectively. There were no intraoperative and postoperative complications. A single dose analgesics was required for one patient, and the other patient used no analgesics after surgery. Oral intake was started 6 hours postoperatively, and the post operative hospital stay was 3 and 4 days, respectively.

Conclusions: The retroperitoneal LESS nephrectomy for singlesystem ectopic ureter with an ipsilateral hypoplastic kidney in children is safe and feasible. In addition to cosmetic benefit, this technique may have some theoretical advantages such as
Table 1. Baseline characteristics and operative outcomes

\begin{tabular}{ccccccccc}
\hline $\begin{array}{c}\text { Case } \\
\text { No. }\end{array}$ & Age & Site & $\begin{array}{c}\text { Inguinal } \\
\text { hernia } \\
\text { occurrence } \\
\text { period after } \\
\text { RRP }\end{array}$ & $\begin{array}{c}\text { Hospital } \\
\text { day }\end{array}$ & $\begin{array}{c}\text { Operation } \\
\text { time }\end{array}$ & $\begin{array}{c}\text { Follow } \\
\text { up } \\
\text { period }\end{array}$ & Complication & Recurrence \\
\hline 1 & 61 & Right & 2 month & 3 & 45 & 15 month & None & - \\
2 & 71 & Right & 3 month & 3 & 35 & 15 month & None & - \\
3 & 76 & Left & 4 month & 3 & 40 & 10 month & None & - \\
4 & 61 & Left & 8 month & 3 & 45 & 11 month & None & - \\
5 & 66 & Left & 7 month & 3 & 43 & 14 month & None & - \\
6 & 59 & Right & 5 month & 3 & 45 & 10 month & None & - \\
7 & 68 & Right & 6 month & 4 & 41 & 12 month & None & . \\
8 & 73 & Left & 6 month & 4 & 42 & 13 month & None & - \\
9 & 74 & Left & 8 month & 3 & 39 & 11 month & None & . \\
10 & 66 & Left & 7 month & 4 & 40 & 10 month & None & - \\
\hline
\end{tabular}

RRP: retropubic radical prostatectomy.

hernia developed $5.60 \pm 2.63$ months after retropubic radical prostatectomy. The mean operative time was $41.50 \pm 4.59 \mathrm{~min}-$ utes. There was no surgical complication and recurrence of inguinal hernia during follow-up.

Conclusions: This study showed the safety and feasibility of inguinal hernia repair using home-made glove single port via previous prostatectomy scar.
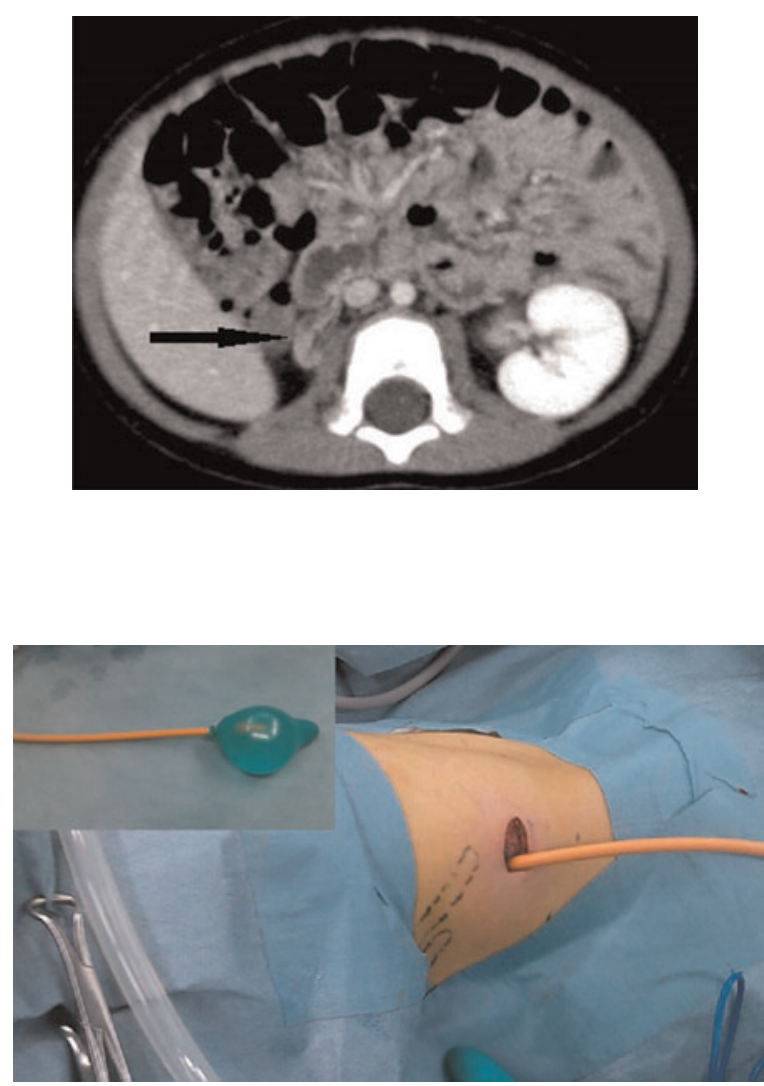

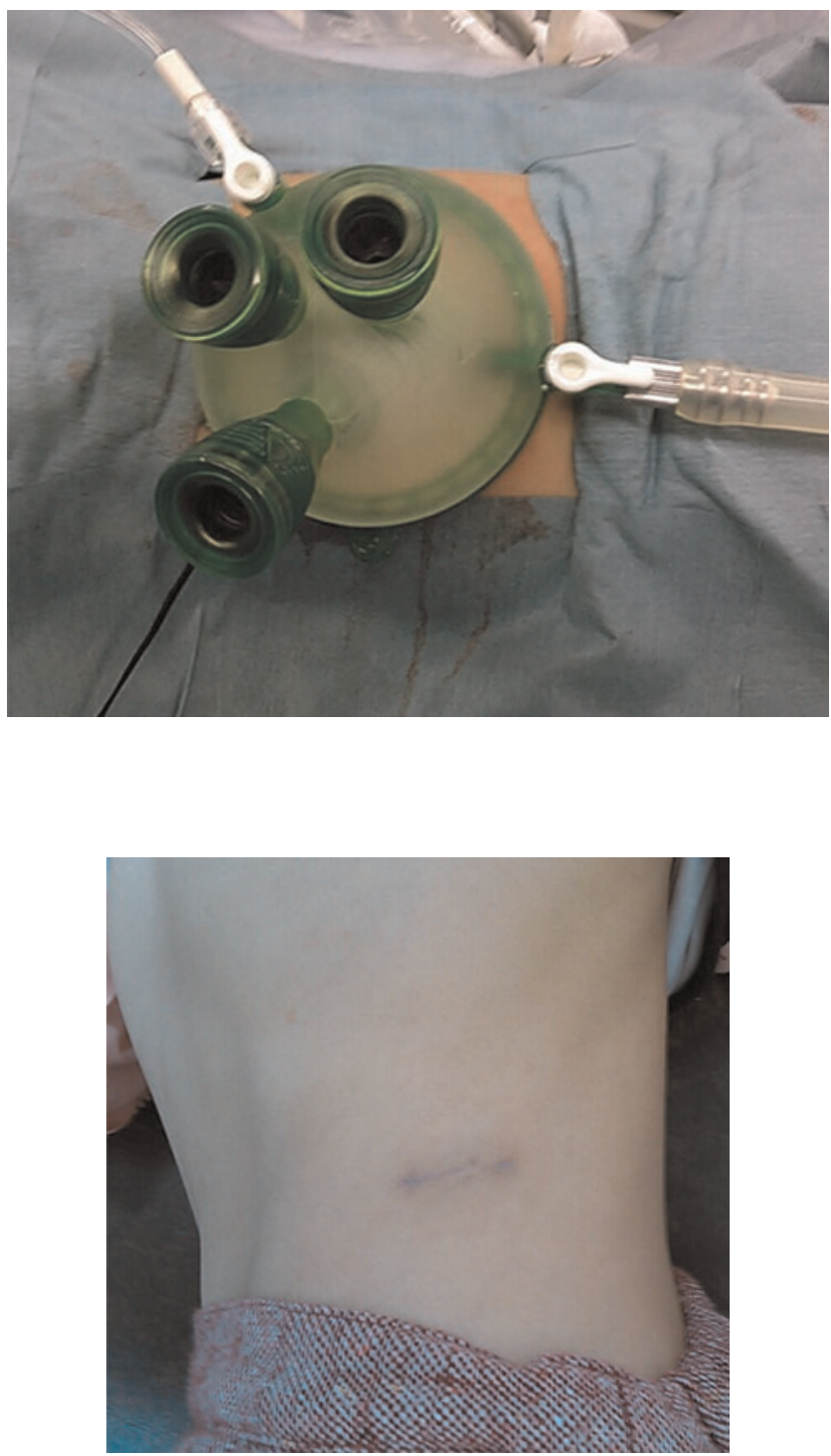

reducing postoperative analgesics, avoiding muscle damage, and minimizing postoperative ileus.

MP19-21 Laparoendoscopic Single-site Repair of Bladder Rupture Using a Home-made Single-port Device: Treatment for a Traumatic Intraperitoneal Bladder Rupture

J Jo, Y Kim, S Park, H Choi, H Moon, K Kim, S Lee

Hanyang University Hospital

Republic of Korea

Introduction \& Objective: We report our experience with a laparoendoscopic single-site (LESS) repair of a bladder rupture using a home-made single-port device. Surgical and multi-port laparoscopic repair of traumatic intraperitoneal bladder rupture cannot be avoid the additional scar on abdominal wall. However, in this study we evaluated the safety and feasibility of the single port laparoscopic traumatic intraperitoneal bladder rupture repair.

Materials and Methods: Among 21 cases of bladder rupture in emergency room at single hospital (2013.03 2015.05), 14 cases are traumatic intraperitoneal bladder rupture patients. 10 cases were done with the single port laparoscopic traumatic intraperitoneal bladder rupture repair. Single-port laparoscopic transabdominal bladder repair were conducted using home-made glove single port through $2 \mathrm{~cm}$ incision of the umbilicus. Using the flexible laparoscopic instruments and rigid instruments, LESS surgery was performed using a procedure similar to conventional laparoscopic surgery. The period of operative time

Fig.1. Application. (A) Home-made single-port device.

(B) Intraperitoneal bladder rupture. (C)

Repair of laceration by LaparoAngle dissectors..
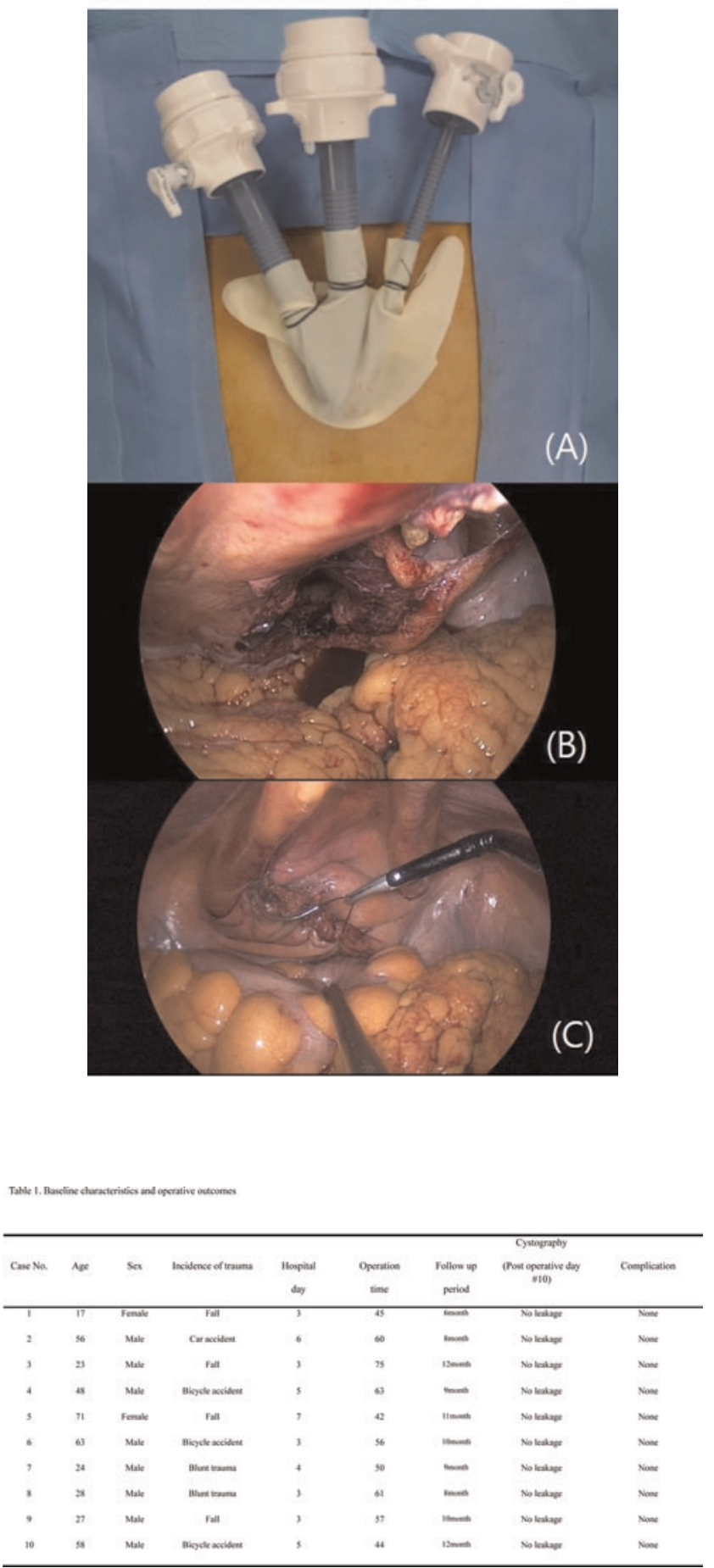
were evaluated. The follow-up time, the complications of bladder repair was also evaluated.

Results: The mean age of 10 patients was $65.58 \pm 5.73$ and all the patients had hemodynamically stable. The mean operative time was $67.58 \pm 6.30$ minutes. All patient did not complain of abdominal pain on the second postoperative day. The urethral Foley catheter was removed 10 days after the operation, at which time a cystography showed no extravasation. There was no surgical complication during follow-up.

Conclusions: LESS may be more efficient and feasible for a traumatic bladder rupture in comparison with conventional laparoscopic surgery if a patient with an intraperitoneal bladder rupture is hemodynamically stable.

MP19-22 Usefulness of laparoendoscopic single site surgery varicocelectomy

Y Yamanaka, S Takada

Osaka Police Hospital

Japan

Introduction \& Objective: To examine the laparoendoscopic single site surgery varicocelectomy in our department.

Materials and Methods: The study included 66 patients who underwent laparoendoscopic single site surgery varicocelectomy from May 2010 to September 2016. The assessment included operative and postoperative semen analysis, postoperative complications and pregnancy rate. The mean follow-up was 5.2 months (range 0.4 to 67.7 ).

Results: The median age was 33 years (range 8-65). 19 patients underwent laparoendoscopic single site surgery varicocelectomy on both sides, 47 patients on one side (46 patients on the left, 1 patient on the right). 49 patients $(74.2 \%)$ were performed for male infertility, and 17 patients $(25.8 \%)$ were performed on scrotal symptoms. The grade of varicocele on the right side was 12 cases of G1, 5 cases of G2, 5 cases of G2 and 2 cases of G3, whereas G1 was 0 cases, G2 was 24 cases, and G3 was 37 cases on the left side. The grade of 4 cases was unknown. The median surgical time was 60 minutes (range 33-187) and the median of the hospital stay was 4 days (range 2-7). Postoperative ileus, abdominal wall scarring hernia, wound infection was experienced 1 case each. All patients disappear varicocele after surgery. Sperm concentration before and after surgery were $14.5 \times 106 / \mathrm{ml}(0-152), 32 \times 106 / \mathrm{ml}(0-107)(\mathrm{p}=<0.001)$, and motility were $49 \%(0-89), 58 \%(0-91)(\mathrm{p}=0.009)$, respectively. Eleven people $(22.4 \%)$ among 49 male infertility admitted natural pregnancy.

Conclusions: In this study, postoperative hydrocele testicle was not observed, and spermatic findings before and after the surgery showed a significant improvement. This procedure is considered to be useful in cases of bilateral varicocele especially.

\section{MPS20: UROTHELIAL CARCINOMA I}

MP20-1 Percutaneous resection of upper urinary tract urothelial carcinoma: a single-surgeon screenshot about a controversial procedure in imperative cases

E De Lorenzis, A Gallioli, L Boeri, S Zanetti, G Sampogna, M Fontana, F Palmisano, M Talso, P Dell'Orto,

F Longo, E Montanari

Department of Urology, Fondazione IRCCS Ca' Granda Ospedale Maggiore Policlinico, Università degli Studi di Milano

Italy

Introduction \& Objective: The experience with percutaneous nephroscopic resection (PNR) of upper tract urothelial carcinoma (UTUC) is mainly limited to sub-groups of patients with imperative indications. In this study we retrospectively analyzed peri-operative and oncological outcomes of our cohort of patients treated with PNR.

Materials and Methods: From September 2015 to October 2016, 6 bipolar PNR for UTUC have been performed in 4 male patients by a single surgeon. All indications to PNR were imperative: 2 solitary kidney, 1 synchronous bilateral UTUC and 1 suspected bilateral UTUC in a patients with a neobladder. Two lesions were multifocal. A history of bladder cancer was present in 2 patients. In all cases was placed a $30 \mathrm{ch}$ renal Amplatz sheath using one shot-dilation technique and a nephrostomy $8 \mathrm{ch}$ tube at the end. Clinical, demographic, peri-operative variables, adiuvant therapy and overall survival were analyzed.

Results: Pre- and peri-operative variables are listed in table 1 . No intraoperative complications occured. We had 3 Clavien grade 2 complications (fever) and one grade I (transient hae- maturia). The final pathology showed an UTUC TaLG in 2 patients, a T1LG in 1 case and one T1HG. We performed 3 secondlook procedures at a mean time of 20,6 days (2 PNR and 1 RIRS): in one case a TaLG was confirmed, in one case the histology was negative and in a patients with previous T1LG was find a TaLG. One patient with a large TaLG in a solitary kidney was submitted to intracavitary therapy with BCG. The patient with a bilateral synchronous T1HG tumor received an adiuvant systemic chemotherapy and subsequently a laparoscopic radical nephroureterectomy on the endoscopic untreated side. One patients showed a TaLG ureteral recurrence 4 months after the PNR. No tumor seeding or

Table 1. Pre- and peri-operative variables

\begin{tabular}{|l|c|}
\hline Mean+/-SD & $\begin{array}{c}\text { Percutaneous nephroscopic resection } \\
\mathbf{n}=6\end{array}$ \\
\hline Age (years) & $72,25+/-4,27$ \\
\hline Charlson Comorbidity Index & $3,25+/-2,2$ \\
\hline Tumor dimension +/- SD (mm) & $32,5+/-12,58$ \\
\hline $\begin{array}{l}\text { Mean pre-operative creatinine } \\
\text { value +/- SD (mg/dl) }\end{array}$ & $1,35+/-0,1$ \\
\hline Operative time (min)* & $138,8+/-50,1$ \\
\hline Lenght of stay (days) & $6,8+/-2,4$ \\
\hline Haemoglobin drop (g/dl) & $1,3+/-1,19$ \\
\hline $\begin{array}{l}\text { Last mean creatinine value }+/- \\
\text { SD (mg/dl)** }\end{array}$ & $1,81+/-0,6$ \\
\hline
\end{tabular}

"including associated procedure (es. cistoscopy, pielography and ureteroscopy)

**one patient underwent radical nephroureterectomy during follow up 
recourse to transient or definitive hemodialysis were observed. At a mean follow-up of $10.75(+/-3,5)$ months all the patients were alive and free from disease.

Conclusions: In very selected patients and in imperative conditions, PNR represents a secure and valid option with conservative and curative intent, even in a multimodal management of UTUC. In this setting an adequate counseling with the patients, a strict follow up and the experience of the surgeon are mandatory.

\section{MP20-2 Endoscopic treatment for upper urinary tract urothelial carcinoma larger than $1 \mathrm{~cm}$}

A Shvero, T Aviv, M Laufer, ZA Dotan, D Zilberman, Y Mor, O Porntoy, J Ramon, H Winkler, N Kleinmann

Department of Urology, Sheba Medical Center, Tel Hashomer, Israel

Israel

Introduction \& Objective: Radical Nephroureterectomy (RNU) is considered the gold standard treatment for upper tract urothelial carcinoma (UTUC) larger than $1 \mathrm{~cm}$. The purpose of this study was to examine surgical and oncological outcome of ureteroscopic (URS) treatment for UTUC larger than $1 \mathrm{~cm}$.

Materials and Methods: A retrospective review was performed of all patients undergoing URS for UTUC at our institution from January 2013 to October 2016. Patients with UTUC larger than $1 \mathrm{~cm}$ were included. Tumor size was determined by pre-operative CT-Urography or retrograde pyelography during URS. Tumors were treated by using a dual laser generator: Neodymium (Nd:YAG) for tumor coagulation and Holmium (Ho:YAG) for tumor resection. Follow-up protocol included URS every 3 months during the first year after initial treatment, every 6 months in the $2^{\text {nd }}$ year, and annually thereafter. Demographic data and tumor characteristics were obtained for all patients. patients' courses were reviewed for tumor recurrence, grade progression and development of locally advanced or metastatic disease.

Results: The cohort included 36 patients. 16 patients had high grade disease on pathology and were referred to RNU. The remaining 20 patients were managed ureteroscopically. Among these patients 13 were males and 7 were females, at a median age of 72 years (range 50-88). Median tumor size was $15 \mathrm{~mm}$ (range 10-30). Location included: kidney 10 (50\%), ureter 8 (40\%) or both $2(10 \%)$. Mean operative time was 72.4 minutes, with no intra-operative or post-operative complications. Six cases required 2 URS to complete the treatment. The mean number of ureteroscopies per patient was 3.4 (1-7). In this cohort, 18 (90\%) patients had low grade disease and were treated with curative intent. 2 patients had high grade disease and refused RNU and underwent palliative URS. Median follow-up time was 11.5 months (range 3-33). 10 patients had local recurrence (50\%) at a mean of 8.1 months after initial surgery. Bladder tumors occurred in 8 patients, 5 of them had a history of bladder tumors. None of the patients in the intend to cure group developed metastatic disease, locally advanced disease, or died of their disease during the follow-up period. One patient had pathological progression and was referred to RNU. One patients with highgrade disease died of his disease after 6 months.

Conclusions: Ureteroscopic treatment of upper tract urothelial carcinoma larger than $1 \mathrm{~cm}$ is surgically feasible, does not involve significant complications and has good short-term oncologic outcome. Strict ureteroscopic surveillance is essential.

\section{MP20-3 Holmium and Thulium transurethral Laser En} Bloc resection of bladder tumors

P Glybochko, Y Alyaev, G Altshuler, A Vinarov, A Dymov, N Sorokin, R Sukhanov, D Enikeev, I Yaroslavskyi, V Vinnichenko, V Lekarev

Sechenov University

Russia

Introduction \& Objective: Current guidelines recommend transurethral resection of the bladder tumor as the gold standard of treatment for NMIBC. However, one of the problems of conventional electroresection of the bladder tumor is that the tumor cells are scattered as the tumor is fragmented so that the possibility of implantation cannot be excluded. Due to high frequency of recurrence after transurethral resection (up 71\%) new approaches to a NMIBC treatment were introduced. The monopolar En Bloc resection of bladder cancer was introduced by Kawada et al. in 1997 to provide larger specimens for pathological analysis, decrease the likelihood of tumor cells scattering during the procedure and their implantation; and thus reduce the incidence of recurrence. However, the risk of conductive obturator nerve stimulation with consecutive accidental perforation of the bladder wall due to muscle contraction and thermal damage of adjacent tissue cannot be eliminated, in particular, when the tumor is located on the side wall of the bladder. Recently, laser En Bloc resection of the bladder tumor was shown to be a safe procedure that helps to avoid the obturator nerve reflex.

Materials and Methods: Since 2015, 43 laser En Bloc resections of bladder tumors were perfomed: 25 with Holmium laser (VersaPulse PowerSuite, Lumenis, Israel) and 18 with the fiber Thulium laser (Urolase, IPG IRE - Polus, Russia). With both lasers, $1.0 \mathrm{~J} / 10 \mathrm{~Hz} / 10 \mathrm{~W}$ settings have been used. Since neither technique nor laser settings were different on Ho and Tm groups, we have combined the groups together. According to the current approach, the principle of En Bloc resection is a superficial circular incision around the base of tumor with a distance of approximately $2-5 \mathrm{~mm}$ from its base. The adequate depth is reached when striated fibers of detrusor muscle are seen. Remaining adhesions and bleeding vessels are coagulated with the laser beam. The tumor is removed by conventional arched loop of resectoscope.

Results: The mean tumor size, cm - 1,81 (0,6-4,5); Operation time, $\min$ - 16,1 (8-27); catheterization time -1 day, Hospitalization - 2 days - 2. Obturator nerve stimulation - 0 ; bladder wall perforation - 0; switch to conventional TURB - 2,3\%, Detrusor muscle was present in 97.7\%. 37 Patients were followed for 12 months; $8(21,6 \%)$ had recurrences, $87.5 \%$ recurrences occurred outside the laser resection field.

Conclusions: Laser (either Holmium or Thulium) En Bloc resection is a safe and effective method of surgical treatment of NMIBC. In comparison to the TURB, Laser En Bloc resection helps to avoid obturator nerve reflex and risk of damage to the bladder as well as provides larger specimens for pathological investigation.

MP20-4 Modified Frailty Index associated with Adverse Outcomes in Patients with Bladder Cancer in Open and Laparoscopic Cystectomies

I Levy, M Finkelstein, MA Palese

Icahn School of Medicine at Mount Sinai

United States

Introduction \& Objective: Rates of frailty in geriatric surgical patients is higher compared to geriatric non-operative patients. 


\begin{tabular}{|c|c|c|c|c|c|}
\hline Characteristic & $\mathrm{mFI}=0$ & $\mathrm{mFI}=1$ & $\mathrm{mFI}=2$ & $\mathrm{mFI} \geq 3$ & P-value \\
\hline $\mathrm{N}$ & $2200(33.4 \%)$ & $3169(48.1 \%)$ & $989(15.0 \%)$ & $234(3.5 \%)$ & \\
\hline Age & $62.96(13.83)$ & $69.39(11.27)$ & $70.00(10.46)$ & $71.85(9.46)$ & $<0.0005$ \\
\hline Sex & & & & & $<0.0005$ \\
\hline Male & $1608(73.1 \%)$ & $2472(78.0 \%)$ & $795(80.4 \%)$ & $187(79.9 \%)$ & \\
\hline Female & $592(26.9 \%)$ & $697(22.0 \%)$ & $194(19.6 \%)$ & $47(20.1 \%)$ & \\
\hline Ethnicity & & & & & 0.0292 \\
\hline White & $1633(74.2 \%)$ & $2447(77.2 \%)$ & $765(77.4 \%)$ & $187(79.9 \%)$ & \\
\hline Non-white & $567(25.8 \%)$ & $722(22.8 \%)$ & $224(22.6 \%)$ & $47(20.1 \%)$ & \\
\hline Smoking Status & & & & & $<0.0005$ \\
\hline Non-smoker & $1639(74.5 \%)$ & $2526(79.7 \%)$ & $756(76.4 \%)$ & $185(79.1 \%)$ & \\
\hline Smoker & $561(25.5 \%)$ & $643(20.3 \%)$ & $233(23.6 \%)$ & $49(20.9 \%)$ & \\
\hline Procedure Type & & & & & $<0.0005$ \\
\hline Laparoscopic & $138(6.3 \%)$ & $125(3.9 \%)$ & $44(4.4 \%)$ & $5(2.1 \%)$ & \\
\hline Open & 2062 (93.7\%) & 3044 (96.1\%) & $945(95.6 \%)$ & $229(97.9 \%)$ & \\
\hline Clavien-Dindo 4 or 5 & $253(11.5 \%)$ & $481(15.2 \%)$ & $163(16.5 \%)$ & $48(20.5 \%)$ & $<0.0005$ \\
\hline 30-day Mortality & $27(1.2 \%)$ & $55(1.7 \%)$ & $22(2.2 \%)$ & $14(6.0 \%)$ & $<0.0005$ \\
\hline
\end{tabular}

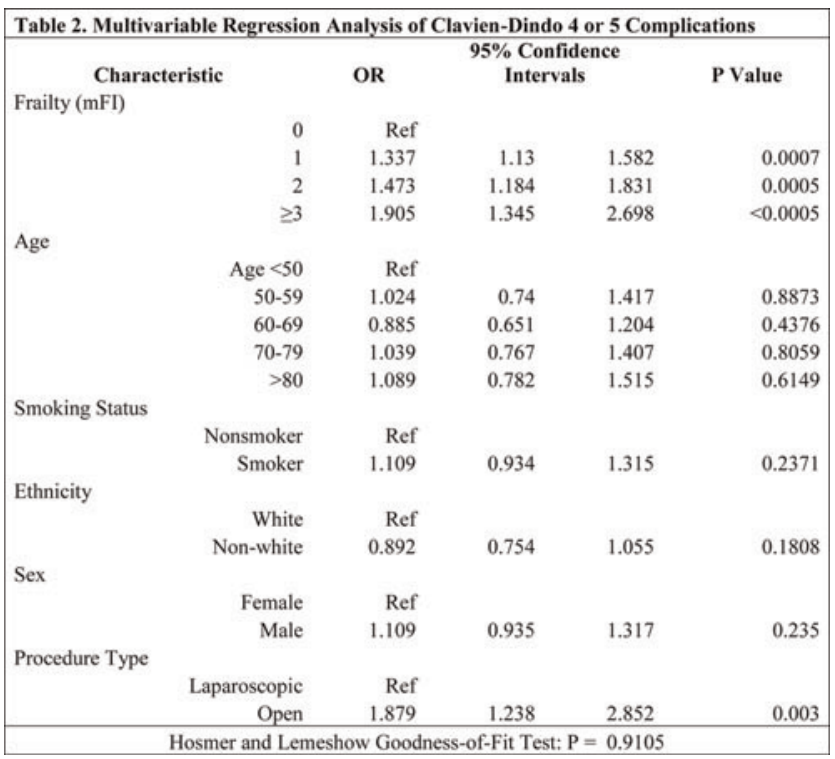

This study aims to determine the effect of frailty on patient outcomes including Clavien-Dindo 4 and 5 (intensive care unitlevel) complications and 30-day mortality for open and laparoscopic cystectomies.

Materials and Methods: Patients with bladder cancer undergoing cystectomies from 2008 to 2014 were queried using the ACSNSQIP database. The mFI was developed using the Canadian Study of Health and Aging Frailty Index (CSHA-FI) as a model. Multivariable regression adjusting for patient demographics of age, sex, ethnicity, smoking status, and whether they underwent open or laparoscopic cystectomy was analyzed to predict ClavienDindo 4 or 5 complications using SAS version 9.22.

Results: 6592 patients undergoing both open and laparoscopic radical cystectomy were queried. $2200(33.4 \%)$ had a $\mathrm{mFI}$ of 0 , $3169(48.1 \%)$ had a mFI of $1,989(15.0 \%)$ had a mFI of 2 , and $234(3.5 \%)$ had a $\mathrm{mFI} \geq 3$. Patients with increasing frailty were older, had a greater percentage of males, whites, and nonsmokers, and had a greater percentage undergo open cystectomies $(\mathrm{P}<0.0005)$. Patients with increasing frailty also experienced increased Clavien-Dindo 4 or 5 complications and 30-day mortality $(\mathrm{P}<0.0005)$ (Table 1$)$. Cystectomy patients with the highest frailty score $(\geq 3)$ had an adjusted odds for Clavien-Dindo 4 or 5 complications of 1.905 (CI: 1.345-2.698, $\mathrm{P}<0.0005$ ) in comparison with non-frail cystectomy patients.
Patients who underwent open cystectomies instead of laparoscopic cystectomies had adjusted odds for Clavien-Dindo 4 or 5 complications of 1.879 (CI: $1.238-2.852, \mathrm{P}<0.005$ ) when only adjusting for frailty (Table 2).

Conclusions: Increasing mFI scores are associated with worsening outcomes for patients undergoing cystectomies; however, patients undergoing laparoscopic cystectomies have significantly reduced odds of adverse outcomes.

MP20-5 Front-firing green-laser assisted laparoscopic partial cystectomy in selected patients with muscle-invasive bladder cancer: technique and initial outcome

K Wu, D He, J Fan

xi' an jiaotong university

China (People's Republic)

Introduction \& Objective: Partial cystectomy, as one of bladder-preserving treatment modalities, has recently drawn great interest in treating muscle-invasive bladder cancer (MIBC). However, when it is performed under laparoscopic view, it could not provide accurate identification of tumor margins unless some assisted techniques are applied. The green-laser has been widely used in treatment of benign prostatic hyperplasia and non-muscle invasive bladder cancer. Here, we describe a novel front-firing green-laser assisted technique in laparoscopic partial cystectomy, allows accurate identification of tumor margins to completely remove the lesion and minimize the damage of normal bladder.

Materials and Methods: Between January 2014 and Oct 2016, 9 patients with a solitary MIBC and request of bladder-preserving treatment were selected. All patients were diagnosed with pelvic CT-scan and cystoscope to assess the extent of primary lesion and the presence of any lymph-node metastases. Bilateral pelvic lymphadenectomy was initially performed before partial cystectomy. Under the direct view of cystoscope, the front-firing green-laser incision was performed $0.5-1 \mathrm{~cm}$ away from the exterior margin of lesion with adequate depth into the fat tissue. Tumors were then en bloc removed via transperitoneal laparoscope under the tracing of laser beam and marked incision.

Results: All the procedure was completed without complications in all 9 patients. The median operating time was 267.1 (240-330) min with a median estimated blood loss of $150(100-300) \mathrm{ml}$. The median hospital stay was 15.6 (12-22) days. All 9 patients were transitional cell carcinoma without lymph-node metastases, and pathological evaluation of tumor margins in 8 patients were all negative but positive in one patient. This patient was subjected to the adjuvant radiotherapy. During the follow-up, no recurrence or metastasis were detected in all 8 patients with negative margins. Conclusions: The use of front-firing green-laser during laparoscopic partial cystectomy is a feasible and safe maneuver to assists the design of accurate incision and minimize the damage of remaining bladder.

MP20-6 Development and Validation of Cystectomy Assessment and Surgical Evaluation Scoring (CASE) for Male Radical Cystectomy

A Hussein, K Sexton, M Meng, A Hosseini, P Wiklund, D Eun, S Daneshmand, B Bochner, J Peabody, R Abaza, E Skinner, R Hautmann, K Guru

Roswell Park Cancer Institute

United States 


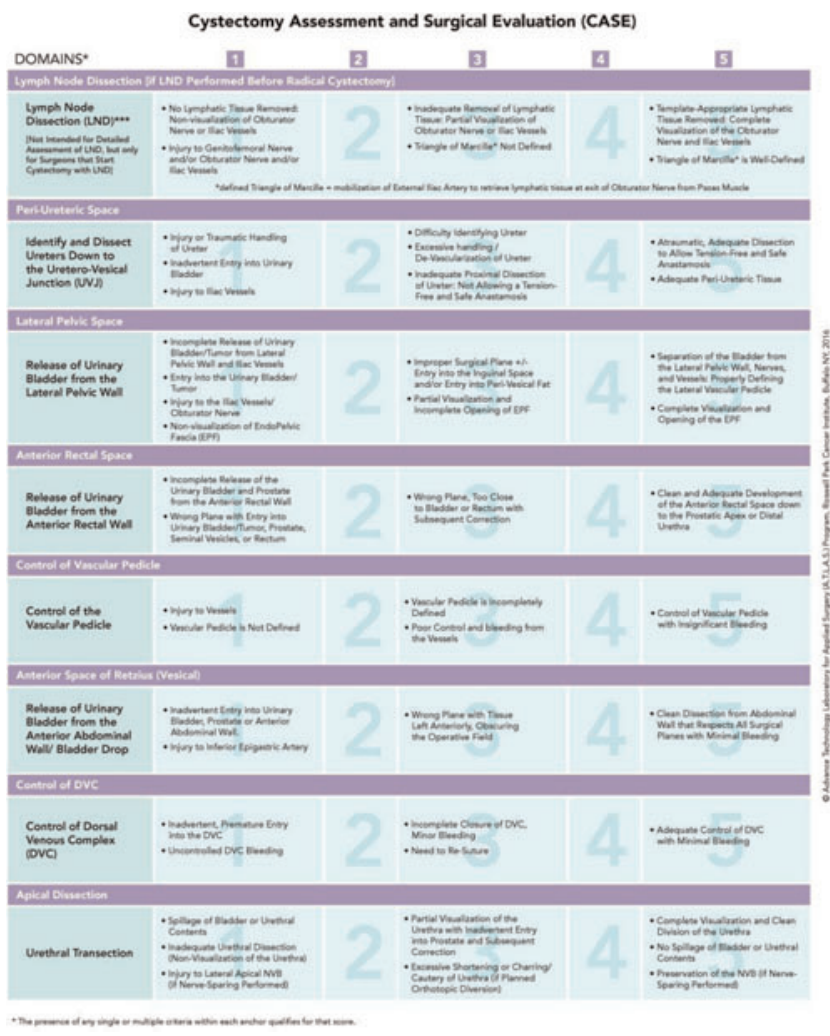

Introduction \& Objective: It is vital to ensure standardization and objective feedback during training and skill acquisition for optimal surgical outcomes and patient safety. We aimed to develop a structured scoring tool: CASE (Cystectomy Assessment \& Surgical Evaluation), that objectively measures and quantifies performance during radical cystectomy (RC) for men

Materials and Methods: A multinational expert panel (11 Surgeons who perform open and/or robot-assisted radical cystectomy-RARC) collaborated towards development and content validation of the male $\mathrm{RC}$ scoring system. The critical steps of male $\mathrm{RC}$ were deconstructed into 9 key domains, where each domain was assessed by 5 anchors evaluating surgical principles, technical proficiency and safety. Content validation was done utilizing the Delphi methodology. Each anchor statement was assessed in terms of 3 aspects: contextual relevance, concordance between language-used and anchor score, and clarity of wording. An independent coordinator collated the comments from the expert panel and computed the Content Validity Index (CVI) for each aspect of each anchor. If CVI was $\geq 0.75$, consensus was reached and the statement was removed from the next round. If consensus was not achieved, the coordinator incorporated the comments from the panel and the updated scoring system was redistributed. This process was repeated until consensus was achieved for all statements. All experts were blinded to each other's assessment.

Results: The expert panel reached consensus after 4 rounds on all aspects including language, relevance of skills assessed, and concordance between the language used and the skill assessed. A ninth domain assessing Disposition of Tissue was removed from the system after the second round. CVI $\geq 0.75$ was achieved in 8 $(11 \%)$ statements in the first round, $44(61 \%)$ statements in the second, $17(24 \%)$ statements in the third and $3(4 \%)$ statements in the $4^{\text {th }}$ round. The final eight domains of the CASE include: Pelvic Lymph Node Dissection, Development of the
Peri-ureteral Space, Lateral Pelvic Space, Anterior Rectal Space, Control of the Vascular Pedicle, Anterior Vesical Space, Control of the Dorsal Venous Complex, and Apical Dissection (Figure 1). Conclusions: We developed and validated a scoring system for $\mathrm{RC}$ that can provide structured feedback for surgical quality assessment, training and feedback. Validation of the scoring system is in process

MP20-7 Analysis of surgeon biometrics during open and robotic radical cystectomy

T Patel, D Hardwick, J Robison, J Mayer

University of South Florida School of Medicine United States

Introduction \& Objective: To determine feasibility in measuring surgeon physical stress during both open radical cystectomy (ORC) and robotic radical cystectomy (RRC). Real-time motion capture and electromyographic (EMG) analysis during live surgery was used to measure markers of physical stress.

Materials and Methods: Under an IRB approved protocol, a single surgeon who routinely performs both ORC and RRC was recruited to participate in study. Analysis was done in two patients, one patient who underwent ORC and the other RRC. Exclusion criteria included BMI $\geq 30$, history of prior radiation, node positive disease, and $>\mathrm{cT} 2$ disease. The diversion was excluded from this study. The Noraxon ${ }^{\circledR}$ Biomechanical Analysis System version 3.8 was used for data analysis. We used 6 MyoMotion Kinematics Analysis Sensors to quantify the amount of joint and segmental motion of the spine, shoulders, and head. Sixteen channels of MyoMuscle EMG sensors were used to measure activation levels, patterns, and fatigue characteristics of key muscle groups. Surgeon strength and endurance of the core musculature was assessed with the Prone Static Plank Test (PSPT) and Modified Biering-Sorensen Test (MBST) before and after surgery each surgery. Validated techniques for motion and EMG data collection and processing were used.

Results: Descriptive analyses were ran for 5 stages of the surgeries representing ureteral dissection (UD), posterior dissection (PD), bladder and prostate pedicle dissection (BPD), dorsal vein ligation (DVL), and lymph node dissection (LND). During ORC, the percentage of time spent in cervical flexion for each stage was $98 \%, 91.8 \%, 87.5 \%, 100 \%$, and $97.1 \%$ respectively. During RRC, $100 \%$ of the time was spent in cervical flexion for all stages. Activation of key msucle groups for both procedures was examined bilaterally across all 5 stages and expressed as a percentage of peak activation in Table 1 . The pre and post open surgery MBST was both $25 \mathrm{~s}$ and the PSPT was $68 \mathrm{~s}$ abd $48 \mathrm{~s}$, respectively. The pre and post robotic surgery MBST was $25.1 \mathrm{~s}$ and $32.4 \mathrm{~s}$, respectively and the PSPT was $59 \mathrm{~s}$ and $51 \mathrm{~s}$, respectively.

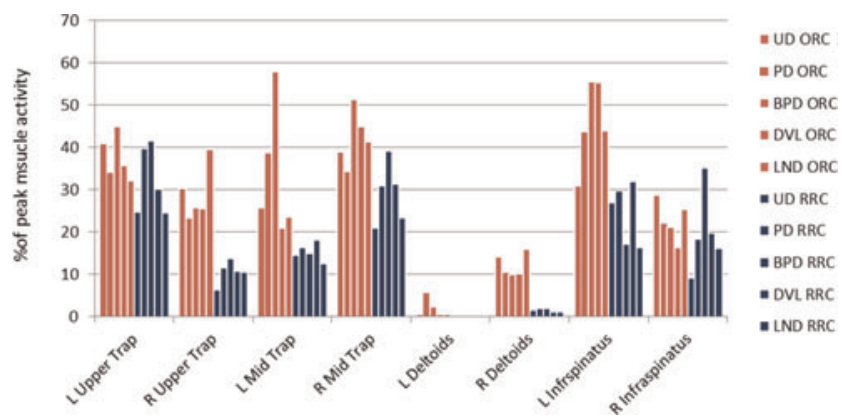


Conclusions: We were able to identify meaningful data using motion and EMG analysis during ORC and RRC. This pilot study will be used to conduct a larger study with multiple surgeons to help identify if there is an ergonomic advantage to RRC over traditional ORC.

MP20-8 Robot-assisted radical cystectomy with intracorporeal urinary diversion in the setting of challenging patient factors

N Ahmadi, D Freitas, C Fay, PW Mekhail, S Chopra, G Cacciamani, IS Gill, M Desai, M Aron

USC Institute of Urology/ USANZ

United States

Introduction \& Objective: Although robot-assisted radical cystectomy (RARC) with intracorporeal urinary diversion (ICUD) has become more common over the past decade, the degree of difficulty and acceptability varies based on patient factors. Many centers reserve the RARC for relatively straightforward cases and the challenging cases are still performed open. The aim of this study was to evaluate the feasibility and safety of RARC with ICUD in the setting of challenging patient factors. Materials and Methods: We retrospectively analyzed 195 patients who underwent RARC with ICUD and bilateral PLND at our institution from July 2010 to December 2015. The patients were grouped into 'challenging' $(n=64,32.8 \%)$ or 'straightforward' $(n=131,67.2 \%)$ groups. 'Challenging' group includes patients with previous open lower abdominal surgery, pelvic radiation, clinical lymphadenopathy, locally advanced disease (T3/T4) or higher body mass index (BMI >35). We compared perioperative and oncological outcomes between the two groups. Results: Orthotopic urinary diversion was performed in $26.6 \%$ $(n=17)$ of 'challenging' group and $36.6 \%(n=48)$ of 'straightforward' group $(\mathrm{p}=0.19)$. There were no differences in median estimated blood loss $(p=0.4)$, operative time ( 450 vs $432 \mathrm{~min}, \mathrm{p}=0.08$ ) and length of stay ( 6.0 vs 6.0 days, $\mathrm{p}=0.9)$ between the two groups, respectively. Transfusion was $0 \%$ in both groups. The proportion of pathological $\mathrm{T}$ stages and positive surgical margin rates showed no significant difference between the two groups. Overall, 30-day complication rate was $76.6 \%$ (high-grade: $18.8 \%$ ) in 'challenging' group and $63.4 \%$ (high-grade: $17.6 \%$ ) in 'straightforward' group $(\mathrm{p}=0.06)$. Moreover, overall 90-day complication rate was $87.5 \%$ (high-grade: $29.7 \%$ ) in 'challenging' group and $77.8 \%$ (high-grade: $24.4 \%)$ in 'straightforward' group $(\mathrm{p}=0.06)$. Although there was a significant difference in renal/metabolic complication rates between the 2 groups (challenging: $17.2 \%$ vs straightforward: $6.1 \%$, $\mathrm{p}=0.014$ ), there were no significant differences in other variables. Analysis of overall and recurrence-free survival demonstrated a trend towards better outcomes in 'straightforward' group, but didn't show a statistical difference $(\mathrm{p}=0.1$ and 0.09$)$

Conclusions: In experienced hands, RARC with ICUD is safe and feasible even in the setting of challenging patient factors, with acceptable perioperative and oncological outcomes. Reproducibility across multiple centers and surgeons is necessary in order to validate these findings

MP20-9 Can Indocyanine Green Fluorescence Angiography Eliminate Ureteral Strictures After Robotic Radical Cystectomy?

N Ahmadi, D Freitas, C Fay, A Berger, M Desai, IS Gill, M Aron
USC Institute of Urology/ USANZ

United States

Introduction \& Objective: Ureteroileal strictures are diagnosed in $3-10 \%$ of patients following radical cystectomy and urinary diversion. Poor vascularity of the distal ureter is a wellknown risk factor. Indocyanine-green (ICG) is an exogenous tracer that can help assess tissue vascularity during robotic surgery. Herein, we report a proof-of-principle with our initial experience in using ICG prior to performing intracorporeal ureteroileal anastomosis during robotic assisted radical cystectomy (RARC).

Materials and Methods: From April 2016 until February 2017, the use of ICG was evaluated in 26 patients who underwent RARC with intracorporeal urinary diversion (IUD). Intravenous ICG (25mg) was administered prior to ureteroileal anastomosis to assess vascularity and the non-perfused distal ureters were excised prior to ureteral spatulation. Anastomosis was performed with 4-0 vicryl sutures over ureteral stents.

Results: Of the 26 patients (52 ureters) who underwent RARCIUD (13 neobladders and 13 ileal conduits), ICG revealed poor distal ureteral enhancement in $13(50 \%)$ patients. Seven $(27 \%)$ patients required bilateral distal ureteral trimming, four $(15 \%)$ patients required left and two $(8 \%)$ patient required right distal ureteral trimming. Median excised ureteral length was $2 \mathrm{~cm}$ (18). Median operative time was 480 minutes (400-625), median estimated blood loss (EBL) was $200 \mathrm{ml}(100-800)$ and median length of stay (LOS) was 5 days (3-9). Twelve (46\%) of patients experienced Low grade (Clavien 1-2) complications. At median follow-up of 5 months (Range 3-12 months), no anastomotic strictures were identified.

Conclusions: Intravenous injection of ICG prior to ureteroileal anastomosis is a useful tool to evaluate distal ureteral vascularity and to identify and excise the non-perfused ureteral segment. Further follow-up is required to confirm long-term benefit of this novel use of ICG. We believe this modification could potentially eliminate ischemic ureteral strictures after RARC.

MP20-10 Lymph node mapping in patients with bladder cancer undergoing laparoscopic radical cystectomy and extended lymphnode dissection.

K Minami, T Harabayashi, N Takada, S Nagamori

department of Urology, Hokkaido Cancer Center Japan

Introduction \& Objective: To evaluate the distribution of extended lymph node dissection (LND) as a nodal staging factor in the treatment of invasive carcinoma of the urinary bladder.

Materials and Methods: A total of 87 patients underwent laparoscopic radical cystectomy with extended LND. The extended LND template was as follows: the proximal limit was at the level of the aortic bifurcation, the distal limits were the deep circumflex iliac vein and Cloquet's LN, and the lateral limit was the genitofemoral nerve. The posterior limit was the internal iliac vessels, including the presacral nodes obturator fossa. Specimens were evaluated as 9 separate regions from designated anatomical locations (1:presacral, 2: right common iliac, 3: left common iliac, 4: right external iliac, 5: left external iliac, 6: right internal iliac, 7: left internal iliac, 8: right obturator, 9: left obturator, Fig.1). The number of lymph nodes (LNs) and presence of positive LNs at each location was prospectively analyzed. 


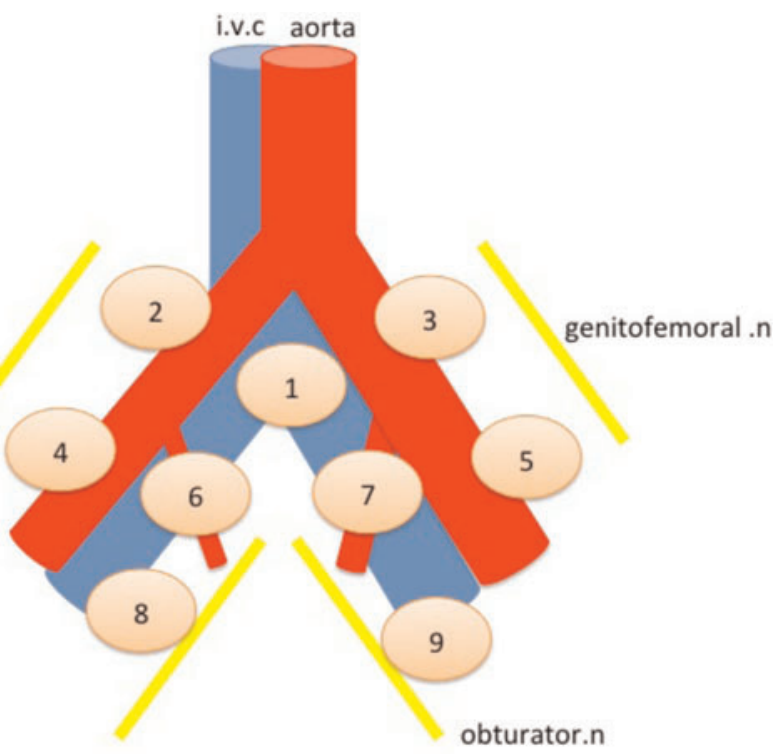

Fig.1 The anatomical locations of the extended LND

Table.1 Summary of lymph node counts and distribution

\begin{tabular}{ll}
\hline & \multicolumn{1}{c}{ total, $n=87$} \\
\hline Total & Median 33 (range, 10-64) \\
Template of Iymph node dissection & \\
presacral & Median 3 (range, 0-9) \\
right common iliac & Median 3 (range, 1-12) \\
left common iliac & Median 2 (range, 0-14) \\
right external iliac & Median 3 (range, 0-13) \\
left external iliac & Median 3 (range, 0-12) \\
right internal iliac & Median 1 (range, 0-8) \\
left internal iliac & Median 1 (range, 0-8) \\
right obturator & Median 7 (range, 2-20) \\
left obturator & Median 6 (range, 1-18) \\
\hline
\end{tabular}

Results: The mean operative time was 342 (230-590) minutes. The estimated blood loss was $282 \mathrm{~mL}(21-760 \mathrm{~mL})$, requiring intraoperative blood transfusion in $10 \%$ of patients. Table 1 shows a summary of node counts. The median number of LNs removed was 33 (range, 10-64). There was no significant difference in the node count between right and left side. In all, 20.0\% (17 patients) of the patients had LNs metastases. The median number of LNs metastases was 2 (1-20). All other patients with LNs metastases above the common iliac bifurcation had more distally located LNs metastases. There were no skip lesions to LNs above the common iliac or presacral area. No Clavien 3-5 lymph node dissection related complications were observed in this study.

Conclusions: Despite the small number of patients, our study demonstrated no major difference in outcomes. Laparoscopic radical cystectomy and lymph node dissection did provide similar pathologic outcomes compared with open and robotic radical cystectomy.

\section{MP20-11 Oncologic Outcomes and Predictive Factors for Recurrence following Robot-assisted Radical Cystectomy for Urothelial Carcinoma: Multicenter Study from Korea}

J Shim, T Kwon, K Rha, Y Lee, J Lee, B Jeong, J Choi, S Kang, S Kang, J Jung

Introduction \& Objective: To evaluate intermediate-term oncologic outcomes, predictive factors for recurrence, and recurrence patterns in a multicenter series of patients treated with robot-assisted radical cystectomy (RARC) for urothelial carcinoma (UC) of the bladder.
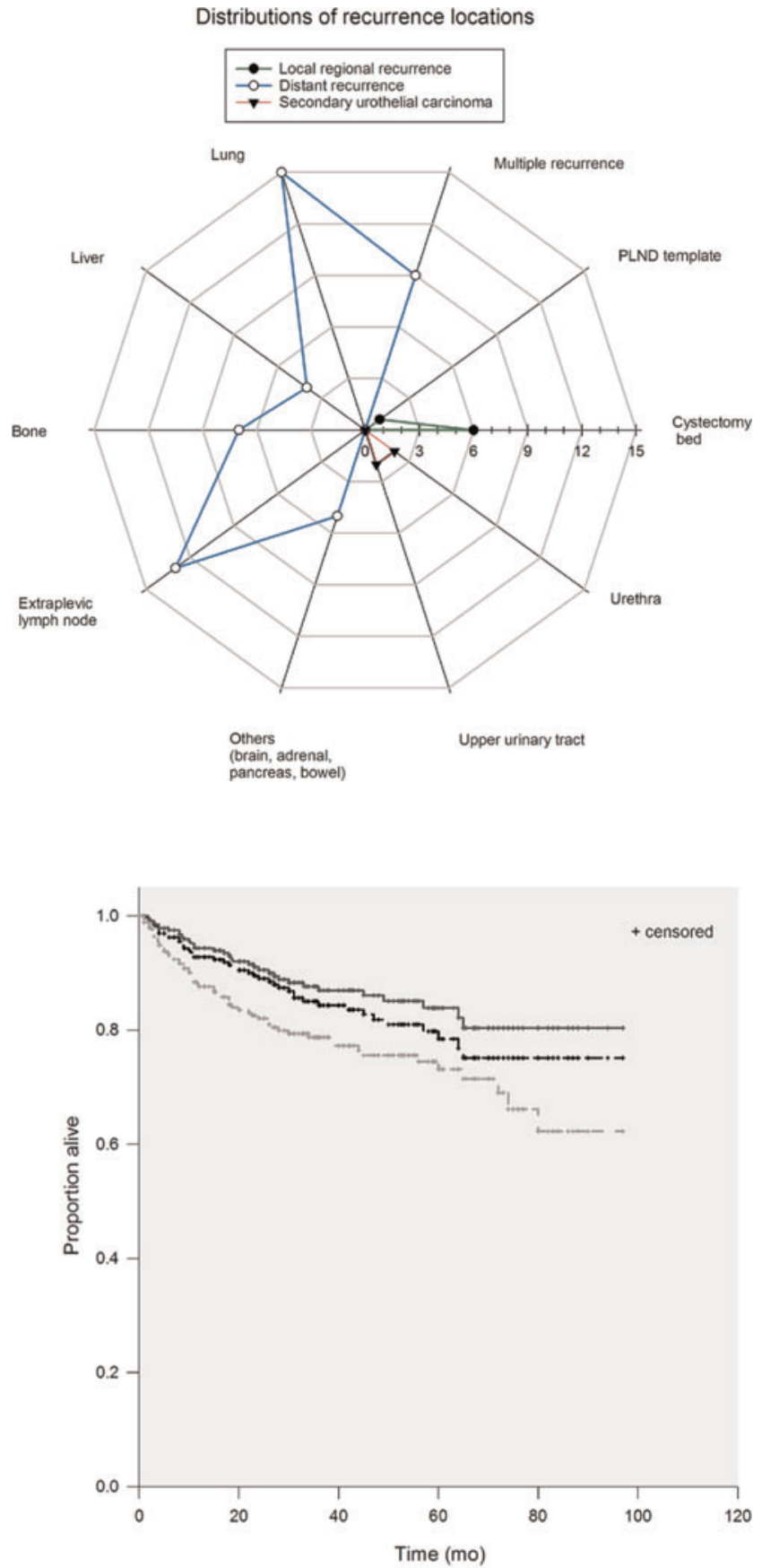

- - Overall survival Cancer-specific survival Recurrence-free survival 
Table 1. Pathologic outcomes

\begin{tabular}{|c|c|c|}
\hline Pathologic outcomes & $n=346$ & IQR \\
\hline \multicolumn{3}{|l|}{ Histologic type, no. (\%) } \\
\hline Urothelial carcinoma & $346(100)$ & \\
\hline \multicolumn{3}{|l|}{ Pathologic tumor stage, no. (\%) } \\
\hline $\mathrm{T} 0 / \mathrm{Ta} / \mathrm{Tis}$ & $53(15.3)$ & \\
\hline $\mathrm{T} 1 / \mathrm{T} 2$ & $184(53.1)$ & \\
\hline $\mathrm{T} 3 / \mathrm{T} 4$ & $109(31.6)$ & \\
\hline Soft tissue margin positive, no. (\%) & $9(2.6)$ & \\
\hline $\mathrm{T} 1 / \mathrm{T} 2$ & $4(1.2)$ & \\
\hline $\mathrm{T} 3 / \mathrm{T} 4$ & $5(1.4)$ & \\
\hline Lymph node yield, Mean \pm SD & $17.1 \pm 12.0$ & $9.0-23.0$ \\
\hline Standard PLND & $10.7 \pm 12.0$ & \\
\hline Extended PLND & $24.0 \pm 11.9$ & \\
\hline Number of patients with LN positive (\%) & $68(19.7)$ & \\
\hline Lymph node density $(\%)$, Mean \pm SD & $25.5 \pm 27.1$ & $7.1-41.9$ \\
\hline Node negative, no. (\%) & $278(80.3)$ & \\
\hline Node density $1-20 \%$, no. (\%) & $47(13.6)$ & \\
\hline Node density $>20 \%$, no. $(\%)$ & $21(6.1)$ & \\
\hline \multicolumn{3}{|l|}{ Pathologic nodal stage, no. (\%) } \\
\hline No & $278(80.3)$ & \\
\hline $\mathrm{N} 1 / \mathrm{N} 2 / \mathrm{N} 3$ & $68(19.7)$ & \\
\hline \multicolumn{3}{|l|}{ Follow-up, mo } \\
\hline Mean \pm SD & $32.9 \pm 25.4$ & $7.0-50.3$ \\
\hline
\end{tabular}

${ }^{\circ}<<0.05$
SD: standard deviation; IQR: interquartile range; PLND: pelvic lymph node dissection; LN: lymph node

SD: standard deviation; IQR: interquartile range; PLND: pelvic lymph node dissection; LN: lymph node

\begin{tabular}{|c|c|c|c|c|c|c|}
\hline \multirow[t]{2}{*}{ Variable } & \multicolumn{3}{|c|}{ 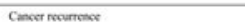 } & \multicolumn{3}{|c|}{ Death from blasler casect } \\
\hline & OR & $955 \mathrm{Cl}$ & Pvalue & OR & $95 \mathrm{SCl}$ & Pvalue \\
\hline \multicolumn{7}{|l|}{ Copporeal type } \\
\hline 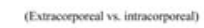 & 0.301 & $0.09-1.03$ & 0.056 & 0.316 & $0.08-1.19$ & 0.059 \\
\hline Positive surpyical manyins & 10916 & $1.90-62.57$ & $0.007 \%$ & 1.844 & $0.29-11.60$ & 0.514 \\
\hline \multicolumn{7}{|l|}{ Type of PLND } \\
\hline (Elended is, tandard) & 2.816 & $1.27-620$ & $0.010^{\circ}$ & 5.935 & $225-15.65$ & $0.000 *$ \\
\hline Number of femoved $L X$ & 0.946 & 0.9-0.99 & $0.010^{\circ}$ & 0.993 & $0.94-1.04$ & 0.776 \\
\hline Numbra of poritive LN & 1.067 & $1.01-1.17$ & $0.026^{\circ}$ & 1.042 & $0.93-1.17$ & 0.484 \\
\hline \multicolumn{7}{|l|}{ Pathobgic tumar stagec } \\
\hline (pT2 of less $1, p T 3 \propto p T 4$ ) & 2.417 & $1.36-430$ & $0.003^{*}$ & 3.504 & $1.80-805$ & $0.000 \%$ \\
\hline \multicolumn{7}{|l|}{ Notal sage } \\
\hline (No $\left.1 \times N_{1} N_{2} N_{3}\right)$ & 1.027 & $039-27$ & 0.957 & 1.019 & $0.31-3,39$ & 0.976 \\
\hline
\end{tabular}

OR: odds ratio; Cl: confidense interval; OBS: orhotopic bladder substitution; PLND; pelvic lymph node dissection; LN: lymph node

Materials and Methods: Between 2007 and 2015, 346 patients underwent RARC at multiple tertiary referral centers in Korea. Descriptive statistics were used for demographics and perioperative variables. Survival and recurrence were estimated with Kaplan-Meier analysis. Logistic regression models were used to determine predictors of recurrence.
Results: Median follow-up was 33 months (interquartile range [IQR]: 7-50). The numbers of patients with organ-confined and lymph node (LN)-positive disease were 237 (68.4\%) and 68 (19.7\%), respectively. LN density (1-20 versus $>20$ ) was $13.6 \%$ and $6.1 \%$, with a median of 17 nodes removed (IQR: 9-23). In logistic regression analysis, type of $\mathrm{LN}$ dissection and pathologic tumor stage were significant predictors of cancer recurrence and death from cancer. Local, distant recurrence and secondary UC occurred in $7(2.0 \%), 53(15.3 \%)$, and $4(1.2 \%)$ patients, respectively. The 5-year overall survival, cancer-specific survival, and recurrence-free survival (RFS) were $78 \%, 84 \%$, and $73 \%$, respectively. At last follow-up, RFS for extended pelvic LN dissection versus standard pelvic LN dissection was $70 \%$ and 47\% ( $P=0.038)$. In addition, at last follow-up, $\mathrm{LN}$ density (0 vs. 1-20 vs. over 20) was $67 \%, 41 \%$, and $29 \%$, respectively $(P<0.001)$.

Conclusions: Patients undergoing RARC in this multi-institutional cohort demonstrated intermediate-term oncologic outcomes, predictive factors for recurrence, and recurrence patterns that were not unusual.

MP20-12 Total intracorporeal robotic radical cystectomy: Does this method have a benefit when compared to the extracorporeal method in the patient outcome?

K Rha, Y Lee, J Lee, B Jeong, J Choi, S Kang, S Kang, J Jeong

Introduction \& Objective: To compare perioperative parameters, complications and oncologic outcomes between robot-

\begin{tabular}{|c|c|c|c|}
\hline Pathologic outcomes & $\begin{array}{l}\text { ECUD } \\
(\mathrm{N}=278)\end{array}$ & $\begin{array}{l}\text { ICUD } \\
(\mathrm{N}=53)\end{array}$ & P-value \\
\hline \multicolumn{4}{|l|}{ Histologic type, no. (\%) } \\
\hline Urothelial carcinoma & $278(100)$ & $53(100)$ & 1.000 \\
\hline Pathologic tumor stage, no. $(\%)$ & & & $0.665^{\dagger}$ \\
\hline $\mathrm{TO} / \mathrm{Ta} / \mathrm{Tis}$ & $38(13.7)$ & $13(24.5)$ & \\
\hline $\mathrm{T} 1 / \mathrm{T} 2$ & $153(55.0)$ & $25(47.2)$ & \\
\hline $\mathrm{T} 3 / \mathrm{T} 4$ & $87(31.3)$ & $15(28.3)$ & \\
\hline High tumor grade & $202(72.7)$ & $53(100)$ & $<0.001^{\star \star}$ \\
\hline Soft tissue margin positive, no. (\%) & $7(2.5)$ & $2(3.8)$ & 0.608 \\
\hline Lymph node yield, Mean \pm SD & $15.1 \pm 12.1$ & $29.8 \pm 13.6$ & $<0.001^{\star \star}$ \\
\hline Number of patients with LN positive (\%) & $53(19.1)$ & $14(26.4)$ & 0.523 \\
\hline Lymph node density $(\%)$, Mean \pm SD & $28.2 \pm 26.7$ & $15.8 \pm 30.6$ & 0.127 \\
\hline Node negative, no. (\%) & $225(80.9)$ & $39(73.6)$ & \\
\hline Node density $1-20 \%$, no. (\%) & $34(12.2)$ & $12(22.6)$ & \\
\hline Node density $>20 \%$, no. $(\%)$ & $19(6.9)$ & $2(3.8)$ & \\
\hline Pathologic nodal stage, no. (\%) & & & $0.175^{\dagger}$ \\
\hline Nx/NO & $227(81.7)$ & $39(73.6)$ & \\
\hline $\mathrm{N} 1 / \mathrm{N} 2 / \mathrm{N} 3$ & $51(18.3)$ & $14(26.4)$ & \\
\hline Recurrence-free survival for $1 \mathrm{yr}(\%)$ & 88.3 & 86.2 & $0.855^{\ddagger}$ \\
\hline
\end{tabular}

${ }^{\prime} P<0.05, " P<0.001,{ }^{\dagger}$ : Pearson chi square test, ${ }^{\ddagger}: \log$ rank test. LN: lymph node 


\begin{tabular}{|c|c|c|c|c|c|}
\hline & $\begin{array}{c}\text { Extracorporeal UD } \\
(\mathrm{N}=278), \mathrm{n}(\%)\end{array}$ & $\begin{array}{c}\text { Intracorporeal UD } \\
(\mathrm{N}=53), \mathrm{n}(\%)\end{array}$ & OR & $95 \% \mathrm{Cl}$ for $\mathrm{OR}, \%$ & Pvalue \\
\hline Infectious & $41(14.7)$ & $8(15.1)$ & 1.028 & $0.452-2.338$ & 1.000 \\
\hline Respiratory & $3(1.0)$ & 0 & . & - & 1.000 \\
\hline Gastrointestinal & 34 (12.2) & $2(3.8)$ & 0.281 & $0.066-1.209$ & 0.090 \\
\hline $\begin{array}{l}\text { Procedure-related } \\
\text { (including transfusion) }\end{array}$ & $42(15.1)$ & $4(7.6)$ & 0.459 & $0.157-1.338$ & 0.193 \\
\hline Gentourinary & $22(7.9)$ & $6(11.3)$ & 1.485 & $0.572-3.859$ & 0.420 \\
\hline Wound-related & $6(2.1)$ & 0 & - & - & 0.595 \\
\hline Nervous & $1(0.4)$ & 0 & - & - & 1.000 \\
\hline Vascular & $2(0.7)$ & 0 & - & . & 1.000 \\
\hline Miscellaneous & $4(1.4)$ & $1(1.9)$ & 1.317 & $0.144-12.023$ & 0.585 \\
\hline
\end{tabular}

- Percentages for complication category do not sum because one patient can have two or more complications

assisted radical cystectomy (RARC) with extracorporeal urinary diversion (ECUD) and intracorporeal urinary diversion (ICUD). Materials and Methods: Between 2007 and 2015, 331 patients underwent RARC with ECUD or ICUD at multi tertiary referral institutions in Korea. Descriptive statistics were used to summarize demographic and perioperative variables. In addition, we analyzed various oncologic outcomes, complications and estimated recurrence-free survival (RFS) using the Kaplan-Meier method.

Results: According to perioperative outcomes, the ICUD group had a significantly longer operation time $(\mathrm{P}<0.001)$. There was no difference in the total estimated blood loss between the two groups, while the surgical transfusion amount was larger in the ICUD group $(\mathrm{P}<0.001)$. Recovery parameters, such as time to flatus, oral intake, and length of hospital stay, were significantly shorter in the ICUD group than in the ECUD group $(\mathrm{P}=0.017$, $\mathrm{P}=0.008$, and $\mathrm{P}=0.046$, respectively). In the analysis of complication type, there were no significant differences between two groups, despite the ICUD group showed trend of decreasing in gastrointestinal complication $(\mathrm{P}=0.090)$. There was no difference in oncologic outcomes such as the positive margin rate $(\mathrm{P}=0.523)$ and the short-term RFS $(\mathrm{P}=0.855)$ between the two groups.

Conclusions: Several perioperative outcomes suggest that ICUD would be surgically impregnable than ECUD. However, the results of this multi-institutional cohort demonstrate the benefits of using an ICUD approach, including a shorter recovery period, despite similar complications, oncologic outcomes, including rate of recurrence.

MP20-13 Sequential robot-assisted bladder diverticulectomy and simple prostatectomy: Technique and surgical outcomes

Z Shen, S Zhong, S Chen, M Zhang

China (People's Republic)

Introduction \& Objective: To present outcomes of sequential robot-assisted bladder diverticulectomy and simple prostatectomy that may be a safe and effective procedure with excellent results.

Materials and Methods: From August 2013 to December 2014, we successfully operated on three men aged from 63 to 78 for large benign prostatic hyperplasia and a secondary bladder diverticulum, by using the robot-assisted bladder diverticulectomy combined with robot-assisted simple prostatectomy approach. The prostate volume was 100,105 , and $120 \mathrm{~mL}$, respectively. The postvoid residual was 100,160 and $300 \mathrm{~mL}$, respectively. The bladder diverticulum volume calculated by transabdominal ultrasonography preoperative was 90, 150 and $280 \mathrm{~mL}$, respectively. All patients underwent follow-up of 6 weeks after the surgery and every 3 months thereafter.

Results: All procedures were successfully performed by robotic surgical system. The operative time was 90, 110 and 130 minutes, respectively. The estimated blood loss was 60, 100 and $120 \mathrm{~mL}$, respectively. The mean hospital stay was 9 days with no intra- or postoperative complications. The average 17-month follow up (range 15-19 Months) confirmed good operative results in all cases.

Conclusions: Bladder outlet obstruction is the main cause of acquired bladder diverticula and is largely due to benign prostatic hyperplasia. Concomitant performance of robot-assisted bladder diverticulectomy along with robot-assisted simple prostatectomy is a safe and effective procedure with excellent results.

MP20-14 The efficacy of hybrid laparoscopic procedures against bladder benign disease

T Hamasaki, Y Endo, J Akatsuka

Introduction \& Objective: It is difficult to understand the situation in the bladder during laparoscopic partial cystectomy. It is safe procedures regard to the anterior wall and apex, however there is a risk of ureter injury with regard to lesions such as near the ureteral orifice. Therefore, we examined the efficacy of the transurethral procedure in laparoscopic surgery.

Materials and Methods: Study performed at Nippon Medical School Main Hospital, Tokyo, Japan. We performed 22 cases of laparoscopic partial cystectomy with benign disease such as bladder endometriosis and bladder diverticulum, from January 2007 to April 2017. All 14 cases of bladder endometriosis were female, and all bladder diverticula 8 men. The effectiveness and safety of this operation were verified.

Results: The average surgical time was 146minutes, ranging from 110-180 minutes. The laparoscopic resection line could be clarified by using a cystoscope in all 24 cases. For seven cases close to the triangle zone, procedures could be performed safely without ureteral damage by using transurethral resection. In addition, we could prevent ureteral orifice from squeezing during suturing the bladder. No relapse was observed during the follow up period.

Conclusions: This procedure not only induces an appropriate resection line in partial cystectomy but also can be done safely by sometimes transurethral resection. There were no disadvantages besides increasing the number of surgeons.

MP20-15 Trans-Urethral Resection of the Bladder Tumour (TURBT) surgery, for the treatment of Non-Muscle Invasive Bladder Cancer (NMIBC), results in changes to selective biochemical parameters

SF Hughes, RM Lamb, P Ella-Tongwiis, I Shergill

University of Chester, BCUHB NW2URC

United Kingdom 
Introduction \& Objective: Currently, there is limited literature on the 'normal' pathophysiological response to TURBT for NMIBC. The aim of the study was to evaluate changes in routine, cellular and molecular biomarkers following TURBT, which could identify 'normal' pathophysiological response for treatment of NMIBC.

Materials and Methods: 28 patients undergoing TURBT for NMIBC were recruited. From patients ( 5 females, 23 males, aged between 65-99 years (median 82 years), venous blood samples were collected pre-operatively (baseline), at 30, 120 and 240 minutes (study 1), and at 1, 3 and 6 months (study 2) post-operatively. Routine haematological biomarkers, such as leukocytes, erythrocytes, haemoglobin, thrombocytes, prothrombin (PT) and partial thromboplastin time (aPTT) were analysed via conventional methods, for assessment of "normal' pathophysiological response to surgery (study 1). Subsequently, cellular and molecular biomarkers, such as MIP-2, EGF, follistatin, VEGFR1, VEGFR2, HER2/neu, IL-6R alpha and TIE-2, which are intrinsically involved in inflammation, growth and angiogenesis were measured, as potential markers of disease recurrence and progression (study 2).

Results: A significant decrease in erythrocytes, leukocytes, haemoglobin, thrombocytes, aPTT, and fibrinogen $(\mathrm{p} \leq 0.05)$ was observed following TURBT, for the treatment of NMBIC. Interestingly, a significant increase was found in PT $(p=0.003)$ following surgery (study 1). MIP-2, FGF, EGF, follistatin, VEGFR1 and VEGFR2 showed increasing concentrations, whilst HER2/neu, IL-6R alpha and TIE-2 showed decreasing concentrations up to 6 months after TURBT (study 2).

Conclusions: We report that several changes to routine and selective molecular and cellular biomarkers occur following TURBT for NMIBC. Analysis of such markers, in larger cohorts, may potentially provide valuable data to identify or predict those patients at risk of cancer recurrence, or those highly likely to develop post-operative complications. This would potentially have significant impact on patients and health care provision.

\section{MP20-16 Percutaneous Resection of Bulky Renal Pelvic Tumor}

M Eshghi, RJ Yau, JH Hillelsohn

New York Medical College

United States

Introduction \& Objective: Large renal pelvic tumors can not be adwquately managed with rigid or flexible ureteroscope. We describe our technique and demonstrate 4 cases of resection.

Materials and Methods: Since 1985 we gradually develped and improved the technique of percutaneous resection of large transitional cell carcinomain the collecting system.

The majority of these cases are patients with solitary kidney, imperative cases due to multiple comorbidities or patients' choce. Majority of these cases are curative depending on the grade and stage of the disease versus palliative casest to avoid hemodilysis. The puncture technique. resection technique, depth of resection, anatomy of collecting system and resction depth will be discussed. More than half of our case undewnt intracavitary chemo or immunotherapy using mitamycin, BCG, interferon and gemcitobin. Results: Considering that we have treated over40 such cases the majority of the patients who expired were free of disease and even those who had resiual disease succumbed mostly to cardio pulmonary complications.

Conclusions: We demonstrate the steps in percutaneous resection of upper tract urothelial carcinoma and technique of post op office intracaviatary chemo and immunotherapy.
MP20-17 Multi-Institutional Evaluation of Quality of Upper Urinary Tract Biopsy Using BIGopsy Forceps, a 2.4F Nitinol Basket, and Piranha Forceps

DJ Lama, S Safiullah, R Youssef, TK Lee, T McDonald, K Sheth, R Hutchinson, J Balani, K Flower, V Margulis, S Savage, J Landman, RM Patel

Department of Urology, University of California, Irvine, Orange, CA

United States

Introduction \& Objective: A number of ureteroscopic devices have been developed to aid the diagnosis of upper urinary tract urothelial carcinoma (UTUC). A recent addition, the back-loaded BIGopsy ${ }^{\circledR}$ biopsy forceps, was developed in order to extract a greater volume of tissue for histopathological diagnosis. In an effort to compare the BIGopsy forceps to contemporary biopsy devices, a retrospective multi-institutional analysis was performed.

Materials and Methods: From 2011-2016, 185 biopsies were performed for suspected UTUC in 142 patients at three institutions. Specimens were collected using 5.9/2.4F BIGopsy forceps (Cook Medical Inc., Bloomington, IN), a 2.4F nitinol basket (Cook Medical Inc., Bloomington, IN) or 3.6/3F Piranha forceps (Boston Scientific Corp., Malborough, Massachusetts). Data included various biopsy characteristics (i.e. endoscopic growth pattern, presence of muscularis propria, lamina propria, intact urothelium, and crush artifact), and concordance between the grade of the biopsy and nephroureterectomy specimen. Experienced GU pathologists, blinded to the device used to obtain the biopsy, completed a subjective Likert-type questionnaire after review of each urothelial specimen. Results: No difference existed between the BIGopsy forceps and $2.4 \mathrm{~F}$ basket in keeping the urothelium intact $(\mathrm{p}=0.186)$ and avoiding crush artifact $(\mathrm{p}=0.349)$ (Figure 1$)$. Both devices outperformed the Piranha forceps for intact urothelium $(p=0.004$ and $\mathrm{p}=0.044$, respectively). Concordance and device performance for papillary and sessile type lesions are shown in Table 1 and 2, respectively; concordance for both types was in the 75$82 \%$ range except for the Piranha biopsy of sessile lesions which was $40 \%$. The pathologist questionnaire indicated no significant

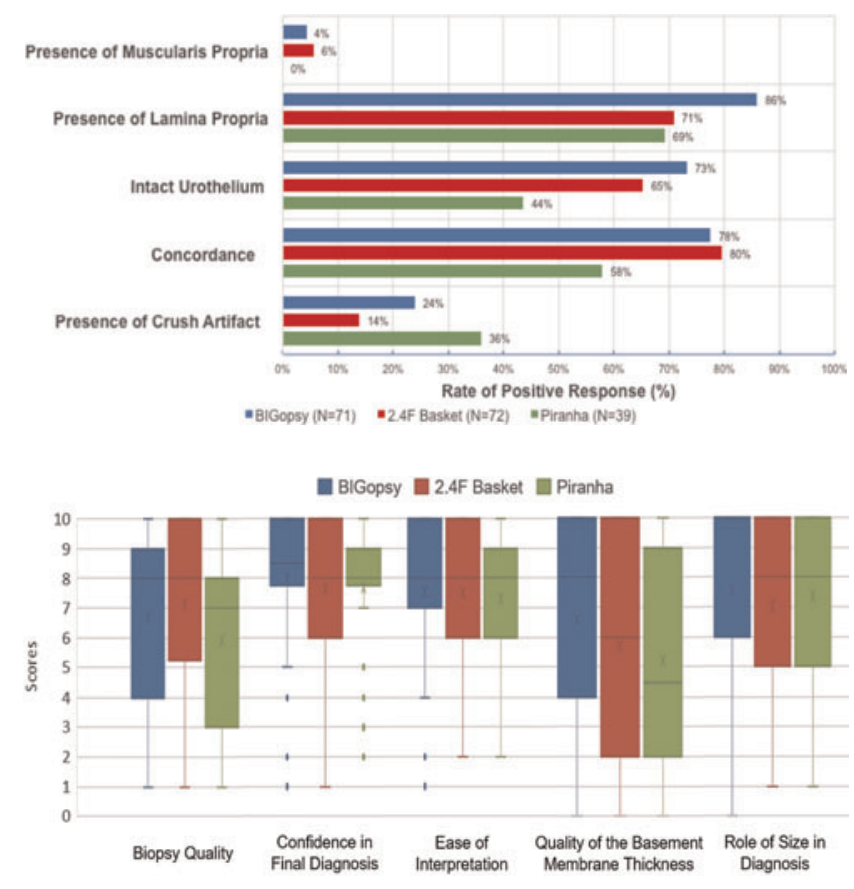




\begin{tabular}{|c|c|c|c|c|c|c|}
\hline & \multicolumn{2}{|c|}{ BIGopsy } & \multicolumn{2}{c|}{ 2.4F Basket } & \multicolumn{2}{c|}{ Piranha } \\
\hline $\begin{array}{l}\text { Device specimens by } \\
\text { histologic appearance }\end{array}$ & $\begin{array}{c}\text { Papillary } \\
(n=36)\end{array}$ & $\begin{array}{c}\text { Sessile } \\
(n=18)\end{array}$ & $\begin{array}{c}\text { Papillary } \\
(n=52)\end{array}$ & $\begin{array}{c}\text { Sessile } \\
(n=9)\end{array}$ & $\begin{array}{c}\text { Papillary } \\
(n=22)\end{array}$ & $\begin{array}{c}\text { Sessile } \\
(n=10)\end{array}$ \\
\hline $\begin{array}{l}\text { Patients undergoing } \\
\text { nephroureterectomy }\end{array}$ & $n=24$ & $n=11$ & $n=34$ & $n=5$ & $n=12$ & $n=5$ \\
\hline Concordant (\%) & $79 \%$ & $82 \%$ & $79 \%$ & $80 \%$ & $75 \%$ & $40 \%$ \\
\hline Not Concordant (\%) & $21 \%$ & $18 \%$ & $21 \%$ & $20 \%$ & $25 \%$ & $60 \%$ \\
\hline
\end{tabular}

\begin{tabular}{|c|c|c|c|c|c|c|}
\hline & $\begin{array}{l}\text { Presence of } \\
\text { Lamina } \\
\text { Propria (\%) }\end{array}$ & p-value & $\begin{array}{l}\text { Presence of } \\
\text { Crush } \\
\text { Artifact (\%) }\end{array}$ & p-value & $\begin{array}{c}\text { Intact } \\
\text { Urothelium } \\
\text { (\%) }\end{array}$ & p-value \\
\hline \multicolumn{7}{|l|}{ Papillary Lesion } \\
\hline $\begin{array}{l}\text { BIGopsy }(n=36) \text { vs. } \\
\text { Basket }(n=52)\end{array}$ & 92 vs. 69 & 0.024 & 17 vs. 12 & 0.708 & 86 vs. 67 & 0.079 \\
\hline $\begin{array}{l}\text { BiGopsy }(n=36) \text { vs. } \\
\text { Piranha }(n=22)\end{array}$ & 92 vs. 64 & 0.022 & 17 vs. 14 & 0.949 & 86 vs. 55 & 0.018 \\
\hline $\begin{array}{l}\text { Basket }(n=52) \text { vs. } \\
\text { Piranha }(n=22)\end{array}$ & 69 vs. 64 & 0.843 & 12 vs. 14 & 0.891 & 67 vs. 55 & 0.436 \\
\hline \multicolumn{7}{|l|}{ Sessile Lesion } \\
\hline $\begin{array}{l}\text { BIGopsy }(n=18) \text { vs. } \\
\text { Basket }(n=9)\end{array}$ & 94 vs. 78 & 0.25 & 44 vs. 44 & 1 & 61 vs. 44 & 0.448 \\
\hline $\begin{array}{l}\text { BIGopsy }(n=18) \text { vs. } \\
\text { Piranha }(n=10)\end{array}$ & 94 vs. 80 & 0.284 & 44 vs. 50 & 1 & 61 vs. 40 & 0.433 \\
\hline $\begin{array}{l}\text { Basket }(n=9) \text { vs. } \\
\text { Piranha }(n=10)\end{array}$ & 78 vs. 80 & 1 & 44 vs. 50 & 1 & 44 vs. 40 & 1 \\
\hline
\end{tabular}

differences between specimens obtained with BIGopsy forceps or the $2.4 \mathrm{~F}$ basket; however, BIGopsy was rated significantly higher than the Piranha forceps for biopsy quality $(p=0.012)$, basement membrane thickness $(\mathrm{p}=0.02)$, and role of biopsy size in accurate diagnosis $(\mathrm{p}=0.043)$ (Figure 2).

Conclusions: BIGopsy forceps was preferentially selected for sessile lesions by experienced urologists and showed similar overall and papillary-specific biopsy performance to the $2.4 \mathrm{~F}$ basket; both devices outperformed the Piranha forceps for diagnosis of upper tract urothelial carcinoma.

MP20-18 A novel transurethral resection technique for superficial bladder tumor: retrograde en bloc resection

K Zhang, J Xing, W Li, Z Wu

Urology Department, the First Affiliated Hospital of Xiamen University

China (People's Republic)

Introduction \& Objective: The aim of this study was to evaluate the safety and efficacy of a novel procedure of retrograde en bloc resection of bladder tumor (RERBT) with conventional monopolar resection electrode for the treatment of superficial bladder tumors.

Materials and Methods: RERBT and conventional TURBT (C-TURBT) was conducted respectively in 40 and 50 patients diagnosed with superficial papillary bladder tumors. In RERBT group, the tumor was en bloc removed retrogradely under direct vision using conventional monopolar electrode. The patients' clinicopathological, intraoperative and postoperative data were compared retrospectively between the RERBT and C-TURBT groups. Results: Of the 90 patients, 40 underwent RERBT and 50 underwent C-TURBT. Both groups were comparable in clinicopathological characteristic. RERBT could be performed as safely and effectively as C-TURBT. There were no significant differences in operative time, surgical complications. The cumulative recurrence of both groups was similar during up to 18 months followup. Detrusor muscle could be identified pathologically in $100 \%$ of

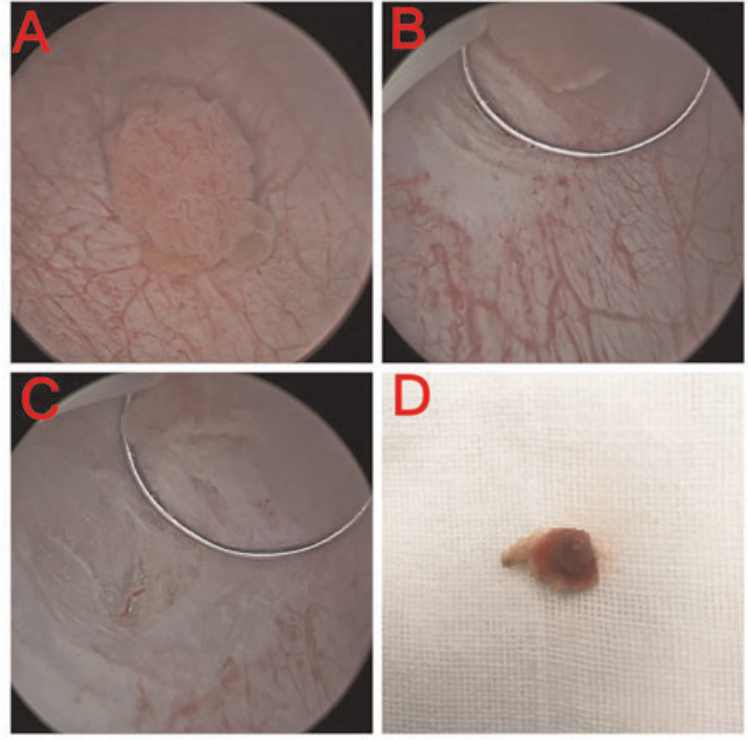

Figure 1. A: $1.5 \mathrm{~cm}$ in diameter of bladder tumor on the right bladder wall. B: Macroscopic normal mucosa about $0.5 \mathrm{~cm}$ away from the tumor base was margined. Then, the bladder mucosa was subsequently cut using a "flash firing" fashion. C: After the deep muscle layer was reach when normal glistening yellow fat is seen between muscle layers, the loop was moved forward along the muscle layer. D: The tumor was resect in one-piece

RERBT tumor specimens and the biopsy of tumor bases, but only in $54 \%$ and $70 \%$ respectively of C-TURBT samples $(\mathrm{p}<0.01)$.

Conclusions: The RERBT technique is feasible and safe for superficial bladder tumors using conventional monopolar resection setting, with the advantages of adequate tumor resection and collecting good quality tumor specimens for pathological diagnosis and staging compared to conventional TURBT.
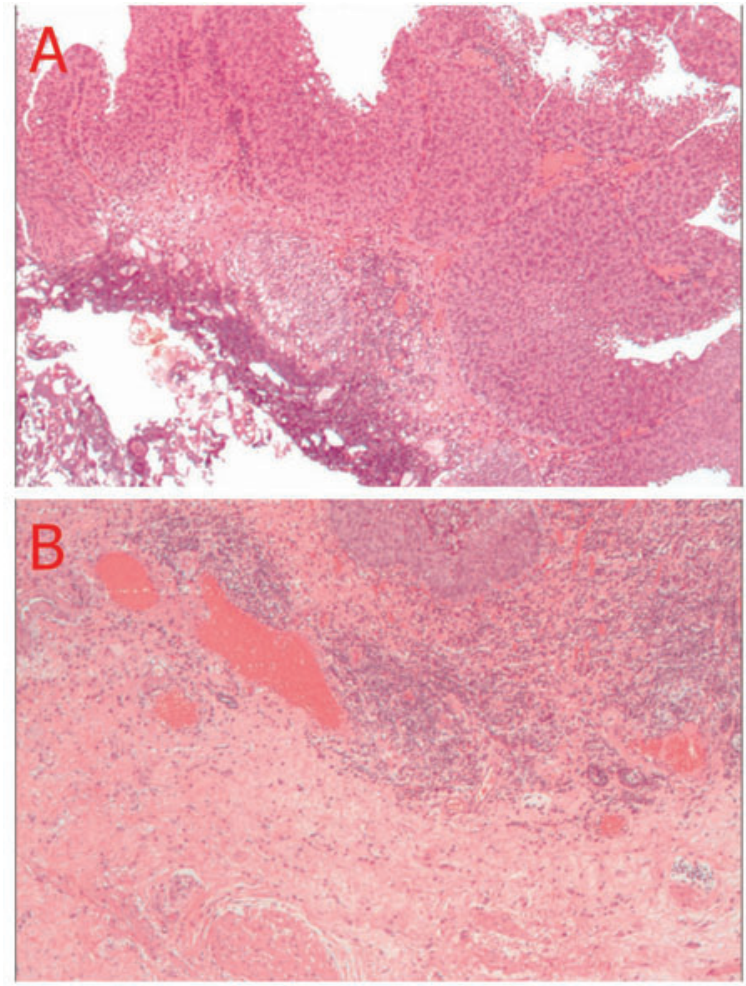

Figure 2. Histologic findings of resected tumors. (A) The lamina propria mucosac was severely charred without muscular propria in C-TURBT group $(\times 40)$. (B) The lamina propria mucosae remained intact and the muscular propria was identified in RERBT group $(\times 40)$. 
Table 1. Clinicopathological characteristics of patients

\begin{tabular}{|c|c|c|c|}
\hline Variable & RERBT (n=40) & C-TURBT $(n=50)$ & p value \\
\hline Age (year) & $60.65 \pm 13.08$ & $60.80 \pm 14.04$ & 0.959 \\
\hline Size & & & 0.650 \\
\hline$\leq 3 \mathrm{~cm}(n)$ & 32 & 38 & \\
\hline$>3 \mathrm{~cm}(\mathrm{n})$ & 8 & 12 & \\
\hline Gender & & & 0.166 \\
\hline Male & 35 & 38 & \\
\hline Female & 5 & 12 & \\
\hline Tumor multiplicity & & & 0.705 \\
\hline Single & 29 & 38 & \\
\hline Multiple & 11 & 12 & \\
\hline Tumor morphology & & & 0.686 \\
\hline Pedunculate & 28 & 33 & \\
\hline Flat & 12 & 17 & \\
\hline Stage & & & 0.119 \\
\hline $\mathrm{Ta}$ & 15 & 27 & \\
\hline $\mathrm{T} 1$ & 25 & 23 & \\
\hline Grade (WHO2004) & & & 0.250 \\
\hline LMP & 9 & 12 & \\
\hline LG & 22 & 23 & \\
\hline HG & 9 & 15 & \\
\hline
\end{tabular}

Table 2. Peri-operative and follow-up data.

\begin{tabular}{|c|c|c|c|}
\hline Variable & RERBT $(n=40)$ & C-TURBT $(n=50)$ & p value \\
\hline Operative time (min) & $36 \pm 11.8$ & $34 \pm 13.6$ & 0.464 \\
\hline Complications & & & 0.7633 \\
\hline Grade I & $1(2.5 \%)$ & $2(4 \%)$ & \\
\hline Grade II & $6(15 \%)$ & $8(16 \%)$ & \\
\hline Grade III & 0 & 0 & \\
\hline Obturator nerve reflex & $9(22 \%)$ & $12(24 \%)$ & 0.867 \\
\hline Bladder perforation & $2(5 \%)$ & $4(8 \%)$ & 0.689 \\
\hline \multicolumn{4}{|l|}{$\begin{array}{l}\text { Presence of the detrusor } \\
\text { muscle }\end{array}$} \\
\hline Tumor specimen & $(40 / 40) 100 \%$ & $(27 / 50) 54 \%$ & 0.000 \\
\hline Tumor base & $(40 / 40) 100 \%$ & $(35 / 50) 70 \%$ & 0.000 \\
\hline Irrigation (day) & $1.16 \pm 0.41$ & $1.22 \pm 0.45$ & 0.518 \\
\hline Catheterization(day) & $4.25 \pm 2.04$ & $4.65 \pm 2.16$ & 0.373 \\
\hline $\begin{array}{l}\text { Postoperative hospital } \\
\text { stay(day) }\end{array}$ & $4.07 \pm 0.57$ & $4.18 \pm 0.59$ & 0.400 \\
\hline $\begin{array}{l}\text { Residual tumor on the } \\
\text { base }\end{array}$ & 0 & $2(5 \%)$ & 0.500 \\
\hline Follow-up(months) & $10.8 \pm 3.9$ & $11.3 \pm 4.22$ & 0.775 \\
\hline \multicolumn{4}{|l|}{ Cumulative recurrence } \\
\hline In field & $2(5 \%)$ & $5(10 \%)$ & 0.455 \\
\hline Out of field & $6(15 \%)$ & $7(14 \%)$ & 0.893 \\
\hline over all & $8(20 \%)$ & $12(24 \%)$ & 0.650 \\
\hline LMP & 0 & 0 & - \\
\hline LG & $1(2.5 \%)$ & $2(4 \%)$ & 0.693 \\
\hline HG & $7(17.5 \%)$ & $10(20 \%)$ & 0.763 \\
\hline
\end{tabular}

RERBT: retrograde en bloc resection of bladder tumor; C-TURBT: conventional transurethral resection of bladder tumor; LMP: papillary urothelia neoplasia of low malignant potential; LG: low grade papillary urothelial carcinoma; HG: high grade papillary urothelial carcinoma
MP20-19 Intraoperative frozen section evaluation of the tumor and its base in patients with $\mathrm{T} 1$ urothelial bladder cancer underwent transurethral resection

Y Shen, H Gan, Q Wang, Y Zhu, D Ye

China (People's Republic)

Introduction \& Objective: Transurethral resection of bladder tumor (TURBt) remains the gold standard for management of bladder cancer, but there is evidence of a high rate of understaging for T1 tumor after primary resection. Muscle in the specimen is important for the accurate bladder staging. However, nearly $40 \%$ resected bladder specimens were failed to be found muscle after the initial TURBt even done by the sophistic urologists. Therefore a second TURBt was justified for correcting the staging error and removing the residual tumors. The frozen section examination can provide a rapid microscopic analysis of a specimen. In this study, we prospectively investigated the tumor base and whether muscle was present in the specimen using frozen section examination, and discussed its value in the initial TURBt.

Materials and Methods: From Jun 2011 to Oct 2016, a total of 95 consecutive patients with T1 bladder cancer were included in this study. A standard TURBt was performed. Once the tumor was removed, the tumor base was either biopsied using cold-cup biopsy forceps or resected. An aliquot of resected tumor as well as the tumor base were both sent for pathological frozen section examination in a separate labeled pot. Then a repeat resection was performed based on the pathological findings either if cancer cells were present in the tumor base or the muscle was present in the specimen. The results, including positive tumor base, presence of muscle in the specimen, tumor stage, residual tumor and concordance between frozen section and paraffin embedded section were compared.

Results: $26(27.4 \%)$ patients had a positive tumor base. $54(56.8 \%)$ tumor bases were found muscle in the specimen while $34(35.8 \%)$ were not found and $7(7.4 \%)$ were difficult to diagnosis because of the over-cauterization of the base specimen. Of 52(54.7\%) patients who underwent a repeat resection, 18(34.6\%) were found residual tumor and $15(27.8 \%)$ were upstaged to muscle invasive tumor. The paraffin embedded section demonstrated 32(61.5\%)specimen were found muscle in patients undergoing the repeat resection. The concordance of muscle in the specimen between the frozen section and paraffin embedded section was $94.7 \%$.

Conclusions: The frozen section examination was justified for the diagnoses of positive tumor base and muscle in the specimen, and it can help to remove the residual tumors and decrease the staging error for T1 urothelial bladder cancer during the initial TURBt.

MP20-20 Transurethral Laser Ablation of Non-Muscle Invasive Bladder Cancer with a Diode Laser: 5 year Outpatient Experience

P James, A Pai, N Arumainayagam, S Agrawal, A Shamsuddin

Ashford and St Peter's Hospitals FT

United Kingdom

Introduction \& Objective: Non-invasive bladder cancer is often recurrent. $5-10 \%$ of patients will have recurrences that are small and few. Treating these recurrences causes morbidity to patients because of the frequent resections under general anaesthesia that are needed to control the disease. The majority of this patient cohort is elderly with major co-morbidities and as such do not fill the criteria for day surgery, often requiring a 1 to 2 day stay in hospital. This project aims to prove the safety and efficacy of receiving outpatient laser treatment under local anaesthetic. 
Laser vaporization of small bladder tumours has several advantages over standard electrocautery techniques. The lack of electrical conduction reduces discomfort to patients, bleeding is almost absent and even patients on anticoagulation therapy can be treated. The European Association of Urology guidelines recognise the use of lasers for treatment of non-muscle invasive bladder tumours, inparticular in co-morbid patients.

Materials and Methods: The Diode laser is a compact, portable and relatively quiet laser. The diode $1470 \mathrm{~nm}(1 \mathrm{~mm}$ depth of penetration) offers improved haemostasis over the Holmium $(0.2 \mathrm{~mm})$ and limits the reported bladder perforation risk with the deeper ND;YAG and diode $980 \mathrm{~mm}$ (5-10mm). As such the diode $1470 \mathrm{~nm}$ may represent the ideal 'urothelial' laser. We kept a prospective dataset of patients receiving TULA treatment over a five year period. Parameters recorded include number of patients/ procedures, patient age, comorbidities, procedure time, pain perception, complications, readmission rates, and patient satisfaction. Results: Between 1st May 2012 and 28th December 2016, there were a total of 454 laser ablations performed on 306 different patients. The median age was 75 (range 24-99 years old). Median procedure time was 10 minutes, mean energy 759J. Out of 306 patients, 192 had pre existing TCC (141 Ta, 34 T1, 4 T2 (prev. DXT), 6 CIS, 7 unknown/ historical NMIBC). 104 were unfit for GA. 102 Laser ablations were conducted whilst the patient was on anticoagulants: (25 aspirin, 22 clopidogrel, 53 warfarin, 1 dabigatran, 1 tinzaparin). No complications were recorded secondary to bleeding. No patients required re-admission.

Out of those questioned, $75 \%$ experienced no pain (19\% mild pain, $6 \%$ moderate pain). All patients opted for repeat outpatientbased TULA.

Conclusions: Bladder cancer can re-occur in up to $50 \%$ of patients over a 5 year period. This often requires multiple procedures and general anaesthetics in patients with multiple medical issues.

The Diode Laser vaporisation of NMI bladder cancer has been proven to be well tolerated, less onerous on patients, and may reduce post operative complications.

MP20-21 Outcomes of Upper Urinary Tract Tumours Following Nephro-ureterectomy Over a 10 Year Period

A Carrera, A Johnstone, N Umez-Eronini, K Qureshi, G Oades, OM Aboumarzouk

Wos trainee

United Kingdom

Introduction \& Objective: Upper urinary tract carcinomas account for $5-10 \%$ of urological carcinomas. The standard treatment for these is a nephro-ureterectomy with either a bladder cuff/pluck. Bladder tumour recurrence following surgery for upper tract urothelial carcinoma is a significant problem and recent studies have found that the instillation of Mitomycin C post-operatively reduces the recurrence rate. We aimed to better evaluate the natural history of nephro-ureterectomy post-operatively in terms of recurrence, mortality rate and the role of Mitomycin C (MMC); specifically in the first 12 months.

Materials and Methods: We carried out a prospective study of nephro-ureterectomy cases performed at our institution between 2007 and 2017. We compared our results to the ODMIT-C trial's MMC arm.

Results: Overall, 106 patients with a mean age of 70 (range 38$86)$ were included. There have been $43(40.6 \%)$ reported cases of bladder recurrence in total and $8(18.6 \%)$ recurrences were within 12 months. There have been $29(27.3 \%)$ deaths overall with $8(27.6 \%)$ cancer specific deaths. The results are presented

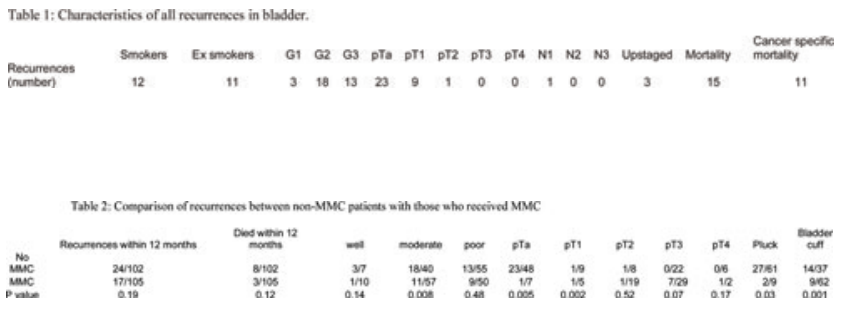

in table 1 . We found no difference between our non-MMC patients when compared to those who had MMC regarding baseline parameters. The results mirrored those of the ODMIT-C (One Dose Mitomycin C) trial (Table 2).

Conclusions: Bladder recurrence and mortality remain important problems following surgery in the long-term outcomes of patients with upper urinary tract urothelial carcinoma. The role of mitomycin $\mathrm{C}$ has been widely discussed and our results are similar to that of the ODMIT-C trial with a significant reduction in recurrence rate.

MP20-22 The usefulness of transurethral resection in one piece (TURBO) for pathological staging of bladder tumor

S Ohtake, J Ohta, K Chiba, K Makiyama, M Yao

department of Urology, Yokohama City University Japan

Introduction \& Objective: We assessed the efficacy of transurethral resection of bladder tumor in one piece (TURBO) as an accurate pathological staging tool for bladder tumor.

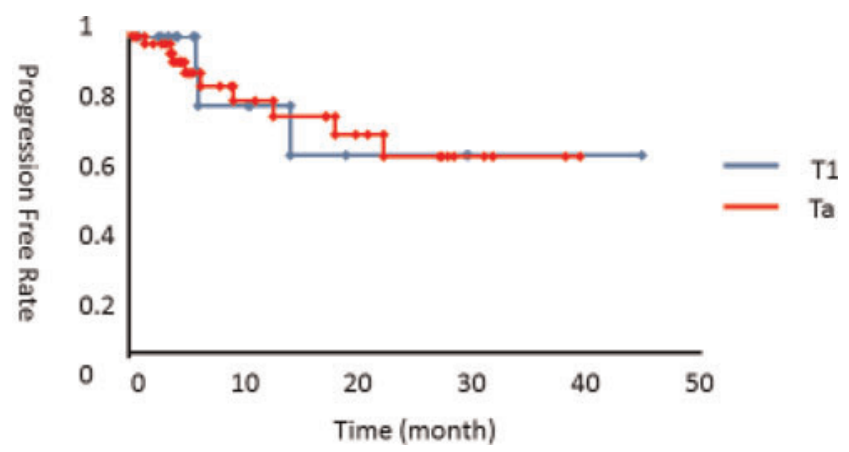

progression free survival

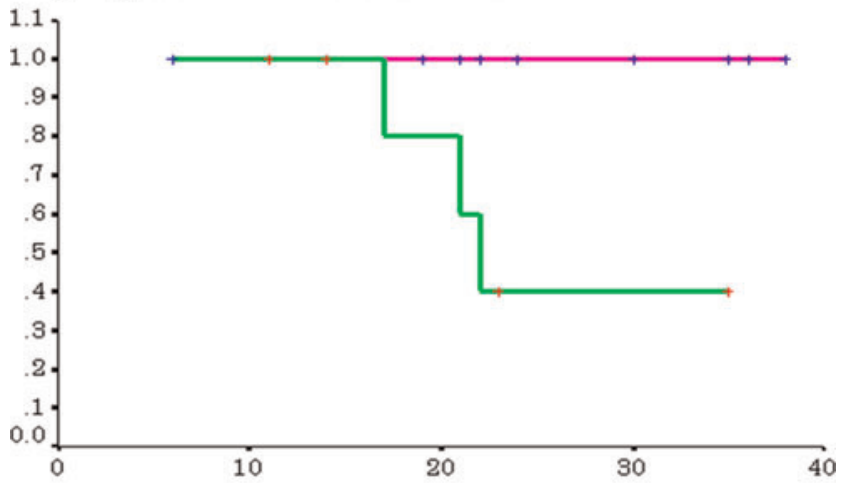

Month 

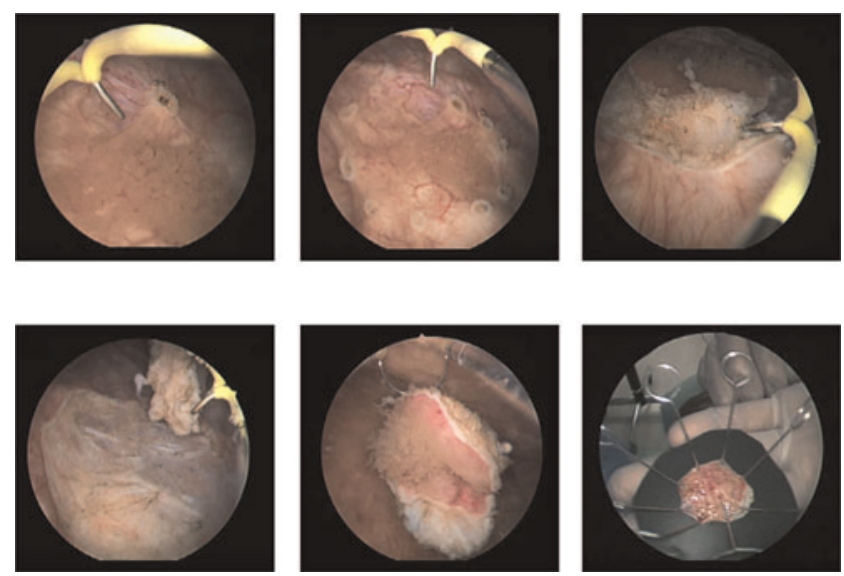

Materials and Methods: One hundred and seventy patients with diagnosed bladder tumor underwent TURBO that was performed either in an en block or in a divisional manner. The resection specimens was evaluated for depth of invasion and presence of tumor in edge. The pT1 specimens were assigned according to the following system, T1A; invasion of connective tissue superficial to the level of the muscularis mucosae, $\mathrm{T} 1 \mathrm{~B}$; invasion to the level of the muscularis mucosae, T1C; invasion through the level of the muscularis mucosae but superficial to the muscularis propria.

Results: Of 58 pT1 patients, portion of muscularis mucosae were identified beneath the tumor base in the specimens in 33 patients. We diagnosed 16 as T1A, 7 as T1B, 10 as T1C. Kaplan-Meier analysis showed that T1A \& T1B significantly correlated with good progression free survival compared with T1C $(p=0.0088)$. There was no patient that was diagnosed as upstaging in $2^{\text {nd }}$ TUR after TURBO. Conclusions: An accurate pathological stage can be assigned by TURBO technique and it may serve as a new tool to predict tumor progression.

MP20-23 En bloc transurethral resection with Hybrid Knife for treatment primary non-muscle-invasive bladder cancer: a single-center, randomized, controlled trial.

J Hu, X Song, X Yu, S Wang

China (People's Republic)
Introduction \& Objective: To evaluate the safety and effectivity y of en bloc transurethral resection with Hybrid Knife as treatment for primary non-muscle-invasive bladder cancer (NMIBC) compared to conventional transurethral resection of bladder tumor Materials and Methods: This was a single-center, randomized, and controlled trial. From March 2016 to September 2016, 86 patients with newly diagnosed NMIBC were enrolled and evaluated with ultrasonography CT scan and cystoscopy. All patients were randomly assigned in a 1:1 ratio to receive either Hybrid Knife En bloc transurethral resect treatment or conventional transurethral resection of bladder tumor (TURBT). The clinical characteristics of the patients in each group, such as age, gender, BMI index and disease type had no significant differences $(\mathrm{P}>0.05)$. Patients with tumor number more than five was ruled out. All patients received intravesical chemotherapy postoperation. After 6-12 month follow-up, patients underwent imaging and cystoscopy examinations. Primary outcome measure was difference of tumor recurrence rate at the end of study.

Results: Major intraoperative or postoperative complications did not occur in all of the patients. Operation time was longer in Hybrid Knife En bloc group than in TURBT group when tumor size more than $3 \mathrm{~cm}$ and tumor number more than three $(45,1 \pm 18.7$ vs. $39.2 \pm 19.1 \mathrm{~min}, \mathrm{P}<0.05)$. Obturator nerve reflection was noted during TURBT in 17 patients, 13 patients was noted during Hybrid Knife En bloc resection $(\mathrm{P}>0.05)$. Number of low risk NMIBC patients was lower in the Hybrid Knife En bloc resection group (17 vs. $25 \mathrm{P}<0.05)$. However, number of moderate and high risk NMIBC patients (T1a, T1b, TaG2, TaG3 Staging) was higher in the Hybrid Knife En bloc resection group ( 26 vs. $18, \mathrm{P}<0.05)$. According to KaplanMeier survival curves, there was no statistical difference in the rate of recurrence in 6-12 months $(\mathrm{P}=0.079)$.

Conclusions: en bloc transurethral resection with Hybrid Knife did not decrease tumor recurrence rate in primary NMIBC for 6-12 months observation. However, T1 tumors were significantly higher among Hybrid Knife group. en bloc resection can reserve tumor margins completely and Clearly than TURBT, which may enable pathologists to distinguish the $T$ stages of bladder cancer more accuracy. Further studies need to be done in future. A multi-center, randomized, prospective, study with a large sample and a long follow-up time is needed to be done in future.

\section{MPS21: LAPAROSCOPIC/ ROBOTIC RENAL SURGERY I}

MP21-1 Withdrawn

MP21-2 Pure Transvaginal Natural Orifice Transluminal Endoscopic Surgery (NOTES) for Nephrectomy: Report of 19 Cases

Z Xiaofeng, Z Guoxi, W Xiaoning, L Dazhi, W Gengqing, X Rihai

Department of Urology, First Affiliated Hospital of Gannan Medical University

China (People's Republic)
Introduction \& Objective: To summarize the initial clinical experience of pure transvaginal NOTES for nephrectomy (PTVNOTES-N), and evaluate its safety and feasibility.

Materials and Methods: From December 2010 to February 2016, nineteen female patients with a mean age of 50.4 yeas (range 27 to 64), were subjected to PTV-NOTES-N in our center. Each patient was married and procreative. The mean BMI was $22.8 \mathrm{~kg} / \mathrm{m}^{2}$, and the mean ASA score was gradeII. Eighteen patients with non-function kidney underwent pure transvaginal NOTES simple nephrectomy (left 4 and right 14). One patient with right renal carcinoma $\left(\mathrm{T}_{1 \mathrm{~b}} \mathrm{~N}_{0} \mathrm{M}_{0}\right)$ underwent pure transvaginal NOTES radical nephrectomy. After induction of general 
anesthesia, the patients were positioned in lithotomy with ipsilateral lumbar at $60^{\circ}$ angle to the floor. A Zou-Port was deployed across the vaginal posterior fornix incision (Except for the initial 3 patients were using a Tri-Port), where the flexible-tip $5.4 \mathrm{~mm}$ $0^{\circ}$ laparoscope, and the lengthened and flexible instruments were all introduced. All the dissection was performed through the transvaginal port. The ureter was identified above its cross over the iliac vessels and ligated using Hem-O-loks. The Gerota's fascia was incised (For the cases of renal tumor and infective renal with perinephric adhesion, the kidney were dissected within an intact Gerota's fascia), and the dissociation of kidney was performed in the order of the inferior pole-lateral-dorsalventral-medial. When the renal hilum was exposed, the renal artery and vein was clipped with Hem-O-lock, respectively. After the upper pole of the kidney was isolated completely, the specimen was removed via the incision of the posterior vaginal fornix. A drainage to pelvic cavity was placed through vagina.

Results: The procedures were successfully performed in all patients without additional trocars except for one, who immediately underwent suprapubic-assisted laparoendoscopic single-site surgery conversion for rectal injury during vaginal entry. The serious postoperative complication occurred in 1 case with right iliac artery thrombosis (Clavien-Dindo IIIa), and was successfully cured with emergency thrombectomy. No other intraoperative complication such as abdominal and pelvic organs injury or massive hemorrhage occurred in successful procedures. The mean operative time was 180 mins. The mean estimated blood loss was $170 \mathrm{ml}$. The mean postoperative visual analogue score (VAS) was 2.1. The mean time for full ambulation was 1.6 days. The patients resumed nutrition on postoperative day 2.2. The pelvic drainage was removed on postoperative 3.5 day, and the mean hospital stay was 6.2 days. There was no scar on the body and the incision of the posterior vaginal fornix healed well. During the 2 to 54 month follow-up period, there were no complications in retrograde infection of pelvic and abdominal cavity, uterine prolapse. 1 case of renal tumor was disease-free survival, no tumor recurrence or metastasis.. A ZouPort was deployed across the vaginal posterior fornix incision (Except for the initial 3 patients were using a Tri-Port), where the flexible-tip $5.4 \mathrm{~mm} 0^{\circ}$ laparoscope, and the lengthened and flexible instruments were all introduced. All the dissection was performed through the transvaginal port. The ureter was identified above its cross over the iliac vessels and ligated using Hem-O-loks. The Gerota's fascia was incised (For the cases of renal tumor and infective renal with perinephric adhesion, the kidney were dissected within an intact Gerota's fascia), and the dissociation of kidney was performed in the order of the inferior pole-lateral-dorsal-ventralmedial. When the renal hilum was exposed, the renal artery and vein was clipped with Hem-O-lock, respectively. After the upper pole of the kidney was isolated completely, the specimen was removed via the incision of the posterior vaginal fornix. A drainage to pelvic cavity was placed through vagina.

Conclusions: Pure transvaginal NOTES for nephrectomy is feasible and effective, it is worthy to be selected in clinic because of many advantages such as less pain, more rapidly recovery and better cosmetic effect. But the technique of PTV-NOTES-N is still full of challenges today. To development of the special instruments is the most important problem to be soluted firstly. other intraoperative complication such as abdominal and pelvic organs injury or massive hemorrhage occurred in successful procedures. The mean operative time was 180 mins. The mean estimated blood loss was $170 \mathrm{ml}$. The mean postoperative visual analogue score (VAS) was 2.1. The mean time for full ambulation was 1.6 days. The patients resumed nutrition on postoperative day 2.2. The pelvic drainage was removed on postoperative 3.5 day, and the mean hospital stay was 6.2 days. There was no scar on the body and the incision of the posterior vaginal fornix healed well. During the 2 to 54 month follow-up period, there were no complications in retrograde infection of pelvic and abdominal cavity, uterine prolapse. 1 case of renal tumor was disease-free survival, no tumor recurrence or metastasis. . A Zou-Port was deployed across the vaginal posterior fornix incision (Except for the initial 3 patients were using a Tri-Port), where the flexible-tip $5.4 \mathrm{~mm}$ $0^{\circ}$ laparoscope, and the lengthened and flexible instruments were all introduced. All the dissection was performed through the transvaginal port. The ureter was identified above its cross over the iliac vessels and ligated using Hem-O-loks. The Gerota's fascia was incised (For the cases of renal tumor and infective renal with perinephric adhesion, the kidney were dissected within an intact Gerota's fascia), and the dissociation of kidney was performed in the order of the inferior polelateral-dorsal-ventral-medial. When the renal hilum was exposed, the renal artery and vein was clipped with Hem-O-lock, respectively. After the upper pole of the kidney was isolated completely, the specimen was removed via the incision of the posterior vaginal fornix. A drainage to pelvic cavity was placed through vagina.

\section{MP21-3 The Incidnece of Adverse Pathological Outcome in Robotic Partial nephrectomy.}

MW Salkini, N Idris, A Lamoshi

West Virginia University

United States

Introduction \& Objective: Robotic partial nephrectomy (RPN) is proven to have similar oncologic outcome in clinically T1(cT1) to open partial nephrectomy. Adverse pathology is known to increase the risk of recurrence in patients after partial nephrectomy. We reviewed our data to assess the incidence of adverse pathological finding.

Materials and Methods: We reviewed prospectively collected data of patients who underwent RPN between August 2009 and March 2017 at our institute. We only included patients with renal mass that is less than $7 \mathrm{~cm}$ and underwent partial nephrectomy. Adverse pathology was defined as pathological exam of the specimen revealing higher stage, $>$ Fuhrman grade 2 disease or sarcomatoid features of the renal cancer.

Results: We found a total of 137 patients with renal mass underwent RPN at our institute. 35 (25.5\%) patient had benign pathology (benign cyst, oncocytoma, angiomyolipoma). 6 patients $(4.3 \%)$ were up staged to T3a and 16 patients $(11.6 \%)$ had pathologically unfavorable masses G3 or G4, and none had sarcomatoid features. A total of 20 patient $(14.6 \%)$ had adverse pathology after robotic partial nephrectomy at our institute.

Conclusions: The incidence of adverse pathology in patient who underwent RPN was $14.6 \%$ at our institute. Longer follow up is needed for patient after RPN to sort out what adverse pathology would translate into recurrence.

MP21-4 Robotic-assisted Laparoscopic Surgeries from the Team of Prof. Shen Zhoujun: 1001 Cases' Experience and Clinical efficacy analysis

Z Shen, S Chen, S Zhong, Y Shen, M Zhang

China (People's Republic)

Introduction \& Objective: To report the results and experience of 1001 cases of robotic-assisted laparoscopic surgeries from the team of Prof. Shen Zhoujun. 
Materials and Methods: From March 2010 to March 2017, 1001 cases of robotic-assisted laparoscopic surgeries were performed by Prof. Shen Zhoujun in Huashan Hospital and Ruijin Hospital, which including 728 cases with upper urinary tract and 273 cases with lower urinary tract. Upper urinary tract operations included 268 adrenalectomies, 67 radical nephrectomies, 206 partial nephrectomies, 20 nephroureterectomies, 4 segmental ureterectomies, 7 simple nephrectomies, 128 lithotomies and pyelourecteroplasties, 1 isthmus disconnection for horseshoe kidney, 1 perirenal lymph duct ligation for chyluria, 24 cystectomies for polycystic disease and 2 retroperitoneal lymphadenectomies. Lower urinary tract operations included 205 radical prostatectomies, 9 simple prostatectomies, 39 radical cystectomies, 14 partial cystectomies, 4 excisions for urachal cyst, 1 pelvic lymphadenectomy for penile carcinoma and 1 vesicovaginal fistula repair.

Results: Two of upper urinary tract operations must be converted to open surgery because of mechanical breakdown during operation and postoperative hemorrhage. All lower urinary tract operations were successful.

Conclusions: Most of urological operations are suitable for robotic surgery, especially pyelourecteroplasty, partial nephrectomy, radical prostatectomy and radical cystectomy. The superiority of robotic surgery can also be demonstrated in greatdiameter adrenalectomy, simple prostatectomy for large volume prostate, and nerve-sparing retroperitoneal lymphadenectomy. Compared with upper urinary tract operations, the advantages of surgeries in lower urinary tract are more obvious.

MP21-5 A Prospective Randomized Trial of Clamped Versus Off-Clamp Robot-Assisted Partial Nephrectomy: Does It Make a Difference?

BG Anderson, K Du, A Potretzke, J Vetter, RS Figenshau

Washington University in Saint Louis

United States

Introduction \& Objective: Robot-assisted partial nephrectomy (RAPN) has become a standard surgical approach for the management of clinical T1a renal masses. During this procedure, most surgeons clamp the renal hilar vessels to induce warm ischemia which facilitates tumor excision and renorrhaphy. To avoid the detrimental impact of warm ischemia on renal function, some surgeons perform RAPN without hilar clamping, or "offclamp." There are no prospective studies that examine whether this method indeed mitigates renal functional impairment. We describe our institutional experience and compare perioperative outcomes and postoperative renal function in a prospective randomized trial.

Materials and Methods: We conducted a prospective trial of patients undergoing RAPN at our institution from 2013 to 2017. We randomized patients to undergo RAPN either with hilar clamping (34 patients) or without hilar clamping (32 patients). Each group was compared across demographics, operative information, perioperative outcomes, and postoperative renal function. We objectively assessed renal function by estimated glomerular filtration rate (eGFR) and renal scintigraphy both preoperatively and at three months postoperatively.

Results: Patients in the clamped and off-clamp groups were similar in age, body mass index, comorbidities, clinical tumor size, nephrometry score, and laterality. More men were randomized to the clamped group $(79.4 \%$ vs $56.3 \%, \mathrm{p}=0.043)$. Off-clamp procedures were lengthier at an average 179.2 minutes compared with 158.2 minutes for clamped procedures $(\mathrm{p}=0.020)$. Otherwise, estimated blood loss, and rates of pelvicalyceal repair, postoperative compli-

\begin{tabular}{|c|c|c|c|}
\hline Variable & Clamped $(n=34)$ & Off-Clamp ( $n=32$ ) & p-value \\
\hline Age in years, mean (SD) & $60.3(11.1)$ & $57.7(9.2)$ & 0.269 \\
\hline Gender, \% & & & 0.043 \\
\hline Male & 79.4 & 56.3 & \\
\hline Female & 20.6 & 43.8 & \\
\hline $\mathrm{BMI}$ in $\mathrm{kg} / \mathrm{m}^{2}$, mean (SD) & $31.2(5.5)$ & $33.1(6.0)$ & 0.130 \\
\hline $\mathrm{CCl}, \%$ & & & 0.325 \\
\hline $\mathrm{CCl}=0$ & 44.1 & 43.8 & \\
\hline $\mathrm{CCl}=1$ & 32.4 & 18.8 & \\
\hline $\mathrm{CCl} \geq 2$ & 23.5 & 37.5 & \\
\hline Clinical tumor size in $\mathrm{cm}$, mean (SD) & $3.0(1.4)$ & $2.8(1.0)$ & 0.496 \\
\hline Nephrometry score, mean (SD) & $7.4(1.9)$ & $6.9(1.9)$ & 0.261 \\
\hline Laterality, \% & & & 0.143 \\
\hline Left & 38.2 & 56.3 & \\
\hline Right & 61.8 & 43.8 & \\
\hline Operative time in minutes, mean (SD) & $158.2(40.7)$ & $179.2(44.0)$ & 0.020 \\
\hline Warm ischemia time in minutes, mean (SD) & $19.6(7.9)$ & $0(-)$ & $<0.001$ \\
\hline Pelvicalyceal repair, \% & 52.9 & 50.0 & 0.811 \\
\hline Estimated blood loss in $\mathrm{mL}$, mean (SD) & $182.6(208.7)$ & $189.5(201.5)$ & 0.943 \\
\hline Complication rate, \% & 14.7 & 15.6 & 0.917 \\
\hline Major complication rate, \% & 8.8 & 3.1 & 0.614 \\
\hline Malignant pathology, \% & 82.4 & 81.3 & 0.908 \\
\hline Positive margin rate, $\%$ & 5.9 & 6.3 & 1.000 \\
\hline Preoperative eGFR in $\mathrm{mL} / \mathrm{min} / 1.73 \mathrm{~m}^{2}$, mean (SD) & $87.5(20.4)$ & $80.3(20.5)$ & 0.373 \\
\hline Postoperative eGFR in mL/min $/ 1.73 \mathrm{~m}^{2}$, mean (SD) & $76.5(18.1)$ & $69.6(21.0)$ & 0.500 \\
\hline Percent change in eGFR, mean (SD) & $-10.2(15.6)$ & $-11.9(17.9)$ & 0.914 \\
\hline Preoperative \% split renal function, mean (SD) & $49.4(4.9)$ & $47.9(6.3)$ & 0.322 \\
\hline Postoperative \% split renal function, mean (SD) & $44.0(8.3)$ & $42.5(7.7)$ & 0.226 \\
\hline Percent change in \% split renal function, mean (SD) & $-11.1(13.4)$ & $-11.0(13.3)$ & 0.642 \\
\hline
\end{tabular}

cations, and positive margins were not different. At a median follow up of three months, no significant differences were seen in change in postoperative eGFR or percent split renal function between the two groups (Table 1).

Conclusions: Off-clamp RAPN results in similar perioperative outcomes when compared with the clamped approach. However, our findings show no benefit in the preservation of renal function.

MP21-6 The experience and skills of retroperitoneal laparoscopic nephron-sparing sugery for giant renal angiomyolipoma

M Qiu

Department of Urology, Peking University Third Hospital, Beijing, China

China (People's Republic)

Introduction \& Objective: To discuss the safety and skills of retroperitoneal laparoscopic nephron-sparing sugery for giant renal angiomyolipoma.

Materials and Methods: 14 cases were involved, including 3 male and 11 female. Left and right were both 7 cases, and age range was 32-68 years (average 49.7 years). The duration of illness was 4 days to 5 years (average 8.6 months). 3 patients with low back pain, 11 cases were found incidentally, physical examination of 5 cases with ipsilateral renal pain. 9 cases underwent ultrasound, which were reported high echo nodules. 2 cases with MRI considering the huge hamartoma, and 14 cases underwent CT were diagnosed as renal hamartoma. 2 cases were hamartoma with hemorrhage. Tumor size was $8-17 \mathrm{~cm}$ (average $10.2 \mathrm{~cm}$ ). Preoperative serum creatinine was normal. After preoperative examination, all cases underwent laparoscopic partial nephrectomy. Results: Surgery was performed successfully, no conversion to open surgery. The operation time was 113-312min (average $176 \mathrm{~min}$ ), and the blood loss was $20-600 \mathrm{ml}$ (average $155 \mathrm{ml}$ ). The renal artery occlusion time was $7-44 \mathrm{~min}$ (mean $26.7 \mathrm{~min}$ ), and postoperative hospitalization time was $4-14 d$ (average $7 \mathrm{~d}$ ). Pathologic report was renal angiomyolipoma. Postoperative pulmonary infection occurred in 1 case, and was improved after symptomatic treatment. Patients were followed up for 3-54 months (average 21.5 months) without recurrence. 
Conclusions: Retroperitoneal laparoscopic nephron-sparing sugery for huge renal angiomyolipoma is safe and effective. Fully exposing the operative field is very important in the operation. Dissociating the tumor to its basilar part, then blocking the renal artery can significantly shorten the operation time. Using barbed absorbable suture to close renal incision wound is safe and effective.

MP21-7 Efficacy of laparoscopic simple nephrectomy for treatment of chronic pain in patients with recurrent ureteropelvic junction obstruction

D Szabo, M Sourial, N François, C Dall, C Miller, DL Zynger, G Box

The Ohio State University Wexner Medical Center United States

Introduction \& Objective: Patients with chronic pain due to recurrent ureteropelvic junction (UPJ) obstruction can be difficult to manage. These patients often have limited reconstructive options and may have reasonable differential renal function of the affected kidney. This study assesses the efficacy of laparoscopic nephrectomy in resolving the pain in those who chose nephrectomy.

Materials and Methods: This is a retrospective review and single surgeon experience comprised of 84 patients who underwent nephrectomy for benign indications (flank pain, recurrent or severe active infections, and/or stone disease) from 2010 through 2015. Resolution of chronic pain postoperatively, as well as other perioperative outcomes, were measured.

Results: Sixty-eight of the $84(81 \%)$ patients presented with flank pain. Pain was thought to be related to recurrent UPJ obstruction in $8 / 68$ patients $(11.8 \%)$. These particular patients underwent an average of $2.4(\mathrm{SD}=1.7)$ procedures prior to nephrectomy and $7 / 8$ $(87.5 \%)$ had prescriptions for narcotics preoperatively. Preoperative nuclear scintigraphy performed in $59 / 68$ pain patients $(86.8 \%)$ with an average function of $22 \%(\mathrm{SD}=15 \%)$ on the affected kidney. Patients with recurrent UPJ obstruction had an average function of $38 \%$ ( $\mathrm{SD}=11 \%)$ with $6 / 8(75.0 \%)$ showing scintigraphic evidence of obstruction (equivocal in 2/8). All 8/8 (100\%) patients with recurrent UPJ obstruction had subjective resolution of their chronic pain, and based on Ohio Automated Rx Reporting System (OARRS) reports, 6/7 (85.7\%) of those taking preoperative narcotics no longer have active narcotic prescriptions (the other takes narcotics for chronic lower back pain). Postoperatively in the patients with recurrent UPJ obstruction, estimated glomerular filtration rate (eGFR) decreased by an average of $4.2 \%(\mathrm{SD}=9.8 \%)$ and serum creatinine ( $\mathrm{SCr}$ ) increased by an average of $18.8 \%$ $(\mathrm{SD}=16.6 \%)$. Overall and major (Clavien $\geq 3 \mathrm{a}$ ) complication rates for all patients at 90 days were $29.4 \%$ and $4.4 \%$, respectively. Overall and major complication rates for the patients with recurrent UPJ obstruction were $12.5 \%$ and $0 \%$, respectively.

Conclusions: Laparoscopic nephrectomy is a safe and effective procedure to relieve chronic flank pain for multiple indications, including select patients with recurrent UPJ obstruction.

MP21-8 Minimally invasive versus open radical nephrectomy for clinical stage $\mathrm{T} 4$ renal masses

ZL Smith, S Johnson, V Packiam, J Rodriguez, S Eggener

The University of Chicago

United States

Introduction \& Objective: Radical nephrectomy $(\mathrm{RN})$ is the mainstay of treatment for clinically localized renal cell carci-

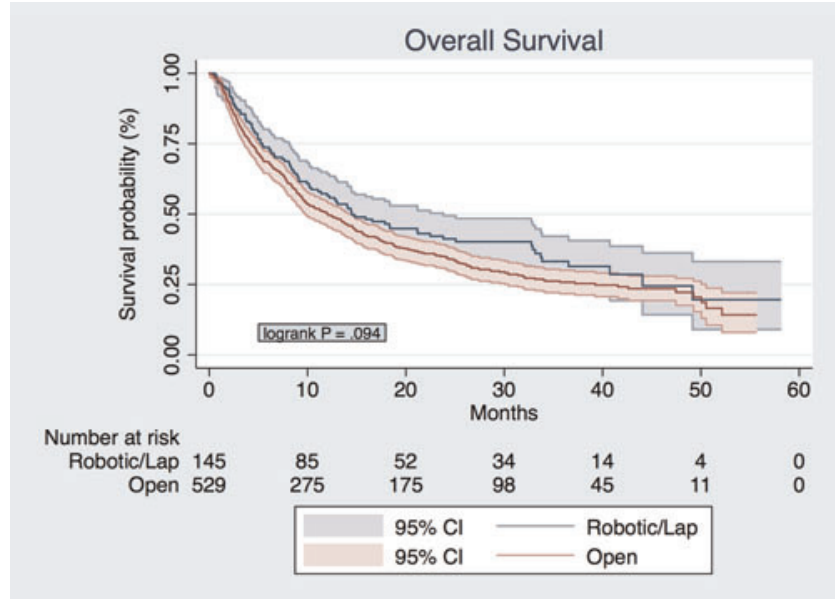

noma (RCC). Little data are available on how minimally invasive RN (MIRN) compares to open radical nephrectomy (ORN) in the setting of clinical stage T4 (cT4) RCC. We sought to evaluate the impact of surgical approach in locally advanced cT4 RCC.

Materials and Methods: We analyzed the National Cancer Data Base (NCDB) participant user files from 2010-2013 to identify patients who underwent RN for cT4 RCC. Using univariable and multivariable analyses, patient and treatment facility characteristics as well as surgical outcomes were compared between MIRN (robotic and laparoscopic) and ORN.

Results: There were 642 (76\%) ORN and 206 (24\%) MIRN performed for cT4 RCC. There was no difference in median age (62 vs 65 years, $\mathrm{p}=0.2$ ) but more patients over 70 were treated with MIRN (38\% vs 26\%, p 0.05). ORN had higher rates of pathological stage T4 (81\% vs $77 \%, \mathrm{p}=0.04)$ and $\mathrm{N} 1(42 \%$ vs $31 \%, \mathrm{p}=0.04)$. Forty-nine (24\%) of MIRN were converted to ORN. Median length of stay (LOS) was shorter for MIRN (4 vs 6 days, $\mathrm{p}<0.01)$. Patients in the ORN group had larger tumors (median 11.2 vs $9.0 \mathrm{~cm}, \mathrm{p}<0.01$ ). Extent of local tissue/organ invasion was similar $(\mathrm{p}=0.1)$, with the most common reason for T4 staging in both groups being colon, duodenum, diaphragm, peritoneum or pancreas invasion. Extent of venous involvement was more advanced in the ORN group $(\mathrm{p}<0.01)$. Lymphadenectomy was performed at similar rates $(37 \%$ vs $31 \%, \mathrm{p}=0.06)$, but nodal yield was greater with ORN (median 5 vs 2 nodes, $\mathrm{p}<0.01$ ). Positive surgical margin (PSM) rate did not differ between groups $(47 \%$ vs $46 \%, \mathrm{p}=0.9)$. Both groups had similar 30-day $(\mathrm{p}=0.8), 90$-day $(\mathrm{p}=0.09)$, and overall $(\mathrm{p}=0.06)$ survival, as well as 30 -day postoperative readmissions $(p=0.9)$. On multivariable analysis, there were no significant predictors of conversion from MIRN to ORN.

Conclusions: MIRN is utilized in $\sim 25 \%$ of all patients with cT4 RCC. Conversion to ORN occurs in $\sim 25 \%$. While hospital stay is shorter, there does not appear to be any difference in PSM rate, readmission rates, or 90-day survival. In appropriately selected patients with cT4 RCC, MIRN appears to be a safe alternative to ORN.

MP21-9 Retroperitoneal versus Transperitoneal RobotAssisted Laparoscopic Partial Nephrectomy: Operative, Oncologic and Late Outcomes

M Lyons, E Stephenson, M Raynor

University of North Carolina at Chapel Hill Department of Urology

United States 
Introduction \& Objective: Retroperitoneoscopic robotic assisted partial nephrectomy has been well established as a feasible method of treating appropriately selected renal masses, however, few studies have evaluated late complications such as neuropathic flank pain and flank hernia. We sought to compare perioperative, oncologic and late outcomes after retroperitoneal and conventional trans-peritoneal robot-assisted partial nephrectomy (rRAPN and tRAPN).

Materials and Methods: We performed a retrospective matched-pair analysis of consecutive patients undergoing rRAPN $(n=42)$ and tRAPN $(n=42)$ performed by a single surgeon from February 2011 to May 2016. Surgical approach was based on patient and tumor characteristics, history of abdominal surgery, and surgeon preference. We compared demographic, tumor, operative and oncologic outcomes as well as late complications, specifically flank hernia and neuropathic pain.

Results: Baseline patient and tumor characteristics were similar between groups. Mean operative time, warm ischemia time, and length of hospital stay and mean follow-up time were not statistically different between groups. Estimated blood loss was significantly lower in the rRAPN group $(113 \mathrm{~mL}$ versus $186 \mathrm{~mL}$, $\mathrm{p}=0.04)$. Oncologic outcomes were similar between groups. Overall complication rates were significantly higher in the rRAPN group (33\% versus $10 \% \mathrm{p}=0.02)$, the majority of which were Clavien-Dindo grade I-II complications (29\% versus 5\%, $\mathrm{p}=0.003 \%$ ). Grade III or higher complications occurred at similar rates in each group (7\% versus $5 \%, \mathrm{p}=0.89)$. Flank hernia was unique to the rRAPN group and occurred in $10 \%$ of patients with 2 of 4 patients requiring surgical repair. Post-operative neuropathic pain was noted in 5 patients (12\%) with 1 patient having persistent pain at time of last follow-up.

Conclusions: Retroperitoneal robot-assisted laparoscopic partial nephrectomy is a safe an efficacious approach to appropriately selected tumors. Unique complications including flank hernia and neuropathic pain must be considered. We have found a significant decrease in flank hernia rates with changes in our access and closure.

MP21-10 The impact of gender difference on the operative time of laparoscopic and robotic partial nephrectomy for T1 renal tumor, and the utility of retroperitoneal fat thickness as a predictor of operative time

K Makiyama, H Ito, K Osaka, Y Yokomizo, N Nakaigawa, M Yao

Dept. Urology, Yokohama City University

Japan

Introduction \& Objective: The present study aimed to investigate the impact of gender difference on operative parameters, especially operative time, of laparoscopic (LPN) and robotic (RAPN) partial nephrectomy for T1 renal tumors.

Materials and Methods: One hundred and eleven (28 female and 83 male), 64 (20 female and 44 male), 7 (0 female 7 male), and 30 (14 female and 16 male) patients with renal tumors suspected to be RCC cT1N0M0 who underwent retroperitoneal, transperitoneal LPN, or retroperitoneal and transperitoneal RAPN, respectively, were analyzed. The influence of gender on operative factors, including retroperitoneal fat tissue thickness determined on CT, was analyzed. The correlation between operative time and gender was evaluated by the unpaired t-test and linear logistic regression model. Results: For both retroperitoneal and transperitoneal LPN and RAPN, the retroperitoneal fat tissue thickness was greater in men than in women. For the retroperitoneal LPN, the operative time was significantly longer in men than in women. In contrast, for the transperitoneal LPN and RAPN, no gender difference was observed in regard to the operative time. For retroperitoneal LPN, linear logistic regression assessment demonstrated that gender, the retroperitoneal fat tissue thickness, and tumor size were significantly correlated with operative time. The coefficient of determination of the prediction model was 0.317 .

Conclusions: The operative time of retroperitoneal LPN was significantly correlated with the gender, the maximum tumor diameter, and retroperitoneal fat tissue thickness. We have developed a prediction model for the operative time of retroperitoneal LPN based on preoperative parameters. Interestingly, for transperitoneal LPN, the gender difference in operative time was not apparent, and predicting operative time may be difficult. The impact of gender difference on the RAPN operative time was unclear due to the limited number of cases.

MP21-11 Trifecta outcomes of robot-assisted partial nephrectomy in solitary kidney - A VCQI database analysis

S Arora, RK Ahlawat, R Abaza, J Adshead, B Challacombe, P Dasgupta, G Gandaglia, D Moon, G Novara, F Porpiglia, U Capitiano, T Yuvaraja, J Porter, A Mottrie, M Bhandari, CG Rogers, H Gill

Vattikuti Urology Institute, Henry Ford Hospital United States

Introduction \& Objective: To analyze the oncological and functional outcomes of robot-assisted partial nephrectomy (RAPN) in renal masses with a solitary kidney in a large multiinstitutional database.

Materials and Methods: A total of 2755 patients in the Vattikuti Global Quality Initiative in robotic urologic surgery (GQI-RUS) database underwent RAPN by 22 surgeons at 14 centers in nine countries. Out of these, 74 underwent RAPN in solitary kidney between 2007 to 2016. A retrospective analysis of the functional and oncological outcomes was done.

Results: All 74 patients underwent RAPN successfully with one conversion to robotic radical nephrectomy. The median (IQR) operative time was 180 (142-230) minutes. Early unclamping was used in 11 cases, while zero-ischemia was used in 12 cases. Trifecta outcomes were achieved in 44/66 (66.7\%) of the patients. Median (IQR) ischemia time was 7.0 (11.95-20) minutes for the entire cohort. Total complication rate was $24.1 \%$ with

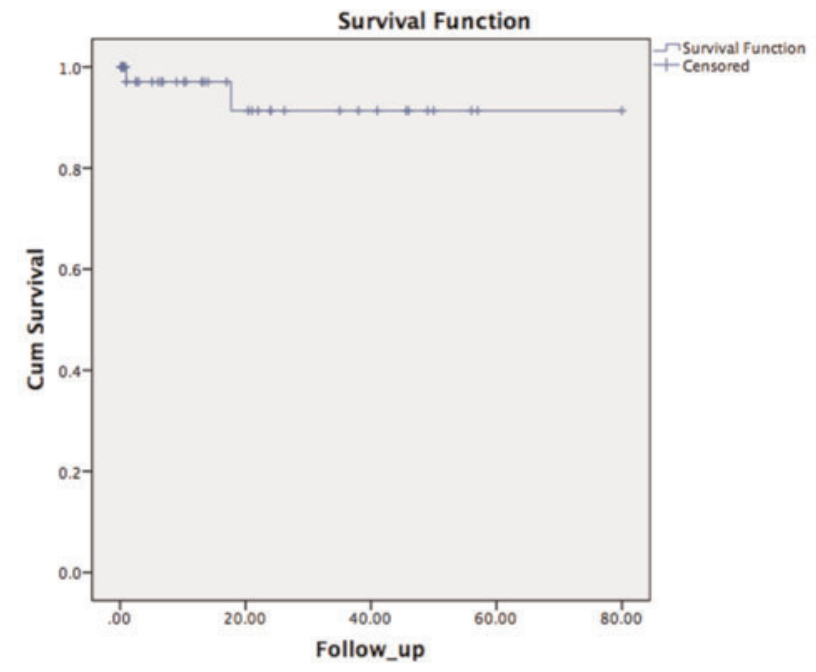

Figure 1-Kaplan Meier survival analysis for death due to any cause after robot assisted partial nephrectomy in 43 patients with renal tumor in a solitary kidney. 


\begin{tabular}{|c|c|}
\hline \multicolumn{2}{|c|}{$\begin{array}{l}\text { Table-Operative and perioperative characteristics of } 74 \text { patients } \\
\text { with solitary kidney who underwent robot assisted partial } \\
\text { nephrectomy at multiple centers in the Vattikuti Global Quality } \\
\text { Initiative in Robotic Urologic Surgery (GQI-RUS) database } \\
\text { between } 2007 \text { and } 2016 \text {. }\end{array}$} \\
\hline Operative time, median (IQR), minutes & $180(142-230)$ \\
\hline Estimated blood loss, median (IQR), ml & $150(100-350)$ \\
\hline \multicolumn{2}{|l|}{ Type of ischemia, $n(\%)$} \\
\hline Warm ischemia & $50(67.6 \%)$ \\
\hline Early unclamping & $11(14.9 \%)$ \\
\hline Zero ischemia & $12(16.2 \%)$ \\
\hline Cold ischemia & $1(1.4 \%)$ \\
\hline \multicolumn{2}{|l|}{ Ischemia time, median (IQR), minutes } \\
\hline Entire cohort & $7.0(11.95-20)$ \\
\hline After excluding zero-ischemia cases & $15.5(8.75-20.0)$ \\
\hline Ischemia time $>25$ minutes, $n(\%)$ & $8(10.8 \%)$ \\
\hline \multicolumn{2}{|l|}{ Intraoperative complications } \\
\hline Gross violation of tumor bed & $3(4.1 \%)$ \\
\hline Major bleeding from tumor bed & $2(2.7 \%)$ \\
\hline Injury to major vessels & $3(4.1 \%)$ \\
\hline Injury to abdominal organs & $1(1.4 \%)$ \\
\hline \multicolumn{2}{|l|}{ Histopathology, $\mathrm{n}(\%)$} \\
\hline Clear cell RCC & $43(58.1 \%)$ \\
\hline Non clear cell RCC & $17(23.1 \%)$ \\
\hline Benign & $12(16.2 \%)$ \\
\hline \multicolumn{2}{|l|}{ Pathological stage } \\
\hline pт1a & $52(83.9 \%)$ \\
\hline pT1b & $9(14.5 \%)$ \\
\hline pT2a & $1(1.6 \%)$ \\
\hline \multicolumn{2}{|l|}{ Fuhrman Grade (Clear cell RCC), $n(\%)$} \\
\hline Grade 1 & $5(8.9 \%)$ \\
\hline Grade 2 & $36(64.3 \%)$ \\
\hline Grade 3 & $15(26.8 \%)$ \\
\hline \multicolumn{2}{|l|}{ Surgical margin } \\
\hline Positive & $4(5.4 \%)$ \\
\hline Negative & $70(94.6 \%)$ \\
\hline \multicolumn{2}{|l|}{ Clavien grade of complications, $n(\%)$} \\
\hline Grade 1 & $5(6.8 \%)$ \\
\hline Grade 2 & $7(9.5 \%)$ \\
\hline Grade 3 & $3(4.1 \%)$ \\
\hline Grade 4 & $2(2.7 \%)$ \\
\hline Grade 5 & $1(1.6 \%)$ \\
\hline
\end{tabular}

Clavien-Dindo grade $1 \& 2$ complications in $16.3 \%$. Positive surgical margins were present in $4(5.4 \%)$. The median drop in estimated GFR (eGFR) at three months was $7 \mathrm{ml} / \mathrm{min} / 1.72 \mathrm{~m}^{2}$ (11.01\%). Median (IQR) follow-up was 10.5 (2.12-24) months. Estimated overall survival was $91.3 \%$ at two years.

Conclusions: Our findings suggest that RAPN is a safe and effective treatment option for renal tumors in solitary kidneys in terms of a trifecta of negative surgical margins, low warm ischemia time, and low operative and perioperative morbidity.

MP21-12 Laparoscopic partial nephrectomy for T1b or greater tumors: comparing the Trifecta outcome of T1a with T1b or greater tumors.

K Otsuka, A Fujimoto, K Hou, T Kato, T Suyama, K Araki, H Masuda, S Kojima, Y Naya

Department of Urology, Teikyo University Medical Center Japan

Introduction \& Objective: "Trifecta" in partial nephrectomy consists of minimal renal function decrease, negative surgical margins and absence of complications. We compare the surgical results of laparoscopic partial nephrectomy (LPN) in terms of Trifecta outcomes between $\mathrm{T} 1 \mathrm{a}$ and $\mathrm{T} 1 \mathrm{~b}$ or greater tumors in single institute.

Materials and Methods: 90 cases of LPN was performed between April 2010 and March 2017. 66 cases were T1a and 24 were $\mathrm{T} 1 \mathrm{~b}$ or greater. 68 were clear, 10 were papillary and 4 were chromophobe, 6 were AML and 2 were complicated cyst in final pathology. All procedure was done by laparoscopy.

Results: In all cases, surgical margin was negative and $\leq 15 \%$ postoperative estimated glomerular filtration rate (e-GFR) decrease were achieved $84.8 \%$ in T1a tumor and $83.3 \%$ in T1b or more tumor. Absence of grade $\geq 2$ Clavien-Dindo complications were achieved $97 \%$ and $91.6 \%$, respectively. Duration of surgery was 193+/-68 min and 257+/-91 min, respectively. Estimated blood loss was $142+/-309 \mathrm{ml}$ and $342+/-402 \mathrm{ml}$, respectively. Tumor recurrence is not observed.

Conclusions: Limitations of this study were the small number of patients and this is a retrospective study. However laparoscopic partial nephrectomy for $\mathrm{T} 1 \mathrm{~b}$ or more tumors is seemed a safe and effective method. Optimal Trifecta outcomes can be achieved.

MP21-13 Impact of selective renal artery clamp during robot-assisted laparoscopic partial nephrectomy on postoperative renal function

F Hongo, Y Inoue, M Kaneko, A Fujihara, Y Naitoh, K Okihara, O Ukimura

Department of Urology, Kyoto Prefectural University of Medicine

Japan

Introduction \& Objective: Prolonged warm ischemia time (WIT) results in irreversible trauma to the kidney. Robot-assisted partial nephrectomy (RAPN) has demonstrated shorter WIT compared to conventional laparoscopic partial nephrectomy (LPN). We assessed the benefit of selective renal arterial clamping for renal function after surgery.

Materials and Methods: RAPN was performed in 54 patients between March 2014 and March 2017. They consisted of 43 males and 11 females. The right kidney was affected in 28 patients, and the left kidney in 26. The mean tumor diameter was $24.5 \mathrm{~mm}$ (11-57). The mean R.E.N.A.L. nephrometry score was 6 (4-10). We used da Vinci Si system. eGFR was examined in 27 patients followed 6 months or more after surgery.

Results: The median console time was 163 minutes (94-301). The median warm ischemic time was 19 minutes (0-39). Furthermore, selective renal artery clamping was performed in 24 patients and off-clamp in 1 patient. There were no intraoperative complications. The results were compared between the selective clamping of 16 cases and total clamping of 11 cases. The rates of decrease in the eGFR after 1 month were statistically different, $11 \%$ in total clamping group (T) and $-9.2 \%$ in selective clamping group (S) (p25min were examined. After 6 months, the rates of decrease in the eGFR were 12.3 and $-17 \%$ in group $\mathrm{T}$ and $\mathrm{S}$, respectively ( $\mathrm{p}$ Conclusions: RAPN could be safely introduced. Selective arterial clamping influenced the eGFR early after surgery, and its long-term effects may be marked in cases with WIT $>25 \mathrm{~min}$.

MP21-14 The impact of the type of renal clamping on the surgical, functional and oncological outcomes of partial nephrectomy

U Boylu, U Yildirim, A Inkaya, B Simsek, E Kucuk

Department of Urology, Health Sciences University Umraniye Training \& Research Hospital, Istanbul, Turkey

Turkey

Introduction \& Objective: We compared the impact of arteryonly vs. artery and vein clamping on the surgical, functional and oncological outcomes of partial nephrectomy. 
Materials and Methods: Of 132 partial nephrectomies performed between 2008 and 2016, a total of 116 (69 robotic, 10 laparoscopic and 37 open) patients with a normal contralateral kidney were included in the study. All cases were performed under warm ischemia. Artery-only $(\mathrm{AO}, \mathrm{n}=44)$ and artery and vein $(A V, n=72)$ groups were compared in terms of tumor size, operation time, warm ischemia time, blood loss, decrease in glomerular filtration rate (GFR), positive surgical margins, and recurrence. Renal functional loss was evaluated with calculated GFR using preoperative and postoperative $6^{\text {th }}$ month creatinine. Results: Tumor size was $4.1 \mathrm{~cm}$ in $\mathrm{AO}$ group and $3.8 \mathrm{~cm}$ in $\mathrm{AV}$ group $(\mathrm{p}=0.3)$. There was no significant difference in operation time between two groups (184 min for AO, $201 \mathrm{~min}$ fo AV, $\mathrm{p}=0.24$ ). Warm ischemia time was $20.5 \mathrm{~min}$ in $\mathrm{AO}$ group and 22.7 min in AV group $(\mathrm{p}=0.15)$. Blood loss was significantly lower in AO group (181 ml) compared to AV group $(331 \mathrm{ml}$, $\mathrm{p}=0.003)$. Decrease in GFR was not statistically significant between $\operatorname{AO}\left(15.1 \mathrm{ml} / \mathrm{min} / 1.73 \mathrm{~m}^{2}\right)$ and $\operatorname{AV}\left(9.5 \mathrm{ml} / \mathrm{min} / 1.73 \mathrm{~m}^{2}\right)$ groups $(\mathrm{p}=0.11)$. Surgical margins were positive in 4 patients in $\mathrm{AO}$ and in 1 patient in AV group $(\mathrm{p}=0.06)$. In a mean of $41-$ month follow-up, there were one recurrence in $\mathrm{AO}$ and 4 recurrences in AV group $(\mathrm{p}=0.64)$.

Conclusions: Artery-only clamping results in less blood loss during partial nephrectomy under warm ischemia when compared to artery and vein clamping. The type of renal clamping does not have any impact on renal functional loss or oncological outcomes.

\section{MP21-15 Intraoperative Ultrasonography in Laparoscopic Partial Nephrectomy for Intrarenal Tumors}

\section{B Qin, H Hu, Q Wang, S Wang}

Department of Urology, Tongji Hospital, Tongji Medical College, Huazhong University of Science and Technology China (People's Republic)

Introduction \& Objective: To evaluate the feasibility and efficacy of intraoperative ultrasonography in laparoscopic partial nephrectomy (LPN) for intrarenal tumors.
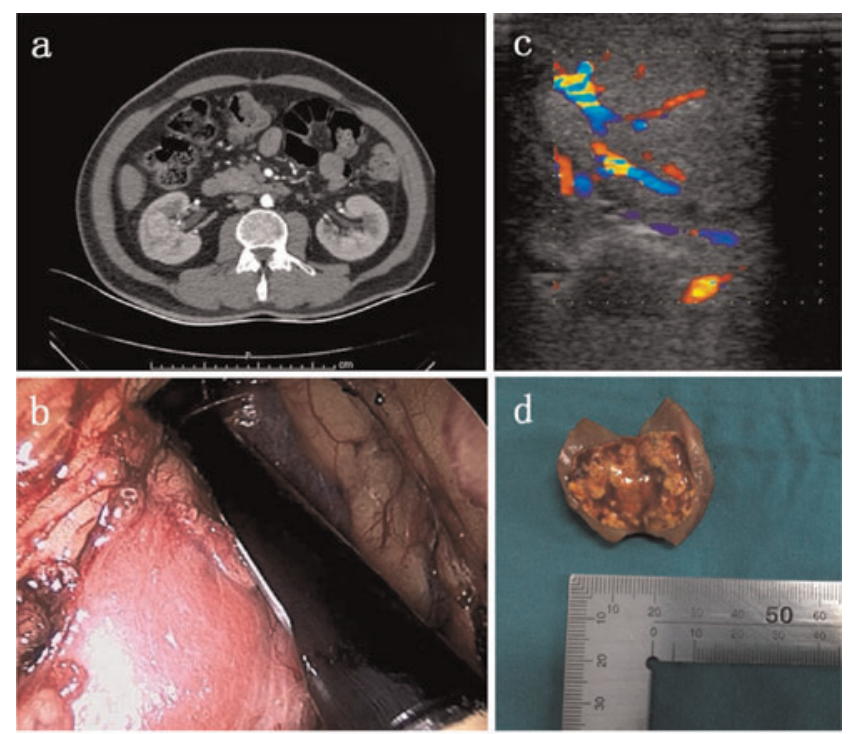

Fig 1. (A) Preoperative CT showing a TIT in right kidney. (B) Screen capture of ILUS with a probe scanning the kidney. (C) ILUS shows the TIT boundary and its blood flow distribution. (D) Excised specimen showing the integrity of TIT.
Table 1. Patients demographics and tumor characteristics

\begin{tabular}{lccc}
\hline & TIT group & Control group & P value \\
\hline Number(n) & 46 & 537 & \\
Sex, n(\%) & & & NS \\
$\quad$ Male & 29 & 363 & NS \\
$\quad$ Female & 17 & 174 & NS \\
Mean age \pm SD(range),years & $51.7(7.3)$ & $53.8(11.6)$ & NS \\
Sides & & & NS \\
$\quad$ Left & 24 & 266 & $<0.05$ \\
$\quad$ Right & 22 & 271 & NS \\
Mean tumor size(SD),cm & $2.42(0.46)$ & $3.29(1.43)$ & \\
Mean (SD) preoperative, & $88.6(13.7)$ & $85.1(13.8)$ & \\
eGFR mL/min/1.73 m2 & & &
\end{tabular}

eGFR, estimated GFR;NS, not significant.

Table 2. Intraoperative and postoperative data of the TIT group and control group.

\begin{tabular}{lccc}
\hline Variable & TIT group & Control group & P value \\
\hline Mean(SD) operation time, min & $127.2(16.0)$ & $120.1(19.2)$ & $<0.05$ \\
Mean(SD) WIT, min & $22.2(6.4)$ & $20.6(4.7)$ & NS \\
Mean(SD) blood loss, mL & $161.3(57.3)$ & $136.6(53.5)$ & $<0.05$ \\
Mean(SD) postoperative & $7.5(2.3)$ & $7.2(1.1)$ & NS \\
hospital stay & & & NS \\
Major complications, n \% & $5 / 46(10.9)$ & $41 / 537(7.6)$ & NS \\
Mean(SD)\% change in eGFR at & $-8.82(4.63)$ & $-8.38(5.21)$ & \\
\multicolumn{1}{c}{ 1-month postop } & & & \\
\hline
\end{tabular}

eGFR, estimated GFR; NS, not significant.

Materials and Methods: All patients who underwent LPN for renal tumors in our institution from January 2010 to October 2016 were assessed retrospectively. Patients were divided into two groups, the first with totally intrarenal tumors (TIT group), defined as a solid renal mass with no exophytic element on both preoperative and intraoperative evaluations, and the second with exophytic tumors (control group). General information and perioperative data of the two groups were compared, including tumor characteristics, operative time, estimated blood loss, warm ischemia time and pathological findings. Intraoperative laparoscopic ultrasonography (ILUS) was used to precisely locate and delineate the TIT border, as well as seeking for other suspected lesions.

Results: We identified 583 patients who underwent LPN in our center, including 46 in the TIT and 537 in the control group. All patients in the TIT group were evaluated by ILUS, and all TIT procedures were successfully performed without conversion to open surgery. The mean tumor sizes in the TIT and control groups were $2.42 \pm 0.46 \mathrm{~cm}$ and $3.29 \pm 1.43 \mathrm{~cm}(\mathrm{p}<0.001)$, respectively. The TIT group's R.E.N.A.L. nephrometry score was higher than that of the control group (median 8.5 vs 6.0, $\mathrm{p}<0.001$ ), and their mean operation times were $127.2 \pm 16.0 \mathrm{~min}$ and $120.1 \pm 19.2 \mathrm{~min}$, respectively. Mean estimated blood loss was lower in the TIT than in the control group $(161.3 \mathrm{ml}$ vs $136.6 \mathrm{ml})$. Mean warm ischemia time differed in the TIT and control groups $(22.2 \pm 6.4$ vs $20.6 \pm 4.7 \mathrm{~min})$, but not significantly. Rates of open conversion and positive margins, as well as rates of major postoperative complications, pathological findings, and 1-month changes in renal function, were similar in the two groups.

Conclusions: Intraoperative ultrasonography is technically feasible and has significant benefits in patients undergoing LPN for TITs. This method may reduce the need for radical nephrectomy in patients with endogenic renal masses. 
MP21-16 Perinephric Drain Placement Is Not Required After Robotic-Assisted Pyeloplasty

N François, M Sourial, C Dall, C Jaeger, G Box

The Ohio State University Wexner Medical Center United States

Introduction \& Objective: Placement of a perinephric drain after pyeloplasty has long been considered dogma but is not evidence based. Several studies have demonstrated safe omission of drain placement following reconstructive renal surgery such as partial nephrectomy. We have transitioned to omitting drains after robotic assisted pyeloplasty (RAP) and are reporting our outcomes.

Materials and Methods: We retrospectively reviewed patient data from a single surgeon who performed RAP between 2009 and 2016, and compared perioperative outcomes between those with and without a perinephric drain placed at the time of surgery, those with primary ureteropelvic junction obstruction (UPJO), and those with non-primary UPJO, defined as either secondary or tertiary.

Results: We identified 83 patients who had undergone RAP with a mean age of 42.2 years (SD 16.1), BMI of $27.7 \mathrm{~kg} / \mathrm{m} 2$ (SD 7.0), and ASA of 2.3 (SD 0.6) at the time of surgery. Overall, 72 patients $(86.7 \%)$ had a primary UPJO while 11 patients $(13.3 \%)$ had a non-primary UPJO. Twenty-eight patients $(33.7 \%)$ had a closed suction perinephric drain placed including 21 of 72 patients $(29.2 \%)$ with a primary UPJO and 7 of 11 patients $(63.6 \%)$ with a non-primary UPJO. All patients had a ureteral stent placed at the time of the operation. Comparing all patients, patients with a primary UPJO, and patients with a non-primary UPJO; the mean length of stay was 1.1 days (SD 0.5)/ 1.1 days (SD 0.5)/ 1.0 days $(\mathrm{SD} 0.4)$ respectively $(\mathrm{p}$-value $=0.6721)$, the mean ureteral stent duration was 40.1 days (SD 1.8)/ 40.6 days (SD 8.2)/ 43.1 days (SD 3.0) respectively $(\mathrm{p}$-value $=0.0864)$, the early complication rate was $16.9 \% / 18.1 \% / 9.1 \%$ respectively ( $\mathrm{p}$-value $=0.6805)$, and the failure rate was $10.8 \% / 6.9 \% / 36.4 \%$ respectively (pvalue $=0.0156)$. Notably, six patients $(21.4 \%)$ who had a drain placed compared to three patients $(5.5 \%)$ who did not have a drain placed failed and required secondary interventions for recurrent UPJ obstruction. There were no reported drain-related complications such as urine leak or intra-abdominal infections.

Conclusions: The incidence of complications that might benefit from drain placement is very low. Perinephric drain placement following RAP did not impact perioperative outcomes. As such it appears omission of a drain is safe.

MP21-17 Comparison of laparoscopic vs robotic partial nephrectomy: a retrospective single center analysis.

A Araki, K Makiyama, K Osaka, K Kondo, N Nakaigawa, M Yao, S Ohtake

Department of Urology, Yokohama City University Japan

Introduction \& Objective: The aim of this study was to evaluate differences in the outcomes of robot-assisted partial nephrectomy (RAPN) and laparoscopic partial nephrectomy (LPN) in our institution.

Materials and Methods: We retrospectively analyzed the medical charts of patients with renal tumors who underwent RAPN $(n=47)$ or LPN $(n=174)$ between 2007 and 2016. Characteristics of patients, Peri-operative data, and complications were reviewed. Results: Demographic characteristics (sex, age, BMI, and laterality) were similar in the two groups. The mean tumor size for RAPN was $30.2 \mathrm{~mm}$ vs $25.2 \mathrm{~mm}$ for LPN $(\mathrm{P}=0.004)$. The mean RENAL ne- phrectomy score for RAPN was 7.04 vs 6.17 for LPN ( $\mathrm{P}=0.0005)$. The mean distance from the tumor to the collecting system or sinus was $3.15 \mathrm{~mm}$ for RAPN vs $4.98 \mathrm{~mm}$ for LPN (P=0.0009). RAPN was associated with a longer operating time (204 vs $182 \mathrm{~min}$, respectively, $\mathrm{P}=0.001$ ) and a higher rate of urinary tract entry than LPN (59.5 vs $33.9 \%$, respectively, $\mathrm{P}=0.001$ ). There were no significant differences in the warm ischemia time, blood loss, accidental tumor incision rate, operative complications, and recurrence rate.

Conclusions: RAPN and LPN provided similar results in terms of the warm ischemia time, blood loss, complications, and recurrence. The RAPN group had longer operation times than the LPN group. In this study, we found that RAPN was safe for operations on larger and nearer tumor to the collecting system or sinus.

MP21-18 ASA Score and the Charlson Comorbidity Index Fail to Discriminate Patients that Have Post-Operative Complications after Robotic Partial Nephrectomy

M Greenstein, K Blum, D Paulucci, D Rosen, B Reddy, R Abaza, D Eun, A Bhandari, A Hemal, K Badani

Department of Urology, Icahn School of Medicine at Mount Sinai

United States

Introduction \& Objective: To evaluate and compare the ability of the American Society of Anesthesiologists (ASA) score, Charlson Comorbidity Index (CCI) and age adjusted CCI to correctly identify patients who experienced a post-operative complication (PC) after robotic partial nephrectomy (RPN).

Materials and Methods: We identified 1,229 patients undergoing RPN from 2006 to 2016 from 5 medical institutions who met eligibility criteria. Total PC were defined as any ClavienDindo complication occurring within 30 days of surgery. The area under receiver operating characteristic curve (AUROC) for any total PC and major (Clavien 3) PCs between the ASA score, CCI and age adjusted CCI was compared using pairwise DeLong's tests for two correlated ROC curves. The association of additional factors with total and major PC was evaluated using univariable and multivariable binary logistic regression models. Results: Overall, 173 PC were identified in 142 patients $(11.4 \%)$. Of these 173 PC, $27.2 \%$ were major and occurred in only $3.7 \%$ of patients overall. The AUROC for total PC was 0.552 for ASA score, 0.513 for CCI and 0.540 for age adjusted CCI and did not significantly differ in discriminability of total PC (lowest pairwise $\mathrm{p}=0.139$ ). The AUROC for major PC was 0.543 for ASA score, 0.528 for $\mathrm{CCI}$ and 0.523 for age adjusted CCI and did not significantly differ in discriminability of major PC (lowest pairwise $\mathrm{p}=0.646$ ). Tumor size $(\mathrm{OR}=1.16, \mathrm{p}=0.013)$, lower baseline eGFR $(\mathrm{OR}=1.01$, $\mathrm{p}=0.003)$, hypertension $(\mathrm{OR}=1.62, \mathrm{p}=0.043)$, coronary artery disease $(\mathrm{CAD} ; \mathrm{OR}=2.28, \mathrm{p}=0.005)$ and upper vs mid pole tumors $(\mathrm{OR}=1.75, \mathrm{p}=0.020)$ were associated with an increased likelihood of total PC No factors were significantly associated with major PC. Conclusions: The ASA score, CCI and age adjusted CCI failed to accurately discriminate patients with total and major PC following RPN. This suggests that RPN is a safe option in patients with greater comorbidity. Hypertension, CAD, lower baseline

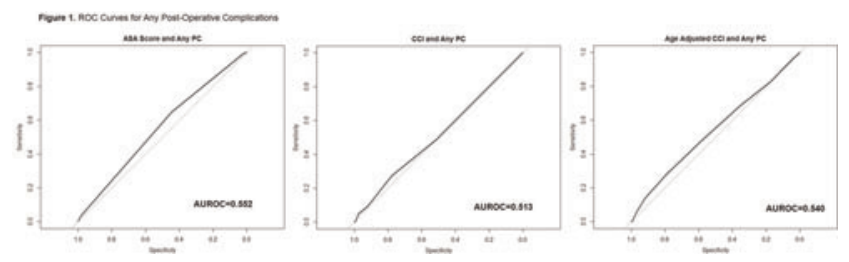


eGFR, tumor size and upper pole tumors were associated with total PC and should be incorporated into risk models for RPN to improve identification and patient counseling of those most likely to experience a PC after RPN.

MP21-19 The Utility of "Composite Tumor Size" in Evaluating Perioperative and Functional Outcome for Multiple versus Solitary Ipsilateral Tumors During Robotic Partial Nephrectomy

K Blum, R Cleary, D Paulucci, R Abaza, D Eun, A Bhandari, A Hemal, K Badani

Department of Urology, Icahn School of Medicine at Mount Sinai

United States

Introduction \& Objective: Ipsilateral multifocal (IMF) renal cell carcinoma carries unique resection challenges leading to removal of additional renal tissue which can increase post-operative complications. In the past, IMF lesions were treated with an open approach, however the utilization of robotic partial nephrectomy (RPN) for IMF is increasing. Despite this trend, the surgical morbidity of IMF lesions in RPN remains poorly characterized. In this study we evaluated peri and postoperative outcomes for IMF compared to solitary masses in the setting of RPN. Materials and Methods: We retrospectively identified 1,061 $(97.3 \%)$ patients with a solitary renal mass and $30(2.7 \%)$ patients with IMF renal masses who underwent RPN between July 2012 and February 2016 from a multi-institutional database.Perioperative and renal functional outcomes were compared using multivariable regression analysis adjusting for surgeon performing the RPN and composite tumor size (sum of the largest tumor diameter for each tumor removed).

Results: No differences in clinical characteristics were found between groups, however composite tumor size was significantly higher in the IMF vs. solitary mass group $(4.6$ vs. $3.0 \mathrm{~cm}, \mathrm{p}<.001)$. In univariable analysis, IMF vs. solitary RPN was associated with longer operative time (185 mins vs 170 mins, $\mathrm{p}=0.010$ ) in addition to a marginally higher warm ischemia time (WIT, $\mathrm{p}=.081$ ) and risk of acute kidney at discharge (AKI, $\mathrm{p}=.078)$. However, when adjusting for composite tumor size in multivariable analysis, no statistically significant differences in operative time $(p=.985)$, WIT $(p=.285)$, $\mathrm{AKI}(\mathrm{p}=.567)$, or any outcome including complications $(\mathrm{p}=.327)$ or renal function (eGFR) at 11.4 months $(=.577)$ were observed. Higher composite tumor size was associated with longer operative time $(\mathrm{p}<0.001)$, longer WIT $(\mathrm{p}<0.001)$, increased likelihood of intraoperative complications $(\mathrm{p}=0.007)$, post-operative complications $(p=0.009)$, reduced likelihood of trifecta achievement $(p<0.001)$, and an increased likelihood of AKI at discharge $(\mathrm{p}<0.001)$.

Conclusions: Robotic partial nephrectomy is safe and feasible for IMF renal masses. Composite tumor size is a new indicator that can be used to predict perioperative outcome during multiple ipsilateral RPN.

MP21-20 Off Clamp Robotic Partial Nephrectomy Does Not Improve Renal Function Outcome for Patients With T1a Tumors Without Impaired Renal Function

D Rosen, K Blum, D Paulucci, R Abaza, D Eun, A Bhandari, A Hemal, K Badani

Department of Urology, Icahn School of Medicine at Mount Sinai

United States

\begin{tabular}{|c|c|c|c|c|c|c|}
\hline \multirow[t]{2}{*}{ Variable } & \multicolumn{3}{|c|}{ All Patients } & \multicolumn{3}{|c|}{ Propensity Score Matched Patients } \\
\hline & $\begin{array}{l}\text { Main Artery } \\
\text { Clamping }\end{array}$ & Off Clamp & $p$ & $\begin{array}{l}\text { Main Artery } \\
\text { Clamping }\end{array}$ & Off Clamp & $p$ \\
\hline $\begin{array}{c}\text { Warm Ischemia } \\
\text { Time }\end{array}$ & $\begin{array}{c}15.0(11.3- \\
19.0)\end{array}$ & $\begin{array}{c}0.0(0.0- \\
0.0)\end{array}$ & $<.001$ & $\begin{array}{c}11.0(9.0- \\
15.0)\end{array}$ & $0.0(0.0-0.0)$ & $<.001$ \\
\hline $\begin{array}{l}\text { Estimated } \\
\text { Blood Loss }\end{array}$ & $\begin{array}{c}50.0(50.0- \\
100.0)\end{array}$ & $\begin{array}{l}100.0 \\
(50.0- \\
168.8)\end{array}$ & .012 & $\begin{array}{c}50.0(50.0- \\
100.0)\end{array}$ & $\begin{array}{c}100.0(50.0- \\
181.3)\end{array}$ & .007 \\
\hline Operative Time & $\begin{array}{c}165.0(134.0- \\
190.0)\end{array}$ & $\begin{array}{l}150.0 \\
(140.1- \\
180.0)\end{array}$ & .635 & $\begin{array}{c}139.5(124.0- \\
180.5)\end{array}$ & $\begin{array}{r}149.0 \\
(142.5- \\
180.0)\end{array}$ & .249 \\
\hline $\begin{array}{l}\text { NNPV } \\
\text { Removed } \\
\text { Positive }\end{array}$ & $\begin{array}{l}7.9(5.0- \\
14.6)\end{array}$ & $\begin{array}{l}7.1(3.5- \\
16.2)\end{array}$ & .711 & $5.9(2.3-8.7)$ & $\begin{array}{c}11.4(5.9- \\
17.2)\end{array}$ & .109 \\
\hline $\begin{array}{l}\text { Surgical Margin } \\
\text { Among } \\
\text { Malignant } \\
\text { Cases } \\
\text { Post-Operative } \\
\text { Complications }\end{array}$ & $13(5.5 \%)$ & $3(7.9 \%)$ & .469 & $3(10.0 \%)$ & $2(8.0 \%)$ & .999 \\
\hline Overall & $40(13.6 \%)$ & $3(5.4 \%)$ & .085 & $7(17.1 \%)$ & $2(5.0 \%)$ & .155 \\
\hline Major & $12(4.1 \%)$ & $1(1.8 \%)$ & .701 & $0(0.0 \%)$ & $1(2.5 \%)$ & .494 \\
\hline $\begin{array}{l}\text { Blood } \\
\text { Transfusion } \\
\text { Length of }\end{array}$ & $0(0.0 \%)$ & $1(1.8 \%)$ & .160 & $0(0.0 \%)$ & $1(2.5 \%)$ & .494 \\
\hline $\begin{array}{c}\text { Hospitalization } \\
\geq 3 \text { Days }\end{array}$ & $68(23.3 \%)$ & $13(22.8 \%)$ & .937 & $10(24.4 \%)$ & $11(26.8 \%)$ & .800 \\
\hline \multicolumn{7}{|l|}{ Discharge } \\
\hline $\begin{array}{l}\% \text { change } \\
\text { eGFR }\end{array}$ & $\begin{array}{c}-10.4 \%(- \\
24.0 \%- \\
2.1 \%)\end{array}$ & $\begin{array}{l}-4.0 \%(- \\
17.1 \% \text { - } \\
9.1 \%)\end{array}$ & .047 & $\begin{array}{l}-3.1 \%(- \\
21.6 \% \text { - } \\
11.1 \%)\end{array}$ & $\begin{array}{c}-7.3 \%(- \\
17.4 \%- \\
7.7 \%)\end{array}$ & .819 \\
\hline AKI & $61(22.9 \%)$ & $3(6.7 \%)$ & .013 & $8(20.5 \%)$ & $3(8.1 \%)$ & .125 \\
\hline Follow-Up & $\begin{array}{l}8.4(6.1- \\
12.2)\end{array}$ & $\begin{array}{l}9.9(6.0- \\
13.8)\end{array}$ & .439 & $7.8(5.9-12.1)$ & $\begin{array}{c}10.2(6.3- \\
13.8)\end{array}$ & .109 \\
\hline $\begin{array}{l}\text { \% change } \\
\text { eGFR }\end{array}$ & $\begin{array}{c}-10.1 \%(- \\
21.1 \%- \\
0.0 \%)\end{array}$ & $\begin{array}{l}-6.2 \%(- \\
13.6 \% \text { - } \\
7.9 \%)\end{array}$ & .048 & $\begin{array}{c}-3.4 \%(- \\
13.5 \%-1.6 \%)\end{array}$ & $\begin{array}{c}-5.1 \%(- \\
12.6 \%- \\
7.9 \%)\end{array}$ & .653 \\
\hline $\begin{array}{l}\text { Progression to } \\
\text { CKD }\end{array}$ & $38(12.9 \%)$ & $6(10.5 \%)$ & .617 & $4(9.8 \%)$ & $4(9.8 \%)$ & .999 \\
\hline
\end{tabular}

Introduction \& Objective: Off-clamp robotic partial nephrectomy (RPN) is intended to better preserve renal function compared to main arterial clamping (MAC) through the reduction of ischemia. However, there is minimal data on whether off-clamp technique extends any benefit in patients undergoing RPN in which this technique is feasible. We examined whether any functional advantage exists to off-clamp RPN in patients with simple tumors and healthy kidneys.

Materials and Methods: 351 T1a patients with bilateral kidneys, eGFR $\geq 60$ and a RENAL Nephrometry Scores of $<10$ underwent RPN were retrospectively identified from a multi-institutional database from five high volume surgeons between 2008 and 2016. Offclamp and MAC patients were 2 to 1 nearest neighbor propensity score matched on tumor size, R.E.N.A.L. score, \% endophytic, tumor location, age, gender, body mass index, comorbidities, baseline eGFR, and surgeon performing the RPN. Preoperative, surgical, and postoperative variables were compared.

Results: After propensity score matching, 41 off-clamp and 82 MAC RPN patients were compared. Off-clamp RPN had a significantly higher estimated blood loss (100 vs. $50 \mathrm{~mL}, \mathrm{p}=.007)$, but no increased rate of transfusion $(\mathrm{p}=.494)$. Baseline demographic or tumor-specific characteristics including tumor size $(p=.707)$ or R.E.N.A.L. Score $(p=.943)$ between both techniques were not statistically different. Rate of complications $(\mathrm{p}=.155)$, positive surgical margins $(\mathrm{p}=.999)$, or non-neoplastic parenchymal volume removed $(\mathrm{p}=.109)$ were similarly not statistically different (Table I). Moreover, no significant differences were observed in rates of acute kidney injury $(\mathrm{p}=.125)$, percent change in eGFR $(\mathrm{p}=.819)$ at discharge, percent change 
in eGFR $(\mathrm{p}=.653)$ or chronic kidney disease stage progression $(\mathrm{p}=.999)$ at median 9.2 months.

Conclusions: In patients with T1a renal tumors without compromised renal function, our data suggest that the use of offclamp RPN marginally increases blood loss without providing functional outcome benefit.

MP21-21 Comparison of Perioperative and Functional Outcomes of Robotic Partial Nephrectomy for cT1a versus cT1b Renal Masses

CR Reynolds, JC Delto, D Paulucci, K Badani, D Eun, R Abaza, J Porter, A Bhandari, A Hemal

Department of Urology, Wake Forest School of Medicine United States

Introduction \& Objective: Partial nephrectomy is increasingly being performed for $\mathrm{T} 1 \mathrm{~b}$ renal masses due to the advantages of nephron-sparing surgery. We report on the clinical and pathologic characteristics of patients with cT1a or cT1b renal masses undergoing robotic partial nephrectomy (RPN) and compare perioperative and functional outcomes between these cohorts in a large multi-institutional study.

Materials and Methods: The present multi-institutional study utilized a prospectively maintained database to identify patients undergoing RPN by 6 surgeons for a solitary clinical T1a $(n=1307$,

Table 1 Demographic and Clinical Characteristics between Patients undergoing RPN for a cT1a or cT1b Renal Mass

\begin{tabular}{|c|c|c|c|}
\hline & сT1a & cT1b & $\mathbf{P}$ \\
\hline $\mathrm{N}$ & $1307(77.6 \%)$ & $377(22.4 \%)$ & \\
\hline Age & $61.0(51.0-68.0 ; 16.0-89.0)$ & $61.0(52.5-67.5 ; 17.0-89.0)$ & .427 \\
\hline Male & $735(56.2 \%)$ & $232(61.5 \%)$ & .067 \\
\hline BMI & $29.3(25.7-34.0 ; 16.5-63.0)$ & $29.2(25.9-34.6 ; 17.0-70.9)$ & .407 \\
\hline Age Adjusted CCI & $3(2-4 ; 0-9)$ & $3(2-4 ; 0-9)$ & .267 \\
\hline HTN & $520(55.6 \%)$ & $164(61.4 \%)$ & .091 \\
\hline DM & $239(18.4 \%)$ & $82(22.0 \%)$ & .120 \\
\hline CAD & $87(9.3 \%)$ & $36(13.5 \%)$ & .047 \\
\hline COPD & $90(6.9 \%)$ & $24(6.4 \%)$ & .741 \\
\hline Baseline eGFR & $81.9(68.8-96.4 ; 12.5-214.3)$ & $81.4(64.2-99.4 ; 4.9-185.1)$ & .412 \\
\hline Baseline CKD $\geq 3$ & $186(14.5 \%)$ & $75(20.2 \%)$ & .008 \\
\hline Previous & $448(52.8 \%)$ & $112(46.9 \%)$ & .103 \\
\hline \multicolumn{4}{|l|}{ Abdominal Surgery } \\
\hline Tumor Size $(\mathrm{cm})$ & $2.5(2.0-3.2 ; 0.6-4.0)$ & $5.0(4.5-5.8 ; 4.03-7.0)$ & $<.001$ \\
\hline RENAL & $7(5-8 ; 4-10)$ & $9(7-9 ; 5-11)$ & $<.001$ \\
\hline \multicolumn{4}{|l|}{ Nephrometry } \\
\hline \multicolumn{4}{|l|}{ Score } \\
\hline Tumor Laterality & & & .747 \\
\hline Right & $674(51.9 \%)$ & $190(50.9 \%)$ & \\
\hline Left & $625(48.1 \%)$ & $183(48.1 \%)$ & \\
\hline Pole & & & .543 \\
\hline Lower & $228(30.3 \%)$ & $75(34.1 \%)$ & \\
\hline Mid & $275(36.6 \%)$ & $74(33.6 \%)$ & \\
\hline Upper & $249(33.1 \%)$ & $71(32.3 \%)$ & \\
\hline Tumor Location & & & .133 \\
\hline Anterior & $425(38.2 \%)$ & $121(37.9 \%)$ & \\
\hline Posterior & $502(45.1 \%)$ & $130(40.8 \%)$ & \\
\hline Lateral & $186(16.7 \%)$ & $68(21.3 \%)$ & \\
\hline Hilar & $137(12.8 \%)$ & $79(24.6 \%)$ & $<.001$ \\
\hline Date of Surgery & $\begin{array}{l}2012(2011-2014 ; 2006- \\
2016)\end{array}$ & $\begin{array}{l}2013(2012-2015 ; 2008- \\
2016)\end{array}$ & $<.001$ \\
\hline Retroperitoneal & $189(14.5 \%)$ & $58(15.4 \%)$ & .655 \\
\hline Clamp Technique & & & .001 \\
\hline Main Artery & $1025(80.3 \%)$ & $325(88.8 \%)$ & \\
\hline Selective & $138(10.8 \%)$ & $24(6.6 \%)$ & \\
\hline Arterial & & & \\
\hline Off-Clamp & $113(8.9 \%)$ & $17(4.6 \%)$ & \\
\hline
\end{tabular}

a. For Categorical Variables: Frequencies presented with percentages in parenthesis; Chi-Squared tests of Independence or Fisher's Exact Tests Performed

b. For Continuous Variables: Medians presented with Interquartile Ranges and Ranges in Parenthesis; Mann-Whitney U Tests performed.

c. $\mathrm{ASA}=$ American Society of Anesthesiologists Score; $\mathrm{BMI}=\mathrm{Body}$ Mass Index; $\mathrm{CCl}$ Charlson Comorbidity Index; CKD = Chronic Kidney Disease; eGFR = Estimated Glomerular Filtration Rates
Table 2. Univariable and Multivariable Comparison of Perioperative and Functional Outcome between Patients undergoing RPN for a cT1a or cT1b Renal Mass

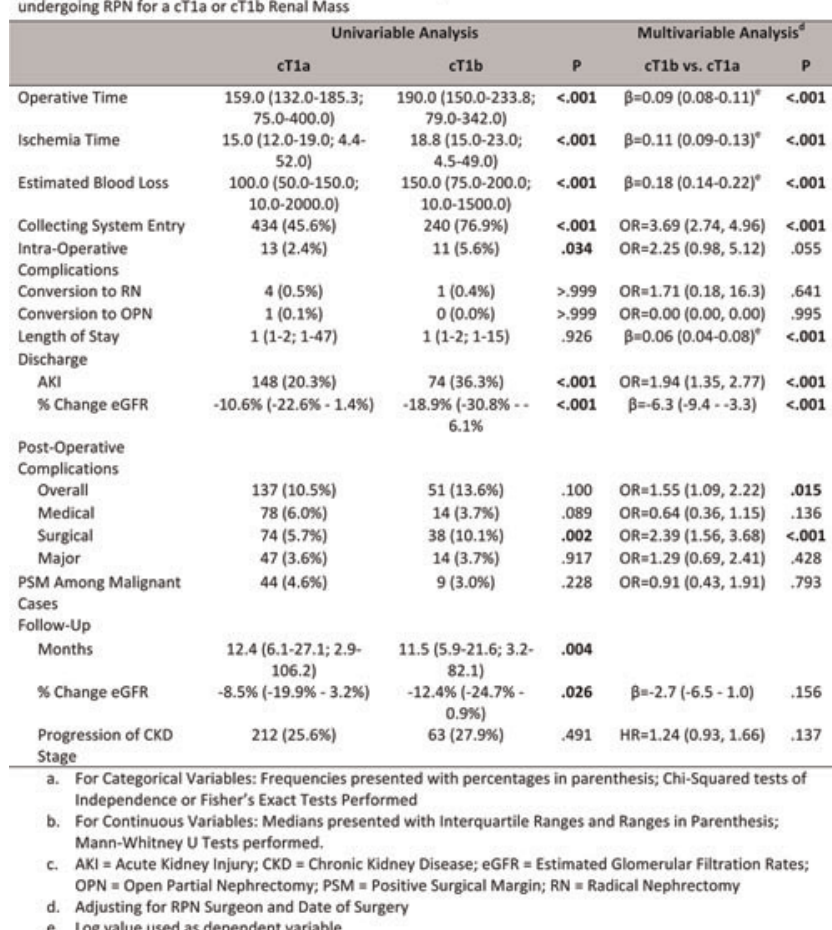

$77.6 \%)$ or $\mathrm{T} 1 \mathrm{~b}(\mathrm{n}=377,22.4 \%)$ renal mass from 2006 to 2016 . Perioperative outcomes in addition to renal function outcome at discharge and at a median follow-up of 12.2 months (IQR: 6.0-25.7; Range: 2.9-106.2) were compared in univariable analysis (MannWhitney U and Chi-squared tests of Independence) and multivariable regression analysis (linear, logistic, cox proportion hazards) adjusting for surgeon performing the RPN and date of surgery.

Results: In univariable analysis, clinical stage T1b was associated with longer operative time $(190.0$ vs. $159.0 ; \mathrm{p}<.001)$, greater warm ischemia time (WIT) (18.8 vs. 15.0 minutes; $\mathrm{p}<.001)$, higher estimated blood loss $(150.0$ vs. $100.0 \mathrm{~mL}$; $\mathrm{p}<.001)$, more intra-operative complications (5.6\% vs. $2.4 \%$, $\mathrm{p}=.034)$, and more surgical post-operative complications $(10.1 \%$ vs. $5.7 \%$; $=.002)$. Results were similar in multivariable analysis with additional findings including more overall postoperative complications $(\mathrm{OR}=1.55, \mathrm{p}=.015)$ and longer length of stay $(\mathrm{p}<.001)$ associated with cT1b masses. No differences in the risk progression of CKD stage at 12.2 months, positive surgical margins, or major post-operative complications requiring invasive and/or ICU management were observed.

Conclusions: Although our study shows a longer operative time, higher WIT, and complication rate for patients undergoing RPN for $\mathrm{cT} 1 \mathrm{~b}$ renal masses, the magnitude of these differences remains small and RPN should be considered in this scenario when anatomically and clinically feasible.

MP21-22 Negative impact of renal hypothermia with icecold saline on renal functional outcome for transperitoneal laparoscopic partial nephrectomy

M Kawamura, T Fujita, M Nishi, D Ishii, K Tabata, K Matsumoto, K Yoshida, M Iwamura

Department of Urology, Kitasato University School of Medicine

Japan 
Introduction \& Objective: Laparoscopic partial nephrectomy (LPN) is a less-invasive surgical procedure for small renal tumors. Although an open surgical procedure traditionally used kidney surface cooling with ice-slush for achieving renal hypothermia during vascular clamping, transperitoneal LPN could not use ice-slush. One technique for renal hypothermia during transperitoneal LPN has been reported as transureteral perfusion of the renal pelvis using ice-cold saline. However, renal functional outcome after transperitoneal LPN using renal hypothermia has not been seriously described. The present study aimed to explore postoperative operation side renal functional outcome with or without renal hypothermia after transperitoneal LPN by dynamic renal scintigraphy.

Materials and Methods: Between July 2006 and December 2014, 68 consecutive patients with small renal tumor received transperitoneal ischemic LPN at our institution. Patients were divided into two groups according to renal hypothermia: (i) hypothermia group and (ii) non-hypothermia group. In hypothermia group, $5 \mathrm{~F}$ ureteral catheter was inserted cystoscopically into the affected renal pelvis for perfusion of ice-cold saline containing indigo carmine dye before laparoscopic procedure. Preoperative, 6 months postoperative, and 12 months postoperative renal functions were assessed by dynamic renal scintigraphy using radio-nuclide technetium Tc 99m-mercaptoacetyl-triglycine. Postoperative operation side renal function was calculated: postoperative/preoperative split renal function (\%). Renal functional decrease between two groups were analyzed.

Results: Hypothermia group included 41 patients $(60.3 \%)$ and non-hypothermia group included 27 patients (39.7\%). In hypothermia group, postoperative operation side renal functions were significantly decreased to $86.2 \%$ at 6 months and $87.9 \%$ at 12 months postoperatively ( $P<0.0001$ and $<0.0001$, respectively). In non-hypothermia group, postoperative operation side renal functions were significantly decreased to $89.8 \%$ at 6 months and $86.2 \%$ at 12 months postoperatively $(P=0.0033$ and 0.0010 , respectively). There was no significant difference of postoperative renal functional decrease between two groups.

Conclusions: Since the no difference of postoperative renal functional decrease between hypothermia group and non-hypothermia group, renal hypothermia with ice-clod saline during transperitoneal LPN has no impact on postoperative operation side renal functional outcome. It was a novel finding using split renal function by dynamic renal scintigraphy.

MP21-23 Renal function and perioperative outcomes of selective versus complete renal arterial clamping during robot-assisted partial nephrectomy using console-integrated real-time three-dimensional image overlay navigation

J Furukawa, N Hinata, K Shigemura, K Harada, T Isihimura, Y Nakano, M Fujisawa

Division of Urology, Kobe University Graduate School of Medicine

Japan

Introduction \& Objective: To preserve a favorable renal function following partial nephrectomy (PN), selective clamping of the arterial branches feeding tumor tissues during $\mathrm{PN}$ has been performed. The objective of this study was to investigate clinical outcomes in patients undergoing selective versus conventional complete renal arterial clamping during robot-assisted partial nephrectomy (RAPN) using an image overlay navigation system.
Materials and Methods: Three-dimensional images were reconstructed from computed tomography with an open source processing software, OsiriX, and were directly visualized on the screen of a da Vinci surgeon's console with TilePro multi-input display functions. Using this real-time imaging system, we performed 56 cases of RAPN incorporating selective arterial clamping (group 1). The renal functional as well as perioperative outcomes in group 1 were compared with those in 110 patients undergoing RAPN with total clamping of the renal artery (group 2) during the same period.

Results: In group 1, tumor resection under selective arterial clamping could be completed in all patients without intraoperative conversion to conventional RAPN with total clamping. There were no significant differences in the tumor size, RENAL nephrometry score, or preoperative estimated glomerular filtration rate (eGFR) between groups 1 and 2. Furthermore, no significant differences were noted in the estimated blood loss, operative time, or warm ischemia time between the 2 groups. Although there was no significant difference in the rate of decrease in eGFR 4 weeks after RAPN between the 2 groups, the rate of decrease in eGFR 1 week after RAPN in group 1 was significantly lower than that in group 2 . The choice of selective or total clamping was also identified as an independent predictor of a postoperative decrease in eGFR by $>20 \%$ at 1 week, but not 4 weeks, after RAPN.

Conclusions: Despite it being on early experience with the initial 56 cases, our precise segmental clamping technique is feasible and safe for performing RAPN, resulting in an improved postoperative renal function, particularly early after surgery.

MP21-24 Prediction of surgical complications in laparoscopic nephrectomy caused by xanthogranulomatous pyelonephritis: how difficult will it be?

AM Cardenas Ortiz, PR Kawano, A Toledo, HA Yamamoto, R Guerra da Silva, JL Amaro, OE Hidetoshi Fugita

\section{UNESP}

Colombia

Introduction \& Objective: Xanthogranulomatous pyelonephritis (XGPN) is considered a relative contraindication to laparoscopic nephrectomy (LN) because of the potential surgical difficulty. The objective of this study is to identify preoperative predictors of surgical difficulty or conversion from laparoscopic to open approach in XGPN based on clinical, laboratory and tomography (CT) parameters.

Materials and Methods: 320 patients from 2006 to 2015 of that underwent transperitoneal laparoscopic nephrectomy for benign conditions, among which $23(7.18 \%)$ had a confirmative histopathological diagnosis of XGPN were further analyzed. Data regarding age, sex, operative time, bleeding, length of hospital stay, radiological exams and complications were evaluated.

Results: Three hundred and twenty patients underwent transperitoneal laparoscopic nephrectomy for benign conditions between 2006 and 2016, among whom 23 (7.18\%) had a confirmatory histopathological diagnosis of XGPN. The mean age was $46 \pm 17,6$ years. Mean BMI was $25 \pm 6,38$. Five cases (21.7\%) had open conversion. Operative time was longer in cases of staghorn stone and open conversion ( $\mathrm{p}:<0,0001)$. Bleeding rate was longer in the presence of staghorn stone (p: 0,006) in patients with conversion to open approach ( $\mathrm{p}:<0.0001)$ and in patients that developed any complication (p: 0,03). There was 
higher hospital length stay in patients that had a conversion of surgical approach (p: $<0,001)$

Conclusions: The laparoscopic treatment of XGPN remains challenging and controversial, due to the technical difficulties posed by the inherent inflammatory process. In our series the bleeding rate is the factor that predict open conversion, higher operative time, higher probability of complication and it is in relation with the presence of staghorn stone. Higher grade complications will be less if novice surgeons are accompanied by advanced laparoscopic surgeons while obtaining the surgical proficiency curve.

\section{MPS22: ONCOLOGY: KIDNEY}

MP22-1 Partial nephrectomy: Publication trends over the last 2 decades

\section{A Pietropaolo, B Rai, C Lockyer, M Hayes, B Somani \\ University Hospital Southampton NHS Foundation Trust United Kingdom}

Introduction \& Objective: Partial nephrectomy (PN) seems to be a rising surgical option for small renal masses. While it started with open partial nephrectomy (OPN), with increase in availability of laparoscopic and robotic surgery, laparoscopic partial nephrectomy (LPN) and robotic partial nephrectomy (RPN) has also seen a steep rise. We wanted to see the publication trends in PN over the last 16-years for all papers published on PubMed.

Materials and Methods: All published papers on partial nephrectomy (PN) including 'open', 'laparoscopic', robotic' and all comparison studies between these options were searched on PubMed over the last 16 years. While review articles were included, total nephrectomy, case reports and those papers that did not have a published abstract were excluded from our analysis. Similarly animal and laboratory studies were also excluded from our analysis. Data was divided into two 8-year periods, period-1 (2000-2007) and period-2 (2008-2015)

Results: A total of 1382 PN procedures were published in the study period (16-years), of which 449 (33\%) were OPN, 459 (33\%) were LPN, 278 (20\%) were RPN, and the remaining 196

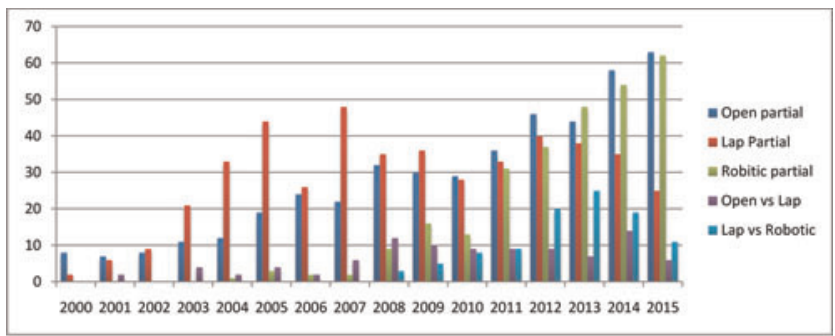

Fig 1

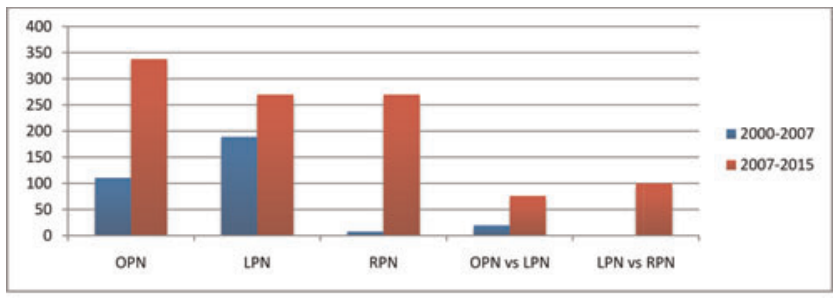

Fig 2
(14\%) were comparative studies between OPN, LPN or RPN (Fig 1).

As a procedure PN had increased more than three times in period-2 (328, period-1; versus 1054, period-2) (Fig 2). Similarly there was a steep rise in all groups of partial nephrectomy especially RPN (from 8 in period- 1 to 270 in period-2). There was also a big rise in published papers on LPN versus RPN in the second time period.

Conclusions: Partial nephrectomy has had a wide uptake with a huge increase in published papers on it. Although there has been a rise in all forms of $\mathrm{PN}$, there has specifically been steep rise in the number of papers on RPN both as cohort and comparative studies.

MP22-2 Robotic versus Open Partial Nephrectomy for Patients with Pre-Existing Chronic Kidney Disease: A Multicenter Analysis

A Bindayi, B Ristau, B Lane, S Berquist, S Dey, S Noyes, K Yim, F Wan, J Proudfoot, R Uzzo, Z Hamilton, IH Derweesh

Department of Urology, UC San Diego Health, La Jolla, California, USA

United States

Introduction \& Objective: Pre-existing chronic kidney disease (CKD) is a definitive indication for nephron-sparing surgery (NSS). Robotic Partial Nephrectomy (RPN) has become the dominant platform for minimally invasive NSS. We compared outcomes of open partial nephrectomy (OPN) vs. RPN in patients with pre-existing $\mathrm{CKD}$, with an emphasis on perioperative and renal functional outcomes.

Materials and Methods: Multicenter retrospective analysis of OPN and RPN in patients with baseline $\geq$ CKD-Stage III [estimated glomerular filtration rate $(\mathrm{eGFR})<60 \mathrm{~mL} / \mathrm{min} / 1.73 \mathrm{~m}^{2}$ ]. Primary outcome was change in eGFR $(\triangle \mathrm{eGFR}, \mathrm{mL} / \mathrm{min} /$ $1.73 \mathrm{~m}^{2}$ ) between preoperative and last follow-up with respect to cT stage (cT1a, cT1b, zcT2). Secondary outcomes included eGFR decline $>50 \%$ and hemodialysis at last follow-up. $\%$, and linear regression (LR) was performed to identify risk factors associated with increasing $\triangle \mathrm{eGFR}$ and multivariable analysis (MVA) was performed for predictors of eGFR decline $>50 \%$.

Results: 525 patients (304 OPN, $221 \mathrm{RPN}$, median follow-up 21.4 months) were analyzed. OPN had larger tumor size (4.7 vs. $3.0 \mathrm{~cm}, \mathrm{p}<0.001$ ) and greater tumor complexity (mean RENAL scores 8.6 vs. $7.8, \mathrm{p}<0.001)$. RPN had lower median estimated blood loss $(\mathrm{p}<0.001)$, ischemia time (RPN 24 vs. OPN 28 minutes, $\mathrm{p}<0.001$ ) and hospital stay (RPN 3 vs. OPN 6 days, $\mathrm{p}<0.001)$. Positive margin $(\mathrm{p}=0.83)$, transfusion $(\mathrm{p}=0.801)$ and 30 -day complications (RPN $15.8 \%$ vs. OPN 20.1\%, p=0.253) were similar. For OPN vs. RPN, mean $\triangle \mathrm{eGFR}$ demonstrated no 
significant difference for cT1a (0.6 vs. $2.43, \mathrm{p}=0.061)$, cT1b $(-3.9$ vs. $0.17, \mathrm{p}=0.184)$, and $\geq \mathrm{cT} 2(-5.3$ vs. $-3.2, \mathrm{p}=0.63)$. GFR decline $>50 \%$ (OPN $7.6 \%$ vs. RPN $4.5 \%, \mathrm{p}=0.202$ ), and hemodialysis (OPN $4.3 \%$ vs. RPN 2.3\%, p=0.235) were similar. LR demonstrated increasing age (Estimate $-0.367, \mathrm{p}=0.007$ ) and increasing RENAL score (Estimate $-1.220, \mathrm{p}=0.043$ ) were significant predictive factors associated with increasing $\triangle \mathrm{eGFR}$. Increasing RENAL score (OR 1.989, $\mathrm{p}=0.002$ ) was the only significant factor associated with eGFR decline $>50 \%$ on MVA. Conclusions: Patients with pre-existing $\geq$ Stage III CKD who undergo RPN and OPN have similar renal functional outcomes when stratified by clinical tumor size. Increasing tumor complexity was the primary risk factor for renal functional decline, suggest that robotic approach should not be a hindrance in appropriately selected patients with CKD and an enhancing renal mass.

MP22-3 Multicenter comparison of robotic and open partial nephrectomy for quality measures controlling for clinical T stage

A Bindayi, M Holden, Z Hamilton, A Larcher, S Dey, M Marshall, K Yim, DS Han, S Noyes, F Wan, U Capitiano, S Stroup, B Lane, IH Derweesh, F Montorsi

Department of Urology, UC San Diego Health, La Jolla, California, USA

United States

Introduction \& Objective: Partial nephrectomy (PN) is standard therapy for small renal masses. Robotic partial nephrectomy (RPN) is being rapidly adopted. Nonetheless, concerns persist about risks of RPN in larger and more complex renal masses with respect margins and renal functional recovery. We compared quality outcomes of RPN and open PN (OPN) in a multicenter international cohort controlling for clinical tumor stage.

Materials and Methods: Multicenter retrospective analysis of OPN and RPN performed from 1990-2016. Primary quality outcome negative margin rate, complication rate, and change in GFR. Other outcomes measured included transfusion rate, estimated blood loss (EBL), change in GFR at last follow up, and return of glomerular filtration rate (GFR) to $>80 \%$ of baseline. Patients were stratified by clinical staging (cT1a, cT1b, and cT2+). Multi-variable analysis (MVA) was performed to identify risk factors associated with quality measures.

Results: 1856 patients were analyzed (mean age 60.6 years, tumor size $3.6 \mathrm{~cm}, 557 \mathrm{RPN} / 1299$ OPN, median follow up 62.0 months). OPN had significantly higher EBL (350 vs. $120 \mathrm{~mL}$, $\mathrm{p}<0.001)$, transfusion rate $(\mathrm{p}<0.001)$, and longer ischemia time (24.1 vs $19.7 \mathrm{~min}, \mathrm{p}<0.001)$. Median hospital stay was shorter for RPN ( 5 vs. 7 days. $p<0.001$ ). There was no difference in rate of negative surgical margin (95.2\% OPN, 94.1\% RPN, $\mathrm{p}=0.302$ ) or lack of perioperative complications $(79.4 \%$ OPN, $82.0 \%$ RPN, $\mathrm{p}=0.204)$. However, rate of return of GFR to $80 \%$ was higher for RPN $(62.9 \%$ vs. $52.0 \%, \mathrm{p}<0.001)$. Change in GFR for OPN vs. RPN by clinical stage revealed cT1a (9.8 vs. 6.7, p $<0.001)$, cT1b (9.6 vs. $7.8, \mathrm{p}=0.172)$, and $\mathrm{cT} 2 \leq(11.5$ vs. $10.3, \mathrm{p}=0.234)$. On MVA for complications, diabetes (OR 1.58, $\mathrm{p}=0.022)$ and coronary artery disease (OR 2.36, $\mathrm{p}>0.001)$ were predictive while increasing preoperative GFR was protective (OR 0.99, $\mathrm{p}=0.032$ ). On MVA for positive margins, hypertension (OR 2.44, $\mathrm{p}=0.006)$ was predictive but increasing BMI was protective (OR 0.94, $\mathrm{p}=0.046$ ). On MVA for GFR return to $>80 \%$ of baseline, worsening outcomes were associated with increasing age $(\mathrm{OR} 0.99, \mathrm{p}=0.04)$ and increasing tumor size (OR 0.91, $\mathrm{p}=0.035$ ).

Conclusions: RPN provides comparable quality outcomes with respect to margin positivity and complication risk while providing benefit in terms of hospital stay and transfusions. RPN may be viewed as the primary surgical option for PN unless contraindicated.

MP22-4 Off-clamp Robot-Assisted partial nephrectomy offered renal functional advantage for selected renal masses

P Mouracade, J Chavali, J Dagenais, MJ Maurice, R Nelson, J Reese, J Kaouk

United States

Introduction \& Objective: Minimization or reducing on-clamp time during PN has led to alternative technical modifications. To compare the outcomes of off-clamp to on-clamp approach during robot-assisted partial nephrectomy (RAPN).

Materials and Methods: Retrospective study of 940 patients who underwent RAPN between 2007 and 2016 for cT1a tumours using on-clamp or off-clamp approaches. We matched the patients in terms of confounding variables. Overall, 309 patients with on-clamp technique were matched to 103 patients with offclamp technique. We compared the clinico-pathological characteristics, perioperative morbidity and late functional outcomes between the propensity score matched groups. Limitations included retrospective analysis.

Results: There were no difference in clinico-pathological characteristics between the 2 matched groups. While operative time $(\mathrm{p}=0.4)$, estimated blood loss $(\mathrm{p}=0.28)$, Clavien grade III-V complications $(p=0.8)$, surgical reoperation $(p=1), 30$-day readmission $(\mathrm{p}=1)$ positive surgical margin $(5.5 \%$ vs. $5.8 \%$, $\mathrm{p}=0.9$ ) were comparable between the 2 groups, there were significant difference in excisional volume loss (median, 7.08 vs. $3.51 \mathrm{~cm} 3, \mathrm{p}<0.01$ ), e-GFR decline (median, -9.7 vs. $-2.2 \mathrm{ml} /$ $\mathrm{min} / 1.73 \mathrm{~m} \mathrm{2}, \mathrm{p}<0.01$ ), percent of e-GFR preservation (median, $87 \%$ vs. $97 \%, \mathrm{p}<0.01$ ), and CKD upstaging (36.5\% vs. $23.3 \%$, $\mathrm{p}=0.01)$ in favour of off-clamp group. Excisional volume loss $(p=0.01)$, off-clamp approach $(p=0.01)$, and age $(p=0.02)$ were predictors of renal function preservation, whereas excisional volume loss $(\mathrm{OR}=1.03, \mathrm{CI} 95 \%$ [1.01-1.1], $\mathrm{p}<0.01)$ predicted CKD upstaging.

Conclusions: RAPN for selected small renal mass using offclamp approach offered renal functional advantage over onclamp. Excisional volume loss, off-clamp approach, and age were independent predictors of renal function preservation. Clinical significance of these findings in various clinical settings will require further investigation.

\section{MP22-5 The Effect of Robotic Assistance on Outcomes for Partial Nephrectomies.}

M Finkelstein, KH Bilal, MA Palese

Icahn School of Medicine at Mount Sinai

United States

Introduction \& Objective: Previous research has attested to the advantages of the Robot-Assisted Laparoscopic Surgical (RALS) approach for partial nephrectomies $(\mathrm{PN})$ over the traditional open 
approach. Such advantages as improved cosmesis, reduced pain, blood loss, and hospital stay, have led a trend to the adoption of RALS in PN. Concurrently, the development of the RALS approach and the necessity for facilities to make expensive capital investments in order to provide such care has resulted in centralization of care. In this study, we look into the benefit of the RALS approach while taking into consideration trends in centralization as well as several other procedure, patient, and physician-level variables in order to eliminate the influence of possible confounders. Materials and Methods: The New York Statewide Planning and Research Cooperative System was queried over the period from 2009 to 2015 for PN (ICD-9 55.4). The American Medical Association Masterfile was used to supplement each procedure with information on an identified urologist and the American Hospital Association Dataviewer was used to supplement each procedure with information on the associated facility. High volume urologists and high volume hospitals were identified such that each accounted for $25 \%$ of all cases. Multivariate analysis was performed to determine adjusted odds ratios, taking into account age, ethnicity, race, insurance, comorbidities, surgeon time from medical school graduation, hospital teaching affiliation, hospital setting, and year in which the procedure was completed.

Results: In the period of interest, 11,357 PN cases, completed at 115 unique facilities, by 403 urologists, were identified. Every increase in urologist volume by 10 was associated with $2 \%$ reduction in long stay (OR 0.980, 95\% CI 0.972-0.988). Every increase in facility volume by 100 was associated with both a $19 \%$ reduction in mortality (OR $0.841,95 \%$ CI $0.782-0.898$ ) and an $11 \%$ reduction in long stay (OR $0.886,95 \%$ CI $0.845-0.926$ ). The use of robotic assistance in laparoscopic surgery was also seen as protective of long stays (OR 0.474, 95\% CI 0.291-0.746) and 30-day readmission (OR 0.664, 95\% CI 0.537-0.818). Additionally congestive heart failure was found to be the single strongest comorbidity associated with 1-year mortality (OR $3.828,95 \%$ CI 1.821-7.486).

Conclusions: When taking into account several factors such as physician and facility volume, this study reveals that the use of RALS in PN is associated with a 53\% reduction in the likelihood of long stays and 34\% reduction in the likelihood of 30-day readmission. Discerning the origin of these benefits is critical to the better understanding of the indications of robotic technology.

MP22-6 5 Year Peri-operative Outcomes of Robot-Assisted Laparoscopic Partial Nephrectomy

A Rasmussen, H Evans, B St. Martin, M Hobart, T Schuler

Division of Urology, University of Alberta

Canada

Introduction \& Objective: The purpose of this study is to review the 90-day peri-operative outcomes of patients undergoing Robot-Assisted Laparoscopic Partial Nephrectomy (RAPN) between 2011 and 2016 at the University of Alberta.

Materials and Methods: Patients who had undergone RAPN for a renal mass in Edmonton between January 2011 and June 2016 were identified using billing codes. The primary outcome was 90-day complication rate. Secondary outcomes included intra-operative blood loss, 90-day transfusion rate, post-operative hemoglobin change, $\mathrm{T}$ stage, margin status, artery clamp type, length of stay, and demographics. Anonymized data were collected from office and hospital charts. Analysis was completed with descriptive statistics.
Results: 232 patients were included in this study. The mean age at time of operation was 57 years with a 2:1 male to female ratio $(67 \%$ vs. $33 \%)$. 56 patients $(24 \%)$ had a BMI $>35$. Average intra-operative blood loss was $302 \mathrm{cc}$ (range 50-5000cc) and $7.8 \%$ of patients required a blood transfusion within 90 days of surgery. 198 masses (85\%) were RCC (pT1-3a) and 34 (15\%) were benign. The positive/indeterminate margin rate was $6.5 \%$. 114 patients $(49 \%)$ had a full clamp of the main renal artery whereas $117(50 \%)$ had a zero ischemia/minimal ischemia approach with either a segmental branch clamped (44\%) or no clamp (6\%). 25 patients $(11 \%)$ experienced a complication within the 90 day post-operative period with $14(6 \%)$ being high grade (Clavien III or greater) complications. The most common complication was delayed renal bleed from arteriovenous fistula or pseduoaneurysm formation with 8 patients requiring angioembolization (segmental artery or main renal artery). The median length of stay was 2 days (mean: 2.4 days, range: 114 days).

Conclusions: RAPN outcomes at the University of Alberta are acceptable when compared with large published series with respect to 90 day peri-operative outcomes. The positive/indeterminate surgical margin rate appears higher than published rates.

MP22-7 Initial experiences of Robotic Laparoendoscopic Single-Site Partial Nephrectomy by da-Vinci $\mathrm{Xi}{ }^{\circledR}$ surgical system

J Na, H Lee, Y Yoon, K Rha, Y Choi, W Han

Department of Urology, Urological Science Institute, Yonsei University College of Medicine

Republic of Korea

Introduction \& Objective: "Single-site surgery" is the term given to various laparoscopic techniques that use a single skin incision around the umbilicus. Although new equipment has been developed for laparoendoscopic single-site surgery (LESS), the surgical instruments have a limited range of motion, and clashing of instruments is a disadvantage. To overcome these difficulties the da-Vincisurgical system has been used for LESS surgery. Here we report our first three clinical experience with Single Site Partial nephrectomy by da-Vinci $\mathrm{Xi}^{\circledR}{ }^{\circledR}$ surgical system (Intuitive Surgical Inc., Sunnyvale, CA, USA)

Materials and Methods: The patient was placed in the conventional flanked kidney position with the ipsilateral side elevated. A $2.2 \mathrm{~cm}$ vertical midline incision was made at the upper border of the umbilicus, and the single site port was inserted. The single site port was established by inserting one 8.5-mm endoscope trocar and two 5-mm accessory trocars through multichannel and securing it to the port. An additional 5-mm trocar was inserted in the midline below the subxiphoid process for liver traction. And 12-mm trocar for assist alongside the single multichannel port was inserted. After the robot was docked dissection was performed until the renal hilum was visible, and the renal vein and artery were exposed. Intraoperative ultrasonography was used to identify the size and margins of the tumor; the ultrasound images were projected onto the console screen to guide tumor resection (TilePro, Intuitive Surgical, Sunnyvale, CA). After clamping the renal pedicles with a laparoscopic bulldog clamp (Aesculap, Tuttlingen, Germany), the mass and surrounding normal tissue were incised by curved scissor. And then the resected renal parenchyma was sutured. 


\begin{tabular}{|c|c|c|c|}
\hline & Patient 111 & Patient \#2 & Patient $\# 3$ \\
\hline Date of op & $2016-12 \cdot 14$ & 2016-12-19 & 2017-03-02 \\
\hline Sex & M & M & M \\
\hline Age(yr) & 45 & 52 & 53 \\
\hline Past History & $\begin{array}{l}\text { HTN, Hyperlipidemia } \\
\text { Partial nephrectomy, Rt. d/t } \\
\text { RCCa, clear cell, pT1 on } \\
\text { Oct, } 2016\end{array}$ & n-s & \begin{tabular}{|l|} 
n-s \\
Preoperative biopsy on Jan, \\
2017
\end{tabular} \\
\hline BMI $\left(\mathrm{kg} / \mathrm{m}^{\wedge} 2\right)$ & 30.4 & 22.2 & 22.6 \\
\hline $\begin{array}{l}\text { Post-op } \\
\text { hospitalization } \\
\text { duration (days) }\end{array}$ & 4 & 7 & 4 \\
\hline $\begin{array}{l}\text { Total op time } \\
(\mathrm{min})\end{array}$ & 229 & 189 & 168 \\
\hline $\begin{array}{l}\text { Console Time } \\
\text { (min) }\end{array}$ & 177 & 133 & 88 \\
\hline $\begin{array}{l}\text { Ischemic Time } \\
\text { (min) }\end{array}$ & 23 & 33 & 25 \\
\hline Suture Time (min) & 18 & 31 & 10 \\
\hline Blood loss (ce) & 100 & 100 & 50 \\
\hline Vessel anatomy & $1 \mathrm{a}, \mathrm{Iv}$ & $1 \mathrm{a}, 2 \mathrm{v}$ & $1 \mathrm{a}, \mathrm{Iv}$ \\
\hline Laterality & Left & Right & Left \\
\hline Position of tumor & $\begin{array}{l}\text { Mid pole, posterior, } \\
\text { exophyting }\end{array}$ & $\begin{array}{l}\text { Lower pole, lateral, } \\
\text { exophyting }\end{array}$ & $\begin{array}{l}\text { Lower pole, anterior, } \\
\text { endophytic }\end{array}$ \\
\hline Pathology & Clear cell type, FG2 & Clear cell type, FG3 & Clear cell type, FG2 \\
\hline $\begin{array}{l}\text { Tumor size on } \\
\text { pathology }\end{array}$ & $2.1 \times 2.0 \mathrm{~cm}$ & $4.3 \times 4.3 \mathrm{~cm}$ & $1.2 \times 1.0 \mathrm{~cm}$ \\
\hline Resection margin & Free from cancer & Free from cancer & Free from cancer \\
\hline
\end{tabular}

Results: Three patients underwent Robotic LESS partial nephrectomy. Mean age at time of surgery was 50 years, average BMI was $25.1 \mathrm{~kg} / \mathrm{m}^{2}$. Average operation time, console time, ischemic time and suture time was $195.3 \mathrm{~min}, 132.7 \mathrm{~min}, 27.0 \mathrm{~min}$, $19.7 \mathrm{~min}$ respectively. Blood loss ranged from 50 to $100 \mathrm{cc}$, and patients were discharged on postoperative day 4 to 7 without any surgical complications. The console time descreased in sequence by $177 \mathrm{~min}$, $133 \mathrm{~min}$ to $88 \mathrm{~min}$. All margins of resected tumors were free of cancer.

Conclusions: Our initial experience with Single Site Partial nephrectomy by da-Vinci $\mathrm{Xi}^{\circledR}$ surgical system demonstrated that the procedure is feasible and safe.

MP22-8 Safety and oncological outcomes of laparoscopic robot-assisted partial nephrectomy (RAPN) for pT1b/pT2a renal cell cancers

S Vamadevan, J Klein, G Wirth, C Iselin

Switzerland

Introduction \& Objective: Robotic assistance has simplified laparoscopic partial nephrectomy for renal cell cancer (RCC). Its indications are constantly being extended to technically more difficult and larger tumors, however possibly at the expense of positive surgical margins and conversion to open surgery. The aim of this study was to assess the safety and oncological

\begin{tabular}{|l|c|c|r|}
\hline \multicolumn{1}{|c|}{ RESULTS } & pT1a & pT1b/pT2a & $p$ \\
\hline Size (cm) & $2.5 \pm 0.7$ & $5.1 \pm 0.9$ & - \\
\hline Age (years) & $59 \pm 13.5$ & $60 \pm 13.7$ & 0.76 \\
\hline Warm ischemia time (min) & $17.9 \pm 5.5$ & $19.5 \pm 4.7$ & 0.1 \\
\hline PADUA tumor scores & $7.7 \pm 1.25$ & $8.95 \pm 1.28$ & 0.003 \\
\hline Blood loss (g/l) & $22.8 \pm 12.8$ & $24.2 \pm 14.5$ & 0.36 \\
\hline Operating time (min) & $253 \pm 85$ & $300 \pm 61$ & 0.004 \\
\hline $\begin{array}{l}\text { Post-operative } \\
\text { complication }\end{array}$ & $26 \%$ & $35 \%$ & 0.22 \\
\hline Surgical margins & $7 \%$ & $0 \%$ & 0.22 \\
\hline Length of stay (days) & $4.3 \pm 2.5$ & $5 \pm 1.9$ & 0.09 \\
\hline
\end{tabular}

outcomes of robotically assisted partial nephrectomy (RAPN) for pT1b/pT2a versus pT1a renal cancers.

Materials and Methods: Analysis of all consecutive RCC treated by RAPN from December 2009 to October 2016 in a single institution by 2 operators. Tumor size, age, warm ischemia time, PADUA tumor scores, blood loss (difference between pre and post operative, day 1 hemoglobin), operative time, postoperative complications (Clavien classification), surgical margins and post-operative length of stay were collected retro prospectively. Pre-operative imaging was reviewed to calculate PADUA scores. Tumor stage was extracted from electronic medical records. Values are means and their statistical significance was verified by using the Student t-test.

Results: 107 patients underwent RAPN; 4 (1 in the pT1a (1.2\%) and 3 in the pT1b/pT2a group (13\%)) required conversion to laparotomy, $\mathrm{p}=0.04)$ were discounted from following analysis. $80 \%(n=83)$ had a pT1a renal cancer and $20 \%(n=20)$ a pT1b/ pT2a. Tumor size was of $2.5 \pm 0.7 \mathrm{~cm}$ in the pT1a group and $5.1 \pm 0.9$ in the $\mathrm{pT} 1 \mathrm{~b} / \mathrm{pT} 2 \mathrm{a}$ group. Patient age was $59 \pm 13.5$ years and $60 \pm 13.7$ respectively $(\mathrm{p}=0.76)$. Warm ischemia time was $17.9 \pm 5.5$ minutes for the pT1a group and $19.5 \pm 4.7$ for the $\mathrm{pT} 1 \mathrm{~b} / \mathrm{pT} 2 \mathrm{a}$ group $(\mathrm{p}=0.10)$. PADUA score was significantly lower in the pT1a group than in the pT1b/pT2a group (56\% low-, $37 \%$ intermediate-, $7 \%$ high risk vs. $15 \%$ low-, $55 \%$ intermediate- and $30 \%$ high risk respectively; $\mathrm{p}<0.003$ ). Post-op day hemoglobin decrease was slightly less in pT1a group with $22.8 \pm 12.8 \mathrm{~g} / 1$ versus $24.2 \pm 14.5$ in $\mathrm{pT} 1 \mathrm{~b} / \mathrm{pT} 2 \mathrm{a}(\mathrm{p}=0.36)$. Operative time was significantly longer for pT1b/pT2a $(300 \pm 61)$ than for pT1a cancers $(253 \pm 85$ minutes; $p=0.004)$. There was no significant difference in post-operative complication rate ( $26 \%$ vs $35 \%$ respectively; $p=0.22)$. $7.2 \%$ of patients had positive surgical margins for pT1a tumors while all patients had negative surgical margins for pT1b/pT2a tumors $(\mathrm{p}=0.22)$. Mean length of stay tended to be shorter in the pT1a group $(4.3 \pm 2.5$ vs. $5 \pm 1.9$ days; however without statistical significance; $\mathrm{p}=0.09$ ).

Conclusions: RAPN offers similar oncologic and minimally invasive outcomes for $\mathrm{pT} 1 \mathrm{~b} / \mathrm{pT} 2 \mathrm{a} \mathrm{RCC}$ in comparison to pT1a cancers, except for the significantly higher risk of conversion to open surgery. Although operative time is slightly longer than for pT1a cancers, the data show that most patients with pT1b/pT2a $\mathrm{RCC}$ benefit from this minimal invasive surgical approach.

\section{MP22-9 Robotic-Assisted Partial Nephrectomy for Renal Angiomyolipoma Larger than $6 \mathrm{~cm}$}

Y Lin, C Ho, T Wu, T Tsai, T Hwang

Shin Kong WHS Hospital

Taiwan (Republic of China) 
Introduction \& Objective: Angiomyolipoma (AML) is a rare benign renal neoplasm. AML larger than $4 \mathrm{~cm}$ posses the risk of spontaneous rupture and requires management. Robotic partial nephrectomy (RAPN) has been proved to be an effective treatment modality for renal tumors. Herein, we report our experience in RAPN for large AML.

Materials and Methods: From June, 2015 to March 2017, a total of 62 RAPN was performed in our hospital. Among the procedures, 8 patients were identified as AML larger than $6 \mathrm{~cm}$. The demographics, perioperative parameters and post-operative details were collected and analyzed retrospectively.

Results: Among the 8 patients, 6 were females and 2 were males. The median age was 54 (21-65) years old. Five tumors were located in the left side and 3 in the right side. The median maximal size of the tumor was $7.5(6-13) \mathrm{cm}$. The median R.E.N.A.L nephrometry score was 7.5 (7-10). Median operative time was 200 (120-300) minutes and median blood loss was 200 (50-450) $\mathrm{ml}$. No intra-operative complication was noted. The median warm ischemia time was $14(0-40)$ minutes. The median Hospital stay was 6.5 (5-9) days. The median preoperative and post-operative creatinine level was $0.72(0.46-1.26)$ and 0.84 (0.56-1.38) $\mathrm{mg} / \mathrm{dL}$, respectively. No postoperative complication and recurrence can be found.

Conclusions: RAPN is a safe and effective surgical procedure for large AML. The complication rate is low. For patients with large ANL, RAPN can be a standard approach.

MP22-10 Development and Validation of an Objective Scoring tool for Minimally Invasive Partial Nephrectomy: Scoring for Partial Nephrectomy (SPaN)

A Hussein, R Abaza, CG Rogers, R Boris, J Porter, M Allaf, K Badani, M Stifelman, J Kaouk, K Guru, D Eun

Roswell Park Cancer Institute United States

Introduction \& Objective: Standardization and objective assessment of surgical performance is crucial to ensure adequacy of training and skill acquisition, which will ultimately ensure optimal surgical outcomes and patient safety. There is lack of objective and procedure-specific tools for comprehensive assessment of minimally invasive partial nephrectomy. We aimed to develop and validate a structured scoring tool for assessment of minimally invasive partial nephrectomy: Scoring for Partial Nephrectomy (SPaN).

Materials and Methods: A national expert panel of 10 robotic surgeons collaborated towards development and content validation of SPaN. The critical steps of partial nephrectomy were deconstructed into 7 key domains, where each domain was assessed by 5 anchors evaluating surgical principles, technical proficiency and safety. Delphi methodology was utilized to validate the content of SPaN. Each anchor statement was assessed in terms of relevance, concordance of language used and the assigned anchor score, and clarity of the wording used. An independent coordinator collected the scores, together with the comments from the expert panel and calculated the Content Validity Index (CVI) for each aspect of each anchor. Consensus was achieved for a certain statement if CVI was $\geq 0.75$, and this statement was removed from the next round. If CVI $<0.75$, the coordinator incorporated the comments from the panel, updated the scoring and redistributed it again to the panel. This process was repeated until consensus was achieved for all statements. All

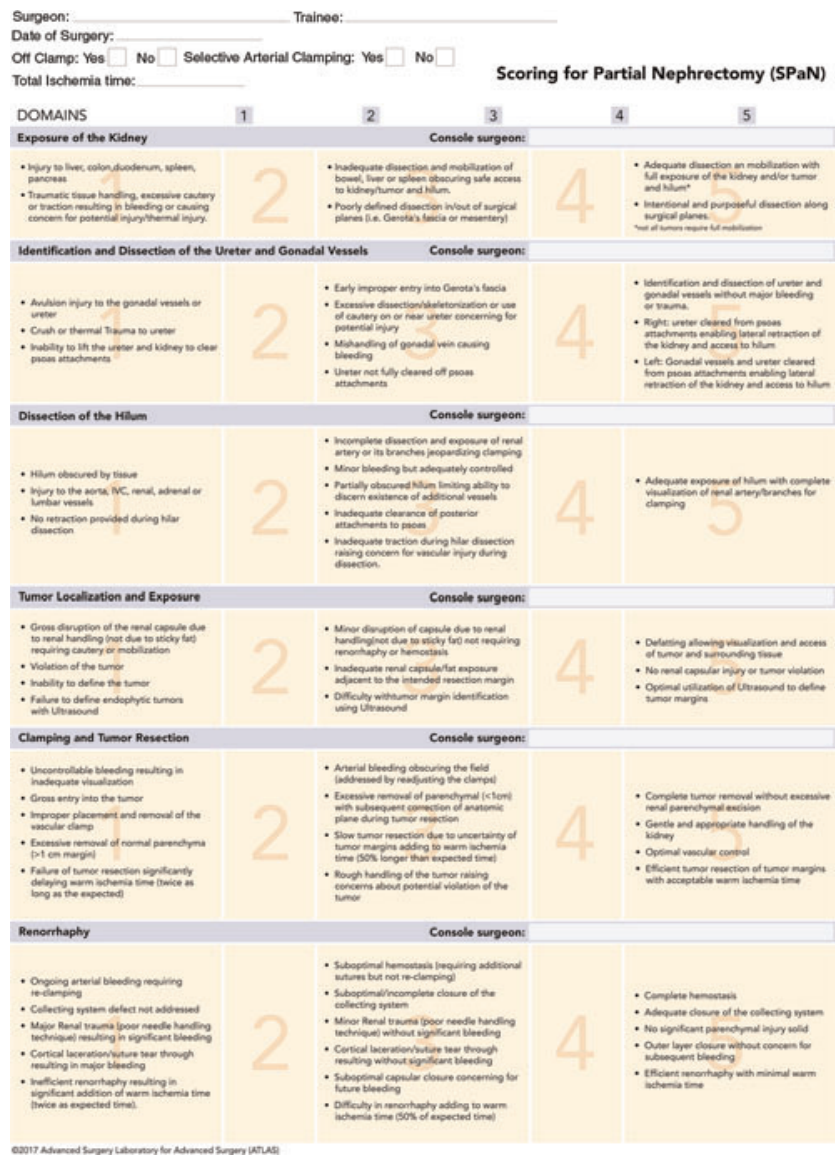

experts were blinded to each other's assessment to minimize "bandwagon" effect.

Results: The expert panel reached consensus after 2 rounds on all aspects. Domain 7 for ischemia time was combined with renorrhaphy based on the panel consensus. CVI $\geq 0.75$ was achieved in $36(67 \%)$ statements in the first round while the consensus for the remaining 18 statements was achieved after the second round. The final six domains of SPaN are: Exposure of the Kidney, Identification and Dissection of the Ureter and Gonadal vessels, Dissection of the Hilum, Tumor Localization and Exposure, Clamping and Tumor Resection, and Renorrhaphy (Figure 1).

Conclusions: We developed and validated a scoring system for minimally invasive partial nephrectomy that can provide structured and objective feedback for assessment of technical skills during minimally invasive partial nephrectomy.

MP22-11 Impact of Positive Surgical Margins on Overall Survival after Partial Nephrectomy, a Matched Comparison Based on the National Cancer Database

C Shum, CD Bahler, CP Sundaram

Khoo Teck Puat Hospital

Singapore

Introduction \& Objective: The impact of positive surgical margins (PSM) in partial nephrectomy (PN) has been a 
controversy. Studies looking at the relationship between PSM and overall survival (OS) are either underpowered or with highly unmatched groups, making it difficult to draw clinically relevant conclusions. We used the National Cancer Database (NCDB) to compare OS between patients with and without PSM, with propensity score matching to avoid confounding by other key covariates.
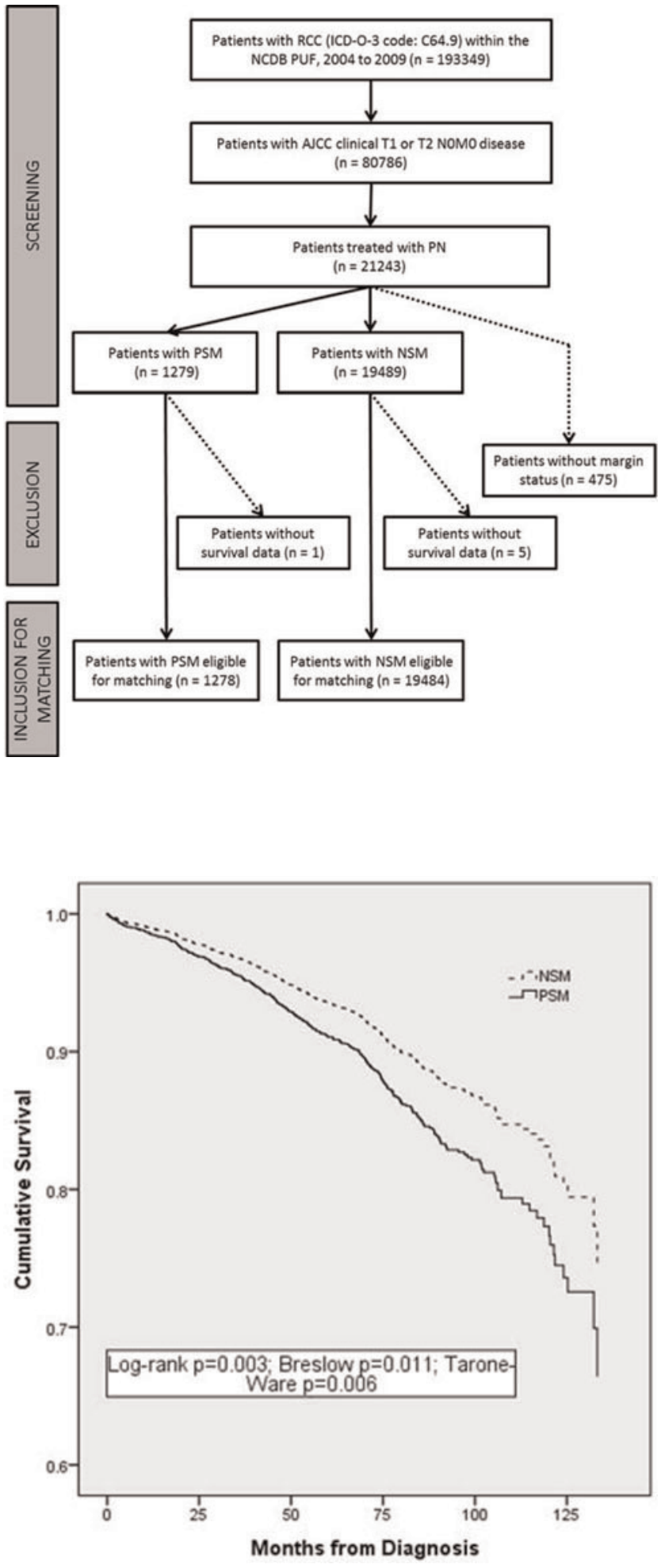

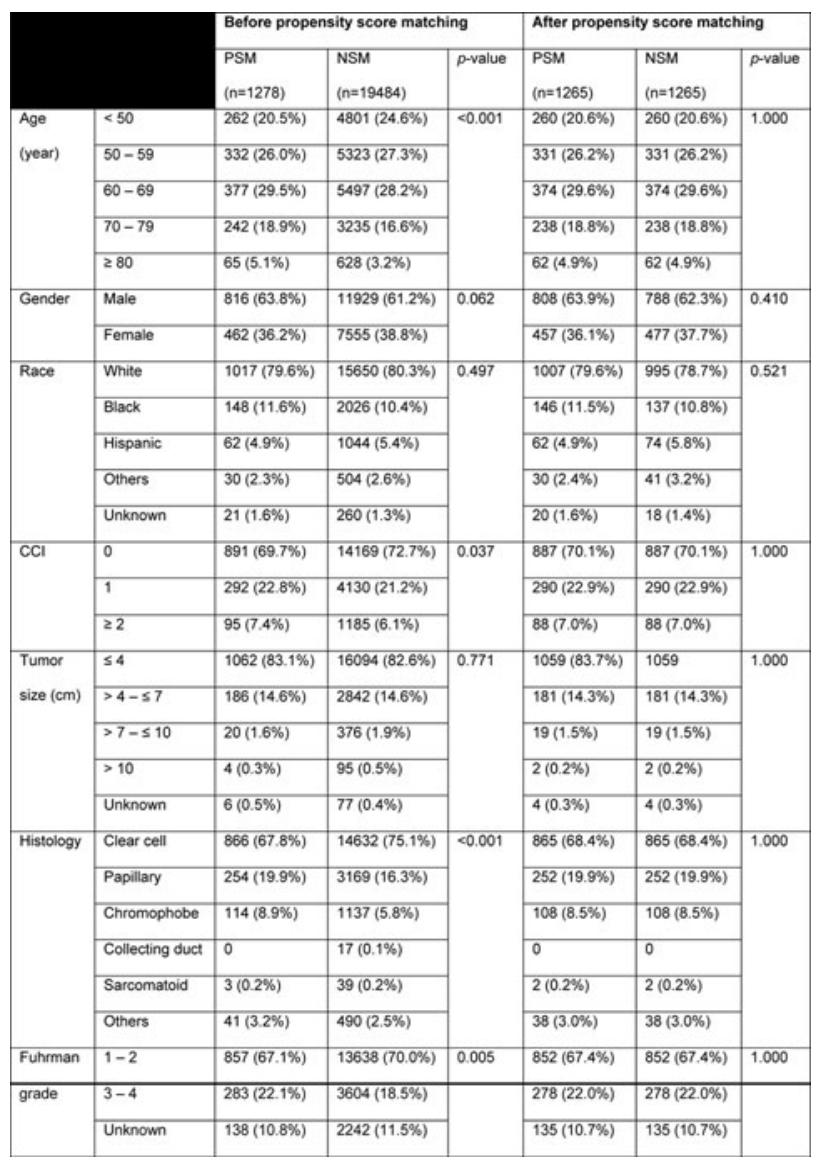

\begin{tabular}{|c|c|c|c|c|}
\hline & HR for mortality & $95 \% \mathrm{Cl}$ & $p$-value \\
\hline \multirow[t]{2}{*}{ Margin status } & NSM & 1.0 (Ref) & & \\
\hline & PSM & 1.393 & $1.138-1.705$ & 0.001 \\
\hline \multirow[t]{5}{*}{ Age (year) } & $<50$ & 1.0 (Ref) & & \\
\hline & $50-59$ & 2.341 & $1.422-3.854$ & 0.001 \\
\hline & $60-69$ & 4.027 & $2.525-6.423$ & $<0.001$ \\
\hline & $70-79$ & 6.721 & $4.214-10.720$ & $<0.001$ \\
\hline & $\geq 80$ & 13.880 & $8.359-23.048$ & $<0.001$ \\
\hline \multirow[t]{2}{*}{ Gender } & Male & 1.0 (Ref) & & \\
\hline & Female & 0.839 & $0.675-1.044$ & 0.116 \\
\hline \multirow[t]{5}{*}{ Race } & White & 1.0 (Ref) & & \\
\hline & Black & 1.176 & $0.842-1.641$ & 0.341 \\
\hline & Hispanic & 1.095 & $0.678-1.769$ & 0.710 \\
\hline & Others & 0.888 & $0.454-1.739$ & 0.729 \\
\hline & Unknown & 0.567 & $0.181-1.771$ & 0.329 \\
\hline \multirow[t]{3}{*}{$\mathrm{CCl}$} & 0 & 1.0 (Ref) & & \\
\hline & 1 & 1.165 & $0.917-1.480$ & 0.211 \\
\hline & 22 & 2.384 & $1.766-3.218$ & $<0.001$ \\
\hline \multirow[t]{5}{*}{ Tumor size $(\mathrm{cm})$} & $\leq 4$ & 1.0 (Ref) & & \\
\hline & $>4-\leq 7$ & 1.592 & $1.237-2.049$ & $<0.001$ \\
\hline & $>7-\leq 10$ & 2.639 & $1.396-4.990$ & 0.003 \\
\hline & $>10$ & 3.548 & $0.490-25.710$ & 0.210 \\
\hline & Unknown & 0.000 & $0-1.008 \times 10^{9}$ & 0.931 \\
\hline \multirow[t]{5}{*}{ Histology } & Clear cell & 1.0 (Ref) & & \\
\hline & \begin{tabular}{|l} 
Papillary \\
\end{tabular} & 0.719 & $0.544-0.949$ & 0.020 \\
\hline & Chromophobe & 0.341 & $0.200-0.580$ & $<0.001$ \\
\hline & Sarcomatoid & 1.724 & $0.238-12.473$ & 0.590 \\
\hline & Others & 0.648 & $0.332-1.264$ & 0.203 \\
\hline \multirow[t]{3}{*}{ Fuhrman grade } & $1-2$ & 1.0 (Ref) & & \\
\hline & $3-4$ & 1.050 & $0.822-1.342$ & 0.694 \\
\hline & Unknown & 1.515 & $1.095-2.097$ & 0.012 \\
\hline
\end{tabular}


Materials and Methods: We identified patients with T1/ T2N0M0 renal cancer treated with PN between 2004 and 2009. These patients were divided into 2 groups based on the presence or absence of PSM. Propensity score matching was used to produce 2 groups similar in age, comorbidity score (CCI), tumor size, histology and grade. Covariates between groups were compared by $\chi^{2}$ test. Cox multiple regression was used to estimate the hazard ratios (HR) of covariates for all-cause mortality. Log-rank, Breslow and Tarone-Ware tests were used to compare OS between matched groups.

Results: After excluding those with missing data on margin status and survival, 1278 patients with PSM and 19484 patients without PSM were eligible for statistical matching, resulting in 2 groups with 1265 patients each, highly similar in age, gender, race, CCI, tumor size, histology and grade (Figure 1 and Table 1). The mean follow-up duration was 70.3 months (median: 72.6 months), with 386 recorded all-cause mortality. Cox multiple regression showed that PSM was associated with a higher risk of all-cause mortality compared to no PSM (HR: 1.393, $\mathrm{p}=0.001$ ). Old age, high CCI and large tumor size were also associated with higher risks, while papillary and chromophobe histology was associated with lower risks (Table 2). At the means of all other covariates, PSM was associated with significantly worse OS, by log-rank, Breslow and Tarone-Ware tests (Figure 2). To the best of our knowledge, our study is the largest matched comparison to determine the impact of PSM on OS after PN.

Conclusions: PSM is associated with significantly worse OS after PN. Every effort should be made to avoid PSM in PN, amidst the various reports that PSM does not affect tumor recurrence and cancer-specific survival.

MP22-12 Comparison of Oncologic Results, Functional Outcomes and Complications after Partial Nephrectomy versus Percutaneous Radiofrequency Ablation in Small sized ( $4 \mathrm{~cm}$ or less) Bosniak III or IV Cystic Renal lesions

W Song, Y Choi, H Sung, H Jeon, B Jeong, S Seo, S Jeon, H Choi, H Lee

samsung medical center

Republic of Korea

Introduction \& Objective: We evaluated the oncologic results, functional outcomes and complications after partial nephrectomy (PN) or percutaneous radiofrequency ablation (RFA) for the treatment of small sized ( $4 \mathrm{~cm}$ or less) Bosniak III or IV cystic renal lesions.

Materials and Methods: We retrospectively reviewed medical records of 135 patients who underwent PN (99) or RFA (36) for small sized ( $4 \mathrm{~cm}$ or less) Bosniak III or IV cystic renal lesions between January 2009 and December 2014. After excluding patients with hereditary cystic disease or less than 12 months of follow-up, 128 (PN, 97; RFA, 31) patients remained for analysis. Pathologic characteristics, tumor violation during surgery, residual tumor, local recurrence and distant metastasis data were collected. Glomerular filtration rate (GFR) was calculated using the Chronic Kidney Disease Epidemiology Collaboration (CKDEPI) equations and checked preoperatively, 1 month, 6 months, and 12 months postoperatively. All complications were graded by Clavien classification system.

Results: The median size of Bosniak III or IV cystic renal lesions was $2.6 \mathrm{~cm}$. Renal cell carcinoma (RCC) accounted for $86.6 \%$ (84/97) of patients in PN group. In RFA group, histologic sub- type was identified in $32.3 \%$ (10/31) of patients, but $90.0 \%(9 / 10)$ of patients revealed RCC. One case of tumor violation occurred in PN group and 2 cases of residual cancer were observed in RFA group. But there were no local recurrence or distance metastasis in both groups during the median follow-up of 34.0 months. Compared with PN group, patients in the RFA group showed a small decrease of percent change of CKD-EPI GFR at 1 month $(-13.6 \%$ vs $-6.8 \%, \mathrm{p}=0.039)$. Perioperative complication rate in PN group was $29.9 \%$ and $22.6 \%$ in RFA group. According to Clavien classification system, Grade IIIa complications rate was $4.1 \%$ in PN group and $6.5 \%$ in RFA group. There were no grade IIIb and IV complications.

Conclusions: The results of our study indicated that percutaneous RFA showed comparable oncologic results and complications and better preservation of renal functions than PN.

MP22-13 Utilization of Renal Biopsy for a Small Renal Mass: A Survey Among American Urologists

RM Patel, S Safiullah, KS Kaler, J Landman, RV Clayman

Department of Urology, University of California, Irvine,

Orange, CA

United States

Introduction \& Objective: Given the limited information regarding the utilization of renal mass biopsy for small renal masses (SRM), we surveyed United States of America (U.S.A.)based urologists to characterize practice patterns and indications to perform a biopsy.

Materials and Methods: Members of the American Urological Association who practice in the U.S.A. were invited to participate in an 11 question web-based survey that was distributed via SurveyMonkey ${ }^{\circledR}$ from December 2016 to January 2017.

Results: A total of 1,019 respondents completed the survey. The respondents equally represented all regions of the U.S.A and most were in private practice (Table 1). Overall, $31.8 \%$ of American urologists would never perform biopsy of a renal mass $\leq 4 \mathrm{~cm}$. Urologists who practiced at an academic hospital were more likely to perform a renal biopsy on both a renal mass $\leq 4 \mathrm{~cm}$ and a renal mass $2-3 \mathrm{~cm}$ compared to private practice and government-based urologists ( $p<0.001$ and $p=0.008$ respectively). Those who saw fewer than 5 small renal masses per year were more likely to never perform a renal biopsy on both a renal mass $\leq 4 \mathrm{~cm}$ and a renal mass $2-3 \mathrm{~cm}$ compared to those who saw more than 5 per year $(\mathrm{p}<0.001)$ (Table 2$)$. Respondents were asked to

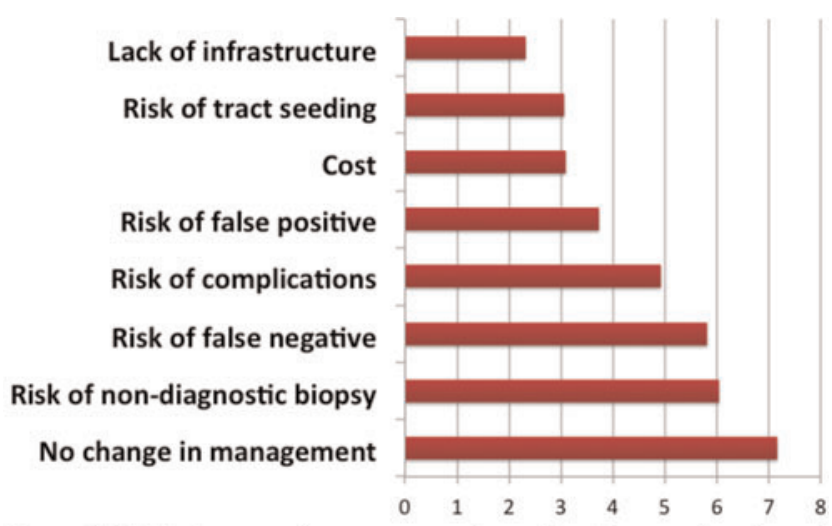

Figure 1: Weighted average for reasons renal mass biopsies are not performed. 


\begin{tabular}{|c|c|c|}
\hline & & Total (\%) \\
\hline \multirow{4}{*}{ Profession } & Urologist & 98.8 \\
\hline & Urology Resident & 0.5 \\
\hline & $\begin{array}{l}\text { Physician } \\
\text { Assistant/Nurse } \\
\text { Practitioner }\end{array}$ & 0.3 \\
\hline & Other & 0.4 \\
\hline \multirow{4}{*}{ Region } & Eastern & 29.5 \\
\hline & Midwestern & 23.4 \\
\hline & Southern & 23.7 \\
\hline & Western & 23.3 \\
\hline \multirow{4}{*}{ Practice Type } & Academic Hospital & 25.3 \\
\hline & Private Hospital & 63.6 \\
\hline & $\begin{array}{l}\text { Veteran's Administration } \\
\text { Hospital (VA) }\end{array}$ & 2.8 \\
\hline & Other & 8.3 \\
\hline \multirow{5}{*}{ Hospital Size } & 0-99 Beds & 7.1 \\
\hline & $100-199$ & 16.1 \\
\hline & $200-299$ & 26.0 \\
\hline & $300-399$ & 18.2 \\
\hline & $400+$ & 32.6 \\
\hline \multirow{4}{*}{$\begin{array}{l}\text { Annual Number of Patients } \\
\text { Treated with a Renal Mass }\end{array}$} & $0-5$ & 10.3 \\
\hline & $5-10$ & 17.0 \\
\hline & $10-15$ & 25.5 \\
\hline & $20+$ & 47.2 \\
\hline
\end{tabular}

Table 1: Demographics of survey respondents

\begin{tabular}{|c|c|c|c|c|}
\hline \multicolumn{5}{|c|}{ Renal mass $\leq 4 \mathrm{~cm}$} \\
\hline & & Never biopsy & $\begin{array}{c}\text { Consider } \\
\text { biopsy }\end{array}$ & $P$ value \\
\hline \multirow[b]{2}{*}{ Practice Type } & Academic (\%) & $19.3 \%$ & $80.7 \%$ & \multirow[b]{2}{*}{$<0.001$} \\
\hline & $\begin{array}{c}\text { Private and } \\
\text { VA (\%) }\end{array}$ & $36.1 \%$ & $63.9 \%$ & \\
\hline \multirow{4}{*}{$\begin{array}{l}\text { \# of patients } \\
\text { seen annually }\end{array}$} & $0-5$ & $57.8 \%$ & $42.2 \%$ & \multirow{4}{*}{$<0.001$} \\
\hline & $5-10$ & $39.5 \%$ & $60.5 \%$ & \\
\hline & $10-15$ & $27.6 \%$ & $72.4 \%$ & \\
\hline & $20+$ & $25.4 \%$ & $74.6 \%$ & \\
\hline \multicolumn{5}{|c|}{ Renal mass $2-3 \mathrm{~cm}$} \\
\hline \multirow[b]{2}{*}{ Practice Type } & Academic (\%) & $28.1 \%$ & $71.9 \%$ & \multirow[b]{2}{*}{0.008} \\
\hline & $\begin{array}{c}\text { Private and } \\
\text { VA (\%) }\end{array}$ & $37.3 \%$ & $62.7 \%$ & \\
\hline \multirow{4}{*}{$\begin{array}{l}\text { \# of patients } \\
\text { seen annually }\end{array}$} & $0-5$ & $55.4 \%$ & $44.6 \%$ & \multirow{4}{*}{$<0.001$} \\
\hline & $5-10$ & $38.0 \%$ & $62.0 \%$ & \\
\hline & $10-15$ & $33.1 \%$ & $66.9 \%$ & \\
\hline & $20+$ & $30.2 \%$ & $69.8 \%$ & \\
\hline
\end{tabular}

Table 2: Frequency of performing biopsy in a small renal mass $\leq 4 \mathrm{~cm}$ and $2-3 \mathrm{~cm}$ based on practice type and number of patients with a renal mass treated annually

rank reasons to not perform a biopsy and the weighted average ranking of each choice is displayed in Figure 1, with most urologists responding that it would not change their management $(\mathrm{p}<0.05)$. Respondents reported that the urologist performs only $2 \%$ of biopsies; however, almost half of the urologists surveyed $(48.3 \%)$ would be interested in learning office-based ultrasoundguided biopsy of a SRM.

Conclusions: In the U.S.A., non-academic urologists and those that see less than 5 cases a year infrequently biopsy a SRM. Currently, interventional radiologists perform almost all SRM biopsies.

MP22-14 Impact of blood transfusions on oncological outcomes of surgically treated localized renal cell carcinoma

M Tsivian, E Tsivian, C Sze, A Schulman, M Abern, E Rampersaud, T Polascik

Duke University Medical Center United States
Introduction \& Objective: Transfusion-induced immunomodulation has been shown to negatively impact the oncological outcomes in several malignancies. However, it is unknown whether there is a clinically significant impact on oncological outcomes in patients with renal cell carcinoma (RCC). In this regard, we set out to assess for associations between perioperative allogeneic blood transfusions (ABTs), overall and cancer-specific survival, and recurrence in patients undergoing surgical treatment for clinically localized RCC.

Materials and Methods: A single center retrospective review of 1056 consecutive patients undergoing surgical treatment (radical or partial nephrectomy) for clinically localized RCC between 2000-2010 was undertaken. The outcome measures were distant and local recurrence-free survival, overall survival, and RCCspecific survival. Kaplan-Meier survival curves were generated. Multivariable proportional regression models adjusted for clinical and pathological characteristics.

Results: On multivariable analyses adjusted for clinical and pathological characteristics, the receipt of ABTs was associated with lower recurrence-free $(\mathrm{HR}=1.86, \mathrm{p}=0.002)$, overall $(\mathrm{HR}=1.83$, $\mathrm{p}=0.016)$ and $\mathrm{RCC}$-specific survival $(\mathrm{HR}=2.12, \mathrm{p}=0.031)$. The negative impact of ABTs was apparent for distant $(\mathrm{HR}=2.24$, $\mathrm{p}<0.001)$ but not local recurrences $(\mathrm{HR}=0.78, \mathrm{p}=0.643)$.

Conclusions: In this study, perioperative ABTs were independently associated with worse oncological outcomes in patients with clinically localized RCC. Receipt of ABT was associated with roughly a 2 -fold increase in the hazard of metastatic progression, all-cause and RCC-specific mortality. Further research is needed on the mechanisms of transfusion-induced immunomodulation, alternative transfusion protocols, and methods for autologous blood transfusion and recovery.

\section{MP22-15 Frozen Sections for Margins During Partial Nephrectomy Do Not Influence Recurrence Rates}

\author{
J Dagenais, P Mouracade, MJ Maurice, O Kara, R Nelson, \\ JS Chavali, J Kaouk \\ United States
}

Introduction \& Objective: Frozen sections are routinely employed to assess margin status during partial nephrectomy for clinically localized renal cell carcinoma; however, their oncologic benefit remains unclear. We wished to determine whether the utilization of frozen sections for this purpose during partial nephrectomy influenced recurrence rates.

Materials and Methods: Retrospective review of 1,090 patients with $(n=172)$ and without $(n=918)$ frozen section during open and robotic partial nephrectomy between 2006 and 2016 for clinically localized renal cell carcinoma at a single institution. Standard follow-up protocols were employed, with imaging used to guide subsequent biopsy for confirmation. Frozen status and patient clinicodemographic and pathologic characteristics were correlated with recurrence using univariate and multivariate competing risk regression analyses with adjustment for all-cause mortality and baseline characteristics. Administrative data was reviewed to calculate costs of frozen sections.

Results: After a median follow-up of 24.8 months, 45/1090 $(4.13 \%)$ patients had recurred. There was no difference in the cumulative incidence of recurrence between patients with and without frozen sections (figure 1; $\chi^{2}=0.001, \mathrm{p}=0.97$ ). On multivariate competing risk analysis, frozen section was not associated with recurrence (table 1; hazard ratio, 1.56; 95\% CI, 0.65-3.76). However, tumor grade (g3-4 vs.1-2: hazard ratio, 


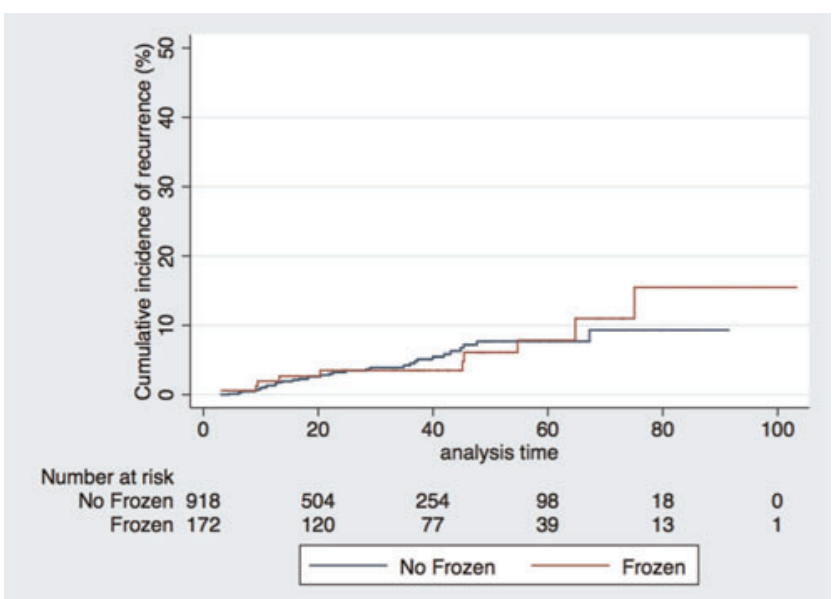

Figure 1: Competing risk models depicting cumulative incidence functions over time for frozen section and no-frozen section cohorts

Table 1: Univariate and multivariate predictors of recurrence

\begin{tabular}{|c|c|c|c|c|c|c|}
\hline \multirow[b]{2}{*}{ Variable } & \multicolumn{3}{|c|}{ Univariate } & \multicolumn{3}{|c|}{ Multivariate } \\
\hline & $\begin{array}{l}\text { Hazard } \\
\text { Ratio }^{a} \\
\text { (HR) }\end{array}$ & $95 \% \mathrm{Cl}$ & $\begin{array}{c}P \\
\text { value }\end{array}$ & $\begin{array}{c}\text { Hazard } \\
\text { Ratio }^{\circ} \\
\text { (HR) }\end{array}$ & $95 \% \mathrm{Cl}$ & $\begin{array}{c}\mathrm{P} \\
\text { value }\end{array}$ \\
\hline Frozen Section & 1.11 & $0.56-2.22$ & 0.76 & 1.56 & $0.65-3.76$ & 0.32 \\
\hline $\begin{array}{l}\text { Age Category } \\
<55 \\
55-69 \\
70+\end{array}$ & $\begin{array}{l}\text { Ref } \\
1.83 \\
1.62\end{array}$ & $\begin{array}{c}- \\
0.86-3.89 \\
0.64-4.10\end{array}$ & $\begin{array}{l}- \\
0.11 \\
0.31\end{array}$ & $\begin{array}{l}\text { Ref } \\
1.27 \\
0.96\end{array}$ & $\begin{array}{c}- \\
0.50-3.21 \\
0.30-3.10\end{array}$ & $\begin{array}{l}- \\
0.62 \\
0.95\end{array}$ \\
\hline Sex (Male) & 1.74 & $0.88-3.43$ & 0.11 & 1.13 & $0.45-2.80$ & 0.80 \\
\hline Race (White) & 3.56 & $0.87-14.6$ & 0.08 & 2.75 & $0.60-12.5$ & 0.19 \\
\hline $\begin{array}{l}\mathrm{CCl}^{\mathrm{C}} \text { Category } \\
0 \\
1-2 \\
3+\end{array}$ & $\begin{array}{l}\text { Ref } \\
1.45 \\
2.71\end{array}$ & $\begin{array}{c}- \\
0.72-2.93 \\
1.29-5.68\end{array}$ & $\begin{array}{c}- \\
0.30 \\
0.009\end{array}$ & $\begin{array}{l}\text { Ref } \\
1.95 \\
2.81\end{array}$ & $\begin{array}{c}- \\
0.80-4.72 \\
0.96-8.20\end{array}$ & $\begin{array}{l}- \\
0.14 \\
0.06\end{array}$ \\
\hline $\begin{array}{l}\text { BMI }{ }^{\text {d }} \text { Category, } \mathrm{kg} / \mathrm{m} \\
<25 \\
25-29 \\
30-34 \\
35+\end{array}$ & $\begin{array}{l}\text { Ref } \\
1.32 \\
0.70 \\
0.77\end{array}$ & $\begin{array}{c}0.59-2.96 \\
0.26-1.87 \\
0.28-2.11\end{array}$ & $\begin{array}{l}0.50 \\
0.48 \\
0.61\end{array}$ & $\begin{array}{l}\text { Ref } \\
1.44 \\
0.85 \\
0.74\end{array}$ & $\begin{array}{c}- \\
0.53-3.90 \\
0.30-3.12 \\
0.23-2.36\end{array}$ & $\begin{array}{l}- \\
0.47 \\
0.81 \\
0.61\end{array}$ \\
\hline Approach (Robotic) & 0.93 & $0.49-1.78$ & 0.83 & 1.26 & $0.56-2.81$ & 0.58 \\
\hline $\begin{array}{l}\text { R.E.N.A.L. Category } \\
\text { Low }(4-6) \\
\text { Moderate }(7-9) \\
\text { High }(10+)\end{array}$ & $\begin{array}{l}\text { Ref } \\
1.85 \\
2.34\end{array}$ & $\begin{array}{c}- \\
0.89-3.85 \\
0.95-5.77\end{array}$ & $\begin{array}{l}0.10 \\
0.06\end{array}$ & $\begin{array}{l}\text { Ref } \\
1.93 \\
1.59\end{array}$ & $\begin{array}{c}- \\
0.89-4.19 \\
0.50-5.05\end{array}$ & $\begin{array}{l}0.09 \\
0.43\end{array}$ \\
\hline Tumor Size, cm & 1.24 & $1.12-1.38$ & $<0.001$ & 1.11 & $0.92-1.34$ & 0.26 \\
\hline $\begin{array}{l}\text { Tumor Grade } \\
1-2 \\
3-4\end{array}$ & $\begin{array}{l}\text { Ref } \\
3.34\end{array}$ & 1.81-6.15 & $<0.001$ & $\begin{array}{l}\text { Ref } \\
2.45\end{array}$ & $\stackrel{-}{1.16-5.14}$ & 0.02 \\
\hline $\begin{array}{l}\text { Tumor Stage } \\
\text { pT1a } \\
\text { pT1b } \\
\text { pT2+ }\end{array}$ & $\begin{array}{l}\text { Ref } \\
1.83 \\
5.63\end{array}$ & $\begin{array}{c}- \\
0.85-3.94 \\
2.92-10.8\end{array}$ & $\begin{array}{c}- \\
0.13 \\
<0.001\end{array}$ & $\begin{array}{l}\text { Ref } \\
1.02 \\
2.86\end{array}$ & $\begin{array}{c}- \\
0.35-2.94 \\
1.13-7.26\end{array}$ & $\begin{array}{l}- \\
0.97 \\
\mathbf{0 . 0 3}\end{array}$ \\
\hline $\begin{array}{l}\text { "Subdistribution haza } \\
\text { with adjustment for all } \\
\text { bubdistribution haza } \\
\text { with adjustment for all- } \\
\text { 'Charlson comorbidity } \\
\text { dBody mass index }\end{array}$ & $\begin{array}{l}\text { tio, esti } \\
\text { se mor } \\
\text { io, esti } \\
\text { se mort }\end{array}$ & $\begin{array}{l}\text { hated using } \\
\text { lity } \\
\text { lated using } \\
\text { lity and bas }\end{array}$ & $\begin{array}{l}\text { Fine and } \\
\text { Fine and } \\
\text { eline pre }\end{array}$ & $\begin{array}{l}\text { ay con } \\
\text { ay con } \\
\text { tors }\end{array}$ & eting risk re & gression \\
\hline
\end{tabular}

2.45; 95\% CI, 1.16-5.14) and stage ( $\geq \mathrm{pT} 2$ vs. pT1a: hazard ratio, $2.86 ; 95 \% \mathrm{CI}, 1.13-7.26)$ were associated with recurrence. The average direct charge per patient undergoing frozen section was $\$ 902$.

Conclusions: Intraoperative frozen section for margins during partial nephrectomy did not predict decreased recurrence rates in a single-institution high volume center. Given the lack of associated benefit, and the added cost, the utilization of frozen section during partial nephrectomy should be limited.

\section{MP22-16 Avoiding Acute Kidney Injury: When to Employ Cold Ischemia}

J Dagenais, MJ Maurice, P Mouracade, O Kara, J Li, R Nelson, E Malkoc, J Kaouk

\section{United States}

Introduction \& Objective: Cold ischemia can optimize renoprotection during extended warm ischemia; however, there is some debate as to exactly when it is beneficial. We thus wished to investigate the relationship between ischemia type (cold vs. warm) and post-operative renal function and obtain standardized estimates of the precise ischemic duration at which point cold ischemia leads to a decreased predicted probability of acute kidney injury (AKI).

Materials and Methods: We retrospectively reviewed 1,354 patients with bilateral kidneys in our single-institution robotic and open PN database. AKI, the primary outcome, was defined as a change in preoperative GFR $>25 \%$ within 72 hours of surgery, or stage 1 of the RIFLE criteria. Using a multivariate logistic regression, the interaction effect of ischemia type (cold vs. warm) and time (continuous) on rates of AKI was estimated. Unadjusted and average adjusted predictions at representative values were calculated, enabling generation of interaction curves. Properties of the curves including their intersection point, partial derivative, and separation point were measured.

Results: There was a significant interaction effect of ischemia type and time on predicted rates of $\mathrm{AKI}$ on univariate $(\mathrm{OR}=1.06$, $\mathrm{p}<0.001)$ and multivariate analyses $(\mathrm{OR}=1.05, \mathrm{p}<0.001)$. Cold ischemia began to predict a decreased risk of AKI at 25.5 minutes, which became statistically significant at 37.1 minutes (Figure 1).

Conclusions: Predicted rates of AKI after PN are highly influenced by the interaction effect of ischemia type and time. After equilibrating warm and cold ischemia groups, we have shown that cold ischemia predicts lower rates of AKI beginning at 25.5 minutes. Strong consideration should be given to employing cold ischemia when clamp time is expected to exceed this threshold.

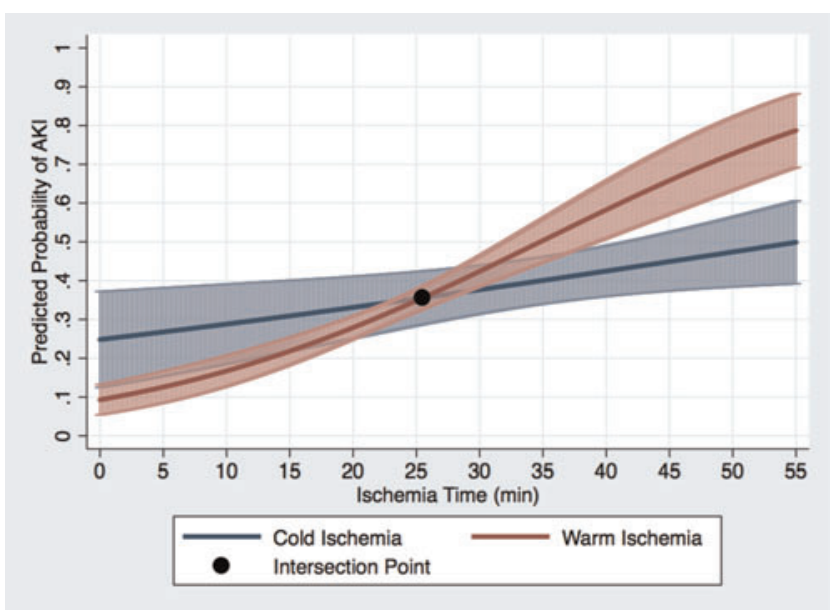

Figure 1: Adjusted predicted probabilities of acute kidney injury (AKI)

${ }^{a}$ Curves represent the average adjusted predicted probabilities of AKI for each ischemia type at representative values of ischemia time ( $6 \mathrm{sec}$ intervals). Model controls for baseline covariates using their population-averaged values. Included covariates: age, sex, race, charlson comorbidity index, BMI, preoperative GFR, R.E.N.A.L. score, maximum tumor diameter, clavien complication grade, and excisional volume loss (log units)

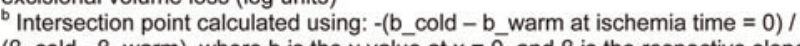
$\left(\beta \_\right.$cold $-\beta \_$warm), where $b$ is the $y$ value at $x=0$, and $\beta$ is the respective slope for each curve. 
MP22-17 How Much is Too Much? Exploring the Relationship between Volume Loss, Oncologic Outcomes, and Renal Dysfunction after Partial Nephrectomy

J Dagenais, P Mouracade, O Kara, JS Chavali, MJ Maurice, J Kaouk

United States

Introduction \& Objective: Limiting excisional volume loss (EVL) during partial nephrectomy $(\mathrm{PN})$ is central to nephron preservation; however, it may come at the cost of worsening oncologic outcomes. Our goal was to investigate this potential tradeoff by examining 10 years of prospectively collected data with greater than 500 patients with long-term outcomes after open or robotic PN. Specifically, we aimed to assess how short- and long-term renal function, surgical margin status, and recurrence rates change as a function of volume loss during PN.

Materials and Methods: We retrospectively reviewed our single-institution PN database to identify patients with clinically localized renal cell carcinoma who underwent open or robotic PN. AKI was defined as a change in preoperative GFR $>25 \%$ within 72 hours of surgery. CKD upstaging was defined as an increase in CKD stage at 3-12 months. Recurrence analyses excluded patients without postoperative cross-sectional imaging. EVL was calculated as the difference between pathologic specimen and tumor volume. Separate multivariate logistic regressions were run to examine the influence of EVL on the outcomes of AKI, CKD upstaging and margin status. A multivariate cox regression was run to examine the influence of EVL on recurrence. Average adjusted predictions at representative values of EVL were determined for graphical representation.

Results: $36.2 \%$ of patients had AKI, 32.9\% had CKD upstaging, $6.3 \%$ had positive margins, and $4.6 \%$ recurred with a median follow-up of 24.8 months [IQR 11.2-44.5] postoperatively. On multivariate analyses, increasing EVL (log units) was associated with significantly increased rates of AKI (OR 1.34, [95\% CI, 1.13-1.58]) and CKD upstaging (OR 1.37 [95\% CI, 1.11-1.69]), and decreased rates of positive margins (OR 0.65 [95\% CI, 0.490.86]), with no association with recurrence (OR 1.31 [95\% CI, 0.95-1.84]) (Figure). Margin status did not independently predict recurrence (OR 2.22 [95\% CI, 0.82-5.98].

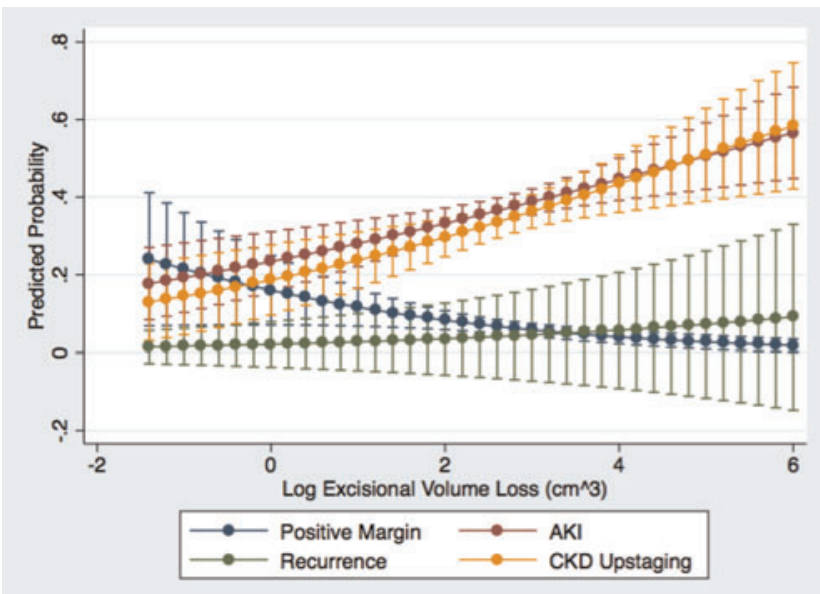

"Each curve represents the average adjusted predicted probability of a given outcome (from 0-1) at representative values of volume loss, while controlling for baseline covariates, using either a multivariate logistic regression (AKI, CKD, positive margin) or multivariate cox regression (recurrence).
Conclusions: This is the first study to our knowledge examining the possible tradeoff between functional and oncologic outcomes after PN. In our high-risk population, very low excisional volumes helped preserve optimal short and long-term renal function with higher positive margins but no effect on recurrence rates during a median follow-up of 25 months. Given the lack of independent influence of margin status on recurrence, minimizing volume loss in PN appears judicious.

\section{MP22-18 Current Trends in Cytoreductive Nephrectomy in the Era of Targeted Molecular Therapy}

Y Phan, S Segaran, J Bell, SY Nakada, A Rane

Royal Shrewsbury Hospital

United Kingdom

Introduction \& Objective: Several factors are considered when determining if a patient is a candidate for cytoreductive nephrectomy $(\mathrm{CN})$. Our aim was to study geographic trends and factors associated with the decision to consider $\mathrm{CN}$.

Materials and Methods: An investigator designed survey was created to assess the rate of $\mathrm{CN}$ being performed around the world and the factors considered when determining patient eligibility. This was distributed to attendees at the World Congress of Endourology 2016 in Cape Town in order to capture an international cohort of urologists. The survey included questions about physician demographics, the timing of $\mathrm{CN}$, what patient factors were considered prior performing $\mathrm{CN}$, and if they followed any guidelines when deciding on $\mathrm{CN}$.

Results: 158 urologists responded to our survey (Asia $=46$, Europe $=35$, Africa $=34$, North America $=29$, South America $=$ 13 and Australia =1). $78(49.4 \%)$ urologists indicated that they follow guidelines for recommending CN. 107 (67.7\%) of respondents stated they perform $\mathrm{CN}$. 64 urologists perform $\mathrm{CN}$ prior to systemic therapy while 20 urologists perform $\mathrm{CN}$ after systemic treatment, and 22 urologists perform $\mathrm{CN}$ before or after systemic treatment. Performance status was the most considered factor while calcium level was the least considered factor when determining eligibility for $\mathrm{CN}$.

Conclusions: This cohort of urologists most commonly consider performance status, age and extent of metastatic disease when determining candidacy for $\mathrm{CN}$; while the grade of the tumour and the calcium level were the least considered. We eagerly await the results of CARMENA and SURTIME trials.

\section{MP22-19 Withdrawn}

MP22-20 Predicting risk of pathological upstaging of clinical $\mathrm{T} 1$ renal masses in the era of renal mass biopsy

ZL Smith, S Johnson, S Eggener

The University of Chicago

United States

Introduction \& Objective: With the growing utilization of renal mass biopsy (RMB), more information is known about renal tumors prior to intervention. We identified patients with renal cell carcinoma (RCC) who were upstaged from cT1 to pT3-4. We compared their outcomes to non-upstaged patients and identified predictors of upstaging. 


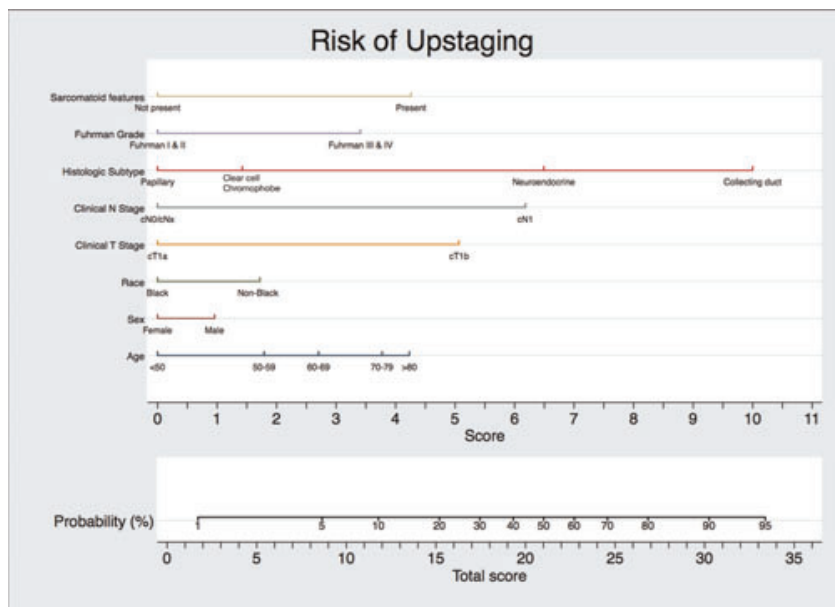

Materials and Methods: Using the National Cancer Database (NCDB) from 2004-2013, we identified patients who underwent partial nephrectomy $(\mathrm{PN})$ or radical nephrectomy $(\mathrm{RN})$ for cT1 RCC. We then compared patients who were upstaged to pT3-4 and to pT1-2 patients. Multivariable logistic regression was used to identify predictors of upstaging and create a predictive nomogram. Cox proportional hazards and Kaplan-Meier methods were used to estimate survival.

Results: A total of 98,936 patients underwent PN or RN for cT1 RCC; 6,409 (6.5\%) were upstaged to pT3-4 (98.4\% pT3, 1.6\% pT4). cT1b masses were upstaged more often than cT1a (11.7\% vs $3.3 \%, \mathrm{p}<0.01)$. On multivariable analysis, age, non-black race, male sex, cT1b, cN1, collecting duct subtype, Fuhrman grade III or $\mathrm{IV}$, and sarcomatoid features were all significant predictors of upstaging (all $\mathrm{p}<0.01)$. These factors were incorporated into a nomogram to assign a predictive score (from 0-35) for risk of pathological upstaging. Upstaged patients were at increased risk of death (HR 2.7, 95\% CI 2.5-2.8), with 5-year overall survival differing significantly $(66.4 \%$ vs $85.4 \%, \mathrm{p}<0.01)$.

Conclusions: Pathological upstaging is rare in cT1 renal masses, but confers a poor prognosis. Multiple risk factors are associated with risk of upstaging, and we have stratified these by their predictive ability. In conjunction with RMB, our nomogram can aid in identifying patients at risk for adverse pathological findings and help stratify their appropriateness for surveillance, treatment, or neoadjuvant trials.

MP22-21 Predictive value of preoperative BMI and total cholesterol in the renal cell carcinoma patients by gender multicenter study

H Jeong, S Kang, H Moon, K Lee, S Choi, Y Park, W Bae, H Cho, U Ha, J Lee, S Kim, S Hong

Department of urology, Seoul St. Mary’s hospital Republic of Korea

Introduction \& Objective: Recently, the effect of preoperative body mass index (BMI) and total cholesterol on prognosis has been reported in patients with RCC but is still controversial and poorly understood. We analyzed the effects of BMI and total cholesterol on the prognosis of RCC.

Materials and Methods: From 1988 to 2015, we retrospectively collected data from 7271 patients surgically treated for
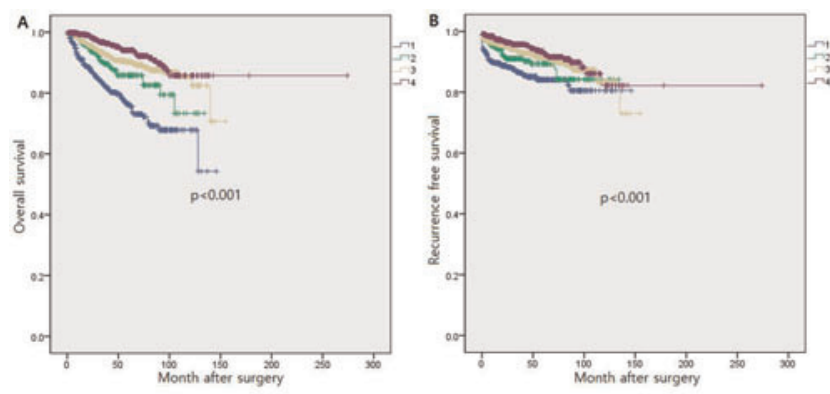

Fig1. Kaplan-Meier survival curves of overall sunvival and recurrence free survival in total groups.

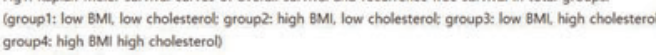

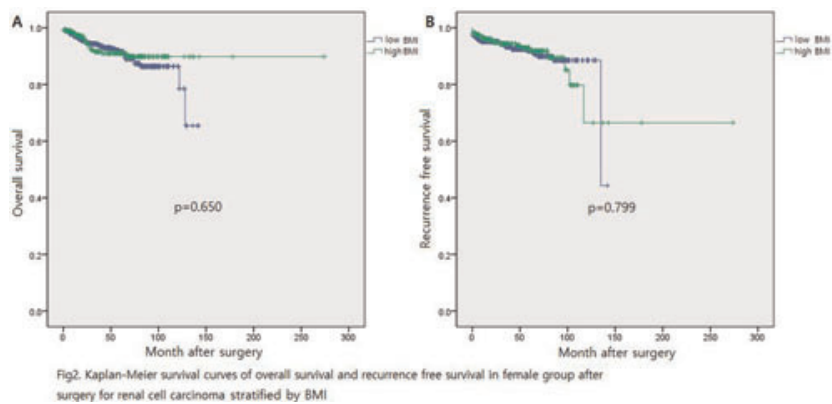

non-metastatic RCC from 8 centers. ROC curve was analyzed to calculate the cut-off value of cholestrol as a predictive factor of RCC. The highest Youden index was shown in 163.5. According to WHO criteria, low BMI and high BMI groups are divided by 25. Kaplan Meyer analysis, Multivariate Cox regression model were performed to identify overall survival (OS), Recurrence free survival (RFS).

Results: Low BMI and low cholesterol group was associated with short OS and RFS $(\mathrm{p}<0.001)$ than any other groups in total cohort. As a subgroup analysis we divided groups by gender. In the male group, the result showed that low BMI and low cholesterol was associated with short OS and RFS similar to total cohort results $(p<0.001)$. But in the female group, the effect of cholesterol on the prognosis was same as male $(\mathrm{p}<0.001)$ but BMI didn't associated with OS and RFS ( $\mathrm{p}=0.650,0.799$ respectively). And on multivariate Cox regression analysis, cholesterol was an independent predictor for OS in both gender groups $(\mathrm{HR}=1.502 \mathrm{p}<0.001, \mathrm{HR}=1.758 \mathrm{p}=0.037$ respectively). However, BMI was a significant prognostic factor in the male group only $(\mathrm{HR}=1.925 \mathrm{p}<0.001)$.

Conclusions: Preoperative BMI and total cholesterol in RCC patients showed statistical significance as OS and RFS predictive factor. But in the subgroup analysis divided by gender, cholesterol was an independent predictor for OS in both groups but BMI had statistical significant only in the male group.

MP22-22 Is There a Role for Nephron-Sparing Cytoreductive Partial Nephrectomy in Renal Cell Carcinoma?

P Heller, L Hockman, L McGuffey, K Murray, N Pokala

University of Missouri

United States 


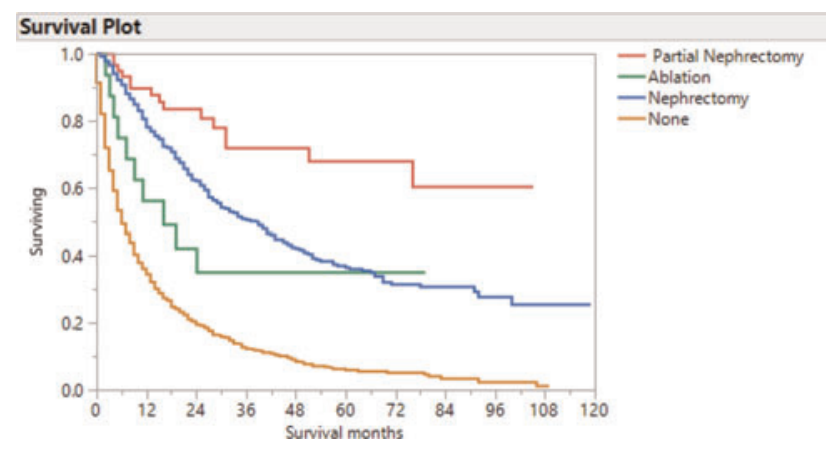

\begin{tabular}{|l|l|l|l|l|l|l|l|l|l|l|}
\hline & \multicolumn{2}{|l|}{$\begin{array}{l}\text { All Patients } \\
\text { (n=18,443) }\end{array}$} & $\begin{array}{l}\text { Partial } \\
\text { nephrectomy } \\
\text { (n=208) }\end{array}$ & \multicolumn{2}{l|}{$\begin{array}{l}\text { Ablation } \\
\text { (n=78) }\end{array}$} & \multicolumn{2}{l|}{$\begin{array}{l}\text { Radical } \\
\text { Nephrectomy } \\
\text { (n=7598) }\end{array}$} & \multicolumn{2}{l}{$\begin{array}{l}\text { No Surgical } \\
\text { Intervention } \\
\text { (n=10,559) }\end{array}$} \\
\cline { 2 - 12 } & OS & CSS & OS & CSS & OS & CSS & OS & CSS & OS & CSS \\
\hline $\begin{array}{l}5 \text {-years } \\
(\%)\end{array}$ & 9.1 & 12.2 & 35.9 & 40.5 & 21.1 & 20.3 & 17.4 & 21 & 2.6 & 4.1 \\
\hline $\begin{array}{l}10 \text {-years } \\
(\%)\end{array}$ & 4.4 & 7.1 & 21.7 & 36.8 & & & 8.6 & 12.4 & 1.0 & 2.0 \\
\hline $\begin{array}{l}\text { 15-years } \\
(\%)\end{array}$ & 2.9 & 5.9 & 8.3 & 18.9 & & & 5.6 & 10.5 & 0.7 & 1.7 \\
\hline
\end{tabular}

Introduction \& Objective: Cytoreductive radical nephrectomy prior to targeted adjuvant therapy can improve survival in select patients with metastatic renal cell carcinoma (mRCC). The aim of our study was to determine if there was a survival benefit in patients who underwent partial nephrectomy in the setting of $\mathrm{mRCC}$ in a large database cohort.

Materials and Methods: SEER 18 database was queried for patients with mRCC from 1933-2013. Patients were excluded if the diagnosis was made at autopsy, multiple primary malignancies were present, or if pathological and/or surgical records were incomplete. Data collected included demographics, tumor stage, type of surgical or non-invasive intervention and survival. A separate comparative analysis was performed for cT1 tumors $(\mathrm{n}=1590)$. Kaplan Meier methods were used to estimate survival. Results: 18,443 patients met inclusion criteria and mean age was 62.7 years. 10,559 patients had no surgical intervention, 7598 underwent radical nephrectomy, 78 underwent ablation and 208 underwent partial nephrectomy. The overall survival (OS) and the cancer specific survival (CSS) of the entire study group is described in Table 1. Comparison of OS and CSS after intervention techniques and no surgical intervention are shown in Table 1. A separate subgroup analysis was completed for the 1590 patients found to have cT1M+disease. 63 underwent partial nephrectomy, 16 underwent ablation, 499 radical nephrectomy and 1012 did not undergo surgical intervention. Patients that underwent partial nephrectomy had improved CSS when compared to patients that underwent ablation or radical nephrectomy $(\mathrm{p}=0.00010)$, Figure 1 .

Conclusions: Patients who undergo partial nephrectomy for metastatic renal cancer have improved survival compared to patients that undergo cytoreductive radical nephrectomy or ablation alone. Partial nephrectomy can be considered a reasonable option even in the setting of metastatic disease. Patient selection and co-morbid conditions must be considered in this setting and most likely is a contributing factor in treatment decision.
MP22-23 Withdrawn

MP22-24 Impact of Hospital Volume on Intermediate to Long-term Patient Survival in Laparoscopic Radical Nephrectomy for Renal Cell Carcinoma

R Hsu, M Barclay, G Lyratzopoulos, V Gnanapragasam, JN Armitage

Academic Urology Group, University of Cambridge United Kingdom

Introduction \& Objective: Centralisation of complex operations is increasing, as are the utilisation of laparoscopic nephrectomy for RCC. Little is known about the impact this may have on patient survival particularly beyond the initial 30 day post-operative period. We performed a population-based study assessing the relationship between hospital volume and survival in RCC patients treated with laparoscopic radical nephrectomy. Materials and Methods: Patients diagnosed with RCC and treated with laparoscopic radical nephrectomy between 2006 and 2010 were identified from the English Hospital Episode Statistics and National Cancer Data Repository. Survival time was calculated from date of surgery until date of death from any cause or until end of 2015.

Hospital volume was defined according to the number of RCC nephrectomies in each hospital each year and was allocated to three volume groups: $<20,20$ to 39 and $\geq 40$ procedures per year. To account for clustering, where risks of death between individuals treated in the same hospital tend to be more similar, shared frailty Cox proportional hazard regression models were fitted to calculate hazard ratios according to hospital volume, adjusting for potential confounders, such as age, sex, number of comorbid conditions, socioeconomic deprivation and year of surgery. We calculated 1-year survival conditional on 30-day survival, 3-year survival conditional on 1-year survival, and 5year survival conditional on 3-year survival. Analyses were stratified for patients in stages T1-T2, and T3-T4.

Results: We analysed data on 3,212 patients, with a median follow up of 6.6 years. T1-T2 patients treated in hospitals in the highest volume category had higher post-procedure 1-year (conditional on 30-day) survival (HR 0.41 compared with patients in the lowest volume category, 95\% CI $0.21-0.82$, $\mathrm{p}=0.01)$. For T3-T4 patients, hospital volume was not associated with 1-year (conditional on 30-day) survival, although this may reflect power limitations in our sample.

Conclusions: Centralisation of RCC nephrectomy may confer a large survival benefit for laparoscopic radical nephrectomy during the first post-operative year for T1 and T2 disease. The effects of this beyond the first year and for T3 and T4 diseases however remain unclear.

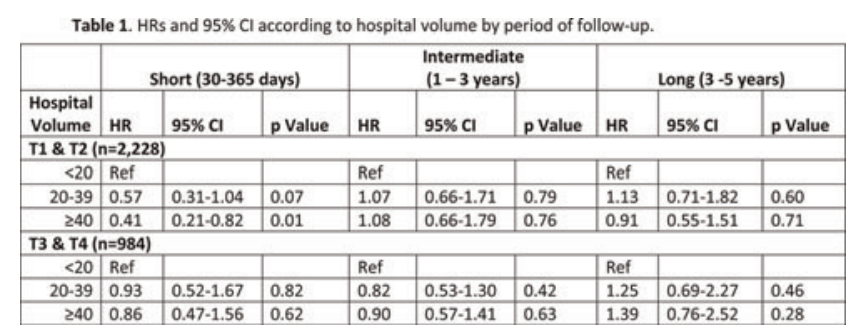




\section{MPS23: IMAGE-GUIDED THERAPY}

MP23-1 Single-session primary high-intensity focused ultrasonography (HIFU) as a treatment for localized prostate cancer: predicting disease-free survival by risk stratification

B Shayegan, T Lee, J Hwang, J Hoogenes, W Orovan

McMaster University

Canada

Introduction \& Objective: High-intensity focused ultrasonography (HIFU) is used with the goal of decreasing toxicity while maintaining efficacy for treatment of organ-confined prostate cancer. We assessed predictors of treatment success in a large cohort of patients for whom HIFU was the primary treatment.

Materials and Methods: We retrospectively analyzed data from 741 patients who underwent a single-session HIFU treatment by a single surgeon using a standardized treatment protocol. Treatment failure was defined as receipt of any salvage therapy or initiation of androgen deprivation. Descriptive statistics and Kaplan-Meier survival analyses were used to assess predictors of treatment failure. Patients with

Results: A total of 521 patients met inclusion criteria. Prior to treatment, mean age was $63.3,91.5 \%$ had a Gleason grade between 5 and 7, and the majority (59\%) had an intermediate D'Amico risk score. The mean PSA was $7.7 \pm 5.6$ and mean PSA density was $0.33 \pm 0.28$. The majority $(70.2 \%)$ had T1c staging. A total of 52 patients (10\%) met the definition of treatment failure. Of the potential predictors for disease-free survival over time, statistical significance was found for PSA density $(0.2 ; \mathrm{p} 10)$ approached significance $(\mathrm{p}=.05)$. Prior TURP was significantly associated with HIFU failure ( $\mathrm{p}$

Conclusions: In this patient sample, lower PSA density and D'Amico risk categorization were significant predictors for disease-free survival post-HIFU. Prior TURP and higher followup PSA levels were each associated with treatment failure. The high treatment success rate $(90 \%)$ in this sample suggests that whole gland HIFU as an alternate treatment method for clinically localized prostate cancer appears promising.

MP23-2 Radiofrequency Ablation of Renal Tumors: 5 year follow-up

G Sung, S Kim

Dong-A University Hospital

Republic of Korea

Introduction \& Objective: The aim of this study was to retrospectively evaluate the long-term results of radiofrequency ablation (RFA) of small renal masses (SRMs).

Materials and Methods: Percutaneous or laparoscopic RFA was performed on 55 renal tumors in 53 patients. The follow-up included physical examination, chest radiography, creatinine, and contrast-enhanced CT or MRI. To confirm pathologic criteria of complete ablation, 35 patients underwent follow-up biopsy. Recurrence was defined as contrast enhancement after 3 months or lesion growth at subsequent imaging or viable cancer cells on follow-up biopsy.

Results: Technical success was achieved in 52/55 renal tumors $(94.5 \%)$. The mean tumor size was $2.25 \mathrm{~cm}$ and the mean follow- up period was 62.5 months. Repeated RFA was necessary in 8 tumors due to incomplete ablation. The overall complication rate occurred in $43 \%$ in 19 patients and the low-grade complications accounted for $95.6 \%$ of the overall complications. Five patients were found to have recurrence at various follow-up intervals and distant metastasis was found in 4 cases and 50 of 53 patients are alive on serial follow-up.

Conclusions: RFA is considered useful treatment for selected patients with SRMs and also for nephron-sparing. Our long-term data suggest excellent therapeutic outcome with RFA, while achieving effective local tumor control.

\section{MP23-3 Interstitial Laser Coagulation of Localized Renal} Cell Carcinoma. Seven Years Follow-up

O Teodorovich, S Naryshkin, G Borisenko, E Rasshchupkina, A Ryazantsev, Y Osmanov, A Kudil, D Kochiev

Central Clinical Hospital 1 JSC RZhD "Russian Railways" Russia

Introduction \& Objective: In addition to partial nephrectomy in the treatment of localized renal cell carcinoma (RCC), widespread various focal techniques for its treatment. We present our results of seven years of experience of interstitial laser coagulation (ILC) as a method of radical treatment of patients with small renal masses (SRM).

Materials and Methods: As a result of the experimental part of the study, an "elementary" volume of coagulation, whose dimensions was $7 \times 5 \mathrm{~mm}$, was determined. Thus, by applying repeated exposure to adjacent tissue, it is possible to coagulate the required tumor volume. Since 2010 , ILC has been applied percutaneously in 38 patients with RCC T1N0M0. In 14 of them with a cytoreductive goal (since 2008, tumor size up to $60 \mathrm{~mm}$, central localization), and in 22 patients with the goal of radical treatment (since 2010, tumor size ranged 5-30 mm, Intraparenchymotic localization). In 4 patients the tumor was located in a single kidney. Before the ILC all patients underwent needle biopsy. ILC was performed using Nd:YAG laser $(1064 \mu \mathrm{m})$ surgical system «Lasurite» operating in the free running mode. Laser radiation delivered through a $600 \mu$ bare fiber by the lumen of the puncture needle. For puncture access the $18 \mathrm{G}$ needles were positioned with stereotactic targeting by ultrasound or MDCT guidance.

The laser operated at the output power $16-24 \mathrm{~W}, 100 \mathrm{~Hz}$ repetition rate, exposure time 10-20s. A single time laser exposure was used to coagulate only a small fraction of tissue which we called "elementary volume" of coagulation, while the required volume of coagulation was achieved by repetitive laser exposure.

The control method is MDCT with bolus contrast. Further results of treatment of patients from the second group will be considered. The follow-up period ranged from 7 till 84 months (median 60 months).

Results: One of the patients is non-compliant. Of the remaining 21 patients in 14 cases $(66,7 \%)$ tumor was fully replaced by scar tissue (max. follow up period - 85 months). Another 6 patients showed a decrease of the size of the tumor and a decrease in blood flow, tumor growth not noted until now. In 1 patient there was no positive dynamics. In 7 cases re-ILC was performed, 
which improved the results obtained after the first procedure. In 1 patient the postoperative period was complicated by hydronephrosis, which required a nephrectomy. In other patients complications were not observed.

Conclusions: The main problem is a precise navigation of puncture needles. ILC of localized kidney tumors by Nd:YAG laser operating in free running mode is quite safe and efficient and it can be used as an independent method of treatment when radical treatment is not feasible.

MP23-4 The course of recurrence following cryoablation for renal tumors

R van der Vijgh, A Montauban van Swijndregt,

B Lagerveld, R Sandkuyl

nefrolithiasis, laparoscopy

Netherlands

Introduction \& Objective: Cryoablation (CA) of renal tumors can be provided both with radiological imaging (IGCA) or laparoscopic (LCA) assistance. In accordance with the EAU guideline on Renal Cell Carcinoma CA can be discussed in patients with cT1a/b renal tumors with many comorbidities, advanced age and/ or a limited life expectancy. The aim of this study is to analyze the disease-specific outcome of patients who had an incomplete cryoablation (IC) of a kidney tumor or a local recurrence (LR).

Materials and Methods: All patients with a CA-treatment between 2006 and 2016 were enrolled. Follow-up was at least multiphase contrast CT or MRI. If on the first follow-up imaging focal contrast staining was still present within the ablation zone IC was diagnosed. If at the first follow-up ablation was considered complete but during subsequent scans a focal staining occurred LR was diagnosed. The course of patients with IC and LR after CA was retrospectively studied.

Results: A total of 259 patients with 289 tumors were treated with LCA $(n=167)$ or IGCA $(n=122)$. In 25 of the tumors $(8.7 \%)$ there was an IC $(n=12)$, or LR $(n=13)$. Of these 19 were treated with IGCA and 6 with LCA. The average time to diagnosis of a LR was 29 months (range 5-128). 20 tumors were treated again with CA, of which 16 successfully. However, one patient in this group developed metastases during follow-up. Of the four not successful re-cryoablations 2 underwent (partial) nephrectomy and 2 deceased due to co-morbidity. 5 of the 25 patients were not subjected to re-cryoablation because of comorbidity $(n=1)$, on own request $(n=1)$, metastases of the renal cell carcinoma $(n=2)$, or indication for nephrectomy $(n=1)$. After re-treatment (cryoablation or (partial) nephrectomy) 18 of the 25 patients $(72 \%)$ were free of disease at an average followup of 32 months (range 1-97). After re-treatment 282 out of the 289 initial tumors $(97.6 \%)$ were radically treated.

Conclusions: In the case of IC or LR after CA for primary cT1a/b renal tumors re-treatment with oncological good results is possible.

MP23-5 Percutaneous US-Guided Cryoablation of the Kidney under Local Anesthesia

P Glybochko, Y Alyaev, L Rapoport, A Amosov, D Tsarichenko, M Enikeev, D Enikeev, D Chinenov, Z Dzhalaev, M Taratkin

I.M. Sechenov First Moscow State Medcal University Clinic of Urology

Russia
Introduction \& Objective: Modern ultrasonographs allow realtime visualization of the process of cryoprobe placement and ice-ball formation. Note that the performance of US-guided cryoablation of renal tumor is restricted to a certain group of patients. The aim of our investigation was to evaluate the quality of US-guided cryoablation as well as to identify the most eligible candidates for this technique.

Materials and Methods: 12 patients with the ages varying between 52 and 75 years who experienced US-guided cryoablation of renal tumor in 2015 were enrolled in the study. The tumor size in 11 patients was up to $3.0 \mathrm{~cm}$ (T1a); in 1 patient, $4.5 \mathrm{~cm}(\mathrm{~T} 1 \mathrm{~b})$. The tumor characteristics were assessed with PADUA score. In 8 patients, it was 6-7 (low); in 3 patients, 8-9 (medium); in 1, 10 (high). For ultrasound examination we used Bk Medical Flex Focus 800. Before surgery, as well as 6 months later, all the patients underwent renal ultrasound dopplerography, CT, and computer-aided 3D-modeling to specify the spatial relations between the surface of the tumor and the elements of the caliceopelvic system. The successful performance of US-guided cryoablation requires that the mass be preferably localized on the posterior or lateral surface of the kidney, in the inferior or median segment without sinus invasion. Such localization provides maximum safety during needle passage, and multipositional control of ice ball formation allows us to visualize the tumor during ultrasonography. We used a SeedNet Gold cryomachine of the third generation and IceSeed and IceRod cryoprobes. In all patients presence of renal cell cancer (RCC) was verified morphologically.

Results: The average cryoablation time was $60 \mathrm{~min}$. Nine operations were performed under spinal anesthesia and three operations under local anesthesia. The hospital stay time extended for an average of two days. The follow-up period lasted eight months on average. According to the ultrasonography findings, after six months the tumor (T1a) in 11 patients reduced in size by an average of $8 \mathrm{~mm}$ and had no blood supply. MSCT with 3Dmodeling revealed tumor reduction, and the attenuation gradient did not exceed $10 \mathrm{HU}$ (initially $200 \mathrm{HU}$ ). There were no postoperative complications exceeding grade I in severity according to Clavien-Dindo scale. The MSCT findings showed stage T1b tumor (PADUA-10) in one patient to reduce in size to $3.7 \mathrm{~cm}$, but the area up to $1.5 \mathrm{~cm}$ with a high attenuation gradient necessitated kidney resection.

Conclusions: We consider percutaneous US-guided cryoablation a method of choice for patients with stage T1a renal tumor localized on the posterior or lateral surface in the inferior or median segment without sinus involvement and PADUA $<9$.

MP23-6 Increased Utilization of Ablation for T1b Renal Cancers with Improved Survival Relative to Observation

M Farrell, J Tasse, S Madassery, B Arslan, S Vourganti

Rush University Medical Center, Department of Urology United States

Introduction \& Objective: Technical limitations and oncologic concerns have traditionally reserved the use of ablation to renal tumors $<4 \mathrm{~cm}$. At our institution, we noted increased use of ablation in large tumors. We investigate the utilization and outcomes for ablation of larger renal tumors in a national dataset.

Materials and Methods: SEER 18 was queried with inclusion criteria of primary site kidney, localized stage, and known renal histology (clear cell, papillary, chromophobe, or RCC). Patients 
before 1998, (when ablation was first recorded), unknown therapy or size, or tumor $>7 \mathrm{~cm}$ were excluded.

Results: Analysis included 84,485 pts with localized RCC. Clinicopathologic data is summarized in Table 1. Ablation was utilized in 4110 pts (4.9\%), extirpation in 73,155 (86.6\%) and observation in $7220(8.5 \%)$. Extirpation was used in younger pts $(60.5 \mathrm{y})$ relative to ablation and observation respectively (67.9 vs

Figure 1. A) Utilization of ablation over time for T1a and T1b renal tumors and B) Kaplan-Meier curve for T1b renal tumors managed by ablation versus observation.

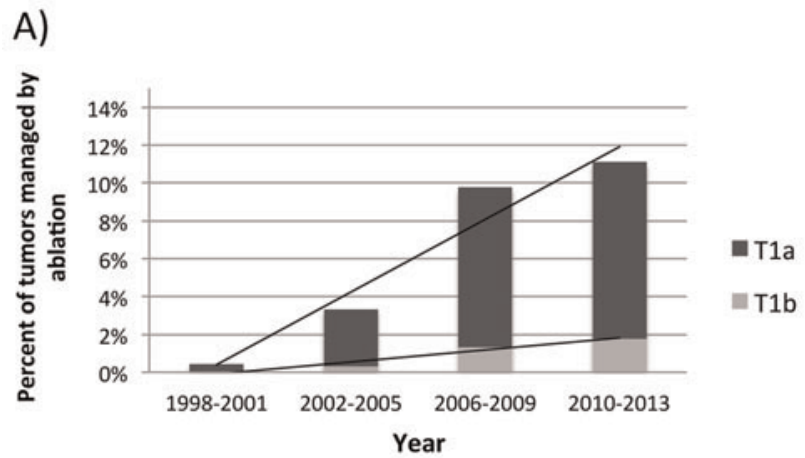

B) T1b renal tumors

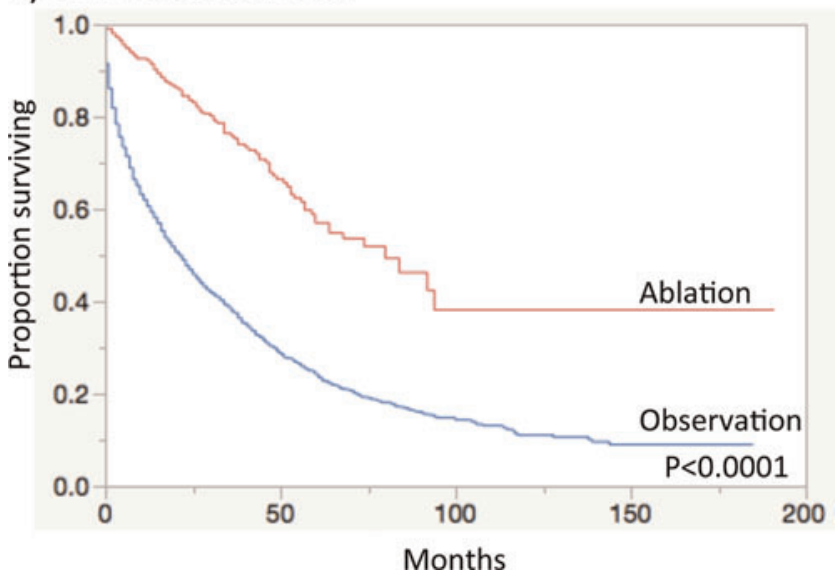

Table 1. Baseline characteristics of patients with Tla and Tlb renal tumors.

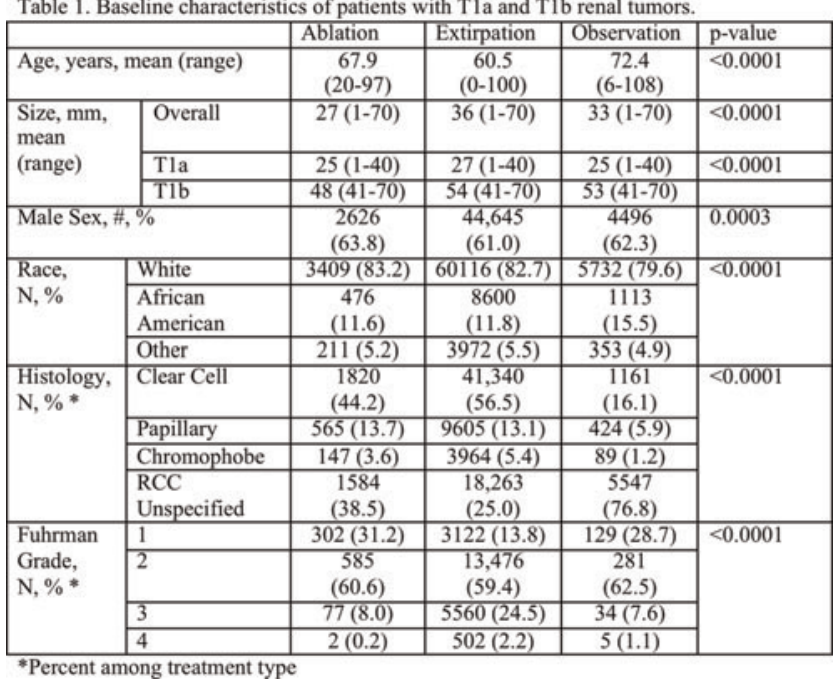

$72.4 \mathrm{y} ; \mathrm{p}<0.001)$. Ablation was increasingly utilized over time with modern era use in $9.3 \%$ of $\mathrm{T} 1 \mathrm{a}$ and $1.8 \%$ of $\mathrm{T} 1 \mathrm{~b}$ tumors (figure 1A). Kaplan Meier analysis revealed longer survival for ablation vs observation for both T1a (99.6 vs $66.3 \mathrm{~m} ; \mathrm{p}<0.001)$ and T1b groups (65.2 vs $40.2 \mathrm{~m}$; p < 0.001; figure 1B). Using Cox hazards models to compare ablation and observation, age (HR $1.04 / \mathrm{yr}$ ), histology (HR 0.85, clear cell), size (HR 1.03/mm) and mode of treatment (HR 4.21 for observation) were significant predictors of survival on univariate analysis - race, tumor grade and sex were not. Multivariable Cox models including all significant predictors showed younger age (HR 1.03/yr), smaller size (HR 1.02/mm) and ablative treatment (HR 2.51 for observation) as significant predictors of survival.

Conclusions: Ablation is increasingly utilized for T1b tumors and may provide improved oncologic control relative to observation ( 2.5 fold improved survival in this data). While this retrospective analysis may be confounded by selection bias, a trial evaluating ablation vs observation in this setting is warranted.

MP23-7 Usefulness of contrast-enhanced ultrasound in the diagnosis of renal masses and its concordance with CT Scan.

G Del Pozo Jiménez, I Castillón Vela, G Rodríguez Reina, D Rengifo Abad, M Rodríguez Monsalve, T Fontanilla Echeveste, J Minaya Bernedo, J Carballido Rodríguez

University Hospital Puerta de Hierro, Majadahonda, Madrid Spain

Introduction \& Objective: The diagnosis of renal mass has been based on contrast enhanced CT Scan and MRI, with conventional ultrasonography being of little use. However, contrastenhanced ultrasound (CEUS) has appeared, and seems to provide greater diagnostic sensitivity.

Our objective is to compare the diagnostic capacity of CT scan and CEUS in the diagnosis of solid and cystic renal masses.

Materials and Methods: Retrospective study of 70 patients with diagnosis of renal lesion, evidenced both in CT and CEUS, between January 2013 and December 2016. All CEUS procedures were performed by two radiologists with expertise in the technique, collecting different variables: lesion enhancement on parenchyma, Doppler etc. Lesions were classified as cystic (Bosniak classification) or solid. Benign lesions were defined as Bosniak II-IIF and malignant as Bosniak III-IV or solid. The diagnostic results of CT and CEUS for renal lesions were compared with the final diagnosis obtained by histopathology (93\%) or image follow-up (7\%). Statistical analysis was performed by measuring the consistency (Kappa index) and validity (sensitivity, specificity, positive and negative predictive values, accuracy) of the study, as well as elaborating an ROC curve and determining the area under the curve.

Results: The diagnosis of cystic lesion was performed in 55.7 and $38.6 \%$ of patients with CT and CEUS respectively, with the mean lesion size being 37.8 and $38.6 \mathrm{~mm}$ for both tests. In 64 patients, surgical excision was performed by radical or partial nephrectomy. Pathologic study was compatible with malignancy in $70 \%$ of cases. The sensitivity, specificity and accuracy of CEUS in the evaluation of renal masses were $93.9 \%, 52.4 \%$ and $81.4 \%$, respectively, versus $71.4 \%, 70 \%$ and $70 \%$ for CT scan. The area under the ROC curve for CEUS and CT were 0.84 and 0.75 , respectively. The Kappa index showed a good concordance between both tests and the pathological report, with indexes of 0.74 and 0.61 for CEUS and CT, respectively. 
Conclusions: CEUS presents greater sensitivity and accuracy, although less specificity than CT scan, in the differential diagnosis of renal mass, being able to contribute to a better therapeutic decision.

MP23-8 High Intensity Focused Ultrasound Kidney Ablation: Pre-Clinical Safety and Efficacy Evaluation in a Porcine Model using a $15 \mathrm{~mm}$ Laparoscopic Probe

S Chopra, IS Gill, A Bove, C Fay, T Shin, K King, V Duddalwar, A Aron, R Chaluisan, J Clanton, J Carr, C Johnson, B Ettinger, A Morris, R Carlson, N Sanghvi, M Carol, R Seip

USC institute of urology

United States

Introduction \& Objective: High intensity focused ultrasound (HIFU) has established itself clinically as a viable, safe, effective, and non-invasive tissue ablation modality. Previous studies have shown that HIFU delivered laparoscopically can be used to ablate kidney tumors, potentially enabling a lower morbidity treatment with faster recovery time as compared to partial nephrectomy procedures. Challenges remain, however, including ensuring full tissue necrosis and consistent energy coupling to the target volume. The objective of this study was to evaluate whether a newly developed laparoscopic HIFU probe is able to address these challenges.

Materials and Methods: A laparoscopic porcine kidney model was used to investigate the safety and efficacy of the new $15 \mathrm{~mm}$ HIFU probe. Under ultrasound guidance, kidneys of 12 pigs were targeted and ablated with HIFU, creating on average 2 ablation zones per kidney of varying sizes and locations in order to quantify the probe's ability to deliver HIFU to any location on the kidney. Efficacy was evaluated via the analysis of ablation volume histology slides, real-time ultrasound images collected during HIFU delivery, and MRI and ultrasound contrast images. Safety was evaluated by surviving a subgroup of the animals $(2 \mathrm{w})$. Gross-pathological data, sonication parameters, and workflow feedback was also collected during the study.

Results: Repeatable lesions could be created at a rate of $0.48 \mathrm{~cm}^{3} / \mathrm{min}$ and average energy densities of $584 \mathrm{cal} / \mathrm{cm}^{3}$. Histological evaluation indicated contiguous ablated volumes using these delivery parameters, extending from the transducer's focal zone to the kidney surface, with a maximum treatment depth of $27 \mathrm{~mm}$. Ablated target volumes ranged from $5.1 \mathrm{~cm}^{3}$ to $24.5 \mathrm{~cm}^{3}$. Conclusions: The results confirm the ability of the new probe to deliver HIFU in a consistent and reliable manner. Initial dose

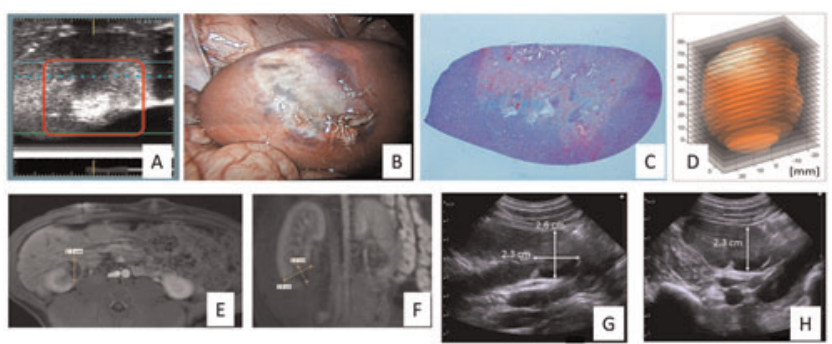

Laparoscopic HIFU kidney ablation under ultrasound guidance (A), immediate post-HIFU ablation volume and histology slide example (B-C), reconstructed lesion volume (white) for efficacy analysis (D), and distant post-HIFU ablation MRI (E-F) and extracorporeal US (G-H) of lesion. requirements for ablating tissue at various depths were also determined. Workflow feedback has resulted in additional system user interface improvements, with all of these results paving the way for a future clinical study.

MP23-9 Analysis of renal function after cryoablation of Small Renal Tumours (SRMs) in patients with solitary kidneys: a European Registry for Renal Cryoablation (EuRECA) multi-institutional study.

K Farrag, T Nielsen, B Lagerveld, F Keeley, G Lughezzani, N Barber, L Hansen, N Buffi, G Guazzoni, J van der Zee, M Ismail, A Emara, L Lund, Østraat, M Borre, S Sriprasad

Darent Valley Hospital

United Kingdom

Introduction \& Objective: Presence of small renal tumours (SRMs) an absolute indication for nephron sparing surgery. The objective of this study was to evaluate the outcomes cryoablation in patients with solitary kidney focusing on renal function from European Registry for Renal Cryoablation (EuRECA) database. Materials and Methods: Of the 808 patients from 8 European centres in the database, 102 patients had SRMs in solitary kidneys. Patient demographics, tumour size and renal function were analysed with a specific focus on estimated glomerular filtration rate (eGFR). Data was collected pre-ablation and 3 months postoperatively.

Results: The mean age of the patients were 65 years (range 49-80 years); mean size of the tumour $2.9 \mathrm{cms}$ (range 1.5 to $7 \mathrm{cms}$ ) and renal cancer was proven by biopsies in 79 patients. The mean preoperative eGFR was 55.0 (SD18.1) and the post -operative eGFR was 51.8 (SD 18.8). The change was $-3.1(-5.2,-1.0)$ $(95 \% \mathrm{CI})$ units, which was statistically significant $(\mathrm{P}=0.004)$. The decrease in the eGFR did not translate to any significant adverse outcome in any of the patients. None of the patients in this cohort has needed any form of renal dialysis at any time after the surgery.

Conclusions: Cryotherapy in solitary kidneys is safe and may be a treatment option when preservation of renal function becomes essential in avoiding dialysis.

MP23-10 Salvage Cryo-Ablation for localized radiorecurrent prostate cancer

A Breda, A Territo, G Basile, F Sanguedolce, P Gavrilov, J Palou, H Villavicencio

Fundació Puigvert, University Autonoma of Barcelona, Spain Spain

Introduction \& Objective: Androgen deprivation treatment (ADT) for locally radio-recurrent prostate cancer (PCa) can cause side effects as well as hormone resistant PCa. In this prospective study, we evaluate the role of Cryo-Ablation (CA), both in the settings of total and hemi-ablation (HCA), as a strategy to delay ADT, as well as a potential curative treatment. Materials and Methods: From January 2010 to June 2016, patients over the age of 65 who developed biochemical failure (BF) according to the Phoenix Criteria after RT were offered CA as a salvage treatment. Inclusion criteria were: PSA $<10 \mathrm{ng} / \mathrm{ml}$, biopsy proven $\mathrm{PCa}$, slow doubling time (DT), organ-confined 
disease according to magnetic resonance (MR) and negative metastatic work up. Total CA was offered to patients with bilateral disease on biopsy and/or MR. HCA was offered to patients with unilateral concordance between MR and biopsy, or in cases of a negative MR with positive unilateral biopsy. All patients were followed with PSA every three months. BF was defined according to the Phoenix Criteria. Furthermore, patients who underwent HCA were offered a protocol biopsy and MR at six months. ADT was offered to patients with a $\mathrm{BF}$ and a rapid $\mathrm{DT}(<$ 6 months) or a PSA $>10$.

Results: 21 patients were recruited. The mean age was $71 \pm 7$. Average pre- CA PSA was $3.5 \mathrm{ng} / \mathrm{ml}$. Pre-operative Gleason score (GS) was $£ 8$ in $48 \%$ (10 cases) of the patients. The GS was indeterminate in 11 patients (52\%). 13 patients received total CA and $8 \mathrm{HCA}$. The mean follow-up was 28 and 26 months with a mean PSA 0.7 and $1.6 \mathrm{ng} / \mathrm{ml}$ in the total CA and HCA groups respectively. There were 6 patients $(29 \%)$ with BF: 4 from the total CA (31\%) and 2 from the HCA (25\%) groups. The mean PSA was $7.2 \mathrm{ng} / \mathrm{ml}$ and none of these patients started ADT. Furthermore, protocol biopsy in the HCA group was positive in 2 patients $(25 \%)$. There were no intra-operative complications. In the CA group, 5 patients developed post-operative complications: Clavien 1 in 4 cases $(31 \%)$ and Clavien 4 in 1 case $(7.7 \%)$. In the HCA group there were Clavien 1 complications in 2 cases $(23 \%)$. As far as continence rate (defined as 0 pads), $8(61 \%)$ and $6(75 \%)$ patients were continent at 1 month from catheter removal in the total CA and HCA groups respectively. Furthermore, 15\% and $32 \%$ of the patients who underwent total and HCA were potent with no medications before treatment. Of these, sexual function was preserved in $30 \%$ and $60 \%$ respectively.

Conclusions: Our data shows that CA may delay ADT in selected patients with radio recurrent $\mathrm{PCa}$, while maintaining a good quality of life. Longer follow-up is still required to establish salvage $\mathrm{CA}$ as a method to cure radio recurrent $\mathrm{PCa}$.

\section{MP23-11 Impact of MRI/Ultrasound Fusion-Guided Biopsy on Prostate Cancer Detection in a Community Based Large Urology Group Practice}

A Van Hoof, N Ruhotina MD, R Kronhaus MD, H James MD, C Pieczonka MD, B Omarbasha MD, DM Albala

Associated Medical Professionals

United States

Introduction \& Objective: Trans-rectal-Ultrasound-guided (TRUS) prostate biopsy for prostate cancer (PCa) detection is associated with an appreciable false-negative rate. Utilization of multiparametric-MRI (mp-MRI) and MRI/US fusion-guided biopsy have been displayed to increase the detection of $\mathrm{PCa}$, particularly intermediate and high risk $\mathrm{PCa}$ with the degree of suspicion on mp-MRI as a strong predictor of final pathology. Much of these findings have been produced in large academic centers, utilizing 3.0T magnets. The present study will assess the impact of targeted MR/US fusion biopsy on the overall rate of $\mathrm{PCa}$ while comparing the rate of PCa detection in targeted ROIs to that of the standard TRUS biopsy in a community based large urology group practice association.

Materials and Methods: All patients receiving a MR/US fusion biopsy underwent mp-MRI using a 1.5T magnet and endorectal coil. PI-RADS scoring was used as the diagnostic criteria for identifying suspicious lesions. $562 \mathrm{MR} / \mathrm{US}$ fusion-guided biopsy procedures performed using the Artemis platform (Eigen/
Hitachi) from September 29th 2015 through March 2nd 2017. The pathological findings from these procedures were compared to that of a historical cohort of 1,562 standard TRUS-biopsies performed at the same practice from January 1st 2014 through December 31st 2014.

Results: PCa detection for all MR/US fusion-biopsies including both standard and targeted cores was $50.4 \%$ (283 of 561). Standard TRUS-biopsy rate of PCa detection was 41.2\% (644 of 1562). In 438 cases both targeted and standard biopsies were performed with targeted biopsy detecting Targeted regions diagnosed 36.3\% less Gleason 6 disease while diagnosing 51.3\% more Gleason $\geq 7$ disease than that of the sextant biopsy cores. Standard extended-sextant biopsy missed 48 cases $(11.0 \%)$ of $\mathrm{PCa}$ that were diagnosed by targeted biopsy cores during the same procedure $26(54.2 \%)$ of which were GS $\geq 7$. The final biopsy pathology resulted in a diagnosis of cancer in $37.1 \%$ (103) of cases with PIRADS 3 lesions, 65.2\% (75) of cases with PIRADS 4 lesions, and 86.7\% (39) of cases with PI-RADS 5 lesions. Of these cases, PCa with Gleason score $\geq 7$ was diagnosed in $36.9 \%$ (38), $62.7 \%$ (47), and $84.6 \%$ (33) of cases respectively.

Conclusions: MR/US fusion-biopsy compared with that of standard biopsy alone was associated with increased detection of PCa. Targeted lesions were associated with increased detection of high/intermediate risk PCa and a decreased detection of low risk PCa. The degree of suspicion on mp-MRI was a strong predictor of overall PCa detection as well as the rate of intermediate and high risk $\mathrm{PC}$ detection.

MP23-12 Predictors of rectourethral fistula after primary, whole gland cryoablation for prostate cancer: Results from the Cryo-On-Line Database (COLD) Registry

A Schulman, K Tay, A Elshafei, E Tsivian, C Sze, T Polascik, J Jones

Duke University Medical Center

United States

Introduction \& Objective: While recto-urethral fistula is a rare complication following salvage cryoablation for radiorecurrent prostate cancer, less is known about the development of rectourethral fistula after primary whole gland cryoablation. We define the incidence and risk factors for recto-urethral fistula in a multicenter, centralized registry.

Materials and Methods: The Cryo-On-Line Data (COLD) Registry was queried for men undergoing primary whole gland cryotherapy between 1990 and 2014 who developed a rectourethral fistula. Patient factors and disease parameters were correlated with the recto-urethral fistula using chi-squared tests for categorical variables and t-test for continuous variables. Variables with $\mathrm{p}<0.25$ were entered into a binary logistic regression with stepwise backward elimination to determine the factors associated with formation of urethral fistula.

Results: We identified 4,102 men who underwent primary whole gland cryotherapy between 1990 and 2014. Median age was 71 years (IQR:66-76). Median PSA was 6.5 (IQR: 4.8-9.8). Available Gleason Score included 8-10:500 pts, 7:1,194 pts and 6:1,601 while pretreatment clinical stage included T1:1539, T2:1,503 T3:328 and T4:20. 1,508 pts received neoadjuvant androgen deprivation. 805 cases were performed at academic centers and 3297 were performed in the private setting. Postoperative recto-urethral fistula was identified in $50(1.2 \%)$ men. 
On univariate analysis, pre-operative Gleason score, pre-operative incontinence and post-operative urinary retention were statistically significant predictors for development of recto-urethral fistula. On multivariate analysis, postoperative urinary retention (OR 7.26, 95\%CI 4.06-13.03, $\mathrm{p}<0.001$ ) pre-operative Gleason score of 7 (OR 1.92, 95\% CI 1.08-3.43, p $1 / 40.027$ ) and pre-operative incontinence (OR 2.95, 95\% CI 1.12- 7.76, $\mathrm{p}^{1} / 40.028$ ) predicted urethral fistula.

Conclusions: In a large, mixed cohort of patients undergoing primary whole gland cryoablation for prostate cancer, the incidence of urethral fistula was very low at $1.2 \%$. The strongest association was present in men with post-procedural urinary retention. Further study regarding instrumentation of the lower tract for retention following cryotherapy in the early post-operative setting is warranted.

MP23-13 Does African-American race impact oncologic outcomes following primary cryotherapy for prostate cancer? A matched study from the Cryo-On-Line Database (COLD) Registry.

A Schulman, M Tsivian, E Tsivian, C Sze, K Tay, A Elshafei, T Polascik, J Jones

Duke University Medical Center

United States

Introduction \& Objective: African-American (AA) men have the highest incidence and disease-specific mortality from prostate cancer of any racial group. Little is known about the impact of race on outcomes following ablative therapies. We examine oncologic outcomes for African-American men undergoing primary cryoablation for prostate cancer.

Materials and Methods: The Cryo-On-Line Data (COLD) Registry was queried for men undergoing primary cryotherapy between 1990 and 2014. A 1:2 cohort of AA men were matched to non-AA men based on age, PSA and Gleason score using the nearest neighbor method. Pretreatment variables and oncologic outcomes including biochemical recurrence (BCR) and control biopsies were examined.

Results: The cohort included 144 AA men matched to 288 nonAA men. Median age $(70: 70, p=0.685)$, median PSA (6.7:6.3, $\mathrm{p}=0.285)$, distribution of Gleason score $(\mathrm{p}=0.82)$ and median gland volume $(33: 32, p=0.74)$ were statistically similar. Patients receiving focal therapy $(10(7 \%): 21(7 \%) \mathrm{p}=0.949)$ and neoadjuvant androgen deprivation $(26(18 \%): 71(25 \%) \mathrm{p}=0.682)$ were similar. The non-AA group had a larger percentage of high risk (31 vs. $21 \%$ ) and lower percentage of low risk (28 vs $36 \%$ ) patients $(\mathrm{p}=0.054)$. Median and interquartile range $(\mathrm{IQR})$ of months of follow-up was equivalent. (AA: 29(11-48) and non-AA: 24(11-58)

Kaplan Meier for any recurrence:

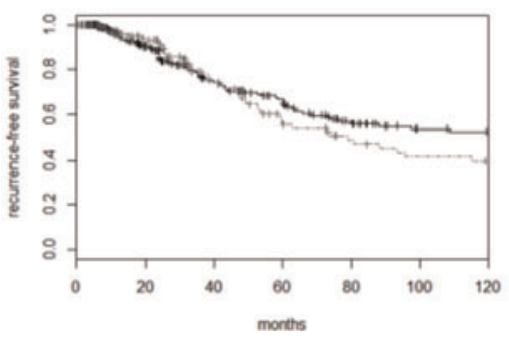

KM log rank tests:

Any recurrence: $\mathrm{P}=0.271$

Phoenix: $\quad 0.394$

Astro: $\quad 0.182$

Biopsy: $\quad 0.665$

Whole gland only,

any recurrence:

0.355 $\mathrm{p}=0.76$.) Rates of BCR Phoenix $(16(12.5 \%): 42(16.6 \%) \mathrm{p}=0.72)$, BCR ASTRO (37(28.9\%):58(22.9\%) $\mathrm{p}=0.212$ and post-treatment positive biopsies $(6 / 28(21 \%): 15 / 52(29 \%) \mathrm{p}=0.676$ were similar. The presence of any recurrence (BCR or positive biopsy) for each group was equivalent (48(33\%): $80(28 \%) \mathrm{p}=0.264$.) [See Graph 1].

Conclusions: A risk-mixed cohort of AA men had non-inferior oncologic outcomes compared to a similar cohort of non-AA men undergoing primary cryoablation for the treatment of prostate cancer. This supports the continued use of cryoablation in AA men.

MP23-14 HIFU focal treatment for unilateral prostate cancer: a comprehensive study of pooled data

S Albisinni, C Melot, K Limani, A Peltier, P Rischmann, RF van Velthoven

\section{JULES BORDET INSTITUTE - ULB BRUSSELS Belgium}

Introduction \& Objective: Although still experimental, focal treatment is being increasingly implemented in the management of prostate cancer (PCa). Aim of the current study was to review available evidence and perform a pooled analysis exploring oncologic and functional results of High Intensity Focal Ultrasound (HIFU) hemiablation and focal therapy for the treatment of unilateral PCa.

Materials and Methods: The National Library of Medicine Database was searched for articles published between January 2007 and April 2017. A wide search was performed including the combination of following words:"HIFU", "prostate", "cancer", "focal". Overall, 167 articles were reviewed. Of these, 5 articles were identified and eligible for the pooled analysis. Data on HIFU hemiablation or focal prostate ablation oncologic and functional results were pooled from these 5 studies that included 296 men with unilateral PCa.

Results: In the 296 analyzed cases, mean age was 68 years (95\%CI 67-70), and mean pre-operative PSA was $6.8 \mathrm{ng} / \mathrm{ml}(5.8$ - 7.7). 3 studies included PCa up to Gleason $7(3+4)$, while 2 studies did include also Gleason 7(4+3). Regarding early complications, low grade Clavien I-II were reported in 20\% (8-32), while high-grade Clavien $\geq$ III were found in $3.3 \%(0-7.9)$. Analyzing oncologic outcomes, mean follow-up was 43 months (38-47) : at one year after HIFU, negative biopsy rate for clinically significant PCa was $87 \%$ (76-95), whilst salvage treatment

Fig 1. Salvage treatment free survival after HIFU hemiablation of the prostate

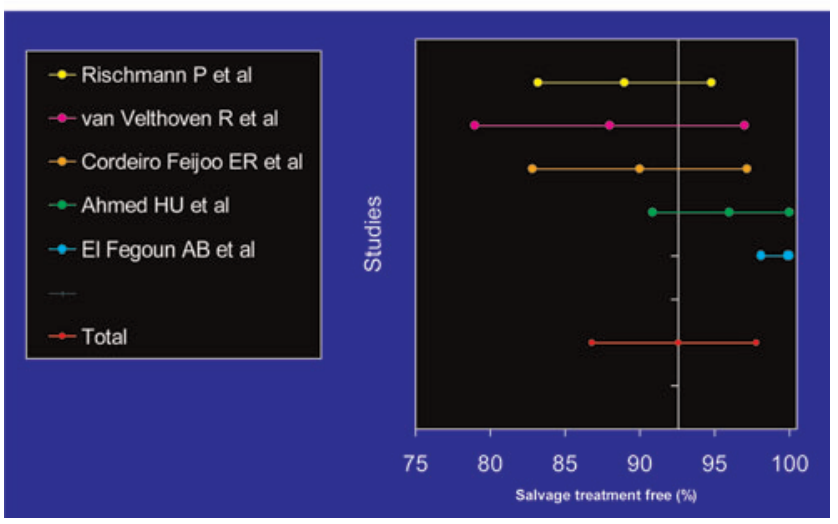


free survival was 93\% (87-98) (Fig 1). Regarding functional outcomes, reported potency rates were $72 \%$ (61-82), and continence $96 \%$ (92-100), although definitions of potency and continence were not homogenous across studies. >studies that included 296 men with unilateral PCa.

Conclusions: In this pooled analysis, HIFU hemiablation and focal treatment of PCa was associated to promising oncologic and functional outcomes. Well constructed and randomizedcontrolled trials are awaited to compare HIFU focal treatment of PCa to current standard of care.

MP23-15 High Intensity Focused Ultrasound: zonal hemiablation for localized prostate cancer, a single center ten years experience

RF van Velthoven, R Diamand, K Limani, S Albisinni, $\mathrm{M}$ Lemort, A Peltier, T Roumeguère

\section{JULES BORDET INSTITUTE - ULB BRUSSELS Belgium}

Introduction \& Objective: Although High Intensity Focused Ultrasound treatment for localized prostate cancer is proposed as an alternative to radical therapies since about two decades, its results remain controversial and hence it is still considered in guidelines as an experimental approach. The aim of the present study is to summarize a retrospective analyse of a prospectively filled database of patients who benefitted of unilateral prostate hemi-ablation between January 2007 and January 2017.

Materials and Methods: Inclusion into the data base supposed absence of any other treatment with curative intent, updated medical records by the patient's urologist. According to D'Amico risk classification, we had 33 patients $(55,38 \%)$ at low risk, 25 patients $(39,68 \%)$ at intermediate risk and 5 patients $(7$, $94 \%)$ at high risk. Mean prostate volume was $33 \mathrm{~cm}^{3}\left(12-87 \mathrm{~cm}^{3}\right)$. A majority of patients were treated with Ablatherm Integrated Imaging ${ }^{\circledR}$.

Results: Nadir PSA was reached after a mean time of 5 months (1-30 months) with a mean value of $1,72 \mathrm{ng} / \mathrm{mL}(0,01-12,2 \mathrm{ng} /$ $\mathrm{mL}$ ). According to Phoenix criteria (nadir $+2 \mathrm{ng} / \mathrm{mL}$ ), biochemical recurrence was seen in 20 patients $(31,75 \%)$ out of which 13 patients $(65 \%)$ went on for a new treatment. We found 7 patients $(11,11 \%)$ that had a secondary HIFU hemi-ablation. 5 of whom on the contralateral side $(7,94 \%)$ and another 1 (1, $59 \%$ ) undergoing a HIFU for the third time. As for functional impairment, 9 patients $(14.29 \%)$ suffered transient stress incontinence (8 Grade I and 1 Grade II), while 5 patients $(7.94 \%)$ still suffer from incontinence (4 Grade I and Grade II). A total of 6 patients $(9.52 \%)$ suffered from a new onset erectile dysfunction that did not allow them to have adequate intercourse currently on medical treatment with oral PDE5 inhibitors while 2 patients $(3.17 \%)$ suffer from persistent erectile dysfunction.

Conclusions: Despite a relatively limited population, our study has the merit of being able to follow over several years the fate of patients who have been treated with HIFU hemi-ablation. Its mean follow-up of 42 months makes it to our knowledge, the study with the longest follow-up for this type of treatment with a follow-up of 10 years for the first patients taken into account ( $50 \%$ of the patients were followed more than 36 months). Treatment of prostate cancer with HIFU hemi-ablation allows satisfactory oncological outcomes albeit at the price of an eventual second treatment in selected cases. With a progressionfree survival of $87.3 \%$ at 10 years and limited side effects, it must be considered in the prostate cancer treatment arsenal. PSA nadir proved to be an important predictor of biochemical recurrence and the need for re-intervention.

\section{MP23-16 MRI Guided TRUS Fusion Prostate Biopsy}

OB Argun, C Obek, MB Tuna, T Doganca, I Tufek, S Keskin, AR Kural

Acibadem University, Department of Urology, Istanbul, Turkey Turkey

Introduction \& Objective: Transrectal ultrasound (TRUS) guided sampling of the prostate with random 10-12 cores remains the standard of care. However, this method possesses significant shortcomings. It has been well documented to be associated with under-sampling of clinically significant cancers along with over-detection of clinically insignificant cancers. Recently, the common use of multiparametric prostate magnetic resonance imaging (mpMRI) has revolutionized the imaging of prostate cancer (PCa). Consequently, MRI guided biopsy techniques have become available. We report our experience with MRI guided TRUS fusion biopsy technique.

Materials and Methods: Patients with a clinical suspicion of prostate cancer and had an abnormal area on MRI underwent MR-TRUS fusion biopsy using the Artemis (Eigen, CA, USA) platform. 3-5 cores were obtained from each target and biopsies were performed under sedation. Twelve core systematic biopsies were obtained in addition to lesion-targeted biopsies. Data was analyzed regarding cancer detection rates.

Results: MRI-TRUS fusion prostate biopsy was performed in 276 consecutive patients who had a total of 582 suspicious lesions detected with mpMRI. Mean age was $61.4( \pm 10.3)$ and mean PSA level was $7.8( \pm 6.7) \mathrm{ng} / \mathrm{ml}$. Overall cancer detection rate was $53.2 \%(147 / 276)$. At $76.1 \%$ of these cancers $(112 / 147)$ Gleason score (GS) was $\geq 7$. Cancer detection rate for PIRADS 3 , 4 and 5 lesions were $10.4 \%, 38.4 \%$, and $77.3 \%$ respectively; GS was $\geq 7$ in $39 \%, 69 \%$ and $90 \%$ with increasing PIRADS score (34-5). Cancer detection rate for systematic biopsy vs. targeted biopsies were $49 \%$ and $46 \%$. Rate of GS $\geq 7$ cancer detection in systematic and targeted biopsies was $64 \%$ and $81 \%$, respectively $(\mathrm{p}<0.05)$. Seventeen of 46 patients with GS 6 cancers at systematic biopsy were upgraded to $\geq 7$ with targeted cores. Five patients with clinically significant cancer $(G S \geq 7)$ would have been missed, if we had performed targeted biopsies only. Infection was observed in 4 patients $(2 \%)$.

Conclusions: Overall cancer detection rate was similar for targeted and systematic random sampling. However, Gleason $\geq 7$ tumor detection was higher and GS 6 cancer detection lower with targeted biopsies. MRI guided TRUS fusion biopsy appears to be a useful tool in fulfilling the main goal of prostate cancer biopsy, which is detecting more clinically significant cancer while avoiding the over-diagnosis of GS 6 cancers.

MP23-17 Renal Mass Thermal Ablation: Cone Beam Computed Tomography, Alone or Combined with Pneumoperitoneum Under Surgeon Control- Description of Technique and Initial Results

EF Kelly, RJ Leveillee

Louisiana State University Shreveport Health

United States 


\begin{tabular}{|l|l|l|}
\hline \multicolumn{3}{|c|}{ Procedural Results } \\
\hline Laparoscopic combined TA & $n=3$ \\
\hline Percutaneous TA & $n=20$ \\
\hline Intra-operative Biopsy & $n=23$ \\
\hline \multicolumn{1}{|c|}{-Number of Cores } & \multicolumn{1}{|c|}{$\bar{x}=4.45$} & $r=3-8$ \\
\hline Pathology Result & $n=7$ Clear Cell \\
\hline \multirow{4}{*}{} & $n=4$ Papillary \\
\cline { 2 - 3 } & $n=5$ Oncocytoma \\
\cline { 2 - 3 } & $n=3$ Clear Cell Papillary \\
\cline { 2 - 3 } & $n=3$ Normal tissue \\
\cline { 2 - 3 } & $n=1$ Other \\
\hline Radiation Exposure (mGy) & $\bar{x}=1007.09$ & $r=364.7-2070.51$ \\
\hline Number of Probes (Cool-tip) & $\bar{x}=1.61$ & $r=1-3$ \\
\hline Number of Cycles & $\bar{x}=1.72$ & $r=1-3$ \\
\hline Intra-operative Complication(s) & $\bar{x}=0$ & $r=0-0$ \\
\hline Post-operative Complication(s) & $n=6$ (all Clavien 1 ) \\
\hline \multirow{2}{*}{ Key: $\bar{x}$ =mean, $r=$ range, $n=$ number of patients } \\
\end{tabular}

\begin{tabular}{|c|c|c|}
\hline \multicolumn{3}{|c|}{ Patient Demographics } \\
\hline Number of Patients & $n=23$ & \\
\hline & \multicolumn{2}{|c|}{18 Male } \\
\hline & \multicolumn{2}{|c|}{6 Female } \\
\hline Age (years) & $\bar{x}=71.1$ & $r=47-85$ \\
\hline BMI & $\bar{x}=27.37$ & $r=20.52-36.58$ \\
\hline ASA Score (1-5) & $\bar{x}=2.91$ & $r=2-4$ \\
\hline Size & $\bar{x}=2.74$ & $r=1.5-4.3$ \\
\hline Laterality & $R=12$ & $\mathrm{~L}=11$ \\
\hline
\end{tabular}

Introduction \& Objective: We report indications and preliminary results for patients undergoing thermal ablation (TA) of small renal masses (SRM) using the cone-beam CT, CBCT, (Siemens Healthcare, Germany) and their "I-Guide" software. This protocol allows 3D treatment planning, probe placement by the surgeon under real-time fluoroscopic guidance, enhanced precision, and decreased radiation exposure. TA, a type of Nephron Sparing Surgery, induces cellular death within the target zone while sparing nearby structures. TA can be delivered via a laparoscopic or percutaneous approach. Laparoscopic exposure is ideal for tumors felt to be inaccessible to the more standard percutaneous approach.

To report the initial results in a cohort where Surgeon performed CBCT TA and renal biopsy (RB) were performed for select patients with SRM.

Materials and Methods: 23 patients were treated between January-October 2016. All patients had general anesthesia. A single surgeon determined the treatment volume, number of probes, trajectory, and energy delivery, performed the biopsy and ablation probe placement. Exclusion criteria were patients who decided to undergo partial nephrectomy or surveillance. Radiofrequency delivered via and internally cooled, insulated probe(s) was employed after core biopsy (18 gauge). Needle Number of ablation cycles, core biopsies, radiation exposure, amount of contrast used, intra-operative complications, and follow-up data were recorded. Pre-operative demographics including age, gender, body mass index, prior biopsy pathology, and tumor size were analyzed. One month post-operative complication events were evaluated using the Clavien-Dindo classification of surgical complications.

Results: All procedures were carried to conclusion and were performed by the Urologic surgeon. Complications were minimal (6), all Clavien 1. See Table $1 \& 2$.
Conclusions: Cone beam CT is a useful adjunct for performing interventional procedures. Due to its ease of use, there is a short learning curve. Urologic surgeons can use the guidance system independently with great accuracy and minimal complications.

MP23-18 Targeted MRI/US Fusion Prostate Biopsy: The First 212 Patients Without a History of Prostate Cancer Relationship Between PIRADS Score and Prostate Cancer Detection Rate: A Single Urologist's Experience.

A Kasraeian, J Cesaretti, J Yellin, A Brochert, B Noell, A Kasraeian

Kasraeian Urology

United States

Introduction \& Objective: Multi-parametric MRI (mpMRI) and targeted MRI/Ultrasound fusion prostate biopsy (tMRI/US FP) are important tools in the diagnosis of prostate cancer (PCA). We report our experience with our first 260 patients. Specifically, we report the relationship between PIRADS score and overall positive biopsy rate as well as detection rate of Gleason 7 or higher PCA in men without a previous history of PCA.

Materials and Methods: Data was prospectively collected and analyzed on 260 men undergoing tMRI/US FPB between September 2014 and December 2016. In all patients, mpMRI was interpreted by a single radiologist using the PIRADS scoring system. 3D rendering of regions of interest was performed prior to tMRI/US FPB performed by a single urologist using the Invivo UroNav System. Concurrent standard 14 core biopsy (14Bx) was performed in all cases.

Results: Combined tMRI/US FP with 14Bx (tMRI/US FP-14Bx) was performed on 260 men between September 2014 and December 2016 of whom 212 presented with rise in PSA or abnormal digital rectal exam and 48 with history of low risk (LR) PCA on active surveillance (AS). Of the 260 men included, mean age was 65 years, mean PSA $7.9 \mathrm{ng} / \mathrm{ml}$ and mean prostate volume 55.6 cc. Overall, 59\% (152/260) of the men biopsied were diagnosed with prostate cancer. Overall cancer detection rate was $55 \%(116 / 212)$ for men without a previous history of PCA and $75 \%(36 / 48)$ for patients on active surveillance $(\mathrm{p}=0.01)$. Of the men without a previous history of PCA, 55\% (116/212) men were diagnosed with PCA using tMRI/US FP-14Bx versus (vs) tMRI/ US FPB alone, $47.2 \%(100 / 212, \mathrm{p}=0.15)$, and 14Bx alone, $42.9 \%$ $(91 / 212, p=0.02)$. In the PCA naïve patients, detection rate of Gleason $\geq 7$ cancers was $37.7 \%$ (80/212) for the combined approach versus $31.6 \%(67 / 212, \mathrm{p}=0.22)$ for fusion alone and $30.2 \%(64 / 212, p=0.12)$ for $14 \mathrm{Bx}$ alone, respectively. Relationship

\begin{tabular}{|l|l|l|l|l|l|l|l}
\hline PIRADS & Number & Any Cancer & $\begin{array}{l}\text { Percentage } \\
\text { Cancer } \\
\text { Detected }\end{array}$ & $\begin{array}{l}\text { Gleason } \\
7,8,9,10\end{array}$ & $\begin{array}{l}\text { Percentage } \\
\text { highest } \\
7,8,9,10\end{array}$ & & \\
\hline 1 & 1 & 1 & & 1 & & & \\
\hline 2 & 13 & 8 & $62 \%$ & 2 & $15 \%$ & & \\
\hline 3 & 16 & 12 & $75 \%$ & 8 & $50 \%$ & & \\
\hline 4 & 8 & 8 & $100 \%$ & 4 & $50 \%$ & & \\
\hline 5 & 4 & 4 & $100 \%$ & 4 & $100 \%$ & & \\
\hline Unassigned & 6 & 3 & $50 \%$ & 2 & $33 \%$ & & \\
\hline total & 48 & 36 & $75 \%$ & 21 & $44 \%$ & & \\
\hline
\end{tabular}

Table 1. An analysis of PIRADS score to cancer incidence and Gleason score $\geq 6$ in 48 men, with a prior history of prostate cancer on AS, who underwent multi-parametric MRI and targeted MRI/UItrasound fusion prostate biopsy. 


\begin{tabular}{|l|l|l|l|l|l|l|l|}
\hline PIRADS & Number & $\begin{array}{l}\text { Any } \\
\text { Cancer }\end{array}$ & $\begin{array}{l}\text { Percentag } \\
\text { e Cancer } \\
\text { detected }\end{array}$ & $\begin{array}{l}\text { Gleason } \\
7,8,9,10\end{array}$ & $\begin{array}{l}\text { Percentag } \\
\text { e highest } \\
7,8,9,10\end{array}$ & & \\
\hline 1 & 1 & 1 & $100 \%$ & & & \\
\hline 2 & 33 & 9 & $27 \%$ & 6 & $18 \%$ & \\
\hline 3 & 76 & 31 & $41 \%$ & 15 & $20 \%$ & \\
\hline 4 & 69 & 46 & $67 \%$ & 34 & $49 \%$ & \\
\hline 5 & 21 & 21 & $100 \%$ & 19 & $90 \%$ & \\
\hline unassigne & 12 & 8 & $67 \%$ & 6 & $50 \%$ & & \\
d & & & & & & & \\
\hline total & 212 & 116 & & & & \\
\hline & & & & & & \\
\hline
\end{tabular}

Table 1. An analysis of PIRADS score to cancer incidence and Gleason score $\geq 6$ in 212 men who underwent multi-parametric MRI and targeted MRI/Ultrasound fusion prostate biopsy. pathological results Gleason score $6(3+3)$ was found in $3(15 \%)$ men, $7(3+4$ or $4+3)$ in $12(60 \%)$ men and $8-10$ in $5(25 \%)$ men. At MR Bx 117 core biopsies were taken from 42 lesions. Mean core number was 2,7 (1-5) for per lesion. Prostate cancer positive core number was $53(45,2 \%)$. In prostate cancer positive patients 62 core biopsies were taken from 21 lesions and total biopsy/ positive core ratio was found $85,4 \%$. At TRUS Bx 456 core biopsies were taken from 38 patients. Mean core number was found 12 for per patient. Prostate cancer positive core number was $27(5,9 \%)$. In prostate cancer positive patients 132 core biopsies were taken and total biopsy/positive core ratio was found $20,4 \%$.

Conclusions: MR targeted prostate biopsy is more efficient than standard biopsy and diagnosing cancer cases fewer cores.

between PIRADS score and overall rate of PCA detected and Gleason Score 7 or higher detected is listed in Table 1.

Conclusions: tMRI/US FP is an important technology which increases the diagnostic yield of prostate biopsy when combined with 14Bx. In our series, the increased positive biopsy rate with combined tMRI/US FP-14Bx was statistically significant when compared to $14 \mathrm{Bx}$ alone. In addition, tMRI/US FP-14Bx found more Gleason 7 or higher PCA in PCA naïve patients. Lastly, PIRADS 4 and 5 lesions on mpMRI were associated with higher rate of both overall and Gleason $\geq 7$ PCA detection.

MP23-19 MR Targeted Prostate Biopsy against Standard Ultrasound Guided Prostate Biopsy. A Single Center Experience : First Results

E Firat, Y ÖZLÜLERDEN, Tuncay, A YAĞCI

Professor

Turkey

Introduction \& Objective: Standart transrectal ultrasound guided biopsy (TRUS Bx) is the currently internationally accepted diagnostic procedure to detect prostate cancer. TRUS Bx has a low sensitivity (about 40\%) so it may lead to repeat biopsies and this situation causes increased complication and infection rates. Multi-parametric MR imaging (mpMRI) has recently emerged as a diagnostic technique and accurately localize significant cancer in the prostate. MR targeted prostate biopsy (MR $\mathrm{Bx}$ ) has been shown to reduce the detection of low-risk cancer and increase the detection rate of intermediate- and high-risk cancer, while using fewer cores. The aim of this study is to evaluate the efficiency of MR targeted prostate biopsy and standard ultrasound guided prostate biopsy.

Materials and Methods: From August 2016 to February 2017, 38 men were enrolled in this study. Multi-parametric MR imaging was performed all patients and lesions were scored according to PIRADS version 2. 42 lesions were detected by $\mathrm{mp}$ MRI. MR targeted and standard prostate biopsies performed during the same session. Age, PSA values, PIRADS scores, pathology results, Gleason scores, total biopsy numbers and positive biopsy numbers were noted.

Results: Mean age was 65.7 (50-84) years and mean PSA value was $13,5(1,35-139,4) \mathrm{ng} / \mathrm{dl}$. A total of $18(47,4 \%)$ men were diagnosed benign pathology. A total of 20 men (52.6\%) were diagnosed with prostate cancer either on standard biopsy, MR targeted biopsy, or both. $8(40 \%)$ patients were diagnosed with only MR Bx, $1(5 \%)$ patient was diagnosed with only TRUS Bx and $11(55 \%)$ patients were diagnosed with both. According to
MP23-20 Geographical variation in the utilization of MRI/ Fusion prostate biopsies among 160 outpatient health care facilities in New York State between 2008-2012.

\section{KH Bilal, M Finkelstein, MA Palese}

Icahn School of Medicine at Mount Sinai

Introduction \& Objective: MRI/Fusion biopsies of prostate have gained significant popularity among urologists due to their accuracy and efficacy. The objective of this study was to observe the geographical variation in the utilization of MRI/Fusion prostate biopsies in New York State between 2008 and 2012.

Materials and Methods: Statewide Planning and Research Cooperative Systems database of New York state was used to study 14,650 patient records from over 160 different outpatient health care facilities. These records were extracted from the database based on CPT codes used for the billing of MRI/Fusion biopsies. Patient records were also stratified based on the geographical location of the facility at which the procedure took place. The data was then plotted over time as well as over geographical space to observe the utilization trends between 20082012.

Results: During the observation period, the annual frequency of MRI/Fusion biopsies increased significantly from 1628 in 2008 to 4694 in $2012(\mathrm{p}<0.05)$ within New York State. It is also interesting to note that while the frequency of MRI/Fusion biopsies increased across the state, this increase was not similar across all regions. Three regions with the greatest utilization of MRI/Fusion biopsies were New York City, Long Island, and

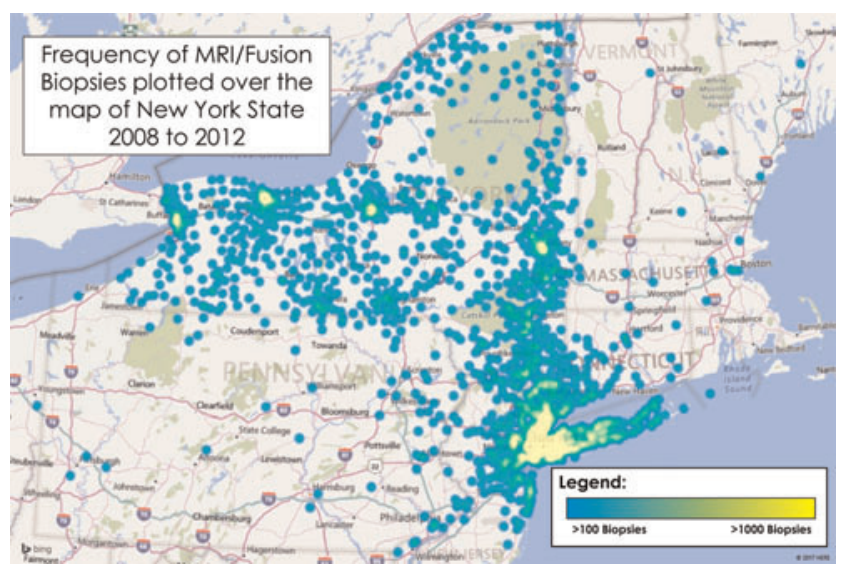
United States 


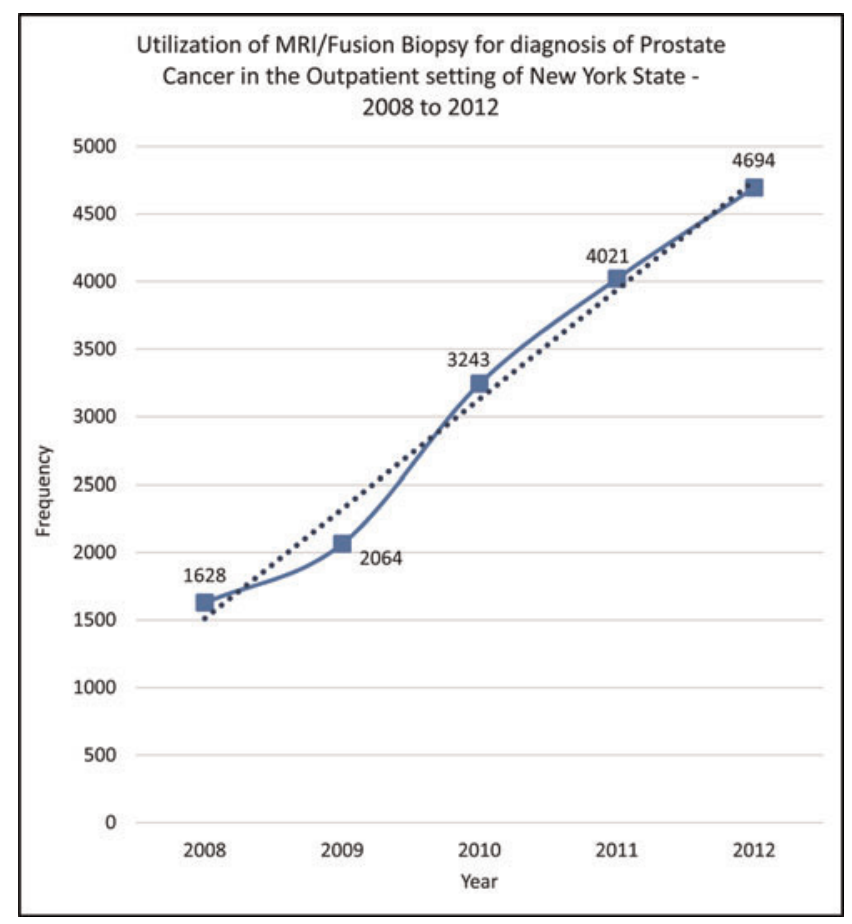

Syracuse with $37.84 \%, 18.87 \%$ and $16.41 \%$ of all cases respectively.

Conclusions: Our results show that the frequency of MRI/Fusion biopsies utilized in New York State has increased steadily and significantly from 1628 to 4694 over the study period of 2008 to 2012. Moreover, using facility zip code data associated with each patient record, we see that this increase was experienced at a greater rate at certain regions in the New York State area. New York City, Long Island, and Syracuse had the greatest rise in MRI/Fusion biopsies.

MP23-21 The Impact of mpMRI on Prostate Cancer Detection With Respect to Prostate Volume

M Glover, R Abboud, A Mahran, C Buzzy, R Thawani, M Wang, V Gulani, L Ponsky, I Jaeger

United States

Introduction \& Objective: It has been reported that prostate volume influences detection of high-grade prostate cancer ( $\mathrm{PCa}$ ) in transrectal ultrasound-guided (TRUS) biopsies. Here, we examined whether prostate volume affects the detection of clinically significant PCa using multiparametric MRI (mpMRI) as compared to TRUS.

Materials and Methods: Patients with a positive TRUS biopsy that underwent an mpMRI within one year of their biopsy $(n=261$, age $68 \pm 7.8$ years, median prostate volume $40.4 \mathrm{cc})$

\begin{tabular}{|c|c|c|c|c|c|c|}
\hline \multirow[b]{2}{*}{ Models } & \multicolumn{2}{|c|}{ Model Outcomes } & \multicolumn{3}{|c|}{ Univariate Analysis } & \multirow[b]{2}{*}{ p value } \\
\hline & Sensitivity & Specificity & Odds Ratio $(95 \% \mathrm{Cl})$ & pvalue & $\operatorname{AUC}^{1}(95 \% \mathrm{CI})$ & \\
\hline$\left.m p M R\right|^{2}$ & $49.2 \%$ & $54.3 \%$ & $0.864(0.499-1.50)$ & 0.602 & $0.482(0.403-0.561)$ & 0.652 \\
\hline TRUS Biopsy? & $56.9 \%$ & $58.6 \%$ & $2.11(1.25-3.57)$ & 0.005 & $0.592(0.519-0.665)$ & 0.015 \\
\hline
\end{tabular}

were studied. Experienced radiologists reported both the PIRADS score and prostate volume for each patient. Biopsies were performed using 6 or 12-core templates and the highest Gleason score was reported. Patients were stratified based on prostate volume ( $\leq 40 \mathrm{cc}$ vs. $>40 \mathrm{cc}$ ). Clinically significant $\mathrm{PCa}$ was defined as PI-RADS 4 or 5 for mpMRI and Gleason Score $\geq 4+3$ for biopsy.

Results: Chi-squared analysis showed that the incidence of clinically significant PCa was dependent on prostate volume for biopsy $(p=0.005)$, but not for mpMRI $(p=0.61)$. The fraction of patients with Gleason scores $\geq 4+3$ were $41.5 \%$ (54/130) and $25.2 \%$ (33/131) for small and large prostates. As for mpMRI, the percentage of patients with PI-RADS 4 or 5 was $74.6 \%(97 / 130)$ for small and 71.8\% (94/131) for large prostates. AUC and logistic regression analysis (Table 1) showed that prostate volume $\leq 40 \mathrm{cc}$ is predictive for clinically significant cancer on TRUS biopsy, not mpMRI.

Conclusions: Unlike TRUS biopsy, MRI was not found to be dependent on prostate volume in diagnosing clinically significant cancer. It is important to be aware of this limitation of TRUS biopsy where clinically significant cancer can be missed in large prostates at a higher rate than small prostates due to undersampling.

MP23-22 Modeling pre-biopsy and mpMRI variables: Avoiding unnecessary prostate biopsies

R Abboud, C Buzzy, A Mahran, M Glover, R Thawani, M Wang, I Jaeger, V Gulani, L Ponsky

\section{UHCMC}

United States

Introduction \& Objective: The National Comprehensive Cancer Network (NCCN) recommends a prostate biopsy for patients with an elevated PSA, abnormal digital rectal exam (DRE) or a previous biopsy with adenocarcinoma, high-grade prostatic intraepithelial neoplasia (HGPIN) or atypical simple acinar proliferation (ASAP). As multi-parametric magnetic resonance imaging (mpMRI) becomes more widely accepted as a viable screening tool for prostate cancer (PCa), it is important for clinicians to consider MRI data when assessing the need for a transrectal ultrasound guided (TRUS) biopsy. This study aims to identify parameters for a positive biopsy in patients with a nonpositive mpMRI.

Materials and Methods: In a retrospective IRB-approved protocol, treatment-naive patients were analyzed with a non-positive mpMRI (PI-RADS $\leq 3$ ) and a TRUS biopsy that occurred within one year of mpMRI $(n=138$, mean age of $64.5 \pm 9.0$ yrs with mean time between MRI and biopsy of $96.2 \pm 95$ days). Following the standard of care, mpMRI's performed six weeks after a biopsy were excluded. Patients were stratified in two groups based on their TRUS biopsy findings for adenocarcinoma. SPSS was used for the analysis.

Results: On univariate analysis, the following variables were predictive of a positive biopsy: small prostate volume $(<45 \mathrm{cc})$, PI-RADS 3 lesions and non-apical lesions on MRI. These variables along with other clinically relevant parameters (Table 1) were modeled using multivariate regression. After backward stepwise elimination, six variables remained in the final model (Table 1, in bold). Area under the curve (AUC) analysis for the Biopsy Predictor Model $(\mathrm{AUC}=0.774)$ returned a value over 1.5 times the NCCN's biopsy guidelines $(\mathrm{AUC}=0.473)$. Our model 


\begin{tabular}{|c|c|c|c|c|c|c|}
\hline \multirow[b]{2}{*}{ Variables } & \multicolumn{2}{|c|}{ Deseriptive Statisties } & \multicolumn{2}{|c|}{ Univariate Analysis } & \multicolumn{2}{|c|}{ Multivariate Analysis } \\
\hline & Negative Biopsy & Positive Biopsy & Odds Ratio $[95 \% \mathrm{Cl}]$ & p Value & Odds Ratio [95\% C] & pValue \\
\hline Prostate Volume ( $(45 \mathrm{ce})$ & $19 / 71(26.8 \times 1)$ & $37 / 67(55.2 \mathrm{2N})$ & $3.38(1.66-6.88)$ & $<0.001^{2}$ & $4.85(2.14-11.0)$ & $<0.001$ \\
\hline Pl-RADS 3 & $28 / 71(39.48)$ & $39 / 67(58.286)$ & $2.14(1.08-4.22)$ & $0.028^{2}$ & $3.06(1.35-6.95)$ & 0.008 \\
\hline Apex Lestion (MRI) & $23 / 71$ (32.486) & $11 / 67(16.489)$ & $0.410(0.181-0.926)$ & $0.032^{4}$ & $0.273(0.103-0.720)$ & 0.009 \\
\hline ASAP or HG-PIN (Biopay) & $3 / 71(4.22 \times 6)$ & $9 / 67(13,4 \times)$ & $3.52(0.909-13.6)$ & $0.068^{4}$ & $4.20(0.972-18.1)$ & 0.055 \\
\hline Race (Africen Americen) & $12 / 71(16.996)$ & $17 / 67(25.45)$ & $1.67(0.729-3.83)$ & $0.2255^{t}$ & $2.49(0.948-6.55)$ & 0.064 \\
\hline Elevated PSA $(\mathrm{ng} / \mathrm{ml})$ & $53 / 71(74.6 \%)$ & $44 / 67(65.756)$ & $0.650(0.312-1.36)$ & $0.250^{1}$ & $0.445(0.185-1.07)$ & 0.070 \\
\hline Personal History, Cancer & $10 / 71(14.18)$ & $4 / 67(5.9776)$ & $2.58(0.769-8.67)$ & $0.125^{t}$ & & \\
\hline Abnormal DRE & $12 / 71(16.9 \times)$ & $17 / 67(25.4 \times 3)$ & $1.67(0.729-3.83)$ & $0.225^{1}$ & & \\
\hline Age of Bliopsy (yrs) & $63.8^{*}( \pm 10.0)$ & $65.3^{*}( \pm 7.92)$ & $1.02(0.980-1.06)$ & $0.324^{2}$ & & \\
\hline
\end{tabular}

has a sensitivity of $93.1 \%$, specificity of $31.0 \%$ and negative predictive value of $81.5 \%$, ultimately improving detection threefold for patients with PCa that were misdiagnosed by NCCN's guidelines. Furthermore, our model reduced unnecessary biopsies by about $17 \%$.

Conclusions: This analysis suggests that alternative clinical parameters, namely apex lesions and prostate volume, should be taken into consideration after a non-positive MRI to determine the need for TRUS biopsy. Clinicians already take into account most of the variables we analyzed; our model offers a quantifiable probability that TRUS biopsy will detect $\mathrm{PCa}$, which can further assist Urologists in their clinical approach.

MP23-23 Prognostic grade group prostate cancer grading system: can multiparametric MRI accurately separate patients with PGG 1, PGG2 and high grade cancer?

K Tay, J Holtz, R Silverman, C Sze, R Gupta, T Polascik

Duke University Medical Center

United States
Introduction \& Objective: Our objective is to determine the accuracy of multiparametric MRI (mpMRI) in predicting pathologic grade of prostate cancer after radical prostatectomy (RP) using the new prognostic grade group (PGG) grading criteria and specifically, whether mpMRI can accurately separate disease into one of two risk categories (low versus higher grade) or one of three risk categories (low, intermediate, or high grade).

Materials and Methods: This retrospective, HIPAA-compliant, IRB-approved study included 140 patients with $\mathrm{PCa}$ who underwent $3 \mathrm{~T}$ mpMRI with endorectal coil and transrectal ultrasound-guided (TRUS-G) biopsy before RP. MpMRI was used to classify lesions using a two-tier (low grade/PGG 1 vs. high grade/ PGG 2-5) or a three-tier system (low grade/PGG 1 vs. intermediate/PGG 2 vs. high grade/PGG 3-5). Accuracy of mpMRI was compared against RP for each classification system. Sensitivity, specificity, positive predictive value (PPV), negative predictive value (NPV), positive likelihood ratio (LR+), and negative likelihood ratio (LR-) of mpMRI for distinguishing low from higher grade disease were also calculated.

Results: The predictive accuracy of mpMRI using the two-tier system is higher than when using three-tier system $(0.77 \& 0.45$, respectively). The sensitivity, specificity, PPV, NPV, LR+, and LR- are $0.94,0.16,0.80,0.45,1.13$ and 0.34 , respectively. There were similar rates of undergrading between mpMRI and TRUSG biopsy compared to RP ( $16 \%$ \& $21 \%$; respectively); rate of overgrading of disease was higher for mpMRI versus TRUS-G biopsy compared to RP ( $42 \%$ \& 17\%, respectively). When mpMRI and TRUS-G biopsy are combined, rate of undergrading is $1.4 \%$ and overgrading is $11 \%$.

Conclusions: The predictive accuracy of mpMRI is higher using a two-tier system versus a three-tier system, suggesting quantitative variables may be necessary to delineate intermediate from high grade disease. Rates of under- and overgrading decreased when mpMRI and TRUS-G biopsy are combined, suggesting these techniques may be complementary in accurately predicting tumor grade.

\section{MPS24: BPH/LUTS OUTCOMES}

\section{MP24-1 Relationship Between Intravesical Prostatic Pro- trusion (Ipp) And Postoperative Outcomes In Patients With Benign Prostatic Hyperplasia}

S Ramani

ST ISABEL

India

Introduction \& Objective: TURP has withstood the test of times in being the gold standard treatment in the management of BPH.a Holmium laser (HoLEP) is currently taken to be the gold standard procedure. TURP still remains the widely used technique for the management of BPH. The main objective of this study was to compare the outcomes of TURP in cases with and without significant IVPP (Intravesical prostatic Protrusion). IPP occurs as the prostate expands into the bladder along the plane of least resistance and is generally caused by enlargement of the median lobe with or without enlargement of the lateral lobes. A high IPP grade can also be a strong outcome predictor after TURP.
Materials and Methods: Patients who were admitted in Kilpauk Medical College and Govt. Royapettah Hospital with lower urinary tract symptoms (LUTS) due to benign prostatic hyperplasia (BPH) with clear evidence of bladder outflow obstruction with a volume $>30 \mathrm{gms}$ were included. They were grouped into three groups based on it Group A - IVPP Group B - IVPP 5 $10 \mathrm{~mm}$ Group C - IVPP $>10 \mathrm{~mm}$ The study design was a prospective clinical study. Preoperative variables (symptom scores, QoL, PVR, uroflowmetry parameters) comparable in between the two groups. Operative variables (operating time, amount of irrigation fluid and blood transfusion required) to be observed and recorded. Postoperative catheterization period and hospital stay (in days) noted.Post operative variables (symptom scores, QOL, PVR, uroflowmetry parameters) to be compared.

Results: Statistical Package for the Social Sciences, version 16.0.0 (SPSS Inc) was used for the statistical analysis. There was no difference with respect to the operative time, hospital stay and amount of irrigation fluid used. The pre op retention was compared between the three groups using Chi Square test and it was high in Group $\mathrm{C}$ when compared to $\mathrm{b}$ and $\mathrm{a}(\mathrm{p}=0.002)$. The $\mathrm{p}$ 
value for the change of QOL was clinically significant $(p=0.47)$. Group $\mathrm{C}$ had a greater betterment in QOL when compared to a and $b$. The change in IPSS was significant between the groups $(\mathrm{p}=0.542)$.

Conclusions: We compared the relationship between the IVPP with the level of obstruction pre operatively. The pre op retention was significantly higher in Group $C(p=0.002)$. The number of patients in group $\mathrm{C}$ who had AUR were more $(50 \%)$ than B (40\%) and A (15\%). There was a clinically significant higher QOL in Group C when compared to $\mathrm{a}$ and $\mathrm{b}$. We expect that together with traditionally accepted preoperative parameters, the IPP can help to predict a better surgical outcome. In conclusion, IPP is an independent parameter for predicting postoperative outcomes in BPH patients who undergo TURP.

\section{PROFILE PLOT IPSS}
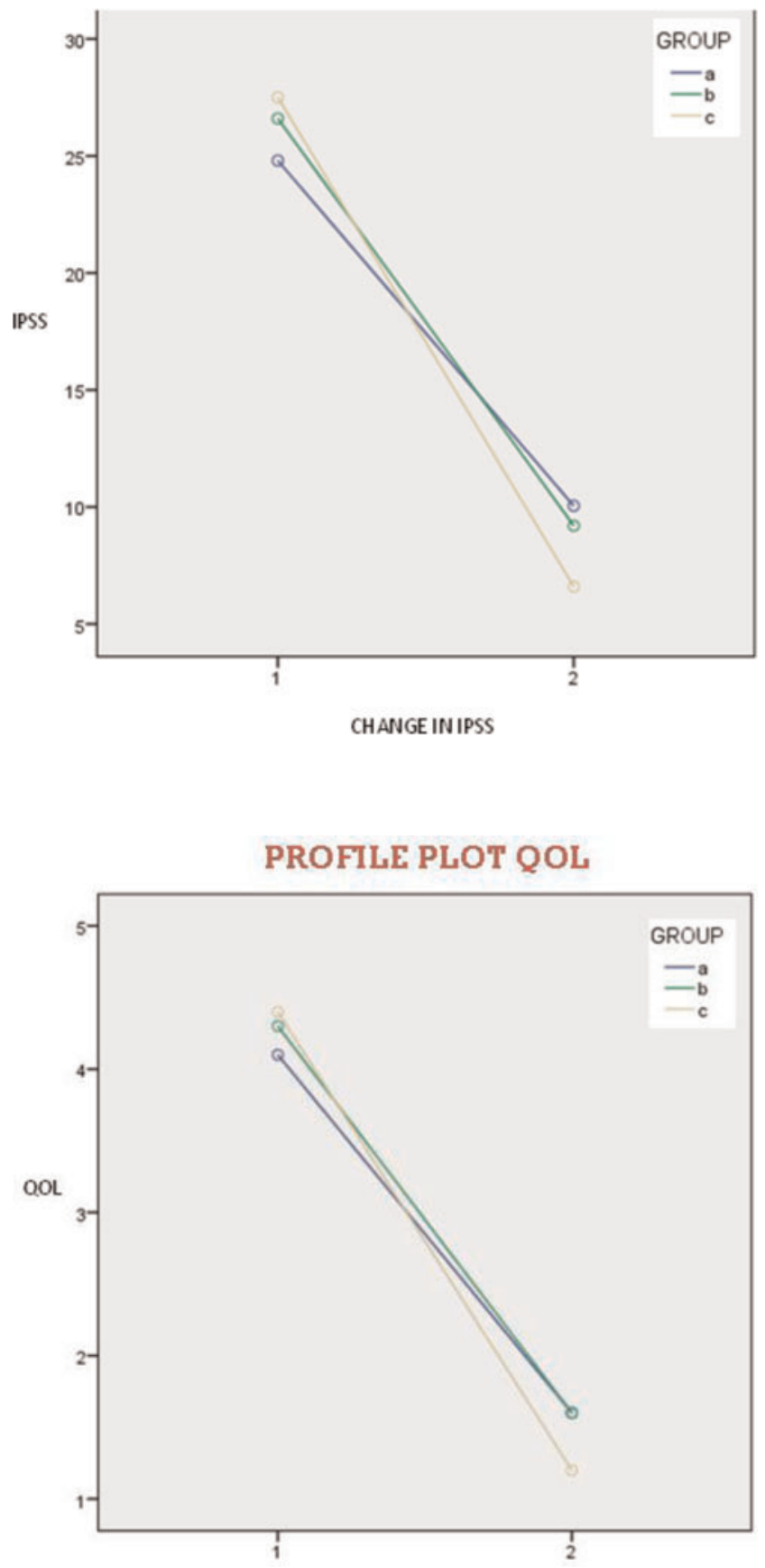

IPSS

\begin{tabular}{|c|c|c|}
\hline GROUP & Pre op IPSS & Post op IPSS \\
\hline a & $24.08 \pm 3.1$ & $10.05 \pm 2.5$ \\
\hline b & $26.6 \pm 2.8$ & $9.02 \pm 3.4$ \\
\hline c & $27.5 \pm 4.5$ & $6.6 \pm 1.3$ \\
\hline
\end{tabular}

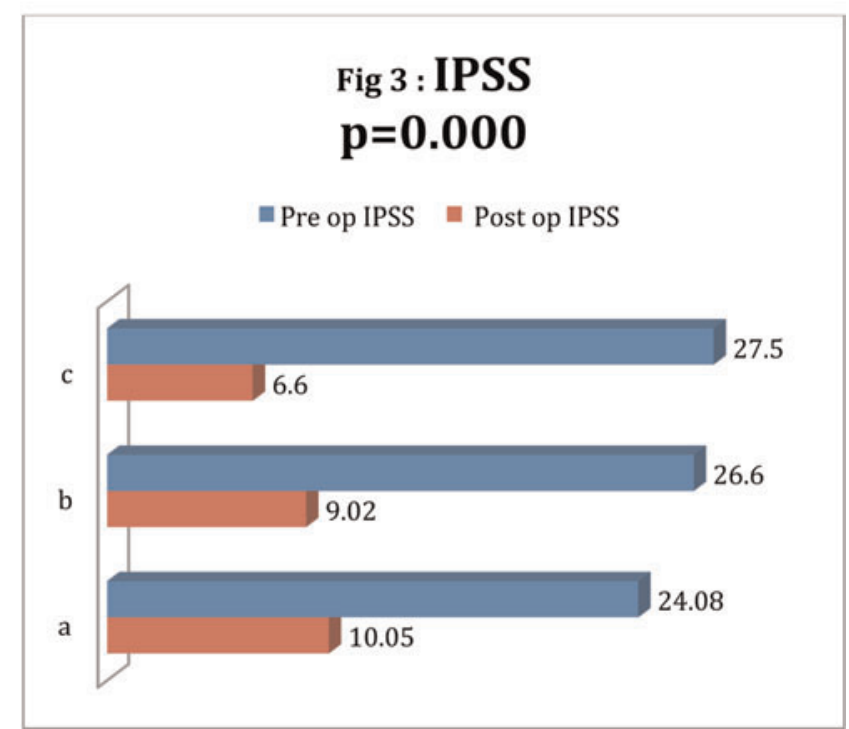

QOL

\begin{tabular}{|c|c|c|}
\hline GROUP & Pre op QOL & Post op QOL \\
\hline a & $4.1 \pm 0.3$ & $1.6 \pm 0.5$ \\
\hline b & $4.3 \pm 0.4$ & $1.6 \pm 0.6$ \\
\hline c & $4.2 \pm 0.4$ & $1.2 \pm 0.4$ \\
\hline P value & 0.09 & 0.047 \\
\hline
\end{tabular}

The QOL before and after surgery was compared between the groups. There was a clinically significant higher QOL in Group $\mathrm{C}$ when compared to $\mathrm{a}$ and $\mathrm{b}$.

Fig 4: QOL

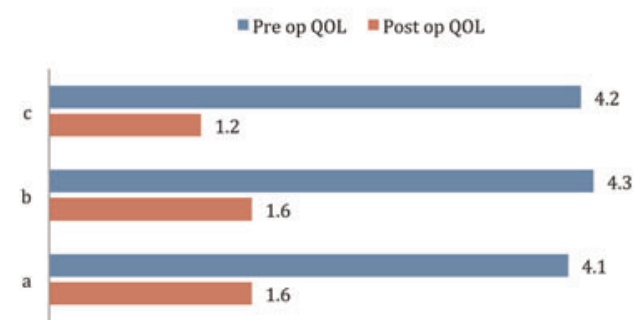

MP24-2 Postoperative dysuria after high- and low-power en-bloc no-touch HoLEP

CM Cracco, CM Scoffone

Department of Urology, Cottolengo Hospital of Torino, Italy Italy 
Introduction \& Objective: HoLEP (Holmium Laser Enucleation of the Prostate) is a safe and effective procedure for benign prostatic obstruction (BPO) treatment. Six years ago we modified the traditional 3-lobe technique into the so-called en-bloc notouch approach, characterized by the enucleation of the adenoma in one single horseshoe-like piece, exploiting the vaporizing effects of the plasma bubble generated around the tip of the laser fiber at a short distance from the tissue. Transient storage symptoms, mostly resolving spontaneously or with medical therapies within 1-3 months, are described among the early complications in $9-59 \%$ of patients who underwent HoLEP and have been correlated also with energy consumption. The aim of the present study was to determine whether postoperative dysuria is somehow influenced by the use of a low-power rather than of a high-power one.

Materials and Methods: 316 patients suffering from BPO (any prostate volume, normal PSA, Qmax 10 , PVR <300 cc) underwent en-bloc no-touch HoLEP in our Department. From January 2012 to May 2015214 consecutive patients underwent highpower HoLEP (group 1) with the 100-120W Versapulse holmium laser (Lumenis), 2J energy setting, $50 \mathrm{~Hz}, 100 \mathrm{~W}$ power. From June 2015 to June 2016102 consecutive patients underwent low-power HoLEP (group 2) for the first 20 cases with the Versapulse holmium laser (Lumenis), then the 50W Auriga XL holmium laser device (Boston Scientific), both 2.2J energy setting, $18 \mathrm{~Hz}$ frequency, long pulse length, almost $40 \mathrm{~W}$ power. Patients demographics and clinical data were prospectively registered. IPSS questionnaires were self-administered before surgery and at 3month follow up, VAS pain evaluation 1 month after surgery.

Results: Age (range 51-87 years) and adenoma weight (range 10-200 grams) were similar in the two groups. Mean energy employed for enucleation was $83.5 \mathrm{~kJ} \pm 32$ d.s. for group 1 , $53.4 \mathrm{~kJ} \pm 23$ d.s. for group $2(\mathrm{p}<0.01)$, with a $\mathrm{kJ} / \mathrm{g}$ ratio $2 \pm 1 \mathrm{vs}$. $1.5 \pm 0.8$. Mean enucleation time was equivalent $(31 \mathrm{~min} \pm 13$ d.s. vs. $27.5 \mathrm{~min} \pm 11 \mathrm{~d}$.s.), mean enucleation efficiency too $(1.64 \mathrm{~g} /$ $\min \pm 0.8$ d.s. vs. $1.7 \mathrm{~g} / \mathrm{min} \pm 1 \mathrm{~d}$.s.). Pre- and postoperative IPSS were similar (pre: $22 \pm 2.4$ d.s. vs. $22 \pm 7$ d.s.; post: $6.5 \pm 5$ d.s. vs. $7.8 \pm 5$ d.s.). Postoperative dysuria had the same incidence $(10 \%)$, but in group 2 mean VAS pain evaluation at 1-month follow up was significantly better $(6.2 \pm 1.5$ d.s. in group 1 , $2.4 \pm 3$ d.s. in group 2).

Conclusions: Low-power en-bloc no-touch HoLEP uses less energy than the high-power approach, with reduced $\mathrm{kJ} / \mathrm{g}$ ratio and similar postoperative dysuria $(10 \%)$, being intensity and duration of the storage symptoms reduced.

\section{MP24-3 Serum prostate specific antigen levels after green laser enucleation of the prostate (GreenLEP). \\ E Rijo, V Misrai, S Tabatabaei, G Ferrari, F Gomez-Sancha \\ Department of Urology, Hospital Quiron Barcelona. Spain}

Introduction \& Objective: Green laser enucleation of the prostate (GreenLEP) is one of the latest techniques in the armory of endoscopic enucleation of the prostate (EEP) for the treatment of benign prostatic hyperplasia (BPH).

Changes in the prostate specific antigen (PSA) after GreenLEP are not well known. To date, analysis of PSA drop after GreenLEP have not been published. The aim of this study is to determine the change of serum PSA following GreenLEP and the relationship between PSA drop and the weight of tissue removed.

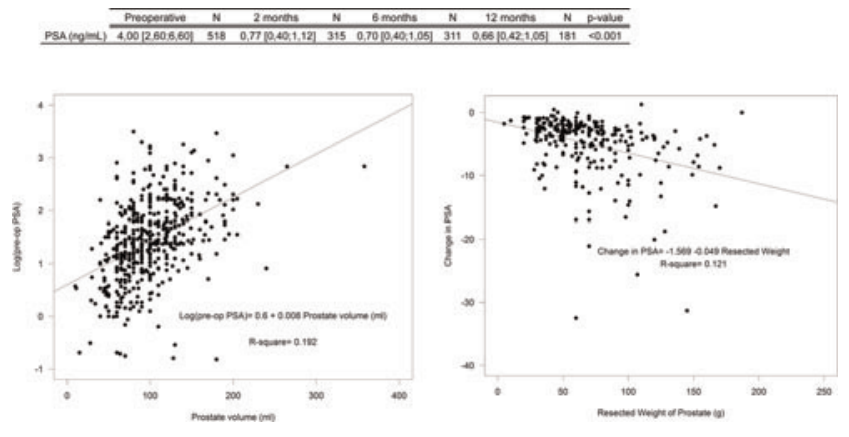

Materials and Methods: Between June 2013 and November 2016, 586 cases of GreenLEP "en bloc" technique were performed at five institutions by five surgeons. Serum PSA levels were measured before and after the procedure at 2, 6 and 12 months. Patients with serum PSA $>20 \mathrm{ng} / \mathrm{mL}$ before surgery or prostate cancer were excluded.

Preoperative TRUS prostate volume and intraoperatively weight of morcellated tissue was recorded. Linear regression analysis was performed for comparing the change in PSA with the weight of morcellated tissue, and comparing preoperative PSA with TRUS volume (data normalization by log transformation of the preoperative PSA prior to linear regression analysis).

Results: The median age of the patients was 68.0 [64.0;73.0], median preoperative PSA level was $4.0 \mathrm{ng} / \mathrm{mL}[2.60 ; 6.61]$. The preoperative TRUS volume averaged 95.0 [70.0;120]. The median 2-month postoperative PSA was $0.77 \mathrm{ng} / \mathrm{mL}$, median 6month PSA 0.70 and 12 -month PSA $0.67 \mathrm{ng} / \mathrm{mL}$.

The median 2-month PSA drop was $3.29 \mathrm{ng} / \mathrm{ml}$ and the median 6-month PSA drop $3.12 \mathrm{ng} / \mathrm{ml}$. The percent reduction in PSA at 2 months was $94.4 \%$.

The log-transformed PSA correlated directly with preoperative TRUS volume $(r=0.4377609, p<0.001)$. Linear regression comparison of the weight of adenoma morcellated with the absolute change in PSA demonstrated that, as the prostatic adenoma increased in size, GreenLEP caused a predictable change in PSA $(r=0.3478639, \mathrm{p}<0.001)$.

Conclusions: GreenLEP dramatically reduces de serum PSA levels $(94.4 \%)$. This dramatic reduction of PSA suggest a complete removal of prostatic adenoma. The results of our study are similar to previous reports on HoLEP PSA drop.

MP24-4 Does a partial prostate resection improve voiding symptoms while shortening the learning curve for HOLEP?

S Bazargani, S Ghodoussipour, A Shah, E Thompson, A Mitra, MD Dunn

UCLA Department of Urology

United States

Introduction \& Objective: Holmium laser enucleation of the prostate (HOLEP) is an ideal option for very large prostate glands that would otherwise be managed by simple prostatectomy. However, a steep learning curve has limited widespread adoption of this procedure. Early in our experience we staged very large glands by removing the median lobe when necessary with enucleation of only one lateral lobe to reduce excessive OR times. These patients were followed to determine if a partial 
Table 1: Characteristics of 84 patients undergoing either complete or partial HOLEP.

\begin{tabular}{|c|c|c|c|}
\hline & $\begin{array}{c}\text { Partial HOLEP } \\
(\mathrm{N}=14)\end{array}$ & $\begin{array}{c}\text { Complete HOLEP } \\
(\mathrm{N}=70)\end{array}$ & $p$ value \\
\hline Age & 69 & 72 & 0.9 \\
\hline BMI & 30 & 29 & 0.4 \\
\hline $\begin{array}{c}\text { Prostate size } \\
(\mathrm{g})\end{array}$ & $93(48-200)$ & $62(35-95)$ & 0.020 \\
\hline $\begin{array}{c}\text { Content about } \\
\text { quality of life } \\
\text { (yes/no) }\end{array}$ & $6(43 \%)$ & $56(80 \%)$ & 0.031 \\
\hline \begin{tabular}{c} 
OR time (Min) \\
\hline
\end{tabular} & 129 & 150 & 0.07 \\
\hline
\end{tabular}

HOLEP adequately reduced obstructing voiding symptoms and whether additional procedures to complete the enucleation were necessary.

Materials and Methods: We performed a retrospective review of 84 patients with bladder outlet obstruction who underwent a HOLEP with a single surgeon between 1/2013-10/2016. We specifically evaluated whether the improved symptoms maintained over time or whether obstructive symptoms re-developed requiring another intervention.

Results: The median follow up time was 18.5 months. Partial HOLEP was done in 14 patients. Compared to those with complete HOLEP, those with a partial HOLEP had larger glands (median 93 vs $62 \mathrm{~g}, \mathrm{p}=0.02$ ) but there was no significant difference in age or BMI (Table 1). Of the partial HOLEPS, 6 patients were content with their symptoms with significant improvement of uroflow parameters and IPSS scores $(\mathrm{p}=0.03)$ at last date of follow up. Eight patients of the 14 patients had persistent symptoms or developed new symptoms such that they required another intervention. One patient developed gross hematuria from the remaining lobe, one patient developed a bladder stone, and the remaining 6 complained of returning obstructive voiding symptoms requiring a completion resection.

Conclusions: While performing a partial HOLEP may maintain lower OR times and assist with the learning curve, the majority of patients will require another surgery within 2 years due to recurrent symptoms or complications such as bleeding and bladder stones. A complete HOLEP should be performed whenever possible to optimize patient outcomes.

MP24-5 The efficacy of fibrin glue injection in the prostatic fossa on decreasing postoperative bleeding following transurethral resection of prostate

M KHORRAMI, K nouri mahdavi

isfahan university of medical sciences

Canada

Introduction \& Objective: To evaluate the efficacy of fibrin glue injection in the prostatic fossa at the end of transurethral resection of prostate (TURP), in decreasing postoperative bleeding in patient with benign prostatic hyperplasia (BPH).

Materials and Methods: In this prospective randomized clinical trial, sixty patients with BPH, who were a candidate for TURP, were randomly divided into two equal groups. In the intervention group, 10cc of fibrin glue was injected in the prostatic fossa at the end of the surgery; through a 5 Fr feeding tube attached to Foley catheter and its tip was proximal to the balloon of catheter. The other thirty patients created the control group. Hemoglobin $(\mathrm{Hb})$ level and lower urinary tract symptoms (LUTS) score were recorded before and 6, 24, $48 \mathrm{~h}$, and 5 days after TURP.

Results: The mean age of the patients and prostate volumes were comparable between the groups. The mean $\mathrm{Hb}$ level before and $6 \mathrm{~h}$ after TURP were not different between the two groups, however, 24 and $48 \mathrm{~h}$ and 5 days after TURP, there was a significant difference as well as a higher decrease in the mean $\mathrm{Hb}$ level of the control group $(\mathrm{P}=0.023)$. The mean LUTS score was not statistically different between the two groups.

Conclusions: Fibrin glue injection in the prostatic after TURP reduces postoperative bleeding without any effect on LUTS score in patients with BPH.

MP24-6 Holmium Laser Enucleation of Prostate v/s Thulium Laser Enucleation of Prostate for Benign Prostatic Obstruction : a Randomized Prospective study.

PK Pattnaik

India

Introduction \& Objective: To compare the efficacy of Holmium and Thulium Laser Enucleation of Prostate in the treatment of benign prostatic obstruction.

Materials and Methods: Between Aug. 2015 and Aug. 2016 a total of 60 patients recruited in this prospective, randomized study, $32 \& 28$ patients underwent Holmium and Thulium Laser Enucleation of Prostate respectively. Both groups were comparable in terms of age $(72+3)$ and TRUS volumes $(71+5 \mathrm{cc})$. Parioperative variables were recorded and both groups were followed up to 3.6 and 12 months with IPSS scores and flow rates.

Results: Patients who underwent Holmium Laser has higher resection time $(55+8 \mathrm{~min})$ and much of gland was resected when compared to patients who underwent Thulium Laser $(36+5 \mathrm{~min})$. In both the groups mean duration of post-operative catheterisation was same $(48+4)$. In the Holmium arm, mean duration of hospital stay was less $(2+1$ days $)$ compared with Thulium Laser duration of hospital stay was less $(3+2)$. The complication rate was more with Holmium Laser (35\%) when compared with Thulium laser (12\%) respectively. At 3,6 and 12 months, improvements observed were comparable with regards to IPSS scores, Qmax and residual volumes.

Conclusions: The efficacy of Holmium Laser is similar standard Thulium Laser but with lesser complications and hospital stay.

MP24-7 Bipolar plasma enucleation versus TURP in medium sized prostates- Is it a possible alternative?

B Geavlete, D Georgescu, R Multescu, C Ene,

C Bulai, P Geavlete

Department of Urology,'Saint John” Emergency Clinical Hospital, Bucharest, România;

Romania

Introduction \& Objective: The aim of this study was to assess the viability of the bipolar plasma enucleation of the prostate (BPEP) by comparison to transurethral resection of the prostate (TURP) in cases of medium size prostates (70-90g).

Materials and Methods: A total of 38 benign prostatic hyperplasia (BPH) patients with an average prostate volume of $73 \mathrm{~mL}$, maximum flow rate (Qmax) below $13 \mathrm{~mL} / \mathrm{s}$ and International 
Prostate Symptom Score (IPSS) over 17 were equally randomized in 2 study arms for BPEP and TURP (19 cases each). All patients were evaluated every 6 months after surgery for a period of one year by IPSS, Qmax, quality of life score (QoL), post-voiding residual urinary volume (PVR) and postoperative prostate volume. Results: BPEP and TURP emphasized similar mean operating times (71.6 versus 67.7 minutes) and resected tissue weights (77.2 versus 75.8 grams). The postoperative hematuria rate ( $10.5 \%$ versus $15.7 \%)$, mean hemoglobin level drop (1.7 versus $1.8 \mathrm{~g} / \mathrm{dL}$ ), catheterization period (1.6 versus 1.8 days) and hospital stay (2.3 versus 2.7 days) were reduced in the BPEP group. The early irritative symptoms' rates were similar subsequent to BPEP and TURP (11.4\% versus $7.1 \%)$. During the one year' follow-up, no statistically significant differences were determined in terms of IPSS, Qmax, QoL, PVR and prostate volume between the two series.

Conclusions: BPEP was characterized by similar surgical efficiency as well as BPH tissue removal capabilities when compared to TURP. Plasma enucleation patients seems to benefit from a superior perioperative safety profile, fewer complications, shorter convalescence period and satisfactory long term symptom scores and voiding parameters.

MP24-8 Report of transurethral resection of prostate in the past 20years:the experience of 3112 cases from single center

\section{Zuo, J Zou, S Lu, Z Zhou, X Wu}

Changzhou No.2 Hospital of Nanjing Medical University China (People's Republic)

Introduction \& Objective: To evaluate the indications, procedure, related technique, complications of prevention and treatment of TURP.

Materials and Methods: The clinical materials were analyzed in 3112 cases with hyperplasia of prostate who accepted TURP surgeries from 1993 January to 2012 December retrospectively. The follow-up time is at least 36 months.

Results: The operative time was $52.0 \pm 22.1 \mathrm{~min}$ (range 20 $185 \mathrm{~min}$ ). Blood transfosion was $10-800 \mathrm{ml}$. The volume of resected prostate was about $40.5 \pm 16.1 \mathrm{ml}$ (range $15-120 \mathrm{ml}$ ). The continuous bladder irrigation persisted for $20.0 \pm 15.1 \mathrm{~h}$ (range $12-48 \mathrm{~h}$ ). The catheterization time was $72.8 \pm 30.1 \mathrm{~h}$ (range 48 $168 \mathrm{~h}$ ) and there was no incontinent patient postoperatively, no conversion to open operation. Two patient died for TUR syndrome postoperatively. 17 cases underwent hemostasia surgeries postoperatively.15 cases were admitted again for operation(TURP) in 3-6 monthes postoperatively. 71 cases accepted TURP again in 6-120 months postoperatively.

Conclusions: TURP is an classical treatment for BPH. There are many advantages such as wide indications, less bleeding, rapid recovery and satifactory efficacy. We think that it is still the "gold treatment standard" for BPH in China.

MP24-9 The Clinical Effect of Bipolar Transurethral Resection in saline of the benign prostate hyperplasia with long term follow-up

S Permpongkosol

Faculty of Medicine, Ramathibodi Hospital, Mahidol

University

Thailand
Introduction \& Objective: Transurethral resection of prostate (TURP) is considered the gold standard for the management of bladder outlet obstruction due to benign prostatic hyperplasia. Our objective is to compare the efficacy and safety of bipolar transurethral resection of the prostate (B-TURP) in the treatment of BPH with prostate gland, $>45 \mathrm{ml}$ and larger than $60 \mathrm{~mL}$ and with long term follow-up. In addition toassess the current treatment of benign prostatic obstruction (BPO) in patients on ongoing oral anticoagulation (OA).

Materials and Methods: From January 2006 to December 2016, 320 Patients with a median age of 70 years and a prostate volume of $42 \mathrm{~cm}^{3}$ were treated with B-TURP by a single Urologist (SP). We retrospectively analyzed the perioperative status of patients' status follow-up at least 6 months and the longest follow-up was 9 years. Post-void residual (PVR) and maximum flow rate (Qmax) were assessed preoperatively and postoperatively. Operative time, length of catheterization and hospitalization and complications were all reported.

Results: The most indication for TURP was failure medication. Perioperative results showed no statistical significance among the groups in terms of catheterization days and the hospitalization length. During the follow-up, PSA, Q max, PVR, and average flow rate were significantly different from pre-operation data $(p<0.005)$. Regarding TURP complications, significant differences were observed in relation to urinary tract infection $(4 \%)$, urethral strictures $(0.6 \%)$. No TUR syndrome was recorded. Most of the patients were not suffered from erectile dysfunction.

Conclusions: Bipolar TURP are safe and effective techniques for BPH management, with a significant reduction of related complications.

\section{MP24-10 Survey of Endourologists: Morcellator Pre-} ferences after Transurethral Prostate Enucleation

K Heinsimer, ME Rivera, J Lingeman, AE Krambeck

United States

Introduction \& Objective: Transurethral enucleation of the prostate for the management of benign prostatic hyperplasia results in excellent patient reported outcomes. Once enucleation is complete, the tissue must be removed from the bladder, typically with morcellation. There are currently two commercially available morcellators in the United States: the Lumenis VersaCut ${ }^{\mathrm{TM}}$ and the Wolf Piranha. We surveyed endourologist to understand their morcellator preference of morcellator.

Materials and Methods: An online survey was sent to endourologists via the Endourological Society email listserv. Data collected included number of years in practice, number of enucleations performed annually, morcellator used, and morcellator preference. Qualities of a morcellator that impacted preference were also assessed on 1-5 Likert scale with 1 being least important and 5 being most important.

Results: A total of 126 urologist responded to the survey. Of these, $56(44.5 \%)$ perform transurethral prostate enucleations, which included: 48 (86\%) holmium, 8(14\%)thulium, $4(7 \%)$ bipolar, $2(4 \%)$ photo, $2(4 \%)$ plasma, $1(2 \%)$ protouch laser, and $1(2 \%)$ diode. For morcellation, more endourologists use the VersaCut $^{\mathrm{TM}}(\mathrm{n}=33,59 \%)$ than the Piranha $(\mathrm{n}=24,43 \%)$. Other methods of morcellation were used by 6 urologists including 4 who use a Storz Resectoscope. More urologist preferred the Wolf $(\mathrm{n}=29,52 \%)$ compared to the Lumenis $(\mathrm{n}=23,41 \%)$. Regarding preference, $44(79 \%)$ use the morcellator they prefer. Reasons for 
not using the preferred device were the cost of operating the device $(18 \%)$ or cost to acquire the device $(3 \%)$. Of those who use the VersaCut ${ }^{\mathrm{TM}}, 27 \%$ prefer the Piranha; while of those who use the Wolf, zero prefer the VersaCut ${ }^{\mathrm{TM}}$. Qualities that impacted the preferences of one morcellator over another, in order of most to least importance were: the preferred device is safer, faster, easier to use, reusable, and less expensive. Of urologists who perform more than 100 enucleations a year, $100 \%$ of them use their preferred morcellator. In this group, 7 (47\%) prefer the Lumenis, 7 $(47 \%)$ prefer the Wolf. Of those who perform $<10$ enucleations per year, $67 \%(n=4)$ prefer the Lumenis, while those who do 11 to 50 cases per year, $67 \%(n=14)$ prefer the Wolf.

Conclusions: Less than half of endourologists surveyed perform transurethral enucleation. Of those, the majority are using their preferred device. Morcellator preference is largely based on safety, efficiency, and ease of use, while cost and being reusable were of lesser importance. Endourologists performing greater than 100 enucleations per year have an equal preference between the Lumenis and Wolf morcellators.

MP24-11 Patient-Centered Goals for Holmium Laser Enucleation of Prostate: A Subjective Evaluation of Successful Surgical Outcome.

K Lee, C Yoon, S Choi, T Oh, T Kim, W LEE

Department of Urology, Pusan National University Hospital, Republic of Korea

Introduction \& Objective: To evaluate the successful surgical outcome using the patient-centered goals in benign prostatic hyperplasia (BPH) patients who underwent Homium Laser Enucleation of the Prostate (HoLEP).

Materials and Methods: From April 2009 to June 2010, 230 consecutive BPH patients scheduled for HoLEP were enrolled and listed their treatment goals for surgery outcomes. On the 3 postoperative months of follow-up, the patients reviewed their original goals and graded its achievement using 5-point goal attainment scale (GAS; -2 to $+2 ;+1$ and +2 was defined as goal achievement). They also completed postoperative satisfaction $(0-100 \%)$, and quality of life (0-6 points) questionnaire.

Results: Of the $182(79.1 \%)$ patients who completed this study, the most common goal was the loss of weak urinary stream $(62.6 \%)$, followed by the loss of residual urine sense $(16.5 \%)$. The goals were achieved in 140 patients $(76.9 \%)$, mean postoperative satisfaction rate was $74.6 \pm 19.5 \%$, and mean QoL scale was $1.8 \pm 1.1$ points. Postoperative satisfaction and QoL were correlated to the GAS significantly $(\mathrm{r}=0.775, \mathrm{p}<0.001$; and $\mathrm{r}=-0.725, \mathrm{p}<0.001$ respectively).

Conclusions: The patients who underwent HoLEP presented successful surgical outcomes according to the patient-centered goal attainment scale which was significantly correlated to postoperative satisfaction and QoL.

\section{MP24-12 Withdrawn}

MP24-13 Postoperative complications in 400 patients undergone endoscopic prostatic surgery with Thullium laser

L Carmignani, G Motta, S Nazzani, M Clementi, C Signorini, E Finkelberg, D Ratti, D Vizziello, S Nazzani

IRCCS Policlinico San Donato

Italy
Introduction \& Objective: Although Trans-Urethral Resection of the Prostate (TURP) is still considered the gold standard of surgical treatment, laser prostatectomy has become increasingly popular due to its reduced peri- and post-operative morbidity, shorter catheterization and hospitalization time. The aim of this study was to report short and long term complications in patients who underwent trans-urethral vapo-enucleation or vaporization of the prostate with Thulium laser (Thuvep/Thuvap).

Materials and Methods: Among the 700 cases of patients undergoing endoscopic procedures with Thullium laser for Benign Prostatic Hyperplasia (BPH) from January 2012 to June 2015, data of 400 patient that underwent Thuvep/thuvap in our centers were collected.

The selection of the population was made after standardization of the technique and after exclusion of the portion of patients operated during the 'learning curve'.

The Follow- up was scheduled at 1 months, 3 months and 12 months. Monitoring of patients consisted in performing a control visit and an uroflowmetric analysis. Instead, in case of suspected urethral stenosis or bladder neck sclerosis, the diagnosis were confirmed by urethrocystoscopy.

Complications such as acute urinary retention, hematuria, transfusion and re-intervention, occured in the first month after the operation. For this reason, the incidence rates of these complications were calculated considering the total population included in the study.

On the contrary, urethral stenosis and bladder neck stenosis occured as late complications and for this reason only patients with one year follow-up were considered.

Results: Only 3 of them required an haemostatic TUR and other 3 required a delayed morcellation.

5 patients required a blood transfusion $(1,25 \%), 3$ of them were under an anti-platelet therapy, 1 was under anti-coagulation therapy and 1 patient only was taking both. 15 patients $(3,75 \%)$ had hematuria and were treated conservatively. Among them, 10 patients were taking an anti-platelet therapy.

In 17 patients $(4,25 \%)$ re-catheterization was performed for Acute Urinary Retention. Bladder neck sclerosis occured in 6 patients $(2,1 \%)$ one year after surgery (among them 2 had undergone Thuvap and 4 Thuvep).

In addition, we reported only 3 cases of UTI $(0,7 \%)$ and 2 cases of bulbar substenosis $(0,6 \%)$. Finally, $1,5 \%$ of all patients had an IPSS greater than 20 at 1 month and $2,1 \%$ of them at one year. Conclusions: Our study seems to suggest that Thullium treatment of Benign Prostatic Hyperplasia (BPH) results in less postoperatory complications than current Gold Standard TURP.

MP24-14 For smaller resection weight of transurethral resection of prostate, could combined incision of bladder neck lead to less acute urinary retention rate after surgery? - a nationwide database study

T Wei, E Huang, H Chung, A Lin

Taipei Veterans General Hospital

Taiwan (Republic of China)

Introduction \& Objective: Acute urinary retention (AUR) after transurethral resection of prostate (TURP) is a complication of great concern, especially for those without Foley indwelled previously. Few studies indicated combined transurethral incision of bladder neck (TUIBN) may be related to better urodynamic results or less bladder neck contracture after TURP. 
However, whether it may reduce post-OP AUR rates is still a question. This article was designed to analyze AUR rates after smaller resection weight TURP in Taiwan according to the claims of the National Health Insurance (NHI) program.

Materials and Methods: In Taiwan, the NHI divides TURPs into four categories according to the resection weight ( $\geqq 5 \sim 15 \mathrm{~g}, \geqq 15 \sim 50 \mathrm{~g}$, and $\geqq 50 \mathrm{~g}$, respectively), and the first group $(<5 \mathrm{~g})$ was defined as "smaller resection weight TURP". AUR was defined as any kind of indwelling catheterization within 2 weeks after TURP. Patients younger than 40 years old were excluded. Patients who received TUIBN or optic or otis urethrotomy within 1 year before TURP and 2 months after were excluded, as well as those who had TURPs within 2 months between each surgery or long admission period (14 days) after TURP. Patients who had diagnosis of prostate or bladder cancer within 3 months peri-operatively were excluded. ICD-9 codes for hypertension (HTN), diabetes mellitus (DM), cerebral vascular disease (CVA), spinal stenosis (SS), and herniated intervertebral disc (HIVD) were used for disease confirmation only when the diagnoses existed at least one year before TURP.

Results: There were 2597 TURPs analyzed, including 2497 TURPs only (group A) and 100 TURPs with combined with TUIBN (group B). The hospitalization days ranged from 3 to 5 days. Total post-OP AUR rate was $9.43 \%$, with $9.69 \%$ and $3.00 \%$ in group $\mathrm{A}$ and $\mathrm{B}(\mathrm{P}=0.022)$. Patient who had had Foley within 2 months before TURP represented $23.64 \%$ of all patients, but pre-OP Foley indwelling was not significantly associated with higher post-OP AUR rate $(11.24 \%)$ than no Foley before TURP $(8.88 \%)(\mathrm{P}=0.083)$. HTN, DM, CVA, SS, and HIVD were not significant risk factors for AUR after TURP. In multivariate analysis, combined TUIBN and younger age are the only two significant factors associated with less AUR rates after smaller resection weight TURPs. $(\mathrm{P}=0.041$ and 0.028 respectively)

Conclusions: In Taiwan, AUR rate is significantly lower in patients receiving combined TUIBN with TURP, regardless of preOP Foley indwelling or other co-morbidities. It implies that for patients with bladder outlet obstruction treated with smaller resection weight TURP, combined TUIBN may be beneficial, especially regarding the post-OP AUR episodes, while the scarring of bladder neck has not yet result in contracture.

MP24-15 Risk factors for unimproved voiding symptoms after holmium laser enucleation of the prostate

B Kim, Y Lee, J Chung, Y Ha, S Choi, J Lee, H Kim, T Kim, E Yoo, T Kwon, S Chung

Kyungpook National University School of Medicine Republic of Korea

Introduction \& Objective: Recently holmium laser enucleation of the prostate (HoLEP) is being performed widely as the operative management of benign prostatic hyperplasia (BPH). HoLEP has been regarded as effective and minimal-invasive procedure for the management of $\mathrm{BPH}$. However, few studies are published about the unimproved voiding symptom after HoLEP. Therefore, we analyzed the risk factors for unimproved voiding symptom after HoLEP.

Materials and Methods: A total of 195 patients underwent HoLEP from January 2012 to June 2015. Patients who were diagnosed with prostate cancer after operation, underwent combine operation for bladder stone or ureter stone, or voiding symptoms after the operation were not evaluated properly due to follow-up loss were excluded. Finally, 127 patients were included and classified into 2 groups according to the improvement of voiding symptoms by international prostate symptom score (IPSS), uroflowmetry (UFM) and post-void residual urine volume (PVR). Age, body mass index (BMI), underlying disease, previous operation history of prostate, medication period for $\mathrm{BPH}$ before the operation, serum prostate-specific antigen (PSA), PSA density, size of prostate, presence of bladder trabeculation and operation time were compared and analyzed using logistic regression analysis.

Results: Of the 127 patients, 24 (19\%) patients showed unimproved voiding symptoms. In these patients, the prostate size $(\mathrm{p}=0.029)$, operation time $(\mathrm{p}=0.005)$ and bladder trabeculation $(\mathrm{p}<0.001)$ were significantly correlated with unimproved voiding symptoms according to univariate analysis. Multivariate analysis showed that the operation time $(\mathrm{p}=0.008, \mathrm{OR}=1.018)$ and bladder trabeculation $(\mathrm{p}<0.001, \mathrm{OR}=16.951)$ were significant risk factors of unimproved voiding symptoms. The cut-off value of operation time was 143.0 (sensitivity $62.5 \%$, specificity $73.8 \%$, AUC 0.658) minutes.

Conclusions: HoLEP is considered to be a safe and effective surgical treatment for $\mathrm{BPH}$, but longer operation time and presence of trabeculation may have negative effects for the improvement of post-operative voiding symptoms.

MP24-16 The introduction of TUEB (Transurethral enucleation with Bipolar) system for large BPH; Initial 40cases

T Yoshida, H Yoshinaga, N Fujimoto

Department of Urology, Fukuoka Wajiro Hospital

Japan

Introduction \& Objective: Large volume of BPH with symptoms refractory to medication indicate for surgical treatment. Transurethral enucleation with bipolar (TUEB) system was developed for enucleation of prostate with a spatula dissecting the avascular area between surgical capsule and adenoma same as procedure of HoLEP. TUEB system does not require expensive instruments such as a LASER system for the hospital already introduced the TURis (transurethral resection in saline) system. TUEB makes it possible to safely enucleate such adenoma with a high degree of efficacy as volumes more than $100 \mathrm{ml}$ and control bleeding with high hemostatic ability. We report the experience of introducing TUEB system, and the advance of operating time, resection volume, symptom scores and complications in the initial 40cases system with large BPH.

Materials and Methods: Fourty patients with large BPH received TUEB in our hospital from January 2015 to December 2016. All TUEB cases were performed by a single surgeon. We show pre-and post-operative data for every patient and display each data: prostate volume, uroflow-metry score, International Prostate Symptom Score (IPSS), Overactive bladder symptom score (OABSS) and Quality of Life Score(QOL score), PSA (ng/ $\mathrm{mL}$ ) and operation time (enucleation and resection time). The specimens of all patients were analyzed by pathologists.

Results: The mean prostate volume was $64 \mathrm{~g}$ (40-130g) and operation time was 140min (90-205min). Catheterization days were 3.3days (2-6days). We experienced the complications; one case of hematuria after restarting of anticoagulant agent, one of urethral stricture requiring urethrotomy and one of perforation of prostate capsule. No transfusion was performed. PSA 
decreased in $10 \%$ of pre-operation level. In two cases prostate cancer were found.

Conclusions: TUEB system is effective for large size of BPH. Resection was performed as expected volumes. A few numbers of adverse effects were observed. TUEB system is costless, effective and safe surgical procedure for BPH.

MP24-17 One out of three men undergoing transurethral resection of the prostate is lost to follow-up: a worrisome picture form the real-life setting

L Boeri, M Fontana, A Gallioli, E De Lorenzis, F Palmisano, S Zanetti, G Sampogna, M Talso, F Longo, F Gadda, P Dell'Orto, E Montanari

Department of Urology, Fondazione IRCCS Ca' Granda Ospedale Maggiore Policlinico, Università degli Studi di Milano

Italy

Introduction \& Objective: Initial follow-up after transurethral resection of the prostate (TURP) for lower urinary symptoms due to benign prostatic hyperplasia (LUTS/BPH) is important to evaluate treatment response and potential treatment-related adverse events. We sought to determine the rate of and factors associated with patient nonadherence to prescribed follow-up after TURP.

Materials and Methods: Data from 154 consecutive patients who underwent TURP from January 2015 to December 2016 were analysed. Demographic information and perioperative data were collected. Comorbidities were scored with the Charlson Comorbidity Index (CCI). Postoperative factors included all complications, as well as procedure-related events (PREs), within 30 days. Patient counselling and follow-up were standardized among the cohort. Prostate specific antigen (PSA), urinary flow rate and postvoiding residual volume were scheduled 3-months after surgery. Patients were considered lost to follow-up (LTF) if they were at least 30 days from their first scheduled follow-up appointment. Phone calls were used to investigate the rate of and reason for being LTF. Descriptive statistics and logistic regression analyses were performed to determine the impact of potential predictors on the rate of compliance with prescribed follow up.

Results: Overall, mean (SD) age was 69.8 (8.5) yrs. Educational status was primary/secondary school and high school/university degree in $70(45.5 \%)$ and $84(54.5 \%)$ patients, respectively. Of 154 patients, $47(30.5 \%)$ were LTF. LTF patients were younger $(\mathrm{p}\langle 0.001)$ and more frequently had lower educational status $(\mathrm{p}=0.07)$ than those who were not LTF. Importantly, patients who experienced a PRE were more likely to follow-up $(p=0.045)$ than those who did not report PREs. Intraoperative characteristics and postoperative outcomes were similar between LTF and no-LTF men. The primary reasons for being LTF were "feeling healed", "concomitant health diseases", "costs" and "forgot the visit" in 27 (58\%), 3 (5\%), 6 (14\%) and $11(23 \%)$ patients, respectively. Multivariable analysis revealed that younger age $(p<0.01)$ and low educational status $(p<0.01)$ were independent predictors of being LTF, after accounting for BMI, $\mathrm{CCI}$ and the presence of PREs.

Conclusions: One out of three patients undergoing TURP fails to return for follow-up. Noncompliance to follow-up was more frequent among young patients with low educational status. On the contrary, patients who experienced a PRE were more likely to follow-up after surgery.
MP24-18 Incidence and predictors of readmission within 30 days of transurethral resection of the prostate: which patients come back?

L Boeri, S Zanetti, G Sampogna, M Fontana, A Gallioli, E De Lorenzis, F Palmisano, F Longo, M Spinelli, F Gadda,

M Ferruti, P Dell'Orto, E Montanari

Department of Urology, Fondazione IRCCS Ca' Granda Ospedale Maggiore Policlinico, Università degli Studi di Milano

Italy

Introduction \& Objective: Hospital readmission rates have come under increased scrutiny due to their significant contribution to increasing medical costs. We aimed to assess the incidence and predictors of 30-day readmission after discharge in a cohort of men treated with transurethral resection of the prostate (TURP) for lower urinary symptoms due to benign prostatic hyperplasia (LUTS/BPH).

Materials and Methods: Data from 160 consecutive patients who underwent TURP from January 2015 to December 2016 were analysed. Demographic information and perioperative data were collected. Intra hospitalization characteristics included length of stay (LOS) and catheterization duration (CD). Comorbidities were scored with the Charlson Comorbidity Index (CCI). The primary outcome was defined as admission to the emergency room (ER) within 30 days of discharge. The number of days of $\mathrm{CD}$ was dichotomized according to the most informative cutoff (MOC) predicting 30-day readmission (3 days). Descriptive statistics and logistic regression analyses were used to test the relationship between covariates and 30-day readmission in the overall population and after stratifying for age at the time of surgery.

Results: Overall, mean (SD) age was 70.1 (8.1) yrs and mean prostate volume was 80 (45.6) ml. Mean LOS and CD were 4.9 (2.5) days and 3.3 (1.6) days, respectively. The overall 30-day ER readmission rate was $14.4 \%$ (23/160 men). However, readmission-requiring hospitalization was reported in only 7 (4.4\%) patients. The most frequent reasons for ER readmission were haematuria $(6.9 \%)$, fever/urinary tract infections $(4.4 \%)$ and acute urinary retention $(3.1 \%)$. Patients who experienced a readmission within 30-days were more likely to be older $(p=0.026)$, to have higher CCI values $(p=0.007)$ and shorter LOS $(p=0.041)$ and CD $(p=0.039)$. In multivariable logistic regression analysis, age (OR 1.1, $\mathrm{p}=0.03)$, CCI (OR 1.46, $\mathrm{p}=0.03)$ and short $\mathrm{CD}(\mathrm{OR} 0.37, \mathrm{p}<0.01)$ were associated with 30-day readmission. However, when analysed according age at the time of surgery, a beneficial effect from a longer $\mathrm{CD}(>\mathrm{MOC})$ was observed only in patients older than 75 years $(p=0.04)$.

Conclusions: We observed a 30-day ER readmission rate of $14.4 \%$ in a cohort of men treated with TURP. Of note, older patients, with high rate of comorbidities and shorter CD were associated with an increased risk of readmission. However, an increase of CD to prevent readmission seemed effective only in patients older than 75 years.

\section{MP24-19 Cost Savings Achieved through Introduction of} HOLEP and Care Pathway

J Johannes, A Lai, A Baccala, J Feliciano, C Georges, M Steinbook, M Voznesensky

United States

Introduction \& Objective: Studies have shown clinical benefits of Holmium Laser Enucleation of the Prostate (HOLEP) over 
TURP or other BPH procedures. Besides learning curve, high capital costs are a barrier to introduction. Our objective is to investigate the impact on length of stay (LOS) as a source of cost savings compared to TURP, in the first 52 cases in a community hospital setting.

Materials and Methods: A prospectively managed database was maintained at the onset of starting a HOLEP program. TURP data from our institution during the same period of time reviewed. All HOLEP patients were managed post-operatively according to a critical care pathway specifically developed to minimize length of stay. Patients underwent 2 hours of continuous bladder irrigation after which a clamping trial was performed. Patients were ambulated with the catheter and clinically evaluated. Patients were discharged home on POD 0 with a catheter if clinically appropriate. Patients were seen in the office 2-3 days post-op for a trial of void. To determine cost savings from decreased LOS, hospital expenses were sourced from the Henry J. Kaiser Family Foundation.

Results: For the first 52 patients, the mean age was 65.7. HOLEP was performed for urinary retention in 32 patients and refractory urinary symptoms in 20. Mean estimated prostate volume was 91.2 grams. Mean resected volume of tissue was 33.5 (range 3$118 \mathrm{cc}$ ) grams with $9.6 \%$ patients having malignant pathology. 3 month outcomes were comparable to larger series with IPSS overall and bother scores improving from 22.5 to $6.9(69 \%)$ and 4.6 to $1.7(63 \%)$ respectively. With our critical care pathway, length of stay averaged 10.7 hours including 6/52 (11.5\%) requiring overnight stays. Mean catheter time was 3.8 days. This compared favorably to the mean TURP LOS during the same period of 36.5 hours. This shows a 25.8 hour LOS benefit with HOLEP. Based on then USA average daily cost of hospitalization of $\$ 2,271$, this decreased LOS potentially generates a health system savings of $\$ 2,441$.

Conclusions: Implementation of a HOLEP program with a postoperative critical care pathway aimed at decreasing hospital length of stay significantly decreases hospital length of stay compared to TURP. The estimated cost savings from this decreased LOS more than offset the capital costs of a HOLEP program. In addition to the superior clinical outcomes, cost savings through short length of stay is another benefit of HOLEP over TURP.

\section{MP24-20 Robotic Transvesical Simple Prostatectomy : USC early experience}

PW Mekhail, A Berger, MW Mekhail, A Abreu, N Ahmadi, A Monish

University of Southern California

United States

Introduction \& Objective: Now with the increase in longevity of men, we have come to see increasing number of benign prostatic hyperplasia(BPH) cases. Management choices for men with symptomatic BPH have significantly increased over the last 2 decades. Yet, despite these developments, open simple prostatectomy (OSP) continues to be the traditional option specially for patients with larger glands $(>100 \mathrm{~g})$ due to the greater volume of adenoma however it is associated with a considerable risk for complication. Laparoscopic simple prostatectomy (LSP) was first performed in 2002, and subsequent series have demonstrated functional outcomes to be equivalent to OSP. However, it's adoption have been hindered due to the technical challenges and
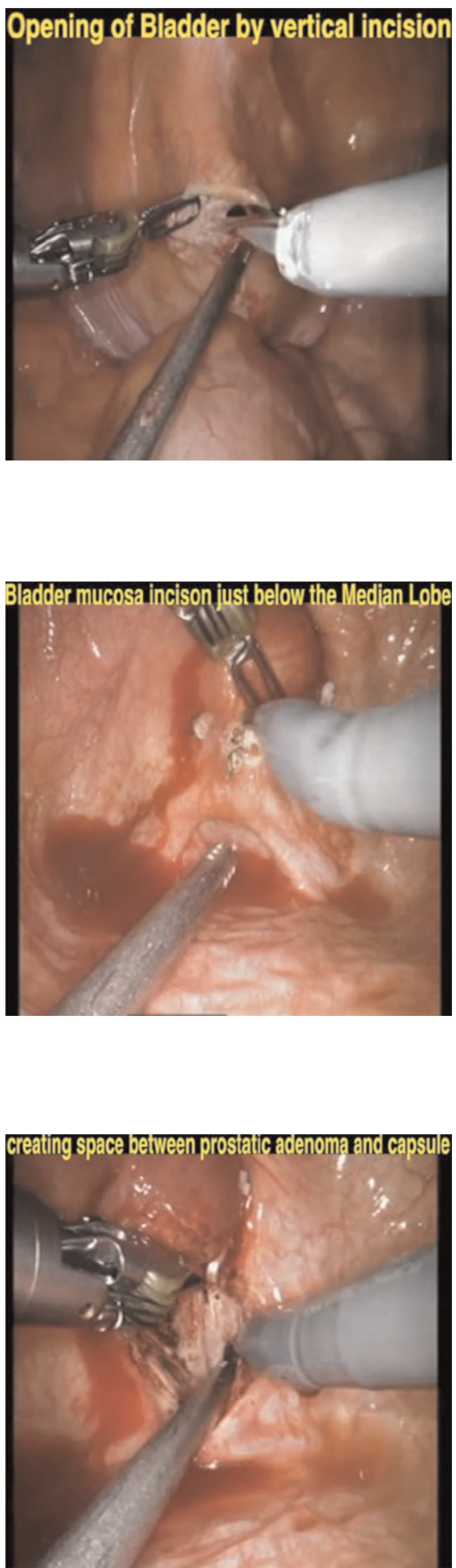

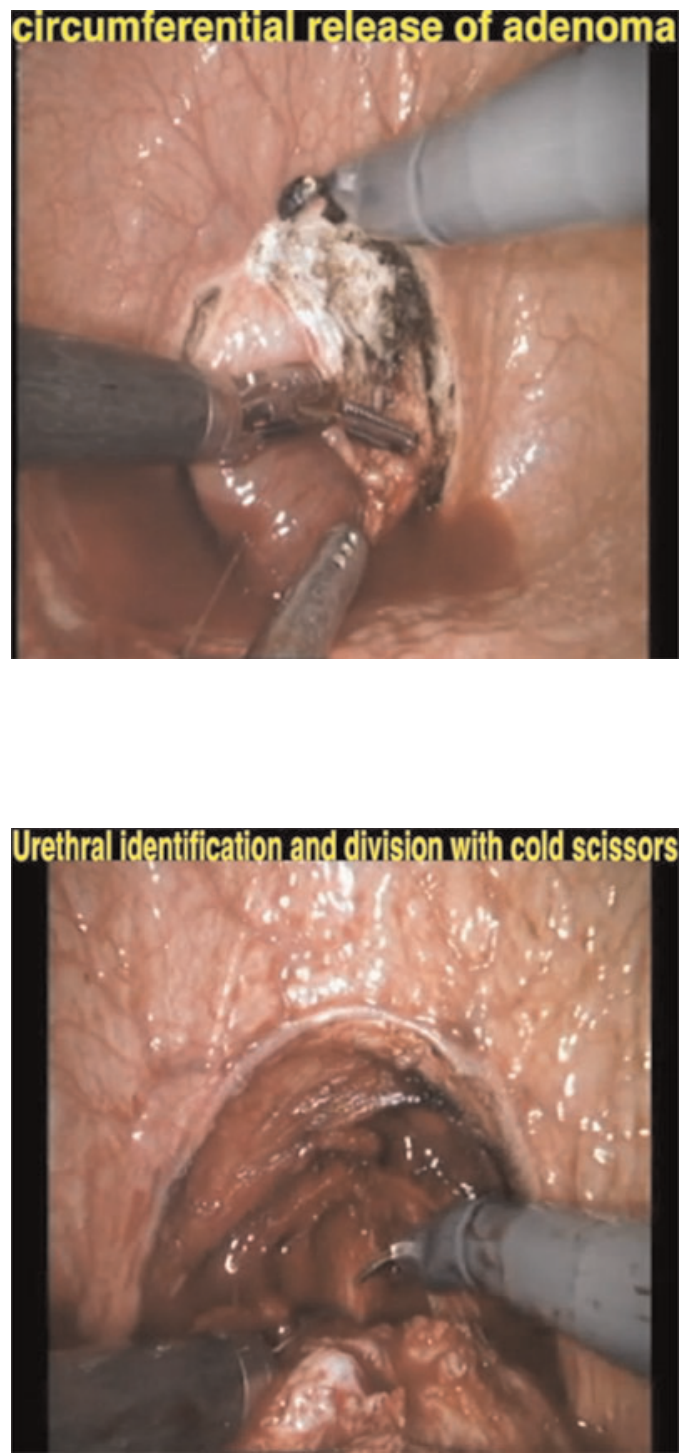

a concomitant robotic diverticulectomy and 5 patients had concomitant cystolithotomy.

Final pathology revealed cancer in 7 patients(5.4\%). 6 patients experienced Clavien 3 complication(clot retention, $n=2)$. Median postoperative IPSS was $7.5(\mathrm{P}<0.001)$, PVR was $6(\mathrm{P}<0.01)$ and Qmax was $19.5(\mathrm{P}<0.001)$. Median follow-up was 7 months (2.61).

Conclusions: RSP is safe, effective and excellent functional outcomes providing a viable option for BPH with Larger glands $(>100)$ with statistically proved better IPS, PVR and Qmax. It provided fewer complication, including reduced blood loss, lesser blood transfusions, shorter hospital stay and low reoperation rates in comparison to open prostatectomy.

MP24-21 Predictors and Recovery of Urinary Incontinence after Holmium Laser Enucleation of Prostate

B Singh, A Sokhal, S Sankhwar

King George's Medical university India

Introduction \& Objective: To investigate potential predictors for postoperative urinary incontinence and its recovery following holmium laser enucleation of prostate.

Materials and Methods: A retrospective analysis of prospectively collected data of 108 patients, who underwent HoLEP, between January 2007 and December 2015 at a tertiary care teaching institution was done. Patient demographics and perioperative variables were analyzed. Patients were assessed at catheter removal,1 week, 1 month, 3 months, 6 months and 12 months postoperatively. Post HoLEP urinary incontinence and its recovery was investigated with reference to patient's age, preoperative acute urinary retention, IPSS/QoL, diabetic status, prostate size, technique of HoLEP (En-bloc versus multilobar enucleation technique), enucleation time, morcellation time, mucosal injury over and distal to sphincter and patient's compliance to Kegel's pelvic exercise. steep learning curve of the laparoscopic approach. The robotic simple prostatectomy(RSP) conceivably overwhelm these challenges by offering stereoscopic three-dimensional (3D) vision and surpassing dexterity to ease the more technically necessitating steps of the simple prostatectomy procedure. Perioperative and short-term functional outcomes are described that show RSP to be feasible, safe, and effective. Here we report the results of our series of RSP.

Materials and Methods: from May 2011 to September 2016, 129 men underwent transvesical RSP at the USC. Baseline demographics, pathology data, postoperative complication, 90d complication and functional outcome were assessed.

Results: Median age was $70 \mathrm{yr}$ (range:52-89), BMI was $28.2 \mathrm{~kg}$ / m2(16.4-48), baseline international Prostate Symptom Score(IPSS) was 27(3-35), prostate volume was $137.5 \mathrm{ml}(27-64.5)$, maximal flow rate(Qmax) was $8 \mathrm{ml} / \mathrm{s}(0-24)$, and preoperative PSA was $6.15 \mathrm{ng} / \mathrm{ml}(1.9-56.3)$. Forty- eight were catheter dependent surgery. Median operative time was 225min(135-400), estimated blood loss was 100ml(10-1000), and hospital stay was 3d(1-21d). No intraoperative complications, no conversion to open surgery and only 1 patient $(0.8 \%)$ required blood transfusion. 6 patients had
Table1. Patient demographics and perioperative parameters.

\begin{tabular}{|l|l|}
\hline Parameters & Value \\
\hline Age; years (Mean \pm SD) & $63.52 \pm 7.965$ \\
\hline AUR; N (\%) & $46(56.4 \%)$ \\
Yes & $62(41.8 \%)$ \\
No & $1.54 \pm 1.42$ \\
\hline LUTS duration; years (Mean \pm SD) & $56.68 \pm 23.26$ \\
\hline Prostate size; grams (Mean \pm SD) & $1.91 \pm .79$ \\
\hline PSA ng/dl (Mean \pm SD) & $18(16.7 \%)$ \\
\hline Diabetes N (\%) & $90(83.4 \%)$ \\
Yes & $12.0 \pm 1.40$ \\
No & $10.90 \pm 1.90$ \\
\hline Preop Hb; gm\% (Mean \pm SD) & $44.76 \pm 8.2$ \\
\hline Post op Hb; gm\% (Mean \pm SD) & \\
\hline Resected prostate weight; gm (Mean \pm & \\
SD) & $60(55.6)$ \\
\hline Technique of HoLEP; N (\%) & $48(44.4)$ \\
Multilobar & $51.56 \pm 25.0$ \\
En-bloc & $22.54 \pm 13.54$ \\
\hline Enucleation time; min (Mean \pm SD) & \\
\hline Morcellation time; min (Mean \pm SD) & $26(24 \%)$ \\
\hline Sphincter mucosal injury; N (\%) & $82(76 \%)$ \\
Yes & $16(14.8 \%)$ \\
No & $4(3.7 \%)$ \\
\hline Complications (\%) & $9(8.3 \%)$ \\
Perforation & $3(2.8 \%)$ \\
Fever & \\
Bladder mucosal injury & \\
\hline
\end{tabular}


Results: Table1 shows patient demographics and perioperative parameters. Mean age, mean prostate volume, mean enucleated weight, enucleation time and morcellation time were 63.52 years, $56.68 \mathrm{~g}, 44.76 \mathrm{~g}, 51.56 \mathrm{~min}$. and $22.54 \mathrm{~min}$. respectively. Postoperative de novo urinary incontinence at catheter removal, 1 week, 1 month, 3 months, 6 months and 12 months was noted in $32(29.6 \%), 30(27.8 \%), 14(13 \%), 8(7.4 \%), 3(2.8 \%)$ and 1 $(0.9 \%)$ patients respectively. On logistic regression analysis, significant predictors of urinary incontinence were higher patient age and mucosal injury over and distal to sphincter.

Conclusions: Post HoLEP urinary incontinence is transient and recovers in most of the patients within 3 months after the procedure. Advanced patient age and intraoperative mucosal injury over and distal to sphincter are significant predictors of post HoLEP urinary incontinence. Compliance to Kegel's pelvic exercise enhance continence recovery.

\section{MPS25: BPH/LUTS I}

\begin{abstract}
MP25-1 Comparison of tissue vaporization rate and efficiency between Lumenis Pulse $120 \mathrm{H}$ Holmium laser and AMS 180W Green Light KTP laser in an Ex-vivo model.
\end{abstract}

\section{SM Kumar}

Beaumont Wayne Hospital, Wayne, Michigan United States

Introduction \& Objective: High powered lasers have significantly increased tissue vaporization efficiency during treatment of BPH (Benign Prostate Hyperplasia) by trans urethral vaporization of the prostate. The purpose of this study was to compare the performance of the Lumenis Pulse120 system (120W Holmium laser) \& XPEEDA fibers versus the GreenLight (180W KTP laser) with MOXY fibers in an ex-vivo model.

Materials and Methods: In order to approximate the prostate composition two different tissues were tested- porcine kidney representing soft tissue and chicken breast as a non-vascularized tissue. XPEEDA fibers were applied in a contact mode with the Lumenis Pulse $120 \mathrm{H}$ at maximum settings of $120 \mathrm{~W}(2 \mathrm{~J}$ at $60 \mathrm{~Hz})$, MOXY Fibers were applied in a non-contact mode with the AMS GreenLight at maximum power settings of $180 \mathrm{~W}$. In order to obtain sufficient data, average of 10 repetitions were done for each individual fiber, utilizing 5 different fibers of XPEEDA or MOXY (total of 50 measurements for each tissue). Vaporization rate was calculated by measuring mass loss/ lasing time ( $\mathrm{g} / \mathrm{min})$, vaporization efficiency was calculated as mass loss/ energy invested ( $\mathrm{g} / \mathrm{Kj})$.

Results: Average vaporization rate was: $1.88 \mathrm{~g} / \mathrm{min}$ for Lumenis Pulse120 laser with XPEEDA fiber and $0.68 \mathrm{~g} / \mathrm{min}$ for the AMS GreenLight laser with MOXY fiber on kidney. Similar results were obtained testing the ablation in chicken breast. In addition, although using lower power of $120 \mathrm{~W}$ compared to $180 \mathrm{~W}$, the vaporization efficiency of the XPEEDA fiber was 4 fold higher than the MOXY ( 0.25 vs 0.06 in both tissues tested).

Conclusions: In these bench test settings the results show a significantly higher vaporization rate using the XPEEDA fibers along with the 120W Holmium laser as compared to the MOXY fiber with Greenlight laser (at maximum180W). Taking into account both vaporization rate and efficiency, the combination of the Lumenis $120 \mathrm{H}$ and the XPEEDA fiber shows superior performance that may have a significant impact on BPH treatment in a clinical setting.

MP25-2 Treatment and outcomes of patients diagnosed with prostate cancer and submitted to Holmium laser enucleation of the prostate (HoLEP) for concomitant benign prostatic hyperplasia.

JE Hunter, C Chemasle
Midcentral Hospital, New Zealand

New Zealand

Introduction \& Objective: To describe the treatment of patients diagnosed with prostate cancer (PCa) and determine if concomitant HoLEP for lower urinary tract symptoms (LUTS) has a positive or detrimental effect on patient's outcome.

Materials and Methods: From 2009 to 2017, 322 patients underwent HoLEP by one consultant in our hospital. We performed a retrospective analysis of these patients to identify those that had been diagnosed with $\mathrm{PCa}$ and reviewed whether there were any complications associated with this.

Results: 48 patients were identified as having PCa at some point. $13(27 \%)$ had a pre-operative diagnosis of $\mathrm{PCa}, 29(60 \%)$ were diagnosed on the HoLEP tissue sample, $6(13 \%)$ were diagnosed post HoLEP procedure. Median time to diagnosis post operatively was 40 months (range 4 - 84 months). Of the 13 patients with a pre-operative diagnosis of PCa: 3 had an old diagnosis with previous external beam radiation treatment (EBRT) requiring HoLEP for prostatic regrowth. 1 was found to have recurrent Gleason 7 and received androgen deprivation therapy (ADT). 6 were having a dis-obstructive HoLEP pre EBRT. 2 were placed on active surveillance (AS) and 2 on watchful waiting (WW). Of the 29 diagnosed on the operative sample: 7 went on to have EBRT, 1 had a radical prostatectomy (RRP), 1 is on AS, and the remaining 19 are/were on WW. 6 patients were diagnosed post HoLEP procedure: 2 of these patients had a RRP, 3 had EBRT, 1 was placed on ADT. In total 22 patients had active treatment. 19 had EBRT, 3 RRP. The median age of those undergoing EBRT was 72 (range 67-77), for RRP it was 66. For those that underwent EBRT, 15 have done well with no longterm problems. 3 have mild incontinence not requiring operative intervention. 1 patient who had EBRT prior to his HoLEP has significant issues with urinary incontinence exacerbated by EBRT. 1 had a stricture requiring dilation. The 3 patients that underwent RRP all had uncomplicated procedures and recovery. Conclusions: Majority of the patients with PCa treated with HoLEP had improved LUTS with few issues. When considering

Table 1: ISUP grade of patients with PCa undergoing HoLEP for LUTS stratified by timing of PCa diagnosis

\begin{tabular}{|l|l|l|l|}
\hline & Pre-HoLEP & HoLEP & Post-HoLEP \\
\hline ISUP 1 & 4 & 16 & 1 \\
\hline ISUP 2 and 3 & 5 & 12 & 2 \\
\hline ISUP 4 and 5 & 4 & 1 & 3 \\
\hline
\end{tabular}


patients for EBRT, if there is evidence of LUTS a dis-obstructive HoLEP pre-treatment should be considered as there is no evidence of increased complications, and a HoLEP post EBRT may be associated with increased complications.

MP25-3 HoLEP performed at low power setting of 30W with an en-bloc enucleation technique

\section{S Minagawa, S Okada, H Morikawa}

Department of Urology, Gyotoku General Hospital Japan

Introduction \& Objective: Holmium laser enucleation of the prostate (HoLEP) is now expected to replace transurethral resection of the prostate (TURP) as the gold standard surgical treatment of benign prostatic hyperplasia (BPH). HoLEP was first reported by Gilling et al. in 1998, the setting of the laser was accomplished by the high-power of $80 \mathrm{~W}(2.0 \mathrm{~J}$ and at a frequency of $40 \mathrm{~Hz}$ ). Therefore, it is mainstream now to use high-power holmium laser more than 80W for HoLEP, it will be the present recognition that is difficulty with the low-power holmium lasers. The low-power holmium laser of the output 30W was widely widespread in Japan, most are used for only trans-ureteral lithotripsy. Therefore, we examined effectiveness and usefulness of HoLEP with 30W lowpower holmium laser with en-bloc technique.

Materials and Methods: Between September 2014 and August 2016, 74 patients with benign prostatic hyperplasia underwent holmium laser enucleation of the prostate with the en-bloc technique in our institution. The enucleation process was performed with the laser set $1.5 \mathrm{~J}$ and at a frequency of $20 \mathrm{~Hz}(30 \mathrm{~W})$ as low-power setting. 44 patients were operated by an experienced surgeon who has performed over 200 HoLEP operations, and 30 patients were performed by inexperienced two surgeons with around 30 HoLEP operation experience. We evaluated the surgical parameters, including total operative time, enucleation time, morcellation time, total energy, and hemoglobin loss.

Results: The mean age and mean preoperative estimated prostate volumes were 73.8 (63-87) years and 94.5 (22-489) $\mathrm{mL}$, respectively. The mean total operating time, mean enucleation time, and mean morcellation time were 91.9 (30-232), 45.4 (13101), and 17.3 (1-106) minutes, respectively. The mean total energy was 50.58 (15.20-90.09) kJ. It was not necessary to increase the output of the laser during enucleation process in all cases. Mean hemoglobin loss was 0.78 (0-2.9) g/dL. One patient required re-hemostasis after surgery, and none of the patients needed blood transfusion during the postoperative period. Superficial bladdermucosal injury was encountered in 1 patient. Other intraoperative complications such as capsular perforation and ureteral orifice injury did not occur in all patients.

Conclusions: HoLEP with en-bloc enucleation technique can be performed without any safety and technical problem with the low-power holmium laser as setting of $1.5 \mathrm{~J}$ and at a frequency of $20 \mathrm{~Hz}$. Thus, this $30 \mathrm{~W}$ low-power setting was thought that it was necessary and sufficient on performing HoLEP. Furthermore, cost of the HoLEP introduction can decrease if it performs HoLEP with a low-power laser.

MP25-4 Metabolic syndrome is predictive of lower urinary tract symptom improvement after holmium laser enucleation of the prostate for benign prostatic obstruction

T Kwon, S Park, S Park, K Moon
Ulsan University Hospital

Republic of Korea

Introduction \& Objective: Current research emphasizes the relationship between benign prostatic hyperplasia (BPH) with/ without lower urinary tract symptoms (LUTS) and metabolic syndrome (MS), with a focus on the worsening of LUTS caused by MS. Therefore, we investigated the effect of MS on patient outcomes who underwent holmium laser enucleation of the prostate (HoLEP) for benign prostatic obstruction.

Materials and Methods: Data from 151 patients who underwent HoLEP by a single surgeon between March 2012 and March 2016 were retrospectively analyzed. Patients with MS were assigned to group $1(n=33)$ and patients without MS in group 2 $(\mathrm{n}=118)$. Clinical characteristics and the International Prostate Symptom Score (IPSS), including quality of life (QoL), peak urinary flow rate (Qmax), and postvoid residual urine (PVR), before surgery and 3 months afterwards were compared between groups. Additionally, predictors of total IPSS improvement after HoLEP were assessed.

Results: Compared with group 2 patients, group 1 patients were older (70.3 vs. 65.2 years old, $\mathrm{p}=0.001$ ). Preoperative data, which included prostate volume, QoL, Qmax, and PVR, were not different between groups. For all patients, both the storage subscore and voiding subscore significantly decreased after surgery $(\mathrm{p}<0.001)$. Postoperative total IPSS and voiding subscore improvement in group 1 were lower than in group 2 (total IPSS improvement 9.2 vs. $12.5, \mathrm{p}=0.042$; voiding subscore improvement 6.6 vs. $8.8, p=0.048)$. Multivariate analysis showed preoperative total IPSS $(\beta=0.79$, CI $0.71-0.94, \mathrm{p}<0.001)$ and number of MS components $(\beta=-0.15$, CI -2.04 to -0.29 , $\mathrm{p}=0.009$ ) were independently associated with total IPSS improvement.

Conclusions: We found that MS was associated with decreased postoperative symptom improvement. Thus, lower urinary tract symptoms after surgery may be a systemic disorder due multiple metabolic risk factors.

MP25-5 En bloc enucleation technique with lateral median approach for safe and quicker operation when performing holmium laser enucleation of the prostate

Y Shibata, S Arai, S Kurihara, T Miyao, Y Miyazawa, R Oki, H Nakayama, H Koike, K Ito, K Suzuki

Japan

Introduction \& Objective: Holmium laser enucleation of the prostate (HoLEP) has become an increasingly popular treatment for symptomatic benign prostatic hyperplasia (BPH). HoLEP allows complete removal of the tissue in the transition zone regardless of the prostate size. Despite the advantages of HoLEP, considerable training and a long learning curve is required to master this surgical technique for safe operation. We perform an en bloc enucleation technique with lateral median approach to median lobe, which enables a surgeon to perform safer and quicker operation.

Materials and Methods: Nearly 400 patients underwent HoLEP in our institution since the introduction of this surgical method. The first ten patients were operated with an ordinary technique introduced by Gilling, et al in 1996, which enucleate the lobes separately starting with the median lobe. We had difficulty in enucleating the median lobe and often caused the prostate capsule perforation into 
retrovesical space near the bladder neck, since the surgical capsule was anatomically very thin and the exfoliation angle was very sharp at that lesion. So we came up with the en bloc enucleation technique with lateral median approach to the median lobe. The procedure consists of following maneuvers: 1) Early encircling mucosal incision at apical adenoma, 2) Lateral approach to bladder neck at upper portion, 3) Lateral to median exfoliation of median lobe at bladder neck, 4) Retrograde exfoliation of hang down adenoma at 12 o'clock position, 5) En bloc downward morcellation of the enucleated adenoma.

Results: Introduction of the technique gave a surgeon clear information on the relation between bladder neck and adenoma to be enucleated. The technique enabled us en bloc enucleation of the prostate adenoma, which helped us to shorten the learning curve when mastering the HoLEP procedure. The daramatic improvement in the efficiency of HoLEP was observed with enucleation efficiency (enucleated gram weight/operation time in minutes) achieving nearly 1.0 gram/minute.

Conclusions: We feel that HoLEP represents a safe and effective treatment for patients with symptomatic BPH and we hope that the presentation of this technique will improve operative times and shorten the learning curve for urologists mastering the HoLEP procedure.

MP25-6 Bipolar plasma enucleation of the prostate (BTUEP) in Benign Prostate Hypertrophy Treatment. Medium-term results

R GENTILE, B GENTILE, L Albanesi, G Rizzo, M Vermiglio, M Buscarini, P Tariciotti, G Mirabile

nuova villa claudia

Italy

Introduction \& Objective: Numerous endoscopic techniques have been described since Iglesias published the results of his modified resectoscope for the treatment of bladder outlet obstruction (BOO) due to benign prostate enlargement (BPE). Classic transurethral resection of the prostate (cTURP) has long been the accepted gold standard to treat symptomatic disease of prostates weighing $30-80 \mathrm{~g}$ (1). A variety of therapeutic solutions and technical innovations have been developed to bring improvements to $\mathrm{BOO}$ endoscopic treatment. The plasma-button bipolar enucleation of the prostate (B-TUEP) is considered a successful treatment option mainly because the large surface creats the conditions for a fast enucleation process, continuous vaporization and concomitant haemostasis $(2,3)$. Aim of this study was to assess safety, efficacy, and medium term durability of B-TUEPfor the treatment of BOO due BPE.

Materials and Methods: Between July 2011 and March 2012, 50 consecutive patients underwent B-TUEP at our institution, by a single surgeon (R.G.). All patients were pre-operatively assessed with maximum urinary flow rate (Qmax), the singlequestion quality of life (QoL), International Prostate Symptoms Score (I.P.S.S.) and the International Index of Erectile Function (IIEF-5) questionnaires, Transrectal Ultrasound gland volume evaluation (TrUS), prostate-specific antigen (PSA)and postvoided residual of urine (PVR). Postoperativeparameters were evaluated and the patients were reassessed at 1-, 3-, 6-,12-, 18-, 24-, and 36-mo follow-up with the same examinations.

Results: We observed a significant improvement occurred at 12, 24 and 36 months in terms of Qmax $(22.3 \pm 4.74 \mathrm{~mL} / \mathrm{s}, 23.2 \pm 0.30 \mathrm{~mL} /$ $\mathrm{sec}$ and $23.6 \pm 1.26 \mathrm{~mL} / \mathrm{sec}$, respectively, p respectively. Two patients (4\%) had persistent $\mathrm{BOO}$ and requiring reoperation. During the 36-month follow up five patients (10\%), developed neoplasms and turned so lost.

Conclusions: After 3-yr follow-up, B-TUEP represents an effective, durable and safe surgical intervention. Voiding parameters such as Qmax, QoL score, IPSS, PVR improved significantly $(\mathrm{p}<0.05)$ from baseline, starting from 3-mo after B-TUEP and continuing during the follow-up, until they reached a plateau that was stable up to the 36-mo visit. The present report adds to the evidence that B-TUEP could be the alternative "size-independent' surgical treatment for symptomatic BPE-related BOO.

MP25-7 Audit on UroLift- a novel minimally invasive technique to treat lower urinary tract symptoms due to benign prostatic enlargement

\section{W Akhter, A Habib, R Kavia, A Habib}

North Wick park hospital, North West London Healthcare NHS trust

United Kingdom

Introduction \& Objective: Transurethral resection of prostate (TURP) is the gold standard treatment to treat patients with lower urinary tract symptoms (LUTS) secondary to benign prostatic enlargement (BPE). UroLift is an emerging minimally invasive technique to treat LUTS without significant side effects and preservation of erectile \& ejaculatory function. The procedure involves the placement of implants that retract the obstructing prostate lobes. NICE recommends that the UroLift system should be considered as an alternative to current surgical procedures for men aged 50 years and older with lower urinary tract symptoms of benign prostatic hyperplasia, who have a prostate of less than $100 \mathrm{ml}$ without an obstructing middle lobe. Aim: To assess the efficacy of UroLift procedure in patients with LUTS due to benign prostatic enlargement in a district general hospital settings. Materials and Methods: The data was collected prospectively from August 2015 to date for 11 patients diagnosed with LUTS due to BPE. We recorded age, pre \& post- operative international prostate score (IPSS), quality of life (QOL), flow test, voided volume, post void residual (PVR), erectile dysfunction (ED), retrograde ejaculation (RE), operating time, \& no. of implants used.

Results: The mean age was 68 (54-74), 7 patients were on tamsulosin, 1 stopped tamsulosin due to side effects \& 3 stopped finasteride due to gynaeocmastia. All procedures performed in general anaesthesia (GA). No. of implants applied was 3-5. The mean prostate size, operating time \& length of hospital stay were 39.1cc (25-66), 19 minutes (8-25), \& 7 hours (6-18) respectively. Post-operatively 3 patients required catheter for urinary retention, 3 experienced dysuria for 2 weeks and one patient complained

Table- 1 (Voiding function)
\begin{tabular}{|l|l|l|l|}
\hline NO. OF PTS (11) & PRE-OPERATIVE & POST-OPERATIVE & $\begin{array}{l}\text { AVERAGE } \\
\text { IMPROVEMENT (\%Pts) }\end{array}$ \\
\hline IPSS & $24.54(11-33)$ & $9.1(4-24)$ & $15.87(72.7 \%)$ \\
\hline QOL & $4.4(3-6)$ & $2(1-4)$ & $2.5(72.7 \%)$ \\
\hline Q-max & $8.6(4.1-18.9)$ & $14.4(5.8-21)$ & $6(63.6 \%)$ \\
\hline VOIDED VOLUME (ml) & $251.8(56-440)$ & $159.1(44-302)$ & $68.75(36.3 \%)$ \\
\hline $\begin{array}{l}\text { POST- VOID RESIDUAL } \\
\text { (ml) }\end{array}$ & $62.6(0-194)$ & $63.25(0-160)$ & $53.3(36.3 \%)$ \\
\hline
\end{tabular}


abdominal pain which settled spontaneously. Table-1 demonstrates voiding function comparison.

Conclusions: Initial experience with UroLift show promising results in the improvement of storage \& voiding LUTS with no adverse effects on sexual functions. Our results are also comparable to published evidence but long term data \& randomised control trials are needed. In addition UroLift as a day case procedure can be cost effective.

MP25-8 Urolift- For the Managment of Obstructive BPH5 year data from LIFT study

S Gange, N Shore, D Bolton, B Cowan, A Cantwell, K McVary, A Te, S Gholami, P Rashid, W Moseley, P Chin, W Dowling, S Freedman, P Incze, K Coffield, S Heron, D Rukstalis, C Roehrborn, J Barkin

University of Toronto

Canada

Introduction \& Objective: The Prostatic Urethral Lift (PUL) has been shown to effectively address lower urinary tract symptoms (LUTS) secondary to benign prostatic hyperplasia $(\mathrm{BPH})$ with minimal side effects. We present the 5 year results of the multi-center, randomized, blinded L.I.F.T. trial of this mechanical approach to BPH.

Materials and Methods: In the L.I.F.T. trial, PUL subjects received implants which were transurethrally placed into the lateral lobes of the prostate. 206 men $>$ age of 50 were randomized to PUL $(\mathrm{N}=140)$ or sham control $(\mathrm{N}=66)$ at 19 centers in North America and Australia. Enrollment criteria included IPSS (International Prostate Symptom Score) $\geq 13$, peak flow (Qmax) $\leq 12 \mathrm{ml} / \mathrm{s}$, and prostate volume $30-80 \mathrm{cc}$. Both control (sham) and active arm patients underwent rigid cystoscopy. Patients and assessors were kept blinded to treatment arm for 3 months. PUL participants were assessed through 5 years via IPSS, quality of life (QOL), BPH Impact Index (BPH II), Qmax, and sexual function questionnaires.

Results: Average symptom reduction in the L.I.F.T. study by 1 month was significant (IPSS: $44 \%$ and QoL: $42 \%, \mathrm{p}<0.001)$ and remained improved through 5 years (IPSS: $38 \%$ and QoL: $54 \%$, $\mathrm{p}<0.001)$. Peak urinary flow rate remained improved $41 \%$ at 5 years. Adverse events were typically mild and transient. No subjects experienced new onset, sustained erectile or ejaculatory dysfunction. Sexual function assessments indicate stable or improved average sexual function scores. For those subjects who

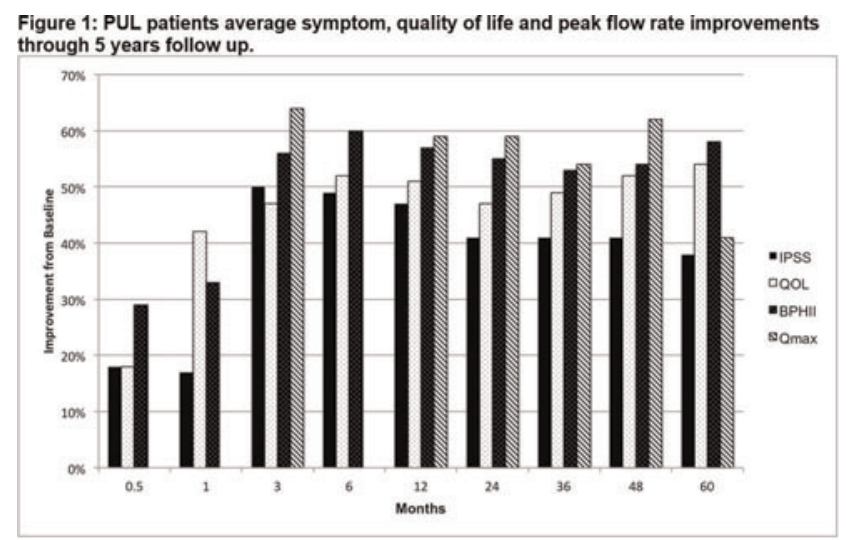

reached their 5 year follow up and were available to assessment, 19 underwent repeat PUL or other BPH procedure.

Conclusions: PUL patients experience rapid recovery with minimal adverse effects. Average symptom response is significant by 2 weeks, and remains stable through 5 years. Erectile and ejaculatory function are preserved. The final long term data from the largest study on the PUL procedure demonstrates that this minimally invasive approach improves symptoms durably.

MP25-9 Predictors of postoperative bacteriuria after Holmium Laser Enucleation of the Prostate (HoLEP)

AM Elmekresh, VH Villarreal, J Farewell, K Doersch, MM El Tayeb, L Tsai

Texas A\&M College of Medicine/Baylor Scott and White United States

Introduction \& Objective: Transient non-bothersome, irritative bladder symptoms are not uncommon following HoLEP, and thus urine culture (UCx) is obtained to differentiate between expected transient irritative symptoms and urinary tract infection (UTI). Our aim is to evaluate the predictors and risk factors of postoperative bacteriuria at $\underline{6}$ weeks post HoLEP in an attempt to decrease the postoperative routine UCx analysis.

Materials and Methods: After IRB approval, a retrospective chart review of 100 patients who had HoLEP between August 2015 and June 2016. It is a protocol at our institution that UCx analysis is performed at the $\underline{6}$ weeks postoperatively. All patients had urine cultures' results preoperatively and $\underline{6}$ weeks postoperatively. Patients' demographics, Preoperative, operative and postoperative characteristics data were obtained and analyzed. Statistical analysis was performed using USPSS and included means with standard deviation, Chi-square test, and Independent T-test when appropriate.

Results: A total of 100 patients were identified, 18 patients in group (A) who had positive postoperative UCx at 6 weeks after HoLEP versus 82 patients in group (B) who had negative postoperative UCx. Patients' demographics were comparable with no statistical significance between both cohorts. $11(61.1 \%)$ vs 22 (26.2\%) patients had preoperative positive UCx in groups (A) and $(B)$, respectively, $(\mathrm{p}=0.052)$. A preoperative history of UTI was noted in $7(38.8 \%)$ vs. $28(34.1 \%)$ patients in groups (A) and (B), respectively, $(\mathrm{P}=0)$. Preoperative Urine retention was present 3 vs. 12 in groups (A) and (B) with a mean length of catheterization (LOC) of 60 vs. 75.58 days, respectively, $(\mathrm{p}=0.708)$. Mean preoperative PVR was 116.45 vs. $145.48 \mathrm{mls}$ in groups $(A)$ and $(B)$, respectively, $(p=0.463)$. Mean preoperative BPH impact index was 5.85 vs. 5.1 in groups (A) and (B), respectively, $(\mathrm{p}=0.439)$. Mean preoperative AUA score is 22.2 vs. 24.48 in groups $(\mathrm{A})$ and $(\mathrm{B})$, respectively, $(\mathrm{P}=0.383)$. Mean OR specimen weight was 68.6 vs. 51.5 gms in groups (A) and $(B)$, respectively, $(\mathrm{p}=0.294)$. Enucleation and morcellation times were comparable with no statistical significance. Postoperative LOC was 1.6 vs. 1.9 in groups (A) and (B), respectively, $(p=0.323)$. Mean postoperative PVR at 6 weeks was 77.5 vs. $45.7 \mathrm{mls}$ in groups $(\mathrm{A})$ and $(\mathrm{B})$, respectively, $(\mathrm{p}=0.014)$. Mean AUA score mean at 6 weeks was comparable at 5.66 vs. 8.2 in groups (A) and (B), respectively, $(\mathrm{p}=0.153)$.

Conclusions: The sole predictor of postoperative bacteriuria is the elevated PVR; we recommend obtaining routine $\mathrm{UCx}$ for patients who presents with PVR above $50 \mathrm{ml}$. 
MP25-10 Low-power versus high-power en-bloc no-touch Holmium Laser Enucleation of the Prostate (HoLEP): comparing feasibility, safety and efficacy

CM Cracco, CM Scoffone

Department of Urology, Cottolengo Hospital of Torino, Italy Italy

Introduction \& Objective: HoLEP (Holmium Laser Enucleation of the Prostate) is a safe and effective procedure for benign prostatic obstruction (BPO) treatment. Six years ago we modified the traditional 3-lobe technique into the so-called en-bloc no-touch approach, enucleating the adenoma in one single horseshoe-like piece (en-bloc) by exploiting the vaporizing effects of the plasma bubble generated around the laser fiber tip (no-touch). After more than 250 procedures with the 100-120W holmium laser device in 2015 we chose to apply a low-power approach, trying to deliver less energy to the capsular plane and minimize postoperative dysuria. The aim of the present work was to assess the feasibility of the low-power approach, comparing its outcomes with those of the traditional high-power HoLEP.

Materials and Methods: 316 patients suffering from symptomatic BPO (any prostate volume, normal PSA, Qmax 10, PVR $<300 \mathrm{cc}$ ) underwent en-bloc no-touch HoLEP in our Department. From 01/2012 to 05/2015 214 consecutive patients underwent high-power HoLEP (group 1) using the 100-120W Versapulse holmium laser (Lumenis), settings $2 \mathrm{~J} \times 50 \mathrm{~Hz}, 100 \mathrm{~W}$ power. From 06/2015 to 06/2016 102 consecutive patients underwent low-power HoLEP (group 2) using the 120W Versapulse holmium laser (Lumenis) for the first 20 patients, then the $50 \mathrm{~W}$ Auriga XL holmium laser device (Boston Scientific), settings $2.2 \mathrm{~J} \times 18 \mathrm{~Hz}$, long pulse length, almost $40 \mathrm{~W}$ power. Patients demographics and clinical data were prospectively registered. Data were correlated using the Pearson correlation test.

Results: Mean age (69.4 years $+/-7.5$ d.s. vs. 67.7 years $+/-8$ d.s.) and adenoma weight (55.3 $\mathrm{g}+/-38.9$ d.s. vs. $45.8 \mathrm{~g}+/-36$ d.s.) were similar in both groups. Energy employed was significantly lower in group $2(83.5 \mathrm{~kJ}+/-32$ d.s. vs. $53.4 \mathrm{~kJ}+/-23$ d.s. $)$ $(\mathrm{p}<0.01)$, with a reduced $\mathrm{kJ} / \mathrm{g}$ ratio $(2+/-1$ d.s. vs. $1.54+/-0.85$ d.s. $)(\mathrm{p}<0.01)$, similar enucleation time $(31 \mathrm{~min}+/-13$ d.s. vs. $27.5 \mathrm{~min}+/-11 \mathrm{~d} . \mathrm{s}$.) and efficiency $(1.64 \mathrm{~g} / \mathrm{min}+/-0.8 \mathrm{~d}$.s. vs. $1.7 \mathrm{~g} / \mathrm{min}+/-1$ d.s.). Pre- and postoperative ( 3 months) IPSS scores sere similar (pre: $22+/-2.4$ d.s. vs. $22+/-7$ d.s.; post: 6.5 $+/-5$ d.s. vs. $7.8+/-5$ d.s.), as well as morcellation time $(9.2 \mathrm{~min}$ $+/-7.6$ d.s. vs. $7.7+/-7.1$ d.s.). Among the complications (Clavien grade 1 and 2), incidence of bleeding (no blood transfusions) $(4.2 \%$ vs. $3 \%)$ and recatheterizations $(4.2 \%$ vs. $3 \%)$ were similar. Long-lasting incontinences of variable entity (mainly mild) $(1.4 \%$ vs. $1.6 \%)$ and of postoperative dysuria (10\%) at 3-month follow up were equivalent.

Conclusions: Low-power en-bloc no-touch HoLEP is feasible, safe and effective as the high-power approach, in the hands of experienced operators, being energy consumption reduced by nearly one third.

MP25-11 The bipolar transurethral enucleation of the prostate (BTUEP) is a safe and effective technique in patients under anticoagulation or antiplatelet therapy

L Boeri, M Fontana, G Sampogna, A Gallioli, E De Lorenzis, F Palmisano, S Zanetti, F Longo, G Albo, F Gadda, P Dell'Orto, E Montanari
Department of Urology, Fondazione IRCCS Ca' Granda Ospedale Maggiore Policlinico, Università degli Studi di Milano

Italy

Introduction \& Objective: Current Guidelines do not provide exact instructions regarding anticoagulation (AC) or antiplatelet (AP) therapy during endoscopic surgery for benign prostatic hyperplasia (BPH). We investigated the safety and efficacy of bipolar transurethral enucleation of the prostate (BTUEP) in a cohort of patients with AC/AP therapy.

Materials and Methods: We analyzed 97 consecutive patients who underwent BTUEP from 2015 to 2017. Data from 24 patients who required AC/AP therapy were compared to those from 63 patients who were not on AC/AP therapy. The AC/AP group included patients whose AP therapy was not interrupted pre-, peri-, and/or postoperatively and patients who underwent perioperative AC bridging with low molecular weight heparin. $\mathrm{Pa}-$ tient's demographics and perioperative data were analysed. Comorbidities were scored with the Charlson Comorbidity Index (CCI). Prostate specific antigen (PSA), urinary flow rate (Qmax) and the International Prostate Symptom Score (IPSS) were collected at baseline and at 3-mos follow up. Complications were scored according to the Clavien-Dindo classification. Descriptive statistics were used to describe the whole cohort.

Results: Overall, mean (SD) PV was 97.1 (40.8) ml; range [60$260 \mathrm{ml}$. No differences in terms of age, preoperative PSA, Qmax, PV and IPSS were observed between the two groups. The $\mathrm{CCI}$ score was higher in AC/AP patients than those in the no AC/ AP group $(p<0.001)$. Intraoperative characteristics were similar between groups. All postoperative outcomes were comparable between groups except for length of hospital stay, which was longer in the AC/AP group (6.3 vs 4.2 days; $p=0.006)$. No differences in catheterization time, hemoglobin drop values or transfusion rates were found between groups. Both cohorts were similar in regard to postoperative PSA value, Qmax and IPSS at 3-months follow up. Complication of Clavien group I and II were reported in $7(8.0 \%), 12(13.8 \%)$ patients respectively with no significant differences between groups.

Conclusions: These findings showed that the use of AC/AP therapy did not adversely affect outcomes of BTUEP except for a slight increase of hospital stay. BTUEP emerged as a safe and effective technique for the surgical treatment of BPH in this population. Overall, keeping in mind that the prevalence of both $\mathrm{BPH}$ and cardiovascular events requiring AC/AP therapy increase with age, finding a safe technique for the surgical treatment of BPH is a major clinical need in this population.

MP25-12 Comparative efficacy and safety of surgical treatments for benign prostate hyperplasia: an application of network meta-analysis

S Huang, C Tseng, K Chien, C Tsai

National Taiwan University hospital yun-in branch urology department

Taiwan (Republic of China)

Introduction \& Objective: In the past 10 years, multiple newnovel methods for surgical treatment of BPH have been developed. To evaluate the efficacy and safety of new-novel methods for surgical treatment of BPH, network meta-analysis has been used. 
Materials and Methods: Pubmed, Embase, Cochrane clinical trial databases, non-restricted to language, were searched from inception to Dec 16, 2016. We included randomized controlled trials to compare vaporization, resection and enucleation of prostate using bipolar, HO-YAG laser, thulium laser, KTP laser or diode laser. We estimated weight mean difference for continuous variables and risk ratio for dichotomous variables by using Generalized linear mixed model. Cochrane Collaboration's risk of bias assessment was applied to appraise the quality of the articles.

Results: 92 trials with 122 articles were included in the analysis. Enucleation methods were superior to resection and vaporization in postop 6 months maximal flow rate (Qmax). The discrepancy enlarged as follow-up time increased from post-op 6 months, 12 months to 36 months. No significant difference was noted between enucleation, vaporization and resection in postop 6 months international prostate symptoms score (IPSS) and Health-related quality of life (HRQOL), however, the discrepancy became significant in post-op 24 and 36 months. Regarding adverse effects, vaporization, especially diode laser, took advantages of enucleation and resection in blood clot tamponade and earlier removal of Foley catheter. Enucleation had less events of urethrae stricture compared to resection and vaporization. With respect to BPH recurrence and reinsertion Foley catheter, enucleation was the best, followed by resection, and vaporization was the worst of the three methods

Conclusions: The efficacy of new-novel method was similar to that of resection in relation to Qmax, IPSS and HRQOL, and it had less side effects compared to that of monopolar resection. Monopolar TURP as the gold standard surgical treatment of BPH is challenged due to the comparable efficacy of new-novel methods with less side effects. Enucleation method seems to be the best method with durable efficacy.

MP25-13 Safety and effectiveness of high-power thulium laser enucleation of the prostate in patients with large glands

\section{J Huang, C Chang, T Lin}

Taiepi Medical University Hospital

Taiwan (Republic of China)

Introduction \& Objective: Holium enucleation and open prostatectomy were the standard treatment for large prostate $(>80 \mathrm{gm})$ according to guidelines. Thulium enucleation is an emerging treatment option for bladder outlet obstruction caused by benign prostate enlargement, which effectively alleviated subjective and objective voiding symptoms with a low rate of complications. However, the application of Thulium enucleation for large prostate lack stronge evidence. To further explore the potential of ThuLEP, this research focused on patients with large glands $(\geq 80 \mathrm{~g})$.

Materials and Methods: We prospectively collected data from 130 patients who underwent ThuLEP by a single surgeon from June 2012 to June 2016. All patients had a prostate gland larger than $80 \mathrm{~g}$, measured using transrectal ultrasonography. The data of ThuLEP from our pilot study were used as the control group. Operative variables, patient profiles, preoperative and postoperative urine flow rates, prostate volume, and the International Prostate Symptom Score (IPSS) were recorded and analyzed using a two-tailed Student's t-test and analysis of variance.

Results: The ages and estimated prostate volume (mean \pm SD) of the patients were $69.68 \pm 15.37$ years $(p=0.3402)$ and $120.97 \pm 44.90 \mathrm{ml}(\mathrm{p}<0.0001)$, respectively. The mean of postvoiding residual urine volume and maximum flow rates before and 3 months after the operation were 204.78/68.60 and 10.19/
Table 1

Baseline characteristics (median \pm standard deviation)

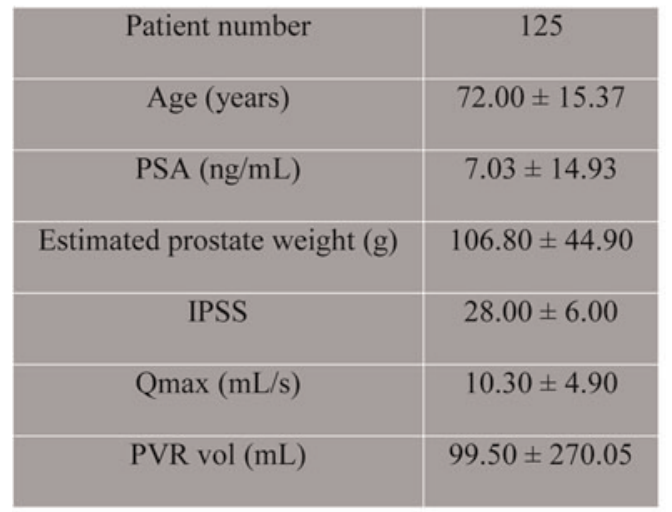

PSA, prostate-specific antigen; IPSS, international prostate symptom score; Qmax, maximum urinary flow rate; PVR, post-void residual volume.

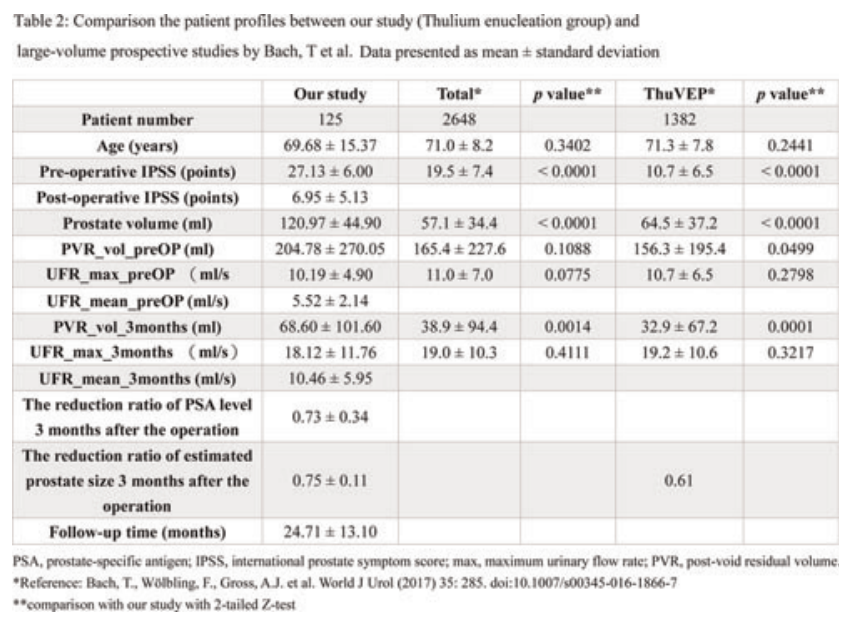

18.12 (in $\mathrm{ml}, \mathrm{ml} / \mathrm{s}$ ), respectively, and were not significantly different with the control group $(p=0.1088,0.0014,0.0775$, $0.4111)$. Preoperative IPSSs in the ThuLEP group and control group were $27.13 \pm 6.00$ and $10.7 \pm 6.5$, respectively, $(p<0.001)$. The reduction ratio of the postoperative prostate volume was $0.75 \pm 0.11$. The mortality rate was $0.7 \%$.

Conclusions: High-power ThuLEP was generally safe and effective. Outcomes were superior in patients with large glands $(\geq 80 \mathrm{ml}$ ). Thus, enucleation using a high-power laser is feasible in patients with large glands and severe symptoms.

MP25-14 How low can we go during holmium laser enucleation of the prostate (HoLEP)? 12-month results from a prospective low-power HoLEP series

C Netsch, B Becker, TR Herrmann, AJ Gross

Asklepios Klinik Barmbek, Department of Urology

Germany 
Introduction \& Objective: To analyze the 12-month outcomes of low-power HoLEP for patients with symptomatic benign prostatic obstruction (BPO).

Materials and Methods: A prospective study was performed from February 2015 to February 2016 at our institution. A total of 54 consecutive patients with symptomatic BPO were treated with HoLEP. All surgeries were performed by two experienced HoLEP surgeons (AJG, CN). A two or three lobe HoLEP technique was done according to prostate anatomy. HoLEP was carried out using a $50 \mathrm{~W}$ Ho:YAG laser (Auriga ${ }^{\circledR}$ XL, Boston Scientific, Ratingen, Germany) at $39.6 \mathrm{~W}(2.2$ Joule, $18 \mathrm{~Hz})$ in combination with a $550 \mu \mathrm{m}$ bare-ended, re-usable laser fibre. All patients were assessed preoperatively by International Prostate Symptom Score (IPSS), Quality of Life (QoL), maximum urinary flow rate (Qmax), post-void residual urine (PVR), PSA, and prostate volume measurement by transrectal ultrasound. The patients were reassessed at 1-, 6-, and 12-month follow-up. The complications were noted and classified according to the modified Clavien classification system. Patient data were expressed as median (interquartile range) or numbers $(\%)$.

Results: Median age was 72.5 (67-77.25) years and the median preoperative prostate volume 74.5 (45-110) $\mathrm{ml}$. 24 (44.7\%) patients presented in urinary retention with an indwelling catheter and $12(22.2 \%)$ patients were treated under ongoing anticoagulant treatment. The median operative time was 65 (41-81) minutes and the enucleation efficiency $1.11(0.82-1.79) \mathrm{g} / \mathrm{min}$, respectively. The median catheterization time was 2 (2-2) days and the median postoperative stay 2 (2-3) days. Clavien 1 (5.7\%), Clavien $2(3.7 \%)$, Clavien $3 \mathrm{a}(3.7 \%)$, and Clavien $3 \mathrm{~b}(5.5 \%)$ complications occurred. One patient treated under ongoing oral anticoagulant therapy needed a blood transfusion $(1.9 \%)$ postoperatively. At 6-month follow-up, median prostate volume (74.5 vs. $15.5 \mathrm{ml})$ and PSA (4.03 vs. $0.54 \mu \mathrm{g} / \mathrm{l})$ had improved significantly compared to baseline $(\mathrm{p} \leq 0.009)$. At 12 -month follow-up, median Qmax (11.9 vs. $29.3 \mathrm{ml} / \mathrm{s})$, PVR (155 vs. $11.15 \mathrm{ml}$ ), IPSS (22 vs. 6), and QoL (5 vs. 1) had improved significantly compared to baseline $(\mathrm{p}<0.001)$. The reoperation rate was zero at 12-month follow-up.

Conclusions: Low-power HoLEP is technically feasible, safe and effective for the treatment of symptomatic BPO. For experienced surgeons, power is less relevant than technique.

MP25-15 Thulium vapoenucleation of the prostate versus holmium laser enucleation of the prostate for the treatment of benign prostatic obstruction: 12-month results of a prospective randomized trial

\section{Netsch, B Becker, TR Herrmann, AJ Gross}

Asklepios Klinik Barmbek, Department of Urology Germany

Introduction \& Objective: To compare the 12-month outcomes of thulium vapoenucleation of the prostate (ThuVEP) with holmium laser enucleation of the prostate (HoLEP) for patients with symptomatic benign prostatic obstruction (BPO).

Materials and Methods: A prospective randomized trial comparing ThuVEP with HoLEP was performed from 02/2015 to 02/ 2016 at our institution. A total of 94 consecutive patients with symptomatic BPO have been enrolled into the study and were prospectively randomized to ThuVEP $(n=48)$ or HoLEP $(n=46)$. All patients were assessed preoperatively by International Prostate Symptom Score (IPSS), Quality of Life (QoL), maximum urinary flow rate (Qmax), post-void residual urine (PVR), PSA, and prostate volume measurement by transrectal ultrasound. The patients were reassessed at 1-, 6-, and 12-month follow-up. The complications were noted and classified according to the modified Clavien classification system. Patient data were expressed as median (interquartile range) or numbers (\%).

Results: There were no statistically significant differences in any baseline characteristics between the groups. Median prostate volume was $80(46.75-100) \mathrm{ml} .43(45.7 \%)$ patients presented in urinary retention with an indwelling catheter. The median operative time was 60 (41-79) minutes without differences between the groups. There were no differences between the groups regarding the median catheter time (2 (2-2) days) and postoperative stay (2 (2-3) days). Clavien 1 (13.8\%), Clavien $2(3.2 \%)$, Clavien $3 \mathrm{a}(2.1 \%)$, and Clavien $3 \mathrm{~b}(4.3 \%)$ complications occurred without differences between the groups. At 6-month follow-up, median prostate volume ( $80 \mathrm{vs} .16 \mathrm{ml}$ ) and PSA (4.14 vs. $0.71 \mu \mathrm{g}$ / 1) had improved significantly compared to baseline $(\mathrm{p}<0.001)$ without differences between the groups. At 12-month follow-up, median Qmax (10.7 vs. 23.3 ml/s), PVR (100 vs. $11.1 \mathrm{ml}$ ), IPSS (20 vs. 5), and QoL (4 vs. 1) had improved significantly $(\mathrm{p}<0.001)$ compared to baseline without significant differences between the groups. The reoperation rate was zero at 12-month follow-up. During 12-month follow-up, an episode of acute urinary retention had occurred in 1 patient $(0.7 \%)$ and $6(6.4 \%)$ patients had developed an urinary tract infection without differences between the groups.

Conclusions: ThuVEP and HoLEP are safe and effective procedures for the treatment of symptomatic BPO. Both procedures give equivalent and satisfactory micturition improvement with low morbidity and sufficient prostate volume reduction.

MP25-16 Sexual outcomes of thulium laser ejaculationsparing surgery (TES) for benign prostatic hyperplasia

L Carmignani, S Nazzani, M Clementi, C Signorini, D Vizziello, G Motta, C Marenghi, P Acquati

Italy

Introduction \& Objective: The aim of this study was to evaluate ejaculation outcomes in patients who underwent transurethral vapoenucleation of the prostate using a thulium laser.

Materials and Methods: A total of 167 patients who underwent thulium vapoenucleation of the prostate (ThuVEP) using an ejaculation-sparing technique were evaluated. Validated questionnaires, including the International Index of Erectile Function-15 and the International Prognostic Scoring System (IPPS), were used to evaluate changes in erectile and ejaculatory functions as well as the effect of lower urinary tract symptoms (LUTS) on quality of life (QoL). Patients were evaluated by using these questionnaires and uroflowmetry preoperatively and at 3 and 6 months postoperatively

Results: Mean age was 69 years, and mean prostate volume was $78.3 \mathrm{~mL}$. All patients were dischargeable after $48 \mathrm{~h}$; only 2 patients were discharged with a urinary catheter in place. A nonsignificant difference in erectile function was observed after surgery, and improvements in postoperative urinary symptoms were found. Ejaculation was preserved in 94 patients (56.3\%) after surgery. A significant improvement in IPPS score was reported at both 3 months (mean score, 8) and 6 months (mean score, 5.2) postoperatively.

Conclusions: Thulium laser ejaculation-sparing surgery reduces LUTS and improves patients' QoL while preserving erectile and ejaculatory functions. 
MP25-17 1000 Cases of Holmium Laser Enucleation of the Prostate: A Single Centre Case Series with 13 years of follow-up

D Whiting, B Penev, M Cynk

Maidstone and Tunbridge Wells NHS Trust

United Kingdom

Introduction \& Objective: Holmium laser enucleation of the prostate (HoLEP) is an established effective treatment option for bladder outflow obstruction secondary to benign prostatic hyperplasia. As a relatively new procedure long-term outcomes for patients undergoing HoLEP are still being evaluated. We describe the outcomes of over 1000 cases in a single centre with up to 13 years of post-operative follow-up.

Materials and Methods: A retrospective review of a prospective database of all HoLEP procedures performed by or under supervision of a single consultant urological surgeon was undertaken. All case notes were reviewed for complications up to March 2017 and recorded in accordance with the Clavien-Dindo classification system. Statistical analysis was performed using a Mann-Whitney U test.

Results: Between December 2003 and March 20171016 cases of HoLEP were performed at our centre. Median patient age was 72 years (range 41-95). Pre-operative acute urinary retention was present in 403 patients $(39.7 \%)$. Median pre-operative flow rate was $8.5 \mathrm{ml} / \mathrm{s}$ (range 1-26.3) $(\mathrm{n}=561)$ and post-operative flow rate

\begin{tabular}{|c|c|}
\hline Complication ( $\mathrm{n}=1016)$ & Number (\%) \\
\hline \multicolumn{2}{|l|}{ Early } \\
\hline \multicolumn{2}{|l|}{ Clavien grade 1: } \\
\hline Failed initial voiding trial & $108(10.6)$ \\
\hline Transient stress incontinence & $66(6.5)$ \\
\hline Frequency and dysuria & $57(5.6)$ \\
\hline Post-operative confusion & $3(0.3)$ \\
\hline \multicolumn{2}{|l|}{ Clavien grade 2: } \\
\hline Urinary tract infection & $54(5.3)$ \\
\hline Secondary haemorrhage & $12(1.2)$ \\
\hline Epididymitis & $7(0.7)$ \\
\hline Blood transfusion & $4(0.4)$ \\
\hline Nausea and vomiting & $2(0.2)$ \\
\hline Chest infection & $1(0.1)$ \\
\hline Chest pain & $1(0.1)$ \\
\hline Supra ventricular tachycardia & $1(0.1)$ \\
\hline \multicolumn{2}{|l|}{ Clavien grade $3 b$ : } \\
\hline Return to theatre & $15(1.5)$ \\
\hline Ureteric obstruction & $2(0.2)$ \\
\hline Conversion to TURP & $2(0.2)$ \\
\hline \multicolumn{2}{|l|}{ Clavien grade $4 a:$} \\
\hline Urinary sepsis & $1(0.1)$ \\
\hline Anuric renal failure & $1(0.1)$ \\
\hline \multicolumn{2}{|l|}{ Clavien grade 5: } \\
\hline $\mathrm{MI}$ and cardiac arrest & $1(0.1)$ \\
\hline \multicolumn{2}{|l|}{ Late } \\
\hline \multicolumn{2}{|l|}{ Clavien grade 2: } \\
\hline Rectoprostatic fistula & $1(0.1)$ \\
\hline \multicolumn{2}{|l|}{ Clavien grade $3 a:$} \\
\hline Submeatal stenosis & $19(1.9)$ \\
\hline \multicolumn{2}{|l|}{ Clavien grade $3 b$ : } \\
\hline Urethral stricture & $49(4.8)$ \\
\hline Redo TURP or HoLEP & $32(3.1)$ \\
\hline Bladder neck stenosis & $13(1.3)$ \\
\hline Stress incontinence & $3(0.3)$ \\
\hline
\end{tabular}

was $19.8 \mathrm{ml} / \mathrm{s}$ (range $1.8-68.4)(\mathrm{n}=695)$. Median pre-operative post-void residual volumes were $264 \mathrm{mls}$ (range 0-5000) $(\mathrm{n}=755)$ and post-operative residual volumes were $70 \mathrm{mls}(0$ 1000) $(\mathrm{n}=737)$. There was a statistically significant improvement in both urinary flow rate and post-void residual volumes $(\mathrm{p}<0.0001) .189$ patients $(23.3 \%)$ were discharged from hospital on the day of surgery; $491(60.6 \%)$ on day $1 ; 85(10.5 \%)$ on day $2 ; 45(5.6 \%)$ stayed 3 days or more $(n=810)$. Post-operative early and late complications are recorded within the table.

Conclusions: HoLEP is an effective and safe treatment for bladder outflow obstruction associated with a short hospital stay and few early and late complications.

MP25-18 Holmium laser enucleation of the prostate is a safe and effective procedure for patients with lower urinary tract symptoms in prostate cancer

D Whiting, B Penev, M Cynk

Maidstone and Tunbridge Wells NHS Trust United Kingdom

Introduction \& Objective: Holmium laser enucleation of the prostate (HoLEP) is used in the treatment of troublesome lower urinary tract symptoms (LUTS) secondary to an enlarged prostate. We evaluated the outcome and complications of HoLEP in patients with concomitant prostate cancer and LUTS.

\begin{tabular}{|c|c|c|}
\hline & Benign ( $n=895)$ & Prostate Cancer $(n=121)$ \\
\hline Age & 72 years (range $41-95$ ) & 74 years (range $55-91$ ) \\
\hline Pre-operative retention & $351(39.2 \%)$ & $52(43.0 \%)$ \\
\hline Pre-operative Qmax & $8.8 \mathrm{ml} / \mathrm{s}$ (range $1-26.3 \mathrm{ml} / \mathrm{s}$ ) & $7.5 \mathrm{ml} / \mathrm{s}(3-17.4 \mathrm{ml} / \mathrm{s})$ \\
\hline Post-operative Qmax & $20.3 \mathrm{ml} / \mathrm{s}(1.8-68.4 \mathrm{ml} / \mathrm{s})$ & $16.5 \mathrm{ml} / \mathrm{s}(2.2-38.7 \mathrm{ml} / \mathrm{s})$ \\
\hline \multicolumn{3}{|l|}{ Early Complications } \\
\hline Clavien grade 1: & $n(\%)$ & $n(\%)$ \\
\hline Failed initial voiding trial & $98(10.9)$ & $10(8.3)$ \\
\hline Transient stress incontinence & $50(5.6)$ & $16(13.2)$ \\
\hline Frequency and dysuria & $55(6.1)$ & $2(1.7)$ \\
\hline Post-operative confusion & $2(0.2)$ & $1(0.8)$ \\
\hline \multicolumn{3}{|l|}{ Clavien grade 2: } \\
\hline Urinary tract infection & $47(5.3)$ & $7(5.8)$ \\
\hline Secondary haemorrhage & $11(1.2)$ & $1(0.8)$ \\
\hline Epididymitis & $7(0.8)$ & $0(0)$ \\
\hline Blood transfusion & $4(0.4)$ & $0(0)$ \\
\hline Nausea and vomiting & $2(0.2)$ & $0(0)$ \\
\hline Chest infection & $1(0.1)$ & $0(0)$ \\
\hline Chest pain & $1(0.1)$ & $0(0)$ \\
\hline Supra ventricular tachycardia & $1(0.1)$ & $0(0)$ \\
\hline \multicolumn{3}{|l|}{ Clavien grade $3 b$ : } \\
\hline Return to theatre & $14(1.6)$ & $1(0.8)$ \\
\hline Ureteric obstruction & $1(0.1)$ & $1(0.8)$ \\
\hline Conversion to TURP & $1(0.1)$ & $1(0.8)$ \\
\hline \multicolumn{3}{|l|}{ Clavien grade $4 a$ : } \\
\hline Urinary sepsis & $1(0.1)$ & $0(0)$ \\
\hline Anuric Renal Failure & $1(0.1)$ & $0(0)$ \\
\hline \multicolumn{3}{|l|}{ Clavien grade 5: } \\
\hline MI and cardiac arrest & $1(0.1)$ & $0(0)$ \\
\hline \multicolumn{3}{|l|}{ Late Complications } \\
\hline \multicolumn{3}{|l|}{ Clavien grade 2: } \\
\hline Rectoprostatic fistula & $0(0)$ & $1(0.8)$ \\
\hline \multicolumn{3}{|l|}{ Clavien grade $3 a:$} \\
\hline Submeatal stenosis & $18(2.0)$ & $1(0.8)$ \\
\hline \multicolumn{3}{|l|}{ Clavien grade $3 b$ : } \\
\hline Urethral stricture & $40(4.5)$ & $9(7.4)$ \\
\hline Redo TURP or HoLEP & $32(3.6)$ & $0(0)$ \\
\hline Bladder neck stenosis & $13(1.5)$ & $0(0)$ \\
\hline Stress incontinence & $2(0.2)$ & $1(0.8)$ \\
\hline
\end{tabular}


Materials and Methods: A retrospective review of a prospective database of all HoLEP procedures performed by or under supervision of a single consultant urological surgeon was undertaken. All case notes were reviewed for complications up to March 2017. The data set was divided into two; benign and prostate cancer. Statistical analysis was performed using a MannWhitney U test, Chi-Squared and Fishers Exact test.

Results: 1016 cases of HoLEP were performed at our centre between December 2003 and March 2017. 121 (11.9\%) had prostate cancer. The results are displayed in the table. There was a statistically significant improvement in urinary flow rate (Qmax) in both groups $(\mathrm{p}<0.01)$. There was a statistically significant difference in the incidence of transient stress incontinence $(p<0.01)$, for all other complications there were no statistically significant differences between the two groups.

Conclusions: HoLEP is associated with a statistically significant higher incidence of transient stress incontinence in patients with prostate cancer. HoLEP is not associated with an increased risk of any other complications in prostate cancer and we propose it is a safe and effective procedure for LUTS in patients both with and without prostate cancer.

MP25-19 Relationship between morcellation efficiency and resected volume of adenoma in holmium laser enucleation of the prostate for patients with benign prostatic hyperplasia

H Kim, K Lee, D Shin, T Kim, S Choi, T Oh, C Yoon, W LEE, J Lee

Pusan National University School of medicine Republic of Korea

Introduction \& Objective: To evaluate the morcellation efficiency according to the resected volume of adenoma from Holmium laser enucleation of the prostate (HoLEP) which was performed in patients with benign prostatic hyperplasia (BPH). Materials and Methods: The present retrospective study was conducted by reviewing the operation record of $438 \mathrm{BPH}$ patients who were treated by HoLEP by two surgeons between July 2012 and June 2014. All patients underwent mechanical morcellation by using VersaCut ${ }^{\circledR}$ morcellator (Lumenis Inc.) once the adenoma wad enucleated by using the VersaPulse ${ }^{\circledR}$ PowerSuite ${ }^{\mathrm{TM}}$ Holmium laser (Lumenis Inc). The inverse (downward) technique was performed during the morcellation to prevent injury of the bladder mucosa. Morcellation efficiency was determined as the morcellated volume of adenoma per minute. To evaluate the correlation between the two variables, morcellation

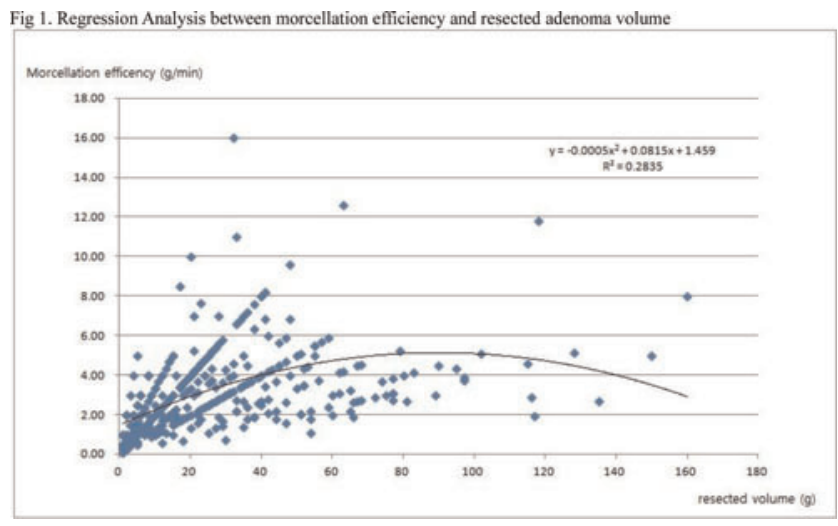

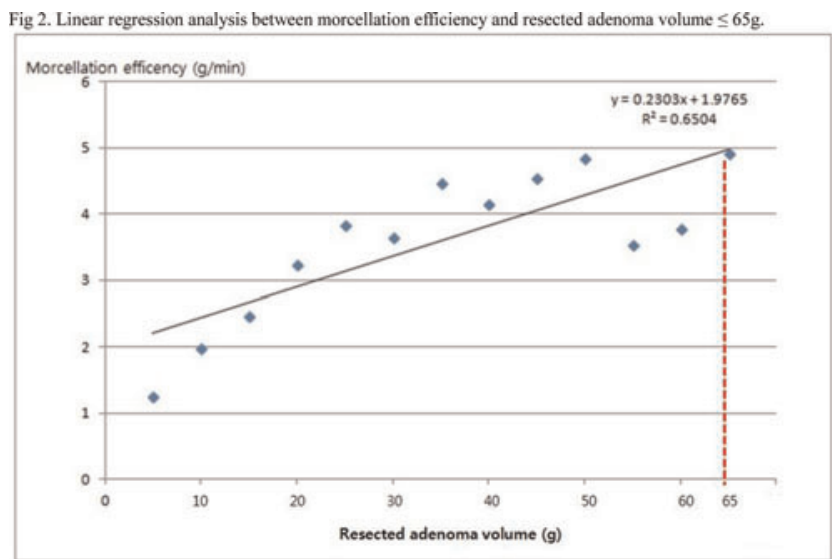

efficiency and volume of resected adenoma, regression analysis was performed. Also, linear correlation between the two variables was evaluated by only taking the morcellation efficiency that increases along with the resected adenoma volume into account.

Results: The mean values of patient's age, prostate volume, and resected volume was $69.2 \pm 7.0$ years, 60.9 $\pm 29.3 \mathrm{~g}$, and $24.7 \pm 25.1 \mathrm{~g}$, respectively. The mean morcellation time was $8.6 \pm 7.9 \mathrm{~min}$ and the mean morcellation efficiency was $2.91 \pm 1.9 \mathrm{~g} / \mathrm{min}$. Morcellation demonstrated increasing efficiency until the volume of resected adenoma reached to $65 \mathrm{~g}$, whereas the efficiency started to decrease from $65 \mathrm{~g}$ of resected adenoma (Fig 1). Positive linear correlation was observed between morcellation efficiency and resected adenoma volume in the patients who had resected adenoma volume $\leq 65 \mathrm{~g}$ (Fig 2).

Conclusions: Morcellation efficiency increases until the resected adenoma volume reaches $65 \mathrm{~g}$ and decreases thereafter.

MP25-20 Clinical significance between resected prostate volume, serum prostate-specific antigen, and lower urinary tract symptoms after Holmium laser enucleation of the prostate

H Kim, K Lee, D Shin, T Kim, S Choi, T Oh, C Yoon, W LEE, J Lee

Pusan National University School of medicine Republic of Korea

Introduction \& Objective: Holmium laser enucleation of the proatate (HoLEP) has resulted the improvement of voiding symptoms, lesser intraoperative bleeding and perioperative complications, and shortening of catheterized duration and hospital stay in benign prostatic hyperplasia (BPH) patients. This study aims to investigate the clinical significance between resected prostate volume, serum prostate-specific antigen (PSA), and lower urinary tract symptoms (LUTS) after HoLEP.

Materials and Methods: Among the total of $586 \mathrm{BPH}$ patients who underwent HoLEP from January 2010 to August 2011, 129 patients who have postoperative follow-up period over a year along with pre- and postoperative PSA and international prostate symptom score questionnaire (IPSS) were enrolled. Age, preoperative prostate volume, resected prostate volume, difference ratio between pre- and postoperative PSA (postoperative PSA divided by preoperative PSA) and between pre- and postoperative IPSS (postoperative IPSS divided by preoperative IPSS) of the enrolled patients were investigated and analyzed. 
Results: The mean age of enrolled patients was 67.5 \pm 7.4 (5184) years. Their mean volume of preoperative and resected prostate was $57.4 \pm 28.8 \mathrm{~g}(18.2-154.0)$ and $24.8 \pm 24.4 \mathrm{~g}(1-158)$, respectively. respectively. Mean preoperative PSA and the mean difference ratio between pre- and postoperative PSA were $4.4 \pm 4.0 \mathrm{ng} / \mathrm{ml} \quad(0.119 \sim 19.34)$ and $0.413 \pm 0.381 \quad(0.001-$ $1.812)$, respectively. Mean pre- and postoperative IPSS were $20.6 \pm 7.2(5-35)$ and $8.5 \pm 6.9(0-35)$, respectively, and the mean difference ratio between the two IPSS scores was $0.476 \pm 0.612$ (0-5.833). Resected prostate volume was significantly correlated with the difference ratio between pre- and postoperative PSA ( $p$ Conclusions: The volume of prostatic tissues resected by HoLEP presented a significant positive correlation with the difference ratio between pre- and postoperative PSA.

MP25-21 A number of Laser procedures are available as effective alternatives to TURP entailing less morbidity and shorter hospital stays. our experience of Thulium Laser Vaporization \& enucleation of prostate and intravesical morcellation.

PK Pattnaik

India

Introduction \& Objective: a number of Laser procedures are available as effective alternatives to TURP entailing less morbidity and shorter hospital stays. We present our experience and results of Thulium Laser Vaporization \& enucleation of prostate and intravesical morcellation.

Materials and Methods: We present a series of 75 cases from July 2015 to July,2016. Patient aged between 60-92 years. Mean size of prostate was 90 grams. Pre-opeatively average uroflow rate was $8 \mathrm{ml} / \mathrm{sec}$. 52 patients had one or the other co-morbidities. Procedure was performed under spinal anaesthesia. 25 patients were on clopidogrel and for these patients procedure was done under general anaesthesia.

Results: average time taken for enucleation was $1.5 \mathrm{~g} / \mathrm{min}$ of resected specimen. Post-operatively no irrigation was used. None of the patients required blood transfusion post-operatively. Catheter was removed after 48 hours in all patients. 15 patients had retention following catheter removal for which they were re-catheterized and catheter was then removed after one week. Average uroflow rate after catheter removal on $2^{\text {nd }}$ post-operative day was $18 \mathrm{ml} / \mathrm{sec}$ and after 6 weeks was $22 \mathrm{ml} / \mathrm{sec}, 6$ patients developed bladder neck contracture which was recognized as early as 3 weeks and bladder neck incision was done. None of the patients had stricture.

Conclusions: Thulium laser vaporization \& enucleation of the prostate is a low risk, minimally invasive treatment option for treatment of BPH. It can be safely performed even in high risk patients and patients on oral anticoagulants. It has less risk of hemorrhage, reduced bladder irrigation and catheter times.

MP25-22 Comparison Of Open And Robot Assisted Adenomectomy For The Treatment Of Large Prostate Glands Due To Benign Prostate Hyperplasia

S Keskin, P Mourmouris, T Doganca, A Skolarikos, I Tufek, OB Argun, MB Tuna, K Andreas, C Obek, AR Kural

Acibadem University, School of Medicine, Department of Urology, Istanbul, Turkey

Turkey
Introduction \& Objective: The purpose of this study was to test the efficacy and safety of robot assisted adenomectomy for benign prostatic hyperplasia (BPH) in comparison with the open approach.

Materials and Methods: This is a retrospective study of 34 patients who underwent adenomectomy for large $(>80 \mathrm{ml})$ prostate adenomas at two different centers by experienced surgeons. Open surgeries were performed at Sismanogleio General Hospital, Athens, and robotic surgeries were performed at Maslak Acıbadem Hospital, Istanbul. Indication for surgery was either incomplete patient satisfaction with medical treatment or necessity for catheterization. A vertical bladder incision was used in open and a vertical vesico-capsular incision in robotic approach. Preoperative, intraoperative and postoperative characteristics, outcomes and complication rates were analyzed and compared between the two groups.

Results: Surgical approach used was open in 10 and robot assisted laparoscopic in 24. Six patients $(60 \%)$ in open and seven patients $(29 \%)$ in robotic arm had preoperative urethral catheter due to relapsed urinary retention. The results of the comparison between the two groups are shown in Table 1. Blood loss was significantly less in the robotic arm $(p=0.02)$ however, operation time was significant longer $(p=0.04)$. Urethral catheter was removed significantly earlier and hospitalization time was significantly less in robotic group. Outcomes based on uroflowmetry and residual urine were similar in both cohorts. No complications $>=2$ according to the Clavien-Dindo score were observed in the robotic arm. In the open group one patient underwent surgical intervention due to bladder rupture (Grade III), one patient developed deep venous thrombosis managed conservatively (Grade II) and two patients required transfusion of 1 unit of blood (Grade II).

Conclusions: Robotic adenomectomy is associated with decreased blood loss, shorter catheter and drainage period, faster hospital discharge along with lower complication rates compared to its open counterpart. It appears to be a safe and feasible alternative for the treatment of large prostate glands due to benign prostate hyperplasia

MP25-23 Enucleation of the prostate and transurethral resection (B-TUEP) vs transvesical prostatectomy for enlarged prostate: initial experience in a single centre with 3year follow-up.

R GENTILE, B GENTILE, L Albanesi, C Falavolti, G Rizzo, M Buscarini, P Tariciotti, G Mirabile

nuova villa claudia

Italy

Introduction \& Objective: Open surgery (i.e. open prostatectomy, OP) or transurethral holmium laser enucleation (HoLEP) is indicated for men with prostates $>80 \mathrm{ml}$. For many years, OP had been the primary treatment option in patients with benign prostatic hyperplasia until it was gradually replaced by transurethral endoscopy. Button bipolar enucleation of the prostate (B-TUEP), a novel personal technique, is a safe and effective procedure for the treatment of bladder obstruction secondary to $\mathrm{BPH}$. Aim of this study was to evaluate, retrospectively, the overall efficacy and safety of B-TUEP vs OP for large BPO. Secondary end points were the intraoperative and perioperative results and whether B-TUEP and OP improved all baseline values. 
Materials and Methods: The clinical data for 240 consecutive patients (120 pts. who underwent OP and 120 pts submitted to BTUEP) for BPH from May 20012 to December 2013 at our centre were retrospectively analysed Indications for surgery were a preoperative International Prostate Symptom Score (IPSS) $\geq 12$ points, Quality of life (QoL) $<4$, maximal urinary flow rate (Qmax) $50 \mathrm{~mL}$, prostate volume $\geq 80$ gr on transrectal ultrasounds and non-responder to therapies. Age, BMI, haemoglobin levels, IPSS, QoL score, International Index of Erectile Function (IIEF-5) Questionnaire, prostate specific antigen (PSA), prostatic volume (PV) and PVR were compared preoperatively between the two groups. Data following a normal distribution are presented as mean \pm standard deviation and were compared using the t-test. Categorical data (percentages) were compared using the chi-square test or Fisher's exact probability test. P-values A total of $211(87.9 \%)$ of 240 patients were followed for 3 years. During the follow-up, we analysed Qmax score, I.P.S.S. score, QoL score, PSA and PVR rate at 1, 3, 6, 12, 18, 24, 30 and 36 mo., IIEF-5 Questionnaire score at 3 mo. and prostatic volume only at $3,12,26$ and 36 mo.

Results: In our experience, B-TUEP and OP techniques showed significantly different results in the intra- and perioperative time. OP procedure required significantly shorter operative time than the B-TUEP ((WMD - $41.5 \mathrm{~min}, \mathrm{p}$ 0.05), catheterization time (WMD $-38.14 \mathrm{~h} \mathrm{p}<0.05)$ and hospital stay (WMD $-16.82 \mathrm{~h}, \mathrm{p}<0.05)$ were significantly shorter in the B-TUEP group. According to Clavien-Dindo Grade System, we observed in the OP group a more number of Grade II-III complications. Both treatments resulted in statistically significant improvements
Table1 Preoperative and postoperative results

\begin{tabular}{|c|c|c|c|}
\hline & Open Surgery & Robotic Surgery & Pvalue \\
\hline Age (years) & $72.40( \pm 3.94)$ & $63.8( \pm 8.06)$ & 0.14 \\
\hline$B M I\left(\mathrm{~kg} / \mathrm{m}^{2}\right)$ & $26.89( \pm 2.43)$ & $28.86( \pm 5.08)$ & 0.35 \\
\hline PVR (preop) (ml) & $380.00( \pm 311.12)$ & $294.50( \pm 234.05)$ & 0.86 \\
\hline $\begin{array}{l}\text { Specimen } \\
\text { Weight(grams) }\end{array}$ & $89.4( \pm 24.59)$ & $113.10( \pm 45.24)$ & 0.13 \\
\hline Operation Time (mins) & $86.50( \pm 17.80)$ & $133.00( \pm 26.14)$ & 0.04 \\
\hline EBL (ml) & $515.00( \pm 284.84)$ & $210.00( \pm 172.88)$ & 0.02 \\
\hline Drain Removal (days) & $1.33( \pm 0.57)$ & $2.66( \pm 1.15)$ & 0.27 \\
\hline Foley Removal (days) & $6.50( \pm 1.3)$ & $3.00( \pm 0.75)$ & 0.01 \\
\hline $\begin{array}{l}\text { Hospitalization Time } \\
\text { (days) }\end{array}$ & $7.88( \pm 1.69)$ & $3.33( \pm 1.22)$ & $<0.001$ \\
\hline Qmax (post-op) (ml/s) & $23.62( \pm 3.66)$ & $17.62( \pm 4.59)$ & 0.24 \\
\hline PVR (post op)ml & $12.22( \pm 13.01)$ & $22.22( \pm 17.22)$ & 0.18 \\
\hline
\end{tabular}

of all parameters evaluated at each postoperative assessment for each parameter at all intervals compared with baseline in both groups $(\mathrm{p}<0.05)$. During the follow-up, there were no significant differences between the groups for Qmax score, QoL score, IIEF5 Questionnaire score, PSA and prostatic volume. A total of 211 $(87.9 \%)$ of 240 patients were followed for 3 years. Re-operations for BOO resulted in exclusion of 9 pts $(7.5 \%)$ in the OP group (2 cases of BNC, 5 cases of residual adenoma, and 2 urethral strictures) and 6 pts (5\%) in B-TUEP group (4 BNC and 2 urethral strictures).

Conclusions: In this study, we showed that B-TUEP has early and intermediate efficacy and safety equivalent to $\mathrm{OP}$ in the treatment of large $\mathrm{BPH}$.

\section{MPS26: LAPAROSCOPIC/ ROBOTIC RENAL SURGERY II}

\begin{abstract}
MP26-1 Diagnostic accuracy of CT angiography in evaluation of vascular anatomy in comparison with intraoperative findings an assessment of $\mathbf{3 9 2}$ patients
\end{abstract}

M Vijayakumar, A Ganpule, A Singh, R Sabnis, M Desai

Muljibhai patel urological hospital nadiad India

Introduction \& Objective: Introduction: Transplantation of kidneys from living related donors is the treatment of choice for patients with end stage renal disease. With less morbidity and early recovery, laparoscopy has become the standard of care for donor nephrectomies. The precise knowledge of vascular anatomy is crucial to a successful outcome. Computed tomography (CT) findings are misleading and less informative in a small number of cases. The reported accuracy of CT angiography in assessing the vascular anatomy is around 85 to $100 \%$. We did a prospective study to assess the diagnostic accuracy of CT angiography in the evaluation of vascular anatomy in comparison with intra operative findings. Aim: To assess the accuracy of CT in predicting the anatomy in patients who underwent laparoscopic donor nephrectomy.

Materials and Methods: 392 patients who underwent laparoscopic donor nephrectomy in our institute between January 2010 and December 2012 were included in our study.

Results: CT scan correlated well with the intra operative findings in most of our patients with good sensitivity and specificity. CT interpreted a case of double renal vein as single and a case of circumaortic vein reported on CT was not detected intra operatively. A case of right side early branching was not detected on CT. A case of a retroaortic branch of renal vein was missed on CT scan. The incidental findings detected on CT scan such as calculi, mass and hemangioma or fibroid can be of help in managing the patient after surgery

Conclusions: Laparoscopic donor nephrectomy requires vital information regarding the vascular anatomy of the kidney. CT scan helps in accurately mapping the vascular anatomy. CT angiography helps in strategic planning of the surgery in terms of port placement and vascular dissection and helps in avoiding vascular complications. The incidental findings detected by CT scan can also help in holistic management of the patient in donor nephrectomy because it is a zero error procedure.

MP26-2 Robotic partial nephrectomy using soft coagulation may reduce the incidence of perioperative hemorrhagic complications

T Yoshida, S Kageyama, M Narita, A Kawauchi

Shiga university of medical science

Japan

Introduction \& Objective: We assessed whether the use of soft coagulation without clamping renal artery in the resection of tumor prevents the incidence of perioperative hemorrhagic 
complications including blood transfusion, bleeding, renal artery pseudoaneurysm and arteriovenous fistula during and after robotic partial nephrectomy.

Materials and Methods: We retrospectively analyzed the data from a total of 34 patients who underwent robotic partial nephrectomy using soft coagulation at Shiga University of Medical Science between October 2015 and April 2017. Tumor resection and hemostasis of the resected surface without renal arterial clamping was performed by use of soft coagulation. Collecting system repair and renorrhaphy with arterial clamping were carried out only if the collecting system was opened.

Results: The mean age of patients was 61.3 (30-80) years. The mean tumor size was 26.3 (7-50) $\mathrm{mm}$. The mean R.E.N.A.L nephrometry score was 6.8 (4-10). The mean tumor-resected time was 38.1 (7-99) min. The mean estimated total blood loss was $62(0-330) \mathrm{mL}$, and none required blood transfusion. There were no cases with postoperative hemorrhagic events including renal artery pseudoaneurysm and arteriovenous fistula.

Conclusions: Robotic partial nephrectomy using soft coagulation may reduce the incidence of perioperative hemorrhagic complications.

\section{MP26-3 European Experience on Robotic Kidney Trans-} plantation

A Breda, A Territo, L Gausa, G Basile, V Tugcu, A Alcaraz, K Decaestecker, M Stockle, P Fornara, G Siena, N Doumerc

Fundació Puigvert, University Autonoma of Barcelona, Spain Spain

Introduction \& Objective: Kidney transplantation (KT) is the preferred treatment for patients with end-stage renal disease. In order to reduce the morbidity of the open surgery, a robotic assisted approach has been recently introduced. We present the results from the ERUS Robotic assisted kidney transplantats (RAKT) group on 111 cases.

Materials and Methods: An ERUS RAKT group was created in March 2016 with the intent of generating prospective data on RAKT. A common prospective recruitment database of RAKT performed at 8 different European Centers was therefore created. Functional outcomes, surgical data and post-operative complications were analyzed.

Results: The patients demographic characteristics were: 42 female and 69 male patients with mean age 41.9 years old (range: 18-67), mean BMI 25,5 kg/m2 (range: 18-35), mean pre-transplantation serum creatinine $579 \mathrm{umol} / \mathrm{L}$ (range: 198 1414), mean GFR $10 \mathrm{ml} / \mathrm{min}$ per $1.73 \mathrm{~m}^{2}$ (range: $4-29$ ). $55 / 111$ were pre-emptive patients. Overall surgical time was $262 \mathrm{~min}$ (range: 130 - 430) with vascular suture time of 40 min (range: 24 - 85), and estimated blood loss $134 \mathrm{ml}$ (range: 40-400 $\mathrm{ml}$ ). Overall ischemia time was $107 \mathrm{~min}$ (range: 53 - 377). The average rewarming time was 53 min (range $35-110$ ). 2 patients were converted to open transplantation for low blood flow at Doppler ultrasound evaluation. On post-operative day 7 , the mean post-transplantation serum creatinine was 180 umol/L (range: 55 - 1326) with a mean GFR $52 \mathrm{ml} / \mathrm{min}$ per $1.73 \mathrm{~m}^{2}$ (range: $4-90$ ). We reported 1 case of wound infection, 3 cases of ileus and 4 cases of bleeding ( 3 of them requiring blood transfusion) managed conservatively. One deep venous thrombosis was recorded and it required anticoagulants drugs. One case lymphocele was reported and it required drainage. 3 cases of transplantectomy were recorded due to massive arte- rial thrombosis. In 5 cases, surgical exploration was performed for intraperitoneal hematoma. Post-operative pain, evaluated with Visual Analog Scale (VAS) score, was optimal. The mean hospital stay was $6 \pm 1$ days (range $4-8$ days). The mean time of ureteral catheter was 30 days (range: $14-110$ ) after the surgery. There were 5 cases of delayed graft function at 1 month. No arterial nor ureteral strictures occurred.

Conclusions: This is the first European muticentric study on RAKT. RAKT appears to be a safe and reproducible surgical procedure in a properly selected group of patient. The potential advantages of RAKT are related to the quality of the vascular anastomosis, the possible low complication rate and the short recovery of the recipients. The success rate in this group is comparable to conventional open KT.

\section{MP26-4 Utilization and Outcomes of Nephroureterectomy for Upper Tract Urothelial Carcinoma by Surgical Ap- proach}

J Rodriguez, ZL Smith, V Packiam, W Boysen, S Johnson, N Smith, A Shalhav, G Steinberg

The University of Chicago

United States

Introduction \& Objective: The effect of surgical approach on outcomes after nephroureterectomy (NU) is poorly defined, particularly given the low incidence of upper tract urothelial carcinoma (UTUC). The purpose of this study is to compare outcomes and survival of open, robotic, and laparoscopic NU (ONU, RNU, LNU) using population-based data.

Materials and Methods: Using the National Cancer Database, we identified patients who underwent NU for UTUC between 2010 and 2013. Demographic and clinicopathologic characteristics were

\begin{tabular}{|c|c|c|c|}
\hline & OR & $95 \% \mathrm{Cl}$ & $\mathbf{P}$ \\
\hline Age & 1.000 & $0.994-1.018$ & 0.347 \\
\hline \multicolumn{4}{|l|}{ Sex (ref male) } \\
\hline Female & 0.921 & $0.756-1.122$ & 0.414 \\
\hline \multicolumn{4}{|l|}{ Race (ref white) } \\
\hline Black & 1.459 & $0.945-2.254$ & 0.088 \\
\hline Other & 0.967 & $0.563-1.660$ & 0.903 \\
\hline Unknown & 0.609 & $0.180-2.060$ & 0.425 \\
\hline \multicolumn{4}{|c|}{ Insurance (ref medicaid/uninsured) } \\
\hline Private & 0.774 & $0.455-1.317$ & 0.345 \\
\hline Medicare & 0.853 & $0.504-1.444$ & 0.553 \\
\hline Unknown/Other & 1.177 & $0.537-2.578$ & 0.684 \\
\hline \multicolumn{4}{|l|}{ Facility Type (ref community) } \\
\hline Comprehensive Cancer Center & 0.850 & $0.580-1.246$ & 0.405 \\
\hline Academic & 0.795 & $0.537-1.176$ & 0.251 \\
\hline Unknown/Other & 1.033 & $0.631-1.691$ & 0.897 \\
\hline \multicolumn{4}{|l|}{ Charleson (ref 0) } \\
\hline 1 & 1.198 & $0.963-1.491$ & 0.105 \\
\hline 2 & 1.010 & $0.713-1.430$ & 0.956 \\
\hline \multicolumn{4}{|l|}{ pT Stage (ref TO/Ta/Tis) } \\
\hline pT1 & 1.148 & $0.767-1.717$ & 0.502 \\
\hline pT2 & 1.723 & $1.150-2.581$ & 0.008 \\
\hline pT3 & 5.020 & $3.580-7.041$ & $<0.001$ \\
\hline pT4 & 23.751 & $15.486-36.4426$ & $<0.001$ \\
\hline \multicolumn{4}{|l|}{ Primary Location (ref renal pelvis) } \\
\hline Ureter & 4.968 & $4.032-6.121$ & $<0.001$ \\
\hline \multicolumn{4}{|l|}{ Who/ISUP Grade (ref low grade) } \\
\hline High Grade & 1.827 & $1.319-2.530$ & $<0.001$ \\
\hline \multicolumn{4}{|l|}{ Surgical Approach (ref open) } \\
\hline Robotic & 0.712 & $0.545-0.930$ & 0.013 \\
\hline Laparoscopic & 0.869 & $0.701-1.077$ & 0.200 \\
\hline
\end{tabular}


compared among the three operative approaches. Multivariate regression analyses were used to determine the impact of approach on performance of lymphadenectomy (LND), positive surgical margins (PSM), and overall survival (OS).

Results: There were 3651 ONU (34\%), 2313 RNU (22\%), and 4746 LNU (44\%) identified for analysis. From 2010-2013, utilization of RNU increased from $14 \%$ to $29 \%$. There were no significant differences in age, sex, race, Charlson score, or insurance status among groups. There were more RNU completed in academic centers compared to ONU and LNU ( $46 \%$ vs $36 \%$ vs $36 \%$; ppT2 tumors among groups $(\mathrm{p}=0.19)$. Patients undergoing ONU had more high-grade tumors than both RNU $(\mathrm{p}=0.02)$ and LNU $(p=0.04)$. On multivariate analysis, age, facility type, pT stage, tumor size, grade, and surgical approach were independent predictors of LND. LND was more likely in RNU (OR 1.46; $\mathrm{p}<0.01)$ and less likely in LNU (OR $0.75 ; \mathrm{p}<0.01)$ compared to ONU. RNU was associated with decreased PSM on multivariate analysis compared to $\mathrm{ONU}(\mathrm{OR}=0.71 ; \mathrm{p}=0.01)$. After adjusting for other factors, OS was not significantly correlated with surgical approach: ONU (reference); RNU (HR 0.89; $\mathrm{p}=0.29$ ); LNU (HR 0.84; $\mathrm{p}=0.06$ ).

Conclusions: In the four years studied, RNU utilization has doubled. RNU is independently associated with higher odds of LND and lower odds of PSM. OS with median follow up of 26 months was not independently affected by surgical approach.

MP26-5 Robotic-Assisted vs. Open Partial Nephrectomy: $A$ retrospective analysis of perioperative and early postoperative outcomes of patients recorded in a national UK registry.

AM Ahmed, A Tsiotras, GD Stewart, JN Armitage

Cambridge University Hospitals NHS Foundation Trust United Kingdom

Introduction \& Objective: The increasing trend for partial nephrectomy $(\mathrm{PN})$ in patients with small, localised renal cell carcinoma has broadened the spectrum and indications for robotic-assisted surgery. We compared the perioperative outcomes of robotic-assisted partial nephrectomy (RAPN) to open partial nephrectomy (OPN) using data from a national UK data registry. Materials and Methods: Data were extracted from the BAUS nephrectomy database, for patients that underwent a PN for a two-year period between January 2014 and December 2015. A retrospective review was performed and parameters that were analysed included patient characteristics, preoperative tumour characteristics, surgical technique, perioperative and early postoperative outcomes. Statistical analysis was performed by ttests and Chi-squared tests where appropriate.

Results: 3264 PN patients were included in the database of which 1009 had a RAPN (30.9\%) and 1269 OPN (38.9\%). During this period, there was a $64 \%$ annual increase in the number of patients that had a RAPN (382 in 2014 to 627 in 2015) and a $2 \%$ decrease in the number of patients that had an OPN (from 641 in 2014 to 628 in 2015). There was an overall annual increase of $13.8 \%$ in the number of patients that had a PN, irrespective of the surgical approach (from 1527 in 2014 to 1734 in 2015). Table 1 summarises the main patient characteristics. Perioperative outcomes for the two groups are presented in Table 2. RAPN patients had fewer postoperative complications, less measured blood loss (MBL), fewer blood transfusions, shorter length of stay (LOS) and fewer ITU admissions than OPN

\begin{tabular}{|c|c|c|c|}
\hline & RAPN & OPN & p value \\
\hline Mean Age (range) & $58(17-85)$ & $58(15-83)$ & 0.31 \\
\hline Male Sex & $605(62.8 \%)$ & $792(62.9 \%)$ & 0.98 \\
\hline Mean BMI & $\begin{array}{c}(n=605) \\
29.3(17.4-49)\end{array}$ & $\begin{array}{c}(n=819) \\
29(11.3-53)\end{array}$ & 0.8 \\
\hline $\begin{array}{l}\text { Preoperative Presentation } \\
\text { Incidental } \\
\text { - Other (e.g. LuTS, } \\
\text { anaemia, flank pain) } \\
\text { - Haematuria }\end{array}$ & $\begin{array}{c}(n=904) \\
673(74.4 \%) \\
121(13.4 \%) \\
110(12.2 \%)\end{array}$ & $\begin{array}{c}(n=1186) \\
857(72.3 \%) \\
206(17.4 \%) \\
130(11 \%)\end{array}$ & 0.57 \\
\hline $\begin{array}{l}\text { Past Surgical History } \\
\text { - None } \\
\text { - Previous laparotomy } \\
\text { - Previous pelvic open or } \\
\text { laparoscopic surgery } \\
\text { - Previous abdominal } \\
\text { laparoscopy } \\
\text { - Previous surgery to } \\
\text { ipsilateral kidney }\end{array}$ & $\begin{array}{c}(n=589) \\
404(71 \%) \\
67(11.8 \%) \\
52(9.1 \%) \\
41(7.2 \%) \\
5(0.88 \%)\end{array}$ & $\begin{array}{c}(n=950) \\
600(63.2 \%) \\
193(20.3 \%) \\
65(6.8 \%) \\
56(5.9 \%) \\
36(3.8 \%)\end{array}$ & 0.09 \\
\hline $\begin{array}{l}\text { ASA Grade } \\
\text { - Grade } 1 \\
: \text { Grade } 2 \\
: \text { Grade } 3 \\
\text { - Grade } 4 \\
\text { Grade } 5\end{array}$ & $\begin{array}{c}(n=904) \\
287(31.7 \%) \\
542(60 \%) \\
74(8.2 \%) \\
1(0.1 \%) \\
-\end{array}$ & $\begin{array}{c}(n=1122) \\
266(23.7 \%) \\
676(60.2 \%) \\
174(15.5 \%) \\
3(0.3 \%) \\
3(0.3 \%)\end{array}$ & 0.16 \\
\hline $\begin{aligned} & \text { Age-related Charlson score } \\
&:<2 \\
&: 2-5 \\
&>5\end{aligned}$ & $\begin{array}{c}(n=795) \\
456(57.4 \%) \\
316(39.7 \%) \\
23(2.9 \%)\end{array}$ & $\begin{array}{c}(n=I I I I) \\
579(52.1 \%) \\
492(44.3 \%) \\
40(3.6 \%)\end{array}$ & 0.56 \\
\hline $\begin{array}{r}\text { Indication for PN } \\
\text { : Elective } \\
\text { - Relative } \\
\text { - Absolute }\end{array}$ & $\begin{array}{c}(n=834) \\
711(85.3 \%) \\
94(11.3 \%) \\
29(3.5 \%)\end{array}$ & $\begin{array}{c}(n=1084) \\
170(69.4 \%) \\
162(14.9 \%) \\
170(15.7 \%)\end{array}$ & $<0.001$ \\
\hline Mean tumour size (in cms) & $\begin{array}{c}(n=714) \\
3.2(1-8.5)\end{array}$ & $\begin{array}{c}(n=942) \\
3.9(0.7-17)\end{array}$ & $<0.001$ \\
\hline
\end{tabular}

\begin{tabular}{|c|c|c|c|}
\hline & RAPN & OPN & p value \\
\hline Ischaemia type & $(n=877)$ & $(n=1043)$ & \multirow{4}{*}{$<0.001$} \\
\hline - Warm & 829 (94.5\%) & $458(43.9 \%)$ & \\
\hline - Cold & $4(0.5 \%)$ & $484(46.4 \%)$ & \\
\hline - Zero & $44(5 \%)$ & $101(9.7 \%)$ & \\
\hline Mean IT (in minutes) & $(n=835)$ & $(n=939)$ & \multirow{4}{*}{$\begin{array}{r}\text { WIT } \\
<0.001\end{array}$} \\
\hline - Warm & $20.7(10-48)$ & $15.9(10-35)$ & \\
\hline - Cold & Not recorded & $27.9(10-71)$ & \\
\hline - Zero & $\mathrm{N} / \mathrm{A}$ & N/A & \\
\hline OT (in hours) & $(n=874)$ & $(n=1122)$ & \multirow{7}{*}{0.04} \\
\hline - $<1$ & $2(0.2 \%)$ & $16(1.4 \%)$ & \\
\hline - $1-2$ & 209 (23.9\%) & $437(38.9 \%)$ & \\
\hline - 2.3 & 455 (52.1\%) & $444(39.6 \%)$ & \\
\hline - 3.4 & $159(18.2 \%)$ & 174 (15.5\%) & \\
\hline - $4-6$ & $46(5.3 \%)$ & $46(4.1 \%)$ & \\
\hline - $>6$ & $3(0.3 \%)$ & $5(0.4 \%)$ & \\
\hline \multirow[t]{2}{*}{ Intraoperative complications } & $(n=940)$ & $(n=1185)$ & \\
\hline & $40(4.3 \%)$ & $68(5.7 \%)$ & 0.55 \\
\hline Mean MBL (in millilitres) & $(n=900)$ & $(n=1092)$ & \multirow{5}{*}{0.07} \\
\hline - $<500$ & $825(91.7 \%)$ & $893(81.8 \%)$ & \\
\hline - $500-1000$ & $60(6.7 \%)$ & $134(12.3 \%)$ & \\
\hline - $\quad 1000-2000$ & $11(1.2 \%)$ & $48(4.4 \%)$ & \\
\hline . $2000-5000$ & $4(0.4 \%)$ & $17(1.6 \%)$ & \\
\hline \multirow{4}{*}{$\begin{array}{c}\text { Units of blood transfused } \\
: \quad \text { Minor }<2 \\
: \quad \text { Moderate 2-6 } \\
\text { - Major }>6\end{array}$} & $(n=920)$ & $(n=1132)$ & \multirow{4}{*}{0.008} \\
\hline & $19(2.1 \%)$ & $54(4.8 \%)$ & \\
\hline & $2(0.2 \%)$ & $18(1.6 \%)$ & \\
\hline & $1(0.1 \%)$ & - & \\
\hline \multirow[t]{2}{*}{ Positive surgical margins } & $(n=775)$ & $(n=907)$ & \\
\hline & $79(10.2 \%)$ & $53(5.8 \%)$ & 0.06 \\
\hline \multirow[t]{2}{*}{ Postoperative complications } & $(n=900)$ & $(n=1128)$ & \\
\hline & $100(11.1 \%)$ & $234(20.7 \%)$ & 0.017 \\
\hline Clavien-Dindo grade & $(n=96)$ & $(n=198)$ & \multirow{8}{*}{$<0.001$} \\
\hline - Grade I & $23(24 \%)$ & $70(35.4 \%)$ & \\
\hline - Grade II & $52(54.2 \%)$ & $94(47.5 \%)$ & \\
\hline - Grade IIIa & $13(13.5 \%)$ & $29(14.6 \%)$ & \\
\hline - Grade IIIb & $4(4.2 \%)$ & - & \\
\hline - Grade IVa & $1(1 \%)$ & $5(2.5 \%)$ & \\
\hline - Grade IVb & $1(1 \%)$ & - & \\
\hline - Grade V & $1(1 \%)$ & - & \\
\hline \multirow[t]{2}{*}{ Median LOS (in days) } & $(n=970)$ & $(n=1151)$ & \multirow[b]{2}{*}{$<0.001$} \\
\hline & $3(1-37)$ & $5(1-77)$ & \\
\hline TTU admissions & $(n=826)$ & $(n=1107)$ & $<0.001$ \\
\hline
\end{tabular}

patients but had a longer warm ischaemia time (WIT) and a longer operating time (OT).

Conclusions: This study provides a contemporary analysis of PN in the UK and demonstrates a move from OPN to RAPN, particularly for elective indications. Increased IT and OT with RAPN are countered by benefits including reduced MBL and shortened LOS. Improved recording of renal nephrometry score and long-term follow-up data will further enhance the utility of this unique data source. 
MP26-6 Retroperitoneal vs. transperitoneal robot assisted partial nephrectomy - an propensity score weighted comparison in a multi-institutional database

S Arora, R Abaza, J Adshead, B Challacombe, RK Ahlawat, P Dasgupta, G Gandaglia, D Moon, G Novara, F Porpiglia,

T Yuvaraja, U Capitiano, A Mottrie, M Bhandari,

CG Rogers, J Porter, H Gill

Vattikuti Urology Institute, Henry Ford Hospital United States

Introduction \& Objective: To compare the retroperitoneal (RP) approach with the transperitoneal (TP) approach for robot assisted partial nephrectomy (RAPN) in both anterior and posterior facing tumors using the Vattikuti Global Quality Initiative in robotic urologic surgery (GQI-RUS) database.

Materials and Methods: 486 patients who underwent RAPN in the prospective arm of GQI-RUS database by 22 surgeons at 14 centres in nine countries. Inverse probability of treatment weighting (IPTW) was done to account for potential selection bias between RP-RAPN and TP-RAPN by adjusting for age,
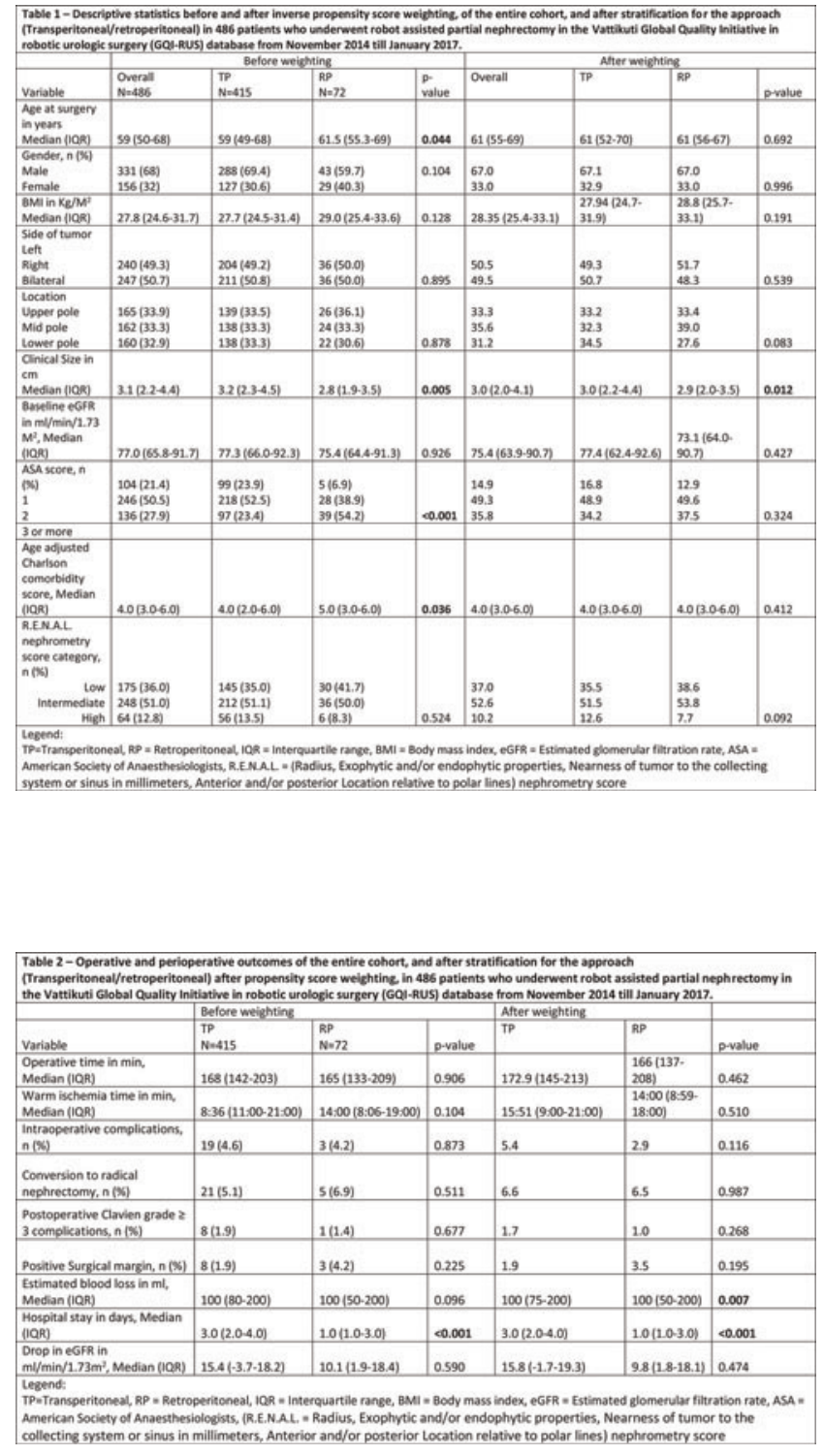

ASA score, age adjusted CCI, and clinical tumor size. Outcomes were compared between the weighted cohorts.

Results: A total of 72 patients underwent RP-RAPN and 415 patients underwent TP-RAPN. All procedures could be completed robotically with 5 conversions to robotic radical nephrectomy, and no open conversion. The hospital stay (median (IQR) 3.0 (2.0-4.0) TP vs. 1.0 (1.0-3.0) RP; p

Conclusions: RP-RAPN is a safe and technically feasible option for nephron sparing surgery in both anterior and posterior renal masses. Both RP-RAPN and TP-RAPN have comparable operative and perioperative outcomes except the hospital stay which is shorter in RP-RAPN.

MP26-7 Subjective perception of nephrometric scores: red tape or clinical necessity

V Malkhasyan, I Semanyakin, D Pushkar

Russia

Introduction \& Objective: One of the main goal of urology nowadays is the development of universal standardized descriptive system (scoring systems SS) for renal masses based on radiologic findings. Many scoring systems such as R.E.N.A.L. and PADUA scores have been introduced into clinical practice, however, this challenge is still valid. The aim of this survey is to evaluate subjective perception of utility and convenience of existing nephrometric scores by urologists.

Materials and Methods: In order to assess subjective perception, utility and convenience of existing nephrometric scores in clinical decision making process we designed a special questionnaire. This form (comprised of 3 parts) was sent to 40 urologists practicing kidney cancer surgery in 6 different cities of Russian Federation. The first part of the questionnaire aimed to evaluate their familiarity with PADUA, R.E.N.A.L. and ABC scores. The second part contained clinical examples of these scoring systems. All urologists involved in survey were offered to rate convenience and clinical utility of all scoring systems using 3 point scale.

Convenience assessment score

1 - score is not easy to use for the busy clinician

2- score is easy to use for the busy clinician

3 - score is most usable for the busy clinician

Utility assessment score

1 - score is useless in clinical practice

2 - score is useful in clinical practice

3 - score is necessary in clinical practice

In the third section of the questionnaire urologists were asked to provide comments what criteria the ideal nephrometric score should meet.

Results: All 40 questionnaires were further analyzed. According to the results of our survey $38(95 \%)$ urologists were familiar with PADUA score and $28(70 \%)$ used it in clinical practice. $35(87.5 \%)$ urologists were familiar with R.E.N.A.L. score and $19(47.5 \%)$ used it in clinical practice. Only 21 $(52.5 \%)$ urologists were familiar with $\mathrm{ABC}$ score and only 8 $(20 \%)$ used it in clinical practice. The total score for PADUA was -87 , median score - 2. For R.E.N.A.L. the total score was 94, median score - 2.5. For ABC the total score was - 112, median score - 3. In terms of clinical utility the total score in PADUA group was - 74, median - 2. In R.E.N.A.L. group the 
total score was 86 , median - 2. For ABC group the total score sum up - 198 point with 2.5 median score.

As the main criteria for the ideal scoring system the majority of urologists suggested that it should create perfect assessment of tumor size and location without viewing images.

Conclusions: Despite the fact that ABC score is the least popular scoring system among Russian urologists most of them perceive it as the most convenient and useful scoring system in clinical practice.

MP26-8 Investigation of the tissue stability of newly introduced polymeric clips for minimally invasive surgery: an ex vivo study

I Kyriazis, P Kallidonis, P Ntasiotis, D Kotsiris, L Amanatides, V Panagopoulos, E Liatsikos

Department of Urology, University of Patras, Greece Greece

Introduction \& Objective: To compare the tissue stability potential of the newly introduced Click'aV ${ }^{\circledR}$ Plus to the established Click'aV $^{\circledR}$ and Hem-o-lok ${ }^{\circledR}$ polymer clips in an ex vivo setting. The Click'aV ${ }^{\circledR}$ Plus has a novel design in the integrated teeth interface.

Materials and Methods: The aim of the tests was to verify the ligating clip stabilization on porcine artery. Freshly harvested porcine arteries were used for the evaluation of the stability of the clips. A clip was set on one side of the artery and a suture (USP 2, polysorb) was placed on the other side. An experimental configuration consisted of a dynamometer with a custom support and clamp unit was used. The support unit allowed the application of force on the clip under manual control of a crank. The clamp unit stabilized the clip. The suture was tied to the dynamometer. One

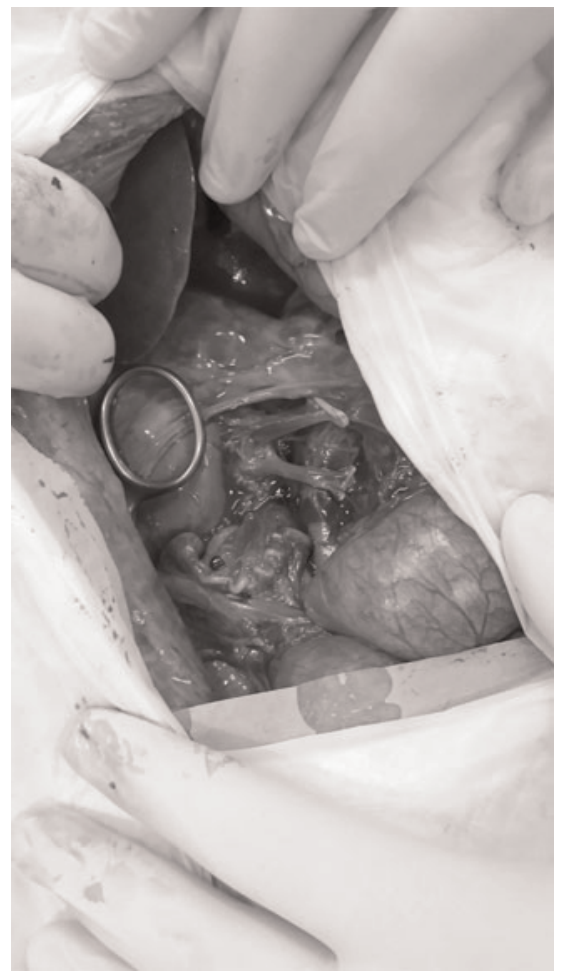

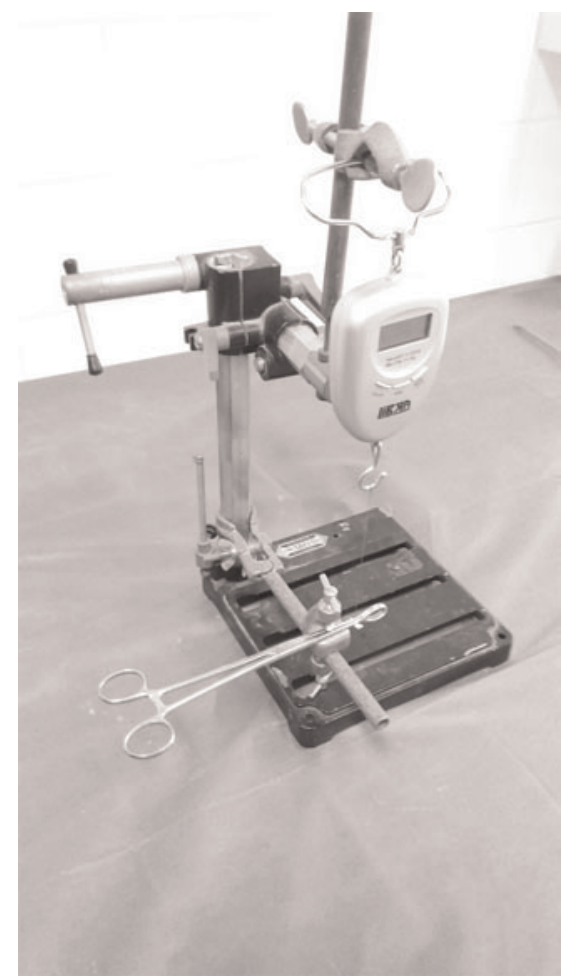

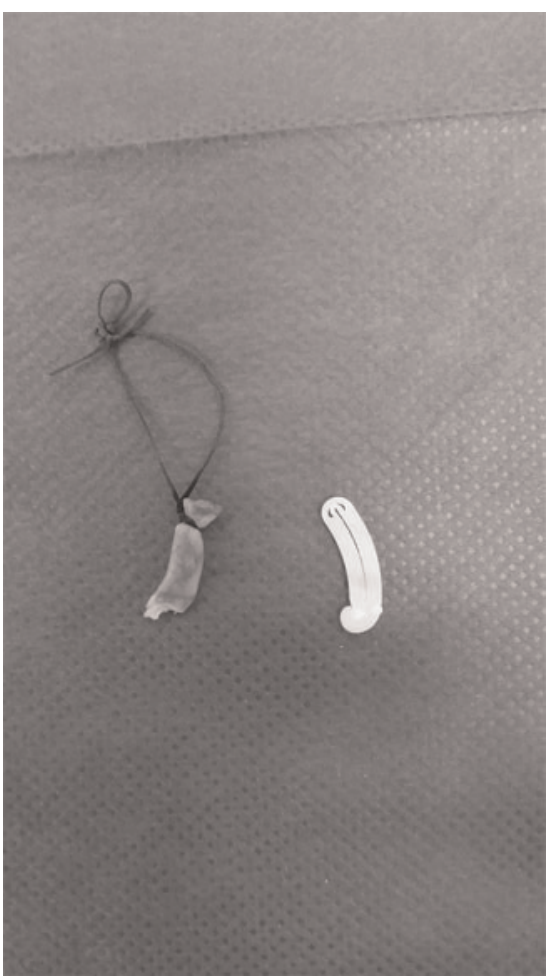

of the investigators slowly rotated the crank and gradually applied higher tension forces on the clip via the suture. Measurements were taken during the process. The highest force value before the artery was slipped though the clip or disrupted was noted. For each clip the measurement was repeated 2 times. 


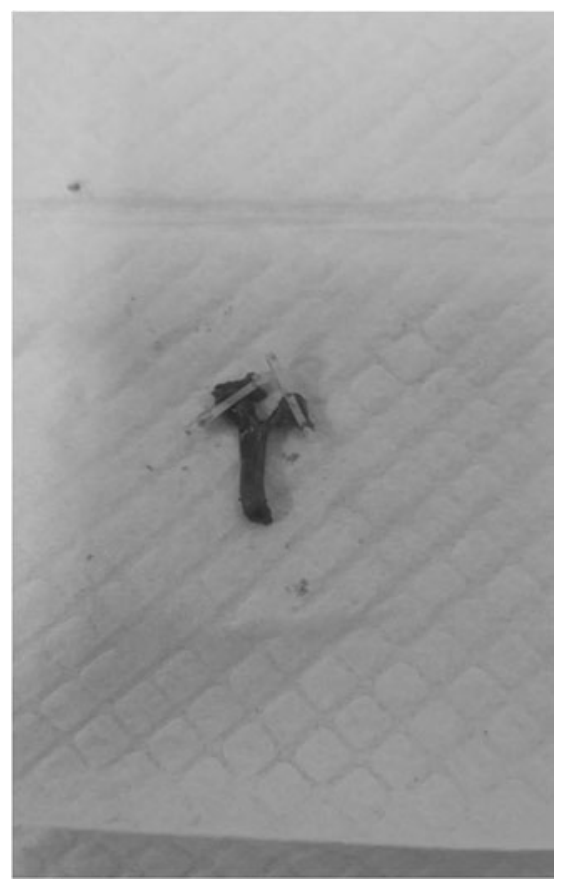

\begin{tabular}{|l|l|l|l|}
\hline Ex vivo/ artery & Click'aV & Click'aV $^{\circ}$ Plus & Hem-o-lok \\
\hline XL size & $10.2 / 9.4 \quad$ & $11.8 / 12.8$ & $14.2 / 12.9$ \\
\hline $\begin{array}{l}\text { Comments } \\
\text { Values in Newton }\end{array}$ & $\begin{array}{l}\text { Artery slipped } \\
\text { through the clip in } \\
\text { both measurements }\end{array}$ & $\begin{array}{l}\text { Artery was cut, in } \\
\text { both measurements }\end{array}$ & $\begin{array}{l}\text { Artery was cut, in } \\
\text { both measurements) }\end{array}$ \\
\hline
\end{tabular}

TABLE 1:Results of the tissue stability test

Results: The table 1 presents the results of the tissue stability test. The artery slipped through the Click'aV ${ }^{\circledR}$ clip in both measurements, showing that the force that was applied was adequate to misplace the clip from the vessel. The artery was eventually cut in all the measurements of the Click'aV® Plus and Hem-o-lok ${ }^{\circledR}$ clips. The latter phenomena practically showed that the clips remained without issues in place even if forces adequate for disruption are applied.

Conclusions: The newly introduced Click'aV®Plus clips remained without issues in place even if forces adequate for disruption are applied.

MP26-9 Comparison of Perioperative Outcomes of Laparoscopic Versus Robotic Assisted Partial Nephrectomy for Patients with Complex Renal Cell Carcinoma: a Single Center Initial Experience from Northwest China

G Zhu, D Wu, W Song, Z Yang, D He

The 1st Affiliated Hospital of Xi' an Jiaotong University China (People's Republic)

Introduction \& Objective: With the three dimensional optics and more flexible wristed instruments, robotic assistant partial nephrectomy (RAPN) may provide advantages than laparoscopic partial nephrectomy (LPN) for treatment of patients with more complex tumors. This study is to compare the perioperative outcomes of patients with complex renal cell carcinoma underwent RAPN or LPN for a single renal mass with the RENAL nephrometry score $\geq 7$.

Materials and Methods: A retrospective analysis was performed for 165 consecutive patients from Northwest China with renal cell carcinoma who underwent either LPN $(n=105)$ or RAPN $(\mathrm{n}=60)$ between January 2015 and April 2017 for a complex renal mass with the RENAL nephrometry score $\geq 7$. In the LNP group, 85 patients received retroperitoneal LPN and the 20 patients received peritoneal LPN. In the RAPN group, 45 patients underwent retroperitoneal RAPN and the 15 patients underwent peritoneal PAPN. The demographic parameters and perioperative outcomes were compared between the two groups, respectively.

Results: There was no significant difference between the two groups with respect to patients' age, gender, laterality, Charlson comorbidity index (CCI), RENAL nephrometry score, tumor size or body mass index. The RAPN group had a slightly higher mean RENAL nephrometry score (7.8 vs. $7.2, \mathrm{p}=0.041)$ and larger mean tumor size ( 4.5 vs. $3.2 \mathrm{~cm}, \mathrm{p}=0.035)$ compared to the LPN group. There were no significant differences with respect to surgical approach, conversion rates, estimated blood loss, transfusion rate, postoperative complications or positive margins between the two groups. The RAPN group had moderate less mean warm ischemic time than the LPN group (18.4 v.s. $22.5 \mathrm{~min}, \mathrm{p}=0.026$ ).

Conclusions: RAPN provides perioperative outcomes comparable to those of LPN for complex renal cell carcinoma with the RENAL nephrometry score $\geq 7$. Our initial experience may indicate that RAPN appears to be a feasible and safe alternative operation to its laparoscopic counterpart for treatment of complex renal tumors with decreased warm ischemia time. A prospective randomized study is needed in the future to confirm our initial findings.

MP26-10 Posterior Hilar Renal Tumors could be treated safely through the Trans-peritoneal Robot-assisted Partial Nephrectomy Approach

m koo, K Chang, A Ali, K Rha

Severance hospital, yonsei medicine Republic of Korea

Introduction \& Objective: Posterior hilar renal tumors are thought to be technically demanding during partial nephrectomy surgery. Our study aimed to evaluate the perioperativeand functional outcomes among patients with these tumors undergoing Trans-peritoneal Robot-assisted Partial Nephrectomy (TRAPN).

Materials and Methods: Ninety-four patients with hilar renal tumors who underwent T-PRPN from September 2006 to December 2015 were analyzed. Patients were divided into two groups according to surfacelocation (anterior $\mathrm{n}=108$ and posterior $\mathrm{n}=117$ ). Primary endpoint was to compare trifecta accomplishment between groups (warm ischemia time $<25 \mathrm{~min}$, positive surgical margin, and 30 days postoperative complication rate). Secondary endpoint was to compare other intraoperative findings, complications rates and early functional outcomes.

Results: Baseline patients' characteristics were comparable between both groups; mean age (51.4 \pm 13.6 vs $54.8 \pm 12.5$, $\mathrm{p}=0.214)$, mean tumor size $(3.3 \pm 1.7 \mathrm{vs} 3.5 \pm 1.6, \mathrm{p}=0.555)$ and mean RENAL nephrometry scores $(7.2 \pm 1.4$ vs $7.7 \pm 1.3$, 


\begin{tabular}{|c|c|c|c|}
\hline Variables & $\begin{array}{l}\text { Anterior hilar } \\
\text { \&tumors }\end{array}$ & $\begin{array}{l}\text { Posterior hilar } \\
\text { \& tumors }\end{array}$ & P-value \\
\hline OT (min) & $4193.2 \pm 94.5$ & $4189.9 \pm 96$ & 40.865 \\
\hline -EBL (ml) & $4422 \pm 558.7$ & $4393.7 \pm 373.4$ & 40.772 \\
\hline - wIT (min) & $925.9=10.1$ & $426.8 \pm 9.4$ & 90.663 \\
\hline Clamp type,n(\%) & 4 & 4 & 4 \\
\hline Non-clamp & $44(8.9)$ & $44(8.2)$ & 40.112 \\
\hline Selective-clamp & $45(11.1)$ & $414(28.6)$ & 4 \\
\hline Total-clamp & $436(80)$ & $431(63.3)$ & \\
\hline Conversionto $\mathrm{RN}, \mathrm{n}(\%)$ & $43(6.7)$ & $41(2)$ & 40227 \\
\hline -Intraoperative injury, $\mathrm{n}(\%)$ & $42(4.4)$ & $40(0)$ & 40.266 \\
\hline - Blood transfusion, $n(\%)$ & $40(0)$ & $41(1)$ & 40.521 \\
\hline - Hospital stay (day), mean(SD) & $4.3 \pm 1.5$ & $45.8 \pm 1.8$ & 40.092 \\
\hline - eGER at 1 month, mean(SD) & $472.7 \pm 14$ & $468.9 \pm 17.4$ & 40.336 \\
\hline + GERR \% preservation mean (\%) & 492.3 & 489.5 & $\hookrightarrow 0.407$ \\
\hline - Acute kidney injury (AKI), n (\%) & $41(2.2)$ & $42(4.1)$ & 40.608 \\
\hline Trifecta achievement, $n(\%)$ & $425(55.6)$ & $426(53.1)$ & 40.838 \\
\hline Causes of trifecta failure, $n(\%)$ & 4 & 4 & 4 \\
\hline PSM & $44(8.9)$ & $42(4.1)$ & 40.421 \\
\hline WIT $>25 \mathrm{~min}$ & $420(44.4)$ & $422(44.9)$ & 40.965 \\
\hline Clavien-Dindo $\geq$ grade $3 \circ$ & $40(0)$ & $42(4.1)$ & 40.357 \\
\hline - Postoperative transfusion, $n(\%)$ & $43(6.7)$ & $41(2)$ & 40.346 \\
\hline
\end{tabular}

$\mathrm{p}=0.65$ ) didn't differ between anterior and posterior surface groups, respectively. Trifecta achievement rate was similar in anterior hilar $(48.9 \%)$ compared to posterior hilar $(44.9 \%)$ tumors $(p=0.428)$, moreover, the remaining intraoperative and perioperative parameters such as; total operative time, estimated blood loss, hospital stay length, intraoperative injury, and perioperative blood transfusion rate showed no significant difference between both groups $(\mathrm{p}=0.865,0.347,0.092,0.226$, and 0.459 , respectively). Regarding functional outcomes, mean eGFR at 5years didn't differ between groups $(82.53 \pm 18.27$ vs85.77 \pm 17.14 , $\mathrm{p}=0.353$ ), as well as, incidence of postoperative acute kidney injury $(\mathrm{p}=0.608)$.

Conclusions: T-PRPN is safe and confers excellent perioperative outcomes for the treatment of posterior hilar renal tumors in the hand of experienced robotic surgeons.

MP26-11 Laparoscopic Cytoreductive Nephrectomy is Associated with Significantly Improved Survival Compared to Open Cytoreductive Nephrectomy or Targeted Therapy Alone

EH Kim, J Vetter, K Du, R Grubb, RS Figenshau

Washington University School of Medicine United States

Introduction \& Objective: In the targeted therapy (TT) era, studies have demonstrated that carefully selected patients with metastatic renal cell carcinoma (mRCC) derive benefit from cytoreductive nephrectomy $(\mathrm{CN})$, while others do not. Although laparoscopic $\mathrm{CN}$ has been demonstrated to have significantly less morbidity than open $\mathrm{CN}$, the association between surgical approach and survival in mRCC has not been examined. We compare the survival outcomes for patients who underwent laparoscopic $\mathrm{CN}$, open $\mathrm{CN}$, and $\mathrm{TT}$ alone at our institution.

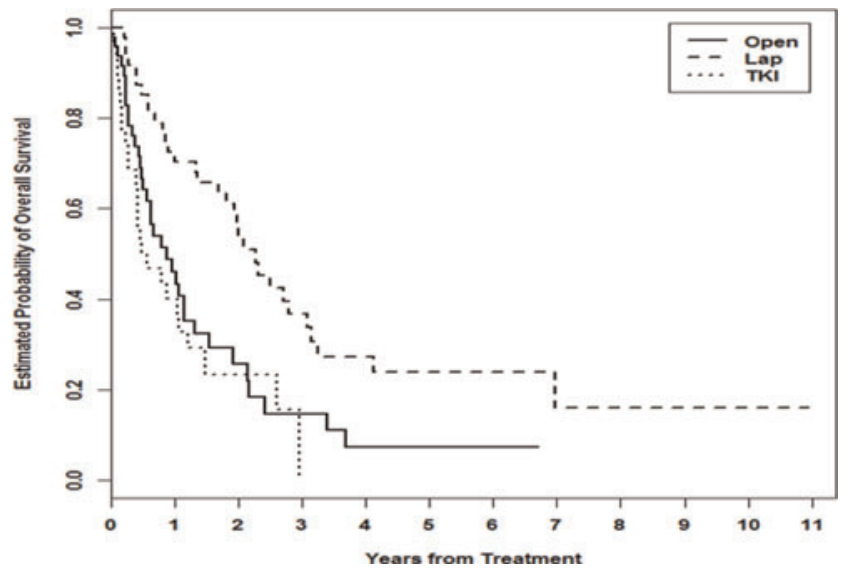

Materials and Methods: We performed a retrospective chart review at our institution for patients who underwent $\mathrm{CN}$ prior to TT (laparoscopic, $\mathrm{n}=48$; open, $\mathrm{n}=48$ ) or who were deemed unfit for surgery and received TT alone $(n=36)$, between January 2007 and December 2012. We recorded preoperative variables for risk stratification (Culp et al. Cancer 2010). We performed Kaplan-Meier estimated survival as well as Cox proportional hazards analyses.

Results: The Kaplan-Meier estimated survival curve is provided in Figure 1. Laparoscopic $\mathrm{CN}$ is associated with significantly greater survival than open CN or TT alone (median survival 26 months versus +1.3 versus $2.6 \pm 1.1$ versus $3.3 \pm 1.2$ factors, respectively; $\mathrm{p}<0.01)$. On multivariate analysis, laparoscopic $\mathrm{CN}$ was an independent predictor of survival (HR 0.51, p <0.01), controlling for preoperative risk factors, while survival was similar between open $\mathrm{CN}$ and TT alone (HR 1.02, $\mathrm{p}=0.94$ ).

Conclusions: In our experience, laparoscopic $\mathrm{CN}$ is a significant predictor of survival in mRCC. Surgeon selection for laparoscopic $\mathrm{CN}$ is biased for patients with improved survival, and may account for clinical variables that are otherwise difficult to quantify. For patients who are not candidates for laparoscopic $\mathrm{CN}$, open $\mathrm{CN}$ does not provide survival benefit over TT alone, while associated with increased morbidity.

MP26-12 Tumor-attaching surface area as the new parameter for extending warm ischemia time in robotic-assisted partial nephrectomy

N Fukami, K Fukaya, M Kusaka, R Shiroki

Japan

Introduction \& Objective: Robotic-assisted partial nephrectomy (RAPN) has been widely recognized as an alternative treatment for small renal tumors. Although the indications for RAPN have expanded to high complexity tumors, one of the major concerns is the warm ischemic time (WIT) on challenging cases. Several renal tumor scoring systems, including with RENAL nephrometry score, PADUA score and C-index, have been used to evaluate the impact of the perioperative outcomes such as WIT and complications, however, those of factors failed to show correlation with perioperative outcomes. The aim of this study was to analyze whether Tumor-attaching surface area (TASA) as the new parameter affecting more than 20 minutes of WIT retrospectively. 
Materials and Methods: Since 2010, 110 cases of renal tumor were treated by RAPN in our institute. One hundred cases without the initial 10 cases were analyzed. TASA was defined as the surgical resection area from preoperative evaluation of CT scans. R, E and N-score component of nephrometry were defined as measured value. We analyzed TASA and components of nephrometry as parameter for more than 20 minutes of WIT.

Results: WIT was stratified WIT $\leqq 20 \mathrm{~min}$ (S-WIT) and WIT $>20$ min (L-WIT). Eighteen out of 100 cases (18.0\%) developed L-WIT. The median WIT in L-WIT and S-WIT were 15 min vs $26 \min (p<0.001)$. For the background factors, patient age, gender, BMI, or procedural approach, clamping method did not show difference between L-WIT and S-WIT. Among nephrometry components, tumor size (median), $33 \mathrm{~mm}$ vs $27 \mathrm{~mm}$ $(p=0.033)$, and $\mathrm{N}$ factor (median), $1 \mathrm{~mm}$ vs $6.7 \mathrm{~mm}(p<0.001)$, were shown to be significantly larger and deeper in L-WIT than S-WIT. TASA (median), $17.5 \mathrm{~cm}^{2}$ vs $7.4 \mathrm{~cm}^{2}(p<0.002)$, was significantly larger on L-WIT compared with S-WIT. Multiple logistic regression analysis demonstrated that only TASA significantly affected WIT (OR: $1.11 ; p=0.045)$.

Conclusions: TASA was detected in the new independent predictor as extension of WIT. This information may be useful in perioperative counseling on patient selection and preparation for RAPN.

MP26-13 The Impact of Marijuana Intake Upon Outcomes of Hand-Assisted Laparoscopic Donor Nepherctomy

D Ruckle, M Keheila, B West, B Mattison, J Thomas, $\mathrm{S}$ Abourbih, A Kore, M De Vera, P Baron, DD Baldwin

Loma Linda University

United States

Introduction \& Objective: There is a current shortage of kidneys available for transplantation. Based on United Network for Organ Sharing recommendations that exclude substance abusers from donation, many transplant institutions refuse live kidney donors who have a history of marijuana use; however, there is no evidence pertaining specifically to the donor or recipient outcomes. This is the first study to investigate the effect of marijuana use by live kidney donors upon outcomes in both donors and recipients. Materials and Methods: A retrospective chart review for living kidney donors and their recipients between January 2000 and May 2016 was performed, stratifying patients based upon prior donor marijuana usage.

Demographics and intra-operative variables were reported and compared for all groups. Outcomes compared included absolute and percent creatinine change and percent glomerular filtration rate (GFR) change in both donors and recipients, stratified by duration of marijuana usage. Baseline values for recipients were calculated based on their 1 week post-op creatinine values. Statistical analysis was performed using the t-test for numerical variables and the chi-square test for categorical variables with $\mathrm{p}$ Results: Of total of 294 renal donor charts reviewed in this study, 31 were marijuana using donors and 263 were non-marijuana using donors. There was no difference in donor preoperative, perioperative, or postoperative outcomes based upon marijuana use ( $p>0.05$ for all comparisons). However, there was a trend toward better preservation of donor GFR at 1 month for marijuana using donors vs. nonmarijuana using donors $(-33.3 \%$ vs. $-38.6 \%$; $\mathrm{p}=0.07)$ respectively. Marijuana kidney recipients and non-marijuana kidney recipients were similar in creatinine change and percent creatinine change at all time periods. At 1 month, marijuana kidney recipients showed a lower percent change in GFR compared to non-marijuana kidney recipients $(+0.9 \%$ vs. $+20.4 \%$; $\mathrm{p}=0.035)$ respectively. However, for all other time points $(6$ months, 1 year, and 5 years), there was no difference in percent GFR change between marijuana kidney recipients and nonmarijuana kidney recipients ( $\mathrm{p}>0.05$ for all comparisons).

Conclusions: There was no difference in renal function between marijuana using donors and non-marijuana using donors and no long-term differences in renal function between non marijuana kidney recipients and marijuana kidney recipients. Considering individuals with a history of marijuana use for live kidney donation could increase the donor pool and yield acceptable outcomes.

MP26-14 Laparoscopic partial nephrectomy for upper pole tumors using pedicle rotation technique

B Guliev, B Komyakov, X Yakubov

North-West medical university by Mechnikov Russia

Introduction \& Objective: Laparoscopic or robot-assisted partial nephrectomy is the standard of care for the treatment of patients with clinical T1 renal masses. Transperitoneal laparoscopic partial nephrectomy (LPN) for upper pole masses, particularly for those located posteriorly, is challenging because of difficult visualization during tumor incision and renorrhaphy. Complete renal mobilization with subsequent pedicle rotation facilitates resection of upper pole renal masses during LPN. We present initial experience of transperitoneal LPN with renal pedicle rotation in patients with upper pole tumors.

Materials and Methods: Retrospective review of all LPNs between October 2008 and December 2016 was performed. We identified 12 patients of all LPN with renal upper pole and posterior tumors. Mean patient age was 52,6 $\pm 11,3$ years (range 4668 years). Mean tumor size was $3.4 \mathrm{~cm}$ (range $2.5-4.8 \mathrm{~cm}$ ). Right kidney tumor was diagnosed in $7(58,3 \%)$ cases, left renal masses - in $5(41,7 \%)$ cases. All 12 patients were underwent LPN using renal pedicle rotation technique. Important steps of this technique included complete kidney mobilization with separating

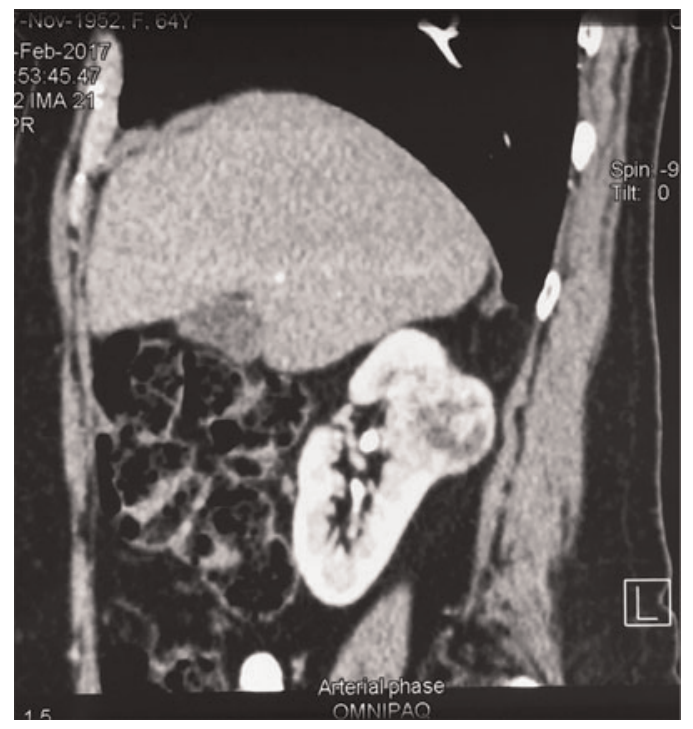



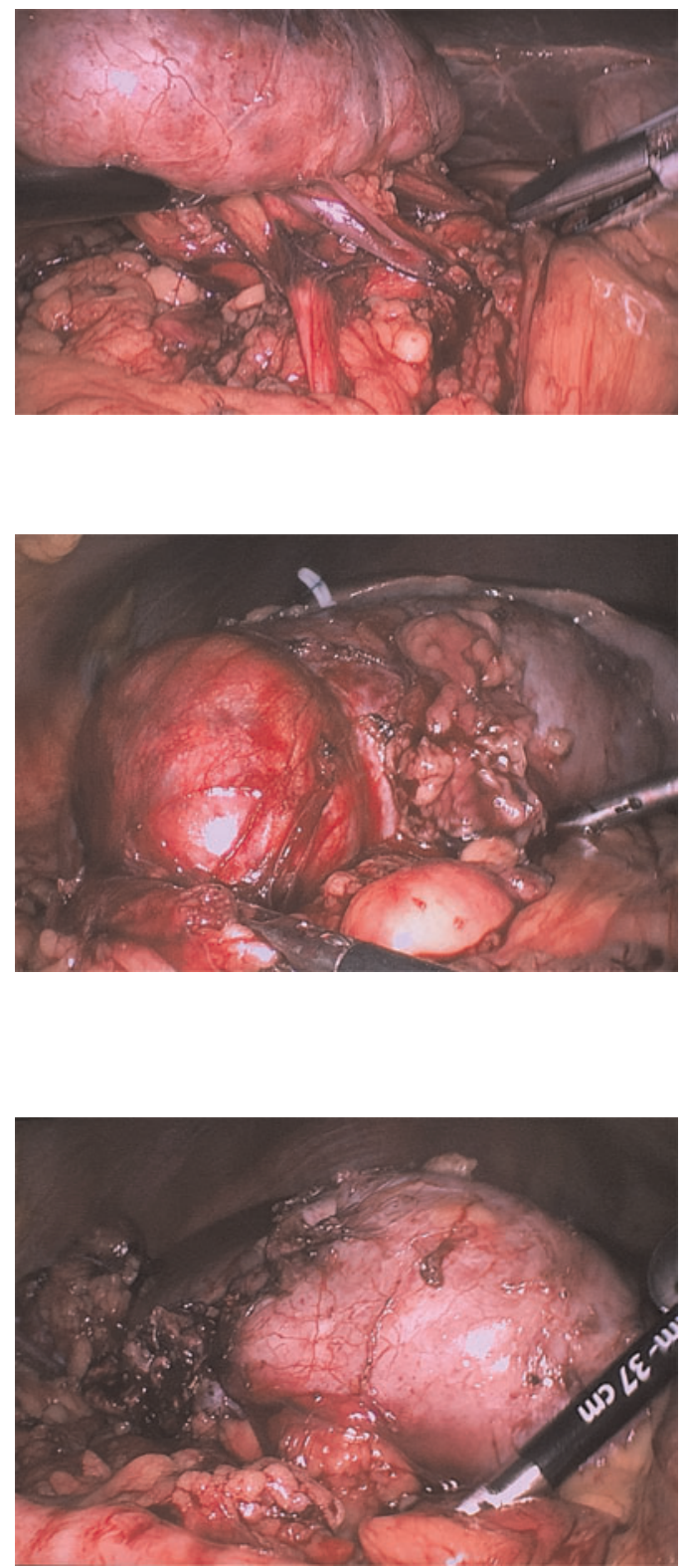

renal artery and vein, rotation the kidney on renal pedicle for a better tumor exposure and its resection (figures 1 - 4).

Results: All 12 surgeries were successful without conversion to radical nephrectomy or open partial nephrectomy. No patients required transfusion, and there were no intraoperative complications. The mean operative time was $120 \pm 35,0 \mathrm{~min}$ (range 90 $-210 \mathrm{~min}$ ), the mean warm ischemia time was $14,5 \pm 7,8 \mathrm{~min}$ (range $10-26 \mathrm{~min}$ ), and the mean estimated blood loss was $120,0 \pm 65,5 \mathrm{~mL}$ (range $60-320 \mathrm{~mL}$ ). Mean postoperative hospital stay was $4.2 \pm 1.5$ days (range $4-8 \mathrm{~d}$ ). No intraoperative complications. Postoperative complications occurred in 3 patients. All patients had negative margins and pathological examination revealed $11(91,7 \%)$ clear cell carcinomas, $1(8,3 \%)$ - epithelial renal angiomyolipoma. During follow up period revealed no local recurrence and distant metastasis.

Conclusions: Transperitoneal LPN with using the kidney pedicle rotation technique is a feasible and safe procedure for a better tumor exposure for incision and suture in renal upper pole posterior tumors.
MP26-15 Use of local anaesthetic infusion systems following laparoscopic nephrectomy and laparoscopic nephrouretectomy

K Parekh, A Sultana, Y Goh, C Jellicoe, V Hariharan, HO Andrews

Department of Urology, Milton Keynes University Hospital United Kingdom

Introduction \& Objective: Morphine causes significant side effects which may prolong post-operative recovery and length of hospital stay (LOS). Local anaesthetic wound infusion systems (LAIS) reduce LOS and pain after open surgery. Our literature search shows no evidence of LAIS use in laparoscopic surgery. Our aim was to assess LAIS use after laparoscopic nephrectomy (LN) and laparoscopic nephrourectomy (LNU) compared with patients treated with morphine via patient controlled analgesia (PCA) and to assess the efficacy of LAIS as a stand-alone post-operative analgesia. Materials and Methods: A retrospective series of patients undergoing LN or LNU from 2014 to 2017 was identified and data collected with a local electronic data management system. Patients were divided into two groups; LAIS (ON-Q* Pain Relief System) with continuous infusion of $0.125 \%$ Bupivacaine $v s$. PCA post-operatively. Post-operatively, all patients received regular paracetamol and where required additional analgesia (morphine, tramadol, codeine) was used. All PCAs and LAIS were removed day 1 post-operatively in the morning unless otherwise indicated. Primary outcomes were post-operative quantity of opiate analgesia used (morphine, tramadol, codeine) in both groups and LOS. Secondary outcomes were age, BMI, co-morbidities, intra- and post-operative analgesia. Statistical analysis was performed using the Mann-Whitney U test.

Results: Thirty-two patients undergoing a LN or LNU during the study period were identified; 16 patients received LAIS and 16 patients received PCA post-operatively. Patient demographics were similar in both groups, median age $(p=0.465)$, BMI $(p=0.250)$ and comorbidities $(p=0.928)$. There was no statistical difference in intra-operative use of opiates $(p=0.112)$, amount of weak opiates (tramadol, codeine) required post-operatively $(p=0.459)$ and LOS $(p=0.250)$. Median quantity of morphine per 24 hours in the PCA group was $26.7 \mathrm{mg}$ (15.9 $54 \mathrm{mg})$ and $3.3 \mathrm{mg}(3.3-15 \mathrm{mg})$ in the LAIS group. Post-operative morphine use was significantly higher in the PCA group compared to LAIS group ( $p=0.00014)$.

Conclusions: Use of LAIS following LN or LNU resulted in significantly lower post-operative morphine requirements compared to PCA thus avoiding side effects and risks associated with cumulative morphine use. Although our study did not show a disparity in LOS, LAIS should be considered a first line treatment after laparoscopic surgery as patients do not require in-hospital monitoring and, therefore, may potentially expedite discharge from hospital.

MP26-16 Does Strict Compliance to the Enhanced Recovery Programme (ERP) affect Outcomes during Laparoscopic Nephrectomy and Nephroureterectomy?

A Sultana, K Parekh, Y Goh, S Ali, HO Andrews

Department of Urology, Milton Keynes University Hospital United Kingdom

Introduction \& Objective: The ERP for urological surgery was developed by the British Association of Urological Surgeons 
(BAUS) in 2015. ERP encompasses a care pathway, through standardised protocols, with improved recovery and reduced hospital stay (LOS). These benefits have led to widespread uptake in the UK, but variations in practice still occur. This study aims to assess compliance with BAUS ERP \& outcomes following laparoscopic nephrectomy (LN) \& nephroureterectomy (LNU).

Materials and Methods: A retrospective study of patients who underwent LN \& LNU at MKUH from January 2015 to December 2016 was carried out. 29 patients $(m=15, f=14)$, mean age of 60.9 $( \pm 16.0)$ years were included. Compliance with ERP recommendations was assessed pre-operatively (PRO), intra-operatively (IOP) and post-operatively (POO). Secondary outcomes included conversion to open surgery (COS); LOS; blood transfusion; Clavien Dindo grade (CDG) of POO complications; and 30 day mortality. Results: All patients were optimised as per BAUS at PRO, except carbohydrate pre-loading (0\%) Analgesia recommendations were adhered to $100 \%, 89.7 \%$ received morphine IOP. $10.3 \%$ received antacids at induction, \& $100 \%$ POO. Antiemetics use was $93.1 \%$ IOP \& 96.6\% POO. All patients had local anaesthesia IOP, $51.7 \%$ had regional blocks. All patients had a urethral catheter inserted, $55.2 \%$ were removed POO day 1. Drains were inserted in $86.2 \%, 73.1 \%$ removed day 1 POO. Only $24.1 \%$ received lactulose POO. Conversion to open surgery $0 \%$; median LOS was 2 days for LN, and 3 for LNU. Blood transfusion $0 \%$; CDG I complications $-34.5 \%$; 30 day mortality $0 \% .3$ patients were readmitted for non-urological reasons.

Conclusions: Strict compliance to BAUS ERP during IOP and POO is low due to the local hospital guidelines on medications, routine use of catheters $\&$ drains, but high in the generic domains with the use of alternative analgesia \& antacids. BAUS ERP encourages individual units to constantly review and audit its practices for the best patient experience. Our outcomes following LN \& LNU are good with short LOS, low complication \& readmission rates without strict adherence to BAUS ERP. We suggest guidelines should include generic prescribing to accommodate variations within local units' drug formularies. The ERP will change from time to time, as more urology units publish their practices and outcomes.

MP26-17 Does Race Impact on Perioperative and Functional Outcomes in Patients Undergoing Robotic Partial Nephrectomy?

CR Reynolds, K Badani, D Paulucci, A Bhandari, D Eun, R Abaza, J Porter, A Hemal

Department of Urology, Wake Forest School of Medicine United States

Introduction \& Objective: We report on the clinical and pathologic characteristics of African American (AAP) and Caucasian patients $(\mathrm{CP})$ undergoing robotic partial nephrectomy (RPN) and determine the association between race and perioperative and functional outcomes.

Materials and Methods: The present multi-institutional study of patients undergoing RPN identified 683 patients who met eligibility criteria including $611(89.5 \%) \mathrm{CP}$ and $72(10.5 \%)$ AAP. Demographic, clinical and pathologic variables were compared between these two cohorts. Perioperative and functional outcome at discharge and at median follow-up of 17.1 months (IQR: $7.4-$ 29.1 Months; Range: 2.9 - 79.8 Months) were also compared using multivariable regression models (linear, logistic, poisson, cox proportion hazards) adjusting for age, gender, BMI, Charlson comorbidity index, clinical stage, baseline eGFR and operating surgeon.

\begin{tabular}{|c|c|c|c|}
\hline & Caucasian & African American & $P$ \\
\hline $\mathrm{N}$ & $611(89.5 \%)$ & $72(10.5 \%)$ & \\
\hline Age & $59.6(12.6)$ & $58.5(11.5)$ & .455 \\
\hline Gender & & & .027 \\
\hline Male & $355(58.1 \%)$ & $32(44.4 \%)$ & \\
\hline Female & $256(41.9 \%)$ & $40(55.6 \%)$ & \\
\hline BMI & $30.2(6.4)$ & $31.4(7.8)$ & .219 \\
\hline ASA Score & & & .612 \\
\hline I & $8(1.4 \%)$ & $1(1.5 \%)$ & \\
\hline II & $208(36.3 \%)$ & $22(33.3 \%)$ & \\
\hline III & $343(59.9 \%)$ & $43(65.2 \%)$ & \\
\hline IV & $143(2.4 \%)$ & $0(0.0 \%)$ & \\
\hline \multicolumn{4}{|l|}{ Comorbidities } \\
\hline $\mathrm{CCI}$ & $0.72(0.99)$ & $0.88(1.09)$ & .225 \\
\hline Obesity & $274(44.9 \%)$ & $33(45.8 \%)$ & .883 \\
\hline Hypertension & $369(61.0 \%)$ & $48(66.7 \%)$ & .349 \\
\hline Hyperlipidemia & $148(24.5 \%)$ & $17(23.6 \%)$ & .874 \\
\hline Diabetes & $128(21.2 \%)$ & $20(27.8 \%)$ & .199 \\
\hline Coronary Artery Disease & $77(12.7 \%)$ & $2(2.8 \%)$ & .013 \\
\hline COPD & $47(7.8 \%)$ & $4(5.6 \%)$ & .501 \\
\hline Baseline eGFR & $80.5(23.8)$ & $89.6(26.2)$ & $<.001$ \\
\hline Baseline CKD Stage & & & .139 \\
\hline I & $202(33.8 \%)$ & $35(49.3 \%)$ & \\
\hline II & $288(48.2 \%)$ & $26(36.6 \%)$ & \\
\hline III & $100(16.8 \%)$ & $10(14.1 \%)$ & \\
\hline IV & $6(1.0 \%)$ & $0(0.0 \%)$ & \\
\hline V & $1(0.2 \%)$ & $0(0.0 \%)$ & \\
\hline Prior Abdominal Surgery & $285(52.0 \%)$ & $35(58.3 \%)$ & .352 \\
\hline Clinical T Stage & & & .015 \\
\hline Tla & $482(79.0 \%)$ & $59(83.1 \%)$ & \\
\hline Tlb & $115(18.9 \%)$ & $7(9.9 \%)$ & \\
\hline $\mathrm{T} 2$ & $13(2.1 \%)$ & $5(7.0 \%)$ & \\
\hline Tumor Size & $3.2(1.5)$ & $3.4(1.9)$ & .428 \\
\hline RENAL Nephrometry Score & $6.7(1.9)$ & $7.1(1.9)$ & .156 \\
\hline \multicolumn{4}{|l|}{ Tumor Location } \\
\hline Right & $321(52.7 \%)$ & $34(47.3 \%)$ & 378 \\
\hline Left & $288(47.3 \%)$ & $38(52.8 \%)$ & .378 \\
\hline Anterior & $222(40.2 \%)$ & $21(32.8 \%)$ & .390 \\
\hline Posterior & $236(42.8 \%)$ & $33(51.6 \%)$ & \\
\hline Lateral & $94(17.0 \%)$ & $10(15.6 \%)$ & \\
\hline Hilar & $72(12.3 \%)$ & $11(16.4 \%)$ & .345 \\
\hline Date of Surgery* & $\begin{array}{c}12 / 2012(1 / 2008- \\
6 / 2016)^{*}\end{array}$ & $\begin{array}{c}12 / 2012(3 / 2008- \\
7 / 2016)\end{array}$ & .282 \\
\hline Clamp Technique & & & .383 \\
\hline Main Artery Clamping & $489(81.5 \%)$ & $58(80.6 \%)$ & \\
\hline Selective Arterial Clamping & $36(6.0 \%)$ & $7(9.7 \%)$ & \\
\hline Off-Clamp & $75(12.5 \%)$ & $7(9.7 \%)$ & \\
\hline \multicolumn{4}{|c|}{$\begin{array}{l}\text { - Continuous Variables: Means presented with standard deviations in parenthesis; }{ }^{*} \\
\text { Medians; Means presented with IQR and Range in parenthesis } \\
\text { - Categorical Variables: Frequencies presented with percentages in parenthesis } \\
\text { - P values in bold indicate statistical significance at } \mathrm{p}<.05 \text { level. }\end{array}$} \\
\hline
\end{tabular}

\begin{tabular}{|c|c|c|c|}
\hline & Caucasian & African American & $\mathrm{P}$ \\
\hline Histology & & & .004 \\
\hline Clear Cell & $264(48.7 \%)$ & $18(28.1 \%)$ & \\
\hline Papillary & $75(13.8 \%)$ & $18(28.1 \%)$ & \\
\hline Chromophobe & $36(6.6 \%)$ & $4(6.2 \%)$ & \\
\hline Other Malignant Histology & $32(5.9 \%)$ & $7(10.9 \%)$ & \\
\hline Benign & $135(24.9 \%)$ & $17(26.6 \%)$ & \\
\hline Fuhrman Grade & & & .941 \\
\hline 1 & $31(16.9 \%)$ & $4(13.8 \%)$ & \\
\hline 2 & $123(67.2 \%)$ & $20(69.0 \%)$ & \\
\hline 3 & $29(15.8 \%)$ & $5(17.2 \%)$ & \\
\hline 4 & $3(1.6 \%)$ & $0(0.0 \%)$ & \\
\hline Pathologic T Stage & & & .382 \\
\hline pTla & $363(80.8 \%)$ & $47(87.0 \%)$ & \\
\hline pTlb & $55(12.2 \%)$ & $6(11.1 \%)$ & \\
\hline pT2 & $9(2.0 \%)$ & $1(1.9 \%)$ & \\
\hline pT3 & $22(4.9 \%)$ & $0(0.0 \%)$ & \\
\hline $\begin{array}{l}\text { - Continuous Variables: } \mathrm{N} \\
\text { Medians; Means presen } \\
\text { - Categorical Variables: } \mathrm{F} \\
\text { - } \quad \text { P values in bold indicate }\end{array}$ & $\begin{array}{l}\text { ans presented } \\
\text { d with IQR and } \\
\text { equencies prese } \\
\text { tatistical signi }\end{array}$ & $\begin{array}{l}\text { ard deviations in pa } \\
\text { parenthesis } \\
\text { percentages in pare } \\
<<05 \text { level. }\end{array}$ & thesis; \\
\hline
\end{tabular}


Table 3. Univariable Comparisons of Perioperative and Functional Outcome of Black and White Patients undergoing RPN

\begin{tabular}{|c|c|c|c|}
\hline & Caucasian & African American & $P$ \\
\hline Operative Time & $160.5(38.2)$ & $172.4(60.4)$ & .440 \\
\hline Ischemia Time & $17.7(7.3)$ & $17.9(6.6)$ & .814 \\
\hline Estimated Blood Loss* & $\begin{array}{l}100.0 ; 132.5(50.0- \\
150.0 ; 10.0-2000.0)\end{array}$ & $\begin{array}{l}50.0 ; 123.7(50.0- \\
150.0 ; 20.0-1200.0)\end{array}$ & .339 \\
\hline \multicolumn{4}{|l|}{ Post-Operative } \\
\hline Length of Stay* & $\begin{array}{l}2.0 ; 2.2(1.0-3.0 ; 1.0- \\
47.0)\end{array}$ & $\begin{array}{l}2.0 ; 2.5(1.0-3.0 \\
1.0-17.0)\end{array}$ & .401 \\
\hline Any Complication & $96(15.9 \%)$ & $7(9.7 \%)$ & .169 \\
\hline Major Complication & $29(4.8 \%)$ & $5(6.9 \%)$ & .394 \\
\hline $\begin{array}{l}\text { Positive Surgical Margin } \\
\text { Discharge }\end{array}$ & $32(7.0 \%)$ & $2(3.8 \%)$ & .562 \\
\hline Acute Kidney Injury & $75(15.1 \%)$ & $10(19.6 \%)$ & .396 \\
\hline$\%$ Change eGFR & $-5.9 \%(19.9)$ & $-11.3 \%(17.5)$ & .063 \\
\hline \multicolumn{4}{|l|}{ Most Recent Follow-Up } \\
\hline Months* & $\begin{array}{l}16.3 ; 20.7(7.3-28.6 ; \\
2.9-79.8)\end{array}$ & $\begin{array}{l}20.5 ; 23.9(10.6- \\
31.5 ; 3.2-69.5)\end{array}$ & .144 \\
\hline Progression of CKD & $104(28.7 \%)$ & $13(31.0 \%)$ & .497 \\
\hline$\%$ Change eGFR & $-8.1 \%(22.2)$ & $-10.9 \%(20.3)$ & .449 \\
\hline
\end{tabular}

Table 4. Multivariable Regression Comparisons of Perioperative and Functional Outcome of Black and White Patients undergoing RPN

\begin{tabular}{|c|c|c|}
\hline & $\begin{array}{l}\text { African American vs. } \\
\text { Caucasian }\end{array}$ & $\mathrm{p}$ \\
\hline Operative Time ${ }^{a}$ & $\beta=7.07(-16.23,30.37)$ & .549 \\
\hline Log Ischemia Time $e^{a^{*}}$ & $\beta=0.02(-0.02,0.07)$ & .333 \\
\hline Log Estimated Blood Loss ${ }^{\mathrm{a}^{*}}$ & $\beta=-0.08(-0.17,0.01)$ & .099 \\
\hline \multicolumn{3}{|l|}{ Post-Operative } \\
\hline Length of Stay ${ }^{b}$ & $\mathrm{e}^{(\beta)}=1.32 ;(1.13,1.55)$ & .001 \\
\hline Any Complication ${ }^{\mathrm{c}}$ & $\mathrm{OR}=0.61(0.23,1.38)$ & .276 \\
\hline Major Complication $^{\mathrm{c}}$ & $\mathrm{OR}=2.03(0.65,5.34)$ & .179 \\
\hline Positive Surgical Margin ${ }^{\mathrm{c}}$ & $\mathrm{OR}=0.66(0.10,2.37)$ & .588 \\
\hline \multicolumn{3}{|l|}{ Discharge } \\
\hline Acute Kidney Injury $^{\mathrm{c}}$ & $\mathrm{OR}=1.13(0.49,2.43)$ & .761 \\
\hline$\%$ Change eGFR ${ }^{a}$ & $\beta=-3.16(-8.71,2.40)$ & .265 \\
\hline \multicolumn{3}{|l|}{ Most Recent Follow-Up } \\
\hline Progression of $\mathrm{CKD}^{\mathrm{d}}$ & $\mathrm{HR}=0.84(0.57,1.52)$ & .573 \\
\hline$\%$ Change eGFR ${ }^{a}$ & $\beta=-1.28(-8.70,6.15)$ & .736 \\
\hline
\end{tabular}

- Measure of association presented with $95 \%$ confidence interval in parenthesis

- Multivariable Regressions adjusted for age, gender, BMI, Charlson comorbidity index, clinical stage, baseline eGFR and operating surgeon

- a: Linear Regression, a*: Linear Regression with Log10 Transformation, b: Poisson Regression, c: Logistic Regression, d: Cox Proportional Hazards Regression

- eGFR=Estimated Glomerular Filtration Rates; $\mathrm{CKD}=$ Chronic Kidney Disease; $\mathrm{HR}=$ Hazards Ratio; $\mathrm{OR}=$ Odds Ratio

Results: AAP tended to be more likely to have prior abdominal surgery, diabetes mellitus, obesity, hypertension and be classified as ASA-3 though these differences were not statistically significant. AAP had more incidence of papillary (28.1\% vs. $13.8 \%, \mathrm{p}=.003)$ and less clear cell $(28.1 \%$ vs. $48.9 \%, \mathrm{p}=.002)$ renal cell carcinoma (RCC). AAP were more likely to have a cT2 tumor $(7.0 \%$ vs. $2.1 \%, \mathrm{p}=.031)$, be female $(55.6 \%$ vs. $41.9 \%$, $\mathrm{p}=.027)$ and have a higher baseline eGFR $(89.6$ vs. $80.5 \mathrm{~mL} /$ $\left.\min / 1.73 \mathrm{~m} 2, \mathrm{p}(\beta)=1.32 ; \mathrm{e}^{(95 \% \mathrm{CI})}=1.13,1.55 ; \mathrm{p}=.001\right)$, but there were no differences in any other perioperative or functional outcome including operative time $(\mathrm{p}=.549), \log$ warm ischemia time $(\mathrm{p}=.333)$, log estimated blood loss $(\mathrm{p}=.099)$, post-operative complications (Any: $\mathrm{p}=.276$; Major: $\mathrm{p}=.179$ ), positive surgical margins $(\mathrm{p}=.588)$, acute kidney injury at discharge $(\mathrm{p}=.761)$, or progression of CKD stage at median follow-up 17.1 months $(\mathrm{HR}=0.84 ; \mathrm{p}=.573)$.

Conclusions: Despite relatively higher medical co-morbidities in AAP, perioperative and renal function outcomes of RPN were not significantly different when stratified by race. However, African American race is associated with a higher rate of papillary RCC which may impact oncologic outcome on long term follow-up. In future studies, larger cohorts with more African American patients would be necessary to validate these findings.

MP26-18 Comparison of Perioperative and Functional Outcomes in Elderly versus Non-Elderly Patients Undergoing Robotic Partial Nephrectomy: A Multi-Institutional Study

CR Reynolds, K Badani, D Paulucci, D Eun, R Abaza, J Porter, A Bhandari, A Hemal

Department of Urology, Wake Forest School of Medicine United States

Introduction \& Objective: We report on the clinical and pathologic characteristics of elderly ( $\geq 70$ years old) and nonelderly patients undergoing robotic partial nephrectomy (RPN) and compare perioperative and functional outcomes between these groups.

Materials and Methods: Our multi-institutional study identified $387(21.0 \%)$ elderly patients ( $\geq 70$ years old) and $1,460(79.0 \%)$ non-elderly patients ( $<70$ years old) who underwent RPN by 6 surgeons between 2006 and 2016. Using Mann-Whitney U and Chi-squared tests of independence, baseline demographics and medical history were compared as were tumor-specific characteristics, perioperative outcome and renal function outcome at discharge and at 12.3 months (IQR: 6.0-25.7; Range: 2.9-106.2). Perioperative and renal function outcome were also compared using multivariable logistic regression models adjusting for R.E.N.A.L. Nephrometry score, date of surgery, and surgeon performing the RPN.

Results: Elderly patients presented with more comorbidities $(\mathrm{p}<.001)$, a lower BMI $(\mathrm{p}<.001)$, lower baseline eGFR $(\mathrm{p}<.001)$, and more prior abdominal surgery $(\mathrm{p}=.011)$. Age $\geq 70$ was associated with more overall $(14.5 \%$ vs. $10.0 \%, \mathrm{p}=.011)$ and medical $(8.5 \%$ vs. $4.7 \%, \mathrm{p}=.003)$ post-operative complications (PC). No clinically significant differences in any other perioperative outcomes including operative time, blood loss, acute kidney injury at discharge, or progression of CKD stage at 12.3 months were observed. Furthermore, in multivariable analysis, results were similar with more medical $\mathrm{PC}(\mathrm{OR}=1.71$; $95 \% \mathrm{CI}=1.06,2.75, \mathrm{p}=.027)$, a marginally higher likelihood of overall $\mathrm{PC}(\mathrm{OR}=1.40 ; 95 \% \mathrm{CI}=0.98,2.02 ; \mathrm{p}=.068)$, and a slightly longer length of stay $(p=.001)$ in elderly patients undergoing RPN.

Conclusions: This analysis shows that RPN is a safe procedure with low complication rates in properly selected elderly patients. The risk of renal functional decline is no greater for this group; thus, RPN remains a viable treatment option for elderly patients diagnosed with a renal mass. 
Table 1. Demographic and Clinical Characteristics of Elderly and Non-Elderly Patients Undergoing RPN

\begin{tabular}{|c|c|c|c|}
\hline & Non-Elderly & Elderly & $\mathbf{P}$ \\
\hline Age & $\begin{array}{l}57(49-64 ; 16- \\
69)\end{array}$ & $74(72-77 ; 70-89)$ & $<.001$ \\
\hline Male & $840(57.5 \%)$ & $237(61.2 \%)$ & .189 \\
\hline BMI & $\begin{array}{l}29.8(25.9-34.7 ; \\
15.7-70.9)\end{array}$ & $\begin{array}{l}28.0(25.2-31.9 ; \\
15.1-51.3)\end{array}$ & $<.001$ \\
\hline Obese & $711(48.9 \%)$ & $144(37.2 \%)$ & $<.001$ \\
\hline $\mathrm{ASA} \geq 3$ & $529(52.7 \%)$ & $185(71.4 \%)$ & $<.001$ \\
\hline \multicolumn{4}{|l|}{$\mathrm{CCI}$} \\
\hline Hypertension & $559(53.1 \%)$ & $212(75.7 \%)$ & $<.001$ \\
\hline & $232(22.1 \%)$ & $100(35.7 \%)$ & $<.001$ \\
\hline \multicolumn{4}{|l|}{ Hyperlipidemia } \\
\hline Diabetes & $263(18.2 \%)$ & $93(24.0 \%)$ & .010 \\
\hline CAD & $88(8.4 \%)$ & $48(17.1 \%)$ & $<.001$ \\
\hline COPD & $\begin{array}{l}88(6.1 \%) \\
138(13.1 \%)\end{array}$ & $\begin{array}{l}36(9.3 \%) \\
27(9.6 \%)\end{array}$ & .025 \\
\hline \multicolumn{4}{|l|}{ Nephrolithiasis } \\
\hline Baseline eGFR & $\begin{array}{l}84.3(69.8-99.5 ; \\
4.9-214.3)\end{array}$ & $\begin{array}{l}69.9(57.2-85.5 \\
12.5-173.7)\end{array}$ & $<.001$ \\
\hline Baseline CKD & & & $<.001$ \\
\hline \multicolumn{4}{|l|}{ Stage } \\
\hline I & $488(41.0 \%)$ & $67(17.5 \%)$ & \\
\hline II & $663(46.3 \%)$ & $193(50.4 \%)$ & \\
\hline III & $172(12.0 \%)$ & $118(30.8 \%)$ & \\
\hline IV & $9(0.6 \%)$ & $4(1.0 \%)$ & \\
\hline V & $1(0.1 \%)$ & $1(0.3 \%)$ & \\
\hline $\begin{array}{l}\text { Prior Abdominal } \\
\text { Surgery }\end{array}$ & $487(50.7 \%)$ & $143(59.8 \%)$ & .011 \\
\hline Solitary Kidney & $25(1.8 \%)$ & $10(2.8 \%)$ & .254 \\
\hline $\begin{array}{l}\text { Multiple Tumors } \\
\text { Removed }\end{array}$ & $32(2.3 \%)$ & $11(3.1 \%)$ & .439 \\
\hline $\begin{array}{l}\text { Dominant Tumor } \\
\text { Size }\end{array}$ & $\begin{array}{l}2.9(2.1-4.0 ; 0.6- \\
14.0)\end{array}$ & $\begin{array}{l}3.0(2.2-3.9 ; 0.9- \\
12.0)\end{array}$ & .543 \\
\hline $\begin{array}{l}\text { RENAL } \\
\text { Nephrometry } \\
\text { Score }\end{array}$ & $7(6-9 ; 4-12)$ & $7(5-8 ; 4-12)$ & .048 \\
\hline Clinical Stage & & & .786 \\
\hline Tla & $1093(75.4 \%)$ & $300(77.7 \%)$ & \\
\hline Tlb & $314(21.7 \%)$ & $76(19.7 \%)$ & \\
\hline $\mathrm{T} 2$ & $42(2.9 \%)$ & $8(2.1 \%)$ & \\
\hline Tumor Laterality & & & .459 \\
\hline Right & $739(51.2 \%)$ & $189(49.1 \%)$ & \\
\hline Left & $704(48.8 \%)$ & $196(50.9 \%)$ & \\
\hline Pole of Tumor & & & .394 \\
\hline Lower & $274(32.2 \%)$ & $68(30.6 \%)$ & \\
\hline Mid & $207(36.1 \%)$ & $73(32.9 \%)$ & \\
\hline Upper & $270(31.7 \%)$ & $81(36.5 \%)$ & \\
\hline $\begin{array}{l}\text { Location of } \\
\text { Tumor }\end{array}$ & & & .397 \\
\hline Anterior & $459(37.5 \%)$ & $135(41.5 \%)$ & \\
\hline Posterior & $542(44.2 \%)$ & $136(41.8 \%)$ & \\
\hline Lateral & $224(18.3 \%)$ & $54(16.6 \%)$ & \\
\hline Hilar & $199(16.6 \%)$ & $38(11.9 \%)$ & .042 \\
\hline Malignant & $1041(76.6 \%)$ & $258(72.5 \%)$ & .106 \\
\hline Date of Surgery & $\begin{array}{l}2012(2006- \\
2016)\end{array}$ & $\begin{array}{l}2013(2007- \\
2016)\end{array}$ & .014 \\
\hline Retroperitoneal & $204(14.0 \%)$ & $56(14.5 \%)$ & .802 \\
\hline Clamp Type & & & .926 \\
\hline Main Artery & $1152(81.0 \%)$ & $302(80.1 \%)$ & \\
\hline Selective & $146(10.3 \%)$ & $41(10.9 \%)$ & \\
\hline Arterial & & & \\
\hline Off Clamp & $125(8.8 \%)$ & $34(9.0 \%)$ & \\
\hline
\end{tabular}

a. P Values in bold indicate statistical significance at $\mathrm{p}<.05$ level.

b. For Categorical Variables: Frequencies presented with percentages in parenthesis; Chi-Squared tests of Independence or Fisher's Exact Tests Performed

c. For Continuous Variables: Medians presented with Interquartile Ranges in Parenthesis; Mann-Whitney U Tests performed.
Table 2. Perioperative and Functional Outcome of Elderly and Non-Elderly Patients Undergoing RPN

\begin{tabular}{|c|c|c|c|c|c|}
\hline & \multicolumn{2}{|c|}{ Univariable Comparisons } & \multirow[b]{2}{*}{$\mathbf{P}$} & \multicolumn{2}{|c|}{ Multivariable Comparisons ${ }^{n}$} \\
\hline & Non-Elderly & Elderly & & & $\mathbf{P}$ \\
\hline $\begin{array}{l}\text { Operative } \\
\text { Time }\end{array}$ & $\begin{array}{l}165(140-200 \\
75-481)\end{array}$ & $\begin{array}{l}163.5(138.3- \\
199 ; 81-333)\end{array}$ & .589 & $\beta=0.01(-0.01,0.03)$ & .206 \\
\hline Ischemia Time & & & .753 & $\beta=-0.00(-0.02,0.02)$ & .848 \\
\hline $\begin{array}{l}\text { Estimated } \\
\text { Blood Loss }\end{array}$ & $\begin{array}{l}100(50-150 ; \\
10-2500)\end{array}$ & $\begin{array}{c}100(50-150 \\
10-1600)\end{array}$ & .320 & $\beta=0.02(-0.03,0.06)$ & .406 \\
\hline $\begin{array}{l}\text { Collecting } \\
\text { System Entry }\end{array}$ & $601(54.4 \%)$ & $152(50.7 \%)$ & .245 & $\mathrm{OR}=1.05(0.76,1.44)$ & .787 \\
\hline $\begin{array}{l}\text { Intra-Operative } \\
\text { Complications }\end{array}$ & $23(3.5 \%)$ & $5(2.8 \%)$ & .629 & $\mathrm{OR}=0.69(0.23,2.05)$ & .503 \\
\hline Length of Stay & $\begin{array}{l}1(1-2 ; 1-47) / \\
1.77(1.67)\end{array}$ & $\begin{array}{c}1(1-2 ; 1-17) / \\
1.99(1.84)\end{array}$ & .027 & $\beta=0.04(0.02,0.06)$ & .001 \\
\hline \multicolumn{6}{|l|}{$\begin{array}{l}\text { Post-Operative } \\
\text { Complications }\end{array}$} \\
\hline Overall & $145(10.0 \%)$ & $56(14.5 \%)$ & .011 & $\mathrm{OR}=1.40(0.98,2.02)$ & .068 \\
\hline Surgical & $90(6.2 \%)$ & $29(7.5 \%)$ & .346 & $\mathrm{OR}=1.09(0.68,1.77)$ & .716 \\
\hline Medical & $68(4.7 \%)$ & $33(8.5 \%)$ & .003 & $\mathrm{OR}=1.71(1.06,2.75)$ & .027 \\
\hline Major & $48(3.3 \%)$ & $16(4.1 \%)$ & .420 & $\mathrm{OR}=1.48(0.82,2.69)$ & .195 \\
\hline PSM & $42(3.8 \%)$ & $16(5.7 \%)$ & .160 & $\mathrm{OR}=1.62(0.86,3.06)$ & .133 \\
\hline \multicolumn{6}{|l|}{ Discharge } \\
\hline $\begin{array}{l}\text { \% Change } \\
\text { eGFR }\end{array}$ & $\begin{array}{l}-13.8 \%(- \\
25.6 \%-0.0 \%\end{array}$ & $\begin{array}{c}-11.7 \%(- \\
23.9 \%-1.0 \%)\end{array}$ & .269 & $\beta=1.17(-1.98,4.33)$ & .467 \\
\hline AKI & $241(26.6 \%)$ & $57(24.9 \%)$ & .599 & $\mathrm{OR}=0.89(0.59,1.34)$ & .591 \\
\hline \multicolumn{6}{|l|}{ Follow-Up } \\
\hline Months & $\begin{array}{l}12.4(6.0-25.9 ; \\
2.9-106.2)\end{array}$ & $\begin{array}{c}11.9(6.0-23.9 ; \\
3.1-96.6)\end{array}$ & .363 & & \\
\hline $\begin{array}{l}\text { \% Change } \\
\text { eGFR }\end{array}$ & $\begin{array}{l}-9.2 \%(-21.1 \% \\
02.1 \%)\end{array}$ & $\begin{array}{c}-22.6 \%(- \\
11.4 \%-2.4 \%)\end{array}$ & .480 & $\beta=0.18(-3.67,4.03)$ & .927 \\
\hline $\begin{array}{l}\text { Progression } \\
\text { of CKD Stage }\end{array}$ & $254(26.3 \%)$ & $58(23.3 \%)$ & .325 & $\mathrm{HR}=1.00(0.73,1.37)$ & .996 \\
\hline \multicolumn{6}{|c|}{$\begin{array}{l}\text { a. Adjusting for R.E.N.A.L. Nephrometry Score, Date of Surgery and Surgeon Performing } \\
\text { RPN }\end{array}$} \\
\hline \multicolumn{6}{|c|}{ b. $P$ values in bold indicate statistical significance at $\mathrm{p}<.05$ level. } \\
\hline \multicolumn{6}{|c|}{ c. Mean also presented with standard deviation in parenthesis } \\
\hline \multicolumn{6}{|c|}{ d. For Categorical Variables: Fi } \\
\hline Squared test & of Independenc & or Fisher's Exa & Tests P & formed & \\
\hline e. For Continu & $s$ Variables: M & ans presented w & th Inter & artile Ranges in Parenth & \\
\hline
\end{tabular}

MP26-19 Feasibility of laparoscopically retrieved adult kidneys in pediatric renal transplantation

S Balaji, A Singh, A Ganpule, R Sabnis, M Desai

Muljibhai Patel Urological Hospital

India

Introduction \& Objective: Renal Transplantation(RT) is the treatment of choice for End Stage Renal Disease(ESRD) in children. We assessed the feasibility and functional outcome of laparoscopically retrieved living adult donor kidneys in pediatric population(age $\leq 16$ years) and analyzed the various factors which can predict outcome of RT

Materials and Methods: 37 renal transplants done in 33 children between 2004 and 2016 were included. We retrospectively recorded age of recipient, native kidney disease, preemptive or dialysis dependency, serum creatinine at 1 month,1year and 3 years, donor age and relation, e GFR and ischemia times and complications from medical records and database. The patients were categorized according to their weight at time of transplantGroup I(10-20kgs), Group II(20-30kgs) and Group III(>30kgs). Success was defined as freedom from dialysis post procedure. Another statistical assessment was done between same groups with outcome being serum creatinine $<1.4 \mathrm{mg} \%$. Multivariate regression analysis was performed to identify the possible prognostic factors affecting living adult donor RT.

Results: Mean age of the recipients was $12.06 \pm 4.2$ years. Overall graft survival was $85.2 \%$ and $81.4 \%$ at 1 and 3 years respectively. There was no statistical difference of graft survival between the groups. On multivariate regression analysis, none of the tested parameters significantly affected the graft outcome or recipient creatinine levels across the groups 
Conclusions: Laparoscopic donor retrieval of adult grafts is feasible for paediatric renal transplantation and outcomes are comparable to open retrieval

MP26-20 Bulb suction drainage is not necessary after routine robotic-assisted partial nephrectomy: A large casecontrol analysis

CA Winter, RB Pickens, WM White

University of Tennessee Medical Center Knoxville United States

Introduction \& Objective: Bulb suction drain placement is routinely done following robotic-assisted partial nephrectomy secondary to concern for post-operative urine leak. We explore the necessity of bulb suction drain placement and the rate of post-operative urine leaks in patients who underwent robotic-assisted partial nephrectomy at our institution.

Materials and Methods: We performed a retrospective chart review to analyze the occurrence of urine leaks following robotic-assisted partial nephrectomy performed by two fellowship trained surgeons between January 2012 to July 2016. Urine leak was defined as drain body fluid creatinine to serum creatinine ratio greater than 5 or any patient with symptomatic urinary ascites within 90 days post-operatively.

Results: Our review included 208 patients who underwent robotic-assisted partial nephrectomy. A total of 124 patients had intra-operative 10 French bulb suction drain placement and 84 patients had drain placement omitted. The mean length of stay for patients who had drain placement versus those who did not was 3.0 and 2.4 days, respectively. In patients who had bulb suction drains, the mean duration the drain was left in place and drain output was 1.7 days and $180.2 \mathrm{~mL}$, respectively. On post-operative day 1 , drain-fluid creatinine (ng/dL) was measured, resulting in a mean drain-fluid creatinine to serum creatinine ratio of 0.97 (range 0.73-3.12). The mean tumor size $(\mathrm{cm})$ and R.E.N.A.L. nephrometry score (range score 4-12) in patients with drain placement vs. those without was $3.3,5.9$ and $2.7,5.2$, respectively. There was a statistically significant difference $(p=.007)$ in R.E.N.A.L. score between the two groups, but was ultimately inconsequential in our population. The collecting system was entered in 61 patients $(29.3 \%$ of cases) resulting in zero urine leaks within this subgroup. No patients were re-admitted 30 to 90 days postoperatively for symptomatic urinary ascites or related complications.

Conclusions: Routine bulb suction drain placement, even in the event of collecting system entry, can safely be omitted following robotic-assisted partial nephrectomy.

\begin{tabular}{|l|l|l|}
\hline Table 1 & $\begin{array}{l}\text { Patients with } \\
\text { drain } \\
\text { (Standard } \\
\text { Deviation) }\end{array}$ & $\begin{array}{l}\text { Patients } \\
\text { without drain } \\
\text { (Standard } \\
\text { Deviation) }\end{array}$ \\
\hline Mean length of stay (days) & 3.0 & 2.4 \\
\hline Mean drain output (mL) & $180.2(190.3)$ & N/A \\
\hline Mean drain output after collecting system entry during resection (mL) & 210.3 & N/A \\
\hline Mean days with drain & 1.7 & N/A \\
\hline Mean tumor size (cm) & $3.3(1.3)$ & $2.7(1.2)$ \\
\hline Mean R.E.N.A.L. score & $5.9(1.9)$ & $5.2(2.0)$ \\
\hline Number of patients with symptomatic post-op urine leak & 0 & 0 \\
\hline
\end{tabular}

MP26-21 Outcomes of robot-assisted partial nephrectomy in high volume centers continue to improve for several years after the initial learning curve

H Gill, T Quinn, R Cho, MJ Maurice, M Allaf, S Bhayani, M Stifelman, J Kaouk, CG Rogers

Vattikuti Urology Institute, Henry Ford Hospital United States

Introduction \& Objective: Little data is available on the learning curve and volume outcome relationship for robot-assisted partial nephrectomy (RPN). The purpose of this study was to evaluate outcomes of RPN by high volume surgeons beyond the initial learning curve.

Materials and Methods: A retrospective database of 1848 RPN procedures performed by 5 high volume surgeons in 5 academic centers from 2007 - 2014 was analyzed. Patient demographics, operative details and perioperative outcomes from each calendar year were compared to identify statistically significant trends.

Results: Over the 8 year study period, there was a significant increase in the median ASA, RENAL nephrometry score and tumor size in the first half of the study as well as an increase in the percentage of RPN performed via the retroperitoneal approach $(p<0.001)$. Despite this, there was a significant decrease in OR time $(\mathrm{p}<0.001)$ and blood transfusion rate $(\mathrm{p}<0.001)$ in the first 4 years after the initial learning curve, before reaching a stable threshold. Estimated blood loss, warm ischemia time and high

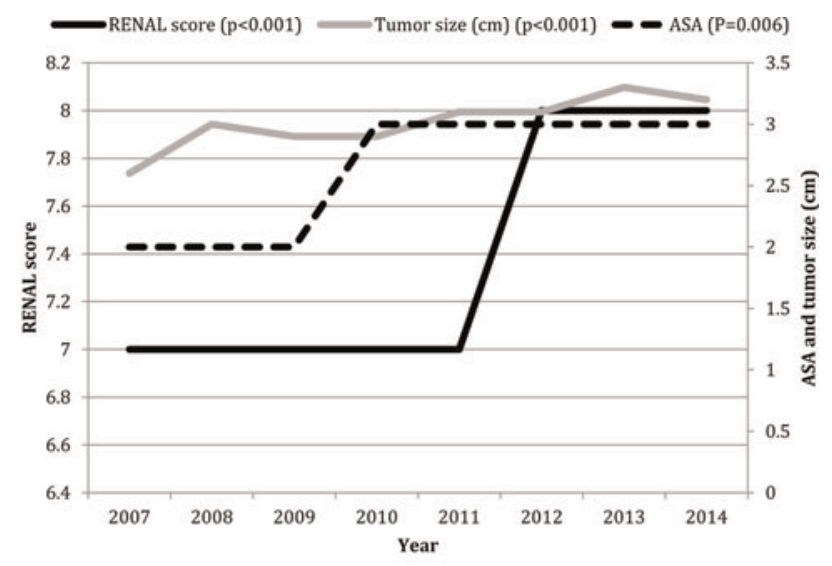

Figure 1. Trends in pre-operative factors of RPN over 8 years. ASA $=$ American Society of Anesthesiologists

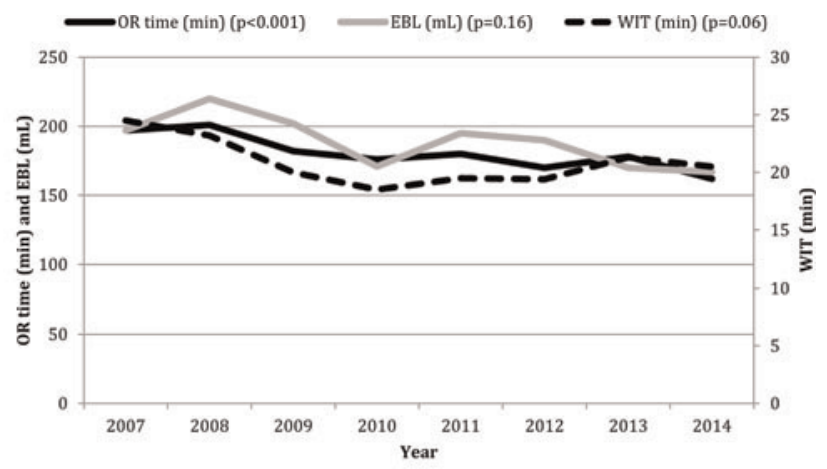

Figure 2. Trends in operative factors of RPN over 8 years. $\mathrm{OR}=$ Operative Time; EBL = Estimated Blood Loss; WIT = Warm Ischemia Time 


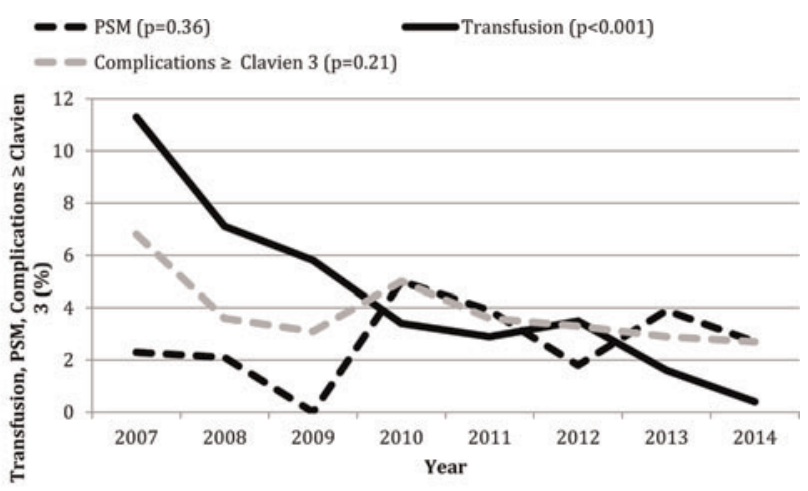

Figure 3. Trends in post-operative factors of RPN over 8 years. PSM $=$ Positive Surgical Margins

Table 1. Pre-operative, perioperative, and post-operative outcomes of RPN. $\mathrm{PN}=$ Robot-Assisted Nephrectomy; $\mathrm{ASA}=$ American Society of Anesthesiologists; $\mathrm{OR}=$ Operative Time; $\mathrm{EBL}$ = Estimated Blood Loss; WIT = Warm Ischemia Time; PSM = Positive Surgical Margins

\begin{tabular}{|c|c|c|c|c|c|c|c|c|c|}
\hline & 2007 & 2008 & 2009 & 2010 & 2011 & 2012 & 2013 & 2014 & p-value \\
\hline $\begin{array}{c}\text { Number of } \\
\text { RPN }\end{array}$ & 44 & 140 & 224 & 259 & 308 & 334 & 311 & 258 & - \\
\hline Median ASA & 2 & 2 & 2 & 3 & 3 & 3 & 3 & 3 & 0.006 \\
\hline $\begin{array}{l}\text { Mean tumor } \\
\text { size (cm) }\end{array}$ & 2.6 & 3.0 & 2.9 & 2.9 & 3.1 & 3.1 & 3.3 & 3.2 & $<0.001$ \\
\hline $\begin{array}{c}\text { Median } \\
\text { RENAL score }\end{array}$ & 7 & 7 & 7 & 7 & 7 & 8 & 8 & 8 & $<0.001$ \\
\hline Mean OR time & 197 & 201 & 182 & 176 & 180 & 170 & 178 & 162 & $<0.001$ \\
\hline $\begin{array}{c}\text { Mean EBL } \\
(\mathrm{mL})\end{array}$ & 197 & 220 & 202 & 171 & 195 & 190 & 170 & 167 & 0.16 \\
\hline $\begin{array}{c}\text { Mean WIT } \\
(\text { min })\end{array}$ & 24.5 & 23.2 & 20 & 18.5 & 19.5 & 19.4 & 21.3 & 20.5 & 0.06 \\
\hline $\begin{array}{l}\text { Retro approach } \\
\mathbf{n}(\%)\end{array}$ & $\stackrel{2}{2}$ & $2.1 \%$ & ${ }_{1.3 \%}^{3}$ & $2.3 \%$ & $\begin{array}{l}16 \\
5.2 \%\end{array}$ & $\begin{array}{c}25 \\
7.5 \%\end{array}$ & $\begin{array}{l}30 \\
9.6 \%\end{array}$ & $\begin{array}{c}33 \\
12.8 \%\end{array}$ & $<0.001$ \\
\hline $\begin{array}{l}\text { PSM } \\
\mathbf{n}(\%)\end{array}$ & $\begin{array}{c}1 \\
2.3 \%\end{array}$ & $2.1 \%$ & $\begin{array}{c}0 \\
0 \%\end{array}$ & $\begin{array}{c}13 \\
5.0 \%\end{array}$ & $\begin{array}{c}12 \\
3.9 \%\end{array}$ & $\begin{array}{c}6 \\
1.8 \%\end{array}$ & $\begin{array}{c}12 \\
3.9 \%\end{array}$ & $2.7 \%$ & 0.36 \\
\hline
\end{tabular}

grade complications (Clavien-Dindo $>3$ ) all decreased over the study period but did not reach statistical significance. Positive surgical margin rate consistently remained low.

Conclusions: Outcomes of RPN performed by high volume surgeons, such as OR time and transfusion rate improved over four years and reached a stable threshold, despite increasing tumor and patient complexity. The use of the retroperitoneal approach increased throughout the study. Our results suggest there may be a longer learning curve to optimize surgical outcomes, even in high volume surgeons.

\section{MP26-22 Laparoscopic Pyelolymphatic Disconnection for} Refractory Chyluria

B Singh, H Pathak, V Kumar, S Sankhwar

King George's Medical university

India

Introduction \& Objective: Filarial or idiopathic chyluria is a debilitating and recurrent disease. Here we present our experience and outcomes of laparoscopic pyelolymphatic disconnection for refractory chyluria.

Materials and Methods: In 24 patients ( $>15$ years of age) with filarial or idiopathic refractory chyluria (after failure of Medical treatment $+\geq 2$ instillation sclerotherapy courses), laparoscopic pyelolymphatic disconnection procedure was performed between March 2010 and April 2016 at 2 tertiary care institutions. Preoperative assessment included clinical examination, ultrasonography, intravenous urography, cystoscopy and retrograde pyelography \pm CT scan, serum albumin, urine test for chyle, cholesterol, triglycerides and albumin. Of these, 23 patients underwent unilateral procedure and 1 underwent bilateral procedure. Transperitoneoscopic procedure included skeletonization of renal artery and vein, nephrolympholysis and upper ureteral lympholysis using 3 or 4 ports. Postoperative assessment included clinical examination, serum albumin, urine test for chyle, cholesterol, triglycerides and albumin at 3,6 and 12 months.

Results: Urine became clear in all patients immediately after surgery. In postoperative period, 4 patients had high ( $>1$ liter/ day) and/or prolonged (> 5days) chylous drain output which settled in 3 weeks in all. Absence of urinary chyle, a significant $(\mathrm{p}<0.05)$ increase in mean serum albumin and a significant $(\mathrm{p}<0.05)$ decrease in mean urinary albumin, triglycerides and cholesterol was noted at 3 month follow up after the procedure. In follow up period, chyluria recurred in 3 patients; in one from ureter at 4 months on operated side, in another at 5 months from unknown site on operated side and in third at 12 months from contralateral unoperated kidney. All these 3 recurrences responded to povidine-iodine instillation sclerotherapy. Thus, laparoscopic pyelolymphatic disconnection alone was curative in $21 / 24(87.5 \%)$ patients and $23 / 25(92 \%)$ renal units at a mean follow up of 44.6 months.

Conclusions: For refractory chyluria, laparoscopic pyelolymphatic disconnection is an effective surgical treatment with low morbidity and offers an early full dietary freedom to these patients.

MP26-23 Bibliometric trends of radical nephrectomy over the last 16 years (2000-2015): A systematic review

\section{A Pietropaolo, B Rai, C Lockyer, M Hayes, B Somani}

University Hospital Southampton NHS Foundation Trust United Kingdom

Introduction \& Objective: Nephrectomy has been a cornerstone for malignant renal tumours although there seems to be a shift from open surgery to laparoscopic and robotic surgery. We wanted to see the bibliometric trends for surgical intervention for all radical nephrectomy procedures over the last 16-years.

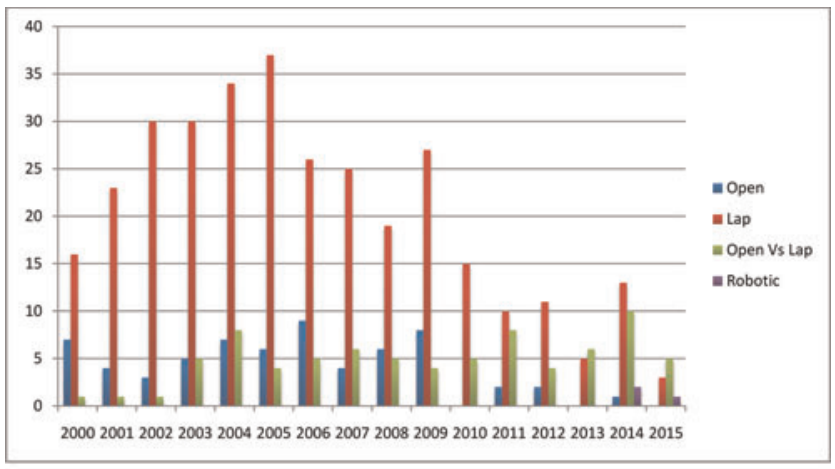

Fig1 


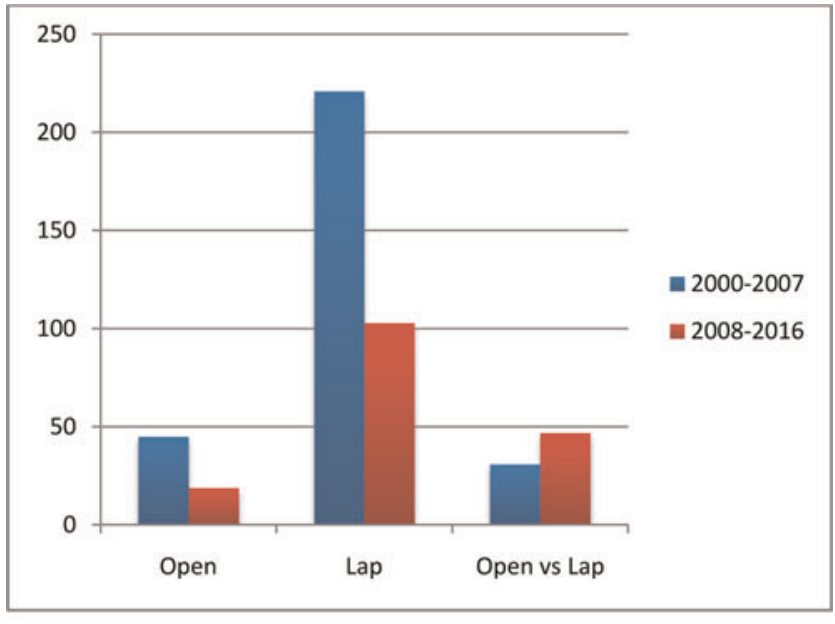

Fig 2

Materials and Methods: All published papers on radical nephrectomy including 'nephrectomy', 'open', 'laparoscopic', robotic' and all comparison studies between these options were searched on PubMed over the last 16 years. We looked at all English language articles on radical nephrectomy over the time period irrespective of the modality of surgical intervention. Patients who underwent Nephroureterectomy, benign nephrectomy and/or paediatric patients were excluded. While review articles were included, partial nephrectomy, case reports and those papers that did not have a published abstract were excluded from our analysis. Similarly animal and laboratory studies were also excluded from our analysis. Data was divided into two 8-year periods, period-1 (2000-2007) and period-2 (2008-2015)

Results: A total of 469 papers on open nephrectomy were published on PubMed over the last 16 years (Fig 1), including 64 (14\%) open nephrectomy, 324 (69\%) laparoscopic nephrectomy, $78(16 \%)$ comparative studies between open and laparoscopic nephrectomy and $3(1 \%)$ robotic nephrectomy. Overall there was a decrease in both open and laparoscopic total nephrectomy in period-2 (Fig 2). The number of open nephrectomy dropped from 45 (period-1) to 19 (period-2), while the number of laparoscopic nephrectomy dropped from 221 (period-1) to 103 (period-2). However, the number of comparative studies between open and laparoscopic nephrectomy rose from 31 (period-1) to 47 (period2) suggesting a move towards achieving a better quality of evidence between these two treatment methods.
Conclusions: While there has been an overall decrease in publications on total nephrectomy over the recent years, this is expected with the rise in partial nephrectomy over the same time period. Laparoscopic total nephrectomy is five times more common than open total nephrectomy and seems to be the gold standard for these procedures.

MP26-24 Laparoscopic nephrectomy in children under 2 years of age

V Izol, N Akdogan, O Yilmaz, F Ok, E Ziyadov, K Karkin

University of Cukurova

Turkey

Turkey

Introduction \& Objective: Laparoscopic nephrectomy $(\mathrm{N})$ or nephroureterectomy (NU) is introduced with increasing frequency in cases with indications in the pediatric age group and introduced to a very young age group with increased experience. In this video, presented are the images from one of our laparascopic $\mathrm{N}$ or NU operations carried out on 15 children under 2 years of age in our clinic for the last 10 years.

Materials and Methods: VCUG obtained for the patient revealed grade 5 hydronephrosis on the left for the 20-month hypoplastic left kidney in the examinations owing to the reason for the recurrent urinary tract infections. Laparoscopic NU was planned for the patient. Transperitoneal entry was performed in the left lumbar position with the veress needle at the pararectal space level. $5 \mathrm{~mm} 30^{\circ}$ laparoscope and standard laparoscopic equipment were employed in the operation.

Results: The mean operative time was $60 \mathrm{~min}$ in the patients under 2 years of age. Mean operation time was 128 (30-220) min, blood loss was minimal. According to modified Clavien classification grade $3 \mathrm{~b}$ complication in 2 patients and grade 2 complications in 2 other patients have been observed. One patient had grade 2 and another patient had grade $3 \mathrm{~b}$ complication during postoperative period. We found that the patients were at the beginning of the learning curve when we took into consideration the time that such complications occurred. The average duration of hospitalization was 55 (28-92) hours. The cosmetic results after surgery were fine and late complications were not observed.

Conclusions: The standard technique is preferred because of early discharge period, low cost and cosmetic benefits with increasing experience in laparoscopy in pediatric patients.

\section{MPS27: NEW TECHNOLOGIES II: STONES AND ROBOTICS} MP27-1 Kidney stone shadow measurements are more accu-
rate than stone measurements with a commercial ultrasound unit

B Dunmire, J Thiel, BW Cunitz, JS Ahn, J Dai, JD Harper, K Sternberg, T Larson, MR Bailey, MD Sorensen

Center for Industrial and Medical Ultrasound, Applied Physics Laboratory, University of Washington United States
Introduction \& Objective: Ultrasound overestimates stone size. Recent publications using a research ultrasound system suggest the posterior acoustic stone shadow may better represent the stone size. In this study, ultrasound images collected, sized, and published (Sternberg et al. Urology 2016) were analyzed for the presence and accuracy of the stone shadow width to determine stone size.

Materials and Methods: This was a retrospective review of data collected at 1 institution where clinical ultrasound and CT were 


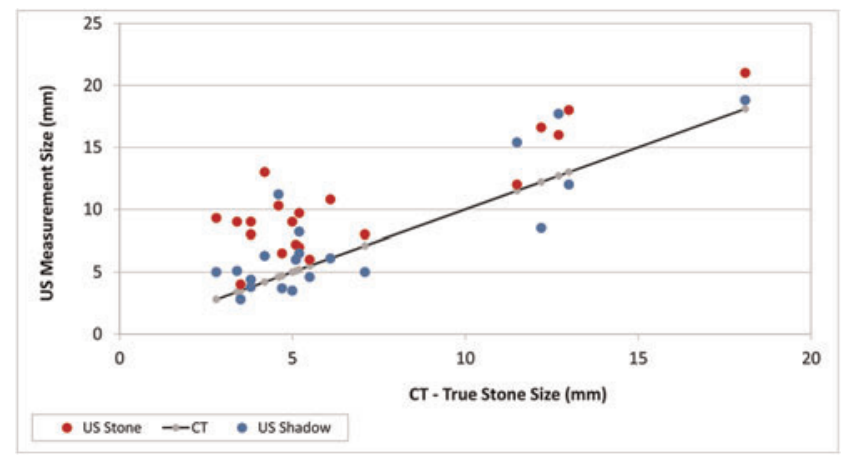

performed within 1 day. True stone size for the largest stone was determined on CT. Corresponding ultrasound stone size was interpreted and reported in an official radiology report. At a second institution blinded to CT measurements, the ultrasound images were assessed for the presence and size of posterior acoustic stone shadows, with all shadow width measurements taken $\sim 1 \mathrm{~cm}$ posterior to the stone due to divergence of stone shadows with greater depths. The accuracy of stone versus shadow size measurements were compared against CT size across three clinical significant size categories ( $\leq 5 \mathrm{~mm}, 5-10 \mathrm{~mm},>10 \mathrm{~mm}$ ).

Results: The original study included 36 subjects from University of Vermont with a stone on US and CT. The stone demonstrated a shadow in 20 cases. $90 \%$ of stones $>5 \mathrm{~mm}$ and $50 \%$ of stones $\leq 5 \mathrm{~mm}$ (as measured on CT) demonstrated an acoustic shadow. When the stone was measured directly with ultrasound, the absolute error was $3.7 \pm 2.3 \mathrm{~mm}$ versus $2.0 \pm 1.7$ based on shadow width. Using the stone shadow measurement, there was a $>2$ fold increase in stones measuring within $1 \mathrm{~mm}$ of the CT measurement. Misclassification of stones for 3 clinically relevant size ranges was reduced from $45 \%$ to $25 \%$ of stones (absolute reduction of 20\%).

Conclusions: Using a commercial ultrasound system, posterior acoustic shadow width was a more accurate measure of stone size when compared to CT than measuring the stone itself. The absence of a shadow suggests the stone is $\leq 5 \mathrm{~mm}$. Ultrasound images were obtained with spatial compounding, which blurs the stone and shadow boundaries. Further optimization of a commercial system is possible to improve the quality and reliability of ultrasound sizing of the stone and shadow. Work supported by NIH P01-DK043881 and NSBRI through NASA NCC 9-58.

MP27-2 The efficiency of dual energy CT in determining urinary calculi composition

O Levi, N Idkedek, A Sidi, A Tsivian, E Surin, E Tavdi, R Bass, D Khunovich

Department of Urology, E.Wolfson Medical Center, Holon, Israel

Israel

Introduction \& Objective: Uric acid calculi are usually diagnosed by their density below 700 Hounsfield Units (HU) on CT scan and absence of radiopaque shadow on abdominal x-ray. However, oxalate calculi may measure less than $700 \mathrm{HU}$-as well. In addition, they are not always identified on x-ray because of small size, location or technical problems. Dual energy CT
(DECT) technology involves two consequent scans with low and high energies, which enables identification of uric acid calculi. However, this technique is not routinely performed because of unavailability of suitable equipment and fear of additional radiation exposure. In our institution a patient with a calculus larger than $3 \mathrm{~mm}$ undergoes further evaluation with DECT. The results are reported in color scheme were red represents uric acid calculus, blue is a non-uric acid calculus and red and blue indicates a mixed calculus. Herein, we report on the accuracy of DECT in identification of calculus composition.

Materials and Methods: Between January 2015 and July 2016 four hundred and sixty eight calculi were scanned by DECT in 284 patients. The average calculus size was $8.8 \mathrm{~mm}$. Fifty six calculi were located in the ureter and the rest in the kidneys. The calculus density in HU was compared to the DECT chemical composition. When biochemical analysis of the calculi was available its results were compared to HU values and DECT findings.

Results: DECT identified 398 calculi $(85 \%)$ as non-uric acid calculi, $58(12 \%)$, as uric acid calculi and $12(3 \%)$ had mixed composition. Density span in HU was 130-640 for uric acid calculi, 100-3710 for non-uric acid calculi and 150-1025 for mixed calculi. All uric acid calculi measured below 700 HU. In 110 calculi with density above 700 HU DECT did not detect any uric acid calculi. In 60/110 (55\%) calculi the diagnosis was confirmed by biochemical stone analysis. In 261 calculi with HU density below 700 , DECT scan has shown $77.8 \%$ to be non-uric acid calculi. In $35 \%$ the results were supported by chemical analysis of the stones. Two hundred and ninety eight calculi were found to be with minimal density below 700HU (suspected mixed composition stone). Of them $96 \%$ were non uric acid calculi and $4 \%$ mixed calculi.

Conclusions: For the purpose of identification of uric acid calculi, DECT scan should be performed for calculi with HU density below 700. There is no role for DECT when calculus measures above $700 \mathrm{HU}$, a value above which no uric stones were detected.

MP27-3 Robot-Assisted Percutaneous Nephrolithotomy Updates on Further Development

M Oo, H Gandhi, K Chong, J Ng, A Goh, Y Tan

Department of Urology, Tan Tock Seng Hospital (Singapore) Singapore

Introduction \& Objective: To make percutaneous access easier in PCNL, we developed a Smart Robotic System (SRS). Here we update the results of our live porcine trials using the SRS.

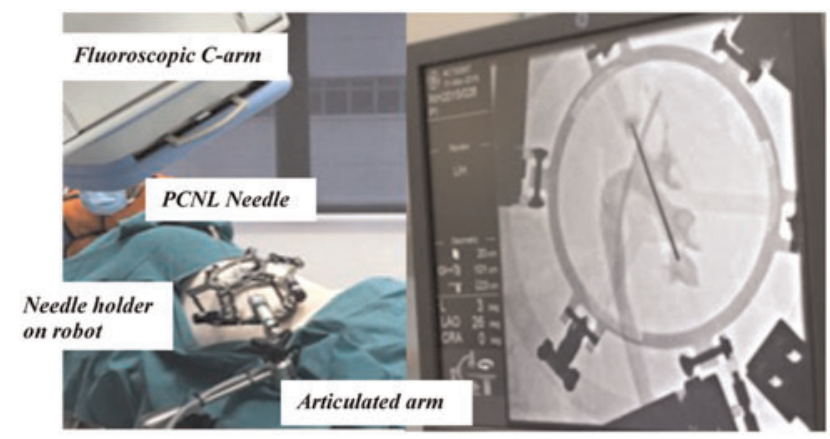

Figure 1: The porcine trial using the smart robotics system (SRS) (Left), with respective fluoroscopic image on the smart robotics system (SRS), needle and kidney (right). 


$\begin{array}{llll}\text { Puncture Type } & \begin{array}{l}\text { Robot-assisted, } \\ \text { mean }( \pm \mathrm{SD})\end{array} & \begin{array}{l}\text { Free-hand, } \\ \text { mean }( \pm \mathrm{SD})\end{array} & \text { P value } \\ \begin{array}{l}\text { Total fluoroscopic } \\ \text { time (secs) }\end{array} & 26.8( \pm 5.8) & 32.6( \pm 25.2) & 0.629 \\ \begin{array}{l}\text { Puncture fluoroscopic } \\ \text { time (secs) }\end{array} & 13.2( \pm 1.6) & 22.0( \pm 19.2) & 0.338 \\ \begin{array}{l}\text { Total radiation dose } \\ \text { (mGy) }\end{array} & 15.2( \pm 13.9) & 15.4( \pm 7.3) & 0.978 \\ \begin{array}{l}\text { Puncture radiation } \\ \text { dose (mGy) }\end{array} & 8.2( \pm 6.8) & 11.2( \pm 7.9) & 0.540 \\ \begin{array}{l}\text { Total time (mins) } \\ \text { Puncture Time (mins) }\end{array} & 3.15( \pm 0.28) & 3.01( \pm 0.47) & 0.587 \\ \begin{array}{l}\text { Alignment time } \\ \text { (mins) }\end{array} & 2.00( \pm 0.12) & 1.43(( \pm 0.38) & 0.387 \\ \end{array}$

Materials and Methods: Our SRS uses an image registration software with a closed loop feedback system to align the puncture needle to the desired calyx using the bullseye technique. The prototype is developed by first testing on a gelatin model to optimize accuracy in targeting. We then attempted percutaneous punctures on a live pig model and compared the results with freehand technique. All punctures were performed by a fellowship trained surgeon. The robotic system was set up as shown in Figure 1. For more accurate targeting we held respiration for up to a minute during the robotic punctures. Data was collected as shown in Table 1.

Results: Initial results for live animal trials are as shown in Table 1. There is less radiation dose and time required during puncture for robotic assistance. Although total duration of procedure and puncture time involved setting up the robotic equipment, this did not result in significant increased time to attaining a successful puncture compared to free-hand technique. The automated alignment by the software and stabilization of needle trajectory during punctures enabled the surgeon to attain access more confidently.

Conclusions: Our robotic system can help surgeons feel more confident, shorten the learning curve, increase the accuracy, potentially reduce the complications and thereby enable more surgeons to adopt this procedure.

MP27-4 Towards intelligent laser lithotripsy: experimental evaluation of o novel laser system for automated stone/ tissue analysis using autofluorescence in real-time

D Schlager, A Miernik, A Brandenburg, J Schütz

University Medical Center Freiburg, Germany Department of Urology, Division of Urotechnology

Germany

Introduction \& Objective: Photonic sciences are considered to gain the same importance in the future as electronics today. A laser system with integrated real-time spectroscopic analysis of targeted structures is not yet available. An "intelligent" laser would be associated with high user-relevant benefits. The aim of the study was to evaluate technical, medical and physical conditions for automated detection of tissue, urinary stone and endoscope components during interventional laser application.

Materials and Methods: Pure samples of urinary stone compositions, native human calculi ( 84 samples), endoscope com- ponents and organic samples (fresh porcine renal parenchyma, collecting system, blood) were analyzed by fluorescence spectroscopy in a dry and a wet model. After acquisition of the spectral information, a data analysis was performed in term of sample differences of signal intensity and emission wavelengths and to determine the most appropriate excitation wavelength. An optimized setup was developed to superimpose fluorescence excitation light to a Holmium laser beam into the treatment fiber. Results: All 84 human kidney stones showed a satisfactory fluorescence signal at $532 \mathrm{~nm}$ excitation. While the amplitude of the signal was found to vary by a factor of 75 , the weakest signal of stone material $(0,038+/-0,043)$ was 3,6-fold larger than the strongest signal of pig kidney tissue $(0,00058+/-0,00058)$. The shape of the spectra was very similar for all stone types. No correlation between stone type and fluorescence amplitude or spectral curve form was observed. Thus, analysis of the amplitude was sufficient for stone/tissue differentiation in real-time.

Conclusions: The study provides information for spectral realtime differentiation of tissue, urinary stones and relevant endoscope components. The data obtained are essential for the construction of a simple intelligent laser system for endourology. This might be a starting point for the development of new medical photonic applications.

MP27-5 Endoscopic clearance lithotripsy devices: bench comparison of stone elimination capacity and drilling speed

MJ Bader, F Strittmatter, A Alghamdi, T Pongratz, M Eisel, C Stief, R Sroka

UroClinic München and Department of Urology, University of Munich

Germany

Introduction \& Objective: Several endoscopic stone fragmentation and clearance lithotripsy systems are currently available. The Swiss LithoClast Master (EMS Switzerland) offers standalone ultrasonic lithotripsy and ultrasonic / pneumatic combination lithotripsy with 2 coaxially mounted probes to transmit ultrasonic vibration and impact generated compression waves. The Shockpulse SE (Olympus Germany) transmits ultrasonic vibration and an ultrasonically generated shock through the same hollow probe. The latest development is the Swiss LithoClast Trilogy (EMS Switzerland) employing an electromagnetic impactor and an ultrasonic lithotripter to deliver ultrasonic vibration and ballistic impact compression waves though the same hollow probe. The objective of this study was to compare stone elimination and drilling speed.

Materials and Methods: Cubical BegoStone (15:3) phantoms of $10 \mathrm{~mm}$ size were used for clearance testing. 10 fragmentation and clearance tests were performed in an underwater hemi-sphere by 5 different operators ( 50 test runs per device). The average stone removal time per operator and per device was recorded and statistical analysis was performed. For the drilling speed test, a free-hand set-up was used. Stones of $15 \mathrm{~mm}$ size were positioned on one side of an underwater balance and lithotripter probes were vertically mounted in direct contact. A weight of $450 \mathrm{~g}$ was placed on the other side of the balance to assure a constant contact pressure. 10 test runs per device were performed. The drilling time until breakthrough or, if no breakthrough occurred, the achieved drilling depth after 1 minute was measured and the resulting drilling speed was calculated. 
Results: The Swiss LithoClast Trilogy was clearing the stone phantoms significantly (Trilogy: $28 \mathrm{sec}$, ShockPulse: $39 \mathrm{sec}$, LithoClast Master ultrasound (US) only: $37 \mathrm{sec}$, LithoClast Master combined: $44 \mathrm{sec}$.). There was no significant difference between the ShockPulse and LithoClast Master with either ultrasound only or combined function. A significant difference $(\mathrm{p}<0.004)$ was found between LithoClast Master used in combination mode vs. ultrasound stand alone. A similar pattern was seen for the drilling speed, where the LithoClast Trilogy outperformed all other lithotripters (Trilogy: $0.65 \mathrm{~mm} / \mathrm{sec}$, ShockPulse: $0.46 \mathrm{~mm} / \mathrm{sec}$, LithoClast Master combined: $0.47 \mathrm{~mm} / \mathrm{sec}$ and LithoClast Master US only: $0.18 \mathrm{~mm} / \mathrm{sec}) .(\mathrm{p}<0.05)$.

Conclusions: The Swiss LithoClast Trilogy was significantly faster than the other lithotripters. Since the other devices use comparable probe sizes and lumen, it seems that the clearance and drill speed advantage of the LithoClast Trilogy is based on the better performance of the electromagnetic impactor.

MP27-6 The safety and efficacy of a new micro-ultrasonic lithotriptor combined with mPCNL in the treatment of renal and upper ureteral stone (a preliminary report of the first 90 cases)

\section{Xiong, X Ye, J Liu, X Huang, L Chen, K Ma}

Peking University People's Hospital

China (People's Republic)

Introduction \& Objective: To evaluate the safety and efficacy of a new micro-ultrasonic lithotriptor $(2 \mathrm{~mm})$ combined with $\mathrm{mPCNL}(16 \mathrm{Fr})$ in the treatment of renal and upper ureteral stones (a preliminary report of the first 90 cases).

Materials and Methods: The total 90 patients with renal and upper ureteral stones who were treated with the micro-ultrasonic lithotriptor combined with mPCNL(mu-mPCNL) between December 2015 and December 2016 in Peking University People's Hospital were retrospectively analyzed. In the study, the duration of operation, intraoperative blood loss, complications, mean hospital stay and residual stone rates were recorded.

Results: All surgeries were successful, the average stone size was $1.9 \mathrm{~cm}$, only 3 patients had residual fragments, the mean operative time was $30.8 \mathrm{~min}$, the SFR of 1st postoperative was $96.7 \%$, the mean hospital stay was 2.4 days, the rate of tubelessPCNL procedure was $84.4 \%$. No patients had blood transfusion or postoperative fever, one patient had perirenal effusion, and recovered by drug treatment, one patient persisting hematuria after withdrawing the nephrostomy tube, and recovered by interventional embolization. All patients were followed up at least one month, no other complication occurred.

Conclusions: The clinical use of the micro-ultrasonic lithotriptor $(2 \mathrm{~mm})$ combined with $\mathrm{mPCNL}(16 \mathrm{Fr})$ in the treatment of renal and upper ureteral stones was safe and efficient, especially for the renal and upper ureteral stones smaller than $2.5 \mathrm{~cm}$.

MP27-7 A Novel Technique For Access During Percutaneous Nephrolithotomy: First Clinical Experience

M Akand, O Kilic, E Altintas, K Bocu, L Civcik, A Buyukaslan, M Koplay

Selcuk University, School of Medicine, Department of Urology Turkey
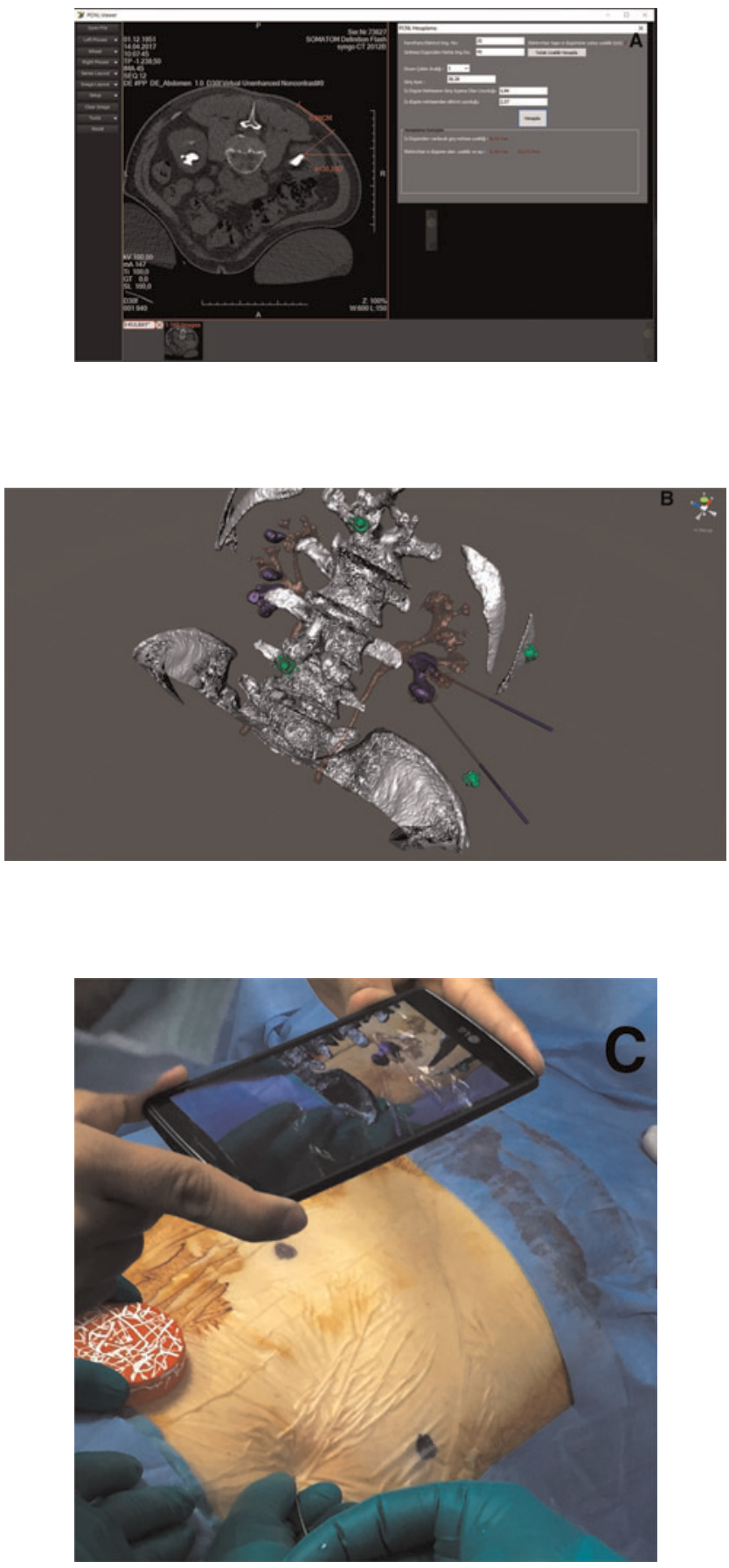

Introduction \& Objective: Determination of the right access point with a correct access angle is the first and the most important step of percutaneous nephrolithotomy (PCNL). Herein, we aimed to present our first clinical experience for determining an optimal access point and angle by means of a novel device which uses software-based mathematical calculation, 3-dimensional (3D) modeling and augmented reality (AR) technology. Materials and Methods: After receiving the institutional review board approval and the informed consents of the patients, a preoperative computed tomography (CT) was performed in 5 patients, who were planned to undergo PCNL for kidney stone. The CT was taken in prone position, where two gel cushions were 
positioned under the patient like in PCNL, 10 minutes after injection of the contrast agent with 4 electrodes placed on the lumbar region of the patient. By using the DICOM objects generated from the CT scans, mathematical and software functions were developed to measure the distance of the stone from the reference electrodes (Figure 1-A). Afterwards, vectoral 3D modeling was performed to calculate the access point and angle. Conversion of the files into FBX/OBJ format provided the data to be used in AR technology. With the specific programs and $\mathrm{AR}$, the $3 \mathrm{D}$ modeling was placed onto the patients' operation field, and calculated access point and a virtual access needle were marked (Figure 1-B/C).

Results: The novel system was tested on 5 patients with a mean age of 47 years. Mean operation duration was 68 min, and mean hospitalization duration was 2.3 days. It was possible to touch the stone successfully with TLA needle in all patients with no flouroscopy. The mean flouroscopy time during the rest of the operation was $2.4 \mathrm{~min}$. None of the patients had any complications. Only 1 patient had a residual stone of $6.5 \mathrm{~mm}$ in the postoperative follow-up with the remaining patients being stone-free.

Legend for Figure 1: A) Axial CT scan of a 67-year-old male patient presenting with bilateral partial staghorn stones. Right PCNL was planned, and access angle and point calculations were performed with the novel software. B) 3D modelling of the right kidney with 2 virtual needle tracts were marked for 2 potential tracts. C) Intraoperative view.

Conclusions: According to the preliminary results, this novel device seems to ensure a correct access point and angle for PCNL. However, further research is required to test its accuracy and safety in detail.

MP27-8 In Vitro Comparison of a Novel Single-probe Dual-energy Lithotripter to Current Generation Devices

D Wollin, B Winship, W Tom, D Radvak, R Jiang, C Scales, M Ferrandino, W Simmons, GM Preminger, M Lipkin

Duke University Medical Center United States

Introduction \& Objective: Current lithotripters for percutaneous renal surgery include the ShockPulse (Olympus) and LithoClast Select (ElectroMedical Systems/Boston Scientific). These dual-energy lithotripters use a combination of ultrasonic fragmentation with impactor function to clear stones more efficiently. The LithoClast Trilogy (ElectroMedical Systems/Boston Scientific) is a novel single-probe, dual-energy lithotripter that utilizes ultrasonic vibration with suction capability along with an electromagnetically-generated impact. Our aim was to compare the stone clearance efficiency of these three devices in an in vitro setting.

Materials and Methods: $1 \mathrm{~cm}^{3}$ cube-shaped Begostone phantoms were created to a hardness that mimics calcium oxalate

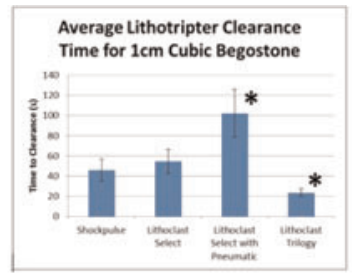

Figure 1: Average Lithotripter Clearance Time for $1 \mathrm{~cm}$ Cubic Begostone $\left({ }^{*}\right.$, significantly different from all other devices, $p<0.01$ monohydrate stones (BegoStone-to-water ratio 5:1). A single stone was placed in a hemispherical silicone support in a water bath. Each lithotripter (ShockPulse, LithoClast Select - with or without pneumatic function - or LithoClast Trilogy) was utilized under direct vision to fragment and suction the phantom utilizing a $300 \mathrm{cc} / \mathrm{min}$ constant suction rate and comparable fragmentation settings. The time to stone clearance for each trial was recorded; 10 trials were performed per device. Statistical analysis was performed with ANOVA.

Results: The LithoClast Select with pneumatic function had the longest clearance time of 138 seconds. The minimum clearance was 18 seconds with the LithoClast Trilogy. When comparing the four treatment methods, there was a difference between groups by one-way ANOVA $\left(\mathrm{F}[3,36]=53.00, \mathrm{p}=2.75 \times 10^{-13}\right)$. Post hoc tests showed that LithoClast Select with pneumatic was significantly slower than all other devices and LithoClast Trilogy was significantly faster than all other devices (all $\mathrm{p}<0.01$ ). LithoClast Select without pneumatic was similar in clearance time to the ShockPulse. (Figure 1)

Conclusions: In an in vitro setting that mimics clinical percutaneous renal surgery, the novel single-probe, dual-energy LithoClast Trilogy was significantly more efficient than current generation dual-energy devices. However, further clinical testing is needed to ensure safety and efficacy in patients.

MP27-9 A Novel Combination Stone Basket and Balloon Device To Treat Ureteral Stones

R Massoudi, TJ Metzner, B Bonneau, T Ngo, R Shinghal, J Leppert

Stanford University School of Medicine

United States

Introduction \& Objective: Ureteral stone extraction devices have not significantly changed in 25 years. The current generation of stone baskets, laser lithotripsy, and ureteral access sheaths

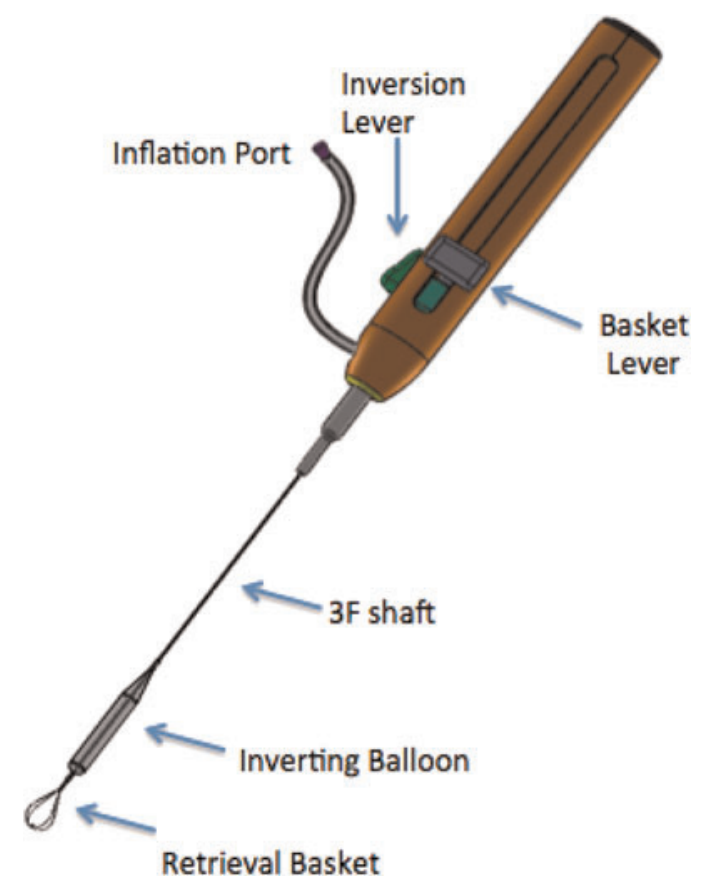


allow for treatment of ureteral stones. We have developed a device that envelopes a stone within a balloon to facilitate safe ureteral stone extraction.

Materials and Methods: The Nautilus device has an outer diameter of 3 French and combines a nitinol stone basket and lowpressure balloon into a single device. After a ureteral stone is visualized through a ureteroscope, the stone is captured in the tipless nitinol basket. The basket is then pulled into the lowpressure balloon (inflated with air to a pressure of 1 atmosphere). The balloon provides a smooth, low-friction covering to safely remove the stone. The device is operated by 2 actuators on a single hand piece. We tested the performance of device prototypes in a porcine model using stone mimics. A ureteroscopy model was created using porcine ureters mounted to an acrylic board. We measured the force required to extract stones ranging from 2 to $7 \mathrm{~mm}$ in size through the ureter with either a traditional stone basket or with the device.

Results: We successfully captured and removed model stones with a diameter of 2 to $5 \mathrm{~mm}$ with the Nautilus device. Larger, irregularly shaped stones were less likely to be captured with the device. Stones extracted with the device required less force (range $0.18 \mathrm{~N}$ to $0.31 \mathrm{~N}$ ) when compared with stones in a standard ureteral stone basket. The force reduction was most pronounced in stenotic and tortuous ureters, and for stones greater than $3 \mathrm{~mm}$ in diameter (mean force reduction $=0.19 \mathrm{~N}, 42 \%$ ).

Conclusions: A combination stone basket and balloon device can reduce the force required to extract ureteral stones.

\section{MP27-10 Acoustic tracking of kidney stones in vitro for} targeting during shock wave lithotripsy

R Cleveland, K Shoar, B Turney

Institute of Biomedical Engineering, University of Oxford United Kingdom

Introduction \& Objective: In shock wave lithotripsy, kidney stones are under continuous motion during treatment and it has been reported than on the order of half the shock waves (SWs) do not impact the stone because of motion. SWs that do not hit the stone likely result in unwanted tissue damage with no therapeutic benefit and if the stone could be tracked in real time then number of mistargeted SWs could be reduced. Tracking the stone with fluoroscopy is not feasible due to radiation dose and using diagnostic ultrasound has not be shown to robust at automatically determining stone location.

Materials and Methods: Here we used an array of twenty-two $500 \mathrm{kHz}$ ultrasound transducers which were placed in a collar designed to mount around a shock wave therapy head. The frequency is much lower then the $3.5-5 \mathrm{MHz}$ used for ultrasound imaging which makes the system here much less sensitive to scattering from tissue but still able to detect reflections from kidney stones. The ultrasound transducers were connected to a Verasonics ultrasound engine which transmitted on one transducer at a time and recorded the echo signal on all elements. The received echoes were analysed using a trilateration algorithm from which the location of targets was calculated on the ultrasound machine. In vitro experiments were carried out with artificial kidney stones made from gypsum cement that were formed in the shape of natural stones. The artificial stones were mounted on a thin stick and translated in a water tank and the ultrasound collar used to determine the stone location. The echo arrival times of echoes from stones was automatically detected using a thresholding algorithm and signals with no detectable stone signal were removed from the processing.

Results: The results showed that the accuracy in determining the location of the stone was better than $4 \mathrm{~mm}$ in the lateral direction and $2 \mathrm{~mm}$ in the axial direction if the stone was within $\pm 15 \mathrm{~mm}$ of the desired focus. This accuracy was retained even if only half the 22 elements were able to detetct an echo. For stones more than $15 \mathrm{~mm}$ from the focus the algorithm could reliable determine they were not in the focus even if the actual position was not accurate. The algorithm was implemented in Matlab and stone location could be determined at least once every second.

Conclusions: The low frequency ultrasound collar described here is capable of tracking kidney stones in real time with an accuracy better than $4 \mathrm{~mm}$. We estimate the time for calculation could be reduced to less than $0.1 \mathrm{~s}$ using more efficient programming language. These results motivate adapting the technology described here for deployment on a clinical lithotripter with the goal of tracking kidney stones and providing a signal to gate SW delivery.

MP27-11 Current disposable ureteroscopes - performance and limitations in a standardized kidney model

\section{Schlager, S Hein, A Miernik, M Schönthaler}

University Medical Center Freiburg, Germany Department of Urology, Division of Urotechnology

Germany

Introduction \& Objective: With increasing fragility of the instruments and rising concerns about sterility of reprocessable scopes, several single use devices for fURS have been introduced. In this study we directly compare currently available disposable digital and fiberoptic flexible ureteroscopes with a contemporary reusable fiberoptic device.

Materials and Methods: LithoVue ${ }^{\mathrm{TM}}$, Uscope, Flexor ${ }^{\circledR} \mathrm{Vue}^{\mathrm{TM}}$ and a standard reusable fiber optic flexible ureteroscope (BOA vision ${ }^{\circledR}$ )were each tested in artificial kidney models. The experimental setup included (a) the visualization of all calices (correct assignment of colored pearls) and (b) the extraction of human calculi from all calices with a common disposable extraction device $\left(\mathrm{NGage}^{\circledR}\right)$. All procedures were performed by 6 experienced surgeons. We recorded successful visualization of calices, stone extraction and times to completion. In addition, the surgeons' workload and performance was determined using the NASA-Task Load Index (NASA-TLX). We referred to a Lickert scale to assess maneuverability, handling, and image quality.

Results: Performances of LithoVue and Uscope in respect to visualization and correct identification of calyces were nearly identical to BOA vision (100\%, $97 \%$ and $100 \%$, respectively). Times to completion were significantly longer using Uscope $(p=0.003)$ and FlexorVue $(p)$. For stone retraction LithoVue performed nearly equal to the reusable scope (97\% vs $95 \%$ stone clearance), while accessibility especially to the lower poles was impeded using Uscope as reflected by the retrieval time per stone ( $73 \mathrm{~s}$ vs. 102s per stone). Image quality was rated best for LithoVue among all devices. Handling and maneuverability within the kidney however were significantly lower among the disposable devices. Workload assessment showed no significant increase when using LithoVue $(p=0.36)$, while a significant increase in workload was measured when using Uscope $(p<0.001)$. Overall performance of FlexorVue was signiicantly lowerin all parameters 
Conclusions: In comparison to currently available disposable ureteroscopes, LithoVue offers overall comparable performance and characteristics to a common reusable device with superior image quality when compared to a common fiber optic scope. Uscope exhibits potential for upper urinary tract stone management but requires further technical improvements. Performance of FlexorVue currently does not offer satisfactory results for routine clinical use.

MP27-12 Linear focus extracorporeal shockwave therapy in the treatment of erectile dysfunction: a multi-center, prospective, randomized, double-blinded, placebo controlled study.

\section{Yang, J Cai, D He}

The First Affiliated Hospital of Xian Jiaotong University China (People's Republic)

Introduction \& Objective: The aim of this study was to investigate the effect of linear focus low-intensity extracorporeal shockwave therapy (LLi-ESWT) in the treatment of erectile dysfunction (ED), and whether the function of cavernosal artery was ameliorated after treatment.

Materials and Methods: This was a multi-center, double-blinded, prospective, randomized, placebo-controlled trial. Sixty ED patients diagnosed using the International Index of Erectile Function-5 (IIEF-5), Erection Hardness Score (EHS) and presence of nocturnal penile tumescence (NPT), were random assigned either into LLiESWT group or the sham group (ratio 2:1). The protocol comprised one treatment session per week for 4 weeks. Patients were followed after 2 months. Follow-up assessment was in the form of IIEF-5, EHS. Prostaglandin injection into the corpus cavernosum was used to induce penile erection and the intra-arterial diameter (IAD), peak systolic velocity (PSV), end-diastolic velocity (EDV), and resistive index (RI) for each cavernosal artery measured by color duplex Doppler ultrasound (CDDU) were performed before treatment and at the follow-up.

Results: 16 patients (80\%) in control group and 33 patients $(82.5 \%)$ in LLiESWT group, total 49patients completed the study. There was no significant difference between these two groups in baseline IIEF-5, EHS, IAD, PSV, EDV and RI. The average IIEF-5, EHS increased significantly in LLiESWT group than in control group. However, in both group, there was no significant difference of the IAD, PSV, EDV and RI before and after treatment, and no significant difference between the two groups at the follow up. No adverse events were reported during the treatment and the follow-up duration in both groups.

Conclusions: This approach was tolerable and effective, suggests that the performance of LLI-ESWT could add a new advanced treatment for ED. However, the function of cavernosal artery was not ameliorated after the treatment, which indicates other mechanism rather than cavernosal artery change plays a role in this process.

MP27-13 Real-Time Doppler Ultrasound Aided Nerve Sparing da Vinci ${ }^{\mathrm{TM}}$ Robot Assisted Radical Prostatectomy Utility of Tandem Dissection of Neurovasular Bundle using a Mini Transducer.

SS Kommu, P Rouse, A Samateh, SH Garnett, PD Rimington

Eastbourne District General Hospital: East Sussex Healthcare NHS Trust

United Kingdom
Introduction \& Objective: Nerve sparing during radical prostatectomy is a crucial step in achieving the ideal of optimal outcomes in the pentafeta in the selected patients. The technique is often performed based on the surgeon's subjective perception of locale of the neurovascular bundle. Herein, We report our initial experience with a mini transducer to permit real-time doppler ultrasound aided nerve sparing during da Vinci ${ }^{\mathrm{TM}}$ Robot Assisted Radical Prostatectomy (RARP). To our knowledge, this is the first report of utility of an intraabdominal mini transducer during RARP.

Materials and Methods: The patient deemed suitable for nerve sparing RARP consented to the procedure. The mini L43K transducer with a frequency range of $12-2 \mathrm{MHz}$ and a footprint of $26 \mathrm{~mm}$ (L43K Hitachi Aloka Medical, Ltd., Japan) was deployed via a $10 \mathrm{ml}$ port following initial dissection of the anterior prostate and ligation of the dorsal vein complex. The transducer head was gripped with the robotic Prograsp $\mathrm{p}^{\mathrm{TM}}$ instrument via a purpose built clip such that scanning ensued without obscuring views from the optics. The flat face of the linear transducer was manipulated with its flexible platform to permit real-time visualization of the neurovascular bundles. In the trapezoid mode the transducer created a wide field of view with a large frequency range ensuring good image quality. Blood flow mapping using eFLOW mode offered fine spatial resolution and increased sensitivity. The transducer also permitted Real-time Tissue Elastography (RTE) providing information on tissue stiffness during the surgical procedure. The neurovascular bundle was dissected with the aid of real-time imaging sent via the built in da Vinci Surgical Systems platform.

Results: The case was successfully completed with preservation of erectile function upon review with no complications and with no discernible increase in the standard operative time.

Conclusions: Real-time doppler ultrasound aided nerve sparing with tandem use of a mini transducer could act as a useful supplementary tool in aiding objective localisation of the neurovascular bundles during RARP. Studies are currently schedule to further validate the efficacy of this novel platform.

MP27-14 No Suture Technique of "Renorraphy" for Laparoscopic Partial Nephrectomy: Novel Use of a New Fibrin Sealant Patch

C Bryson, D Singh

Yale New Haven Hospital

United States

Introduction \& Objective: Incidental renal tumor detection is increasing with the widespread use of cross-sectional imaging. If surgery is indicated and feasible, nephron-sparing removal of these tumors is preferable to preserve long-term renal functioning. The key clinical issue with partial nephrectomy is performing a safe and thorough cancer operation while minimizing the warm ischemia threat to the kidney and obtaining good hemostasis. To that end, fibrin sealant patches are agents made from purified human protein which can be directly applied to surgical sites to aid in hemostasis, and their use in intraabdominal surgery is growing. As of the initiation, of this study, this product was not FDA approved for renal use and this was undertaken as a novel, feasibility study. We describe a case series of nine patients who underwent a successful laparoscopic partial nephrectomy with the use of a fibrin sealant patch as the main hemostatic agent.

Materials and Methods: Nine patients underwent a laparoscopic partial nephrectomy for ten lesions which used a fibrin sealant patch for hemostasis intraoperatively by a single surgeon at a single institution. Their demographic data, warm ischemia 
time, operative time, estimated blood loss, tumor characteristics, secondary hemostatic agents, length of stay, and complications were retrospectively analyzed.

Results: A fibrin sealant patch alone was effective for hemostasis without any other agents in six cases. Two of these cases did use suture material to close the collecting system. The four remaining cases used argon beam, a surgical bolster, suture material, or other fibrin products to aid in hemostasis. No patients required blood products. The average lesion size was $2.6 \mathrm{~cm}$ (range 1.3$4.5 \mathrm{~cm}$ ). Seven of the lesions were $>50 \%$ exophytic. No lesions were entirely endophytic. The average clamp time was 12.2 minutes (range 7-19). Length of stay was on average 4.5 days (range 2-8). There were no postoperative complications related to the fibrin sealant patches.

Conclusions: In appropriately selected patients, this fibrin patch may be a useful adjunct which may reduce warm ischemia time while obtaining adequate hemostasis. This simple technique may also broaden the applicability of laparoscopic partial nephrectomy. Further research and evaluation is required to establish the use of this patch on a more diverse set of renal tumors.

MP27-15 Investigation of the artery sealing efficacy of newly introduced polymeric clips for minimally invasive surgery: an in vivo study and histologic evaluation

I Kyriazis, P Kallidonis, M Ozsoy, P Ntasiotis, D Kotsiris, T Vrettos, P Ravazoula, E Liatsikos

Department of Urology, University of Patras, Greece Greece

Introduction \& Objective: Histologic evaluation of the effect of the Click'aV®Plus and Click'aV®clips on the porcine arteries and veins. The Click'aV®Plus has a novel design in the integrated teeth interface and is available in extra-large size.

Materials and Methods: A pig was operated by a laparoscopic approach and a nephrectomy on the one side took place. During the nephrectomy, a Click'aV®Plus and a Click'aV® clips were placed on the renal artery with a few $\mathrm{mm}$ distance between them. Similarly the same clips were placed on the renal vein the same way. The kidney was removed and the pig was sacrificed 3 days later. The specimens were resected and underwent HematoxylinEosin staining. An experienced pathologist examined slices from the site of the clip and between the clips.

Results: The artery between the clips did not have any specific lesions except for some endothelial atrophy. The tissue surrounding the vessel has intermediate acute inflammation. Similar findings were observed for the Click'aV®clips. In the case of Click'aV®Plus, Intermediate acute inflammation tissue is present in the arterial wall and the surrounding tissue was noted. The vein between the clips had cellular damage at the muscular layer due to the presence of acute inflammation. The tissue surrounding the vessel has severe acute inflammation. The Click'aV®clip exhibited mild acute inflammation in the tissue surrounding the vessel. The Click'aV®Plus clip was related to necrosis of the vascular wall and intermediate acute inflammation of the tissue surrounding the vein. Intermediate inflammation was observed in the vascular wall.

Conclusions: The Click'aV®Plus clip is related to inflammatory reaction while the Click'aV®clip induced a less prominent reaction. These findings could be also related to the process of preparing the vessels intraoperatively. A longer follow-up time may provide a clearer perspective on the impact of the observed inflammation to the outcome of surgery and more importantly in the development of any adverse events related to the clip.
MP27-16 Utility of an Electronic Procedural Prostate Mapping Chart (e-PPMC ${ }^{\mathrm{TM}}$ ) in Robot Assisted Radical Prostatectomy

SS Kommu, A Samateh, P Rouse, J Wilson, SH Garnett, RA Persad, PD Rimington, (S Academic Research Group

Eastbourne District General Hospital: East Sussex Healthcare NHS Trust

United Kingdom

Introduction \& Objective: Robot Assisted Radical Prostatectomy(RARP) is a major operation involving complex surgical extirpation and reconstruction. Herein, we describe and test the utility of an individualized and specialized electronic platform that permits optimal communication of the patient's details throughout the journey of RARP using an Electronic Procedural Prostate Mapping Chart(e-PPMC $\left.{ }^{\mathrm{TM}}\right)$.

Materials and Methods: The e-PPMC ${ }^{\mathrm{TM}}$ is a novel platform which involves a detailed summary of the patient including performance status and preoperative histopathology superimposed onto a map of the prostate. The details are exhibited in a simple format for seamless communication at all stages of the patient's journey including intraoperative real-time dissection. The platform also depicts MRI and biopsy findings coupled with nomograms including those described by Partin and Briganti. Fifty RARP patients were enrolled utilizing the e-PPMC ${ }^{\mathrm{TM}}$. The individual e-PPMC ${ }^{\mathrm{TM}}$, which is a signature of the patient's disease process, was shown on an electronic tablet format. Utility was analyzed at three key stages of (A)time of consent for procedure,(B)intraoperatively and (C)follow-up clinic appointment.Patients were evaluated by questionnaires addressing key questions and the intraoperative utility was tested as part of a Fellowship Training Programme in RARP.

Results: In Stage A, all patients felt they understood their individual disease process and were able to explain their understanding of T3a and apical disease where applicable. They were able to also explain their understanding of Gleason Score and reason for or against lymph node dissection and nerve sparing. Those with significant apical disease and T3a disease were primed about the potential likelihood of positive margins. In Stage B, the intraoperative utility was put to test in a study involving the Robotic Fellow and two senior trainers. The fellow did not impact on total operative time (mean 135minutes), estimated blood loss (median $<50 \mathrm{mls}$ ), complication rate(One Clavien grade $\geq 2$ complications) nor hospital stay(median 28 hours). In Stage C, the patients who had positive margins were able to reproduce their understanding based on the e-PPMC ${ }^{\mathrm{TM}}$.

Conclusions: The e-PPMC ${ }^{\mathrm{TM}}$ is a useful supplementary tool for improved communication before, during and after RARP. The tool also aids in communicating final histological findings at the first clinic visit following the procedure. Furthermore, it can be used for helping to tailor the specific approach preoperatively. Studies are currently underway to couple the e-PPMC ${ }^{\mathrm{TM}}$ with 3-D printout of the prostate.

MP27-17 Perineal Approaching Robotic Radical Prostatectomy Our Experience: First 15 cases

V Tugcu, O Akca, A Simsek, I Yigitbasi, S Sahin, A Tasci

Bakirkoy Sadi Konuk Training and Research Hospital Center, Turkey

Turkey 
Introduction \& Objective: Minimally invasive techniques are increasingly being developed and preferred. Many new techniques have been developed in radical prostatectomy operations. Perineal approaching robotic radical prostatectomy is a new technique.

Materials and Methods: Between November 2016 and February 2017, 15 patients underwent perineal approaching robotic radical prostatectomy with 3 arms XI Da Vinci robotic system because of prostate canser.

Results: The mean age of the patients was $60,2 \pm 7,8$. The mean body mass index of the patients was found to be $28.8 \pm 1,9$. All patients had a Charlson score of 2. Four patients had previous major abdominal surgeries. Preoperative mean PSA value was found to be $7,3 \pm 2,4$. The mean prostate volume was $40,8 \pm 12,4$ cc. Preoperative pathology of a patient was Gleason $4+4$ adenocarcinoma and preoperative pathology of 5 patients was Gleason $3+4$. Preoperative pathology of the other patients was Gleason $3+3$ adenocarcinoma. We performed multiparametric magnetic resonance imaging for all patients. Multiparametric magnetic resonance imaging of the patient with preoperative pathology gleason $4+4$ adenocarcinoma was suspected to have right seminal vesicle involvement, whereas extraprostatic spreading was not detected on multiparametric magnetic resonance imaging of other patients. One patient underwent open surgery due to carbon dioxide retention. İn our series postoperative wound infection developed in the perineal region of a patient. Daily wound care was followed. No additional treatment needed. The mean time of postoperative catheterization was $9,4 \pm 3,1$ days. Six patients were full continent after urethral catheter removing. Continence recovery was $\% 66$ at end of first week, was \% 80 at the end of first month.. PSA recurrence was not detected in any postoperative patients.

Conclusions: Perineal approaching robotic radical prostatectomy is a technique that can be applied safely in experienced centers. Advantages include early mobilization and early return to daily life. There is a need for further research in this area.

MP27-18 Usefulness of Endowrist One Vessel Sealer instrument and Endowrist Clip Applier for da Vinci Surgical System in robot-assisted radical prostatectomy

K Tanaka, M Matsumoto, Y Takechi

Kita-Harima Medical Center

Japan

Introduction \& Objective: To evaluate the efficacy of Endowrist One Vessel Sealer instrument and Endowrist Clip Applier for robot-assisted radical prostatectomy (RARP), we performed retrospective analysis.

Materials and Methods: In 98 cases of RARP, we used Endowrist One Vessel Sealer for 34 cases (group 1) and Ligasure for 64 cases (group 2). We compared 2 groups in perioperative

Table 1 : Comparison of Endowrist One Vessel Sealer instrument and conventional method

\begin{tabular}{lccc}
\hline & Group 1 & Group 2 & P \\
\hline $\mathrm{N}$ & 34 & 64 & \\
Operative time (min) & $288.2 \pm 40.5$ & $299.2 \pm 54.3$ & 0.2719 \\
Console time (min) & $213.9 \pm 32.3$ & $225.8 \pm 47.1$ & 0.1959 \\
Estimated blood loss (ml) & $120.6 \pm 297.6$ & $123.9 \pm 157.9$ & 0.9412 \\
Positve surgical margin rate (\%) & 17.6 & 12.5 & 0.5498 \\
Number of lymphnode & $15.9 \pm 6.6$ & $10.2 \pm 6.7$ & $<0.01$ \\
\hline
\end{tabular}

Table 2 : Comparison of Endowrist Clip Applier and conventional method

\begin{tabular}{lccc}
\hline & Group 1 & Group 2 & $\mathrm{p}$ \\
\hline $\mathrm{N}$ & 15 & 19 & \\
Operative time (min) & $284.9 \pm 37.9$ & $298.2 \pm 51.4$ & 0.4088 \\
Console time (min) & $210.5 \pm 32.3$ & $229.1 \pm 54.8$ & 0.2568 \\
Estimated blood loss (ml) & $60.5 \pm 88.6$ & $134.4 \pm 141.7$ & 0.0873 \\
Positve surgical margin rate (\%) & 26.7 & 10.5 & 0.3696 \\
\hline
\end{tabular}

parameters and pathological results. In 34 nerve-sparing cases, we used Endowrist Clip Applier for 15 cases (group 3) and laparoscopic clip applier for 19 cases (group 4). We compared group 3 and group 4 in perioperative parameters and pathological results.

Results: There was no significant difference in operative time, console time, estimated blood loss and positive surgical margin rate between group 1 and group 2 (Table 1). The number of lymphnode was significant higher in group 1 . There was either no significant difference in operative time, console time, estimated blood loss and positive surgical margin rate between group 3 and group 4 (Table 2).

Conclusions: Endowrist One Vessel Sealer instrument and Endowrist Clip Applier were equal to conventional method in perioperative data in RARP. As for lymphnode dissection, Endowrist One Vessel sealer instrument seemed to be better than the conventional method.

MP27-19 Percutaneous Externally Assembled Laparoscopic Versus Traditional Laparoscopic Instruments: A Prospective Study of Cosmesis and Pain

I Kelly, M Pierce, S Abourbih, H Wagner, J Cheng, DD Baldwin

Loma Linda University

United States

Introduction \& Objective: A novel Percutaneous Externally Assembled Laparoscopic (PEAL) paradigm was developed to decrease incisional pain and laparoscopic scars. The aim of this study is to compare post-operative cosmesis and pain between the PEAL instrument site and traditional 5 and $12 \mathrm{~mm}$ port sites. Materials and Methods: Six healthy kidney donors consented to use of the PEAL instruments for laparoscopic donor

\begin{tabular}{|l|c|c|}
\hline & Mean & $\begin{array}{c}\text { p-value Compared } \\
\text { to PEAL }\end{array}$ \\
\hline Patient Rated Cosmesis & & - \\
\hline PEAL Site & 10.0 & $<0.01$ \\
\hline 5 mm Port Site & 8.8 & $<0.01$ \\
\hline 12 mm Port Site & 8.8 & $<0.01$ \\
\hline Hand Assist Site & 7.0 & \\
\hline Physician Rated Cosmesis & & - \\
\hline PEAL Site & 10.0 & 0.03 \\
\hline 5 mm Port Site & 8.0 & $<0.01$ \\
\hline 12 mm Port Site & 7.0 & $<0.01$ \\
\hline Hand Assist Site & 4.3 & \\
\hline Scar Size & & - \\
\hline PEAL Site & 3.2 & 0.02 \\
\hline 5 mm Port Site & 14.3 & $<0.01$ \\
\hline 12 mm Port Site & 21.3 & $<0.01$ \\
\hline Hand Assist Site & 59.7 & \\
\hline
\end{tabular}

Table 1: Cosmesis and Scar Size Comparison 
nephrectomy from July 2016 to March 2017. Nephrectomies were performed using the hand-assisted technique with a PEAL instrument replacing one of the traditional laparoscopic ports, along with an additional $12 \mathrm{~mm}$ working port and a $5 \mathrm{~mm}$ port for the laparoscopic camera. The primary outcome was post-operative comparative cosmesis between scars as determined by the patient and surgeon, with each patient acting as their own control. Secondary outcomes included scar length and immediate postoperative pain. Cosmesis was rated using a Likert scale from 1 to 10 with 10 being the best cosmesis. Pain was rated on a numeric rating scale from 0 to 10 , with 10 being the worst pain. One-way ANOVA was calculated with a p-value $<0.05$ considered significant.

Results: Each patient had a $2.96 \mathrm{~mm}$ PEAL instrument site, $5 \mathrm{~mm}$ and $12 \mathrm{~mm}$ traditional laparoscopic ports, and an $8 \mathrm{~cm}$ periumbilical midline hand-assist incision allowing a direct comparison of relative pain and cosmesis without inter-patient variability. There were no complications. At mean follow-up of 6.8 months, both patients and surgeon significantly preferred the PEAL site as the most cosmetically appealing, followed by the $5 \mathrm{~mm}, 12 \mathrm{~mm}$, and hand-assist sites, (Table 1). Scar length was also significantly smaller for the PEAL site compared to each other incision (Table 1) Additionally, immediate post-operative pain was lower at the PEAL site, followed by the $12 \mathrm{~mm}, 5 \mathrm{~mm}$, and hand-assist ports, respectively, but this did not reach significance (2.0, 4.3, 4.0, and 5.3, respectively).

Conclusions: The PEAL incisions show significantly improved cosmesis and scar size compared to traditional 5 and $12 \mathrm{~mm}$ laparoscopic ports. Use of PEAL instruments will reduce the invasiveness of laparoscopic surgery.

MP27-20 A Pilot Experience in Using A Digital App To Follow-Up Prostate Cancer Patients in Shropshire, UK

Y Phan, A Loh, A Anandakumar, S Umranikar, A Elves

Royal Shrewsbury Hospital

United Kingdom

Introduction \& Objective: It is not uncommon for patients with cancer to experience physical, mental and social distress, forming a significant burden that has a negative impact on their quality of life. We have piloted a digital app called VitruCare in our hospital in order to address these issues in patients with prostate cancer. More importantly, the app also serves as a communication tool between the hospital medical team and the patients.

Materials and Methods: Patients with prostate cancer were invited to use VitruCare in our pilot study. 53 users were followed prospectively. Data on various domains such as "My Goals", "My Lifestyle", "My Priorities", "My Diaries", and "How Do I Feel Today" were analysed retrospectively.

Results: The users of this application have a median age of 72.5 years old. $14 \%$ have nodal or bone metastasis, and median time since treatment is 48 months. $60 \%$ have completed the lifestyle questionnaire and "How Do I Feel Today" trackers. $20 \%$ of the users who completed the lifestyle questionnaire reported anxiety. $42 \%$ have used the diary function and $47 \%$ have used the secured messaging function. Usage of the lifestyle questionnaire, "How Do I Feel Today" trackers, secured messaging and diary functions does not appear to be age related. Patients who have been treated and further away from treatment in time are more likely to be used the app.
Conclusions: The level of engagement in this pilot study reflects the willingness of patients to utilize this innovative app that has the potential to monitor the well-being of patients with prostate cancer out with the constraints of a fixed clinic appointment.

MP27-21 Impact of a Novel Wireless Hands-Free Audio System on Communication during Robotic Surgery: A Prospective Randomized Comparison

A Thomas, M Keheila, J Thomas, B West, B Mattison, D Ruckle, M Pierce, P Hogue, J W. Cheng, H Wagner, S Abourbih, DD Baldwin

United States

Introduction \& Objective: Intraoperative miscommunication is one of the most frequently identified causes of medical errors. Miscommunication may occur in a robotic surgery where room noise is high and current robotic communication systems may be hampered by disruptive feedback. The purpose of this study was to compare the efficacy of a novel wireless communication system (WCS) to the traditional commercially available robotic communication system.

Materials and Methods: Five subjects were randomly positioned in a simulated robotic surgery at the robotic console, assistant console, circulating nurse, anesthesia workspace, and bedside assistant positions. 120 surgical phrases were read from the primary robotic console using the standard Da Vinci Si speakers and 120 phrases were also read through the WCS. The reader was blinded to the system in use. The primary endpoint was the number of correctly recorded phrases. Secondary endpoints included the clarity and effectiveness of the systems which were evaluated using a Likert scale (1-10). Statistical analysis was performed with a $\mathrm{p}<0.05$ considered significant.

Results: A total of 240 phrases were recorded. The WCS had significantly increased number of correct phrases compared to the Da Vinci Si speakers at the bedside assistant (96 vs. 78 total correct; $\mathrm{p}=0.009$ ), the anesthesia workspace (101 vs. 69 total correct; $\mathrm{p}<0.001$ ), and the circulating nurse positions (96 vs. 71 total correct; $\mathrm{p}<0.001)$, respectively. The assistant robotic console did not display any significant differences between the two systems $(p=0.43)$. The assistant robotic console had significantly increased number of correct phrases compared to the bedside position, anesthesia workspace, and circulating nurse position during use of the conventional robotic communication system ( $p<0.05$ for all). In contrast, there were no significant differences in the number of correct phrases recorded between any positions when using the WCS. The median Likert scores for overall clarity and effectiveness were both higher for the wireless system compared to the conventional speaker system ( 7 vs. 4 for both, respectively $\mathrm{p}<0.05$ ).

Conclusions: The use of the WCS resulted in enhanced accuracy, clarity and effectiveness of communication between the console surgeon and all operating room positions except the assistant robotic console position. Implementation of a similar system could reduce the number of miscommunication events and improve the safety and efficiency of robotic surgery.

MP27-22 A Robotic Machine-assisted Transperineal Prostate Biopsy System - Safety profile review

J Kwok, D Yong, C Wong, K Chong

National Healthcare Group - Tan Tock Seng Hospital Singapore 
Introduction \& Objective: We review the safety profile of a robotic machine-assisted ultrasound system for performing transperineal prostate biopsies that allows stability and accurate localization of up to $1 \mathrm{~mm}$. This localisation allows accurate rebiopsy for cancer active surveillance, accuracy to surgical planning for nerve-sparing radical prostatectomy, and open doors to accurate focal therapy options.

Materials and Methods: The AmaKris-SR1 robotic machineassisted ultrasound-guided system developed by Abacus Global Technology Pte Ltd uses standard 18G biopsy needles for transperineal entry. It is coupled with transrectal ultrasoundguided prostate imaging with specific 3D spatial localization in $\mathrm{x}, \mathrm{y}, \mathrm{z}$ co-ordinates with mapping of the prostate. Biopsy needle insertion is done by the procedurist to obtain prostate biopsies with accurate automated localization ( $\mathrm{x}, \mathrm{y}, \mathrm{z}$ coordinates to within $1 \mathrm{~mm}$ ), with the aid of robotic assistance helping to position the trajectory of the needle. (Diagram 1) After institutional ethics board approval, between 18th April 2016 and 4th October 2016, patients enrolled were followed up for 30-day post-biopsy for complications to evaluate its safety profile.

Results: Gross hematuria occurred in 6 of 19 patients (31\%) which were self-limiting, and did not require bladder washouts or blood transfusions. Acute retention of urine (ARU) occurred in two patients $(10.5 \%)$, of which one patient underwent concurrent cystolitholapaxy. One patient developed sepsis (5.26\%) and was managed in the general ward setting. He did not have hypotension or cardiovascular sequelae, and was discharged well. Significantly, there were no reports of rectal bleeding or hematospermia.

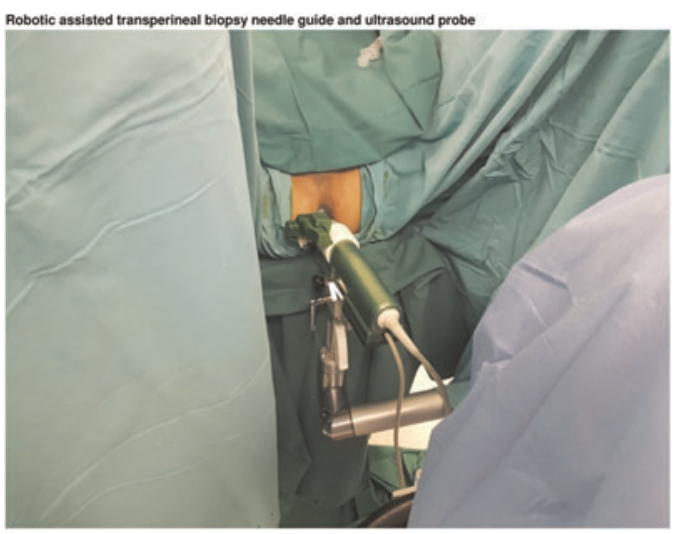

Conclusions: In this small pilot study, there was no rectal bleeding or hematopermia. Similar rates of ARU and selflimiting gross hematuria occurred. Due to a small sample size, one case contributed to our sepsis rate, which may not be representative. This is compared to internationally reported sepsis rates of 0.3 to $3.1 \%$. With this automated robotic-assisted targeting device, its safety profile is acceptable to be used for more accurate prostate biopsies with significant applications in prostate cancer detection and management.

\section{MPS28: LAPAROSCOPIC AND ROBOTIC SURGERY (BENIGN)}

\section{MP28-1 Robotic Surgery for Benign Urologic Conditions}

S Chopra, R Azhar, L Medina, R Sotelo, PW Mekhail, A Abreu, G Cacciamani, S Nagaraj, C Beckler, J Cai, M Desai, M Aron, IS Gill, A Berger

\section{USC DEPARTMENT OF UROLOGY United States}

Introduction \& Objective: To describe our experience using robotics for the treatment of benign urologic conditions.

Materials and Methods: Robotic urologic procedures from 2010-2013 were retrospectively analyzed $\mathrm{f}$. In total, 1645 robotic urologic procedures were identified in our prospectively maintained databases during this time frame. Cases of interest were then classified as benign/non-malignant based on the final pathology report and/or diagnosis of a benign disease (e.g., sacrocolpopexy). Robotic cases of interest included the following: adrenalectomy, pyeloplasty, renal artery aneurysm repair, simple nephrectomy, ureteral reimplant, ileal ureter, sacrocolpopexy, and simple prostatectomy.

Results: A total of 196 cases were classified as benign. A total of 193 cases ( $11.7 \%$ of the 1645 robotic cases) were included in the analysis. Nine adrenalectomies, 75 robotic pyeloplasties, 42 robotic simple prostatectomies, 32 simple nephrectomies, 19 robotic sacrocolpopexies, 10 robotic ureteral reimplants, 4 renal artery aneurysm repairs, and 2 robotic ileal ureters were included in this study. Three cases were con- verted to open or pure laparoscopic approach. One $(0.5 \%)$ intra-operative blood transfusion was required. All surgical specimens were benign on final pathology. Complication rate was $8.6 \%(n=16)$ within 30 days and $3.8 \%(n=7) 30$ 90 days after surgery. Follow-up ranged from 3 month to 48 months. At the final follow up visit, all patients reported to be doing well and had no complaints concerning their urologic condition.

Conclusions: Our recent data demonstrate that robotic surgery is a safe and feasible alternative surgical option for treating patients with benign urologic conditions.

MP28-2 Ease of suturing during robotic assisted pyeloplasty and reimplantation impacts total operative time: Results of a prospective study on the learning curve of robotic pediatric urology procedures

N Stern, R Clark, A Shukla, S Dave

Western University

Canada

Introduction \& Objective: The transition to robotic assisted procedures in pediatric urology leads to an increase in operative times, which impacts health care costs and resources. Attempts to 


\begin{tabular}{|c|c|c|}
\hline \multicolumn{3}{|l|}{ Table 1. Baseline Characteristics } \\
\hline & Robot Assisted Ureteral Reimplantation & Robotic Assisted Pyeloplasty \\
\hline $\begin{array}{c}\text { Number of } \\
\text { patients }\end{array}$ & 27 & 19 \\
\hline $\begin{array}{c}\text { Gender } \\
\text { (male:female) }\end{array}$ & $20: 7$ & $2: 17$ \\
\hline $\begin{array}{c}\text { Mean age } \\
\text { (months) }\end{array}$ & $95.6(6-222)$ & $61.5(6-129)$ \\
\hline $\begin{array}{c}\text { Mean follow up } \\
\text { (months) }\end{array}$ & $23.9(6-36)$ & $23.8(6-39)$ \\
\hline Success rate (\%) & 100 & 100 \\
\hline \begin{tabular}{c} 
Complications \\
\hline
\end{tabular} & $\begin{array}{c}\text { One Urinoma requiring nephrostomy and } \\
\text { prolonged stenting, One post-op UT1 }\end{array}$ & $\begin{array}{c}\text { One Delayed ureter injury requiring stenting. } \\
\text { One intraoperative acute kidney injury }\end{array}$ \\
\hline
\end{tabular}

\begin{tabular}{|c|c|c|c|c|c|c|}
\hline \multicolumn{10}{|c|}{ Table 2. Operative times } \\
\hline & Robot Assisted Ureteral Reimplantation & \multicolumn{3}{|c|}{ Robotic Assisted Pyeloplasty } \\
\hline Mean time (min) & First quartile & Last quartile & Average & First quartile & Last quartile & Average \\
\hline $\begin{array}{c}\text { Total operative } \\
\text { time }\end{array}$ & 211.4 & $160.4^{*}$ & 178.2 & 218.2 & 159 & 188.8 \\
\hline $\begin{array}{c}\text { Port placement } \\
\text { and closure }\end{array}$ & 46.9 & $35.5^{*}$ & 42.2 & 41 & 34.6 & 37.8 \\
\hline Dissection & 36 & 29.7 & 31.2 & 80.6 & $42.4^{*}$ & 42.9 \\
\hline $\begin{array}{c}\text { Dismember pelvis } \\
\text { and ureter } \\
\text { spatulation }\end{array}$ & 18.6 & 15 & 16.48 & - & - & - \\
\hline $\begin{array}{c}\text { Submucosal tunnel } \\
\text { creation }\end{array}$ & -- & - & - & 39.8 & 32.4 & 38.6 \\
\hline Suturing & 110 & $65.6^{*}$ & 79.1 & 80.6 & $42.4^{*}$ & 60.1 \\
\hline $\begin{array}{c}\text { Resident console } \\
\text { time }\end{array}$ & 5.3 & $18.9^{*}$ & 16.6 & 0 & $19.8^{*}$ & 16.2 \\
\hline
\end{tabular}

reduce the learning curve of robotic surgery in adults have focused on structured training to improve operative times at each step, but this work has not extended to pediatric urology. The objectives of this study are to investigate the learning curve and operative times of a single surgeon transitioning from laparoscopic pyeloplasty to robotic assisted pyeloplasty (RAP) and from open ureteric reimplantation to robotic assisted extra-vesical ureteral reimplantation (RUR) and to determine where efficiency gains are found early in the learning curve.

Materials and Methods: This prospective cohort study includes all RAP and RUR procedures performed using a 3-port technique between July 2013-December 2016. Both operations were sectioned into discrete operative steps: ports insertion and closure, dissection of the ureteropelvic junction (RAP) or ureter (RUR), dismemberment of the renal pelvis and spatulation (RAP) or creation of the submucosal tunnel (RUR), ureteropelvic anastomosis (RAP) or closure of the submucosal detrusor tunnel (RUR) recorded by a trained unbiased coder. The primary outcome was the trends of total and step specific operative times. Success was defined as resolution or significant decrease in the grade of hydronephrosis for the RAP group and resolution of UTIs off antibiotic prophylaxis or negative VCUG in the RUR group. Operative times were compared using student's t-test by the first and last quartiles.

Results: Please see attached tables

Conclusions: A reduction in intracorporeal suturing time leads to the greatest efficiency gain when transitioning to RAP and RUR. At our center, despite a relatively low volume of robotic cases (14/year), this efficiency was achieved within the first 20 procedures for both RAP and RUR. This study will aid the design of structured residency training programs to improve the operative learning curve for novice surgeons initiating RAP and RUR.
MP28-3 Laparoscopic management of complicated urachal remnants

H Jeong

Department of Urology, Wonkwang University School of Medicine

Republic of Korea

Introduction \& Objective: Managing persistent and symptomatic urachal anomalies requires wide surgical excision of all anomalous tissue with a cuff of bladder tissue via the open approach. We report our 7 cases with laparoscopic complete removal of symptomatic urachal remnants with or without a cuff of bladder tissue expecting a lesser invasiveness, lower morbidity and report the feasibility of this approach with the efficacy and outcomes.

Materials and Methods: Seven patients with a mean age of 36.5 years who had symptomatic urachal diseases performed laparoscopic excision between July 2004 and July 2012. Using four ports, the urachal remnant was dissected transperitoneally and then removed via the umbilicus port. The clinical results of laparoscopic urachal remnant excision as a minimal invasive surgery, the perioperative records and pathologic results were evaluated retrospectively

Results: There were no intraoperative or postoperative complications. Mean surgery time lasted 2.7 hours. Mean hospital stay was 14.6 days. The patients with bladder cuff resection had a longer admission, Foley catheterization period (14.4, 11 days). Pathological evaluations were 6 cases of infected urachal cyst, 1 cases of infected urachal sinus, 1 case of urachal adenocarcinoma. We have not found postoperative complications including any symptom recurrence with or without infection, voiding difficulty during a mean follow-up of 46.3 months.

Conclusions: The perioperative surgical outcomes achieve infection control and symptomatic relief, and additionally good cosmesis. Laparoscopic complete removal of symptomatic urachal remnants with or without a cuff of bladder tissue seems to be a safe, effective, better cosmetic alternative adding the advantages of a minimally invasive approach.

MP28-4 Three Dimensional Laparoscopic Ureterolithotomy: New Treatment Option for Large Upper Ureteral Stones

\section{J Kang, J Jeon}

Chinjujeil hospital

Republic of Korea

Introduction \& Objective: We compared the outcomes of two dimensional (2D) laparoscopic ureterolithotomy versus three dimensional (3D) laparoscopic ureterolithotomy in patients with an upper ureteral stone larger than $10 \mathrm{~mm}$.

Materials and Methods: Between March 2013 and June 2016, a total of 102 patients underwent transperitoneal laparoscopic ureterolithotomy for upper ureteral stone larger than $10 \mathrm{~mm}$. In all cases, ureteroscopic stone removal was attempted, however they were not successful. Sixty-eight of them underwent two dimensional (2D), while 34 underwent three dimensional (3D) laparoscopic ureterolithotomy. Patient demography, overall success rate, intraoperative blood loss, mean operation time, duration of hospital stay and complications were compared.

Results: Significant differences were not observed in terms of stone size (1.7 vs $1.6 \mathrm{~cm}, \mathrm{p}=0.2)$, overall success rate $(99 \%$ vs $97 \%, \mathrm{p}=1.0)$, hemoglobin change (1.0 vs $0.8, \mathrm{p}=0.4)$ and 
duration of hospital stay (3.1 vs 3.0 days, $p=0.5$ ). No blood transfusion and abdominal organ injuries were reported in both groups. However, there was statistically significant difference in the mean operation time ( 140 vs 95 minutes, $\mathrm{p}<0.05)$.

Conclusions: Three dimensional (3D) laparoscopic ureterolithotomy is effective and safe method of operation in the treatment of upper ureteral stones. It can reduce the operation time compared to two dimensional (2D) laparoscopic ureterolithotomy.

MP28-5 Comparison of Umbilicus-sparing laparoscopic approach Versus Open approach for treating symptomatic Urachal remnants in adults

J Hu, Z Liu

China (People's Republic)

Introduction \& Objective: The traditional surgical approach for removing a symptomatic urachal remnant is via a lower midline laparotomy and infraumbilical incision or a laparoscopic approach with umbilicoplasty. We reviewed our experience with umbilicus-sparing laparoscopic urachal remnant excision in a single-center study and evaluated its efficacy and outcomes versus open approach.

Materials and Methods: This study was a retrospective study. Between March 2012 and September 2016, thirty-two consecutive patients with symptomatic urachal remnants who underwent the umbilicus-sparing laparoscopic approach $(n=17$, USLA group) or open approach ( $\mathrm{n}=15$, OA group). The efficacy, recovery, and long-term outcomes were reviewed.

Results: The clinical characteristics of the patients in each group, such as age, gender, BMI index and disease type had no significant differences $(p>0.05)$. No significant difference was found in the surgical procedure times $[76 \mathrm{~min}(53 \sim 113 \mathrm{~min})$ versus $69 \mathrm{~min}(49 \sim 103 \mathrm{~min}), \mathrm{p}=0.089]$ and intraoperative blood loss [29.4 $\mathrm{mL}(15 \sim 70 \mathrm{~mL})$ versus $32.2 \mathrm{ml}(20 \sim 75 \mathrm{ml})$, $\mathrm{p}=0.068$ ] between the USLA groups and OA groups. But the mean postoperative hospital stay [3.6day ( $3 \sim 8$ day)) versus 5.1day ( $4 \sim 9$ day), $\mathrm{p}=0.021]$ and the time of full recovery [12.9day ( $8 \sim 18$ day) versus 16.8 day $(11 \sim 23$ day $), p<0.01$ ], the USLA group were both significantly shorter than that of the OA group. Incisional infection was postoperative observed in one of the 15 patients $(6.7 \%)$ after receiving open approach. No infected recurrence and malignant transformation had occurred at a mean follow-up of 32.1 months and 33.7 months in USLA groups and OA groups, respectively.

Conclusions: To minimize the morbidity of radical excision, umbilicus-sparing and postoperative recovery, laparoscopic management of benign urachal remnants in adults is a safe and efficacious alternative with superior cosmetic outcomes compared to an open approach or umbilicoplasty.

MP28-6 Transperitoneal Laparoscopic Pyeloplasty for Retrocaval Ureter: Single Surgeon's Experience and Literature Review

T Oh, J Lee, S Park, I Seo

Republic of Korea

Introduction \& Objective: We present surgical techniques and operative results of laparoscopic pyeloplasty for patients with
Table 1. Results of Published Papers for Retrocaval Ureter: Clinical and Laparoscopic Parameters

\begin{tabular}{|c|c|c|c|c|c|c|c|c|c|c|}
\hline & No. pts & $\begin{array}{l}\text { Mean } \\
\text { age } \\
\text { (years) }\end{array}$ & $\begin{array}{l}\text { Sex } \\
\text { (malef } \\
\text { fermale) }\end{array}$ & $\begin{array}{l}\text { Pits with } \\
\text { Flank pain }\end{array}$ & Approach & $\begin{array}{l}\text { Op time } \\
\text { (minuses) }\end{array}$ & $\begin{array}{l}\text { slood loss } \\
\text { (mel) }\end{array}$ & \begin{tabular}{|l|} 
Complic \\
stion
\end{tabular} & $\begin{array}{l}\text { Hospied } \\
\text { stay } \\
\text { (dersy) }\end{array}$ & $\begin{array}{l}\text { Success } \\
\text { rase (x) }\end{array}$ \\
\hline Liv E et al. & 9 & \begin{tabular}{ll|}
344 & \\
$(27-53)$. & \\
\end{tabular} & $6 / 3$ & 9 & $R P$ & $\begin{array}{l}103 \\
189 \cdot 110)\end{array}$ & $\begin{array}{l}\text { Not } \\
\text { Neported }\end{array}$ & No & $\begin{array}{l}7 \\
(6.9)\end{array}$ & 100 \\
\hline $\begin{array}{l}\text { Ding GQ et } \\
\alpha\end{array}$ & 9 & $\begin{array}{l}35 \\
(19-47)\end{array}$ & $9 / 0$ & 6 & $T P$ & $\begin{array}{l}135 \\
00-250)\end{array}$ & $\begin{array}{l}\text { Minimal } \\
(<60) \text {. }\end{array}$ & \begin{tabular}{l|}
1 (urine \\
leakage)
\end{tabular} & $\begin{array}{l}7.3 \\
(7-10)\end{array}$ & 100 \\
\hline Chen $\mathrm{S}$ et at & 5 & $\begin{array}{l}29.4 \\
16-47\end{array}$ & $4 / 1$ & 5 & RP & $\begin{array}{l}902 \\
(81-126)\end{array}$ & $\begin{array}{l}383 \\
(21-50)\end{array}$ & No & 6.5 & 100 \\
\hline Chen $Z$ et at & 12 & $\begin{array}{l}35 \\
\text { a9-45). }\end{array}$ & $8 / 4$ & 10 & RP & $\begin{array}{l}112 \\
(89 \cdot 158)\end{array}$ & $\begin{array}{l}35 \\
(21-60)\end{array}$ & No & 6 & 100 \\
\hline Li HZ en al. & 10 & $\begin{array}{l}31 \\
(9-48)\end{array}$ & $6 / 4$ & 7 & No & $\begin{array}{l}82 \\
(60-110)\end{array}$ & $\begin{array}{l}\text { Manimal } \\
(<10) .\end{array}$ & No & $\begin{array}{l}6.5 \\
(5-7)\end{array}$ & 100 \\
\hline$X_{u}$ Df et at. & 7 & $\begin{array}{l}38 \\
\text { (18.50) }\end{array}$ & 70 & 6 & RP & $\begin{array}{l}128.6 \\
(97-189)\end{array}$ & $\begin{array}{l}20 \\
\text { a15-50). }\end{array}$ & No & $\begin{array}{l}6.5 \\
(5.92)\end{array}$ & 100 \\
\hline $\begin{array}{l}\text { Simfoecosh N } \\
t \pi \text { al }\end{array}$ & 6 & \begin{tabular}{l|l|}
31 & \\
$(16-50)$ \\
\end{tabular} & $3 / 3$ & 4 & TP & $\begin{array}{l}180 \\
a 50-2100\end{array}$ & $\begin{array}{l}\text { minimal } \\
\text { (< so) }\end{array}$ & No & 4 & 100 \\
\hline Our study & 7 & \begin{tabular}{l|l|}
3633 \\
$(16-66)$
\end{tabular} & $5 / 2$ & 5 & $T P$ & $\begin{array}{l}1718 \\
(97-240)\end{array}$ & $\begin{array}{l}1843 \\
(33-332 .\end{array}$ & No & $\begin{array}{l}9.2 \\
(6-14)\end{array}$ & 100 \\
\hline Total & 65 & $\begin{array}{l}34.0 \\
(9-66)\end{array}$ & $48 / 17$ & 52 & $\begin{array}{l}\text { RP 43 } \\
\text { TP 22 }\end{array}$ & $\begin{array}{l}1222 \\
(60-250)\end{array}$ & & & $\begin{array}{l}6.6 \\
(3 \cdot 14)\end{array}$ & 100 \\
\hline
\end{tabular}

retrocaval ureters in a single surgeon's experience, and review similar papers.

Materials and Methods: Seven patients with retrocaval ureters were enrolled in this study from April 2005 to October 2016. The mean age of 5 males and 2 females was 36.3 years old. The mean body mass index was 22.1. The chief complaint was flank pain ( $\mathrm{n}=5$ patients); the remaining cases were detected incidentally. All patients showed hydronephrosis and typical fish-hook deformity of the retrocaval ureter on imaging studies. Three patients showed an obstructed pattern on the renal scan. A single surgeon performed laparoscopic pyeloplasties with transperitoneal approaches including double-J ureteral stent insertions. The operative and follow-up results were checked and compared with published papers.

Results: All operations were successfully completed without conversion to open surgery. The mean operative time was 171.8 minutes (range, $97-240$ minutes). The estimated blood loss was $184.3 \mathrm{~mL}$ (range, 50-332 mL). No operative complications were encountered. There were no obstruction and symptom after the mean follow-up of 16.3 months (range, 6-30 months). We found 7 papers from PUBMED, which had more than five cases of laparoscopic reconstruction of the retrocaval ureter. We reviewed and summarized the clinical and operative parameters from the 7 papers and our data.

Conclusions: Our results show that transperitoneal laparoscopic pyeloplasty is a safe and effective treatment. Data from published papers and ours summarize clinical parameters of the retrocaval ureter, and suggest that the laparoscopic reconstruction can be considered as the standard treatment for the retrocaval ureter.

MP28-7 Clips offer a safe alternative to staples for vascular control of the renal vessels during laparoscopic donor nephrectomy

R Sowerby, T Kroczak, D Ghiculete, JY Lee, R D’A. Honey, K Pace, M Ordon

University of Toronto

Canada

Introduction \& Objective: Vascular control of the renal vessels during laparoscopic donor nephrectomy is controversial. Despite 
some authors concluding that using non-transfixion techniques (titanium or polymer locking clips) instead of transfixion techniques (staples or sutures) to control the renal artery is "both legally indefensible and morally intolerable" (1), many centres continue to use clips. The study objective was to review our 10year experience using clips to control the renal vessels and determine the safety of this practice.

Materials and Methods: We performed a retrospective review of all patients who underwent a laparoscopic donor nephrectomy at our centre (01/2007-08/2016). We recorded details of vascular control and complications. The primary outcome was complications or failures associated with vascular control of the renal vessels, which included clip dislodgement or crossing, conversion to open, additional procedures for bleeding, and blood transfusions. Secondary outcomes were changes in haemoglobin $(\mathrm{Hb})$ and warm ischemia time.

Results: We included 388 patients with 444 renal arteries and 393 renal veins. 374 renal arteries $(96.4 \%)$ were controlled with 3 titanium clips. There were $4(0.36 \%)$ events where one of the three titanium clips placed on a renal artery failed with none causing bleeding. These included 1 crossed clip requiring placement of a 4th titanium clip and 3 clip dislodgements requiring suturing distal to the clip (1), placing another titanium clip (1), and no intervention (1). 364 renal veins (93.8\%) were controlled with 2 polymer locking clips, with $1(0.27 \%)$ clip dislodgement causing no bleeding and requiring no intervention. There were no conversions to open or secondary procedures due to bleeding, blood transfusions, or donor deaths. Median warm ischemia time was 160 seconds and the median change in $\mathrm{Hb}(\mathrm{g} /$ L) was -18 (pre-operative to post-operative) and +1 (post-operative to discharge).

Conclusions: During laparoscopic donor nephrectomy, using 3 titanium clips on the renal artery(s) and 2 polymer locking clips on the renal vein(s) is safe and provides excellent vascular control. (1) Friedman AL, Peters TG, Ratner LE. Regulatory failure contributing to deaths of live kidney donors. American Journal of Transplantation. 2012 Apr 1;12(4):829-34.

MP28-8 Laparoscopic Donor Nephrectomy In Patients With A Circumaortic Left Renal Vein: Feasibility And Outcomes

A Hsieh, J Stites, G Portman, R Munver

Hackensack University Medical Center United States

Introduction \& Objective: Laparoscopic donor nephrectomy offers many advantages over open donor nephrectomy. However, the procedure is more technically difficult in patients with anomalous renal vasculature such as a left circumaortic renal vein (CARV). This study demonstrates our experience and results in patients with a circumaortic renal vein.

Materials and Methods: We retrospectively analyzed all laparoscopic donor nephrectomies performed by a single surgeon in patients with a CARV between 2003 and 2017. Study variables included operative time, estimated blood loss, warm ischemia time, length of hospitalization, donor complications, and graft function.

Results: A total of 10 patients with a circumaortic left renal vein underwent laparoscopic donor nephrectomy. The cohort included 6 males and 4 females with a mean age of 36.4 (range 28-51) and mean BMI of $29 \mathrm{~kg} / \mathrm{m}^{2}$ (range 25-33). The mean operative time was 239 min (range 187-315), estimated blood loss $127 \mathrm{~mL}$ (range 50-250), warm ischemia time $163 \mathrm{sec}$ (range 92-236), and length of hospital stay was 2.3 days (range 2-3). There were no conversions to an open procedure. There was 1 intraoperative complication that involved a stapler misfire on the retroaortic portion of the renal vein that was salvaged with intracorporeal suturing. There was 1 minor postoperative complication (Clavien I-II) that involved a $1 \mathrm{~cm}$ superficial skin separation at the extraction site that was managed conservatively. Graft function after 1 and 2 years was $100 \%$.

Conclusions: Laparoscopic donor nephrectomy performed in patients with a circumaortic left renal vein yields comparable results in terms of graft survival to the reported rates of donor nephrectomies with normal renal vasculature. In our experience, the presence of a circumaortic renal vein does not preclude renal donation as this procedure is a safe and effective method of kidney procurement in this patient population.

\section{MP28-9 Robotic Kidney Transplantation: Bakirkoy Ex- perience}

V Tugcu, S Sahin, AH Yavuzsan, I Yigitbasi, FG Akbay, S Apaydin

Bakirkoy Sadi Konuk Training and Research Hospital Center, Turkey

Turkey

Introduction \& Objective: As minimal invasive treatments develop, most urologists have preferred minimally invasive methods in recent years.Various authors proposed minimally invasive transplantation techniques and successful outcomes. we initiated robotic kidney transplantation (RKT) in our clinic in December 2015. In this report, we aimed to present the first 45 cases of a center with high robotic urology experience.

Materials and Methods: Starting from January 2016, we performed 45 RKTs in Bakirkoy Dr. Sadi Konuk Teaching and Research Hospital. Before surgery, the whole robotic procedure was thoroughly explained to the patient and informed consent was taken.

Results: We performed RKT in 28 male and 17 female patients. Mean patient age was 37.2 \pm 11.1 . Mean body mass index of the patients was $23.2 \pm 3.2$. Mean preoperative creatinine level was $5.8 \pm 1.8 \mathrm{mg} / \mathrm{dl}$. Mean preoperative dialysis durations ranged from 0 to 5 years. Mean operative time was $261.2 \pm 45.7$ minutes. Mean warm ischemia and re-warming durations were $1.83 \pm 0.5$ minutes and $52.51 \pm 17.89$ minutes. We used a mean of $218.8 \pm 3.8 \mathrm{cc}$ of ice slush. Mean blood loss was $184.4 \pm 53.2$ cc. Mean incision length was $5.16 \pm 0.7 \mathrm{~cm}$. We did not need any necessity for open conversion nor did not encounter any intraoperative complications. Two patients in our cohort suffered from ileus and had to be treated with exploratory laparotomy following consults of general surgeons; which happened to be because of paralysis caused by extensive use of ice slush. We decreased the amount of ice slush used and did not encounter any ileus following those two early operations. One of the most encountered complications, lymphocele, did not occur in our series. Mean drain withdrawal time was $3.42 \pm 0.8$ days. Patients had a hospital stay of $9.02 \pm 2.9$ days. At discharge, they had a creatinine level of a mean $1.6 \pm 0.89 \mathrm{mg} / \mathrm{dl}$ and glomerular filtration rate of $56.6 \pm 24.1$. Fourty four patients have a follow up of one month following surgery and they had a mean creatinine and glomerular filtration rate of $1.3 \pm 0.8 \mathrm{mg} / \mathrm{dl}$ and $69.4 \pm 23.7$, respectively. Thirty eight patients have a follow up of 6 months following surgery and they had a mean creatinine and glomerular filtration rate of $1.3 \pm 0.4 \mathrm{mg} / \mathrm{dl}$ and $68.4 \pm 22.9$, respectively. 
Conclusions: With transplantation being performed via a minimal invasive approach, more patients may be able to have kidney transplant and this important health issue may decrease significantly. However, complexity of this operation should limit the surgeons. One should have great robotic experience before attempting this kind of surgery.

MP28-10 Laparoscopic orchiopexy for bilateral high intra-abdominal testes in adult patients, single vs two stages

A hammady, M Elbadry

minia university

Egypt

Introduction \& Objective: management of the high intra abdominal testis laparoscopically is still a matter of controversy in pediatric as well as adult group of patients. Laparoscopic FowlerStephens orchidopexy (FSO) is performed either by single stage (clipping and division of the spermatic vessel and proceed for orchiopexy at the same time) or by two stages FSO (clipping and division of the spermatic vessel only and performing laparoscopic release of the testis along with orchiopexy 3-6 months later ). To study the outcome of Laparoscopic single stage and two stages FSO for the management of high intra abdominal testes in adult group of patients.

Materials and Methods: Our Study included 35 patients (70 testes), all of them had bilateral non palpable abdominal testes and laparoscopic surgical procedures (One stage FSO was done for 18 patients ( 36 testicles) and Two stages FSO was done for 17 patients (34 testicles) were performed at our department during the period between December 2014 and March 2016. Their mean age was 31.2 years. The comparative criteria include; Time of surgery, postoperative complications, and testicular viability.

Results: Testicular Doppler study revealed 2 atrophied testes in 2 different patients of one stage F.S.O. (5.5\%), one atrophied testis among two stages FSO group (5.8\%), this difference was statistically insignificant. The operative time difference was significant between both groups $(86.6 \pm 10.1 \mathrm{~min}$ for one stage vs $122.7 \pm 13.5$ min for two stages FSO).

Conclusions: Single-stage FSO avoids repeated anesthesia, the incidence of testicular atrophy is very comparable with twostages FSO and shorter operative time, making it more optimal than two stages FSO.

MP28-11 Rectourinary Fistulas: Laparoscopic and Robotic Management and Outcomes

R Sotelo, L Medina, FZ Husain, G Cacciamani, IS Gill

\section{USC DEPARTMENT OF UROLOGY}

United States

Introduction \& Objective: To discuss the management of rectourinary fistulas (RFs), and share our experience and outcomes of 30 patients using a minimally invasive approach.

Materials and Methods: The data of 30 patients who underwent treatment for RFs between 2001 to 2017 was retrospectively reviewed. The patients were managed by a single surgeon (RS). Follow-up ranged between 3 months and 15 years.

Results: Of the 30 cases, 3 patients had colovesical fistulas (CVFs), 19 had rectovesical fistulas (RVFs), and 8 had rectourethral fistulas
(RUF). For the colovesical group, (average age 47-year-old), all cases were a result of a complicated course of diverticulitis. all 3 cases were managed by performing a laparoscopic sigmoidectomy with fistula resection. There were no reported complications and all procedures had successful outcomes. In the RVF group, 2 patients were females (mean age of 69 years) in whom the fistula developed as a consequence of radiation treatments for uterine cancer. For the males in the RVF group (mean age of 64 years), $70.6 \%$ of fistulas (12 of 17 cases) occurred after prostatectomies. The remaining cases were due to low anterior colon resections ( 2 cases), intraoperative trauma (1 case), external beam radiotherapy (EBRT) (1 case), and inflammatory bowel disease (IBD) (1 case). Of these RVF patients, 5 had previous failed attempts at other institutions, all patients reached the criteria for successful repair with a transabdominal/transvesical approach. Two complications were reported (2 Urinary tract infections). In the RUF group patients (mean age of 73 years). Of these patients, $62.5 \%$ (5 cases) fistulas developed following surgical procedures: simple prostatectomy ( 1 case), transurethral resection of prostate ( 1 case), low colonic resections ( 2 cases), and perianal fistula correction (1 case).. In this group, 1 patient had previous failed repair attempts. Fistulas in the 3 remaining patients in the RUF group were a consequence of cryotherapy ( 2 patients) and high intensity focused ultrasound (HIFU) (1 patient). The choice in management was a robotic or laparoscopic prostatectomy with primary closure of the rectal defect and tissue interposition, normally using the omentum. One patient had a pelvic abscess that was drained and subsequently treated with prolonged antiobiotic therapy. The repair success rate in all patients was $100 \%$. Conclusions: Minimally invasive surgery can be used to perform rectourinary fistula repairs with excellent success rates with the proper training and experience.

\section{MP28-12 Report of pneumoscopic vesicovaginal fistula} repair.

H Choi, J Bae, H Chung, S Bae

Konkuk University School of Medicine

Republic of Korea

Introduction \& Objective: We report the feasibility and effectiveness of pneumovesicoscopic vesicovaginal fistula repair. Materials and Methods: The patient was 51 years old and undergone transabdominal hysteretomy with bilateral salpingo-oophrectomy 4 weeks ago. Cystoscopy was performed in modified lithotomy position using insufflation of gas and the fistula was inspected in detail. Fistulas were located superior to the trigone. A $5 \mathrm{~mm}$ endoscopic port was placed into the bladder under cystoscopic view in the midline between the umbilicus and pubic symphysis and two more working ports were placed $5 \mathrm{~cm}$ laterally. The vagina was packed with gauze to prevent gas leakage. A circum fistula incision was made by laparoscopic scissors and the bladder dissected away from the underlying vagina. The edges of the fistula were excised and after adequate dissection, the vagina was sutured vertically and the bladder sutured horizontally using 4-0 vicryl. The bladder was catheterised.

Results: The whole procedure was accomplished without intraoperative or postoperative complication. The operative time was 135 minutes, and blood loss was minimal. The patient was discharged 7 days after the operation and catheters were removed after 15 days following surgery. At follow-up visit after 3 months, no recurrence of VVF was noted. 
Conclusions: Pneumoscopic vesicovaginal fistula repair is technically safe and feasible with no recurrence of fistula. Considering the main advantages of less morbidity and safety in non peritoneal approach, our vesicoscopic technique may be an alternative option of surgical methods of vesicovaginal fistula.

MP28-13 Robot Assisted Transplant Allograft Nephrectomy Series: A Novel Approach for a Challenging Operation

R Nunez, S McAdams, HM Abdul-Muhsin, G Kelli, KL Stern, N Katariya, EP Castle

United States

Introduction \& Objective: Transplant nephrectomy has historically been performed in an open fashion by transplant surgeons and carries morbidity up to $50 \%$ with mortality up to $7 \%$. To date, there is a single reported case of robot assisted transplant allograft nephrectomy from a deceased donor kidney. We herein present our series of robotic assisted transplant nephrectomy (RTN) and provide a comparison to the open transplant

Materials and Methods: All patients who underwent robotic allograft nephrectomy at Mayo Clinic Arizona were included. Patients were not excluded for undergoing a concurrent procedure. All RTN were performed by a single Urologist (EPC) in conjunction with a single Transplant surgeon (NNK) via a transperitoneal approach utilizing a dual console Da Vinci Robotic Si/Xi surgical system. Study design was retrospective and observational. For comparison, we included a group of consecutive patients that have undergone open transplant nephrectomy (OTN) at our institution since 2011. Variables analyzed included: demographics (age, BMI, ASA), comorbidities, transplant related (time from transplant to transplant nephrectomy, living related or deceased donor transplants), operative variables (operative time, estimated blood loss and additional procedures performed) peri-operative variables (length of stay (LOS), drain duration, Foley catheter duration, and hemoglobin change), and 30-day Clavien-Dindo complications. All variables were analyzed by non-parametric tests with commercially available software (SPSS vs, 21, Chicago, Illinois).

Results: Six patients underwent RTN between 10/31/2014 until 4/31/2016 and twelve patients underwent OTN from May 2011 until April 2016. Patient demographics were similar for the two groups. The time from transplant to transplant nephrectomy was a median of 5.9 years (range: 0.3 - 40) for RTN and 3.4 years (range: $0.1-24$ ) for OTN. The majority of transplants were from deceased donors $(66 \%)$ in both groups. The operating time was longer for RTN with a median of 306 minutes (range: 178 - 532) compared to 91 minutes (range: 24 - 167) for the OTN group. Of note, in two of the six RTN cases bilateral laparoscopic native nephrectomies were performed and in a third case a robotic nephrectomy and a lymph node biopsy by plastic surgery was performed. When excluding patients with multiple procedures, the operative times for RTN were median 275 minutes (range: 178 - 292). In the RTN group there were no intraoperative complications or conversions to open nephrectomy. In the OTN group, there was one internal iliac injury. Estimated blood loss was less for RTN at median $150 \mathrm{~mL}$ (range: 100 - 400) compared to $250 \mathrm{~mL}$ (range: $25-2500$ ) for OTN, with transfusion rates of $16 \%$ and $34 \%$ respectively. Drains were utilized in $84 \%$ RTN and $66 \%$ of OTN cases, for a median of 2 days in each group. For RTN there were two major complications (two grade IIIa for small bowel obstruction) and one minor complication (grade II for blood transfusion). For OTN there were two major complications (two grade IIIb for return to OR) and four minor complications (grade II).

Conclusions: In this first reported series of robotic transabdominal allograft nephrectomy we demonstrate the safety and feasibility of the use of robotic technology for transplant nephrectomy. Although open transplant nephrectomy appears to be associated with a shorter operative time, robot assisted radical nephrectomy resulted in lower blood loss with a lower transfusion rate, and shorter LOS. This is a small series that includes our learning curve and we anticipate outcomes of the robotic approach to transplant nephrectomy will improve.

MP28-14 Prospective Evaluation of surgical and functional outcomes of Robot-assisted sacrocolpopexy and sacrocervicopexy in the management of vault prolapse

\section{S Arakere, D Dubey, RK Ahlawat, A Jain}

Fortis escorts kidney and urology institute India

Introduction \& Objective: To evaluate the long term surgical and functional outcomes following robotic assisted sacrocolpopexy and sacrocervicopexy.

Materials and Methods: This was a prospective study of 45 patients who underwent robotic sacrocolpopexy/sacrocervicopexy. Comparison of preoperative and 12-month postoperative objective and subjective assessments via the Pelvic Organ Prolapse Quantification (POP-Q), the Pelvic Floor Distress Inventory, Short Form 20 (PFDI-20); the Pelvic Floor Impact Questionnaire, Short Form 7 (PFIQ-7); and the Pelvic Organ Prolapse/Urinary Incontinence Sexual Questionnaire 12 (PISQ12) were made. Anatomical success was defined as POP-Q stage 0 or 1 at all postoperative intervals. To be considered a "clinical cure," a given patient had to have all POP-Q points $\leq 0$, apical POP-Q point $\mathrm{C} \leq 5$, no reported pelvic organ prolapse symptoms on the PFDI-20, and no reoperation for prolapse at all postoperative intervals.

Results: 40 patients completed the 1-year follow-up. The PFDI20 mean score improved from 94.4 at baseline to 30 at 12 months $(\mathrm{p}<0.0001)$; PFIQ-7 scores improved from 65 to 7 ( $\mathrm{p}<0.0001)$; and PISQ-12 scores improved from 34.3 to $36.6(\mathrm{p}<0.0001)$. The "anatomical success" rate was $85 \%$ and the "clinical cure" rate was $90 \%$.

Conclusions: Robotic assisted sacrocolpopexy and sacrocervicopexy are effective, efficient, and safe procedures.

MP28-15 Comparing Traditional Three Port and Single Site Laparoscopic Inguinal Hernia Repair in Children

C Tong, J Van Batavia, D Chu, A Srinivasan

Albert Einstein Medical Center

United States

Introduction \& Objective: In the US, 4\% of children are diagnosed with inguinal hernias. Laparoscopic herniorrhaphy has been considered comparable to the open technique. While traditional laparoscopic herniorrhaphy involves three separate 


\begin{tabular}{|c|c|c|c|}
\hline Variable & Single-Site $(n=44)$ & Three-Port $(n=23)$ & p-value \\
\hline Median age, years [IQR] & $5.52[3.00-7.53]$ & $5.14[2.88-7.51]$ & 0.98 \\
\hline Male:female & $41: 3$ & $22: 1$ & 1.0 \\
\hline \multicolumn{4}{|l|}{ Hernias } \\
\hline $\begin{array}{l}\text { Single, } \%(n) \\
\text { Bilateral, \% (n) }\end{array}$ & $\begin{array}{l}89 \%(39) \\
11 \%(5)\end{array}$ & $\begin{array}{c}100 \%(23) \\
0 \%(0)\end{array}$ & 0.16 \\
\hline Median duration of surgery, minutes [IQR] & $73[58-93]$ & $74.5[64.97 .5]$ & 0.02 \\
\hline Ap patients with postop follow-up, \% (n) & $68.2 \%(30)$ & $78 \%(18)$ & 0.57 \\
\hline Median follow-up, months [IQR] & $3.1[2.70-7.30]$ & $3.0[2.21-15.96]$ & 0.42 \\
\hline Immediate postop complaints, $\%$ (n) & $13.6 \%(6)$ & $0 \%(0)$ & 0.09 \\
\hline \multicolumn{4}{|l|}{ Intermediate adverse outcomes } \\
\hline Hernia recurrence, $\boldsymbol{\psi}(n)$ & $2.3 \%(1)$ & $0 \%(0)$ & 1.0 \\
\hline Contralateral hydrocele, $\%(n)$ & $0 \%(0)$ & $4.3 \%(1)$ & 1.0 \\
\hline \multicolumn{4}{|l|}{ Presence of bilateral descended testes at } \\
\hline Success, $\%(n)$ & $97.8 \%(43)$ & $95.7 \%(22)$ & 1.0 \\
\hline
\end{tabular}

skin incisions for ports, advancing technology has made single site (SS) laparoscopic herniorrhaphy possible. We thus sought to compare the safety and efficacy of traditional 3-port to SS laparoscopic herniorrhaphy.

Materials and Methods: We retrospectively reviewed our IRBapproved registry for initial laparoscopic herniorrhaphies at our tertiary care center between 4/2013 and 10/2016. Surgeries were performed by a single surgeon using the peritoneal leaflet closure technique. All surgeries were performed using the 3 port approach until mid-2014 when the SS port system was adopted. Outcomes of operative success, operative duration, and complications were compared using univariable tests.

Results: Of 67 children (63 boys) with median age 5.3 years (range 1.5-16.7), 3-port and SS laparoscopy were used in 23 $(34 \%)$ and $44(66 \%)$ children, respectively, including 5 children in the SS cohort who underwent bilateral herniorrhaphy. Operative success at a median follow-up of 3 months were $95.7 \%$ and $97.8 \%$, respectively, for the 3-port and SS groups $(\mathrm{p}=1.0)$. Median operative durations were 74.5 and 73 minutes for the 3 -port and SS groups, respectively $(\mathrm{p}=0.02)$. Postoperatively, the two groups had comparable postoperative complaints $(0 \%$ in the 3-port and $13.6 \%$ in the SS) and intermediate-term complications: 1 boy (4\%) in the 3-port group developed a contralateral hydrocele (despite a closed ring at surgery) and 1 boy ( $2 \%$ ) in the SS group had a hernia recurrence from inguinal floor weakness requiring open modified Bassini repair.

Conclusions: SS laparoscopic herniorrhaphy appears as safe and effective as the traditional 3-port system with comparable outcomes and complications. Success rate was greater than $95 \%$ in both groups.

MP28-16 The Use of Intraureteral and Intraurinary diversion Indocyanine Green During Robotic Ureteroenteric Reimplantation for the Management of Benign Anastomotic Strictures

Z Lee, A Asghar, B Waldorf, D Eun

Temple University Hospital

United States

Introduction \& Objective: Definitive surgical management of benign anastomotic strictures after radical cystectomy and ureteroenteric diversion formation is technically challenging given the reconstructed anatomy and reoperative surgical field. We describe the use of intraureteral and intraurinary diversion indocyanine green and subsequent visualization under near-infrared fluorescence during robotic ureteroenteric reimplantation.
Materials and Methods: We retrospectively reviewed 7 patients who underwent robotic ureteroenteric reimplantation by a single surgeon between March 2013 and March 2017. Indocyanine green (25 milligrams in 10 milliliters of distilled water) was injected antegrade into the lumen of the ureter via a percutaneous nephrostomy tube, and retrograde into the lumen of the urinary diversion via a Foley catheter. All patients consented to off-label use of indocyanine green. At follow up, all patients were assessed for: clinical success, the absence of flank pain attributable to ureteral pathology; and radiological success, the presence of reflux on loopogram and/or the absence of obstruction on renal scan.

Results: Six of $7(85.7 \%)$ patients previously underwent an ileal conduit creation and $1 / 7(14.3 \%)$ patients previously underwent a Studer orthotopic neobladder. The median time to development of an anastomotic stricture was 6.7 (range 1.4-74.0) months, and the median stricture length was 2.5 (range 1.0-5.0) centimeters. Four of $7(57.1 \%)$ patients underwent a Bricker-type reimplant, $1 / 7(14.3 \%)$ patients underwent a Wallace-type reimplant for bilateral anastomotic strictures, 1/7 (14.3\%) patients underwent a left to right transureteroureterostomy, and 1/7 (14.3\%) patients underwent an appendiceal ureteral replacement. Visualization of indocyanine green under near-infrared fluorescence allowed for precise identification of the ureter and urinary diversion, which fluoresced green; and localization of the anastomotic stricture margins, which poorly fluoresced green or did not fluoresce at all. The median OR time was 205 (range 176-388) minutes. At a median follow up of 22 (range 2-36) months, 5/7 (71.4\%) repairs were clinically and radiologically successful.

Conclusions: Intraureteral and intraurinary diversion injection of indocyanine green may be used as a real-time, intraoperative contrast agent to facilitate identification of the ureter, urinary diversion, and anastomotic stricture. Despite this, robotic ureteroenteric reimplantation remains technically challenging and should be performed at experienced centers.

\section{MP28-17 Robotic Ureterolithotomy for Management of Large, Impacted Ureteral Calculi}

WR Lai, R Thomas

Department of Urology, Tulane University School of Medicine United States

Introduction \& Objective: Endourological techniques are currently the gold standard for the contemporary management of renal and ureteral calculi. Ureteroscopic treatment of larger ureteral calculi greater than $1 \mathrm{~cm}$ may require multiple procedures and lead to retained residual stone fragments and associated fibrosis/stricture of the ureter. These stones are often impacted in the ureter and can be removed in a single procedure with ureterolithotomy. With the proliferation of robotics, the learning curve associated with laparoscopic suturing is minimized. We present our experience with robot-assisted laparoscopic ureterolithotomy from 2009 to 2016.

Materials and Methods: A retrospective review of robotic ureterolithotomy was performed at a single institution. Patient demographics and pre-operative information were collected. Retrograde pyelogram is performed prior to positioning the patient for the robotic portion of the procedure. A ureteral catheter is advanced to the level of the ureteral calculus and secured to the Foley catheter. Robotic ureterolithotomy is performed by the 
transperitoneal approach, with trocar configuration depending on the location of the ureteral calculus. The ureter is opened longitudinally over the ureteral calculus with round-tip scissors. After removal of the ureteral calculus, the ureteral catheter is exchanged for a double-pigtail ureteral stent over a guidewire in retrograde fashion. The ureterotomy is closed with 4-0 polyglactin suture. The ureteral stents were removed in 6 weeks post-procedure. If there is an associated ureteral stricture distal to the ureteral calculus, a concomitant ureteral reconstruction procedure is performed depending on the location of the stricture.

Results: There were 12 robotic ureterolithotomies performed. One was associated with concomitant ureteroneocystostomy. Mean age 55.8. Mean body index 29.2. Median estimated blood loss $40 \mathrm{ml}$. Median operative time $149 \mathrm{~min}$, including cystoscopy time. Median post-op length of stay 1 day. Median stone diameter $17.5 \mathrm{~mm}$. Median stone weight $858 \mathrm{mg}$. All were mixed calcium-based stones. One patient presented back to the hospital within 90 days of surgery with an obstructing contralateral ureteral calculus that was managed with staged ureteroscopy. One patient developed a recurrent ureteral calculus with associated ureteral stricture in the ipsilateral ureter and was managed with retrograde Holmium laser ureteroscopic endoureterotomy 55 months later. No other ureteral strictures were associated with the ureterolithotomies to date.

Conclusions: Robotic ureterolithotomy can be safely performed with minimal morbidity to remove large ureteral calculi in a single procedure.

MP28-18 A novel technique of access of peritoneal cavity and creation of tunnel in Laparoscopic Orchidopexy with Points of Technique

H Singh, A Kumar, S Singh, A Pal, J Kushwaha

Super Specialty Cancer Institute, C.G City, Lucknow, and King George's Medical University

India

Introduction \& Objective: Diagnostic Laparoscopy (DL) \& Laparoscopic Orchidopexy (LO) has proved to be the best available procedure for diagnosis and management of impalpable undescended testes. Creation of tunnel in open technique is usually simpler but in LO tunnel creation is little cumbersome usually using haemostatic forceps from below or dissecting forceps from above in grown up children and adults where pneumoperitoneum is usually lost and procedure becomes blind quiet often. We are using this novel technique to create more precise safe and better controlled access and tunnel. We are presenting our initial experience with points of technique.

Materials and Methods: Twenty patients age from 5 to $28 \mathrm{yrs}$ with low impalpable testes are included in study from 2013 to 16 in Dept. of Surgery KGMU, Lucknow. We used standard three port technique, after DL, cases suitable for single stage LO were taken up and testis mobilisation was done with standard technique. Then scrotal subdartos pouch created and a PCNL guide is passed through pouch via base of scrotum superolaterally to pubic tubercle into the peritoneal cavity through neohiatus between medial and lateral ligament. Tract dilated with serial PCNLdilators and finally Lap-trocar passed and laparoscopic forceps used to hold Gubraanaculam and testis is brought down to scrotum and placed in subdartos pouch.

Results: LO was done in 20 cases using this novel technique, access \& tunnel creation was more precise, simpler, smooth and without any complication
Conclusions: LO in grown up children and adults using this novel technique of access to peritoneal cavity and tunnel creation is more precise smooth, simpler and safe with better control on pneumoperitoneum and better visualisation.

MP28-19 Critical Analysis Of Laparoscopic Donor Nephrectomy In Jehovah's Witnesses

A Hsieh, J Stites, G Portman, R Munver

Hackensack University Medical Center United States

Introduction \& Objective: This personal preference for "bloodless surgery" is perhaps most well known among the Jehovah's Witness religious group, which prohibits whole blood transfusions based on their beliefs. Transplant programs may be hesitant to consider Jehovah's Witnesses as potential kidney donors because of the perceived risk involved with blood transfusion refusal. The present study examines outcomes of laparoscopic donor nephrectomy in living donors that are Jehovah's Witnesses.

Materials and Methods: We retrospectively analyzed all laparoscopic donor nephrectomies performed on Jehovah's Witness patients at a single institutions between 2003 and 2017. Study variables include estimated blood loss, warm ischemia time, length of hospitalization, donor operating room time, donor complications, and graft function. Inclusion criteria included patients that underwent donor nephrectomy via a laparoscopic approach. Perioperative outcomes were analyzed in this patient cohort.

Results: A total of 11 kidneys were procured of which 9 were performed laparoscopically. The cohort included 4 males and 5 females with a mean age of 35 (range 24-48). Mean operative time was 207 minutes (range 135-239), estimated blood loss was $79 \mathrm{~mL}$ (range 50-200), warm ischemia time was 115 seconds (90-155), and length of hospital stay was 2 days (range 1-3 days). No cases were converted to an open procedure and graft function at 2 years was $100 \%$. When compared to a control group of transfusion-consenting patients, there were no statistical differences with regards to mean operative time, estimated blood loss, warm ischemia time, length of hospital stay, and graft function.

Conclusions: This study demonstrates that laparoscopic donor nephrectomy in renal donors refusing blood transfusion yield results comparable to the control group. Preoperative planning, selection, and perioperative techniques can optimize patient variables to allow Jehovah's Witnesses to donate kidneys without compromising donor or graft outcomes.

MP28-20 Migration of Left Renal Vein Endovascular Stents for Nutcracker Syndrome

S Chen, L Tian, K Yang, H Zhang, W Jin, M Li

China (People's Republic)

Introduction \& Objective: Endovascular stenting for nutcracker syndrome is more appealing due to its minimally invasive nature. The clinical implications of migration are significant and can lead to thrombosis, vessel trauma, embolization, and its most disastrous consequence-rupture. The aim of our study was 
to identify the risk factors and management of stent migration after endovascular stenting for nutcracker syndrome

Materials and Methods: Ninety patients undergoing endovascular stenting for nutcracker syndrome from October 1998 through October 2013 were retrospectively analyzed. Follow-up was performed by clinical examinations and duplex ultrasound at $1,3,6$ and 12 months, and yearly thereafter or as symptoms recurred. In that case, or if changes were noted on duplex ultrasound, computed tomographic angiography was performed.

Results: Seven patients (7.8\%) showed stent migration. Mean time at the detection of migration was 2.4 months. Migration direction was proximal in five cases. Five patients developed recurrent symptoms. The angle between left renal vein and inferior vena cava was 112 and 95 degrees in migrated group and normal group, respectively (P0.05) . Both failed percutaneous removal and subsequent surgery were conducted in two patients, only surgery in two patients, and watch waiting in three patients. Follow-up revealed that the outcome of five patients were good except for two patients with recurrent symptom.

Conclusions: Follow-up is recommended once every two weeks within two months. Undersized stent, oversize angle, and educational purposes might lead to stent migration. Exact location of stent placement and Valsalva maneuver might reduce the risk of stent migration. Stent migration with recurrent symptom requires management, especially migration to the heart. Percutaneous removal of migrated stents may be difficult, thus open surgery may be required.

\section{MP28-21 Withdrawn}

MP28-22 Bilateral laparoscopic varicocelectomy associated with bilateral inguinal hernioplasty in the same procedure: a controlled prostective study.

J DA CRUZ, L Novaes, O De Campos, M DA SILVA,

R SAKATA, R DOS SANTOS, L SAVIO,

F Mota Filho, C PASSEROTTI

GERMAN HOSPITAL OSWALDO CRUZ, SAO PAULO, BRAZIL

Brazil

Introduction \& Objective: With the advent of minimally invasive surgery it is possible to treat more than one condition with the same access and without significantly increasing the morbidity of the procedure. The objective of this study was to compare the performance of bilateral laparoscopic varicocelectomy with bilateral laparoscopic inguinal hernioplasty in the same procedure against laparoscopic bilateral inguinal hernioplasty in order to assess if these multiple procedures could be performed without incresing operative time or worsening postoperative recovery.

Materials and Methods: Ten patients with bilateral inguinal hernia underwent videolaparoscopic bilateral inguinal hernia by pre-peritoneal transabdominal technique (Group I) and compared to ten patients with bilateral inguinal hernia associated with bilateral varicocele submitted to bilateral laparoscopic varicocelectomy and bilateral inguinal hernioplasty in the same procedure (Group II). The total operative time, complications and post-operative pain were assessed and compared between the two groups.

Results: There was no difference between groups total operative time $(\mathrm{p}=0.381)$, pain in post-operative day $1(\mathrm{p}=0.748)$, pain in post-operative day $7 \quad(\mathrm{p}=0.318)$, pain 1 month after surgery $(p=0.496)$, pain 6 months after surgery $(p=>.999)$ and complications $(p=0.305)$. There was only one complication (spermatic cord hematoma) in Group I and none in Group II.

Conclusions: Performing bilateral laparoscopic varicocelectomy together with bilateral laparoscopic inguinal hernioplasty is a safe option, with good results for the treatment of a patient with bilateral varicocele associated with bilateral inguinal hernia.

MP28-23 Case series study of transvesical and transurethral repair of vesicovaginal fistula

C Huang

China Medical University Hospital

Taiwan (Republic of China)

Introduction \& Objective: Iatrogenic vesicovaginal fistulas are rare but always creat bothersome problems for the patients and the surgeons. The two major management methods are transvaginal and transabdominal approach. The later include opening, laparoscopic or robot-assisted laparosopic repair of the vesicovaginal fistula. Here, we would like to introduce a effective,
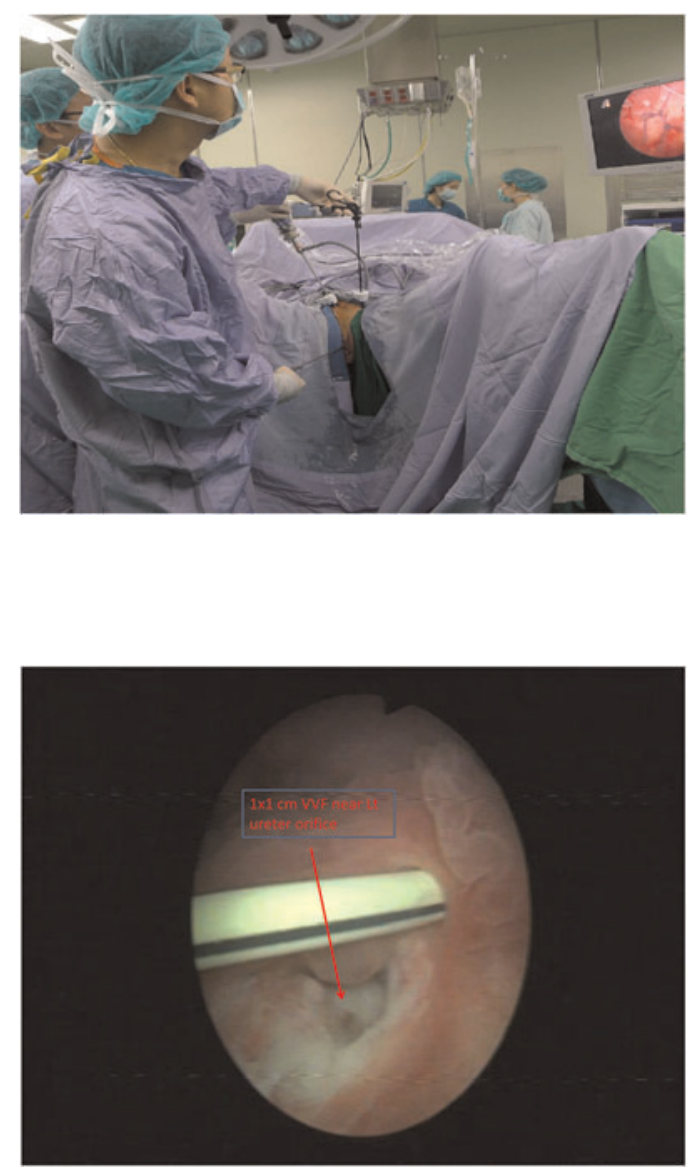

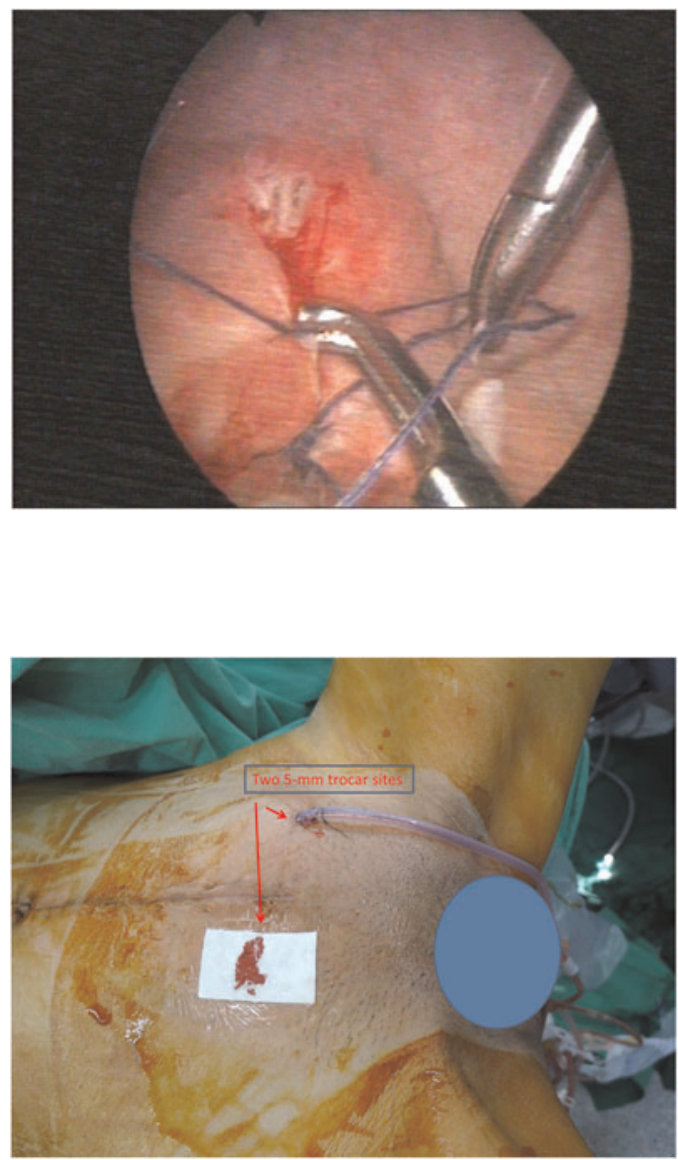

alternative approach method via transvesical and transurethral laparoscopic technique to repair the vasicovaginal fistulas.

Materials and Methods: Two middle-aged females patients suffered from sustained urine leakage from vagina for 2 years and 2 months following gynecological surgery for endocervix adenocarcinoma and ovary cancer, respectively. Under generalized anesthesia, we created two 5-mm trocar wound over bilateral suprapubic area and these two trocars were inserted into urinary bladder under the direct vision guiding from cystoscopy. The urinary bladder was expanded with distilled water. Under the 70-degree cystoscopic veiw, the vesicovaginal fistula less than $1.5 \mathrm{~cm}$ in size were trimmed and closed with interrupted whole-layer suture. One suprapubic Foley was left and kept in bladder for 3-4 weeks. Before removing it, cystogram exam was done to make sure than the fistula had been completely healed.

Results: Both of the two cases got successful vesicovaginal fistula repair with no more urine leakage from vagina after removing the suprapubic Foley. The mean operation time (skin to skin) is 120 minuts with minimal blood loss. The mean hospital course is 4 days. The mean post-operative suprapubic Foley indwelling time is 28 days. There were no any perioperative complications but only one case developed UTI after cystogram exam.

Conclusions: Transurethral laparoscopic repair is feasible and effective for small vesicovaginal fistula. This minimal invasive approach method keeps away from dissection of the pelvic adhesive previous operative scar and simplify the repair. But due to the limited cases experience, the further larged-scaled study is needed to compare it's successful rate to the traditional method.

MP28-24 Robotic vs. Open Ureteroneocystotomy: The Novice Single Surgeon Experience

\author{
B Shah, N Manimala, J Lockhart, L Wiegand \\ University of South Florida \\ United States
}

Introduction \& Objective: Ureteroneocystostomy is commonly used for management of mid to distal benign ureteral stricture disease. Newer studies have shown intraoperative and post-operative benefits of robot-assisted approaches over open approaches. However, there is a learning curve associated with robotic surgery compared to classic open surgery teachings. The aim of this study is to present our single surgeon experience to determine if these benefits still hold true early in the career of a novice surgeon.

Materials and Methods: An IRB approved single surgeon procedure database containing 2,598 cases performed by a novice surgeon in his first two years of practice between 04/10/ 2015-04/10/2017 were analyzed, identifying 15 ureteral re-implantations, 10 performed with robot assistance and 5 performed open. Student T-tests and Fishers Exact Tests were used to analyze continuous and categorical variables, respectively, between the two groups.

Results: Demographic, preoperative, intraoperative, and postoperative data was compared between the 10 patients who underwent robot assisted surgery vs. the 5 patients who underwent open surgery. All patients were preferentially scheduled for robotic surgery except in the cases where the robot was not available or the patient would benefit from open surgery over laparoscopic surgery. The majority of ureteral strictures were secondary to ureteral injury during an obstetrics or gynecologic procedure $(53 \%)$ or repeated endoscopic procedures for management of nephrolithiasis (20\%). Median procedure time was surprisingly longer by 16 minutes for open cases compared to robotic cases, however, this difference was not statistically significant ( $270 \mathrm{~min}$ vs. $286 \mathrm{~min}, \mathrm{p}=0.799)$. A statistical difference favoring robotic surgery was seen in operative blood loss $(50 \mathrm{~mL}$ vs. $200 \mathrm{~mL}, \mathrm{P}<0.001)$, median post-operative days for advancement to regular diet (post-op day 0 vs. post-op day 4 , $\mathrm{p}<0.001$ ), and median hospital days ( 2 vs. $5, \mathrm{p}=0.003$ ). There were clavien-dindo class 1 complications in each group and no grade 2-5 complications were seen. The most common reason for extended hospital stay in the open group was prolonged ileus with delayed return in tolerance to a regular diet. With a median follow up of about 80 days for both groups, no stricture reoccurrences were seen.

Conclusions: The most common causes of ureteral injury resulting in need for ureteral reimplantation were obstetrics and gynecologic procedures followed by endoscopic procedures for nephrolithiasis. A clear difference in length of hospital stay, time until tolerance of a regular diet, and intraoperative blood loss was found favoring robot assisted laparoscopic surgery over open surgery. 


\section{MPS29: FUNCTIONAL UROLOGY (BPH/INCONTINENCE)}

MP29-1 Is preoperative urinary flow a predictive value of postoperative acute urinary retention rate?

L Carmignani, G Motta, M Clementi, D Vizziello, C Signorini, C Marenghi, D Ratti, S Nazzani

IRCCS Policlinico San donato Italy

Introduction \& Objective: The purpose of this study is to assess postoperative acute urinary retention probability after Thulium Laser Vapoenucleation (ThuVEP) for the treatment of benign prostate hyperplasia/lower urinary tract symptoms (BPH/LUTS) in patients with different pre-operative maximum flow urinary rate.

Materials and Methods: A total of 365 patients were treated with ThuVEP (thullium:YAG laser 120 or $150 \mathrm{~W}, 800$ or $1000 \mathrm{~nm}$ bare fiber) for removal of obstructing benign hyperplastic tissue from January 2012 to August 2016. All patients were divided in three groups: Group A Flow max $\geq 10 \mathrm{ml} / \mathrm{sec}$, Group B $5 \leq$ Flow $\max <10 \mathrm{ml} / \mathrm{sec}$, and Group C Flow max $<5 \mathrm{ml} / \mathrm{sec}$. Patient with an indwelling catheter or without a preoperative flow were excluded. Thuvep was performed, and the follow-up outcome was investigated 3 months after surgery. We considered mean prostate volume $(\mathrm{ml})$ operative time $(\mathrm{min})$, catheterization time (hours), hospitalization (days) and acute urinary retention rate (AUR) (\%). A total of 269 patients completed the 3 month postoperative assessment

Results: Group A included 37 patients, Group B 156 and Group C 66; mean preoperative prostate volume was respectively $82.37 \pm 43.5 \mathrm{ml}, 72.52 \pm 43.05$ and $84.67 \pm 43.02$. ( $\mathrm{P}>0.05)$. Mean catheterization time was $37 \pm 26$ hours for Group A, $35 \pm 26$ hours for Group B and $42 \pm 25$ hours for Group C. $(\mathrm{P}>0.05)$ Mean operative times were respectively 1:19 $\pm 40 \mathrm{~min}$, $1: 18 \pm 40 \mathrm{~min}$ and $1: 28 \pm 36 \mathrm{~min}$. $(\mathrm{P}>0.05)$. Mean hospitalization time was $2.44 \pm 1.58$ days for Group A, $2.55 \pm 1.53$ for Group B and $2.86 \pm 1.56$ for group $\mathrm{C}$. $(\mathrm{P}>0.05)$ In three months follow up we observed 4 AUR (11\%) in group A, $13(8.3 \%)$ in group B and $5(7.5 \%)$ in group $\mathrm{C}(\mathrm{P}=0.3323)$.

Conclusions: In ThuVep, the preoperative maximum flow can't predict AUR probability.

MP29-2 Preoperative Voiding Efficacy Could Predict Postoperative Alpha blocker Usage after Transurethral Resection of The Prostate

C Hsu, W Cheng, C Chang, C Huang, Y Chiu, S Hong, A Chiu

Division of Urology, Department of Surgery, Zhongxiao

Branch, Taipei City Hospital

Taiwan (Republic of China)

Introduction \& Objective: Some patients with benign prostatic enlargement (BPE) have to take alpha blockers to control their lower urinary tract symptoms after transurethral resection of the prostate (TURP). In the present study, we tried to find out noninvasive predictors of postoperative alpha blocker usage after TURP.

Materials and Methods: From January $1^{\text {st }}, 2011$ to December $31^{\text {st }}, 2016$, patients who received TURP for BPE in our community-based hospital were included. Those who didn't have complete preoperative evaluation, lost follow-up postoperatively, or received palliative TURP for prostate cancer were excluded. Preoperative voiding efficacy, defined as voided volume on uroflowmetry divided by total volume (voided volume on uroflowmetry plus residual urine on bladder ultrasound), as well as maximum flow rate (Qmax), mean flow rate (Qmean) on uroflowmetry, serum prostatic specific antigen (PSA), prostate size estimated by transrectal ultrasound and weight of resected prostatic tissue were compared between patients who needed alpha blockers for more than three months postoperatively and those who didn't. Fisher exact test, Mann-Whitney U test, multiple regression analysis and receiver operating characteristic (ROC) curve were used for statistical analysis.

Results: There were 41 patients included for analysis, and 19 patients $(46.3 \%)$ had to take alpha blockers after TURP. The demographic data were shown in the Table. Patients who needed postoperative alpha blockers tended to be older $(p<0.01)$ and had worse preoperative voiding efficacy $(61.7 \%$ vs. $74.6 \%, p=0.02)$. There were no statistical differences when it comes to voided volume, Qmax, Qmean, serum PSA level, prostate size and weight of resected prostatic tissue. Voiding efficacy remained significant after multivariate analysis $(p=0.028)$. A cutoff level of voiding efficacy to predict postoperative alpha blockers usage was less than $63.3 \%(\mathrm{AUC}=0.713, p=0.01$, sensitivity $81.82 \%$, and specificity $=52.63 \%)$.

Conclusions: Preoperative voiding efficacy less than $63.3 \%$ could predict post-TURP alpha blockers usage. It could help patient counseling before TURP.

\begin{tabular}{|c|c|c|c|}
\hline Alpha-blockers use after TURP & YES & No & Pvalue \\
\hline Numbers & $19(46.3 \%)$ & $22(53.7 \%)$ & \\
\hline Age (years) & & & $<0.01$ \\
\hline$<70$ & $7(36.8 \%)$ & $13(63.6 \%)$ & \\
\hline$\geqq 70$ & $12(63.2 \%)$ & $8(36.4 \%)$ & \\
\hline Voiding Efficacy(\%) & $61.7 \%$ & $74.6 \%$ & 0.02 \\
\hline Voiding Volume $(\mathrm{ml})$ & 168.5 & 191.7 & 0.40 \\
\hline Total Volume (ml) & 267.1 & 255.5 & 0.49 \\
\hline $\mathrm{Qmax}(\mathrm{ml} / \mathrm{s})$ & 10.2 & 12.1 & 0.60 \\
\hline Qmean (ml/s) & 4.0 & 5.4 & 0.44 \\
\hline PSA (ng/ml) & 7.02 & 8.72 & 0.89 \\
\hline Prostate Size $\left(\mathrm{cm}^{3}\right)$ & 74.81 & 61.46 & 0.19 \\
\hline Resected Prostate (gram) & 36.2 & 22.5 & 0.11 \\
\hline
\end{tabular}

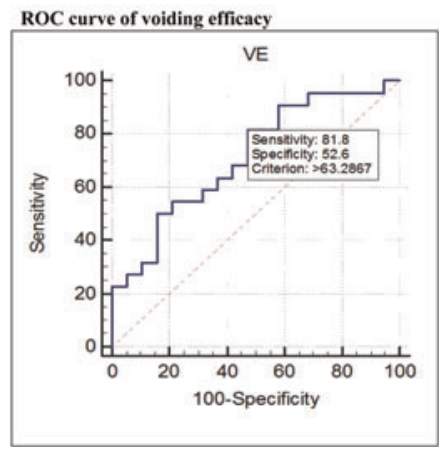


MP29-3 What are uro-geriatric factors that impact decision making in the elderly, with a refractory urinary retention, after a multidisciplinary team board?

C Rambaud, S Gonfrier, C Arlaud, B Tibi, R Prader, O Guérin, M Durand, P Regnier

Departement of Urology, Andrology and Renal transplantation, Hospital Pasteur II, CHU Nice

France

Introduction \& Objective: The indwelling urinary catheter (IUC) is over used on the elderly, with a risk of complications. Alternative treatments exist but underused due to difficulties of decision making. The aim of the study is to analyze factors that influence decision-making by a multidisciplinary team board, after a comprehensive geriatric assessment (CGA), in the elderly with a refractory urinary retention.

Materials and Methods: A standardized multidisciplinary team board was established for patients, over $70 \mathrm{y}$, with a refractory urinary retention, over a 1-Year period in 2016. Decision making for each patient was made after a CGA. Uro-geriatric and demographic clinical data were collected. We collected the alternative technics and their respective success rates (defined by the lack of urinary retention at 7 days).

Results: Ninety-seven were enrolled with a mean age of $87 \mathrm{y}$ with $37 \%$ of women. Alternative technics were offered in 58,8\% $(n=57)$, including 30 catheter withdrawals, 13 thermo-expandable intra-prostatic stents, 15 prostatic photovaporizations. The global success rates of alternative technics were $93 \%(n=53)$. In univariate analyzes, predictive factors of IUC were neurologic comorbidities (HR:3.1 [1.6-7.7], $\mathrm{p}=0.014$ ), an important dependence (ADL <2) (HR:5.5 [2.2-14], p<0,001) and an older age (HR:1.1 [1.1-1.2], $\mathrm{p}=0.017)$. In multivariate analyzes, the factor was the dependence (ADL <2) (HR:5 [1.9-12.9], $\mathrm{p}=0.001)$. Compare to photovaporizations of the prostate, incontinentation's surgeries were significantly proposed for older patients (86y vs $81,4 \mathrm{y}, \mathrm{p}=0,026)$, more dependent $(\mathrm{ADL}<2$, $83.3 \%$ vs $13.3 \%, \mathrm{p}<0.001)$, with neuroleptic treatments $(50 \%$ vs $13.3 \%, \mathrm{p}=0.038)$ and a risk to tear off the catheter $(83.3 \%$ vs $20 \%, \mathrm{p}=0.001)$.

Conclusions: Dependence appears to be an important factor influencing decision making in the elderly with urinary retention. A multidisciplinary analysis offers a better chance to screen patients and propose personalized treatments adapted to their profile.

MP29-4 Patient Reported Outcomes via Online Questionnaire (PROVOQ) - A Novel, Automated Approach for tracking Patient reported 30 day continence following Robot-Assisted Radical Prostatectomy (RARP).

LM Huynh, TE Ahlering

University of California, Irvine - Department of Urology United States

Introduction \& Objective: The average survival following radical prostatectomy (RP) is 22 years which underscores the critical need to address improvements in the recovery of urinary continence and potency. The primary limiting factor addressing these problems is the challenge of securing reliable, time-sensitive patient outcomes that surgeons can use to track and improve their outcomes and accurately transmit to their patients.
We developed a novel, automated HIPAA-compliant system to assess surgeon's early urinary continence rates.

Materials and Methods: Under an approved IRB protocol, an automated email survey is sent 30 days following catheter removal asking about their current pad status (none, security, 1, 2 or more). Patients' responses are populated in real-time into three databases and reminders are automatically sent if no response is received within two days, with up to three reminders (a total grace period of 6 days). Response rate was compared to our historic control group who was given a pre-stamped and addressed "pad free" postcard (PFC) at discharge, to be mailed to the PI when "pad-free" status is achieved. Additionally, patients were asked to fill out daily urinary pad usage logs (DUPL) with instructions to return results at 30-days. An "intervention" (i.e. phone call or email) was instituted if a spontaneous response was not received. Long term outcomes were similarly assessed at 3, 9, 15 and 24 months post-RP.

Results: In the control group, collecting PFCs and DUPLs yielded a response rate of $78 \%$ (39/50) within $30 \pm 6$ days; in contrast, the automated system yielded 94\% (47/50) without intervention $(\mathrm{p}=0.022)$. Further, the control group required paper data to be entered individually into our database(s). We estimated that subsequent phone "interventions" in the control group required approximately 30 minutes per patient plus an additional 15 minutes per patient to upload individual patient PFC or DUPL responses to the databases. This sums to about 15 hours of data acquisition and entry per 50 patients.

Conclusions: PROVOQ captured greater than $90 \%$ of follow-up data with high fidelity and little intervention from study coordinators. With widespread implementation, PROVOQ presents as an opportunity to integrate short and long term outcomes at potentially significantly higher rates with dramatically reduced costs in a HIPAA compliant manner.

MP29-5 Analysis and Predictors of Urinary Retention for Idiopathic Overactive Bladder treated with Intravesical Onabotulinumtoxin A Injection

Y Lu, T Kuo, L Ng

Singapore General Hospital

Singapore

Introduction \& Objective: One of the most common adverse events of Intravesical Onabotulinumtoxin A Injection (BoNTA) injection is urinary retention. The purpose of this study was to find the rate of urinary retention after treatment with BoNTA for medication refractory idiopathic overactive bladder $(\mathrm{OAB})$ symptoms and determine whether or not there are factors that predict this outcome.

Table 1. Patient characteristics ( $\mathrm{N}=43$ )

\begin{tabular}{llll}
\hline \multicolumn{3}{c}{ Table 1. Patient characteristics $(\mathbf{N}=43)$} \\
\hline \multirow{2}{*}{ Demographics } & Characteristics & Patients $(\mathbf{n})$ & $\%$ \\
\hline \multirow{4}{*}{ Age } & Male & 11 & 25.6 \\
& Female & 32 & 74.4 \\
\hline \multirow{2}{*}{ Race } & $<50$ & 8 & 18.6 \\
& $\geq 50$ & 35 & 81.4 \\
& Mean & $60.7 \pm 14.8$ & \\
& Chinese & 34 & 79.1 \\
& Malay & 1 & 2.32 \\
BMI & Indian & 4 & 9.30 \\
Cormobidities & Others & 4 & 9.30 \\
\hline
\end{tabular}


Table 2. Complications ( $\mathrm{N}=43$ )

\begin{tabular}{lll}
\hline & Patient $(\mathbf{n})$ & $\%$ \\
\hline Retention & 10 & 23.3 \\
UTI & 7 & 16.3 \\
Sepsis & 1 & 2.32 \\
\hline
\end{tabular}

Table 3. Univariate analysis of postop retention after receiving $100 \mathrm{u}$ Botox A

\begin{tabular}{lll}
\hline & Mean & P-value \\
\hline Age & $60.7 \pm 14.8$ & 0.753 \\
Gender & & 0.196 \\
DM & $13.3 \pm 8.5$ & 0.343 \\
Qmax & $33.5 \pm 17.3$ & $0.016^{*}$ \\
Pdet@Qmax & $31.7 \pm 19.0$ & 0.228 \\
Opening Pdet & $217.5 \pm 96.5$ & 0.131 \\
Max Capacity & $16.8 \pm 26.6$ & 0.879 \\
Preop RU (urodynamic) & & 0.149 \\
\hline${ }^{*}$ p<0.05 & &
\end{tabular}

${ }^{*} \mathrm{p}<0.05$

\begin{tabular}{llll}
\multicolumn{4}{l}{ Table 4. Multivariate analysis of postop retention after receiving 100u Botox A } \\
\hline Age & Odds ratio & $95 \% \mathrm{Cl}$ & P-value \\
Gender & 0.899 & $0.805-1.004$ & 0.059 \\
DM & 0.107 & $0.008-1.444$ & 0.092 \\
Qmax & 0.303 & $0.019-4.736$ & 0.395 \\
Pdet@Qmax & 0.697 & $0.507-0.957$ & $0.026^{*}$ \\
Opening Pdet & 1.011 & $0.906-1.130$ & 0.840 \\
Max Capacity & 1.056 & $0.952-1.171$ & 0.301 \\
Preop RU (urodynamic) & 0.982 & $0.943-1.023$ & 0.383 \\
\hline *p 0.05 & & &
\end{tabular}

${ }^{*} p<0.05$

Materials and Methods: Patients who had BoNTA treatment in urology department of Singapore General Hospital between the years 2013 - 2015 for medication refractory idiopathic OAB symptoms were retrospectively reviewed. Demographic data, urodynamic data and post BoNTA treatment adverse events were collected. The primary outcome measure was post procedure urinary retention. Statistical significance was assessed with multivariate logistic regression.

Results: Based on inclusion and exclusion criteria, 43 patients were identified. Mean age was $60.7 \pm 14.8$ years and $74.4 \%$ of the patients were women. The rate of urinary retention was $23.3 \%$. The rate of urinary tract infection was $16.3 \%$. For patients receiving $100 \mathrm{u}$ BoNTA treatment, univariate and multivariate analysis revealed that Qmax (OR 0.697, 95\% CI 0.507-0.957, $\mathrm{p}=0.026$ ) were associated with post procedure urinary retention. Conclusions: Post procedure urinary retention rate after BoNTA treatment for medication refractory idiopathic $\mathrm{OAB}$ was $23 \%$ in this study. Lower Qmax is associated with post procedure urinary retention.

MP29-6 A change in UTI incidence in our intradetrusor Botox patient cohort following changes in pre-operative protocol and antibiotic prophylaxis

A Brewin, T Nitkunan, E White, R Walker

United Kingdom
Introduction \& Objective: The incidence of UTI following intradetrusor Botox ${ }^{\circledR}$ in our Trust was previously found to be $33 \%$. This led to changes in antibiotic prophylaxis and procedural protocol. Oral co-amoxiclav is now given for local anaesthetic cases. The protocol involves a urine specimen sent for culture 2 weeks prior, and an on the day check for UTI symptoms and urine dipstick. The aim of this re-audit was to determine whether the UTI rate had altered since these changes.

Materials and Methods: Data was gathered from the 42 patients who were planned to receive intradetrusor Botox ${ }^{\circledR}$ between January and June 2016. Information from our Botox ${ }^{\circledR}$ database, clinic letters, operation notes and microbiology results were reviewed.

Results: One of the 42 planned procedures was cancelled due to UTI symptoms and positive urine dipstick on procedural day. 24/ $41(59 \%)$ were under local. Idiopathic:neurogenic cases were 29:12. 10/37 (27\%) pre-procedure urine cultures were positive $\left(>10^{\wedge} 8 \mathrm{cfu} / 1\right)$. The percentage of all organisms sensitive to Trimethoprim was $78 \%$. All pre-procedure UTIs were treated with appropriate antibiotics. 8/41 $(20 \%)$ patients had a proven UTI following intradetrusor Botox ${ }^{\circledR}$. The percentage of all organisms sensitive to Trimethoprim was $43 \%$. A positive urine culture was unrelated to clean intermittent self-catheterisation or diabetes.

Conclusions: There has been a reduction of post-Botox ${ }^{\circledR}$ UTI incidence from $33 \%$ to $20 \%$ following changes in the protocol. Changes in bacterial resistance patterns are seen post Botox ${ }^{\circledR}$ and this warrants further study.

MP29-7 A prospective evaluation of a modified cervicosacropexy /vagino-sacropexy procedure in treatment of female patients with refractory urge/mixed urinary incontinence

A Kumar, G Kumar, N Kumar, M Patel, P Gupta

Department of Urology and Renal Transplant, VMMC and Safdarjang Hospital, New Delhi, India

India

Introduction \& Objective: There is no cure for mixed/urge urinary incontinence till date. We performed the first study in Asia prospectively evaluating the safety and efficacy of a new modified cervico-sacropexy(CESA) /vagino-sacropexy(VESA) in treatment of female patients with refractory urge/mixed urinary incontinence.

Materials and Methods: The study was prospectively conducted from February 2014 to December 2016 at our institute. All consecutive patients undergoing CESA/VASA for refractory urge/mixed urinary incontinence were included. All these patients had no response to anticholinergic medications and intravesical botulinum toxin therapy. All patients were evaluated preoperatively using detailed history, physical examination, ultrasound abdomen and pelvis, and urodynamics study. A specially designed polyvinylidene fluoride mesh was placed anteriorly on uterus/vaginal vault(post-hysterectomy), laterally through uterosacral ligaments and posteriorly to sacrum at $\mathrm{S} 2$ vertebra level. The demographic, peri-operative and follow data(including urodynamics study at 3 and 6 months postoperatively) were analysed. The efficacy was measured as cure (absence of all subjective and objective incontinence symptoms.), improvement(in both subjectively and objectively) and failure.

Results: The study included 115 patients. The mixed and urge urinary incontinence were found in $63(54.7 \%)$ and $52(45.3 \%)$ respectively. The mean age and BMI were 47.1 years and 
$29.3 \mathrm{~kg} / \mathrm{m} 2$ respectively. In mixed urinary incontinence group, the mean valsalva leak point pressure was $71.3 \mathrm{~mm} \mathrm{Hg}$. Further, mild cystocele and mild uterine prolapse were present in 23 $(36.3 \%)$ patients and $21(31.3 \%)$ patients in mixed group. The mean operating time and estimated blood loss were $75.7 \mathrm{~min}$ and $81.4 \mathrm{ml}$ respectively. There were no major intra or peri-operative complications. At mean follow up of 24.7 months,98 (85.2\%) patients were cured $-53(84.1 \%)$ in mixed incontinence group and $45(86.5 \%)$ in urge incontinence group. The other 9 patients $(7.8 \%)$ showed improvement after surgery. There was no improvement in 8 patients $(6.9 \%)$ after surgery.

Conclusions: In female patients with refractory mixed/urge urinary incontinence, CESA/VASA was safe and effective. However, a large prospective randomized controlled trial with longer follow up is required to further validate these results.

MP29-8 The efficacy of mid-urethral sling in female stress urinary incontinence with overactive bladder

H Lee, J Seo

Cheil General Hospital \& Women's Healthcare Center, Dankook University College of Medicine

Republic of Korea

Introduction \& Objective: Many patients suffer from urinary stress incontinence and overactive bladder $(\mathrm{OAB})$, and who

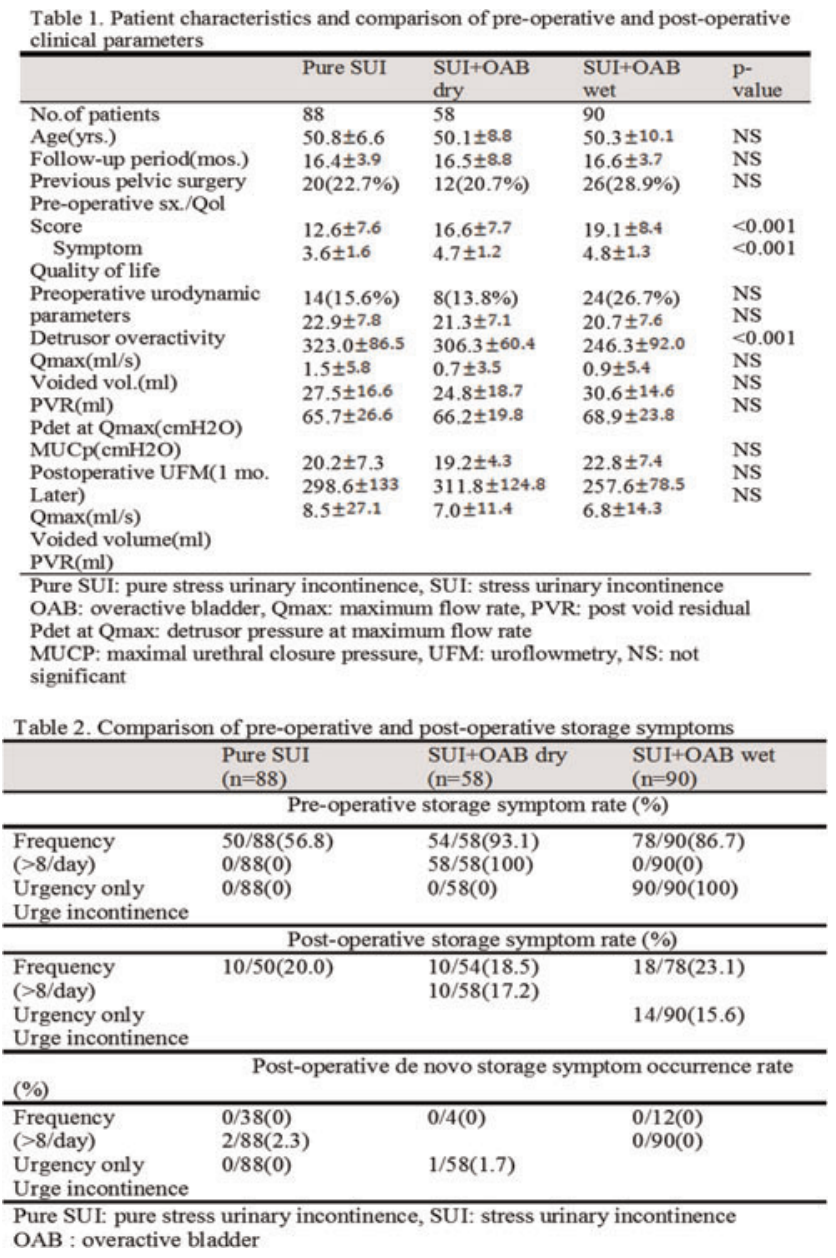

present with urinary stress incontinence have concomitant $\mathrm{OAB}$. Aim of this study is to investigate the efficacy of synthetic mid-urethral slings in female urinary stress incontinence patients with $\mathrm{OAB}$.

Materials and Methods: A total of 295 women with urinary stress incontinence were enrolled. The patients were divided into three groups: pure urinary stress incontinence (group 1), urinary stress incontinence with $\mathrm{OAB}$ dry (group 2) and urinary stress incontinence with OAB wet (group 3). Out of those 295, only the patients with a follow-up of at least 12 months were included in this study, yielding 236 patients. All women were assigned midurethral slings by the same surgeon. All questionnaires by phone were used to evaluate the postoperative improvement of urinary stress incontinence and storage symptoms. Statistical analysis was performed using a Student's t test, a one-way ANOVA, and a chi-square test.

Results: There were significant differences in pre-operative symptom score, quality of life score, and pre-operative voided volume among the 3 groups. (Table 1) There were no significant differences among the 3 groups in terms of the cure rate for the stress component (group 1,88.6\%; group 2, 86.2\%; group 3, $86.7 \%, \mathrm{p}=0.943) .80 .0 \%$ of Group 1 patients improved in frequency, but 2 patients $(2.3 \%)$ complained about de novo urgency. In group 2, $81.5 \%$ and $82.7 \%$ improved in frequency and urgency, respectively, but one patient $(1.7 \%)$ complained about de novo urge incontinence. In Group 3, 76.9\% and $84.4 \%$ improved in frequency and urge incontinence, respectively. Limitation include the retrospective design, relatively short follow up period, and improvement of symptoms was not assessed separately.

Conclusions: $\mathrm{OAB}$ symptom was improved after mid-urethral sling operation without any treatment for OAB symptom. Midurethral slings are simple, safe, and highly-effective in treating urinary stress incontinence with $\mathrm{OAB}$ in $1^{\text {st }}$ line treatment.

MP29-9 Recurrent urinary retention: 6 months follow-up of elderly patients who benefited an alternative treatment to the indwelling catheter after a multidisciplinary team board.

C Rambaud, S Gonfrier, C Arlaud, J Fallot, O Guérin, M Durand, P Regnier

Departement of Urology, Andrology and Renal transplantation, Hospital Pasteur II, CHU Nice

France

Introduction \& Objective: The indwelling urinary catheter (IUC) is over used on the elderly. The aim is to analyze the success rate of alternative treatments (AT) to the IUC at 6 months follow up.

Materials and Methods: A standardized multidisciplinary team board was established to screen patients over $70 \mathrm{y}$ who can benefit from an alternative treatment option to IUC, using comprehensive geriatric assessment. We evaluated success of AT (defined by the lack of urinary retention at 7 days, 1, 3 and 6 months) and overall death rates.

Results: Sixty-one patients were enrolled with a mean age of $87 y$ (women: 42.6\%). Demographic and clinical characteristics of patients are listed in Table 1. Alternative technics were offered to $62 \%(\mathrm{n}=38)$, including 23 catheter withdrawals, 7 thermo-expandable intra-prostatic stents, 8 prostatic photovaporizations. Overall, the success rate was $92.1 \%$ at 7 days and raised up to 


\begin{tabular}{|c|c|c|c|}
\hline & $\begin{array}{c}\text { Alternative } \\
\text { technics } n=38\end{array}$ & $I U C n=23$ & $p$ value \\
\hline \multicolumn{4}{|l|}{ Characteristics } \\
\hline Age (years) & 85.3 & 89.3 & 0.021 \\
\hline Male $n(\%)$ & $20(52,6)$ & $15(65,2)$ & 0.335 \\
\hline \multicolumn{4}{|l|}{ Geriatric Assessment } \\
\hline Neurologic comorbidities n (\%) & $20(52.6)$ & $18(78.9)$ & 0.045 \\
\hline Dementia n (\%) & $13(34.2)$ & $14(60.9)$ & 0.042 \\
\hline Parkinson's diseasen (\%) & $1(2.6)$ & $3(13)$ & 0.111 \\
\hline Others & $6(15.8)$ & $3(13)$ & 0.769 \\
\hline Diabetes & $7(18.4)$ & $8(34.8)$ & 0.150 \\
\hline Autonomy (ADL $<$ ) & $15(39.5)$ & $18(78.3)$ & 0.003 \\
\hline Cognitive impairment $n(\%)$ & $22(57.9)$ & $18(78.3)$ & 0.105 \\
\hline Risk of agitation $n(\%)$ & $13(34.2)$ & $7(30.4)$ & 0.761 \\
\hline \multicolumn{4}{|l|}{ Urologic assessment } \\
\hline Urologic comorbidities n (\%) & $7(18.4)$ & $10(43.5)$ & 0.758 \\
\hline \multicolumn{4}{|l|}{ Urologic treatment $n(\%)$} \\
\hline Alpha-blockers & $21(55.3)$ & $14(60.9)$ & 0.668 \\
\hline Women & 3 & 1 & \\
\hline Men & 18 & 13 & \\
\hline 5-alpha-reductase-inhibitors & $7(18.4)$ & $3(13)$ & 0.582 \\
\hline Plant extract & $5(13.2)$ & $1(4.3)$ & 0.263 \\
\hline \multicolumn{4}{|l|}{ Urinary incontinence $n(\%)$} \\
\hline Continent & $21(55.5)$ & $17(47.8)$ & 0.145 \\
\hline Incontinent & $3(7.9)$ & $10(43.5)$ & 0.001 \\
\hline
\end{tabular}

$100 \%$ at 1, 3 and 6-month follow-up. The IUC group patients were significantly older $(89.3 \mathrm{vs} 85.3 \mathrm{y}, \mathrm{p}=0.021)$, very dependent (ADL $<2,78.3 \%$ vs $39.5 \%, \mathrm{p}=0.03$ ) and with neurologic comorbidities $(78.3 \%$ vs $52.6 \%, \mathrm{p}=0.045)$. The global rate of death of the cohort were at 1, 3 and 6 months: $6.6 \%(n=4), 21.3 \%$ $(\mathrm{n}=13), 36 \%(\mathrm{n}=22)$. At 6 months, the rate of death in the IUC group was higher $(65 \%$ vs $18.4 \%, p=0.01)$. In univariate analyzes, predictive factors of a 6-months death were neurologic comorbidities (HR:4,3 [1.2-14.9], $\mathrm{p}=0.023$ ), a dependence (ADL <2) (HR:4.9 [1.5-16]) and the IUC (HR:5.5 [1.8-17], $\mathrm{p}=0.003)$. In multivariate analyzes, the factors were a dependence $(\mathrm{ADL}<2)(\mathrm{HR}: 3,9[1.1-13.4], \mathrm{p}=0.034)$ and the IUC (HR: 4.4 [1.4-14.5], $\mathrm{p}=0.014)$.

Conclusions: The multidisciplinary analysis may offer a better chance to deal with IUC in elderly people with a steady global success rate of $62 \%$ catheter withdrawals at 6 months. The elevated rate of death in IUC group highlight the frailty of dependent patients and data is needed to report the relation with IUC.

MP29-10 Efficacy and safety of OnabotulinumtoxinA in patients with overactive bladder according to site of injection: A systematic review and meta-analysis

J Jo, S Park, H Choi, H Moon, S Lee, K Kim, Y Kim

Hanyang University Hospital

Republic of Korea

Introduction \& Objective: We conducted this study to assess the efficacy and safety of OnabotulinumtoxinA according to site of injection for treating overactive bladder.

Materials and Methods: A systematic literature review was performed to locate randomized controlled trials of OnabotulinumtoxinA for treatment of neurogenic detrusor overactive bladder and idiopathic overactive bladder in adults. We searched databases such as MEDLINE, EMBASE, and the Cochrane Controlled Trials Register using the Ovid platform. This metaanalysis was based on Cochrane Review Methods.
A
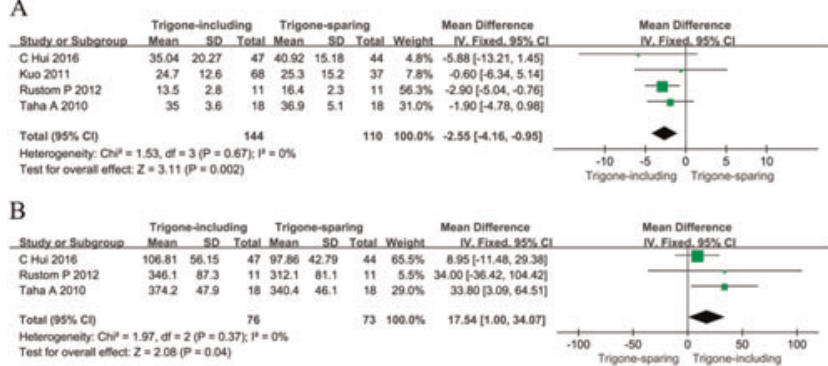

C

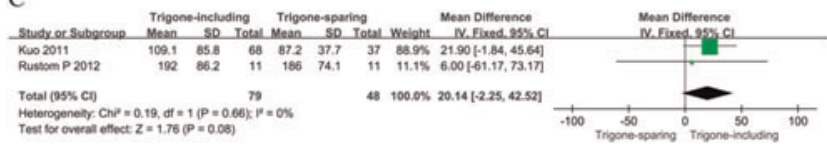

D

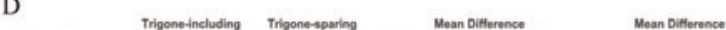

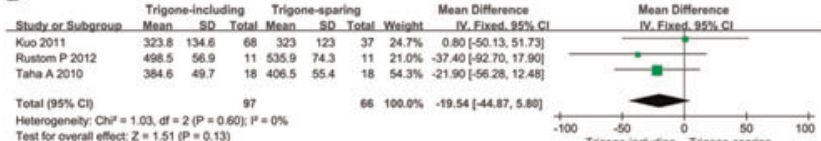

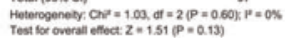
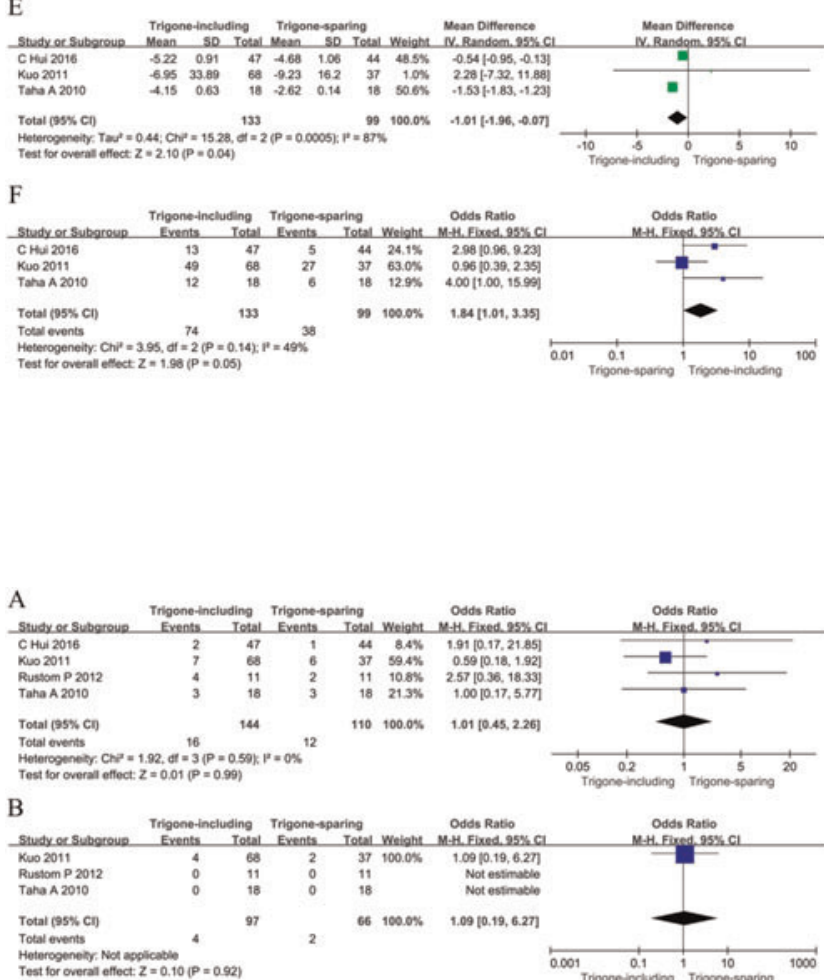

C

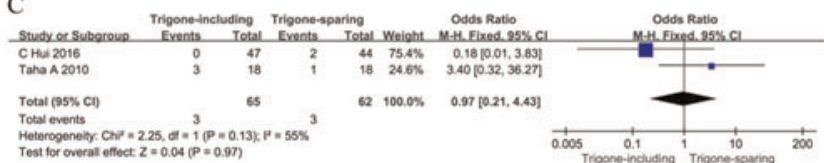

D

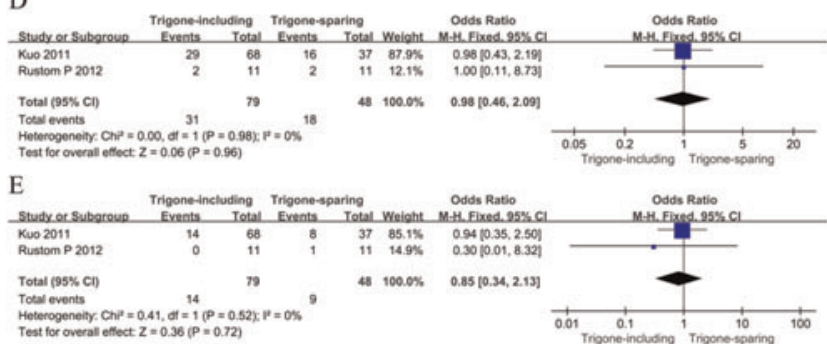


Results: Seven studies (339 participants) were included. Trigone-including intradetrusor injection gave rise to a lower detrusor pressure $\left(\mathrm{WMD}=-2.55 \mathrm{cnH}_{2} \mathrm{O}, 95 \% \mathrm{CI}=-4.16\right.$ to -0.95 , $\left.\mathrm{P}=0.002, \mathrm{I}^{2}=0 \%\right)$ and higher volume at first desire to void $\left(\mathrm{WMD}=17.54 \mathrm{ml}, 95 \% \mathrm{CI}=1.00\right.$ to $34.07, \mathrm{P}=0.04, \mathrm{I}^{2}=0 \%$ ) than trigone-sparing intradetrusor injection. Incontinence episodes were also significantly less frequent after trigone-including intradetrusor injection (WMD $=-1.01$ numbers per day, 95\% $\mathrm{CI}=-1.96$ to $\left.-0.07, \mathrm{P}=0.04, \mathrm{I}^{2}=87 \%\right)$. There were no differences in efficacy according to injection site between intradetrusor and suburothelial injection, or in safety in terms of the incidence of vesico-ureteral reflux, hematuria, general weakness, bladder discomfort, large postvoid residual, and urinary tract infection.

Conclusions: Trigone-including OnabotulinumtoxinA injection is superior to trigone-sparing injection in terms of efficacy, as measured by detrusor presser, higher volume at first desire to voidand episodes of incontinence, without any difference in complications. However, the depth of injection does not influence the efficacy or safety of OnabotulinumtoxinA injections.

MP29-11 Relationship between pelvic muscle contractility and ability of immediate continence after robot-assisted radial prostatectomy.

K Park, J Huh, Y Kim, S Kim

Republic of Korea

Introduction \& Objective: To analyze the clinical parameters correlated with early recovery of urinary continence after radical prostatectomy, focusing especially on urethral mobility during pelvic contraction at catheter removal.

Materials and Methods: At the post-op 7 days, a cystography was performed in 67 men (median age 65 years, range 55-76) who had undergone robot assisted laparoscopic prostatectomy.

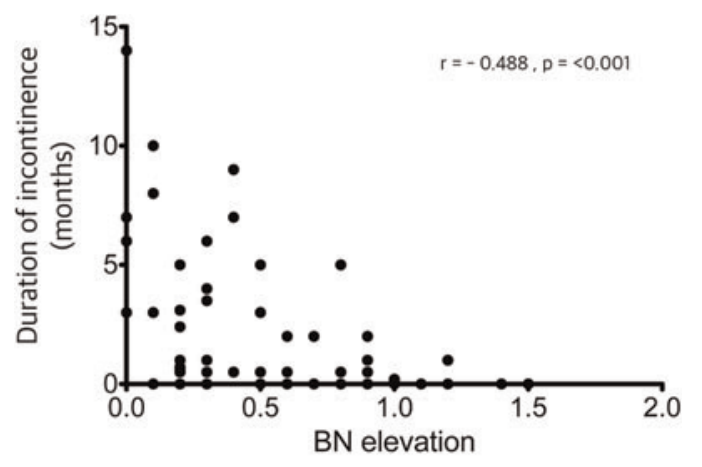

Time to recovery post op incontinence

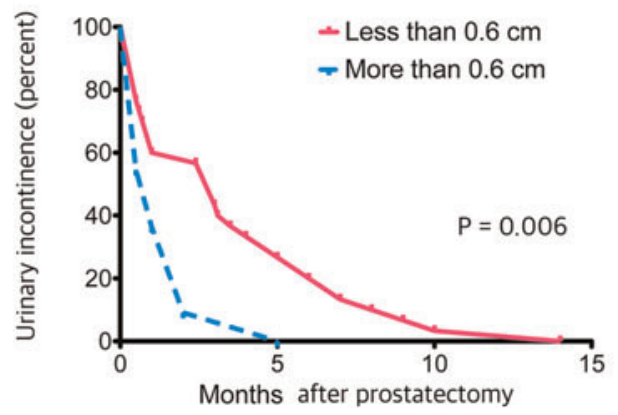

The vertical length of bladder neck movement between resting and contraction was measured. The correlation between rate of incontinence recovery on post op 3 months and various clinical parameters, including age, prostate volume, International Prostate Symptom Score, quality of life score and the length of urethral movement was analyzed.

Results: An inverse correlation was observed between length of urethral movement on day 7 and recovery rate on post op 3months urinary incontinence (defined as [number of patients with continence/ total number of patients on post op 3months] ( $\mathrm{r}=-0.488$, $P<0.05)$. Classifying patients into two groups according to the length of urethral movement of $6 \mathrm{~mm}$ gave the best 2017-0504accuracy for the prediction of postoperative recovery of urinary incontinence with receiver operating characteristic analysis. A statistically significant difference was observed between group 1 (length $>=6 \mathrm{~mm})$ and group $2(<6 \mathrm{~mm})$ for the leakage rate until 3 months after the catheter was removed $(P<0.01)$. Urethral movement at the post-op 7 days was significantly correlated with post-op 3 months recovery of continence in the two groups $(P<0.05)$.

Conclusions: Urethral mobility after robot assisted laparoscopic prostatectomy, which can be easily evaluated using cystography, may be a good predictor of post-op 3 months recovery of urinary continence.

MP29-12 A Prospective, Multi-Institutional Assessment of 30-Day Pad-Free Continence Predicting Long-term Continence Following Robot Assisted Radical Prostatectomy (RARP)

LM Huynh, R Derderian, D Skarecky, TE Ahlering

University of California, Irvine - Department of Urology United States

Figure 1: 30-Day Continence Rates Correspond Directly with Overall Continence Rates Across Four Institutions

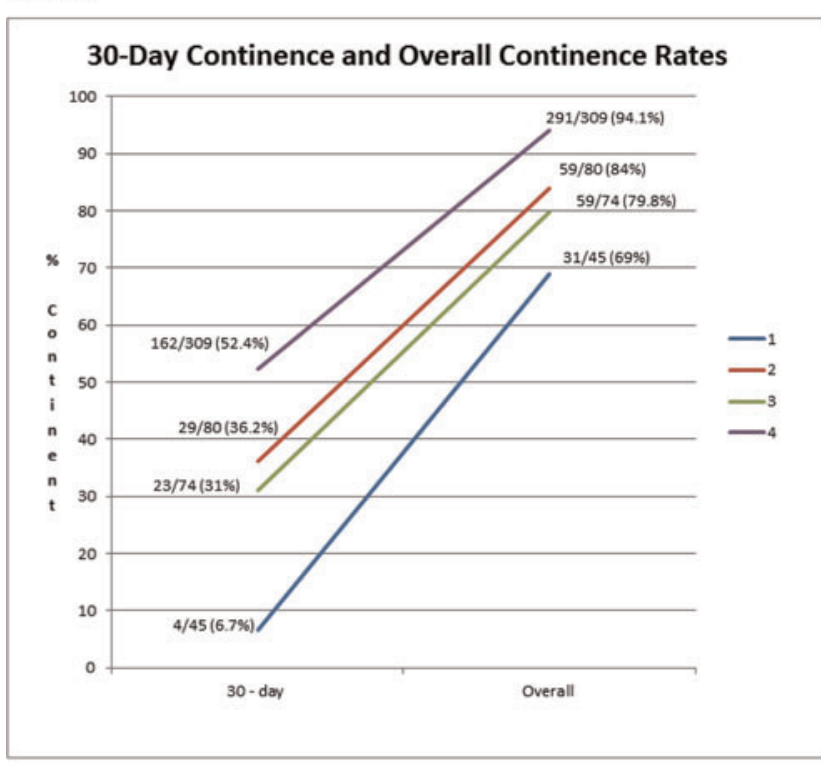

Slopes:

Site 1: 0.185

Site 2: 0.1426

Site 3: 0.1456

Site 4: 0.12447 
Introduction \& Objective: Critical to, and at the same time, limiting assessment of long term (1-year) urinary outcomes recovery is procuring accurate data in a high proportion of patients. Both interest and diligence of patients and study sites profoundly impact outcomes data collection. Additionally, early as opposed to long term follow-up is much easier to acquire. In 2010, we instituted a program to optimize the capture of 30-day "pad-free" continence via a combination of stamped and preaddressed pad-free status postcards (PFC) and daily urinary pad logs (DUPL) to assess prediction of 1-year pad-free status rate. Materials and Methods: Complete data was collected prospectively from four high volume institutions whose patients $(\mathrm{n}=508)$ underwent RARP. At discharge, patients were given stamped and pre-addressed PFC; to assess internal consistency, they were also given a DUPL to track daily pad use with instructions to fax results. Pad-free status was also separately assessed via self-administered validated questionnaires at 6 and 12 months. A contracted third party called all "non-responders" (day 32-35) to document pad status.

Results: A third party, independent audit was performed posthoc to internally assess response rates and outcome validity. The data was confirmed via internal audit. The 30-day response rates varied very slightly for the separate institutions: $66.5 \%$ for the PFC and $70.4 \%$ for the DUPL. The correlation in pad-free dates from the PFC and DUPL was 0.98 (p For the entire cohort, padfree status at 30-days was $43 \%$ (218/508) and at 1 year $88 \%$ (447/ 508). Figure 1 displays individual site rates, which varied between the 4 sites. Remarkably the slopes (pts/mo) of recovery were reasonably consistent (range $3.73-5.55$ ). Notably, there were no cross-over events.

Conclusions: Clinical outcomes following RP are difficult to assess, as confirmation requires 1-2 years. This delay is further complicated by the discipline and expense associated with comprehensive data collection. As a single predictor of overall continence at 1 year, 30-day continence yields congruent rates of recovery at the four institutions studied. In utilizing the predictive capability of 30-day continence, surgeons can not only project their patients' recovery, but also use the resultant model to monitor their individual outcomes.

\section{MP29-13 Outcomes of transobturator tape procedures in women with mixed urinary incontinence}

K Oh, K OH, S Kim, Y Cho, M Kim, H Chung, E Hwang, S Jung, T Kang, K Park, D Kwon

Republic of Korea

Introduction \& Objective: Treatment of mixed urinary incontinence (MUI) is often challenging, as a single modality may be inadequate for alleviating both the urge and stress component. The aim of our study was to investigate subjective and objective outcomes after a transobturator tape procedure (TOT) in women with MUI.

Materials and Methods: We prospectively analyzed the surgical outcomes in 178 patients with stress urinary incontinence or MUI who underwent TOT. Overall objective cure of incontinence and subjective symptom improvement (Incontinence Quality of Life: I-QoL; Overactive bladder-quality of life symptom score short form: OAB-q SF; and American Urological Association [AUA] symptom score) were compared in women with stress incontinence only (group I), MUI with predominant stress leakage (group II), and MUI with predominant urge incontinence (group III). All subjects underwent routine office evaluation and subjective assessment for incontinence and $\mathrm{OAB}$ symptoms preoperatively and at 3 months postoperatively.

Results: Preoperatively, 37\% had stress incontinence only, 36\% had MUI with predominant stress leakage, and 27\% had MUI with predominant urge incontinence. Mean patient age was $59 \pm 10$ years. The overall incontinence cure rate was similar between groups (group I: $98.1 \%$, group II: $96.9 \%$, group III: $95.5 \% ; \mathrm{p}=0.02)$. After incontinence surgery, I-QoL, OAB-q SF (symptom bother), OAB-q SF (heath- related quality of life [HRQoL]), and AUA (QoL) symptom scores were improved $(\mathrm{p}=0.015, \mathrm{p}=0.032, \mathrm{p}=0.029, \mathrm{p}=0.021$, respectively). Groups I and II showed significant improvement in subjective outcomes compared to group III.

Conclusions: Women with MUI with predominant stress leakage showed a better objective and subjective outcome than those with predominant urge incontinence, following the TOT procedure. The TOT procedure effectively achieves incontinence cure and improves the quality of life of patients with MUI who have predominant urge incontinence.

MP29-14 Minisling single incision tape for treatment of female stress incontinence, is it better?!

M Elbadry, A essam, A hammady, m gamal, m abdelmalek

minia university

Egypt

Introduction \& Objective: Introduction: stress urinary incontinence in female has a great impact in quality of life. there are many modalities of surgical repair ; minisling single incision tape is recently added to the urethral slings with better result and less hospital stay.

Study objective: in our study we will provide a prospective comparative study between minisling single incision tape and the ordinary transobturator one.

Materials and Methods: 123 female patient from January 2016 to November 2016 is randomly categorized into 2 groups by a computer software, group A (62 patients) using minisling tape, group B (61 patients) using the ordinary tape at Minia Uneversity hospital with mean age $45 \mathrm{y} \pm 10 \mathrm{y}$.

Results: the follow up mean was 333 days. patients show significant improvement post operatively, post operatively 49 patients $(79 \%)$ in group A are continent, 9 patients $(14 \%)$ are incontinent but still better than pre operatively, 4 patients $(7 \%)$ still presented with leakage, meanwhile in group B 50 patients $(82 \%)$ are continent, 8 patients $(13 \%)$ with improved symptoms, 3 patients $(5 \%)$ are still incontinent. the post-operative complications such as urinary tract infection, pain are insignificant in both groups.

Conclusions: minisling single incision vaginal tape is a good effective valuable method of stress urinary incontinence surgical repair in female, it gives better results with less morbidity.

MP29-15 Does Preoperative Prostate Volume Predictive For Medical Therapy Continuation After TURP?

M Zor, E Kaya, T Ebiloglu, A Coguplugil, S Bedir, S Bedir

Gulhane Training and Research Hospital

Turkey 
Table 1. The summary of additional diagnostic work-up according to groups.

\begin{tabular}{|c|c|c|c|}
\hline & & Group 1 (n:5) & Group 2 (n:14) \\
\hline \multirow{6}{*}{ 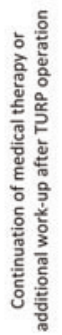 } & $\alpha$-adrenergic blockers & 2 & 5 \\
\hline & anticholinergics & - & 2 \\
\hline & $\begin{array}{l}\alpha \text {-adrenergic blocker + } \\
\text { anticholinergic }\end{array}$ & 1 & 2 \\
\hline & $5 \alpha$-reductase inhibitor & 1 & - \\
\hline & $\begin{array}{l}\alpha \text {-adrenergic blocker }+5 \alpha \text {-reductase } \\
\text { inhibitor }\end{array}$ & - & 1 \\
\hline & Cystoscopy / cystometry & 1 & 4 \\
\hline
\end{tabular}

Introduction \& Objective: A small proportion of patients who underwent TURP for benign prostate hyperplasia has persistent lower urinary tract symptoms and needs continuing medical treatment. In our study, we aimed to evaluate whether or not is there any relationship between preoperative prostate volume and postoperative persistent LUTS and continuing medical treatment.

Materials and Methods: The patients were divided into two groups (group 1: prostate volume $\geq 80 \mathrm{ml}$, and group 2: prostate volume $<80 \mathrm{ml}$ ) according to prostate volume. Preoperative PSA levels, prostate volume, comorbidities, postoperative cystoscopy and cystometry necessity and postoperative medical therapy continuation were evaluated and compared between groups.

Results: A total of 87 patients included to the study. Prostate volume was $\geq 80 \mathrm{ml}$ in 26 patients (group1), and $<80 \mathrm{ml}$ in 61 patients (Group 2). Of 87 patients 19 (22\%) had persistent LUTS and underwent cystoscopy, cystometry or were restarted medical therapy. The data summerized in table 1. Despite there were no statistical significance, LUTS were seen more frequently in patients who had prostate volume less than $80 \mathrm{ml}$ and underwent TURP.

Conclusions: Despite high volume and prospective studies are warranted for determining the risk factors for postoperative LUTS following TURP operations, our study demonstrates that TURP operations may be more pleasing in larger prostates.

MP29-16 A Prospective, Multi-Institutional Assessment of 90-Day Percent Fullness - Predictive Modeling of Potency Recovery Post-Robot Assisted Radical Prostatectomy (RARP)

LM Huynh, D Skarecky, TE Ahlering

University of California, Irvine - Department of Urology United States

Introduction \& Objective: The International Index of Erectile Function-5 (IIEF-5) is the most utilized validated questionnaire to assess potency in men following RARP. At 90 days post-RP, $60-90 \%$ of men report scores $<15$, poorly discriminating partial return of erections or (more importantly) the ultimate likelihood of potency recovery. The present study evaluates a novel patientreported \% fullness scale compared to preoperative erections at 90 days as a predictor of 1 year potency.

Materials and Methods: Clinico-pathological data from five high-volume surgeons whose patients underwent RARP were prospectively collected. 59 of 199 men with baseline IIEF-5 1-14 were excluded. The novel $\%$ fullness scale is "how firm are your
Figure 1: Percent Fullness Tertiles for Men of All Ages with a Preoperative IIEF-5 15-25, Recovery of Potency as Defined as an IIEF-5 15-25.

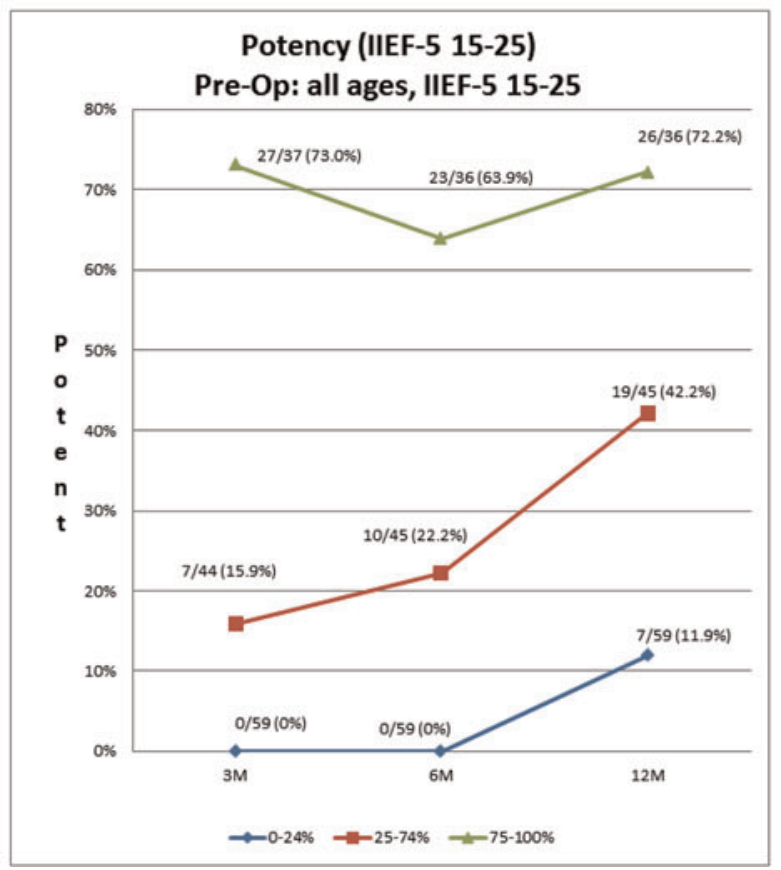

erections compared to before surgery?" is scored from $0-100 \%$ and was collected at 3,6, and 12 months along with standard IIEF-5. The primary outcome was utility of 90-day $\%$ fullness to predict potency status at 1 year, as defined as an IIEF-5 15-25. Results: 140 men with baseline IIEF-5 15-25 were included in the analysis. At 90-days, $106(75.7 \%)$ had not recovered potency defined as an IIEF-5 $<15$ and $91.1 \%$ of these 112 reported IIEF$5<10$. At one year, 24 of the $106(22.6 \%)$ recovered potency. In distinction, in the Figure utilizing \% fullness at 90 days, the lowest tertile (0-24\%) included 59/140 (42\%) of men and predicts only $7 / 59(11.9 \%)$ will recovery potency at 1 year. The second tertile $(25-74 \%)$ included $44 / 140$ (31.4\%), with $19 / 44$ $(42.2 \%)$ recovering potency at 1 year. Finally, the highest tertile included 36/140 (25.7\%), of which 26/36 (72.2\%) recovered potency be 1 year. Overall, 90 -day $\%$ fullness tertiles were highly predictive of 1 year potency recovery (p-value: $0.0001,95 \% \mathrm{CI}$ : 3.394 to 38.277, OR: 11.397). Correlation (r) between \% fullness tertiles and IIEF-5 were $0.28,0.52$, and 0.47 in the $0-24 \%, 25$ $74 \%$, and $75-100 \%$ tertiles, respectively.

Conclusions: An IIEF-5 below 15 at 90 days is a poor indicator of who will and will not recover long-term potency. For men with IIEF-5 $<15$ at 90 -days, $0-24 \%$ fullness predicts only a $13 \%$ recovery rate at 1 year whereas the recovery rate is $42 \%$ in men with $25-74 \%$ fullness. $\%$ fullness accurately estimates 1 -year recovery and discerns the need for early therapeutic intervention.

MP29-17 Urodynamic Testing in Patients with Lower Urinary Tract Symptoms Prior to Robotic Assisted Laparoscopic Prostatectomy Helps Guide Postoperative Use of Anticholinergic Therapy for Early Return of Continence

S Razdan, LA Barrueto, JP Chiou, S Razdan

University of Miami Miller School of Medicine

United States 
Introduction \& Objective: OBJECTIVES: To evaluate the efficacy of preoperative urodynamic testing in patients with detrusor instability and sensory urgency undergoing robotic assisted laparoscopic prostatectomy (RALP) in predicting the need for postoperative anticholinergic therapy for early return of continence.

Materials and Methods: PATIENTS AND METHODS: We retrospectively studied the records of 180 patients with irritative lower urinary tract symptoms (LUTS) who were prospectively chosen to undergo urodynamic testing prior to RALP. Ninety patients with abnormal findings of detrusor instability or sensory urgency on urodynamics were divided into two groups. Group A consisted of 45 patients who received three months of anticholinergic therapy upon Foley catheter removal after surgery, while Group B consisted of 45 patients who did not receive anticholinergic therapy. Group $\mathrm{C}$ consisted of ninety patients who had normal urodynamic results and did not receive anticholinergic therapy. All groups underwent our maximal urethral length preservation (MULP) technique to maximize postoperative return of urinary continence. Patients were followed 10 days, 3 months, and 6 months after surgery and their degree of continence was measured. A 1-month telephonic interview was also conducted. Incontinence was defined as the use of at least one small liner.

Results: There was a significant difference in return to continence between Group A and Group B. Average number of days until return of urinary continence in Group A was $22 \pm 3$ whereas it was $54 \pm 8$ in group B $(\mathrm{p}<.05)$. There was no significant difference in average number of days until return of continence between Group A and Group C $(20 \pm 3$ days, p<.05). By the $3-$ month follow up, $95 \%$ of all patients had return of urinary continence. By the 6-month follow up, $99 \%$ of all patients had return of continence.

Conclusions: In our large series of men undergoing RALP, we noted that patients with LUTS were at increased risk of developing postoperative delayed return of urinary continence. This prompted us to use urodynamic testing prior to RALP to detect those patients with LUTS who had sensory urgency or detrusor instability and help guide our postoperative treatment. The use of postoperative anticholinergics can help hasten return of urinary continence in these men when compared to men who did not receive postoperative anticholinergics. Interestingly, we noted that patients with a preoperative diagnosis of type II diabetes mellitus were more likely to have detrusor instability and sensory urgency on urodynamics.

\section{MP29-18 Withdrawn}

MP29-19 Dehydrated Human Amniotic Membrane and Early Return of Erectile Function Post Robotic-Assisted Laparoscopic Prostatectomy: Update from a High Volume Surgeon

S Razdan, JP Chiou, LA Barrueto, S Razdan

University of Miami Miller School of Medicine United States

Introduction \& Objective: OBJECTIVES: To evaluate the efficacy of dehydrated human amniotic membrane (dHAM) in achieving early return of erectile function post robotic-assisted laparoscopic prostatectomy (RALP).
Materials and Methods: PATIENTS AND METHODS: We retrospectively studied the time to achieve erectile function in two groups of men undergoing RALP for localized prostate cancer. Group A consisted of 300 patients who had dHAM placed on bilateral neurovascular bundles (NVBs). Adherence to the NVB was facilitated by use of human fibrin sealant spray. Group B consisted of 300 patients who did not receive dHAM. Bilateral NVB preservation was carried out in both groups by our modified technique. Both groups had equivalent preoperative quality of erections as measured by SHIM score.

Our primary endpoint was time to first erection.

Results: There were no significant differences in preoperative quality of erections between Group A $(\mathrm{SHIM}=22)$ and Group B $(\mathrm{SHIM}=21)(\mathrm{p}$ value $=.215)$. Patients in Group A achieved early return of erectile function, with $90 \%$ of patients having erections within a month. Group A had a mean time to first erection of $18 \pm 5$ days, while Group B had a mean time to first erection of $47 \pm 10$ days $(\mathrm{p}<.05)$.

Conclusions: While meticulous neurovascular bundle preservation remains an important factor in early return of erectile function, the use of dHAM can help achieve even earlier onset of erections. Our previous study in 2012 was the first in the world to show that dHAM can help hasten onset of erections post RALP when used to cover bilateral NVBs. Our new technique of using human fibrin sealant spray to secure the membranes in place over the NVBs seems to have greatly accelerated the time to first erection from our prior groundbreaking study by maximizing surface area contact between the membrane and the NVB.

MP29-20 Improved urinary function following robotic radical prostatectomy with a concurrent retropubic vascularised fascial sling (RoboSling)

S Leslie, R Thanigasalam, P Sved, A Vasilaras, N Jeffery, P Bergamin, G McClintock, E Lim, M Winter, N Ahmadi, M Desai, M Aron, IS Gill

Australia

Introduction \& Objective: Urinary incontinence following radical prostatectomy remains a deterrent for many patients when deciding on treatment for their localized prostate cancer. Although in the long-term continence rates exceed 90\%, early continence rates are much lower, ranging between $30 \%$ to $50 \%$ at 6 weeks. We describe a novel technique to improve early and late urinary function by using an autologous sling of vascularized fascia (RoboSling) placed underneath the urethrovesical anastomosis at the time of robotic assisted radical prostatectomy (RARP).

Materials and Methods: This is a prospective, non randomised cohort study comparing the urinary function outcomes between patients who underwent a standard RARP operation and those who in addition to RARP had a RoboSling procedure performed concurrently. Between January 2016 and December 2016, 42 patients underwent RARP, 21 without a RoboSling and 21 with a RoboSling. Data was collected on pad usage as well as clinical and oncological outcomes. The RoboSling technique consists of mobilising a rectangular, vascularized flap of peritoneum from the posterior aspect of the bladder. This is performed at the beginning of the operation. Following removal of the prostate, the flap is tunnelled underneath the bladder to rest over the rectum. It is then incorporated into the Rocco stitch with a 3-0 v-loc suture. Following the urethrovesical anastomosis, separate 3-0 v-loc 
sutures are placed through the corners of the flap on each side. The sutures are then passed through the pubic bone and the RoboSling tensioned providing a hammock underneath the urethra and bladder neck.

Results: Mean age of the 42 patients was 67. Mean follow-up was 6 months. For patients without the RoboSling, 0 pad usage at 2 weeks, 6 weeks, 3 months and 6 months was $11 \%$, $32 \%, 40 \%$ and $62 \%$ respectively. For patients with the RoboSling, 0 pad usage at 2 weeks, 6 weeks, 3 months and 6 months was $18 \%, 42 \%, 49 \%$ and $71 \%$ respectively. Two patients in the RoboSling group experienced a post-operative urinary tract infection. No patients experienced urinary retention, although 2 patients in the RoboSling group described mild straining on urination. However, these symptoms were self-limiting.

Conclusions: Patients undergoing a RoboSling procedure at the time of robotic radical prostatectomy experienced an earlier return to continence compared to the control arm. There were no Clavien III or IV complications in this patient cohort demonstrating safety of the procedure. A randomized controlled, blinded study is now underway to further assess the merits of this novel technique.

MP29-21 An Evaluation of Functional Outcomes after Robot-Assisted Radical Prostatectomy in patients with large prostates $(\geq 60 \mathrm{~g})$

Y Law, M Tan, J Du, H Huang, S Ho

Department of Urology, Singapore General Hospital Singapore

Introduction \& Objective: Robotic-assisted laparoscopic radical prostatectomy (RALP) has been widely accepted as a form of treatment for prostate cancer. Incidentally, some of these prostate cancer patients may have large prostates and therefore experience lower urinary tract symptoms (LUTS). We aim to review the functional outcomes of patients who have large prostates weighting $\geq 60 \mathrm{~g}$ and have undergone RALP.

Materials and Methods: We retrospectively analyzed 75 patients with prostate weights $\geq 60 \mathrm{~g}$ who had undergone RALP from July 2004 to April 2013. Their functional outcomes were compared pre and post procedure using the International Prostate Symptom Score (IPSS) including a measure of quality of life (QoL). Uroflow studies measured mean peak flow rates (Qmax) and post voided residual volumes (PVR) The SPSS (ver. 12) was used. Statistical analysis was carried out using the independent sample $t$-test and chi-square test. $\mathrm{P}<0.05$ indicated statistical significance.

Results: The baseline IPSS compared against 3, 6 and 12 months post operation were 8.18 vs $8.26(n=39), 8.52$ vs $5.55(n=29)$ and 6.69 vs $3.92(\mathrm{n}=24)$ respectively. The baseline QoL compared against 3, 6 and 12 months post operation were 2.12 vs 2.15 $(n=39), 2.03$ vs $1.79(n=29)$ and 1.67 vs $1.41(n=24)$ respectively. Preoperative Qmax improved from 12 to $18.79 \mathrm{ml} / \mathrm{s}$ postoperatively $(\mathrm{n}=27)$ and pre-operative PVR improved from 23.41 to $2.89 \mathrm{ml}$ post-operatively $(\mathrm{n}=27)(\mathrm{p}<0.05)$ Percentage of continent patients at baseline, 3,6 and 12 months were $98.5 \%$, $63.2 \%, 82 \%$ and $88.9 \%$ respectively.

Conclusions: There was overall improvement in the IPSS, QOL, Qmax and PVR values post RALP for patients with prostate weight $\geq 60 \mathrm{~g}$. There is interval improvement in both IPSS and QoL at 3 months vs 6 months and 3 months vs 12 months

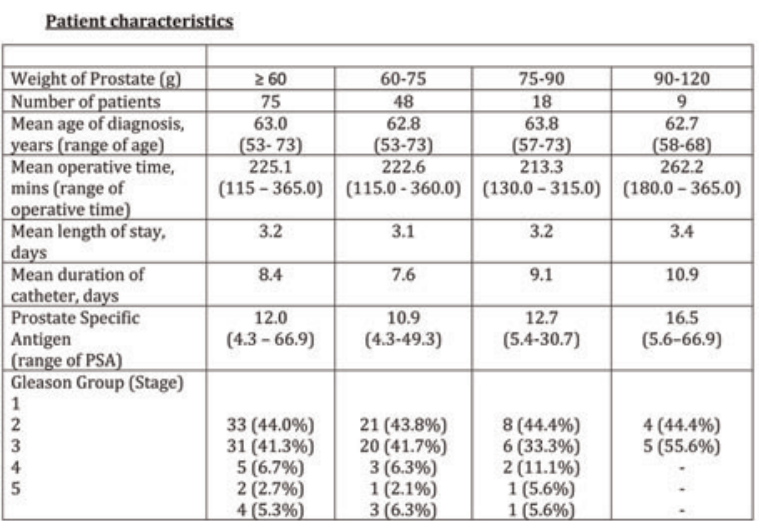
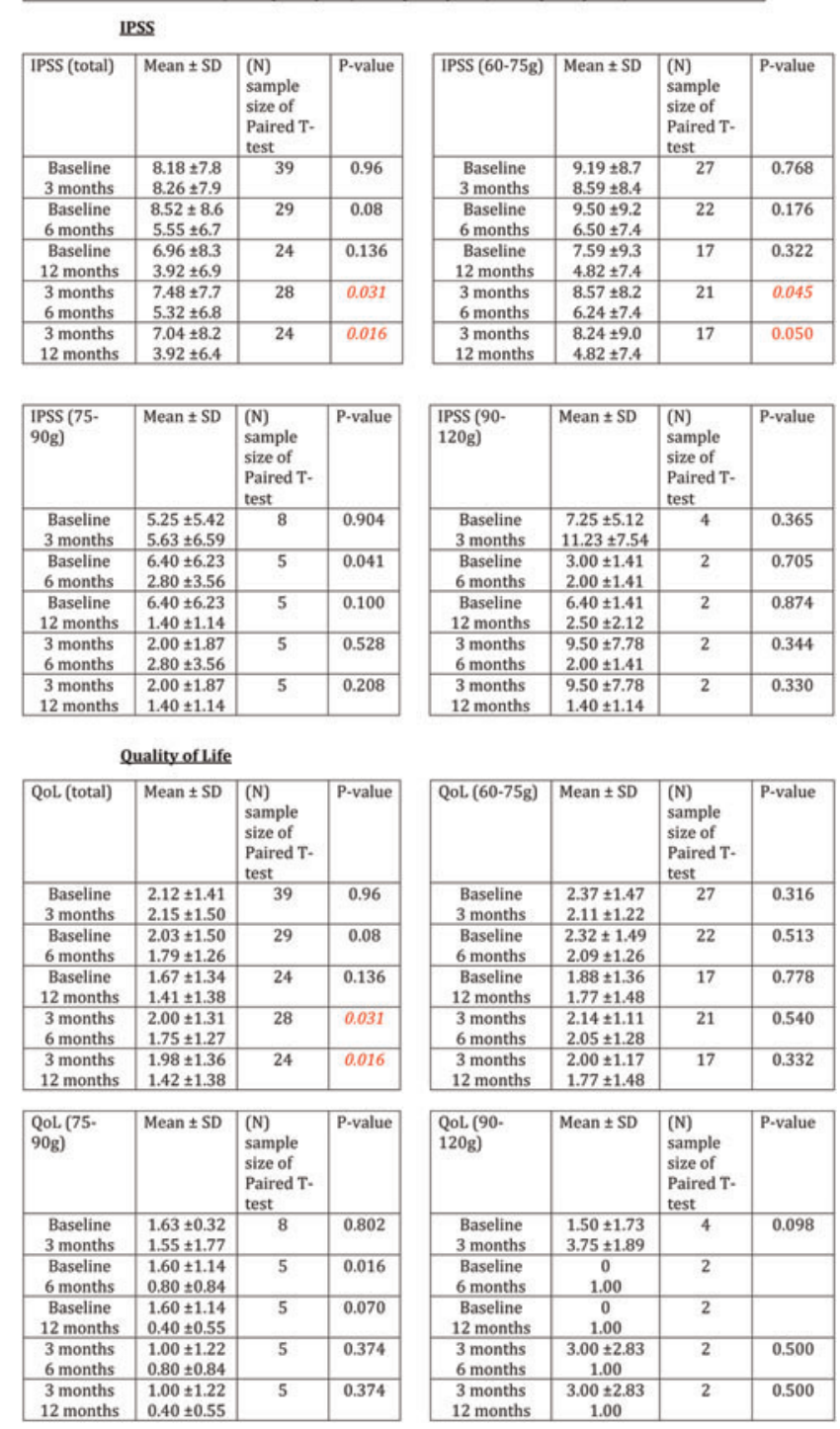

$(\mathrm{p}<0.05)$. However, when the IPSS scores/QoL scores at baseline were compared individually with post-operative values obtained at 3,6,12 month intervals the improvement did not reach statistical significance. IPSS is a subjective questionnaire whereas uroflow was an objective measure. Although there was statistically significant improvement in uroflow parameters, patients may not have reported an improvement of IPSS values correspondingly. 

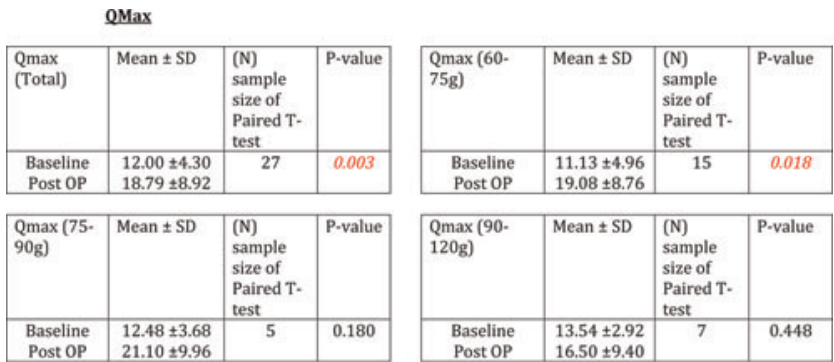

PVB
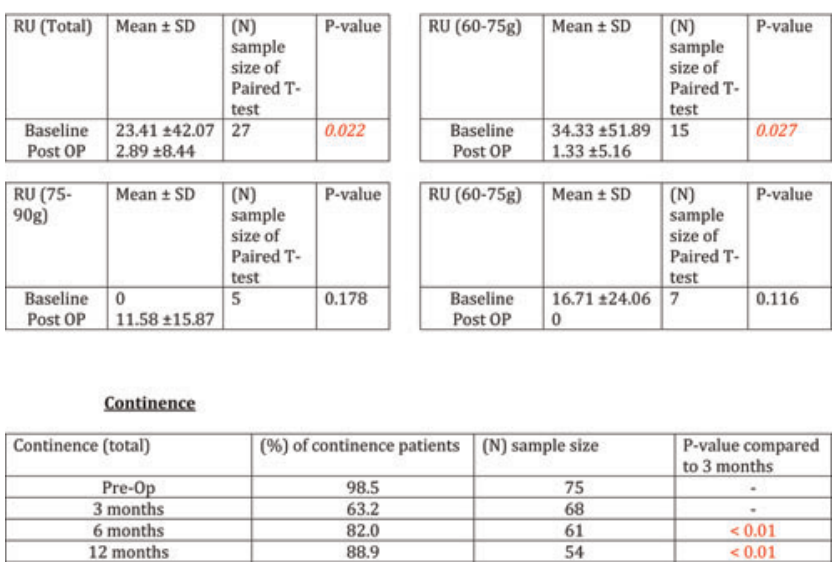

MP29-22 Improvement of Immediate Continence Control after Minimally Invasive Radical Prostatectomy - from anterior to posterior, from robotic-assisted to pure laparoscopic

K Chiu

ivision of Urology, Department of Surgery, Taichung Veterans General Hospital, Taichung, Taiwan

Taiwan (Republic of China)

Introduction \& Objective: Post-operative incontinence was still a main drawback for patients underwent minimally invasive radical prostatectomy, not only laparoscopic radical prostatectomy (LRP) but also robotic-assisted radical prostatectomy (RaRP). Several papers published suggested more than 200 cases for overcoming the steep learning curve of these kinds of surgeries to achieve high quality of post-operative continence control. We present the experience of a single surgeon about continence recovery both on LRP and RaRP.

Materials and Methods: From Mar. 2004 to Feb. 2017, totally 180 patients received minimally invasive radical prostatectomy (both LRP and RaRP) performed by a single surgeon were retrospectively evaluated about their continence control by questionnaires conducted at out-patient clinics on 1 week, 1 month and 3 months after the procedures, respectively. The methods of operation as well as the reconstruction techniques used for regaining continence control were compared. The continence control was defined as no pad use on any time.

Results: The most useful single technique to improve early continence control was Retzius-sparing radical prostatectomy not only for LRP but also for RaRP. As many techniques used for continence reconstruction guaranteed a better continence recovery.

Conclusions: Improvement of surgical techniques would offer patients early continence control for less experienced surgeons. If possible, starting with Retzius-sparing radical prostatectomy could offer better chance of continence control.
MP29-23 Robitic-Assisted Sacrocolpopexy For Pelvic Organ Prolapse: Technique And Early Outcomes

PW Mekhail, FZ Husain, C Sevilla, MW Mekhail, IS Gill, A Monish, L Rodriguez

University of Southern California

United States

Introduction \& Objective: Robotic-assisted sacrocolpopexy (RAS) is an emerging alternative to the traditional open and laparoscopic approaches. We report our single-center experience in patients who underwent RAS for pelvic organ prolapse.

Materials and Methods: A retrospective chart review was performed of 29 patients with grade 3-4 or symptomatic grade 2 pelvic organ prolapse, between July 2010 and December 2015, who underwent RAS at our institution. The measured perioperative outcomes included operative time (OT), estimated blood loss (EBL), post-operative transfusion rate, length of hospital stay (LOS), and early (30 day) and late (90 day) complication data. Continuous variables were shown as mean, standard deviation (SD), and minimum and maximum values (range). Categorical variables were shown as frequencies and percentages.

Results: The mean (range) patient age and BMI was 62 years (39 - 83) and 28 (20 - 41), respectively. Pre-operative symptoms reported were urinary incontinence in 5 patients $(17.2 \%)$, pelvic pressure in $25(86.2 \%)$, constipation in $6(20.6 \%)$, and urinary tract infections (UTIs) in 7 (24\%). A cystocele was present in 23 cases $(79.3 \%)$, apical prolapse in $18(62 \%)$, and rectocele in 17 $(58.6 \%) .14$ patients $(46.7 \%)$ had both a rectocele and cystocele. The mean (range) OT was 246 minutes (150 - 360) and EBL was $105 \mathrm{~mL}$ (50 - 600). No perioperative transfusions were needed. Concomitant procedures performed were urethral sling placement $(40 \%)$, transvaginal rectocele repair $(38 \%)$ and 1 laparoscopic left radical nephrectomy. The mean (range) LOS was 1.6 days $(1-5)$. The mean follow-up was 4.6 months (1-16). Early minor (Clavien II) postoperative complications occurred in 3 patients $(10.3 \%)$. Late minor postoperative complications (Clavien II) occurred in 2 patients $(6.8 \%)$. No major complications occurred. A grade I POP relapse occurred during the first month in 3 patients and during the first 3 months in another 3 patients. Mesh erosion occurred in only 1 patient, after 3 months. Conclusions: RAS is a safe and effective treatment option for pelvic organ prolapse, with an acceptable morbidity.

MP29-24 Technical development of a transvaginal probe using near infrared light to monitor pelvic floor muscle hemodynamics bilaterally

EG Deegan, L Stothers, D Lazare, AJ Macnab

International Collaboration on Repair Discoveries (ICORD) and Dept. of Experimental Medicine, UBC

Canada

Introduction \& Objective: Near infrared spectroscopy (NIRS) is a noninvasive optical method for measuring oxygenation and hemodynamics in muscle in real time. Light in the NIR spectrum penetrates into tissue and detects variations in the concentration of the chromophores oxygenated $(\mathrm{O} 2 \mathrm{Hb})$ and deoxygenated hemoglobin $(\mathrm{HHb})$ from which changes in tissue blood volume and oxygen supply and demand are derived. This allows physiologic parameters related to muscle function to be quantified in response to contraction. 
Assessment of pelvic floor muscle (PFM) function is central to managing urinary incontinence (UI) / lower urinary tract symptoms (LUTS) but currently lacks quantifiable physiologic measures. Objectives: (1) to develop a transvaginal probe incorporating NIRS technology able to assess changes in $\mathrm{O} 2 \mathrm{Hb}$ and $\mathrm{HHb}$ in the right and left PFM and (2) to compare NIRS derived parameters in continent controls vs cases with LUTS due to neurogenic disease.

Materials and Methods: A prototype transvaginal probe was designed and used to monitor female volunteers during conduct of standardized exercise protocol during rest, maximal voluntary, sustained and fast repeated PFM contractions. Data captured at $10 \mathrm{~Hz}$ included $\mathrm{O} 2 \mathrm{Hb}, \mathrm{HHb}$ and their sum total hemoglobin $(\mathrm{tHb})$ included right and left sided channel recordings to allow detection of asymmetric muscle function. Changes in relative $\mathrm{O} 2 \mathrm{Hb}, \mathrm{HHb}$ and $\mathrm{tHb}$ concentrations were compared from rest to peak contraction and validated methods used to determine oxygen consumption during exercise and resaturation rate in response to contraction.

Results: The transvaginal probe incorporated miniature fiberoptic cables inside a disposable optically inert plastic speculum. NIR light (wavelengths) 766nm, 861 nm, 906nm and $971 \mathrm{~nm}$ generated by a 4 channel Oxymon Mk III (Artinis Medical Systems) were transmitted to paired NIR emitter and detector optodes secured bilaterally with a $20 \mathrm{~mm}$ inter optode distance to yield a tissue penetration depth of $10 \mathrm{~mm}$. The probe was well tolerated during collections $(\mathrm{N}=10$ graphs of controls and 3 cases) chromophore changes were noise free and correlate with published NIRS muscle data.

An example graph derived is shown in figure one.

Conclusions: This pilot study demonstrates the feasibility of using a transvaginal NIR probe to measure novel physiologic data in the context of PFM exercise. This provides new insight into how symmetry and coordination may contribute to PFM function.

\section{MPS30: BPH/LUTS II}

MP30-1 The WATER Study - A Phase III Blinded Randomized Trial of Aquablation vs. Transurethral Resection of the Prostate for the treatment of Benign Prostatic Hyperplasia

P Gilling, C Roehrborn

Bay of Plenty District Health Board

New Zealand

Introduction \& Objective: Prostate resection for patients with LUTS remains the most common procedure for surgical treatment of BPH. A prospective single-arm multicenter trial using Aquablation at 3 centers in Australia and New Zealand with 1-year follow-up on 21 men with a similar profile to WATER has been previously presented. We compared the safety and efficacy of prostate ablation using a highvelocity waterjet (Aquablation) with TURP in the WATER study.

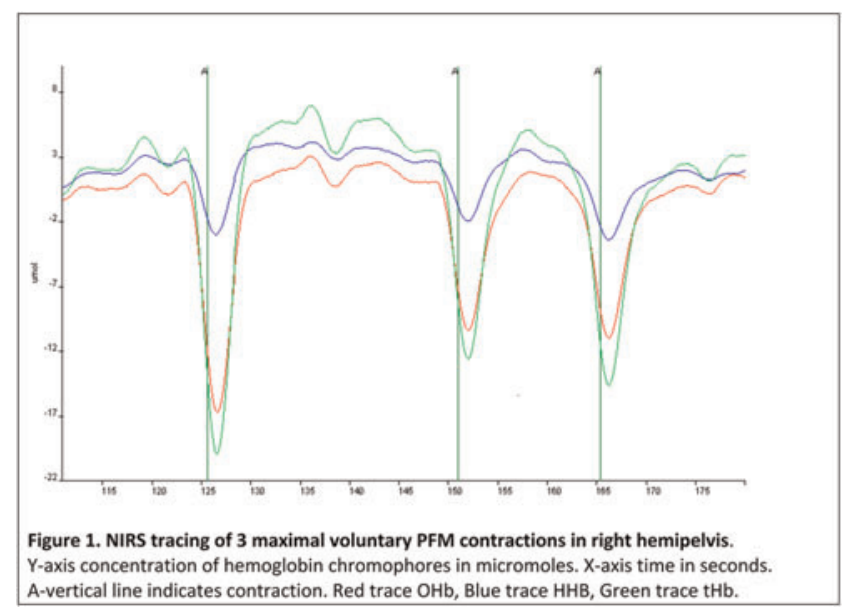

Materials and Methods: In this randomized, double-blind, multicenter phase III trial, patients with moderate-to-severe LUTS related to BPH were assigned to standard TURP or Aquablation. The trial had a co-primary safety and efficacy endpoint designed to show non-inferiority. With a minimum enrollment of 177, the estimated power for safety was $99 \%$ and efficacy was $80 \%$. The primary safety endpoint was the occurrence of Clavien-Dindo Grade 1 or higher peri-operative complications at 3 months. The primary efficacy endpoint was the reduction at 6 months in IPSS score.

Results: The geographic enrollment from the International and US sites was 91 and 93 subjects, respectively. The baseline demographic profile (Table 1), and mean prostate volume (T: $52 \mathrm{~mL}$ vs. A: $54 \mathrm{~mL}, \mathrm{p}=0.31$ ) were similar in both arms. Mean operative time was equivalent between the two groups (T: 35.5 vs. A:32.8 minutes, $\mathrm{p}=0.28$ ), but mean resection time was significantly less in the Aquablation group (28 vs. 4 minutes, $\mathrm{p}<.0001)$. The length of hospital stay was similar for both arms, a mean of 1.4 days. At 3 months, Qmax significantly improved in both groups (T: $9.1 \pm 2.7$ to $20.1 \pm 11.4$ vs. A: $9.4 \pm 3.0$ to $20.9 \pm 13.4, \mathrm{p}=\mathrm{NS})$.

Conclusions: Preliminary analyses demonstrate equal early efficacy of Aquablation and TURP however ablation/resection time was significantly shorter for Aquablation.

Table 1

\begin{tabular}{lrrrrrrr}
\multicolumn{1}{c}{ Aquablation } & \multicolumn{7}{c}{ TURP } \\
\hline & $\mathbf{N}$ & Mean & SD & N & Mean & SD & P value \\
\hline Age & 117 & 66.0 & 7.3 & 67 & 65.8 & 7.2 & 0.8596 \\
BMI & 117 & 28.4 & 4.1 & 67 & 28.2 & 4.5 & 0.7941 \\
& & & & & & & \\
IPSS & 116 & 22.9 & 6.0 & 65 & 22.2 & 6.1 & 0.4276 \\
IPSS QoL & 116 & 4.8 & 1.1 & 65 & 4.8 & 1.0 & 0.8009 \\
MSHQ & 108 & 7.4 & 4.1 & 61 & 8.2 & 4.0 & 0.2155 \\
SHIM & 115 & 13.7 & 8.6 & 64 & 13.4 & 9.4 & 0.8243 \\
\hline
\end{tabular}


MP30-2 Green laser (532-nm) enucleation of the prostate (GreenLEP) en bloc technique. initial experience, and shortterm outcomes.

E Rijo, J Lorente, O Bielsa, F Gomez-Sancha

Department of Urology, Hospital Quiron Barcelona. Spain

Introduction \& Objective: All transurethral endoscopic enucleation of the prostate (EEP) techniques (HoLEP, ThuLEP, ThuVEP, DiLEP and bipolar enucleation) represent alternatives to open prostatectomy and TURP for the treatment of lower urinary tract symptoms secondary to benign prostatic hyperplasia (BPH).

Green laser "en bloc" enucleation of the prostate (GreenLEP) followed by mechanical morcellation is one of the latest techniques in the armory of EEP.

Nowadays many urologists seem to be unaware that EEP is feasible with 532-nm laser.

The aim of this study is to report our initial experience with this novel technique, assesment of feasibility and short-term outcomes.

Materials and Methods: Sixty-one patients underwent GreenLEP by a single surgeon (E.R.) using the technique described by Fernando Gomez-Sancha between Sept 2015 and Oct 2016 at our institution with prostate volumes between 50 and $195 \mathrm{cc}$. All patients were evaluated preoperatively and after surgery (at 1, 3, and 6 months) by PSA, prostate volume, IPSS, QOL score, Qmax, and PVR, and all the complications were recorded. The surgery was performed with a 532-nm lithium triborate laser (GreenLight XPS 180W device; American Medical Systems, Minnetonka, MN), 2090 side-fire laser fiber and a Piranha morcellation system (Richard Wolf GmbH, Germany).

Results: The mean operative time was 63 minutes and the mean amount of retrieved prostate tissue was 64 gr. [50.0;84.0]. There was no need for blood transfusion. Capsular perforation occurred in five cases. We did not have any case of bladder injuries related to the morcellation procedure. The postoperative catheterization

\begin{tabular}{l|c}
\hline & {$[\mathrm{ALL}]$} \\
\hline \hline Age & $\mathrm{N}=61$ \\
PSA (ng/ml) & $68.0[65.0 ; 72.0]$ \\
IPSS & $3.68[2.79 ; 4.37]$ \\
QoL & $24.0[21.0 ; 27.0]$ \\
Qmax (ml/s) & $5.00[4.00 ; 5.00]$ \\
PVR (ml) & $9.00[7.75 ; 10.7]$ \\
Prostate volume (ml) & $90.0[75.0 ; 115]$ \\
Days of stay & $90.0[75.0 ; 115]$ \\
intervention time (min) & $2.00[2.00 ; 2.00]$ \\
Capsular perforation & $63.0[59.0 ; 70.0]$ \\
Specimen weight (gr) & $5(8.20 \%)$ \\
Catheterization time (days) & $64.0[50.0 ; 84.0]$ \\
Complication & $2.00[2.00 ; 2.00]$ \\
Clavien: & $9(14.8 \%)$ \\
I & \\
II & $2(22.2 \%)$ \\
IIIb & $2(22.2 \%)$ \\
Clavien minor $(\mathrm{I} / \mathrm{ll})$ & $5(55.6 \%)$ \\
Clavien major $(\mathrm{III} / \mathrm{IV})$ & $4(6.56 \%)$ \\
IPSS post & $5(8.20 \%)$ \\
QoL post & $5.00[4.00 ; 6.00]$ \\
Qmax post (ml/s) & $2.00[1.00 ; 2.00]$ \\
PVR post (ml) & $28.0[24.0 ; 32.0]$ \\
1 month SUI & $0.00[0.00 ; 10.0]$ \\
3 months SUl & $6(9.84 \%)$ \\
6 months SUI & $3(4.92 \%)$ \\
2 months PSA (ng/ml) & $0(0.00 \%)$ \\
6 months PSA (ng/ml) & $1.02[0.92 ; 1.45]$ \\
Tissue removed (\%) & $0.88[0.66 ; 1.05]$ \\
PSA drop & $73.5(7.85)$ \\
PSA decrease (\%) & $2.62(1.19)$ \\
\hline & $96.1(2.14)$ \\
\hline
\end{tabular}

time (same as hospital stay) was 48 hours, except in two cases: one of incomplete morcellation because of bleeding and requiring an endoscopic second look (morcellation for residual adenoma), and the other required open surgery conversion for removal of the enucleated adenoma (poor vision because of venous bleeding caused by surgical perforation of the capsule during enucleation). Transient urinary incontinence was present in three cases and was completely resolved in 2 months. Three months later, all the parameters evaluated preoperatively showed significant improvement.

Conclusions: GreenLEP represents a safe alternative enucleation technique for the complete removal of en bloc (one lobe) adenomatous prostate tissue. EEP is feasible with a 532-nm sidefiring fiber. It should be emphasized that green laser is not only useful for classic vaporization, but also for EEP.

These promising results warrant further studies to assess longterm outcomes.

MP30-3 Modern management of benign prostatic hyperplasia in large glands: a review of a single center with high volume open, robotic, and laser prostatectomy.

S Mehta, G Gaunay, S Smith, Z Okeke, L Richstone, P Samson

Smith Institute for Urology

United States

Introduction \& Objective: The traditional management of large prostates has been the open suprapubic prostatectomy (OSP). The stress of this procedure led to the development of minimally invasive procedures: robotic suprapubic prostatectomy (RSP) and laser enucleation of the prostate (LEP). Our institution is the first to compare all three modalities.

Materials and Methods: With IRB approval, the medical records of all patients undergoing OSP, RSP, or LEP between January 2008 and March 2017 were reviewed. RSP was performed on the da Vinci system. LEP was performed using either the holmium or thulium (Vela XL) lasers. We summarized demographics, perioperative outcomes assessed at follow-up, and complications encountered during or after hospitalization. Data were analyzed by ANOVA using SAS 9.4.

Results: In total, 69 OSP, 38 RSP, and 134 LEP procedures were performed in the study time-frame, with an average follow-up of 12,5 , and 5 months respectively. OSP patients tended to be older than RSP and LEP patients ( 73 vs. 68 and 70 years respectively). LEP patients in our study tended to have smaller prostates than OSP and RSP (97 vs. 165 and 137 gm respectively), although this did not reach statistical significance. Otherwise ASA, BMI, and preoperative hemoglobin, maximum flow rate, and post-void residuals were the same. Operative time was significantly higher for RSP compared to OSP and LEP (203 vs. 130 and 136 minutes respectively). Foley duration and analgesic requirement favored LEP in comparison with OSP and RSP (4 vs. 11 and 12 days; 5 vs. 29 and 20 morphine equivalents respectively). Furthermore, all OSP patients were given a patient-controlled analgesic while neither RSP nor LEP patients needed one. Length of stay was significantly higher in OSP as opposed to RSP or LEP (6 vs. 3 and 2 days respectively).

Conclusions: Our contemporary analysis reveal that all three reviewed modalities produce equivalent functional outcomes. Patients should be counseled on unique issues associated with each, such as the need for PCA in OSP or the risk of strictures in LEP. Multi-institutional studies may help identify if any modality is superior to another. 
MP30-4 Evaluating the learning curve for robotic assisted simple prostatectomy

I Sorokin, BA Johnson, N Singla, C Roehrborn, J Gahan

United States

Introduction \& Objective: Robotic assisted simple prostatectomy (RASP) and Holmium laser enucleation of the prostate (HoLEP) have equivalent outcomes for treating large volume prostates. However, RASP incorporates skills familiar to surgeons trained in robotics and may be better suited for wider adoption into urological practice and residency teaching programs. Prior studies have shown the HoLEP learning curve to be around 20-60 cases. Our objective was to evaluate the learning curve for RASP.

Materials and Methods: A retrospective review of 94 consecutive cases performed by 2 surgeons from 2014 to 2017 was conducted. Cases by each surgeon were divided into cohorts of 10 cases to assess changes in means analyzed by Analysis of Variance (ANOVA). Learning curve was evaluated by assessing trends in percentage of prostate tissue yield, percentage PSA reduction, estimated blood loss (EBL), and operating room time. Scatter plots of cases with a logarithmic best-fit line were drawn to analyze the learning curve.

Results: Surgeon 1 performed 57 cases while surgeon 2 performed 37 cases. The mean age of the entire cohort was 69.4 years and mean prostate volume was $135 \mathrm{~cm} 3$. Percentage tissue yield demonstrated a plateau after 10 cases for surgeon 2 while staying relatively even for surgeon 1. EBL demonstrated a decreasing trend with a large decrease over the first 10 cases and improvement still evident beyond 40 cases. The average EBL in the first 10 cases for both surgeons was $462.5 \mathrm{~mL}$ vs. $200 \mathrm{~mL}$ for their last 10 cases $(\mathrm{p}=0.01)$. Percentage of PSA reduction and operating room time stayed consistent between both surgeons with a plateau of the best-fit line evident after the first few cases. Neither operating room time nor percentage of PSA reduction time showed any statistically significant difference between the cohort groups. All factors considered together, a learning curve of 5-10 cases was observed graphically. Conclusions: Between our two-surgeon cohort, the learning curve for RASP appears to be much shorter compared to HoLEP. This is likely based on the surgeon's prior experience with other various robotic procedures.

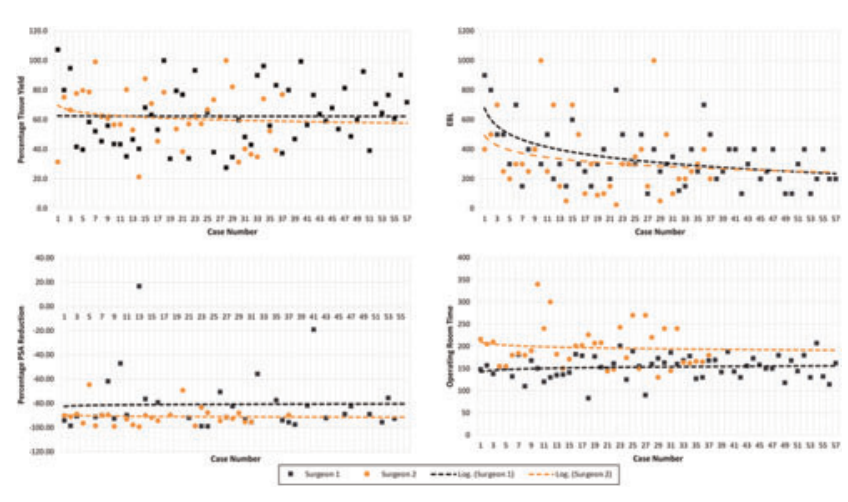

MP30-5 Benign prostatic hyperplasia in octuagenarians: a thulium laser vaporization experience

L Carmignani, C Signorini, M Clementi, D Vizziello, G Motta, S Nazzani, S Picozzi, R Stubinski

IRCCS Policlinico San donato

Italy
Introduction \& Objective: Geriatric patients with benign prostatic hyperplasia (BPH) are often treated with an indwelling urinary catheter, worsening quality of life with high risk of urinary tract infections. We evaluated the outcomes of patients $>80$ years treated with thulium laser vaporization of the prostate (ThuVAP).

Materials and Methods: We enrolled 78 patients (age $\geq 80$ years) treated with ThuVAP at two medical centers. Bedfast patients or with cognitive impairment or neurogenic bladder were excluded. We evaluated the following criteria: age, ASA score, antiplatelet and anticoagulation therapy, International Prostate Symptom (IPSS) and quality of life (QoL) scores, maximum urinary flow rate (Qmax), and post-void residual (PVR) before surgery and postoperatively at 3 and 6 months. Surgery-related complications were recorded.

Results: Mean age was 82.8 years, $20 \%$ of patients had an ASA score $>2,25$ had an indwelling catheter, and 18 were previously ineligible for surgery. 17 patients were taking antiplatelet medication, 6 patients were assuming anticoagulation therapy. Before surgery, mean IPSS scores were 19.3, respectively. At 3 and 6 months, the mean scores improved to 8.1 and 6.5, respectively. Preoperatively, the mean Qmax and PVR values were 6.8 and 169.5, respectively. Postoperatively, the values improved to 16.7 and 16.2 and 102.3 and 86.4, at 3 and 6 months, respectively. No intraoperative complications occurred. Blood transfusion was necessary in one case. One month after surgery 5 patients had an indwelling catheter.

Conclusions: ThuVAP may reduce LUTS and improve IPSS scores in geriatric patients also with an indwelling catheter.

MP30-6 Safety and feasibility of thulium laser transrethral resection of prostate for benign prostatic enlargement surgery in overweight and obese patients.

L Carmignani, D Vizziello, M Clementi, A Conti, E De Lorenzis, P Acquati

IRCCS Policlinico San donato Italy

Introduction \& Objective: We aimed to determine safety and feasibility of thulium laser transurethral vapoenucleation of prostate (ThuVEP) for treatment of obese patients affected by benign prostatic hyperplasia (BPH).

Materials and Methods: We retrospectively analysed data of 452 patients with BPH who underwent ThuVEP from February 2012 to March 2016 at our Institution. Patients were divided in three groups according to BMI: Normal weight (18.5 30; group 3 ), for a total of 412 patients evaluable for this study. Preoperative total serum prostate-specific antigen (PSA), digital rectal examination of the prostate, transrectal ultrasound (TRUS), renal ultrasound, urine culture, and uroflowmetry, International Prognostic Scoring System (IPSS) score, Quality of Life (QoL) score were analysed. Post-operative complications, hospital stay and days of catheterization, questionnaires and uroflowmetry at one and three months after surgery, were evaluated. Preoperative data, surgical outcomes, complication rate and clinical outcomes were compared between groups.

Results: The mean age of patients was $68.8+-7.934$. The preoperative mean IPSS among group was $19.02+-6.575,20.06+-$ $6.989,19.53+-8.246$ respectively. At one and three months of follow up, this value was $8.63+-5.342,8.04+-4.583,7.67+-5.581$ and 5.88+-4.77, 5.59+-4.802, 6.47+-5.594 respectivley (all p 
between groups $>0.05$ ). There was no statistically significant difference among three groups as for hospital stay and days of catheterization (all $\mathrm{p}>0,05)$.

Conclusions: Compared with normal weight population, overweight and obese patients did not show a statistically significant difference about complication rate and clinical outcomes. Our results described ThuVEP safe and feasible treatment option even in patients overweight and substantially enlarged prostate.

\section{MP30-7 Comparison of the urinary outcomes of thuvap in two groups of patients with a prostate volume $<80 \mathrm{ml}$ and $>80 \mathrm{ml}$ (preliminary data). \\ L Carmignani, M Clementi, D Vizziello, G Motta, E Finkelberg, D Ratti, S Picozzi \\ IRCCS Policlinico San donato Italy}

Introduction \& Objective: Transurethral vaporization of the prostate (ThuVAP) is a new technique for the surgical treatment of men affected by benign prostatic hyperplasia (BPH). It is as effective as TURP and simple prostatectomy, considered the gold standard therapy for prostate of small volumes $(30-80 \mathrm{ml})$ and large volumes $(>80 \mathrm{ml})$ respectively. Moreover, it offers many advantages in terms of complications. However, data in literature regarding ThuVAP are still lacking. The aim of this study is to compare urinary outcomes of this technique in two groups of patients with prostate volume $80 \mathrm{ml}$.

Materials and Methods: We retrospectively analysed prospectively collected data of 128 patients with BPH, who underwent ThuVAP from February 2012 to June 2016 at our Institution. Patients were divided in two groups according to prostate volume: 76 80ml (group B). Surgical indications were decided according to EAU Guidelines. Preoperative data of total serum prostate-specific antigen (PSA), digital rectal examination of the prostate, transrectal ultrasound (TRUS), renal ultrasound, urine culture, and uroflowmetry and International Prognostic Scoring System (IPSS) score were collected. We also evaluated operation time and hospital stay. Post-operative maximum flow (Qmax) and IPSS were analysed at 3 months after surgery.

Results: The median age of patients was 69 years (interquartile range [IQR]: 64-75) for group A and 71 years (IQR 66-75) for group B. The preoperative median IPSS between groups was 20 (IQR 11-27), 27 (IQR 19.5-29), respectively. At 3- months follow up, this value was 6 (IQR 4.25-9), 6 (IQR 2.5-7.75), respectively. The preoperative median Qmax was 8.6 (IQR 6.711.1), 8.25 (IQR 5.9-10.4), respectively. Al 3-months follow up, this value was 15 (IQR 10.87-19.9), 19 (IQR 13.6-23.85), respectively. The median operative time was 50 minutes (IQR 4065 ) for group A and 70 minutes (IQR 50-95) for group B. The median hospital stay was 2 days (IQR 2-3) for group A and 3 days (IQR 2-3) for group B. The most frequent complications were acute urinary retention, urinary tract infection and macrohaematuria. In group A, $11.84 \%$ of patients had minor complications (Clavien I/II) and $2.63 \%$ had a major complication (Clavien III); in group B, $21.15 \%$ of patients had minor complications and no one had major complications.

Conclusions: Our results describe ThuVAP as an effective and feasible treatment option even in patients with large prostate volumes, even if with a slightly increased risk of minor complications.
MP30-8 Thulium laser prostate enucleation in refractory urinary retention: operative and functional outcomes in a large cohort of patients

L Carmignani, S Maruccia, D Vizziello, E Finkelberg,

D Ratti, A Saccà, A Pastore, F Pisano, M Schirinzi

IRCCS Policlinico San donato

Italy

Introduction \& Objective: The aim of the study is to evaluate the functional and operative results of thulium laser enucleation of the prostate (ThuLEP) in patients with indwelling catheters for refractory urinary retention.

Materials and Methods: Patients with indwelling catheter, undergoing ThuLEP for benign prostate hyperplasia, were prospectively enrolled. Every episode of urinary retention was treated with urinary catheter positioning followed by at least 2 attempts of catheter removal. Patients were investigated with flowmetry and the self-administered International Prostate Symptom Score questionnaire at 30 and 90 days following ThuLEP.

Results: Three hundred eighty-one patients underwent ThuLEP, and 99 of these had indwelling catheters, but only 93 (24\%) were eligible according to the inclusion criteria. In 46 patients the bladder catheter was removed on the first postoperative day, in 31 patients on the second postoperative day, in 5 patients on the third postoperative day, in 6 patients on the fourth postoperative day, in 1 patient on the fifth postoperative day, and in 2 patients each on the sixth and seventh postoperative days. The average hospital stay was $2.3( \pm 1.7)$ days. No patients undergoing ThuLEP, at the 90-day follow-up, required further catheterization. Flowmetry showed significant improvement in all parameters, and the mean International Prostate Symptom Score dropped from 21.33 preoperatively to $3.2(\mathrm{P}=.004)$ at 90 days postoperatively.

Conclusions: This prospective study shows that ThuLEP is a safe and effective approach in refractory urinary retention patients. In our case series, no patients required postoperative intermittent catheterization. All functional outcomes investigated reported a statistical significant improvement. (c) 2016 Elsevier Inc.

\section{MP30-9 Thulium laser vaporization: how many grams per} minute?

L Carmignani, M Clementi, G Motta, C Signorini, D Vizziello, E Finkelberg, S Maruccia, S Nazzani

IRCCS Policlinico San donato

Italy

Introduction \& Objective: Vaporization of prostate with Thulium laser is always more frequently used because of the simplicity of the surgical performance. Some authors referred that this technique would require much more time than enucleation, in particular for prostate of high volumes. Otherwise in the last years laser producers increased the power of laser and the fiber diameter so that the surgeon can vaporize a more amount of tissue in the unit of time. The aim of our study is to determinate in the most accurate way the amount of prostate tissue vaporized per minute at our institution.

Materials and Methods: Data from 40 patients underwent to Thuvap in our center were analyzed. Surgical interventions were 
performed by a single surgeon using Thulium lase 200 Watt and an end-fire fiber of $800 \mu \mathrm{m}$. All patients underwent transrectal ultrasound of prostate to determine prostate and adenoma volumes before surgery. Data of energy delivered and time of energy distribution were registered during surgery. IPSS and uroflowmetry were performed at 1 and 6 months after surgery. Evaluation of the tissue vaporized per minute is done dividing adenoma volume by minutes of laser distribution.

Results: From data analyzed we deduced that the amount of tissue vaporized is $1,8 \mathrm{~g} / \mathrm{min}$. Mean-IPSS of patients is 7,38 at 1 month after surgery and mean Q-max of $18,81 \mathrm{ml} / \mathrm{s}$. At 6 months mean- IPSS is 7,5 and Qmax 20,71. This allows to predict surgical times to optimize timing of surgery and it can be helpful for the anesthesist during surgery.

Conclusions: The high energy of new lasers and the increase of fiber dimensions allow a rapid, safe and effective vaporization, with no necessity of prostate morcellation for patient's benefit.

MP30-10 Can thulium vaporization of the prostate be considered as safe and effective as thulium vapoenucleation also for prostates larger than $80 \mathrm{ml}$ ? Preliminary results from a single institution

L Carmignani, M Clementi, S Nazzani, C Signorini, D Vizziello, S Picozzi, C Marenghi, D Ratti, R Stubinski, G Motta

IRCCS Policlinico San donato Italy

Introduction \& Objective: Minimally invasive laser technologies for BPO treatment are spreading because they can offer many advantages and less complications compared to simple prostatectomy and TURP. Thulium laser can be used both for prostate vaporization (ThuVap) and vapoenucleation (ThuVep) procedures. The aim of this study is to compare the efficacy and safety of ThuVap and ThuVep in a group of patients with a prostate volume larger than $>80 \mathrm{ml}$.

Materials and Methods: Between February 2012 and June 2016 , a total of 160 patients with a prostate volume $>80 \mathrm{ml}$ underwent ThuVep (group A - no. 108) or ThuVap (group B - no. 52 ) in our centre. Surgical indications were decided according to EAU Guidelines, the choice of the technique was based on relevant surgeons' skills. Preoperative assessment included digital rectal examination, prostate volume measurement via transrectal ultrasound (TRUS), uroflowmetry, international prostate symptom score (IPSS), quality of life (QoL), prostate specific antigen (PSA), blood analysis, urinalysis and urine culture. We also evaluated operation time, catheterization time and hospital stay. IPSS, QoL and maximum flow rate (Qmax) were recorded to assess the functional outcome 3 months after surgery. All data are expressed as median and interquartile range. Complications are expressed according to the Clavien classification system (CCS).

Results: The mean age was 68 in group A (62-74) and 71 in group B (66-75). The median prostate volume was $106 \mathrm{ml}$ (90-131.5) for group A and $109.5 \mathrm{ml}$ (95-151.25) for ThuVap patients. The median operation time was $1.35 \mathrm{~h}$ for ThuVep (including morcellation time) and $1.10 \mathrm{~h}$ for ThuVap. Median catheterization time was $27.5 \mathrm{~h}(22-43)$ in group A and $41 \mathrm{~h}$ (2848 ) in group B. The hospital stay was 2 days (2-3) for ThuVep and 3 days (2-3) for ThuVap group. The functional outcome for patients which underwent ThuVep and ThuVap are respectively
IPSS: 4 (2-6) and 6 (2.5-7.75), QoL: 1(0-1) for both, Qmax: $20.6 \mathrm{ml} / \mathrm{sec}(16.6-25.92)$ and $19 \mathrm{ml} / \mathrm{sec}(13.6-23.85)$. Minor complications (Clavien I/II) occurred in $16,66 \%$ in group A and $21.15 \%$ in group B. Major complications (Clavien 3a) occurred only in the ThuVep group in $4.63 \%$ of patients. One Clavien 4 occurred in the group A.

Conclusions: Compared to ThuVep, which is known to be a sizeindependent technique, ThuVap has similar functional outcomes at 3 months after surgery but a lower rate of major complications. ThuVap is a safe and effective technique for the treatment of symptomatic BPO also in patients with prostate volumes $>80 \mathrm{ml}$.

MP30-11 Simultaneous Percutaneous Cystolithotripsy with TURP is the gold standard for management of large prostates with big bladder stones.

\section{Raghavendran, K Kumar}

Apollo BGS Hospitals, Mysore

India

Introduction \& Objective: $67 \%$ of Bladder stone patients have Prostatic enlargement. They need cystolithotripsy (CL) either percutaneously (PCCL) or transurethrally (TUCL), preferably simultaneously with TURP. The purpose of this study was to find which CL is better. In percutaneous procedure, the loss of tract is a factor especially when the stone is big and we define our first refinement (Guidewire exit) to eliminate this problem. The second problem faced during the simultaneous procedures is the continuous drainage provided by 30Fr Amplatz sheath may empty the bladder making TURP difficult. Our second
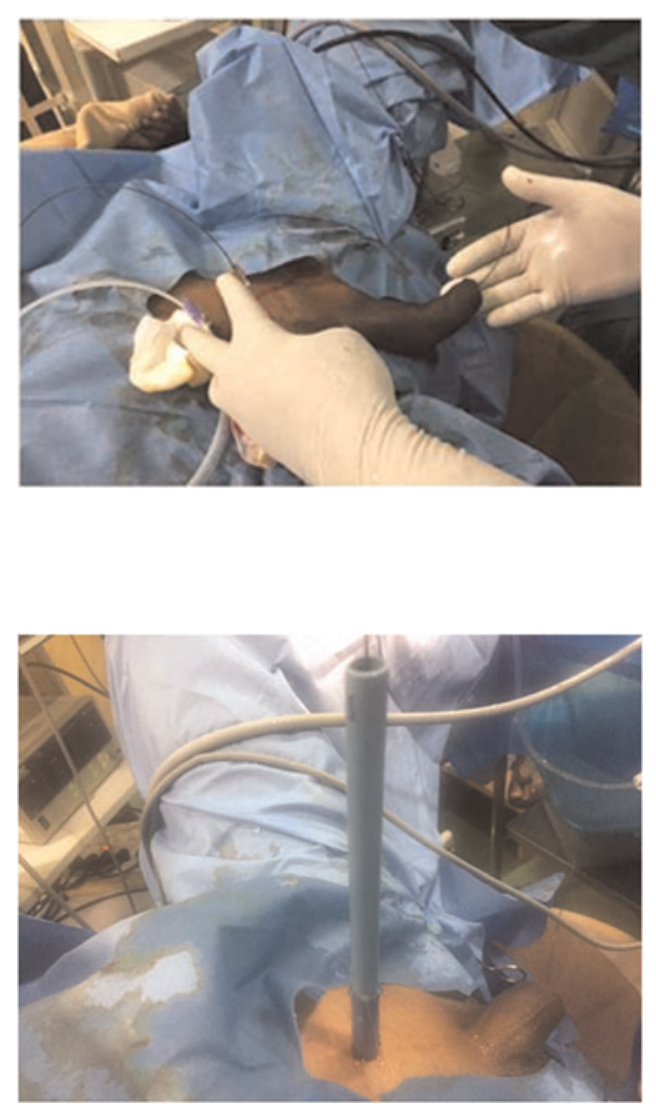
refinement (intermittent blocking and decompression with dilator) was to nullify this and thus make the technique of PCCL the procedure of choice..

Materials and Methods: 38 patients underwent TUCL (19) or PCCL (19) with simultaneous TURP. Initially the stone was removed followed by TURP. TUCL was performed with $26 \mathrm{Fr}$ Nephroscope with pneumatic lithotripter and stone extraction achieved. There were 2 refinements in the PCCL technique. The initial guidewire through the suprapubic percutaneous route was brought out through the penile urethra. The 2 nd refinement was the suprapubic amplatz being blocked with dilator and intermittent decompression achieved by removing dilator.

Results: Both the groups were comparable pre-operatively. The average prostate size was more than $60 \mathrm{gm}$. The average stone size was $4.2 \mathrm{~cm}$. The operating and resection time was significantly lower in the PCCL group. Visibility was better in the PCCL group. Resected tissue was significantly more in the PCCL group. There was no loss of access or tract in the PCCL group. The TUCL group had more urethral strictures. Retained fragments were more in the TUCL group ( 3 cases) and needed an additional procedure.

Conclusions: CL can be combined safely with TURP. Guidewire exit through penile urethra helps in maintaining the percutaneous tract for a long time. Intermittent decompression by using the amplatz and dilator helps in making PCCL faster, safer and more effective than TUCL with lesser morbidity.

\section{MP30-12 Does The Volume Effect of Prostatic Adenoma in Prostate Exist?: a Prospective Study}

J Oh, K Kim, T Kim, C Kim, K Kim, H Jung, S Yoon

Republic of Korea

Introduction \& Objective: During Holmium laser enucleation of the prostate (HoLEP) procedure, spatial recovery was often found after enucleation of prostatic adenoma. In the aspect of prostatic anatomy, peripheral zone of prostate may be pressed by solid, tense adenoma. However, there was no evidence to prove it. Our study was aimed to measure difference between preoperative estimated peripheral zone volume and postoperative remnant specimen using preoperatively and postoperatively ultrasonographic measurement and analyzed the correlation between difference of pre- and post- operative peripheral zone volume and density of enucleated adenoma.

Materials and Methods: All patients were prospectively enrolled who underwent HoLEP by single surgeon ( JKO) between 2013 and 2015. Preoperative and postoperative prostate ultrasonography examination were performed by single examiner

\begin{tabular}{|c|c|}
\hline Number (n) & 116 \\
\hline Age (vears) & $69.8=9.2$ \\
\hline \multicolumn{2}{|l|}{ Ultrasonographic parameters } \\
\hline total prostate volume (ml) & $57.2=23.0$ \\
\hline transitional zone volume $(\mathrm{m})$ & $280=163$ \\
\hline calculated periphral zone volume (ml) & $292=99$ \\
\hline postoperative residual prostate volume (mi) & $30.8=10.7$ \\
\hline calculated enuclested volume (mi) & $26.4=155$ \\
\hline \multicolumn{2}{|l|}{ Postoperative specimen parameters } \\
\hline weight of enuclested prostate (g) & $20.6=15.4$ \\
\hline volume of enucleated prostate $(\mathrm{ml})$ & $209 \div 159$ \\
\hline Ratio between weight and volume of specimen & $1.0 \neq 0.2$ \\
\hline Difference between calculated peripheral zone volume and postoperative residual prostate volume $(m)$ & $1.6 \pm 4.2$ \\
\hline
\end{tabular}

(JKO) during operations and weight and volume of enucleated adenoma were measured. We compared preoperative peripheral zone volume with postoperative residual prostate volume using ultrasonography and also analyzed the correlation coefficients between preoperative periphral zone volume and postoperative parameters.

Results: Total 116 patients with a mean age $( \pm$ SD) of $69.8( \pm 9.2)$ years were analyzed. Mean preoperative adenoma volume was $28.0( \pm 16.3) \mathrm{ml}$ and mean weight and volume of adenoma were $20.6( \pm 15.4) \mathrm{g}$ and $20.9( \pm 15.9) \mathrm{ml}$, respectively. Mean enucleated adenoma density was $1.0( \pm 0.2) \mathrm{g} / \mathrm{ml}$ and mean calculated peripheral zone volume and postoperative residual prostate volume were $29.2( \pm 9.9)$ and $30.8( \pm 10.7) \mathrm{ml}$, respectively. Difference between postoperative residual volume and preoperative peripheral zone volue was $1.6( \pm 10.7) \mathrm{ml}$. Difference between pre- and post- operative peripheral zone volume using ultrasonography showed no significant correlation with the density of prostatic adenoma (Pearson correlation coefficient 0.071 , p-value 0.451 ).

Conclusions: Our study showed there was difference between pre- and post- operative peripheral zone volume after HoLEP. However, it has no signficant relationship among prostatic tissue factors including density of prostatic adenoma.

\section{MP30-13 Laser Technologies in Surgical Treatment of Benign Prostatic Hyperplasia}

D Enikeev, P Glybochko, G Altshuler, A Vinarov, L Rapoport, M Enikeev, N Sorokin, A Dymov, R Sukhanov, O Khamraev, M Taratkin

I.M. Sechenov First Moscow State Medcal University Clinic of Urology

Russia

Introduction \& Objective: Lasers have been used for medical purposes since the mid-1960s. Over the last decades, they have undergone significant changes. Medical laser equipment has ceased to be science fiction and is currently an essential tool for medical practitioners in many branches of medicine. Aim of our study was to evaluate effectiveness of thulium and holmium laser enucleation of the prostate.

Materials and Methods: From 2013 to 2015, 489 patients with the mean age of 67.5 years and mean prostate volume of 90.1 (50-260) $\mathrm{cm}^{3}$ underwent holmium laser enucleation. From 2016 to 2017 , we have performed up to 153 thulium laser enucleations of enlarged prostate. The mean patient age was 66.8 (54-83) years (tab.1). All patients were followed up for median of 6 month.

Results: There were no significant difference between HoLEP and ThuLEP effectiveness ( $p>0.05)$ (tab.2.). Mean weights of resected tissue after HoLEP (71.2 \pm 38.2$)$ and ThuLEP (68.4 \pm 31.6$)$ were comparable $(p=0.1)$. However mean length of surgery was significantly shorter after ThuLEP $(79.6 \pm 29.4)$ than after HoLEP $(105,7 \pm 33,2) \quad(p<0,01)$. However, after thulium enucleation bleeding in postoperative period occurred in 1 case $(0.6 \%)$, and after holmium laser enucleation in 5 cases. The rate of urinary

Tab.1. Preoperative characteristics
\begin{tabular}{|c|l|l|l|l|}
\hline & IPSS & QoL & Qmax, $(\mathrm{ml} / \mathrm{s})$ & $\begin{array}{l}\text { Post-void residual } \\
\text { volume }(\mathrm{ml})\end{array}$ \\
\hline HoLEP & $21.3 \pm 4.0$ & $4.1 \pm 0.5$ & $6.8 \pm 2.1$ & $65.1 \pm 33.5$ \\
\hline ThuLEP & $23.2 \pm 2.2$ & $4.8 \pm 0.8$ & $7.6 \pm 2.4$ & $80.5 \pm 30.6$ \\
\hline
\end{tabular}


Tab.2. Postoperative characteristics

Tab.2. Postoperative characteristics
\begin{tabular}{|l|l|l|}
\hline & HoLEP $(\mathrm{n}=489)$ & ThuLEP $(\mathrm{n}=153)$ \\
\hline I-PSS & $11.1 \pm 2.8$ & $8.8 \pm 3.9$ \\
\hline QoL & $2.8 \pm 0.9$ & $1.8 \pm 1.1$ \\
\hline Qmax (ml/s) & $18.5 \pm 5.1$ & $17.2 \pm 3.3$ \\
\hline $\begin{array}{l}\text { Post-void residual volume } \\
(\mathrm{ml})\end{array}$ & $15.1 \pm 15.9$ & $10.5 \pm 10.7$ \\
\hline $\begin{array}{l}\text { Decrease in prostate volume } \\
(\%)\end{array}$ & 81 & 86 \\
\hline
\end{tabular}

Conclusion: Laser enucleation techniques are among the most advanced and efficient options for management of LUTS secondary to BPH. Holmium enucleation of the prostate currently heads the list of prostatic hyperplasia management techniques. The newest addition - thulium enucleation demonstrates efficacy comparable to that of holmium enucleation.

incontinence was 7.7\% (38 cases) after HoLEP and 5.9\% (9 cases) after ThuLEP. After both techniques we observed significant decrease in prostate volume.

Conclusions: Laser enucleation techniques are among the most advanced and efficient options for management of LUTS secondary to BPH. Holmium enucleation of the prostate currently heads the list of prostatic hyperplasia management techniques. The newest addition - thulium enucleation - demonstrates efficacy comparable to that of holmium enucleation.

MP30-14 Outcome and complications of Holmium Laser Enucleation of the Prostate in patients on anticoagulation

K Guest, T Smith, M Cynk

Maidstone and Tunbridge Wells NHS Trust

United Kingdom

Introduction \& Objective: Holmium Laser Enucleation of the Prostate (HoLEP) has emerged as an alternative to Transurethral Resection of the Prostate (TURP) for the surgical management of bladder outflow obstruction secondary to benign prostatic enlargement. A key advantage over TURP is the reduced risk of significant bleeding complications. Patients on anticoagulation pose a difficult challenge in this type of surgery. The objective of this study was to evaluate the outcomes of HoLEP in patients on anticoagulation in a large single centre UK series.

Materials and Methods: We retrospectively analysed a prospective database of all HoLEP procedures performed under the care of a single consultant urological surgeon between December 2003 and March 2017. We identified patients who remained on anticoagulation in the peri-operative period. Those included were either on anti-platelet therapy or anticoagulants such as warfarin bridged with low molecular weight heparin (LMWH) or heparin infusion. Outcome measures including post-operative flow rate (FR), post-void residual volume (PVR) and international prostate symptom score (IPSS) were compared between anticoagulated and non-anticoagulated patients using the MannWhitney U test. Complications were listed in accordance with the Clavien-Dindo classification system.

Results: 1034 HoLEP procedures were performed over a 13-year period. Of these, 57 patients remained on anticoagulants perioperatively (age range 63-95 years). 29 were bridged with prophylactic-dose LMWH, 11 with treatment-dose LMWH and 5 with heparin infusion. Of those on anti-platelets, 7 remained on clopidogrel, 3 on aspirin and 2 on dual anti-platelets. Median FR of $19.3 \mathrm{ml} / \mathrm{s}$ (range $7.9-40.1$ ), PVR of $46 \mathrm{ml}$ (range $0-465$ ) and
IPSS of 0 (range 0-32) were found in the anticoagulated group. There was no statistically significant difference in FR $(p=0.77)$, PVR $(p=0.13)$ or IPSS $(p=0.72)$ between the 2 groups. Secondary haemorrhage and need for blood transfusion were slightly more prominent in the anticoagulated group (7\% and $1.8 \%$, respectively) than the non-anticoagulated group $(0.9 \%$ and $0.3 \%$, respectively), although the number of these remains very low. Only one anticoagulated patient had significant bleeding requiring return to theatre. Other complications appeared comparable across both groups.

Conclusions: HoLEP is safe and effective in patients on anticoagulants. Aspirin and clopidogrel do not need to be stopped prior to surgery. Our practice is to temporarily stop warfarin in patients with atrial fibrillation, and bridge with LMWH in those with prosthetic heart valves. There is an increased risk of secondary bleeding in anticoagulated patients, but overall the complication rate is low.

MP30-15 A simple technique of tissue collection after morcellation during holmium laser enucleation of prostate

PN Maheshwari

Fortis Hospital Mulund, Mumbai, India India

Introduction \& Objective: To present a simple method for collection of tissue after morcellation during holmium laser enucleation of prostate (HoLEP) and to compare it to the standard collection method.

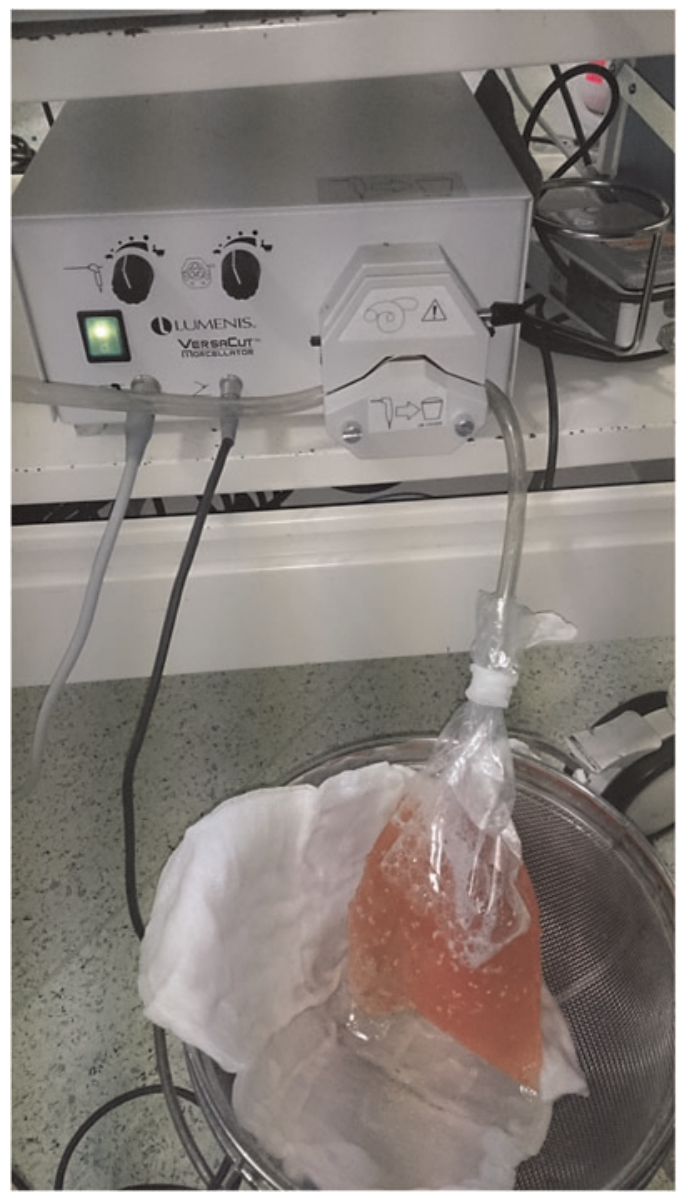




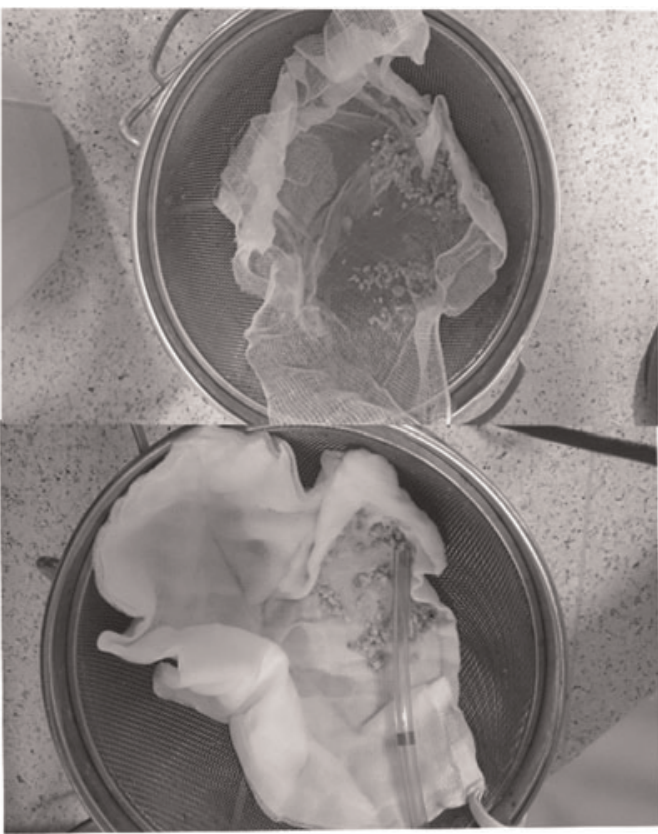

Materials and Methods: HoLEP is a standard surgical treatment for benign enlargement of prostate in many centers around the world. Morcellation is an important last step of the procedure by which the enucleated tissue is crushed in the bladder and removed transurethrally. The commonly used Lumenis VersaCut ${ }^{\mathrm{TM}}$ morcellator does not have a post-morcellation tissue collection system. Routinely the efflux from exit tube is strained using a sieve and a gauze piece to collect the morcellated tissue. This carries risk of tissue loss, spillage, and an added effort to scrape the tissue stuck to the gauze piece. We present a novel technique of tissue collection, where a perforated double-layered polythene bag is attached to the exit tube. The tissue gets collected in the bag while the fluid gets drained off the perforations. The paramedical staffs were asked to compare their experiences of the two techniques for the ease of set-up, risk of spillage, and ease of tissue collection.

Results: The presented technique was successfully used in 60 patients. Although both the techniques are easy to set-up, the staff favored the polythene-bag technique due to the low risk of spillage and ease of tissue collection.

Conclusions: The presented technique of using a ubiquitous polythene bag is simple, cheap and an efficient technique of morcellated tissue collection after HoLEP.

MP30-16 Feasibility, Safety, and Efficacy of Transurethral Thulium Laser Prostatectomy in the Outpatient Setting: A Single Center Experience in the United States

T Cisu, D Sobel, RT Grunert

University of Vermont College of Medicine United States

Introduction \& Objective: Thulium laser prostatectomy is a minimally invasive procedure for the treatment of benign prostatic hyperplasia (BPH). In an effort to seek out procedures that minimize hospital stay while maintaining superior hemostasis, this study details an initial experience utilizing thulium laser vaporization in the outpatient setting.

\section{AUA Symptom Scores}

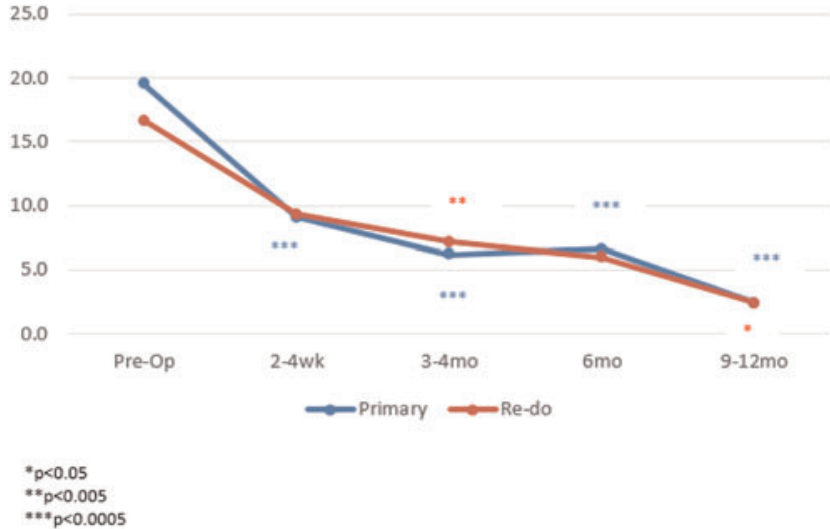

Materials and Methods: A retrospective chart review of patients who underwent thulium laser vapo-enucleation between 2014 and 2016 was performed. AUA symptom scores, PSA reduction, duration of catheterization, and postoperative complications were analyzed. All procedures were performed by a single surgeon at a single institution utilizing a 150-watt laser.

Results: 100 patients were included in the analysis. 7 patients were anticoagulated and 7 different patients had concurrent cystolithalopaxies performed. 21 patients were redo procedures from prior TURPs or laser prostatectomies. Of the primary cases, the mean prostate size was $55 \mathrm{gm}$ (range 15-167gm). 96 patients were able to be discharge as outpatients the day of surgery. The mean pre-operative AUA symptom scores were 19.5 and 16.7 for primary and re-do, respectively. The mean AUA symptom scores were significantly reduced to 6.2 and 7.2 , respectively $(\mathrm{p}<0.005$ for both), at 3-month follow-up and further reduced to 2.4 and 2.4 ( $\mathrm{p}<0.05$ for both), respectively, at 12-month follow-up (Figure 1). For all patients, the mean preoperative PSA was $4.0 \mathrm{ng}$ / $\mathrm{mL}$ (range 0.2-25.6). Mean postoperative PSA was $1.40 \mathrm{ng} / \mathrm{mL}$ (range 0.1-6.6). Ejaculatory function was preserved in 47 patients $(46.5 \%)$ and worsening erectile function in 9 patients (8.91\%). Transient short-term stress incontinence occurred in 4 patients and resolved in all. Nearly half of patients $(48.9 \%)$ required two or fewer days of post-op catheterization, with a mean of 2.80 days (range 0-14 days). All patients passed voiding trials following catheter removal. Postoperatively, of the 12 patients with postoperative hematuria, 4/7 of the anti-coagulated patients went into clot retention requiring admission and continuous bladder irrigation.

Conclusions: This single surgeon experience demonstrates that thulium laser vaporization of the prostate is a safe and effective alternative for the treatment of BPH in the outpatient setting with results comparable to the traditional inpatient electrosurgical methods. Anti-coagulated are at an increased risk for delayed bleeding and may require prolonged catheterization and more reduced post-op activity than non-anticoagulated patients to reduce this risk.

MP30-17 Robotic assisted versus open simple prostatectomy for benign prostatic hyperplasia in large glands: a propensity score matched comparison

I Sorokin, BA Johnson, V Sundaram, N Singla, V Margulis, C Roehrborn, J Gahan

UT Southwestern

United States 
Table 2 - Peri- and post-operative patient outcomes before and after propensity score matching

\begin{tabular}{|c|c|c|c|c|c|c|}
\hline & \multicolumn{3}{|l|}{ All patients } & \multicolumn{3}{|c|}{ Propensity-score matched patients } \\
\hline & $\operatorname{RASP}(n=64)$ & OSP $(n=103)$ & p-value & $\operatorname{RASP}(n=59)$ & OSP (n=59) & $\begin{array}{l}\mathrm{p} \text { - } \\
\text { value }\end{array}$ \\
\hline $\begin{array}{l}\text { Operative time, mins, (tSD), } \\
\text { mean }\end{array}$ & $160.9( \pm 32.4)$ & $94.5( \pm 16.7)$ & $<0.001$ & $\begin{array}{l}161.4( \pm \\
31.3)\end{array}$ & $93.0( \pm 18.0)$ & $<0.001$ \\
\hline EBL, mL, (tSD), mean & \begin{tabular}{|l|}
$327.9( \pm$ \\
$192.5)$
\end{tabular} & $596.7( \pm 292.6)$ & $<0.001$ & $\begin{array}{l}338.9( \pm \\
194.8)\end{array}$ & $\begin{array}{l}587.3( \pm \\
287.5)\end{array}$ & $<0.001$ \\
\hline \% Hct drop, ( \pm SD), mean & $12.3( \pm 7.6)$ & $22.2( \pm 13.6)$ & $<0.001$ & $12.3( \pm 7.8)$ & $19.5( \pm 14.1)$ & 0.001 \\
\hline Transfusion rate (\%) & $2(3.1)$ & $9(8.7)$ & 0.207 & $2(3.4)$ & $4(6.8)$ & 0.679 \\
\hline $\begin{array}{l}\text { Catheterization time, day, } \\
( \pm S D) \text {, mean }\end{array}$ & $5.7( \pm 2.6)$ & $3.3( \pm 3.5)$ & $<0.001$ & $5.7( \pm 2.7)$ & $3.1( \pm 3.1)$ & $<0.001$ \\
\hline LOS, day, ( \pm SD), mean & $1.5( \pm 1.2)$ & $2.7( \pm 1.5)$ & $<0.001$ & $1.5( \pm 1.2)$ & $2.6( \pm 1.5)$ & $<0.001$ \\
\hline $\begin{array}{l}\text { Specimen weight, gm, } \\
( \pm S D) \text {, mean }\end{array}$ & $81.3( \pm 36)$ & $103.8( \pm 49.1)$ & 0.002 & $82.9( \pm 35.6)$ & $91.8( \pm 41.7)$ & 0.217 \\
\hline $\begin{array}{l}\text { \% PV reduction, (ISD), } \\
\text { mean }\end{array}$ & $61.5( \pm 19.8)$ & $71.3( \pm 26.8)$ & 0.015 & $61.4( \pm 19.9)$ & $65.2( \pm 27.9)$ & 0.405 \\
\hline $\begin{array}{l}\text { PSA post-op, ng/ml, (tSD), } \\
\text { mean }\end{array}$ & $0.9( \pm 0.8)$ & $0.7( \pm 1.1)$ & 0.364 & $0.9( \pm 0.8)$ & $0.7( \pm 0.8)$ & 0.44 \\
\hline Conversion to open (\%) & $0(0)$ & - & - & $0(0)$ & - & - \\
\hline Incontinence (\%) & $1(1.6)$ & $3(2.9)$ & 0.999 & $1(1.7)$ & $1(1.7)$ & 0.999 \\
\hline $\begin{array}{l}\text { No. Catheter dependent } \\
\text { post-op (X) }\end{array}$ & $0(0)$ & $3(2.9)$ & 0.287 & $0(0)$ & $2(3.4)$ & 0.496 \\
\hline PVR, mL, (IQR), median & $7(0-57)$ & $32(0-84)$ & 0.201 & $3.5(0.55)$ & $48(18-111)$ & 0.007 \\
\hline $\begin{array}{l}\text { Qmax post-op, ml/s, (tSD), } \\
\text { mean }\end{array}$ & $22.4( \pm 9.9)$ & $20.7( \pm 10.6)$ & 0.377 & $22.4( \pm 9.9)$ & $20.1( \pm 11.9)$ & 0.36 \\
\hline IPSS post-op, (tSD), mean & $7.3( \pm 5.7)$ & $6.9( \pm 5.1)$ & 0.705 & $7.3( \pm 5.8)$ & $7.1( \pm 5.9)$ & 0.884 \\
\hline Qol post-op, ( \pm SD), mean & $1.3( \pm 1.3)$ & $1.3( \pm 1.2)$ & 0.068 & $1.3( \pm 1.4)$ & $1.6( \pm 1.3)$ & 0.601 \\
\hline
\end{tabular}

Table 3 - Change in PSA and functional outcomes in propensity score matched cohort

\begin{tabular}{|l|l|l|l|}
\hline & RASP & OSP & p-value \\
\hline Percentage $\triangle$ PSA, ng/ml & $-87.7 \%$ & $-90.7 \%$ & 0.44 \\
\hline$\Delta$ PSS & -11.5 & -10.8 & 0.884 \\
\hline$\Delta$ Qmax, ml/s & +12.6 & +11.5 & 0.36 \\
\hline$\Delta$ QoL & -2.6 & -2.3 & 0.601 \\
\hline $\begin{array}{l}\text { Percentage } \Delta \text { Num Catheter } \\
\text { dependent }\end{array}$ & $-100 \%$ & $-94.4 \%$ & 0.496 \\
\hline
\end{tabular}

Introduction \& Objective: Open simple prostatectomy (OSP) remains the standard treatment for large glands causing lower urinary tract symptoms but may be associated with significant blood loss and prolonged hospital stay. Robotic assisted simple prostatectomy (RASP) provides a less invasive alternative that may reduce the morbidity associated with the open approach without compromising outcomes. Our objective was to report the largest comparative analysis of robotic versus open simple prostatectomy for large volume prostate glands.

Materials and Methods: We retrospectively reviewed 103 patients that underwent OSP and 64 patients that underwent RASP from 2012-2016 at a single institution. A propensity score matched analysis was performed with 5 covariates including age, body mass index, race, Charlson-comorbidity index, and prostate volume. Perioperative, postoperative, and functional outcomes were compared between groups.

Results: There was no statistically significant difference between groups for all pre-operative demographic variables after propensity score matching. RASP compared to OSP showed a significant shorter average length of stay (1.5 vs. 2.6 days, $\mathrm{p}<0.001$ ), but longer mean operative time (161 vs. 93 mins, $\mathrm{p}<0.001)$. The robotic approach was also associated with a lower estimated blood loss $(339$ vs. $587 \mathrm{~mL}, \mathrm{p}<0.001)$ and lower percentage hematocrit drop ( 12.3 vs. $19.5 \%, \mathrm{p}=0.001)$. Two patients required blood transfusions in the robot group compared to 4 in the open group, but this was not significant $(p=0.271)$. Improvements in Qmax, IPSS, Quality of life, PVR, and post-operative prostate-specific antigen levels were similar before and after surgery for both groups, but no different between groups. There was no difference in complications between groups.
Conclusions: RASP is a safe and effective treatment for the surgical management of benign prostatic hyperplasia. It provides similar outcomes to the open approach; however, offers the advantage of reduced length of stay and blood loss.

MP30-18 Robot-assisted simple prostatectomy for large volume benign prostatic hyperplasia: Initial single-center experience in China

Z Shen, S Chen, S Zhong, M Zhang

China (People's Republic)

Introduction \& Objective: To evaluate the safe, feasibility and reproducibility of a robot-assisted simple prostatectomy (RASP) in cases of symptomatic large volume benign prostatic hyperplasia.

Materials and Methods: From Jan 2014 to July 2015, 16 consecutive patients underwent RASP for large volume benign prostatic hyperplasia. The median age of patients was 69 years (range 62-79 years). The median prostate volume on preoperative transrectal ultrasonography was $98.3(80-130) \mathrm{mL}$. The media prostate-specific antigen (PSA) value $3.69(1.2-6.9) \mathrm{ng} /$ $\mathrm{mL}$. The median preoperative International Prostate Symptom Score (IPSS), quality of life(QOL), maximum flow rate (Qmax), and postvoid residual (PVR) were 22.9, $4.8,8.9 \mathrm{~mL} / \mathrm{s}$, and $78 \mathrm{~mL}$, respectively.

Results: All procedures were successfully performed by RASP. There were no intraoperative complications and no conversions to open surgery. The median operation time was 92.5 minutes (range 80-110 minutes). The media estimated blood loss was $125.5 \mathrm{~mL}$. Intraoperative blood transfusion was required in one patient $(6.3 \%)$. The media specimen weight on pathological examination was $86 \mathrm{~g}$, and the average amount of removed tissue was $83.5 \%$. The media (range) hospital stay was 5.1 days (range 4-7 days) while the media (range) Foley catheter duration was 7.9 days (range 6-10 days). Early functional outcomes demonstrated significant improvement from baseline with a $48 \%$ reduction in media IPSS, a 67\% reduction in media QOL, a 230\% increase in media Qmax, and an $84 \%$ reduction in media PVR $(\mathrm{P}<0.005)$. This study is limited by small sample size and short follow-up period.

Conclusions: Our technique of RASP is safe, feasible and reproducible procedure with outcome advantages when compared with the open or with other minimally invasive techniques (laser or laparoscopy). Good functional outcomes suggest it is a viable option for symptomatic large volume benign prostatic hyperplasia and can be used for patients requiring concomitant procedures.

MP30-19 Holmium enucleation of prostate in patients taking anti platelets

H Pothiyedath, KM Ramaswami

\section{METROMED INSTITUTE OF ADVANCED UROLOGY AND RENAL TRANSPLANT \\ India}

Introduction \& Objective: (HoLEP) hasbeen proved to be as effective as transurethral resection of prostate (TURP) for the treatment of benign prostatic hyperplasia (BPH). Oral 
anticoagulation $(\mathrm{OA})$ is considered a strict contraindication to transurethral resection of theprostate (TURP). In recent years, however, safe and effective surgical alternatives such as holmium laser enucleation of the prostate (HoLEP) have emerged. Materials and Methods: A prospective study was done including all patients who underwent HoLEP at our institution from December 2014 to February 2017. Follow up period ranged from 1 month to 24 months. A pulsed high power 100Watts Holmium Laser with $26 \mathrm{~F}$ resectoscopesheath was used for enucleation and tissue was removed by transurethral morcellation. All patients were preoperatively assessed with ultrasound prostate volume estimation, maximum urine flow rate (Q Max), post void residual urine volume (PVR) assessment and International Prostate Symptom Score(IPSS). Various intra, peri and post operative parameters were assessed and patients followed up. Fifteen patients were taking Aspirin tablet, 7 were on clopidogrel tablet and another 7 patients were on tablet warfarin who were substituted with unfractionatedheparin perioperatively. These medications were not stopped prior to surgery.

Results: 1128 patients were involved in the study. Mean prostate volume was $62 \pm 34 \mathrm{cc}$. Enucleation time was $55 \pm 22.9 \mathrm{~min}$ and morcellation time $17.3 \pm 14 \mathrm{~min}$, whilst resected weight was $40 \pm 27.5 \mathrm{~g}$. Catheter time was $36 \pm 14.7 \mathrm{~h}$ and hospital stay was $48 \pm 26 \mathrm{~h}$. Mean serum hemoglobin and sodium did not drop significantly from baseline after the procedure. Maximum size of gland operated was $350 \mathrm{~g}$ which was completed in three sittings. High risk cardiac patients were managed successfully with HoLEP without any major complications. Out of 29 patients on anticoagulant medications, 4 patients had mild hematuria which was managed conservatively without stopping antiplatelet medications. A significant improvement occurred in Q max, PVR and IPSS at follow-up compared with baseline $(\mathrm{p}<0.05) .12$ percent of patients complained of irritative urinary symptoms, Transient stress incontinence was reported in 7 patients which settled by itself in 3 months. Persistent stress leak was reported in 1 patient at 6 months follow up.

Conclusions: HoLEP represents an effective and safe surgical intervention for $\mathrm{BPH}$, even in patients taking oral anticoagulants. The risk of thrombosis involved with stoppage of antiplatelet medications can be significantly avoided in patients undergoing HoLEP. There was no significant increase in complications related to bleeding.

\section{MPS31: LAPAROSCOPIC/ROBOTIC MISCELLANEOUS ONCOLOGY}

MP31-1 Can the Use of Robotics Reduce the High Morbidity of Inguinal Lymphadenectomy?

R Patel, K Radadia, V Kosherkov, EA Singer, S Elsamra

Rutgers Robert Wood Johnson Medical School United States

Introduction \& Objective: Inguinal lymphadenectomy improves survival in many cancers, but carries a high morbidity. We report updated results of robotic inguinal lymphadenectomy (RILND) technique in comparison to open inguinal lymphadenectomy (OILND) performed at our institution.

Materials and Methods: A retrospective review identified 18 patients who underwent an ILND between 2010 and 2016. 12 patients underwent an OILND, while 6 underwent a RILND. Four fellowship-trained surgeons who were well established in practice performed all OILND. In contrast, all RILND were performed by a fellowship-trained surgeon who was fascicle with robotics but was just starting to perform this operation via a minimally invasive technique. Baseline characteristics, operative data, lymph node (LN) yield, and 30-day complications were assessed. $P<0.05$ was considered statistically significant.

Results: Fifteen limbs underwent an OILND, and ten underwent a RILND. The baseline characteristics were similar across both groups. Mean operative time per limb for RILND was 161.7 minutes vs 123.9 minutes for OILND, $P=0.09$. Blood loss was lower for the RILND cohort (45.8 cc vs. $78.6 \mathrm{cc}, P=0.23$ ). Average length of stay was similar across both cohorts. LN yield per limb was higher for RILND in comparison to OILND, 9.5 vs. $5.9, P=0.07$. Nodal metastasis rate was $1.1 \%$ in the RILND vs. $11.4 \%$ in OILND, $P<0.05$. All patients were discharged with a drain in place. Average drain removal time was faster for RILND at a mean of 17 days. Complications were observed in $53.3 \%$ of limbs that underwent open surgery and in $30 \%$ of limbs that underwent a RILND $(P<0.05)$. No Clavien III or higher complications were seen in limbs that underwent RILND, while $20 \%$ of OILND had Clavien III or higher complications, $P=0.25$. Specific complications for the RILND included lymphedema, transient neuropraxia and cellulitis. The complications for the OILND included cellulitis, chronic lymphedema, seromas requiring drainage and hematomas requiring operative intervention. Other patient characteristics are highlighted in Table 1.

\begin{tabular}{|c|c|c|c|}
\hline & $\begin{array}{l}\text { Robotic ILND } \\
\quad(\mathrm{n}=10)\end{array}$ & $\begin{array}{c}\text { Open ILND } \\
(\mathbf{n}=\mathbf{1 5})\end{array}$ & $\boldsymbol{P}$ \\
\hline \multicolumn{4}{|l|}{ Baseline } \\
\hline Age (years) & 52 & 62.3 & 0.15 \\
\hline BMI & 28.8 & 28.3 & 0.89 \\
\hline Pathology, N (\%) & & & 0.24 \\
\hline$<=\mathrm{T} 2$ & $5(50)$ & $6(40)$ & \\
\hline $\mathrm{T} 3+$ & $5(50)$ & $8(60)$ & \\
\hline \multicolumn{4}{|l|}{ Operative } \\
\hline $\begin{array}{l}\text { Operative Time } \\
\text { (mins per Limb) }\end{array}$ & 161.7 & 123.9 & 0.09 \\
\hline $\begin{array}{l}\text { Estimated Blood } \\
\text { Loss (cc) }\end{array}$ & 45.8 & 78.6 & 0.23 \\
\hline $\begin{array}{l}\text { Time for } \\
\text { Drainage (days) }\end{array}$ & 17 & 18.7 & 0.72 \\
\hline $\begin{array}{l}\text { Length of stay } \\
\text { (days) }\end{array}$ & 1 & 1.25 & 0.60 \\
\hline Number of LN & 9.5 & 5.9 & 0.07 \\
\hline $\begin{array}{c}\text { Complications } \\
N(\%)\end{array}$ & & & $<0.05$ \\
\hline $\mathrm{I} / \mathrm{II}$ & $3(30)$ & $5(33.3)$ & \\
\hline III/IV & 0 & $3(20)$ & \\
\hline
\end{tabular}


Conclusions: RILND is a new minimally invasive approach. This preliminary study suggests that RILND can reduce morbidity compared to open surgery while having similar operative time, EBL, and nodal yield.

MP31-2 Perioperative Outcomes And Complication Rates Following Robot Assisted Retroperitoneal Lymph Node Dissection For Testicular Cancer: A Multi-Institutional Update

HM Abdul-Muhsin, M Marshall, KL Stern, S Stroup, M Woods, J L'esperance, EP Castle

Mayo Clinic Arizona

United States

Introduction \& Objective: Retroperitoneal Lymph node dissection (RPLND) is an established treatment option for metastatic testicular cancer. The robotic approach has the potential to decrease the morbidity associated with this procedure and to enhance the recovery while maintaining safe oncological outcomes. The objective of this study is to evaluate the perioperative outcomes and postoperative complication rates for robot assisted retroperitoneal lymph node dissection (RA-RPLND) in a large multi-Institutional cohort.

Materials and Methods: Retroperitoneal Lymph node dissection (RPLND) is an established treatment option for metastatic testicular cancer. The robotic approach has the potential to decrease the morbidity associated with this procedure and to enhance the recovery while maintaining safe oncological outcomes. The objective of this study is to evaluate the perioperative outcomes and postoperative complication rates for robot assisted retroperitoneal lymph node dissection (RA-RPLND) in a large multi-Institutional cohort. Results: There were 105 patients who underwent RA-RPLND. The mean patients' age was 29years ( $\mathrm{SD} \pm 13.5$ ), mean BMI was $26.4 \mathrm{Kg} / \mathrm{m} 2$ (SD \pm 10.3 ). Bilateral Full template dissection was performed in $79(75.2 \%)$ patients. Nerve sparing was attempted in $67(63.8 \%)$ patients. There were $61(58.6 \%)$ patients who underwent primary RA-RPLND compared to 43 (41.3\%) patients who received previous chemotherapy. Mean operative time was $339 \mathrm{~min}$ (SD \pm 291 ). Estimated blood loss was $255 \mathrm{ml}$ (SD \pm 481$)$. Length of postoperative hospital stay was 2.5 days (SD \pm 3.9$)$. There were $7(6.6 \%)$ conversions to open RPLND. Postoperatively, there were 27 total complications (Grade $\mathrm{I}=19$, $\mathrm{II}=5, \mathrm{III}=2, \mathrm{IV}=1$ ). From oncological point of view, mean lymph node (LN) yield was $26.8 \mathrm{LNs}$ (SD \pm 14.7 ) with positive LN identified in 37 patients (35.2\%). Among the primary RA-RPLND patients who had positive LNs, there were eight patients who received adjuvant chemotherapy. There were seven recurrences (6.6\%) identified at a median follow up of 15 months (IQR 3-31.3). Conclusions: This study demonstrates that RA-RPLND procedure is safe, reproducible. it may provide improved morbidity and less convalescence.

MP31-3 Development of a Robotic Surgery Program at Johns Hopkins Aramco Healthcare: An International Collaboration

T Tartir, K Taheini, M Johnson, J Ziemba, M Allaf

United States

Introduction \& Objective: Robotic surgery has a known learning curve, and complications have been reported to occur with greater frequency early in the adoption of this technology. A robust tutorial program, coupled with experienced mentorship, may flatten this learning curve, ultimately improving patient outcomes. Herein, we review the implementation of a focused program to introduce robotic surgery via an international health system partnership.

Materials and Methods: Johns Hopkins Aramco Healthcare is a joint venture between Johns Hopkins Medicine and Saudi Aramco, inaugurated in 2014. One of its early priorities was the establishment of a robotic surgery program. We developed a curriculum that included didactic lectures, surgical simulation, observerships, and ultimately mentored surgical procedures. The training was multi-disciplinary, and the entire robotic surgical team was included: nursing, anesthesia, as well as the surgeons and surgical assistants. Surgical cases were tracked in a prospectively maintained database, and this was assessed for clinical outcomes and complications.

Results: Between December 2015 and January 2017, 30 robotic surgical cases were performed. Procedures performed were: adrenalectomy (5), cyst decortication (2), nephrectomy (2), partial nephrectomy (8), pyeloplasty (3), and robotic assisted laparoscopic prostatectomy (10). There were three conversions to an open procedure, which were all then successfully completed. One complication occurred, a pulmonary embolus, which was managed medically without sequelae.

Conclusions: A multidisciplinary educational program, coupled with longitudinal mentorship, can simplify the introduction of a robotic surgery program and permit the rapid achievement of acceptable results. Additional benefits of our collaboration include leveraging supply chain synergies, which simplifies device acquisition.

MP31-4 "Video Endoscopic Inguinal Lymphadenectomy (VEIL) vs Conventional Inguinal Lymphadenectomy (CIL) - a comparative evaluation in cases of Carcinoma Penis"

H Singh, S Jain, A Kumar, A Pal

Super Specialty Cancer Institute, C.G City, Lucknow, and King George's Medical University

India

Introduction \& Objective: Presence and magnitude of the inguinal nodal metastases are the most important determinants of the oncologic outcome in patients with penile cancer. Conventional open inguinal lymph node dissection (CIL) is associated with major complications. Video Endoscopic Inguinal Lymphadenectomy (VEIL) is a novel technique in which we try to remove diseased lymph nodes by endoscopic surgery. We plan to do prospective comparative study of VEIL v/s CIL in patients having carcinoma penis with clinically impalpable as well as palpable low volume inguinal lymph nodes.

Materials and Methods: 20 patients of CA penis 12 with impalpable ILN and 8 with low volume palpable ILN were enrolled. CIL was performed on one side and VEIL on other side of each patient. Results of CIL and VEIL were compared and results of VEIL in two groups (palpable and impalpable) were compared. Results: OT time for VEIL was 139mins (impalpable group138mins, palpable group- 140mins). Intra operative complications were $0 \%$. LN yield was 9.3 /dissection with positivity of $10 \%$ (IPLN- 9.1(8\%), PLN-9.5(12.5\%)). Complications rate were $20 \%$ (IPLN-16.6\%, PLN- 25\%). Drainage required was for 4.3 days with output of $80 \mathrm{ml}$ (IPLN- 4.2, PLN- 4.3). 
Conclusions: In our early experience, VEIL was a safe and feasible technique in patients with penile carcinoma with non palpable and palpable low volume inguinal lymph nodes. It allows the removal of inguinal lymph nodes within the same limits as in conventional surgical dissection and potentially reduces surgical morbidity with comparable oncological outcome.

\section{MP31-5 Minimally Invasive Inguinal Lymphadenectomy}

N Jung, H Smith, C Keel, A Singh

University of TN College of Medicine Chattanooga/Erlanger Health System

United States

Introduction \& Objective: Inguinal lymphadenectomies (ILN) are fraught with significant morbidity including poor wound healing, deep vein thrombosis and lymphedema. These complications have significant sequelae and impose significant costs to the patient and the health care system. Minimally invasive surgical approaches may be able to limit complications and decrease morbidity. We present our initial experience with minimally invasive ILN.

Materials and Methods: This is a retrospective review of a prospectively maintained IRB approved database that includes all robotic and laparoscopic ILN at our institution from September 2013 until present. A total of 8 patients were identified. Data was collected on demographics, pathology, estimated blood loss (EBL), operative time, length of stay (LOS) and complications.

Results: All cases were completed successfully with minimally invasive techniques. Patients median age was 44 and ranged from 22-61 years old. The median BMI of the cohort was 24.8 and ranged from 20.4-40.2. Four of the cases were for sentinel lymph node positive melanoma. Three of the cases were for penile cancer with clinically negative lymph node disease. One of the cases was for squamous cell carcinoma that presented with a $5.2 \mathrm{~cm}$ inguinal lymph node. (Figure 1) In this group the average EBL was $42 \mathrm{cc}$ with a range of $0-100 \mathrm{ml}$. The average node count of the cohort was 7.2 nodes and ranged from $1-14$ nodes. Patient LOS averaged 1.6 days and ranged from 0-4 days. Operative time averaged 177 minutes and ranged from 120-231 minutes. Saphenous vein was spared in all cases. Average follow up to date is 24 months. The only complications were one Clavien grade II port site cellulitis and one lymphocele.

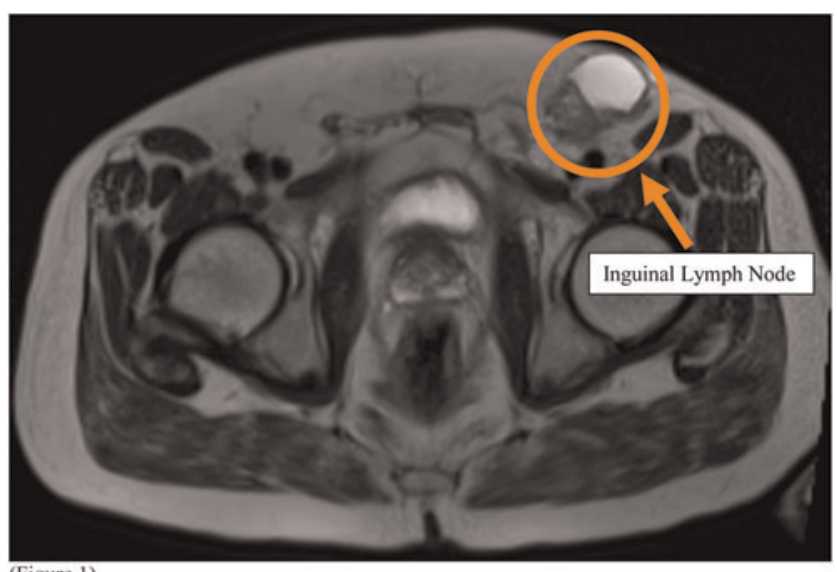

Conclusions: Our initial experience with minimally invasive ILN has demonstrated that this approach minimizes morbidity. This approach also decreases the patient's length of stay when compared to the classic open ILN in the literature. Further research into the oncological efficacy and long term outcome of this approach is needed.

MP31-6 Salvage Robotic Retroperitoneal Lymphadenectomy for Prostate Cancer Nodal Recurrence: technical aspects and results

\section{F Mota Filho, J DA CRUZ, R Santos, M Nunes, R Sakata,} C PASSEROTTI

Clinique Saint-Augustin, Bordeaux-France

Brazil

Introduction \& Objective: Prostate cancer is the most common tumor in age men. Beside treatment advances, approximately $40 \%$ of the treated patients could develop biochemical recurrence after initial treatment. Positron emission tomography (PET-CT) with 68Ga-Prostatic Specific Membrane Antigen (PSMA) could early identify patients with only lymph node disease who may represent a particular group who have, probably, more favorable outcome than men with bone or visceral
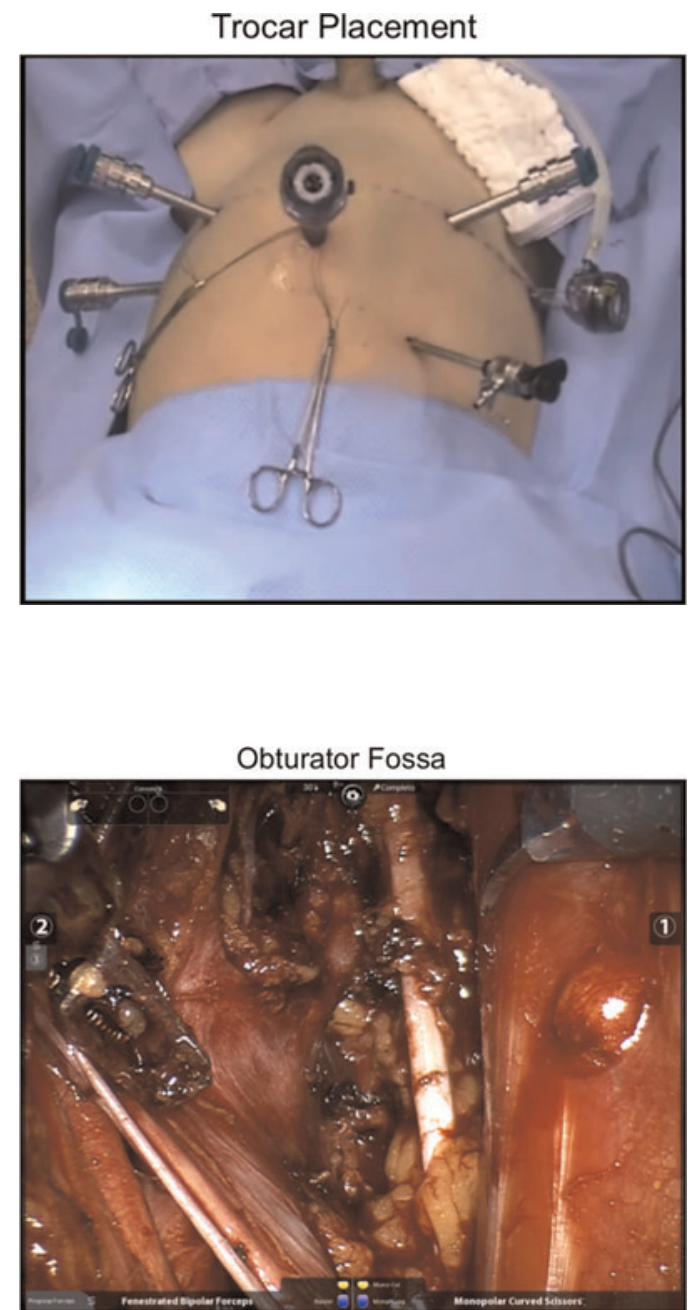

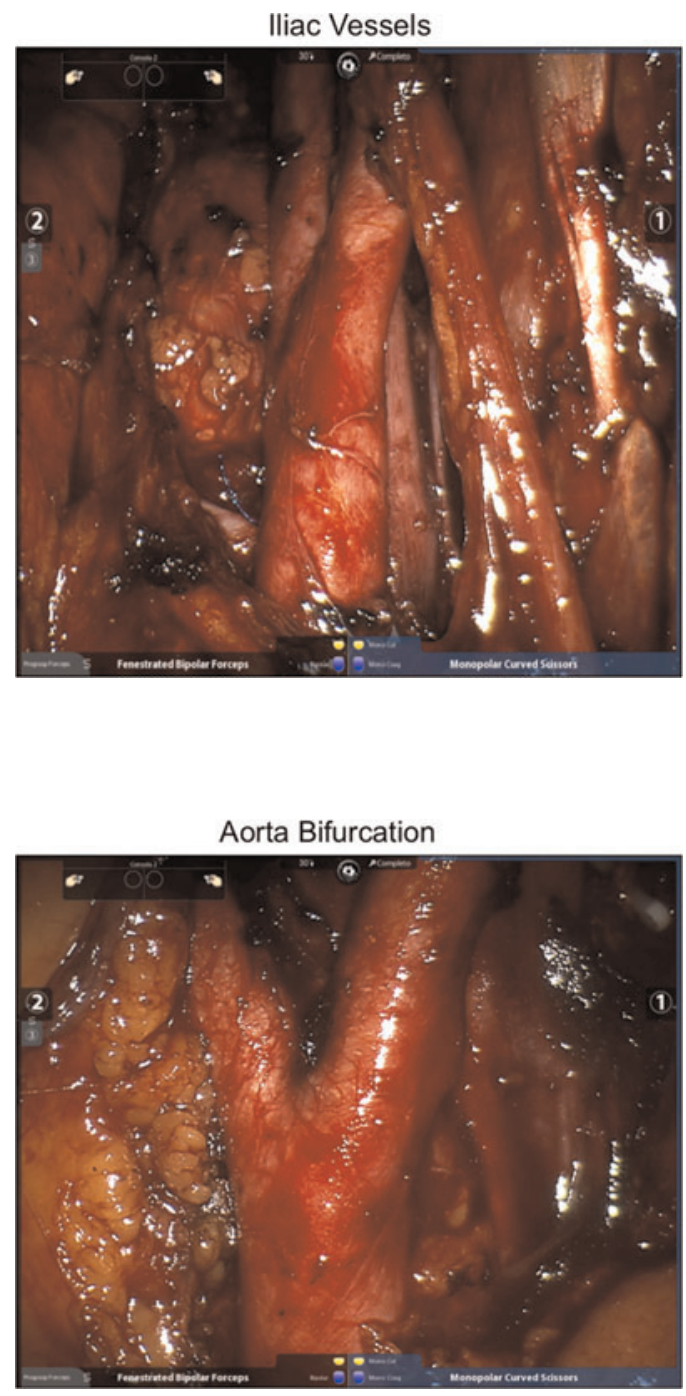

organs involvement. Previous data found favorable cancer control outcomes after Salvage Lymph Node Dissection (SLND), might postponing hormonal therapy or clinical progression in roughly one-third of these patients. Objective: To report results of 10 cases submitted a Robotic Retroperitoneal SLND (RSLND) and discuss technical aspects.

Materials and Methods: Study group comprised 10 prostate cancer patients with only lymph node recurrence diagnosed by PET-CT/PSMA: 5 had Robot-Assisted Radical Prostatectomy (RALP) and 5 had open surgery for previous treatment. Trocar placement was the same of the RALP and our dissection template extends from bellow mesenteric inferior artery at aorta bifurcation cranially up to obturator fossa distally, removing completely fatty-lymphatic tissue from vascular branches (figure 1).

Results: Median time until RSLND was 58 months after primary treatment. Median operative time and estimated blood loss was 120 minutes and $100 \mathrm{~mL}$, respectively. No patient had open conversion, blood transfusion or intraoperative complication. The mean number of removed nodes per patient was 46 and mean number of positive nodes per patient was 3 . At 2 months postoperatively, the median (range) PSA level was 0.106 (0.0030.76), which reflects an median PSA level reduction of $89 \%$. Table 1 compiled all the results.

\begin{tabular}{|c|c|}
\hline Variable & Value \\
\hline $\begin{array}{l}\text { Median (range) } \\
\text { Age } \\
\text { ASA Classification } \\
\text { PSA level at initial diagnosis, ng/mL } \\
\text { PSA level at RSLND, ng/mL }\end{array}$ & $\begin{array}{c}65(49-75) \\
2(2-2) \\
7(4-23.2) \\
0.70(0.24-14)\end{array}$ \\
\hline $\begin{array}{l}\text { Prior Radioterapy, } \mathrm{n} / \mathrm{N} \\
\text { Adjuvant } \\
\text { Salvage }\end{array}$ & $\begin{array}{l}10 / 10 \\
6 \\
4\end{array}$ \\
\hline $\begin{array}{l}\text { Primary Gleason Score, } \mathrm{n}(\%) \\
3+3 \\
3+4 \\
4+3 \\
3+5 \\
5+3 \\
4+5\end{array}$ & $\begin{array}{l}1(10 \%) \\
1(10 \%) \\
1(10 \%) \\
1(10 \%) \\
1(10 \%) \\
4(40 \%)\end{array}$ \\
\hline $\begin{array}{l}\text { Median (range) } \\
\text { Operative time, minutes } \\
\text { Estimated blood loss, mL }\end{array}$ & $\begin{array}{l}120(95-140) \\
100(50-150)\end{array}$ \\
\hline $\begin{array}{l}\text { n/N } \\
\text { Open Conversion } \\
\text { Blood transfusion } \\
\text { Intraoperative complications }\end{array}$ & $\begin{array}{l}0 / 10 \\
0 / 10 \\
0 / 10\end{array}$ \\
\hline Patients with positive Lymph Nodes at RSLND (\%) & $8(80 \%)$ \\
\hline $\begin{array}{l}\text { Mean, Median, (Range) } \\
\text { No. Lymph Nodes Removed/Patient } \\
\text { No. Lymph Nodes Positive/Patient }\end{array}$ & $\begin{array}{c}46 ; 36 ;(10-89) \\
3 ; 3 ;(0-7)\end{array}$ \\
\hline Postoperative PSA Level, ng/mL, median, (Range) & $0.106(0.003-0.76)$ \\
\hline$\%$ decrease in PSA level, median & $89 \%$ \\
\hline
\end{tabular}

Conclusions: The initial brazilian series of RSLND was presented, demonstrate safe and feasible to reduce morbity and improve number of lymphonodes removed. Salvage lymphadenectomy could improve oncological outcomes, but randomized controlled trials and longer follow up is necessary

MP31-7 Comparison of Surgical Outcomes of Laparoscopic Versus Robotic Assisted Repair of Vesicovaginal Fistula: Initial Experience From Northwest China

G Zhu, D Wu, W Song, Z Yang, D He

The 1st Affiliated Hospital of Xi' an Jiaotong University China (People's Republic)

Introduction \& Objective: Vesicovaginal fistula (VVF) can appear 1 to 6 weeks after gynecologic or obstetric surgeries, and it still remains a challenge to surgeons; however, controversy still exists over the ideal approach and time to repair. With the development of minimally invasive laparoscopic or robotic surgery, it is possible to repair some of these fistulas by using those less incisional approaches. This study is to compare the perioperative 
outcomes of patients with single VVF underwent laparoscopic repair (LR) or robotic assisted repair (RAR) via transabdominal extravesical approach.

Materials and Methods: A retrospective analysis was performed for 56 consecutive patients with VVF who underwent either LR $(n=30)$ or RAR $(n=26)$ between May 2014 and April 2017. All the patients were diagnosed as post-hysterectomy $(n=48)$ or post-myomectomy $(n=8) V V F$, which was located as a single fistula between the posterior wall of the bladder and the anterior wall of the vagina. The vaginoscopy and cystoscopy were routinely preformed for each patient, and one Foley catheter was preoperatively indwelled to the bladder through the fistula from the vagina with a balloon in the bladder side of the fistula. The demographic parameters and surgical outcomes were compared between the two groups, respectively.

Results: Fistula repair was successful in all the cases with no conversion. The mean operation time of RAR group was less than LR group (100.5 v.s. $156.2 \mathrm{~min}, \mathrm{p}<0.001)$. The mean drainage time of RAR group was less than LR group (4.5 v.s. 6.2 days, $p=0.002$ ). There were no significant differences with respect to the estimated blood loss, transfusion rate, length of hospital stay, postoperative complications and recurrent rate between the two groups. The Foley catheter was removed from the bladder around 3 weeks postoperatively after voiding cystourethrography for all the patients. With the mean follow-up time of 3.5 months, these patients continued to void normally without any recurrence of VVF.

Conclusions: These results may indicate that RAR provides surgical outcomes comparable to those of LR for the treatment of VVF, which is located between the posterior wall of the bladder and the anterior wall of the vagina. Our initial experience suggests that robotic assisted VVF repair is feasible with shorter operation and drainage time than the laparoscopic approach. The minimally invasive robotic assisted VVF repair via transabdominal extravesical approach may be a more attractive option for some patients with VVF.

MP31-8 Post-Chemotherapy Laparoscopic Retroperitoneal Lymph Node Dissection for Select Stage II and III Mixed Malignant Germ-Cell Testicular Tumors

EH Kim, K Du, RS Figenshau

Washington University in Saint Louis United States

Introduction \& Objective: The gold standard for treatment of residual disease following chemotherapy for stage II and III testicular cancer remains open retroperitoneal lymph node dissection (RPLND). Due to the significant morbidity associated with open RPLND, laparoscopic approaches have been developed and demonstrated to be safe and effective in select cases. However, outcomes for post-chemotherapy laparoscopic RPLND (L-RPLND) for mixed malignant germ-cell testicular tumors (MMGCT) are limited in the literature.

Materials and Methods: We performed retrospective chart review for patients who underwent L-RPLND at our institution for MMGCT from May 2006 to October 2016. Patient clinical data, perioperative and survival outcomes were recorded.

Results: A total of $24 \mathrm{~L}-\mathrm{RPLND}$ were performed in the postchemotherapy setting, $33 \%(8 / 24)$ of which were bilateral template. Robot assistance was utilized in a minority of cases (5/ $24=21 \%$ ). Patient clinical data and outcomes are summarized in Table 1. All perioperative outcomes were significantly inferior
Table 1. Clinical data and outcomes

\begin{tabular}{|c|c|c|c|}
\hline & $\begin{array}{l}\text { Bilateral Template } \\
n=8\end{array}$ & $\begin{array}{l}\text { Unilateral Template } \\
\qquad n=16\end{array}$ & p-value \\
\hline Stage Group (\%) & & & 0.67 \\
\hline ॥ & $3(38 \%)$ & $9(56 \%)$ & \\
\hline III & $5(62 \%)$ & $7(44 \%)$ & \\
\hline Mean size of dominant node (SD) & $3.0(1.8) \mathrm{cm}$ & $2.9(1.9) \mathrm{cm}$ & 0.86 \\
\hline Robot-assisted (\%) & $2(25 \%)$ & $3(19 \%)$ & 1.0 \\
\hline \multicolumn{4}{|l|}{ Perioperative Outcomes } \\
\hline Mean Operative Time (SD) & $373(72)$ minutes & 290 (55) minutes & $<0.01$ \\
\hline Mean Estimated Blood Loss (SD) & $930(1140) \mathrm{mL}$ & $172(123) \mathrm{mL}$ & 0.01 \\
\hline Open Conversions (\%) & $4(50 \%)$ & $0(0 \%)$ & $<0.01$ \\
\hline Mean Length of Stay (SD) & $3.3(2.1)$ days & $1.2(0.5)$ days & $<0.01$ \\
\hline Complications (\%) & & & $<0.01$ \\
\hline Clavien I & 1 , ileus $(12 \%)$ & 1, lymphocele $(6 \%)$ & \\
\hline Clavien II & 2 , transfusion $(25 \%)$ & 1 , C.diff colitis $(6 \%)$ & \\
\hline Clavien III & $\begin{array}{l}\text { 1, IVC filter (12\%); } 2 \text {, } \\
\text { lymphocele drain (25\%) }\end{array}$ & $0(0 \%)$ & \\
\hline Pathology (\%) & & & 0.10 \\
\hline Viable germ cell tumor & $1(12 \%)$ & $1(6 \%)$ & \\
\hline Mature teratoma & $3(38 \%)$ & $12(75 \%)$ & \\
\hline Fibrosis / necrosis & $4(50 \%)$ & $3(19 \%)$ & \\
\hline \multicolumn{4}{|l|}{ Oncologic Outcomes } \\
\hline Mean Lymph Node Yield (SD) & $19.4(10.6)$ & $23.7(12.3)$ & 0.44 \\
\hline Mean Follow-up (SD) & 35.1 (35.4) months & $43.3(38.4)$ months & 0.62 \\
\hline Recurrences (\%) & $0(0 \%)$ & $2(13 \%)$ & 0.54 \\
\hline
\end{tabular}

for the bilateral group, including operative time, estimated blood loss, open conversion rate, complications, and length of hospital stay. Of note, the open conversion rate was $50 \%$ for the bilateral group versus $0 \%$ for the unilateral group. Two recurrences were noted after unilateral L-RPLND, at 3.4 and 67 months. Both patients had mature teratoma diagnosed on RPLND pathology. The early recurrence following right L-RPLND was managed with open bilateral RPLND with aortic resection and reconstruction; the patient ultimately died 31 months following initial L-RPLND. The patient with late recurrence following left L-RPLND had no evidence of disease at 48 month follow-up, and then was lost to follow-up until he re-presented with a pathologic compression fracture in the setting of diffuse metastatic disease at 67 months. He was placed on hospice and died 69 months following initial L-RPLND.

Conclusions: In our experience, L-RPLND for stage II and III MMGCT is safe and effective in the post-chemotherapy setting for select patients. Bilateral L-RPLND was associated with inferior perioperative outcomes, but similar oncologic outcomes to unilateral L-RPLND. Given the relatively high rate of conversion, patients requiring bilateral RPLND should be considered for an open operation.

MP31-9 Robotic-assisted radical prostatectomy associated with robotic-assisted cholecystetomy in the same procedure: case series report and review of literature.

J Queiroz, P Califano, M Belkovsky, J DA CRUZ, R SAKATA, M DA SILVA, M Srougi, W Nahas, C PASSEROTTI

\section{GERMAN HOSPITAL OSWALDO CRUZ, SAO PAULO, BRAZIL \\ Brazil}

Introduction \& Objective: With the advent of robotic surgery it became much easier to surgically treat various diseases and eventually more than 1 disease in the same surgical intervention. The objective of this study is to describe a case series of roboticassisted radical prostatectomy associated with robotic-assisted cholecystectomy in the same intervention. 
Materials and Methods: From 2008 to 2017, six patients undergoing robotic-assisted radical prostatectomy associated with robotic-assisted cholecystectomy in the same robotic session in our institution. Demographic, intra and post-operative data of these patients was collected. In addition, a literature review was performed to assessed the current published data on the subject. Results: Patients average age were $61.3 \pm 10.1$ years old, mean operative time was $232 \pm 35 \mathrm{~min}$, estimated blood loss of all patients were less than $100 \mathrm{cc}$. All patients were discharged $48 \mathrm{hrs}$ after surgery, there were no intra-operative or post-operative complications.

Conclusions: Robotic-assisted cholecystectomy at the same surgical time as robotic-assisted radical prostatectomy is a safe and effective option, with good results for the treatment of patients with prostate cancer associated with gallbladder stones.

MP31-10 Results of a Center for Robotic Surgery in Brazil: A nine years experience.

J Queiroz, P Califano, M Belkovsky, J DA CRUZ, R SAKATA, M DA SILVA, M Srougi, W Nahas, C PASSEROTTI

GERMAN HOSPITAL OSWALDO CRUZ, SAO PAULO, BRAZIL

Brazil

Introduction \& Objective: Robotic surgery is increasingly present in the reality of the treatment of urological diseases in Brazil, since it is a formidable tool for the treatment of several affections. The purpose of this study is to describe the results of a robotic surgery center in Brazil.

Materials and Methods: Data from all robotic urologic surgeries at the Center for Robotic Surgery of the Hospital Alemão Oswaldo Cruz from June 2008 to January 2016 were collected. Data regarding surgical time, aspirated blood loss, complications according to Clavien-Dindo's classification and time were tabulated and analyzed.

Results: During the study period, 704 robotic surgeries were performed, of which 603 were radical prostatectomies, 41 were partial or total nephrectomies, 25 were simple prostatectomies, 13 pyeloplasties, 7 pelvicolithotomies, 6 retroperitoneal tumor exercises, 5 ureteral reimplants, 4 partial cystectomies. The mean total operative time was $289 \pm 59 \mathrm{~min}$, mean aspirated blood loss was $232 \pm 190 \mathrm{ml}$, the number of complications was $22(3 \%) ; 18$ (2.5\%) complications were Grade I, $3(0.4 \%)$ complications Grade II and $1(0.1 \%)$ complication Grade III. The mean length of hospital stay was $2.9 \pm 1.3$ days

Conclusions: Robotic surgery in a specialized center presents good results, with small blood loss, few complication rates and short hospitalizations.

MP31-11 The utility of axial abdominal imaging after partial nephrectomy for $\mathrm{T} 1$ renal cell carcinoma surveillance

I Sorokin, BA Johnson, N Canvasser, V Margulis, Y Lotan, G Raj, A Sagalowsky, J Gahan, J Cadeddu

UT Southwestern

United States

Introduction \& Objective: The overall recurrence rate of T1 renal cell carcinoma is low. We evaluated abdominal imaging
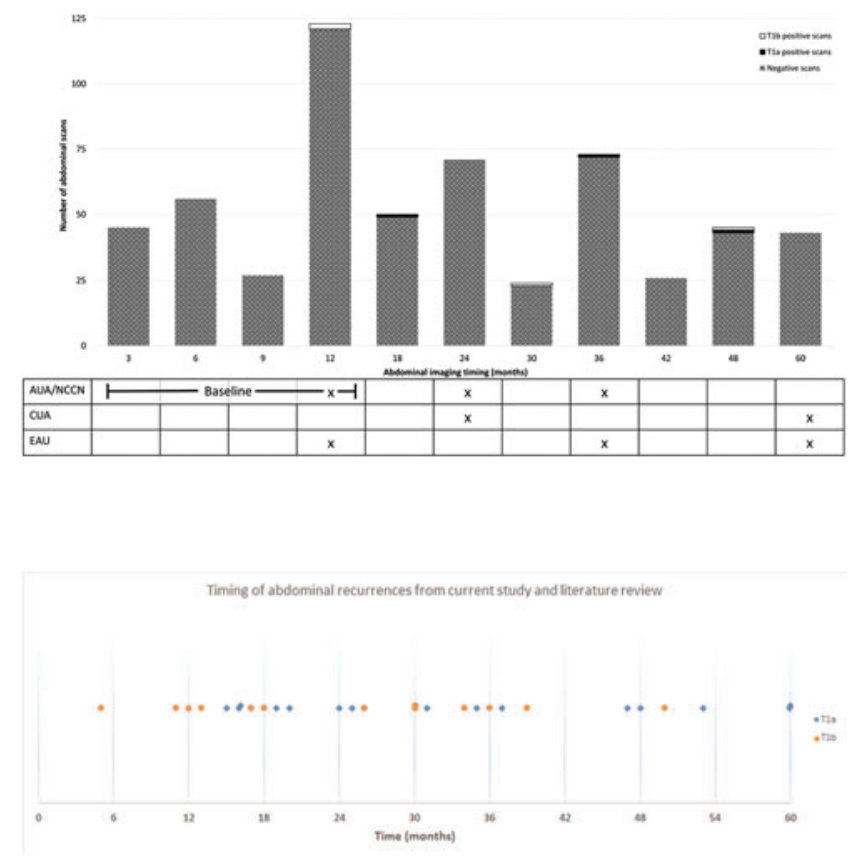

after partial nephrectomy based on current guidelines for T1 renal cell carcinoma surveillance.

Materials and Methods: We retrospectively reviewed T1 renal cell carcinoma patients who underwent partial nephrectomy between 2006-2012 followed by abdominal imaging at our institution. Primary and secondary outcomes were incidence and timing of imaging diagnosed abdominal recurrences, respectively. A literature review was conducted to summarize prior reports of incidence and timing of recurrences after PN for T1 disease.

Results: In total, $160 \mathrm{~T} 1 \mathrm{a}$ and $37 \mathrm{~T} 1 \mathrm{~b}$ patients underwent partial nephrectomy. Seven patients had an abdominal recurrence: 3 patients with both local and distant recurrences, and 4 patients with a metachronous contralateral kidney recurrence. The incidence of an abdominal recurrence detected by imaging was higher in $\mathrm{T} 1 \mathrm{~b}$ compared to T1a groups $(10.8 \%$ vs. $1.9 \%$, $\mathrm{p}=0.024)$. Although not significant, the median time to recurrence occurred earlier in T1b vs. T1a (13 vs. 37 months, $\mathrm{p}=0.480$ ), and both groups had recurrences after 3 years of suggested guideline surveillance. In the literature combined with the present study, the time to median recurrence for $\mathrm{T} 1 \mathrm{~b}$ vs. T1a was 24 vs. 29 months, respectively $(\mathrm{p}=0.226)$.

Conclusions: Recurrences detected by abdominal imaging occurred earlier and more frequently in T1b compared to T1a patients. Future recommendations for surveillance strategies after $\mathrm{PN}$ should distinguish T1a from T1b with less intense frequency of imaging for T1a. A longer period of surveillance should be considered since recurrences can occur beyond 3 years.

MP31-12 Effect of robot-assisted laparoscopic urological surgeries on the modern philosophy of nursing

X Peng, X Wang, S Zhong, Z Shen, Q Ding

China (People's Republic)

Introduction \& Objective: To summarize the measures and effectiveness of perioperative nursing in patients undergoing Da 
Vinci robot-assisted laparoscopic urological surgeries, and to evaluate the effect of robotic surgeries on the modern philosophy of nursing.

Materials and Methods: The clinical data of 720 patients undergoing Da Vinci robot-assisted laparoscopic urological surgerieswere retrospectively analyzed. Upper urinary tract operations are 512 cases and lower urinary tract operations are 208 cases. The upper urinary tract operations include adrenalectomy (214), partial nephrectomy (106), nephrolithotomy or ureterolithotomy (75), pyeloureteroplasty (42), radical nephrectomy (27), renal cyst unroofing (23), nephroureterectomy (9), paraganglioma resection (9), double kidney resection (5), and ligation of renal artery aneurysms (2). The lower urinary tract operations include radical prostatectomy (122), radical cystectomy (38), partial cystectomy (28), vesical diverticulectomy (7), urachal cyst resection (5), retroperitoneal lymph node dissection (4 cases), high ligation of spermatic vein (2), and repair of vesicovagina fistula (2). Preoperative care focused on psychological nursing and preoperative preparations. Postoperative care included psychological nursing, strict monitoring of vital signs, respiratory care, operative incision and drain tube nursing, diet nursing, early functional exercise, and close observation to prevent the postoperative complications. The operation duration, blood loss, recovery time of intestinal function, time of out-of-bed activity, time of removing drainage tube and catheter, and postoperative hospital stays were compared between the first 360 surgeries and after 360 surgeries.

Results: Compared with the first 360 surgeries, the after 360 surgeries have shorter operation duration (101.7 \pm 68.2 minVS.130.9 $\pm 71.6 \mathrm{~min})$, less blood loss $(192.9 \pm 216.2 \mathrm{ml} \mathrm{VS}$. $253.4 \pm 261.5 \mathrm{ml}$ ), shorter recovery time of intestinal function $(0.6 \pm 0.4 \mathrm{~d}$ VS. $1.0 \pm 0.9 \mathrm{~d})$, shorter time of out-of-bed activity $(2.0 \pm 2.5 \mathrm{~d}$ VS. $3.3 \pm 3.1 \mathrm{~d})$, shorter time of removing drainage tube $(5.1 \pm 2.2 \mathrm{~d}$ VS. $7.5 \pm 3.0 \mathrm{~d})$ and catheter $(5.8 \pm 4.2 \mathrm{~d}$ VS. $9.2 \pm 5.1 \mathrm{~d})$, and shorter postoperative hospital stays $(7.1 \pm 3.8 \mathrm{~d}$ VS. $12.0 \pm 4.3 \mathrm{~d}$ ).

Conclusions: Da Vinci robot-assisted laparoscopic urological surgeries have obvious advantages with less trauma, less bleeding, satisfactory results, fewer complications and faster postoperative recovery. The can reduce significantly the difficulty and workload of nursing care and improve nursing care quality and efficiency.

MP31-13 Repeat Robot Partial Nephrectomy: Initial Experience

S Chopra, R Ahar, C Beckler, L Medina, R Sotelo, G Cacciamani, A Abreu, J Cai, M Desai, M Aron, IS Gill, A Berger

USC institute of urology

United States

Introduction \& Objective: Robotic partial nephrectomy (RPN) is gaining more popularity given the unique features offered by robotics. However, nephron-sparing surgery (NSS) can be challenging for patients who have undergone a prior kidney tumor local therapy and have developed a new or recurrent tumor in the same kidney. Herein, we evaluate our experience with repeat RPN (R-RPN) and use matched-pair analysis to compare our results to those obtained in a primary RPN (P-RPN) series at our institution.

Materials and Methods: Patients were identified through a prospectively maintained database approved by the institutional review board at our institution. R-RPN cases were performed at our institution between November 2010 and July 2013. R-RPN patients were matched 1:1 to P-RPN patients according to age, baseline GFR, tumor size, tumor location, and surgery type (clamped vs. unclamped). Preoperative, operative, and postoperative details and outcomes were compared. Complications were coded according to the Clavien system

Results: A total of 20 cases (10 R-RPN vs. 10 P-RPN) were analyzed, and a total of 30 tumors were removed (10 P-RPN and 20 R-RPN). All robotic procedures were completed successfully without any intra-operative complications. The demographical features that were significantly different (Median (Range)) between the groups were the American Society of Anesthesiologists score (2 (2-3) vs. 3 (2-4), p

Conclusions: R-RPN is a safe and feasible procedure in appropriately selected cases. When compared with P-RPN, our experience with R-RPN demonstrated similar perioperative and oncologic outcomes

\section{MP31-14 Robotic-Assisted Partial Nephrectomy for Hilar} Tumors: Initial Experience.

M Santomauro, S Chopra, L Medina, R Sotelo, G Cacciamani, A Abreu, C Metcalfe, M Aron, M Desai, IS Gill, A Berger

USC institute of urology

United States

Introduction \& Objective: To compare hilar v. nonhilar tumors using perioperative outcome data in patients undergoing a robotic partial nephrectomy (RPN) at our institution

Materials and Methods: Retrospectively reviewed a prospectively managed database of 487 patients that underwent an RPN at USC from 2009 to 2016. Perioperative data including demographic and follow-up data was registered

Results: Of the 487 patients, 55 underwent a robotic partial nephrectomy for a hilar tumor. Mean tumor size for hilar tumors, $3.6 \mathrm{~cm}$, was larger compared to non-hilar tumors, $3.3 \mathrm{~cm}$, $(\mathrm{p}=0.02)$. Also, RENAL score showed significant differences: 8.7 for Hilar and 7.2 for Non Hilar tumors (p: 3, male ratio, and BMI. Perioperative variables were similar in regards to EBL, operative time, warm ischemia time, hospital stay and transfusion rate. Overall, the complication rate was $11.29 \%$ with minor complication rate (Clavien <iii) $p=$ "" $2.46 \% .<=$ "" at = " "

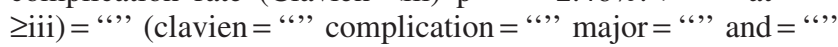
$8.82 \%="$ " ">

Conclusions: Despite a preconceived notion that hilar tumors are associated with a greater degree of complexity, our experience demonstrates that RAPN is a safe and efficacious procedure for hilar tumors and is associated with a similar outcome profile when compared to nonhilar tumors in patients undergoing RPN at a high-volume institution.

\section{MP31-15 The early experience of laparoscopic retroperi-} toneal nephrectomy in Mongolia

A Sankjaajamts, D Batbold, T Zev, M Munkhbaatar, S Avirmed, J Jodov

Mongolian National University of Medical Sciences Mongolia 
Introduction \& Objective: Laparoscopic surgery is safe and feasible technique for renal surgery. Many renal surgeries are performed by either two approaches that transperitoneal or retroperitoneal. Surgically retroperitoneal laparoscopy might be advantageous over transperitoneal approach due to safe port placement, visceral handling with lesser risk of injury, more rapid access to the renal pedicle and easer renal artery control. However Laparoscopic retroperitoneal renal surgery is far from routine in most Hospital in Mongolia.

Materials and Methods: Here we present our experience with laparoscopic retroperitoneal renal surgery in 20 patients. All patient in prone position, 3 or 4 trocars and 30 degree telescope ENDOCAMELEON (OLYMPUS, JAPAN) was used. Our laparoscopic surgeries was under the general anesthesia. Two $12 \mathrm{~mm}$ balloon trocars and one $5 \mathrm{~mm}$ trocars were used. This surgeries was performed in Ulaanbaatar Songdo hospital and Hospital number-2, between 2016 Jan-2016 Dec.

Results: A total 20 patients who underwent laparoscopic retroperitoneal renal surgery. Mean operation time was 180 minutes. Estimated blood loss $<50 \mathrm{ml}$. The average length of hospitalization was 4 days. There were 12 patients with renal cell carcinoma, 7 patients with severe hydronephrosis, 1 patient with large renal cyst, 1 patient with renal pelvic stone. No complication occurred in the postoperative course as the all patient showed good recovery and there was no bleeding

Conclusions: Literature said that it is possible to state that retroperitoneoscopy can be the technique of choice for accessing and carrying out all the surgery of the upper urinary tract. Therefore laparoscopic renal surgery can be performed efficiently and effectively with transperitoneal or retroperitoneal approach. We hope our input will be changed our surgical techniques targeted to retroperitoneal approach. This is the first reported data in Mongolia to our knowledge. But this study has a limitations due to number of cases.

MP31-16 Transition from retroperitoneal to transperitoneal robotic-assisted laparoscopic partial nephrectomy :the learning curve for experienced surgeon in the robotic surgery era

W Liao, C Zhang, L Diao, Q Yang, X Yao

tianjin medical university cancer institute\&hospital China (People's Republic)

Introduction \& Objective: In China, most urology surgeons are familiar with the retroperitoneal laparoscopic surgery, even some experienced surgeons never done the transperitoneal laparoscopic renal surgery. robotic-assisted laparoscopic partial nephrectomy (RALPN) is more commonly performed with transperitoneal approach. Here we assessed the learning curve during the transition from retroperitoneal to transperitoneal RALPN.

Materials and Methods: From January 2016 to February 2017,39 patients with kindey neoplasm underwent either transperitoneal RALPN (t-RALPN) or retroperitoneal RALPN (rRALPN) by the same surgeon (have worked $>20$ years, done laparoscopic partial nephrectomy $>50$ /year, but never done any transperitoneal minimally invasive surgery) were retrospectively analyzed. The learning curve was defined as the number of cases required to consistently perform t-RALPN with shorter average operative times (OT) and warm ischemia times (WIT), as compared to the r-RALPN.

Results: 14 patients underwent t-RALPN and 25 patients underwent r-RALPN. The 2 groups had comparable demographics
Table 1: Demographics and Perioperative data

\begin{tabular}{|c|c|c|c|}
\hline & $t$-RALPN & r-RALPN & P value \\
\hline $\mathrm{N}$ & 14 & 25 & \\
\hline Age (mean \pm SD) & $53.2 \pm 10.9$ & $53.1 \pm 8.68$ & 0.977 \\
\hline \multicolumn{4}{|l|}{ Sex } \\
\hline Male & 8 & 19 & 0.287 \\
\hline Female & 6 & 6 & \\
\hline BMI $\left(\mathrm{kg} / \mathrm{m}^{2}\right) \quad($ mean \pm SD $)$ & $23.7 \pm 1.87$ & $24.8 \pm 1.57$ & 0.065 \\
\hline ASA Score (mean \pm SD) & $2.5 \pm 0.5$ & $2.4 \pm 0.8$ & 0.172 \\
\hline $\begin{array}{l}\text { Tumor Size (mm) (mean } \pm \\
\text { SD) }\end{array}$ & $3.8 \pm 0.54$ & $3.1 \pm 0.68$ & 0.005 \\
\hline Tumor Side & & & 0.685 \\
\hline Left & 5 & 12 & \\
\hline Right & 9 & 13 & \\
\hline Tumor Location & & & 0.083 \\
\hline Anterior & 12 & 14 & \\
\hline Posterior & 2 & 11 & \\
\hline Nephrometry scores & $8.3 \pm 1.0$ & $7.6 \pm 1.0$ & 0.052 \\
\hline $\begin{array}{l}\text { operative time (min), } \\
\quad(\text { mean } \pm S D)\end{array}$ & 171. $6 \pm 26.8$ & $190.8 \pm 50.8$ & 0.198 \\
\hline $\begin{array}{l}\text { Warm ischemia time (min), } \\
(\text { mean } \pm S D)\end{array}$ & $25.7 \pm 5.7$ & $25.2 \pm 7.1$ & 0.818 \\
\hline $\begin{array}{l}\text { Estimated blood loss }(\mathrm{ml}) \text {, } \\
(\text { mean } \pm \text { SD) }\end{array}$ & $64.3 \pm 46.2$ & $95.0 \pm 106.3$ & 0.312 \\
\hline conversion number $(\mathrm{N})$ & 1 & 3 & \\
\hline $\begin{array}{l}\text { post-operation Hospital } \\
\text { stay (d), (mean } \pm S D)\end{array}$ & $8.2 \pm 1.2$ & $8.3 \pm 1.2$ & 0.869 \\
\hline
\end{tabular}

and perioperative results except the median tumor diametes(tgroup: $3.8 \mathrm{vs} \mathrm{R}$-group: $3.1 \mathrm{~cm} \mathrm{p}=0.005$ ). No patients in either group had a positive surgical margin or need transfusion. complication rates were comparable between the two groups, conversion to open surgey (t-group: 1 case, r-group: 3 cases). There was a downward trend in both OT and WIT during the t-RALPN learning curve. After the first 5 t-RALPN cases, the average OT reached the average OT and WIT of the last cases. The average OT of the first 5 RPN patients was 173.6minutes, compared with the average OT of the r-RALPN patients of 190.8 minutes $(\mathrm{P}=0.468)$.

Conclusions: In China, It may be easiler to start the RALPN through the retroperitoneal approach for surgeons who never done transperitoneal minimally invasive surgery. t-RALPN achieved a similar OT and WIT as r-RALPN after 5 procedures. Both transperitoneal and retroperitoneal approach of RALPN were effective and safety for kidney neoplasm.

MP31-17 The clinical application of retroperitoneal laparoscopic surgery combined with mini-flank incision "hybrid surgery" for partial nephrectomy of complex renal tumor

X Ye, J Liu, T Xu, X Huang

Urology Department

China (People's Republic)

Introduction \& Objective: To evaluate the clinical effect and safety of retroperitoneal laparoscopic surgery combined with mini-flank incision (hybrid surgery) in complex renal tumors with partial nephrectomy.

Materials and Methods: Between April 2015 and December 2016, the clinical data from 16 patients with renal tumors who were performed the hybrid surgery, including 10 male and 6 female patients, were retrospectively reviewed. The average age was $50.2 \pm 10.7$ years, 9 located in left side and 7 in right side, the mean tumor size was $(6.1 \pm 1.0) \mathrm{cm}$, the mean R.E.N.A.L. score was $9.3 \pm 1.3$. All the patients received the hybrid surgery, the first step was to prepare the renal artery with retroperitoneal 
laparoscopy, and the kidney and tumor should be adequately separated, and then the incision about $10 \mathrm{~cm}$ was be done under the costal arch, the renal artery was clamped with ice mud surrounded, and then removed the tumor and closed the wound. The operative time, cold ischemia time, estimate blood loss, operative and post-operative complications and short-term (3 months) renal function.

Results: All the 16 patients were successfully performed hybrid surgery. 1 patient received a second surgery to for acute postoperative bleeding. Mean operative time was $(164.9 \pm 23.6) \mathrm{min}$, mean cold ischemia was $(32.4 \pm 6.2)$ min, mean estimated blood loss was $(204 \pm 125.1) \mathrm{ml}, 3$ patients accepted blood transfusion, 1 patient accepted dressing change for the infected wound and recovered. No other complications happened. The mean 1 day postoperative serum creatinine level was (126.35 \pm 26.4$) \mathrm{umol} / \mathrm{L}$. During follow-up period from 3 to 20 months, no local recurrence or metastasis occurred.

Conclusions: nephron sparing surgery in complex renal tumor is difficult, however, this hybrid surgery is safe and effective, which is worthy of further application and spreading.

MP31-18 Cystic versus Solid Masses: Is There a Difference in Perioperative Outcome During Robotic Partial Nephrectomy?

K Blum, D Paulucci, R Abaza, A Bhandari, JC Delto, K Badani

Department of Urology, Icahn School of Medicine at Mount Sinai

United States

Introduction \& Objective: Partial nephrectomy is a well-established treatment for both solid and suspicious cystic masses. We sought to determine if there is a difference in perioperative and postoperative outcomes during robotic PN (RPN) for cystic versus solid masses.

Materials and Methods: We identified 253 patients who underwent RPN for a cT1 renal mass from 2013 to 2016 with complete data on the type of lesion (solid or cystic) from our multi-institutional database. Lesions were pre-operatively classified as solid or cystic according to radiology reports. Perioperative and renal functional outcome at discharge and at median follow-up 6.9 months were compared in multivariable analysis between patients with solid $(\mathrm{n}=198,84.3 \%)$ and cystic lesions $(\mathrm{n}=37,15.7 \%)$.

Results: Baseline characteristics including age (Average: 60.1 years, $\mathrm{p}=.427$ ), baseline eGFR (Average: $82.9 \mathrm{~mL} / \mathrm{min} / 1.73 \mathrm{~m}^{2}$,

\begin{tabular}{clll} 
Malignant Pathology & $158(80.2 \%)$ & $29(78.4 \%)$ & .799 \\
Clear Cell RCC & $108(54.8 \%)$ & $14(37.8 \%)$ & .058 \\
Papillary RCC & $25(12.7 \%)$ & $6(16.2 \%)$ & .597 \\
Chromophobe & $7(3.6 \%)$ & $2(5.4 \%)$ & .637 \\
RCC & & & \\
Cystic RCC & $10(5.1 \%)$ & $4(10.8 \%)$ & .246 \\
Angiomyolipoma & $11(5.6 \%)$ & $1(2.7 \%)$ & .697 \\
Oncocytoma & $19(9.6 \%)$ & $2(5.4 \%)$ & .543 \\
Benign Cyst & $8(4.1 \%)$ & $5(13.5 \%)$ & .037 \\
Mixed RCC & $9(4.6 \%)$ & $3(8.1 \%)$ & .410 \\
Fuhrman Grade & & & .823 \\
1 & $16(10.8 \%)$ & $4(16.0 \%)$ & .497 \\
2 & $97(65.5 \%)$ & $16(64.0 \%)$ & .881 \\
3 & $33(22.3 \%)$ & $5(20.0 \%)$ & .797 \\
4 & $2(1.4 \%)$ & $0(0.0 \%)$ & $>.999$ \\
\hline
\end{tabular}

a. $\mathrm{ASA}=$ American Society of Anesthesiologists Score; $\mathrm{BMI}=$ Body Mass Index; $\mathrm{CCI}=$ Charlson Comorbidity Index; COPD: Chronic Obstructive Pulmonary Disease eGFR $=$ Estimated Glomerular Filtration Rates
Table 1. Baseline Demographic, Clinical and Pathologic Characteristics of Patients Undergoing RPN for a Solid or Cystic Lesion

\begin{tabular}{|c|c|c|c|}
\hline & Solid & Cystic & $\mathbf{p}$ \\
\hline $\mathbf{N}$ & $198(84.3 \%)$ & $37(15.7 \%)$ & \\
\hline Age & $59.8(24.0-82.0)$ & $61.7(31.0-86.0)$ & .427 \\
\hline Male & $119(60.1 \%)$ & $23(62.2 \%)$ & .814 \\
\hline BMI & $32.7(16.5-70.9)$ & $30.8(18.6-52.2)$ & .143 \\
\hline $\mathbf{A S A} \geq \mathbf{3}$ & $63(39.1 \%)$ & $19(55.9 \%)$ & .072 \\
\hline Age Adjusted CCI & $3(0-9)$ & $4(0-7)$ & .249 \\
\hline Hypertension & $108(54.5 \%)$ & $24(64.9 \%)$ & .246 \\
\hline Diabetes Mellitus & $55(27.8 \%)$ & $8(21.6 \%)$ & .438 \\
\hline Hyperlipidemia & $71(35.9 \%)$ & $16(43.2 \%)$ & .393 \\
\hline Coronary Artery & $19(9.6 \%)$ & $1(2.7 \%)$ & .215 \\
\hline \multicolumn{4}{|l|}{ Disease } \\
\hline COPD & $5(2.5 \%)$ & $0(0.0 \%)$ & $>.999$ \\
\hline Nephrolithiasis & $29(14.7 \%)$ & $4(10.8 \%)$ & .538 \\
\hline Baseline eGFR & $83.1(33.3-214.3)$ & $81.6(26.1-159.3)$ & .749 \\
\hline Baseline CKD $\geq 3$ & $33(17.1 \%)$ & $7(18.9 \%)$ & .789 \\
\hline Solitary Kidney & $3(1.5 \%)$ & $0(0.0 \%)$ & $>.999$ \\
\hline Tumor Laterality & & & .227 \\
\hline Right Kidney & $107(54.0 \%)$ & $91(45.9 \%)$ & \\
\hline Left Kidney & $16(43.2 \%)$ & $21(56.8 \%)$ & \\
\hline Tumor Size $(\mathrm{cm})$ & $3.3(0.9-7.0)$ & $3.1(1.4-6.4)$ & .377 \\
\hline Clinical Stage & & & .594 \\
\hline T1a & $147(74.2 \%)$ & $29(78.4 \%)$ & \\
\hline T1b & $51(25.8 \%)$ & $8(21.6 \%)$ & \\
\hline RENAL Nephrometry & $7(4-11)$ & $7(4-11)$ & .748 \\
\hline \multicolumn{4}{|c|}{ Score } \\
\hline Pole of Tumor & & & .815 \\
\hline Upper & $53(35.3 \%)$ & $12(41.4 \%)$ & \\
\hline Mid & $42(28.0 \%)$ & $7(24.1 \%)$ & \\
\hline Lower & $55(36.7 \%)$ & $10(34.5 \%)$ & \\
\hline Tumor Location & & & .529 \\
\hline Anterior & $40(27.6 \%)$ & $11(35.5 \%)$ & \\
\hline Posterior & $70(48.3 \%)$ & $15(48.4 \%)$ & \\
\hline Lateral & $35(24.1 \%)$ & $5(16.1 \%)$ & \\
\hline Percent Endophytic & & & .519 \\
\hline $100 \%$ & $63(39.1 \%)$ & $10(28.6 \%)$ & \\
\hline$>\mathbf{5 0} \%$ & $76(47.2 \%)$ & $20(57.1 \%)$ & \\
\hline$<\mathbf{5 0} \%$ & $22(13.7 \%)$ & $5(14.3 \%)$ & \\
\hline Hilar & $36(21.4 \%)$ & $7(20.0 \%)$ & .851 \\
\hline Date of Surgery & & & .871 \\
\hline Clamp Type & & & .007 \\
\hline Main Artery & $152(84.4 \%)$ & $29(82.9 \%)$ & \\
\hline Selective Arterial & $23(12.8 \%)$ & $1(2.9 \%)$ & \\
\hline Off-Clamp & $5(2.8 \%)$ & $5(14.3 \%)$ & \\
\hline
\end{tabular}

$\mathrm{p}=.749$ ) and tumor size (Average: $3.3 \mathrm{~cm}, \mathrm{p}=.377$ ) were similar, as were rates of malignancy $(78.4 \%$ vs. $80.2 \%, p=.799)$. No difference in any outcome for cystic vs. solid masses including operative time $(b=-0.09 ; 95 \% \mathrm{CI}=-0.23,0.03 ; \mathrm{p}=.119)$, ischemia time $(b=-1.01 ; 95 \% C I=-2.54,0.52 ; p=.195)$, blood loss $(\mathrm{p}=.784)$, post-operative complication $(\mathrm{p}=.879)$, length of stay $(\mathrm{p}=.349)$, positive surgical margins $(\mathrm{p}=.993)$, non-neoplastic parenchymal volume removed $(\mathrm{p}=.926)$, acute kidney injury at discharge $(\mathrm{p}=.953)$ or progression of CKD stage at 6.9 months $(\mathrm{HR}=1.75 ; 95 \% \mathrm{CI}=0.85,3.61 ; \mathrm{p}=.129)$ were observed.

Conclusions: Cystic compared to solid masses on imaging proved to have an equivalent rate of malignant pathology. During RPN, the type of mass removed does not appear to influence the volume of normal kidney removed or any perioperative or short term renal functional outcome.

MP31-19 Off-clamping robot-assisted partial nephrectomy and re-recognition of HuaShan standard

Z Shen, S Zhong, Z Fang, Z Wu, S Chen, M Zhang, X Zhang, C Feng, S Mao, Q Ding

China (People's Republic) 
Introduction \& Objective: To explore the application of HuaShan nephrometry score (HSNS) in prediction of zero-ischemia time for off-clamping robot-assisted partial nephrectomy (RAPN). Materials and Methods: The data of 24 patients with renal tumor managed by off-clamping RAPN and 57 patients managed by clamping RAPN during January 2014 to December 2016 were retrospectively analyzed, of them 13 were males and 11 were females, with ranging 24-84 (55.5 \pm 27.5$)$ years old. The average tumor diameter was $3.2 \pm 0.8 \mathrm{~cm}$. 15 tumors were on the left side, 9 were on the right and 2 bilateral; the dorsal and ventral tumors were 10 and 14 respectively. We conducted a comparative study of evaluating the renal tumors in off-clamping RAPN and clamping RAPN by using HSNS.

Results: SHNS of 24 patients in off-clamping RAPN group were all less than 10, while the SHNS in clamping RAPN group were range from 11 to 24 . There were significant differences in renal warm ischemia time, intraoperative blood loss and hospital stay $(P<0.05)$, and meanwhile, there was no significant differences in operative time and postoperative complications $(P>0.05)$ in the two groups.

Conclusions: As to RAPN, patients with high HSNS had longer renal warm ischemia time, more intraoperative blood loss and longer hospital stay. Based on our experience, patients with SHNS $<10$ are recommended to undergo off-clamping RAPN, SHNS ranging from 11 to 24 may undergo clamping RAPN, and SHNS > 25 may undergo RAPN. Hence, HSNS can better assess the risk and difficulty of RAPN.

MP31-20 Outcome of near-infrared fluorescence imaging with indocyanine green in robot-assisted partial nephrectomy on different tumor morphology: preliminary 1st case series in Japan.

S Hisasue, H Takeuchi, S Ohta, T Kubota, Y Mitsui

Japan

Introduction \& Objective: Robot-assisted partial nephrectomy (RAPN) has been a gold standard for the surgery of small renal cell carcinoma for several years. The shape of renal cell carcinoma is usually round; however, some tumors show oval or irregular shapes. We demonstrate the preliminary outcome of near-infrared fluorescence imaging (NIFI) with indocyanine green (ICG) in RAPN on different tumor morphology.

Materials and Methods: We evaluated 9 patients who underwent RAPN with da Vinci Xi (Intuitive Surgical Inc., Sunnyvale,

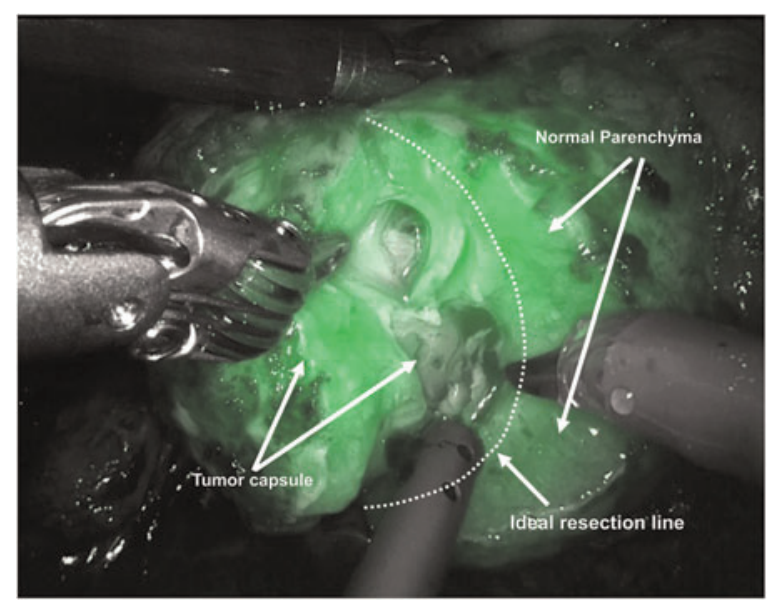

CA, USA). We assessed R.E.N.A.L nephrometry score and tumor morphology with 3D images made by multidetector computed tomography data using AZE VirtualPlace (AZE Inc., Tokyo, Japan). We divided tumors into round, oval, and irregular shape from 3D images. We injected ICG $1 \mathrm{ml}$ intravenously, then clumped renal artery, and started tumor resection with $5 \mathrm{~mm}$ tumor margin. We confirm the resection site with NIFI (Firefly ${ }^{\mathrm{TM}}$, Intuitive Surgical Inc., Sunnyvale, CA, USA) intraoperatively on occasion.

Results: Eight patients were male, and 1 patient was female. Affected side of kidney was right in 3 and left in 6. RAPN was carried out with intraperitoneal approach in 7 and retroperitoneal approach in 2. Median patient age was 69 years (range: $55-75$ ), median tumor size was $39.2 \mathrm{~mm}$ (range: $10.0-46.6 \mathrm{~mm}$ ), median R.E.N.A.L nephrometry score was 8 (range: 6-10), median console time was $125 \mathrm{~min}$. (range: 96-180), median total operative time was 165 min. (range: 141-286), median warm-ischemic time was $18 \mathrm{~min}$. (range: 12-35), and median blood loss was $100 \mathrm{ml}$ (range: 10-180). Tumor shapes were round in 4, oval in 2, and irregular in 3. Histopathological results in all patients were clear cell carcinoma, pT1a, and surgical margins were all negative. Intraoperative exposure of tumor capsule was confirmed in 4 with NIFI. Two of 3 irregular shaped tumors were exposed. The mean tumor diameter was $42.2 \mathrm{~mm}$ in the exposure group, and $30.4 \mathrm{~mm}$ in the non-exposure group $(\mathrm{p}=0.059)$.

Conclusions: From the current preliminary outcome, NIFI with ICG may be helpful for RAPN in the irregular shaped or relatively large tumors.

\section{MPS32: EDUCATION/ SIMULATION II}

MP32-1 Urology Residents' Experience and Attitude Towards Surgical Simulation: Presenting Our Four Year Experience With A Multi Institutional, Multi Modality Simulation Model

A Chow, BA Sherer, E Yura, S Kielb, E Kocjancic, S Eggener, T Turk, S Park, S Psutka, M Abern,

K Latchamsetty, C Coogan

Rush University Medical Center

United States
Introduction \& Objective: Surgical simulation with authentic and high fidelity simulators is increasingly used to improve surgical technical skills, decrease learning curve and to improve surgical outcomes and patient safety. Although surgical simulation is not an Acceditation Council for Graduate Medical Education (ACGME) requirement for Urology residency, a recent survey suggest that Urology program directors are in favor of incorporating its use into residency training. Furthermore, there is limited data in the literature regarding resident's perception and opinions about surgical simulation. This study 


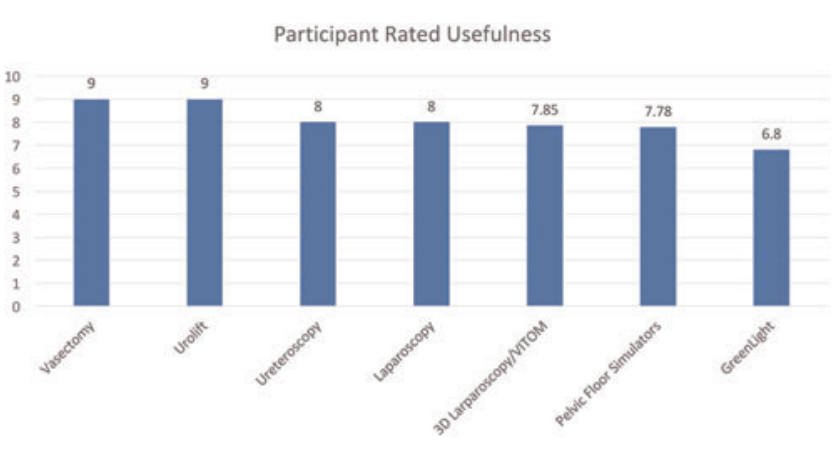

presents our four-year experience with a multi-modality, multiinstitutional workshop model of urologic simulation for resident education.

Materials and Methods: Residents from six area urology programs rotated through simulation stations in four consecutive sessions from 2014 to 2017. Workshops included greenlight photovaporization of the prostate, endoscopic stone extraction, laparoscopic peg transfer, 3D laparoscopy rope pass, transobturator sling placement, intravesical injection, HD video system trainer (VITOM), vasectomy, and Urolift. Expert faculty members provided teaching assistance, objective scoring, and verbal feedback. Participants completed a non-validated questionnaire evaluating utility of the workshop and soliciting suggestions for improvement.

Results: 63 of 75 (84\%) participants (PGY 1-6) completed the exit questionnaire. On a scale from 1-10, median rating of each exercise's usefulness ranged from 7.5 to 9 . Cumulative median scores of the course remained high over the four years: time limit $(9 / 10)$, faculty instruction $(9 / 10)$, ease of use $(9 / 10)$, face validity $(8 / 10)$ and overall course (9.10). On multivariate analysis, there was no difference in rating of domains between post-graduate years. Most participants $(67 \%)$ believe that simulation training should be a requirement of Urology residency. 63 (97\%) participants viewed the lab as beneficial to their education.

Conclusions: The multi-institutional, multi-modality workshop model is a novel design that offers valuable training experience for residents. High ratings of usefulness for each exercise demonstrated excellent face validity provided by the course. Most participating residents believe that this model is a valuable training experience should be a requirement for Urology residency.

\section{MP32-2 Assessment of Urology Postgraduate Trainees'} Competencies in Flexible Ureteroscopic Stone Extraction

M Aloosh, F Couture, N Fahmy, M Elhilali, S Andonian

Division of Urology, McGill University

Canada

Introduction \& Objective: To assess flexible ureteroscopic stone-extraction skill of urology postgraduate trainees (PGTs) at an Objective Structured Clinical Examination (OSCE) and to determine whether previous experience in the operating theatre or practice on the simulator correlated with performance.

Materials and Methods: After obtaining ethics approval, PGTs from post-graduate years (PGYs) 3 to 5 were recruited from all four Quebec urology training programs during an OSCE. After a short orientation to the UroMentor simulator, PGTs were asked

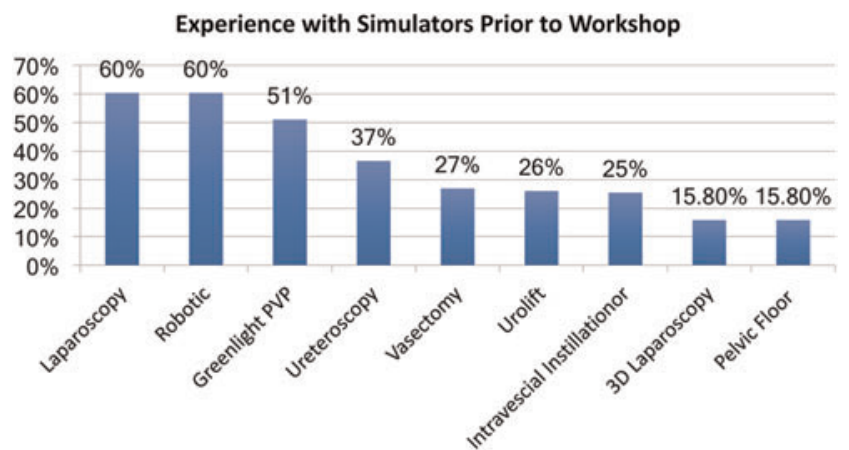

to perform Task 10 for 15 minutes, where two small stones from the left proximal ureter and renal pelvis were extracted using a basket. Competency of PGTs in performing the task was assessed using objective assessment from the simulator and subjective evaluations using Ureteroscopy-Global Rating Scale (URSGRS). Simulator performance reports and URS-GRS scores were analyzed.

Results: Thirty PGTs (9 PGY-3, 11 PGY-4, 10 PGY-5) participated in this study. PGTs had performed a mean of 277.9 cystoscopies, 55.9 semi-rigid and 45.7 flexible ureteroscopies prior to the study. Mean URS-GRS score of the participants was $20.0 \pm 4.4$, mean operative time was $10.9 \pm 2.1$ minutes, mean fluoroscopy time was $7.0 \pm 4.9$ seconds, and mean number of traumas was $10.8 \pm 3.8$. Using norm-referenced method with 3 experts, cut-off score of 19 on the URS-GRS was determined to indicate competency. Sixty percent (18/30) of PGTs were competent. Mean URS-GRS score of the 8 PGTs who had practiced on the simulator was significantly higher than the mean score of those who did not practice $(24.6 \pm 3.0$ vs $18.3 \pm 3.6, \mathrm{p}<0.001)$. Previous experience in the operating theatre and PGY level did not correlate with performance.

Conclusions: This study confirmed the feasibility of incorporating the UroMentor at OSCEs to assess competency of urology PGTs in ureteroscopic stone extraction skills. PGTs who practiced on the simulator scored significantly higher than those who did not practice.

MP32-3 Impact of resident trainee involvement on robot assisted radical prostatectomy outcomes.

E Schommer, K Tonkovich, D Thiel

Mayo Clinic Florida

United States

Introduction \& Objective: We examined peri-operative outcomes of robot assisted radical prostatectomy (RARP) and their association with resident involvement in different steps of RARP.

Materials and Methods: RARP was divided into 7 steps: bladder takedown (BTD), endopelvic fascia, bladder neck (BN), seminal vesicle/vas deferens (sv/vas), pedicle/nerve sparing, apex, and anastomosis. 372 RARP performed by a single surgeon from July 2007 to February 2015 were analyzed. Resident console time (RCT) during each of the 7 key steps was recorded. Peri-operative variables were compared between surgeon-only (SO) and resident-involved (RI) cases. Outcomes of trainee performance on key steps were compared with two sample t-test or Wilcoxon rank sum test. 
Results: Residents performed on the console for 232 of 372 cases (62.4\%). Preoperative BMI (mean 28.4), PSA (mean 6.7), Gleason score (6-7 88.7\%, 8-10 11.3\%), ASA score (mean 2.4), AUA score (mean 8.9) and SHIM score (mean 15.9) were similar between SO and RI cases. 2nd year resident status $(\mathrm{P}<0.0001)$ and pre-operative PSA $>10(\mathrm{P}=0.0093)$ were associated with no RCT. Estimated blood loss $(\mathrm{P}=0.09)$, transfusion $(\mathrm{P}=0.11)$, and complications $(\mathrm{P}=.33)$ were no different between $\mathrm{SO}$ and RI cases. Mean OR time (ORT) was less for the SO cases (190.4 vs. $206.4 \mathrm{~min}, \mathrm{P}=0.003)$. There was no difference in positive margins $(\mathrm{P}=0.79)$, length of stay (LOS) $(\mathrm{P}=0.30)$, catheter days $(\mathrm{P}=0.17)$, readmission $(\mathrm{P}=0.33)$, reoperation $(\mathrm{P}=0.73)$, home with drain $(\mathrm{P}=0.88)$, or undetectable 6 month PSA $(\mathrm{P}=0.07)$ when comparing $\mathrm{SO}$ to RI cases. Residents performing BN step had no effect on $\mathrm{BN}$ margins $(\mathrm{P}=0.73)$, prolonged catheterization $(\mathrm{P}=0.62)$, home with drain $(\mathrm{P}=0.4121)$, or 6 month undetectable PSA $(\mathrm{P}=0.1070)$. ORT was not affected by resident performing anastomosis $(\mathrm{P}=0.08)$ or sv/vas $(\mathrm{P}=0.82)$, but was significantly prolonged if BTD performed by trainee (233.0 vs. 191.7min, $\mathrm{P}<0.0001$ ). Residents performing anastomosis had no effect on prolonged catheter time $(\mathrm{P}=0.62)$, home with drain $(\mathrm{P}=0.41)$, or LOS $(\mathrm{P}=0.20)$. Residents were more likely to be involved in at least one portion of the case $(74.0 \%$ vs. $56.6 \%$, $\mathrm{P}=0.0012)$, in $\mathrm{BN}(63.4 \%$ vs. $30.6 \% \mathrm{P}<0.0001)$, and anastomosis $(48.0 \%$ vs. $15.3 \%, \mathrm{P}<0.0001)$ following purchase of Mimic simulator (Mimic Technologies, Inc., Seattle, WA, USA) in January 2012.

Conclusions: Supervised RCT in RARP does not affect perioperative outcomes but prolongs ORT compared to SO cases with BTD step adding the most ORT. Robotic simulation appears to have increased RCT in various steps of RARP.

MP32-4 Learning curve associated with the WHO Surgical Safety Checklist: A simulation based evaluation.

F Dar, T Abe, P Amnattrakul, A Aydin, N Raison, S Khan, P Dasgupta, K Ahmed

MRC Centre for Transplantation, King's College London United Kingdom

Introduction \& Objective: It has been established that the use of the WHO Surgical Safety Checklist specifically has reduced morbidity and mortality in surgery today. Utilising simulation-

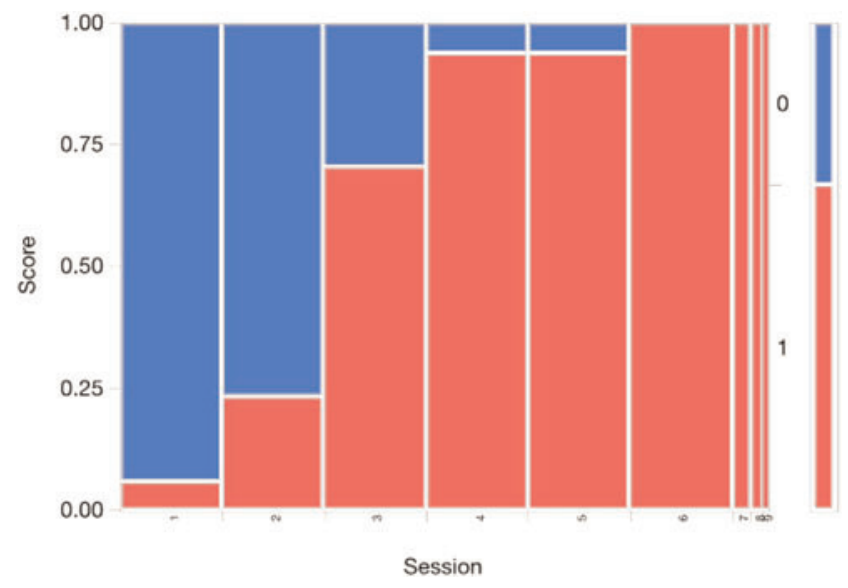

based training, the aim of this study is to assess the learning curve associated with conducting the WHO Checklist.

Materials and Methods: This prospective observational study recruited novice medical students $(n=19)$ as participants, focusing on the successful completion of the WHO checklist whilst performing ureteroscopy within a full immersion simulation environment. After an initial didactic and video-based lecture, participants proceeded to run through the WHO checklist independently and go on to perform the procedure. All sessions were done in a virtual operative theatre, with an acting anesthetist and scrub nurse present. Assessment of this specific component was done via a task completion based checklist.

Results: After training session one, two students were unable to continue. The remaining seventeen students $(\mathrm{M} / \mathrm{F}=10 / 7$, median age of 22) completed 6 training sessions during a median of 21 days (range, 10-32). Graphical representation of the data displaying the mean completion rate of the WHO checklist for each session clearly indicates a plateau phase after the fourth session, where successful independent completion of the WHO checklist is achieved by $16 / 17$ participants with all participants completing the checklist by the sixth session. (Figure 1)

Conclusions: As the WHO checklist becomes more integral to daily surgical practice, safe and effective utilisation of it should be ensured. This study suggests a learning curve to the checklist which may be used to establish the need for familiarisation with it prior to utilisation.

MP32-5 Urology Resident Workload Profiles when Performing Live Porcine Nephrectomies and Robotic Surgery Simulator Training Modules: Update

T Rogers, G Ogaya-Pinies, E Hernandez, H Palayapalayam, V Patel

United States

Introduction \& Objective: The field of robotic surgery continues to grow and requires the mastery of complex surgical technology. The fact that the technology involved in robotic surgery is evolving quickly and there is a steep learning curve requires that trainees become proficient at a faster pace. This leads to the increased utilization of simulation training tools. In pursuit of improving resident training in robotics the Southeastern Section of the American Urological Association (SES AUA) puts on an annual robotic training course for residents. In January 2016 the resident workload profiles for the 2015 course were evaluated and showed that residents experienced similar levels of workload when performing the virtual reality training modules compared to live porcine surgical training. This is an update to that study with two more years of resident data.

Materials and Methods: Residents from the 14 Urology programs across the SES AUA were invited to the 2-day training course over a 3 -year period $(2015,2016,2017) .71$ residents in total have participated in the course. On day 1 the course faculty present didactic lectures to the residents. On day 2 the group of residents are divided into 2 groups. Half of the trainees perform virtual reality simulator training modules on the Mimic da VinciTrainer (MdVT, Mimic Technologies, Inc., Seattle, WA, USA) for 4 hours. At the same time the other half of the residents perform radical and partial nephrectomies on a live porcine model using the da Vinci Si robot (Intuitive Surgical Inc., Sunnyvale, CA, USA). After 4 hours the groups switch and perform the opposite task. All of the participating residents were asked to 
complete the NASA-TLX questionnaire following each training module.

Results: The NASA-TLX data was collected and evaluated for each year and for both the animal and simulator models. The means for each TLX scale were analyzed across both interfaces for the 3-year period of 2015-2017. There workload profiles were similar across 5 of the 6 TLX scales. The only scale that showed a significant difference was frustration. Frustration was significantly higher for the simulation model when compared to the animal model for the 3-year period, $t(2)=4.43, p=0.011$. The study published in 2016 based on the 2015 course showed the same results.

Conclusions: The resident workload profiles are similar for both live animal and MdVT training models as in pertains to robotic surgery. This shows that each model is equally difficult. The residents are experiencing high mental demand and high effort for both training models. However, over a 3-year period the residents remain significantly more frustrated with the simulator, MdVT, training method when compared to live porcine surgery.

MP32-6 Ureteroscopy simulation training under full immersion decreases mental workload in novice operators.

T Abe, F Dar, P Amnattrakul, A Aydin, N Raison, N Shinohara, S Khan, K Ahmed, P Dasgupta

Department of Urology, Hokkaido University Hospital, Sapporo, Japan

Japan

Introduction \& Objective: In general, difficult procedures result in a higher mental workload, which may increase fatigue and surgical errors. Our study aimed at investigating the effect of ureteroscopy simulation training under a full immersion, high fidelity setting focusing on performance and mental workload in novice operators. .

Materials and Methods: Nineteen medical students voluntary participated in the present simulation study. After didactic and video-based lecture, they underwent a dry-box training of renal stone case including a rigid cystoscope part (Task-1, performing a WHO checklist, assembling a scope, insertion of guidewire and access sheath after examining a bladder) and a flexible ureteroscopy part (Task-2, catching a stone located in upper calyx by a basket after inspecting an upper, middle, and lower calyx). Training was performed in a virtual operative theater, with the two collaborators doing roles of anesthetist or scrub nurse. Technical skills were assessed by one of the collaborators (an experienced urologist) onsite using an OSATS score at each training session, and mental workload was subjectively evaluated by National Aeronautics and Space Administration Task Load Index (NASA-TLX) questionnaire after each training session.

Results: After the $1^{\text {st }}$ training session, two students withdraw, and the remaining seventeen students completed a minimum of 6 training sessions ( $\mathrm{M}: \mathrm{F}=10: 7$, median age of 22) during a median of 21 days (range, 10-32). In both Task 1 and 2, OSATS score was improving (MANOVA model, task 1: $\mathrm{p}<0.0001$, task 2: $\mathrm{p}<0.0001$ ), and NASA-TLX score was decreasing (task $1: \mathrm{p}=0.00005$, task $2: \mathrm{p}=0.0028)$ during the training sessions (Figure 1).

Conclusions: Repeated simulation trainings of ureterosocpy under a full immersion setting reduced mental workload as well as skill acquisition.

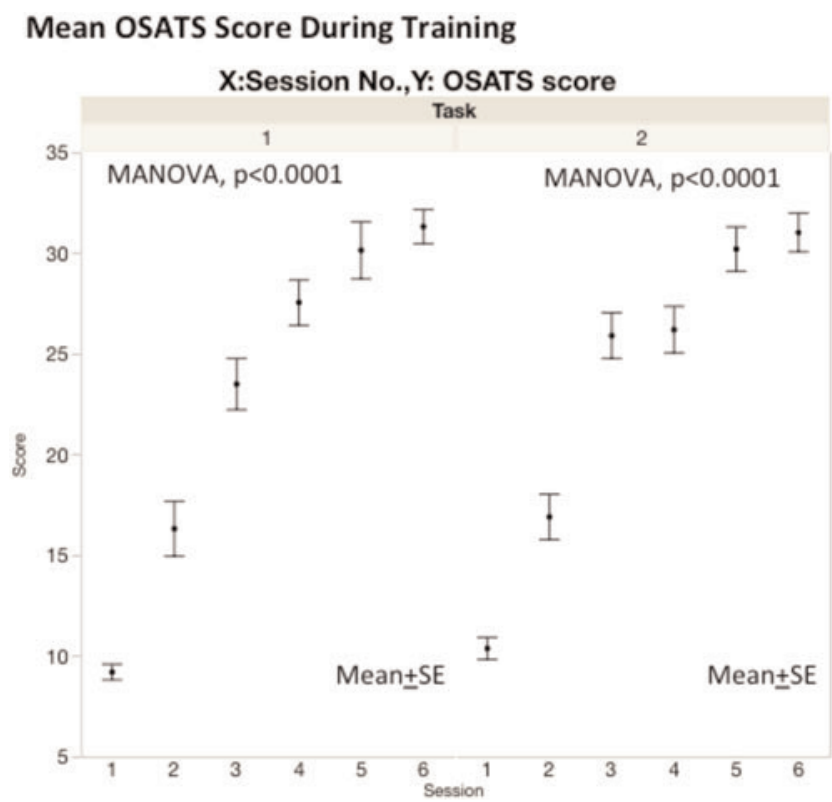

\section{Mean NASA TLX Score During Training}

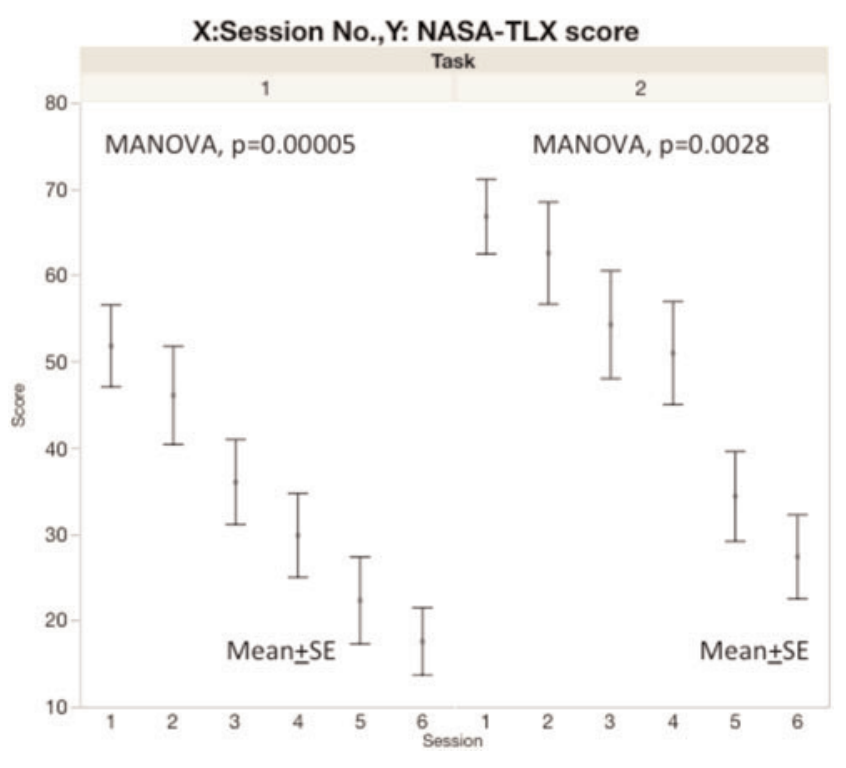

MP32-7 Effectiveness of Procedural Virtual Reality Simulation in Surgical Training

P Harrison, N Raison, K Ahmed

Kings College London

United Kingdom

Introduction \& Objective: Basic virtual reality (VR) simulation training is an effective method of robotic surgical skills training. Procedural VR has not been investigated to the same extent. The purpose of this narrative review was to establish the effectiveness of procedural virtual reality (VR) training.

Materials and Methods: A review was conducted of articles investigating procedural virtual reality training against a control or another type of simulation training with a measured outcome 
assessment. Articles published until January 2017 were considered. Ovid was used to search EMBASE, MEDLINE and PsycINFO databases.

Results: This review included 20 relevant studies which reported on procedural VR training in various specialities. 6 studies assessed the outcome of procedural VR training on a VR simulator, 6 using a porcine model, 1 using fresh frozen cadavers and virtual reality, 5 in the operating room (OR) with real patients and 2 in the OR using models. A statistically significant improvement was seen when comparing procedural VR training groups against a control in 2 studies when assessed in VR, 2 studies when assessed in porcine models and in 4 studies when assessed with live patients in the OR. 8 studies reported that no difference was found between procedural VR training and other types of simulation training or a control. Other types of simulation were found to be more effective than procedural VR training in 3 studies.

Conclusions: Procedural VR training allows a surgical trainee to develop key surgical skills and is an effective training tool. Procedural VR training may be more effective for trainees who have already acquired basic surgical skills from another type of simulation such as basic VR or bench top models. Additional studies investigating both basic and procedural VR simulation may provide evidence for when (i.e. what stage in training) will a trainee surgeon gain the most from procedural VR training. Further validation of procedural VR modules will allow further implementation of this type of simulation into surgical curriculum.

MP32-8 Acceptability of the Fundamentals of Laparoscopic Surgery (FLS) programme tasks for Urology trainees

J Hendry, L Kerr, C McIlhenny

NHS Forth Valley

United Kingdom

Introduction \& Objective: The Fundamentals of Laparoscopic Surgery (FLS) is a well validated training and assessment programme for laparoscopic skills. Although widely recognised, especially in general surgical training in the USA, FLS has no established role in urology training in the UK. We sought to assess the utility and acceptability of FLS tasks for urology trainees.

Materials and Methods: Twelve urology trainees (ST3- ST6) undertook FLS skills tasks of peg transfer, pattern cutting and intracorporeal suturing with repetitions throughout a training day. Pre task questionnaires of laparoscopic competence and operative experience were recorded. Post task questionnaires assessed usefulness of FLS tasks, acceptability of proficiency levels and potential future role for FLS in training and assessment. Improvements in peg transfer time were charted by participants undertaking a minimum of 10 repetitions and completion times recorded.

Results: There were seven ST3 - ST4 and five ST5-ST7 trainees with increasing years of training having no benefit to baseline peg transfer task times (103.7s vs. 117.1s, ttest 0.11). When split by previous laparoscopic simulation training those trainees with less than three hours exposure had non-significant but poorer trending times compared to those with greater than three hours simulation training (156.7s vs. $143.3 \mathrm{~s}$, ttest 0.51 .) This was reflected in final repetition timings where those with previous simulation training showed greater improvements $(113.5 \mathrm{~s}$ vs 84.2s, ttest 0.002). All trainees felt the FLS tasks improved their laparoscopic surgical skills with $92 \%$ rating this as transferable to theatre. Trainees found the proficiency levels of all tasks acceptable and would recommend FLS task training to others. Eleven (92\%) trainees would wish regular FLS training incorporated into the training programme.

Conclusions: The FLS tasks trialled here are acceptable to urology trainees and exposure to tasks improves self-rated ability with laparoscopic skills. Improvements in laparoscopic skills tasks relates to previous simulation exposure rather than stage of training. With simulation and performance assessment an increasing focus in surgical education, further use of the FLS programme in urology is warranted and would be welcomed by trainees.

MP32-9 Evaluating the benefits of using objective benchmarks in a virtual reality training curriculum, for robotic and laparoscopic surgery.

W Watkinson, N Raison, K Ahmed, P Harrison

King's College London

United Kingdom

Introduction \& Objective: Using virtual reality (VR) simulators has shown benefits within robotic/laparoscopic training, however the most effective form is yet to be established. Objective benchmarks are pre-determined scores participants must achieve on an exercise to progress. Aim: to perform a systematic review, evaluating the benefits of objective benchmarks in VR simulation for Robotic/Laparoscopic surgical training.

Materials and Methods: A systematic search of the OVID database (Embase, Ovid MEDLINE(R) and Psych Info) was performed; data extraction was done according to specific inclusion and exclusion criteria.

Results: 20 studies were included for analysis; 9 RCT, 11 nonrandomised trials. The analysis demonstrates after using a VR curriculum with objective benchmarks, participants tested on an outcome assessment significantly outperform participants who used VR curriculum without objective benchmarks/ didn't use VR. Once completing a VR curriculum using objective benchmarks, participants can successfully transfer their skills to the theatre, performing surgical operations, as demonstrated in 6 studies the intervention outperformed the control for surgical assessment. The analysis establishes expert benchmarks as beneficial, yet disparity in terms of attainability of proficiency targets within the curriculum was highlighted, emphasising lack of consensus in terms of defining expert benchmarks and optimal targets for proficiency.

Conclusions: Using a VR curriculum with objective benchmarks provides a more effective training curriculum than a VR curriculum without benchmarks, providing surgical competency that can be transferred safely to a clinical setting. Clarity is required for defining the most suitable objective benchmarks within a VR curriculum, with or without restrictions such as time and maximum attempts.

MP32-10 Use of Google Trends to Track Online Behavior and Interest in Kidney Stone Surgery

C Tong, PC Dreher, E Ghiraldi, J Friedlander

Hahnemann University Hospital/Drexel College of Medicine United States 


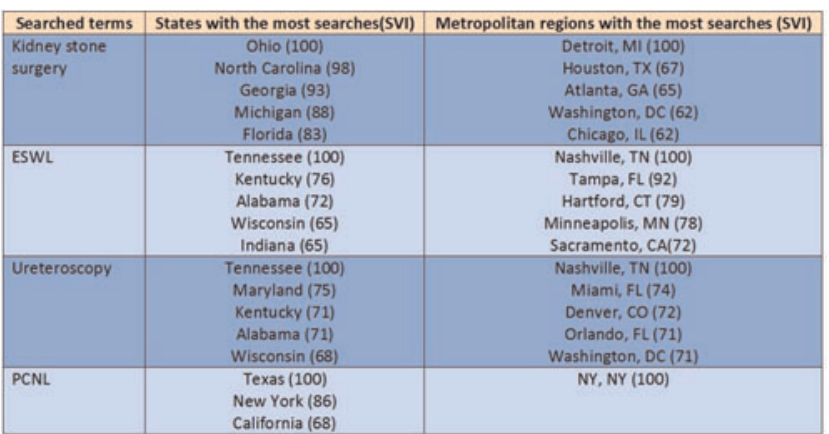

Introduction \& Objective: Internet search trends data is a unique resource for monitoring online health information-seeking behavior. Analysis of these data has resulted in a new research discipline termed infodemiology, the study of the determinants and distribution of health information. We utilized Google trends to explore patient interest and search inquiries into surgical treatment options of kidney stones. We sampled the population's interest in surgical modalities and examined the relative frequency of searches across the U.S.

Materials and Methods: We queried Google Trends via the Google Insights for Search (http://google.com/trends) using terms related to kidney stone surgical treatments within the U.S. from 2011 to 2016. "Kidney stone surgery" was used for lay terminology and identified to be the most common term. The Search Volume Index (SVI) graph for the U.S. was recorded as well as regional distribution and related queries. For specific surgical treatment modalities, the following medical terminology was used: percutaneous nephrolithotomy (PCNL), extracorporeal shockwave lithotripsy (ESWL), ureteroscopy, laser lithotripsy, and laparoscopic nephrolithotomy. Each query was then compared analyzed to assess changes in the interest in these search terms over time.

Results: The research trends for the search "kidney stone surgery" remained fairly constant over time. Specific surgical modality yielded similar stable search trends, but at a lower frequency than "kidney stone surgery." ESWL had the highest SVI and laparoscopic nephrolithotomy had the lowest. The top 5 US states with greatest search volume for the term "kidney stone surgery" was Ohio, North Carolina, Georgia, Michigan and Florida, whereas the top 5 metropolitan regions were Detroit, Houston, Atlanta, Washington DC and Chicago.

Conclusions: Google Trends data is a useful tool to analyze online health information-seeking behavior of Internet users and may help direct resource use and patient education. Despite an increase in stone prevalence over the past decade, search rates have remained relatively constant. High relative search volume was seen from states within and outside the traditional stone belt, suggesting a possible changing trend.

MP32-11 Construct and Content Validity of the Visual Prostate Symptom Score Following Inclusion of Pictograms for Urgency and Urgency Incontinence by men with $\mathrm{Ob}$ structive Lower urinary Tract Symptoms in a Low and Middle-income Country (LMIC)

L Stothers, AJ Macnab, R Mukisa

Department of Urologic Sciences UBC and Peter Wall Institute of Advanced Studies

Canada

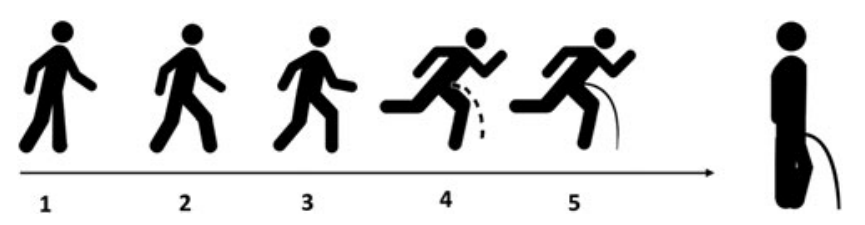

Introduction \& Objective: Worldwide estimates indicate 1.1 billion men will be affected by lower urinary tract symptoms (LUTS) and or bladder outlet obstruction (BOO) by 2018; the greatest burden will be in developing regions where literacy and language are barriers to symptom reporting. The Visual Prostate Symptom Score (VPSS) addresses limited ability to read or write by using pictograms to measure frequency, nocturia, weak stream and quality of life, but does not include a scale for either overactive bladder $(\mathrm{OAB})$ or urgency urinary incontinence (UUI). Objectives: Evaluate construct and content validity of measures for $\mathrm{OAB}$ and UUI by: (1) evaluating patient understanding of each pictogram (2) comparing VPSS pictogram descriptions to IPSS responses (3) quantifying impressions of pictogram usefulness.

Materials and Methods: 3 versions of an OAB and UUI pictogram were developed. Men presenting with LUTS to a rural medical clinic in Uganda completed: the VPSS, IPSS and OAB and UUI pictograms without and with assistance; described what they understood image sequences to represent and rated usefulness on a visual analogue scale. Statistical analysis: Student's t, Fisher's exact, and Spearman's correlation tests.

Results: 136 scores: men, mean age 69, range 45-93 yrs with educational level: no schooling 20\%, grade 1-4 $62 \%$ and grade 5$1218 \%$. The VPSS was completed without assistance in $94 \%$ $(\mathrm{p}<0.05)$ while only 2 using the IPSS independently. Analysis by level of education completed indicated those with; no schooling all required assistance with IPSS vs 6\% with VPSS ( $<<0.05$ ); grade $1-4100 \%$ vs $14 \%$ and higher grades $40 \%$ vs $0 \%$. Within existing VPSS images: 94\%, independent of education, had inherent recognition of weak stream, $75 \%$ for frequency and $42 \%$ for nocturia. Feedback indicated (1) overall VPSS comprehension and patient reporting was improved by increasing image size and contrast detail for the frequency and nocturia pictograms and (2) subjects valued the ability to report OAB UUI symptoms.

Likert scale measures indicated that of the 3 sequences for OAB UUI evaluated the most readily understood sequence is that shown in fig 1 .

Conclusions: Construct validity related to immediate recognition was greatest with the most simplified version depicting $O A B$ UUI (fig 1). Increasing image size and clarity and inclusion OAB UUI parameters to the existing VPSS are important constructs to future measurement of men's LUTS globally.

MP32-12 Safety of live surgery in urology. Propensity scored matched analysis

G Ogaya-Pinies, H Palayapalayam, E Hernandez, X Bonet, T Rogers, C Jenson, V Patel

Global Robotic Institute. Celebration Health/ Florida Hospital United States

United States

Introduction \& Objective: Live surgery events (LSE), have become one of the most attended activities at surgical meetings, 
Table 1. Preoperative Variables

\begin{tabular}{|c|c|c|c|}
\hline Variable & Live Surgery & Control & P Value \\
\hline Total patients & 36 & 108 & .. \\
\hline $\begin{array}{l}\text { BMI (kg/m2) } \\
\text { (median) (IQR) }\end{array}$ & $26(24-29)$ & $26(24-28)$ & 0.4142 \\
\hline $\begin{array}{l}\text { Age (years) (median) } \\
\text { (IQR) }\end{array}$ & $55(50-62)$ & $59(53-65)$ & 0.0159 \\
\hline \multicolumn{4}{|l|}{ Comorbidities } \\
\hline Diabetes & $1(2.77 \%)$ & $5(4.63 \%)$ & \\
\hline HTN & $14(38.89 \%)$ & $46(42.59 \%)$ & 0.5635 \\
\hline CAD & $2(5.56 \%)$ & $6(5.55 \%)$ & \\
\hline $\begin{array}{l}\text { PSA (ng/mL) } \\
\text { (median) (IQR) }\end{array}$ & $5.4(4.2-8.5)$ & $4.7(3.8-6.6)$ & 0.1087 \\
\hline \multicolumn{4}{|l|}{ Preop Gleason score } \\
\hline$\leq 6$ & $20(55.56 \%)$ & $62(57.41 \%)$ & \\
\hline 7 & $16(44.44 \%)$ & $46(42.59 \%)$ & 0.8459 \\
\hline$\geq 8$ & $0(0 \%)$ & $0(0 \%)$ & \\
\hline \multicolumn{4}{|l|}{ Clinical Stage (cT) } \\
\hline cT1 & $33(91.67 \%)$ & $103(95.37 \%)$ & \\
\hline cT2 & $3(8.33 \%)$ & $5(6.63 \%)$ & 0.4008 \\
\hline cT3 & $0(0 \%)$ & $0(0 \%)$ & \\
\hline SHIM (median)(IQR) & $19.7(16-25)$ & $20.7(15-25)$ & 0.4308 \\
\hline SHIM $\geq 21$ & 21 (patients) & 67 & \\
\hline SHIM $<21$ & 15 (patients) & 41 & \\
\hline AUASS (median)(IQR) & $6.7(3.1-11)$ & $8.6(3-11.7)$ & 0.1421 \\
\hline \multicolumn{4}{|l|}{ D'Amico class } \\
\hline Low risk & $22(61.11 \%)$ & $62(57.41 \%)$ & \\
\hline Int risk & $14(38.89 \%)$ & 46 (42.59\%) & 0.6963 \\
\hline High risk & - & - & \\
\hline
\end{tabular}

Table 2. Intraoperative Outcomes

\begin{tabular}{lccc}
\hline \multicolumn{1}{c}{ Variable } & $\begin{array}{c}\text { Live } \\
\text { Surgery }\end{array}$ & Control & P value \\
\hline $\begin{array}{l}\text { Operative time (min) } \\
\text { (median) (IQR) }\end{array}$ & $136(118-151)$ & $122(99-140)$ & 0.0036 \\
$\begin{array}{l}\text { Console time (min) } \\
\text { (median) (IQR) }\end{array}$ & $73(70-79)$ & $77(73-87)$ & 0.0402 \\
$\begin{array}{l}\text { Estimated Blood Loss } \\
\text { (mL) (median) (IQR) }\end{array}$ & $120(80-150)$ & $110(60-150)$ & 0.2175 \\
$\begin{array}{l}\text { Nerve Sparing } \\
\text { Partial }\end{array}$ & $6(16.6 \%)$ & $31(28.70 \%)$ & \\
$\quad$ Full & $30(83.3 \%)$ & $77(71.30 \%)$ & 0.1892 \\
$\begin{array}{l}\text { Lymphadenectomy } \\
\quad \text { Yes }\end{array}$ & $7(19.44 \%)$ & $51(47.22 \%)$ & 0.0033 \\
$\quad$ No & $29(80.56 \%)$ & $57(52.77 \%)$ & \\
\hline
\end{tabular}

providing a unique opportunity for the audience to observe in real time, the decision-making process by skilled and experienced surgeons. However, there is an ongoing discussion on whether patients who are treated during LSE are at a higher risk for complications.

Materials and Methods: From January 2008 through April 2016, >9000 patients underwent RARP at our institution, performed by a single surgeon. From this group, 36 patients underwent live RARP transmission, via video link from our institution to an external congress. A propensity-matched analysis was conducted, in a 1:3 proportion, comparing outcomes of live transmission cases to those operated under regular circumstances. Data were prospectively collected in a customized database and retrospectively analyzed. The groups were compared by using the Student's t-test and analysis of variance (ANOVA) for continuous factors, two-tailed Fisher exact test for categorical factors and a " $p$ " value of

Results: There were no significant demographic differences between the two groups regarding the comorbidities, clinical tumor stage, pre-operative PSA, biopsy Gleason score, body mass index (BMI), pre-operative SHIM score and AUA
Table 3. Post-Operative Outcomes

\begin{tabular}{|c|c|c|c|}
\hline Variable & $\begin{array}{c}\text { Live } \\
\text { Surgery }\end{array}$ & Control & $P$ value \\
\hline $\begin{array}{l}\text { Length hospital stay } \\
\text { (days) (median) (IQR) }\end{array}$ & $1.1(1.0-1.4)$ & $1.2(1.0-1.8)$ & 0.5977 \\
\hline $\begin{array}{l}\text { Indwelling Foley } \\
\text { cath§ (days) (median) } \\
\text { (IQR) }\end{array}$ & $5(4-7)$ & $5(4-6)$ & 0.9168 \\
\hline $\begin{array}{l}\text { Complications } \\
\text { Clavien grade 1-2 } \\
\text { Clavien grade 3-4 }\end{array}$ & $\begin{array}{l}0 \\
0\end{array}$ & $\begin{array}{c}4^{*}(3.70 \%) \\
0\end{array}$ & 0.2415 \\
\hline $\begin{array}{l}30 \text { days hospital } \\
\text { readmission }\end{array}$ & 0 & 0 & - \\
\hline $\begin{array}{l}\text { Number of ptף } \\
\text { reached potency }(\%)\end{array}$ & $27(69.44 \%)$ & $73(67.59 \%)$ & 0.4034 \\
\hline $\begin{array}{l}\text { Time to potency } \\
\text { (days) (median) (IQR) }\end{array}$ & $120(10-210)$ & $135(21-250)$ & 0.6199 \\
\hline $\begin{array}{l}\text { Number of pt } \\
\text { reached continence } \\
(\%)\end{array}$ & $35(97.22 \%)$ & $105(97.22 \%)$ & 1.000 \\
\hline $\begin{array}{l}\text { Time to continence } \\
\text { (days) (median) (IQR) }\end{array}$ & $50(14-100)$ & $60(21-120)$ & 0.5965 \\
\hline $\begin{array}{l}\text { Follow-up (months) } \\
\text { (median) (IQR) }\end{array}$ & $31(15-50)$ & $28(9-45)$ & 0.5027 \\
\hline $\begin{array}{l}\text { Number of ptI with } \\
\text { BCR }(\%)\end{array}$ & $1(2.77 \%)$ & $4(3.71 \%)$ & 0.7927 \\
\hline
\end{tabular}

Table 4. Pathological Outcomes

\begin{tabular}{lccc}
\hline \multicolumn{1}{c}{ Variable } & $\begin{array}{c}\text { Live } \\
\text { Surgery }\end{array}$ & Control & P Value \\
& & & \\
\hline $\begin{array}{l}\text { Prostate Weight } \\
\text { (gr)(median) (IQR) }\end{array}$ & $48(42-60)$ & $50(36-72)$ & 0.3909 \\
Positive Surgical & & & \\
Margins & & & \\
Negative & $33(91.67 \%)$ & $98(90.74 \%)$ & 1.0000 \\
Positive & $3(8.33 \%)$ & $10(9.26 \%)$ & \\
Pathological Stage & & & \\
pT2a \& b & $3(8.33 \%)$ & $14(12.96 \%)$ & \\
pT2c & $30(83.33 \%)$ & $79(73.15 \%)$ & \\
pT3a & $2(5.55 \%)$ & $15(13.89 \%)$ & 0.1418 \\
pT3b & $1(2.78 \%)$ & $0(0 \%)$ & \\
Final Gleason Score & $11(30.56 \%)$ & $39(36.11 \%)$ & $0 . .4918$ \\
56 & $25(69.44 \%)$ & $68(62.96 \%)$ & \\
7 & $0(0 \%)$ & $3(2.78 \%)$ & \\
$\geq 8$ & $7(19.44 \%)$ & $51(47.22 \%)$ & 0.0033 \\
Lymph node pathology & $0(0 \%)$ & $0(0 \%)$ & \\
pN0 & $29(80.56 \%)$ & $57(52.78 \%)$ & \\
pN1 & & & \\
pNx & & & \\
\hline
\end{tabular}

symptoms score and D'Amico class. The only significant difference in the demographic was the age Fifty-five y.o (interquartile range (IQR): 50-62) was the median age for the LS group vs. 59 y.o (IQR: 53-65) for the control group $(\mathrm{p}=0.016)$. The console median time was shorter for the LS group $73 \mathrm{~min}$ (IQR: 70-79) compared to the $77 \mathrm{~min}$ (IQR: 73-87), of the control group $(p=0.0402)$. No major complications were reported in any of the groups, and only 4 minor complications were observed in the control group $(\mathrm{p}=0.2415)$. After a median follow-up of 31 months \pm (IQR: 18-50), only one patient $(2.77 \%)$ in the live surgery group presented biochemical recurrence vs. four $(3.71 \%)$ in the control group $(\mathrm{p}=0.7927)$. No differences were seen in the continence rate, $97.22 \%$ for both 
groups, and no differences were observed in the potency rate for either group: $69.44 \%$ for the LS group vs. $67.59 \%$ in the control group $(\mathrm{p}=0.4034)$.

Conclusions: In this series of live transmission of RARP, the perioperative results (oncological/ functional outcomes and complications) were similar to those found in daily practice. These findings suggest that LS of this procedure in selected patients and at selected centers is safe. Further evaluation is necessary of the results of other surgeons at other centers

MP32-13 Role of personality in postoperative health-related quality of life following ureteroscopic stone extraction with stent insertion

R Davis, NJ Farber, P Modi, S Elsamra, E Olweny

Rutgers Robert Wood Johnson Medical School United States

Introduction \& Objective: Ureteral stents are known to cause significant health-related quality of life (HRQoL) impairment in a majority of patients. Postoperative HRQoL has been shown to be influenced by personality traits in a variety of clinical settings, but this has not previously been investigated following ureteroscopic stone extraction with stent insertion (URS).

Materials and Methods: Adult patients scheduled for URS were prospectively recruited. Those with indwelling stents or history of stenting within the preceding 6 months were excluded. Personality traits were preoperatively assessed using the validated Ten Item Personality Inventory (TIPI), which scores the 'Big Five' personality traits i.e. extraversion, agreeableness, conscientiousness, emotional stability and openness on a 1 to 7 scale. Postoperative HRQoL was assessed using the Ureteral Stent Symptom Questionnaire (USSQ), administered with the stent in situ (USSQ 1) and after stent removal (USSQ 2). Correlations between personality traits and USSQ scores were evaluated using Kendall's tau test. Multivariable logistic regression was used to examine predictors of HRQoL impairment.

Results: 52 patients completed both the TIPI and USSQ 1, of whom 33 completed the USSQ 2. The majority were female $(62 \%)$, white $(67 \%)$, with at least a high school diploma (97\%). With the stent in situ, openness significantly correlated with composite pain index $(\mathrm{r}=0.20, \mathrm{p}=0.03)$ and general health index $(r=0.23, p=0.017)$. Females but not males with higher openness scores were more likely to report flank or loin pain due to the stent $(\mathrm{p}=0.02)$. With the stent removed, a moderate but non-significant correlation between openness and composite pain index persisted $(\mathrm{r}=0.30, \mathrm{p}=0.09)$. On multivariable analysis, high agreeableness and low emotional stability scores were significant independent predictors of composite urine index with the stent in situ, while low agreeableness and high conscientiousness were significant predictors of urine index score with the stent removed. Female gender was a significant predictor of urine index with the stent removed, as well as of general health index with and without the stent $(\mathrm{p}<0.05$ in each case).

Conclusions: Patient-reported HRQoL after URS appears to be influenced by personality traits such as openness, emotional stability, agreeableness and conscientiousness, as well as female gender. Pending further evaluation, these findings could have future implications in individualizing postoperative care after URS so as to optimize patient QOL.
MP32-14 A comparison of Twitter usage between general urologists and endourologists

A Ludvigson, J Ingimarsson

Maine Medical Center

United States

Introduction \& Objective: Twitter is a powerful tool for practicing clinicians and institutions, allowing direct interaction with patients and other healthcare professionals on a large scale. We compared posts from the top 100 urology accounts to the top 100 endourology accounts to examine endourology's Twitter impact and patterns of usage.

Materials and Methods: The online commercial Twitter analytics tool Followerwonk was used to compile a list of the top 100 accounts on Twitter with "urology," "urologist," or "urological" in the username or account description, sorted by number of followers. This procedure was repeated for the same variations of "endourology." An open-source Python script was used to download all public posts for the past year for each account. Data analysis was carried out with IBM SPSS Statistics v. 23.

Results: The number of followers for the top 100 urology accounts ranged from 53,495 to 1,870 , with a mean of 6,464 and median of 3,170 . The number of followers for the top 100 endourology accounts was much lower, with a range from 2,973 to 56 , a mean of 395.67 , and a median of 235 . 31,885 posts from urology accounts were available for analysis over the past year, compared to 20,384 for endourology. Urology and endourology posts had the same proportions of retweets: $45.46 \%$ vs. $45.25 \%$, respectively. Endourology had a greater percentage of direct replies to other users than general urology: $22.22 \%$ vs. $11.86 \%$, respectively. Urology accounts tweeted a higher percentage of posts with links: $36.74 \%$ vs. $29.87 \%$, respectively. Urology accounts used more hashtags than endourology accounts: $47.11 \%$ of posts vs. $43.83 \%$, respectively. All of these differences were statistically significant with $\mathrm{p}$ values less than 0.0001 , except for proportion of retweets.

Conclusions: General urologists and endourologists use Twitter in significantly different ways. Endourology accounts tended to communicate with individual users with greater frequency, and post fewer links. This implies endourologsts use Twitter to interact directly with other members of the community more often, rather than posting news items or announcements. However, endourology accounts are followed by far fewer users compared to

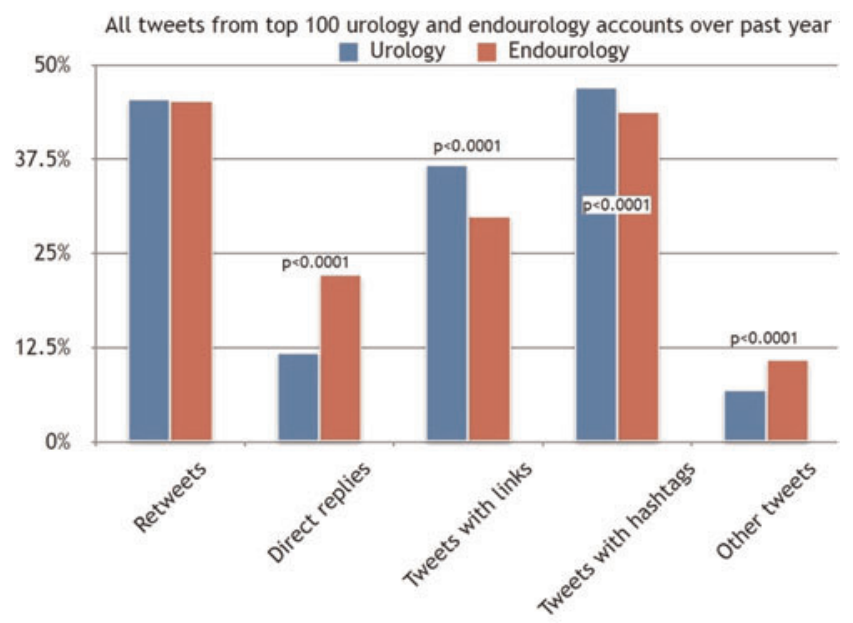


general urology and post less often. These data suggest that although endourologists are engaged on Twitter, there is more work to be done to improve the impact and reach of their accounts.

MP32-15 Differences in practice patterns among LatinAmerican urologists with or without endourology training.

BO Manzo, E Lozada, F Daels, M Bertacchi, C Vicentini, A Rasguido, M Cabrera, P Gonzalez, W Pereyra, J Blasco, G Manzo, C Velazquez, A Rodriguez, E Aleman, A Etienne

Endourology Department at Hospital Regional de Alta Especialidad del Bajío

Mexico

Introduction \& Objective: It has been proved that there is a direct relationship between surgeon's or hospital volumen procedures and the postoperative outcomes in several urological procedures such as cistoprosatectomy, radical prostatectomy as well as percutaneous nephrolithotomy (NLPC). Percutaneous nephrolithotomy practice patterns around the world have been published as a result of a survey sent to members of the Endourological Society. However differences in percutaneous nephrolithotomy practice patterns among urologists based on endourological or lithiasis training in latin America have not been published.

Objective: To know practice patterns on percutaneous nephrolithotomy among urologists in Latin America with or without endourology or lithiasis training.

Materials and Methods: A 27 items survey was created on Surveymonkey ${ }^{\circledR}$ web platform about percutaneous nephrolithotomy for the treatment of kidney stones and was sent via e-mail and other electronic media to 3000 urologists from 15 different Latin American countries. For analysis of results, two groups were created: (group 1 endourology trained urologists and group 2: those without training) so then they were compared with the test X2 with the program SPSS version 20 for Windows.

Results: A total of 331 participants were obtained with a response rate of $11 \%$. Of the total number of participants, $221(66.7 \%)$ had an endourology training and $106(33.2 \%)$ did not. $91.9 \%$ of group 1 performed NLPC and $63.2 \%$ of group $2(\mathrm{p}=.0005)$. Preoperative tomography was used in $85.1 \%$ and $58.5 \%$ (p 0.0005) while preoperative nephrolitometry scales were used $12.7 \%$ and $4.7 \%(\mathrm{p}$ $.0005)$ respectively. Combined management were the practice pattern in $45.2 \%$ and $32.1 \%$ (p 0.0005) and multiple percutaneous tract realization was $68.3 \%$ and $38.7 \%$ (p 0.0005) respectively. Minimally invasive percutaneous nephrolithotomy was performed in $19.9 \%$ and $5.7 \%$ (p 0.0005) in each group.

Conclusions: There are statistically significant differences in the practice patterns of percutaneous nephrolithotomy between Latin-American urologists trained in endourology and those not trained. Preoperative tomography, use of nephrolitometric scales, minimally invasive percutaneous nephrolithotomy, combined management (endoscopic and percutaneous) and a greater percentage of multiple percutaneous tract are most commonly performed by trained urologists.

MP32-16 Comparison of Evidence Quality Underlying Surgical Subspecialty Guidelines in Urology, Orthopedics and Otolaryngology

SG Antoine, AC Small, DJ Robins, MJ Lipsky, JM McKiernan, OD Shah
NewYork-Presbyterian Hospital / Columbia University College of Physicians and Surgeons

United States

Introduction \& Objective: Surgical subspecialty societies release clinical practice guidelines to provide topic-specific, evidence-based recommendations to clinicians. Guidelines can shape clinical practice patterns. We aimed to critically analyze the evidence quality and prevalence of opinions within urology, orthopedic and otolaryngology guidelines.

Materials and Methods: Guidelines issued through 2016 were extracted from the American Urological Association (AUA), American Academy of Orthopedic Surgeons (AAOS), and American Academy of Otolaryngology-Head and Neck Surgery (AAO-HNS) websites. Statements were characterized by whether or not they were evidence-based, the strength of the recommendation, and the quality of evidence included. "Clinical principal" statements were excluded from analysis. Statement strength and evidence quality were graded as "high," "moderate," and "low" due to variable nomenclature between societies. Comparisons were performed with Fisher's exact test and t-tests with significance $\mathrm{p}<0.05$.

Results: The AUA has published the most guideline documents/ statements (24/558 AUA, 18/330 AAOS, 13/163 AAO-HNS). "Clinical principal" statements were excluded from analysis (64 AUA, 7 AAOS, 0 AAO-HNS). Significantly fewer of the AUA guideline statements were evidence-based $(66.8 \%)$ than AAOS $(80.5 \%, \mathrm{p}<0.001)$ or AAO-HNS $(99.8 \%, \mathrm{p}<0.001)$. The proportion of statements with a "high" strength was not significantly

\begin{tabular}{|l|r|r|}
\hline \multicolumn{3}{|c}{ Tabla1. Participants by country } \\
\hline Country & N & Percentage \\
\hline Mexico & 84 & $25.4 \%$ \\
\hline Argentina & 48 & $14.5 \%$ \\
\hline Brazil & 35 & $10.6 \%$ \\
\hline Colombia & 20 & $6 \%$ \\
\hline Uruguay & 18 & $5.4 \%$ \\
\hline Peru & 16 & $4.8 \%$ \\
\hline Dominican Republic & 11 & $3.3 \%$ \\
\hline Ecuador & 4 & $1.2 \%$ \\
\hline Paraguay & 15 & $4.5 \%$ \\
\hline Venezuela & 34 & $10.3 \%$ \\
\hline Chile & 12 & $3.6 \%$ \\
\hline Nicaragua & 15 & $4.5 \%$ \\
\hline El Salvador & 1 & $0.3 \%$ \\
\hline Guatemala & 9 & $2.7 \%$ \\
\hline
\end{tabular}

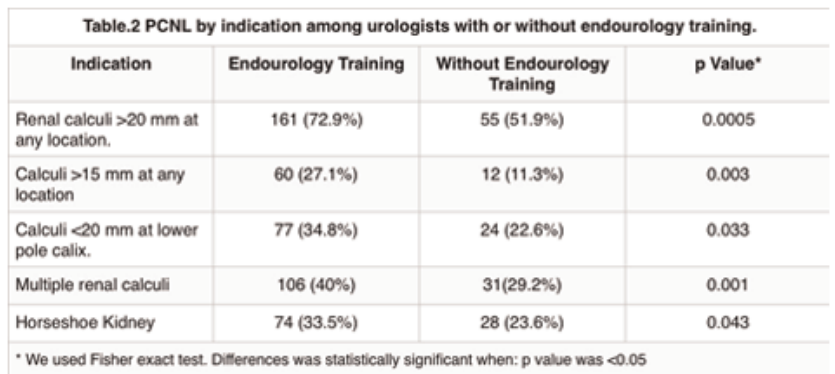




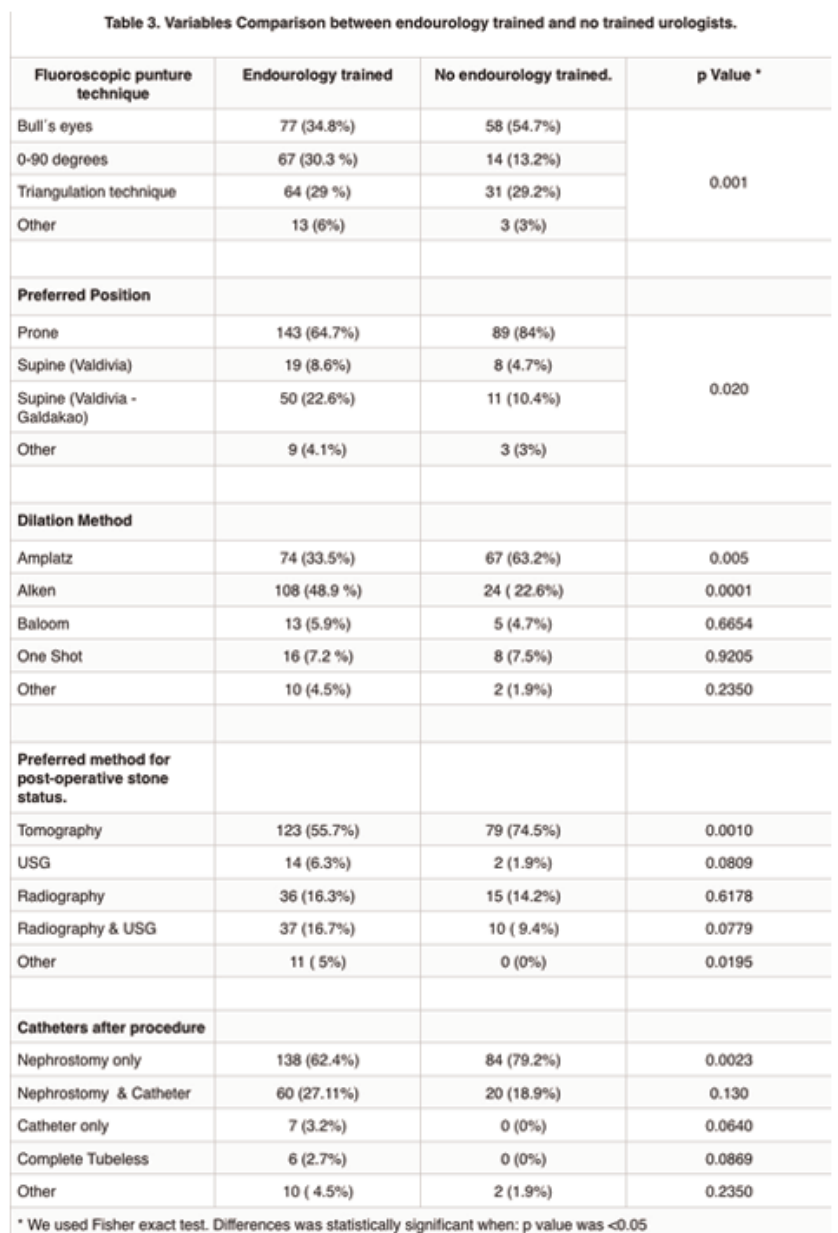

- We used Fisher exact test. Differences was statistically signilicant when: p value was $<0.05$

Table: Comparison of Evidence Quality Underlying Surgical Subspecialty Guidelines in Urology (AUA), Orthopedics (AAOS) and Otolaryngology (AAO-HNS)

\begin{tabular}{|c|c|c|c|c|c|c|c|c|}
\hline & \multicolumn{2}{|c|}{$\begin{array}{l}\text { Urology } \\
\text { (AUA) }\end{array}$} & \multicolumn{3}{|c|}{$\begin{array}{l}\text { Orthopedics } \\
\text { (AAOS) }\end{array}$} & \multicolumn{3}{|c|}{$\begin{array}{l}\text { Otolaryngology } \\
\text { (AAO-HNS) }\end{array}$} \\
\hline & n & $\%$ & $\mathbf{n}$ & $\%$ & $\begin{array}{l}\text { P(AUA } \\
\text { vs. } \\
\text { AAOS) }\end{array}$ & n & $\%$ & $\begin{array}{l}\text { p (AUA } \\
\text { vs. AAO } \\
\text { HNS) }\end{array}$ \\
\hline \multicolumn{9}{|l|}{ Baseline Characteristics } \\
\hline Total guideline documents & 24 & & 18 & & & 13 & & \\
\hline Total statements & 558 & & 330 & & & 163 & & \\
\hline $\begin{array}{l}\text { Total statements (excluding } \\
\text { clinical principals) }\end{array}$ & 494 & & 323 & & & 163 & & \\
\hline $\begin{array}{l}\text { Statements per guideline } \\
\text { (mean } \pm \text { SD) }\end{array}$ & $\begin{array}{l}20.6 \pm \\
11.15\end{array}$ & & $\begin{array}{c}17.9 \pm \\
9.2\end{array}$ & & 0.419 & $\begin{array}{c}12.5 \pm \\
3.3\end{array}$ & & $0.016^{\circ}$ \\
\hline \multicolumn{9}{|l|}{ Statement Strength } \\
\hline Evidence-based statements & 330 & $66.8 \%$ & 260 & $80.5 \%$ & $<0.001^{*}$ & 161 & $98.8 \%$ & $<0.001^{*}$ \\
\hline $\begin{array}{l}\text { Non-evidence based } \\
\text { statements }\end{array}$ & 164 & $33.2 \%$ & 63 & $19.5 \%$ & $<0.001^{*}$ & 2 & $1.2 \%$ & $<0.001^{*}$ \\
\hline High & 73 & $14.8 \%$ & 55 & $17.0 \%$ & 0.841 & 29 & $17.8 \%$ & 0.343 \\
\hline Moderate & 189 & $38.3 \%$ & 83 & $25.7 \%$ & $<0.001^{*}$ & 102 & $62.6 \%$ & 0.205 \\
\hline Low & 68 & $13.8 \%$ & 122 & $37.8 \%$ & $<0.001^{*}$ & 30 & $18.4 \%$ & 0.633 \\
\hline \multicolumn{9}{|l|}{ Evidence Quality } \\
\hline $\begin{array}{l}\text { Total statements with } \\
\text { evidence quality designated }\end{array}$ & 330 & $55.6 \%$ & 323 & $97.9 \%$ & $<0.001^{*}$ & 163 & $100.0 \%$ & $6<0.001^{*}$ \\
\hline High & 9 & $1.5 \%$ & 55 & $16.7 \%$ & $<0.001^{*}$ & 26 & $16.0 \%$ & $<0.001^{\circ}$ \\
\hline Moderate & 73 & $12.3 \%$ & 83 & $25.2 \%$ & 0.773 & 131 & $80.4 \%$ & $<0.001^{\circ}$ \\
\hline Low & 156 & $26.3 \%$ & 122 & $37.0 \%$ & $<0.001^{*}$ & 4 & $2.5 \%$ & $<0.001^{*}$ \\
\hline
\end{tabular}

" $p<0.05$ different across specialties (14.8\% AUA vs. $17 \%$ AAOS, $p=0.431$; and $17.8 \%$ AAO-HNS, $\mathrm{p}=0.383$ ). However, only $2.7 \%$ of the AUA guidelines were based on "high" quality evidence, which was less than the AAOS $(17 \%, \mathrm{p}<0.001)$ and the AAO-HNS $(16 \%, \mathrm{p}<0.001)$.

Conclusions: Although the AUA publishes many more guideline statements than the AAOS or AAO-HNS, a smaller proportion of those statements are evidence-based and they tend to be developed using lower quality evidence. The AUA invests significant resources into the development of these guidelines, and may be more willing to provide guidance across a wider range of subjects where evidence is lacking or unavailable. Urologists should continue to conduct high quality research to improve the quality of these guidelines.

MP32-17 A critical analysis of evidence and opinions within the American Urological Association's nephrolithiasis Clinical Practice Guidelines

AC Small, MJ Lipsky, DJ Robins, SG Antoine, BE Muller, JM McKiernan, OD Shah

NewYork-Presbyterian Hospital / Columbia University Medical Center

United States

Introduction \& Objective: The American Urological Association (AUA) releases clinical practice guidelines to provide topicspecific, evidence based recommendations to clinicians. Multiple studies have shown that urologists tend to follow guidelines and that they can impact practice patterns. As such, it is important to critically review guidelines and the evidence upon which they are based. We sought to analyze the evidence and opinions that form the basis of AUA clinical practice guidelines and compare nephrolithiasis (NL) guidelines to the other urologic subspecialties.

Materials and Methods: Guidelines through 2016 were extracted from AUAnet.org. NL and other urologic guidelines were analyzed. Statements that were "standards," "recommendations," or "options" were defined as evidence based, and statements that were "clinical principles" or "expert opinions" were defined as non-evidence based. Descriptive statistics and comparisons of evidence-based and non-evidence based statements were performed with Fisher's exact test with $\mathrm{p}<0.05$.

Results: A total of 24 currently available guideline documents including 558 guideline statements were analyzed (TABLE).

Table: AUA Clinical Practice Guideline characteristics for nephrolithiasis vs. other urologic guidelines

\begin{tabular}{|c|c|c|c|c|c|}
\hline & \multicolumn{2}{|c|}{$\begin{array}{l}\text { Nephrolithiasis } \\
\text { guidelines }\end{array}$} & \multicolumn{2}{|c|}{$\begin{array}{l}\text { Other urologic } \\
\text { guidelines }\end{array}$} & $\mathbf{p}$ \\
\hline Total guideline documents & \multicolumn{2}{|c|}{2} & \multicolumn{2}{|c|}{22} & - \\
\hline Total statements & \multicolumn{2}{|c|}{83} & \multicolumn{2}{|c|}{475} & - \\
\hline Statements per guideline (mean \pm SD) & \multicolumn{2}{|c|}{$41.5 \pm 20.5$} & \multicolumn{2}{|c|}{$21.6 \pm 10.3$} & 0.022 \\
\hline Evidence based statements & 52 & $62.7 \%$ & 350 & $73.7 \%$ & 0.047 \\
\hline \multicolumn{5}{|l|}{ Statement type } & \multirow{6}{*}{$<0.001$} \\
\hline Standard & 8 & $9.6 \%$ & 86 & $18.1 \%$ & \\
\hline Recommendation & 43 & $51.8 \%$ & 183 & $38.5 \%$ & \\
\hline Option & 1 & $1.2 \%$ & 81 & $17.1 \%$ & \\
\hline Clinical Principle & 10 & $12.0 \%$ & 54 & $11.4 \%$ & \\
\hline Expert opinion & 21 & $25.3 \%$ & 71 & $14.9 \%$ & \\
\hline \multicolumn{5}{|l|}{ Evidence letter grade } & \multirow{5}{*}{$<0.001$} \\
\hline Letter grade designated & 52 & $62.7 \%$ & 184 & $38.7 \%$ & \\
\hline Grade-A & 2 & $2.4 \%$ & 7 & $1.5 \%$ & \\
\hline Grade-B & 25 & $30.1 \%$ & 48 & $10.1 \%$ & \\
\hline Grade-C & 25 & $30.1 \%$ & 129 & $27.2 \%$ & \\
\hline
\end{tabular}


There are two guidelines on NL which had significantly more statements per guideline than other urologic topics (NL $41.5 \pm 20.5$ vs. other $21.6 \pm 10.3, p=0.022)$. Overall, other urologic guidelines had a higher proportion of evidence based statements than NL guidelines (NL $62.7 \%$ vs. other $73.7 \%$, $\mathrm{p}=0.047$ ). The highest proportion of evidence based statements was in the pediatrics guidelines ( $97 \%$ of 58 statements) and the lowest was in trauma/reconstruction (61\% of 71 statements). Likewise, NL had fewer "standard" statements $(9.6 \%$ vs. $18.1 \%)$ and more "expert opinion" statements $(25.3 \%$ vs. $14.9 \%)$ than other urologic guidelines $(\mathrm{p}<0.001)$. Among evi- dence-based statements, NL had significantly higher evidence grade designations than other urologic guidelines (Grade-A $2.4 \%$ vs. $1.5 \%$; Grade-B $30.1 \%$ vs. $10.1 \%$; Grade-C $30.1 \%$ vs. $27.2 \%$, $\mathrm{p}<0.001)$.

Conclusions: The majority of AUA clinical practice guideline statements are evidence based, but the quality of evidence tends to be low. NL guidelines are less evidence-based than other urologic guidelines, but the evidence is of higher quality. Urology practitioners should continue to strive to conduct high quality research to improve the quality of these guidelines.

\section{MPS33: NON-SURGICAL AND MEDICAL MANAGEMENT OF KIDNEY STONES II}

\author{
Hypocitraturia \\ N Patel, R Ward, J Calle, E Remer, M Monga \\ Cleveland Clinic Foundation \\ United States
}

MP33-1 Vascular Disease and Kidney stones: Abdomina Aortic Calcifications Are Associated with Low Urine pH and

Introduction \& Objective: Vascular calcifications are associated with nephrolithiasis. While studies have demonstrated correlations with vascular disease and formation of calcium stones in kidney stone formers (KSF), an etiologic link has remained elusive. As a non-contrast CT scan (NCCT) is typically part of a stone evaluation, our objective was to evaluate the association of NCCT-based assessment of abdominal aortic calcifications (AAC) with 24-hour urine parameters and stone composition in KSF.

Materials and Methods: $97 \mathrm{KSF}$ were included who had CT imaging and 24-hour urine studies. For each patient, semi-automated CT software was utilized to provide an AAC Agaston score from the celiac axis to the aortic bifurcation. Univariate analysis was performed to compare patients with or without AAC. Multivariate logistic regression was performed to assess for variables associated with 24-hour urine parameters and stone composition.

Results: Presence of AAC was associated with hypertension, diabetes, peripheral vascular disease and coronary artery disease. Patient with any AAC showed lower 24-hour urine citrate (399 vs $593 \mathrm{mg}$ /day, p < 0.001) and lower 24-hour urine $\mathrm{pH}(5.862$ vs $6.328, \mathrm{p}=0.003)$. When controlling for age, system comorbidities, the presence of AAC was associated with low urine $\mathrm{pH}<6(\mathrm{OR} 2.86, \mathrm{p}=0.032)$ and hypocitraturia $<320 \mathrm{mg} / \mathrm{d}$ (OR 4.37, $\mathrm{p}=0.005)$. Receiver operating characteristic curve showed increasing AAC was associated with low urine $\mathrm{pH}(\mathrm{AUC}$ 0.683, $\mathrm{p}=0.002)$ and uric acid stone formation (AUC 0.698, p=0.045).

Conclusions: NCCT-based diagnosis of AAC is associated with low urine $\mathrm{pH}$, hypocitraturia and uric acid stone formation. The presence of AAC could be an additional prognosticator for the utility of alkalinization therapy.

\section{MP33-2 Diabetes and Stone Risk: A Matter of Glycemic} Control?

\section{SL Best, K Maciolek, KL Penniston}

University of Wisconsin School of Medicine and Public Health United States

Introduction \& Objective: Patients with diabetes mellitus (DM) have lower urine $\mathrm{pH}$ and a higher prevalence of uric acid calculi. It is unknown if glycemic control improves urinary risk factors for stones. We examined the association of hemoglobin A1c (HgbA1c), a measure of long-term DM control, with $24 \mathrm{hr}$ urine risk factors for uric acid and calcium calculi.

Materials and Methods: With IRB approval, we identified 176 stone formers (SFs) with a total of $40824 \mathrm{~h}$ urine collections and HgbA1c measures obtained within 3 months of each urine collection. Patients were separated by glycemic status: normoglycemic (NG, HgbA1c < 6.5) and hyperglycemic ( $\mathrm{HG}$, $\mathrm{HgbA1c} \geq 6.5)$; $24 \mathrm{~h}$ urine parameters were compared. The NG

Table 1: Comparison of urine parameters in normoglycemic $(\mathrm{HgbA1} \mathrm{c}<6.5)$ and hyperglycemic $(\mathrm{HgbA} 1 \mathrm{c} \geq 6.5)$ stone formers.

\begin{tabular}{|l|c|c|c|}
\hline & $\begin{array}{c}\text { HgbA1c }<6.5 \\
(\mathbf{n = 1 8 4})\end{array}$ & $\begin{array}{c}\text { HgbA1c } \geq 6.5 \\
(\mathbf{n = 2 2 4})\end{array}$ & p-value $^{*}$ \\
\hline HgbA1c (\%) & $6.0(5.5-6.2)$ & $7.4(7.0-8.3)$ & - \\
\hline $\mathrm{Ca}(\mathrm{mg} / \mathrm{d})$ & $201(122-292)$ & $198(117-330)$ & 0.83 \\
\hline $\mathrm{Ox}(\mathrm{mg} / \mathrm{d})$ & $39(31-500)$ & $45(35-60)$ & 0.26 \\
\hline $\mathrm{UA}(\mathrm{mg} / \mathrm{d})$ & $557(429-770)$ & $587(407-783)$ & 0.91 \\
\hline $\mathrm{Cit}(\mathrm{mg} / \mathrm{d})$ & $608(357-812)$ & $696(369-1150)$ & $<0.01$ \\
\hline $\mathrm{pH}$ & $6.3(5.8-6.8)$ & $5.9(5.5-6.5)$ & $<0.01$ \\
\hline $\mathrm{Vol}(\mathrm{L} / \mathrm{d})$ & $2.0(1.4-2.7)$ & $1.9(1.5-2.4)$ & 0.08 \\
\hline $\mathrm{Na}(\mathrm{mmol} / \mathrm{d})$ & $152(110-203)$ & $185(133-234)$ & 0.061 \\
\hline Creat $(\mathrm{mg} / \mathrm{d})$ & $1456(1125-1875)$ & $1515(1146-1955)$ & 0.32 \\
\hline $\mathrm{Ca}$ Ox SS & $1.6(1.1-2.3)$ & $1.8(1.2-2.7)$ & 0.28 \\
\hline Brush SS & $1.5(0.61-2.8)$ & $0.92(0.28-1.8)$ & $\mathbf{0 . 0 1}$ \\
\hline UA SS & $0.66(0.19-1.7)$ & $1.3(0.49-2.7)$ & $<0.01$ \\
\hline
\end{tabular}

Values reported as median (IQR).

$\mathrm{Ca}=$ calcium, $\mathrm{Ox}=$ oxalate, $\mathrm{UA}=$ uric acid, $\mathrm{Cit}=$ citrate, $\mathrm{Vol}=$ volume, $\mathrm{Na}=$ sodium, Creat $=$ creatinine, $\mathrm{SS}=$ supersaturation, Brush $=$ brushite ${ }^{*} p<0.05$ indicates that $\mathrm{HgbA1c}$ correlates with the urine parameter on multivariate analysis adjusting for BMI, gender, thiazide use and potassium citrate use. 
Table 2: Comparison of urine parameters in normoglycemic stone formers (HgbA1c < 6.5) without and with a history of diabetes mellitus.

\begin{tabular}{|l|c|c|c|}
\hline & $\begin{array}{c}\text { HgbA1c }<6.5 \\
\text { - DM }\end{array}$ & $\begin{array}{c}\text { HgbA1c } \geq 6.5 \\
\text { + DM }\end{array}$ & p-value* \\
\hline HgbA1c (\%) & $5.7(5.4-6.0)$ & $6.2(5.8-6.3)$ & - \\
\hline $\mathrm{Ca}(\mathrm{mg} / \mathrm{d})$ & $182(114-240)$ & $222(140-320)$ & 0.062 \\
\hline Ox (mg/d) & $36(30-46)$ & $40(32-51)$ & 0.75 \\
\hline $\mathrm{UA}(\mathrm{mg} / \mathrm{d})$ & $542(432-735)$ & $575(425-788)$ & 0.85 \\
\hline $\mathrm{Cit}(\mathrm{mg} / \mathrm{d})$ & $586(390-786)$ & $621(300-844)$ & 0.58 \\
\hline $\mathrm{pH}$ & $6.4(5.9-6.7)$ & $6.3(5.8-6.8)$ & 0.84 \\
\hline $\mathrm{Vol}(\mathrm{L} / \mathrm{d})$ & $1.9(1.4-2.7)$ & $2.0(1.4-2.8)$ & 0.88 \\
\hline $\mathrm{Na}(\mathrm{mmol} / \mathrm{d})$ & $142(102-192)$ & $159(120-221)$ & 0.47 \\
\hline Creat (mg/d) & $1439(1083-1844)$ & $1460(1219-1906)$ & 0.38 \\
\hline Ca Ox SS & $1.5(0.94-2.1)$ & $1.8(1.2-2.4)$ & 0.14 \\
\hline Brush SS & $1.4(0.53-2.6)$ & $1.4(0.82-3.0)$ & 0.36 \\
\hline UA SS & $0.6(0.22-1.4)$ & $0.72(0.18-1.9)$ & 0.82 \\
\hline
\end{tabular}

Values reported as median (IQR).

$\mathrm{Ca}=$ calcium, $\mathrm{Ox}=$ oxalate, $\mathrm{UA}=$ uric acid, $\mathrm{Cit}=$ citrate, $\mathrm{Vol}=$ volume $\mathrm{Na}=$ sodium, Creat $=$ creatinine, $\mathrm{SS}=$ supersaturation, Brush $=$ brushite ${ }^{*} p<0.05$ indicates that history of DM correlates with the urine parameter on multivariate analysis adjusting for BMI, gender, thiazide use and potassium citrate use.

cohort was divided into SF with no history of DM and those with well-controlled DM, characterized by HgbA1c $<6.5$ near the time of their 24-h urine collection. Variables were analyzed using chi squared, Welch's t-test and multivariate regression adjusting for BMI, age, gender, and for thiazide and potassium citrate use.

Results: Patients in the HG group were older (62 vs $60 y$, $\mathrm{p}=0.005)$ with higher BMI ( 34 vs $32, \mathrm{p}=0.003)$ and prevalence of thiazide use (46\% vs $36 \%, \mathrm{p}=0.04)$. Hyperglycemia was found to correlate with lower urine $\mathrm{pH}$ and brushite SS as well as higher uric acid supersaturation (Table 1). Normoglycemic SFs with well-controlled DM had similar urinary uric acid stone risk factors as SFs without DM (Table 2). History of DM did not correlate with any urine parameter on multivariate analysis in normoglycemic SF ( $p>0.05)$.

Conclusions: Successful glycemic control is associated with reduced urinary risk factors for uric acid stone formation. With better glycemic control, patients with DM had equivalent risk factors for uric acid stones to those without DM. Our findings support the promotion of glycemic control as a component of medical management of uric acid stone disease. Future studies will confirm whether glycemic control reduces stone formation in an individual.

\section{MP33-3 Current Use of Medical Expulsive Therapy Among Endourologists}

DC Fedrigon, RK Jain, S Sivalingam

Cleveland Clinic Foundation

United States

Introduction \& Objective: To characterize current practice patterns and perspectives among endourologists on medical expulsive therapy (MET) for the treatment of acute ureteral calculi. Materials and Methods: An online survey was administered to Endourological Society members. Respondents' MET usage and index case management (i.e. afebrile patient with a ureteral calculus $<10 \mathrm{~mm}$ and adequately controlled pain) were compared based on international status, practice setting, interval since training completion, and endourological fellowship training.
Statistical analysis was performed using SPSS via Pearson chisquare and Student's t-tests.

Results: Of 237 complete responses, $65 \%$ were international, $61 \%$ were academic, $66 \%$ had $>10$ years in practice, and $71 \%$ were endourology fellowship-trained. For the index patient $70 \%$ preferred MET as the initial approach and $88 \%$ reported using MET for patients with ureteral calculi with no significant differences between the international, academic, practice length, and fellowship trained groups. Respondents within 10 years of training were significantly more likely to choose MET (82\% vs $64 \%, \mathrm{p}=0.006$ ). While $82 \%$ of respondents were aware of the SUSPEND trial which discouraged MET, $70 \%$ of them reported that the results had not altered their use of MET. Nearly $85 \%$ of respondents recommended more research on MET and $90 \%$ reported being aware of the current AUA guidelines on the surgical management of stones. Mean MET prescription length was $19.9 \pm 10.3$ days for all respondents. MET prescriptions were significantly longer for respondents who were US-based (22.9 vs 18.1 days, $\mathrm{p}=0.001$ ), in academic settings (22.3 vs 16.1 days, $\mathrm{p}<0.001$ ), and within 10 years of training (23.9 vs 17.7 days, $\mathrm{p}<0.001)$. Respondents utilized MET more often for distal stones as compared to mid-ureter or proximal stones $(98 \%$ vs $59 \%$ vs $44 \%$ respectively). For stones sized $5-8 \mathrm{~mm}, 8-10 \mathrm{~mm}$, and $>10 \mathrm{~mm}, 76 \%, 33 \%$ and $8 \%$ of respondents reported using MET, respectively. US-based respondents were more likely to use MET for proximal and mid-ureteral stones (68\% vs $43 \%$, $\mathrm{p}<0.001)$ as well as for stones $>10 \mathrm{~mm}(13 \%$ vs $4 \%, \mathrm{p}=0.009)$. Conclusions: Medical expulsive therapy continues to be the preferred initial approach for patients with ureteral calculi $<10 \mathrm{~mm}$ among endourologists despite conflicting data in the literature. While the current AUA practice guidelines are followed by the majority of respondents, it is notable that our survey suggests MET is being used more liberally than the criteria established in the guidelines, specifically in proximal and mid ureteral stones.

MP33-4 Assessment of Adherence to Metabolic Evaluation Guidelines in Patients with Nephrolithiasis - Results of a National Multi-centric study

S Harmouch, H Abou-Haidar, S Andonian, JY Lee, T Schuler, A Lantz, BH Chew, N Bhojani

Division of Urology, Université de Montréal Canada

Introduction \& Objective: The rate of recurrence after a single stone episode is estimated to be $50 \%$ at 5 years making the role of stone prevention a high priority. The AUA and CUA Guidelines recommend that clinicians perform metabolic testing in high-risk and recurrent stone formers. However, adherence to these guidelines has not been confirmed. The purpose of this study was to evaluate metabolic work-up patterns in a Canadian population, to assess not only its use, but also patients' understanding of their disease and their interest in following a diet or treatment to prevent future stone events.

Materials and Methods: Patients presenting to participating hospitals for shock wave lithotripsy were administered a short questionnaire to identify risk factors of stone disease recurrence and to assess the use of metabolic workup evaluations for patients who require it according to the CUA and AUA guidelines. Furthermore, patients were asked if they received explanations about the results of their metabolic workup, if they understood 
Table 1. Baseline Characteristics of participants

\begin{tabular}{|c|c|}
\hline Total number of patients & 202.0 \\
\hline Age (median, years) & 56.0 \\
\hline \multicolumn{2}{|l|}{ Sex (\%) } \\
\hline Female & 45.0 \\
\hline Male & 55.0 \\
\hline \multicolumn{2}{|l|}{ Referring urologist (\%) } \\
\hline Community-based center & 49.5 \\
\hline Academic center & 50.5 \\
\hline Unknown & 0.5 \\
\hline \multicolumn{2}{|l|}{ Indications for metabolic evaluation (\%) } \\
\hline Multiple/Bilateral stones & 62.4 \\
\hline Non-calcium stones & 2.0 \\
\hline Family history of stones & 28.2 \\
\hline Single kidney/CKD & 2.0 \\
\hline Poor kidney function & 5.9 \\
\hline Stones during pregnancy & 2.5 \\
\hline Systemic disease & 10.4 \\
\hline Intestinal surgery & 3.5 \\
\hline Occupation with risk for public safety & 2.0 \\
\hline Percentage of patients with $>1$ indication & 76.0 \\
\hline Cause of stone disease explained (\%) & 19.3 \\
\hline Patients interested in more information about disease (\%) & 87.1 \\
\hline Patients interested in following a diet or taking medications for disease (\%) & 83.7 \\
\hline \multicolumn{2}{|l|}{ Metabolic evaluation } \\
\hline Received when indication present (\%) & 27.7 \\
\hline Explained when received (\%) & 60.7 \\
\hline Understood by patient (\%) & 63.6 \\
\hline \multicolumn{2}{|l|}{ Type of prescribing physician } \\
\hline Urologist & 62.5 \\
\hline Nephrologist & 25.0 \\
\hline Family doctor & 16.1 \\
\hline \multicolumn{2}{|l|}{ Date of metabolic evaluation } \\
\hline$\leq 1$ year & 53.6 \\
\hline $1-5$ years & 16.1 \\
\hline $5-10$ years & 5.3 \\
\hline$\geq 10$ years & 23.2 \\
\hline
\end{tabular}

these explanations when given, and if they were interested in following a diet or treatment to prevent future stone events. Statistical analyses were used to calculate percentages of patients for each of the preceding categories.

Results: Results were obtained for 202 patients (Table 1). 76\% of participants had an indication to get a metabolic evaluation, and an additional $17.8 \%$ of patients were interested in knowing more about their disease. Amongst patients with any kind of indication for a metabolic workup, only $27.7 \%$ had one performed $23.7 \%$ in community-based centers and $31.3 \%$ in academic centers). $62.5 \%$ of metabolic evaluations were prescribed by a urologist. $60.7 \%$ of patients who had a metabolic evaluation received explanations about their results; however, only $63.6 \%$ of participants understood these explanations. $87.1 \%$ of the participants are interested in having more explanations about their kidney stones disease, and $83.7 \%$ are interested in following a diet or taking a medication to prevent further stone disease.

Conclusions: Results of this study demonstrate that adherence to CUA and AUA guidelines is suboptimal, and that explanations given to patients about their stone disease may not be adequate. The majority of patients with nephrolithiasis are interested in knowing more about their stone disease, and are willing to follow a diet or a treatment to prevent future stone events.

MP33-5 Metformin Use and 24-hour Urine Abnormalities in Urinary Stone Formers

MJ Wardenburg, BK Carew, BJ Otto, VY Bird, VG Bird

Department of Urology, University of Florida, Gainesville, Florida

United States
Table 1: Demographics of patients

\begin{tabular}{|c|c|c|c|}
\hline & Diet Controlled $(n=23)$ & Metformin $(n=44)$ & 'p-value \\
\hline Age, mean (SD) & $58.2(11.5)$ & $61.8(9.6)$ & 0.172 \\
\hline Male (\%) & $15(65.2)$ & $24(54.5)$ & 0.4 \\
\hline Hemoglobin A1C, mean (SD) & $6.6(0.3)$ & $6.6(0.9)$ & 0.994 \\
\hline BMI (kg/m2), mean (SD) & $31.4(7.1)$ & $33.3(6.4)$ & 0.262 \\
\hline $\operatorname{CAD}(\%)$ & $2(8.7)$ & $6(13.6)$ & 0.554 \\
\hline HTN (\%) & $15(65.2)$ & $29(65.9)$ & 0.955 \\
\hline $\mathrm{HL}(\%)$ & $10(43.5)$ & $28(63.6)$ & 0.114 \\
\hline CKD $(\%)$ & $3(13)$ & $6(13.6)$ & 0.946 \\
\hline Bone disease (\%) & $0(0)$ & $1(2.3)$ & 1 \\
\hline Primary Stone Type & & & 0.569 \\
\hline Calcium Oxalate (\%) & $11(55)$ & $20(62.5)$ & \\
\hline Calcium Phosphate (\%) & $2(10)$ & $2(6.3)$ & \\
\hline Uric Acid (\%) & $7(35)$ & $8(25)$ & \\
\hline Struvite (\%) & $0(0)$ & $2(6.3)$ & \\
\hline
\end{tabular}

Table 2: Mean \pm SD 24 hour urine variables

\begin{tabular}{lccc}
\hline Group & Diet Controlled $(\mathrm{n}=23)$ & Metformin $(\mathrm{n}=44)$ & $\mathrm{p}$ value' \\
\hline Oxalate $(\mathrm{mg} /$ day) & $33.6 \pm 14.5$ & $40.8 \pm 16.5$ & 0.082 \\
Calcium (mg/day) & $155.2 \pm 98.86$ & $211.2 \pm 148.9$ & 0.109 \\
Citrate (mg/day) & $392.3 \pm 282.1$ & $542.1 \pm 377.2$ & 0.099 \\
Uric acid ( $\mathrm{g} /$ day) & $0.59 \pm 0.25$ & $0.60 \pm 0.26$ & 0.854 \\
Sodium (mmol/day) & $150.6 \pm 89.1$ & $180.6 \pm 97.1$ & 0.223 \\
Urine pH & $5.73 \pm 0.47$ & $5.88 \pm 0.54$ & 0.284 \\
Urine volume (L/day) & $1.51 \pm 0.64$ & $1.79 \pm 0.72$ & 0.124 \\
Cr/kg (mg/kg/day) & $16.7 \pm 5.4$ & $17.7 \pm 4.9$ & 0.459 \\
SS CaOX & $6.79 \pm 3.15$ & $7.96 \pm 4.15$ & 0.24 \\
SS CaP & $0.79 \pm 0.77$ & $1.14 \pm 1.21$ & 0.205 \\
\hline Cr, creatinine; SS CaOX, supersaturation calcium oxalate; SS CaP, supersaturation calcium phosphate &
\end{tabular}

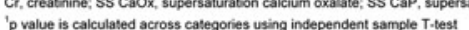

Introduction \& Objective: Diabetes is associated with increased risk of nephrolithiasis. It is unclear how medications used to treat diabetes affects that risk. We evaluated effects of metformin on 24-hour urine parameters in a cohort of diabetic urinary stone formers.

Materials and Methods: Methods: Retrospective review of 494 patients who underwent initial 24-hour urine analysis between 07/2010 and 01/2015 was performed. 98 (19.8\%) diabetics were identified and medical records reviewed for use of metformin or other diabetes medications at time of urine collection. Patient demographics, urine parameters, and stone composition were compared between diabetics on metformin and diet-only controlled diabetics using either t-test or chi-square test for continuous and categorical variables, respectively.

Results: There were no significant differences in age, gender, BMI, hemoglobin A1c, comorbidities, and stone type between 
the metformin group $(n=44)$ and the diet-only group $(n=23)$ (Table 1). Mean urine oxalate and calcium levels trended higher in the metformin group ( $40.8 \mathrm{mg} /$ day vs. $33.6 \mathrm{mg} / \mathrm{day} ; \mathrm{p}=0.082$, and $211.2 \mathrm{mg} /$ day vs. $155.2 \mathrm{mg} /$ day; $\mathrm{p}=0.109$ ) (Table 2). Hyperuricosuria $(27.3 \%$ vs. $17.4 \%$; $=0.547)$, hyperoxaluria $(43.2 \%$ vs. $30.4 \% ; \mathrm{p}=0.309)$ and hypercalciuria $(38.6 \%$ vs. $17.4 \% ; \mathrm{p}=0.099)$ were more frequent in the metformin group, although this did not reach statistical significance (Table 3). Hypocitraturia was significantly less common in the metformin group (50\% vs. $78.3 \%$; $\mathrm{p}=0.025$ ).

Conclusions: Metformin users had no significant differences in mean 24-hour urine values compared to diet-only controlled diabetics, however they did have more abnormal testing results and significantly less hypocitraturia.

\section{MP33-6 The Association of Hemoglobin A1C and Urinary Oxalate in Stone Formers}

K Wood, J Knight, R Holmes, D Assimos, R Oster, M Colaco

University of Alabama at Birmingham

United States

Introduction \& Objective: Greater body mass index (BMI) and type 2 diabetes mellitus are associated with kidney stone risk. Increased urinary excretion of oxalate (Uox) has been correlated to increasing BMI. Our objectives were to determine if this association is linked to hemoglobin A1C levels (A1C).

Materials and Methods: We retrospectively reviewed 1,428 twenty-four hour urine collections gathered from a single institution from 2004-2015 from two urologists. 665 unique non cystinuric adult stoneformers (SF) with complete data including $\mathrm{BMI}$, age, gender, A1C levels, and Uox were then analyzed using ANOVA, Chisquared, and linear regression analyses.

Results: Average age of SF was 49.9 years. $46 \%$ of SF were female. Average BMI was 29.2 (Underweight 1.4\%, Normal weight $26.5 \%$, Overweight $35.8 \%$, Obese $27.7 \%$, Morbidly Obese $8.6 \%)$. Greater BMI correlated with increased Uox (mg/d) $(\mathrm{p} \leq 0.0001, \mathrm{r}=0.245)$ and remained significant for both males $(\mathrm{p} \leq 0.001, \mathrm{r}=0.30)$ and females $(\mathrm{p} \leq 0.001, \mathrm{r}=0.195)$. The positive correlation between BMI and Uox was also seen in both African American and Caucasian subjects $(r=0.34, p=0.02$ and $\mathrm{r}=0.20, \mathrm{p} \leq 0.005)$. A significant positive correlation between $\mathrm{A} 1 \mathrm{C}$ and Uox was demonstrated $(\mathrm{r}=0.24, \mathrm{p} \leq 0.009)$.

Conclusions: Among SFs, there is a positive correlation between BMI and Uox as well as A1C and Uox. These relationships may explain associations between both obesity and diabetes and the development of kidney stones.

MP33-7 Medical expulsive therapy for distal ureteral stones: Tamsulosin versus silodosin in the Turkish Population

E Arda, B Cakiroglu, E Akdeniz

Trakya University

Turkey

Introduction \& Objective: Was to contribute, to the limited number of studies comparing tamsulosin and silodosin in the treatment of distal ureteral Stones, with a higher patient population.
Materials and Methods: Between January 2010 and January 2016, patients with renal colic at the urology emergency clinic who were diagnosed with ureteral stones and followed up with conservative treatment were retrospectively screened. According to the inclusion-exclusion criteria patients were divided into three groups. Group 1: 150 patients followed with watchfull waiting; Group 2: 156 patients who received $0.4 \mathrm{mg}$ of tamsulosin daily; Group 3: 159 patients who received 8mg of silodosin daily. The side effects of drugs used in the treatment, the duration of stone reduction and expulsion rates were evaluated and compared separately.

Results: A total of 465 patients were included to the study. No statistically significant difference was found in terms of age, gender and stone size among the groups. Patient characteristics and results are shown in Table 1. Differences of stone expulsion rate between the groups at the first week werefound non-significant using the Chi-square test $(\mathrm{p}=0.155)$. The stone expulsion rates after two weeks were found significantly different between Group 1 vs. Group 2 and Group 1 vs. Group 3. Stone expulsion rate after three weeks were found significantly different between Group 1 vs. Group 2 and Group 1 vs. Group 3.

Conclusions: According to our results, no statistically significant superiority between tamsulosin and silodosin was shown in the treatment of distal ureteral stones in the Turkish population.

\section{MP33-8 Nephrolithiasis is Associated With Nonalcoholic Fatty Liver Disease}

A Weiss, E Ghiraldi, J Gaughan, J Friedlander

Albert Einstein Medical Center

United States

Introduction \& Objective: Nephrolithiasis and nonalcoholic fatty liver disease (NAFLD) have common metabolic risk factors, however little is known regarding the association between the two conditions. We sought to investigate the association between NAFLD and nephrolithiasis at the population level using data from the National Health and Nutrition Examination Survey (NHANES) III.

Materials and Methods: We analyzed data from the NHANES III, conducted from 1988 to 1994, and compared nephrolithiasis and hepatic ultrasound data. Participants with evidence of liver disease not due to NAFLD (positive hepatitis B surface antigen or anti-hepatitis $\mathrm{C}$ virus test with positive HCV RNA, excessive alcohol use defined as $>14$ drinks/week in women and $>21$ drinks/week in men, and iron overload defined as transferrin saturation $>50 \%$,) were excluded. Participants aged 20-74 years were classified into those with NAFLD (defined as moderate or severe hepatic steatosis based on ultrasound analysis, in the absence of other causes of liver disease; $n=2498$ ) and those without NAFLD (controls; $n=9361$ ). The risk of nephrolithiasis due to NAFLD was estimated using logistic regression with a propensity score (age, BMI, diabetes mellitus, elevated liver enzymes, gender, GFR, gout, HTN, race, and smoking status) to adjust the odds ratio.

Results: Individuals with NAFLD were older $(48.7 \pm 15.3$ vs. $43.3 \pm 16.0$ years, $\mathrm{p}<0.001)$, and predominantly female $(51 \%)$. Compared to controls, individuals with NAFLD were more likely to be Caucasian (74.3\% vs. $65.4 \%)$ and less likely to be African American (21.5\% vs. $31.5 \%$ ). They also had a higher prevalence of components of metabolic syndrome (higher BMI, $30.9 \pm 6.4$ vs. $27.2 \pm 5.2, p<0.001$, higher prevalence of diabetes mellitus $(15.1 \%$ vs. $6.3 \%, \mathrm{p}<0.0001)$, and higher prevalence of 
HTN (35.7\% vs. $23.6 \%, \mathrm{p}<0.0001)$ ), respectively. Individuals with NAFLD also had a higher prevalence of gout $(3.92 \%$ vs. $2.0 \%, \mathrm{p}<0.0001)$. After adjusting for major clinical, demographic, and metabolic factors, NAFLD was found to be independently associated with nephrolithiasis (odds ratio, 1.286; $95 \%$ CI, 1.024-1.614).

Conclusions: In a large population based analysis NAFLD was found to be independently associated with an increased risk of nephrolithiasis.

\section{MP33-9 Patient Perceptions of Non-Obstructing Kidney Stones and Pain}

DW Smith, MS Borofsky

University of Minnesota

United States

Introduction \& Objective: There is a general sentiment within the urologic community that in the absence of obstruction, small renal stones should not cause pain. However, several prior studies investigating this question have found that pain often does improve when such stones are removed. Given the lack of consensus, management of the patient with flank pain in the setting of nonobstructing renal stones is challenging, with high potential for patient frustration given lack of a diagnosis or explanation for their symptoms. Web based resources, particularly message boards, have become popular for similar medical questions that lack clarity in etiology and treatment. We sought to assess online postings from patients reportin nonobstructing stones and pain to assess attitudes and experiences with this controversial scenario.

Materials and Methods: Publically accessable online resources including medical websites, forums, social media postings, and support groups were searched to identify articles and threads specific to nonobstructing stones and pain. Fourty three unique patient reported experiences describing pain in the setting of small non-obstructing renal stones were identified. Entries were characterized with respect to attitudes about diagnosis, medical consultation patterns, interventions, and outcomes.

Results: A majority of patients $(36 / 43,83.7 \%)$ noted disappointment or anger as a result of being told their stone was unlikely to be causing symptoms. 67.4\% (29/43) reported consultation with more than one provider; only $3(6.9 \%)$ reported being offered surgical intervention. Notably, all patients who underwent surgical treatment reported improvement of pain after stone removal.

Conclusions: Patients with nonobstructing stones and flank pain pose a clinical challenge. Reports of anger, dissapointment and mulitple consultations with providers are common among publically available patient reported experiences on the internet. Awareness regarding attitudes and sentiments in this unique population may be helpful when counseling such patients and highlights the need for further investigation into the therapeutic effect of stone removal in such circumstances.

MP33-10 A pilot study comparing virtual and real consultation(out patient) for stone patients : Future of Endourology stone clinic

HG Thummar, K Thummar, N Zinto, N T

sterling

India

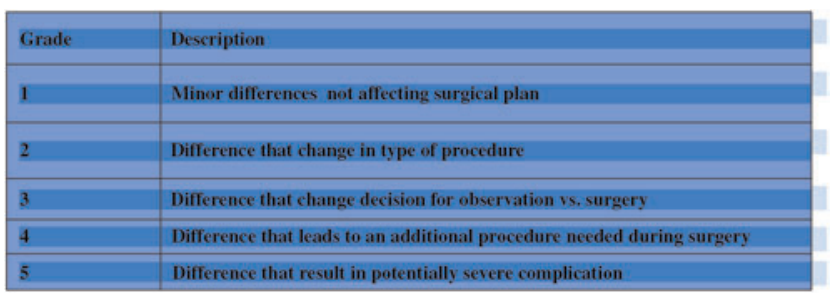

Introduction \& Objective: Healthcare professionals are among the last, service providers to not use Internet technology to communicate with the people they serve. As much as patients are able to communicate with their doctors, and physicians, medical professionals will also be able to transmit data between each other; overall helping the patients' well-being. Many a times, it's very difficult for patients to come from far places for stone clinic for consultation wasting a lot of time. With increased use of mobile phones, Internet and with increased skill of fast communication through handy available cell phone to almost everyone, its possible to use virtual consultation. Uncomplicated stone patients do not need detail clinical examination most of the time. Can we use these advantages in current era in providing health care at every corner of world without compromising quality of care? We could not find literature to address this issue. Aim of our study was to assess feasibility and accuracy of e-consultation in stone clinic and compare with standard consultation defining good practice and inform its implementation in relation to clinician-patient consultations via whatsapp and similar virtual media.

Materials and Methods: We included 42 uncomplicated stone patients who presented to endourology clinic during Jan 2016 to June 2016 according our inclusion criteria. First we did e-consultation using whatsapp and other virtual media and assessed clinical history, biochemical profile and imaging with images and communicated through media and made a provisional diagnosis and decided management plan. Then we did real consultation as standard practice and decided management plan. We compared diagnosis and management plans in this both consultations virtual and real. We graded in five grades according to difference in diagnosis and management plan. We assessed 5-point likert scale also for virtual and real consultation feedback.

Results: There was no significant difference in diagnosis or management plan in virtual Vs. real consultation. There was grade 1 change in 3 patients, grade 2 and 3 change in one patient. Five point likert scale score difference was not significantly different.

Conclusions: Based on this pilot study, virtual consultation (econsultation) for kidney stone patients is feasible and accurate in selected group of patients without compromising quality of care and may be a future of endourology clinic. However, further study with large number of patients are required to define its role in future.

MP33-11 Conservative Management of Staghorn Calculi: Is it Safe?

T Nikonow Morgan, M Shahait, A Maganty, A Nevo, MC Ost, S Jackman, T Averch, MJ Semins

UPMC

United States 
Introduction \& Objective: To describe the clinical characteristics, infectious and kidney function patterns, and overall outcomes in a cohort of patients with staghorn calculi treated conservatively

Materials and Methods: Staghorn calculi managed non-operatively between January 2009 and January 2017 were identified. A retrospective analysis of demographics, comorbidities, renal function, clinical characteristics, treatments, and outcomes was completed

Results: Twenty-nine patients were identified (16 male, 13 female). Mean age was 71 (IQR 61-81). Mean follow-up was 24 months (IQR 1-34). Fifty nine percent (17/29) had complete staghorn calculi with $21 \%(6 / 29)$ bilateral. Mean body mass index was 29.4 (IQR 24.8-31.7). Of the 29 patients, 14 were treated conservatively due to comorbidities, 12 refused treatments, and 3 were due to aberrant anatomy. Additionally 3 patients had advanced age ( $>85$ years old) and 3 were on anti-coagulation. The Age-Adjusted Charlson Comorbidity Index Score (CCI) demonstrated 7 high-risk patients with a CCI of 6 or 7 and 3 patients with a CCI $>8$. Overall, kidney function remained clinically stable for all patients; none of the patients in the study required hemodialysis. During follow up, $38 \%$ of patients became symptomatic. Two patients reported having flank pain, 5 reported gross hematuria, and 6 had urinary tract infections. No patients in the cohort developed an abscess, nor were any patients on daily prophylactic antibiotics. There was only 1 related admission for a complication of a staghorn stone during the study; this was for pyelonephritis. There were two deaths during the study period. One death was unrelated cardiac death and the other was from urosepsis in a noncompliant patient

Conclusions: Our study shows that conservative treatment of staghorn stone in frail patients is feasible with plausible outcome. Future efforts should be directed toward standardization of this approach utilizing frailty scores and outcome measurements.

MP33-12 A UK Snapshot of Loin Pain / Urolithiasis outcomes from June 2015 to June 2016

T Marsden, B Turney, M Bultitude, F Keeley, S Gordon, SO Irving, W Finch, B Grey, O Wiseman, A Rogers, N Shrotri, D Smith

University College London Hospitals United Kingdom

Introduction \& Objective: We aimed to provide a "snapshot" of UK practice for the diagnosis, management and one year follow up of patients presenting acutely with loin pain in whom a diagnosis of urolithiasis was made.

Materials and Methods: Radiology records were used to identify all emergency CTKUBs performed in June 2015 across ten UK hospitals. Of 827 patients, $408(49.3 \%)$ had urolithiasis confirmed; the remaining 419 patients $(50.7 \%)$ were stone-free. Results: Stone bearing patients were significantly more likely be male, have hydronephrosis, a history of stone disease (all $\mathrm{p}\langle 0.0001)$ and to have haematuria $(\mathrm{p}\langle 0.001)$. The majority of patients with ureteric colic were accounted for by distal (42.6\%) and proximal $(16.7 \%)$ ureteric stones; $25.9 \%(\mathrm{n}=106)$ had renal stones only.

Most patients (84\%) were managed conservatively; $31 \%$ received MET. Non-admitted patients (56.8\% of the cohort) had smaller stones (median $4 \mathrm{~mm}$, mean $4.5 \mathrm{~mm}$ ) than admitted patients (median $5 \mathrm{~mm}$, mean $6.7 \mathrm{~mm}$ ). In turn, patients admitted but

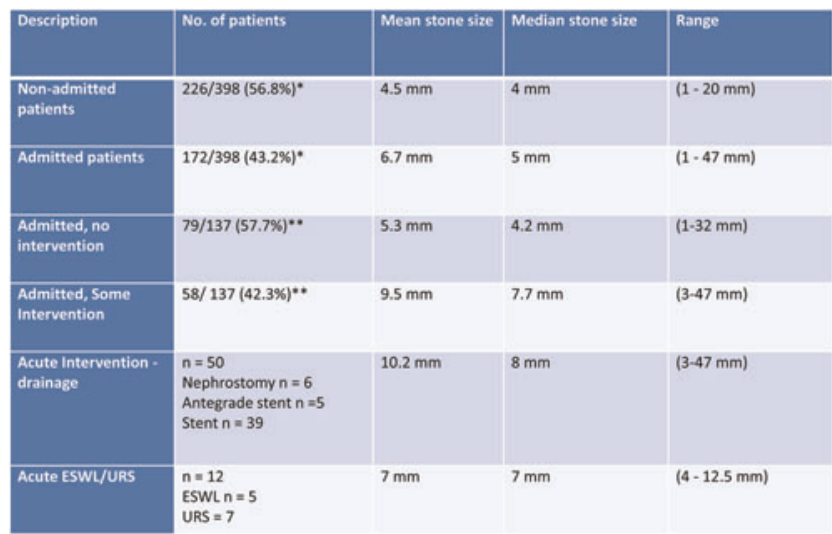

Table 1.: Acute admission and intervention
intervention data for 35 admitted patients.

not requiring intervention had smaller stones (median $4.2 \mathrm{~mm}$ and mean $5.3 \mathrm{~mm}$ ) than those who needed intervention (median $7.7 \mathrm{~mm}$, mean $9.5 \mathrm{~mm}$ ). Of those requiring intervention, $80.6 \%$ underwent emergency drainage with a JJ stent or nephrostomy insertion, $11.3 \%$ had primary URS and $8 \%$ acute ESWL. Patients who had emergency drainage tended to have the largest stones (median $8 \mathrm{~mm}$, mean $10.2 \mathrm{~mm}$ ). These data are summarised in Table 1. Stone size also predicted the likelihood of early representation ranging from $4.1 \%$ for stones $1-4 \mathrm{~mm}$ to $15.3 \%$ for stones $7-10 \mathrm{~mm}$ during the first six weeks after the acute episode. Conclusions: As is consistent with the literature, around 50\% of emergency CTKUBs were positive for stones and most patients were managed conservatively. Those with larger stones were more likely to be admitted, require intervention (especially emergency drainage), re-present to emergency services and take longer to be stone and tube free than patients with smaller stones. This suggests that definitive management of patients with larger stones (i.e.) $7 \mathrm{~mm}$ ) should be planned as soon as feasible to reduce both their uncertainty and tube-related morbidity.

\section{MP33-13 Systemic Biomineralization in Kidney Stone Formers}

BA Sherer, A Fernandez, DT Tzou, S Ho, ML Stoller

\section{UCSF}

United States

Introduction \& Objective: Emerging data has revealed that patients with nephrolithiasis are at increased risk for atherosclerosis, coronary artery disease, and stroke. While nephrolithiasis occurs within a broad spectrum of human pathologic biomineralization, the overall association of nephrolithiasis with mineralization processes at non-renal, non-osseous sites is poorly understood. The objective of this study is to characterize the overall burden of systemic biomineralization in known kidney stone formers (SFs) compared to non stone formers (NSFs).

Materials and Methods: The presence and quantity of biomineralization at 9 non-renal anatomic locations (listed in results) was determined by a blinded, retrospective review of clinical non-contrast computed tomography (CT) scans of the abdomen/ pelvis in known SFs $(n=120$, mean age 51.7) and were compared to a gender and age-matched control group of NSFs (renal 


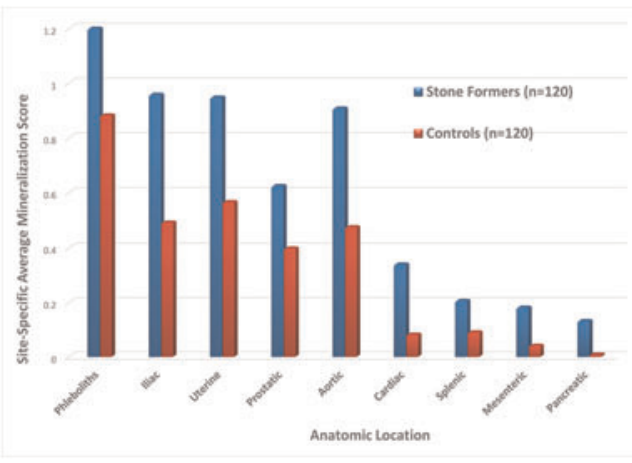

transplant donors, $\mathrm{n}=120$, mean age 51.6). Each anatomic site of interest was assigned a mineralization score based on calculated CT volume of visible calcification $(0=$ none $)(1=\leq 0.5 \mathrm{~cm} 3)$ $(2=0.5 \mathrm{~cm} 3$ to $1.0 \mathrm{~cm} 3)(3=\geq 1.0 \mathrm{~cm} 3)$. Patients were also evaluated for age, gender, BMI, comorbidities, and stone type.

Results: The average systemic mineralization score was significantly higher in stone formers (4.68) compared to controls (2.56). SFs were more likely than NSFs to have mineral densities at all locations, including the heart (19.2\% vs $7.5 \%)$, aorta (48.3\% vs $26.7 \%)$, iliac arteries (51.7\% vs 30.0\%), spleen/splenic artery (15\% vs $5.0 \%)$, mesentery ( $12.5 \%$ vs $4.7 \%)$, pelvic phleboliths ( $77.0 \%$ vs $52.5 \%$ ), uterus ( $46.4 \%$ vs $45.0 \%)$, prostate $(65.5 \%$ vs $52.0 \%)$, and pancreas $(9.2 \%$ vs $0.8 \%)$. Site-specific mineralization scores were significantly higher in SFs at all anatomic sites. SFs also had higher degree of obesity (BMI 30.4 vs 27.5) and higher rates of comorbidities including diabetes, hypertension, and hyperlipidemia.

Conclusions: SFs have increased mineralization at many anatomic sites compared to NSFs. Understanding nephrolithiasis within the context of systemic biomineralization may help to better elucidate underlying mechanisms of nephrolithiasis and other pathologic biomineralization processes.

\section{MP33-14 Rates of Spontaneous Ureteral Stone Passage After Ureteral Stent Placement}

J Kuebker, J Robles, N Miller, S Herrell, RS Hsi

Vanderbilt University Medical Center United States

Introduction \& Objective: Definitive management of ureteral calculi consists of either a trial of spontaneous passage or surgical intervention, most often with ureteroscopy (URS) or shockwave lithotripsy. As a temporizing solution, ureteral stents are often initially placed necessitating a subsequent procedure. While stone passage with a concurrent ureteral stent is thought to be uncommon, a resultant "negative" URS is admittedly unsatisfactory. There is a paucity of data describing this occurrence. Thus, we sought to report the frequency and predictors of spontaneous ureteral stone passage after stent placement.

Materials and Methods: A retrospective review was performed querying records from January 2015 to February 2017 at a tertiary nephrolithiasis referral center to identify all adult, nonpregnant patients who underwent ureteral stent placement for a single ureteral stone. Subjects were identified by CPT code 52332 (ureteral stent placement) and an ICD9/10 diagnosis code of calculous disease (N20.1, N20.2, 592.1, 592.0). All records and imaging were individually reviewed. Ureteral stones were defined as the entire stone being contained in the ureter. Stones located at the UPJ were excluded. Univariate analysis of gender, age, BMI, stone diameter, alpha blocker use, UTI presence, and time from stent to URS or CT were analyzed to determine associations with spontaneous stone passage.

Results: A total of 153 subjects met the inclusion criteria. Mean \pm SD age, BMI, and maximum stone diameter were $50.5 \pm 16.9$ years, $30.2 \pm 6.6 \mathrm{~kg} / \mathrm{m}^{2}$, and $6.5 \pm 2.2 \mathrm{~mm}$ respectively. Subsequent to stent placement, 150 patients underwent URS and 3 had a CT only (no URS). Overall, 8/150 evaluated with URS and $2 / 3$ who underwent CT showed interval passage for an overall passage rate of $6.6 \%$. Gender, age, BMI, laterality, alpha blocker use, and presence of a UTI at presentation were not associated with passage. Size and location differed significantly in patients where stones passed with stent in place $(\mathrm{p}<.001$ and $\mathrm{p}=.003)$. Passage rates for stones $<3 \mathrm{~mm}, 3-$ $4.9 \mathrm{~mm}$, and $5-7 \mathrm{~mm}$ were $50 \%, 11.8 \%$, and $5.6 \%$, respectively. No stones $>7 \mathrm{~mm}$ passed, and when these cases were excluded the passage rate was $10.8 \%$. Distal stones were much more likely to pass $(18.4 \%)$ compared to mid $(4 \%)$ and proximal $(2.2 \%)$. When distal stones $>7 \mathrm{~mm}$ were excluded, the passage rate was $23.3 \%$.

Conclusions: Passage of ureteral stones with a concurrent stent in place occurs in half of stones $<3 \mathrm{~mm}$ and 1 of 9 stones $3-5 \mathrm{~mm}$ in size. Patients with small distal stones may be candidates for interval imaging before staged definitive intervention.

MP33-15 Assessing the Need for Technology to Promote Fluid Consumption in Patients with Nephrolithiasis

N Streeper, K Lehman, D Conroy

Penn State Hershey Medical Center United States

Introduction \& Objective: The benefit of increased fluid intake on stone prevention has been well documented. Unfortunately, patients' adherence to recommendations is commonly below $50 \%$. Although simple and inexpensive, it can be challenging because patients have limited support once they leave clinic. Smartphone applications (apps) offer a new opportunity to support adherence to these recommendations that often require lifestyle behavior modifications. The purpose of this study is to evaluate whether our patients with kidney stones would be interested in utilizing technology to aid with compliance to recommendations and which tools may be useful in the design of a disease-specific app or device.

Materials and Methods: Adult patients with a diagnosis of kidney stones were recruited from our outpatient clinics. With IRB approval, patients completed a 54-item questionnaire to evaluate stone history, current utilization of apps for kidney stone prevention, interest in technology to increase fluid intake and tools that patients would like to include in the design of a diseasespecific app.

Results: There were a total of 94 patients (44 male: 50 female) with a mean age of $51.8 \pm 13.9$. Patients reported being willing to make lifestyle changes to prevent kidney stones (96.8\%). The recommendation to increase fluid intake for stone prevention was recalled by the majority of the patients (93.4\%). Although, $35.6 \%$ of the patients admitted to following that recommendation half of the time or less, and $70.2 \%$ do not monitor their fluid 
intake. The majority of patients $(94.9 \%)$ had never installed an app to help with increasing fluid consumption, however $86.1 \%$ of patients would be interested in using an app or device to aid in complying with recommendations. Tools that patients would most commonly like to see incorporated included: automated notification triggered by technology when lapse in preventive behavior was detected, educational material, self-monitoring tools for tracking behavior and scheduler with reminders. The majority of patients (72.2\%) believed that an app or device could improve their compliance to recommendations.

Conclusions: Patients are interested in utilizing smartphone apps or devices to improve adherence to increased fluid intake recommendation for stone prevention. Tailoring a disease-specific application or device to meet the needs of this patient population is an important first step. Future work is necessary to determine the clinical efficacy of such technology in the prevention of kidney stones.

\section{MP33-16 Withdrawn}

MP33-17 Gender disparity in urolithiasis - fact or fiction? GN Tundo, VM Pais

Dartmouth Hitchcock Medical Center United States

Introduction \& Objective: Although urolithiasis affects both sexes, conventional teaching has proposed that men are up to $3 \mathrm{x}$ more likely to suffer from kidney stones. However clinical practice refutes such disparity, particularly among working-aged adults, and small studies have also suggested erosion of such a gender gap. As stone disease has been shown to increase work absenteeism, the significant financial burden this may impose warrants further evaluation of the epidemiology of stone disease in working and reproductive-aged adults. We therefore sought to examine the relationship between gender and stone prevalence among U.S. adults $<50$ years of age.

Materials and Methods: We analyzed the weighted, nationally representative cohort of U.S. adults from the 2007-2012 National Health and Nutrition Examination Survey. Weighted proportions and multivariate logistic regression of the cohort and pertinent subgroups were assessed to determine the prevalence and odds of nephrolithiasis using survey analytic codes with Stata version 13.1.

Results: The cohort of 17,658 subjects - weighted to represent the nationwide U.S. population of 218,828,951 adults - was $48.1 \%$ male and $51.9 \%$ female. Among all adults, men had a significantly higher stone prevalence of $9.8 \%$ (vs $7.7 \%$ for women, $\mathrm{p}=0.002)$. The cohort of those less than 50 years of age represented 123,976,786 subjects (49\% male and 51\% female). When restricted to those under age 50 , there was no difference in stone prevalence $(6.3 \%$ male vs. $6.4 \%$ female, $p=0.85)$. On multivariate logistic regression among the entire cohort, men were $37 \%$ more likely to have stones when adjusting for diabetes, obesity, ethnicity and age (OR 1.37, p <0.001). However, looking at those under the age of 50, there was no difference in stone prevalence (OR 1.0, $\mathrm{p}=0.98$ ).

Conclusions: Among working and reproductive-aged U.S. adults less than 50 years of age, the much-touted gender disparity in stone prevalence is not present. Prior assessments of genderbased prevalence of stones may have failed to specifically assess this economically critical demographic, or there may in fact be a change in epidemiology. Recognition that women are just as likely as men to form stones in this population suggests the need for continued efforts to better elucidate the pathophysiology of stones in women.

\section{MPS34: UROTHELIAL CARCINOMA II}

\begin{abstract}
MP34-1 Positive Predictive Value of CT Urography for Upper Tract Urothelial Carcinoma Diagnosis using Diagnostic Ureteroscopy as the Reference Standard
\end{abstract}

TC Chang, I Mintz, J Ben-Chaim, S Conti, J Leppert, S Barnes, JC Liao, M Sofer

Department of Urology, Stanford University United States

Introduction \& Objective: Computed tomographic urography (CTU) is the imaging modality of choice for assessing clinical findings suspicious for upper tract urothelial carcinoma (UTUC). However, diagnostic ureteroscopy (DURS) is often negative when pathological findings on CTU support the diagnosis of UTUC. The aim of this study was to assess the positive predictive value (PPV) of CTU for identifying UTUC compared to DURS as the reference standard.

Materials and Methods: The study group comprised 79 consecutive patients from 2 academic institutions. They presented with CTU findings of upper tract wall thickening, hydronephrosis, a filling defect and/or contrast enhancement diagnosed in consensus by 2 radiologists as suspicious for UTUC. DURS, with either wash cytology when endoscopically negative or bi- opsies when endoscopically positive, was used as the reference standard. The results of DURS were classified as UTUC, benign lesions (BL) and no pathological findings (NPF). PPV was calculated and statistical analysis was conducted. The results of DURS were classified as UTUC, benign lesions (BL) and no pathological findings (NPF). The PPV of CTU for diagnosing UTUC was calculated as compared to DURS.

Results: The combination of CTU findings had a higher PPV compared to solitary CTU findings for the detection of UTUC (65\% vs $42 \%$, respectively; $\mathrm{p}<0.05$; OR 2.5 , 95\% CI $1.007-$

\begin{tabular}{|l|c|c|c|}
\hline CTU finding & PPV for BL & PPV for UTUC & Overall PPV \\
\hline Wall thickness & $14 \%$ & $29 \%$ & $43 \%$ \\
\hline Hydronephrosis & $60 \%$ & N/A (no cases) & $60 \%$ \\
\hline SOL & $6 \%$ & $59 \%$ & $65 \%$ \\
\hline Enhancement & $50 \%$ & N/A (no cases) & $50 \%$ \\
\hline$\geq 2$ CTU findings & $18 \%$ & $65 \%$ & $82 \%$ \\
\hline $\begin{array}{l}\text { Wall thickness and other CTU } \\
\text { findings }\end{array}$ & $55 \%$ & $27 \%$ & $82 \%$ \\
\hline $\begin{array}{l}\text { Hydronephrosis and other CTU } \\
\text { findings }\end{array}$ & $22 \%$ & $44 \%$ & $66 \%$ \\
\hline SOL and other CTU findings & $13 \%$ & $79 \%$ & $92 \%$ \\
\hline $\begin{array}{l}\text { Enhancement and other CTU } \\
\text { findings }\end{array}$ & N/A (no cases) & $68 \%$ & $68 \%$ \\
\hline SOL and contrast enhancement & N/A (no cases) & $93 \%$ & $93 \%$ \\
\hline
\end{tabular}


6.28), as well as for overall endoscopic pathological findings (82\% vs 60\%, respectively; $\mathrm{p}<0.05$; OR $3.1,95 \%$ CI 1.07-9.02). Arbitrary stratification of solitary CTU findings as minor UTUC predictors (PPV <50\%: thickening, hydronephrosis and enhancement) and major UTUC predictors (PPV $\geq 50 \%$ : filling defect) resulted in significantly better prediction for the major group ( $<<0.0$; OR 7.2, 95\% IC 1.39-38.15).

Conclusions: The best PPV of CTU diagnosis of UTUC is achieved based on a combination of findings, with filling defect appearing to be the most significant among them. In the absence of filling defect, other CTU findings, such as thickening, hydronephrosis and enhancement, are not predictive for UTUC. We suggest that the need for DURS in these cases should be reconsidered in correlation with other data (e.g., cytology, biomarkers, history of heavy smoking, recurrent hematuria, etc.). DURS remains the diagnostic standard for deciding whether or not to proceed to nephroureterectomy.

MP34-2 Isolated red patches seen during endoscopic surveillance of bladder cancer - how often should we biopsy?

N Nkwam, S Momcilovic, S Trecarten, A Bazo, G Mann, B Sherwood, R Parkinson

University Hospials of Leicester NHS Trust, Leicester, United Kingdom

United Kingdom

Introduction \& Objective: Red patches in the bladder are often seen during endoscopic surveillance of bladder cancer at cystoscopy, particularly in patients who have had intravesical BCG treatment. However, it is difficult to distinguish BCG artefact from malignancy, namely carcinoma in-situ (CIS) in the absence of narrow band imaging or photodynamic diagnostics. Therefore, can we safely assume that histologically benign persistent red patches biopsied previously within a certain timeframe will remain benign entities? Our objectives are to establish whether the regular biopsy of red patches seen during endoscopic surveillance for bladder cancer is worthwhile and determine a suitable time frame for repeat biopsy of prior histologically benign persistent red patches in patients on endoscopic surveillance for bladder cancer.

Materials and Methods: 4,805 flexible cystoscopy (FC) reports over a 12-month period (January - December 2015) were retrospectively reviewed at a UK tertiary teaching hospital and those undergoing cystoscopic surveillance for bladder cancer and found to have solitary red patches at flexible cystoscopy were included in the study. A proportion of these cases had biopsies taken which underwent histopathological analysis.

Results: 241 flexible cystoscopies performed on 183 patients on endoscopic surveillance for bladder cancer had red patches and of these, $120(49.8 \%)$ had a history of intravesical BCG therapy. Eighty-five patients $(35.3 \%)$ underwent biopsy of the red patch. Malignancy was found in 20 biopsies $(23.5 \%)$, of which, 11 out of $20(55 \%)$ were CIS. Sixteen of these recurrences had been biopsied previously of which $11(68.8 \%)$ were benign at last biopsy, 6 of which in the last 12 months. The remaining four recurrences had no previous biopsy. Eleven out of sixteen $(68.8 \%)$ of recurrences were found in patients who had been biopsied within the last 12 months. No cases of malignancy were identified in patients with low-risk bladder cancer.

Conclusions: We recommend the biopsy of all red patches found during endoscopic surveillance of patients with intermediate/ high risk bladder cancer due to the significant incidence of malignant recurrence identified, particularly if no biopsy has been performed within the previous 12 months due to the high yield of malignant recurrence identified. This is independent of previous biopsy histology.

\section{MP34-3 Withdrawn}

MP34-4 Oncologic outcomes of kidney sparing surgery using endoscopic management of upper urinary tract urothelial carcinoma

C Shen, Y Tsai, C Ou, W Yang

Department of Urology, National Cheng Kung University Hospital

Taiwan (Republic of China)

Introduction \& Objective: While radical nephroureterectomy remains standard of care for upper tract urothelial carcinoma (UTUC), it is now proposed that by using kidney sparing surgery the patients could benefit from avoiding morbidity of nephrectomy and the risk of developing chronic kidney disease or dialysis-dependent renal failure. Therefore, the nephron-sparing approach may be preferable in selected patients. We've examined the oncologic outcomes of endoscopic management of UTUC from 2004 to 2017.

Materials and Methods: A total of 22 patients who received endoscopic management of urothelial carcinoma at a single center in Taiwan were included from April 2004 to February 2017. Endoscopic approaches including semirigid ureteroscopic tumor ablation, percutaneous nephrostomy tumor ablations were

\section{Survival proportions: Survival of Progression free}

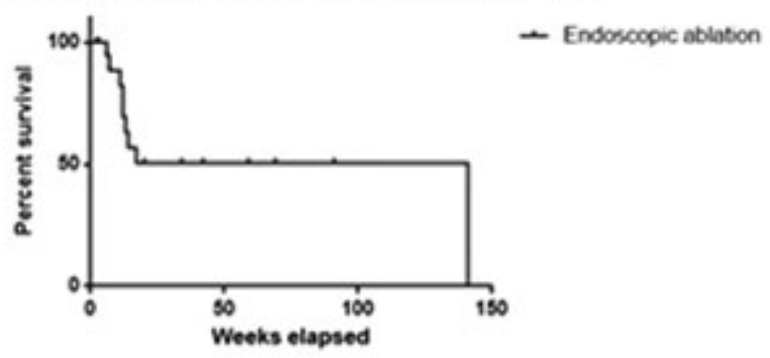

Survival proportions: Survival of Overall

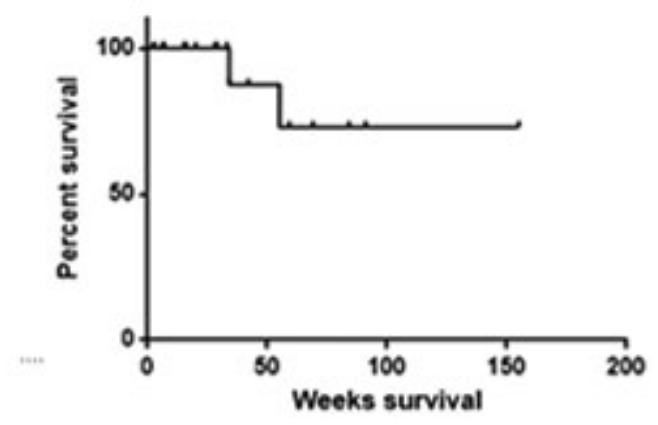

Figure 1: Progression free survival and overall survival of UTuc following endoscopic treatment? 
Survival proportions: Survival of HG progression free

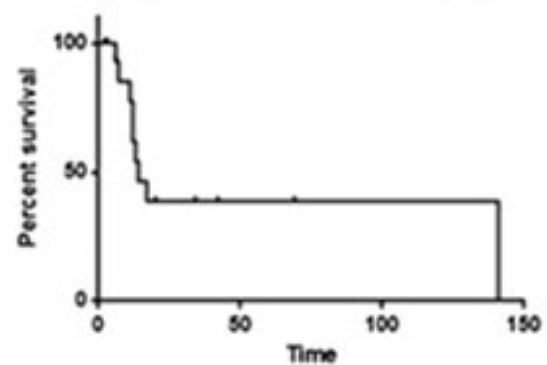

Survival proportions: Survival of LG progression free

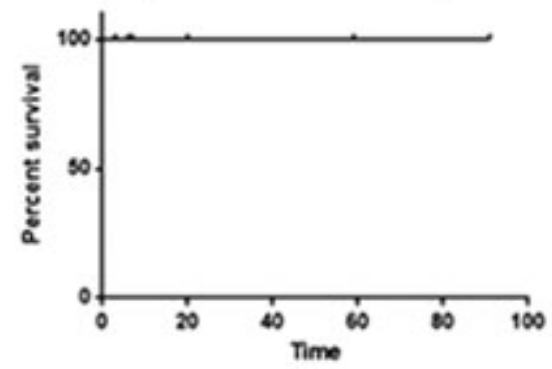

Figure 2: Progression fret sumive of high grade and low grade UnuC following endoscopic treatment .

Survival proportions: Survival of High grade Overall

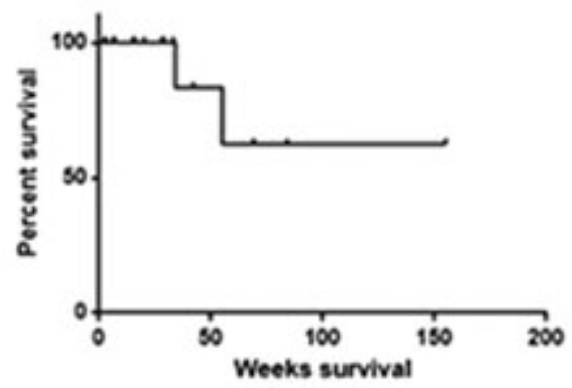

Survival proportions: Survival of Low grade Overall

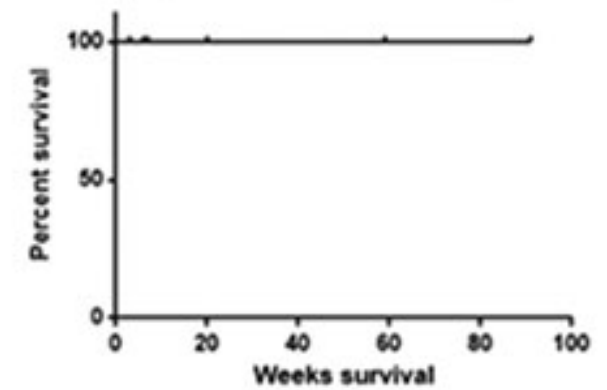

Figure 3: Overall survival of high grade and low grade UTUC following endoscopic treatment-

applied to these patients. Tumors were first biopsied then treated with fulguration, the neodymium:YAG laser and/or the holmium:YAG laser afterwards. Patients were treated and then followed on a stringent postoperative endoscopic and image protocol.

\begin{tabular}{|c|c|}
\hline 4h & \\
\hline 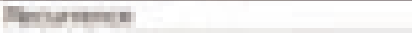 & E \\
\hline Fintar & ITharl| \\
\hline 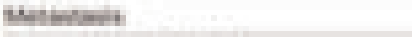 & E \\
\hline 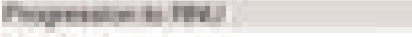 & 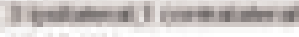 \\
\hline Hint ing & 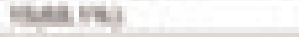 \\
\hline 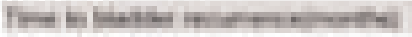 & AIII \\
\hline 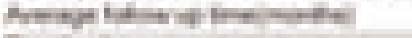 & Fill \\
\hline 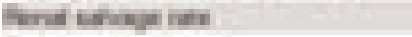 & $I 1=7$ \\
\hline 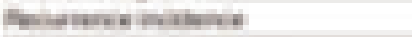 & Ellatin \\
\hline 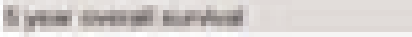 & AHE \\
\hline 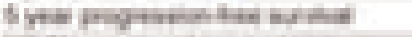 & Hin \\
\hline 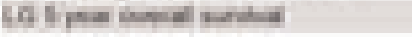 & ming \\
\hline 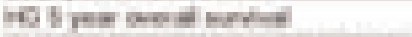 & 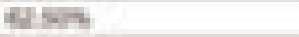 \\
\hline 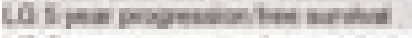 & 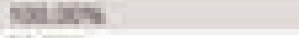 \\
\hline 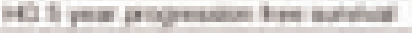 & Hent \\
\hline
\end{tabular}

\begin{tabular}{|c|c|}
\hline Patient characteristics & no. \\
\hline Age & 67.86 \\
\hline \multicolumn{2}{|l|}{ Gender } \\
\hline Female & $12(54 \%)$ \\
\hline Male & $10(46 \%)$ \\
\hline \multicolumn{2}{|l|}{ Stage } \\
\hline рTO & $2(9.0 \%)$ \\
\hline pTa & $11(50.0 \%)$ \\
\hline pT1 & $8(36.3 \%)$ \\
\hline pT2 & 0 \\
\hline рT3 & $1(4.5 \%)$ \\
\hline \multicolumn{2}{|l|}{ Grade } \\
\hline High & 16 \\
\hline Low & 6 \\
\hline Pre-operative serum creatinine & 1.91 \\
\hline Post-operative serum creatinine & 3.65 \\
\hline
\end{tabular}

Results: Of the 22 patients (10 males and 12 females) included, the mean age was $67.86 \pm 12.49$ years. Tumor pathological staging was T0 $(n=2)$, Ta $(n=11), T 1(n=8), T 2(n=0)$, and T3 $(n=1)$. Histopathology grading was low grade $(n=6)$ and high grade $(n=16) .15$ patients $(71.4 \%)$ had multifocal urothelial carcinoma by the time of endoscopic management. 9 patients had cancer progression, of which, 5 patients had bladder recurrence $(22.7 \%), 1$ had distant metastasis $(4.7 \%)$, and 4 had progression and finally underwent radical nephroureterectomy (18.1\%). No patients had local recurrence. The mean time to bladder recurrence was 16.37 months (6-31 months); the mean follow-up time was 34.75 months (2-155 months). The 5-year overall survival rate was $72.91 \%$ (19/21); 2 patients were lost to follow-up, and no patient expired. The mean 5-year progression-free survival was $50.59 \%$. The mean preoperative creatinine level was $1.91 \mathrm{mg} / \mathrm{dL}$, and at 12 months after operation it was $3.65 \mathrm{mg} / \mathrm{dL}$. Conclusions: In our experience, endoscopic management of UTUC is a feasible approach. Despite the risk of local recurrence, patients receiving endoscopic management had favorable postoperative outcomes with low rate of progression to nephroureterectomy, low local recurrence rate, and high overall survival. Besides, the benefit of renal function preservation should also be considered with this modality. It is therefore, a reasonable approach to treat selected patients with a meticulous and stringent postoperative follow up schedule. 
MP34-5 Ureteropelvic junction involvement does not predict advanced disease or clinical outcomes for patients with upper urinary tract urothelial carcinoma isolated to the ureter

K Wymer, D Gearman, A Toussi, V Sharma, T Miest, B Leibovich, M Tollefson

Mayo Clinic

United States

Introduction \& Objective: The prognostic importance of upper urinary tract urothelial carcinoma (UTUC) location is unclear. Multiple prior studies have compared renal pelvis versus ureteral involvement with varying results. However, to the best of our knowledge, the significance of location within the ureter has not yet been evaluated. Specifically, owing to the thinner muscularis layer within the ureteropelvic junction (UPJ), it may be more vulnerable to advanced disease.

Materials and Methods: We reviewed data from 395 patients who underwent radical nephrouretercomy at the Mayo Clinic from 1995-2009. Patients with non-urothelial carcinoma or UTUC involving the renal pelvis were excluded. Of the remaining 118 patients, we excluded an additional 17 who received neoadjuvant therapy. We therefore included 101 patients in our analysis. Primary tumor location was recorded. For patients with multifocal disease, the location of highest stage disease was used. Tumor characteristics, recurrence free survival, and overall survival were compared between patients with UTUC involving the UPJ and those without.

Results: We found no significant difference in tumor size or the incidence of high grade, advanced (T3/T4 or N+), or multifocal disease between the two groups (Table 1). Tumors involving the UPJ were significantly less likely to be $>3 \mathrm{~cm}(\mathrm{p}=0.02)$; however, this did not remain significant when controlling for tumor grade and stage $(\mathrm{p}=0.5)$. In regards to clinical outcomes, there

Figure 1. Recurrence-free and overall survival by UPJ involvement.

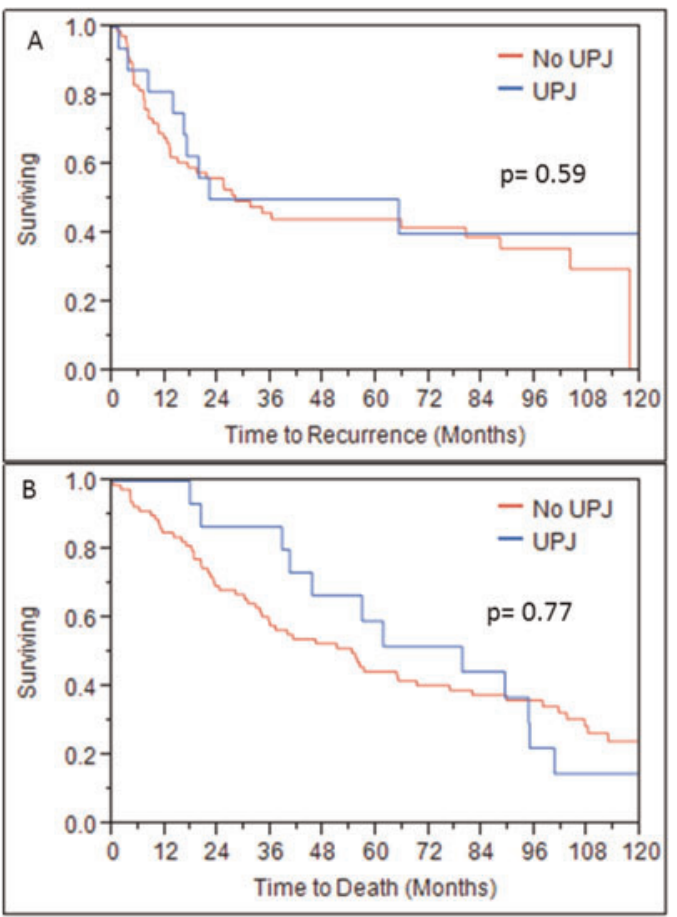

Table 1. Tumor characteristics by UPJ involvement

\begin{tabular}{|c|c|c|c|}
\hline & UPJ $(n=18)$ & No UPJ $(n=83)$ & p value \\
\hline \% Grade (No.) & & & 0.55 \\
\hline High Grade & $44.4(8)$ & $55.4(46)$ & \\
\hline Low Grade & $55.6(10)$ & $43.4(36)$ & \\
\hline Unknown & $0(0)$ & $1.2(1)$ & \\
\hline \% Stage (No.) & & & 0.66 \\
\hline $\mathrm{T} 3 / \mathrm{T} 4$ or $\mathrm{N}+$ & $27.8(5)$ & $22.9(19)$ & \\
\hline $\mathrm{Ta} / \mathrm{Tis} / \mathrm{T} 1 / \mathrm{T} 2$ and $\mathrm{N}-$ & $72.2(13)$ & $77.1(64)$ & \\
\hline Unknown & $0(0)$ & $0(0)$ & \\
\hline \% Size (No.) & & & $0.03^{*}$ \\
\hline$>3 \mathrm{~cm}$ & $16.6(3)$ & $41(34)$ & \\
\hline$<3 \mathrm{~cm}$ & $77.8(14)$ & $44.5(37)$ & \\
\hline Unknown & $5.6(1)$ & $14.5(12)$ & \\
\hline \% Multifocal (No.) & & & 0.92 \\
\hline Multifocal & $22.2(4)$ & $24.1(20)$ & \\
\hline Unifocal & $11.1(2)$ & $13.3(11)$ & \\
\hline Unknown & $66.7(12)$ & $62.7(52)$ & \\
\hline
\end{tabular}

was no significant difference in recurrence free survival or overall survival (Figure 1) between the two groups.

Conclusions: For patients with UTUC isolated to the ureter, disease location does not correlate with advanced disease, disease recurrence, or overall survival.

MP34-6 Does retrograde Double-J stenting plays a role for tumor seeding in upper urinary tract in cases of bladder urothelial malignancy?

A Sokhal, R Aeron, S Sankhwar, S Goel

king George Medical College

India

Introduction \& Objective: Urinary bladder cancer is among the leading cause of cancer related mortality in the developed world. Majority of early recurrence post cystectomy is experienced within 1-2 years and most commonly involves lung, liver or bone metastasis. Patients may present with signs and symptoms of renal failure, owing to the lower ureteral obstruction.

Materials and Methods: This was a retrospective study where records of 748 patients, diagnosed as bladder mass between January 2007 to December 2015 in the Department Of Urology, King George Medical College, Lucknow were studied. Out of 748 patients 36 patients underwent double J stenting for management of renal failure in presence of bladder mass and were included in the study. Patients having histology other than transitional cell carcinoma (TCC) of urinary bladder, having diagnosed concurrent upper tract TCC and those having incomplete 


\begin{tabular}{|l|l|}
\hline Variables & Patients (N=36) \\
\hline Age (Mean; SD) years & $48.01 \pm 18.2$ (37-70) \\
\hline Sex & \\
Male & 34 \\
Female & 2 \\
\hline BMI Kg/m ${ }^{2}$ & $22.6 \pm 4.2$ \\
\hline $\begin{array}{l}\text { Tobacco intake } \\
\text { Yes }\end{array}$ & 32 \\
No & 4 \\
\hline Comorbidity & \\
Diabetes & 7 \\
Hypertension & 5 \\
\hline Serum creatinine mg/dl (Mean; range) & $4.8(2.2-11.9)$ \\
\hline $\begin{array}{l}\text { Tumor location } \\
\text { Right }\end{array}$ & 19 \\
Left & 14 \\
Both side & 3 \\
\hline Histology & \\
High grade & 28 \\
Low grade & 8 \\
\hline Tumor stage & \\
pT1 & pT2 \\
pT3 & 2 \\
\hline Concomitant CIS & 4 \\
Yes & 30 \\
No & \\
\hline Mean follow up (Mean; range), months & $27.6 \pm 4.5$ (17-61) \\
\hline Upper tract TCC at 12 months & 2 \\
Yes & 34 \\
No & \\
\hline & 32 \\
\hline
\end{tabular}

records and follow up were excluded. Patients were analysed at follow-up visit at 3, 6 and 12 months.

Results: The mean age of the patients was $48.01 \pm 18.2$ (37-70) years. Male were $94.4 \%$. Patients were having deranged serum creatinine ranging from $2.2-11.9 \mathrm{mg} / \mathrm{dl}$. High grade was present in 28 patients $(77.8 \%)$. Patients were followed regularly with mean follow up of 27 months. At the end of 1 year, 2 patients $(5.55 \%)$ were diagnosed to have upper tract transitional cell carcinoma.

Conclusions: Urinary bladder transitional cell carcinoma has a higher tendancy to get implanted in the upper tract. However insertion of a DJ stent for renal recovery in a bladder cancer, does not increase significantly the risk of tumour implanted to the upper tract leading to upper tract TCC.

MP34-7 Tumor Location and Its Association with NonOrgan Confined Upper Tract Urothelial Carcinoma

D Gearman, A Toussi, V Sharma, T Miest, B Leibovich, M Tollefson

Mayo Clinic

United States

Introduction \& Objective: Tumor location has been identified as an important prognostic factor in patients with upper tract urothelial carcinoma (UTUC). However, controversy exists regarding which location (ureter vs. renal pelvis) is associated worse oncologic outcomes. We report our outcomes on tumor location and its association with non-organ confined disease using a large single institution database.

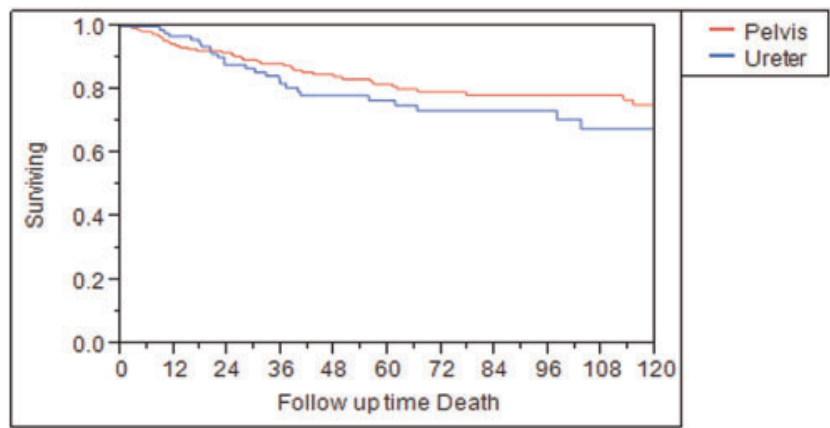

Materials and Methods: Between 1995 and 2009, we retrospectively reviewed and identified 395 patients who underwent radical nephroureterectomy (RNU) for UTUC at Mayo Clinic. We excluded patients with metastatic urothelial carcinoma (UC), preoperative chemotherapy, and tumors located within both renal pelvis and ureter, leaving 343 patients for analysis. Forward stepwise selection with a threshold of 0.1 and 0.25 was used to identify factors for multivariable analysis. Kaplan Meier analysis and Cox Proportional hazard logistic models were utilized.

Results: Patient population characteristics were similar between the two groups. Median follow-up was 34.3 months (IQR 6.990.4 months). $32.8 \%$ of patients with renal pelvis tumors had non-organ confined disease (pT3/4 and/or pN+) vs. $23.7 \%$ of patients with ureteral tumors $(\mathrm{p}=0.08)$. When controlling for pathologic grade, tumor size $(>3 \mathrm{~cm})$, and tumor multifocality, patients with renal pelvis tumors are 2.5 times more likely to have non-organ confined disease at the time of $\mathrm{RNU}(\mathrm{p}=0.009)$. Interestingly, tumor location did not impact cancer specific mortality $(\mathrm{p}=0.34)$.

Conclusions: Tumors located within the renal pelvis are associated with higher rates of non-organ confined disease. However, tumor location did not affect cancer-specific survival. Tumor location, along with other pre-operative factors such as pathologic grade, tumor size, and multifocality can be used to risk stratify patients for best initial treatment strategy.

MP34-8 NBI re-transurethral resection (reNBITURBT) for pT1HG non-muscle invasive bladder cancer : does it improve detection rate? Preliminary experience in single centre

R GENTILE, M Buscarini, M Vermiglio, B GENTILE, L Albanesi

nuova villa claudia

Italy

Introduction \& Objective: Bladder cancer has the highest incidence among all urogenital cancers, $80 \%$ of which are initially diagnosed at Ta or T1 stage or are classified as non-muscle invasive, also called no-muscle invasive bladder cancer. At pathological examination the tumour stage of $9-49 \%$ of resected lesions is understimated. EAU guidelines recommend repeat resection. The aim of this study was to evaluate the efficacy in identifying persistent bladder cancer following classic re-c White Light Transurethral Bladder Tumours (WL TURBT) regarding to re-NBITURBT.

Materials and Methods: From June 2010 to April 2012, 797 patients, 423 male and 374 female, affected by primitives or 
recurrences or suspicious bladder lesions, were underwent to WL plus NBI cystoscopy and subsequently to WL Bipolar Gyrus PK cTURBT. We observed the presence of at least one cancer in 512 patients and of them only $474(92,5 \%)$ were evaluated. We have identified 127 patients with pT1HG lesions $(26,03 \%)$. A WL repeat TUBT (re cWLTURBT) was performed 4-6 weeks after initial cWLTURBT in all the patients. At this point, a NBI recTURBT was carried out to confirm what had been seen in the white ligh resection, and to report suspicious areas with NBI light. The re-cWLTURBT was performed by the same surgeon who was involved in the initial cWLTURBT. The average of the follow-up was at 24 (16-38) months.

Results: Out of the total 127 patients treated with WL plus NBI re-cTURBT, positive tumors were found in 24 patients, and the total positive rate was $18.81 \%$. Using only re WL cTURBT, we identified 19 patients $(79.1 \%$ ) with bladder tumours, while only 5 pts. were visible by re-NBI cTURBT( $20.8 \%$, Z test $>20 \%$ ). According to logistic-regression model analysis, multiple tumors in WLTURBT $(\mathrm{p}<0.01)$ and high-graded tumor $(\mathrm{p}<0.05)$ were independent risks for positive tumors in WL re-cTURBT than multiple tumours $(\mathrm{p}<0,05)$, high-graded tumor $(\mathrm{p}<0.05)$ and tumor in $\mathrm{T} 1$ stage $(\mathrm{P}<0,05)$ were independent risks for positive tumors, in the NBI re cTURBT.

Conclusions: The authors concluded that a high rate of residual tumor presence would be found in the repeated WLcTURBT for urothelial bladder carcinoma $(18,8 \%, \mathrm{Z}$ test $>10 \%)$ and transurethral resection could increase the pathological stage accuracy. Further, re -NBIcTURBT can be a safe option, recommended in non-muscle invasive bladder cancer patients with high risks such as multiple tumor, T1 tumor or high grade carcinoma to increase detecion persistent bladder neoplasms rate.

\section{MP34-9 Withdrawn}

MP34-10 The impact of narrow band imaging (NBI) in the detection of bladder tumour in transitional cell carcinoma of the bladder: A randomized crossover controlled trial from a tertiary care centre in India

P Mukherjee, R Manojkumar, C Beri, J L, S Kumar, N Kekre, A Devasia

department of urology, christian medical college, vellore India

Introduction \& Objective: Complete trans-urethral resection of all visible tumours is the cornerstone of treatment of bladder cancer. White light (WL), although used as the standard light source, has limited specificity and sensitivity. The aim of the study was to determine the impact of narrow band imaging (NBI) in detecting tumours during resection of bladder cancer.

Materials and Methods: This was a single centre prospective interventional study with a randomized control crossover trial design. Patients with bladder tumours were randomized into two arms where they were resected under WL first followed by NBI in ARM A, or NBI followed by WL in ARM B. The number of additional tumours detected by the second light source, in both arms, was analyzed. Of 178 patients screened, 110 were randomized. There were 54 in ARM A and 56 in ARM B

Results: The number of tumors identified at the second look was 20 out of $54(37 \%)$ in ARM A. That is, NBI was able to pick up $37 \%$ percent of tumors not identified by white light. In contrast, the number of tumors picked up in ARM B was 5 out of $56(9 \%)$. That is, only $9 \%$ of tumors were identified by WL at the second look. This difference of $28 \%$ (95\% CI: $14 \%$ - 44\%) was statistically significant $(\mathrm{p}<.001)$ After resection with the first light, suspicious lesions were identified in 25 instances with the second light. Twenty were identified in ARM A, (Second light source being narrow band) Of these, 9 had tumors or CIS detected in them on histopathology. Five were identified with ARM B (Second light source being white light). None of these were malignant on histopathology. Nine additional tumours were detected by the second light source, all of which were detected in ARM A (WL followed by NBI). Therefore NBI had an odds ratio of 23 in detecting additional tumours. Of the thirteen instances of Carcinoma in situ (CIS) detected in all the resections during the study, 10 were detected by NBI (Odds Ratio 1.3).

Conclusions: Narrow band imaging is superior to white light in the detection of tumours, therefore allowing a more complete resection. NBI is also superior to WL for detecting carcinoma in situ.

MP34-11 Observing the trends in treatment options for urothelial carcinoma in the inpatient setting of New York State hospitals - between 1994-2012

KH Bilal, DS Ali, M Finkelstein, MA Palese

Icahn School of Medicine at Mount Sinai United States

Introduction \& Objective: This project studied the utilization trends of Trans Urethral Resection of Bladder Tumor (TURB, ICD9-57.49) and Radical Cystectomy (RC, ICD.9-57.71) for patients diagnosed with urothelial carcinoma in the state of New York, between 1994-2012.

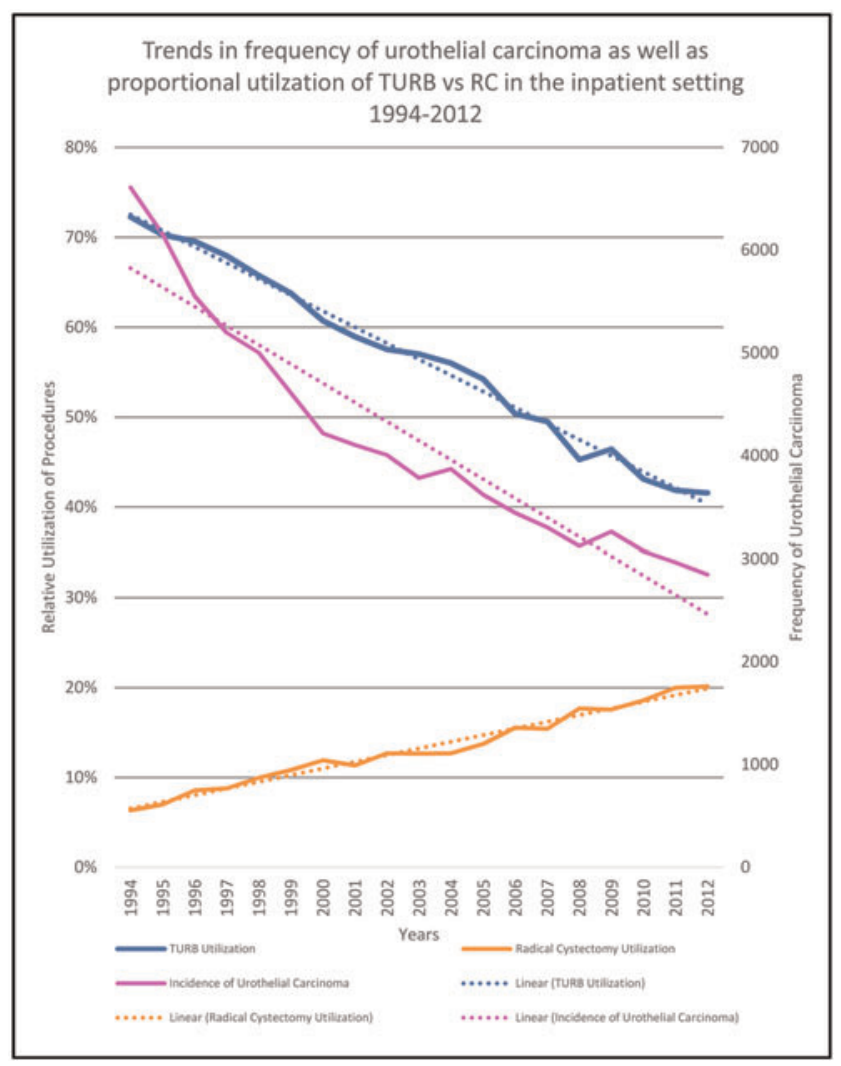


Materials and Methods: Statewide Planning and Research Cooperative Systems database of New York state was used to study 78,837 inpatient records of individuals diagnosed with urothelial carcinoma between 1994-2012 in New York State. These records were stratified based on the treatment of choice using ICD-9 procedure codes. The two most common treatment options were TURB and RC. The utilization trends were then plotted for each year between 1994-2012. Chi-square statistical analyses was conducted to assess the significance of the trends observed for both procedures and the treatment of urothelial carcinoma in the inpatient setting.

Results: During the observation period, the annual frequency of urothelial carcinoma treated in the inpatient setting decreased significantly from 6610 in 1994 to 2850 in $2012(n=9460$, $\mathrm{p}<0.04)$ within New York State. It is interesting to note that the proportion of urothelial carcinoma cases that were treated with TURB decreased significantly from $72 \%$ in 1994 to $42 \%$ in 2012 $(\mathrm{n}=5962, \mathrm{p}<0.05)$ in the inpatient setting. Moreover, within the same study period, the proportion of urothelial carcinomas that were treated with radical cystectomy increased significantly from $6 \%$ in 1994 to $21 \%$ in $2012(n=994, p<0.05)$.

Conclusions: Our results show that the frequency of urothelial carcinoma cases treated in the inpatient setting has steadily declined over the study period. We also observed a significant decrease in the proportion of cases treated with TURB between 1994 and 2012. During the same time, a significant increase was observed in the proportion of cases of urothelial carcinoma treated with radical cystectomy. Possible explanations for the trends observed include the fact that TURB is now utilized more in the outpatient urology setting. Invasive and more complex cases are likely to be admitted to the inpatient setting and are now significantly more likely to undergo radical cystectomy rather than TURB for the treatment of urothelial carcinoma.

MP34-12 Endoscopic Management of Upper Tract Urothelial Cancer: Improved Prediction of Invasive Cancer Using a Ureteroscopic Scoring Model

S Jeon, H Sung, Y Choi, H Jeon, B Jeong, S Seo, H Lee, H Choi

samsung medical center

Republic of Korea

Introduction \& Objective: There is a lack of evidence for the role of tumor depth in predicting upper tract urothelial cancer (UTUC), which is likely due to the difficulty in obtaining representative lamina muscularis tissue. We assumed that invasive UTUC should be excluded from conservative management. The current study aimed to investigate the clinical and ureteroscopic factors associated with UTUC, and to establish a model for the improved prediction of invasive UTUC using a newly proposed ureteroscopic scoring system.

Materials and Methods: We analyzed tumor depth and grade from ureteroscopic biopsies in 172 patients who underwent imaging studies, urine cytology, and radical nephroureterectomy. Invasive UTUC was defined as muscle-invasive or non-organ confined tumors (T2 or greater and/or pathologically confirmed lymphadenopathy). Ureteroscopic scoring was defined as the sum of T-staging (stromal invasion) and the grade (high). Scores of 0,1 , and 2 were assigned depending on the risk factors. The predictive accuracy of two models was compared by measuring area under the curve (AUC). Ureteroscopic scoring, hydronephrosis, local invasion on imaging, and positive urine cytology were integrated to measure the risk of muscle-invasive or nonorgan-confined UTUC. Using risk factors, a nomogram for predicting invasive UTUC was created.
Results: In the multivariate analysis, lamina propria invasion was a significant factor associated with an increased risk of invasive UTUC. Positive urine cytology, hydronephrosis, and local invasion on imaging were also significant. Presence of a highgrade tumor was not significant due to interaction with lamina propria invasion $(\mathrm{P}<0.001)$. In the ureteroscopic scoring model, the odds ratio of invasive UTUC was significantly related to the ureteroscopic scoring number $(30.9 \%$ (56/81), $66.7 \%$ (14/42), and $83.7 \%(41 / 49)$ according to the sum of risk factors 0 to 2 , respectively, $(\mathrm{P}<0.001)$. Positive predictive value (PPV) for invasive UTUC was increased in relation to the number of risk factors including urine cytology, hydronephrosis, local invasion on imaging, and any abnormal ureteroscopic finding (lamina propria invasion or presence of high-grade tumors). The PPV gradually increased as follows: $6.3 \%, 33.3 \%, 52.1 \%$, $81.6 \%$, to $92.9 \%$ for 0 to 4 positive risk factors, respectively $(\mathrm{P}<0.001)$.

Conclusions: When lamina propria invasion and presence of a high-grade tumor were incorporated, our novel ureteroscopic scoring model was highly predictive of invasive UTUC.

MP34-13 Financial Benefit of TULA (Transurethral Laser Ablation) for Recurrent Non-Muscle Invasive Bladder Cancer.

P James, A Pai, A Chetwood, T Smith, N Arumainayagam, A Shamsuddin, S Agrawal

Ashford and St Peter's Hospitals FT

United Kingdom

Introduction \& Objective: 7,700 people are diagnosed with bladder cancer each year in the UK. The management of those with low grade NMI bladder cancer costs over 35 million pounds per year. Bladder cancer is often recurrent and 5-10\% of patients will have recurrences that are small and few. Current practice within our Trust is to manage these recurrences under general anaesthetic with a TURBT procedure or cystodiathermy. This paper presents the financial case to adopt the use of a Diode laser to treat suitable patients.

Materials and Methods: We performed a resource comparison between TURBT vs TULA. The strategic rationale for TULA took into account our Tariff (the amount remunerated from Clinical Commissioning Groups (CCGs) for services provided), the release of Operating Theatre time and Bed Days, Staffing Levels, Capital Expenditure and Consumables.

Results: Activity Numbers: In 2015/16 we performed 132 TURBT procedures. Total bed days assigned to TURBT amounted to 282 (average of 2.1 days per case). The procedure involves GA and use of a fully staffed operating theatre. It takes approximately 90 minutes. 50 of these cases (35-40\%) were deemed suitable for outpatient treatment with the Diode Laser.

Financials: TURBT has a $16 / 17$ national tariff of $£ 1608$. Outpatient laser procedures have a $16 / 17$ tariff of $£ 979$. If 50 cases can be converted from IP (at £1608) to OP(£979) then the saving to commissioners would be about $£ 31,450$

Release of Theatre Time and Beds: Total surgical time for TURBT is 90 minutes. TULA takes about 30 minutes. Converting 50 cases from IP to OP would release 50 hours of theatre time. The average cost of theatre time is estimated at $£ 940$ per hour. Releasing 50 hours of time would release $£ 47,000$. Additionally, converting 50 cases from IP to OP would release about 100 bed days per year. The average cost is estimated at $£ 245$ a day. This would release $£ 24,500$. In total, $£ 71,500$ worth of theatre time and ward resources would be released. Allowing for 
the reduction in income of $£ 31,450$, this represents a release of about $£ 40,000$ for the Trust due to lower treatment costs. Allowing for the cost of single-use laser fibres (at $£ 160$ per patient) and nursing staff costs of $£ 8,000$ a year for the outpatient procedure, the net benefit to the Trust is about $£ 24,000$ a year.

Conclusions: The financial case for TULA of NMI Bladder Cancer is strong. This procedure has the potential to alleviate demand on theatre time and hospital beds, reduce commissioners' costs, and also improve the financial efficiency of our Trust.

MP34-14 Is suitable today to use only the WLTURBT for a correct stadiation of a non muscle invasive bladder cancer. Preliminary experience in high-flow centre with the use of WL TURBT plus repeat NBI TURBT

R GENTILE, M Vermiglio, M Buscarini, B GENTILE, L Albanesi, P Tariciotti

nuova villa claudia

Italy

Introduction \& Objective: A single centre, prospective trial was performed trying to assess the impact of NBI TURBT( repeat NBI TURBT) in cases of non-muscle invasive bladder tumors (NMIBT) by comparison to the standard approach (WLTURBT). Our goal was to determine the superiority of the new method in terms of detection rates and subsequent postoperative treatment changes.

Materials and Methods: A total of 797 NMIBT suspected consecutive cases were enrolled in the study. The inclusion criteria were represented by hematuria, positive urinary cytology and/or ultrasound suspicion of bladder tumors. All patients underwent WLC and NBI cystoscopy Standard transurethral resection of bladder tumors was performed for all lesions visible in WL (WL TURBT) and following, the same surgeon, performed NBI guided resection on the margins and the bottom (repeat NBI TURBT).

Results: The overall persistent NMIBT detection rates were significantly improved for the NBI evaluation (454 lesions in 303 pts on the margins, 201 lesions in 151 pts on the bottom and 129 lesion margins plus bottom) ((Tab 1, 2 and 3). Regarding to positive with WL or NBI initial cystoscopy we observed a significant improvement following repeat NBI TURBT detection rate $(\mathrm{p}<0,05)$. Additional tumors were diagnosed by NBI repeat TURBT in a significantly higher proportion of CIS, pTa, pT1 tumours(see the table). As a result of these supplementary findings, the postoperative treatment was significantly improved in a substantial proportion of cases.

Conclusions: NBI cystoscopy represents a valuable diagnostic alternative in NMIBT patients, with significant improvement of tumor visual accuracy as well as detection rates. This approach provided a substantial amelioration to the risk category stratification and subsequent bladder cancer therapeutic management.

MP34-15 Template-based laparoscopic lymphadenectomy combined with laparoscopic nephroureterectomy for renal pelvic and proximal ureter cancer: examination of safety and adequacy

K Araki, K Ohotsuka, A Fujimoto, K Hou, T Kato, T Suyama, H Masuda, S Kojima, Y Naya, T Ichikawa

Teikyo University Chiba Medical Center Japan
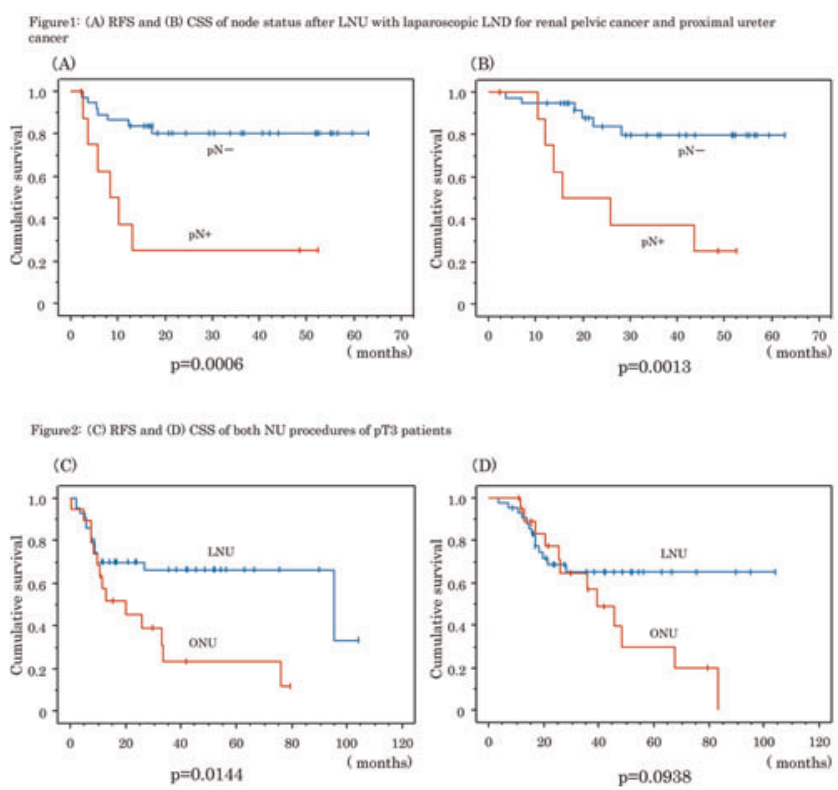

Introduction \& Objective: To assess the quality and dequacy of laparoscopic template- based lymph node dissection (LND) combined with laparoscopic nephroureterectomy (LNU) for renal pelvic cancer and proximal ureter cancer.

Materials and Methods: A total of 219 patients who had undergone nephroureterectomy for upper urinary tract cancer (UUTC) from 2007 to 2016 were identified. Of these, 53 patients were treated with laparoscopic template based LND with LNU. We examined the quality and adequacy of this procedure.

Results: The time needed for LND was $50.1 \pm 21.0 \mathrm{~min}$ (right side, $63.5 \pm 16.7 \mathrm{~min}$, left side, $42.6 \pm 19.5 \mathrm{~min}[\mathrm{p}=0.030])$. The number of dissected lymph nodes was $13.8 \pm 6.3$ (right side, $12.9 \pm 5.9$, left side, $14.5 \pm 6.3[\mathrm{p}=0.3178])$. No major complications were observed. There was a statistically significant difference in recurrence free survival (FRS) and cancer specific survival (CSS) between node negative and node positive patients ( $p=0.0016$ and, $p=0.0009$ for RFS and CSS, respectively).

Conclusions: Our procedure of laparoscopic LND combined with LNU is sufficient in terms of quality and adequacy.

MP34-16 Oncologic Outcomes Following Minimally-Invasive Radical Nephroureterectomy for Upper Tract Urothelial Carcinoma

A Toussi, T Miest, G Chow, B Leibovich, M Tollefson

United States

Introduction \& Objective: Open radical nephroureterectomy (ORNU) with excision of the bladder cuff remains the standard treatment for upper tract urothelial carcinoma (UTUC). Meanwhile, evidence to support the use of a minimally invasive approach has been variable with conflicting outcomes. Herein, we report oncologic outcomes for three different surgical approaches to radical nephroureterectomy (RNU) using a large single-center database.

Materials and Methods: We reviewed 524 patients who underwent RNU at Mayo Clinic between 1995 and 2009. Patients 


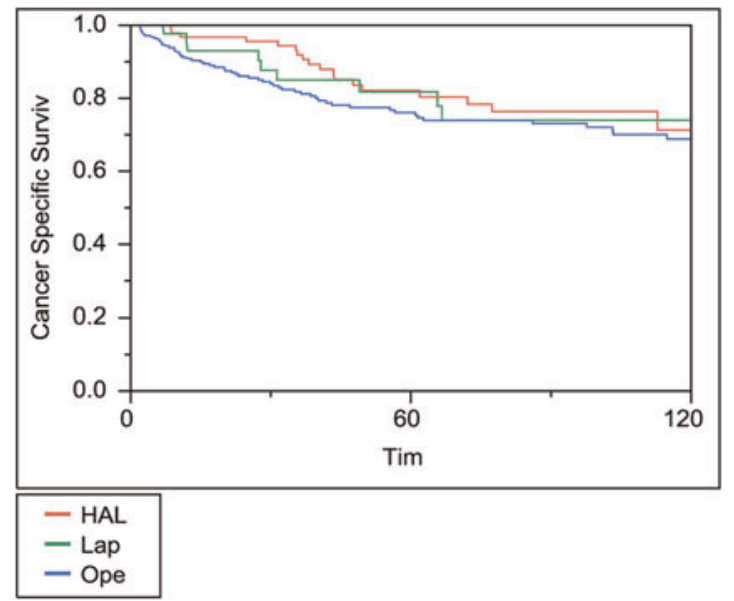

were divided into open (ORNU), laparoscopic (LRNU) and hand-assist laparoscopic (HaRNU) Patients with metastatic disease at RNU, neoadjuvant chemotherapy, and non-urothelial primary were excluded. Multivariable Cox Proportional regression modeling and Kaplan-Meier analysis was used for statistical analysis.

Results: Median age at RNU was 73 years (IQR 65.2, 78.6) and $67.8 \%(\mathrm{n}=283)$ were male. A total of $63 \%, 26 \%$ and $11 \%$ underwent ORNU, HaRNU and LRNU, respectively. Median follow up time was 43 months (IQR 17.2, 100.2). Lymphadenectomy was performed most commonly during ORNU (28.7\%) compared to HaRNU $(24.9 \%)$ and LRNU $(8.7 \%)(\mathrm{p}<0.01)$. For HaRNU, LRNU and ORNU there was no statistically significant difference in 10 -year CSM (28.7\% vs $26.0 \%$ vs $31.2 \%$; $\mathrm{p}=0.37$ ) (Figure 1) or 5 -year RFS (36.8\% vs $52.9 \%$ vs $42.2 \%$; $\mathrm{p}=0.11$ ), respectively. On multivariable analysis features independently associated with increased CSM included smoking history (HR 2.78; $<<0.01$ ), high tumor grade (HR 5.65; p <0.001), pT2 or higher (HR 2.93; p<0.01), lymph node positivity (HR 3.59; $\mathrm{p}=0.02)$ and tumor size $>3 \mathrm{~cm}(\mathrm{HR} 2.27 ; \mathrm{p}=0.02)$. There was no increased risk of CSM associated with HaRNU vs ORNU (HR $0.48 ; \mathrm{p}=0.06$ ) or LRNU vs ORNU (HR 1.14; $\mathrm{p}=0.80$ ).

Conclusions: Surgical approach was not significantly associated with cancer-specific mortality. As such, a minimally-invasive approach appears to be an oncologically safe option for appropriately selected patients with UTUC.

MP34-17 The use of homemade device for hand-assisted retroperitoneoscopic nephroureterectomy

T Tai

National Cheng Kung University Hospital

Taiwan (Republic of China)

Introduction \& Objective: To conduct hand-assisted retroperitoneoscopic nephroureterectomy with homemade design hand port device (modified from our previous design).

Materials and Methods: Twenty patients with upper tract urothelial cancer received hand-assisted retroperitoneoscopic nephroureterectomy. The procedures were carried out using a homemade hand-assist device comprising a medium sized Alexis wound retractor and surgical gloves. The Alexis wound retractor was positioned through a $7 \mathrm{~cm}$ Gibson incision ready for use. The

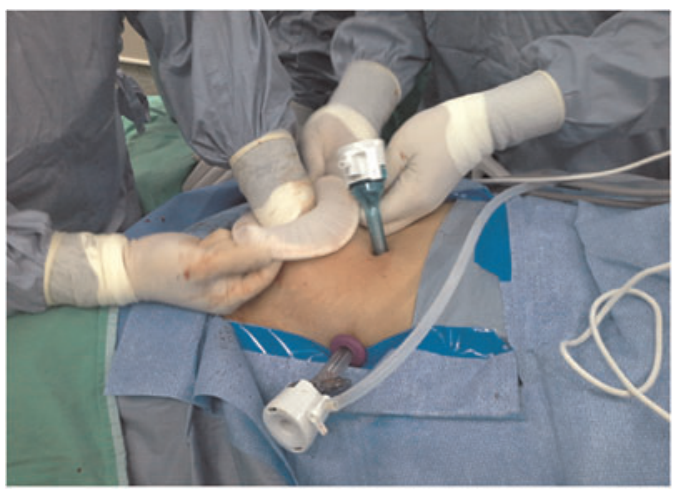

surgeon inserted his double-gloved, non-dominant hand into the retroperitoneal space via the wound retractor. During the procedure, the cuff of outer surgical glove was turned outside-in, and snapped onto the external ring of the Alexis wound. Gently grasp the white retractor ring and cuff of surgical glove at 3 and 9 o'clock. Roll outward until desired retractor is achieved to prevent the surgical glove slipping from the external ring of Alexis wound retractor during pneumoretroperitoneum (new modification). We successfully created pneumoretroperitoneum by insufflating with carbon dioxide at $15 \mathrm{~mm} \mathrm{Hg}$. The procedure was carried out through a $7 \mathrm{~cm}$ Gibson incision, and two additional laparoscopic ports

Results: All procedures were performed without complication. The mean estimated blood loss was $100 \mathrm{~mL}$. The mean operation time was 95 minutes.

Conclusions: Our preliminary results show that carrying out hand-assisted retroperitoneoscopic nephroureterectomy using a homemade hand-assist device is safe and feasible. Homemade hand-assist device offers a cost reduction for the hand assisted laparoscopic procedure compared with commercially available devices.

MP34-18 Does the Xi da Vinci robot reduce operative time of nephro-ureterctomy comparaired with the Si system?

L Vouga, G Wirth, C Iselin

Urology

Switzerland

Introduction \& Objective: Robot-assistance during radical nephro-ureterectomy has allowed adopting a purely laparoscopic approach, rendering a lower quadrant incision obsolete. One major drawback of the Da Vinci Si system was the need to redock the robot during the procedure. This step can be avoided with the newer generation of the robotic system (Xi), which encompasses a larger operative field. The aim of this study was to compare feasibility and outcomes of robot-assisted, radical nephro-ureterectomy (RRNU) in all consecutive patients treated either with the Si or Xi system in a single center.

Materials and Methods: All patients treated between April 2014 and October 2016 by on resident an two surgeons well over their learning curves (>300 and 500 cases of robot-assisted surgery in both) were included. Indication for surgery, operating time, surgical margins, complications according to ClavienDindo and hospital stay were collected prospectively. Standard parametric and non-parametric tests were employed to compare outcomes. 
Results: Thirteen patients underwent RRNU, 6 with the Si system requiring robot redocking, 7 with the $\mathrm{Xi}$ system. In 8 of the 13 procedures, a resident in training performed part of the operation. Side distribution was even (6 left and 7 right). Indications were tumors of the upper urinary tract in 11 patients, atrophic kidney with massively dilated ureters due to endometriosis and tuberculosis in the remaining two. Median operative time was significantly shorter in patients operated with the $\mathrm{Xi}$ system (303 min (IQR 297-365) versus $383 \mathrm{~min}$ (337-436); $\mathrm{p}=0.036$ ). All operations were completed as planned apart for one patient in whom distal ureterectomy was not performed due to multiple adhesions caused by a previous urinomas. In all other cancer patients, a bladder cuff was removed. No urine spillage occurred. Tumor stage demographics was: 5 pTa, 1 pT1, 1 pT2, 4 pT3. Surgical margins were all negative. Complication rates were similar in both groups and were of low grade: no complication Clavien $\geq 3,2$ complications Clavien 1 in both groups, 1 complication Clavien 2 in the Xi group. Median hospital stay was similar in both groups (4.5 (IQR 4-6.6) versus 6 (IQR 4.58.75 days; $p=0.17$ with $\mathrm{Si}$ and $\mathrm{Xi}$, respectively).

Conclusions: In this series of consecutively treated patients, the $\mathrm{Xi}$ system achieved slightly shorter operative times, however with statistical significance. This may be attributed to the fact that repositioning and redocking of the robot became obsolete with the $\mathrm{Xi}$ system. Procedures were safe and yielded only minor complications, whitnessing the minimally invasive benefit of the technique.

\section{MP34-19 Rigid Ureteroscopic resection of bulky ureteral tumor}

JH Hillelsohn, M Eshghi, A Schulman, NH Patel, SA Fullerton

New York Medical College

United States

Introduction \& Objective: Bulky urothelial tumors of the ureter typically need a rigid ureteroscopy (uretero-resectoscope) in order to be adequately staged and treated. Changes in rigid ureteroscopic design have allowed larger lesions that typically would need to be treated with a nephroureterectomy to be approached in a retrograde fashion.

Materials and Methods: We will review 25 cases selected from our experience in the last 10 years of bulkly urothelial tumors treated in a retrograde fashion with a uretero-resectoscope.

Results: We plan to show in outr video, techniques and tips for resection of bulky ureteral tumors. Specifically we will review the rigid uretero-resectoscopes available in practice and outline when each should be used. We will also outline the technique for resection depending on the location of the tumor.

Conclusions: Our brief video presentation will show that bulky ureteral tumors can be treated successfully with a minimally invasive approach avoiding more invasive surgical intervention in many cases.

MP34-20 Clinical Assessment Of The Durability Of A Single-Use Digital Flexible Ureteroscope For The Treatment Of Large Stone Burden Or Extensive Upper Tract Urothelial Carcinoma

G Portman, J Stites, B Press, R Munver

Hackensack University Medical Center

United States
Introduction \& Objective: Flexible ureteroscopy is an important procedure in endourology for both stone treatment and upper tract urothelial carcinoma diagnosis and treatment. However, there are instances when using a reusable digital ureteroscope can subject it to significant stress and damage, risking the need for future repair. A single-use digital flexible ureteroscope has been developed, which may potentially preserve the life of reusable digital ureteroscopes. The purpose of this study was to evaluate the durability of a single-use digital flexible ureteroscope (LithoVue ${ }^{\mathrm{TM}}$ Single-use Digital Flexible Ureteroscope, Boston Scientific Corporation, Marlborough, MA) in patients with large stone burden or for the treatment of extensive upper tract urothelial carcinoma.

Materials and Methods: A retrospective review was performed of all cases utilizing LithoVue ${ }^{\mathrm{TM}}$ Single-use Digital Flexible Ureteroscope at a single institution. The inclusion criteria for this study were a large stone burden $(\geq 1.5 \mathrm{~cm})$ or extensive ureteral/ renal urothelial carcinoma with expected operative duration (> 1 hour). Perioperative data were analyzed, including number of scopes used, postoperative visual inspection, image quality, and deflection.

Results: A total of 18 ureteroscopy procedures met inclusion criteria which included 20 renal units. Mean operative time was 74 minutes (range 42-117). Ureteroscopy was performed for treatment of 15 patients with ureteral stones/renal stones with a mean stone burden of $2.2 \mathrm{~cm}$ (range $1.5-3.0 \mathrm{~cm}$ ), and 3 patients with ureteral/renal pelvis or calyceal tumors. Ureteral access sheaths were used for 17 of renal units, and a ureteral stent was left in $100 \%$ of renal units. The mean Holmium:YAG energy usage was $18.3 \mathrm{~kJ}$ (range 6.6-40.1). There were no major surgical complications and 1 case of technical equipment malfunction (monitor flickering) that did not preclude completion of the case. Visual inspection revealed electrocautery damage to the tip of the ureteroscope in 3 cases, but without compromise of the digital image during the case. Scope deflection in both directions was preserved at the conclusion of all cases.

Conclusions: The LithoVue ${ }^{\mathrm{TM}}$ Single-use Digital Flexible Ureteroscope was used successfully for complex ureteroscopic cases in place of a reusable ureteroscope, and proved to be durable in all cases. A single-use ureteroscope may be a reasonable alternative to subjecting the more expensive reusable ureteroscope to extensive stress and damage, while potentially resulting in cost savings.

MP34-21 Partial cystectomy for bladder urothelial cancer: single medical center experience

L Tsai, P Hsiao, Y Chi-Rei, G Chen, S Chou

Department of Urology, China Medical University Hospital, Taichung, Taiwan

Taiwan (Republic of China)

Introduction \& Objective: Radical cystectomy with pelvic lymph node dissection remains the gold standard of invasive bladder urothelial carcinoma. However, radical cystectomy with urinary diversion carries a high risk of perioperative complication and has a major impact on each patient's quality of life. Partial cystectomy is though a shorter, less adverse effect compared with the radical operation and do not require urinary diversion. Recent studies also reported acceptable oncologic outcomes in carefully selected patients while offering improved quality of life. We retrospectively reviewed and analyzed all cases in a single medical center. 
Materials and Methods: Patients who underwent partial cystectomy for primary bladder cancer from 2001 to 2016 were identified from our database. Clinical, pathological and followup data were reviewed.

Results: A total 22 patients received partial cystectomy due to bladder urothelial cancer were included in the study. There were 18 males and 4 females patients. Median age was 62 years old. The tumor positions were lateral, anterior, posterior, dome, bladder neck and just near UO: 8, 2, 6, 6, 3 and 2, respectively. Nineteen patients were categorized into clinical stage T2NOM0 and other 3 patients were staged as clinical stage T2N1M1, T1N0M0, and TaN0M0. Neoadjuvant chemotherapy was performed on 9 patients. Fourteen patients received open method operation while 3 and 5 patients received laparoscopic and robotic surgery, respectively. The average blood loss during the operation was $163 \mathrm{ml}$. Ten patients reach ypT0 on the final pathology report. Bladder recurrence was noted in 8 patients during follow-up including five Tcis and three Ta bladder tumors. There were two patients developed lymph metastasis or visceral metastasis. Mean recurrence-free survival for these patients were $14 \pm 13$ months. One of the 22 patients died of bladder cancer with multiple visceral metastases. Whether patients received neoadjuvant chemotherapy or achieved final pathology stage ypT0 seem reduced bladder recurrence rate but not to a significant level $(\mathrm{P}=0.09,0.4$, respectively). Several risk factors may lead to recurrences such as multiple tumors, the tumor located in diverticulum or ureteral orifice.

Conclusions: Patients treated with partial cystectomy for primary bladder cancer had satisfactory cancer control and favorable perioperative morbidity consistent with other contemporary reports. There was still no consensus about patient selection criteria for partial cystectomy. Prospective, randomized studies including patients selection and comparison between bladder sparing treatment modalities and radical cystectomy are needed in the future.

MP34-22 Initial experience with laparoscopic radical cystectomy and evaluation of operative and oncologic outcomes

K Ohba, T Kondo, K Araki, H Nakanishi, Y Sagara, T Matsuo, Y Miyata, H Sakai

Department of Urology and Renal Transplantation, Nagasaki University Hospital

Japan

Introduction \& Objective: Radical cystectomy is the standard treatment for muscle-invasive urothelial carcinoma (UC). However, high complication and mortality rates have been reported. Although laparoscopic radical cystectomy (LRC) is a minimally invasive procedure, it is technically challenging. Furthermore, little information is available regarding the perioperative and oncologic outcomes of LRC. We assessed our technique and outcomes of LRC and urinary diversion for the management of UC. Materials and Methods: We retrospectively evaluated 26 consecutive patients who underwent LRC for treatment of UC from March 2014 to March 2017 at a single institution. The LRC procedure was started by placing five ports and positioning the patient with the head down at an angle of $30^{\circ}$. After assessing the pelvic organ anatomy, the bladder was removed with the prostate or uterus. The obturator; external, internal, and common iliac; and presacral lymph nodes were dissected. Urinary diversions were performed via a minilaparotomy technique.
Results: The median patient age was 79.0 (range, 62-84) years, the median body mass index was 23.31 (range, 17.44-27.69) kg/ $\mathrm{m} 2$, and the median Charlson comorbidity index was 3.33. Prior to LRC, 19 patients underwent neoadjuvant chemotherapy, 10 underwent low abdominal open surgery, and 1 underwent radiation therapy. The median operative time was 420 (range, 286570) min, and the median estimated blood loss volume was 475 (range, 130-1150) ml. Nine patients underwent urethral resection, and one patient underwent neobladder construction. The pathological stage was $\leq \mathrm{pT} 2$ in 9 patients and $\geq \mathrm{pT} 3$ in 17 . Three patients had lymph node involvement. The estimated 2-year cancer-specific survival rate was 64\%. Major postoperative complications were ureteral stenosis in one patient and ileal anastomosis failure in another.

Conclusions: LRC appears to be a favorable procedure with a low complication rate and acceptable operative and oncologic outcomes in patients with UC.

\section{MP34-23 Comparison of Monopolar and Bipolar Trans- urethral Resection of Bladder Tumor: A Randomized Controlled Trial}

SK Singh, M Pragatheeswarane, SK Devana, RS Mavuduru, N Kakkar, S Kumar, AK Mandal

Post Graduate Institute of Medical Education \& Research, Chandigarh, India

India

Introduction \& Objective: Monopolar Transurethral resection of Bladder Tumor (TURBT) is considered the gold standard in the surgical treatment of non-muscle invasive bladder tumors. It is associated with obturator jerk, bladder perforation and charring of the tissue. The study was conducted to compare the above limitations of Monopolar TURBT (MTURBT) with Bipolar TURBT (BTURBT).

Materials and Methods: The patients undergoing TURBT from July 2015 and October 2016 were randomized into two groups. Block randomization was performed with each block comprising of 10. Allocation was concealed in sealed envelope and implemented by operating room technician. Patients, pathologist and statistician were blinded regarding the allocation. All patients were operated under regional anaesthesia. BTURBT was performed by using a bipolar electrocautery with a 26 Fr resectoscope. For BTURBT power settings with automatic adjustment were used delivering 160 to $200 \mathrm{~W}$ for cutting and 100 to $120 \mathrm{~W}$ for coagulation. MTURBT was performed by using a standard 26 Fr resectoscope in the power setting with energy levels of 70 to $90 \mathrm{~W}$ for cutting and 30 to $60 \mathrm{~W}$ for coagulation. The intraoperative and postoperative events were noted. The TURBT chips were evaluated for both cautery artefacts as well as for thermal damage and were categorized from Grade 0 to 3 depending on degree of involvement of the specimen with artefact and thermal damage.

Results: Total 75 patients underwent BTURBT and 70 underwent MTURBT. Both the groups were comparable for demographic profiles including age, gender, co morbidities, ASA grade, tumor size, tumor location and resection time. The incidence of obturator jerk was not significantly higher in MTURBT group $\{8 / 70(11 \%)$ Vs $13 / 75(17 \%), p=0.325\}$. The bladder perforation rate was also not significantly higher (4/70 Vs $1 / 75$, $\mathrm{p}=0.192$ ). The cautery artefacts was not observed in significantly high proportion of patients in bipolar group (22/75 Vs 3/ 
$70, p<0.001)$. Grade 3 thermal damage was observed in significantly high proportion of patients in Monopolar group (12/70 Vs $3 / 75, \mathrm{p}=0.024)$

Conclusions: Bipolar TURBT is not superior to monopolar TURBT and is not devoid of obturator jerk and bladder perfo- ration. The less cautery artefacts and thermal damage may be an added advantage of Bipolar TURBT which may help the pathologist in better interpretation of histopathological grading and invasion of bladder tumor. However, its clinical significance is not established as yet.

\section{MPS35: ENDOSCOPY AND RECONSTRUCTIVE SURGERY}

\section{MP35-1 Laparoscopic assisted ileal ureter}

B Komyakov, B Guliev, A Shipilov

North-West medical university by Mechnikov Russia

Introduction \& Objective: The intestinal ureteral substitution is an acceptable procedure in patients with long ureteral defects. The aim of this study is to evaluate the long-term results and effectiveness of laparoscopic ileal ureteral replacement.

Materials and Methods: From 2001 to 2016, 90 patients underwent ileal ureteral substitution at our clinic. 12 (13,3\%) patients underwent laparoscopic reconstruction of ureter. There were $62(68,9 \%)$ women and $28(31,1 \%)$ men with a median age of 50,5 $\pm 9,5$ years (range from 18 to 69 years). The main causes of ureteral strictures were: retroperitoneal fibrosis following radiation therapy, colorectal and gynecological cancer surgery. To this study included the results of ileal ureteral substitution in 27 patients. In 15 patients we used open, in 12 patients used laparoscopic approach. Technique of operation (mobilization of ureter, ileal resection, creation of ureteroileal and ileobladder anastomoses) was similar in the both groups. We performed comparative analysis parameters, such as operative time, blood loss, narcotic analgesic, hospital stay and time to convalescence. Outpatient visits occurred at 3 and 6 months postoperatively and at least annually thereafter.

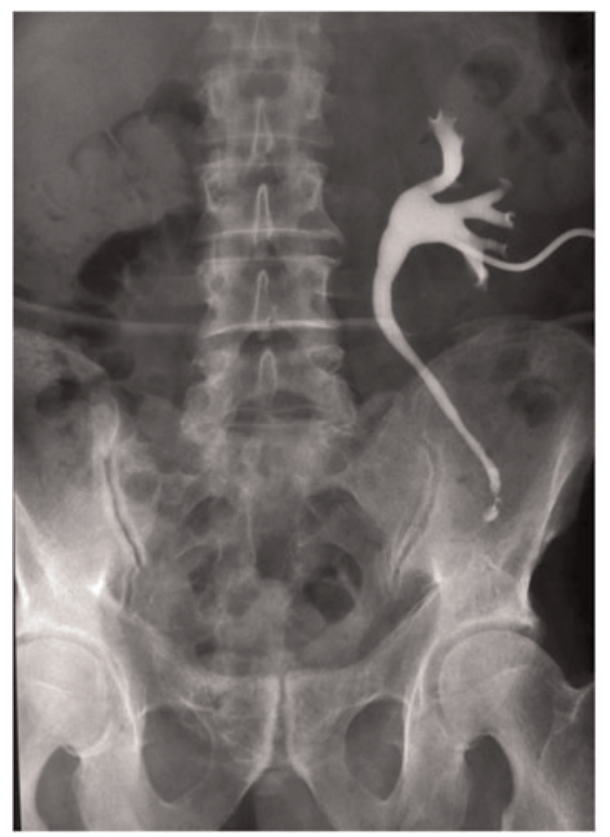

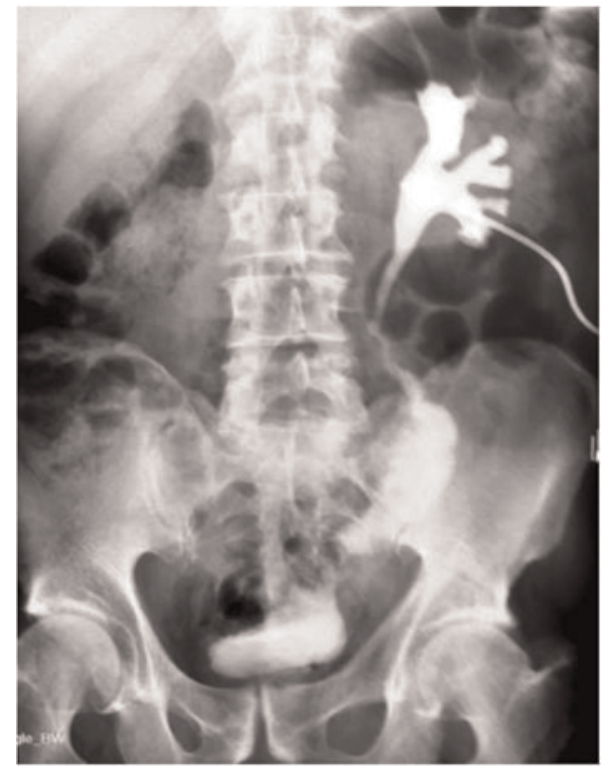

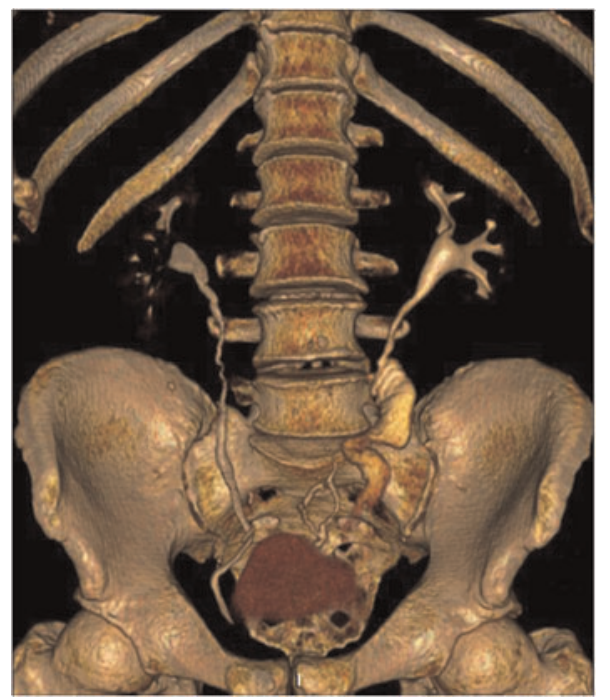

Results: No conversion in patients who were underwent laparoscopic ileal replacement of ureter. Operative time was longer for $70 \mathrm{~min}$ in laparoscopic group, but this difference wasn't significantly $(\mathrm{p}<0,05)$. Narcotic analgesic use was dramatically less for the laparoscopic group than for the open group (40 mg 
versus $240 \mathrm{mg}, \mathrm{p}<0,05)$. Hospital stay was significantly less in laparoscopic group $(6 \mathrm{~d}$ versus $9 \mathrm{~d}, \mathrm{p}<0,05)$. Preoperative and postoperative serum creatinine, bicarbonate and chloride levels were unchanged for the laparoscopic and open groups. Moderate metabolic acidosis developed in 1 patient in open group. Time to convalescence was significantly less for the laparoscopic group (4 weeks versus 5,5 weeks, $\mathrm{p}<0,05$ ). Figures $1-3$ demonstrate results of laparoscopic ileal substitution by ileum in patient with extended stricture of left ureter.

Conclusions: Ileal ureteral substitution using laparoscopic approach is an effective and minimally invasive procedure. Compared with open procedures this technique allows to decrease trauma of surgery, hospital stay and time convalescence.

MP35-2 Five year outcomes data utilizing delayed barbed absorbable suture for robotic sacrocolpopexy

\section{S Castellucci}

Manatee Hospital, Bradenton Florida United States

Introduction \& Objective: Pelvic organ prolapse (POP), is the herniation of the pelvic organs to or beyond the vaginal walls that impact daily activities, sexual function, and exercise. Abdominal sacrocolpopexy is currently considered the gold standard for repair due to superior outcomes and lower recurrence rates. The utilization of barbed delayed absorbable sutures for repair have been discussed but there is limited data available. We now present 5-year outcomes data of robotic sacrocolpopexy for pelvic organ prolapse using the V-loc barbed suture in comparison to interrupted sutures utilizing Gortex sutures.

Materials and Methods: This study includes a total of 45 patients with symptomatic grade 3 or 4 POP defects classified according to the Baden-Walker classification system. The repair was completed through RSC between 2012 and 2017 giving up to 5 year follow up data. Procedures were completed by the same physician (SAC), at the same facility Manatee Memorial Hospital in Bradenton, FL. The first 5 patients had their procedure completed utilizing Gortex interrupted sutures, whereas the remaining 40 were completed utilizing the barbed V-loc suture. All demographic characteristics were recorded including age, race, body mass index (BMI), and prior abdominal surgeries. Patients charts were also reviewed to assess for any need for further POP procedures. All procedure was performed using the Boston Scientific Upsylon Y-mesh.

Results: There was no statistical significance seen when evaluating age, race, BMI, or prior abdominal surgeries. Out of the 5 patients who had their procedure completed with Gortex interrupted sutures, 1 (20\%) did have recurrence of their prolapse that was apical whereas out of the 40 patients who had their procedure completed with V-loc sutures, 1 (2.5\%) had recurrence of predominant rectocele prolapse requiring retreatment. The patient with recurrence and had her procedure completed with the Gortex sutures was predominantly apical and anterior, whereas the patient completed with the V-loc was predominantly rectocele. This did demonstrate statistical significance with $\mathrm{P}<0.05$. Eight $(5.6 \%)$ patients underwent concomitant hysterectomy, and $15(30 \%)$ underwent anti-incontinence surgery and there was no statistical significance between the groups. Average mesh suturing times between the groups were statistically significant $(\mathrm{P}<0.05)$ with the patients having Gortex sutures averaging $50+/-12.4$, and the $\mathrm{V}$-loc group averaging $20+/-5.4$. Follow-up ranges from 3 months to 60 months with a mean follow-up time of 22 months.
Conclusions: This study is the first to look into 5 year efficacy utilizing the Gortex sutures in comparison to the V-loc delayed absorbable sutures. According to our results utilization of the Vloc sutures shows statistical significance in decreasing operative time for mesh suturing and for recurrence. Therefore these delayed absorbable sutures are both safe and effective for RSC procedures with 5 year data. Utilization of this technique can improve operative times without adverse patient consequences.

MP35-3 Minimally Invasive Renal Artery Aneurysm Repair: Initial Case Series

S Chopra, A Abreu, C Fay, A Aron, M Desai, M Aron, R Sotelo, IS Gill

USC institute of urology

United States

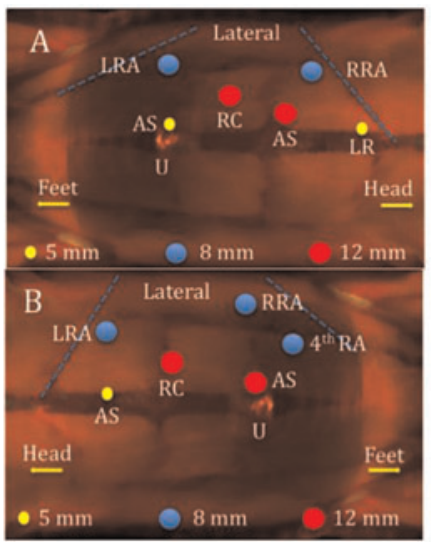

Figure 1: Port placement for robotic RAA repair for right sided (A) and left sided (B) surgery.

Legends: RRA - right robotic arm, LRA - left robotic arm, AS - assistant port, RC robotic camera port, $\mathrm{LR}$ - liver retractor port, $\mathrm{U}$ - umbilicus
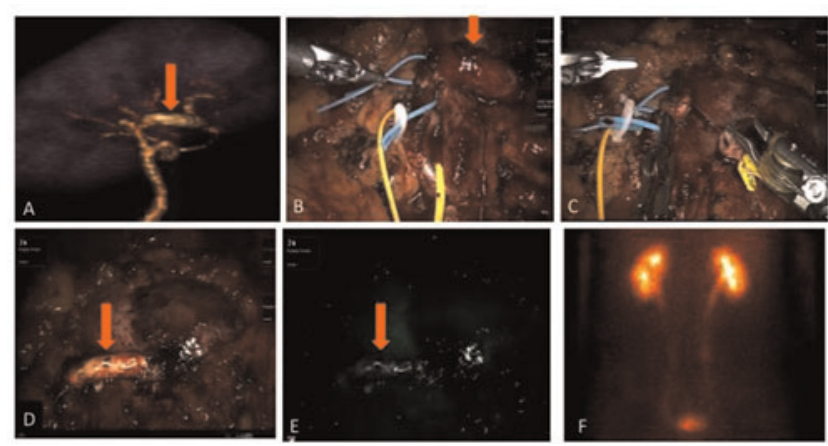

Figure 2: Composite showing robotic interposition graft repair of a renal artery aneurysm. A - 3D reconstruction of CT scan, B - Operative view showing the dissected aneurysm and feeding vessels, C-after excision of the aneurysmal section of the artery, D - repair with interposition graft, $\mathrm{E}$ - use of ICG to show perfusion in the kidney after completed repair, F - nuclear scan showing excellent perfusion and excretion from both kidneys during followup 
Introduction \& Objective: Renal artery aneurysm (RAA) is a rare clinical condition and is traditionally managed with endovascular techniques when treatment is indicated. There are few reports within the literature demonstrating minimally invasive techniques for surgical intervention. Herein, we report our initial experience of minimally invasive RAA repair.

Materials and Methods: Six patients of RAA were included in this study of which five had a complex RAA involving the main renal artery, and one patient had multiple aneurysms. Five patients underwent reconstruction robotically with one patient undergoing a robotic graft interposition.

Results: Median (range) aneurysm size was $2.4 \mathrm{~cm}$ (1.8-3.0). Median (range) operative time was 4.0 hours (3.5-6.0) with a median (range) warm ischemia time of $31.0 \mathrm{~min}$ (3-32). Median (range) estimated blood loss was 125 (50-400) and no patient required an intra-operative blood transfusion. There were no open conversions and no intra-operative complications. Median (range) length of hospital stay was 3 days (2-6). At a median (range) follow up of 31.5 months (1-67), all patients report to be doing well with no post-operative complications.

Conclusions: Our experience demonstrates that minimally invasive techniques can be safe and effective in the treatment of such rare cases as RAA.

MP35-4 Robotic uteretal reimplantation for uretero-enteric anastomotic strictures

N Ahmadi, C Fay, D Freitas, A Berger, L Misuraca, S Guaglianone, M Ferriero, G Simone, M Desai, A Goh, M Gallucci, M Aron

USC Institute of Urology/ USANZ

United States

Introduction \& Objective: Uretero-enteric anastomotic strictures occur in about $2-10 \%$ of patients treated with radical cystectomy and urinary diversion (UD). In this study we report the outcomes of robotic ureteral reimplantation for ureteroenteric anastomotic strictures in patients previously treated with robot assisted radical cystectomy (RARC) and UDs

Materials and Methods: From April 2013 to July 201612 patients underwent robotic ureteral reimplantation in three tertiary referral centers. Out of 12 patients, 7 had orthotopic neobladder, 4 ileal conduit and 1 Indiana pouch. All patients had prior RARC and all but one had intracorporeal UD.

Surgical steps include a careful ureteral dissection on the surface of the ureter/s to avoid injuring the iliac vessels, spatulation of the ureters, JJ stent insertion and finally uretero-ileal anastomosis.

Baseline, perioperative and early functional outcomes data are reported.

Results: Mean time from RARC to uretero-anastomotic stricture diagnosis was 174 days (33-674). Mean stricture length was $2 \mathrm{~cm}$ (range 0.5-3), median operative time was 201 minutes (83-310) and median length of stay was 2 days (2-12).

All cases were completed robotically. Intraoperative blood loss was negligible. Four patients experienced a Clavien grade II complication (urinary tract infection requiring antibiotics). At a median follow-up of 320 days (55-907) no recurrences occurred. Conclusions: Robotic ureteral reimplantation for uretero-enteric strictures is a safe and highly effective procedure. Given the suboptimal success rate of endoscopic treatment, robotic repair has become a first treatment option in our centers
MP35-5 Management of Recto-Urethral Fistulas after Focal Treatment for Prostate Cancer

R Sotelo, L Medina, D Hatcher, L Doumanian, IS Gill

\section{USC DEPARTMENT OF UROLOGY}

United States

Introduction \& Objective: With the increased usage of focal modalities for the treatment of prostate cancer (PCa), 50\% of rectourethral fistulas (RUF) are now associated with some form of radiation. In these cases, it may be beneficial to perform a cystoprostatectomy and ileal conduit creation with concomitant closure of the rectum. In addition, there are alternative management approaches such as prostatectomy with the use of omental flaps, bladder closure with placement of a suprapubic catheter, or creation of a catheterizable stoma. In this video, we demonstrate the management of two complex cases of RUF after focal treatment for PCa. Materials and Methods: Case 1: An 83 year old man underwent cryotherapy for $\mathrm{PCa}$. On post-operative day 14, he presented with urine per rectum. Cystoscopy confirmed the presence of a RUF. Urinary and fecal diversions were unsuccessful. Three months later, robotic surgical repair was performed. Case 2: An 85 year old man underwent salvage therapy for PCa with High-Intensity Focused Ultrasound (HIFU) after previous treatment with external beam radiation therapy (EBRT). Two months postoperatively, he presented with urine per rectum. A computerized tomography (CT) scan confirmed the presence of a RUF. Robotic surgical repair was subsequently performed.

Results: Both patients underwent robotic-assisted laparoscopic RUF repair, including salvage prostatectomy, rectal defect closure, and omental flap placement. In the first case, the next step was completion of a vesico-urethral anastomosis, and, in the second case, the next step was closure of the bladder neck and a suprapubic tube placement. Success was confirmed with imaging studies and no reported symptoms of recurrence at 4 and 8 weeks. One pelvic abscess was reported at week 9 in case 2 and it was resolved with prolonged antibiotic therapy.

Conclusions: The robotic system is useful for the treatment of a complicated RUF. The performance of a prostatectomy in these cases is necessary to achieve proper dissection planes and a successful posterior rectal defect closure.

MP35-6 Sexual dysfunction and abdominal aortic aneurysm surgical repair: incidence and consequences for clinical practice

P Regnier, F Lareyre, R Haider, F Roustan, Y Bodokh, P Treacy, B Tibi, R Prader, J Amiel, D Chevallier, M Durand

Departement of Urology, Andrology and Renal transplantation, Hospital Pasteur II, CHU Nice

France

Introduction \& Objective: Open repair (OR) is the gold standard techniqueof aortic abdominal aneurysm (AAA) surgery. Endovascular aneurysm repair (EVAR) is a less invasive modality of traitment established. While major outcomes have been well studied, the impact of AAA treatment on sexual dysfunction (SD) has been less described. The aim of our review was to summarize current knowledge on SD occurrence in context of AAA surgical repair.

Materials and Methods: We carried out a systematic review according to PRISMA criteria via Pubmed research from 2002 to 2016 of any studies dealing with SD and AAA repair based on key 


\begin{tabular}{|c|c|c|c|c|c|c|}
\hline \multirow[t]{2}{*}{ References } & \multirow{2}{*}{$\begin{array}{l}\text { AAA } \\
\text { surgery } \\
\text { technique }\end{array}$} & \multirow{2}{*}{\begin{tabular}{|l}
$\begin{array}{l}\text { Follow up } \\
\text { (months) }\end{array}$ \\
\end{tabular}} & \multicolumn{3}{|c|}{ Population (n) } & \multirow{2}{*}{$\begin{array}{l}\text { Methodology to } \\
\text { assess ED }\end{array}$} \\
\hline & & & \begin{tabular}{|l}
$\mathbf{O R}$ \\
\end{tabular} & EVAR & HALS & \\
\hline $\begin{array}{l}\text { Mald et al } \\
\text { 2015 }\end{array}$ & EVAR VS OR & 12 & 30 & 70 & 1 & KEED questionnaire \\
\hline $\begin{array}{l}\text { Becqueremin et al } \\
2011\end{array}$ & EVAR V 0 OR & 36 & 149 & 150 & 1 & Interview \\
\hline $\begin{array}{l}\text { Verouxo et at. } \\
2010\end{array}$ & EVAR vS HALS & 12 & 50 & & 50 & IIEF \\
\hline 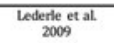 & \begin{tabular}{|l|l|} 
EVAR v & $0 R$ \\
\end{tabular} & 21 & 429 & 439 & 1 & IIEF \\
\hline $\begin{array}{l}\text { Prinssen et al } \\
2004\end{array}$ & EVAR vS OR & 12 & 76 & 77 & 1 & $\begin{array}{l}\text { Scale adppted from the } \\
\text { Medical Outcomes Strudy }\end{array}$ \\
\hline $\begin{array}{c}\text { Limn etal } \\
2002\end{array}$ & 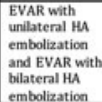 & 11 & $f$ & 12 & & $\begin{array}{l}\text { - Interview } \\
\text { - Pentile brachial index. }\end{array}$ \\
\hline
\end{tabular}

Table 1: Summary of studies on sexual dysfunction in context of AAA surgical repair EVAR: Endovascular aneurysm repair. OR: open repair. HALS: hand-assisted laparoscopic repair. SD: sexual dysfunction. ED: erectile dysfunction. RE: retrograde ejaculation.

HA: hypogastric artery. PBI: penile brachial index. Preop: preoperative. Post op: postoperative.
MP35-7 The Early Outcomes of Ureteral Reconstruction in Iatrogenic Ureteral Injury and Non-Iatrogenic Ureteral Surgeries

\section{Tseng, P Chow, K Huang}

National Taiwan University Hospital

Taiwan (Republic of China)

Introduction \& Objective: To compare the outcomes between iatrogenic ureteral injury and non-iatrogenic ureteral surgeries through ureteroureterostomy (UU) and ureteroneocystostomy (UNC) with or without psoas hitch or Boari flap.

Materials and Methods: We retrospectively reviewed a series of patients who underwent ureteral reconstructions from 2012 to 2015 in a single tertiary referral center. Patient data, hospital days, complications, ureteral stents dependence, preoperative and postoperative estimated glomerular filtration rate (eGFR), and imaging studies regarding hydronephrosis were assessed.

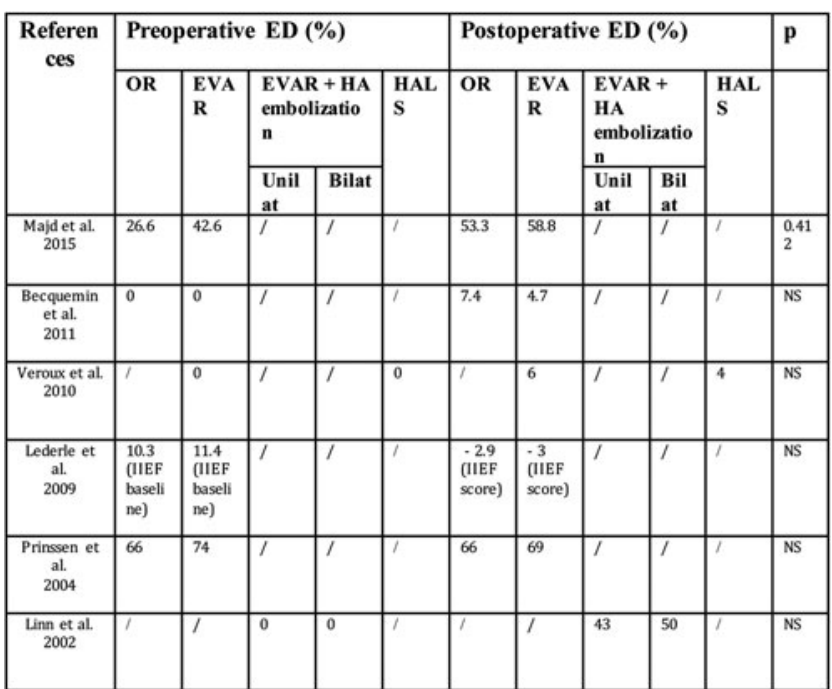

Table 2: Preoperative and postoperative erectile dysfunction results EVAR: Endovascular aneurysm repair. OR: open repair. HALS: hand-assisted laparoscopic repair. ED: erectile dysfunction.

HA: hypogastric artery. Unilat: unilateral. Bilat: bilateral. Preop: preoperative. Post op: postoperative

words: erectile dysfunction, sexual dysfunction, impotence, aortic aneurysm repair, EVAR, open aortic aneurysm repair. SD included erectile dysfunction (ED) and retrograde ejaculation (RE). ED was assessed only by interview, IIEF score and Keed score. Results: Overall 59 studies were eligible, 30 were reviewed of which 6prospectiveswere analyzed, comparing SD after OR, EVAR, laparoscopic repair (LR)(Table 1). Only originals articles were assessed. SD is a frequent preoperative AAA surgery disease, andincreasesone year after OR, EVAR and LR. There is not statistical difference according to each techniquein contrast of retrospectives studies (Table 2). Concerning EVAR, bilateral hypogastric artery (HA) embolization is more frequently associated with SD than unilateral embolization.

Conclusions: SD is a poorly evaluated AAA surgery complication, but affects quality of life. According to these prospectives studies, all techniques have an impact on sexual function, without statistic difference.

\begin{tabular}{|c|c|c|c|}
\hline \multicolumn{4}{|c|}{ Patient characteristics } \\
\hline \multicolumn{2}{|c|}{ Age (y) (median, Q25-Q75) } & 47.0 & $(33.5-58.0)$ \\
\hline \multirow[t]{2}{*}{ Gender } & Male & 19 & (31.1\%) \\
\hline & Female & 42 & $(68.9 \%)$ \\
\hline \multirow[t]{3}{*}{ BMI } & $<24$ & 31 & $56.4 \%$ \\
\hline & Overweight & 19 & $34.5 \%$ \\
\hline & $>28$ & 5 & $9.1 \%$ \\
\hline \multirow[t]{2}{*}{ Hypertension } & yes & 12 & $(19.7 \%)$ \\
\hline & no & 49 & $(80.3 \%)$ \\
\hline \multirow[t]{2}{*}{ Diabetes Mellitus } & yes & 2 & $(3.3 \%)$ \\
\hline & no & 59 & $(96.7 \%)$ \\
\hline \multirow[t]{2}{*}{ Anemia } & YES & 30 & $(49.2 \%)$ \\
\hline & NO & 31 & $(50.8 \%)$ \\
\hline
\end{tabular}

\begin{tabular}{|c|c|c|c|}
\hline \multicolumn{2}{|c|}{ Patient characteristics (continue) } & $\mathrm{N}(\%)$ & (\%) \\
\hline \multicolumn{4}{|l|}{$\begin{array}{l}\text { Pre-op eGFR } \\
\left(\mathrm{mL} / \mathrm{min} / 1.73 \mathrm{~m}^{2}\right)\end{array}$} \\
\hline \multicolumn{2}{|l|}{$>$ or $=60$} & 50 & $82 \%$ \\
\hline \multicolumn{2}{|l|}{$<60$} & 11 & $18 \%$ \\
\hline \multirow[t]{3}{*}{ Lesion laterilaty } & Right & 31 & $50.8 \%$ \\
\hline & Left & 28 & $45.9 \%$ \\
\hline & Bilateral & 2 & $3.3 \%$ \\
\hline \multirow[t]{3}{*}{ Lesion loccation } & Upper & 9 & $14.8 \%$ \\
\hline & Middle & 4 & $6.6 \%$ \\
\hline & Lower & 48 & $78.7 \%$ \\
\hline \multirow[t]{5}{*}{ Etiology } & latrogenic & 30 & $49.2 \%$ \\
\hline & Malignancy & 9 & $14.8 \%$ \\
\hline & Benign stricture & 20 & $32.8 \%$ \\
\hline & Ectopic ureter & 1 & $1.6 \%$ \\
\hline & VUR & 1 & $1.6 \%$ \\
\hline
\end{tabular}




\begin{tabular}{|c|c|c|}
\hline & \multicolumn{2}{|l|}{ Median } \\
\hline $\begin{array}{l}\text { Hospital stay (Day) } \\
\text { (median ,Q25-Q75) }\end{array}$ & 16 & $(11.0-23.0)$ \\
\hline Op time (min) & 288.0 & $(195.50-343.75)$ \\
\hline Estimated blood loss (ml) & 100 & $(50-275)$ \\
\hline Foley days & 9 & $(6.0-12.0)$ \\
\hline Drain days & 9 & $(7-11.2)$ \\
\hline Post OP DBJ days & 67.5 & $(52.0-90.25)$ \\
\hline & Yes & No \\
\hline Pre-operaion DBJ & $15(24.6 \%)$ & $46(75.4 \%)$ \\
\hline Pre-operation PCN & $13(21.3 \%)$ & $48(78.7 \%)$ \\
\hline Pre-OP hydronephrosis & $42(68.9 \%)$ & $19(31.1 \%)$ \\
\hline Post op long term stent & $14(23.0 \%)$ & $47(77.0 \%)$ \\
\hline
\end{tabular}

Statistics analyses were done for evaluating surgical and functional outcomes.

Results: A total of 61 patients underwent ureteral reconstruction at our institution with a median 16.4 months (IQR 10.8-21.7 months) follow-up. Thirty-one (50.8\%) patients had iatrogenic ureteral injuries and thirty (49.2\%) patients had pre-planned ureteral reconstruction surgery. Hydronephrosis progression was significantly higher $(\mathrm{p}=0.038)$ in iatrogenic group $(25.8 \%)$ than non-iatrogenic group (6\%). No significant difference was found in long-term stenosis rate $(\mathrm{p}=0.497)$ and deterioration of eGFR $(\mathrm{p}=0.973)$. Overall, $77 \%$ patients were free from long-term ureteral stenting and $93 \%$ had stable or improved renal function. Conclusions: We found both surgical and functional outcomes were equivalent between iatrogenic ureteral injury group and non-iatrogenic ureteral surgery group. The higher proportion of postoperative hydronephrosis would not affect the final excellent renal function.

\section{MP35-8 The Outcome of Robotic Boari Flap}

\section{MW Salkini, N Idris \\ West Virginia University United States}

Introduction \& Objective: Ureteroneocystostomy with creation of Boari flap represents a good option to substitute for the loss of the distal ureter in both benign and malignant conditions. Traditionally the procedure is performed through a large midline or Gibson incision. Utilizing the robotic daVinci surgical sytem made it feasible to achieve the objectives of the procedure with minimally invasive approach. We aim to report on our first series of robotic assisted ureteral substitution with Boari flap.

Materials and Methods: Between September 2009 and March, 2017, the de Vinci Si robotic system was used to reconstruct 13 distal ureters for 4 benign and 9 malignant conditions, in our institute. We utilized the $5 \mathrm{~W}$ shaped trocar placement in 11 cases and added sixth trocar for assistance in 3 cases. The remaining ureteral end was spatulated and reimplanted end to end to the
Boari flap. Utilizing the daVinci robotic sytem we implanted 6 right ureters and 7 left ureters into the robotically created Boari Flap. Negative margin was insured in all the malignant cases. Results: The average patient age was 67 years (ranging from 3479). We had 6 males and 7 females patients. All cases were completed robotically with no conversion, the patients were followed for an average of 35 months (ranging from 3 months to 72 months). The ureters were patent in all of the 4 (\% 30) patients who had benign ureteral stricture. However, 3 patients (\%23) who had lower ureteral TCC developed ureterovesical anastomosis stricture.. All of the strictures were due to high grade TCC on the initial and final pathology after nephroureterectomy with excision of the flap. One patient with history of high grade TCC of the lower ureter developed multifocal high grade TCC in the renal pelvis on the same side 3 years later.

Conclusions: Robotic reconstruction of the lower ureter with Boari flap is feasible and has an acceptable oncologic outcome. High grade TCC of the lower ureter is predictor of recurrence at the anastomosis. Longer follow up is needed especially in cases of malignancy.

MP35-9 The outcome of conservative management of unilateral asymptomatic Pelvi-ureteric junction (APUJO) Obstruction

\author{
ME Hassan, M Smith, K Subramonian
}

Queen Elizabeth Hospital Birmingham United Kingdom

Introduction \& Objective: The decision making process for managing asymptomatic PUJO poses a dilemma as there are various options that can be tailored to each patient. In this cohort study, we investigate the safety and suitability of conservative management of APUJO

Materials and Methods: In our tertiary institution, a cohort of APUJO patients were followed up annually by clinical history, blood tests and nuclear medicine scans. This database was analysed with regards to demographics, Blood results; including creatinine, eGFR and Haemoglobin (Hb) and MAG3/DMSA compared using Student's t test.

Results: Our cohort consisted of 31 patients (12 males and 19 females) with an average age of 64 (41-91) years. Average follow up - 4.8 (1 to 9) years. ASA grades II and III (84\%). Mode of presentation- incidental finding (61\%), pain (19\%) and $20 \%$ presented with haematuria, UTI and pyelonephritis. Follow up, 29 (94\%) patients remained asymptomatic, one- minimally symptomatic and another had UTIs that settled with antibiotics. Nineteen patients (61\%) PUJ obstruction on the right side and $\mathrm{n}=12(39 \%)$ on the left. Each patient had an average of 4 MAG3 renograms during the study. The percentage of function reported on MAG3 during presentation was compared with the MAG3 report at the last date of follow up, there was no significant change $P(0.1931)$ in renal function during the follow up period for the majority of patients except three, who had a deterioration in their function. One patient needed intervention \& was managed with a long term stent. One patient was managed conservatively for 4 years until she had heart surgery, then was fit for Pyeloplasty. PUJO was detected at a certain point but it has been there for many years before with minimal change in renal function.

Patients were seen in clinic annually and there was no adverse events or mortality related to the PUJ obstruction. Two patients 
deceased during the follow up period due to other causes, age 83 and 77 years. There was no significant change in $\mathrm{Hb}-P(0.9222)$, creatinine $P(0.7752)$ and eGFR $P(0.6663)$ during the follow up period.

Conclusions: Patients with asymptomatic PUJ obstruction can be safely monitored over a long period of time. There should be close monitoring of the patients with regular MAG3 renograms and blood tests. Although PUJO was detected at a certain point in time, the condition may have been there for many years before it became symptomatic or started to affect the renal function. The patients should be counselled about all the management options during their follow up visits.

MP35-10 Feasibility and safety of ileal neobladder with simplified catheterization and irrigation protocol

K Kim, D Lee

Department of Urology, Mokdong Hospital, Ewha Womans University College of Medicine

Republic of Korea

Introduction \& Objective: We evaluated the feasibility and safety of ileal neobladder reconstruction with simplified catheterization and irrigation protocol.

Materials and Methods: From January 2012 to September 2016, 214 patients who underwent radical cystectomy with Studer neobladder were included in this study. In this period, we developed surgical protocol and patients were divided by 3 groups according to catheterization status and manual irrigation protocol. From January 2012 to July 2014, 60 patients (Group 1) underwent surgery without cystostomy. These patients had Levin tube, ureteral stents and urethral catheter, and were treated 3 times of routine manual irrigation a day. From July 2014 to December 2015, 80 patients (Group 2) had only urethral catheter without cystostomy tube, Levin tube and ureteral stents. They were treated with routine manual irrigation. From January 2016 to September 2016, 74 patients (Group 3) had only urethral catheter and were treated with simplified irrigation protocol. With simplified irrigation protocol, patients and caregivers were educated to squeeze urethral catheter to prevent mucous obstruction and manual irrigation was performed only when urine output was less than $100 \mathrm{cc}$ for 3 hours. Perioperative outcomes and postoperative complication were compared between 3 groups.

Results: While operative time was significantly decreased in patients who did not have ureteral stents, day to regular diet and

\begin{tabular}{|c|c|c|c|c|}
\hline & $\begin{array}{l}\text { Group 1 } \\
(n=60)\end{array}$ & $\begin{array}{c}\text { Group 2 } \\
(\mathrm{n}=80)\end{array}$ & $\begin{array}{c}\text { Group } 3 \\
(n=74)\end{array}$ & $P$ value \\
\hline \multicolumn{5}{|l|}{ Perioperative Outcomes } \\
\hline Operative time ( $\mathrm{min})$, mean $\pm \mathrm{SD}$ & $307.3 \pm 43.4$ & $284.6 \pm 45.8$ & $290.8 \pm 39.5$ & 0.008 \\
\hline Estimated blood loss (ml), mean \pm SD & $689.6 \pm 290.8$ & $568.5 \pm 270.5$ & $209.2 \pm 24.3$ & $<0.001$ \\
\hline Transfusion, $n(\%)$ & $27(45.0)$ & $12(15.0)$ & $9(12.2)$ & $<0.001$ \\
\hline Days to regular diet, mean $\pm S D$ & $10.3 \pm 7.2$ & $9.8 \pm 6.6$ & $9.3 \pm 5.4$ & 0.653 \\
\hline Hospital stay (days) & $20.2 \pm 10.1$ & $19.2 \pm 7.5$ & $17.7 \pm 6.3$ & 0.189 \\
\hline \multicolumn{5}{|l|}{ Postoperative complications } \\
\hline Clavien-Dindo $\geq 3, n(\%)$ & $4(6.7)$ & $6(7.5)$ & $7(9.5)$ & 0.824 \\
\hline 30-day readmission, $n(\%)$ & $8(13.3)$ & $14(17.5)$ & $9(12.2)$ & 0.615 \\
\hline Febrile UTI within 30 days, $n$ (\%) & $10(16.7)$ & $22(27.5)$ & $12(16.2)$ & 0.152 \\
\hline Urinary leakage, n (\%) & $1(1.7)$ & $1(1.3)$ & $4(5.4)$ & 0.243 \\
\hline Postoperative ileus, n (\%) & $16(26.7)$ & $23(28.8)$ & $16(21.6)$ & 0.588 \\
\hline Ureteral stricture, $n$ (\%) & $3(5.0)$ & $4(5.0)$ & $3(4.1)$ & 0.953 \\
\hline
\end{tabular}

hospital stay were not significantly different between three groups. The rates of major complication (Clavien-Dindo grade 3 or greater) were $6.7 \%, 7.5 \%$ and $9.5 \%$ in group 1,2 and 3 , respectively $(\mathrm{P}=0.824)$. Also, the rate of urinary leakage, postoperative ileus, readmission within 30 days after discharge and febrile urinary tract infection within 30 days after surgery was not significantly different (Table). Of 74 patients in group 3, 52 patients $(71.3 \%)$ were not treated with additional manual irrigation until removal of urethral catheter. Of 22 patients treated with additional manual irrigation for mucous obstruction, 3 times or greater of manual irrigation was performed in 8 patients $(10.8 \%)$

Conclusions: Simplified catheterization and irrigation protocol did not adversely affect perioperative outcomes and postoperative complication. With simplified irrigation protocol, additional irrigation was not necessary in approximately $70 \%$ of patients.

MP35-11 A Preoperative Mathematical Model to Estimate Available Tissue Length for Ureteral Reimplantation by Different Methods of Bladder Mobilization

MA Perez, GL Lloyd

Denver VA/ University of Colorado Department of SurgeryUrology

United States

Introduction \& Objective: Ureteral injury occurs after pelvic surgery at a rate that has been estimated to range from $0.2-9 \%$ of cases; other causes of iatrogenic ureteral obstruction include endoscopic surgery, malignancy, radiation and external trauma. These injuries are commonly reconstructed with ureteral reimplantation; depending on the level of injury more complex reconstructions of the bladder may be required, such as Boari flap, to bridge distances to viable proximal ureter. Accurate preoperative estimate of the amount of ureteral length available after bladder mobilization, with or without advancement flap, is desirable. We sought to create a graphical tool to estimate the distance able to be generated from the base of the bladder after maximal mobilization toward ureteral reimplantation, based on bladder volume (BV).

Materials and Methods: Starting assumptions included:

1. A fully mobilized bladder conformationally represents a sphere

2. a maximally mobilized bladder would yield a length for simple direct reimplantation equivalent to $1 / 2$ circumference.

3. Boari flap designed as commonly cited with a basewidth to length ratio of $1: 1$; with a $4 \mathrm{~cm}$ wide base as commonly suggested and thus would optimally add $4 \mathrm{~cm}$ to the length generated from a mobilized bladder.

A curve was generated by solving $\mathrm{v}=4 / 3 \pi(\mathrm{c} / 2 \pi)^{3}$ for $1 / 2$ circumference from bladder volume (Fig 1).

Results: Figure 1 demonstrates this relationship between bladder volume and available length for simple direct reimplantation. Boari flap reimplantation adds $4 \mathrm{~cm}$ of additional potential length at the cost of roughly an additional $8 \mathrm{~cm}$ of sutured repair of the bladder.

Conclusions: We present a mathematical model for the estimation of maximal generated length from bladder for ureteral reimplantation in the setting of distal ureteral loss. We believe that knowledge and usage of this model will prove beneficial in 


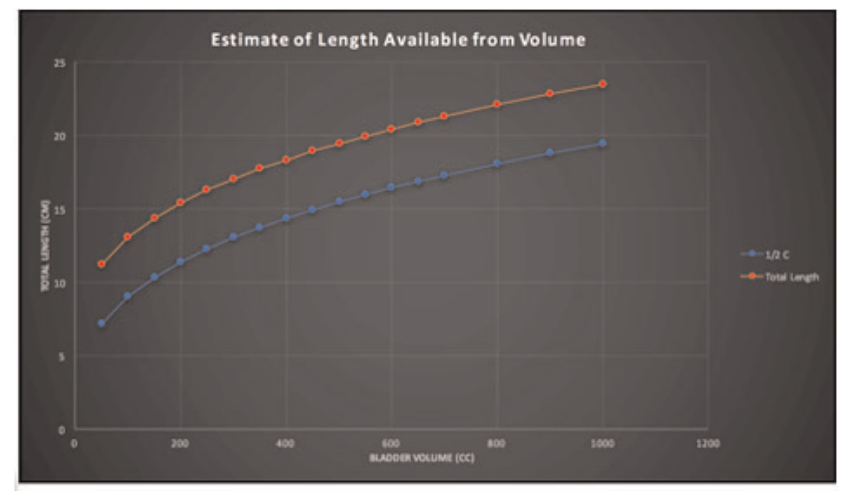

Figure 2. Estimate of Length Available from Volume. 1/2 circumference calculated from $c=2 \pi \sqrt[3]{\frac{3 v}{4 \pi}}$ (blue curve). Total length calculated by solving for $1 / 2 \mathrm{C}$ then adding an additional $4 \mathrm{~cm}$ to original length (orange curve).

complex and difficult reconstructions for accurate surgical planning. This mathematical model awaits validation in vivo, and suffers from limitations including the inability to estimate additional length from bladder compliance, as well as limitation of mobilization from fixation at the contralateral vascular pedicle. Bladder volume provides a useful estimate of available tissue for preoperative planning in the setting of ureteral loss of length.

MP35-12 Robotic reconstruction of ureteroenteric stricture after urinary diversion

AC Weinberg, D Volkin, W Huang, L Zhao

NYU Department of Urology

United States

Introduction \& Objective: Ureteroenteric strictures (UES) are difficult complications after urinary diversion surgery. Endoscopic management may be unsuccessful. Open surgical revision is difficult with significant morbidity. We present our experience with robotic reconstruction of UES, highlighting the benefit of near-infrared technology (Firefly ${ }^{\mathrm{TM}}$ ) to define the extent of diseased tissue.

Materials and Methods: We retrospectively reviewed 10 patients who underwent robotic UES repair at our institution between July 2015 and January 2017. Indication for the procedure was hydroureteronephrosis, pain, or worsening renal failure. On follow-up, patients were assessed for clinical success by absence of ureteral obstruction on cross sectional imaging. All patients have nephrostomy tube placed prior to the OR. Following docking of the surgical robot, the ureteroscope is passed ante-

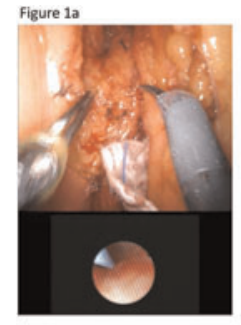

Identification of ureteroenteric stricture
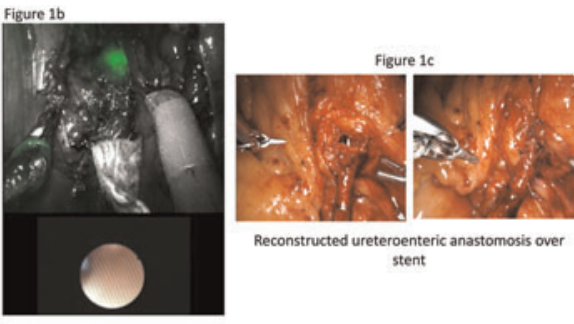

\begin{tabular}{|l|l|}
\hline & Median, Percentage \\
\hline Number of patients & 10 \\
\hline Age at surgery & 73 years (57-78) \\
\hline Gender & Male 100\% \\
\hline Time to reconstruction & 8 Months (5-298) \\
\hline Type of urinary diversion & $60 \%$ Neobladder \\
& $40 \%$-lleal Conduit \\
\hline Previous management & $50 \%$-Balloon dilation, stent placement \\
& $20 \%$-Laser incision, stent placement \\
& $20 \%$-Chronic nephrostomy tube \\
& $10 \%$-No treatment \\
\hline Side & $70 \%$-Left \\
& $30 \%$-Right \\
\hline Length of stricture & $2 \mathrm{~cm}$ (1-3) \\
\hline Surgery time & 353 min (270-480) \\
\hline Type of reconstruction & $50 \%$-Bowel Advancement Flap \\
& $50 \%$-Direct Anastomosis \\
\hline Pathology of stricture & $100 \%$ benign \\
\hline Length of stay & 3 days (2-5) \\
\hline Time with stent & 36 days (20-71) \\
\hline Resolution on imaging & $80 \%$-Complete resolution \\
& $20 \%$-Mild residual hydro \\
\hline
\end{tabular}

grade to the level of the UES and if possible a wire is placed across the stricture. We utilize Firefly ${ }^{\mathrm{TM}}$ technology to help guide use to the stricture (Figure 1a, 1b). Ureteral viability was identified the aid of intravascular injection of indocyanine green (ICG). The ischemic segment of diseased ureter was excised, and the healthy ureter was spatulated and reanastomosed over a stent to the bowel segment (Figure 1c).

Results: $70 \%$ of patient presented with a left UES, six patients had previous neobladder urinary diversion, average time from cystectomy to UES repair was 8 months (5-298). 90\% of patients had previously undergone an endoscopic procedure; the majority was balloon dilation with stent placement. All patients underwent robotic UES repair without conversion to open surgery. The median length of stricture was $1.0 \mathrm{~cm}$ (range 1.0-3.0 cm), operative time was $353 \mathrm{~min}$ (range $270-480 \mathrm{~min}$ ), estimated blood loss was $50 \mathrm{ml}$ (range $15-100 \mathrm{ml}$ ), and length of stay was 3 days (range 2-5). There were no intraoperative complications. All stents were removed in the office at about 5 weeks. At a median follow-up of 3 months (range 1-27), 100\% of cases were clinically and $80 \%$ of cases were radiologically successful.

Conclusions: Robotic repair of ureteroenteric strictures is a feasible, effective and durable technique for managing a complex reconstructive problem.

MP35-13 Comparative outcomes of laparoscopic and open ureteroneocystoneostomy

B Guliev, B Komyakov, V Ochelenko, A Shipilov

North-West medical university by Mechnikov

Russia

Introduction \& Objective: Ureteroneocystostomy can be used for the treatment of patients with strictures of pelvic ureter. Over the last decade, laparoscopy more commonly uses as a minimally invasive approach for upper urinary tract reconstruction. The aim of this study is to present our experience with laparoscopic 
ureteroneocystosctomy (LUNC) and compare with open ureteroneocystostomy (OUNC).

Materials and Methods: Thirty-two patients who had undergone LUNC from 2010 to 2016 were identified and included in the analysis. Of 124 eligible patients who had undergone OUNC from 1996 to 2010, 45 were included for comparison to allow matched comparison based on the surgical technique used. Patients with bilateral surgeries, ureteroneocystostomy as part of other procedure, and correction of vesicoureteral reflux were excluded from the study. Perioperative and postoperative data including demographics, surgical outcomes, and clinical and radiographic findings at postoperative follow-up were considered in the comparative analysis. The significant difference between variables was evaluated using the Wilcoxon test for continuous and Fisher test for categorical variables.

Results: $14(43,7 \%)$ patients in the laparoscopic group underwent primary ureterocystostomy vs $21(46.6 \%)$ patients in the OUNC group. $8(25 \%)$ patients underwent LUNC with a Boari flap compared with $12(26,7 \%)$ in the open group. Psoas hitch technique used in $10(31,3 \%)$ patients in laparoscopic group compared with $12(26,7 \%)$ in the open group $(\mathrm{P}=0.1)$. Operative time was significantly longer in the laparoscopic group (median 210 vs $160 \mathrm{~min}, \mathrm{P}=0.0008$ ). Patients after LUNC had a shorter hospital stay (median 3 vs 6 days, $\mathrm{P}=0.0004$ ), less estimated blood loss ( 80 vs $150 \mathrm{~mL}, \mathrm{P}=<0.0002$ ), and less narcotic pain requirement (morphine equivalent, $\mathrm{mg} 102.5$ vs $280, \mathrm{P}=0.0001$ ). There as no significant difference in the rate of reoperation between groups: LUNC 2/32 (6,3\%) vs OUNC 4/45 (8,9\%) $\mathrm{P}=0.8$. Conclusions: LUNC provides significant shorter hospital stay, less narcotic pain requirement, and less blood loss than ONUC. Advantages of the laparoscopic approach for dissection and suturing can be useful for reconstructive procedures in patients with strictures of pelvic ureter

MP35-14 Perceptions and practice patterns of holmium laser goggles in endourological procedures: an unnecessary evil?

N Paterson, R Fitzpatrick, J Denstedt, B Blew, J Watterson

The University of Ottawa

Canada

Introduction \& Objective: The holmium laser is used increasingly for a wide array of urological procedures. The safety policies governing the use of this laser in the operating theater vary greatly across centers. Laser safety goggles are mandatory at many centers for individuals within the nominal hazard zone, as set out by the institution. Recent ex vivo studies suggest standard eyewear may be equally as effective, with better visibility and less financial burden. We sought to evaluate the perceptions and practice patterns in laser safety and goggle usage.

Materials and Methods: A brief 24 question survey was sent out via e-mail to an international email list of 2000 urologists that were members of the Endourological Society. The data was collected anonymously using Survey Monkey. All data was analysed using SPSS.

Results: 264 (14\%) urologists completed the survey representing a confidence interval of $95 \%$ with a margin of error of $5.5 \% .34 \%$ worked in the community while $63 \%$ worked at an academic institution. $70 \%$ were fellowship trained and of those, $84 \%$ had endourologic/minimally invasive surgery fellowships. 97\% routinely used the holmium laser, with the most common uses being lithotripsy (99\%), laser incision $(71 \%)$, tumor ablation
(58\%) and prostate ablation (26\%). $76 \%$ had received formal laser safety training while only $65 \%$ have formal institutional laser safety policies. $40 \%$ of the respondents wore laser safety glasses in the operating room. There were no witnessed eye injuries reported among the cohort. $70 \%$ of surgeons felt that laser safety goggles may impair their vision. 50\% did not know at what distance an activated laser has the potential to risk eye injury. When presented with the information that regular eye glasses may be as effective as laser goggles for preventing injuries, the overwhelming majority $(86 \%)$ would choose regular eye wear. Conclusions: Laser safety goggle usage varies greatly worldwide. Despite policies mandating laser goggle usage for all personnel within an operating theater, there is no evidence that this prevents injuries. With over two thirds of surgeons suggesting that goggles impair their vision, and recent literature suggesting regular eye wear is equivalent to prevent harm, policies should be adjusted to better match the potential hazards inherent to the holmium laser. This would be cost saving to hospitals.

MP35-15 Clinical Observation of Endoscopic Surgery for Hemorrhagic Cystitis after Hematopoietic Stem Cell Transplantation

J Liu, B Yang, T Xu, X Wang, X Huang

Peking University International Hospital

China (People's Republic)

Introduction \& Objective: To investigate the therapy and outcome of endoscopic surgery for hemorrhagic cystitis after hematopoietic stem cell transplantation

Materials and Methods: From December 2014 to February 2016, 9 patients with severe hemorrhagic cystitis(HC) after Hematopoietic stem cell transplantation(HSCT) received endoscopic surgery. We analyzed their medical records and summarized the clinical features and outcome, which had taken the recent literature into account.

Results: Among the 9 patients, 6 patients were male, the left 3 were women, with the average age 36.6 years (16-57 years). 14 endoscopic operations were performed on the 9 patients, with the average operation time $83.5 \mathrm{~min}(42-227 \mathrm{~min})$, without preoperative complications. Among the 9 patients, 7 patients were cured by endoscopic surgery, of the 7 patients, 4 patients received one operation, 2 patients were cured upon two operations for hematuria again, 1 patient underwent three operations due to the repeated bladder tamponade, 2 patients died of pulmonary infection without relation to operation.

Conclusions: The endoscopic surgery is effective and safe for HC patients after HSCT, but the indication of surgery is the key point for the success of surgery.

MP35-16 Partial-tickness-endopyelotomy for failed pyeloplasty and factors effecting the success rate

T Ebiloglu, E Kaya, M Zor, S Sarıkaya, B Topuz, B Kopru, $S$ Bedir

Etimesgut Military Hospital

Turkey

Introduction \& Objective: ?To detect the success rates of partial-tickness-endopyelotomies after failed pyeloplasty and factors effecting these success rates. 
Table. Factors affecting the endopyelotomy success after failed pyeloplasty.

\begin{tabular}{lc}
\hline Factors & $\mathrm{P}^{*}$ value \\
\hline Gender & 0.972 \\
Time lag from first operation & 0.075 \\
Preop. grade of hydronephrosis & 0.497 \\
Pain relief after preop. JJ catheter & 0.157 \\
insertion & \\
\hline
\end{tabular}

* Enter Logistic Regression

Materials and Methods: -Between 2007 and 2017, 12 patients had endopyelotomy after failed pyeloplasty. We analyzed the post operational 1 year results. Endopyelotomy was applied without reaching the peri-ureteral fat tissue (partial-ticknessendopyelotomy). A JJ catheter was applied for all patients at the end of all procedures. Total symptomatic relief was defined as success, partial symptomatic relief was defined as partial success, no symptomatic relief was defined as failure.

Results: There were 7 (58.3\%) males and $5(42,7 \%)$ females. Seven $(58.3 \%)$ patients had right sided uretero-pelvic stenosis(UPS), and $5(42,7 \%)$ patients had left sided UPS. Patients mean age was 29.3(20-43). The mean time after first operation was 2.22(0.25-13) years. All patients had pain at related side. $\operatorname{Ten}(83.3 \%)(2$ missing) patients had obstruction at IVP; $3(25 \%)$ had grade $2,5(41.7 \%)$ had grade 3 , and $2(16.7 \%$ ) had grade 4 hydonephrosis at USG(2 missing); 11(91.7\%)(1 missing) didn't have respond to IV diuretic at DTPA renal Scintigraphy. After operations $5(58.3 \%)$ patients had obstruction at IVP $(\mathrm{p}=0.564)$; $1(8.3 \%)$ had no hydronephrosis, $1(8.3 \%)$ had grade $2,6(50 \%)$ had grade 3, at USG(4 missing) ( $\mathrm{p}=0.04)$; and 8(66.7\%) didn't have respond to IV diuretic at DTPA renal Scintigraphy (2 missing) $(\mathrm{p}=0.058)$. Six patients had failure $(50 \%), 5(41.7 \%)$ patients had partial success, and $1(8.3 \%)$ patient had success $(\mathrm{p}=0.174)$. Factor effecting the success rate was depicted in table.

Conclusions: - Partial-tickness-endopyelotomy was not a good alternative after failed pyeloplasty. We also didn't detect any factors affecting the success rate of endopyelotomies.

MP35-17 Transperitoneal Laparoscopic Pyeloplasty Associated Rigid Nephroscopy in the Treatment of Ureteropelvic Junction Obstruction and Renal Calculi

J Liu, X Ye, L Xiong, X Huang, T Xu

Urology Department

China (People's Republic)

Introduction \& Objective: The treatment of ureteropelvic junction obstruction (UPJO) and concomitant calculus is a complex prosess. We present our experience with using rigid nephroscopy for renal calculi removal during laparoscopic pyeloplasy for UPJO.

Materials and Methods: From January 2014 to February 2017, 12 patients with UPJO and renal stone had transperitoneal laparoscopic pyeloplasy at our institution; all of those had been simultaneously treated with rigid nephroscopy for renal stone removal. For stone extraction, a rigid nepgroscopy or ureteroscope was passed through an assistant trocar under laparoscopic vision directly into a small hole previously created pyelotomy. The stones were extracted using a rigid forceps or stone basket
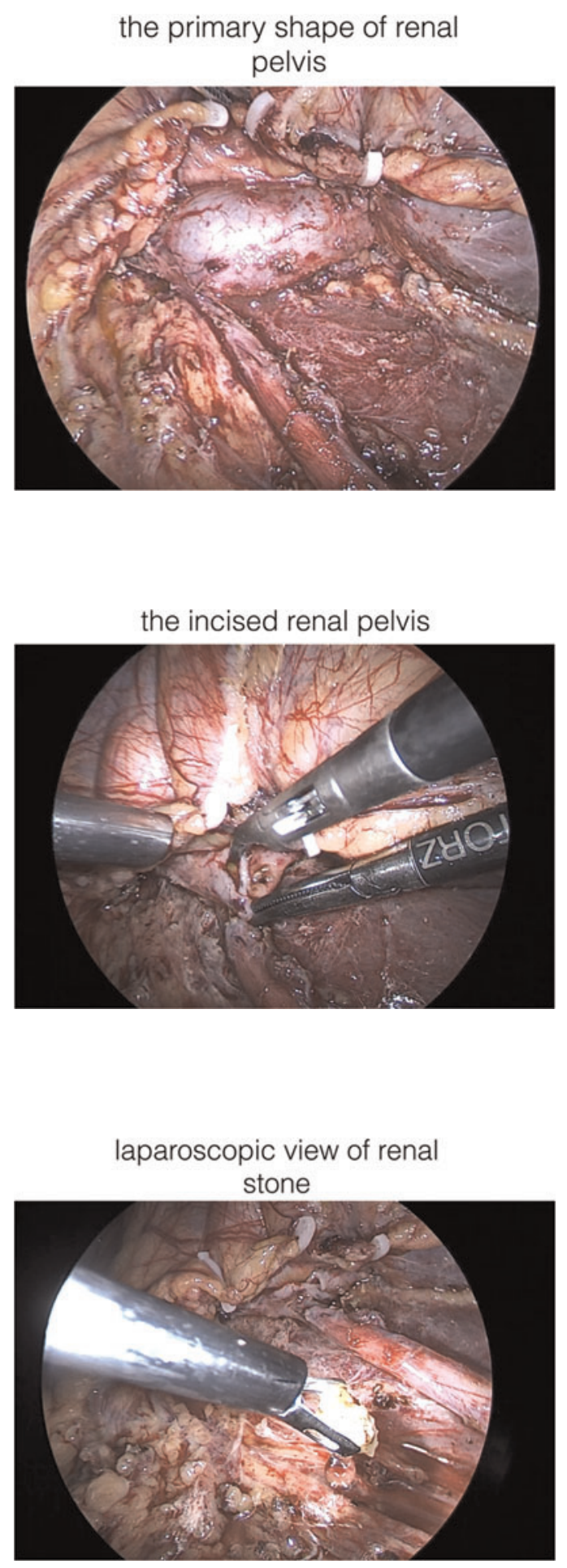

through the rigid nepgroscopy. Then transperitoneal laparoscopic dismembered Anderson-Hynes pyeloplasty was operated. Results: The mean operative time was 152 minutes, the mean time for nephrolithiasis removal by rigid nephroscopy was 26.5 minutes, the number of stones was 1 to 55. Complete stone clearance was achieved in 11 of 12 patients. Residual calculi in 1 patient were removed with a single session of extracorporeal shock wave lithotripsy. At the mean follow-up of 11.4 months, no patients had obstruction or recurrent stones. 
stones extracted by a forceps through the rigid nephroscopy

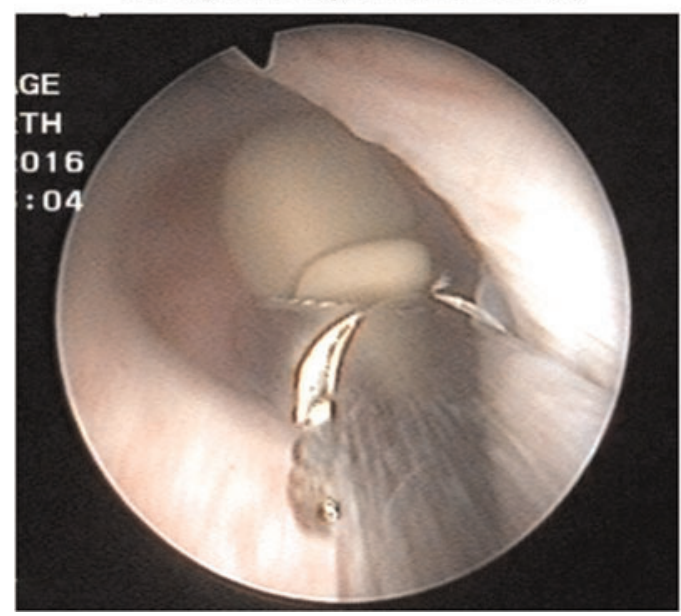

\section{the view after pyeloplasty}

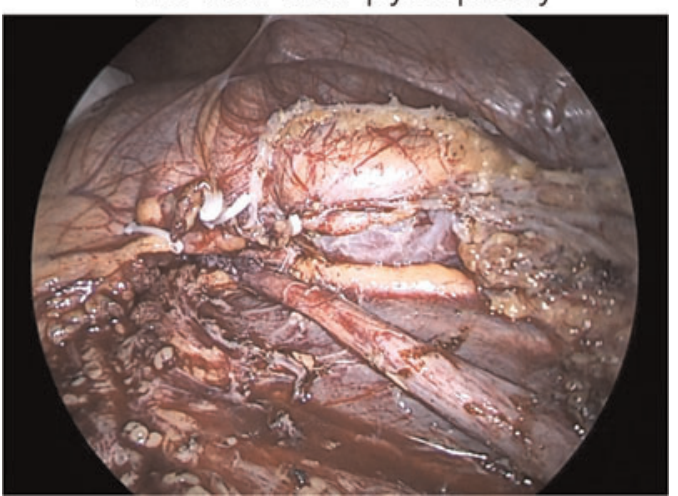

Conclusions: Our data suggest that the use of a concomitant rigid nephroscope during laparoscopic pyeloplasy is a safe and minimally invasive option for the treatment of UPJO complicated with renal calculi.

MP35-18 Percutaneous ureteroscopy laser unroofing-a minimally invasive approach for renal cyst treatment

J Hu, X Yu, S Wang, Z Ye

China (People's Republic)

Introduction \& Objective: To assess the safety and efficacy of a novel technology referred to as percutaneous ureteroscopy laser unroofing in the management of simple renal cysts.

Materials and Methods: From November 2014 to October 2016, 71 patients having surgical indications with renal cysts were enrolled and evaluated with ultrasonography and CT scan. Of all the 71 patients, include 6 patients with parapelvic cysts and 5 patients with renal cyst complicated with ipsilateral renal calculi. All of the patients received combined spinal and epidural analgesia or paravertebral nerve block anesthesia. Patients were

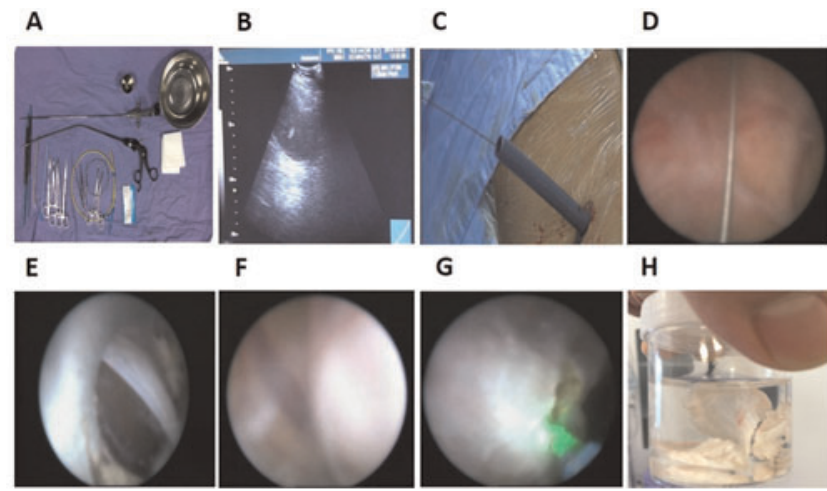

A

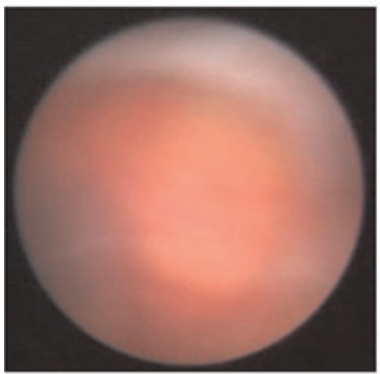

C

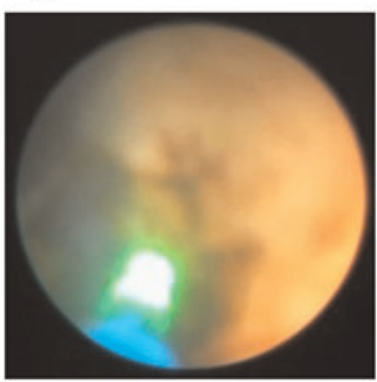

B

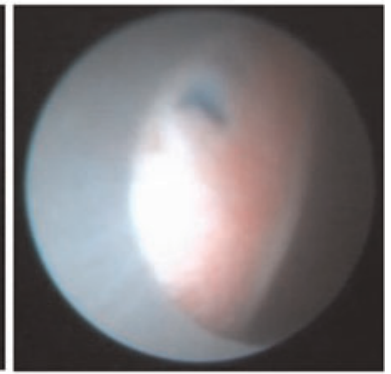

D

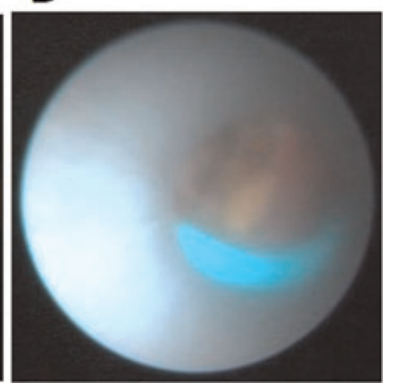

A

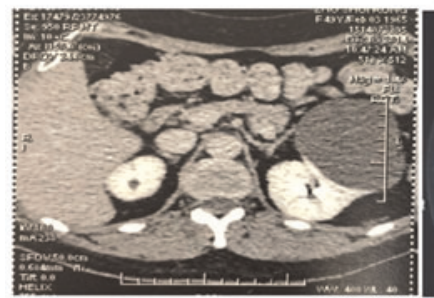

C

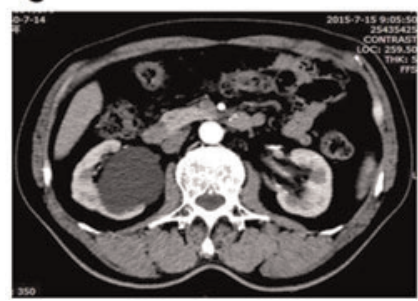

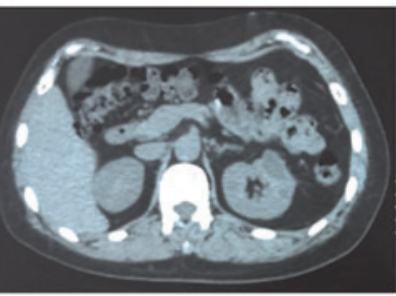

D

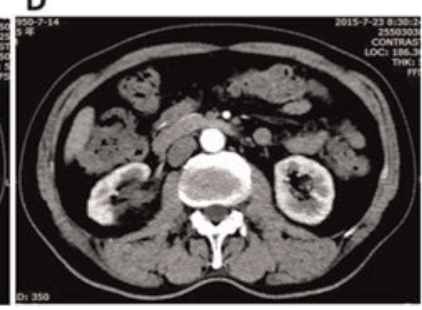


placed in the prone position for percutaneous puncture and tract dilation. Under ultrasound guidance, an eighteen-gauge needle was placed inside the cyst cavity, and a guidewire was introduced followed by sequential dilation up to $28 \mathrm{~F}$. The extra-parenchymal portion of cyst wall was dissociated as much as possible from perirenal adipose tissue with an ureteroscope and irrigation pump pressure, then incised the collapsed cyst wall using either a Thulium or Holmium laser, and a pathological examination was performed. Complicated with ipsilateral renal calculi were treated simultaneously. For parapelvic cysts patients, laser was used to incise cyst wall towards identified pelvis to create a permanent communication between the cyst and adjacent renal collecting system. One end of a F6 double-J stent was inserted into the cyst cavity at the end to prevent auto-closure for at most two months. $22 \mathrm{~F}$ nephrostomy tube was left in renal pelvis for a duration of two weeks. More than $50 \%$ reduction in cyst volume was considered a success. The perioperative complications, hospitalization days and the effective rate of surgery were evaluated.

Results: All operations were conducted without intraoperative complications. The hospital stay after the surgery was 2-4 days (mean 2.5 days). Mean of 11.7 months follow-up, the results showed that the cyst was completely resolved in 53 patients, its size was reduced to less than $50 \%$ in 15 patients, and treatment failed in only 3 anterior cyst patients.

Conclusions: Percutaneous ureteroscopy laser unroofing is an effective and less invasive alternative for treatment of renal cysts in selected patients with a solitary, larger posterior or parapelvic renal cyst.

MP35-19 Intracorporeal excisional tapering of megaureters: An easy task with Robot assistance.

SK Singh, RS Mavuduru, GS Bora, SK Devana, K Parmar, AK Mandal

Post Graduate Institute of Medical Education \& Research, Chandigarh, India

India

Introduction \& Objective: Megaureters requiring intracorporeal ureteral tapering for re-implantation is challenging laparoscopically. After dismembering the ureterovesical junction (UVJ), it is cumbersome to maintain the orientation for excision and suturing. We describe two cases, which were successfully managed by intracorporeal excision and tapering and re-implantation with Robotic assistance.

Materials and Methods: Of two patients, one 52 year, female presented with urosepsis and uremic symptoms. Initially managed by bilateral percutaneous nephrostomy. Nephrostogram, done revealed bilateral obstructed megaureters. She had no voiding symptoms and her Urodynamic studies were normal. Patient then underwent bilateral ureteric reimplantation. The second patient, 27 year male presented with right loin pain and on evaluation was found to have obstructed megaureter. Both patients underwent robotic assisted ureteric reimplantation using DaVinciSi. Both were positioned in steep trendelenburg position and the Robot was docked in between the two legs (Pelvic Docking). Four arms were used. Pelvic ureter was mobilised till UVJ. The fourth arm was helpful in retracting the bladder caudally to keep the ureter straight and taut. Using a Fenestrated bipolar, the ureter was held medially and excisional tapering of distal $5 \mathrm{~cm}$ length was done with scissors', keeping intact the longitudinal leash of vessels anterolaterally. Following this the edges of the ureter were approximated using continuous sutures.
The ureterovesical junction was dismembered and ureteric reimplantation was performed posterolaterally extravesically using modified Lich-Gregoir technique.

Results: Operative time for bilateral re-implantation was 180 minutes, awhile for unilateral re-implantation was 110 minutes. The drains were removed on postoperative day 3 and the patients were discharged. Per urethral catheter was removed at one week and Double'J' stents at 6 weeks. Perioperative period was uneventful. Conclusions: Robotic assistance makes the intracoporeal ureteric excisional tapering and re-implantation easy with successful outcomes in cases with obstructed megaureters.

MP35-20 Cluster analysis identifies three urodynamic patterns in patients with orthotopic neobladder reconstruction

\section{K Kim, D Lee}

Department of Urology, Mokdong Hospital, Ewha Womans University College of Medicine

Republic of Korea

Introduction \& Objective: While previous studies have described the urodynamic parameters of orthotopic neobladders, we have limited information on the characterization of urodynamic or voiding patterns in patients with orthotopic neobladder. In this study, we aimed to classify patients with orthotopic neobladder according to urodynamic parameters using cluster analysis and investigated urinary functional outcomes in each group.

Materials and Methods: From January 2012 to November 2015, 142 patients with bladder cancer underwent radical cystectomy and orthotopic neobladder reconstruction at our institute. Of the 142 patients, 103 with complete urodynamic data and information on urinary functional outcomes were included in this study. K-means clustering was performed with urodynamic parameters which included maximal cystometric capacity, residual volume, maximal flow rate, compliance, and detrusor pressure at maximum flow rate. Three groups emerged by cluster analysis. Urodynamic parameters and urinary function outcomes were compared between three groups.

Results: Group $1(n=44)$ had ideal urodynamic parameters with a mean maximal bladder capacity of $513.3 \mathrm{ml}$ and mean residual urine volume of $33.1 \mathrm{ml}$. Group $2(\mathrm{n}=42)$ was characterized by small bladder capacity with low compliance. Patients in group 2 had higher rates of daytime incontinence and nighttime incontinence than patients in group 1 . Group $3(n=17)$ was characterized by large residual urine volume with high compliance. When we examined gender differences in urodynamics and functional outcomes, residual urine volume and the rate of daytime incontinence were only marginally significant. However, females were significantly more likely to belong to group 2 or $3(\mathrm{P}=0.003)$. In multivariate analysis to identify factors associated with group 1 which has the most ideal urodynamic pattern, age (OR 0.95, $\mathrm{P}=0.017)$ and male gender (OR 7.57, $\mathrm{P}=0.003$ ) were identified as significant factors.

Conclusions: While patients with ileal neobladder present with various voiding symptoms, three urodynamic patterns were identified by cluster analysis. Approximately half of patients had ideal urodynamic parameters. The other two groups were characterized by large residual urine and small capacity bladder with low compliance. Young age and male gender appear to have a favorable impact on urodynamic and voiding outcomes in patients undergoing orthotopic neobladder reconstruction. 
MP35-21 Percutaneous Nephroscopic Pancreatic Necrosectomy: A urologist's gift to pancreatic surgeon

V Jain, N Aggarwal, A Arora

Institute of Liver and Biliary Sciences, New Delhi, INDIA India

Introduction \& Objective: Pancreatic abscess and necrosis are devastating complications of acute severe pancreatitis. Customarily these complications have been managed by placement of percutaneous drains in the abscess cavities. The patients who do not respond to drainage alone require open necrosectomy. We recently started doing pancreatic necrosectomy in a manner exactly similar to our PCNL.

Materials and Methods: Pancreatic necrosectomy was done using 27 Fr nephroscope in a manner similar to standard PCNL. The transperitoneal pre-placed drain tract was dilated using Amplatz dilators over the guidewire and Alken rod. The Amplatz sheath placed in the necrotic cavity and using the nephroscope and forceps the cavity was completely cleaned of pus and all the necrotic material.

Results: This procedure was done in seven patients who did not respond to per-cutaneous drain placement. There were six males and 1 female, in the age range of 28-52 years. All patients underwent the procedure as described above. One patient required two sittings for two different cavities. All patients responded well to treatment as their fever disappeared and counts normalised. None of them required ICU care which was a norm after open pancreatic necrosectomy and avoidance of a large laparotomy scar was an obvious benefit.

Conclusions: Percutaneous Nephroscopic Pancreatic Necrosectomy is a minimally-invasive treatment option for the management of pancreatic necrosis, which does not respond to percutaneous drainage alone. It is safe and viable. It is done in a manner exactly similar to PCNL in a pre-placed tract by a urologist who is well-versed in this procedure. It has significantly less morbidity as compared to standard open necrosectomy and it can be repeated as many number of times required to completely drain the cavities.

In 1990s, nephroscope revolutionized the management of large renal stones and a new era of minimally invasive treatment of stones began in the life of urologist. In 2010s, the same nephroscope is going the change the management of pancreatic necrosis and new era of minimally invasive management is likely to begin in the management of this devastating complication of acute necrotizing pancreatitis.

MP35-22 The effect of hyaluronic acid/carboxymethylcellulose instillation to prevent urethral stricture after transurethral bladder surgery

S Choi, S Yoon, J Do, S Lee, S Jeh, J Hyun, D Seo, C Lee, K Chung, S Kam, J Hwa

Department of Urology, Gyeongsang National University Hospital

Republic of Korea

Introduction \& Objective: To evaluate the effects of GUARDIXSL (hyaluronic acid/carboxymethylcellulose) instillation on the occurrence of urethral stricture after transurethral bladder surgery. Materials and Methods: From January 2011 to June 2014, we retrospectively investigated records of patients who underwent transurethral bladder tumor surgery in our hospital. Among 174 patient, 74 patients received GUARDIX-SL instillation (Group A) and 100 patient did not (Group B). Each patient was evaluated
Table 1. Baseline characteristics and surgical outcomes

\begin{tabular}{lccc}
\hline & Group A $(\mathrm{n}=74)$ & Group B $(\mathrm{n}=100)$ & p-value \\
\hline Age $($ years $)$ & $68.47 \pm 11.66$ & $67.60 \pm 11.32$ & 0.620 \\
BMI $\left(\mathrm{kg} / \mathrm{m}^{2}\right)$ & $23.16 \pm 2.96$ & $23.85 \pm 3.62$ & 0.180 \\
DM & $11(14.86 \%)$ & $19(19.00 \%)$ & $0.475^{\mathrm{a}}$ \\
HTN & $30(40.54 \%)$ & $43(43.00 \%)$ & $0.745^{\mathrm{a}}$ \\
Prostate volume $(\mathrm{CC})$ & $27.35 \pm 10.40$ & $27.19 \pm 6.63$ & 0.902 \\
IPSS total & $14.63 \pm 6.76$ & $13.09 \pm 6.38$ & 0.126 \\
Obstructive subscore & $9.16 \pm 4.27$ & $8.31 \pm 4.06$ & 0.183 \\
Irritative subscore & $5.47 \pm 2.97$ & $4.78 \pm 2.74$ & 0.114 \\
QoL & $2.57 \pm 1.16$ & $2.32 \pm 1.13$ & 0.159 \\
Voided volume $(\mathrm{mL})$ & $225.20 \pm 66.17$ & $230.34 \pm 100.59$ & 0.481 \\
Qmax (mL/sec) & $20.17 \pm 6.69$ & $21.92 \pm 7.95$ & 0.128 \\
PVR (mL) & $20.23 \pm 25.88$ & $21.60 \pm 27.59$ & 0.740 \\
BCG & $56(75.67 \%)$ & $76(76.00 \%)$ & $0.961^{\mathrm{a}}$ \\
Urethral stricture & $2(2.70 \%)$ & $11(11.0 \%)$ & $0.040^{\mathrm{a}}$ \\
\hline
\end{tabular}

Continuous variables were analyzed by using Student $t$-test and categorical variables were analyzed by using ${ }^{2} \mathrm{Chi}$-square test.

$\mathrm{BMI}=$ body mass index, $\mathrm{DM}=$ diabetes mellitus, $\mathrm{HTN}=$ hypertention, $\mathrm{IPSS}=$ International Prostate Symptom Score, QoL $=$ Quality of Life, $\mathrm{Qmax}=\operatorname{maximal}$ flow rate, PVR= postvoiding residual urine volume, $\mathrm{BCG}=$ bacillus Calmette-Guérin

Table 2. Univariate logistic regression analysis of potential factors for incidence of urethral stricture.

\begin{tabular}{lcc}
\hline & Univariate HR $(95 \% \mathrm{CI})$ & p-value \\
\hline Age & $1.023(0.970-1.080)$ & 0.401 \\
BMI & $1.031(0.876-1.213)$ & 0.716 \\
DM & $2.308(0.661-8.058)$ & 0.190 \\
HTN & $0.593(0.175-2.004)$ & 0.400 \\
Prostate volume & $1.028(0.978-1.082)$ & 0.278 \\
GUARDIX-SL instillation & $0.225(0.048-1.047)$ & 0.057 \\
BCG instillation & $1.066(0.279-4.068)$ & 0.926 \\
\hline
\end{tabular}

$\mathrm{BMI}=$ body mass index, $\mathrm{DM}=$ diabetes mellitus, $\mathrm{HTN}=$ hypertention, $\mathrm{BCG}=$ bacillus Calmette-Guérin

at preoperation, postoperative 12 weeks. Baseline characteristics were compared and the effectiveness of GUARDIX-SL was evaluated by the International Prostate Symptom Score (IPSS), uroflowmetry parameters.

Results: Baseline characteristics of two group were not significantly different. Urethral stricture occurrence were $2(2.7 \%)$ in Group A and $11(11.0 \%)$ in Group B and significantly different $(\mathrm{p}=0.040)$ (Table 1). IPSS total, obstructive subscore, irritative subscore and Quality of life (QoL) were significantly increased at 12 weeks from baseline in Group B ( $p=0.023,0.030,0.029$ and 0.011 , respectively). Maximal flow rate was significantly decreased at the same period $(\mathrm{p}=0.018)$. However, univariate and 
Table 3. Multivariate logistic regression analysis of potential factors for incidence of urethral stricture.

\begin{tabular}{lcc}
\hline & Multivariate HR $(95 \% \mathrm{CI})$ & p-value \\
\hline Age & $1.033(0.970-1.099)$ & 0.363 \\
BMI & $1.028(0.855-1.247)$ & 0.765 \\
DM & $2.258(0.605-9.193)$ & 0.217 \\
HTN & $0.430(0.113-1.629)$ & 0.214 \\
Prostate volume & $1.038(0.975-1.104)$ & 0.240 \\
GUARDIX-SL injection & $0.215(0.044-1.049)$ & 0.057 \\
BCG instillation & $1.359(0.303-6.101)$ & 0.689 \\
\hline
\end{tabular}

$\mathrm{BMI}=$ body mass index, $\mathrm{DM}=$ diabetes mellitus, $\mathrm{HTN}=$ hypertention, $\mathrm{BCG}=$ bacillus Calmette-Guérin

multivariate logistic regression analysis showed that GUARDIX-SL instillation was not significant protective factor for urethral stricture occurrence $(\mathrm{p}=0.057$ and 0.057) (Table 2).

Conclusions: During transurethral bladder tumor surgery, GUARDIX-SL instillation decreased the occurrence of urethral stricture, however it was not significant protective factor. Further well designed studies are needed.

MP35-23 Use of Guidewire during Placement of Prophylactic Ureteral Localization Stents (PULSe) for Colorectal Surgery (CRS) Cases Decreases Urologic-induced Operative Complications

R Pathak, G Broderick, R Pak, S Petrou, P Young, E Schommer, D Thiel

Mayo Clinic

United States
Introduction \& Objective: PULSe aids in intraoperative localization and detection of suspected ureteral injury during complex CRS cases. We previously reported the incidence of urologic-induced Clavien grade III complications of PULSe placement at our institution from July 2013 to June 2014 is estimated at $4 \%$. As a quality health initiative, we sought to compare a modification of technique, mandatory use of guidewire assistance during PULSe placement to reduce urologic-induced complications in this patient cohort.

Materials and Methods: Following results of the above study, we made guidewire usage during PULSe placement mandatory at our institution. We reviewed all patients who underwent cystoscopy and PULSe placement at the time of CRS over a 12month period (July 2015 to June 2016). Bilateral 5 French $\times 70$ $\mathrm{cm}$ TigerTail (Bard Medical Division, Covington, GA) PULSe devices were placed with use of guidewire. Flouroscopy was not used. We compared this patient cohort to our prior cohort from July 2013 to June 2014 with the following variables: age, BMI, American Society of Anesthesiologists (ASA) score, pre-operative creatinine, post-operative creatinine, pre/post-operative creatinine difference, and Clavien III urologic-induced complications.

Results: 132 patients with a mean age and BMI of 55.78 (18-89) and 27.02, respectively underwent bilateral PULSe placement with mandatory use of guidewire. Mean pre- and post-procedural creatinine levels were 0.91 and 1.04 , respectively with a mean pre/post procedural creatinine difference of 0.09. No Clavien III complications were encountered in the contemporary cohort, compared to the prior incidence of $4 \%(\mathrm{p}<0.001)$. Moreover, post-operative creatinine and pre/post-procedural creatinine difference also favored the contemporary cohort $(\mathrm{p}<0.022$ and $\mathrm{p}<0.003$, respectively).

Conclusions: Mandatory use of guidewire prior to PULSe placement reduced our incidence of urologic-induced Clavien III complications to zero. Benefits were also observed in post-operative and pre/post procedural renal function with use of guidewire.

\section{VIDEO SESSIONS}

\section{VS1: URETEROSCOPY}

V1-1 Accept the challenge: A staged approach for impacted upper ureteric calculi making task difficult to Easy !

HG Thummar, N Zinto, N T, K Thummar, J Vyas

\section{Sterling}

India

Introduction \& Objective: it's a challenging task to manage large burden upper ureteric stone because it can be associated with severe hydronephrosis, compromised renal function, and infection or pyonephrosis. It makes all modality difficult whether performed antegrade or retrograde, because the large volume in the narrow confines of the ureter does not lend itself to bulk extraction, as can be done for renal stones. We present the results of our technique of retrograde ureteroscopy combined with antegrade irrigation after staging the procedure.

Materials and Methods: We identified 65 patients from 2009 to 2016 who presented with hydronephrosis due to high volume ureteral stones without renal stones and underwent percutaneous nephrostomy (PCN) for decompression. The patients then underwent retrograde ureteroscopy, lithotripsy, and stone extraction (URSL) with our novel technique at a separate session. Irrigation was performed with normal saline using a low-pressure system (gravity at $10 \mathrm{~cm} \mathrm{H} 2 \mathrm{O}$ ) through the PCN. Data regarding stone size/location, degree of hydronephrosis, presence of infection, OR time, EBL, stone-free rate (SFR) and efficiency quotients (EQ), complications, and need for ancillary procedures were analyzed. Results: We identified 65 patients from 2009 to 2016 who presented with hydronephrosis due to high volume ureteral stones without renal stones and underwent percutaneous nephrostomy (PCN) for decompression. The patients then underwent retrograde ureteroscopy, lithotripsy, and stone extraction (URSL) with our novel technique at a separate session. Irrigation was performed with normal saline using a low-pressure system (gravity at $10 \mathrm{~cm}$ $\mathrm{H} 2 \mathrm{O}$ ) through the PCN. Data regarding stone size/location, degree of hydronephrosis, presence of infection, OR time, EBL, stone- 
free rate (SFR) and efficiency quotients (EQ), complications, and need for ancillary procedures were analyzed.

Conclusions: Staged treatment of large volume ureteral stones with PCN placement followed by retrograde URSL with continuous antegrade irrigation is a safe, effective technique with high success and low morbidity.

\section{V1-2 Ureteroscopic Management of a Large Upper Tract Urothelial Carcinoma}

K Scotland, N Kleinmann, L Hubbard, SG Hubosky, DH Bagley

Thomas Jefferson University Hospital

United States

Introduction \& Objective: Upper tract urothelial carcinoma is a relatively rare form of cancer, accounting for approximately $5 \%$ of urothelial tumors and $8 \%$ of renal tumors. Radical nephroureterectomy has traditionally been considered the standard treatment for UTUC. However, the risk of developing chronic kidney disease and contralateral recurrence has raised the need for techniques for renal preservation. Ureteroscopic management of UTUC has achieved favorable treatment outcomes and renal preservation rates in patients with solitary kidneys and renal insufficiency. It has also been applied in carefully selected patients with a normal contralateral kidney. Here we present the ureteroscopic management of a large upper tract tumor.

Materials and Methods: Ureteroscopy was performed using a "no touch technique", with the urinary tract visualized prior to guide wire insertion. Biopsy was obtained using a 2.4 French flat wire basket. A combination of neodymium: yttrium-aluminum-garnet (YAG) and holmium: YAG was used to treat the tumor. Retrograde pyelography and direct vision with ureteroscopy were used to confirm complete intraoperative tumor ablation.

Results: Ureteroscopy with laser treatment resulted in the clearance of a large urothelial tumor involving the entire upper pole of the left kidney.

Conclusions: Uretersocopy is a viable option for the management of large low grade upper tract urothelial carcinoma.

\section{V1-3 Vaporization of ureteral fibroepithelioma}

A Basiri, M Dadpour, A Vali

Urology and Nephrology Research Center (UNRC) Iran

Introduction \& Objective: Fibroepithelial polyps of ureter are rare benign lesions of urinary tract. We present a 42 year old woman with a fibro-epithelial polyp of right ureter caused severe hydronephrosis and pain.

Materials and Methods: evaluation include intra venous pyelogram and magnetic resonance pyelography showed a tumor in distal part of right ureter and diagnosis was confirmed by ureteroscopy and biopsy. we used 8 french ureteroscope and laser probe to vaporize a benign sessile polyp of ureter with no stalk.

Results: In the beginning of the surgery we were not able to pass the polyp and go to proximal part of ureter by ureteroscope. After passing two guide wire we started to vaporize the polyp by laser. After vaporization of most volume of that, finally we passed and reached the proximal part of ureter and pelvis. we placed 2 double $\mathrm{J}$ stents and finished the surgery and candidated the patient to ureteroscopy 6 weeks later. After 6 weeks the patient underwent ureteroscopy and vaporization of the rest part of the polyp and in the final there was not any obstruction in the ureter.

Conclusions: Endoscopic vaporization by laser is a good choice for benign polyp of ureter cause obstruction and hydronephrosis.

\section{V1-4 Malignant Ureteral Obstruction: Management with Tandem Ureteral Stenting}

G Gaunay, P Samson, AD Smith, Z Okeke

Smith Institute for Urology

United States

Introduction \& Objective: Malignant ureteral obstruction (MUO) is a common, challenging pathology resulting from intrinsic obstruction or extrinsic compression due to urologic or non-urologic cancers. Complex ureteral reconstruction is often precluded and treatment commonly takes the form of percutaneous nephrostomy or a form of indwelling stent. Tandem ureteral stents (TUS) a side effect profile superior to that of metallic stents while resisting occlusion better than a single polymeric stent.

Materials and Methods: A case is presented of a 63-year-old male patient with recurrent colonic adenocarcinoma following primary resection with retroperitoneal adenopathy leading to left ureteral obstruction and renal insufficiency. After consideration of all management options, TUS placement was decided upon.

Results: A guidewire is advanced through the ureteral orifice proximally into the renal collecting system under fluoroscopic guidance. A retrograde pyelogram may be obtained prior to wire advancement to better ascertain the degree of obstruction. Balloon dilation of the distal ureter was carried out to enable subsequent stent advancement. A second guidewire was advanced through a dual-lumen catheter advanced over the indwelling wire. With two wires in position, two appropriate length 6 French ureteral stents were inserted over the wires. Care was taken to insert stents symmetrically, as one unit, as friction between the stents may lead to inadvertent disproportionate proximal placement if asymmetrically advanced. Pushers were then introduced and similarly advanced. Stents were deployed once the pushers reached the pubic symphysis. Final fluoroscopic images were obtained to confirm proximal and distal curl formation.

Conclusions: TUS are a highly effective management option for select patients with MUO demonstrating excellent resistance to occlusion and tolerable side effect profile. Urologists should consider this easily reproducible management option when dealing with MUO.

\section{V1-5 Using flexible ureteroscopy to treat a complex biliary stone in a multidisciplinar aproach}

RS Campos, G Guimarães, C Zurstrassen, R Oliveira, J Kawaoka Matushita, B Benigno, S Zequi

\section{AC Camargo Cancer Center}

Brazil

Introduction \& Objective: Endoscopic retrograde cholangiophancreatography (ERCP) has become a widely avaiable and routine procedureon the management of common bile duct stones (CBDS) with more than $90 \%$ sucess rateS. However, ERCP cannot be performed effectively in all patients. Factors such as variability in the anatomy of the ampulla of vater, the 
presence of duodenal diverticuli or surgical procedures involving the stomach such as Rouxen-Y anastomosis may limit sucess. Options for those patients are limited. Technological development of endo-urological materials and new sources of energy to disintegrate urinary calculi, associated with interventional radiology technics, present as promising alternatives. The authors aim to describe a single experience with percutaneous treatment of complex biliary calculi, using conventional endourologic techniques and materials associated with interventional radiology techniques.

Materials and Methods: 43 year old female, diagnosed with pancreatic head adenocarcinoma and liver metastasis. She had undergone biliary drainage stent in October 2014, using biliary stent $8 \times 60 \mathrm{~mm}$. Submitted to a new liver drainage due to tumor ingrowth through the stent mesh after 5 months. On July, 2015, she underwent a new hospitalization due to cholangitis secondary to tumor progression through the stent mesh and a stone in the biliary tract. The first attempt tobreak the stone using angioplasty balloon and internal/external biliary drain with has failed. A new approach after four weeks with a contralateral puncture using two angioplasty balloons. As this procedure was unsuccesful as well, we decided to keep the patient in internal-external biliary drainage. Due to the failure of percutaneous treatment, we decided to combine the treatment with endourologic technique by placing an access sheath $14 \mathrm{~F}$ and subsequent passage of flexible ureteroscopy. The lithotripsy was performed using a laser fiber 270 microns ( $-8 \mathrm{hz}$ and $01 \mathrm{~J})$. Stone fragments were removed with a tipless basket $(2,2 \mathrm{~F})$. Subsequently, the stenosis was dilated with a ballon and a stent was implanted through the prior transpapillary stent in the middle/distal common bile duct.

Results: A final cholangiography showed no residual stone fragments and the contrast medium could pass easily. Post procedure, this patient developed pancreatitis that resolved on the third day, when she was discharged. She could restarted chemotherapy seventeen days later.

Conclusions: The association of interventional radiology with endourologic technichs can offer an effective alternative to treat such complex biliary stones.

\section{V1-6 Ureteroscopy Utilizing Coagulum and ClearPetra Access Sheath}

ME Rivera, J Lingeman, AE Krambeck

Department of Urology, Mayo Clinic (Rochester, MN) United States

Introduction \& Objective: We present a 60 year old male with a significant past medical history of urosepsis with pseudomonas aeruginosa. The patient carries a diagnosis of multiple sclerosis with neurogenic bowel and bladder. The bladder is managed with an indwelling suprapubic catheter. The patient has undergone multiple previous percutaneous nephrolithotomies and ureteroscopies. He presents with computed tomography (CT) imaging demonstrating a large amount of bilateral stone burden.

Materials and Methods: After two weeks of pre-operative intravenous antibiotics the patient undergoes a primary percutaneous nephrolithotomy. CT imaging on post-operative day (POD) 1 demonstrates a large residual calculus in a parallel calyx in the right kidney. Due to the known infectious nature of the patient's stone disease and the fragility of the stone the patient is consented for a right ureteroscopy performed on POD 3.

Results: Due to the known fragile nature of the patient's stone we decide to utilize a suction access sheath to render the patient as stone free as possible. An outflow nipple is attached to allow for closed suction and suction settings are between $150-250 \mathrm{~mm}$
$\mathrm{Hg}$. Ureteroscopy is performed and the stone identified. Laser dusting settings are $0.8 \mathrm{~J}$ and $30 \mathrm{~Hz}$ due to composition of the patients stone. Once lasering is completed the stone fragments are basket extracted. We then utilize 8cc of the patient's blood and inject it through the ureteroscope into the calyx of interest and the renal pelvis. After waiting a period of 5 minutes to allow the blood to coagulate, we begin to basket extract coagulum with attached stone fragments. As we remove stone with the basket small fragments flow alongside the ureteroscope and are suctioned into the collection container. After all clot is removed, repeat ureteroscopy demonstrates excellent stone clearance. The patient does well and was dismissed on POD 7.

Conclusions: We demonstrate the utility of a suction access sheath, coupled with patient derived coagulum to assist with basket extraction of a fragile, infected renal calculus.

\section{V1-7 Robotic assisted retrograde intra-renal surgery for large kidney stone using Avicenna Roboflex}

A Patel, N Tokatli, V Tugcu, S Bedir, J Klein, A Atmaca, M Imamoglu, K Sarica, A Kabakci, J Rassweiler, R Saglam

Spire Roding

United Kingdom

Introduction \& Objective: International guidelines universally recommend PCNL as the treatment of choice for large renal stones. Guidelines by their very nature reflect past practice trends and serve to limit maverick practice. In the past decade, many centers have reported on classical retrograde intra-renal surgery for large renal stones $(>2 \mathrm{~cm})$ with limited single-stage success (due to the interplay of many factors), and better outcomes after multiple stages. Unfortunately, stone size is a poor surrogate for stone volume, while few publications utilize a composite matrix of stone volume, hardness + composition, for outcome prediction.

Materials and Methods: This video demonstrates the step-bystep technique and advantages of Avicenna Roboflex robotic assisted retrograde intra-renal surgery (RA-RIRS), the latest iteration of master-slave robotics in this field. Due to reduced invasiveness, the patient, a 50 year old Iraqi general surgeon, preferred this approach over PCNL. Non-contrast CT showed a large $(2941.5 \mathrm{~mm} 3)$ left renal stone, with 1076 bone window Hounsfield units. Our previous studies have shown: a) an upper stone volume limit of $4000 \mathrm{~mm} 3$ with HU of 1000 for RA-RIRS, yielding $89 \%$ single-session stone free rate; b) that ureteral access sheath size $>11 / 13 \mathrm{Fr}$ allows $35 \mathrm{ml} / \mathrm{min}$. irrigation without raising intra-renal pressure $>25 \mathrm{~cm}$ water, optimizing safety and vision, while allowing irrigant and dust efflux compared to smaller sheaths.

Results: The RA-RIRS procedure was uncomplicated. Optimized efficiency led to single stage complete stone fragmentation (stone dusting time $\times$ minutes, fragmentation efficiency $\mathrm{mm} 3 / \mathrm{min}$ ). RA-RIRS benefits to the surgeon included improved comfort + ergonomics, touch-screen adjustable endoscope motion scaling, laser fiber positioning, control of respiratory excursion + irrigation flow, ability to remotely operate a readied laser and fluoroscopic $\mathrm{C}$-arm, and less fatigue (no lead protection required), cumulatively culminating in $50 \%$ greater control of all procedural aspects compared to manual RIRS.

Conclusions: The Avicenna Roboflex allows safe, efficient single stage treatment of renal stones larger than is recommended by current international guidelines. Key design features optimize patient safety and instrument longevity significantly compared to manual operation and by reducing surgeon fatigue, optimizes potential for efficiency, and releases expensive staff resources, to 
yield cost savings in several OR domains, especially when multiple such procedures are planned in a day's operating

V1-8 Ultrasound-assisted ureteroscopy with direct vision stent placement during pregnancy.

RJ Yau, A Schulman, NH Patel, C Fox, M Zhang, M Eshghi

New York Medical College

United States

Introduction \& Objective: Pregnancy precludes the use of fluoroscopy during ureteroscopy. The absence of external imaging makes confirmation of correct stent placement challenging in this scenario. We highlight direct vision stent placement that ensures accurate stent deployment in the abscence of fluoroscopy.

Materials and Methods: The patient is a 28 year old female in the second trimester with kinking of the right ureter and urolithiasis. We present a video that demonstrates direct vision stent placement following ureteroscopy and lithotripsy.

Results: Ureteroscopy and lithotripsy were successfully performed and the stent was accurately deployed in a gravid female with complicated proximal ureteral anatomy. The video highlights the technique. Of note, an experienced assistant is typically required for this procedure.

Conclusions: Direct vision stent placement is feasible with new generation flexible digital ureteroscopes. It is particularly useful in cases the preclude the use of fluoroscopy.

V1-9 Flexible Ureteroscopic Management of Renal Caliceal Diverticular Stones

Y Yoon, J Na, J Jung, J Jo, H Lee, W Han, S Park

Department of Urology, Hanyang University College of

Medicine

Republic of Korea

Introduction \& Objective: Caliceal diverticulum is an abnormality in the renal parenchyma that results in nonsecretory, urothelial-lined cavity which is filled with urine or small stones. Although mostly asymptomatic, some diverticulum need treatment because of hematuria, infection and flank pain. Herein we report our initial experiences of flexible ureteroscopic managements of renal caliceal diverticular stones.

Materials and Methods: A total of 4 patients underwent flexible ureteroscopic management of renal caliceal diverticular stones from March through June 2016 in our institution by a single surgeon. Flexible ureteroscopic surgery was offered to all patients with symptomatic renal diverticular stones regardless of location and size. Perioperative and postoperative outcomes were described and intraoperative difficulties were noted.

Results: Three diverticuli were in the upper pole and one was in the mid pole. We could approach every diverticulum with flexible ureteroscopes after retrograde pyelography and diverticular neck was easily incised with a 200-um holmium laser probe. The mean operation time 70.5 minutes and the mean estimated blood loss was $13.3 \mathrm{ml}$. All stones in the diverticulum were clusters of tiny stones, and none of them was needed fragmentation before removal. Every patient could leave hospital the day after surgery. There was no intraoperative or postoperative complication. However, one of the patients visited emergency room just after removal of double-J catheter because of the $4 \mathrm{~mm}$-sized remnant stone. The stone ingredient analysis showed that Carbone Oxalate monohydrate was major component of diverticular stones. Conclusions: Our experience with flexible ureteroscopic management of renal caliceal diverticular stones shows that the procedure is feasible. The flexible ureteroscope showed a high success rate, low morbidity, and a short hospital stay, therefore it could be more efficacious and safer than SWL and PCNL.

\section{V1-10 Flexible ureteroscopic Management of En- docalyceal Diverticular Calculi}

\section{A Al Aown}

Armed Forces Hospital Southern Region, Khamis Mushyt Saudi Arabia

Introduction \& Objective: Calyceal diverticula are uncommon urine-containing cavities within the renal parenchyma that communicate with the collecting system through a narrow channel. Diverticula that are located deep within renal parenchyma can be difficult to manage due to challenges in identifying the patent communication of the diverticulum to the collecting system and the potential blurring of the endoscopic vision due to significant hemorrhage from surrounding parenchyma. This report aims to demonstrate an endoscopic approach for the diagnosis and management of calyceal diverticular calculi

Materials and Methods: a 45-year-old female who initially presented with left flank pain and recurrent urinary tract infections. CT urography revealed an $8 \mathrm{~mm}$ left intra-parenchymal lower renal calyceal diverticulum stone. She had a previous history of unsuccessful ESWL. A ureteral catheter was placed cystoscopically to allow contrast injection (retrograde pyelography) for the localization of the diverticular opening to the renal collecting system. During the procedure, a flexible digital ureteroscope was inserted over a guide wire. Inspection of the pelvicalyceal system revealed no stone and the calyceal diverticulum was suspected. A second injection of contrast took place and diverticular opening has been located. A guide wire was inserted through the diverticular opening followed by flexible ureteroscopic dilatation and the stone could be eventually visualized. Laser litrotripsy was performed to fragment the stone.

Results: Operative time was 90 minutes. There were no intraoperative or post-operative complications. Intraoperative blood loss was insignificant. The patient was discharged on the same day. Post-operatively, no complications were encountered. The symptoms of flank pain and recurrent urinary tract infections were resolved during the follow-up period of 12 months. Followup CT urography at 6 months showed no residual stones in the diverticulum.

Conclusions: In this report, we demonstrate a safe and reproducible way for the management of a symptomatic calyceal diverticular stone. Essential technical tricks during the presented endoscopic approach include the placement of a ureteral catheter, performance of multiple retrograde pyelographies and the good visualization of the pelvicalyceal system with the use of new generation of digital flexible ureteroscopes. Careful preoperative imaging and high degree of suspicion are also essential. 


\section{VS2: PCNL}

\section{V2-1 Withdrawn}

V2-2 The new generation super-mini percutaneous nephrolithotomy (SMP) system: a step-by-step guide

G Zeng, W Zhu, Y Liu, J Fan

Department of Urology, Minimally Invasive Surgery Center, the First Affiliated Hospital of Guangzhou Medical University. Guangzhou Institute of Urology. Guangdong Key Laboratory of Urology

China (People's Republic)

Introduction \& Objective: To present our novel miniaturized endoscopic system and describe a step-by-step guide for successful implementation of the super-mini percutaneous nephrolithotomy (SMP).

Materials and Methods:

- The new generation SMP endoscopic system consists of (i) a 40,000-pixel super-mini nephroscope with an 8.0 $\mathrm{F}$ outer diameter and $7.5 \mathrm{~F}$ inner diameter dismountable sheath (ii) and a newly designed irrigationsuction sheath available in either $12 \mathrm{~F}$ or $14 \mathrm{~F}$.

- The irrigation-suction sheath is a two-layered metal structure. The key feature of the irrigation-suction sheath is to allow irrigation and suction respectively (the inflow through the space between the two layers of the sheath, the outflow through the central lumen of the sheath). This property could improve irrigation and stone clearance despite extremely reduced instrument dimension.

- A total of 59 patients with renal stones underwent new generation SMP between April 2016 and December 2016. The percutaneous tract dilatation was carried out to $14 \mathrm{~F}$. Cutting lithotripsy was performed using either holmium laser or pneumatic lithotripter. Stone fragments were sucked out by vacuum suctioning through the sheath. A nephrostomy tube or Double-J stent was placed only if clinically indicated. Low-dose CT was performed to assess the stone-free status on the morning after the procedure.

\section{Results:}

- The mean stone burden was $2.4 \mathrm{~cm} .9$ of the 59 patients had diabetes, and 5 had hypertension.

- SMP was completed successfully in all patients with a mean operative duration of 32.9 min and a mean 1.3 $\mathrm{gm} / \mathrm{dL}$ hemoglobin decrease. The stone-free rate was $91.5 \%$.

- Complications occurred in $5.1 \%$ of the patients, all of them were Clavien I (minor fever managed by antipyretic therapy), no transfusions were needed.

Conclusions: The new generation SMP system is safe, feasible, and efficient for managing moderate-size renal calculi with advantages of a small percutaneous tract, minimal risk of bleeding, high efficacy in stone clearance, improved visual field, short operative time and easiness to operate.
V2-3 Complete Calcified ureteral stent: a combined onesession approach

F Torricelli, R Berjeaut, L Laferreira, F Vicentini, G Marchini, A Danilovic, W Nahas, M Srougi, E Mazzucchi

Section of Endourology, Department of Urology, Hospital das Clinicas, University of Sao Paulo Medical School

Brazil

Introduction \& Objective: Double J incrustation is one of the most serious complications of forgotten ureteral stents. Studies have demonstrated that higher the degree of calcification, poorer the surgical outcome. Nephroureterectomy and cystolithotomy have been described as possible treatments to FECal V forgotten stents. Yet, the best surgical approach is not a consensus among urologists. Combined percutaneous and transurethral access is an option, however it is a challenging procedure. The aim of this video is to demonstrate a successful one-session approach to a complete calcified ureteral stent, preserving the affected kidney without complications.

Materials and Methods: 33-year-old male, presented at our service with an increased urinary frequency, afebrile UTI and left lumbar pain. He underwent an ureterolithotripsy with ureteral stenting three years ago. After that intervention, he was lost to follow-up. A non-contrast CT scan revealed a complete calcified ureteral stent (FECal V). After careful pre-operative planning, patient was submitted to a combined one-step approach including left semi-rigid ureteroscopy, left percutaneous nephrolithotomy (PCNL) and an open cystolithotomy in supine decubitus. This video demonstrates the surgical steps of the procedure for a FECal V (completely calcified) stent removal.

Results: Operative time was 240 minutes. The calcified stent was completely removed. A 6 Fr ureteral catheter and a 16 Fr nephrostomy tube were left at the end of the procedure. No double $\mathrm{J}$ stent was used in order to avoid the same kind of complication. The patient had no peri or postoperative complications. A CT scan was performed in the first postoperative day and revealed two small residual fragments $(7 \mathrm{~mm}$ and $6 \mathrm{~mm})$. The ureteral catheter was removed on first POD and nephrostomy tube on the second POD. Patient was discharged from hospital on the third POD. The patient is now scheduled to a flexible ureteroscopy to treat the small residual fragments.

Conclusions: Forgotten ureteral stent is a surgical challenge, requiring multiple approaches and advanced urological techniques. Our video illustrates that a less invasive and combined one-step procedure to preserve the kidney is both safe and feasible, when performed by an experienced surgeon.

\section{V2-4 Micro-Nephrolithotomy in a transplanted kidney: a peculiar indication for a niche procedure}

S Zanetti, G Sampogna, M Talso, E De Lorenzis, L Boeri, A Gallioli, F Palmisano, M Fontana, I Oliva, F Longo, C Beretta, M Ferraresso, E Montanari

Department of Urology, Fondazione IRCCS Ca' Granda Ospedale Maggiore Policlinico, Università degli Studi di Milano

Italy 
Introduction \& Objective: Micro-percutaneous nephrolithotomy (Micro-PCNL) is a minimally invasive technique proposed by Mahesh Desai as a one-step PCNL. It's performed using a 3 components $4.85 \mathrm{Fr}$ all-seeing needle. The three-way port allows to connect the irrigation and to introduce a fiber optic and a laser fiber. Micro-PCNL is described as a promising treatment for solitary renal stones, with volume $<1000 \mathrm{~mm}^{3}$ and low density, regardless of stone location. Besides this, the Microperc set has recently been described for new indications such as ureteric, bladder and urethral stones and for treating noncalculus diseases like posterior urethral valves and vesico-ureteric reflux. In this video we present one more possible application to treat a stone in a just transplanted kidney.

Materials and Methods: We present the case of a 64 years old man with End Stage Renal Disease needing a kidney transplant from a cadaveric donor. During pre-transplant ultrasonographic evaluation, a $7 \mathrm{~mm}$ stone was found in the kidney.

During the transplant procedure, after vascular anastomosis, we proceeded with the stone removal. Before suturing the transplanted ureter to the bladder, a flexible ureteroscopy was attempted through a 10-12 Fr ureteral access sheath but the access to the narrow calyx hosting the stone was not possible. Then, we decided to convert to a Micro-nephrolithotomy using the MicroPerc set. We punctured the kidney under Ultrasonographic control with the $4.85 \mathrm{Fr}$ needle getting access to the target calix. After visualizing the stone, we proceeded with Homium laser lithotripsy using the dusting setting until obtaining fragments $<1 \mathrm{~mm}$. At the end, a double $\mathrm{J}$ stent was placed ad the transplant procedure was concluded.

Results: We didn't register any intra- or post-operative complication. 1 month after surgery the double $\mathrm{J}$ stent was removed uneventfully.

The Ultrasound examination at 45 days showed the absence of residual fragments and the collecting didn't appear dilated.

Conclusions: MicroPCNL is a technique with very strict and selective indications in kidney stone disease.

Nevertheless, its applicability in many different urological fields and in challenging situations makes it very attractive and it should be part of the armamentarium of the skilled endourologist.

\section{V2-5 Laser Assisted Retrograde Ureteroscopy for Percu- taneous Nephrostomy: A New and Safer Technique for Percutaneous Nephrolithotomy.}

CA Uribe, HD Osorio, JA Benavides, CH Martinez

CES University, Medellin, Colombia

Colombia

Introduction \& Objective: We describe a new option to allow direct vision of the indicated calyx to puncture with the use of flexible ureteroscope in order to develop a percutaneous tract using holmium laser fiber.

Materials and Methods: We present a case of a 48-year-old woman with right renal colic. The computed tomography (CT) scan showed a $2.6 \mathrm{~cm}$ staghorn stone in the right kidney. Under general anesthesia, the patient is placed in prone position. Flexible ureteroscopy is carried out. With the use of holmium laser the selected renal papillae is identified. The laser fiber is exteriorized through the kidney to the skin for an accurate guided puncture.

Results: A laser assisted retrograde ureteroscopy for percutaneous nephrostomy was performed in a patient with right kidney stone. Retrograde contrast and air are injected (pyelography) for calyx anatomy evaluation and the most posterior calyx is chosen. Then, retrograde holmium fiber is passed to the selected calyx and the laser is fired continuously through the papillae. The green light through the skin revealed the ideal spot for the access. We use the holmium laser fiber as a guide to pass the needle. Once the needle is inside, the laser fiber is removed and urine is observed in the needle showing proper access in the selected calyx.

Guidewire is inserted and passed to the bladder using a tipless nitinol basket. After this, double lumen catheter is inserted and safety wire is passed. Dilation of the tract is performed all the time with retrograde endoscopic control. After dilation is complete, a 30 Fr Amplatz sheath is inserted and stone fragmentation with holmium laser is completed. Finally, stone fragments are removed using a nitinol basket.

Conclusions: Percutaneous retrograde laser puncture allows an accurate location of the calyx, less bleeding risk and less radiation for the patient and the surgeon. The procedure is performed under direct vision, giving an adequate percutaneous tract and offering a safe access with less potential complications.

V2-6 A novel modification of the ureteral access sheath for its use as a percutaneous access sheath with a pediatric cystoscope in minipercutaneous nephrolithotomy. 'Preliminary results'.

BO Manzo, J Sanchez, C Salazar, G Manzo, H Sanchez, R Maldonado, M Vanzzini, M Badillo

Endourology Department at Hospital Regional de Alta Especialidad del Bajío

Mexico

Introduction \& Objective: The birth of minimally invasive percutaneous nephrolithotomy (Miniperc) has produced a decrease in comorbidities and complications of conventional NLPC. The percutaneous Amplatz sheath is a key piece in conventional NLPC since it offers a stationary, independent percutaneous access to the nephroscope and at the same time offers a free entrance and exit, however in the case of miniperc, it is difficult to find a percutaneous access sheath that offers same advantages as an Amplatz sheath because every mini-nephroscope commercially available has his own percutaneous sheath. For this reason, we modified ureteral access sheath to fulfill

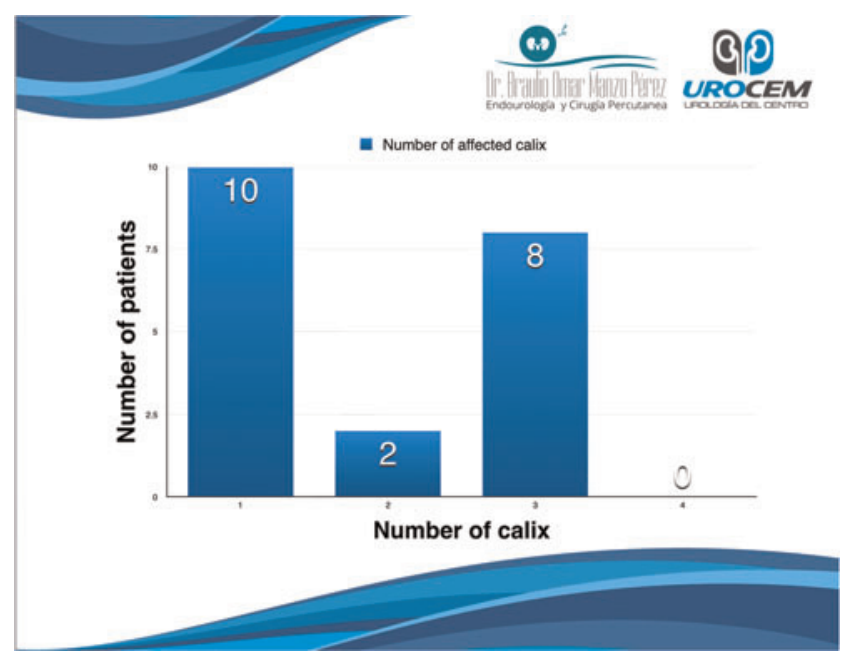



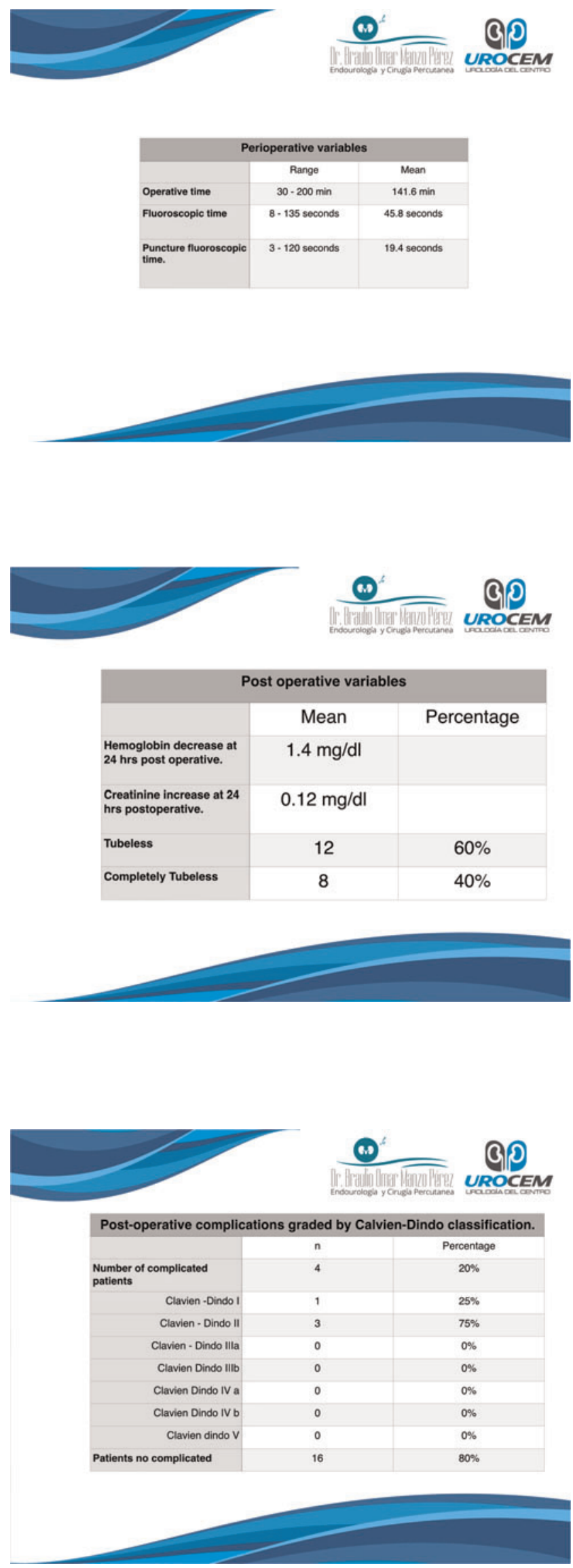

the function of a percutaneous one and enable its use with a pediatric cystoscope for minimally invasive percutaneous nephrolithotomy. To present our modification to the ureteral access sheath for its use as a percutaneous access sheath with a pediatric cystoscope for mini-percutaneous nephrolithotomy and to show its safety and efficacy.

Materials and Methods: We prospectively evaluated 18 patients and 20 renal units with renal stones that were undergone to Miniperc (14-15fr) with a pediatric cystoscope and Holmium laser lithotripsy, with the modification of the ureteral access sheath as a percutaneous access sheath during October 2015 May 2016.

Results:A total of 18 patients and 20 renal units were undergone to miniperc, 10 left and 10 right renal units. Mean age was 35.9 years, $9(50 \%)$ women and $9(50 \%)$ men. The stone burden was $50.24-2479 \mathrm{~mm} 2$ (mean $671 \mathrm{~mm} 2$ ), there were a calculi mean of 1.55 and density of $1162 \mathrm{UH}$. Surgical time was 30-280 min(mean $141 \mathrm{~min}$ ). Mean fluoroscopic time of 8-135 min. (mean: 45.8 seconds). $100 \%$ of surgical events was completed with adequate endoscopic vision. There were no tract losses and 4 patients had postoperative complications: Clavien Dindo I (1) and II (3), that were not related to the ureteral access sheath. Stone free rate was $45 \%$.

Conclusions: Our modification to the ureteral access sheath is effective and safe to perform minimally invasive percutaneous nephrolithotomy with a pediatric cystoscope in the absence of a pediatric nephroscope. A prospective randomized study is necessary to corroborate our results using them as a basis and justification for such research.

V2-7 Improvement of surgical outcomes after preoperative rehearsal using $3 \mathrm{D}$ printed patient specific simulation for percutaneous nephrolithotomy (PCNL)

\section{A Ghazi, E Erturk}

University of Rochester

United States

Introduction \& Objective: Surgical simulation is known to enhance technical skills and this effect is typically strongest for novices because complex procedures such as the PCNL have a steep learning curve. While the benefit of simulation is evident for cases when residents participate, it is unclear how preoperative rehearsal can impact patient safety in the hands of experienced practitioners. We present our initial experience using a high fidelity 3D printed simulation for surgical rehearsal in patients undergoing a PCNL.

Materials and Methods: A total of 20 consecutive patients underwent PCNL by a single Urologist. Before the live procedure, 10 patient-specific simulations were performed for preoperative rehearsal. These models were created by converting

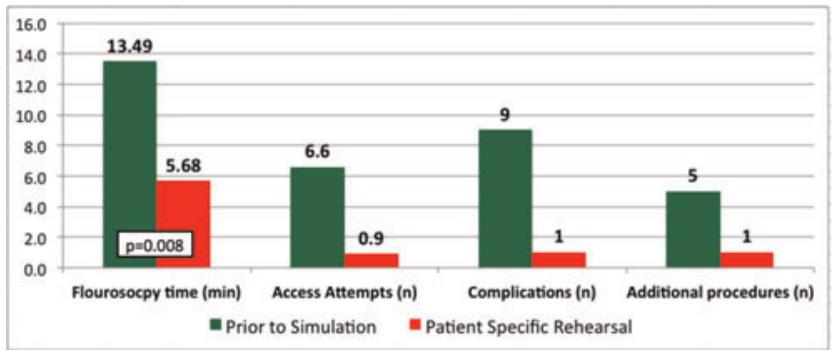


DICOM images into stereolithography files and 3D printing components for assembly. In addition to the patient's pelvicalyceal system and staghorn calculi, their kidney, spine, and posterior abdominal wall were also fabricated to create a complete procedural simulation. All steps of a PCNL were practiced including fluoroscopic access, nephroscopy, and lithotripsy. Procedure specific metrics such as radiation time and number of needle attempts were collected for live and simulation events. Outcomes from the first 10 patients without prior rehearsal were compared to the next 10 patients with preoperative practice.

Results: The mean fluoroscopy time was significantly lower in the rehearsal group (13.5 vs 5.7 mins, $\mathrm{p}=0.008$ ). The average combined time of fluoroscopy for simulation and live cases was still lower than the mean time for unrehearsed group (9.63 vs 113.5 mins, $p=0.09)$. Similarly, the average number of percutaneous needle access attempts was lower in the rehearsed group ( 0.9 vs 6.6 attempts, $\mathrm{p}<0.001)$. The total number of complications and additional procedures was higher in the unrehearsed group ( 9 vs 1 and 5 vs 1, respectively). There were no differences in mean patient age, body mass index, or stone size between the two groups (Table 1).

Conclusions: This study demonstrated that patient-specific rehearsal improved surgeon performance for a complex endourological procedure. There was a significant reduction in fluoroscopy time, percutaneous needle access attempts, additional procedures and complications, with an improved stone clearance rate. There was no significant increase in radiation dose to the surgeon despite rehearsal. Advances in 3D printing technology permit it's routine use for simulation of complicated operations and ability to directly impact patient outcomes.

V2-8 A New Twist on an Old Technique: EndoscopicGuided Lawson Retrograde Nephrostomy Access for Percutaneous Nephrolithotomy in Prone Position

KS Kaler, W Kim

Department of Urology, University of California, Irvine United States

Introduction \& Objective: Retrograde percutaneous access was first introduced by Lawson et al (J. Urol, 1983), and later modified by Hosking ( $\mathrm{J}$. Urol, 1986) performed in the modified lithotomy position. Recently, Kawahara et al (BJUI, 2011) and Wynberg et al (J. Endo, 2012) modified this further using ureteroscopy to guide the Lawson puncture wire in the modified lithotomy position. We have further modified this technique by performing the procedure in the prone split-leg position. Here we present our technique.

Materials and Methods: In patients undergoing percutaneous nephrolithotomy, preoperative CT scans are carefully reviewed for surgical planning. Patients are carefully selected using a subcostal upper pole posterior calyx as the target, have sagittal CT tract length of less than $10 \mathrm{~cm}$, no retrorenal organs on imaging, and no contraindications to prone position. The Lawson retrograde puncture wire and sheath is then inserted through the ureteroscope and advanced through the selected upper pole posterior calyx under fluoroscopic and endoscopic guidance. The Lawson wire is advanced towards the skin posteriorly and exchanged for a standard $260 \mathrm{~cm}$ exchange wire using a 5-French or 8-French ureteral catheter. Subsequently standard nephrostomy sheath is placed following balloon dilation.
Results: This technique was utilized successfully in 4/4 patients with favorable stone and anatomic characteristics. We currently have a $100 \%$ stone-free rate on follow-up CT imaging with in 1 week. There were no post-operative complications in our limited series.

Conclusions: Endoscopic-guided Lawson retrograde nephrostomy access for percutaneous nephrolithotomy in prone position, in this very limited series, is safe, feasible, and efficient. This technique needs to be further studied.

\section{V2-9 Supine Percutaneous Nephrolithotomy for Cystine Staghorn with Urolith Nanopulse}

O Angerri, E Emiliani, F Sánchez-Martin, F Millan, $\mathrm{H}$ Villavicencio

\section{FUNDACIO PUIGVERT}

Spain

Introduction \& Objective: Complete Cystine staghorn it's always a challenging surgery. Supine percutaneous nephrolithotomy (PCNL) it's our standard treatment for this situation. We use a new intracorporeal lithotripsy device, called Urolith Nanopulse to achieve stone free status for this patient.

Materials and Methods: Urolith Nanopulse it's a novel lithotripsy model that it uses electrical pulses to break stones at a substantially higher voltage and a very fast discharge time (nanosecond range). As compared with electrohydraulic lithotripters, nanopulse is more energy efficient in urinary stone fragmentation, because it acts directly on the stone and not via shock wave generated in the liquid.

Results: In Valdivia supine position, cystoscopy was performed with the patient under general anesthesia. An ureteropelvic occlusion ballon catheter $6 \mathrm{fr} / 75 \mathrm{~cm} / 0.4 \mathrm{ml}$ is positioned. Keeping supine position the percutaneous tract was established. Puncture of the inferior calix using fluoroscope with $0^{\circ}-90^{\circ}$ degrees technique. Dilatation of the percutaneous access tract is achieved using balloon dilatator to accommodate a $24 \mathrm{Fr}$ sheath. A $18 \mathrm{Fr}$ nephroscope was inserted using a $6 \mathrm{~F}$ probe with Urolith Nanopulse. We set the energy $1 \mathrm{~J}$ and $5 \mathrm{HZ}$. As we know cystine stones are very hard but since the beginning of the surgery we see fragments with Urolith Nanopulse easily. We complete the extraction of the stones with a $4.5 \mathrm{~F}$ Nitinol basket. At the end of the surgery we introduce a flexible Nephroscope to look all the calyx. A $18 \mathrm{Fr}$ nephrostomy tube and double $\mathrm{J}$ with a bladder catheter were left in place with minimal hematuria. The patient was discharged at 3rd day.

Conclusions: Urolith Nanopulse has proved to be an intracorporeal lithotripter able to break even the hardest stones without complications.

\section{V2-10 Double negative pressure technique for stone clearance in MPCNL}

D Lai, X Li, Y He, G Xu

Fifth affiliated Hospital, Guangzhou Medical University

China (People's Republic)

Introduction \& Objective: To explore the clinical outcome of using double negative pressure technique for stone clearance in minimally invasive percutaneous nephrolithotomy (MPCNL).

Materials and Methods: From March 2016 to March 2017, 100 patients who underwent MPCNL combined with negative pressure 
sheath (ClearPetra) and connecting negative pressure system to nephroscope (Wolf, designed by Li Xun) in the Fifth Affiliated Hospital of Guangzhou Medical Universitywas retrospectively analyzed. The operative time, stone composition, stone clearance rate, preoperative and postoperative hemoglobin level and creatinine were recored. Complications such as postoperative fever, hemorrhage, sepsis were analyzed.

Results: All operations were performed successfully. The mean diameter of the calculi was $3.5 \mathrm{~cm}(5.5-7.0 \mathrm{~cm})$. The mean operation time was $46 \mathrm{~min}$ (25-72min). Postoperative hospital stay was 5 to 8 days. The stone clearance rate was $90 \%(90 / 100)$. Significant hemorrhage was not found after operation and no cases underwent super selective renal artery embolization. 5 cases $(5 \%)$ had postoperative fever.

Conclusions: Using double negative pressure technique for stone clearance in MPCNL can significantly shorten the operative time and reduce the rate of complications.

\section{V2-11 Totally Tubeless Outpatient Minimally Invasive} Percutaneous Nephrolithotomy (MIP)

J Davalos, JE Abbott, U Nagele

University of Maryland Baltimore Washington Medical Center United States

Introduction \& Objective: The gold standard treatment for renal stone burden $>20 \mathrm{~mm}$ is percutaneous nephrolithotomy. Recently, there has been a movement toward doing these procedures with a smaller tract size, minimizing renal drain tubes, and in an outpatient setting. This video demonstrates achievement all of these recent advancements in a healthy young patient. Materials and Methods: A 24 year-old female patient with symptomatic kidney stones was referred to our institution for percutaneous surgical treatment. CT imaging showed $20 \mathrm{~mm}$ of lower pole renal stone burden with an additional $5 \mathrm{~mm}$ stone in a branching lower pole calyx. Right side MIP was planned. In prone position, renal access was obtained under fluoroscopic guidance. Karl Storz 15.5/16.5 Fr sheath was placed into the lower pole calyx. Renoscopy was performed with Karl Storz 12 Fr rigid nephroscope. Stones were fragmented with Holmium:YAG laser using a 200micron fiber and all calculi were passively extracted from the kidney with the vacuum cleaner effect. ${ }^{1}$ Results: Complete stone clearance was achieved. Procedure was performed totally tubeless (without stent or nephrostomy) and the patient was discharged 108 minutes after surgery completion. Conclusions: Totally tubeless outpatient minimally invasive percutaneous nephrolithotomy is safe and feasible. Care should be exercised in patient selection for these cases. 1. Nichlas AP, Schilling D, Bader MJ. The vacuum cleaner effect in minimally invasive percutaneous nephrolitholapaxy. World J Urol. 2015;33(11):1847-53

\section{V2-12 Percutanous Exctraction Of Forgotten Calcified} D-J Catheter At Supine Valdivia Position

F ATEŞ, M Temel, BAŞAL, E malkoç, S Çakmak, $T$ ŞENKUL

Turkey Health Sciences University

Turkey

Introduction \& Objective: A 56 years old man with endoscopic ureteral stone treatment history within 3 years ago presented with macroscopic hematuria to our hospital. Ultrasonography and CT was performed and calcified D-J catheter with bladder and kidney stones and grade 2 hydronephrozis were detected on the right side. We performed laser lithotripsy and percutanous nephrolithotomy (PNL) and D-J catheter extraction consecutively. Materials and Methods: First laser lithotripsy was performed at lithotomy position to the distal calcified part of D-J catheter. Then ureterescopy was done and no calcification was obtained at ureteral part of D-J catheter. PNL with pneumatic lithotripter and extraction of D-J catheter were performed at supine Valdivia position and $14 \mathrm{f}$ nephrostomy catheter was placed.

Results: Operation period was 120 minutes an blood loss was minimal. No complication was occurred. Urethral catheter and nephrostomy catheter were removed at postoperative fist and second days respectively. Before removing nephrostomy catheter antegrade pyelography was performed to check the ureteral passage. The patient was discharge at third day postoperatively. Conclusions: Combination of PNL at supine Valdivia position with laser lithotripsy is a safe and feasible methot for the treatment of forgotten calcified D-J catheters.

\section{V2-13 Percutaneous Electrovaporization of Large Volume} Upper Tract Transitional Cell Carcinoma

T Tran, M Gupta

Icahn School of Medicine at Mount Sinai United States

Introduction \& Objective: Kidney-sparing surgery for upper tract transitional cell carcinoma can be performed in carefully selected candidates. Resection of large volume upper tract transitional cell carcinoma in the kidney is often performed using a percutaneous technique. Previously described methods for percutaneous resection include the use of laser as well as bipolar and monopolar loops. Here, we describe our technique for percutaneous resection using the bipolar vaporizing button.

Materials and Methods: Following placement of a retrograde occlusion device at the ureteropelvic junction, a retrograde pyelogram is performed. Access in the prone position is achieved. Dilation of the tract to allow for placement of a 30French access sheath is performed. The loop or biopsy forceps can be used for debulking of tumor and clot as well as performing a deep-tissue staging biopsy. The bipolar vaporizing button is then used for ablation and resection of the remaining tumor. A 20-French nephrostomy tube is left in place postoperatively.

Results: Use of the bipolar vaporizing button was noted to have several advantages over other contemporary methods for percutaneous resection. In comparison to the laser, resection was more timely and hemostasis was more readily achieved. While resection with the loop remained an efficient alternative, use of the vaporizing button allowed for better hemostasis while performing resection through tumor tissue or parenchyma.

Conclusions: The bipolar vaporizing button allows for safe and efficient percutaneous resection of upper tract transitional cell carcinoma.

V2-14 ESUT educational video on fluoroscopic guided puncture in PCNL: All techniques step by step

I Kyriazis, E Liatsikos, O Sopilidis, P Kallidonis, M Vasilas

Department of Urology, University of Patras

Greece 
Introduction \& Objective: Kidney puncture during percutaneous nephrolithotomy (PCNL) is regarded as one of the most demanding aspects of the procedure and only a minority of urologists perform this step without assistance by a radiologist. Currently a wide variation of fluoroscopic guided techniques is available in clinical practice. In this work we describe the most common fluoroscopic guided access techniques in a step-by-step manner aiming to assist on the standardization of their technique and terminology.

Materials and Methods: A high quality animation video was created for each of the respective fluoroscopic techniques focusing into the parallel projection of external surgical maneuvers and their effect in the 3 dimensional space of the kidney.

Results: Four predominant fluoroscopic guided percutaneous access techniques are available each with different advantages and limitations. Monoplanar access is employed when a stablesingle axis fluoroscopic generator is available and is mostly based on surgeons' experience. Biplanar access employs a second fluoroscopy axis to access puncture's depth. Bull's eye technique follows a coaxial to fluoroscopy puncture path and is associated with the shorter learning curve at the cost of increased hand radiation exposure. Hybrid and conventional triangulate techniques use target projection by two fluoroscopic planes to define the exact localization of target in space and access it through a third puncture site.

Conclusions: Fluoroscopic guidance during PCNL puncture is a very efficient method of access establishment. Percutaneous surgeon should be familiar with all available variations of fluoroscopic approach in order to be ready to adopt puncture on any given scenario.

\section{VS3: ROBOTIC UPPER TRACT SURGERY FOR CANCER I}

\author{
V3-1 Robotic partial nephrectomy involving in- \\ tracorporeal cold ischemia for post cryoablation failure \\ Z Hamilton, M Holden, O Raheem, IH Derweesh \\ Department of Urology, UC San Diego Health, La Jolla, \\ California, USA \\ United States
}

Introduction \& Objective: The reference standard treatment for small renal masses is partial nephrectomy, and the robotic approach has become rapidly adopted in this setting. However, minimally invasive approach to partial nephrectomy can be complicated by previous surgery or percutaneous renal ablative therapy. The ideal approach to partial nephrectomy is unknown in this setting. We present a surgical video of a 62 year old female with a previous left renal cryoablation, who developed an ipsilateral recurrent left renal mass in the previous ablative zone. We performed a robotic partial nephrectomy utilizing intracorporeal cold ischemia. Our surgery is technically innovative in that we introduce a method for providing cold ischemia through a minimally invasive robotic approach in the setting of cryotherapy failure.

Materials and Methods: The Da Vinci Xi (Intuitive Inc, Sunnyvale, CA, USA) robotic platform was utilized with a 4 arm approach. Robotic ports were placed in a linear configuration lateral to the rectus muscle and an assistant port near the umbilicus. An additional $15 \mathrm{~mm}$ accessory port is placed near the $12^{\text {th }}$ rib for introduction of ice slush. Modified $20 \mathrm{~mL}$ syringes are used to deploy ice slush through the $15 \mathrm{~mm}$ accessory port. The renal artery is clamped and the tumor completely excised. Renorrhaphy was performed with adequate hemostasis.

Results: Estimated blood loss was 50mL Cold ischemia time was 83 minutes. Final pathology revealed clear cell renal cell carcinoma, Fuhrman grade 2, $2.0 \mathrm{~cm}$, with negative margins, pT1a. Follow up renal functional studies showed a GFR $>60 \mathrm{~mL} / \mathrm{min} /$ $1.73 \mathrm{~m}^{2}$ at 6 month follow up.

Conclusions: Robotic partial nephrectomy in the setting of percutaneous ablative failure is technically feasible and can be performed with the addition of ice slush cold ischemia. Further study is requisite to confirm these results.
V3-2 Minimally Invasive Nephron-Sparing Surgical Management of Multifocal Renal Masses in the Hereditary Kidney Cancer Syndrome Patient

KL Stern, MA Zell, EP Castle

Mayo Clinic Arizona

United States

Introduction \& Objective: Patients with hereditary kidney cancer syndromes such as von Hippel-Lindau syndrome, are at risk for multifocal, recurrent, bilateral renal masses, often presenting at a young age. This presents a challenge for surgical management, with minimally-invasive nephron-sparing procedures of utmost importance. Additionally, the associated syndromic co-morbidities can present unique challenges with surgery in previously-operated fields.

Materials and Methods: A 29 year old male with von HippelLindau syndrome was referred to our clinic for management of multiple bilateral renal masses. His past medical and surgical history was significant for Whipple procedure performed for a benign neuroendocrine tumor at the head of the pancreas as well as several other stigmata of VHL elsewhere in the body, including paratesticular cystadenomas and cerebellar hemangioblastomas. Abdominal MRI was significant for as many as 10 masses in the left kidney that ranged between 0.3 to $1.7 \mathrm{~cm}$, as well as multiple masses in the right renal hilum/sinus measuring up to $3.5 \mathrm{~cm}$. Given their location they were not amenable to ablative therapy and a complete nephrectomy was recommended at an outside institution. Nuclear medicine kidney scan showed normal split function ( $48 \% \mathrm{~L}, 52 \% \mathrm{R})$. Preoperative creatinine was $0.9 \mathrm{mg} / \mathrm{dL}$. In November 2016 he underwent a right robotassisted partial nephrectomy on four tumors. Three separate solid tumors in the renal sinus and hilum were removed on-clamp, with total clamp time of 25 minutes and 26 seconds. A fourth tumor was removed in the medial upper aspect of the kidney off clamp. Estimated blood loss was 1L. Total operative time was 3 hours, with 2.5 hours of console time, including 30 minutes of adhesiolysis.

Results: The patient was discharged home on postoperative day number 4 after an uncomplicated postoperative course. His hemoglobin nadir was $10.8 \mathrm{~g} / \mathrm{dL}$. Creatinine at discharge was 
$1.0 \mathrm{mg} / \mathrm{dL}$ and at two week follow-up was $0.9 \mathrm{mg} / \mathrm{dL}$. On follow up visits, the patient's incisions were well-healed. His postoperative creatinine was unchanged from prior to surgery. Final pathology determined all four masses to be clear cell RCC, Furman grade 3 , with negative margins.

Conclusions: Complex partial nephrectomies of centrally-located renal masses are technically feasible and safe and can provide excellent oncologic and nephron-sparing outcomes in patients with hereditary kidney cancer syndromes.

V3-3 Transperitoneal Robotic Assisted Laparoscopic Partial Nephrectomy in a Patient With Severe Scoliosis

C Shen, $\mathrm{C} \mathrm{Hu}, \mathrm{C} \mathrm{Ou}$

Department of Urology, National Cheng Kung University Hospital

Taiwan (Republic of China)

Introduction \& Objective: We report a case of robotic assisted laparoscopic partial nephrectomy in a patient with left kidney renal cell carcinoma and a severe left convex lumbar scoliosis. To our knowledge, this is the first article that describes a transperitoneal robotic assisted laparoscopic partial nephrectomy in the presence of severe spinal deformities and altered visceral and vascular abdominal anatomy.
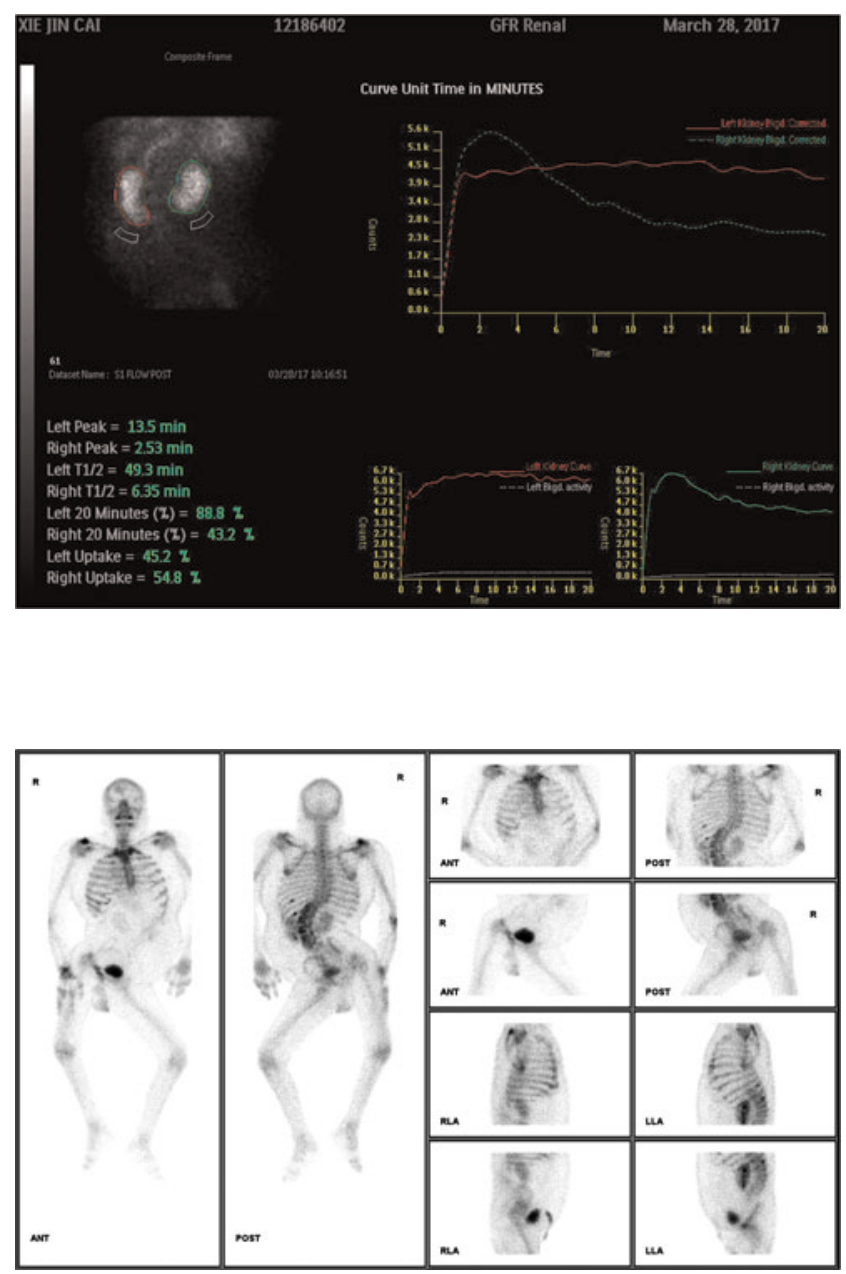
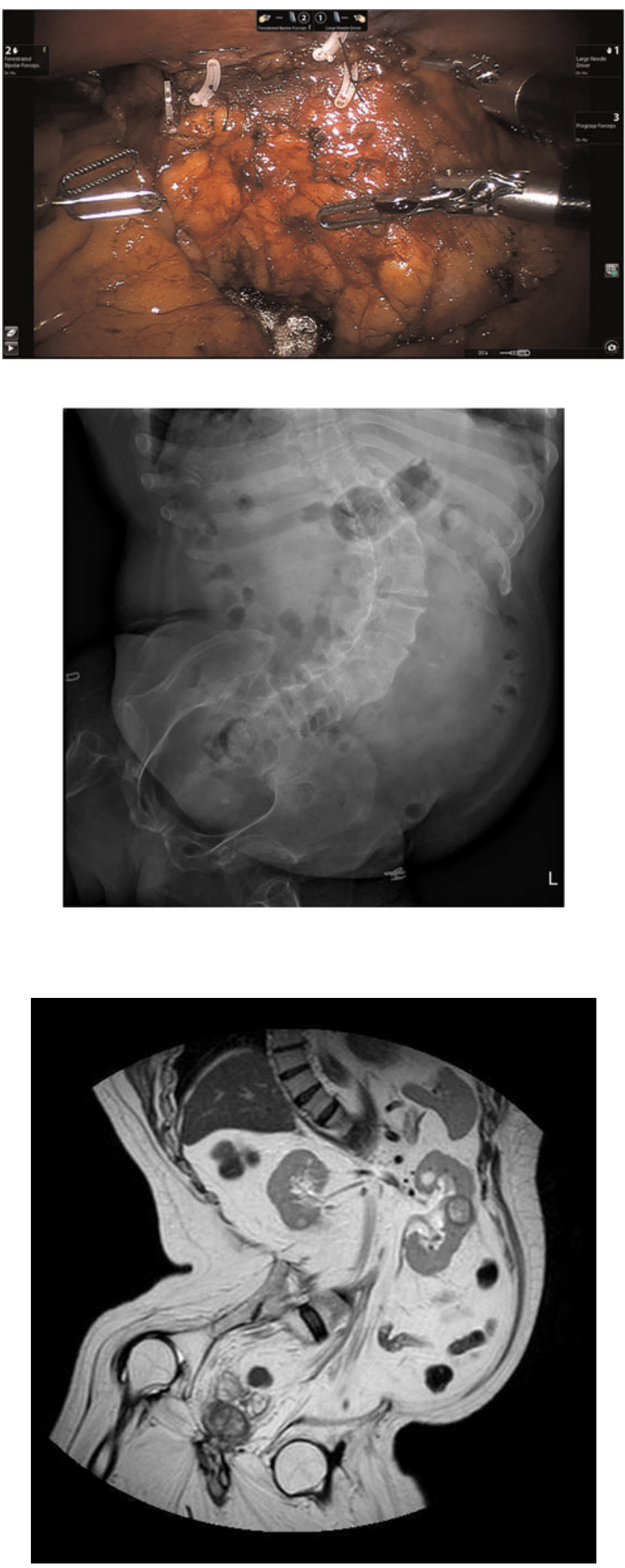

Materials and Methods: After serial meticulous preoperative imaging including computed tomography and magnetic resonance imaging, the surgical procedure was conducted by an experienced robotic assisted laparoscopic surgeon. The scoliosis made it impossible to use the psoas muscle as a landmark for the 
dissection of the renal hilum, neither can we explore and suspend the ureter first using caudocranial dissection of the left renal hilum. Thus after dissecting line of Toldt and exposure of left Gerota fascia, we directly identified left renal vein and renal artery within a rather limited operating space. Left renal tumor was identified via sonography then excised with left renal pedicle clamping. Tumor margin sent during the operation yielded negative result for malignancy.

Results: The surgeon was able to complete the transperitoneal robotic assisted laparoscopic partial nephrectomy without complications.

Conclusions: With altered visceral and vascular anatomy in scoliosis patients, robotic assisted laparoscopic partial nephrectomy is a feasible approach to prevent major vascular and visceral injury in this challenging surgical procedure while ensuring patient safety and cancer control.

\section{V3-4 Robot assisted radical nephrectomy and inferior vena cava thrombectomy: surgical technique, perioperative and oncologic outcomes}

G Simone, L Misuraca, G Tuderti, D Hatcher, M Ferriero, D Andre Luis, F Minisola, M Aron, S Guaglianone, M Desai, IS Gill, M Gallucci

Regina Elena National Cancer Institute Rome Italy

Introduction \& Objective: In this video we highlight surgical steps of a right radical nephrectomy and level IIIb inferior vena cava (IVC) thrombectomy using an occluding balloon Fogarty catheter to control the upper boundary of IVC thrombus under transesophageal ultrasound guidance.

Materials and Methods: Key surgical steps are higlighted in the video.

Preoperative arterial embolization was performed. A right template retroperitoneal lymph node dissection was performed and all lumbar veins were secured to achieve a complete IVC control; the left renal vein and the distal IVC segment were prepared and encircled with Roummel Tourniquet. Short hepatic veins were secured with Ligasure and the posterior aspect of the IVC was prepared. Proximal IVC was encircled and right renal vein was stapled.

The distal IVC and left renal vein Tourniquets were cinched down and a small cavotomy was performed to insert a Fogarty catheter with an occluding balloon tip. Once the balloon was inflated and distally attracted, cavotomy was performed and the thrombus progressively mobilized and secured into an endocatch bag. The balloon was deflated to restore IVC flow throw the major hepatic veins. The cava was sutured with a 3/0 Visi black monocryl suture.

Thirty-five consecutive patients with renal tumor and IVC thrombus were treated between July 2011 and September 2016 in two tertiary referral centers; perioperative and oncologic data were reported.

Results: Fogarty catheter was successfully used in 7 (20\%) cases. Open conversion was necessary in one case $(2.8 \%)$. Median operative time was 300 minutes. Ten patients (28.6\%) required blood transfusion (Clavien grade 2); one patient $(2.8 \%)$ had a Clavien grade 3 a complication (gastroscopy); two patients (5.7\%) had Clavien grade 3b complications (reintervention due to bleeding from adrenal gland and subphrenic ascess requiring drainage, respectively); one patient (2.8\%) experienced a PRESS syndrome requiring ICU admission (Clavien 4a).
Out of 13 patients who underwent cytoreductive nephrectomy and IVC thrombectomy, only one patient died of disease progression 14 months postoperatively. Both 2-yr cancer specific and overall survival rates in this subpopulation were $88.9 \%$.

Twenty-two patients received surgery with curative intent and 5 of these experienced disease recurrence: $2-y r$ metastasis free, cancer specific and overall survival rates were $56 \%, 100 \%$ and $94.4 \%$, respectively.

Conclusions: The increasing experience with robotic surgery has made nephrectomy and IVC thrombectomy a feasible and safe treatment option in tertiary referral centers, associated with favourable perioperative outcomes and encouraging short term oncologic outcomes.

V3-5 Robotic Assisted Repair of Diaphragmatic Injury with Patch during Complex Partial Nephrectomy

J Bloom, C White, M Stifelman

National Cancer Institute

United States

Introduction \& Objective: This video demonstrates our experience with using a Gore-Tex patch to repair a diaphragmatic injury that was encountered during a robotic assisted partial nephrectomy on a kidney that had significant scar tissue from a prior open surgery.

Materials and Methods: Our patient is a 70 year old female with a solitary left kidney and had previously undergone an open retroperitoneal partial nephrectomy. While mobilizing the lateral portion of the kidney robotically, we encountered a great deal of scar tissue and bleeding from the prior operative site. At this time, we recognized a diaphragmatic injury.

Results: The patient remained stable and there were no issues from anesthesia. We decided to finish mobilizing the kidney and performing the partial nephrectomy. After this was complete, we used a piece of Gore-Tex that was measured to the size of the defect. Using prolene sutures this graft was fixed over the defect and reinforced. A chest tube was placed immediately after the procedure, she did well and had a routine postoperative course.

Conclusions: An injury to the pleura or diaphragm of a patient can be encountered during a partial nephrectomy. Robotic surgeons should possess the skills to both recognize the injury as well as the strategies for repair.

V3-6 Surgical Navigation using Intuitive Image-to-Patient Registration for Robot-Assisted Partial Nephrectomy: Clinical Feasibility study

S Kobayashi, B Cho, K Tatsugami, M Hashizume, M Eto

Department of Advanced Medical Initiatives Faculty of Medical Sciences, Kyushu University

Japan

Introduction \& Objective: Robot-assisted renal partial nephrectomy (RAPN) for a small renal tumor has become a standard treatment option. Although surgeons require more information like real-time 3D images from preoperative images due to the increasing difficulty of surgery, such as a renal hilus tumor or a complete endophytic renal tumor, there are few 
reports on surgical navigation specialized for robotic surgery. We focused on the extension of Tile Pro ${ }^{\circledR}$ and started to develop the surgical navigation system using 3D images and optical tracking system. To apply surgical navigation with automatic tracking system to RAPN efficaciously and to evaluate its effectiveness clinically.

Materials and Methods: We manually performed segmentation of kidney, renal vessels, urine, a tumor from enhanced computed tomography and reconstructed as 3D image using 3D slicer. To prepare for registration, optical tracking markers were attached to an operating table and da Vinci's endoscope, and we performed calibration between the camera coordinate system and the optical tracking system. To synchronize between 3D image and endoscopic image, we conducted intuitively manual registration preoperatively. Patients who performed RAPN without surgical navigation (WOSN $n=26$ ) and with surgical navigation (WSN n =22) from June 2013 to December 2016 were identified as the object of this study. The surgical process was classified in detail and surgical time was assessed. And complications, renal function were evaluated.

Results: Only surgical approach was significantly different between WOSN group and WSN group $(\mathrm{p}=0.004)$. Age, sex, body mass index, RENAL nephremetry score and tumor site were not significantly different. Median console time was $1 \mathrm{hr} 50 \mathrm{~min} 1 \mathrm{ss}$ (WOSN) vs $1 \mathrm{hr} 40 \mathrm{~min} 12 \mathrm{~s}(\mathrm{WSN})(p=0.172)$, median time to reach renal hilus was $22 \mathrm{~min} 29 \mathrm{ss}$ vs $5 \min 55 \mathrm{ss}(\mathrm{p}=0.0024)$, median time to identify renal artery was 5 min36ss vs 1 min40ss $(\mathrm{p}=0.016)$, median time to exfoliate renal artery and support with vessel tape was $9 \min 16 \mathrm{ss}$ vs $6 \min 7 \mathrm{ss}(\mathrm{p}=0.040)$, median time to suture renal parenchyma was $23 \min 37 \mathrm{ss}$ vs $6 \mathrm{~min} 27 \mathrm{ss}$ $(\mathrm{p}<0.0001)$. Complications, renal function and pathological outcomes were not significantly different. On multivariable analysis, time to renal hilus $(\mathrm{p}=0.0293)$ and to suture renal parenchyma $(p<0.0001)$ were strong positively associate with surgical navigation.

Conclusions: We proposed the surgical navigation with automatic tracking system and clinical application for RAPN. It is suggested that the surgical navigation is strong factors associated with time to reach renal hilus and time to suture renal parenchyma at RAPN.

V3-7 Optimization of Blood Loss During Robotic IVC Thrombectomy - Is the Robotic Approach Feasible in the Jehovah's Witness?

P Heller, A Jones, C Johans, K Murray, N Pokala

University of Missouri

United States

Introduction \& Objective: There have been various series describing the feasibility and safety of robotic-assisted laparoscopic inferior vena cava thrombectomy with appropriate patient selection. Management of these tumors via a robotic approach may decrease blood loss; however, still have a high risk of significant intraoperative blood loss. With appropriate strategies the operation can be performed in Jehovah's witnesses who refuse transfusion. We present our experience of three patients, one of whom was a Jehovah 's Witness that refused blood transfusion, who underwent a complete intracorporeal robotic-assisted laparoscopic IVC tumor thrombectomy, and describe our strategies and surgical technique to optimize intraoperative blood loss.

\begin{tabular}{|c|c|c|c|}
\hline Patient No. & 1 & 2 & 3 \\
\hline Age (yrs.) & 60 & 58 & 60 \\
\hline ECOG & 0 & 0 & 1 \\
\hline Gender & $\mathrm{F}$ & M & M \\
\hline $\mathrm{BMI}(\mathrm{Kg} / \mathrm{m} 2)$ & 35 & 39 & 25 \\
\hline ASA Class & 3 & 3 & 3 \\
\hline Renal Tumor Size & 12 & 9.6 & 14.5 \\
\hline Laterality & Rt & Rt & Rt \\
\hline $\begin{array}{l}\text { Tumor Thrombus Level } \\
\text { (Mayo Classification) }\end{array}$ & ॥ & $1 / 11$ & ॥ \\
\hline IVC Thrombus Length $(\mathrm{cm})$ & 4.7 & 2 & 3 \\
\hline Preexisting Mets & Yes ${ }^{-}$ & None (Concurrent colon mass) & Yes \\
\hline Neoadjuvant Chemotherapy & None & None & None \\
\hline $\begin{array}{c}\text { Preoperative Renal } \\
\text { Emobolization }\end{array}$ & Y & N & Y \\
\hline Console Time (min) & 556 & 268 & 320 \\
\hline EBL & 400 & 900 & 300 \\
\hline Hospital Stay & 7 & 8 & 13 \\
\hline Intraoperative Complications & $\mathrm{N}$ & N & $\mathrm{N}$ \\
\hline Postoperative Complications & None & Clavien IIIa* & Clavien Illa \\
\hline Histology & RCC & RCC & RCC \\
\hline Tumor Grade & 4 & 4 & 4 \\
\hline PTNM Stage & PT3bNOMx & PT3aNOMx & PT3bNOMx \\
\hline Months Followup & 8 & 9 & 5 \\
\hline Current Status & Alive/NED & Aliveł & Alivet \\
\hline
\end{tabular}

$\infty$ Subsequently biopsied with pathology showing necrotizing granulomas with sarcomatoid features. fDeveloped $\mathrm{mRCC}$ to the lung and started on Sutent 3 months after surgery. Experienced disease progression and switched to cabozatinib 6 months post-op with regression of metastatic disease. Started on FOLFOX for colon CA months after surgey for metastatic colon cancer.

† Experienced progression of lung and liver mets and was started on nivolumab.

*Developed sepsis from infected seroma and acute respiratory failure requiring intubation.

+ Experienced a post-operative STEMI requiring cardiac stenting.

Materials and Methods: Three patients underwent completely intra-corporeal robotic-assisted laparoscopic IVC tumor thrombectomy for level II IVC tumor thrombi. Retrospective chart review was performed.

Results: Three cases were performed (NP) completely robotically without the need for conversion to open. Mean renal tumor size was $12 \mathrm{~cm}$ (range $9.6-14.5 \mathrm{~cm}$ ) and mean tumor venous thrombus length was $3.23 \mathrm{~cm}$ (range $2-4.7 \mathrm{~cm}$ ). Two patients presented with metastatic disease. One patient underwent robotic-assisted laparoscopic subtotal colectomy for a transverse colon mass on the same OR date. Mean console time was 388 minutes (range 268-556 minutes), estimated blood loss $533 \mathrm{ml}$ (range $300-900 \mathrm{cc}$ ), hospital stay 9.3 days (range $7-13$ days). No intraoperative complications occurred. Two Clavien IIIa postoperative complications occurred. At a mean of 7.33 months of followup, all patients are still alive.

Conclusions: Robotic IVC thrombectomy is feasible and safe with appropriate preoperative planning and patient selection.

V3-8 Da Vinci Robotic Assisted Laparoscopic Hemi-nephrectomy for Upper pole high risk tumors.

\section{Y T B, S Waigankar, A Pednekar, T Kaushik}

Kokilaben Dhirubhai Ambani Hospital and Research Institute India

Introduction \& Objective: Da Vinci Si Robot assisted laparoscopic Heminephrectomy is gaining popularity in managing high risk upper polar renal tumours. It reduces the technical difficulty of parenchymal suturing during renorrhaphy. We aim to present the technique and outcomes of Laparoscopic (robot assisted) heminephrectomy. 
Materials and Methods: We analyzed 12 heminephrectomies (5-Left and 7-right sided) done from 2012 to 2017 for 12 Renal tumors (8-incidental \& 4-hematuria) (All upper pole tumors) in 12 male patients. Our surgical approach included colon reflection, hilar dissection, tumour identification, tumour scoring, arterial clamping, tumour dissection, inner and outer renorrhaphy with PCS closure, declamping and specimen retrieval

Results: The mean age was 42 years (24-54). The median RENAL Nephrometry score was $10 \mathrm{P}$ with angiography showing single renal artery. The mean age \& eGFR of the patient was 42 years \& $112 \mathrm{ml} / \mathrm{min} / 1.73 \mathrm{~m}^{2}$ respectively. The mean console time, warm ischemia time, blood loss \& hospitalization time was $143 \mathrm{mins}, 26 \mathrm{mins}, 70 \mathrm{ml}$ and 5 days respectively. Intra-operative and post-operative period were uneventful. On histopathology all tumour were Conventional Clear cell renal carcinomas. The patients were discharged on postoperative day 3 . At 1 and 3 months of follow-up the patient were disease-free with minimal change in eGFR.

Conclusions: In the hands of experienced surgeons, RAPN is a safe and reproducible approach for the treatment of challenging renal tumors. It is also effective in renal function preservation and oncologic control at an early follow-up interval. However more number of patients with long term outcomes data is necessary.

V3-9 Robotic Radical Nephrectomy with Level II Inferior Vena Cava Thrombectomy

\section{A Beksac, J Sfakianos, K Badani}

Icahn School of Medicine at Mount Sinai United States

Introduction \& Objective: In this video, we present a case of transperitoneal radical nephrectomy with level II inferior vena cava (IVC) thrombectomy. The patient is a 78 year old female with intermittent history of hematuria. Abdominal MRI revealed $7.8 \times 7.1 \times 6.7 \mathrm{~cm}$ upper pole mass with level II inferior vena cava (IVC) thrombus. Chest imaging did not reveal any evidence of metastases.

Materials and Methods: The patient was positioned in the full flank position. Liver was retracted cephalad with the self-retaining grasper and the colon was reflected medially by incising the white line of Toldt. The duodenum was reflected medially until the undersurface of IVC and aorta were exposed. Gonadal vein and ureter were identified and dissected proximally to the renal vein. Vena cava and renal vein were circumferentially dissected to the proximal and distal of the thrombus. The right renal artery was identified in the inter-aortocaval space and clipped. Intraoperative ultrasound was used to delineate the extent of the tumor thrombus. Rummel tourniquets were utilized along with bulldog clamps for venous control. Vena cava was incised and the thrombus was removed. IVC was reconstructed and closed with 4-0 prolene sutures. Tourniquets were released from the renal vein, suprarenal IVC and infrarenal IVC respectively. The renal artery was taken with Endo-GIA stapler. Posterior attachments were lysed and specimen was removed.

Results: Surgery was completed in 2:10 hours, with an estimated blood loss of $100 \mathrm{~mL}$. IVC occlusion time was 16 minutes. Drain was removed at day 1 and patient was discharged without complications. Pathology revealed grade III clear cell carcinoma and negative surgical margins.

Conclusions: This video demonstrates a stepwise technique for transperitoneal IVC thrombectomy. IVC thrombectomy is a reproducible robotic operation in experienced centers.
V3-10 Robotic Assisted Laparoscopic Right Radical Nephrectomy with Right Template Retroperitoneal Lymph Node Dissection

\author{
AB Edwards, J Messer
}

University of Louisville

United States

Introduction \& Objective: Robotic Assisted Laparoscopic Surgery has been increasingly utilized for more complex operative interventions with the potential benefit of improved convalescence and decreased blood loss.

Materials and Methods: We present a 80 year old female with a $6 \mathrm{~cm}$ right renal mass and a $5 \mathrm{~cm}$ paracaval lymph node discovered on CT scan for the evaluation of right sided flank pain treated with a Robotic Assisted Laparoscopic Right Radical Nephrectomy with Right Template Retroperitoneal Lymph Node Dissection.

Results: Total Operative time was 142 minutes, with an estimated blood loss of $250 \mathrm{~mL}$. The patient was discharged home on postopertaive day number one. Final Pathology revealed a pT4aN1 Clear Cell Renal Cell Carcinoma with negative margins, and one 5 cn metastatic lymph node out of 37 total lymph nodes removed. Conclusions: The robotic platform can be utilized to manage renal cell carcinoma even in the setting of metastatic adenopathy with comparable short term pathologic outcomes.

V3-11 Robotic Assisted Laparoscopic Partial Nephrectomy on a complex upper pole posterior endophytic tumor

AB Edwards, J Messer

University of Louisville

United States

Introduction \& Objective: Nephron Sparing Surgery is being used in increasingly complex renal lesions with the potential benefit of renal preservation, and similar oncologic outcomes for small renal masses. The Robotic Assisted Laparoscopic Approach has the potential for improved convalescence and decreased blood loss.

Materials and Methods: We present a Robotic Asssited Laparoscopic Partial Nephrectomy on an endophytic posterior upper pole right renal mass on an incidentally discovered $3.3 \mathrm{~cm}$ mass, on a 75 year old female with stage II chronic kidney disease.

Results: Total operative time was 110 minutes with warm ischemia time of 32 minutes, and estimated blood loss of $350 \mathrm{~mL}$. The patient was discharged home on postoperative day number 2 with final pathology revealing a pT3a margin negative furhman grade II clear cell renal cell carcinoma.

Conclusions: Robotic Assisted Laparoscopic partial nephrectomy can be applied safely to complex renal masses, with equivalent pathologic outcomes, with acceptable warm ischemia times.

\section{V3-12 Robotic Partial Nephrectomy: 4-Arm Retro- peritoneal Approach}

H Sawkar, N Patel, D Hatcher, FZ Husain, S Ghodoussipour, M Aron

\section{United States}

Introduction \& Objective: Retroperitoneal access for partial nephrectomy has long been a useful approach for postero-medial kidney tumors, however there is a relative lack of familiarity with 
this technique. Our video goes over a step by step approach for performing a successful retroperitoneal partial nephrectomy using the DaVinci Si and Xi Robotic systems.

Materials and Methods: Patients are positioned in the full 90 degree flank position with the back of the patient at the very edge of the table. Both arms are extended anteriorly and supported on double arm boards or modifications thereof. The table is fully flexed to increase space between the lower ribs and the iliac crest. A five trocar access is performed, which includes a camera port, 3 working robotic arms and one assistant port. The robot is then docked over the patient's ipsilateral shoulder.

Results: We present a sample case: a 52 year old male with a $4.7 \mathrm{~cm}$ right sided postero-medial tumor abutting the renal hilum. In a systematic fashion, the renal artery was exposed, the was tumor defatted and excised, and the renal defect was closed. There were no intraoperative complications and the blood loss was approximately $100 \mathrm{cc}$. The patient had an uneventful postoperative course and was discharged on postoperative day 2.

Conclusions: In conclusion, robotic assisted retroperitoneal partial nephrectomy is an effective approach for posterior renal tumors. The techniques outlined in this video are a good starting point for familiarizing a surgeon with this procedure.

V3-13 Robotic partial nephrectomy in complex renal tumors

S Arakere, RK Ahlawat, A Mandhani, A Mallya, I Banerjee, F Zafer, T Jindal

Fortis Escorts Kidney and Urology Institute India

Introduction \& Objective: Complex renal tumors may preclude robotic partial nephrectomy in some patients. We describe our technique, illustrated with video, of robotic partial nephrectomy for challenging renal tumors, including hilar, endophytic, and multiple tumors.

Materials and Methods: Robotic assistance was used to resect 38 tumors in 34 patients (mean age 48.2 years, range 30-57 years). All patients had complex tumor features on preoperative imaging, including hilar tumors (28 patients), endophytic tumors (seven patients), and/or multiple tumors (four patients).

Results: Robotic partial nephrectomy procedures were performed successfully without complications. Hilar clamping was utilized with a mean warm ischemia time of 29 minutes (range 20-30 minutes). Mean blood loss was $200 \mathrm{ml}$ (range 100$350 \mathrm{ml}$ ). All patients had negative surgical margins. Mean index tumor size was $3.8 \mathrm{~cm}$ (range $2.4-6 \mathrm{~cm}$ ). Mean hospital stay was 2.5 days. At three months follow-up, no patients experienced perioperative complications or a statistically significant change in serum creatinine or estimated glomerular filtration rate and there was no evidence of tumor recurrence.

Conclusions: Robotic partial nephrectomy is a safe and feasible approach for select patients with complex renal tumors. Robotic assistance facilitates tumor resection and renal reconstruction, offering a minimally invasive surgical option for select patients with complex tumors who might otherwise require open surgery.

V3-14 Robot-assisted Level II Inferior Vena Cava tumor thrombectomy with Metastasectomy for left sided renal tumor: Step-by-Step Technique

S Arakere, RK Ahlawat, A Mandhani, A Mallya, I Banerjee, F Zafer, T Jindal

Fortis Escorts Kidney and Urology Institute India

Introduction \& Objective: Robotic approach for level II IVC tumor thrombectomy has commonly been described for right sided tumor with IVC thrombus. There are only few reports of the management of left renal vein and IVC thrombus described involving embolization of left renal artery followed by stapling of left renal vein through the thrombus and then tackling the IVC thrombus. We describe Robotic technique wherein the need for preoperative embolization of artery and the need to staple the renal vein was avoided. Materials and Methods: 62 years old female with BMI of 35 presented with $10 \times 7 \mathrm{~cm}$ left renal tumor and level II IVC thrombus ( $3 \mathrm{~cm}$ above the renal vein). Diameter of left renal vein containing tumor thrombus was $4.5 \mathrm{cms}$. Using Davinci Xi robot left renal artery was ligated and left renal vein was mobilized carefully to the medial most extent. Patient was then placed in right lateral decubitus and robot was re docked. Sequential clamps were applied after clipping the lumbar and hepatic veins. Cavotomy was performed where the left renal vein entered the IVC and thrombus was removed. Patient was then placed again in left lateral decubitus and the procedure was completed by laparoscopy.

Results: Total operative time was 4.5 hours, estimated blood loss was $300 \mathrm{ml}$ with hospital stay of 5 days. Warm ischemia time for right kidney was $13 \mathrm{~min}$. Patient was up and about on the first post-operative day. Pathology revealed Clear cell RCC with focal rhabdoid and sarcomatoid differentiation. Surgical/vascular margin was negative. Liver nodule showed metastatic deposits with rhabdoid differentiation.

Conclusions: Robotic assisted IVC tumour thrombectomy for left sided tumor necessitates a higher technical expertise. It helps in avoiding major morbidity of an open surgery in patients with high BMI.

\section{VS4: BPH SURGERY AND OUTCOMES}

\author{
V4-1 Transurethral resection of prostate with Prostato- \\ Hemostatic Catheter \\ S Yang, D Kim, W Kim, J Kang \\ Gang-Nam Cha Hospital \\ Republic of Korea
}

Introduction \& Objective: While transurethral resection prostate (TURP) is still considered the golden standard for surgical treatment for benign prostate hyperplasia $(\mathrm{BPH})$, postoperative massive hematuria has still been problematic complications of TURP (Stefanie Petrou Binder, 2012). Therefore, most patients have to stay at a ward at least one or two night for continuous 
bladder irrigation and observation. In this circumstance, we consider if a patient does not demand continuous bladder irrigation after surgery of TURP, a patient will not need to stay 1 or 2 days in hospital. It can lower healthcare fee and time for patient and hospital as well.

Materials and Methods: Prospectively, we have done TURP surgery with Prostato-Hemostatic Catheter (PC Catheter) for 108 patients who have bulky size of prostate from $40 \mathrm{cc}$ to $100 \mathrm{cc}$ without continuous bladder irrigation. We utilized bipolar TUR machine made by Karl Storz, PC catheter and hemostats named Green-Plast which consists of prothrombin, thrombin and fibrin. Results: Among of 158 cases of TURP with PC catheter, 11 patients suffered urinary difficulty and demanded continuous bladder irrigation after removal of the catheter in the post-operative second day. 7 patients experienced hematuria, getting transfusion of packed RBC.

Conclusions: Although we have never tried TURP surgery without staying at ward and continuous bladder irrigation, through these results, we figured out that hemostats on resected prostatic fossa is helpful for stopping bleeding. We consider that TURP with PC catheter and hemostats will be performed in outpatient surgery center. The patient could go to home with the catheter and receive removal of the catheter in outpatient department.

V4-2 Evaluation of the ProSac: a novel device to facilitate safety and efficiency of tissue morcellation during holmium laser enucleation of the prostate

I Sorokin, H Beardsley, J Antonelli, BA Johnson, J Cadeddu, J Gahan

United States

Introduction \& Objective: Morcellation of prostate adenoma after holmium laser enucleation (HoLEP) is both time consuming and potentially morbid. We previously described a novel polyethylene sack (PercSac) that was shown to improve the efficiency of cystolitholapaxy in a porcine bladder. This device has been redesigned to be stronger with augmented geometry to accommodate enucleated prostate tissue. Our objective was to evaluate the ability of this device to capture and facilitate morcellation of prostate adenoma.

Materials and Methods: Heat fixed chicken breast was used to mimic enucleated prostatic adenoma. Approximately 30 grams of tissue was placed in a $1200 \mathrm{~mL}$ container and morcellated for 5 minutes using a 26 Fr rigid nephroscope. Four trials were performed with the device and 4 trials were performed without as a control. Functionality, safety (number of bag perforations) and morcellation efficiency (calculated in grams of tissue morcellated per minute) were evaluated.

Results: The device was able to capture up to 30 grams of tissue with good closure while maintaining good distention and visualization during morcellation. During 3 of the trials with the device, the morcellator caught the bag causing a small rent, but no perforation. Mean morcellation efficiency was $2.97 \mathrm{gm} / \mathrm{min}$ with the ProSac compared to $2.50 \mathrm{gm} / \mathrm{min}$ without the device $(\mathrm{p}=0.189)$.

Conclusions: The ProSac is a novel device that can provide additional safety during morcellation after HoLEP. Initial testing demonstrates that the ProSac does not impair morcellation efficiency and may actually enhance it. Further testing with more sophisticated models and refinements are needed to validate this device.
V4-3 The Efficacy and Safety of an Improved Transurethral Enucleation for the Treatment of Benign Prostatic Hyperplasia

Y Xu, M Luo, T Xie, S Li, B Huang

Shanghai People's Tenth Hosiptal

China (People's Republic)

Introduction \& Objective: To compare the clinical curative effect and safety of a improved transurethral enucleation (iTUEP) for benign prostatic hyperplasia (BPH) with conventional transurethral enucleation of prostate (TUEP) and transurethral resection of prostate (TURP).
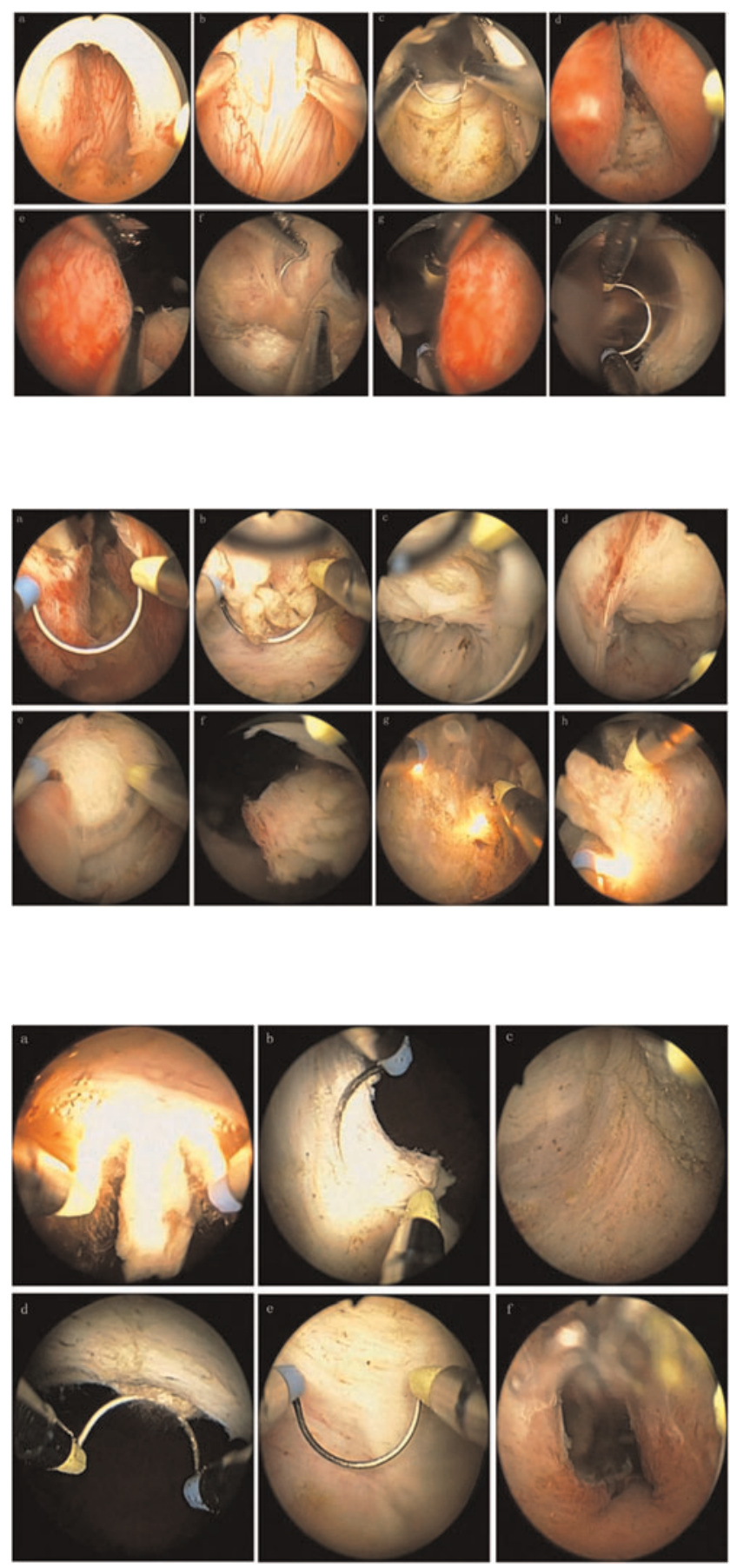
Table 1. Patient's characteristic

\begin{tabular}{l|l|l|l}
\hline & iTUEP & $\begin{array}{l}\text { TUEP } \\
\text { P-value* }\end{array}$ & $\begin{array}{l}\text { TURP } \\
\text { P-value* }\end{array}$ \\
\hline Patients & 62 & 57 & 54 \\
\hline Mean age(y) & $69.03 \pm 10.06$ & $64.07 \pm 9.78$ & $65.70 \pm 10.17$ \\
& & 0.061 & 0.1610 \\
\hline Prostate volume $(\mathrm{cm} 3)$ & $87.69 \pm 26.71$ & $88.78 \pm 28.18$ & $89.89 \pm 24.30$ \\
& & 0.8795 & 0.7097 \\
\hline PSA (ng/ml) & $2.46 \pm 1.33$ & $2.74 \pm 1.49$ & $2.27 \pm 1.44$ \\
& & 0.4489 & 0.5595 \\
\hline Residual volume (ml) & $147.06 \pm 76.21$ & $170.30 \pm 68.16$ & $180.75 \pm 78.98$ \\
& & 0.2259 & 0.0664 \\
\hline Baseline Qmáx $(\mathrm{ml} / \mathrm{s})$ & $8.59 \pm 2.63$ & $8.11 \pm 2.94$ & $7.55 \pm 2.45$ \\
& & 0.5108 & 0.0806 \\
\hline Baseline IPSS & $25.69 \pm 4.58$ & $24.48 \pm 5.81$ & $23.75 \pm 5.35$ \\
& & 0.3749 & 0.1019 \\
\hline Baseline QOL & $4.81 \pm 0.98$ & $4.40 \pm 0.993$ & $4.59 \pm 1.17$ \\
& & 0.1171 & 0.3897
\end{tabular}

Prostate volume calculation formula: $\mathrm{V}=0.52 * \mathrm{~L} * \mathrm{H} * \mathrm{~W}$ ( $\mathrm{L}$ : front and back diameter, $\mathrm{H}$ : up and down diameter, W: Left and right diameter, used ultrasound to measure), PSA: prostatic specific antigen, IPSS: international prostate symptom score, Qmáx: Maximum urinary flow rate, QOL: Quality of Life.

*: compared with iTUEP group.

Table 2. Clinical data

\begin{tabular}{|c|c|c|c|}
\hline & ITUEP & \begin{tabular}{|l|} 
TUEP \\
P-value*
\end{tabular} & \begin{tabular}{|l|} 
TURP \\
P-value*
\end{tabular} \\
\hline Operating time (min) & $47.6 \pm 5.7$ & $\begin{array}{l}66.1 \pm 6.4 \\
<0.0001\end{array}$ & \begin{tabular}{|l|}
$47.9 \pm 7.9$ \\
0.8554 \\
\end{tabular} \\
\hline Morcellating time ( $\mathrm{min}$ ) & $19.9 \pm 3.4$ & $\begin{array}{l}21.0 \pm 3.6 \\
0.2331\end{array}$ & $\mathrm{~N}$ \\
\hline Hospital stay (h) & $3.3 \pm 1.2$ & \begin{tabular}{|l|}
$3.6 \pm 1.2$ \\
0.3428 \\
\end{tabular} & $\begin{array}{l}5.3 \pm 2.0 \\
<0.0001 \\
\end{array}$ \\
\hline Catheter indwelling time $(\mathrm{h})$ & $1.7 \pm 0.8$ & $\begin{array}{l}2.1 \pm 0.9 \\
0.076\end{array}$ & \begin{tabular}{|l|l|}
$3.5 \pm 1.3$ \\
$<0.0001$ \\
\end{tabular} \\
\hline $\begin{array}{l}\text { Postoperative bladder washing } \\
\text { time (h) }\end{array}$ & $58.7 \pm 8.0$ & $\begin{array}{l}60.3 \pm 7.8 \\
0.4421 \\
\end{array}$ & $\begin{array}{l}68.0 \pm 8.7 \\
<0.0001\end{array}$ \\
\hline 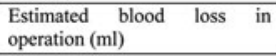 & $68.8 \pm 16.8$ & \begin{tabular}{|l|}
$64.0 \pm 13.3$ \\
0.2350 \\
\end{tabular} & $\begin{array}{l}133.2 \pm 29.4 \\
<0.0001 \\
\end{array}$ \\
\hline weight of removed prostate $(\mathrm{g})$ & $60.3 \pm 7.8$ & $\begin{array}{l}58.7 \pm 8.0 \\
0.4411\end{array}$ & $\begin{array}{l}46.6 \pm 8.0 \\
<0.0001 \\
\end{array}$ \\
\hline postoperative urethral stricture & $0 / 32$ & $0 / 27$ & $3 / 44$ \\
\hline Postoperative blood transfusion & $0 / 32$ & $0 / 27$ & $3 / 44$ \\
\hline
\end{tabular}

Materials and Methods: $173 \mathrm{BPH}$ patients treated in our hospital by different surgical procedures from July 2014 to July 2015 were included for retrospective analysis (62 cases of iTUEP, 57 cases of TUEP, and 54 cases of TURP). All the operations were done by the same doctor. We compared surgical duration, intraoperative blood loss, postoperative bladder washing time, duration of urinary catheterization, length of hospital day, and incidence of complications at 6 months postoperatively. For intergroup comparisons, normally distributed data were analyzed using the t-test and are presented as the mean \pm standard deviation.

Results: There were no significant differences in preoperative parameters among the three groups. There were significant improvements between pre- and postoperative international prostatic symptom score, quality of life, maximum flow rate, and post-void residual urine in each group $(p<0.05)$. Intraoperative blood loss, postoperative bladder washing time, postoperative complications, and length of hospital stay were significantly reduced in the iTUEP group as compared to the TURP group $(p<0.05)$. Surgical duration was significantly shorter for the iTUEP group than for the TUEP group $(p<0.05)$.

Conclusions: iTUEP is as efficacious as TURP and TUEP for the treatment of BPH. iTUEP is superior to TURP in terms of safety, blood loss, recovery time, and complication rate, and is superior to TUEP in regards to surgical duration.
V4-4 A novel technique for robotic prostatic adenomectomy: an evolution of transdouglas robotic prostatectomy

B de Concilio, G Zeccolini, M Justich, F Vedovo, A Celia

San Bassano Hospital, Urology Department

Italy

Introduction \& Objective: Transdouglas robotic assisted prostatectomy (usually known as "Bocciardi" Technique) is effective technique in the treatment of prostate cancer. Robotic prostate adenomectomy has nowadays an unclear role in the treatment of prostatic enlargement because of the leading role of endoscopic treatment. Only few reports are known about the use of robotic surgery for prostate benign enlargement. Transdouglas approach has been tested in order to perform prostatic adenomectomy for severe benign prostatic enlargement with urinary flow outlet obstruction.

Materials and Methods: 67 years old patient had for several years severe urinary flow outlet obstruction. Combination medical therapy is not effective. Transrectal Ultrasound scan detects 130 gr enalarged prostate with middle lobe. IPSS is 30 . Robotic assisted adenomectomy is scheduled. Four $8 \mathrm{~mm}$ robotic trocars and one $12 \mathrm{~mm}$ trocar for the assistant are placed, as during robotic assisted radical prostatectomy. Transdouglas approach is performed in order to perform bladder neck sparing adenomectomy. The video shows the opening of the prostate capsule from below, according to the access to the prostate described by Bocciardi. The adenoma, together with the middle lobe, is split by the capsule from the base to the veru montanum. The adenomectomy so performed by transdouglas access is easy and quick. Blood loss is almost undetectable because the dissection is anatomical, helped by great vision and assisted by bipolar haemostasis. After the enucleation of the adenoma, the bladder neck is sutured to the prostatic capsule and then it is closed by double layer watertight suture. Peritoneum is sutured. A drainage is put in the abdominal cavity. Whole Operation time is $120 \mathrm{~min}$. Blood loss is $80 \mathrm{cc}$. There is no perioperative or post-operative complication. Catheter is removed after 4 days. Uroflowmetry shows a peak flow of $30 \mathrm{ml} / \mathrm{sec}$. Pathological examination is negative for tumor. After 60 days IPSS is 8 .

Results: 5 procedures according to this novel technique have been performed. The mean prostatic volume was $125 \mathrm{cc}$. Mean operation time was $90 \mathrm{~min}$. Average blood loss was $85 \mathrm{cc}$. Average follow-up is 180 days. The mean preoperative IPSS is 27. The mean post-operative IPSS 10 . No complication occurred. The patients were discharged after average 4 days. Pathological examination was always negative for tumor.

Conclusions: Robotic Transdouglas prostate adenomectomy is safe and effective minimally invasive treatment for benign prostatic enlargement. It is a novel technique to peform bladder neck sparing prostatic adenomectomy and could be one more field of application of robotic technology.

V4-5 How to shorten your learning curve in transurethral diode laser enucleation of prostate?

Y Zhang, M Liu, J Wang

Beijing Hospital

China (People's Republic)

Introduction \& Objective: Transurethral laser enucleation of prostate is the focus in the treatment of $\mathrm{BPH}$. Traditional 
methods of HoLEP (holmium laser enucleation of prostate) is difficult. The method with diode laser will be a good way to learn and the modified one can make doctors operate easily. As the same time it can shorten the learning curve obviously.

Materials and Methods: This study included 32 men with LUTS who need to receive TURP between March 2016 and January 2017. The modified operation method of DiLEP (diode laser enucleation of prostate)was used to carry out in all cases. Data of operations was recorded.

Results: A total of 32 men were included and the average age was $60.7 \pm 4.3$ years old. The average volume of prostate was $70.1 \pm 41.6 \mathrm{ml}(35 \sim 150 \mathrm{ml})$. The average operation time and estimate blood loss were $55.2 \pm 50.8 \mathrm{~min}$ and $37.1 \pm 62.5 \mathrm{ml}$. The irrigation time and catheter time were $8.1 \pm 3.4$ hours and $4 \pm 1.2$ days. There is no permanent incontinence and construction of outside orifice of urethra happened in two of 32 cases.

Conclusions: This study showed that the modified DiLEP is a safety and feasible method and easy to carry out. The shorter learning curve can be observed contrast to other enucleation methods.

V4-6 The role of Holmium laser enucleation of the prostate to alleviate PSA uncertainties

C Chemasle, JE Hunter, R Fong

Midcentral Hospital, New Zealand

New Zealand

Introduction \& Objective: Screening for prostate cancer (PCa) is still a topic of hot debate. However, there are still a significant number of patients referred for abnormal PSA who are found to have lower urinary tract symptoms (LUTS) related to benign prostatic hyperplasia $(\mathrm{BPH})$. We retrospectively reviewed a series of 322 Holmium laser enucleations of the prostate (HoLEP) to assess if this technique could alleviate concerns about raised PSA. Materials and Methods: We have performed 322 HoLEP procedures between February 2009 and March 2017. Data presented includes: Patient age, histology on trans-rectal biopsies before/ after HoLEP and HoLEP specimen, enucleated tissue weight, PSA before and after HoLEP, and PSA density. Study data is presented as median values. The Wilcoxon's rank sum test was used to analyse the data.

Results: We identified 199 patients who had a PSA of 4 or more before their HoLEP. 2 groups of patients were compared, those who underwent a pre-operative biopsy (56) and those that did not (143). Among the 56 who had a biopsy before their procedure, 11 were positive for cancer (yield 19.6\%). 1 was diagnosed with PCa on HoLEP tissue and 4 were diagnosed post HoLEP. Among the 143 patients not biopsied before HoLEP, 22 (15.4\%) had an incidental finding of $\mathrm{PCa}$ in the enucleated specimen and 2 diagnosed post HoLEP.

Table 1: Comparison between those patients that underwent a biopsy preoperatively to those that did not (median values Wilcoxon analysis). From this we can see we were performing biopsies on a significantly younger cohort than those who proceeded straight to HoLEP. This reflects the respective management strategies for the different age groups. The preoperative PSA and PSA density is also much higher in the group that were biopsied. The two groups had very similar postoperative PSA levels.

Conclusions: HoLEP produces a significant PSA diminution in patients presenting with abnormal PSA and LUTS. This might have a role in alleviating uncertainties and avoiding unnecessary or non-informative pre-operative biopsies.
V4-7 Aquablation of the Prostate for the Surgical Treatment of Benign Prostatic Hyperplasia

\section{A Thomas}

Department of Urology, Princess of Wales Hospital, Bridgend United Kingdom

Introduction \& Objective: Aquablation is a new surgical procedure designed to treat LUTS due to BPH. The surgical technique combines intra-procedural ultrasound image guidance and robotics for a controlled resection of the prostate utilizing a heatfree waterjet. To report the definitive method of performing Aquablation using the techniques developed and refined as part the Phase III WATER Study randomizing Aquablation to TURP. Materials and Methods: The AquaBeam System (PROCEPT BioRobotics, Redwood Shores, CA) is an image-guided robotic system delivering Aquablation, a precise waterjet therapy for the targeted heat-free removal of prostate tissue. Utilizing real-time transrectal ultrasound imaging as well as an integrated cystoscope, the AquaBeam handpiece is positioned along the length of the prostate. Using ultrasound guidance, the surgeon plans the depth and angle of the tissue resection in the transverse view, and in the sagittal view, draws a treatment contour that conforms to the shape of the adenoma while sparing the bladder neck and verumontanum. The AquaBeam System automatically translates the markers into the defined resection volume. Once surgical mapping is complete, the surgeon initiates the Aquablation treatment using a foot pedal. The console pump delivers a highvelocity waterjet orthogonally to the length of the handpiece at various flow rates based on the required depth of penetration. The AquaBeam System enables a precise and controlled resection of the prostate in accordance with the surgeon's planned contour and the average resection time is less than 5 minutes. Cautery can be used for hemostasis; however, hemostasis can also be achieved by positioning the balloon of a Foley catheter inside the prostatic cavity to tamponade the bleeding, thus eliminating the need for cautery.

Results: Over 200 patients have been treated using Aquablation in early clinical research as well as the WATER Study. The technology and procedural technique has continued to advance and evolve including the approach to hemostasis utilizing the Foley catheter.

Conclusions: The combination of surgical mapping and a highvelocity waterjet with robotic guidance considerably shorten the resection time and may reduce complications. The results of the Phase I and Phase II study have been previously presented, demonstrating that Aquablation appears to be safe and effective. The 6-month results from the WATER Study, a prospective randomized double-blind clinical trial comparing Aquablation to standard TURP, will provide conclusive results compared to TURP.

\section{V4-8 Tips and Tricks for Safe Morcellation after Prostate Enucleation}

D Whiting, M Cynk

Maidstone and Tunbridge Wells NHS Trust United Kingdom

Introduction \& Objective: Holmium laser enucleation of the prostate (HoLEP) is being increasingly used as an effective treatment option for bladder outflow obstruction secondary to benign prostatic hyperplasia. However, reports of bladder mucosal 
injuries secondary to morcellation of the prostatic adenoma following enucleation remain a concern for some urologists. We reviewed the experience in a single centre over 13 years and suggest some tips and tricks to perform a safe and quick morcellation.

Materials and Methods: A retrospective review of a prospective database of all HoLEP procedures performed by or under supervision of a single consultant urological surgeon was undertaken. All case notes were reviewed for morcellation injury. Data was recorded for morcellation time, retrieval time and specimen weight.

Results: Between December 2003 and March 20171016 cases of HoLEP were performed at our centre. Median patient age was 72 years (range 41-95).

Median time required for morcellation was 10 minutes (range 1-120) $(\mathrm{n}=525)$. In these patients the median specimen weight was 30 grams (range 0.7-256) $(\mathrm{n}=502)$. In $36(6.9 \%)$ cases additional time was required for retrieval of remaining prostatic adenoma using a cold curette; median time for retrieval was 5 minutes (range 1-65).

Of the 1016 cases performed there were no incidences of bladder mucosal injury.

Conclusions: Our case series demonstrates that morcellation can be performed quickly and safely. These are some of the tips and tricks that are used at our centre.

1. Make a smooth transition to morcellation without emptying the bladder to prevent bleeding that could interfere with vision during morcellation

2. Keep the bladder full and therefore away from your morcellator by using two ports of irrigation and ensuring the irrigation fluid does not run out

3. If the adenoma stops "dancing" it can become polished and difficult to remove so drop it and re-engage

4. Flush the inner blade should the suction stop working

5. Slow down morcellation when pieces before small to prevent them falling off the morcellator

6. Use a cold curette to remove any small polished pieces

7. Always check the prostatic fossa for any hiding pieces of prostatic adenoma

By following these tips and tricks morcellation after prostate enucleation can be performed quickly and safely.

V4-9 The evolution of Green laser (532-nm) techniques in the treatment of benign prostatic obstruction: Not only for PVP.

E Rijo, J Lorente, O Bielsa, F Gomez-Sancha

Department of Urology, Hospital Quiron Barcelona. Spain

Introduction \& Objective: The first human trials with $60-\mathrm{W}$ green laser (KTP/532-nm) used for the treatment of lower urinary tract symptoms (LUTS) secondary to benign prostatic hyperplasia (BPH) were conducted in 1998. The term "photoselective vaporization of the prostate" (PVP) was introduced by Professor Reza Malek.

PVP is an established, safe and effective alternative to TURP for the treatment of $\mathrm{BPH}$ obstruction.

Nowadays the majority of urologists are unaware of other approaches and techniques with green laser, apart from PVP.

- To demonstrate the evolution of techniques for the treatment of benign prostatic obstruction with green laser (532-nm).
- Summary of advantages and disadvantages of each technique.

Materials and Methods: We review the main techniques for the treatment of LUTS secondary to BPH with green laser (532-nm), highlighting the advantages and disadvantages of each technique Results: Over the last 20 years green laser technology has undergone a radical evolution leading to variations of the console crystal, increased power and modification of the fiber resulting in a more efficient procedure.

The "anatomic" photoselective vaporization of the prostate, photoselective vapoenucleation of the prostate, ejaculation-preserving photoselective vaporization of the prostate and green laser enucleation of the prostate (GreenLEP) represent different techniques that have evolved from classic PVP to improve its outcome.

Conclusions: In the last two decades green laser technology and techniques have experienced a tremendous evolution in the field of minimally invasive surgery. The evolution from a "classic PVP" to anatomic PVP, vapoenucleation and finally enucleation, offers many treatment options depending on the prostate gland size and the status of the patient.

The green laser $(532 \mathrm{~nm})$ has evolved as a safe, effective and appropriate source of energy for the treatment of benign prostatic obstruction.

\section{V4-10 Thulium Laser Enucleation of the Prostate (Thu-} LEP): First Experience.

P Glybochko, G Altshuler, A Vinarov, L Rapoport, M Enikeev, D Enikeev, N Sorokin, A Dymov, O Khamraev, R Sukhanov, M Taratkin, V Vinnichenko

I.M. Sechenov First Moscow State Medcal University Clinic of Urology

Russia

Introduction \& Objective: Thulium laser enucleation of the prostate (ThuLEP) refers to endoscopic enucleation of prostatic adenoma with the help of thulium laser. Compared to conventional laser surgery techniques, ThuLEP is characterized by lesser depth of laser energy penetration due to the wavelength of thulium laser $(1.94 \mathrm{~nm})$ matching that of the water absorption peak in tissue, and highly efficient blood vessel coagulation.

Our aim was to evaluate and describe the first results of ThuLEP, as well as its effects on voiding parameters and the quality of life.

Materials and Methods: The study included 153 patients aged 54 to 85 years (mean age 68.2 years) who underwent ThuLEP. The mean prostate volume was $95.2 \pm 45.3 \mathrm{~cm} 3(45-250 \mathrm{~cm} 3)$. In all patients, IPSS, QoL, Qmax, and post-void residual urine volume were evaluated prior to ThuLEP and six month after the surgery. All manipulations were performed by three certified surgeons skilled in holmium laser enucleation of the prostate (600 cases). The surgeries were performed using novel equipment (Urolase, Russia), a fiber thulium laser with two wavelengths of $1.94 \mathrm{~nm}$ for surgery and 1.55 for coagulation, power of $120 \mathrm{~W}$, frequency more than $2000 \mathrm{~Hz}$, and energy up to $8 \mathrm{~J}$.

Results: Mean surgery duration was $79.6 \pm 29.4 \mathrm{~min}$, mean enucleation duration was $36.6 \pm 20.8 \mathrm{~min}$, and mean morcellation duration was $39.6 \pm 19.1 \mathrm{~min}$. The mean enucleated tissue weight was $76.5 \pm 35.0 \mathrm{~g}$. In all patients, we noted significant IPSS improvement $(23.2 \pm 2.2$ prior to ThuLEP and 7.8 \pm 2.9 post-ThuLEP $[\mathrm{p}<0.05])$ and QoL index improvement $(4.8 \pm 0.8$ prior to ThuLEP and $1.8 \pm 1.1$ post-ThuLEP $[\mathrm{p}<0.05])$. Mean Qmax 
before and after the surgery was $7.6 \pm 2.4 \mathrm{ml} / \mathrm{s}$ and $18.2 \pm 3.3 \mathrm{ml} / \mathrm{s}$ respectively $(\mathrm{p}<0.05)$. Mean post-void residual volume was $80.5 \pm 30.6 \mathrm{ml}$ prior to the surgery and $10.8 \pm 8.1 \mathrm{ml}$ one month after ThuLEP $(\mathrm{p}<0.05)$. In two cases $(1.3 \%)$, we observed intraoperative bleeding from the prostatic venous sinus which prevented single-step morcellation. The hemorrhage was managed by Foley catheter placement and pressing the distended balloon against the site of the enucleation. Morcellation was performed two days after the surgery. One patient $(0.6 \%)$ developed postoperative bleeding which prompted cystoscopy and coagulation of the damaged blood vessel.

Conclusions: Thulium laser enucleation of the prostate enables significant improvement of voiding parameters and the quality of life. ThuLEP has proven to be an effective treatment option with low risk of complications. We are planning to undertake further studies of this technique and continue monitoring the patients after ThuLEP.

V4-11 Photoselective Vapoenucleation of the prostate using $180 \mathrm{~W}$ green laser (532-nm).

E Rijo, F Gomez-Sancha

Department of Urology, Hospital Quiron Barcelona. Spain

Introduction \& Objective: PVP is an established, safe and effective alternative to TURP for the treatment of BPH obstruction in small to medium glands.

For large prostates $(>80 \mathrm{ml}$.) open prostatectomy or Holmium laser enucleation of the prostate (HoLEP) remains the prefered choice of treatment. The Vapoenucleation of the prostate using green laser is an alternative hybrid technique between vaporization and enucleation. Consists in vaporizing the lateral lobes and enucleating the median lobe.

The advantages compared with PVP are: fairly fast operation, low re-operation rates, suitable for large glands and provides pathology specimen.

Green laser vapoenucleation is not a synonym of green laser enucleation of the prostate (GreenLEP), there are totally different techniques. The aim of this video is to demonstrate the green laser vapoenucleation technique step-by-step.

Materials and Methods: The surgery was performed with a 532-nm lithium triborate laser (GreenLight $^{\mathrm{TM}}$ XPS 180W, American Medical Systems, Minnetonka, Minnesota, USA), MoXy ${ }^{\mathrm{TM}}$ side-fire laser fiber at power settings: $180 \mathrm{~W}$ for cutting, $35 \mathrm{~W}$ for coagulation and a Piranha ${ }^{\mathrm{TM}}$ morcellation system (Richard Wolf GmbH, Germany). Case Report:

A 68-year-old patient presented with lower urinary tract symptoms secondary to benign prostatic obstruction. The prostate volume was $88 \mathrm{~mL}$, PSA of $3.4 \mathrm{ng} / \mathrm{mL}$, peak urinary flow rate (Qmax) was $7 \mathrm{~mL} / \mathrm{sec}$, postvoid residual volumen (PVR) of $160 \mathrm{~mL}$ and international prostate symptom score (IPSS) of 24.

It was decided to proceed with green laser vapoenucleation technique in this case.

Results: The total operative time was 72 minutes, total energy employed was $354 \mathrm{~kJ}$ and pathology report showed no evidence of prostate cancer $(35 \mathrm{~g})$.

The bladder catheter was removed at 24 hours postsurgery and the patient was discharged 48 hours postoperative without complication.

Three months later all the parameters showed significant improvement (PSA: $1.02 \mathrm{ng} / \mathrm{mL}$, IPSS: 4, Qmax: $43 \mathrm{~mL} / \mathrm{seg}$ and PVR: $15 \mathrm{~mL})$.
Conclusions: Green laser vapoenucleation of the prostate represents a safe alternative technique for the complete removal of adenomatous prostate tissue, regardless of gland size, it is particularly advantageous for the treatment of large prostates.

This technique can also be used as an intermediate step during the learning curve of GreenLEP.

These promising results warrant further studies to assess longterm outcomes.

V4-12 En-bloc TURBT (E-TURBT) with collins loop: a new endoscopic treatment in the bladder tumour

R GENTILE, B GENTILE, G Mirabile, L Albanesi, G Rizzo, $\mathrm{P}$ Tariciotti

nuova villa claudia

Italy

Introduction \& Objective: Bladder cancer is the second most common malignancy of urologic. The bladder tumour's incidence rates increased with the aging population and new socioeconomic challenges. The standard treatment for non-muscle invasive bladder cancer (NMIBC) is the classic transurethral tumour's resection using resection loops (cTURBT). Recurrence is seen in 50-70\% of non-muscle invasive bladder cancer, mostly during the 1 st year. Inadequacy of cTURBT is not only judged by absence of detrusor muscle in an initial specimen, but also by the rate of recurrence. To avoid all this, different concepts of en bloc resection of bladder tumors have been developed. We know that the resection of tumors is contrary to all oncological surgical principles. We want show a new technique, an en-bloc removal of bladder tumour (E-TURBT) with Collins loop with plasmakinetic ESG 400 system.

Materials and Methods: From 2014 we perfomed E-TURBT. In the first time we used buttom loop but from the last 2 year we prefer bipolar Collins loop, because it's more fine and accurate. Goals of new treatment modalities are reduction of perioperative and postoperative comorbidities, better pathological work-up of the specimens and increased recurrence-free survival. We analized the hemoglobin pre and post ETURBT, hospitalization days and complication during the treatment, in the hospital and at home.

Results: We not have complication during the ETURBT. The mediam hospitalitation days was 1,5 days. The hemostasis was better during the treatment and after the removing thee catheter. The value of hemoglobin was stable after the treatment. In all operation' piece was present the mussle layer. so the pathologist is able to orient the surgical specimen and determine, in pT1HG, the presence and involvement of the muscularis mucosae, by evaluating the substaging

Conclusions: The advantages using E-TURBT with plasmakinetic ESG 400 system are a more precise cutting line as well as better hemostasis. The en bloc thecnique can to send a operation piece with the mussle layer, so the pathologist can determine the presence and involvement of the muscularis mucosae, by evaluating the substaging The concept of removing a bladder tumor by cTURBT should be changed to a technique of en-bloc resection to provide better cancer control and long term outcome in non-muscle invasive bladder cancer.

V4-13 Transurethral resection of a tumor in a Bladder Diverticulum

N Younes, K Al-rumaihi, A Badawi, K Khalafalla, T Ahmed

Hamad Medical Corporation

Qatar 
Introduction \& Objective: We reviewed a clinical case of Transurethral resection of a tumor in a bladder diverticulum. Materials and Methods: A 64-year-old male Presented to the Emergency Department with a complaint of recurrent episodes of Terminal Painless gross hematuria for the past 2 months with no associated clots, No History of Lower urinary tract symptoms, Dysuria, UTI or Abdominal pain.

Patient is known Diabetic, Hypertensive and history of Coronary artery disease on Aspirin No past surgical history of medical importance was noted. On Examination, He was in a Good General Condition, Vitally stable, Afebrile Abdomen exam showed a Soft, Lax, non-tender abdomen, no palpable bladder mass by bimanual examination. Digital rectal Exam showed Mild enlarged, non-tender, soft, mobile, smooth prostate He had normal blood work including Renal function test. Patient underwent a CT-scan with IV contrast showing an exophytic soft tissue density lesion arising from the posterior aspect of the bladder wall, on the left side- likely representing a neoplastic pathology arising from a pre-existing bladder diverticulum and no signs of uretic obstruction noted. Flexible Cystoscopy was done for the patient and revealed a Normal urethra \& prostate, a diverticulum at the right lateral bladder wall which was free of tumor and Another Diverticulum at left lateral bladder with a suspicious growth mixed with clots within it. Methods: Available options: First step: Biopsy and Transurethral resection of the tumor. Second step: according to the histopathology results

1. Intravesical therapy

2. Diverticulectomy

3. Partial cystectomy

4. Radical cystectomy

Up to this moment, there is no Guidelines on the management of bladder diverticular tumors. In this case, a biopsy and TURBT was done for the diverticular tumor revealing a papillary urothelial carcinoma(TA High grade). Decision was taken for another Cold cut biopsy and fulguration of the rest of tumor which showed on histopathology a urothelial mucosa with a focus of carcinoma in situ (T Cis).

Results: Patient had an uneventful Postoperative period on both settings and was discharged with no further complications on the same day of admission.

Conclusions: TURBT and Intravesical therapy in a bladder diverticulum is feasible and precautions should be more towards the Diverticular bed to avoid perforation during the TRUBT by using an Angled Loop during resection. Follow up plan:
Intravesical BCG (6 weeks induction \& 12 months Maintenance), Flexible cystoscopy every 3 months and repeat CT scan after 3 months.

\section{V4-14 A New Method of Suprapubic Cystotomy \\ LM Wyner \\ Marshall University Joan C. Edwards School of Medicine United States}

Introduction \& Objective: Suprapubic cystotomy, or suprapubic tube placement, may be achieved using a variety of techniques. A new method is described utilizing easily obtainable equipment. The only requirement is that the urethra be able to tolerate passage of a curved Van Buren sound.

Materials and Methods: The equipment needed is: a minor surgical set, a cystoscopy tray, an 18 French curved Van Buren sound, and an 18 French Foley catheter whose tip has been cut off just distal to the balloon. The patient is placed in dorsal lithotomy position. The genitalia and lower abdomen are sterilely prepped and draped. Cystoscopy is performed to rule-out intravesical pathology, and also to partially fill the bladder to allow for a clean entry into the dome. The sound is passed per urethra, and its tip palpated in the suprapubic region. This area is cut down with the cautery, until the tip is exposed. The cut end of the catheter is wedged firmly onto the tip of the sound, and the sound-catheter assembly is then pulled antegrade, until the tip of the catheter exits the urethral meatus. The assembly is then disengaged, and the catheter tip is followed back into the bladder with the cystoscope, while the catheter is withdrawn retrograde, so that the catheter balloon may be inflated in the bladder lumen under direct vision, prior to affixing the catheter to the skin closure sutures.

Results: The catheter may be exchanged over a wire prior to the establishment of a tract, if necessary, as it is functioning essentially as a Councill catheter. Two helpful steps in the procedure are: (1) Make sure the catheter balloon has not been damaged by cutting off the catheter tip, and (2) Lubricate the entire catheter prior to pulling the sound-catheter assembly antegrade through the urethra.

Conclusions: The method described above is easy to perform, and does not rely on more complicated instruments, such as the suprapubic trocar or Lowsley tractor, which may be prone to breakage or misplacement in a busy operating room.

\section{VS5: ADRENAL AND BENIGN UPPER TRACT SURGERY}

\section{V5-1 Retroperitoneal laparoscopic adrenalectomy for large adrenal pheochromocytoma}

R Shimizu, Y Kimura, T Yumioka, N Yamaguchi, H Iwamoto, S Morizane, K Hikita, M Honda, A Takenaka

Division of Urology, Department of Surgery, Tottori University Faculty of medicine, Yonago, Tottori, Japan Japan

Introduction \& Objective: Generally, it is recommended that laparoscopic adrenalectomy should be done for pheochromocytoma less than $6 \mathrm{~cm}$-diameter. However, the indication is controversial. It is reported that retroperitoneal laparoscopic adrenalectomy is useful in large pheochromocytoma, because it is possible to treat the feeding artery from the dorsal side in the early process. In this video, we will present Retroperitoneal laparoscopic adrenalectomy for two patients with large adrenal tumors.

Materials and Methods: We experienced two patients who had undergone retroperitoneal adrenalectomy for large adrenal tumors. Case 1 is a 76 - year - old female who complained of headache, palpitations and sweating. An abdominal tumor was 
found with the ultrasonography. Abdominal CT showed a 7-cmmass in the right adrenal gland. Blood test and urinalysis showed high values of catecholamine. And MIBG scintigraphy showed accumulation of right adrenal gland. Based on the diagnosis of pheochromocytoma, retroperitoneal adrenalectomy was performed. Case 2 is a 69 - year - old man coincidentally showing a $12-\mathrm{cm}$ tumor in right adrenal gland by CT. Urinalysis showed a mild elevation of catecholamine. Since it was as large as $12 \mathrm{~cm}$, laparoscopy was expected to be difficult, but if we were able to process tumor vessels, we thought that it was possible with a small incision even in case of laparotomy transition. Therefore we would chose the retroperitoneal approach.

Results: In case 1 the pneumoperitoneum time was 134 minutes and the amount of bleeding was small. There was no significant change in blood pressure during the operation. In case 2 due to adhesion we had to perform an open operation with a small incision. Pneumoperitoneum time was 180 minutes, operation time was 280 minutes, bleeding volume was $550 \mathrm{ml}$.

Conclusions: We experienced two patients who had undergone retroperitoneal adrenalectomy for large adrenal tumors. pheochromocytomas markedly develope tumor vessels. In the retroperitoneal approach, tumor blood vessels can be easily treated in an early process and it decreases the bleeding and enables safer operation. We suggest that retroperitoneal laparoscopic surgery is useful for large adrenal tumors.

V5-2 Robotic partial adrenalectomy for symptomatic aldosterone-secreting adenomas: technique and outcomes

G Simone, G Tuderti, L Misuraca, A Celia, B de Concilio, A Stigliano, F Minisola, M Ferriero, G Romeo, S Guaglianone, M Gallucci

Regina Elena National Cancer Institute Rome Italy

Introduction \& Objective: Partial adrenalectomy for functioning adrenal masses is significantly underused. Preliminary experiences suggested the effectiveness of partial adrenalectomy for functioning adrenal adenomas.

We describe surgical technique and present perioperative and functional outcomes of a two center series including nine symptomatic aldosterone-secreting adenomas treated with robotic partial adrenalectomy (RPA).

Materials and Methods: From June 2014 to October 2016 RPA was performed in 9 consecutive patients with symptomatic aldosterone-secreting adrenal adenomas.

Surgical steps include: Incision of Gerota's fascia at the level of the upper pole of the kidney and exposure of the adrenal gland; careful dissection of the medial aspect of the gland, preserving adrenal vessels with a selective control of vessels feeding the adrenal mass; progressive dissection of the mass with a pure enucleation technique in order to maximize the amount of adrenal parenchyma spared; specimen retrieval into an endocatch bag; hemostasis and closure of adrenal defect with a sliding clip technique.

Two cases are demonstrated in the video.

Baseline, perioperative and early functional outcomes data are reported.

Results: All cases were completed robotically. Intraoperative blood loss was negligible, postoperative course was uneventful in all cases, except for 1 patient who required antibiotic therapy for post-operative fever (Clavien grade 2 complication). Median hospital stay was 3 days (IQR: 2-3).
Patients became normotensive immediately after surgery. Aldosterone and plasmatic renin activity levels decreased and returned within the normal range after surgery as well.

Conclusions: Robotic Partial Adrenalectomy is a safe and feasible technique. Thanks to surgical skills acquired with partial nephrectomy, an increasing adoption of adrenal sparing surgery among minimally-invasive urologists is likely to be anticipated.

\section{V5-3 Robot assisted Partial Adrenalectomy for adrenal tumours - Our initial experience}

\section{Y T B, S Waigankar, A Padegaonkar, A Pednekar}

Kokilaben Dhirubhai Ambani Hospital and Research Institute India

Introduction \& Objective: Recent reports in literature have described adrenal-sparing surgery as a surgical option for patients with hereditary syndromes, small benign lesions or bilateral tumors to preserve unaffected adrenal tissue. We report our early outcomes \& experience of robot assisted partial adrenalectomy (RAPA) technique.

Materials and Methods: We performed 5 RAPA procedures on 4 patients presenting with bilateral pheochromocytoma in one patient (two sittings), two patients with Cohn's syndrome and one patient with adrenal adenoma at our institute between 2012 and 2017. Surgical approach included transperitoneal approach, exposure of adrenal gland, careful excision of mass, and haemostasis by clips, cautery and suturing the cut edge.

Results: Mean age of the patients was 32 years (24-48), mean console time was $80 \mathrm{mts}$ (60-130), mean blood loss of $60 \mathrm{ml}$ (30100), mean hospitalization of 3 days (2-5). Patient with pheochromocytoma had hypertension during surgery which was controlled. There were no other intra- or post-operative complications. Frozen section was not done. Functional assessment was normal in all other patients except one with adrenal adenoma in whom studies were not performed. At median follow up of 18 months (5-32), none of the patient had biochemical or local recurrences.

Conclusions: RAPA is feasible, safe and provides encouraging functional and oncologic outcomes in patients with a adrenal lesion/s. It may decrease the risk of the development of adrenal insufficiency. Our experience showed good short term outcomes. However more number of patients with long term outcomes data is necessary.

V5-4 Management of brisk hemorrhage during robotic right adrenalectomy for a $9 \mathrm{~cm}$ pheochromocytoma.

A Schulman, RJ Yau, NH Patel, MS Choudhury, M Eshghi, JL Phillips

Duke University Medical Center United States

Introduction \& Objective: Acute hemorrhage is a feared complication of minimally invasive adrenalectomy. In this video, we present brisk bleeding during robotic-assisted right adrenalectomy for a $9 \mathrm{~cm}$ pheochromocytoma.

Materials and Methods: The patient has a $9 \mathrm{~cm}$ right adrenal tumor with elevated plasma free metanephrines and imaging features consistent with a pheochromocytoma. 
During ligation of the adrenal vein, brisk bleeding is encountered from a rent in the friable capsule.

Results: Multiple maneuvers are undertaken to control the bleeding including application of vessel sealer and oversewing the defect.

The video illustrates several key points during this critical event including judicious use of suction, use of emergency 'rescue' sutures and constant awareness of visual landmarks in the absence of visualization. Ultimately, hemostasis is obtained following complete removal of the tumor.

Conclusions: Rapid bleeding during minimally-invasive adrenalectomy remains a significant risk. Several maneuvers illustrated in the video may be useful during this scenario.

V5-5 Robotic-assisted laparoscopic partial adrenalectomy for a unilateral pheochromocytoma in suspected von Hippel-Lindau disease

R Lee, L Giusto, B Waldorf, E Gewirtz, D Eun

Temple University Hospital

United States

Introduction \& Objective: Pheochromocytomas are catecholamine producing tumors of the adrenal medulla. In von HippelLindau (VHL) disease, a familial cancer syndrome affecting multiple organs, patients develop predominantly norepinephrine-secreting pheochromocytomas resulting in hypertension, flushing, and hyperhidrosis. These patients are prone to bilateral pheochromocytomas, CNS hemangioblastomas, and clear cell renal cell carcinoma. Because morbidity of bilateral adrenalectomy, adrenal cortical sparing surgery has been proposed to prevent adrenal insufficiency. Minimally invasive techniques have been utilized for partial adrenalectomy, however the reporting of robotic assisted techniques remains minimal. Here, we present a case of a unilateral adrenal mass in a patient with suspected VHL disease and describe our technique for roboticassisted partial adrenalectomy.

Materials and Methods: A 47-year-old female presented with multiple episodes of chest pain, palpitations, sweating, and flushing. Workup included biochemical evaluation and triple phase CT. CT revealed a left $2.9 \mathrm{~cm}$ enhancing adrenal mass and laboratory values showed elevations in plasma and urine metanephrines. Given the patient's history of retinal hemangioblastoma and newly diagnosed pheochromocytoma, a diagnosis of VHL disease was suspected. Due to the risk of ipsilateral or contralateral recurrence in VHL disease, the patient opted for robot-assisted partial adrenalectomy. Patient was prescribed phenoxybenzamine preoperatively.

Results: Blood pressure (BP) was monitored with an arterial catheter and fluctuations in BP were adequately controlled with beta blockade. Continuous infusion of BP medication was not required. Console time was 76 minutes with an estimated blood loss of $100 \mathrm{~mL}$. Warm ischemia time was 21 minutes. The patient was discharged on post-operative day 1 . Pathology revealed $3 \mathrm{~cm}$ pheochromocytoma, confirmed by immunohistochemical stains. Surgical margins were negative. Patient reported full resolution of symptoms at 3-week post operative follow up. Post-operative catecholamine levels are pending.

Conclusions: Here we demonstrate the safety and efficacy of robotic-assisted partial adrenalectomy in select patients for unilateral pheochromocytomas. Adrenal cortical sparing surgery is a viable option in patients with VHL disease.
V5-6 Transperitoneal Laparoscopic Left Adrenalectomy in Patients with Ectopic Pelvic Kidney: A Live Surgery Experience

Y Aslan, O Guzel, I Aykanat, M Balci, A Tuncel

University of Health Sciences, Ankara Numune Research and Training Hospital, Dept. of Urology, Ankara-Turkey

Turkey

Introduction \& Objective: In this video, we are presenting a successfully treated a case underwent transperitoneal left laparoscopic adrenalectomy with a left pelvic kidney perform during the "Laparoscopic Adrenalectomy Course"' within the scientific program of Turkish Endourological Society.

Materials and Methods: A left adrenal mass was detected incidentally in a 57-yr- old male patient. In triphasic enhanced computerized abdominal tomography, $24 \times 22 \mathrm{~mm}$ in diameter mass was detected. The density was 27 Hounsfield Unit (HU) in unenhanced series and $88 \mathrm{HU}$ in portal phase. The washout rate was $35 \%$. In addition, the left kidney located in the pelvic region and abnormal vascular structures localized in adrenal gland. In biochemical analyses, subclinic Cushing Syndrome was detected (24 hours urine cortisol level: 76.05 (3.5-45) $\mu \mathrm{g} /$ day, night salivary cortisol level: $27.07(0.2-2.2) \mathrm{ng} / \mathrm{mL})$. The patient underwent transperitoneal left laparoscopic adrenalectomy.

Results: The operation was started with three trocars (one was $12 \mathrm{~mm}$ and two was $11 \mathrm{~mm}$ ) and then additional one trochar $(5 \mathrm{~mm})$ was placed for bowel retraction. During surgery, it was difficult to find the left adrenal gland and mass because the left kidney placed at ectopic region. During the operation, firstly the tale of pancreas was diagnosed. Secondly, the left adrenal gland was found to slightly lower than its original localization. The mass and inferior body of adrenal gland were dissected blunt and sharp dissection. It was seen that left adrenal vein drained into the lateral direction of the left renal vein. Thirtly, the adrenal vein ligated with using vessel sealing device and the gland was removed. There was no per-operative and postoperative complications. The patient was discharged on the second day postoperatively. The histopathological examination revealed oncocytic adenoma.

Conclusions: The adrenal gland locates original anatomic region in patient with ipsilateral ectopic kidney. Vascular abnormalities could be seen. Laparoscopic surgery is a good option which provides better anatomic vision in that patients.

\section{V5-7 robotic-assisted excision of a large Right Adrenal Cyst}

H Alenezi, A Albaghli, A Alenezi, F Abul

Ministry of health - Adan hospital

Kuwait

Introduction \& Objective: Large adrenal masses and cysts were considered as a relative contraindication for minimally invasive surgery by many authorities. However, since the introduction of the robotic platform, complex surgeries have been performed by successfully without increased complications. Herein we describe the performance of robotic excision of large adrenal cyst. Materials and Methods: 36-year-old male presented with recurrent right upper quadrant pain and abdominal distension for one year. Patient has no history of hypertension and is otherwise healthy. Radiological investigations revealed a $12-\mathrm{cm}$ right simple adrenal cyst compressing the right kidney and renal hilum 
inferiorly. Full functional adrenal workup was negative. Patient was consented for robotic excision of his large right adrenal cyst. Results: Operative time was 132 minutes. There were no intraoperative complications and blood loss was minimal. Patient had uneventful postoperative recovery and was discharged to home on day 2 postoperative. Patient had complete resolution of his preoperative symptoms. Followup abdominal ultrasound 2 months postoperatively revealed no residual masses.

Conclusions: Robotic excision of large simple adrenal cysts is feasible and offers patients the advantages of short convalescence and hospital stay with reduced pain.

\section{V5-8 Withdrawn}

V5-9 Double blessing - Laparoscopic partial nephrectomy with nephrolithotomy for a complex lower polar cyst with lower calyceal partial staghorn calculus

S Kallappan, M Ramalingam, S Nachimuthu

Urology Clinic

India

Introduction \& Objective: Removal of a lower calyceal partial staghorn calculus in the presence of a lower polar cyst abutting the calyx is a challenge. Among the options lower polar partial nephrectomy with nephrolithotomy is a feasible double blessing Materials and Methods: 74 year old gentleman presented with a complex right lower polar cyst with a partial staghorn calculus involving the lower calyx. The cyst was abutting the wall of the lower calyx. With the patient in the right kidney position dissection of the hilum was done. After obtaining vascular control partial nephrectomy was done. During the procedure the lower calyx opened up and the calculus could be seen. The calyx was removed and the calyceal defect was closed. Ureteral stent and drain were placed.

Results: The procedure was completed in 190 minutes. The warm ischemia time was 30 minutes. Patient was discharged on the 5 th post operative day. Drain was removed prior to discharge. Ureteric stent was removed after 6 weeks.

Conclusions: Laparoscopic partial nephrectomy with nephrolithotomy is the ideal option for a complex lower polar cyst with lower calyceal partial staghorn calculus

V5-10 Percutaneous Externally Assembled Laparoscopic Instruments to Decrease the Invasiveness of Hand-Assisted Laparoscopic Donor Nephrectomy

J Thomas, S Abourbih, M Pierce, J Cheng, H Wagner, DD Baldwin

\section{Loma Linda University Medical Center}

United States

Introduction \& Objective: Kidney donation is an altruistic act that provides no benefit to the donor. Because of this, donor surgeons strive to decrease the invasiveness and morbidity associated with donor nephrectomy. In this video, we demonstrate the use of a novel percutaneously assembled laparoscopic (PEAL) instrument as a method to reduce the invasiveness of donor nephrectomy.

Materials and Methods: Using the PEAL instruments, a 32 year-old hispanic female underwent a hand-assisted laparoscopic donor nephrectomy. Preoperative evaluation revealed a single left renal vein, and an early branching renal artery $(7 \mathrm{~mm}$ from the aorta), which added additional complexity to the case. A PEAL instrument was used in place of a conventional laparoscopic port to decrease the morbidity of the procedure. The peal instrument consists of a reusable handle, $3 \mathrm{~mm}$ shaft, and interchangeable $5 \mathrm{~mm}$ instrument tips. The shaft was inserted without a trocar through a $3 \mathrm{~mm}$ incision in the anterior axillary line, just below the costal margin. The PEAL introducer tip was brought through a Gelport placed at the umbilicus and exchanged for a fenestrated grasper that was used for retraction during the case. One $5 \mathrm{~mm}$ instrument trocar was placed subcostally in the midclavicular line for use of a $5 \mathrm{~mm}$ endo-eye camera. A single $12 \mathrm{~mm}$ mid-clavicular port was placed at the lower border of the Gelport for insertion of a stapling device.

Results: This video will demonstrate how the PEAL instrument was utilized to decrease the invasiveness of the procedure. The PEAL instrument was used for lateral retraction, applying pressure to bleeding points, and for maintaining counter traction. Total operative time was 225 minutes. A healthy kidney with a single artery and vein was implanted into the recipient without complication. The patient rated the postoperative pain at the PEAL site as a 2/10 on a Likert scale, which was the lowest of her incisions. In comparison the pain score of the $5 \mathrm{~mm}$ was a $3 / 10$, the $12 \mathrm{~mm}$ was a 5/10 and the Gelport incision was a 6/10 using the Likert scale. The patient was discharged on post-operative day 1 with no complications. Postoperative donor creatinine was $1.0 \mathrm{mg} / \mathrm{dL}$ at the time of discharge. There was immediate graft function in the recipient with a nadir creatinine of $0.7 \mathrm{mg} / \mathrm{dL}$ on post-operative day 3 .

Conclusions: This video presents the use of the novel PEAL instrument paradigm to decrease the invasiveness and improve the cosmesis of hand-assisted laparoscopic donor nephrectomy. Use of these instruments could decrease donor morbidity and reduce a potential obstacle for kidney donation.

V5-11 Laparo-endoscopic single site (LESS) right nephrectomy

R Bass, O Levi, A Sidi, A Tsivian

Department of Urologic Surgery, E.Wolfson M.C., Holon, Israel

Israel

Introduction \& Objective: In recent years we implemented an innovative laparoscopic method - a complete nephrectomy (radical and simple) performed through a single port. This method requires special laparoscopic equipment and advanced skills. Application of this approach may improve the recovery process, cosmetic results and minimize complications. We present here a video that describes the steps of right simple nephrectomy through a single port in a patient with nonfunctioning kidney.

Materials and Methods: From a prospective collected database we identified 20 patients who underwent complete nephrectomy using LESS approach. The procedures were performed through a single entry port, usually in the umbilicus with application of special equipment.

Results: The patient's age range from 7 to 83 years, 10 - females, 10 nephrectomies from each side. The average surgery time 140.3 minutes. There were no conversions to open surgery or conventional laparoscopy. In one case, an additional trocar was inserted to extract large specimen. There were no postoperative complications. 
The video demonstrates the steps of simple nephrectomy using LESS.

Conclusions: Laparo-Endoscopic Single Site (LESS) Nephrectomy is a feasible procedure with a acceptable length of surgery. However, the complexity of the approach and the special surgical equipment require accumulation of experience in general laparoscopy, therefore it is recommended to perform these surgeries at selected medical centers.

\section{V5-12 Da Vinci Si Robotic Assisted Laparoscopic Excision} Of Retroperitoneal Mass (Paraganglioma)

Y T B, S Waigankar, A Padegaonkar, K Chakradhar, S Sharma

Kokilaben Dhirubhai Ambani Hospital and Research Institute India

Introduction \& Objective: Extra-adrenal pheochromocytoma is a rare entity. Robotic surgery has been utilized for excision of these masses which help to overcome the limitations of laparoscopic surgery w.r.t. range of movements. The aim is to present the technique of robot assisted laparoscopic excision of retroperitoneal mass (Paraganglioma).

Materials and Methods: $37 / \mathrm{F}$ married since 13years presented with uncontrolled hypertension since 5 months. She has been taking 3 anti-hypertensive medications since 12 years after being diagnosed for pregnancy induced hypertension. She has h/o undergone laparoscopic cholecystectomy and LSCS in the past. The investigations revealed a high level of free plasma metanephrine (3099 pg/ml) \& Chromogranin A (124 ng/ml). The CT scan revealed a well-defined, lobulated, inhomogenously enhancing lesion $(\sim 6-7 \mathrm{cms})$ in the left para-aortic region. After presurgical counselling and review by the anaesthesiologist, she underwent Laparoscopic (Robotic assisted) excision of the retroperitoneal mass.

Results: Laparoscopic (Robotic assisted) excision of the retroperitoneal mass took around took 360mins (Console time: 180mins). Total blood loss was $200 \mathrm{ml}$. Intra-operative and Postoperative period was uneventful and total hospital stay was 7 days. Patient was discharged on single antihypertensive medication.

Conclusions: Robot-assisted laparoscopic surgical system provides a novel, minimally invasive solution for treating secreting extraadrenal pheochromocytomas. Furthermore, this technique reduces stimulation of the tumor and prevents or minimizes complications.
V5-13 Interdisciplinary Approach to Dermoid Cyst of the Spine: How Can Urologists Help Neurosurgeons?

R Mauck, L Zhao, N Singla, C Hatchette

UTSW

United States

Introduction \& Objective: Spinal intradural dermoid cysts are rare benign lesions that can produce local mass effect. Traditionally treatment has been open removal of symptomatic lesions by neurosurgeons. However, local recurrence is common due to the difficulty of removing all elements surgically, especially in delicate spaces around the cord and nerve roots. In the present video, we report a case of a spinal dermoid cyst presenting with a tethered cord-like syndrome including fecal incontinence and perineal and lower extremity numbness. We highlight how the endoscopic skills of urologists can be used to treat this difficult neurosurgical problem. To our knowledge, flexible endoscopy in the intradural space has not been previously described in humans. Materials and Methods: After multidisciplinary discussions, we devised a plan to use a 16 Fr flexible cystoscope with lowpressure gravity saline irrigation to access the spinal canal via incision of the pseudomeningocele. Given the soft texture of the dermoid cysts, a 5-6 Fr Penumbra thromboembolectomy device was felt to be suitable to suction the tumor and was inserted through the cystoscope for real-time visualization of cyst removal. Continuous neuromonitoring was used to identify any changes in intracranial pressure. The neurosurgery team was adequately prepared to convert to an open surgery in the event of neurological changes.

Results: By post-operative day 1, the patient reported complete resolution of her neurological presenting symptoms, and she was discharged uneventfully by post-operative day 3. A post-operative MRI demonstrated almost complete resection of the lesion. At 3-year follow-up, the patient was found to have some regrowth of dermoid cyst in the lumbar region on MRI. However, her symptoms have remained unchanged since her surgery, and she continues to be followed conservatively. Should her symptoms worsen, repeat endoscopic resection can be considered.

Conclusions: For the first time, we report the successful utilization of endoscopy in the intradural space. Indeed, urologic technologies and skills have potential novel applications in nonurologic surgeries. Interdisciplinary cooperation can provide effective treatment options that would not otherwise be possible. Patient selection and cautious planning play an important role in devising innovative surgical approaches. In this particular case, the patient benefited from thoughtful collaboration between urologists and neurosurgeons.

\section{VS6: PEDIATRICS AND TRANSPLANTATION}

\section{V6-1 Pediatric Robotic Pyeloplasty in a Duplex Kidney \\ DY Yang, CF Granberg, R Avant, P Casale, PC Gargollo}

Mayo Clinic, Rochester, MN

United States

Introduction \& Objective: Upper urinary tract duplication is seen in $0.5 \%-0.9 \%$ of patients. Classically, the upper pole moiety is associated with ureterocele or ectopic ureter while the lower pole moiety is associated with vesicoureteral reflux. Duplicated systems are not associated with increased rates of ureteropelvic junction (UPJ) obstruction. Although up to $7 \%$ of patients with UPJ obstruction are found to have ipsilateral duplicated systems. We present a case of pediatric robotic pyeloplasty in a patient with a prior heminephrectomy.

Materials and Methods: A 14 year old female presented with right flank pain. As an infant she was found to have a duplicated right kidney with a nonfunctioning upper pole. She underwent an 
upper pole nephroureterectomy. Following surgery, she remained asymptomatic until re-presenting to the clinic with worsening flank pain over the last month. Imaging was performed demonstrating a right lower pole and MAG3 lasix renogram demonstrated obstruction. She elected to proceed with right robotic pyeloplasty.

Results: Following an extensive dissection of the renal pelvis, the robotic pyeloplasty was performed without complication. Blood loss was minimal and she was dismissed from the hospital on post-operative day 1 . She was participating in competitive athletics at 2 weeks.

Conclusions: Robotic pyeloplasty is a safe and effective treatment for UPJO in duplex kidneys. When considering minimally invasive options, the precision, dexterity, and advanced imaging is valuable in a previously operated field.

V6-2 Robot Assisted Laparoscopic Bilateral Ureteral Reimplantation and Left Tailoring Procedure for a 2-Year-Old Boy Patient: Right Ureterovesical Stricture and Left Ureterovesical Reflux

Y Ozgok, S Yalcin

Agri Military Hospital, Department of Urology, Agri, Turkey Turkey

Introduction \& Objective: Robotic assisted laparoscopic extravesical ureteral reimplantation is an alternative choice for both ureterovesical reflux and ureterovesical stricture treatments in pediatric population. Herewith this video-absract we would like to share our experience of bilateral extravesical ureteral reimplantation.

Materials and Methods: 2-year-old boy patient referred to our clinic with right ureterovesical stricture and grade 4 vesicoureteral reflux. Following necessary evaluation, patient was gone robot assisted bilateral extravesical ureteral reimplantation and left tailoring procedure. Bilateral D-J stents were placed into both ureters. Peritoneum was closed after the operation and drain was left in the abdominal cavity.

Results: Whole operation time was 125 minutes, estimated blood loss was $15 \mathrm{cc}$. No complication was recorded during and after the operation. Drain, urethral catheter and the D-J stents were removed 24 hours, 7 days and 30 days, sincerely.

Conclusions: Robotic assisted laparoscopic extravesical ureteral reimplantation is an alternative safe and efficacious choice for both ureterovesical reflux and ureterovesical stricture treatments in pediatric population.

V6-3 Hybrid Lparoendoscopic Single Site Ureteroureterostomy With Transmesentric Approach For Congenital Miduretral Stricture

K Kobayashi, K Johnin, M Narita, A Kawauchi

Shiga university of medical science

Japan

Introduction \& Objective: Congenital midureteric stricture (CMUS) which develops from obstructive lesion between PUJ and UVJ is rare obstructive uropathy. No standardized terminology for midureteral stricture disease has been developed or proposed, and there are only a few case reports of laparoscopic management of CMUS.

Here, we present our experience with Hybrid Lparoendoscopic Single Site(LESS) ureterouretrostomy with transmesentric approach for CMUS..
Materials and Methods: The patient was placed in the lateral flank position. A $20 \mathrm{~mm}$ vertical incision was made at the umbilicus for insertion of the LESS port. Under visual guidance, an additional 5-mm port was placed in the lower abdominal region.

Longitudinal incision was made in the mesentery and peritoneum overlying the ureter.

After the ureter was isolated from surrounding tissue, a traction suture of the proximal ureter was placed from the abdominal wall. The ureter was divided at the level of stenosis. The distal ureter was spatulated on the lateral aspect.

The posterior anastomosis was created intermittently using a 5-0 monofilament suture. Double $\mathbf{J}$ stent was placed from bladder to proximal ureter. The anterior anastomosis was created in the same manner. Finally, mesentery was repaired with Interrupted suture. Results: The Operation was taken 238 minutes with minimal blood loss. No major complications occurred, and the hospital stay was 5 days. After one months from operation, Double J Stent was removed. she has progressed favourably after the operation with no recurrence of left flank pain.

Conclusions: Hybrid LESS ureterouretrostomy with transmesentric approach for CMUS is technically feasible, safe, and provides excellent cosmetic outocome.

\section{V6-4 Laparoscopic Radical Nephrectomy for Prenatally} Diagnosed Wilms Tumor

A Toussi, CF Granberg, PC Gargollo

\section{United States}

Introduction \& Objective: Wilms' tumor is the most common primary malignant solid renal tumor of the kidney in childhood. However, neonatal incidence is extremely low and prenatal detection is even more uncommon. In fact, according to the National Wilms' Tumor Study $1-3$, only $0.12 \%$ of all Wilms' tumors occurred in patients younger than 30 days old. Traditionally, the surgical approach in the neonates has been via an open incision. We present a case of prenatally diagnosed Wilms' tumor and offer insight into a contemporary and minimally-invasive surgical approach.

Materials and Methods: We present a case of prenatally diagnosed Wilms' tumor, subsequent operative approach and management.

Results: The patient was referred to our institution with an abnormal prenatal renal ultrasound. CT scan after delivery showed a multifocal enhancing left renal mass. She underwent an uncomplicated left laparoscopic radical nephrectomy and retroperitoneal lymph node sampling using a novel hidden incision endoscopic surgery (HIdES) technique. Final pathology revealed favorable histology Stage II Wilms' Tumor. She received adjuvant chemotherapy with Vincristine and Dactinomycin based on the EE4A regimen.

Conclusions: We highlight the diagnostic pathway, peri-operative management, and surgical approach in this case report. Minimally-invasive approach is a safe and viable surgical option in highly selected patients in the neonatal period.

V6-5 Robot-assisted left pyeloplasty in a pediatric patient with gastrostomy tube and severe scoliosis

JP Joseph, CF Granberg, PC Gargollo

Department of Urology, Mayo Clinic

United States 
Introduction \& Objective: Robot-assisted pyeloplasty is performed routinely in the setting of pediatric ureteropelvic junction (UPJ) obstruction. However, published experience is limited in the setting of patients with prior abdominal surgery and significant anatomic complexity. We present our experience with robot-assisted left pyeloplasty in an 11 year-old male with percutaneous gastrostomy tube and severe thoracolumbar scoliosis.

Materials and Methods: Our patient presented with episodic left flank pain, nausea, and vomiting. CT scan and diuretic renography demonstrated findings consistent with left UPJ obstruction. The decision was made to proceed with robot-assisted left pyeloplasty. The previously described hidden incision endoscopic surgery (HIdES) technique was employed in our approach to port placement. Specifically, ports were inferior to the pfannenstiel line with the exception of an infraumbilical working port at the site of laparoscopic access.

Results: Operative time was 116 minutes, estimated blood loss was $5 \mathrm{~mL}$. The patient was discharged on post-operative day one. The indwelling ureteral stent was removed three weeks postoperatively, and ultrasound three months post-operatively demonstrated near complete resolution of the left hydronephrosis.

Conclusions: In anatomically complex pediatric patients with UPJ obstruction, robot-assisted pyeloplasty may be feasible and safe in select cases. In our experience, the HIdES technique afforded a safe inferior point of access, without compromise to triangulation or technical ergonomics.

\section{V6-6 Robotic Assisted Laparoscopic Pyelopyelostomy of a} Pediatric Upper Pole UPJ Obstruction

DK Agarwal, IJ Sobol, K Eeg, CF Granberg, PC Gargollo

Department of Urology, Mayo Clinic, Rochester, MN United States

Introduction \& Objective: We demonstrate a robotic assisted laparoscopic left upper to lower pole pyelopyelostomy using HiDES port placement for a 14 year old patient with upper pole UPJ obstruction in a duplicated system with high confluence of the upper and lower pole moieties.

Materials and Methods: A robotic assisted laparoscopic left upper to lower pole pyelopyelostomy was performed using the da Vinci ${ }^{\circledR}$ Xi Surgical System (Intuitive Surgical, Sunnyvale, CA) with HiDES port placement. A ureteral stent was placed during the procedure and removed one month postoperatively.

Results: The surgery was successfully performed with no immediate postoperative complications. Total robotic time was 2 hours and 23 minutes. The patient continues to do well after stent removal.

Conclusions: A robotic pyelopyelostomy is an option to manage UPJ obstruction in a renal duplication with high confluence of upper and lower pole moieties. The HiDES technique for port placement is an effective method to optimize cosmesis without compromising surgical access to the kidney.

V6-7 Second Stage Fowler-stephens Orchiopexy Using Only Novel Percutaneous Externally Assembled Laparoscopic (Peal) Instruments: a Video Presentation

J Lee, C Bautista, I Kelly, S Abourbih, M T Chau, H Wagner, J Cheng, M Pierce, M Keheila, DD Baldwin

Loma Linda University

United States
Introduction \& Objective: Laparoscopy is the gold standard for diagnosis and treatment of boys with non-palpable testicles. In an effort to reduce the invasiveness of laparoscopic orchiopexy, various strategies have been employed including use of laparoendoscopic single site surgery (LESS) and needlescopic surgery. Needlescopic instruments have limited functionality due to their small size and are more prone to intrabdominal organ injury. LESS has been criticized when used in children due to the requirement of a much larger $3 \mathrm{~cm}$ incision. We have previously demonstrated substitution of a single novel percutaneous externally assembled laparoscopic (PEAL) instrument for a traditional laparoscopic instrument during a FowlerStephens orchiopexy. First generation instrument tips were limited to a grasper and shears, so only one PEAL instrument could be used. In contrast, second generation instruments include a wider variety of instrumentation. In this video we demonstrate the use of second generation PEAL tools for all working instruments used during a second stage FowlerStephens orchiopexy.

Materials and Methods: The PEAL surgical paradigm is composed of a reusable handpiece with a disposable $2.96 \mathrm{~mm}$ instrument shaft and interchangeable disposable $5 \mathrm{~mm}$ instrument tips. Second generation instruments have a simpler attachment mechanism. This video demonstrates how the shaft is placed directly through the abdomen without a trocar and then brought out through the camera port for attachment of the working tip. The tip is then brought back into the abdomen to perform the surgery and then removed in the same fashion. Due to their small size and because they are assembled externally, they produce an essentially scarless outcome. Only PEAL working instruments were used for the performance of a second stage Fowler-Stephens orchiopexy.

Results: Using this innovative new technique, a 12 year-old child underwent a successful second stage left Fowler-Stephens orchiopexy. Operative time was 105 minutes. Blood loss was minimal. He was discharged the day of surgery with no complications. At follow up, the objective cosmetic results for both PEAL sites were excellent, and the testis was palpable in the scrotum and well-positioned.

Conclusions: Due to its improved cosmesis and ease of performance, the PEAL surgical paradigm continues to shows promise in reducing the invasiveness of pediatric Fowler-Stephens orchiopexy, maximizing cosmesis and minimizing morbidity.

\section{V6-8 Hidden Incision Endoscopic Surgery (HIdES) Partial Cystectomy}

\section{Gearman, CF Granberg, PC Gargollo}

Mayo Clinic

United States

Introduction \& Objective: Hidden incision endoscopic sugery is a minimally invasive technique that allows the ability to hide inicisions in Pfannenstiel line. We outline this technique in a child with a urachal anomaly.

Materials and Methods: We employed the HIdES technique to perform a partial cystectomy in a child with a urachal anomaly Results: We performed a partial cystectomy showing the utility of the HIdES technique.

Conclusions: This technique can be safely applied to a number of pediatric sugeries. We outline the use in a partial cystectomy for a urachal anomaly. 
V6-9 Robotic Assisted Tapered Distal End-to-Side Uretero-Ureterostomy for Duplex Kidney with Upper Pole Obstruction

L Ni, S Dave

Western University

Canada

Introduction \& Objective: In Duplex anomalies, the upper pole system is often associated with obstruction at the uretero-vesical junction (UVJ) associated with an ureterocele or intrinsic UVJ obstruction. Surgical options include upper pole heminephrectomy, distal uretero-ureterostomy (U-U) or simple ligation of the upper pole ureter. This video demonstrates a robotic-assisted distal U-U for left duplex kidney with upper pole UVJ obstruction without a ureterocele.

Materials and Methods: A 5-year-old boy presented with urinary frequency with no previous history of urinary tract infections. An incidental ultrasound revealed left duplex kidney with upper pole Grade IV and lower pole grade II hydronephrosis with a dilated upper pole ureter showing a distal tapered segment as it entered the bladder in an abnormal distal insertion. A MAG 3 Lasix renogram showed minimal function of the upper pole moiety with a T-1/2 drainage time of $38 \mathrm{~min}$ for the upper pole ureter suggesting UVJ obstruction. A voiding cystourethrogram did not show any evidence of vesicoureteral reflux to either moiety. After discussing the pros and cons of each approach and factoring in possible complications and implications of retaining a poorly functioning upper pole, the parents decided to proceed with a distal robotic-assisted U-U.

Results: The patient underwent a robotic-assisted distal end-toside upper pole to lower pole U-U with tapering of the upper pole ureter. A double-J stent was placed across the anastomosis into the bladder. A Foley catheter was left intraoperatively and removed at 24 hours' post-op and the patient was discharged home on post-operative day two. The stent was removed 6 weeks after the procedure. Follow up at 4 months' post stent removal showed resolution of lower moiety hydronephrosis and distal hydroureter and decrease of the upper moiety hydronephrosis.

Conclusions: Robotic assisted distal U-U is a viable and effective minimally invasive option for surgical correction of duplex systems with upper pole UVJ obstruction.

V6-10 Recurrent Bladder Stones in a Patient with Prior Bladder Exstrophy Repair

J Han, JM Shields, A Rabley, VG Bird

SUNY Downstate

United States

Introduction \& Objective: Bladder exstrophy-epispadias complex is a rare congenital anomaly presenting with protrusion of the urinary bladder through an abdominal wall defect. Repair of bladder exstrophy is usually performed during the first days of life. Subsequent bladder calculi in such patients occur at a rate of approximately $15 \%$ and are associated with infeciton, foreign bodies, and urinary stasis. Surgical repair involves dissecting free the exposed urothelium from the mucocutaneous junction and then approximating the freed urotheial edges to close the bladder. We present the first reported case of a bladder calculus resulting from hair-bearing epithelium serving as a nidus.

Materials and Methods: This is a case of a 24 year old male with a history of prior bladder exstrophy repair as an infant who was referred from an outside hospital for recurrent bladder stones which appeared to be "attached to hairs". The passage of these stones was first noted at the age of 16 years old. Some stones were voided spontaneously while others were removed endoscopically. The patient voids spontaneously and does not catheterize.

Results: Upon entry into the bladder one can clearly see a well-demarcated scar consistent with a history of bladder exstrophy repair. Hairs can also clearly be seen proturding from the scar tissue. The hair follicles and scar were ablated using a 365 micron laser fiber and a holmium laser. The patient tolerated the procedure well and follow-up endoscopy revealed evidence of recurrent hairs or stones. While a number of reports exist in the literature regarding formation of bladder calculi from a hair nidus, theses reports all realte to hair introduced during catheterization.

Conclusions: This case is the first reported incidence of a bladder calculus resulting from hair-bearing epithelium serving as a nidus. This case emphasizes the need for close follow-up and continued surveillance in patients with a history of bladder exstrophy.

V6-11 Robotic Kidney Transplant with grafts having multiple renal arteries - Technical nuances

FA Zafar, A Mallya, S Arakere, I Banerjee, P Ghosh, A Mandhani, RK Ahlawat

Fortis Escorts Kidney and Urology Institute India

Introduction \& Objective: Kidney Transplant with multiple renal arteries is technically challenging. In grafts with lower polar accessory artery, there are additional concerns regarding the ureteric blood supply. In this setting, higher incidences of vascular and urological complications have been reported in some studies. Hence, there is a hesitation in using grafts with multiple arteries. In the current practice, excluding the otherwise suitable live donors only on the ground of technical challenges no longer seems to be acceptable. This becomes particularly more important in developing countries like India, where cadaveric donor program is still in its infancy and where living donor nephrectomy contributes to the majority of graft pool. We present a video highlighting our technique and experience in Robotic kidney transplants using grafts with multiple arteries.

Materials and Methods: 160 out of a total of 881 patients underwent Robotic Kidney transplant from February 2013 to January 2017. Multiple renal arteries were assessed preoperatively by studying CT renal angiographic 3D reconstruction images and also on the bench after graft retrieval. Depending on the location and size of graft vessels, decision was taken to make pantaloons, anastomose vessel separately to inferior epigastric or external iliac or sacrifice it if very insignificant.

Results: Patients were divided into two groups based upon the number of renal arteries in the graft. Out of 160 grafts, 128 grafts $(76.8 \%)$ had a single renal artery (SRA) while 32 patients (23.2\%) had multiple renal arteries (MRA). 30 allografts had 2 renal arteries while 2 grafts had 3 renal arteries. The mean age for MRA group was 37.5 and for SRA group was 39.08. Mean BMI was 25.95 and 24.7 in MRA \& SRA group respectively. The mean warm ischemia time (WIT) in MRA group was $166 \mathrm{sec}-$ onds, which was significantly higher than that in SRA group (147.8 seconds), $p$ value 0.005 . The mean total ischemia time in 
patients receiving graft with multiple renal arteries was significantly higher as compared to the patients receiving grafts with single renal artery (79.8 minutes in MRA vs. $65.2 \mathrm{~min}$ in SRA, $p$ value 0.0001$)$. Blood loss was found to be similar in the two groups. The mean creatinine levels at discharge $(1.17 \mathrm{mg} / \mathrm{dl}$ in MRA group and $1.27 \mathrm{mg} / \mathrm{dl}$ in SRA group) and at 3 months $(1.18 \mathrm{mg} / \mathrm{dl}$ in MRA group and $1.26 \mathrm{mg} / \mathrm{dl}$ in SRA group) were comparable.

Conclusions: Multiple renal arteries, albeit technically challenging, are not a contraindication for robotic kidney transplant. The outcomes are comparable with grafts having single renal artery

\section{V6-12 A Simple Iced Renal Jacket during Renal Trans- plantation}

FA Zafar, S Arakere, A Mallya, I Banerjee, T Jindal, A Gupta, A Mandhani, RK Ahlawat

Fortis Escorts Kidney and Urology Institute India

Introduction \& Objective: In Renal Transplant, positioning and handling the kidney during transplant can be cumbersome. In Robotic kidney transplant, atraumatic handling of the graft can be a challenge due to lack of haptic feedback. We present out technique of open and robotic kidney transplant using a gauze jacket for easy handling of the graft.

Materials and Methods: Records of patients who underwent 771 open and 160 patients who underwent robotic renal recipient allograftingbetween January 2013 and December 2016 were reviewed retrospectively. In the open technique a renal jacket was made using folded gauze with an opening cut out in the center to deliver the graft hilum through it after the graft has been perfused with cold ringer's lactate and then place finely grated fluffy ice made of frozen Ringer's lactate over the graft. The jacket hangs the graft over the recipient vessels and is held in place by using two Kelly clamps thus keeping the surgeon's and assistant's hands free. For the robotic transplant we modified this technique where the graft is kept in ice filled jacket and the flaps are sutured together using interrupted silk suture with the suture at the upper pole kept longer for identification. Ice is also kept over the dropped bladder. This helps in maintaining a low intracorporeal graft temperature and also helps in maneuvering the graft during anastomosis.

Results: Documented graft temperatureswere $18.4(14-26){ }^{\circ} \mathrm{C}$ for open and 20.3(15.6-27) ${ }^{\circ} \mathrm{C}$ for robotic transplant

Conclusions: The iced renal jacket is inexpensive, helps in tailoring the cold ischemia time, increases the surgeon's convenience and allows for easy maneuverability of the graft.

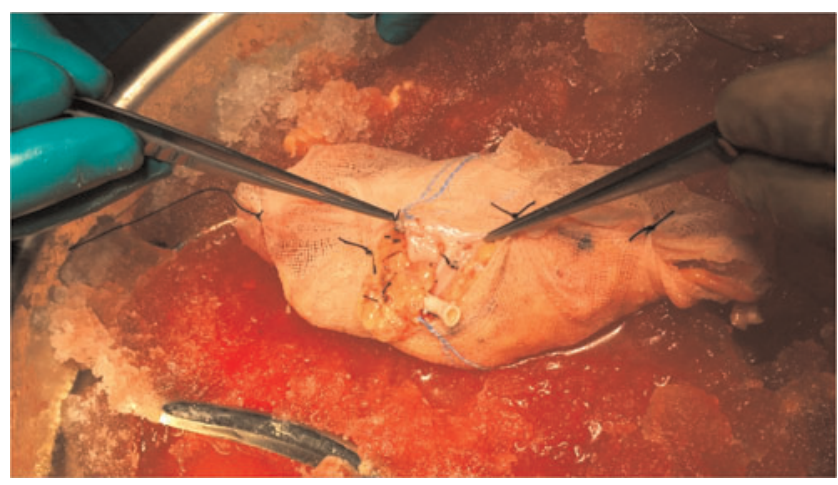

V6-13 Robotic kidney transplantation in a patient previously treated with robotic radical prostatectomy

A Breda, A Territo, G Basile, I Schwartzmann, L Gausa, O Rodriguez, J Gaya, J Ponce de Leon, H Villavicencio

Fundació Puigvert, University Autonoma of Barcelona, Spain Spain

Introduction \& Objective: Kidney transplantation is the preferred treatment for patients with end-stage renal disease. In order to reduce the morbidity of the open surgery, a robotic assisted approach has been recently introduced. The robotic surgery allows the performance of kidney transplantation under optimal operative conditions while maintaining the safety and the functional results of the open approach.

Materials and Methods: We present the case of a wife donating to her husband affected by end stage renal disease due to polycystic kidney disease with a past surgical history of robotic radical prostatectomy for adenocarcinoma. Gleason score 3+4, Stage pT2c.

Results: A RAKT was successfully performed. Console surgical time was 260 min with 40 min for vascular suture. The kidney started to produce urine intra-operatively. The patient presented an uneventful recovery with serum creatinine of $1.5 \mathrm{mg} / \mathrm{dl}$ on postoperative day 7. No surgical complications were recorded.

Conclusions: Robotic kidney transplantation is a feasible technique also in patients previously treated with robotic radical prostatectomy. The advantages of technique are related to the quality of the vascular anastomosis and the low complication rate.

\section{V6-14 Robot Assisted Ureteric Reimplantation of Trans- planted Kidney}

\section{A Beksac, S Prasad, J Sfakianos, K Badani}

Icahn School of Medicine at Mount Sinai United States

Introduction \& Objective: Recently, the rate of renal transplantation has increased in the US. Renal transplantation has been performed on more challenging patients, including prior pelvic surgery, radiation and ureteral anatomy that can result in increased rates of postoperative complications. Herein, we report a case of ureteral stricture of a transplanted kidney, with prior history of pelvic radiation.

Materials and Methods: The patient is a 69 year old man with a prior history of prostate cancer treated with radiotherapy. During transplantation, right uretero-ureterostomy was performed due history of pelvic radiation. Patient developed a distal ureteral stricture postoperatively. Nephrostomy tube was placed, followed by ureteral balloon dilation and subsequent double $\mathrm{J}$ stent (DJS) placement. Patient failed these endoscopic treatments and ureteral reconstruction was recommended. The patient was positioned in the steep Trendelenburg position. The bowel overlying the transplanted kidney were lysed and released. The peritoneum overlying the kidney transplant was carefully dissected and indocyanine green was injected through the nephrostomy tube to help identify the renal pelvis and ureter. There was significant periureteral fibrosis. Ureter was carefully dissected to avoid devascularization, and later dissected from the adjacent fibrotic tissue all the way to the strictured segment distally. Ureter was dissected proximally to the hilum to allow mobilization. Ureter was clamped with hem-o-lock clip distally. 
The bladder was then mobilized to allow a tension-free anostomosis. The ureter was spatulated and anostomosis was performed with 4.0 vicryl sutures. Before the anostomosis was completed DJS was inserted into the transplanted kidney ureter.

Results: Total operative time was 2:00 hours, with an estimated blood loss of $30 \mathrm{~mL}$. Jackson-Pratt drain was removed and patient was discharged postoperative day 1 , foley was removed at day 7 and stent was removed at week 4. Creatinine was stable after DJS removal and nephrostomy clamping. Subsequently, nephrostomy was removed. Follow up on week 8 showed his creatinine at 1.18 .

Conclusions: Our experience was encouraging and confirmed the feasibility and safety of this approach to ureteral reconstruction of transplant kidneys.

\section{VS7: NEW TECHNIQUES IN ROBOTIC SURGERY}

\section{V7-1 Robotic Pelvic Exenteration (Cystoprostatectomy and Combined Abdominoperineal Resection) with Double Barreled Wet Colostomy}

N Velazquez, W Huang, M Bernstein, M Bjurlin

NYU Langone Medical Center

United States

Introduction \& Objective: Patients with locally advanced pelvic carcinoma or severe radiation sequelae may be managed with pelvic exenteration which requires both fecal and urinary diversion. Double barreled wet colostomy (DBWC) represents a simultaneous urinary and fecal diversion through a single ostomy. This procedure has been described through an open surgical approach, however literature is lacking on the technique and safety of a robotic approach. We show our combined robotic cystoprostatectomy and abdomnioperineal resection with creation of a DBWC. To our knowledge this is the first description of the combined procedure done robotically.

Materials and Methods: Using a high definition recording system and iMovie software with narrative and annotative editing, we created a video of our technique for robotic pelvic exenteration with DBWC using the Surgical Intuitive Da Vinci Xi robot. Lithotomy position is used to access the perineum. 4 robotic and 1 assistant ports are placed allowing access to the descending colon and pelvic structures. Key steps include: mobilizing the sigmoid colon and rectum from the pelvic sidewall to the splenic flexure; bowel transection at the mid descending colon; bilateral pelvic lymph node dissection (PLND) from the bifurcation of the aorta and including the common iliac vessels, external iliac obturator, and hypogastric lymph nodes, including the triangle of Marcille; transecting the bladder pedicles; dropping the bladder and entering the space of Retzius; dissecting the endopelvic fascia and lateral prostate; transecting the dorsal vein complex and urethra; dissecting the posterior plane of the rectum along the sacrum; en bloc removal of the specimen via the perineal incision; creating a ureteroenteric anastomosis with double $\mathrm{J}$ ureteral stents placement in the urinary reservoir; and wet colostomy site creation $10-15 \mathrm{~cm}$ from the colonic suture line and brought to the skin to complete the DBWC.

Results: Operative time was 480 minutes and estimated blood loss was $250 \mathrm{~mL}$. Final pathology demonstrated pT4N1 Gleason 9 prostate cancer and invasion of the rectum, bladder and pelvic wall. Postoperatively the DBWC functions well, without complication of ascending pyelonephritis or electrolyte imbalance.

Conclusions: This video presents our technique of robotic cystoprostatectomy and abdomnioperineal resection with creation of a DBWC in the first patient managed in this fashion at our institution. Robotic pelvic exenteration and DBWC is feasible and safe for locally advanced pelvic malignancies. This allows for excision of the bladder, prostate, sigmoid colon, rectum, and anus en bloc while performing an extensive PLND. Early follow up shows good functional outcomes.

\section{V7-2 The Novel Use of a Magnetic Retraction Device in Robotic Urologic Surgery}

P Samson, P Mistry, L Kavoussi, L Richstone

Smith Institute for Urology

United States

Introduction \& Objective: The advancement of robotic technology has revolutionized surgical treatment for prostate cancer. Although the robot has facilitated surgery in narrow working spaces, it still has its shortcomings such as the collision of robotic and laparoscopic instruments both inside and outside the patient. Magnetic anchoring and guidance system (MAGS) technology provides extra tools within the patient without the use of additional trocars or bulky instruments. MAGS has not yet been described in robotic urologic surgery.

Materials and Methods: We present two robotic urologic cases in which we used a magnetic retractor device. The magnetic retractor is inserted through the air-seal trocar and deployed with a laparoscopic handheld device by the bedside assistant. The retractor is then coupled to a handheld magnet that is placed outside of the patient's abdominal wall in the desired position. The first case is a 56-year-old male who was found to have Gleason 7 prostate cancer on prostate biopsy. He elected for nerve-sparing robotic-assisted laparoscopic prostatectomy (RALP). The second case is a 56-year-old male with recurrent urinary tract infections and a history of transurethral microwave thermotherapy of the prostate who presented with two large bladder diverticula. He elected for a transurethral button vaporization of the prostate and robotic-assisted laparoscopic bladder diverticulectomy (RABD) in the same operative setting.

Results: In the RALP, the magnetic retractor provided anterior retraction of the vasa deferentia during the dissection of the seminal vesicles. The magnetic retractor was then used to retract the vasa and seminal vesicles to facilitate posterior dissection of the prostate while the fourth robotic arm provided cephalad retraction of the bladder. During the dissection of the neurovascular bundles and prostatic pedicles, the magnetic retractor was helpful in pulling the prostate medially without the use of the fourth robotic arm. There were no intraoperative complications. In the RABD, the magnetic retractor was used for additional 
retraction of the bladder in angles the robotic fourth arm was not able to provide. There were no intraoperative complications. Conclusions: The use of a magnetic retraction device facilitates certain steps in robotic-assisted laparoscopic prostatectomy, and may be useful in other robotic urologic surgeries.

V7-3 Retroperitoneal Robot-assisted Laparoscopic Partial Nephrectomy

M Lyons, J Vukina, M McKibben, M Raynor

University of North Carolina at Chapel Hill Department of Urology

United States

Introduction \& Objective: Retroperitoneoscopic robotic assisted partial nephrectomy has been well established as a feasible method of treating appropriately selected renal masses, however, few studies have evaluated late complications such as neuropathic flank pain and flank hernia. We sought to compare perioperative, oncologic and late outcomes after retroperitoneal and conventional trans-peritoneal robot-assisted partial nephrectomy (rRAPN and tRAPN).

Materials and Methods: We performed a retrospective matched-pair analysis of consecutive patients undergoing rRAPN $(n=42)$ and tRAPN $(n=42)$ performed by a single surgeon from February 2011 to May 2016. Surgical approach was based on patient and tumor characteristics, history of abdominal surgery, and surgeon preference. We compared demographic, tumor, operative and oncologic outcomes as well as late complications, specifically flank hernia and neuropathic pain.

Results: Baseline patient and tumor characteristics were similar between groups. Mean operative time, warm ischemia time, and length of hospital stay and mean follow-up time were not statistically different between groups. Estimated blood loss was significantly lower in the rRAPN group $(113 \mathrm{~mL}$ versus $186 \mathrm{~mL}$, $\mathrm{p}=0.04$ ). Oncologic outcomes were similar between groups. Overall complication rates were significantly higher in the rRAPN group $(33 \%$ versus $10 \% \mathrm{p}=0.02)$, the majority of which were Clavien-Dindo grade I-II complications (29\% versus $5 \%$, $\mathrm{p}=0.003 \%$ ). Grade III or higher complications occurred at similar rates in each group $(7 \%$ versus $5 \%, \mathrm{p}=0.89)$. Flank hernia was unique to the rRAPN group and occurred in $10 \%$ of patients with 2 of 4 patients requiring surgical repair. Post-operative neuropathic pain was noted in 5 patients (12\%) with 1 patient having persistent pain at time of last follow-up.

Conclusions: Retroperitoneal robot-assisted laparoscopic partial nephrectomy is a safe an efficacious approach to appropriately selected tumors. Unique complications including flank hernia and neuropathic pain must be considered. We have found a significant decrease in flank hernia rates with changes in our access and closure.

V7-4 Retroperitoneal robotic partial nephrectomy for solitary horseshoe kidney

Z Hamilton, M Holden, O Raheem, IH Derweesh

Department of Urology, UC San Diego Health, La Jolla, California, USA

United States

Introduction \& Objective: The standard treatment for localized small renal masses is partial nephrectomy and robotic partial nephrectomy has become rapidly adopted in this setting. The retroperitoneal approach provides advantages for posterior tumors. We present a surgical video of a 74 year old male with a clinical T1a, $3.7 \mathrm{~cm}$, left renal mass, in a solitary left horseshoe kidney who underwent robotic retroperitoneal partial nephrectomy with cold ischemic technique. Our surgery is technically innovative in that we utilize a method for providing cold ischemia through a minimally invasive robotic procedure with a retroperitoneal approach.

Materials and Methods: The Da Vinci Si (Intuitive Inc, Sunnyvale, CA, USA) robotic platform was utilized with a 4-arm approach. Four robotic ports were placed in a linear configuration transverse to the $12^{\text {th }}$ rib and an assistant port was in the lower quadrant. An additional $12 \mathrm{~mm}$ accessory port was placed near the $12^{\text {th }}$ rib for introduction of ice slush. Modified $20 \mathrm{~mL}$ syringes are used to deploy ice slush through the $12 \mathrm{~mm}$ accessory port. The renal artery is clamped and the tumor completely excised. Renorrhaphy was performed with adequate hemostasis.

Results: Estimated blood loss was $100 \mathrm{~mL}$ Cold ischemia time was 20 minutes. Patient was discharged home on postoperative day 3. No complications were noted after surgery. Final pathology revealed clear cell renal cell carcinoma, Fuhrman grade $2,3.5 \mathrm{~cm}$, with negative margins, pT1a.

Conclusions: Robotic retroperitoneal partial nephrectomy of T1 renal masses can be performed with the addition of cold ischemia for a solitary renal mass, even with anatomic variability.

V7-5 Robot Assisted Laparoscopic Retroperitoneal lymph node dissection using the Da Vinci Si: Lateral and Anterior Approaches

M Ferretti, M Stifelman, G Lovallo, M Ahmed

New York Medical College

United States

Introduction \& Objective: Laparoscopic and Robotic retroperitoneal lymph node dissection has been described for the management of testicular cancer. Here we present our technique utilizing both anterior and lateral approaches.

Materials and Methods: Robot assisted laparoscopic retroperitoneal lymph node dissection is performed at our institution utilizing the Da Vinci Si system. Both lateral and anterior approaches may be employed.

Results: Robot assisted laparoscopic retroperitoneal lymph node dissection is performed at our instituttion in select cases in the management of testicular cacer.

Conclusions: Robot assisted laparoscopic retroperitoneal lymph node dissection is safe and effective in the management of testicular cancer.

V7-6 Robotic Assisted Lymph Node Dissection in the Management of Penile Cancer

J Bloom, M Ferretti, G Lovallo, M Ahmed

National Cancer Institute

United States

Introduction \& Objective: We present our experience with the management of multiple suspicious lymph nodes, in both the groin and pelvis, in a patient with penile cancer. Robotic assisted 
procedures are becoming the standard of care for many urologic conditions and this approach could be utilized for penile cancer lymphadectomies to limit morbidity.

Materials and Methods: Our patient is a 30 year old man who underwent a partial penectomy for high grade T2 penile cancer. Imaging revealed lymphadenopathy in the left inguinal region and bilateral pelvic nodes. We performed a roboticassisted bilateral pelvic lymphadectomy and ingunal lymphadenectomy robotically for both diagnostic and therapeutic purposes in one setting.

Results: We first performed a bilateral pelvic lymphadenctomy in the standard fashion and sent frozen sections which subsequently came back negative. The robot was then repositioned on the left side of the patient in order to perform the inguinal lymphadenectomy. This superficial inguinal node dissection was performed, skeletonizing the saphenous vein and inuinal ligament. A 10 french Jackson Pratt drain was left in place. The total time for these procedures was two hours and 43 minutes. The patient tolerated the procedure well and all nodes were negative. The patient was able to be discharged home on postoperative day 1.

Conclusions: Robotic-assisted pelvic and inguinal lymph node dissections can be safely performed in one session by experienced robotic surgeons. This technology can limit the morbidity of the standard open procedure and can be performed in a relatively short amount of time. Our patient did well after the procedure and required little time in the hospital post operatively.

\section{V7-7 Use of Immunofluorescence for Ureteral Identifica- tion During Robotic Surgery}

\section{JA Albersheim-Carter, WB Gaertner, CA Dixon, CJ Weight}

University of Minnesota Medical School

United States

Introduction \& Objective: Iatrogenic ureteral injury is a serious complication associated with colorectal and gynecologic surgery. Incidence of ureteral injury ranges from $0.44 \%-0.59 \%$ in colorectal surgery, $0.2 \%-2.5 \%$ in routine gynecologic surgery, and $10 \%-30 \%$ in radical, malignant gynecologic surgery. Prophylactic intraoperative ureteral stents - either regular or lighted may help identify iatrogenic injuries after they occur, or even help prevent them. Urologists are often asked to place these stents at the beginning of surgical cases, with as many as $4.2 \%$ of laparoscopic colectomies requiring stent placement, translating into thousands of cases in the US each year. However, an increasing number of colorectal and gynecologic surgeries are performed either laparoscopically or robotically, in which haptic feedback is limited and stents may not help prevent ureteral injury. In this video abstract, we describe the use of indocyanine green (ICG) dye in the visualization of ureters during robotic surgery to prevent ureteral injury.

Materials and Methods: With patients in dorsal lithotomy position, place a cone-tipped catheter. Next, inject $3 \mathrm{~mL}$ of ICG solution at a concentration of $2.5 \mathrm{mg} / \mathrm{mL}$ in each ureter, followed by a $5 \mathrm{~mL}$ flush of sterile saline, to adequately load the ureters with immunofluorescent dye. Once the surgeon is approaching the area of the ureter, he or she may switch to Firefly NIRF mode. This allows for up to 3 hours of ureteral visualization on da Vinci $\mathrm{Xi}$ or $\mathrm{Si}$.

Results: This technique has been performed in 7 patients at our institution, with no complications or ill effects. The cost of ICG is approximately $\$ 300.00$ per case in addition to urology service fees; the cost of routine ureteral stents are approximtaely $\$ 170$ per case in addition to urology service fees; and the cost of lighted stents are approximately $\$ 750$ per case in addition to urology service fees.

Conclusions: This described method of using ICG is more cost-effective than lighted stents, and more useful for ureteral visualization than open-ended stents placed prior to surgery. In addition, ICG has already been used successfully by urologists for ureteral reconstruction surgeries and could be explored as a tool for more optimal visualization during robotic ureteral reimplantation.

\section{V7-8 Robotic Assisted Extended Pelvic Lymph Node Dissection Including Presacral Area: tips and tricks}

RJ Yau, M Stifelman, G Lovallo, M Ahmed

New York Medical College

United States

Introduction \& Objective: Lymph node dissection during radical cystectomy is both diagnostic and therapeutic. An incomplete node dissection can leave cancer behind and have a negative impact on patient survival. Therefore, meticulous node dissection must be performed regardless of open or robotic approach. In this video, we present our technique on performing a meticulous lymph node dissection up to the bifurcation of the aorta in a quick and efficient manner.

Materials and Methods: The video presentation was created by editing portions of the various robotic assisted radical cystectomies. All patient identifiers were removed in compliance with HIPAA. The video editing software was Adobe Premiere.

Results: The first step is to place the trochars in a supraumbilical plane. This port position allows for proper visualization of the common iliac vessels. The first step of the dissection is to approach the ureter by staying in the retroperitoneal space by exposing the side wall. When performing anterior lymph node dissection, it is important to keep the instruments parallel to vessels to prevent perforation. The goal of a complete lymph node dissection is to be able to lift the vessels free from nodal attachments. The first anterior branch of the internal iliac artery is the umbilical artery which gives rise to the superior vesical artery and medial umbilical ligament. This can be ligated during the node dissection to aide in the posterior approach to the radical cystectomy. We do not routinely use clips during the node dissection because it does not decrease lymphocele formation and it adds to operative time. In our experience, this technique averages 30 to 40 lymph nodes per case.

Conclusions: This systematic technique can reliably guide the surgeon to the aortic bifurcation and is useful in all surgery requiring extended lymph node dissection. It is our preferred approach to radical cystectomy because the exposure, efficiency, and lymph node yield are excellent.

\section{V7-9 Robotic Assisted Laparoscopic Inguinal Lymphade-} nectomy for Invasive Penile Cancer

\author{
P Knoll, V Ramakrishnan, A Rogman, TM FitzGibbon, \\ J Messer
}

University of Louisville

United States 
Introduction \& Objective: Open inguinal lymphadenectomy for invasive penile cancer is acknowledged as a morbid procedure. We present a 73 year-old-male who underwent a robotic assisted laparoscopic inguinal lymph node dissection due to increasing evidence that this technique has potential for decreased morbidity and similar nodal yield compared to the open procedure.

Materials and Methods: We present our technique for robotic assisted inguinal lymphadenectomy. Three $8 \mathrm{~mm}$ robotic ports and one $12 \mathrm{~mm}$ assistant port was inserted into each upper thigh. The saphenous vein and its tributaries were spared, and $10 \mathrm{~mm}$ Hem-o-lok clips were used for lymphostasis. Superficial and deep lymph nodes were harvested. Bilateral 19 French drains were placed in femoral space at the conclusion of the procedure. Results: Operative time was 180 minutes for the pelvic and inguinal lymphadenectomy including docking and undocking of the robot. There was an estimated blood loss of $100 \mathrm{cc}$. The patient was discharged on post-operative day two after an uneventful hospital course with bilateral drains in place. Final pathology was pT3N1Mx. There was one lymph node positive for disease in the right inguinal lymph node packet. Four lymph nodes were harvested from the left inguinal region and five lymph nodes on the right. Twenty- two pelvic lymph nodes were harvested and all were negative for disease. On postoperative day 18 , the patient had no signs of lymphedema, wound necrosis, or DVT, and the bilateral drains were removed.

Conclusions: Robotic assisted laparoscopic inguinal lymphadenectomy can be performed in an efficient manner with reduced morbidity and adequate lymph node dissection.

V7-10 Robotic-assisted laparoscopic excision of a post chemotherapy, pelvic rhabdomyosarcoma in a child

LF Sávio, R Ivanovic, J DA CRUZ, R Sakata, M Nunes, R Maia, C PASSEROTTI

GERMAN HOSPITAL OSWALDO CRUZ, SAO PAULO, BRAZIL

Brazil

Introduction \& Objective: Rhabdomyosarcoma (RMS) is a rare tumor that mostly children, accounting for only $3 \%$ of malignant neoplasms in this age group. About 15 to $20 \%$ of all RMS arise from the genitourinary tract. Radiation therapy and chemotherapy are important neo adjuvant and/or adjuvant therapies to prevent treatment failure. We present a novel approach for the management of RMS using robotic-assisted laparoscopic surgery to remove a residual RMS tumor following chemotherapy.

Materials and Methods: A 24 months old male child presented with a history of lower urinary tract symptoms and a palpable abdominal mass. Pelvic MRI demonstrated a $10 \mathrm{~cm}$ soft mass with irregular borders. An ultrasound guided needle biopsy was subsequently performed, demonstrating an embryonary rhabdomyosarcoma. Chemotherapy with vincristine, topotecan and etoposide was instituted for 4 months, resulting in tumor regression and leaving a residual $1.4 \mathrm{~cm}$ mass after re-assessing the mass through MRI. Robotic-assisted laparoscopic excision was then performed. The child was placed in the supine position. A $12 \mathrm{~mm}$ umbilical port was used for the camera and two additional $8 \mathrm{~mm}$ ports were placed for the robot arms. A $5 \mathrm{~mm}$ port was used by the assistant to pass sutures, provide suction/irrigation and retraction.

Results: The procedure had none intraoperative complications. Access to the abdominal cavity was performed without any in- cident. The tumor was dissected and removed preserving its' margins. There was no evidence of positive macroscopic surgical margins. Estimated blood loss of less than 50cc. No blood transfusion was necessary and neither the use of vasoactive drugs. The patient did not require ICU support and was discharged home on the 3nd post-operative day. Final pathological examination and an immunohistochemistry with desmin and myogenin confirmed the diagnosis of a pelvic embryonary RMS. In the 5 years of follow-up, the patient has remained asymptomatic for urinary symptoms and without radiological findings of tumor recurrence.

Conclusions: The robotic-assisted laparoscopic approach provides a safe and effective means of removing a pelvic tumor.

\section{V7-11 Novel Use of Fluorescence Lymphangiography During Robotic Groin Dissection for Penile Cancer}

M Bjurlin, L Zhao, D Volkin, A Kenigsberg, A Mass, S Taneja, W Huang

NYU Department of Urology

United States

Introduction \& Objective: Our objective is to describe a novel technique of robotic inguinal lymphadenectomy with near infrared fluorescence imaging (NIRF) using indocyanine green (ICG) to facilitate lymph node identification during robotic groin dissection for penile cancer.

Materials and Methods: The patient is placed in lithotomy position with access to the groin. 3 robotic ports and 1 assist port are placed in a $\mathrm{V}$ configuration below the tip of femoral triangle. Intradermal ICG is injected at the base of the penis $(0.5 \mathrm{ml}$ of $2 \mathrm{mg} / \mathrm{kg}$ concentration in normal saline) and the lymphatic channels and nodes are visualized using NIRF in the robotic console approximately 15 minutes after injection. The surgical template established in the open approach is then replicated utilizing NIRF to ensure complete resection of the affected nodes.

Results: A total of 10 groin dissections in 5 patients have been completed using this technique with an average lymph node yield of 7 per groin (range 5-13 lymph nodes). Mean operative time per groin was 207 minutes (range 164 minutes - 258 minutes) and estimated blood loss was $38 \mathrm{ml}$ (range $25-50 \mathrm{ml}$ ). Mean length of hospital stay was 1.8 days (range $0-4$ days). Identification of the lymphatic drainage pattern from the superficial to deep groin nodes to pelvic nodes underneath the inguinal ligament was identified in all patients. With a mean follow-up of 10 months (range 3-16 months), there have been no post-operative infections, lymphatic leaks, wound breakdown, or necrosis. Pathologically involved lymph nodes were identified using NIRF.

Conclusions: Our novel technique of robotic inguinal lymphadenectomy with fluorescence lymphangiography allows for identification and excision of both superficial and deep groin nodes with a significant reduction in morbidity compared to the open approach. Prospective studies are required to ensure longterm efficacy and results of this procedure.

V7-12 Initial Report of Robotic Laparoendoscopic SingleSite Partial Nephrectomy by da-Vinci $\mathrm{Xi}{ }^{\circledR}$ surgical system for Renal Tumor $>\mathbf{4 c m}$

H Lee, Y Yoon, J Na, K Rha, Y Choi, W Han

Department of Urology, NHIS ILSAN Hospital

Republic of Korea 
Introduction \& Objective: "Single-site surgery" is the term given to various laparoscopic techniques that use a single skin incision around the umbilicus. Although new equipment has been developed for LESS, the surgical instruments have a limited range of motion, and clashing of instruments is a disadvantage. To overcome these difficulties the da-Vinci surgical system has been used for LESS surgery. Then we report our first clinical experience with Single Site Partial nephrectomy by da-Vinci $\mathrm{Xi}{ }^{\circledR}$ surgical system (Intuitive Surgical Inc., Sunnyvale, CA, USA)

Materials and Methods: The patient was placed in the conventional flanked kidney position with the ipsilateral side elevated. About $2.2 \mathrm{~cm}$ vertical midline incision was made at the upper border of the umbilicus, and the single site port was inserted. The single site port was established by inserting one $8.5-\mathrm{mm}$ endoscope trocar and two 5-mm accessory trocars through multichannel and securing it to the port. After placement of the device, the same care as with any laparoscopic procedure was exercised in the entry and exit of instruments to prevent undue injury to the intra-abdominal organs. An additional 5-mm trocar was inserted in the midline below the subxiphoid process for liver traction. And 12-mm trocar for assist alongside the single multichannel port was inserted. After the robot was docked, the bowel was mobilized to the iliac area, and the descending colon was swept medially using blunt dissection to expose the gonadal vessels and ureter. The ureter was dissected medially to the region of the iliac vessel. Cephalocaudal dissection was performed around the kidney until the posterior space of the kidney was reached. The kidney was elevated until the renal hilum was visible. The renal vein and artery were exposed. And peri-renal fat was completely removed. Intraoperative ultrasonography was used to identify the size and margins of the tumor; the ultrasound images were projected onto the console screen to guide tumor resection (TilePro, Intuitive Surgical, Sunnyvale, CA). After clamping the renal pedicles with a laparoscopic bulldog clamp (Aesculap, Tuttlingen, Germany), the mass and surrounding normal tissue were incised by curved scissor. And then the resected renal parenchyma was sutured.

Results: The patient was a 52-year-old male. The patient's CT scan showed about $4.7 \mathrm{~cm}$ heterogeneous enhancing mass in right kidney suspicious renal cell carcinoma. The console time was 120 minutes and warm ischemic time was 30minutes. Estimated blood loss of whole procedure was $100 \mathrm{ml}$, and there was no intraoperative or postoperative complication. The pathological result was clear cell type renal cell carcinoma, Fuhrman nuclear grade 3, and resection margin was free of carcinoma.

Conclusions: Our initial experience with Single Site Partial nephrectomy by da-Vinci $\mathrm{Xi}{ }^{\circledR}$ surgical system demonstrated that the procedure is feasible and safe.

V7-13 Novel Robotic Approach to Inguinal and Pelvic Lymphadenectomy for Metastatic Melanoma

N Jung, H Smith, A Hyde, A Valle, C Keel

University of TN College of Medicine Chattanooga/Erlanger Health System

United States

Introduction \& Objective: To describe a new approach to pelvic lymphadenectomy designed to reduce complication rates.
Materials and Methods: This case describes the treatment of a 44 year old male requiring an inguinal lymph node dissection. This case was approached robotically in order to minimize complications. This video describes and demonstrates the robotic technique, as well as, the patient's clinical course and follow-up under the care of one surgeon at a large, academic hospital.

Results: Complete resection of the inguinal lymph nodes using the Da'Vinci Xi robot resulted in no complications with no signs of recurrence at follow-up.

Conclusions: Continued expectations to provide exceptional oncological care while minimizing morbidity have lead surgeons to novel treatment approaches for inguinal lymphadenectomy. Open inguinal lymph node dissections continue to have significant rates of complications. The vast majority of complications are due to tissue necrosis, wound dehiscence, seroma formation, and lymphedema. Previously published rates of seroma formation are as high as $32 \%$ for the open technique, while the instance of lymphedema may be as high as $40 \%$. Wound infections also comprise a significant portion of morbidity at $24 \%$ and skin flap complications are reported at $52 \%$. With this technique our cohort experienced one total Clavien grade II complication (20\%) and had an average length of stay under two days which is similar to other minimally invasive techniques. Our oncological outcomes with this technique have been equivalent to the open approach to date. No patients have had recurrent disease. Our lymph node count per side ranges from 1-54; however, $60 \%$ of the groins contain 6 or greater lymph nodes. Robotic inguinal lymph node dissection represents a promising alternative to the traditional open technique with the possibility of lower overall morbidity. Additional studies should be conducted evaluating the oncologic efficacy of this approach.

\section{V7-14 Robotic Metast Resection}

RJ Leveillee, D Diaz, G Leveillee, S Unsal

Bethesda Health Systems

United States

Introduction \& Objective: Renal Cell Carcinoma (RCC) is often treated with Radical Nephrectomy $(\mathrm{RN})$ for $>$ cT1b masses. Isolated metastatic recurrence has been traditionally treated with "Metastatectomy" - with durable local control and potential increase in Cancer specific survival. Aggressive open surgical resection has been the mainstay with a few isolated reports of laparoscopic retroperitoneal mass/lymph node or adrenal resection. These cases can be challenging due to previous surgery with loss of anatomic landmarks and extensive scar tissue. This video will demonstrate the step-by-step approach of resection of a $5 \mathrm{~cm}$ Para-aortic mass biopsy proven to be metastatic RCC utilizing the DaVinci Xi Robotic Platform.

Materials and Methods: An 81year-old male underwent DaVinci assisted LEFT RN two years prior for $5.8 \mathrm{~cm} \mathrm{RCC}$, Grade 2, RCC, (pT1bN0Mx). Medical history includes Atrial Fibrillation (on rivaroxaban) and obesity. Abdominal Imaging every 6 months- reveals an enlarging $5 \mathrm{~cm}$ paraortic mass first detected at 23 months. Positron Emission Tomography scan was inconclusive. A needle biopsy confirmed RCC recurrence. Exploration and resection of the mass and para-aortic lymphadenectomy was performed utilizing the DaVinci Xi (Intuitive surgical, SunnyVale, CA, USA). 
Results: Patient positioning, trocar placement, methods of dissection and mass excision are demonstrated. The operative time was 310 minutes and estimated blood loss was $150 \mathrm{ml}$. There were no Intra-operative complications. Length of stay was 3 days.

Conclusions: Solitary Metastatic renal cancer masses can be effectively resected. With Careful planning and cautious dissection in a previously operated field, visceral organ as well as major vascular injuries can be avoided. The advantages of the DaVinci Xi including improved 3D vision, dexterity, and precision (such as for hemostatic clip application) make it a technically more feasible operation with low morbidity.

\section{VS8: LAPAROSCOPIC UPPER TRACT SURGERY FOR CANCER}

\author{
V8-1 Clampless laparoscopic partial nephrectomy for hi- \\ lar tumor \\ R Sanseverino, G Napodano, O Intilla, U Di Mauro, G Molisso, \\ T Realfonso \\ Dept. of Urology Umberto I Hospital \\ Italy
}

Introduction \& Objective: Nephron sparing surgery is now reference standard for many T1 renal tumors. Although hilar clamping creates bloodless operative field, it necessarily imposes kidney ischemic injury. "Zero ischemia" partial nephrectomy allows to eliminate ischemia during nephron sparing surgery. It is possible to realize a clampless LPN also for the treatment of hilar tumors

Materials and Methods: A 65 years old male presented at our institution with hilar tumor of left kidney $(55 \mathrm{~mm})$. Renal score was $11 \mathrm{~h}$ and $\mathrm{C}$ index was 1.2 . A transperitoneal approach was performed and hilar vessels are prepared in event that bulldog clamping may subsequently be needed. Intraoperative monitoring includes electrocardiogram, central venous pressure (CVP), electroencephalographic bispectral (BIS) index (BIS monitor ${ }^{\mathrm{TM}}$ ), NICOM (non invasive cardiac output monitoring), urinary Foley catheter. A controlled hypotension, to carefully lower the mean arterial pressure (MAP) while maintaining excellent systemic perfusion, is maintened at approximately $60 \mathrm{mmHg}$. To induce hypotension, the doses of inhalational isoflurane is increased. The renal lesion is excised using Ligasure ${ }^{\mathrm{tm}}$. Calyceal suture was performed with Monocryl ${ }^{\text {tm.. }}$ Renal parenchyma was repaired with Vicryl ${ }^{\mathrm{TM}}$ sutures arrested with absorbable clips and Hem-Olok ${ }^{\mathrm{TM}}$. Hemopatch and Floseal were applied to resection bed.

Results: BMI, ASA score and tumor size were 26, II and 55mm, respectively. Operative time, blood loss, $\Delta \mathrm{Hb}$ were $185 \mathrm{~min}, 400 \mathrm{ml}$, $2.8 \mathrm{gr} / \mathrm{dl}$, respectively. No transfusion was required. The procedure was performed without clamping. Hospital stay was 6 days. No postoperative complications occurred. Histological evaluation revealed RCC pT1b, Furhman 2 with negative surgical margins

Conclusions: Clampless laparoscopic partial nephrectomy represents a safe and reproducible technique that allow to sparing renal parenchyma and preserve renal function also in challenging case as hilar tumor

V8-2 Laparoscopic inferior vena cava thrombectomy with double loop tourniquet in $\mathrm{RCC}$

S Hong, H Jeong, S Cho, S Kang, H Moon, K Lee, S Choi, Y Park, W Bae, H Cho, U Ha, J Lee, S Kim

Department of urology, Seoul St. Mary’s hospital Republic of Korea
Introduction \& Objective: Renal cell carcinoma (RCC) with vascular involvement in renal vein or inferior vena cava (IVC) can be seen $4 \%$ to $36 \%$ of cases. Despite advances in laparoscopic surgery, IVC thrombectomy using laparoscopy is still a technically challenging. In our study we report a case of IVC thrombectomy with double loop tourniquet technique.

Materials and Methods: 61-years old female presented with incidental renal mass. The CT scan showed $8 \mathrm{~cm}$ sized exophytic, markedly heteorgenous enhancing mass involves right lower pole, extending to the right renal vein and infradiaphragmatic IVC. There were multiple slightly enlarged lymph nodes in the retrocaval and aortocaval space. All procedures were performed transperitoneally. Bowel was mobilized and renal artery was ligated. Thrombus was managed by sequential clamping of the lower IVC, contralateral renal vein, and cephalad IVC, along with mobilization of the IVC and occlusion of lumbar veins, allowing for vascular isolation. Vascular clamping was performed with double-looped vessel loops and clips around vessels, "double loop tourniquet". Radical nephrectomy was completed with standard laparoscopy.

Results: The operative time was 140 minutes and IVC clamping time was 17 minutes. Estimated blood loss was 200cc. Hemodynamics were stable during IVC clamping and there were no perioperative complications. The patient was discharged well on POD 5. The pathology showed pT3bNOM0, Fuhrman grade was III, and cell type was clear cell RCC. Surgical margin was negative.

Conclusions: Laparoscopic IVC thrombectomy is still a complex and technically demanding surgery. Double loop tourniquet technique is safe and effective method for laparoscopic IVC thrombectomy to improve the perioperavie outcomes in selected patients.

V8-3 Laparoscopic Partial Nephrectomy Concomitant with Isthmusectomy for Isthmus Renal Cell Carcinoma in the Horseshoe Kidney

$\mathrm{RC} \mathrm{Wu}, \mathrm{V}$ Lin

E-Da hospital

Taiwan (Republic of China)

Introduction \& Objective: Tumors at the isthmus of a horseshoe kidney (HSK) can be easily mistaken for other intra-abdominal diseases and require thorough investigation. Meanwhile, there have been only a few reported cases presenting renal cell carcinoma in HSK to date. We report on a case that was diagnosed with renal cell carcinoma confined in the isthmus of a horseshoe kidney and treated by laparoscopic partial nephrectomy. 
Materials and Methods: A 40-year-old woman who presented with epigastralgia was finally diagnosed with renal cell carcinoma in the isthmus of a horseshoe kidney by enhanced computed tomography (CT). Initial investigations by non-enhanced CT scan lead to the suspicion of foreign bodies (fish bones) in the duodenum but nothing was discovered under endoscopy. Further enhanced CT revealed a tumor located at the isthmus of the HSK. Arteriography was done to identify the blood supply of the kidneys and tumor.

Results: Laparoscopic partial nephrectomy was successfully performed and the final pathology analysis revealed a pT1a multilocular cystic renal cell carcinoma.

Conclusions: Laparoscopic surgery with an experienced team has demonstrated to be a valuable treatment in the management of horseshoe kidneys with renal cell carcinoma confined in the isthmus, which should be differentiated from intra-abdominal lesions.

\section{V8-4 Clampless laparoscopic partial nephrectomy with tumor enucleation \\ P Kallidonis, D Kotsiris, P Ntasiotis, I Kyriazis, V Panagopoulos, E Liatsikos \\ Department of Urology, University of Patras, Greece Greece}

Introduction \& Objective: The purpose of this study is to describe and highlight the results of clampless laparoscopic partial nephrectomy with tumor enucleation in patients with renal tumors. The indications for clampless partial nephrectomy are the same as for the partial nephrectomy but the tumor has to be exophytic and there is no contraindication for sizes $>4 \mathrm{~cm}$.

Materials and Methods: The operation was performed to twenty three patients with mean age 63.3636 years old(42-78) with renal tumors that fullfilled the criteria for partial nephrectomy. After entering the abdomen with the Hasson technique, pneumoperitoneum is created and 2-3 additional trocars are placed. Then the bowel is medially mobilized and the Toldt's line is dissected. Furthermore, the upper pole is mobilized with sharp and blunt dissection and then the same procedure for the lower pole and the ureter is performed. The renal artery is afterwards prepared so that in case of bleeding during the procedure, its rapid detection and clamping is allowed. The correct plain for the excision of the tumor is the pseudocapsule between the tumor and the renal parenchyma. When the dissection is performed on this capsule, the bleeding from the renal parenchyma is limited. The ultrasonic scissors and the bipolar forceps are used for the dissection. Hemostasis at the site of the renal lesion after the excision of the tumor is possible with the use of the bipolar forceps. Sliding clip renorrhapy is performed. A first deep layer is performed initially. This layer aims into limiting the bleeding from the bed of the lesion and to close any communications to the pelvicalyceal system. The second layer approximate or closes the opening of the lesion and provides additional hemostasis. Finally, the kidney is sutured to its attachments and returns to its anatomical position and the tumor is removed with a laparoscopic bag.

Results: Twenty two out of twenty three surgeries were completed without any intraoperative complication or need for blood transfusion. Only in one patient open radical nephrectomy was performed due to excessive bleeding. One patient presented urinoma due a clot in ureter and another a pseudoaneurysm. The mean operative time was $139.2727 \mathrm{~min}(90-178)$ and the average tumor size was $4.0368 \mathrm{~cm}(2.05-5.72)$. There was no increase to creatinine levels observed (mean increase -0.01818) and the mean Hemoglobin drop was $1.3619 \operatorname{gr}(0.1-3.2)$. The functional outcome was excellent at least during the short follow-up period (3-6 months).

Conclusions: Clampless laparoscopic partial nephrectomy with tumor enucleation is a safe and feasible procedure for experienced urologists and provides satisfactory postoperative functional results.

V8-5 3 -D Laparoscopic transperitoneal partial nephrectomy for clinical T1b renal tumors: A prospective evaluation

A Kumar, G Kumar, N Kumar, M Patel, P Gupta

Department of Urology and Renal Transplant, VMMC and Safdarjang Hospital, New Delhi, India India

Introduction \& Objective: The role of partial nephrectomy in clinical T1b renal tumors is still not established. The laparoscopic partial nephrectomy for clinical T1b renal tumors is a technically challenging procedure. We prospectively evaluate the feasibility, safety, efficacy and long term oncological results of 3 D laparoscopic transperitoneal partial nephrectomy(LPN) in clinical T1b renal tumors.

Materials and Methods: All consecutive patients undergoing LPN for a clinical T1b renal tumors and normal contralateral kidney by a single surgeon between June 2011 and May 2016 at our institution were included. The various clinical data including patients demographic profile, intraoperative and postoperative data, complications and follow up were recorded and analyzed. We are presenting video of one such case.

Results: A total of 53 patients were included in the study. The mean age was 51 years with mean preoperative serum creatinine and estimated glomerular filtration rate (GFR) were $0.91 \mathrm{mg} / \mathrm{dl}$ and $73.1 \mathrm{ml} / \mathrm{min} / 1.73 \mathrm{~m}^{2}$ respectively. The mean tumor size was $5.1 \mathrm{~cm}$. The tumor was mesorenal in $7(13.2 \%)$ patients, superior polar in $25(47.2 \%)$ patients and inferior polar in $21(39.6 \%)$ patients. The tumor growth pattern was cortical in $29(54.7 \%)$ patients and corticomedullar in 24(45.3\%) patients. The mean operating time and estimated blood loss were $129.3 \mathrm{~min}$ and 147. $1 \mathrm{ml}$ respectively. The mean ischemia time was $21.3 \mathrm{~min}$. Three $(5.6 \%)$ patients were converted to open. The blood transfusion was required in $5(9.4 \%)$ patients. The mean hospital stay and mean convalescence period were 3.3 days and 1.39 weeks respectively. The positive surgical margins was seen in $1(1.8 \%)$ patient. In histopathology, renal cell carcinoma was found in $92.4 \%$ and oncocytoma in $7.6 \%$ patients. The intraoperative and postoperative complications were $5.6 \%$ and $7.5 \%$ respectively and mainly Clavien 1 and 2 only. The mean estimated GFR at 1 year was not significantly lesser than preoperative value( $\mathrm{p}=0.71$ ). At mean follow up of 47.1 months, there was no local or distal recurrence.

Conclusions: 3-D Laparoscopic transperitoneal partial nephrectomy for clinical stage T1b renal tumors is feasible, effective with preservation of renal function, and has acceptable complications with good long term survival. However, it is a technically challenging procedure and should be done by surgeons of significant laparoscopic expertise. 
V8-6 Laparoscopic partial nephrectomy for posterior hilar tumors: technique and clinical outcomes

H Hirabayashi, R Hattori, H Kawanishi, J Nagayama, H Matsui, T Sano, S Suzuki, K Suzuki, K Kato

Japanese Red Cross Nagoya Daiichi Hospital Japan

Introduction \& Objective: Posterior renal hilar tumors, due to their anatomic location, pose some technical challenges for laparoscopic partial nephrectomy (LPN). We demonstrate our technique and clinical outcomes of LPN for posterior hilar tumors.

Materials and Methods: Seven patients with a posterior renal hilar tumor received LPN at our institution from November 2015 to February 2017. Our surgical technique involves transperitoneal approach, full kidney mobilization to flip the kidney medially for access to its posterior aspect, and the following procedures to the maximum extent possible before artery clamping: intrasinusal dissection upon the intrarenal collecting system and segmental vessels, ligation and division of the vessels supplying the tumor, parenchymal incision through the hilum, and circumferential cortical incision around the tumor with electrocautery. Following main or segmental artery clamping, the tumor excision is completed and renorrhaphy is performed. Results: Median tumor size was $32 \mathrm{~mm}$ (range 23 to 55). R.E.N.A.L. nephrometry score was 8 in 4 patients, 9 in 2 patients, 10 in 1 patient. 5 patients underwent surgeries with main renal artery clamping, and 2 underwent surgeries with segmental artery clamping. Median warm ischemia time was 18 minutes (range 10 to 25), median estimated blood loss was $150 \mathrm{~mL}$ (range 0 to 275). Histopathology confirmed renal cell carcinoma with negative margins in all patients. Postoperative urine leakage was detected in 2 patients. Median change in eGFR during postoperative 1, 3 and 6 months were $-11 \%,-9 \%$ and $-8 \%$, respectively.

Conclusions: For posterior renal hilar tumors, the described technique is safe and allows a reduction of warm ischemia time and preservation of renal function.

V8-7 Percutaneous Renal Endoscopy and Resection of Urothelial Carcinoma of the Renal Pelvis Performed Under Local Anesthetic in High Risk Patient

JE Abbott, J Davalos

University of Maryland Baltimore Washington Medical Center United States

Introduction \& Objective: Nephroureterectomy is the gold standard treatment in managing upper tract urothelial carcinoma (UTUC). However, there is an emerging role of nephron-sparing endoscopic treatment, either percutaneous or ureteroscopic, especially in low grade (LG) tumors. Many patients with urothelial carcinoma are in the later stages of life and often have numerous comorbidities. Many are at high risk for even undergoing general anesthesia typically required for surgical intervention. We are presenting a case or percutaneous excision and ablation of LG UTUC in a high-risk patient successfully performed under local anesthetic.

Materials and Methods: An 82 year-old male with history of cardiomyopathy, coronary artery disease, permanent atrial fibrillation s/p AICD placement on Coumadin, congestive heart failure with left systolic function of $35 \%, \mathrm{O} 2$ dependent COPD, severe pulmonary hypertension, thoracic aortic aneurysm diabetes and alcoholism referred to our institution with newly diagnosed bulky right renal pelvis LG UTUC causing a symptomatic hydronephrosis. He was medically determined to be at significantly high risk for undergoing general anesthetic with recommendations against any significant surgical procedure. In prone position, under a paravertebral regional anesthetic block, we performed percutaneous excision and ablation of the renal pelvis tumor. Renal access was achieved with fluoroscopic and endoscopic needle guidance and a standard 30/34 Fr access sheath was placed.

Results: Procedure was tolerated well. All tumor was excised in an antegrade fashion. Tumor pathology demonstrated LG UTUC. Patient remains under close surveillance.

Conclusions: Percutaneous renal endoscopy and resection of upper tract urothelial cancer is safe and feasible under paravertebral regional anesthetic and may be considered in patients at high risk for undergoing general anesthetic.

V8-8 Complete laparoscopic approach for upper tract transitional cell carcinoma

M Vijayakumar, A Ganpule, A Singh, R Sabnis, M Desai

Muljibhai patel urological hospital nadiad

India

Introduction \& Objective: Introduction: Laparocopic nephroureterectomy and removal of bladder cuff can be done with either open or endoscopic or complete laparoscopic approach. Complete laparoscopic approach is technically challenging. However with certain modifications, the steep learning curve can be reduced maintaining oncological efficacy Aim: In this video we describe our suggested modifications of doing complete laparoscopic nephroureterectomy with bladder cuff removal

Materials and Methods: This video highlights our technique of doing laparoscopic nephroureterectomy $\bullet$ The essential points of steps include -1 . completing the nephroureterectomy and bladder cuff excision in two separate positions -2 . Port placement according to site selection which would be common for both the nephroureterectomy and bladder cuff excision with no new ports -3 . Adjuncts to be used for minimizing number of ports during bladder cuff excision like silk on straight needle Results: In a total number of 15 patients the mean operative time was $187 \mathrm{~min}$ with a hemoglobin drop of $1.6 \mathrm{gm}$ with total hospital stay of 4 days. The PUC removal was after an average of 6.2 days. There were no intra operative or post-operative (3 months) complications

Conclusions: Complete laparoscopic approach for upper tract TCC is a safe approach with less morbidity. The number of ports can be reduced by the above mentioned technique

V8-9 Transvescicoscopic bladder cuff excision in laparoscopic nephroureterectomy: Initial Experience

Y Yoon, S Kim, J Na, H Lee, S Park, W Han

Department of Urology, Hanyang University College of Medicine

Republic of Korea

Introduction \& Objective: To describe the management of the distal ureter during laparoscopic radical nephroureterectomy with the transvesical laparoscopic approach. 
Materials and Methods: The patient was placed in the modified lithotomy position with abducted thighs. The procedure started with cystoscopic examination of the bladder and ureteral orifices. After a small skin incision, a 5-mm-diameter trocar was introduced into the bladder dome. The anterior bladder wall was suspended to the abdominal wall to prevent the trocar from slipping out and 2 more 3-mm-diameter trocars were placed. The ureter was mobilized with hook electrocautery and dissected ureteral end was placed in extravesical space. Bladder was repaired with 4-0 vicryl. Then patient was placed in lateral position and conventional laparoscopic radical nephroureterectomy was performed. While dissection of distal ureter, the distal end of ureter was smoothly removed from perivesical space without any difficulty. Results: The patient was a 61-year-old female. The patient's CT scan showed right proximal ureteral mass and the result of previously performed ureteroscopic biopsy was transitional cell carcinoma, low grade. The operation time of transvesical bladder cuff excision was 50 minutes, and laparoscopic nephroureterectomy was performed for 120 minutes. Estimated blood loss of whole procedure was $300 \mathrm{ml}$ and there was no intraoperative or postoperative complication. Foley catheter was removed at 1 week. After 6 months postoperatively, there's no evidence of recurrence or metastasis.

Conclusions: Our initial experience with transvesicoscopic bladder cuff excision in laparoscopic radical nephroureterectomy demonstrated that the procedure is feasible and safe.

\section{V8-10 Percutaneous Resection of Bulky Renal Pelvis Tumors}

RJ Yau, M Eshghi

New York Medical College

United States

Introduction \& Objective: Little is known about urothelial cell carcinoma of the upper tract compared to the lower urinary tract counterpart. Due to the relative uncommon incidence of upper tract urothelial cell carcinoma (UTUC), no randomized control trials have been performed. Most of the guidelines in treatment of UTUC are borrowed from the literature on bladder cancer. Staging of UTUC is the best indicator of prognosis but it cannot be obtained without radical nephroureterectomy, which is the gold standard. In the setting of conservative endourologic management, tumor grade is often used as a substitute. Our objective is to present a case report of one of our patients managed conservatively for high tumor burden UTUC. We will discuss the indications for conservative management and the surgical techniques for percutaneous resection of UTUC.

Materials and Methods: The video presentation was created by performing a literature review, researching the medical record, and video editing portions of the operation. All patient identifiers were removed in compliance with HIPAA. The video editing software was Adobe Premiere.

Results: Our 74 year old patient was found to have high tumor burden in his renal pelvis in his solitary kidney. He had a previous radical nephrectomy for RCC over two decades ago. He underwent successful percutaneous resection of the bulky renal pelvis tumor as described in the video. The pathology of biopsy specimens revealed low grade UC. The second look percutanous operation showed that the first surgery had grossly cleared the tumor. However, tissue resection of previous resected sites returned as high grade UCC. He received mitomycin C through the nephrostomy tube. He elected not to undergo radical nephroureterectomy of his solitary kidney because he did not want to go into renal failure and be on dialysis. He currently is managed with routine retrograde ureteroscopy and laser fulguration for recurrent tumors and remains metastasis free.

Conclusions: Indications for conservative management of UTUC include functional or anatomical solitary kidney, severe medical comorbidities, and the patient's decision. When faced with bulky renal tumor and conservative endoscopic management is indicated, percutaneous resection is feasible option with good efficacy. Routine monitoring post operatively is mandatory since this is not the standard of care and recurrence is common.

V8-11 A prospective evaluation of 3-D laparoscopic transperitoneal radical nephrectomy for large renal tumors(clinical T2N0M0)

A Kumar, G Kumar, N Kumar, M Patel, P Gupta

Department of Urology and Renal Transplant, VMMC and Safdarjang Hospital, New Delhi, India

India

Introduction \& Objective: To prospectively evaluate the feasibility, safety and long term results of 3-D laparoscopic transperitoneal radical nephrectomy(LRN) in large renal tumors(clinical T2N0M0).

Materials and Methods: All consecutive patients undergoing 3-D laparoscopic radical nephrectomy $(\mathrm{LRN})$ for a clinical stage T2N0M0 by a single surgeon between January 2012 and December 2016 at our institution were included. The various clinical data including patients demographic profile, intraoperative and postoperative data, complications and follow up were recorded and analyzed. Results: A total of 51 patients were included in the study. The mean tumor size was $7.9 \mathrm{~cm}$. Limited hilar lymphadenectomy was performed in $38(74.5 \%)$ patients. Concomitant lymphadenectomy was performed in $15(29.4 \%)$ patients. The mean operating time and mean estimated blood loss were $179.3 \mathrm{~min}$ and $193.1 \mathrm{ml}$ respectively. In $3(5.8 \%)$ patients, conversion to open was required. The blood transfusion was required in 5(9.8\%) patients. The mean hospital stay and mean convalescence period were 3.5 days and 1.49 weeks respectively. The intraoperative and postoperative complications were seen in $3(5.8 \%$ - bleeding: 1 , bowel injury:1) and 5(7.8\% - wound infection :1, delayed bleeding:1, atelectasis:1, ileus:1) respectively. The renal cell carcinoma and oncocytoma were found on histopathological examination in $48(94.1 \%)$ and 3(5.9\%) patients respectively. At mean follow up of 49.1 months, there were 2 distant metastases(brain -1, lung -1). The 5-year overall, cancer-specific and recurrence free survival were $92.3 \%, 94.1 \%$ and $96.1 \%$ respectively.

Conclusions: The LRN for for large renal tumors(clinical T2N0M0), using a 3-D Laparoscopy system is technically feasible, safe, effective with good long term survival outcomes. However, this technically challenging procedure should be attempted by surgeons of significant expertise.

\section{V8-12 Renal Cell Carcinoma in a patient with total situs inversus}

P Patki, A Raman, C Tanabalan

Royal Free Hospital \& Barts Health London. UK United Kingdom

Introduction \& Objective: Total Situs Inversus has an incidence of $1 / 8000$ to $1 / 20000$ in the general population. Previously there have only been 5 documented cases of renal cell carcinoma in patients with total situs inversus and these have all been managed 
with open radical or partial nephrectomy. We add our case to the literature and present a video of the first laparoscopic radical nephrectomy in a patient with RCC and total situs inversus.

Materials and Methods: With meticulous preoperative planning including cross sectional imaging, ultrasound marking of the correct side on the day of the operation and again on induction of anaesthesia laparoscopic radical nephrectomy is feasible and safe in patients with total situs inversus.

Results: Our patient underwent a lapraoscopic radical nephrectomy and had complete tumour removal in a safe and controlled manner with no complication and an uneventful post operative recovery.

Conclusions: Laparoscopic radical nephrectomy is feasible and safe in patients with total situs inversus, and should be considered as the treatment of choice for these rare cases.

\section{V8-13 Partial nephrectomy for bilateral synchronus renal} cancer

R Sanseverino, G Napodano, O Intilla, U Di Mauro, G Molisso, T Realfonso

Dept. of Urology Umberto I Hospital Italy

Introduction \& Objective: We report a case of a patient affected by bilateral renal cancer who underwent a two-stage laparoscopic partial nephrectomy.

Materials and Methods: A 65 years old male presented bilateral renal tumors: a right mesorenal $(42 \mathrm{~mm})$ and a left hylar $(38 \mathrm{~mm})$ lesions. RENAL score of tumors was $8 \mathrm{p}$ and $9 \mathrm{p}$, respectively. Renography revealed a GFR $77 \mathrm{ml} / \mathrm{min}$ (rught $40 \mathrm{ml} / \mathrm{min}$; left $37 \mathrm{ml} / \mathrm{min}$ ). The video shows two surgical procedure. In the first, a right transperitoneal approach was performed; the tumour is identified. The renal artery was clamped with bull dog. The tumour was excised. Renorraphy was perfomed Vicryl ${ }^{\mathrm{TM}}$ sutures secured with Hem-O-lock clips. The artery was unclamped. A Floseal $^{\mathrm{TM}}$ was applied on the resected renal surface. In the second procedure, a left transperitoneal approach was performed; the tumour is identified. The renal artery was clamped with bull dog. The tumour was excised. Renorraphy was perfomed Vicryl ${ }^{\mathrm{TM}}$ sutures secured with absorbable clips. The artery was unclamped. A Floseal ${ }^{\mathrm{TM}}$ was applied on the resected renal surface

Results: Warm ischemia time of both procedures was 20 and 23 minutes, respectively. No postoperative complications occurred. Histological evaluation revealed RCC (pT1b and pT1a)

Conclusions: Bilateral laparoscopic partial nephrectomy is a feasible and reproducible procedure that allows a good oncological control of bilateral synchronous renal cancer
V8-14 Laparoscopic nephroureterectomy for urothelial tumor in horseshoe kidney.

G Del Pozo Jiménez, I Castillón Vela, J Diez Diez, A Souto Souto, J Carballido Rodríguez

University Hospital Puerta de Hierro, Majadahonda, Madrid Spain

Introduction \& Objective: Horseshoe kidney (HK) is the most frequent renal fusion anomaly and presents a greater predisposition for tumor pathology. The upper urinary tract urothelial tumor is described in a few cases, comprising 5-7\% of the renal tumors of the HK. The standard treatment has been open nephroureterectomy due to HK abnormal anatomy and vascularization that make surgery complex. However, laparoscopic approach is currently advocated.

The objective is to evaluate the usefulness of the laparoscopic approach in the treatment of HK disease.

Materials and Methods: We present a 70-year-old male with a $2.5 \mathrm{~cm}$ filling defect in the upper calyx group (UCG) of the right kidney on CT urography. Urine cytologies showed atypia and a inflammatory component. With the working diagnosis of UUT urothelial carcinoma flexible ureterorenoscopy was performed and confirmed the diagnosis: $2 \mathrm{~cm}$ solid lesion at the entrance of the UCG, not amenable for conservative treatment. In accordance with these findings and after vascular CT scan, transperitoneal laparoscopic nephroureterectomy was performed. It was technically difficult due to the anomalous vascularization the HK presented and because of the difficulties for division of the isthmus, since it is quite broad and thick, able to control not amenable for control with a stapler. It required section and suture in two planes with a 0 absorbable suture. The ureter was released laparoscopically down to its entrance in the bladder and the open sugrgery bladder cuff was performed.

Results: There were no intraoperative complications with a surgical time of 280 minutes. Hospital stay was seven days. Blood transfusion was necessary in the immediate postoperative period (Clavien II). Drains were withdrawn on the third and fourth day. Postoperative renal function stabilized on a creatinine value of $1.2 \mathrm{mg} / \mathrm{dl}$ at discharge. There were no postoperative complications. The pathological report was compatible with $3 \mathrm{~cm}$ UUT urothelial carcinoma located in renal pelvis and UCG, pT2G3.

Conclusions: Laparoscopic approach for $\mathrm{HK}$ is possible, obtaining the advantages of laparoscopy and maintaining the oncological requirements. Nevertheless, we believe we must be careful and plan surgery very well, with a good vascular reconstruction and be prepared for different approaches to the renal isthmus.

\section{VS9: ROBOTIC LOWER TRACT SURGERY FOR CANCER}

V9-1 Our Technique of Retzius sparing Robot assisted laparoscopic radical prostatectomy: initial results

I Banerjee, A Mallya, S Arakere, FA Zafar, A Mandhani, RK Ahlawat, T Jindal

Fortis escorts kidney and urology institute India
Introduction \& Objective: A major component of trifecta of robot assisted laparoscopic radical prostatectomy (RALP) is continence. The Retzius sparing approach have achieved excellent early continence. In this video we describe our surgical technique of Retzius sparing RALP and initial results.

Materials and Methods: We modified the conventional Retzius sparing robotic prostatectomy technique of Bocciardi in that, we 
used 30 degrees up telescope instead of 0 degree for prostate dissection. For lymphadenectomy 30 degrees down telescope was used. As compared to the conventional Retzius sparing approach we did not put suspensory sling sutures in prostate for retraction; that can put undue stretch over the neurovascular bundles. Instead, prograsp forceps were used judiciously for better control over retraction. We divided the vas deferences late: after identifying neurovascular bundles and completing posterior dissection. This allowed early identification and preservation of neurovascular bundles.

Results: We operated 7 patients since January 2017. Three out of 7 patients were pathological stage T3b and 4 out of 7 patients were in Gleason grade group 3 or higher. Mean console time and blood loss were $110.71 \pm 18.35$ minute and $142.85 \pm 83.80 \mathrm{ml}$ respectively. Urethral catheter was removed on $10.9 \pm 1.3$ days. Considering 0 pad definition 3 out of $7(42.8 \%$ ) patients were continent at 1 month. Including patients using 1 security pad $5(71.42 \%)$ patients were continent at 1 month. Multifocal margin positivity was present in a single stage pT3b disease patient out of total 2 patients with margin positivity.

Conclusions: Our technique of Retzius-sparing RALP can achieve excellent early continence at 1 month. Proper case selection is very important for an acceptable oncological outcome.

V9-2 Anatomic robot assisted radical cystectomy in female: step by step technique

G Simone, S Guaglianone, L Misuraca, F Minisola, G Tuderti, M Ferriero, M Gallucci

Regina Elena National Cancer Institute Rome Italy

Introduction \& Objective: Robot assisted radical cystectomy in female for bladder cancer is a challenging urologic surgical procedure. We describe step by step surgical technique and present perioperative outcomes of a single patientwhounderwent a robot assisted radical cystectomy (RARC) with totally intracorporeal orthotopic neobladder (iON).

Materials and Methods: A 66 yr-old female patient with a cT1/ N0/M0 high grade BCG refractory recurrent bladder cancer, underwent RARC and iON. Key steps of surgery include: the ligation of gonadic pedicles, meticulous dissection of the umbilical and uterine artery and the ureter, dissection of the bladder pedicle, opening of the vagina and creation of the plane between vagina and bladder. Cut of the urethra and securing the Foley catheter with the entire specimen placed into an Endocatch bag to minimize any urine spillage. Removal of the specimen into an endocatch bag through the vagina. Extended pelvic lymph node dissection. Suture of the vagina and creation of a peritoneal flap as posterior neobladder support.

Results: The procedure was successfully completed. Operative time was 295 minutes, EBL was $250 \mathrm{~mL}$, time to flatus was 3 days, time to bowel was 7 days. Hemoglobin and creatinine at discharge were $10.3 \mathrm{~g} / \mathrm{dL}$ and $0.76 \mathrm{mg} / \mathrm{dL}$, respectively. The hospital stay was 8 days. The pathologic stage was pT0 pN0. The number of nodes removed was 26 . Postoperative course was uneventful. The patient recovered daytime continence 45 days after surgery.

Conclusions: A meticulous dissection of vascular suppliers of the bladder, a natural orifice specimen retrieval and the ease of posterior neobladder support thanks to a perfect vision of the small pelvis anatomic structures may contribute to minimize invasiveness and to improve perioperative outcomes of radical cystectomy in female patients.

\section{V9-3 Nerve Sparing Technique During Robot Assisted Retroperitoneal Lymph Node Dissection in Testicular Cancer}

HM Abdul-Muhsin, M Marshall, J L'esperance, M Woods, S Stroup, EP Castle

Mayo Clinic Arizona

United States

Introduction \& Objective: Several anatomic studies demonstrated the importance of nerve sparing during retroperitoneal lymph node dissection (RPLND) in ejaculatory function preservation. The anatomic course of the nerves in relation to great vessels and lymphatic structures is well established and the technique is well described in open surgery. However, little is reported regarding the nerve sparing in robot assisted RPLND. The aim of this video is to provide an instructional guide to nerve sparing during robot assisted RPLND

Materials and Methods: The evolution of this technique took place through the collaboration of three tertiary training centers since early 2008. The procedure was performed through a transperitoneal approach in lithotomy position. This video will highlight the technical aspects of this procedure. These are: (1) Patient positioning. (2)Robotic ports placement. (3) Proper exposure and bowel retraction. (4) Regional anatomy of the sympathetic chain ganglia and the post-ganglionic plexus in relation to great vessels and the lymphatic structures.

Results: Proper bowel retraction is extremely important and can be best achieved through steep Trendelenburg position and retraction suture where the transected edge of the peritoneum is sutured to the anterior abdominal wall. Given the anatomy of the nerves in relation to the great vessels a split and role technique can be safely performed anterior to the inferior vena cava (IVC) since all nerves and ganglia lie posterior to it. This relationship also highlights the importance of complete mobilization of the IVC to expose these nerves and the lymph nodes. This is feasible and can be completed robotically after careful and secure ligation of the lumbar veins. Division of the inferior mesenteric vessels can be safely performed to facilitate the case and gain more exposure with no sequelae. Dissection of the interaortocaval and preaortic areas should be done with extreme care due to abundance of post ganglionic sympathetic nerves in these areas. The nerves can be preserved by splitting and rolling the surround tissue with minimal use of cautery. Dissection can start at the aortic bifurcation where the hypogastric plexus is located superficially and carried out in a cranial direction.

Conclusions: In conclusion we think that proper exposure is extremely important. Complete mobilization of the IVC should not be avoided and oncological outcomes should not be compromised to preserve ejaculatory function.

V9-4 Robot assisted radical cystectomy and cutaneous ureterostomy is a new paradigm in managing select cases of muscle invasive bladder cancers.

S Arakere, RK Ahlawat, A Mandhani, A Mallya, I Banerjee, F Zafer, T Jindal

Fortis escorts kidney and urology institute India

Introduction \& Objective: Ileal conduit is associated with metabolic complications, prolonged ileus, intestinal obstruction, 
and ureteroileal strictures. We describe robot assisted cystectomy and cutaneous ureterostomy as an alternative form of urinary diversion to avoid such complications in select patients with muscle invasive bladder cancer.

Materials and Methods: Robot assisted radical cystectomy was done with few modifications to mobilize ureters. Transureteroureterostomy was done over the stents and one ureter was brought out at anterior axillary line between iliac crest and lower rib cage, instead of spino-umbilical line. Wide mouthed stoma was made taking a ' $V$ ' shaped skin flap into spatulated ureteral end to prevent stomal stenosis.

Results: Both the patients did well with functioning stoma at 1 and 3 months of follow up. A modified ureterostomy bag was applied 4 weeks after the removal of double J stent. None of the patients had any problem related to urostomy application. Console time was 180 and 220 minutes. Blood loss was 400 and 550 cc. Patients had an uneventful and faster postoperative recovery. Conclusions: Modified lateral cutaneous ureterostomy avoids use of bowel and its related complications in select patients of muscle invasive bladder cancer.

V9-5 Robot assisted laparoscopic nerve sparing cystoprostatectomy: Intracorporeal Studer pouch formation

Y Ozgok, S Yalcin

Agri Military Hospital, Department of Urology, Agri, Turkey Turkey

Introduction \& Objective: Robot assisted laparoscopic nerve sparing cystoprostatectomy contains three main steps of the operation. While first two steps are the extirpative parts;intracorporeal Studer pouch formation is the reconstructive part of the surgery. Herewith this video-abstract we would like tos hare our experience on robotic Studer pouch formation.

Materials and Methods: Studer pouch formation is started with suturing the proximal part of the membraneus remnant urethra to the antimesenteric border of the wall of the ileal segment where $1-\mathrm{cm}$ opening is prepared on. A double-armed 3/0 quill suture is used for the anostomosis. An estimated $20-\mathrm{cm}$ segment on the right and a $30-\mathrm{cm}$ segment on the left side of the urethroileal anastomosis are assigned. $30-\mathrm{cm}$ ileal segment is not disturbed starting from the ileocecal valv and left attached to the caecum. As the ileo-urethral anastomosis is held in place, laparoscopic intestinal staplers are placed perpendicular across the intestinal wall. Proximal and distal ends of the ileum are put together, and a side-to-side ileoileostomy is accomplished with the use of laparoscopic intestinal staplers. Sparing the distal 10-cm segment as the afferent loop, the antimesenteric border of the remaining ileal segment is incised all the way long. Closure of the posterior wall is performed first. Medial aspects of the opened ileal segments are put together with interrupted sutures followed by a running suture of $3 / 0 \mathrm{~V}$-loc resulting in a completed posterior wall anastomosis. Anterior wall anastomosis is accomplished using a running 3/0 V-loc leaving the proximal redundant wall on the left open. Ureteral stents are then introduced in the midline through the abdominal wall into the peritoneal cavity, taken up into the pouch, then to the level of the ureteroileal anastomosis. Finally, the redundant ileal wall of the pouch is closed on itself with another running 3/0 V-loc while ureteral stents are kept and stablized between these sutures. Later, uretero-ileal anastomoses were performed on each side of the pouch seperately. Watertightness of the created Studer pouch is tested, filling it with $150 \mathrm{~mL}$ of saline.
Results: Robot assisted laparoscopic Studer pouch formation, urethro-ileal and uretero-ileal anostomoses took nearly 2,5 hours. No complication was recorded during or after the surgery. Estimated blood loss was less than $10 \mathrm{cc}$.

Conclusions: Robot assisted laparoscopic nerve sparing cystoprostatectomy, extended lymph node dissection and urinary diversion is safe and reproducible method. Patient benefits excellent short-term surgical and pathological outcomes, satisfactory functional results and the advantages of the minimal invasive surgery.

V9-6 Robot-assisted radical retrograde cystectomy with vaginal preservation and the ideal neobladder

T Yumioka, S Morizane, R Shimizu, Y Kimura, N Yamaguchi, H Iwamoto, P Tsounapi, K Hikita, M Honda, A Takenaka

Totter University Faculty of Medicine/Division of Urology/

Japan

Japan

Introduction \& Objective: The gold standard treatment for invasive bladder tumors is radical cystectomy. Urinary diversion is very important for quality of life. In the ileal neobladder in women, the clinical issue is hypercontinence, which has many proposed causes. One of the causes is an overly acute neocystourethral angle because of the downward migration of the ileal neobladder. To prevent this issue, preservation of the vagina appears to be necessary. In addition, for open radical cystectomy in women, a retrograde procedure with vagina preservation is difficult because of severe bleeding due to the lack of clear dissection plane between vagina and urethra. We attempted robotassisted retrograde radical cystectomy with vaginal preservation and extracorporeal urinary diversion for the ileal neobladder for a patient with a invasive bladder tumor.

Materials and Methods: The patient was a 59-year-old female. She had been experiencing gross hematuria since 1 month and underwent transurethral resection of the bladder tumor (TURBT). A histopathological examination revealed an invasive squamous cell carcinoma, INF $\gamma$, and pT2 or more. Bone and CT scans revealed no metastasis. We diagnosed an invasive bladder tumor, T2NOM0. One month after TURBT, we conducted robotassisted radical cystectomy with vaginal preservation. To preserve the vagina, we peeled off the bladder and vagina using a retrograde approach. After cystectomy, the patient cart was docked out, and a midline incision of $8 \mathrm{~cm}$ in the abdominal region was performed. We conducted ileal neobladder reconstruction using the modified Studer's procedure. The patient cart was redocked, and bladder urethral anastomosis was performed by the surgeon using the robot console.

Results: The surgeon's console time was $299 \mathrm{~min}$, and the estimated blood loss was $350 \mathrm{ml}$. Furthermore, the extracted volume of the bladder and uterus was $250 \mathrm{~g}$. Seventeen days after the surgery, the urethral catheter was removed. Histopathology confirmed T0 and no lymph node metastasis. At her discharge, she had no residual volume and no neobladdervaginal fistula.

Conclusions: Robot-assisted radical cystectomy with vaginal preservation using a retrograde approach can be safely performed. The early urinary function and oncologic outcome is good; however, long-term follow up is required to better assess urinary function of the neobladder and oncologic outcome. It is suggested that robot-assisted radical retrograde cystectomy is an appropriate procedure for ileal neobladder in women. 
V9-7 Robotic assisted en-bolic excision of tumor with prostate and rectum involvement

C Yang, L Tsai, P Hsiao, C Chang

Department of Urology, China Medical University Hospital Taiwan (Republic of China)

Introduction \& Objective: Gastrointestianl Stromal Tumor (Gist) of rectum with prostate invasion is very rare. Opens surgical resection of this deep location in pelvic cavity is challenge due to difficult control of distal prostate and rectum. Here we report an unusual Gist with anterior rectum and prostate invasion and treated by robotic assisted tumor excision with anterior rectum and entire prostate resection.

Materials and Methods: A 63 year-old male presented with LUTs. DRE revealed a big bulging mass from left prostate fossa. Serum PSA $0.63 \mathrm{nh} / \mathrm{ml}$. TRUS guide biopsy confirmed GIST. MRI reveled a tumor arising from anterior rectum with left prostate invasion. Following 6 months imanib treament. Robotic assisted radical prostatectomy with tumor and anterior rectal wall resection was carried out smoothly. The right side neurovascular bundles was preserved. Anterior rectal wall was two-layer closed Results: Operation time $400 \mathrm{~min}$ and blood loss 150ml. Pathology reported all surgical margin were free from tumor invasion. Prophylatic ileostomy was closed two months after surgery. He is continent sexual function return partially. Tumor free for one year follow up.

Conclusions: Rectal Gist with prostate invasion can be treated efficiently by robotic assisted en-bolic resection with radical prostatectomy and rectal wall excision

V9-8 A Novel Modified Urethro-vesical Anastomosis Technique: Prevention Of Urethral Retraction With Stay Sutures (PURS) During Robot Assisted Radical Prostatectomy

OB Argun, MB Tuna, T Doganca, I Tufek, C Obek, AR Kural

Acibadem University, Department of Urology, Istanbul, Turkey Turkey

Introduction \& Objective: In this video a modified vesicourethral anastomosis technique during robot assisted radical prostatectomy is presented.

Materials and Methods: After controlling the dorsal venous complex with two Bulldog clamps, apical dissection was ensued. Bulldog clamps assured a bloodless apical dissection. Urethra was freed and then incised at the anterior aspect. Bulldog clamps were removed, the dorsal vein selectively sutured with $2 / 0$ polyglactin and anterior reconstruction was performed. Two 3/0 barbed sutures were passed from the posterior aspect of the urethra at 5 o'clock and 7 o'clock positions outside in and served as stay sutures, preventing the perineal retraction of the urethra. Hence anastomosis sutures could be placed very precisely through the urethral complex, avoiding injury to the external urinary sphincter. Subsequently, the urethra was transected and prostatectomy completed. Modified posterior reconstruction suture was placed with $2 / 0$ polyglactin suture and bladder neck and urethra were approximated. The 7 o'clock position suture was initially taken and passed through bladder neck first inside out then outside in. The same was repeated with the 5'o clock suture. Both sutures were pulled and bladder neck and urethra were approximated to form the base of the anastomosis. Anastomosis was continued with the 7'o clock suture clockwise passing through the bladder neck outside in and through the urethra inside out. Sutures were passed through urethra, not external urinary sphincter complex. Anastomosis proceeded with the five o'clock anastomosis suture running counter-clockwise and again passing through the bladder neck outside in and through the urethra inside out and the anastomosis was completed. Bladder was filled and water-tight anastomosis confirmed.

Results: Modified anastomosis was performed in consecutive 60 patients. This cohort of patients was compared with 60 matchpaired patients operated with the standard continuous running anastomosis technique. Both post catheter removal $1^{\text {st }}$ week, postoperative $1^{\text {st }}$ month and 3rd month continence rates were significantly superior in modified anastomosis group. There was no significant difference between the groups regarding complication and recatheterization rates.

Conclusions: Novel modified urethro-vesical anastomosis technique depicted in this video with prevention of urethral retraction with stay sutures - PURS - during robot assisted radical prostatectomy significantly improves early urinary control.

V9-9 Intracorporeal partly stapled 'Padua Ileal Bladder' using robotic staplers: surgical technique, perioperative and early functional outcomes of a prospective single center series

G Simone, G Salvatore, M Francesco, M Ferriero, L Misuraca, G Tuderti, G Romeo, M Gallucci

\section{Regina Elena National Cancer Institute Rome} Italy

Introduction \& Objective: Robot assisted radical cystectomy (RARC) with totally intracorporeal orthotopic neobladders is a challenging surgical procedure. The potentially increased risk of neobladders stone formation consequent to the use of staplers to create the neobladders is still a matter of debate. Robotic staplers have been recently made commercially available. In this prospective study (www.clinicaltrials.gov NCT02665156) we assessed the feasibility, safety and time efficiency of RARC with intracorporeal partly stapled "Padua Ileal Bladder" using robotic staplers.

Materials and Methods: Twenty-two consecutive patients with muscle invasive or high grade bladder cancer were treated between March 2016 and October 2016. Baseline, perioperative and follow-up data were prospectively collected and maintained into an IRB approved database. Key steps of surgery include: selection of 45 centimeters of ileum and division of the distal and proximal part of the ileum using robotic staplers; detubularization of the ileal loop; creation of the neo-bladder neck with one stapler load; double folding of the proximal ileal loop using twothree stapler loads; hand-sewing of the posterior neobladders wall with barbed suture; uretero-ileal anastomoses on JJ stents with a modified split-nipple technique; urethroneobladder anastomos is performed according to Van Velthoven; hand-sewing of the anterior neobladders wall with barbed suture.

Results: All procedures were successfully completed robotically. Median total operative time was 270 minutes (IQR:255295). One patient $(4,5 \%)$ had wound infection (Clavien grade 1$)$, three patients (13.6\%) had Clavien grade 2 complications (blood pack trasfusion, urinary tract infection requiring antibiotics, hypoxaemia requiring oxygen treatment), one patient $(4.5 \%)$ needed urethral catheter replacement in the OR (Clavien grade $3 b)$ and one patient $(4.5 \%)$ had acute kidney failure requiring temporary dialysis (Clavien grade $4 a$ ). Median hospital stay was 
9 days (IQR 8-11). Three patients (13.5\%) required readmission after discharge (Candidaemia requiring medical treatment [Clavien grade 2] and nephrostomy tube insertion in two patients [Clavien 3a]). Overall complication rate was $40.1 \%$ and overall severe complication incidence was $18.2 \% ; 59.5 \%$ of patients did not experience any complication. At a median follow-up of 3 months, no patients developed recurrence, daytime continence rate was $59 \%$.

Conclusions: We first report safety and time efficiency in the use of robotic staplers to create orthotopic neobladder. This preliminary report highlights feasibility of this technique and favorable perioperative and functional outcomes.

V9-10 Video Demonstration: Robotic-assisted Laparoscopic Radical Cystoprostatectomy with Neobladder formation-Modified Karolinska Technique: Surgical technique \& Outcomes.

Y T B, S Waigankar, A Pednekar, V Wagaskar

Kokilaben Dhirubhai Ambani Hospital and Research Institute India

Introduction \& Objective: At present, robotic assisted laparoscopic orthotopic neobladder is emerging as the preferred and challenging method of urinary diversion. We share our singleinstitute experience of Robotic assisted neobladder reconstruction.

Materials and Methods: Robotic assistance was used to construct a studer pouch in 20 cases of muscle invasive bladder cancers using the Modified Karolinska technique. Steps of Technique includes Isolation of terminal ileum loops, Urethralneobladder and enteric anastomosis, Ileal detubularisation, Posterior wall Construction, Anterior wall folding and construction, Ureteroenteric anastomosis (Brickers), Ureteric stent and suprapubic catheter, Final anterior wall completion. Extended pelvic lymph node dissection was done in all cases. The follow-up period was 6months to one year.

Results: Mean operating time was 190.4 minutes (range 180 to 220), blood loss was $460 \mathrm{cc}$ (range 200 to 600) and hospital stay was 10 days. Clavien-Dindo Complications [Minor (Grade I \& II) \& Major (Grade III and above)] were $11 \%$ and $6 \%$ respectively upto 30days and $6 \%$ and $1 \%$ respectively between 30 90 days. Mean nodal yield was 24 (range 15to 30). Final diagnosis showed pT1-pT2 and pT3 - pT4 in $8 \%$ and $14 \%$ of specimens with a node positivity rate of $7 \%$ and no positive surgical margins. During follow-up there was no evidence of tumor recurrence. The day-time continence and potency at 12 months was $72 \%$ and $80 \%$ respectively.

Conclusions: This technique has good functional and oncological outcomes and appears to be a viable alternative to an open approach, offering patients the advantages of a minimally invasive approach.

V9-11 Robot assisted laparoscopic nerve sparing cystoprostatectomy: Experience on the patient with muscle invasive transitional cell carsinoma treated previously by trimodal therapy

S Yalcin, Y Ozgok, A Canda

Agri Military Hospital, Department of Urology, Agri, Turkey Turkey
Introduction \& Objective: Robot assisted laparoscopic neurovascular bundle sparing (NVBs) radical cystoprostatectomy (RC), bilateral extended lymph node dissection with intracorporeal Studer pouch construction is one of the well accepted modalities in the literature. Herewith this video-abstract we would like to share the NVBs RC experience of ours using robot.

Materials and Methods: 51-year-old patient who had muscle invasive transitional cell carsinoma had the treatment of TURBT followed radiotherapy delivered to the bladder with chemotherapy. Due to detection of recurrance in the follow-up period, patient was referred to our clinic.

Robot assisted laparoscopic NVBs RC, bilateral extended lymph node dissection with intracorporeal Studer pouch construction was planned. Five ports configuration was used. A 12$\mathrm{mm}$ camera port, a $12-\mathrm{mm}$ and a $15-\mathrm{mm}$ assistant ports and 38 $\mathrm{mm}$ robotic ports were used. One of the robot ports was used inside the $15-\mathrm{mm}$ assistant port. Whole procedure was performed with a 0 -degree lens. Initially, the patient was placed in a deep, 30-degree, Trendelenburg position. Dissections of both ureters without damaging their adventitia were performed first. After a sufficient length was obtained, ureters are double clipped and cut where they enter the bladder, and the distal parts were sent for frozen section analysis. Then, posterior dissection of the prostate was performed. Seminal vesicles are identified and the Denonvilliers fascia is opened in the midline. The prostate is dissected off the rectum and NVBs on each side. Lateral bladder pedicles are developed and severed with a vessel sealing system (LigaSure). The endopelvic fascia is opened. Subsequently, the urachus is contained within the surgical specimen, incising lateral to the medial umblical ligaments up to the level of the umbilicus on the anterior abdomen. Cystectomy is completed with dorsal venous complex ligation using the $0 \mathrm{~V}$-loc suture. Sampling of the urethra for frozen section analysis is performed. Specimen has taken into the retrieval bag.

Results: Operation time was nearly 2 hours for the robotic cystectoprostatectomy procedure. Estimated blood loss was less than $30 \mathrm{cc}$. No complication was recorded during and after the operation.

Conclusions: Although robot assisted laparoscopic NVBs radical cystoprostatectomy, bilateral extended lymph node dissection with intracorporeal Studer pouch construction is a complex procedure, it can be performed with excellent short-term surgical and pathological outcomes and satisfactory functional results after considerable experience gained.

V9-12 Robot assisted radical prostatectomy for prostate cancer with more than 100 gram prostate: Technique and outcomes

H Palayapalayam Ganapathi, G Ogaya-Pinies, E Hernandez, T Rogers, V Patel

Global Robotics Institute at Florida Hospital Celebration Health, University of Central Florida United States

Introduction \& Objective: Benign prostatic hyperplasia (BPH) is seen in more than $30 \%$ of men over 60 years age. It is not uncommon to encounter men with $\mathrm{BPH}$ having significant prostate cancer seeking treatment. Radical prostatectomy is technically challenging in very large prostate. Few prior studies considered more than 70 gram prostate as large size and reported inconsistent influence of prostate volume on oncological and 
functional outcomes. We present our experience of robot assisted laparoscopic radical prostatectomy (RALP) in prostate weighing more than 100 gram.

Materials and Methods: We retrospectively reviewed our IRB approved prostate cancer database. RALP specimen weighed more than 100 gram in 183 men (Group 1) and 70 to 100 gram in 647 men (group 2). We compared demographic, operative, oncologic and functional outcomes between these two groups. In this video we describe the technical nuances during RALP for very large prostate with prostate cancer and present their outcomes. Early ligation of dorsal venous complex (DVC) reduces venous blood loss during further dissection. Proper identification of bladder neck and anterior entry favors recognition of median lobe. After visualizing bilateral ureteric orifice, constant upward traction of median lobe by fourth arm is important to enter proper posterior plane. Fish-mouth reconstruction of bladder neck helps watertight vesico-urethral anastomosis and urinary continence.

Results: Patient profile, operative parameters, oncologic and functional outcomes are shown in table 1. D'Amico risk classification was comparable in both groups. Operative time and estimated blood loss was higher in group 1 (>100 gram size). Chance of achieving bilateral full nerve spare was less in group 1 . Higher incidence of extra capsular extension was observed in group 2 but positive surgical margin was similar between groups. At 12 months more than $95 \%$ achieved continence and there was no difference in biochemical recurrence, continence and potency between groups.

Conclusions: Very large prostate size has slightly longer operative time and more blood loss. Larger size decreases chance of bilateral full NS. But, oncological and functional outcomes are not compromised by prostate size in experienced surgeons hands.

V9-13 Robot assisted partial cystectomy with bilateral vesico-ureteric junction resection and reimplantation for a large paraganglioma involving urinary bladder trigone

J Stolzenburg, H Do, A Dietel, R Ganzer, I Kyriazis, E Liatsikos, P Kallidonis, A Ravichandran-Chandra,

V Arthanareeswaran

Department of Urology, University of Patras, Greece Greece

Introduction \& Objective: Paragangliomas are neuroendocrine tumors of extra adrenal origin. Although rare, urinary bladder is the most common locale for paragangliomas within the genitourinary system. In this video, we report the possibility of managing a patient with paraganglioma involving the bladder trigone using robot assisted surgery.

Materials and Methods: We describe the operative technique of robot assisted partial cystectomy with bilateral vesico-ureteric junction resection and reimplantation for a large paraganglioma involving urinary bladder trigone.

Results: After complete mobilization of urinary bladder, the tumor was initially resected without disturbing the anatomical vesico-ureteric junctions. Intraoperative frozen section(IOFS) revealed presence of tumour in the margins and further resection, including the bladder outlet and both vesico-ureteric junctions, were performed. The re-resected tumour margins were free from tumour on IOFS. The bladder outlet was completely reconstructed and the tumour free margins of the bladder were closed primarily. Both ureters were then re-implanted using the classical technique described by Politano-Leadbetter with two separate incisions on the lateral surface of bladder. Although extensive resection of the trigone including the bladder outlet and ureteric orifices were done, postoperative

Conclusions: In conclusion, paraganglioma involving the bladder trigone can be effectively managed using robot assisted surgery with bilateral reimplantation of ureters following extensive resection. IOFS is an absolute necessity to identify tumor free margins.

V9-14 Experience of robot-assisted radical prostatectomy (RARP) in renal transplant recipients

T Nishikimi, H Kobayashi, H Yamada, R Ishida, Y Yamauchi, K Hattori

\section{Nagoya Daini Red Cross Hospital Department of Urology Japan}

Introduction \& Objective: With the recent increase in renal transplant recipients, diagnosis of prostate cancer after renal transplantation has also become more frequent. Our experience with robot-assisted radical prostatectomy (RARP) in two renal transplant recipients is presented in this video.

Materials and Methods: Standard RARP employs four ports, including a camera for the robot and two extra ports for an assistant. We shifted the standard RARP port configuration to the left by $2 \mathrm{~cm}$ for renal transplant recipients.

Results: Case 1A 72-year-old man with an 18-year dialysis history underwent renal transplantation from a deceased donor 5 years previously. Biopsy was conducted due to a high PSA level $(9.73 \mathrm{ng} / \mathrm{mL})$ and prostate cancer $(\mathrm{GS} 4+4=8)$ was detected at 6/12 sites (left: 6/6). RARP was performed with a diagnosis of cT2aNOM0 disease.

Case 2 A 59-year-old man with a 1-year dialysis history underwent renal transplantation 11 years previously. Biopsy was conducted due to a high PSA level $(6.21 \mathrm{ng} / \mathrm{mL})$ and prostate cancer (GS4 $+5=9$ ) was detected at 1/12 sites (left: 1/6). RARP was performed with a diagnosis of $\mathrm{cT} 1 \mathrm{cN} 0 \mathrm{M} 0$ disease.

Discussion: The following precautions are required when RARP is performed in renal transplant recipients. (1) It should be recognized that lymph node dissection will be difficult on the side of renal transplantation. (2) The ports should be placed carefully because the transplanted kidney protrudes considerably into the operating field. (3) Prevesical detachment should be minimized and the site of the ureteral stent should be selected carefully in consideration of migration of the transplanted ureter. (4) Protective maneuvers are required because the tissues are fragile due to dialysis and oral steroid therapy. (5) Information should be collected, such as the time and method of transplantation as well as the center where it was done, because of individual differences in the severity of pelvic cavity adhesions and migration of the transplanted ureter.

Conclusions: RARP was possible without serious complications in our two patients, suggesting that RARP can be an effective option for renal transplant recipients. 


\section{VS10: LAPAROSCOPIC AND ROBOTIC RECONSTRUCTIVE SURGERY}

V10-1 An unusual case of bladder mass and its 3-D laparoscopic management

A Kumar, G Kumar, N Kumar, M Patel, P Gupta

Department of Urology and Renal Transplant, VMMC and Safdarjang Hospital, New Delhi, India India

Introduction \& Objective: Various Non- neoplastic and inflammatory disorders can manifest as a focal bladder mass and mimic malignancy. Primary Dermoid cyst as bladder mass is very rare and approximately 9 cases have been cited in literature. We are presenting the first reported case of primary Dermoid cyst as bladder mass which was managed laparoscopically. which to best of our knowledge has not been published before

Materials and Methods: A 51 year old female presented with history of irritative LUTS for last 1 year with no history of hematuria, tobacco use and comorbidities. The physical examination was normal. The kidney function tests were normal. The ultrasound showed a $4 \times 3 \mathrm{~cm}$ mass in anterior wall of urinary bladder. The urine cytology for malignant cells was negative. The CT abdomen showed a heterogeneous mass measuring $4 \times 3 \mathrm{~cm}$ in size in right lateral and anterior wall of urinary bladder with calcification and hypodense area in the mass without perivesical extension and regional lymphadenopathy with normal adnexa bilaterally. The patient underwent cystoscopy and transurethral resection biopsy. The cystoscopy showed greyish white mass with hair follicles with soft yellowish necrotic tissue arising from the dome of bladder. The histopathology showed keratinised stratified squamous epithelium with presence of hair follicles, adipocytes and mucous glands in the subepithelium, with no evidence of malignancy. Therefore, the final diagnosis of benign primary urinary bladder dermoid cyst was made and patient was planned for 3-D laparoscopic transperitoneal partial cystectomy. We are presenting video of that case.

Results: The operating time was $70 \mathrm{~min}$ and estimated blood loss was $40 \mathrm{ml}$. There were no intraoperative and postoperative complications. On postoperative day 2, drain was removed and patient was discharged. The foley catheter was removed on $7^{\text {th }}$ postoperative day. At 2 year follow up, patient is asymptomatic. The cystoscopy done at 3 monthly intervals upto 2 years showed no tumor recurrence. The CT abdomen at 1 year and 2 year follow up are normal.

Conclusions: If a "mass with peripheral calcification with hair growth" is found on anterior wall or dome of bladder, a dermoid cyst should be considered as a differential diagnosis. Primary Dermoid cyst of urinary bladder can be managed safely and effectively by 3-D laparoscopic partial cystectomy.

V10-2 Lessons learnt from 8 successful early laparoscopic repair of vesicovaginal fistula

S Kallappan, M Ramalingam, S Nachimuthu, C Dinakaran

Urology Clinic

India
Introduction \& Objective: Gynecological causes fo vesicovaginal fistulae are common nowadays. Early repair was deferred in obstetric vesicovaginal fistulae as results were poor due to poor tissue viability. Laparoscopic repair was done successfully in 8 gynecological vesicovaginal fistulae.

Materials and Methods: Eight women developed vesicovaginal fistulae following hysterectomy. Early repair was planned after confirming the absence of urinary infection. Cystoscopy was done to examine the fistula to confirm the absence of slough. Bilateral ureteral catheters or stents were placed. Laparoscopy was done using 5 ports.

Lessons learnt to make the procedure successful are as follows.

Foley balloon with about $40 \mathrm{ml}$ in the baloon was used to prevent gas leak from vagina.

As it was an early repair the adhesions were flimsy. The wall of the bladder was not fibrosed unduely and could be dissected sharply. Bladder was bivalved and the fistula was excised with a $5 \mathrm{~mm}$ margin. The bladder wall was held apart with a suture through the abdominal wall. The vaginal wall could be seperated well from the bladder wall. Barbed sutures were used to maintain tension. Omental flap was brought inbetween the baldder and vagina. Urethal foley was left in along with an abdominal drain.

Results: The time taken for the surgery was between 150 to 180 minutes. Patients were in the hospital for 5 days. Drain was removed before discharge. Catheter was removed on the 21 st day. None of the eight patients had a recurrence of the fistula.

Conclusions: Early laparoscopic repair of gynecological vesico vaginal fistula is feasible and reduces the distress in the patients.

V10-3 Laparoscopic repair of vesicouterine fistula using TACHOSILTM sponge

R Sanseverino, G Napodano, O Intilla, U Di Mauro, G Molisso, T Realfonso

Dept. of Urology Umberto I Hospital Italy

Introduction \& Objective: Vesicouterine fistulas (VUF) represent about $4 \%$ of all urogenital fistulas. The most common causes of fistulas are surgical, obstetric and related to radiation therapy. Depending on their localization, we distinguish corporeal and cervical fistula. We show a case of a patient affected by a corporeal vesicouterine fistula repaired by laparoscopic approach.

Materials and Methods: A 35 years old female presented at our institution with a corporeal vesicouterine fistula resulted from a previous Caesarean section. The patient complained of Youssef syndrome (amenorrhea, menouria, urinary continence). Cystography and cystoscopy revealed vesicouterine fistula. After positioning bilateral ureteral catheter, a transperitoneal approach is performed. After resection of the fistolosous tract, bladder is repaired with a double layer suture. A Taschosil ${ }^{\text {TM }}$ sponge is applied on uterin surface. A drain is placed in peritoneal cavity. 
Results: Surgical procedure time and fluid loss are $150 \mathrm{~min}$ and $150 \mathrm{ml}$, respectively. Catheter is removed after 10 days. No relapse of fistula occurred

Conclusions: Corporeal vesicouterine fistulas are very infrequent and present with a classical Yousef syndrome. Laparascopic repair of vesicouterine fistulas is a safe and reproducible technique offering excellent results

V10-4 Transvesical laparoendoscopic single-site surgery to repair a vesicovaginal fistula in man after female to male sex reassignment

M Borowik, M Przudzik, M Roslan

Department of Urology, University of Warmia and Mazury, Olsztyn, Poland

Poland

Introduction \& Objective: The prevalence of transsexualism is approximately 1 in 20000 in the general population. A female/ male sex realignment surgery (SRS) is performed on transsexuals with masculinizing genitoplasty (hysterectomy, vaginectomy, metoidioplasty and neophalloplasty). Usually, such patients need several complex procedures that are not free of complications. A rare complication after SRS is a vesicovaginal fistula (VVF). The aim of the study is to present the case of a transgender man with a VVF that was repaired with percutaneous transvesical laparoendoscopic single-site surgery - the T-LESS technique.

Materials and Methods: In February 2017, we performed a TLESS repair for a vesicovaginal fistula in a 67 year old man, who underwent several operations from 2012 to 2016 because of transsexualism. After vaginectomy a vesicovaginal fistula occurred that was operated on unsuccessfully in 2015. In our center, the recurrent fistula between the bladder and the $3-\mathrm{cm}$ long remnant of the vagina was diagnosed. Because there was not enough space in the vagina to perform the Latzko operation, we decided to use the T-LESS technique. The procedure was performed through a single-port device (Tri-Port+, Olympus, Germany) that was introduced under cystoscopic control directly into the bladder via a $1.5 \mathrm{~cm}$ incision made $2-5 \mathrm{~cm}$ above the pubic symphysis. A pneumovesicum was established with carbon dioxide up to a pressure of $14 \mathrm{~mm} \mathrm{Hg}$. A standard 10$\mathrm{mm}$, rigid, $0^{\circ}$ videolaparoscope and straight laparoscopic instruments were used. The fistula was separated from the bladder mucosa by a circular cut using a hook electrode. Both the vaginal wall and the bladder defect were closed tightly with absorbable 3/0 running sutures (V-Loc ${ }^{\mathrm{TM}} 90$ Absorbable Wound Closure Device, Covidien, Norwalk, CT, USA). The rectus sheath and skin incision were closed with one and two stitches, respectively. Ureteral catheters were left in place for five days, and the 18 F Foley catheter was left for three weeks. Results: The operative time was $170 \mathrm{~min}$. The procedure was completed with no extra port insertion. No blood loss or complications were observed. The postoperative hospital stay was five days. In a 12-week follow-up, no urine leakage was observed, and the laboratory results were all within the normal range. Vaginal examination revealed no leaks and complete closure of the fistula.

Conclusions: Our positive experience with this approach shows that the T-LESS access for VVF repair in transsexual man is feasible, safe and efficacious. The method can be considered particularly in patients with recurrent fistulas or presenting a small vaginal remnant, in whom the standard procedures are likely to result in failure.

\section{V10-5 Trans-urethral laparoscopic suture of the bladder post major trauma \\ P Grange, C Kouriefs, J Makanjuola, A Khan \\ United Kingdom}

Introduction \& Objective: We present our technique of transurethral laparoscopy to repair an intra-peritoneal bladder rupture using a single hand laparoscopic technique with gas cystoscopy, in a female patient with multiple co-morbidities who sustained multiple injuries (pelvic, abdominal) following a fall from a height.

Materials and Methods: A $5 \mathrm{~mm}$ laparoscopic port inserted into the urethra and continuous $\mathrm{CO} 2$ gas Insufflation of the bladder with a pressure of $12-15 \mathrm{mmHg}$. A $5 \mathrm{~mm}$ EndoEye 30 degree laparoscope is used to visualise the bladder and identify the bladder perforation. We performed a single hand suture technique using a monofilament suture. Forehand and backhand mattress sutures are places and the the Grange-mignot extracorporal knot is used to close the defect. A Microfrance knot pusher used to secure the knots and cut the suture.

Results: A foley catheter was inserted post procedure and a cystogram was performed to confirm no urinary leak day 10 post repair before the foley catheter could be safely removed.

Conclusions: We believe this technique is safe and an alternative to traditional open or trans-peritoneal laparoscopy to repair an intra-peritoneal bladder rupture

V10-6 Laparoscopic Repair of a Vesicosigmoid Fistula Secondary to Holmium Laser Enucleation of the Prostate

J Kang, J Jeon

Chinjujeil hospital

Republic of Korea

Introduction \& Objective: Holmium Laser Enucleation of the Prostate(HoLEP) consists of two consecutive procedures, enucleation of a prostatic adenoma and intravesical morcellation of the enucleated prostatic tissue. Morcellation of the resected adenoma should ideally be performed with a fully distended bladder. However, when visualization is inadequate or when the procedure is performed by an inexperienced surgeon, this step can be dangerous.

Materials and Methods: A 68-year-old male patient was referred for treatment of a vesicosigmoid fistula secondary to HoLEP. Three days prior, the patient had undergone a HoLEP for benign prostatic hyperplasia in the urology department of another hospital. He complained of lower abdominal pain and dirty stool-like material in his urine starting from 3 days after his surgery. A CT scan and colonoscopic examination were performed, on the basis of which a fistula between the posterior wall of the bladder and the sigmoid colon was identified. During the colonoscopy, a small fistula opening with surrounding edema was noted in the sigmoid colon at $20 \mathrm{~cm}$ from the anal verge.

Results: We performed emergency laparoscopic repair of the vesicosigmoid fistula. The sigmoid colon was tightly attached to the posterior wall of the bladder. However, no fecal material was 
present in the peritoneal cavity. After detaching the colon from the bladder, the presence of a small fistula opening was confirmed. The fistula tract was resected, and interrupted sutures were used to repair the bladder; after which primary repair with wedge resection of the inflamed sigmoid colon was performed. Four days after the operation, the patient complained of lower abdominal pain and had a follow-up CT scan showing bowel wall edema with large amounts of fluid collected in the abdominal cavity. Laparoscopic exploration was performed, and leakage from the primary repair site in the sigmoid colon was noted. Segmental resection and primary closure of the sigmoid colon were performed. Ten days after the second operation, the patient was discharged without any complications. At a follow-up examination 2 months after discharge, the patient reported that he was able to void well, without incontinence. His bowel habits and defecation were also satisfactory.

Conclusions: When performing a HoLEP, meticulous control of the morcellator and clear visualization are crucial.

V10-7 Amniotic Mesenchymal Stem Cells in the Management of Recurrent Urethral Stricture

O Alalao, K Al-rumaihi, N Younes, A Alansari

\section{HAMAD MEDICAL CORPORATION} Qatar

Introduction \& Objective: The next major advance in urology will likely come from the fields of tissue engineering and stem cell therapy.

This has moved some to consider this a standard reconstructive tool and use this process regularly. The final advancement not yet reached is the use of stem cells for urethral reconstruction. Stem cells are unique in that they can regenerate and self-renew and differentiate into a number of different cell types, including all layers of a structure such as the urethra. Stem cells have been effectively used in other urologic applications including voiding dysfunction, urinary incontinence, and erectile dysfunction. These cells are referred to as secretomes and function to encourage cell growth and differentiation. The cells used are mesenchymal stem cells, and while performing some secretory functions, the cells do not engraft into the injured tissue and cannot completely regenerate the affected body structures. If these unique stem cells or secretomes can be used to better heal damaged tissues or structures, such as the urethra, significantly less invasive procedures could be used to reconstruct urethral strictures or stenosis.

Materials and Methods: Surgigraft ${ }^{\mathrm{TM}}$ by Synergy Biologics is a chorion free regenerative tissue matrix comprised of a single layer of cuboidal epithelial cells attached to several biologic reservoir layers including basement, compact, spongy, and fibroblast layers. Epithelial cells manufacture multiple vasoactive peptides, growth factors, cytokines and extracellular matrix (ECM) proteins. These biological factors may reside in the epithelium or may be transported and accumulated in the basement membrane where they act as a reservoir from which the amnion exerts its therapeutic effects following transplantation.

Surgigraft was applied in its liquid form and injected in the bulbar-urethra for 8 cases of recurrent urethral stricture after their first recurrence.

Patient age was between 30-50 years, all of them had unknown etiology of their strictures, non of them were trauma cases.

Results: We are presenting here one of the 8 cases done at Hamad Medical Corporation, all the cases were followed for 6 months, after their elective visual internal uretherotomy then the graft injection.

All cases had 2 months of weekly urethral dilation after the procedure and examined after 6 months with visual flexible cystoscopy with resolution of the stricture.

Conclusions: Therapies still awaiting transition from the laboratory to the clinical setting are the use of stem cells and secretomes. With each progression in the field of urethral reconstruction, the ultimate goal remains to create a successful, durable outcome, while maximizing patient quality of life.

\section{V10-8 Robotic Assisted Repair of Parastomal Hernia}

PW Mekhail, MW Mekhail, D Hatcher, A Monish

University of Southern California

United States

Introduction \& Objective: Parastomal hernia is a common and vexing problem after ileal conduit urinary diversion that can cause problems with the stoma appliance resulting in leakage,
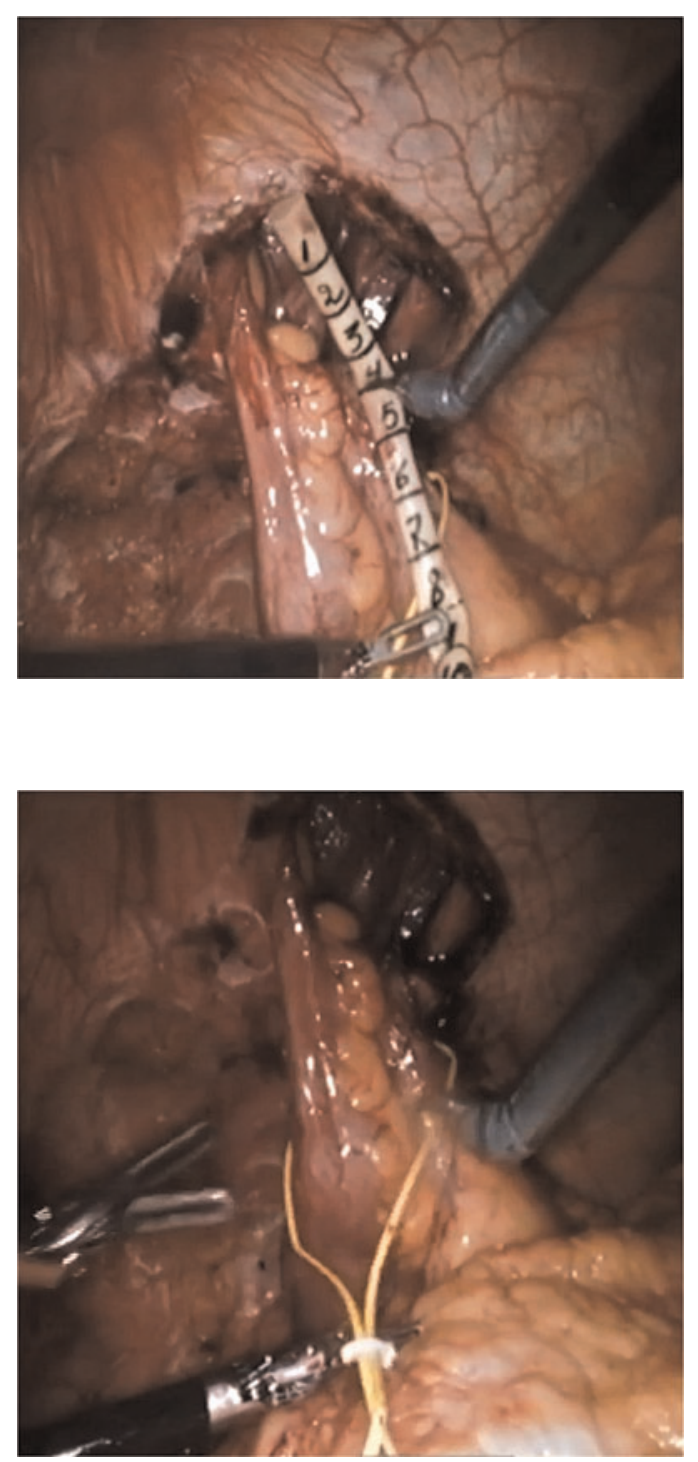


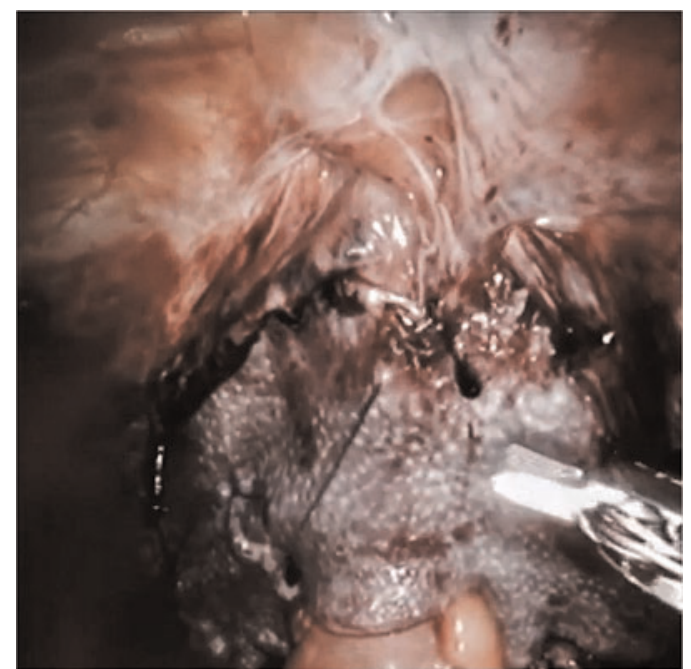

odor and impairment of quality of life. Historically these hernias have been managed open surgically, which requires considerable dissection of the abdominal wall for mesh placement, and may also require relocating the stoma to the opposite side. Needles to say, the morbidity of an open parastomal hernia repair can be considerable and recurrence rates can be approximately $30 \%$.

Materials and Methods: Here we present our technique of robotic parastomal hernia repair with a biologic mesh. We used a biologic mesh with a key-hole method in order to avoid risk of erosion into the bowel segment.

We compare the outcome with another contemporary patient who had open repair of parastomal hernia.

Results: Robotic repair was completed successfully in the 63 year old morbidly obese lady (BMI 36). Operating time was 3 hours and blood loss was $10 \mathrm{~mL}$. Length of hospital stay was 2 days and at the last follow up at 3 months, there was no evidence of recurrence and her stoma appliance issues have resolved.

The other patient who had open repair had a hospital stay of 4 days and developed a recurrence within 6 months requiring a second open repair and relocation of the stoma.
Conclusions: Robotic repair of parastomal hernia is safe and effective. It is less invasive than open surgery and is likely to have a shorter hospital stay period.

V10-9 Laparoscopic radical cystectomy: delayed ureteral intersection.

D Perlin, I Aleksandrov, V Zipunnikov, I Dymkov

\section{GBUZ VOUNC}

Russia

Introduction \& Objective: The gold standard for treatment of localized muscle-invasive, bladder cancer is open radical cystectomy (ORC). However, in the recent years numerous clinics introduce a laparoscopic approach which seems lead to a faster recovery, shorter hospital stay and more rapid return to daily activities. Prolonged blocking of the outflow of urine may decrease renal function; we used a delayed crossing of the ureters. Previously performed a complete discharge of the bladder, ligation of the bladders pedicles and the intersection of the urethra. Further, ligation and excision of the ureters from the bladder were performed.

Materials and Methods: In the report were included 35 patients with bladder cancer, who have been underwent laparoscopic radical cystectomy (LRC) in a single institute between April 2013 and March 2016. Only patients have been submitted to full intracorporal ileal conduits were included.

Results: Twenty seven men and eight women underwent LRC. The mean operative time was 378 minutes, the mean blood loss was $285 \mathrm{ml}$., the mean length of hospital stay was 12.4 days. The postoperative complication rate was $11.4 \%$. However, the majority of the patients successfully treated with minimally invasive procedures. Generally, our results were similar to other reported studies.

Conclusions: Laparoscopic radical cystectomy is a safe and efficient modality of treatment of bladder cancer. The delayed intersection of the ureters allows preserving the kidney function. In order to prevent the spread of urine through the abdominal cavity, the external urethral orifice was sutured. However, it needed more procedures and longer period for providing LRC an alternative to ORC.

\section{VS11: ROBOTIC UPPER TRACT SURGERY FOR CANCER II}

V11-1 Robot assisted partial nephrectomy for multiple renal tumors - A Vattikuti Collective Quality Initiative database analysis

S Arora, RK Ahlawat, J Adshead, R Abaza, B Challacombe, P Dasgupta, G Gandaglia, D Moon, F Porpiglia, T Yuvaraja, U Capitiano, J Porter, A Mottrie, M Bhandari, CG Rogers, H Gill

Vattikuti Urology Institute, Henry Ford Hospital United States

Introduction \& Objective: This video shows the technique and outcomes of robot assisted partial nephrectomy (RAPN) for multiple tumors in a single operative session.
Materials and Methods: Retrospective analysis of the Vattikuti Collective Quality Initiative prospective database was done. A total of 501 patients underwent RAPN from November 2014 till January 2017 at 14 institutions in nine countries. Out of these, 37 patients underwent RAPN for multiple tumors. The representative video shows a case with multiple renal tumors operated via the transperitoneal approach using warm ischemia.

Results: A total of 81 tumors were excised in 37 patients (mean [SD], 2.19[0.66] tumors per patient) with no conversion to open surgery and one conversion to robotic radical nephrectomy. The median [IQR] tumor size was 3.2 [2.2-5.0] cm. Retroperitoneal and transperitoneal approach were used in $5(13.5 \%)$ and 32 $(86.5 \%)$ of the patients, respectively. Zero ischemia was used in $5(13.5 \%)$ patients. Median [IQR] operative time was 156.0 
[127.0-180.0] min. Median [IQR] estimated blood loss was 150 [50-200] ml. No patients had Clavien-Dindo grade $\geq 3$ complications. Seven $(18.9 \%)$ patients had grade 1-2 complications. One patient $(2.7 \%)$ had a positive surgical margin, and the median [IQR] hospital stay was 3 (1.5-4.0) days.

Conclusions: RAPN can be safely performed for multiple tumors in the same kidney in a single operative session. The procedure has a low morbidity and excellent outcomes in a multiinstitutional setting.

\section{V11-2 Concurrent robotic kidney and general surgery procedures}

H Gill, M Franco, D Kwon, CG Rogers

Vattikuti Urology Institute, Henry Ford Hospital United States

Introduction \& Objective: When kidney tumors are found incidentally during evaluation of a general surgery condition, patients may request concurrent robotic surgeries to address both issues simultaneously. This video shows two such cases.

Materials and Methods: Robotic nephrectomy and cholecystectomy A 46-year-old female with right upper quadrant pain was found to have cholelithiasis and a $7.1 \mathrm{~cm}$ right renal mass. The procedure was performed with the daVinci Si with 4-arm approach and flank position with no redocking. Ports were placed along with a $5 \mathrm{~mm}$ subxiphoid port for a liver retractor. The upper pole attachments of the kidney were mobilized, facilitating exposure of the gallbladder. The nephrectomy was done first and hilar vessels were ligated with specimen being placed in a retrieval bag. Attention was turned to the cholecystectomy and the cystic duct and artery were dissected and ligated with robotic hemlock clips. The remaining gallbladder attachments were freed. Robotic partial nephrectomy and partial gastrectomy A 66-year-old female with GI symptoms was found to have a $3.1 \mathrm{~cm}$ enhancing mass consistent with a GIST tumor and a $4 \mathrm{~cm}$ renal mass of the left lower pole. The procedure was performed with the daVinci Si with 4-arm transabdominal approach in flank position and no redocking. Ports were placed with a Gelpoint for the assistant port to allow intraoperative assessment of the specimens. The partial gastrectomy was performed first, using the robotic vessel sealer to expose the gastric fundus. The endoGIA stapler was used to remove the lesion at its base, and removed through the Gelpoint. Partial nephrectomy was performed next. The colon was mobilized and the renal hilar vessels were dissected. Intraoperative ultrasound was used to delineate tumor margins and the tumor was excised.

Results: Robotic nephrectomy and cholecystectomy OR time was 170 minutes, EBL of 30cc and 1 day stay. Final pathology confirmed a $7.1 \mathrm{~cm}$ chromophobe renal cell carcinoma (RCC) with sarcomatoid differentiation (pT2a). Gallbladder pathology showed acute cholecystitis and cholelithiasis. Robotic partial nephrectomy and partial gastrectomy OR time was 150 minutes, EBL of 200cc and a stay of 3 days. Final pathology confirmed a $4 \mathrm{~cm}$ papillary RCC type 1 (pT1a), grade 3 . Partial gastrectomy pathology confirmed a $3.1 \mathrm{~cm}$ GIST tumor. We performed an additional two cases. All procedures documented had negative margins and no complications.

Conclusions: We demonstrate the feasibility of concurrent robotic kidney procedures in conjunction with robotic general surgery procedures. With careful planning and proper technique, robotic kidney and robotic general surgery procedures may be performed simultaneously with favourable outcomes.
V11-3 Robotic Minimal Margin Enucleo-resection Technique of Left Renal Tumor

H tavukcu, O Aytac, H Kulaksızoglu, F Atug

ISTANBUL BILIM UNIVERSITY

Turkey

Introduction \& Objective: The main goal of partial nephrectomy is the achieving the negative surgical margin. Minimal enucleo-resection technique depends on the resection of the $1 \mathrm{~mm}$ renal tissue around renal tumor in partial nephrectomy. In this video we present a case minimal enucleo-resection robotic partial nephrectomy.

Materials and Methods: Fifty four years old male patient admitted to our clinic with incidentally diagnosed renal tumor with $18 \times 17 \mathrm{~mm}$ in lower pole of left kidney. Patient was positioned to right decubitus for robotic partial nephrectomy. The colon was medialized after an incision on the Toldt line and retroperitoneal space was entered. For hilus dissection left ureter was found inferiorly. Main renal artery and vein were dissected, renal artery was suspended with a vessel tape. The margins of tumor was scored with monopolar cautery and excision line was marked. Than renal artery was clamped with a bulldog clamp. The tumor was excised by cold scissors with $1 \mathrm{~mm}$ renal parenchyma around tumor as a minimal margin enucleo-resection technique. The tumor bed was sutured with 3/0 v-lock suture. Renography was performed with sliding weck-clip technique by $2 / 0$ vicryl. The duration of ischemia was $14 \mathrm{~min}$, operative time was $95 \mathrm{~min}$. for nephrectomy, total hemorrhage was $20 \mathrm{ml}$. No serious complication was seen on perioperative period. The surgical drain was removed postoperative day 1 and patient was discharged at the same time

Results: The pathologic examination was reported as clear cell variant of renal cell carcinoma with negative surgical margins.

Conclusions: Minimal enucleo-resection technique in robotic partial nephrectomy is a new and important(feasible) technique in decreasing the ischemic trauma and as well as achieving the negative surgical margin.

V11-4 Robotically-assisted retroperitoneal lymph node dissection in stage IIa seminoma

S Tadtayev, E Mayer, D Nicol

Royal Free Hospital

United Kingdom

Introduction \& Objective: The robotically assisted approach has been increasingly used for retroperitoneal surgery in highly selected patients with metastatic germ cell tumours as an alternative to radiotherapy and to reduce the burden of the chemotherapeutic treatment. We present our experience in templateguided lymph node dissection in a patient with a stage IIa seminoma of the left testicle.

Materials and Methods: Patient underwent left orchidectomy in October 2016 which yielded diagnosis of $36 \mathrm{~mm}$ classical seminoma. Staging investigations in the form of FDG PET identified $10 \mathrm{~mm}$ FDG-avid lymph node in the left paraaortic region, just below the level of the left renal vein. The operation was performed with the patient in the lateral position and Da Vinci Xi surgical robot in four arm configuration.

Results: Recovery was uneventful and discharge home was achieved on the 1st post-operative day. Final histological analysis demonstrated metastatic seminoma $<5 \%$ of the lymph node 
by volume, which corresponded to the area of FDG activity. There was a further node in the left spermatic cord replaced with metastatic seminoma to $35 \%$ of the volume.

Conclusions: Robotically assisted retroperitoneal lymph node dissection has become an option for a selected group of patients with stage IIa seminoma as an alternative to radiotherapy. It allows for prompt convalescence and early return to work. Both template-guided retroperitoneal lymph node dissection and radiotherapy are followed by adjuvant BEP chemotherapy, according to the local protocol.

V11-5 'Hilum First': A Novel Technique to Radical Nephrectomy for Large Renal Masses

M Ahmed, G Lovallo, C White

Hackensack University Medical Center United States

Introduction \& Objective: We describe our novel technique for robotic radical nephrectomy for very large renal masses larger than fifteen $\mathrm{cm}$.

Materials and Methods: 11 patients underwent robotic radical nephrectomy using the novel technique of "Hilum First" which is demonstrated in our video.

Results: All patients underwent radical nephrectomy in less than one hour. Median operative time was 47 minutes. All margins were negative.

Conclusions: Robotic radical nephrectomy using a "Hilum First" approach is feasible and can be performed in less than one hour for patients with very large renal masses.

\section{V11-6 Single-Site Robotic Partial Nephrectomy}

M Holden, Z Hamilton, IH Derweesh

Department of Urology, UC San Diego Health, La Jolla, California

United States

Introduction \& Objective: Nephron sparing surgery is the treatment of choice for the majority of clinical T1 renal masses, Initial The robotic approach has gained popularity as a minimally invasive option for such lesions. However, despite the advances in articulation and ergonomics that the robotic approach affords, there is still room for the application of laparoscopic principles. In particular, the single-site approach to pure laparoscopy demonstrated reduced postoperative pain scores and remains widely used for other procedures. The application of this technique to robotic procedures has been limited by instrument clashing as well as bedside clearances at the robotic arms. However, with contemporary access devices and an anatomically favorable lesion, the single-site approach can be performed using the robotic platform. We present a case of single site robotic-assisted laparoscopic partial nephrectomy, to demonstrate the safety and efficacy of this approach

Materials and Methods: A 71 year-old patient was incidentally diagnosed with an enhancing $1.0 \mathrm{~cm}$ left exophytic renal mass during an evaluation for refractory hypertension. Given the size, location, and history of hypertension, the patient was offered partial nephrectomy. Due to favorable anatomy and lesion morphology, this was performed using a single-site robotic approach. A $6 \mathrm{~cm}$ periumbilical incision accommodated a GelPOINT access device (Applied Medical) that was used to deploy
3 robotic trocars (DaVinci Xi, Intuitive Surgical) and a $12 \mathrm{~mm}$ assistant port.

Results: Dissection proceeded smoothly, and the renal hilum was exposed, including an accessory lower pole artery. After localizing the mass with intraoperative ultrasound, bulldog clamps were placed on the renal arteries and the mass was resected. Renorrhapy was performed in two layers using 3-0 monocryl. Total clamp time was 17 minutes of warm ischemia. Estimated blood loss was $50 \mathrm{~mL}$. Creatinine was 0.80 (vs 0.84 preoperatively) and patient was discharged on postoperative day \#1 with minimal discomfort. Final pathology revealed a $1.4 \times 1.3 \times 1.2$ oncocytoma with negative margins.

Conclusions: More renal masses are being treated with partial nephrectomy, often via robotic approach. In well-selected patients, the single-site robotic approach can extend the benefits of minimally invasive surgery without compromising safety or oncologic outcome.

\section{V11-7 Serial Hem-o-lok Application for Robotic Partial (SHARP) Nephrectomy}

\section{JS Hwong, SL Chang, R Korets}

UCLA Institute of Urologic Oncology

United States

Introduction \& Objective: Partial nephrectomy has become the standard of care for the management of small renal masses. Multiple studies have demonstrated the clear detrimental effect of warm ischemia on renal function after partial nephrectomy. The main factors affecting post-surgical renal function are the baseline renal function of the patient preoperatively, the amount of healthy renal parenchyma remaining postoperatively, and the total duration of warm ischemia needed during resection. By developing techniques for off-clamp partial nephrectomy, ischemic injury to healthy parenchyma can be completed avoided. In this video, we demonstrate a novel off-clamp technique for the resection of small exophytic renal masses, thereby avoiding ischemic injury while maintaining visualization and hemostasis during tumor resection.

Materials and Methods: The patient is a 55 year-old male with incidentally-detected bilateral renal masses. Physical examination and laboratory tests are unremarkable. Initial surgical management focuses on the larger $4.5 \mathrm{~cm}$ right renal mass. Crosssectional imaging demonstrates an exophytic mass at the posterior upper pole of the right kidney with an adjacent $7 \mathrm{~cm}$ benignappearing simple renal cyst.

Results: We utilize a robot-assisted retroperitoneoscopic approach with the patient in a full flank position. All three arms of the robot system are used, as well as a $12 \mathrm{~mm}$ port placed for the bedside assistant. The retroperitoneal space is developed with a balloon dissector, placed at the site of the laparoscopic camera port. Gerota's fascia is incised, and hilar vessels are dissected for easy access in the event of excessive bleeding. After the mass is exposed, the adjacent benign-appearing cyst is decorticated. Offclamp resection of the exophytic mass progresses with excellent hemostasis using serial application of 0-Vicryl sutures with dual sliding Hem-o-lok clips for compression of the parenchyma. Pathology demonstrates a pT1b papillary renal cell carcinoma with negative surgical margins.

Conclusions: Off-clamp resection of small exophytic masses can be safely performed with the serial application of Hem-o-lok clips. Decortication of benign-appearing cysts adjacent to the mass may facilitate this technique. 
V11-8 Dilated Lumbar Veins due to Cement Application to lomber vertebrae in a metastatic renal cell cancer patient

O Aytac, H tavukcu, H Kulaksizoglu, F Atug

ISTANBUL BILIM UNIVERSITY

Turkey

Introduction \& Objective: Bone cement application for bone fractures is one of the most common procedures in orthopaedic surgery. In this video we present a case concomitant robotic radical nephrectomy, after bone cement application to vertebrae for metastatic renal tumour.

Materials and Methods: Eighty three years old female patient admitted to our clinic with back pain. Radiologic evaluation revealed right kidney tumour and vertebral metastasis on lomber vertebrae. The patient iniatilly had spine surgery with cement application to fractured and high risk for fracture vertebral bones on prone position. Then patient was repositioned to left decubitus for robotic nephrectomy. The colon was medialized after an incision on the Toldt line and retroperitoneal space was entered. For hilus dissection right ureter was found inferiorly. After controlling the right gonadal vein, accessory lomber vein was dissected which was more dilated than as usual. This vein could have been damage easily if no safe and clear dissection was performed. After controlling this vein with large hem-o-lok clips, renal hilus dissection was performed. First branch of renal artery and then main renal artery and vein were controlled and cut. Total operation time was $90 \mathrm{~min}$. for nephrectomy, total hemorrhage was $100 \mathrm{ml}$. No serious complication was seen on perioperative period.

Results: The nehrectomy pathology was reported as clear cell variant of renal cell carcinoma with Fuhrman grade. Patient had free of back pain after the second month of operation. The vertebral biopsy was also metastasis of the renal tumor.

Conclusions: Concomitan cement application followed by robotic radical surgery has not been reported previously. Cement use seems to have can affect of dilated venous structures. This should be cautioned in this type of surgery.

V11-9 Laparoscopic resection of retroperitoneal malignant tumors.

D Perlin, I Aleksandrov, I Dymkov, V Zipunnikov

GBUZ VOUNC

Russia

Introduction \& Objective: Retroperitoneal tumors are a rare finding and most masses are of malignant origin. We share our experience of laparoscopic management of retroperitoneal tumors.

Materials and Methods: We evaluated four patients operated on in our hospital in Volgograd from January 2008. One man and three women underwent laparoscopic transperitoneal resection of retroperitoneal tumors. The mean tumor size was $8.7 \mathrm{~cm}$ (range, 4.2 to $12.5 \mathrm{~cm}$ ).

Results: The mean intra-operational time was 185 min (range, 87 to $260 \mathrm{~min}$ ). The mean hospitalization period was 12 days (range, 9 to 16 days). In three patients liposarcoma was detected, in one leiomyosarcoma. The local recurrence is revealed in one case.

Conclusions: The laparoscopic approach is technically safe and successful in maintaining oncologic principles and avoiding recurrences in retroperitoneal malignant masses, representing a feasible alternative to open surgery.

\section{V11-10 Robot Assisted Inferior Vena Cavoscopy}

HM Abdul-Muhsin, M Marshall, A Chau, RJ Fowl, S Stroup, J L'esperance, M Woods, EP Castle

Mayo Clinic Arizona

United States

Introduction \& Objective: Conventional therapy of inferior vena cava tumor invasion in retroperitoneal malignancies involves caval resection with or without vascular grafting. Assessment of the extent of tumor invasion relies on preoperative (CT and MRI) and intraoperative (US) imaging. However, these imaging modalities cannot differentiate between bland and tumor thrombi. In this video we are presenting a technique for direct visualization of the inferior vena cava (IVC) lumen to assess for tumor invasion during a robot assisted retroperitoneal lymph node dissection for testicular cancer.

Materials and Methods: A 29 year old male patient with metastatic non-seminomatous mixed germ cell tumor was found to have a persistent metastatic retroperitoneal lymph node mass following four cycles of BEP chemotherapy. Preoperative imaging demonstrated a $6 \mathrm{~cm}$ interaortocaval mass with adjacent filling defect in the IVC. Preoperative imaging could not differentiate between tumor invasion vs bland thrombus.

Results: After induction of general anesthesia, the patient was placed in a modified lithotomy position. Standard sterile prep and drape was performed. Six robotic ports were inserted in the bilateral lower abdomen (two $12 \mathrm{~mm}$, four $8 \mathrm{~mm}$ ). He was placed in steep Trendelenburg. The robotic surgical system was docked. The small bowel was retracted cephalad and to the left upper quadrant. The retroperitoneal space was developed. Retroperitoneal lymph node dissection was started in a regular manner. The large mass was encountered in the interaortocaval region and was adherent to the IVC wall. It was dissected free from all attachments except the IVC wall. Proximal and distal control was obtained with vessel loops. A small venotomy was made on the IVC anterior wall just distal to the mass. A flexible cystoscope was introduced through one of the assistant ports and into the IVC lumen. Examination of the IVC showed multiple synechiae and vascular webs consistent with organized and recanalized bland thrombus with no evidence of tumor invasion. Given these findings no caval resection was needed. These webs were excised and the remaining part of the wall reconstructed, preserving more than $50 \%$ of the IVC circumference. The remaining part of the dissection was completed in the conventional manner. Operative time was $383 \mathrm{~min}$, EBL was $150 \mathrm{ml}$, and LOS was one day. Postoperatively patient had smooth recovery with no postoperative complication. Postoperative pathology was consistent with a bland thrombus. Follow up imaging showed preserved IVC circumference and no filling defects.

Conclusions: Direct visualization of the IVC lumen is feasible using conventional endoscopic instruments and robotic approach. This helps avoid a large venotomy or graft placement and completion of the retroperitoneal lymph node dissection in a minimally invasive manner.

V11-11 Marriage of Dyna-Computed Tomography Guidance During Laparoscopy for Needle Placement and Thermal Ablation: A Feasibility Study

EF Kelly, RJ Leveillee

Louisiana State University Shreveport Health United States 
Introduction \& Objective: We report the initial cases of small renal masses (SRM) treated by laparoscopic thermal ablation (TA), in a hybrid operating room, using a fixed angiographic system (DynaCT, Artis Q zeego system, Siemens Healthcare $\mathrm{GMbH}$, Forchheim, Germany). Patients who are not ideal candidates for partial nephrectomy are often offered percutaneous CT-guided TA performed by interventional radiologists (IR) with or without Urologic assistance. In special circumstances the tumor may be located too close to vital structures whereby collateral damage may occur. One option in these situations is instillation of isosmotic fluid to displace bowel (Hydrodissection). Alternatively, we propose laparoscopic exposure and simultaneous 3D imaging. The laparoscopic protocol is ideal for patients with anterior tumors located within $1 \mathrm{~cm}$ of surrounding vital tissue (excluding the liver). Coupling laparoscopic exposure with the Dyna-CT technology provides the advantage of enhanced precision of needle placement and decreased radiation exposure to the patient and staff as compared to conventional methods of targeting while avoiding damage to surrounding structures.

To demonstrate the indications and preliminary results of TA performed by coupling laparoscopic exposure with 3D CT targeting.

Materials and Methods: 3 patients with anterior SRM were chosen for inclusion. This was a proof of concept study. Patient 1 had an anterior renal mass and lymphoma treated previously by radiation and chemotherapy precluding laparoscopic nephrec-

\begin{tabular}{|l|c|c|c|c|}
\hline \multicolumn{5}{|c|}{ Procedural Results } \\
\hline & Patient A & Patient B & Patient C & Average \\
\hline Intra-operative Blopsy & Yes & Yes & Yes & - \\
\hline Pathology Result & Inconclusive & CCPST RCC & CC RCC & - \\
\hline Radiation Exposure (mGy) & 1007.82 & 432.16 & 696.09 & 712.02 \\
\hline Contrast (cc) & 75 & 75 & 0 & 50 \\
\hline \# of Probes (Cool-tip) & 1 & 1 & 2 & 1.33 \\
\hline \# of Cycles & 1 & 2 & 3 & 2 \\
\hline Intra-operative Complication(s) & 0 & 0 & 0 & 0 \\
\hline Post-Operative Complication(s) & 0 & 0 & 1 & 0.33 \\
\hline \multicolumn{1}{|c|}{ Clavien Grade } & - & - & 1 & - \\
\hline Follow-up (days) & 208 & 131 & 47 & 128.67 \\
\hline \multicolumn{1}{|c|}{ Key: PRT1 - CCPST - Clear Cell Papillary Subtype, CC-Clear Cell, RCC- Renal Cell Carcinoma }
\end{tabular}

tomy. Patient 2 (BMI 21) had virtually no perirenal fat and a bowel adherent mass. Patient 3 had severe coronary artery disease, on anticoagulation, and a tumor adjacent to the spleen, pancreas, and duodenum. Patient and tumor data were attained and peri/post-operative complication events were recorded.

Results: Please see tables below.

Conclusions: TA for SRM when indicated can provide alternatives for select patients when vital structures are in close proximity to the tumor ablation zone.

\section{VS12: ROBOTIC LOWER TRACT SURGERY FOR BENIGN DISEASE}

\author{
V12-1 Mullerian Duct abnormalities: A Robotic Approach \\ to Surgical Excision \\ P Sturch, (S Academic Research Group, A Samateh, S Mackie, \\ PD Rimington \\ Eastbourne District General Hospital; East Sussex Healthcare \\ NHS Trust \\ United Kingdom
}

Introduction \& Objective: Persistent Mullerian Duct Syndrome is a rare condition in which a phenotypically normal 46XY male presents with retained Mullerian duct structures. Management is focused in surgical removal of these structures for symptomatic relief and the risk potential malignant change.

We present a case of a 33 year old male presenting with unilateral renal agenesis, a large pelvic cyst and groin cyst and discuss the points of technique learned from the robotic assisted excision.

Materials and Methods: A 5-port was used and the cyst was approached posteriorly to mobilise it from the sigmoid colon.

The cyst was drained to facilitate dissection away from the rectum. Right ureter identified and preserved during lateral dissection. Left redundant ureter identified and clipped and cut.

Further lateral and posterior dissection progressed tot he pelvic floor. Anterior aspect of the cyst densely adherent to the bladder - carefully dissected off Cyst dissected off posterior urethra but urethra opened and repaired with anastomotic suture

Results: ASA I Total operation time: 195mins Estimated blood loss: 300ml Discharge: Day 2 post op with catheter and suc- cessful outpatient removal of catheter Post-operative complications: nil Transfusion: No

Histology: Benign Seminal vesicle cyst with Vas adherant to wall

Conclusions: A robotic-assisted approach to excision of Mullerian duct remnants facilitates the preservation of closely associated pelvic organs with the aim of preserving potency and continence.

\section{V12-2 Robotic Hutch Diverticulectomy \& Ureteric Re-} implant with Vas Preservation

\section{R Catterwell, C Brown, R Thurairaja}

King's College Hospital \& Guy's Hospital, London United Kingdom

Introduction \& Objective: This video presents the surgical technique of robotic hutch diverticulum resection involving ureteric re-implantation with preservation of the ipsilateral vas. Materials and Methods: A 25 year old male was referred with a 12 month history left iliac fossa pain associated with bladder distention. There was no history of urinary tract infection or voiding dysfunction. Imaging demonstrated a large bladder diverticulum intimately related to the distal left ureter. Video urodynamical studies were unremmarkable, inparticular there was no vesico-ureteric reflux. Cystoscopic evaluation confirmed a hutch type diverticulum containing the left ureteric orifice. 

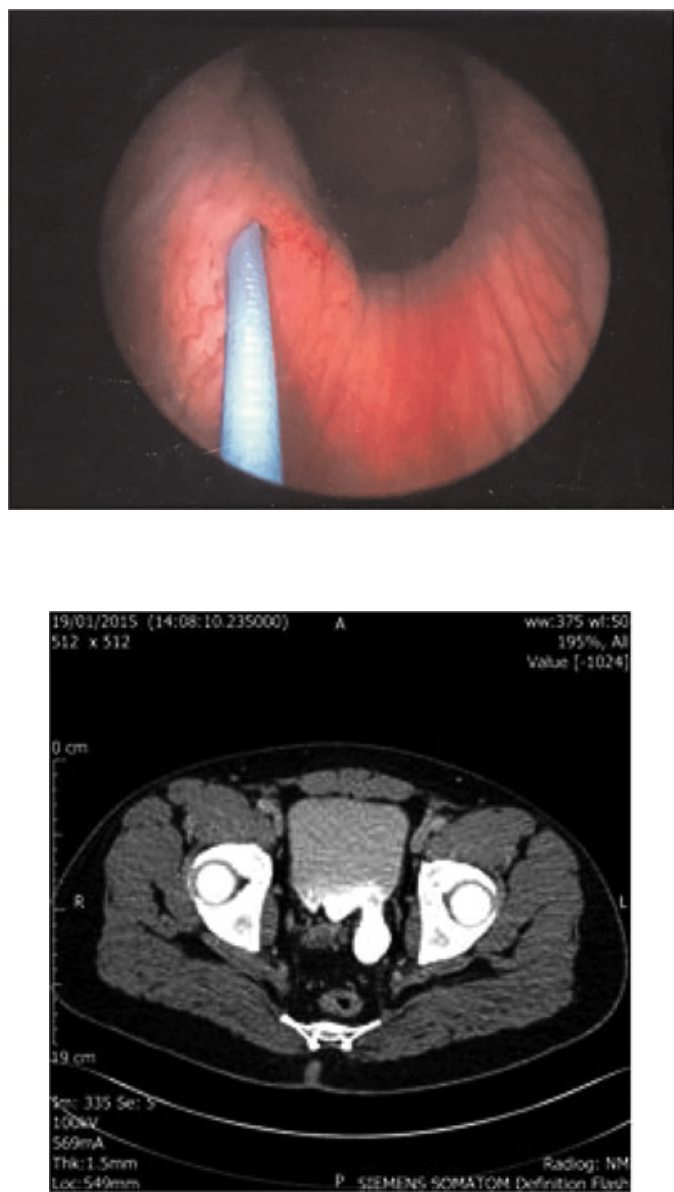

Robotic diverticulectomy was performed requiring ureteric reimplant. Considering the patients age and fertility concerns conservation of the left vas was desirable.

Results: The patient was pre-stented and placed in a $15-20^{\circ}$ Trendelenburg position. A six port, transperitoneal approach with four active robotic arms was utilized. The left ureter was identified at the pelvic brim and dissected distally to the bladder. The vas was identified and preserved. The bladder was released from the anterior peritoneum lateral to the medial umbilical ligament on the ipsilateral side only. The diverticular sac was identified and dissected from the surrounding tissue. The distal ureter was confirmed to be intimately related to the medial border of the diverticulum and diverticulectomy without ureteric reimplant not feasible. The distal ureter was divided with preservation of maximal ureteric length. Diverticulectomy was completed with a double layer continuous closure of the bladder defect. The bladder was then filled and a cystotomy created at the ipsilateral dome. The spatulated ureter was re-implanted utilizing two 4/0 PDS anchoring sutures and a 4/0 PDS watertight, continuous uretero-vesical anastomosis. A drain and urethral catheter were left insitu. Abdominal drain was removed day two post surgery and the patient discharged the following day following day. Ureteric stent was removed four weeks following the procedure. There has been complete resolution of the patient's symptoms.

Conclusions: Robotic diverticulectomy can be safely perfomed including cases where ureteric resection and re-impantation is required. In such patients preservation of the ipsilateral vas is possible.
V12-3 Robotic Assisted Laparoscopic Transvesical Repair of Rectovesical Fistula Following Open Radical Prostatectomy: Use of Allograft Human Dermis Interposition

E Capoccia, LA Deane, P Whelan

Rush University Medical Center

United States

Introduction \& Objective: Rectovesical fistula repair is a rare occurrence following radical prostatectomy. Several operative approaches to fistula repair have been described. In this video, we describe a novel technique using a robotic approach with allograft human dermis interposition.

Materials and Methods: We started the procedure by doing a cystoscopy and identifying the fistula. Bilateral ureteral stents were then placed. Ports were placed, each slightly more cephalad than traditional for robotic prostatectomy. The colon was retracted laterally using a dual blade atrial retractor. Dense scarring was encountered during the initial dissection between the bladder and rectum and we thus decided to open the bladder along the posterior midline. Three unidirectionally barbed stay sutures were placed to maintain access to the operative field. The fistula was circumferentially delineated with curved monopolar scissors. A plane was developed between the rectum and bladder with Potts scissors. The rectum was repaired with a 3-0 Vicryl continuous suture. The repair was tested using a colorectal anastomotic evaluator which showed a small leak which was reinforced with 40 Monocryl. Since the retrovesical space was densely scarred and we opted not to split the trigone, a free interposition graft was deemed necessary. We opted to us a small patch of allograft human dermis which was tacked in place using 4-0 Vicryl. The detrusor and bladder mucosa were then approximated in a tension free fashion over the graft with 3-0 unidirectionally barbed suture. The stay sutures were removed and the bladder was closed in two layers, again using 3-0 unidirectionally barbed suture.

Results: The JP drain was removed on postoperative day 1 and he was discharged on postoperative day 2. A cystogram was performed 3 weeks later showing a normal bladder without evidence of leak or communication with the rectum. The patients has good urinary continence. Colostomy takedown occurred 7 weeks postoperatively with excellent return of bowel function.

Conclusions: This video demonstrates the feasibility of robotic assisted rectovesical fistula repair. Since we did not split the trigone and the rectovesical space was densely scarred, an omental interposition was not feasible. We therefore opted to use allograft human dermis to reinforce the repair. In appropriately selected patients, a robotic approach to rectovesical fistula repair may allow a minimally invasive option for patients with this complex clinical entity, providing superb visibility of the pathology, the ability for precise suture placement and avoid the potential morbidities of an abdominal, perineal or parasacral incision, with or without muscular flap interposition.

\section{V12-4 Extravesical robotic-assisted bladder diverticu- lectomy with radical prostatectomy}

H Palayapalayam Ganapathi, G Ogaya-Pinies, E Hernandez, T Rogers, V Patel

Global Robotics Institute at Florida Hospital Celebration Health, University of Central Florida United States

Introduction \& Objective: Urinary bladder diverticula are often asymptomatic. Occasionally they need surgical correction if 
highly symptomatic or narrow mouthed. Robotic-assisted bladder diverticulectomy offers minimal access option with good water-tight bladder closure similar to open procedure. It is not uncommon to see large bladder diverticulum in patients evaluated for radical prostatectomy. We present our experience of robotic-assisted bladder diverticulectomy (RABD) concomitantly performed with robotic-assisted radical prostatectomy (RALP) in symptomatic patients.

Materials and Methods: During evaluation for prostate cancer, six men had associated symptomatic bladder diverticulum (moderate to severe AUA symptom score, recurrent urinary tract infection) and treated simultaneously. Standard six ports placed similar to RALP. Robot was side docked to allow intra-operative flexible cystoscopy. This helped to identify/ protect ureteric orifices and guided in dissection of diverticulum.

Results: All six extra-vesical RADB procedures completed successfully followed by RALP. There was no intra-operative complication or conversion to open procedure. One patient required ureteric stenting due to orifice close to diverticular neck. Mean operative time was 55 minutes for RABD part and 70 minutes for RALP part. Mean estimated blood loss was $175 \mathrm{ml}$. No significant complication related to surgery reported in follow up. Post operative cystogram showed no leak at bladder closure site / vesico-urethral anastomotic site and catheter removed in 7 days. None of the diverticular mucosa had malignant changes. At median follow up of 6 months, all six men regained urinary continence (using 0-1 pad) and AUA symptom score decreased to mild symptoms only.

Conclusions: Concomitant RABD and RALP are technically feasible and safe when indicated. Side docking with intra-operative flexible cystoscopy helps to protect ureteric orifices and guide in dissection.

V12-5 Purely intracorporeal robotic augmentation ileocystoplasty in ketamine cystitis

I Chen, J Lin, J Tsai, C Yu, T Wu, S Jeng

Kaohsiung Veterans General Hospital

Taiwan (Republic of China)

Introduction \& Objective: To present our initial experience of robotic augmentation ileocystoplasty for a patient with ketamine cystitis.

Materials and Methods: A 32-year-old lady, with a medical history of ketamine abuse for less than one year, presented with severe storage symptoms, dysuria and difficulty in voiding for more than 1 year, and was found to have ketamine cystitis at some local clinic. She had undergone hydrodistention 5 times, but her symptoms weren't ameliorated. Since conservative treatment failed and her maximal bladder capacity under anesthesia was only $90 \mathrm{~mL}$, augmentation enterocystoplasty was recommended. With the patient placed in the steep Trendelenburg position, five trocars were placed similar to radical cystectomy. The bladder was dropped first by dividing the urachus and medical umbilical ligament bilaterally; then entered the space of Retzius. An approximate $30-35 \mathrm{~cm}$ segment of bowel was harvested using Endo GIA staplers and ileal continuity was reestablished with a side-to-side anastomosis using the Endo GIA device. The staple lines of the chosen segment was excised on each end, and the segment was opened along the antimesenteric border. The sides of the segment were approximated to form a U-shape patch using 2-0 V-Loc sutures. Thereafter, the bladder was distended with saline and bivalved at the midsagittal line.
The bottom of the $\mathbf{U}$ was initially sutured to the posterior bladder neck, in a running continuous fashion with 2-0 V-Loc sutures, beginning posteriorly and ending anteriorly. The integrity of the completed augmentation was assured by irrigating the bladder via the Foley catheter, and eventually a pelvic drain was positioned.

Results: The operative time was 300 minutes, and the estimated blood loss was $10 \mathrm{~mL}$. There were no intraoperative or postoperative complications. The pelvic drain was removed on postoperative day 5 . No urine leakage was affirmed by cystography and the Foley catheter was removed on postoperative day 15.

Conclusions: Robot-assisted augmentation ileocystoplasty provides rapid postoperative convalescence and good aesthetics. It can be a good option for treating emerging ketamine cystitis.

V12-6 Robot assisted Boari flap ureteroneocystostomy using Politano-Leadbetter approach in a patient with distal stricture in duplex ureters

J Stolzenburg, H Do, A Dietel, R Ganzer, I Kyriazis, E Liatsikos, P Kallidonis, V Arthanareeswaran

Department of Urology, University of Patras, Greece Greece

Introduction \& Objective: Ureteral duplication is the most common renal abnormality, occurring in approximately $1 \%$ of the population. Treatment of strictures in duplex ureters is challenged by the altered anatomy of this congenital defect. In this video we present our experience in managing a patient with a stricture in the distal part of duplex ureters.

Materials and Methods: We demonstrate our robot assisted surgical technique of Boari flap ureteroneocystostomy using Politano-Leadbetter approach in a patient with distal stricture in duplex ureters.

Results: The duplex ureters are mobilized caudally until the site of stricture is identified and is transected proximal to the stricture making sure the distal ends of the intact ureters are well perfused and free from fibrosis. The bladder should be completely mobilized, including the contralateral side, to allow a tension free anastomosis. The appropriate dimensions of the flap are planned such that the cephalad side of the Boari flap has adequate width to wrap around the duplex ureters. The psoas hitch is then performed to bridge the length of resected ureters and to enable anatomical positioning of the ureters thereby preventing undue kinking. The duplex ureters are implanted replicating the technique described by Politano and Leadbetter for open surgical approach. A double J stent is placed in each ureter leaving enough length to allow the pigtail to be positioned in the bladder rather than the Boari flap. The bladder opening is closed using running 3-0 V-loc suture following tubularization of the Boari flap.

Conclusions: Robotic assisted Boari flap ureteroneocystostomy is feasible. The maneuverability and flexibility of the DaVinci instruments helps the surgeon to perform these fine steps precisely.

\section{V12-7 Robot Assisted Transplant Ureteroneocystostomy}

HM Abdul-Muhsin, K Gross, KL Stern, N Katariya, EP Castle

Mayo Clinic Arizona

United States

Introduction \& Objective: Anastomotic ureteral strictures are one of the most common complications after kidney transplantation. 
Early diagnosis and management is key in preserving graft function. Definite surgical intervention provides durable cure and has a high success rate. However, it is technically challenging and associated with more morbidity due the dense fibrotic tissue around the transplanted ureter. In this video we will describe our technique in performing robot assisted transplant ureteroneocystostomy with a brief description of outcomes in a case series

Materials and Methods: These procedures were performed through the collaboration of our urology and transplantation surgical teams. The procedure was performed through a transperitoneal approach in lithotomy position. The ports were placed in a similar location to robot assisted radical prostatectomy. This video will highlight the technical aspects of this procedure. These are: (1) Identification of the transplanted ureter. (2) Proximal and distal dissection of the ureter. (3) Techniques of approximation of the distal end of the transplanted ureter to the bladder (4) Urethrovesical anastomosis

Results: Location of the transplanted ureter is variable and can be identified after careful dissection of the paravesical space. This should not be confused with medial the umbilical ligament, vas difference and native ureter. Preoperative stenting and intermittent filling of the bladder during dissection can help identify the ureter. It is important to ensure complete excision of the stenotic ureter to prevent recurrence. Previous balder wall defect should be closed in a water tight fashion to prevent urine leak. A stented, spatulated, tension free, water tight anastomosis should be done with absorbable sutures. In order to bridge the gap between the distal end of the ureter and the bladder the same techniques that are used in non-transplant patients can still be utilized. This includes psoas hitch, release of the contralateral pedicle in addition to release of the transplanted kidney from the anterior abdominal wall. The plane between the transplanted kidney and the anterior abdominal wall is an avascular plane that can be relatively easily to develop. This technique was successfully implemented in three cases with complete resolution of obstruction, no anastomotic leakage and no complications. All patents were discharged on day 1 with a foley catheter for a week. A nephrostogram was performed at 7 days. The stent was removed in 4-6 weeks.

Conclusions: Robot assisted ureteroneocystostomy can be performed with acceptable initial results and the robotic platform provide benefits of early recovery

V12-8 Robotic Assisted Extravesical Ureteric Reimplantation with Psoas Hitch for Ureterovesical Junction Obstruction

\section{Ni, S Dave}

Western University

Canada

Introduction \& Objective: Obstructed primary megaureters are commonly encountered in pediatric urological practice and a significant proportion of these children are managed conservatively and discharged after stable ultrasound imaging and unequivocal MAG3 renograms over the first few years of life. Herein we present a young adult who presented with symptomatic uretero-vesical junction (UVJ) obstruction with no prior history of urological issues.

Materials and Methods: A 21-year-old female presented with recurrent left-sided flank pain and no documented urinary tract infections (UTI's) or stones. Work-up revealed an absent left ureteric jet on ultrasound and a distal hydroureter on CT scan. A retrograde left pyelogram showed dilated lower ureter with a tapered distal segment (rat-tail deformity) suggestive of a primary obstructed megaureter. MAG 3 renogram showed preserved left renal function and isotope hold-up in the left distal ureter. A trial of double-J stent placement led to resolution of left-sided flank pain but was associated with stent related bladder spasms. Surgical consent for left ureteric reimplantation was obtained after discussing the possible etiology of her symptoms and the small likelihood of persistent symptoms post-operatively.

Results: The patient underwent a robotic-assisted left ureteric reimplantation after excision of the distal adynamic segment. Given a short ureteric length despite mobilization, a psoas hitch had to be performed to allow extravesical ureteric reimplantation with a short detrusor tunnel. Since our patient did not have a prior history of UTI's, we chose to construct a relatively short detrusor tunnel accepting a risk of vesicoureteral reflux, while decreasing the risk of a recurrent UVJ obstruction. She was discharged on post-operative day three and her stent was removed at six weeks. At nine months' follow-up, she is pain free with no UTI's. Ultrasound shows resolution of left hydroureter and a normal left ureteric jet.

Conclusions: Obstructed megaureters can present with symptoms in adulthood. Robotic assisted dismembered extra vesical ureteric reimplantation with excision of the adynamic segment is an effective surgical option to treat these patients in a minimally invasive manner.

\section{V12-9 Combined Holmium Laser Enucleation of the Prostate and Robotic Diverticulectomy}

\section{KL Stern, WA Critchlow, MR Humphreys}

\section{Mayo Clinic Arizona}

United States

Introduction \& Objective: Chronic bladder outlet obstruction from prostatic hyperplasia is a known cause of bladder diverticula, which can lead to urinary retention, infection, stones, ureteral obstruction, and rupture. Small diverticula often require no treatment; larger or symptomatic diverticula are often managed by diverticulectomy via either an open, laparoscopic, or robot-assisted approach. We present a patient with chronic urinary retention on self-intermittent catheterization and a $7.5 \mathrm{~cm}$ by $8.5 \mathrm{~cm}$ by $8.5 \mathrm{~cm}$ bladder diverticulum that was treated with a combined holmium laser enucleation of the prostate and roboticassisted diverticulectomy. The preoperative prostate size was estimated at $116 \mathrm{~cm} 3$ with a significant median lobe.

Materials and Methods: We first performed a HoLEP in our standard trilobar fashion. The diverticular mouth was close, but not involving, the left ureteral orifice. After enucleation, the median lobe was able to be grasped and placed into the diverticulum. The lateral lobes were too large to place into the diverticulum cystoscopically, and were therefore placed into the bladder. Meticulous hemostasis was achieved. We then placed an open ended ureteral catheter into the left ureteral orifice. Continuous bladder irrigation was run until the point the bladder was subsequently opened. The patient was re-prepped and draped and the DaVinci Xi robot was docked and ports were placed using a standard robotic prostatectomy template. We traced the left ureter to its insertion into the bladder. We dissected the 
diverticulum off the pelvic sidewall and obturator fossa. We transected the diverticular neck and identified the lateral lobes in the bladder. These were grasped, removed, and placed into the diverticulum. The cystotomy was then closed in two layers and the diverticulum, containing the three prostate lobes, was placed in an EndoCatch bag and removed through the supraumbilical incision. The ureteral catheter was removed prior to extubation. Results: The patient was discharged on postoperative day two with a Foley catheter. His Jackson-Pratt drain was removed prior to discharge after a drain creatinine was found to be consistent with serum creatinine. His Foley catheter was removed on postoperative day ten after a cystogram demonstrated no contrast extravasation. Postoperative post void residual was negligible.

Conclusions: We present a minimally invasive approach to simultaneously treat a large bladder diverticulum and chronic bladder outlet obstruction secondary to significant prostatic hyperplasia under a single anesthetic with minimal postoperative morbidity.

\section{V12-10 Robotic Assisted Ileal Ureter and Ileal Bladder Augmentation}

TA Perkins, J Messer

University of Louisville

United States

Introduction \& Objective: Long-term effects of radiation can cause multiple urologic complications. We present a 32-year-old female with history of stage IIIB cervical cancer who developed a right ureteral stricture near the level of the iliac vessels, as well as a compromised bladder capacity $(300 \mathrm{~mL}$ under anesthesia at $40 \mathrm{~cm}$ of water), secondary to radiation.

Materials and Methods: We present our technique for roboticassisted ileal ureter reconstruction and ileal bladder augmentation. We utilize a $30 \mathrm{~cm}$ segment of ileum to create the ileal bladder augmentation as well as the ileal ureter.

Results: Total operative time was 367 minutes with estimated blood loss of $200 \mathrm{ml}$. The patient was on an enhanced recovery pathway. Regular diet was resumed on postoperative day 4 . Patient was discharged home on postoperative day 6 with foley catheter and ureteral stent in place. Foley catheter was removed on postoperative day 15 after obtaining a normal cystogram. Ureteral stent was removed 7 weeks following surgery after obtaining a normal retrograde pyelogram.

Conclusions: Management of radiation induced ureteral strictures with concomitant radiation cystitis can be complex. Due to potential compromise of bladder capacity, standard operative reconstruction may be difficult. A robotic-assisted ileal bladder augmentation with ileal ureter reconstruction may be a good option for select patients.

V12-11 New technologies for old procedures when FireFly technologie improves Robotic Bladder Diverticulectomy

F Vedovo, B de Concilio, G Zeccolini, M Justich, A Celia

Azienda Sanitaria Universitaria Integrata di Trieste Italy

Introduction \& Objective: Several techniques have been described to aid in the intra-operative identification of the bladder diverticula. The video shows the peculiar advantage of using Firefly Fluorescence Imaging da Vinci System during bladder diverticula detection and dissection.

Materials and Methods: Patient is placed in the lithotomic position and $30^{\circ}$ Trendelenburg. Supraumbilical camera trocar is inserted with the Hasson technique. We use a four-arm robotic approach and a 5 to 6 ports placement consisting of: one 8-mm camera port, three 8-mm robotic ports and one to two assistant ports. The robotic ports run parallel at $14 \mathrm{~cm}$ from the pubic bone. Pneumoperitoneum is established at $12 \mathrm{Hg} \mathrm{mm}$. The bladder is accessed via a transperitoneal route. We perform a flexible cystoscopy with the Firefly Fluorescence Imaging System on for the diverticulum detection. The peritoneum over the bladder is then incised to expose the diverticulum. We use this near-infrared technology also as a guide in the diverticulum dissection. Using sharp and blunt dissection, the diverticulum is resected to its neck. Completion of diverticulectomy and hydraulic tightness test. Drainage placement in the Retzius space and peritoneum reconstruction.

Results: Several approaches have been described for intra-operative diverticulum identification and its dissections: Parra used a cystoscopic transillumination of diverticulum; Das proposed the use of a Foley $50 \mathrm{~mL}$ balloon inserted in the diverticulum, while Nadler used a balloon catheter, made from a surgical glove, placed in the diverticulum and bloated with $180 \mathrm{cc}$ saline solution. We present our technique in which transperitoneal bladder diverticulectomy is performed under the Firefly guidance that provide real-time, image-guided identification of key anatomical landmarks.

Conclusions: In our experience, intra-operative use of Firefly Fluorescence Imaging da Vinci System makes identification and dissection of the diverticulum rapid, safe and effective with no additional cost, even in disadvantageous anatomic conditions such as lateral-posterior diverticula.

V12-12 Intraureteral indocyanine green (ICG) application for ureter identification during robotic surgery

I Tufek, OB Argun, MB Tuna, T Doganca, C Obek, S Keskin, AR Kural

Acibadem University, School of Medicine, Department of Urology, Istanbul, Turkey

Turkey

Introduction \& Objective: In this video intraureteral indocyanine green (ICG) application during robotic ureteral reconstruction is presented.

Materials and Methods: First patient had retroperitoneal fibrosis. PET-CT revealed high SUV value of the tissue surrounding right ureter. There was right grade II hydronephrosis on retrograde pyelography. Robotic ureterolysis was planned for ureteral release and histopathological evaluation of surrounding tissue. Open-end catheter was inserted. The procedure was performed with daVinci Xi system in modified left lateral decubitus position. Following colon medialization, ureter was identified. ICG dissolved in $10 \mathrm{ml}$. distilled water was applied via open-end catheter and open-end catheter clamped. Fire-fly mode was operated and ureteral segments captured inside the fibrotic tissue was delineated. Ureter was freed. Fibrotic tissue around the ureter was excised ad sent for histopathological evaluation. Colonic serosa was used for ureteral intraperitonealization. A $4.8 \mathrm{~F} \mathrm{JJ}$ catheter was placed. 
Second patient had an unsuccessful right ureterorenosopy at another center. Flexible ureterorenoscopy revealed right upper ureteral stricture and submucosal stone fragments. Robotic ureteroureteral anastomosis was planned. The procedure was performed with daVinci Xi system in the same position. Colon was medialized and right kidney was identified. Ureter was freed. Following release of the periureteric fibrotic tissue, ICG was applied via open-end catheter. Fire-fly mode was operated and ureteral stricture identified. Periureteral stone at the level of the ureteral stricture was removed. Following ureteral transection, proximal stenotic segment was resected and the kidney mobilized. Then distal stenotic segment was resected. Following ureteral spatulation, ureteroureteral anastomosis was performed with $4 / 0$ polyglactin. Open-end catheter was used to confirm the patency of the ureteral lumen during anastomosis. After completion of the anastomosis, ureter was retroperitonealised. Retrograde $4.8 \mathrm{~F} \mathrm{JJ}$ catheter was inserted.

Results: Histopathological evaluation of the fibrotic tissue in first patient revealed no malignancy. Postoperative period was uneventful in both patients. In the first patient JJ catheter was removed at postoperative $4^{\text {th }}$ week. Postoperative $6^{\text {th }}$ month PET CT revealed decreased SUV value of the soft tissue around the ureter. JJ catheter was removed at postoperative $6^{\text {th }}$ week in the second patient. Right kidney was normal on postoperative $2^{\text {nd }}$ month CT pyelography.

Conclusions: Intraureteral ICG administration facilitates identification of ureter during robotic ureteral reconstruction.

V12-13 Robotic Salvage Prostatectomy for Radiation Induced Posterior Urethral Stenosis

D Volkin, AC Weinberg, L Zhao

NYU Department of Urology

United States

Introduction \& Objective: Posterior urethral stenosis after radiation therapy is a difficult clinical problem, with endoscopic modalities offering limited success. Historically, treatment involved open reconstruction, which is associated with significant morbidity and risk of incontinence. We present a novel surgical technique to treat patients with symptomatic posterior urethral stenosis using a space of Retzius-sparing approach.

Materials and Methods: A 60-year-old man with a history of brachytherapy and external beam radiation therapy for prostate cancer developed a posterior urethral stenosis that was refractory to endoscopic therapy. He had severe obstructive lower urinary tract symptoms and was referred to our institution for further management. He elected to undergo robotic reconstruction. In order to maximize continence preservation, we chose a space of Retzius-sparing approach. Intraoperatively, a $2 \mathrm{~cm}$ posterior urethral stenosis was excised circumferentially and an anastomosis was created between the bladder neck and the membranous urethra.

Results: The surgery was successfully completed with no intraoperative or immediate postoperative complications. The patient was discharged on the first postoperative day. His Foley catheter was removed 14 days after surgery and the suprapubic tube was removed at 21 days. At 2 month follow up, the patient had resolution of his preoperative lower urinary tract symptoms and had 1 pad per day incontinence.

Conclusions: Robotic salvage prostatectomy with a space of Retzius sparing approach is a viable option for treating posterior urethral stenosis. Long-term outcomes in a larger cohort of patients need to be elucidated.

\section{V12-14 Management of Complex Urinary Fistula}

\section{Ahmed, L DiGiorgio}

Hackensack University Medical Center United States

Introduction \& Objective: Urinary fistula after gyencologic surgery is a difficult problem with varied outcomes.

Materials and Methods: We propose our robotic technique with regenerative matrix as a treatment option.

Results: We demonstrate a novel succesful surgical technique for this complicated problem.

Conclusions: Robotic vesicovaginal fistula repair with a regenerative matrix is a good option for managing this disease process.

\section{VS13: ROBOTIC UPPER TRACT SURGERY FOR BENIGN DISEASE}

V13-1 Robotic Pyelolithotomy For The Intact Removal of a Complete Staghorn Stone: A Feasible Approach Even After Previous Open Pyelolithotomy.

A Chow, R Yong, P Tsambarlis, LA Deane

Rush University Medical Center

United States

Introduction \& Objective: Complete Staghorn stone removal can pose a challenge to the endourologist. We describe a robotic approach for complete removal of a recurrent staghorn stone after previous open pyelolithtomy.
Materials and Methods: The patient is placed in a left modified flank position with mild flexion. 4 ports were placed: $12 \mathrm{~mm}$ port for camera paramedian to the left of the midline, $8 \mathrm{~mm}$ robotic port left lower quadrant at the level of the umbilicus, $8 \mathrm{~mm}$ robotic port midclavicular line 2 finger breaths below the costal margin, $12 \mathrm{~mm}$ Airseal assistant port paramedian infraumbilical. The white line of Toldt was incised and the colon was mobilized medially. Anterior Gerota's fascia was opened and tacked to the lateral abdominal wall exposing renal pelvis and parenchyma. Intraoperative ultrasound confirmed the underlying stone. The ureter was identified and followed proximally to the renal pelvis. Dissection was performed through scar tissue at the renal pelvis. 
A V-shaped Gil-Vernet pyelolithotomy incision was made and extended onto the superior and inferior caliceal infundibula. Da Vinci Prograsp forceps were used to manipulate the stone out of the renal pelvis. Pressure was held on the renal pelvis with minilaps for hemostasis. The collecting system was inspected and irrigated using the robotic lens. The pyelotomy was closed with 40 Monocryl suture on a TF needle in 2 lengths of suture, superiorly and inferiorly. The sutures were tied in tension-free watertight mucosal-to-mucosal apposition fashion. Gerota's fascia was closed over the renal pelvis. The colon was tacked to the white line of Toldt. The specimen was retrieved through a mini-Pfannenstiehl incision via specimen bag. Flexible cystoscopy was performed for retrograde pyelogram and insertion of a double $\mathrm{J}$ stent. Results: We used this approach for complete intact removal of a staghorn stone following open pyelolithotomy. The patient was discharged on postoperative day 1 and seen in clinic 5 weeks later for stent removal.

Conclusions: Robotic pyelolithotomy is a minimally invasive alternative that can be offered to patients with complete staghorn stones even after major open stone surgery. It is necessary to have pre-operative imaging to understand the calyceal anatomy to free the stone. Complete visualization of calyces to the papillae after stone removal is possible and the renal pelvis can be closed in an efficient manner.

V13-2 Technique of robot-assisted laparoscopic upper moiety partial nephrectomy in the management of complicated duplex collecting systems

D Yong, K Png

National Healthcare Group - Tan Tock Seng Hospital Singapore

Introduction \& Objective: Upper moiety partial nephrectomy may be indicated in the management of poor-functioning moiety due to repeated infection. This can be achieved with minimally invasive techniques using pure laparoscopy or robotic assistance, depending on the complexity of anatomy.

Materials and Methods: Our patient had a complete duplex collecting system and underwent robot assisted laparoscopic upper moiety partial nephrectomy. Her history of presentation and preoperative imaging are included in this video. The techniques used to ensure adequate hemostasis were also demonstrated.

Results: Partial nephrectomies of the upper moiety were performed in two separate patients. Estimated blood loss was $100 \mathrm{mls}$ and console time was 255 mins for the robot-assisted case. There were no intraoperative complications and the patient was discharged on post op day two. However she presented 6 weeks later with a small collection in the resection bed requiring percutaneous aspiration.

Conclusions: Argon plasma coagulation, selective vascular clamping or suture over-sewing are useful adjuncts to hemostasis. Upper moiety partial nephrectomy can be performed with either laparoscopic or robotic assistance, depending on the complexity of the anatomy.

V13-3 Robotic partial nephrectomy for renal congenital anomalies in adults: tips, tricks and outcomes

M Sourial, N François, C Miller, DL Zynger, G Box

The Ohio State University Wexner Medical Center United States
Introduction \& Objective: Although they usually present in childhood, renal congenital anomalies may present in adulthood. The literature is sparse on outcomes of adult patients treated with robotic partial nephrectomy for benign congenital renal anomalies.

Materials and Methods: Retrospective chart review of patients who underwent robotic partial nephrectomy for symptomatic benign renal congenital anomalies between 2010 and 2016 . Demographic, perioperative outcomes, and resolution of presenting symptom was abstracted from patient charts.

Results: Six patients underwent a robotic partial nephrectomy for various benign renal congenital anomalies: atrophic upper pole/stone (2), ectopic ureter (2), calyceal diverticulum (2). Median (IQR) age was 35 years $(33$ - 37). Median (IQR) operating time and blood loss was 247 minutes $(218$ - 271), and $125 \mathrm{~mL}(100-187.5)$, respectively. Median (IQR) length of stay was 36 hours $(35-38.5)$. The presenting symptom [pain (4), infection (1), and incontinence (1)] resolved in all patients after the procedure, with full preservation of renal function. One patient had a postoperative meatal stenosis that required a meatotomy.

Conclusions: Robotic partial nephrectomy for symptomatic renal congenital anomalies is a safe and effective procedure at relieving the presenting symptom.

\section{V13-4 Robot-assisted laparoscopic extravascular stent for nutcracker syndrome}

I Sorokin, BA Johnson, J Nelson, J Rectenwald, J Cadeddu

UT Southwestern

United States

Introduction \& Objective: Minimally invasive treatment options are a safe and feasible alternative for treatment of nutcracker syndrome. Endovascular stenting has shown promising long-term resolution of symptoms but can be complicated by stent migration or thrombosis. Laparoscopic extravascular stent placement has shown promising results with the potential to avoid these complications. We report the first case of extravascular stent placement using the robotic approach for the treatment of nutcracker syndrome.

Materials and Methods: The da Vinci Si robot system was used to perform the procedure. The left renal vein was completely dissected out from its origin on the IVC to the lateral insertion of the gonadal vein. A $2 \mathrm{~cm}$ Polytetrafluoroethylene (ePTFE) externally supported (ringed) vascular graft was split longitudinally and wrapped around the left renal vein. The edges of the split graft were re-approximated with interrupted 4-0 Vicryl sutures. The graft was also secured to the adventitia of the aorta to prevent migration.

Results: The estimated blood loss was minimal and total procedure time was 118 minutes. Post-operative course was uncomplicated and patient noted that her pain was relieved on day 1. Relieving the LRV compression also resolved her symptoms of pelvic congestion and no further procedures on the gonadal vein were required.

Conclusions: Extravascular stenting of the left renal vein appears to be a safe and effective alternative treatment for nutcracker syndrome. It avoids the potential high risk complications of venous reconstruction or migration/thrombosis related to endovascular stenting. The robotic approach offers several advantages over laparoscopy with improved visualization and precise suturing around critical sutures. 
V13-5 Surgical Management of Chronic Flank Pain in Autosomal Dominant Polycystic Kidney Disease: Robotic Cyst Decortication and Renal Denervation

B Norris, J Borin

NYUMC

United States

Introduction \& Objective: Patients with autosomal dominant polycystic kidney disease (ADPKD) often suffer with chronic pain in the back, abdomen and flank, abdominal fullness and early satiety. The pain can be quite disabling and often impacts activities of daily living and quality of life. The pain arises from cystic compression of the renal capsule and parenchyma; signals are transmitted through afferent sensory nerves surrounding the renal artery. Renal cyst decortication and renal denervation is often reserved for patients who have failed other conservative measures and had previously had success with renal cyst aspiration. Renal cyst decortication has been well studied and it appears to have lasting pain control for up to 10 years afterwards. Our objective was to demonstrate the use of the robot in bilateral renal cyst decortication and renal denervation in the management of chronic pain from ADPKD. We were able to perform a bilateral procedure with minimal repositioning of the patient.

Materials and Methods: After general anesthesia, the patient was placed in a modified left lateral decubitus position. A small gel roll was placed under his left flank, a larger gel roll under the right flank to facilitate transitioning from the right side to the left side. Ports were placed midline to allow bilateral dissection. The $\mathrm{Da}$ Vinci Xi robot was docked. The right renal hilum was dissected out and renal denervation performed by skeletonizing the right renal artery and dissecting any medial attachments from the hilum to the lower pole. Gerota's fascia was removed and all visible cysts were then aspirated and decorticated, taking care to avoid spilling any cyst fluid. The large gel roll was then removed from the right flank and the table rotated to the right. Using the same midline ports and an additional left lower quadrant port, left sided denervation and cyst decortication was performed. There were 40 cysts decorticated on the right and 28 on the left.

Results: The operating time was 5 hours. Estimated blood loss was $50 \mathrm{mls}$. The patient was discharged home day on postoperative day one. There were no postoperative complications and he reported immediate resolution of his chronic flank pain and continues to be pain free 2 months post operatively.

Conclusions: Bilateral robotic assisted cyst decortication and renal denervation can be safely and effectively employed in the management of chronic pain in autosomal dominant polycystic kidney disease when conservative measures have failed.

V13-6 Cutting for Stones: Robotic assisted Nephrolithotomy and Pyelolithotomy

B Norris, J Borin

NYUMC

United States

Introduction \& Objective: Prior to the laparoscopic and robotic era, patients presenting with large renal and ureteral calculi that had failed, or were not suitable for endoscopic or percutaneous measures, were committed to open stone surgery. With the advent of minimally invasive surgery, robotic assisted stone surgery is emerging as an alternative to percutaneous nephrolithotomy (PCNL). Laparoscopic and robotic pyelolithotomy and nephrolithotomy have been shown to be an effective approach with less bleeding and sepsis compared to PCNL for large renal calculi. Our objective was to demonstrate the surgical technique of robotic assisted nephrolithotomy and pyelolithotomy in the management of nephrolithiasis.

Materials and Methods: Robotic assisted stone surgery was performed via a transperitoneal approach by a single surgeon on 3 patients. Robotic pyelolithotomy was performed in 2 patients and a robotic nephrolithotomy performed for an excluded calyceal diverticulum containing multiple stones.

Results: Patient 1 had a large staghorn calculus. Patient 2 had a large $2.6 \times 1.9 \mathrm{~cm}$ calculus in the right renal pelvis with hydronephrosis. Patient 3 had a calyceal diverticulum containing multiple small stones and had failed an endoscopic and percutaneous approach. All patients had an uncomplicated postoperative course and were discharged home day 1 .

Conclusions: Robotic assisted pyelolithotomy and nephrolithotomy can be safely used in the management of renal calculi as an alternative to PCNL or when PCNL has previously failed.

\section{V13-7 Early Robotic Repair of a Catastrophic Iatrogenic Ureteral Injury}

A Radtke, K Jacobsohn

Medical College of Wisconsin

United States

Introduction \& Objective: Traditional conservative management of ureteral injury includes stent placement, nephrostomy tube, and/or delayed repair. These approaches, however, are not always possible. We present a case which demonstrates the feasibility of early robotic repair of a catastrophic iatrogenic ureteral injury.

Materials and Methods: A 73-year-old male was transferred to our institution 24 hours after reported ureteral avulsion during a ureteroscopy for stone disease. The injury could not be stented and attempts at a nephrostomy tube failed. We performed our own retrograde pyelogram and identified what appeared to be a devastating injury to the mid ureter. We were fortunate to pass a wire across the injuries, but given the extent of the damage, elected to perform immediate robotic primary repair. A 4-port laparoscopic robotic approach was taken to identify the left kidney and subsequently the left ureter. Gentle dissection around the ureter identified two separate full thickness injuries. The ureter was repaired using running absorbable suture in a tension free, watertight fashion. A drain was placed in the vicinity of the repair bed.

Results: We were able to successfully identify and repair two separate full thickness injuries to the mid left ureter. The patient was ultimately discharged on postoperative day 3 with a stent and foley catheter. He underwent cystoscopy with stent removal and retrograde pyelogram 4 weeks after surgery, which demonstrated a patent ureter. Renal ultrasound at 6 weeks demonstrated no hydronephrosis, and the patient remains asymptomatic.

Conclusions: Early robotic repair is a safe and effective management of catastrophic iatrogenic ureteral injury. The approach and principles of reconstruction are similar to already established techniques. The initial increased risk of proceeding to a more invasive treatment modality is likely offset by a potentially decreased rate of ureteral stricture. Further investigation of this approach as well as evaluation of long term outcomes will be critical to establishing this as a standard option for management of iatrogenic ureteral injuries. 
V13-8 Robotic Single Port Pielolithotomy: an alternative technique for stone burden calculi

LF Sávio, J DA CRUZ, F Mota Filho, R Sakata, M Nunes, R Maia, C PASSEROTTI

\section{GERMAN HOSPITAL OSWALDO CRUZ, SAO PAULO, BRAZIL \\ Brazil}

Introduction \& Objective: Lately different minimally invasive techniques have demonstrated comparable results for management of renal stones. Robotic-assisted single-port laparoendoscopic pielolithotomy represents one step beyond minimally invasive surgery. The aim of this study is to demonstrate the stepby-step components for completing a successful single port pielolithotomy.

Materials and Methods: We present a case of a 50-year-old man with symptomatic renal calculi, who presented with lower abdominal pain and hematuria. His past medical history and physical examination was otherwise not significant. CT-scan revealed a $2 \mathrm{~cm}$ calculi in the renal pelvis, a $1.2 \mathrm{~cm}$ stone in the inferior calyx and two $3 \mathrm{~mm}$ stones in the superior calyxes. His laboratory exams were in the normal range. All main current options were offered to him including percutaneous nephrolithotripsy, laparoscopic pielolithotomy, robotic-assisted pielolithotomy and single-port robotic-assisted pielolithotomy. The patient chose the single port technique. As far as the authors know, this is the first description of a single-port technique for the treatment of multiple and large calculi.

Results: The patient was placed in 60 degrees over his right side. He was firmly attached to the table. A single umbilical $2 \mathrm{~cm}$ incision was performed with Hasson's technique and a Gelport ${ }^{\circledR}$ was placed. Cavity inspection for adhesions was made and the imaginary line between the umbilical incision and the kidney was used for robot docking. The first two robotic arms were placed and the robot was placed over the left shoulder for placing the remaining arms. The Mattox maneuver was performed for exposing the left kidney and the left ureter was identified and dissected until the renal pelvis. A hitch-stitch was made from the abdominal wall, through the kidney and out of the abdominal wall. A two-centimeter incision was made in the renal pelvis. Using the robotic arms, the main calculi was extracted without difficult. After clearing the pelvis, a flexible cystoscope was passed through the trocar removing the remaining fragments.

Conclusions: We describe a new option for large and multiple renal calculi treatment. It seems a viable and safe option with apparent lower risks of bleeding and for better parenchymal sparring. Further studies may unveil other indications for this approach and its rightful place as a therapeutically tool.

\section{V13-9 Robotic Assisted Left Renal Vein Transposition}

HM Abdul-Muhsin, A Chau, C Martin, S Money, EP Castle

Mayo Clinic Arizona

United States

Introduction \& Objective: We present a video of a robotic assisted left renal vein transposition to treat a patient with renal nutcracker syndrome (RNS).

Materials and Methods: A healthy 19 year old female with a BMI $19 \mathrm{~kg} / \mathrm{m}^{2}$ presented with a 2 year history abdominal pain without a discernable etiology. She underwent an extensive work up including abdominal duplex ultrasound (US), computed tomography (CT), magnetic resonance imaging, endoscopy, and psychiatric evaluation due to discordance in her symptomatology and radiographic findings. A follow up CT later demonstrated worsening compression of her left renal vein by the superior mesenteric artery (SMA). A left renal venogram demonstrated a $70 \%$ stenosis, and a pressure gradient of $3 \mathrm{mmHg}$. Multiple large venous lumbar collaterals were also seen. Intravascular US identified vascular webs and confirmed chronic venous compression. Results: After induction of general anesthesia, the patient was placed in a modified lithotomy position. Standard sterile prep and drape was performed. Six robotic ports were inserted in the bilateral lower abdomen (two $12 \mathrm{~mm}$, four $8 \mathrm{~mm}$ ). She was placed in steep Trendelenburg. The robotic surgical system was docked and connected to the ports. The small bowel was retracted cephalad and to the left upper quadrant. The base of the small bowel mesentery was incised. The retroperitoneal space was opened between the SMA and infrarenal abdominal aorta. The left renal vein was mobilized. The left adrenal and gonadal veins were divided with a vessel sealing device and a vascular stapler respectively. Vascular control of the suprarenal inferior vena cava (IVC), infrarenal IVC, and right renal vein were obtained with vessel loops. Lumbar veins were ligated and divided selectively. Intravenous heparin was given. The left renal vein was clamped near the hilum with a bulldog clamp. The renal vein was then transected at the confluence with the IVC with a small cuff of the IVC and closed with 5-0 prolene suture in running fashion. Vascular webs were excised from the intima of the renal vein. The left renal vein was then re-anastomosed to the IVC more distally in a tension free end-to-side fashion with a running 5-0 prolene suture. The patient was discharged on post-operative day 1. At her one week postoperative visit, she demonstrated a full recovery from her surgery and reported significant improvement in her abdominal pain.

Conclusions: Robotic assisted left renal vein transposition is a feasible surgical technique to treat patients with this condition.

\section{V13-10 Robot Assisted Partial Nephrectomies - 12 Tumors, 1 Kidney}

KL Stern, G Grimbsy, C Chen, T Ho, EP Castle

Mayo Clinic Arizona

United States

Introduction \& Objective: An angiomyolipoma is a benign renal mass composed of blood vessels, smooth muscle and fat. Angiomyolipomas occur in up to $60 \%$ of patients with tuberous sclerosis, an autosomal dominant disease caused by a mutation in the TSC gene on chromosome 9 or chromosome 16. The angiomyolipomas in patients with tuberous sclerosis are often multiple and bilateral. Other manifestations of the disease include hamartomas, adenoma sebaceum, lymphangioleiomyomatosis and cardiac rhabdomyomas. The risk of hemorrhage with angiomyolipomas increases with tumors larger than 4 centimeters. Elective treatment includes arterial embolization, ablation or nephron-sparing excision, if possible. Everolimus, a mTOR inhibitor, is also FDA approved to shrink angiomyolipomas in patients with tuberous sclerosis.

Materials and Methods: An 18 year-old female was referred to our clinic for evaluation of multiple bilateral angiomyolipomas. She had been on Everolimus for several years and had undergone several unsuccessful embolizations at an outside facility. MRI of the abdomen showed more than 30 angiomyolipomas in the left kidney and more than 20 in the right kidney. The largest tumors 
in the left kidney measure 4 centimeters, 2.7 centimeters, and 2 centimeters. A renal scan indicated relatively equal kidney function. The patient went to the operating room for robot assisted left partial nephrectomies. Twelve tumors were enucleated without hilar clamping. The 2 largest tumor defects were closed with a sliding clip technique renorrhaphy. Hemostatic matrix was placed for additional hemostasis. It should be noted that the enucleations went well without much bleeding and we speculate that the Everolimus may have helped with this, although it was held for 10 days prior to surgery. Total operative time was 3 hours and total robot console time was 2.5 hours.

Results: Twelve tumors were excised with the largest measuring almost 5 centimeters. There were no intraoperative or postoperative complications. Her hemoglobin nadir was $10.2 \mathrm{~g} / \mathrm{dL}$ from a preoperative value of $11.7 \mathrm{~g} / \mathrm{dL}$. Postoperatively her creatinine stayed stable at $0.6 \mathrm{mg} / \mathrm{dL}$. She was discharged home on postoperative day 2 .

Conclusions: Robot partial nephrectomy for multiple angiomyolipomas is a feasible, safe, and effective option in patients with tuberous sclerosis who wish to preserve renal function while pursuing surgical management.

\section{V13-11 Robotic-assisted Laparoscopic Calyceal Diverti- culectomy}

N Jung, H Smith, D Turner, J Class, D Butler, C Keel, A Singh

University of TN College of Medicine Chattanooga/Erlanger

Health System

United States

Introduction \& Objective: A calyceal diverticulum is a cystic cavity within the kidney that is lined by transitional epithelium and communicates with a calyx, or less commonly, with the renal pelvis. A calyceal diverticulum forms as a result of the failure of the degeneration of the ureteric bud. $0.2-0.5 \%$ are congenital; $40 \%$ are associated with calculi. This is a video demonstrating a robot assisted laparoscopic calyceal diverticulectomy.

Materials and Methods: This video demonstrates two cases of robotic calyceal diverticulectomy the first patient is a 19 year-old caucasian female and the second patient is a 25 year-old caucasian female. Who both had sudden onset of flank pain and abdominal pain. Initial work up was consistent with infection; however on repeat imaging, both patients were found to have a calyceal diverticulum. Both patients underwent a robot assisted laparoscopic diverticulectomy. Initial dissection was similar to a partial nephrectomy with mobilization of the large bowel and exposure of the renal hilum. The diverticulum is then incised, drained, and excised to the infundibulum to prevent recurrence. Any remaining urothelium was fulgurated.

Results: Both patients who underwent a robot assisted laparoscopic calyceal diverticulectomy are complication and re-admission free to date.

Conclusions: Robotic-assisted laparoscopic calyceal diverticulectomy is a feasible and safe option in the management of large persistent calyceal diverticulum.

V13-12 Robot Assisted Ureteroureterostomy for Management of Recurrent Proximal Ureteral Stricture

WR Lai, R Thomas

Department of Urology, Tulane University School of Medicine United States
Introduction \& Objective: Surgical management of recurrent proximal ureteral strictures has historically been performed with open surgery with associated incisional morbidity. We have previously demonstrated the feasibility and durability of robotic ureteroureterostomy for short length proximal ureteral strictures. In the case of recurrent ureteral stricture from open surgery, this can be salvaged with robotic surgery. In this video, we present a case of a robotic redo ureteroureterostomy for a recurrent proximal ureteral stricture.

Materials and Methods: The case is a 39 year old male who previously underwent an open left proximal ureteroureterostomy one decade ago at an outside facility for a proximal ureteral stricture secondary to recurrent ureterolithiasis. He developed recurrent left flank pain and was noted on CT imaging to have left hydronephrosis, and nuclear medicine renogram with increased drainage time. A retrograde pyelogram showed a recurrent left proximal ureteral stricture surrounded by multiple metal surgical clips. He elected for a left robotic redo ureteroureterostomy. During ureterolysis, the old metal clips from prior surgery were noted to be tightly adhered to periureteral tissue and removed. The strictured segment was incised longitudinally to expose the posterior ureteral plate. Surrounding fibrotic tissue was excised from the ureter. Relaxing incisions were performed on the posterior ureteral plate in anticipation of performing a modified Heineke-Miculicz closure. Nephrolysis, pelviolysis, and downward nephropexy were performed to achieve a tension-free ureteral anastomosis. The ureteral stent was placed in retrograde manner. These steps are illustrated in further detail in the video. Results: Length of the proximal ureteral stricture was $3 \mathrm{~cm}$. Six metal clips were removed. Total operative time was $352 \mathrm{~min}$, including cystoscopy and anesthesia times. Estimated blood loss was $25 \mathrm{ml}$. There were no intraoperative complications. Foley catheter was removed on post-op day 2 . He was discharged home on post-op day 2 . The surgical drain was removed 1 week postop. The ureteral stent was removed 8 weeks post-op. He continues to do well with no recurrence of left flank pain.

Conclusions: A redo proximal ureteroureterostomy procedure can be performed safely with the robotic platform. Keys to the success of this procedure include maintaining an adequate vascular supply to the ureter, removing foreign bodies which contributed to the fibrosis of the recurrent stricture, and performing a downward nephropexy as indicated to ensure a tension-free anastomosis.

V13-13 Expanding the spectrum of Robotic Pyeloplasty: bilateral ureteropelvic junction obstruction with multiple large calyceal stones on one side and crossing vessels in the other

A Mallya, I Banerjee, S Arakere, FA Zafar, A Mandhani, RK Ahlawat

Fortis Escorts Kidney and Urology Institute India

Introduction \& Objective: Minimally Invasive Pyeloplasty is widely accepted as the standard of care for surgical management of pelvic-ureteric junction obstruction (PUJO)

Bilateral Pyeloplasty using robotic technology in the same sitting has gained in popularity. We present a case of simultaneous bilateral robot assisted pyeloplasty (RAP) with multiple large stone extraction from several calyces in the left kidney and transposition of PUJ on right side due to crossing vessels. 
Materials and Methods: A 44 year old male with a body mass index of 28, presented with mild left sided flank pain. Imaging revealed bilateral hydronephrosis with four $1.5-2 \mathrm{~cm}$ spherical calyceal calculi on the left side. Diuretic nuclear scan (DTPA) demonstrated bilateral obstructed curves. Patient was worked up for bilateral RAP with retrieval of calculi in the same sitting. Under general anesthesia, bilateral retrograde pyelography with Double J stent placement was done. In lateral position, left sided procedure was performed first with two $8 \mathrm{~mm}$ ports in midline, one assistant port just below umbilicus and one $8 \mathrm{~mm}$ port in iliac fossa at the level of mid clavicular line. Proceeding through the routine steps of pyeloplasty, pelvis was opened. Retrieval of stones was done totally with robotic assistance utilising versatile features of the $8 \mathrm{~mm}$ Da Vinci Xi endoscope and robotic wristed instruments to grasp the calculi after a thorough search in each calyx. After stone retrieval the remaining steps were completed and stones retrieved using an endobag via the assistant port. The contralateral side was tackled through the same midline ports with an additional $8 \mathrm{~mm}$ port in right iliac fossa after undocking and redocking on the opposite side. We found crossing vessels in front of the PUJ and hence dismemberment and transposition of the PUJ anterior to the vessels was performed.

Results: Total operative time was 220 minutes, console time was170 minutes. Time for stone extraction and retrieval was 25 minutes. Orals were started after 6 hours. Perurethral catheter removed on POD1 and drains removed on POD2 and discharged on the same day. DJ stents removed were removed after 4 weeks Conclusions: Bilateral Robotic pyeloplasty can be accomplished with midline ports and one additional port in either iliac fossa. Stones in the setting of PUJO even upto $2 \mathrm{~cm}$ in size can be retrieved with the help of robotic instruments without resorting to any other conventional methods of stone retreival(URS, Nephroscope, lithotripters and accessories). Features of the Robotic endoscope and instruments have distinct advantages in this setting and can find further application
V13-14 Robotic Assisted Laparoscopic Ureteroplasty for a Recurrent Proximal Ureteral Stricture with Buccal Mucosal Graft

TM FitzGibbon, V Ramakrishnan, P Knoll, J Messer

University of Louisville Department of Urology

United States

Introduction \& Objective: There are limited options for the treatment of recurrent ureteral strictures. Definitive repair with open ureteroplasty has the potential to be a technically challenging procedure. The use of the Robotic Platform with magnification has the potential to alleviate some of the technical complexities associated with this.

Materials and Methods: In this case, we present a 69-year-old woman who underwent robotic-assisted laparoscopic ureteroplasty with buccal mucosa graft for treatment of a recurrent proximal ureteral stricture after a prior dismembered ureteroureterostomy approximately 20 years earlier. The stricture was associated with a T1/2 of 65 minutes and symmetrical renal function on a MAG3 Lasix Renal Scan. Due to the patients comorbid interstitial cystitis decision was made to proceed with buccal mucosal grafting. She was managed with a nephrostomy tube prior to surgery.

Results: Operative time was 201 minutes for the surgery, including harvest of the buccal mucosal graft as well as docking and undocking the robot. There was an estimated blood loss of 50cc. The patient had an uneventful hospital course and had percutaneous drain and nephrostomy tube removed prior to discharge on post-operative day two. Six weeks post-operatively the repair was patent and the ureteral stent was removed.

Conclusions: Robotic Assisted Laparoscopic Ureteroplasty can be utilized in select cases demonstrating the ability to use graft material, and result in acceptable outcomes with minimal patient morbididty. 